NISTIR 7317

\title{
Free Space Optics Communication System Testing in Smoke and Fire Environments
}

Alexander Maranghides

William Mell

William D. Walton

Erik L. Johnsson

Nelson P. Bryner

U.S. Department of Commerce

Technology Administration

Building and Fire Research Laboratory

National Institute of Standards

And Technology

Gaithersburg, MD 20899

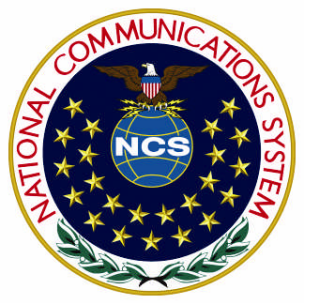

National

Communications

System

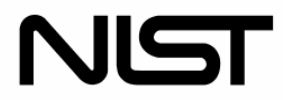

National Institute of Standards and Technology

Technology Administration, U.S. Department of Commerce 
NISTIR 7317

\title{
Free Space Optics Communication System Testing in Smoke and Fire Environments
}

\author{
Alexander Maranghides \\ William Mell \\ William D. Walton \\ Erik L. Johnsson \\ Nelson P. Bryner
}

U.S. Department of Commerce

Technology Administration

Building and Fire Research Laboratory

National Institute of Standards

And Technology

Gaithersburg, MD 20899
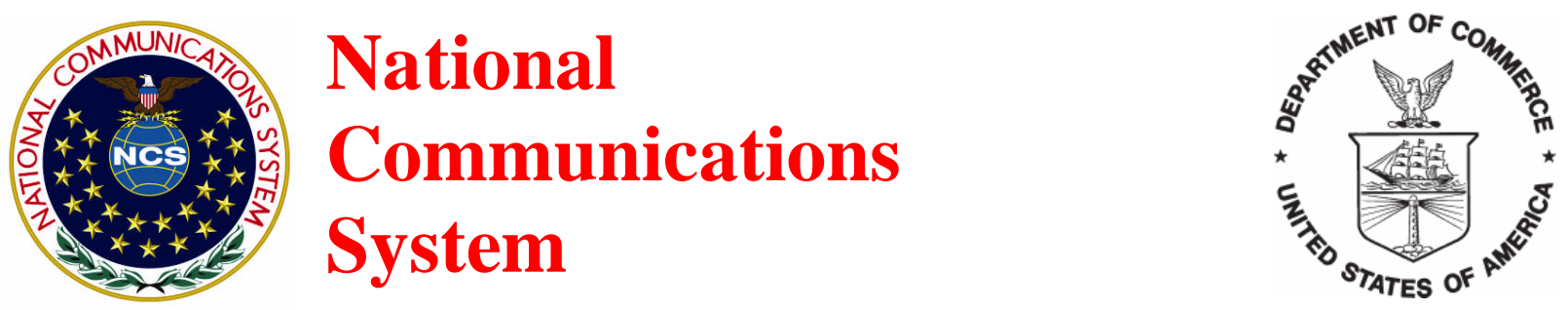

National Communication System

Dr. Peter Fonash, Deputy Manager

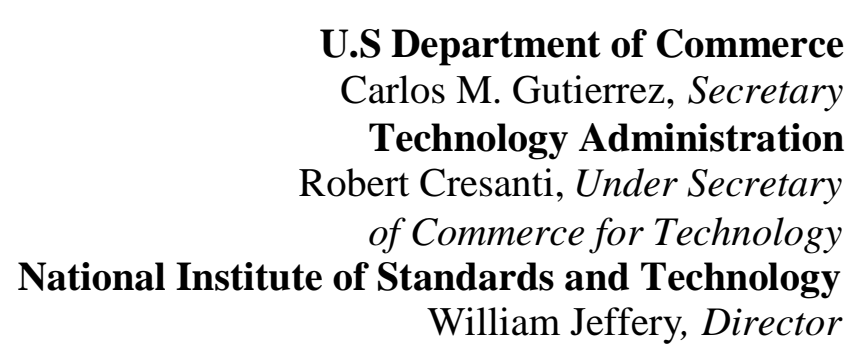


Disclaimer:

Certain commercial equipment, instruments, or materials are identified in this document. Such identification does not imply recommendation or endorsement by the National Institute of Standards and Technology, nor does it imply that the products identified are necessarily the best available for the purpose. 


\section{Table of Contents}

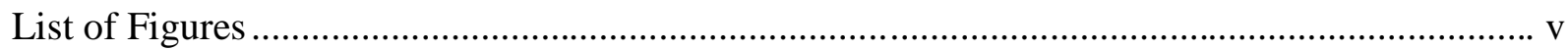

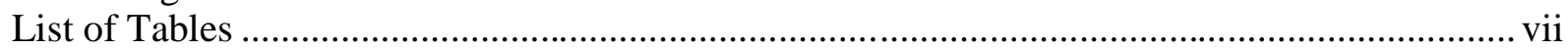

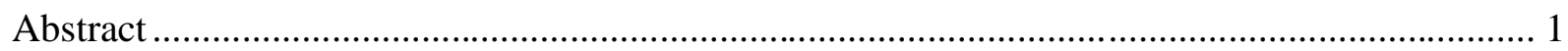

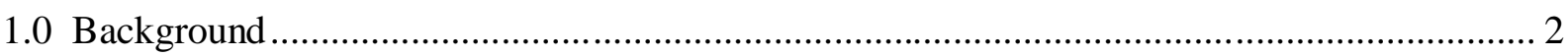

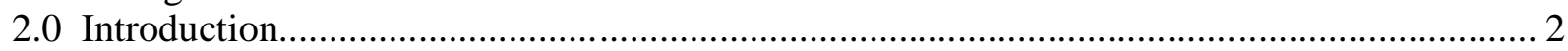

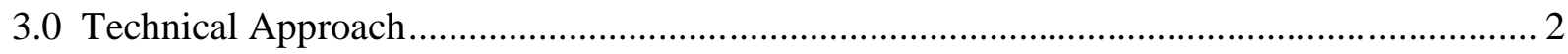

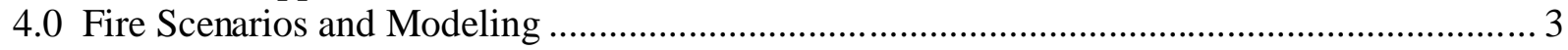

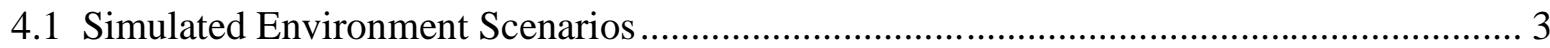

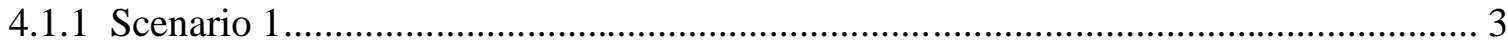

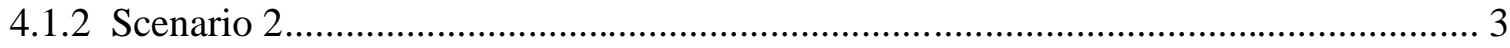

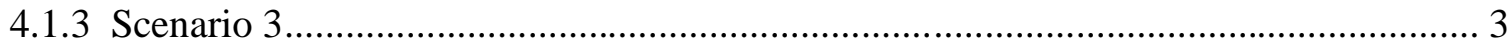

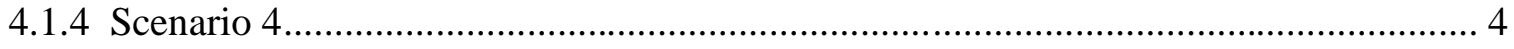

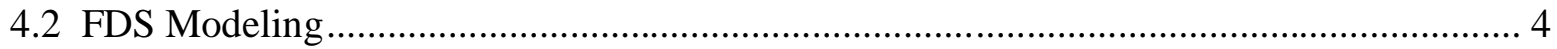

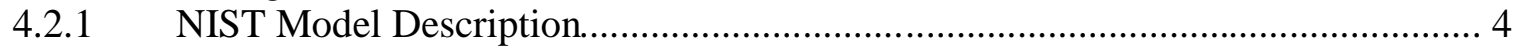

4.2.2 Application of Model to Fire and Smoke Scenarios .................................................. 5

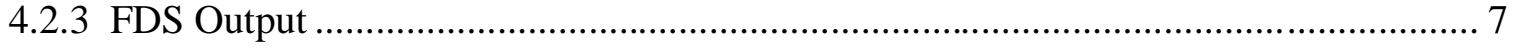

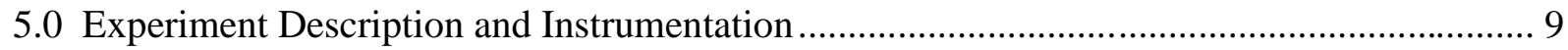

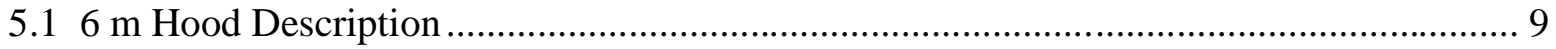

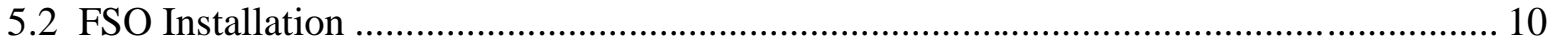

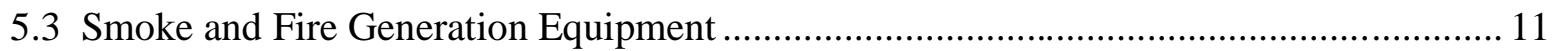

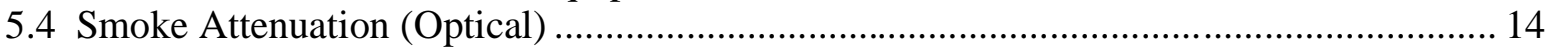

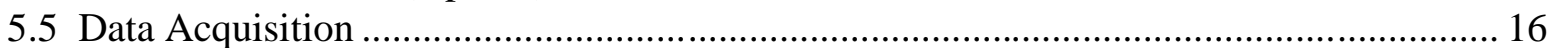

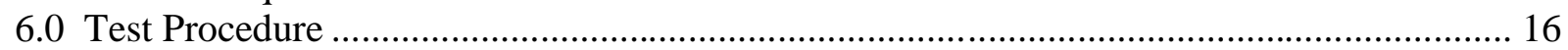

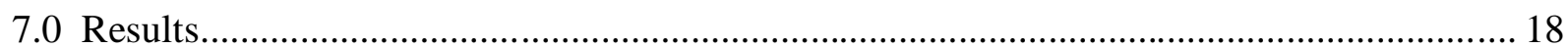

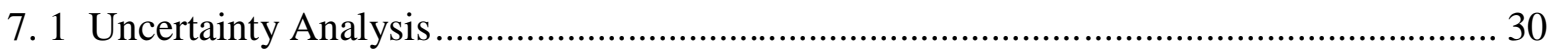

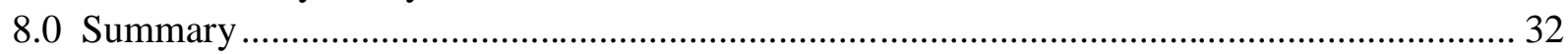

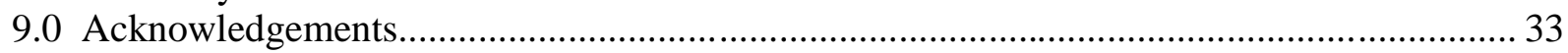

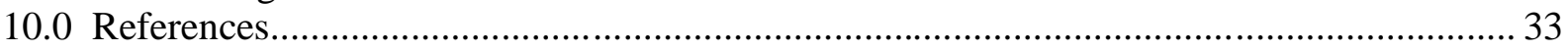

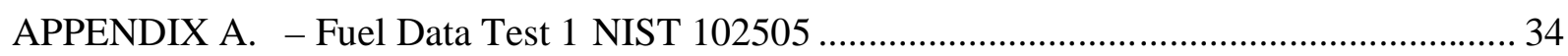

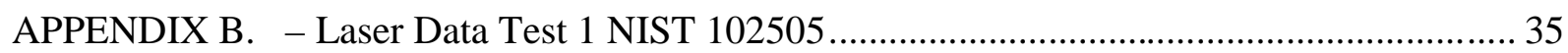

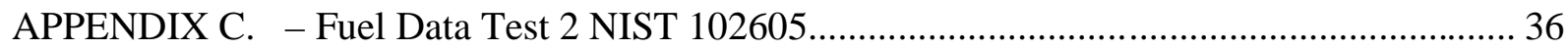

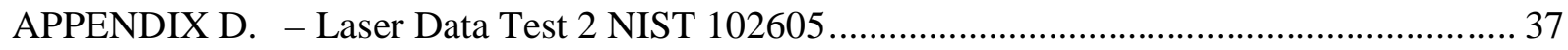

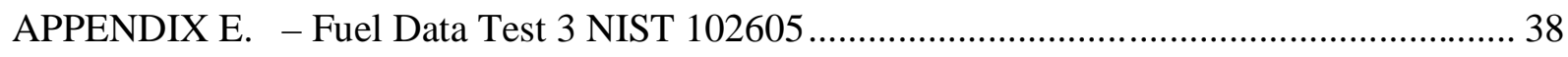

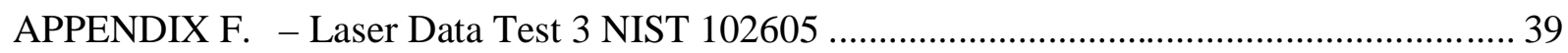

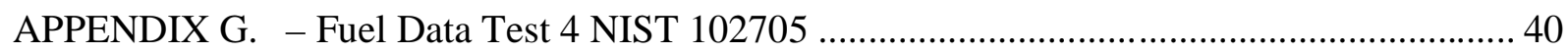

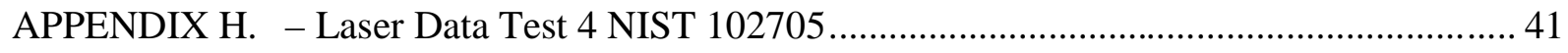




\section{List of Figures}

Figure 1. An outdoor crude oil pool fire experiment conducted by NIST..............................5

Figure 2. Example of a tanker spill simulation with four surrounding buildings...................... 6

Figure 3. Example of a diesel tanker spill with sixteen surrounding buildings ........................... 7

Figure 4. Height versus time averaged transmittance for the $7 \mathrm{~m} \mathrm{x} 7 \mathrm{~m}$ pool fire simulation... 8

Figure 5. Height versus time averaged transmittance for the $30 \mathrm{~m}(\mathrm{X}) \times 20 \mathrm{~m}(\mathrm{Y})$ pool fire

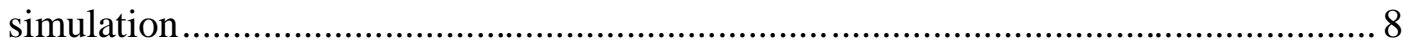

Figure 6. Large Fire Laboratory exhaust hoods and duct system .......................................... 10

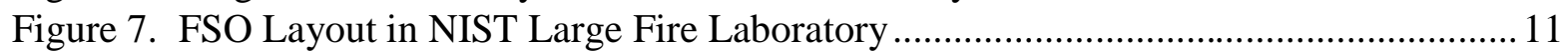

Figure 8. Schematic drawing of LFL spray burner hardware ............................................... 12

Figure 9. Fire with smoke focused by small hood (Scenarios 1, 2 and 3) ...............................13

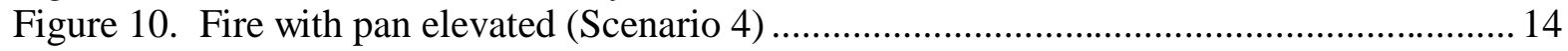

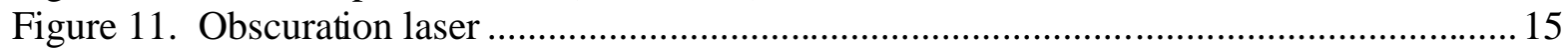

Figure 12a. Sample laser detector calibration plot showing transmittance versus the laser detector signal (transmittance ranging from 0.0 to 1.0 ) ..........................17

Figure 12b. Sample laser detector calibration plot showing transmittance versus the laser detector signal (transmittance ranging from 0.0 to 0.012 ) .................... 17

Figure 13. Test 1 transmittance plotted versus time from ignition (11:26:23 EDT)

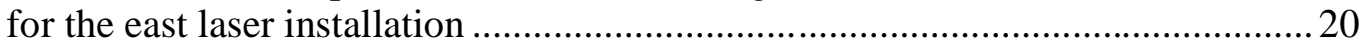

Figure 14. Test 1 transmittance plotted versus time from ignition (11:26:23 EDT)

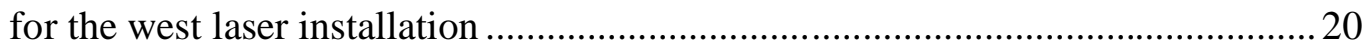

Figure 15. Test 1 transmittance plotted versus time from ignition (11:26:23 EDT)

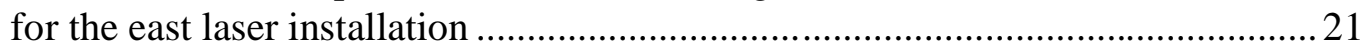

Figure 16. Test 1 transmittance plotted versus time from ignition (11:26:23 EDT)

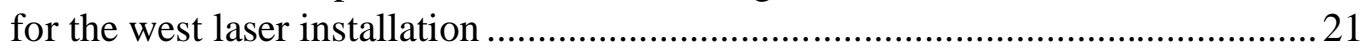

Figure 17. Test 2 transmittance plotted versus time from ignition (9:47:01 EDT) for the east laser installation ......................................................................... 22

Figure 18. Test 2 transmittance plotted versus time from ignition (9:47:01 EDT) for the west laser installation .................................................................. 22

Figure 19. Test 2 transmittance plotted versus time from ignition (9:47:01 EDT) for the east laser installation ................................................................... 23

Figure 20. Test 2 transmittance plotted versus time from ignition (9:47:01 EDT)

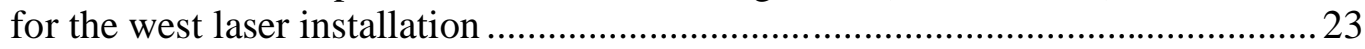

Figure 21. Test 3 transmittance plotted versus time from ignition (14:57:20 EDT)

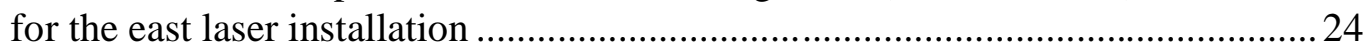

Figure 22. Test 3 transmittance plotted versus time from ignition (14:57:20 EDT) for the west laser installation

Figure 23. Test 3 transmittance plotted versus time from ignition (14:57:20 EDT) for the east laser installation

Figure 24. Test 3 transmittance plotted versus time from ignition (14:57:20 EDT)

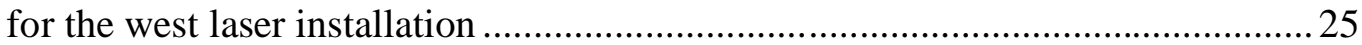

Figure 25. Test 4 transmittance plotted versus time from ignition (14:20:46 EDT) for the east laser installation

Figure 26. Test 4 transmittance plotted versus time from ignition (14:20:46 EDT) for the west laser installation 


\section{List of Figures, continued}

Figure 27. Test 4 transmittance plotted versus time from ignition (14:20:46 EDT)

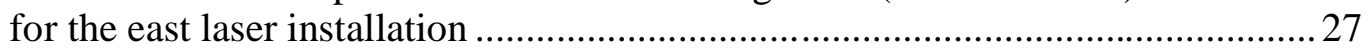

Figure 28. Test 4 transmittance plotted versus time from ignition (14:20:46 EDT)

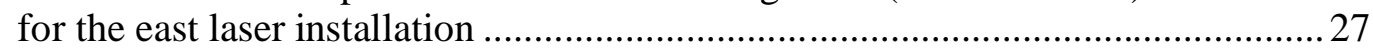

Figure 29. Test 1 diesel fuel flow rate plotted versus time from ignition $(11: 26: 23$ EDT) ....... 28

Figure 30. Test 2 diesel fuel flow rate plotted versus time from ignition $(9: 47: 01$ EDT) ........ 28

Figure 31. Test 3 diesel fuel flow rate plotted versus time from ignition (14:57:20 EDT)......29

Figure 32. Test 4 diesel fuel flow rate plotted versus time from ignition (14:20:46 EDT)...... 29 


\section{List of Tables}

Table 1. Summary of scenarios considered (all with no wind) ....................................................

Table 2. Predicted target transmittance values

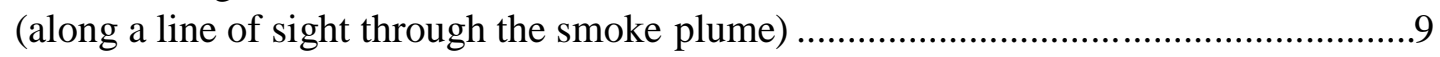

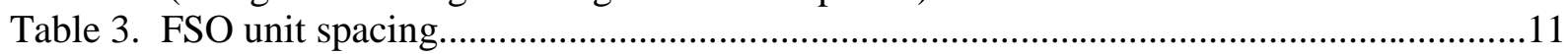

Table 4. Decrease in Laser Light Intensity at Pathlengths from $1 \mathrm{~m}$ to $30 \mathrm{~m} \ldots \ldots \ldots \ldots \ldots \ldots \ldots$

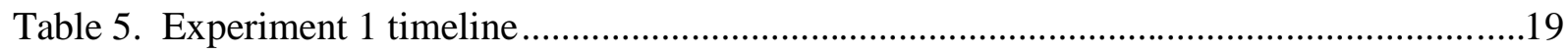

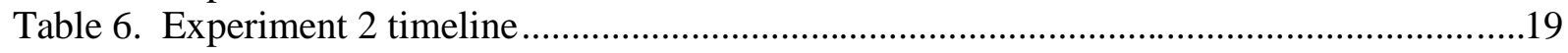

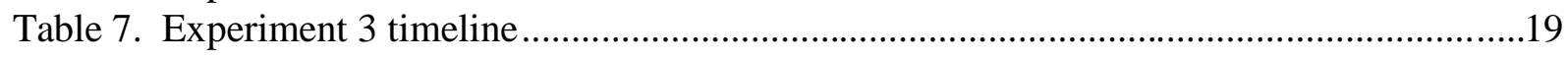

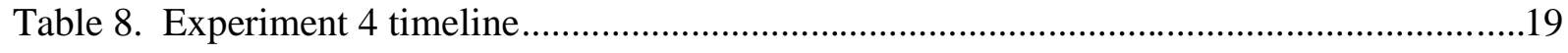

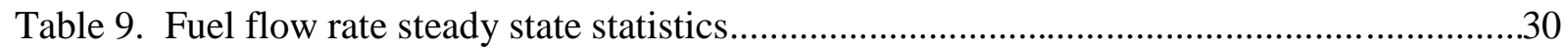

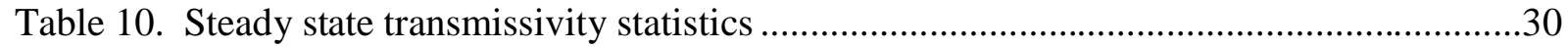

Table 11. Uncertainty in instrument location, fuel flow rate, and transmittance...............31 


\title{
Free Space Optics Communication System Testing in Smoke and Fire Environments
}

\author{
by \\ Alexander Maranghides, William Mell, William D. Walton, Erik L. Johnsson, and \\ Nelson P. Bryner
}

\begin{abstract}
Free-Space Optics, also known as "FSO" or "Optical Wireless", use laser light to transmit a digital signal, data, voice, or video information, between two transceivers. These laser-based systems require unobstructed line of sight to properly operate. FSO system performance, signal intensity and integrity, is related to beam obscuration from environmental conditions including the presence of smoke and flames. The National Communications System (NCS), the telecommunications sector specific agency under the Department of Homeland Security is interested in quantifying the performance of FSO units. In order to assess whether smoke and flames affect FSO performance, a preliminary evaluation was conducted by NCS and the National Institute of Standards and Technology (NIST). The evaluation used both computer modeling and a limited set of indoor experiments. Several obscuration scenarios based on the smoke from realistic diesel fuel fires of varying sizes were jointly developed. The NIST Fire Dynamics Simulator (FDS), a computational fluid dynamics computer fire model, was used to predict the levels of smoke obscuration for the different realistic fire scenarios at a specified distance above the fire. The FDS predictions were used to design the laboratory experiments. Even though the laboratory fires were smaller than those in the original scenarios, the laboratory configurations could produce similar smoke concentrations. The fire experiments were conducted in the NIST, Building and Fire Research Laboratory, Large Fire Laboratory in Gaithersburg, Maryland. NCS provided the FSO units and NIST set up instrumentation to characterize smoke obscuration. The smoke obscuration measurements showed that the target transmittance levels were achieved and that desired smoke obscurations could be generated over prolonged durations. The performance of the FSO units was assessed and reported by NCS.
\end{abstract}

KEY WORDS: computer fire modeling, diesel fuel fires, fire tests, free space optics, optical wireless, smoke measurement, smoke obscuration 


\subsection{Background}

Free-Space Optics, also known as "FSO" or "Optical Wireless", can be used to transmit optical data, voice and video information. FSO can be used in the event of an emergency as an alternative to the dial tone system. Like fiber optic cable based systems, FSO communications systems use laser light to transmit a digital signal between two transceivers. However, unlike fiber, the laser light is transmitted through the air (free-space) instead of through a glass fiber. In order for the signal to be transmitted and received without significant loss, there must be an unobstructed line of sight between each FSO unit. Signal attenuation from smoke and fire can affect ssystem performance.

\subsection{Introduction}

FSO units require unobstructed line of sight to operate properly, but weather conditions such as clouds, fog, rain, and particulates such as smoke and dust, can interfere or attenuate signal intensity and integrity. FSO can be used in the event of an emergency as an alternative to the dial tone system. The National Communications System (NCS), an agency of the Department of Homeland Security (DHS) is interested in quantifying the performance of FSO units. In order to assess whether smoke and flames affect FSO performance, a preliminary evaluation was conducted by NCS and the National Institute of Standards and Technology (NIST).

The purpose of this report is to document the NIST contributions to the fire scenario design and the particulars of the model simulations. Additionally this report documents the conducted experiments and the performance of the NIST instrumentation. NIST did not collect any FSO performance data and therefore cannot comment on the behavior of the FSO units. The performance of the FSO units is characterized and documented separately by NCS.

\subsection{Technical Approach}

The technical approach included computer modeling and a limited set of indoor/laboratory experiments. The five step process is listed below:

- Development of realistic fire scenarios

- Modeling of realistic fire scenarios

- Interpretation of model output

- Design and instrumentation of laboratory experiments

- Execution of laboratory experiments

Several obscuration scenarios based on the smoke from realistic diesel fuel fires of varying sizes were jointly developed between NIST and NCS. The NIST Fire Dynamics Simulator (FDS), a computational fluid dynamics computer fire model, was used to simulate the levels of smoke obscuration for four different fire scenarios at a specified distance above the fire. These scenarios generally represented a large diesel fuel fire at ground level with the FSO units mounted on the top of relatively tall buildings. As the smoke plume rose from the fire, the smoke concentration was diluted by air that was entrained into the plume. Fire experiments were conducted in the NIST, Building and Fire Research Laboratory, Large Fire Laboratory in Gaithersburg, Maryland. The fires that could safely be used during the experimental phase of this effort were significantly 
smaller that the realistic full scale fire scenarios. The FDS predictions were therefore used to design smaller laboratory fires that produced similar smoke concentrations. This was accomplished by placing the FSO units at a lower elevation, and thus closer to the fire, than would be the case in an actual installation. In the laboratory experiments, FSO units provided by the NCS were installed such that the laser beams passed through the smoke. Neutral density filters were used to attenuate the signal intensity and simulate the longer path lengths encountered in real life FSO installations. Obscuration through the smoke plume was measured by NIST using calibrated lasers in order to document the behavior of the plume. The NIST measurements were not used as telltales of FSO unit performance. They were instead used to link the laboratory scale experiments to the model predictions of real scale events and to permit experimental repeatability.

\subsection{Fire Scenarios and Modeling}

\subsection{Simulated Environment Scenarios}

The purpose of this effort was to develop realistic fire and smoke generation scenarios. Smoke concentrations generated from diesel fuel spill accidents and intentional releases (terrorist attacks) were assessed. NIST and NCS generated four realistic diesel fuel spill scenarios. Aspects of these scenarios are listed in Table 1.

\subsubsection{Scenario 1}

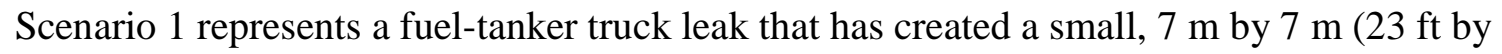
$23 \mathrm{ft}$ ), diesel fuel spill and is burning at street level at an intersection. FSO line of sight is through the smoke plume $46 \mathrm{~m}(150 \mathrm{ft})$ above the spill. The laboratory scale simulation of this consisted of a diesel fuel fire near floor level with the smoke plume rising up to the height of the communications beam path and measurements, $3.7 \mathrm{~m}(12 \mathrm{ft})$ above the floor. The laboratory simulation was designed to achieve transmittance levels $\left(\mathrm{I} / \mathrm{I}_{\mathrm{o}}\right)$ comparable to those predicted by the model at $46 \mathrm{~m}(150 \mathrm{ft})$ above the spill.

\subsubsection{Scenario 2}

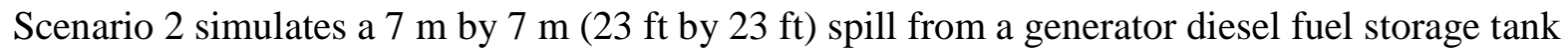
on the roof of a building in the path of the FSO beam. The roof top spill is $23 \mathrm{~m}$ (75 ft) above

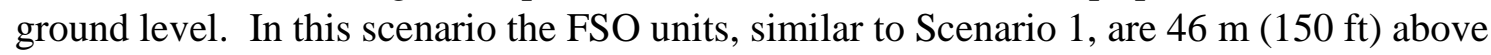
ground level. The FSO line of sight is through the smoke plume $23 \mathrm{~m}(75 \mathrm{ft})$ above the spill. The laboratory scale recreation of this scenario consisted of a diesel fuel fire near floor level with the smoke plume rising up to the height of the communications beam path and measurements, $3.7 \mathrm{~m}$ (12 ft) above the floor. The laboratory simulation of this scenario was designed to achieve transmittance levels $\left(\mathrm{I} / \mathrm{I}_{\mathrm{o}}\right)$ comparable to those predicted by the model $23 \mathrm{~m}(75 \mathrm{ft})$ above the spill.

\subsubsection{Scenario 3}

Scenario 3 represents a large $30 \mathrm{~m}$ by $20 \mathrm{~m}$ ( $98 \mathrm{ft}$ by $66 \mathrm{ft}$ ) diesel fuel tanker truck spill fire at ground level. In this scenario the FSO units are placed $46 \mathrm{~m}(150 \mathrm{ft})$ above ground level. The laboratory scale simulation of this scenario also consisted of a diesel fuel fire near floor level with the smoke plume rising up to the height of the communications beam path and 


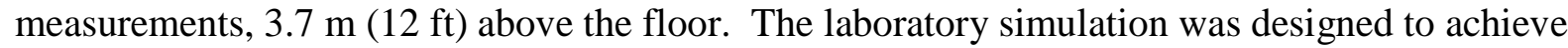
transmittance $\left(\mathrm{I} / \mathrm{I}_{\mathrm{o}}\right)$ levels comparable to those predicted by the model $46 \mathrm{~m}(150 \mathrm{ft})$ above the fuel spill.

\subsubsection{Scenario 4}

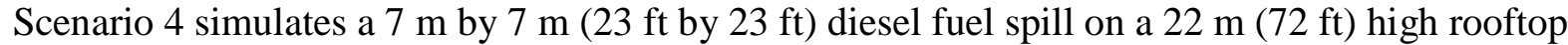
from a generator diesel fuel storage tank. In this scenario, the FSO units are $23 \mathrm{~m} \mathrm{(75} \mathrm{ft)} \mathrm{above}$

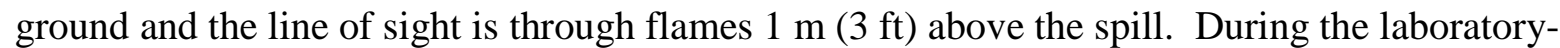
scale simulation the burner was raised and positioned so the communication and measurement beams crossed through the flames as well as the smoke.

Table 1. Summary of scenarios considered (all with no wind)

\begin{tabular}{|l|l|l|l|}
\hline Scenario & Fuel Spill Size & Fire Location & FSO Height Above Ground Level \\
\hline 1 & $\begin{array}{l}7 \mathrm{~m} \times 7 \mathrm{~m} \\
(23 \mathrm{ft} \times 23 \mathrm{ft})\end{array}$ & $\begin{array}{l}\text { on ground, at street } \\
\text { intersection }\end{array}$ & $46 \mathrm{~m}(150 \mathrm{ft})$ \\
\hline 2 & $\begin{array}{l}7 \mathrm{~m} \times 7 \mathrm{~m} \\
(23 \mathrm{ft} \times 23 \mathrm{ft})\end{array}$ & $\begin{array}{l}\text { on top of building; } 23 \mathrm{~m} \\
(75 \mathrm{feet}) \text { elevation }\end{array}$ & $46 \mathrm{~m}(150 \mathrm{ft})$ \\
\hline 3 & $\begin{array}{l}30 \mathrm{~m} \times 20 \mathrm{~m} \\
(98 \mathrm{ft} \times 66 \mathrm{ft})\end{array}$ & $\begin{array}{l}\text { on ground, at street } \\
\text { intersection }\end{array}$ & $46 \mathrm{~m}(150 \mathrm{ft})$ \\
\hline 4 & $\begin{array}{l}7 \mathrm{~m} \times 7 \mathrm{~m} \\
(23 \mathrm{ft} \times 23 \mathrm{ft})\end{array}$ & $\begin{array}{l}\text { on top of building; } 22 \mathrm{~m} \\
(72 \text { feet) elevation }\end{array}$ & $23 \mathrm{~m} \mathrm{(75ft)}$ \\
\hline
\end{tabular}

\subsection{FDS Modeling}

The primary purpose of the modeling effort, using the NIST developed fire model FDS, was to provide a basis for comparing the relative transmittance across the above defined four different scenarios. Additionally, the model generated transmittance values were used to guide the design of the experiments conducted in the NIST Large Fire Laboratory (see Section 4.0). The above defined four scenarios were used to calculate the expected obscuration as a function of height from the fuel spills. Even though obscuration is dependent on wavelength, the computer modeling was used to illustrate, in this first phase of the FSO technology evaluation, the range of obscurations than can be achieved. Future simulations of laboratory and field experiments can be used to better determine if the simulated smoke obscurations are accurate in an absolute sense and to also reduce the associated uncertainties.

\subsubsection{NIST Model Description}

Over the past 25 years NIST has developed a fire and smoke-transport model FDS. Time dependent, three-dimensional, simulations of both structural fires and outdoor fires are possible. FDS uses the methods of computational fluid dynamics (CFD) to solve the equations governing the fluid motion, combustion, and heat transfer. Throughout the course of its development, experiments conducted in NIST's Large Fire Laboratory, and elsewhere, have been used to evaluate and further refine the modeling approach. FDS is currently used by hundreds of fire 
protection engineers around the world. Both a technical manual (McGrattan, 2004) and a users' guide (McGrattan and Forney, 2004) are available on the web and the program itself can be downloaded. A visualization package for FDS output, called Smokeview, has also been developed at NIST (Forney and McGrattan, 2004).

\subsubsection{Application of Model to Fire and Smoke Scenarios}

Users of FDS can easily specify the wind and both the location and the required thermo-chemical specifications of a liquid fuel. In the exploratory simulation study conducted here, the fuel is assumed to be diesel, and there is no ambient wind. The smoke yield and burning rate of the diesel fuel were based on full-scale field experiments conducted by NIST in the 1990s (Walton et al., 1994). These experiments included both diesel and crude oil fuels. The smoke yield is the fraction of fuel mass, consumed in the gas phase combustion, which is converted to smoke $(11 \%$ here). The burning rate is the rate at which fuel gases are produced at the surface of the liquid diesel pool $\left(0.054 \mathrm{~kg} / \mathrm{s} \mathrm{m}^{2}\right.$ here $)$. An example of an experiment, in this case a $15.2 \mathrm{~m}$ by $15.2 \mathrm{~m}$ $(50 \mathrm{ft}$ by $50 \mathrm{ft}$ ) crude oil pool fire, is shown in Fig. 1. Note the influence of the ambient wind, which bends the plume to the left.

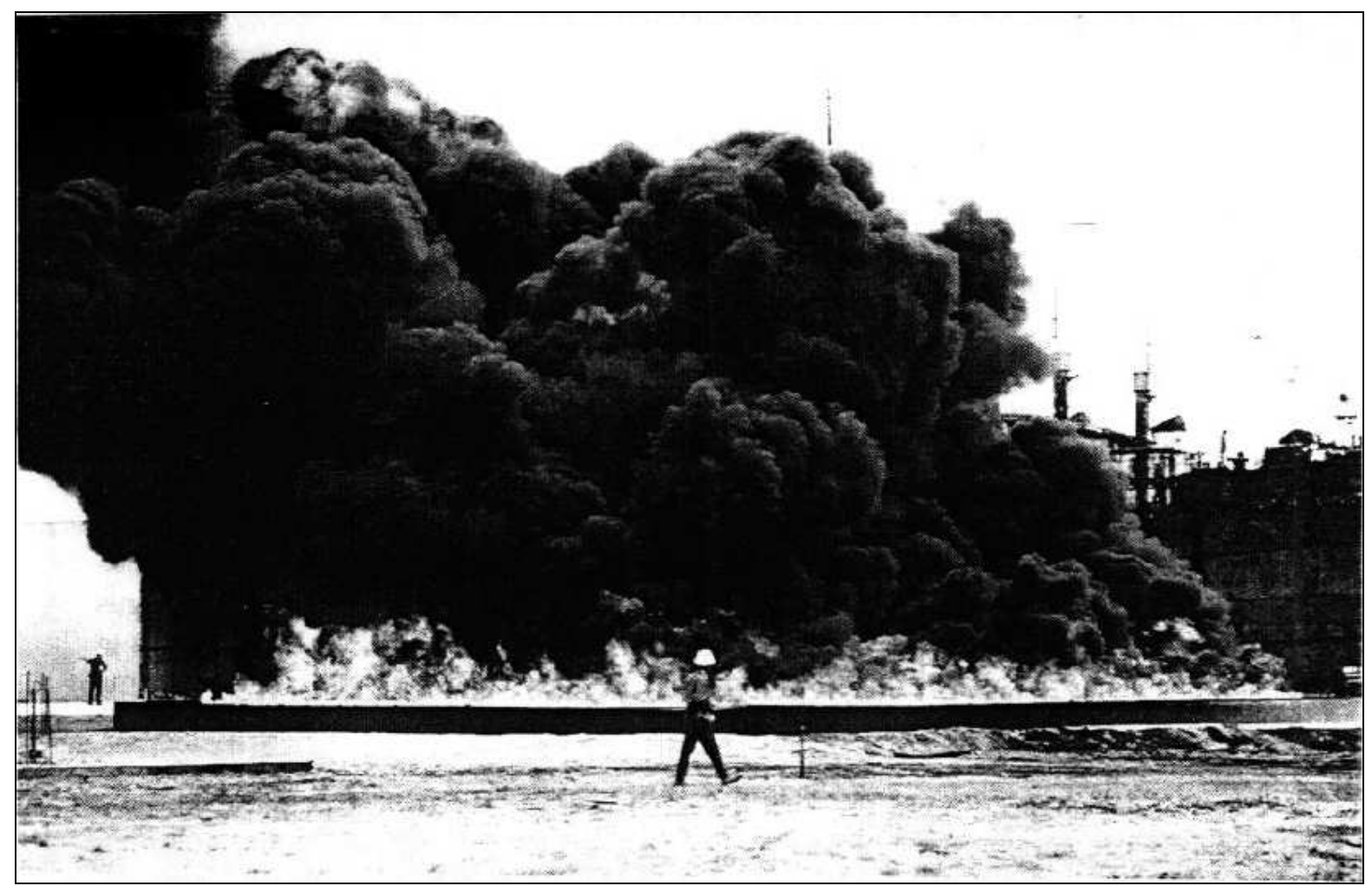

Figure 1. An outdoor crude oil pool fire experiment conducted by NIST. The size of the base of the pool is $15.2 \mathrm{~m} \times 15.2 \mathrm{~m}$. 
A number of computer simulations were made to test the influence of the computational grid resolution, the presence of surrounding buildings, and overall computational domain size on smoke plume behavior and soot concentrations. At this time, all buildings were assumed to be of similar height. For example, Figures 2 and 3 show the tanker spill scenario with either four buildings (Fig. 2) or sixteen buildings (Fig. 3) surrounding the pool fire. In Fig. 2, the dimensions of the pool are $30 \mathrm{~m}$ by $20 \mathrm{~m}$ in the $\mathrm{x}$ and y directions, respectively. The buildings are separated by $22 \mathrm{~m}$, are $50 \mathrm{~m} \times 50 \mathrm{~m}$ in plan area, and $18 \mathrm{~m}$ tall. The dimensions of computational domain are $100 \mathrm{~m}$ x $100 \mathrm{~m}$ x $100 \mathrm{~m}$ in the $\mathrm{x}, \mathrm{y}, \mathrm{z}$ directions, respectively. In Fig. 3 , the size and separation of the buildings are identical to those in Fig. 2, however, the dimensions of the computational domain are $150 \mathrm{~m} \times 150 \mathrm{~m} \times 100 \mathrm{~m}$, in the $\mathrm{x}, \mathrm{y}$, and $\mathrm{z}$ directions, respectively. While it is not possible to see from these figures, a more detailed analysis of the results showed there was very little difference in the smoke concentrations between these two simulations. This suggested that it is not necessary, at least with zero wind, to include sixteen buildings in the simulations.

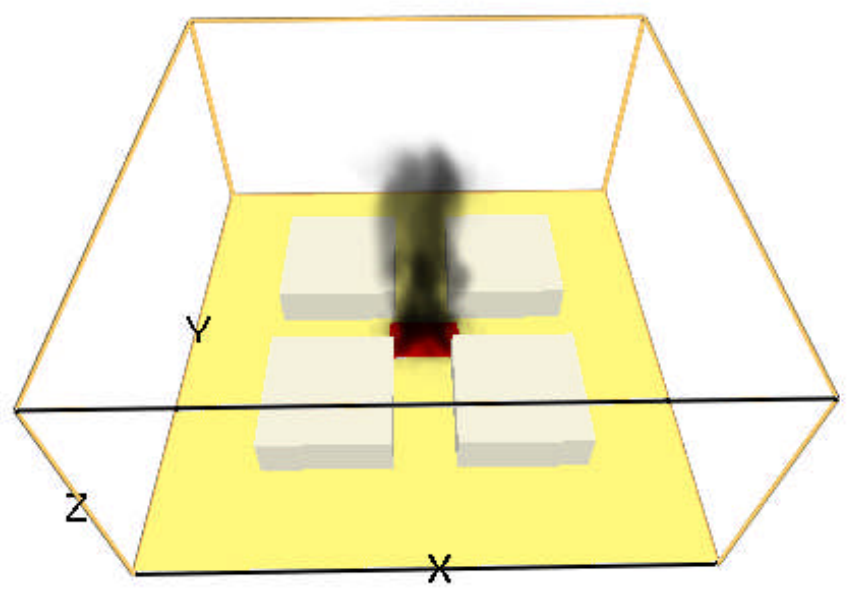

Figure 2. Example of a diesel tanker spill simulation with four surrounding buildings. The spill occurs at a roadway intersection 


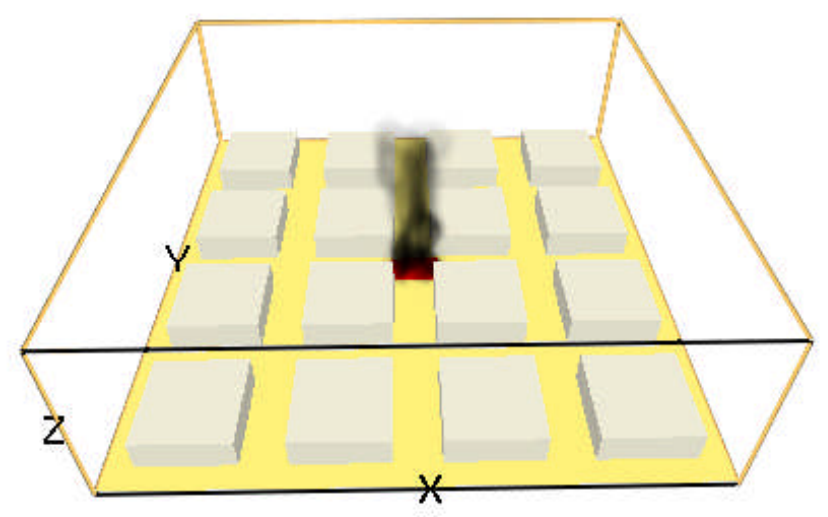

Figure 3. Example of a diesel tanker spill simulation with sixteen surrounding buildings.

\subsubsection{FDS Output}

Table 2 lists values of the time-averaged transmittance across the plume for the four scenarios considered here. The transmittance is defined in Equation (1). The smoke concentration measurement is based on the strong light extinction characteristic of flame-generated smoke. This measurement is based on Bouguer's Law, which relates the ratio of the transmitted and incident intensities to the mass concentration of smoke $\mathrm{M}_{\mathrm{s}}$, the path length through the smoke, $\mathrm{L}$, and the specific extinction coefficient $\sigma_{\mathrm{s}}$ via the following expression:

$$
\frac{I}{I_{0}}=\exp \left(-\sigma_{s} M_{s} L\right)
$$

The value selected for the specific extinction coefficient $\sigma_{\mathrm{s}}$ was $9.5 \mathrm{~m}^{2} / \mathrm{g}$ at $633 \mathrm{~nm}$ (Mulholland, G. et al 2002). The $633 \mathrm{~nm}$ wavelength was selected specifically to allow comparison of the model output with the transmittance data collected during the experiments (see section 5.4).

Model output transmittance values were time averaged over a number of time intervals ( $1 \mathrm{~min}$ to $3 \mathrm{~min}$ ) and compared to ensure the resulting value was not dependent on the averaging window. Line plots of the transmittance across the plume as a function of the height above the surface of the diesel pool are also shown. The $7 \mathrm{~m} \times 7 \mathrm{~m}(23 \mathrm{ft} \times 23 \mathrm{ft})$ case is shown in Fig. 4 and the $30 \mathrm{~m}$

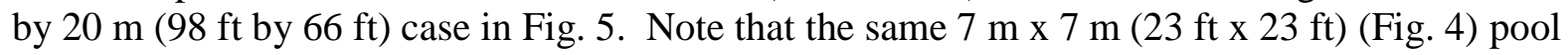
fire simulation, with the pool fire located on the ground, was used to obtain transmittance values for all three scenarios involving the $7 \mathrm{~m} \mathrm{x} 7 \mathrm{~m}(23 \mathrm{ft} \times 23 \mathrm{ft})$ pool fire. In particular, for scenario 4 , which has the pool fire on top of a building, transmittance values were obtained from simulations with the pool fire on the ground. This was done because exploratory simulations showed that in the absence of wind, because of the conservation of mass, it made no significant difference in the transmittance values if the fire was located on the ground or rooftop. Both Figures 4 and 5 show that transmittance increases as one moves away from the surface of the burning liquid fuel. 


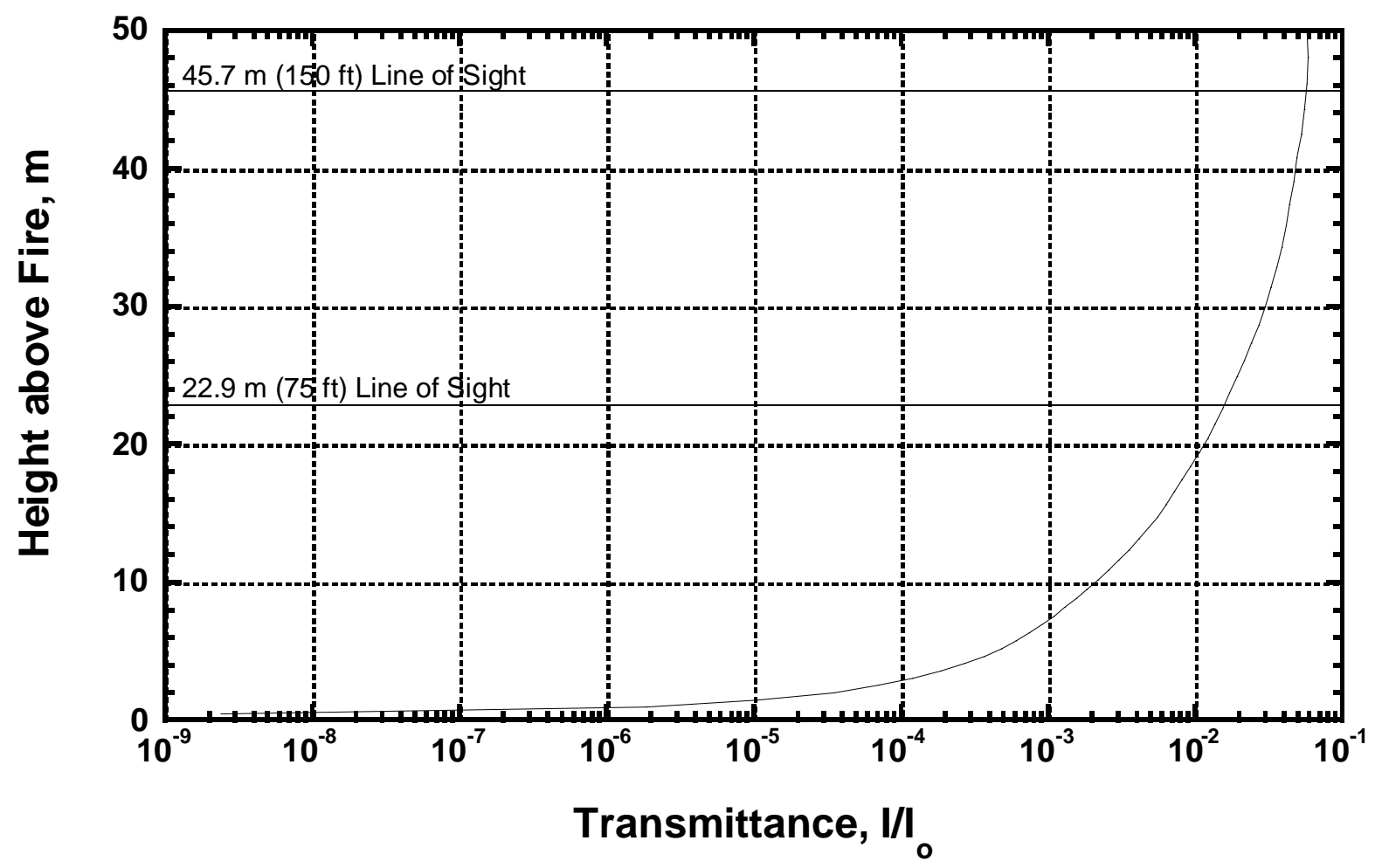

Figure 4. Height versus time averaged centerline transmittance for the $7 \mathrm{~m} \mathbf{x} \mathbf{m}$ pool fire simulation

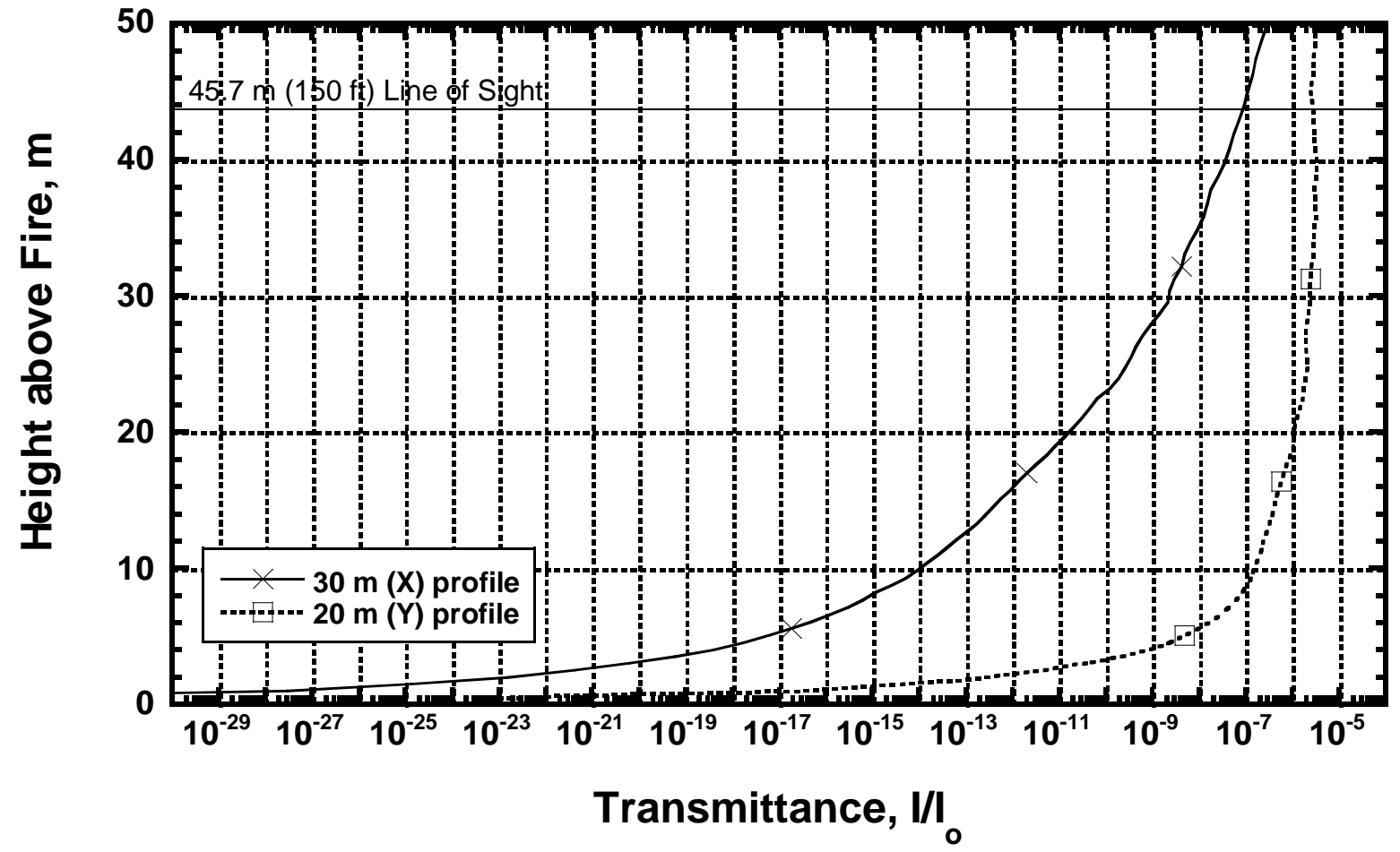

Figure 5. Height versus time averaged centerline transmittance for the $30 \mathrm{~m}$ (X) $\times 20 \mathrm{~m}(\mathrm{Y})$ pool fire simulation 
The pool fire scenarios simulated with FDS in this study are summarized in Table 1. The time averages of the predicted transmittance across the smoke plume for each scenario are listed in Table 2. These transmittance values were computed for beams at different heights, corresponding to the FSO locations listed in Table 1. The simulations provided a basis for comparing the relative transmittance (which is a function of the smoke mass concentration and the path length) across the different scenarios. Through this comparison scenario 4 was identified as having the most severe smoke obscuration.

Table 2. Predicted target transmittance values (along a line of sight through the smoke plume)

\begin{tabular}{|c|c|c|c|}
\hline Scenario & Spill, $\mathrm{m} \times \mathrm{m}$ & Height, $\mathrm{m}(\mathrm{ft})$ & Target Transmittance $\left(\mathrm{I} / \mathrm{I}_{\mathrm{o}}\right)$ \\
\hline 1 & $7 \times 7$ & $46(150)$ & 0.06 \\
\hline 2 & $7 \times 7$ & $23(75)$ & 0.016 \\
\hline 3 & $30 \times 20$ & $46(150)$ & $1.4 \times 10^{-7}$ (across 30 m dimension) \\
& & & $1.4 \times 10^{-6}$ (across 20 m dimension) \\
\hline 4 & $7 \times 7$ & $1(3)$ & $1.9 \times 10^{-6}$ \\
\hline
\end{tabular}

\subsection{Experiment Description and Instrumentation}

The primary objective of the experiments was to assess the performance of the FSO units in different levels of smoke and intermittent flames. Four indoor experiments were conducted in the NIST Large Fire Laboratory (LFL).

\section{$5.16 \mathrm{~m}$ Hood Description}

The LFL High Bay test area is nominally $9 \mathrm{~m}(30 \mathrm{ft})$ wide and $37 \mathrm{~m}(120 \mathrm{ft})$ long. Two hoods,

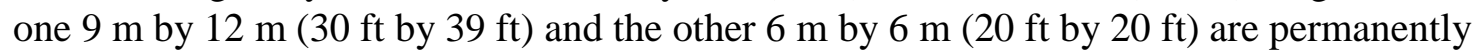
installed in the facility. Side and top view diagrams of the hoods and duct systems are shown in Fig. 6.

The FSO experiments were conducted under the $6 \mathrm{~m}$ by $6 \mathrm{~m}(20 \mathrm{ft}$ by $20 \mathrm{ft})$ hood. The test area beneath the hood is open on three sides. The fourth side is relatively close to the north wall of

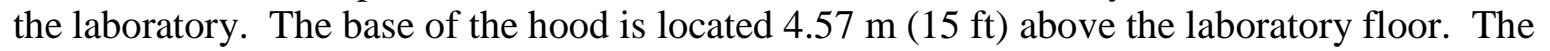
interior surface of the hood is coated with a fibrous cement mix to provide thermal insulation of

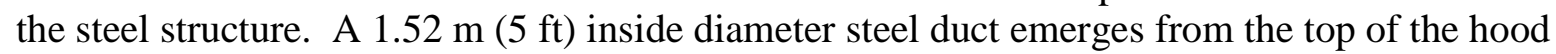
and immediately turns to become horizontal, running under the high bay roof. Immediately downstream of this are inlets for ducts from smaller calorimeter hoods. These were sealed off to preclude dilution of the captured plume gases by the six-meter hood. The duct then makes a right angle turn upward, passes through the roof and makes a second right angle turn to run along the top of the roof. The entire length of the duct is thermally insulated to minimize condensation of plume moisture. 


\subsection{FSO Installation}

Four FSO units from different manufacturers were installed in the east end of the High Bay test area and four corresponding FSO units were installed in the west end of the test area. Each of the units was aligned to face the corresponding unit at the opposite end of the area with the beams crossing above the center of the fire. FSO units, placed at each end of the High Bay, consisted of a receiver and one or more transmitters. FSO unit locations are shown in Fig. 7.

A flexible hood skirt normally used to extend the sides of the hood downward was raised to permit the FSO units to send data at an average height of $3.9 \mathrm{~m}(12.7 \mathrm{ft})$ above the floor. The face-to-face path length of the FSO units is given in Table 3. The placement of the FSO units was selected by NCS and the FSO units were installed by each respective manufacture. Variations in unit spacing distances are accounted for by NCS in the performance assessment of the FSO.
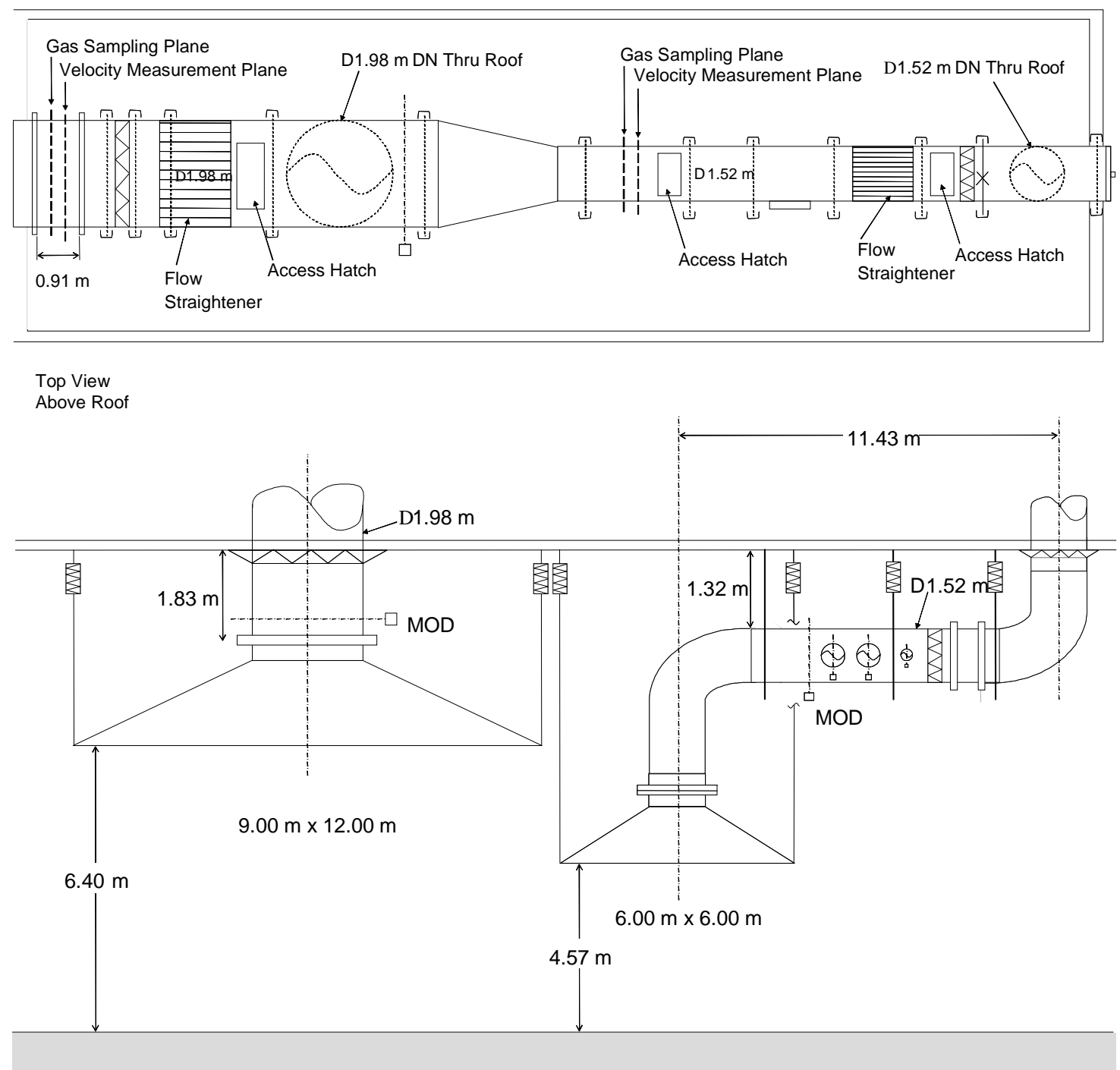

Figure 6. Large Fire Laboratory exhaust hoods and duct system 
Table 3. FSO unit spacing

\begin{tabular}{|c|c|c|}
\hline FSO Unit & Face-to-Face Distance & $\begin{array}{c}\text { Total Combined Uncertainty in Face } \\
\text { to Face Distance }\end{array}$ \\
\hline 1 & $30.62 \mathrm{~m}(100.44 \mathrm{ft})$ & $30.62 \mathrm{~m}(100.44 \mathrm{ft}) \pm 0.5 \mathrm{~m}(1.64 \mathrm{ft})$ \\
\hline 2 & $30.80 \mathrm{~m}(101.06 \mathrm{ft})$ & $30.80 \mathrm{~m}(101.06 \mathrm{ft}) \pm 0.5 \mathrm{~m}(1.64 \mathrm{ft})$ \\
\hline 3 & $31.09 \mathrm{~m}(102.00 \mathrm{ft})$ & $31.09 \mathrm{~m}(102.00 \mathrm{ft}) \pm 0.5 \mathrm{~m}(1.64 \mathrm{ft})$ \\
\hline 4 & $30.98 \mathrm{~m}(101.36 \mathrm{ft})$ & $30.98 \mathrm{~m}(101.36 \mathrm{ft}) \pm 0.5 \mathrm{~m}(1.64 \mathrm{ft})$ \\
\hline
\end{tabular}

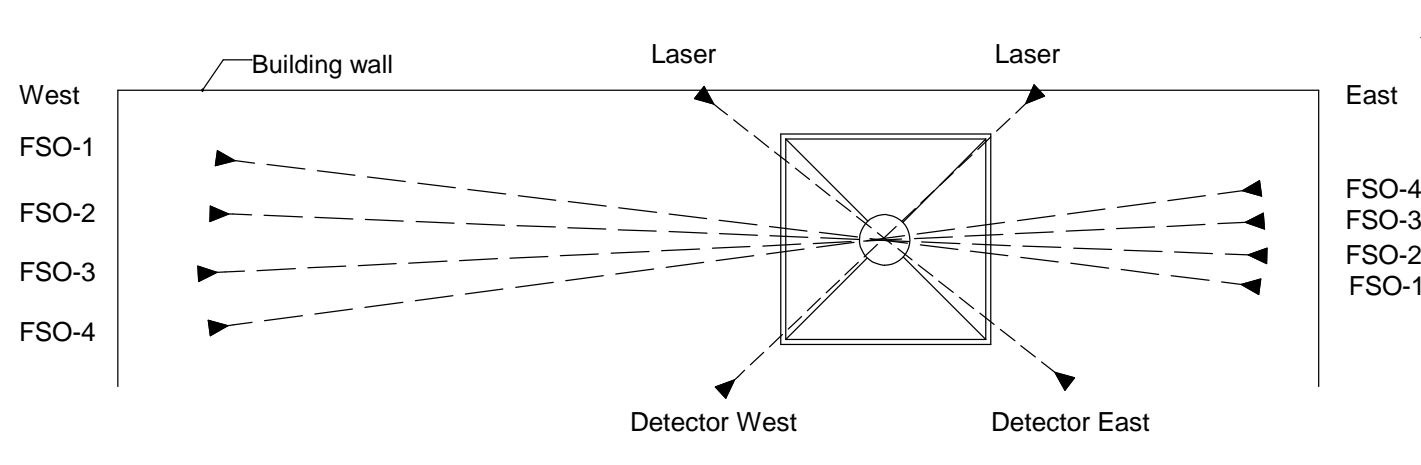

Plan View

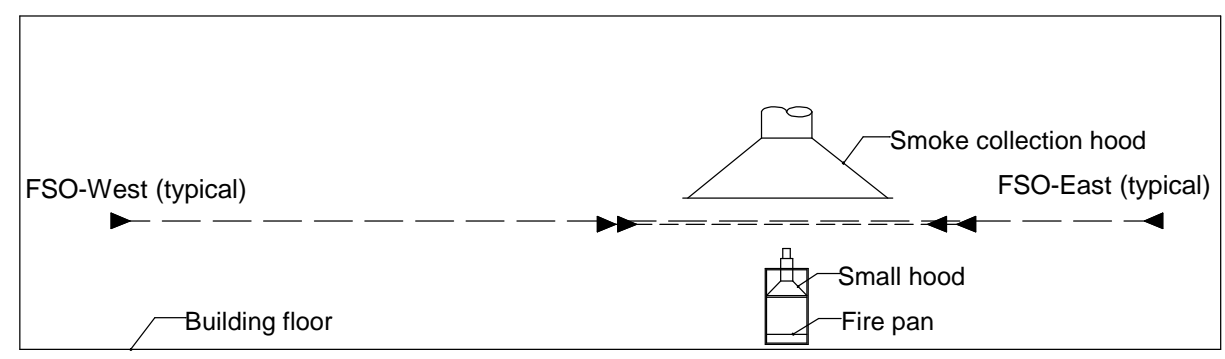

Elevation View

Figure 7. FSO Layout in NIST Large Fire Laboratory

\subsection{Smoke and Fire Generation Equipment}

The fire for the experiments was a diesel fuel pool fire with the fuel delivered to the test area using a liquid fuel spray system. The liquid spray system consisted of a fuel storage facility, a liquid pump, a turbine flow meter, and a spray nozzle mounted above a catch pan. A schematic drawing of the spray burner hardware is shown in Fig. 8. The fuel was stored in $190 \mathrm{~L}$ (50 gal) drums that were mounted on a rack in a steel shed located outside of the main building. A positive displacement pump, controlled by a variable frequency driver, was used to deliver the required fuel flow rate. The frequency driver and the emergency shut-off solenoid were both manually controlled. 
A dual rotor turbine flow meter measured the volumetric flow rate in the range of $0.06 \mathrm{~L} / \mathrm{min}$ to $11 \mathrm{~L} / \mathrm{min}$. The burner assembly was water cooled and insulated with approximately $50 \mathrm{~mm}$ of mineral wool to prevent drift in the fuel flow rate.

At the burner exit, a stainless steel, coarse atomization, spray nozzle was used to disperse the fuel. The nozzle had a $90^{\circ}$ full-cone spray pattern and was mounted $449 \mathrm{~mm}$ (13.8 in) above a $1.4 \mathrm{~m}$ by $1.4 \mathrm{~m}$ (54 in by 54 in) steel pan discharging down towards the pan. The pan was mounted under a small hood with a short stack extending above the hood. The small hood can be seen in Fig. 7. The small hood and stack were used to collect and focus the smoke in the area were the FSO laser beams crossed above the fire. Although the small hood altered the smoke plume from the fire, the objective of the experiments was to obtain a specified smoke obscuration in the laser path of the FSO units. Figure 9 shows the fire with the smoke flowing through and around the small hood.

For Scenario 4 the small hood and stack were lowered and the pan was placed on the small hood support frame $2.9 \mathrm{~m}(9.6 \mathrm{ft})$ above the floor. The fuel nozzle remained $449 \mathrm{~mm}(13.8 \mathrm{in})$ above the pan. At that elevation the laser beams from the FSO units passed through both flames and smoke from the diesel fuel fire. Figure 10 shows the elevated fire with FSO units in the background.

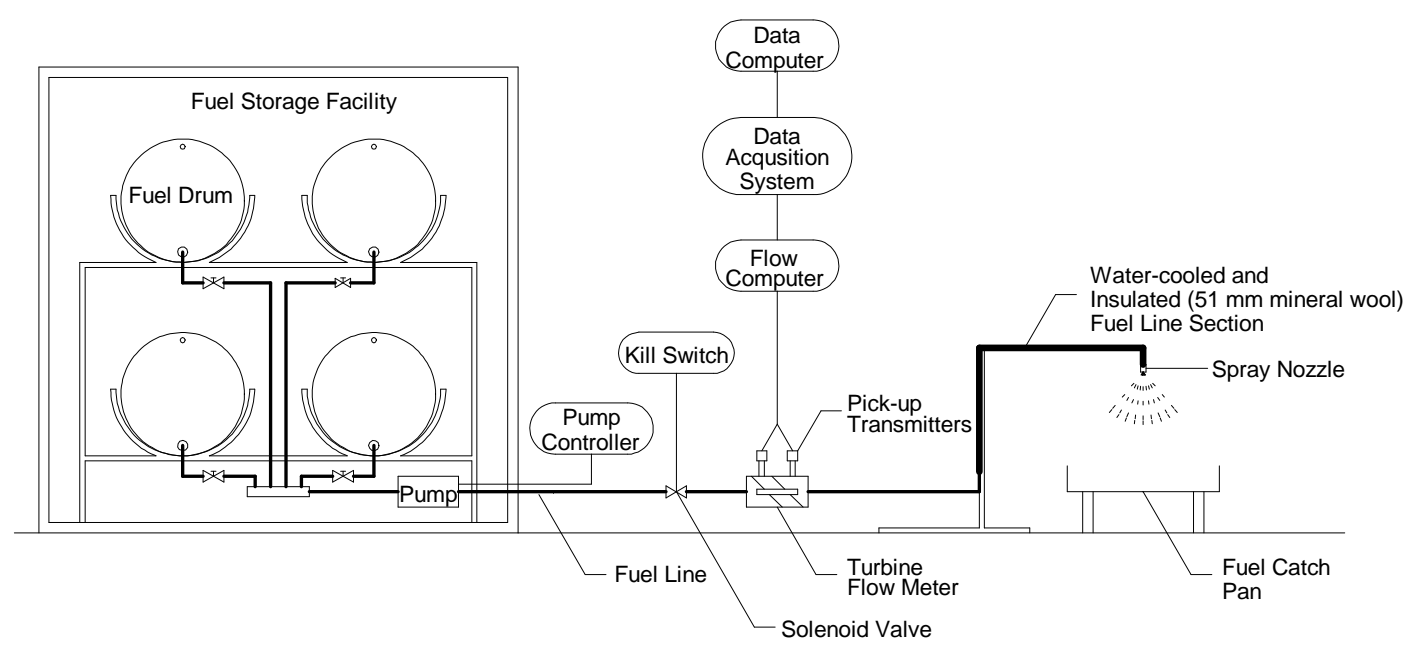

Figure 8. Schematic drawing of LFL spray burner hardware 


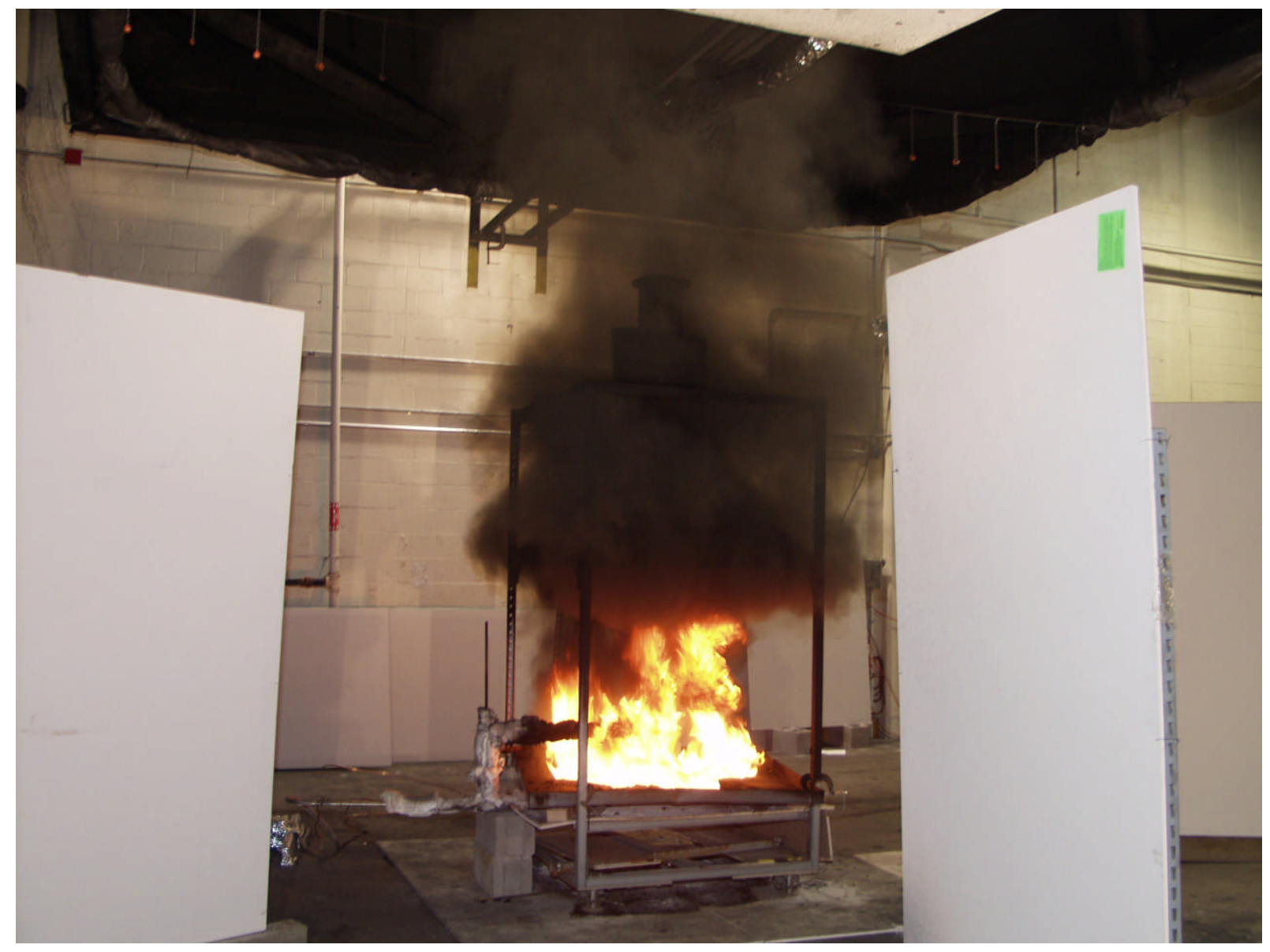

Figure 9. Fire with smoke focused by small hood (Scenarios 1, 2 and 3) 


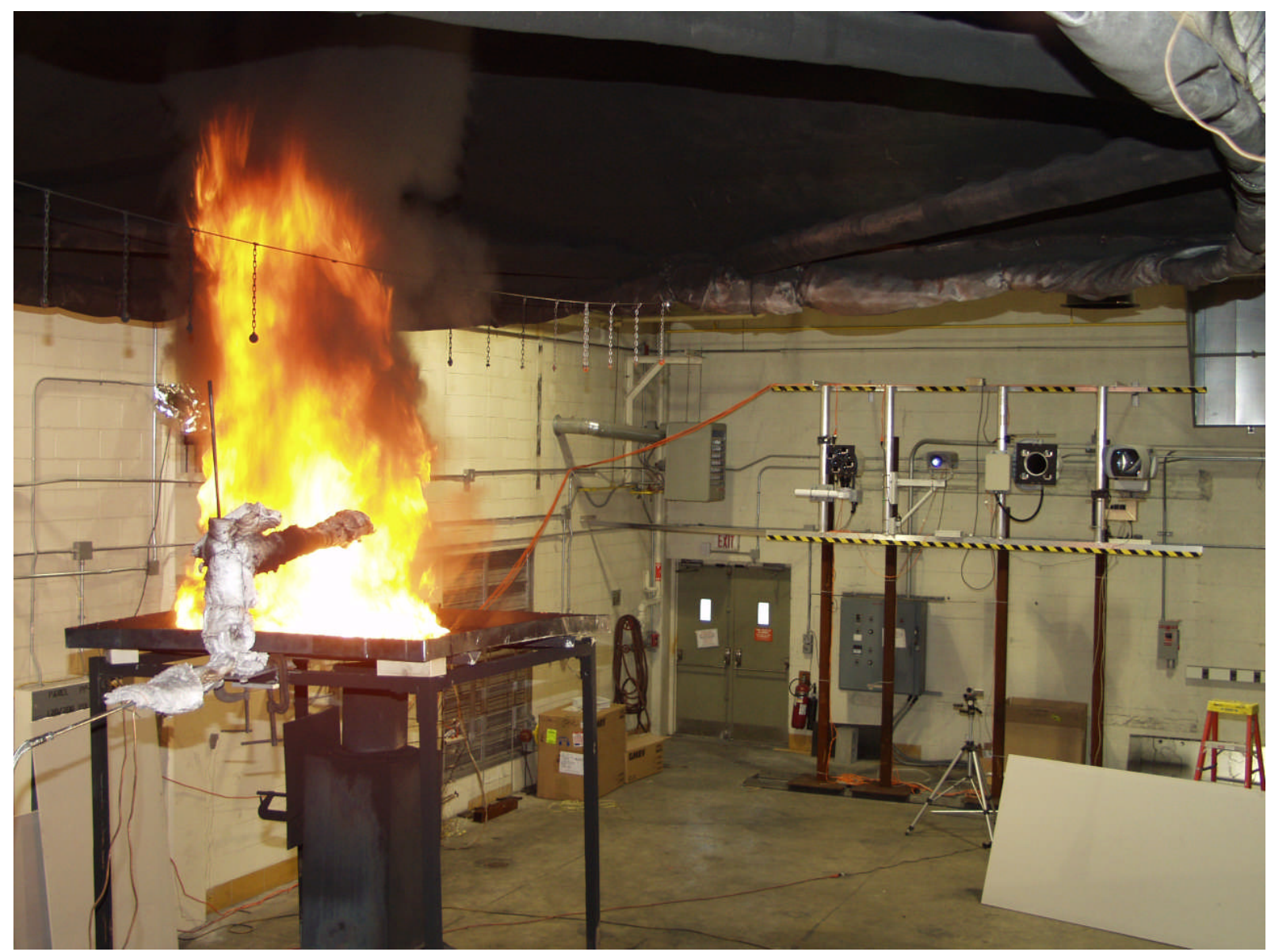

Figure 10. Fire with pan elevated (Scenario 4)

\subsection{Smoke Attenuation (Optical)}

Two soot extinction measurements with calibrated lasers were used to determine smoke plume obscuration. The NIST measurements were not used as telltales of FSO unit performance as they operated at different frequencies and did not go thought the exact same sections of the plume. They were instead used to link the laboratory scale experiments to the model predictions of real scale events and to permit experimental repeatability. The basic instrument components were a visible laser diode with a wavelength of $650 \mathrm{~nm}$, output power of $4.5 \mathrm{~mW}$, and a beam area of $4.0 \mathrm{~mm}$ by $0.6 \mathrm{~mm}$. Figure 7 shows the position of the NIST lasers and detectors. A fast response silicon photo detector with an active area of $3.6 \mathrm{~mm}$ by $3.6 \mathrm{~mm}$ was used to measure the laser output. In front of the detector were a $650 \mathrm{~nm} \pm 10 \mathrm{~nm}$ bandpass filter to block other emission sources and a 220 grit glass diffuser to evenly spread the laser beam over the detector. Figure 11 shows a laser installed in the experiment area. FSO operating wavelengths ranges from $785 \mathrm{~nm}$ to $1550 \mathrm{~nm}$.

The two lasers were mounted $3.8 \mathrm{~m}(12.4 \mathrm{ft})$ above the floor with the laser beams at approximately right angles to each other. The laser beams were at approximately $45^{\circ}$ angles to the beams of the FSO lasers as shown in Fig. 7. The path length for the east laser was $13.1 \mathrm{~m}$ 
$(43.0 \mathrm{ft})$ and for the west laser was $12.1 \mathrm{~m}(39.8 \mathrm{ft})$. The measured laser output was calibrated with neutral density filters prior to the experiments.

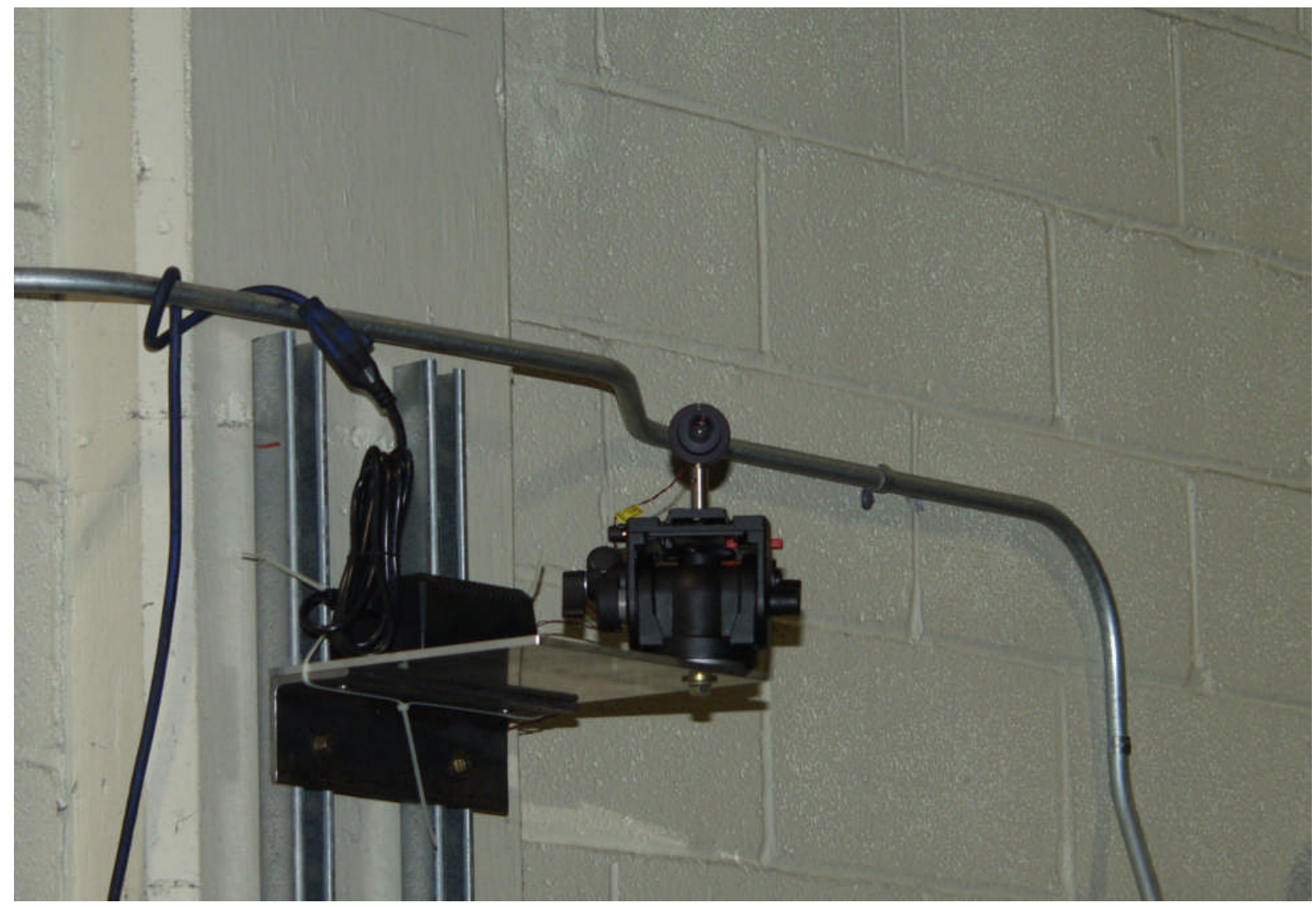

Figure 11. Obscuration laser

The smoke concentration measurement, as discussed in section 3.2.2, is based on the strong light extinction characteristic of flame-generated smoke. The ability to infer mass concentration from a light extinction measurement is made possible by the discovery that $\sigma_{s}$ is nearly universal for post-flame smoke produced from over-ventilated fires. A $\sigma_{s}$ value of $9.5 \mathrm{~m}^{2} / \mathrm{g}$ at a wavelength of $633 \mathrm{~nm}$ was selected as it was representative of heavy hydrocarbons (Mulholland 1992). This is the same wavelength selected for FDS model transmittance predictions (see section 4.2.2). The basic qualitative ideas that support this universality are that soot from all flames is basically carbon in the form of agglomerates with primary sphere sizes much smaller than the wavelength of light and a fractal dimension less than two. For these conditions the light absorption cross section is proportional to the mass and is the dominant contribution to the light-extinction coefficient. There will be a smaller contribution from the light scattering cross-section that depends on the agglomerate size.

Solving Equation (1) for the mass concentration, one obtains the following result:

$$
M_{s}=\frac{\ln \left(I_{0} / I\right)}{\sigma_{s} L}
$$


Equation (2) can be used to calculate the mass concentration $\left(M_{s}\right)$ if the transmittance, pathlength, and specific extinction coefficient are known. On the other hand if the mass concentration and the specific extinction coefficient are known, Equation (1) can be used to compute the decrease in the light intensity per pathlength. For example assuming a specific extinction coefficient of $9.5 \mathrm{~m}^{2} / \mathrm{g}$ and a soot mass concentration of $0.01 \mathrm{~g} / \mathrm{m}^{3}$, the decrease of laser light intensity ranges from $9 \%$ to $94 \%$ over path lengths of $1 \mathrm{~m}$ to $30 \mathrm{~m}$, respectively (Table 4).

Table 4. Decrease in Laser Light Intensity at Pathlengths from $1 \mathrm{~m}$ to $30 \mathrm{~m}$.

\begin{tabular}{|c|c|c|c|}
\hline $\begin{array}{c}\text { Pathlength } \\
(\mathrm{m})\end{array}$ & $\begin{array}{c}\text { Transmittance } \\
\mathrm{I} / \mathrm{I}_{\mathrm{o}}\end{array}$ & $\begin{array}{c}\text { Decrease in Laser } \\
\text { Light Intensity, } \\
{\left[\left(1-\mathrm{I} / \mathrm{I}_{\mathrm{o}}\right) * 100\right]}\end{array}$ & $\begin{array}{c}\text { Total Combined Uncertainty in } \\
\text { Decrease in Laser Light Intensity }\end{array}$ \\
\hline 1 & .91 & $9 \%$ & $9 \% \pm 0.9 \%$ \\
\hline 5 & .62 & $38 \%$ & $38 \% \pm 3.8 \%$ \\
\hline 10 & .39 & $61 \%$ & $85 \% \pm 6.1 \%$ \\
\hline 20 & .15 & $85 \%$ & $94 \% \pm 9.4 \%$ \\
\hline 30 & .06 & $94 \%$ & $\mathrm{~g} / \mathrm{m}^{3}$ \\
\hline
\end{tabular}

\subsection{Data Acquisition}

In addition to the communications link data monitoring by the sponsor guest researchers, there were two data acquisition systems utilized by NIST. The NIST Data Acquisition system (DAQ) was utilized for monitoring the fuel flow. A portable data acquisition system was used to measure the output from the calibrated lasers. A voltage "marker" signal was used to synchronize both data acquisition systems. Sampling rates were $1 \mathrm{~Hz}$ for the fuel flow DAQ and $0.5 \mathrm{~Hz}$ for the portable system.

\subsection{Test Procedure}

Prior to the start of each experiment, the lasers used to measure the smoke obscuration were calibrated using neutral density filters. Filters with optical densities of $0.1,0.2,0.3,0.4,0.5,0.6$, 1.0, 2.0, 3.0, and 4.0 corresponding to a transmittance $\left(\mathrm{I} / \mathrm{I}_{0}\right)$ of $0.79,0.63,0.50,0.40,0.32,0.25$, $0.10,0.01,0.001$, and 0.0001 were used. Figure 12 shows an example of the transmittance versus voltage for a laser. The transmittance varied almost linearly with voltage as seen by solid lines on Figure 12. A linear variation was used to process the data collected during testing.

The detector output was found to drift slightly during the course of an experiment. This drift was likely due to temperature changes in the detector. To accommodate this drift, the output of the detector, with no obscuration, was measured just prior to the experiment and just afterwards. The drift was assumed to take place at a constant rate during the course of the experiment. Therefore, a linear correction was applied so that the detector output at the end of the experiment would be equal to the output at the beginning of the experiment. The correction for detector drift was applied prior to the conversion of detector voltage to transmittance. 


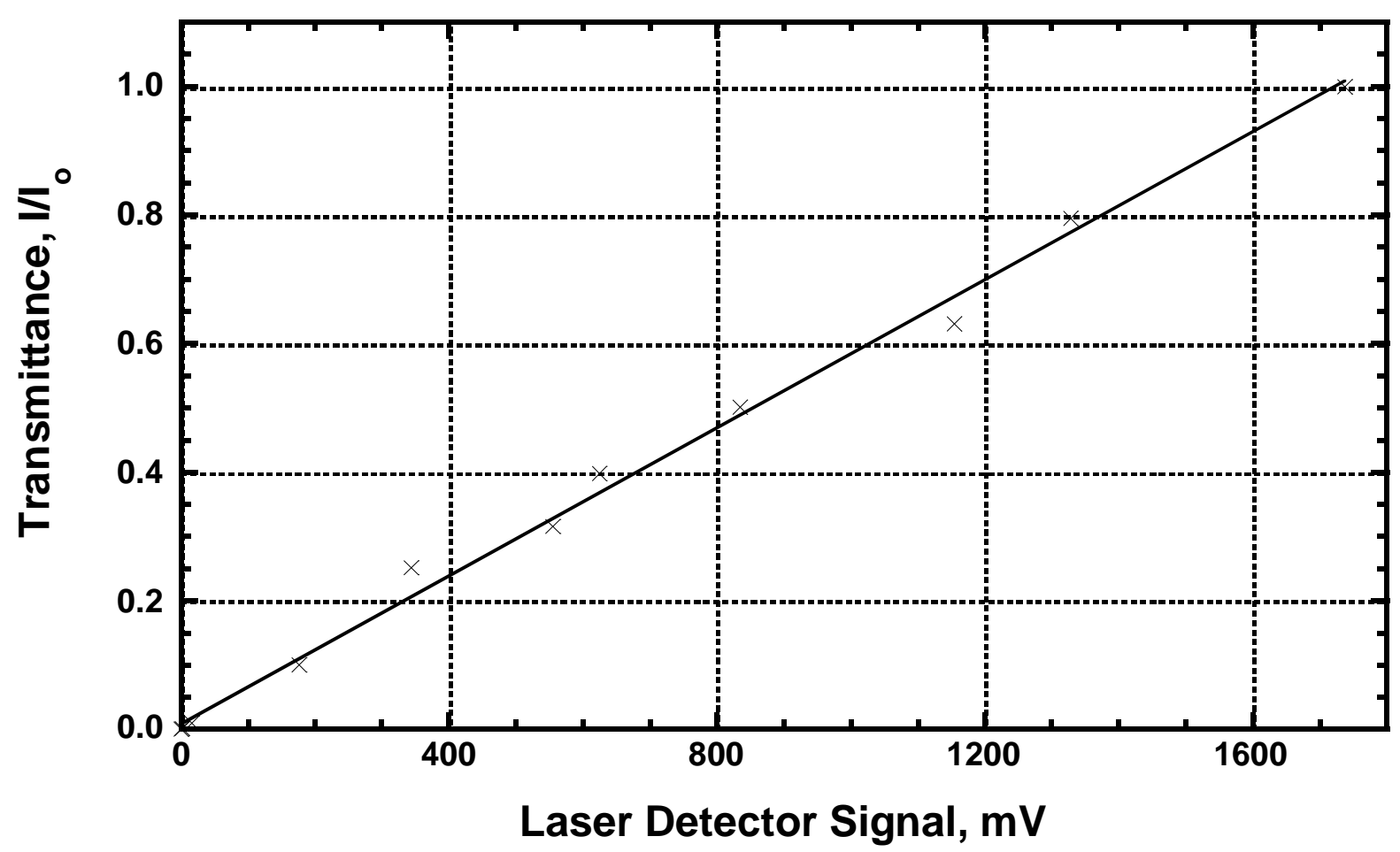

Figure 12a. Sample laser detector calibration plot showing transmittance versus the laser detector signal (transmittance ranging from 0.0 to 1.0)

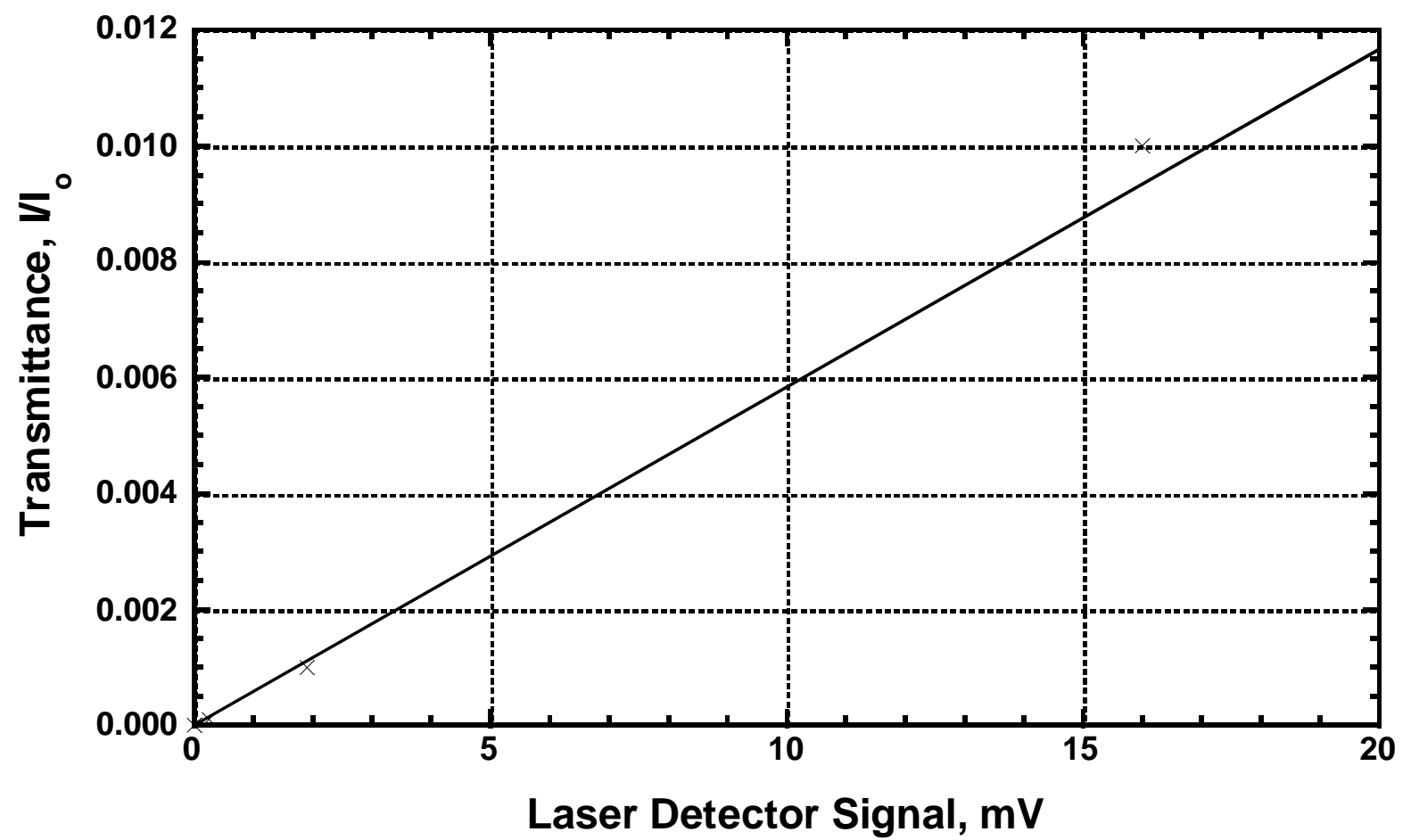

Figure 12b. Sample laser detector calibration plot showing transmittance versus the laser detector signal (transmittance ranging from 0.0 to 0.012 ) 
A small pilot flame was ignited prior to the experiment to serve as an igniter for the diesel fuel. The experiment began when the fuel flow was started and the fire was allowed to reach steady burning in the pan. The transmission output from the calibrated lasers was examined and the fuel flow rate adjusted to achieve the desired obscuration in the smoke above the fire. Once the obscuration values reached the desired target values, the FSO measurements were initiated.

\subsection{Results}

Results and timelines for the four experiments are listed below. Tables 5 through 8 contain the experiment timelines. Figures 13 through 28 show the transmittance for each experiment plotted versus time from ignition. The first pair of plots for each experiment uses the full $y$-axis while the second pair focuses on the range of interest for the particular experiment. All data shown are averaged over 10 s intervals. Figures 29 through 32 show the diesel fuel flow rate versus time from ignition for each experiment.

\section{Experiment 1}

The fuel was ignited at 11:26:23 EDT. The target obscuration value $\mathrm{I} / \mathrm{I}_{\mathrm{o}}$ of 0.1 was reached 3525 $\mathrm{s}$ after ignition. The target obscuration period ended $17092 \mathrm{~s}$ after ignition. Experiment 1 ended and the fuel secured $17158 \mathrm{~s}$ after ignition.

\section{Experiment 2}

The fuel was ignited at 9:47:01 EDT. The target obscuration value $\mathrm{I} / \mathrm{I}_{\mathrm{o}}$ of 0.01 was reached $399 \mathrm{~s}$ after ignition. The target obscuration period ended $8691 \mathrm{~s}$ after ignition. Experiment 2 was terminated and the fuel secured $8692 \mathrm{~s}$ after ignition.

\section{Experiment 3}

The fuel was ignited at 14:57:20 EDT. A fuel distribution system problem early in the experiment caused the momentary reduction in obscuration. The first target obscuration value $\mathrm{I} / \mathrm{I}_{\mathrm{o}}$ of 0.001 was reached $3039 \mathrm{~s}$ after ignition. The first target obscuration period ended $4831 \mathrm{~s}$ after ignition. The second target obscuration value $\mathrm{I} / \mathrm{I}_{\mathrm{o}}$ of 0.01 was reached $4846 \mathrm{~s}$ after ignition and ended $5795 \mathrm{~s}$. Experiment 2 was then terminated and the fuel secured $8692 \mathrm{~s}$ after ignition.

\section{Experiment 4}

In this experiment, the fire pan was raised and the FSO beams were crossing a region of intermittent flames. The observed large fluctuations in transmittance were therefore expected. The fuel was ignited at 14:20:46 EDT and the fuel flow was adjusted in order obtain intermittent flames at the FSO beam crossing region. This was achieved at $1937 \mathrm{~s}$ after ignition. The fuel flow remained at the same level until $5337 \mathrm{~s}$ after ignition. At $5442 \mathrm{~s}$ the experiments was terminated and the fuel was secured. 
Table 5. Experiment 1 timeline

\begin{tabular}{|c|c|l|}
\hline Time (EDT) & Time from Ignition (s) & \multicolumn{1}{|c|}{ Event } \\
\hline $11: 26: 23$ & 0 & Fuel ignited \\
\hline $12: 25: 08$ & 3525 & Target obscuration value reached \\
\hline $16: 11: 15$ & 17092 & Target obscuration period ended \\
\hline $16: 12: 21$ & 17158 & Fuel stopped \\
\hline
\end{tabular}

Table 6. Experiment 2 timeline

\begin{tabular}{|c|c|l|}
\hline Time (EDT) & Time from Ignition (s) & \multicolumn{1}{|c|}{ Event } \\
\hline $9: 47: 01$ & 0 & Fuel ignited \\
\hline $9: 53: 40$ & 399 & Target obscuration value reached \\
\hline $12: 11: 52$ & 8691 & Target obscuration period ended \\
\hline $12: 11: 53$ & 8692 & Fuel stopped \\
\hline
\end{tabular}

Table 7. Experiment 3 timeline

\begin{tabular}{|c|c|l|}
\hline Time (EDT) & Time from Ignition (s) & \multicolumn{1}{|c|}{ Event } \\
\hline $14: 57: 20$ & 0 & Fuel ignited \\
\hline $15: 47: 59$ & 3039 & $1^{\text {st }}$ target obscuration value reached \\
\hline $16: 17: 51$ & 4831 & $1^{\text {st }}$ target obscuration period ended \\
\hline $16: 18: 06$ & 4846 & $2^{\text {nd }}$ target obscuration value reached \\
\hline $16: 33: 55$ & 5795 & $2^{\text {nd }}$ target obscuration period ended \\
\hline $16: 33: 58$ & 5798 & Fuel stopped \\
\hline
\end{tabular}

Table 8. Experiment 4 timeline

\begin{tabular}{|c|c|l|}
\hline Time (EDT) & Time from Ignition (s) & \multicolumn{1}{|c|}{ Event } \\
\hline $14: 20: 46$ & 0 & Fuel ignited \\
\hline $14: 53: 03$ & 1937 & Target fuel flow reached \\
\hline $15: 49: 43$ & 5337 & Target fuel flow period ended \\
\hline $15: 51: 28$ & 5442 & Fuel stopped \\
\hline
\end{tabular}




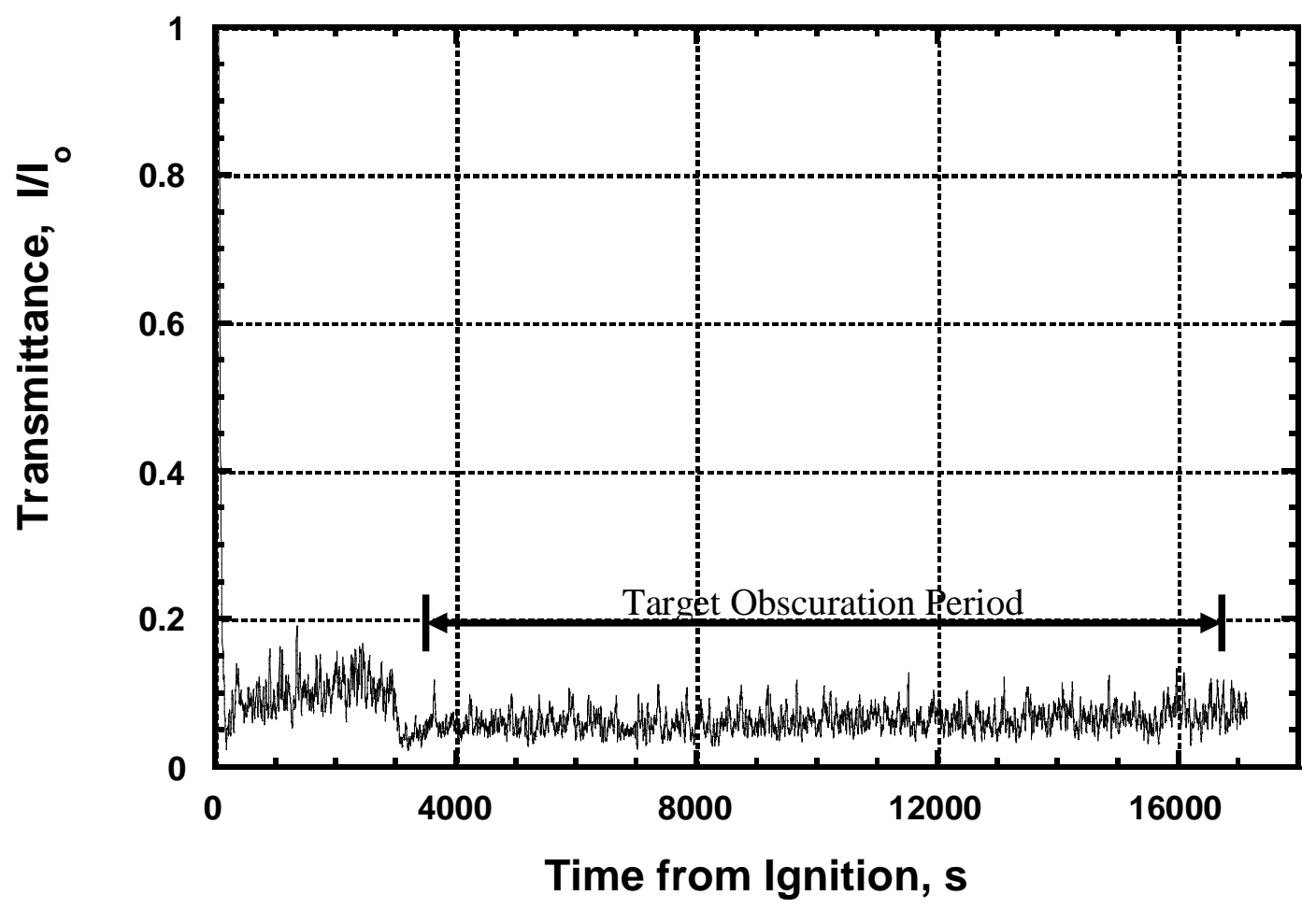

Figure 13. Test 1 transmittance plotted versus time from ignition (11:26:23 EDT) for the east laser installation

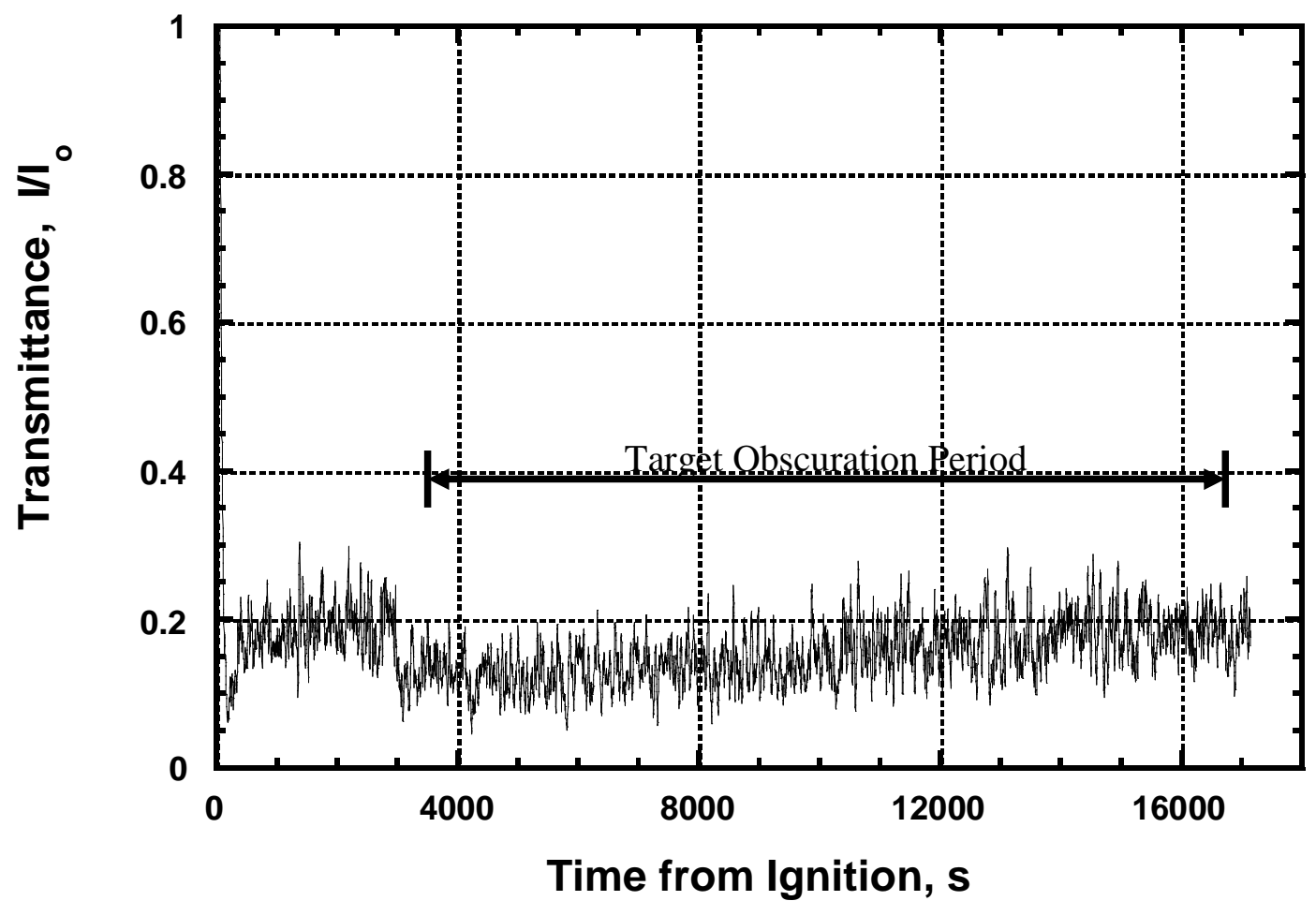

Figure 14. Test 1 transmittance plotted versus time from ignition (11:26:23 EDT) for the west laser installation 


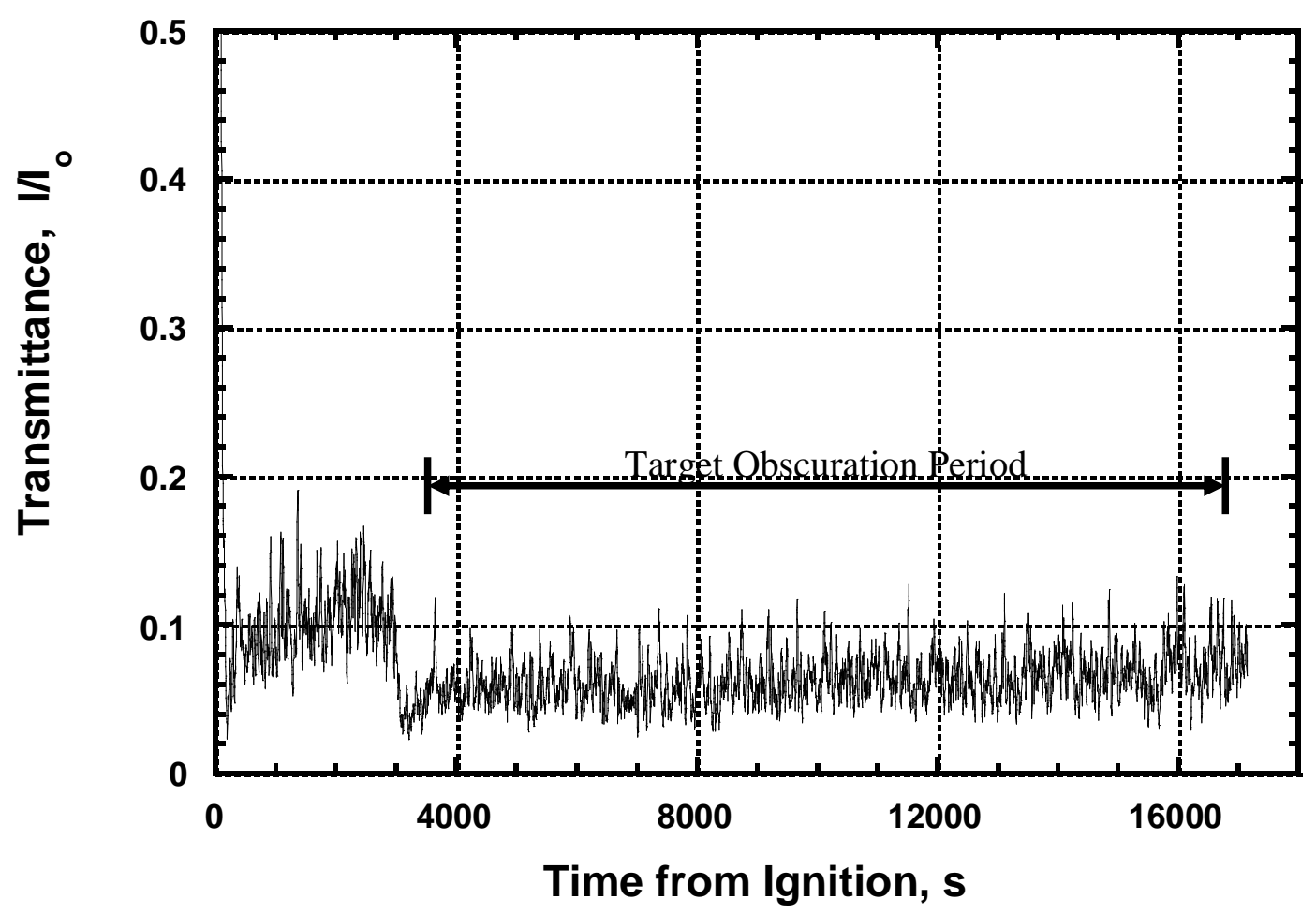

Figure 15. Test 1 transmittance plotted versus time from ignition (11:26:23 EDT) for the east laser installation (expanded scale)

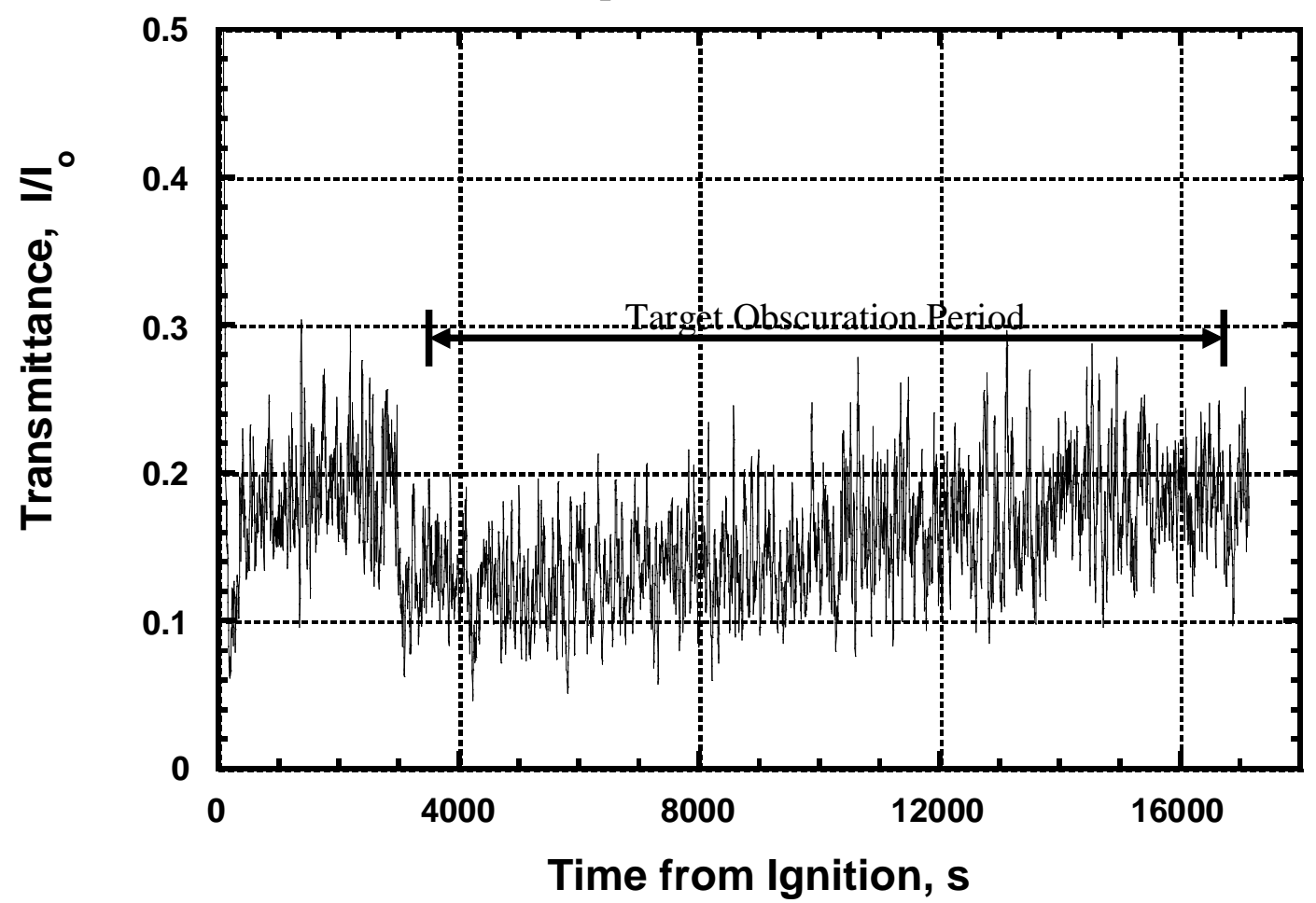

Figure 16. Test 1 transmittance plotted versus time from ignition (11:26:23 EDT) for the west laser installation (expanded scale) 


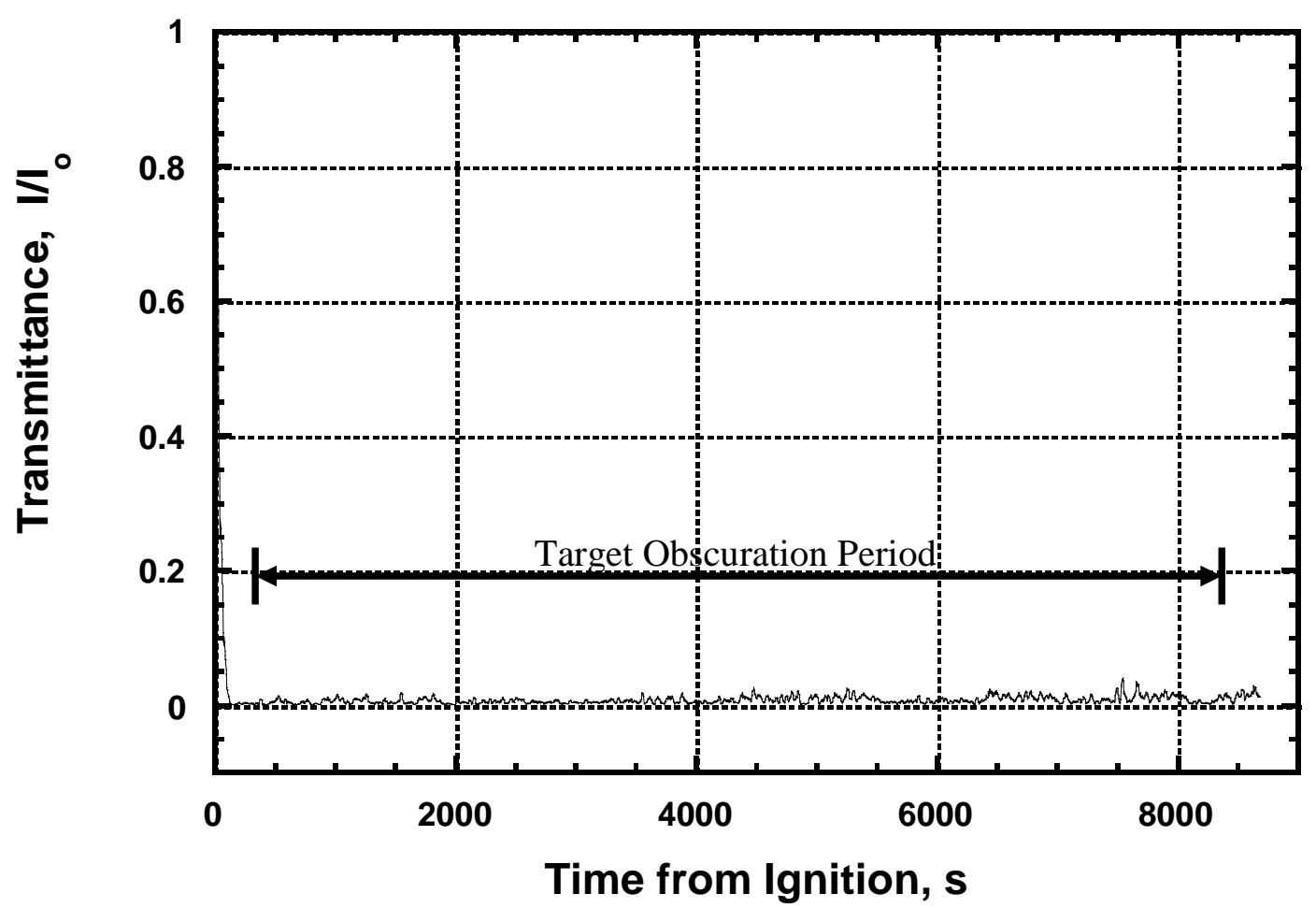

Figure 17. Test 2 transmittance plotted versus time from ignition (9:47:01 EDT) for the east laser installation

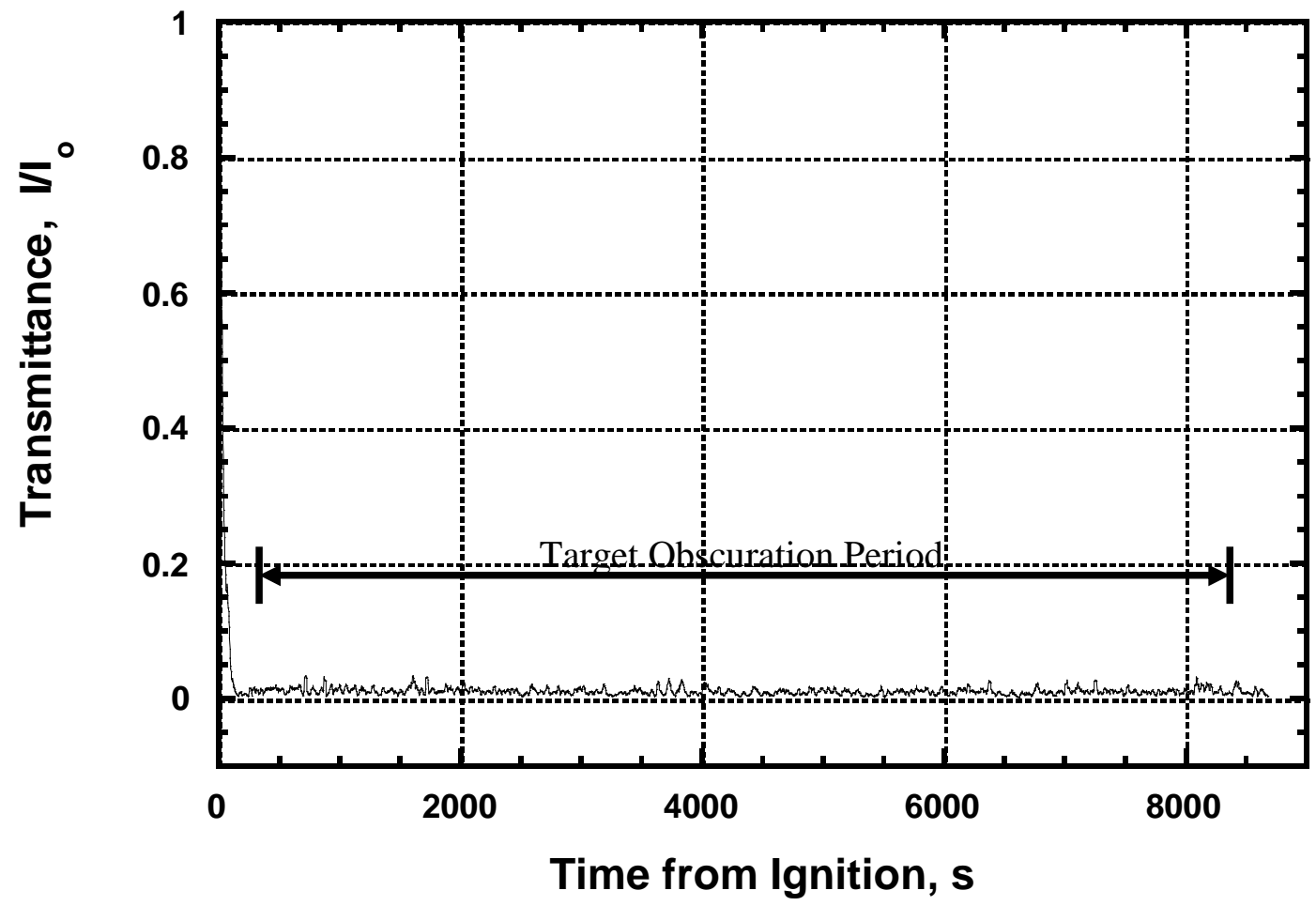

Figure 18. Test 2 transmittance plotted versus time from ignition (9:47:01 EDT) for the west laser installation 


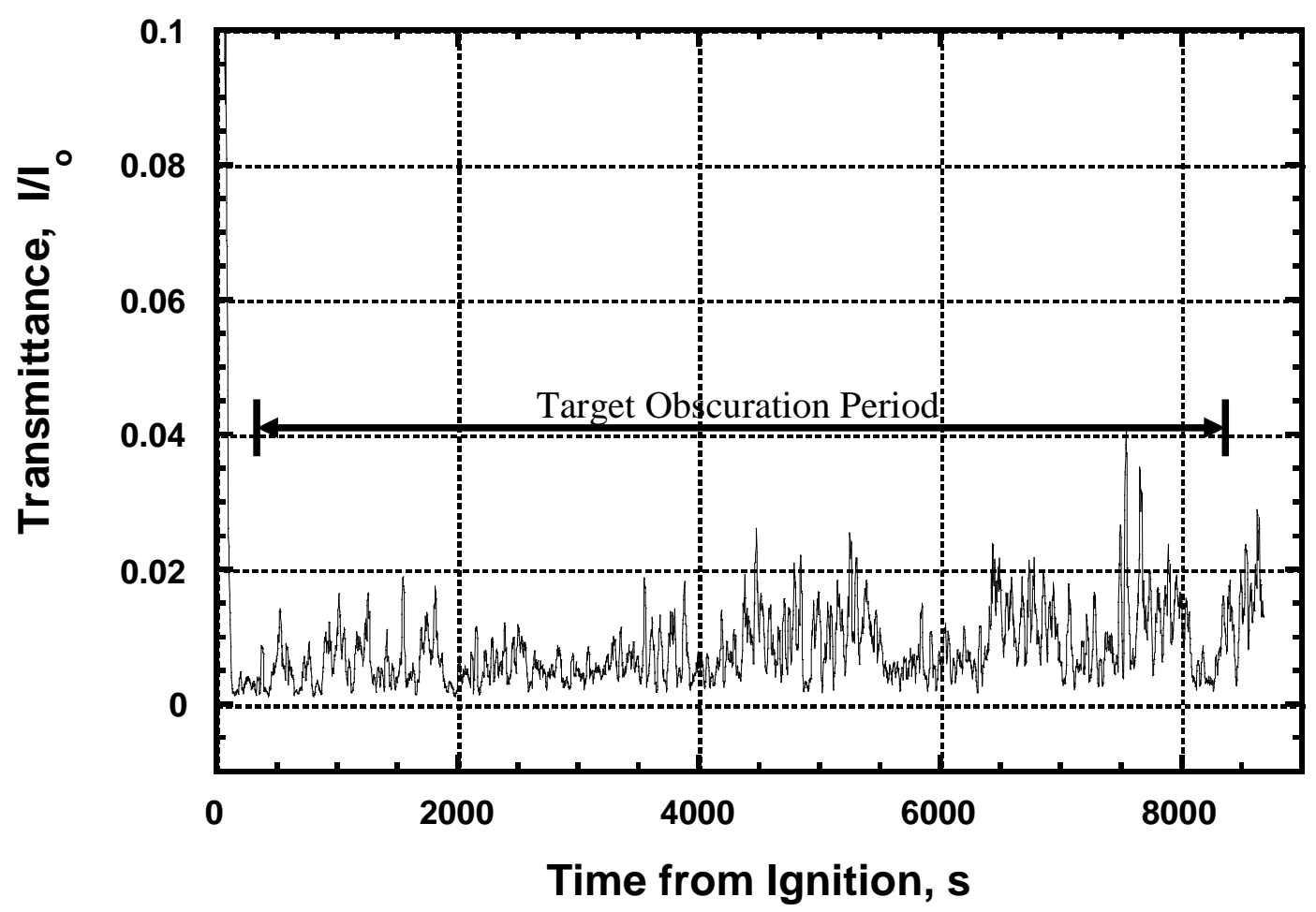

Figure 19. Test 2 transmittance plotted versus time from ignition (9:47:01 EDT) for the east laser installation (expanded scale)

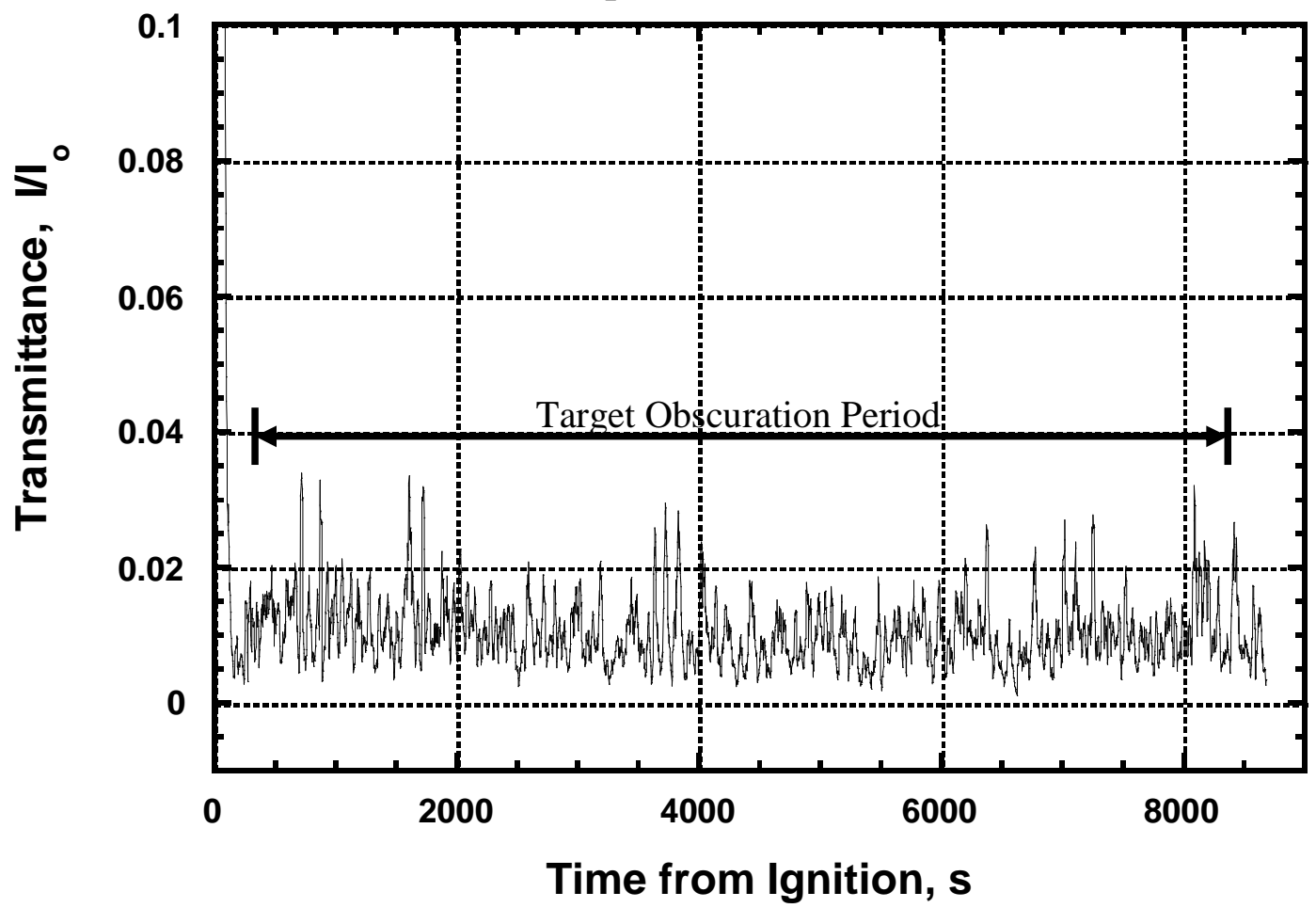

Figure 20. Test 2 transmittance plotted versus time from ignition (9:47:01 EDT) for the west laser installation (expanded scale) 


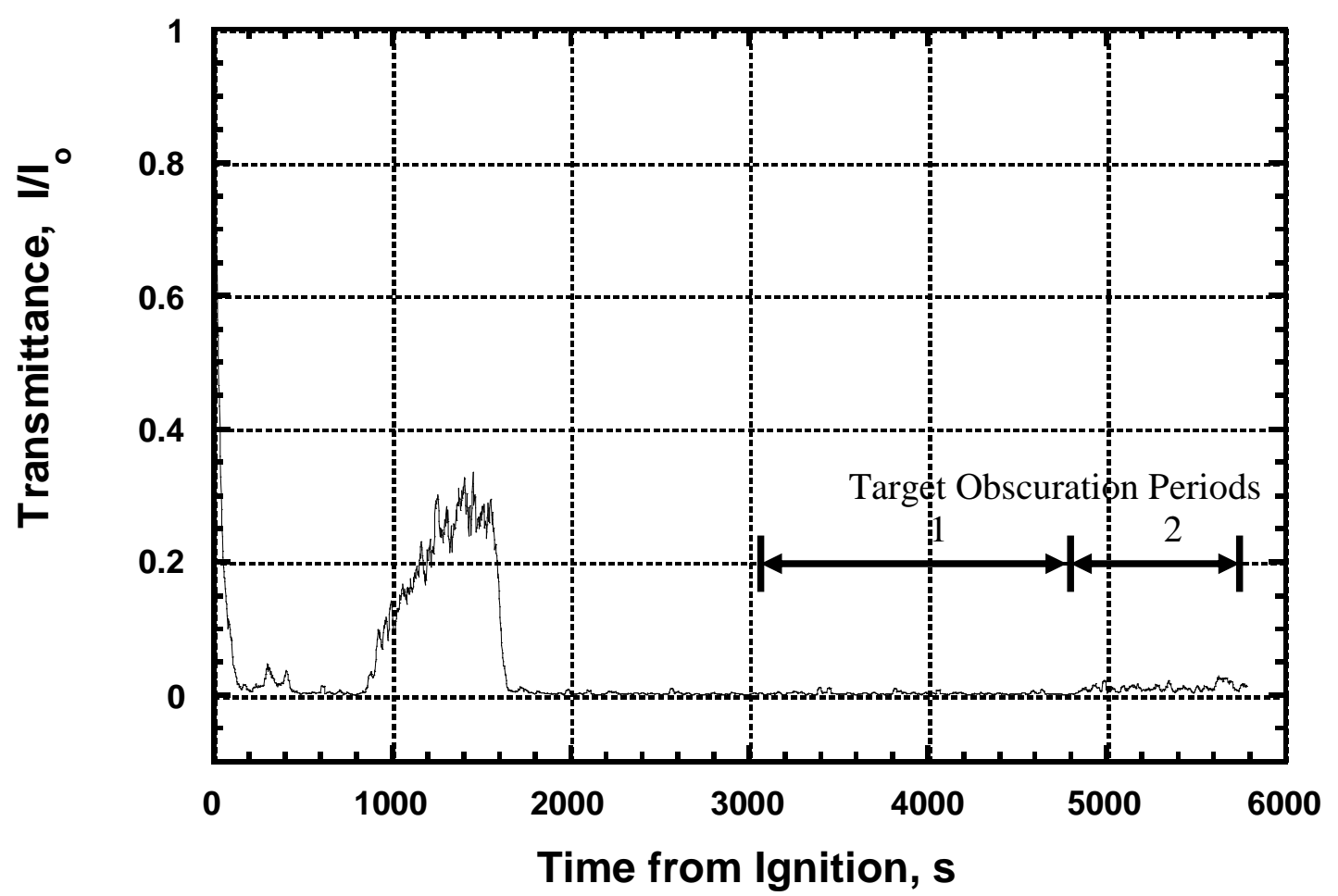

Figure 21. Test 3 transmittance plotted versus time from ignition (14:57:20 EDT) for the east laser installation

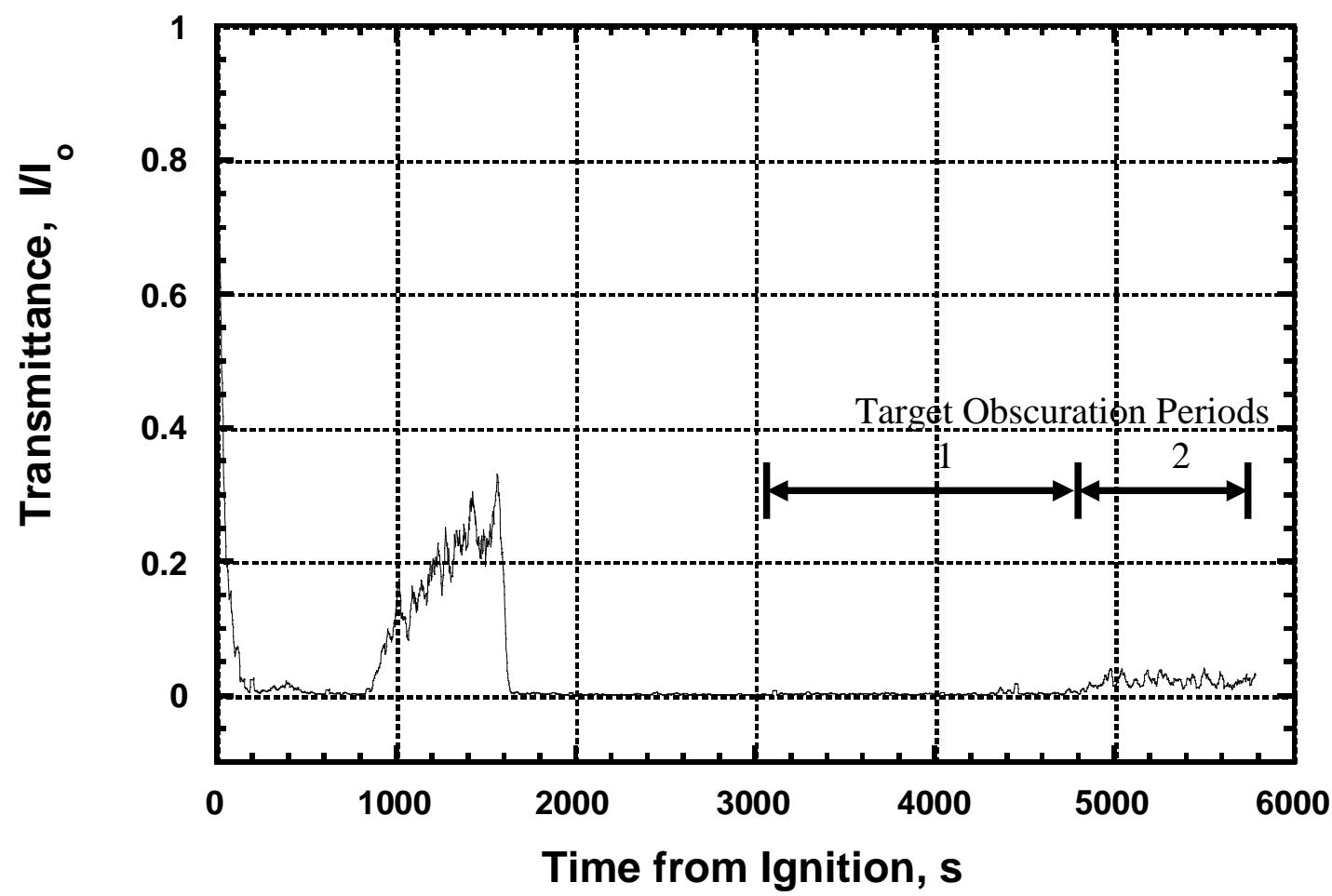

Figure 22. Test 3 transmittance plotted versus time from ignition (14:57:20 EDT) for the west laser installation 


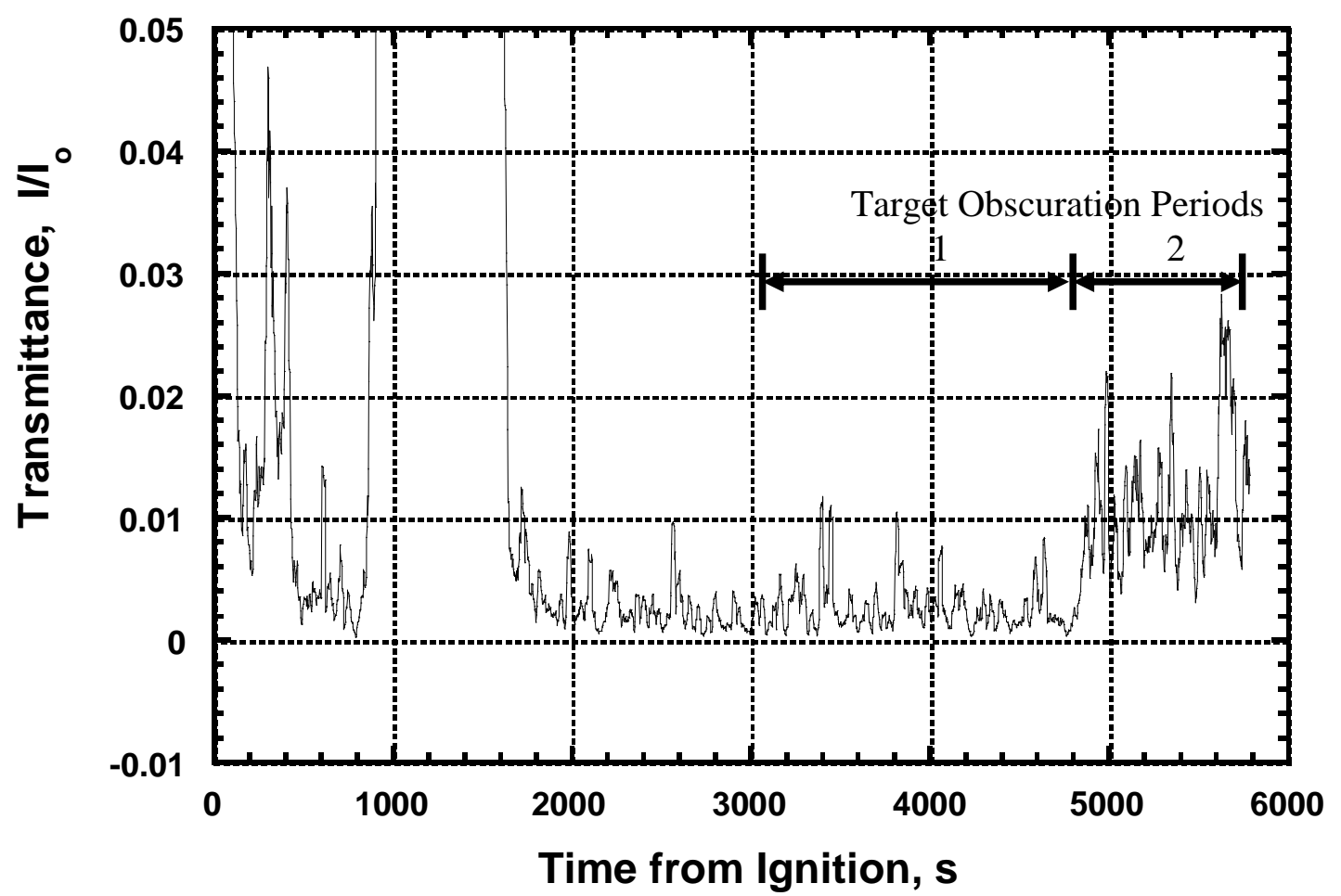

Figure 23. Test 3 transmittance plotted versus time from ignition (14:57:20 EDT) for the east laser installation (expanded scale)

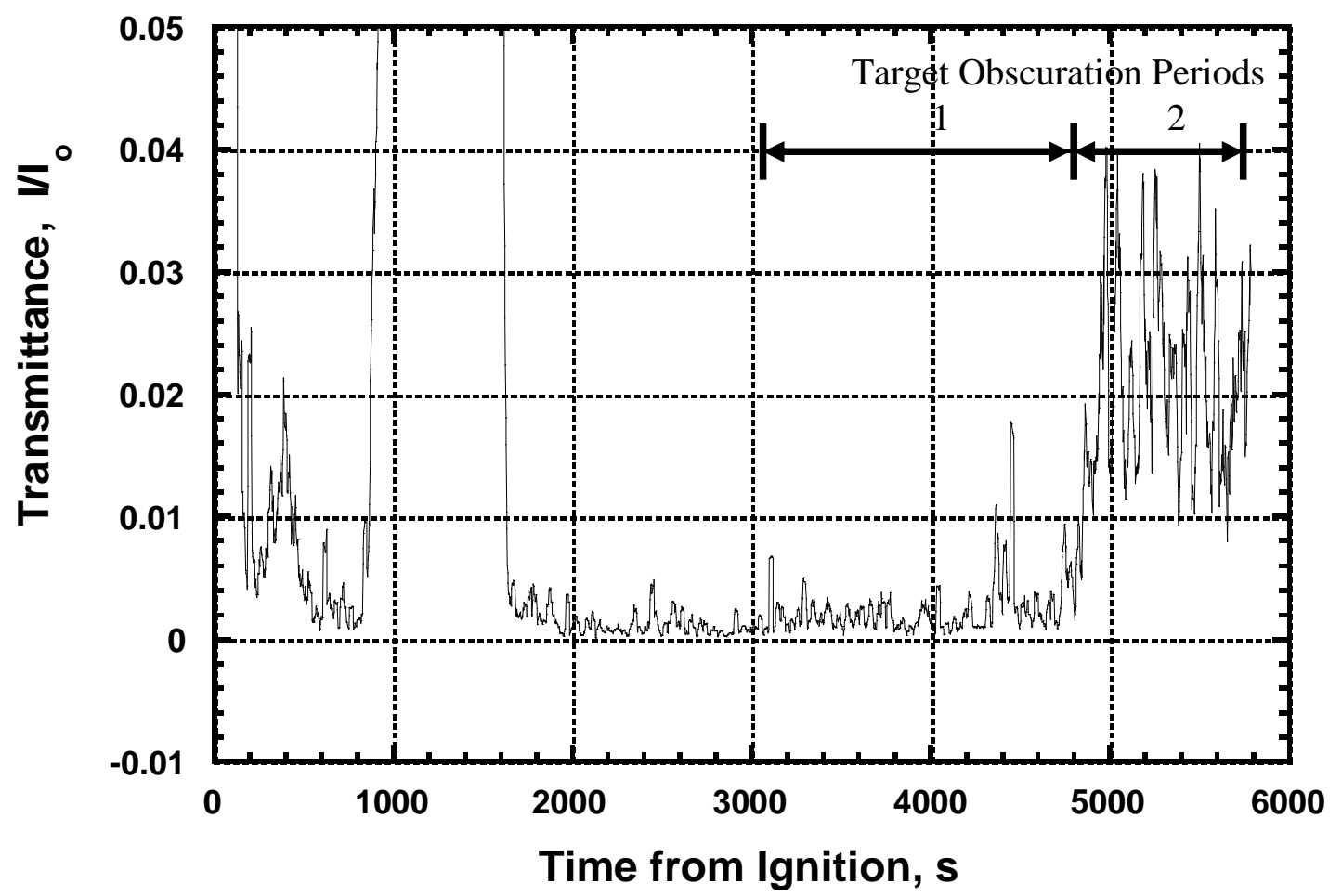

Figure 24. Test 3 transmittance plotted versus time from ignition (14:57:20 EDT) for the west laser installation (expanded scale) 


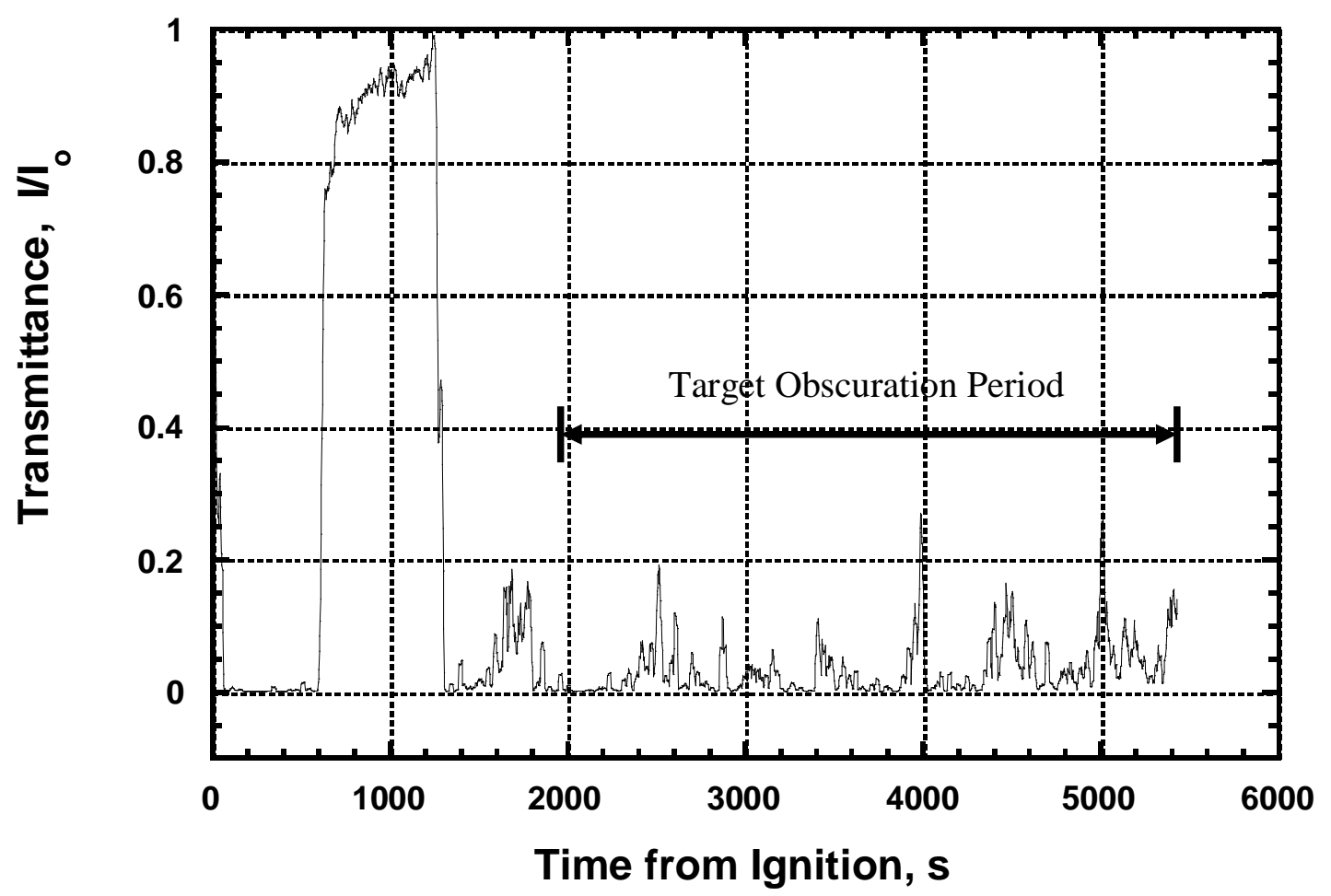

Figure 25. Test 4 transmittance plotted versus time from ignition (14:20:46 EDT) for the east laser installation

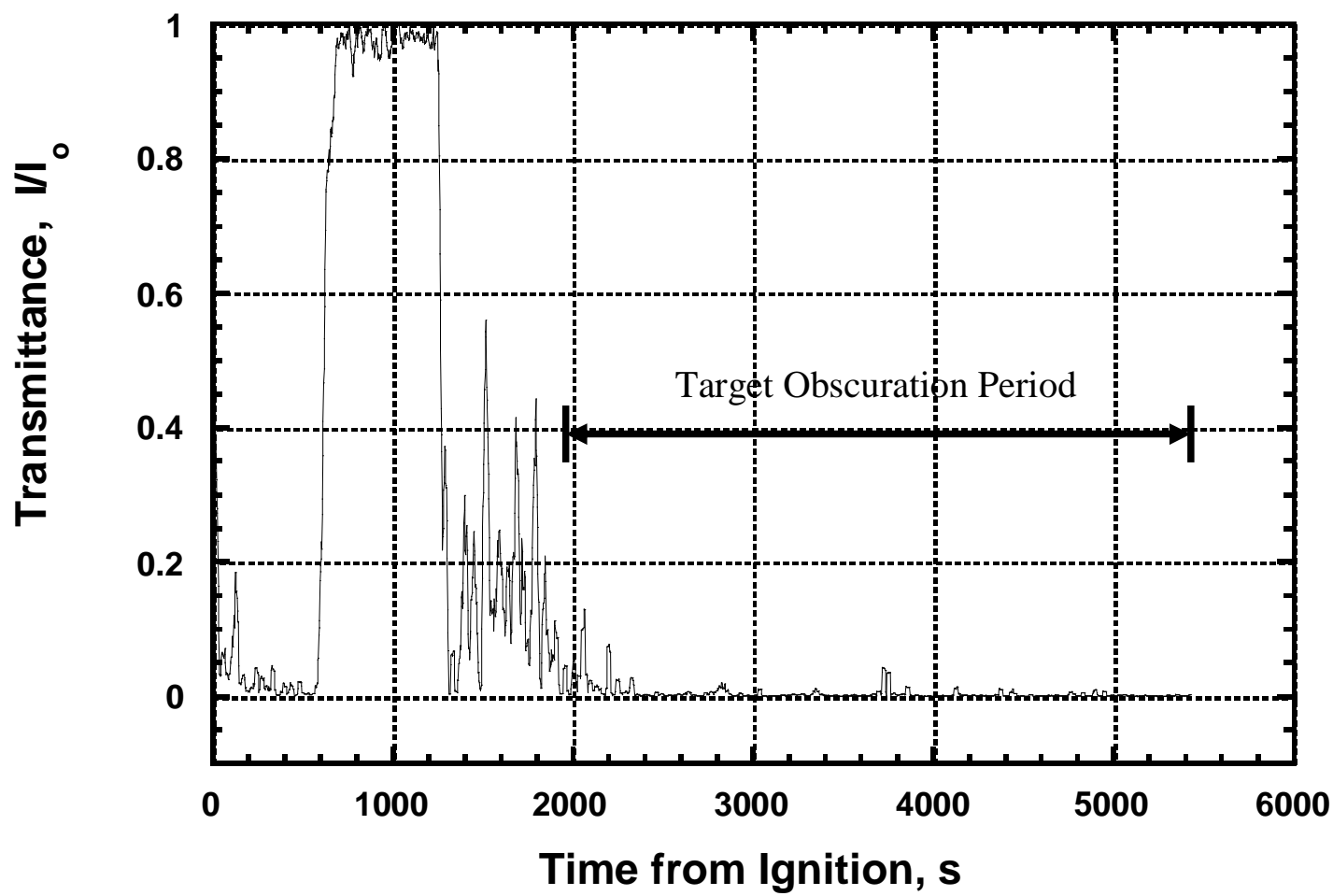

Figure 26. Test 4 transmittance plotted versus time from ignition (14:20:46 EDT) for the west laser installation 


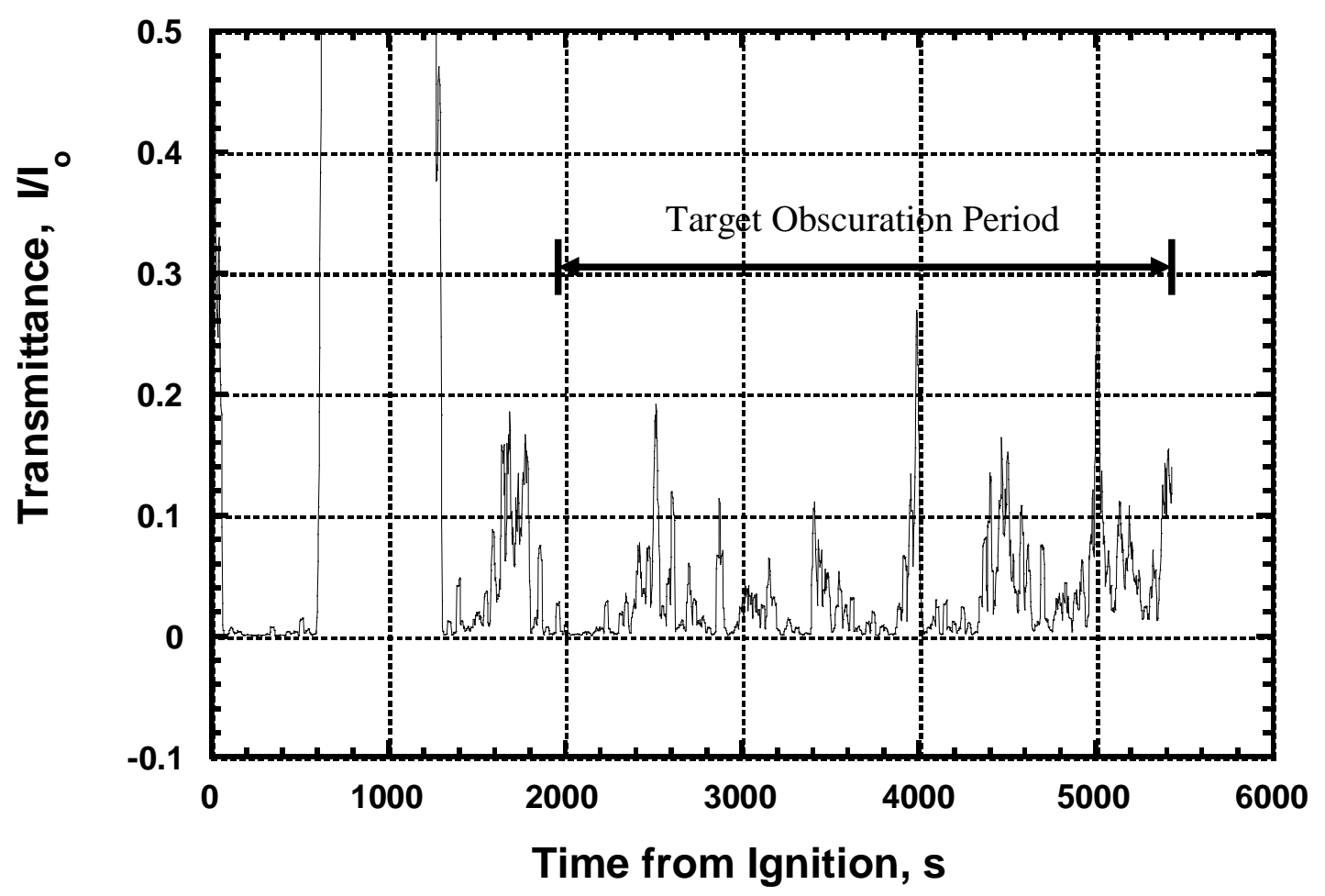

Figure 27. Test 4 transmittance plotted versus time from ignition (14:20:46 EDT) for the east laser installation (expanded scale)

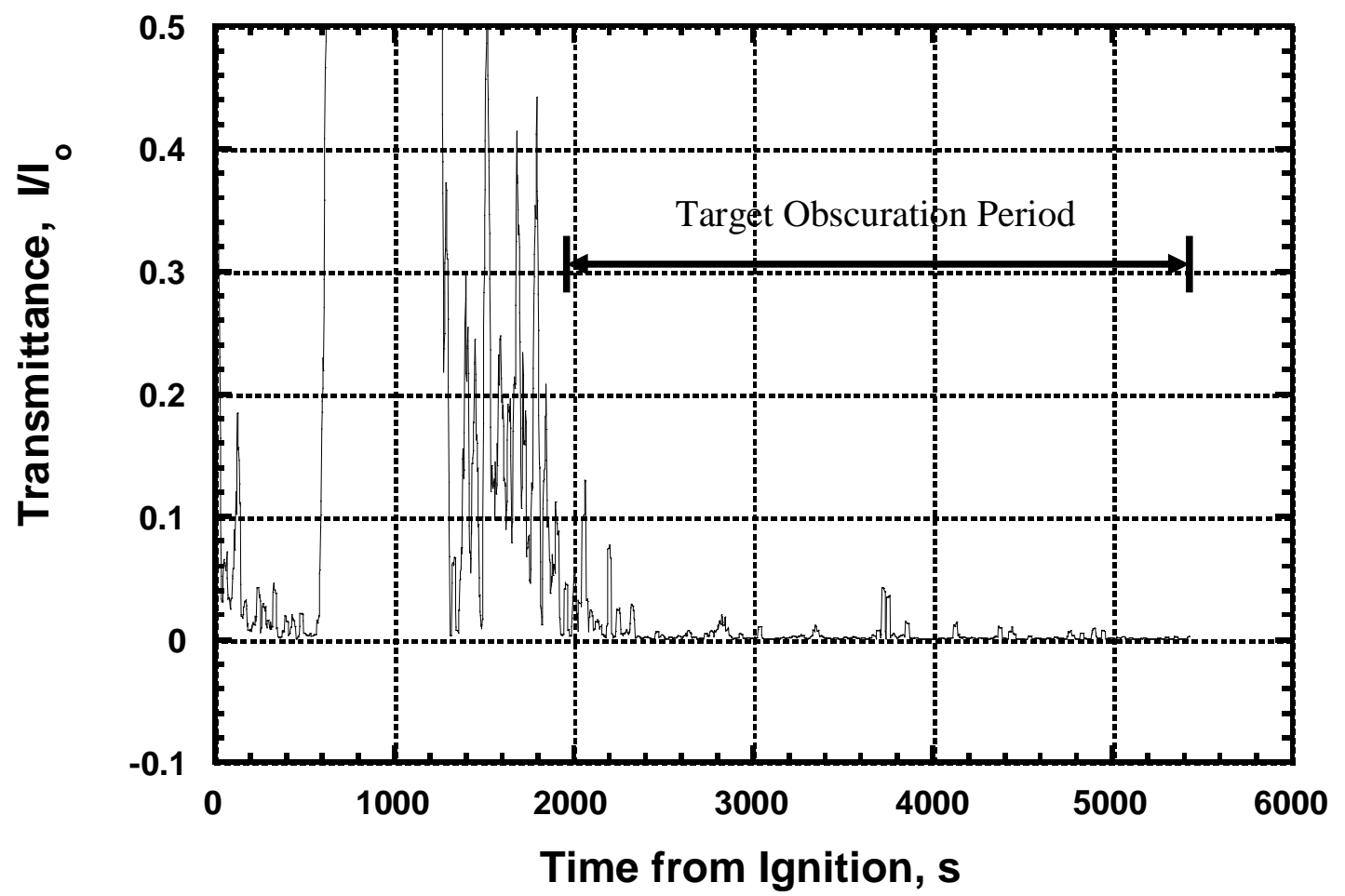

Figure 28. Test 4 transmittance plotted versus time from ignition (14:20:46 EDT) for the east laser installation (expanded scale) 


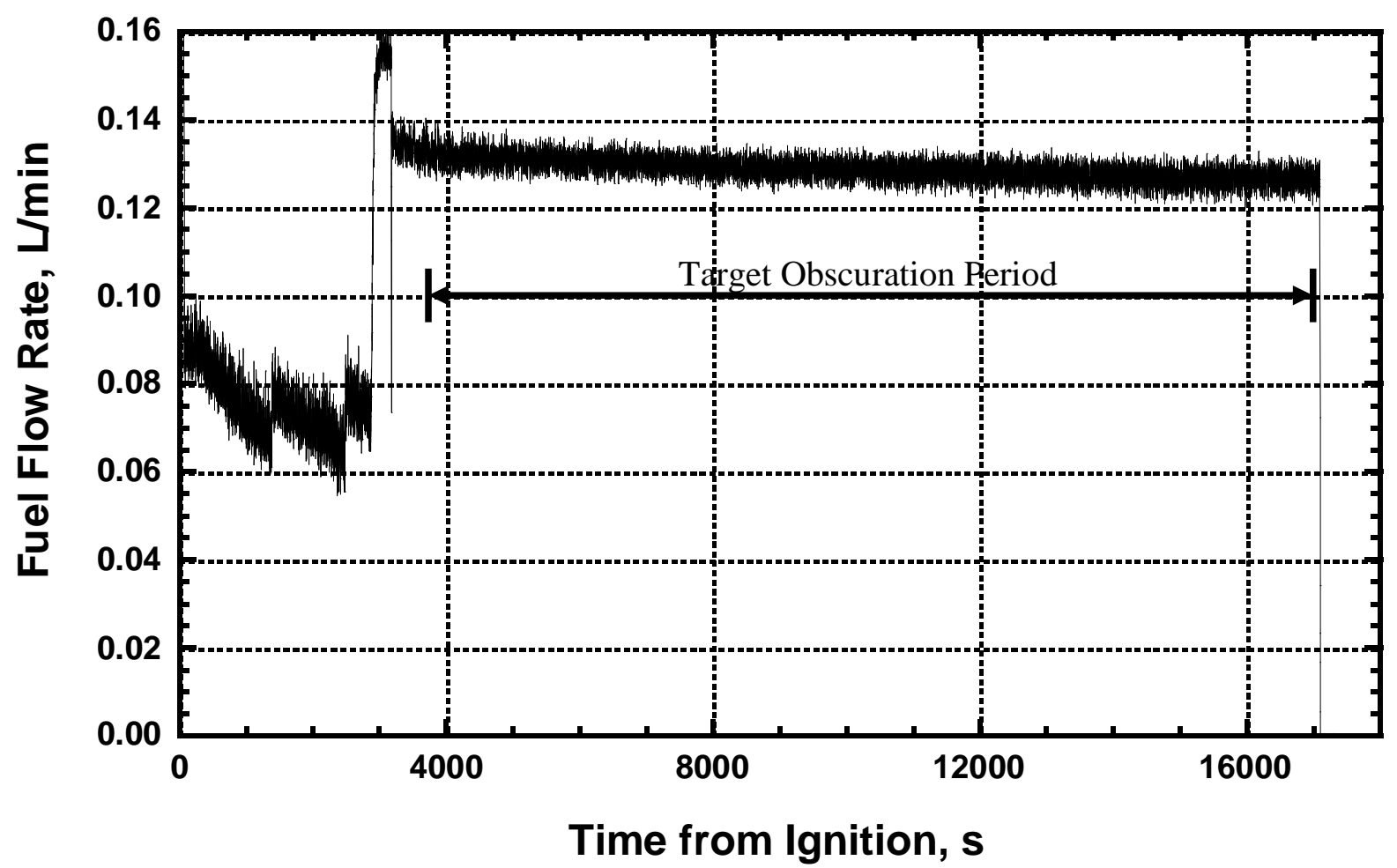

Figure 29. Test 1 diesel fuel flow rate plotted versus time from ignition (11:26:23 EDT)

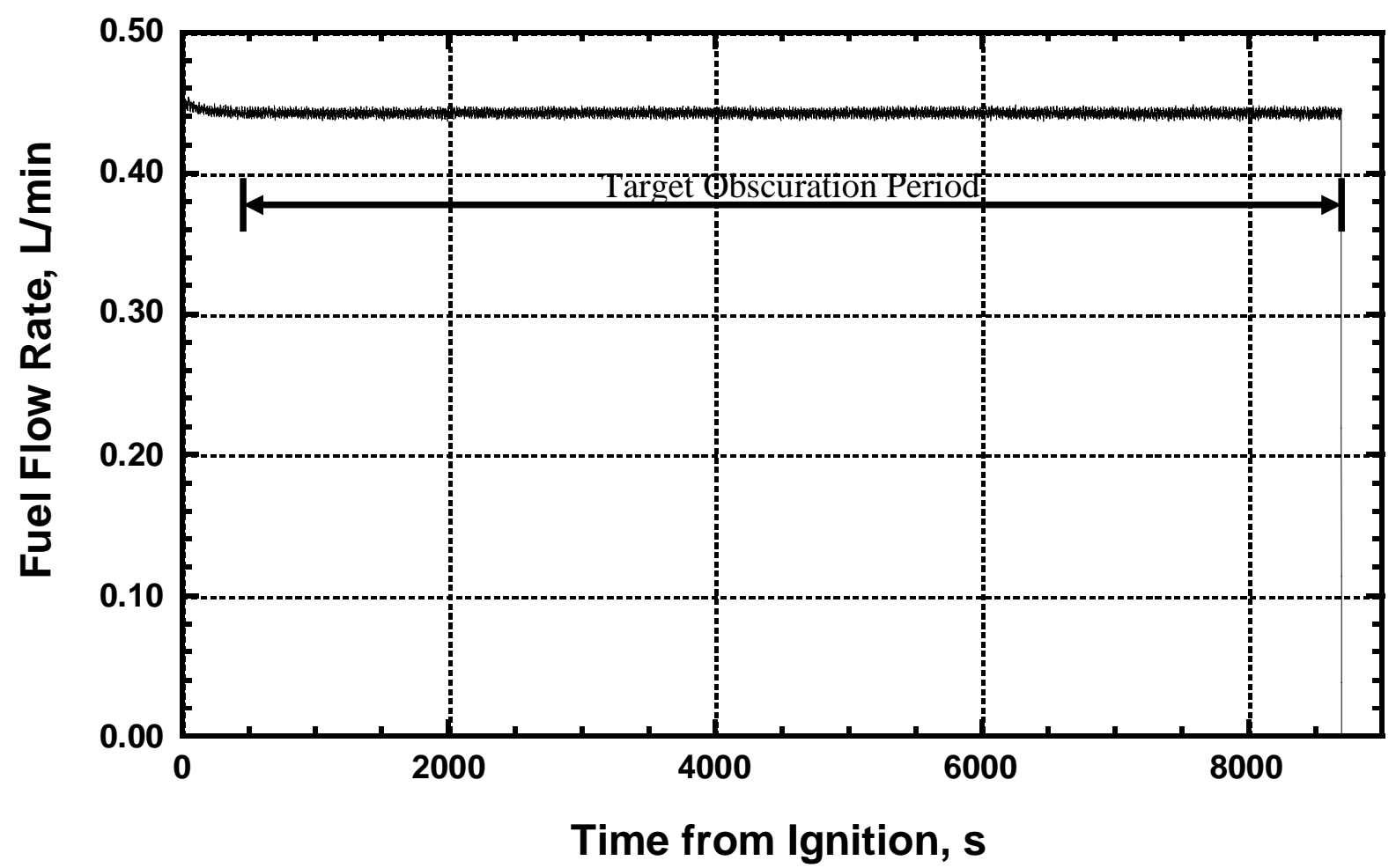

Figure 30. Test 2 diesel fuel flow rate plotted versus time from ignition (9:47:01 EDT) 


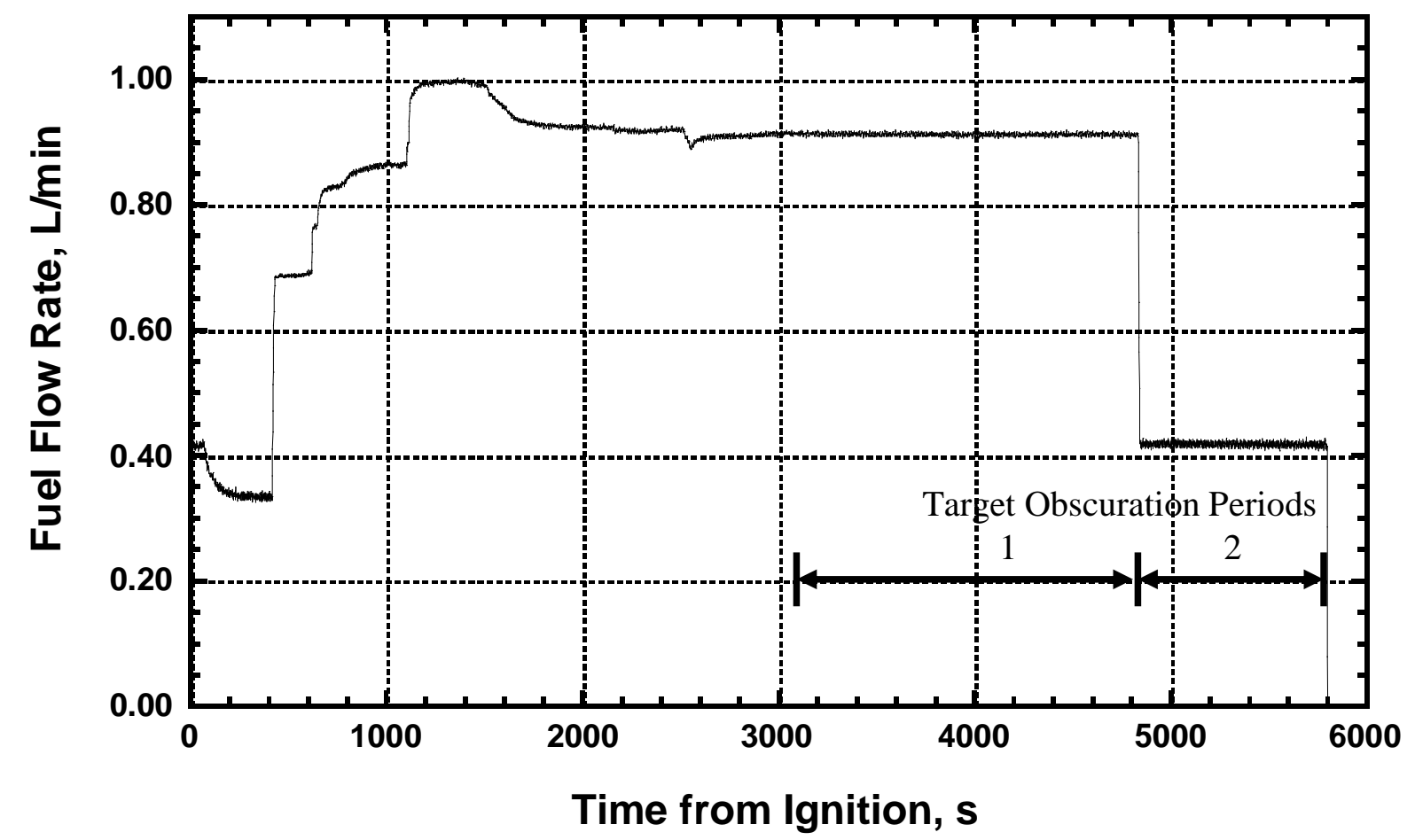

Figure 31. Test 3 diesel fuel flow rate plotted versus time from ignition (14:57:20 EDT)

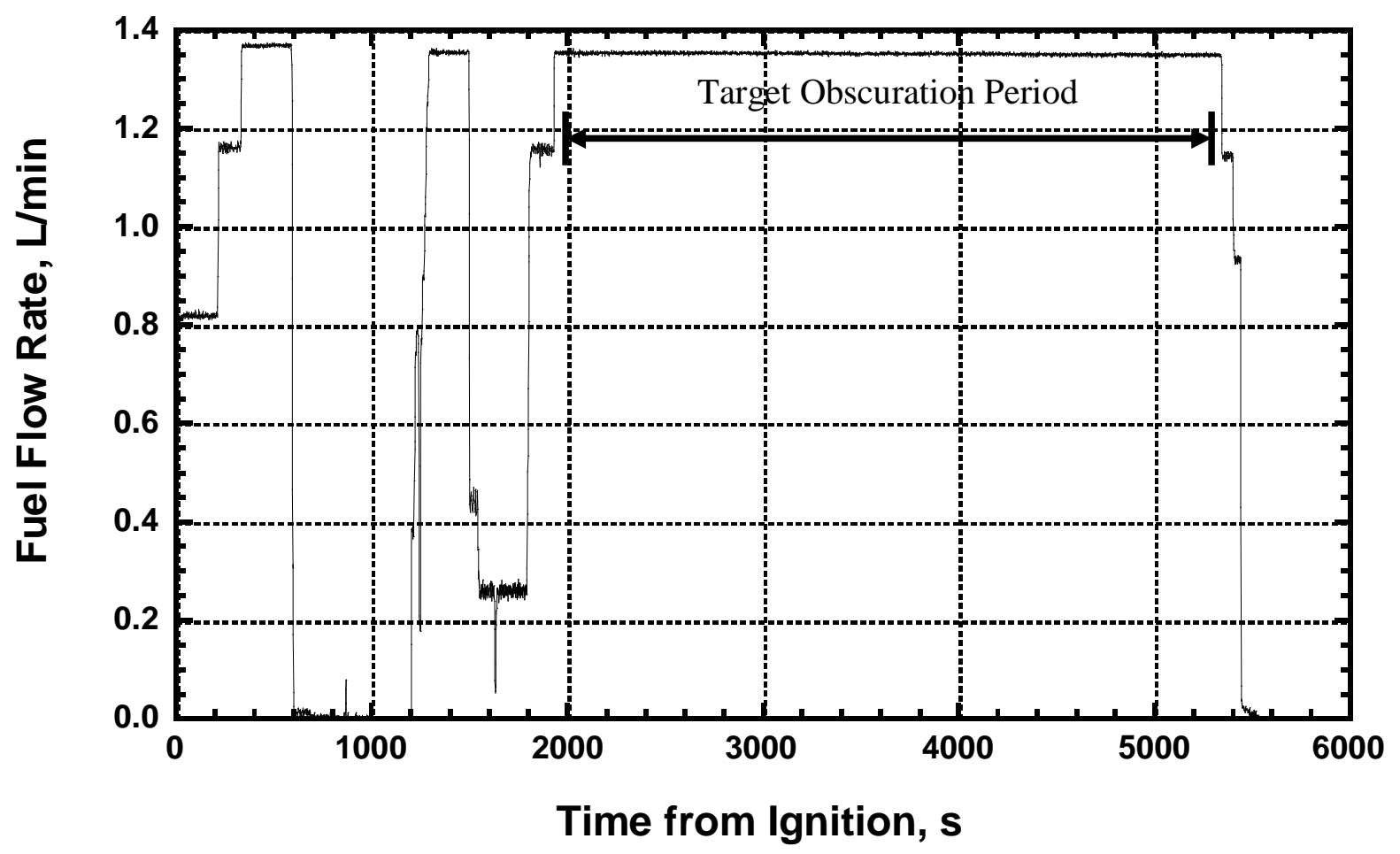

Figure 32. Test 4 diesel fuel flow rate plotted versus time from ignition (14:20:46 EDT) 
Table 9. Fuel flow rate steady state statistics

\begin{tabular}{|c|c|c|c|c|}
\hline $\begin{array}{c}\text { Experiment } \\
\text { Number }\end{array}$ & $\begin{array}{c}\text { Initiation of } \\
\text { Steady State } \\
\text { Window (EDT) }\end{array}$ & $\begin{array}{c}\text { Termination of } \\
\text { Steady State } \\
\text { Window (EDT) }\end{array}$ & $\begin{array}{c}\text { Average Fuel } \\
\text { Flow Rate } \\
(1 / \text { min) }\end{array}$ & $\begin{array}{c}\text { Fuel Flow Rate } \\
\text { Standard } \\
\text { Deviation } \\
(1 / \text { min })\end{array}$ \\
\hline 1 & $12: 25: 08$ & $16: 11: 15$ & 0.1297 & 0.0025 \\
\hline 2 & $9: 53: 40$ & $12: 11: 53$ & 0.4428 & 0.0021 \\
\hline 3A & $15: 47: 59$ & $16: 17: 51$ & 0.9128 & 0.0024 \\
\hline 4 & $16: 18: 06$ & $16: 33: 55$ & 0.4182 & 0.0039 \\
\hline & $14: 53: 03$ & $15: 49: 43$ & 1.3515 & 0.0023 \\
\hline
\end{tabular}

Table 10. Steady state transmissivity statistics

\begin{tabular}{|c|c|c|c|c|}
\hline \multirow[b]{2}{*}{$\begin{array}{l}\text { Experiment } \\
\text { Number }\end{array}$} & \multicolumn{2}{|c|}{ East Station } & \multicolumn{2}{|c|}{ West Station } \\
\hline & $\begin{array}{c}\text { Average } \\
\text { Transmittance } \\
\left(\mathrm{I} / \mathrm{I}_{\mathrm{O}}\right)\end{array}$ & $\begin{array}{c}\text { Transmittance } \\
\text { Standard } \\
\text { Deviation }\end{array}$ & $\begin{array}{c}\text { Average } \\
\text { Transmittance } \\
\left(\mathrm{I} / \mathrm{I}_{0}\right)\end{array}$ & $\begin{array}{c}\text { Transmittance } \\
\text { Standard } \\
\text { Deviation }\end{array}$ \\
\hline 1 & 0.0637 & 0.0425 & 0.1540 & 0.0858 \\
\hline 2 & 0.0083 & 0.0110 & 0.0105 & 0.0139 \\
\hline $3 \mathrm{~A}$ & 0.0027 & 0.0056 & 0.0025 & 0.0058 \\
\hline $3 \mathrm{~B}$ & 0.0113 & 0.0129 & 0.0215 & 0.0204 \\
\hline 4 & 0.0332 & 0.0825 & 0.0055 & 0.0381 \\
\hline
\end{tabular}

Table 10 lists the east and west station laser transmittances, including the calculated standard deviations. The target transmittance value for scenario 1 was 0.1 , and the average measurements were 0.0637 and 0.1540 . For scenario 2, the target transmittance was 0.01 and the average measured values ranged from 0.0083 to 0.0105 . The target value for scenario 3 was 0.001 and the average transmittance values that could be achieved in the LFL setting (experiment 3a) were 0.0027 and 0.0025 . The above mentioned three scenarios were designed to span transmittance values across three orders of magnitude. The data collected demonstrate that the test series achieved the desired decrease in transmittance.

\section{1 Uncertainty Analysis}

There are different components of uncertainty in the instrument location, fuel flow rate, and transmittance data reported here. Uncertainties are grouped into two categories according to the method used to estimate them. Type A uncertainties are evaluated by statistical methods, and Type B are evaluated by other means [Taylor and Kuyatt, 1994]. Type B analysis of systematic uncertainties involves estimating the upper $(+a)$ and lower $(-a)$ limits for the quantity in question such that the probability that the value would be in the interval $( \pm a)$ is essentially 100 percent. After estimating uncertainties by either Type A or B analysis, the uncertainties are combined in quadrature to yield the combined standard uncertainty. Multiplying the combined standard uncertainty by a coverage factor of two results in the total expanded uncertainty that corresponds to a $95 \%$ confidence interval $(2 \mathrm{~F})$. 
Components of uncertainty are tabulated in Table 11. Some of these components, such as the zero and calibration elements, are derived from instrument specifications. Other components, such as incident and transmitted intensities, and pulse count light extinction measurements incorporate previous experience. For the positioning of the instrumentation, this resulted in an estimate of $\pm 11 \%$ total expanded uncertainty. Calibration of the fuel flow is routinely completed using graduated cylinder and stopwatch. Combining the calibration and pulse count uncertainty with other components resulted in a total expanded uncertainty of $\pm 5 \%$ total expanded uncertainty for the fuel flow rate.

Estimating the uncertainty in the experimental transmittance required the uncertainties in the initial or calibration of the laser, the incident intensity of the source laser throughout each test, and the transmitted intensity as sensed by the photodetector. The incident intensity component includes an estimate of the drift of the source laser throughout the test period. The photodetector exhibited much less drift as it tracked the transmitted intensity, however, as the smoke plume moved back and forth, the laser would have traversed different pathlengths. The transmittance is the ratio of the incident intensity to the transmitted intensity and whether the absorption and scattering of the light (causing the change in transmittance) occurs in $1.2 \mathrm{~m}$ or $1.6 \mathrm{~m}$ (of pathlength) does not change the transmittance. The unsteady nature of the smoke plume resulted in a wider range of transmittances around the design setpoint which was undesirable from an experimental perspective. These fluctuations in plume width or cross-section are undesirable and introduce changes in transmittance, offsets from the design transmittance setpoint, but do not affect the uncertainty in the transmittance measurements. However, real smoke plumes do exhibit unsteady pulsing or "puffing" behavior and while the unsteady behavior does add to the analysis complexity, the changing plume diameter may be more representative of real fire plumes. The uncertainty components in laser source and detection resulted in a total expanded uncertainty range of $-20 \%$ to $+14 \%$. During the development of each smoke exposure scenario, target transmittances were also calculated using Equation 1. Estimating the uncertainty in the target or model transmittance required the uncertainties in smoke extinction coefficient, $\sigma$, smoke mass concentration, and pathlength. Unlike the experimental transmittances, the model transmittances (for a given extinction coefficient and mass concentration) are a direct function of pathlength. A pathlength of $1.6 \mathrm{~m}$ will scatter or absorb approximately $30 \%$ more light than $1.2 \mathrm{~m}$ because the $1.6 \mathrm{~m}$ path is about $30 \%$ longer. These components resulted in a total expanded uncertainty range of $-30 \%$ to $+24 \%$.

Table 11. Uncertainty in instrument location, fuel flow rate, and transmittance.

\begin{tabular}{|l|c|c|c|}
\hline & $\begin{array}{c}\text { Component } \\
\text { Standard } \\
\text { Uncertainty }\end{array}$ & $\begin{array}{c}\text { Combined } \\
\text { Standard } \\
\text { Uncertainty }\end{array}$ & $\begin{array}{c}\text { Total } \\
\text { Expanded } \\
\text { Uncertainty }\end{array}$ \\
\hline $\begin{array}{c}\text { Instrument Location } \\
\text { Position }\end{array}$ & $\pm 5 \%$ & & \\
Repeatability $^{1}$ & $\pm 2 \%$ & $\pm 6 \%$ & $\pm 11 \%$ \\
Random $^{1}$ & $\pm 2 \%$ & & \\
\hline $\begin{array}{c}\text { Fuel Flow Rate } \\
\text { Calibration }\end{array}$ & $\pm 1 \%$ & $\pm 3 \%$ & $\pm 5 \%$ \\
Pulse Count & $\pm 1 \%$ & & \\
\hline
\end{tabular}




\begin{tabular}{|c|c|c|c|}
\hline $\begin{array}{l}\text { Repeatability } \\
\text { Random }^{1}\end{array}$ & $\begin{array}{l} \pm 1 \% \\
\pm 2 \% \\
\end{array}$ & & \\
\hline $\begin{array}{l}\text { Transmittance } \\
\text { (Experimental) } \\
\text { Calibration } \\
\text { Incident Intensity } \\
\text { Transmitted Intensity } \\
\text { Repeatability }^{1} \\
\text { Random }^{1} \\
\end{array}$ & $\begin{array}{c} \pm 1 \% \\
-8 \% \text { to }+5 \% \\
-5 \% \text { to }+5 \% \\
\pm 2 \% \\
\pm 2 \% \\
\end{array}$ & $-10 \%$ to $+7 \%$ & $-20 \%$ to $+14 \%$ \\
\hline $\begin{array}{l}\text { Transmittance (Model) } \\
\text { Extinction Coef. ( } \sigma \text { ) } \\
\text { Mass Concentration } \\
\text { Pathlength } \\
\text { Repeatability }^{1} \\
\text { Random }^{1}\end{array}$ & $\begin{array}{l} \pm 2 \% \\
\pm 3 \% \\
\pm 5 \% \\
\pm 0.5 \% \\
\pm 0.5 \%\end{array}$ & $-15 \%$ to $+12 \%$ & $-30 \%$ to $+24 \%$ \\
\hline
\end{tabular}

Notes: 1. Random and repeatability evaluated as Type A, other components as Type B.

\subsection{Summary}

Free-Space Optics (also known as "FSO" or "Optical Wireless") utilizes laser-based optical technology to transmit data, voice and video information over distances of up to several kilometers. In order maintain a communications link, these optical systems require unobstructed line of sight between the transmitter and the receiver units. FSO system performance, including signal intensity and integrity, is degraded by beam obscuration, absorption, and scattering from weather conditions and/or the presence of smoke.

The National Institute of Standards and Technology (NIST) Fire Dynamics Simulator (FDS), was used to model and estimate the smoke obscuration that would result from several different fire scenarios. The smoke obscuration was predicted at different locations above each fire. FDS model results were then used to design medium scale fire tests which could effectively simulate the amount of smoke obscuration that were predicted for the large fire scenarios. A set of four medium scale experiments was conducted in the NIST, Building and Fire Research Laboratory, Large Fire Laboratory in Gaithersburg, Maryland to assess the performance of FSO units with different levels of signal obscuration. Three experiments were conducted (Scenarios 1, 2 and 3) with the FSO beams transmitting through different levels of smoke and one experiment (Scenario 4) was conducted with the FSO units transmitting through flames and smoke. The NIST calibrated lasers were used to characterize the transmittance across the plume and flames as a function of time. While this test series collected a limited set of transmittance data, the burns resulted in transmittances ranging from of 0.1540 to 0.0025 , which represents a $94.6 \%$, $99 \%$, and $99.75 \%$ decrease in transmitted intensity, respectively.

Although the target transmittance values were achieved in the Large Fire Laboratory, significantly lower values can be expected in locations near large outdoor burns or over longer path lengths through a smoke plume. FDS simulations, for the large fuel spills, predicted very low transmittance values near the fuel surface. Among many other factors, the effects of wind 
and plume lean can in many situations significantly reduce transmittance values. Additional work is needed to validate FDS for large outdoor fire plumes and to assess the transmittance values in such settings. The information in this report can be used by NCS to begin assessing the performance of FSO in environments with varying degrees of smoke obscuration.

\subsection{Acknowledgements}

William Ryan of the NCS and Rodney Marshburn and Quinn Wu of Booz Allen Hamilton provided critical input in the development of the fire scenarios and NCS and NIST teams.

\subsection{References}

Bryant, R. A.; Hamins, A.; Johnsson, E. L.; Grove, B. S.; Maranghides, A.; Mulholland, G. W.; Ohlemiller, T. J.; Guthrie, W. F., The NIST 3 Megawatt Quantitative Heat Release Rate Facility, NIST SP1007, 2003.

Bundy, M; Maranghides, A.; Hamins, A.; and Mulholland, G. The NIST Large Fire Laboratory Liquid Spray Burner, NISTIR in progress.

Forney, G.P. and McGrattan, K.B. (2004) User's Guide for Smokeview Version 4: A Tool for Visualizing Fire Dynamics Simulation Data, NISTIR Special Publication, 1017, http://fire.nist.gov/bfrlpubs/

Hamins, A., others, NIST Draft report on project 5: Reconstruction of the Fires in the World Trade Center Towers, NIST NCSTAR 1-5B (Draft), Sept. 2005.

McGrattan, K.B. (2004) Fire Dynamics Simulator (Version 4), "Technical Reference Guide", NISTIR Special Publication 1018, McGrattan , K., editor, http://fire.nist.gov/bfrlpubs/

McGrattan, K.G. and Forney, G. (2004) Fire Dynamics Simulator (Version 4), "Users Guide", NISTIR Special Publication 1019, http://fire.nist.gov/bfrlpubs/

Mulholland, G. "The emission and optical properties of smoke" in Recent Advances in Flame Retardancy of Polymeric Materials, Vol. 3, pp. 22-29 (1992)

Taylor, B. N. and C. E. Kuyatt, Guidelines For Evaluating and Expressing the Uncertainty of NIST Measurement Results. National Institute of Standards and Technology (U.S.) NIST-TN 1297; September 1994.

Walton, W.D., McElroy, J., Twilley, W.H., Hiltabrand, R.R. (1994) Smoke Measurements Using a Helicopter Transported Sampling Package, Proceedings of the $17^{\text {th }}$ Artic and Marine Oil Spill Program (AMOP) Technical Seminar, Vol. 1, June 8-10, 1994, Vancouver, British Columbia, also available at http://fire.nist.gov/bfrlpubs/ 
APPENDIX A. - Fuel Data Test 1 NIST 102505

Appendix $A$ is fuel data from Test 1 in tabular format and is approximately 330 pages in length. Fuel data is included with electronic version of report on compact disk, but not routinely included with paper version of report. 
Appendix A - Fuel Data Test 1 NIST102505_1.xls

Time from Ignition, s EDT Time Liq Flow, $1 / \mathrm{min}$

$\begin{array}{rll}0 & 11: 26: 23 & 0.1583 \\ 1 & 11: 26: 24 & 0.2916 \\ 2 & 11: 26: 25 & 0.2942 \\ 3 & 11: 26: 26 & 0.1528 \\ 4 & 11: 26: 27 & 0.2089 \\ 5 & 11: 26: 28 & 0.2188 \\ 6 & 11: 26: 29 & 0.2245 \\ 7 & 11: 26: 30 & 0.2228 \\ 8 & 11: 26: 31 & 0.2226 \\ 9 & 11: 26: 32 & 0.2171 \\ 10 & 11: 26: 33 & 0.2199 \\ 11 & 11: 26: 34 & 0.2180 \\ 12 & 11: 26: 35 & 0.2155 \\ 13 & 11: 26: 36 & 0.2129 \\ 14 & 11: 26: 37 & 0.2167 \\ 15 & 11: 26: 38 & 0.2224 \\ 16 & 11: 26: 39 & 0.2191 \\ 17 & 11: 26: 40 & 0.2179 \\ 18 & 11: 26: 41 & 0.2182 \\ 19 & 11: 26: 42 & 0.2193 \\ 20 & 11: 26: 43 & 0.2205 \\ 21 & 11: 26: 44 & 0.2213 \\ 22 & 11: 26: 45 & 0.2261 \\ 23 & 11: 26: 46 & 0.2176 \\ 24 & 11: 26: 47 & 0.2259 \\ 25 & 11: 26: 48 & 0.2234 \\ 26 & 11: 26: 49 & 0.2222 \\ 27 & 11: 26: 50 & 0.2214 \\ 28 & 11: 26: 51 & 0.2277 \\ 29 & 11: 26: 52 & 0.2191 \\ 30 & 11: 26: 53 & 0.2280 \\ 31 & 11: 26: 54 & 0.2233 \\ 32 & 11: 26: 55 & 0.2318 \\ 33 & 11: 26: 56 & 0.2217 \\ 34 & 11: 26: 57 & 0.2239 \\ 35 & 11: 26: 58 & 0.2171 \\ 36 & 11: 26: 59 & 0.2292 \\ 37 & 11: 27: 00 & 0.2218 \\ 38 & 11: 27: 01 & 0.2240 \\ 39 & 11: 27: 02 & 0.2218 \\ 40 & 11: 27: 03 & 0.2250 \\ 41 & 11: 27: 04 & 0.2250 \\ 42 & 11: 27: 05 & 0.2218 \\ 43 & 11: 27: 06 & 0.2210 \\ 44 & 11: 27: 07 & 0.2214 \\ 45 & 11: 27: 08 & 0.2191 \\ 46 & 11: 27: 09 & 0.2178 \\ 47 & 11: 27: 10 & 0.2248 \\ 48 & 11: 27: 11 & 0.2219 \\ 49 & 11: 27: 12 & 0.2284 \\ 50 & 11: 27: 13 & 0.2220\end{array}$


Appendix A - Fuel Data Test 1 NIST102505_1.xls

\begin{tabular}{|c|c|c|}
\hline 51 & $11: 27: 14$ & 0.2250 \\
\hline 52 & $11: 27: 15$ & 0.2233 \\
\hline 53 & $11: 27: 16$ & 0.2212 \\
\hline 54 & $11: 27: 17$ & 0.2182 \\
\hline 55 & $11: 27: 18$ & 0.2229 \\
\hline 56 & $11: 27: 19$ & 0.2228 \\
\hline 57 & $11: 27: 20$ & 0.2230 \\
\hline 58 & $11: 27: 21$ & 0.2197 \\
\hline 59 & $11: 27: 22$ & 0.2218 \\
\hline 60 & $11: 27: 23$ & 0.2221 \\
\hline 61 & $11: 27: 24$ & 0.2215 \\
\hline 62 & $11: 27: 25$ & 0.2203 \\
\hline 63 & $11: 27: 26$ & 0.2203 \\
\hline 64 & $11: 27: 27$ & 0.2262 \\
\hline 65 & $11: 27: 28$ & 0.2235 \\
\hline 66 & $11: 27: 29$ & 0.2230 \\
\hline 67 & $11: 27: 30$ & 0.2229 \\
\hline 68 & $11: 27: 31$ & 0.1990 \\
\hline 69 & $11: 27: 32$ & 0.1789 \\
\hline 70 & $11: 27: 33$ & 0.1495 \\
\hline 71 & $11: 27: 34$ & 0.1287 \\
\hline 72 & $11: 27: 35$ & 0.1027 \\
\hline 73 & $11: 27: 36$ & 0.0974 \\
\hline 74 & $11: 27: 37$ & 0.0827 \\
\hline 75 & $11: 27: 38$ & 0.0897 \\
\hline 76 & $11: 27: 39$ & 0.0826 \\
\hline 77 & $11: 27: 40$ & 0.0874 \\
\hline 78 & $11: 27: 41$ & 0.0827 \\
\hline 79 & $11: 27: 42$ & 0.0845 \\
\hline 80 & $11: 27: 43$ & 0.0908 \\
\hline 81 & $11: 27: 44$ & 0.0890 \\
\hline 82 & $11: 27: 45$ & 0.0986 \\
\hline 83 & $11: 27: 46$ & 0.0917 \\
\hline 84 & $11: 27: 47$ & 0.0980 \\
\hline 85 & $11: 27: 48$ & 0.0835 \\
\hline 86 & $11: 27: 49$ & 0.0940 \\
\hline 87 & $11: 27: 50$ & 0.0843 \\
\hline 88 & $11: 27: 51$ & 0.0866 \\
\hline 89 & $11: 27: 52$ & 0.0867 \\
\hline 90 & $11: 27: 53$ & 0.0877 \\
\hline 91 & $11: 27: 54$ & 0.0879 \\
\hline 92 & $11: 27: 55$ & 0.0790 \\
\hline 93 & $11: 27: 56$ & 0.0908 \\
\hline 94 & $11: 27: 57$ & 0.0878 \\
\hline 95 & $11: 27: 58$ & 0.0953 \\
\hline 96 & $11: 27: 59$ & 0.0839 \\
\hline 97 & $11: 28: 00$ & 0.0927 \\
\hline 98 & $11: 28: 01$ & 0.0844 \\
\hline 99 & 11:28:02 & 0.0874 \\
\hline 100 & $11: 28: 03$ & 0.0885 \\
\hline 101 & $11: 28: 04$ & 0.0849 \\
\hline 102 & $11: 28: 05$ & 0.0895 \\
\hline
\end{tabular}


Appendix A - Fuel Data Test 1 NIST102505_1.xls

$\begin{array}{lll}103 & 11: 28: 06 & 0.0830 \\ 104 & 11: 28: 07 & 0.0912 \\ 105 & 11: 28: 08 & 0.0882 \\ 106 & 11: 28: 09 & 0.0894 \\ 107 & 11: 28: 10 & 0.0839 \\ 108 & 11: 28: 11 & 0.0882 \\ 109 & 11: 28: 12 & 0.0917 \\ 110 & 11: 28: 13 & 0.0934 \\ 111 & 11: 28: 14 & 0.0908 \\ 112 & 11: 28: 15 & 0.0905 \\ 113 & 11: 28: 16 & 0.0935 \\ 114 & 11: 28: 17 & 0.0868 \\ 115 & 11: 28: 18 & 0.0914 \\ 116 & 11: 28: 19 & 0.0867 \\ 117 & 11: 28: 20 & 0.0877 \\ 118 & 11: 28: 21 & 0.0840 \\ 119 & 11: 28: 22 & 0.0850 \\ 120 & 11: 28: 23 & 0.0870 \\ 121 & 11: 28: 24 & 0.0855 \\ 122 & 11: 28: 25 & 0.0900 \\ 123 & 11: 28: 26 & 0.0894 \\ 124 & 11: 28: 27 & 0.0967 \\ 125 & 11: 28: 28 & 0.0922 \\ 126 & 11: 28: 29 & 0.0977 \\ 127 & 11: 28: 30 & 0.0845 \\ 128 & 11: 28: 31 & 0.0906 \\ 129 & 11: 28: 32 & 0.0832 \\ 130 & 11: 28: 33 & 0.0853 \\ 131 & 11: 28: 34 & 0.0902 \\ 132 & 11: 28: 35 & 0.0898 \\ 133 & 11: 28: 36 & 0.0930 \\ 134 & 11: 28: 37 & 0.0809 \\ 135 & 11: 28: 38 & 0.0881 \\ 136 & 11: 28: 39 & 0.0861 \\ 137 & 11: 28: 40 & 0.0909 \\ 138 & 11: 28: 41 & 0.0858 \\ 139 & 11: 28: 42 & 0.0881 \\ 140 & 11: 28: 43 & 0.0899 \\ 141 & 11: 28: 44 & 0.0892 \\ 142 & 11: 28: 45 & 0.0865 \\ 143 & 11: 28: 46 & 0.0836 \\ 144 & 11: 28: 47 & 0.0922 \\ 145 & 11: 28: 48 & 0.0831 \\ 146 & 11: 28: 49 & 0.0895 \\ 147 & 11: 28: 50 & 0.0865 \\ 148 & 11: 28: 51 & 0.0894 \\ 149 & 11: 28: 52 & 0.0818 \\ 150 & 11: 28: 53 & 0.0883 \\ 151 & 11: 28: 54 & 0.0895 \\ 152 & 11: 28: 55 & 0.0922 \\ 153 & 11: 28: 56 & 0.0866 \\ 154 & 11: 28: 57 & 0.0858\end{array}$


Appendix A - Fuel Data Test 1 NIST102505_1.xls

$\begin{array}{lll}155 & 11: 28: 58 & 0.0894 \\ 156 & 11: 28: 59 & 0.0875 \\ 157 & 11: 29: 00 & 0.0936 \\ 158 & 11: 29: 01 & 0.0890 \\ 159 & 11: 29: 02 & 0.0905 \\ 160 & 11: 29: 03 & 0.0870 \\ 161 & 11: 29: 04 & 0.0869 \\ 162 & 11: 29: 05 & 0.0813 \\ 163 & 11: 29: 06 & 0.0858 \\ 164 & 11: 29: 07 & 0.0854 \\ 165 & 11: 29: 08 & 0.0817 \\ 166 & 11: 29: 09 & 0.0905 \\ 167 & 11: 29: 10 & 0.0831 \\ 168 & 11: 29: 11 & 0.0916 \\ 169 & 11: 29: 12 & 0.0852 \\ 170 & 11: 29: 13 & 0.0958 \\ 171 & 11: 29: 14 & 0.0862 \\ 172 & 11: 29: 15 & 0.0908 \\ 173 & 11: 29: 16 & 0.0842 \\ 174 & 11: 29: 17 & 0.0862 \\ 175 & 11: 29: 18 & 0.0877 \\ 176 & 11: 29: 19 & 0.0861 \\ 177 & 11: 29: 20 & 0.0902 \\ 178 & 11: 29: 21 & 0.0890 \\ 179 & 11: 29: 22 & 0.0978 \\ 180 & 11: 29: 23 & 0.0853 \\ 181 & 11: 29: 24 & 0.0937 \\ 182 & 11: 29: 25 & 0.0881 \\ 183 & 11: 29: 26 & 0.0896 \\ 184 & 11: 29: 27 & 0.0876 \\ 185 & 11: 29: 28 & 0.0855 \\ 186 & 11: 29: 29 & 0.0880 \\ 187 & 11: 29: 30 & 0.0811 \\ 188 & 11: 29: 31 & 0.0892 \\ 189 & 11: 29: 32 & 0.0860 \\ 190 & 11: 29: 33 & 0.0937 \\ 191 & 11: 29: 34 & 0.0860 \\ 192 & 11: 29: 35 & 0.0923 \\ 193 & 11: 29: 36 & 0.0810 \\ 194 & 11: 29: 37 & 0.0895 \\ 195 & 11: 29: 38 & 0.0876 \\ 196 & 11: 29: 39 & 0.0865 \\ 197 & 11: 29: 40 & 0.0961 \\ 198 & 11: 29: 41 & 0.0871 \\ 199 & 11: 29: 42 & 0.0921 \\ 200 & 11: 29: 43 & 0.0817 \\ 201 & 11: 29: 44 & 0.0897 \\ 202 & 11: 29: 45 & 0.0826 \\ 203 & 11: 29: 46 & 0.0875 \\ 204 & 11: 29: 47 & 0.0856 \\ 205 & 11: 29: 48 & 0.0917 \\ 206 & 11: 29: 49 & 0.0877\end{array}$


Appendix A - Fuel Data Test 1 NIST102505_1.xls

$\begin{array}{lll}207 & 11: 29: 50 & 0.0846 \\ 208 & 11: 29: 51 & 0.0906 \\ 209 & 11: 29: 52 & 0.0816 \\ 210 & 11: 29: 53 & 0.0861 \\ 211 & 11: 29: 54 & 0.0835 \\ 212 & 11: 29: 55 & 0.0886 \\ 213 & 11: 29: 56 & 0.0828 \\ 214 & 11: 29: 57 & 0.0889 \\ 215 & 11: 29: 58 & 0.0868 \\ 216 & 11: 29: 59 & 0.0893 \\ 217 & 11: 30: 00 & 0.0864 \\ 218 & 11: 30: 01 & 0.0837 \\ 219 & 11: 30: 02 & 0.0893 \\ 220 & 11: 30: 03 & 0.0843 \\ 221 & 11: 30: 04 & 0.0928 \\ 222 & 11: 30: 05 & 0.0890 \\ 223 & 11: 30: 06 & 0.0956 \\ 224 & 11: 30: 07 & 0.0894 \\ 225 & 11: 30: 08 & 0.0898 \\ 226 & 11: 30: 09 & 0.0891 \\ 227 & 11: 30: 10 & 0.0875 \\ 228 & 11: 30: 11 & 0.0847 \\ 229 & 11: 30: 12 & 0.0864 \\ 230 & 11: 30: 13 & 0.0981 \\ 231 & 11: 30: 14 & 0.0857 \\ 232 & 11: 30: 15 & 0.0922 \\ 233 & 11: 30: 16 & 0.0869 \\ 234 & 11: 30: 17 & 0.0965 \\ 235 & 11: 30: 18 & 0.0838 \\ 236 & 11: 30: 19 & 0.0882 \\ 237 & 11: 30: 20 & 0.0854 \\ 238 & 11: 30: 21 & 0.0839 \\ 239 & 11: 30: 22 & 0.0845 \\ 240 & 11: 30: 23 & 0.0806 \\ 241 & 11: 30: 24 & 0.0907 \\ 242 & 11: 30: 25 & 0.0898 \\ 243 & 11: 30: 26 & 0.0933 \\ 244 & 11: 30: 27 & 0.0889 \\ 245 & 11: 30: 28 & 0.0884 \\ 246 & 11: 30: 29 & 0.0853 \\ 247 & 11: 30: 30 & 0.0857 \\ 248 & 11: 30: 31 & 0.0847 \\ 249 & 11: 30: 32 & 0.0881 \\ 250 & 11: 30: 33 & 0.0914 \\ 251 & 11: 30: 34 & 0.0873 \\ 252 & 11: 30: 35 & 0.0994 \\ 253 & 11: 30: 36 & 0.0913 \\ 254 & 11: 30: 37 & 0.0963 \\ 255 & 11: 30: 38 & 0.0853 \\ 256 & 11: 30: 39 & 0.0888 \\ 257 & 11: 30: 40 & 0.0885 \\ 258 & 11: 30: 41 & 0.0885\end{array}$


Appendix A - Fuel Data Test 1 NIST102505_1.xIs

$\begin{array}{lll}259 & 11: 30: 42 & 0.0907 \\ 260 & 11: 30: 43 & 0.0910 \\ 261 & 11: 30: 44 & 0.0935 \\ 262 & 11: 30: 45 & 0.0840 \\ 263 & 11: 30: 46 & 0.0910 \\ 264 & 11: 30: 47 & 0.0878 \\ 265 & 11: 30: 48 & 0.0916 \\ 266 & 11: 30: 49 & 0.0829 \\ 267 & 11: 30: 50 & 0.0878 \\ 268 & 11: 30: 51 & 0.0905 \\ 269 & 11: 30: 52 & 0.0889 \\ 270 & 11: 30: 53 & 0.0879 \\ 271 & 11: 30: 54 & 0.0819 \\ 272 & 11: 30: 55 & 0.0921 \\ 273 & 11: 30: 56 & 0.0826 \\ 274 & 11: 30: 57 & 0.0905 \\ 275 & 11: 30: 58 & 0.0850 \\ 276 & 11: 30: 59 & 0.0914 \\ 277 & 11: 31: 00 & 0.0859 \\ 278 & 11: 31: 01 & 0.0908 \\ 279 & 11: 31: 02 & 0.0838 \\ 280 & 11: 31: 03 & 0.0858 \\ 281 & 11: 31: 04 & 0.0837 \\ 282 & 11: 31: 05 & 0.0808 \\ 283 & 11: 31: 06 & 0.0897 \\ 284 & 11: 31: 07 & 0.0855 \\ 285 & 11: 31: 08 & 0.0945 \\ 286 & 11: 31: 09 & 0.0835 \\ 287 & 11: 31: 10 & 0.0907 \\ 288 & 11: 31: 11 & 0.0898 \\ 289 & 11: 31: 12 & 0.0923 \\ 290 & 11: 31: 13 & 0.0894 \\ 291 & 11: 31: 14 & 0.0839 \\ 292 & 11: 31: 15 & 0.0904 \\ 293 & 11: 31: 16 & 0.0849 \\ 294 & 11: 31: 17 & 0.0893 \\ 295 & 11: 31: 18 & 0.0819 \\ 296 & 11: 31: 19 & 0.0888 \\ 297 & 11: 31: 20 & 0.0801 \\ 298 & 11: 31: 21 & 0.0869 \\ 299 & 11: 31: 22 & 0.0860 \\ 300 & 11: 31: 23 & 0.0875 \\ 301 & 11: 31: 24 & 0.0899 \\ 302 & 11: 31: 25 & 0.0852 \\ 303 & 11: 31: 26 & 0.0912 \\ 304 & 11: 31: 27 & 0.0841 \\ 305 & 11: 31: 28 & 0.0931 \\ 306 & 11: 31: 29 & 0.0820 \\ 307 & 11: 31: 30 & 0.0923 \\ 308 & 11: 31: 31 & 0.0862 \\ 309 & 11: 31: 32 & 0.0932 \\ 310 & 11: 31: 33 & 0.0872\end{array}$


Appendix A - Fuel Data Test 1 NIST102505_1.xls

$\begin{array}{lll}311 & 11: 31: 34 & 0.0860 \\ 312 & 11: 31: 35 & 0.0898 \\ 313 & 11: 31: 36 & 0.0860 \\ 314 & 11: 31: 37 & 0.0920 \\ 315 & 11: 31: 38 & 0.0827 \\ 316 & 11: 31: 39 & 0.0987 \\ 317 & 11: 31: 40 & 0.0878 \\ 318 & 11: 31: 41 & 0.0979 \\ 319 & 11: 31: 42 & 0.0835 \\ 320 & 11: 31: 43 & 0.0907 \\ 321 & 11: 31: 44 & 0.0828 \\ 322 & 11: 31: 45 & 0.0840 \\ 323 & 11: 31: 46 & 0.0835 \\ 324 & 11: 31: 47 & 0.0837 \\ 325 & 11: 31: 48 & 0.0875 \\ 326 & 11: 31: 49 & 0.0792 \\ 327 & 11: 31: 50 & 0.0905 \\ 328 & 11: 31: 51 & 0.0904 \\ 329 & 11: 31: 52 & 0.0939 \\ 330 & 11: 31: 53 & 0.0863 \\ 331 & 11: 31: 54 & 0.0856 \\ 332 & 11: 31: 55 & 0.0878 \\ 333 & 11: 31: 56 & 0.0885 \\ 334 & 11: 31: 57 & 0.0865 \\ 335 & 11: 31: 58 & 0.0821 \\ 336 & 11: 31: 59 & 0.0877 \\ 337 & 11: 32: 00 & 0.0829 \\ 338 & 11: 32: 01 & 0.0895 \\ 339 & 11: 32: 02 & 0.0844 \\ 340 & 11: 32: 03 & 0.0940 \\ 341 & 11: 32: 04 & 0.0842 \\ 342 & 11: 32: 05 & 0.0887 \\ 343 & 11: 32: 06 & 0.0831 \\ 344 & 11: 32: 07 & 0.0842 \\ 345 & 11: 32: 08 & 0.0864 \\ 346 & 11: 32: 09 & 0.0836 \\ 347 & 11: 32: 10 & 0.0878 \\ 348 & 11: 32: 11 & 0.0847 \\ 349 & 11: 32: 12 & 0.0945 \\ 350 & 11: 32: 13 & 0.0878 \\ 351 & 11: 32: 14 & 0.0944 \\ 352 & 11: 32: 15 & 0.0833 \\ 353 & 11: 32: 16 & 0.0889 \\ 354 & 11: 32: 17 & 0.0884 \\ 355 & 11: 32: 18 & 0.0836 \\ 356 & 11: 32: 19 & 0.0853 \\ 357 & 11: 32: 20 & 0.0839 \\ 358 & 11: 32: 21 & 0.0934 \\ 359 & 11: 32: 22 & 0.0830 \\ 360 & 11: 32: 23 & 0.0921 \\ 361 & 11: 32: 24 & 0.0849 \\ 362 & 11: 32: 25 & 0.0896\end{array}$


Appendix A - Fuel Data Test 1 NIST102505_1.xls

$\begin{array}{lll}363 & 11: 32: 26 & 0.0834 \\ 364 & 11: 32: 27 & 0.0843 \\ 365 & 11: 32: 28 & 0.0833 \\ 366 & 11: 32: 29 & 0.0809 \\ 367 & 11: 32: 30 & 0.0857 \\ 368 & 11: 32: 31 & 0.0865 \\ 369 & 11: 32: 32 & 0.0943 \\ 370 & 11: 32: 33 & 0.0895 \\ 371 & 11: 32: 34 & 0.0940 \\ 372 & 11: 32: 35 & 0.0885 \\ 373 & 11: 32: 36 & 0.0879 \\ 374 & 11: 32: 37 & 0.0858 \\ 375 & 11: 32: 38 & 0.0841 \\ 376 & 11: 32: 39 & 0.0872 \\ 377 & 11: 32: 40 & 0.0818 \\ 378 & 11: 32: 41 & 0.0865 \\ 379 & 11: 32: 42 & 0.0814 \\ 380 & 11: 32: 43 & 0.0862 \\ 381 & 11: 32: 44 & 0.0801 \\ 382 & 11: 32: 45 & 0.0864 \\ 383 & 11: 32: 46 & 0.0811 \\ 384 & 11: 32: 47 & 0.0872 \\ 385 & 11: 32: 48 & 0.0825 \\ 386 & 11: 32: 49 & 0.0820 \\ 387 & 11: 32: 50 & 0.0848 \\ 388 & 11: 32: 51 & 0.0814 \\ 389 & 11: 32: 52 & 0.0905 \\ 390 & 11: 32: 53 & 0.0796 \\ 391 & 11: 32: 54 & 0.0901 \\ 392 & 11: 32: 55 & 0.0831 \\ 393 & 11: 32: 56 & 0.0905 \\ 394 & 11: 32: 57 & 0.0795 \\ 396 & 11: 32: 59 & 0.0895 \\ 396 & 11: 32: 59 & 0.0800 \\ 397 & 11: 33: 00 & 0.0779 \\ 398 & 11: 33: 01 & 0.0875 \\ 399 & 11: 33: 02 & 0.0825 \\ 400 & 11: 33: 03 & 0.0880 \\ 401 & 11: 33: 04 & 0.0835 \\ 402 & 11: 33: 05 & 0.0918 \\ 403 & 11: 33: 06 & 0.0820 \\ 404 & 11: 33: 07 & 0.0897 \\ 405 & 11: 33: 08 & 0.0850 \\ 406 & 11: 33: 09 & 0.0859 \\ 407 & 11: 33: 10 & 0.0850 \\ 408 & 11: 33: 11 & 0.0805 \\ 409 & 11: 33: 12 & 0.0886 \\ 410 & 11: 33: 13 & 0.0828 \\ 411 & 11: 33: 14 & 0.0906 \\ 412 & 11: 33: 15 & 0.0798 \\ 413 & 11: 33: 16 & 0.0899 \\ 414 & 11: 33: 17 & 0.0799\end{array}$


Appendix A - Fuel Data Test 1 NIST102505_1.xls

$\begin{array}{lll}415 & 11: 33: 18 & 0.0842 \\ 416 & 11: 33: 19 & 0.0789 \\ 417 & 11: 33: 20 & 0.0881 \\ 418 & 11: 33: 21 & 0.0853 \\ 419 & 11: 33: 22 & 0.0789 \\ 420 & 11: 33: 23 & 0.0930 \\ 421 & 11: 33: 24 & 0.0889 \\ 422 & 11: 33: 25 & 0.0929 \\ 423 & 11: 33: 26 & 0.0819 \\ 424 & 11: 33: 27 & 0.0953 \\ 425 & 11: 33: 28 & 0.0839 \\ 426 & 11: 33: 29 & 0.0867 \\ 427 & 11: 33: 30 & 0.0845 \\ 428 & 11: 33: 31 & 0.0857 \\ 429 & 11: 33: 32 & 0.0870 \\ 430 & 11: 33: 33 & 0.0794 \\ 431 & 11: 33: 34 & 0.0849 \\ 432 & 11: 33: 35 & 0.0832 \\ 433 & 11: 33: 36 & 0.0924 \\ 434 & 11: 33: 37 & 0.0816 \\ 435 & 11: 33: 38 & 0.0930 \\ 436 & 11: 33: 39 & 0.0884 \\ 437 & 11: 33: 40 & 0.0856 \\ 438 & 11: 33: 41 & 0.0842 \\ 439 & 11: 33: 42 & 0.0797 \\ 440 & 11: 33: 43 & 0.0821 \\ 441 & 11: 33: 44 & 0.0755 \\ 442 & 11: 33: 45 & 0.0847 \\ 443 & 11: 33: 46 & 0.0811 \\ 444 & 11: 33: 47 & 0.0874 \\ 445 & 11: 33: 48 & 0.0801 \\ 446 & 11: 33: 49 & 0.0860 \\ 447 & 11: 33: 50 & 0.0824 \\ 448 & 11: 33: 51 & 0.0829 \\ 449 & 11: 33: 52 & 0.0830 \\ 450 & 11: 33: 53 & 0.0813 \\ 451 & 11: 33: 54 & 0.0872 \\ 452 & 11: 33: 55 & 0.0855 \\ 453 & 11: 33: 56 & 0.0910 \\ 454 & 11: 33: 57 & 0.0822 \\ 455 & 11: 33: 58 & 0.0888 \\ 456 & 11: 33: 59 & 0.0801 \\ 457 & 11: 34: 00 & 0.0827 \\ 458 & 11: 34: 01 & 0.0773 \\ 459 & 11: 34: 02 & 0.0819 \\ 460 & 11: 34: 03 & 0.0826 \\ 461 & 11: 34: 04 & 0.0778 \\ 462 & 11: 34: 05 & 0.0905 \\ 463 & 11: 34: 06 & 0.0869 \\ 464 & 11: 34: 07 & 0.0940 \\ 465 & 11: 34: 08 & 0.0810 \\ 466 & 11: 34: 09 & 0.0878\end{array}$


Appendix A - Fuel Data Test 1 NIST102505_1.xIs

$\begin{array}{lll}467 & 11: 34: 10 & 0.0842 \\ 468 & 11: 34: 11 & 0.0836 \\ 469 & 11: 34: 12 & 0.0854 \\ 470 & 11: 34: 13 & 0.0824 \\ 471 & 11: 34: 14 & 0.0884 \\ 472 & 11: 34: 15 & 0.0819 \\ 473 & 11: 34: 16 & 0.0874 \\ 474 & 11: 34: 17 & 0.0766 \\ 475 & 11: 34: 18 & 0.0874 \\ 476 & 11: 34: 19 & 0.0772 \\ 477 & 11: 34: 20 & 0.0830 \\ 478 & 11: 34: 21 & 0.0817 \\ 479 & 11: 34: 22 & 0.0856 \\ 480 & 11: 34: 23 & 0.0831 \\ 481 & 11: 34: 24 & 0.0812 \\ 482 & 11: 34: 25 & 0.0861 \\ 483 & 11: 34: 26 & 0.0775 \\ 484 & 11: 34: 27 & 0.0851 \\ 485 & 11: 34: 28 & 0.0810 \\ 486 & 11: 34: 29 & 0.0840 \\ 487 & 11: 34: 30 & 0.0767 \\ 488 & 11: 34: 31 & 0.0830 \\ 489 & 11: 34: 32 & 0.0861 \\ 490 & 11: 34: 33 & 0.0869 \\ 491 & 11: 34: 34 & 0.0861 \\ 492 & 11: 34: 35 & 0.0798 \\ 493 & 11: 34: 36 & 0.0868 \\ 494 & 11: 34: 37 & 0.0785 \\ 495 & 11: 34: 38 & 0.0862 \\ 496 & 11: 34: 39 & 0.0782 \\ 497 & 11: 34: 40 & 0.0876 \\ 498 & 11: 34: 41 & 0.0805 \\ 499 & 11: 34: 42 & 0.0871 \\ 500 & 11: 34: 43 & 0.0783 \\ 501 & 11: 34: 44 & 0.0808 \\ 502 & 11: 34: 45 & 0.0828 \\ 503 & 11: 34: 46 & 0.0797 \\ 504 & 11: 34: 47 & 0.0847 \\ 505 & 11: 34: 48 & 0.0813 \\ 506 & 11: 34: 49 & 0.0874 \\ 507 & 11: 34: 50 & 0.0809 \\ 508 & 11: 34: 51 & 0.0850 \\ 509 & 11: 34: 52 & 0.0816 \\ 510 & 11: 34: 53 & 0.0854 \\ 511 & 11: 34: 54 & 0.0813 \\ 512 & 11: 34: 55 & 0.0821 \\ 513 & 11: 34: 56 & 0.0862 \\ 514 & 11: 34: 57 & 0.0780 \\ 515 & 11: 34: 58 & 0.0841 \\ 516 & 11: 34: 59 & 0.0850 \\ 517 & 11: 35: 00 & 0.0920 \\ 518 & 11: 35: 01 & 0.0795\end{array}$


Appendix A - Fuel Data Test 1 NIST102505_1.xIs

$\begin{array}{lll}519 & 11: 35: 02 & 0.0920 \\ 520 & 11: 35: 03 & 0.0866 \\ 521 & 11: 35: 04 & 0.0884 \\ 522 & 11: 35: 05 & 0.0837 \\ 523 & 11: 35: 06 & 0.0816 \\ 524 & 11: 35: 07 & 0.0827 \\ 525 & 11: 35: 08 & 0.0741 \\ 526 & 11: 35: 09 & 0.0849 \\ 527 & 11: 35: 10 & 0.0806 \\ 528 & 11: 35: 11 & 0.0869 \\ 529 & 11: 35: 12 & 0.0794 \\ 530 & 11: 35: 13 & 0.0853 \\ 531 & 11: 35: 14 & 0.0798 \\ 532 & 11: 35: 15 & 0.0824 \\ 533 & 11: 35: 16 & 0.0805 \\ 534 & 11: 35: 17 & 0.0791 \\ 535 & 11: 35: 18 & 0.0817 \\ 536 & 11: 35: 19 & 0.0775 \\ 537 & 11: 35: 20 & 0.0889 \\ 538 & 11: 35: 21 & 0.0863 \\ 539 & 11: 35: 22 & 0.0915 \\ 540 & 11: 35: 23 & 0.0850 \\ 541 & 11: 35: 24 & 0.0850 \\ 542 & 11: 35: 25 & 0.0842 \\ 543 & 11: 35: 26 & 0.0829 \\ 544 & 11: 35: 27 & 0.0808 \\ 545 & 11: 35: 28 & 0.0777 \\ 546 & 11: 35: 29 & 0.0849 \\ 547 & 11: 35: 30 & 0.0771 \\ 548 & 11: 35: 31 & 0.0840 \\ 549 & 11: 35: 32 & 0.0811 \\ 550 & 11: 35: 33 & 0.0861 \\ 551 & 11: 35: 34 & 0.0769 \\ 552 & 11: 35: 35 & 0.0830 \\ 553 & 11: 35: 36 & 0.0773 \\ 554 & 11: 35: 37 & 0.0778 \\ 555 & 11: 35: 38 & 0.0837 \\ 556 & 11: 35: 39 & 0.0801 \\ 557 & 11: 35: 40 & 0.0871 \\ 558 & 11: 35: 41 & 0.0763 \\ 559 & 11: 35: 42 & 0.0864 \\ 560 & 11: 35: 43 & 0.0761 \\ 561 & 11: 35: 44 & 0.0818 \\ 562 & 11: 35: 45 & 0.0824 \\ 563 & 11: 35: 46 & 0.0836 \\ 564 & 11: 35: 47 & 0.0850 \\ 565 & 11: 35: 48 & 0.0786 \\ 566 & 11: 35: 49 & 0.0889 \\ 567 & 11: 35: 50 & 0.0825 \\ 568 & 11: 35: 51 & 0.0906 \\ 569 & 11: 35: 52 & 0.0789 \\ 570 & 11: 35: 53 & 0.0896\end{array}$


Appendix A - Fuel Data Test 1 NIST102505_1.xIs

$\begin{array}{lll}571 & 11: 35: 54 & 0.0787 \\ 572 & 11: 35: 55 & 0.0842 \\ 573 & 11: 35: 56 & 0.0747 \\ 574 & 11: 35: 57 & 0.0801 \\ 575 & 11: 35: 58 & 0.0781 \\ 576 & 11: 35: 59 & 0.0734 \\ 577 & 11: 36: 00 & 0.0843 \\ 578 & 11: 36: 01 & 0.0800 \\ 579 & 11: 36: 02 & 0.0875 \\ 580 & 11: 36: 03 & 0.0781 \\ 581 & 11: 36: 04 & 0.0869 \\ 582 & 11: 36: 05 & 0.0802 \\ 583 & 11: 36: 06 & 0.0847 \\ 584 & 11: 36: 07 & 0.0790 \\ 585 & 11: 36: 08 & 0.0823 \\ 586 & 11: 36: 09 & 0.0868 \\ 587 & 11: 36: 10 & 0.0826 \\ 588 & 11: 36: 11 & 0.0865 \\ 589 & 11: 36: 12 & 0.0794 \\ 590 & 11: 36: 13 & 0.0860 \\ 591 & 11: 36: 14 & 0.0770 \\ 592 & 11: 36: 15 & 0.0853 \\ 593 & 11: 36: 16 & 0.0781 \\ 594 & 11: 36: 17 & 0.0805 \\ 595 & 11: 36: 18 & 0.0778 \\ 596 & 11: 36: 19 & 0.0795 \\ 597 & 11: 36: 20 & 0.0819 \\ 598 & 11: 36: 21 & 0.0736 \\ 599 & 11: 36: 22 & 0.0818 \\ 600 & 11: 36: 23 & 0.0811 \\ 601 & 11: 36: 24 & 0.0852 \\ 602 & 11: 36: 25 & 0.0772 \\ 603 & 11: 36: 26 & 0.0806 \\ 604 & 11: 36: 27 & 0.0783 \\ 605 & 11: 36: 28 & 0.0814 \\ 606 & 11: 36: 29 & 0.0783 \\ 607 & 11: 36: 30 & 0.0786 \\ 608 & 11: 36: 31 & 0.0846 \\ 609 & 11: 36: 32 & 0.0753 \\ 610 & 11: 36: 33 & 0.0855 \\ 611 & 11: 36: 34 & 0.0790 \\ 612 & 11: 36: 35 & 0.0884 \\ 613 & 11: 36: 36 & 0.0774 \\ 614 & 11: 36: 37 & 0.0819 \\ 615 & 11: 36: 38 & 0.0792 \\ 616 & 11: 36: 39 & 0.0778 \\ 617 & 11: 36: 40 & 0.0770 \\ 618 & 11: 36: 41 & 0.0748 \\ 619 & 11: 36: 42 & 0.0825 \\ 620 & 11: 36: 43 & 0.0765 \\ 621 & 11: 36: 44 & 0.0865 \\ 622 & 11: 36: 45 & 0.0841\end{array}$


Appendix A - Fuel Data Test 1 NIST102505_1.xIs

$\begin{array}{lll}623 & 11: 36: 46 & 0.0893 \\ 624 & 11: 36: 47 & 0.0816 \\ 625 & 11: 36: 48 & 0.0793 \\ 626 & 11: 36: 49 & 0.0825 \\ 627 & 11: 36: 50 & 0.0793 \\ 628 & 11: 36: 51 & 0.0798 \\ 629 & 11: 36: 52 & 0.0772 \\ 630 & 11: 36: 53 & 0.0812 \\ 631 & 11: 36: 54 & 0.0751 \\ 632 & 11: 36: 55 & 0.0801 \\ 633 & 11: 36: 56 & 0.0799 \\ 634 & 11: 36: 57 & 0.0848 \\ 635 & 11: 36: 58 & 0.0808 \\ 636 & 11: 36: 59 & 0.0816 \\ 637 & 11: 37: 00 & 0.0868 \\ 638 & 11: 37: 01 & 0.0785 \\ 639 & 11: 37: 02 & 0.0840 \\ 640 & 11: 37: 03 & 0.0770 \\ 641 & 11: 37: 04 & 0.0869 \\ 642 & 11: 37: 05 & 0.0761 \\ 643 & 11: 37: 06 & 0.0826 \\ 644 & 11: 37: 07 & 0.0754 \\ 645 & 11: 37: 08 & 0.0798 \\ 646 & 11: 37: 09 & 0.0789 \\ 647 & 11: 37: 10 & 0.0761 \\ 648 & 11: 37: 11 & 0.0834 \\ 649 & 11: 37: 12 & 0.0830 \\ 650 & 11: 37: 13 & 0.0916 \\ 651 & 11: 37: 14 & 0.0826 \\ 652 & 11: 37: 15 & 0.0917 \\ 653 & 11: 37: 16 & 0.0810 \\ 654 & 11: 37: 17 & 0.0904 \\ 655 & 11: 37: 18 & 0.0786 \\ 656 & 11: 37: 19 & 0.0809 \\ 657 & 11: 37: 20 & 0.0781 \\ 658 & 11: 37: 21 & 0.0750 \\ 659 & 11: 37: 22 & 0.0837 \\ 660 & 11: 37: 23 & 0.0788 \\ 661 & 11: 37: 24 & 0.0847 \\ 662 & 11: 37: 25 & 0.0746 \\ 663 & 11: 37: 26 & 0.0869 \\ 664 & 11: 37: 27 & 0.0792 \\ 665 & 11: 37: 28 & 0.0881 \\ 666 & 11: 37: 29 & 0.0794 \\ 667 & 11: 37: 30 & 0.0820 \\ 668 & 11: 37: 31 & 0.0795 \\ 669 & 11: 37: 32 & 0.0774 \\ 670 & 11: 37: 33 & 0.0811 \\ 671 & 11: 37: 34 & 0.0795 \\ 672 & 11: 37: 35 & 0.0839 \\ 673 & 11: 37: 36 & 0.0743 \\ 674 & 11: 37: 37 & 0.0842\end{array}$


Appendix A - Fuel Data Test 1 NIST102505_1.xIs

$\begin{array}{lll}675 & 11: 37: 38 & 0.0805 \\ 676 & 11: 37: 39 & 0.0821 \\ 677 & 11: 37: 40 & 0.0820 \\ 678 & 11: 37: 41 & 0.0809 \\ 679 & 11: 37: 42 & 0.0889 \\ 680 & 11: 37: 43 & 0.0785 \\ 681 & 11: 37: 44 & 0.0819 \\ 682 & 11: 37: 45 & 0.0745 \\ 683 & 11: 37: 46 & 0.0834 \\ 684 & 11: 37: 47 & 0.0728 \\ 685 & 11: 37: 48 & 0.0806 \\ 686 & 11: 37: 49 & 0.0735 \\ 687 & 11: 37: 50 & 0.0789 \\ 688 & 11: 37: 51 & 0.0791 \\ 689 & 11: 37: 52 & 0.0757 \\ 690 & 11: 37: 53 & 0.0836 \\ 691 & 11: 37: 54 & 0.0791 \\ 692 & 11: 37: 55 & 0.0870 \\ 693 & 11: 37: 56 & 0.0759 \\ 694 & 11: 37: 57 & 0.0882 \\ 695 & 11: 37: 58 & 0.0787 \\ 696 & 11: 37: 59 & 0.0836 \\ 697 & 11: 38: 00 & 0.0747 \\ 698 & 11: 38: 01 & 0.0794 \\ 699 & 11: 38: 02 & 0.0762 \\ 700 & 11: 38: 03 & 0.0714 \\ 701 & 11: 38: 04 & 0.0766 \\ 702 & 11: 38: 05 & 0.0757 \\ 703 & 11: 38: 06 & 0.0849 \\ 704 & 11: 38: 07 & 0.0771 \\ 705 & 11: 38: 08 & 0.0823 \\ 706 & 11: 38: 09 & 0.0811 \\ 707 & 11: 38: 10 & 0.0803 \\ 708 & 11: 38: 11 & 0.0774 \\ 709 & 11: 38: 12 & 0.0772 \\ 710 & 11: 38: 13 & 0.0813 \\ 711 & 11: 38: 14 & 0.0748 \\ 712 & 11: 38: 15 & 0.0802 \\ 713 & 11: 38: 16 & 0.0759 \\ 714 & 11: 38: 17 & 0.0840 \\ 715 & 11: 38: 18 & 0.0726 \\ 716 & 11: 38: 19 & 0.0819 \\ 717 & 11: 38: 20 & 0.0809 \\ 718 & 11: 38: 21 & 0.0812 \\ 719 & 11: 38: 22 & 0.0791 \\ 720 & 11: 38: 23 & 0.0762 \\ 721 & 11: 38: 24 & 0.0800 \\ 722 & 11: 38: 25 & 0.0767 \\ 723 & 11: 38: 26 & 0.0825 \\ 724 & 11: 38: 27 & 0.0735 \\ 725 & 11: 38: 28 & 0.0815 \\ 726 & 11: 38: 29 & 0.0728\end{array}$


Appendix A - Fuel Data Test 1 NIST102505_1.xIs

$\begin{array}{lll}727 & 11: 38: 30 & 0.0767 \\ 728 & 11: 38: 31 & 0.0775 \\ 729 & 11: 38: 32 & 0.0774 \\ 730 & 11: 38: 33 & 0.0772 \\ 731 & 11: 38: 34 & 0.0702 \\ 732 & 11: 38: 35 & 0.0837 \\ 733 & 11: 38: 36 & 0.0789 \\ 734 & 11: 38: 37 & 0.0815 \\ 735 & 11: 38: 38 & 0.0745 \\ 736 & 11: 38: 39 & 0.0822 \\ 737 & 11: 38: 40 & 0.0755 \\ 738 & 11: 38: 41 & 0.0783 \\ 739 & 11: 38: 42 & 0.0796 \\ 740 & 11: 38: 43 & 0.0780 \\ 741 & 11: 38: 44 & 0.0797 \\ 742 & 11: 38: 45 & 0.0699 \\ 743 & 11: 38: 46 & 0.0821 \\ 744 & 11: 38: 47 & 0.0783 \\ 745 & 11: 38: 48 & 0.0867 \\ 746 & 11: 38: 49 & 0.0736 \\ 747 & 11: 38: 50 & 0.0816 \\ 748 & 11: 38: 51 & 0.0673 \\ 749 & 11: 38: 52 & 0.0734 \\ 750 & 11: 38: 53 & 0.0779 \\ 751 & 11: 38: 54 & 0.0754 \\ 752 & 11: 38: 55 & 0.0816 \\ 753 & 11: 38: 56 & 0.0724 \\ 754 & 11: 38: 57 & 0.0891 \\ 755 & 11: 38: 58 & 0.0797 \\ 756 & 11: 38: 59 & 0.0881 \\ 757 & 11: 39: 00 & 0.0735 \\ 758 & 11: 39: 01 & 0.0792 \\ 759 & 11: 39: 02 & 0.0731 \\ 760 & 11: 39: 03 & 0.0748 \\ 761 & 11: 39: 04 & 0.0734 \\ 762 & 11: 39: 05 & 0.0725 \\ 763 & 11: 39: 06 & 0.0787 \\ 764 & 11: 39: 07 & 0.0726 \\ 765 & 11: 39: 08 & 0.0820 \\ 766 & 11: 39: 09 & 0.0800 \\ 767 & 11: 39: 10 & 0.0869 \\ 768 & 11: 39: 11 & 0.0756 \\ 769 & 11: 39: 12 & 0.0824 \\ 770 & 11: 39: 13 & 0.0817 \\ 771 & 11: 39: 14 & 0.0760 \\ 772 & 11: 39: 15 & 0.0755 \\ 773 & 11: 39: 16 & 0.0735 \\ 774 & 11: 39: 17 & 0.0779 \\ 775 & 11: 39: 18 & 0.0717 \\ 776 & 11: 39: 19 & 0.0773 \\ 777 & 11: 39: 20 & 0.0770 \\ 778 & 11: 39: 21 & 0.0790\end{array}$


Appendix A - Fuel Data Test 1 NIST102505_1.xIs

$\begin{array}{lll}779 & 11: 39: 22 & 0.0749 \\ 780 & 11: 39: 23 & 0.0706 \\ 781 & 11: 39: 24 & 0.0776 \\ 782 & 11: 39: 25 & 0.0764 \\ 783 & 11: 39: 26 & 0.0791 \\ 784 & 11: 39: 27 & 0.0706 \\ 785 & 11: 39: 28 & 0.0863 \\ 786 & 11: 39: 29 & 0.0752 \\ 787 & 11: 39: 30 & 0.0843 \\ 788 & 11: 39: 31 & 0.0730 \\ 789 & 11: 39: 32 & 0.0863 \\ 790 & 11: 39: 33 & 0.0815 \\ 791 & 11: 39: 34 & 0.0757 \\ 792 & 11: 39: 35 & 0.0755 \\ 793 & 11: 39: 36 & 0.0720 \\ 794 & 11: 39: 37 & 0.0822 \\ 795 & 11: 39: 38 & 0.0741 \\ 796 & 11: 39: 39 & 0.0793 \\ 797 & 11: 39: 40 & 0.0719 \\ 798 & 11: 39: 41 & 0.0803 \\ 799 & 11: 39: 42 & 0.0760 \\ 800 & 11: 39: 43 & 0.0754 \\ 801 & 11: 39: 44 & 0.0735 \\ 802 & 11: 39: 45 & 0.0745 \\ 803 & 11: 39: 46 & 0.0758 \\ 804 & 11: 39: 47 & 0.0683 \\ 805 & 11: 39: 48 & 0.0777 \\ 806 & 11: 39: 49 & 0.0794 \\ 807 & 11: 39: 50 & 0.0861 \\ 808 & 11: 39: 51 & 0.0787 \\ 809 & 11: 39: 52 & 0.0756 \\ 810 & 11: 39: 53 & 0.0778 \\ 811 & 11: 39: 54 & 0.0784 \\ 812 & 11: 39: 55 & 0.0796 \\ 813 & 11: 39: 56 & 0.0716 \\ 814 & 11: 39: 57 & 0.0800 \\ 815 & 11: 39: 58 & 0.0739 \\ 816 & 11: 39: 59 & 0.0816 \\ 817 & 11: 40: 00 & 0.0708 \\ 818 & 11: 40: 01 & 0.0783 \\ 819 & 11: 40: 02 & 0.0728 \\ 820 & 11: 40: 03 & 0.0776 \\ 821 & 11: 40: 04 & 0.0777 \\ 822 & 11: 40: 05 & 0.0749 \\ 823 & 11: 40: 06 & 0.0773 \\ 824 & 11: 40: 07 & 0.0761 \\ 825 & 11: 40: 08 & 0.0848 \\ 826 & 11: 40: 09 & 0.0754 \\ 827 & 11: 40: 10 & 0.0817 \\ 828 & 11: 40: 11 & 0.0761 \\ 829 & 11: 40: 12 & 0.0780 \\ 830 & 11: 40: 13 & 0.0759\end{array}$


Appendix A - Fuel Data Test 1 NIST102505_1.xls

$\begin{array}{lll}831 & 11: 40: 14 & 0.0745 \\ 832 & 11: 40: 15 & 0.0784 \\ 833 & 11: 40: 16 & 0.0732 \\ 834 & 11: 40: 17 & 0.0784 \\ 835 & 11: 40: 18 & 0.0685 \\ 836 & 11: 40: 19 & 0.0790 \\ 837 & 11: 40: 20 & 0.0703 \\ 838 & 11: 40: 21 & 0.0763 \\ 839 & 11: 40: 22 & 0.0750 \\ 840 & 11: 40: 23 & 0.0774 \\ 841 & 11: 40: 24 & 0.0773 \\ 842 & 11: 40: 25 & 0.0717 \\ 843 & 11: 40: 26 & 0.0780 \\ 844 & 11: 40: 27 & 0.0749 \\ 845 & 11: 40: 28 & 0.0827 \\ 846 & 11: 40: 29 & 0.0711 \\ 847 & 11: 40: 30 & 0.0821 \\ 848 & 11: 40: 31 & 0.0723 \\ 849 & 11: 40: 32 & 0.0781 \\ 850 & 11: 40: 33 & 0.0703 \\ 851 & 11: 40: 34 & 0.0789 \\ 852 & 11: 40: 35 & 0.0793 \\ 853 & 11: 40: 36 & 0.0740 \\ 854 & 11: 40: 37 & 0.0766 \\ 855 & 11: 40: 38 & 0.0727 \\ 856 & 11: 40: 39 & 0.0772 \\ 857 & 11: 40: 40 & 0.0715 \\ 858 & 11: 40: 41 & 0.0753 \\ 859 & 11: 40: 42 & 0.0724 \\ 860 & 11: 40: 43 & 0.0797 \\ 861 & 11: 40: 44 & 0.0740 \\ 862 & 11: 40: 45 & 0.0738 \\ 863 & 11: 40: 46 & 0.0740 \\ 864 & 11: 40: 47 & 0.0691 \\ 865 & 11: 40: 48 & 0.0759 \\ 866 & 11: 40: 49 & 0.0752 \\ 867 & 11: 40: 50 & 0.0821 \\ 868 & 11: 40: 51 & 0.0744 \\ 869 & 11: 40: 52 & 0.0752 \\ 870 & 11: 40: 53 & 0.0744 \\ 871 & 11: 40: 54 & 0.0757 \\ 872 & 11: 40: 55 & 0.0777 \\ 873 & 11: 40: 56 & 0.0708 \\ 874 & 11: 40: 57 & 0.0792 \\ 875 & 11: 40: 58 & 0.0736 \\ 876 & 11: 40: 59 & 0.0837 \\ 877 & 11: 41: 00 & 0.0716 \\ 878 & 11: 41: 01 & 0.0829 \\ 879 & 11: 41: 02 & 0.0756 \\ 880 & 11: 41: 03 & 0.0784 \\ 881 & 11: 41: 04 & 0.0718 \\ 882 & 11: 41: 05 & 0.0726\end{array}$


Appendix A - Fuel Data Test 1 NIST102505_1.xIs

$\begin{array}{lll}883 & 11: 41: 06 & 0.0762 \\ 884 & 11: 41: 07 & 0.0729 \\ 885 & 11: 41: 08 & 0.0796 \\ 886 & 11: 41: 09 & 0.0772 \\ 887 & 11: 41: 10 & 0.0843 \\ 888 & 11: 41: 11 & 0.0738 \\ 889 & 11: 41: 12 & 0.0742 \\ 890 & 11: 41: 13 & 0.0707 \\ 891 & 11: 41: 14 & 0.0736 \\ 892 & 11: 41: 15 & 0.0745 \\ 893 & 11: 41: 16 & 0.0730 \\ 894 & 11: 41: 17 & 0.0780 \\ 895 & 11: 41: 18 & 0.0708 \\ 896 & 11: 41: 19 & 0.0786 \\ 897 & 11: 41: 20 & 0.0726 \\ 898 & 11: 41: 21 & 0.0875 \\ 899 & 11: 41: 22 & 0.0773 \\ 900 & 11: 41: 23 & 0.0820 \\ 901 & 11: 41: 24 & 0.0703 \\ 902 & 11: 41: 25 & 0.0747 \\ 903 & 11: 41: 26 & 0.0722 \\ 904 & 11: 41: 27 & 0.0676 \\ 905 & 11: 41: 28 & 0.0770 \\ 906 & 11: 41: 29 & 0.0742 \\ 907 & 11: 41: 30 & 0.0809 \\ 908 & 11: 41: 31 & 0.0742 \\ 909 & 11: 41: 32 & 0.0853 \\ 910 & 11: 41: 33 & 0.0725 \\ 911 & 11: 41: 34 & 0.0755 \\ 912 & 11: 41: 35 & 0.0680 \\ 913 & 11: 41: 36 & 0.0713 \\ 914 & 11: 41: 37 & 0.0777 \\ 915 & 11: 41: 38 & 0.0742 \\ 916 & 11: 41: 39 & 0.0839 \\ 917 & 11: 41: 40 & 0.0757 \\ 918 & 11: 41: 41 & 0.0838 \\ 919 & 11: 41: 42 & 0.0708 \\ 920 & 11: 41: 43 & 0.0794 \\ 921 & 11: 41: 44 & 0.0717 \\ 922 & 11: 41: 45 & 0.0722 \\ 923 & 11: 41: 46 & 0.0696 \\ 924 & 11: 41: 47 & 0.0691 \\ 925 & 11: 41: 48 & 0.0736 \\ 926 & 11: 41: 49 & 0.0668 \\ 927 & 11: 41: 50 & 0.0775 \\ 928 & 11: 41: 51 & 0.0772 \\ 929 & 11: 41: 52 & 0.0839 \\ 930 & 11: 41: 53 & 0.0696 \\ 931 & 11: 41: 54 & 0.0747 \\ 932 & 11: 41: 55 & 0.0710 \\ 933 & 11: 41: 56 & 0.0787 \\ 934 & 11: 41: 57 & 0.0764\end{array}$


Appendix A - Fuel Data Test 1 NIST102505_1.xIs

$\begin{array}{lll}935 & 11: 41: 58 & 0.0711 \\ 936 & 11: 41: 59 & 0.0752 \\ 937 & 11: 42: 00 & 0.0719 \\ 938 & 11: 42: 01 & 0.0749 \\ 939 & 11: 42: 02 & 0.0730 \\ 940 & 11: 42: 03 & 0.0762 \\ 941 & 11: 42: 04 & 0.0719 \\ 942 & 11: 42: 05 & 0.0699 \\ 943 & 11: 42: 06 & 0.0765 \\ 944 & 11: 42: 07 & 0.0750 \\ 945 & 11: 42: 08 & 0.0767 \\ 946 & 11: 42: 09 & 0.0697 \\ 947 & 11: 42: 10 & 0.0808 \\ 948 & 11: 42: 11 & 0.0683 \\ 949 & 11: 42: 12 & 0.0753 \\ 950 & 11: 42: 13 & 0.0689 \\ 951 & 11: 42: 14 & 0.0756 \\ 952 & 11: 42: 15 & 0.0741 \\ 953 & 11: 42: 16 & 0.0716 \\ 954 & 11: 42: 17 & 0.0757 \\ 955 & 11: 42: 18 & 0.0718 \\ 956 & 11: 42: 19 & 0.0803 \\ 957 & 11: 42: 20 & 0.0725 \\ 958 & 11: 42: 21 & 0.0880 \\ 959 & 11: 42: 22 & 0.0736 \\ 960 & 11: 42: 23 & 0.0809 \\ 961 & 11: 42: 24 & 0.0678 \\ 962 & 11: 42: 25 & 0.0745 \\ 963 & 11: 42: 26 & 0.0694 \\ 964 & 11: 42: 27 & 0.0687 \\ 965 & 11: 42: 28 & 0.0734 \\ 966 & 11: 42: 29 & 0.0692 \\ 967 & 11: 42: 30 & 0.0758 \\ 968 & 11: 42: 31 & 0.0681 \\ 969 & 11: 42: 32 & 0.0811 \\ 970 & 11: 42: 33 & 0.0765 \\ 971 & 11: 42: 34 & 0.0778 \\ 972 & 11: 42: 35 & 0.0718 \\ 973 & 11: 42: 36 & 0.0732 \\ 974 & 11: 42: 37 & 0.0775 \\ 975 & 11: 42: 38 & 0.0704 \\ 976 & 11: 42: 39 & 0.0769 \\ 977 & 11: 42: 40 & 0.0680 \\ 978 & 11: 42: 41 & 0.0769 \\ 979 & 11: 42: 42 & 0.0678 \\ 980 & 11: 42: 43 & 0.0731 \\ 981 & 11: 42: 44 & 0.0706 \\ 982 & 11: 42: 45 & 0.0774 \\ 983 & 11: 42: 46 & 0.0732 \\ 984 & 11: 42: 47 & 0.0694 \\ 985 & 11: 42: 48 & 0.0771 \\ 986 & 11: 42: 49 & 0.0722\end{array}$


Appendix A - Fuel Data Test 1 NIST102505_1.xIs

$\begin{array}{rll}987 & 11: 42: 50 & 0.0796 \\ 988 & 11: 42: 51 & 0.0682 \\ 989 & 11: 42: 52 & 0.0816 \\ 990 & 11: 42: 53 & 0.0735 \\ 991 & 11: 42: 54 & 0.0778 \\ 992 & 11: 42: 55 & 0.0674 \\ 993 & 11: 42: 56 & 0.0788 \\ 994 & 11: 42: 57 & 0.0749 \\ 995 & 11: 42: 58 & 0.0683 \\ 996 & 11: 42: 59 & 0.0697 \\ 997 & 11: 43: 00 & 0.0663 \\ 998 & 11: 43: 01 & 0.0764 \\ 999 & 11: 43: 02 & 0.0655 \\ 1000 & 11: 43: 03 & 0.0795 \\ 1001 & 11: 43: 04 & 0.0729 \\ 1002 & 11: 43: 05 & 0.0752 \\ 1003 & 11: 43: 06 & 0.0685 \\ 1004 & 11: 43: 07 & 0.0679 \\ 1005 & 11: 43: 08 & 0.0750 \\ 1006 & 11: 43: 09 & 0.0713 \\ 1007 & 11: 43: 10 & 0.0774 \\ 1008 & 11: 43: 11 & 0.0739 \\ 1009 & 11: 43: 12 & 0.0795 \\ 1010 & 11: 43: 13 & 0.0700 \\ 1011 & 11: 43: 14 & 0.0691 \\ 1012 & 11: 43: 15 & 0.0712 \\ 1013 & 11: 43: 16 & 0.0740 \\ 1014 & 11: 43: 17 & 0.0727 \\ 1015 & 11: 43: 18 & 0.0677 \\ 1016 & 11: 43: 19 & 0.0776 \\ 1017 & 11: 43: 20 & 0.0695 \\ 1018 & 11: 43: 21 & 0.0798 \\ 1019 & 11: 43: 22 & 0.0712 \\ 1020 & 11: 43: 23 & 0.0779 \\ 1021 & 11: 43: 24 & 0.0727 \\ 1022 & 11: 43: 25 & 0.0753 \\ 1023 & 11: 43: 26 & 0.0695 \\ 1024 & 11: 43: 27 & 0.0689 \\ 1025 & 11: 43: 28 & 0.0718 \\ 1026 & 11: 43: 29 & 0.0640 \\ 1027 & 11: 43: 30 & 0.0731 \\ 1028 & 11: 43: 31 & 0.0720 \\ 1029 & 11: 43: 32 & 0.0765 \\ 1030 & 11: 43: 33 & 0.0643 \\ 1031 & 11: 43: 34 & 0.0711 \\ 1032 & 11: 43: 35 & 0.0720 \\ 1033 & 11: 43: 36 & 0.0736 \\ 1034 & 11: 43: 37 & 0.0697 \\ 1035 & 11: 43: 38 & 0.0695 \\ 1036 & 11: 43: 39 & 0.0731 \\ 1037 & 11: 43: 40 & 0.0657 \\ 1038 & 11: 43: 41 & 0.0753\end{array}$


Appendix A - Fuel Data Test 1 NIST102505_1.xIs

$\begin{array}{lll}1039 & 11: 43: 42 & 0.0689 \\ 1040 & 11: 43: 43 & 0.0827 \\ 1041 & 11: 43: 44 & 0.0686 \\ 1042 & 11: 43: 45 & 0.0765 \\ 1043 & 11: 43: 46 & 0.0664 \\ 1044 & 11: 43: 47 & 0.0672 \\ 1045 & 11: 43: 48 & 0.0678 \\ 1046 & 11: 43: 49 & 0.0650 \\ 1047 & 11: 43: 50 & 0.0729 \\ 1048 & 11: 43: 51 & 0.0725 \\ 1049 & 11: 43: 52 & 0.0809 \\ 1050 & 11: 43: 53 & 0.0763 \\ 1051 & 11: 43: 54 & 0.0795 \\ 1052 & 11: 43: 55 & 0.0731 \\ 1053 & 11: 43: 56 & 0.0699 \\ 1054 & 11: 43: 57 & 0.0754 \\ 1055 & 11: 43: 58 & 0.0727 \\ 1056 & 11: 43: 59 & 0.0734 \\ 1057 & 11: 44: 00 & 0.0653 \\ 1058 & 11: 44: 01 & 0.0728 \\ 1059 & 11: 44: 02 & 0.0660 \\ 1060 & 11: 44: 03 & 0.0700 \\ 1061 & 11: 44: 04 & 0.0707 \\ 1062 & 11: 44: 05 & 0.0757 \\ 1063 & 11: 44: 06 & 0.0728 \\ 1064 & 11: 44: 07 & 0.0667 \\ 1065 & 11: 44: 08 & 0.0732 \\ 1066 & 11: 44: 09 & 0.0712 \\ 1067 & 11: 44: 10 & 0.0792 \\ 1068 & 11: 44: 11 & 0.0676 \\ 1069 & 11: 44: 12 & 0.0796 \\ 1070 & 11: 44: 13 & 0.0738 \\ 1071 & 11: 44: 14 & 0.0795 \\ 1072 & 11: 44: 15 & 0.0686 \\ 1073 & 11: 44: 16 & 0.0733 \\ 1074 & 11: 44: 17 & 0.0736 \\ 1075 & 11: 44: 18 & 0.0674 \\ 1076 & 11: 44: 19 & 0.0719 \\ 1077 & 11: 44: 20 & 0.0680 \\ 1078 & 11: 44: 21 & 0.0740 \\ 1079 & 11: 44: 22 & 0.0640 \\ 1080 & 11: 44: 23 & 0.0718 \\ 1081 & 11: 44: 24 & 0.0749 \\ 1082 & 11: 44: 25 & 0.0774 \\ 1083 & 11: 44: 26 & 0.0738 \\ 1084 & 11: 44: 27 & 0.0749 \\ 1085 & 11: 44: 28 & 0.0786 \\ 1086 & 11: 44: 29 & 0.0705 \\ 1087 & 11: 44: 30 & 0.0775 \\ 1088 & 11: 44: 31 & 0.0671 \\ 1089 & 11: 44: 32 & 0.0784 \\ 1090 & 11: 44: 33 & 0.0660\end{array}$


Appendix A - Fuel Data Test 1 NIST102505_1.xls

$\begin{array}{lll}1091 & 11: 44: 34 & 0.0717 \\ 1092 & 11: 44: 35 & 0.0671 \\ 1093 & 11: 44: 36 & 0.0774 \\ 1094 & 11: 44: 37 & 0.0739 \\ 1095 & 11: 44: 38 & 0.0703 \\ 1096 & 11: 44: 39 & 0.0753 \\ 1097 & 11: 44: 40 & 0.0713 \\ 1098 & 11: 44: 41 & 0.0768 \\ 1099 & 11: 44: 42 & 0.0670 \\ 1100 & 11: 44: 43 & 0.0703 \\ 1101 & 11: 44: 44 & 0.0709 \\ 1102 & 11: 44: 45 & 0.0736 \\ 1103 & 11: 44: 46 & 0.0751 \\ 1104 & 11: 44: 47 & 0.0752 \\ 1105 & 11: 44: 48 & 0.0787 \\ 1106 & 11: 44: 49 & 0.0702 \\ 1107 & 11: 44: 50 & 0.0762 \\ 1108 & 11: 44: 51 & 0.0675 \\ 1109 & 11: 44: 52 & 0.0781 \\ 1110 & 11: 44: 53 & 0.0678 \\ 1111 & 11: 44: 54 & 0.0735 \\ 1112 & 11: 44: 55 & 0.0705 \\ 1113 & 11: 44: 56 & 0.0720 \\ 1114 & 11: 44: 57 & 0.0700 \\ 1115 & 11: 44: 58 & 0.0662 \\ 1116 & 11: 44: 59 & 0.0784 \\ 1117 & 11: 45: 00 & 0.0744 \\ 1118 & 11: 45: 01 & 0.0849 \\ 1119 & 11: 45: 02 & 0.0689 \\ 1120 & 11: 45: 03 & 0.0779 \\ 1121 & 11: 45: 04 & 0.0689 \\ 1122 & 11: 45: 05 & 0.0727 \\ 1123 & 11: 45: 06 & 0.0616 \\ 1124 & 11: 45: 07 & 0.0656 \\ 1125 & 11: 45: 08 & 0.0682 \\ 1126 & 11: 45: 09 & 0.0642 \\ 1127 & 11: 45: 10 & 0.0752 \\ 1128 & 11: 45: 11 & 0.0682 \\ 1129 & 11: 45: 12 & 0.0771 \\ 1130 & 11: 45: 13 & 0.0680 \\ 1131 & 11: 45: 14 & 0.0771 \\ 1132 & 11: 45: 15 & 0.0753 \\ 1133 & 11: 45: 16 & 0.0759 \\ 1134 & 11: 45: 17 & 0.0734 \\ 1135 & 11: 45: 18 & 0.0680 \\ 1136 & 11: 45: 19 & 0.0745 \\ 1137 & 11: 45: 20 & 0.0677 \\ 1138 & 11: 45: 21 & 0.0719 \\ 1139 & 11: 45: 22 & 0.0636 \\ 1140 & 11: 45: 23 & 0.0725 \\ 1141 & 11: 45: 24 & 0.0635 \\ 1142 & 11: 45: 25 & 0.0690\end{array}$


Appendix A - Fuel Data Test 1 NIST102505_1.xIs

$\begin{array}{lll}1143 & 11: 45: 26 & 0.0730 \\ 1144 & 11: 45: 27 & 0.0734 \\ 1145 & 11: 45: 28 & 0.0740 \\ 1146 & 11: 45: 29 & 0.0649 \\ 1147 & 11: 45: 30 & 0.0747 \\ 1148 & 11: 45: 31 & 0.0665 \\ 1149 & 11: 45: 32 & 0.0746 \\ 1150 & 11: 45: 33 & 0.0651 \\ 1151 & 11: 45: 34 & 0.0722 \\ 1152 & 11: 45: 35 & 0.0632 \\ 1153 & 11: 45: 36 & 0.0672 \\ 1154 & 11: 45: 37 & 0.0740 \\ 1155 & 11: 45: 38 & 0.0671 \\ 1156 & 11: 45: 39 & 0.0770 \\ 1157 & 11: 45: 40 & 0.0684 \\ 1158 & 11: 45: 41 & 0.0832 \\ 1159 & 11: 45: 42 & 0.0716 \\ 1160 & 11: 45: 43 & 0.0810 \\ 1161 & 11: 45: 44 & 0.0656 \\ 1162 & 11: 45: 45 & 0.0724 \\ 1163 & 11: 45: 46 & 0.0711 \\ 1164 & 11: 45: 47 & 0.0704 \\ 1165 & 11: 45: 48 & 0.0712 \\ 1166 & 11: 45: 49 & 0.0701 \\ 1167 & 11: 45: 50 & 0.0791 \\ 1168 & 11: 45: 51 & 0.0689 \\ 1169 & 11: 45: 52 & 0.0727 \\ 1170 & 11: 45: 53 & 0.0687 \\ 1171 & 11: 45: 54 & 0.0731 \\ 1172 & 11: 45: 55 & 0.0665 \\ 1173 & 11: 45: 56 & 0.0679 \\ 1174 & 11: 45: 57 & 0.0705 \\ 1175 & 11: 45: 58 & 0.0655 \\ 1176 & 11: 45: 59 & 0.0728 \\ 1177 & 11: 46: 00 & 0.0673 \\ 1178 & 11: 46: 01 & 0.0785 \\ 1179 & 11: 46: 02 & 0.0702 \\ 1180 & 11: 46: 03 & 0.0804 \\ 1181 & 11: 46: 04 & 0.0665 \\ 1182 & 11: 46: 05 & 0.0734 \\ 1183 & 11: 46: 06 & 0.0686 \\ 1184 & 11: 46: 07 & 0.0663 \\ 1185 & 11: 46: 08 & 0.0664 \\ 1186 & 11: 46: 09 & 0.0658 \\ 1187 & 11: 46: 10 & 0.0736 \\ 1188 & 11: 46: 11 & 0.0664 \\ 1189 & 11: 46: 12 & 0.0779 \\ 1190 & 11: 46: 13 & 0.0712 \\ 1191 & 11: 46: 14 & 0.0788 \\ 1192 & 11: 46: 15 & 0.0692 \\ 1193 & 11: 46: 16 & 0.0708 \\ 1194 & 11: 46: 17 & 0.0719\end{array}$


Appendix A - Fuel Data Test 1 NIST102505_1.xls

$\begin{array}{lll}1195 & 11: 46: 18 & 0.0645 \\ 1196 & 11: 46: 19 & 0.0705 \\ 1197 & 11: 46: 20 & 0.0639 \\ 1198 & 11: 46: 21 & 0.0725 \\ 1199 & 11: 46: 22 & 0.0681 \\ 1200 & 11: 46: 23 & 0.0735 \\ 1201 & 11: 46: 24 & 0.0703 \\ 1202 & 11: 46: 25 & 0.0750 \\ 1203 & 11: 46: 26 & 0.0727 \\ 1204 & 11: 46: 27 & 0.0658 \\ 1205 & 11: 46: 28 & 0.0717 \\ 1206 & 11: 46: 29 & 0.0672 \\ 1207 & 11: 46: 30 & 0.0773 \\ 1208 & 11: 46: 31 & 0.0657 \\ 1209 & 11: 46: 32 & 0.0757 \\ 1210 & 11: 46: 33 & 0.0660 \\ 1211 & 11: 46: 34 & 0.0739 \\ 1212 & 11: 46: 35 & 0.0626 \\ 1213 & 11: 46: 36 & 0.0692 \\ 1214 & 11: 46: 37 & 0.0668 \\ 1215 & 11: 46: 38 & 0.0627 \\ 1216 & 11: 46: 39 & 0.0769 \\ 1217 & 11: 46: 40 & 0.0637 \\ 1218 & 11: 46: 41 & 0.0687 \\ 1219 & 11: 46: 42 & 0.0652 \\ 1220 & 11: 46: 43 & 0.0762 \\ 1221 & 11: 46: 44 & 0.0656 \\ 1222 & 11: 46: 45 & 0.0684 \\ 1223 & 11: 46: 46 & 0.0658 \\ 1224 & 11: 46: 47 & 0.0672 \\ 1225 & 11: 46: 48 & 0.0710 \\ 1226 & 11: 46: 49 & 0.0606 \\ 1227 & 11: 46: 50 & 0.0738 \\ 1228 & 11: 46: 51 & 0.0691 \\ 1229 & 11: 46: 52 & 0.0736 \\ 1230 & 11: 46: 53 & 0.0727 \\ 1231 & 11: 46: 54 & 0.0678 \\ 1232 & 11: 46: 55 & 0.0675 \\ 1233 & 11: 46: 56 & 0.0694 \\ 1234 & 11: 46: 57 & 0.0703 \\ 1235 & 11: 46: 58 & 0.0628 \\ 1236 & 11: 46: 59 & 0.0714 \\ 1237 & 11: 47: 00 & 0.0680 \\ 1238 & 11: 47: 01 & 0.0744 \\ 1239 & 11: 47: 02 & 0.0660 \\ 1240 & 11: 47: 03 & 0.0721 \\ 1241 & 11: 47: 04 & 0.0679 \\ 1242 & 11: 47: 05 & 0.0694 \\ 1243 & 11: 47: 06 & 0.0679 \\ 1244 & 11: 47: 07 & 0.0695 \\ 1245 & 11: 47: 08 & 0.0767 \\ 1246 & 11: 47: 09 & 0.0664\end{array}$


Appendix A - Fuel Data Test 1 NIST102505_1.xIs

$\begin{array}{lll}1247 & 11: 47: 10 & 0.0736 \\ 1248 & 11: 47: 11 & 0.0656 \\ 1249 & 11: 47: 12 & 0.0751 \\ 1250 & 11: 47: 13 & 0.0638 \\ 1251 & 11: 47: 14 & 0.0683 \\ 1252 & 11: 47: 15 & 0.0653 \\ 1253 & 11: 47: 16 & 0.0718 \\ 1254 & 11: 47: 17 & 0.0720 \\ 1255 & 11: 47: 18 & 0.0654 \\ 1256 & 11: 47: 19 & 0.0700 \\ 1257 & 11: 47: 20 & 0.0657 \\ 1258 & 11: 47: 21 & 0.0711 \\ 1259 & 11: 47: 22 & 0.0660 \\ 1260 & 11: 47: 23 & 0.0684 \\ 1261 & 11: 47: 24 & 0.0702 \\ 1262 & 11: 47: 25 & 0.0716 \\ 1263 & 11: 47: 26 & 0.0720 \\ 1264 & 11: 47: 27 & 0.0649 \\ 1265 & 11: 47: 28 & 0.0694 \\ 1266 & 11: 47: 29 & 0.0650 \\ 1267 & 11: 47: 30 & 0.0736 \\ 1268 & 11: 47: 31 & 0.0669 \\ 1269 & 11: 47: 32 & 0.0793 \\ 1270 & 11: 47: 33 & 0.0669 \\ 1271 & 11: 47: 34 & 0.0737 \\ 1272 & 11: 47: 35 & 0.0683 \\ 1273 & 11: 47: 36 & 0.0689 \\ 1274 & 11: 47: 37 & 0.0710 \\ 1275 & 11: 47: 38 & 0.0628 \\ 1276 & 11: 47: 39 & 0.0667 \\ 1277 & 11: 47: 40 & 0.0636 \\ 1278 & 11: 47: 41 & 0.0745 \\ 1279 & 11: 47: 42 & 0.0632 \\ 1280 & 11: 47: 43 & 0.0720 \\ 1281 & 11: 47: 44 & 0.0673 \\ 1282 & 11: 47: 45 & 0.0690 \\ 1283 & 11: 47: 46 & 0.0637 \\ 1284 & 11: 47: 47 & 0.0626 \\ 1285 & 11: 47: 48 & 0.0716 \\ 1286 & 11: 47: 49 & 0.0645 \\ 1287 & 11: 47: 50 & 0.0710 \\ 1288 & 11: 47: 51 & 0.0675 \\ 1289 & 11: 47: 52 & 0.0706 \\ 1290 & 11: 47: 53 & 0.0670 \\ 1291 & 11: 47: 54 & 0.0659 \\ 1292 & 11: 47: 55 & 0.0669 \\ 1293 & 11: 47: 56 & 0.0693 \\ 1294 & 11: 47: 57 & 0.0716 \\ 1295 & 11: 47: 58 & 0.0687 \\ 1296 & 11: 47: 59 & 0.0828 \\ 1297 & 11: 48: 00 & 0.0718 \\ 1298 & 11: 48: 01 & 0.0743\end{array}$


Appendix A - Fuel Data Test 1 NIST102505_1.xIs

$\begin{array}{lll}1299 & 11: 48: 02 & 0.0637 \\ 1300 & 11: 48: 03 & 0.0707 \\ 1301 & 11: 48: 04 & 0.0691 \\ 1302 & 11: 48: 05 & 0.0703 \\ 1303 & 11: 48: 06 & 0.0705 \\ 1304 & 11: 48: 07 & 0.0674 \\ 1305 & 11: 48: 08 & 0.0749 \\ 1306 & 11: 48: 09 & 0.0667 \\ 1307 & 11: 48: 10 & 0.0717 \\ 1308 & 11: 48: 11 & 0.0648 \\ 1309 & 11: 48: 12 & 0.0710 \\ 1310 & 11: 48: 13 & 0.0647 \\ 1311 & 11: 48: 14 & 0.0667 \\ 1312 & 11: 48: 15 & 0.0646 \\ 1313 & 11: 48: 16 & 0.0678 \\ 1314 & 11: 48: 17 & 0.0706 \\ 1315 & 11: 48: 18 & 0.0624 \\ 1316 & 11: 48: 19 & 0.0719 \\ 1317 & 11: 48: 20 & 0.0650 \\ 1318 & 11: 48: 21 & 0.0762 \\ 1319 & 11: 48: 22 & 0.0647 \\ 1320 & 11: 48: 23 & 0.0700 \\ 1321 & 11: 48: 24 & 0.0674 \\ 1322 & 11: 48: 25 & 0.0662 \\ 1323 & 11: 48: 26 & 0.0691 \\ 1324 & 11: 48: 27 & 0.0642 \\ 1325 & 11: 48: 28 & 0.0734 \\ 1326 & 11: 48: 29 & 0.0650 \\ 1327 & 11: 48: 30 & 0.0730 \\ 1328 & 11: 48: 31 & 0.0688 \\ 1329 & 11: 48: 32 & 0.0692 \\ 1330 & 11: 48: 33 & 0.0638 \\ 1331 & 11: 48: 34 & 0.0645 \\ 1332 & 11: 48: 35 & 0.0686 \\ 1333 & 11: 48: 36 & 0.0659 \\ 1334 & 11: 48: 37 & 0.0728 \\ 1335 & 11: 48: 38 & 0.0648 \\ 1336 & 11: 48: 39 & 0.0790 \\ 1337 & 11: 48: 40 & 0.0680 \\ 1338 & 11: 48: 41 & 0.0752 \\ 1339 & 11: 48: 42 & 0.0626 \\ 1340 & 11: 48: 43 & 0.0708 \\ 1341 & 11: 48: 44 & 0.0639 \\ 1342 & 11: 48: 45 & 0.0649 \\ 1343 & 11: 48: 46 & 0.0705 \\ 1344 & 11: 48: 47 & 0.0653 \\ 1345 & 11: 48: 48 & 0.0742 \\ 1346 & 11: 48: 49 & 0.0626 \\ 1347 & 11: 48: 50 & 0.0761 \\ 1348 & 11: 48: 51 & 0.0687 \\ 1349 & 11: 48: 52 & 0.0757 \\ 1350 & 11: 48: 53 & 0.0626\end{array}$


Appendix A - Fuel Data Test 1 NIST102505_1.xls

$\begin{array}{lll}1351 & 11: 48: 54 & 0.0685 \\ 1352 & 11: 48: 55 & 0.0687 \\ 1353 & 11: 48: 56 & 0.0608 \\ 1354 & 11: 48: 57 & 0.0674 \\ 1355 & 11: 48: 58 & 0.0675 \\ 1356 & 11: 48: 59 & 0.0714 \\ 1357 & 11: 49: 00 & 0.0598 \\ 1358 & 11: 49: 01 & 0.0733 \\ 1359 & 11: 49: 02 & 0.0650 \\ 1360 & 11: 49: 03 & 0.0683 \\ 1361 & 11: 49: 04 & 0.0648 \\ 1362 & 11: 49: 05 & 0.0632 \\ 1363 & 11: 49: 06 & 0.0705 \\ 1364 & 11: 49: 07 & 0.0641 \\ 1365 & 11: 49: 08 & 0.0722 \\ 1366 & 11: 49: 09 & 0.0634 \\ 1367 & 11: 49: 10 & 0.0710 \\ 1368 & 11: 49: 11 & 0.0622 \\ 1369 & 11: 49: 12 & 0.0648 \\ 1370 & 11: 49: 13 & 0.0596 \\ 1371 & 11: 49: 14 & 0.0626 \\ 1372 & 11: 49: 15 & 0.0670 \\ 1373 & 11: 49: 16 & 0.0601 \\ 1374 & 11: 49: 17 & 0.0725 \\ 1375 & 11: 49: 18 & 0.0682 \\ 1376 & 11: 49: 19 & 0.0737 \\ 1377 & 11: 49: 20 & 0.0647 \\ 1378 & 11: 49: 21 & 0.0671 \\ 1379 & 11: 49: 22 & 0.0665 \\ 1380 & 11: 49: 23 & 0.0695 \\ 1381 & 11: 49: 24 & 0.0647 \\ 1382 & 11: 49: 25 & 0.0611 \\ 1383 & 11: 49: 26 & 0.0654 \\ 1384 & 11: 49: 27 & 0.0615 \\ 1385 & 11: 49: 28 & 0.0740 \\ 1386 & 11: 49: 29 & 0.0750 \\ 1387 & 11: 49: 30 & 0.0777 \\ 1388 & 11: 49: 31 & 0.0724 \\ 1389 & 11: 49: 32 & 0.0775 \\ 1390 & 11: 49: 33 & 0.0734 \\ 1391 & 11: 49: 34 & 0.0848 \\ 1392 & 11: 49: 35 & 0.0757 \\ 1393 & 11: 49: 36 & 0.0830 \\ 1394 & 11: 49: 37 & 0.0710 \\ 1395 & 11: 49: 38 & 0.0824 \\ 1396 & 11: 49: 39 & 0.0775 \\ 1397 & 11: 49: 40 & 0.0869 \\ 1398 & 11: 49: 41 & 0.0749 \\ 1399 & 11: 49: 42 & 0.0794 \\ 1400 & 11: 49: 43 & 0.0823 \\ 1401 & 11: 49: 44 & 0.0837 \\ 1402 & 11: 49: 45 & 0.0782\end{array}$


Appendix A - Fuel Data Test 1 NIST102505_1.xls

$\begin{array}{lll}1403 & 11: 49: 46 & 0.0756 \\ 1404 & 11: 49: 47 & 0.0827 \\ 1405 & 11: 49: 48 & 0.0782 \\ 1406 & 11: 49: 49 & 0.0772 \\ 1407 & 11: 49: 50 & 0.0733 \\ 1408 & 11: 49: 51 & 0.0748 \\ 1409 & 11: 49: 52 & 0.0739 \\ 1410 & 11: 49: 53 & 0.0746 \\ 1411 & 11: 49: 54 & 0.0714 \\ 1412 & 11: 49: 55 & 0.0810 \\ 1413 & 11: 49: 56 & 0.0781 \\ 1414 & 11: 49: 57 & 0.0803 \\ 1415 & 11: 49: 58 & 0.0757 \\ 1416 & 11: 49: 59 & 0.0803 \\ 1417 & 11: 50: 00 & 0.0819 \\ 1418 & 11: 50: 01 & 0.0768 \\ 1419 & 11: 50: 02 & 0.0730 \\ 1420 & 11: 50: 03 & 0.0762 \\ 1421 & 11: 50: 04 & 0.0691 \\ 1422 & 11: 50: 05 & 0.0827 \\ 1423 & 11: 50: 06 & 0.0778 \\ 1424 & 11: 50: 07 & 0.0814 \\ 1425 & 11: 50: 08 & 0.0734 \\ 1426 & 11: 50: 09 & 0.0800 \\ 1427 & 11: 50: 10 & 0.0721 \\ 1428 & 11: 50: 11 & 0.0817 \\ 1429 & 11: 50: 12 & 0.0701 \\ 1430 & 11: 50: 13 & 0.0813 \\ 1431 & 11: 50: 14 & 0.0735 \\ 1432 & 11: 50: 15 & 0.0818 \\ 1433 & 11: 50: 16 & 0.0742 \\ 1434 & 11: 50: 17 & 0.0883 \\ 1435 & 11: 50: 18 & 0.0776 \\ 1436 & 11: 50: 19 & 0.0858 \\ 1437 & 11: 50: 20 & 0.0710 \\ 1438 & 11: 50: 21 & 0.0795 \\ 1439 & 11: 50: 22 & 0.0783 \\ 1440 & 11: 50: 23 & 0.0771 \\ 1441 & 11: 50: 24 & 0.0744 \\ 1442 & 11: 50: 25 & 0.0748 \\ 1443 & 11: 50: 26 & 0.0806 \\ 1444 & 11: 50: 27 & 0.0804 \\ 1445 & 11: 50: 28 & 0.0757 \\ 1446 & 11: 50: 29 & 0.0718 \\ 1447 & 11: 50: 30 & 0.0772 \\ 1448 & 11: 50: 31 & 0.0781 \\ 1449 & 11: 50: 32 & 0.0760 \\ 1450 & 11: 50: 33 & 0.0735 \\ 1451 & 11: 50: 34 & 0.0783 \\ 1452 & 11: 50: 35 & 0.0775 \\ 1453 & 11: 50: 36 & 0.0785 \\ 1454 & 11: 50: 37 & 0.0719\end{array}$


Appendix A - Fuel Data Test 1 NIST102505_1.xIs

$\begin{array}{lll}1455 & 11: 50: 38 & 0.0738 \\ 1456 & 11: 50: 39 & 0.0783 \\ 1457 & 11: 50: 40 & 0.0798 \\ 1458 & 11: 50: 41 & 0.0740 \\ 1459 & 11: 50: 42 & 0.0802 \\ 1460 & 11: 50: 43 & 0.0738 \\ 1461 & 11: 50: 44 & 0.0798 \\ 1462 & 11: 50: 45 & 0.0764 \\ 1463 & 11: 50: 46 & 0.0809 \\ 1464 & 11: 50: 47 & 0.0736 \\ 1465 & 11: 50: 48 & 0.0814 \\ 1466 & 11: 50: 49 & 0.0764 \\ 1467 & 11: 50: 50 & 0.0754 \\ 1468 & 11: 50: 51 & 0.0738 \\ 1469 & 11: 50: 52 & 0.0759 \\ 1470 & 11: 50: 53 & 0.0721 \\ 1471 & 11: 50: 54 & 0.0834 \\ 1472 & 11: 50: 55 & 0.0744 \\ 1473 & 11: 50: 56 & 0.0811 \\ 1474 & 11: 50: 57 & 0.0717 \\ 1475 & 11: 50: 58 & 0.0794 \\ 1476 & 11: 50: 59 & 0.0698 \\ 1477 & 11: 51: 00 & 0.0769 \\ 1478 & 11: 51: 01 & 0.0765 \\ 1479 & 11: 51: 02 & 0.0819 \\ 1480 & 11: 51: 03 & 0.0740 \\ 1481 & 11: 51: 04 & 0.0794 \\ 1482 & 11: 51: 05 & 0.0761 \\ 1483 & 11: 51: 06 & 0.0837 \\ 1484 & 11: 51: 07 & 0.0789 \\ 1485 & 11: 51: 08 & 0.0862 \\ 1486 & 11: 51: 09 & 0.0752 \\ 1487 & 11: 51: 10 & 0.0757 \\ 1488 & 11: 51: 11 & 0.0716 \\ 1489 & 11: 51: 12 & 0.0718 \\ 1490 & 11: 51: 13 & 0.0706 \\ 1491 & 11: 51: 14 & 0.0748 \\ 1492 & 11: 51: 15 & 0.0790 \\ 1493 & 11: 51: 16 & 0.0825 \\ 1494 & 11: 51: 17 & 0.0797 \\ 1495 & 11: 51: 18 & 0.0749 \\ 1496 & 11: 51: 19 & 0.0807 \\ 1497 & 11: 51: 20 & 0.0760 \\ 1498 & 11: 51: 21 & 0.0808 \\ 1499 & 11: 51: 22 & 0.0726 \\ 1500 & 11: 51: 23 & 0.0834 \\ 1501 & 11: 51: 24 & 0.0784 \\ 1502 & 11: 51: 25 & 0.0838 \\ 1503 & 11: 51: 26 & 0.0735 \\ 1504 & 11: 51: 27 & 0.0828 \\ 1505 & 11: 51: 28 & 0.0759 \\ 1506 & 11: 51: 29 & 0.0760\end{array}$


Appendix A - Fuel Data Test 1 NIST102505_1.xls

\begin{tabular}{lll}
1507 & $11: 51: 30$ & 0.0733 \\
1508 & $11: 51: 31$ & 0.0761 \\
1509 & $11: 51: 32$ & 0.0705 \\
1510 & $11: 51: 33$ & 0.0834 \\
1511 & $11: 51: 34$ & 0.0753 \\
1512 & $11: 51: 35$ & 0.0839 \\
1513 & $11: 51: 36$ & 0.0702 \\
1514 & $11: 51: 37$ & 0.0834 \\
1515 & $11: 51: 38$ & 0.0737 \\
1516 & $11: 51: 39$ & 0.0791 \\
1517 & $11: 51: 40$ & 0.0723 \\
1518 & $11: 51: 41$ & 0.0805 \\
1519 & $11: 51: 42$ & 0.0768 \\
1520 & $11: 51: 43$ & 0.0817 \\
1521 & $11: 51: 44$ & 0.0706 \\
1522 & $11: 51: 45$ & 0.0786 \\
1523 & $11: 51: 46$ & 0.0809 \\
1524 & $11: 51: 47$ & 0.0811 \\
1525 & $11: 51: 48$ & 0.0779 \\
1526 & $11: 51: 49$ & 0.0698 \\
1527 & $11: 51: 50$ & 0.0737 \\
1528 & $11: 51: 51$ & 0.0746 \\
1529 & $11: 51: 52$ & 0.0725 \\
1530 & $11: 51: 53$ & 0.0752 \\
1531 & $11: 51: 54$ & 0.0734 \\
1532 & $11: 51: 55$ & 0.0799 \\
1533 & $11: 51: 56$ & 0.0784 \\
1534 & $11: 51: 57$ & 0.0766 \\
1535 & $11: 51: 58$ & 0.0751 \\
1536 & $11: 51: 59$ & 0.0724 \\
1537 & $11: 52: 00$ & 0.0800 \\
1538 & $11: 52: 01$ & 0.0751 \\
1539 & $11: 52: 02$ & 0.0822 \\
1540 & $11: 52: 03$ & 0.0712 \\
1541 & $11: 52: 04$ & 0.0783 \\
1542 & $11: 52: 05$ & 0.0760 \\
1543 & $11: 52: 06$ & 0.0779 \\
1544 & $11: 52: 07$ & 0.0683 \\
1545 & $11: 52: 08$ & 0.0764 \\
1546 & $11: 52: 09$ & 0.0770 \\
1547 & $11: 52: 10$ & 0.0824 \\
1548 & $11: 52: 11$ & 0.0717 \\
1549 & $11: 52: 12$ & 0.0774 \\
1550 & $11: 52: 13$ & 0.0776 \\
1551 & $11: 52: 14$ & 0.0796 \\
1552 & $11: 52: 15$ & 0.0729 \\
1553 & $11: 52: 16$ & 0.0812 \\
1554 & $11: 52: 17$ & 0.0718 \\
1555 & $11: 52: 18$ & 0.0820 \\
1556 & $11: 52: 19$ & 0.0737 \\
1557 & $11: 52: 20$ & 0.0788 \\
1558 & $11: 52: 21$ & 0.0722 \\
& & \\
\hline
\end{tabular}


Appendix A - Fuel Data Test 1 NIST102505_1.xIs

$\begin{array}{lll}1559 & 11: 52: 22 & 0.0804 \\ 1560 & 11: 52: 23 & 0.0810 \\ 1561 & 11: 52: 24 & 0.0813 \\ 1562 & 11: 52: 25 & 0.0768 \\ 1563 & 11: 52: 26 & 0.0715 \\ 1564 & 11: 52: 27 & 0.0722 \\ 1565 & 11: 52: 28 & 0.0766 \\ 1566 & 11: 52: 29 & 0.0713 \\ 1567 & 11: 52: 30 & 0.0754 \\ 1568 & 11: 52: 31 & 0.0692 \\ 1569 & 11: 52: 32 & 0.0783 \\ 1570 & 11: 52: 33 & 0.0765 \\ 1571 & 11: 52: 34 & 0.0781 \\ 1572 & 11: 52: 35 & 0.0734 \\ 1573 & 11: 52: 36 & 0.0751 \\ 1574 & 11: 52: 37 & 0.0724 \\ 1575 & 11: 52: 38 & 0.0728 \\ 1576 & 11: 52: 39 & 0.0738 \\ 1577 & 11: 52: 40 & 0.0752 \\ 1578 & 11: 52: 41 & 0.0747 \\ 1579 & 11: 52: 42 & 0.0731 \\ 1580 & 11: 52: 43 & 0.0746 \\ 1581 & 11: 52: 44 & 0.0678 \\ 1582 & 11: 52: 45 & 0.0826 \\ 1583 & 11: 52: 46 & 0.0739 \\ 1584 & 11: 52: 47 & 0.0816 \\ 1585 & 11: 52: 48 & 0.0688 \\ 1586 & 11: 52: 49 & 0.0779 \\ 1587 & 11: 52: 50 & 0.0744 \\ 1588 & 11: 52: 51 & 0.0807 \\ 1589 & 11: 52: 52 & 0.0694 \\ 1590 & 11: 52: 53 & 0.0807 \\ 1591 & 11: 52: 54 & 0.0726 \\ 1592 & 11: 52: 55 & 0.0800 \\ 1593 & 11: 52: 56 & 0.0691 \\ 1594 & 11: 52: 57 & 0.0756 \\ 1595 & 11: 52: 58 & 0.0787 \\ 1596 & 11: 52: 59 & 0.0795 \\ 1597 & 11: 53: 00 & 0.0753 \\ 1598 & 11: 53: 01 & 0.0776 \\ 1599 & 11: 53: 02 & 0.0731 \\ 1600 & 11: 53: 03 & 0.0749 \\ 1601 & 11: 53: 04 & 0.0685 \\ 1602 & 11: 53: 05 & 0.0734 \\ 1603 & 11: 53: 06 & 0.0678 \\ 1604 & 11: 53: 07 & 0.0752 \\ 1605 & 11: 53: 08 & 0.0714 \\ 1606 & 11: 53: 09 & 0.0768 \\ 1607 & 11: 53: 10 & 0.0732 \\ 1608 & 11: 53: 11 & 0.0786 \\ 1609 & 11: 53: 12 & 0.0766 \\ 1610 & 11: 53: 13 & 0.0755\end{array}$


Appendix A - Fuel Data Test 1 NIST102505_1.xls

\begin{tabular}{lll}
1611 & $11: 53: 14$ & 0.0771 \\
1612 & $11: 53: 15$ & 0.0695 \\
1613 & $11: 53: 16$ & 0.0756 \\
1614 & $11: 53: 17$ & 0.0744 \\
1615 & $11: 53: 18$ & 0.0753 \\
1616 & $11: 53: 19$ & 0.0767 \\
1617 & $11: 53: 20$ & 0.0697 \\
1618 & $11: 53: 21$ & 0.0731 \\
1619 & $11: 53: 22$ & 0.0722 \\
1620 & $11: 53: 23$ & 0.0680 \\
1621 & $11: 53: 24$ & 0.0729 \\
1622 & $11: 53: 25$ & 0.0669 \\
1623 & $11: 53: 26$ & 0.0799 \\
1624 & $11: 53: 27$ & 0.0774 \\
1625 & $11: 53: 28$ & 0.0839 \\
1626 & $11: 53: 29$ & 0.0760 \\
1627 & $11: 53: 30$ & 0.0850 \\
1628 & $11: 53: 31$ & 0.0731 \\
1629 & $11: 53: 32$ & 0.0812 \\
1630 & $11: 53: 33$ & 0.0692 \\
1631 & $11: 53: 34$ & 0.0831 \\
1632 & $11: 53: 35$ & 0.0741 \\
1633 & $11: 53: 36$ & 0.0782 \\
1634 & $11: 53: 37$ & 0.0690 \\
1635 & $11: 53: 38$ & 0.0782 \\
1636 & $11: 53: 39$ & 0.0766 \\
1637 & $11: 53: 40$ & 0.0800 \\
1638 & $11: 53: 41$ & 0.0696 \\
1639 & $11: 53: 42$ & 0.0735 \\
1640 & $11: 53: 43$ & 0.0756 \\
1641 & $11: 53: 44$ & 0.0796 \\
1642 & $11: 53: 45$ & 0.0773 \\
1643 & $11: 53: 46$ & 0.0736 \\
1644 & $11: 53: 47$ & 0.0716 \\
1645 & $11: 53: 48$ & 0.0753 \\
1646 & $11: 53: 49$ & 0.0732 \\
1647 & $11: 53: 50$ & 0.0755 \\
1648 & $11: 53: 51$ & 0.0725 \\
1649 & $11: 53: 52$ & 0.0775 \\
1650 & $11: 53: 53$ & 0.0726 \\
1651 & $11: 53: 54$ & 0.0770 \\
1652 & $11: 53: 55$ & 0.0738 \\
1653 & $11: 53: 56$ & 0.0735 \\
1654 & $11: 53: 57$ & 0.0768 \\
1655 & $11: 53: 58$ & 0.0744 \\
1656 & $11: 53: 59$ & 0.0770 \\
1657 & $11: 54: 00$ & 0.0667 \\
1658 & $11: 54: 01$ & 0.0740 \\
1659 & $11: 54: 02$ & 0.0733 \\
1660 & $11: 54: 03$ & 0.0741 \\
1661 & $11: 54: 04$ & 0.0697 \\
1662 & $11: 54: 05$ & 0.0706 \\
& & \\
\hline
\end{tabular}


Appendix A - Fuel Data Test 1 NIST102505_1.xIs

$\begin{array}{lll}1663 & 11: 54: 06 & 0.0685 \\ 1664 & 11: 54: 07 & 0.0780 \\ 1665 & 11: 54: 08 & 0.0757 \\ 1666 & 11: 54: 09 & 0.0788 \\ 1667 & 11: 54: 10 & 0.0710 \\ 1668 & 11: 54: 11 & 0.0791 \\ 1669 & 11: 54: 12 & 0.0670 \\ 1670 & 11: 54: 13 & 0.0750 \\ 1671 & 11: 54: 14 & 0.0701 \\ 1672 & 11: 54: 15 & 0.0796 \\ 1673 & 11: 54: 16 & 0.0696 \\ 1674 & 11: 54: 17 & 0.0799 \\ 1675 & 11: 54: 18 & 0.0734 \\ 1676 & 11: 54: 19 & 0.0813 \\ 1677 & 11: 54: 20 & 0.0736 \\ 1678 & 11: 54: 21 & 0.0750 \\ 1679 & 11: 54: 22 & 0.0730 \\ 1680 & 11: 54: 23 & 0.0777 \\ 1681 & 11: 54: 24 & 0.0778 \\ 1682 & 11: 54: 25 & 0.0757 \\ 1683 & 11: 54: 26 & 0.0743 \\ 1684 & 11: 54: 27 & 0.0752 \\ 1685 & 11: 54: 28 & 0.0710 \\ 1686 & 11: 54: 29 & 0.0774 \\ 1687 & 11: 54: 30 & 0.0714 \\ 1688 & 11: 54: 31 & 0.0757 \\ 1689 & 11: 54: 32 & 0.0745 \\ 1690 & 11: 54: 33 & 0.0760 \\ 1691 & 11: 54: 34 & 0.0772 \\ 1692 & 11: 54: 35 & 0.0766 \\ 1693 & 11: 54: 36 & 0.0731 \\ 1694 & 11: 54: 37 & 0.0710 \\ 1695 & 11: 54: 38 & 0.0727 \\ 1696 & 11: 54: 39 & 0.0768 \\ 1697 & 11: 54: 40 & 0.0774 \\ 1698 & 11: 54: 41 & 0.0688 \\ 1699 & 11: 54: 42 & 0.0807 \\ 1700 & 11: 54: 43 & 0.0760 \\ 1701 & 11: 54: 44 & 0.0812 \\ 1702 & 11: 54: 45 & 0.0697 \\ 1703 & 11: 54: 46 & 0.0792 \\ 1704 & 11: 54: 47 & 0.0709 \\ 1705 & 11: 54: 48 & 0.0804 \\ 1706 & 11: 54: 49 & 0.0653 \\ 1707 & 11: 54: 50 & 0.0714 \\ 1708 & 11: 54: 51 & 0.0727 \\ 1709 & 11: 54: 52 & 0.0748 \\ 1710 & 11: 54: 53 & 0.0706 \\ 1711 & 11: 54: 54 & 0.0744 \\ 1712 & 11: 54: 55 & 0.0672 \\ 1713 & 11: 54: 56 & 0.0764 \\ 1714 & 11: 54: 57 & 0.0688\end{array}$


Appendix A - Fuel Data Test 1 NIST102505_1.xIs

$\begin{array}{lll}1715 & 11: 54: 58 & 0.0790 \\ 1716 & 11: 54: 59 & 0.0674 \\ 1717 & 11: 55: 00 & 0.0796 \\ 1718 & 11: 55: 01 & 0.0732 \\ 1719 & 11: 55: 02 & 0.0815 \\ 1720 & 11: 55: 03 & 0.0710 \\ 1721 & 11: 55: 04 & 0.0772 \\ 1722 & 11: 55: 05 & 0.0737 \\ 1723 & 11: 55: 06 & 0.0741 \\ 1724 & 11: 55: 07 & 0.0765 \\ 1725 & 11: 55: 08 & 0.0749 \\ 1726 & 11: 55: 09 & 0.0735 \\ 1727 & 11: 55: 10 & 0.0714 \\ 1728 & 11: 55: 11 & 0.0754 \\ 1729 & 11: 55: 12 & 0.0718 \\ 1730 & 11: 55: 13 & 0.0740 \\ 1731 & 11: 55: 14 & 0.0732 \\ 1732 & 11: 55: 15 & 0.0702 \\ 1733 & 11: 55: 16 & 0.0707 \\ 1734 & 11: 55: 17 & 0.0697 \\ 1735 & 11: 55: 18 & 0.0704 \\ 1736 & 11: 55: 19 & 0.0759 \\ 1737 & 11: 55: 20 & 0.0711 \\ 1738 & 11: 55: 21 & 0.0799 \\ 1739 & 11: 55: 22 & 0.0688 \\ 1740 & 11: 55: 23 & 0.0780 \\ 1741 & 11: 55: 24 & 0.0698 \\ 1742 & 11: 55: 25 & 0.0724 \\ 1743 & 11: 55: 26 & 0.0703 \\ 1744 & 11: 55: 27 & 0.0722 \\ 1745 & 11: 55: 28 & 0.0696 \\ 1746 & 11: 55: 29 & 0.0778 \\ 1747 & 11: 55: 30 & 0.0677 \\ 1748 & 11: 55: 31 & 0.0734 \\ 1749 & 11: 55: 32 & 0.0722 \\ 1750 & 11: 55: 33 & 0.0797 \\ 1751 & 11: 55: 34 & 0.0716 \\ 1752 & 11: 55: 35 & 0.0832 \\ 1753 & 11: 55: 36 & 0.0686 \\ 1754 & 11: 55: 37 & 0.0768 \\ 1755 & 11: 55: 38 & 0.0713 \\ 1756 & 11: 55: 39 & 0.0792 \\ 1757 & 11: 55: 40 & 0.0721 \\ 1758 & 11: 55: 41 & 0.0767 \\ 1759 & 11: 55: 42 & 0.0731 \\ 1760 & 11: 55: 43 & 0.0769 \\ 1761 & 11: 55: 44 & 0.0706 \\ 1762 & 11: 55: 45 & 0.0727 \\ 1763 & 11: 55: 46 & 0.0788 \\ 1764 & 11: 55: 47 & 0.0738 \\ 1765 & 11: 55: 48 & 0.0694 \\ 1766 & 11: 55: 49 & 0.0707\end{array}$


Appendix A - Fuel Data Test 1 NIST102505_1.xIs

$\begin{array}{lll}1767 & 11: 55: 50 & 0.0792 \\ 1768 & 11: 55: 51 & 0.0743 \\ 1769 & 11: 55: 52 & 0.0731 \\ 1770 & 11: 55: 53 & 0.0697 \\ 1771 & 11: 55: 54 & 0.0683 \\ 1772 & 11: 55: 55 & 0.0721 \\ 1773 & 11: 55: 56 & 0.0700 \\ 1774 & 11: 55: 57 & 0.0682 \\ 1775 & 11: 55: 58 & 0.0722 \\ 1776 & 11: 55: 59 & 0.0759 \\ 1777 & 11: 56: 00 & 0.0794 \\ 1778 & 11: 56: 01 & 0.0715 \\ 1779 & 11: 56: 02 & 0.0782 \\ 1780 & 11: 56: 03 & 0.0651 \\ 1781 & 11: 56: 04 & 0.0823 \\ 1782 & 11: 56: 05 & 0.0777 \\ 1783 & 11: 56: 06 & 0.0761 \\ 1784 & 11: 56: 07 & 0.0660 \\ 1785 & 11: 56: 08 & 0.0787 \\ 1786 & 11: 56: 09 & 0.0740 \\ 1787 & 11: 56: 10 & 0.0750 \\ 1788 & 11: 56: 11 & 0.0721 \\ 1789 & 11: 56: 12 & 0.0691 \\ 1790 & 11: 56: 13 & 0.0684 \\ 1791 & 11: 56: 14 & 0.0748 \\ 1792 & 11: 56: 15 & 0.0640 \\ 1793 & 11: 56: 16 & 0.0707 \\ 1794 & 11: 56: 17 & 0.0737 \\ 1795 & 11: 56: 18 & 0.0813 \\ 1796 & 11: 56: 19 & 0.0698 \\ 1797 & 11: 56: 20 & 0.0791 \\ 1798 & 11: 56: 21 & 0.0657 \\ 1799 & 11: 56: 22 & 0.0674 \\ 1800 & 11: 56: 23 & 0.0781 \\ 1801 & 11: 56: 24 & 0.0737 \\ 1802 & 11: 56: 25 & 0.0740 \\ 1803 & 11: 56: 26 & 0.0722 \\ 1804 & 11: 56: 27 & 0.0712 \\ 1805 & 11: 56: 28 & 0.0730 \\ 1806 & 11: 56: 29 & 0.0714 \\ 1807 & 11: 56: 30 & 0.0730 \\ 1808 & 11: 56: 31 & 0.0774 \\ 1809 & 11: 56: 32 & 0.0687 \\ 1810 & 11: 56: 33 & 0.0714 \\ 1811 & 11: 56: 34 & 0.0722 \\ 1812 & 11: 56: 35 & 0.0751 \\ 1813 & 11: 56: 36 & 0.0721 \\ 1814 & 11: 56: 37 & 0.0730 \\ 1815 & 11: 56: 38 & 0.0726 \\ 1816 & 11: 56: 39 & 0.0750 \\ 1817 & 11: 56: 40 & 0.0701 \\ 1818 & 11: 56: 41 & 0.0743\end{array}$


Appendix A - Fuel Data Test 1 NIST102505_1.xIs

$\begin{array}{lll}1819 & 11: 56: 42 & 0.0652 \\ 1820 & 11: 56: 43 & 0.0777 \\ 1821 & 11: 56: 44 & 0.0705 \\ 1822 & 11: 56: 45 & 0.0779 \\ 1823 & 11: 56: 46 & 0.0671 \\ 1824 & 11: 56: 47 & 0.0774 \\ 1825 & 11: 56: 48 & 0.0691 \\ 1826 & 11: 56: 49 & 0.0790 \\ 1827 & 11: 56: 50 & 0.0663 \\ 1828 & 11: 56: 51 & 0.0758 \\ 1829 & 11: 56: 52 & 0.0715 \\ 1830 & 11: 56: 53 & 0.0789 \\ 1831 & 11: 56: 54 & 0.0703 \\ 1832 & 11: 56: 55 & 0.0748 \\ 1833 & 11: 56: 56 & 0.0756 \\ 1834 & 11: 56: 57 & 0.0735 \\ 1835 & 11: 56: 58 & 0.0745 \\ 1836 & 11: 56: 59 & 0.0740 \\ 1837 & 11: 57: 00 & 0.0700 \\ 1838 & 11: 57: 01 & 0.0760 \\ 1839 & 11: 57: 02 & 0.0654 \\ 1840 & 11: 57: 03 & 0.0701 \\ 1841 & 11: 57: 04 & 0.0677 \\ 1842 & 11: 57: 05 & 0.0728 \\ 1843 & 11: 57: 06 & 0.0721 \\ 1844 & 11: 57: 07 & 0.0726 \\ 1845 & 11: 57: 08 & 0.0707 \\ 1846 & 11: 57: 09 & 0.0730 \\ 1847 & 11: 57: 10 & 0.0772 \\ 1848 & 11: 57: 11 & 0.0730 \\ 1849 & 11: 57: 12 & 0.0731 \\ 1850 & 11: 57: 13 & 0.0699 \\ 1851 & 11: 57: 14 & 0.0753 \\ 1852 & 11: 57: 15 & 0.0797 \\ 1853 & 11: 57: 16 & 0.0825 \\ 1854 & 11: 57: 17 & 0.0690 \\ 1855 & 11: 57: 18 & 0.0793 \\ 1856 & 11: 57: 19 & 0.0664 \\ 1857 & 11: 57: 20 & 0.0770 \\ 1858 & 11: 57: 21 & 0.0680 \\ 1859 & 11: 57: 22 & 0.0719 \\ 1860 & 11: 57: 23 & 0.0749 \\ 1861 & 11: 57: 24 & 0.0772 \\ 1862 & 11: 57: 25 & 0.0697 \\ 1863 & 11: 57: 26 & 0.0697 \\ 1864 & 11: 57: 27 & 0.0672 \\ 1865 & 11: 57: 28 & 0.0728 \\ 1866 & 11: 57: 29 & 0.0671 \\ 1867 & 11: 57: 30 & 0.0727 \\ 1868 & 11: 57: 31 & 0.0723 \\ 1869 & 11: 57: 32 & 0.0737 \\ 1870 & 11: 57: 33 & 0.0688\end{array}$


Appendix A - Fuel Data Test 1 NIST102505_1.xIs

$\begin{array}{lll}1871 & 11: 57: 34 & 0.0700 \\ 1872 & 11: 57: 35 & 0.0692 \\ 1873 & 11: 57: 36 & 0.0795 \\ 1874 & 11: 57: 37 & 0.0709 \\ 1875 & 11: 57: 38 & 0.0736 \\ 1876 & 11: 57: 39 & 0.0678 \\ 1877 & 11: 57: 40 & 0.0683 \\ 1878 & 11: 57: 41 & 0.0767 \\ 1879 & 11: 57: 42 & 0.0726 \\ 1880 & 11: 57: 43 & 0.0751 \\ 1881 & 11: 57: 44 & 0.0730 \\ 1882 & 11: 57: 45 & 0.0750 \\ 1883 & 11: 57: 46 & 0.0714 \\ 1884 & 11: 57: 47 & 0.0707 \\ 1885 & 11: 57: 48 & 0.0738 \\ 1886 & 11: 57: 49 & 0.0729 \\ 1887 & 11: 57: 50 & 0.0696 \\ 1888 & 11: 57: 51 & 0.0703 \\ 1889 & 11: 57: 52 & 0.0662 \\ 1890 & 11: 57: 53 & 0.0709 \\ 1891 & 11: 57: 54 & 0.0692 \\ 1892 & 11: 57: 55 & 0.0737 \\ 1893 & 11: 57: 56 & 0.0665 \\ 1894 & 11: 57: 57 & 0.0732 \\ 1895 & 11: 57: 58 & 0.0692 \\ 1896 & 11: 57: 59 & 0.0799 \\ 1897 & 11: 58: 00 & 0.0686 \\ 1898 & 11: 58: 01 & 0.0726 \\ 1899 & 11: 58: 02 & 0.0680 \\ 1900 & 11: 58: 03 & 0.0731 \\ 1901 & 11: 58: 04 & 0.0626 \\ 1902 & 11: 58: 05 & 0.0722 \\ 1903 & 11: 58: 06 & 0.0681 \\ 1904 & 11: 58: 07 & 0.0744 \\ 1905 & 11: 58: 08 & 0.0627 \\ 1906 & 11: 58: 09 & 0.0690 \\ 1907 & 11: 58: 10 & 0.0631 \\ 1908 & 11: 58: 11 & 0.0729 \\ 1909 & 11: 58: 12 & 0.0695 \\ 1910 & 11: 58: 13 & 0.0748 \\ 1911 & 11: 58: 14 & 0.0690 \\ 1912 & 11: 58: 15 & 0.0755 \\ 1913 & 11: 58: 16 & 0.0631 \\ 1914 & 11: 58: 17 & 0.0681 \\ 1915 & 11: 58: 18 & 0.0685 \\ 1916 & 11: 58: 19 & 0.0732 \\ 1917 & 11: 58: 20 & 0.0711 \\ 1918 & 11: 58: 21 & 0.0704 \\ 1919 & 11: 58: 22 & 0.0743 \\ 1920 & 11: 58: 23 & 0.0662 \\ 1921 & 11: 58: 24 & 0.0747 \\ 1922 & 11: 58: 25 & 0.0685\end{array}$


Appendix A - Fuel Data Test 1 NIST102505_1.xIs

$\begin{array}{lll}1923 & 11: 58: 26 & 0.0703 \\ 1924 & 11: 58: 27 & 0.0661 \\ 1925 & 11: 58: 28 & 0.0691 \\ 1926 & 11: 58: 29 & 0.0682 \\ 1927 & 11: 58: 30 & 0.0754 \\ 1928 & 11: 58: 31 & 0.0719 \\ 1929 & 11: 58: 32 & 0.0754 \\ 1930 & 11: 58: 33 & 0.0699 \\ 1931 & 11: 58: 34 & 0.0768 \\ 1932 & 11: 58: 35 & 0.0692 \\ 1933 & 11: 58: 36 & 0.0792 \\ 1934 & 11: 58: 37 & 0.0654 \\ 1935 & 11: 58: 38 & 0.0788 \\ 1936 & 11: 58: 39 & 0.0677 \\ 1937 & 11: 58: 40 & 0.0776 \\ 1938 & 11: 58: 41 & 0.0666 \\ 1939 & 11: 58: 42 & 0.0816 \\ 1940 & 11: 58: 43 & 0.0704 \\ 1941 & 11: 58: 44 & 0.0765 \\ 1942 & 11: 58: 45 & 0.0618 \\ 1943 & 11: 58: 46 & 0.0725 \\ 1944 & 11: 58: 47 & 0.0740 \\ 1945 & 11: 58: 48 & 0.0768 \\ 1946 & 11: 58: 49 & 0.0707 \\ 1947 & 11: 58: 50 & 0.0689 \\ 1948 & 11: 58: 51 & 0.0724 \\ 1949 & 11: 58: 52 & 0.0702 \\ 1950 & 11: 58: 53 & 0.0668 \\ 1951 & 11: 58: 54 & 0.0666 \\ 1952 & 11: 58: 55 & 0.0717 \\ 1953 & 11: 58: 56 & 0.0700 \\ 1954 & 11: 58: 57 & 0.0726 \\ 1955 & 11: 58: 58 & 0.0668 \\ 1956 & 11: 58: 59 & 0.0782 \\ 1957 & 11: 59: 00 & 0.0716 \\ 1958 & 11: 59: 01 & 0.0742 \\ 1959 & 11: 59: 02 & 0.0654 \\ 1960 & 11: 59: 03 & 0.0714 \\ 1961 & 11: 59: 04 & 0.0710 \\ 1962 & 11: 59: 05 & 0.0735 \\ 1963 & 11: 59: 06 & 0.0671 \\ 1964 & 11: 59: 07 & 0.0698 \\ 1965 & 11: 59: 08 & 0.0651 \\ 1966 & 11: 59: 09 & 0.0706 \\ 1967 & 11: 59: 10 & 0.0680 \\ 1968 & 11: 59: 11 & 0.0767 \\ 1969 & 11: 59: 12 & 0.0668 \\ 1970 & 11: 59: 13 & 0.0766 \\ 1971 & 11: 59: 14 & 0.0668 \\ 1972 & 11: 59: 15 & 0.0754 \\ 1973 & 11: 59: 16 & 0.0655 \\ 1974 & 11: 59: 17 & 0.0789\end{array}$


Appendix A - Fuel Data Test 1 NIST102505_1.xIs

$\begin{array}{lll}1975 & 11: 59: 18 & 0.0760 \\ 1976 & 11: 59: 19 & 0.0737 \\ 1977 & 11: 59: 20 & 0.0687 \\ 1978 & 11: 59: 21 & 0.0669 \\ 1979 & 11: 59: 22 & 0.0672 \\ 1980 & 11: 59: 23 & 0.0708 \\ 1981 & 11: 59: 24 & 0.0636 \\ 1982 & 11: 59: 25 & 0.0699 \\ 1983 & 11: 59: 26 & 0.0670 \\ 1984 & 11: 59: 27 & 0.0744 \\ 1985 & 11: 59: 28 & 0.0731 \\ 1986 & 11: 59: 29 & 0.0698 \\ 1987 & 11: 59: 30 & 0.0697 \\ 1988 & 11: 59: 31 & 0.0655 \\ 1989 & 11: 59: 32 & 0.0710 \\ 1990 & 11: 59: 33 & 0.0748 \\ 1991 & 11: 59: 34 & 0.0739 \\ 1992 & 11: 59: 35 & 0.0728 \\ 1993 & 11: 59: 36 & 0.0702 \\ 1994 & 11: 59: 37 & 0.0733 \\ 1995 & 11: 59: 38 & 0.0755 \\ 1996 & 11: 59: 39 & 0.0703 \\ 1997 & 11: 59: 40 & 0.0736 \\ 1998 & 11: 59: 41 & 0.0640 \\ 1999 & 11: 59: 42 & 0.0776 \\ 2000 & 11: 59: 43 & 0.0729 \\ 2001 & 11: 59: 44 & 0.0822 \\ 2002 & 11: 59: 45 & 0.0677 \\ 2003 & 11: 59: 46 & 0.0770 \\ 2004 & 11: 59: 47 & 0.0653 \\ 2005 & 11: 59: 48 & 0.0718 \\ 2006 & 11: 59: 49 & 0.0645 \\ 2007 & 11: 59: 50 & 0.0761 \\ 2008 & 11: 59: 51 & 0.0669 \\ 2009 & 11: 59: 52 & 0.0764 \\ 2010 & 11: 59: 53 & 0.0637 \\ 2011 & 11: 59: 54 & 0.0736 \\ 2012 & 11: 59: 55 & 0.0723 \\ 2013 & 11: 59: 56 & 0.0731 \\ 2014 & 11: 59: 57 & 0.0661 \\ 2015 & 11: 59: 58 & 0.0731 \\ 2016 & 11: 59: 59 & 0.0691 \\ 2017 & 12: 00: 00 & 0.0722 \\ 2018 & 12: 00: 01 & 0.0726 \\ 2019 & 12: 00: 02 & 0.0685 \\ 2020 & 12: 00: 03 & 0.0689 \\ 2021 & 12: 00: 04 & 0.0745 \\ 2022 & 12: 00: 05 & 0.0660 \\ 2023 & 12: 00: 06 & 0.0669 \\ 2024 & 12: 00: 07 & 0.0658 \\ 2025 & 12: 00: 08 & 0.0709 \\ 2026 & 12: 00: 09 & 0.0688\end{array}$


Appendix A - Fuel Data Test 1 NIST102505_1.xIs

$\begin{array}{lll}2027 & 12: 00: 10 & 0.0768 \\ 2028 & 12: 00: 11 & 0.0697 \\ 2029 & 12: 00: 12 & 0.0694 \\ 2030 & 12: 00: 13 & 0.0700 \\ 2031 & 12: 00: 14 & 0.0653 \\ 2032 & 12: 00: 15 & 0.0657 \\ 2033 & 12: 00: 16 & 0.0678 \\ 2034 & 12: 00: 17 & 0.0724 \\ 2035 & 12: 00: 18 & 0.0646 \\ 2036 & 12: 00: 19 & 0.0701 \\ 2037 & 12: 00: 20 & 0.0685 \\ 2038 & 12: 00: 21 & 0.0764 \\ 2039 & 12: 00: 22 & 0.0672 \\ 2040 & 12: 00: 23 & 0.0794 \\ 2041 & 12: 00: 24 & 0.0632 \\ 2042 & 12: 00: 25 & 0.0756 \\ 2043 & 12: 00: 26 & 0.0683 \\ 2044 & 12: 00: 27 & 0.0789 \\ 2045 & 12: 00: 28 & 0.0650 \\ 2046 & 12: 00: 29 & 0.0702 \\ 2047 & 12: 00: 30 & 0.0750 \\ 2048 & 12: 00: 31 & 0.0740 \\ 2049 & 12: 00: 32 & 0.0695 \\ 2050 & 12: 00: 33 & 0.0719 \\ 2051 & 12: 00: 34 & 0.0644 \\ 2052 & 12: 00: 35 & 0.0738 \\ 2053 & 12: 00: 36 & 0.0697 \\ 2054 & 12: 00: 37 & 0.0731 \\ 2055 & 12: 00: 38 & 0.0691 \\ 2056 & 12: 00: 39 & 0.0731 \\ 2057 & 12: 00: 40 & 0.0736 \\ 2058 & 12: 00: 41 & 0.0751 \\ 2059 & 12: 00: 42 & 0.0713 \\ 2060 & 12: 00: 43 & 0.0682 \\ 2061 & 12: 00: 44 & 0.0766 \\ 2062 & 12: 00: 45 & 0.0686 \\ 2063 & 12: 00: 46 & 0.0723 \\ 2064 & 12: 00: 47 & 0.0764 \\ 2065 & 12: 00: 48 & 0.0731 \\ 2066 & 12: 00: 49 & 0.0730 \\ 2067 & 12: 00: 50 & 0.0752 \\ 2068 & 12: 00: 51 & 0.0649 \\ 2069 & 12: 00: 52 & 0.0722 \\ 2070 & 12: 00: 53 & 0.0615 \\ 2071 & 12: 00: 54 & 0.0749 \\ 2072 & 12: 00: 55 & 0.0662 \\ 2073 & 12: 00: 56 & 0.0767 \\ 2074 & 12: 00: 57 & 0.0661 \\ 2075 & 12: 00: 58 & 0.0698 \\ 2077 & 12: 00: 59 & 0.0685 \\ 2078 & 12: 01: 00 & 0.0717 \\ & & 0.0653\end{array}$


Appendix A - Fuel Data Test 1 NIST102505_1.xIs

$\begin{array}{lll}2079 & 12: 01: 02 & 0.0710 \\ 2080 & 12: 01: 03 & 0.0662 \\ 2081 & 12: 01: 04 & 0.0722 \\ 2082 & 12: 01: 05 & 0.0641 \\ 2083 & 12: 01: 06 & 0.0713 \\ 2084 & 12: 01: 07 & 0.0615 \\ 2085 & 12: 01: 08 & 0.0733 \\ 2086 & 12: 01: 09 & 0.0762 \\ 2087 & 12: 01: 10 & 0.0719 \\ 2088 & 12: 01: 11 & 0.0699 \\ 2089 & 12: 01: 12 & 0.0667 \\ 2090 & 12: 01: 13 & 0.0668 \\ 2091 & 12: 01: 14 & 0.0671 \\ 2092 & 12: 01: 15 & 0.0606 \\ 2093 & 12: 01: 16 & 0.0618 \\ 2094 & 12: 01: 17 & 0.0661 \\ 2095 & 12: 01: 18 & 0.0703 \\ 2096 & 12: 01: 19 & 0.0680 \\ 2097 & 12: 01: 20 & 0.0695 \\ 2098 & 12: 01: 21 & 0.0681 \\ 2099 & 12: 01: 22 & 0.0725 \\ 2100 & 12: 01: 23 & 0.0681 \\ 2101 & 12: 01: 24 & 0.0723 \\ 2102 & 12: 01: 25 & 0.0731 \\ 2103 & 12: 01: 26 & 0.0716 \\ 2104 & 12: 01: 27 & 0.0783 \\ 2105 & 12: 01: 28 & 0.0659 \\ 2106 & 12: 01: 29 & 0.0734 \\ 2107 & 12: 01: 30 & 0.0635 \\ 2108 & 12: 01: 31 & 0.0744 \\ 2109 & 12: 01: 32 & 0.0663 \\ 2110 & 12: 01: 33 & 0.0715 \\ 2111 & 12: 01: 34 & 0.0645 \\ 2112 & 12: 01: 35 & 0.0681 \\ 2113 & 12: 01: 36 & 0.0688 \\ 2114 & 12: 01: 37 & 0.0728 \\ 2115 & 12: 01: 38 & 0.0652 \\ 2116 & 12: 01: 39 & 0.0710 \\ 2117 & 12: 01: 40 & 0.0674 \\ 2118 & 12: 01: 41 & 0.0724 \\ 2119 & 12: 01: 42 & 0.0596 \\ 2120 & 12: 01: 43 & 0.0676 \\ 2121 & 12: 01: 44 & 0.0732 \\ 2122 & 12: 01: 45 & 0.0741 \\ 2123 & 12: 01: 46 & 0.0590 \\ 2124 & 12: 01: 47 & 0.0649 \\ 2125 & 12: 01: 48 & 0.0623 \\ 2126 & 12: 01: 49 & 0.0741 \\ 2127 & 12: 01: 50 & 0.0665 \\ 2128 & 12: 01: 51 & 0.0674 \\ 2129 & 12: 01: 52 & 0.0625 \\ 2130 & 12: 01: 53 & 0.0683\end{array}$


Appendix A - Fuel Data Test 1 NIST102505_1.xls

$\begin{array}{lll}2131 & 12: 01: 54 & 0.0720 \\ 2132 & 12: 01: 55 & 0.0718 \\ 2133 & 12: 01: 56 & 0.0687 \\ 2134 & 12: 01: 57 & 0.0685 \\ 2135 & 12: 01: 58 & 0.0642 \\ 2136 & 12: 01: 59 & 0.0669 \\ 2137 & 12: 02: 00 & 0.0735 \\ 2138 & 12: 02: 01 & 0.0638 \\ 2139 & 12: 02: 02 & 0.0769 \\ 2140 & 12: 02: 03 & 0.0737 \\ 2141 & 12: 02: 04 & 0.0782 \\ 2142 & 12: 02: 05 & 0.0644 \\ 2143 & 12: 02: 06 & 0.0704 \\ 2144 & 12: 02: 07 & 0.0674 \\ 2145 & 12: 02: 08 & 0.0715 \\ 2146 & 12: 02: 09 & 0.0654 \\ 2147 & 12: 02: 10 & 0.0667 \\ 2148 & 12: 02: 11 & 0.0653 \\ 2149 & 12: 02: 12 & 0.0781 \\ 2150 & 12: 02: 13 & 0.0667 \\ 2151 & 12: 02: 14 & 0.0757 \\ 2152 & 12: 02: 15 & 0.0616 \\ 2153 & 12: 02: 16 & 0.0747 \\ 2154 & 12: 02: 17 & 0.0707 \\ 2155 & 12: 02: 18 & 0.0824 \\ 2156 & 12: 02: 19 & 0.0660 \\ 2157 & 12: 02: 20 & 0.0692 \\ 2158 & 12: 02: 21 & 0.0731 \\ 2159 & 12: 02: 22 & 0.0734 \\ 2160 & 12: 02: 23 & 0.0717 \\ 2161 & 12: 02: 24 & 0.0683 \\ 2162 & 12: 02: 25 & 0.0666 \\ 2163 & 12: 02: 26 & 0.0750 \\ 2164 & 12: 02: 27 & 0.0673 \\ 2165 & 12: 02: 28 & 0.0655 \\ 2166 & 12: 02: 29 & 0.0651 \\ 2167 & 12: 02: 30 & 0.0677 \\ 2168 & 12: 02: 31 & 0.0697 \\ 2169 & 12: 02: 32 & 0.0701 \\ 2170 & 12: 02: 33 & 0.0736 \\ 2171 & 12: 02: 34 & 0.0689 \\ 2172 & 12: 02: 35 & 0.0750 \\ 2173 & 12: 02: 36 & 0.0690 \\ 2174 & 12: 02: 37 & 0.0749 \\ 2175 & 12: 02: 38 & 0.0643 \\ 2176 & 12: 02: 39 & 0.0769 \\ 2177 & 12: 02: 40 & 0.0684 \\ 2178 & 12: 02: 41 & 0.0789 \\ 2179 & 12: 02: 42 & 0.0653 \\ 2180 & 12: 02: 43 & 0.0692 \\ 2181 & 12: 02: 44 & 0.0675 \\ 2182 & 12: 02: 45 & 0.0689\end{array}$


Appendix A - Fuel Data Test 1 NIST102505_1.xls

$\begin{array}{lll}2183 & 12: 02: 46 & 0.0628 \\ 2184 & 12: 02: 47 & 0.0673 \\ 2185 & 12: 02: 48 & 0.0619 \\ 2186 & 12: 02: 49 & 0.0764 \\ 2187 & 12: 02: 50 & 0.0665 \\ 2188 & 12: 02: 51 & 0.0784 \\ 2189 & 12: 02: 52 & 0.0644 \\ 2190 & 12: 02: 53 & 0.0748 \\ 2191 & 12: 02: 54 & 0.0669 \\ 2192 & 12: 02: 55 & 0.0705 \\ 2193 & 12: 02: 56 & 0.0722 \\ 2194 & 12: 02: 57 & 0.0692 \\ 2195 & 12: 02: 58 & 0.0714 \\ 2196 & 12: 02: 59 & 0.0728 \\ 2197 & 12: 03: 00 & 0.0612 \\ 2198 & 12: 03: 01 & 0.0688 \\ 2199 & 12: 03: 02 & 0.0667 \\ 2200 & 12: 03: 03 & 0.0698 \\ 2201 & 12: 03: 04 & 0.0687 \\ 2202 & 12: 03: 05 & 0.0654 \\ 2203 & 12: 03: 06 & 0.0703 \\ 2204 & 12: 03: 07 & 0.0638 \\ 2205 & 12: 03: 08 & 0.0726 \\ 2206 & 12: 03: 09 & 0.0674 \\ 2207 & 12: 03: 10 & 0.0721 \\ 2208 & 12: 03: 11 & 0.0629 \\ 2209 & 12: 03: 12 & 0.0789 \\ 2210 & 12: 03: 13 & 0.0661 \\ 2211 & 12: 03: 14 & 0.0705 \\ 2212 & 12: 03: 15 & 0.0710 \\ 2213 & 12: 03: 16 & 0.0700 \\ 2214 & 12: 03: 17 & 0.0655 \\ 2215 & 12: 03: 18 & 0.0800 \\ 2216 & 12: 03: 19 & 0.0667 \\ 2217 & 12: 03: 20 & 0.0724 \\ 2218 & 12: 03: 21 & 0.0614 \\ 2219 & 12: 03: 22 & 0.0640 \\ 2220 & 12: 03: 23 & 0.0639 \\ 2221 & 12: 03: 24 & 0.0699 \\ 2222 & 12: 03: 25 & 0.0645 \\ 2223 & 12: 03: 26 & 0.0723 \\ 2224 & 12: 03: 27 & 0.0636 \\ 2225 & 12: 03: 28 & 0.0762 \\ 2226 & 12: 03: 29 & 0.0630 \\ 2227 & 12: 03: 30 & 0.0716 \\ 2228 & 12: 03: 31 & 0.0626 \\ 2229 & 12: 03: 32 & 0.0677 \\ 2230 & 12: 03: 33 & 0.0689 \\ 2231 & 12: 03: 34 & 0.0703 \\ 2232 & 12: 03: 35 & 0.0674 \\ 2233 & 12: 03: 36 & 0.0684 \\ 2234 & 12: 03: 37 & 0.0745\end{array}$


Appendix A - Fuel Data Test 1 NIST102505_1.xIs

$\begin{array}{lll}2235 & 12: 03: 38 & 0.0646 \\ 2236 & 12: 03: 39 & 0.0692 \\ 2237 & 12: 03: 40 & 0.0644 \\ 2238 & 12: 03: 41 & 0.0656 \\ 2239 & 12: 03: 42 & 0.0681 \\ 2240 & 12: 03: 43 & 0.0695 \\ 2241 & 12: 03: 44 & 0.0743 \\ 2242 & 12: 03: 45 & 0.0743 \\ 2243 & 12: 03: 46 & 0.0685 \\ 2244 & 12: 03: 47 & 0.0734 \\ 2245 & 12: 03: 48 & 0.0668 \\ 2246 & 12: 03: 49 & 0.0751 \\ 2247 & 12: 03: 50 & 0.0600 \\ 2248 & 12: 03: 51 & 0.0736 \\ 2249 & 12: 03: 52 & 0.0727 \\ 2250 & 12: 03: 53 & 0.0779 \\ 2251 & 12: 03: 54 & 0.0666 \\ 2252 & 12: 03: 55 & 0.0672 \\ 2253 & 12: 03: 56 & 0.0684 \\ 2254 & 12: 03: 57 & 0.0728 \\ 2255 & 12: 03: 58 & 0.0613 \\ 2256 & 12: 03: 59 & 0.0717 \\ 2257 & 12: 04: 00 & 0.0641 \\ 2258 & 12: 04: 01 & 0.0807 \\ 2259 & 12: 04: 02 & 0.0704 \\ 2260 & 12: 04: 03 & 0.0760 \\ 2261 & 12: 04: 04 & 0.0659 \\ 2262 & 12: 04: 05 & 0.0700 \\ 2263 & 12: 04: 06 & 0.0693 \\ 2264 & 12: 04: 07 & 0.0653 \\ 2265 & 12: 04: 08 & 0.0665 \\ 2266 & 12: 04: 09 & 0.0632 \\ 2267 & 12: 04: 10 & 0.0680 \\ 2268 & 12: 04: 11 & 0.0732 \\ 2269 & 12: 04: 12 & 0.0632 \\ 2270 & 12: 04: 13 & 0.0647 \\ 2271 & 12: 04: 14 & 0.0657 \\ 2272 & 12: 04: 15 & 0.0653 \\ 2273 & 12: 04: 16 & 0.0723 \\ 2274 & 12: 04: 17 & 0.0646 \\ 2275 & 12: 04: 18 & 0.0722 \\ 2276 & 12: 04: 19 & 0.0647 \\ 2277 & 12: 04: 20 & 0.0747 \\ 2278 & 12: 04: 21 & 0.0645 \\ 2279 & 12: 04: 22 & 0.0705 \\ 2280 & 12: 04: 23 & 0.0590 \\ 2281 & 12: 04: 24 & 0.0679 \\ 2282 & 12: 04: 25 & 0.0693 \\ 2283 & 12: 04: 26 & 0.0680 \\ 2284 & 12: 04: 27 & 0.0617 \\ 2285 & 12: 04: 28 & 0.0667 \\ 2286 & 12: 04: 29 & 0.0636\end{array}$


Appendix A - Fuel Data Test 1 NIST102505_1.xIs

$\begin{array}{lll}2287 & 12: 04: 30 & 0.0745 \\ 2288 & 12: 04: 31 & 0.0638 \\ 2289 & 12: 04: 32 & 0.0726 \\ 2290 & 12: 04: 33 & 0.0609 \\ 2291 & 12: 04: 34 & 0.0723 \\ 2292 & 12: 04: 35 & 0.0621 \\ 2293 & 12: 04: 36 & 0.0677 \\ 2294 & 12: 04: 37 & 0.0633 \\ 2295 & 12: 04: 38 & 0.0758 \\ 2296 & 12: 04: 39 & 0.0690 \\ 2297 & 12: 04: 40 & 0.0750 \\ 2298 & 12: 04: 41 & 0.0658 \\ 2299 & 12: 04: 42 & 0.0688 \\ 2300 & 12: 04: 43 & 0.0704 \\ 2301 & 12: 04: 44 & 0.0671 \\ 2302 & 12: 04: 45 & 0.0675 \\ 2303 & 12: 04: 46 & 0.0623 \\ 2304 & 12: 04: 47 & 0.0723 \\ 2305 & 12: 04: 48 & 0.0643 \\ 2306 & 12: 04: 49 & 0.0660 \\ 2307 & 12: 04: 50 & 0.0681 \\ 2308 & 12: 04: 51 & 0.0652 \\ 2309 & 12: 04: 52 & 0.0661 \\ 2310 & 12: 04: 53 & 0.0717 \\ 2311 & 12: 04: 54 & 0.0631 \\ 2312 & 12: 04: 55 & 0.0693 \\ 2313 & 12: 04: 56 & 0.0648 \\ 2314 & 12: 04: 57 & 0.0776 \\ 2315 & 12: 04: 58 & 0.0662 \\ 2316 & 12: 04: 59 & 0.0752 \\ 2317 & 12: 05: 00 & 0.0580 \\ 2318 & 12: 05: 01 & 0.0742 \\ 2319 & 12: 05: 02 & 0.0665 \\ 2320 & 12: 05: 03 & 0.0717 \\ 2321 & 12: 05: 04 & 0.0596 \\ 2322 & 12: 05: 05 & 0.0730 \\ 2323 & 12: 05: 06 & 0.0655 \\ 2324 & 12: 05: 07 & 0.0694 \\ 2325 & 12: 05: 08 & 0.0679 \\ 2326 & 12: 05: 09 & 0.0644 \\ 2327 & 12: 05: 10 & 0.0605 \\ 2328 & 12: 05: 11 & 0.0701 \\ 2329 & 12: 05: 12 & 0.0640 \\ 2330 & 12: 05: 13 & 0.0677 \\ 2331 & 12: 05: 14 & 0.0656 \\ 2332 & 12: 05: 15 & 0.0743 \\ 2333 & 12: 05: 16 & 0.0716 \\ 2334 & 12: 05: 17 & 0.0663 \\ 2335 & 12: 05: 18 & 0.0678 \\ 2336 & 12: 05: 19 & 0.0626 \\ 2337 & 12: 05: 20 & 0.0713 \\ 2338 & 12: 05: 21 & 0.0671\end{array}$


Appendix A - Fuel Data Test 1 NIST102505_1.xls

$\begin{array}{lll}2339 & 12: 05: 22 & 0.0690 \\ 2340 & 12: 05: 23 & 0.0613 \\ 2341 & 12: 05: 24 & 0.0699 \\ 2342 & 12: 05: 25 & 0.0670 \\ 2343 & 12: 05: 26 & 0.0633 \\ 2344 & 12: 05: 27 & 0.0594 \\ 2345 & 12: 05: 28 & 0.0642 \\ 2346 & 12: 05: 29 & 0.0620 \\ 2347 & 12: 05: 30 & 0.0701 \\ 2348 & 12: 05: 31 & 0.0610 \\ 2349 & 12: 05: 32 & 0.0655 \\ 2350 & 12: 05: 33 & 0.0642 \\ 2351 & 12: 05: 34 & 0.0750 \\ 2352 & 12: 05: 35 & 0.0648 \\ 2353 & 12: 05: 36 & 0.0681 \\ 2354 & 12: 05: 37 & 0.0597 \\ 2355 & 12: 05: 38 & 0.0683 \\ 2356 & 12: 05: 39 & 0.0626 \\ 2357 & 12: 05: 40 & 0.0709 \\ 2358 & 12: 05: 41 & 0.0612 \\ 2359 & 12: 05: 42 & 0.0717 \\ 2360 & 12: 05: 43 & 0.0646 \\ 2361 & 12: 05: 44 & 0.0704 \\ 2362 & 12: 05: 45 & 0.0658 \\ 2363 & 12: 05: 46 & 0.0646 \\ 2364 & 12: 05: 47 & 0.0694 \\ 2365 & 12: 05: 48 & 0.0653 \\ 2366 & 12: 05: 49 & 0.0648 \\ 2367 & 12: 05: 50 & 0.0662 \\ 2368 & 12: 05: 51 & 0.0732 \\ 2369 & 12: 05: 52 & 0.0673 \\ 2370 & 12: 05: 53 & 0.0666 \\ 2371 & 12: 05: 54 & 0.0547 \\ 2372 & 12: 05: 55 & 0.0708 \\ 2373 & 12: 05: 56 & 0.0661 \\ 2374 & 12: 05: 57 & 0.0618 \\ 2375 & 12: 05: 58 & 0.0554 \\ 2376 & 12: 05: 59 & 0.0691 \\ 2377 & 12: 06: 00 & 0.0652 \\ 2378 & 12: 06: 01 & 0.0669 \\ 2379 & 12: 06: 02 & 0.0667 \\ 2380 & 12: 06: 03 & 0.0667 \\ 2381 & 12: 06: 04 & 0.0621 \\ 2382 & 12: 06: 05 & 0.0692 \\ 2383 & 12: 06: 06 & 0.0592 \\ 2384 & 12: 06: 07 & 0.0714 \\ 2385 & 12: 06: 08 & 0.0642 \\ 2386 & 12: 06: 09 & 0.0676 \\ 2387 & 12: 06: 10 & 0.0579 \\ 2388 & 12: 06: 11 & 0.0635 \\ 2390 & 12: 06: 12 & 0.0664 \\ & 12: 06: 13 & 0.0680\end{array}$


Appendix A - Fuel Data Test 1 NIST102505_1.xIs

$\begin{array}{lll}2391 & 12: 06: 14 & 0.0611 \\ 2392 & 12: 06: 15 & 0.0637 \\ 2393 & 12: 06: 16 & 0.0601 \\ 2394 & 12: 06: 17 & 0.0700 \\ 2395 & 12: 06: 18 & 0.0653 \\ 2396 & 12: 06: 19 & 0.0696 \\ 2397 & 12: 06: 20 & 0.0556 \\ 2398 & 12: 06: 21 & 0.0634 \\ 2399 & 12: 06: 22 & 0.0626 \\ 2400 & 12: 06: 23 & 0.0661 \\ 2401 & 12: 06: 24 & 0.0624 \\ 2402 & 12: 06: 25 & 0.0643 \\ 2403 & 12: 06: 26 & 0.0647 \\ 2404 & 12: 06: 27 & 0.0654 \\ 2405 & 12: 06: 28 & 0.0675 \\ 2406 & 12: 06: 29 & 0.0677 \\ 2407 & 12: 06: 30 & 0.0682 \\ 2408 & 12: 06: 31 & 0.0684 \\ 2409 & 12: 06: 32 & 0.0692 \\ 2410 & 12: 06: 33 & 0.0584 \\ 2411 & 12: 06: 34 & 0.0723 \\ 2412 & 12: 06: 35 & 0.0652 \\ 2413 & 12: 06: 36 & 0.0734 \\ 2414 & 12: 06: 37 & 0.0593 \\ 2415 & 12: 06: 38 & 0.0741 \\ 2416 & 12: 06: 39 & 0.0627 \\ 2417 & 12: 06: 40 & 0.0747 \\ 2418 & 12: 06: 41 & 0.0550 \\ 2419 & 12: 06: 42 & 0.0720 \\ 2420 & 12: 06: 43 & 0.0639 \\ 2421 & 12: 06: 44 & 0.0751 \\ 2422 & 12: 06: 45 & 0.0611 \\ 2423 & 12: 06: 46 & 0.0715 \\ 2424 & 12: 06: 47 & 0.0665 \\ 2425 & 12: 06: 48 & 0.0666 \\ 2426 & 12: 06: 49 & 0.0659 \\ 2427 & 12: 06: 50 & 0.0644 \\ 2428 & 12: 06: 51 & 0.0672 \\ 2429 & 12: 06: 52 & 0.0634 \\ 2430 & 12: 06: 53 & 0.0663 \\ 2431 & 12: 06: 54 & 0.0651 \\ 2432 & 12: 06: 55 & 0.0604 \\ 2433 & 12: 06: 56 & 0.0647 \\ 2434 & 12: 06: 57 & 0.0676 \\ 2435 & 12: 06: 58 & 0.0638 \\ 2436 & 12: 06: 59 & 0.0687 \\ 2437 & 12: 07: 00 & 0.0613 \\ 2438 & 12: 07: 01 & 0.0729 \\ 2439 & 12: 07: 02 & 0.0656 \\ 2440 & 12: 07: 03 & 0.0672 \\ 2441 & 12: 07: 04 & 0.0592 \\ 2442 & 12: 07: 05 & 0.0724\end{array}$


Appendix A - Fuel Data Test 1 NIST102505_1.xIs

$\begin{array}{lll}2443 & 12: 07: 06 & 0.0640 \\ 2444 & 12: 07: 07 & 0.0708 \\ 2445 & 12: 07: 08 & 0.0600 \\ 2446 & 12: 07: 09 & 0.0630 \\ 2447 & 12: 07: 10 & 0.0646 \\ 2448 & 12: 07: 11 & 0.0707 \\ 2449 & 12: 07: 12 & 0.0641 \\ 2450 & 12: 07: 13 & 0.0754 \\ 2451 & 12: 07: 14 & 0.0614 \\ 2452 & 12: 07: 15 & 0.0701 \\ 2453 & 12: 07: 16 & 0.0613 \\ 2454 & 12: 07: 17 & 0.0643 \\ 2455 & 12: 07: 18 & 0.0637 \\ 2456 & 12: 07: 19 & 0.0644 \\ 2457 & 12: 07: 20 & 0.0617 \\ 2458 & 12: 07: 21 & 0.0672 \\ 2459 & 12: 07: 22 & 0.0601 \\ 2460 & 12: 07: 23 & 0.0674 \\ 2461 & 12: 07: 24 & 0.0582 \\ 2462 & 12: 07: 25 & 0.0636 \\ 2463 & 12: 07: 26 & 0.0702 \\ 2464 & 12: 07: 27 & 0.0654 \\ 2465 & 12: 07: 28 & 0.0669 \\ 2466 & 12: 07: 29 & 0.0601 \\ 2467 & 12: 07: 30 & 0.0752 \\ 2468 & 12: 07: 31 & 0.0682 \\ 2469 & 12: 07: 32 & 0.0616 \\ 2470 & 12: 07: 33 & 0.0555 \\ 2471 & 12: 07: 34 & 0.0663 \\ 2472 & 12: 07: 35 & 0.0619 \\ 2473 & 12: 07: 36 & 0.0629 \\ 2474 & 12: 07: 37 & 0.0577 \\ 2475 & 12: 07: 38 & 0.0612 \\ 2476 & 12: 07: 39 & 0.0628 \\ 2477 & 12: 07: 40 & 0.0680 \\ 2478 & 12: 07: 41 & 0.0641 \\ 2479 & 12: 07: 42 & 0.0692 \\ 2480 & 12: 07: 43 & 0.0554 \\ 2481 & 12: 07: 44 & 0.0736 \\ 2482 & 12: 07: 45 & 0.0632 \\ 2483 & 12: 07: 46 & 0.0732 \\ 2484 & 12: 07: 47 & 0.0581 \\ 2485 & 12: 07: 48 & 0.0761 \\ 2486 & 12: 07: 49 & 0.0715 \\ 2487 & 12: 07: 50 & 0.0785 \\ 2488 & 12: 07: 51 & 0.0739 \\ 2489 & 12: 07: 52 & 0.0732 \\ 2490 & 12: 07: 53 & 0.0708 \\ 2491 & 12: 07: 54 & 0.0843 \\ 2492 & 12: 07: 55 & 0.0776 \\ 2493 & 12: 07: 56 & 0.0865 \\ 2494 & 12: 07: 57 & 0.0732\end{array}$


Appendix A - Fuel Data Test 1 NIST102505_1.xIs

$\begin{array}{lll}2495 & 12: 07: 58 & 0.0752 \\ 2496 & 12: 07: 59 & 0.0803 \\ 2497 & 12: 08: 00 & 0.0755 \\ 2498 & 12: 08: 01 & 0.0774 \\ 2499 & 12: 08: 02 & 0.0730 \\ 2500 & 12: 08: 03 & 0.0724 \\ 2501 & 12: 08: 04 & 0.0825 \\ 2502 & 12: 08: 05 & 0.0807 \\ 2503 & 12: 08: 06 & 0.0769 \\ 2504 & 12: 08: 07 & 0.0739 \\ 2505 & 12: 08: 08 & 0.0672 \\ 2506 & 12: 08: 09 & 0.0802 \\ 2507 & 12: 08: 10 & 0.0781 \\ 2508 & 12: 08: 11 & 0.0808 \\ 2509 & 12: 08: 12 & 0.0755 \\ 2510 & 12: 08: 13 & 0.0765 \\ 2511 & 12: 08: 14 & 0.0760 \\ 2512 & 12: 08: 15 & 0.0792 \\ 2513 & 12: 08: 16 & 0.0773 \\ 2514 & 12: 08: 17 & 0.0830 \\ 2515 & 12: 08: 18 & 0.0704 \\ 2516 & 12: 08: 19 & 0.0764 \\ 2517 & 12: 08: 20 & 0.0815 \\ 2518 & 12: 08: 21 & 0.0779 \\ 2519 & 12: 08: 22 & 0.0790 \\ 2520 & 12: 08: 23 & 0.0834 \\ 2521 & 12: 08: 24 & 0.0731 \\ 2522 & 12: 08: 25 & 0.0786 \\ 2523 & 12: 08: 26 & 0.0832 \\ 2524 & 12: 08: 27 & 0.0765 \\ 2525 & 12: 08: 28 & 0.0766 \\ 2526 & 12: 08: 29 & 0.0761 \\ 2527 & 12: 08: 30 & 0.0782 \\ 2528 & 12: 08: 31 & 0.0779 \\ 2529 & 12: 08: 32 & 0.0908 \\ 2530 & 12: 08: 33 & 0.0770 \\ 2531 & 12: 08: 34 & 0.0843 \\ 2532 & 12: 08: 35 & 0.0769 \\ 2533 & 12: 08: 36 & 0.0737 \\ 2534 & 12: 08: 37 & 0.0722 \\ 2535 & 12: 08: 38 & 0.0804 \\ 2536 & 12: 08: 39 & 0.0754 \\ 2537 & 12: 08: 40 & 0.0833 \\ 2538 & 12: 08: 41 & 0.0684 \\ 2539 & 12: 08: 42 & 0.0805 \\ 2540 & 12: 08: 43 & 0.0783 \\ 2541 & 12: 08: 44 & 0.0729 \\ 2542 & 12: 08: 45 & 0.0737 \\ 2543 & 12: 08: 46 & 0.0713 \\ 2544 & 12: 08: 47 & 0.0736 \\ 2545 & 12: 08: 48 & 0.0780 \\ 2546 & 12: 08: 49 & 0.0774\end{array}$


Appendix A - Fuel Data Test 1 NIST102505_1.xIs

$\begin{array}{lll}2547 & 12: 08: 50 & 0.0776 \\ 2548 & 12: 08: 51 & 0.0838 \\ 2549 & 12: 08: 52 & 0.0705 \\ 2550 & 12: 08: 53 & 0.0800 \\ 2551 & 12: 08: 54 & 0.0781 \\ 2552 & 12: 08: 55 & 0.0787 \\ 2553 & 12: 08: 56 & 0.0737 \\ 2554 & 12: 08: 57 & 0.0750 \\ 2555 & 12: 08: 58 & 0.0729 \\ 2556 & 12: 08: 59 & 0.0857 \\ 2557 & 12: 09: 00 & 0.0795 \\ 2558 & 12: 09: 01 & 0.0785 \\ 2559 & 12: 09: 02 & 0.0788 \\ 2560 & 12: 09: 03 & 0.0749 \\ 2561 & 12: 09: 04 & 0.0731 \\ 2562 & 12: 09: 05 & 0.0834 \\ 2563 & 12: 09: 06 & 0.0747 \\ 2564 & 12: 09: 07 & 0.0819 \\ 2565 & 12: 09: 08 & 0.0804 \\ 2566 & 12: 09: 09 & 0.0686 \\ 2567 & 12: 09: 10 & 0.0796 \\ 2568 & 12: 09: 11 & 0.0761 \\ 2569 & 12: 09: 12 & 0.0854 \\ 2570 & 12: 09: 13 & 0.0731 \\ 2571 & 12: 09: 14 & 0.0732 \\ 2572 & 12: 09: 15 & 0.0780 \\ 2573 & 12: 09: 16 & 0.0805 \\ 2574 & 12: 09: 17 & 0.0763 \\ 2575 & 12: 09: 18 & 0.0857 \\ 2576 & 12: 09: 19 & 0.0683 \\ 2577 & 12: 09: 20 & 0.0711 \\ 2578 & 12: 09: 21 & 0.0751 \\ 2579 & 12: 09: 22 & 0.0771 \\ 2580 & 12: 09: 23 & 0.0758 \\ 2581 & 12: 09: 24 & 0.0835 \\ 2582 & 12: 09: 25 & 0.0709 \\ 2583 & 12: 09: 26 & 0.0769 \\ 2584 & 12: 09: 27 & 0.0805 \\ 2585 & 12: 09: 28 & 0.0783 \\ 2586 & 12: 09: 29 & 0.0799 \\ 2587 & 12: 09: 30 & 0.0796 \\ 2588 & 12: 09: 31 & 0.0748 \\ 2589 & 12: 09: 32 & 0.0816 \\ 2590 & 12: 09: 33 & 0.0808 \\ 2591 & 12: 09: 34 & 0.0797 \\ 2592 & 12: 09: 35 & 0.0767 \\ 2593 & 12: 09: 36 & 0.0703 \\ 2594 & 12: 09: 37 & 0.0826 \\ 2595 & 12: 09: 38 & 0.0732 \\ 2596 & 12: 09: 39 & 0.0802 \\ 2597 & 12: 09: 40 & 0.0708 \\ 2598 & 12: 09: 41 & 0.0728\end{array}$


Appendix A - Fuel Data Test 1 NIST102505_1.xIs

$\begin{array}{lll}2599 & 12: 09: 42 & 0.0744 \\ 2600 & 12: 09: 43 & 0.0792 \\ 2601 & 12: 09: 44 & 0.0740 \\ 2602 & 12: 09: 45 & 0.0832 \\ 2603 & 12: 09: 46 & 0.0724 \\ 2604 & 12: 09: 47 & 0.0786 \\ 2605 & 12: 09: 48 & 0.0754 \\ 2606 & 12: 09: 49 & 0.0744 \\ 2607 & 12: 09: 50 & 0.0805 \\ 2608 & 12: 09: 51 & 0.0714 \\ 2609 & 12: 09: 52 & 0.0681 \\ 2610 & 12: 09: 53 & 0.0781 \\ 2611 & 12: 09: 54 & 0.0793 \\ 2612 & 12: 09: 55 & 0.0725 \\ 2613 & 12: 09: 56 & 0.0802 \\ 2614 & 12: 09: 57 & 0.0681 \\ 2615 & 12: 09: 58 & 0.0709 \\ 2616 & 12: 09: 59 & 0.0804 \\ 2617 & 12: 10: 00 & 0.0830 \\ 2618 & 12: 10: 01 & 0.0734 \\ 2619 & 12: 10: 02 & 0.0817 \\ 2620 & 12: 10: 03 & 0.0703 \\ 2621 & 12: 10: 04 & 0.0743 \\ 2622 & 12: 10: 05 & 0.0773 \\ 2623 & 12: 10: 06 & 0.0781 \\ 2624 & 12: 10: 07 & 0.0732 \\ 2625 & 12: 10: 08 & 0.0793 \\ 2626 & 12: 10: 09 & 0.0791 \\ 2627 & 12: 10: 10 & 0.0745 \\ 2628 & 12: 10: 11 & 0.0766 \\ 2629 & 12: 10: 12 & 0.0843 \\ 2630 & 12: 10: 13 & 0.0760 \\ 2631 & 12: 10: 14 & 0.0715 \\ 2632 & 12: 10: 15 & 0.0731 \\ 2633 & 12: 10: 16 & 0.0686 \\ 2634 & 12: 10: 17 & 0.0778 \\ 2635 & 12: 10: 18 & 0.0738 \\ 2636 & 12: 10: 19 & 0.0799 \\ 2637 & 12: 10: 20 & 0.0753 \\ 2638 & 12: 10: 21 & 0.0750 \\ 2639 & 12: 10: 22 & 0.0759 \\ 2640 & 12: 10: 23 & 0.0764 \\ 2641 & 12: 10: 24 & 0.0719 \\ 2642 & 12: 10: 25 & 0.0746 \\ 2643 & 12: 10: 26 & 0.0729 \\ 2644 & 12: 10: 27 & 0.0814 \\ 2645 & 12: 10: 28 & 0.0759 \\ 2646 & 12: 10: 29 & 0.0818 \\ 2647 & 12: 10: 30 & 0.0719 \\ 2648 & 12: 10: 31 & 0.0764 \\ 2649 & 12: 10: 32 & 0.0761 \\ 2650 & 12: 10: 33 & 0.0730\end{array}$


Appendix A - Fuel Data Test 1 NIST102505_1.xIs

$\begin{array}{lll}2651 & 12: 10: 34 & 0.0760 \\ 2652 & 12: 10: 35 & 0.0763 \\ 2653 & 12: 10: 36 & 0.0709 \\ 2654 & 12: 10: 37 & 0.0761 \\ 2655 & 12: 10: 38 & 0.0821 \\ 2656 & 12: 10: 39 & 0.0723 \\ 2657 & 12: 10: 40 & 0.0760 \\ 2658 & 12: 10: 41 & 0.0670 \\ 2659 & 12: 10: 42 & 0.0741 \\ 2660 & 12: 10: 43 & 0.0818 \\ 2661 & 12: 10: 44 & 0.0829 \\ 2662 & 12: 10: 45 & 0.0767 \\ 2663 & 12: 10: 46 & 0.0737 \\ 2664 & 12: 10: 47 & 0.0742 \\ 2665 & 12: 10: 48 & 0.0843 \\ 2666 & 12: 10: 49 & 0.0736 \\ 2667 & 12: 10: 50 & 0.0781 \\ 2668 & 12: 10: 51 & 0.0700 \\ 2669 & 12: 10: 52 & 0.0745 \\ 2670 & 12: 10: 53 & 0.0837 \\ 2671 & 12: 10: 54 & 0.0791 \\ 2672 & 12: 10: 55 & 0.0790 \\ 2673 & 12: 10: 56 & 0.0766 \\ 2674 & 12: 10: 57 & 0.0698 \\ 2675 & 12: 10: 58 & 0.0775 \\ 2676 & 12: 10: 59 & 0.0754 \\ 2677 & 12: 11: 00 & 0.0736 \\ 2678 & 12: 11: 01 & 0.0831 \\ 2679 & 12: 11: 02 & 0.0693 \\ 2680 & 12: 11: 03 & 0.0732 \\ 2681 & 12: 11: 04 & 0.0758 \\ 2682 & 12: 11: 05 & 0.0730 \\ 2683 & 12: 11: 06 & 0.0673 \\ 2684 & 12: 11: 07 & 0.0771 \\ 2685 & 12: 11: 08 & 0.0682 \\ 2686 & 12: 11: 09 & 0.0886 \\ 2687 & 12: 11: 10 & 0.0766 \\ 2688 & 12: 11: 11 & 0.0781 \\ 2689 & 12: 11: 12 & 0.0730 \\ 2690 & 12: 11: 13 & 0.0705 \\ 2691 & 12: 11: 14 & 0.0689 \\ 2692 & 12: 11: 15 & 0.0754 \\ 2693 & 12: 11: 16 & 0.0689 \\ 2694 & 12: 11: 17 & 0.0710 \\ 2695 & 12: 11: 18 & 0.0752 \\ 2696 & 12: 11: 19 & 0.0696 \\ 2697 & 12: 11: 20 & 0.0779 \\ 2698 & 12: 11: 21 & 0.0702 \\ 2699 & 12: 11: 22 & 0.0785 \\ 2700 & 12: 11: 23 & 0.0710 \\ 2701 & 12: 11: 24 & 0.0724 \\ 2702 & 12: 11: 25 & 0.0746\end{array}$


Appendix A - Fuel Data Test 1 NIST102505_1.xls

$\begin{array}{lll}2703 & 12: 11: 26 & 0.0774 \\ 2704 & 12: 11: 27 & 0.0743 \\ 2705 & 12: 11: 28 & 0.0768 \\ 2706 & 12: 11: 29 & 0.0675 \\ 2707 & 12: 11: 30 & 0.0801 \\ 2708 & 12: 11: 31 & 0.0687 \\ 2709 & 12: 11: 32 & 0.0767 \\ 2710 & 12: 11: 33 & 0.0722 \\ 2711 & 12: 11: 34 & 0.0704 \\ 2712 & 12: 11: 35 & 0.0720 \\ 2713 & 12: 11: 36 & 0.0772 \\ 2714 & 12: 11: 37 & 0.0728 \\ 2715 & 12: 11: 38 & 0.0789 \\ 2716 & 12: 11: 39 & 0.0688 \\ 2717 & 12: 11: 40 & 0.0723 \\ 2718 & 12: 11: 41 & 0.0759 \\ 2719 & 12: 11: 42 & 0.0735 \\ 2720 & 12: 11: 43 & 0.0787 \\ 2721 & 12: 11: 44 & 0.0712 \\ 2722 & 12: 11: 45 & 0.0717 \\ 2723 & 12: 11: 46 & 0.0734 \\ 2724 & 12: 11: 47 & 0.0755 \\ 2725 & 12: 11: 48 & 0.0705 \\ 2726 & 12: 11: 49 & 0.0731 \\ 2727 & 12: 11: 50 & 0.0670 \\ 2728 & 12: 11: 51 & 0.0762 \\ 2729 & 12: 11: 52 & 0.0715 \\ 2730 & 12: 11: 53 & 0.0819 \\ 2731 & 12: 11: 54 & 0.0644 \\ 2732 & 12: 11: 55 & 0.0782 \\ 2733 & 12: 11: 56 & 0.0739 \\ 2734 & 12: 11: 57 & 0.0720 \\ 2735 & 12: 11: 58 & 0.0705 \\ 2736 & 12: 11: 59 & 0.0768 \\ 2737 & 12: 12: 00 & 0.0717 \\ 2738 & 12: 12: 01 & 0.0731 \\ 2739 & 12: 12: 02 & 0.0782 \\ 2740 & 12: 12: 03 & 0.0770 \\ 2741 & 12: 12: 04 & 0.0702 \\ 2742 & 12: 12: 05 & 0.0762 \\ 2743 & 12: 12: 06 & 0.0772 \\ 2744 & 12: 12: 07 & 0.0675 \\ 2745 & 12: 12: 08 & 0.0768 \\ 2746 & 12: 12: 09 & 0.0780 \\ 2747 & 12: 12: 10 & 0.0751 \\ 2748 & 12: 12: 11 & 0.0751 \\ 2749 & 12: 12: 12 & 0.0767 \\ 2750 & 12: 12: 13 & 0.0673 \\ 2751 & 12: 12: 14 & 0.0825 \\ 2752 & 12: 12: 15 & 0.0683 \\ 2753 & 12: 12: 16 & 0.0785 \\ 2754 & 12: 12: 17 & 0.0716\end{array}$


Appendix A - Fuel Data Test 1 NIST102505_1.xls

$\begin{array}{lll}2755 & 12: 12: 18 & 0.0776 \\ 2756 & 12: 12: 19 & 0.0744 \\ 2757 & 12: 12: 20 & 0.0746 \\ 2758 & 12: 12: 21 & 0.0736 \\ 2759 & 12: 12: 22 & 0.0711 \\ 2760 & 12: 12: 23 & 0.0736 \\ 2761 & 12: 12: 24 & 0.0827 \\ 2762 & 12: 12: 25 & 0.0738 \\ 2763 & 12: 12: 26 & 0.0788 \\ 2764 & 12: 12: 27 & 0.0720 \\ 2765 & 12: 12: 28 & 0.0722 \\ 2766 & 12: 12: 29 & 0.0788 \\ 2767 & 12: 12: 30 & 0.0709 \\ 2768 & 12: 12: 31 & 0.0783 \\ 2769 & 12: 12: 32 & 0.0706 \\ 2770 & 12: 12: 33 & 0.0743 \\ 2771 & 12: 12: 34 & 0.0706 \\ 2772 & 12: 12: 35 & 0.0743 \\ 2773 & 12: 12: 36 & 0.0695 \\ 2774 & 12: 12: 37 & 0.0739 \\ 2775 & 12: 12: 38 & 0.0708 \\ 2776 & 12: 12: 39 & 0.0815 \\ 2777 & 12: 12: 40 & 0.0720 \\ 2778 & 12: 12: 41 & 0.0799 \\ 2779 & 12: 12: 42 & 0.0715 \\ 2780 & 12: 12: 43 & 0.0717 \\ 2781 & 12: 12: 44 & 0.0730 \\ 2782 & 12: 12: 45 & 0.0832 \\ 2783 & 12: 12: 46 & 0.0767 \\ 2784 & 12: 12: 47 & 0.0721 \\ 2785 & 12: 12: 48 & 0.0716 \\ 2786 & 12: 12: 49 & 0.0685 \\ 2787 & 12: 12: 50 & 0.0717 \\ 2788 & 12: 12: 51 & 0.0772 \\ 2789 & 12: 12: 52 & 0.0739 \\ 2790 & 12: 12: 53 & 0.0724 \\ 2791 & 12: 12: 54 & 0.0737 \\ 2792 & 12: 12: 55 & 0.0702 \\ 2793 & 12: 12: 56 & 0.0810 \\ 2794 & 12: 12: 57 & 0.0744 \\ 2795 & 12: 12: 58 & 0.0748 \\ 2796 & 12: 12: 59 & 0.0695 \\ 2797 & 12: 13: 00 & 0.0720 \\ 2798 & 12: 13: 01 & 0.0693 \\ 2799 & 12: 13: 02 & 0.0805 \\ 2800 & 12: 13: 03 & 0.0758 \\ 2801 & 12: 13: 04 & 0.0756 \\ 2802 & 12: 13: 05 & 0.0700 \\ 2803 & 12: 13: 06 & 0.0695 \\ 2804 & 12: 13: 07 & 0.0733 \\ 2805 & 12: 13: 08 & 0.0741 \\ 2806 & 12: 13: 09 & 0.0754\end{array}$


Appendix A - Fuel Data Test 1 NIST102505_1.xIs

$\begin{array}{lll}2807 & 12: 13: 10 & 0.0783 \\ 2808 & 12: 13: 11 & 0.0698 \\ 2809 & 12: 13: 12 & 0.0776 \\ 2810 & 12: 13: 13 & 0.0766 \\ 2811 & 12: 13: 14 & 0.0725 \\ 2812 & 12: 13: 15 & 0.0767 \\ 2813 & 12: 13: 16 & 0.0686 \\ 2814 & 12: 13: 17 & 0.0744 \\ 2815 & 12: 13: 18 & 0.0792 \\ 2816 & 12: 13: 19 & 0.0777 \\ 2817 & 12: 13: 20 & 0.0727 \\ 2818 & 12: 13: 21 & 0.0761 \\ 2819 & 12: 13: 22 & 0.0680 \\ 2820 & 12: 13: 23 & 0.0823 \\ 2821 & 12: 13: 24 & 0.0735 \\ 2822 & 12: 13: 25 & 0.0738 \\ 2823 & 12: 13: 26 & 0.0726 \\ 2824 & 12: 13: 27 & 0.0729 \\ 2825 & 12: 13: 28 & 0.0692 \\ 2826 & 12: 13: 29 & 0.0781 \\ 2827 & 12: 13: 30 & 0.0679 \\ 2828 & 12: 13: 31 & 0.0707 \\ 2829 & 12: 13: 32 & 0.0746 \\ 2830 & 12: 13: 33 & 0.0677 \\ 2831 & 12: 13: 34 & 0.0717 \\ 2832 & 12: 13: 35 & 0.0724 \\ 2833 & 12: 13: 36 & 0.0737 \\ 2834 & 12: 13: 37 & 0.0741 \\ 2835 & 12: 13: 38 & 0.0742 \\ 2836 & 12: 13: 39 & 0.0699 \\ 2837 & 12: 13: 40 & 0.0793 \\ 2838 & 12: 13: 41 & 0.0697 \\ 2839 & 12: 13: 42 & 0.0809 \\ 2840 & 12: 13: 43 & 0.0659 \\ 2841 & 12: 13: 44 & 0.0751 \\ 2842 & 12: 13: 45 & 0.0743 \\ 2843 & 12: 13: 46 & 0.0730 \\ 2844 & 12: 13: 47 & 0.0648 \\ 2845 & 12: 13: 48 & 0.0692 \\ 2846 & 12: 13: 49 & 0.0708 \\ 2847 & 12: 13: 50 & 0.0791 \\ 2848 & 12: 13: 51 & 0.0731 \\ 2849 & 12: 13: 52 & 0.0799 \\ 2850 & 12: 13: 53 & 0.0645 \\ 2851 & 12: 13: 54 & 0.0736 \\ 2852 & 12: 13: 55 & 0.0768 \\ 2853 & 12: 13: 56 & 0.0765 \\ 2854 & 12: 13: 57 & 0.0699 \\ 2855 & 12: 13: 58 & 0.0708 \\ 2857 & 12: 13: 59 & 0.0673 \\ 2858 & 12: 14: 00 & 0.0662 \\ & & 0.0760\end{array}$


Appendix A - Fuel Data Test 1 NIST102505_1.xIs

$\begin{array}{lll}2859 & 12: 14: 02 & 0.0734 \\ 2860 & 12: 14: 03 & 0.0804 \\ 2861 & 12: 14: 04 & 0.0757 \\ 2862 & 12: 14: 05 & 0.0696 \\ 2863 & 12: 14: 06 & 0.0668 \\ 2864 & 12: 14: 07 & 0.0809 \\ 2865 & 12: 14: 08 & 0.0694 \\ 2866 & 12: 14: 09 & 0.0768 \\ 2867 & 12: 14: 10 & 0.0682 \\ 2868 & 12: 14: 11 & 0.0716 \\ 2869 & 12: 14: 12 & 0.0766 \\ 2870 & 12: 14: 13 & 0.0726 \\ 2871 & 12: 14: 14 & 0.0695 \\ 2872 & 12: 14: 15 & 0.0747 \\ 2873 & 12: 14: 16 & 0.0648 \\ 2874 & 12: 14: 17 & 0.0779 \\ 2875 & 12: 14: 18 & 0.0770 \\ 2876 & 12: 14: 19 & 0.0738 \\ 2877 & 12: 14: 20 & 0.0833 \\ 2878 & 12: 14: 21 & 0.0739 \\ 2879 & 12: 14: 22 & 0.0799 \\ 2880 & 12: 14: 23 & 0.0767 \\ 2881 & 12: 14: 24 & 0.0761 \\ 2882 & 12: 14: 25 & 0.0767 \\ 2883 & 12: 14: 26 & 0.0816 \\ 2884 & 12: 14: 27 & 0.0805 \\ 2885 & 12: 14: 28 & 0.0915 \\ 2886 & 12: 14: 29 & 0.0876 \\ 2887 & 12: 14: 30 & 0.0980 \\ 2888 & 12: 14: 31 & 0.0898 \\ 2889 & 12: 14: 32 & 0.0922 \\ 2890 & 12: 14: 33 & 0.0951 \\ 2891 & 12: 14: 34 & 0.0969 \\ 2892 & 12: 14: 35 & 0.0979 \\ 2893 & 12: 14: 36 & 0.1028 \\ 2894 & 12: 14: 37 & 0.1007 \\ 2895 & 12: 14: 38 & 0.1089 \\ 2896 & 12: 14: 39 & 0.1122 \\ 2897 & 12: 14: 40 & 0.1075 \\ 2898 & 12: 14: 41 & 0.1153 \\ 2899 & 12: 14: 42 & 0.1071 \\ 2900 & 12: 14: 43 & 0.1192 \\ 2901 & 12: 14: 44 & 0.1122 \\ 2902 & 12: 14: 45 & 0.1196 \\ 2903 & 12: 14: 46 & 0.1138 \\ 2904 & 12: 14: 47 & 0.1262 \\ 2905 & 12: 14: 48 & 0.1230 \\ 2906 & 12: 14: 49 & 0.1240 \\ 2907 & 12: 14: 50 & 0.1267 \\ 2909 & 12: 14: 51 & 0.1246 \\ 2910 & 12: 14: 52 & 0.1290 \\ & & 0.1310\end{array}$


Appendix A - Fuel Data Test 1 NIST102505_1.xls

$\begin{array}{lll}2911 & 12: 14: 54 & 0.1304 \\ 2912 & 12: 14: 55 & 0.1312 \\ 2913 & 12: 14: 56 & 0.1296 \\ 2914 & 12: 14: 57 & 0.1294 \\ 2915 & 12: 14: 58 & 0.1323 \\ 2916 & 12: 14: 59 & 0.1310 \\ 2917 & 12: 15: 00 & 0.1371 \\ 2918 & 12: 15: 01 & 0.1326 \\ 2919 & 12: 15: 02 & 0.1436 \\ 2920 & 12: 15: 03 & 0.1367 \\ 2921 & 12: 15: 04 & 0.1384 \\ 2922 & 12: 15: 05 & 0.1387 \\ 2923 & 12: 15: 06 & 0.1390 \\ 2924 & 12: 15: 07 & 0.1430 \\ 2925 & 12: 15: 08 & 0.1395 \\ 2926 & 12: 15: 09 & 0.1431 \\ 2927 & 12: 15: 10 & 0.1429 \\ 2928 & 12: 15: 11 & 0.1412 \\ 2929 & 12: 15: 12 & 0.1428 \\ 2930 & 12: 15: 13 & 0.1503 \\ 2931 & 12: 15: 14 & 0.1422 \\ 2932 & 12: 15: 15 & 0.1483 \\ 2933 & 12: 15: 16 & 0.1444 \\ 2934 & 12: 15: 17 & 0.1441 \\ 2935 & 12: 15: 18 & 0.1463 \\ 2936 & 12: 15: 19 & 0.1458 \\ 2937 & 12: 15: 20 & 0.1472 \\ 2938 & 12: 15: 21 & 0.1459 \\ 2939 & 12: 15: 22 & 0.1503 \\ 2940 & 12: 15: 23 & 0.1506 \\ 2941 & 12: 15: 24 & 0.1499 \\ 2942 & 12: 15: 25 & 0.1464 \\ 2943 & 12: 15: 26 & 0.1474 \\ 2944 & 12: 15: 27 & 0.1485 \\ 2945 & 12: 15: 28 & 0.1491 \\ 2946 & 12: 15: 29 & 0.1480 \\ 2947 & 12: 15: 30 & 0.1510 \\ 2948 & 12: 15: 31 & 0.1464 \\ 2949 & 12: 15: 32 & 0.1541 \\ 2950 & 12: 15: 33 & 0.1513 \\ 2951 & 12: 15: 34 & 0.1517 \\ 2952 & 12: 15: 35 & 0.1501 \\ 2953 & 12: 15: 36 & 0.1476 \\ 2954 & 12: 15: 37 & 0.1533 \\ 2955 & 12: 15: 38 & 0.1462 \\ 2956 & 12: 15: 39 & 0.1507 \\ 2957 & 12: 15: 40 & 0.1438 \\ 2958 & 12: 15: 41 & 0.1527 \\ 2959 & 12: 15: 42 & 0.1476 \\ 2960 & 12: 15: 43 & 0.1498 \\ 2961 & 12: 15: 44 & 0.1500 \\ 2962 & 12: 15: 45 & 0.1498\end{array}$


Appendix A - Fuel Data Test 1 NIST102505_1.xls

$\begin{array}{lll}2963 & 12: 15: 46 & 0.1546 \\ 2964 & 12: 15: 47 & 0.1530 \\ 2965 & 12: 15: 48 & 0.1569 \\ 2966 & 12: 15: 49 & 0.1472 \\ 2967 & 12: 15: 50 & 0.1538 \\ 2968 & 12: 15: 51 & 0.1500 \\ 2969 & 12: 15: 52 & 0.1546 \\ 2970 & 12: 15: 53 & 0.1498 \\ 2971 & 12: 15: 54 & 0.1516 \\ 2972 & 12: 15: 55 & 0.1519 \\ 2973 & 12: 15: 56 & 0.1528 \\ 2974 & 12: 15: 57 & 0.1541 \\ 2975 & 12: 15: 58 & 0.1520 \\ 2976 & 12: 15: 59 & 0.1532 \\ 2977 & 12: 16: 00 & 0.1524 \\ 2978 & 12: 16: 01 & 0.1551 \\ 2979 & 12: 16: 02 & 0.1506 \\ 2980 & 12: 16: 03 & 0.1539 \\ 2981 & 12: 16: 04 & 0.1536 \\ 2982 & 12: 16: 05 & 0.1520 \\ 2983 & 12: 16: 06 & 0.1491 \\ 2984 & 12: 16: 07 & 0.1499 \\ 2985 & 12: 16: 08 & 0.1497 \\ 2986 & 12: 16: 09 & 0.1534 \\ 2987 & 12: 16: 10 & 0.1513 \\ 2988 & 12: 16: 11 & 0.1544 \\ 2989 & 12: 16: 12 & 0.1508 \\ 2990 & 12: 16: 13 & 0.1530 \\ 2991 & 12: 16: 14 & 0.1555 \\ 2992 & 12: 16: 15 & 0.1501 \\ 2993 & 12: 16: 16 & 0.1548 \\ 2994 & 12: 16: 17 & 0.1505 \\ 2995 & 12: 16: 18 & 0.1561 \\ 2996 & 12: 16: 19 & 0.1525 \\ 2997 & 12: 16: 20 & 0.1559 \\ 2998 & 12: 16: 21 & 0.1518 \\ 2999 & 12: 16: 22 & 0.1562 \\ 3000 & 12: 16: 23 & 0.1561 \\ 3001 & 12: 16: 24 & 0.1553 \\ 3002 & 12: 16: 25 & 0.1553 \\ 3003 & 12: 16: 26 & 0.1553 \\ 3004 & 12: 16: 27 & 0.1535 \\ 3005 & 12: 16: 28 & 0.1578 \\ 3006 & 12: 16: 29 & 0.1555 \\ 3007 & 12: 16: 30 & 0.1528 \\ 3008 & 12: 16: 31 & 0.1553 \\ 3009 & 12: 16: 32 & 0.1498 \\ 3010 & 12: 16: 33 & 0.1561 \\ 3011 & 12: 16: 34 & 0.1554 \\ 3012 & 12: 16: 35 & 0.1570 \\ 3013 & 12: 16: 36 & 0.1575 \\ 3014 & 12: 16: 37 & 0.1550\end{array}$


Appendix A - Fuel Data Test 1 NIST102505_1.xIs

$\begin{array}{lll}3015 & 12: 16: 38 & 0.1545 \\ 3016 & 12: 16: 39 & 0.1558 \\ 3017 & 12: 16: 40 & 0.1556 \\ 3018 & 12: 16: 41 & 0.1581 \\ 3019 & 12: 16: 42 & 0.1540 \\ 3020 & 12: 16: 43 & 0.1546 \\ 3021 & 12: 16: 44 & 0.1560 \\ 3022 & 12: 16: 45 & 0.1517 \\ 3023 & 12: 16: 46 & 0.1555 \\ 3024 & 12: 16: 47 & 0.1553 \\ 3025 & 12: 16: 48 & 0.1559 \\ 3026 & 12: 16: 49 & 0.1564 \\ 3027 & 12: 16: 50 & 0.1566 \\ 3028 & 12: 16: 51 & 0.1544 \\ 3029 & 12: 16: 52 & 0.1590 \\ 3030 & 12: 16: 53 & 0.1554 \\ 3031 & 12: 16: 54 & 0.1554 \\ 3032 & 12: 16: 55 & 0.1563 \\ 3033 & 12: 16: 56 & 0.1524 \\ 3034 & 12: 16: 57 & 0.1590 \\ 3035 & 12: 16: 58 & 0.1558 \\ 3036 & 12: 16: 59 & 0.1585 \\ 3037 & 12: 17: 00 & 0.1511 \\ 3038 & 12: 17: 01 & 0.1574 \\ 3039 & 12: 17: 02 & 0.1582 \\ 3040 & 12: 17: 03 & 0.1564 \\ 3041 & 12: 17: 04 & 0.1543 \\ 3042 & 12: 17: 05 & 0.1530 \\ 3043 & 12: 17: 06 & 0.1576 \\ 3044 & 12: 17: 07 & 0.1552 \\ 3045 & 12: 17: 08 & 0.1579 \\ 3046 & 12: 17: 09 & 0.1521 \\ 3047 & 12: 17: 10 & 0.1569 \\ 3048 & 12: 17: 11 & 0.1573 \\ 3049 & 12: 17: 12 & 0.1571 \\ 3050 & 12: 17: 13 & 0.1546 \\ 3051 & 12: 17: 14 & 0.1533 \\ 3052 & 12: 17: 15 & 0.1554 \\ 3053 & 12: 17: 16 & 0.1567 \\ 3054 & 12: 17: 17 & 0.1543 \\ 3055 & 12: 17: 18 & 0.1567 \\ 3056 & 12: 17: 19 & 0.1536 \\ 3057 & 12: 17: 20 & 0.1556 \\ 3058 & 12: 17: 21 & 0.1565 \\ 3059 & 12: 17: 22 & 0.1543 \\ 3060 & 12: 17: 23 & 0.1593 \\ 3061 & 12: 17: 24 & 0.1515 \\ 3062 & 12: 17: 25 & 0.1594 \\ 3063 & 12: 17: 26 & 0.1533 \\ 3064 & 12: 17: 27 & 0.1569 \\ 3065 & 12: 17: 28 & 0.1511 \\ 3066 & 12: 17: 29 & 0.1562\end{array}$


Appendix A - Fuel Data Test 1 NIST102505_1.xls

$\begin{array}{lll}3067 & 12: 17: 30 & 0.1559 \\ 3068 & 12: 17: 31 & 0.1538 \\ 3069 & 12: 17: 32 & 0.1547 \\ 3070 & 12: 17: 33 & 0.1543 \\ 3071 & 12: 17: 34 & 0.1551 \\ 3072 & 12: 17: 35 & 0.1561 \\ 3073 & 12: 17: 36 & 0.1568 \\ 3074 & 12: 17: 37 & 0.1551 \\ 3075 & 12: 17: 38 & 0.1555 \\ 3076 & 12: 17: 39 & 0.1526 \\ 3077 & 12: 17: 40 & 0.1588 \\ 3078 & 12: 17: 41 & 0.1536 \\ 3079 & 12: 17: 42 & 0.1564 \\ 3080 & 12: 17: 43 & 0.1525 \\ 3081 & 12: 17: 44 & 0.1575 \\ 3082 & 12: 17: 45 & 0.1551 \\ 3083 & 12: 17: 46 & 0.1557 \\ 3084 & 12: 17: 47 & 0.1571 \\ 3085 & 12: 17: 48 & 0.1525 \\ 3086 & 12: 17: 49 & 0.1584 \\ 3087 & 12: 17: 50 & 0.1524 \\ 3088 & 12: 17: 51 & 0.1566 \\ 3089 & 12: 17: 52 & 0.1511 \\ 3090 & 12: 17: 53 & 0.1600 \\ 3091 & 12: 17: 54 & 0.1537 \\ 3092 & 12: 17: 55 & 0.1561 \\ 3093 & 12: 17: 56 & 0.1521 \\ 3094 & 12: 17: 57 & 0.1541 \\ 3095 & 12: 17: 58 & 0.1575 \\ 3096 & 12: 17: 59 & 0.1550 \\ 3097 & 12: 18: 00 & 0.1599 \\ 3098 & 12: 18: 01 & 0.1520 \\ 3099 & 12: 18: 02 & 0.1547 \\ 3100 & 12: 18: 03 & 0.1563 \\ 3101 & 12: 18: 04 & 0.1545 \\ 3102 & 12: 18: 05 & 0.1528 \\ 3103 & 12: 18: 06 & 0.1538 \\ 3104 & 12: 18: 07 & 0.1519 \\ 3105 & 12: 18: 08 & 0.1555 \\ 3106 & 12: 18: 09 & 0.1556 \\ 3107 & 12: 18: 10 & 0.1573 \\ 3108 & 12: 18: 11 & 0.1555 \\ 3109 & 12: 18: 12 & 0.1571 \\ 3110 & 12: 18: 13 & 0.1551 \\ 3111 & 12: 18: 14 & 0.1536 \\ 3112 & 12: 18: 15 & 0.1573 \\ 3113 & 12: 18: 16 & 0.1490 \\ 3114 & 12: 18: 17 & 0.1593 \\ 3115 & 12: 18: 18 & 0.1557 \\ 3116 & 12: 18: 19 & 0.1596 \\ 3117 & 12: 18: 20 & 0.1517 \\ 3118 & 12: 18: 21 & 0.1575\end{array}$


Appendix A - Fuel Data Test 1 NIST102505_1.xls

$\begin{array}{lll}3119 & 12: 18: 22 & 0.1553 \\ 3120 & 12: 18: 23 & 0.1565 \\ 3121 & 12: 18: 24 & 0.1554 \\ 3122 & 12: 18: 25 & 0.1516 \\ 3123 & 12: 18: 26 & 0.1572 \\ 3124 & 12: 18: 27 & 0.1539 \\ 3125 & 12: 18: 28 & 0.1567 \\ 3126 & 12: 18: 29 & 0.1543 \\ 3127 & 12: 18: 30 & 0.1542 \\ 3128 & 12: 18: 31 & 0.1531 \\ 3129 & 12: 18: 32 & 0.1553 \\ 3130 & 12: 18: 33 & 0.1536 \\ 3131 & 12: 18: 34 & 0.1545 \\ 3132 & 12: 18: 35 & 0.1565 \\ 3133 & 12: 18: 36 & 0.1595 \\ 3134 & 12: 18: 37 & 0.1543 \\ 3135 & 12: 18: 38 & 0.1540 \\ 3136 & 12: 18: 39 & 0.1520 \\ 3137 & 12: 18: 40 & 0.1521 \\ 3138 & 12: 18: 41 & 0.1567 \\ 3139 & 12: 18: 42 & 0.1520 \\ 3140 & 12: 18: 43 & 0.1557 \\ 3141 & 12: 18: 44 & 0.1516 \\ 3142 & 12: 18: 45 & 0.1556 \\ 3143 & 12: 18: 46 & 0.1546 \\ 3144 & 12: 18: 47 & 0.1548 \\ 3145 & 12: 18: 48 & 0.1553 \\ 3146 & 12: 18: 49 & 0.1515 \\ 3147 & 12: 18: 50 & 0.1559 \\ 3148 & 12: 18: 51 & 0.1537 \\ 3149 & 12: 18: 52 & 0.1562 \\ 3150 & 12: 18: 53 & 0.1549 \\ 3151 & 12: 18: 54 & 0.1528 \\ 3152 & 12: 18: 55 & 0.1532 \\ 3153 & 12: 18: 56 & 0.1559 \\ 3154 & 12: 18: 57 & 0.1514 \\ 3155 & 12: 18: 58 & 0.1568 \\ 3156 & 12: 18: 59 & 0.1524 \\ 3157 & 12: 19: 00 & 0.1571 \\ 3158 & 12: 19: 01 & 0.1538 \\ 3159 & 12: 19: 02 & 0.1540 \\ 3160 & 12: 19: 03 & 0.1562 \\ 3161 & 12: 19: 04 & 0.1500 \\ 3162 & 12: 19: 05 & 0.1547 \\ 3163 & 12: 19: 06 & 0.1554 \\ 3164 & 12: 19: 07 & 0.1542 \\ 3165 & 12: 19: 08 & 0.1548 \\ 3166 & 12: 19: 09 & 0.1561 \\ 3167 & 12: 19: 10 & 0.1525 \\ 3168 & 12: 19: 11 & 0.1596 \\ 3169 & 12: 19: 12 & 0.1547 \\ 3170 & 12: 19: 13 & 0.1552\end{array}$


Appendix A - Fuel Data Test 1 NIST102505_1.xls

$\begin{array}{lll}3171 & 12: 19: 14 & 0.1565 \\ 3172 & 12: 19: 15 & 0.1518 \\ 3173 & 12: 19: 16 & 0.1562 \\ 3174 & 12: 19: 17 & 0.1553 \\ 3175 & 12: 19: 18 & 0.1546 \\ 3176 & 12: 19: 19 & 0.1518 \\ 3177 & 12: 19: 20 & 0.1539 \\ 3178 & 12: 19: 21 & 0.1249 \\ 3179 & 12: 19: 22 & 0.0735 \\ 3180 & 12: 19: 23 & 0.1125 \\ 3181 & 12: 19: 24 & 0.1277 \\ 3182 & 12: 19: 25 & 0.1354 \\ 3183 & 12: 19: 26 & 0.1376 \\ 3184 & 12: 19: 27 & 0.1380 \\ 3185 & 12: 19: 28 & 0.1391 \\ 3186 & 12: 19: 29 & 0.1349 \\ 3187 & 12: 19: 30 & 0.1410 \\ 3188 & 12: 19: 31 & 0.1341 \\ 3189 & 12: 19: 32 & 0.1387 \\ 3190 & 12: 19: 33 & 0.1380 \\ 3191 & 12: 19: 34 & 0.1418 \\ 3192 & 12: 19: 35 & 0.1396 \\ 3193 & 12: 19: 36 & 0.1389 \\ 3194 & 12: 19: 37 & 0.1400 \\ 3195 & 12: 19: 38 & 0.1359 \\ 3196 & 12: 19: 39 & 0.1382 \\ 3197 & 12: 19: 40 & 0.1312 \\ 3198 & 12: 19: 41 & 0.1378 \\ 3199 & 12: 19: 42 & 0.1328 \\ 3200 & 12: 19: 43 & 0.1364 \\ 3201 & 12: 19: 44 & 0.1328 \\ 3202 & 12: 19: 45 & 0.1361 \\ 3203 & 12: 19: 46 & 0.1325 \\ 3204 & 12: 19: 47 & 0.1349 \\ 3205 & 12: 19: 48 & 0.1351 \\ 3206 & 12: 19: 49 & 0.1344 \\ 3207 & 12: 19: 50 & 0.1340 \\ 3208 & 12: 19: 51 & 0.1319 \\ 3209 & 12: 19: 52 & 0.1361 \\ 3210 & 12: 19: 53 & 0.1341 \\ 3211 & 12: 19: 54 & 0.1349 \\ 3212 & 12: 19: 55 & 0.1329 \\ 3213 & 12: 19: 56 & 0.1404 \\ 3214 & 12: 19: 57 & 0.1337 \\ 3215 & 12: 19: 58 & 0.1371 \\ 3216 & 12: 19: 59 & 0.1347 \\ 3217 & 12: 20: 00 & 0.1377 \\ 3218 & 12: 20: 01 & 0.1339 \\ 3219 & 12: 20: 02 & 0.1367 \\ 3220 & 12: 20: 03 & 0.1385 \\ 3221 & 12: 20: 04 & 0.1381 \\ 3222 & 12: 20: 05 & 0.1383\end{array}$


Appendix A - Fuel Data Test 1 NIST102505_1.xls

$\begin{array}{lll}3223 & 12: 20: 06 & 0.1355 \\ 3224 & 12: 20: 07 & 0.1384 \\ 3225 & 12: 20: 08 & 0.1333 \\ 3226 & 12: 20: 09 & 0.1359 \\ 3227 & 12: 20: 10 & 0.1348 \\ 3228 & 12: 20: 11 & 0.1370 \\ 3229 & 12: 20: 12 & 0.1346 \\ 3230 & 12: 20: 13 & 0.1367 \\ 3231 & 12: 20: 14 & 0.1358 \\ 3232 & 12: 20: 15 & 0.1359 \\ 3233 & 12: 20: 16 & 0.1345 \\ 3234 & 12: 20: 17 & 0.1324 \\ 3235 & 12: 20: 18 & 0.1335 \\ 3236 & 12: 20: 19 & 0.1303 \\ 3237 & 12: 20: 20 & 0.1366 \\ 3238 & 12: 20: 21 & 0.1325 \\ 3239 & 12: 20: 22 & 0.1373 \\ 3240 & 12: 20: 23 & 0.1325 \\ 3241 & 12: 20: 24 & 0.1395 \\ 3242 & 12: 20: 25 & 0.1353 \\ 3243 & 12: 20: 26 & 0.1372 \\ 3244 & 12: 20: 27 & 0.1334 \\ 3245 & 12: 20: 28 & 0.1344 \\ 3246 & 12: 20: 29 & 0.1348 \\ 3247 & 12: 20: 30 & 0.1318 \\ 3248 & 12: 20: 31 & 0.1368 \\ 3249 & 12: 20: 32 & 0.1325 \\ 3250 & 12: 20: 33 & 0.1362 \\ 3251 & 12: 20: 34 & 0.1319 \\ 3252 & 12: 20: 35 & 0.1358 \\ 3253 & 12: 20: 36 & 0.1334 \\ 3254 & 12: 20: 37 & 0.1375 \\ 3255 & 12: 20: 38 & 0.1371 \\ 3256 & 12: 20: 39 & 0.1361 \\ 3257 & 12: 20: 40 & 0.1340 \\ 3258 & 12: 20: 41 & 0.1329 \\ 3259 & 12: 20: 42 & 0.1345 \\ 3260 & 12: 20: 43 & 0.1326 \\ 3261 & 12: 20: 44 & 0.1340 \\ 3262 & 12: 20: 45 & 0.1309 \\ 3263 & 12: 20: 46 & 0.1345 \\ 3264 & 12: 20: 47 & 0.1311 \\ 3265 & 12: 20: 48 & 0.1354 \\ 3266 & 12: 20: 49 & 0.1359 \\ 3267 & 12: 20: 50 & 0.1374 \\ 3268 & 12: 20: 51 & 0.1317 \\ 3269 & 12: 20: 52 & 0.1330 \\ 3270 & 12: 20: 53 & 0.1296 \\ 3273 & 12: 20: 54 & 0.1306 \\ 3274 & 12: 20: 55 & 0.1349 \\ & 12: 20: 57 & 0.1331 \\ 325 & 0.1343\end{array}$


Appendix A - Fuel Data Test 1 NIST102505_1.xls

$\begin{array}{lll}3275 & 12: 20: 58 & 0.1310 \\ 3276 & 12: 20: 59 & 0.1351 \\ 3277 & 12: 21: 00 & 0.1314 \\ 3278 & 12: 21: 01 & 0.1367 \\ 3279 & 12: 21: 02 & 0.1355 \\ 3280 & 12: 21: 03 & 0.1369 \\ 3281 & 12: 21: 04 & 0.1339 \\ 3282 & 12: 21: 05 & 0.1341 \\ 3283 & 12: 21: 06 & 0.1365 \\ 3284 & 12: 21: 07 & 0.1350 \\ 3285 & 12: 21: 08 & 0.1351 \\ 3286 & 12: 21: 09 & 0.1331 \\ 3287 & 12: 21: 10 & 0.1345 \\ 3288 & 12: 21: 11 & 0.1300 \\ 3289 & 12: 21: 12 & 0.1366 \\ 3290 & 12: 21: 13 & 0.1336 \\ 3291 & 12: 21: 14 & 0.1379 \\ 3292 & 12: 21: 15 & 0.1332 \\ 3293 & 12: 21: 16 & 0.1363 \\ 3294 & 12: 21: 17 & 0.1330 \\ 3295 & 12: 21: 18 & 0.1345 \\ 3296 & 12: 21: 19 & 0.1339 \\ 3297 & 12: 21: 20 & 0.1336 \\ 3298 & 12: 21: 21 & 0.1333 \\ 3299 & 12: 21: 22 & 0.1314 \\ 3300 & 12: 21: 23 & 0.1361 \\ 3301 & 12: 21: 24 & 0.1310 \\ 3302 & 12: 21: 25 & 0.1353 \\ 3303 & 12: 21: 26 & 0.1305 \\ 3304 & 12: 21: 27 & 0.1368 \\ 3305 & 12: 21: 28 & 0.1339 \\ 3306 & 12: 21: 29 & 0.1338 \\ 3307 & 12: 21: 30 & 0.1335 \\ 3308 & 12: 21: 31 & 0.1328 \\ 3309 & 12: 21: 32 & 0.1339 \\ 3310 & 12: 21: 33 & 0.1299 \\ 3311 & 12: 21: 34 & 0.1346 \\ 3312 & 12: 21: 35 & 0.1310 \\ 3313 & 12: 21: 36 & 0.1349 \\ 3314 & 12: 21: 37 & 0.1322 \\ 3315 & 12: 21: 38 & 0.1369 \\ 3316 & 12: 21: 39 & 0.1355 \\ 3317 & 12: 21: 40 & 0.1405 \\ 3318 & 12: 21: 41 & 0.1368 \\ 3319 & 12: 21: 42 & 0.1371 \\ 3320 & 12: 21: 43 & 0.1329 \\ 3321 & 12: 21: 44 & 0.1346 \\ 3322 & 12: 21: 45 & 0.1326 \\ 3323 & 12: 21: 46 & 0.1314 \\ 3324 & 12: 21: 47 & 0.1342 \\ 3325 & 12: 21: 48 & 0.1329 \\ 3326 & 12: 21: 49 & 0.1377\end{array}$


Appendix A - Fuel Data Test 1 NIST102505_1.xls

$\begin{array}{lll}3327 & 12: 21: 50 & 0.1312 \\ 3328 & 12: 21: 51 & 0.1343 \\ 3329 & 12: 21: 52 & 0.1301 \\ 3330 & 12: 21: 53 & 0.1361 \\ 3331 & 12: 21: 54 & 0.1361 \\ 3332 & 12: 21: 55 & 0.1363 \\ 3333 & 12: 21: 56 & 0.1354 \\ 3334 & 12: 21: 57 & 0.1311 \\ 3335 & 12: 21: 58 & 0.1359 \\ 3336 & 12: 21: 59 & 0.1332 \\ 3337 & 12: 22: 00 & 0.1351 \\ 3338 & 12: 22: 01 & 0.1313 \\ 3339 & 12: 22: 02 & 0.1383 \\ 3340 & 12: 22: 03 & 0.1362 \\ 3341 & 12: 22: 04 & 0.1385 \\ 3342 & 12: 22: 05 & 0.1378 \\ 3343 & 12: 22: 06 & 0.1385 \\ 3344 & 12: 22: 07 & 0.1374 \\ 3345 & 12: 22: 08 & 0.1344 \\ 3346 & 12: 22: 09 & 0.1341 \\ 3347 & 12: 22: 10 & 0.1325 \\ 3348 & 12: 22: 11 & 0.1346 \\ 3349 & 12: 22: 12 & 0.1305 \\ 3350 & 12: 22: 13 & 0.1341 \\ 3351 & 12: 22: 14 & 0.1292 \\ 3352 & 12: 22: 15 & 0.1345 \\ 3353 & 12: 22: 16 & 0.1327 \\ 3354 & 12: 22: 17 & 0.1355 \\ 3355 & 12: 22: 18 & 0.1312 \\ 3356 & 12: 22: 19 & 0.1339 \\ 3357 & 12: 22: 20 & 0.1337 \\ 3358 & 12: 22: 21 & 0.1332 \\ 3359 & 12: 22: 22 & 0.1364 \\ 3360 & 12: 22: 23 & 0.1344 \\ 3361 & 12: 22: 24 & 0.1400 \\ 3362 & 12: 22: 25 & 0.1363 \\ 3363 & 12: 22: 26 & 0.1377 \\ 3364 & 12: 22: 27 & 0.1345 \\ 3365 & 12: 22: 28 & 0.1395 \\ 3366 & 12: 22: 29 & 0.1338 \\ 3367 & 12: 22: 30 & 0.1392 \\ 3368 & 12: 22: 31 & 0.1396 \\ 3369 & 12: 22: 32 & 0.1404 \\ 3370 & 12: 22: 33 & 0.1371 \\ 3371 & 12: 22: 34 & 0.1361 \\ 3372 & 12: 22: 35 & 0.1387 \\ 3373 & 12: 22: 36 & 0.1327 \\ 3374 & 12: 22: 37 & 0.1350 \\ 3375 & 12: 22: 38 & 0.1306 \\ 3376 & 12: 22: 39 & 0.1371 \\ 3377 & 12: 22: 40 & 0.1316 \\ 3378 & 12: 22: 41 & 0.1355\end{array}$


Appendix A - Fuel Data Test 1 NIST102505_1.xls

$\begin{array}{lll}3379 & 12: 22: 42 & 0.1335 \\ 3380 & 12: 22: 43 & 0.1370 \\ 3381 & 12: 22: 44 & 0.1341 \\ 3382 & 12: 22: 45 & 0.1349 \\ 3383 & 12: 22: 46 & 0.1356 \\ 3384 & 12: 22: 47 & 0.1337 \\ 3385 & 12: 22: 48 & 0.1324 \\ 3386 & 12: 22: 49 & 0.1316 \\ 3387 & 12: 22: 50 & 0.1351 \\ 3388 & 12: 22: 51 & 0.1306 \\ 3389 & 12: 22: 52 & 0.1345 \\ 3390 & 12: 22: 53 & 0.1319 \\ 3391 & 12: 22: 54 & 0.1350 \\ 3392 & 12: 22: 55 & 0.1312 \\ 3393 & 12: 22: 56 & 0.1365 \\ 3394 & 12: 22: 57 & 0.1343 \\ 3395 & 12: 22: 58 & 0.1358 \\ 3396 & 12: 22: 59 & 0.1294 \\ 3397 & 12: 23: 00 & 0.1310 \\ 3398 & 12: 23: 01 & 0.1322 \\ 3399 & 12: 23: 02 & 0.1308 \\ 3400 & 12: 23: 03 & 0.1349 \\ 3401 & 12: 23: 04 & 0.1354 \\ 3402 & 12: 23: 05 & 0.1370 \\ 3403 & 12: 23: 06 & 0.1322 \\ 3404 & 12: 23: 07 & 0.1344 \\ 3405 & 12: 23: 08 & 0.1332 \\ 3406 & 12: 23: 09 & 0.1358 \\ 3407 & 12: 23: 10 & 0.1349 \\ 3408 & 12: 23: 11 & 0.1355 \\ 3409 & 12: 23: 12 & 0.1359 \\ 3410 & 12: 23: 13 & 0.1350 \\ 3411 & 12: 23: 14 & 0.1341 \\ 3412 & 12: 23: 15 & 0.1324 \\ 3413 & 12: 23: 16 & 0.1335 \\ 3414 & 12: 23: 17 & 0.1292 \\ 3415 & 12: 23: 18 & 0.1340 \\ 3416 & 12: 23: 19 & 0.1324 \\ 3417 & 12: 23: 20 & 0.1360 \\ 3418 & 12: 23: 21 & 0.1307 \\ 3419 & 12: 23: 22 & 0.1350 \\ 3420 & 12: 23: 23 & 0.1326 \\ 3421 & 12: 23: 24 & 0.1331 \\ 3422 & 12: 23: 25 & 0.1349 \\ 3423 & 12: 23: 26 & 0.1323 \\ 3424 & 12: 23: 27 & 0.1347 \\ 3425 & 12: 23: 28 & 0.1312 \\ 3426 & 12: 23: 29 & 0.1366 \\ 3427 & 12: 23: 30 & 0.1312 \\ 3428 & 12: 23: 31 & 0.1347 \\ 3429 & 12: 23: 32 & 0.1318 \\ 3430 & 12: 23: 33 & 0.1358\end{array}$


Appendix A - Fuel Data Test 1 NIST102505_1.xls

$\begin{array}{lll}3431 & 12: 23: 34 & 0.1328 \\ 3432 & 12: 23: 35 & 0.1336 \\ 3433 & 12: 23: 36 & 0.1360 \\ 3434 & 12: 23: 37 & 0.1342 \\ 3435 & 12: 23: 38 & 0.1333 \\ 3436 & 12: 23: 39 & 0.1315 \\ 3437 & 12: 23: 40 & 0.1362 \\ 3438 & 12: 23: 41 & 0.1322 \\ 3439 & 12: 23: 42 & 0.1342 \\ 3440 & 12: 23: 43 & 0.1306 \\ 3441 & 12: 23: 44 & 0.1355 \\ 3442 & 12: 23: 45 & 0.1343 \\ 3443 & 12: 23: 46 & 0.1385 \\ 3444 & 12: 23: 47 & 0.1383 \\ 3445 & 12: 23: 48 & 0.1392 \\ 3446 & 12: 23: 49 & 0.1354 \\ 3447 & 12: 23: 50 & 0.1364 \\ 3448 & 12: 23: 51 & 0.1362 \\ 3449 & 12: 23: 52 & 0.1344 \\ 3450 & 12: 23: 53 & 0.1355 \\ 3451 & 12: 23: 54 & 0.1309 \\ 3452 & 12: 23: 55 & 0.1346 \\ 3453 & 12: 23: 56 & 0.1320 \\ 3454 & 12: 23: 57 & 0.1341 \\ 3455 & 12: 23: 58 & 0.1299 \\ 3456 & 12: 23: 59 & 0.1337 \\ 3457 & 12: 24: 00 & 0.1313 \\ 3458 & 12: 24: 01 & 0.1342 \\ 3459 & 12: 24: 02 & 0.1340 \\ 3460 & 12: 24: 03 & 0.1349 \\ 3461 & 12: 24: 04 & 0.1352 \\ 3462 & 12: 24: 05 & 0.1313 \\ 3463 & 12: 24: 06 & 0.1364 \\ 3464 & 12: 24: 07 & 0.1303 \\ 3465 & 12: 24: 08 & 0.1360 \\ 3466 & 12: 24: 09 & 0.1325 \\ 3467 & 12: 24: 10 & 0.1386 \\ 3468 & 12: 24: 11 & 0.1342 \\ 3469 & 12: 24: 12 & 0.1354 \\ 3470 & 12: 24: 13 & 0.1330 \\ 3471 & 12: 24: 14 & 0.1347 \\ 3472 & 12: 24: 15 & 0.1326 \\ 3473 & 12: 24: 16 & 0.1311 \\ 3474 & 12: 24: 17 & 0.1348 \\ 3475 & 12: 24: 18 & 0.1321 \\ 3476 & 12: 24: 19 & 0.1341 \\ 3477 & 12: 24: 20 & 0.1295 \\ 3478 & 12: 24: 21 & 0.1336 \\ 3479 & 12: 24: 22 & 0.1294 \\ 3480 & 12: 24: 23 & 0.1334 \\ 3481 & 12: 24: 24 & 0.1328 \\ 3482 & 12: 24: 25 & 0.1348\end{array}$


Appendix A - Fuel Data Test 1 NIST102505_1.xIs

$\begin{array}{lll}3483 & 12: 24: 26 & 0.1339 \\ 3484 & 12: 24: 27 & 0.1325 \\ 3485 & 12: 24: 28 & 0.1325 \\ 3486 & 12: 24: 29 & 0.1323 \\ 3487 & 12: 24: 30 & 0.1332 \\ 3488 & 12: 24: 31 & 0.1315 \\ 3489 & 12: 24: 32 & 0.1339 \\ 3490 & 12: 24: 33 & 0.1303 \\ 3491 & 12: 24: 34 & 0.1318 \\ 3492 & 12: 24: 35 & 0.1300 \\ 3493 & 12: 24: 36 & 0.1356 \\ 3494 & 12: 24: 37 & 0.1293 \\ 3495 & 12: 24: 38 & 0.1348 \\ 3496 & 12: 24: 39 & 0.1323 \\ 3497 & 12: 24: 40 & 0.1346 \\ 3498 & 12: 24: 41 & 0.1325 \\ 3499 & 12: 24: 42 & 0.1332 \\ 3500 & 12: 24: 43 & 0.1355 \\ 3501 & 12: 24: 44 & 0.1324 \\ 3502 & 12: 24: 45 & 0.1325 \\ 3503 & 12: 24: 46 & 0.1297 \\ 3504 & 12: 24: 47 & 0.1347 \\ 3505 & 12: 24: 48 & 0.1308 \\ 3506 & 12: 24: 49 & 0.1375 \\ 3507 & 12: 24: 50 & 0.1356 \\ 3508 & 12: 24: 51 & 0.1391 \\ 3509 & 12: 24: 52 & 0.1352 \\ 3510 & 12: 24: 53 & 0.1367 \\ 3511 & 12: 24: 54 & 0.1353 \\ 3512 & 12: 24: 55 & 0.1346 \\ 3513 & 12: 24: 56 & 0.1372 \\ 3514 & 12: 24: 57 & 0.1359 \\ 3515 & 12: 24: 58 & 0.1361 \\ 3516 & 12: 24: 59 & 0.1326 \\ 3517 & 12: 25: 00 & 0.1396 \\ 3518 & 12: 25: 01 & 0.1315 \\ 3519 & 12: 25: 02 & 0.1349 \\ 3520 & 12: 25: 03 & 0.1342 \\ 3521 & 12: 25: 04 & 0.1368 \\ 3522 & 12: 25: 05 & 0.1346 \\ 3523 & 12: 25: 06 & 0.1352 \\ 3524 & 12: 25: 07 & 0.1383 \\ 3525 & 12: 25: 08 & 0.1329 \\ 3526 & 12: 25: 09 & 0.1335 \\ 3527 & 12: 25: 10 & 0.1294 \\ 3528 & 12: 25: 11 & 0.1326 \\ 3529 & 12: 25: 12 & 0.1288 \\ 3530 & 12: 25: 13 & 0.1339 \\ 3531 & 12: 25: 14 & 0.1327 \\ 3532 & 12: 25: 15 & 0.1356 \\ 3533 & 12: 25: 16 & 0.1326 \\ 3534 & 12: 25: 17 & 0.1349\end{array}$


Appendix A - Fuel Data Test 1 NIST102505_1.xls

$\begin{array}{lll}3535 & 12: 25: 18 & 0.1324 \\ 3536 & 12: 25: 19 & 0.1329 \\ 3537 & 12: 25: 20 & 0.1329 \\ 3538 & 12: 25: 21 & 0.1324 \\ 3539 & 12: 25: 22 & 0.1331 \\ 3540 & 12: 25: 23 & 0.1305 \\ 3541 & 12: 25: 24 & 0.1340 \\ 3542 & 12: 25: 25 & 0.1325 \\ 3543 & 12: 25: 26 & 0.1345 \\ 3544 & 12: 25: 27 & 0.1304 \\ 3545 & 12: 25: 28 & 0.1348 \\ 3546 & 12: 25: 29 & 0.1326 \\ 3547 & 12: 25: 30 & 0.1342 \\ 3548 & 12: 25: 31 & 0.1331 \\ 3549 & 12: 25: 32 & 0.1329 \\ 3550 & 12: 25: 33 & 0.1353 \\ 3551 & 12: 25: 34 & 0.1322 \\ 3552 & 12: 25: 35 & 0.1344 \\ 3553 & 12: 25: 36 & 0.1323 \\ 3554 & 12: 25: 37 & 0.1355 \\ 3555 & 12: 25: 38 & 0.1300 \\ 3556 & 12: 25: 39 & 0.1340 \\ 3557 & 12: 25: 40 & 0.1330 \\ 3558 & 12: 25: 41 & 0.1348 \\ 3559 & 12: 25: 42 & 0.1328 \\ 3560 & 12: 25: 43 & 0.1341 \\ 3561 & 12: 25: 44 & 0.1327 \\ 3562 & 12: 25: 45 & 0.1320 \\ 3563 & 12: 25: 46 & 0.1306 \\ 3564 & 12: 25: 47 & 0.1300 \\ 3565 & 12: 25: 48 & 0.1315 \\ 3566 & 12: 25: 49 & 0.1278 \\ 3567 & 12: 25: 50 & 0.1352 \\ 3568 & 12: 25: 51 & 0.1320 \\ 3569 & 12: 25: 52 & 0.1352 \\ 3570 & 12: 25: 53 & 0.1316 \\ 3571 & 12: 25: 54 & 0.1348 \\ 3572 & 12: 25: 55 & 0.1319 \\ 3573 & 12: 25: 56 & 0.1338 \\ 3574 & 12: 25: 57 & 0.1289 \\ 3575 & 12: 25: 58 & 0.1314 \\ 3576 & 12: 25: 59 & 0.1309 \\ 3577 & 12: 26: 00 & 0.1284 \\ 3578 & 12: 26: 01 & 0.1335 \\ 3579 & 12: 26: 02 & 0.1296 \\ 3580 & 12: 26: 03 & 0.1348 \\ 3581 & 12: 26: 04 & 0.1312 \\ 3582 & 12: 26: 05 & 0.1353 \\ 3583 & 12: 26: 06 & 0.1326 \\ 3584 & 12: 26: 07 & 0.1343 \\ 3585 & 12: 26: 08 & 0.1313 \\ 3586 & 12: 26: 09 & 0.1345\end{array}$


Appendix A - Fuel Data Test 1 NIST102505_1.xIs

$\begin{array}{lll}3587 & 12: 26: 10 & 0.1330 \\ 3588 & 12: 26: 11 & 0.1317 \\ 3589 & 12: 26: 12 & 0.1320 \\ 3590 & 12: 26: 13 & 0.1291 \\ 3591 & 12: 26: 14 & 0.1321 \\ 3592 & 12: 26: 15 & 0.1305 \\ 3593 & 12: 26: 16 & 0.1353 \\ 3594 & 12: 26: 17 & 0.1332 \\ 3595 & 12: 26: 18 & 0.1364 \\ 3596 & 12: 26: 19 & 0.1354 \\ 3597 & 12: 26: 20 & 0.1364 \\ 3598 & 12: 26: 21 & 0.1344 \\ 3599 & 12: 26: 22 & 0.1335 \\ 3600 & 12: 26: 23 & 0.1344 \\ 3601 & 12: 26: 24 & 0.1311 \\ 3602 & 12: 26: 25 & 0.1319 \\ 3603 & 12: 26: 26 & 0.1269 \\ 3604 & 12: 26: 27 & 0.1324 \\ 3605 & 12: 26: 28 & 0.1288 \\ 3606 & 12: 26: 29 & 0.1336 \\ 3607 & 12: 26: 30 & 0.1319 \\ 3608 & 12: 26: 31 & 0.1348 \\ 3609 & 12: 26: 32 & 0.1318 \\ 3610 & 12: 26: 33 & 0.1316 \\ 3611 & 12: 26: 34 & 0.1337 \\ 3612 & 12: 26: 35 & 0.1325 \\ 3613 & 12: 26: 36 & 0.1321 \\ 3614 & 12: 26: 37 & 0.1308 \\ 3615 & 12: 26: 38 & 0.1320 \\ 3616 & 12: 26: 39 & 0.1288 \\ 3617 & 12: 26: 40 & 0.1347 \\ 3618 & 12: 26: 41 & 0.1305 \\ 3619 & 12: 26: 42 & 0.1345 \\ 3620 & 12: 26: 43 & 0.1310 \\ 3621 & 12: 26: 44 & 0.1348 \\ 3622 & 12: 26: 45 & 0.1317 \\ 3623 & 12: 26: 46 & 0.1330 \\ 3624 & 12: 26: 47 & 0.1335 \\ 3625 & 12: 26: 48 & 0.1319 \\ 3626 & 12: 26: 49 & 0.1336 \\ 3627 & 12: 26: 50 & 0.1307 \\ 3628 & 12: 26: 51 & 0.1335 \\ 3629 & 12: 26: 52 & 0.1302 \\ 3630 & 12: 26: 53 & 0.1353 \\ 3631 & 12: 26: 54 & 0.1336 \\ 3632 & 12: 26: 55 & 0.1334 \\ 3633 & 12: 26: 56 & 0.1302 \\ 3634 & 12: 26: 57 & 0.1334 \\ 3635 & 12: 26: 58 & 0.1355 \\ 3636 & 12: 26: 59 & 0.1360 \\ 3637 & 12: 27: 00 & 0.1338 \\ 3638 & 12: 27: 01 & 0.1321\end{array}$


Appendix A - Fuel Data Test 1 NIST102505_1.xIs

$\begin{array}{lll}3639 & 12: 27: 02 & 0.1327 \\ 3640 & 12: 27: 03 & 0.1309 \\ 3641 & 12: 27: 04 & 0.1330 \\ 3642 & 12: 27: 05 & 0.1313 \\ 3643 & 12: 27: 06 & 0.1331 \\ 3644 & 12: 27: 07 & 0.1285 \\ 3645 & 12: 27: 08 & 0.1362 \\ 3646 & 12: 27: 09 & 0.1329 \\ 3647 & 12: 27: 10 & 0.1361 \\ 3648 & 12: 27: 11 & 0.1378 \\ 3649 & 12: 27: 12 & 0.1371 \\ 3650 & 12: 27: 13 & 0.1359 \\ 3651 & 12: 27: 14 & 0.1343 \\ 3652 & 12: 27: 15 & 0.1365 \\ 3653 & 12: 27: 16 & 0.1343 \\ 3654 & 12: 27: 17 & 0.1376 \\ 3655 & 12: 27: 18 & 0.1347 \\ 3656 & 12: 27: 19 & 0.1392 \\ 3657 & 12: 27: 20 & 0.1338 \\ 3658 & 12: 27: 21 & 0.1378 \\ 3659 & 12: 27: 22 & 0.1352 \\ 3660 & 12: 27: 23 & 0.1356 \\ 3661 & 12: 27: 24 & 0.1313 \\ 3662 & 12: 27: 25 & 0.1333 \\ 3663 & 12: 27: 26 & 0.1305 \\ 3664 & 12: 27: 27 & 0.1297 \\ 3665 & 12: 27: 28 & 0.1321 \\ 3666 & 12: 27: 29 & 0.1300 \\ 3667 & 12: 27: 30 & 0.1337 \\ 3668 & 12: 27: 31 & 0.1276 \\ 3669 & 12: 27: 32 & 0.1342 \\ 3670 & 12: 27: 33 & 0.1295 \\ 3671 & 12: 27: 34 & 0.1374 \\ 3672 & 12: 27: 35 & 0.1383 \\ 3673 & 12: 27: 36 & 0.1363 \\ 3674 & 12: 27: 37 & 0.1347 \\ 3675 & 12: 27: 38 & 0.1348 \\ 3676 & 12: 27: 39 & 0.1364 \\ 3677 & 12: 27: 40 & 0.1306 \\ 3678 & 12: 27: 41 & 0.1321 \\ 3679 & 12: 27: 42 & 0.1272 \\ 3680 & 12: 27: 43 & 0.1335 \\ 3681 & 12: 27: 44 & 0.1307 \\ 3682 & 12: 27: 45 & 0.1344 \\ 3683 & 12: 27: 46 & 0.1330 \\ 3684 & 12: 27: 47 & 0.1348 \\ 3685 & 12: 27: 48 & 0.1327 \\ 3686 & 12: 27: 49 & 0.1320 \\ 3687 & 12: 27: 50 & 0.1331 \\ 3688 & 12: 27: 51 & 0.1322 \\ 3689 & 12: 27: 52 & 0.1327 \\ 3690 & 12: 27: 53 & 0.1297\end{array}$


Appendix A - Fuel Data Test 1 NIST102505_1.xls

$\begin{array}{lll}3691 & 12: 27: 54 & 0.1334 \\ 3692 & 12: 27: 55 & 0.1307 \\ 3693 & 12: 27: 56 & 0.1361 \\ 3694 & 12: 27: 57 & 0.1333 \\ 3695 & 12: 27: 58 & 0.1376 \\ 3696 & 12: 27: 59 & 0.1344 \\ 3697 & 12: 28: 00 & 0.1392 \\ 3698 & 12: 28: 01 & 0.1403 \\ 3699 & 12: 28: 02 & 0.1397 \\ 3700 & 12: 28: 03 & 0.1375 \\ 3701 & 12: 28: 04 & 0.1358 \\ 3702 & 12: 28: 05 & 0.1370 \\ 3703 & 12: 28: 06 & 0.1327 \\ 3704 & 12: 28: 07 & 0.1346 \\ 3705 & 12: 28: 08 & 0.1319 \\ 3706 & 12: 28: 09 & 0.1357 \\ 3707 & 12: 28: 10 & 0.1326 \\ 3708 & 12: 28: 11 & 0.1391 \\ 3709 & 12: 28: 12 & 0.1350 \\ 3710 & 12: 28: 13 & 0.1385 \\ 3711 & 12: 28: 14 & 0.1350 \\ 3712 & 12: 28: 15 & 0.1369 \\ 3713 & 12: 28: 16 & 0.1347 \\ 3714 & 12: 28: 17 & 0.1343 \\ 3715 & 12: 28: 18 & 0.1369 \\ 3716 & 12: 28: 19 & 0.1347 \\ 3717 & 12: 28: 20 & 0.1364 \\ 3718 & 12: 28: 21 & 0.1303 \\ 3719 & 12: 28: 22 & 0.1368 \\ 3720 & 12: 28: 23 & 0.1307 \\ 3721 & 12: 28: 24 & 0.1344 \\ 3722 & 12: 28: 25 & 0.1309 \\ 3723 & 12: 28: 26 & 0.1333 \\ 3724 & 12: 28: 27 & 0.1319 \\ 3725 & 12: 28: 28 & 0.1317 \\ 3726 & 12: 28: 29 & 0.1344 \\ 3727 & 12: 28: 30 & 0.1326 \\ 3728 & 12: 28: 31 & 0.1328 \\ 3729 & 12: 28: 32 & 0.1281 \\ 3730 & 12: 28: 33 & 0.1319 \\ 3731 & 12: 28: 34 & 0.1273 \\ 3732 & 12: 28: 35 & 0.1348 \\ 3733 & 12: 28: 36 & 0.1332 \\ 3734 & 12: 28: 37 & 0.1366 \\ 3735 & 12: 28: 38 & 0.1311 \\ 3736 & 12: 28: 39 & 0.1337 \\ 3737 & 12: 28: 40 & 0.1308 \\ 3738 & 12: 28: 41 & 0.1320 \\ 3739 & 12: 28: 42 & 0.1312 \\ 3740 & 12: 28: 43 & 0.1309 \\ 3741 & 12: 28: 44 & 0.1320 \\ 3742 & 12: 28: 45 & 0.1292\end{array}$


Appendix A - Fuel Data Test 1 NIST102505_1.xls

$\begin{array}{lll}3743 & 12: 28: 46 & 0.1342 \\ 3744 & 12: 28: 47 & 0.1299 \\ 3745 & 12: 28: 48 & 0.1344 \\ 3746 & 12: 28: 49 & 0.1299 \\ 3747 & 12: 28: 50 & 0.1350 \\ 3748 & 12: 28: 51 & 0.1322 \\ 3749 & 12: 28: 52 & 0.1327 \\ 3750 & 12: 28: 53 & 0.1307 \\ 3751 & 12: 28: 54 & 0.1321 \\ 3752 & 12: 28: 55 & 0.1329 \\ 3753 & 12: 28: 56 & 0.1300 \\ 3754 & 12: 28: 57 & 0.1326 \\ 3755 & 12: 28: 58 & 0.1288 \\ 3756 & 12: 28: 59 & 0.1319 \\ 3757 & 12: 29: 00 & 0.1270 \\ 3758 & 12: 29: 01 & 0.1326 \\ 3759 & 12: 29: 02 & 0.1295 \\ 3760 & 12: 29: 03 & 0.1330 \\ 3761 & 12: 29: 04 & 0.1321 \\ 3762 & 12: 29: 05 & 0.1322 \\ 3763 & 12: 29: 06 & 0.1311 \\ 3764 & 12: 29: 07 & 0.1301 \\ 3765 & 12: 29: 08 & 0.1326 \\ 3766 & 12: 29: 09 & 0.1299 \\ 3767 & 12: 29: 10 & 0.1311 \\ 3768 & 12: 29: 11 & 0.1296 \\ 3769 & 12: 29: 12 & 0.1333 \\ 3770 & 12: 29: 13 & 0.1291 \\ 3771 & 12: 29: 14 & 0.1345 \\ 3772 & 12: 29: 15 & 0.1323 \\ 3773 & 12: 29: 16 & 0.1354 \\ 3774 & 12: 29: 17 & 0.1302 \\ 3775 & 12: 29: 18 & 0.1334 \\ 3776 & 12: 29: 19 & 0.1315 \\ 3777 & 12: 29: 20 & 0.1329 \\ 3778 & 12: 29: 21 & 0.1327 \\ 3779 & 12: 29: 22 & 0.1321 \\ 3780 & 12: 29: 23 & 0.1328 \\ 3781 & 12: 29: 24 & 0.1315 \\ 3782 & 12: 29: 25 & 0.1370 \\ 3783 & 12: 29: 26 & 0.1344 \\ 3784 & 12: 29: 27 & 0.1361 \\ 3785 & 12: 29: 28 & 0.1312 \\ 3786 & 12: 29: 29 & 0.1338 \\ 3787 & 12: 29: 30 & 0.1321 \\ 3788 & 12: 29: 31 & 0.1334 \\ 3789 & 12: 29: 32 & 0.1310 \\ 3790 & 12: 29: 33 & 0.1306 \\ 3791 & 12: 29: 34 & 0.1316 \\ 3792 & 12: 29: 35 & 0.1292 \\ 3793 & 12: 29: 36 & 0.1342 \\ 3794 & 12: 29: 37 & 0.1318\end{array}$


Appendix A - Fuel Data Test 1 NIST102505_1.xIs

$\begin{array}{lll}3795 & 12: 29: 38 & 0.1372 \\ 3796 & 12: 29: 39 & 0.1328 \\ 3797 & 12: 29: 40 & 0.1351 \\ 3798 & 12: 29: 41 & 0.1317 \\ 3799 & 12: 29: 42 & 0.1326 \\ 3800 & 12: 29: 43 & 0.1322 \\ 3801 & 12: 29: 44 & 0.1330 \\ 3802 & 12: 29: 45 & 0.1327 \\ 3803 & 12: 29: 46 & 0.1287 \\ 3804 & 12: 29: 47 & 0.1352 \\ 3805 & 12: 29: 48 & 0.1317 \\ 3806 & 12: 29: 49 & 0.1348 \\ 3807 & 12: 29: 50 & 0.1294 \\ 3808 & 12: 29: 51 & 0.1338 \\ 3809 & 12: 29: 52 & 0.1312 \\ 3810 & 12: 29: 53 & 0.1326 \\ 3811 & 12: 29: 54 & 0.1317 \\ 3812 & 12: 29: 55 & 0.1340 \\ 3813 & 12: 29: 56 & 0.1318 \\ 3814 & 12: 29: 57 & 0.1319 \\ 3815 & 12: 29: 58 & 0.1347 \\ 3816 & 12: 29: 59 & 0.1312 \\ 3817 & 12: 30: 00 & 0.1319 \\ 3818 & 12: 30: 01 & 0.1287 \\ 3819 & 12: 30: 02 & 0.1332 \\ 3820 & 12: 30: 03 & 0.1296 \\ 3821 & 12: 30: 04 & 0.1354 \\ 3822 & 12: 30: 05 & 0.1319 \\ 3823 & 12: 30: 06 & 0.1349 \\ 3824 & 12: 30: 07 & 0.1301 \\ 3825 & 12: 30: 08 & 0.1338 \\ 3826 & 12: 30: 09 & 0.1311 \\ 3827 & 12: 30: 10 & 0.1321 \\ 3828 & 12: 30: 11 & 0.1315 \\ 3829 & 12: 30: 12 & 0.1340 \\ 3830 & 12: 30: 13 & 0.1340 \\ 3831 & 12: 30: 14 & 0.1280 \\ 3832 & 12: 30: 15 & 0.1347 \\ 3833 & 12: 30: 16 & 0.1327 \\ 3834 & 12: 30: 17 & 0.1379 \\ 3835 & 12: 30: 18 & 0.1326 \\ 3836 & 12: 30: 19 & 0.1393 \\ 3837 & 12: 30: 20 & 0.1351 \\ 3838 & 12: 30: 21 & 0.1348 \\ 3839 & 12: 30: 22 & 0.1312 \\ 3840 & 12: 30: 23 & 0.1317 \\ 3841 & 12: 30: 24 & 0.1319 \\ 3842 & 12: 30: 25 & 0.1298 \\ 3843 & 12: 30: 26 & 0.1310 \\ 3844 & 12: 30: 27 & 0.1285 \\ 3845 & 12: 30: 28 & 0.1315 \\ 3846 & 12: 30: 29 & 0.1275\end{array}$


Appendix A - Fuel Data Test 1 NIST102505_1.xIs

$\begin{array}{lll}3847 & 12: 30: 30 & 0.1339 \\ 3848 & 12: 30: 31 & 0.1304 \\ 3849 & 12: 30: 32 & 0.1344 \\ 3850 & 12: 30: 33 & 0.1299 \\ 3851 & 12: 30: 34 & 0.1326 \\ 3852 & 12: 30: 35 & 0.1296 \\ 3853 & 12: 30: 36 & 0.1288 \\ 3854 & 12: 30: 37 & 0.1294 \\ 3855 & 12: 30: 38 & 0.1295 \\ 3856 & 12: 30: 39 & 0.1319 \\ 3857 & 12: 30: 40 & 0.1262 \\ 3858 & 12: 30: 41 & 0.1324 \\ 3860 & 12: 30: 43 & 0.1291 \\ 3860 & 12: 30: 43 & 0.1333 \\ 3861 & 12: 30: 44 & 0.1302 \\ 3862 & 12: 30: 45 & 0.1327 \\ 3863 & 12: 30: 46 & 0.1310 \\ 3864 & 12: 30: 47 & 0.1313 \\ 3865 & 12: 30: 48 & 0.1336 \\ 3866 & 12: 30: 49 & 0.1337 \\ 3867 & 12: 30: 50 & 0.1350 \\ 3868 & 12: 30: 51 & 0.1321 \\ 3869 & 12: 30: 52 & 0.1375 \\ 3870 & 12: 30: 53 & 0.1319 \\ 3871 & 12: 30: 54 & 0.1357 \\ 3872 & 12: 30: 55 & 0.1333 \\ 3873 & 12: 30: 56 & 0.1390 \\ 3874 & 12: 30: 57 & 0.1350 \\ 3875 & 12: 30: 58 & 0.1382 \\ 3876 & 12: 30: 59 & 0.1357 \\ 3877 & 12: 31: 00 & 0.1353 \\ 3878 & 12: 31: 01 & 0.1313 \\ 3879 & 12: 31: 02 & 0.1333 \\ 3880 & 12: 31: 03 & 0.1322 \\ 3881 & 12: 31: 04 & 0.1287 \\ 3882 & 12: 31: 05 & 0.1338 \\ 3883 & 12: 31: 06 & 0.1333 \\ 3884 & 12: 31: 07 & 0.1378 \\ 3885 & 12: 31: 08 & 0.1320 \\ 3886 & 12: 31: 09 & 0.1365 \\ 3887 & 12: 31: 10 & 0.1337 \\ 3888 & 12: 31: 11 & 0.1357 \\ 3889 & 12: 31: 12 & 0.1317 \\ 3890 & 12: 31: 13 & 0.1326 \\ 3891 & 12: 31: 14 & 0.1317 \\ 3892 & 12: 31: 15 & 0.1307 \\ 3893 & 12: 31: 16 & 0.1346 \\ 3894 & 12: 31: 17 & 0.1303 \\ 3895 & 12: 31: 18 & 0.1336 \\ 3896 & 12: 31: 19 & 0.1292 \\ 3897 & 12: 31: 20 & 0.1338 \\ 3898 & 12: 31: 21 & 0.1295\end{array}$


Appendix A - Fuel Data Test 1 NIST102505_1.xIs

$\begin{array}{lll}3899 & 12: 31: 22 & 0.1335 \\ 3900 & 12: 31: 23 & 0.1313 \\ 3901 & 12: 31: 24 & 0.1332 \\ 3902 & 12: 31: 25 & 0.1314 \\ 3903 & 12: 31: 26 & 0.1290 \\ 3904 & 12: 31: 27 & 0.1341 \\ 3905 & 12: 31: 28 & 0.1317 \\ 3906 & 12: 31: 29 & 0.1342 \\ 3907 & 12: 31: 30 & 0.1287 \\ 3908 & 12: 31: 31 & 0.1324 \\ 3909 & 12: 31: 32 & 0.1281 \\ 3910 & 12: 31: 33 & 0.1327 \\ 3911 & 12: 31: 34 & 0.1301 \\ 3912 & 12: 31: 35 & 0.1342 \\ 3913 & 12: 31: 36 & 0.1317 \\ 3914 & 12: 31: 37 & 0.1333 \\ 3915 & 12: 31: 38 & 0.1334 \\ 3916 & 12: 31: 39 & 0.1325 \\ 3917 & 12: 31: 40 & 0.1354 \\ 3918 & 12: 31: 41 & 0.1327 \\ 3919 & 12: 31: 42 & 0.1364 \\ 3920 & 12: 31: 43 & 0.1309 \\ 3921 & 12: 31: 44 & 0.1333 \\ 3922 & 12: 31: 45 & 0.1288 \\ 3923 & 12: 31: 46 & 0.1345 \\ 3924 & 12: 31: 47 & 0.1308 \\ 3925 & 12: 31: 48 & 0.1334 \\ 3926 & 12: 31: 49 & 0.1305 \\ 3927 & 12: 31: 50 & 0.1328 \\ 3928 & 12: 31: 51 & 0.1321 \\ 3929 & 12: 31: 52 & 0.1310 \\ 3930 & 12: 31: 53 & 0.1346 \\ 3931 & 12: 31: 54 & 0.1318 \\ 3932 & 12: 31: 55 & 0.1336 \\ 3933 & 12: 31: 56 & 0.1270 \\ 3934 & 12: 31: 57 & 0.1327 \\ 3935 & 12: 31: 58 & 0.1286 \\ 3936 & 12: 31: 59 & 0.1324 \\ 3937 & 12: 32: 00 & 0.1303 \\ 3938 & 12: 32: 01 & 0.1328 \\ 3939 & 12: 32: 02 & 0.1292 \\ 3940 & 12: 32: 03 & 0.1318 \\ 3941 & 12: 32: 04 & 0.1331 \\ 3942 & 12: 32: 05 & 0.1311 \\ 3943 & 12: 32: 06 & 0.1319 \\ 3944 & 12: 32: 07 & 0.1295 \\ 3945 & 12: 32: 08 & 0.1312 \\ 3946 & 12: 32: 09 & 0.1271 \\ 3947 & 12: 32: 10 & 0.1344 \\ 3948 & 12: 32: 11 & 0.1305 \\ 3949 & 12: 32: 12 & 0.1341 \\ 3950 & 12: 32: 13 & 0.1296\end{array}$


Appendix A - Fuel Data Test 1 NIST102505_1.xls

$\begin{array}{lll}3951 & 12: 32: 14 & 0.1337 \\ 3952 & 12: 32: 15 & 0.1300 \\ 3953 & 12: 32: 16 & 0.1315 \\ 3954 & 12: 32: 17 & 0.1308 \\ 3955 & 12: 32: 18 & 0.1304 \\ 3956 & 12: 32: 19 & 0.1308 \\ 3957 & 12: 32: 20 & 0.1274 \\ 3958 & 12: 32: 21 & 0.1327 \\ 3959 & 12: 32: 22 & 0.1285 \\ 3960 & 12: 32: 23 & 0.1325 \\ 3961 & 12: 32: 24 & 0.1291 \\ 3962 & 12: 32: 25 & 0.1343 \\ 3963 & 12: 32: 26 & 0.1320 \\ 3964 & 12: 32: 27 & 0.1344 \\ 3965 & 12: 32: 28 & 0.1336 \\ 3966 & 12: 32: 29 & 0.1338 \\ 3967 & 12: 32: 30 & 0.1342 \\ 3968 & 12: 32: 31 & 0.1317 \\ 3969 & 12: 32: 32 & 0.1369 \\ 3970 & 12: 32: 33 & 0.1322 \\ 3971 & 12: 32: 34 & 0.1337 \\ 3972 & 12: 32: 35 & 0.1278 \\ 3973 & 12: 32: 36 & 0.1330 \\ 3974 & 12: 32: 37 & 0.1294 \\ 3975 & 12: 32: 38 & 0.1329 \\ 3976 & 12: 32: 39 & 0.1302 \\ 3977 & 12: 32: 40 & 0.1333 \\ 3978 & 12: 32: 41 & 0.1310 \\ 3979 & 12: 32: 42 & 0.1306 \\ 3980 & 12: 32: 43 & 0.1323 \\ 3981 & 12: 32: 44 & 0.1302 \\ 3982 & 12: 32: 45 & 0.1324 \\ 3983 & 12: 32: 46 & 0.1288 \\ 3984 & 12: 32: 47 & 0.1327 \\ 3985 & 12: 32: 48 & 0.1287 \\ 3986 & 12: 32: 49 & 0.1323 \\ 3987 & 12: 32: 50 & 0.1307 \\ 3988 & 12: 32: 51 & 0.1356 \\ 3989 & 12: 32: 52 & 0.1299 \\ 3990 & 12: 32: 53 & 0.1331 \\ 3991 & 12: 32: 54 & 0.1321 \\ 3992 & 12: 32: 55 & 0.1320 \\ 3993 & 12: 32: 56 & 0.1305 \\ 3994 & 12: 32: 57 & 0.1299 \\ 3995 & 12: 32: 58 & 0.1318 \\ 3996 & 12: 32: 59 & 0.1284 \\ 3997 & 12: 33: 00 & 0.1325 \\ 3998 & 12: 33: 01 & 0.1295 \\ 3999 & 12: 33: 02 & 0.1338 \\ 4000 & 12: 33: 03 & 0.1307 \\ 4001 & 12: 33: 04 & 0.1325 \\ 4002 & 12: 33: 05 & 0.1298\end{array}$


Appendix A - Fuel Data Test 1 NIST102505_1.xls

$\begin{array}{lll}4003 & 12: 33: 06 & 0.1305 \\ 4004 & 12: 33: 07 & 0.1301 \\ 4005 & 12: 33: 08 & 0.1303 \\ 4006 & 12: 33: 09 & 0.1318 \\ 4007 & 12: 33: 10 & 0.1292 \\ 4008 & 12: 33: 11 & 0.1317 \\ 4009 & 12: 33: 12 & 0.1277 \\ 4010 & 12: 33: 13 & 0.1324 \\ 4011 & 12: 33: 14 & 0.1273 \\ 4012 & 12: 33: 15 & 0.1324 \\ 4013 & 12: 33: 16 & 0.1297 \\ 4014 & 12: 33: 17 & 0.1320 \\ 4015 & 12: 33: 18 & 0.1320 \\ 4016 & 12: 33: 19 & 0.1322 \\ 4017 & 12: 33: 20 & 0.1325 \\ 4018 & 12: 33: 21 & 0.1313 \\ 4019 & 12: 33: 22 & 0.1352 \\ 4020 & 12: 33: 23 & 0.1317 \\ 4021 & 12: 33: 24 & 0.1346 \\ 4022 & 12: 33: 25 & 0.1310 \\ 4023 & 12: 33: 26 & 0.1360 \\ 4024 & 12: 33: 27 & 0.1315 \\ 4025 & 12: 33: 28 & 0.1354 \\ 4026 & 12: 33: 29 & 0.1363 \\ 4027 & 12: 33: 30 & 0.1370 \\ 4028 & 12: 33: 31 & 0.1344 \\ 4029 & 12: 33: 32 & 0.1325 \\ 4030 & 12: 33: 33 & 0.1353 \\ 4031 & 12: 33: 34 & 0.1314 \\ 4032 & 12: 33: 35 & 0.1318 \\ 4033 & 12: 33: 36 & 0.1298 \\ 4034 & 12: 33: 37 & 0.1333 \\ 4035 & 12: 33: 38 & 0.1298 \\ 4036 & 12: 33: 39 & 0.1332 \\ 4037 & 12: 33: 40 & 0.1320 \\ 4038 & 12: 33: 41 & 0.1374 \\ 4039 & 12: 33: 42 & 0.1339 \\ 4040 & 12: 33: 43 & 0.1369 \\ 4041 & 12: 33: 44 & 0.1342 \\ 4042 & 12: 33: 45 & 0.1357 \\ 4043 & 12: 33: 46 & 0.1365 \\ 4044 & 12: 33: 47 & 0.1333 \\ 4045 & 12: 33: 48 & 0.1322 \\ 4046 & 12: 33: 49 & 0.1280 \\ 4047 & 12: 33: 50 & 0.1329 \\ 4048 & 12: 33: 51 & 0.1284 \\ 4049 & 12: 33: 52 & 0.1332 \\ 4050 & 12: 33: 53 & 0.1288 \\ 4051 & 12: 33: 54 & 0.1336 \\ 4052 & 12: 33: 55 & 0.1308 \\ 4053 & 12: 33: 56 & 0.1324 \\ 4054 & 12: 33: 57 & 0.1332\end{array}$


Appendix A - Fuel Data Test 1 NIST102505_1.xls

$\begin{array}{lll}4055 & 12: 33: 58 & 0.1314 \\ 4056 & 12: 33: 59 & 0.1321 \\ 4057 & 12: 34: 00 & 0.1293 \\ 4058 & 12: 34: 01 & 0.1322 \\ 4059 & 12: 34: 02 & 0.1310 \\ 4060 & 12: 34: 03 & 0.1345 \\ 4061 & 12: 34: 04 & 0.1302 \\ 4062 & 12: 34: 05 & 0.1367 \\ 4063 & 12: 34: 06 & 0.1318 \\ 4064 & 12: 34: 07 & 0.1356 \\ 4065 & 12: 34: 08 & 0.1334 \\ 4066 & 12: 34: 09 & 0.1357 \\ 4067 & 12: 34: 10 & 0.1329 \\ 4068 & 12: 34: 11 & 0.1316 \\ 4069 & 12: 34: 12 & 0.1292 \\ 4070 & 12: 34: 13 & 0.1290 \\ 4071 & 12: 34: 14 & 0.1311 \\ 4072 & 12: 34: 15 & 0.1268 \\ 4073 & 12: 34: 16 & 0.1329 \\ 4074 & 12: 34: 17 & 0.1288 \\ 4075 & 12: 34: 18 & 0.1333 \\ 4076 & 12: 34: 19 & 0.1303 \\ 4077 & 12: 34: 20 & 0.1342 \\ 4078 & 12: 34: 21 & 0.1311 \\ 4079 & 12: 34: 22 & 0.1317 \\ 4080 & 12: 34: 23 & 0.1299 \\ 4081 & 12: 34: 24 & 0.1301 \\ 4082 & 12: 34: 25 & 0.1299 \\ 4083 & 12: 34: 26 & 0.1270 \\ 4084 & 12: 34: 27 & 0.1332 \\ 4085 & 12: 34: 28 & 0.1297 \\ 4086 & 12: 34: 29 & 0.1341 \\ 4087 & 12: 34: 30 & 0.1310 \\ 4088 & 12: 34: 31 & 0.1363 \\ 4089 & 12: 34: 32 & 0.1330 \\ 4090 & 12: 34: 33 & 0.1348 \\ 4091 & 12: 34: 34 & 0.1320 \\ 4092 & 12: 34: 35 & 0.1318 \\ 4093 & 12: 34: 36 & 0.1333 \\ 4094 & 12: 34: 37 & 0.1319 \\ 4095 & 12: 34: 38 & 0.1352 \\ 4096 & 12: 34: 39 & 0.1310 \\ 4097 & 12: 34: 40 & 0.1332 \\ 4098 & 12: 34: 41 & 0.1306 \\ 4099 & 12: 34: 42 & 0.1372 \\ 4100 & 12: 34: 43 & 0.1327 \\ 4101 & 12: 34: 44 & 0.1361 \\ 4102 & 12: 34: 45 & 0.1362 \\ 4103 & 12: 34: 46 & 0.1347 \\ 4104 & 12: 34: 47 & 0.1317 \\ 4105 & 12: 34: 48 & 0.1300 \\ 4106 & 12: 34: 49 & 0.1333\end{array}$


Appendix A - Fuel Data Test 1 NIST102505_1.xls

$\begin{array}{lll}4107 & 12: 34: 50 & 0.1304 \\ 4108 & 12: 34: 51 & 0.1332 \\ 4109 & 12: 34: 52 & 0.1277 \\ 4110 & 12: 34: 53 & 0.1335 \\ 4111 & 12: 34: 54 & 0.1287 \\ 4112 & 12: 34: 55 & 0.1320 \\ 4113 & 12: 34: 56 & 0.1284 \\ 4114 & 12: 34: 57 & 0.1329 \\ 4115 & 12: 34: 58 & 0.1290 \\ 4116 & 12: 34: 59 & 0.1319 \\ 4117 & 12: 35: 00 & 0.1326 \\ 4118 & 12: 35: 01 & 0.1324 \\ 4119 & 12: 35: 02 & 0.1318 \\ 4120 & 12: 35: 03 & 0.1309 \\ 4121 & 12: 35: 04 & 0.1318 \\ 4122 & 12: 35: 05 & 0.1279 \\ 4123 & 12: 35: 06 & 0.1323 \\ 4124 & 12: 35: 07 & 0.1300 \\ 4125 & 12: 35: 08 & 0.1338 \\ 4126 & 12: 35: 09 & 0.1285 \\ 4127 & 12: 35: 10 & 0.1338 \\ 4128 & 12: 35: 11 & 0.1309 \\ 4129 & 12: 35: 12 & 0.1335 \\ 4130 & 12: 35: 13 & 0.1291 \\ 4131 & 12: 35: 14 & 0.1303 \\ 4132 & 12: 35: 15 & 0.1295 \\ 4133 & 12: 35: 16 & 0.1284 \\ 4134 & 12: 35: 17 & 0.1333 \\ 4135 & 12: 35: 18 & 0.1293 \\ 4136 & 12: 35: 19 & 0.1319 \\ 4137 & 12: 35: 20 & 0.1275 \\ 4138 & 12: 35: 21 & 0.1325 \\ 4139 & 12: 35: 22 & 0.1286 \\ 4140 & 12: 35: 23 & 0.1314 \\ 4141 & 12: 35: 24 & 0.1310 \\ 4142 & 12: 35: 25 & 0.1347 \\ 4143 & 12: 35: 26 & 0.1307 \\ 4144 & 12: 35: 27 & 0.1307 \\ 4145 & 12: 35: 28 & 0.1306 \\ 4146 & 12: 35: 29 & 0.1287 \\ 4147 & 12: 35: 30 & 0.1326 \\ 4148 & 12: 35: 31 & 0.1303 \\ 4149 & 12: 35: 32 & 0.1325 \\ 4150 & 12: 35: 33 & 0.1285 \\ 4151 & 12: 35: 34 & 0.1338 \\ 4152 & 12: 35: 35 & 0.1312 \\ 4153 & 12: 35: 36 & 0.1345 \\ 4154 & 12: 35: 37 & 0.1288 \\ 4155 & 12: 35: 38 & 0.1328 \\ 4156 & 12: 35: 39 & 0.1308 \\ 4157 & 12: 35: 40 & 0.1293 \\ 4158 & 12: 35: 41 & 0.1329\end{array}$


Appendix A - Fuel Data Test 1 NIST102505_1.xIs

$\begin{array}{lll}4159 & 12: 35: 42 & 0.1305 \\ 4160 & 12: 35: 43 & 0.1334 \\ 4161 & 12: 35: 44 & 0.1290 \\ 4162 & 12: 35: 45 & 0.1333 \\ 4163 & 12: 35: 46 & 0.1295 \\ 4164 & 12: 35: 47 & 0.1336 \\ 4165 & 12: 35: 48 & 0.1320 \\ 4166 & 12: 35: 49 & 0.1332 \\ 4167 & 12: 35: 50 & 0.1318 \\ 4168 & 12: 35: 51 & 0.1307 \\ 4169 & 12: 35: 52 & 0.1317 \\ 4170 & 12: 35: 53 & 0.1300 \\ 4171 & 12: 35: 54 & 0.1301 \\ 4172 & 12: 35: 55 & 0.1283 \\ 4173 & 12: 35: 56 & 0.1313 \\ 4174 & 12: 35: 57 & 0.1272 \\ 4175 & 12: 35: 58 & 0.1308 \\ 4176 & 12: 35: 59 & 0.1297 \\ 4177 & 12: 36: 00 & 0.1340 \\ 4178 & 12: 36: 01 & 0.1288 \\ 4179 & 12: 36: 02 & 0.1327 \\ 4180 & 12: 36: 03 & 0.1301 \\ 4181 & 12: 36: 04 & 0.1302 \\ 4182 & 12: 36: 05 & 0.1296 \\ 4183 & 12: 36: 06 & 0.1298 \\ 4184 & 12: 36: 07 & 0.1312 \\ 4185 & 12: 36: 08 & 0.1268 \\ 4186 & 12: 36: 09 & 0.1326 \\ 4187 & 12: 36: 10 & 0.1297 \\ 4188 & 12: 36: 11 & 0.1329 \\ 4189 & 12: 36: 12 & 0.1316 \\ 4190 & 12: 36: 13 & 0.1332 \\ 4191 & 12: 36: 14 & 0.1317 \\ 4192 & 12: 36: 15 & 0.1321 \\ 4193 & 12: 36: 16 & 0.1325 \\ 4194 & 12: 36: 17 & 0.1313 \\ 4195 & 12: 36: 18 & 0.1318 \\ 4196 & 12: 36: 19 & 0.1290 \\ 4197 & 12: 36: 20 & 0.1321 \\ 4198 & 12: 36: 21 & 0.1273 \\ 4199 & 12: 36: 22 & 0.1307 \\ 4200 & 12: 36: 23 & 0.1295 \\ 4201 & 12: 36: 24 & 0.1326 \\ 4202 & 12: 36: 25 & 0.1295 \\ 4203 & 12: 36: 26 & 0.1336 \\ 4204 & 12: 36: 27 & 0.1314 \\ 4205 & 12: 36: 28 & 0.1338 \\ 4206 & 12: 36: 29 & 0.1294 \\ 4207 & 12: 36: 30 & 0.1299 \\ 4208 & 12: 36: 31 & 0.1303 \\ 4209 & 12: 36: 32 & 0.1322 \\ 4210 & 12: 36: 33 & 0.1342\end{array}$


Appendix A - Fuel Data Test 1 NIST102505_1.xls

$\begin{array}{lll}4211 & 12: 36: 34 & 0.1328 \\ 4212 & 12: 36: 35 & 0.1333 \\ 4213 & 12: 36: 36 & 0.1265 \\ 4214 & 12: 36: 37 & 0.1323 \\ 4215 & 12: 36: 38 & 0.1296 \\ 4216 & 12: 36: 39 & 0.1335 \\ 4217 & 12: 36: 40 & 0.1336 \\ 4218 & 12: 36: 41 & 0.1311 \\ 4219 & 12: 36: 42 & 0.1299 \\ 4220 & 12: 36: 43 & 0.1287 \\ 4221 & 12: 36: 44 & 0.1350 \\ 4222 & 12: 36: 45 & 0.1314 \\ 4223 & 12: 36: 46 & 0.1349 \\ 4224 & 12: 36: 47 & 0.1297 \\ 4225 & 12: 36: 48 & 0.1326 \\ 4226 & 12: 36: 49 & 0.1282 \\ 4227 & 12: 36: 50 & 0.1330 \\ 4228 & 12: 36: 51 & 0.1299 \\ 4229 & 12: 36: 52 & 0.1349 \\ 4230 & 12: 36: 53 & 0.1300 \\ 4231 & 12: 36: 54 & 0.1299 \\ 4232 & 12: 36: 55 & 0.1330 \\ 4233 & 12: 36: 56 & 0.1311 \\ 4234 & 12: 36: 57 & 0.1323 \\ 4235 & 12: 36: 58 & 0.1290 \\ 4236 & 12: 36: 59 & 0.1348 \\ 4237 & 12: 37: 00 & 0.1331 \\ 4238 & 12: 37: 01 & 0.1365 \\ 4239 & 12: 37: 02 & 0.1308 \\ 4240 & 12: 37: 03 & 0.1382 \\ 4241 & 12: 37: 04 & 0.1354 \\ 4242 & 12: 37: 05 & 0.1371 \\ 4243 & 12: 37: 06 & 0.1305 \\ 4244 & 12: 37: 07 & 0.1323 \\ 4245 & 12: 37: 08 & 0.1308 \\ 4246 & 12: 37: 09 & 0.1299 \\ 4247 & 12: 37: 10 & 0.1305 \\ 4248 & 12: 37: 11 & 0.1292 \\ 4249 & 12: 37: 12 & 0.1310 \\ 4250 & 12: 37: 13 & 0.1286 \\ 4251 & 12: 37: 14 & 0.1331 \\ 4252 & 12: 37: 15 & 0.1303 \\ 4253 & 12: 37: 16 & 0.1345 \\ 4254 & 12: 37: 17 & 0.1302 \\ 4255 & 12: 37: 18 & 0.1327 \\ 4256 & 12: 37: 19 & 0.1319 \\ 4257 & 12: 37: 20 & 0.1337 \\ 4258 & 12: 37: 21 & 0.1323 \\ 4259 & 12: 37: 22 & 0.1328 \\ 4260 & 12: 37: 23 & 0.1328 \\ 4261 & 12: 37: 24 & 0.1293 \\ 4262 & 12: 37: 25 & 0.1344\end{array}$


Appendix A - Fuel Data Test 1 NIST102505_1.xls

$\begin{array}{lll}4263 & 12: 37: 26 & 0.1328 \\ 4264 & 12: 37: 27 & 0.1341 \\ 4265 & 12: 37: 28 & 0.1293 \\ 4266 & 12: 37: 29 & 0.1337 \\ 4267 & 12: 37: 30 & 0.1329 \\ 4268 & 12: 37: 31 & 0.1343 \\ 4269 & 12: 37: 32 & 0.1311 \\ 4270 & 12: 37: 33 & 0.1319 \\ 4271 & 12: 37: 34 & 0.1303 \\ 4272 & 12: 37: 35 & 0.1293 \\ 4273 & 12: 37: 36 & 0.1357 \\ 4274 & 12: 37: 37 & 0.1321 \\ 4275 & 12: 37: 38 & 0.1344 \\ 4276 & 12: 37: 39 & 0.1309 \\ 4277 & 12: 37: 40 & 0.1359 \\ 4278 & 12: 37: 41 & 0.1310 \\ 4279 & 12: 37: 42 & 0.1333 \\ 4280 & 12: 37: 43 & 0.1311 \\ 4281 & 12: 37: 44 & 0.1330 \\ 4282 & 12: 37: 45 & 0.1305 \\ 4283 & 12: 37: 46 & 0.1293 \\ 4284 & 12: 37: 47 & 0.1327 \\ 4285 & 12: 37: 48 & 0.1300 \\ 4286 & 12: 37: 49 & 0.1321 \\ 4287 & 12: 37: 50 & 0.1283 \\ 4288 & 12: 37: 51 & 0.1312 \\ 4289 & 12: 37: 52 & 0.1283 \\ 4290 & 12: 37: 53 & 0.1322 \\ 4291 & 12: 37: 54 & 0.1314 \\ 4292 & 12: 37: 55 & 0.1339 \\ 4293 & 12: 37: 56 & 0.1294 \\ 4294 & 12: 37: 57 & 0.1319 \\ 4295 & 12: 37: 58 & 0.1304 \\ 4296 & 12: 37: 59 & 0.1294 \\ 4297 & 12: 38: 00 & 0.1301 \\ 4298 & 12: 38: 01 & 0.1302 \\ 4299 & 12: 38: 02 & 0.1349 \\ 4300 & 12: 38: 03 & 0.1281 \\ 4301 & 12: 38: 04 & 0.1338 \\ 4302 & 12: 38: 05 & 0.1306 \\ 4303 & 12: 38: 06 & 0.1348 \\ 4304 & 12: 38: 07 & 0.1308 \\ 4305 & 12: 38: 08 & 0.1341 \\ 4306 & 12: 38: 09 & 0.1307 \\ 4307 & 12: 38: 10 & 0.1304 \\ 4308 & 12: 38: 11 & 0.1311 \\ 4309 & 12: 38: 12 & 0.1298 \\ 4310 & 12: 38: 13 & 0.1336 \\ 4311 & 12: 38: 14 & 0.1296 \\ 4312 & 12: 38: 15 & 0.1330 \\ 4313 & 12: 38: 16 & 0.1287 \\ 4314 & 12: 38: 17 & 0.1319\end{array}$


Appendix A - Fuel Data Test 1 NIST102505_1.xls

$\begin{array}{lll}4315 & 12: 38: 18 & 0.1283 \\ 4316 & 12: 38: 19 & 0.1325 \\ 4317 & 12: 38: 20 & 0.1295 \\ 4318 & 12: 38: 21 & 0.1320 \\ 4319 & 12: 38: 22 & 0.1336 \\ 4320 & 12: 38: 23 & 0.1342 \\ 4321 & 12: 38: 24 & 0.1344 \\ 4322 & 12: 38: 25 & 0.1317 \\ 4323 & 12: 38: 26 & 0.1371 \\ 4324 & 12: 38: 27 & 0.1327 \\ 4325 & 12: 38: 28 & 0.1348 \\ 4326 & 12: 38: 29 & 0.1282 \\ 4327 & 12: 38: 30 & 0.1334 \\ 4328 & 12: 38: 31 & 0.1298 \\ 4329 & 12: 38: 32 & 0.1329 \\ 4330 & 12: 38: 33 & 0.1308 \\ 4331 & 12: 38: 34 & 0.1325 \\ 4332 & 12: 38: 35 & 0.1289 \\ 4333 & 12: 38: 36 & 0.1305 \\ 4334 & 12: 38: 37 & 0.1323 \\ 4335 & 12: 38: 38 & 0.1322 \\ 4336 & 12: 38: 39 & 0.1335 \\ 4337 & 12: 38: 40 & 0.1310 \\ 4338 & 12: 38: 41 & 0.1345 \\ 4339 & 12: 38: 42 & 0.1300 \\ 4340 & 12: 38: 43 & 0.1324 \\ 4341 & 12: 38: 44 & 0.1318 \\ 4342 & 12: 38: 45 & 0.1344 \\ 4343 & 12: 38: 46 & 0.1316 \\ 4344 & 12: 38: 47 & 0.1321 \\ 4345 & 12: 38: 48 & 0.1324 \\ 4346 & 12: 38: 49 & 0.1312 \\ 4347 & 12: 38: 50 & 0.1305 \\ 4348 & 12: 38: 51 & 0.1282 \\ 4349 & 12: 38: 52 & 0.1323 \\ 4350 & 12: 38: 53 & 0.1280 \\ 4351 & 12: 38: 54 & 0.1317 \\ 4352 & 12: 38: 55 & 0.1297 \\ 4353 & 12: 38: 56 & 0.1331 \\ 4354 & 12: 38: 57 & 0.1291 \\ 4355 & 12: 38: 58 & 0.1334 \\ 4356 & 12: 38: 59 & 0.1317 \\ 4357 & 12: 39: 00 & 0.1347 \\ 4358 & 12: 39: 01 & 0.1313 \\ 4359 & 12: 39: 02 & 0.1301 \\ 4360 & 12: 39: 03 & 0.1296 \\ 4361 & 12: 39: 04 & 0.1282 \\ 4362 & 12: 39: 05 & 0.1342 \\ 4363 & 12: 39: 06 & 0.1313 \\ 4364 & 12: 39: 07 & 0.1361 \\ 4365 & 12: 39: 08 & 0.1307 \\ 4366 & 12: 39: 09 & 0.1365\end{array}$


Appendix A - Fuel Data Test 1 NIST102505_1.xls

$\begin{array}{lll}4367 & 12: 39: 10 & 0.1339 \\ 4368 & 12: 39: 11 & 0.1368 \\ 4369 & 12: 39: 12 & 0.1315 \\ 4370 & 12: 39: 13 & 0.1333 \\ 4371 & 12: 39: 14 & 0.1306 \\ 4372 & 12: 39: 15 & 0.1302 \\ 4373 & 12: 39: 16 & 0.1298 \\ 4374 & 12: 39: 17 & 0.1288 \\ 4375 & 12: 39: 18 & 0.1313 \\ 4376 & 12: 39: 19 & 0.1274 \\ 4377 & 12: 39: 20 & 0.1327 \\ 4378 & 12: 39: 21 & 0.1300 \\ 4379 & 12: 39: 22 & 0.1336 \\ 4380 & 12: 39: 23 & 0.1300 \\ 4381 & 12: 39: 24 & 0.1338 \\ 4382 & 12: 39: 25 & 0.1295 \\ 4383 & 12: 39: 26 & 0.1306 \\ 4384 & 12: 39: 27 & 0.1286 \\ 4385 & 12: 39: 28 & 0.1288 \\ 4386 & 12: 39: 29 & 0.1300 \\ 4387 & 12: 39: 30 & 0.1273 \\ 4388 & 12: 39: 31 & 0.1330 \\ 4389 & 12: 39: 32 & 0.1306 \\ 4390 & 12: 39: 33 & 0.1335 \\ 4391 & 12: 39: 34 & 0.1293 \\ 4392 & 12: 39: 35 & 0.1357 \\ 4393 & 12: 39: 36 & 0.1333 \\ 4394 & 12: 39: 37 & 0.1352 \\ 4395 & 12: 39: 38 & 0.1309 \\ 4396 & 12: 39: 39 & 0.1321 \\ 4397 & 12: 39: 40 & 0.1310 \\ 4398 & 12: 39: 41 & 0.1295 \\ 4399 & 12: 39: 42 & 0.1314 \\ 4400 & 12: 39: 43 & 0.1305 \\ 4401 & 12: 39: 44 & 0.1319 \\ 4402 & 12: 39: 45 & 0.1278 \\ 4403 & 12: 39: 46 & 0.1331 \\ 4404 & 12: 39: 47 & 0.1301 \\ 4405 & 12: 39: 48 & 0.1347 \\ 4406 & 12: 39: 49 & 0.1316 \\ 4407 & 12: 39: 50 & 0.1340 \\ 4408 & 12: 39: 51 & 0.1303 \\ 4409 & 12: 39: 52 & 0.1304 \\ 4410 & 12: 39: 53 & 0.1325 \\ 4411 & 12: 39: 54 & 0.1309 \\ 4412 & 12: 39: 55 & 0.1310 \\ 4413 & 12: 39: 56 & 0.1268 \\ 4414 & 12: 39: 57 & 0.1328 \\ 4415 & 12: 39: 58 & 0.1311 \\ 4416 & 12: 39: 59 & 0.1356 \\ 4417 & 12: 40: 00 & 0.1304 \\ 4418 & 12: 40: 01 & 0.1371\end{array}$


Appendix A - Fuel Data Test 1 NIST102505_1.xls

$\begin{array}{lll}4419 & 12: 40: 02 & 0.1303 \\ 4420 & 12: 40: 03 & 0.1323 \\ 4421 & 12: 40: 04 & 0.1322 \\ 4422 & 12: 40: 05 & 0.1310 \\ 4423 & 12: 40: 06 & 0.1314 \\ 4424 & 12: 40: 07 & 0.1283 \\ 4425 & 12: 40: 08 & 0.1321 \\ 4426 & 12: 40: 09 & 0.1282 \\ 4427 & 12: 40: 10 & 0.1315 \\ 4428 & 12: 40: 11 & 0.1298 \\ 4429 & 12: 40: 12 & 0.1338 \\ 4430 & 12: 40: 13 & 0.1331 \\ 4431 & 12: 40: 14 & 0.1366 \\ 4432 & 12: 40: 15 & 0.1369 \\ 4433 & 12: 40: 16 & 0.1362 \\ 4434 & 12: 40: 17 & 0.1327 \\ 4435 & 12: 40: 18 & 0.1310 \\ 4436 & 12: 40: 19 & 0.1328 \\ 4437 & 12: 40: 20 & 0.1300 \\ 4438 & 12: 40: 21 & 0.1326 \\ 4439 & 12: 40: 22 & 0.1306 \\ 4440 & 12: 40: 23 & 0.1334 \\ 4441 & 12: 40: 24 & 0.1297 \\ 4442 & 12: 40: 25 & 0.1342 \\ 4443 & 12: 40: 26 & 0.1334 \\ 4444 & 12: 40: 27 & 0.1337 \\ 4445 & 12: 40: 28 & 0.1305 \\ 4446 & 12: 40: 29 & 0.1313 \\ 4447 & 12: 40: 30 & 0.1329 \\ 4448 & 12: 40: 31 & 0.1315 \\ 4449 & 12: 40: 32 & 0.1314 \\ 4450 & 12: 40: 33 & 0.1296 \\ 4451 & 12: 40: 34 & 0.1316 \\ 4452 & 12: 40: 35 & 0.1279 \\ 4453 & 12: 40: 36 & 0.1329 \\ 4454 & 12: 40: 37 & 0.1304 \\ 4455 & 12: 40: 38 & 0.1343 \\ 4456 & 12: 40: 39 & 0.1296 \\ 4457 & 12: 40: 40 & 0.1331 \\ 4458 & 12: 40: 41 & 0.1302 \\ 4459 & 12: 40: 42 & 0.1309 \\ 4460 & 12: 40: 43 & 0.1291 \\ 4461 & 12: 40: 44 & 0.1328 \\ 4462 & 12: 40: 45 & 0.1334 \\ 4463 & 12: 40: 46 & 0.1328 \\ 4464 & 12: 40: 47 & 0.1344 \\ 4465 & 12: 40: 48 & 0.1303 \\ 4466 & 12: 40: 49 & 0.1328 \\ 4467 & 12: 40: 50 & 0.1290 \\ 4468 & 12: 40: 51 & 0.1329 \\ 4469 & 12: 40: 52 & 0.1320 \\ 4470 & 12: 40: 53 & 0.1341\end{array}$


Appendix A - Fuel Data Test 1 NIST102505_1.xls

$\begin{array}{lll}4471 & 12: 40: 54 & 0.1310 \\ 4472 & 12: 40: 55 & 0.1320 \\ 4473 & 12: 40: 56 & 0.1308 \\ 4474 & 12: 40: 57 & 0.1299 \\ 4475 & 12: 40: 58 & 0.1314 \\ 4476 & 12: 40: 59 & 0.1292 \\ 4477 & 12: 41: 00 & 0.1309 \\ 4478 & 12: 41: 01 & 0.1284 \\ 4479 & 12: 41: 02 & 0.1321 \\ 4480 & 12: 41: 03 & 0.1299 \\ 4481 & 12: 41: 04 & 0.1340 \\ 4482 & 12: 41: 05 & 0.1304 \\ 4483 & 12: 41: 06 & 0.1330 \\ 4484 & 12: 41: 07 & 0.1295 \\ 4485 & 12: 41: 08 & 0.1308 \\ 4486 & 12: 41: 09 & 0.1301 \\ 4487 & 12: 41: 10 & 0.1288 \\ 4488 & 12: 41: 11 & 0.1329 \\ 4489 & 12: 41: 12 & 0.1274 \\ 4490 & 12: 41: 13 & 0.1330 \\ 4491 & 12: 41: 14 & 0.1298 \\ 4492 & 12: 41: 15 & 0.1348 \\ 4493 & 12: 41: 16 & 0.1307 \\ 4494 & 12: 41: 17 & 0.1357 \\ 4495 & 12: 41: 18 & 0.1317 \\ 4496 & 12: 41: 19 & 0.1326 \\ 4497 & 12: 41: 20 & 0.1346 \\ 4498 & 12: 41: 21 & 0.1339 \\ 4499 & 12: 41: 22 & 0.1348 \\ 4500 & 12: 41: 23 & 0.1311 \\ 4501 & 12: 41: 24 & 0.1352 \\ 4502 & 12: 41: 25 & 0.1323 \\ 4503 & 12: 41: 26 & 0.1361 \\ 4504 & 12: 41: 27 & 0.1303 \\ 4505 & 12: 41: 28 & 0.1353 \\ 4506 & 12: 41: 29 & 0.1314 \\ 4507 & 12: 41: 30 & 0.1336 \\ 4508 & 12: 41: 31 & 0.1337 \\ 4509 & 12: 41: 32 & 0.1351 \\ 4510 & 12: 41: 33 & 0.1334 \\ 4511 & 12: 41: 34 & 0.1338 \\ 4512 & 12: 41: 35 & 0.1357 \\ 4513 & 12: 41: 36 & 0.1317 \\ 4514 & 12: 41: 37 & 0.1325 \\ 4515 & 12: 41: 38 & 0.1287 \\ 4516 & 12: 41: 39 & 0.1316 \\ 4517 & 12: 41: 40 & 0.1279 \\ 4518 & 12: 41: 41 & 0.1331 \\ 4519 & 12: 41: 42 & 0.1315 \\ 4520 & 12: 41: 43 & 0.1341 \\ 4521 & 12: 41: 44 & 0.1314 \\ 4522 & 12: 41: 45 & 0.1320\end{array}$


Appendix A - Fuel Data Test 1 NIST102505_1.xls

$\begin{array}{lll}4523 & 12: 41: 46 & 0.1339 \\ 4524 & 12: 41: 47 & 0.1310 \\ 4525 & 12: 41: 48 & 0.1310 \\ 4526 & 12: 41: 49 & 0.1281 \\ 4527 & 12: 41: 50 & 0.1310 \\ 4528 & 12: 41: 51 & 0.1266 \\ 4529 & 12: 41: 52 & 0.1319 \\ 4530 & 12: 41: 53 & 0.1295 \\ 4531 & 12: 41: 54 & 0.1342 \\ 4532 & 12: 41: 55 & 0.1298 \\ 4533 & 12: 41: 56 & 0.1337 \\ 4534 & 12: 41: 57 & 0.1314 \\ 4535 & 12: 41: 58 & 0.1330 \\ 4536 & 12: 41: 59 & 0.1305 \\ 4537 & 12: 42: 00 & 0.1319 \\ 4538 & 12: 42: 01 & 0.1309 \\ 4539 & 12: 42: 02 & 0.1269 \\ 4540 & 12: 42: 03 & 0.1331 \\ 4541 & 12: 42: 04 & 0.1315 \\ 4542 & 12: 42: 05 & 0.1350 \\ 4543 & 12: 42: 06 & 0.1307 \\ 4544 & 12: 42: 07 & 0.1372 \\ 4545 & 12: 42: 08 & 0.1332 \\ 4546 & 12: 42: 09 & 0.1367 \\ 4547 & 12: 42: 10 & 0.1318 \\ 4548 & 12: 42: 11 & 0.1324 \\ 4549 & 12: 42: 12 & 0.1305 \\ 4550 & 12: 42: 13 & 0.1294 \\ 4551 & 12: 42: 14 & 0.1323 \\ 4552 & 12: 42: 15 & 0.1310 \\ 4553 & 12: 42: 16 & 0.1350 \\ 4554 & 12: 42: 17 & 0.1315 \\ 4555 & 12: 42: 18 & 0.1351 \\ 4556 & 12: 42: 19 & 0.1310 \\ 4557 & 12: 42: 20 & 0.1346 \\ 4558 & 12: 42: 21 & 0.1349 \\ 4559 & 12: 42: 22 & 0.1363 \\ 4560 & 12: 42: 23 & 0.1325 \\ 4561 & 12: 42: 24 & 0.1321 \\ 4562 & 12: 42: 25 & 0.1292 \\ 4563 & 12: 42: 26 & 0.1289 \\ 4564 & 12: 42: 27 & 0.1322 \\ 4565 & 12: 42: 28 & 0.1293 \\ 4566 & 12: 42: 29 & 0.1320 \\ 4567 & 12: 42: 30 & 0.1270 \\ 4568 & 12: 42: 31 & 0.1327 \\ 4569 & 12: 42: 32 & 0.1288 \\ 4570 & 12: 42: 33 & 0.1321 \\ 4571 & 12: 42: 34 & 0.1316 \\ 4572 & 12: 42: 35 & 0.1344 \\ 4573 & 12: 42: 36 & 0.1334 \\ 4574 & 12: 42: 37 & 0.1324\end{array}$


Appendix A - Fuel Data Test 1 NIST102505_1.xls

$\begin{array}{lll}4575 & 12: 42: 38 & 0.1362 \\ 4576 & 12: 42: 39 & 0.1330 \\ 4577 & 12: 42: 40 & 0.1330 \\ 4578 & 12: 42: 41 & 0.1277 \\ 4579 & 12: 42: 42 & 0.1330 \\ 4580 & 12: 42: 43 & 0.1287 \\ 4581 & 12: 42: 44 & 0.1329 \\ 4582 & 12: 42: 45 & 0.1296 \\ 4583 & 12: 42: 46 & 0.1336 \\ 4584 & 12: 42: 47 & 0.1309 \\ 4585 & 12: 42: 48 & 0.1319 \\ 4586 & 12: 42: 49 & 0.1330 \\ 4587 & 12: 42: 50 & 0.1303 \\ 4588 & 12: 42: 51 & 0.1304 \\ 4589 & 12: 42: 52 & 0.1283 \\ 4590 & 12: 42: 53 & 0.1325 \\ 4591 & 12: 42: 54 & 0.1286 \\ 4592 & 12: 42: 55 & 0.1313 \\ 4593 & 12: 42: 56 & 0.1301 \\ 4594 & 12: 42: 57 & 0.1358 \\ 4595 & 12: 42: 58 & 0.1320 \\ 4596 & 12: 42: 59 & 0.1337 \\ 4597 & 12: 43: 00 & 0.1326 \\ 4598 & 12: 43: 01 & 0.1335 \\ 4599 & 12: 43: 02 & 0.1316 \\ 4600 & 12: 43: 03 & 0.1312 \\ 4601 & 12: 43: 04 & 0.1320 \\ 4602 & 12: 43: 05 & 0.1289 \\ 4603 & 12: 43: 06 & 0.1312 \\ 4604 & 12: 43: 07 & 0.1287 \\ 4605 & 12: 43: 08 & 0.1328 \\ 4606 & 12: 43: 09 & 0.1278 \\ 4607 & 12: 43: 10 & 0.1341 \\ 4608 & 12: 43: 11 & 0.1297 \\ 4609 & 12: 43: 12 & 0.1332 \\ 4610 & 12: 43: 13 & 0.1343 \\ 4611 & 12: 43: 14 & 0.1339 \\ 4612 & 12: 43: 15 & 0.1327 \\ 4613 & 12: 43: 16 & 0.1303 \\ 4614 & 12: 43: 17 & 0.1328 \\ 4615 & 12: 43: 18 & 0.1282 \\ 4616 & 12: 43: 19 & 0.1311 \\ 4617 & 12: 43: 20 & 0.1273 \\ 4618 & 12: 43: 21 & 0.1321 \\ 4619 & 12: 43: 22 & 0.1297 \\ 4620 & 12: 43: 23 & 0.1328 \\ 4621 & 12: 43: 24 & 0.1334 \\ 4622 & 12: 43: 25 & 0.1319 \\ 4623 & 12: 43: 26 & 0.1292 \\ 4624 & 12: 43: 27 & 0.1301 \\ 4625 & 12: 43: 28 & 0.1312 \\ 4626 & 12: 43: 29 & 0.1290\end{array}$


Appendix A - Fuel Data Test 1 NIST102505_1.xls

$\begin{array}{lll}4627 & 12: 43: 30 & 0.1320 \\ 4628 & 12: 43: 31 & 0.1292 \\ 4629 & 12: 43: 32 & 0.1340 \\ 4630 & 12: 43: 33 & 0.1303 \\ 4631 & 12: 43: 34 & 0.1343 \\ 4632 & 12: 43: 35 & 0.1320 \\ 4633 & 12: 43: 36 & 0.1361 \\ 4634 & 12: 43: 37 & 0.1342 \\ 4635 & 12: 43: 38 & 0.1369 \\ 4636 & 12: 43: 39 & 0.1369 \\ 4637 & 12: 43: 40 & 0.1349 \\ 4638 & 12: 43: 41 & 0.1349 \\ 4639 & 12: 43: 42 & 0.1325 \\ 4640 & 12: 43: 43 & 0.1343 \\ 4641 & 12: 43: 44 & 0.1294 \\ 4642 & 12: 43: 45 & 0.1311 \\ 4643 & 12: 43: 46 & 0.1291 \\ 4644 & 12: 43: 47 & 0.1323 \\ 4645 & 12: 43: 48 & 0.1311 \\ 4646 & 12: 43: 49 & 0.1319 \\ 4647 & 12: 43: 50 & 0.1319 \\ 4648 & 12: 43: 51 & 0.1325 \\ 4649 & 12: 43: 52 & 0.1302 \\ 4650 & 12: 43: 53 & 0.1298 \\ 4651 & 12: 43: 54 & 0.1315 \\ 4652 & 12: 43: 55 & 0.1298 \\ 4653 & 12: 43: 56 & 0.1318 \\ 4654 & 12: 43: 57 & 0.1298 \\ 4655 & 12: 43: 58 & 0.1324 \\ 4656 & 12: 43: 59 & 0.1274 \\ 4657 & 12: 44: 00 & 0.1335 \\ 4658 & 12: 44: 01 & 0.1313 \\ 4659 & 12: 44: 02 & 0.1342 \\ 4660 & 12: 44: 03 & 0.1312 \\ 4661 & 12: 44: 04 & 0.1326 \\ 4662 & 12: 44: 05 & 0.1308 \\ 4663 & 12: 44: 06 & 0.1298 \\ 4664 & 12: 44: 07 & 0.1328 \\ 4665 & 12: 44: 08 & 0.1288 \\ 4666 & 12: 44: 09 & 0.1321 \\ 4667 & 12: 44: 10 & 0.1279 \\ 4668 & 12: 44: 11 & 0.1326 \\ 4669 & 12: 44: 12 & 0.1281 \\ 4670 & 12: 44: 13 & 0.1323 \\ 4671 & 12: 44: 14 & 0.1327 \\ 4672 & 12: 44: 15 & 0.1339 \\ 4673 & 12: 44: 16 & 0.1317 \\ 4674 & 12: 44: 17 & 0.1332 \\ 4675 & 12: 44: 18 & 0.1335 \\ 4676 & 12: 44: 19 & 0.1322 \\ 4677 & 12: 44: 20 & 0.1346 \\ 4678 & 12: 44: 21 & 0.1295\end{array}$


Appendix A - Fuel Data Test 1 NIST102505_1.xls

$\begin{array}{lll}4679 & 12: 44: 22 & 0.1340 \\ 4680 & 12: 44: 23 & 0.1296 \\ 4681 & 12: 44: 24 & 0.1337 \\ 4682 & 12: 44: 25 & 0.1295 \\ 4683 & 12: 44: 26 & 0.1337 \\ 4684 & 12: 44: 27 & 0.1313 \\ 4685 & 12: 44: 28 & 0.1322 \\ 4686 & 12: 44: 29 & 0.1314 \\ 4687 & 12: 44: 30 & 0.1322 \\ 4688 & 12: 44: 31 & 0.1288 \\ 4689 & 12: 44: 32 & 0.1287 \\ 4690 & 12: 44: 33 & 0.1309 \\ 4691 & 12: 44: 34 & 0.1305 \\ 4692 & 12: 44: 35 & 0.1333 \\ 4693 & 12: 44: 36 & 0.1294 \\ 4694 & 12: 44: 37 & 0.1349 \\ 4695 & 12: 44: 38 & 0.1309 \\ 4696 & 12: 44: 39 & 0.1334 \\ 4697 & 12: 44: 40 & 0.1319 \\ 4698 & 12: 44: 41 & 0.1366 \\ 4699 & 12: 44: 42 & 0.1324 \\ 4700 & 12: 44: 43 & 0.1327 \\ 4701 & 12: 44: 44 & 0.1327 \\ 4702 & 12: 44: 45 & 0.1321 \\ 4703 & 12: 44: 46 & 0.1342 \\ 4704 & 12: 44: 47 & 0.1310 \\ 4705 & 12: 44: 48 & 0.1366 \\ 4706 & 12: 44: 49 & 0.1312 \\ 4707 & 12: 44: 50 & 0.1333 \\ 4708 & 12: 44: 51 & 0.1295 \\ 4709 & 12: 44: 52 & 0.1329 \\ 4710 & 12: 44: 53 & 0.1302 \\ 4711 & 12: 44: 54 & 0.1324 \\ 4712 & 12: 44: 55 & 0.1315 \\ 4713 & 12: 44: 56 & 0.1312 \\ 4714 & 12: 44: 57 & 0.1305 \\ 4715 & 12: 44: 58 & 0.1275 \\ 4716 & 12: 44: 59 & 0.1347 \\ 4717 & 12: 45: 00 & 0.1295 \\ 4718 & 12: 45: 01 & 0.1325 \\ 4719 & 12: 45: 02 & 0.1262 \\ 4720 & 12: 45: 03 & 0.1327 \\ 4721 & 12: 45: 04 & 0.1280 \\ 4722 & 12: 45: 05 & 0.1317 \\ 4723 & 12: 45: 06 & 0.1308 \\ 4724 & 12: 45: 07 & 0.1317 \\ 4725 & 12: 45: 08 & 0.1304 \\ 4726 & 12: 45: 09 & 0.1291 \\ 4727 & 12: 45: 10 & 0.1324 \\ 4728 & 12: 45: 11 & 0.1296 \\ 4729 & 12: 45: 12 & 0.1301 \\ 4730 & 12: 45: 13 & 0.1283\end{array}$


Appendix A - Fuel Data Test 1 NIST102505_1.xls

$\begin{array}{lll}4731 & 12: 45: 14 & 0.1327 \\ 4732 & 12: 45: 15 & 0.1279 \\ 4733 & 12: 45: 16 & 0.1342 \\ 4734 & 12: 45: 17 & 0.1310 \\ 4735 & 12: 45: 18 & 0.1337 \\ 4736 & 12: 45: 19 & 0.1313 \\ 4737 & 12: 45: 20 & 0.1333 \\ 4738 & 12: 45: 21 & 0.1315 \\ 4739 & 12: 45: 22 & 0.1297 \\ 4740 & 12: 45: 23 & 0.1325 \\ 4741 & 12: 45: 24 & 0.1290 \\ 4742 & 12: 45: 25 & 0.1320 \\ 4743 & 12: 45: 26 & 0.1291 \\ 4744 & 12: 45: 27 & 0.1325 \\ 4745 & 12: 45: 28 & 0.1294 \\ 4746 & 12: 45: 29 & 0.1323 \\ 4747 & 12: 45: 30 & 0.1313 \\ 4748 & 12: 45: 31 & 0.1330 \\ 4749 & 12: 45: 32 & 0.1308 \\ 4750 & 12: 45: 33 & 0.1326 \\ 4751 & 12: 45: 34 & 0.1339 \\ 4752 & 12: 45: 35 & 0.1324 \\ 4753 & 12: 45: 36 & 0.1316 \\ 4754 & 12: 45: 37 & 0.1292 \\ 4755 & 12: 45: 38 & 0.1317 \\ 4756 & 12: 45: 39 & 0.1274 \\ 4757 & 12: 45: 40 & 0.1310 \\ 4758 & 12: 45: 41 & 0.1295 \\ 4759 & 12: 45: 42 & 0.1331 \\ 4760 & 12: 45: 43 & 0.1287 \\ 4761 & 12: 45: 44 & 0.1333 \\ 4762 & 12: 45: 45 & 0.1312 \\ 4763 & 12: 45: 46 & 0.1323 \\ 4764 & 12: 45: 47 & 0.1296 \\ 4765 & 12: 45: 48 & 0.1304 \\ 4766 & 12: 45: 49 & 0.1304 \\ 4767 & 12: 45: 50 & 0.1279 \\ 4768 & 12: 45: 51 & 0.1336 \\ 4769 & 12: 45: 52 & 0.1291 \\ 4770 & 12: 45: 53 & 0.1328 \\ 4771 & 12: 45: 54 & 0.1276 \\ 4772 & 12: 45: 55 & 0.1329 \\ 4773 & 12: 45: 56 & 0.1308 \\ 4774 & 12: 45: 57 & 0.1331 \\ 4775 & 12: 45: 58 & 0.1314 \\ 4776 & 12: 45: 59 & 0.1304 \\ 4777 & 12: 46: 00 & 0.1308 \\ 4778 & 12: 46: 01 & 0.1290 \\ 4779 & 12: 46: 02 & 0.1316 \\ 4780 & 12: 46: 03 & 0.1272 \\ 4781 & 12: 46: 04 & 0.1300 \\ 4782 & 12: 46: 05 & 0.1267\end{array}$


Appendix A - Fuel Data Test 1 NIST102505_1.xls

$\begin{array}{lll}4783 & 12: 46: 06 & 0.1312 \\ 4784 & 12: 46: 07 & 0.1281 \\ 4785 & 12: 46: 08 & 0.1336 \\ 4786 & 12: 46: 09 & 0.1298 \\ 4787 & 12: 46: 10 & 0.1330 \\ 4788 & 12: 46: 11 & 0.1323 \\ 4789 & 12: 46: 12 & 0.1322 \\ 4790 & 12: 46: 13 & 0.1317 \\ 4791 & 12: 46: 14 & 0.1295 \\ 4792 & 12: 46: 15 & 0.1347 \\ 4793 & 12: 46: 16 & 0.1308 \\ 4794 & 12: 46: 17 & 0.1337 \\ 4795 & 12: 46: 18 & 0.1293 \\ 4796 & 12: 46: 19 & 0.1333 \\ 4797 & 12: 46: 20 & 0.1291 \\ 4798 & 12: 46: 21 & 0.1329 \\ 4799 & 12: 46: 22 & 0.1340 \\ 4800 & 12: 46: 23 & 0.1336 \\ 4801 & 12: 46: 24 & 0.1324 \\ 4802 & 12: 46: 25 & 0.1303 \\ 4803 & 12: 46: 26 & 0.1324 \\ 4804 & 12: 46: 27 & 0.1298 \\ 4805 & 12: 46: 28 & 0.1313 \\ 4806 & 12: 46: 29 & 0.1296 \\ 4807 & 12: 46: 30 & 0.1318 \\ 4808 & 12: 46: 31 & 0.1290 \\ 4809 & 12: 46: 32 & 0.1332 \\ 4810 & 12: 46: 33 & 0.1313 \\ 4811 & 12: 46: 34 & 0.1341 \\ 4812 & 12: 46: 35 & 0.1291 \\ 4813 & 12: 46: 36 & 0.1323 \\ 4814 & 12: 46: 37 & 0.1304 \\ 4815 & 12: 46: 38 & 0.1300 \\ 4816 & 12: 46: 39 & 0.1298 \\ 4817 & 12: 46: 40 & 0.1284 \\ 4818 & 12: 46: 41 & 0.1313 \\ 4819 & 12: 46: 42 & 0.1271 \\ 4820 & 12: 46: 43 & 0.1322 \\ 4821 & 12: 46: 44 & 0.1305 \\ 4822 & 12: 46: 45 & 0.1342 \\ 4823 & 12: 46: 46 & 0.1320 \\ 4824 & 12: 46: 47 & 0.1368 \\ 4825 & 12: 46: 48 & 0.1333 \\ 4826 & 12: 46: 49 & 0.1347 \\ 4827 & 12: 46: 50 & 0.1342 \\ 4828 & 12: 46: 51 & 0.1333 \\ 4829 & 12: 46: 52 & 0.1355 \\ 4830 & 12: 46: 53 & 0.1323 \\ 4831 & 12: 46: 54 & 0.1366 \\ 4832 & 12: 46: 55 & 0.1305 \\ 4833 & 12: 46: 56 & 0.1334 \\ 4834 & 12: 46: 57 & 0.1291\end{array}$


Appendix A - Fuel Data Test 1 NIST102505_1.xls

$\begin{array}{lll}4835 & 12: 46: 58 & 0.1343 \\ 4836 & 12: 46: 59 & 0.1306 \\ 4837 & 12: 47: 00 & 0.1332 \\ 4838 & 12: 47: 01 & 0.1328 \\ 4839 & 12: 47: 02 & 0.1318 \\ 4840 & 12: 47: 03 & 0.1310 \\ 4841 & 12: 47: 04 & 0.1299 \\ 4842 & 12: 47: 05 & 0.1335 \\ 4843 & 12: 47: 06 & 0.1280 \\ 4844 & 12: 47: 07 & 0.1306 \\ 4845 & 12: 47: 08 & 0.1266 \\ 4846 & 12: 47: 09 & 0.1328 \\ 4847 & 12: 47: 10 & 0.1296 \\ 4848 & 12: 47: 11 & 0.1333 \\ 4849 & 12: 47: 12 & 0.1311 \\ 4850 & 12: 47: 13 & 0.1338 \\ 4851 & 12: 47: 14 & 0.1301 \\ 4852 & 12: 47: 15 & 0.1316 \\ 4853 & 12: 47: 16 & 0.1295 \\ 4854 & 12: 47: 17 & 0.1284 \\ 4855 & 12: 47: 18 & 0.1309 \\ 4856 & 12: 47: 19 & 0.1290 \\ 4857 & 12: 47: 20 & 0.1313 \\ 4858 & 12: 47: 21 & 0.1276 \\ 4859 & 12: 47: 22 & 0.1335 \\ 4860 & 12: 47: 23 & 0.1314 \\ 4861 & 12: 47: 24 & 0.1335 \\ 4862 & 12: 47: 25 & 0.1295 \\ 4863 & 12: 47: 26 & 0.1322 \\ 4864 & 12: 47: 27 & 0.1309 \\ 4865 & 12: 47: 28 & 0.1314 \\ 4866 & 12: 47: 29 & 0.1300 \\ 4867 & 12: 47: 30 & 0.1299 \\ 4868 & 12: 47: 31 & 0.1331 \\ 4869 & 12: 47: 32 & 0.1330 \\ 4870 & 12: 47: 33 & 0.1356 \\ 4871 & 12: 47: 34 & 0.1319 \\ 4872 & 12: 47: 35 & 0.1355 \\ 4873 & 12: 47: 36 & 0.1319 \\ 4874 & 12: 47: 37 & 0.1368 \\ 4875 & 12: 47: 38 & 0.1324 \\ 4876 & 12: 47: 39 & 0.1344 \\ 4877 & 12: 47: 40 & 0.1314 \\ 4878 & 12: 47: 41 & 0.1325 \\ 4879 & 12: 47: 42 & 0.1318 \\ 4880 & 12: 47: 43 & 0.1308 \\ 4881 & 12: 47: 44 & 0.1354 \\ 4882 & 12: 47: 45 & 0.1315 \\ 4883 & 12: 47: 46 & 0.1342 \\ 4884 & 12: 47: 47 & 0.1305 \\ 4885 & 12: 47: 48 & 0.1342 \\ 4886 & 12: 47: 49 & 0.1304\end{array}$


Appendix A - Fuel Data Test 1 NIST102505_1.xls

$\begin{array}{lll}4887 & 12: 47: 50 & 0.1327 \\ 4888 & 12: 47: 51 & 0.1312 \\ 4889 & 12: 47: 52 & 0.1335 \\ 4890 & 12: 47: 53 & 0.1318 \\ 4891 & 12: 47: 54 & 0.1311 \\ 4892 & 12: 47: 55 & 0.1334 \\ 4893 & 12: 47: 56 & 0.1305 \\ 4894 & 12: 47: 57 & 0.1326 \\ 4895 & 12: 47: 58 & 0.1273 \\ 4896 & 12: 47: 59 & 0.1339 \\ 4897 & 12: 48: 00 & 0.1291 \\ 4898 & 12: 48: 01 & 0.1324 \\ 4899 & 12: 48: 02 & 0.1302 \\ 4900 & 12: 48: 03 & 0.1380 \\ 4901 & 12: 48: 04 & 0.1342 \\ 4902 & 12: 48: 05 & 0.1337 \\ 4903 & 12: 48: 06 & 0.1305 \\ 4904 & 12: 48: 07 & 0.1310 \\ 4905 & 12: 48: 08 & 0.1310 \\ 4906 & 12: 48: 09 & 0.1285 \\ 4907 & 12: 48: 10 & 0.1286 \\ 4908 & 12: 48: 11 & 0.1264 \\ 4909 & 12: 48: 12 & 0.1317 \\ 4910 & 12: 48: 13 & 0.1269 \\ 4911 & 12: 48: 14 & 0.1321 \\ 4912 & 12: 48: 15 & 0.1341 \\ 4913 & 12: 48: 16 & 0.1362 \\ 4914 & 12: 48: 17 & 0.1335 \\ 4915 & 12: 48: 18 & 0.1332 \\ 4916 & 12: 48: 19 & 0.1329 \\ 4917 & 12: 48: 20 & 0.1336 \\ 4918 & 12: 48: 21 & 0.1337 \\ 4919 & 12: 48: 22 & 0.1313 \\ 4920 & 12: 48: 23 & 0.1322 \\ 4921 & 12: 48: 24 & 0.1293 \\ 4922 & 12: 48: 25 & 0.1342 \\ 4923 & 12: 48: 26 & 0.1329 \\ 4924 & 12: 48: 27 & 0.1327 \\ 4925 & 12: 48: 28 & 0.1320 \\ 4926 & 12: 48: 29 & 0.1338 \\ 4927 & 12: 48: 30 & 0.1339 \\ 4928 & 12: 48: 31 & 0.1333 \\ 4929 & 12: 48: 32 & 0.1312 \\ 4930 & 12: 48: 33 & 0.1309 \\ 4931 & 12: 48: 34 & 0.1321 \\ 4932 & 12: 48: 35 & 0.1292 \\ 4933 & 12: 48: 36 & 0.1309 \\ 4934 & 12: 48: 37 & 0.1280 \\ 4935 & 12: 48: 38 & 0.1320 \\ 4936 & 12: 48: 39 & 0.1281 \\ 4937 & 12: 48: 40 & 0.1341 \\ 4938 & 12: 48: 41 & 0.1303\end{array}$


Appendix A - Fuel Data Test 1 NIST102505_1.xIs

$\begin{array}{lll}4939 & 12: 48: 42 & 0.1327 \\ 4940 & 12: 48: 43 & 0.1308 \\ 4941 & 12: 48: 44 & 0.1320 \\ 4942 & 12: 48: 45 & 0.1313 \\ 4943 & 12: 48: 46 & 0.1293 \\ 4944 & 12: 48: 47 & 0.1334 \\ 4945 & 12: 48: 48 & 0.1291 \\ 4946 & 12: 48: 49 & 0.1322 \\ 4947 & 12: 48: 50 & 0.1267 \\ 4948 & 12: 48: 51 & 0.1322 \\ 4949 & 12: 48: 52 & 0.1283 \\ 4950 & 12: 48: 53 & 0.1319 \\ 4951 & 12: 48: 54 & 0.1301 \\ 4952 & 12: 48: 55 & 0.1327 \\ 4953 & 12: 48: 56 & 0.1310 \\ 4954 & 12: 48: 57 & 0.1309 \\ 4955 & 12: 48: 58 & 0.1313 \\ 4956 & 12: 48: 59 & 0.1290 \\ 4957 & 12: 49: 00 & 0.1314 \\ 4958 & 12: 49: 01 & 0.1294 \\ 4959 & 12: 49: 02 & 0.1314 \\ 4960 & 12: 49: 03 & 0.1271 \\ 4961 & 12: 49: 04 & 0.1329 \\ 4962 & 12: 49: 05 & 0.1302 \\ 4963 & 12: 49: 06 & 0.1340 \\ 4964 & 12: 49: 07 & 0.1290 \\ 4965 & 12: 49: 08 & 0.1321 \\ 4966 & 12: 49: 09 & 0.1317 \\ 4967 & 12: 49: 10 & 0.1289 \\ 4968 & 12: 49: 11 & 0.1313 \\ 4969 & 12: 49: 12 & 0.1296 \\ 4970 & 12: 49: 13 & 0.1311 \\ 4971 & 12: 49: 14 & 0.1272 \\ 4972 & 12: 49: 15 & 0.1332 \\ 4973 & 12: 49: 16 & 0.1284 \\ 4974 & 12: 49: 17 & 0.1339 \\ 4975 & 12: 49: 18 & 0.1313 \\ 4976 & 12: 49: 19 & 0.1349 \\ 4977 & 12: 49: 20 & 0.1334 \\ 4978 & 12: 49: 21 & 0.1335 \\ 4979 & 12: 49: 22 & 0.1341 \\ 4980 & 12: 49: 23 & 0.1339 \\ 4981 & 12: 49: 24 & 0.1348 \\ 4982 & 12: 49: 25 & 0.1303 \\ 4983 & 12: 49: 26 & 0.1354 \\ 4984 & 12: 49: 27 & 0.1288 \\ 4985 & 12: 49: 28 & 0.1321 \\ 4986 & 12: 49: 29 & 0.1302 \\ 4987 & 12: 49: 30 & 0.1331 \\ 4988 & 12: 49: 31 & 0.1321 \\ 4989 & 12: 49: 32 & 0.1331 \\ 4990 & 12: 49: 33 & 0.1346\end{array}$


Appendix A - Fuel Data Test 1 NIST102505_1.xIs

$\begin{array}{lll}4991 & 12: 49: 34 & 0.1313 \\ 4992 & 12: 49: 35 & 0.1301 \\ 4993 & 12: 49: 36 & 0.1276 \\ 4994 & 12: 49: 37 & 0.1336 \\ 4995 & 12: 49: 38 & 0.1296 \\ 4996 & 12: 49: 39 & 0.1325 \\ 4997 & 12: 49: 40 & 0.1281 \\ 4998 & 12: 49: 41 & 0.1318 \\ 4999 & 12: 49: 42 & 0.1280 \\ 5000 & 12: 49: 43 & 0.1318 \\ 5001 & 12: 49: 44 & 0.1311 \\ 5002 & 12: 49: 45 & 0.1341 \\ 5003 & 12: 49: 46 & 0.1321 \\ 5004 & 12: 49: 47 & 0.1315 \\ 5005 & 12: 49: 48 & 0.1321 \\ 5006 & 12: 49: 49 & 0.1293 \\ 5007 & 12: 49: 50 & 0.1308 \\ 5008 & 12: 49: 51 & 0.1274 \\ 5009 & 12: 49: 52 & 0.1313 \\ 5010 & 12: 49: 53 & 0.1267 \\ 5011 & 12: 49: 54 & 0.1315 \\ 5012 & 12: 49: 55 & 0.1311 \\ 5013 & 12: 49: 56 & 0.1338 \\ 5014 & 12: 49: 57 & 0.1320 \\ 5015 & 12: 49: 58 & 0.1335 \\ 5016 & 12: 49: 59 & 0.1338 \\ 5017 & 12: 50: 00 & 0.1319 \\ 5018 & 12: 50: 01 & 0.1325 \\ 5019 & 12: 50: 02 & 0.1324 \\ 5020 & 12: 50: 03 & 0.1343 \\ 5021 & 12: 50: 04 & 0.1298 \\ 5022 & 12: 50: 05 & 0.1341 \\ 5023 & 12: 50: 06 & 0.1306 \\ 5024 & 12: 50: 07 & 0.1349 \\ 5025 & 12: 50: 08 & 0.1314 \\ 5026 & 12: 50: 09 & 0.1355 \\ 5027 & 12: 50: 10 & 0.1326 \\ 5028 & 12: 50: 11 & 0.1328 \\ 5029 & 12: 50: 12 & 0.1332 \\ 5030 & 12: 50: 13 & 0.1315 \\ 5031 & 12: 50: 14 & 0.1323 \\ 5032 & 12: 50: 15 & 0.1294 \\ 5033 & 12: 50: 16 & 0.1346 \\ 5034 & 12: 50: 17 & 0.1298 \\ 5035 & 12: 50: 18 & 0.1322 \\ 5036 & 12: 50: 19 & 0.1282 \\ 5037 & 12: 50: 20 & 0.1326 \\ 5038 & 12: 50: 21 & 0.1300 \\ 5039 & 12: 50: 22 & 0.1320 \\ 5040 & 12: 50: 23 & 0.1324 \\ 5041 & 12: 50: 24 & 0.1331 \\ 5042 & 12: 50: 25 & 0.1330\end{array}$


Appendix A - Fuel Data Test 1 NIST102505_1.xIs

$\begin{array}{lll}5043 & 12: 50: 26 & 0.1322 \\ 5044 & 12: 50: 27 & 0.1342 \\ 5045 & 12: 50: 28 & 0.1315 \\ 5046 & 12: 50: 29 & 0.1341 \\ 5047 & 12: 50: 30 & 0.1313 \\ 5048 & 12: 50: 31 & 0.1336 \\ 5049 & 12: 50: 32 & 0.1287 \\ 5050 & 12: 50: 33 & 0.1319 \\ 5051 & 12: 50: 34 & 0.1324 \\ 5052 & 12: 50: 35 & 0.1340 \\ 5053 & 12: 50: 36 & 0.1323 \\ 5054 & 12: 50: 37 & 0.1305 \\ 5055 & 12: 50: 38 & 0.1319 \\ 5056 & 12: 50: 39 & 0.1290 \\ 5057 & 12: 50: 40 & 0.1325 \\ 5058 & 12: 50: 41 & 0.1293 \\ 5059 & 12: 50: 42 & 0.1337 \\ 5060 & 12: 50: 43 & 0.1286 \\ 5061 & 12: 50: 44 & 0.1327 \\ 5062 & 12: 50: 45 & 0.1320 \\ 5063 & 12: 50: 46 & 0.1345 \\ 5064 & 12: 50: 47 & 0.1330 \\ 5065 & 12: 50: 48 & 0.1337 \\ 5066 & 12: 50: 49 & 0.1335 \\ 5067 & 12: 50: 50 & 0.1306 \\ 5068 & 12: 50: 51 & 0.1307 \\ 5069 & 12: 50: 52 & 0.1292 \\ 5070 & 12: 50: 53 & 0.1324 \\ 5071 & 12: 50: 54 & 0.1286 \\ 5072 & 12: 50: 55 & 0.1314 \\ 5073 & 12: 50: 56 & 0.1288 \\ 5074 & 12: 50: 57 & 0.1313 \\ 5075 & 12: 50: 58 & 0.1295 \\ 5076 & 12: 50: 59 & 0.1327 \\ 5077 & 12: 51: 00 & 0.1323 \\ 5078 & 12: 51: 01 & 0.1320 \\ 5079 & 12: 51: 02 & 0.1309 \\ 5080 & 12: 51: 03 & 0.1288 \\ 5081 & 12: 51: 04 & 0.1315 \\ 5082 & 12: 51: 05 & 0.1279 \\ 5083 & 12: 51: 06 & 0.1301 \\ 5084 & 12: 51: 07 & 0.1262 \\ 5085 & 12: 51: 08 & 0.1311 \\ 5086 & 12: 51: 09 & 0.1267 \\ 5087 & 12: 51: 10 & 0.1315 \\ 5088 & 12: 51: 11 & 0.1322 \\ 5089 & 12: 51: 12 & 0.1344 \\ 5090 & 12: 51: 13 & 0.1295 \\ 5091 & 12: 51: 14 & 0.1311 \\ 5092 & 12: 51: 15 & 0.1294 \\ 5093 & 12: 51: 16 & 0.1293 \\ 5094 & 12: 51: 17 & 0.1310\end{array}$


Appendix A - Fuel Data Test 1 NIST102505_1.xls

$\begin{array}{lll}5095 & 12: 51: 18 & 0.1317 \\ 5096 & 12: 51: 19 & 0.1331 \\ 5097 & 12: 51: 20 & 0.1293 \\ 5098 & 12: 51: 21 & 0.1346 \\ 5099 & 12: 51: 22 & 0.1299 \\ 5100 & 12: 51: 23 & 0.1334 \\ 5101 & 12: 51: 24 & 0.1313 \\ 5102 & 12: 51: 25 & 0.1355 \\ 5103 & 12: 51: 26 & 0.1312 \\ 5104 & 12: 51: 27 & 0.1317 \\ 5105 & 12: 51: 28 & 0.1332 \\ 5106 & 12: 51: 29 & 0.1313 \\ 5107 & 12: 51: 30 & 0.1332 \\ 5108 & 12: 51: 31 & 0.1296 \\ 5109 & 12: 51: 32 & 0.1348 \\ 5110 & 12: 51: 33 & 0.1302 \\ 5111 & 12: 51: 34 & 0.1329 \\ 5112 & 12: 51: 35 & 0.1305 \\ 5113 & 12: 51: 36 & 0.1348 \\ 5114 & 12: 51: 37 & 0.1317 \\ 5115 & 12: 51: 38 & 0.1344 \\ 5116 & 12: 51: 39 & 0.1341 \\ 5117 & 12: 51: 40 & 0.1354 \\ 5118 & 12: 51: 41 & 0.1337 \\ 5119 & 12: 51: 42 & 0.1323 \\ 5120 & 12: 51: 43 & 0.1342 \\ 5121 & 12: 51: 44 & 0.1302 \\ 5122 & 12: 51: 45 & 0.1313 \\ 5123 & 12: 51: 46 & 0.1291 \\ 5124 & 12: 51: 47 & 0.1322 \\ 5125 & 12: 51: 48 & 0.1290 \\ 5126 & 12: 51: 49 & 0.1319 \\ 5127 & 12: 51: 50 & 0.1302 \\ 5128 & 12: 51: 51 & 0.1326 \\ 5129 & 12: 51: 52 & 0.1297 \\ 5130 & 12: 51: 53 & 0.1306 \\ 5131 & 12: 51: 54 & 0.1317 \\ 5132 & 12: 51: 55 & 0.1296 \\ 5133 & 12: 51: 56 & 0.1305 \\ 5134 & 12: 51: 57 & 0.1277 \\ 5135 & 12: 51: 58 & 0.1309 \\ 5136 & 12: 51: 59 & 0.1263 \\ 5137 & 12: 52: 00 & 0.1322 \\ 5138 & 12: 52: 01 & 0.1296 \\ 5139 & 12: 52: 02 & 0.1341 \\ 5140 & 12: 52: 03 & 0.1298 \\ 5141 & 12: 52: 04 & 0.1324 \\ 5142 & 12: 52: 05 & 0.1303 \\ 5143 & 12: 52: 06 & 0.1296 \\ 5144 & 12: 52: 07 & 0.1302 \\ 5145 & 12: 52: 08 & 0.1296 \\ 5146 & 12: 52: 09 & 0.1314\end{array}$


Appendix A - Fuel Data Test 1 NIST102505_1.xls

$\begin{array}{lll}5147 & 12: 52: 10 & 0.1282 \\ 5148 & 12: 52: 11 & 0.1336 \\ 5149 & 12: 52: 12 & 0.1304 \\ 5150 & 12: 52: 13 & 0.1352 \\ 5151 & 12: 52: 14 & 0.1319 \\ 5152 & 12: 52: 15 & 0.1354 \\ 5153 & 12: 52: 16 & 0.1317 \\ 5154 & 12: 52: 17 & 0.1323 \\ 5155 & 12: 52: 18 & 0.1302 \\ 5156 & 12: 52: 19 & 0.1306 \\ 5157 & 12: 52: 20 & 0.1296 \\ 5158 & 12: 52: 21 & 0.1281 \\ 5159 & 12: 52: 22 & 0.1315 \\ 5160 & 12: 52: 23 & 0.1296 \\ 5161 & 12: 52: 24 & 0.1320 \\ 5162 & 12: 52: 25 & 0.1277 \\ 5163 & 12: 52: 26 & 0.1339 \\ 5164 & 12: 52: 27 & 0.1304 \\ 5165 & 12: 52: 28 & 0.1331 \\ 5166 & 12: 52: 29 & 0.1307 \\ 5167 & 12: 52: 30 & 0.1315 \\ 5168 & 12: 52: 31 & 0.1299 \\ 5169 & 12: 52: 32 & 0.1291 \\ 5170 & 12: 52: 33 & 0.1305 \\ 5171 & 12: 52: 34 & 0.1282 \\ 5172 & 12: 52: 35 & 0.1313 \\ 5173 & 12: 52: 36 & 0.1277 \\ 5174 & 12: 52: 37 & 0.1325 \\ 5175 & 12: 52: 38 & 0.1296 \\ 5176 & 12: 52: 39 & 0.1317 \\ 5177 & 12: 52: 40 & 0.1290 \\ 5178 & 12: 52: 41 & 0.1310 \\ 5179 & 12: 52: 42 & 0.1288 \\ 5180 & 12: 52: 43 & 0.1311 \\ 5181 & 12: 52: 44 & 0.1325 \\ 5182 & 12: 52: 45 & 0.1305 \\ 5183 & 12: 52: 46 & 0.1309 \\ 5184 & 12: 52: 47 & 0.1283 \\ 5185 & 12: 52: 48 & 0.1305 \\ 5186 & 12: 52: 49 & 0.1279 \\ 5187 & 12: 52: 50 & 0.1322 \\ 5188 & 12: 52: 51 & 0.1308 \\ 5189 & 12: 52: 52 & 0.1334 \\ 5190 & 12: 52: 53 & 0.1316 \\ 5191 & 12: 52: 54 & 0.1349 \\ 5192 & 12: 52: 55 & 0.1324 \\ 5193 & 12: 52: 56 & 0.1325 \\ 5194 & 12: 52: 57 & 0.1309 \\ 5195 & 12: 52: 58 & 0.1284 \\ 5196 & 12: 52: 59 & 0.1307 \\ 5197 & 12: 53: 00 & 0.1275 \\ 5198 & 12: 53: 01 & 0.1334\end{array}$


Appendix A - Fuel Data Test 1 NIST102505_1.xls

$\begin{array}{lll}5199 & 12: 53: 02 & 0.1313 \\ 5200 & 12: 53: 03 & 0.1323 \\ 5201 & 12: 53: 04 & 0.1285 \\ 5202 & 12: 53: 05 & 0.1322 \\ 5203 & 12: 53: 06 & 0.1311 \\ 5204 & 12: 53: 07 & 0.1328 \\ 5205 & 12: 53: 08 & 0.1309 \\ 5206 & 12: 53: 09 & 0.1314 \\ 5207 & 12: 53: 10 & 0.1307 \\ 5208 & 12: 53: 11 & 0.1285 \\ 5209 & 12: 53: 12 & 0.1312 \\ 5210 & 12: 53: 13 & 0.1287 \\ 5211 & 12: 53: 14 & 0.1313 \\ 5212 & 12: 53: 15 & 0.1269 \\ 5213 & 12: 53: 16 & 0.1342 \\ 5214 & 12: 53: 17 & 0.1314 \\ 5215 & 12: 53: 18 & 0.1338 \\ 5216 & 12: 53: 19 & 0.1297 \\ 5217 & 12: 53: 20 & 0.1318 \\ 5218 & 12: 53: 21 & 0.1291 \\ 5219 & 12: 53: 22 & 0.1288 \\ 5220 & 12: 53: 23 & 0.1292 \\ 5221 & 12: 53: 24 & 0.1290 \\ 5222 & 12: 53: 25 & 0.1307 \\ 5223 & 12: 53: 26 & 0.1266 \\ 5224 & 12: 53: 27 & 0.1331 \\ 5225 & 12: 53: 28 & 0.1297 \\ 5226 & 12: 53: 29 & 0.1327 \\ 5227 & 12: 53: 30 & 0.1297 \\ 5228 & 12: 53: 31 & 0.1333 \\ 5229 & 12: 53: 32 & 0.1316 \\ 5230 & 12: 53: 33 & 0.1317 \\ 5231 & 12: 53: 34 & 0.1326 \\ 5232 & 12: 53: 35 & 0.1324 \\ 5233 & 12: 53: 36 & 0.1329 \\ 5234 & 12: 53: 37 & 0.1303 \\ 5235 & 12: 53: 38 & 0.1348 \\ 5236 & 12: 53: 39 & 0.1305 \\ 5237 & 12: 53: 40 & 0.1335 \\ 5238 & 12: 53: 41 & 0.1305 \\ 5239 & 12: 53: 42 & 0.1341 \\ 5240 & 12: 53: 43 & 0.1314 \\ 5241 & 12: 53: 44 & 0.1333 \\ 5242 & 12: 53: 45 & 0.1311 \\ 5243 & 12: 53: 46 & 0.1323 \\ 5244 & 12: 53: 47 & 0.1310 \\ 5245 & 12: 53: 48 & 0.1296 \\ 5246 & 12: 53: 49 & 0.1334 \\ 5247 & 12: 53: 50 & 0.1295 \\ 5248 & 12: 53: 51 & 0.1313 \\ 5249 & 12: 53: 52 & 0.1255 \\ 5250 & 12: 53: 53 & 0.1328\end{array}$


Appendix A - Fuel Data Test 1 NIST102505_1.xIs

$\begin{array}{lll}5251 & 12: 53: 54 & 0.1286 \\ 5252 & 12: 53: 55 & 0.1311 \\ 5253 & 12: 53: 56 & 0.1296 \\ 5254 & 12: 53: 57 & 0.1330 \\ 5255 & 12: 53: 58 & 0.1315 \\ 5256 & 12: 53: 59 & 0.1303 \\ 5257 & 12: 54: 00 & 0.1326 \\ 5258 & 12: 54: 01 & 0.1296 \\ 5259 & 12: 54: 02 & 0.1305 \\ 5260 & 12: 54: 03 & 0.1269 \\ 5261 & 12: 54: 04 & 0.1303 \\ 5262 & 12: 54: 05 & 0.1264 \\ 5263 & 12: 54: 06 & 0.1327 \\ 5264 & 12: 54: 07 & 0.1303 \\ 5265 & 12: 54: 08 & 0.1339 \\ 5266 & 12: 54: 09 & 0.1289 \\ 5267 & 12: 54: 10 & 0.1309 \\ 5268 & 12: 54: 11 & 0.1341 \\ 5269 & 12: 54: 12 & 0.1319 \\ 5270 & 12: 54: 13 & 0.1312 \\ 5271 & 12: 54: 14 & 0.1290 \\ 5272 & 12: 54: 15 & 0.1328 \\ 5273 & 12: 54: 16 & 0.1291 \\ 5274 & 12: 54: 17 & 0.1316 \\ 5275 & 12: 54: 18 & 0.1284 \\ 5276 & 12: 54: 19 & 0.1325 \\ 5277 & 12: 54: 20 & 0.1295 \\ 5278 & 12: 54: 21 & 0.1334 \\ 5279 & 12: 54: 22 & 0.1320 \\ 5280 & 12: 54: 23 & 0.1334 \\ 5281 & 12: 54: 24 & 0.1309 \\ 5282 & 12: 54: 25 & 0.1319 \\ 5283 & 12: 54: 26 & 0.1316 \\ 5284 & 12: 54: 27 & 0.1297 \\ 5285 & 12: 54: 28 & 0.1323 \\ 5286 & 12: 54: 29 & 0.1298 \\ 5287 & 12: 54: 30 & 0.1321 \\ 5288 & 12: 54: 31 & 0.1280 \\ 5289 & 12: 54: 32 & 0.1336 \\ 5290 & 12: 54: 33 & 0.1303 \\ 5291 & 12: 54: 34 & 0.1334 \\ 5292 & 12: 54: 35 & 0.1294 \\ 5293 & 12: 54: 36 & 0.1327 \\ 5294 & 12: 54: 37 & 0.1304 \\ 5295 & 12: 54: 38 & 0.1295 \\ 5296 & 12: 54: 39 & 0.1314 \\ 5297 & 12: 54: 40 & 0.1315 \\ 5298 & 12: 54: 41 & 0.1308 \\ 5299 & 12: 54: 42 & 0.1266 \\ 5300 & 12: 54: 43 & 0.1322 \\ 5301 & 12: 54: 44 & 0.1284 \\ 5302 & 12: 54: 45 & 0.1326\end{array}$


Appendix A - Fuel Data Test 1 NIST102505_1.xIs

$\begin{array}{lll}5303 & 12: 54: 46 & 0.1321 \\ 5304 & 12: 54: 47 & 0.1345 \\ 5305 & 12: 54: 48 & 0.1314 \\ 5306 & 12: 54: 49 & 0.1328 \\ 5307 & 12: 54: 50 & 0.1325 \\ 5308 & 12: 54: 51 & 0.1308 \\ 5309 & 12: 54: 52 & 0.1308 \\ 5310 & 12: 54: 53 & 0.1294 \\ 5311 & 12: 54: 54 & 0.1301 \\ 5312 & 12: 54: 55 & 0.1273 \\ 5313 & 12: 54: 56 & 0.1306 \\ 5314 & 12: 54: 57 & 0.1291 \\ 5315 & 12: 54: 58 & 0.1336 \\ 5316 & 12: 54: 59 & 0.1295 \\ 5317 & 12: 55: 00 & 0.1338 \\ 5318 & 12: 55: 01 & 0.1305 \\ 5319 & 12: 55: 02 & 0.1318 \\ 5320 & 12: 55: 03 & 0.1305 \\ 5321 & 12: 55: 04 & 0.1317 \\ 5322 & 12: 55: 05 & 0.1333 \\ 5323 & 12: 55: 06 & 0.1298 \\ 5324 & 12: 55: 07 & 0.1311 \\ 5325 & 12: 55: 08 & 0.1274 \\ 5326 & 12: 55: 09 & 0.1319 \\ 5327 & 12: 55: 10 & 0.1283 \\ 5328 & 12: 55: 11 & 0.1332 \\ 5329 & 12: 55: 12 & 0.1307 \\ 5330 & 12: 55: 13 & 0.1333 \\ 5331 & 12: 55: 14 & 0.1309 \\ 5332 & 12: 55: 15 & 0.1320 \\ 5333 & 12: 55: 16 & 0.1308 \\ 5334 & 12: 55: 17 & 0.1293 \\ 5335 & 12: 55: 18 & 0.1316 \\ 5336 & 12: 55: 19 & 0.1291 \\ 5337 & 12: 55: 20 & 0.1322 \\ 5338 & 12: 55: 21 & 0.1277 \\ 5339 & 12: 55: 22 & 0.1333 \\ 5340 & 12: 55: 23 & 0.1296 \\ 5341 & 12: 55: 24 & 0.1326 \\ 5342 & 12: 55: 25 & 0.1321 \\ 5343 & 12: 55: 26 & 0.1357 \\ 5344 & 12: 55: 27 & 0.1345 \\ 5345 & 12: 55: 28 & 0.1304 \\ 5346 & 12: 55: 29 & 0.1334 \\ 5347 & 12: 55: 30 & 0.1298 \\ 5348 & 12: 55: 31 & 0.1322 \\ 5349 & 12: 55: 32 & 0.1284 \\ 5350 & 12: 55: 33 & 0.1315 \\ 5351 & 12: 55: 34 & 0.1273 \\ 5352 & 12: 55: 35 & 0.1312 \\ 5353 & 12: 55: 36 & 0.1316 \\ 5354 & 12: 55: 37 & 0.1326\end{array}$


Appendix A - Fuel Data Test 1 NIST102505_1.xIs

$\begin{array}{lll}5355 & 12: 55: 38 & 0.1315 \\ 5356 & 12: 55: 39 & 0.1306 \\ 5357 & 12: 55: 40 & 0.1311 \\ 5358 & 12: 55: 41 & 0.1293 \\ 5359 & 12: 55: 42 & 0.1303 \\ 5360 & 12: 55: 43 & 0.1279 \\ 5361 & 12: 55: 44 & 0.1307 \\ 5362 & 12: 55: 45 & 0.1273 \\ 5363 & 12: 55: 46 & 0.1299 \\ 5364 & 12: 55: 47 & 0.1290 \\ 5365 & 12: 55: 48 & 0.1323 \\ 5366 & 12: 55: 49 & 0.1314 \\ 5367 & 12: 55: 50 & 0.1342 \\ 5368 & 12: 55: 51 & 0.1339 \\ 5369 & 12: 55: 52 & 0.1318 \\ 5370 & 12: 55: 53 & 0.1307 \\ 5371 & 12: 55: 54 & 0.1291 \\ 5372 & 12: 55: 55 & 0.1325 \\ 5373 & 12: 55: 56 & 0.1289 \\ 5374 & 12: 55: 57 & 0.1313 \\ 5375 & 12: 55: 58 & 0.1287 \\ 5376 & 12: 55: 59 & 0.1319 \\ 5377 & 12: 56: 00 & 0.1281 \\ 5378 & 12: 56: 01 & 0.1329 \\ 5379 & 12: 56: 02 & 0.1316 \\ 5380 & 12: 56: 03 & 0.1331 \\ 5381 & 12: 56: 04 & 0.1322 \\ 5382 & 12: 56: 05 & 0.1308 \\ 5383 & 12: 56: 06 & 0.1336 \\ 5384 & 12: 56: 07 & 0.1300 \\ 5385 & 12: 56: 08 & 0.1302 \\ 5386 & 12: 56: 09 & 0.1268 \\ 5387 & 12: 56: 10 & 0.1315 \\ 5388 & 12: 56: 11 & 0.1274 \\ 5389 & 12: 56: 12 & 0.1316 \\ 5390 & 12: 56: 13 & 0.1306 \\ 5391 & 12: 56: 14 & 0.1336 \\ 5392 & 12: 56: 15 & 0.1306 \\ 5393 & 12: 56: 16 & 0.1327 \\ 5394 & 12: 56: 17 & 0.1329 \\ 5395 & 12: 56: 18 & 0.1328 \\ 5396 & 12: 56: 19 & 0.1316 \\ 5397 & 12: 56: 20 & 0.1320 \\ 5398 & 12: 56: 21 & 0.1340 \\ 5399 & 12: 56: 22 & 0.1314 \\ 5400 & 12: 56: 23 & 0.1349 \\ 5401 & 12: 56: 24 & 0.1311 \\ 5402 & 12: 56: 25 & 0.1332 \\ 5403 & 12: 56: 26 & 0.1295 \\ 5404 & 12: 56: 27 & 0.1352 \\ 5405 & 12: 56: 28 & 0.1333 \\ 5406 & 12: 56: 29 & 0.1360\end{array}$


Appendix A - Fuel Data Test 1 NIST102505_1.xIs

$\begin{array}{lll}5407 & 12: 56: 30 & 0.1341 \\ 5408 & 12: 56: 31 & 0.1343 \\ 5409 & 12: 56: 32 & 0.1319 \\ 5410 & 12: 56: 33 & 0.1313 \\ 5411 & 12: 56: 34 & 0.1328 \\ 5412 & 12: 56: 35 & 0.1274 \\ 5413 & 12: 56: 36 & 0.1311 \\ 5414 & 12: 56: 37 & 0.1291 \\ 5415 & 12: 56: 38 & 0.1319 \\ 5416 & 12: 56: 39 & 0.1288 \\ 5417 & 12: 56: 40 & 0.1334 \\ 5418 & 12: 56: 41 & 0.1337 \\ 5419 & 12: 56: 42 & 0.1351 \\ 5420 & 12: 56: 43 & 0.1337 \\ 5421 & 12: 56: 44 & 0.1320 \\ 5422 & 12: 56: 45 & 0.1333 \\ 5423 & 12: 56: 46 & 0.1304 \\ 5424 & 12: 56: 47 & 0.1326 \\ 5425 & 12: 56: 48 & 0.1291 \\ 5426 & 12: 56: 49 & 0.1318 \\ 5427 & 12: 56: 50 & 0.1283 \\ 5428 & 12: 56: 51 & 0.1323 \\ 5429 & 12: 56: 52 & 0.1303 \\ 5430 & 12: 56: 53 & 0.1320 \\ 5431 & 12: 56: 54 & 0.1286 \\ 5432 & 12: 56: 55 & 0.1320 \\ 5433 & 12: 56: 56 & 0.1332 \\ 5434 & 12: 56: 57 & 0.1317 \\ 5435 & 12: 56: 58 & 0.1311 \\ 5436 & 12: 56: 59 & 0.1300 \\ 5437 & 12: 57: 00 & 0.1313 \\ 5438 & 12: 57: 01 & 0.1274 \\ 5439 & 12: 57: 02 & 0.1307 \\ 5440 & 12: 57: 03 & 0.1293 \\ 5441 & 12: 57: 04 & 0.1321 \\ 5442 & 12: 57: 05 & 0.1310 \\ 5443 & 12: 57: 06 & 0.1344 \\ 5444 & 12: 57: 07 & 0.1351 \\ 5445 & 12: 57: 08 & 0.1351 \\ 5446 & 12: 57: 09 & 0.1330 \\ 5447 & 12: 57: 10 & 0.1327 \\ 5448 & 12: 57: 11 & 0.1333 \\ 5449 & 12: 57: 12 & 0.1302 \\ 5450 & 12: 57: 13 & 0.1322 \\ 5451 & 12: 57: 14 & 0.1292 \\ 5452 & 12: 57: 15 & 0.1324 \\ 5453 & 12: 57: 16 & 0.1285 \\ 5454 & 12: 57: 17 & 0.1322 \\ 5455 & 12: 57: 18 & 0.1332 \\ 5456 & 12: 57: 19 & 0.1335 \\ 5457 & 12: 57: 20 & 0.1318 \\ 5458 & 12: 57: 21 & 0.1306\end{array}$


Appendix A - Fuel Data Test 1 NIST102505_1.xIs

$\begin{array}{lll}5459 & 12: 57: 22 & 0.1323 \\ 5460 & 12: 57: 23 & 0.1299 \\ 5461 & 12: 57: 24 & 0.1325 \\ 5462 & 12: 57: 25 & 0.1297 \\ 5463 & 12: 57: 26 & 0.1343 \\ 5464 & 12: 57: 27 & 0.1297 \\ 5465 & 12: 57: 28 & 0.1337 \\ 5466 & 12: 57: 29 & 0.1318 \\ 5467 & 12: 57: 30 & 0.1348 \\ 5468 & 12: 57: 31 & 0.1282 \\ 5469 & 12: 57: 32 & 0.1326 \\ 5470 & 12: 57: 33 & 0.1300 \\ 5471 & 12: 57: 34 & 0.1304 \\ 5472 & 12: 57: 35 & 0.1299 \\ 5473 & 12: 57: 36 & 0.1327 \\ 5474 & 12: 57: 37 & 0.1332 \\ 5475 & 12: 57: 38 & 0.1313 \\ 5476 & 12: 57: 39 & 0.1330 \\ 5477 & 12: 57: 40 & 0.1299 \\ 5478 & 12: 57: 41 & 0.1339 \\ 5479 & 12: 57: 42 & 0.1301 \\ 5480 & 12: 57: 43 & 0.1346 \\ 5481 & 12: 57: 44 & 0.1324 \\ 5482 & 12: 57: 45 & 0.1357 \\ 5483 & 12: 57: 46 & 0.1337 \\ 5484 & 12: 57: 47 & 0.1329 \\ 5485 & 12: 57: 48 & 0.1310 \\ 5486 & 12: 57: 49 & 0.1293 \\ 5487 & 12: 57: 50 & 0.1311 \\ 5488 & 12: 57: 51 & 0.1284 \\ 5489 & 12: 57: 52 & 0.1313 \\ 5490 & 12: 57: 53 & 0.1268 \\ 5491 & 12: 57: 54 & 0.1339 \\ 5492 & 12: 57: 55 & 0.1307 \\ 5493 & 12: 57: 56 & 0.1345 \\ 5494 & 12: 57: 57 & 0.1306 \\ 5495 & 12: 57: 58 & 0.1327 \\ 5496 & 12: 57: 59 & 0.1305 \\ 5497 & 12: 58: 00 & 0.1309 \\ 5498 & 12: 58: 01 & 0.1330 \\ 5499 & 12: 58: 02 & 0.1305 \\ 5500 & 12: 58: 03 & 0.1322 \\ 5501 & 12: 58: 04 & 0.1281 \\ 5502 & 12: 58: 05 & 0.1322 \\ 5503 & 12: 58: 06 & 0.1280 \\ 5504 & 12: 58: 07 & 0.1321 \\ 5505 & 12: 58: 08 & 0.1307 \\ 5506 & 12: 58: 09 & 0.1332 \\ 5507 & 12: 58: 10 & 0.1310 \\ 5508 & 12: 58: 11 & 0.1321 \\ 5509 & 12: 58: 12 & 0.1326 \\ 5510 & 12: 58: 13 & 0.1302\end{array}$


Appendix A - Fuel Data Test 1 NIST102505_1.xls

$\begin{array}{lll}5511 & 12: 58: 14 & 0.1299 \\ 5512 & 12: 58: 15 & 0.1278 \\ 5513 & 12: 58: 16 & 0.1318 \\ 5514 & 12: 58: 17 & 0.1288 \\ 5515 & 12: 58: 18 & 0.1313 \\ 5516 & 12: 58: 19 & 0.1311 \\ 5517 & 12: 58: 20 & 0.1341 \\ 5518 & 12: 58: 21 & 0.1313 \\ 5519 & 12: 58: 22 & 0.1346 \\ 5520 & 12: 58: 23 & 0.1323 \\ 5521 & 12: 58: 24 & 0.1335 \\ 5522 & 12: 58: 25 & 0.1295 \\ 5523 & 12: 58: 26 & 0.1297 \\ 5524 & 12: 58: 27 & 0.1303 \\ 5525 & 12: 58: 28 & 0.1270 \\ 5526 & 12: 58: 29 & 0.1315 \\ 5527 & 12: 58: 30 & 0.1277 \\ 5528 & 12: 58: 31 & 0.1313 \\ 5529 & 12: 58: 32 & 0.1278 \\ 5530 & 12: 58: 33 & 0.1322 \\ 5531 & 12: 58: 34 & 0.1291 \\ 5532 & 12: 58: 35 & 0.1309 \\ 5533 & 12: 58: 36 & 0.1311 \\ 5534 & 12: 58: 37 & 0.1310 \\ 5535 & 12: 58: 38 & 0.1326 \\ 5536 & 12: 58: 39 & 0.1314 \\ 5537 & 12: 58: 40 & 0.1359 \\ 5538 & 12: 58: 41 & 0.1316 \\ 5539 & 12: 58: 42 & 0.1328 \\ 5540 & 12: 58: 43 & 0.1272 \\ 5541 & 12: 58: 44 & 0.1332 \\ 5542 & 12: 58: 45 & 0.1290 \\ 5543 & 12: 58: 46 & 0.1322 \\ 5544 & 12: 58: 47 & 0.1309 \\ 5545 & 12: 58: 48 & 0.1332 \\ 5546 & 12: 58: 49 & 0.1311 \\ 5547 & 12: 58: 50 & 0.1310 \\ 5548 & 12: 58: 51 & 0.1319 \\ 5549 & 12: 58: 52 & 0.1296 \\ 5550 & 12: 58: 53 & 0.1324 \\ 5551 & 12: 58: 54 & 0.1272 \\ 5552 & 12: 58: 55 & 0.1326 \\ 5553 & 12: 58: 56 & 0.1294 \\ 5554 & 12: 58: 57 & 0.1336 \\ 5555 & 12: 58: 58 & 0.1305 \\ 5556 & 12: 58: 59 & 0.1358 \\ 5557 & 12: 59: 00 & 0.1320 \\ 5558 & 12: 59: 01 & 0.1324 \\ 5559 & 12: 59: 02 & 0.1338 \\ 5560 & 12: 59: 03 & 0.1330 \\ 5561 & 12: 59: 04 & 0.1345 \\ 5562 & 12: 59: 05 & 0.1302\end{array}$


Appendix A - Fuel Data Test 1 NIST102505_1.xIs

$\begin{array}{lll}5563 & 12: 59: 06 & 0.1335 \\ 5564 & 12: 59: 07 & 0.1307 \\ 5565 & 12: 59: 08 & 0.1336 \\ 5566 & 12: 59: 09 & 0.1296 \\ 5567 & 12: 59: 10 & 0.1344 \\ 5568 & 12: 59: 11 & 0.1314 \\ 5569 & 12: 59: 12 & 0.1331 \\ 5570 & 12: 59: 13 & 0.1345 \\ 5571 & 12: 59: 14 & 0.1323 \\ 5572 & 12: 59: 15 & 0.1309 \\ 5573 & 12: 59: 16 & 0.1299 \\ 5574 & 12: 59: 17 & 0.1305 \\ 5575 & 12: 59: 18 & 0.1318 \\ 5576 & 12: 59: 19 & 0.1325 \\ 5577 & 12: 59: 20 & 0.1299 \\ 5578 & 12: 59: 21 & 0.1334 \\ 5579 & 12: 59: 22 & 0.1307 \\ 5580 & 12: 59: 23 & 0.1324 \\ 5581 & 12: 59: 24 & 0.1298 \\ 5582 & 12: 59: 25 & 0.1348 \\ 5583 & 12: 59: 26 & 0.1326 \\ 5584 & 12: 59: 27 & 0.1334 \\ 5585 & 12: 59: 28 & 0.1305 \\ 5586 & 12: 59: 29 & 0.1305 \\ 5587 & 12: 59: 30 & 0.1304 \\ 5588 & 12: 59: 31 & 0.1281 \\ 5589 & 12: 59: 32 & 0.1321 \\ 5590 & 12: 59: 33 & 0.1285 \\ 5591 & 12: 59: 34 & 0.1332 \\ 5592 & 12: 59: 35 & 0.1298 \\ 5593 & 12: 59: 36 & 0.1338 \\ 5594 & 12: 59: 37 & 0.1308 \\ 5595 & 12: 59: 38 & 0.1332 \\ 5596 & 12: 59: 39 & 0.1313 \\ 5597 & 12: 59: 40 & 0.1318 \\ 5598 & 12: 59: 41 & 0.1296 \\ 5599 & 12: 59: 42 & 0.1297 \\ 5600 & 12: 59: 43 & 0.1312 \\ 5601 & 12: 59: 44 & 0.1294 \\ 5602 & 12: 59: 45 & 0.1311 \\ 5603 & 12: 59: 46 & 0.1287 \\ 5604 & 12: 59: 47 & 0.1344 \\ 5605 & 12: 59: 48 & 0.1317 \\ 5606 & 12: 59: 49 & 0.1335 \\ 5607 & 12: 59: 50 & 0.1304 \\ 5608 & 12: 59: 51 & 0.1339 \\ 5609 & 12: 59: 52 & 0.1322 \\ 5610 & 12: 59: 53 & 0.1321 \\ 5611 & 12: 59: 54 & 0.1297 \\ 5612 & 12: 59: 55 & 0.1295 \\ 5613 & 12: 59: 56 & 0.1320 \\ 5614 & 12: 59: 57 & 0.1300\end{array}$


Appendix A - Fuel Data Test 1 NIST102505_1.xIs

$\begin{array}{lll}5615 & 12: 59: 58 & 0.1324 \\ 5616 & 12: 59: 59 & 0.1295 \\ 5617 & 13: 00: 00 & 0.1323 \\ 5618 & 13: 00: 01 & 0.1301 \\ 5619 & 13: 00: 02 & 0.1332 \\ 5620 & 13: 00: 03 & 0.1348 \\ 5621 & 13: 00: 04 & 0.1357 \\ 5622 & 13: 00: 05 & 0.1337 \\ 5623 & 13: 00: 06 & 0.1302 \\ 5624 & 13: 00: 07 & 0.1328 \\ 5625 & 13: 00: 08 & 0.1301 \\ 5626 & 13: 00: 09 & 0.1324 \\ 5627 & 13: 00: 10 & 0.1288 \\ 5628 & 13: 00: 11 & 0.1316 \\ 5629 & 13: 00: 12 & 0.1278 \\ 5630 & 13: 00: 13 & 0.1315 \\ 5631 & 13: 00: 14 & 0.1312 \\ 5632 & 13: 00: 15 & 0.1336 \\ 5633 & 13: 00: 16 & 0.1309 \\ 5634 & 13: 00: 17 & 0.1316 \\ 5635 & 13: 00: 18 & 0.1322 \\ 5636 & 13: 00: 19 & 0.1314 \\ 5637 & 13: 00: 20 & 0.1308 \\ 5638 & 13: 00: 21 & 0.1284 \\ 5639 & 13: 00: 22 & 0.1316 \\ 5640 & 13: 00: 23 & 0.1283 \\ 5641 & 13: 00: 24 & 0.1319 \\ 5642 & 13: 00: 25 & 0.1295 \\ 5643 & 13: 00: 26 & 0.1339 \\ 5644 & 13: 00: 27 & 0.1314 \\ 5645 & 13: 00: 28 & 0.1334 \\ 5646 & 13: 00: 29 & 0.1303 \\ 5647 & 13: 00: 30 & 0.1320 \\ 5648 & 13: 00: 31 & 0.1284 \\ 5649 & 13: 00: 32 & 0.1290 \\ 5650 & 13: 00: 33 & 0.1320 \\ 5651 & 13: 00: 34 & 0.1298 \\ 5652 & 13: 00: 35 & 0.1324 \\ 5653 & 13: 00: 36 & 0.1273 \\ 5654 & 13: 00: 37 & 0.1318 \\ 5655 & 13: 00: 38 & 0.1280 \\ 5656 & 13: 00: 39 & 0.1311 \\ 5657 & 13: 00: 40 & 0.1293 \\ 5658 & 13: 00: 41 & 0.1331 \\ 5659 & 13: 00: 42 & 0.1306 \\ 5660 & 13: 00: 43 & 0.1309 \\ 5661 & 13: 00: 44 & 0.1322 \\ 5662 & 13: 00: 45 & 0.1305 \\ 5663 & 13: 00: 46 & 0.1311 \\ 5664 & 13: 00: 47 & 0.1279 \\ 5665 & 13: 00: 48 & 0.1314 \\ 5666 & 13: 00: 49 & 0.1272\end{array}$


Appendix A - Fuel Data Test 1 NIST102505_1.xIs

$\begin{array}{lll}5667 & 13: 00: 50 & 0.1318 \\ 5668 & 13: 00: 51 & 0.1309 \\ 5669 & 13: 00: 52 & 0.1341 \\ 5670 & 13: 00: 53 & 0.1325 \\ 5671 & 13: 00: 54 & 0.1327 \\ 5672 & 13: 00: 55 & 0.1320 \\ 5673 & 13: 00: 56 & 0.1306 \\ 5674 & 13: 00: 57 & 0.1318 \\ 5675 & 13: 00: 58 & 0.1295 \\ 5676 & 13: 00: 59 & 0.1326 \\ 5677 & 13: 01: 00 & 0.1286 \\ 5678 & 13: 01: 01 & 0.1314 \\ 5679 & 13: 01: 02 & 0.1275 \\ 5680 & 13: 01: 03 & 0.1320 \\ 5681 & 13: 01: 04 & 0.1289 \\ 5682 & 13: 01: 05 & 0.1327 \\ 5683 & 13: 01: 06 & 0.1325 \\ 5684 & 13: 01: 07 & 0.1325 \\ 5685 & 13: 01: 08 & 0.1318 \\ 5686 & 13: 01: 09 & 0.1295 \\ 5687 & 13: 01: 10 & 0.1331 \\ 5688 & 13: 01: 11 & 0.1309 \\ 5689 & 13: 01: 12 & 0.1325 \\ 5690 & 13: 01: 13 & 0.1290 \\ 5691 & 13: 01: 14 & 0.1336 \\ 5692 & 13: 01: 15 & 0.1290 \\ 5693 & 13: 01: 16 & 0.1331 \\ 5694 & 13: 01: 17 & 0.1328 \\ 5695 & 13: 01: 18 & 0.1349 \\ 5696 & 13: 01: 19 & 0.1321 \\ 5697 & 13: 01: 20 & 0.1325 \\ 5698 & 13: 01: 21 & 0.1334 \\ 5699 & 13: 01: 22 & 0.1306 \\ 5700 & 13: 01: 23 & 0.1322 \\ 5701 & 13: 01: 24 & 0.1299 \\ 5702 & 13: 01: 25 & 0.1335 \\ 5703 & 13: 01: 26 & 0.1293 \\ 5704 & 13: 01: 27 & 0.1322 \\ 5705 & 13: 01: 28 & 0.1286 \\ 5706 & 13: 01: 29 & 0.1324 \\ 5707 & 13: 01: 30 & 0.1297 \\ 5708 & 13: 01: 31 & 0.1313 \\ 5709 & 13: 01: 32 & 0.1337 \\ 5710 & 13: 01: 33 & 0.1321 \\ 5711 & 13: 01: 34 & 0.1313 \\ 5712 & 13: 01: 35 & 0.1298 \\ 5713 & 13: 01: 36 & 0.1342 \\ 5714 & 13: 01: 37 & 0.1303 \\ 5715 & 13: 01: 38 & 0.1341 \\ 5716 & 13: 01: 39 & 0.1301 \\ 5717 & 13: 01: 40 & 0.1342 \\ 5718 & 13: 01: 41 & 0.1295\end{array}$


Appendix A - Fuel Data Test 1 NIST102505_1.xIs

$\begin{array}{lll}5719 & 13: 01: 42 & 0.1339 \\ 5720 & 13: 01: 43 & 0.1327 \\ 5721 & 13: 01: 44 & 0.1344 \\ 5722 & 13: 01: 45 & 0.1307 \\ 5723 & 13: 01: 46 & 0.1330 \\ 5724 & 13: 01: 47 & 0.1312 \\ 5725 & 13: 01: 48 & 0.1293 \\ 5726 & 13: 01: 49 & 0.1303 \\ 5727 & 13: 01: 50 & 0.1287 \\ 5728 & 13: 01: 51 & 0.1317 \\ 5729 & 13: 01: 52 & 0.1263 \\ 5730 & 13: 01: 53 & 0.1330 \\ 5731 & 13: 01: 54 & 0.1280 \\ 5732 & 13: 01: 55 & 0.1318 \\ 5733 & 13: 01: 56 & 0.1316 \\ 5734 & 13: 01: 57 & 0.1328 \\ 5735 & 13: 01: 58 & 0.1318 \\ 5736 & 13: 01: 59 & 0.1321 \\ 5737 & 13: 02: 00 & 0.1295 \\ 5738 & 13: 02: 01 & 0.1308 \\ 5739 & 13: 02: 02 & 0.1330 \\ 5740 & 13: 02: 03 & 0.1282 \\ 5741 & 13: 02: 04 & 0.1324 \\ 5742 & 13: 02: 05 & 0.1286 \\ 5743 & 13: 02: 06 & 0.1326 \\ 5744 & 13: 02: 07 & 0.1286 \\ 5745 & 13: 02: 08 & 0.1327 \\ 5746 & 13: 02: 09 & 0.1301 \\ 5747 & 13: 02: 10 & 0.1316 \\ 5748 & 13: 02: 11 & 0.1317 \\ 5749 & 13: 02: 12 & 0.1336 \\ 5750 & 13: 02: 13 & 0.1321 \\ 5751 & 13: 02: 14 & 0.1316 \\ 5752 & 13: 02: 15 & 0.1338 \\ 5753 & 13: 02: 16 & 0.1303 \\ 5754 & 13: 02: 17 & 0.1335 \\ 5755 & 13: 02: 18 & 0.1317 \\ 5756 & 13: 02: 19 & 0.1361 \\ 5757 & 13: 02: 20 & 0.1314 \\ 5758 & 13: 02: 21 & 0.1357 \\ 5759 & 13: 02: 22 & 0.1323 \\ 5760 & 13: 02: 23 & 0.1341 \\ 5761 & 13: 02: 24 & 0.1320 \\ 5762 & 13: 02: 25 & 0.1315 \\ 5763 & 13: 02: 26 & 0.1311 \\ 5764 & 13: 02: 27 & 0.1284 \\ 5765 & 13: 02: 28 & 0.1313 \\ 5766 & 13: 02: 29 & 0.1275 \\ 5767 & 13: 02: 30 & 0.1314 \\ 5768 & 13: 02: 31 & 0.1299 \\ 5769 & 13: 02: 32 & 0.1349 \\ 5770 & 13: 02: 33 & 0.1322\end{array}$


Appendix A - Fuel Data Test 1 NIST102505_1.xls

$\begin{array}{lll}5771 & 13: 02: 34 & 0.1354 \\ 5772 & 13: 02: 35 & 0.1347 \\ 5773 & 13: 02: 36 & 0.1357 \\ 5774 & 13: 02: 37 & 0.1331 \\ 5775 & 13: 02: 38 & 0.1327 \\ 5776 & 13: 02: 39 & 0.1328 \\ 5777 & 13: 02: 40 & 0.1305 \\ 5778 & 13: 02: 41 & 0.1329 \\ 5779 & 13: 02: 42 & 0.1302 \\ 5780 & 13: 02: 43 & 0.1309 \\ 5781 & 13: 02: 44 & 0.1267 \\ 5782 & 13: 02: 45 & 0.1323 \\ 5783 & 13: 02: 46 & 0.1310 \\ 5784 & 13: 02: 47 & 0.1337 \\ 5785 & 13: 02: 48 & 0.1307 \\ 5786 & 13: 02: 49 & 0.1327 \\ 5787 & 13: 02: 50 & 0.1327 \\ 5788 & 13: 02: 51 & 0.1309 \\ 5789 & 13: 02: 52 & 0.1300 \\ 5790 & 13: 02: 53 & 0.1279 \\ 5791 & 13: 02: 54 & 0.1302 \\ 5792 & 13: 02: 55 & 0.1262 \\ 5793 & 13: 02: 56 & 0.1309 \\ 5794 & 13: 02: 57 & 0.1274 \\ 5795 & 13: 02: 58 & 0.1326 \\ 5796 & 13: 02: 59 & 0.1292 \\ 5797 & 13: 03: 00 & 0.1329 \\ 5798 & 13: 03: 01 & 0.1301 \\ 5799 & 13: 03: 02 & 0.1311 \\ 5800 & 13: 03: 03 & 0.1291 \\ 5801 & 13: 03: 04 & 0.1296 \\ 5802 & 13: 03: 05 & 0.1298 \\ 5803 & 13: 03: 06 & 0.1274 \\ 5804 & 13: 03: 07 & 0.1318 \\ 5805 & 13: 03: 08 & 0.1281 \\ 5806 & 13: 03: 09 & 0.1317 \\ 5807 & 13: 03: 10 & 0.1276 \\ 5808 & 13: 03: 11 & 0.1309 \\ 5809 & 13: 03: 12 & 0.1301 \\ 5810 & 13: 03: 13 & 0.1320 \\ 5811 & 13: 03: 14 & 0.1329 \\ 5812 & 13: 03: 15 & 0.1330 \\ 5813 & 13: 03: 16 & 0.1316 \\ 5814 & 13: 03: 17 & 0.1303 \\ 5815 & 13: 03: 18 & 0.1336 \\ 5816 & 13: 03: 19 & 0.1293 \\ 5817 & 13: 03: 20 & 0.1310 \\ 5818 & 13: 03: 21 & 0.1268 \\ 5819 & 13: 03: 22 & 0.1335 \\ 5820 & 13: 03: 23 & 0.1295 \\ 5821 & 13: 03: 24 & 0.1317 \\ 5822 & 13: 03: 25 & 0.1301\end{array}$


Appendix A - Fuel Data Test 1 NIST102505_1.xIs

$\begin{array}{lll}5823 & 13: 03: 26 & 0.1334 \\ 5824 & 13: 03: 27 & 0.1308 \\ 5825 & 13: 03: 28 & 0.1301 \\ 5826 & 13: 03: 29 & 0.1309 \\ 5827 & 13: 03: 30 & 0.1299 \\ 5828 & 13: 03: 31 & 0.1314 \\ 5829 & 13: 03: 32 & 0.1282 \\ 5830 & 13: 03: 33 & 0.1315 \\ 5831 & 13: 03: 34 & 0.1271 \\ 5832 & 13: 03: 35 & 0.1320 \\ 5833 & 13: 03: 36 & 0.1311 \\ 5834 & 13: 03: 37 & 0.1326 \\ 5835 & 13: 03: 38 & 0.1310 \\ 5836 & 13: 03: 39 & 0.1317 \\ 5837 & 13: 03: 40 & 0.1322 \\ 5838 & 13: 03: 41 & 0.1298 \\ 5839 & 13: 03: 42 & 0.1311 \\ 5840 & 13: 03: 43 & 0.1277 \\ 5841 & 13: 03: 44 & 0.1330 \\ 5842 & 13: 03: 45 & 0.1297 \\ 5843 & 13: 03: 46 & 0.1324 \\ 5844 & 13: 03: 47 & 0.1301 \\ 5845 & 13: 03: 48 & 0.1342 \\ 5846 & 13: 03: 49 & 0.1315 \\ 5847 & 13: 03: 50 & 0.1332 \\ 5848 & 13: 03: 51 & 0.1338 \\ 5849 & 13: 03: 52 & 0.1322 \\ 5850 & 13: 03: 53 & 0.1307 \\ 5851 & 13: 03: 54 & 0.1285 \\ 5852 & 13: 03: 55 & 0.1328 \\ 5853 & 13: 03: 56 & 0.1300 \\ 5854 & 13: 03: 57 & 0.1326 \\ 5855 & 13: 03: 58 & 0.1282 \\ 5856 & 13: 03: 59 & 0.1315 \\ 5857 & 13: 04: 00 & 0.1291 \\ 5858 & 13: 04: 01 & 0.1319 \\ 5859 & 13: 04: 02 & 0.1308 \\ 5860 & 13: 04: 03 & 0.1327 \\ 5861 & 13: 04: 04 & 0.1299 \\ 5862 & 13: 04: 05 & 0.1319 \\ 5863 & 13: 04: 06 & 0.1327 \\ 5864 & 13: 04: 07 & 0.1316 \\ 5865 & 13: 04: 08 & 0.1308 \\ 5866 & 13: 04: 09 & 0.1287 \\ 5867 & 13: 04: 10 & 0.1307 \\ 5868 & 13: 04: 11 & 0.1261 \\ 5869 & 13: 04: 12 & 0.1325 \\ 5870 & 13: 04: 13 & 0.1289 \\ 5871 & 13: 04: 14 & 0.1317 \\ 5872 & 13: 04: 15 & 0.1297 \\ 5873 & 13: 04: 16 & 0.1317 \\ 5874 & 13: 04: 17 & 0.1283\end{array}$


Appendix A - Fuel Data Test 1 NIST102505_1.xIs

$\begin{array}{lll}5875 & 13: 04: 18 & 0.1293 \\ 5876 & 13: 04: 19 & 0.1307 \\ 5877 & 13: 04: 20 & 0.1294 \\ 5878 & 13: 04: 21 & 0.1319 \\ 5879 & 13: 04: 22 & 0.1281 \\ 5880 & 13: 04: 23 & 0.1316 \\ 5881 & 13: 04: 24 & 0.1278 \\ 5882 & 13: 04: 25 & 0.1311 \\ 5883 & 13: 04: 26 & 0.1300 \\ 5884 & 13: 04: 27 & 0.1336 \\ 5885 & 13: 04: 28 & 0.1302 \\ 5886 & 13: 04: 29 & 0.1324 \\ 5887 & 13: 04: 30 & 0.1318 \\ 5888 & 13: 04: 31 & 0.1311 \\ 5889 & 13: 04: 32 & 0.1294 \\ 5890 & 13: 04: 33 & 0.1292 \\ 5891 & 13: 04: 34 & 0.1299 \\ 5892 & 13: 04: 35 & 0.1267 \\ 5893 & 13: 04: 36 & 0.1328 \\ 5894 & 13: 04: 37 & 0.1284 \\ 5895 & 13: 04: 38 & 0.1331 \\ 5896 & 13: 04: 39 & 0.1307 \\ 5897 & 13: 04: 40 & 0.1335 \\ 5898 & 13: 04: 41 & 0.1322 \\ 5899 & 13: 04: 42 & 0.1320 \\ 5900 & 13: 04: 43 & 0.1325 \\ 5901 & 13: 04: 44 & 0.1318 \\ 5902 & 13: 04: 45 & 0.1325 \\ 5903 & 13: 04: 46 & 0.1316 \\ 5904 & 13: 04: 47 & 0.1324 \\ 5905 & 13: 04: 48 & 0.1292 \\ 5906 & 13: 04: 49 & 0.1332 \\ 5907 & 13: 04: 50 & 0.1300 \\ 5908 & 13: 04: 51 & 0.1334 \\ 5909 & 13: 04: 52 & 0.1295 \\ 5910 & 13: 04: 53 & 0.1341 \\ 5911 & 13: 04: 54 & 0.1312 \\ 5912 & 13: 04: 55 & 0.1316 \\ 5913 & 13: 04: 56 & 0.1284 \\ 5914 & 13: 04: 57 & 0.1281 \\ 5915 & 13: 04: 58 & 0.1287 \\ 5916 & 13: 04: 59 & 0.1282 \\ 5917 & 13: 05: 00 & 0.1343 \\ 5918 & 13: 05: 01 & 0.1295 \\ 5919 & 13: 05: 02 & 0.1321 \\ 5920 & 13: 05: 03 & 0.1281 \\ 5921 & 13: 05: 04 & 0.1326 \\ 5922 & 13: 05: 05 & 0.1305 \\ 5923 & 13: 05: 06 & 0.1324 \\ 5924 & 13: 05: 07 & 0.1283 \\ 5925 & 13: 05: 08 & 0.1309 \\ 5926 & 13: 05: 09 & 0.1275\end{array}$


Appendix A - Fuel Data Test 1 NIST102505_1.xIs

$\begin{array}{lll}5927 & 13: 05: 10 & 0.1270 \\ 5928 & 13: 05: 11 & 0.1309 \\ 5929 & 13: 05: 12 & 0.1277 \\ 5930 & 13: 05: 13 & 0.1308 \\ 5931 & 13: 05: 14 & 0.1267 \\ 5932 & 13: 05: 15 & 0.1312 \\ 5933 & 13: 05: 16 & 0.1283 \\ 5934 & 13: 05: 17 & 0.1310 \\ 5935 & 13: 05: 18 & 0.1293 \\ 5936 & 13: 05: 19 & 0.1336 \\ 5937 & 13: 05: 20 & 0.1312 \\ 5938 & 13: 05: 21 & 0.1315 \\ 5939 & 13: 05: 22 & 0.1315 \\ 5940 & 13: 05: 23 & 0.1297 \\ 5941 & 13: 05: 24 & 0.1299 \\ 5942 & 13: 05: 25 & 0.1276 \\ 5943 & 13: 05: 26 & 0.1314 \\ 5944 & 13: 05: 27 & 0.1265 \\ 5945 & 13: 05: 28 & 0.1313 \\ 5946 & 13: 05: 29 & 0.1310 \\ 5947 & 13: 05: 30 & 0.1344 \\ 5948 & 13: 05: 31 & 0.1316 \\ 5949 & 13: 05: 32 & 0.1350 \\ 5950 & 13: 05: 33 & 0.1301 \\ 5951 & 13: 05: 34 & 0.1290 \\ 5952 & 13: 05: 35 & 0.1293 \\ 5953 & 13: 05: 36 & 0.1278 \\ 5954 & 13: 05: 37 & 0.1303 \\ 5955 & 13: 05: 38 & 0.1263 \\ 5956 & 13: 05: 39 & 0.1317 \\ 5957 & 13: 05: 40 & 0.1267 \\ 5958 & 13: 05: 41 & 0.1314 \\ 5959 & 13: 05: 42 & 0.1290 \\ 5960 & 13: 05: 43 & 0.1331 \\ 5961 & 13: 05: 44 & 0.1295 \\ 5962 & 13: 05: 45 & 0.1327 \\ 5963 & 13: 05: 46 & 0.1309 \\ 5964 & 13: 05: 47 & 0.1301 \\ 5965 & 13: 05: 48 & 0.1307 \\ 5966 & 13: 05: 49 & 0.1295 \\ 5967 & 13: 05: 50 & 0.1301 \\ 5968 & 13: 05: 51 & 0.1266 \\ 5969 & 13: 05: 52 & 0.1308 \\ 5970 & 13: 05: 53 & 0.1282 \\ 5971 & 13: 05: 54 & 0.1317 \\ 5972 & 13: 05: 55 & 0.1300 \\ 5973 & 13: 05: 56 & 0.1330 \\ 5974 & 13: 05: 57 & 0.1330 \\ 5975 & 13: 05: 58 & 0.1336 \\ 5976 & 13: 05: 59 & 0.1312 \\ 5977 & 13: 06: 00 & 0.1310 \\ 5978 & 13: 06: 01 & 0.1333\end{array}$


Appendix A - Fuel Data Test 1 NIST102505_1.xIs

$\begin{array}{lll}5979 & 13: 06: 02 & 0.1303 \\ 5980 & 13: 06: 03 & 0.1328 \\ 5981 & 13: 06: 04 & 0.1304 \\ 5982 & 13: 06: 05 & 0.1337 \\ 5983 & 13: 06: 06 & 0.1298 \\ 5984 & 13: 06: 07 & 0.1352 \\ 5985 & 13: 06: 08 & 0.1318 \\ 5986 & 13: 06: 09 & 0.1340 \\ 5987 & 13: 06: 10 & 0.1316 \\ 5988 & 13: 06: 11 & 0.1335 \\ 5989 & 13: 06: 12 & 0.1313 \\ 5990 & 13: 06: 13 & 0.1286 \\ 5991 & 13: 06: 14 & 0.1296 \\ 5992 & 13: 06: 15 & 0.1276 \\ 5993 & 13: 06: 16 & 0.1306 \\ 5994 & 13: 06: 17 & 0.1264 \\ 5995 & 13: 06: 18 & 0.1328 \\ 5996 & 13: 06: 19 & 0.1290 \\ 5997 & 13: 06: 20 & 0.1326 \\ 5998 & 13: 06: 21 & 0.1325 \\ 5999 & 13: 06: 22 & 0.1326 \\ 6000 & 13: 06: 23 & 0.1310 \\ 6001 & 13: 06: 24 & 0.1291 \\ 6002 & 13: 06: 25 & 0.1315 \\ 6003 & 13: 06: 26 & 0.1289 \\ 6004 & 13: 06: 27 & 0.1303 \\ 6005 & 13: 06: 28 & 0.1264 \\ 6006 & 13: 06: 29 & 0.1321 \\ 6007 & 13: 06: 30 & 0.1272 \\ 6008 & 13: 06: 31 & 0.1313 \\ 6009 & 13: 06: 32 & 0.1290 \\ 6010 & 13: 06: 33 & 0.1330 \\ 6011 & 13: 06: 34 & 0.1301 \\ 6012 & 13: 06: 35 & 0.1332 \\ 6013 & 13: 06: 36 & 0.1304 \\ 6014 & 13: 06: 37 & 0.1303 \\ 6015 & 13: 06: 38 & 0.1301 \\ 6016 & 13: 06: 39 & 0.1292 \\ 6017 & 13: 06: 40 & 0.1313 \\ 6018 & 13: 06: 41 & 0.1271 \\ 6019 & 13: 06: 42 & 0.1326 \\ 6020 & 13: 06: 43 & 0.1283 \\ 6021 & 13: 06: 44 & 0.1327 \\ 6022 & 13: 06: 45 & 0.1302 \\ 6023 & 13: 06: 46 & 0.1333 \\ 6024 & 13: 06: 47 & 0.1309 \\ 6025 & 13: 06: 48 & 0.1324 \\ 6026 & 13: 06: 49 & 0.1307 \\ 6027 & 13: 06: 50 & 0.1304 \\ 6028 & 13: 06: 51 & 0.1305 \\ 6029 & 13: 06: 52 & 0.1274 \\ 6030 & 13: 06: 53 & 0.1332\end{array}$


Appendix A - Fuel Data Test 1 NIST102505_1.xIs

$\begin{array}{lll}6031 & 13: 06: 54 & 0.1286 \\ 6032 & 13: 06: 55 & 0.1320 \\ 6033 & 13: 06: 56 & 0.1295 \\ 6034 & 13: 06: 57 & 0.1330 \\ 6035 & 13: 06: 58 & 0.1281 \\ 6036 & 13: 06: 59 & 0.1314 \\ 6037 & 13: 07: 00 & 0.1326 \\ 6038 & 13: 07: 01 & 0.1321 \\ 6039 & 13: 07: 02 & 0.1312 \\ 6040 & 13: 07: 03 & 0.1289 \\ 6041 & 13: 07: 04 & 0.1310 \\ 6042 & 13: 07: 05 & 0.1281 \\ 6043 & 13: 07: 06 & 0.1315 \\ 6044 & 13: 07: 07 & 0.1291 \\ 6045 & 13: 07: 08 & 0.1310 \\ 6046 & 13: 07: 09 & 0.1276 \\ 6047 & 13: 07: 10 & 0.1317 \\ 6048 & 13: 07: 11 & 0.1331 \\ 6049 & 13: 07: 12 & 0.1348 \\ 6050 & 13: 07: 13 & 0.1326 \\ 6051 & 13: 07: 14 & 0.1314 \\ 6052 & 13: 07: 15 & 0.1340 \\ 6053 & 13: 07: 16 & 0.1298 \\ 6054 & 13: 07: 17 & 0.1311 \\ 6055 & 13: 07: 18 & 0.1286 \\ 6056 & 13: 07: 19 & 0.1313 \\ 6057 & 13: 07: 20 & 0.1271 \\ 6058 & 13: 07: 21 & 0.1330 \\ 6059 & 13: 07: 22 & 0.1303 \\ 6060 & 13: 07: 23 & 0.1344 \\ 6061 & 13: 07: 24 & 0.1304 \\ 6062 & 13: 07: 25 & 0.1324 \\ 6063 & 13: 07: 26 & 0.1322 \\ 6064 & 13: 07: 27 & 0.1326 \\ 6065 & 13: 07: 28 & 0.1318 \\ 6066 & 13: 07: 29 & 0.1299 \\ 6067 & 13: 07: 30 & 0.1305 \\ 6068 & 13: 07: 31 & 0.1272 \\ 6069 & 13: 07: 32 & 0.1308 \\ 6070 & 13: 07: 33 & 0.1287 \\ 6071 & 13: 07: 34 & 0.1331 \\ 6072 & 13: 07: 35 & 0.1283 \\ 6073 & 13: 07: 36 & 0.1332 \\ 6074 & 13: 07: 37 & 0.1300 \\ 6075 & 13: 07: 38 & 0.1306 \\ 6076 & 13: 07: 39 & 0.1329 \\ 6077 & 13: 07: 40 & 0.1311 \\ 6078 & 13: 07: 41 & 0.1321 \\ 6079 & 13: 07: 42 & 0.1296 \\ 6080 & 13: 07: 43 & 0.1328 \\ 6081 & 13: 07: 44 & 0.1281 \\ 6082 & 13: 07: 45 & 0.1317\end{array}$


Appendix A - Fuel Data Test 1 NIST102505_1.xIs

$\begin{array}{lll}6083 & 13: 07: 46 & 0.1274 \\ 6084 & 13: 07: 47 & 0.1332 \\ 6085 & 13: 07: 48 & 0.1283 \\ 6086 & 13: 07: 49 & 0.1335 \\ 6087 & 13: 07: 50 & 0.1325 \\ 6088 & 13: 07: 51 & 0.1330 \\ 6089 & 13: 07: 52 & 0.1310 \\ 6090 & 13: 07: 53 & 0.1305 \\ 6091 & 13: 07: 54 & 0.1311 \\ 6092 & 13: 07: 55 & 0.1291 \\ 6093 & 13: 07: 56 & 0.1294 \\ 6094 & 13: 07: 57 & 0.1264 \\ 6095 & 13: 07: 58 & 0.1311 \\ 6096 & 13: 07: 59 & 0.1272 \\ 6097 & 13: 08: 00 & 0.1324 \\ 6098 & 13: 08: 01 & 0.1291 \\ 6099 & 13: 08: 02 & 0.1327 \\ 6100 & 13: 08: 03 & 0.1287 \\ 6101 & 13: 08: 04 & 0.1304 \\ 6102 & 13: 08: 05 & 0.1291 \\ 6103 & 13: 08: 06 & 0.1284 \\ 6104 & 13: 08: 07 & 0.1324 \\ 6105 & 13: 08: 08 & 0.1291 \\ 6106 & 13: 08: 09 & 0.1316 \\ 6107 & 13: 08: 10 & 0.1264 \\ 6108 & 13: 08: 11 & 0.1309 \\ 6109 & 13: 08: 12 & 0.1274 \\ 6110 & 13: 08: 13 & 0.1308 \\ 6111 & 13: 08: 14 & 0.1307 \\ 6112 & 13: 08: 15 & 0.1327 \\ 6113 & 13: 08: 16 & 0.1305 \\ 6114 & 13: 08: 17 & 0.1300 \\ 6115 & 13: 08: 18 & 0.1327 \\ 6116 & 13: 08: 19 & 0.1304 \\ 6117 & 13: 08: 20 & 0.1306 \\ 6118 & 13: 08: 21 & 0.1284 \\ 6119 & 13: 08: 22 & 0.1311 \\ 6120 & 13: 08: 23 & 0.1263 \\ 6121 & 13: 08: 24 & 0.1306 \\ 6122 & 13: 08: 25 & 0.1300 \\ 6123 & 13: 08: 26 & 0.1338 \\ 6124 & 13: 08: 27 & 0.1299 \\ 6125 & 13: 08: 28 & 0.1317 \\ 6126 & 13: 08: 29 & 0.1341 \\ 6127 & 13: 08: 30 & 0.1345 \\ 6128 & 13: 08: 31 & 0.1326 \\ 6129 & 13: 08: 32 & 0.1319 \\ 6130 & 13: 08: 33 & 0.1330 \\ 6131 & 13: 08: 34 & 0.1299 \\ 6132 & 13: 08: 35 & 0.1340 \\ 6133 & 13: 08: 36 & 0.1298 \\ 6134 & 13: 08: 37 & 0.1334\end{array}$


Appendix A - Fuel Data Test 1 NIST102505_1.xIs

$\begin{array}{lll}6135 & 13: 08: 38 & 0.1306 \\ 6136 & 13: 08: 39 & 0.1360 \\ 6137 & 13: 08: 40 & 0.1320 \\ 6138 & 13: 08: 41 & 0.1334 \\ 6139 & 13: 08: 42 & 0.1336 \\ 6140 & 13: 08: 43 & 0.1328 \\ 6141 & 13: 08: 44 & 0.1316 \\ 6142 & 13: 08: 45 & 0.1296 \\ 6143 & 13: 08: 46 & 0.1346 \\ 6144 & 13: 08: 47 & 0.1293 \\ 6145 & 13: 08: 48 & 0.1328 \\ 6146 & 13: 08: 49 & 0.1280 \\ 6147 & 13: 08: 50 & 0.1333 \\ 6148 & 13: 08: 51 & 0.1301 \\ 6149 & 13: 08: 52 & 0.1334 \\ 6150 & 13: 08: 53 & 0.1316 \\ 6151 & 13: 08: 54 & 0.1328 \\ 6152 & 13: 08: 55 & 0.1314 \\ 6153 & 13: 08: 56 & 0.1293 \\ 6154 & 13: 08: 57 & 0.1302 \\ 6155 & 13: 08: 58 & 0.1294 \\ 6156 & 13: 08: 59 & 0.1316 \\ 6157 & 13: 09: 00 & 0.1282 \\ 6158 & 13: 09: 01 & 0.1331 \\ 6159 & 13: 09: 02 & 0.1298 \\ 6160 & 13: 09: 03 & 0.1344 \\ 6161 & 13: 09: 04 & 0.1307 \\ 6162 & 13: 09: 05 & 0.1327 \\ 6163 & 13: 09: 06 & 0.1286 \\ 6164 & 13: 09: 07 & 0.1308 \\ 6165 & 13: 09: 08 & 0.1327 \\ 6166 & 13: 09: 09 & 0.1296 \\ 6167 & 13: 09: 10 & 0.1318 \\ 6168 & 13: 09: 11 & 0.1293 \\ 6169 & 13: 09: 12 & 0.1332 \\ 6170 & 13: 09: 13 & 0.1289 \\ 6171 & 13: 09: 14 & 0.1320 \\ 6172 & 13: 09: 15 & 0.1305 \\ 6173 & 13: 09: 16 & 0.1337 \\ 6174 & 13: 09: 17 & 0.1310 \\ 6175 & 13: 09: 18 & 0.1345 \\ 6176 & 13: 09: 19 & 0.1334 \\ 6177 & 13: 09: 20 & 0.1334 \\ 6178 & 13: 09: 21 & 0.1313 \\ 6179 & 13: 09: 22 & 0.1314 \\ 6180 & 13: 09: 23 & 0.1328 \\ 6181 & 13: 09: 24 & 0.1301 \\ 6182 & 13: 09: 25 & 0.1321 \\ 6183 & 13: 09: 26 & 0.1296 \\ 6184 & 13: 09: 27 & 0.1340 \\ 6185 & 13: 09: 28 & 0.1301 \\ 6186 & 13: 09: 29 & 0.1358\end{array}$


Appendix A - Fuel Data Test 1 NIST102505_1.xIs

$\begin{array}{lll}6187 & 13: 09: 30 & 0.1320 \\ 6188 & 13: 09: 31 & 0.1342 \\ 6189 & 13: 09: 32 & 0.1317 \\ 6190 & 13: 09: 33 & 0.1334 \\ 6191 & 13: 09: 34 & 0.1327 \\ 6192 & 13: 09: 35 & 0.1323 \\ 6193 & 13: 09: 36 & 0.1340 \\ 6194 & 13: 09: 37 & 0.1289 \\ 6195 & 13: 09: 38 & 0.1329 \\ 6196 & 13: 09: 39 & 0.1277 \\ 6197 & 13: 09: 40 & 0.1328 \\ 6198 & 13: 09: 41 & 0.1289 \\ 6199 & 13: 09: 42 & 0.1325 \\ 6200 & 13: 09: 43 & 0.1308 \\ 6201 & 13: 09: 44 & 0.1322 \\ 6202 & 13: 09: 45 & 0.1316 \\ 6203 & 13: 09: 46 & 0.1300 \\ 6204 & 13: 09: 47 & 0.1329 \\ 6205 & 13: 09: 48 & 0.1295 \\ 6206 & 13: 09: 49 & 0.1306 \\ 6207 & 13: 09: 50 & 0.1275 \\ 6208 & 13: 09: 51 & 0.1324 \\ 6209 & 13: 09: 52 & 0.1278 \\ 6210 & 13: 09: 53 & 0.1322 \\ 6211 & 13: 09: 54 & 0.1303 \\ 6212 & 13: 09: 55 & 0.1326 \\ 6213 & 13: 09: 56 & 0.1312 \\ 6214 & 13: 09: 57 & 0.1331 \\ 6215 & 13: 09: 58 & 0.1327 \\ 6216 & 13: 09: 59 & 0.1307 \\ 6217 & 13: 10: 00 & 0.1306 \\ 6218 & 13: 10: 01 & 0.1280 \\ 6219 & 13: 10: 02 & 0.1308 \\ 6220 & 13: 10: 03 & 0.1262 \\ 6221 & 13: 10: 04 & 0.1306 \\ 6222 & 13: 10: 05 & 0.1281 \\ 6223 & 13: 10: 06 & 0.1325 \\ 6224 & 13: 10: 07 & 0.1281 \\ 6225 & 13: 10: 08 & 0.1330 \\ 6226 & 13: 10: 09 & 0.1304 \\ 6227 & 13: 10: 10 & 0.1311 \\ 6228 & 13: 10: 11 & 0.1276 \\ 6229 & 13: 10: 12 & 0.1276 \\ 6230 & 13: 10: 13 & 0.1297 \\ 6231 & 13: 10: 14 & 0.1286 \\ 6232 & 13: 10: 15 & 0.1323 \\ 6233 & 13: 10: 16 & 0.1306 \\ 6234 & 13: 10: 17 & 0.1327 \\ 6235 & 13: 10: 18 & 0.1285 \\ 6236 & 13: 10: 19 & 0.1316 \\ 6237 & 13: 10: 20 & 0.1311 \\ 6238 & 13: 10: 21 & 0.1333\end{array}$


Appendix A - Fuel Data Test 1 NIST102505_1.xIs

$\begin{array}{lll}6239 & 13: 10: 22 & 0.1296 \\ 6240 & 13: 10: 23 & 0.1323 \\ 6241 & 13: 10: 24 & 0.1315 \\ 6242 & 13: 10: 25 & 0.1304 \\ 6243 & 13: 10: 26 & 0.1308 \\ 6244 & 13: 10: 27 & 0.1304 \\ 6245 & 13: 10: 28 & 0.1311 \\ 6246 & 13: 10: 29 & 0.1269 \\ 6247 & 13: 10: 30 & 0.1312 \\ 6248 & 13: 10: 31 & 0.1291 \\ 6249 & 13: 10: 32 & 0.1330 \\ 6250 & 13: 10: 33 & 0.1288 \\ 6251 & 13: 10: 34 & 0.1322 \\ 6252 & 13: 10: 35 & 0.1326 \\ 6253 & 13: 10: 36 & 0.1329 \\ 6254 & 13: 10: 37 & 0.1315 \\ 6255 & 13: 10: 38 & 0.1300 \\ 6256 & 13: 10: 39 & 0.1316 \\ 6257 & 13: 10: 40 & 0.1278 \\ 6258 & 13: 10: 41 & 0.1311 \\ 6259 & 13: 10: 42 & 0.1284 \\ 6260 & 13: 10: 43 & 0.1321 \\ 6261 & 13: 10: 44 & 0.1289 \\ 6262 & 13: 10: 45 & 0.1331 \\ 6263 & 13: 10: 46 & 0.1312 \\ 6264 & 13: 10: 47 & 0.1318 \\ 6265 & 13: 10: 48 & 0.1304 \\ 6266 & 13: 10: 49 & 0.1303 \\ 6267 & 13: 10: 50 & 0.1318 \\ 6268 & 13: 10: 51 & 0.1284 \\ 6269 & 13: 10: 52 & 0.1303 \\ 6270 & 13: 10: 53 & 0.1262 \\ 6271 & 13: 10: 54 & 0.1295 \\ 6272 & 13: 10: 55 & 0.1252 \\ 6273 & 13: 10: 56 & 0.1317 \\ 6274 & 13: 10: 57 & 0.1297 \\ 6275 & 13: 10: 58 & 0.1331 \\ 6276 & 13: 10: 59 & 0.1288 \\ 6277 & 13: 11: 00 & 0.1319 \\ 6278 & 13: 11: 01 & 0.1283 \\ 6279 & 13: 11: 02 & 0.1276 \\ 6280 & 13: 11: 03 & 0.1328 \\ 6281 & 13: 11: 04 & 0.1293 \\ 6282 & 13: 11: 05 & 0.1324 \\ 6283 & 13: 11: 06 & 0.1287 \\ 6284 & 13: 11: 07 & 0.1321 \\ 6285 & 13: 11: 08 & 0.1285 \\ 6286 & 13: 11: 09 & 0.1309 \\ 6287 & 13: 11: 10 & 0.1297 \\ 6288 & 13: 11: 11 & 0.1333 \\ 6289 & 13: 11: 12 & 0.1300 \\ 6290 & 13: 11: 13 & 0.1312\end{array}$


Appendix A - Fuel Data Test 1 NIST102505_1.xIs

$\begin{array}{lll}6291 & 13: 11: 14 & 0.1310 \\ 6292 & 13: 11: 15 & 0.1293 \\ 6293 & 13: 11: 16 & 0.1303 \\ 6294 & 13: 11: 17 & 0.1290 \\ 6295 & 13: 11: 18 & 0.1312 \\ 6296 & 13: 11: 19 & 0.1269 \\ 6297 & 13: 11: 20 & 0.1329 \\ 6298 & 13: 11: 21 & 0.1303 \\ 6299 & 13: 11: 22 & 0.1335 \\ 6300 & 13: 11: 23 & 0.1297 \\ 6301 & 13: 11: 24 & 0.1321 \\ 6302 & 13: 11: 25 & 0.1299 \\ 6303 & 13: 11: 26 & 0.1306 \\ 6304 & 13: 11: 27 & 0.1292 \\ 6305 & 13: 11: 28 & 0.1285 \\ 6306 & 13: 11: 29 & 0.1294 \\ 6307 & 13: 11: 30 & 0.1269 \\ 6308 & 13: 11: 31 & 0.1328 \\ 6309 & 13: 11: 32 & 0.1273 \\ 6310 & 13: 11: 33 & 0.1303 \\ 6311 & 13: 11: 34 & 0.1276 \\ 6312 & 13: 11: 35 & 0.1311 \\ 6313 & 13: 11: 36 & 0.1282 \\ 6314 & 13: 11: 37 & 0.1306 \\ 6315 & 13: 11: 38 & 0.1316 \\ 6316 & 13: 11: 39 & 0.1298 \\ 6317 & 13: 11: 40 & 0.1317 \\ 6318 & 13: 11: 41 & 0.1296 \\ 6319 & 13: 11: 42 & 0.1326 \\ 6320 & 13: 11: 43 & 0.1273 \\ 6321 & 13: 11: 44 & 0.1315 \\ 6322 & 13: 11: 45 & 0.1289 \\ 6323 & 13: 11: 46 & 0.1326 \\ 6324 & 13: 11: 47 & 0.1302 \\ 6325 & 13: 11: 48 & 0.1335 \\ 6326 & 13: 11: 49 & 0.1334 \\ 6327 & 13: 11: 50 & 0.1314 \\ 6328 & 13: 11: 51 & 0.1307 \\ 6329 & 13: 11: 52 & 0.1293 \\ 6330 & 13: 11: 53 & 0.1322 \\ 6331 & 13: 11: 54 & 0.1285 \\ 6332 & 13: 11: 55 & 0.1307 \\ 6333 & 13: 11: 56 & 0.1273 \\ 6334 & 13: 11: 57 & 0.1312 \\ 6335 & 13: 11: 58 & 0.1272 \\ 6336 & 13: 11: 59 & 0.1318 \\ 6337 & 13: 12: 00 & 0.1307 \\ 6338 & 13: 12: 01 & 0.1334 \\ 6339 & 13: 12: 02 & 0.1299 \\ 6340 & 13: 12: 03 & 0.1319 \\ 6341 & 13: 12: 04 & 0.1303 \\ 6342 & 13: 12: 05 & 0.1297\end{array}$


Appendix A - Fuel Data Test 1 NIST102505_1.xls

$\begin{array}{lll}6343 & 13: 12: 06 & 0.1304 \\ 6344 & 13: 12: 07 & 0.1283 \\ 6345 & 13: 12: 08 & 0.1294 \\ 6346 & 13: 12: 09 & 0.1269 \\ 6347 & 13: 12: 10 & 0.1324 \\ 6348 & 13: 12: 11 & 0.1295 \\ 6349 & 13: 12: 12 & 0.1324 \\ 6350 & 13: 12: 13 & 0.1289 \\ 6351 & 13: 12: 14 & 0.1317 \\ 6352 & 13: 12: 15 & 0.1314 \\ 6353 & 13: 12: 16 & 0.1325 \\ 6354 & 13: 12: 17 & 0.1301 \\ 6355 & 13: 12: 18 & 0.1308 \\ 6356 & 13: 12: 19 & 0.1325 \\ 6357 & 13: 12: 20 & 0.1301 \\ 6358 & 13: 12: 21 & 0.1320 \\ 6359 & 13: 12: 22 & 0.1285 \\ 6360 & 13: 12: 23 & 0.1303 \\ 6361 & 13: 12: 24 & 0.1296 \\ 6362 & 13: 12: 25 & 0.1302 \\ 6363 & 13: 12: 26 & 0.1306 \\ 6364 & 13: 12: 27 & 0.1321 \\ 6365 & 13: 12: 28 & 0.1301 \\ 6366 & 13: 12: 29 & 0.1297 \\ 6367 & 13: 12: 30 & 0.1310 \\ 6368 & 13: 12: 31 & 0.1288 \\ 6369 & 13: 12: 32 & 0.1306 \\ 6370 & 13: 12: 33 & 0.1280 \\ 6371 & 13: 12: 34 & 0.1306 \\ 6372 & 13: 12: 35 & 0.1257 \\ 6373 & 13: 12: 36 & 0.1311 \\ 6374 & 13: 12: 37 & 0.1312 \\ 6375 & 13: 12: 38 & 0.1341 \\ 6376 & 13: 12: 39 & 0.1321 \\ 6377 & 13: 12: 40 & 0.1330 \\ 6378 & 13: 12: 41 & 0.1327 \\ 6379 & 13: 12: 42 & 0.1324 \\ 6380 & 13: 12: 43 & 0.1306 \\ 6381 & 13: 12: 44 & 0.1275 \\ 6382 & 13: 12: 45 & 0.1329 \\ 6383 & 13: 12: 46 & 0.1289 \\ 6384 & 13: 12: 47 & 0.1309 \\ 6385 & 13: 12: 48 & 0.1262 \\ 6386 & 13: 12: 49 & 0.1316 \\ 6387 & 13: 12: 50 & 0.1284 \\ 6388 & 13: 12: 51 & 0.1335 \\ 6389 & 13: 12: 52 & 0.1310 \\ 6390 & 13: 12: 53 & 0.1329 \\ 6391 & 13: 12: 54 & 0.1298 \\ 6392 & 13: 12: 55 & 0.1307 \\ 6393 & 13: 12: 56 & 0.1313 \\ 6394 & 13: 12: 57 & 0.1284\end{array}$


Appendix A - Fuel Data Test 1 NIST102505_1.xls

$\begin{array}{lll}6395 & 13: 12: 58 & 0.1311 \\ 6396 & 13: 12: 59 & 0.1298 \\ 6397 & 13: 13: 00 & 0.1326 \\ 6398 & 13: 13: 01 & 0.1276 \\ 6399 & 13: 13: 02 & 0.1321 \\ 6400 & 13: 13: 03 & 0.1298 \\ 6401 & 13: 13: 04 & 0.1336 \\ 6402 & 13: 13: 05 & 0.1292 \\ 6403 & 13: 13: 06 & 0.1321 \\ 6404 & 13: 13: 07 & 0.1301 \\ 6405 & 13: 13: 08 & 0.1302 \\ 6406 & 13: 13: 09 & 0.1317 \\ 6407 & 13: 13: 10 & 0.1302 \\ 6408 & 13: 13: 11 & 0.1316 \\ 6409 & 13: 13: 12 & 0.1290 \\ 6410 & 13: 13: 13 & 0.1320 \\ 6411 & 13: 13: 14 & 0.1293 \\ 6412 & 13: 13: 15 & 0.1327 \\ 6413 & 13: 13: 16 & 0.1297 \\ 6414 & 13: 13: 17 & 0.1341 \\ 6415 & 13: 13: 18 & 0.1307 \\ 6416 & 13: 13: 19 & 0.1316 \\ 6417 & 13: 13: 20 & 0.1295 \\ 6418 & 13: 13: 21 & 0.1296 \\ 6419 & 13: 13: 22 & 0.1301 \\ 6420 & 13: 13: 23 & 0.1282 \\ 6421 & 13: 13: 24 & 0.1333 \\ 6422 & 13: 13: 25 & 0.1298 \\ 6423 & 13: 13: 26 & 0.1326 \\ 6424 & 13: 13: 27 & 0.1291 \\ 6425 & 13: 13: 28 & 0.1328 \\ 6426 & 13: 13: 29 & 0.1293 \\ 6427 & 13: 13: 30 & 0.1311 \\ 6428 & 13: 13: 31 & 0.1308 \\ 6429 & 13: 13: 32 & 0.1307 \\ 6430 & 13: 13: 33 & 0.1296 \\ 6431 & 13: 13: 34 & 0.1281 \\ 6432 & 13: 13: 35 & 0.1317 \\ 6433 & 13: 13: 36 & 0.1285 \\ 6434 & 13: 13: 37 & 0.1309 \\ 6435 & 13: 13: 38 & 0.1270 \\ 6436 & 13: 13: 39 & 0.1316 \\ 6437 & 13: 13: 40 & 0.1287 \\ 6438 & 13: 13: 41 & 0.1329 \\ 6439 & 13: 13: 42 & 0.1310 \\ 6440 & 13: 13: 43 & 0.1347 \\ 6441 & 13: 13: 44 & 0.1312 \\ 6442 & 13: 13: 45 & 0.1318 \\ 6443 & 13: 13: 46 & 0.1305 \\ 6444 & 13: 13: 47 & 0.1291 \\ 6445 & 13: 13: 48 & 0.1304 \\ 6446 & 13: 13: 49 & 0.1290\end{array}$


Appendix A - Fuel Data Test 1 NIST102505_1.xls

$\begin{array}{lll}6447 & 13: 13: 50 & 0.1306 \\ 6448 & 13: 13: 51 & 0.1263 \\ 6449 & 13: 13: 52 & 0.1310 \\ 6450 & 13: 13: 53 & 0.1294 \\ 6451 & 13: 13: 54 & 0.1328 \\ 6452 & 13: 13: 55 & 0.1290 \\ 6453 & 13: 13: 56 & 0.1321 \\ 6454 & 13: 13: 57 & 0.1285 \\ 6455 & 13: 13: 58 & 0.1286 \\ 6456 & 13: 13: 59 & 0.1312 \\ 6457 & 13: 14: 00 & 0.1286 \\ 6458 & 13: 14: 01 & 0.1303 \\ 6459 & 13: 14: 02 & 0.1262 \\ 6460 & 13: 14: 03 & 0.1332 \\ 6461 & 13: 14: 04 & 0.1297 \\ 6462 & 13: 14: 05 & 0.1342 \\ 6463 & 13: 14: 06 & 0.1303 \\ 6464 & 13: 14: 07 & 0.1353 \\ 6465 & 13: 14: 08 & 0.1326 \\ 6466 & 13: 14: 09 & 0.1332 \\ 6467 & 13: 14: 10 & 0.1299 \\ 6468 & 13: 14: 11 & 0.1304 \\ 6469 & 13: 14: 12 & 0.1304 \\ 6470 & 13: 14: 13 & 0.1283 \\ 6471 & 13: 14: 14 & 0.1302 \\ 6472 & 13: 14: 15 & 0.1278 \\ 6473 & 13: 14: 16 & 0.1307 \\ 6474 & 13: 14: 17 & 0.1266 \\ 6475 & 13: 14: 18 & 0.1325 \\ 6476 & 13: 14: 19 & 0.1300 \\ 6477 & 13: 14: 20 & 0.1330 \\ 6478 & 13: 14: 21 & 0.1293 \\ 6479 & 13: 14: 22 & 0.1307 \\ 6480 & 13: 14: 23 & 0.1300 \\ 6481 & 13: 14: 24 & 0.1319 \\ 6482 & 13: 14: 25 & 0.1315 \\ 6483 & 13: 14: 26 & 0.1301 \\ 6484 & 13: 14: 27 & 0.1319 \\ 6485 & 13: 14: 28 & 0.1281 \\ 6486 & 13: 14: 29 & 0.1338 \\ 6487 & 13: 14: 30 & 0.1308 \\ 6488 & 13: 14: 31 & 0.1318 \\ 6489 & 13: 14: 32 & 0.1306 \\ 6490 & 13: 14: 33 & 0.1322 \\ 6491 & 13: 14: 34 & 0.1330 \\ 6492 & 13: 14: 35 & 0.1320 \\ 6493 & 13: 14: 36 & 0.1307 \\ 6494 & 13: 14: 37 & 0.1291 \\ 6495 & 13: 14: 38 & 0.1307 \\ 6496 & 13: 14: 39 & 0.1272 \\ 6497 & 13: 14: 40 & 0.1301 \\ 6498 & 13: 14: 41 & 0.1276\end{array}$


Appendix A - Fuel Data Test 1 NIST102505_1.xIs

$\begin{array}{lll}6499 & 13: 14: 42 & 0.1313 \\ 6500 & 13: 14: 43 & 0.1273 \\ 6501 & 13: 14: 44 & 0.1324 \\ 6502 & 13: 14: 45 & 0.1320 \\ 6503 & 13: 14: 46 & 0.1349 \\ 6504 & 13: 14: 47 & 0.1322 \\ 6505 & 13: 14: 48 & 0.1329 \\ 6506 & 13: 14: 49 & 0.1329 \\ 6507 & 13: 14: 50 & 0.1310 \\ 6508 & 13: 14: 51 & 0.1330 \\ 6509 & 13: 14: 52 & 0.1315 \\ 6510 & 13: 14: 53 & 0.1343 \\ 6511 & 13: 14: 54 & 0.1285 \\ 6512 & 13: 14: 55 & 0.1355 \\ 6513 & 13: 14: 56 & 0.1306 \\ 6514 & 13: 14: 57 & 0.1333 \\ 6515 & 13: 14: 58 & 0.1298 \\ 6516 & 13: 14: 59 & 0.1310 \\ 6517 & 13: 15: 00 & 0.1303 \\ 6518 & 13: 15: 01 & 0.1296 \\ 6519 & 13: 15: 02 & 0.1314 \\ 6520 & 13: 15: 03 & 0.1279 \\ 6521 & 13: 15: 04 & 0.1290 \\ 6522 & 13: 15: 05 & 0.1260 \\ 6523 & 13: 15: 06 & 0.1295 \\ 6524 & 13: 15: 07 & 0.1256 \\ 6525 & 13: 15: 08 & 0.1311 \\ 6526 & 13: 15: 09 & 0.1306 \\ 6527 & 13: 15: 10 & 0.1331 \\ 6528 & 13: 15: 11 & 0.1321 \\ 6529 & 13: 15: 12 & 0.1342 \\ 6530 & 13: 15: 13 & 0.1303 \\ 6531 & 13: 15: 14 & 0.1299 \\ 6532 & 13: 15: 15 & 0.1300 \\ 6533 & 13: 15: 16 & 0.1286 \\ 6534 & 13: 15: 17 & 0.1302 \\ 6535 & 13: 15: 18 & 0.1283 \\ 6536 & 13: 15: 19 & 0.1326 \\ 6537 & 13: 15: 20 & 0.1295 \\ 6538 & 13: 15: 21 & 0.1317 \\ 6539 & 13: 15: 22 & 0.1283 \\ 6540 & 13: 15: 23 & 0.1322 \\ 6541 & 13: 15: 24 & 0.1300 \\ 6542 & 13: 15: 25 & 0.1309 \\ 6543 & 13: 15: 26 & 0.1308 \\ 6544 & 13: 15: 27 & 0.1325 \\ 6545 & 13: 15: 28 & 0.1332 \\ 6546 & 13: 15: 29 & 0.1293 \\ 6547 & 13: 15: 30 & 0.1330 \\ 6548 & 13: 15: 31 & 0.1286 \\ 6549 & 13: 15: 32 & 0.1326 \\ 6550 & 13: 15: 33 & 0.1304\end{array}$


Appendix A - Fuel Data Test 1 NIST102505_1.xIs

$\begin{array}{lll}6551 & 13: 15: 34 & 0.1324 \\ 6552 & 13: 15: 35 & 0.1303 \\ 6553 & 13: 15: 36 & 0.1319 \\ 6554 & 13: 15: 37 & 0.1324 \\ 6555 & 13: 15: 38 & 0.1333 \\ 6556 & 13: 15: 39 & 0.1317 \\ 6557 & 13: 15: 40 & 0.1291 \\ 6558 & 13: 15: 41 & 0.1315 \\ 6559 & 13: 15: 42 & 0.1292 \\ 6560 & 13: 15: 43 & 0.1320 \\ 6561 & 13: 15: 44 & 0.1289 \\ 6562 & 13: 15: 45 & 0.1336 \\ 6563 & 13: 15: 46 & 0.1299 \\ 6564 & 13: 15: 47 & 0.1349 \\ 6565 & 13: 15: 48 & 0.1312 \\ 6566 & 13: 15: 49 & 0.1344 \\ 6567 & 13: 15: 50 & 0.1325 \\ 6568 & 13: 15: 51 & 0.1319 \\ 6569 & 13: 15: 52 & 0.1298 \\ 6570 & 13: 15: 53 & 0.1283 \\ 6571 & 13: 15: 54 & 0.1316 \\ 6572 & 13: 15: 55 & 0.1295 \\ 6573 & 13: 15: 56 & 0.1306 \\ 6574 & 13: 15: 57 & 0.1256 \\ 6575 & 13: 15: 58 & 0.1326 \\ 6576 & 13: 15: 59 & 0.1290 \\ 6577 & 13: 16: 00 & 0.1318 \\ 6578 & 13: 16: 01 & 0.1308 \\ 6579 & 13: 16: 02 & 0.1330 \\ 6580 & 13: 16: 03 & 0.1307 \\ 6581 & 13: 16: 04 & 0.1297 \\ 6582 & 13: 16: 05 & 0.1312 \\ 6583 & 13: 16: 06 & 0.1288 \\ 6584 & 13: 16: 07 & 0.1306 \\ 6585 & 13: 16: 08 & 0.1267 \\ 6586 & 13: 16: 09 & 0.1318 \\ 6587 & 13: 16: 10 & 0.1257 \\ 6588 & 13: 16: 11 & 0.1310 \\ 6589 & 13: 16: 12 & 0.1301 \\ 6590 & 13: 16: 13 & 0.1326 \\ 6591 & 13: 16: 14 & 0.1300 \\ 6592 & 13: 16: 15 & 0.1301 \\ 6593 & 13: 16: 16 & 0.1323 \\ 6594 & 13: 16: 17 & 0.1318 \\ 6595 & 13: 16: 18 & 0.1319 \\ 6596 & 13: 16: 19 & 0.1285 \\ 6597 & 13: 16: 20 & 0.1334 \\ 6598 & 13: 16: 21 & 0.1288 \\ 6599 & 13: 16: 22 & 0.1318 \\ 6600 & 13: 16: 23 & 0.1295 \\ 6601 & 13: 16: 24 & 0.1332 \\ 6602 & 13: 16: 25 & 0.1312\end{array}$


Appendix A - Fuel Data Test 1 NIST102505_1.xIs

$\begin{array}{lll}6603 & 13: 16: 26 & 0.1342 \\ 6604 & 13: 16: 27 & 0.1330 \\ 6605 & 13: 16: 28 & 0.1329 \\ 6606 & 13: 16: 29 & 0.1318 \\ 6607 & 13: 16: 30 & 0.1288 \\ 6608 & 13: 16: 31 & 0.1324 \\ 6609 & 13: 16: 32 & 0.1288 \\ 6610 & 13: 16: 33 & 0.1319 \\ 6611 & 13: 16: 34 & 0.1276 \\ 6612 & 13: 16: 35 & 0.1320 \\ 6613 & 13: 16: 36 & 0.1277 \\ 6614 & 13: 16: 37 & 0.1308 \\ 6615 & 13: 16: 38 & 0.1302 \\ 6616 & 13: 16: 39 & 0.1337 \\ 6617 & 13: 16: 40 & 0.1310 \\ 6618 & 13: 16: 41 & 0.1319 \\ 6619 & 13: 16: 42 & 0.1313 \\ 6620 & 13: 16: 43 & 0.1295 \\ 6621 & 13: 16: 44 & 0.1328 \\ 6622 & 13: 16: 45 & 0.1300 \\ 6623 & 13: 16: 46 & 0.1324 \\ 6624 & 13: 16: 47 & 0.1285 \\ 6625 & 13: 16: 48 & 0.1336 \\ 6626 & 13: 16: 49 & 0.1300 \\ 6627 & 13: 16: 50 & 0.1322 \\ 6628 & 13: 16: 51 & 0.1309 \\ 6629 & 13: 16: 52 & 0.1319 \\ 6630 & 13: 16: 53 & 0.1304 \\ 6631 & 13: 16: 54 & 0.1295 \\ 6632 & 13: 16: 55 & 0.1312 \\ 6633 & 13: 16: 56 & 0.1291 \\ 6634 & 13: 16: 57 & 0.1304 \\ 6635 & 13: 16: 58 & 0.1275 \\ 6636 & 13: 16: 59 & 0.1305 \\ 6637 & 13: 17: 00 & 0.1266 \\ 6638 & 13: 17: 01 & 0.1325 \\ 6639 & 13: 17: 02 & 0.1304 \\ 6640 & 13: 17: 03 & 0.1337 \\ 6641 & 13: 17: 04 & 0.1293 \\ 6642 & 13: 17: 05 & 0.1331 \\ 6643 & 13: 17: 06 & 0.1311 \\ 6644 & 13: 17: 07 & 0.1305 \\ 6645 & 13: 17: 08 & 0.1300 \\ 6646 & 13: 17: 09 & 0.1298 \\ 6647 & 13: 17: 10 & 0.1311 \\ 6648 & 13: 17: 11 & 0.1277 \\ 6649 & 13: 17: 12 & 0.1305 \\ 6650 & 13: 17: 13 & 0.1273 \\ 6651 & 13: 17: 14 & 0.1314 \\ 6652 & 13: 17: 15 & 0.1272 \\ 6653 & 13: 17: 16 & 0.1321 \\ 6654 & 13: 17: 17 & 0.1307\end{array}$


Appendix A - Fuel Data Test 1 NIST102505_1.xIs

$\begin{array}{lll}6655 & 13: 17: 18 & 0.1322 \\ 6656 & 13: 17: 19 & 0.1298 \\ 6657 & 13: 17: 20 & 0.1304 \\ 6658 & 13: 17: 21 & 0.1316 \\ 6659 & 13: 17: 22 & 0.1289 \\ 6660 & 13: 17: 23 & 0.1311 \\ 6661 & 13: 17: 24 & 0.1290 \\ 6662 & 13: 17: 25 & 0.1314 \\ 6663 & 13: 17: 26 & 0.1268 \\ 6664 & 13: 17: 27 & 0.1315 \\ 6665 & 13: 17: 28 & 0.1319 \\ 6666 & 13: 17: 29 & 0.1335 \\ 6667 & 13: 17: 30 & 0.1303 \\ 6668 & 13: 17: 31 & 0.1313 \\ 6669 & 13: 17: 32 & 0.1321 \\ 6670 & 13: 17: 33 & 0.1303 \\ 6671 & 13: 17: 34 & 0.1302 \\ 6672 & 13: 17: 35 & 0.1278 \\ 6673 & 13: 17: 36 & 0.1305 \\ 6674 & 13: 17: 37 & 0.1280 \\ 6675 & 13: 17: 38 & 0.1312 \\ 6676 & 13: 17: 39 & 0.1285 \\ 6677 & 13: 17: 40 & 0.1313 \\ 6678 & 13: 17: 41 & 0.1308 \\ 6679 & 13: 17: 42 & 0.1322 \\ 6680 & 13: 17: 43 & 0.1327 \\ 6681 & 13: 17: 44 & 0.1316 \\ 6682 & 13: 17: 45 & 0.1307 \\ 6683 & 13: 17: 46 & 0.1285 \\ 6684 & 13: 17: 47 & 0.1311 \\ 6685 & 13: 17: 48 & 0.1277 \\ 6686 & 13: 17: 49 & 0.1308 \\ 6687 & 13: 17: 50 & 0.1272 \\ 6688 & 13: 17: 51 & 0.1325 \\ 6689 & 13: 17: 52 & 0.1294 \\ 6690 & 13: 17: 53 & 0.1351 \\ 6691 & 13: 17: 54 & 0.1314 \\ 6692 & 13: 17: 55 & 0.1339 \\ 6693 & 13: 17: 56 & 0.1325 \\ 6694 & 13: 17: 57 & 0.1329 \\ 6695 & 13: 17: 58 & 0.1320 \\ 6696 & 13: 17: 59 & 0.1302 \\ 6697 & 13: 18: 00 & 0.1357 \\ 6698 & 13: 18: 01 & 0.1306 \\ 6699 & 13: 18: 02 & 0.1325 \\ 6700 & 13: 18: 03 & 0.1264 \\ 6701 & 13: 18: 04 & 0.1322 \\ 6702 & 13: 18: 05 & 0.1286 \\ 6703 & 13: 18: 06 & 0.1322 \\ 6704 & 13: 18: 07 & 0.1315 \\ 6705 & 13: 18: 08 & 0.1321 \\ 6706 & 13: 18: 09 & 0.1309\end{array}$


Appendix A - Fuel Data Test 1 NIST102505_1.xIs

$\begin{array}{lll}6707 & 13: 18: 10 & 0.1290 \\ 6708 & 13: 18: 11 & 0.1327 \\ 6709 & 13: 18: 12 & 0.1293 \\ 6710 & 13: 18: 13 & 0.1317 \\ 6711 & 13: 18: 14 & 0.1275 \\ 6712 & 13: 18: 15 & 0.1323 \\ 6713 & 13: 18: 16 & 0.1270 \\ 6714 & 13: 18: 17 & 0.1311 \\ 6715 & 13: 18: 18 & 0.1295 \\ 6716 & 13: 18: 19 & 0.1318 \\ 6717 & 13: 18: 20 & 0.1302 \\ 6718 & 13: 18: 21 & 0.1316 \\ 6719 & 13: 18: 22 & 0.1321 \\ 6720 & 13: 18: 23 & 0.1308 \\ 6721 & 13: 18: 24 & 0.1300 \\ 6722 & 13: 18: 25 & 0.1272 \\ 6723 & 13: 18: 26 & 0.1307 \\ 6724 & 13: 18: 27 & 0.1266 \\ 6725 & 13: 18: 28 & 0.1300 \\ 6726 & 13: 18: 29 & 0.1270 \\ 6727 & 13: 18: 30 & 0.1308 \\ 6728 & 13: 18: 31 & 0.1289 \\ 6729 & 13: 18: 32 & 0.1320 \\ 6730 & 13: 18: 33 & 0.1303 \\ 6731 & 13: 18: 34 & 0.1314 \\ 6732 & 13: 18: 35 & 0.1286 \\ 6733 & 13: 18: 36 & 0.1290 \\ 6734 & 13: 18: 37 & 0.1282 \\ 6735 & 13: 18: 38 & 0.1259 \\ 6736 & 13: 18: 39 & 0.1312 \\ 6737 & 13: 18: 40 & 0.1287 \\ 6738 & 13: 18: 41 & 0.1316 \\ 6739 & 13: 18: 42 & 0.1260 \\ 6740 & 13: 18: 43 & 0.1315 \\ 6741 & 13: 18: 44 & 0.1287 \\ 6742 & 13: 18: 45 & 0.1316 \\ 6743 & 13: 18: 46 & 0.1307 \\ 6744 & 13: 18: 47 & 0.1304 \\ 6745 & 13: 18: 48 & 0.1303 \\ 6746 & 13: 18: 49 & 0.1291 \\ 6747 & 13: 18: 50 & 0.1319 \\ 6748 & 13: 18: 51 & 0.1286 \\ 6749 & 13: 18: 52 & 0.1322 \\ 6750 & 13: 18: 53 & 0.1285 \\ 6751 & 13: 18: 54 & 0.1335 \\ 6752 & 13: 18: 55 & 0.1292 \\ 6753 & 13: 18: 56 & 0.1324 \\ 6754 & 13: 18: 57 & 0.1319 \\ 6755 & 13: 18: 58 & 0.1329 \\ 6756 & 13: 18: 59 & 0.1305 \\ 6757 & 13: 19: 00 & 0.1297 \\ 6758 & 13: 19: 01 & 0.1309\end{array}$


Appendix A - Fuel Data Test 1 NIST102505_1.xls

$\begin{array}{lll}6759 & 13: 19: 02 & 0.1285 \\ 6760 & 13: 19: 03 & 0.1313 \\ 6761 & 13: 19: 04 & 0.1276 \\ 6762 & 13: 19: 05 & 0.1325 \\ 6763 & 13: 19: 06 & 0.1267 \\ 6764 & 13: 19: 07 & 0.1310 \\ 6765 & 13: 19: 08 & 0.1272 \\ 6766 & 13: 19: 09 & 0.1320 \\ 6767 & 13: 19: 10 & 0.1288 \\ 6768 & 13: 19: 11 & 0.1317 \\ 6769 & 13: 19: 12 & 0.1314 \\ 6770 & 13: 19: 13 & 0.1308 \\ 6771 & 13: 19: 14 & 0.1297 \\ 6772 & 13: 19: 15 & 0.1288 \\ 6773 & 13: 19: 16 & 0.1306 \\ 6774 & 13: 19: 17 & 0.1266 \\ 6775 & 13: 19: 18 & 0.1293 \\ 6776 & 13: 19: 19 & 0.1268 \\ 6777 & 13: 19: 20 & 0.1319 \\ 6778 & 13: 19: 21 & 0.1297 \\ 6779 & 13: 19: 22 & 0.1327 \\ 6780 & 13: 19: 23 & 0.1332 \\ 6781 & 13: 19: 24 & 0.1338 \\ 6782 & 13: 19: 25 & 0.1316 \\ 6783 & 13: 19: 26 & 0.1305 \\ 6784 & 13: 19: 27 & 0.1314 \\ 6785 & 13: 19: 28 & 0.1291 \\ 6786 & 13: 19: 29 & 0.1317 \\ 6787 & 13: 19: 30 & 0.1286 \\ 6788 & 13: 19: 31 & 0.1314 \\ 6789 & 13: 19: 32 & 0.1279 \\ 6790 & 13: 19: 33 & 0.1309 \\ 6791 & 13: 19: 34 & 0.1293 \\ 6792 & 13: 19: 35 & 0.1330 \\ 6793 & 13: 19: 36 & 0.1299 \\ 6794 & 13: 19: 37 & 0.1325 \\ 6795 & 13: 19: 38 & 0.1309 \\ 6796 & 13: 19: 39 & 0.1306 \\ 6797 & 13: 19: 40 & 0.1296 \\ 6798 & 13: 19: 41 & 0.1287 \\ 6799 & 13: 19: 42 & 0.1308 \\ 6800 & 13: 19: 43 & 0.1272 \\ 6801 & 13: 19: 44 & 0.1320 \\ 6802 & 13: 19: 45 & 0.1289 \\ 6803 & 13: 19: 46 & 0.1327 \\ 6804 & 13: 19: 47 & 0.1292 \\ 6805 & 13: 19: 48 & 0.1333 \\ 6806 & 13: 19: 49 & 0.1309 \\ 6807 & 13: 19: 50 & 0.1320 \\ 6808 & 13: 19: 51 & 0.1282 \\ 6809 & 13: 19: 52 & 0.1294 \\ 6810 & 13: 19: 53 & 0.1289\end{array}$


Appendix A - Fuel Data Test 1 NIST102505_1.xIs

$\begin{array}{lll}6811 & 13: 19: 54 & 0.1267 \\ 6812 & 13: 19: 55 & 0.1314 \\ 6813 & 13: 19: 56 & 0.1298 \\ 6814 & 13: 19: 57 & 0.1315 \\ 6815 & 13: 19: 58 & 0.1279 \\ 6816 & 13: 19: 59 & 0.1342 \\ 6817 & 13: 20: 00 & 0.1314 \\ 6818 & 13: 20: 01 & 0.1350 \\ 6819 & 13: 20: 02 & 0.1318 \\ 6820 & 13: 20: 03 & 0.1324 \\ 6821 & 13: 20: 04 & 0.1296 \\ 6822 & 13: 20: 05 & 0.1281 \\ 6823 & 13: 20: 06 & 0.1310 \\ 6824 & 13: 20: 07 & 0.1276 \\ 6825 & 13: 20: 08 & 0.1296 \\ 6826 & 13: 20: 09 & 0.1275 \\ 6827 & 13: 20: 10 & 0.1309 \\ 6828 & 13: 20: 11 & 0.1269 \\ 6829 & 13: 20: 12 & 0.1318 \\ 6830 & 13: 20: 13 & 0.1290 \\ 6831 & 13: 20: 14 & 0.1325 \\ 6832 & 13: 20: 15 & 0.1298 \\ 6833 & 13: 20: 16 & 0.1309 \\ 6834 & 13: 20: 17 & 0.1289 \\ 6835 & 13: 20: 18 & 0.1278 \\ 6836 & 13: 20: 19 & 0.1323 \\ 6837 & 13: 20: 20 & 0.1288 \\ 6838 & 13: 20: 21 & 0.1312 \\ 6839 & 13: 20: 22 & 0.1271 \\ 6840 & 13: 20: 23 & 0.1324 \\ 6841 & 13: 20: 24 & 0.1296 \\ 6842 & 13: 20: 25 & 0.1325 \\ 6843 & 13: 20: 26 & 0.1293 \\ 6844 & 13: 20: 27 & 0.1312 \\ 6845 & 13: 20: 28 & 0.1289 \\ 6846 & 13: 20: 29 & 0.1285 \\ 6847 & 13: 20: 30 & 0.1318 \\ 6848 & 13: 20: 31 & 0.1294 \\ 6849 & 13: 20: 32 & 0.1320 \\ 6850 & 13: 20: 33 & 0.1292 \\ 6851 & 13: 20: 34 & 0.1323 \\ 6852 & 13: 20: 35 & 0.1261 \\ 6853 & 13: 20: 36 & 0.1304 \\ 6854 & 13: 20: 37 & 0.1314 \\ 6855 & 13: 20: 38 & 0.1331 \\ 6856 & 13: 20: 39 & 0.1307 \\ 6857 & 13: 20: 40 & 0.1337 \\ 6858 & 13: 20: 41 & 0.1329 \\ 6859 & 13: 20: 42 & 0.1324 \\ 6860 & 13: 20: 43 & 0.1309 \\ 6861 & 13: 20: 44 & 0.1288 \\ 6862 & 13: 20: 45 & 0.1315\end{array}$


Appendix A - Fuel Data Test 1 NIST102505_1.xIs

$\begin{array}{lll}6863 & 13: 20: 46 & 0.1299 \\ 6864 & 13: 20: 47 & 0.1338 \\ 6865 & 13: 20: 48 & 0.1312 \\ 6866 & 13: 20: 49 & 0.1344 \\ 6867 & 13: 20: 50 & 0.1301 \\ 6868 & 13: 20: 51 & 0.1332 \\ 6869 & 13: 20: 52 & 0.1307 \\ 6870 & 13: 20: 53 & 0.1317 \\ 6871 & 13: 20: 54 & 0.1303 \\ 6872 & 13: 20: 55 & 0.1296 \\ 6873 & 13: 20: 56 & 0.1296 \\ 6874 & 13: 20: 57 & 0.1271 \\ 6875 & 13: 20: 58 & 0.1318 \\ 6876 & 13: 20: 59 & 0.1290 \\ 6877 & 13: 21: 00 & 0.1317 \\ 6878 & 13: 21: 01 & 0.1273 \\ 6879 & 13: 21: 02 & 0.1323 \\ 6880 & 13: 21: 03 & 0.1293 \\ 6881 & 13: 21: 04 & 0.1328 \\ 6882 & 13: 21: 05 & 0.1305 \\ 6883 & 13: 21: 06 & 0.1312 \\ 6884 & 13: 21: 07 & 0.1297 \\ 6885 & 13: 21: 08 & 0.1284 \\ 6886 & 13: 21: 09 & 0.1334 \\ 6887 & 13: 21: 10 & 0.1285 \\ 6888 & 13: 21: 11 & 0.1313 \\ 6889 & 13: 21: 12 & 0.1267 \\ 6890 & 13: 21: 13 & 0.1343 \\ 6891 & 13: 21: 14 & 0.1283 \\ 6892 & 13: 21: 15 & 0.1314 \\ 6893 & 13: 21: 16 & 0.1297 \\ 6894 & 13: 21: 17 & 0.1321 \\ 6895 & 13: 21: 18 & 0.1302 \\ 6896 & 13: 21: 19 & 0.1290 \\ 6897 & 13: 21: 20 & 0.1322 \\ 6898 & 13: 21: 21 & 0.1291 \\ 6899 & 13: 21: 22 & 0.1311 \\ 6900 & 13: 21: 23 & 0.1284 \\ 6901 & 13: 21: 24 & 0.1331 \\ 6902 & 13: 21: 25 & 0.1293 \\ 6903 & 13: 21: 26 & 0.1340 \\ 6904 & 13: 21: 27 & 0.1321 \\ 6905 & 13: 21: 28 & 0.1349 \\ 6906 & 13: 21: 29 & 0.1317 \\ 6907 & 13: 21: 30 & 0.1317 \\ 6908 & 13: 21: 31 & 0.1317 \\ 6909 & 13: 21: 32 & 0.1305 \\ 6910 & 13: 21: 33 & 0.1298 \\ 6911 & 13: 21: 34 & 0.1298 \\ 6912 & 13: 21: 35 & 0.1325 \\ 6913 & 13: 21: 36 & 0.1283 \\ 6914 & 13: 21: 37 & 0.1307\end{array}$


Appendix A - Fuel Data Test 1 NIST102505_1.xls

$\begin{array}{lll}6915 & 13: 21: 38 & 0.1289 \\ 6916 & 13: 21: 39 & 0.1313 \\ 6917 & 13: 21: 40 & 0.1292 \\ 6918 & 13: 21: 41 & 0.1326 \\ 6919 & 13: 21: 42 & 0.1321 \\ 6920 & 13: 21: 43 & 0.1331 \\ 6921 & 13: 21: 44 & 0.1307 \\ 6922 & 13: 21: 45 & 0.1306 \\ 6923 & 13: 21: 46 & 0.1313 \\ 6924 & 13: 21: 47 & 0.1293 \\ 6925 & 13: 21: 48 & 0.1314 \\ 6926 & 13: 21: 49 & 0.1289 \\ 6927 & 13: 21: 50 & 0.1307 \\ 6928 & 13: 21: 51 & 0.1258 \\ 6929 & 13: 21: 52 & 0.1304 \\ 6930 & 13: 21: 53 & 0.1303 \\ 6931 & 13: 21: 54 & 0.1333 \\ 6932 & 13: 21: 55 & 0.1297 \\ 6933 & 13: 21: 56 & 0.1327 \\ 6934 & 13: 21: 57 & 0.1297 \\ 6935 & 13: 21: 58 & 0.1282 \\ 6936 & 13: 21: 59 & 0.1298 \\ 6937 & 13: 22: 00 & 0.1293 \\ 6938 & 13: 22: 01 & 0.1302 \\ 6939 & 13: 22: 02 & 0.1282 \\ 6940 & 13: 22: 03 & 0.1336 \\ 6941 & 13: 22: 04 & 0.1300 \\ 6942 & 13: 22: 05 & 0.1331 \\ 6943 & 13: 22: 06 & 0.1298 \\ 6944 & 13: 22: 07 & 0.1330 \\ 6945 & 13: 22: 08 & 0.1300 \\ 6946 & 13: 22: 09 & 0.1313 \\ 6947 & 13: 22: 10 & 0.1300 \\ 6948 & 13: 22: 11 & 0.1295 \\ 6949 & 13: 22: 12 & 0.1305 \\ 6950 & 13: 22: 13 & 0.1268 \\ 6951 & 13: 22: 14 & 0.1307 \\ 6952 & 13: 22: 15 & 0.1288 \\ 6953 & 13: 22: 16 & 0.1322 \\ 6954 & 13: 22: 17 & 0.1282 \\ 6955 & 13: 22: 18 & 0.1319 \\ 6956 & 13: 22: 19 & 0.1296 \\ 6957 & 13: 22: 20 & 0.1321 \\ 6958 & 13: 22: 21 & 0.1286 \\ 6959 & 13: 22: 22 & 0.1290 \\ 6960 & 13: 22: 23 & 0.1278 \\ 6961 & 13: 22: 24 & 0.1278 \\ 6962 & 13: 22: 25 & 0.1326 \\ 6963 & 13: 22: 26 & 0.1279 \\ 6964 & 13: 22: 27 & 0.1317 \\ 6965 & 13: 22: 28 & 0.1287 \\ 6966 & 13: 22: 29 & 0.1317\end{array}$


Appendix A - Fuel Data Test 1 NIST102505_1.xls

$\begin{array}{lll}6967 & 13: 22: 30 & 0.1278 \\ 6968 & 13: 22: 31 & 0.1309 \\ 6969 & 13: 22: 32 & 0.1316 \\ 6970 & 13: 22: 33 & 0.1337 \\ 6971 & 13: 22: 34 & 0.1322 \\ 6972 & 13: 22: 35 & 0.1301 \\ 6973 & 13: 22: 36 & 0.1339 \\ 6974 & 13: 22: 37 & 0.1302 \\ 6975 & 13: 22: 38 & 0.1326 \\ 6976 & 13: 22: 39 & 0.1280 \\ 6977 & 13: 22: 40 & 0.1335 \\ 6978 & 13: 22: 41 & 0.1288 \\ 6979 & 13: 22: 42 & 0.1317 \\ 6980 & 13: 22: 43 & 0.1288 \\ 6981 & 13: 22: 44 & 0.1326 \\ 6982 & 13: 22: 45 & 0.1301 \\ 6983 & 13: 22: 46 & 0.1310 \\ 6984 & 13: 22: 47 & 0.1283 \\ 6985 & 13: 22: 48 & 0.1303 \\ 6986 & 13: 22: 49 & 0.1303 \\ 6987 & 13: 22: 50 & 0.1280 \\ 6988 & 13: 22: 51 & 0.1320 \\ 6989 & 13: 22: 52 & 0.1305 \\ 6990 & 13: 22: 53 & 0.1312 \\ 6991 & 13: 22: 54 & 0.1268 \\ 6992 & 13: 22: 55 & 0.1314 \\ 6994 & 13: 22: 57 & 0.1311 \\ 6994 & 13: 22: 57 & 0.1330 \\ 6995 & 13: 22: 58 & 0.1298 \\ 6996 & 13: 22: 59 & 0.1321 \\ 6997 & 13: 23: 00 & 0.1297 \\ 6998 & 13: 23: 01 & 0.1294 \\ 6999 & 13: 23: 02 & 0.1336 \\ 7000 & 13: 23: 03 & 0.1301 \\ 7001 & 13: 23: 04 & 0.1321 \\ 7002 & 13: 23: 05 & 0.1279 \\ 7003 & 13: 23: 06 & 0.1319 \\ 7004 & 13: 23: 07 & 0.1273 \\ 7005 & 13: 23: 08 & 0.1327 \\ 7006 & 13: 23: 09 & 0.1311 \\ 7007 & 13: 23: 10 & 0.1336 \\ 7008 & 13: 23: 11 & 0.1303 \\ 7009 & 13: 23: 12 & 0.1298 \\ 7010 & 13: 23: 13 & 0.1304 \\ 7011 & 13: 23: 14 & 0.1289 \\ 7012 & 13: 23: 15 & 0.1305 \\ 7013 & 13: 23: 16 & 0.1269 \\ 7014 & 13: 23: 17 & 0.1309 \\ 7015 & 13: 23: 18 & 0.1266 \\ 7016 & 13: 23: 19 & 0.1305 \\ 7017 & 13: 23: 20 & 0.1275 \\ 7018 & 13: 23: 21 & 0.1312\end{array}$


Appendix A - Fuel Data Test 1 NIST102505_1.xls

$\begin{array}{lll}7019 & 13: 23: 22 & 0.1285 \\ 7020 & 13: 23: 23 & 0.1303 \\ 7021 & 13: 23: 24 & 0.1317 \\ 7022 & 13: 23: 25 & 0.1302 \\ 7023 & 13: 23: 26 & 0.1287 \\ 7024 & 13: 23: 27 & 0.1272 \\ 7025 & 13: 23: 28 & 0.1299 \\ 7026 & 13: 23: 29 & 0.1263 \\ 7027 & 13: 23: 30 & 0.1297 \\ 7028 & 13: 23: 31 & 0.1272 \\ 7029 & 13: 23: 32 & 0.1317 \\ 7030 & 13: 23: 33 & 0.1283 \\ 7031 & 13: 23: 34 & 0.1325 \\ 7032 & 13: 23: 35 & 0.1323 \\ 7033 & 13: 23: 36 & 0.1337 \\ 7034 & 13: 23: 37 & 0.1323 \\ 7035 & 13: 23: 38 & 0.1316 \\ 7036 & 13: 23: 39 & 0.1326 \\ 7037 & 13: 23: 40 & 0.1292 \\ 7038 & 13: 23: 41 & 0.1316 \\ 7039 & 13: 23: 42 & 0.1285 \\ 7040 & 13: 23: 43 & 0.1320 \\ 7041 & 13: 23: 44 & 0.1279 \\ 7042 & 13: 23: 45 & 0.1312 \\ 7043 & 13: 23: 46 & 0.1292 \\ 7044 & 13: 23: 47 & 0.1319 \\ 7045 & 13: 23: 48 & 0.1300 \\ 7046 & 13: 23: 49 & 0.1308 \\ 7047 & 13: 23: 50 & 0.1315 \\ 7048 & 13: 23: 51 & 0.1318 \\ 7049 & 13: 23: 52 & 0.1302 \\ 7050 & 13: 23: 53 & 0.1278 \\ 7051 & 13: 23: 54 & 0.1317 \\ 7052 & 13: 23: 55 & 0.1278 \\ 7053 & 13: 23: 56 & 0.1308 \\ 7054 & 13: 23: 57 & 0.1270 \\ 7055 & 13: 23: 58 & 0.1327 \\ 7056 & 13: 23: 59 & 0.1292 \\ 7057 & 13: 24: 00 & 0.1322 \\ 7058 & 13: 24: 01 & 0.1324 \\ 7059 & 13: 24: 02 & 0.1311 \\ 7060 & 13: 24: 03 & 0.1290 \\ 7061 & 13: 24: 04 & 0.1291 \\ 7062 & 13: 24: 05 & 0.1312 \\ 7063 & 13: 24: 06 & 0.1281 \\ 7064 & 13: 24: 07 & 0.1307 \\ 7065 & 13: 24: 08 & 0.1269 \\ 7066 & 13: 24: 09 & 0.1306 \\ 7067 & 13: 24: 10 & 0.1276 \\ 7068 & 13: 24: 11 & 0.1319 \\ 7069 & 13: 24: 12 & 0.1308 \\ 7070 & 13: 24: 13 & 0.1330\end{array}$


Appendix A - Fuel Data Test 1 NIST102505_1.xls

$\begin{array}{lll}7071 & 13: 24: 14 & 0.1287 \\ 7072 & 13: 24: 15 & 0.1315 \\ 7073 & 13: 24: 16 & 0.1302 \\ 7074 & 13: 24: 17 & 0.1296 \\ 7075 & 13: 24: 18 & 0.1300 \\ 7076 & 13: 24: 19 & 0.1288 \\ 7077 & 13: 24: 20 & 0.1313 \\ 7078 & 13: 24: 21 & 0.1260 \\ 7079 & 13: 24: 22 & 0.1322 \\ 7080 & 13: 24: 23 & 0.1292 \\ 7081 & 13: 24: 24 & 0.1329 \\ 7082 & 13: 24: 25 & 0.1289 \\ 7083 & 13: 24: 26 & 0.1330 \\ 7084 & 13: 24: 27 & 0.1293 \\ 7085 & 13: 24: 28 & 0.1297 \\ 7086 & 13: 24: 29 & 0.1307 \\ 7087 & 13: 24: 30 & 0.1288 \\ 7088 & 13: 24: 31 & 0.1304 \\ 7089 & 13: 24: 32 & 0.1262 \\ 7090 & 13: 24: 33 & 0.1318 \\ 7091 & 13: 24: 34 & 0.1282 \\ 7092 & 13: 24: 35 & 0.1323 \\ 7093 & 13: 24: 36 & 0.1288 \\ 7094 & 13: 24: 37 & 0.1336 \\ 7095 & 13: 24: 38 & 0.1308 \\ 7096 & 13: 24: 39 & 0.1328 \\ 7097 & 13: 24: 40 & 0.1322 \\ 7098 & 13: 24: 41 & 0.1314 \\ 7099 & 13: 24: 42 & 0.1285 \\ 7100 & 13: 24: 43 & 0.1277 \\ 7101 & 13: 24: 44 & 0.1313 \\ 7102 & 13: 24: 45 & 0.1265 \\ 7103 & 13: 24: 46 & 0.1291 \\ 7104 & 13: 24: 47 & 0.1276 \\ 7105 & 13: 24: 48 & 0.1308 \\ 7106 & 13: 24: 49 & 0.1295 \\ 7107 & 13: 24: 50 & 0.1323 \\ 7108 & 13: 24: 51 & 0.1310 \\ 7109 & 13: 24: 52 & 0.1330 \\ 7110 & 13: 24: 53 & 0.1284 \\ 7111 & 13: 24: 54 & 0.1290 \\ 7112 & 13: 24: 55 & 0.1304 \\ 7113 & 13: 24: 56 & 0.1305 \\ 7114 & 13: 24: 57 & 0.1325 \\ 7115 & 13: 24: 58 & 0.1305 \\ 7116 & 13: 24: 59 & 0.1318 \\ 7117 & 13: 25: 00 & 0.1270 \\ 7118 & 13: 25: 01 & 0.1323 \\ 7119 & 13: 25: 02 & 0.1303 \\ 7120 & 13: 25: 03 & 0.1333 \\ 7121 & 13: 25: 04 & 0.1294 \\ 7122 & 13: 25: 05 & 0.1318\end{array}$


Appendix A - Fuel Data Test 1 NIST102505_1.xls

$\begin{array}{lll}7123 & 13: 25: 06 & 0.1313 \\ 7124 & 13: 25: 07 & 0.1303 \\ 7125 & 13: 25: 08 & 0.1296 \\ 7126 & 13: 25: 09 & 0.1286 \\ 7127 & 13: 25: 10 & 0.1297 \\ 7128 & 13: 25: 11 & 0.1255 \\ 7129 & 13: 25: 12 & 0.1316 \\ 7130 & 13: 25: 13 & 0.1284 \\ 7131 & 13: 25: 14 & 0.1326 \\ 7132 & 13: 25: 15 & 0.1284 \\ 7133 & 13: 25: 16 & 0.1312 \\ 7134 & 13: 25: 17 & 0.1290 \\ 7135 & 13: 25: 18 & 0.1276 \\ 7136 & 13: 25: 19 & 0.1311 \\ 7137 & 13: 25: 20 & 0.1285 \\ 7138 & 13: 25: 21 & 0.1299 \\ 7139 & 13: 25: 22 & 0.1252 \\ 7140 & 13: 25: 23 & 0.1317 \\ 7141 & 13: 25: 24 & 0.1279 \\ 7142 & 13: 25: 25 & 0.1318 \\ 7143 & 13: 25: 26 & 0.1282 \\ 7144 & 13: 25: 27 & 0.1323 \\ 7145 & 13: 25: 28 & 0.1273 \\ 7146 & 13: 25: 29 & 0.1308 \\ 7147 & 13: 25: 30 & 0.1310 \\ 7148 & 13: 25: 31 & 0.1292 \\ 7149 & 13: 25: 32 & 0.1285 \\ 7150 & 13: 25: 33 & 0.1283 \\ 7151 & 13: 25: 34 & 0.1301 \\ 7152 & 13: 25: 35 & 0.1264 \\ 7153 & 13: 25: 36 & 0.1292 \\ 7154 & 13: 25: 37 & 0.1262 \\ 7155 & 13: 25: 38 & 0.1312 \\ 7156 & 13: 25: 39 & 0.1275 \\ 7157 & 13: 25: 40 & 0.1324 \\ 7158 & 13: 25: 41 & 0.1288 \\ 7159 & 13: 25: 42 & 0.1304 \\ 7160 & 13: 25: 43 & 0.1291 \\ 7161 & 13: 25: 44 & 0.1306 \\ 7162 & 13: 25: 45 & 0.1318 \\ 7163 & 13: 25: 46 & 0.1294 \\ 7164 & 13: 25: 47 & 0.1310 \\ 7165 & 13: 25: 48 & 0.1278 \\ 7166 & 13: 25: 49 & 0.1308 \\ 7167 & 13: 25: 50 & 0.1273 \\ 7168 & 13: 25: 51 & 0.1308 \\ 7169 & 13: 25: 52 & 0.1294 \\ 7171 & 13: 25: 53 & 0.1320 \\ 7171 & 13: 25: 54 & 0.1301 \\ 7172 & 13: 25: 55 & 0.1297 \\ 7173 & 13: 25: 56 & 0.1319 \\ 7174 & 13: 25: 57 & 0.1290\end{array}$


Appendix A - Fuel Data Test 1 NIST102505_1.xls

$\begin{array}{lll}7175 & 13: 25: 58 & 0.1304 \\ 7176 & 13: 25: 59 & 0.1283 \\ 7177 & 13: 26: 00 & 0.1335 \\ 7178 & 13: 26: 01 & 0.1289 \\ 7179 & 13: 26: 02 & 0.1324 \\ 7180 & 13: 26: 03 & 0.1296 \\ 7181 & 13: 26: 04 & 0.1323 \\ 7182 & 13: 26: 05 & 0.1269 \\ 7183 & 13: 26: 06 & 0.1318 \\ 7184 & 13: 26: 07 & 0.1299 \\ 7185 & 13: 26: 08 & 0.1302 \\ 7186 & 13: 26: 09 & 0.1285 \\ 7187 & 13: 26: 10 & 0.1303 \\ 7188 & 13: 26: 11 & 0.1303 \\ 7189 & 13: 26: 12 & 0.1291 \\ 7190 & 13: 26: 13 & 0.1335 \\ 7191 & 13: 26: 14 & 0.1302 \\ 7192 & 13: 26: 15 & 0.1337 \\ 7193 & 13: 26: 16 & 0.1291 \\ 7194 & 13: 26: 17 & 0.1352 \\ 7195 & 13: 26: 18 & 0.1323 \\ 7196 & 13: 26: 19 & 0.1338 \\ 7197 & 13: 26: 20 & 0.1312 \\ 7198 & 13: 26: 21 & 0.1324 \\ 7199 & 13: 26: 22 & 0.1298 \\ 7200 & 13: 26: 23 & 0.1281 \\ 7201 & 13: 26: 24 & 0.1311 \\ 7202 & 13: 26: 25 & 0.1282 \\ 7203 & 13: 26: 26 & 0.1321 \\ 7204 & 13: 26: 27 & 0.1262 \\ 7205 & 13: 26: 28 & 0.1322 \\ 7206 & 13: 26: 29 & 0.1297 \\ 7207 & 13: 26: 30 & 0.1335 \\ 7208 & 13: 26: 31 & 0.1293 \\ 7209 & 13: 26: 32 & 0.1319 \\ 7210 & 13: 26: 33 & 0.1283 \\ 7211 & 13: 26: 34 & 0.1283 \\ 7212 & 13: 26: 35 & 0.1293 \\ 7213 & 13: 26: 36 & 0.1286 \\ 7214 & 13: 26: 37 & 0.1305 \\ 7215 & 13: 26: 38 & 0.1270 \\ 7216 & 13: 26: 39 & 0.1315 \\ 7217 & 13: 26: 40 & 0.1265 \\ 7218 & 13: 26: 41 & 0.1306 \\ 7219 & 13: 26: 42 & 0.1302 \\ 7220 & 13: 26: 43 & 0.1316 \\ 7221 & 13: 26: 44 & 0.1293 \\ 7222 & 13: 26: 45 & 0.1292 \\ 7223 & 13: 26: 46 & 0.1322 \\ 7224 & 13: 26: 47 & 0.1307 \\ 7226 & 13: 26: 48 & 0.1315 \\ 7226 & 13: 26: 49 & 0.1293\end{array}$


Appendix A - Fuel Data Test 1 NIST102505_1.xls

$\begin{array}{lll}7227 & 13: 26: 50 & 0.1326 \\ 7228 & 13: 26: 51 & 0.1251 \\ 7229 & 13: 26: 52 & 0.1303 \\ 7230 & 13: 26: 53 & 0.1280 \\ 7231 & 13: 26: 54 & 0.1323 \\ 7232 & 13: 26: 55 & 0.1287 \\ 7233 & 13: 26: 56 & 0.1330 \\ 7234 & 13: 26: 57 & 0.1296 \\ 7235 & 13: 26: 58 & 0.1307 \\ 7236 & 13: 26: 59 & 0.1272 \\ 7237 & 13: 27: 00 & 0.1289 \\ 7238 & 13: 27: 01 & 0.1289 \\ 7239 & 13: 27: 02 & 0.1264 \\ 7240 & 13: 27: 03 & 0.1308 \\ 7241 & 13: 27: 04 & 0.1264 \\ 7242 & 13: 27: 05 & 0.1307 \\ 7243 & 13: 27: 06 & 0.1270 \\ 7244 & 13: 27: 07 & 0.1315 \\ 7245 & 13: 27: 08 & 0.1274 \\ 7246 & 13: 27: 09 & 0.1303 \\ 7247 & 13: 27: 10 & 0.1308 \\ 7248 & 13: 27: 11 & 0.1307 \\ 7249 & 13: 27: 12 & 0.1308 \\ 7250 & 13: 27: 13 & 0.1284 \\ 7251 & 13: 27: 14 & 0.1337 \\ 7252 & 13: 27: 15 & 0.1296 \\ 7253 & 13: 27: 16 & 0.1316 \\ 7254 & 13: 27: 17 & 0.1270 \\ 7255 & 13: 27: 18 & 0.1322 \\ 7256 & 13: 27: 19 & 0.1281 \\ 7257 & 13: 27: 20 & 0.1339 \\ 7258 & 13: 27: 21 & 0.1330 \\ 7259 & 13: 27: 22 & 0.1335 \\ 7260 & 13: 27: 23 & 0.1307 \\ 7261 & 13: 27: 24 & 0.1290 \\ 7262 & 13: 27: 25 & 0.1314 \\ 7263 & 13: 27: 26 & 0.1290 \\ 7264 & 13: 27: 27 & 0.1308 \\ 7265 & 13: 27: 28 & 0.1274 \\ 7266 & 13: 27: 29 & 0.1308 \\ 7267 & 13: 27: 30 & 0.1258 \\ 7268 & 13: 27: 31 & 0.1307 \\ 7269 & 13: 27: 32 & 0.1284 \\ 7270 & 13: 27: 33 & 0.1325 \\ 7271 & 13: 27: 34 & 0.1282 \\ 7272 & 13: 27: 35 & 0.1313 \\ 7273 & 13: 27: 36 & 0.1314 \\ 7274 & 13: 27: 37 & 0.1314 \\ 7275 & 13: 27: 38 & 0.1301 \\ 7276 & 13: 27: 39 & 0.1287 \\ 7277 & 13: 27: 40 & 0.1298 \\ 7278 & 13: 27: 41 & 0.1261\end{array}$


Appendix A - Fuel Data Test 1 NIST102505_1.xls

$\begin{array}{lll}7279 & 13: 27: 42 & 0.1300 \\ 7280 & 13: 27: 43 & 0.1277 \\ 7281 & 13: 27: 44 & 0.1312 \\ 7282 & 13: 27: 45 & 0.1284 \\ 7283 & 13: 27: 46 & 0.1321 \\ 7284 & 13: 27: 47 & 0.1320 \\ 7285 & 13: 27: 48 & 0.1331 \\ 7286 & 13: 27: 49 & 0.1306 \\ 7287 & 13: 27: 50 & 0.1313 \\ 7288 & 13: 27: 51 & 0.1330 \\ 7289 & 13: 27: 52 & 0.1315 \\ 7290 & 13: 27: 53 & 0.1333 \\ 7291 & 13: 27: 54 & 0.1301 \\ 7292 & 13: 27: 55 & 0.1308 \\ 7293 & 13: 27: 56 & 0.1272 \\ 7294 & 13: 27: 57 & 0.1316 \\ 7295 & 13: 27: 58 & 0.1293 \\ 7296 & 13: 27: 59 & 0.1326 \\ 7297 & 13: 28: 00 & 0.1291 \\ 7298 & 13: 28: 01 & 0.1328 \\ 7299 & 13: 28: 02 & 0.1303 \\ 7300 & 13: 28: 03 & 0.1303 \\ 7301 & 13: 28: 04 & 0.1336 \\ 7302 & 13: 28: 05 & 0.1301 \\ 7303 & 13: 28: 06 & 0.1311 \\ 7304 & 13: 28: 07 & 0.1269 \\ 7305 & 13: 28: 08 & 0.1330 \\ 7306 & 13: 28: 09 & 0.1280 \\ 7307 & 13: 28: 10 & 0.1315 \\ 7308 & 13: 28: 11 & 0.1278 \\ 7309 & 13: 28: 12 & 0.1312 \\ 7310 & 13: 28: 13 & 0.1286 \\ 7311 & 13: 28: 14 & 0.1288 \\ 7313 & 13: 28: 15 & 0.1316 \\ 7313 & 13: 28: 16 & 0.1296 \\ 7314 & 13: 28: 17 & 0.1311 \\ 7315 & 13: 28: 18 & 0.1279 \\ 7316 & 13: 28: 19 & 0.1320 \\ 7317 & 13: 28: 20 & 0.1290 \\ 7318 & 13: 28: 21 & 0.1327 \\ 7319 & 13: 28: 22 & 0.1284 \\ 7320 & 13: 28: 23 & 0.1335 \\ 7321 & 13: 28: 24 & 0.1301 \\ 7322 & 13: 28: 25 & 0.1330 \\ 7323 & 13: 28: 26 & 0.1292 \\ 7324 & 13: 28: 27 & 0.1309 \\ 7325 & 13: 28: 28 & 0.1296 \\ 7326 & 13: 28: 29 & 0.1278 \\ 7327 & 13: 28: 30 & 0.1292 \\ 7328 & 13: 28: 31 & 0.1281 \\ 7329 & 13: 28: 32 & 0.1303 \\ 7330 & 13: 28: 33 & 0.1264\end{array}$


Appendix A - Fuel Data Test 1 NIST102505_1.xls

$\begin{array}{lll}7331 & 13: 28: 34 & 0.1319 \\ 7332 & 13: 28: 35 & 0.1293 \\ 7333 & 13: 28: 36 & 0.1323 \\ 7334 & 13: 28: 37 & 0.1286 \\ 7335 & 13: 28: 38 & 0.1314 \\ 7336 & 13: 28: 39 & 0.1285 \\ 7337 & 13: 28: 40 & 0.1289 \\ 7338 & 13: 28: 41 & 0.1283 \\ 7339 & 13: 28: 42 & 0.1293 \\ 7340 & 13: 28: 43 & 0.1316 \\ 7341 & 13: 28: 44 & 0.1291 \\ 7342 & 13: 28: 45 & 0.1335 \\ 7343 & 13: 28: 46 & 0.1305 \\ 7344 & 13: 28: 47 & 0.1328 \\ 7345 & 13: 28: 48 & 0.1285 \\ 7346 & 13: 28: 49 & 0.1319 \\ 7347 & 13: 28: 50 & 0.1317 \\ 7348 & 13: 28: 51 & 0.1328 \\ 7349 & 13: 28: 52 & 0.1308 \\ 7350 & 13: 28: 53 & 0.1294 \\ 7351 & 13: 28: 54 & 0.1315 \\ 7352 & 13: 28: 55 & 0.1280 \\ 7353 & 13: 28: 56 & 0.1317 \\ 7354 & 13: 28: 57 & 0.1274 \\ 7355 & 13: 28: 58 & 0.1326 \\ 7356 & 13: 28: 59 & 0.1282 \\ 7357 & 13: 29: 00 & 0.1325 \\ 7358 & 13: 29: 01 & 0.1293 \\ 7359 & 13: 29: 02 & 0.1330 \\ 7360 & 13: 29: 03 & 0.1304 \\ 7361 & 13: 29: 04 & 0.1305 \\ 7362 & 13: 29: 05 & 0.1322 \\ 7363 & 13: 29: 06 & 0.1291 \\ 7364 & 13: 29: 07 & 0.1295 \\ 7365 & 13: 29: 08 & 0.1277 \\ 7366 & 13: 29: 09 & 0.1322 \\ 7367 & 13: 29: 10 & 0.1279 \\ 7368 & 13: 29: 11 & 0.1312 \\ 7369 & 13: 29: 12 & 0.1285 \\ 7370 & 13: 29: 13 & 0.1314 \\ 7371 & 13: 29: 14 & 0.1285 \\ 7372 & 13: 29: 15 & 0.1302 \\ 7373 & 13: 29: 16 & 0.1296 \\ 7374 & 13: 29: 17 & 0.1300 \\ 7375 & 13: 29: 18 & 0.1304 \\ 7376 & 13: 29: 19 & 0.1279 \\ 7377 & 13: 29: 20 & 0.1330 \\ 7378 & 13: 29: 21 & 0.1294 \\ 7379 & 13: 29: 22 & 0.1329 \\ 7380 & 13: 29: 23 & 0.1271 \\ 7381 & 13: 29: 24 & 0.1318 \\ 7382 & 13: 29: 25 & 0.1288\end{array}$


Appendix A - Fuel Data Test 1 NIST102505_1.xls

$\begin{array}{lll}7383 & 13: 29: 26 & 0.1323 \\ 7384 & 13: 29: 27 & 0.1293 \\ 7385 & 13: 29: 28 & 0.1312 \\ 7386 & 13: 29: 29 & 0.1286 \\ 7387 & 13: 29: 30 & 0.1294 \\ 7388 & 13: 29: 31 & 0.1291 \\ 7389 & 13: 29: 32 & 0.1307 \\ 7390 & 13: 29: 33 & 0.1318 \\ 7391 & 13: 29: 34 & 0.1286 \\ 7392 & 13: 29: 35 & 0.1304 \\ 7393 & 13: 29: 36 & 0.1275 \\ 7394 & 13: 29: 37 & 0.1307 \\ 7395 & 13: 29: 38 & 0.1287 \\ 7396 & 13: 29: 39 & 0.1316 \\ 7397 & 13: 29: 40 & 0.1304 \\ 7398 & 13: 29: 41 & 0.1312 \\ 7399 & 13: 29: 42 & 0.1295 \\ 7400 & 13: 29: 43 & 0.1282 \\ 7401 & 13: 29: 44 & 0.1324 \\ 7402 & 13: 29: 45 & 0.1286 \\ 7403 & 13: 29: 46 & 0.1312 \\ 7404 & 13: 29: 47 & 0.1273 \\ 7405 & 13: 29: 48 & 0.1327 \\ 7406 & 13: 29: 49 & 0.1273 \\ 7407 & 13: 29: 50 & 0.1317 \\ 7408 & 13: 29: 51 & 0.1297 \\ 7409 & 13: 29: 52 & 0.1337 \\ 7410 & 13: 29: 53 & 0.1316 \\ 7412 & 13: 29: 54 & 0.1324 \\ 7412 & 13: 29: 55 & 0.1327 \\ 7413 & 13: 29: 56 & 0.1298 \\ 7414 & 13: 29: 57 & 0.1301 \\ 7415 & 13: 29: 58 & 0.1281 \\ 7416 & 13: 29: 59 & 0.1306 \\ 7417 & 13: 30: 00 & 0.1266 \\ 7418 & 13: 30: 01 & 0.1307 \\ 7419 & 13: 30: 02 & 0.1280 \\ 7420 & 13: 30: 03 & 0.1309 \\ 7421 & 13: 30: 04 & 0.1278 \\ 7422 & 13: 30: 05 & 0.1315 \\ 7423 & 13: 30: 06 & 0.1307 \\ 7424 & 13: 30: 07 & 0.1311 \\ 7425 & 13: 30: 08 & 0.1285 \\ 7426 & 13: 30: 09 & 0.1289 \\ 7427 & 13: 30: 10 & 0.1300 \\ 7428 & 13: 30: 11 & 0.1275 \\ 7429 & 13: 30: 12 & 0.1306 \\ 7430 & 13: 30: 13 & 0.1264 \\ 7431 & 13: 30: 14 & 0.1301 \\ 7432 & 13: 30: 15 & 0.1275 \\ 7433 & 13: 30: 16 & 0.1321 \\ 7434 & 13: 30: 17 & 0.1289\end{array}$


Appendix A - Fuel Data Test 1 NIST102505_1.xls

$\begin{array}{lll}7435 & 13: 30: 18 & 0.1319 \\ 7436 & 13: 30: 19 & 0.1283 \\ 7437 & 13: 30: 20 & 0.1301 \\ 7438 & 13: 30: 21 & 0.1280 \\ 7439 & 13: 30: 22 & 0.1271 \\ 7440 & 13: 30: 23 & 0.1302 \\ 7441 & 13: 30: 24 & 0.1277 \\ 7442 & 13: 30: 25 & 0.1294 \\ 7443 & 13: 30: 26 & 0.1264 \\ 7444 & 13: 30: 27 & 0.1312 \\ 7445 & 13: 30: 28 & 0.1277 \\ 7446 & 13: 30: 29 & 0.1324 \\ 7447 & 13: 30: 30 & 0.1307 \\ 7448 & 13: 30: 31 & 0.1312 \\ 7449 & 13: 30: 32 & 0.1298 \\ 7450 & 13: 30: 33 & 0.1288 \\ 7451 & 13: 30: 34 & 0.1319 \\ 7452 & 13: 30: 35 & 0.1290 \\ 7453 & 13: 30: 36 & 0.1323 \\ 7454 & 13: 30: 37 & 0.1291 \\ 7455 & 13: 30: 38 & 0.1328 \\ 7456 & 13: 30: 39 & 0.1278 \\ 7457 & 13: 30: 40 & 0.1327 \\ 7458 & 13: 30: 41 & 0.1297 \\ 7459 & 13: 30: 42 & 0.1337 \\ 7460 & 13: 30: 43 & 0.1302 \\ 7461 & 13: 30: 44 & 0.1328 \\ 7462 & 13: 30: 45 & 0.1303 \\ 7463 & 13: 30: 46 & 0.1304 \\ 7464 & 13: 30: 47 & 0.1303 \\ 7465 & 13: 30: 48 & 0.1304 \\ 7466 & 13: 30: 49 & 0.1314 \\ 7467 & 13: 30: 50 & 0.1276 \\ 7468 & 13: 30: 51 & 0.1323 \\ 7469 & 13: 30: 52 & 0.1295 \\ 7470 & 13: 30: 53 & 0.1322 \\ 7471 & 13: 30: 54 & 0.1289 \\ 7472 & 13: 30: 55 & 0.1339 \\ 7473 & 13: 30: 56 & 0.1316 \\ 7474 & 13: 30: 57 & 0.1328 \\ 7475 & 13: 30: 58 & 0.1312 \\ 7476 & 13: 30: 59 & 0.1310 \\ 7477 & 13: 31: 00 & 0.1305 \\ 7478 & 13: 31: 01 & 0.1276 \\ 7479 & 13: 31: 02 & 0.1299 \\ 7480 & 13: 31: 03 & 0.1276 \\ 7481 & 13: 31: 04 & 0.1315 \\ 7482 & 13: 31: 05 & 0.1260 \\ 7483 & 13: 31: 06 & 0.1322 \\ 7484 & 13: 31: 07 & 0.1289 \\ 7485 & 13: 31: 08 & 0.1313 \\ 7486 & 13: 31: 09 & 0.1299\end{array}$


Appendix A - Fuel Data Test 1 NIST102505_1.xls

$\begin{array}{lll}7487 & 13: 31: 10 & 0.1310 \\ 7488 & 13: 31: 11 & 0.1284 \\ 7489 & 13: 31: 12 & 0.1288 \\ 7490 & 13: 31: 13 & 0.1309 \\ 7491 & 13: 31: 14 & 0.1293 \\ 7492 & 13: 31: 15 & 0.1310 \\ 7493 & 13: 31: 16 & 0.1290 \\ 7494 & 13: 31: 17 & 0.1320 \\ 7495 & 13: 31: 18 & 0.1279 \\ 7496 & 13: 31: 19 & 0.1329 \\ 7497 & 13: 31: 20 & 0.1303 \\ 7498 & 13: 31: 21 & 0.1340 \\ 7499 & 13: 31: 22 & 0.1308 \\ 7500 & 13: 31: 23 & 0.1332 \\ 7501 & 13: 31: 24 & 0.1309 \\ 7502 & 13: 31: 25 & 0.1299 \\ 7503 & 13: 31: 26 & 0.1335 \\ 7504 & 13: 31: 27 & 0.1298 \\ 7505 & 13: 31: 28 & 0.1308 \\ 7506 & 13: 31: 29 & 0.1255 \\ 7507 & 13: 31: 30 & 0.1313 \\ 7508 & 13: 31: 31 & 0.1288 \\ 7509 & 13: 31: 32 & 0.1323 \\ 7510 & 13: 31: 33 & 0.1280 \\ 7511 & 13: 31: 34 & 0.1323 \\ 7512 & 13: 31: 35 & 0.1289 \\ 7513 & 13: 31: 36 & 0.1298 \\ 7514 & 13: 31: 37 & 0.1293 \\ 7515 & 13: 31: 38 & 0.1282 \\ 7516 & 13: 31: 39 & 0.1297 \\ 7517 & 13: 31: 40 & 0.1267 \\ 7518 & 13: 31: 41 & 0.1314 \\ 7519 & 13: 31: 42 & 0.1255 \\ 7520 & 13: 31: 43 & 0.1305 \\ 7521 & 13: 31: 44 & 0.1279 \\ 7522 & 13: 31: 45 & 0.1315 \\ 7523 & 13: 31: 46 & 0.1287 \\ 7524 & 13: 31: 47 & 0.1310 \\ 7525 & 13: 31: 48 & 0.1306 \\ 7526 & 13: 31: 49 & 0.1303 \\ 7527 & 13: 31: 50 & 0.1308 \\ 7528 & 13: 31: 51 & 0.1281 \\ 7529 & 13: 31: 52 & 0.1321 \\ 7530 & 13: 31: 53 & 0.1292 \\ 7531 & 13: 31: 54 & 0.1319 \\ 7533 & 13: 31: 55 & 0.1292 \\ 7533 & 13: 31: 56 & 0.1323 \\ 7534 & 13: 31: 57 & 0.1264 \\ 7535 & 13: 31: 58 & 0.1309 \\ 7536 & 13: 31: 59 & 0.1309 \\ 7537 & 13: 32: 00 & 0.1322 \\ 7538 & 13: 32: 01 & 0.1297\end{array}$


Appendix A - Fuel Data Test 1 NIST102505_1.xls

$\begin{array}{lll}7539 & 13: 32: 02 & 0.1309 \\ 7540 & 13: 32: 03 & 0.1330 \\ 7541 & 13: 32: 04 & 0.1310 \\ 7542 & 13: 32: 05 & 0.1324 \\ 7543 & 13: 32: 06 & 0.1301 \\ 7544 & 13: 32: 07 & 0.1327 \\ 7545 & 13: 32: 08 & 0.1275 \\ 7546 & 13: 32: 09 & 0.1312 \\ 7547 & 13: 32: 10 & 0.1288 \\ 7548 & 13: 32: 11 & 0.1336 \\ 7549 & 13: 32: 12 & 0.1295 \\ 7550 & 13: 32: 13 & 0.1322 \\ 7551 & 13: 32: 14 & 0.1303 \\ 7552 & 13: 32: 15 & 0.1298 \\ 7553 & 13: 32: 16 & 0.1321 \\ 7554 & 13: 32: 17 & 0.1299 \\ 7555 & 13: 32: 18 & 0.1313 \\ 7556 & 13: 32: 19 & 0.1272 \\ 7557 & 13: 32: 20 & 0.1323 \\ 7558 & 13: 32: 21 & 0.1284 \\ 7559 & 13: 32: 22 & 0.1320 \\ 7560 & 13: 32: 23 & 0.1297 \\ 7561 & 13: 32: 24 & 0.1330 \\ 7562 & 13: 32: 25 & 0.1314 \\ 7563 & 13: 32: 26 & 0.1306 \\ 7564 & 13: 32: 27 & 0.1288 \\ 7565 & 13: 32: 28 & 0.1281 \\ 7566 & 13: 32: 29 & 0.1302 \\ 7567 & 13: 32: 30 & 0.1266 \\ 7568 & 13: 32: 31 & 0.1315 \\ 7569 & 13: 32: 32 & 0.1267 \\ 7570 & 13: 32: 33 & 0.1303 \\ 7571 & 13: 32: 34 & 0.1267 \\ 7572 & 13: 32: 35 & 0.1310 \\ 7573 & 13: 32: 36 & 0.1269 \\ 7574 & 13: 32: 37 & 0.1289 \\ 7575 & 13: 32: 38 & 0.1316 \\ 7576 & 13: 32: 39 & 0.1312 \\ 7577 & 13: 32: 40 & 0.1302 \\ 7578 & 13: 32: 41 & 0.1293 \\ 7579 & 13: 32: 42 & 0.1338 \\ 7580 & 13: 32: 43 & 0.1295 \\ 7581 & 13: 32: 44 & 0.1316 \\ 7582 & 13: 32: 45 & 0.1277 \\ 7583 & 13: 32: 46 & 0.1335 \\ 7584 & 13: 32: 47 & 0.1303 \\ 7585 & 13: 32: 48 & 0.1339 \\ 7586 & 13: 32: 49 & 0.1317 \\ 7587 & 13: 32: 50 & 0.1328 \\ 7588 & 13: 32: 51 & 0.1298 \\ 7589 & 13: 32: 52 & 0.1305 \\ 7590 & 13: 32: 53 & 0.1298\end{array}$


Appendix A - Fuel Data Test 1 NIST102505_1.xls

$\begin{array}{lll}7591 & 13: 32: 54 & 0.1295 \\ 7592 & 13: 32: 55 & 0.1313 \\ 7593 & 13: 32: 56 & 0.1304 \\ 7594 & 13: 32: 57 & 0.1323 \\ 7595 & 13: 32: 58 & 0.1280 \\ 7596 & 13: 32: 59 & 0.1335 \\ 7597 & 13: 33: 00 & 0.1305 \\ 7598 & 13: 33: 01 & 0.1326 \\ 7599 & 13: 33: 02 & 0.1297 \\ 7600 & 13: 33: 03 & 0.1322 \\ 7601 & 13: 33: 04 & 0.1300 \\ 7602 & 13: 33: 05 & 0.1301 \\ 7603 & 13: 33: 06 & 0.1298 \\ 7604 & 13: 33: 07 & 0.1280 \\ 7605 & 13: 33: 08 & 0.1302 \\ 7606 & 13: 33: 09 & 0.1264 \\ 7607 & 13: 33: 10 & 0.1299 \\ 7608 & 13: 33: 11 & 0.1269 \\ 7609 & 13: 33: 12 & 0.1305 \\ 7610 & 13: 33: 13 & 0.1276 \\ 7611 & 13: 33: 14 & 0.1328 \\ 7612 & 13: 33: 15 & 0.1308 \\ 7613 & 13: 33: 16 & 0.1322 \\ 7614 & 13: 33: 17 & 0.1290 \\ 7615 & 13: 33: 18 & 0.1314 \\ 7616 & 13: 33: 19 & 0.1313 \\ 7617 & 13: 33: 20 & 0.1306 \\ 7618 & 13: 33: 21 & 0.1319 \\ 7619 & 13: 33: 22 & 0.1291 \\ 7620 & 13: 33: 23 & 0.1322 \\ 7621 & 13: 33: 24 & 0.1279 \\ 7622 & 13: 33: 25 & 0.1312 \\ 7623 & 13: 33: 26 & 0.1295 \\ 7624 & 13: 33: 27 & 0.1320 \\ 7625 & 13: 33: 28 & 0.1300 \\ 7627 & 13: 33: 29 & 0.1314 \\ 7627 & 13: 33: 30 & 0.1302 \\ 7628 & 13: 33: 31 & 0.1292 \\ 7629 & 13: 33: 32 & 0.1293 \\ 7630 & 13: 33: 33 & 0.1279 \\ 7631 & 13: 33: 34 & 0.1302 \\ 7632 & 13: 33: 35 & 0.1253 \\ 7633 & 13: 33: 36 & 0.1320 \\ 7634 & 13: 33: 37 & 0.1277 \\ 7635 & 13: 33: 38 & 0.1322 \\ 7636 & 13: 33: 39 & 0.1311 \\ 7637 & 13: 33: 40 & 0.1333 \\ 7638 & 13: 33: 41 & 0.1308 \\ 7639 & 13: 33: 42 & 0.1297 \\ 7640 & 13: 33: 43 & 0.1314 \\ 7641 & 13: 33: 44 & 0.1280 \\ 7642 & 13: 33: 45 & 0.1302\end{array}$


Appendix A - Fuel Data Test 1 NIST102505_1.xIs

$\begin{array}{lll}7643 & 13: 33: 46 & 0.1272 \\ 7644 & 13: 33: 47 & 0.1324 \\ 7645 & 13: 33: 48 & 0.1275 \\ 7646 & 13: 33: 49 & 0.1307 \\ 7647 & 13: 33: 50 & 0.1283 \\ 7648 & 13: 33: 51 & 0.1322 \\ 7649 & 13: 33: 52 & 0.1281 \\ 7650 & 13: 33: 53 & 0.1309 \\ 7651 & 13: 33: 54 & 0.1318 \\ 7652 & 13: 33: 55 & 0.1300 \\ 7653 & 13: 33: 56 & 0.1296 \\ 7654 & 13: 33: 57 & 0.1273 \\ 7655 & 13: 33: 58 & 0.1311 \\ 7656 & 13: 33: 59 & 0.1280 \\ 7657 & 13: 34: 00 & 0.1307 \\ 7658 & 13: 34: 01 & 0.1285 \\ 7659 & 13: 34: 02 & 0.1312 \\ 7660 & 13: 34: 03 & 0.1265 \\ 7661 & 13: 34: 04 & 0.1311 \\ 7662 & 13: 34: 05 & 0.1304 \\ 7663 & 13: 34: 06 & 0.1320 \\ 7664 & 13: 34: 07 & 0.1297 \\ 7665 & 13: 34: 08 & 0.1315 \\ 7666 & 13: 34: 09 & 0.1309 \\ 7667 & 13: 34: 10 & 0.1294 \\ 7668 & 13: 34: 11 & 0.1312 \\ 7669 & 13: 34: 12 & 0.1288 \\ 7670 & 13: 34: 13 & 0.1314 \\ 7671 & 13: 34: 14 & 0.1256 \\ 7672 & 13: 34: 15 & 0.1319 \\ 7673 & 13: 34: 16 & 0.1286 \\ 7674 & 13: 34: 17 & 0.1315 \\ 7675 & 13: 34: 18 & 0.1298 \\ 7676 & 13: 34: 19 & 0.1309 \\ 7677 & 13: 34: 20 & 0.1300 \\ 7678 & 13: 34: 21 & 0.1302 \\ 7680 & 13: 34: 22 & 0.1323 \\ 7680 & 13: 34: 23 & 0.1296 \\ 7681 & 13: 34: 24 & 0.1313 \\ 7682 & 13: 34: 25 & 0.1282 \\ 7683 & 13: 34: 26 & 0.1336 \\ 7684 & 13: 34: 27 & 0.1288 \\ 7685 & 13: 34: 28 & 0.1322 \\ 7686 & 13: 34: 29 & 0.1298 \\ 7687 & 13: 34: 30 & 0.1332 \\ 7688 & 13: 34: 31 & 0.1305 \\ 7689 & 13: 34: 32 & 0.1293 \\ 7690 & 13: 34: 33 & 0.1308 \\ 7691 & 13: 34: 34 & 0.1295 \\ 7692 & 13: 34: 35 & 0.1304 \\ 7693 & 13: 34: 36 & 0.1273 \\ 7694 & 13: 34: 37 & 0.1313\end{array}$


Appendix A - Fuel Data Test 1 NIST102505_1.xls

$\begin{array}{lll}7695 & 13: 34: 38 & 0.1261 \\ 7696 & 13: 34: 39 & 0.1299 \\ 7697 & 13: 34: 40 & 0.1270 \\ 7698 & 13: 34: 41 & 0.1313 \\ 7699 & 13: 34: 42 & 0.1276 \\ 7700 & 13: 34: 43 & 0.1310 \\ 7701 & 13: 34: 44 & 0.1303 \\ 7702 & 13: 34: 45 & 0.1309 \\ 7703 & 13: 34: 46 & 0.1292 \\ 7704 & 13: 34: 47 & 0.1291 \\ 7705 & 13: 34: 48 & 0.1304 \\ 7706 & 13: 34: 49 & 0.1274 \\ 7707 & 13: 34: 50 & 0.1294 \\ 7708 & 13: 34: 51 & 0.1268 \\ 7709 & 13: 34: 52 & 0.1299 \\ 7710 & 13: 34: 53 & 0.1256 \\ 7711 & 13: 34: 54 & 0.1313 \\ 7712 & 13: 34: 55 & 0.1291 \\ 7713 & 13: 34: 56 & 0.1319 \\ 7714 & 13: 34: 57 & 0.1282 \\ 7715 & 13: 34: 58 & 0.1310 \\ 7716 & 13: 34: 59 & 0.1281 \\ 7717 & 13: 35: 00 & 0.1272 \\ 7718 & 13: 35: 01 & 0.1304 \\ 7719 & 13: 35: 02 & 0.1280 \\ 7720 & 13: 35: 03 & 0.1303 \\ 7721 & 13: 35: 04 & 0.1275 \\ 7722 & 13: 35: 05 & 0.1332 \\ 7723 & 13: 35: 06 & 0.1302 \\ 7724 & 13: 35: 07 & 0.1322 \\ 7725 & 13: 35: 08 & 0.1284 \\ 7726 & 13: 35: 09 & 0.1327 \\ 7727 & 13: 35: 10 & 0.1300 \\ 7728 & 13: 35: 11 & 0.1299 \\ 7729 & 13: 35: 12 & 0.1278 \\ 7730 & 13: 35: 13 & 0.1273 \\ 7731 & 13: 35: 14 & 0.1295 \\ 7732 & 13: 35: 15 & 0.1261 \\ 7733 & 13: 35: 16 & 0.1310 \\ 7734 & 13: 35: 17 & 0.1264 \\ 7735 & 13: 35: 18 & 0.1310 \\ 7736 & 13: 35: 19 & 0.1290 \\ 7737 & 13: 35: 20 & 0.1325 \\ 7738 & 13: 35: 21 & 0.1292 \\ 7739 & 13: 35: 22 & 0.1295 \\ 7741 & 13: 35: 23 & 0.1296 \\ 7741 & 13: 35: 24 & 0.1299 \\ 7742 & 13: 35: 25 & 0.1292 \\ 7743 & 13: 35: 26 & 0.1285 \\ 7744 & 13: 35: 27 & 0.1321 \\ 7745 & 13: 35: 28 & 0.1278 \\ 7746 & 13: 35: 29 & 0.1296\end{array}$


Appendix A - Fuel Data Test 1 NIST102505_1.xIs

$\begin{array}{lll}7747 & 13: 35: 30 & 0.1276 \\ 7748 & 13: 35: 31 & 0.1304 \\ 7749 & 13: 35: 32 & 0.1286 \\ 7750 & 13: 35: 33 & 0.1303 \\ 7751 & 13: 35: 34 & 0.1294 \\ 7752 & 13: 35: 35 & 0.1290 \\ 7753 & 13: 35: 36 & 0.1279 \\ 7754 & 13: 35: 37 & 0.1262 \\ 7755 & 13: 35: 38 & 0.1293 \\ 7756 & 13: 35: 39 & 0.1259 \\ 7757 & 13: 35: 40 & 0.1283 \\ 7758 & 13: 35: 41 & 0.1249 \\ 7759 & 13: 35: 42 & 0.1292 \\ 7760 & 13: 35: 43 & 0.1269 \\ 7761 & 13: 35: 44 & 0.1311 \\ 7762 & 13: 35: 45 & 0.1311 \\ 7763 & 13: 35: 46 & 0.1311 \\ 7764 & 13: 35: 47 & 0.1304 \\ 7765 & 13: 35: 48 & 0.1288 \\ 7766 & 13: 35: 49 & 0.1310 \\ 7767 & 13: 35: 50 & 0.1277 \\ 7768 & 13: 35: 51 & 0.1297 \\ 7769 & 13: 35: 52 & 0.1261 \\ 7770 & 13: 35: 53 & 0.1301 \\ 7771 & 13: 35: 54 & 0.1266 \\ 7772 & 13: 35: 55 & 0.1308 \\ 7773 & 13: 35: 56 & 0.1299 \\ 7774 & 13: 35: 57 & 0.1319 \\ 7775 & 13: 35: 58 & 0.1287 \\ 7776 & 13: 35: 59 & 0.1299 \\ 7777 & 13: 36: 00 & 0.1291 \\ 7778 & 13: 36: 01 & 0.1277 \\ 7779 & 13: 36: 02 & 0.1291 \\ 7780 & 13: 36: 03 & 0.1276 \\ 7781 & 13: 36: 04 & 0.1301 \\ 7782 & 13: 36: 05 & 0.1254 \\ 7783 & 13: 36: 06 & 0.1299 \\ 7784 & 13: 36: 07 & 0.1293 \\ 7785 & 13: 36: 08 & 0.1315 \\ 7786 & 13: 36: 09 & 0.1267 \\ 7787 & 13: 36: 10 & 0.1304 \\ 7788 & 13: 36: 11 & 0.1275 \\ 7789 & 13: 36: 12 & 0.1278 \\ 7790 & 13: 36: 13 & 0.1282 \\ 7791 & 13: 36: 14 & 0.1272 \\ 7792 & 13: 36: 15 & 0.1302 \\ 7793 & 13: 36: 16 & 0.1285 \\ 7794 & 13: 36: 17 & 0.1324 \\ 7795 & 13: 36: 18 & 0.1303 \\ 7796 & 13: 36: 19 & 0.1325 \\ 7797 & 13: 36: 20 & 0.1283 \\ 7798 & 13: 36: 21 & 0.1322\end{array}$


Appendix A - Fuel Data Test 1 NIST102505_1.xIs

$\begin{array}{lll}7799 & 13: 36: 22 & 0.1290 \\ 7800 & 13: 36: 23 & 0.1305 \\ 7801 & 13: 36: 24 & 0.1305 \\ 7802 & 13: 36: 25 & 0.1295 \\ 7803 & 13: 36: 26 & 0.1295 \\ 7804 & 13: 36: 27 & 0.1279 \\ 7805 & 13: 36: 28 & 0.1295 \\ 7806 & 13: 36: 29 & 0.1243 \\ 7807 & 13: 36: 30 & 0.1288 \\ 7808 & 13: 36: 31 & 0.1282 \\ 7809 & 13: 36: 32 & 0.1318 \\ 7810 & 13: 36: 33 & 0.1287 \\ 7811 & 13: 36: 34 & 0.1323 \\ 7812 & 13: 36: 35 & 0.1302 \\ 7813 & 13: 36: 36 & 0.1307 \\ 7814 & 13: 36: 37 & 0.1273 \\ 7815 & 13: 36: 38 & 0.1279 \\ 7816 & 13: 36: 39 & 0.1284 \\ 7817 & 13: 36: 40 & 0.1262 \\ 7818 & 13: 36: 41 & 0.1316 \\ 7819 & 13: 36: 42 & 0.1264 \\ 7820 & 13: 36: 43 & 0.1306 \\ 7821 & 13: 36: 44 & 0.1267 \\ 7822 & 13: 36: 45 & 0.1335 \\ 7823 & 13: 36: 46 & 0.1307 \\ 7824 & 13: 36: 47 & 0.1340 \\ 7825 & 13: 36: 48 & 0.1309 \\ 7826 & 13: 36: 49 & 0.1318 \\ 7827 & 13: 36: 50 & 0.1314 \\ 7828 & 13: 36: 51 & 0.1287 \\ 7829 & 13: 36: 52 & 0.1317 \\ 7830 & 13: 36: 53 & 0.1287 \\ 7831 & 13: 36: 54 & 0.1310 \\ 7832 & 13: 36: 55 & 0.1264 \\ 7833 & 13: 36: 56 & 0.1319 \\ 7834 & 13: 36: 57 & 0.1280 \\ 7835 & 13: 36: 58 & 0.1313 \\ 7836 & 13: 36: 59 & 0.1305 \\ 7837 & 13: 37: 00 & 0.1312 \\ 7838 & 13: 37: 01 & 0.1293 \\ 7839 & 13: 37: 02 & 0.1281 \\ 7840 & 13: 37: 03 & 0.1311 \\ 7841 & 13: 37: 04 & 0.1293 \\ 7842 & 13: 37: 05 & 0.1319 \\ 7843 & 13: 37: 06 & 0.1274 \\ 7844 & 13: 37: 07 & 0.1314 \\ 7845 & 13: 37: 08 & 0.1265 \\ 7846 & 13: 37: 09 & 0.1300 \\ 7847 & 13: 37: 10 & 0.1277 \\ 7848 & 13: 37: 11 & 0.1326 \\ 7849 & 13: 37: 12 & 0.1307 \\ 7850 & 13: 37: 13 & 0.1329\end{array}$


Appendix A - Fuel Data Test 1 NIST102505_1.xls

$\begin{array}{lll}7851 & 13: 37: 14 & 0.1315 \\ 7852 & 13: 37: 15 & 0.1315 \\ 7853 & 13: 37: 16 & 0.1298 \\ 7854 & 13: 37: 17 & 0.1294 \\ 7855 & 13: 37: 18 & 0.1337 \\ 7856 & 13: 37: 19 & 0.1287 \\ 7857 & 13: 37: 20 & 0.1317 \\ 7858 & 13: 37: 21 & 0.1283 \\ 7859 & 13: 37: 22 & 0.1312 \\ 7860 & 13: 37: 23 & 0.1274 \\ 7861 & 13: 37: 24 & 0.1321 \\ 7862 & 13: 37: 25 & 0.1328 \\ 7863 & 13: 37: 26 & 0.1330 \\ 7864 & 13: 37: 27 & 0.1303 \\ 7865 & 13: 37: 28 & 0.1302 \\ 7866 & 13: 37: 29 & 0.1317 \\ 7867 & 13: 37: 30 & 0.1291 \\ 7868 & 13: 37: 31 & 0.1307 \\ 7869 & 13: 37: 32 & 0.1284 \\ 7870 & 13: 37: 33 & 0.1325 \\ 7871 & 13: 37: 34 & 0.1277 \\ 7872 & 13: 37: 35 & 0.1337 \\ 7873 & 13: 37: 36 & 0.1305 \\ 7874 & 13: 37: 37 & 0.1341 \\ 7875 & 13: 37: 38 & 0.1301 \\ 7876 & 13: 37: 39 & 0.1322 \\ 7877 & 13: 37: 40 & 0.1294 \\ 7878 & 13: 37: 41 & 0.1280 \\ 7879 & 13: 37: 42 & 0.1296 \\ 7880 & 13: 37: 43 & 0.1282 \\ 7881 & 13: 37: 44 & 0.1302 \\ 7882 & 13: 37: 45 & 0.1250 \\ 7883 & 13: 37: 46 & 0.1317 \\ 7884 & 13: 37: 47 & 0.1289 \\ 7885 & 13: 37: 48 & 0.1336 \\ 7886 & 13: 37: 49 & 0.1290 \\ 7887 & 13: 37: 50 & 0.1320 \\ 7888 & 13: 37: 51 & 0.1283 \\ 7889 & 13: 37: 52 & 0.1290 \\ 7890 & 13: 37: 53 & 0.1276 \\ 7891 & 13: 37: 54 & 0.1281 \\ 7892 & 13: 37: 55 & 0.1312 \\ 7893 & 13: 37: 56 & 0.1265 \\ 7895 & 13: 37: 57 & 0.1320 \\ 7895 & 13: 37: 58 & 0.1273 \\ 7896 & 13: 37: 59 & 0.1299 \\ 7897 & 13: 38: 00 & 0.1266 \\ 7898 & 13: 38: 01 & 0.1305 \\ 7899 & 13: 38: 02 & 0.1279 \\ 7900 & 13: 38: 03 & 0.1292 \\ 7901 & 13: 38: 04 & 0.1305 \\ 7902 & 13: 38: 05 & 0.1312\end{array}$


Appendix A - Fuel Data Test 1 NIST102505_1.xls

$\begin{array}{lll}7903 & 13: 38: 06 & 0.1316 \\ 7904 & 13: 38: 07 & 0.1284 \\ 7905 & 13: 38: 08 & 0.1320 \\ 7906 & 13: 38: 09 & 0.1296 \\ 7907 & 13: 38: 10 & 0.1324 \\ 7908 & 13: 38: 11 & 0.1283 \\ 7909 & 13: 38: 12 & 0.1330 \\ 7910 & 13: 38: 13 & 0.1288 \\ 7911 & 13: 38: 14 & 0.1323 \\ 7912 & 13: 38: 15 & 0.1323 \\ 7913 & 13: 38: 16 & 0.1340 \\ 7914 & 13: 38: 17 & 0.1317 \\ 7915 & 13: 38: 18 & 0.1295 \\ 7916 & 13: 38: 19 & 0.1316 \\ 7917 & 13: 38: 20 & 0.1281 \\ 7918 & 13: 38: 21 & 0.1298 \\ 7919 & 13: 38: 22 & 0.1259 \\ 7920 & 13: 38: 23 & 0.1309 \\ 7921 & 13: 38: 24 & 0.1265 \\ 7922 & 13: 38: 25 & 0.1298 \\ 7923 & 13: 38: 26 & 0.1277 \\ 7924 & 13: 38: 27 & 0.1314 \\ 7925 & 13: 38: 28 & 0.1277 \\ 7926 & 13: 38: 29 & 0.1298 \\ 7927 & 13: 38: 30 & 0.1300 \\ 7928 & 13: 38: 31 & 0.1288 \\ 7929 & 13: 38: 32 & 0.1283 \\ 7930 & 13: 38: 33 & 0.1272 \\ 7931 & 13: 38: 34 & 0.1298 \\ 7932 & 13: 38: 35 & 0.1272 \\ 7933 & 13: 38: 36 & 0.1296 \\ 7934 & 13: 38: 37 & 0.1266 \\ 7935 & 13: 38: 38 & 0.1303 \\ 7936 & 13: 38: 39 & 0.1277 \\ 7937 & 13: 38: 40 & 0.1323 \\ 7938 & 13: 38: 41 & 0.1318 \\ 7939 & 13: 38: 42 & 0.1339 \\ 7940 & 13: 38: 43 & 0.1294 \\ 7941 & 13: 38: 44 & 0.1290 \\ 7942 & 13: 38: 45 & 0.1273 \\ 7943 & 13: 38: 46 & 0.1262 \\ 7944 & 13: 38: 47 & 0.1320 \\ 7945 & 13: 38: 48 & 0.1268 \\ 7946 & 13: 38: 49 & 0.1301 \\ 7947 & 13: 38: 50 & 0.1262 \\ 7948 & 13: 38: 51 & 0.1307 \\ 7949 & 13: 38: 52 & 0.1273 \\ 7950 & 13: 38: 53 & 0.1295 \\ 7951 & 13: 38: 54 & 0.1306 \\ 7952 & 13: 38: 55 & 0.1294 \\ 7953 & 13: 38: 56 & 0.1296 \\ 7954 & 13: 38: 57 & 0.1290\end{array}$


Appendix A - Fuel Data Test 1 NIST102505_1.xIs

$\begin{array}{lll}7955 & 13: 38: 58 & 0.1307 \\ 7956 & 13: 38: 59 & 0.1278 \\ 7957 & 13: 39: 00 & 0.1298 \\ 7958 & 13: 39: 01 & 0.1251 \\ 7959 & 13: 39: 02 & 0.1292 \\ 7960 & 13: 39: 03 & 0.1251 \\ 7961 & 13: 39: 04 & 0.1296 \\ 7962 & 13: 39: 05 & 0.1308 \\ 7963 & 13: 39: 06 & 0.1327 \\ 7964 & 13: 39: 07 & 0.1278 \\ 7965 & 13: 39: 08 & 0.1294 \\ 7966 & 13: 39: 09 & 0.1274 \\ 7967 & 13: 39: 10 & 0.1260 \\ 7968 & 13: 39: 11 & 0.1289 \\ 7969 & 13: 39: 12 & 0.1282 \\ 7970 & 13: 39: 13 & 0.1310 \\ 7971 & 13: 39: 14 & 0.1271 \\ 7972 & 13: 39: 15 & 0.1305 \\ 7973 & 13: 39: 16 & 0.1266 \\ 7974 & 13: 39: 17 & 0.1301 \\ 7975 & 13: 39: 18 & 0.1291 \\ 7976 & 13: 39: 19 & 0.1318 \\ 7977 & 13: 39: 20 & 0.1296 \\ 7978 & 13: 39: 21 & 0.1300 \\ 7979 & 13: 39: 22 & 0.1300 \\ 7980 & 13: 39: 23 & 0.1281 \\ 7981 & 13: 39: 24 & 0.1294 \\ 7982 & 13: 39: 25 & 0.1272 \\ 7983 & 13: 39: 26 & 0.1304 \\ 7984 & 13: 39: 27 & 0.1259 \\ 7985 & 13: 39: 28 & 0.1315 \\ 7986 & 13: 39: 29 & 0.1270 \\ 7987 & 13: 39: 30 & 0.1303 \\ 7988 & 13: 39: 31 & 0.1286 \\ 7989 & 13: 39: 32 & 0.1306 \\ 7990 & 13: 39: 33 & 0.1292 \\ 7991 & 13: 39: 34 & 0.1281 \\ 7992 & 13: 39: 35 & 0.1284 \\ 7993 & 13: 39: 36 & 0.1283 \\ 7994 & 13: 39: 37 & 0.1291 \\ 7995 & 13: 39: 38 & 0.1253 \\ 7996 & 13: 39: 39 & 0.1307 \\ 7997 & 13: 39: 40 & 0.1261 \\ 7998 & 13: 39: 41 & 0.1297 \\ 7999 & 13: 39: 42 & 0.1257 \\ 8000 & 13: 39: 43 & 0.1307 \\ 8001 & 13: 39: 44 & 0.1269 \\ 8002 & 13: 39: 45 & 0.1295 \\ 8003 & 13: 39: 46 & 0.1296 \\ 8004 & 13: 39: 47 & 0.1287 \\ 8005 & 13: 39: 48 & 0.1283 \\ 8006 & 13: 39: 49 & 0.1260\end{array}$


Appendix A - Fuel Data Test 1 NIST102505_1.xIs

$\begin{array}{lll}8007 & 13: 39: 50 & 0.1304 \\ 8008 & 13: 39: 51 & 0.1270 \\ 8009 & 13: 39: 52 & 0.1297 \\ 8010 & 13: 39: 53 & 0.1276 \\ 8011 & 13: 39: 54 & 0.1314 \\ 8012 & 13: 39: 55 & 0.1288 \\ 8013 & 13: 39: 56 & 0.1321 \\ 8014 & 13: 39: 57 & 0.1316 \\ 8015 & 13: 39: 58 & 0.1316 \\ 8016 & 13: 39: 59 & 0.1287 \\ 8017 & 13: 40: 00 & 0.1275 \\ 8018 & 13: 40: 01 & 0.1304 \\ 8019 & 13: 40: 02 & 0.1284 \\ 8020 & 13: 40: 03 & 0.1305 \\ 8021 & 13: 40: 04 & 0.1268 \\ 8022 & 13: 40: 05 & 0.1297 \\ 8023 & 13: 40: 06 & 0.1259 \\ 8024 & 13: 40: 07 & 0.1301 \\ 8025 & 13: 40: 08 & 0.1296 \\ 8026 & 13: 40: 09 & 0.1315 \\ 8027 & 13: 40: 10 & 0.1288 \\ 8028 & 13: 40: 11 & 0.1301 \\ 8029 & 13: 40: 12 & 0.1297 \\ 8030 & 13: 40: 13 & 0.1288 \\ 8031 & 13: 40: 14 & 0.1291 \\ 8032 & 13: 40: 15 & 0.1280 \\ 8033 & 13: 40: 16 & 0.1296 \\ 8034 & 13: 40: 17 & 0.1251 \\ 8035 & 13: 40: 18 & 0.1285 \\ 8036 & 13: 40: 19 & 0.1291 \\ 8037 & 13: 40: 20 & 0.1318 \\ 8038 & 13: 40: 21 & 0.1301 \\ 8039 & 13: 40: 22 & 0.1315 \\ 8040 & 13: 40: 23 & 0.1323 \\ 8041 & 13: 40: 24 & 0.1314 \\ 8042 & 13: 40: 25 & 0.1306 \\ 8043 & 13: 40: 26 & 0.1278 \\ 8044 & 13: 40: 27 & 0.1304 \\ 8045 & 13: 40: 28 & 0.1269 \\ 8046 & 13: 40: 29 & 0.1302 \\ 8047 & 13: 40: 30 & 0.1270 \\ 8048 & 13: 40: 31 & 0.1318 \\ 8049 & 13: 40: 32 & 0.1289 \\ 8050 & 13: 40: 33 & 0.1345 \\ 8051 & 13: 40: 34 & 0.1301 \\ 8052 & 13: 40: 35 & 0.1317 \\ 8053 & 13: 40: 36 & 0.1308 \\ 8054 & 13: 40: 37 & 0.1311 \\ 8055 & 13: 40: 38 & 0.1302 \\ 8056 & 13: 40: 39 & 0.1283 \\ 8057 & 13: 40: 40 & 0.1288 \\ 8058 & 13: 40: 41 & 0.1273\end{array}$


Appendix A - Fuel Data Test 1 NIST102505_1.xIs

$\begin{array}{lll}8059 & 13: 40: 42 & 0.1312 \\ 8060 & 13: 40: 43 & 0.1260 \\ 8061 & 13: 40: 44 & 0.1322 \\ 8062 & 13: 40: 45 & 0.1278 \\ 8063 & 13: 40: 46 & 0.1317 \\ 8064 & 13: 40: 47 & 0.1305 \\ 8065 & 13: 40: 48 & 0.1316 \\ 8066 & 13: 40: 49 & 0.1297 \\ 8067 & 13: 40: 50 & 0.1281 \\ 8068 & 13: 40: 51 & 0.1313 \\ 8069 & 13: 40: 52 & 0.1285 \\ 8070 & 13: 40: 53 & 0.1305 \\ 8071 & 13: 40: 54 & 0.1270 \\ 8072 & 13: 40: 55 & 0.1307 \\ 8073 & 13: 40: 56 & 0.1253 \\ 8074 & 13: 40: 57 & 0.1311 \\ 8075 & 13: 40: 58 & 0.1281 \\ 8076 & 13: 40: 59 & 0.1323 \\ 8077 & 13: 41: 00 & 0.1283 \\ 8078 & 13: 41: 01 & 0.1316 \\ 8079 & 13: 41: 02 & 0.1285 \\ 8080 & 13: 41: 03 & 0.1279 \\ 8081 & 13: 41: 04 & 0.1294 \\ 8082 & 13: 41: 05 & 0.1285 \\ 8083 & 13: 41: 06 & 0.1286 \\ 8084 & 13: 41: 07 & 0.1252 \\ 8085 & 13: 41: 08 & 0.1297 \\ 8086 & 13: 41: 09 & 0.1267 \\ 8087 & 13: 41: 10 & 0.1302 \\ 8088 & 13: 41: 11 & 0.1270 \\ 8089 & 13: 41: 12 & 0.1307 \\ 8090 & 13: 41: 13 & 0.1286 \\ 8091 & 13: 41: 14 & 0.1296 \\ 8092 & 13: 41: 15 & 0.1270 \\ 8093 & 13: 41: 16 & 0.1266 \\ 8094 & 13: 41: 17 & 0.1277 \\ 8095 & 13: 41: 18 & 0.1250 \\ 8096 & 13: 41: 19 & 0.1307 \\ 8097 & 13: 41: 20 & 0.1285 \\ 8098 & 13: 41: 21 & 0.1313 \\ 8099 & 13: 41: 22 & 0.1278 \\ 8100 & 13: 41: 23 & 0.1328 \\ 8101 & 13: 41: 24 & 0.1292 \\ 8102 & 13: 41: 25 & 0.1310 \\ 8103 & 13: 41: 26 & 0.1292 \\ 8104 & 13: 41: 27 & 0.1311 \\ 8105 & 13: 41: 28 & 0.1287 \\ 8106 & 13: 41: 29 & 0.1272 \\ 8107 & 13: 41: 30 & 0.1301 \\ 8108 & 13: 41: 31 & 0.1278 \\ 8109 & 13: 41: 32 & 0.1297 \\ 8110 & 13: 41: 33 & 0.1258\end{array}$


Appendix A - Fuel Data Test 1 NIST102505_1.xls

$\begin{array}{lll}8111 & 13: 41: 34 & 0.1309 \\ 8112 & 13: 41: 35 & 0.1277 \\ 8113 & 13: 41: 36 & 0.1313 \\ 8114 & 13: 41: 37 & 0.1276 \\ 8115 & 13: 41: 38 & 0.1303 \\ 8116 & 13: 41: 39 & 0.1287 \\ 8117 & 13: 41: 40 & 0.1283 \\ 8118 & 13: 41: 41 & 0.1308 \\ 8119 & 13: 41: 42 & 0.1284 \\ 8120 & 13: 41: 43 & 0.1307 \\ 8121 & 13: 41: 44 & 0.1262 \\ 8122 & 13: 41: 45 & 0.1314 \\ 8123 & 13: 41: 46 & 0.1264 \\ 8124 & 13: 41: 47 & 0.1300 \\ 8125 & 13: 41: 48 & 0.1266 \\ 8126 & 13: 41: 49 & 0.1308 \\ 8127 & 13: 41: 50 & 0.1276 \\ 8128 & 13: 41: 51 & 0.1300 \\ 8129 & 13: 41: 52 & 0.1301 \\ 8130 & 13: 41: 53 & 0.1292 \\ 8131 & 13: 41: 54 & 0.1290 \\ 8132 & 13: 41: 55 & 0.1281 \\ 8133 & 13: 41: 56 & 0.1297 \\ 8134 & 13: 41: 57 & 0.1251 \\ 8135 & 13: 41: 58 & 0.1291 \\ 8136 & 13: 41: 59 & 0.1264 \\ 8137 & 13: 42: 00 & 0.1300 \\ 8138 & 13: 42: 01 & 0.1262 \\ 8139 & 13: 42: 02 & 0.1317 \\ 8140 & 13: 42: 03 & 0.1291 \\ 8141 & 13: 42: 04 & 0.1311 \\ 8142 & 13: 42: 05 & 0.1282 \\ 8143 & 13: 42: 06 & 0.1291 \\ 8145 & 13: 42: 07 & 0.1280 \\ 8145 & 13: 42: 08 & 0.1262 \\ 8146 & 13: 42: 09 & 0.1305 \\ 8147 & 13: 42: 10 & 0.1265 \\ 8148 & 13: 42: 11 & 0.1299 \\ 8149 & 13: 42: 12 & 0.1259 \\ 8150 & 13: 42: 13 & 0.1326 \\ 8151 & 13: 42: 14 & 0.1297 \\ 8152 & 13: 42: 15 & 0.1329 \\ 8153 & 13: 42: 16 & 0.1312 \\ 8154 & 13: 42: 17 & 0.1335 \\ 8155 & 13: 42: 18 & 0.1315 \\ 8156 & 13: 42: 19 & 0.1297 \\ 8157 & 13: 42: 20 & 0.1332 \\ 8158 & 13: 42: 21 & 0.1289 \\ 8159 & 13: 42: 22 & 0.1297 \\ 8160 & 13: 42: 23 & 0.1257 \\ 8161 & 13: 42: 24 & 0.1317 \\ 8162 & 13: 42: 25 & 0.1285\end{array}$


Appendix A - Fuel Data Test 1 NIST102505_1.xIs

$\begin{array}{lll}8163 & 13: 42: 26 & 0.1313 \\ 8164 & 13: 42: 27 & 0.1294 \\ 8165 & 13: 42: 28 & 0.1315 \\ 8166 & 13: 42: 29 & 0.1301 \\ 8167 & 13: 42: 30 & 0.1291 \\ 8168 & 13: 42: 31 & 0.1295 \\ 8169 & 13: 42: 32 & 0.1281 \\ 8170 & 13: 42: 33 & 0.1295 \\ 8171 & 13: 42: 34 & 0.1272 \\ 8172 & 13: 42: 35 & 0.1301 \\ 8173 & 13: 42: 36 & 0.1257 \\ 8174 & 13: 42: 37 & 0.1293 \\ 8175 & 13: 42: 38 & 0.1292 \\ 8176 & 13: 42: 39 & 0.1313 \\ 8177 & 13: 42: 40 & 0.1276 \\ 8178 & 13: 42: 41 & 0.1305 \\ 8179 & 13: 42: 42 & 0.1300 \\ 8180 & 13: 42: 43 & 0.1301 \\ 8181 & 13: 42: 44 & 0.1286 \\ 8182 & 13: 42: 45 & 0.1267 \\ 8183 & 13: 42: 46 & 0.1306 \\ 8184 & 13: 42: 47 & 0.1268 \\ 8185 & 13: 42: 48 & 0.1309 \\ 8186 & 13: 42: 49 & 0.1284 \\ 8187 & 13: 42: 50 & 0.1325 \\ 8188 & 13: 42: 51 & 0.1287 \\ 8189 & 13: 42: 52 & 0.1325 \\ 8190 & 13: 42: 53 & 0.1308 \\ 8191 & 13: 42: 54 & 0.1320 \\ 8192 & 13: 42: 55 & 0.1303 \\ 8193 & 13: 42: 56 & 0.1309 \\ 8194 & 13: 42: 57 & 0.1320 \\ 8195 & 13: 42: 58 & 0.1297 \\ 8196 & 13: 42: 59 & 0.1318 \\ 8197 & 13: 43: 00 & 0.1296 \\ 8198 & 13: 43: 01 & 0.1320 \\ 8199 & 13: 43: 02 & 0.1273 \\ 8200 & 13: 43: 03 & 0.1327 \\ 8201 & 13: 43: 04 & 0.1304 \\ 8202 & 13: 43: 05 & 0.1337 \\ 8203 & 13: 43: 06 & 0.1288 \\ 8204 & 13: 43: 07 & 0.1323 \\ 8205 & 13: 43: 08 & 0.1301 \\ 8206 & 13: 43: 09 & 0.1297 \\ 8207 & 13: 43: 10 & 0.1283 \\ 8208 & 13: 43: 11 & 0.1278 \\ 8209 & 13: 43: 12 & 0.1307 \\ 8210 & 13: 43: 13 & 0.1262 \\ 8211 & 13: 43: 14 & 0.1313 \\ 8212 & 13: 43: 15 & 0.1270 \\ 8213 & 13: 43: 16 & 0.1311 \\ 8214 & 13: 43: 17 & 0.1289\end{array}$


Appendix A - Fuel Data Test 1 NIST102505_1.xls

$\begin{array}{lll}8215 & 13: 43: 18 & 0.1317 \\ 8216 & 13: 43: 19 & 0.1294 \\ 8217 & 13: 43: 20 & 0.1296 \\ 8218 & 13: 43: 21 & 0.1307 \\ 8219 & 13: 43: 22 & 0.1291 \\ 8220 & 13: 43: 23 & 0.1303 \\ 8221 & 13: 43: 24 & 0.1279 \\ 8222 & 13: 43: 25 & 0.1321 \\ 8223 & 13: 43: 26 & 0.1275 \\ 8224 & 13: 43: 27 & 0.1298 \\ 8225 & 13: 43: 28 & 0.1267 \\ 8226 & 13: 43: 29 & 0.1305 \\ 8227 & 13: 43: 30 & 0.1274 \\ 8228 & 13: 43: 31 & 0.1303 \\ 8229 & 13: 43: 32 & 0.1311 \\ 8230 & 13: 43: 33 & 0.1305 \\ 8231 & 13: 43: 34 & 0.1295 \\ 8232 & 13: 43: 35 & 0.1275 \\ 8233 & 13: 43: 36 & 0.1308 \\ 8234 & 13: 43: 37 & 0.1279 \\ 8235 & 13: 43: 38 & 0.1299 \\ 8236 & 13: 43: 39 & 0.1253 \\ 8237 & 13: 43: 40 & 0.1289 \\ 8238 & 13: 43: 41 & 0.1254 \\ 8239 & 13: 43: 42 & 0.1328 \\ 8240 & 13: 43: 43 & 0.1292 \\ 8241 & 13: 43: 44 & 0.1319 \\ 8242 & 13: 43: 45 & 0.1292 \\ 8243 & 13: 43: 46 & 0.1307 \\ 8244 & 13: 43: 47 & 0.1286 \\ 8245 & 13: 43: 48 & 0.1289 \\ 8246 & 13: 43: 49 & 0.1308 \\ 8247 & 13: 43: 50 & 0.1304 \\ 8248 & 13: 43: 51 & 0.1335 \\ 8249 & 13: 43: 52 & 0.1279 \\ 8250 & 13: 43: 53 & 0.1336 \\ 8251 & 13: 43: 54 & 0.1285 \\ 8252 & 13: 43: 55 & 0.1314 \\ 8253 & 13: 43: 56 & 0.1284 \\ 8254 & 13: 43: 57 & 0.1313 \\ 8255 & 13: 43: 58 & 0.1290 \\ 8256 & 13: 43: 59 & 0.1276 \\ 8257 & 13: 44: 00 & 0.1318 \\ 8258 & 13: 44: 01 & 0.1292 \\ 8259 & 13: 44: 02 & 0.1309 \\ 8260 & 13: 44: 03 & 0.1271 \\ 8261 & 13: 44: 04 & 0.1323 \\ 8262 & 13: 44: 05 & 0.1275 \\ 8263 & 13: 44: 06 & 0.1314 \\ 8264 & 13: 44: 07 & 0.1282 \\ 8265 & 13: 44: 08 & 0.1325 \\ 8266 & 13: 44: 09 & 0.1294\end{array}$


Appendix A - Fuel Data Test 1 NIST102505_1.xls

$\begin{array}{lll}8267 & 13: 44: 10 & 0.1302 \\ 8268 & 13: 44: 11 & 0.1303 \\ 8270 & 13: 44: 12 & 0.1293 \\ 8270 & 13: 44: 13 & 0.1297 \\ 8271 & 13: 44: 14 & 0.1276 \\ 8272 & 13: 44: 15 & 0.1319 \\ 8273 & 13: 44: 16 & 0.1278 \\ 8274 & 13: 44: 17 & 0.1306 \\ 8275 & 13: 44: 18 & 0.1281 \\ 8276 & 13: 44: 19 & 0.1313 \\ 8277 & 13: 44: 20 & 0.1288 \\ 8278 & 13: 44: 21 & 0.1313 \\ 8279 & 13: 44: 22 & 0.1296 \\ 8280 & 13: 44: 23 & 0.1306 \\ 8281 & 13: 44: 24 & 0.1289 \\ 8282 & 13: 44: 25 & 0.1283 \\ 8283 & 13: 44: 26 & 0.1302 \\ 8284 & 13: 44: 27 & 0.1276 \\ 8285 & 13: 44: 28 & 0.1301 \\ 8287 & 13: 44: 29 & 0.1272 \\ 8287 & 13: 44: 30 & 0.1302 \\ 8288 & 13: 44: 31 & 0.1266 \\ 8289 & 13: 44: 32 & 0.1324 \\ 8290 & 13: 44: 33 & 0.1291 \\ 8291 & 13: 44: 34 & 0.1318 \\ 8292 & 13: 44: 35 & 0.1296 \\ 8293 & 13: 44: 36 & 0.1309 \\ 8294 & 13: 44: 37 & 0.1281 \\ 8295 & 13: 44: 38 & 0.1276 \\ 8296 & 13: 44: 39 & 0.1300 \\ 8297 & 13: 44: 40 & 0.1278 \\ 8298 & 13: 44: 41 & 0.1301 \\ 8299 & 13: 44: 42 & 0.1254 \\ 8300 & 13: 44: 43 & 0.1305 \\ 8301 & 13: 44: 44 & 0.1270 \\ 8302 & 13: 44: 45 & 0.1302 \\ 8303 & 13: 44: 46 & 0.1278 \\ 8304 & 13: 44: 47 & 0.1318 \\ 8305 & 13: 44: 48 & 0.1293 \\ 8306 & 13: 44: 49 & 0.1299 \\ 8307 & 13: 44: 50 & 0.1301 \\ 8308 & 13: 44: 51 & 0.1280 \\ 8309 & 13: 44: 52 & 0.1287 \\ 8310 & 13: 44: 53 & 0.1266 \\ 8311 & 13: 44: 54 & 0.1294 \\ 8312 & 13: 44: 55 & 0.1255 \\ 8313 & 13: 44: 56 & 0.1304 \\ 8314 & 13: 44: 57 & 0.1283 \\ 8315 & 13: 44: 58 & 0.1318 \\ 8316 & 13: 44: 59 & 0.1304 \\ 8317 & 13: 45: 00 & 0.1301 \\ 8318 & 13: 45: 01 & 0.1319\end{array}$


Appendix A - Fuel Data Test 1 NIST102505_1.xIs

$\begin{array}{lll}8319 & 13: 45: 02 & 0.1312 \\ 8320 & 13: 45: 03 & 0.1294 \\ 8321 & 13: 45: 04 & 0.1273 \\ 8322 & 13: 45: 05 & 0.1310 \\ 8323 & 13: 45: 06 & 0.1269 \\ 8324 & 13: 45: 07 & 0.1306 \\ 8326 & 13: 45: 08 & 0.1267 \\ 8326 & 13: 45: 09 & 0.1312 \\ 8327 & 13: 45: 10 & 0.1277 \\ 8328 & 13: 45: 11 & 0.1306 \\ 8329 & 13: 45: 12 & 0.1302 \\ 8330 & 13: 45: 13 & 0.1319 \\ 8331 & 13: 45: 14 & 0.1291 \\ 8332 & 13: 45: 15 & 0.1296 \\ 8333 & 13: 45: 16 & 0.1301 \\ 8334 & 13: 45: 17 & 0.1279 \\ 8335 & 13: 45: 18 & 0.1288 \\ 8336 & 13: 45: 19 & 0.1261 \\ 8337 & 13: 45: 20 & 0.1298 \\ 8338 & 13: 45: 21 & 0.1268 \\ 8339 & 13: 45: 22 & 0.1308 \\ 8340 & 13: 45: 23 & 0.1298 \\ 8341 & 13: 45: 24 & 0.1321 \\ 8342 & 13: 45: 25 & 0.1274 \\ 8343 & 13: 45: 26 & 0.1300 \\ 8344 & 13: 45: 27 & 0.1277 \\ 8345 & 13: 45: 28 & 0.1279 \\ 8346 & 13: 45: 29 & 0.1293 \\ 8347 & 13: 45: 30 & 0.1292 \\ 8348 & 13: 45: 31 & 0.1309 \\ 8350 & 13: 45: 32 & 0.1270 \\ 8351 & 13: 45: 33 & 0.1298 \\ 8352 & 13: 45: 34 & 0.1264 \\ 8353 & 13: 45: 35 & 0.1310 \\ 8354 & 13: 45: 36 & 0.1299 \\ 8355 & 13: 45: 37 & 0.1325 \\ 8356 & 13: 45: 38 & 0.1307 \\ 8357 & 13: 45: 39 & 0.1297 \\ 8358 & 13: 45: 40 & 0.1306 \\ 8359 & 13: 45: 41 & 0.1287 \\ 8360 & 13: 45: 42 & 0.1286 \\ 8361 & 13: 45: 43 & 0.1276 \\ 8362 & 13: 45: 44 & 0.1285 \\ 8363 & 13: 45: 45 & 0.1249 \\ 8364 & 13: 45: 46 & 0.1310 \\ 8365 & 13: 45: 47 & 0.1281 \\ 8366 & 13: 45: 48 & 0.1327 \\ 8367 & 13: 45: 49 & 0.1276 \\ 8368 & 13: 45: 50 & 0.1312 \\ 8369 & 13: 45: 51 & 0.1307 \\ 8370 & 13: 45: 52 & 0.1311 \\ 8371 & 13: 45: 53 & 0.1287\end{array}$


Appendix A - Fuel Data Test 1 NIST102505_1.xIs

$\begin{array}{lll}8372 & 13: 45: 55 & 0.1296 \\ 8373 & 13: 45: 55 & 0.1309 \\ 8374 & 13: 45: 56 & 0.1281 \\ 8375 & 13: 45: 57 & 0.1297 \\ 8376 & 13: 45: 58 & 0.1267 \\ 8377 & 13: 45: 59 & 0.1306 \\ 8378 & 13: 46: 00 & 0.1267 \\ 8379 & 13: 46: 01 & 0.1303 \\ 8380 & 13: 46: 02 & 0.1293 \\ 8381 & 13: 46: 03 & 0.1314 \\ 8382 & 13: 46: 04 & 0.1294 \\ 8383 & 13: 46: 05 & 0.1306 \\ 8384 & 13: 46: 06 & 0.1295 \\ 8385 & 13: 46: 07 & 0.1276 \\ 8386 & 13: 46: 08 & 0.1293 \\ 8387 & 13: 46: 09 & 0.1274 \\ 8388 & 13: 46: 10 & 0.1289 \\ 8389 & 13: 46: 12 & 0.1244 \\ 8390 & 13: 46: 12 & 0.1298 \\ 8391 & 13: 46: 13 & 0.1293 \\ 8392 & 13: 46: 14 & 0.1320 \\ 8393 & 13: 46: 15 & 0.1300 \\ 8394 & 13: 46: 16 & 0.1325 \\ 8395 & 13: 46: 17 & 0.1321 \\ 8396 & 13: 46: 18 & 0.1316 \\ 8397 & 13: 46: 19 & 0.1291 \\ 8398 & 13: 46: 20 & 0.1277 \\ 8399 & 13: 46: 21 & 0.1295 \\ 8400 & 13: 46: 22 & 0.1276 \\ 8401 & 13: 46: 23 & 0.1303 \\ 8402 & 13: 46: 24 & 0.1271 \\ 8403 & 13: 46: 25 & 0.1301 \\ 8404 & 13: 46: 26 & 0.1286 \\ 8405 & 13: 46: 27 & 0.1315 \\ 8406 & 13: 46: 28 & 0.1306 \\ 8407 & 13: 46: 29 & 0.1312 \\ 8408 & 13: 46: 30 & 0.1294 \\ 8409 & 13: 46: 31 & 0.1271 \\ 8410 & 13: 46: 32 & 0.1312 \\ 8411 & 13: 46: 33 & 0.1290 \\ 8412 & 13: 46: 34 & 0.1309 \\ 8413 & 13: 46: 35 & 0.1276 \\ 8414 & 13: 46: 36 & 0.1323 \\ 8415 & 13: 46: 37 & 0.1288 \\ 8416 & 13: 46: 38 & 0.1324 \\ 8417 & 13: 46: 39 & 0.1309 \\ 8418 & 13: 46: 40 & 0.1331 \\ 8419 & 13: 46: 41 & 0.1267 \\ 8420 & 13: 46: 42 & 0.1294 \\ 8421 & 13: 46: 43 & 0.1266 \\ 8422 & 13: 46: 44 & 0.1261 \\ 8423 & 13: 46: 45 & 0.1289\end{array}$


Appendix A - Fuel Data Test 1 NIST102505_1.xIs

$\begin{array}{lll}8424 & 13: 46: 46 & 0.1269 \\ 8425 & 13: 46: 47 & 0.1288 \\ 8426 & 13: 46: 48 & 0.1239 \\ 8427 & 13: 46: 49 & 0.1293 \\ 8428 & 13: 46: 50 & 0.1263 \\ 8429 & 13: 46: 51 & 0.1293 \\ 8430 & 13: 46: 52 & 0.1269 \\ 8431 & 13: 46: 53 & 0.1290 \\ 8432 & 13: 46: 54 & 0.1280 \\ 8433 & 13: 46: 55 & 0.1282 \\ 8434 & 13: 46: 56 & 0.1289 \\ 8435 & 13: 46: 57 & 0.1253 \\ 8436 & 13: 46: 58 & 0.1295 \\ 8437 & 13: 46: 59 & 0.1270 \\ 8438 & 13: 47: 00 & 0.1317 \\ 8439 & 13: 47: 01 & 0.1270 \\ 8440 & 13: 47: 02 & 0.1302 \\ 8441 & 13: 47: 03 & 0.1272 \\ 8442 & 13: 47: 04 & 0.1310 \\ 8443 & 13: 47: 05 & 0.1285 \\ 8444 & 13: 47: 06 & 0.1287 \\ 8445 & 13: 47: 07 & 0.1293 \\ 8446 & 13: 47: 08 & 0.1286 \\ 8447 & 13: 47: 09 & 0.1285 \\ 8448 & 13: 47: 10 & 0.1281 \\ 8449 & 13: 47: 11 & 0.1313 \\ 8450 & 13: 47: 12 & 0.1266 \\ 8451 & 13: 47: 13 & 0.1320 \\ 8452 & 13: 47: 14 & 0.1288 \\ 8453 & 13: 47: 15 & 0.1323 \\ 8454 & 13: 47: 16 & 0.1285 \\ 8455 & 13: 47: 17 & 0.1335 \\ 8456 & 13: 47: 18 & 0.1308 \\ 8457 & 13: 47: 19 & 0.1298 \\ 8458 & 13: 47: 20 & 0.1304 \\ 8459 & 13: 47: 21 & 0.1292 \\ 8460 & 13: 47: 22 & 0.1298 \\ 8461 & 13: 47: 23 & 0.1259 \\ 8462 & 13: 47: 24 & 0.1294 \\ 8463 & 13: 47: 25 & 0.1261 \\ 8464 & 13: 47: 26 & 0.1305 \\ 8465 & 13: 47: 27 & 0.1272 \\ 8466 & 13: 47: 28 & 0.1324 \\ 8467 & 13: 47: 29 & 0.1289 \\ 8468 & 13: 47: 30 & 0.1307 \\ 8469 & 13: 47: 31 & 0.1273 \\ 8470 & 13: 47: 32 & 0.1286 \\ 8471 & 13: 47: 33 & 0.1261 \\ 8472 & 13: 47: 34 & 0.1247 \\ 8473 & 13: 47: 35 & 0.1301 \\ 8474 & 13: 47: 36 & 0.1266 \\ 8475 & 13: 47: 37 & 0.1295\end{array}$


Appendix A - Fuel Data Test 1 NIST102505_1.xIs

$\begin{array}{lll}8476 & 13: 47: 38 & 0.1253 \\ 8477 & 13: 47: 39 & 0.1307 \\ 8478 & 13: 47: 40 & 0.1261 \\ 8479 & 13: 47: 41 & 0.1315 \\ 8480 & 13: 47: 42 & 0.1294 \\ 8481 & 13: 47: 43 & 0.1315 \\ 8482 & 13: 47: 44 & 0.1302 \\ 8483 & 13: 47: 45 & 0.1298 \\ 8484 & 13: 47: 46 & 0.1305 \\ 8485 & 13: 47: 47 & 0.1275 \\ 8486 & 13: 47: 48 & 0.1291 \\ 8487 & 13: 47: 49 & 0.1268 \\ 8488 & 13: 47: 50 & 0.1317 \\ 8489 & 13: 47: 51 & 0.1281 \\ 8490 & 13: 47: 52 & 0.1328 \\ 8491 & 13: 47: 53 & 0.1314 \\ 8492 & 13: 47: 54 & 0.1331 \\ 8493 & 13: 47: 55 & 0.1303 \\ 8494 & 13: 47: 56 & 0.1303 \\ 8495 & 13: 47: 57 & 0.1307 \\ 8496 & 13: 47: 58 & 0.1285 \\ 8497 & 13: 47: 59 & 0.1296 \\ 8498 & 13: 48: 00 & 0.1256 \\ 8499 & 13: 48: 01 & 0.1313 \\ 8500 & 13: 48: 02 & 0.1261 \\ 8501 & 13: 48: 03 & 0.1298 \\ 8502 & 13: 48: 04 & 0.1250 \\ 8503 & 13: 48: 05 & 0.1303 \\ 8504 & 13: 48: 06 & 0.1295 \\ 8505 & 13: 48: 07 & 0.1346 \\ 8506 & 13: 48: 08 & 0.1310 \\ 8507 & 13: 48: 09 & 0.1316 \\ 8508 & 13: 48: 10 & 0.1282 \\ 8509 & 13: 48: 11 & 0.1264 \\ 8510 & 13: 48: 12 & 0.1298 \\ 8511 & 13: 48: 13 & 0.1271 \\ 8512 & 13: 48: 14 & 0.1275 \\ 8513 & 13: 48: 15 & 0.1245 \\ 8514 & 13: 48: 16 & 0.1289 \\ 8515 & 13: 48: 17 & 0.1259 \\ 8516 & 13: 48: 18 & 0.1309 \\ 8517 & 13: 48: 19 & 0.1292 \\ 8518 & 13: 48: 20 & 0.1307 \\ 8519 & 13: 48: 21 & 0.1296 \\ 8520 & 13: 48: 22 & 0.1309 \\ 8521 & 13: 48: 23 & 0.1308 \\ 8522 & 13: 48: 24 & 0.1285 \\ 8523 & 13: 48: 25 & 0.1294 \\ 8524 & 13: 48: 26 & 0.1259 \\ 8525 & 13: 48: 27 & 0.1297 \\ 8526 & 13: 48: 28 & 0.1262 \\ 8527 & 13: 48: 29 & 0.1304\end{array}$


Appendix A - Fuel Data Test 1 NIST102505_1.xIs

$\begin{array}{lll}8528 & 13: 48: 30 & 0.1291 \\ 8529 & 13: 48: 31 & 0.1312 \\ 8530 & 13: 48: 32 & 0.1281 \\ 8531 & 13: 48: 33 & 0.1302 \\ 8532 & 13: 48: 34 & 0.1313 \\ 8533 & 13: 48: 35 & 0.1292 \\ 8534 & 13: 48: 36 & 0.1285 \\ 8535 & 13: 48: 37 & 0.1266 \\ 8536 & 13: 48: 38 & 0.1312 \\ 8537 & 13: 48: 39 & 0.1281 \\ 8538 & 13: 48: 40 & 0.1311 \\ 8539 & 13: 48: 41 & 0.1278 \\ 8540 & 13: 48: 42 & 0.1316 \\ 8541 & 13: 48: 43 & 0.1283 \\ 8542 & 13: 48: 44 & 0.1313 \\ 8543 & 13: 48: 45 & 0.1312 \\ 8544 & 13: 48: 46 & 0.1311 \\ 8545 & 13: 48: 47 & 0.1300 \\ 8546 & 13: 48: 48 & 0.1280 \\ 8547 & 13: 48: 49 & 0.1293 \\ 8548 & 13: 48: 50 & 0.1278 \\ 8549 & 13: 48: 51 & 0.1299 \\ 8550 & 13: 48: 52 & 0.1267 \\ 8551 & 13: 48: 53 & 0.1302 \\ 8552 & 13: 48: 54 & 0.1266 \\ 8553 & 13: 48: 55 & 0.1328 \\ 8554 & 13: 48: 56 & 0.1299 \\ 8555 & 13: 48: 57 & 0.1337 \\ 8556 & 13: 48: 58 & 0.1289 \\ 8557 & 13: 48: 59 & 0.1325 \\ 8558 & 13: 49: 00 & 0.1299 \\ 8559 & 13: 49: 01 & 0.1300 \\ 8560 & 13: 49: 02 & 0.1324 \\ 8561 & 13: 49: 03 & 0.1301 \\ 8562 & 13: 49: 04 & 0.1317 \\ 8563 & 13: 49: 05 & 0.1282 \\ 8564 & 13: 49: 06 & 0.1304 \\ 8565 & 13: 49: 07 & 0.1254 \\ 8566 & 13: 49: 08 & 0.1306 \\ 8567 & 13: 49: 09 & 0.1298 \\ 8568 & 13: 49: 10 & 0.1323 \\ 8569 & 13: 49: 11 & 0.1308 \\ 8570 & 13: 49: 12 & 0.1314 \\ 8571 & 13: 49: 13 & 0.1313 \\ 8572 & 13: 49: 14 & 0.1286 \\ 8573 & 13: 49: 15 & 0.1289 \\ 8574 & 13: 49: 16 & 0.1269 \\ 8575 & 13: 49: 17 & 0.1302 \\ 8576 & 13: 49: 18 & 0.1256 \\ 8577 & 13: 49: 19 & 0.1294 \\ 8578 & 13: 49: 20 & 0.1275 \\ 8579 & 13: 49: 21 & 0.1308\end{array}$


Appendix A - Fuel Data Test 1 NIST102505_1.xIs

$\begin{array}{lll}8580 & 13: 49: 22 & 0.1292 \\ 8581 & 13: 49: 23 & 0.1311 \\ 8582 & 13: 49: 24 & 0.1317 \\ 8583 & 13: 49: 25 & 0.1300 \\ 8584 & 13: 49: 26 & 0.1276 \\ 8585 & 13: 49: 27 & 0.1268 \\ 8586 & 13: 49: 28 & 0.1300 \\ 8587 & 13: 49: 29 & 0.1265 \\ 8588 & 13: 49: 30 & 0.1287 \\ 8589 & 13: 49: 31 & 0.1263 \\ 8590 & 13: 49: 32 & 0.1300 \\ 8591 & 13: 49: 33 & 0.1268 \\ 8592 & 13: 49: 34 & 0.1306 \\ 8593 & 13: 49: 35 & 0.1310 \\ 8594 & 13: 49: 36 & 0.1326 \\ 8595 & 13: 49: 37 & 0.1296 \\ 8596 & 13: 49: 38 & 0.1311 \\ 8597 & 13: 49: 39 & 0.1304 \\ 8598 & 13: 49: 40 & 0.1297 \\ 8599 & 13: 49: 41 & 0.1313 \\ 8600 & 13: 49: 42 & 0.1288 \\ 8601 & 13: 49: 43 & 0.1306 \\ 8602 & 13: 49: 44 & 0.1260 \\ 8603 & 13: 49: 45 & 0.1318 \\ 8604 & 13: 49: 46 & 0.1299 \\ 8605 & 13: 49: 47 & 0.1324 \\ 8606 & 13: 49: 48 & 0.1270 \\ 8607 & 13: 49: 49 & 0.1315 \\ 8608 & 13: 49: 50 & 0.1286 \\ 8609 & 13: 49: 51 & 0.1299 \\ 8610 & 13: 49: 52 & 0.1298 \\ 8611 & 13: 49: 53 & 0.1285 \\ 8612 & 13: 49: 54 & 0.1300 \\ 8613 & 13: 49: 55 & 0.1266 \\ 8614 & 13: 49: 56 & 0.1317 \\ 8615 & 13: 49: 57 & 0.1265 \\ 8616 & 13: 49: 58 & 0.1310 \\ 8617 & 13: 49: 59 & 0.1287 \\ 8618 & 13: 50: 00 & 0.1327 \\ 8619 & 13: 50: 01 & 0.1284 \\ 8620 & 13: 50: 02 & 0.1293 \\ 8621 & 13: 50: 03 & 0.1299 \\ 8622 & 13: 50: 04 & 0.1293 \\ 8623 & 13: 50: 05 & 0.1291 \\ 8624 & 13: 50: 06 & 0.1273 \\ 8625 & 13: 50: 07 & 0.1305 \\ 8626 & 13: 50: 08 & 0.1254 \\ 8627 & 13: 50: 09 & 0.1288 \\ 8628 & 13: 50: 10 & 0.1260 \\ 8629 & 13: 50: 11 & 0.1309 \\ 8630 & 13: 50: 12 & 0.1267 \\ 8631 & 13: 50: 13 & 0.1318\end{array}$


Appendix A - Fuel Data Test 1 NIST102505_1.xIs

$\begin{array}{lll}8632 & 13: 50: 14 & 0.1293 \\ 8633 & 13: 50: 15 & 0.1304 \\ 8634 & 13: 50: 16 & 0.1277 \\ 8635 & 13: 50: 17 & 0.1283 \\ 8636 & 13: 50: 18 & 0.1292 \\ 8637 & 13: 50: 19 & 0.1262 \\ 8638 & 13: 50: 20 & 0.1302 \\ 8639 & 13: 50: 21 & 0.1279 \\ 8640 & 13: 50: 22 & 0.1310 \\ 8641 & 13: 50: 23 & 0.1262 \\ 8642 & 13: 50: 24 & 0.1325 \\ 8643 & 13: 50: 25 & 0.1283 \\ 8644 & 13: 50: 26 & 0.1315 \\ 8645 & 13: 50: 27 & 0.1296 \\ 8646 & 13: 50: 28 & 0.1298 \\ 8647 & 13: 50: 29 & 0.1295 \\ 8648 & 13: 50: 30 & 0.1260 \\ 8649 & 13: 50: 31 & 0.1296 \\ 8650 & 13: 50: 32 & 0.1274 \\ 8651 & 13: 50: 33 & 0.1290 \\ 8651 & 13: 50: 34 & 0.1250 \\ 8652 & 13: 50: 35 & 0.1303 \\ 8653 & 13: 50: 36 & 0.1269 \\ 8654 & 13: 50: 37 & 0.1299 \\ 8655 & 13: 50: 38 & 0.1286 \\ 8656 & 13: 50: 39 & 0.1295 \\ 8657 & 13: 50: 40 & 0.1291 \\ 8658 & 13: 50: 41 & 0.1289 \\ 8659 & 13: 50: 42 & 0.1304 \\ 8660 & 13: 50: 43 & 0.1280 \\ 8661 & 13: 50: 44 & 0.1311 \\ 8662 & 13: 50: 45 & 0.1276 \\ 8663 & 13: 50: 46 & 0.1293 \\ 8664 & 13: 50: 47 & 0.1254 \\ 8665 & 13: 50: 48 & 0.1303 \\ 8666 & 13: 50: 49 & 0.1298 \\ 8667 & 13: 50: 50 & 0.1332 \\ 8668 & 13: 50: 51 & 0.1301 \\ 8669 & 13: 50: 52 & 0.1332 \\ 8670 & 13: 50: 53 & 0.1320 \\ 8671 & 13: 50: 54 & 0.1304 \\ 8672 & 13: 50: 55 & 0.1266 \\ 8673 & 13: 50: 56 & 0.1264 \\ 8674 & 13: 50: 57 & 0.1296 \\ 8675 & 13: 50: 58 & 0.1243 \\ 8676 & 13: 50: 59 & 0.1303 \\ 8677 & 13: 51: 00 & 0.1283 \\ 8678 & 13: 51: 01 & 0.1315 \\ 8679 & 13: 51: 02 & 0.1288 \\ 8680 & 13: 51: 03 & 0.1336 \\ 8681 & 13: 51: 04 & 0.1297 \\ 8682 & 13: 51: 05 & 0.1320\end{array}$


Appendix A - Fuel Data Test 1 NIST102505_1.xIs

$\begin{array}{lll}8683 & 13: 51: 06 & 0.1296 \\ 8684 & 13: 51: 07 & 0.1306 \\ 8685 & 13: 51: 08 & 0.1296 \\ 8686 & 13: 51: 09 & 0.1261 \\ 8687 & 13: 51: 10 & 0.1309 \\ 8688 & 13: 51: 11 & 0.1271 \\ 8689 & 13: 51: 12 & 0.1301 \\ 8690 & 13: 51: 13 & 0.1249 \\ 8691 & 13: 51: 14 & 0.1306 \\ 8692 & 13: 51: 15 & 0.1280 \\ 8693 & 13: 51: 16 & 0.1307 \\ 8694 & 13: 51: 17 & 0.1267 \\ 8695 & 13: 51: 18 & 0.1287 \\ 8696 & 13: 51: 19 & 0.1273 \\ 8697 & 13: 51: 20 & 0.1258 \\ 8698 & 13: 51: 21 & 0.1294 \\ 8699 & 13: 51: 22 & 0.1292 \\ 8700 & 13: 51: 23 & 0.1315 \\ 8701 & 13: 51: 24 & 0.1272 \\ 8702 & 13: 51: 25 & 0.1314 \\ 8703 & 13: 51: 26 & 0.1288 \\ 8704 & 13: 51: 27 & 0.1322 \\ 8705 & 13: 51: 28 & 0.1279 \\ 8706 & 13: 51: 29 & 0.1321 \\ 8707 & 13: 51: 30 & 0.1294 \\ 8708 & 13: 51: 31 & 0.1292 \\ 8709 & 13: 51: 32 & 0.1280 \\ 8710 & 13: 51: 33 & 0.1280 \\ 8711 & 13: 51: 34 & 0.1291 \\ 8712 & 13: 51: 35 & 0.1261 \\ 8713 & 13: 51: 36 & 0.1297 \\ 8714 & 13: 51: 37 & 0.1272 \\ 8715 & 13: 51: 38 & 0.1304 \\ 8716 & 13: 51: 39 & 0.1263 \\ 8717 & 13: 51: 40 & 0.1322 \\ 8718 & 13: 51: 41 & 0.1286 \\ 8719 & 13: 51: 42 & 0.1311 \\ 8720 & 13: 51: 43 & 0.1297 \\ 8721 & 13: 51: 44 & 0.1299 \\ 8722 & 13: 51: 45 & 0.1306 \\ 8723 & 13: 51: 46 & 0.1277 \\ 8724 & 13: 51: 47 & 0.1317 \\ 8725 & 13: 51: 48 & 0.1272 \\ 8726 & 13: 51: 49 & 0.1299 \\ 8727 & 13: 51: 50 & 0.1260 \\ 8728 & 13: 51: 51 & 0.1308 \\ 8729 & 13: 51: 52 & 0.1279 \\ 8730 & 13: 51: 53 & 0.1315 \\ 8731 & 13: 51: 54 & 0.1301 \\ 8732 & 13: 51: 55 & 0.1317 \\ 8733 & 13: 51: 56 & 0.1293 \\ 8734 & 13: 51: 57 & 0.1281\end{array}$


Appendix A - Fuel Data Test 1 NIST102505_1.xls

$\begin{array}{lll}8735 & 13: 51: 58 & 0.1307 \\ 8736 & 13: 51: 59 & 0.1282 \\ 8737 & 13: 52: 00 & 0.1305 \\ 8738 & 13: 52: 01 & 0.1261 \\ 8739 & 13: 52: 02 & 0.1301 \\ 8740 & 13: 52: 03 & 0.1274 \\ 8741 & 13: 52: 04 & 0.1318 \\ 8742 & 13: 52: 05 & 0.1311 \\ 8743 & 13: 52: 06 & 0.1343 \\ 8744 & 13: 52: 07 & 0.1294 \\ 8745 & 13: 52: 08 & 0.1309 \\ 8746 & 13: 52: 09 & 0.1293 \\ 8747 & 13: 52: 10 & 0.1295 \\ 8748 & 13: 52: 11 & 0.1300 \\ 8749 & 13: 52: 12 & 0.1281 \\ 8750 & 13: 52: 13 & 0.1300 \\ 8751 & 13: 52: 14 & 0.1250 \\ 8752 & 13: 52: 15 & 0.1287 \\ 8753 & 13: 52: 16 & 0.1272 \\ 8754 & 13: 52: 17 & 0.1311 \\ 8755 & 13: 52: 18 & 0.1271 \\ 8756 & 13: 52: 19 & 0.1320 \\ 8757 & 13: 52: 20 & 0.1286 \\ 8758 & 13: 52: 21 & 0.1314 \\ 8759 & 13: 52: 22 & 0.1318 \\ 8760 & 13: 52: 23 & 0.1302 \\ 8761 & 13: 52: 24 & 0.1316 \\ 8762 & 13: 52: 25 & 0.1277 \\ 8763 & 13: 52: 26 & 0.1330 \\ 8764 & 13: 52: 27 & 0.1279 \\ 8765 & 13: 52: 28 & 0.1324 \\ 8766 & 13: 52: 29 & 0.1298 \\ 8767 & 13: 52: 30 & 0.1317 \\ 8768 & 13: 52: 31 & 0.1284 \\ 8769 & 13: 52: 32 & 0.1302 \\ 8770 & 13: 52: 33 & 0.1296 \\ 8771 & 13: 52: 34 & 0.1289 \\ 8772 & 13: 52: 35 & 0.1268 \\ 8773 & 13: 52: 36 & 0.1269 \\ 8774 & 13: 52: 37 & 0.1313 \\ 8775 & 13: 52: 38 & 0.1272 \\ 8776 & 13: 52: 39 & 0.1299 \\ 8777 & 13: 52: 40 & 0.1272 \\ 8778 & 13: 52: 41 & 0.1303 \\ 8779 & 13: 52: 42 & 0.1282 \\ 8780 & 13: 52: 43 & 0.1312 \\ 8781 & 13: 52: 44 & 0.1293 \\ 8782 & 13: 52: 45 & 0.1320 \\ 8783 & 13: 52: 46 & 0.1287 \\ 8784 & 13: 52: 47 & 0.1298 \\ 8785 & 13: 52: 48 & 0.1293 \\ 8786 & 13: 52: 49 & 0.1276\end{array}$


Appendix A - Fuel Data Test 1 NIST102505_1.xIs

$\begin{array}{lll}8787 & 13: 52: 50 & 0.1294 \\ 8788 & 13: 52: 51 & 0.1272 \\ 8789 & 13: 52: 52 & 0.1295 \\ 8790 & 13: 52: 53 & 0.1263 \\ 8791 & 13: 52: 54 & 0.1304 \\ 8792 & 13: 52: 55 & 0.1295 \\ 8793 & 13: 52: 56 & 0.1333 \\ 8794 & 13: 52: 57 & 0.1306 \\ 8795 & 13: 52: 58 & 0.1311 \\ 8796 & 13: 52: 59 & 0.1318 \\ 8797 & 13: 53: 00 & 0.1302 \\ 8798 & 13: 53: 01 & 0.1304 \\ 8799 & 13: 53: 02 & 0.1281 \\ 8800 & 13: 53: 03 & 0.1304 \\ 8801 & 13: 53: 04 & 0.1258 \\ 8802 & 13: 53: 05 & 0.1291 \\ 8803 & 13: 53: 06 & 0.1282 \\ 8804 & 13: 53: 07 & 0.1315 \\ 8805 & 13: 53: 08 & 0.1279 \\ 8806 & 13: 53: 09 & 0.1310 \\ 8807 & 13: 53: 10 & 0.1304 \\ 8808 & 13: 53: 11 & 0.1305 \\ 8809 & 13: 53: 12 & 0.1283 \\ 8810 & 13: 53: 13 & 0.1276 \\ 8811 & 13: 53: 14 & 0.1285 \\ 8812 & 13: 53: 15 & 0.1263 \\ 8813 & 13: 53: 16 & 0.1282 \\ 8814 & 13: 53: 17 & 0.1254 \\ 8815 & 13: 53: 18 & 0.1291 \\ 8816 & 13: 53: 19 & 0.1263 \\ 8817 & 13: 53: 20 & 0.1306 \\ 8818 & 13: 53: 21 & 0.1307 \\ 8819 & 13: 53: 22 & 0.1310 \\ 8820 & 13: 53: 23 & 0.1289 \\ 8821 & 13: 53: 24 & 0.1288 \\ 8822 & 13: 53: 25 & 0.1299 \\ 8823 & 13: 53: 26 & 0.1285 \\ 8824 & 13: 53: 27 & 0.1303 \\ 8825 & 13: 53: 28 & 0.1285 \\ 8826 & 13: 53: 29 & 0.1306 \\ 8827 & 13: 53: 30 & 0.1255 \\ 8828 & 13: 53: 31 & 0.1298 \\ 8829 & 13: 53: 32 & 0.1283 \\ 8830 & 13: 53: 33 & 0.1309 \\ 8831 & 13: 53: 34 & 0.1274 \\ 8832 & 13: 53: 35 & 0.1304 \\ 8833 & 13: 53: 36 & 0.1297 \\ 8834 & 13: 53: 37 & 0.1300 \\ 8835 & 13: 53: 38 & 0.1275 \\ 8836 & 13: 53: 39 & 0.1273 \\ 8837 & 13: 53: 40 & 0.1281 \\ 8838 & 13: 53: 41 & 0.1258\end{array}$


Appendix A - Fuel Data Test 1 NIST102505_1.xIs

$\begin{array}{lll}8839 & 13: 53: 42 & 0.1301 \\ 8840 & 13: 53: 43 & 0.1260 \\ 8841 & 13: 53: 44 & 0.1303 \\ 8842 & 13: 53: 45 & 0.1267 \\ 8843 & 13: 53: 46 & 0.1332 \\ 8844 & 13: 53: 47 & 0.1300 \\ 8845 & 13: 53: 48 & 0.1307 \\ 8846 & 13: 53: 49 & 0.1284 \\ 8847 & 13: 53: 50 & 0.1297 \\ 8848 & 13: 53: 51 & 0.1290 \\ 8849 & 13: 53: 52 & 0.1268 \\ 8850 & 13: 53: 53 & 0.1313 \\ 8851 & 13: 53: 54 & 0.1270 \\ 8852 & 13: 53: 55 & 0.1299 \\ 8853 & 13: 53: 56 & 0.1267 \\ 8854 & 13: 53: 57 & 0.1302 \\ 8855 & 13: 53: 58 & 0.1269 \\ 8856 & 13: 53: 59 & 0.1297 \\ 8857 & 13: 54: 00 & 0.1292 \\ 8858 & 13: 54: 01 & 0.1312 \\ 8859 & 13: 54: 02 & 0.1286 \\ 8860 & 13: 54: 03 & 0.1290 \\ 8861 & 13: 54: 04 & 0.1296 \\ 8862 & 13: 54: 05 & 0.1272 \\ 8863 & 13: 54: 06 & 0.1280 \\ 8864 & 13: 54: 07 & 0.1247 \\ 8865 & 13: 54: 08 & 0.1288 \\ 8866 & 13: 54: 09 & 0.1262 \\ 8867 & 13: 54: 10 & 0.1307 \\ 8868 & 13: 54: 11 & 0.1303 \\ 8869 & 13: 54: 12 & 0.1339 \\ 8870 & 13: 54: 13 & 0.1286 \\ 8871 & 13: 54: 14 & 0.1310 \\ 8872 & 13: 54: 15 & 0.1288 \\ 8873 & 13: 54: 16 & 0.1291 \\ 8874 & 13: 54: 17 & 0.1293 \\ 8875 & 13: 54: 18 & 0.1287 \\ 8876 & 13: 54: 19 & 0.1309 \\ 8877 & 13: 54: 20 & 0.1263 \\ 8878 & 13: 54: 21 & 0.1300 \\ 8879 & 13: 54: 22 & 0.1274 \\ 8880 & 13: 54: 23 & 0.1315 \\ 8881 & 13: 54: 24 & 0.1263 \\ 8882 & 13: 54: 25 & 0.1301 \\ 8883 & 13: 54: 26 & 0.1304 \\ 8884 & 13: 54: 27 & 0.1307 \\ 8885 & 13: 54: 28 & 0.1292 \\ 8886 & 13: 54: 29 & 0.1270 \\ 8887 & 13: 54: 30 & 0.1290 \\ 8888 & 13: 54: 31 & 0.1257 \\ 8889 & 13: 54: 32 & 0.1280 \\ 8890 & 13: 54: 33 & 0.1238\end{array}$


Appendix A - Fuel Data Test 1 NIST102505_1.xls

$\begin{array}{lll}8891 & 13: 54: 34 & 0.1287 \\ 8892 & 13: 54: 35 & 0.1247 \\ 8893 & 13: 54: 36 & 0.1309 \\ 8894 & 13: 54: 37 & 0.1271 \\ 8895 & 13: 54: 38 & 0.1308 \\ 8896 & 13: 54: 39 & 0.1277 \\ 8897 & 13: 54: 40 & 0.1295 \\ 8898 & 13: 54: 41 & 0.1264 \\ 8899 & 13: 54: 42 & 0.1250 \\ 8900 & 13: 54: 43 & 0.1281 \\ 8901 & 13: 54: 44 & 0.1264 \\ 8902 & 13: 54: 45 & 0.1292 \\ 8903 & 13: 54: 46 & 0.1243 \\ 8904 & 13: 54: 47 & 0.1320 \\ 8905 & 13: 54: 48 & 0.1285 \\ 8906 & 13: 54: 49 & 0.1307 \\ 8907 & 13: 54: 50 & 0.1282 \\ 8908 & 13: 54: 51 & 0.1306 \\ 8909 & 13: 54: 52 & 0.1274 \\ 8910 & 13: 54: 53 & 0.1267 \\ 8911 & 13: 54: 54 & 0.1272 \\ 8912 & 13: 54: 55 & 0.1257 \\ 8913 & 13: 54: 56 & 0.1283 \\ 8914 & 13: 54: 57 & 0.1233 \\ 8915 & 13: 54: 58 & 0.1290 \\ 8916 & 13: 54: 59 & 0.1249 \\ 8917 & 13: 55: 00 & 0.1284 \\ 8918 & 13: 55: 01 & 0.1265 \\ 8919 & 13: 55: 02 & 0.1290 \\ 8920 & 13: 55: 03 & 0.1272 \\ 8921 & 13: 55: 04 & 0.1288 \\ 8922 & 13: 55: 05 & 0.1292 \\ 8923 & 13: 55: 06 & 0.1273 \\ 8924 & 13: 55: 07 & 0.1296 \\ 8925 & 13: 55: 08 & 0.1262 \\ 8926 & 13: 55: 09 & 0.1305 \\ 8927 & 13: 55: 10 & 0.1259 \\ 8928 & 13: 55: 11 & 0.1300 \\ 8929 & 13: 55: 12 & 0.1286 \\ 8930 & 13: 55: 13 & 0.1319 \\ 8931 & 13: 55: 14 & 0.1282 \\ 8932 & 13: 55: 15 & 0.1300 \\ 8933 & 13: 55: 16 & 0.1301 \\ 8934 & 13: 55: 17 & 0.1296 \\ 8935 & 13: 55: 18 & 0.1289 \\ 8936 & 13: 55: 19 & 0.1273 \\ 8937 & 13: 55: 20 & 0.1285 \\ 8938 & 13: 55: 21 & 0.1255 \\ 8939 & 13: 55: 22 & 0.1302 \\ 8940 & 13: 55: 23 & 0.1272 \\ 8941 & 13: 55: 24 & 0.1318 \\ 8942 & 13: 55: 25 & 0.1278\end{array}$


Appendix A - Fuel Data Test 1 NIST102505_1.xIs

$\begin{array}{lll}8943 & 13: 55: 26 & 0.1321 \\ 8944 & 13: 55: 27 & 0.1287 \\ 8945 & 13: 55: 28 & 0.1288 \\ 8946 & 13: 55: 29 & 0.1260 \\ 8947 & 13: 55: 30 & 0.1277 \\ 8948 & 13: 55: 31 & 0.1305 \\ 8949 & 13: 55: 32 & 0.1261 \\ 8950 & 13: 55: 33 & 0.1313 \\ 8951 & 13: 55: 34 & 0.1263 \\ 8952 & 13: 55: 35 & 0.1313 \\ 8953 & 13: 55: 36 & 0.1278 \\ 8954 & 13: 55: 37 & 0.1301 \\ 8955 & 13: 55: 38 & 0.1269 \\ 8956 & 13: 55: 39 & 0.1307 \\ 8957 & 13: 55: 40 & 0.1293 \\ 8958 & 13: 55: 41 & 0.1297 \\ 8959 & 13: 55: 42 & 0.1278 \\ 8960 & 13: 55: 43 & 0.1277 \\ 8961 & 13: 55: 44 & 0.1289 \\ 8962 & 13: 55: 45 & 0.1258 \\ 8963 & 13: 55: 46 & 0.1299 \\ 8964 & 13: 55: 47 & 0.1261 \\ 8965 & 13: 55: 48 & 0.1305 \\ 8966 & 13: 55: 49 & 0.1274 \\ 8967 & 13: 55: 50 & 0.1316 \\ 8968 & 13: 55: 51 & 0.1283 \\ 8969 & 13: 55: 52 & 0.1310 \\ 8970 & 13: 55: 53 & 0.1273 \\ 8971 & 13: 55: 54 & 0.1282 \\ 8972 & 13: 55: 55 & 0.1268 \\ 8973 & 13: 55: 56 & 0.1254 \\ 8974 & 13: 55: 57 & 0.1300 \\ 8975 & 13: 55: 58 & 0.1289 \\ 8976 & 13: 55: 59 & 0.1296 \\ 8977 & 13: 56: 00 & 0.1240 \\ 8978 & 13: 56: 01 & 0.1302 \\ 8979 & 13: 56: 02 & 0.1264 \\ 8980 & 13: 56: 03 & 0.1295 \\ 8981 & 13: 56: 04 & 0.1275 \\ 8982 & 13: 56: 05 & 0.1296 \\ 8983 & 13: 56: 06 & 0.1291 \\ 8984 & 13: 56: 07 & 0.1277 \\ 8985 & 13: 56: 08 & 0.1311 \\ 8986 & 13: 56: 09 & 0.1272 \\ 8987 & 13: 56: 10 & 0.1308 \\ 8988 & 13: 56: 11 & 0.1258 \\ 8989 & 13: 56: 12 & 0.1309 \\ 8990 & 13: 56: 13 & 0.1268 \\ 8991 & 13: 56: 14 & 0.1321 \\ 8992 & 13: 56: 15 & 0.1289 \\ 8993 & 13: 56: 16 & 0.1326 \\ 8994 & 13: 56: 17 & 0.1298\end{array}$


Appendix A - Fuel Data Test 1 NIST102505_1.xIs

$\begin{array}{lll}8995 & 13: 56: 18 & 0.1308 \\ 8996 & 13: 56: 19 & 0.1291 \\ 8997 & 13: 56: 20 & 0.1292 \\ 8998 & 13: 56: 21 & 0.1301 \\ 8999 & 13: 56: 22 & 0.1257 \\ 9000 & 13: 56: 23 & 0.1303 \\ 9001 & 13: 56: 24 & 0.1263 \\ 9002 & 13: 56: 25 & 0.1304 \\ 9003 & 13: 56: 26 & 0.1265 \\ 9004 & 13: 56: 27 & 0.1302 \\ 9005 & 13: 56: 28 & 0.1288 \\ 9006 & 13: 56: 29 & 0.1316 \\ 9007 & 13: 56: 30 & 0.1315 \\ 9008 & 13: 56: 31 & 0.1292 \\ 9009 & 13: 56: 32 & 0.1305 \\ 9010 & 13: 56: 33 & 0.1280 \\ 9011 & 13: 56: 34 & 0.1329 \\ 9012 & 13: 56: 35 & 0.1293 \\ 9013 & 13: 56: 36 & 0.1318 \\ 9014 & 13: 56: 37 & 0.1261 \\ 9015 & 13: 56: 38 & 0.1309 \\ 9016 & 13: 56: 39 & 0.1277 \\ 9017 & 13: 56: 40 & 0.1313 \\ 9018 & 13: 56: 41 & 0.1285 \\ 9019 & 13: 56: 42 & 0.1322 \\ 9020 & 13: 56: 43 & 0.1287 \\ 9021 & 13: 56: 44 & 0.1290 \\ 9022 & 13: 56: 45 & 0.1285 \\ 9023 & 13: 56: 46 & 0.1280 \\ 9024 & 13: 56: 47 & 0.1295 \\ 9025 & 13: 56: 48 & 0.1271 \\ 9026 & 13: 56: 49 & 0.1301 \\ 9027 & 13: 56: 50 & 0.1257 \\ 9028 & 13: 56: 51 & 0.1291 \\ 9029 & 13: 56: 52 & 0.1288 \\ 9030 & 13: 56: 53 & 0.1320 \\ 9031 & 13: 56: 54 & 0.1282 \\ 9032 & 13: 56: 55 & 0.1317 \\ 9033 & 13: 56: 56 & 0.1306 \\ 9034 & 13: 56: 57 & 0.1308 \\ 9035 & 13: 56: 58 & 0.1292 \\ 9036 & 13: 56: 59 & 0.1292 \\ 9037 & 13: 57: 00 & 0.1295 \\ 9038 & 13: 57: 01 & 0.1267 \\ 9039 & 13: 57: 02 & 0.1288 \\ 9040 & 13: 57: 03 & 0.1253 \\ 9041 & 13: 57: 04 & 0.1303 \\ 9042 & 13: 57: 05 & 0.1258 \\ 9043 & 13: 57: 06 & 0.1311 \\ 9044 & 13: 57: 07 & 0.1279 \\ 9045 & 13: 57: 08 & 0.1308 \\ 9046 & 13: 57: 09 & 0.1281\end{array}$


Appendix A - Fuel Data Test 1 NIST102505_1.xls

$\begin{array}{lll}9047 & 13: 57: 10 & 0.1286 \\ 9048 & 13: 57: 11 & 0.1291 \\ 9049 & 13: 57: 12 & 0.1259 \\ 9050 & 13: 57: 13 & 0.1308 \\ 9051 & 13: 57: 14 & 0.1270 \\ 9052 & 13: 57: 15 & 0.1297 \\ 9053 & 13: 57: 16 & 0.1255 \\ 9054 & 13: 57: 17 & 0.1300 \\ 9055 & 13: 57: 18 & 0.1261 \\ 9056 & 13: 57: 19 & 0.1294 \\ 9057 & 13: 57: 20 & 0.1294 \\ 9058 & 13: 57: 21 & 0.1308 \\ 9059 & 13: 57: 22 & 0.1309 \\ 9060 & 13: 57: 23 & 0.1297 \\ 9061 & 13: 57: 24 & 0.1328 \\ 9062 & 13: 57: 25 & 0.1283 \\ 9063 & 13: 57: 26 & 0.1310 \\ 9064 & 13: 57: 27 & 0.1257 \\ 9065 & 13: 57: 28 & 0.1311 \\ 9066 & 13: 57: 29 & 0.1270 \\ 9067 & 13: 57: 30 & 0.1304 \\ 9068 & 13: 57: 31 & 0.1278 \\ 9069 & 13: 57: 32 & 0.1303 \\ 9070 & 13: 57: 33 & 0.1269 \\ 9071 & 13: 57: 34 & 0.1288 \\ 9072 & 13: 57: 35 & 0.1292 \\ 9073 & 13: 57: 36 & 0.1278 \\ 9074 & 13: 57: 37 & 0.1295 \\ 9075 & 13: 57: 38 & 0.1264 \\ 9076 & 13: 57: 39 & 0.1311 \\ 9077 & 13: 57: 40 & 0.1266 \\ 9078 & 13: 57: 41 & 0.1295 \\ 9079 & 13: 57: 42 & 0.1263 \\ 9080 & 13: 57: 43 & 0.1317 \\ 9081 & 13: 57: 44 & 0.1271 \\ 9082 & 13: 57: 45 & 0.1295 \\ 9083 & 13: 57: 46 & 0.1298 \\ 9084 & 13: 57: 47 & 0.1290 \\ 9085 & 13: 57: 48 & 0.1290 \\ 9086 & 13: 57: 49 & 0.1270 \\ 9087 & 13: 57: 50 & 0.1305 \\ 9088 & 13: 57: 51 & 0.1268 \\ 9089 & 13: 57: 52 & 0.1288 \\ 9090 & 13: 57: 53 & 0.1258 \\ 9091 & 13: 57: 54 & 0.1318 \\ 9092 & 13: 57: 55 & 0.1275 \\ 9093 & 13: 57: 56 & 0.1312 \\ 9094 & 13: 57: 57 & 0.1296 \\ 9095 & 13: 57: 58 & 0.1324 \\ 9096 & 13: 57: 59 & 0.1282 \\ 9097 & 13: 58: 00 & 0.1285 \\ 9098 & 13: 58: 01 & 0.1290\end{array}$


Appendix A - Fuel Data Test 1 NIST102505_1.xls

$\begin{array}{lll}9099 & 13: 58: 02 & 0.1280 \\ 9100 & 13: 58: 03 & 0.1303 \\ 9101 & 13: 58: 04 & 0.1269 \\ 9102 & 13: 58: 05 & 0.1314 \\ 9103 & 13: 58: 06 & 0.1271 \\ 9104 & 13: 58: 07 & 0.1304 \\ 9105 & 13: 58: 08 & 0.1276 \\ 9106 & 13: 58: 09 & 0.1326 \\ 9107 & 13: 58: 10 & 0.1304 \\ 9108 & 13: 58: 11 & 0.1307 \\ 9109 & 13: 58: 12 & 0.1314 \\ 9110 & 13: 58: 13 & 0.1306 \\ 9111 & 13: 58: 14 & 0.1302 \\ 9112 & 13: 58: 15 & 0.1273 \\ 9113 & 13: 58: 16 & 0.1313 \\ 9114 & 13: 58: 17 & 0.1268 \\ 9115 & 13: 58: 18 & 0.1305 \\ 9116 & 13: 58: 19 & 0.1269 \\ 9117 & 13: 58: 20 & 0.1329 \\ 9118 & 13: 58: 21 & 0.1298 \\ 9119 & 13: 58: 22 & 0.1340 \\ 9120 & 13: 58: 23 & 0.1319 \\ 9121 & 13: 58: 24 & 0.1327 \\ 9122 & 13: 58: 25 & 0.1293 \\ 9123 & 13: 58: 26 & 0.1297 \\ 9124 & 13: 58: 27 & 0.1305 \\ 9125 & 13: 58: 28 & 0.1280 \\ 9126 & 13: 58: 29 & 0.1300 \\ 9127 & 13: 58: 30 & 0.1272 \\ 9128 & 13: 58: 31 & 0.1300 \\ 9129 & 13: 58: 32 & 0.1260 \\ 9130 & 13: 58: 33 & 0.1311 \\ 9131 & 13: 58: 34 & 0.1288 \\ 9132 & 13: 58: 35 & 0.1329 \\ 9133 & 13: 58: 36 & 0.1288 \\ 9134 & 13: 58: 37 & 0.1308 \\ 9135 & 13: 58: 38 & 0.1287 \\ 9136 & 13: 58: 39 & 0.1287 \\ 9137 & 13: 58: 40 & 0.1298 \\ 9138 & 13: 58: 41 & 0.1275 \\ 9139 & 13: 58: 42 & 0.1302 \\ 9140 & 13: 58: 43 & 0.1253 \\ 9141 & 13: 58: 44 & 0.1312 \\ 9142 & 13: 58: 45 & 0.1278 \\ 9143 & 13: 58: 46 & 0.1313 \\ 9144 & 13: 58: 47 & 0.1280 \\ 9145 & 13: 58: 48 & 0.1316 \\ 9146 & 13: 58: 49 & 0.1287 \\ 9147 & 13: 58: 50 & 0.1283 \\ 9148 & 13: 58: 51 & 0.1259 \\ 9149 & 13: 58: 52 & 0.1275 \\ 9150 & 13: 58: 53 & 0.1305\end{array}$


Appendix A - Fuel Data Test 1 NIST102505_1.xls

$\begin{array}{lll}9151 & 13: 58: 54 & 0.1259 \\ 9152 & 13: 58: 55 & 0.1306 \\ 9153 & 13: 58: 56 & 0.1260 \\ 9154 & 13: 58: 57 & 0.1299 \\ 9155 & 13: 58: 58 & 0.1266 \\ 9156 & 13: 58: 59 & 0.1306 \\ 9157 & 13: 59: 00 & 0.1292 \\ 9158 & 13: 59: 01 & 0.1324 \\ 9159 & 13: 59: 02 & 0.1282 \\ 9160 & 13: 59: 03 & 0.1293 \\ 9161 & 13: 59: 04 & 0.1286 \\ 9162 & 13: 59: 05 & 0.1269 \\ 9163 & 13: 59: 06 & 0.1313 \\ 9164 & 13: 59: 07 & 0.1273 \\ 9165 & 13: 59: 08 & 0.1293 \\ 9166 & 13: 59: 09 & 0.1247 \\ 9167 & 13: 59: 10 & 0.1320 \\ 9168 & 13: 59: 11 & 0.1288 \\ 9169 & 13: 59: 12 & 0.1315 \\ 9170 & 13: 59: 13 & 0.1276 \\ 9171 & 13: 59: 14 & 0.1306 \\ 9172 & 13: 59: 15 & 0.1272 \\ 9173 & 13: 59: 16 & 0.1275 \\ 9174 & 13: 59: 17 & 0.1282 \\ 9175 & 13: 59: 18 & 0.1262 \\ 9176 & 13: 59: 19 & 0.1289 \\ 9177 & 13: 59: 20 & 0.1247 \\ 9178 & 13: 59: 21 & 0.1305 \\ 9179 & 13: 59: 22 & 0.1257 \\ 9180 & 13: 59: 23 & 0.1289 \\ 9181 & 13: 59: 24 & 0.1280 \\ 9182 & 13: 59: 25 & 0.1305 \\ 9183 & 13: 59: 26 & 0.1283 \\ 9184 & 13: 59: 27 & 0.1295 \\ 9185 & 13: 59: 28 & 0.1303 \\ 9186 & 13: 59: 29 & 0.1282 \\ 9187 & 13: 59: 30 & 0.1288 \\ 9188 & 13: 59: 31 & 0.1264 \\ 9189 & 13: 59: 32 & 0.1303 \\ 9190 & 13: 59: 33 & 0.1275 \\ 9191 & 13: 59: 34 & 0.1312 \\ 9192 & 13: 59: 35 & 0.1277 \\ 9193 & 13: 59: 36 & 0.1311 \\ 9194 & 13: 59: 37 & 0.1282 \\ 9195 & 13: 59: 38 & 0.1313 \\ 9196 & 13: 59: 39 & 0.1311 \\ 9197 & 13: 59: 40 & 0.1295 \\ 9198 & 13: 59: 41 & 0.1280 \\ 9199 & 13: 59: 42 & 0.1273 \\ 9200 & 13: 59: 43 & 0.1300 \\ 9201 & 13: 59: 44 & 0.1266 \\ 9202 & 13: 59: 45 & 0.1294\end{array}$


Appendix A - Fuel Data Test 1 NIST102505_1.xIs

$\begin{array}{lll}9203 & 13: 59: 46 & 0.1269 \\ 9204 & 13: 59: 47 & 0.1300 \\ 9205 & 13: 59: 48 & 0.1274 \\ 9206 & 13: 59: 49 & 0.1301 \\ 9207 & 13: 59: 50 & 0.1302 \\ 9208 & 13: 59: 51 & 0.1304 \\ 9209 & 13: 59: 52 & 0.1286 \\ 9210 & 13: 59: 53 & 0.1278 \\ 9211 & 13: 59: 54 & 0.1286 \\ 9212 & 13: 59: 55 & 0.1270 \\ 9213 & 13: 59: 56 & 0.1276 \\ 9214 & 13: 59: 57 & 0.1246 \\ 9215 & 13: 59: 58 & 0.1298 \\ 9216 & 13: 59: 59 & 0.1267 \\ 9217 & 14: 00: 00 & 0.1304 \\ 9218 & 14: 00: 01 & 0.1289 \\ 9219 & 14: 00: 02 & 0.1326 \\ 9220 & 14: 00: 03 & 0.1294 \\ 9221 & 14: 00: 04 & 0.1310 \\ 9222 & 14: 00: 05 & 0.1307 \\ 9223 & 14: 00: 06 & 0.1282 \\ 9224 & 14: 00: 07 & 0.1283 \\ 9225 & 14: 00: 08 & 0.1256 \\ 9226 & 14: 00: 09 & 0.1307 \\ 9227 & 14: 00: 10 & 0.1262 \\ 9228 & 14: 00: 11 & 0.1307 \\ 9229 & 14: 00: 12 & 0.1269 \\ 9230 & 14: 00: 13 & 0.1332 \\ 9231 & 14: 00: 14 & 0.1289 \\ 9232 & 14: 00: 15 & 0.1337 \\ 9233 & 14: 00: 16 & 0.1299 \\ 9234 & 14: 00: 17 & 0.1319 \\ 9235 & 14: 00: 18 & 0.1305 \\ 9236 & 14: 00: 19 & 0.1297 \\ 9237 & 14: 00: 20 & 0.1325 \\ 9238 & 14: 00: 21 & 0.1293 \\ 9239 & 14: 00: 22 & 0.1321 \\ 9240 & 14: 00: 23 & 0.1283 \\ 9241 & 14: 00: 24 & 0.1329 \\ 9242 & 14: 00: 25 & 0.1274 \\ 9243 & 14: 00: 26 & 0.1320 \\ 9244 & 14: 00: 27 & 0.1277 \\ 9245 & 14: 00: 28 & 0.1309 \\ 9246 & 14: 00: 29 & 0.1270 \\ 9247 & 14: 00: 30 & 0.1291 \\ 9248 & 14: 00: 31 & 0.1264 \\ 9249 & 14: 00: 32 & 0.1252 \\ 9250 & 14: 00: 33 & 0.1285 \\ 9251 & 14: 00: 34 & 0.1268 \\ 9252 & 14: 00: 35 & 0.1302 \\ 9254 & 14: 00: 36 & 0.1268 \\ & 14: 00: 37 & 0.1327\end{array}$


Appendix A - Fuel Data Test 1 NIST102505_1.xIs

\begin{tabular}{|c|c|c|}
\hline 9255 & $14: 00: 38$ & 0.1286 \\
\hline 9256 & $14: 00: 39$ & 0.1323 \\
\hline 9257 & $14: 00: 40$ & 0.1295 \\
\hline 9258 & $14: 00: 41$ & 0.1321 \\
\hline 9259 & $14: 00: 42$ & 0.1288 \\
\hline 9260 & $14: 00: 43$ & 0.1281 \\
\hline 9261 & $14: 00: 44$ & 0.1309 \\
\hline 9262 & $14: 00: 45$ & 0.1282 \\
\hline 9263 & $14: 00: 46$ & 0.1308 \\
\hline 9264 & 14:00:47 & 0.1269 \\
\hline 9265 & $14: 00: 48$ & 0.1313 \\
\hline 9266 & $14: 00: 49$ & 0.1270 \\
\hline 9267 & $14: 00: 50$ & 0.1298 \\
\hline 9268 & $14: 00: 51$ & 0.1260 \\
\hline 9269 & $14: 00: 52$ & 0.1304 \\
\hline 9270 & $14: 00: 53$ & 0.1268 \\
\hline 9271 & $14: 00: 54$ & 0.1306 \\
\hline 9272 & $14: 00: 55$ & 0.1308 \\
\hline 9273 & $14: 00: 56$ & 0.1327 \\
\hline 9274 & $14: 00: 57$ & 0.1306 \\
\hline 9275 & $14: 00: 58$ & 0.1300 \\
\hline 9276 & $14: 00: 59$ & 0.1317 \\
\hline 9277 & $14: 01: 00$ & 0.1274 \\
\hline 9278 & $14: 01: 01$ & 0.1326 \\
\hline 9279 & 14:01:02 & 0.1295 \\
\hline 9280 & $14: 01: 03$ & 0.1331 \\
\hline 9281 & $14: 01: 04$ & 0.1286 \\
\hline 9282 & 14:01:05 & 0.1337 \\
\hline 9283 & $14: 01: 06$ & 0.1303 \\
\hline 9284 & $14: 01: 07$ & 0.1319 \\
\hline 9285 & $14: 01: 08$ & 0.1290 \\
\hline 9286 & 14:01:09 & 0.1296 \\
\hline 9287 & $14: 01: 10$ & 0.1283 \\
\hline 9288 & $14: 01: 11$ & 0.1275 \\
\hline 9289 & $14: 01: 12$ & 0.1312 \\
\hline 9290 & $14: 01: 13$ & 0.1267 \\
\hline 9291 & $14: 01: 14$ & 0.1307 \\
\hline 9292 & $14: 01: 15$ & 0.1266 \\
\hline 9293 & $14: 01: 16$ & 0.1319 \\
\hline 9294 & $14: 01: 17$ & 0.1280 \\
\hline 9295 & $14: 01: 18$ & 0.1310 \\
\hline 9296 & $14: 01: 19$ & 0.1316 \\
\hline 9297 & $14: 01: 20$ & 0.1305 \\
\hline 9298 & $14: 01: 21$ & 0.1272 \\
\hline 9299 & 14:01:22 & 0.1265 \\
\hline 9300 & $14: 01: 23$ & 0.1306 \\
\hline 9301 & $14: 01: 24$ & 0.1283 \\
\hline 9302 & $14: 01: 25$ & 0.1299 \\
\hline 9303 & $14: 01: 26$ & 0.1257 \\
\hline 9304 & $14: 01: 27$ & 0.1292 \\
\hline 9305 & $14: 01: 28$ & 0.1266 \\
\hline 9306 & $14: 01: 29$ & 0.1305 \\
\hline
\end{tabular}


Appendix A - Fuel Data Test 1 NIST102505_1.xIs

$\begin{array}{lll}9307 & 14: 01: 30 & 0.1301 \\ 9308 & 14: 01: 31 & 0.1308 \\ 9309 & 14: 01: 32 & 0.1301 \\ 9310 & 14: 01: 33 & 0.1285 \\ 9311 & 14: 01: 34 & 0.1308 \\ 9312 & 14: 01: 35 & 0.1300 \\ 9313 & 14: 01: 36 & 0.1319 \\ 9314 & 14: 01: 37 & 0.1277 \\ 9315 & 14: 01: 38 & 0.1316 \\ 9316 & 14: 01: 39 & 0.1275 \\ 9317 & 14: 01: 40 & 0.1307 \\ 9318 & 14: 01: 41 & 0.1279 \\ 9319 & 14: 01: 42 & 0.1337 \\ 9320 & 14: 01: 43 & 0.1311 \\ 9321 & 14: 01: 44 & 0.1322 \\ 9322 & 14: 01: 45 & 0.1323 \\ 9323 & 14: 01: 46 & 0.1321 \\ 9324 & 14: 01: 47 & 0.1300 \\ 9325 & 14: 01: 48 & 0.1301 \\ 9326 & 14: 01: 49 & 0.1315 \\ 9327 & 14: 01: 50 & 0.1277 \\ 9328 & 14: 01: 51 & 0.1309 \\ 9329 & 14: 01: 52 & 0.1281 \\ 9330 & 14: 01: 53 & 0.1304 \\ 9331 & 14: 01: 54 & 0.1266 \\ 9332 & 14: 01: 55 & 0.1310 \\ 9333 & 14: 01: 56 & 0.1307 \\ 9334 & 14: 01: 57 & 0.1322 \\ 9335 & 14: 01: 58 & 0.1297 \\ 9336 & 14: 01: 59 & 0.1304 \\ 9337 & 14: 02: 00 & 0.1296 \\ 9338 & 14: 02: 01 & 0.1280 \\ 9339 & 14: 02: 02 & 0.1300 \\ 9340 & 14: 02: 03 & 0.1276 \\ 9341 & 14: 02: 04 & 0.1310 \\ 9342 & 14: 02: 05 & 0.1258 \\ 9343 & 14: 02: 06 & 0.1302 \\ 9344 & 14: 02: 07 & 0.1291 \\ 9345 & 14: 02: 08 & 0.1315 \\ 9346 & 14: 02: 09 & 0.1295 \\ 9347 & 14: 02: 10 & 0.1307 \\ 9348 & 14: 02: 11 & 0.1306 \\ 9349 & 14: 02: 12 & 0.1296 \\ 9350 & 14: 02: 13 & 0.1302 \\ 9351 & 14: 02: 14 & 0.1291 \\ 9352 & 14: 02: 15 & 0.1305 \\ 9353 & 14: 02: 16 & 0.1261 \\ 9354 & 14: 02: 17 & 0.1316 \\ 9355 & 14: 02: 18 & 0.1286 \\ 9356 & 14: 02: 19 & 0.1305 \\ 9357 & 14: 02: 20 & 0.1254 \\ 9358 & 14: 02: 21 & 0.1308\end{array}$


Appendix A - Fuel Data Test 1 NIST102505_1.xIs

$\begin{array}{lll}9359 & 14: 02: 22 & 0.1299 \\ 9360 & 14: 02: 23 & 0.1301 \\ 9361 & 14: 02: 24 & 0.1281 \\ 9362 & 14: 02: 25 & 0.1271 \\ 9363 & 14: 02: 26 & 0.1291 \\ 9364 & 14: 02: 27 & 0.1261 \\ 9365 & 14: 02: 28 & 0.1290 \\ 9366 & 14: 02: 29 & 0.1273 \\ 9367 & 14: 02: 30 & 0.1327 \\ 9368 & 14: 02: 31 & 0.1288 \\ 9369 & 14: 02: 32 & 0.1322 \\ 9370 & 14: 02: 33 & 0.1318 \\ 9371 & 14: 02: 34 & 0.1333 \\ 9372 & 14: 02: 35 & 0.1271 \\ 9373 & 14: 02: 36 & 0.1294 \\ 9374 & 14: 02: 37 & 0.1278 \\ 9375 & 14: 02: 38 & 0.1264 \\ 9376 & 14: 02: 39 & 0.1306 \\ 9377 & 14: 02: 40 & 0.1267 \\ 9378 & 14: 02: 41 & 0.1302 \\ 9379 & 14: 02: 42 & 0.1247 \\ 9380 & 14: 02: 43 & 0.1296 \\ 9381 & 14: 02: 44 & 0.1262 \\ 9382 & 14: 02: 45 & 0.1296 \\ 9383 & 14: 02: 46 & 0.1286 \\ 9384 & 14: 02: 47 & 0.1323 \\ 9385 & 14: 02: 48 & 0.1281 \\ 9386 & 14: 02: 49 & 0.1296 \\ 9387 & 14: 02: 50 & 0.1284 \\ 9388 & 14: 02: 51 & 0.1259 \\ 9389 & 14: 02: 52 & 0.1297 \\ 9390 & 14: 02: 53 & 0.1283 \\ 9391 & 14: 02: 54 & 0.1299 \\ 9392 & 14: 02: 55 & 0.1253 \\ 9393 & 14: 02: 56 & 0.1297 \\ 9394 & 14: 02: 57 & 0.1291 \\ 9395 & 14: 02: 58 & 0.1321 \\ 9396 & 14: 02: 59 & 0.1296 \\ 9397 & 14: 03: 00 & 0.1320 \\ 9398 & 14: 03: 01 & 0.1307 \\ 9399 & 14: 03: 02 & 0.1312 \\ 9400 & 14: 03: 03 & 0.1302 \\ 9401 & 14: 03: 04 & 0.1285 \\ 9402 & 14: 03: 05 & 0.1297 \\ 9403 & 14: 03: 06 & 0.1252 \\ 9404 & 14: 03: 07 & 0.1291 \\ 9405 & 14: 03: 08 & 0.1272 \\ 9406 & 14: 03: 09 & 0.1304 \\ 9407 & 14: 03: 10 & 0.1263 \\ 9408 & 14: 03: 11 & 0.1315 \\ 9409 & 14: 03: 12 & 0.1301 \\ 9410 & 14: 03: 13 & 0.1308\end{array}$


Appendix A - Fuel Data Test 1 NIST102505_1.xls

$\begin{array}{lll}9411 & 14: 03: 14 & 0.1286 \\ 9412 & 14: 03: 15 & 0.1276 \\ 9413 & 14: 03: 16 & 0.1293 \\ 9414 & 14: 03: 17 & 0.1265 \\ 9415 & 14: 03: 18 & 0.1282 \\ 9416 & 14: 03: 19 & 0.1256 \\ 9417 & 14: 03: 20 & 0.1304 \\ 9418 & 14: 03: 21 & 0.1270 \\ 9419 & 14: 03: 22 & 0.1311 \\ 9420 & 14: 03: 23 & 0.1277 \\ 9421 & 14: 03: 24 & 0.1311 \\ 9422 & 14: 03: 25 & 0.1276 \\ 9423 & 14: 03: 26 & 0.1302 \\ 9424 & 14: 03: 27 & 0.1292 \\ 9425 & 14: 03: 28 & 0.1295 \\ 9426 & 14: 03: 29 & 0.1311 \\ 9427 & 14: 03: 30 & 0.1284 \\ 9428 & 14: 03: 31 & 0.1301 \\ 9429 & 14: 03: 32 & 0.1261 \\ 9430 & 14: 03: 33 & 0.1336 \\ 9431 & 14: 03: 34 & 0.1296 \\ 9432 & 14: 03: 35 & 0.1327 \\ 9433 & 14: 03: 36 & 0.1293 \\ 9434 & 14: 03: 37 & 0.1322 \\ 9435 & 14: 03: 38 & 0.1300 \\ 9436 & 14: 03: 39 & 0.1303 \\ 9437 & 14: 03: 40 & 0.1307 \\ 9438 & 14: 03: 41 & 0.1287 \\ 9439 & 14: 03: 42 & 0.1297 \\ 9440 & 14: 03: 43 & 0.1258 \\ 9441 & 14: 03: 44 & 0.1303 \\ 9442 & 14: 03: 45 & 0.1263 \\ 9443 & 14: 03: 46 & 0.1306 \\ 9444 & 14: 03: 47 & 0.1274 \\ 9445 & 14: 03: 48 & 0.1322 \\ 9446 & 14: 03: 49 & 0.1284 \\ 9447 & 14: 03: 50 & 0.1303 \\ 9448 & 14: 03: 51 & 0.1281 \\ 9449 & 14: 03: 52 & 0.1286 \\ 9450 & 14: 03: 53 & 0.1270 \\ 9451 & 14: 03: 54 & 0.1251 \\ 9452 & 14: 03: 55 & 0.1301 \\ 9453 & 14: 03: 56 & 0.1262 \\ 9454 & 14: 03: 57 & 0.1297 \\ 9455 & 14: 03: 58 & 0.1249 \\ 9456 & 14: 03: 59 & 0.1302 \\ 9457 & 14: 04: 00 & 0.1266 \\ 9458 & 14: 04: 01 & 0.1291 \\ 9459 & 14: 04: 02 & 0.1287 \\ 9460 & 14: 04: 03 & 0.1300 \\ 9461 & 14: 04: 04 & 0.1282 \\ 9462 & 14: 04: 05 & 0.1277\end{array}$


Appendix A - Fuel Data Test 1 NIST102505_1.xIs

$\begin{array}{lll}9463 & 14: 04: 06 & 0.1298 \\ 9464 & 14: 04: 07 & 0.1269 \\ 9465 & 14: 04: 08 & 0.1283 \\ 9466 & 14: 04: 09 & 0.1247 \\ 9467 & 14: 04: 10 & 0.1295 \\ 9468 & 14: 04: 11 & 0.1253 \\ 9469 & 14: 04: 12 & 0.1322 \\ 9470 & 14: 04: 13 & 0.1287 \\ 9471 & 14: 04: 14 & 0.1320 \\ 9472 & 14: 04: 15 & 0.1291 \\ 9473 & 14: 04: 16 & 0.1300 \\ 9474 & 14: 04: 17 & 0.1273 \\ 9475 & 14: 04: 18 & 0.1267 \\ 9476 & 14: 04: 19 & 0.1289 \\ 9477 & 14: 04: 20 & 0.1270 \\ 9478 & 14: 04: 21 & 0.1294 \\ 9479 & 14: 04: 22 & 0.1245 \\ 9480 & 14: 04: 23 & 0.1318 \\ 9481 & 14: 04: 24 & 0.1297 \\ 9482 & 14: 04: 25 & 0.1331 \\ 9483 & 14: 04: 26 & 0.1278 \\ 9484 & 14: 04: 27 & 0.1326 \\ 9485 & 14: 04: 28 & 0.1293 \\ 9486 & 14: 04: 29 & 0.1306 \\ 9487 & 14: 04: 30 & 0.1303 \\ 9488 & 14: 04: 31 & 0.1296 \\ 9489 & 14: 04: 32 & 0.1317 \\ 9490 & 14: 04: 33 & 0.1281 \\ 9491 & 14: 04: 34 & 0.1330 \\ 9492 & 14: 04: 35 & 0.1283 \\ 9493 & 14: 04: 36 & 0.1323 \\ 9494 & 14: 04: 37 & 0.1302 \\ 9495 & 14: 04: 38 & 0.1325 \\ 9496 & 14: 04: 39 & 0.1292 \\ 9497 & 14: 04: 40 & 0.1299 \\ 9498 & 14: 04: 41 & 0.1303 \\ 9499 & 14: 04: 42 & 0.1295 \\ 9500 & 14: 04: 43 & 0.1278 \\ 9501 & 14: 04: 44 & 0.1279 \\ 9502 & 14: 04: 45 & 0.1307 \\ 9503 & 14: 04: 46 & 0.1269 \\ 9504 & 14: 04: 47 & 0.1302 \\ 9505 & 14: 04: 48 & 0.1271 \\ 9506 & 14: 04: 49 & 0.1309 \\ 9507 & 14: 04: 50 & 0.1270 \\ 9508 & 14: 04: 51 & 0.1322 \\ 9509 & 14: 04: 52 & 0.1298 \\ 9510 & 14: 04: 53 & 0.1306 \\ 9511 & 14: 04: 54 & 0.1276 \\ 9512 & 14: 04: 55 & 0.1291 \\ 9513 & 14: 04: 56 & 0.1290 \\ 9514 & 14: 04: 57 & 0.1264\end{array}$


Appendix A - Fuel Data Test 1 NIST102505_1.xIs

$\begin{array}{lll}9515 & 14: 04: 58 & 0.1292 \\ 9516 & 14: 04: 59 & 0.1268 \\ 9517 & 14: 05: 00 & 0.1301 \\ 9518 & 14: 05: 01 & 0.1257 \\ 9519 & 14: 05: 02 & 0.1299 \\ 9520 & 14: 05: 03 & 0.1275 \\ 9521 & 14: 05: 04 & 0.1310 \\ 9522 & 14: 05: 05 & 0.1270 \\ 9523 & 14: 05: 06 & 0.1292 \\ 9524 & 14: 05: 07 & 0.1275 \\ 9525 & 14: 05: 08 & 0.1269 \\ 9526 & 14: 05: 09 & 0.1292 \\ 9527 & 14: 05: 10 & 0.1287 \\ 9528 & 14: 05: 11 & 0.1297 \\ 9529 & 14: 05: 12 & 0.1273 \\ 9530 & 14: 05: 13 & 0.1328 \\ 9531 & 14: 05: 14 & 0.1290 \\ 9532 & 14: 05: 15 & 0.1325 \\ 9533 & 14: 05: 16 & 0.1291 \\ 9534 & 14: 05: 17 & 0.1325 \\ 9535 & 14: 05: 18 & 0.1310 \\ 9536 & 14: 05: 19 & 0.1312 \\ 9537 & 14: 05: 20 & 0.1325 \\ 9538 & 14: 05: 21 & 0.1289 \\ 9539 & 14: 05: 22 & 0.1299 \\ 9540 & 14: 05: 23 & 0.1255 \\ 9541 & 14: 05: 24 & 0.1312 \\ 9542 & 14: 05: 25 & 0.1265 \\ 9543 & 14: 05: 26 & 0.1322 \\ 9544 & 14: 05: 27 & 0.1277 \\ 9545 & 14: 05: 28 & 0.1329 \\ 9546 & 14: 05: 29 & 0.1278 \\ 9547 & 14: 05: 30 & 0.1307 \\ 9548 & 14: 05: 31 & 0.1304 \\ 9549 & 14: 05: 32 & 0.1299 \\ 9550 & 14: 05: 33 & 0.1300 \\ 9551 & 14: 05: 34 & 0.1294 \\ 9552 & 14: 05: 35 & 0.1312 \\ 9553 & 14: 05: 36 & 0.1262 \\ 9554 & 14: 05: 37 & 0.1288 \\ 9555 & 14: 05: 38 & 0.1262 \\ 9556 & 14: 05: 39 & 0.1307 \\ 9557 & 14: 05: 40 & 0.1262 \\ 9558 & 14: 05: 41 & 0.1315 \\ 9559 & 14: 05: 42 & 0.1290 \\ 9560 & 14: 05: 43 & 0.1306 \\ 9561 & 14: 05: 44 & 0.1289 \\ 9562 & 14: 05: 45 & 0.1284 \\ 9563 & 14: 05: 46 & 0.1281 \\ 9564 & 14: 05: 47 & 0.1267 \\ 9565 & 14: 05: 48 & 0.1287 \\ 9566 & 14: 05: 49 & 0.1258\end{array}$


Appendix A - Fuel Data Test 1 NIST102505_1.xIs

$\begin{array}{lll}9567 & 14: 05: 50 & 0.1298 \\ 9568 & 14: 05: 51 & 0.1248 \\ 9569 & 14: 05: 52 & 0.1302 \\ 9570 & 14: 05: 53 & 0.1275 \\ 9571 & 14: 05: 54 & 0.1304 \\ 9572 & 14: 05: 55 & 0.1268 \\ 9573 & 14: 05: 56 & 0.1284 \\ 9574 & 14: 05: 57 & 0.1253 \\ 9575 & 14: 05: 58 & 0.1256 \\ 9576 & 14: 05: 59 & 0.1294 \\ 9577 & 14: 06: 00 & 0.1266 \\ 9578 & 14: 06: 01 & 0.1296 \\ 9579 & 14: 06: 02 & 0.1249 \\ 9580 & 14: 06: 03 & 0.1294 \\ 9581 & 14: 06: 04 & 0.1261 \\ 9582 & 14: 06: 05 & 0.1304 \\ 9583 & 14: 06: 06 & 0.1277 \\ 9584 & 14: 06: 07 & 0.1312 \\ 9585 & 14: 06: 08 & 0.1286 \\ 9586 & 14: 06: 09 & 0.1280 \\ 9587 & 14: 06: 10 & 0.1302 \\ 9588 & 14: 06: 11 & 0.1287 \\ 9589 & 14: 06: 12 & 0.1306 \\ 9590 & 14: 06: 13 & 0.1270 \\ 9591 & 14: 06: 14 & 0.1319 \\ 9592 & 14: 06: 15 & 0.1277 \\ 9593 & 14: 06: 16 & 0.1308 \\ 9594 & 14: 06: 17 & 0.1291 \\ 9595 & 14: 06: 18 & 0.1337 \\ 9596 & 14: 06: 19 & 0.1307 \\ 9597 & 14: 06: 20 & 0.1299 \\ 9598 & 14: 06: 21 & 0.1293 \\ 9599 & 14: 06: 22 & 0.1298 \\ 9600 & 14: 06: 23 & 0.1283 \\ 9601 & 14: 06: 24 & 0.1263 \\ 9602 & 14: 06: 25 & 0.1305 \\ 9603 & 14: 06: 26 & 0.1262 \\ 9604 & 14: 06: 27 & 0.1294 \\ 9605 & 14: 06: 28 & 0.1273 \\ 9606 & 14: 06: 29 & 0.1313 \\ 9607 & 14: 06: 30 & 0.1264 \\ 9608 & 14: 06: 31 & 0.1309 \\ 9609 & 14: 06: 32 & 0.1291 \\ 9610 & 14: 06: 33 & 0.1313 \\ 9611 & 14: 06: 34 & 0.1280 \\ 9612 & 14: 06: 35 & 0.1288 \\ 9613 & 14: 06: 36 & 0.1297 \\ 9614 & 14: 06: 37 & 0.1275 \\ 9615 & 14: 06: 38 & 0.1291 \\ 9616 & 14: 06: 39 & 0.1268 \\ 9617 & 14: 06: 40 & 0.1289 \\ 9618 & 14: 06: 41 & 0.1241\end{array}$


Appendix A - Fuel Data Test 1 NIST102505_1.xIs

$\begin{array}{lll}9619 & 14: 06: 42 & 0.1304 \\ 9620 & 14: 06: 43 & 0.1274 \\ 9621 & 14: 06: 44 & 0.1317 \\ 9622 & 14: 06: 45 & 0.1280 \\ 9623 & 14: 06: 46 & 0.1310 \\ 9624 & 14: 06: 47 & 0.1287 \\ 9625 & 14: 06: 48 & 0.1282 \\ 9626 & 14: 06: 49 & 0.1295 \\ 9627 & 14: 06: 50 & 0.1290 \\ 9628 & 14: 06: 51 & 0.1301 \\ 9629 & 14: 06: 52 & 0.1269 \\ 9630 & 14: 06: 53 & 0.1316 \\ 9631 & 14: 06: 54 & 0.1280 \\ 9632 & 14: 06: 55 & 0.1312 \\ 9633 & 14: 06: 56 & 0.1271 \\ 9634 & 14: 06: 57 & 0.1326 \\ 9635 & 14: 06: 58 & 0.1291 \\ 9636 & 14: 06: 59 & 0.1300 \\ 9637 & 14: 07: 00 & 0.1288 \\ 9638 & 14: 07: 01 & 0.1289 \\ 9639 & 14: 07: 02 & 0.1299 \\ 9640 & 14: 07: 03 & 0.1268 \\ 9641 & 14: 07: 04 & 0.1310 \\ 9642 & 14: 07: 05 & 0.1266 \\ 9643 & 14: 07: 06 & 0.1305 \\ 9644 & 14: 07: 07 & 0.1266 \\ 9645 & 14: 07: 08 & 0.1311 \\ 9646 & 14: 07: 09 & 0.1275 \\ 9647 & 14: 07: 10 & 0.1301 \\ 9648 & 14: 07: 11 & 0.1303 \\ 9649 & 14: 07: 12 & 0.1292 \\ 9650 & 14: 07: 13 & 0.1282 \\ 9651 & 14: 07: 14 & 0.1268 \\ 9652 & 14: 07: 15 & 0.1309 \\ 9653 & 14: 07: 16 & 0.1277 \\ 9654 & 14: 07: 17 & 0.1299 \\ 9655 & 14: 07: 18 & 0.1266 \\ 9656 & 14: 07: 19 & 0.1307 \\ 9657 & 14: 07: 20 & 0.1270 \\ 9658 & 14: 07: 21 & 0.1309 \\ 9659 & 14: 07: 22 & 0.1299 \\ 9660 & 14: 07: 23 & 0.1322 \\ 9661 & 14: 07: 24 & 0.1300 \\ 9662 & 14: 07: 25 & 0.1291 \\ 9663 & 14: 07: 26 & 0.1295 \\ 9664 & 14: 07: 27 & 0.1275 \\ 9665 & 14: 07: 28 & 0.1308 \\ 9666 & 14: 07: 29 & 0.1276 \\ 9667 & 14: 07: 30 & 0.1318 \\ 9668 & 14: 07: 31 & 0.1275 \\ 9669 & 14: 07: 32 & 0.1306 \\ 9670 & 14: 07: 33 & 0.1279\end{array}$


Appendix A - Fuel Data Test 1 NIST102505_1.xIs

$\begin{array}{lll}9671 & 14: 07: 34 & 0.1321 \\ 9672 & 14: 07: 35 & 0.1285 \\ 9673 & 14: 07: 36 & 0.1297 \\ 9674 & 14: 07: 37 & 0.1300 \\ 9675 & 14: 07: 38 & 0.1280 \\ 9676 & 14: 07: 39 & 0.1296 \\ 9677 & 14: 07: 40 & 0.1271 \\ 9678 & 14: 07: 41 & 0.1318 \\ 9679 & 14: 07: 42 & 0.1256 \\ 9680 & 14: 07: 43 & 0.1300 \\ 9681 & 14: 07: 44 & 0.1254 \\ 9682 & 14: 07: 45 & 0.1335 \\ 9683 & 14: 07: 46 & 0.1303 \\ 9684 & 14: 07: 47 & 0.1317 \\ 9685 & 14: 07: 48 & 0.1286 \\ 9686 & 14: 07: 49 & 0.1314 \\ 9687 & 14: 07: 50 & 0.1297 \\ 9688 & 14: 07: 51 & 0.1286 \\ 9689 & 14: 07: 52 & 0.1289 \\ 9690 & 14: 07: 53 & 0.1284 \\ 9691 & 14: 07: 54 & 0.1297 \\ 9692 & 14: 07: 55 & 0.1251 \\ 9693 & 14: 07: 56 & 0.1285 \\ 9694 & 14: 07: 57 & 0.1257 \\ 9695 & 14: 07: 58 & 0.1300 \\ 9696 & 14: 07: 59 & 0.1261 \\ 9697 & 14: 08: 00 & 0.1309 \\ 9698 & 14: 08: 01 & 0.1284 \\ 9699 & 14: 08: 02 & 0.1298 \\ 9700 & 14: 08: 03 & 0.1274 \\ 9701 & 14: 08: 04 & 0.1270 \\ 9702 & 14: 08: 05 & 0.1282 \\ 9703 & 14: 08: 06 & 0.1265 \\ 9704 & 14: 08: 07 & 0.1298 \\ 9705 & 14: 08: 08 & 0.1276 \\ 9706 & 14: 08: 09 & 0.1303 \\ 9707 & 14: 08: 10 & 0.1267 \\ 9708 & 14: 08: 11 & 0.1318 \\ 9709 & 14: 08: 12 & 0.1291 \\ 9710 & 14: 08: 13 & 0.1316 \\ 9711 & 14: 08: 14 & 0.1290 \\ 9712 & 14: 08: 15 & 0.1308 \\ 9713 & 14: 08: 16 & 0.1287 \\ 9714 & 14: 08: 17 & 0.1264 \\ 9715 & 14: 08: 18 & 0.1283 \\ 9716 & 14: 08: 19 & 0.1277 \\ 9717 & 14: 08: 20 & 0.1295 \\ 9718 & 14: 08: 21 & 0.1243 \\ 9719 & 14: 08: 22 & 0.1307 \\ 9720 & 14: 08: 23 & 0.1278 \\ 9721 & 14: 08: 24 & 0.1314 \\ 9722 & 14: 08: 25 & 0.1273\end{array}$


Appendix A - Fuel Data Test 1 NIST102505_1.xIs

$\begin{array}{lll}9723 & 14: 08: 26 & 0.1309 \\ 9724 & 14: 08: 27 & 0.1286 \\ 9725 & 14: 08: 28 & 0.1295 \\ 9726 & 14: 08: 29 & 0.1282 \\ 9727 & 14: 08: 30 & 0.1292 \\ 9728 & 14: 08: 31 & 0.1286 \\ 9729 & 14: 08: 32 & 0.1246 \\ 9730 & 14: 08: 33 & 0.1301 \\ 9731 & 14: 08: 34 & 0.1253 \\ 9732 & 14: 08: 35 & 0.1295 \\ 9733 & 14: 08: 36 & 0.1267 \\ 9734 & 14: 08: 37 & 0.1302 \\ 9735 & 14: 08: 38 & 0.1270 \\ 9736 & 14: 08: 39 & 0.1289 \\ 9737 & 14: 08: 40 & 0.1306 \\ 9738 & 14: 08: 41 & 0.1292 \\ 9739 & 14: 08: 42 & 0.1308 \\ 9740 & 14: 08: 43 & 0.1279 \\ 9741 & 14: 08: 44 & 0.1317 \\ 9742 & 14: 08: 45 & 0.1277 \\ 9743 & 14: 08: 46 & 0.1304 \\ 9744 & 14: 08: 47 & 0.1270 \\ 9745 & 14: 08: 48 & 0.1312 \\ 9746 & 14: 08: 49 & 0.1290 \\ 9747 & 14: 08: 50 & 0.1323 \\ 9748 & 14: 08: 51 & 0.1314 \\ 9749 & 14: 08: 52 & 0.1318 \\ 9750 & 14: 08: 53 & 0.1297 \\ 9751 & 14: 08: 54 & 0.1298 \\ 9752 & 14: 08: 55 & 0.1305 \\ 9753 & 14: 08: 56 & 0.1273 \\ 9754 & 14: 08: 57 & 0.1288 \\ 9755 & 14: 08: 58 & 0.1250 \\ 9756 & 14: 08: 59 & 0.1293 \\ 9757 & 14: 09: 00 & 0.1252 \\ 9758 & 14: 09: 01 & 0.1305 \\ 9759 & 14: 09: 02 & 0.1283 \\ 9760 & 14: 09: 03 & 0.1307 \\ 9761 & 14: 09: 04 & 0.1286 \\ 9762 & 14: 09: 05 & 0.1304 \\ 9763 & 14: 09: 06 & 0.1281 \\ 9764 & 14: 09: 07 & 0.1267 \\ 9765 & 14: 09: 08 & 0.1274 \\ 9766 & 14: 09: 09 & 0.1269 \\ 9767 & 14: 09: 10 & 0.1304 \\ 9768 & 14: 09: 11 & 0.1260 \\ 9769 & 14: 09: 12 & 0.1313 \\ 9770 & 14: 09: 13 & 0.1275 \\ 9771 & 14: 09: 14 & 0.1310 \\ 9772 & 14: 09: 15 & 0.1301 \\ 9773 & 14: 09: 16 & 0.1314 \\ 9774 & 14: 09: 17 & 0.1305\end{array}$


Appendix A - Fuel Data Test 1 NIST102505_1.xIs

$\begin{array}{lll}9775 & 14: 09: 18 & 0.1286 \\ 9776 & 14: 09: 19 & 0.1295 \\ 9777 & 14: 09: 20 & 0.1276 \\ 9778 & 14: 09: 21 & 0.1294 \\ 9779 & 14: 09: 22 & 0.1274 \\ 9780 & 14: 09: 23 & 0.1302 \\ 9781 & 14: 09: 24 & 0.1246 \\ 9782 & 14: 09: 25 & 0.1296 \\ 9783 & 14: 09: 26 & 0.1285 \\ 9784 & 14: 09: 27 & 0.1315 \\ 9785 & 14: 09: 28 & 0.1279 \\ 9786 & 14: 09: 29 & 0.1300 \\ 9787 & 14: 09: 30 & 0.1319 \\ 9788 & 14: 09: 31 & 0.1294 \\ 9789 & 14: 09: 32 & 0.1284 \\ 9790 & 14: 09: 33 & 0.1268 \\ 9791 & 14: 09: 34 & 0.1327 \\ 9792 & 14: 09: 35 & 0.1275 \\ 9793 & 14: 09: 36 & 0.1301 \\ 9794 & 14: 09: 37 & 0.1270 \\ 9795 & 14: 09: 38 & 0.1314 \\ 9796 & 14: 09: 39 & 0.1270 \\ 9797 & 14: 09: 40 & 0.1302 \\ 9798 & 14: 09: 41 & 0.1277 \\ 9799 & 14: 09: 42 & 0.1304 \\ 9800 & 14: 09: 43 & 0.1282 \\ 9801 & 14: 09: 44 & 0.1280 \\ 9802 & 14: 09: 45 & 0.1291 \\ 9803 & 14: 09: 46 & 0.1272 \\ 9804 & 14: 09: 47 & 0.1296 \\ 9805 & 14: 09: 48 & 0.1266 \\ 9806 & 14: 09: 49 & 0.1295 \\ 9807 & 14: 09: 50 & 0.1245 \\ 9808 & 14: 09: 51 & 0.1294 \\ 9809 & 14: 09: 52 & 0.1267 \\ 9810 & 14: 09: 53 & 0.1306 \\ 9811 & 14: 09: 54 & 0.1277 \\ 9812 & 14: 09: 55 & 0.1311 \\ 9813 & 14: 09: 56 & 0.1296 \\ 9814 & 14: 09: 57 & 0.1286 \\ 9815 & 14: 09: 58 & 0.1280 \\ 9816 & 14: 09: 59 & 0.1276 \\ 9817 & 14: 10: 00 & 0.1295 \\ 9818 & 14: 10: 01 & 0.1252 \\ 9819 & 14: 10: 02 & 0.1310 \\ 9820 & 14: 10: 03 & 0.1274 \\ 9821 & 14: 10: 04 & 0.1327 \\ 9822 & 14: 10: 05 & 0.1284 \\ 9823 & 14: 10: 06 & 0.1316 \\ 9824 & 14: 10: 07 & 0.1279 \\ 9825 & 14: 10: 08 & 0.1294 \\ 9826 & 14: 10: 09 & 0.1256\end{array}$


Appendix A - Fuel Data Test 1 NIST102505_1.xIs

$\begin{array}{lll}9827 & 14: 10: 10 & 0.1271 \\ 9828 & 14: 10: 11 & 0.1288 \\ 9829 & 14: 10: 12 & 0.1258 \\ 9830 & 14: 10: 13 & 0.1303 \\ 9831 & 14: 10: 14 & 0.1255 \\ 9832 & 14: 10: 15 & 0.1297 \\ 9833 & 14: 10: 16 & 0.1267 \\ 9834 & 14: 10: 17 & 0.1310 \\ 9835 & 14: 10: 18 & 0.1275 \\ 9836 & 14: 10: 19 & 0.1299 \\ 9837 & 14: 10: 20 & 0.1300 \\ 9838 & 14: 10: 21 & 0.1294 \\ 9839 & 14: 10: 22 & 0.1287 \\ 9840 & 14: 10: 23 & 0.1273 \\ 9841 & 14: 10: 24 & 0.1295 \\ 9842 & 14: 10: 25 & 0.1267 \\ 9843 & 14: 10: 26 & 0.1283 \\ 9844 & 14: 10: 27 & 0.1276 \\ 9845 & 14: 10: 28 & 0.1316 \\ 9846 & 14: 10: 29 & 0.1287 \\ 9847 & 14: 10: 30 & 0.1314 \\ 9848 & 14: 10: 31 & 0.1311 \\ 9849 & 14: 10: 32 & 0.1313 \\ 9850 & 14: 10: 33 & 0.1298 \\ 9851 & 14: 10: 34 & 0.1281 \\ 9852 & 14: 10: 35 & 0.1280 \\ 9853 & 14: 10: 36 & 0.1273 \\ 9854 & 14: 10: 37 & 0.1299 \\ 9855 & 14: 10: 38 & 0.1266 \\ 9856 & 14: 10: 39 & 0.1302 \\ 9857 & 14: 10: 40 & 0.1259 \\ 9858 & 14: 10: 41 & 0.1306 \\ 9859 & 14: 10: 42 & 0.1285 \\ 9860 & 14: 10: 43 & 0.1335 \\ 9861 & 14: 10: 44 & 0.1295 \\ 9862 & 14: 10: 45 & 0.1317 \\ 9863 & 14: 10: 46 & 0.1291 \\ 9864 & 14: 10: 47 & 0.1286 \\ 9865 & 14: 10: 48 & 0.1320 \\ 9866 & 14: 10: 49 & 0.1292 \\ 9867 & 14: 10: 50 & 0.1309 \\ 9868 & 14: 10: 51 & 0.1270 \\ 9869 & 14: 10: 52 & 0.1314 \\ 9870 & 14: 10: 53 & 0.1271 \\ 9871 & 14: 10: 54 & 0.1308 \\ 9872 & 14: 10: 55 & 0.1287 \\ 9873 & 14: 10: 56 & 0.1316 \\ 9874 & 14: 10: 57 & 0.1291 \\ 9875 & 14: 10: 58 & 0.1292 \\ 9876 & 14: 10: 59 & 0.1311 \\ 9877 & 14: 11: 00 & 0.1287 \\ 9878 & 14: 11: 01 & 0.1304\end{array}$


Appendix A - Fuel Data Test 1 NIST102505_1.xIs

$\begin{array}{lll}9879 & 14: 11: 02 & 0.1263 \\ 9880 & 14: 11: 03 & 0.1304 \\ 9881 & 14: 11: 04 & 0.1270 \\ 9882 & 14: 11: 05 & 0.1308 \\ 9883 & 14: 11: 06 & 0.1268 \\ 9884 & 14: 11: 07 & 0.1321 \\ 9885 & 14: 11: 08 & 0.1283 \\ 9886 & 14: 11: 09 & 0.1323 \\ 9887 & 14: 11: 10 & 0.1303 \\ 9888 & 14: 11: 11 & 0.1312 \\ 9889 & 14: 11: 12 & 0.1292 \\ 9890 & 14: 11: 13 & 0.1272 \\ 9891 & 14: 11: 14 & 0.1279 \\ 9892 & 14: 11: 15 & 0.1268 \\ 9893 & 14: 11: 16 & 0.1282 \\ 9894 & 14: 11: 17 & 0.1244 \\ 9895 & 14: 11: 18 & 0.1301 \\ 9896 & 14: 11: 19 & 0.1271 \\ 9897 & 14: 11: 20 & 0.1313 \\ 9898 & 14: 11: 21 & 0.1274 \\ 9899 & 14: 11: 22 & 0.1305 \\ 9900 & 14: 11: 23 & 0.1279 \\ 9901 & 14: 11: 24 & 0.1291 \\ 9902 & 14: 11: 25 & 0.1276 \\ 9903 & 14: 11: 26 & 0.1289 \\ 9904 & 14: 11: 27 & 0.1300 \\ 9905 & 14: 11: 28 & 0.1279 \\ 9906 & 14: 11: 29 & 0.1319 \\ 9907 & 14: 11: 30 & 0.1286 \\ 9908 & 14: 11: 31 & 0.1297 \\ 9909 & 14: 11: 32 & 0.1269 \\ 9910 & 14: 11: 33 & 0.1309 \\ 9911 & 14: 11: 34 & 0.1296 \\ 9912 & 14: 11: 35 & 0.1320 \\ 9913 & 14: 11: 36 & 0.1287 \\ 9914 & 14: 11: 37 & 0.1304 \\ 9915 & 14: 11: 38 & 0.1304 \\ 9916 & 14: 11: 39 & 0.1284 \\ 9917 & 14: 11: 40 & 0.1284 \\ 9918 & 14: 11: 41 & 0.1264 \\ 9919 & 14: 11: 42 & 0.1287 \\ 9920 & 14: 11: 43 & 0.1245 \\ 9921 & 14: 11: 44 & 0.1317 \\ 9922 & 14: 11: 45 & 0.1280 \\ 9923 & 14: 11: 46 & 0.1320 \\ 9924 & 14: 11: 47 & 0.1285 \\ 9925 & 14: 11: 48 & 0.1314 \\ 9926 & 14: 11: 49 & 0.1286 \\ 9927 & 14: 11: 50 & 0.1282 \\ 9928 & 14: 11: 51 & 0.1302 \\ 9929 & 14: 11: 52 & 0.1281 \\ 9930 & 14: 11: 53 & 0.1295\end{array}$


Appendix A - Fuel Data Test 1 NIST102505_1.xls

$\begin{array}{lll}9931 & 14: 11: 54 & 0.1247 \\ 9932 & 14: 11: 55 & 0.1298 \\ 9933 & 14: 11: 56 & 0.1255 \\ 9934 & 14: 11: 57 & 0.1302 \\ 9935 & 14: 11: 58 & 0.1282 \\ 9936 & 14: 11: 59 & 0.1337 \\ 9937 & 14: 12: 00 & 0.1290 \\ 9938 & 14: 12: 01 & 0.1305 \\ 9939 & 14: 12: 02 & 0.1280 \\ 9940 & 14: 12: 03 & 0.1279 \\ 9941 & 14: 12: 04 & 0.1297 \\ 9942 & 14: 12: 05 & 0.1251 \\ 9943 & 14: 12: 06 & 0.1292 \\ 9944 & 14: 12: 07 & 0.1256 \\ 9945 & 14: 12: 08 & 0.1307 \\ 9946 & 14: 12: 09 & 0.1266 \\ 9947 & 14: 12: 10 & 0.1313 \\ 9948 & 14: 12: 11 & 0.1268 \\ 9949 & 14: 12: 12 & 0.1284 \\ 9950 & 14: 12: 13 & 0.1261 \\ 9951 & 14: 12: 14 & 0.1283 \\ 9952 & 14: 12: 15 & 0.1275 \\ 9953 & 14: 12: 16 & 0.1255 \\ 9954 & 14: 12: 17 & 0.1295 \\ 9955 & 14: 12: 18 & 0.1252 \\ 9956 & 14: 12: 19 & 0.1288 \\ 9957 & 14: 12: 20 & 0.1263 \\ 9958 & 14: 12: 21 & 0.1296 \\ 9959 & 14: 12: 22 & 0.1261 \\ 9960 & 14: 12: 23 & 0.1301 \\ 9961 & 14: 12: 24 & 0.1276 \\ 9962 & 14: 12: 25 & 0.1277 \\ 9963 & 14: 12: 26 & 0.1264 \\ 9964 & 14: 12: 27 & 0.1265 \\ 9965 & 14: 12: 28 & 0.1287 \\ 9966 & 14: 12: 29 & 0.1256 \\ 9967 & 14: 12: 30 & 0.1300 \\ 9968 & 14: 12: 31 & 0.1262 \\ 9969 & 14: 12: 32 & 0.1301 \\ 9970 & 14: 12: 33 & 0.1266 \\ 9971 & 14: 12: 34 & 0.1316 \\ 9972 & 14: 12: 35 & 0.1278 \\ 9973 & 14: 12: 36 & 0.1290 \\ 9974 & 14: 12: 37 & 0.1287 \\ 9975 & 14: 12: 38 & 0.1276 \\ 9976 & 14: 12: 39 & 0.1297 \\ 9977 & 14: 12: 40 & 0.1260 \\ 9978 & 14: 12: 41 & 0.1293 \\ 9981 & 14: 12: 42 & 0.1251 \\ 9982 & 14: 12: 43 & 0.1289 \\ & 14: 12: 45 & 0.1255 \\ 9.1308\end{array}$


Appendix A - Fuel Data Test 1 NIST102505_1.xIs

$\begin{array}{rll}9983 & 14: 12: 46 & 0.1284 \\ 9984 & 14: 12: 47 & 0.1327 \\ 9985 & 14: 12: 48 & 0.1299 \\ 9986 & 14: 12: 49 & 0.1310 \\ 9987 & 14: 12: 50 & 0.1288 \\ 9988 & 14: 12: 51 & 0.1284 \\ 9989 & 14: 12: 52 & 0.1288 \\ 9990 & 14: 12: 53 & 0.1272 \\ 9991 & 14: 12: 54 & 0.1300 \\ 9992 & 14: 12: 55 & 0.1251 \\ 9993 & 14: 12: 56 & 0.1312 \\ 9994 & 14: 12: 57 & 0.1276 \\ 9995 & 14: 12: 58 & 0.1320 \\ 9996 & 14: 12: 59 & 0.1289 \\ 9997 & 14: 13: 00 & 0.1323 \\ 9998 & 14: 13: 01 & 0.1281 \\ 9999 & 14: 13: 02 & 0.1270 \\ 10000 & 14: 13: 03 & 0.1302 \\ 10001 & 14: 13: 04 & 0.1275 \\ 10002 & 14: 13: 05 & 0.1300 \\ 10003 & 14: 13: 06 & 0.1257 \\ 10004 & 14: 13: 07 & 0.1313 \\ 10005 & 14: 13: 08 & 0.1261 \\ 10006 & 14: 13: 09 & 0.1304 \\ 10007 & 14: 13: 10 & 0.1279 \\ 10008 & 14: 13: 11 & 0.1306 \\ 10009 & 14: 13: 12 & 0.1275 \\ 10010 & 14: 13: 13 & 0.1303 \\ 10011 & 14: 13: 14 & 0.1316 \\ 10012 & 14: 13: 15 & 0.1281 \\ 10013 & 14: 13: 16 & 0.1272 \\ 10014 & 14: 13: 17 & 0.1256 \\ 10015 & 14: 13: 18 & 0.1292 \\ 10016 & 14: 13: 19 & 0.1241 \\ 10017 & 14: 13: 20 & 0.1286 \\ 10018 & 14: 13: 21 & 0.1261 \\ 10019 & 14: 13: 22 & 0.1301 \\ 10020 & 14: 13: 23 & 0.1255 \\ 10021 & 14: 13: 24 & 0.1304 \\ 10022 & 14: 13: 25 & 0.1276 \\ 10023 & 14: 13: 26 & 0.1290 \\ 10024 & 14: 13: 27 & 0.1258 \\ 10025 & 14: 13: 28 & 0.1260 \\ 10026 & 14: 13: 29 & 0.1267 \\ 10027 & 14: 13: 30 & 0.1260 \\ 10028 & 14: 13: 31 & 0.1310 \\ 10029 & 14: 13: 32 & 0.1251 \\ 10030 & 14: 13: 33 & 0.1288 \\ 10031 & 14: 13: 34 & 0.1269 \\ 10032 & 14: 13: 35 & 0.1308 \\ 10033 & 14: 13: 36 & 0.1263 \\ 10034 & 14: 13: 37 & 0.1309\end{array}$


Appendix A - Fuel Data Test 1 NIST102505_1.xIs

$\begin{array}{lll}10035 & 14: 13: 38 & 0.1293 \\ 10036 & 14: 13: 39 & 0.1303 \\ 10037 & 14: 13: 40 & 0.1284 \\ 10038 & 14: 13: 41 & 0.1290 \\ 10039 & 14: 13: 42 & 0.1280 \\ 10040 & 14: 13: 43 & 0.1265 \\ 10041 & 14: 13: 44 & 0.1320 \\ 10042 & 14: 13: 45 & 0.1275 \\ 10043 & 14: 13: 46 & 0.1308 \\ 10044 & 14: 13: 47 & 0.1292 \\ 10045 & 14: 13: 48 & 0.1336 \\ 10046 & 14: 13: 49 & 0.1298 \\ 10047 & 14: 13: 50 & 0.1318 \\ 10048 & 14: 13: 51 & 0.1312 \\ 10049 & 14: 13: 52 & 0.1318 \\ 10050 & 14: 13: 53 & 0.1282 \\ 10051 & 14: 13: 54 & 0.1288 \\ 10052 & 14: 13: 55 & 0.1284 \\ 10053 & 14: 13: 56 & 0.1236 \\ 10054 & 14: 13: 57 & 0.1303 \\ 10055 & 14: 13: 58 & 0.1261 \\ 10056 & 14: 13: 59 & 0.1311 \\ 10057 & 14: 14: 00 & 0.1272 \\ 10058 & 14: 14: 01 & 0.1326 \\ 10059 & 14: 14: 02 & 0.1269 \\ 10060 & 14: 14: 03 & 0.1291 \\ 10061 & 14: 14: 04 & 0.1304 \\ 10062 & 14: 14: 05 & 0.1290 \\ 10063 & 14: 14: 06 & 0.1275 \\ 10064 & 14: 14: 07 & 0.1261 \\ 10065 & 14: 14: 08 & 0.1285 \\ 10066 & 14: 14: 09 & 0.1242 \\ 10067 & 14: 14: 10 & 0.1278 \\ 10068 & 14: 14: 11 & 0.1248 \\ 10069 & 14: 14: 12 & 0.1292 \\ 10070 & 14: 14: 13 & 0.1274 \\ 10071 & 14: 14: 14 & 0.1326 \\ 10072 & 14: 14: 15 & 0.1291 \\ 10073 & 14: 14: 16 & 0.1301 \\ 10074 & 14: 14: 17 & 0.1269 \\ 10075 & 14: 14: 18 & 0.1277 \\ 10076 & 14: 14: 19 & 0.1272 \\ 10077 & 14: 14: 20 & 0.1261 \\ 10078 & 14: 14: 21 & 0.1302 \\ 10079 & 14: 14: 22 & 0.1271 \\ 10080 & 14: 14: 23 & 0.1307 \\ 10081 & 14: 14: 24 & 0.1270 \\ 10082 & 14: 14: 25 & 0.1321 \\ 10083 & 14: 14: 26 & 0.1283 \\ 10084 & 14: 14: 27 & 0.1310 \\ 10085 & 14: 14: 28 & 0.1298 \\ 10086 & 14: 14: 29 & 0.1304\end{array}$


Appendix A - Fuel Data Test 1 NIST102505_1.xls

$\begin{array}{lll}10087 & 14: 14: 30 & 0.1283 \\ 10088 & 14: 14: 31 & 0.1276 \\ 10089 & 14: 14: 32 & 0.1304 \\ 10090 & 14: 14: 33 & 0.1279 \\ 10091 & 14: 14: 34 & 0.1294 \\ 10092 & 14: 14: 35 & 0.1243 \\ 10093 & 14: 14: 36 & 0.1300 \\ 10094 & 14: 14: 37 & 0.1278 \\ 10095 & 14: 14: 38 & 0.1315 \\ 10096 & 14: 14: 39 & 0.1264 \\ 10097 & 14: 14: 40 & 0.1315 \\ 10098 & 14: 14: 41 & 0.1271 \\ 10099 & 14: 14: 42 & 0.1288 \\ 10100 & 14: 14: 43 & 0.1282 \\ 10101 & 14: 14: 44 & 0.1270 \\ 10102 & 14: 14: 45 & 0.1297 \\ 10103 & 14: 14: 46 & 0.1254 \\ 10104 & 14: 14: 47 & 0.1310 \\ 10105 & 14: 14: 48 & 0.1248 \\ 10106 & 14: 14: 49 & 0.1288 \\ 10107 & 14: 14: 50 & 0.1249 \\ 10108 & 14: 14: 51 & 0.1305 \\ 10109 & 14: 14: 52 & 0.1284 \\ 10110 & 14: 14: 53 & 0.1308 \\ 10111 & 14: 14: 54 & 0.1312 \\ 10112 & 14: 14: 55 & 0.1310 \\ 10113 & 14: 14: 56 & 0.1275 \\ 10114 & 14: 14: 57 & 0.1269 \\ 10115 & 14: 14: 58 & 0.1291 \\ 10116 & 14: 14: 59 & 0.1262 \\ 10117 & 14: 15: 00 & 0.1307 \\ 10118 & 14: 15: 01 & 0.1274 \\ 10119 & 14: 15: 02 & 0.1317 \\ 10120 & 14: 15: 03 & 0.1271 \\ 10121 & 14: 15: 04 & 0.1323 \\ 10122 & 14: 15: 05 & 0.1280 \\ 10123 & 14: 15: 06 & 0.1299 \\ 10124 & 14: 15: 07 & 0.1286 \\ 10125 & 14: 15: 08 & 0.1291 \\ 10126 & 14: 15: 09 & 0.1300 \\ 10127 & 14: 15: 10 & 0.1273 \\ 10128 & 14: 15: 11 & 0.1305 \\ 10129 & 14: 15: 12 & 0.1265 \\ 10130 & 14: 15: 13 & 0.1295 \\ 10131 & 14: 15: 14 & 0.1266 \\ 10132 & 14: 15: 15 & 0.1300 \\ 10133 & 14: 15: 16 & 0.1298 \\ 10134 & 14: 15: 17 & 0.1317 \\ 10135 & 14: 15: 18 & 0.1287 \\ 10136 & 14: 15: 19 & 0.1309 \\ 10137 & 14: 15: 20 & 0.1306 \\ 10138 & 14: 15: 21 & 0.1297\end{array}$


Appendix A - Fuel Data Test 1 NIST102505_1.xls

$\begin{array}{lll}10139 & 14: 15: 22 & 0.1298 \\ 10140 & 14: 15: 23 & 0.1284 \\ 10141 & 14: 15: 24 & 0.1295 \\ 10142 & 14: 15: 25 & 0.1260 \\ 10143 & 14: 15: 26 & 0.1314 \\ 10144 & 14: 15: 27 & 0.1283 \\ 10145 & 14: 15: 28 & 0.1317 \\ 10146 & 14: 15: 29 & 0.1259 \\ 10147 & 14: 15: 30 & 0.1316 \\ 10148 & 14: 15: 31 & 0.1271 \\ 10149 & 14: 15: 32 & 0.1290 \\ 10150 & 14: 15: 33 & 0.1271 \\ 10151 & 14: 15: 34 & 0.1291 \\ 10152 & 14: 15: 35 & 0.1287 \\ 10153 & 14: 15: 36 & 0.1281 \\ 10154 & 14: 15: 37 & 0.1310 \\ 10155 & 14: 15: 38 & 0.1276 \\ 10156 & 14: 15: 39 & 0.1317 \\ 10157 & 14: 15: 40 & 0.1272 \\ 10158 & 14: 15: 41 & 0.1336 \\ 10159 & 14: 15: 42 & 0.1297 \\ 10160 & 14: 15: 43 & 0.1323 \\ 10161 & 14: 15: 44 & 0.1311 \\ 10162 & 14: 15: 45 & 0.1323 \\ 10163 & 14: 15: 46 & 0.1300 \\ 10164 & 14: 15: 47 & 0.1285 \\ 10165 & 14: 15: 48 & 0.1320 \\ 10166 & 14: 15: 49 & 0.1275 \\ 10167 & 14: 15: 50 & 0.1301 \\ 10168 & 14: 15: 51 & 0.1271 \\ 10169 & 14: 15: 52 & 0.1315 \\ 10170 & 14: 15: 53 & 0.1280 \\ 10171 & 14: 15: 54 & 0.1310 \\ 10172 & 14: 15: 55 & 0.1304 \\ 10173 & 14: 15: 56 & 0.1322 \\ 10174 & 14: 15: 57 & 0.1299 \\ 10175 & 14: 15: 58 & 0.1306 \\ 10176 & 14: 15: 59 & 0.1303 \\ 10177 & 14: 16: 00 & 0.1276 \\ 10178 & 14: 16: 01 & 0.1289 \\ 10179 & 14: 16: 02 & 0.1265 \\ 10180 & 14: 16: 03 & 0.1301 \\ 10181 & 14: 16: 04 & 0.1252 \\ 10182 & 14: 16: 05 & 0.1296 \\ 10183 & 14: 16: 06 & 0.1297 \\ 10184 & 14: 16: 07 & 0.1316 \\ 10185 & 14: 16: 08 & 0.1270 \\ 10186 & 14: 16: 09 & 0.1306 \\ 10187 & 14: 16: 10 & 0.1301 \\ 10188 & 14: 16: 11 & 0.1301 \\ 10189 & 14: 16: 12 & 0.1296 \\ 10190 & 14: 16: 13 & 0.1277\end{array}$


Appendix A - Fuel Data Test 1 NIST102505_1.xIs

$\begin{array}{lll}10191 & 14: 16: 14 & 0.1301 \\ 10192 & 14: 16: 15 & 0.1275 \\ 10193 & 14: 16: 16 & 0.1307 \\ 10194 & 14: 16: 17 & 0.1274 \\ 10195 & 14: 16: 18 & 0.1307 \\ 10196 & 14: 16: 19 & 0.1275 \\ 10197 & 14: 16: 20 & 0.1292 \\ 10198 & 14: 16: 21 & 0.1292 \\ 10199 & 14: 16: 22 & 0.1308 \\ 10200 & 14: 16: 23 & 0.1271 \\ 10201 & 14: 16: 24 & 0.1282 \\ 10202 & 14: 16: 25 & 0.1303 \\ 10203 & 14: 16: 26 & 0.1272 \\ 10204 & 14: 16: 27 & 0.1295 \\ 10205 & 14: 16: 28 & 0.1260 \\ 10206 & 14: 16: 29 & 0.1298 \\ 10207 & 14: 16: 30 & 0.1264 \\ 10208 & 14: 16: 31 & 0.1297 \\ 10209 & 14: 16: 32 & 0.1278 \\ 10210 & 14: 16: 33 & 0.1311 \\ 10211 & 14: 16: 34 & 0.1275 \\ 10212 & 14: 16: 35 & 0.1291 \\ 10213 & 14: 16: 36 & 0.1287 \\ 10214 & 14: 16: 37 & 0.1268 \\ 10215 & 14: 16: 38 & 0.1294 \\ 10216 & 14: 16: 39 & 0.1262 \\ 10217 & 14: 16: 40 & 0.1298 \\ 10218 & 14: 16: 41 & 0.1276 \\ 10219 & 14: 16: 42 & 0.1312 \\ 10220 & 14: 16: 43 & 0.1280 \\ 10221 & 14: 16: 44 & 0.1313 \\ 10222 & 14: 16: 45 & 0.1286 \\ 10223 & 14: 16: 46 & 0.1308 \\ 10224 & 14: 16: 47 & 0.1319 \\ 10225 & 14: 16: 48 & 0.1306 \\ 10226 & 14: 16: 49 & 0.1273 \\ 10227 & 14: 16: 50 & 0.1265 \\ 10228 & 14: 16: 51 & 0.1301 \\ 10229 & 14: 16: 52 & 0.1273 \\ 10230 & 14: 16: 53 & 0.1292 \\ 10231 & 14: 16: 54 & 0.1263 \\ 10232 & 14: 16: 55 & 0.1301 \\ 10233 & 14: 16: 56 & 0.1277 \\ 10234 & 14: 16: 57 & 0.1310 \\ 10235 & 14: 16: 58 & 0.1297 \\ 10236 & 14: 16: 59 & 0.1306 \\ 10237 & 14: 17: 00 & 0.1269 \\ 10238 & 14: 17: 01 & 0.1297 \\ 10239 & 14: 17: 02 & 0.1306 \\ 10240 & 14: 17: 03 & 0.1281 \\ 10241 & 14: 17: 04 & 0.1294 \\ 10242 & 14: 17: 05 & 0.1273\end{array}$


Appendix A - Fuel Data Test 1 NIST102505_1.xls

$\begin{array}{lll}10243 & 14: 17: 06 & 0.1307 \\ 10244 & 14: 17: 07 & 0.1258 \\ 10245 & 14: 17: 08 & 0.1303 \\ 10246 & 14: 17: 09 & 0.1294 \\ 10247 & 14: 17: 10 & 0.1339 \\ 10248 & 14: 17: 11 & 0.1301 \\ 10249 & 14: 17: 12 & 0.1314 \\ 10250 & 14: 17: 13 & 0.1323 \\ 10251 & 14: 17: 14 & 0.1311 \\ 10252 & 14: 17: 15 & 0.1302 \\ 10253 & 14: 17: 16 & 0.1278 \\ 10254 & 14: 17: 17 & 0.1299 \\ 10255 & 14: 17: 18 & 0.1262 \\ 10256 & 14: 17: 19 & 0.1302 \\ 10257 & 14: 17: 20 & 0.1276 \\ 10258 & 14: 17: 21 & 0.1322 \\ 10259 & 14: 17: 22 & 0.1281 \\ 10260 & 14: 17: 23 & 0.1332 \\ 10261 & 14: 17: 24 & 0.1291 \\ 10262 & 14: 17: 25 & 0.1300 \\ 10263 & 14: 17: 26 & 0.1310 \\ 10264 & 14: 17: 27 & 0.1299 \\ 10265 & 14: 17: 28 & 0.1318 \\ 10266 & 14: 17: 29 & 0.1277 \\ 10267 & 14: 17: 30 & 0.1313 \\ 10268 & 14: 17: 31 & 0.1269 \\ 10269 & 14: 17: 32 & 0.1303 \\ 10270 & 14: 17: 33 & 0.1275 \\ 10271 & 14: 17: 34 & 0.1311 \\ 10272 & 14: 17: 35 & 0.1279 \\ 10273 & 14: 17: 36 & 0.1328 \\ 10274 & 14: 17: 37 & 0.1320 \\ 10275 & 14: 17: 38 & 0.1315 \\ 10276 & 14: 17: 39 & 0.1283 \\ 10277 & 14: 17: 40 & 0.1279 \\ 10278 & 14: 17: 41 & 0.1293 \\ 10279 & 14: 17: 42 & 0.1275 \\ 10280 & 14: 17: 43 & 0.1312 \\ 10281 & 14: 17: 44 & 0.1280 \\ 10282 & 14: 17: 45 & 0.1320 \\ 10283 & 14: 17: 46 & 0.1274 \\ 10284 & 14: 17: 47 & 0.1329 \\ 10285 & 14: 17: 48 & 0.1262 \\ 10286 & 14: 17: 49 & 0.1291 \\ 10287 & 14: 17: 50 & 0.1296 \\ 10288 & 14: 17: 51 & 0.1286 \\ 10289 & 14: 17: 52 & 0.1284 \\ 10290 & 14: 17: 53 & 0.1268 \\ 10291 & 14: 17: 54 & 0.1299 \\ 10292 & 14: 17: 55 & 0.1256 \\ 10293 & 14: 17: 56 & 0.1275 \\ 10294 & 14: 17: 57 & 0.1250\end{array}$


Appendix A - Fuel Data Test 1 NIST102505_1.xls

$\begin{array}{lll}10295 & 14: 17: 58 & 0.1290 \\ 10296 & 14: 17: 59 & 0.1253 \\ 10297 & 14: 18: 00 & 0.1300 \\ 10298 & 14: 18: 01 & 0.1302 \\ 10299 & 14: 18: 02 & 0.1320 \\ 10300 & 14: 18: 03 & 0.1279 \\ 10301 & 14: 18: 04 & 0.1296 \\ 10302 & 14: 18: 05 & 0.1292 \\ 10303 & 14: 18: 06 & 0.1286 \\ 10304 & 14: 18: 07 & 0.1306 \\ 10305 & 14: 18: 08 & 0.1293 \\ 10306 & 14: 18: 09 & 0.1307 \\ 10307 & 14: 18: 10 & 0.1251 \\ 10308 & 14: 18: 11 & 0.1303 \\ 10309 & 14: 18: 12 & 0.1263 \\ 10310 & 14: 18: 13 & 0.1300 \\ 10311 & 14: 18: 14 & 0.1275 \\ 10312 & 14: 18: 15 & 0.1299 \\ 10313 & 14: 18: 16 & 0.1281 \\ 10314 & 14: 18: 17 & 0.1277 \\ 10315 & 14: 18: 18 & 0.1298 \\ 10316 & 14: 18: 19 & 0.1257 \\ 10317 & 14: 18: 20 & 0.1293 \\ 10318 & 14: 18: 21 & 0.1268 \\ 10319 & 14: 18: 22 & 0.1316 \\ 10320 & 14: 18: 23 & 0.1265 \\ 10321 & 14: 18: 24 & 0.1304 \\ 10322 & 14: 18: 25 & 0.1275 \\ 10323 & 14: 18: 26 & 0.1309 \\ 10324 & 14: 18: 27 & 0.1300 \\ 10325 & 14: 18: 28 & 0.1304 \\ 10326 & 14: 18: 29 & 0.1283 \\ 10327 & 14: 18: 30 & 0.1280 \\ 10328 & 14: 18: 31 & 0.1297 \\ 10329 & 14: 18: 32 & 0.1256 \\ 10330 & 14: 18: 33 & 0.1299 \\ 10331 & 14: 18: 34 & 0.1244 \\ 10332 & 14: 18: 35 & 0.1286 \\ 10333 & 14: 18: 36 & 0.1251 \\ 10334 & 14: 18: 37 & 0.1295 \\ 10335 & 14: 18: 38 & 0.1265 \\ 10336 & 14: 18: 39 & 0.1291 \\ 10337 & 14: 18: 40 & 0.1283 \\ 10338 & 14: 18: 41 & 0.1283 \\ 10339 & 14: 18: 42 & 0.1257 \\ 10340 & 14: 18: 43 & 0.1245 \\ 10341 & 14: 18: 44 & 0.1296 \\ 10342 & 14: 18: 45 & 0.1259 \\ 10343 & 14: 18: 46 & 0.1283 \\ 10344 & 14: 18: 47 & 0.1266 \\ 10345 & 14: 18: 48 & 0.1305 \\ 10346 & 14: 18: 49 & 0.1273\end{array}$


Appendix A - Fuel Data Test 1 NIST102505_1.xls

$\begin{array}{lll}10347 & 14: 18: 50 & 0.1310 \\ 10348 & 14: 18: 51 & 0.1304 \\ 10349 & 14: 18: 52 & 0.1324 \\ 10350 & 14: 18: 53 & 0.1282 \\ 10351 & 14: 18: 54 & 0.1295 \\ 10352 & 14: 18: 55 & 0.1298 \\ 10353 & 14: 18: 56 & 0.1296 \\ 10354 & 14: 18: 57 & 0.1316 \\ 10355 & 14: 18: 58 & 0.1299 \\ 10356 & 14: 18: 59 & 0.1319 \\ 10357 & 14: 19: 00 & 0.1263 \\ 10358 & 14: 19: 01 & 0.1312 \\ 10359 & 14: 19: 02 & 0.1287 \\ 10360 & 14: 19: 03 & 0.1324 \\ 10361 & 14: 19: 04 & 0.1288 \\ 10362 & 14: 19: 05 & 0.1317 \\ 10363 & 14: 19: 06 & 0.1300 \\ 10364 & 14: 19: 07 & 0.1296 \\ 10365 & 14: 19: 08 & 0.1323 \\ 10366 & 14: 19: 09 & 0.1284 \\ 10367 & 14: 19: 10 & 0.1300 \\ 10368 & 14: 19: 11 & 0.1248 \\ 10369 & 14: 19: 12 & 0.1309 \\ 10370 & 14: 19: 13 & 0.1262 \\ 10371 & 14: 19: 14 & 0.1310 \\ 10372 & 14: 19: 15 & 0.1283 \\ 10373 & 14: 19: 16 & 0.1324 \\ 10374 & 14: 19: 17 & 0.1281 \\ 10375 & 14: 19: 18 & 0.1288 \\ 10376 & 14: 19: 19 & 0.1290 \\ 10377 & 14: 19: 20 & 0.1280 \\ 10378 & 14: 19: 21 & 0.1291 \\ 10379 & 14: 19: 22 & 0.1262 \\ 10380 & 14: 19: 23 & 0.1294 \\ 10381 & 14: 19: 24 & 0.1250 \\ 10382 & 14: 19: 25 & 0.1296 \\ 10383 & 14: 19: 26 & 0.1280 \\ 10384 & 14: 19: 27 & 0.1304 \\ 10385 & 14: 19: 28 & 0.1271 \\ 10386 & 14: 19: 29 & 0.1303 \\ 10387 & 14: 19: 30 & 0.1302 \\ 10388 & 14: 19: 31 & 0.1297 \\ 10389 & 14: 19: 32 & 0.1276 \\ 10390 & 14: 19: 33 & 0.1263 \\ 10391 & 14: 19: 34 & 0.1289 \\ 10392 & 14: 19: 35 & 0.1255 \\ 10393 & 14: 19: 36 & 0.1271 \\ 10394 & 14: 19: 37 & 0.1248 \\ 10395 & 14: 19: 38 & 0.1301 \\ 10396 & 14: 19: 39 & 0.1266 \\ 10397 & 14: 19: 40 & 0.1307 \\ 10398 & 14: 19: 41 & 0.1283\end{array}$


Appendix A - Fuel Data Test 1 NIST102505_1.xIs

$\begin{array}{lll}10399 & 14: 19: 42 & 0.1308 \\ 10400 & 14: 19: 43 & 0.1286 \\ 10401 & 14: 19: 44 & 0.1304 \\ 10402 & 14: 19: 45 & 0.1280 \\ 10403 & 14: 19: 46 & 0.1259 \\ 10404 & 14: 19: 47 & 0.1300 \\ 10405 & 14: 19: 48 & 0.1267 \\ 10406 & 14: 19: 49 & 0.1291 \\ 10407 & 14: 19: 50 & 0.1243 \\ 10408 & 14: 19: 51 & 0.1296 \\ 10409 & 14: 19: 52 & 0.1264 \\ 10410 & 14: 19: 53 & 0.1288 \\ 10411 & 14: 19: 54 & 0.1276 \\ 10412 & 14: 19: 55 & 0.1303 \\ 10413 & 14: 19: 56 & 0.1278 \\ 10414 & 14: 19: 57 & 0.1283 \\ 10415 & 14: 19: 58 & 0.1284 \\ 10416 & 14: 19: 59 & 0.1258 \\ 10417 & 14: 20: 00 & 0.1272 \\ 10418 & 14: 20: 01 & 0.1240 \\ 10419 & 14: 20: 02 & 0.1294 \\ 10420 & 14: 20: 03 & 0.1246 \\ 10421 & 14: 20: 04 & 0.1297 \\ 10422 & 14: 20: 05 & 0.1284 \\ 10423 & 14: 20: 06 & 0.1318 \\ 10424 & 14: 20: 07 & 0.1274 \\ 10425 & 14: 20: 08 & 0.1299 \\ 10426 & 14: 20: 09 & 0.1259 \\ 10427 & 14: 20: 10 & 0.1258 \\ 10428 & 14: 20: 11 & 0.1279 \\ 10429 & 14: 20: 12 & 0.1259 \\ 10430 & 14: 20: 13 & 0.1290 \\ 10431 & 14: 20: 14 & 0.1242 \\ 10432 & 14: 20: 15 & 0.1301 \\ 10433 & 14: 20: 16 & 0.1251 \\ 10434 & 14: 20: 17 & 0.1283 \\ 10435 & 14: 20: 18 & 0.1275 \\ 10436 & 14: 20: 19 & 0.1305 \\ 10437 & 14: 20: 20 & 0.1282 \\ 10438 & 14: 20: 21 & 0.1278 \\ 10439 & 14: 20: 22 & 0.1299 \\ 10440 & 14: 20: 23 & 0.1274 \\ 10441 & 14: 20: 24 & 0.1305 \\ 10442 & 14: 20: 25 & 0.1256 \\ 10443 & 14: 20: 26 & 0.1311 \\ 10444 & 14: 20: 27 & 0.1269 \\ 10445 & 14: 20: 28 & 0.1306 \\ 10446 & 14: 20: 29 & 0.1272 \\ 10447 & 14: 20: 30 & 0.1310 \\ 10448 & 14: 20: 31 & 0.1280 \\ 10449 & 14: 20: 32 & 0.1289 \\ 10450 & 14: 20: 33 & 0.1286\end{array}$


Appendix A - Fuel Data Test 1 NIST102505_1.xIs

$\begin{array}{lll}10451 & 14: 20: 34 & 0.1284 \\ 10452 & 14: 20: 35 & 0.1290 \\ 10453 & 14: 20: 36 & 0.1274 \\ 10454 & 14: 20: 37 & 0.1298 \\ 10455 & 14: 20: 38 & 0.1258 \\ 10456 & 14: 20: 39 & 0.1309 \\ 10457 & 14: 20: 40 & 0.1288 \\ 10458 & 14: 20: 41 & 0.1300 \\ 10459 & 14: 20: 42 & 0.1251 \\ 10460 & 14: 20: 43 & 0.1308 \\ 10461 & 14: 20: 44 & 0.1284 \\ 10462 & 14: 20: 45 & 0.1300 \\ 10463 & 14: 20: 46 & 0.1271 \\ 10464 & 14: 20: 47 & 0.1273 \\ 10465 & 14: 20: 48 & 0.1283 \\ 10466 & 14: 20: 49 & 0.1256 \\ 10467 & 14: 20: 50 & 0.1294 \\ 10468 & 14: 20: 51 & 0.1265 \\ 10469 & 14: 20: 52 & 0.1294 \\ 10470 & 14: 20: 53 & 0.1246 \\ 10471 & 14: 20: 54 & 0.1318 \\ 10472 & 14: 20: 55 & 0.1287 \\ 10473 & 14: 20: 56 & 0.1312 \\ 10474 & 14: 20: 57 & 0.1283 \\ 10475 & 14: 20: 58 & 0.1295 \\ 10476 & 14: 20: 59 & 0.1265 \\ 10477 & 14: 21: 00 & 0.1250 \\ 10478 & 14: 21: 01 & 0.1290 \\ 10479 & 14: 21: 02 & 0.1272 \\ 10480 & 14: 21: 03 & 0.1310 \\ 10481 & 14: 21: 04 & 0.1251 \\ 10482 & 14: 21: 05 & 0.1316 \\ 10483 & 14: 21: 06 & 0.1253 \\ 10484 & 14: 21: 07 & 0.1292 \\ 10485 & 14: 21: 08 & 0.1278 \\ 10486 & 14: 21: 09 & 0.1306 \\ 10487 & 14: 21: 10 & 0.1282 \\ 10488 & 14: 21: 11 & 0.1269 \\ 10489 & 14: 21: 12 & 0.1300 \\ 10490 & 14: 21: 13 & 0.1290 \\ 10491 & 14: 21: 14 & 0.1305 \\ 10492 & 14: 21: 15 & 0.1259 \\ 10493 & 14: 21: 16 & 0.1296 \\ 10494 & 14: 21: 17 & 0.1241 \\ 10495 & 14: 21: 18 & 0.1290 \\ 10496 & 14: 21: 19 & 0.1284 \\ 10497 & 14: 21: 20 & 0.1318 \\ 10498 & 14: 21: 21 & 0.1281 \\ 10499 & 14: 21: 22 & 0.1302 \\ 10500 & 14: 21: 23 & 0.1280 \\ 10501 & 14: 21: 24 & 0.1280 \\ 10502 & 14: 21: 25 & 0.1266\end{array}$


Appendix A - Fuel Data Test 1 NIST102505_1.xIs

$\begin{array}{lll}10503 & 14: 21: 26 & 0.1251 \\ 10504 & 14: 21: 27 & 0.1282 \\ 10505 & 14: 21: 28 & 0.1250 \\ 10506 & 14: 21: 29 & 0.1309 \\ 10507 & 14: 21: 30 & 0.1266 \\ 10508 & 14: 21: 31 & 0.1300 \\ 10509 & 14: 21: 32 & 0.1273 \\ 10510 & 14: 21: 33 & 0.1317 \\ 10511 & 14: 21: 34 & 0.1278 \\ 10512 & 14: 21: 35 & 0.1297 \\ 10513 & 14: 21: 36 & 0.1289 \\ 10514 & 14: 21: 37 & 0.1282 \\ 10515 & 14: 21: 38 & 0.1287 \\ 10516 & 14: 21: 39 & 0.1279 \\ 10517 & 14: 21: 40 & 0.1289 \\ 10518 & 14: 21: 41 & 0.1246 \\ 10519 & 14: 21: 42 & 0.1296 \\ 10520 & 14: 21: 43 & 0.1285 \\ 10521 & 14: 21: 44 & 0.1319 \\ 10522 & 14: 21: 45 & 0.1281 \\ 10523 & 14: 21: 46 & 0.1314 \\ 10524 & 14: 21: 47 & 0.1315 \\ 10525 & 14: 21: 48 & 0.1301 \\ 10526 & 14: 21: 49 & 0.1275 \\ 10527 & 14: 21: 50 & 0.1261 \\ 10528 & 14: 21: 51 & 0.1297 \\ 10529 & 14: 21: 52 & 0.1263 \\ 10530 & 14: 21: 53 & 0.1298 \\ 10531 & 14: 21: 54 & 0.1254 \\ 10532 & 14: 21: 55 & 0.1290 \\ 10533 & 14: 21: 56 & 0.1247 \\ 10534 & 14: 21: 57 & 0.1305 \\ 10535 & 14: 21: 58 & 0.1287 \\ 10536 & 14: 21: 59 & 0.1299 \\ 10537 & 14: 22: 00 & 0.1278 \\ 10538 & 14: 22: 01 & 0.1294 \\ 10539 & 14: 22: 02 & 0.1292 \\ 10540 & 14: 22: 03 & 0.1281 \\ 10541 & 14: 22: 04 & 0.1291 \\ 10542 & 14: 22: 05 & 0.1276 \\ 10543 & 14: 22: 06 & 0.1296 \\ 10544 & 14: 22: 07 & 0.1243 \\ 10545 & 14: 22: 08 & 0.1305 \\ 10546 & 14: 22: 09 & 0.1277 \\ 10547 & 14: 22: 10 & 0.1314 \\ 10548 & 14: 22: 11 & 0.1260 \\ 10549 & 14: 22: 12 & 0.1304 \\ 10550 & 14: 22: 13 & 0.1281 \\ 10551 & 14: 22: 14 & 0.1282 \\ 10552 & 14: 22: 15 & 0.1276 \\ 10553 & 14: 22: 16 & 0.1286 \\ 10554 & 14: 22: 17 & 0.1299\end{array}$


Appendix A - Fuel Data Test 1 NIST102505_1.xIs

$\begin{array}{lll}10555 & 14: 22: 18 & 0.1268 \\ 10556 & 14: 22: 19 & 0.1314 \\ 10557 & 14: 22: 20 & 0.1279 \\ 10558 & 14: 22: 21 & 0.1299 \\ 10559 & 14: 22: 22 & 0.1259 \\ 10560 & 14: 22: 23 & 0.1326 \\ 10561 & 14: 22: 24 & 0.1297 \\ 10562 & 14: 22: 25 & 0.1310 \\ 10563 & 14: 22: 26 & 0.1274 \\ 10564 & 14: 22: 27 & 0.1287 \\ 10565 & 14: 22: 28 & 0.1290 \\ 10566 & 14: 22: 29 & 0.1277 \\ 10567 & 14: 22: 30 & 0.1295 \\ 10568 & 14: 22: 31 & 0.1269 \\ 10569 & 14: 22: 32 & 0.1291 \\ 10570 & 14: 22: 33 & 0.1240 \\ 10571 & 14: 22: 34 & 0.1304 \\ 10572 & 14: 22: 35 & 0.1294 \\ 10573 & 14: 22: 36 & 0.1314 \\ 10574 & 14: 22: 37 & 0.1266 \\ 10575 & 14: 22: 38 & 0.1305 \\ 10576 & 14: 22: 39 & 0.1283 \\ 10577 & 14: 22: 40 & 0.1278 \\ 10578 & 14: 22: 41 & 0.1286 \\ 10579 & 14: 22: 42 & 0.1272 \\ 10580 & 14: 22: 43 & 0.1280 \\ 10581 & 14: 22: 44 & 0.1231 \\ 10582 & 14: 22: 45 & 0.1291 \\ 10583 & 14: 22: 46 & 0.1248 \\ 10584 & 14: 22: 47 & 0.1295 \\ 10585 & 14: 22: 48 & 0.1276 \\ 10586 & 14: 22: 49 & 0.1318 \\ 10587 & 14: 22: 50 & 0.1273 \\ 10588 & 14: 22: 51 & 0.1303 \\ 10589 & 14: 22: 52 & 0.1275 \\ 10590 & 14: 22: 53 & 0.1266 \\ 10591 & 14: 22: 54 & 0.1281 \\ 10592 & 14: 22: 55 & 0.1274 \\ 10593 & 14: 22: 56 & 0.1285 \\ 10594 & 14: 22: 57 & 0.1250 \\ 10595 & 14: 22: 58 & 0.1284 \\ 10596 & 14: 22: 59 & 0.1271 \\ 10597 & 14: 23: 00 & 0.1305 \\ 10598 & 14: 23: 01 & 0.1271 \\ 10599 & 14: 23: 02 & 0.1289 \\ 10600 & 14: 23: 03 & 0.1280 \\ 10601 & 14: 23: 04 & 0.1282 \\ 10602 & 14: 23: 05 & 0.1265 \\ 10603 & 14: 23: 06 & 0.1254 \\ 10604 & 14: 23: 07 & 0.1299 \\ 10605 & 14: 23: 08 & 0.1267 \\ 10606 & 14: 23: 09 & 0.1301\end{array}$


Appendix A - Fuel Data Test 1 NIST102505_1.xIs

$\begin{array}{lll}10607 & 14: 23: 10 & 0.1268 \\ 10608 & 14: 23: 11 & 0.1290 \\ 10609 & 14: 23: 12 & 0.1252 \\ 10610 & 14: 23: 13 & 0.1297 \\ 10611 & 14: 23: 14 & 0.1294 \\ 10612 & 14: 23: 15 & 0.1292 \\ 10613 & 14: 23: 16 & 0.1285 \\ 10614 & 14: 23: 17 & 0.1261 \\ 10615 & 14: 23: 18 & 0.1329 \\ 10616 & 14: 23: 19 & 0.1283 \\ 10617 & 14: 23: 20 & 0.1293 \\ 10618 & 14: 23: 21 & 0.1244 \\ 10619 & 14: 23: 22 & 0.1312 \\ 10620 & 14: 23: 23 & 0.1276 \\ 10621 & 14: 23: 24 & 0.1304 \\ 10622 & 14: 23: 25 & 0.1268 \\ 10623 & 14: 23: 26 & 0.1310 \\ 10624 & 14: 23: 27 & 0.1295 \\ 10625 & 14: 23: 28 & 0.1290 \\ 10626 & 14: 23: 29 & 0.1291 \\ 10627 & 14: 23: 30 & 0.1286 \\ 10628 & 14: 23: 31 & 0.1295 \\ 10629 & 14: 23: 32 & 0.1269 \\ 10630 & 14: 23: 33 & 0.1292 \\ 10631 & 14: 23: 34 & 0.1245 \\ 10632 & 14: 23: 35 & 0.1295 \\ 10633 & 14: 23: 36 & 0.1272 \\ 10634 & 14: 23: 37 & 0.1312 \\ 10635 & 14: 23: 38 & 0.1273 \\ 10636 & 14: 23: 39 & 0.1325 \\ 10637 & 14: 23: 40 & 0.1297 \\ 10638 & 14: 23: 41 & 0.1299 \\ 10639 & 14: 23: 42 & 0.1301 \\ 10640 & 14: 23: 43 & 0.1280 \\ 10641 & 14: 23: 44 & 0.1296 \\ 10642 & 14: 23: 45 & 0.1252 \\ 10643 & 14: 23: 46 & 0.1301 \\ 10644 & 14: 23: 47 & 0.1260 \\ 10645 & 14: 23: 48 & 0.1318 \\ 10646 & 14: 23: 49 & 0.1280 \\ 10647 & 14: 23: 50 & 0.1328 \\ 10648 & 14: 23: 51 & 0.1288 \\ 10649 & 14: 23: 52 & 0.1305 \\ 10650 & 14: 23: 53 & 0.1284 \\ 10651 & 14: 23: 54 & 0.1283 \\ 10652 & 14: 23: 55 & 0.1272 \\ 10653 & 14: 23: 56 & 0.1249 \\ 10654 & 14: 23: 57 & 0.1308 \\ 10655 & 14: 23: 58 & 0.1280 \\ 10656 & 14: 23: 59 & 0.1314 \\ 10657 & 14: 24: 00 & 0.1264 \\ 10658 & 14: 24: 01 & 0.1318\end{array}$


Appendix A - Fuel Data Test 1 NIST102505_1.xIs

$\begin{array}{lll}10659 & 14: 24: 02 & 0.1274 \\ 10660 & 14: 24: 03 & 0.1308 \\ 10661 & 14: 24: 04 & 0.1292 \\ 10662 & 14: 24: 05 & 0.1295 \\ 10663 & 14: 24: 06 & 0.1293 \\ 10664 & 14: 24: 07 & 0.1287 \\ 10665 & 14: 24: 08 & 0.1318 \\ 10666 & 14: 24: 09 & 0.1275 \\ 10667 & 14: 24: 10 & 0.1290 \\ 10668 & 14: 24: 11 & 0.1256 \\ 10669 & 14: 24: 12 & 0.1295 \\ 10670 & 14: 24: 13 & 0.1243 \\ 10671 & 14: 24: 14 & 0.1312 \\ 10672 & 14: 24: 15 & 0.1280 \\ 10673 & 14: 24: 16 & 0.1313 \\ 10674 & 14: 24: 17 & 0.1290 \\ 10675 & 14: 24: 18 & 0.1296 \\ 10676 & 14: 24: 19 & 0.1271 \\ 10677 & 14: 24: 20 & 0.1263 \\ 10678 & 14: 24: 21 & 0.1303 \\ 10679 & 14: 24: 22 & 0.1273 \\ 10680 & 14: 24: 23 & 0.1291 \\ 10681 & 14: 24: 24 & 0.1261 \\ 10682 & 14: 24: 25 & 0.1306 \\ 10683 & 14: 24: 26 & 0.1267 \\ 10684 & 14: 24: 27 & 0.1292 \\ 10685 & 14: 24: 28 & 0.1294 \\ 10686 & 14: 24: 29 & 0.1296 \\ 10687 & 14: 24: 30 & 0.1271 \\ 10688 & 14: 24: 31 & 0.1278 \\ 10689 & 14: 24: 32 & 0.1280 \\ 10690 & 14: 24: 33 & 0.1270 \\ 10691 & 14: 24: 34 & 0.1285 \\ 10692 & 14: 24: 35 & 0.1263 \\ 10693 & 14: 24: 36 & 0.1288 \\ 10694 & 14: 24: 37 & 0.1261 \\ 10695 & 14: 24: 38 & 0.1308 \\ 10696 & 14: 24: 39 & 0.1297 \\ 10697 & 14: 24: 40 & 0.1327 \\ 10698 & 14: 24: 41 & 0.1274 \\ 10699 & 14: 24: 42 & 0.1306 \\ 10700 & 14: 24: 43 & 0.1305 \\ 10701 & 14: 24: 44 & 0.1295 \\ 10702 & 14: 24: 45 & 0.1282 \\ 10703 & 14: 24: 46 & 0.1280 \\ 10704 & 14: 24: 47 & 0.1305 \\ 10705 & 14: 24: 48 & 0.1260 \\ 10706 & 14: 24: 49 & 0.1280 \\ 10707 & 14: 24: 50 & 0.1246 \\ 10708 & 14: 24: 51 & 0.1297 \\ 10709 & 14: 24: 52 & 0.1263 \\ 10710 & 14: 24: 53 & 0.1315\end{array}$


Appendix A - Fuel Data Test 1 NIST102505_1.xls

$\begin{array}{lll}10711 & 14: 24: 54 & 0.1297 \\ 10712 & 14: 24: 55 & 0.1317 \\ 10713 & 14: 24: 56 & 0.1282 \\ 10714 & 14: 24: 57 & 0.1293 \\ 10715 & 14: 24: 58 & 0.1283 \\ 10716 & 14: 24: 59 & 0.1259 \\ 10717 & 14: 25: 00 & 0.1302 \\ 10718 & 14: 25: 01 & 0.1265 \\ 10719 & 14: 25: 02 & 0.1301 \\ 10720 & 14: 25: 03 & 0.1261 \\ 10721 & 14: 25: 04 & 0.1310 \\ 10722 & 14: 25: 05 & 0.1281 \\ 10723 & 14: 25: 06 & 0.1303 \\ 10724 & 14: 25: 07 & 0.1286 \\ 10725 & 14: 25: 08 & 0.1302 \\ 10726 & 14: 25: 09 & 0.1275 \\ 10727 & 14: 25: 10 & 0.1262 \\ 10728 & 14: 25: 11 & 0.1283 \\ 10729 & 14: 25: 12 & 0.1286 \\ 10730 & 14: 25: 13 & 0.1297 \\ 10731 & 14: 25: 14 & 0.1245 \\ 10732 & 14: 25: 15 & 0.1309 \\ 10733 & 14: 25: 16 & 0.1264 \\ 10734 & 14: 25: 17 & 0.1303 \\ 10735 & 14: 25: 18 & 0.1278 \\ 10736 & 14: 25: 19 & 0.1316 \\ 10737 & 14: 25: 20 & 0.1276 \\ 10738 & 14: 25: 21 & 0.1275 \\ 10739 & 14: 25: 22 & 0.1294 \\ 10740 & 14: 25: 23 & 0.1278 \\ 10741 & 14: 25: 24 & 0.1303 \\ 10742 & 14: 25: 25 & 0.1260 \\ 10743 & 14: 25: 26 & 0.1302 \\ 10744 & 14: 25: 27 & 0.1260 \\ 10745 & 14: 25: 28 & 0.1301 \\ 10746 & 14: 25: 29 & 0.1260 \\ 10747 & 14: 25: 30 & 0.1314 \\ 10748 & 14: 25: 31 & 0.1277 \\ 10749 & 14: 25: 32 & 0.1313 \\ 10750 & 14: 25: 33 & 0.1280 \\ 10751 & 14: 25: 34 & 0.1290 \\ 10752 & 14: 25: 35 & 0.1267 \\ 10753 & 14: 25: 36 & 0.1258 \\ 10754 & 14: 25: 37 & 0.1310 \\ 10755 & 14: 25: 38 & 0.1269 \\ 10756 & 14: 25: 39 & 0.1294 \\ 10757 & 14: 25: 40 & 0.1240 \\ 10758 & 14: 25: 41 & 0.1304 \\ 10759 & 14: 25: 42 & 0.1258 \\ 10760 & 14: 25: 43 & 0.1296 \\ 10761 & 14: 25: 44 & 0.1277 \\ 10762 & 14: 25: 45 & 0.1295\end{array}$


Appendix A - Fuel Data Test 1 NIST102505_1.xIs

$\begin{array}{lll}10763 & 14: 25: 46 & 0.1281 \\ 10764 & 14: 25: 47 & 0.1270 \\ 10765 & 14: 25: 48 & 0.1287 \\ 10766 & 14: 25: 49 & 0.1264 \\ 10767 & 14: 25: 50 & 0.1277 \\ 10768 & 14: 25: 51 & 0.1247 \\ 10769 & 14: 25: 52 & 0.1282 \\ 10770 & 14: 25: 53 & 0.1229 \\ 10771 & 14: 25: 54 & 0.1294 \\ 10772 & 14: 25: 55 & 0.1261 \\ 10773 & 14: 25: 56 & 0.1307 \\ 10774 & 14: 25: 57 & 0.1260 \\ 10775 & 14: 25: 58 & 0.1296 \\ 10776 & 14: 25: 59 & 0.1277 \\ 10777 & 14: 26: 00 & 0.1272 \\ 10778 & 14: 26: 01 & 0.1270 \\ 10779 & 14: 26: 02 & 0.1263 \\ 10780 & 14: 26: 03 & 0.1279 \\ 10781 & 14: 26: 04 & 0.1246 \\ 10782 & 14: 26: 05 & 0.1310 \\ 10783 & 14: 26: 06 & 0.1277 \\ 10784 & 14: 26: 07 & 0.1313 \\ 10785 & 14: 26: 08 & 0.1283 \\ 10786 & 14: 26: 09 & 0.1322 \\ 10787 & 14: 26: 10 & 0.1273 \\ 10788 & 14: 26: 11 & 0.1286 \\ 10789 & 14: 26: 12 & 0.1290 \\ 10790 & 14: 26: 13 & 0.1279 \\ 10791 & 14: 26: 14 & 0.1284 \\ 10792 & 14: 26: 15 & 0.1271 \\ 10793 & 14: 26: 16 & 0.1279 \\ 10794 & 14: 26: 17 & 0.1240 \\ 10795 & 14: 26: 18 & 0.1308 \\ 10796 & 14: 26: 19 & 0.1272 \\ 10797 & 14: 26: 20 & 0.1313 \\ 10798 & 14: 26: 21 & 0.1271 \\ 10799 & 14: 26: 22 & 0.1313 \\ 10800 & 14: 26: 23 & 0.1275 \\ 10801 & 14: 26: 24 & 0.1278 \\ 10802 & 14: 26: 25 & 0.1282 \\ 10803 & 14: 26: 26 & 0.1267 \\ 10804 & 14: 26: 27 & 0.1294 \\ 10805 & 14: 26: 28 & 0.1262 \\ 10806 & 14: 26: 29 & 0.1303 \\ 10807 & 14: 26: 30 & 0.1255 \\ 10808 & 14: 26: 31 & 0.1300 \\ 10809 & 14: 26: 32 & 0.1276 \\ 10810 & 14: 26: 33 & 0.1311 \\ 10811 & 14: 26: 34 & 0.1266 \\ 10812 & 14: 26: 35 & 0.1318 \\ 10813 & 14: 26: 36 & 0.1302 \\ 10814 & 14: 26: 37 & 0.1294\end{array}$


Appendix A - Fuel Data Test 1 NIST102505_1.xIs

$\begin{array}{lll}10815 & 14: 26: 38 & 0.1268 \\ 10816 & 14: 26: 39 & 0.1262 \\ 10817 & 14: 26: 40 & 0.1280 \\ 10818 & 14: 26: 41 & 0.1240 \\ 10819 & 14: 26: 42 & 0.1283 \\ 10820 & 14: 26: 43 & 0.1260 \\ 10821 & 14: 26: 44 & 0.1297 \\ 10822 & 14: 26: 45 & 0.1255 \\ 10823 & 14: 26: 46 & 0.1298 \\ 10824 & 14: 26: 47 & 0.1278 \\ 10825 & 14: 26: 48 & 0.1299 \\ 10826 & 14: 26: 49 & 0.1256 \\ 10827 & 14: 26: 50 & 0.1272 \\ 10828 & 14: 26: 51 & 0.1264 \\ 10829 & 14: 26: 52 & 0.1242 \\ 10830 & 14: 26: 53 & 0.1292 \\ 10831 & 14: 26: 54 & 0.1289 \\ 10832 & 14: 26: 55 & 0.1306 \\ 10833 & 14: 26: 56 & 0.1254 \\ 10834 & 14: 26: 57 & 0.1307 \\ 10835 & 14: 26: 58 & 0.1281 \\ 10836 & 14: 26: 59 & 0.1323 \\ 10837 & 14: 27: 00 & 0.1281 \\ 10838 & 14: 27: 01 & 0.1313 \\ 10839 & 14: 27: 02 & 0.1290 \\ 10840 & 14: 27: 03 & 0.1289 \\ 10841 & 14: 27: 04 & 0.1326 \\ 10842 & 14: 27: 05 & 0.1290 \\ 10843 & 14: 27: 06 & 0.1294 \\ 10844 & 14: 27: 07 & 0.1239 \\ 10845 & 14: 27: 08 & 0.1300 \\ 10846 & 14: 27: 09 & 0.1254 \\ 10847 & 14: 27: 10 & 0.1304 \\ 10848 & 14: 27: 11 & 0.1277 \\ 10849 & 14: 27: 12 & 0.1318 \\ 10850 & 14: 27: 13 & 0.1280 \\ 10851 & 14: 27: 14 & 0.1291 \\ 10852 & 14: 27: 15 & 0.1282 \\ 10853 & 14: 27: 16 & 0.1284 \\ 10854 & 14: 27: 17 & 0.1287 \\ 10855 & 14: 27: 18 & 0.1258 \\ 10856 & 14: 27: 19 & 0.1314 \\ 10857 & 14: 27: 20 & 0.1260 \\ 10858 & 14: 27: 21 & 0.1299 \\ 10859 & 14: 27: 22 & 0.1271 \\ 10860 & 14: 27: 23 & 0.1298 \\ 10861 & 14: 27: 24 & 0.1263 \\ 10862 & 14: 27: 25 & 0.1294 \\ 10863 & 14: 27: 26 & 0.1295 \\ 10864 & 14: 27: 27 & 0.1292 \\ 10865 & 14: 27: 28 & 0.1280 \\ 10866 & 14: 27: 29 & 0.1275\end{array}$


Appendix A - Fuel Data Test 1 NIST102505_1.xIs

$\begin{array}{lll}10867 & 14: 27: 30 & 0.1290 \\ 10868 & 14: 27: 31 & 0.1259 \\ 10869 & 14: 27: 32 & 0.1293 \\ 10870 & 14: 27: 33 & 0.1260 \\ 10871 & 14: 27: 34 & 0.1300 \\ 10872 & 14: 27: 35 & 0.1260 \\ 10873 & 14: 27: 36 & 0.1314 \\ 10874 & 14: 27: 37 & 0.1273 \\ 10875 & 14: 27: 38 & 0.1289 \\ 10876 & 14: 27: 39 & 0.1251 \\ 10877 & 14: 27: 40 & 0.1265 \\ 10878 & 14: 27: 41 & 0.1271 \\ 10879 & 14: 27: 42 & 0.1252 \\ 10880 & 14: 27: 43 & 0.1293 \\ 10881 & 14: 27: 44 & 0.1247 \\ 10882 & 14: 27: 45 & 0.1279 \\ 10883 & 14: 27: 46 & 0.1247 \\ 10884 & 14: 27: 47 & 0.1324 \\ 10885 & 14: 27: 48 & 0.1291 \\ 10886 & 14: 27: 49 & 0.1325 \\ 10887 & 14: 27: 50 & 0.1302 \\ 10888 & 14: 27: 51 & 0.1308 \\ 10889 & 14: 27: 52 & 0.1280 \\ 10890 & 14: 27: 53 & 0.1260 \\ 10891 & 14: 27: 54 & 0.1299 \\ 10892 & 14: 27: 55 & 0.1266 \\ 10893 & 14: 27: 56 & 0.1289 \\ 10894 & 14: 27: 57 & 0.1234 \\ 10895 & 14: 27: 58 & 0.1305 \\ 10896 & 14: 27: 59 & 0.1275 \\ 10897 & 14: 28: 00 & 0.1311 \\ 10898 & 14: 28: 01 & 0.1282 \\ 10899 & 14: 28: 02 & 0.1300 \\ 10900 & 14: 28: 03 & 0.1278 \\ 10901 & 14: 28: 04 & 0.1260 \\ 10902 & 14: 28: 05 & 0.1267 \\ 10903 & 14: 28: 06 & 0.1259 \\ 10904 & 14: 28: 07 & 0.1280 \\ 10905 & 14: 28: 08 & 0.1244 \\ 10906 & 14: 28: 09 & 0.1297 \\ 10907 & 14: 28: 10 & 0.1251 \\ 10908 & 14: 28: 11 & 0.1293 \\ 10909 & 14: 28: 12 & 0.1256 \\ 10910 & 14: 28: 13 & 0.1299 \\ 10911 & 14: 28: 14 & 0.1284 \\ 10912 & 14: 28: 15 & 0.1294 \\ 10913 & 14: 28: 16 & 0.1301 \\ 10914 & 14: 28: 17 & 0.1299 \\ 10915 & 14: 28: 18 & 0.1299 \\ 10916 & 14: 28: 19 & 0.1271 \\ 10917 & 14: 28: 20 & 0.1311 \\ 10918 & 14: 28: 21 & 0.1260\end{array}$


Appendix A - Fuel Data Test 1 NIST102505_1.xIs

$\begin{array}{lll}10919 & 14: 28: 22 & 0.1291 \\ 10920 & 14: 28: 23 & 0.1258 \\ 10921 & 14: 28: 24 & 0.1306 \\ 10922 & 14: 28: 25 & 0.1267 \\ 10923 & 14: 28: 26 & 0.1294 \\ 10924 & 14: 28: 27 & 0.1282 \\ 10925 & 14: 28: 28 & 0.1294 \\ 10926 & 14: 28: 29 & 0.1273 \\ 10927 & 14: 28: 30 & 0.1296 \\ 10928 & 14: 28: 31 & 0.1300 \\ 10929 & 14: 28: 32 & 0.1287 \\ 10930 & 14: 28: 33 & 0.1300 \\ 10931 & 14: 28: 34 & 0.1249 \\ 10932 & 14: 28: 35 & 0.1293 \\ 10933 & 14: 28: 36 & 0.1275 \\ 10934 & 14: 28: 37 & 0.1310 \\ 10935 & 14: 28: 38 & 0.1254 \\ 10936 & 14: 28: 39 & 0.1302 \\ 10937 & 14: 28: 40 & 0.1284 \\ 10938 & 14: 28: 41 & 0.1285 \\ 10939 & 14: 28: 42 & 0.1269 \\ 10940 & 14: 28: 43 & 0.1272 \\ 10941 & 14: 28: 44 & 0.1284 \\ 10942 & 14: 28: 45 & 0.1259 \\ 10943 & 14: 28: 46 & 0.1281 \\ 10944 & 14: 28: 47 & 0.1246 \\ 10945 & 14: 28: 48 & 0.1289 \\ 10946 & 14: 28: 49 & 0.1248 \\ 10947 & 14: 28: 50 & 0.1321 \\ 10948 & 14: 28: 51 & 0.1284 \\ 10949 & 14: 28: 52 & 0.1297 \\ 10950 & 14: 28: 53 & 0.1278 \\ 10951 & 14: 28: 54 & 0.1283 \\ 10952 & 14: 28: 55 & 0.1266 \\ 10953 & 14: 28: 56 & 0.1243 \\ 10954 & 14: 28: 57 & 0.1297 \\ 10955 & 14: 28: 58 & 0.1295 \\ 10956 & 14: 28: 59 & 0.1307 \\ 10957 & 14: 29: 00 & 0.1263 \\ 10958 & 14: 29: 01 & 0.1287 \\ 10959 & 14: 29: 02 & 0.1284 \\ 10960 & 14: 29: 03 & 0.1315 \\ 10961 & 14: 29: 04 & 0.1288 \\ 10962 & 14: 29: 05 & 0.1291 \\ 10963 & 14: 29: 06 & 0.1285 \\ 10964 & 14: 29: 07 & 0.1279 \\ 10965 & 14: 29: 08 & 0.1274 \\ 10966 & 14: 29: 09 & 0.1257 \\ 10967 & 14: 29: 10 & 0.1299 \\ 10968 & 14: 29: 11 & 0.1261 \\ 10969 & 14: 29: 12 & 0.1303 \\ 10970 & 14: 29: 13 & 0.1273\end{array}$


Appendix A - Fuel Data Test 1 NIST102505_1.xls

$\begin{array}{lll}10971 & 14: 29: 14 & 0.1314 \\ 10972 & 14: 29: 15 & 0.1274 \\ 10973 & 14: 29: 16 & 0.1307 \\ 10974 & 14: 29: 17 & 0.1301 \\ 10975 & 14: 29: 18 & 0.1297 \\ 10976 & 14: 29: 19 & 0.1272 \\ 10977 & 14: 29: 20 & 0.1289 \\ 10978 & 14: 29: 21 & 0.1300 \\ 10979 & 14: 29: 22 & 0.1278 \\ 10980 & 14: 29: 23 & 0.1299 \\ 10981 & 14: 29: 24 & 0.1268 \\ 10982 & 14: 29: 25 & 0.1308 \\ 10983 & 14: 29: 26 & 0.1257 \\ 10984 & 14: 29: 27 & 0.1316 \\ 10985 & 14: 29: 28 & 0.1283 \\ 10986 & 14: 29: 29 & 0.1313 \\ 10987 & 14: 29: 30 & 0.1280 \\ 10988 & 14: 29: 31 & 0.1306 \\ 10989 & 14: 29: 32 & 0.1284 \\ 10990 & 14: 29: 33 & 0.1269 \\ 10991 & 14: 29: 34 & 0.1293 \\ 10992 & 14: 29: 35 & 0.1272 \\ 10993 & 14: 29: 36 & 0.1280 \\ 10994 & 14: 29: 37 & 0.1242 \\ 10995 & 14: 29: 38 & 0.1313 \\ 10996 & 14: 29: 39 & 0.1276 \\ 10997 & 14: 29: 40 & 0.1305 \\ 10998 & 14: 29: 41 & 0.1260 \\ 10999 & 14: 29: 42 & 0.1301 \\ 11000 & 14: 29: 43 & 0.1279 \\ 11001 & 14: 29: 44 & 0.1281 \\ 11002 & 14: 29: 45 & 0.1261 \\ 11003 & 14: 29: 46 & 0.1267 \\ 11004 & 14: 29: 47 & 0.1293 \\ 11005 & 14: 29: 48 & 0.1263 \\ 11006 & 14: 29: 49 & 0.1310 \\ 11007 & 14: 29: 50 & 0.1281 \\ 11008 & 14: 29: 51 & 0.1313 \\ 11009 & 14: 29: 52 & 0.1280 \\ 11010 & 14: 29: 53 & 0.1331 \\ 11011 & 14: 29: 54 & 0.1291 \\ 11012 & 14: 29: 55 & 0.1319 \\ 11013 & 14: 29: 56 & 0.1300 \\ 11014 & 14: 29: 57 & 0.1309 \\ 11015 & 14: 29: 58 & 0.1276 \\ 11016 & 14: 29: 59 & 0.1258 \\ 11017 & 14: 30: 00 & 0.1301 \\ 11018 & 14: 30: 01 & 0.1274 \\ 11019 & 14: 30: 02 & 0.1305 \\ 11020 & 14: 30: 03 & 0.1246 \\ 11021 & 14: 30: 04 & 0.1314 \\ 11022 & 14: 30: 05 & 0.1286\end{array}$


Appendix A - Fuel Data Test 1 NIST102505_1.xls

$\begin{array}{lll}11023 & 14: 30: 06 & 0.1318 \\ 11024 & 14: 30: 07 & 0.1270 \\ 11025 & 14: 30: 08 & 0.1299 \\ 11026 & 14: 30: 09 & 0.1261 \\ 11027 & 14: 30: 10 & 0.1266 \\ 11028 & 14: 30: 11 & 0.1283 \\ 11029 & 14: 30: 12 & 0.1259 \\ 11030 & 14: 30: 13 & 0.1292 \\ 11031 & 14: 30: 14 & 0.1242 \\ 11032 & 14: 30: 15 & 0.1299 \\ 11033 & 14: 30: 16 & 0.1256 \\ 11034 & 14: 30: 17 & 0.1294 \\ 11035 & 14: 30: 18 & 0.1268 \\ 11036 & 14: 30: 19 & 0.1307 \\ 11037 & 14: 30: 20 & 0.1267 \\ 11038 & 14: 30: 21 & 0.1279 \\ 11039 & 14: 30: 22 & 0.1286 \\ 11040 & 14: 30: 23 & 0.1275 \\ 11041 & 14: 30: 24 & 0.1272 \\ 11042 & 14: 30: 25 & 0.1249 \\ 11043 & 14: 30: 26 & 0.1277 \\ 11044 & 14: 30: 27 & 0.1229 \\ 11045 & 14: 30: 28 & 0.1283 \\ 11046 & 14: 30: 29 & 0.1288 \\ 11047 & 14: 30: 30 & 0.1314 \\ 11048 & 14: 30: 31 & 0.1280 \\ 11049 & 14: 30: 32 & 0.1312 \\ 11050 & 14: 30: 33 & 0.1310 \\ 11051 & 14: 30: 34 & 0.1309 \\ 11052 & 14: 30: 35 & 0.1285 \\ 11053 & 14: 30: 36 & 0.1289 \\ 11054 & 14: 30: 37 & 0.1310 \\ 11055 & 14: 30: 38 & 0.1272 \\ 11056 & 14: 30: 39 & 0.1284 \\ 11057 & 14: 30: 40 & 0.1246 \\ 11058 & 14: 30: 41 & 0.1292 \\ 11059 & 14: 30: 42 & 0.1255 \\ 11060 & 14: 30: 43 & 0.1317 \\ 11061 & 14: 30: 44 & 0.1287 \\ 11062 & 14: 30: 45 & 0.1315 \\ 11063 & 14: 30: 46 & 0.1285 \\ 11064 & 14: 30: 47 & 0.1299 \\ 11065 & 14: 30: 48 & 0.1276 \\ 11066 & 14: 30: 49 & 0.1257 \\ 11067 & 14: 30: 50 & 0.1285 \\ 11068 & 14: 30: 51 & 0.1271 \\ 11069 & 14: 30: 52 & 0.1283 \\ 11070 & 14: 30: 53 & 0.1234 \\ 11071 & 14: 30: 54 & 0.1308 \\ 11072 & 14: 30: 55 & 0.1270 \\ 11073 & 14: 30: 56 & 0.1304 \\ 11074 & 14: 30: 57 & 0.1281\end{array}$


Appendix A - Fuel Data Test 1 NIST102505_1.xls

$\begin{array}{lll}11075 & 14: 30: 58 & 0.1309 \\ 11076 & 14: 30: 59 & 0.1281 \\ 11077 & 14: 31: 00 & 0.1274 \\ 11078 & 14: 31: 01 & 0.1269 \\ 11079 & 14: 31: 02 & 0.1263 \\ 11080 & 14: 31: 03 & 0.1286 \\ 11081 & 14: 31: 04 & 0.1245 \\ 11082 & 14: 31: 05 & 0.1301 \\ 11083 & 14: 31: 06 & 0.1245 \\ 11084 & 14: 31: 07 & 0.1299 \\ 11085 & 14: 31: 08 & 0.1270 \\ 11086 & 14: 31: 09 & 0.1329 \\ 11087 & 14: 31: 10 & 0.1297 \\ 11088 & 14: 31: 11 & 0.1312 \\ 11089 & 14: 31: 12 & 0.1265 \\ 11090 & 14: 31: 13 & 0.1287 \\ 11091 & 14: 31: 14 & 0.1277 \\ 11092 & 14: 31: 15 & 0.1261 \\ 11093 & 14: 31: 16 & 0.1293 \\ 11094 & 14: 31: 17 & 0.1261 \\ 11095 & 14: 31: 18 & 0.1290 \\ 11096 & 14: 31: 19 & 0.1248 \\ 11097 & 14: 31: 20 & 0.1317 \\ 11098 & 14: 31: 21 & 0.1281 \\ 11099 & 14: 31: 22 & 0.1317 \\ 11100 & 14: 31: 23 & 0.1276 \\ 11101 & 14: 31: 24 & 0.1301 \\ 11102 & 14: 31: 25 & 0.1265 \\ 11103 & 14: 31: 26 & 0.1263 \\ 11104 & 14: 31: 27 & 0.1269 \\ 11105 & 14: 31: 28 & 0.1263 \\ 11106 & 14: 31: 29 & 0.1301 \\ 11107 & 14: 31: 30 & 0.1261 \\ 11108 & 14: 31: 31 & 0.1324 \\ 11109 & 14: 31: 32 & 0.1276 \\ 11110 & 14: 31: 33 & 0.1309 \\ 11111 & 14: 31: 34 & 0.1273 \\ 11112 & 14: 31: 35 & 0.1324 \\ 11113 & 14: 31: 36 & 0.1281 \\ 11114 & 14: 31: 37 & 0.1295 \\ 11115 & 14: 31: 38 & 0.1278 \\ 11116 & 14: 31: 39 & 0.1281 \\ 11117 & 14: 31: 40 & 0.1292 \\ 11118 & 14: 31: 41 & 0.1253 \\ 11119 & 14: 31: 42 & 0.1293 \\ 11120 & 14: 31: 43 & 0.1267 \\ 11121 & 14: 31: 44 & 0.1303 \\ 11122 & 14: 31: 45 & 0.1260 \\ 11123 & 14: 31: 46 & 0.1309 \\ 11124 & 14: 31: 47 & 0.1279 \\ 11125 & 14: 31: 48 & 0.1315 \\ 11126 & 14: 31: 49 & 0.1286\end{array}$


Appendix A - Fuel Data Test 1 NIST102505_1.xls

$\begin{array}{lll}11127 & 14: 31: 50 & 0.1303 \\ 11128 & 14: 31: 51 & 0.1257 \\ 11129 & 14: 31: 52 & 0.1254 \\ 11130 & 14: 31: 53 & 0.1292 \\ 11131 & 14: 31: 54 & 0.1276 \\ 11132 & 14: 31: 55 & 0.1286 \\ 11133 & 14: 31: 56 & 0.1234 \\ 11134 & 14: 31: 57 & 0.1295 \\ 11135 & 14: 31: 58 & 0.1246 \\ 11136 & 14: 31: 59 & 0.1288 \\ 11137 & 14: 32: 00 & 0.1295 \\ 11138 & 14: 32: 01 & 0.1332 \\ 11139 & 14: 32: 02 & 0.1296 \\ 11140 & 14: 32: 03 & 0.1292 \\ 11141 & 14: 32: 04 & 0.1280 \\ 11142 & 14: 32: 05 & 0.1267 \\ 11143 & 14: 32: 06 & 0.1290 \\ 11144 & 14: 32: 07 & 0.1264 \\ 11145 & 14: 32: 08 & 0.1300 \\ 11146 & 14: 32: 09 & 0.1255 \\ 11147 & 14: 32: 10 & 0.1301 \\ 11148 & 14: 32: 11 & 0.1272 \\ 11149 & 14: 32: 12 & 0.1319 \\ 11150 & 14: 32: 13 & 0.1262 \\ 11151 & 14: 32: 14 & 0.1292 \\ 11152 & 14: 32: 15 & 0.1300 \\ 11153 & 14: 32: 16 & 0.1287 \\ 11154 & 14: 32: 17 & 0.1288 \\ 11155 & 14: 32: 18 & 0.1252 \\ 11156 & 14: 32: 19 & 0.1311 \\ 11157 & 14: 32: 20 & 0.1266 \\ 11158 & 14: 32: 21 & 0.1298 \\ 11159 & 14: 32: 22 & 0.1249 \\ 11160 & 14: 32: 23 & 0.1290 \\ 11161 & 14: 32: 24 & 0.1256 \\ 11162 & 14: 32: 25 & 0.1294 \\ 11163 & 14: 32: 26 & 0.1280 \\ 11164 & 14: 32: 27 & 0.1285 \\ 11165 & 14: 32: 28 & 0.1288 \\ 11166 & 14: 32: 29 & 0.1287 \\ 11167 & 14: 32: 30 & 0.1308 \\ 11168 & 14: 32: 31 & 0.1275 \\ 11169 & 14: 32: 32 & 0.1298 \\ 11170 & 14: 32: 33 & 0.1244 \\ 11171 & 14: 32: 34 & 0.1307 \\ 11172 & 14: 32: 35 & 0.1266 \\ 11173 & 14: 32: 36 & 0.1296 \\ 11174 & 14: 32: 37 & 0.1265 \\ 11175 & 14: 32: 38 & 0.1301 \\ 11176 & 14: 32: 39 & 0.1283 \\ 11177 & 14: 32: 40 & 0.1298 \\ 11178 & 14: 32: 41 & 0.1283\end{array}$


Appendix A - Fuel Data Test 1 NIST102505_1.xIs

$\begin{array}{lll}11179 & 14: 32: 42 & 0.1275 \\ 11180 & 14: 32: 43 & 0.1288 \\ 11181 & 14: 32: 44 & 0.1253 \\ 11182 & 14: 32: 45 & 0.1303 \\ 11183 & 14: 32: 46 & 0.1254 \\ 11184 & 14: 32: 47 & 0.1289 \\ 11185 & 14: 32: 48 & 0.1246 \\ 11186 & 14: 32: 49 & 0.1317 \\ 11187 & 14: 32: 50 & 0.1291 \\ 11188 & 14: 32: 51 & 0.1334 \\ 11189 & 14: 32: 52 & 0.1314 \\ 11190 & 14: 32: 53 & 0.1321 \\ 11191 & 14: 32: 54 & 0.1286 \\ 11192 & 14: 32: 55 & 0.1280 \\ 11193 & 14: 32: 56 & 0.1305 \\ 11194 & 14: 32: 57 & 0.1278 \\ 11195 & 14: 32: 58 & 0.1299 \\ 11196 & 14: 32: 59 & 0.1256 \\ 11197 & 14: 33: 00 & 0.1320 \\ 11198 & 14: 33: 01 & 0.1273 \\ 11199 & 14: 33: 02 & 0.1316 \\ 11200 & 14: 33: 03 & 0.1304 \\ 11201 & 14: 33: 04 & 0.1326 \\ 11202 & 14: 33: 05 & 0.1295 \\ 11203 & 14: 33: 06 & 0.1301 \\ 11204 & 14: 33: 07 & 0.1294 \\ 11205 & 14: 33: 08 & 0.1269 \\ 11206 & 14: 33: 09 & 0.1298 \\ 11207 & 14: 33: 10 & 0.1269 \\ 11208 & 14: 33: 11 & 0.1302 \\ 11209 & 14: 33: 12 & 0.1255 \\ 11210 & 14: 33: 13 & 0.1291 \\ 11211 & 14: 33: 14 & 0.1266 \\ 11212 & 14: 33: 15 & 0.1303 \\ 11213 & 14: 33: 16 & 0.1265 \\ 11214 & 14: 33: 17 & 0.1290 \\ 11215 & 14: 33: 18 & 0.1290 \\ 11216 & 14: 33: 19 & 0.1283 \\ 11217 & 14: 33: 20 & 0.1275 \\ 11218 & 14: 33: 21 & 0.1264 \\ 11219 & 14: 33: 22 & 0.1299 \\ 11220 & 14: 33: 23 & 0.1259 \\ 11221 & 14: 33: 24 & 0.1282 \\ 11222 & 14: 33: 25 & 0.1264 \\ 11223 & 14: 33: 26 & 0.1304 \\ 11224 & 14: 33: 27 & 0.1255 \\ 11225 & 14: 33: 28 & 0.1313 \\ 11226 & 14: 33: 29 & 0.1275 \\ 11227 & 14: 33: 30 & 0.1301 \\ 11228 & 14: 33: 31 & 0.1279 \\ 11229 & 14: 33: 32 & 0.1298 \\ 11230 & 14: 33: 33 & 0.1303\end{array}$


Appendix A - Fuel Data Test 1 NIST102505_1.xls

$\begin{array}{lll}11231 & 14: 33: 34 & 0.1273 \\ 11232 & 14: 33: 35 & 0.1293 \\ 11233 & 14: 33: 36 & 0.1262 \\ 11234 & 14: 33: 37 & 0.1306 \\ 11235 & 14: 33: 38 & 0.1256 \\ 11236 & 14: 33: 39 & 0.1282 \\ 11237 & 14: 33: 40 & 0.1256 \\ 11238 & 14: 33: 41 & 0.1298 \\ 11239 & 14: 33: 42 & 0.1273 \\ 11240 & 14: 33: 43 & 0.1287 \\ 11241 & 14: 33: 44 & 0.1289 \\ 11242 & 14: 33: 45 & 0.1281 \\ 11243 & 14: 33: 46 & 0.1284 \\ 11244 & 14: 33: 47 & 0.1261 \\ 11245 & 14: 33: 48 & 0.1297 \\ 11246 & 14: 33: 49 & 0.1270 \\ 11247 & 14: 33: 50 & 0.1305 \\ 11248 & 14: 33: 51 & 0.1268 \\ 11249 & 14: 33: 52 & 0.1305 \\ 11250 & 14: 33: 53 & 0.1287 \\ 11251 & 14: 33: 54 & 0.1295 \\ 11252 & 14: 33: 55 & 0.1299 \\ 11253 & 14: 33: 56 & 0.1306 \\ 11254 & 14: 33: 57 & 0.1271 \\ 11255 & 14: 33: 58 & 0.1270 \\ 11256 & 14: 33: 59 & 0.1301 \\ 11257 & 14: 34: 00 & 0.1261 \\ 11258 & 14: 34: 01 & 0.1280 \\ 11259 & 14: 34: 02 & 0.1241 \\ 11260 & 14: 34: 03 & 0.1294 \\ 11261 & 14: 34: 04 & 0.1265 \\ 11262 & 14: 34: 05 & 0.1297 \\ 11263 & 14: 34: 06 & 0.1290 \\ 11264 & 14: 34: 07 & 0.1304 \\ 11265 & 14: 34: 08 & 0.1285 \\ 11266 & 14: 34: 09 & 0.1274 \\ 11267 & 14: 34: 10 & 0.1297 \\ 11268 & 14: 34: 11 & 0.1278 \\ 11269 & 14: 34: 12 & 0.1301 \\ 11270 & 14: 34: 13 & 0.1283 \\ 11271 & 14: 34: 14 & 0.1301 \\ 11272 & 14: 34: 15 & 0.1233 \\ 11273 & 14: 34: 16 & 0.1287 \\ 11274 & 14: 34: 17 & 0.1274 \\ 11275 & 14: 34: 18 & 0.1302 \\ 11276 & 14: 34: 19 & 0.1256 \\ 11277 & 14: 34: 20 & 0.1311 \\ 11278 & 14: 34: 21 & 0.1284 \\ 11279 & 14: 34: 22 & 0.1284 \\ 11280 & 14: 34: 23 & 0.1297 \\ 11281 & 14: 34: 24 & 0.1278 \\ 11282 & 14: 34: 25 & 0.1295\end{array}$


Appendix A - Fuel Data Test 1 NIST102505_1.xls

$\begin{array}{lll}11283 & 14: 34: 26 & 0.1261 \\ 11284 & 14: 34: 27 & 0.1304 \\ 11285 & 14: 34: 28 & 0.1247 \\ 11286 & 14: 34: 29 & 0.1286 \\ 11287 & 14: 34: 30 & 0.1278 \\ 11288 & 14: 34: 31 & 0.1303 \\ 11289 & 14: 34: 32 & 0.1281 \\ 11290 & 14: 34: 33 & 0.1286 \\ 11291 & 14: 34: 34 & 0.1295 \\ 11292 & 14: 34: 35 & 0.1286 \\ 11293 & 14: 34: 36 & 0.1287 \\ 11294 & 14: 34: 37 & 0.1262 \\ 11295 & 14: 34: 38 & 0.1310 \\ 11296 & 14: 34: 39 & 0.1252 \\ 11297 & 14: 34: 40 & 0.1293 \\ 11298 & 14: 34: 41 & 0.1270 \\ 11299 & 14: 34: 42 & 0.1315 \\ 11300 & 14: 34: 43 & 0.1275 \\ 11301 & 14: 34: 44 & 0.1327 \\ 11302 & 14: 34: 45 & 0.1297 \\ 11303 & 14: 34: 46 & 0.1316 \\ 11304 & 14: 34: 47 & 0.1277 \\ 11305 & 14: 34: 48 & 0.1295 \\ 11306 & 14: 34: 49 & 0.1298 \\ 11307 & 14: 34: 50 & 0.1263 \\ 11308 & 14: 34: 51 & 0.1309 \\ 11309 & 14: 34: 52 & 0.1277 \\ 11310 & 14: 34: 53 & 0.1308 \\ 11311 & 14: 34: 54 & 0.1260 \\ 11312 & 14: 34: 55 & 0.1299 \\ 11313 & 14: 34: 56 & 0.1273 \\ 11314 & 14: 34: 57 & 0.1313 \\ 11315 & 14: 34: 58 & 0.1277 \\ 11316 & 14: 34: 59 & 0.1301 \\ 11317 & 14: 35: 00 & 0.1286 \\ 11318 & 14: 35: 01 & 0.1273 \\ 11319 & 14: 35: 02 & 0.1286 \\ 11320 & 14: 35: 03 & 0.1273 \\ 11321 & 14: 35: 04 & 0.1289 \\ 11322 & 14: 35: 05 & 0.1237 \\ 11323 & 14: 35: 06 & 0.1305 \\ 11324 & 14: 35: 07 & 0.1272 \\ 11325 & 14: 35: 08 & 0.1306 \\ 11326 & 14: 35: 09 & 0.1274 \\ 11327 & 14: 35: 10 & 0.1304 \\ 11328 & 14: 35: 11 & 0.1266 \\ 11329 & 14: 35: 12 & 0.1254 \\ 11330 & 14: 35: 13 & 0.1255 \\ 11331 & 14: 35: 14 & 0.1251 \\ 11332 & 14: 35: 15 & 0.1274 \\ 11333 & 14: 35: 16 & 0.1227 \\ 11334 & 14: 35: 17 & 0.1284\end{array}$


Appendix A - Fuel Data Test 1 NIST102505_1.xIs

$\begin{array}{lll}11335 & 14: 35: 18 & 0.1243 \\ 11336 & 14: 35: 19 & 0.1281 \\ 11337 & 14: 35: 20 & 0.1261 \\ 11338 & 14: 35: 21 & 0.1311 \\ 11339 & 14: 35: 22 & 0.1265 \\ 11340 & 14: 35: 23 & 0.1297 \\ 11341 & 14: 35: 24 & 0.1277 \\ 11342 & 14: 35: 25 & 0.1267 \\ 11343 & 14: 35: 26 & 0.1289 \\ 11344 & 14: 35: 27 & 0.1274 \\ 11345 & 14: 35: 28 & 0.1293 \\ 11346 & 14: 35: 29 & 0.1246 \\ 11347 & 14: 35: 30 & 0.1288 \\ 11348 & 14: 35: 31 & 0.1256 \\ 11349 & 14: 35: 32 & 0.1305 \\ 11350 & 14: 35: 33 & 0.1269 \\ 11351 & 14: 35: 34 & 0.1308 \\ 11352 & 14: 35: 35 & 0.1283 \\ 11353 & 14: 35: 36 & 0.1287 \\ 11354 & 14: 35: 37 & 0.1273 \\ 11355 & 14: 35: 38 & 0.1289 \\ 11356 & 14: 35: 39 & 0.1293 \\ 11357 & 14: 35: 40 & 0.1270 \\ 11358 & 14: 35: 41 & 0.1322 \\ 11359 & 14: 35: 42 & 0.1290 \\ 11360 & 14: 35: 43 & 0.1332 \\ 11361 & 14: 35: 44 & 0.1276 \\ 11362 & 14: 35: 45 & 0.1339 \\ 11363 & 14: 35: 46 & 0.1308 \\ 11364 & 14: 35: 47 & 0.1321 \\ 11365 & 14: 35: 48 & 0.1273 \\ 11366 & 14: 35: 49 & 0.1284 \\ 11367 & 14: 35: 50 & 0.1272 \\ 11368 & 14: 35: 51 & 0.1270 \\ 11369 & 14: 35: 52 & 0.1322 \\ 11370 & 14: 35: 53 & 0.1262 \\ 11371 & 14: 35: 54 & 0.1298 \\ 11372 & 14: 35: 55 & 0.1252 \\ 11373 & 14: 35: 56 & 0.1317 \\ 11374 & 14: 35: 57 & 0.1277 \\ 11375 & 14: 35: 58 & 0.1314 \\ 11376 & 14: 35: 59 & 0.1289 \\ 11377 & 14: 36: 00 & 0.1310 \\ 11378 & 14: 36: 01 & 0.1286 \\ 11379 & 14: 36: 02 & 0.1263 \\ 11380 & 14: 36: 03 & 0.1287 \\ 11381 & 14: 36: 04 & 0.1273 \\ 11382 & 14: 36: 05 & 0.1306 \\ 11383 & 14: 36: 06 & 0.1257 \\ 11384 & 14: 36: 07 & 0.1302 \\ 11385 & 14: 36: 08 & 0.1252 \\ 11386 & 14: 36: 09 & 0.1307\end{array}$


Appendix A - Fuel Data Test 1 NIST102505_1.xIs

$\begin{array}{lll}11387 & 14: 36: 10 & 0.1265 \\ 11388 & 14: 36: 11 & 0.1298 \\ 11389 & 14: 36: 12 & 0.1271 \\ 11390 & 14: 36: 13 & 0.1304 \\ 11391 & 14: 36: 14 & 0.1278 \\ 11392 & 14: 36: 15 & 0.1271 \\ 11393 & 14: 36: 16 & 0.1275 \\ 11394 & 14: 36: 17 & 0.1275 \\ 11395 & 14: 36: 18 & 0.1294 \\ 11396 & 14: 36: 19 & 0.1247 \\ 11397 & 14: 36: 20 & 0.1308 \\ 11398 & 14: 36: 21 & 0.1269 \\ 11399 & 14: 36: 22 & 0.1303 \\ 11400 & 14: 36: 23 & 0.1265 \\ 11401 & 14: 36: 24 & 0.1300 \\ 11402 & 14: 36: 25 & 0.1264 \\ 11403 & 14: 36: 26 & 0.1277 \\ 11404 & 14: 36: 27 & 0.1262 \\ 11405 & 14: 36: 28 & 0.1268 \\ 11406 & 14: 36: 29 & 0.1292 \\ 11407 & 14: 36: 30 & 0.1278 \\ 11408 & 14: 36: 31 & 0.1313 \\ 11409 & 14: 36: 32 & 0.1277 \\ 11410 & 14: 36: 33 & 0.1304 \\ 11411 & 14: 36: 34 & 0.1254 \\ 11412 & 14: 36: 35 & 0.1295 \\ 11413 & 14: 36: 36 & 0.1266 \\ 11414 & 14: 36: 37 & 0.1291 \\ 11415 & 14: 36: 38 & 0.1295 \\ 11416 & 14: 36: 39 & 0.1290 \\ 11417 & 14: 36: 40 & 0.1291 \\ 11418 & 14: 36: 41 & 0.1266 \\ 11419 & 14: 36: 42 & 0.1322 \\ 11420 & 14: 36: 43 & 0.1271 \\ 11421 & 14: 36: 44 & 0.1290 \\ 11422 & 14: 36: 45 & 0.1241 \\ 11423 & 14: 36: 46 & 0.1311 \\ 11424 & 14: 36: 47 & 0.1262 \\ 11425 & 14: 36: 48 & 0.1305 \\ 11426 & 14: 36: 49 & 0.1293 \\ 11427 & 14: 36: 50 & 0.1316 \\ 11428 & 14: 36: 51 & 0.1278 \\ 11429 & 14: 36: 52 & 0.1272 \\ 11430 & 14: 36: 53 & 0.1279 \\ 11431 & 14: 36: 54 & 0.1274 \\ 11432 & 14: 36: 55 & 0.1298 \\ 11433 & 14: 36: 56 & 0.1256 \\ 11434 & 14: 36: 57 & 0.1316 \\ 11435 & 14: 36: 58 & 0.1248 \\ 11436 & 14: 36: 59 & 0.1291 \\ 11437 & 14: 37: 00 & 0.1270 \\ 11438 & 14: 37: 01 & 0.1307\end{array}$


Appendix A - Fuel Data Test 1 NIST102505_1.xls

$\begin{array}{lll}11439 & 14: 37: 02 & 0.1268 \\ 11440 & 14: 37: 03 & 0.1284 \\ 11441 & 14: 37: 04 & 0.1302 \\ 11442 & 14: 37: 05 & 0.1279 \\ 11443 & 14: 37: 06 & 0.1283 \\ 11444 & 14: 37: 07 & 0.1266 \\ 11445 & 14: 37: 08 & 0.1308 \\ 11446 & 14: 37: 09 & 0.1266 \\ 11447 & 14: 37: 10 & 0.1303 \\ 11448 & 14: 37: 11 & 0.1289 \\ 11449 & 14: 37: 12 & 0.1329 \\ 11450 & 14: 37: 13 & 0.1269 \\ 11451 & 14: 37: 14 & 0.1306 \\ 11452 & 14: 37: 15 & 0.1305 \\ 11453 & 14: 37: 16 & 0.1318 \\ 11454 & 14: 37: 17 & 0.1278 \\ 11455 & 14: 37: 18 & 0.1280 \\ 11456 & 14: 37: 19 & 0.1287 \\ 11457 & 14: 37: 20 & 0.1278 \\ 11458 & 14: 37: 21 & 0.1308 \\ 11459 & 14: 37: 22 & 0.1277 \\ 11460 & 14: 37: 23 & 0.1309 \\ 11461 & 14: 37: 24 & 0.1271 \\ 11462 & 14: 37: 25 & 0.1322 \\ 11463 & 14: 37: 26 & 0.1290 \\ 11464 & 14: 37: 27 & 0.1320 \\ 11465 & 14: 37: 28 & 0.1303 \\ 11466 & 14: 37: 29 & 0.1308 \\ 11467 & 14: 37: 30 & 0.1274 \\ 11468 & 14: 37: 31 & 0.1264 \\ 11469 & 14: 37: 32 & 0.1297 \\ 11470 & 14: 37: 33 & 0.1274 \\ 11471 & 14: 37: 34 & 0.1284 \\ 11472 & 14: 37: 35 & 0.1226 \\ 11473 & 14: 37: 36 & 0.1297 \\ 11474 & 14: 37: 37 & 0.1271 \\ 11475 & 14: 37: 38 & 0.1311 \\ 11476 & 14: 37: 39 & 0.1262 \\ 11477 & 14: 37: 40 & 0.1306 \\ 11478 & 14: 37: 41 & 0.1276 \\ 11479 & 14: 37: 42 & 0.1284 \\ 11480 & 14: 37: 43 & 0.1267 \\ 11481 & 14: 37: 44 & 0.1287 \\ 11482 & 14: 37: 45 & 0.1279 \\ 11483 & 14: 37: 46 & 0.1262 \\ 11484 & 14: 37: 47 & 0.1312 \\ 11485 & 14: 37: 48 & 0.1251 \\ 11486 & 14: 37: 49 & 0.1290 \\ 11487 & 14: 37: 50 & 0.1272 \\ 11488 & 14: 37: 51 & 0.1305 \\ 11489 & 14: 37: 52 & 0.1264 \\ 11490 & 14: 37: 53 & 0.1284\end{array}$


Appendix A - Fuel Data Test 1 NIST102505_1.xIs

$\begin{array}{lll}11491 & 14: 37: 54 & 0.1307 \\ 11492 & 14: 37: 55 & 0.1308 \\ 11493 & 14: 37: 56 & 0.1296 \\ 11494 & 14: 37: 57 & 0.1268 \\ 11495 & 14: 37: 58 & 0.1307 \\ 11496 & 14: 37: 59 & 0.1276 \\ 11497 & 14: 38: 00 & 0.1278 \\ 11498 & 14: 38: 01 & 0.1244 \\ 11499 & 14: 38: 02 & 0.1305 \\ 11500 & 14: 38: 03 & 0.1264 \\ 11501 & 14: 38: 04 & 0.1317 \\ 11502 & 14: 38: 05 & 0.1274 \\ 11503 & 14: 38: 06 & 0.1298 \\ 11504 & 14: 38: 07 & 0.1276 \\ 11505 & 14: 38: 08 & 0.1285 \\ 11506 & 14: 38: 09 & 0.1273 \\ 11507 & 14: 38: 10 & 0.1272 \\ 11508 & 14: 38: 11 & 0.1296 \\ 11509 & 14: 38: 12 & 0.1263 \\ 11510 & 14: 38: 13 & 0.1300 \\ 11511 & 14: 38: 14 & 0.1259 \\ 11512 & 14: 38: 15 & 0.1310 \\ 11513 & 14: 38: 16 & 0.1257 \\ 11514 & 14: 38: 17 & 0.1291 \\ 11515 & 14: 38: 18 & 0.1284 \\ 11516 & 14: 38: 19 & 0.1301 \\ 11517 & 14: 38: 20 & 0.1293 \\ 11518 & 14: 38: 21 & 0.1290 \\ 11519 & 14: 38: 22 & 0.1323 \\ 11520 & 14: 38: 23 & 0.1291 \\ 11521 & 14: 38: 24 & 0.1312 \\ 11522 & 14: 38: 25 & 0.1264 \\ 11523 & 14: 38: 26 & 0.1318 \\ 11524 & 14: 38: 27 & 0.1274 \\ 11525 & 14: 38: 28 & 0.1307 \\ 11526 & 14: 38: 29 & 0.1291 \\ 11527 & 14: 38: 30 & 0.1303 \\ 11528 & 14: 38: 31 & 0.1275 \\ 11529 & 14: 38: 32 & 0.1284 \\ 11530 & 14: 38: 33 & 0.1295 \\ 11531 & 14: 38: 34 & 0.1275 \\ 11532 & 14: 38: 35 & 0.1285 \\ 11533 & 14: 38: 36 & 0.1259 \\ 11534 & 14: 38: 37 & 0.1298 \\ 11535 & 14: 38: 38 & 0.1257 \\ 11536 & 14: 38: 39 & 0.1288 \\ 11537 & 14: 38: 40 & 0.1252 \\ 11538 & 14: 38: 41 & 0.1296 \\ 11539 & 14: 38: 42 & 0.1258 \\ 11540 & 14: 38: 43 & 0.1281 \\ 11541 & 14: 38: 44 & 0.1279 \\ 11542 & 14: 38: 45 & 0.1288\end{array}$


Appendix A - Fuel Data Test 1 NIST102505_1.xIs

$\begin{array}{lll}11543 & 14: 38: 46 & 0.1269 \\ 11544 & 14: 38: 47 & 0.1269 \\ 11545 & 14: 38: 48 & 0.1279 \\ 11546 & 14: 38: 49 & 0.1261 \\ 11547 & 14: 38: 50 & 0.1294 \\ 11548 & 14: 38: 51 & 0.1264 \\ 11549 & 14: 38: 52 & 0.1307 \\ 11550 & 14: 38: 53 & 0.1259 \\ 11551 & 14: 38: 54 & 0.1310 \\ 11552 & 14: 38: 55 & 0.1285 \\ 11553 & 14: 38: 56 & 0.1316 \\ 11554 & 14: 38: 57 & 0.1268 \\ 11555 & 14: 38: 58 & 0.1291 \\ 11556 & 14: 38: 59 & 0.1257 \\ 11557 & 14: 39: 00 & 0.1256 \\ 11558 & 14: 39: 01 & 0.1291 \\ 11559 & 14: 39: 02 & 0.1266 \\ 11560 & 14: 39: 03 & 0.1281 \\ 11561 & 14: 39: 04 & 0.1232 \\ 11562 & 14: 39: 05 & 0.1302 \\ 11563 & 14: 39: 06 & 0.1265 \\ 11564 & 14: 39: 07 & 0.1303 \\ 11565 & 14: 39: 08 & 0.1277 \\ 11566 & 14: 39: 09 & 0.1309 \\ 11567 & 14: 39: 10 & 0.1270 \\ 11568 & 14: 39: 11 & 0.1269 \\ 11569 & 14: 39: 12 & 0.1283 \\ 11570 & 14: 39: 13 & 0.1270 \\ 11571 & 14: 39: 14 & 0.1280 \\ 11572 & 14: 39: 15 & 0.1255 \\ 11573 & 14: 39: 16 & 0.1275 \\ 11574 & 14: 39: 17 & 0.1234 \\ 11575 & 14: 39: 18 & 0.1291 \\ 11576 & 14: 39: 19 & 0.1282 \\ 11577 & 14: 39: 20 & 0.1318 \\ 11578 & 14: 39: 21 & 0.1291 \\ 11579 & 14: 39: 22 & 0.1316 \\ 11580 & 14: 39: 23 & 0.1301 \\ 11581 & 14: 39: 24 & 0.1303 \\ 11582 & 14: 39: 25 & 0.1271 \\ 11583 & 14: 39: 26 & 0.1278 \\ 11584 & 14: 39: 27 & 0.1289 \\ 11585 & 14: 39: 28 & 0.1253 \\ 11586 & 14: 39: 29 & 0.1316 \\ 11587 & 14: 39: 30 & 0.1258 \\ 11588 & 14: 39: 31 & 0.1295 \\ 11589 & 14: 39: 32 & 0.1267 \\ 11590 & 14: 39: 33 & 0.1318 \\ 11591 & 14: 39: 34 & 0.1284 \\ 11592 & 14: 39: 35 & 0.1291 \\ 11593 & 14: 39: 36 & 0.1291 \\ 11594 & 14: 39: 37 & 0.1298\end{array}$


Appendix A - Fuel Data Test 1 NIST102505_1.xIs

$\begin{array}{lll}11595 & 14: 39: 38 & 0.1293 \\ 11596 & 14: 39: 39 & 0.1275 \\ 11597 & 14: 39: 40 & 0.1310 \\ 11598 & 14: 39: 41 & 0.1266 \\ 11599 & 14: 39: 42 & 0.1302 \\ 11600 & 14: 39: 43 & 0.1254 \\ 11601 & 14: 39: 44 & 0.1311 \\ 11602 & 14: 39: 45 & 0.1268 \\ 11603 & 14: 39: 46 & 0.1288 \\ 11604 & 14: 39: 47 & 0.1273 \\ 11605 & 14: 39: 48 & 0.1280 \\ 11606 & 14: 39: 49 & 0.1273 \\ 11607 & 14: 39: 50 & 0.1254 \\ 11608 & 14: 39: 51 & 0.1302 \\ 11609 & 14: 39: 52 & 0.1288 \\ 11610 & 14: 39: 53 & 0.1291 \\ 11611 & 14: 39: 54 & 0.1248 \\ 11612 & 14: 39: 55 & 0.1309 \\ 11613 & 14: 39: 56 & 0.1282 \\ 11614 & 14: 39: 57 & 0.1321 \\ 11615 & 14: 39: 58 & 0.1275 \\ 11616 & 14: 39: 59 & 0.1316 \\ 11617 & 14: 40: 00 & 0.1289 \\ 11618 & 14: 40: 01 & 0.1302 \\ 11619 & 14: 40: 02 & 0.1274 \\ 11620 & 14: 40: 03 & 0.1277 \\ 11621 & 14: 40: 04 & 0.1285 \\ 11622 & 14: 40: 05 & 0.1251 \\ 11623 & 14: 40: 06 & 0.1295 \\ 11624 & 14: 40: 07 & 0.1248 \\ 11625 & 14: 40: 08 & 0.1302 \\ 11626 & 14: 40: 09 & 0.1276 \\ 11627 & 14: 40: 10 & 0.1312 \\ 11628 & 14: 40: 11 & 0.1272 \\ 11629 & 14: 40: 12 & 0.1304 \\ 11630 & 14: 40: 13 & 0.1302 \\ 11631 & 14: 40: 14 & 0.1286 \\ 11632 & 14: 40: 15 & 0.1271 \\ 11633 & 14: 40: 16 & 0.1254 \\ 11634 & 14: 40: 17 & 0.1298 \\ 11635 & 14: 40: 18 & 0.1257 \\ 11636 & 14: 40: 19 & 0.1294 \\ 11637 & 14: 40: 20 & 0.1256 \\ 11638 & 14: 40: 21 & 0.1289 \\ 11639 & 14: 40: 22 & 0.1249 \\ 11640 & 14: 40: 23 & 0.1283 \\ 11641 & 14: 40: 24 & 0.1290 \\ 11642 & 14: 40: 25 & 0.1299 \\ 11643 & 14: 40: 26 & 0.1273 \\ 11644 & 14: 40: 27 & 0.1267 \\ 11645 & 14: 40: 28 & 0.1291 \\ 11646 & 14: 40: 29 & 0.1261\end{array}$


Appendix A - Fuel Data Test 1 NIST102505_1.xIs

$\begin{array}{lll}11647 & 14: 40: 30 & 0.1274 \\ 11648 & 14: 40: 31 & 0.1244 \\ 11649 & 14: 40: 32 & 0.1276 \\ 11650 & 14: 40: 33 & 0.1267 \\ 11651 & 14: 40: 34 & 0.1307 \\ 11652 & 14: 40: 35 & 0.1286 \\ 11654 & 14: 40: 37 & 0.1320 \\ 11654 & 14: 40: 37 & 0.1257 \\ 11655 & 14: 40: 38 & 0.1280 \\ 11656 & 14: 40: 39 & 0.1283 \\ 11657 & 14: 40: 40 & 0.1285 \\ 11658 & 14: 40: 41 & 0.1298 \\ 11659 & 14: 40: 42 & 0.1285 \\ 11660 & 14: 40: 43 & 0.1285 \\ 11661 & 14: 40: 44 & 0.1238 \\ 11662 & 14: 40: 45 & 0.1290 \\ 11663 & 14: 40: 46 & 0.1266 \\ 11664 & 14: 40: 47 & 0.1306 \\ 11665 & 14: 40: 48 & 0.1260 \\ 11666 & 14: 40: 49 & 0.1291 \\ 11667 & 14: 40: 50 & 0.1297 \\ 11668 & 14: 40: 51 & 0.1301 \\ 11669 & 14: 40: 52 & 0.1277 \\ 11670 & 14: 40: 53 & 0.1265 \\ 11671 & 14: 40: 54 & 0.1281 \\ 11672 & 14: 40: 55 & 0.1249 \\ 11673 & 14: 40: 56 & 0.1272 \\ 11674 & 14: 40: 57 & 0.1235 \\ 11675 & 14: 40: 58 & 0.1301 \\ 11676 & 14: 40: 59 & 0.1254 \\ 11677 & 14: 41: 00 & 0.1303 \\ 11678 & 14: 41: 01 & 0.1278 \\ 11679 & 14: 41: 02 & 0.1310 \\ 11680 & 14: 41: 03 & 0.1282 \\ 11681 & 14: 41: 04 & 0.1304 \\ 11682 & 14: 41: 05 & 0.1288 \\ 11683 & 14: 41: 06 & 0.1271 \\ 11684 & 14: 41: 07 & 0.1299 \\ 11685 & 14: 41: 08 & 0.1284 \\ 11686 & 14: 41: 09 & 0.1318 \\ 11687 & 14: 41: 10 & 0.1260 \\ 11688 & 14: 41: 11 & 0.1329 \\ 11689 & 14: 41: 12 & 0.1263 \\ 11690 & 14: 41: 13 & 0.1300 \\ 11691 & 14: 41: 14 & 0.1277 \\ 11692 & 14: 41: 15 & 0.1289 \\ 11693 & 14: 41: 16 & 0.1300 \\ 11694 & 14: 41: 17 & 0.1297 \\ 11695 & 14: 41: 18 & 0.1315 \\ 11696 & 14: 41: 19 & 0.1282 \\ 11697 & 14: 41: 20 & 0.1309 \\ 11698 & 14: 41: 21 & 0.1248\end{array}$


Appendix A - Fuel Data Test 1 NIST102505_1.xIs

$\begin{array}{lll}11699 & 14: 41: 22 & 0.1300 \\ 11700 & 14: 41: 23 & 0.1259 \\ 11701 & 14: 41: 24 & 0.1308 \\ 11702 & 14: 41: 25 & 0.1269 \\ 11703 & 14: 41: 26 & 0.1313 \\ 11704 & 14: 41: 27 & 0.1279 \\ 11705 & 14: 41: 28 & 0.1280 \\ 11706 & 14: 41: 29 & 0.1266 \\ 11707 & 14: 41: 30 & 0.1269 \\ 11708 & 14: 41: 31 & 0.1276 \\ 11709 & 14: 41: 32 & 0.1257 \\ 11710 & 14: 41: 33 & 0.1293 \\ 11711 & 14: 41: 34 & 0.1244 \\ 11712 & 14: 41: 35 & 0.1286 \\ 11713 & 14: 41: 36 & 0.1263 \\ 11714 & 14: 41: 37 & 0.1294 \\ 11715 & 14: 41: 38 & 0.1263 \\ 11716 & 14: 41: 39 & 0.1289 \\ 11717 & 14: 41: 40 & 0.1287 \\ 11718 & 14: 41: 41 & 0.1282 \\ 11719 & 14: 41: 42 & 0.1270 \\ 11720 & 14: 41: 43 & 0.1255 \\ 11721 & 14: 41: 44 & 0.1317 \\ 11722 & 14: 41: 45 & 0.1286 \\ 11723 & 14: 41: 46 & 0.1308 \\ 11724 & 14: 41: 47 & 0.1270 \\ 11725 & 14: 41: 48 & 0.1324 \\ 11726 & 14: 41: 49 & 0.1291 \\ 11727 & 14: 41: 50 & 0.1302 \\ 11728 & 14: 41: 51 & 0.1289 \\ 11729 & 14: 41: 52 & 0.1295 \\ 11730 & 14: 41: 53 & 0.1277 \\ 11731 & 14: 41: 54 & 0.1274 \\ 11732 & 14: 41: 55 & 0.1264 \\ 11733 & 14: 41: 56 & 0.1263 \\ 11734 & 14: 41: 57 & 0.1289 \\ 11735 & 14: 41: 58 & 0.1251 \\ 11736 & 14: 41: 59 & 0.1301 \\ 11737 & 14: 42: 00 & 0.1241 \\ 11738 & 14: 42: 01 & 0.1276 \\ 11739 & 14: 42: 02 & 0.1251 \\ 11740 & 14: 42: 03 & 0.1295 \\ 11741 & 14: 42: 04 & 0.1256 \\ 11742 & 14: 42: 05 & 0.1290 \\ 11743 & 14: 42: 06 & 0.1281 \\ 11744 & 14: 42: 07 & 0.1289 \\ 11745 & 14: 42: 08 & 0.1282 \\ 11746 & 14: 42: 09 & 0.1275 \\ 11747 & 14: 42: 10 & 0.1284 \\ 11748 & 14: 42: 11 & 0.1249 \\ 11749 & 14: 42: 12 & 0.1299 \\ 11750 & 14: 42: 13 & 0.1272\end{array}$


Appendix A - Fuel Data Test 1 NIST102505_1.xls

$\begin{array}{lll}11751 & 14: 42: 14 & 0.1303 \\ 11752 & 14: 42: 15 & 0.1263 \\ 11753 & 14: 42: 16 & 0.1318 \\ 11754 & 14: 42: 17 & 0.1303 \\ 11755 & 14: 42: 18 & 0.1313 \\ 11756 & 14: 42: 19 & 0.1272 \\ 11757 & 14: 42: 20 & 0.1271 \\ 11758 & 14: 42: 21 & 0.1277 \\ 11759 & 14: 42: 22 & 0.1252 \\ 11760 & 14: 42: 23 & 0.1303 \\ 11761 & 14: 42: 24 & 0.1276 \\ 11762 & 14: 42: 25 & 0.1320 \\ 11763 & 14: 42: 26 & 0.1269 \\ 11764 & 14: 42: 27 & 0.1315 \\ 11765 & 14: 42: 28 & 0.1280 \\ 11766 & 14: 42: 29 & 0.1324 \\ 11767 & 14: 42: 30 & 0.1287 \\ 11768 & 14: 42: 31 & 0.1313 \\ 11769 & 14: 42: 32 & 0.1299 \\ 11770 & 14: 42: 33 & 0.1280 \\ 11771 & 14: 42: 34 & 0.1315 \\ 11772 & 14: 42: 35 & 0.1288 \\ 11773 & 14: 42: 36 & 0.1300 \\ 11774 & 14: 42: 37 & 0.1259 \\ 11775 & 14: 42: 38 & 0.1306 \\ 11776 & 14: 42: 39 & 0.1266 \\ 11777 & 14: 42: 40 & 0.1301 \\ 11778 & 14: 42: 41 & 0.1280 \\ 11779 & 14: 42: 42 & 0.1311 \\ 11780 & 14: 42: 43 & 0.1290 \\ 11781 & 14: 42: 44 & 0.1282 \\ 11782 & 14: 42: 45 & 0.1287 \\ 11783 & 14: 42: 46 & 0.1265 \\ 11784 & 14: 42: 47 & 0.1294 \\ 11785 & 14: 42: 48 & 0.1270 \\ 11786 & 14: 42: 49 & 0.1304 \\ 11787 & 14: 42: 50 & 0.1247 \\ 11788 & 14: 42: 51 & 0.1292 \\ 11789 & 14: 42: 52 & 0.1289 \\ 11790 & 14: 42: 53 & 0.1308 \\ 11791 & 14: 42: 54 & 0.1276 \\ 11792 & 14: 42: 55 & 0.1286 \\ 11793 & 14: 42: 56 & 0.1294 \\ 11794 & 14: 42: 57 & 0.1290 \\ 11795 & 14: 42: 58 & 0.1276 \\ 11796 & 14: 42: 59 & 0.1259 \\ 11797 & 14: 43: 00 & 0.1290 \\ 11798 & 14: 43: 01 & 0.1262 \\ 11799 & 14: 43: 02 & 0.1289 \\ 11800 & 14: 43: 03 & 0.1256 \\ 11801 & 14: 43: 04 & 0.1302 \\ 11802 & 14: 43: 05 & 0.1279\end{array}$


Appendix A - Fuel Data Test 1 NIST102505_1.xls

$\begin{array}{lll}11803 & 14: 43: 06 & 0.1306 \\ 11804 & 14: 43: 07 & 0.1301 \\ 11805 & 14: 43: 08 & 0.1317 \\ 11806 & 14: 43: 09 & 0.1290 \\ 11807 & 14: 43: 10 & 0.1282 \\ 11808 & 14: 43: 11 & 0.1281 \\ 11809 & 14: 43: 12 & 0.1265 \\ 11810 & 14: 43: 13 & 0.1288 \\ 11811 & 14: 43: 14 & 0.1268 \\ 11812 & 14: 43: 15 & 0.1293 \\ 11813 & 14: 43: 16 & 0.1250 \\ 11814 & 14: 43: 17 & 0.1298 \\ 11815 & 14: 43: 18 & 0.1281 \\ 11816 & 14: 43: 19 & 0.1318 \\ 11817 & 14: 43: 20 & 0.1279 \\ 11818 & 14: 43: 21 & 0.1308 \\ 11819 & 14: 43: 22 & 0.1292 \\ 11820 & 14: 43: 23 & 0.1290 \\ 11821 & 14: 43: 24 & 0.1281 \\ 11822 & 14: 43: 25 & 0.1274 \\ 11823 & 14: 43: 26 & 0.1291 \\ 11824 & 14: 43: 27 & 0.1242 \\ 11825 & 14: 43: 28 & 0.1306 \\ 11826 & 14: 43: 29 & 0.1273 \\ 11827 & 14: 43: 30 & 0.1309 \\ 11828 & 14: 43: 31 & 0.1259 \\ 11829 & 14: 43: 32 & 0.1313 \\ 11830 & 14: 43: 33 & 0.1279 \\ 11831 & 14: 43: 34 & 0.1286 \\ 11832 & 14: 43: 35 & 0.1266 \\ 11833 & 14: 43: 36 & 0.1277 \\ 11834 & 14: 43: 37 & 0.1295 \\ 11835 & 14: 43: 38 & 0.1274 \\ 11836 & 14: 43: 39 & 0.1284 \\ 11837 & 14: 43: 40 & 0.1249 \\ 11838 & 14: 43: 41 & 0.1296 \\ 11839 & 14: 43: 42 & 0.1246 \\ 11840 & 14: 43: 43 & 0.1301 \\ 11841 & 14: 43: 44 & 0.1269 \\ 11842 & 14: 43: 45 & 0.1301 \\ 11843 & 14: 43: 46 & 0.1283 \\ 11844 & 14: 43: 47 & 0.1301 \\ 11845 & 14: 43: 48 & 0.1278 \\ 11846 & 14: 43: 49 & 0.1272 \\ 11847 & 14: 43: 50 & 0.1278 \\ 11848 & 14: 43: 51 & 0.1263 \\ 11849 & 14: 43: 52 & 0.1277 \\ 11850 & 14: 43: 53 & 0.1227 \\ 11851 & 14: 43: 54 & 0.1283 \\ 11852 & 14: 43: 55 & 0.1289 \\ 11853 & 14: 43: 56 & 0.1310 \\ 11854 & 14: 43: 57 & 0.1277\end{array}$


Appendix A - Fuel Data Test 1 NIST102505_1.xls

$\begin{array}{lll}11855 & 14: 43: 58 & 0.1321 \\ 11856 & 14: 43: 59 & 0.1316 \\ 11857 & 14: 44: 00 & 0.1291 \\ 11858 & 14: 44: 01 & 0.1278 \\ 11859 & 14: 44: 02 & 0.1259 \\ 11860 & 14: 44: 03 & 0.1296 \\ 11861 & 14: 44: 04 & 0.1266 \\ 11862 & 14: 44: 05 & 0.1288 \\ 11863 & 14: 44: 06 & 0.1258 \\ 11864 & 14: 44: 07 & 0.1295 \\ 11865 & 14: 44: 08 & 0.1270 \\ 11866 & 14: 44: 09 & 0.1296 \\ 11867 & 14: 44: 10 & 0.1288 \\ 11868 & 14: 44: 11 & 0.1296 \\ 11869 & 14: 44: 12 & 0.1278 \\ 11870 & 14: 44: 13 & 0.1267 \\ 11871 & 14: 44: 14 & 0.1279 \\ 11872 & 14: 44: 15 & 0.1261 \\ 11873 & 14: 44: 16 & 0.1277 \\ 11874 & 14: 44: 17 & 0.1237 \\ 11875 & 14: 44: 18 & 0.1281 \\ 11876 & 14: 44: 19 & 0.1236 \\ 11877 & 14: 44: 20 & 0.1295 \\ 11878 & 14: 44: 21 & 0.1261 \\ 11879 & 14: 44: 22 & 0.1312 \\ 11880 & 14: 44: 23 & 0.1272 \\ 11881 & 14: 44: 24 & 0.1297 \\ 11882 & 14: 44: 25 & 0.1267 \\ 11883 & 14: 44: 26 & 0.1253 \\ 11884 & 14: 44: 27 & 0.1302 \\ 11885 & 14: 44: 28 & 0.1263 \\ 11886 & 14: 44: 29 & 0.1276 \\ 11887 & 14: 44: 30 & 0.1224 \\ 11888 & 14: 44: 31 & 0.1284 \\ 11889 & 14: 44: 32 & 0.1232 \\ 11890 & 14: 44: 33 & 0.1284 \\ 11891 & 14: 44: 34 & 0.1254 \\ 11892 & 14: 44: 35 & 0.1296 \\ 11893 & 14: 44: 36 & 0.1265 \\ 11894 & 14: 44: 37 & 0.1264 \\ 11895 & 14: 44: 38 & 0.1297 \\ 11896 & 14: 44: 39 & 0.1280 \\ 11897 & 14: 44: 40 & 0.1290 \\ 11898 & 14: 44: 41 & 0.1268 \\ 11899 & 14: 44: 42 & 0.1298 \\ 11900 & 14: 44: 43 & 0.1256 \\ 11901 & 14: 44: 44 & 0.1299 \\ 11902 & 14: 44: 45 & 0.1281 \\ 11903 & 14: 44: 46 & 0.1320 \\ 11904 & 14: 44: 47 & 0.1280 \\ 11905 & 14: 44: 48 & 0.1319 \\ 11906 & 14: 44: 49 & 0.1321\end{array}$


Appendix A - Fuel Data Test 1 NIST102505_1.xls

$\begin{array}{lll}11907 & 14: 44: 50 & 0.1298 \\ 11908 & 14: 44: 51 & 0.1264 \\ 11909 & 14: 44: 52 & 0.1262 \\ 11910 & 14: 44: 53 & 0.1294 \\ 11911 & 14: 44: 54 & 0.1258 \\ 11912 & 14: 44: 55 & 0.1290 \\ 11913 & 14: 44: 56 & 0.1264 \\ 11914 & 14: 44: 57 & 0.1299 \\ 11915 & 14: 44: 58 & 0.1262 \\ 11916 & 14: 44: 59 & 0.1302 \\ 11917 & 14: 45: 00 & 0.1283 \\ 11918 & 14: 45: 01 & 0.1308 \\ 11919 & 14: 45: 02 & 0.1274 \\ 11920 & 14: 45: 03 & 0.1285 \\ 11921 & 14: 45: 04 & 0.1288 \\ 11922 & 14: 45: 05 & 0.1276 \\ 11923 & 14: 45: 06 & 0.1291 \\ 11924 & 14: 45: 07 & 0.1270 \\ 11925 & 14: 45: 08 & 0.1281 \\ 11926 & 14: 45: 09 & 0.1244 \\ 11927 & 14: 45: 10 & 0.1313 \\ 11928 & 14: 45: 11 & 0.1267 \\ 11929 & 14: 45: 12 & 0.1303 \\ 11930 & 14: 45: 13 & 0.1279 \\ 11931 & 14: 45: 14 & 0.1297 \\ 11932 & 14: 45: 15 & 0.1287 \\ 11933 & 14: 45: 16 & 0.1271 \\ 11934 & 14: 45: 17 & 0.1282 \\ 11935 & 14: 45: 18 & 0.1258 \\ 11936 & 14: 45: 19 & 0.1281 \\ 11937 & 14: 45: 20 & 0.1239 \\ 11938 & 14: 45: 21 & 0.1291 \\ 11939 & 14: 45: 22 & 0.1248 \\ 11940 & 14: 45: 23 & 0.1281 \\ 11941 & 14: 45: 24 & 0.1261 \\ 11942 & 14: 45: 25 & 0.1325 \\ 11943 & 14: 45: 26 & 0.1278 \\ 11944 & 14: 45: 27 & 0.1315 \\ 11945 & 14: 45: 28 & 0.1298 \\ 11946 & 14: 45: 29 & 0.1295 \\ 11947 & 14: 45: 30 & 0.1277 \\ 11948 & 14: 45: 31 & 0.1264 \\ 11949 & 14: 45: 32 & 0.1284 \\ 11950 & 14: 45: 33 & 0.1244 \\ 11951 & 14: 45: 34 & 0.1301 \\ 11952 & 14: 45: 35 & 0.1259 \\ 11953 & 14: 45: 36 & 0.1302 \\ 11954 & 14: 45: 37 & 0.1263 \\ 11955 & 14: 45: 38 & 0.1308 \\ 11956 & 14: 45: 39 & 0.1281 \\ 11957 & 14: 45: 40 & 0.1286 \\ 11958 & 14: 45: 41 & 0.1285\end{array}$


Appendix A - Fuel Data Test 1 NIST102505_1.xIs

$\begin{array}{lll}11959 & 14: 45: 42 & 0.1276 \\ 11960 & 14: 45: 43 & 0.1279 \\ 11961 & 14: 45: 44 & 0.1257 \\ 11962 & 14: 45: 45 & 0.1290 \\ 11963 & 14: 45: 46 & 0.1244 \\ 11964 & 14: 45: 47 & 0.1286 \\ 11965 & 14: 45: 48 & 0.1266 \\ 11966 & 14: 45: 49 & 0.1301 \\ 11967 & 14: 45: 50 & 0.1262 \\ 11968 & 14: 45: 51 & 0.1294 \\ 11969 & 14: 45: 52 & 0.1307 \\ 11970 & 14: 45: 53 & 0.1295 \\ 11971 & 14: 45: 54 & 0.1273 \\ 11972 & 14: 45: 55 & 0.1262 \\ 11973 & 14: 45: 56 & 0.1290 \\ 11974 & 14: 45: 57 & 0.1254 \\ 11975 & 14: 45: 58 & 0.1275 \\ 11976 & 14: 45: 59 & 0.1248 \\ 11977 & 14: 46: 00 & 0.1296 \\ 11978 & 14: 46: 01 & 0.1267 \\ 11979 & 14: 46: 02 & 0.1306 \\ 11980 & 14: 46: 03 & 0.1301 \\ 11981 & 14: 46: 04 & 0.1322 \\ 11982 & 14: 46: 05 & 0.1285 \\ 11983 & 14: 46: 06 & 0.1300 \\ 11984 & 14: 46: 07 & 0.1295 \\ 11985 & 14: 46: 08 & 0.1292 \\ 11986 & 14: 46: 09 & 0.1304 \\ 11987 & 14: 46: 10 & 0.1281 \\ 11988 & 14: 46: 11 & 0.1300 \\ 11989 & 14: 46: 12 & 0.1251 \\ 11990 & 14: 46: 13 & 0.1318 \\ 11991 & 14: 46: 14 & 0.1288 \\ 11992 & 14: 46: 15 & 0.1317 \\ 11993 & 14: 46: 16 & 0.1281 \\ 11994 & 14: 46: 17 & 0.1311 \\ 11995 & 14: 46: 18 & 0.1286 \\ 11996 & 14: 46: 19 & 0.1292 \\ 11997 & 14: 46: 20 & 0.1279 \\ 11998 & 14: 46: 21 & 0.1262 \\ 11999 & 14: 46: 22 & 0.1277 \\ 12000 & 14: 46: 23 & 0.1260 \\ 12001 & 14: 46: 24 & 0.1285 \\ 12002 & 14: 46: 25 & 0.1235 \\ 12003 & 14: 46: 26 & 0.1299 \\ 12004 & 14: 46: 27 & 0.1272 \\ 12005 & 14: 46: 28 & 0.1311 \\ 12006 & 14: 46: 29 & 0.1263 \\ 12007 & 14: 46: 30 & 0.1310 \\ 12008 & 14: 46: 31 & 0.1269 \\ 12009 & 14: 46: 32 & 0.1277 \\ 12010 & 14: 46: 33 & 0.1300\end{array}$


Appendix A - Fuel Data Test 1 NIST102505_1.xls

$\begin{array}{lll}12011 & 14: 46: 34 & 0.1265 \\ 12012 & 14: 46: 35 & 0.1288 \\ 12013 & 14: 46: 36 & 0.1268 \\ 12014 & 14: 46: 37 & 0.1295 \\ 12015 & 14: 46: 38 & 0.1267 \\ 12016 & 14: 46: 39 & 0.1317 \\ 12017 & 14: 46: 40 & 0.1265 \\ 12018 & 14: 46: 41 & 0.1322 \\ 12019 & 14: 46: 42 & 0.1286 \\ 12020 & 14: 46: 43 & 0.1310 \\ 12021 & 14: 46: 44 & 0.1281 \\ 12022 & 14: 46: 45 & 0.1285 \\ 12023 & 14: 46: 46 & 0.1282 \\ 12024 & 14: 46: 47 & 0.1250 \\ 12025 & 14: 46: 48 & 0.1290 \\ 12026 & 14: 46: 49 & 0.1269 \\ 12027 & 14: 46: 50 & 0.1304 \\ 12028 & 14: 46: 51 & 0.1256 \\ 12029 & 14: 46: 52 & 0.1316 \\ 12030 & 14: 46: 53 & 0.1276 \\ 12031 & 14: 46: 54 & 0.1310 \\ 12032 & 14: 46: 55 & 0.1267 \\ 12033 & 14: 46: 56 & 0.1298 \\ 12034 & 14: 46: 57 & 0.1271 \\ 12035 & 14: 46: 58 & 0.1274 \\ 12036 & 14: 46: 59 & 0.1284 \\ 12037 & 14: 47: 00 & 0.1277 \\ 12038 & 14: 47: 01 & 0.1284 \\ 12039 & 14: 47: 02 & 0.1243 \\ 12040 & 14: 47: 03 & 0.1306 \\ 12041 & 14: 47: 04 & 0.1267 \\ 12042 & 14: 47: 05 & 0.1303 \\ 12043 & 14: 47: 06 & 0.1275 \\ 12044 & 14: 47: 07 & 0.1330 \\ 12045 & 14: 47: 08 & 0.1299 \\ 12046 & 14: 47: 09 & 0.1301 \\ 12047 & 14: 47: 10 & 0.1304 \\ 12048 & 14: 47: 11 & 0.1285 \\ 12049 & 14: 47: 12 & 0.1305 \\ 12050 & 14: 47: 13 & 0.1267 \\ 12051 & 14: 47: 14 & 0.1307 \\ 12052 & 14: 47: 15 & 0.1260 \\ 12053 & 14: 47: 16 & 0.1298 \\ 12054 & 14: 47: 17 & 0.1282 \\ 12055 & 14: 47: 18 & 0.1308 \\ 12056 & 14: 47: 19 & 0.1264 \\ 12057 & 14: 47: 20 & 0.1289 \\ 12058 & 14: 47: 21 & 0.1293 \\ 12059 & 14: 47: 22 & 0.1288 \\ 12060 & 14: 47: 23 & 0.1271 \\ 12061 & 14: 47: 24 & 0.1269 \\ 12062 & 14: 47: 25 & 0.1300\end{array}$


Appendix A - Fuel Data Test 1 NIST102505_1.xls

$\begin{array}{lll}12063 & 14: 47: 26 & 0.1261 \\ 12064 & 14: 47: 27 & 0.1277 \\ 12065 & 14: 47: 28 & 0.1253 \\ 12066 & 14: 47: 29 & 0.1292 \\ 12067 & 14: 47: 30 & 0.1261 \\ 12068 & 14: 47: 31 & 0.1284 \\ 12069 & 14: 47: 32 & 0.1278 \\ 12070 & 14: 47: 33 & 0.1286 \\ 12071 & 14: 47: 34 & 0.1266 \\ 12072 & 14: 47: 35 & 0.1253 \\ 12073 & 14: 47: 36 & 0.1285 \\ 12074 & 14: 47: 37 & 0.1241 \\ 12075 & 14: 47: 38 & 0.1271 \\ 12076 & 14: 47: 39 & 0.1241 \\ 12077 & 14: 47: 40 & 0.1294 \\ 12078 & 14: 47: 41 & 0.1256 \\ 12079 & 14: 47: 42 & 0.1289 \\ 12080 & 14: 47: 43 & 0.1271 \\ 12081 & 14: 47: 44 & 0.1295 \\ 12082 & 14: 47: 45 & 0.1269 \\ 12083 & 14: 47: 46 & 0.1257 \\ 12084 & 14: 47: 47 & 0.1296 \\ 12085 & 14: 47: 48 & 0.1270 \\ 12086 & 14: 47: 49 & 0.1293 \\ 12087 & 14: 47: 50 & 0.1242 \\ 12088 & 14: 47: 51 & 0.1294 \\ 12089 & 14: 47: 52 & 0.1247 \\ 12090 & 14: 47: 53 & 0.1294 \\ 12091 & 14: 47: 54 & 0.1278 \\ 12092 & 14: 47: 55 & 0.1299 \\ 12093 & 14: 47: 56 & 0.1274 \\ 12094 & 14: 47: 57 & 0.1291 \\ 12095 & 14: 47: 58 & 0.1278 \\ 12096 & 14: 47: 59 & 0.1277 \\ 12097 & 14: 48: 00 & 0.1280 \\ 12098 & 14: 48: 01 & 0.1266 \\ 12099 & 14: 48: 02 & 0.1272 \\ 12100 & 14: 48: 03 & 0.1230 \\ 12101 & 14: 48: 04 & 0.1286 \\ 12102 & 14: 48: 05 & 0.1264 \\ 12103 & 14: 48: 06 & 0.1293 \\ 12104 & 14: 48: 07 & 0.1245 \\ 12105 & 14: 48: 08 & 0.1288 \\ 12106 & 14: 48: 09 & 0.1276 \\ 12107 & 14: 48: 10 & 0.1288 \\ 12108 & 14: 48: 11 & 0.1263 \\ 12109 & 14: 48: 12 & 0.1277 \\ 12110 & 14: 48: 13 & 0.1287 \\ 12111 & 14: 48: 14 & 0.1255 \\ 12112 & 14: 48: 15 & 0.1299 \\ 12113 & 14: 48: 16 & 0.1247 \\ 12114 & 14: 48: 17 & 0.1285\end{array}$


Appendix A - Fuel Data Test 1 NIST102505_1.xls

$\begin{array}{lll}12115 & 14: 48: 18 & 0.1271 \\ 12116 & 14: 48: 19 & 0.1326 \\ 12117 & 14: 48: 20 & 0.1279 \\ 12118 & 14: 48: 21 & 0.1307 \\ 12119 & 14: 48: 22 & 0.1269 \\ 12120 & 14: 48: 23 & 0.1296 \\ 12121 & 14: 48: 24 & 0.1278 \\ 12122 & 14: 48: 25 & 0.1267 \\ 12123 & 14: 48: 26 & 0.1303 \\ 12124 & 14: 48: 27 & 0.1261 \\ 12125 & 14: 48: 28 & 0.1292 \\ 12126 & 14: 48: 29 & 0.1243 \\ 12127 & 14: 48: 30 & 0.1306 \\ 12128 & 14: 48: 31 & 0.1276 \\ 12129 & 14: 48: 32 & 0.1315 \\ 12130 & 14: 48: 33 & 0.1266 \\ 12131 & 14: 48: 34 & 0.1310 \\ 12132 & 14: 48: 35 & 0.1262 \\ 12133 & 14: 48: 36 & 0.1272 \\ 12134 & 14: 48: 37 & 0.1296 \\ 12135 & 14: 48: 38 & 0.1266 \\ 12136 & 14: 48: 39 & 0.1285 \\ 12137 & 14: 48: 40 & 0.1241 \\ 12138 & 14: 48: 41 & 0.1306 \\ 12139 & 14: 48: 42 & 0.1256 \\ 12140 & 14: 48: 43 & 0.1287 \\ 12141 & 14: 48: 44 & 0.1250 \\ 12142 & 14: 48: 45 & 0.1300 \\ 12143 & 14: 48: 46 & 0.1289 \\ 12144 & 14: 48: 47 & 0.1314 \\ 12145 & 14: 48: 48 & 0.1315 \\ 12146 & 14: 48: 49 & 0.1296 \\ 12147 & 14: 48: 50 & 0.1276 \\ 12148 & 14: 48: 51 & 0.1264 \\ 12149 & 14: 48: 52 & 0.1311 \\ 12150 & 14: 48: 53 & 0.1265 \\ 12151 & 14: 48: 54 & 0.1307 \\ 12152 & 14: 48: 55 & 0.1281 \\ 12153 & 14: 48: 56 & 0.1316 \\ 12154 & 14: 48: 57 & 0.1285 \\ 12155 & 14: 48: 58 & 0.1326 \\ 12156 & 14: 48: 59 & 0.1314 \\ 12157 & 14: 49: 00 & 0.1324 \\ 12158 & 14: 49: 01 & 0.1283 \\ 12159 & 14: 49: 02 & 0.1291 \\ 12160 & 14: 49: 03 & 0.1295 \\ 12161 & 14: 49: 04 & 0.1271 \\ 12162 & 14: 49: 05 & 0.1281 \\ 12163 & 14: 49: 06 & 0.1246 \\ 12164 & 14: 49: 07 & 0.1291 \\ 12165 & 14: 49: 08 & 0.1250 \\ 12166 & 14: 49: 09 & 0.1303\end{array}$


Appendix A - Fuel Data Test 1 NIST102505_1.xIs

$\begin{array}{lll}12167 & 14: 49: 10 & 0.1268 \\ 12168 & 14: 49: 11 & 0.1323 \\ 12169 & 14: 49: 12 & 0.1296 \\ 12170 & 14: 49: 13 & 0.1316 \\ 12171 & 14: 49: 14 & 0.1293 \\ 12172 & 14: 49: 15 & 0.1289 \\ 12173 & 14: 49: 16 & 0.1326 \\ 12174 & 14: 49: 17 & 0.1283 \\ 12175 & 14: 49: 18 & 0.1297 \\ 12176 & 14: 49: 19 & 0.1243 \\ 12177 & 14: 49: 20 & 0.1299 \\ 12178 & 14: 49: 21 & 0.1258 \\ 12179 & 14: 49: 22 & 0.1292 \\ 12180 & 14: 49: 23 & 0.1267 \\ 12181 & 14: 49: 24 & 0.1296 \\ 12182 & 14: 49: 25 & 0.1280 \\ 12183 & 14: 49: 26 & 0.1280 \\ 12184 & 14: 49: 27 & 0.1286 \\ 12185 & 14: 49: 28 & 0.1271 \\ 12186 & 14: 49: 29 & 0.1287 \\ 12187 & 14: 49: 30 & 0.1253 \\ 12188 & 14: 49: 31 & 0.1295 \\ 12189 & 14: 49: 32 & 0.1247 \\ 12190 & 14: 49: 33 & 0.1285 \\ 12191 & 14: 49: 34 & 0.1260 \\ 12192 & 14: 49: 35 & 0.1310 \\ 12193 & 14: 49: 36 & 0.1276 \\ 12194 & 14: 49: 37 & 0.1290 \\ 12195 & 14: 49: 38 & 0.1291 \\ 12196 & 14: 49: 39 & 0.1271 \\ 12197 & 14: 49: 40 & 0.1271 \\ 12198 & 14: 49: 41 & 0.1251 \\ 12199 & 14: 49: 42 & 0.1305 \\ 12200 & 14: 49: 43 & 0.1277 \\ 12201 & 14: 49: 44 & 0.1300 \\ 12202 & 14: 49: 45 & 0.1256 \\ 12203 & 14: 49: 46 & 0.1304 \\ 12204 & 14: 49: 47 & 0.1278 \\ 12205 & 14: 49: 48 & 0.1313 \\ 12206 & 14: 49: 49 & 0.1298 \\ 12207 & 14: 49: 50 & 0.1326 \\ 12208 & 14: 49: 51 & 0.1285 \\ 12209 & 14: 49: 52 & 0.1286 \\ 12210 & 14: 49: 53 & 0.1282 \\ 12211 & 14: 49: 54 & 0.1266 \\ 12212 & 14: 49: 55 & 0.1284 \\ 12213 & 14: 49: 56 & 0.1248 \\ 12214 & 14: 49: 57 & 0.1283 \\ 12215 & 14: 49: 58 & 0.1250 \\ 12216 & 14: 49: 59 & 0.1286 \\ 12217 & 14: 50: 00 & 0.1274 \\ 12218 & 14: 50: 01 & 0.1304\end{array}$


Appendix A - Fuel Data Test 1 NIST102505_1.xls

$\begin{array}{lll}12219 & 14: 50: 02 & 0.1276 \\ 12220 & 14: 50: 03 & 0.1285 \\ 12221 & 14: 50: 04 & 0.1297 \\ 12222 & 14: 50: 05 & 0.1283 \\ 12223 & 14: 50: 06 & 0.1271 \\ 12224 & 14: 50: 07 & 0.1264 \\ 12225 & 14: 50: 08 & 0.1264 \\ 12226 & 14: 50: 09 & 0.1224 \\ 12227 & 14: 50: 10 & 0.1281 \\ 12228 & 14: 50: 11 & 0.1253 \\ 12229 & 14: 50: 12 & 0.1293 \\ 12230 & 14: 50: 13 & 0.1283 \\ 12231 & 14: 50: 14 & 0.1320 \\ 12232 & 14: 50: 15 & 0.1317 \\ 12233 & 14: 50: 16 & 0.1301 \\ 12234 & 14: 50: 17 & 0.1275 \\ 12235 & 14: 50: 18 & 0.1268 \\ 12236 & 14: 50: 19 & 0.1300 \\ 12237 & 14: 50: 20 & 0.1273 \\ 12238 & 14: 50: 21 & 0.1292 \\ 12239 & 14: 50: 22 & 0.1259 \\ 12240 & 14: 50: 23 & 0.1294 \\ 12241 & 14: 50: 24 & 0.1254 \\ 12242 & 14: 50: 25 & 0.1305 \\ 12243 & 14: 50: 26 & 0.1274 \\ 12244 & 14: 50: 27 & 0.1309 \\ 12245 & 14: 50: 28 & 0.1287 \\ 12246 & 14: 50: 29 & 0.1321 \\ 12247 & 14: 50: 30 & 0.1283 \\ 12248 & 14: 50: 31 & 0.1267 \\ 12249 & 14: 50: 32 & 0.1302 \\ 12250 & 14: 50: 33 & 0.1267 \\ 12251 & 14: 50: 34 & 0.1288 \\ 12252 & 14: 50: 35 & 0.1261 \\ 12253 & 14: 50: 36 & 0.1312 \\ 12254 & 14: 50: 37 & 0.1269 \\ 12255 & 14: 50: 38 & 0.1295 \\ 12256 & 14: 50: 39 & 0.1293 \\ 12257 & 14: 50: 40 & 0.1294 \\ 12258 & 14: 50: 41 & 0.1264 \\ 12259 & 14: 50: 42 & 0.1282 \\ 12260 & 14: 50: 43 & 0.1285 \\ 12261 & 14: 50: 44 & 0.1291 \\ 12262 & 14: 50: 45 & 0.1297 \\ 12263 & 14: 50: 46 & 0.1288 \\ 12264 & 14: 50: 47 & 0.1306 \\ 12265 & 14: 50: 48 & 0.1255 \\ 12266 & 14: 50: 49 & 0.1307 \\ 12267 & 14: 50: 50 & 0.1277 \\ 12268 & 14: 50: 51 & 0.1328 \\ 12269 & 14: 50: 52 & 0.1277 \\ 12270 & 14: 50: 53 & 0.1324\end{array}$


Appendix A - Fuel Data Test 1 NIST102505_1.xls

$\begin{array}{lll}12271 & 14: 50: 54 & 0.1293 \\ 12272 & 14: 50: 55 & 0.1297 \\ 12273 & 14: 50: 56 & 0.1314 \\ 12274 & 14: 50: 57 & 0.1283 \\ 12275 & 14: 50: 58 & 0.1283 \\ 12276 & 14: 50: 59 & 0.1244 \\ 12277 & 14: 51: 00 & 0.1300 \\ 12278 & 14: 51: 01 & 0.1257 \\ 12279 & 14: 51: 02 & 0.1305 \\ 12280 & 14: 51: 03 & 0.1275 \\ 12281 & 14: 51: 04 & 0.1328 \\ 12282 & 14: 51: 05 & 0.1285 \\ 12283 & 14: 51: 06 & 0.1300 \\ 12284 & 14: 51: 07 & 0.1271 \\ 12285 & 14: 51: 08 & 0.1278 \\ 12286 & 14: 51: 09 & 0.1267 \\ 12287 & 14: 51: 10 & 0.1249 \\ 12288 & 14: 51: 11 & 0.1291 \\ 12289 & 14: 51: 12 & 0.1251 \\ 12290 & 14: 51: 13 & 0.1293 \\ 12291 & 14: 51: 14 & 0.1261 \\ 12292 & 14: 51: 15 & 0.1300 \\ 12293 & 14: 51: 16 & 0.1248 \\ 12294 & 14: 51: 17 & 0.1291 \\ 12295 & 14: 51: 18 & 0.1298 \\ 12296 & 14: 51: 19 & 0.1306 \\ 12297 & 14: 51: 20 & 0.1277 \\ 12298 & 14: 51: 21 & 0.1281 \\ 12299 & 14: 51: 22 & 0.1272 \\ 12300 & 14: 51: 23 & 0.1260 \\ 12301 & 14: 51: 24 & 0.1283 \\ 12302 & 14: 51: 25 & 0.1257 \\ 12303 & 14: 51: 26 & 0.1301 \\ 12304 & 14: 51: 27 & 0.1269 \\ 12305 & 14: 51: 28 & 0.1298 \\ 12306 & 14: 51: 29 & 0.1255 \\ 12307 & 14: 51: 30 & 0.1292 \\ 12308 & 14: 51: 31 & 0.1293 \\ 12309 & 14: 51: 32 & 0.1295 \\ 12310 & 14: 51: 33 & 0.1269 \\ 12311 & 14: 51: 34 & 0.1265 \\ 12312 & 14: 51: 35 & 0.1300 \\ 12313 & 14: 51: 36 & 0.1276 \\ 12314 & 14: 51: 37 & 0.1291 \\ 12315 & 14: 51: 38 & 0.1259 \\ 12316 & 14: 51: 39 & 0.1293 \\ 12317 & 14: 51: 40 & 0.1244 \\ 12318 & 14: 51: 41 & 0.1298 \\ 12319 & 14: 51: 42 & 0.1282 \\ 12320 & 14: 51: 43 & 0.1318 \\ 12321 & 14: 51: 44 & 0.1273 \\ 12322 & 14: 51: 45 & 0.1297\end{array}$


Appendix A - Fuel Data Test 1 NIST102505_1.xls

$\begin{array}{lll}12323 & 14: 51: 46 & 0.1281 \\ 12324 & 14: 51: 47 & 0.1272 \\ 12325 & 14: 51: 48 & 0.1291 \\ 12326 & 14: 51: 49 & 0.1276 \\ 12327 & 14: 51: 50 & 0.1292 \\ 12328 & 14: 51: 51 & 0.1244 \\ 12329 & 14: 51: 52 & 0.1294 \\ 12330 & 14: 51: 53 & 0.1262 \\ 12331 & 14: 51: 54 & 0.1306 \\ 12332 & 14: 51: 55 & 0.1269 \\ 12333 & 14: 51: 56 & 0.1309 \\ 12334 & 14: 51: 57 & 0.1279 \\ 12335 & 14: 51: 58 & 0.1269 \\ 12336 & 14: 51: 59 & 0.1289 \\ 12337 & 14: 52: 00 & 0.1267 \\ 12338 & 14: 52: 01 & 0.1277 \\ 12339 & 14: 52: 02 & 0.1246 \\ 12340 & 14: 52: 03 & 0.1308 \\ 12341 & 14: 52: 04 & 0.1253 \\ 12342 & 14: 52: 05 & 0.1299 \\ 12343 & 14: 52: 06 & 0.1268 \\ 12344 & 14: 52: 07 & 0.1315 \\ 12345 & 14: 52: 08 & 0.1282 \\ 12346 & 14: 52: 09 & 0.1310 \\ 12347 & 14: 52: 10 & 0.1277 \\ 12348 & 14: 52: 11 & 0.1294 \\ 12349 & 14: 52: 12 & 0.1270 \\ 12350 & 14: 52: 13 & 0.1269 \\ 12351 & 14: 52: 14 & 0.1313 \\ 12352 & 14: 52: 15 & 0.1274 \\ 12353 & 14: 52: 16 & 0.1313 \\ 12354 & 14: 52: 17 & 0.1271 \\ 12355 & 14: 52: 18 & 0.1309 \\ 12356 & 14: 52: 19 & 0.1271 \\ 12357 & 14: 52: 20 & 0.1294 \\ 12358 & 14: 52: 21 & 0.1277 \\ 12359 & 14: 52: 22 & 0.1294 \\ 12360 & 14: 52: 23 & 0.1275 \\ 12361 & 14: 52: 24 & 0.1272 \\ 12362 & 14: 52: 25 & 0.1301 \\ 12363 & 14: 52: 26 & 0.1277 \\ 12364 & 14: 52: 27 & 0.1304 \\ 12365 & 14: 52: 28 & 0.1253 \\ 12366 & 14: 52: 29 & 0.1292 \\ 12367 & 14: 52: 30 & 0.1252 \\ 12368 & 14: 52: 31 & 0.1290 \\ 12369 & 14: 52: 32 & 0.1254 \\ 12370 & 14: 52: 33 & 0.1296 \\ 12371 & 14: 52: 34 & 0.1271 \\ 12372 & 14: 52: 35 & 0.1286 \\ 12373 & 14: 52: 36 & 0.1283 \\ 12374 & 14: 52: 37 & 0.1268\end{array}$


Appendix A - Fuel Data Test 1 NIST102505_1.xls

$\begin{array}{lll}12375 & 14: 52: 38 & 0.1281 \\ 12376 & 14: 52: 39 & 0.1269 \\ 12377 & 14: 52: 40 & 0.1271 \\ 12378 & 14: 52: 41 & 0.1232 \\ 12379 & 14: 52: 42 & 0.1302 \\ 12380 & 14: 52: 43 & 0.1264 \\ 12381 & 14: 52: 44 & 0.1307 \\ 12382 & 14: 52: 45 & 0.1273 \\ 12383 & 14: 52: 46 & 0.1294 \\ 12384 & 14: 52: 47 & 0.1266 \\ 12385 & 14: 52: 48 & 0.1274 \\ 12386 & 14: 52: 49 & 0.1252 \\ 12387 & 14: 52: 50 & 0.1254 \\ 12388 & 14: 52: 51 & 0.1279 \\ 12389 & 14: 52: 52 & 0.1253 \\ 12390 & 14: 52: 53 & 0.1299 \\ 12391 & 14: 52: 54 & 0.1266 \\ 12392 & 14: 52: 55 & 0.1285 \\ 12393 & 14: 52: 56 & 0.1237 \\ 12394 & 14: 52: 57 & 0.1297 \\ 12395 & 14: 52: 58 & 0.1256 \\ 12396 & 14: 52: 59 & 0.1268 \\ 12397 & 14: 53: 00 & 0.1293 \\ 12398 & 14: 53: 01 & 0.1286 \\ 12399 & 14: 53: 02 & 0.1284 \\ 12400 & 14: 53: 03 & 0.1255 \\ 12401 & 14: 53: 04 & 0.1298 \\ 12402 & 14: 53: 05 & 0.1268 \\ 12403 & 14: 53: 06 & 0.1295 \\ 12404 & 14: 53: 07 & 0.1243 \\ 12405 & 14: 53: 08 & 0.1314 \\ 12406 & 14: 53: 09 & 0.1273 \\ 12407 & 14: 53: 10 & 0.1311 \\ 12408 & 14: 53: 11 & 0.1271 \\ 12409 & 14: 53: 12 & 0.1300 \\ 12410 & 14: 53: 13 & 0.1263 \\ 12411 & 14: 53: 14 & 0.1262 \\ 12412 & 14: 53: 15 & 0.1279 \\ 12413 & 14: 53: 16 & 0.1263 \\ 12414 & 14: 53: 17 & 0.1283 \\ 12415 & 14: 53: 18 & 0.1235 \\ 12416 & 14: 53: 19 & 0.1291 \\ 12417 & 14: 53: 20 & 0.1242 \\ 12418 & 14: 53: 21 & 0.1285 \\ 12419 & 14: 53: 22 & 0.1275 \\ 12420 & 14: 53: 23 & 0.1302 \\ 12421 & 14: 53: 24 & 0.1295 \\ 12422 & 14: 53: 25 & 0.1310 \\ 12423 & 14: 53: 26 & 0.1298 \\ 12424 & 14: 53: 27 & 0.1285 \\ 12425 & 14: 53: 28 & 0.1295 \\ 12426 & 14: 53: 29 & 0.1257\end{array}$


Appendix A - Fuel Data Test 1 NIST102505_1.xls

$\begin{array}{lll}12427 & 14: 53: 30 & 0.1299 \\ 12428 & 14: 53: 31 & 0.1250 \\ 12429 & 14: 53: 32 & 0.1296 \\ 12430 & 14: 53: 33 & 0.1260 \\ 12431 & 14: 53: 34 & 0.1297 \\ 12432 & 14: 53: 35 & 0.1251 \\ 12433 & 14: 53: 36 & 0.1292 \\ 12434 & 14: 53: 37 & 0.1292 \\ 12435 & 14: 53: 38 & 0.1288 \\ 12436 & 14: 53: 39 & 0.1268 \\ 12437 & 14: 53: 40 & 0.1250 \\ 12438 & 14: 53: 41 & 0.1306 \\ 12439 & 14: 53: 42 & 0.1267 \\ 12440 & 14: 53: 43 & 0.1283 \\ 12441 & 14: 53: 44 & 0.1226 \\ 12442 & 14: 53: 45 & 0.1302 \\ 12443 & 14: 53: 46 & 0.1265 \\ 12444 & 14: 53: 47 & 0.1303 \\ 12445 & 14: 53: 48 & 0.1267 \\ 12446 & 14: 53: 49 & 0.1298 \\ 12447 & 14: 53: 50 & 0.1286 \\ 12448 & 14: 53: 51 & 0.1279 \\ 12449 & 14: 53: 52 & 0.1282 \\ 12450 & 14: 53: 53 & 0.1264 \\ 12451 & 14: 53: 54 & 0.1273 \\ 12452 & 14: 53: 55 & 0.1256 \\ 12453 & 14: 53: 56 & 0.1280 \\ 12454 & 14: 53: 57 & 0.1240 \\ 12455 & 14: 53: 58 & 0.1299 \\ 12456 & 14: 53: 59 & 0.1263 \\ 12457 & 14: 54: 00 & 0.1311 \\ 12458 & 14: 54: 01 & 0.1263 \\ 12459 & 14: 54: 02 & 0.1304 \\ 12460 & 14: 54: 03 & 0.1291 \\ 12461 & 14: 54: 04 & 0.1305 \\ 12462 & 14: 54: 05 & 0.1283 \\ 12463 & 14: 54: 06 & 0.1288 \\ 12464 & 14: 54: 07 & 0.1288 \\ 12465 & 14: 54: 08 & 0.1250 \\ 12466 & 14: 54: 09 & 0.1305 \\ 12467 & 14: 54: 10 & 0.1251 \\ 12468 & 14: 54: 11 & 0.1298 \\ 12469 & 14: 54: 12 & 0.1262 \\ 12470 & 14: 54: 13 & 0.1330 \\ 12471 & 14: 54: 14 & 0.1297 \\ 12472 & 14: 54: 15 & 0.1315 \\ 12473 & 14: 54: 16 & 0.1282 \\ 12474 & 14: 54: 17 & 0.1289 \\ 12475 & 14: 54: 18 & 0.1283 \\ 12476 & 14: 54: 19 & 0.1272 \\ 12477 & 14: 54: 20 & 0.1317 \\ 12478 & 14: 54: 21 & 0.1265\end{array}$


Appendix A - Fuel Data Test 1 NIST102505_1.xIs

$\begin{array}{lll}12479 & 14: 54: 22 & 0.1307 \\ 12480 & 14: 54: 23 & 0.1282 \\ 12481 & 14: 54: 24 & 0.1313 \\ 12482 & 14: 54: 25 & 0.1272 \\ 12483 & 14: 54: 26 & 0.1334 \\ 12484 & 14: 54: 27 & 0.1297 \\ 12485 & 14: 54: 28 & 0.1303 \\ 12486 & 14: 54: 29 & 0.1246 \\ 12487 & 14: 54: 30 & 0.1259 \\ 12488 & 14: 54: 31 & 0.1273 \\ 12489 & 14: 54: 32 & 0.1252 \\ 12490 & 14: 54: 33 & 0.1291 \\ 12491 & 14: 54: 34 & 0.1249 \\ 12492 & 14: 54: 35 & 0.1290 \\ 12493 & 14: 54: 36 & 0.1257 \\ 12494 & 14: 54: 37 & 0.1297 \\ 12495 & 14: 54: 38 & 0.1256 \\ 12496 & 14: 54: 39 & 0.1284 \\ 12497 & 14: 54: 40 & 0.1291 \\ 12498 & 14: 54: 41 & 0.1278 \\ 12499 & 14: 54: 42 & 0.1258 \\ 12500 & 14: 54: 43 & 0.1260 \\ 12501 & 14: 54: 44 & 0.1281 \\ 12502 & 14: 54: 45 & 0.1255 \\ 12503 & 14: 54: 46 & 0.1283 \\ 12504 & 14: 54: 47 & 0.1242 \\ 12505 & 14: 54: 48 & 0.1294 \\ 12506 & 14: 54: 49 & 0.1260 \\ 12507 & 14: 54: 50 & 0.1320 \\ 12508 & 14: 54: 51 & 0.1273 \\ 12509 & 14: 54: 52 & 0.1292 \\ 12510 & 14: 54: 53 & 0.1283 \\ 12511 & 14: 54: 54 & 0.1287 \\ 12512 & 14: 54: 55 & 0.1268 \\ 12513 & 14: 54: 56 & 0.1255 \\ 12514 & 14: 54: 57 & 0.1301 \\ 12515 & 14: 54: 58 & 0.1259 \\ 12516 & 14: 54: 59 & 0.1282 \\ 12517 & 14: 55: 00 & 0.1234 \\ 12518 & 14: 55: 01 & 0.1300 \\ 12519 & 14: 55: 02 & 0.1268 \\ 12520 & 14: 55: 03 & 0.1285 \\ 12521 & 14: 55: 04 & 0.1253 \\ 12522 & 14: 55: 05 & 0.1293 \\ 12523 & 14: 55: 06 & 0.1274 \\ 12524 & 14: 55: 07 & 0.1274 \\ 12525 & 14: 55: 08 & 0.1291 \\ 12526 & 14: 55: 09 & 0.1271 \\ 12527 & 14: 55: 10 & 0.1278 \\ 12528 & 14: 55: 11 & 0.1257 \\ 12529 & 14: 55: 12 & 0.1285 \\ 12530 & 14: 55: 13 & 0.1235\end{array}$


Appendix A - Fuel Data Test 1 NIST102505_1.xls

$\begin{array}{lll}12531 & 14: 55: 14 & 0.1294 \\ 12532 & 14: 55: 15 & 0.1265 \\ 12533 & 14: 55: 16 & 0.1307 \\ 12534 & 14: 55: 17 & 0.1285 \\ 12535 & 14: 55: 18 & 0.1290 \\ 12536 & 14: 55: 19 & 0.1290 \\ 12537 & 14: 55: 20 & 0.1286 \\ 12538 & 14: 55: 21 & 0.1275 \\ 12539 & 14: 55: 22 & 0.1277 \\ 12540 & 14: 55: 23 & 0.1292 \\ 12541 & 14: 55: 24 & 0.1253 \\ 12542 & 14: 55: 25 & 0.1284 \\ 12543 & 14: 55: 26 & 0.1251 \\ 12544 & 14: 55: 27 & 0.1294 \\ 12545 & 14: 55: 28 & 0.1247 \\ 12546 & 14: 55: 29 & 0.1296 \\ 12547 & 14: 55: 30 & 0.1296 \\ 12548 & 14: 55: 31 & 0.1305 \\ 12549 & 14: 55: 32 & 0.1259 \\ 12550 & 14: 55: 33 & 0.1285 \\ 12551 & 14: 55: 34 & 0.1289 \\ 12552 & 14: 55: 35 & 0.1276 \\ 12553 & 14: 55: 36 & 0.1316 \\ 12554 & 14: 55: 37 & 0.1267 \\ 12555 & 14: 55: 38 & 0.1305 \\ 12556 & 14: 55: 39 & 0.1275 \\ 12558 & 14: 55: 41 & 0.1311 \\ 12558 & 14: 55: 41 & 0.1279 \\ 12559 & 14: 55: 42 & 0.1297 \\ 12560 & 14: 55: 43 & 0.1297 \\ 12561 & 14: 55: 44 & 0.1283 \\ 12562 & 14: 55: 45 & 0.1255 \\ 12563 & 14: 55: 46 & 0.1252 \\ 12564 & 14: 55: 47 & 0.1298 \\ 12565 & 14: 55: 48 & 0.1263 \\ 12566 & 14: 55: 49 & 0.1287 \\ 12567 & 14: 55: 50 & 0.1250 \\ 12568 & 14: 55: 51 & 0.1300 \\ 12569 & 14: 55: 52 & 0.1266 \\ 12570 & 14: 55: 53 & 0.1316 \\ 12571 & 14: 55: 54 & 0.1281 \\ 12572 & 14: 55: 55 & 0.1315 \\ 12573 & 14: 55: 56 & 0.1281 \\ 12574 & 14: 55: 57 & 0.1301 \\ 12575 & 14: 55: 58 & 0.1277 \\ 12576 & 14: 55: 59 & 0.1261 \\ 12577 & 14: 56: 00 & 0.1283 \\ 12578 & 14: 56: 01 & 0.1257 \\ 12579 & 14: 56: 02 & 0.1293 \\ 12580 & 14: 56: 03 & 0.1237 \\ 12581 & 14: 56: 04 & 0.1311 \\ 12582 & 14: 56: 05 & 0.1272\end{array}$


Appendix A - Fuel Data Test 1 NIST102505_1.xIs

$\begin{array}{lll}12583 & 14: 56: 06 & 0.1315 \\ 12584 & 14: 56: 07 & 0.1267 \\ 12585 & 14: 56: 08 & 0.1296 \\ 12586 & 14: 56: 09 & 0.1273 \\ 12587 & 14: 56: 10 & 0.1278 \\ 12588 & 14: 56: 11 & 0.1275 \\ 12589 & 14: 56: 12 & 0.1272 \\ 12590 & 14: 56: 13 & 0.1288 \\ 12591 & 14: 56: 14 & 0.1253 \\ 12592 & 14: 56: 15 & 0.1291 \\ 12593 & 14: 56: 16 & 0.1261 \\ 12594 & 14: 56: 17 & 0.1306 \\ 12595 & 14: 56: 18 & 0.1270 \\ 12596 & 14: 56: 19 & 0.1328 \\ 12597 & 14: 56: 20 & 0.1286 \\ 12598 & 14: 56: 21 & 0.1296 \\ 12599 & 14: 56: 22 & 0.1297 \\ 12600 & 14: 56: 23 & 0.1293 \\ 12601 & 14: 56: 24 & 0.1293 \\ 12602 & 14: 56: 25 & 0.1254 \\ 12603 & 14: 56: 26 & 0.1292 \\ 12604 & 14: 56: 27 & 0.1248 \\ 12605 & 14: 56: 28 & 0.1287 \\ 12606 & 14: 56: 29 & 0.1238 \\ 12607 & 14: 56: 30 & 0.1290 \\ 12608 & 14: 56: 31 & 0.1267 \\ 12609 & 14: 56: 32 & 0.1303 \\ 12610 & 14: 56: 33 & 0.1270 \\ 12611 & 14: 56: 34 & 0.1286 \\ 12612 & 14: 56: 35 & 0.1268 \\ 12613 & 14: 56: 36 & 0.1280 \\ 12614 & 14: 56: 37 & 0.1291 \\ 12615 & 14: 56: 38 & 0.1281 \\ 12616 & 14: 56: 39 & 0.1294 \\ 12617 & 14: 56: 40 & 0.1247 \\ 12618 & 14: 56: 41 & 0.1321 \\ 12619 & 14: 56: 42 & 0.1287 \\ 12620 & 14: 56: 43 & 0.1313 \\ 12621 & 14: 56: 44 & 0.1266 \\ 12622 & 14: 56: 45 & 0.1309 \\ 12623 & 14: 56: 46 & 0.1282 \\ 12624 & 14: 56: 47 & 0.1284 \\ 12625 & 14: 56: 48 & 0.1256 \\ 12626 & 14: 56: 49 & 0.1264 \\ 12627 & 14: 56: 50 & 0.1288 \\ 12628 & 14: 56: 51 & 0.1251 \\ 12629 & 14: 56: 52 & 0.1270 \\ 12630 & 14: 56: 53 & 0.1252 \\ 12631 & 14: 56: 54 & 0.1306 \\ 12632 & 14: 56: 55 & 0.1256 \\ 12633 & 14: 56: 56 & 0.1309 \\ 12634 & 14: 56: 57 & 0.1284\end{array}$


Appendix A - Fuel Data Test 1 NIST102505_1.xIs

$\begin{array}{lll}12635 & 14: 56: 58 & 0.1308 \\ 12636 & 14: 56: 59 & 0.1289 \\ 12637 & 14: 57: 00 & 0.1279 \\ 12638 & 14: 57: 01 & 0.1290 \\ 12639 & 14: 57: 02 & 0.1269 \\ 12640 & 14: 57: 03 & 0.1288 \\ 12641 & 14: 57: 04 & 0.1259 \\ 12642 & 14: 57: 05 & 0.1298 \\ 12643 & 14: 57: 06 & 0.1249 \\ 12644 & 14: 57: 07 & 0.1293 \\ 12645 & 14: 57: 08 & 0.1275 \\ 12646 & 14: 57: 09 & 0.1307 \\ 12647 & 14: 57: 10 & 0.1280 \\ 12648 & 14: 57: 11 & 0.1273 \\ 12649 & 14: 57: 12 & 0.1280 \\ 12650 & 14: 57: 13 & 0.1271 \\ 12651 & 14: 57: 14 & 0.1267 \\ 12652 & 14: 57: 15 & 0.1245 \\ 12653 & 14: 57: 16 & 0.1261 \\ 12654 & 14: 57: 17 & 0.1221 \\ 12655 & 14: 57: 18 & 0.1281 \\ 12656 & 14: 57: 19 & 0.1257 \\ 12657 & 14: 57: 20 & 0.1294 \\ 12658 & 14: 57: 21 & 0.1247 \\ 12659 & 14: 57: 22 & 0.1308 \\ 12660 & 14: 57: 23 & 0.1280 \\ 12661 & 14: 57: 24 & 0.1300 \\ 12662 & 14: 57: 25 & 0.1263 \\ 12663 & 14: 57: 26 & 0.1269 \\ 12664 & 14: 57: 27 & 0.1291 \\ 12665 & 14: 57: 28 & 0.1261 \\ 12666 & 14: 57: 29 & 0.1283 \\ 12667 & 14: 57: 30 & 0.1260 \\ 12668 & 14: 57: 31 & 0.1287 \\ 12669 & 14: 57: 32 & 0.1238 \\ 12670 & 14: 57: 33 & 0.1302 \\ 12671 & 14: 57: 34 & 0.1265 \\ 12672 & 14: 57: 35 & 0.1294 \\ 12673 & 14: 57: 36 & 0.1266 \\ 12674 & 14: 57: 37 & 0.1279 \\ 12675 & 14: 57: 38 & 0.1284 \\ 12676 & 14: 57: 39 & 0.1250 \\ 12677 & 14: 57: 40 & 0.1288 \\ 12678 & 14: 57: 41 & 0.1256 \\ 12679 & 14: 57: 42 & 0.1292 \\ 12680 & 14: 57: 43 & 0.1249 \\ 12681 & 14: 57: 44 & 0.1304 \\ 12682 & 14: 57: 45 & 0.1269 \\ 12683 & 14: 57: 46 & 0.1298 \\ 12684 & 14: 57: 47 & 0.1270 \\ 12685 & 14: 57: 48 & 0.1301 \\ 12686 & 14: 57: 49 & 0.1273\end{array}$


Appendix A - Fuel Data Test 1 NIST102505_1.xls

$\begin{array}{lll}12687 & 14: 57: 50 & 0.1278 \\ 12688 & 14: 57: 51 & 0.1283 \\ 12689 & 14: 57: 52 & 0.1274 \\ 12690 & 14: 57: 53 & 0.1287 \\ 12691 & 14: 57: 54 & 0.1234 \\ 12692 & 14: 57: 55 & 0.1274 \\ 12693 & 14: 57: 56 & 0.1266 \\ 12694 & 14: 57: 57 & 0.1300 \\ 12695 & 14: 57: 58 & 0.1259 \\ 12696 & 14: 57: 59 & 0.1300 \\ 12697 & 14: 58: 00 & 0.1295 \\ 12698 & 14: 58: 01 & 0.1290 \\ 12699 & 14: 58: 02 & 0.1269 \\ 12700 & 14: 58: 03 & 0.1262 \\ 12701 & 14: 58: 04 & 0.1277 \\ 12702 & 14: 58: 05 & 0.1248 \\ 12703 & 14: 58: 06 & 0.1267 \\ 12704 & 14: 58: 07 & 0.1238 \\ 12705 & 14: 58: 08 & 0.1285 \\ 12706 & 14: 58: 09 & 0.1258 \\ 12707 & 14: 58: 10 & 0.1302 \\ 12708 & 14: 58: 11 & 0.1292 \\ 12709 & 14: 58: 12 & 0.1320 \\ 12710 & 14: 58: 13 & 0.1279 \\ 12711 & 14: 58: 14 & 0.1288 \\ 12712 & 14: 58: 15 & 0.1284 \\ 12713 & 14: 58: 16 & 0.1274 \\ 12714 & 14: 58: 17 & 0.1287 \\ 12715 & 14: 58: 18 & 0.1269 \\ 12716 & 14: 58: 19 & 0.1298 \\ 12717 & 14: 58: 20 & 0.1247 \\ 12718 & 14: 58: 21 & 0.1286 \\ 12719 & 14: 58: 22 & 0.1256 \\ 12720 & 14: 58: 23 & 0.1305 \\ 12721 & 14: 58: 24 & 0.1261 \\ 12722 & 14: 58: 25 & 0.1302 \\ 12723 & 14: 58: 26 & 0.1286 \\ 12724 & 14: 58: 27 & 0.1285 \\ 12725 & 14: 58: 28 & 0.1267 \\ 12726 & 14: 58: 29 & 0.1265 \\ 12727 & 14: 58: 30 & 0.1277 \\ 12728 & 14: 58: 31 & 0.1234 \\ 12729 & 14: 58: 32 & 0.1270 \\ 12730 & 14: 58: 33 & 0.1278 \\ 12731 & 14: 58: 34 & 0.1308 \\ 12732 & 14: 58: 35 & 0.1262 \\ 12733 & 14: 58: 36 & 0.1306 \\ 12734 & 14: 58: 37 & 0.1298 \\ 12735 & 14: 58: 38 & 0.1294 \\ 12736 & 14: 58: 39 & 0.1265 \\ 12737 & 14: 58: 40 & 0.1267 \\ 12738 & 14: 58: 41 & 0.1289\end{array}$


Appendix A - Fuel Data Test 1 NIST102505_1.xIs

$\begin{array}{lll}12739 & 14: 58: 42 & 0.1269 \\ 12740 & 14: 58: 43 & 0.1286 \\ 12741 & 14: 58: 44 & 0.1265 \\ 12742 & 14: 58: 45 & 0.1288 \\ 12743 & 14: 58: 46 & 0.1237 \\ 12744 & 14: 58: 47 & 0.1295 \\ 12745 & 14: 58: 48 & 0.1268 \\ 12746 & 14: 58: 49 & 0.1305 \\ 12747 & 14: 58: 50 & 0.1269 \\ 12748 & 14: 58: 51 & 0.1299 \\ 12749 & 14: 58: 52 & 0.1270 \\ 12750 & 14: 58: 53 & 0.1271 \\ 12751 & 14: 58: 54 & 0.1309 \\ 12752 & 14: 58: 55 & 0.1267 \\ 12753 & 14: 58: 56 & 0.1277 \\ 12754 & 14: 58: 57 & 0.1232 \\ 12755 & 14: 58: 58 & 0.1295 \\ 12756 & 14: 58: 59 & 0.1248 \\ 12757 & 14: 59: 00 & 0.1305 \\ 12758 & 14: 59: 01 & 0.1280 \\ 12759 & 14: 59: 02 & 0.1292 \\ 12760 & 14: 59: 03 & 0.1282 \\ 12761 & 14: 59: 04 & 0.1292 \\ 12762 & 14: 59: 05 & 0.1271 \\ 12763 & 14: 59: 06 & 0.1263 \\ 12764 & 14: 59: 07 & 0.1278 \\ 12765 & 14: 59: 08 & 0.1247 \\ 12766 & 14: 59: 09 & 0.1285 \\ 12767 & 14: 59: 10 & 0.1244 \\ 12768 & 14: 59: 11 & 0.1287 \\ 12769 & 14: 59: 12 & 0.1266 \\ 12770 & 14: 59: 13 & 0.1304 \\ 12771 & 14: 59: 14 & 0.1255 \\ 12772 & 14: 59: 15 & 0.1299 \\ 12773 & 14: 59: 16 & 0.1287 \\ 12774 & 14: 59: 17 & 0.1288 \\ 12775 & 14: 59: 18 & 0.1269 \\ 12776 & 14: 59: 19 & 0.1278 \\ 12777 & 14: 59: 20 & 0.1284 \\ 12778 & 14: 59: 21 & 0.1248 \\ 12779 & 14: 59: 22 & 0.1297 \\ 12780 & 14: 59: 23 & 0.1260 \\ 12781 & 14: 59: 24 & 0.1304 \\ 12782 & 14: 59: 25 & 0.1260 \\ 12783 & 14: 59: 26 & 0.1316 \\ 12784 & 14: 59: 27 & 0.1280 \\ 12785 & 14: 59: 28 & 0.1283 \\ 12786 & 14: 59: 29 & 0.1286 \\ 12787 & 14: 59: 30 & 0.1283 \\ 12788 & 14: 59: 31 & 0.1272 \\ 12789 & 14: 59: 32 & 0.1255 \\ 12790 & 14: 59: 33 & 0.1305\end{array}$


Appendix A - Fuel Data Test 1 NIST102505_1.xIs

$\begin{array}{lll}12791 & 14: 59: 34 & 0.1259 \\ 12792 & 14: 59: 35 & 0.1293 \\ 12793 & 14: 59: 36 & 0.1251 \\ 12794 & 14: 59: 37 & 0.1296 \\ 12795 & 14: 59: 38 & 0.1253 \\ 12796 & 14: 59: 39 & 0.1286 \\ 12797 & 14: 59: 40 & 0.1278 \\ 12798 & 14: 59: 41 & 0.1310 \\ 12799 & 14: 59: 42 & 0.1283 \\ 12800 & 14: 59: 43 & 0.1287 \\ 12801 & 14: 59: 44 & 0.1279 \\ 12802 & 14: 59: 45 & 0.1257 \\ 12803 & 14: 59: 46 & 0.1283 \\ 12804 & 14: 59: 47 & 0.1248 \\ 12805 & 14: 59: 48 & 0.1291 \\ 12806 & 14: 59: 49 & 0.1251 \\ 12807 & 14: 59: 50 & 0.1303 \\ 12808 & 14: 59: 51 & 0.1269 \\ 12809 & 14: 59: 52 & 0.1295 \\ 12810 & 14: 59: 53 & 0.1271 \\ 12811 & 14: 59: 54 & 0.1298 \\ 12812 & 14: 59: 55 & 0.1264 \\ 12813 & 14: 59: 56 & 0.1281 \\ 12814 & 14: 59: 57 & 0.1282 \\ 12815 & 14: 59: 58 & 0.1287 \\ 12816 & 14: 59: 59 & 0.1299 \\ 12817 & 15: 00: 00 & 0.1260 \\ 12818 & 15: 00: 01 & 0.1297 \\ 12819 & 15: 00: 02 & 0.1269 \\ 12820 & 15: 00: 03 & 0.1299 \\ 12821 & 15: 00: 04 & 0.1249 \\ 12822 & 15: 00: 05 & 0.1317 \\ 12823 & 15: 00: 06 & 0.1292 \\ 12824 & 15: 00: 07 & 0.1304 \\ 12825 & 15: 00: 08 & 0.1266 \\ 12826 & 15: 00: 09 & 0.1274 \\ 12827 & 15: 00: 10 & 0.1273 \\ 12828 & 15: 00: 11 & 0.1254 \\ 12829 & 15: 00: 12 & 0.1270 \\ 12830 & 15: 00: 13 & 0.1245 \\ 12831 & 15: 00: 14 & 0.1282 \\ 12832 & 15: 00: 15 & 0.1234 \\ 12833 & 15: 00: 16 & 0.1288 \\ 12834 & 15: 00: 17 & 0.1277 \\ 12835 & 15: 00: 18 & 0.1315 \\ 12836 & 15: 00: 19 & 0.1269 \\ 12837 & 15: 00: 20 & 0.1291 \\ 12838 & 15: 00: 21 & 0.1266 \\ 12839 & 15: 00: 22 & 0.1264 \\ 12840 & 15: 00: 23 & 0.1287 \\ 12841 & 15: 00: 24 & 0.1270 \\ 12842 & 15: 00: 25 & 0.1294\end{array}$


Appendix A - Fuel Data Test 1 NIST102505_1.xIs

$\begin{array}{lll}12843 & 15: 00: 26 & 0.1243 \\ 12844 & 15: 00: 27 & 0.1302 \\ 12845 & 15: 00: 28 & 0.1274 \\ 12846 & 15: 00: 29 & 0.1298 \\ 12847 & 15: 00: 30 & 0.1260 \\ 12848 & 15: 00: 31 & 0.1302 \\ 12849 & 15: 00: 32 & 0.1281 \\ 12850 & 15: 00: 33 & 0.1289 \\ 12851 & 15: 00: 34 & 0.1265 \\ 12852 & 15: 00: 35 & 0.1269 \\ 12853 & 15: 00: 36 & 0.1288 \\ 12854 & 15: 00: 37 & 0.1253 \\ 12855 & 15: 00: 38 & 0.1276 \\ 12856 & 15: 00: 39 & 0.1254 \\ 12857 & 15: 00: 40 & 0.1290 \\ 12858 & 15: 00: 41 & 0.1249 \\ 12859 & 15: 00: 42 & 0.1321 \\ 12860 & 15: 00: 43 & 0.1272 \\ 12861 & 15: 00: 44 & 0.1287 \\ 12862 & 15: 00: 45 & 0.1289 \\ 12863 & 15: 00: 46 & 0.1284 \\ 12864 & 15: 00: 47 & 0.1269 \\ 12865 & 15: 00: 48 & 0.1266 \\ 12866 & 15: 00: 49 & 0.1295 \\ 12867 & 15: 00: 50 & 0.1251 \\ 12868 & 15: 00: 51 & 0.1285 \\ 12869 & 15: 00: 52 & 0.1253 \\ 12870 & 15: 00: 53 & 0.1294 \\ 12871 & 15: 00: 54 & 0.1247 \\ 12872 & 15: 00: 55 & 0.1296 \\ 12873 & 15: 00: 56 & 0.1296 \\ 12874 & 15: 00: 57 & 0.1316 \\ 12875 & 15: 00: 58 & 0.1276 \\ 12876 & 15: 00: 59 & 0.1288 \\ 12877 & 15: 01: 00 & 0.1274 \\ 12878 & 15: 01: 01 & 0.1257 \\ 12879 & 15: 01: 02 & 0.1276 \\ 12880 & 15: 01: 03 & 0.1254 \\ 12881 & 15: 01: 04 & 0.1292 \\ 12882 & 15: 01: 05 & 0.1242 \\ 12883 & 15: 01: 06 & 0.1300 \\ 12884 & 15: 01: 07 & 0.1277 \\ 12885 & 15: 01: 08 & 0.1307 \\ 12886 & 15: 01: 09 & 0.1263 \\ 12887 & 15: 01: 10 & 0.1293 \\ 12888 & 15: 01: 11 & 0.1245 \\ 12889 & 15: 01: 12 & 0.1248 \\ 12890 & 15: 01: 13 & 0.1279 \\ 12891 & 15: 01: 14 & 0.1255 \\ 12892 & 15: 01: 15 & 0.1280 \\ 12893 & 15: 01: 16 & 0.1225 \\ 12894 & 15: 01: 17 & 0.1306\end{array}$


Appendix A - Fuel Data Test 1 NIST102505_1.xIs

$\begin{array}{lll}12895 & 15: 01: 18 & 0.1272 \\ 12896 & 15: 01: 19 & 0.1321 \\ 12897 & 15: 01: 20 & 0.1265 \\ 12898 & 15: 01: 21 & 0.1311 \\ 12899 & 15: 01: 22 & 0.1280 \\ 12900 & 15: 01: 23 & 0.1294 \\ 12901 & 15: 01: 24 & 0.1271 \\ 12902 & 15: 01: 25 & 0.1296 \\ 12903 & 15: 01: 26 & 0.1282 \\ 12904 & 15: 01: 27 & 0.1247 \\ 12905 & 15: 01: 28 & 0.1279 \\ 12906 & 15: 01: 29 & 0.1232 \\ 12907 & 15: 01: 30 & 0.1276 \\ 12908 & 15: 01: 31 & 0.1259 \\ 12909 & 15: 01: 32 & 0.1296 \\ 12910 & 15: 01: 33 & 0.1262 \\ 12911 & 15: 01: 34 & 0.1291 \\ 12912 & 15: 01: 35 & 0.1299 \\ 12913 & 15: 01: 36 & 0.1281 \\ 12914 & 15: 01: 37 & 0.1271 \\ 12915 & 15: 01: 38 & 0.1259 \\ 12916 & 15: 01: 39 & 0.1295 \\ 12917 & 15: 01: 40 & 0.1266 \\ 12918 & 15: 01: 41 & 0.1289 \\ 12919 & 15: 01: 42 & 0.1253 \\ 12920 & 15: 01: 43 & 0.1290 \\ 12921 & 15: 01: 44 & 0.1255 \\ 12922 & 15: 01: 45 & 0.1281 \\ 12923 & 15: 01: 46 & 0.1262 \\ 12924 & 15: 01: 47 & 0.1290 \\ 12925 & 15: 01: 48 & 0.1266 \\ 12926 & 15: 01: 49 & 0.1279 \\ 12927 & 15: 01: 50 & 0.1278 \\ 12928 & 15: 01: 51 & 0.1264 \\ 12929 & 15: 01: 52 & 0.1278 \\ 12930 & 15: 01: 53 & 0.1260 \\ 12931 & 15: 01: 54 & 0.1281 \\ 12932 & 15: 01: 55 & 0.1235 \\ 12933 & 15: 01: 56 & 0.1297 \\ 12934 & 15: 01: 57 & 0.1266 \\ 12935 & 15: 01: 58 & 0.1306 \\ 12936 & 15: 01: 59 & 0.1252 \\ 12937 & 15: 02: 00 & 0.1298 \\ 12938 & 15: 02: 01 & 0.1279 \\ 12939 & 15: 02: 02 & 0.1295 \\ 12940 & 15: 02: 03 & 0.1278 \\ 12941 & 15: 02: 04 & 0.1283 \\ 12942 & 15: 02: 05 & 0.1303 \\ 12943 & 15: 02: 06 & 0.1267 \\ 12944 & 15: 02: 07 & 0.1303 \\ 12945 & 15: 02: 08 & 0.1270 \\ 12946 & 15: 02: 09 & 0.1307\end{array}$


Appendix A - Fuel Data Test 1 NIST102505_1.xIs

$\begin{array}{lll}12947 & 15: 02: 10 & 0.1258 \\ 12948 & 15: 02: 11 & 0.1327 \\ 12949 & 15: 02: 12 & 0.1296 \\ 12950 & 15: 02: 13 & 0.1299 \\ 12951 & 15: 02: 14 & 0.1256 \\ 12952 & 15: 02: 15 & 0.1271 \\ 12953 & 15: 02: 16 & 0.1270 \\ 12954 & 15: 02: 17 & 0.1250 \\ 12955 & 15: 02: 18 & 0.1272 \\ 12956 & 15: 02: 19 & 0.1238 \\ 12957 & 15: 02: 20 & 0.1275 \\ 12958 & 15: 02: 21 & 0.1239 \\ 12959 & 15: 02: 22 & 0.1297 \\ 12960 & 15: 02: 23 & 0.1283 \\ 12961 & 15: 02: 24 & 0.1288 \\ 12962 & 15: 02: 25 & 0.1272 \\ 12963 & 15: 02: 26 & 0.1281 \\ 12964 & 15: 02: 27 & 0.1279 \\ 12965 & 15: 02: 28 & 0.1244 \\ 12966 & 15: 02: 29 & 0.1276 \\ 12967 & 15: 02: 30 & 0.1242 \\ 12968 & 15: 02: 31 & 0.1293 \\ 12969 & 15: 02: 32 & 0.1245 \\ 12970 & 15: 02: 33 & 0.1273 \\ 12971 & 15: 02: 34 & 0.1245 \\ 12972 & 15: 02: 35 & 0.1293 \\ 12973 & 15: 02: 36 & 0.1260 \\ 12974 & 15: 02: 37 & 0.1280 \\ 12975 & 15: 02: 38 & 0.1276 \\ 12976 & 15: 02: 39 & 0.1262 \\ 12977 & 15: 02: 40 & 0.1274 \\ 12978 & 15: 02: 41 & 0.1253 \\ 12979 & 15: 02: 42 & 0.1278 \\ 12980 & 15: 02: 43 & 0.1255 \\ 12981 & 15: 02: 44 & 0.1303 \\ 12982 & 15: 02: 45 & 0.1279 \\ 12983 & 15: 02: 46 & 0.1315 \\ 12984 & 15: 02: 47 & 0.1273 \\ 12985 & 15: 02: 48 & 0.1322 \\ 12986 & 15: 02: 49 & 0.1310 \\ 12987 & 15: 02: 50 & 0.1307 \\ 12988 & 15: 02: 51 & 0.1289 \\ 12989 & 15: 02: 52 & 0.1282 \\ 12990 & 15: 02: 53 & 0.1298 \\ 12991 & 15: 02: 54 & 0.1256 \\ 12992 & 15: 02: 55 & 0.1287 \\ 12993 & 15: 02: 56 & 0.1258 \\ 12994 & 15: 02: 57 & 0.1290 \\ 12995 & 15: 02: 58 & 0.1258 \\ 12996 & 15: 02: 59 & 0.1317 \\ 12997 & 15: 03: 00 & 0.1274 \\ 12998 & 15: 03: 01 & 0.1305\end{array}$


Appendix A - Fuel Data Test 1 NIST102505_1.xIs

$\begin{array}{lll}12999 & 15: 03: 02 & 0.1279 \\ 13000 & 15: 03: 03 & 0.1291 \\ 13001 & 15: 03: 04 & 0.1277 \\ 13002 & 15: 03: 05 & 0.1264 \\ 13003 & 15: 03: 06 & 0.1268 \\ 13004 & 15: 03: 07 & 0.1249 \\ 13005 & 15: 03: 08 & 0.1296 \\ 13006 & 15: 03: 09 & 0.1243 \\ 13007 & 15: 03: 10 & 0.1308 \\ 13008 & 15: 03: 11 & 0.1269 \\ 13009 & 15: 03: 12 & 0.1312 \\ 13010 & 15: 03: 13 & 0.1283 \\ 13011 & 15: 03: 14 & 0.1297 \\ 13012 & 15: 03: 15 & 0.1256 \\ 13013 & 15: 03: 16 & 0.1248 \\ 13014 & 15: 03: 17 & 0.1284 \\ 13015 & 15: 03: 18 & 0.1257 \\ 13016 & 15: 03: 19 & 0.1278 \\ 13017 & 15: 03: 20 & 0.1226 \\ 13018 & 15: 03: 21 & 0.1295 \\ 13019 & 15: 03: 22 & 0.1267 \\ 13020 & 15: 03: 23 & 0.1320 \\ 13021 & 15: 03: 24 & 0.1274 \\ 13022 & 15: 03: 25 & 0.1320 \\ 13023 & 15: 03: 26 & 0.1295 \\ 13024 & 15: 03: 27 & 0.1280 \\ 13025 & 15: 03: 28 & 0.1262 \\ 13026 & 15: 03: 29 & 0.1270 \\ 13027 & 15: 03: 30 & 0.1283 \\ 13028 & 15: 03: 31 & 0.1250 \\ 13029 & 15: 03: 32 & 0.1280 \\ 13030 & 15: 03: 33 & 0.1250 \\ 13031 & 15: 03: 34 & 0.1297 \\ 13032 & 15: 03: 35 & 0.1251 \\ 13033 & 15: 03: 36 & 0.1301 \\ 13034 & 15: 03: 37 & 0.1260 \\ 13035 & 15: 03: 38 & 0.1297 \\ 13036 & 15: 03: 39 & 0.1278 \\ 13037 & 15: 03: 40 & 0.1284 \\ 13038 & 15: 03: 41 & 0.1267 \\ 13039 & 15: 03: 42 & 0.1266 \\ 13040 & 15: 03: 43 & 0.1304 \\ 13041 & 15: 03: 44 & 0.1270 \\ 13042 & 15: 03: 45 & 0.1286 \\ 13043 & 15: 03: 46 & 0.1246 \\ 13044 & 15: 03: 47 & 0.1289 \\ 13045 & 15: 03: 48 & 0.1244 \\ 13046 & 15: 03: 49 & 0.1298 \\ 13047 & 15: 03: 50 & 0.1280 \\ 13048 & 15: 03: 51 & 0.1308 \\ 13049 & 15: 03: 52 & 0.1288 \\ 13050 & 15: 03: 53 & 0.1292\end{array}$


Appendix A - Fuel Data Test 1 NIST102505_1.xIs

$\begin{array}{lll}13051 & 15: 03: 54 & 0.1278 \\ 13052 & 15: 03: 55 & 0.1262 \\ 13053 & 15: 03: 56 & 0.1286 \\ 13054 & 15: 03: 57 & 0.1267 \\ 13055 & 15: 03: 58 & 0.1288 \\ 13056 & 15: 03: 59 & 0.1244 \\ 13057 & 15: 04: 00 & 0.1301 \\ 13058 & 15: 04: 01 & 0.1267 \\ 13059 & 15: 04: 02 & 0.1313 \\ 13060 & 15: 04: 03 & 0.1272 \\ 13061 & 15: 04: 04 & 0.1309 \\ 13062 & 15: 04: 05 & 0.1277 \\ 13063 & 15: 04: 06 & 0.1292 \\ 13064 & 15: 04: 07 & 0.1278 \\ 13065 & 15: 04: 08 & 0.1275 \\ 13066 & 15: 04: 09 & 0.1287 \\ 13067 & 15: 04: 10 & 0.1253 \\ 13068 & 15: 04: 11 & 0.1313 \\ 13069 & 15: 04: 12 & 0.1273 \\ 13070 & 15: 04: 13 & 0.1317 \\ 13071 & 15: 04: 14 & 0.1279 \\ 13072 & 15: 04: 15 & 0.1337 \\ 13073 & 15: 04: 16 & 0.1273 \\ 13074 & 15: 04: 17 & 0.1284 \\ 13075 & 15: 04: 18 & 0.1268 \\ 13076 & 15: 04: 19 & 0.1284 \\ 13077 & 15: 04: 20 & 0.1273 \\ 13078 & 15: 04: 21 & 0.1270 \\ 13079 & 15: 04: 22 & 0.1277 \\ 13080 & 15: 04: 23 & 0.1232 \\ 13081 & 15: 04: 24 & 0.1280 \\ 13082 & 15: 04: 25 & 0.1252 \\ 13083 & 15: 04: 26 & 0.1292 \\ 13084 & 15: 04: 27 & 0.1239 \\ 13085 & 15: 04: 28 & 0.1307 \\ 13086 & 15: 04: 29 & 0.1264 \\ 13087 & 15: 04: 30 & 0.1277 \\ 13088 & 15: 04: 31 & 0.1272 \\ 13089 & 15: 04: 32 & 0.1269 \\ 13090 & 15: 04: 33 & 0.1289 \\ 13091 & 15: 04: 34 & 0.1248 \\ 13092 & 15: 04: 35 & 0.1299 \\ 13093 & 15: 04: 36 & 0.1252 \\ 13094 & 15: 04: 37 & 0.1285 \\ 13095 & 15: 04: 38 & 0.1248 \\ 13096 & 15: 04: 39 & 0.1290 \\ 13097 & 15: 04: 40 & 0.1256 \\ 13098 & 15: 04: 41 & 0.1276 \\ 13099 & 15: 04: 42 & 0.1285 \\ 13100 & 15: 04: 43 & 0.1308 \\ 13101 & 15: 04: 44 & 0.1284 \\ 13102 & 15: 04: 45 & 0.1267\end{array}$


Appendix A - Fuel Data Test 1 NIST102505_1.xIs

$\begin{array}{lll}13103 & 15: 04: 46 & 0.1312 \\ 13104 & 15: 04: 47 & 0.1287 \\ 13105 & 15: 04: 48 & 0.1301 \\ 13106 & 15: 04: 49 & 0.1258 \\ 13107 & 15: 04: 50 & 0.1302 \\ 13108 & 15: 04: 51 & 0.1273 \\ 13109 & 15: 04: 52 & 0.1304 \\ 13110 & 15: 04: 53 & 0.1294 \\ 13111 & 15: 04: 54 & 0.1297 \\ 13112 & 15: 04: 55 & 0.1285 \\ 13113 & 15: 04: 56 & 0.1271 \\ 13114 & 15: 04: 57 & 0.1270 \\ 13115 & 15: 04: 58 & 0.1267 \\ 13116 & 15: 04: 59 & 0.1290 \\ 13117 & 15: 05: 00 & 0.1258 \\ 13118 & 15: 05: 01 & 0.1283 \\ 13119 & 15: 05: 02 & 0.1233 \\ 13120 & 15: 05: 03 & 0.1282 \\ 13121 & 15: 05: 04 & 0.1279 \\ 13122 & 15: 05: 05 & 0.1306 \\ 13123 & 15: 05: 06 & 0.1269 \\ 13124 & 15: 05: 07 & 0.1284 \\ 13125 & 15: 05: 08 & 0.1280 \\ 13126 & 15: 05: 09 & 0.1280 \\ 13127 & 15: 05: 10 & 0.1251 \\ 13128 & 15: 05: 11 & 0.1259 \\ 13129 & 15: 05: 12 & 0.1285 \\ 13130 & 15: 05: 13 & 0.1245 \\ 13131 & 15: 05: 14 & 0.1281 \\ 13132 & 15: 05: 15 & 0.1254 \\ 13133 & 15: 05: 16 & 0.1301 \\ 13134 & 15: 05: 17 & 0.1259 \\ 13135 & 15: 05: 18 & 0.1305 \\ 13136 & 15: 05: 19 & 0.1266 \\ 13137 & 15: 05: 20 & 0.1295 \\ 13138 & 15: 05: 21 & 0.1260 \\ 13139 & 15: 05: 22 & 0.1267 \\ 13140 & 15: 05: 23 & 0.1262 \\ 13141 & 15: 05: 24 & 0.1243 \\ 13142 & 15: 05: 25 & 0.1298 \\ 13143 & 15: 05: 26 & 0.1272 \\ 13144 & 15: 05: 27 & 0.1314 \\ 13145 & 15: 05: 28 & 0.1255 \\ 13146 & 15: 05: 29 & 0.1301 \\ 13147 & 15: 05: 30 & 0.1273 \\ 13148 & 15: 05: 31 & 0.1318 \\ 13149 & 15: 05: 32 & 0.1274 \\ 13150 & 15: 05: 33 & 0.1313 \\ 13151 & 15: 05: 34 & 0.1260 \\ 13152 & 15: 05: 35 & 0.1259 \\ 13153 & 15: 05: 36 & 0.1280 \\ 13154 & 15: 05: 37 & 0.1248\end{array}$


Appendix A - Fuel Data Test 1 NIST102505_1.xls

$\begin{array}{lll}13155 & 15: 05: 38 & 0.1265 \\ 13156 & 15: 05: 39 & 0.1227 \\ 13157 & 15: 05: 40 & 0.1277 \\ 13158 & 15: 05: 41 & 0.1244 \\ 13159 & 15: 05: 42 & 0.1288 \\ 13160 & 15: 05: 43 & 0.1285 \\ 13161 & 15: 05: 44 & 0.1312 \\ 13162 & 15: 05: 45 & 0.1271 \\ 13163 & 15: 05: 46 & 0.1285 \\ 13164 & 15: 05: 47 & 0.1270 \\ 13165 & 15: 05: 48 & 0.1273 \\ 13166 & 15: 05: 49 & 0.1291 \\ 13167 & 15: 05: 50 & 0.1266 \\ 13168 & 15: 05: 51 & 0.1280 \\ 13169 & 15: 05: 52 & 0.1238 \\ 13170 & 15: 05: 53 & 0.1286 \\ 13171 & 15: 05: 54 & 0.1274 \\ 13172 & 15: 05: 55 & 0.1306 \\ 13173 & 15: 05: 56 & 0.1266 \\ 13174 & 15: 05: 57 & 0.1292 \\ 13175 & 15: 05: 58 & 0.1281 \\ 13176 & 15: 05: 59 & 0.1281 \\ 13177 & 15: 06: 00 & 0.1267 \\ 13178 & 15: 06: 01 & 0.1273 \\ 13179 & 15: 06: 02 & 0.1281 \\ 13180 & 15: 06: 03 & 0.1237 \\ 13181 & 15: 06: 04 & 0.1293 \\ 13182 & 15: 06: 05 & 0.1259 \\ 13183 & 15: 06: 06 & 0.1306 \\ 13184 & 15: 06: 07 & 0.1253 \\ 13185 & 15: 06: 08 & 0.1305 \\ 13186 & 15: 06: 09 & 0.1262 \\ 13187 & 15: 06: 10 & 0.1287 \\ 13188 & 15: 06: 11 & 0.1274 \\ 13189 & 15: 06: 12 & 0.1268 \\ 13190 & 15: 06: 13 & 0.1275 \\ 13191 & 15: 06: 14 & 0.1242 \\ 13192 & 15: 06: 15 & 0.1290 \\ 13193 & 15: 06: 16 & 0.1249 \\ 13194 & 15: 06: 17 & 0.1279 \\ 13195 & 15: 06: 18 & 0.1232 \\ 13196 & 15: 06: 19 & 0.1293 \\ 13197 & 15: 06: 20 & 0.1259 \\ 13198 & 15: 06: 21 & 0.1290 \\ 13199 & 15: 06: 22 & 0.1272 \\ 13200 & 15: 06: 23 & 0.1281 \\ 13201 & 15: 06: 24 & 0.1254 \\ 13202 & 15: 06: 25 & 0.1255 \\ 13203 & 15: 06: 26 & 0.1281 \\ 13204 & 15: 06: 27 & 0.1238 \\ 13205 & 15: 06: 28 & 0.1264 \\ 13206 & 15: 06: 29 & 0.1244\end{array}$


Appendix A - Fuel Data Test 1 NIST102505_1.xIs

$\begin{array}{lll}13207 & 15: 06: 30 & 0.1293 \\ 13208 & 15: 06: 31 & 0.1262 \\ 13209 & 15: 06: 32 & 0.1318 \\ 13210 & 15: 06: 33 & 0.1278 \\ 13211 & 15: 06: 34 & 0.1299 \\ 13212 & 15: 06: 35 & 0.1278 \\ 13213 & 15: 06: 36 & 0.1295 \\ 13214 & 15: 06: 37 & 0.1288 \\ 13215 & 15: 06: 38 & 0.1275 \\ 13216 & 15: 06: 39 & 0.1281 \\ 13217 & 15: 06: 40 & 0.1254 \\ 13218 & 15: 06: 41 & 0.1281 \\ 13219 & 15: 06: 42 & 0.1248 \\ 13220 & 15: 06: 43 & 0.1293 \\ 13221 & 15: 06: 44 & 0.1276 \\ 13222 & 15: 06: 45 & 0.1305 \\ 13223 & 15: 06: 46 & 0.1256 \\ 13224 & 15: 06: 47 & 0.1297 \\ 13225 & 15: 06: 48 & 0.1264 \\ 13226 & 15: 06: 49 & 0.1270 \\ 13227 & 15: 06: 50 & 0.1279 \\ 13228 & 15: 06: 51 & 0.1270 \\ 13229 & 15: 06: 52 & 0.1286 \\ 13230 & 15: 06: 53 & 0.1235 \\ 13231 & 15: 06: 54 & 0.1291 \\ 13232 & 15: 06: 55 & 0.1267 \\ 13233 & 15: 06: 56 & 0.1296 \\ 13234 & 15: 06: 57 & 0.1244 \\ 13235 & 15: 06: 58 & 0.1306 \\ 13236 & 15: 06: 59 & 0.1263 \\ 13237 & 15: 07: 00 & 0.1283 \\ 13238 & 15: 07: 01 & 0.1251 \\ 13239 & 15: 07: 02 & 0.1259 \\ 13240 & 15: 07: 03 & 0.1276 \\ 13241 & 15: 07: 04 & 0.1261 \\ 13242 & 15: 07: 05 & 0.1295 \\ 13243 & 15: 07: 06 & 0.1279 \\ 13244 & 15: 07: 07 & 0.1302 \\ 13245 & 15: 07: 08 & 0.1254 \\ 13246 & 15: 07: 09 & 0.1314 \\ 13247 & 15: 07: 10 & 0.1267 \\ 13248 & 15: 07: 11 & 0.1314 \\ 13249 & 15: 07: 12 & 0.1294 \\ 13250 & 15: 07: 13 & 0.1309 \\ 13251 & 15: 07: 14 & 0.1272 \\ 13252 & 15: 07: 15 & 0.1270 \\ 13253 & 15: 07: 16 & 0.1282 \\ 13254 & 15: 07: 17 & 0.1263 \\ 13255 & 15: 07: 18 & 0.1281 \\ 13256 & 15: 07: 19 & 0.1235 \\ 13257 & 15: 07: 20 & 0.1297 \\ 13258 & 15: 07: 21 & 0.1274\end{array}$


Appendix A - Fuel Data Test 1 NIST102505_1.xls

$\begin{array}{lll}13259 & 15: 07: 22 & 0.1296 \\ 13260 & 15: 07: 23 & 0.1251 \\ 13261 & 15: 07: 24 & 0.1300 \\ 13262 & 15: 07: 25 & 0.1297 \\ 13263 & 15: 07: 26 & 0.1278 \\ 13264 & 15: 07: 27 & 0.1262 \\ 13265 & 15: 07: 28 & 0.1255 \\ 13266 & 15: 07: 29 & 0.1289 \\ 13267 & 15: 07: 30 & 0.1252 \\ 13268 & 15: 07: 31 & 0.1280 \\ 13269 & 15: 07: 32 & 0.1247 \\ 13270 & 15: 07: 33 & 0.1292 \\ 13271 & 15: 07: 34 & 0.1260 \\ 13272 & 15: 07: 35 & 0.1294 \\ 13273 & 15: 07: 36 & 0.1293 \\ 13274 & 15: 07: 37 & 0.1280 \\ 13275 & 15: 07: 38 & 0.1289 \\ 13276 & 15: 07: 39 & 0.1281 \\ 13277 & 15: 07: 40 & 0.1293 \\ 13278 & 15: 07: 41 & 0.1277 \\ 13279 & 15: 07: 42 & 0.1296 \\ 13280 & 15: 07: 43 & 0.1252 \\ 13281 & 15: 07: 44 & 0.1304 \\ 13282 & 15: 07: 45 & 0.1254 \\ 13283 & 15: 07: 46 & 0.1295 \\ 13284 & 15: 07: 47 & 0.1259 \\ 13285 & 15: 07: 48 & 0.1307 \\ 13286 & 15: 07: 49 & 0.1278 \\ 13287 & 15: 07: 50 & 0.1294 \\ 13288 & 15: 07: 51 & 0.1289 \\ 13289 & 15: 07: 52 & 0.1307 \\ 13290 & 15: 07: 53 & 0.1275 \\ 13291 & 15: 07: 54 & 0.1265 \\ 13292 & 15: 07: 55 & 0.1289 \\ 13293 & 15: 07: 56 & 0.1265 \\ 13294 & 15: 07: 57 & 0.1307 \\ 13295 & 15: 07: 58 & 0.1257 \\ 13296 & 15: 07: 59 & 0.1294 \\ 13297 & 15: 08: 00 & 0.1263 \\ 13298 & 15: 08: 01 & 0.1311 \\ 13299 & 15: 08: 02 & 0.1284 \\ 13300 & 15: 08: 03 & 0.1304 \\ 13301 & 15: 08: 04 & 0.1278 \\ 13302 & 15: 08: 05 & 0.1282 \\ 13303 & 15: 08: 06 & 0.1288 \\ 13304 & 15: 08: 07 & 0.1260 \\ 13305 & 15: 08: 08 & 0.1300 \\ 13306 & 15: 08: 09 & 0.1253 \\ 13307 & 15: 08: 10 & 0.1291 \\ 13308 & 15: 08: 11 & 0.1244 \\ 13309 & 15: 08: 12 & 0.1319 \\ 13310 & 15: 08: 13 & 0.1279\end{array}$


Appendix A - Fuel Data Test 1 NIST102505_1.xls

$\begin{array}{lll}13311 & 15: 08: 14 & 0.1315 \\ 13312 & 15: 08: 15 & 0.1285 \\ 13313 & 15: 08: 16 & 0.1301 \\ 13314 & 15: 08: 17 & 0.1253 \\ 13315 & 15: 08: 18 & 0.1255 \\ 13316 & 15: 08: 19 & 0.1284 \\ 13317 & 15: 08: 20 & 0.1283 \\ 13318 & 15: 08: 21 & 0.1301 \\ 13319 & 15: 08: 22 & 0.1237 \\ 13320 & 15: 08: 23 & 0.1316 \\ 13321 & 15: 08: 24 & 0.1278 \\ 13322 & 15: 08: 25 & 0.1324 \\ 13323 & 15: 08: 26 & 0.1268 \\ 13324 & 15: 08: 27 & 0.1307 \\ 13325 & 15: 08: 28 & 0.1272 \\ 13326 & 15: 08: 29 & 0.1283 \\ 13327 & 15: 08: 30 & 0.1259 \\ 13328 & 15: 08: 31 & 0.1274 \\ 13329 & 15: 08: 32 & 0.1279 \\ 13330 & 15: 08: 33 & 0.1255 \\ 13331 & 15: 08: 34 & 0.1292 \\ 13332 & 15: 08: 35 & 0.1248 \\ 13333 & 15: 08: 36 & 0.1295 \\ 13334 & 15: 08: 37 & 0.1254 \\ 13335 & 15: 08: 38 & 0.1304 \\ 13336 & 15: 08: 39 & 0.1259 \\ 13337 & 15: 08: 40 & 0.1292 \\ 13338 & 15: 08: 41 & 0.1288 \\ 13339 & 15: 08: 42 & 0.1283 \\ 13340 & 15: 08: 43 & 0.1277 \\ 13341 & 15: 08: 44 & 0.1253 \\ 13342 & 15: 08: 45 & 0.1305 \\ 13343 & 15: 08: 46 & 0.1262 \\ 13344 & 15: 08: 47 & 0.1293 \\ 13345 & 15: 08: 48 & 0.1246 \\ 13346 & 15: 08: 49 & 0.1285 \\ 13347 & 15: 08: 50 & 0.1244 \\ 13348 & 15: 08: 51 & 0.1276 \\ 13349 & 15: 08: 52 & 0.1280 \\ 13350 & 15: 08: 53 & 0.1291 \\ 13351 & 15: 08: 54 & 0.1278 \\ 13352 & 15: 08: 55 & 0.1273 \\ 13353 & 15: 08: 56 & 0.1295 \\ 13354 & 15: 08: 57 & 0.1278 \\ 13355 & 15: 08: 58 & 0.1297 \\ 13356 & 15: 08: 59 & 0.1249 \\ 13357 & 15: 09: 00 & 0.1303 \\ 13358 & 15: 09: 01 & 0.1257 \\ 13359 & 15: 09: 02 & 0.1295 \\ 13360 & 15: 09: 03 & 0.1282 \\ 13361 & 15: 09: 04 & 0.1321 \\ 13362 & 15: 09: 05 & 0.1296\end{array}$


Appendix A - Fuel Data Test 1 NIST102505_1.xIs

$\begin{array}{lll}13363 & 15: 09: 06 & 0.1282 \\ 13364 & 15: 09: 07 & 0.1264 \\ 13365 & 15: 09: 08 & 0.1268 \\ 13366 & 15: 09: 09 & 0.1277 \\ 13367 & 15: 09: 10 & 0.1243 \\ 13368 & 15: 09: 11 & 0.1304 \\ 13369 & 15: 09: 12 & 0.1249 \\ 13370 & 15: 09: 13 & 0.1284 \\ 13371 & 15: 09: 14 & 0.1242 \\ 13372 & 15: 09: 15 & 0.1297 \\ 13373 & 15: 09: 16 & 0.1245 \\ 13374 & 15: 09: 17 & 0.1283 \\ 13375 & 15: 09: 18 & 0.1267 \\ 13376 & 15: 09: 19 & 0.1286 \\ 13377 & 15: 09: 20 & 0.1257 \\ 13378 & 15: 09: 21 & 0.1252 \\ 13379 & 15: 09: 22 & 0.1282 \\ 13380 & 15: 09: 23 & 0.1250 \\ 13381 & 15: 09: 24 & 0.1259 \\ 13382 & 15: 09: 25 & 0.1225 \\ 13383 & 15: 09: 26 & 0.1277 \\ 13384 & 15: 09: 27 & 0.1235 \\ 13385 & 15: 09: 28 & 0.1290 \\ 13386 & 15: 09: 29 & 0.1276 \\ 13387 & 15: 09: 30 & 0.1315 \\ 13388 & 15: 09: 31 & 0.1276 \\ 13389 & 15: 09: 32 & 0.1282 \\ 13390 & 15: 09: 33 & 0.1280 \\ 13391 & 15: 09: 34 & 0.1261 \\ 13392 & 15: 09: 35 & 0.1271 \\ 13393 & 15: 09: 36 & 0.1254 \\ 13394 & 15: 09: 37 & 0.1285 \\ 13395 & 15: 09: 38 & 0.1228 \\ 13396 & 15: 09: 39 & 0.1280 \\ 13397 & 15: 09: 40 & 0.1268 \\ 13398 & 15: 09: 41 & 0.1302 \\ 13399 & 15: 09: 42 & 0.1251 \\ 13400 & 15: 09: 43 & 0.1292 \\ 13401 & 15: 09: 44 & 0.1271 \\ 13402 & 15: 09: 45 & 0.1279 \\ 13403 & 15: 09: 46 & 0.1255 \\ 13404 & 15: 09: 47 & 0.1255 \\ 13405 & 15: 09: 48 & 0.1267 \\ 13406 & 15: 09: 49 & 0.1245 \\ 13407 & 15: 09: 50 & 0.1298 \\ 13408 & 15: 09: 51 & 0.1249 \\ 13409 & 15: 09: 52 & 0.1288 \\ 13410 & 15: 09: 53 & 0.1253 \\ 13411 & 15: 09: 54 & 0.1302 \\ 13412 & 15: 09: 55 & 0.1267 \\ 13413 & 15: 09: 56 & 0.1281 \\ 13414 & 15: 09: 57 & 0.1283\end{array}$


Appendix A - Fuel Data Test 1 NIST102505_1.xls

$\begin{array}{lll}13415 & 15: 09: 58 & 0.1270 \\ 13416 & 15: 09: 59 & 0.1265 \\ 13417 & 15: 10: 00 & 0.1241 \\ 13418 & 15: 10: 01 & 0.1283 \\ 13419 & 15: 10: 02 & 0.1274 \\ 13420 & 15: 10: 03 & 0.1291 \\ 13421 & 15: 10: 04 & 0.1235 \\ 13422 & 15: 10: 05 & 0.1284 \\ 13423 & 15: 10: 06 & 0.1240 \\ 13424 & 15: 10: 07 & 0.1279 \\ 13425 & 15: 10: 08 & 0.1264 \\ 13426 & 15: 10: 09 & 0.1309 \\ 13427 & 15: 10: 10 & 0.1272 \\ 13428 & 15: 10: 11 & 0.1287 \\ 13429 & 15: 10: 12 & 0.1282 \\ 13430 & 15: 10: 13 & 0.1260 \\ 13431 & 15: 10: 14 & 0.1253 \\ 13432 & 15: 10: 15 & 0.1236 \\ 13433 & 15: 10: 16 & 0.1276 \\ 13434 & 15: 10: 17 & 0.1245 \\ 13435 & 15: 10: 18 & 0.1287 \\ 13436 & 15: 10: 19 & 0.1277 \\ 13437 & 15: 10: 20 & 0.1323 \\ 13438 & 15: 10: 21 & 0.1267 \\ 13439 & 15: 10: 22 & 0.1298 \\ 13440 & 15: 10: 23 & 0.1263 \\ 13441 & 15: 10: 24 & 0.1260 \\ 13442 & 15: 10: 25 & 0.1278 \\ 13443 & 15: 10: 26 & 0.1280 \\ 13444 & 15: 10: 27 & 0.1289 \\ 13445 & 15: 10: 28 & 0.1232 \\ 13446 & 15: 10: 29 & 0.1287 \\ 13447 & 15: 10: 30 & 0.1258 \\ 13448 & 15: 10: 31 & 0.1291 \\ 13449 & 15: 10: 32 & 0.1245 \\ 13450 & 15: 10: 33 & 0.1308 \\ 13451 & 15: 10: 34 & 0.1279 \\ 13452 & 15: 10: 35 & 0.1283 \\ 13453 & 15: 10: 36 & 0.1268 \\ 13454 & 15: 10: 37 & 0.1290 \\ 13455 & 15: 10: 38 & 0.1281 \\ 13456 & 15: 10: 39 & 0.1243 \\ 13457 & 15: 10: 40 & 0.1272 \\ 13458 & 15: 10: 41 & 0.1216 \\ 13459 & 15: 10: 42 & 0.1277 \\ 13460 & 15: 10: 43 & 0.1259 \\ 13461 & 15: 10: 44 & 0.1295 \\ 13462 & 15: 10: 45 & 0.1254 \\ 13463 & 15: 10: 46 & 0.1289 \\ 13464 & 15: 10: 47 & 0.1273 \\ 13465 & 15: 10: 48 & 0.1284 \\ 13466 & 15: 10: 49 & 0.1260\end{array}$


Appendix A - Fuel Data Test 1 NIST102505_1.xls

$\begin{array}{lll}13467 & 15: 10: 50 & 0.1272 \\ 13468 & 15: 10: 51 & 0.1288 \\ 13469 & 15: 10: 52 & 0.1262 \\ 13470 & 15: 10: 53 & 0.1300 \\ 13471 & 15: 10: 54 & 0.1271 \\ 13472 & 15: 10: 55 & 0.1284 \\ 13473 & 15: 10: 56 & 0.1256 \\ 13474 & 15: 10: 57 & 0.1304 \\ 13475 & 15: 10: 58 & 0.1290 \\ 13476 & 15: 10: 59 & 0.1312 \\ 13477 & 15: 11: 00 & 0.1270 \\ 13478 & 15: 11: 01 & 0.1287 \\ 13479 & 15: 11: 02 & 0.1277 \\ 13480 & 15: 11: 03 & 0.1260 \\ 13481 & 15: 11: 04 & 0.1283 \\ 13482 & 15: 11: 05 & 0.1261 \\ 13483 & 15: 11: 06 & 0.1286 \\ 13484 & 15: 11: 07 & 0.1242 \\ 13485 & 15: 11: 08 & 0.1304 \\ 13486 & 15: 11: 09 & 0.1265 \\ 13487 & 15: 11: 10 & 0.1301 \\ 13488 & 15: 11: 11 & 0.1269 \\ 13489 & 15: 11: 12 & 0.1289 \\ 13490 & 15: 11: 13 & 0.1257 \\ 13491 & 15: 11: 14 & 0.1256 \\ 13492 & 15: 11: 15 & 0.1291 \\ 13493 & 15: 11: 16 & 0.1263 \\ 13494 & 15: 11: 17 & 0.1282 \\ 13495 & 15: 11: 18 & 0.1238 \\ 13496 & 15: 11: 19 & 0.1285 \\ 13497 & 15: 11: 20 & 0.1241 \\ 13498 & 15: 11: 21 & 0.1276 \\ 13499 & 15: 11: 22 & 0.1247 \\ 13500 & 15: 11: 23 & 0.1289 \\ 13501 & 15: 11: 24 & 0.1259 \\ 13502 & 15: 11: 25 & 0.1260 \\ 13503 & 15: 11: 26 & 0.1290 \\ 13504 & 15: 11: 27 & 0.1273 \\ 13505 & 15: 11: 28 & 0.1296 \\ 13506 & 15: 11: 29 & 0.1242 \\ 13507 & 15: 11: 30 & 0.1283 \\ 13508 & 15: 11: 31 & 0.1251 \\ 13509 & 15: 11: 32 & 0.1304 \\ 13510 & 15: 11: 33 & 0.1269 \\ 13511 & 15: 11: 34 & 0.1316 \\ 13512 & 15: 11: 35 & 0.1275 \\ 13513 & 15: 11: 36 & 0.1298 \\ 13514 & 15: 11: 37 & 0.1269 \\ 13515 & 15: 11: 38 & 0.1287 \\ 13516 & 15: 11: 39 & 0.1271 \\ 13517 & 15: 11: 40 & 0.1245 \\ 13518 & 15: 11: 41 & 0.1289\end{array}$


Appendix A - Fuel Data Test 1 NIST102505_1.xls

$\begin{array}{lll}13519 & 15: 11: 42 & 0.1259 \\ 13520 & 15: 11: 43 & 0.1279 \\ 13521 & 15: 11: 44 & 0.1230 \\ 13522 & 15: 11: 45 & 0.1285 \\ 13523 & 15: 11: 46 & 0.1252 \\ 13524 & 15: 11: 47 & 0.1285 \\ 13525 & 15: 11: 48 & 0.1266 \\ 13526 & 15: 11: 49 & 0.1293 \\ 13527 & 15: 11: 50 & 0.1266 \\ 13528 & 15: 11: 51 & 0.1275 \\ 13529 & 15: 11: 52 & 0.1284 \\ 13530 & 15: 11: 53 & 0.1268 \\ 13531 & 15: 11: 54 & 0.1285 \\ 13532 & 15: 11: 55 & 0.1266 \\ 13533 & 15: 11: 56 & 0.1288 \\ 13534 & 15: 11: 57 & 0.1240 \\ 13535 & 15: 11: 58 & 0.1293 \\ 13536 & 15: 11: 59 & 0.1269 \\ 13537 & 15: 12: 00 & 0.1306 \\ 13538 & 15: 12: 01 & 0.1247 \\ 13539 & 15: 12: 02 & 0.1289 \\ 13540 & 15: 12: 03 & 0.1271 \\ 13541 & 15: 12: 04 & 0.1274 \\ 13542 & 15: 12: 05 & 0.1270 \\ 13543 & 15: 12: 06 & 0.1270 \\ 13544 & 15: 12: 07 & 0.1276 \\ 13545 & 15: 12: 08 & 0.1230 \\ 13546 & 15: 12: 09 & 0.1289 \\ 13547 & 15: 12: 10 & 0.1258 \\ 13548 & 15: 12: 11 & 0.1279 \\ 13549 & 15: 12: 12 & 0.1240 \\ 13550 & 15: 12: 13 & 0.1313 \\ 13551 & 15: 12: 14 & 0.1275 \\ 13552 & 15: 12: 15 & 0.1297 \\ 13553 & 15: 12: 16 & 0.1275 \\ 13554 & 15: 12: 17 & 0.1282 \\ 13555 & 15: 12: 18 & 0.1273 \\ 13556 & 15: 12: 19 & 0.1263 \\ 13557 & 15: 12: 20 & 0.1278 \\ 13558 & 15: 12: 21 & 0.1228 \\ 13559 & 15: 12: 22 & 0.1295 \\ 13560 & 15: 12: 23 & 0.1271 \\ 13561 & 15: 12: 24 & 0.1291 \\ 13562 & 15: 12: 25 & 0.1244 \\ 13563 & 15: 12: 26 & 0.1302 \\ 13564 & 15: 12: 27 & 0.1273 \\ 13565 & 15: 12: 28 & 0.1284 \\ 13566 & 15: 12: 29 & 0.1264 \\ 13567 & 15: 12: 30 & 0.1272 \\ 13568 & 15: 12: 31 & 0.1283 \\ 13569 & 15: 12: 32 & 0.1262 \\ 13570 & 15: 12: 33 & 0.1289\end{array}$


Appendix A - Fuel Data Test 1 NIST102505_1.xIs

$\begin{array}{lll}13571 & 15: 12: 34 & 0.1261 \\ 13572 & 15: 12: 35 & 0.1301 \\ 13573 & 15: 12: 36 & 0.1258 \\ 13574 & 15: 12: 37 & 0.1318 \\ 13575 & 15: 12: 38 & 0.1276 \\ 13576 & 15: 12: 39 & 0.1306 \\ 13577 & 15: 12: 40 & 0.1284 \\ 13578 & 15: 12: 41 & 0.1289 \\ 13579 & 15: 12: 42 & 0.1257 \\ 13580 & 15: 12: 43 & 0.1246 \\ 13581 & 15: 12: 44 & 0.1267 \\ 13582 & 15: 12: 45 & 0.1257 \\ 13583 & 15: 12: 46 & 0.1276 \\ 13584 & 15: 12: 47 & 0.1225 \\ 13585 & 15: 12: 48 & 0.1296 \\ 13586 & 15: 12: 49 & 0.1263 \\ 13587 & 15: 12: 50 & 0.1292 \\ 13588 & 15: 12: 51 & 0.1240 \\ 13589 & 15: 12: 52 & 0.1279 \\ 13590 & 15: 12: 53 & 0.1259 \\ 13591 & 15: 12: 54 & 0.1267 \\ 13592 & 15: 12: 55 & 0.1263 \\ 13593 & 15: 12: 56 & 0.1273 \\ 13594 & 15: 12: 57 & 0.1293 \\ 13595 & 15: 12: 58 & 0.1264 \\ 13596 & 15: 12: 59 & 0.1312 \\ 13597 & 15: 13: 00 & 0.1276 \\ 13598 & 15: 13: 01 & 0.1308 \\ 13599 & 15: 13: 02 & 0.1259 \\ 13600 & 15: 13: 03 & 0.1312 \\ 13601 & 15: 13: 04 & 0.1280 \\ 13602 & 15: 13: 05 & 0.1307 \\ 13603 & 15: 13: 06 & 0.1268 \\ 13604 & 15: 13: 07 & 0.1288 \\ 13605 & 15: 13: 08 & 0.1279 \\ 13606 & 15: 13: 09 & 0.1265 \\ 13607 & 15: 13: 10 & 0.1264 \\ 13608 & 15: 13: 11 & 0.1234 \\ 13609 & 15: 13: 12 & 0.1278 \\ 13610 & 15: 13: 13 & 0.1230 \\ 13611 & 15: 13: 14 & 0.1296 \\ 13612 & 15: 13: 15 & 0.1264 \\ 13613 & 15: 13: 16 & 0.1302 \\ 13614 & 15: 13: 17 & 0.1264 \\ 13615 & 15: 13: 18 & 0.1287 \\ 13616 & 15: 13: 19 & 0.1242 \\ 13617 & 15: 13: 20 & 0.1246 \\ 13618 & 15: 13: 21 & 0.1287 \\ 13619 & 15: 13: 22 & 0.1258 \\ 13620 & 15: 13: 23 & 0.1258 \\ 13621 & 15: 13: 24 & 0.1221 \\ 13622 & 15: 13: 25 & 0.1290\end{array}$


Appendix A - Fuel Data Test 1 NIST102505_1.xIs

$\begin{array}{lll}13623 & 15: 13: 26 & 0.1241 \\ 13624 & 15: 13: 27 & 0.1281 \\ 13625 & 15: 13: 28 & 0.1257 \\ 13626 & 15: 13: 29 & 0.1287 \\ 13627 & 15: 13: 30 & 0.1264 \\ 13628 & 15: 13: 31 & 0.1263 \\ 13629 & 15: 13: 32 & 0.1282 \\ 13630 & 15: 13: 33 & 0.1259 \\ 13631 & 15: 13: 34 & 0.1275 \\ 13632 & 15: 13: 35 & 0.1255 \\ 13633 & 15: 13: 36 & 0.1288 \\ 13634 & 15: 13: 37 & 0.1240 \\ 13635 & 15: 13: 38 & 0.1295 \\ 13636 & 15: 13: 39 & 0.1262 \\ 13637 & 15: 13: 40 & 0.1297 \\ 13638 & 15: 13: 41 & 0.1235 \\ 13639 & 15: 13: 42 & 0.1282 \\ 13640 & 15: 13: 43 & 0.1251 \\ 13641 & 15: 13: 44 & 0.1256 \\ 13642 & 15: 13: 45 & 0.1251 \\ 13643 & 15: 13: 46 & 0.1240 \\ 13644 & 15: 13: 47 & 0.1257 \\ 13645 & 15: 13: 48 & 0.1228 \\ 13646 & 15: 13: 49 & 0.1269 \\ 13647 & 15: 13: 50 & 0.1226 \\ 13648 & 15: 13: 51 & 0.1270 \\ 13649 & 15: 13: 52 & 0.1255 \\ 13650 & 15: 13: 53 & 0.1298 \\ 13651 & 15: 13: 54 & 0.1277 \\ 13652 & 15: 13: 55 & 0.1284 \\ 13653 & 15: 13: 56 & 0.1254 \\ 13654 & 15: 13: 57 & 0.1262 \\ 13655 & 15: 13: 58 & 0.1270 \\ 13656 & 15: 13: 59 & 0.1239 \\ 13657 & 15: 14: 00 & 0.1282 \\ 13658 & 15: 14: 01 & 0.1238 \\ 13659 & 15: 14: 02 & 0.1283 \\ 13660 & 15: 14: 03 & 0.1249 \\ 13661 & 15: 14: 04 & 0.1306 \\ 13662 & 15: 14: 05 & 0.1276 \\ 13663 & 15: 14: 06 & 0.1292 \\ 13664 & 15: 14: 07 & 0.1249 \\ 13665 & 15: 14: 08 & 0.1272 \\ 13666 & 15: 14: 09 & 0.1264 \\ 13667 & 15: 14: 10 & 0.1244 \\ 13668 & 15: 14: 11 & 0.1295 \\ 13669 & 15: 14: 12 & 0.1265 \\ 13670 & 15: 14: 13 & 0.1285 \\ 13671 & 15: 14: 14 & 0.1246 \\ 13672 & 15: 14: 15 & 0.1320 \\ 13673 & 15: 14: 16 & 0.1278 \\ 13674 & 15: 14: 17 & 0.1323\end{array}$


Appendix A - Fuel Data Test 1 NIST102505_1.xls

$\begin{array}{lll}13675 & 15: 14: 18 & 0.1287 \\ 13676 & 15: 14: 19 & 0.1314 \\ 13677 & 15: 14: 20 & 0.1284 \\ 13678 & 15: 14: 21 & 0.1286 \\ 13679 & 15: 14: 22 & 0.1268 \\ 13680 & 15: 14: 23 & 0.1267 \\ 13681 & 15: 14: 24 & 0.1270 \\ 13682 & 15: 14: 25 & 0.1245 \\ 13683 & 15: 14: 26 & 0.1295 \\ 13684 & 15: 14: 27 & 0.1256 \\ 13685 & 15: 14: 28 & 0.1299 \\ 13686 & 15: 14: 29 & 0.1248 \\ 13687 & 15: 14: 30 & 0.1307 \\ 13688 & 15: 14: 31 & 0.1264 \\ 13689 & 15: 14: 32 & 0.1284 \\ 13690 & 15: 14: 33 & 0.1256 \\ 13691 & 15: 14: 34 & 0.1272 \\ 13692 & 15: 14: 35 & 0.1268 \\ 13693 & 15: 14: 36 & 0.1255 \\ 13694 & 15: 14: 37 & 0.1298 \\ 13695 & 15: 14: 38 & 0.1263 \\ 13696 & 15: 14: 39 & 0.1291 \\ 13697 & 15: 14: 40 & 0.1242 \\ 13698 & 15: 14: 41 & 0.1292 \\ 13699 & 15: 14: 42 & 0.1276 \\ 13700 & 15: 14: 43 & 0.1312 \\ 13701 & 15: 14: 44 & 0.1265 \\ 13702 & 15: 14: 45 & 0.1288 \\ 13703 & 15: 14: 46 & 0.1254 \\ 13704 & 15: 14: 47 & 0.1261 \\ 13705 & 15: 14: 48 & 0.1284 \\ 13706 & 15: 14: 49 & 0.1282 \\ 13707 & 15: 14: 50 & 0.1305 \\ 13708 & 15: 14: 51 & 0.1255 \\ 13709 & 15: 14: 52 & 0.1304 \\ 13710 & 15: 14: 53 & 0.1265 \\ 13711 & 15: 14: 54 & 0.1287 \\ 13712 & 15: 14: 55 & 0.1272 \\ 13713 & 15: 14: 56 & 0.1297 \\ 13714 & 15: 14: 57 & 0.1272 \\ 13715 & 15: 14: 58 & 0.1279 \\ 13716 & 15: 14: 59 & 0.1271 \\ 13717 & 15: 15: 00 & 0.1274 \\ 13718 & 15: 15: 01 & 0.1286 \\ 13719 & 15: 15: 02 & 0.1264 \\ 13720 & 15: 15: 03 & 0.1279 \\ 13721 & 15: 15: 04 & 0.1236 \\ 13722 & 15: 15: 05 & 0.1300 \\ 13723 & 15: 15: 06 & 0.1266 \\ 13724 & 15: 15: 07 & 0.1305 \\ 13725 & 15: 15: 08 & 0.1265 \\ 13726 & 15: 15: 09 & 0.1303\end{array}$


Appendix A - Fuel Data Test 1 NIST102505_1.xls

$\begin{array}{lll}13727 & 15: 15: 10 & 0.1279 \\ 13728 & 15: 15: 11 & 0.1293 \\ 13729 & 15: 15: 12 & 0.1272 \\ 13730 & 15: 15: 13 & 0.1273 \\ 13731 & 15: 15: 14 & 0.1270 \\ 13732 & 15: 15: 15 & 0.1242 \\ 13733 & 15: 15: 16 & 0.1301 \\ 13734 & 15: 15: 17 & 0.1253 \\ 13735 & 15: 15: 18 & 0.1293 \\ 13736 & 15: 15: 19 & 0.1250 \\ 13737 & 15: 15: 20 & 0.1302 \\ 13738 & 15: 15: 21 & 0.1275 \\ 13739 & 15: 15: 22 & 0.1289 \\ 13740 & 15: 15: 23 & 0.1284 \\ 13741 & 15: 15: 24 & 0.1279 \\ 13742 & 15: 15: 25 & 0.1274 \\ 13743 & 15: 15: 26 & 0.1249 \\ 13744 & 15: 15: 27 & 0.1290 \\ 13745 & 15: 15: 28 & 0.1242 \\ 13746 & 15: 15: 29 & 0.1283 \\ 13747 & 15: 15: 30 & 0.1255 \\ 13748 & 15: 15: 31 & 0.1294 \\ 13749 & 15: 15: 32 & 0.1254 \\ 13750 & 15: 15: 33 & 0.1284 \\ 13751 & 15: 15: 34 & 0.1290 \\ 13752 & 15: 15: 35 & 0.1285 \\ 13753 & 15: 15: 36 & 0.1260 \\ 13754 & 15: 15: 37 & 0.1246 \\ 13755 & 15: 15: 38 & 0.1306 \\ 13756 & 15: 15: 39 & 0.1271 \\ 13757 & 15: 15: 40 & 0.1292 \\ 13758 & 15: 15: 41 & 0.1255 \\ 13759 & 15: 15: 42 & 0.1302 \\ 13760 & 15: 15: 43 & 0.1259 \\ 13761 & 15: 15: 44 & 0.1292 \\ 13762 & 15: 15: 45 & 0.1263 \\ 13763 & 15: 15: 46 & 0.1295 \\ 13764 & 15: 15: 47 & 0.1272 \\ 13765 & 15: 15: 48 & 0.1265 \\ 13766 & 15: 15: 49 & 0.1283 \\ 13767 & 15: 15: 50 & 0.1271 \\ 13768 & 15: 15: 51 & 0.1288 \\ 13769 & 15: 15: 52 & 0.1259 \\ 13770 & 15: 15: 53 & 0.1289 \\ 13771 & 15: 15: 54 & 0.1239 \\ 13772 & 15: 15: 55 & 0.1291 \\ 13773 & 15: 15: 56 & 0.1276 \\ 13774 & 15: 15: 57 & 0.1311 \\ 13775 & 15: 15: 58 & 0.1256 \\ 13776 & 15: 15: 59 & 0.1304 \\ 13777 & 15: 16: 00 & 0.1296 \\ 13778 & 15: 16: 01 & 0.1288\end{array}$


Appendix A - Fuel Data Test 1 NIST102505_1.xls

$\begin{array}{lll}13779 & 15: 16: 02 & 0.1272 \\ 13780 & 15: 16: 03 & 0.1267 \\ 13781 & 15: 16: 04 & 0.1274 \\ 13782 & 15: 16: 05 & 0.1242 \\ 13783 & 15: 16: 06 & 0.1282 \\ 13784 & 15: 16: 07 & 0.1247 \\ 13785 & 15: 16: 08 & 0.1289 \\ 13786 & 15: 16: 09 & 0.1242 \\ 13787 & 15: 16: 10 & 0.1317 \\ 13788 & 15: 16: 11 & 0.1272 \\ 13789 & 15: 16: 12 & 0.1292 \\ 13790 & 15: 16: 13 & 0.1270 \\ 13791 & 15: 16: 14 & 0.1273 \\ 13792 & 15: 16: 15 & 0.1262 \\ 13793 & 15: 16: 16 & 0.1246 \\ 13794 & 15: 16: 17 & 0.1292 \\ 13795 & 15: 16: 18 & 0.1250 \\ 13796 & 15: 16: 19 & 0.1289 \\ 13797 & 15: 16: 20 & 0.1251 \\ 13798 & 15: 16: 21 & 0.1290 \\ 13799 & 15: 16: 22 & 0.1238 \\ 13800 & 15: 16: 23 & 0.1298 \\ 13801 & 15: 16: 24 & 0.1280 \\ 13802 & 15: 16: 25 & 0.1287 \\ 13803 & 15: 16: 26 & 0.1265 \\ 13804 & 15: 16: 27 & 0.1249 \\ 13805 & 15: 16: 28 & 0.1287 \\ 13806 & 15: 16: 29 & 0.1259 \\ 13807 & 15: 16: 30 & 0.1273 \\ 13808 & 15: 16: 31 & 0.1253 \\ 13809 & 15: 16: 32 & 0.1295 \\ 13810 & 15: 16: 33 & 0.1250 \\ 13811 & 15: 16: 34 & 0.1303 \\ 13812 & 15: 16: 35 & 0.1259 \\ 13813 & 15: 16: 36 & 0.1303 \\ 13814 & 15: 16: 37 & 0.1266 \\ 13815 & 15: 16: 38 & 0.1307 \\ 13816 & 15: 16: 39 & 0.1259 \\ 13817 & 15: 16: 40 & 0.1257 \\ 13818 & 15: 16: 41 & 0.1270 \\ 13819 & 15: 16: 42 & 0.1254 \\ 13820 & 15: 16: 43 & 0.1271 \\ 13821 & 15: 16: 44 & 0.1230 \\ 13822 & 15: 16: 45 & 0.1286 \\ 13823 & 15: 16: 46 & 0.1250 \\ 13824 & 15: 16: 47 & 0.1289 \\ 13825 & 15: 16: 48 & 0.1274 \\ 13826 & 15: 16: 49 & 0.1295 \\ 13827 & 15: 16: 50 & 0.1256 \\ 13828 & 15: 16: 51 & 0.1295 \\ 13829 & 15: 16: 52 & 0.1278 \\ 13830 & 15: 16: 53 & 0.1272\end{array}$


Appendix A - Fuel Data Test 1 NIST102505_1.xIs

$\begin{array}{lll}13831 & 15: 16: 54 & 0.1274 \\ 13832 & 15: 16: 55 & 0.1262 \\ 13833 & 15: 16: 56 & 0.1280 \\ 13834 & 15: 16: 57 & 0.1235 \\ 13835 & 15: 16: 58 & 0.1294 \\ 13836 & 15: 16: 59 & 0.1262 \\ 13837 & 15: 17: 00 & 0.1305 \\ 13838 & 15: 17: 01 & 0.1264 \\ 13839 & 15: 17: 02 & 0.1309 \\ 13840 & 15: 17: 03 & 0.1277 \\ 13841 & 15: 17: 04 & 0.1269 \\ 13842 & 15: 17: 05 & 0.1269 \\ 13843 & 15: 17: 06 & 0.1259 \\ 13844 & 15: 17: 07 & 0.1288 \\ 13845 & 15: 17: 08 & 0.1255 \\ 13846 & 15: 17: 09 & 0.1298 \\ 13847 & 15: 17: 10 & 0.1242 \\ 13848 & 15: 17: 11 & 0.1285 \\ 13849 & 15: 17: 12 & 0.1267 \\ 13850 & 15: 17: 13 & 0.1301 \\ 13851 & 15: 17: 14 & 0.1257 \\ 13852 & 15: 17: 15 & 0.1294 \\ 13853 & 15: 17: 16 & 0.1276 \\ 13854 & 15: 17: 17 & 0.1282 \\ 13855 & 15: 17: 18 & 0.1251 \\ 13856 & 15: 17: 19 & 0.1251 \\ 13857 & 15: 17: 20 & 0.1273 \\ 13858 & 15: 17: 21 & 0.1244 \\ 13859 & 15: 17: 22 & 0.1272 \\ 13860 & 15: 17: 23 & 0.1250 \\ 13861 & 15: 17: 24 & 0.1290 \\ 13862 & 15: 17: 25 & 0.1260 \\ 13863 & 15: 17: 26 & 0.1304 \\ 13864 & 15: 17: 27 & 0.1291 \\ 13865 & 15: 17: 28 & 0.1291 \\ 13866 & 15: 17: 29 & 0.1263 \\ 13867 & 15: 17: 30 & 0.1258 \\ 13868 & 15: 17: 31 & 0.1275 \\ 13869 & 15: 17: 32 & 0.1261 \\ 13870 & 15: 17: 33 & 0.1285 \\ 13871 & 15: 17: 34 & 0.1266 \\ 13872 & 15: 17: 35 & 0.1303 \\ 13873 & 15: 17: 36 & 0.1250 \\ 13874 & 15: 17: 37 & 0.1310 \\ 13875 & 15: 17: 38 & 0.1273 \\ 13876 & 15: 17: 39 & 0.1303 \\ 13877 & 15: 17: 40 & 0.1260 \\ 13878 & 15: 17: 41 & 0.1304 \\ 13879 & 15: 17: 42 & 0.1267 \\ 13880 & 15: 17: 43 & 0.1259 \\ 13881 & 15: 17: 44 & 0.1265 \\ 13882 & 15: 17: 45 & 0.1263\end{array}$


Appendix A - Fuel Data Test 1 NIST102505_1.xls

$\begin{array}{lll}13883 & 15: 17: 46 & 0.1267 \\ 13884 & 15: 17: 47 & 0.1222 \\ 13885 & 15: 17: 48 & 0.1300 \\ 13886 & 15: 17: 49 & 0.1262 \\ 13887 & 15: 17: 50 & 0.1299 \\ 13888 & 15: 17: 51 & 0.1256 \\ 13889 & 15: 17: 52 & 0.1295 \\ 13890 & 15: 17: 53 & 0.1261 \\ 13891 & 15: 17: 54 & 0.1283 \\ 13892 & 15: 17: 55 & 0.1250 \\ 13893 & 15: 17: 56 & 0.1269 \\ 13894 & 15: 17: 57 & 0.1273 \\ 13895 & 15: 17: 58 & 0.1257 \\ 13896 & 15: 17: 59 & 0.1289 \\ 13897 & 15: 18: 00 & 0.1263 \\ 13898 & 15: 18: 01 & 0.1293 \\ 13899 & 15: 18: 02 & 0.1242 \\ 13900 & 15: 18: 03 & 0.1294 \\ 13901 & 15: 18: 04 & 0.1272 \\ 13902 & 15: 18: 05 & 0.1307 \\ 13903 & 15: 18: 06 & 0.1268 \\ 13904 & 15: 18: 07 & 0.1300 \\ 13905 & 15: 18: 08 & 0.1263 \\ 13906 & 15: 18: 09 & 0.1255 \\ 13907 & 15: 18: 10 & 0.1285 \\ 13908 & 15: 18: 11 & 0.1261 \\ 13909 & 15: 18: 12 & 0.1277 \\ 13910 & 15: 18: 13 & 0.1236 \\ 13911 & 15: 18: 14 & 0.1300 \\ 13912 & 15: 18: 15 & 0.1263 \\ 13913 & 15: 18: 16 & 0.1314 \\ 13914 & 15: 18: 17 & 0.1265 \\ 13915 & 15: 18: 18 & 0.1303 \\ 13916 & 15: 18: 19 & 0.1269 \\ 13917 & 15: 18: 20 & 0.1273 \\ 13918 & 15: 18: 21 & 0.1239 \\ 13919 & 15: 18: 22 & 0.1250 \\ 13920 & 15: 18: 23 & 0.1267 \\ 13921 & 15: 18: 24 & 0.1232 \\ 13922 & 15: 18: 25 & 0.1298 \\ 13923 & 15: 18: 26 & 0.1248 \\ 13924 & 15: 18: 27 & 0.1283 \\ 13925 & 15: 18: 28 & 0.1249 \\ 13926 & 15: 18: 29 & 0.1295 \\ 13927 & 15: 18: 30 & 0.1257 \\ 13928 & 15: 18: 31 & 0.1306 \\ 13929 & 15: 18: 32 & 0.1289 \\ 13930 & 15: 18: 33 & 0.1291 \\ 13931 & 15: 18: 34 & 0.1261 \\ 13932 & 15: 18: 35 & 0.1265 \\ 13933 & 15: 18: 36 & 0.1279 \\ 13934 & 15: 18: 37 & 0.1241\end{array}$


Appendix A - Fuel Data Test 1 NIST102505_1.xIs

$\begin{array}{lll}13935 & 15: 18: 38 & 0.1289 \\ 13936 & 15: 18: 39 & 0.1251 \\ 13937 & 15: 18: 40 & 0.1294 \\ 13938 & 15: 18: 41 & 0.1258 \\ 13939 & 15: 18: 42 & 0.1302 \\ 13940 & 15: 18: 43 & 0.1275 \\ 13941 & 15: 18: 44 & 0.1263 \\ 13942 & 15: 18: 45 & 0.1264 \\ 13943 & 15: 18: 46 & 0.1262 \\ 13944 & 15: 18: 47 & 0.1251 \\ 13945 & 15: 18: 48 & 0.1240 \\ 13946 & 15: 18: 49 & 0.1284 \\ 13947 & 15: 18: 50 & 0.1267 \\ 13948 & 15: 18: 51 & 0.1292 \\ 13949 & 15: 18: 52 & 0.1253 \\ 13950 & 15: 18: 53 & 0.1303 \\ 13951 & 15: 18: 54 & 0.1269 \\ 13952 & 15: 18: 55 & 0.1297 \\ 13953 & 15: 18: 56 & 0.1255 \\ 13954 & 15: 18: 57 & 0.1297 \\ 13955 & 15: 18: 58 & 0.1279 \\ 13956 & 15: 18: 59 & 0.1285 \\ 13957 & 15: 19: 00 & 0.1276 \\ 13958 & 15: 19: 01 & 0.1268 \\ 13959 & 15: 19: 02 & 0.1270 \\ 13960 & 15: 19: 03 & 0.1228 \\ 13961 & 15: 19: 04 & 0.1276 \\ 13962 & 15: 19: 05 & 0.1253 \\ 13963 & 15: 19: 06 & 0.1292 \\ 13964 & 15: 19: 07 & 0.1238 \\ 13965 & 15: 19: 08 & 0.1297 \\ 13966 & 15: 19: 09 & 0.1265 \\ 13967 & 15: 19: 10 & 0.1285 \\ 13968 & 15: 19: 11 & 0.1254 \\ 13969 & 15: 19: 12 & 0.1265 \\ 13970 & 15: 19: 13 & 0.1260 \\ 13971 & 15: 19: 14 & 0.1246 \\ 13972 & 15: 19: 15 & 0.1282 \\ 13973 & 15: 19: 16 & 0.1253 \\ 13974 & 15: 19: 17 & 0.1310 \\ 13975 & 15: 19: 18 & 0.1260 \\ 13976 & 15: 19: 19 & 0.1305 \\ 13977 & 15: 19: 20 & 0.1272 \\ 13978 & 15: 19: 21 & 0.1307 \\ 13979 & 15: 19: 22 & 0.1262 \\ 13980 & 15: 19: 23 & 0.1288 \\ 13981 & 15: 19: 24 & 0.1262 \\ 13982 & 15: 19: 25 & 0.1258 \\ 13983 & 15: 19: 26 & 0.1268 \\ 13984 & 15: 19: 27 & 0.1259 \\ 13985 & 15: 19: 28 & 0.1275 \\ 13986 & 15: 19: 29 & 0.1230\end{array}$


Appendix A - Fuel Data Test 1 NIST102505_1.xls

$\begin{array}{lll}13987 & 15: 19: 30 & 0.1281 \\ 13988 & 15: 19: 31 & 0.1258 \\ 13989 & 15: 19: 32 & 0.1291 \\ 13990 & 15: 19: 33 & 0.1233 \\ 13991 & 15: 19: 34 & 0.1287 \\ 13992 & 15: 19: 35 & 0.1260 \\ 13993 & 15: 19: 36 & 0.1257 \\ 13994 & 15: 19: 37 & 0.1258 \\ 13995 & 15: 19: 38 & 0.1264 \\ 13996 & 15: 19: 39 & 0.1284 \\ 13997 & 15: 19: 40 & 0.1248 \\ 13998 & 15: 19: 41 & 0.1300 \\ 13999 & 15: 19: 42 & 0.1260 \\ 14000 & 15: 19: 43 & 0.1292 \\ 14001 & 15: 19: 44 & 0.1251 \\ 14002 & 15: 19: 45 & 0.1314 \\ 14003 & 15: 19: 46 & 0.1282 \\ 14004 & 15: 19: 47 & 0.1289 \\ 14005 & 15: 19: 48 & 0.1253 \\ 14006 & 15: 19: 49 & 0.1270 \\ 14007 & 15: 19: 50 & 0.1259 \\ 14008 & 15: 19: 51 & 0.1248 \\ 14009 & 15: 19: 52 & 0.1281 \\ 14010 & 15: 19: 53 & 0.1247 \\ 14011 & 15: 19: 54 & 0.1287 \\ 14012 & 15: 19: 55 & 0.1245 \\ 14013 & 15: 19: 56 & 0.1304 \\ 14014 & 15: 19: 57 & 0.1269 \\ 14015 & 15: 19: 58 & 0.1292 \\ 14016 & 15: 19: 59 & 0.1264 \\ 14017 & 15: 20: 00 & 0.1283 \\ 14018 & 15: 20: 01 & 0.1238 \\ 14019 & 15: 20: 02 & 0.1258 \\ 14020 & 15: 20: 03 & 0.1291 \\ 14021 & 15: 20: 04 & 0.1268 \\ 14022 & 15: 20: 05 & 0.1277 \\ 14023 & 15: 20: 06 & 0.1238 \\ 14024 & 15: 20: 07 & 0.1286 \\ 14025 & 15: 20: 08 & 0.1244 \\ 14026 & 15: 20: 09 & 0.1293 \\ 14027 & 15: 20: 10 & 0.1275 \\ 14028 & 15: 20: 11 & 0.1301 \\ 14029 & 15: 20: 12 & 0.1275 \\ 14030 & 15: 20: 13 & 0.1277 \\ 14031 & 15: 20: 14 & 0.1265 \\ 14032 & 15: 20: 15 & 0.1257 \\ 14033 & 15: 20: 16 & 0.1261 \\ 14034 & 15: 20: 17 & 0.1244 \\ 14035 & 15: 20: 18 & 0.1277 \\ 14036 & 15: 20: 19 & 0.1233 \\ 14037 & 15: 20: 20 & 0.1285 \\ 14038 & 15: 20: 21 & 0.1276\end{array}$


Appendix A - Fuel Data Test 1 NIST102505_1.xIs

$\begin{array}{lll}14039 & 15: 20: 22 & 0.1324 \\ 14040 & 15: 20: 23 & 0.1278 \\ 14041 & 15: 20: 24 & 0.1316 \\ 14042 & 15: 20: 25 & 0.1276 \\ 14043 & 15: 20: 26 & 0.1273 \\ 14044 & 15: 20: 27 & 0.1268 \\ 14045 & 15: 20: 28 & 0.1251 \\ 14046 & 15: 20: 29 & 0.1279 \\ 14047 & 15: 20: 30 & 0.1242 \\ 14048 & 15: 20: 31 & 0.1278 \\ 14049 & 15: 20: 32 & 0.1252 \\ 14050 & 15: 20: 33 & 0.1284 \\ 14051 & 15: 20: 34 & 0.1248 \\ 14052 & 15: 20: 35 & 0.1292 \\ 14053 & 15: 20: 36 & 0.1284 \\ 14054 & 15: 20: 37 & 0.1291 \\ 14055 & 15: 20: 38 & 0.1260 \\ 14056 & 15: 20: 39 & 0.1270 \\ 14057 & 15: 20: 40 & 0.1279 \\ 14058 & 15: 20: 41 & 0.1249 \\ 14059 & 15: 20: 42 & 0.1257 \\ 14060 & 15: 20: 43 & 0.1223 \\ 14061 & 15: 20: 44 & 0.1280 \\ 14062 & 15: 20: 45 & 0.1256 \\ 14063 & 15: 20: 46 & 0.1297 \\ 14064 & 15: 20: 47 & 0.1275 \\ 14065 & 15: 20: 48 & 0.1310 \\ 14066 & 15: 20: 49 & 0.1267 \\ 14067 & 15: 20: 50 & 0.1284 \\ 14068 & 15: 20: 51 & 0.1263 \\ 14069 & 15: 20: 52 & 0.1274 \\ 14070 & 15: 20: 53 & 0.1285 \\ 14071 & 15: 20: 54 & 0.1274 \\ 14072 & 15: 20: 55 & 0.1286 \\ 14073 & 15: 20: 56 & 0.1236 \\ 14074 & 15: 20: 57 & 0.1274 \\ 14075 & 15: 20: 58 & 0.1265 \\ 14076 & 15: 20: 59 & 0.1297 \\ 14077 & 15: 21: 00 & 0.1240 \\ 14078 & 15: 21: 01 & 0.1303 \\ 14079 & 15: 21: 02 & 0.1272 \\ 14080 & 15: 21: 03 & 0.1282 \\ 14081 & 15: 21: 04 & 0.1264 \\ 14082 & 15: 21: 05 & 0.1272 \\ 14083 & 15: 21: 06 & 0.1285 \\ 14084 & 15: 21: 07 & 0.1265 \\ 14085 & 15: 21: 08 & 0.1285 \\ 14086 & 15: 21: 09 & 0.1255 \\ 14087 & 15: 21: 10 & 0.1308 \\ 14088 & 15: 21: 11 & 0.1275 \\ 14089 & 15: 21: 12 & 0.1308 \\ 14090 & 15: 21: 13 & 0.1292\end{array}$


Appendix A - Fuel Data Test 1 NIST102505_1.xls

$\begin{array}{lll}14091 & 15: 21: 14 & 0.1308 \\ 14092 & 15: 21: 15 & 0.1279 \\ 14093 & 15: 21: 16 & 0.1268 \\ 14094 & 15: 21: 17 & 0.1279 \\ 14095 & 15: 21: 18 & 0.1270 \\ 14096 & 15: 21: 19 & 0.1278 \\ 14097 & 15: 21: 20 & 0.1254 \\ 14098 & 15: 21: 21 & 0.1282 \\ 14099 & 15: 21: 22 & 0.1227 \\ 14100 & 15: 21: 23 & 0.1275 \\ 14101 & 15: 21: 24 & 0.1257 \\ 14102 & 15: 21: 25 & 0.1300 \\ 14103 & 15: 21: 26 & 0.1261 \\ 14104 & 15: 21: 27 & 0.1284 \\ 14105 & 15: 21: 28 & 0.1278 \\ 14106 & 15: 21: 29 & 0.1263 \\ 14107 & 15: 21: 30 & 0.1264 \\ 14108 & 15: 21: 31 & 0.1240 \\ 14109 & 15: 21: 32 & 0.1263 \\ 14110 & 15: 21: 33 & 0.1217 \\ 14111 & 15: 21: 34 & 0.1276 \\ 14112 & 15: 21: 35 & 0.1247 \\ 14113 & 15: 21: 36 & 0.1286 \\ 14114 & 15: 21: 37 & 0.1230 \\ 14115 & 15: 21: 38 & 0.1301 \\ 14116 & 15: 21: 39 & 0.1253 \\ 14117 & 15: 21: 40 & 0.1274 \\ 14118 & 15: 21: 41 & 0.1268 \\ 14119 & 15: 21: 42 & 0.1267 \\ 14120 & 15: 21: 43 & 0.1279 \\ 14121 & 15: 21: 44 & 0.1247 \\ 14122 & 15: 21: 45 & 0.1291 \\ 14123 & 15: 21: 46 & 0.1245 \\ 14124 & 15: 21: 47 & 0.1288 \\ 14125 & 15: 21: 48 & 0.1261 \\ 14126 & 15: 21: 49 & 0.1299 \\ 14127 & 15: 21: 50 & 0.1249 \\ 14128 & 15: 21: 51 & 0.1289 \\ 14129 & 15: 21: 52 & 0.1276 \\ 14130 & 15: 21: 53 & 0.1279 \\ 14131 & 15: 21: 54 & 0.1267 \\ 14132 & 15: 21: 55 & 0.1257 \\ 14133 & 15: 21: 56 & 0.1289 \\ 14134 & 15: 21: 57 & 0.1266 \\ 14135 & 15: 21: 58 & 0.1281 \\ 14136 & 15: 21: 59 & 0.1260 \\ 14137 & 15: 22: 00 & 0.1313 \\ 14138 & 15: 22: 01 & 0.1265 \\ 14139 & 15: 22: 02 & 0.1311 \\ 14140 & 15: 22: 03 & 0.1296 \\ 14141 & 15: 22: 04 & 0.1325 \\ 14142 & 15: 22: 05 & 0.1285\end{array}$


Appendix A - Fuel Data Test 1 NIST102505_1.xls

$\begin{array}{lll}14143 & 15: 22: 06 & 0.1294 \\ 14144 & 15: 22: 07 & 0.1287 \\ 14145 & 15: 22: 08 & 0.1279 \\ 14146 & 15: 22: 09 & 0.1280 \\ 14147 & 15: 22: 10 & 0.1260 \\ 14148 & 15: 22: 11 & 0.1276 \\ 14149 & 15: 22: 12 & 0.1240 \\ 14150 & 15: 22: 13 & 0.1310 \\ 14151 & 15: 22: 14 & 0.1276 \\ 14152 & 15: 22: 15 & 0.1324 \\ 14153 & 15: 22: 16 & 0.1265 \\ 14154 & 15: 22: 17 & 0.1312 \\ 14155 & 15: 22: 18 & 0.1284 \\ 14156 & 15: 22: 19 & 0.1284 \\ 14157 & 15: 22: 20 & 0.1261 \\ 14158 & 15: 22: 21 & 0.1256 \\ 14159 & 15: 22: 22 & 0.1278 \\ 14160 & 15: 22: 23 & 0.1247 \\ 14161 & 15: 22: 24 & 0.1295 \\ 14162 & 15: 22: 25 & 0.1260 \\ 14163 & 15: 22: 26 & 0.1307 \\ 14164 & 15: 22: 27 & 0.1254 \\ 14165 & 15: 22: 28 & 0.1307 \\ 14166 & 15: 22: 29 & 0.1260 \\ 14167 & 15: 22: 30 & 0.1285 \\ 14168 & 15: 22: 31 & 0.1246 \\ 14169 & 15: 22: 32 & 0.1258 \\ 14170 & 15: 22: 33 & 0.1264 \\ 14171 & 15: 22: 34 & 0.1230 \\ 14172 & 15: 22: 35 & 0.1282 \\ 14173 & 15: 22: 36 & 0.1261 \\ 14174 & 15: 22: 37 & 0.1298 \\ 14175 & 15: 22: 38 & 0.1255 \\ 14176 & 15: 22: 39 & 0.1305 \\ 14177 & 15: 22: 40 & 0.1246 \\ 14178 & 15: 22: 41 & 0.1283 \\ 14179 & 15: 22: 42 & 0.1282 \\ 14180 & 15: 22: 43 & 0.1286 \\ 14181 & 15: 22: 44 & 0.1280 \\ 14182 & 15: 22: 45 & 0.1266 \\ 14183 & 15: 22: 46 & 0.1300 \\ 14184 & 15: 22: 47 & 0.1267 \\ 14185 & 15: 22: 48 & 0.1278 \\ 14186 & 15: 22: 49 & 0.1228 \\ 14187 & 15: 22: 50 & 0.1287 \\ 14188 & 15: 22: 51 & 0.1243 \\ 14189 & 15: 22: 52 & 0.1279 \\ 14190 & 15: 22: 53 & 0.1251 \\ 14191 & 15: 22: 54 & 0.1288 \\ 14192 & 15: 22: 55 & 0.1270 \\ 14193 & 15: 22: 56 & 0.1271 \\ 14194 & 15: 22: 57 & 0.1267\end{array}$


Appendix A - Fuel Data Test 1 NIST102505_1.xls

$\begin{array}{lll}14195 & 15: 22: 58 & 0.1278 \\ 14196 & 15: 22: 59 & 0.1295 \\ 14197 & 15: 23: 00 & 0.1256 \\ 14198 & 15: 23: 01 & 0.1288 \\ 14199 & 15: 23: 02 & 0.1231 \\ 14200 & 15: 23: 03 & 0.1299 \\ 14201 & 15: 23: 04 & 0.1264 \\ 14202 & 15: 23: 05 & 0.1297 \\ 14203 & 15: 23: 06 & 0.1271 \\ 14204 & 15: 23: 07 & 0.1295 \\ 14205 & 15: 23: 08 & 0.1260 \\ 14206 & 15: 23: 09 & 0.1259 \\ 14207 & 15: 23: 10 & 0.1281 \\ 14208 & 15: 23: 11 & 0.1255 \\ 14209 & 15: 23: 12 & 0.1274 \\ 14210 & 15: 23: 13 & 0.1224 \\ 14211 & 15: 23: 14 & 0.1290 \\ 14212 & 15: 23: 15 & 0.1244 \\ 14213 & 15: 23: 16 & 0.1285 \\ 14214 & 15: 23: 17 & 0.1256 \\ 14215 & 15: 23: 18 & 0.1299 \\ 14216 & 15: 23: 19 & 0.1273 \\ 14217 & 15: 23: 20 & 0.1279 \\ 14218 & 15: 23: 21 & 0.1264 \\ 14219 & 15: 23: 22 & 0.1253 \\ 14220 & 15: 23: 23 & 0.1282 \\ 14221 & 15: 23: 24 & 0.1234 \\ 14222 & 15: 23: 25 & 0.1278 \\ 14223 & 15: 23: 26 & 0.1227 \\ 14224 & 15: 23: 27 & 0.1283 \\ 14225 & 15: 23: 28 & 0.1252 \\ 14226 & 15: 23: 29 & 0.1317 \\ 14227 & 15: 23: 30 & 0.1284 \\ 14228 & 15: 23: 31 & 0.1314 \\ 14229 & 15: 23: 32 & 0.1294 \\ 14230 & 15: 23: 33 & 0.1288 \\ 14231 & 15: 23: 34 & 0.1266 \\ 14232 & 15: 23: 35 & 0.1241 \\ 14233 & 15: 23: 36 & 0.1283 \\ 14234 & 15: 23: 37 & 0.1258 \\ 14235 & 15: 23: 38 & 0.1286 \\ 14236 & 15: 23: 39 & 0.1238 \\ 14237 & 15: 23: 40 & 0.1300 \\ 14238 & 15: 23: 41 & 0.1250 \\ 14239 & 15: 23: 42 & 0.1286 \\ 14240 & 15: 23: 43 & 0.1276 \\ 14241 & 15: 23: 44 & 0.1297 \\ 14242 & 15: 23: 45 & 0.1261 \\ 14243 & 15: 23: 46 & 0.1261 \\ 14244 & 15: 23: 47 & 0.1283 \\ 14245 & 15: 23: 48 & 0.1257 \\ 14246 & 15: 23: 49 & 0.1275\end{array}$


Appendix A - Fuel Data Test 1 NIST102505_1.xls

$\begin{array}{lll}14247 & 15: 23: 50 & 0.1251 \\ 14248 & 15: 23: 51 & 0.1286 \\ 14249 & 15: 23: 52 & 0.1251 \\ 14250 & 15: 23: 53 & 0.1293 \\ 14251 & 15: 23: 54 & 0.1267 \\ 14252 & 15: 23: 55 & 0.1305 \\ 14253 & 15: 23: 56 & 0.1267 \\ 14254 & 15: 23: 57 & 0.1285 \\ 14255 & 15: 23: 58 & 0.1279 \\ 14256 & 15: 23: 59 & 0.1274 \\ 14257 & 15: 24: 00 & 0.1276 \\ 14258 & 15: 24: 01 & 0.1270 \\ 14259 & 15: 24: 02 & 0.1274 \\ 14260 & 15: 24: 03 & 0.1221 \\ 14261 & 15: 24: 04 & 0.1280 \\ 14262 & 15: 24: 05 & 0.1258 \\ 14263 & 15: 24: 06 & 0.1289 \\ 14264 & 15: 24: 07 & 0.1233 \\ 14265 & 15: 24: 08 & 0.1298 \\ 14266 & 15: 24: 09 & 0.1270 \\ 14267 & 15: 24: 10 & 0.1276 \\ 14268 & 15: 24: 11 & 0.1263 \\ 14269 & 15: 24: 12 & 0.1284 \\ 14270 & 15: 24: 13 & 0.1282 \\ 14271 & 15: 24: 14 & 0.1270 \\ 14272 & 15: 24: 15 & 0.1299 \\ 14273 & 15: 24: 16 & 0.1261 \\ 14274 & 15: 24: 17 & 0.1297 \\ 14275 & 15: 24: 18 & 0.1246 \\ 14276 & 15: 24: 19 & 0.1307 \\ 14277 & 15: 24: 20 & 0.1274 \\ 14278 & 15: 24: 21 & 0.1314 \\ 14279 & 15: 24: 22 & 0.1275 \\ 14280 & 15: 24: 23 & 0.1285 \\ 14281 & 15: 24: 24 & 0.1255 \\ 14282 & 15: 24: 25 & 0.1253 \\ 14283 & 15: 24: 26 & 0.1297 \\ 14284 & 15: 24: 27 & 0.1256 \\ 14285 & 15: 24: 28 & 0.1291 \\ 14286 & 15: 24: 29 & 0.1234 \\ 14287 & 15: 24: 30 & 0.1313 \\ 14288 & 15: 24: 31 & 0.1275 \\ 14289 & 15: 24: 32 & 0.1306 \\ 14290 & 15: 24: 33 & 0.1257 \\ 14291 & 15: 24: 34 & 0.1294 \\ 14292 & 15: 24: 35 & 0.1265 \\ 14293 & 15: 24: 36 & 0.1278 \\ 14294 & 15: 24: 37 & 0.1261 \\ 14295 & 15: 24: 38 & 0.1265 \\ 14296 & 15: 24: 39 & 0.1279 \\ 14297 & 15: 24: 40 & 0.1248 \\ 14298 & 15: 24: 41 & 0.1306\end{array}$


Appendix A - Fuel Data Test 1 NIST102505_1.xls

$\begin{array}{lll}14299 & 15: 24: 42 & 0.1265 \\ 14300 & 15: 24: 43 & 0.1298 \\ 14301 & 15: 24: 44 & 0.1242 \\ 14302 & 15: 24: 45 & 0.1304 \\ 14303 & 15: 24: 46 & 0.1272 \\ 14304 & 15: 24: 47 & 0.1287 \\ 14305 & 15: 24: 48 & 0.1251 \\ 14306 & 15: 24: 49 & 0.1266 \\ 14307 & 15: 24: 50 & 0.1248 \\ 14308 & 15: 24: 51 & 0.1235 \\ 14309 & 15: 24: 52 & 0.1277 \\ 14310 & 15: 24: 53 & 0.1240 \\ 14311 & 15: 24: 54 & 0.1289 \\ 14312 & 15: 24: 55 & 0.1246 \\ 14313 & 15: 24: 56 & 0.1294 \\ 14314 & 15: 24: 57 & 0.1266 \\ 14315 & 15: 24: 58 & 0.1302 \\ 14316 & 15: 24: 59 & 0.1246 \\ 14317 & 15: 25: 00 & 0.1272 \\ 14318 & 15: 25: 01 & 0.1239 \\ 14319 & 15: 25: 02 & 0.1240 \\ 14320 & 15: 25: 03 & 0.1268 \\ 14321 & 15: 25: 04 & 0.1247 \\ 14322 & 15: 25: 05 & 0.1272 \\ 14323 & 15: 25: 06 & 0.1212 \\ 14324 & 15: 25: 07 & 0.1288 \\ 14325 & 15: 25: 08 & 0.1268 \\ 14326 & 15: 25: 09 & 0.1305 \\ 14327 & 15: 25: 10 & 0.1256 \\ 14328 & 15: 25: 11 & 0.1300 \\ 14329 & 15: 25: 12 & 0.1268 \\ 14330 & 15: 25: 13 & 0.1285 \\ 14331 & 15: 25: 14 & 0.1273 \\ 14332 & 15: 25: 15 & 0.1280 \\ 14333 & 15: 25: 16 & 0.1273 \\ 14334 & 15: 25: 17 & 0.1239 \\ 14335 & 15: 25: 18 & 0.1283 \\ 14336 & 15: 25: 19 & 0.1234 \\ 14337 & 15: 25: 20 & 0.1282 \\ 14338 & 15: 25: 21 & 0.1243 \\ 14339 & 15: 25: 22 & 0.1303 \\ 14340 & 15: 25: 23 & 0.1269 \\ 14341 & 15: 25: 24 & 0.1294 \\ 14342 & 15: 25: 25 & 0.1293 \\ 14343 & 15: 25: 26 & 0.1285 \\ 14344 & 15: 25: 27 & 0.1262 \\ 14345 & 15: 25: 28 & 0.1241 \\ 14346 & 15: 25: 29 & 0.1286 \\ 14347 & 15: 25: 30 & 0.1252 \\ 14348 & 15: 25: 31 & 0.1294 \\ 14349 & 15: 25: 32 & 0.1247 \\ 14350 & 15: 25: 33 & 0.1284\end{array}$


Appendix A - Fuel Data Test 1 NIST102505_1.xIs

$\begin{array}{lll}14351 & 15: 25: 34 & 0.1254 \\ 14352 & 15: 25: 35 & 0.1289 \\ 14353 & 15: 25: 36 & 0.1263 \\ 14354 & 15: 25: 37 & 0.1295 \\ 14355 & 15: 25: 38 & 0.1289 \\ 14356 & 15: 25: 39 & 0.1276 \\ 14357 & 15: 25: 40 & 0.1236 \\ 14358 & 15: 25: 41 & 0.1243 \\ 14359 & 15: 25: 42 & 0.1268 \\ 14360 & 15: 25: 43 & 0.1233 \\ 14361 & 15: 25: 44 & 0.1276 \\ 14362 & 15: 25: 45 & 0.1269 \\ 14363 & 15: 25: 46 & 0.1288 \\ 14364 & 15: 25: 47 & 0.1256 \\ 14365 & 15: 25: 48 & 0.1306 \\ 14366 & 15: 25: 49 & 0.1302 \\ 14367 & 15: 25: 50 & 0.1313 \\ 14368 & 15: 25: 51 & 0.1278 \\ 14369 & 15: 25: 52 & 0.1279 \\ 14370 & 15: 25: 53 & 0.1289 \\ 14371 & 15: 25: 54 & 0.1267 \\ 14372 & 15: 25: 55 & 0.1275 \\ 14373 & 15: 25: 56 & 0.1243 \\ 14374 & 15: 25: 57 & 0.1284 \\ 14375 & 15: 25: 58 & 0.1246 \\ 14376 & 15: 25: 59 & 0.1284 \\ 14377 & 15: 26: 00 & 0.1265 \\ 14378 & 15: 26: 01 & 0.1311 \\ 14379 & 15: 26: 02 & 0.1270 \\ 14380 & 15: 26: 03 & 0.1307 \\ 14381 & 15: 26: 04 & 0.1274 \\ 14382 & 15: 26: 05 & 0.1274 \\ 14383 & 15: 26: 06 & 0.1268 \\ 14384 & 15: 26: 07 & 0.1260 \\ 14385 & 15: 26: 08 & 0.1262 \\ 14386 & 15: 26: 09 & 0.1230 \\ 14387 & 15: 26: 10 & 0.1280 \\ 14388 & 15: 26: 11 & 0.1258 \\ 14389 & 15: 26: 12 & 0.1295 \\ 14390 & 15: 26: 13 & 0.1233 \\ 14391 & 15: 26: 14 & 0.1289 \\ 14392 & 15: 26: 15 & 0.1273 \\ 14393 & 15: 26: 16 & 0.1296 \\ 14394 & 15: 26: 17 & 0.1259 \\ 14395 & 15: 26: 18 & 0.1270 \\ 14396 & 15: 26: 19 & 0.1269 \\ 14397 & 15: 26: 20 & 0.1241 \\ 14398 & 15: 26: 21 & 0.1285 \\ 14399 & 15: 26: 22 & 0.1247 \\ 14400 & 15: 26: 23 & 0.1297 \\ 14401 & 15: 26: 24 & 0.1257 \\ 14402 & 15: 26: 25 & 0.1315\end{array}$


Appendix A - Fuel Data Test 1 NIST102505_1.xls

$\begin{array}{lll}14403 & 15: 26: 26 & 0.1271 \\ 14404 & 15: 26: 27 & 0.1290 \\ 14405 & 15: 26: 28 & 0.1294 \\ 14406 & 15: 26: 29 & 0.1299 \\ 14407 & 15: 26: 30 & 0.1288 \\ 14408 & 15: 26: 31 & 0.1260 \\ 14409 & 15: 26: 32 & 0.1290 \\ 14410 & 15: 26: 33 & 0.1250 \\ 14411 & 15: 26: 34 & 0.1287 \\ 14412 & 15: 26: 35 & 0.1249 \\ 14413 & 15: 26: 36 & 0.1288 \\ 14414 & 15: 26: 37 & 0.1247 \\ 14415 & 15: 26: 38 & 0.1290 \\ 14416 & 15: 26: 39 & 0.1293 \\ 14417 & 15: 26: 40 & 0.1268 \\ 14418 & 15: 26: 41 & 0.1253 \\ 14419 & 15: 26: 42 & 0.1258 \\ 14420 & 15: 26: 43 & 0.1303 \\ 14421 & 15: 26: 44 & 0.1259 \\ 14422 & 15: 26: 45 & 0.1275 \\ 14423 & 15: 26: 46 & 0.1225 \\ 14424 & 15: 26: 47 & 0.1279 \\ 14425 & 15: 26: 48 & 0.1229 \\ 14426 & 15: 26: 49 & 0.1278 \\ 14427 & 15: 26: 50 & 0.1248 \\ 14428 & 15: 26: 51 & 0.1286 \\ 14429 & 15: 26: 52 & 0.1264 \\ 14430 & 15: 26: 53 & 0.1269 \\ 14431 & 15: 26: 54 & 0.1270 \\ 14432 & 15: 26: 55 & 0.1253 \\ 14433 & 15: 26: 56 & 0.1268 \\ 14434 & 15: 26: 57 & 0.1241 \\ 14435 & 15: 26: 58 & 0.1284 \\ 14436 & 15: 26: 59 & 0.1246 \\ 14437 & 15: 27: 00 & 0.1290 \\ 14438 & 15: 27: 01 & 0.1258 \\ 14439 & 15: 27: 02 & 0.1303 \\ 14440 & 15: 27: 03 & 0.1258 \\ 14441 & 15: 27: 04 & 0.1291 \\ 14442 & 15: 27: 05 & 0.1250 \\ 14443 & 15: 27: 06 & 0.1272 \\ 14444 & 15: 27: 07 & 0.1269 \\ 14445 & 15: 27: 08 & 0.1263 \\ 14446 & 15: 27: 09 & 0.1281 \\ 14447 & 15: 27: 10 & 0.1264 \\ 14448 & 15: 27: 11 & 0.1290 \\ 14449 & 15: 27: 12 & 0.1250 \\ 14450 & 15: 27: 13 & 0.1272 \\ 14451 & 15: 27: 14 & 0.1249 \\ 14452 & 15: 27: 15 & 0.1301 \\ 14453 & 15: 27: 16 & 0.1264 \\ 14454 & 15: 27: 17 & 0.1266\end{array}$


Appendix A - Fuel Data Test 1 NIST102505_1.xIs

$\begin{array}{lll}14455 & 15: 27: 18 & 0.1270 \\ 14456 & 15: 27: 19 & 0.1266 \\ 14457 & 15: 27: 20 & 0.1252 \\ 14458 & 15: 27: 21 & 0.1239 \\ 14459 & 15: 27: 22 & 0.1256 \\ 14460 & 15: 27: 23 & 0.1222 \\ 14461 & 15: 27: 24 & 0.1276 \\ 14462 & 15: 27: 25 & 0.1246 \\ 14463 & 15: 27: 26 & 0.1288 \\ 14464 & 15: 27: 27 & 0.1239 \\ 14465 & 15: 27: 28 & 0.1305 \\ 14466 & 15: 27: 29 & 0.1277 \\ 14467 & 15: 27: 30 & 0.1296 \\ 14468 & 15: 27: 31 & 0.1259 \\ 14469 & 15: 27: 32 & 0.1284 \\ 14470 & 15: 27: 33 & 0.1278 \\ 14471 & 15: 27: 34 & 0.1253 \\ 14472 & 15: 27: 35 & 0.1287 \\ 14473 & 15: 27: 36 & 0.1255 \\ 14474 & 15: 27: 37 & 0.1300 \\ 14475 & 15: 27: 38 & 0.1247 \\ 14476 & 15: 27: 39 & 0.1317 \\ 14477 & 15: 27: 40 & 0.1273 \\ 14478 & 15: 27: 41 & 0.1313 \\ 14479 & 15: 27: 42 & 0.1271 \\ 14480 & 15: 27: 43 & 0.1284 \\ 14481 & 15: 27: 44 & 0.1257 \\ 14482 & 15: 27: 45 & 0.1243 \\ 14483 & 15: 27: 46 & 0.1277 \\ 14484 & 15: 27: 47 & 0.1258 \\ 14485 & 15: 27: 48 & 0.1266 \\ 14486 & 15: 27: 49 & 0.1222 \\ 14487 & 15: 27: 50 & 0.1295 \\ 14488 & 15: 27: 51 & 0.1257 \\ 14489 & 15: 27: 52 & 0.1281 \\ 14490 & 15: 27: 53 & 0.1230 \\ 14491 & 15: 27: 54 & 0.1287 \\ 14492 & 15: 27: 55 & 0.1253 \\ 14493 & 15: 27: 56 & 0.1253 \\ 14494 & 15: 27: 57 & 0.1234 \\ 14495 & 15: 27: 58 & 0.1236 \\ 14496 & 15: 27: 59 & 0.1260 \\ 14497 & 15: 28: 00 & 0.1224 \\ 14498 & 15: 28: 01 & 0.1274 \\ 14499 & 15: 28: 02 & 0.1233 \\ 14500 & 15: 28: 03 & 0.1280 \\ 14501 & 15: 28: 04 & 0.1256 \\ 14502 & 15: 28: 05 & 0.1311 \\ 14503 & 15: 28: 06 & 0.1264 \\ 14504 & 15: 28: 07 & 0.1291 \\ 14505 & 15: 28: 08 & 0.1265 \\ 14506 & 15: 28: 09 & 0.1279\end{array}$


Appendix A - Fuel Data Test 1 NIST102505_1.xIs

$\begin{array}{lll}14507 & 15: 28: 10 & 0.1259 \\ 14508 & 15: 28: 11 & 0.1242 \\ 14509 & 15: 28: 12 & 0.1285 \\ 14510 & 15: 28: 13 & 0.1256 \\ 14511 & 15: 28: 14 & 0.1280 \\ 14512 & 15: 28: 15 & 0.1230 \\ 14513 & 15: 28: 16 & 0.1296 \\ 14514 & 15: 28: 17 & 0.1260 \\ 14515 & 15: 28: 18 & 0.1310 \\ 14516 & 15: 28: 19 & 0.1271 \\ 14517 & 15: 28: 20 & 0.1298 \\ 14518 & 15: 28: 21 & 0.1247 \\ 14519 & 15: 28: 22 & 0.1274 \\ 14520 & 15: 28: 23 & 0.1282 \\ 14521 & 15: 28: 24 & 0.1253 \\ 14522 & 15: 28: 25 & 0.1264 \\ 14523 & 15: 28: 26 & 0.1227 \\ 14524 & 15: 28: 27 & 0.1268 \\ 14525 & 15: 28: 28 & 0.1224 \\ 14526 & 15: 28: 29 & 0.1289 \\ 14527 & 15: 28: 30 & 0.1256 \\ 14528 & 15: 28: 31 & 0.1308 \\ 14529 & 15: 28: 32 & 0.1265 \\ 14530 & 15: 28: 33 & 0.1282 \\ 14531 & 15: 28: 34 & 0.1257 \\ 14532 & 15: 28: 35 & 0.1250 \\ 14533 & 15: 28: 36 & 0.1264 \\ 14534 & 15: 28: 37 & 0.1260 \\ 14535 & 15: 28: 38 & 0.1272 \\ 14536 & 15: 28: 39 & 0.1225 \\ 14537 & 15: 28: 40 & 0.1295 \\ 14538 & 15: 28: 41 & 0.1245 \\ 14539 & 15: 28: 42 & 0.1282 \\ 14540 & 15: 28: 43 & 0.1254 \\ 14541 & 15: 28: 44 & 0.1291 \\ 14542 & 15: 28: 45 & 0.1272 \\ 14543 & 15: 28: 46 & 0.1267 \\ 14544 & 15: 28: 47 & 0.1268 \\ 14545 & 15: 28: 48 & 0.1258 \\ 14546 & 15: 28: 49 & 0.1281 \\ 14547 & 15: 28: 50 & 0.1251 \\ 14548 & 15: 28: 51 & 0.1284 \\ 14549 & 15: 28: 52 & 0.1221 \\ 14550 & 15: 28: 53 & 0.1287 \\ 14551 & 15: 28: 54 & 0.1270 \\ 14552 & 15: 28: 55 & 0.1301 \\ 14553 & 15: 28: 56 & 0.1252 \\ 14554 & 15: 28: 57 & 0.1298 \\ 14555 & 15: 28: 58 & 0.1280 \\ 14556 & 15: 28: 59 & 0.1283 \\ 14557 & 15: 29: 00 & 0.1265 \\ 14558 & 15: 29: 01 & 0.1269\end{array}$


Appendix A - Fuel Data Test 1 NIST102505_1.xls

$\begin{array}{lll}14559 & 15: 29: 02 & 0.1262 \\ 14560 & 15: 29: 03 & 0.1218 \\ 14561 & 15: 29: 04 & 0.1285 \\ 14562 & 15: 29: 05 & 0.1254 \\ 14563 & 15: 29: 06 & 0.1299 \\ 14564 & 15: 29: 07 & 0.1249 \\ 14565 & 15: 29: 08 & 0.1309 \\ 14566 & 15: 29: 09 & 0.1262 \\ 14567 & 15: 29: 10 & 0.1286 \\ 14568 & 15: 29: 11 & 0.1257 \\ 14569 & 15: 29: 12 & 0.1275 \\ 14570 & 15: 29: 13 & 0.1272 \\ 14571 & 15: 29: 14 & 0.1248 \\ 14572 & 15: 29: 15 & 0.1288 \\ 14573 & 15: 29: 16 & 0.1242 \\ 14574 & 15: 29: 17 & 0.1280 \\ 14575 & 15: 29: 18 & 0.1244 \\ 14576 & 15: 29: 19 & 0.1293 \\ 14577 & 15: 29: 20 & 0.1243 \\ 14578 & 15: 29: 21 & 0.1311 \\ 14579 & 15: 29: 22 & 0.1275 \\ 14580 & 15: 29: 23 & 0.1281 \\ 14581 & 15: 29: 24 & 0.1250 \\ 14582 & 15: 29: 25 & 0.1272 \\ 14583 & 15: 29: 26 & 0.1272 \\ 14584 & 15: 29: 27 & 0.1244 \\ 14585 & 15: 29: 28 & 0.1247 \\ 14586 & 15: 29: 29 & 0.1224 \\ 14587 & 15: 29: 30 & 0.1271 \\ 14588 & 15: 29: 31 & 0.1237 \\ 14589 & 15: 29: 32 & 0.1292 \\ 14590 & 15: 29: 33 & 0.1243 \\ 14591 & 15: 29: 34 & 0.1297 \\ 14592 & 15: 29: 35 & 0.1261 \\ 14593 & 15: 29: 36 & 0.1278 \\ 14594 & 15: 29: 37 & 0.1236 \\ 14595 & 15: 29: 38 & 0.1233 \\ 14596 & 15: 29: 39 & 0.1261 \\ 14597 & 15: 29: 40 & 0.1243 \\ 14598 & 15: 29: 41 & 0.1262 \\ 14599 & 15: 29: 42 & 0.1220 \\ 14600 & 15: 29: 43 & 0.1287 \\ 14601 & 15: 29: 44 & 0.1235 \\ 14602 & 15: 29: 45 & 0.1273 \\ 14603 & 15: 29: 46 & 0.1261 \\ 14604 & 15: 29: 47 & 0.1303 \\ 14605 & 15: 29: 48 & 0.1282 \\ 14606 & 15: 29: 49 & 0.1281 \\ 14607 & 15: 29: 50 & 0.1288 \\ 14608 & 15: 29: 51 & 0.1272 \\ 14609 & 15: 29: 52 & 0.1291 \\ 14610 & 15: 29: 53 & 0.1258\end{array}$


Appendix A - Fuel Data Test 1 NIST102505_1.xls

$\begin{array}{lll}14611 & 15: 29: 54 & 0.1297 \\ 14612 & 15: 29: 55 & 0.1247 \\ 14613 & 15: 29: 56 & 0.1297 \\ 14614 & 15: 29: 57 & 0.1282 \\ 14615 & 15: 29: 58 & 0.1320 \\ 14616 & 15: 29: 59 & 0.1267 \\ 14617 & 15: 30: 00 & 0.1311 \\ 14618 & 15: 30: 01 & 0.1266 \\ 14619 & 15: 30: 02 & 0.1261 \\ 14620 & 15: 30: 03 & 0.1258 \\ 14621 & 15: 30: 04 & 0.1263 \\ 14622 & 15: 30: 05 & 0.1279 \\ 14623 & 15: 30: 06 & 0.1247 \\ 14624 & 15: 30: 07 & 0.1284 \\ 14625 & 15: 30: 08 & 0.1244 \\ 14626 & 15: 30: 09 & 0.1269 \\ 14627 & 15: 30: 10 & 0.1239 \\ 14628 & 15: 30: 11 & 0.1290 \\ 14629 & 15: 30: 12 & 0.1241 \\ 14630 & 15: 30: 13 & 0.1302 \\ 14631 & 15: 30: 14 & 0.1279 \\ 14632 & 15: 30: 15 & 0.1272 \\ 14633 & 15: 30: 16 & 0.1258 \\ 14634 & 15: 30: 17 & 0.1252 \\ 14635 & 15: 30: 18 & 0.1270 \\ 14636 & 15: 30: 19 & 0.1221 \\ 14637 & 15: 30: 20 & 0.1265 \\ 14638 & 15: 30: 21 & 0.1235 \\ 14639 & 15: 30: 22 & 0.1282 \\ 14640 & 15: 30: 23 & 0.1248 \\ 14641 & 15: 30: 24 & 0.1296 \\ 14642 & 15: 30: 25 & 0.1294 \\ 14643 & 15: 30: 26 & 0.1282 \\ 14644 & 15: 30: 27 & 0.1251 \\ 14645 & 15: 30: 28 & 0.1266 \\ 14646 & 15: 30: 29 & 0.1283 \\ 14647 & 15: 30: 30 & 0.1246 \\ 14648 & 15: 30: 31 & 0.1257 \\ 14649 & 15: 30: 32 & 0.1221 \\ 14650 & 15: 30: 33 & 0.1276 \\ 14651 & 15: 30: 34 & 0.1237 \\ 14652 & 15: 30: 35 & 0.1278 \\ 14653 & 15: 30: 36 & 0.1277 \\ 14654 & 15: 30: 37 & 0.1285 \\ 14655 & 15: 30: 38 & 0.1268 \\ 14656 & 15: 30: 39 & 0.1266 \\ 14657 & 15: 30: 40 & 0.1264 \\ 14658 & 15: 30: 41 & 0.1258 \\ 14659 & 15: 30: 42 & 0.1274 \\ 14660 & 15: 30: 43 & 0.1248 \\ 14661 & 15: 30: 44 & 0.1258 \\ 14662 & 15: 30: 45 & 0.1212\end{array}$


Appendix A - Fuel Data Test 1 NIST102505_1.xIs

$\begin{array}{lll}14663 & 15: 30: 46 & 0.1270 \\ 14664 & 15: 30: 47 & 0.1276 \\ 14665 & 15: 30: 48 & 0.1301 \\ 14666 & 15: 30: 49 & 0.1266 \\ 14667 & 15: 30: 50 & 0.1288 \\ 14668 & 15: 30: 51 & 0.1288 \\ 14669 & 15: 30: 52 & 0.1274 \\ 14670 & 15: 30: 53 & 0.1249 \\ 14671 & 15: 30: 54 & 0.1245 \\ 14672 & 15: 30: 55 & 0.1288 \\ 14673 & 15: 30: 56 & 0.1248 \\ 14674 & 15: 30: 57 & 0.1273 \\ 14675 & 15: 30: 58 & 0.1239 \\ 14676 & 15: 30: 59 & 0.1281 \\ 14677 & 15: 31: 00 & 0.1240 \\ 14678 & 15: 31: 01 & 0.1267 \\ 14679 & 15: 31: 02 & 0.1241 \\ 14680 & 15: 31: 03 & 0.1289 \\ 14681 & 15: 31: 04 & 0.1262 \\ 14682 & 15: 31: 05 & 0.1258 \\ 14683 & 15: 31: 06 & 0.1250 \\ 14684 & 15: 31: 07 & 0.1246 \\ 14685 & 15: 31: 08 & 0.1258 \\ 14686 & 15: 31: 09 & 0.1234 \\ 14687 & 15: 31: 10 & 0.1266 \\ 14688 & 15: 31: 11 & 0.1241 \\ 14689 & 15: 31: 12 & 0.1291 \\ 14690 & 15: 31: 13 & 0.1264 \\ 14691 & 15: 31: 14 & 0.1300 \\ 14692 & 15: 31: 15 & 0.1261 \\ 14693 & 15: 31: 16 & 0.1284 \\ 14694 & 15: 31: 17 & 0.1268 \\ 14695 & 15: 31: 18 & 0.1265 \\ 14696 & 15: 31: 19 & 0.1263 \\ 14697 & 15: 31: 20 & 0.1249 \\ 14698 & 15: 31: 21 & 0.1282 \\ 14699 & 15: 31: 22 & 0.1245 \\ 14700 & 15: 31: 23 & 0.1282 \\ 14701 & 15: 31: 24 & 0.1249 \\ 14702 & 15: 31: 25 & 0.1305 \\ 14703 & 15: 31: 26 & 0.1262 \\ 14704 & 15: 31: 27 & 0.1306 \\ 14705 & 15: 31: 28 & 0.1284 \\ 14706 & 15: 31: 29 & 0.1304 \\ 14707 & 15: 31: 30 & 0.1272 \\ 14708 & 15: 31: 31 & 0.1275 \\ 14709 & 15: 31: 32 & 0.1311 \\ 14710 & 15: 31: 33 & 0.1276 \\ 14711 & 15: 31: 34 & 0.1295 \\ 14712 & 15: 31: 35 & 0.1255 \\ 14713 & 15: 31: 36 & 0.1294 \\ 14714 & 15: 31: 37 & 0.1253\end{array}$


Appendix A - Fuel Data Test 1 NIST102505_1.xls

$\begin{array}{lll}14715 & 15: 31: 38 & 0.1280 \\ 14716 & 15: 31: 39 & 0.1264 \\ 14717 & 15: 31: 40 & 0.1293 \\ 14718 & 15: 31: 41 & 0.1264 \\ 14719 & 15: 31: 42 & 0.1254 \\ 14720 & 15: 31: 43 & 0.1240 \\ 14721 & 15: 31: 44 & 0.1246 \\ 14722 & 15: 31: 45 & 0.1254 \\ 14723 & 15: 31: 46 & 0.1223 \\ 14724 & 15: 31: 47 & 0.1278 \\ 14725 & 15: 31: 48 & 0.1232 \\ 14726 & 15: 31: 49 & 0.1279 \\ 14727 & 15: 31: 50 & 0.1240 \\ 14728 & 15: 31: 51 & 0.1289 \\ 14729 & 15: 31: 52 & 0.1248 \\ 14730 & 15: 31: 53 & 0.1284 \\ 14731 & 15: 31: 54 & 0.1282 \\ 14732 & 15: 31: 55 & 0.1271 \\ 14733 & 15: 31: 56 & 0.1269 \\ 14734 & 15: 31: 57 & 0.1257 \\ 14735 & 15: 31: 58 & 0.1287 \\ 14736 & 15: 31: 59 & 0.1255 \\ 14737 & 15: 32: 00 & 0.1283 \\ 14738 & 15: 32: 01 & 0.1243 \\ 14739 & 15: 32: 02 & 0.1289 \\ 14740 & 15: 32: 03 & 0.1246 \\ 14741 & 15: 32: 04 & 0.1284 \\ 14742 & 15: 32: 05 & 0.1261 \\ 14743 & 15: 32: 06 & 0.1294 \\ 14744 & 15: 32: 07 & 0.1256 \\ 14745 & 15: 32: 08 & 0.1253 \\ 14746 & 15: 32: 09 & 0.1260 \\ 14747 & 15: 32: 10 & 0.1253 \\ 14748 & 15: 32: 11 & 0.1261 \\ 14749 & 15: 32: 12 & 0.1221 \\ 14750 & 15: 32: 13 & 0.1283 \\ 14751 & 15: 32: 14 & 0.1231 \\ 14752 & 15: 32: 15 & 0.1287 \\ 14753 & 15: 32: 16 & 0.1256 \\ 14754 & 15: 32: 17 & 0.1325 \\ 14755 & 15: 32: 18 & 0.1283 \\ 14756 & 15: 32: 19 & 0.1294 \\ 14757 & 15: 32: 20 & 0.1256 \\ 14758 & 15: 32: 21 & 0.1272 \\ 14759 & 15: 32: 22 & 0.1273 \\ 14760 & 15: 32: 23 & 0.1250 \\ 14761 & 15: 32: 24 & 0.1250 \\ 14762 & 15: 32: 25 & 0.1225 \\ 14763 & 15: 32: 26 & 0.1270 \\ 14764 & 15: 32: 27 & 0.1231 \\ 14765 & 15: 32: 28 & 0.1288 \\ 14766 & 15: 32: 29 & 0.1248\end{array}$


Appendix A - Fuel Data Test 1 NIST102505_1.xls

$\begin{array}{lll}14767 & 15: 32: 30 & 0.1287 \\ 14768 & 15: 32: 31 & 0.1240 \\ 14769 & 15: 32: 32 & 0.1267 \\ 14770 & 15: 32: 33 & 0.1236 \\ 14771 & 15: 32: 34 & 0.1224 \\ 14772 & 15: 32: 35 & 0.1248 \\ 14773 & 15: 32: 36 & 0.1228 \\ 14774 & 15: 32: 37 & 0.1257 \\ 14775 & 15: 32: 38 & 0.1210 \\ 14776 & 15: 32: 39 & 0.1263 \\ 14777 & 15: 32: 40 & 0.1219 \\ 14778 & 15: 32: 41 & 0.1269 \\ 14779 & 15: 32: 42 & 0.1265 \\ 14780 & 15: 32: 43 & 0.1292 \\ 14781 & 15: 32: 44 & 0.1278 \\ 14782 & 15: 32: 45 & 0.1271 \\ 14783 & 15: 32: 46 & 0.1275 \\ 14784 & 15: 32: 47 & 0.1254 \\ 14785 & 15: 32: 48 & 0.1262 \\ 14786 & 15: 32: 49 & 0.1222 \\ 14787 & 15: 32: 50 & 0.1284 \\ 14788 & 15: 32: 51 & 0.1245 \\ 14789 & 15: 32: 52 & 0.1279 \\ 14790 & 15: 32: 53 & 0.1256 \\ 14791 & 15: 32: 54 & 0.1301 \\ 14792 & 15: 32: 55 & 0.1265 \\ 14793 & 15: 32: 56 & 0.1285 \\ 14795 & 15: 32: 58 & 0.1270 \\ 14795 & 15: 32: 58 & 0.1277 \\ 14796 & 15: 32: 59 & 0.1275 \\ 14797 & 15: 33: 00 & 0.1268 \\ 14798 & 15: 33: 01 & 0.1278 \\ 14799 & 15: 33: 02 & 0.1230 \\ 14800 & 15: 33: 03 & 0.1287 \\ 14801 & 15: 33: 04 & 0.1266 \\ 14802 & 15: 33: 05 & 0.1321 \\ 14803 & 15: 33: 06 & 0.1262 \\ 14804 & 15: 33: 07 & 0.1306 \\ 14805 & 15: 33: 08 & 0.1268 \\ 14806 & 15: 33: 09 & 0.1285 \\ 14807 & 15: 33: 10 & 0.1248 \\ 14808 & 15: 33: 11 & 0.1260 \\ 14809 & 15: 33: 12 & 0.1273 \\ 14810 & 15: 33: 13 & 0.1244 \\ 14811 & 15: 33: 14 & 0.1270 \\ 14812 & 15: 33: 15 & 0.1243 \\ 14813 & 15: 33: 16 & 0.1284 \\ 14814 & 15: 33: 17 & 0.1242 \\ 14815 & 15: 33: 18 & 0.1285 \\ 14816 & 15: 33: 19 & 0.1243 \\ 14817 & 15: 33: 20 & 0.1293 \\ 14818 & 15: 33: 21 & 0.1256\end{array}$


Appendix A - Fuel Data Test 1 NIST102505_1.xIs

$\begin{array}{lll}14819 & 15: 33: 22 & 0.1274 \\ 14820 & 15: 33: 23 & 0.1232 \\ 14821 & 15: 33: 24 & 0.1252 \\ 14822 & 15: 33: 25 & 0.1293 \\ 14823 & 15: 33: 26 & 0.1258 \\ 14824 & 15: 33: 27 & 0.1292 \\ 14825 & 15: 33: 28 & 0.1251 \\ 14826 & 15: 33: 29 & 0.1289 \\ 14827 & 15: 33: 30 & 0.1252 \\ 14828 & 15: 33: 31 & 0.1303 \\ 14829 & 15: 33: 32 & 0.1285 \\ 14830 & 15: 33: 33 & 0.1291 \\ 14831 & 15: 33: 34 & 0.1261 \\ 14832 & 15: 33: 35 & 0.1264 \\ 14833 & 15: 33: 36 & 0.1275 \\ 14834 & 15: 33: 37 & 0.1247 \\ 14835 & 15: 33: 38 & 0.1252 \\ 14836 & 15: 33: 39 & 0.1233 \\ 14837 & 15: 33: 40 & 0.1274 \\ 14838 & 15: 33: 41 & 0.1234 \\ 14839 & 15: 33: 42 & 0.1283 \\ 14840 & 15: 33: 43 & 0.1272 \\ 14841 & 15: 33: 44 & 0.1307 \\ 14842 & 15: 33: 45 & 0.1282 \\ 14843 & 15: 33: 46 & 0.1266 \\ 14844 & 15: 33: 47 & 0.1271 \\ 14845 & 15: 33: 48 & 0.1271 \\ 14846 & 15: 33: 49 & 0.1283 \\ 14847 & 15: 33: 50 & 0.1256 \\ 14848 & 15: 33: 51 & 0.1284 \\ 14849 & 15: 33: 52 & 0.1235 \\ 14850 & 15: 33: 53 & 0.1278 \\ 14851 & 15: 33: 54 & 0.1257 \\ 14852 & 15: 33: 55 & 0.1299 \\ 14853 & 15: 33: 56 & 0.1250 \\ 14854 & 15: 33: 57 & 0.1297 \\ 14855 & 15: 33: 58 & 0.1290 \\ 14856 & 15: 33: 59 & 0.1289 \\ 14857 & 15: 34: 00 & 0.1251 \\ 14858 & 15: 34: 01 & 0.1251 \\ 14859 & 15: 34: 02 & 0.1266 \\ 14860 & 15: 34: 03 & 0.1241 \\ 14861 & 15: 34: 04 & 0.1251 \\ 14862 & 15: 34: 05 & 0.1231 \\ 14863 & 15: 34: 06 & 0.1293 \\ 14864 & 15: 34: 07 & 0.1261 \\ 14865 & 15: 34: 08 & 0.1298 \\ 14866 & 15: 34: 09 & 0.1281 \\ 14867 & 15: 34: 10 & 0.1315 \\ 14868 & 15: 34: 11 & 0.1271 \\ 14869 & 15: 34: 12 & 0.1295 \\ 14870 & 15: 34: 13 & 0.1265\end{array}$


Appendix A - Fuel Data Test 1 NIST102505_1.xIs

$\begin{array}{lll}14871 & 15: 34: 14 & 0.1248 \\ 14872 & 15: 34: 15 & 0.1281 \\ 14873 & 15: 34: 16 & 0.1264 \\ 14874 & 15: 34: 17 & 0.1270 \\ 14875 & 15: 34: 18 & 0.1219 \\ 14876 & 15: 34: 19 & 0.1279 \\ 14877 & 15: 34: 20 & 0.1247 \\ 14878 & 15: 34: 21 & 0.1289 \\ 14879 & 15: 34: 22 & 0.1245 \\ 14880 & 15: 34: 23 & 0.1295 \\ 14881 & 15: 34: 24 & 0.1275 \\ 14882 & 15: 34: 25 & 0.1278 \\ 14883 & 15: 34: 26 & 0.1255 \\ 14884 & 15: 34: 27 & 0.1260 \\ 14885 & 15: 34: 28 & 0.1264 \\ 14886 & 15: 34: 29 & 0.1235 \\ 14887 & 15: 34: 30 & 0.1291 \\ 14888 & 15: 34: 31 & 0.1242 \\ 14889 & 15: 34: 32 & 0.1283 \\ 14890 & 15: 34: 33 & 0.1251 \\ 14891 & 15: 34: 34 & 0.1298 \\ 14892 & 15: 34: 35 & 0.1265 \\ 14893 & 15: 34: 36 & 0.1262 \\ 14894 & 15: 34: 37 & 0.1268 \\ 14895 & 15: 34: 38 & 0.1271 \\ 14896 & 15: 34: 39 & 0.1268 \\ 14897 & 15: 34: 40 & 0.1234 \\ 14898 & 15: 34: 41 & 0.1267 \\ 14899 & 15: 34: 42 & 0.1229 \\ 14900 & 15: 34: 43 & 0.1282 \\ 14901 & 15: 34: 44 & 0.1243 \\ 14902 & 15: 34: 45 & 0.1310 \\ 14903 & 15: 34: 46 & 0.1258 \\ 14904 & 15: 34: 47 & 0.1294 \\ 14905 & 15: 34: 48 & 0.1278 \\ 14906 & 15: 34: 49 & 0.1288 \\ 14907 & 15: 34: 50 & 0.1260 \\ 14908 & 15: 34: 51 & 0.1267 \\ 14909 & 15: 34: 52 & 0.1291 \\ 14910 & 15: 34: 53 & 0.1260 \\ 14911 & 15: 34: 54 & 0.1276 \\ 14912 & 15: 34: 55 & 0.1244 \\ 14913 & 15: 34: 56 & 0.1303 \\ 14914 & 15: 34: 57 & 0.1256 \\ 14915 & 15: 34: 58 & 0.1275 \\ 14916 & 15: 34: 59 & 0.1241 \\ 14917 & 15: 35: 00 & 0.1289 \\ 14918 & 15: 35: 01 & 0.1253 \\ 14919 & 15: 35: 02 & 0.1283 \\ 14920 & 15: 35: 03 & 0.1276 \\ 14921 & 15: 35: 04 & 0.1270 \\ 14922 & 15: 35: 05 & 0.1271\end{array}$


Appendix A - Fuel Data Test 1 NIST102505_1.xls

$\begin{array}{lll}14923 & 15: 35: 06 & 0.1248 \\ 14924 & 15: 35: 07 & 0.1269 \\ 14925 & 15: 35: 08 & 0.1230 \\ 14926 & 15: 35: 09 & 0.1281 \\ 14927 & 15: 35: 10 & 0.1257 \\ 14928 & 15: 35: 11 & 0.1285 \\ 14929 & 15: 35: 12 & 0.1244 \\ 14930 & 15: 35: 13 & 0.1283 \\ 14931 & 15: 35: 14 & 0.1271 \\ 14932 & 15: 35: 15 & 0.1262 \\ 14933 & 15: 35: 16 & 0.1254 \\ 14934 & 15: 35: 17 & 0.1242 \\ 14935 & 15: 35: 18 & 0.1271 \\ 14936 & 15: 35: 19 & 0.1239 \\ 14937 & 15: 35: 20 & 0.1257 \\ 14938 & 15: 35: 21 & 0.1219 \\ 14939 & 15: 35: 22 & 0.1272 \\ 14940 & 15: 35: 23 & 0.1240 \\ 14941 & 15: 35: 24 & 0.1277 \\ 14942 & 15: 35: 25 & 0.1289 \\ 14943 & 15: 35: 26 & 0.1291 \\ 14944 & 15: 35: 27 & 0.1260 \\ 14945 & 15: 35: 28 & 0.1254 \\ 14946 & 15: 35: 29 & 0.1279 \\ 14947 & 15: 35: 30 & 0.1268 \\ 14948 & 15: 35: 31 & 0.1289 \\ 14949 & 15: 35: 32 & 0.1255 \\ 14950 & 15: 35: 33 & 0.1287 \\ 14951 & 15: 35: 34 & 0.1228 \\ 14952 & 15: 35: 35 & 0.1280 \\ 14953 & 15: 35: 36 & 0.1267 \\ 14954 & 15: 35: 37 & 0.1299 \\ 14955 & 15: 35: 38 & 0.1240 \\ 14956 & 15: 35: 39 & 0.1309 \\ 14957 & 15: 35: 40 & 0.1277 \\ 14958 & 15: 35: 41 & 0.1282 \\ 14959 & 15: 35: 42 & 0.1304 \\ 14960 & 15: 35: 43 & 0.1264 \\ 14961 & 15: 35: 44 & 0.1270 \\ 14962 & 15: 35: 45 & 0.1220 \\ 14963 & 15: 35: 46 & 0.1277 \\ 14964 & 15: 35: 47 & 0.1256 \\ 14965 & 15: 35: 48 & 0.1293 \\ 14966 & 15: 35: 49 & 0.1248 \\ 14967 & 15: 35: 50 & 0.1296 \\ 14968 & 15: 35: 51 & 0.1246 \\ 14969 & 15: 35: 52 & 0.1284 \\ 14970 & 15: 35: 53 & 0.1264 \\ 14971 & 15: 35: 54 & 0.1286 \\ 14972 & 15: 35: 55 & 0.1269 \\ 14973 & 15: 35: 56 & 0.1259 \\ 14974 & 15: 35: 57 & 0.1268\end{array}$


Appendix A - Fuel Data Test 1 NIST102505_1.xIs

$\begin{array}{lll}14975 & 15: 35: 58 & 0.1231 \\ 14976 & 15: 35: 59 & 0.1282 \\ 14977 & 15: 36: 00 & 0.1259 \\ 14978 & 15: 36: 01 & 0.1284 \\ 14979 & 15: 36: 02 & 0.1253 \\ 14980 & 15: 36: 03 & 0.1286 \\ 14981 & 15: 36: 04 & 0.1270 \\ 14982 & 15: 36: 05 & 0.1277 \\ 14983 & 15: 36: 06 & 0.1237 \\ 14984 & 15: 36: 07 & 0.1252 \\ 14985 & 15: 36: 08 & 0.1269 \\ 14986 & 15: 36: 09 & 0.1241 \\ 14987 & 15: 36: 10 & 0.1261 \\ 14988 & 15: 36: 11 & 0.1233 \\ 14989 & 15: 36: 12 & 0.1283 \\ 14990 & 15: 36: 13 & 0.1247 \\ 14991 & 15: 36: 14 & 0.1282 \\ 14992 & 15: 36: 15 & 0.1259 \\ 14993 & 15: 36: 16 & 0.1283 \\ 14994 & 15: 36: 17 & 0.1277 \\ 14995 & 15: 36: 18 & 0.1273 \\ 14996 & 15: 36: 19 & 0.1257 \\ 14997 & 15: 36: 20 & 0.1263 \\ 14998 & 15: 36: 21 & 0.1271 \\ 14999 & 15: 36: 22 & 0.1242 \\ 15000 & 15: 36: 23 & 0.1285 \\ 15001 & 15: 36: 24 & 0.1237 \\ 15002 & 15: 36: 25 & 0.1285 \\ 15003 & 15: 36: 26 & 0.1264 \\ 15004 & 15: 36: 27 & 0.1301 \\ 15005 & 15: 36: 28 & 0.1248 \\ 15006 & 15: 36: 29 & 0.1299 \\ 15007 & 15: 36: 30 & 0.1266 \\ 15008 & 15: 36: 31 & 0.1272 \\ 15009 & 15: 36: 32 & 0.1258 \\ 15010 & 15: 36: 33 & 0.1253 \\ 15011 & 15: 36: 34 & 0.1256 \\ 15012 & 15: 36: 35 & 0.1239 \\ 15013 & 15: 36: 36 & 0.1269 \\ 15014 & 15: 36: 37 & 0.1234 \\ 15015 & 15: 36: 38 & 0.1283 \\ 15016 & 15: 36: 39 & 0.1241 \\ 15017 & 15: 36: 40 & 0.1306 \\ 15018 & 15: 36: 41 & 0.1255 \\ 15019 & 15: 36: 42 & 0.1267 \\ 15020 & 15: 36: 43 & 0.1241 \\ 15021 & 15: 36: 44 & 0.1281 \\ 15022 & 15: 36: 45 & 0.1264 \\ 15023 & 15: 36: 46 & 0.1252 \\ 15024 & 15: 36: 47 & 0.1285 \\ 15025 & 15: 36: 48 & 0.1244 \\ 15026 & 15: 36: 49 & 0.1265\end{array}$


Appendix A - Fuel Data Test 1 NIST102505_1.xIs

$\begin{array}{lll}15027 & 15: 36: 50 & 0.1219 \\ 15028 & 15: 36: 51 & 0.1273 \\ 15029 & 15: 36: 52 & 0.1235 \\ 15030 & 15: 36: 53 & 0.1278 \\ 15031 & 15: 36: 54 & 0.1262 \\ 15032 & 15: 36: 55 & 0.1275 \\ 15033 & 15: 36: 56 & 0.1260 \\ 15034 & 15: 36: 57 & 0.1259 \\ 15035 & 15: 36: 58 & 0.1280 \\ 15036 & 15: 36: 59 & 0.1264 \\ 15037 & 15: 37: 00 & 0.1273 \\ 15038 & 15: 37: 01 & 0.1239 \\ 15039 & 15: 37: 02 & 0.1297 \\ 15040 & 15: 37: 03 & 0.1246 \\ 15041 & 15: 37: 04 & 0.1281 \\ 15042 & 15: 37: 05 & 0.1236 \\ 15043 & 15: 37: 06 & 0.1290 \\ 15044 & 15: 37: 07 & 0.1263 \\ 15045 & 15: 37: 08 & 0.1263 \\ 15046 & 15: 37: 09 & 0.1267 \\ 15047 & 15: 37: 10 & 0.1257 \\ 15048 & 15: 37: 11 & 0.1269 \\ 15049 & 15: 37: 12 & 0.1249 \\ 15050 & 15: 37: 13 & 0.1287 \\ 15051 & 15: 37: 14 & 0.1253 \\ 15052 & 15: 37: 15 & 0.1310 \\ 15053 & 15: 37: 16 & 0.1252 \\ 15054 & 15: 37: 17 & 0.1318 \\ 15055 & 15: 37: 18 & 0.1272 \\ 15056 & 15: 37: 19 & 0.1318 \\ 15057 & 15: 37: 20 & 0.1283 \\ 15058 & 15: 37: 21 & 0.1298 \\ 15059 & 15: 37: 22 & 0.1260 \\ 15060 & 15: 37: 23 & 0.1269 \\ 15061 & 15: 37: 24 & 0.1293 \\ 15062 & 15: 37: 25 & 0.1260 \\ 15063 & 15: 37: 26 & 0.1276 \\ 15064 & 15: 37: 27 & 0.1241 \\ 15065 & 15: 37: 28 & 0.1295 \\ 15066 & 15: 37: 29 & 0.1272 \\ 15067 & 15: 37: 30 & 0.1314 \\ 15068 & 15: 37: 31 & 0.1292 \\ 15069 & 15: 37: 32 & 0.1313 \\ 15070 & 15: 37: 33 & 0.1254 \\ 15071 & 15: 37: 34 & 0.1277 \\ 15072 & 15: 37: 35 & 0.1248 \\ 15073 & 15: 37: 36 & 0.1239 \\ 15074 & 15: 37: 37 & 0.1285 \\ 15075 & 15: 37: 38 & 0.1247 \\ 15076 & 15: 37: 39 & 0.1269 \\ 15077 & 15: 37: 40 & 0.1228 \\ 15078 & 15: 37: 41 & 0.1280\end{array}$


Appendix A - Fuel Data Test 1 NIST102505_1.xIs

$\begin{array}{lll}15079 & 15: 37: 42 & 0.1232 \\ 15080 & 15: 37: 43 & 0.1264 \\ 15081 & 15: 37: 44 & 0.1271 \\ 15082 & 15: 37: 45 & 0.1308 \\ 15083 & 15: 37: 46 & 0.1265 \\ 15084 & 15: 37: 47 & 0.1266 \\ 15085 & 15: 37: 48 & 0.1283 \\ 15086 & 15: 37: 49 & 0.1261 \\ 15087 & 15: 37: 50 & 0.1260 \\ 15088 & 15: 37: 51 & 0.1234 \\ 15089 & 15: 37: 52 & 0.1267 \\ 15090 & 15: 37: 53 & 0.1226 \\ 15091 & 15: 37: 54 & 0.1278 \\ 15092 & 15: 37: 55 & 0.1262 \\ 15093 & 15: 37: 56 & 0.1308 \\ 15094 & 15: 37: 57 & 0.1251 \\ 15095 & 15: 37: 58 & 0.1299 \\ 15096 & 15: 37: 59 & 0.1268 \\ 15097 & 15: 38: 00 & 0.1288 \\ 15098 & 15: 38: 01 & 0.1282 \\ 15099 & 15: 38: 02 & 0.1268 \\ 15100 & 15: 38: 03 & 0.1270 \\ 15101 & 15: 38: 04 & 0.1227 \\ 15102 & 15: 38: 05 & 0.1275 \\ 15103 & 15: 38: 06 & 0.1256 \\ 15104 & 15: 38: 07 & 0.1287 \\ 15105 & 15: 38: 08 & 0.1242 \\ 15106 & 15: 38: 09 & 0.1303 \\ 15107 & 15: 38: 10 & 0.1265 \\ 15108 & 15: 38: 11 & 0.1287 \\ 15109 & 15: 38: 12 & 0.1245 \\ 15110 & 15: 38: 13 & 0.1252 \\ 15111 & 15: 38: 14 & 0.1268 \\ 15112 & 15: 38: 15 & 0.1241 \\ 15113 & 15: 38: 16 & 0.1276 \\ 15114 & 15: 38: 17 & 0.1242 \\ 15115 & 15: 38: 18 & 0.1301 \\ 15116 & 15: 38: 19 & 0.1268 \\ 15117 & 15: 38: 20 & 0.1311 \\ 15118 & 15: 38: 21 & 0.1262 \\ 15119 & 15: 38: 22 & 0.1310 \\ 15120 & 15: 38: 23 & 0.1293 \\ 15121 & 15: 38: 24 & 0.1297 \\ 15122 & 15: 38: 25 & 0.1263 \\ 15123 & 15: 38: 26 & 0.1264 \\ 15124 & 15: 38: 27 & 0.1275 \\ 15125 & 15: 38: 28 & 0.1242 \\ 15126 & 15: 38: 29 & 0.1264 \\ 15127 & 15: 38: 30 & 0.1229 \\ 15128 & 15: 38: 31 & 0.1280 \\ 15129 & 15: 38: 32 & 0.1242 \\ 15130 & 15: 38: 33 & 0.1292\end{array}$


Appendix A - Fuel Data Test 1 NIST102505_1.xIs

$\begin{array}{lll}15131 & 15: 38: 34 & 0.1272 \\ 15132 & 15: 38: 35 & 0.1295 \\ 15133 & 15: 38: 36 & 0.1255 \\ 15134 & 15: 38: 37 & 0.1262 \\ 15135 & 15: 38: 38 & 0.1275 \\ 15136 & 15: 38: 39 & 0.1249 \\ 15137 & 15: 38: 40 & 0.1269 \\ 15138 & 15: 38: 41 & 0.1249 \\ 15139 & 15: 38: 42 & 0.1291 \\ 15140 & 15: 38: 43 & 0.1248 \\ 15141 & 15: 38: 44 & 0.1275 \\ 15142 & 15: 38: 45 & 0.1254 \\ 15143 & 15: 38: 46 & 0.1286 \\ 15144 & 15: 38: 47 & 0.1264 \\ 15145 & 15: 38: 48 & 0.1258 \\ 15146 & 15: 38: 49 & 0.1257 \\ 15147 & 15: 38: 50 & 0.1245 \\ 15148 & 15: 38: 51 & 0.1270 \\ 15149 & 15: 38: 52 & 0.1235 \\ 15150 & 15: 38: 53 & 0.1271 \\ 15151 & 15: 38: 54 & 0.1224 \\ 15152 & 15: 38: 55 & 0.1264 \\ 15153 & 15: 38: 56 & 0.1246 \\ 15154 & 15: 38: 57 & 0.1303 \\ 15155 & 15: 38: 58 & 0.1259 \\ 15156 & 15: 38: 59 & 0.1278 \\ 15157 & 15: 39: 00 & 0.1246 \\ 15158 & 15: 39: 01 & 0.1257 \\ 15159 & 15: 39: 02 & 0.1257 \\ 15160 & 15: 39: 03 & 0.1256 \\ 15161 & 15: 39: 04 & 0.1266 \\ 15162 & 15: 39: 05 & 0.1212 \\ 15163 & 15: 39: 06 & 0.1281 \\ 15164 & 15: 39: 07 & 0.1253 \\ 15165 & 15: 39: 08 & 0.1295 \\ 15166 & 15: 39: 09 & 0.1250 \\ 15167 & 15: 39: 10 & 0.1297 \\ 15168 & 15: 39: 11 & 0.1268 \\ 15169 & 15: 39: 12 & 0.1285 \\ 15170 & 15: 39: 13 & 0.1265 \\ 15171 & 15: 39: 14 & 0.1259 \\ 15172 & 15: 39: 15 & 0.1276 \\ 15173 & 15: 39: 16 & 0.1245 \\ 15174 & 15: 39: 17 & 0.1257 \\ 15175 & 15: 39: 18 & 0.1224 \\ 15176 & 15: 39: 19 & 0.1286 \\ 15177 & 15: 39: 20 & 0.1239 \\ 15178 & 15: 39: 21 & 0.1300 \\ 15179 & 15: 39: 22 & 0.1265 \\ 15180 & 15: 39: 23 & 0.1285 \\ 15181 & 15: 39: 24 & 0.1252 \\ 15182 & 15: 39: 25 & 0.1271\end{array}$


Appendix A - Fuel Data Test 1 NIST102505_1.xIs

$\begin{array}{lll}15183 & 15: 39: 26 & 0.1263 \\ 15184 & 15: 39: 27 & 0.1248 \\ 15185 & 15: 39: 28 & 0.1263 \\ 15186 & 15: 39: 29 & 0.1241 \\ 15187 & 15: 39: 30 & 0.1287 \\ 15188 & 15: 39: 31 & 0.1243 \\ 15189 & 15: 39: 32 & 0.1293 \\ 15190 & 15: 39: 33 & 0.1253 \\ 15191 & 15: 39: 34 & 0.1279 \\ 15192 & 15: 39: 35 & 0.1274 \\ 15193 & 15: 39: 36 & 0.1288 \\ 15194 & 15: 39: 37 & 0.1256 \\ 15195 & 15: 39: 38 & 0.1240 \\ 15196 & 15: 39: 39 & 0.1265 \\ 15197 & 15: 39: 40 & 0.1246 \\ 15198 & 15: 39: 41 & 0.1263 \\ 15199 & 15: 39: 42 & 0.1216 \\ 15200 & 15: 39: 43 & 0.1281 \\ 15201 & 15: 39: 44 & 0.1241 \\ 15202 & 15: 39: 45 & 0.1280 \\ 15203 & 15: 39: 46 & 0.1262 \\ 15204 & 15: 39: 47 & 0.1301 \\ 15205 & 15: 39: 48 & 0.1270 \\ 15206 & 15: 39: 49 & 0.1283 \\ 15207 & 15: 39: 50 & 0.1255 \\ 15208 & 15: 39: 51 & 0.1254 \\ 15209 & 15: 39: 52 & 0.1272 \\ 15210 & 15: 39: 53 & 0.1240 \\ 15211 & 15: 39: 54 & 0.1283 \\ 15212 & 15: 39: 55 & 0.1250 \\ 15213 & 15: 39: 56 & 0.1294 \\ 15214 & 15: 39: 57 & 0.1248 \\ 15215 & 15: 39: 58 & 0.1300 \\ 15216 & 15: 39: 59 & 0.1257 \\ 15217 & 15: 40: 00 & 0.1306 \\ 15218 & 15: 40: 01 & 0.1268 \\ 15219 & 15: 40: 02 & 0.1291 \\ 15220 & 15: 40: 03 & 0.1260 \\ 15221 & 15: 40: 04 & 0.1265 \\ 15222 & 15: 40: 05 & 0.1295 \\ 15223 & 15: 40: 06 & 0.1270 \\ 15224 & 15: 40: 07 & 0.1307 \\ 15225 & 15: 40: 08 & 0.1243 \\ 15226 & 15: 40: 09 & 0.1309 \\ 15227 & 15: 40: 10 & 0.1275 \\ 15228 & 15: 40: 11 & 0.1313 \\ 15229 & 15: 40: 12 & 0.1260 \\ 15230 & 15: 40: 13 & 0.1308 \\ 15231 & 15: 40: 14 & 0.1274 \\ 15232 & 15: 40: 15 & 0.1274 \\ 15233 & 15: 40: 16 & 0.1257 \\ 15234 & 15: 40: 17 & 0.1265\end{array}$


Appendix A - Fuel Data Test 1 NIST102505_1.xIs

$\begin{array}{lll}15235 & 15: 40: 18 & 0.1275 \\ 15236 & 15: 40: 19 & 0.1236 \\ 15237 & 15: 40: 20 & 0.1259 \\ 15238 & 15: 40: 21 & 0.1244 \\ 15239 & 15: 40: 22 & 0.1298 \\ 15240 & 15: 40: 23 & 0.1253 \\ 15241 & 15: 40: 24 & 0.1300 \\ 15242 & 15: 40: 25 & 0.1301 \\ 15243 & 15: 40: 26 & 0.1319 \\ 15244 & 15: 40: 27 & 0.1263 \\ 15245 & 15: 40: 28 & 0.1271 \\ 15246 & 15: 40: 29 & 0.1244 \\ 15247 & 15: 40: 30 & 0.1232 \\ 15248 & 15: 40: 31 & 0.1278 \\ 15249 & 15: 40: 32 & 0.1239 \\ 15250 & 15: 40: 33 & 0.1284 \\ 15251 & 15: 40: 34 & 0.1234 \\ 15252 & 15: 40: 35 & 0.1276 \\ 15253 & 15: 40: 36 & 0.1234 \\ 15254 & 15: 40: 37 & 0.1276 \\ 15255 & 15: 40: 38 & 0.1261 \\ 15256 & 15: 40: 39 & 0.1290 \\ 15257 & 15: 40: 40 & 0.1240 \\ 15258 & 15: 40: 41 & 0.1268 \\ 15259 & 15: 40: 42 & 0.1264 \\ 15260 & 15: 40: 43 & 0.1242 \\ 15261 & 15: 40: 44 & 0.1271 \\ 15262 & 15: 40: 45 & 0.1247 \\ 15263 & 15: 40: 46 & 0.1287 \\ 15264 & 15: 40: 47 & 0.1241 \\ 15265 & 15: 40: 48 & 0.1299 \\ 15266 & 15: 40: 49 & 0.1254 \\ 15267 & 15: 40: 50 & 0.1293 \\ 15268 & 15: 40: 51 & 0.1246 \\ 15269 & 15: 40: 52 & 0.1272 \\ 15270 & 15: 40: 53 & 0.1238 \\ 15271 & 15: 40: 54 & 0.1245 \\ 15272 & 15: 40: 55 & 0.1285 \\ 15273 & 15: 40: 56 & 0.1260 \\ 15274 & 15: 40: 57 & 0.1272 \\ 15275 & 15: 40: 58 & 0.1242 \\ 15276 & 15: 40: 59 & 0.1289 \\ 15277 & 15: 41: 00 & 0.1254 \\ 15278 & 15: 41: 01 & 0.1282 \\ 15279 & 15: 41: 02 & 0.1253 \\ 15280 & 15: 41: 03 & 0.1298 \\ 15281 & 15: 41: 04 & 0.1250 \\ 15282 & 15: 41: 05 & 0.1283 \\ 15283 & 15: 41: 06 & 0.1251 \\ 15284 & 15: 41: 07 & 0.1240 \\ 15285 & 15: 41: 08 & 0.1280 \\ 15286 & 15: 41: 09 & 0.1250\end{array}$


Appendix A - Fuel Data Test 1 NIST102505_1.xIs

$\begin{array}{lll}15287 & 15: 41: 10 & 0.1260 \\ 15288 & 15: 41: 11 & 0.1222 \\ 15289 & 15: 41: 12 & 0.1291 \\ 15290 & 15: 41: 13 & 0.1251 \\ 15291 & 15: 41: 14 & 0.1290 \\ 15292 & 15: 41: 15 & 0.1265 \\ 15293 & 15: 41: 16 & 0.1291 \\ 15294 & 15: 41: 17 & 0.1257 \\ 15295 & 15: 41: 18 & 0.1267 \\ 15296 & 15: 41: 19 & 0.1284 \\ 15297 & 15: 41: 20 & 0.1254 \\ 15298 & 15: 41: 21 & 0.1279 \\ 15299 & 15: 41: 22 & 0.1235 \\ 15300 & 15: 41: 23 & 0.1288 \\ 15301 & 15: 41: 24 & 0.1245 \\ 15302 & 15: 41: 25 & 0.1283 \\ 15303 & 15: 41: 26 & 0.1247 \\ 15304 & 15: 41: 27 & 0.1291 \\ 15305 & 15: 41: 28 & 0.1250 \\ 15306 & 15: 41: 29 & 0.1268 \\ 15307 & 15: 41: 30 & 0.1276 \\ 15308 & 15: 41: 31 & 0.1275 \\ 15309 & 15: 41: 32 & 0.1266 \\ 15310 & 15: 41: 33 & 0.1257 \\ 15311 & 15: 41: 34 & 0.1268 \\ 15312 & 15: 41: 35 & 0.1214 \\ 15313 & 15: 41: 36 & 0.1279 \\ 15314 & 15: 41: 37 & 0.1251 \\ 15315 & 15: 41: 38 & 0.1290 \\ 15316 & 15: 41: 39 & 0.1248 \\ 15317 & 15: 41: 40 & 0.1298 \\ 15318 & 15: 41: 41 & 0.1261 \\ 15319 & 15: 41: 42 & 0.1273 \\ 15320 & 15: 41: 43 & 0.1253 \\ 15321 & 15: 41: 44 & 0.1267 \\ 15322 & 15: 41: 45 & 0.1263 \\ 15323 & 15: 41: 46 & 0.1257 \\ 15324 & 15: 41: 47 & 0.1280 \\ 15325 & 15: 41: 48 & 0.1236 \\ 15326 & 15: 41: 49 & 0.1274 \\ 15327 & 15: 41: 50 & 0.1254 \\ 15328 & 15: 41: 51 & 0.1283 \\ 15329 & 15: 41: 52 & 0.1248 \\ 15330 & 15: 41: 53 & 0.1269 \\ 15331 & 15: 41: 54 & 0.1254 \\ 15332 & 15: 41: 55 & 0.1268 \\ 15333 & 15: 41: 56 & 0.1231 \\ 15334 & 15: 41: 57 & 0.1236 \\ 15335 & 15: 41: 58 & 0.1269 \\ 15336 & 15: 41: 59 & 0.1243 \\ 15337 & 15: 42: 00 & 0.1260 \\ 15338 & 15: 42: 01 & 0.1234\end{array}$


Appendix A - Fuel Data Test 1 NIST102505_1.xIs

$\begin{array}{lll}15339 & 15: 42: 02 & 0.1283 \\ 15340 & 15: 42: 03 & 0.1246 \\ 15341 & 15: 42: 04 & 0.1296 \\ 15342 & 15: 42: 05 & 0.1270 \\ 15343 & 15: 42: 06 & 0.1305 \\ 15344 & 15: 42: 07 & 0.1263 \\ 15345 & 15: 42: 08 & 0.1277 \\ 15346 & 15: 42: 09 & 0.1260 \\ 15347 & 15: 42: 10 & 0.1253 \\ 15348 & 15: 42: 11 & 0.1290 \\ 15349 & 15: 42: 12 & 0.1246 \\ 15350 & 15: 42: 13 & 0.1280 \\ 15351 & 15: 42: 14 & 0.1234 \\ 15352 & 15: 42: 15 & 0.1304 \\ 15353 & 15: 42: 16 & 0.1259 \\ 15354 & 15: 42: 17 & 0.1302 \\ 15355 & 15: 42: 18 & 0.1267 \\ 15356 & 15: 42: 19 & 0.1305 \\ 15357 & 15: 42: 20 & 0.1274 \\ 15358 & 15: 42: 21 & 0.1262 \\ 15359 & 15: 42: 22 & 0.1269 \\ 15360 & 15: 42: 23 & 0.1267 \\ 15361 & 15: 42: 24 & 0.1266 \\ 15362 & 15: 42: 25 & 0.1227 \\ 15363 & 15: 42: 26 & 0.1284 \\ 15364 & 15: 42: 27 & 0.1241 \\ 15365 & 15: 42: 28 & 0.1290 \\ 15366 & 15: 42: 29 & 0.1253 \\ 15367 & 15: 42: 30 & 0.1298 \\ 15368 & 15: 42: 31 & 0.1257 \\ 15369 & 15: 42: 32 & 0.1263 \\ 15370 & 15: 42: 33 & 0.1257 \\ 15371 & 15: 42: 34 & 0.1265 \\ 15372 & 15: 42: 35 & 0.1274 \\ 15373 & 15: 42: 36 & 0.1253 \\ 15374 & 15: 42: 37 & 0.1280 \\ 15375 & 15: 42: 38 & 0.1247 \\ 15376 & 15: 42: 39 & 0.1281 \\ 15377 & 15: 42: 40 & 0.1253 \\ 15378 & 15: 42: 41 & 0.1278 \\ 15379 & 15: 42: 42 & 0.1248 \\ 15380 & 15: 42: 43 & 0.1291 \\ 15381 & 15: 42: 44 & 0.1276 \\ 15382 & 15: 42: 45 & 0.1285 \\ 15383 & 15: 42: 46 & 0.1261 \\ 15384 & 15: 42: 47 & 0.1252 \\ 15385 & 15: 42: 48 & 0.1286 \\ 15386 & 15: 42: 49 & 0.1253 \\ 15387 & 15: 42: 50 & 0.1270 \\ 15388 & 15: 42: 51 & 0.1229 \\ 15389 & 15: 42: 52 & 0.1293 \\ 15390 & 15: 42: 53 & 0.1244\end{array}$


Appendix A - Fuel Data Test 1 NIST102505_1.xls

$\begin{array}{lll}15391 & 15: 42: 54 & 0.1284 \\ 15392 & 15: 42: 55 & 0.1254 \\ 15393 & 15: 42: 56 & 0.1323 \\ 15394 & 15: 42: 57 & 0.1273 \\ 15395 & 15: 42: 58 & 0.1298 \\ 15396 & 15: 42: 59 & 0.1264 \\ 15397 & 15: 43: 00 & 0.1268 \\ 15398 & 15: 43: 01 & 0.1281 \\ 15399 & 15: 43: 02 & 0.1261 \\ 15400 & 15: 43: 03 & 0.1264 \\ 15401 & 15: 43: 04 & 0.1209 \\ 15402 & 15: 43: 05 & 0.1291 \\ 15403 & 15: 43: 06 & 0.1263 \\ 15404 & 15: 43: 07 & 0.1306 \\ 15405 & 15: 43: 08 & 0.1248 \\ 15406 & 15: 43: 09 & 0.1289 \\ 15407 & 15: 43: 10 & 0.1252 \\ 15408 & 15: 43: 11 & 0.1254 \\ 15409 & 15: 43: 12 & 0.1257 \\ 15410 & 15: 43: 13 & 0.1241 \\ 15411 & 15: 43: 14 & 0.1265 \\ 15412 & 15: 43: 15 & 0.1243 \\ 15413 & 15: 43: 16 & 0.1289 \\ 15414 & 15: 43: 17 & 0.1247 \\ 15415 & 15: 43: 18 & 0.1290 \\ 15416 & 15: 43: 19 & 0.1261 \\ 15417 & 15: 43: 20 & 0.1304 \\ 15418 & 15: 43: 21 & 0.1264 \\ 15419 & 15: 43: 22 & 0.1269 \\ 15420 & 15: 43: 23 & 0.1279 \\ 15421 & 15: 43: 24 & 0.1279 \\ 15422 & 15: 43: 25 & 0.1279 \\ 15423 & 15: 43: 26 & 0.1234 \\ 15424 & 15: 43: 27 & 0.1285 \\ 15425 & 15: 43: 28 & 0.1245 \\ 15426 & 15: 43: 29 & 0.1275 \\ 15427 & 15: 43: 30 & 0.1224 \\ 15428 & 15: 43: 31 & 0.1292 \\ 15429 & 15: 43: 32 & 0.1254 \\ 15430 & 15: 43: 33 & 0.1294 \\ 15431 & 15: 43: 34 & 0.1266 \\ 15432 & 15: 43: 35 & 0.1276 \\ 15433 & 15: 43: 36 & 0.1251 \\ 15434 & 15: 43: 37 & 0.1258 \\ 15435 & 15: 43: 38 & 0.1270 \\ 15436 & 15: 43: 39 & 0.1244 \\ 15437 & 15: 43: 40 & 0.1263 \\ 15438 & 15: 43: 41 & 0.1240 \\ 15439 & 15: 43: 42 & 0.1288 \\ 15440 & 15: 43: 43 & 0.1246 \\ 15441 & 15: 43: 44 & 0.1307 \\ 15442 & 15: 43: 45 & 0.1265\end{array}$


Appendix A - Fuel Data Test 1 NIST102505_1.xIs

$\begin{array}{lll}15443 & 15: 43: 46 & 0.1297 \\ 15444 & 15: 43: 47 & 0.1245 \\ 15445 & 15: 43: 48 & 0.1271 \\ 15446 & 15: 43: 49 & 0.1262 \\ 15447 & 15: 43: 50 & 0.1273 \\ 15448 & 15: 43: 51 & 0.1286 \\ 15449 & 15: 43: 52 & 0.1283 \\ 15450 & 15: 43: 53 & 0.1293 \\ 15451 & 15: 43: 54 & 0.1241 \\ 15452 & 15: 43: 55 & 0.1290 \\ 15453 & 15: 43: 56 & 0.1261 \\ 15454 & 15: 43: 57 & 0.1302 \\ 15455 & 15: 43: 58 & 0.1263 \\ 15456 & 15: 43: 59 & 0.1303 \\ 15457 & 15: 44: 00 & 0.1292 \\ 15458 & 15: 44: 01 & 0.1280 \\ 15459 & 15: 44: 02 & 0.1252 \\ 15460 & 15: 44: 03 & 0.1252 \\ 15461 & 15: 44: 04 & 0.1281 \\ 15462 & 15: 44: 05 & 0.1247 \\ 15463 & 15: 44: 06 & 0.1262 \\ 15464 & 15: 44: 07 & 0.1231 \\ 15465 & 15: 44: 08 & 0.1279 \\ 15466 & 15: 44: 09 & 0.1237 \\ 15467 & 15: 44: 10 & 0.1302 \\ 15468 & 15: 44: 11 & 0.1258 \\ 15469 & 15: 44: 12 & 0.1299 \\ 15470 & 15: 44: 13 & 0.1267 \\ 15471 & 15: 44: 14 & 0.1272 \\ 15472 & 15: 44: 15 & 0.1250 \\ 15473 & 15: 44: 16 & 0.1236 \\ 15474 & 15: 44: 17 & 0.1290 \\ 15475 & 15: 44: 18 & 0.1257 \\ 15476 & 15: 44: 19 & 0.1286 \\ 15477 & 15: 44: 20 & 0.1240 \\ 15478 & 15: 44: 21 & 0.1298 \\ 15479 & 15: 44: 22 & 0.1258 \\ 15480 & 15: 44: 23 & 0.1305 \\ 15481 & 15: 44: 24 & 0.1260 \\ 15482 & 15: 44: 25 & 0.1281 \\ 15483 & 15: 44: 26 & 0.1236 \\ 15484 & 15: 44: 27 & 0.1251 \\ 15485 & 15: 44: 28 & 0.1254 \\ 15486 & 15: 44: 29 & 0.1248 \\ 15487 & 15: 44: 30 & 0.1260 \\ 15488 & 15: 44: 31 & 0.1231 \\ 15489 & 15: 44: 32 & 0.1275 \\ 15490 & 15: 44: 33 & 0.1227 \\ 15491 & 15: 44: 34 & 0.1287 \\ 15492 & 15: 44: 35 & 0.1249 \\ 15493 & 15: 44: 36 & 0.1287 \\ 15494 & 15: 44: 37 & 0.1246\end{array}$


Appendix A - Fuel Data Test 1 NIST102505_1.xIs

$\begin{array}{lll}15495 & 15: 44: 38 & 0.1274 \\ 15496 & 15: 44: 39 & 0.1234 \\ 15497 & 15: 44: 40 & 0.1253 \\ 15498 & 15: 44: 41 & 0.1281 \\ 15499 & 15: 44: 42 & 0.1268 \\ 15500 & 15: 44: 43 & 0.1275 \\ 15501 & 15: 44: 44 & 0.1242 \\ 15502 & 15: 44: 45 & 0.1276 \\ 15503 & 15: 44: 46 & 0.1250 \\ 15504 & 15: 44: 47 & 0.1284 \\ 15505 & 15: 44: 48 & 0.1260 \\ 15506 & 15: 44: 49 & 0.1312 \\ 15507 & 15: 44: 50 & 0.1262 \\ 15508 & 15: 44: 51 & 0.1297 \\ 15509 & 15: 44: 52 & 0.1272 \\ 15510 & 15: 44: 53 & 0.1274 \\ 15511 & 15: 44: 54 & 0.1266 \\ 15512 & 15: 44: 55 & 0.1251 \\ 15513 & 15: 44: 56 & 0.1269 \\ 15514 & 15: 44: 57 & 0.1210 \\ 15515 & 15: 44: 58 & 0.1261 \\ 15516 & 15: 44: 59 & 0.1259 \\ 15517 & 15: 45: 00 & 0.1299 \\ 15518 & 15: 45: 01 & 0.1267 \\ 15519 & 15: 45: 02 & 0.1299 \\ 15520 & 15: 45: 03 & 0.1274 \\ 15521 & 15: 45: 04 & 0.1273 \\ 15522 & 15: 45: 05 & 0.1254 \\ 15523 & 15: 45: 06 & 0.1248 \\ 15524 & 15: 45: 07 & 0.1288 \\ 15525 & 15: 45: 08 & 0.1249 \\ 15526 & 15: 45: 09 & 0.1278 \\ 15527 & 15: 45: 10 & 0.1232 \\ 15528 & 15: 45: 11 & 0.1286 \\ 15529 & 15: 45: 12 & 0.1238 \\ 15530 & 15: 45: 13 & 0.1275 \\ 15531 & 15: 45: 14 & 0.1273 \\ 15532 & 15: 45: 15 & 0.1286 \\ 15533 & 15: 45: 16 & 0.1250 \\ 15534 & 15: 45: 17 & 0.1260 \\ 15535 & 15: 45: 18 & 0.1287 \\ 15536 & 15: 45: 19 & 0.1288 \\ 15537 & 15: 45: 20 & 0.1284 \\ 15538 & 15: 45: 21 & 0.1239 \\ 15539 & 15: 45: 22 & 0.1272 \\ 15540 & 15: 45: 23 & 0.1214 \\ 15541 & 15: 45: 24 & 0.1274 \\ 15542 & 15: 45: 25 & 0.1285 \\ 15543 & 15: 45: 26 & 0.1316 \\ 15544 & 15: 45: 27 & 0.1276 \\ 15545 & 15: 45: 28 & 0.1290 \\ 15546 & 15: 45: 29 & 0.1287\end{array}$


Appendix A - Fuel Data Test 1 NIST102505_1.xIs

$\begin{array}{lll}15547 & 15: 45: 30 & 0.1273 \\ 15548 & 15: 45: 31 & 0.1265 \\ 15549 & 15: 45: 32 & 0.1257 \\ 15550 & 15: 45: 33 & 0.1286 \\ 15551 & 15: 45: 34 & 0.1248 \\ 15552 & 15: 45: 35 & 0.1284 \\ 15553 & 15: 45: 36 & 0.1255 \\ 15554 & 15: 45: 37 & 0.1288 \\ 15555 & 15: 45: 38 & 0.1237 \\ 15556 & 15: 45: 39 & 0.1303 \\ 15557 & 15: 45: 40 & 0.1284 \\ 15558 & 15: 45: 41 & 0.1304 \\ 15559 & 15: 45: 42 & 0.1267 \\ 15560 & 15: 45: 43 & 0.1277 \\ 15561 & 15: 45: 44 & 0.1277 \\ 15562 & 15: 45: 45 & 0.1251 \\ 15563 & 15: 45: 46 & 0.1259 \\ 15564 & 15: 45: 47 & 0.1230 \\ 15565 & 15: 45: 48 & 0.1275 \\ 15566 & 15: 45: 49 & 0.1227 \\ 15567 & 15: 45: 50 & 0.1293 \\ 15568 & 15: 45: 51 & 0.1276 \\ 15569 & 15: 45: 52 & 0.1319 \\ 15570 & 15: 45: 53 & 0.1265 \\ 15571 & 15: 45: 54 & 0.1295 \\ 15572 & 15: 45: 55 & 0.1265 \\ 15573 & 15: 45: 56 & 0.1264 \\ 15574 & 15: 45: 57 & 0.1270 \\ 15575 & 15: 45: 58 & 0.1250 \\ 15576 & 15: 45: 59 & 0.1266 \\ 15577 & 15: 46: 00 & 0.1215 \\ 15578 & 15: 46: 01 & 0.1278 \\ 15579 & 15: 46: 02 & 0.1228 \\ 15580 & 15: 46: 03 & 0.1283 \\ 15581 & 15: 46: 04 & 0.1255 \\ 15582 & 15: 46: 05 & 0.1283 \\ 15583 & 15: 46: 06 & 0.1261 \\ 15584 & 15: 46: 07 & 0.1264 \\ 15585 & 15: 46: 08 & 0.1263 \\ 15586 & 15: 46: 09 & 0.1246 \\ 15587 & 15: 46: 10 & 0.1253 \\ 15588 & 15: 46: 11 & 0.1242 \\ 15589 & 15: 46: 12 & 0.1271 \\ 15590 & 15: 46: 13 & 0.1219 \\ 15591 & 15: 46: 14 & 0.1283 \\ 15592 & 15: 46: 15 & 0.1249 \\ 15593 & 15: 46: 16 & 0.1291 \\ 15594 & 15: 46: 17 & 0.1240 \\ 15595 & 15: 46: 18 & 0.1301 \\ 15596 & 15: 46: 19 & 0.1261 \\ 15597 & 15: 46: 20 & 0.1262 \\ 15598 & 15: 46: 21 & 0.1257\end{array}$


Appendix A - Fuel Data Test 1 NIST102505_1.xIs

$\begin{array}{lll}15599 & 15: 46: 22 & 0.1269 \\ 15600 & 15: 46: 23 & 0.1275 \\ 15601 & 15: 46: 24 & 0.1244 \\ 15602 & 15: 46: 25 & 0.1278 \\ 15603 & 15: 46: 26 & 0.1251 \\ 15604 & 15: 46: 27 & 0.1296 \\ 15605 & 15: 46: 28 & 0.1248 \\ 15606 & 15: 46: 29 & 0.1305 \\ 15607 & 15: 46: 30 & 0.1264 \\ 15608 & 15: 46: 31 & 0.1290 \\ 15609 & 15: 46: 32 & 0.1263 \\ 15610 & 15: 46: 33 & 0.1274 \\ 15611 & 15: 46: 34 & 0.1263 \\ 15612 & 15: 46: 35 & 0.1254 \\ 15613 & 15: 46: 36 & 0.1271 \\ 15614 & 15: 46: 37 & 0.1241 \\ 15615 & 15: 46: 38 & 0.1282 \\ 15616 & 15: 46: 39 & 0.1241 \\ 15617 & 15: 46: 40 & 0.1301 \\ 15618 & 15: 46: 41 & 0.1258 \\ 15619 & 15: 46: 42 & 0.1296 \\ 15620 & 15: 46: 43 & 0.1259 \\ 15621 & 15: 46: 44 & 0.1301 \\ 15622 & 15: 46: 45 & 0.1286 \\ 15623 & 15: 46: 46 & 0.1296 \\ 15624 & 15: 46: 47 & 0.1280 \\ 15625 & 15: 46: 48 & 0.1262 \\ 15626 & 15: 46: 49 & 0.1271 \\ 15627 & 15: 46: 50 & 0.1221 \\ 15628 & 15: 46: 51 & 0.1274 \\ 15629 & 15: 46: 52 & 0.1245 \\ 15630 & 15: 46: 53 & 0.1291 \\ 15631 & 15: 46: 54 & 0.1255 \\ 15632 & 15: 46: 55 & 0.1304 \\ 15633 & 15: 46: 56 & 0.1290 \\ 15634 & 15: 46: 57 & 0.1290 \\ 15635 & 15: 46: 58 & 0.1250 \\ 15636 & 15: 46: 59 & 0.1249 \\ 15637 & 15: 47: 00 & 0.1271 \\ 15638 & 15: 47: 01 & 0.1250 \\ 15639 & 15: 47: 02 & 0.1263 \\ 15640 & 15: 47: 03 & 0.1233 \\ 15641 & 15: 47: 04 & 0.1267 \\ 15642 & 15: 47: 05 & 0.1228 \\ 15643 & 15: 47: 06 & 0.1290 \\ 15644 & 15: 47: 07 & 0.1259 \\ 15645 & 15: 47: 08 & 0.1301 \\ 15646 & 15: 47: 09 & 0.1269 \\ 15647 & 15: 47: 10 & 0.1291 \\ 15648 & 15: 47: 11 & 0.1281 \\ 15649 & 15: 47: 12 & 0.1273 \\ 15650 & 15: 47: 13 & 0.1271\end{array}$


Appendix A - Fuel Data Test 1 NIST102505_1.xIs

$\begin{array}{lll}15651 & 15: 47: 14 & 0.1256 \\ 15652 & 15: 47: 15 & 0.1274 \\ 15653 & 15: 47: 16 & 0.1218 \\ 15654 & 15: 47: 17 & 0.1282 \\ 15655 & 15: 47: 18 & 0.1248 \\ 15656 & 15: 47: 19 & 0.1293 \\ 15657 & 15: 47: 20 & 0.1245 \\ 15658 & 15: 47: 21 & 0.1298 \\ 15659 & 15: 47: 22 & 0.1268 \\ 15660 & 15: 47: 23 & 0.1275 \\ 15661 & 15: 47: 24 & 0.1254 \\ 15662 & 15: 47: 25 & 0.1262 \\ 15663 & 15: 47: 26 & 0.1282 \\ 15664 & 15: 47: 27 & 0.1244 \\ 15665 & 15: 47: 28 & 0.1264 \\ 15666 & 15: 47: 29 & 0.1240 \\ 15667 & 15: 47: 30 & 0.1280 \\ 15668 & 15: 47: 31 & 0.1240 \\ 15669 & 15: 47: 32 & 0.1295 \\ 15670 & 15: 47: 33 & 0.1258 \\ 15671 & 15: 47: 34 & 0.1285 \\ 15672 & 15: 47: 35 & 0.1250 \\ 15673 & 15: 47: 36 & 0.1280 \\ 15674 & 15: 47: 37 & 0.1261 \\ 15675 & 15: 47: 38 & 0.1257 \\ 15676 & 15: 47: 39 & 0.1282 \\ 15677 & 15: 47: 40 & 0.1240 \\ 15678 & 15: 47: 41 & 0.1269 \\ 15679 & 15: 47: 42 & 0.1223 \\ 15680 & 15: 47: 43 & 0.1289 \\ 15681 & 15: 47: 44 & 0.1243 \\ 15682 & 15: 47: 45 & 0.1280 \\ 15683 & 15: 47: 46 & 0.1262 \\ 15684 & 15: 47: 47 & 0.1285 \\ 15685 & 15: 47: 48 & 0.1247 \\ 15686 & 15: 47: 49 & 0.1268 \\ 15687 & 15: 47: 50 & 0.1269 \\ 15688 & 15: 47: 51 & 0.1257 \\ 15689 & 15: 47: 52 & 0.1272 \\ 15690 & 15: 47: 53 & 0.1239 \\ 15691 & 15: 47: 54 & 0.1288 \\ 15692 & 15: 47: 55 & 0.1242 \\ 15693 & 15: 47: 56 & 0.1287 \\ 15694 & 15: 47: 57 & 0.1252 \\ 15695 & 15: 47: 58 & 0.1301 \\ 15696 & 15: 47: 59 & 0.1272 \\ 15697 & 15: 48: 00 & 0.1287 \\ 15698 & 15: 48: 01 & 0.1281 \\ 15699 & 15: 48: 02 & 0.1275 \\ 15700 & 15: 48: 03 & 0.1275 \\ 15701 & 15: 48: 04 & 0.1249 \\ 15702 & 15: 48: 05 & 0.1292\end{array}$


Appendix A - Fuel Data Test 1 NIST102505_1.xls

$\begin{array}{lll}15703 & 15: 48: 06 & 0.1242 \\ 15704 & 15: 48: 07 & 0.1275 \\ 15705 & 15: 48: 08 & 0.1246 \\ 15706 & 15: 48: 09 & 0.1297 \\ 15707 & 15: 48: 10 & 0.1249 \\ 15708 & 15: 48: 11 & 0.1309 \\ 15709 & 15: 48: 12 & 0.1283 \\ 15710 & 15: 48: 13 & 0.1301 \\ 15711 & 15: 48: 14 & 0.1264 \\ 15712 & 15: 48: 15 & 0.1267 \\ 15713 & 15: 48: 16 & 0.1265 \\ 15714 & 15: 48: 17 & 0.1248 \\ 15715 & 15: 48: 18 & 0.1259 \\ 15716 & 15: 48: 19 & 0.1227 \\ 15717 & 15: 48: 20 & 0.1273 \\ 15718 & 15: 48: 21 & 0.1230 \\ 15719 & 15: 48: 22 & 0.1298 \\ 15720 & 15: 48: 23 & 0.1257 \\ 15721 & 15: 48: 24 & 0.1296 \\ 15722 & 15: 48: 25 & 0.1269 \\ 15723 & 15: 48: 26 & 0.1288 \\ 15724 & 15: 48: 27 & 0.1262 \\ 15725 & 15: 48: 28 & 0.1244 \\ 15726 & 15: 48: 29 & 0.1285 \\ 15727 & 15: 48: 30 & 0.1255 \\ 15728 & 15: 48: 31 & 0.1276 \\ 15729 & 15: 48: 32 & 0.1240 \\ 15730 & 15: 48: 33 & 0.1286 \\ 15731 & 15: 48: 34 & 0.1244 \\ 15732 & 15: 48: 35 & 0.1279 \\ 15733 & 15: 48: 36 & 0.1275 \\ 15734 & 15: 48: 37 & 0.1280 \\ 15735 & 15: 48: 38 & 0.1237 \\ 15736 & 15: 48: 39 & 0.1246 \\ 15737 & 15: 48: 40 & 0.1265 \\ 15738 & 15: 48: 41 & 0.1241 \\ 15739 & 15: 48: 42 & 0.1262 \\ 15740 & 15: 48: 43 & 0.1236 \\ 15741 & 15: 48: 44 & 0.1286 \\ 15742 & 15: 48: 45 & 0.1248 \\ 15743 & 15: 48: 46 & 0.1309 \\ 15744 & 15: 48: 47 & 0.1282 \\ 15745 & 15: 48: 48 & 0.1303 \\ 15746 & 15: 48: 49 & 0.1262 \\ 15747 & 15: 48: 50 & 0.1273 \\ 15748 & 15: 48: 51 & 0.1270 \\ 15749 & 15: 48: 52 & 0.1259 \\ 15750 & 15: 48: 53 & 0.1267 \\ 15751 & 15: 48: 54 & 0.1256 \\ 15752 & 15: 48: 55 & 0.1286 \\ 15753 & 15: 48: 56 & 0.1240 \\ 15754 & 15: 48: 57 & 0.1278\end{array}$


Appendix A - Fuel Data Test 1 NIST102505_1.xIs

$\begin{array}{lll}15755 & 15: 48: 58 & 0.1248 \\ 15756 & 15: 48: 59 & 0.1301 \\ 15757 & 15: 49: 00 & 0.1267 \\ 15758 & 15: 49: 01 & 0.1296 \\ 15759 & 15: 49: 02 & 0.1271 \\ 15760 & 15: 49: 03 & 0.1267 \\ 15761 & 15: 49: 04 & 0.1259 \\ 15762 & 15: 49: 05 & 0.1247 \\ 15763 & 15: 49: 06 & 0.1255 \\ 15764 & 15: 49: 07 & 0.1212 \\ 15765 & 15: 49: 08 & 0.1293 \\ 15766 & 15: 49: 09 & 0.1255 \\ 15767 & 15: 49: 10 & 0.1295 \\ 15768 & 15: 49: 11 & 0.1241 \\ 15769 & 15: 49: 12 & 0.1303 \\ 15770 & 15: 49: 13 & 0.1255 \\ 15771 & 15: 49: 14 & 0.1278 \\ 15772 & 15: 49: 15 & 0.1266 \\ 15773 & 15: 49: 16 & 0.1269 \\ 15774 & 15: 49: 17 & 0.1286 \\ 15775 & 15: 49: 18 & 0.1261 \\ 15776 & 15: 49: 19 & 0.1296 \\ 15777 & 15: 49: 20 & 0.1244 \\ 15778 & 15: 49: 21 & 0.1285 \\ 15779 & 15: 49: 22 & 0.1253 \\ 15780 & 15: 49: 23 & 0.1315 \\ 15781 & 15: 49: 24 & 0.1271 \\ 15782 & 15: 49: 25 & 0.1292 \\ 15783 & 15: 49: 26 & 0.1283 \\ 15784 & 15: 49: 27 & 0.1297 \\ 15785 & 15: 49: 28 & 0.1260 \\ 15786 & 15: 49: 29 & 0.1259 \\ 15787 & 15: 49: 30 & 0.1301 \\ 15788 & 15: 49: 31 & 0.1258 \\ 15789 & 15: 49: 32 & 0.1273 \\ 15790 & 15: 49: 33 & 0.1230 \\ 15791 & 15: 49: 34 & 0.1302 \\ 15792 & 15: 49: 35 & 0.1270 \\ 15793 & 15: 49: 36 & 0.1317 \\ 15794 & 15: 49: 37 & 0.1289 \\ 15795 & 15: 49: 38 & 0.1318 \\ 15796 & 15: 49: 39 & 0.1285 \\ 15797 & 15: 49: 40 & 0.1278 \\ 15798 & 15: 49: 41 & 0.1264 \\ 15799 & 15: 49: 42 & 0.1265 \\ 15800 & 15: 49: 43 & 0.1277 \\ 15801 & 15: 49: 44 & 0.1269 \\ 15802 & 15: 49: 45 & 0.1299 \\ 15803 & 15: 49: 46 & 0.1234 \\ 15804 & 15: 49: 47 & 0.1286 \\ 15805 & 15: 49: 48 & 0.1234 \\ 15806 & 15: 49: 49 & 0.1277\end{array}$


Appendix A - Fuel Data Test 1 NIST102505_1.xIs

$\begin{array}{lll}15807 & 15: 49: 50 & 0.1250 \\ 15808 & 15: 49: 51 & 0.1277 \\ 15809 & 15: 49: 52 & 0.1267 \\ 15810 & 15: 49: 53 & 0.1269 \\ 15811 & 15: 49: 54 & 0.1282 \\ 15812 & 15: 49: 55 & 0.1259 \\ 15813 & 15: 49: 56 & 0.1253 \\ 15814 & 15: 49: 57 & 0.1242 \\ 15815 & 15: 49: 58 & 0.1288 \\ 15816 & 15: 49: 59 & 0.1234 \\ 15817 & 15: 50: 00 & 0.1285 \\ 15818 & 15: 50: 01 & 0.1241 \\ 15819 & 15: 50: 02 & 0.1298 \\ 15820 & 15: 50: 03 & 0.1252 \\ 15821 & 15: 50: 04 & 0.1296 \\ 15822 & 15: 50: 05 & 0.1262 \\ 15823 & 15: 50: 06 & 0.1270 \\ 15824 & 15: 50: 07 & 0.1269 \\ 15825 & 15: 50: 08 & 0.1270 \\ 15826 & 15: 50: 09 & 0.1275 \\ 15827 & 15: 50: 10 & 0.1245 \\ 15828 & 15: 50: 11 & 0.1292 \\ 15829 & 15: 50: 12 & 0.1254 \\ 15830 & 15: 50: 13 & 0.1285 \\ 15831 & 15: 50: 14 & 0.1247 \\ 15832 & 15: 50: 15 & 0.1320 \\ 15833 & 15: 50: 16 & 0.1281 \\ 15834 & 15: 50: 17 & 0.1298 \\ 15835 & 15: 50: 18 & 0.1284 \\ 15836 & 15: 50: 19 & 0.1290 \\ 15837 & 15: 50: 20 & 0.1302 \\ 15838 & 15: 50: 21 & 0.1258 \\ 15839 & 15: 50: 22 & 0.1287 \\ 15840 & 15: 50: 23 & 0.1247 \\ 15841 & 15: 50: 24 & 0.1290 \\ 15842 & 15: 50: 25 & 0.1264 \\ 15843 & 15: 50: 26 & 0.1311 \\ 15844 & 15: 50: 27 & 0.1231 \\ 15845 & 15: 50: 28 & 0.1297 \\ 15846 & 15: 50: 29 & 0.1274 \\ 15847 & 15: 50: 30 & 0.1293 \\ 15848 & 15: 50: 31 & 0.1243 \\ 15849 & 15: 50: 32 & 0.1248 \\ 15850 & 15: 50: 33 & 0.1261 \\ 15851 & 15: 50: 34 & 0.1239 \\ 15852 & 15: 50: 35 & 0.1273 \\ 15853 & 15: 50: 36 & 0.1235 \\ 15854 & 15: 50: 37 & 0.1268 \\ 15855 & 15: 50: 38 & 0.1226 \\ 15856 & 15: 50: 39 & 0.1277 \\ 15857 & 15: 50: 40 & 0.1227 \\ 15858 & 15: 50: 41 & 0.1262\end{array}$


Appendix A - Fuel Data Test 1 NIST102505_1.xIs

$\begin{array}{lll}15859 & 15: 50: 42 & 0.1284 \\ 15860 & 15: 50: 43 & 0.1272 \\ 15861 & 15: 50: 44 & 0.1263 \\ 15862 & 15: 50: 45 & 0.1250 \\ 15863 & 15: 50: 46 & 0.1290 \\ 15864 & 15: 50: 47 & 0.1260 \\ 15865 & 15: 50: 48 & 0.1275 \\ 15866 & 15: 50: 49 & 0.1228 \\ 15867 & 15: 50: 50 & 0.1297 \\ 15868 & 15: 50: 51 & 0.1251 \\ 15869 & 15: 50: 52 & 0.1282 \\ 15870 & 15: 50: 53 & 0.1272 \\ 15871 & 15: 50: 54 & 0.1294 \\ 15872 & 15: 50: 55 & 0.1251 \\ 15873 & 15: 50: 56 & 0.1270 \\ 15874 & 15: 50: 57 & 0.1266 \\ 15875 & 15: 50: 58 & 0.1261 \\ 15876 & 15: 50: 59 & 0.1271 \\ 15877 & 15: 51: 00 & 0.1244 \\ 15878 & 15: 51: 01 & 0.1287 \\ 15879 & 15: 51: 02 & 0.1227 \\ 15880 & 15: 51: 03 & 0.1274 \\ 15881 & 15: 51: 04 & 0.1260 \\ 15882 & 15: 51: 05 & 0.1303 \\ 15883 & 15: 51: 06 & 0.1261 \\ 15884 & 15: 51: 07 & 0.1296 \\ 15885 & 15: 51: 08 & 0.1283 \\ 15886 & 15: 51: 09 & 0.1281 \\ 15887 & 15: 51: 10 & 0.1250 \\ 15888 & 15: 51: 11 & 0.1247 \\ 15889 & 15: 51: 12 & 0.1264 \\ 15890 & 15: 51: 13 & 0.1224 \\ 15891 & 15: 51: 14 & 0.1272 \\ 15892 & 15: 51: 15 & 0.1246 \\ 15893 & 15: 51: 16 & 0.1287 \\ 15894 & 15: 51: 17 & 0.1246 \\ 15895 & 15: 51: 18 & 0.1297 \\ 15896 & 15: 51: 19 & 0.1280 \\ 15897 & 15: 51: 20 & 0.1299 \\ 15898 & 15: 51: 21 & 0.1272 \\ 15899 & 15: 51: 22 & 0.1265 \\ 15900 & 15: 51: 23 & 0.1287 \\ 15901 & 15: 51: 24 & 0.1255 \\ 15902 & 15: 51: 25 & 0.1265 \\ 15903 & 15: 51: 26 & 0.1218 \\ 15904 & 15: 51: 27 & 0.1282 \\ 15905 & 15: 51: 28 & 0.1241 \\ 15906 & 15: 51: 29 & 0.1281 \\ 15907 & 15: 51: 30 & 0.1247 \\ 15908 & 15: 51: 31 & 0.1288 \\ 15909 & 15: 51: 32 & 0.1259 \\ 15910 & 15: 51: 33 & 0.1276\end{array}$


Appendix A - Fuel Data Test 1 NIST102505_1.xIs

$\begin{array}{lll}15911 & 15: 51: 34 & 0.1265 \\ 15912 & 15: 51: 35 & 0.1263 \\ 15913 & 15: 51: 36 & 0.1271 \\ 15914 & 15: 51: 37 & 0.1241 \\ 15915 & 15: 51: 38 & 0.1284 \\ 15916 & 15: 51: 39 & 0.1243 \\ 15917 & 15: 51: 40 & 0.1280 \\ 15918 & 15: 51: 41 & 0.1241 \\ 15919 & 15: 51: 42 & 0.1302 \\ 15920 & 15: 51: 43 & 0.1264 \\ 15921 & 15: 51: 44 & 0.1299 \\ 15922 & 15: 51: 45 & 0.1273 \\ 15923 & 15: 51: 46 & 0.1279 \\ 15924 & 15: 51: 47 & 0.1259 \\ 15925 & 15: 51: 48 & 0.1251 \\ 15926 & 15: 51: 49 & 0.1297 \\ 15927 & 15: 51: 50 & 0.1259 \\ 15928 & 15: 51: 51 & 0.1274 \\ 15929 & 15: 51: 52 & 0.1227 \\ 15930 & 15: 51: 53 & 0.1294 \\ 15931 & 15: 51: 54 & 0.1259 \\ 15932 & 15: 51: 55 & 0.1281 \\ 15933 & 15: 51: 56 & 0.1264 \\ 15934 & 15: 51: 57 & 0.1294 \\ 15935 & 15: 51: 58 & 0.1273 \\ 15936 & 15: 51: 59 & 0.1287 \\ 15937 & 15: 52: 00 & 0.1285 \\ 15938 & 15: 52: 01 & 0.1281 \\ 15939 & 15: 52: 02 & 0.1289 \\ 15940 & 15: 52: 03 & 0.1259 \\ 15941 & 15: 52: 04 & 0.1303 \\ 15942 & 15: 52: 05 & 0.1251 \\ 15943 & 15: 52: 06 & 0.1298 \\ 15944 & 15: 52: 07 & 0.1286 \\ 15945 & 15: 52: 08 & 0.1308 \\ 15946 & 15: 52: 09 & 0.1254 \\ 15947 & 15: 52: 10 & 0.1284 \\ 15948 & 15: 52: 11 & 0.1276 \\ 15949 & 15: 52: 12 & 0.1284 \\ 15950 & 15: 52: 13 & 0.1264 \\ 15951 & 15: 52: 14 & 0.1276 \\ 15952 & 15: 52: 15 & 0.1290 \\ 15953 & 15: 52: 16 & 0.1253 \\ 15954 & 15: 52: 17 & 0.1288 \\ 15955 & 15: 52: 18 & 0.1257 \\ 15956 & 15: 52: 19 & 0.1288 \\ 15957 & 15: 52: 20 & 0.1241 \\ 15958 & 15: 52: 21 & 0.1295 \\ 15959 & 15: 52: 22 & 0.1259 \\ 15960 & 15: 52: 23 & 0.1291 \\ 15961 & 15: 52: 24 & 0.1243 \\ 15962 & 15: 52: 25 & 0.1266\end{array}$


Appendix A - Fuel Data Test 1 NIST102505_1.xIs

$\begin{array}{lll}15963 & 15: 52: 26 & 0.1267 \\ 15964 & 15: 52: 27 & 0.1257 \\ 15965 & 15: 52: 28 & 0.1271 \\ 15966 & 15: 52: 29 & 0.1250 \\ 15967 & 15: 52: 30 & 0.1284 \\ 15968 & 15: 52: 31 & 0.1232 \\ 15969 & 15: 52: 32 & 0.1285 \\ 15970 & 15: 52: 33 & 0.1247 \\ 15971 & 15: 52: 34 & 0.1301 \\ 15972 & 15: 52: 35 & 0.1247 \\ 15973 & 15: 52: 36 & 0.1282 \\ 15974 & 15: 52: 37 & 0.1233 \\ 15975 & 15: 52: 38 & 0.1239 \\ 15976 & 15: 52: 39 & 0.1280 \\ 15977 & 15: 52: 40 & 0.1251 \\ 15978 & 15: 52: 41 & 0.1259 \\ 15979 & 15: 52: 42 & 0.1199 \\ 15980 & 15: 52: 43 & 0.1292 \\ 15981 & 15: 52: 44 & 0.1263 \\ 15982 & 15: 52: 45 & 0.1296 \\ 15983 & 15: 52: 46 & 0.1229 \\ 15984 & 15: 52: 47 & 0.1273 \\ 15985 & 15: 52: 48 & 0.1272 \\ 15986 & 15: 52: 49 & 0.1266 \\ 15987 & 15: 52: 50 & 0.1265 \\ 15988 & 15: 52: 51 & 0.1257 \\ 15989 & 15: 52: 52 & 0.1254 \\ 15990 & 15: 52: 53 & 0.1227 \\ 15991 & 15: 52: 54 & 0.1250 \\ 15992 & 15: 52: 55 & 0.1204 \\ 15993 & 15: 52: 56 & 0.1275 \\ 15994 & 15: 52: 57 & 0.1251 \\ 15995 & 15: 52: 58 & 0.1290 \\ 15996 & 15: 52: 59 & 0.1242 \\ 15997 & 15: 53: 00 & 0.1278 \\ 15998 & 15: 53: 01 & 0.1281 \\ 15999 & 15: 53: 02 & 0.1269 \\ 16000 & 15: 53: 03 & 0.1238 \\ 16001 & 15: 53: 04 & 0.1240 \\ 16002 & 15: 53: 05 & 0.1286 \\ 16003 & 15: 53: 06 & 0.1250 \\ 16004 & 15: 53: 07 & 0.1270 \\ 16005 & 15: 53: 08 & 0.1249 \\ 16006 & 15: 53: 09 & 0.1283 \\ 16007 & 15: 53: 10 & 0.1246 \\ 16008 & 15: 53: 11 & 0.1284 \\ 16009 & 15: 53: 12 & 0.1267 \\ 16010 & 15: 53: 13 & 0.1280 \\ 16011 & 15: 53: 14 & 0.1263 \\ 16012 & 15: 53: 15 & 0.1259 \\ 16013 & 15: 53: 16 & 0.1273 \\ 16014 & 15: 53: 17 & 0.1260\end{array}$


Appendix A - Fuel Data Test 1 NIST102505_1.xIs

$\begin{array}{lll}16015 & 15: 53: 18 & 0.1272 \\ 16016 & 15: 53: 19 & 0.1256 \\ 16017 & 15: 53: 20 & 0.1273 \\ 16018 & 15: 53: 21 & 0.1220 \\ 16019 & 15: 53: 22 & 0.1275 \\ 16020 & 15: 53: 23 & 0.1265 \\ 16021 & 15: 53: 24 & 0.1310 \\ 16022 & 15: 53: 25 & 0.1262 \\ 16023 & 15: 53: 26 & 0.1303 \\ 16024 & 15: 53: 27 & 0.1265 \\ 16025 & 15: 53: 28 & 0.1269 \\ 16026 & 15: 53: 29 & 0.1258 \\ 16027 & 15: 53: 30 & 0.1259 \\ 16028 & 15: 53: 31 & 0.1247 \\ 16029 & 15: 53: 32 & 0.1220 \\ 16030 & 15: 53: 33 & 0.1282 \\ 16031 & 15: 53: 34 & 0.1234 \\ 16032 & 15: 53: 35 & 0.1267 \\ 16033 & 15: 53: 36 & 0.1239 \\ 16034 & 15: 53: 37 & 0.1293 \\ 16035 & 15: 53: 38 & 0.1243 \\ 16036 & 15: 53: 39 & 0.1291 \\ 16037 & 15: 53: 40 & 0.1256 \\ 16038 & 15: 53: 41 & 0.1258 \\ 16039 & 15: 53: 42 & 0.1253 \\ 16040 & 15: 53: 43 & 0.1248 \\ 16041 & 15: 53: 44 & 0.1249 \\ 16042 & 15: 53: 45 & 0.1215 \\ 16043 & 15: 53: 46 & 0.1279 \\ 16044 & 15: 53: 47 & 0.1247 \\ 16045 & 15: 53: 48 & 0.1290 \\ 16046 & 15: 53: 49 & 0.1233 \\ 16047 & 15: 53: 50 & 0.1286 \\ 16048 & 15: 53: 51 & 0.1252 \\ 16049 & 15: 53: 52 & 0.1267 \\ 16050 & 15: 53: 53 & 0.1238 \\ 16051 & 15: 53: 54 & 0.1266 \\ 16052 & 15: 53: 55 & 0.1274 \\ 16053 & 15: 53: 56 & 0.1246 \\ 16054 & 15: 53: 57 & 0.1275 \\ 16055 & 15: 53: 58 & 0.1233 \\ 16056 & 15: 53: 59 & 0.1276 \\ 16057 & 15: 54: 00 & 0.1247 \\ 16058 & 15: 54: 01 & 0.1286 \\ 16059 & 15: 54: 02 & 0.1226 \\ 16060 & 15: 54: 03 & 0.1276 \\ 16061 & 15: 54: 04 & 0.1277 \\ 16062 & 15: 54: 05 & 0.1280 \\ 16063 & 15: 54: 06 & 0.1238 \\ 16064 & 15: 54: 07 & 0.1258 \\ 16065 & 15: 54: 08 & 0.1266 \\ 16066 & 15: 54: 09 & 0.1241\end{array}$


Appendix A - Fuel Data Test 1 NIST102505_1.xIs

$\begin{array}{lll}16067 & 15: 54: 10 & 0.1278 \\ 16068 & 15: 54: 11 & 0.1234 \\ 16069 & 15: 54: 12 & 0.1278 \\ 16070 & 15: 54: 13 & 0.1249 \\ 16071 & 15: 54: 14 & 0.1306 \\ 16072 & 15: 54: 15 & 0.1247 \\ 16073 & 15: 54: 16 & 0.1282 \\ 16074 & 15: 54: 17 & 0.1269 \\ 16075 & 15: 54: 18 & 0.1272 \\ 16076 & 15: 54: 19 & 0.1262 \\ 16077 & 15: 54: 20 & 0.1254 \\ 16078 & 15: 54: 21 & 0.1286 \\ 16079 & 15: 54: 22 & 0.1237 \\ 16080 & 15: 54: 23 & 0.1271 \\ 16081 & 15: 54: 24 & 0.1249 \\ 16082 & 15: 54: 25 & 0.1292 \\ 16083 & 15: 54: 26 & 0.1258 \\ 16084 & 15: 54: 27 & 0.1294 \\ 16085 & 15: 54: 28 & 0.1277 \\ 16086 & 15: 54: 29 & 0.1277 \\ 16087 & 15: 54: 30 & 0.1245 \\ 16088 & 15: 54: 31 & 0.1252 \\ 16089 & 15: 54: 32 & 0.1285 \\ 16090 & 15: 54: 33 & 0.1260 \\ 16091 & 15: 54: 34 & 0.1283 \\ 16092 & 15: 54: 35 & 0.1246 \\ 16093 & 15: 54: 36 & 0.1296 \\ 16094 & 15: 54: 37 & 0.1245 \\ 16095 & 15: 54: 38 & 0.1280 \\ 16096 & 15: 54: 39 & 0.1248 \\ 16097 & 15: 54: 40 & 0.1293 \\ 16098 & 15: 54: 41 & 0.1274 \\ 16099 & 15: 54: 42 & 0.1271 \\ 16100 & 15: 54: 43 & 0.1277 \\ 16101 & 15: 54: 44 & 0.1274 \\ 16102 & 15: 54: 45 & 0.1273 \\ 16103 & 15: 54: 46 & 0.1247 \\ 16104 & 15: 54: 47 & 0.1282 \\ 16105 & 15: 54: 48 & 0.1235 \\ 16106 & 15: 54: 49 & 0.1281 \\ 16107 & 15: 54: 50 & 0.1248 \\ 16108 & 15: 54: 51 & 0.1295 \\ 16109 & 15: 54: 52 & 0.1244 \\ 16110 & 15: 54: 53 & 0.1305 \\ 16111 & 15: 54: 54 & 0.1275 \\ 16112 & 15: 54: 55 & 0.1287 \\ 16113 & 15: 54: 56 & 0.1249 \\ 16114 & 15: 54: 57 & 0.1265 \\ 16115 & 15: 54: 58 & 0.1261 \\ 16116 & 15: 54: 59 & 0.1244 \\ 16117 & 15: 55: 00 & 0.1266 \\ 16118 & 15: 55: 01 & 0.1229\end{array}$


Appendix A - Fuel Data Test 1 NIST102505_1.xls

$\begin{array}{lll}16119 & 15: 55: 02 & 0.1286 \\ 16120 & 15: 55: 03 & 0.1245 \\ 16121 & 15: 55: 04 & 0.1296 \\ 16122 & 15: 55: 05 & 0.1256 \\ 16123 & 15: 55: 06 & 0.1273 \\ 16124 & 15: 55: 07 & 0.1282 \\ 16125 & 15: 55: 08 & 0.1282 \\ 16126 & 15: 55: 09 & 0.1256 \\ 16127 & 15: 55: 10 & 0.1264 \\ 16128 & 15: 55: 11 & 0.1295 \\ 16129 & 15: 55: 12 & 0.1254 \\ 16130 & 15: 55: 13 & 0.1272 \\ 16131 & 15: 55: 14 & 0.1236 \\ 16132 & 15: 55: 15 & 0.1284 \\ 16133 & 15: 55: 16 & 0.1254 \\ 16134 & 15: 55: 17 & 0.1288 \\ 16135 & 15: 55: 18 & 0.1272 \\ 16136 & 15: 55: 19 & 0.1292 \\ 16137 & 15: 55: 20 & 0.1261 \\ 16138 & 15: 55: 21 & 0.1262 \\ 16139 & 15: 55: 22 & 0.1248 \\ 16140 & 15: 55: 23 & 0.1247 \\ 16141 & 15: 55: 24 & 0.1264 \\ 16142 & 15: 55: 25 & 0.1245 \\ 16143 & 15: 55: 26 & 0.1274 \\ 16144 & 15: 55: 27 & 0.1221 \\ 16145 & 15: 55: 28 & 0.1286 \\ 16146 & 15: 55: 29 & 0.1260 \\ 16147 & 15: 55: 30 & 0.1305 \\ 16148 & 15: 55: 31 & 0.1253 \\ 16149 & 15: 55: 32 & 0.1301 \\ 16150 & 15: 55: 33 & 0.1267 \\ 16151 & 15: 55: 34 & 0.1270 \\ 16152 & 15: 55: 35 & 0.1272 \\ 16153 & 15: 55: 36 & 0.1261 \\ 16154 & 15: 55: 37 & 0.1279 \\ 16155 & 15: 55: 38 & 0.1249 \\ 16156 & 15: 55: 39 & 0.1287 \\ 16157 & 15: 55: 40 & 0.1251 \\ 16158 & 15: 55: 41 & 0.1288 \\ 16159 & 15: 55: 42 & 0.1245 \\ 16160 & 15: 55: 43 & 0.1292 \\ 16161 & 15: 55: 44 & 0.1267 \\ 16162 & 15: 55: 45 & 0.1289 \\ 16163 & 15: 55: 46 & 0.1245 \\ 16164 & 15: 55: 47 & 0.1252 \\ 16165 & 15: 55: 48 & 0.1288 \\ 16166 & 15: 55: 49 & 0.1258 \\ 16167 & 15: 55: 50 & 0.1280 \\ 16168 & 15: 55: 51 & 0.1255 \\ 16169 & 15: 55: 52 & 0.1300 \\ 16170 & 15: 55: 53 & 0.1253\end{array}$


Appendix A - Fuel Data Test 1 NIST102505_1.xIs

$\begin{array}{lll}16171 & 15: 55: 54 & 0.1297 \\ 16172 & 15: 55: 55 & 0.1282 \\ 16173 & 15: 55: 56 & 0.1328 \\ 16174 & 15: 55: 57 & 0.1264 \\ 16175 & 15: 55: 58 & 0.1294 \\ 16176 & 15: 55: 59 & 0.1254 \\ 16177 & 15: 56: 00 & 0.1258 \\ 16178 & 15: 56: 01 & 0.1268 \\ 16179 & 15: 56: 02 & 0.1249 \\ 16180 & 15: 56: 03 & 0.1279 \\ 16181 & 15: 56: 04 & 0.1248 \\ 16182 & 15: 56: 05 & 0.1296 \\ 16183 & 15: 56: 06 & 0.1259 \\ 16184 & 15: 56: 07 & 0.1309 \\ 16185 & 15: 56: 08 & 0.1248 \\ 16186 & 15: 56: 09 & 0.1294 \\ 16187 & 15: 56: 10 & 0.1289 \\ 16188 & 15: 56: 11 & 0.1298 \\ 16189 & 15: 56: 12 & 0.1251 \\ 16190 & 15: 56: 13 & 0.1266 \\ 16191 & 15: 56: 14 & 0.1270 \\ 16192 & 15: 56: 15 & 0.1239 \\ 16193 & 15: 56: 16 & 0.1285 \\ 16194 & 15: 56: 17 & 0.1243 \\ 16195 & 15: 56: 18 & 0.1293 \\ 16196 & 15: 56: 19 & 0.1245 \\ 16197 & 15: 56: 20 & 0.1299 \\ 16198 & 15: 56: 21 & 0.1260 \\ 16199 & 15: 56: 22 & 0.1279 \\ 16200 & 15: 56: 23 & 0.1246 \\ 16201 & 15: 56: 24 & 0.1258 \\ 16202 & 15: 56: 25 & 0.1262 \\ 16203 & 15: 56: 26 & 0.1249 \\ 16204 & 15: 56: 27 & 0.1272 \\ 16205 & 15: 56: 28 & 0.1229 \\ 16206 & 15: 56: 29 & 0.1275 \\ 16207 & 15: 56: 30 & 0.1228 \\ 16208 & 15: 56: 31 & 0.1294 \\ 16209 & 15: 56: 32 & 0.1256 \\ 16210 & 15: 56: 33 & 0.1280 \\ 16211 & 15: 56: 34 & 0.1250 \\ 16212 & 15: 56: 35 & 0.1277 \\ 16213 & 15: 56: 36 & 0.1256 \\ 16214 & 15: 56: 37 & 0.1246 \\ 16215 & 15: 56: 38 & 0.1260 \\ 16216 & 15: 56: 39 & 0.1242 \\ 16217 & 15: 56: 40 & 0.1272 \\ 16218 & 15: 56: 41 & 0.1227 \\ 16219 & 15: 56: 42 & 0.1286 \\ 16220 & 15: 56: 43 & 0.1252 \\ 16221 & 15: 56: 44 & 0.1287 \\ 16222 & 15: 56: 45 & 0.1269\end{array}$


Appendix A - Fuel Data Test 1 NIST102505_1.xIs

$\begin{array}{lll}16223 & 15: 56: 46 & 0.1282 \\ 16224 & 15: 56: 47 & 0.1278 \\ 16225 & 15: 56: 48 & 0.1276 \\ 16226 & 15: 56: 49 & 0.1270 \\ 16227 & 15: 56: 50 & 0.1252 \\ 16228 & 15: 56: 51 & 0.1283 \\ 16229 & 15: 56: 52 & 0.1232 \\ 16230 & 15: 56: 53 & 0.1271 \\ 16231 & 15: 56: 54 & 0.1230 \\ 16232 & 15: 56: 55 & 0.1282 \\ 16233 & 15: 56: 56 & 0.1222 \\ 16234 & 15: 56: 57 & 0.1288 \\ 16235 & 15: 56: 58 & 0.1273 \\ 16236 & 15: 56: 59 & 0.1301 \\ 16237 & 15: 57: 00 & 0.1246 \\ 16238 & 15: 57: 01 & 0.1263 \\ 16239 & 15: 57: 02 & 0.1263 \\ 16240 & 15: 57: 03 & 0.1241 \\ 16241 & 15: 57: 04 & 0.1281 \\ 16242 & 15: 57: 05 & 0.1235 \\ 16243 & 15: 57: 06 & 0.1288 \\ 16244 & 15: 57: 07 & 0.1245 \\ 16245 & 15: 57: 08 & 0.1290 \\ 16246 & 15: 57: 09 & 0.1244 \\ 16247 & 15: 57: 10 & 0.1288 \\ 16248 & 15: 57: 11 & 0.1253 \\ 16249 & 15: 57: 12 & 0.1286 \\ 16250 & 15: 57: 13 & 0.1250 \\ 16251 & 15: 57: 14 & 0.1255 \\ 16252 & 15: 57: 15 & 0.1275 \\ 16253 & 15: 57: 16 & 0.1261 \\ 16254 & 15: 57: 17 & 0.1271 \\ 16255 & 15: 57: 18 & 0.1217 \\ 16256 & 15: 57: 19 & 0.1289 \\ 16257 & 15: 57: 20 & 0.1250 \\ 16258 & 15: 57: 21 & 0.1289 \\ 16259 & 15: 57: 22 & 0.1261 \\ 16260 & 15: 57: 23 & 0.1301 \\ 16261 & 15: 57: 24 & 0.1265 \\ 16262 & 15: 57: 25 & 0.1275 \\ 16263 & 15: 57: 26 & 0.1263 \\ 16264 & 15: 57: 27 & 0.1270 \\ 16265 & 15: 57: 28 & 0.1281 \\ 16266 & 15: 57: 29 & 0.1252 \\ 16267 & 15: 57: 30 & 0.1293 \\ 16268 & 15: 57: 31 & 0.1243 \\ 16269 & 15: 57: 32 & 0.1284 \\ 16270 & 15: 57: 33 & 0.1253 \\ 16271 & 15: 57: 34 & 0.1297 \\ 16272 & 15: 57: 35 & 0.1245 \\ 16273 & 15: 57: 36 & 0.1276 \\ 16274 & 15: 57: 37 & 0.1260\end{array}$


Appendix A - Fuel Data Test 1 NIST102505_1.xIs

$\begin{array}{lll}16275 & 15: 57: 38 & 0.1277 \\ 16276 & 15: 57: 39 & 0.1242 \\ 16277 & 15: 57: 40 & 0.1245 \\ 16278 & 15: 57: 41 & 0.1274 \\ 16279 & 15: 57: 42 & 0.1246 \\ 16280 & 15: 57: 43 & 0.1278 \\ 16281 & 15: 57: 44 & 0.1248 \\ 16282 & 15: 57: 45 & 0.1281 \\ 16283 & 15: 57: 46 & 0.1228 \\ 16284 & 15: 57: 47 & 0.1279 \\ 16285 & 15: 57: 48 & 0.1254 \\ 16286 & 15: 57: 49 & 0.1285 \\ 16287 & 15: 57: 50 & 0.1253 \\ 16288 & 15: 57: 51 & 0.1249 \\ 16289 & 15: 57: 52 & 0.1275 \\ 16290 & 15: 57: 53 & 0.1261 \\ 16291 & 15: 57: 54 & 0.1260 \\ 16292 & 15: 57: 55 & 0.1228 \\ 16293 & 15: 57: 56 & 0.1280 \\ 16294 & 15: 57: 57 & 0.1259 \\ 16295 & 15: 57: 58 & 0.1284 \\ 16296 & 15: 57: 59 & 0.1260 \\ 16297 & 15: 58: 00 & 0.1301 \\ 16298 & 15: 58: 01 & 0.1263 \\ 16299 & 15: 58: 02 & 0.1278 \\ 16300 & 15: 58: 03 & 0.1274 \\ 16301 & 15: 58: 04 & 0.1268 \\ 16302 & 15: 58: 05 & 0.1266 \\ 16303 & 15: 58: 06 & 0.1258 \\ 16304 & 15: 58: 07 & 0.1287 \\ 16305 & 15: 58: 08 & 0.1252 \\ 16306 & 15: 58: 09 & 0.1280 \\ 16307 & 15: 58: 10 & 0.1253 \\ 16308 & 15: 58: 11 & 0.1282 \\ 16309 & 15: 58: 12 & 0.1240 \\ 16310 & 15: 58: 13 & 0.1269 \\ 16311 & 15: 58: 14 & 0.1285 \\ 16312 & 15: 58: 15 & 0.1291 \\ 16313 & 15: 58: 16 & 0.1265 \\ 16314 & 15: 58: 17 & 0.1248 \\ 16315 & 15: 58: 18 & 0.1271 \\ 16316 & 15: 58: 19 & 0.1267 \\ 16317 & 15: 58: 20 & 0.1260 \\ 16318 & 15: 58: 21 & 0.1217 \\ 16319 & 15: 58: 22 & 0.1291 \\ 16320 & 15: 58: 23 & 0.1243 \\ 16321 & 15: 58: 24 & 0.1284 \\ 16322 & 15: 58: 25 & 0.1246 \\ 16323 & 15: 58: 26 & 0.1294 \\ 16324 & 15: 58: 27 & 0.1253 \\ 16325 & 15: 58: 28 & 0.1300 \\ 16326 & 15: 58: 29 & 0.1282\end{array}$


Appendix A - Fuel Data Test 1 NIST102505_1.xIs

$\begin{array}{lll}16327 & 15: 58: 30 & 0.1278 \\ 16328 & 15: 58: 31 & 0.1261 \\ 16329 & 15: 58: 32 & 0.1250 \\ 16330 & 15: 58: 33 & 0.1274 \\ 16331 & 15: 58: 34 & 0.1225 \\ 16332 & 15: 58: 35 & 0.1282 \\ 16333 & 15: 58: 36 & 0.1264 \\ 16334 & 15: 58: 37 & 0.1312 \\ 16335 & 15: 58: 38 & 0.1254 \\ 16336 & 15: 58: 39 & 0.1298 \\ 16337 & 15: 58: 40 & 0.1258 \\ 16338 & 15: 58: 41 & 0.1273 \\ 16339 & 15: 58: 42 & 0.1252 \\ 16340 & 15: 58: 43 & 0.1254 \\ 16341 & 15: 58: 44 & 0.1269 \\ 16342 & 15: 58: 45 & 0.1250 \\ 16343 & 15: 58: 46 & 0.1288 \\ 16344 & 15: 58: 47 & 0.1250 \\ 16345 & 15: 58: 48 & 0.1296 \\ 16346 & 15: 58: 49 & 0.1253 \\ 16347 & 15: 58: 50 & 0.1310 \\ 16348 & 15: 58: 51 & 0.1248 \\ 16349 & 15: 58: 52 & 0.1276 \\ 16350 & 15: 58: 53 & 0.1283 \\ 16351 & 15: 58: 54 & 0.1276 \\ 16352 & 15: 58: 55 & 0.1265 \\ 16353 & 15: 58: 56 & 0.1245 \\ 16354 & 15: 58: 57 & 0.1297 \\ 16355 & 15: 58: 58 & 0.1254 \\ 16356 & 15: 58: 59 & 0.1265 \\ 16357 & 15: 59: 00 & 0.1226 \\ 16358 & 15: 59: 01 & 0.1285 \\ 16359 & 15: 59: 02 & 0.1229 \\ 16360 & 15: 59: 03 & 0.1294 \\ 16361 & 15: 59: 04 & 0.1279 \\ 16362 & 15: 59: 05 & 0.1309 \\ 16363 & 15: 59: 06 & 0.1270 \\ 16364 & 15: 59: 07 & 0.1282 \\ 16365 & 15: 59: 08 & 0.1274 \\ 16366 & 15: 59: 09 & 0.1260 \\ 16367 & 15: 59: 10 & 0.1266 \\ 16368 & 15: 59: 11 & 0.1249 \\ 16369 & 15: 59: 12 & 0.1274 \\ 16370 & 15: 59: 13 & 0.1223 \\ 16371 & 15: 59: 14 & 0.1284 \\ 16372 & 15: 59: 15 & 0.1250 \\ 16373 & 15: 59: 16 & 0.1304 \\ 16374 & 15: 59: 17 & 0.1252 \\ 16375 & 15: 59: 18 & 0.1305 \\ 16376 & 15: 59: 19 & 0.1257 \\ 16377 & 15: 59: 20 & 0.1269 \\ 16378 & 15: 59: 21 & 0.1261\end{array}$


Appendix A - Fuel Data Test 1 NIST102505_1.xIs

$\begin{array}{lll}16379 & 15: 59: 22 & 0.1268 \\ 16380 & 15: 59: 23 & 0.1284 \\ 16381 & 15: 59: 24 & 0.1239 \\ 16382 & 15: 59: 25 & 0.1305 \\ 16383 & 15: 59: 26 & 0.1257 \\ 16384 & 15: 59: 27 & 0.1299 \\ 16385 & 15: 59: 28 & 0.1251 \\ 16386 & 15: 59: 29 & 0.1312 \\ 16387 & 15: 59: 30 & 0.1273 \\ 16388 & 15: 59: 31 & 0.1298 \\ 16389 & 15: 59: 32 & 0.1272 \\ 16390 & 15: 59: 33 & 0.1277 \\ 16391 & 15: 59: 34 & 0.1289 \\ 16392 & 15: 59: 35 & 0.1252 \\ 16393 & 15: 59: 36 & 0.1284 \\ 16394 & 15: 59: 37 & 0.1238 \\ 16395 & 15: 59: 38 & 0.1288 \\ 16396 & 15: 59: 39 & 0.1241 \\ 16397 & 15: 59: 40 & 0.1290 \\ 16398 & 15: 59: 41 & 0.1247 \\ 16399 & 15: 59: 42 & 0.1289 \\ 16400 & 15: 59: 43 & 0.1245 \\ 16401 & 15: 59: 44 & 0.1281 \\ 16402 & 15: 59: 45 & 0.1245 \\ 16403 & 15: 59: 46 & 0.1235 \\ 16404 & 15: 59: 47 & 0.1268 \\ 16405 & 15: 59: 48 & 0.1257 \\ 16406 & 15: 59: 49 & 0.1278 \\ 16407 & 15: 59: 50 & 0.1234 \\ 16408 & 15: 59: 51 & 0.1303 \\ 16409 & 15: 59: 52 & 0.1268 \\ 16410 & 15: 59: 53 & 0.1302 \\ 16411 & 15: 59: 54 & 0.1261 \\ 16412 & 15: 59: 55 & 0.1308 \\ 16413 & 15: 59: 56 & 0.1266 \\ 16414 & 15: 59: 57 & 0.1274 \\ 16415 & 15: 59: 58 & 0.1245 \\ 16416 & 15: 59: 59 & 0.1246 \\ 16417 & 16: 00: 00 & 0.1267 \\ 16418 & 16: 00: 01 & 0.1236 \\ 16419 & 16: 00: 02 & 0.1270 \\ 16420 & 16: 00: 03 & 0.1238 \\ 16421 & 16: 00: 04 & 0.1280 \\ 16422 & 16: 00: 05 & 0.1236 \\ 16423 & 16: 00: 06 & 0.1298 \\ 16424 & 16: 00: 07 & 0.1249 \\ 16425 & 16: 00: 08 & 0.1292 \\ 16426 & 16: 00: 09 & 0.1259 \\ 16427 & 16: 00: 10 & 0.1275 \\ 16428 & 16: 00: 11 & 0.1247 \\ 16429 & 16: 00: 12 & 0.1261 \\ 16430 & 16: 00: 13 & 0.1280\end{array}$


Appendix A - Fuel Data Test 1 NIST102505_1.xIs

$\begin{array}{lll}16431 & 16: 00: 14 & 0.1244 \\ 16432 & 16: 00: 15 & 0.1268 \\ 16433 & 16: 00: 16 & 0.1239 \\ 16434 & 16: 00: 17 & 0.1277 \\ 16435 & 16: 00: 18 & 0.1236 \\ 16436 & 16: 00: 19 & 0.1288 \\ 16437 & 16: 00: 20 & 0.1263 \\ 16438 & 16: 00: 21 & 0.1283 \\ 16439 & 16: 00: 22 & 0.1254 \\ 16440 & 16: 00: 23 & 0.1249 \\ 16441 & 16: 00: 24 & 0.1267 \\ 16442 & 16: 00: 25 & 0.1245 \\ 16443 & 16: 00: 26 & 0.1247 \\ 16444 & 16: 00: 27 & 0.1226 \\ 16445 & 16: 00: 28 & 0.1281 \\ 16446 & 16: 00: 29 & 0.1257 \\ 16447 & 16: 00: 30 & 0.1299 \\ 16448 & 16: 00: 31 & 0.1249 \\ 16449 & 16: 00: 32 & 0.1291 \\ 16450 & 16: 00: 33 & 0.1253 \\ 16451 & 16: 00: 34 & 0.1285 \\ 16452 & 16: 00: 35 & 0.1245 \\ 16453 & 16: 00: 36 & 0.1245 \\ 16454 & 16: 00: 37 & 0.1254 \\ 16455 & 16: 00: 38 & 0.1273 \\ 16456 & 16: 00: 39 & 0.1279 \\ 16457 & 16: 00: 40 & 0.1238 \\ 16458 & 16: 00: 41 & 0.1294 \\ 16459 & 16: 00: 42 & 0.1269 \\ 16460 & 16: 00: 43 & 0.1279 \\ 16461 & 16: 00: 44 & 0.1229 \\ 16462 & 16: 00: 45 & 0.1295 \\ 16463 & 16: 00: 46 & 0.1260 \\ 16464 & 16: 00: 47 & 0.1284 \\ 16465 & 16: 00: 48 & 0.1244 \\ 16466 & 16: 00: 49 & 0.1261 \\ 16467 & 16: 00: 50 & 0.1274 \\ 16468 & 16: 00: 51 & 0.1247 \\ 16469 & 16: 00: 52 & 0.1269 \\ 16470 & 16: 00: 53 & 0.1229 \\ 16471 & 16: 00: 54 & 0.1277 \\ 16472 & 16: 00: 55 & 0.1236 \\ 16473 & 16: 00: 56 & 0.1294 \\ 16474 & 16: 00: 57 & 0.1238 \\ 16475 & 16: 00: 58 & 0.1308 \\ 16476 & 16: 00: 59 & 0.1284 \\ 16477 & 16: 01: 00 & 0.1291 \\ 16478 & 16: 01: 01 & 0.1248 \\ 16479 & 16: 01: 02 & 0.1250 \\ 16480 & 16: 01: 03 & 0.1273 \\ 16481 & 16: 01: 04 & 0.1242 \\ 16482 & 16: 01: 05 & 0.1260\end{array}$


Appendix A - Fuel Data Test 1 NIST102505_1.xIs

$\begin{array}{lll}16483 & 16: 01: 06 & 0.1231 \\ 16484 & 16: 01: 07 & 0.1283 \\ 16485 & 16: 01: 08 & 0.1234 \\ 16486 & 16: 01: 09 & 0.1292 \\ 16487 & 16: 01: 10 & 0.1251 \\ 16488 & 16: 01: 11 & 0.1290 \\ 16489 & 16: 01: 12 & 0.1251 \\ 16490 & 16: 01: 13 & 0.1281 \\ 16491 & 16: 01: 14 & 0.1265 \\ 16492 & 16: 01: 15 & 0.1258 \\ 16493 & 16: 01: 16 & 0.1256 \\ 16494 & 16: 01: 17 & 0.1242 \\ 16495 & 16: 01: 18 & 0.1263 \\ 16496 & 16: 01: 19 & 0.1215 \\ 16497 & 16: 01: 20 & 0.1281 \\ 16498 & 16: 01: 21 & 0.1252 \\ 16499 & 16: 01: 22 & 0.1297 \\ 16500 & 16: 01: 23 & 0.1264 \\ 16501 & 16: 01: 24 & 0.1303 \\ 16502 & 16: 01: 25 & 0.1268 \\ 16503 & 16: 01: 26 & 0.1283 \\ 16504 & 16: 01: 27 & 0.1255 \\ 16505 & 16: 01: 28 & 0.1264 \\ 16506 & 16: 01: 29 & 0.1275 \\ 16507 & 16: 01: 30 & 0.1250 \\ 16508 & 16: 01: 31 & 0.1275 \\ 16509 & 16: 01: 32 & 0.1255 \\ 16510 & 16: 01: 33 & 0.1289 \\ 16511 & 16: 01: 34 & 0.1249 \\ 16512 & 16: 01: 35 & 0.1314 \\ 16513 & 16: 01: 36 & 0.1255 \\ 16514 & 16: 01: 37 & 0.1283 \\ 16515 & 16: 01: 38 & 0.1272 \\ 16516 & 16: 01: 39 & 0.1266 \\ 16517 & 16: 01: 40 & 0.1260 \\ 16518 & 16: 01: 41 & 0.1247 \\ 16519 & 16: 01: 42 & 0.1295 \\ 16520 & 16: 01: 43 & 0.1258 \\ 16521 & 16: 01: 44 & 0.1288 \\ 16522 & 16: 01: 45 & 0.1257 \\ 16523 & 16: 01: 46 & 0.1307 \\ 16524 & 16: 01: 47 & 0.1251 \\ 16525 & 16: 01: 48 & 0.1299 \\ 16526 & 16: 01: 49 & 0.1278 \\ 16527 & 16: 01: 50 & 0.1282 \\ 16528 & 16: 01: 51 & 0.1248 \\ 16529 & 16: 01: 52 & 0.1251 \\ 16530 & 16: 01: 53 & 0.1275 \\ 16531 & 16: 01: 54 & 0.1257 \\ 16532 & 16: 01: 55 & 0.1274 \\ 16533 & 16: 01: 56 & 0.1229 \\ 16534 & 16: 01: 57 & 0.1268\end{array}$


Appendix A - Fuel Data Test 1 NIST102505_1.xIs

$\begin{array}{lll}16535 & 16: 01: 58 & 0.1225 \\ 16536 & 16: 01: 59 & 0.1272 \\ 16537 & 16: 02: 00 & 0.1239 \\ 16538 & 16: 02: 01 & 0.1283 \\ 16539 & 16: 02: 02 & 0.1261 \\ 16540 & 16: 02: 03 & 0.1267 \\ 16541 & 16: 02: 04 & 0.1266 \\ 16542 & 16: 02: 05 & 0.1261 \\ 16543 & 16: 02: 06 & 0.1249 \\ 16544 & 16: 02: 07 & 0.1232 \\ 16545 & 16: 02: 08 & 0.1254 \\ 16546 & 16: 02: 09 & 0.1223 \\ 16547 & 16: 02: 10 & 0.1271 \\ 16548 & 16: 02: 11 & 0.1237 \\ 16549 & 16: 02: 12 & 0.1286 \\ 16550 & 16: 02: 13 & 0.1239 \\ 16551 & 16: 02: 14 & 0.1286 \\ 16552 & 16: 02: 15 & 0.1279 \\ 16553 & 16: 02: 16 & 0.1299 \\ 16554 & 16: 02: 17 & 0.1249 \\ 16555 & 16: 02: 18 & 0.1252 \\ 16556 & 16: 02: 19 & 0.1249 \\ 16557 & 16: 02: 20 & 0.1224 \\ 16558 & 16: 02: 21 & 0.1263 \\ 16559 & 16: 02: 22 & 0.1217 \\ 16560 & 16: 02: 23 & 0.1276 \\ 16561 & 16: 02: 24 & 0.1244 \\ 16562 & 16: 02: 25 & 0.1306 \\ 16563 & 16: 02: 26 & 0.1259 \\ 16564 & 16: 02: 27 & 0.1284 \\ 16565 & 16: 02: 28 & 0.1284 \\ 16566 & 16: 02: 29 & 0.1283 \\ 16567 & 16: 02: 30 & 0.1260 \\ 16568 & 16: 02: 31 & 0.1243 \\ 16569 & 16: 02: 32 & 0.1276 \\ 16570 & 16: 02: 33 & 0.1245 \\ 16571 & 16: 02: 34 & 0.1267 \\ 16572 & 16: 02: 35 & 0.1227 \\ 16573 & 16: 02: 36 & 0.1290 \\ 16574 & 16: 02: 37 & 0.1243 \\ 16575 & 16: 02: 38 & 0.1281 \\ 16576 & 16: 02: 39 & 0.1258 \\ 16577 & 16: 02: 40 & 0.1285 \\ 16578 & 16: 02: 41 & 0.1244 \\ 16579 & 16: 02: 42 & 0.1258 \\ 16580 & 16: 02: 43 & 0.1279 \\ 16581 & 16: 02: 44 & 0.1254 \\ 16582 & 16: 02: 45 & 0.1271 \\ 16583 & 16: 02: 46 & 0.1228 \\ 16584 & 16: 02: 47 & 0.1271 \\ 16585 & 16: 02: 48 & 0.1219 \\ 16586 & 16: 02: 49 & 0.1283\end{array}$


Appendix A - Fuel Data Test 1 NIST102505_1.xIs

$\begin{array}{lll}16587 & 16: 02: 50 & 0.1251 \\ 16588 & 16: 02: 51 & 0.1303 \\ 16589 & 16: 02: 52 & 0.1248 \\ 16590 & 16: 02: 53 & 0.1292 \\ 16591 & 16: 02: 54 & 0.1254 \\ 16592 & 16: 02: 55 & 0.1255 \\ 16593 & 16: 02: 56 & 0.1261 \\ 16594 & 16: 02: 57 & 0.1267 \\ 16595 & 16: 02: 58 & 0.1274 \\ 16596 & 16: 02: 59 & 0.1227 \\ 16597 & 16: 03: 00 & 0.1288 \\ 16598 & 16: 03: 01 & 0.1256 \\ 16599 & 16: 03: 02 & 0.1290 \\ 16600 & 16: 03: 03 & 0.1230 \\ 16601 & 16: 03: 04 & 0.1294 \\ 16602 & 16: 03: 05 & 0.1256 \\ 16603 & 16: 03: 06 & 0.1262 \\ 16604 & 16: 03: 07 & 0.1232 \\ 16605 & 16: 03: 08 & 0.1262 \\ 16606 & 16: 03: 09 & 0.1255 \\ 16607 & 16: 03: 10 & 0.1234 \\ 16608 & 16: 03: 11 & 0.1273 \\ 16609 & 16: 03: 12 & 0.1227 \\ 16610 & 16: 03: 13 & 0.1273 \\ 16611 & 16: 03: 14 & 0.1240 \\ 16612 & 16: 03: 15 & 0.1287 \\ 16613 & 16: 03: 16 & 0.1240 \\ 16614 & 16: 03: 17 & 0.1283 \\ 16615 & 16: 03: 18 & 0.1271 \\ 16616 & 16: 03: 19 & 0.1271 \\ 16617 & 16: 03: 20 & 0.1246 \\ 16618 & 16: 03: 21 & 0.1249 \\ 16619 & 16: 03: 22 & 0.1263 \\ 16620 & 16: 03: 23 & 0.1238 \\ 16621 & 16: 03: 24 & 0.1277 \\ 16622 & 16: 03: 25 & 0.1241 \\ 16623 & 16: 03: 26 & 0.1275 \\ 16624 & 16: 03: 27 & 0.1240 \\ 16625 & 16: 03: 28 & 0.1289 \\ 16626 & 16: 03: 29 & 0.1285 \\ 16627 & 16: 03: 30 & 0.1292 \\ 16628 & 16: 03: 31 & 0.1254 \\ 16629 & 16: 03: 32 & 0.1274 \\ 16630 & 16: 03: 33 & 0.1269 \\ 16631 & 16: 03: 34 & 0.1271 \\ 16632 & 16: 03: 35 & 0.1276 \\ 16633 & 16: 03: 36 & 0.1266 \\ 16634 & 16: 03: 37 & 0.1285 \\ 16635 & 16: 03: 38 & 0.1240 \\ 16636 & 16: 03: 39 & 0.1304 \\ 16637 & 16: 03: 40 & 0.1260 \\ 16638 & 16: 03: 41 & 0.1318\end{array}$


Appendix A - Fuel Data Test 1 NIST102505_1.xls

$\begin{array}{lll}16639 & 16: 03: 42 & 0.1273 \\ 16640 & 16: 03: 43 & 0.1305 \\ 16641 & 16: 03: 44 & 0.1264 \\ 16642 & 16: 03: 45 & 0.1277 \\ 16643 & 16: 03: 46 & 0.1252 \\ 16644 & 16: 03: 47 & 0.1260 \\ 16645 & 16: 03: 48 & 0.1263 \\ 16646 & 16: 03: 49 & 0.1229 \\ 16647 & 16: 03: 50 & 0.1295 \\ 16648 & 16: 03: 51 & 0.1252 \\ 16649 & 16: 03: 52 & 0.1300 \\ 16650 & 16: 03: 53 & 0.1249 \\ 16651 & 16: 03: 54 & 0.1299 \\ 16652 & 16: 03: 55 & 0.1263 \\ 16653 & 16: 03: 56 & 0.1293 \\ 16654 & 16: 03: 57 & 0.1242 \\ 16655 & 16: 03: 58 & 0.1248 \\ 16656 & 16: 03: 59 & 0.1247 \\ 16657 & 16: 04: 00 & 0.1226 \\ 16658 & 16: 04: 01 & 0.1277 \\ 16659 & 16: 04: 02 & 0.1251 \\ 16660 & 16: 04: 03 & 0.1290 \\ 16661 & 16: 04: 04 & 0.1245 \\ 16662 & 16: 04: 05 & 0.1313 \\ 16663 & 16: 04: 06 & 0.1266 \\ 16664 & 16: 04: 07 & 0.1303 \\ 16665 & 16: 04: 08 & 0.1257 \\ 16666 & 16: 04: 09 & 0.1291 \\ 16667 & 16: 04: 10 & 0.1249 \\ 16668 & 16: 04: 11 & 0.1250 \\ 16669 & 16: 04: 12 & 0.1255 \\ 16670 & 16: 04: 13 & 0.1252 \\ 16671 & 16: 04: 14 & 0.1255 \\ 16672 & 16: 04: 15 & 0.1222 \\ 16673 & 16: 04: 16 & 0.1282 \\ 16674 & 16: 04: 17 & 0.1240 \\ 16675 & 16: 04: 18 & 0.1278 \\ 16676 & 16: 04: 19 & 0.1246 \\ 16677 & 16: 04: 20 & 0.1294 \\ 16678 & 16: 04: 21 & 0.1267 \\ 16679 & 16: 04: 22 & 0.1267 \\ 16680 & 16: 04: 23 & 0.1272 \\ 16681 & 16: 04: 24 & 0.1249 \\ 16682 & 16: 04: 25 & 0.1253 \\ 16683 & 16: 04: 26 & 0.1216 \\ 16684 & 16: 04: 27 & 0.1283 \\ 16685 & 16: 04: 28 & 0.1239 \\ 16686 & 16: 04: 29 & 0.1281 \\ 16687 & 16: 04: 30 & 0.1250 \\ 16688 & 16: 04: 31 & 0.1310 \\ 16689 & 16: 04: 32 & 0.1261 \\ 16690 & 16: 04: 33 & 0.1289\end{array}$


Appendix A - Fuel Data Test 1 NIST102505_1.xIs

$\begin{array}{lll}16691 & 16: 04: 34 & 0.1256 \\ 16692 & 16: 04: 35 & 0.1287 \\ 16693 & 16: 04: 36 & 0.1264 \\ 16694 & 16: 04: 37 & 0.1256 \\ 16695 & 16: 04: 38 & 0.1252 \\ 16696 & 16: 04: 39 & 0.1240 \\ 16697 & 16: 04: 40 & 0.1286 \\ 16698 & 16: 04: 41 & 0.1228 \\ 16699 & 16: 04: 42 & 0.1274 \\ 16700 & 16: 04: 43 & 0.1254 \\ 16701 & 16: 04: 44 & 0.1304 \\ 16702 & 16: 04: 45 & 0.1250 \\ 16703 & 16: 04: 46 & 0.1287 \\ 16704 & 16: 04: 47 & 0.1253 \\ 16705 & 16: 04: 48 & 0.1273 \\ 16706 & 16: 04: 49 & 0.1275 \\ 16707 & 16: 04: 50 & 0.1261 \\ 16708 & 16: 04: 51 & 0.1266 \\ 16709 & 16: 04: 52 & 0.1212 \\ 16710 & 16: 04: 53 & 0.1273 \\ 16711 & 16: 04: 54 & 0.1253 \\ 16712 & 16: 04: 55 & 0.1293 \\ 16713 & 16: 04: 56 & 0.1228 \\ 16714 & 16: 04: 57 & 0.1294 \\ 16715 & 16: 04: 58 & 0.1259 \\ 16716 & 16: 04: 59 & 0.1284 \\ 16717 & 16: 05: 00 & 0.1272 \\ 16718 & 16: 05: 01 & 0.1273 \\ 16719 & 16: 05: 02 & 0.1291 \\ 16720 & 16: 05: 03 & 0.1251 \\ 16721 & 16: 05: 04 & 0.1301 \\ 16722 & 16: 05: 05 & 0.1244 \\ 16723 & 16: 05: 06 & 0.1284 \\ 16724 & 16: 05: 07 & 0.1250 \\ 16725 & 16: 05: 08 & 0.1298 \\ 16726 & 16: 05: 09 & 0.1246 \\ 16727 & 16: 05: 10 & 0.1275 \\ 16728 & 16: 05: 11 & 0.1270 \\ 16729 & 16: 05: 12 & 0.1276 \\ 16730 & 16: 05: 13 & 0.1268 \\ 16731 & 16: 05: 14 & 0.1246 \\ 16732 & 16: 05: 15 & 0.1293 \\ 16733 & 16: 05: 16 & 0.1253 \\ 16734 & 16: 05: 17 & 0.1284 \\ 16735 & 16: 05: 18 & 0.1237 \\ 16736 & 16: 05: 19 & 0.1288 \\ 16737 & 16: 05: 20 & 0.1256 \\ 16738 & 16: 05: 21 & 0.1295 \\ 16739 & 16: 05: 22 & 0.1269 \\ 16740 & 16: 05: 23 & 0.1288 \\ 16741 & 16: 05: 24 & 0.1252 \\ 16742 & 16: 05: 25 & 0.1256\end{array}$


Appendix A - Fuel Data Test 1 NIST102505_1.xIs

$\begin{array}{lll}16743 & 16: 05: 26 & 0.1257 \\ 16744 & 16: 05: 27 & 0.1268 \\ 16745 & 16: 05: 28 & 0.1271 \\ 16746 & 16: 05: 29 & 0.1239 \\ 16747 & 16: 05: 30 & 0.1298 \\ 16748 & 16: 05: 31 & 0.1254 \\ 16749 & 16: 05: 32 & 0.1291 \\ 16750 & 16: 05: 33 & 0.1243 \\ 16751 & 16: 05: 34 & 0.1314 \\ 16752 & 16: 05: 35 & 0.1281 \\ 16753 & 16: 05: 36 & 0.1305 \\ 16754 & 16: 05: 37 & 0.1252 \\ 16755 & 16: 05: 38 & 0.1279 \\ 16756 & 16: 05: 39 & 0.1276 \\ 16757 & 16: 05: 40 & 0.1261 \\ 16758 & 16: 05: 41 & 0.1280 \\ 16759 & 16: 05: 42 & 0.1249 \\ 16760 & 16: 05: 43 & 0.1300 \\ 16761 & 16: 05: 44 & 0.1253 \\ 16762 & 16: 05: 45 & 0.1308 \\ 16763 & 16: 05: 46 & 0.1262 \\ 16764 & 16: 05: 47 & 0.1307 \\ 16765 & 16: 05: 48 & 0.1265 \\ 16766 & 16: 05: 49 & 0.1294 \\ 16767 & 16: 05: 50 & 0.1248 \\ 16768 & 16: 05: 51 & 0.1261 \\ 16769 & 16: 05: 52 & 0.1294 \\ 16770 & 16: 05: 53 & 0.1262 \\ 16771 & 16: 05: 54 & 0.1263 \\ 16772 & 16: 05: 55 & 0.1234 \\ 16773 & 16: 05: 56 & 0.1298 \\ 16774 & 16: 05: 57 & 0.1254 \\ 16775 & 16: 05: 58 & 0.1291 \\ 16776 & 16: 05: 59 & 0.1273 \\ 16777 & 16: 06: 00 & 0.1281 \\ 16778 & 16: 06: 01 & 0.1275 \\ 16779 & 16: 06: 02 & 0.1263 \\ 16780 & 16: 06: 03 & 0.1244 \\ 16781 & 16: 06: 04 & 0.1256 \\ 16782 & 16: 06: 05 & 0.1283 \\ 16783 & 16: 06: 06 & 0.1244 \\ 16784 & 16: 06: 07 & 0.1285 \\ 16785 & 16: 06: 08 & 0.1235 \\ 16786 & 16: 06: 09 & 0.1289 \\ 16787 & 16: 06: 10 & 0.1251 \\ 16788 & 16: 06: 11 & 0.1290 \\ 16789 & 16: 06: 12 & 0.1247 \\ 16790 & 16: 06: 13 & 0.1296 \\ 16791 & 16: 06: 14 & 0.1262 \\ 16792 & 16: 06: 15 & 0.1272 \\ 16793 & 16: 06: 16 & 0.1245 \\ 16794 & 16: 06: 17 & 0.1252\end{array}$


Appendix A - Fuel Data Test 1 NIST102505_1.xIs

$\begin{array}{lll}16795 & 16: 06: 18 & 0.1259 \\ 16796 & 16: 06: 19 & 0.1239 \\ 16797 & 16: 06: 20 & 0.1291 \\ 16798 & 16: 06: 21 & 0.1241 \\ 16799 & 16: 06: 22 & 0.1282 \\ 16800 & 16: 06: 23 & 0.1256 \\ 16801 & 16: 06: 24 & 0.1307 \\ 16802 & 16: 06: 25 & 0.1264 \\ 16803 & 16: 06: 26 & 0.1281 \\ 16804 & 16: 06: 27 & 0.1229 \\ 16805 & 16: 06: 28 & 0.1268 \\ 16806 & 16: 06: 29 & 0.1244 \\ 16807 & 16: 06: 30 & 0.1245 \\ 16808 & 16: 06: 31 & 0.1292 \\ 16809 & 16: 06: 32 & 0.1243 \\ 16810 & 16: 06: 33 & 0.1276 \\ 16811 & 16: 06: 34 & 0.1231 \\ 16812 & 16: 06: 35 & 0.1290 \\ 16813 & 16: 06: 36 & 0.1259 \\ 16814 & 16: 06: 37 & 0.1314 \\ 16815 & 16: 06: 38 & 0.1277 \\ 16816 & 16: 06: 39 & 0.1305 \\ 16817 & 16: 06: 40 & 0.1269 \\ 16818 & 16: 06: 41 & 0.1278 \\ 16819 & 16: 06: 42 & 0.1302 \\ 16820 & 16: 06: 43 & 0.1278 \\ 16821 & 16: 06: 44 & 0.1288 \\ 16822 & 16: 06: 45 & 0.1234 \\ 16823 & 16: 06: 46 & 0.1290 \\ 16824 & 16: 06: 47 & 0.1242 \\ 16825 & 16: 06: 48 & 0.1279 \\ 16826 & 16: 06: 49 & 0.1245 \\ 16827 & 16: 06: 50 & 0.1298 \\ 16828 & 16: 06: 51 & 0.1259 \\ 16829 & 16: 06: 52 & 0.1265 \\ 16830 & 16: 06: 53 & 0.1263 \\ 16831 & 16: 06: 54 & 0.1266 \\ 16832 & 16: 06: 55 & 0.1267 \\ 16833 & 16: 06: 56 & 0.1242 \\ 16834 & 16: 06: 57 & 0.1274 \\ 16835 & 16: 06: 58 & 0.1233 \\ 16836 & 16: 06: 59 & 0.1281 \\ 16837 & 16: 07: 00 & 0.1253 \\ 16838 & 16: 07: 01 & 0.1289 \\ 16839 & 16: 07: 02 & 0.1238 \\ 16840 & 16: 07: 03 & 0.1291 \\ 16841 & 16: 07: 04 & 0.1283 \\ 16842 & 16: 07: 05 & 0.1290 \\ 16843 & 16: 07: 06 & 0.1245 \\ 16844 & 16: 07: 07 & 0.1264 \\ 16845 & 16: 07: 08 & 0.1260 \\ 16846 & 16: 07: 09 & 0.1243\end{array}$


Appendix A - Fuel Data Test 1 NIST102505_1.xIs

$\begin{array}{lll}16847 & 16: 07: 10 & 0.1269 \\ 16848 & 16: 07: 11 & 0.1243 \\ 16849 & 16: 07: 12 & 0.1284 \\ 16850 & 16: 07: 13 & 0.1234 \\ 16851 & 16: 07: 14 & 0.1280 \\ 16852 & 16: 07: 15 & 0.1275 \\ 16853 & 16: 07: 16 & 0.1311 \\ 16854 & 16: 07: 17 & 0.1285 \\ 16855 & 16: 07: 18 & 0.1260 \\ 16856 & 16: 07: 19 & 0.1273 \\ 16857 & 16: 07: 20 & 0.1265 \\ 16858 & 16: 07: 21 & 0.1277 \\ 16859 & 16: 07: 22 & 0.1248 \\ 16860 & 16: 07: 23 & 0.1284 \\ 16861 & 16: 07: 24 & 0.1235 \\ 16862 & 16: 07: 25 & 0.1283 \\ 16863 & 16: 07: 26 & 0.1267 \\ 16864 & 16: 07: 27 & 0.1282 \\ 16865 & 16: 07: 28 & 0.1244 \\ 16866 & 16: 07: 29 & 0.1287 \\ 16867 & 16: 07: 30 & 0.1284 \\ 16868 & 16: 07: 31 & 0.1279 \\ 16869 & 16: 07: 32 & 0.1245 \\ 16870 & 16: 07: 33 & 0.1250 \\ 16871 & 16: 07: 34 & 0.1260 \\ 16872 & 16: 07: 35 & 0.1235 \\ 16873 & 16: 07: 36 & 0.1277 \\ 16874 & 16: 07: 37 & 0.1246 \\ 16875 & 16: 07: 38 & 0.1297 \\ 16876 & 16: 07: 39 & 0.1245 \\ 16877 & 16: 07: 40 & 0.1301 \\ 16878 & 16: 07: 41 & 0.1259 \\ 16879 & 16: 07: 42 & 0.1297 \\ 16880 & 16: 07: 43 & 0.1252 \\ 16881 & 16: 07: 44 & 0.1285 \\ 16882 & 16: 07: 45 & 0.1240 \\ 16883 & 16: 07: 46 & 0.1230 \\ 16884 & 16: 07: 47 & 0.1276 \\ 16885 & 16: 07: 48 & 0.1249 \\ 16886 & 16: 07: 49 & 0.1275 \\ 16887 & 16: 07: 50 & 0.1224 \\ 16888 & 16: 07: 51 & 0.1286 \\ 16889 & 16: 07: 52 & 0.1229 \\ 16890 & 16: 07: 53 & 0.1269 \\ 16891 & 16: 07: 54 & 0.1285 \\ 16892 & 16: 07: 55 & 0.1310 \\ 16893 & 16: 07: 56 & 0.1270 \\ 16894 & 16: 07: 57 & 0.1275 \\ 16895 & 16: 07: 58 & 0.1286 \\ 16896 & 16: 07: 59 & 0.1260 \\ 16897 & 16: 08: 00 & 0.1271 \\ 16898 & 16: 08: 01 & 0.1227\end{array}$


Appendix A - Fuel Data Test 1 NIST102505_1.xIs

$\begin{array}{lll}16899 & 16: 08: 02 & 0.1298 \\ 16900 & 16: 08: 03 & 0.1250 \\ 16901 & 16: 08: 04 & 0.1293 \\ 16902 & 16: 08: 05 & 0.1249 \\ 16903 & 16: 08: 06 & 0.1300 \\ 16904 & 16: 08: 07 & 0.1262 \\ 16905 & 16: 08: 08 & 0.1269 \\ 16906 & 16: 08: 09 & 0.1269 \\ 16907 & 16: 08: 10 & 0.1263 \\ 16908 & 16: 08: 11 & 0.1275 \\ 16909 & 16: 08: 12 & 0.1240 \\ 16910 & 16: 08: 13 & 0.1269 \\ 16911 & 16: 08: 14 & 0.1234 \\ 16912 & 16: 08: 15 & 0.1287 \\ 16913 & 16: 08: 16 & 0.1246 \\ 16914 & 16: 08: 17 & 0.1305 \\ 16915 & 16: 08: 18 & 0.1269 \\ 16916 & 16: 08: 19 & 0.1287 \\ 16917 & 16: 08: 20 & 0.1262 \\ 16918 & 16: 08: 21 & 0.1291 \\ 16919 & 16: 08: 22 & 0.1247 \\ 16920 & 16: 08: 23 & 0.1245 \\ 16921 & 16: 08: 24 & 0.1270 \\ 16922 & 16: 08: 25 & 0.1255 \\ 16923 & 16: 08: 26 & 0.1268 \\ 16924 & 16: 08: 27 & 0.1219 \\ 16925 & 16: 08: 28 & 0.1289 \\ 16926 & 16: 08: 29 & 0.1261 \\ 16927 & 16: 08: 30 & 0.1290 \\ 16928 & 16: 08: 31 & 0.1232 \\ 16929 & 16: 08: 32 & 0.1296 \\ 16930 & 16: 08: 33 & 0.1264 \\ 16931 & 16: 08: 34 & 0.1266 \\ 16932 & 16: 08: 35 & 0.1238 \\ 16933 & 16: 08: 36 & 0.1249 \\ 16934 & 16: 08: 37 & 0.1265 \\ 16935 & 16: 08: 38 & 0.1235 \\ 16936 & 16: 08: 39 & 0.1277 \\ 16937 & 16: 08: 40 & 0.1225 \\ 16938 & 16: 08: 41 & 0.1280 \\ 16939 & 16: 08: 42 & 0.1254 \\ 16940 & 16: 08: 43 & 0.1307 \\ 16941 & 16: 08: 44 & 0.1242 \\ 16942 & 16: 08: 45 & 0.1295 \\ 16943 & 16: 08: 46 & 0.1275 \\ 16944 & 16: 08: 47 & 0.1289 \\ 16945 & 16: 08: 48 & 0.1250 \\ 16946 & 16: 08: 49 & 0.1261 \\ 16947 & 16: 08: 50 & 0.1271 \\ 16948 & 16: 08: 51 & 0.1239 \\ 16949 & 16: 08: 52 & 0.1250 \\ 16950 & 16: 08: 53 & 0.1216\end{array}$


Appendix A - Fuel Data Test 1 NIST102505_1.xIs

$\begin{array}{lll}16951 & 16: 08: 54 & 0.1270 \\ 16952 & 16: 08: 55 & 0.1261 \\ 16953 & 16: 08: 56 & 0.1307 \\ 16954 & 16: 08: 57 & 0.1279 \\ 16955 & 16: 08: 58 & 0.1307 \\ 16956 & 16: 08: 59 & 0.1248 \\ 16957 & 16: 09: 00 & 0.1281 \\ 16958 & 16: 09: 01 & 0.1268 \\ 16959 & 16: 09: 02 & 0.1259 \\ 16960 & 16: 09: 03 & 0.1285 \\ 16961 & 16: 09: 04 & 0.1272 \\ 16962 & 16: 09: 05 & 0.1293 \\ 16963 & 16: 09: 06 & 0.1237 \\ 16964 & 16: 09: 07 & 0.1287 \\ 16965 & 16: 09: 08 & 0.1248 \\ 16966 & 16: 09: 09 & 0.1298 \\ 16967 & 16: 09: 10 & 0.1255 \\ 16968 & 16: 09: 11 & 0.1286 \\ 16969 & 16: 09: 12 & 0.1254 \\ 16970 & 16: 09: 13 & 0.1265 \\ 16971 & 16: 09: 14 & 0.1253 \\ 16972 & 16: 09: 15 & 0.1265 \\ 16973 & 16: 09: 16 & 0.1272 \\ 16974 & 16: 09: 17 & 0.1230 \\ 16975 & 16: 09: 18 & 0.1272 \\ 16976 & 16: 09: 19 & 0.1251 \\ 16977 & 16: 09: 20 & 0.1275 \\ 16978 & 16: 09: 21 & 0.1258 \\ 16979 & 16: 09: 22 & 0.1278 \\ 16980 & 16: 09: 23 & 0.1276 \\ 16981 & 16: 09: 24 & 0.1271 \\ 16982 & 16: 09: 25 & 0.1245 \\ 16983 & 16: 09: 26 & 0.1246 \\ 16984 & 16: 09: 27 & 0.1269 \\ 16985 & 16: 09: 28 & 0.1245 \\ 16986 & 16: 09: 29 & 0.1252 \\ 16987 & 16: 09: 30 & 0.1208 \\ 16988 & 16: 09: 31 & 0.1274 \\ 16989 & 16: 09: 32 & 0.1234 \\ 16990 & 16: 09: 33 & 0.1270 \\ 16991 & 16: 09: 34 & 0.1244 \\ 16992 & 16: 09: 35 & 0.1299 \\ 16993 & 16: 09: 36 & 0.1272 \\ 16994 & 16: 09: 37 & 0.1280 \\ 16995 & 16: 09: 38 & 0.1265 \\ 16996 & 16: 09: 39 & 0.1255 \\ 16997 & 16: 09: 40 & 0.1269 \\ 16998 & 16: 09: 41 & 0.1247 \\ 16999 & 16: 09: 42 & 0.1284 \\ 17000 & 16: 09: 43 & 0.1241 \\ 17001 & 16: 09: 44 & 0.1287 \\ 17002 & 16: 09: 45 & 0.1263\end{array}$


Appendix A - Fuel Data Test 1 NIST102505_1.xls

$\begin{array}{lll}17003 & 16: 09: 46 & 0.1296 \\ 17004 & 16: 09: 47 & 0.1237 \\ 17005 & 16: 09: 48 & 0.1277 \\ 17006 & 16: 09: 49 & 0.1257 \\ 17007 & 16: 09: 50 & 0.1274 \\ 17008 & 16: 09: 51 & 0.1244 \\ 17009 & 16: 09: 52 & 0.1246 \\ 17010 & 16: 09: 53 & 0.1270 \\ 17011 & 16: 09: 54 & 0.1231 \\ 17012 & 16: 09: 55 & 0.1267 \\ 17013 & 16: 09: 56 & 0.1241 \\ 17014 & 16: 09: 57 & 0.1283 \\ 17015 & 16: 09: 58 & 0.1239 \\ 17016 & 16: 09: 59 & 0.1284 \\ 17017 & 16: 10: 00 & 0.1279 \\ 17018 & 16: 10: 01 & 0.1269 \\ 17019 & 16: 10: 02 & 0.1234 \\ 17020 & 16: 10: 03 & 0.1244 \\ 17021 & 16: 10: 04 & 0.1273 \\ 17022 & 16: 10: 05 & 0.1259 \\ 17023 & 16: 10: 06 & 0.1276 \\ 17024 & 16: 10: 07 & 0.1240 \\ 17025 & 16: 10: 08 & 0.1289 \\ 17026 & 16: 10: 09 & 0.1252 \\ 17027 & 16: 10: 10 & 0.1278 \\ 17028 & 16: 10: 11 & 0.1266 \\ 17029 & 16: 10: 12 & 0.1312 \\ 17030 & 16: 10: 13 & 0.1267 \\ 17031 & 16: 10: 14 & 0.1287 \\ 17032 & 16: 10: 15 & 0.1269 \\ 17033 & 16: 10: 16 & 0.1258 \\ 17034 & 16: 10: 17 & 0.1272 \\ 17035 & 16: 10: 18 & 0.1231 \\ 17036 & 16: 10: 19 & 0.1263 \\ 17037 & 16: 10: 20 & 0.1215 \\ 17038 & 16: 10: 21 & 0.1281 \\ 17039 & 16: 10: 22 & 0.1251 \\ 17040 & 16: 10: 23 & 0.1291 \\ 17041 & 16: 10: 24 & 0.1253 \\ 17042 & 16: 10: 25 & 0.1295 \\ 17043 & 16: 10: 26 & 0.1256 \\ 17044 & 16: 10: 27 & 0.1269 \\ 17045 & 16: 10: 28 & 0.1260 \\ 17046 & 16: 10: 29 & 0.1264 \\ 17047 & 16: 10: 30 & 0.1257 \\ 17048 & 16: 10: 31 & 0.1227 \\ 17049 & 16: 10: 32 & 0.1288 \\ 17050 & 16: 10: 33 & 0.1237 \\ 17051 & 16: 10: 34 & 0.1286 \\ 17052 & 16: 10: 35 & 0.1250 \\ 17053 & 16: 10: 36 & 0.1303 \\ 17054 & 16: 10: 37 & 0.1261\end{array}$


Appendix A - Fuel Data Test 1 NIST102505_1.xls

$\begin{array}{lll}17055 & 16: 10: 38 & 0.1278 \\ 17056 & 16: 10: 39 & 0.1248 \\ 17057 & 16: 10: 40 & 0.1269 \\ 17058 & 16: 10: 41 & 0.1278 \\ 17059 & 16: 10: 42 & 0.1236 \\ 17060 & 16: 10: 43 & 0.1268 \\ 17061 & 16: 10: 44 & 0.1225 \\ 17062 & 16: 10: 45 & 0.1269 \\ 17063 & 16: 10: 46 & 0.1243 \\ 17064 & 16: 10: 47 & 0.1286 \\ 17065 & 16: 10: 48 & 0.1244 \\ 17066 & 16: 10: 49 & 0.1274 \\ 17067 & 16: 10: 50 & 0.1282 \\ 17068 & 16: 10: 51 & 0.1282 \\ 17069 & 16: 10: 52 & 0.1273 \\ 17070 & 16: 10: 53 & 0.1252 \\ 17071 & 16: 10: 54 & 0.1299 \\ 17072 & 16: 10: 55 & 0.1257 \\ 17073 & 16: 10: 56 & 0.1263 \\ 17074 & 16: 10: 57 & 0.1225 \\ 17075 & 16: 10: 58 & 0.1293 \\ 17076 & 16: 10: 59 & 0.1253 \\ 17077 & 16: 11: 00 & 0.1293 \\ 17078 & 16: 11: 01 & 0.1251 \\ 17079 & 16: 11: 02 & 0.1300 \\ 17080 & 16: 11: 03 & 0.1256 \\ 17081 & 16: 11: 04 & 0.1259 \\ 17082 & 16: 11: 05 & 0.1247 \\ 17083 & 16: 11: 06 & 0.1247 \\ 17084 & 16: 11: 07 & 0.1265 \\ 17085 & 16: 11: 08 & 0.1228 \\ 17086 & 16: 11: 09 & 0.1277 \\ 17087 & 16: 11: 10 & 0.1230 \\ 17088 & 16: 11: 11 & 0.1278 \\ 17089 & 16: 11: 12 & 0.1248 \\ 17090 & 16: 11: 13 & 0.1307 \\ 17091 & 16: 11: 14 & 0.1265 \\ 17092 & 16: 11: 15 & 0.1306 \\ 17093 & 16: 11: 16 & 0.1192 \\ 17094 & 16: 11: 17 & 0.0722 \\ 17095 & 16: 11: 18 & 0.0343 \\ 17096 & 16: 11: 19 & 0.0235 \\ 17097 & 16: 11: 20 & 0.0169 \\ 17098 & 16: 11: 21 & 0.0055 \\ 17099 & 16: 11: 22 & 0.0003 \\ 17100 & 16: 11: 23 & 0.0004 \\ 17101 & 16: 11: 24 & 0.0005 \\ 17102 & 16: 11: 25 & 0.0002 \\ 17103 & 16: 11: 26 & 0.0001 \\ 17104 & 16: 11: 27 & 0.0002 \\ 17105 & 16: 11: 28 & 0.0004 \\ 17106 & 16: 11: 29 & 0.0004\end{array}$


Appendix A - Fuel Data Test 1 NIST102505_1.xls

\begin{tabular}{lll}
17107 & $16: 11: 30$ & 0.0001 \\
17108 & $16: 11: 31$ & 0.0001 \\
17109 & $16: 11: 32$ & 0.0003 \\
17110 & $16: 11: 33$ & 0.0004 \\
17111 & $16: 11: 34$ & 0.0001 \\
17112 & $16: 11: 35$ & 0.0001 \\
17113 & $16: 11: 36$ & 0.0002 \\
17114 & $16: 11: 37$ & 0.0004 \\
17115 & $16: 11: 38$ & 0.0003 \\
17116 & $16: 11: 39$ & 0.0001 \\
17117 & $16: 11: 40$ & 0.0001 \\
17118 & $16: 11: 41$ & 0.0003 \\
17119 & $16: 11: 42$ & 0.0004 \\
17120 & $16: 11: 43$ & 0.0002 \\
17121 & $16: 11: 44$ & 0.0000 \\
17122 & $16: 11: 45$ & 0.0002 \\
17123 & $16: 11: 46$ & 0.0004 \\
17124 & $16: 11: 47$ & 0.0003 \\
17125 & $16: 11: 48$ & 0.0001 \\
17126 & $16: 11: 49$ & 0.0001 \\
17127 & $16: 11: 50$ & 0.0003 \\
17128 & $16: 11: 51$ & 0.0004 \\
17129 & $16: 11: 52$ & 0.0002 \\
17130 & $16: 11: 53$ & 0.0001 \\
17131 & $16: 11: 54$ & 0.0002 \\
17132 & $16: 11: 55$ & 0.0004 \\
17133 & $16: 11: 56$ & 0.0003 \\
17134 & $16: 11: 57$ & 0.0001 \\
17135 & $16: 11: 58$ & 0.0001 \\
17136 & $16: 11: 59$ & 0.0004 \\
17137 & $16: 12: 00$ & 0.0004 \\
17138 & $16: 12: 01$ & 0.0002 \\
17139 & $16: 12: 02$ & 0.0001 \\
17140 & $16: 12: 03$ & 0.0002 \\
17141 & $16: 12: 04$ & 0.0004 \\
17142 & $16: 12: 05$ & 0.0003 \\
17143 & $16: 12: 06$ & 0.0001 \\
17144 & $16: 12: 07$ & 0.0001 \\
17145 & $16: 12: 08$ & 0.0003 \\
17146 & $16: 12: 09$ & 0.0003 \\
17147 & $16: 12: 10$ & 0.0002 \\
17148 & $16: 12: 11$ & 0.0001 \\
17149 & $16: 12: 12$ & 0.0002 \\
17150 & $16: 12: 13$ & 0.0005 \\
17151 & $16: 12: 14$ & 0.0002 \\
17152 & $16: 12: 15$ & 0.0001 \\
17153 & $16: 12: 16$ & 0.0001 \\
17154 & $16: 12: 17$ & 0.0004 \\
17155 & $16: 12: 18$ & 0.0003 \\
17156 & $16: 12: 19$ & 0.0002 \\
17157 & $16: 12: 20$ & 0.0001 \\
17158 & $16: 12: 21$ & 0.0002 \\
\hline & &
\end{tabular}


APPENDIX B. - Laser Data Test 1 NIST 102505

Appendix $B$ is laser data from Test 1 in tabular format and is approximately 166 pages in length. Fuel data is included with electronic version of report on compact disk, but not routinely included with paper version of report. 
Appendix B - Laser Data Test 1 NIST102505_1.xls

Time from Ignition, s EDT Time $\quad$ //lo East $\quad$ //lo West

$\begin{array}{rlll}0 & 11: 26: 23 & 1.0249 & 1.0140 \\ 2 & 11: 26: 25 & 1.0352 & 1.0053 \\ 4 & 11: 26: 27 & 0.9837 & 0.9942 \\ 6 & 11: 26: 29 & 1.0016 & 0.9995 \\ 8 & 11: 26: 31 & 0.9708 & 0.9841 \\ 10 & 11: 26: 33 & 0.9719 & 0.9946 \\ 12 & 11: 26: 35 & 1.0000 & 0.9971 \\ 14 & 11: 26: 37 & 0.9963 & 1.0144 \\ 16 & 11: 26: 39 & 1.0330 & 0.9727 \\ 18 & 11: 26: 41 & 1.0137 & 1.0095 \\ 20 & 11: 26: 43 & 1.0044 & 1.0242 \\ 22 & 11: 26: 45 & 0.9644 & 0.9719 \\ 24 & 11: 26: 47 & 0.9767 & 1.0171 \\ 26 & 11: 26: 49 & 1.0139 & 0.9971 \\ 28 & 11: 26: 51 & 1.0071 & 0.9942 \\ 30 & 11: 26: 53 & 0.9660 & 1.0124 \\ 32 & 11: 26: 55 & 0.9435 & 1.0112 \\ 34 & 11: 26: 57 & 0.9951 & 1.0009 \\ 36 & 11: 26: 59 & 0.9573 & 0.9880 \\ 38 & 11: 27: 01 & 1.0063 & 0.9974 \\ 40 & 11: 27: 03 & 1.0256 & 1.0166 \\ 42 & 11: 27: 05 & 1.0084 & 0.9941 \\ 44 & 11: 27: 07 & 1.0162 & 1.0041 \\ 46 & 11: 27: 09 & 0.9890 & 0.9883 \\ 48 & 11: 27: 11 & 1.0030 & 1.0009 \\ 50 & 11: 27: 13 & 0.9953 & 0.9865 \\ 52 & 11: 27: 15 & 1.0276 & 1.0067 \\ 54 & 11: 27: 17 & 0.9855 & 1.0238 \\ 56 & 11: 27: 19 & 1.0088 & 1.0364 \\ 58 & 11: 27: 21 & 0.9963 & 1.0034 \\ 60 & 11: 27: 23 & 1.0033 & 0.9760 \\ 62 & 11: 27: 25 & 1.0087 & 0.9839 \\ 64 & 11: 27: 27 & 1.0219 & 0.9933 \\ 66 & 11: 27: 29 & 0.9513 & 0.9954 \\ 68 & 11: 27: 31 & 1.0246 & 0.9886 \\ 70 & 11: 27: 33 & 0.8991 & 0.9432 \\ 72 & 11: 27: 35 & 0.7537 & 0.7385 \\ 74 & 11: 27: 37 & 0.9088 & 0.8456 \\ 76 & 11: 27: 39 & 0.9364 & 0.6799 \\ 78 & 11: 27: 41 & 0.8425 & 0.6347 \\ 80 & 11: 27: 43 & 0.5967 & 0.6497 \\ 82 & 11: 27: 45 & 0.6286 & 0.7257 \\ 84 & 11: 27: 47 & 0.6022 & 0.4621 \\ 86 & 11: 27: 49 & 0.4487 & 0.5336 \\ 88 & 11: 27: 51 & 0.4743 & 0.5240 \\ 90 & 11: 27: 53 & 0.4501 & 0.4871 \\ 92 & 11: 27: 55 & 0.3628 & 0.4763 \\ 94 & 11: 27: 57 & 0.3520 & 0.4988 \\ 96 & 11: 27: 59 & 0.4209 & 0.4237 \\ 100 & 11: 28: 01 & 0.4741 & 0.3467 \\ & & 0.3987 & 0.4053\end{array}$

B - 1 


$\begin{array}{llll}102 & 11: 28: 05 & 0.2943 & 0.4280 \\ 104 & 11: 28: 07 & 0.4007 & 0.3206 \\ 106 & 11: 28: 09 & 0.3813 & 0.3903 \\ 108 & 11: 28: 11 & 0.1643 & 0.2890 \\ 110 & 11: 28: 13 & 0.2607 & 0.2479 \\ 112 & 11: 28: 15 & 0.1493 & 0.2288 \\ 114 & 11: 28: 17 & 0.1588 & 0.2447 \\ 116 & 11: 28: 19 & 0.1958 & 0.2243 \\ 118 & 11: 28: 21 & 0.1392 & 0.3139 \\ 120 & 11: 28: 23 & 0.1228 & 0.1440 \\ 122 & 11: 28: 25 & 0.1627 & 0.2099 \\ 124 & 11: 28: 27 & 0.1427 & 0.1707 \\ 126 & 11: 28: 29 & 0.2699 & 0.1092 \\ 128 & 11: 28: 31 & 0.1273 & 0.1771 \\ 130 & 11: 28: 33 & 0.1501 & 0.2311 \\ 132 & 11: 28: 35 & 0.2553 & 0.1731 \\ 134 & 11: 28: 37 & 0.1192 & 0.1477 \\ 136 & 11: 28: 39 & 0.0743 & 0.1003 \\ 138 & 11: 28: 41 & 0.1175 & 0.1097 \\ 140 & 11: 28: 43 & 0.1349 & 0.1392 \\ 142 & 11: 28: 45 & 0.1241 & 0.1669 \\ 144 & 11: 28: 47 & 0.2273 & 0.2084 \\ 146 & 11: 28: 49 & 0.1197 & 0.2537 \\ 148 & 11: 28: 51 & 0.1705 & 0.2220 \\ 150 & 11: 28: 53 & 0.0612 & 0.2240 \\ 152 & 11: 28: 55 & 0.0975 & 0.0622 \\ 154 & 11: 28: 57 & 0.0658 & 0.0266 \\ 156 & 11: 28: 59 & 0.1368 & 0.0884 \\ 158 & 11: 29: 01 & 0.1094 & 0.1146 \\ 160 & 11: 29: 03 & 0.0597 & 0.1650 \\ 162 & 11: 29: 05 & 0.1056 & 0.1313 \\ 164 & 11: 29: 07 & 0.0582 & 0.0717 \\ 166 & 11: 29: 09 & 0.0280 & 0.1537 \\ 168 & 11: 29: 11 & 0.0545 & 0.1038 \\ 170 & 11: 29: 13 & 0.0523 & 0.0985 \\ 172 & 11: 29: 15 & 0.0280 & 0.0816 \\ 174 & 11: 29: 17 & 0.0229 & 0.0435 \\ 176 & 11: 29: 19 & 0.0524 & 0.0368 \\ 178 & 11: 29: 21 & 0.1204 & 0.0890 \\ 180 & 11: 29: 23 & 0.0305 & 0.0545 \\ 182 & 11: 29: 25 & 0.0070 & 0.0468 \\ 184 & 11: 29: 27 & 0.0149 & 0.0515 \\ 186 & 11: 29: 29 & 0.0226 & 0.0718 \\ 188 & 11: 29: 31 & 0.0232 & 0.1178 \\ 190 & 11: 29: 33 & 0.0325 & 0.1368 \\ 192 & 11: 29: 35 & 0.0274 & 0.0275 \\ 194 & 11: 29: 37 & 0.0180 & 0.0148 \\ 196 & 11: 29: 39 & 0.0370 & 0.0629 \\ 198 & 11: 29: 41 & 0.0467 & 0.0598 \\ 200 & 11: 29: 43 & 0.0149 & 0.0504 \\ 202 & 11: 29: 45 & 0.0171 & 0.0348 \\ 204 & 11: 29: 47 & 0.1754 & 0.1576\end{array}$




$\begin{array}{llll}206 & 11: 29: 49 & 0.0829 & 0.0115 \\ 208 & 11: 29: 51 & 0.0706 & 0.1556 \\ 210 & 11: 29: 53 & 0.0484 & 0.0762 \\ 212 & 11: 29: 55 & 0.0224 & 0.0437 \\ 214 & 11: 29: 57 & 0.0338 & 0.0613 \\ 216 & 11: 29: 59 & 0.0277 & 0.0290 \\ 218 & 11: 30: 01 & 0.0301 & 0.1390 \\ 220 & 11: 30: 03 & 0.0555 & 0.2013 \\ 222 & 11: 30: 05 & 0.0132 & 0.1117 \\ 224 & 11: 30: 07 & 0.0615 & 0.0627 \\ 226 & 11: 30: 09 & 0.0430 & 0.0993 \\ 228 & 11: 30: 11 & 0.0842 & 0.0691 \\ 230 & 11: 30: 13 & 0.0472 & 0.0848 \\ 232 & 11: 30: 15 & 0.0552 & 0.2801 \\ 234 & 11: 30: 17 & 0.2151 & 0.1751 \\ 236 & 11: 30: 19 & 0.0648 & 0.0771 \\ 238 & 11: 30: 21 & 0.0660 & 0.0432 \\ 240 & 11: 30: 23 & 0.0610 & 0.0616 \\ 242 & 11: 30: 25 & 0.0694 & 0.1211 \\ 244 & 11: 30: 27 & 0.0606 & 0.1309 \\ 246 & 11: 30: 29 & 0.0807 & 0.1897 \\ 248 & 11: 30: 31 & 0.0338 & 0.0495 \\ 250 & 11: 30: 33 & 0.0400 & 0.0754 \\ 252 & 11: 30: 35 & 0.0328 & 0.0704 \\ 254 & 11: 30: 37 & 0.0202 & 0.0604 \\ 256 & 11: 30: 39 & 0.0243 & 0.0372 \\ 258 & 11: 30: 41 & 0.0301 & 0.1613 \\ 260 & 11: 30: 43 & 0.0351 & 0.1057 \\ 262 & 11: 30: 45 & 0.0785 & 0.0521 \\ 264 & 11: 30: 47 & 0.0460 & 0.4629 \\ 266 & 11: 30: 49 & 0.0770 & 0.1082 \\ 268 & 11: 30: 51 & 0.0470 & 0.0499 \\ 270 & 11: 30: 53 & 0.0437 & 0.0451 \\ 272 & 11: 30: 55 & 0.0645 & 0.1620 \\ 274 & 11: 30: 57 & 0.1018 & 0.0248 \\ 276 & 11: 30: 59 & 0.1189 & 0.0968 \\ 278 & 11: 31: 01 & 0.0164 & 0.0440 \\ 280 & 11: 31: 03 & 0.1655 & 0.1476 \\ 282 & 11: 31: 05 & 0.0500 & 0.0898 \\ 284 & 11: 31: 07 & 0.0790 & 0.0440 \\ 286 & 11: 31: 09 & 0.0850 & 0.0553 \\ 288 & 11: 31: 11 & 0.2414 & 0.1666 \\ 290 & 11: 31: 13 & 0.1544 & 0.1412 \\ 292 & 11: 31: 15 & 0.0422 & 0.0738 \\ 294 & 11: 31: 17 & 0.0688 & 0.1499 \\ 296 & 11: 31: 19 & 0.0981 & 0.1494 \\ 298 & 11: 31: 21 & 0.0713 & 0.1076 \\ 300 & 11: 31: 23 & 0.0834 & 0.0735 \\ 302 & 11: 31: 25 & 0.1190 & 0.1270 \\ 304 & 11: 31: 27 & 0.0261 & 0.1531 \\ 306 & 11: 31: 29 & 0.0762 & 0.0945 \\ 308 & 11: 31: 31 & 0.0181 & 0.0392\end{array}$


Appendix B - Laser Data Test 1 NIST102505_1.xls

$\begin{array}{llll}310 & 11: 31: 33 & 0.0570 & 0.0454 \\ 312 & 11: 31: 35 & 0.0561 & 0.1191 \\ 314 & 11: 31: 37 & 0.0330 & 0.1178 \\ 316 & 11: 31: 39 & 0.1260 & 0.0762 \\ 318 & 11: 31: 41 & 0.0249 & 0.3149 \\ 320 & 11: 31: 43 & 0.0211 & 0.0627 \\ 322 & 11: 31: 45 & 0.0221 & 0.2042 \\ 324 & 11: 31: 47 & 0.0597 & 0.1290 \\ 326 & 11: 31: 49 & 0.1497 & 0.2371 \\ 328 & 11: 31: 51 & 0.0678 & 0.0541 \\ 330 & 11: 31: 53 & 0.1239 & 0.0366 \\ 332 & 11: 31: 55 & 0.0264 & 0.0767 \\ 334 & 11: 31: 57 & 0.0872 & 0.0331 \\ 336 & 11: 31: 59 & 0.0852 & 0.0704 \\ 338 & 11: 32: 01 & 0.1070 & 0.1463 \\ 340 & 11: 32: 03 & 0.0967 & 0.1096 \\ 342 & 11: 32: 05 & 0.0505 & 0.1249 \\ 344 & 11: 32: 07 & 0.1192 & 0.2567 \\ 346 & 11: 32: 09 & 0.0267 & 0.0836 \\ 348 & 11: 32: 11 & 0.3514 & 0.1263 \\ 350 & 11: 32: 13 & 0.0540 & 0.0610 \\ 352 & 11: 32: 15 & 0.1321 & 0.2232 \\ 354 & 11: 32: 17 & 0.1342 & 0.1911 \\ 356 & 11: 32: 19 & 0.0844 & 0.2481 \\ 358 & 11: 32: 21 & 0.0849 & 0.3157 \\ 360 & 11: 32: 23 & 0.1481 & 0.3102 \\ 362 & 11: 32: 25 & 0.0440 & 0.2242 \\ 364 & 11: 32: 27 & 0.2746 & 0.1230 \\ 366 & 11: 32: 29 & 0.1145 & 0.1411 \\ 368 & 11: 32: 31 & 0.1075 & 0.1242 \\ 370 & 11: 32: 33 & 0.1503 & 0.0778 \\ 372 & 11: 32: 35 & 0.0862 & 0.0610 \\ 374 & 11: 32: 37 & 0.0342 & 0.1145 \\ 376 & 11: 32: 39 & 0.2053 & 0.1503 \\ 378 & 11: 32: 41 & 0.0522 & 0.1824 \\ 380 & 11: 32: 43 & 0.1237 & 0.2019 \\ 382 & 11: 32: 45 & 0.1160 & 0.1464 \\ 384 & 11: 32: 47 & 0.1076 & 0.5858 \\ 386 & 11: 32: 49 & 0.1757 & 0.1835 \\ 388 & 11: 32: 51 & 0.0796 & 0.0543 \\ 390 & 11: 32: 53 & 0.1002 & 0.2304 \\ 392 & 11: 32: 55 & 0.2639 & 0.1423 \\ 394 & 11: 32: 57 & 0.1337 & 0.1343 \\ 396 & 11: 32: 59 & 0.1051 & 0.1646 \\ 398 & 11: 33: 01 & 0.0596 & 0.1684 \\ 400 & 11: 33: 03 & 0.0401 & 0.1107 \\ 402 & 11: 33: 05 & 0.0198 & 0.1908 \\ 404 & 11: 33: 07 & 0.1273 & 0.3160 \\ 406 & 11: 33: 09 & 0.1586 & 0.2174 \\ 408 & 11: 33: 11 & 0.1168 & 0.1715 \\ 410 & 11: 33: 13 & 0.0789 & 0.1383 \\ 412 & 11: 33: 15 & 0.1080 & 0.3957\end{array}$

B - 4 


$\begin{array}{llll}414 & 11: 33: 17 & 0.0818 & 0.1729 \\ 416 & 11: 33: 19 & 0.0802 & 0.2543 \\ 418 & 11: 33: 21 & 0.0716 & 0.3886 \\ 420 & 11: 33: 23 & 0.0755 & 0.0944 \\ 422 & 11: 33: 25 & 0.0451 & 0.0709 \\ 424 & 11: 33: 27 & 0.0915 & 0.1097 \\ 426 & 11: 33: 29 & 0.1763 & 0.1708 \\ 428 & 11: 33: 31 & 0.0693 & 0.0860 \\ 430 & 11: 33: 33 & 0.1004 & 0.1584 \\ 432 & 11: 33: 35 & 0.1322 & 0.2051 \\ 434 & 11: 33: 37 & 0.0294 & 0.1334 \\ 436 & 11: 33: 39 & 0.0460 & 0.1869 \\ 438 & 11: 33: 41 & 0.1235 & 0.3931 \\ 440 & 11: 33: 43 & 0.0888 & 0.1168 \\ 442 & 11: 33: 45 & 0.1411 & 0.1388 \\ 444 & 11: 33: 47 & 0.1430 & 0.2383 \\ 446 & 11: 33: 49 & 0.0892 & 0.1693 \\ 448 & 11: 33: 51 & 0.0670 & 0.1237 \\ 450 & 11: 33: 53 & 0.0706 & 0.0657 \\ 452 & 11: 33: 55 & 0.0602 & 0.1096 \\ 454 & 11: 33: 57 & 0.0958 & 0.1995 \\ 456 & 11: 33: 59 & 0.0414 & 0.1179 \\ 458 & 11: 34: 01 & 0.0685 & 0.2080 \\ 460 & 11: 34: 03 & 0.0677 & 0.1271 \\ 462 & 11: 34: 05 & 0.0799 & 0.1158 \\ 464 & 11: 34: 07 & 0.1383 & 0.0940 \\ 466 & 11: 34: 09 & 0.1033 & 0.0801 \\ 468 & 11: 34: 11 & 0.1377 & 0.2053 \\ 470 & 11: 34: 13 & 0.0806 & 0.0879 \\ 472 & 11: 34: 15 & 0.0542 & 0.1393 \\ 474 & 11: 34: 17 & 0.0516 & 0.1556 \\ 476 & 11: 34: 19 & 0.0333 & 0.1008 \\ 478 & 11: 34: 21 & 0.0314 & 0.1546 \\ 480 & 11: 34: 23 & 0.1111 & 0.1892 \\ 482 & 11: 34: 25 & 0.0544 & 0.1445 \\ 484 & 11: 34: 27 & 0.0830 & 0.1201 \\ 486 & 11: 34: 29 & 0.0401 & 0.3494 \\ 488 & 11: 34: 31 & 0.0518 & 0.1348 \\ 490 & 11: 34: 33 & 0.0717 & 0.1271 \\ 492 & 11: 34: 35 & 0.1062 & 0.0393 \\ 494 & 11: 34: 37 & 0.0342 & 0.1069 \\ 496 & 11: 34: 39 & 0.1192 & 0.1820 \\ 498 & 11: 34: 41 & 0.1304 & 0.1932 \\ 500 & 11: 34: 43 & 0.0819 & 0.1380 \\ 502 & 11: 34: 45 & 0.0961 & 0.1454 \\ 504 & 11: 34: 47 & 0.0814 & 0.1570 \\ 506 & 11: 34: 49 & 0.0697 & 0.1232 \\ 508 & 11: 34: 51 & 0.0582 & 0.1700 \\ 510 & 11: 34: 53 & 0.0614 & 0.1134 \\ 512 & 11: 34: 55 & 0.0910 & 0.1095 \\ 514 & 11: 34: 57 & 0.0811 & 0.1715 \\ 516 & 11: 34: 59 & 0.0805 & 0.1552\end{array}$




$\begin{array}{llll}518 & 11: 35: 01 & 0.0548 & 0.1347 \\ 520 & 11: 35: 03 & 0.0437 & 0.0843 \\ 522 & 11: 35: 05 & 0.0514 & 0.1113 \\ 524 & 11: 35: 07 & 0.1182 & 0.2418 \\ 526 & 11: 35: 09 & 0.0429 & 0.1517 \\ 528 & 11: 35: 11 & 0.1032 & 0.3004 \\ 530 & 11: 35: 13 & 0.0651 & 0.2785 \\ 532 & 11: 35: 15 & 0.0566 & 0.2403 \\ 534 & 11: 35: 17 & 0.0525 & 0.1194 \\ 536 & 11: 35: 19 & 0.0863 & 0.2760 \\ 538 & 11: 35: 21 & 0.0798 & 0.3990 \\ 540 & 11: 35: 23 & 0.0570 & 0.2374 \\ 542 & 11: 35: 25 & 0.1848 & 0.2025 \\ 544 & 11: 35: 27 & 0.0389 & 0.1110 \\ 546 & 11: 35: 29 & 0.0586 & 0.1383 \\ 548 & 11: 35: 31 & 0.1233 & 0.1874 \\ 550 & 11: 35: 33 & 0.0330 & 0.1459 \\ 552 & 11: 35: 35 & 0.0572 & 0.1387 \\ 554 & 11: 35: 37 & 0.2716 & 0.2202 \\ 556 & 11: 35: 39 & 0.0651 & 0.1030 \\ 558 & 11: 35: 41 & 0.2051 & 0.2194 \\ 560 & 11: 35: 43 & 0.0740 & 0.2516 \\ 562 & 11: 35: 45 & 0.0536 & 0.1496 \\ 564 & 11: 35: 47 & 0.0470 & 0.1235 \\ 566 & 11: 35: 49 & 0.0695 & 0.0853 \\ 568 & 11: 35: 51 & 0.0669 & 0.0636 \\ 570 & 11: 35: 53 & 0.1810 & 0.3041 \\ 572 & 11: 35: 55 & 0.0673 & 0.1906 \\ 574 & 11: 35: 57 & 0.0764 & 0.0949 \\ 576 & 11: 35: 59 & 0.0774 & 0.4822 \\ 578 & 11: 36: 01 & 0.0800 & 0.1644 \\ 580 & 11: 36: 03 & 0.0897 & 0.1126 \\ 582 & 11: 36: 05 & 0.1114 & 0.3044 \\ 584 & 11: 36: 07 & 0.0462 & 0.1134 \\ 586 & 11: 36: 09 & 0.0904 & 0.1861 \\ 588 & 11: 36: 11 & 0.0361 & 0.1697 \\ 590 & 11: 36: 13 & 0.1172 & 0.3442 \\ 592 & 11: 36: 15 & 0.1813 & 0.1017 \\ 594 & 11: 36: 17 & 0.0291 & 0.1009 \\ 596 & 11: 36: 19 & 0.1246 & 0.1990 \\ 598 & 11: 36: 21 & 0.0609 & 0.5033 \\ 600 & 11: 36: 23 & 0.1248 & 0.0695 \\ 602 & 11: 36: 25 & 0.0870 & 0.0688 \\ 604 & 11: 36: 27 & 0.1130 & 0.1677 \\ 606 & 11: 36: 29 & 0.0484 & 0.0828 \\ 608 & 11: 36: 31 & 0.1048 & 0.1083 \\ 610 & 11: 36: 33 & 0.1003 & 0.1476 \\ 612 & 11: 36: 35 & 0.0430 & 0.2804 \\ 614 & 11: 36: 37 & 0.0754 & 0.1415 \\ 616 & 11: 36: 39 & 0.1565 & 0.1171 \\ 618 & 11: 36: 41 & 0.0501 & 0.1747 \\ 620 & 11: 36: 43 & 0.0307 & 0.1162\end{array}$




$\begin{array}{llll}622 & 11: 36: 45 & 0.0529 & 0.1834 \\ 624 & 11: 36: 47 & 0.0343 & 0.1815 \\ 626 & 11: 36: 49 & 0.0887 & 0.0956 \\ 628 & 11: 36: 51 & 0.0534 & 0.1277 \\ 630 & 11: 36: 53 & 0.0333 & 0.1911 \\ 632 & 11: 36: 55 & 0.1529 & 0.0837 \\ 634 & 11: 36: 57 & 0.0536 & 0.0995 \\ 636 & 11: 36: 59 & 0.0766 & 0.1750 \\ 638 & 11: 37: 01 & 0.0358 & 0.1482 \\ 640 & 11: 37: 03 & 0.0859 & 0.2769 \\ 642 & 11: 37: 05 & 0.2617 & 0.1619 \\ 644 & 11: 37: 07 & 0.0959 & 0.3183 \\ 646 & 11: 37: 09 & 0.0556 & 0.0829 \\ 648 & 11: 37: 11 & 0.1004 & 0.1774 \\ 650 & 11: 37: 13 & 0.1801 & 0.1836 \\ 652 & 11: 37: 15 & 0.0577 & 0.1480 \\ 654 & 11: 37: 17 & 0.0581 & 0.1742 \\ 656 & 11: 37: 19 & 0.0221 & 0.1424 \\ 658 & 11: 37: 21 & 0.0288 & 0.2345 \\ 660 & 11: 37: 23 & 0.0472 & 0.0582 \\ 662 & 11: 37: 25 & 0.0495 & 0.0755 \\ 664 & 11: 37: 27 & 0.0820 & 0.1630 \\ 666 & 11: 37: 29 & 0.1514 & 0.3226 \\ 668 & 11: 37: 31 & 0.0982 & 0.1383 \\ 670 & 11: 37: 33 & 0.0734 & 0.1203 \\ 672 & 11: 37: 35 & 0.0660 & 0.1929 \\ 674 & 11: 37: 37 & 0.0481 & 0.1979 \\ 676 & 11: 37: 39 & 0.1005 & 0.1243 \\ 678 & 11: 37: 41 & 0.1050 & 0.1460 \\ 680 & 11: 37: 43 & 0.0482 & 0.2968 \\ 682 & 11: 37: 45 & 0.0644 & 0.3206 \\ 684 & 11: 37: 47 & 0.1236 & 0.1541 \\ 686 & 11: 37: 49 & 0.0685 & 0.1218 \\ 688 & 11: 37: 51 & 0.1857 & 0.1781 \\ 690 & 11: 37: 53 & 0.1434 & 0.1304 \\ 692 & 11: 37: 55 & 0.1193 & 0.2246 \\ 694 & 11: 37: 57 & 0.0757 & 0.1509 \\ 696 & 11: 37: 59 & 0.1757 & 0.3386 \\ 698 & 11: 38: 01 & 0.0709 & 0.1124 \\ 700 & 11: 38: 03 & 0.1314 & 0.0835 \\ 702 & 11: 38: 05 & 0.0741 & 0.1575 \\ 704 & 11: 38: 07 & 0.0984 & 0.3040 \\ 706 & 11: 38: 09 & 0.0350 & 0.0840 \\ 708 & 11: 38: 11 & 0.1194 & 0.1774 \\ 710 & 11: 38: 13 & 0.0401 & 0.1559 \\ 712 & 11: 38: 15 & 0.0430 & 0.1308 \\ 714 & 11: 38: 17 & 0.0264 & 0.2855 \\ 716 & 11: 38: 19 & 0.0616 & 0.1742 \\ 718 & 11: 38: 21 & 0.1556 & 0.0515 \\ 720 & 11: 38: 23 & 0.0711 & 0.0905 \\ 722 & 11: 38: 25 & 0.0288 & 0.0824 \\ 724 & 11: 38: 27 & 0.0823 & 0.1086\end{array}$




$\begin{array}{llll}726 & 11: 38: 29 & 0.1429 & 0.3142 \\ 728 & 11: 38: 31 & 0.0713 & 0.1431 \\ 730 & 11: 38: 33 & 0.1382 & 0.1865 \\ 732 & 11: 38: 35 & 0.1463 & 0.2996 \\ 734 & 11: 38: 37 & 0.2194 & 0.2274 \\ 736 & 11: 38: 39 & 0.0894 & 0.1257 \\ 738 & 11: 38: 41 & 0.1092 & 0.1429 \\ 740 & 11: 38: 43 & 0.0813 & 0.1454 \\ 742 & 11: 38: 45 & 0.0959 & 0.2368 \\ 744 & 11: 38: 47 & 0.1619 & 0.2064 \\ 746 & 11: 38: 49 & 0.0245 & 0.1297 \\ 748 & 11: 38: 51 & 0.1311 & 0.1725 \\ 750 & 11: 38: 53 & 0.0691 & 0.1417 \\ 752 & 11: 38: 55 & 0.0619 & 0.1198 \\ 754 & 11: 38: 57 & 0.0926 & 0.1977 \\ 756 & 11: 38: 59 & 0.1680 & 0.1619 \\ 758 & 11: 39: 01 & 0.0588 & 0.1053 \\ 760 & 11: 39: 03 & 0.0471 & 0.0807 \\ 762 & 11: 39: 05 & 0.0429 & 0.2445 \\ 764 & 11: 39: 07 & 0.0877 & 0.1290 \\ 766 & 11: 39: 09 & 0.0245 & 0.0988 \\ 768 & 11: 39: 11 & 0.2425 & 0.1406 \\ 770 & 11: 39: 13 & 0.0734 & 0.0563 \\ 772 & 11: 39: 15 & 0.0195 & 0.1585 \\ 774 & 11: 39: 17 & 0.0586 & 0.3021 \\ 776 & 11: 39: 19 & 0.0673 & 0.2195 \\ 778 & 11: 39: 21 & 0.0278 & 0.2989 \\ 780 & 11: 39: 23 & 0.0409 & 0.1796 \\ 782 & 11: 39: 25 & 0.1248 & 0.2671 \\ 784 & 11: 39: 27 & 0.0666 & 0.1414 \\ 786 & 11: 39: 29 & 0.0437 & 0.1374 \\ 788 & 11: 39: 31 & 0.0321 & 0.3163 \\ 790 & 11: 39: 33 & 0.1332 & 0.2464 \\ 792 & 11: 39: 35 & 0.1435 & 0.1274 \\ 794 & 11: 39: 37 & 0.0927 & 0.1187 \\ 796 & 11: 39: 39 & 0.1381 & 0.1320 \\ 798 & 11: 39: 41 & 0.0785 & 0.0582 \\ 800 & 11: 39: 43 & 0.1254 & 0.1858 \\ 802 & 11: 39: 45 & 0.0640 & 0.3126 \\ 804 & 11: 39: 47 & 0.1400 & 0.2181 \\ 806 & 11: 39: 49 & 0.1003 & 0.1978 \\ 808 & 11: 39: 51 & 0.0343 & 0.1423 \\ 810 & 11: 39: 53 & 0.1408 & 0.1679 \\ 812 & 11: 39: 55 & 0.0614 & 0.1954 \\ 814 & 11: 39: 57 & 0.0683 & 0.1758 \\ 816 & 11: 39: 59 & 0.0633 & 0.2171 \\ 818 & 11: 40: 01 & 0.0205 & 0.2087 \\ 820 & 11: 40: 03 & 0.0932 & 0.2530 \\ 822 & 11: 40: 05 & 0.0521 & 0.3255 \\ 824 & 11: 40: 07 & 0.0464 & 0.0543 \\ 826 & 11: 40: 09 & 0.0829 & 0.2844 \\ 828 & 11: 40: 11 & 0.0456 & 0.1529\end{array}$




$\begin{array}{llll}830 & 11: 40: 13 & 0.0745 & 0.1616 \\ 832 & 11: 40: 15 & 0.0346 & 0.1165 \\ 834 & 11: 40: 17 & 0.0508 & 0.1872 \\ 836 & 11: 40: 19 & 0.1173 & 0.2283 \\ 838 & 11: 40: 21 & 0.1154 & 0.4725 \\ 840 & 11: 40: 23 & 0.1405 & 0.2363 \\ 842 & 11: 40: 25 & 0.1451 & 0.2496 \\ 844 & 11: 40: 27 & 0.0972 & 0.2395 \\ 846 & 11: 40: 29 & 0.1205 & 0.2041 \\ 848 & 11: 40: 31 & 0.2263 & 0.2160 \\ 850 & 11: 40: 33 & 0.0882 & 0.4177 \\ 852 & 11: 40: 35 & 0.0676 & 0.1479 \\ 854 & 11: 40: 37 & 0.1078 & 0.1429 \\ 856 & 11: 40: 39 & 0.0621 & 0.2258 \\ 858 & 11: 40: 41 & 0.0788 & 0.1343 \\ 860 & 11: 40: 43 & 0.1340 & 0.0877 \\ 862 & 11: 40: 45 & 0.0763 & 0.2052 \\ 864 & 11: 40: 47 & 0.0851 & 0.1136 \\ 866 & 11: 40: 49 & 0.0808 & 0.1293 \\ 868 & 11: 40: 51 & 0.0515 & 0.1232 \\ 870 & 11: 40: 53 & 0.0465 & 0.3160 \\ 872 & 11: 40: 55 & 0.0533 & 0.2385 \\ 874 & 11: 40: 57 & 0.1580 & 0.1013 \\ 876 & 11: 40: 59 & 0.1233 & 0.1082 \\ 878 & 11: 41: 01 & 0.0681 & 0.1437 \\ 880 & 11: 41: 03 & 0.0407 & 0.2512 \\ 882 & 11: 41: 05 & 0.0873 & 0.1616 \\ 884 & 11: 41: 07 & 0.1038 & 0.3615 \\ 886 & 11: 41: 09 & 0.0918 & 0.1657 \\ 888 & 11: 41: 11 & 0.1067 & 0.1285 \\ 890 & 11: 41: 13 & 0.0515 & 0.2932 \\ 892 & 11: 41: 15 & 0.0227 & 0.2075 \\ 894 & 11: 41: 17 & 0.0434 & 0.1057 \\ 896 & 11: 41: 19 & 0.0619 & 0.3678 \\ 898 & 11: 41: 21 & 0.0536 & 0.1527 \\ 900 & 11: 41: 23 & 0.1239 & 0.2514 \\ 902 & 11: 41: 25 & 0.1567 & 0.0792 \\ 904 & 11: 41: 27 & 0.0550 & 0.1470 \\ 906 & 11: 41: 29 & 0.2644 & 0.3634 \\ 908 & 11: 41: 31 & 0.2941 & 0.1488 \\ 910 & 11: 41: 33 & 0.3272 & 0.1225 \\ 912 & 11: 41: 35 & 0.0484 & 0.0777 \\ 914 & 11: 41: 37 & 0.1047 & 0.0770 \\ 916 & 11: 41: 39 & 0.1024 & 0.1283 \\ 918 & 11: 41: 41 & 0.0963 & 0.1303 \\ 920 & 11: 41: 43 & 0.0690 & 0.1735 \\ 922 & 11: 41: 45 & 0.2346 & 0.1166 \\ 924 & 11: 41: 47 & 0.0618 & 0.2910 \\ 926 & 11: 41: 49 & 0.0518 & 0.1327 \\ 928 & 11: 41: 51 & 0.0410 & 0.1861 \\ 930 & 11: 41: 53 & 0.1231 & 0.1799 \\ 932 & 11: 41: 55 & 0.0351 & 0.1273\end{array}$




$\begin{array}{rlll}934 & 11: 41: 57 & 0.0514 & 0.1372 \\ 936 & 11: 41: 59 & 0.0621 & 0.1742 \\ 938 & 11: 42: 01 & 0.0840 & 0.1325 \\ 940 & 11: 42: 03 & 0.0516 & 0.1725 \\ 942 & 11: 42: 05 & 0.0131 & 0.1197 \\ 944 & 11: 42: 07 & 0.1549 & 0.2034 \\ 946 & 11: 42: 09 & 0.0876 & 0.1683 \\ 948 & 11: 42: 11 & 0.0409 & 0.2659 \\ 950 & 11: 42: 13 & 0.1115 & 0.0998 \\ 952 & 11: 42: 15 & 0.0292 & 0.1386 \\ 954 & 11: 42: 17 & 0.0413 & 0.1259 \\ 956 & 11: 42: 19 & 0.1253 & 0.1292 \\ 958 & 11: 42: 21 & 0.1105 & 0.2134 \\ 960 & 11: 42: 23 & 0.1383 & 0.1489 \\ 962 & 11: 42: 25 & 0.0435 & 0.0798 \\ 964 & 11: 42: 27 & 0.0376 & 0.2885 \\ 966 & 11: 42: 29 & 0.0456 & 0.2075 \\ 968 & 11: 42: 31 & 0.0697 & 0.0691 \\ 970 & 11: 42: 33 & 0.1094 & 0.1525 \\ 972 & 11: 42: 35 & 0.1322 & 0.1848 \\ 974 & 11: 42: 37 & 0.0584 & 0.0723 \\ 976 & 11: 42: 39 & 0.0916 & 0.2769 \\ 978 & 11: 42: 41 & 0.1092 & 0.1494 \\ 980 & 11: 42: 43 & 0.1352 & 0.0876 \\ 982 & 11: 42: 45 & 0.0928 & 0.2647 \\ 984 & 11: 42: 47 & 0.1734 & 0.0692 \\ 986 & 11: 42: 49 & 0.1009 & 0.1219 \\ 988 & 11: 42: 51 & 0.0782 & 0.2757 \\ 990 & 11: 42: 53 & 0.0509 & 0.0966 \\ 992 & 11: 42: 55 & 0.0230 & 0.1336 \\ 994 & 11: 42: 57 & 0.0962 & 0.2994 \\ 996 & 11: 42: 59 & 0.1687 & 0.0965 \\ 998 & 11: 43: 01 & 0.0655 & 0.2289 \\ 1000 & 11: 43: 03 & 0.0683 & 0.1008 \\ 1002 & 11: 43: 05 & 0.1316 & 0.0893 \\ 1004 & 11: 43: 07 & 0.0686 & 0.1437 \\ 1006 & 11: 43: 09 & 0.0499 & 0.1342 \\ 1008 & 11: 43: 11 & 0.0565 & 0.2252 \\ 1010 & 11: 43: 13 & 0.0259 & 0.1681 \\ 1012 & 11: 43: 15 & 0.1340 & 0.2908 \\ 1014 & 11: 43: 17 & 0.0675 & 0.0659 \\ 1016 & 11: 43: 19 & 0.0792 & 0.2447 \\ 1018 & 11: 43: 21 & 0.0625 & 0.2650 \\ 1020 & 11: 43: 23 & 0.0490 & 0.1545 \\ 1022 & 11: 43: 25 & 0.0578 & 0.1031 \\ 1024 & 11: 43: 27 & 0.0671 & 0.1372 \\ 1026 & 11: 43: 29 & 0.0898 & 0.2590 \\ 1028 & 11: 43: 31 & 0.0562 & 0.1603 \\ 1030 & 11: 43: 33 & 0.0338 & 0.1034 \\ 1032 & 11: 43: 35 & 0.0556 & 0.0620 \\ 1034 & 11: 43: 37 & 0.1318 & 0.1128 \\ 1036 & 11: 43: 39 & 0.1565 & 0.1505\end{array}$




$\begin{array}{llll}1038 & 11: 43: 41 & 0.1093 & 0.3782 \\ 1040 & 11: 43: 43 & 0.1107 & 0.1306 \\ 1042 & 11: 43: 45 & 0.2008 & 0.0561 \\ 1044 & 11: 43: 47 & 0.0967 & 0.2228 \\ 1046 & 11: 43: 49 & 0.1790 & 0.2212 \\ 1048 & 11: 43: 51 & 0.0798 & 0.1102 \\ 1050 & 11: 43: 53 & 0.0572 & 0.1116 \\ 1052 & 11: 43: 55 & 0.0740 & 0.2981 \\ 1054 & 11: 43: 57 & 0.0217 & 0.1665 \\ 1056 & 11: 43: 59 & 0.0168 & 0.1833 \\ 1058 & 11: 44: 01 & 0.1447 & 0.1750 \\ 1060 & 11: 44: 03 & 0.0424 & 0.1301 \\ 1062 & 11: 44: 05 & 0.0421 & 0.0807 \\ 1064 & 11: 44: 07 & 0.0804 & 0.2506 \\ 1066 & 11: 44: 09 & 0.1347 & 0.1170 \\ 1068 & 11: 44: 11 & 0.1799 & 0.2420 \\ 1070 & 11: 44: 13 & 0.0478 & 0.0785 \\ 1072 & 11: 44: 15 & 0.3098 & 0.2241 \\ 1074 & 11: 44: 17 & 0.1577 & 0.1172 \\ 1076 & 11: 44: 19 & 0.2178 & 0.0683 \\ 1078 & 11: 44: 21 & 0.2670 & 0.1409 \\ 1080 & 11: 44: 23 & 0.1306 & 0.2974 \\ 1082 & 11: 44: 25 & 0.1586 & 0.2185 \\ 1084 & 11: 44: 27 & 0.0413 & 0.1191 \\ 1086 & 11: 44: 29 & 0.1131 & 0.3082 \\ 1088 & 11: 44: 31 & 0.1121 & 0.1732 \\ 1090 & 11: 44: 33 & 0.1508 & 0.1506 \\ 1092 & 11: 44: 35 & 0.1272 & 0.1744 \\ 1094 & 11: 44: 37 & 0.0827 & 0.1417 \\ 1096 & 11: 44: 39 & 0.0809 & 0.1816 \\ 1098 & 11: 44: 41 & 0.0862 & 0.1117 \\ 1100 & 11: 44: 43 & 0.0763 & 0.3135 \\ 1102 & 11: 44: 45 & 0.1232 & 0.3030 \\ 1104 & 11: 44: 47 & 0.0271 & 0.2027 \\ 1106 & 11: 44: 49 & 0.0968 & 0.2142 \\ 1108 & 11: 44: 51 & 0.1353 & 0.1470 \\ 1110 & 11: 44: 53 & 0.1416 & 0.1254 \\ 1112 & 11: 44: 55 & 0.0469 & 0.0557 \\ 1114 & 11: 44: 57 & 0.1910 & 0.2653 \\ 1116 & 11: 44: 59 & 0.0393 & 0.1826 \\ 1118 & 11: 45: 01 & 0.0420 & 0.2860 \\ 1120 & 11: 45: 03 & 0.0850 & 0.0864 \\ 1122 & 11: 45: 05 & 0.2675 & 0.1563 \\ 1124 & 11: 45: 07 & 0.2628 & 0.1662 \\ 1126 & 11: 45: 09 & 0.4108 & 0.2512 \\ 1128 & 11: 45: 11 & 0.1180 & 0.2112 \\ 1130 & 11: 45: 13 & 0.0728 & 0.2166 \\ 1132 & 11: 45: 15 & 0.0462 & 0.1463 \\ 1134 & 11: 45: 17 & 0.1232 & 0.1533 \\ 1136 & 11: 45: 19 & 0.0516 & 0.1995 \\ 1138 & 11: 45: 21 & 0.0435 & 0.2130 \\ 1140 & 11: 45: 23 & 0.0578 & 0.1373\end{array}$




$\begin{array}{llll}1142 & 11: 45: 25 & 0.0343 & 0.1523 \\ 1144 & 11: 45: 27 & 0.0821 & 0.1408 \\ 1146 & 11: 45: 29 & 0.0271 & 0.0731 \\ 1148 & 11: 45: 31 & 0.1859 & 0.0943 \\ 1150 & 11: 45: 33 & 0.2334 & 0.0740 \\ 1152 & 11: 45: 35 & 0.0940 & 0.1346 \\ 1154 & 11: 45: 37 & 0.0725 & 0.1205 \\ 1156 & 11: 45: 39 & 0.0570 & 0.1578 \\ 1158 & 11: 45: 41 & 0.0354 & 0.2783 \\ 1160 & 11: 45: 43 & 0.1729 & 0.2260 \\ 1162 & 11: 45: 45 & 0.0487 & 0.1999 \\ 1164 & 11: 45: 47 & 0.1037 & 0.1369 \\ 1166 & 11: 45: 49 & 0.0420 & 0.2028 \\ 1168 & 11: 45: 51 & 0.0384 & 0.2149 \\ 1170 & 11: 45: 53 & 0.1176 & 0.1070 \\ 1172 & 11: 45: 55 & 0.0744 & 0.1787 \\ 1174 & 11: 45: 57 & 0.1128 & 0.2007 \\ 1176 & 11: 45: 59 & 0.0805 & 0.2405 \\ 1178 & 11: 46: 01 & 0.1626 & 0.3393 \\ 1180 & 11: 46: 03 & 0.2324 & 0.3806 \\ 1182 & 11: 46: 05 & 0.1130 & 0.0817 \\ 1184 & 11: 46: 07 & 0.1759 & 0.1866 \\ 1186 & 11: 46: 09 & 0.1182 & 0.3418 \\ 1188 & 11: 46: 11 & 0.0933 & 0.1189 \\ 1190 & 11: 46: 13 & 0.0643 & 0.1230 \\ 1192 & 11: 46: 15 & 0.0786 & 0.1089 \\ 1194 & 11: 46: 17 & 0.1318 & 0.1227 \\ 1196 & 11: 46: 19 & 0.0390 & 0.1458 \\ 1198 & 11: 46: 21 & 0.0420 & 0.2653 \\ 1200 & 11: 46: 23 & 0.0488 & 0.2217 \\ 1202 & 11: 46: 25 & 0.0437 & 0.3030 \\ 1204 & 11: 46: 27 & 0.1713 & 0.1071 \\ 1206 & 11: 46: 29 & 0.0434 & 0.0540 \\ 1208 & 11: 46: 31 & 0.0920 & 0.0806 \\ 1210 & 11: 46: 33 & 0.0927 & 0.0842 \\ 1212 & 11: 46: 35 & 0.2321 & 0.2195 \\ 1214 & 11: 46: 37 & 0.1654 & 0.4078 \\ 1216 & 11: 46: 39 & 0.0673 & 0.1150 \\ 1218 & 11: 46: 41 & 0.1261 & 0.3112 \\ 1220 & 11: 46: 43 & 0.0499 & 0.1993 \\ 1222 & 11: 46: 45 & 0.1511 & 0.4181 \\ 1224 & 11: 46: 47 & 0.0743 & 0.1762 \\ 1226 & 11: 46: 49 & 0.1523 & 0.1805 \\ 1228 & 11: 46: 51 & 0.1222 & 0.1708 \\ 1230 & 11: 46: 53 & 0.1242 & 0.2001 \\ 1232 & 11: 46: 55 & 0.0934 & 0.2465 \\ 1234 & 11: 46: 57 & 0.0548 & 0.1984 \\ 1236 & 11: 46: 59 & 0.1625 & 0.1886 \\ 1238 & 11: 47: 01 & 0.1263 & 0.2428 \\ 1240 & 11: 47: 03 & 0.0582 & 0.1490 \\ 1242 & 11: 47: 05 & 0.0571 & 0.1816 \\ 1244 & 11: 47: 07 & 0.1562 & 0.1554\end{array}$




$\begin{array}{llll}1246 & 11: 47: 09 & 0.1112 & 0.1094 \\ 1248 & 11: 47: 11 & 0.1393 & 0.1490 \\ 1250 & 11: 47: 13 & 0.0760 & 0.2523 \\ 1252 & 11: 47: 15 & 0.0582 & 0.1098 \\ 1254 & 11: 47: 17 & 0.0775 & 0.0792 \\ 1256 & 11: 47: 19 & 0.0784 & 0.1686 \\ 1258 & 11: 47: 21 & 0.0887 & 0.2528 \\ 1260 & 11: 47: 23 & 0.0589 & 0.1213 \\ 1262 & 11: 47: 25 & 0.0930 & 0.1155 \\ 1264 & 11: 47: 27 & 0.0658 & 0.1911 \\ 1266 & 11: 47: 29 & 0.1276 & 0.1332 \\ 1268 & 11: 47: 31 & 0.0603 & 0.0805 \\ 1270 & 11: 47: 33 & 0.0813 & 0.2461 \\ 1272 & 11: 47: 35 & 0.0494 & 0.2029 \\ 1274 & 11: 47: 37 & 0.0726 & 0.2642 \\ 1276 & 11: 47: 39 & 0.0455 & 0.1387 \\ 1278 & 11: 47: 41 & 0.0502 & 0.0738 \\ 1280 & 11: 47: 43 & 0.0585 & 0.1277 \\ 1282 & 11: 47: 45 & 0.0283 & 0.1175 \\ 1284 & 11: 47: 47 & 0.0236 & 0.0714 \\ 1286 & 11: 47: 49 & 0.0777 & 0.4704 \\ 1288 & 11: 47: 51 & 0.0485 & 0.0618 \\ 1290 & 11: 47: 53 & 0.0748 & 0.2143 \\ 1292 & 11: 47: 55 & 0.0500 & 0.1699 \\ 1294 & 11: 47: 57 & 0.0715 & 0.3713 \\ 1296 & 11: 47: 59 & 0.0810 & 0.1088 \\ 1298 & 11: 48: 01 & 0.0674 & 0.0899 \\ 1300 & 11: 48: 03 & 0.0410 & 0.2157 \\ 1302 & 11: 48: 05 & 0.0846 & 0.2302 \\ 1304 & 11: 48: 07 & 0.1124 & 0.1938 \\ 1306 & 11: 48: 09 & 0.1275 & 0.2776 \\ 1308 & 11: 48: 11 & 0.0967 & 0.1524 \\ 1310 & 11: 48: 13 & 0.0630 & 0.1331 \\ 1312 & 11: 48: 15 & 0.0784 & 0.2291 \\ 1314 & 11: 48: 17 & 0.1340 & 0.1542 \\ 1316 & 11: 48: 19 & 0.1052 & 0.2178 \\ 1318 & 11: 48: 21 & 0.0823 & 0.1087 \\ 1320 & 11: 48: 23 & 0.0433 & 0.2046 \\ 1322 & 11: 48: 25 & 0.0588 & 0.0784 \\ 1324 & 11: 48: 27 & 0.1608 & 0.2623 \\ 1326 & 11: 48: 29 & 0.1042 & 0.2058 \\ 1328 & 11: 48: 31 & 0.0642 & 0.0609 \\ 1330 & 11: 48: 33 & 0.0689 & 0.1968 \\ 1332 & 11: 48: 35 & 0.0795 & 0.1806 \\ 1334 & 11: 48: 37 & 0.0581 & 0.2771 \\ 1336 & 11: 48: 39 & 0.1488 & 0.2008 \\ 1338 & 11: 48: 41 & 0.0741 & 0.1915 \\ 1340 & 11: 48: 43 & 0.0505 & 0.1568 \\ 1342 & 11: 48: 45 & 0.0307 & 0.1680 \\ 1344 & 11: 48: 47 & 0.0937 & 0.0650 \\ 1346 & 11: 48: 49 & 0.0906 & 0.0880 \\ 1348 & 11: 48: 51 & 0.1639 & 0.1533\end{array}$




$\begin{array}{llll}1350 & 11: 48: 53 & 0.0296 & 0.0972 \\ 1352 & 11: 48: 55 & 0.1647 & 0.1519 \\ 1354 & 11: 48: 57 & 0.1703 & 0.1140 \\ 1356 & 11: 48: 59 & 0.1587 & 0.1254 \\ 1358 & 11: 49: 01 & 0.1606 & 0.0702 \\ 1360 & 11: 49: 03 & 0.3214 & 0.0384 \\ 1362 & 11: 49: 05 & 0.3412 & 0.0772 \\ 1364 & 11: 49: 07 & 0.1683 & 0.0716 \\ 1366 & 11: 49: 09 & 0.2570 & 0.2775 \\ 1368 & 11: 49: 11 & 0.1095 & 0.2382 \\ 1370 & 11: 49: 13 & 0.0687 & 0.1107 \\ 1372 & 11: 49: 15 & 0.1725 & 0.2431 \\ 1374 & 11: 49: 17 & 0.0849 & 0.5357 \\ 1376 & 11: 49: 19 & 0.2525 & 0.4773 \\ 1378 & 11: 49: 21 & 0.0666 & 0.2613 \\ 1380 & 11: 49: 23 & 0.1055 & 0.3126 \\ 1382 & 11: 49: 25 & 0.0353 & 0.2589 \\ 1384 & 11: 49: 27 & 0.0678 & 0.2418 \\ 1386 & 11: 49: 29 & 0.1780 & 0.3605 \\ 1388 & 11: 49: 31 & 0.0941 & 0.2259 \\ 1390 & 11: 49: 33 & 0.0229 & 0.2185 \\ 1392 & 11: 49: 35 & 0.0931 & 0.2010 \\ 1394 & 11: 49: 37 & 0.0951 & 0.1699 \\ 1396 & 11: 49: 39 & 0.1193 & 0.0716 \\ 1398 & 11: 49: 41 & 0.1214 & 0.2582 \\ 1400 & 11: 49: 43 & 0.1235 & 0.0845 \\ 1402 & 11: 49: 45 & 0.2208 & 0.1774 \\ 1404 & 11: 49: 47 & 0.1273 & 0.1046 \\ 1406 & 11: 49: 49 & 0.1356 & 0.1432 \\ 1408 & 11: 49: 51 & 0.2275 & 0.1444 \\ 1410 & 11: 49: 53 & 0.0776 & 0.3048 \\ 1412 & 11: 49: 55 & 0.1117 & 0.3282 \\ 1414 & 11: 49: 57 & 0.1036 & 0.2016 \\ 1416 & 11: 49: 59 & 0.2463 & 0.2991 \\ 1418 & 11: 50: 01 & 0.1215 & 0.1554 \\ 1420 & 11: 50: 03 & 0.1054 & 0.1652 \\ 1422 & 11: 50: 05 & 0.2204 & 0.0898 \\ 1424 & 11: 50: 07 & 0.1338 & 0.3123 \\ 1426 & 11: 50: 09 & 0.0296 & 0.1208 \\ 1428 & 11: 50: 11 & 0.0439 & 0.1901 \\ 1430 & 11: 50: 13 & 0.0803 & 0.1155 \\ 1432 & 11: 50: 15 & 0.0621 & 0.2281 \\ 1434 & 11: 50: 17 & 0.0454 & 0.3395 \\ 1436 & 11: 50: 19 & 0.0708 & 0.2623 \\ 1438 & 11: 50: 21 & 0.0665 & 0.3512 \\ 1440 & 11: 50: 23 & 0.0785 & 0.2842 \\ 1442 & 11: 50: 25 & 0.0677 & 0.3834 \\ 1444 & 11: 50: 27 & 0.1510 & 0.2456 \\ 1446 & 11: 50: 29 & 0.0744 & 0.2113 \\ 1448 & 11: 50: 31 & 0.0919 & 0.1158 \\ 1450 & 11: 50: 33 & 0.1182 & 0.0590 \\ 1452 & 11: 50: 35 & 0.1013 & 0.1891\end{array}$




$\begin{array}{llll}1454 & 11: 50: 37 & 0.0877 & 0.1356 \\ 1456 & 11: 50: 39 & 0.1209 & 0.1058 \\ 1458 & 11: 50: 41 & 0.0801 & 0.2101 \\ 1460 & 11: 50: 43 & 0.0884 & 0.2900 \\ 1462 & 11: 50: 45 & 0.1269 & 0.3324 \\ 1464 & 11: 50: 47 & 0.0914 & 0.0989 \\ 1466 & 11: 50: 49 & 0.2226 & 0.1172 \\ 1468 & 11: 50: 51 & 0.0393 & 0.1658 \\ 1470 & 11: 50: 53 & 0.0879 & 0.2543 \\ 1472 & 11: 50: 55 & 0.0476 & 0.0769 \\ 1474 & 11: 50: 57 & 0.1589 & 0.1855 \\ 1476 & 11: 50: 59 & 0.0421 & 0.1551 \\ 1478 & 11: 51: 01 & 0.0578 & 0.0812 \\ 1480 & 11: 51: 03 & 0.1005 & 0.0888 \\ 1482 & 11: 51: 05 & 0.2212 & 0.1088 \\ 1484 & 11: 51: 07 & 0.1754 & 0.2233 \\ 1486 & 11: 51: 09 & 0.1226 & 0.0626 \\ 1488 & 11: 51: 11 & 0.1000 & 0.1462 \\ 1490 & 11: 51: 13 & 0.0623 & 0.1058 \\ 1492 & 11: 51: 15 & 0.0466 & 0.3055 \\ 1494 & 11: 51: 17 & 0.0650 & 0.1930 \\ 1496 & 11: 51: 19 & 0.1922 & 0.2303 \\ 1498 & 11: 51: 21 & 0.1232 & 0.2044 \\ 1500 & 11: 51: 23 & 0.0778 & 0.1365 \\ 1502 & 11: 51: 25 & 0.0599 & 0.0993 \\ 1504 & 11: 51: 27 & 0.0940 & 0.1825 \\ 1506 & 11: 51: 29 & 0.1290 & 0.2496 \\ 1508 & 11: 51: 31 & 0.1589 & 0.2879 \\ 1510 & 11: 51: 33 & 0.0312 & 0.2407 \\ 1512 & 11: 51: 35 & 0.0528 & 0.2932 \\ 1514 & 11: 51: 37 & 0.1320 & 0.2747 \\ 1516 & 11: 51: 39 & 0.1808 & 0.1541 \\ 1518 & 11: 51: 41 & 0.0994 & 0.1084 \\ 1520 & 11: 51: 43 & 0.0852 & 0.1468 \\ 1522 & 11: 51: 45 & 0.1362 & 0.0724 \\ 1524 & 11: 51: 47 & 0.0699 & 0.1128 \\ 1526 & 11: 51: 49 & 0.0488 & 0.0842 \\ 1528 & 11: 51: 51 & 0.1077 & 0.0895 \\ 1530 & 11: 51: 53 & 0.1074 & 0.0757 \\ 1532 & 11: 51: 55 & 0.0896 & 0.1665 \\ 1534 & 11: 51: 57 & 0.0219 & 0.2073 \\ 1536 & 11: 51: 59 & 0.0546 & 0.1252 \\ 1538 & 11: 52: 01 & 0.1110 & 0.0816 \\ 1540 & 11: 52: 03 & 0.2126 & 0.1651 \\ 1542 & 11: 52: 05 & 0.1458 & 0.4808 \\ 1544 & 11: 52: 07 & 0.0444 & 0.1715 \\ 1546 & 11: 52: 09 & 0.2430 & 0.2472 \\ 1548 & 11: 52: 11 & 0.0549 & 0.3989 \\ 1550 & 11: 52: 13 & 0.1166 & 0.2562 \\ 1552 & 11: 52: 15 & 0.1569 & 0.1938 \\ 1554 & 11: 52: 17 & 0.0820 & 0.2150 \\ 1556 & 11: 52: 19 & 0.0989 & 0.1011\end{array}$


Appendix B - Laser Data Test 1 NIST102505_1.xls

$\begin{array}{llll}1558 & 11: 52: 21 & 0.0865 & 0.1656 \\ 1560 & 11: 52: 23 & 0.1258 & 0.1676 \\ 1562 & 11: 52: 25 & 0.0675 & 0.1620 \\ 1564 & 11: 52: 27 & 0.0368 & 0.0816 \\ 1566 & 11: 52: 29 & 0.0762 & 0.2062 \\ 1568 & 11: 52: 31 & 0.0607 & 0.1743 \\ 1570 & 11: 52: 33 & 0.1847 & 0.2376 \\ 1572 & 11: 52: 35 & 0.0720 & 0.1255 \\ 1574 & 11: 52: 37 & 0.0463 & 0.3304 \\ 1576 & 11: 52: 39 & 0.0521 & 0.1890 \\ 1578 & 11: 52: 41 & 0.0603 & 0.2499 \\ 1580 & 11: 52: 43 & 0.0671 & 0.2790 \\ 1582 & 11: 52: 45 & 0.1572 & 0.3573 \\ 1584 & 11: 52: 47 & 0.1467 & 0.1611 \\ 1586 & 11: 52: 49 & 0.1522 & 0.2242 \\ 1588 & 11: 52: 51 & 0.0453 & 0.1898 \\ 1590 & 11: 52: 53 & 0.0777 & 0.1614 \\ 1592 & 11: 52: 55 & 0.1243 & 0.1405 \\ 1594 & 11: 52: 57 & 0.0490 & 0.1309 \\ 1596 & 11: 52: 59 & 0.0632 & 0.4347 \\ 1598 & 11: 53: 01 & 0.1381 & 0.1918 \\ 1600 & 11: 53: 03 & 0.1308 & 0.0884 \\ 1602 & 11: 53: 05 & 0.0316 & 0.1410 \\ 1604 & 11: 53: 07 & 0.0478 & 0.1563 \\ 1606 & 11: 53: 09 & 0.0221 & 0.2304 \\ 1608 & 11: 53: 11 & 0.1089 & 0.3420 \\ 1610 & 11: 53: 13 & 0.1636 & 0.1350 \\ 1612 & 11: 53: 15 & 0.1289 & 0.1329 \\ 1614 & 11: 53: 17 & 0.1749 & 0.2221 \\ 1616 & 11: 53: 19 & 0.0704 & 0.0444 \\ 1618 & 11: 53: 21 & 0.0773 & 0.1329 \\ 1620 & 11: 53: 23 & 0.1058 & 0.1339 \\ 1622 & 11: 53: 25 & 0.0755 & 0.1304 \\ 1624 & 11: 53: 27 & 0.1242 & 0.2483 \\ 1626 & 11: 53: 29 & 0.1030 & 0.1816 \\ 1628 & 11: 53: 31 & 0.0478 & 0.1305 \\ 1630 & 11: 53: 33 & 0.0340 & 0.1228 \\ 1632 & 11: 53: 35 & 0.1006 & 0.1325 \\ 1634 & 11: 53: 37 & 0.0909 & 0.2361 \\ 1636 & 11: 53: 39 & 0.0566 & 0.1756 \\ 1638 & 11: 53: 41 & 0.0770 & 0.1135 \\ 1640 & 11: 53: 43 & 0.1152 & 0.2355 \\ 1642 & 11: 53: 45 & 0.0928 & 0.2216 \\ 1644 & 11: 53: 47 & 0.0782 & 0.2913 \\ 1646 & 11: 53: 49 & 0.0418 & 0.1892 \\ 1648 & 11: 53: 51 & 0.0898 & 0.3262 \\ 1650 & 11: 53: 53 & 0.0298 & 0.1369 \\ 1652 & 11: 53: 55 & 0.2965 & 0.2149 \\ 1654 & 11: 53: 57 & 0.0562 & 0.1777 \\ 1656 & 11: 53: 59 & 0.0495 & 0.2379 \\ 1658 & 11: 54: 01 & 0.1568 & 0.1598 \\ 1660 & 11: 54: 03 & 0.1343 & 0.1179\end{array}$




$\begin{array}{llll}1662 & 11: 54: 05 & 0.1657 & 0.2424 \\ 1664 & 11: 54: 07 & 0.0472 & 0.1470 \\ 1666 & 11: 54: 09 & 0.0828 & 0.1267 \\ 1668 & 11: 54: 11 & 0.0461 & 0.1446 \\ 1670 & 11: 54: 13 & 0.0642 & 0.2297 \\ 1672 & 11: 54: 15 & 0.1424 & 0.1598 \\ 1674 & 11: 54: 17 & 0.1770 & 0.1158 \\ 1676 & 11: 54: 19 & 0.1738 & 0.1434 \\ 1678 & 11: 54: 21 & 0.1158 & 0.1996 \\ 1680 & 11: 54: 23 & 0.0383 & 0.1126 \\ 1682 & 11: 54: 25 & 0.0494 & 0.2050 \\ 1684 & 11: 54: 27 & 0.1428 & 0.4393 \\ 1686 & 11: 54: 29 & 0.0724 & 0.1687 \\ 1688 & 11: 54: 31 & 0.0329 & 0.0998 \\ 1690 & 11: 54: 33 & 0.4496 & 0.3269 \\ 1692 & 11: 54: 35 & 0.2574 & 0.2296 \\ 1694 & 11: 54: 37 & 0.1095 & 0.1312 \\ 1696 & 11: 54: 39 & 0.1154 & 0.0844 \\ 1698 & 11: 54: 41 & 0.1152 & 0.2748 \\ 1700 & 11: 54: 43 & 0.1064 & 0.1680 \\ 1702 & 11: 54: 45 & 0.0476 & 0.2076 \\ 1704 & 11: 54: 47 & 0.0859 & 0.0908 \\ 1706 & 11: 54: 49 & 0.1021 & 0.1419 \\ 1708 & 11: 54: 51 & 0.1101 & 0.2425 \\ 1710 & 11: 54: 53 & 0.0975 & 0.2104 \\ 1712 & 11: 54: 55 & 0.0790 & 0.2390 \\ 1714 & 11: 54: 57 & 0.1379 & 0.1503 \\ 1716 & 11: 54: 59 & 0.0569 & 0.2077 \\ 1718 & 11: 55: 01 & 0.0639 & 0.1101 \\ 1720 & 11: 55: 03 & 0.0938 & 0.1004 \\ 1722 & 11: 55: 05 & 0.0619 & 0.1785 \\ 1724 & 11: 55: 07 & 0.0548 & 0.2178 \\ 1726 & 11: 55: 09 & 0.2132 & 0.2569 \\ 1728 & 11: 55: 11 & 0.1307 & 0.3602 \\ 1730 & 11: 55: 13 & 0.0891 & 0.4164 \\ 1732 & 11: 55: 15 & 0.1155 & 0.2666 \\ 1734 & 11: 55: 17 & 0.1723 & 0.1211 \\ 1736 & 11: 55: 19 & 0.1322 & 0.0631 \\ 1738 & 11: 55: 21 & 0.2098 & 0.2388 \\ 1740 & 11: 55: 23 & 0.0531 & 0.2163 \\ 1742 & 11: 55: 25 & 0.1118 & 0.3552 \\ 1744 & 11: 55: 27 & 0.1546 & 0.2668 \\ 1746 & 11: 55: 29 & 0.0645 & 0.3139 \\ 1748 & 11: 55: 31 & 0.0410 & 0.3070 \\ 1750 & 11: 55: 33 & 0.2963 & 0.2711 \\ 1752 & 11: 55: 35 & 0.1838 & 0.1651 \\ 1754 & 11: 55: 37 & 0.2036 & 0.1464 \\ 1756 & 11: 55: 39 & 0.2040 & 0.1060 \\ 1758 & 11: 55: 41 & 0.1472 & 0.3766 \\ 1760 & 11: 55: 43 & 0.0584 & 0.2429 \\ 1762 & 11: 55: 45 & 0.0644 & 0.2945 \\ 1764 & 11: 55: 47 & 0.0347 & 0.3022 \\ & & & \end{array}$




$\begin{array}{llll}1766 & 11: 55: 49 & 0.0985 & 0.4430 \\ 1768 & 11: 55: 51 & 0.0451 & 0.1598 \\ 1770 & 11: 55: 53 & 0.0662 & 0.0689 \\ 1772 & 11: 55: 55 & 0.0957 & 0.2742 \\ 1774 & 11: 55: 57 & 0.0820 & 0.3193 \\ 1776 & 11: 55: 59 & 0.0805 & 0.2493 \\ 1778 & 11: 56: 01 & 0.1167 & 0.1266 \\ 1780 & 11: 56: 03 & 0.0879 & 0.2032 \\ 1782 & 11: 56: 05 & 0.1096 & 0.1832 \\ 1784 & 11: 56: 07 & 0.1097 & 0.1201 \\ 1786 & 11: 56: 09 & 0.0343 & 0.0940 \\ 1788 & 11: 56: 11 & 0.0536 & 0.4882 \\ 1790 & 11: 56: 13 & 0.0628 & 0.2372 \\ 1792 & 11: 56: 15 & 0.0771 & 0.1540 \\ 1794 & 11: 56: 17 & 0.1032 & 0.2923 \\ 1796 & 11: 56: 19 & 0.1075 & 0.1324 \\ 1798 & 11: 56: 21 & 0.0955 & 0.1280 \\ 1800 & 11: 56: 23 & 0.0834 & 0.1008 \\ 1802 & 11: 56: 25 & 0.0398 & 0.1745 \\ 1804 & 11: 56: 27 & 0.0654 & 0.1371 \\ 1806 & 11: 56: 29 & 0.0344 & 0.1943 \\ 1808 & 11: 56: 31 & 0.0712 & 0.1440 \\ 1810 & 11: 56: 33 & 0.1672 & 0.2249 \\ 1812 & 11: 56: 35 & 0.1663 & 0.1537 \\ 1814 & 11: 56: 37 & 0.1172 & 0.1882 \\ 1816 & 11: 56: 39 & 0.1364 & 0.2324 \\ 1818 & 11: 56: 41 & 0.0624 & 0.0703 \\ 1820 & 11: 56: 43 & 0.1051 & 0.1972 \\ 1822 & 11: 56: 45 & 0.1001 & 0.1242 \\ 1824 & 11: 56: 47 & 0.0698 & 0.2710 \\ 1826 & 11: 56: 49 & 0.0905 & 0.0919 \\ 1828 & 11: 56: 51 & 0.1573 & 0.2926 \\ 1830 & 11: 56: 53 & 0.1030 & 0.1288 \\ 1832 & 11: 56: 55 & 0.1083 & 0.1484 \\ 1834 & 11: 56: 57 & 0.0381 & 0.1117 \\ 1836 & 11: 56: 59 & 0.0291 & 0.1521 \\ 1838 & 11: 57: 01 & 0.0596 & 0.2603 \\ 1840 & 11: 57: 03 & 0.0961 & 0.1589 \\ 1842 & 11: 57: 05 & 0.0458 & 0.3063 \\ 1844 & 11: 57: 07 & 0.0981 & 0.1549 \\ 1846 & 11: 57: 09 & 0.0914 & 0.1590 \\ 1848 & 11: 57: 11 & 0.0646 & 0.1103 \\ 1850 & 11: 57: 13 & 0.0871 & 0.1756 \\ 1852 & 11: 57: 15 & 0.2103 & 0.2881 \\ 1854 & 11: 57: 17 & 0.1197 & 0.2364 \\ 1856 & 11: 57: 19 & 0.0755 & 0.2403 \\ 1858 & 11: 57: 21 & 0.1486 & 0.2324 \\ 1860 & 11: 57: 23 & 0.0577 & 0.1393 \\ 1862 & 11: 57: 25 & 0.1336 & 0.1823 \\ 1864 & 11: 57: 27 & 0.1461 & 0.1880 \\ 1866 & 11: 57: 29 & 0.0543 & 0.1228 \\ 1868 & 11: 57: 31 & 0.1574 & 0.1547\end{array}$




$\begin{array}{llll}1870 & 11: 57: 33 & 0.1538 & 0.3307 \\ 1872 & 11: 57: 35 & 0.1351 & 0.0862 \\ 1874 & 11: 57: 37 & 0.0666 & 0.1113 \\ 1876 & 11: 57: 39 & 0.1214 & 0.2263 \\ 1878 & 11: 57: 41 & 0.1155 & 0.3030 \\ 1880 & 11: 57: 43 & 0.0581 & 0.2317 \\ 1882 & 11: 57: 45 & 0.1602 & 0.2503 \\ 1884 & 11: 57: 47 & 0.0557 & 0.1784 \\ 1886 & 11: 57: 49 & 0.1343 & 0.2703 \\ 1888 & 11: 57: 51 & 0.1310 & 0.1595 \\ 1890 & 11: 57: 53 & 0.0688 & 0.1897 \\ 1892 & 11: 57: 55 & 0.0781 & 0.1371 \\ 1894 & 11: 57: 57 & 0.0735 & 0.0656 \\ 1896 & 11: 57: 59 & 0.1919 & 0.1033 \\ 1898 & 11: 58: 01 & 0.0855 & 0.2493 \\ 1900 & 11: 58: 03 & 0.1088 & 0.1504 \\ 1902 & 11: 58: 05 & 0.1732 & 0.1148 \\ 1904 & 11: 58: 07 & 0.1840 & 0.2395 \\ 1906 & 11: 58: 09 & 0.0716 & 0.2315 \\ 1908 & 11: 58: 11 & 0.0781 & 0.1758 \\ 1910 & 11: 58: 13 & 0.0542 & 0.3267 \\ 1912 & 11: 58: 15 & 0.0451 & 0.2133 \\ 1914 & 11: 58: 17 & 0.0846 & 0.3238 \\ 1916 & 11: 58: 19 & 0.1114 & 0.1733 \\ 1918 & 11: 58: 21 & 0.1111 & 0.0686 \\ 1920 & 11: 58: 23 & 0.1489 & 0.1648 \\ 1922 & 11: 58: 25 & 0.0432 & 0.2712 \\ 1924 & 11: 58: 27 & 0.0642 & 0.1342 \\ 1926 & 11: 58: 29 & 0.0716 & 0.1780 \\ 1928 & 11: 58: 31 & 0.0771 & 0.1563 \\ 1930 & 11: 58: 33 & 0.0950 & 0.2669 \\ 1932 & 11: 58: 35 & 0.1181 & 0.1969 \\ 1934 & 11: 58: 37 & 0.0938 & 0.4248 \\ 1936 & 11: 58: 39 & 0.0533 & 0.2116 \\ 1938 & 11: 58: 41 & 0.0672 & 0.1557 \\ 1940 & 11: 58: 43 & 0.0774 & 0.1621 \\ 1942 & 11: 58: 45 & 0.0457 & 0.2139 \\ 1944 & 11: 58: 47 & 0.1763 & 0.2835 \\ 1946 & 11: 58: 49 & 0.0965 & 0.0585 \\ 1948 & 11: 58: 51 & 0.0652 & 0.1107 \\ 1950 & 11: 58: 53 & 0.0893 & 0.1861 \\ 1952 & 11: 58: 55 & 0.1142 & 0.2002 \\ 1954 & 11: 58: 57 & 0.1817 & 0.2636 \\ 1956 & 11: 58: 59 & 0.0562 & 0.2486 \\ 1958 & 11: 59: 01 & 0.0981 & 0.1302 \\ 1960 & 11: 59: 03 & 0.0677 & 0.3883 \\ 1962 & 11: 59: 05 & 0.0941 & 0.4464 \\ 1964 & 11: 59: 07 & 0.1679 & 0.2277 \\ 1966 & 11: 59: 09 & 0.1135 & 0.1530 \\ 1968 & 11: 59: 11 & 0.0919 & 0.1503 \\ 1970 & 11: 59: 13 & 0.0769 & 0.1492 \\ 1972 & 11: 59: 15 & 0.0992 & 0.1222 \\ & & & \end{array}$




$\begin{array}{llll}1974 & 11: 59: 17 & 0.2593 & 0.3082 \\ 1976 & 11: 59: 19 & 0.0815 & 0.3630 \\ 1978 & 11: 59: 21 & 0.0615 & 0.1160 \\ 1980 & 11: 59: 23 & 0.2244 & 0.3385 \\ 1982 & 11: 59: 25 & 0.1108 & 0.0802 \\ 1984 & 11: 59: 27 & 0.0432 & 0.0755 \\ 1986 & 11: 59: 29 & 0.0969 & 0.0874 \\ 1988 & 11: 59: 31 & 0.0828 & 0.0994 \\ 1990 & 11: 59: 33 & 0.0267 & 0.1412 \\ 1992 & 11: 59: 35 & 0.2359 & 0.2515 \\ 1994 & 11: 59: 37 & 0.1160 & 0.2571 \\ 1996 & 11: 59: 39 & 0.1795 & 0.1607 \\ 1998 & 11: 59: 41 & 0.1696 & 0.1816 \\ 2000 & 11: 59: 43 & 0.1470 & 0.2489 \\ 2002 & 11: 59: 45 & 0.0725 & 0.1082 \\ 2004 & 11: 59: 47 & 0.1938 & 0.0893 \\ 2006 & 11: 59: 49 & 0.1603 & 0.1814 \\ 2008 & 11: 59: 51 & 0.1338 & 0.2472 \\ 2010 & 11: 59: 53 & 0.0742 & 0.1605 \\ 2012 & 11: 59: 55 & 0.0840 & 0.0933 \\ 2014 & 11: 59: 57 & 0.0955 & 0.1911 \\ 2016 & 11: 59: 59 & 0.2204 & 0.3192 \\ 2018 & 12: 00: 01 & 0.0844 & 0.1453 \\ 2020 & 12: 00: 03 & 0.1447 & 0.0565 \\ 2022 & 12: 00: 05 & 0.1388 & 0.1532 \\ 2024 & 12: 00: 07 & 0.1702 & 0.1308 \\ 2026 & 12: 00: 09 & 0.1094 & 0.3330 \\ 2028 & 12: 00: 11 & 0.1120 & 0.0918 \\ 2030 & 12: 00: 13 & 0.1520 & 0.2533 \\ 2032 & 12: 00: 15 & 0.3081 & 0.3820 \\ 2034 & 12: 00: 17 & 0.1553 & 0.1498 \\ 2036 & 12: 00: 19 & 0.1222 & 0.1081 \\ 2038 & 12: 00: 21 & 0.0682 & 0.3280 \\ 2040 & 12: 00: 23 & 0.0541 & 0.2013 \\ 2042 & 12: 00: 25 & 0.0384 & 0.2428 \\ 2044 & 12: 00: 27 & 0.0963 & 0.1036 \\ 2046 & 12: 00: 29 & 0.1073 & 0.2015 \\ 2048 & 12: 00: 31 & 0.1173 & 0.1636 \\ 2050 & 12: 00: 33 & 0.0474 & 0.2856 \\ 2052 & 12: 00: 35 & 0.1040 & 0.1540 \\ 2054 & 12: 00: 37 & 0.1308 & 0.1071 \\ 2056 & 12: 00: 39 & 0.2353 & 0.0693 \\ 2058 & 12: 00: 41 & 0.1556 & 0.1643 \\ 2060 & 12: 00: 43 & 0.0324 & 0.2369 \\ 2062 & 12: 00: 45 & 0.1762 & 0.2266 \\ 2064 & 12: 00: 47 & 0.1556 & 0.0770 \\ 2066 & 12: 00: 49 & 0.1537 & 0.2022 \\ 2068 & 12: 00: 51 & 0.0886 & 0.1284 \\ 2070 & 12: 00: 53 & 0.1002 & 0.2589 \\ 2072 & 12: 00: 55 & 0.0825 & 0.2035 \\ 2074 & 12: 00: 57 & 0.1800 & 0.0608 \\ 2076 & 12: 00: 59 & 0.0176 & 0.0879\end{array}$


Appendix B - Laser Data Test 1 NIST102505_1.xls

$\begin{array}{llll}2078 & 12: 01: 01 & 0.0976 & 0.2485 \\ 2080 & 12: 01: 03 & 0.1690 & 0.2983 \\ 2082 & 12: 01: 05 & 0.1009 & 0.3036 \\ 2084 & 12: 01: 07 & 0.1088 & 0.1335 \\ 2086 & 12: 01: 09 & 0.0448 & 0.1289 \\ 2088 & 12: 01: 11 & 0.0996 & 0.1399 \\ 2090 & 12: 01: 13 & 0.0481 & 0.2900 \\ 2092 & 12: 01: 15 & 0.1947 & 0.1162 \\ 2094 & 12: 01: 17 & 0.0473 & 0.1630 \\ 2096 & 12: 01: 19 & 0.0872 & 0.1349 \\ 2098 & 12: 01: 21 & 0.1247 & 0.1698 \\ 2100 & 12: 01: 23 & 0.1047 & 0.2635 \\ 2102 & 12: 01: 25 & 0.1070 & 0.0775 \\ 2104 & 12: 01: 27 & 0.1526 & 0.0687 \\ 2106 & 12: 01: 29 & 0.0370 & 0.2199 \\ 2108 & 12: 01: 31 & 0.0795 & 0.2380 \\ 2110 & 12: 01: 33 & 0.0468 & 0.1973 \\ 2112 & 12: 01: 35 & 0.0703 & 0.0579 \\ 2114 & 12: 01: 37 & 0.1712 & 0.1905 \\ 2116 & 12: 01: 39 & 0.1847 & 0.1708 \\ 2118 & 12: 01: 41 & 0.1038 & 0.1429 \\ 2120 & 12: 01: 43 & 0.2228 & 0.1930 \\ 2122 & 12: 01: 45 & 0.0942 & 0.0717 \\ 2124 & 12: 01: 47 & 0.2338 & 0.1700 \\ 2126 & 12: 01: 49 & 0.1139 & 0.1724 \\ 2128 & 12: 01: 51 & 0.0842 & 0.0979 \\ 2130 & 12: 01: 53 & 0.0611 & 0.2389 \\ 2132 & 12: 01: 55 & 0.1216 & 0.1295 \\ 2134 & 12: 01: 57 & 0.2380 & 0.1549 \\ 2136 & 12: 01: 59 & 0.0745 & 0.0540 \\ 2138 & 12: 02: 01 & 0.1234 & 0.2424 \\ 2140 & 12: 02: 03 & 0.0620 & 0.1389 \\ 2142 & 12: 02: 05 & 0.1813 & 0.2387 \\ 2144 & 12: 02: 07 & 0.0616 & 0.2422 \\ 2146 & 12: 02: 09 & 0.1299 & 0.2394 \\ 2148 & 12: 02: 11 & 0.2329 & 0.1711 \\ 2150 & 12: 02: 13 & 0.0713 & 0.1738 \\ 2152 & 12: 02: 15 & 0.1656 & 0.1778 \\ 2154 & 12: 02: 17 & 0.1625 & 0.1861 \\ 2156 & 12: 02: 19 & 0.0753 & 0.0436 \\ 2158 & 12: 02: 21 & 0.0838 & 0.5283 \\ 2160 & 12: 02: 23 & 0.0878 & 0.2737 \\ 2162 & 12: 02: 25 & 0.1075 & 0.2707 \\ 2164 & 12: 02: 27 & 0.0598 & 0.2152 \\ 2166 & 12: 02: 29 & 0.1973 & 0.1723 \\ 2168 & 12: 02: 31 & 0.1148 & 0.2100 \\ 2170 & 12: 02: 33 & 0.1182 & 0.0833 \\ 2172 & 12: 02: 35 & 0.0495 & 0.3009 \\ 2174 & 12: 02: 37 & 0.0152 & 0.1815 \\ 2176 & 12: 02: 39 & 0.0593 & 0.2359 \\ 2178 & 12: 02: 41 & 0.0320 & 0.1565 \\ 2180 & 12: 02: 43 & 0.1378 & 0.1591\end{array}$




$\begin{array}{llll}2182 & 12: 02: 45 & 0.0414 & 0.1434 \\ 2184 & 12: 02: 47 & 0.0708 & 0.2122 \\ 2186 & 12: 02: 49 & 0.0868 & 0.4101 \\ 2188 & 12: 02: 51 & 0.0889 & 0.4886 \\ 2190 & 12: 02: 53 & 0.0489 & 0.3952 \\ 2192 & 12: 02: 55 & 0.0421 & 0.2638 \\ 2194 & 12: 02: 57 & 0.0929 & 0.1803 \\ 2196 & 12: 02: 59 & 0.0849 & 0.2870 \\ 2198 & 12: 03: 01 & 0.0692 & 0.2037 \\ 2200 & 12: 03: 03 & 0.2148 & 0.1347 \\ 2202 & 12: 03: 05 & 0.0881 & 0.3833 \\ 2204 & 12: 03: 07 & 0.0474 & 0.3160 \\ 2206 & 12: 03: 09 & 0.0341 & 0.1619 \\ 2208 & 12: 03: 11 & 0.1344 & 0.0914 \\ 2210 & 12: 03: 13 & 0.1628 & 0.1495 \\ 2212 & 12: 03: 15 & 0.1548 & 0.0996 \\ 2214 & 12: 03: 17 & 0.1801 & 0.3368 \\ 2216 & 12: 03: 19 & 0.1086 & 0.1615 \\ 2218 & 12: 03: 21 & 0.0842 & 0.1487 \\ 2220 & 12: 03: 23 & 0.0957 & 0.3570 \\ 2222 & 12: 03: 25 & 0.0561 & 0.1383 \\ 2224 & 12: 03: 27 & 0.1554 & 0.2948 \\ 2226 & 12: 03: 29 & 0.1216 & 0.1256 \\ 2228 & 12: 03: 31 & 0.0414 & 0.1416 \\ 2230 & 12: 03: 33 & 0.1408 & 0.2560 \\ 2232 & 12: 03: 35 & 0.0903 & 0.3833 \\ 2234 & 12: 03: 37 & 0.0853 & 0.1674 \\ 2236 & 12: 03: 39 & 0.1269 & 0.3936 \\ 2238 & 12: 03: 41 & 0.1009 & 0.1771 \\ 2240 & 12: 03: 43 & 0.0576 & 0.2860 \\ 2242 & 12: 03: 45 & 0.0327 & 0.0953 \\ 2244 & 12: 03: 47 & 0.0826 & 0.2048 \\ 2246 & 12: 03: 49 & 0.0458 & 0.3027 \\ 2248 & 12: 03: 51 & 0.0896 & 0.1791 \\ 2250 & 12: 03: 53 & 0.1386 & 0.0838 \\ 2252 & 12: 03: 55 & 0.0849 & 0.1916 \\ 2254 & 12: 03: 57 & 0.2212 & 0.2123 \\ 2256 & 12: 03: 59 & 0.0816 & 0.2008 \\ 2258 & 12: 04: 01 & 0.1884 & 0.2992 \\ 2260 & 12: 04: 03 & 0.1348 & 0.1645 \\ 2262 & 12: 04: 05 & 0.1539 & 0.1252 \\ 2264 & 12: 04: 07 & 0.0647 & 0.3413 \\ 2266 & 12: 04: 09 & 0.2354 & 0.1459 \\ 2268 & 12: 04: 11 & 0.0769 & 0.1329 \\ 2270 & 12: 04: 13 & 0.2588 & 0.1847 \\ 2272 & 12: 04: 15 & 0.1599 & 0.3220 \\ 2274 & 12: 04: 17 & 0.0654 & 0.1283 \\ 2276 & 12: 04: 19 & 0.1001 & 0.1462 \\ 2278 & 12: 04: 21 & 0.0693 & 0.1849 \\ 2280 & 12: 04: 23 & 0.0566 & 0.1542 \\ 2282 & 12: 04: 25 & 0.1323 & 0.0536 \\ 2284 & 12: 04: 27 & 0.2683 & 0.3344\end{array}$




$\begin{array}{llll}2286 & 12: 04: 29 & 0.0968 & 0.1059 \\ 2288 & 12: 04: 31 & 0.0437 & 0.1843 \\ 2290 & 12: 04: 33 & 0.0734 & 0.2828 \\ 2292 & 12: 04: 35 & 0.0906 & 0.2999 \\ 2294 & 12: 04: 37 & 0.2315 & 0.1529 \\ 2296 & 12: 04: 39 & 0.1082 & 0.2418 \\ 2298 & 12: 04: 41 & 0.3807 & 0.2294 \\ 2300 & 12: 04: 43 & 0.0871 & 0.2350 \\ 2302 & 12: 04: 45 & 0.1025 & 0.1286 \\ 2304 & 12: 04: 47 & 0.1142 & 0.2271 \\ 2306 & 12: 04: 49 & 0.0596 & 0.1208 \\ 2308 & 12: 04: 51 & 0.0942 & 0.1273 \\ 2310 & 12: 04: 53 & 0.1294 & 0.1256 \\ 2312 & 12: 04: 55 & 0.1194 & 0.2903 \\ 2314 & 12: 04: 57 & 0.0405 & 0.2717 \\ 2316 & 12: 04: 59 & 0.1631 & 0.2906 \\ 2318 & 12: 05: 01 & 0.0891 & 0.1612 \\ 2320 & 12: 05: 03 & 0.0562 & 0.1316 \\ 2322 & 12: 05: 05 & 0.3738 & 0.1009 \\ 2324 & 12: 05: 07 & 0.1821 & 0.3021 \\ 2326 & 12: 05: 09 & 0.0593 & 0.2843 \\ 2328 & 12: 05: 11 & 0.1644 & 0.1680 \\ 2330 & 12: 05: 13 & 0.0827 & 0.1554 \\ 2332 & 12: 05: 15 & 0.0913 & 0.3297 \\ 2334 & 12: 05: 17 & 0.3163 & 0.1537 \\ 2336 & 12: 05: 19 & 0.0960 & 0.2647 \\ 2338 & 12: 05: 21 & 0.2324 & 0.0649 \\ 2340 & 12: 05: 23 & 0.0772 & 0.2240 \\ 2342 & 12: 05: 25 & 0.0413 & 0.1258 \\ 2344 & 12: 05: 27 & 0.3183 & 0.1371 \\ 2346 & 12: 05: 29 & 0.2117 & 0.1461 \\ 2348 & 12: 05: 31 & 0.0837 & 0.1011 \\ 2350 & 12: 05: 33 & 0.0706 & 0.1714 \\ 2352 & 12: 05: 35 & 0.0646 & 0.1873 \\ 2354 & 12: 05: 37 & 0.0714 & 0.1016 \\ 2356 & 12: 05: 39 & 0.2021 & 0.1845 \\ 2358 & 12: 05: 41 & 0.0396 & 0.2447 \\ 2360 & 12: 05: 43 & 0.1230 & 0.2649 \\ 2362 & 12: 05: 45 & 0.0687 & 0.1072 \\ 2364 & 12: 05: 47 & 0.0587 & 0.2120 \\ 2366 & 12: 05: 49 & 0.0387 & 0.1076 \\ 2368 & 12: 05: 51 & 0.0985 & 0.2699 \\ 2370 & 12: 05: 53 & 0.1323 & 0.1256 \\ 2372 & 12: 05: 55 & 0.2263 & 0.2735 \\ 2374 & 12: 05: 57 & 0.0524 & 0.1333 \\ 2376 & 12: 05: 59 & 0.0389 & 0.0941 \\ 2378 & 12: 06: 01 & 0.1129 & 0.1940 \\ 2380 & 12: 06: 03 & 0.2296 & 0.4162 \\ 2382 & 12: 06: 05 & 0.1535 & 0.1143 \\ 2384 & 12: 06: 07 & 0.0824 & 0.1580 \\ 2386 & 12: 06: 09 & 0.1185 & 0.1967 \\ 2388 & 12: 06: 11 & 0.1932 & 0.2071\end{array}$




$\begin{array}{llll}2390 & 12: 06: 13 & 0.0284 & 0.4161 \\ 2392 & 12: 06: 15 & 0.0763 & 0.1986 \\ 2394 & 12: 06: 17 & 0.0682 & 0.3707 \\ 2396 & 12: 06: 19 & 0.1482 & 0.2417 \\ 2398 & 12: 06: 21 & 0.3102 & 0.4821 \\ 2400 & 12: 06: 23 & 0.0844 & 0.2355 \\ 2402 & 12: 06: 25 & 0.0996 & 0.1743 \\ 2404 & 12: 06: 27 & 0.1764 & 0.1481 \\ 2406 & 12: 06: 29 & 0.0616 & 0.1244 \\ 2408 & 12: 06: 31 & 0.0999 & 0.3695 \\ 2410 & 12: 06: 33 & 0.0767 & 0.1710 \\ 2412 & 12: 06: 35 & 0.3706 & 0.2428 \\ 2414 & 12: 06: 37 & 0.1480 & 0.0917 \\ 2416 & 12: 06: 39 & 0.1530 & 0.1052 \\ 2418 & 12: 06: 41 & 0.2027 & 0.0847 \\ 2420 & 12: 06: 43 & 0.2254 & 0.1837 \\ 2422 & 12: 06: 45 & 0.1205 & 0.1363 \\ 2424 & 12: 06: 47 & 0.1028 & 0.0947 \\ 2426 & 12: 06: 49 & 0.1671 & 0.0815 \\ 2428 & 12: 06: 51 & 0.0449 & 0.1356 \\ 2430 & 12: 06: 53 & 0.1079 & 0.1282 \\ 2432 & 12: 06: 55 & 0.0959 & 0.2292 \\ 2434 & 12: 06: 57 & 0.0847 & 0.1822 \\ 2436 & 12: 06: 59 & 0.1150 & 0.1526 \\ 2438 & 12: 07: 01 & 0.0784 & 0.2233 \\ 2440 & 12: 07: 03 & 0.0740 & 0.1407 \\ 2442 & 12: 07: 05 & 0.1159 & 0.0572 \\ 2444 & 12: 07: 07 & 0.0840 & 0.1519 \\ 2446 & 12: 07: 09 & 0.0566 & 0.2005 \\ 2448 & 12: 07: 11 & 0.1876 & 0.2563 \\ 2450 & 12: 07: 13 & 0.1799 & 0.2189 \\ 2452 & 12: 07: 15 & 0.1712 & 0.2181 \\ 2454 & 12: 07: 17 & 0.1112 & 0.2421 \\ 2456 & 12: 07: 19 & 0.1638 & 0.3048 \\ 2458 & 12: 07: 21 & 0.1180 & 0.1267 \\ 2460 & 12: 07: 23 & 0.1810 & 0.4152 \\ 2462 & 12: 07: 25 & 0.1424 & 0.1179 \\ 2464 & 12: 07: 27 & 0.3790 & 0.3096 \\ 2466 & 12: 07: 29 & 0.1377 & 0.1786 \\ 2468 & 12: 07: 31 & 0.0595 & 0.2006 \\ 2470 & 12: 07: 33 & 0.0700 & 0.1167 \\ 2472 & 12: 07: 35 & 0.1237 & 0.1465 \\ 2474 & 12: 07: 37 & 0.0524 & 0.2096 \\ 2476 & 12: 07: 39 & 0.0678 & 0.1913 \\ 2478 & 12: 07: 41 & 0.1414 & 0.1669 \\ 2480 & 12: 07: 43 & 0.1590 & 0.1904 \\ 2482 & 12: 07: 45 & 0.2706 & 0.0826 \\ 2484 & 12: 07: 47 & 0.2312 & 0.1173 \\ 2486 & 12: 07: 49 & 0.1805 & 0.1368 \\ 2488 & 12: 07: 51 & 0.1123 & 0.1271 \\ 2490 & 12: 07: 53 & 0.1543 & 0.2216 \\ 2492 & 12: 07: 55 & 0.0691 & 0.1694\end{array}$




$\begin{array}{llll}2494 & 12: 07: 57 & 0.0770 & 0.2494 \\ 2496 & 12: 07: 59 & 0.1038 & 0.2109 \\ 2498 & 12: 08: 01 & 0.0397 & 0.1573 \\ 2500 & 12: 08: 03 & 0.1466 & 0.1392 \\ 2502 & 12: 08: 05 & 0.0996 & 0.2136 \\ 2504 & 12: 08: 07 & 0.0490 & 0.2401 \\ 2506 & 12: 08: 09 & 0.1559 & 0.2456 \\ 2508 & 12: 08: 11 & 0.0522 & 0.1767 \\ 2510 & 12: 08: 13 & 0.0690 & 0.1220 \\ 2512 & 12: 08: 15 & 0.1689 & 0.1647 \\ 2514 & 12: 08: 17 & 0.0516 & 0.3696 \\ 2516 & 12: 08: 19 & 0.0437 & 0.0762 \\ 2518 & 12: 08: 21 & 0.0908 & 0.1252 \\ 2520 & 12: 08: 23 & 0.0951 & 0.1882 \\ 2522 & 12: 08: 25 & 0.0872 & 0.3926 \\ 2524 & 12: 08: 27 & 0.0416 & 0.5315 \\ 2526 & 12: 08: 29 & 0.1094 & 0.4664 \\ 2528 & 12: 08: 31 & 0.0630 & 0.1602 \\ 2530 & 12: 08: 33 & 0.1953 & 0.1575 \\ 2532 & 12: 08: 35 & 0.0625 & 0.2725 \\ 2534 & 12: 08: 37 & 0.0553 & 0.0600 \\ 2536 & 12: 08: 39 & 0.0681 & 0.0742 \\ 2538 & 12: 08: 41 & 0.0995 & 0.1335 \\ 2540 & 12: 08: 43 & 0.2160 & 0.0627 \\ 2542 & 12: 08: 45 & 0.0215 & 0.1442 \\ 2544 & 12: 08: 47 & 0.1410 & 0.0485 \\ 2546 & 12: 08: 49 & 0.1455 & 0.2821 \\ 2548 & 12: 08: 51 & 0.2674 & 0.1580 \\ 2550 & 12: 08: 53 & 0.1061 & 0.0837 \\ 2552 & 12: 08: 55 & 0.0964 & 0.3439 \\ 2554 & 12: 08: 57 & 0.0718 & 0.0789 \\ 2556 & 12: 08: 59 & 0.1824 & 0.1085 \\ 2558 & 12: 09: 01 & 0.0536 & 0.1831 \\ 2560 & 12: 09: 03 & 0.2200 & 0.2179 \\ 2562 & 12: 09: 05 & 0.1041 & 0.0646 \\ 2564 & 12: 09: 07 & 0.1791 & 0.4954 \\ 2566 & 12: 09: 09 & 0.1449 & 0.1133 \\ 2568 & 12: 09: 11 & 0.0413 & 0.3919 \\ 2570 & 12: 09: 13 & 0.1789 & 0.2520 \\ 2572 & 12: 09: 15 & 0.1665 & 0.2951 \\ 2574 & 12: 09: 17 & 0.1938 & 0.2037 \\ 2576 & 12: 09: 19 & 0.0607 & 0.4262 \\ 2578 & 12: 09: 21 & 0.3151 & 0.1383 \\ 2580 & 12: 09: 23 & 0.0511 & 0.0998 \\ 2582 & 12: 09: 25 & 0.0912 & 0.1649 \\ 2584 & 12: 09: 27 & 0.1079 & 0.1984 \\ 2586 & 12: 09: 29 & 0.0377 & 0.1398 \\ 2588 & 12: 09: 31 & 0.1071 & 0.0771 \\ 2590 & 12: 09: 33 & 0.1064 & 0.2812 \\ 2592 & 12: 09: 35 & 0.0560 & 0.0839 \\ 2594 & 12: 09: 37 & 0.1535 & 0.0790 \\ 2596 & 12: 09: 39 & 0.0884 & 0.0902\end{array}$


Appendix B - Laser Data Test 1 NIST102505_1.xls

$\begin{array}{llll}2598 & 12: 09: 41 & 0.2240 & 0.0805 \\ 2600 & 12: 09: 43 & 0.0837 & 0.1668 \\ 2602 & 12: 09: 45 & 0.0373 & 0.1046 \\ 2604 & 12: 09: 47 & 0.1765 & 0.0557 \\ 2606 & 12: 09: 49 & 0.0563 & 0.3386 \\ 2608 & 12: 09: 51 & 0.0651 & 0.1607 \\ 2610 & 12: 09: 53 & 0.0936 & 0.2187 \\ 2612 & 12: 09: 55 & 0.0738 & 0.1732 \\ 2614 & 12: 09: 57 & 0.0263 & 0.2229 \\ 2616 & 12: 09: 59 & 0.0890 & 0.2609 \\ 2618 & 12: 10: 01 & 0.0898 & 0.1552 \\ 2620 & 12: 10: 03 & 0.1153 & 0.1259 \\ 2622 & 12: 10: 05 & 0.1513 & 0.1933 \\ 2624 & 12: 10: 07 & 0.0934 & 0.1338 \\ 2626 & 12: 10: 09 & 0.0549 & 0.0825 \\ 2628 & 12: 10: 11 & 0.1196 & 0.2047 \\ 2630 & 12: 10: 13 & 0.1163 & 0.0712 \\ 2632 & 12: 10: 15 & 0.2643 & 0.0757 \\ 2634 & 12: 10: 17 & 0.1764 & 0.1723 \\ 2636 & 12: 10: 19 & 0.0417 & 0.2428 \\ 2638 & 12: 10: 21 & 0.0262 & 0.0827 \\ 2640 & 12: 10: 23 & 0.1028 & 0.2532 \\ 2642 & 12: 10: 25 & 0.1308 & 0.2832 \\ 2644 & 12: 10: 27 & 0.1145 & 0.1904 \\ 2646 & 12: 10: 29 & 0.0270 & 0.3736 \\ 2648 & 12: 10: 31 & 0.1517 & 0.1817 \\ 2650 & 12: 10: 33 & 0.0872 & 0.1418 \\ 2652 & 12: 10: 35 & 0.2008 & 0.0847 \\ 2654 & 12: 10: 37 & 0.0669 & 0.1277 \\ 2656 & 12: 10: 39 & 0.1334 & 0.1457 \\ 2658 & 12: 10: 41 & 0.0730 & 0.0790 \\ 2660 & 12: 10: 43 & 0.0814 & 0.0500 \\ 2662 & 12: 10: 45 & 0.0605 & 0.2075 \\ 2664 & 12: 10: 47 & 0.1031 & 0.2283 \\ 2666 & 12: 10: 49 & 0.0340 & 0.1006 \\ 2668 & 12: 10: 51 & 0.1680 & 0.1680 \\ 2670 & 12: 10: 53 & 0.0620 & 0.0840 \\ 2672 & 12: 10: 55 & 0.0395 & 0.1160 \\ 2674 & 12: 10: 57 & 0.0549 & 0.1240 \\ 2676 & 12: 10: 59 & 0.0563 & 0.1037 \\ 2678 & 12: 11: 01 & 0.0692 & 0.0749 \\ 2680 & 12: 11: 03 & 0.1090 & 0.1535 \\ 2682 & 12: 11: 05 & 0.0753 & 0.2064 \\ 2684 & 12: 11: 07 & 0.1535 & 0.1560 \\ 2686 & 12: 11: 09 & 0.0715 & 0.1097 \\ 2688 & 12: 11: 11 & 0.0813 & 0.1765 \\ 2690 & 12: 11: 13 & 0.1900 & 0.1778 \\ 2692 & 12: 11: 15 & 0.1479 & 0.2205 \\ 2694 & 12: 11: 17 & 0.1043 & 0.0644 \\ 2696 & 12: 11: 19 & 0.0780 & 0.1626 \\ 2698 & 12: 11: 21 & 0.0878 & 0.1777 \\ 2700 & 12: 11: 23 & 0.0646 & 0.1893\end{array}$




$\begin{array}{llll}2702 & 12: 11: 25 & 0.1348 & 0.1317 \\ 2704 & 12: 11: 27 & 0.1259 & 0.1745 \\ 2706 & 12: 11: 29 & 0.0616 & 0.1485 \\ 2708 & 12: 11: 31 & 0.0500 & 0.0860 \\ 2710 & 12: 11: 33 & 0.0649 & 0.0556 \\ 2712 & 12: 11: 35 & 0.1583 & 0.1213 \\ 2714 & 12: 11: 37 & 0.0745 & 0.2685 \\ 2716 & 12: 11: 39 & 0.0808 & 0.2115 \\ 2718 & 12: 11: 41 & 0.1312 & 0.2191 \\ 2720 & 12: 11: 43 & 0.0367 & 0.1963 \\ 2722 & 12: 11: 45 & 0.2532 & 0.1336 \\ 2724 & 12: 11: 47 & 0.1516 & 0.2952 \\ 2726 & 12: 11: 49 & 0.0838 & 0.1660 \\ 2728 & 12: 11: 51 & 0.0900 & 0.2665 \\ 2730 & 12: 11: 53 & 0.1110 & 0.1991 \\ 2732 & 12: 11: 55 & 0.1184 & 0.3928 \\ 2734 & 12: 11: 57 & 0.0682 & 0.1903 \\ 2736 & 12: 11: 59 & 0.0603 & 0.2753 \\ 2738 & 12: 12: 01 & 0.1532 & 0.1743 \\ 2740 & 12: 12: 03 & 0.0500 & 0.2107 \\ 2742 & 12: 12: 05 & 0.1707 & 0.2838 \\ 2744 & 12: 12: 07 & 0.1320 & 0.2208 \\ 2746 & 12: 12: 09 & 0.1415 & 0.1290 \\ 2748 & 12: 12: 11 & 0.1136 & 0.1807 \\ 2750 & 12: 12: 13 & 0.1094 & 0.1338 \\ 2752 & 12: 12: 15 & 0.0130 & 0.0782 \\ 2754 & 12: 12: 17 & 0.0487 & 0.1935 \\ 2756 & 12: 12: 19 & 0.1071 & 0.1325 \\ 2758 & 12: 12: 21 & 0.1456 & 0.0716 \\ 2760 & 12: 12: 23 & 0.1782 & 0.1297 \\ 2762 & 12: 12: 25 & 0.1435 & 0.0802 \\ 2764 & 12: 12: 27 & 0.0573 & 0.2292 \\ 2766 & 12: 12: 29 & 0.2521 & 0.1602 \\ 2768 & 12: 12: 31 & 0.0607 & 0.2609 \\ 2770 & 12: 12: 33 & 0.1578 & 0.3015 \\ 2772 & 12: 12: 35 & 0.0494 & 0.3149 \\ 2774 & 12: 12: 37 & 0.2699 & 0.7085 \\ 2776 & 12: 12: 39 & 0.1055 & 0.2073 \\ 2778 & 12: 12: 41 & 0.1466 & 0.1925 \\ 2780 & 12: 12: 43 & 0.0915 & 0.0970 \\ 2782 & 12: 12: 45 & 0.1220 & 0.0722 \\ 2784 & 12: 12: 47 & 0.1041 & 0.1868 \\ 2786 & 12: 12: 49 & 0.0913 & 0.2231 \\ 2788 & 12: 12: 51 & 0.0235 & 0.1140 \\ 2790 & 12: 12: 53 & 0.0637 & 0.2350 \\ 2792 & 12: 12: 55 & 0.0699 & 0.1807 \\ 2794 & 12: 12: 57 & 0.0630 & 0.2068 \\ 2796 & 12: 12: 59 & 0.1705 & 0.2671 \\ 2798 & 12: 13: 01 & 0.0226 & 0.3008 \\ 2800 & 12: 13: 03 & 0.1312 & 0.2363 \\ 2802 & 12: 13: 05 & 0.0718 & 0.5828 \\ 2804 & 12: 13: 07 & 0.0984 & 0.2778\end{array}$




$\begin{array}{llll}2806 & 12: 13: 09 & 0.0515 & 0.0890 \\ 2808 & 12: 13: 11 & 0.0626 & 0.1829 \\ 2810 & 12: 13: 13 & 0.2110 & 0.2327 \\ 2812 & 12: 13: 15 & 0.1294 & 0.1138 \\ 2814 & 12: 13: 17 & 0.0921 & 0.1817 \\ 2816 & 12: 13: 19 & 0.0592 & 0.3342 \\ 2818 & 12: 13: 21 & 0.1692 & 0.2874 \\ 2820 & 12: 13: 23 & 0.1087 & 0.1384 \\ 2822 & 12: 13: 25 & 0.0571 & 0.2215 \\ 2824 & 12: 13: 27 & 0.1106 & 0.4535 \\ 2826 & 12: 13: 29 & 0.0263 & 0.1494 \\ 2828 & 12: 13: 31 & 0.0961 & 0.1545 \\ 2830 & 12: 13: 33 & 0.0798 & 0.1656 \\ 2832 & 12: 13: 35 & 0.1456 & 0.1018 \\ 2834 & 12: 13: 37 & 0.0260 & 0.2532 \\ 2836 & 12: 13: 39 & 0.0785 & 0.3694 \\ 2838 & 12: 13: 41 & 0.0521 & 0.2615 \\ 2840 & 12: 13: 43 & 0.0325 & 0.1011 \\ 2842 & 12: 13: 45 & 0.0517 & 0.1825 \\ 2844 & 12: 13: 47 & 0.0498 & 0.3028 \\ 2846 & 12: 13: 49 & 0.0526 & 0.1582 \\ 2848 & 12: 13: 51 & 0.2495 & 0.2723 \\ 2850 & 12: 13: 53 & 0.0397 & 0.1638 \\ 2852 & 12: 13: 55 & 0.0770 & 0.2688 \\ 2854 & 12: 13: 57 & 0.0887 & 0.1515 \\ 2856 & 12: 13: 59 & 0.0938 & 0.1196 \\ 2858 & 12: 14: 01 & 0.1120 & 0.2041 \\ 2860 & 12: 14: 03 & 0.1597 & 0.2600 \\ 2862 & 12: 14: 05 & 0.0990 & 0.2212 \\ 2864 & 12: 14: 07 & 0.1463 & 0.1919 \\ 2866 & 12: 14: 09 & 0.0601 & 0.1319 \\ 2868 & 12: 14: 11 & 0.1978 & 0.0950 \\ 2870 & 12: 14: 13 & 0.0492 & 0.1385 \\ 2872 & 12: 14: 15 & 0.1065 & 0.2954 \\ 2874 & 12: 14: 17 & 0.1449 & 0.2673 \\ 2876 & 12: 14: 19 & 0.0493 & 0.2883 \\ 2878 & 12: 14: 21 & 0.0839 & 0.1463 \\ 2880 & 12: 14: 23 & 0.1150 & 0.1998 \\ 2882 & 12: 14: 25 & 0.1054 & 0.4437 \\ 2884 & 12: 14: 27 & 0.0479 & 0.1147 \\ 2886 & 12: 14: 29 & 0.1509 & 0.2412 \\ 2888 & 12: 14: 31 & 0.1081 & 0.2253 \\ 2890 & 12: 14: 33 & 0.0990 & 0.2307 \\ 2892 & 12: 14: 35 & 0.0472 & 0.1187 \\ 2894 & 12: 14: 37 & 0.0101 & 0.1468 \\ 2896 & 12: 14: 39 & 0.1710 & 0.1949 \\ 2898 & 12: 14: 41 & 0.1283 & 0.1939 \\ 2900 & 12: 14: 43 & 0.1108 & 0.2703 \\ 2902 & 12: 14: 45 & 0.1315 & 0.1219 \\ 2904 & 12: 14: 47 & 0.1270 & 0.2536 \\ 2906 & 12: 14: 49 & 0.2552 & 0.3539 \\ 2908 & 12: 14: 51 & 0.1072 & 0.1480\end{array}$




$\begin{array}{llll}2910 & 12: 14: 53 & 0.1230 & 0.1697 \\ 2912 & 12: 14: 55 & 0.0228 & 0.1468 \\ 2914 & 12: 14: 57 & 0.1881 & 0.1757 \\ 2916 & 12: 14: 59 & 0.0792 & 0.2482 \\ 2918 & 12: 15: 01 & 0.0917 & 0.1458 \\ 2920 & 12: 15: 03 & 0.0567 & 0.1628 \\ 2922 & 12: 15: 05 & 0.0915 & 0.1207 \\ 2924 & 12: 15: 07 & 0.1591 & 0.1911 \\ 2926 & 12: 15: 09 & 0.1664 & 0.2204 \\ 2928 & 12: 15: 11 & 0.0645 & 0.2427 \\ 2930 & 12: 15: 13 & 0.1128 & 0.3733 \\ 2932 & 12: 15: 15 & 0.0574 & 0.0992 \\ 2934 & 12: 15: 17 & 0.1369 & 0.2475 \\ 2936 & 12: 15: 19 & 0.1203 & 0.2974 \\ 2938 & 12: 15: 21 & 0.1121 & 0.3973 \\ 2940 & 12: 15: 23 & 0.0778 & 0.1292 \\ 2942 & 12: 15: 25 & 0.1845 & 0.0796 \\ 2944 & 12: 15: 27 & 0.0722 & 0.1078 \\ 2946 & 12: 15: 29 & 0.1180 & 0.0866 \\ 2948 & 12: 15: 31 & 0.1725 & 0.1408 \\ 2950 & 12: 15: 33 & 0.1425 & 0.0867 \\ 2952 & 12: 15: 35 & 0.1695 & 0.1881 \\ 2954 & 12: 15: 37 & 0.1461 & 0.0283 \\ 2956 & 12: 15: 39 & 0.0490 & 0.1120 \\ 2958 & 12: 15: 41 & 0.0419 & 0.3112 \\ 2960 & 12: 15: 43 & 0.0784 & 0.2397 \\ 2962 & 12: 15: 45 & 0.0874 & 0.5604 \\ 2964 & 12: 15: 47 & 0.0624 & 0.2950 \\ 2966 & 12: 15: 49 & 0.0488 & 0.1613 \\ 2968 & 12: 15: 51 & 0.0500 & 0.0777 \\ 2970 & 12: 15: 53 & 0.0574 & 0.1874 \\ 2972 & 12: 15: 55 & 0.0330 & 0.1663 \\ 2974 & 12: 15: 57 & 0.1337 & 0.1586 \\ 2976 & 12: 15: 59 & 0.0745 & 0.2431 \\ 2978 & 12: 16: 01 & 0.0411 & 0.3037 \\ 2980 & 12: 16: 03 & 0.1787 & 0.1528 \\ 2982 & 12: 16: 05 & 0.1270 & 0.1072 \\ 2984 & 12: 16: 07 & 0.0245 & 0.2153 \\ 2986 & 12: 16: 09 & 0.0675 & 0.1024 \\ 2988 & 12: 16: 11 & 0.0411 & 0.1611 \\ 2990 & 12: 16: 13 & 0.0810 & 0.1052 \\ 2992 & 12: 16: 15 & 0.2258 & 0.0747 \\ 2994 & 12: 16: 17 & 0.1734 & 0.2133 \\ 2996 & 12: 16: 19 & 0.0766 & 0.1170 \\ 2998 & 12: 16: 21 & 0.0747 & 0.1635 \\ 3000 & 12: 16: 23 & 0.0514 & 0.1116 \\ 3002 & 12: 16: 25 & 0.0782 & 0.0774 \\ 3004 & 12: 16: 27 & 0.0567 & 0.0919 \\ 3006 & 12: 16: 29 & 0.1355 & 0.0758 \\ 3008 & 12: 16: 31 & 0.0597 & 0.2067 \\ 3010 & 12: 16: 33 & 0.0712 & 0.2737 \\ 3012 & 12: 16: 35 & 0.0820 & 0.1028\end{array}$




$\begin{array}{llll}3014 & 12: 16: 37 & 0.0242 & 0.1722 \\ 3016 & 12: 16: 39 & 0.1103 & 0.0898 \\ 3018 & 12: 16: 41 & 0.1357 & 0.1052 \\ 3020 & 12: 16: 43 & 0.0590 & 0.1000 \\ 3022 & 12: 16: 45 & 0.0776 & 0.2861 \\ 3024 & 12: 16: 47 & 0.0291 & 0.1272 \\ 3026 & 12: 16: 49 & 0.0258 & 0.0690 \\ 3028 & 12: 16: 51 & 0.0090 & 0.1078 \\ 3030 & 12: 16: 53 & 0.0520 & 0.0970 \\ 3032 & 12: 16: 55 & 0.1211 & 0.1147 \\ 3034 & 12: 16: 57 & 0.0637 & 0.1752 \\ 3036 & 12: 16: 59 & 0.0169 & 0.0722 \\ 3038 & 12: 17: 01 & 0.0474 & 0.0503 \\ 3040 & 12: 17: 03 & 0.0511 & 0.0659 \\ 3042 & 12: 17: 05 & 0.0861 & 0.0289 \\ 3044 & 12: 17: 07 & 0.0449 & 0.3071 \\ 3046 & 12: 17: 09 & 0.0788 & 0.0665 \\ 3048 & 12: 17: 11 & 0.1267 & 0.2098 \\ 3050 & 12: 17: 13 & 0.0514 & 0.0585 \\ 3052 & 12: 17: 15 & 0.0492 & 0.0539 \\ 3054 & 12: 17: 17 & 0.0258 & 0.1374 \\ 3056 & 12: 17: 19 & 0.0766 & 0.0684 \\ 3058 & 12: 17: 21 & 0.0861 & 0.0716 \\ 3060 & 12: 17: 23 & 0.0146 & 0.0723 \\ 3062 & 12: 17: 25 & 0.0439 & 0.0792 \\ 3064 & 12: 17: 27 & 0.0366 & 0.0888 \\ 3066 & 12: 17: 29 & 0.0249 & 0.1083 \\ 3068 & 12: 17: 31 & 0.0313 & 0.0275 \\ 3070 & 12: 17: 33 & 0.0435 & 0.1452 \\ 3072 & 12: 17: 35 & 0.0220 & 0.2054 \\ 3074 & 12: 17: 37 & 0.0147 & 0.1243 \\ 3076 & 12: 17: 39 & 0.0333 & 0.1774 \\ 3078 & 12: 17: 41 & 0.0510 & 0.1075 \\ 3080 & 12: 17: 43 & 0.0745 & 0.1116 \\ 3082 & 12: 17: 45 & 0.0229 & 0.1974 \\ 3084 & 12: 17: 47 & 0.0369 & 0.0997 \\ 3086 & 12: 17: 49 & 0.0141 & 0.0546 \\ 3088 & 12: 17: 51 & 0.0136 & 0.0491 \\ 3090 & 12: 17: 53 & 0.0219 & 0.0975 \\ 3092 & 12: 17: 55 & 0.0844 & 0.0836 \\ 3094 & 12: 17: 57 & 0.0511 & 0.0977 \\ 3096 & 12: 17: 59 & 0.0364 & 0.0655 \\ 3098 & 12: 18: 01 & 0.0215 & 0.0299 \\ 3100 & 12: 18: 03 & 0.0456 & 0.0191 \\ 3102 & 12: 18: 05 & 0.0884 & 0.0329 \\ 3104 & 12: 18: 07 & 0.0125 & 0.0719 \\ 3106 & 12: 18: 09 & 0.0112 & 0.0821 \\ 3108 & 12: 18: 11 & 0.0321 & 0.0838 \\ 3110 & 12: 18: 13 & 0.0226 & 0.0808 \\ 3112 & 12: 18: 15 & 0.0187 & 0.0879 \\ 3114 & 12: 18: 17 & 0.0088 & 0.0764 \\ 3116 & 12: 18: 19 & 0.0502 & 0.3384\end{array}$




$\begin{array}{llll}3118 & 12: 18: 21 & 0.0504 & 0.1230 \\ 3120 & 12: 18: 23 & 0.0497 & 0.1287 \\ 3122 & 12: 18: 25 & 0.0152 & 0.0687 \\ 3124 & 12: 18: 27 & 0.0241 & 0.0702 \\ 3126 & 12: 18: 29 & 0.0349 & 0.1801 \\ 3128 & 12: 18: 31 & 0.0107 & 0.0819 \\ 3130 & 12: 18: 33 & 0.0801 & 0.1062 \\ 3132 & 12: 18: 35 & 0.0845 & 0.1812 \\ 3134 & 12: 18: 37 & 0.0218 & 0.1506 \\ 3136 & 12: 18: 39 & 0.0263 & 0.1081 \\ 3138 & 12: 18: 41 & 0.0891 & 0.1277 \\ 3140 & 12: 18: 43 & 0.0265 & 0.1285 \\ 3142 & 12: 18: 45 & 0.0312 & 0.1586 \\ 3144 & 12: 18: 47 & 0.0281 & 0.1051 \\ 3146 & 12: 18: 49 & 0.0245 & 0.1597 \\ 3148 & 12: 18: 51 & 0.0239 & 0.0782 \\ 3150 & 12: 18: 53 & 0.0150 & 0.0295 \\ 3152 & 12: 18: 55 & 0.0405 & 0.1221 \\ 3154 & 12: 18: 57 & 0.0775 & 0.0908 \\ 3156 & 12: 18: 59 & 0.0289 & 0.1361 \\ 3158 & 12: 19: 01 & 0.0596 & 0.0550 \\ 3160 & 12: 19: 03 & 0.0669 & 0.1813 \\ 3162 & 12: 19: 05 & 0.0397 & 0.1917 \\ 3164 & 12: 19: 07 & 0.0847 & 0.2569 \\ 3166 & 12: 19: 09 & 0.0375 & 0.1987 \\ 3168 & 12: 19: 11 & 0.0163 & 0.0688 \\ 3170 & 12: 19: 13 & 0.0075 & 0.1004 \\ 3172 & 12: 19: 15 & 0.0608 & 0.0872 \\ 3174 & 12: 19: 17 & 0.1212 & 0.1170 \\ 3176 & 12: 19: 19 & 0.1276 & 0.1779 \\ 3178 & 12: 19: 21 & 0.0112 & 0.0812 \\ 3180 & 12: 19: 23 & 0.0142 & 0.0208 \\ 3182 & 12: 19: 25 & 0.0339 & 0.0601 \\ 3184 & 12: 19: 27 & 0.1039 & 0.0710 \\ 3186 & 12: 19: 29 & 0.0457 & 0.1578 \\ 3188 & 12: 19: 31 & 0.0399 & 0.2216 \\ 3190 & 12: 19: 33 & 0.0294 & 0.0497 \\ 3192 & 12: 19: 35 & 0.0123 & 0.0128 \\ 3194 & 12: 19: 37 & 0.0403 & 0.1439 \\ 3196 & 12: 19: 39 & 0.0095 & 0.0273 \\ 3198 & 12: 19: 41 & 0.0522 & 0.0128 \\ 3200 & 12: 19: 43 & 0.0408 & 0.0862 \\ 3202 & 12: 19: 45 & 0.0207 & 0.0482 \\ 3204 & 12: 19: 47 & 0.0272 & 0.0945 \\ 3206 & 12: 19: 49 & 0.0160 & 0.0675 \\ 3208 & 12: 19: 51 & 0.0133 & 0.0918 \\ 3210 & 12: 19: 53 & 0.0440 & 0.2271 \\ 3212 & 12: 19: 55 & 0.0297 & 0.0594 \\ 3214 & 12: 19: 57 & 0.0064 & 0.0584 \\ 3216 & 12: 19: 59 & 0.0164 & 0.0942 \\ 3218 & 12: 20: 01 & 0.0146 & 0.0530 \\ 3220 & 12: 20: 03 & 0.0282 & 0.1275\end{array}$




$\begin{array}{llll}3222 & 12: 20: 05 & 0.0381 & 0.1982 \\ 3224 & 12: 20: 07 & 0.0230 & 0.1217 \\ 3226 & 12: 20: 09 & 0.0474 & 0.0983 \\ 3228 & 12: 20: 11 & 0.0792 & 0.3696 \\ 3230 & 12: 20: 13 & 0.0329 & 0.0825 \\ 3232 & 12: 20: 15 & 0.0207 & 0.1188 \\ 3234 & 12: 20: 17 & 0.0765 & 0.2292 \\ 3236 & 12: 20: 19 & 0.0573 & 0.2014 \\ 3238 & 12: 20: 21 & 0.0056 & 0.2651 \\ 3240 & 12: 20: 23 & 0.0358 & 0.2069 \\ 3242 & 12: 20: 25 & 0.0127 & 0.1404 \\ 3244 & 12: 20: 27 & 0.0148 & 0.1406 \\ 3246 & 12: 20: 29 & 0.0172 & 0.1032 \\ 3248 & 12: 20: 31 & 0.0578 & 0.1636 \\ 3250 & 12: 20: 33 & 0.0190 & 0.1616 \\ 3252 & 12: 20: 35 & 0.0280 & 0.3807 \\ 3254 & 12: 20: 37 & 0.0281 & 0.1260 \\ 3256 & 12: 20: 39 & 0.0418 & 0.0622 \\ 3258 & 12: 20: 41 & 0.0677 & 0.3046 \\ 3260 & 12: 20: 43 & 0.0430 & 0.0815 \\ 3262 & 12: 20: 45 & 0.0509 & 0.2734 \\ 3264 & 12: 20: 47 & 0.0514 & 0.1208 \\ 3266 & 12: 20: 49 & 0.0353 & 0.2724 \\ 3268 & 12: 20: 51 & 0.0238 & 0.1347 \\ 3270 & 12: 20: 53 & 0.0307 & 0.0779 \\ 3272 & 12: 20: 55 & 0.0230 & 0.1075 \\ 3274 & 12: 20: 57 & 0.0508 & 0.0859 \\ 3276 & 12: 20: 59 & 0.0512 & 0.1916 \\ 3278 & 12: 21: 01 & 0.0385 & 0.1585 \\ 3280 & 12: 21: 03 & 0.0324 & 0.0870 \\ 3282 & 12: 21: 05 & 0.0519 & 0.0806 \\ 3284 & 12: 21: 07 & 0.0697 & 0.1221 \\ 3286 & 12: 21: 09 & 0.0404 & 0.0919 \\ 3288 & 12: 21: 11 & 0.0892 & 0.0579 \\ 3290 & 12: 21: 13 & 0.0152 & 0.2179 \\ 3292 & 12: 21: 15 & 0.0518 & 0.1721 \\ 3294 & 12: 21: 17 & 0.0098 & 0.1050 \\ 3296 & 12: 21: 19 & 0.0182 & 0.1691 \\ 3298 & 12: 21: 21 & 0.0246 & 0.0868 \\ 3300 & 12: 21: 23 & 0.0304 & 0.0872 \\ 3302 & 12: 21: 25 & 0.0531 & 0.0731 \\ 3304 & 12: 21: 27 & 0.0279 & 0.0704 \\ 3306 & 12: 21: 29 & 0.0221 & 0.1399 \\ 3308 & 12: 21: 31 & 0.0607 & 0.1529 \\ 3310 & 12: 21: 33 & 0.1016 & 0.1551 \\ 3312 & 12: 21: 35 & 0.0416 & 0.2108 \\ 3314 & 12: 21: 37 & 0.0591 & 0.0759 \\ 3316 & 12: 21: 39 & 0.0393 & 0.1417 \\ 3318 & 12: 21: 41 & 0.0405 & 0.1027 \\ 3320 & 12: 21: 43 & 0.0362 & 0.0784 \\ 3322 & 12: 21: 45 & 0.0324 & 0.0509 \\ 3324 & 12: 21: 47 & 0.0411 & 0.1323\end{array}$


Appendix B - Laser Data Test 1 NIST102505_1.xls

$\begin{array}{llll}3326 & 12: 21: 49 & 0.0271 & 0.0498 \\ 3328 & 12: 21: 51 & 0.1502 & 0.1385 \\ 3330 & 12: 21: 53 & 0.0566 & 0.1728 \\ 3332 & 12: 21: 55 & 0.1162 & 0.0697 \\ 3334 & 12: 21: 57 & 0.0462 & 0.0879 \\ 3336 & 12: 21: 59 & 0.0567 & 0.0346 \\ 3338 & 12: 22: 01 & 0.0665 & 0.0736 \\ 3340 & 12: 22: 03 & 0.0880 & 0.0360 \\ 3342 & 12: 22: 05 & 0.0657 & 0.1885 \\ 3344 & 12: 22: 07 & 0.0463 & 0.0823 \\ 3346 & 12: 22: 09 & 0.0114 & 0.0496 \\ 3348 & 12: 22: 11 & 0.0538 & 0.2409 \\ 3350 & 12: 22: 13 & 0.0316 & 0.1591 \\ 3352 & 12: 22: 15 & 0.0347 & 0.1077 \\ 3354 & 12: 22: 17 & 0.0808 & 0.0758 \\ 3356 & 12: 22: 19 & 0.0272 & 0.1767 \\ 3358 & 12: 22: 21 & 0.0213 & 0.0469 \\ 3360 & 12: 22: 23 & 0.0388 & 0.1387 \\ 3362 & 12: 22: 25 & 0.0301 & 0.1129 \\ 3364 & 12: 22: 27 & 0.0598 & 0.1177 \\ 3366 & 12: 22: 29 & 0.1277 & 0.0720 \\ 3368 & 12: 22: 31 & 0.0461 & 0.2361 \\ 3370 & 12: 22: 33 & 0.0435 & 0.0972 \\ 3372 & 12: 22: 35 & 0.0325 & 0.0294 \\ 3374 & 12: 22: 37 & 0.0310 & 0.0710 \\ 3376 & 12: 22: 39 & 0.0426 & 0.0801 \\ 3378 & 12: 22: 41 & 0.0590 & 0.1703 \\ 3380 & 12: 22: 43 & 0.0340 & 0.1218 \\ 3382 & 12: 22: 45 & 0.0106 & 0.0780 \\ 3384 & 12: 22: 47 & 0.0297 & 0.0512 \\ 3386 & 12: 22: 49 & 0.0258 & 0.0945 \\ 3388 & 12: 22: 51 & 0.0752 & 0.1970 \\ 3390 & 12: 22: 53 & 0.0114 & 0.0750 \\ 3392 & 12: 22: 55 & 0.0591 & 0.0955 \\ 3394 & 12: 22: 57 & 0.0615 & 0.1754 \\ 3396 & 12: 22: 59 & 0.0653 & 0.1233 \\ 3398 & 12: 23: 01 & 0.0335 & 0.0454 \\ 3400 & 12: 23: 03 & 0.0834 & 0.2111 \\ 3402 & 12: 23: 05 & 0.0314 & 0.1024 \\ 3404 & 12: 23: 07 & 0.0527 & 0.0811 \\ 3406 & 12: 23: 09 & 0.0423 & 0.2840 \\ 3408 & 12: 23: 11 & 0.0898 & 0.3311 \\ 3410 & 12: 23: 13 & 0.0569 & 0.1648 \\ 3412 & 12: 23: 15 & 0.0512 & 0.2177 \\ 3414 & 12: 23: 17 & 0.0453 & 0.0674 \\ 3416 & 12: 23: 19 & 0.0211 & 0.3209 \\ 3418 & 12: 23: 21 & 0.0680 & 0.0293 \\ 3420 & 12: 23: 23 & 0.0716 & 0.0534 \\ 3422 & 12: 23: 25 & 0.0180 & 0.0545 \\ 3424 & 12: 23: 27 & 0.0298 & 0.0391 \\ 3428 & 12: 23: 29 & 0.0415 & 0.1437 \\ 12: 23: 31 & 0.0186 & 0.1859\end{array}$

B - 33 


$\begin{array}{llll}3430 & 12: 23: 33 & 0.0301 & 0.1997 \\ 3432 & 12: 23: 35 & 0.0029 & 0.1265 \\ 3434 & 12: 23: 37 & 0.0590 & 0.1405 \\ 3436 & 12: 23: 39 & 0.0115 & 0.1573 \\ 3438 & 12: 23: 41 & 0.0104 & 0.0717 \\ 3440 & 12: 23: 43 & 0.0381 & 0.2880 \\ 3442 & 12: 23: 45 & 0.0359 & 0.2512 \\ 3444 & 12: 23: 47 & 0.0538 & 0.2647 \\ 3446 & 12: 23: 49 & 0.0735 & 0.1008 \\ 3448 & 12: 23: 51 & 0.0252 & 0.0683 \\ 3450 & 12: 23: 53 & 0.0287 & 0.0389 \\ 3452 & 12: 23: 55 & 0.0791 & 0.1199 \\ 3454 & 12: 23: 57 & 0.0450 & 0.1156 \\ 3456 & 12: 23: 59 & 0.0106 & 0.1464 \\ 3458 & 12: 24: 01 & 0.0197 & 0.0897 \\ 3460 & 12: 24: 03 & 0.0806 & 0.1766 \\ 3462 & 12: 24: 05 & 0.0668 & 0.1122 \\ 3464 & 12: 24: 07 & 0.0223 & 0.1011 \\ 3466 & 12: 24: 09 & 0.0339 & 0.1408 \\ 3468 & 12: 24: 11 & 0.0327 & 0.0489 \\ 3470 & 12: 24: 13 & 0.0785 & 0.1663 \\ 3472 & 12: 24: 15 & 0.0834 & 0.0869 \\ 3474 & 12: 24: 17 & 0.0122 & 0.1012 \\ 3476 & 12: 24: 19 & 0.0771 & 0.1931 \\ 3478 & 12: 24: 21 & 0.0504 & 0.2491 \\ 3480 & 12: 24: 23 & 0.0772 & 0.1203 \\ 3482 & 12: 24: 25 & 0.0162 & 0.0965 \\ 3484 & 12: 24: 27 & 0.0478 & 0.0829 \\ 3486 & 12: 24: 29 & 0.0576 & 0.1923 \\ 3488 & 12: 24: 31 & 0.0211 & 0.3041 \\ 3490 & 12: 24: 33 & 0.0063 & 0.0642 \\ 3492 & 12: 24: 35 & 0.0290 & 0.1697 \\ 3494 & 12: 24: 37 & 0.0178 & 0.1361 \\ 3496 & 12: 24: 39 & 0.0647 & 0.1717 \\ 3498 & 12: 24: 41 & 0.0307 & 0.2368 \\ 3500 & 12: 24: 43 & 0.0503 & 0.1356 \\ 3502 & 12: 24: 45 & 0.0966 & 0.2131 \\ 3504 & 12: 24: 47 & 0.2510 & 0.2331 \\ 3506 & 12: 24: 49 & 0.0419 & 0.1912 \\ 3508 & 12: 24: 51 & 0.0145 & 0.2915 \\ 3510 & 12: 24: 53 & 0.0272 & 0.2384 \\ 3512 & 12: 24: 55 & 0.0091 & 0.0492 \\ 3514 & 12: 24: 57 & 0.0506 & 0.0743 \\ 3516 & 12: 24: 59 & 0.0390 & 0.3206 \\ 3518 & 12: 25: 01 & 0.0477 & 0.1194 \\ 3520 & 12: 25: 03 & 0.0210 & 0.1292 \\ 3522 & 12: 25: 05 & 0.1028 & 0.0749 \\ 3524 & 12: 25: 07 & 0.0814 & 0.1346 \\ 3528 & 12: 25: 09 & 0.0264 & 0.1200 \\ 3530 & 12: 25: 11 & 0.0295 & 0.0868 \\ & 12: 25: 15 & 0.0237 & 0.1222 \\ & & & 0.1402\end{array}$




$\begin{array}{llll}3534 & 12: 25: 17 & 0.0840 & 0.3759 \\ 3536 & 12: 25: 19 & 0.0506 & 0.0861 \\ 3538 & 12: 25: 21 & 0.0600 & 0.1312 \\ 3540 & 12: 25: 23 & 0.0185 & 0.1115 \\ 3542 & 12: 25: 25 & 0.0881 & 0.3080 \\ 3544 & 12: 25: 27 & 0.0085 & 0.1229 \\ 3546 & 12: 25: 29 & 0.0141 & 0.1894 \\ 3548 & 12: 25: 31 & 0.0675 & 0.0829 \\ 3550 & 12: 25: 33 & 0.0245 & 0.0961 \\ 3552 & 12: 25: 35 & 0.1774 & 0.1613 \\ 3554 & 12: 25: 37 & 0.0606 & 0.0753 \\ 3556 & 12: 25: 39 & 0.0227 & 0.0787 \\ 3558 & 12: 25: 41 & 0.1214 & 0.1136 \\ 3560 & 12: 25: 43 & 0.0292 & 0.2524 \\ 3562 & 12: 25: 45 & 0.0611 & 0.1174 \\ 3564 & 12: 25: 47 & 0.0423 & 0.0880 \\ 3566 & 12: 25: 49 & 0.0321 & 0.0521 \\ 3568 & 12: 25: 51 & 0.0237 & 0.0861 \\ 3570 & 12: 25: 53 & 0.0713 & 0.1228 \\ 3572 & 12: 25: 55 & 0.1002 & 0.1871 \\ 3574 & 12: 25: 57 & 0.0520 & 0.1929 \\ 3576 & 12: 25: 59 & 0.0338 & 0.0657 \\ 3578 & 12: 26: 01 & 0.0543 & 0.0769 \\ 3580 & 12: 26: 03 & 0.0562 & 0.1408 \\ 3582 & 12: 26: 05 & 0.1551 & 0.2185 \\ 3584 & 12: 26: 07 & 0.0904 & 0.1221 \\ 3586 & 12: 26: 09 & 0.0143 & 0.0938 \\ 3588 & 12: 26: 11 & 0.0242 & 0.1418 \\ 3590 & 12: 26: 13 & 0.0347 & 0.1440 \\ 3592 & 12: 26: 15 & 0.0540 & 0.0703 \\ 3594 & 12: 26: 17 & 0.0795 & 0.2453 \\ 3596 & 12: 26: 19 & 0.1134 & 0.0640 \\ 3598 & 12: 26: 21 & 0.1043 & 0.0758 \\ 3600 & 12: 26: 23 & 0.0453 & 0.0529 \\ 3602 & 12: 26: 25 & 0.0577 & 0.0872 \\ 3604 & 12: 26: 27 & 0.0492 & 0.2905 \\ 3606 & 12: 26: 29 & 0.0259 & 0.1890 \\ 3608 & 12: 26: 31 & 0.0398 & 0.0672 \\ 3610 & 12: 26: 33 & 0.0320 & 0.2579 \\ 3612 & 12: 26: 35 & 0.0769 & 0.1102 \\ 3614 & 12: 26: 37 & 0.1371 & 0.0930 \\ 3616 & 12: 26: 39 & 0.0193 & 0.1686 \\ 3618 & 12: 26: 41 & 0.0334 & 0.3358 \\ 3620 & 12: 26: 43 & 0.1246 & 0.1808 \\ 3622 & 12: 26: 45 & 0.1187 & 0.0298 \\ 3624 & 12: 26: 47 & 0.0763 & 0.1186 \\ 3626 & 12: 26: 49 & 0.0872 & 0.0753 \\ 3628 & 12: 26: 51 & 0.0373 & 0.0835 \\ 3630 & 12: 26: 53 & 0.0249 & 0.0453 \\ 3632 & 12: 26: 55 & 0.0175 & 0.1211 \\ 3634 & 12: 26: 57 & 0.2304 & 0.1349 \\ 3636 & 12: 26: 59 & 0.0119 & 0.1566\end{array}$




$\begin{array}{llll}3638 & 12: 27: 01 & 0.2144 & 0.5204 \\ 3640 & 12: 27: 03 & 0.0429 & 0.1919 \\ 3642 & 12: 27: 05 & 0.2124 & 0.1041 \\ 3644 & 12: 27: 07 & 0.0412 & 0.1829 \\ 3646 & 12: 27: 09 & 0.1322 & 0.0539 \\ 3648 & 12: 27: 11 & 0.0485 & 0.0387 \\ 3650 & 12: 27: 13 & 0.2095 & 0.1505 \\ 3652 & 12: 27: 15 & 0.1077 & 0.1159 \\ 3654 & 12: 27: 17 & 0.0411 & 0.0943 \\ 3656 & 12: 27: 19 & 0.1650 & 0.2811 \\ 3658 & 12: 27: 21 & 0.0227 & 0.0412 \\ 3660 & 12: 27: 23 & 0.0148 & 0.1317 \\ 3662 & 12: 27: 25 & 0.0956 & 0.0587 \\ 3664 & 12: 27: 27 & 0.0340 & 0.1562 \\ 3666 & 12: 27: 29 & 0.0673 & 0.1774 \\ 3668 & 12: 27: 31 & 0.0683 & 0.1507 \\ 3670 & 12: 27: 33 & 0.1172 & 0.1494 \\ 3672 & 12: 27: 35 & 0.0878 & 0.3481 \\ 3674 & 12: 27: 37 & 0.0678 & 0.2378 \\ 3676 & 12: 27: 39 & 0.0497 & 0.0966 \\ 3678 & 12: 27: 41 & 0.0663 & 0.0750 \\ 3680 & 12: 27: 43 & 0.0199 & 0.1529 \\ 3682 & 12: 27: 45 & 0.0387 & 0.0403 \\ 3684 & 12: 27: 47 & 0.0537 & 0.1367 \\ 3686 & 12: 27: 49 & 0.0620 & 0.1902 \\ 3688 & 12: 27: 51 & 0.0679 & 0.1432 \\ 3690 & 12: 27: 53 & 0.0797 & 0.0386 \\ 3692 & 12: 27: 55 & 0.0197 & 0.0930 \\ 3694 & 12: 27: 57 & 0.0698 & 0.0169 \\ 3696 & 12: 27: 59 & 0.1333 & 0.1192 \\ 3698 & 12: 28: 01 & 0.0509 & 0.2479 \\ 3700 & 12: 28: 03 & 0.0189 & 0.1575 \\ 3702 & 12: 28: 05 & 0.0452 & 0.1351 \\ 3704 & 12: 28: 07 & 0.0499 & 0.1786 \\ 3706 & 12: 28: 09 & 0.0468 & 0.1420 \\ 3708 & 12: 28: 11 & 0.0474 & 0.2463 \\ 3710 & 12: 28: 13 & 0.0630 & 0.2037 \\ 3712 & 12: 28: 15 & 0.0667 & 0.0500 \\ 3714 & 12: 28: 17 & 0.0148 & 0.1222 \\ 3716 & 12: 28: 19 & 0.0472 & 0.1055 \\ 3718 & 12: 28: 21 & 0.0026 & 0.1458 \\ 3720 & 12: 28: 23 & 0.0787 & 0.0871 \\ 3722 & 12: 28: 25 & 0.0969 & 0.1624 \\ 3724 & 12: 28: 27 & 0.0394 & 0.0661 \\ 3726 & 12: 28: 29 & 0.0910 & 0.1505 \\ 3728 & 12: 28: 31 & 0.0241 & 0.0864 \\ 3730 & 12: 28: 33 & 0.0436 & 0.1348 \\ 3732 & 12: 28: 35 & 0.0169 & 0.1789 \\ 3734 & 12: 28: 37 & 0.0952 & 0.0982 \\ 3736 & 12: 28: 39 & 0.0835 & 0.0898 \\ 3738 & 12: 28: 41 & 0.0622 & 0.1112 \\ 3740 & 12: 28: 43 & 0.0304 & 0.1361\end{array}$




$\begin{array}{llll}3742 & 12: 28: 45 & 0.0261 & 0.1096 \\ 3744 & 12: 28: 47 & 0.0783 & 0.0828 \\ 3746 & 12: 28: 49 & 0.0204 & 0.0437 \\ 3748 & 12: 28: 51 & 0.1051 & 0.1164 \\ 3750 & 12: 28: 53 & 0.0199 & 0.1233 \\ 3752 & 12: 28: 55 & 0.0466 & 0.1327 \\ 3754 & 12: 28: 57 & 0.0633 & 0.2300 \\ 3756 & 12: 28: 59 & 0.0472 & 0.1015 \\ 3758 & 12: 29: 01 & 0.0176 & 0.1604 \\ 3760 & 12: 29: 03 & 0.0497 & 0.1348 \\ 3762 & 12: 29: 05 & 0.0203 & 0.1208 \\ 3764 & 12: 29: 07 & 0.0387 & 0.1027 \\ 3766 & 12: 29: 09 & 0.0205 & 0.0933 \\ 3768 & 12: 29: 11 & 0.0515 & 0.1489 \\ 3770 & 12: 29: 13 & 0.0734 & 0.1472 \\ 3772 & 12: 29: 15 & 0.0295 & 0.1240 \\ 3774 & 12: 29: 17 & 0.0275 & 0.2636 \\ 3776 & 12: 29: 19 & 0.0761 & 0.2040 \\ 3778 & 12: 29: 21 & 0.0506 & 0.2102 \\ 3780 & 12: 29: 23 & 0.0914 & 0.1404 \\ 3782 & 12: 29: 25 & 0.0435 & 0.1534 \\ 3784 & 12: 29: 27 & 0.0732 & 0.0953 \\ 3786 & 12: 29: 29 & 0.0690 & 0.2073 \\ 3788 & 12: 29: 31 & 0.0688 & 0.1349 \\ 3790 & 12: 29: 33 & 0.0764 & 0.1238 \\ 3792 & 12: 29: 35 & 0.0167 & 0.1132 \\ 3794 & 12: 29: 37 & 0.0565 & 0.1945 \\ 3796 & 12: 29: 39 & 0.0279 & 0.0571 \\ 3798 & 12: 29: 41 & 0.0301 & 0.0778 \\ 3800 & 12: 29: 43 & 0.0508 & 0.1464 \\ 3802 & 12: 29: 45 & 0.0581 & 0.1258 \\ 3804 & 12: 29: 47 & 0.1298 & 0.1249 \\ 3806 & 12: 29: 49 & 0.0271 & 0.0809 \\ 3808 & 12: 29: 51 & 0.0609 & 0.3548 \\ 3810 & 12: 29: 53 & 0.0426 & 0.1761 \\ 3812 & 12: 29: 55 & 0.0416 & 0.1828 \\ 3814 & 12: 29: 57 & 0.0108 & 0.0350 \\ 3816 & 12: 29: 59 & 0.0251 & 0.2389 \\ 3818 & 12: 30: 01 & 0.0527 & 0.1103 \\ 3820 & 12: 30: 03 & 0.0366 & 0.1248 \\ 3822 & 12: 30: 05 & 0.0531 & 0.0768 \\ 3824 & 12: 30: 07 & 0.0338 & 0.0580 \\ 3826 & 12: 30: 09 & 0.0706 & 0.0238 \\ 3828 & 12: 30: 11 & 0.0737 & 0.0339 \\ 3830 & 12: 30: 13 & 0.0655 & 0.0781 \\ 3832 & 12: 30: 15 & 0.0242 & 0.1052 \\ 3834 & 12: 30: 17 & 0.0447 & 0.2128 \\ 3836 & 12: 30: 19 & 0.0543 & 0.0806 \\ 3838 & 12: 30: 21 & 0.2008 & 0.0530 \\ 3840 & 12: 30: 23 & 0.0355 & 0.0697 \\ 3842 & 12: 30: 25 & 0.0547 & 0.1534 \\ 3844 & 12: 30: 27 & 0.0187 & 0.0683\end{array}$




$\begin{array}{llll}3846 & 12: 30: 29 & 0.0645 & 0.1011 \\ 3848 & 12: 30: 31 & 0.1382 & 0.2841 \\ 3850 & 12: 30: 33 & 0.0614 & 0.1324 \\ 3852 & 12: 30: 35 & 0.0264 & 0.1878 \\ 3854 & 12: 30: 37 & 0.0543 & 0.1622 \\ 3856 & 12: 30: 39 & 0.0675 & 0.1084 \\ 3858 & 12: 30: 41 & 0.0278 & 0.1025 \\ 3860 & 12: 30: 43 & 0.0360 & 0.1306 \\ 3862 & 12: 30: 45 & 0.0535 & 0.4238 \\ 3864 & 12: 30: 47 & 0.0183 & 0.3640 \\ 3866 & 12: 30: 49 & 0.0349 & 0.1916 \\ 3868 & 12: 30: 51 & 0.0342 & 0.0983 \\ 3870 & 12: 30: 53 & 0.0652 & 0.1073 \\ 3872 & 12: 30: 55 & 0.0636 & 0.1028 \\ 3874 & 12: 30: 57 & 0.0274 & 0.0616 \\ 3876 & 12: 30: 59 & 0.0861 & 0.0981 \\ 3878 & 12: 31: 01 & 0.0704 & 0.0914 \\ 3880 & 12: 31: 03 & 0.0593 & 0.2690 \\ 3882 & 12: 31: 05 & 0.0341 & 0.1160 \\ 3884 & 12: 31: 07 & 0.0378 & 0.0637 \\ 3886 & 12: 31: 09 & 0.0529 & 0.0464 \\ 3888 & 12: 31: 11 & 0.0360 & 0.1950 \\ 3890 & 12: 31: 13 & 0.0424 & 0.0388 \\ 3892 & 12: 31: 15 & 0.0443 & 0.1383 \\ 3894 & 12: 31: 17 & 0.1021 & 0.1277 \\ 3896 & 12: 31: 19 & 0.0848 & 0.1297 \\ 3898 & 12: 31: 21 & 0.0973 & 0.1186 \\ 3900 & 12: 31: 23 & 0.0533 & 0.2039 \\ 3902 & 12: 31: 25 & 0.0427 & 0.0707 \\ 3904 & 12: 31: 27 & 0.0547 & 0.1195 \\ 3906 & 12: 31: 29 & 0.0159 & 0.1501 \\ 3908 & 12: 31: 31 & 0.0140 & 0.1122 \\ 3910 & 12: 31: 33 & 0.0516 & 0.2350 \\ 3912 & 12: 31: 35 & 0.1745 & 0.1161 \\ 3914 & 12: 31: 37 & 0.0538 & 0.0649 \\ 3916 & 12: 31: 39 & 0.0470 & 0.0751 \\ 3918 & 12: 31: 41 & 0.0315 & 0.0244 \\ 3920 & 12: 31: 43 & 0.0802 & 0.1576 \\ 3922 & 12: 31: 45 & 0.0298 & 0.2462 \\ 3924 & 12: 31: 47 & 0.1214 & 0.0840 \\ 3926 & 12: 31: 49 & 0.0285 & 0.1098 \\ 3928 & 12: 31: 51 & 0.0733 & 0.0419 \\ 3930 & 12: 31: 53 & 0.0370 & 0.1097 \\ 3932 & 12: 31: 55 & 0.1130 & 0.0835 \\ 3934 & 12: 31: 57 & 0.0254 & 0.1314 \\ 3936 & 12: 31: 59 & 0.0991 & 0.0782 \\ 3938 & 12: 32: 01 & 0.0621 & 0.1657 \\ 3940 & 12: 32: 03 & 0.0615 & 0.1322 \\ 3942 & 12: 32: 05 & 0.0669 & 0.1513 \\ 3944 & 12: 32: 07 & 0.0608 & 0.0877 \\ 3946 & 12: 32: 09 & 0.0172 & 0.1102 \\ 3948 & 12: 32: 11 & 0.0419 & 0.0945\end{array}$




$\begin{array}{llll}3950 & 12: 32: 13 & 0.1165 & 0.2123 \\ 3952 & 12: 32: 15 & 0.0967 & 0.0820 \\ 3954 & 12: 32: 17 & 0.1037 & 0.0482 \\ 3956 & 12: 32: 19 & 0.0589 & 0.1219 \\ 3958 & 12: 32: 21 & 0.0415 & 0.0739 \\ 3960 & 12: 32: 23 & 0.1282 & 0.1909 \\ 3962 & 12: 32: 25 & 0.0388 & 0.1135 \\ 3964 & 12: 32: 27 & 0.0261 & 0.2174 \\ 3966 & 12: 32: 29 & 0.1206 & 0.1208 \\ 3968 & 12: 32: 31 & 0.0376 & 0.0672 \\ 3970 & 12: 32: 33 & 0.0377 & 0.1655 \\ 3972 & 12: 32: 35 & 0.0607 & 0.1294 \\ 3974 & 12: 32: 37 & 0.0518 & 0.1295 \\ 3976 & 12: 32: 39 & 0.0252 & 0.0433 \\ 3978 & 12: 32: 41 & 0.0447 & 0.1427 \\ 3980 & 12: 32: 43 & 0.0526 & 0.1338 \\ 3982 & 12: 32: 45 & 0.0580 & 0.2187 \\ 3984 & 12: 32: 47 & 0.0520 & 0.1364 \\ 3986 & 12: 32: 49 & 0.0804 & 0.2015 \\ 3988 & 12: 32: 51 & 0.0354 & 0.2213 \\ 3990 & 12: 32: 53 & 0.0576 & 0.0457 \\ 3992 & 12: 32: 55 & 0.0897 & 0.1621 \\ 3994 & 12: 32: 57 & 0.1289 & 0.1155 \\ 3996 & 12: 32: 59 & 0.0372 & 0.1383 \\ 3998 & 12: 33: 01 & 0.0369 & 0.0209 \\ 4000 & 12: 33: 03 & 0.0279 & 0.1482 \\ 4002 & 12: 33: 05 & 0.0692 & 0.0394 \\ 4004 & 12: 33: 07 & 0.0377 & 0.0689 \\ 4006 & 12: 33: 09 & 0.0802 & 0.0740 \\ 4008 & 12: 33: 11 & 0.0389 & 0.1324 \\ 4010 & 12: 33: 13 & 0.0255 & 0.1481 \\ 4012 & 12: 33: 15 & 0.1295 & 0.1028 \\ 4014 & 12: 33: 17 & 0.0564 & 0.1533 \\ 4016 & 12: 33: 19 & 0.0518 & 0.1061 \\ 4018 & 12: 33: 21 & 0.0783 & 0.0917 \\ 4020 & 12: 33: 23 & 0.0359 & 0.0900 \\ 4022 & 12: 33: 25 & 0.2203 & 0.1574 \\ 4024 & 12: 33: 27 & 0.0202 & 0.0846 \\ 4026 & 12: 33: 29 & 0.1068 & 0.1048 \\ 4028 & 12: 33: 31 & 0.0476 & 0.0754 \\ 4030 & 12: 33: 33 & 0.0567 & 0.0726 \\ 4032 & 12: 33: 35 & 0.0491 & 0.0768 \\ 4034 & 12: 33: 37 & 0.0477 & 0.1768 \\ 4036 & 12: 33: 39 & 0.0241 & 0.0875 \\ 4038 & 12: 33: 41 & 0.0458 & 0.1344 \\ 4040 & 12: 33: 43 & 0.0407 & 0.2404 \\ 4042 & 12: 33: 45 & 0.0091 & 0.1498 \\ 4044 & 12: 33: 47 & 0.0386 & 0.1610 \\ 4046 & 12: 33: 49 & 0.0278 & 0.0766 \\ 4048 & 12: 33: 51 & 0.0566 & 0.0874 \\ 4050 & 12: 33: 53 & 0.0312 & 0.1610 \\ 4052 & 12: 33: 55 & 0.0470 & 0.0405\end{array}$




$\begin{array}{llll}4054 & 12: 33: 57 & 0.1871 & 0.0697 \\ 4056 & 12: 33: 59 & 0.0755 & 0.1034 \\ 4058 & 12: 34: 01 & 0.0779 & 0.2149 \\ 4060 & 12: 34: 03 & 0.0267 & 0.1576 \\ 4062 & 12: 34: 05 & 0.0293 & 0.2185 \\ 4064 & 12: 34: 07 & 0.0084 & 0.1845 \\ 4066 & 12: 34: 09 & 0.0283 & 0.0780 \\ 4068 & 12: 34: 11 & 0.0434 & 0.1232 \\ 4070 & 12: 34: 13 & 0.0433 & 0.2334 \\ 4072 & 12: 34: 15 & 0.0777 & 0.2171 \\ 4074 & 12: 34: 17 & 0.0328 & 0.1442 \\ 4076 & 12: 34: 19 & 0.0444 & 0.2101 \\ 4078 & 12: 34: 21 & 0.0270 & 0.1136 \\ 4080 & 12: 34: 23 & 0.0813 & 0.0719 \\ 4082 & 12: 34: 25 & 0.1032 & 0.1134 \\ 4084 & 12: 34: 27 & 0.0186 & 0.1266 \\ 4086 & 12: 34: 29 & 0.0386 & 0.0748 \\ 4088 & 12: 34: 31 & 0.0192 & 0.1164 \\ 4090 & 12: 34: 33 & 0.0638 & 0.1646 \\ 4092 & 12: 34: 35 & 0.0222 & 0.0968 \\ 4094 & 12: 34: 37 & 0.0293 & 0.0835 \\ 4096 & 12: 34: 39 & 0.0116 & 0.2797 \\ 4098 & 12: 34: 41 & 0.0267 & 0.2427 \\ 4100 & 12: 34: 43 & 0.0616 & 0.1314 \\ 4102 & 12: 34: 45 & 0.0555 & 0.1175 \\ 4104 & 12: 34: 47 & 0.0506 & 0.3334 \\ 4106 & 12: 34: 49 & 0.0238 & 0.0983 \\ 4108 & 12: 34: 51 & 0.0793 & 0.0344 \\ 4110 & 12: 34: 53 & 0.0958 & 0.3760 \\ 4112 & 12: 34: 55 & 0.0269 & 0.1262 \\ 4114 & 12: 34: 57 & 0.0176 & 0.1086 \\ 4116 & 12: 34: 59 & 0.0468 & 0.1487 \\ 4118 & 12: 35: 01 & 0.0331 & 0.2373 \\ 4120 & 12: 35: 03 & 0.0821 & 0.2742 \\ 4122 & 12: 35: 05 & 0.0271 & 0.0813 \\ 4124 & 12: 35: 07 & 0.0122 & 0.1721 \\ 4126 & 12: 35: 09 & 0.1178 & 0.1313 \\ 4128 & 12: 35: 11 & 0.0539 & 0.3044 \\ 4130 & 12: 35: 13 & 0.0357 & 0.1292 \\ 4132 & 12: 35: 15 & 0.0793 & 0.0335 \\ 4134 & 12: 35: 17 & 0.0322 & 0.0907 \\ 4136 & 12: 35: 19 & 0.0029 & 0.1825 \\ 4138 & 12: 35: 21 & 0.0189 & 0.0946 \\ 4140 & 12: 35: 23 & 0.0521 & 0.1213 \\ 4142 & 12: 35: 25 & 0.0907 & 0.0509 \\ 4144 & 12: 35: 27 & 0.0601 & 0.1577 \\ 4146 & 12: 35: 29 & 0.1200 & 0.1304 \\ 4148 & 12: 35: 31 & 0.0096 & 0.1126 \\ 4150 & 12: 35: 33 & 0.0520 & 0.1194 \\ 4152 & 12: 35: 35 & 0.0381 & 0.0787 \\ 4154 & 12: 35: 37 & 0.0601 & 0.1683 \\ 4156 & 12: 35: 39 & 0.0293 & 0.1824\end{array}$




$\begin{array}{llll}4158 & 12: 35: 41 & 0.0307 & 0.1766 \\ 4160 & 12: 35: 43 & 0.0110 & 0.0884 \\ 4162 & 12: 35: 45 & 0.0705 & 0.0394 \\ 4164 & 12: 35: 47 & 0.1441 & 0.1291 \\ 4166 & 12: 35: 49 & 0.0334 & 0.1824 \\ 4168 & 12: 35: 51 & 0.0639 & 0.0197 \\ 4170 & 12: 35: 53 & 0.0682 & 0.0412 \\ 4172 & 12: 35: 55 & 0.1078 & 0.2414 \\ 4174 & 12: 35: 57 & 0.0873 & 0.0410 \\ 4176 & 12: 35: 59 & 0.0927 & 0.1314 \\ 4178 & 12: 36: 01 & 0.0158 & 0.1397 \\ 4180 & 12: 36: 03 & 0.0671 & 0.0579 \\ 4182 & 12: 36: 05 & 0.0262 & 0.0421 \\ 4184 & 12: 36: 07 & 0.0552 & 0.0454 \\ 4186 & 12: 36: 09 & 0.1057 & 0.0511 \\ 4188 & 12: 36: 11 & 0.0633 & 0.1318 \\ 4190 & 12: 36: 13 & 0.0390 & 0.0882 \\ 4192 & 12: 36: 15 & 0.0200 & 0.0792 \\ 4194 & 12: 36: 17 & 0.0250 & 0.0491 \\ 4196 & 12: 36: 19 & 0.0595 & 0.2308 \\ 4198 & 12: 36: 21 & 0.0354 & 0.1802 \\ 4200 & 12: 36: 23 & 0.0716 & 0.0399 \\ 4202 & 12: 36: 25 & 0.1288 & 0.2073 \\ 4204 & 12: 36: 27 & 0.0956 & 0.1047 \\ 4206 & 12: 36: 29 & 0.0154 & 0.0491 \\ 4208 & 12: 36: 31 & 0.0223 & 0.2136 \\ 4210 & 12: 36: 33 & 0.0466 & 0.1205 \\ 4212 & 12: 36: 35 & 0.0393 & 0.0303 \\ 4214 & 12: 36: 37 & 0.0485 & 0.0743 \\ 4216 & 12: 36: 39 & 0.0396 & 0.1919 \\ 4218 & 12: 36: 41 & 0.0307 & 0.1302 \\ 4220 & 12: 36: 43 & 0.0386 & 0.0523 \\ 4222 & 12: 36: 45 & 0.0110 & 0.0300 \\ 4224 & 12: 36: 47 & 0.1156 & 0.0222 \\ 4226 & 12: 36: 49 & 0.1301 & 0.0780 \\ 4228 & 12: 36: 51 & 0.1121 & 0.0646 \\ 4230 & 12: 36: 53 & 0.0749 & 0.0152 \\ 4232 & 12: 36: 55 & 0.1578 & 0.0251 \\ 4234 & 12: 36: 57 & 0.1054 & 0.0437 \\ 4236 & 12: 36: 59 & 0.1067 & 0.0544 \\ 4238 & 12: 37: 01 & 0.0514 & 0.0715 \\ 4240 & 12: 37: 03 & 0.0824 & 0.0499 \\ 4242 & 12: 37: 05 & 0.0644 & 0.0980 \\ 4244 & 12: 37: 07 & 0.0708 & 0.0158 \\ 4246 & 12: 37: 09 & 0.0762 & 0.1331 \\ 4248 & 12: 37: 11 & 0.0822 & 0.1642 \\ 4250 & 12: 37: 13 & 0.1126 & 0.0657 \\ 4252 & 12: 37: 15 & 0.0593 & 0.0962 \\ 4254 & 12: 37: 17 & 0.0633 & 0.0368 \\ 4256 & 12: 37: 19 & 0.0539 & 0.0825 \\ 4258 & 12: 37: 21 & 0.0526 & 0.0510 \\ 4260 & 12: 37: 23 & 0.1009 & 0.1981\end{array}$


Appendix B - Laser Data Test 1 NIST102505_1.xls

$\begin{array}{llll}4262 & 12: 37: 25 & 0.0288 & 0.0450 \\ 4264 & 12: 37: 27 & 0.1208 & 0.0636 \\ 4266 & 12: 37: 29 & 0.1474 & 0.1293 \\ 4268 & 12: 37: 31 & 0.1881 & 0.0506 \\ 4270 & 12: 37: 33 & 0.0468 & 0.0303 \\ 4272 & 12: 37: 35 & 0.0248 & 0.0545 \\ 4274 & 12: 37: 37 & 0.0600 & 0.1079 \\ 4276 & 12: 37: 39 & 0.1034 & 0.1790 \\ 4278 & 12: 37: 41 & 0.0329 & 0.1014 \\ 4280 & 12: 37: 43 & 0.0456 & 0.0533 \\ 4282 & 12: 37: 45 & 0.0527 & 0.0192 \\ 4284 & 12: 37: 47 & 0.0358 & 0.0339 \\ 4286 & 12: 37: 49 & 0.0325 & 0.0316 \\ 4288 & 12: 37: 51 & 0.0287 & 0.1496 \\ 4290 & 12: 37: 53 & 0.0431 & 0.1056 \\ 4292 & 12: 37: 55 & 0.0406 & 0.1312 \\ 4294 & 12: 37: 57 & 0.0370 & 0.0405 \\ 4296 & 12: 37: 59 & 0.0651 & 0.0199 \\ 4298 & 12: 38: 01 & 0.0639 & 0.1398 \\ 4300 & 12: 38: 03 & 0.0450 & 0.1645 \\ 4302 & 12: 38: 05 & 0.1146 & 0.1048 \\ 4304 & 12: 38: 07 & 0.0148 & 0.0916 \\ 4306 & 12: 38: 09 & 0.0497 & 0.1233 \\ 4308 & 12: 38: 11 & 0.0265 & 0.1739 \\ 4310 & 12: 38: 13 & 0.0410 & 0.2099 \\ 4312 & 12: 38: 15 & 0.0331 & 0.1088 \\ 4314 & 12: 38: 17 & 0.0665 & 0.0396 \\ 4316 & 12: 38: 19 & 0.0136 & 0.1190 \\ 4318 & 12: 38: 21 & 0.0588 & 0.1342 \\ 4320 & 12: 38: 23 & 0.0737 & 0.1828 \\ 4322 & 12: 38: 25 & 0.1051 & 0.1222 \\ 4324 & 12: 38: 27 & 0.0788 & 0.0793 \\ 4326 & 12: 38: 29 & 0.0118 & 0.1078 \\ 4328 & 12: 38: 31 & 0.0632 & 0.2111 \\ 4330 & 12: 38: 33 & 0.0271 & 0.0892 \\ 4332 & 12: 38: 35 & 0.0794 & 0.0625 \\ 4334 & 12: 38: 37 & 0.0576 & 0.0325 \\ 4336 & 12: 38: 39 & 0.0260 & 0.1508 \\ 4338 & 12: 38: 41 & 0.0548 & 0.0937 \\ 4340 & 12: 38: 43 & 0.0751 & 0.0520 \\ 4342 & 12: 38: 45 & 0.0410 & 0.0498 \\ 4344 & 12: 38: 47 & 0.2102 & 0.0531 \\ 4346 & 12: 38: 49 & 0.0540 & 0.0569 \\ 4348 & 12: 38: 51 & 0.0952 & 0.1176 \\ 4350 & 12: 38: 53 & 0.1653 & 0.1723 \\ 4352 & 12: 38: 55 & 0.0330 & 0.1825 \\ 4354 & 12: 38: 57 & 0.0365 & 0.1299 \\ 4356 & 12: 38: 59 & 0.0550 & 0.0818 \\ 4358 & 12: 39: 01 & 0.0201 & 0.0456 \\ 4360 & 12: 39: 03 & 0.0371 & 0.1091 \\ 4362 & 12: 39: 05 & 0.0677 & 0.1736 \\ 4364 & 12: 39: 07 & 0.0804 & 0.1747\end{array}$




$\begin{array}{llll}4366 & 12: 39: 09 & 0.0504 & 0.0805 \\ 4368 & 12: 39: 11 & 0.0407 & 0.1578 \\ 4370 & 12: 39: 13 & 0.0200 & 0.2025 \\ 4372 & 12: 39: 15 & 0.0318 & 0.0391 \\ 4374 & 12: 39: 17 & 0.1072 & 0.1239 \\ 4376 & 12: 39: 19 & 0.0166 & 0.0782 \\ 4378 & 12: 39: 21 & 0.0437 & 0.2266 \\ 4380 & 12: 39: 23 & 0.0940 & 0.0724 \\ 4382 & 12: 39: 25 & 0.0231 & 0.2017 \\ 4384 & 12: 39: 27 & 0.0251 & 0.0922 \\ 4386 & 12: 39: 29 & 0.1400 & 0.0807 \\ 4388 & 12: 39: 31 & 0.0305 & 0.1985 \\ 4390 & 12: 39: 33 & 0.1352 & 0.1557 \\ 4392 & 12: 39: 35 & 0.0557 & 0.0850 \\ 4394 & 12: 39: 37 & 0.0224 & 0.0878 \\ 4396 & 12: 39: 39 & 0.1194 & 0.1189 \\ 4398 & 12: 39: 41 & 0.0558 & 0.2135 \\ 4400 & 12: 39: 43 & 0.0267 & 0.1400 \\ 4402 & 12: 39: 45 & 0.0220 & 0.0839 \\ 4404 & 12: 39: 47 & 0.0288 & 0.1023 \\ 4406 & 12: 39: 49 & 0.0192 & 0.1160 \\ 4408 & 12: 39: 51 & 0.0381 & 0.1462 \\ 4410 & 12: 39: 53 & 0.0689 & 0.1380 \\ 4412 & 12: 39: 55 & 0.0560 & 0.2305 \\ 4414 & 12: 39: 57 & 0.0307 & 0.0405 \\ 4416 & 12: 39: 59 & 0.1654 & 0.3775 \\ 4418 & 12: 40: 01 & 0.0883 & 0.1145 \\ 4420 & 12: 40: 03 & 0.0393 & 0.1399 \\ 4422 & 12: 40: 05 & 0.1226 & 0.0386 \\ 4424 & 12: 40: 07 & 0.0215 & 0.0981 \\ 4426 & 12: 40: 09 & 0.0245 & 0.1101 \\ 4428 & 12: 40: 11 & 0.0385 & 0.1423 \\ 4430 & 12: 40: 13 & 0.0852 & 0.1712 \\ 4432 & 12: 40: 15 & 0.0873 & 0.1381 \\ 4434 & 12: 40: 17 & 0.0928 & 0.1302 \\ 4436 & 12: 40: 19 & 0.0697 & 0.1883 \\ 4438 & 12: 40: 21 & 0.0376 & 0.1012 \\ 4440 & 12: 40: 23 & 0.0277 & 0.1034 \\ 4442 & 12: 40: 25 & 0.0644 & 0.1356 \\ 4444 & 12: 40: 27 & 0.0698 & 0.1868 \\ 4446 & 12: 40: 29 & 0.0295 & 0.0505 \\ 4448 & 12: 40: 31 & 0.0252 & 0.0546 \\ 4450 & 12: 40: 33 & 0.0545 & 0.1489 \\ 4452 & 12: 40: 35 & 0.0496 & 0.3174 \\ 4454 & 12: 40: 37 & 0.1041 & 0.1349 \\ 4456 & 12: 40: 39 & 0.1112 & 0.1834 \\ 4458 & 12: 40: 41 & 0.0632 & 0.0877 \\ 4460 & 12: 40: 43 & 0.0570 & 0.0739 \\ 4462 & 12: 40: 45 & 0.0238 & 0.1182 \\ 4464 & 12: 40: 47 & 0.0997 & 0.2232 \\ 4466 & 12: 40: 49 & 0.0257 & 0.1578 \\ 4468 & 12: 40: 51 & 0.0673 & 0.1833\end{array}$




$\begin{array}{llll}4470 & 12: 40: 53 & 0.0400 & 0.0653 \\ 4472 & 12: 40: 55 & 0.0410 & 0.1245 \\ 4474 & 12: 40: 57 & 0.0223 & 0.1684 \\ 4476 & 12: 40: 59 & 0.0550 & 0.1755 \\ 4478 & 12: 41: 01 & 0.1305 & 0.1059 \\ 4480 & 12: 41: 03 & 0.0596 & 0.0632 \\ 4482 & 12: 41: 05 & 0.0370 & 0.0959 \\ 4484 & 12: 41: 07 & 0.0372 & 0.1184 \\ 4486 & 12: 41: 09 & 0.0166 & 0.1269 \\ 4488 & 12: 41: 11 & 0.0173 & 0.0763 \\ 4490 & 12: 41: 13 & 0.0141 & 0.0752 \\ 4492 & 12: 41: 15 & 0.0258 & 0.1604 \\ 4494 & 12: 41: 17 & 0.1434 & 0.1466 \\ 4496 & 12: 41: 19 & 0.0651 & 0.1057 \\ 4498 & 12: 41: 21 & 0.0954 & 0.4104 \\ 4500 & 12: 41: 23 & 0.0604 & 0.1835 \\ 4502 & 12: 41: 25 & 0.0623 & 0.0872 \\ 4504 & 12: 41: 27 & 0.0562 & 0.0977 \\ 4506 & 12: 41: 29 & 0.0110 & 0.0861 \\ 4508 & 12: 41: 31 & 0.0455 & 0.0934 \\ 4510 & 12: 41: 33 & 0.0830 & 0.1421 \\ 4512 & 12: 41: 35 & 0.0660 & 0.2801 \\ 4514 & 12: 41: 37 & 0.0074 & 0.2035 \\ 4516 & 12: 41: 39 & 0.0302 & 0.0921 \\ 4518 & 12: 41: 41 & 0.0696 & 0.0897 \\ 4520 & 12: 41: 43 & 0.1283 & 0.2860 \\ 4522 & 12: 41: 45 & 0.0180 & 0.0858 \\ 4524 & 12: 41: 47 & 0.0302 & 0.0791 \\ 4526 & 12: 41: 49 & 0.0392 & 0.1216 \\ 4528 & 12: 41: 51 & 0.0470 & 0.0864 \\ 4530 & 12: 41: 53 & 0.0306 & 0.1761 \\ 4532 & 12: 41: 55 & 0.0986 & 0.0618 \\ 4534 & 12: 41: 57 & 0.0223 & 0.0553 \\ 4536 & 12: 41: 59 & 0.0382 & 0.1615 \\ 4538 & 12: 42: 01 & 0.0758 & 0.2025 \\ 4540 & 12: 42: 03 & 0.0188 & 0.0671 \\ 4542 & 12: 42: 05 & 0.0170 & 0.0846 \\ 4544 & 12: 42: 07 & 0.0318 & 0.1240 \\ 4546 & 12: 42: 09 & 0.0409 & 0.1803 \\ 4548 & 12: 42: 11 & 0.1005 & 0.0337 \\ 4550 & 12: 42: 13 & 0.0381 & 0.1892 \\ 4552 & 12: 42: 15 & 0.0930 & 0.0995 \\ 4554 & 12: 42: 17 & 0.0897 & 0.2167 \\ 4556 & 12: 42: 19 & 0.0588 & 0.1789 \\ 4558 & 12: 42: 21 & 0.0296 & 0.1562 \\ 4560 & 12: 42: 23 & 0.0724 & 0.3920 \\ 4562 & 12: 42: 25 & 0.0184 & 0.0688 \\ 4564 & 12: 42: 27 & 0.0216 & 0.1994 \\ 4566 & 12: 42: 29 & 0.1040 & 0.0631 \\ 4568 & 12: 42: 31 & 0.0189 & 0.0627 \\ 4570 & 12: 42: 33 & 0.0313 & 0.0536 \\ 4572 & 12: 42: 35 & 0.0309 & 0.1346\end{array}$




$\begin{array}{llll}4574 & 12: 42: 37 & 0.0236 & 0.0376 \\ 4576 & 12: 42: 39 & 0.0762 & 0.0536 \\ 4578 & 12: 42: 41 & 0.0274 & 0.1216 \\ 4580 & 12: 42: 43 & 0.0800 & 0.1515 \\ 4582 & 12: 42: 45 & 0.0937 & 0.2199 \\ 4584 & 12: 42: 47 & 0.0278 & 0.1114 \\ 4586 & 12: 42: 49 & 0.1344 & 0.0659 \\ 4588 & 12: 42: 51 & 0.0604 & 0.2806 \\ 4590 & 12: 42: 53 & 0.0596 & 0.0231 \\ 4592 & 12: 42: 55 & 0.0562 & 0.0484 \\ 4594 & 12: 42: 57 & 0.0075 & 0.1039 \\ 4596 & 12: 42: 59 & 0.0628 & 0.0756 \\ 4598 & 12: 43: 01 & 0.1461 & 0.0991 \\ 4600 & 12: 43: 03 & 0.1268 & 0.0928 \\ 4602 & 12: 43: 05 & 0.0809 & 0.3932 \\ 4604 & 12: 43: 07 & 0.0433 & 0.0742 \\ 4606 & 12: 43: 09 & 0.0407 & 0.2218 \\ 4608 & 12: 43: 11 & 0.0126 & 0.0631 \\ 4610 & 12: 43: 13 & 0.0408 & 0.1335 \\ 4612 & 12: 43: 15 & 0.0230 & 0.0722 \\ 4614 & 12: 43: 17 & 0.0354 & 0.0937 \\ 4616 & 12: 43: 19 & 0.0381 & 0.1531 \\ 4618 & 12: 43: 21 & 0.0702 & 0.1650 \\ 4620 & 12: 43: 23 & 0.0211 & 0.0569 \\ 4622 & 12: 43: 25 & 0.1175 & 0.0966 \\ 4624 & 12: 43: 27 & 0.0433 & 0.1502 \\ 4626 & 12: 43: 29 & 0.0522 & 0.1283 \\ 4628 & 12: 43: 31 & 0.0842 & 0.1658 \\ 4630 & 12: 43: 33 & 0.0688 & 0.1467 \\ 4632 & 12: 43: 35 & 0.0522 & 0.0714 \\ 4634 & 12: 43: 37 & 0.1171 & 0.0776 \\ 4636 & 12: 43: 39 & 0.0945 & 0.0300 \\ 4638 & 12: 43: 41 & 0.0229 & 0.1515 \\ 4640 & 12: 43: 43 & 0.0244 & 0.1525 \\ 4642 & 12: 43: 45 & 0.0873 & 0.0583 \\ 4644 & 12: 43: 47 & 0.0273 & 0.1707 \\ 4646 & 12: 43: 49 & 0.0074 & 0.0782 \\ 4648 & 12: 43: 51 & 0.0131 & 0.1209 \\ 4650 & 12: 43: 53 & 0.0388 & 0.1738 \\ 4652 & 12: 43: 55 & 0.0244 & 0.0640 \\ 4654 & 12: 43: 57 & 0.1471 & 0.1997 \\ 4656 & 12: 43: 59 & 0.0481 & 0.0877 \\ 4658 & 12: 44: 01 & 0.1529 & 0.1495 \\ 4660 & 12: 44: 03 & 0.0499 & 0.1916 \\ 4662 & 12: 44: 05 & 0.1044 & 0.2503 \\ 4664 & 12: 44: 07 & 0.0983 & 0.0791 \\ 4666 & 12: 44: 09 & 0.0622 & 0.2360 \\ 4668 & 12: 44: 11 & 0.0539 & 0.1197 \\ 4670 & 12: 44: 13 & 0.0318 & 0.2014 \\ 4672 & 12: 44: 15 & 0.0224 & 0.1850 \\ 4674 & 12: 44: 17 & 0.0823 & 0.0589 \\ 4676 & 12: 44: 19 & 0.0345 & 0.0950\end{array}$




$\begin{array}{llll}4678 & 12: 44: 21 & 0.0524 & 0.1090 \\ 4680 & 12: 44: 23 & 0.1198 & 0.0634 \\ 4682 & 12: 44: 25 & 0.0660 & 0.2868 \\ 4684 & 12: 44: 27 & 0.0357 & 0.0895 \\ 4686 & 12: 44: 29 & 0.0663 & 0.2042 \\ 4688 & 12: 44: 31 & 0.0554 & 0.0688 \\ 4690 & 12: 44: 33 & 0.0227 & 0.1420 \\ 4692 & 12: 44: 35 & 0.0280 & 0.0875 \\ 4694 & 12: 44: 37 & 0.0370 & 0.0786 \\ 4696 & 12: 44: 39 & 0.0559 & 0.1462 \\ 4698 & 12: 44: 41 & 0.0942 & 0.1104 \\ 4700 & 12: 44: 43 & 0.0252 & 0.0411 \\ 4702 & 12: 44: 45 & 0.0558 & 0.0340 \\ 4704 & 12: 44: 47 & 0.0527 & 0.0614 \\ 4706 & 12: 44: 49 & 0.0247 & 0.0408 \\ 4708 & 12: 44: 51 & 0.1391 & 0.1149 \\ 4710 & 12: 44: 53 & 0.0833 & 0.0378 \\ 4712 & 12: 44: 55 & 0.0513 & 0.0602 \\ 4714 & 12: 44: 57 & 0.0384 & 0.0613 \\ 4716 & 12: 44: 59 & 0.1100 & 0.2678 \\ 4718 & 12: 45: 01 & 0.0323 & 0.0976 \\ 4720 & 12: 45: 03 & 0.0283 & 0.0750 \\ 4722 & 12: 45: 05 & 0.0122 & 0.0323 \\ 4724 & 12: 45: 07 & 0.0281 & 0.0511 \\ 4726 & 12: 45: 09 & 0.0178 & 0.1128 \\ 4728 & 12: 45: 11 & 0.0529 & 0.1094 \\ 4730 & 12: 45: 13 & 0.0803 & 0.1747 \\ 4732 & 12: 45: 15 & 0.1259 & 0.1456 \\ 4734 & 12: 45: 17 & 0.0355 & 0.3326 \\ 4736 & 12: 45: 19 & 0.0699 & 0.0647 \\ 4738 & 12: 45: 21 & 0.0429 & 0.2150 \\ 4740 & 12: 45: 23 & 0.0572 & 0.0582 \\ 4742 & 12: 45: 25 & 0.1011 & 0.3068 \\ 4744 & 12: 45: 27 & 0.0619 & 0.0620 \\ 4746 & 12: 45: 29 & 0.0994 & 0.1738 \\ 4748 & 12: 45: 31 & 0.1310 & 0.1322 \\ 4750 & 12: 45: 33 & 0.0353 & 0.3188 \\ 4752 & 12: 45: 35 & 0.0888 & 0.1447 \\ 4754 & 12: 45: 37 & 0.0295 & 0.0516 \\ 4756 & 12: 45: 39 & 0.0755 & 0.1420 \\ 4758 & 12: 45: 41 & 0.0698 & 0.1536 \\ 4760 & 12: 45: 43 & 0.0557 & 0.1412 \\ 4762 & 12: 45: 45 & 0.0175 & 0.0566 \\ 4764 & 12: 45: 47 & 0.0467 & 0.0400 \\ 4766 & 12: 45: 49 & 0.0394 & 0.1291 \\ 4768 & 12: 45: 51 & 0.0962 & 0.1439 \\ 4770 & 12: 45: 53 & 0.0589 & 0.0345 \\ 4772 & 12: 45: 55 & 0.0858 & 0.0425 \\ 4774 & 12: 45: 57 & 0.0442 & 0.1043 \\ 4776 & 12: 45: 59 & 0.1272 & 0.1411 \\ 4778 & 12: 46: 01 & 0.0154 & 0.0385 \\ 4780 & 12: 46: 03 & 0.1138 & 0.1238\end{array}$




$\begin{array}{llll}4782 & 12: 46: 05 & 0.0631 & 0.0916 \\ 4784 & 12: 46: 07 & 0.0195 & 0.0931 \\ 4786 & 12: 46: 09 & 0.0752 & 0.0524 \\ 4788 & 12: 46: 11 & 0.0478 & 0.0617 \\ 4790 & 12: 46: 13 & 0.0545 & 0.0713 \\ 4792 & 12: 46: 15 & 0.0464 & 0.0426 \\ 4794 & 12: 46: 17 & 0.0385 & 0.3143 \\ 4796 & 12: 46: 19 & 0.0348 & 0.4257 \\ 4798 & 12: 46: 21 & 0.1592 & 0.2551 \\ 4800 & 12: 46: 23 & 0.0639 & 0.0599 \\ 4802 & 12: 46: 25 & 0.1008 & 0.1126 \\ 4804 & 12: 46: 27 & 0.0808 & 0.1890 \\ 4806 & 12: 46: 29 & 0.0209 & 0.0582 \\ 4808 & 12: 46: 31 & 0.0344 & 0.0917 \\ 4810 & 12: 46: 33 & 0.0559 & 0.0631 \\ 4812 & 12: 46: 35 & 0.0484 & 0.0805 \\ 4814 & 12: 46: 37 & 0.0740 & 0.0795 \\ 4816 & 12: 46: 39 & 0.0420 & 0.1421 \\ 4818 & 12: 46: 41 & 0.0195 & 0.1282 \\ 4820 & 12: 46: 43 & 0.0239 & 0.0942 \\ 4822 & 12: 46: 45 & 0.0731 & 0.1198 \\ 4824 & 12: 46: 47 & 0.0150 & 0.1737 \\ 4826 & 12: 46: 49 & 0.0654 & 0.0860 \\ 4828 & 12: 46: 51 & 0.0588 & 0.2036 \\ 4830 & 12: 46: 53 & 0.0521 & 0.0909 \\ 4832 & 12: 46: 55 & 0.0311 & 0.0935 \\ 4834 & 12: 46: 57 & 0.1929 & 0.1200 \\ 4836 & 12: 46: 59 & 0.0249 & 0.1103 \\ 4838 & 12: 47: 01 & 0.0464 & 0.1001 \\ 4840 & 12: 47: 03 & 0.0299 & 0.0380 \\ 4842 & 12: 47: 05 & 0.0367 & 0.1219 \\ 4844 & 12: 47: 07 & 0.0303 & 0.1031 \\ 4846 & 12: 47: 09 & 0.0428 & 0.1330 \\ 4848 & 12: 47: 11 & 0.0322 & 0.1186 \\ 4850 & 12: 47: 13 & 0.0732 & 0.1627 \\ 4852 & 12: 47: 15 & 0.0313 & 0.0683 \\ 4854 & 12: 47: 17 & 0.0138 & 0.0668 \\ 4856 & 12: 47: 19 & 0.0239 & 0.1524 \\ 4858 & 12: 47: 21 & 0.1169 & 0.0533 \\ 4860 & 12: 47: 23 & 0.0487 & 0.0936 \\ 4862 & 12: 47: 25 & 0.0526 & 0.0460 \\ 4864 & 12: 47: 27 & 0.0734 & 0.1058 \\ 4866 & 12: 47: 29 & 0.0385 & 0.0949 \\ 4868 & 12: 47: 31 & 0.0078 & 0.0909 \\ 4870 & 12: 47: 33 & 0.1102 & 0.1095 \\ 4872 & 12: 47: 35 & 0.1067 & 0.1961 \\ 4874 & 12: 47: 37 & 0.0373 & 0.1119 \\ 4876 & 12: 47: 39 & 0.0528 & 0.3040 \\ 4878 & 12: 47: 41 & 0.0757 & 0.2698 \\ 4880 & 12: 47: 43 & 0.0267 & 0.2682 \\ 4882 & 12: 47: 45 & 0.1973 & 0.3098 \\ 4884 & 12: 47: 47 & 0.0691 & 0.0552\end{array}$




$\begin{array}{llll}4886 & 12: 47: 49 & 0.1233 & 0.1668 \\ 4888 & 12: 47: 51 & 0.0821 & 0.0871 \\ 4890 & 12: 47: 53 & 0.0398 & 0.0802 \\ 4892 & 12: 47: 55 & 0.0392 & 0.1447 \\ 4894 & 12: 47: 57 & 0.0349 & 0.1238 \\ 4896 & 12: 47: 59 & 0.0325 & 0.0439 \\ 4898 & 12: 48: 01 & 0.0565 & 0.2397 \\ 4900 & 12: 48: 03 & 0.0148 & 0.1627 \\ 4902 & 12: 48: 05 & 0.0831 & 0.1157 \\ 4904 & 12: 48: 07 & 0.0631 & 0.1806 \\ 4906 & 12: 48: 09 & 0.0345 & 0.1580 \\ 4908 & 12: 48: 11 & 0.0690 & 0.1637 \\ 4910 & 12: 48: 13 & 0.0778 & 0.1284 \\ 4912 & 12: 48: 15 & 0.1328 & 0.0700 \\ 4914 & 12: 48: 17 & 0.0512 & 0.0788 \\ 4916 & 12: 48: 19 & 0.0524 & 0.0722 \\ 4918 & 12: 48: 21 & 0.1359 & 0.1629 \\ 4920 & 12: 48: 23 & 0.0464 & 0.1454 \\ 4922 & 12: 48: 25 & 0.0960 & 0.0836 \\ 4924 & 12: 48: 27 & 0.1615 & 0.1118 \\ 4926 & 12: 48: 29 & 0.0358 & 0.0608 \\ 4928 & 12: 48: 31 & 0.0901 & 0.1491 \\ 4930 & 12: 48: 33 & 0.1077 & 0.0357 \\ 4932 & 12: 48: 35 & 0.1363 & 0.0547 \\ 4934 & 12: 48: 37 & 0.0846 & 0.0899 \\ 4936 & 12: 48: 39 & 0.0573 & 0.0984 \\ 4938 & 12: 48: 41 & 0.0249 & 0.0946 \\ 4940 & 12: 48: 43 & 0.2204 & 0.0474 \\ 4942 & 12: 48: 45 & 0.0599 & 0.1558 \\ 4944 & 12: 48: 47 & 0.0480 & 0.0732 \\ 4946 & 12: 48: 49 & 0.0168 & 0.0703 \\ 4948 & 12: 48: 51 & 0.1332 & 0.1054 \\ 4950 & 12: 48: 53 & 0.0783 & 0.0672 \\ 4952 & 12: 48: 55 & 0.0274 & 0.1379 \\ 4954 & 12: 48: 57 & 0.0650 & 0.0792 \\ 4956 & 12: 48: 59 & 0.0731 & 0.0719 \\ 4958 & 12: 49: 01 & 0.0383 & 0.1776 \\ 4960 & 12: 49: 03 & 0.0411 & 0.1249 \\ 4962 & 12: 49: 05 & 0.0524 & 0.1709 \\ 4964 & 12: 49: 07 & 0.0225 & 0.0937 \\ 4966 & 12: 49: 09 & 0.0246 & 0.1180 \\ 4968 & 12: 49: 11 & 0.0312 & 0.0769 \\ 4970 & 12: 49: 13 & 0.0330 & 0.1967 \\ 4972 & 12: 49: 15 & 0.0580 & 0.0732 \\ 4974 & 12: 49: 17 & 0.0490 & 0.1134 \\ 4976 & 12: 49: 19 & 0.0772 & 0.0626 \\ 4978 & 12: 49: 21 & 0.0856 & 0.2247 \\ 4980 & 12: 49: 23 & 0.0346 & 0.0595 \\ 4982 & 12: 49: 25 & 0.0263 & 0.0578 \\ 4984 & 12: 49: 27 & 0.0826 & 0.2482 \\ 4986 & 12: 49: 29 & 0.0240 & 0.1660 \\ 4988 & 12: 49: 31 & 0.0169 & 0.0396\end{array}$




$\begin{array}{llll}4990 & 12: 49: 33 & 0.0303 & 0.2814 \\ 4992 & 12: 49: 35 & 0.1424 & 0.2742 \\ 4994 & 12: 49: 37 & 0.0806 & 0.2380 \\ 4996 & 12: 49: 39 & 0.1282 & 0.0991 \\ 4998 & 12: 49: 41 & 0.0684 & 0.0798 \\ 5000 & 12: 49: 43 & 0.0791 & 0.1883 \\ 5002 & 12: 49: 45 & 0.0453 & 0.1068 \\ 5004 & 12: 49: 47 & 0.0833 & 0.1076 \\ 5006 & 12: 49: 49 & 0.0136 & 0.2912 \\ 5008 & 12: 49: 51 & 0.0240 & 0.1218 \\ 5010 & 12: 49: 53 & 0.0305 & 0.3184 \\ 5012 & 12: 49: 55 & 0.0419 & 0.1074 \\ 5014 & 12: 49: 57 & 0.0514 & 0.1659 \\ 5016 & 12: 49: 59 & 0.0443 & 0.0568 \\ 5018 & 12: 50: 01 & 0.0520 & 0.1129 \\ 5020 & 12: 50: 03 & 0.0238 & 0.1467 \\ 5022 & 12: 50: 05 & 0.0959 & 0.0629 \\ 5024 & 12: 50: 07 & 0.0221 & 0.0546 \\ 5026 & 12: 50: 09 & 0.0473 & 0.1533 \\ 5028 & 12: 50: 11 & 0.0294 & 0.1183 \\ 5030 & 12: 50: 13 & 0.0297 & 0.1523 \\ 5032 & 12: 50: 15 & 0.0904 & 0.0452 \\ 5034 & 12: 50: 17 & 0.0245 & 0.0339 \\ 5036 & 12: 50: 19 & 0.1565 & 0.0709 \\ 5038 & 12: 50: 21 & 0.0897 & 0.0918 \\ 5040 & 12: 50: 23 & 0.0502 & 0.1936 \\ 5042 & 12: 50: 25 & 0.0819 & 0.0505 \\ 5044 & 12: 50: 27 & 0.0322 & 0.0887 \\ 5046 & 12: 50: 29 & 0.0399 & 0.0643 \\ 5048 & 12: 50: 31 & 0.0483 & 0.0467 \\ 5050 & 12: 50: 33 & 0.0483 & 0.0515 \\ 5052 & 12: 50: 35 & 0.0481 & 0.0798 \\ 5054 & 12: 50: 37 & 0.0335 & 0.1119 \\ 5056 & 12: 50: 39 & 0.0532 & 0.1641 \\ 5058 & 12: 50: 41 & 0.0385 & 0.1383 \\ 5060 & 12: 50: 43 & 0.0241 & 0.0730 \\ 5062 & 12: 50: 45 & 0.0398 & 0.2008 \\ 5064 & 12: 50: 47 & 0.0325 & 0.1569 \\ 5066 & 12: 50: 49 & 0.1134 & 0.1442 \\ 5068 & 12: 50: 51 & 0.0890 & 0.0830 \\ 5070 & 12: 50: 53 & 0.0225 & 0.2189 \\ 5072 & 12: 50: 55 & 0.0321 & 0.1213 \\ 5074 & 12: 50: 57 & 0.0107 & 0.0654 \\ 5076 & 12: 50: 59 & 0.0376 & 0.0852 \\ 5078 & 12: 51: 01 & 0.1078 & 0.0481 \\ 5080 & 12: 51: 03 & 0.0434 & 0.1513 \\ 5082 & 12: 51: 05 & 0.0477 & 0.0876 \\ 5084 & 12: 51: 07 & 0.0549 & 0.0884 \\ 5086 & 12: 51: 09 & 0.0331 & 0.2819 \\ 5088 & 12: 51: 11 & 0.0668 & 0.1405 \\ 5090 & 12: 51: 13 & 0.0510 & 0.1732 \\ 5092 & 12: 51: 15 & 0.0709 & 0.1615\end{array}$




$\begin{array}{llll}5094 & 12: 51: 17 & 0.0634 & 0.0774 \\ 5096 & 12: 51: 19 & 0.0991 & 0.1171 \\ 5098 & 12: 51: 21 & 0.0880 & 0.1593 \\ 5100 & 12: 51: 23 & 0.1166 & 0.1438 \\ 5102 & 12: 51: 25 & 0.0285 & 0.1173 \\ 5104 & 12: 51: 27 & 0.0271 & 0.0794 \\ 5106 & 12: 51: 29 & 0.0865 & 0.0474 \\ 5108 & 12: 51: 31 & 0.0645 & 0.0799 \\ 5110 & 12: 51: 33 & 0.0610 & 0.0624 \\ 5112 & 12: 51: 35 & 0.1115 & 0.0612 \\ 5114 & 12: 51: 37 & 0.0580 & 0.1548 \\ 5116 & 12: 51: 39 & 0.0496 & 0.0886 \\ 5118 & 12: 51: 41 & 0.0586 & 0.1360 \\ 5120 & 12: 51: 43 & 0.1068 & 0.0357 \\ 5122 & 12: 51: 45 & 0.0676 & 0.0615 \\ 5124 & 12: 51: 47 & 0.0908 & 0.0425 \\ 5126 & 12: 51: 49 & 0.0278 & 0.0341 \\ 5128 & 12: 51: 51 & 0.0490 & 0.0936 \\ 5130 & 12: 51: 53 & 0.0673 & 0.1137 \\ 5132 & 12: 51: 55 & 0.0868 & 0.1060 \\ 5134 & 12: 51: 57 & 0.1816 & 0.2340 \\ 5136 & 12: 51: 59 & 0.0431 & 0.0404 \\ 5138 & 12: 52: 01 & 0.0518 & 0.0324 \\ 5140 & 12: 52: 03 & 0.1212 & 0.1759 \\ 5142 & 12: 52: 05 & 0.0541 & 0.2519 \\ 5144 & 12: 52: 07 & 0.0761 & 0.0806 \\ 5146 & 12: 52: 09 & 0.0571 & 0.1180 \\ 5148 & 12: 52: 11 & 0.0474 & 0.0919 \\ 5150 & 12: 52: 13 & 0.0327 & 0.0664 \\ 5152 & 12: 52: 15 & 0.0278 & 0.1449 \\ 5154 & 12: 52: 17 & 0.0221 & 0.1726 \\ 5156 & 12: 52: 19 & 0.0313 & 0.1163 \\ 5158 & 12: 52: 21 & 0.0269 & 0.1099 \\ 5160 & 12: 52: 23 & 0.0466 & 0.1747 \\ 5162 & 12: 52: 25 & 0.0679 & 0.3051 \\ 5164 & 12: 52: 27 & 0.0230 & 0.0436 \\ 5166 & 12: 52: 29 & 0.1192 & 0.0526 \\ 5168 & 12: 52: 31 & 0.1097 & 0.0701 \\ 5170 & 12: 52: 33 & 0.0787 & 0.1605 \\ 5172 & 12: 52: 35 & 0.0743 & 0.0453 \\ 5174 & 12: 52: 37 & 0.0527 & 0.0178 \\ 5176 & 12: 52: 39 & 0.0189 & 0.0699 \\ 5178 & 12: 52: 41 & 0.0445 & 0.0557 \\ 5180 & 12: 52: 43 & 0.0308 & 0.0532 \\ 5182 & 12: 52: 45 & 0.0209 & 0.1908 \\ 5184 & 12: 52: 47 & 0.0758 & 0.0885 \\ 5186 & 12: 52: 49 & 0.1042 & 0.1271 \\ 5188 & 12: 52: 51 & 0.0211 & 0.0602 \\ 5190 & 12: 52: 53 & 0.0616 & 0.0679 \\ 5192 & 12: 52: 55 & 0.0261 & 0.0732 \\ 5194 & 12: 52: 57 & 0.0091 & 0.0712 \\ 5196 & 12: 52: 59 & 0.0213 & 0.0483\end{array}$




$\begin{array}{llll}5198 & 12: 53: 01 & 0.0195 & 0.0887 \\ 5200 & 12: 53: 03 & 0.0452 & 0.1021 \\ 5202 & 12: 53: 05 & 0.0564 & 0.1046 \\ 5204 & 12: 53: 07 & 0.0211 & 0.0821 \\ 5206 & 12: 53: 09 & 0.0321 & 0.0661 \\ 5208 & 12: 53: 11 & 0.0154 & 0.0535 \\ 5210 & 12: 53: 13 & 0.0244 & 0.0700 \\ 5212 & 12: 53: 15 & 0.0368 & 0.2448 \\ 5214 & 12: 53: 17 & 0.0473 & 0.1220 \\ 5216 & 12: 53: 19 & 0.1027 & 0.0352 \\ 5218 & 12: 53: 21 & 0.0444 & 0.1278 \\ 5220 & 12: 53: 23 & 0.0215 & 0.1130 \\ 5222 & 12: 53: 25 & 0.0176 & 0.0904 \\ 5224 & 12: 53: 27 & 0.0347 & 0.0560 \\ 5226 & 12: 53: 29 & 0.1967 & 0.2008 \\ 5228 & 12: 53: 31 & 0.1688 & 0.1286 \\ 5230 & 12: 53: 33 & 0.0357 & 0.1576 \\ 5232 & 12: 53: 35 & 0.1707 & 0.1261 \\ 5234 & 12: 53: 37 & 0.0578 & 0.1071 \\ 5236 & 12: 53: 39 & 0.0192 & 0.0466 \\ 5238 & 12: 53: 41 & 0.0474 & 0.0702 \\ 5240 & 12: 53: 43 & 0.0664 & 0.0789 \\ 5242 & 12: 53: 45 & 0.0043 & 0.1484 \\ 5244 & 12: 53: 47 & 0.0192 & 0.2422 \\ 5246 & 12: 53: 49 & 0.0412 & 0.1775 \\ 5248 & 12: 53: 51 & 0.0704 & 0.0100 \\ 5250 & 12: 53: 53 & 0.0312 & 0.0896 \\ 5252 & 12: 53: 55 & 0.0616 & 0.0634 \\ 5254 & 12: 53: 57 & 0.0616 & 0.0818 \\ 5256 & 12: 53: 59 & 0.0768 & 0.0976 \\ 5258 & 12: 54: 01 & 0.0204 & 0.0561 \\ 5260 & 12: 54: 03 & 0.0651 & 0.0490 \\ 5262 & 12: 54: 05 & 0.2715 & 0.0751 \\ 5264 & 12: 54: 07 & 0.1374 & 0.1753 \\ 5266 & 12: 54: 09 & 0.0229 & 0.0708 \\ 5268 & 12: 54: 11 & 0.0177 & 0.1710 \\ 5270 & 12: 54: 13 & 0.0499 & 0.0719 \\ 5272 & 12: 54: 15 & 0.0659 & 0.1638 \\ 5274 & 12: 54: 17 & 0.0343 & 0.0405 \\ 5276 & 12: 54: 19 & 0.0344 & 0.1532 \\ 5278 & 12: 54: 21 & 0.0552 & 0.1927 \\ 5280 & 12: 54: 23 & 0.0067 & 0.0806 \\ 5282 & 12: 54: 25 & 0.0654 & 0.2424 \\ 5284 & 12: 54: 27 & 0.0199 & 0.1720 \\ 5286 & 12: 54: 29 & 0.0260 & 0.0795 \\ 5288 & 12: 54: 31 & 0.0427 & 0.2458 \\ 5290 & 12: 54: 33 & 0.0275 & 0.0939 \\ 5292 & 12: 54: 35 & 0.0172 & 0.1927 \\ 5294 & 12: 54: 37 & 0.0655 & 0.0795 \\ 5296 & 12: 54: 39 & 0.0108 & 0.0763 \\ 5298 & 12: 54: 41 & 0.0177 & 0.0819 \\ 5300 & 12: 54: 43 & 0.0531 & 0.1353\end{array}$




$\begin{array}{llll}5302 & 12: 54: 45 & 0.0204 & 0.0629 \\ 5304 & 12: 54: 47 & 0.0712 & 0.1414 \\ 5306 & 12: 54: 49 & 0.1205 & 0.1410 \\ 5308 & 12: 54: 51 & 0.0468 & 0.1467 \\ 5310 & 12: 54: 53 & 0.0233 & 0.0159 \\ 5312 & 12: 54: 55 & 0.0327 & 0.2934 \\ 5314 & 12: 54: 57 & 0.0135 & 0.1012 \\ 5316 & 12: 54: 59 & 0.0549 & 0.1681 \\ 5318 & 12: 55: 01 & 0.0394 & 0.1594 \\ 5320 & 12: 55: 03 & 0.0474 & 0.1499 \\ 5322 & 12: 55: 05 & 0.0314 & 0.0747 \\ 5324 & 12: 55: 07 & 0.0483 & 0.2674 \\ 5326 & 12: 55: 09 & 0.0592 & 0.3569 \\ 5328 & 12: 55: 11 & 0.0246 & 0.1856 \\ 5330 & 12: 55: 13 & 0.0260 & 0.1491 \\ 5332 & 12: 55: 15 & 0.0926 & 0.2486 \\ 5334 & 12: 55: 17 & 0.0346 & 0.1088 \\ 5336 & 12: 55: 19 & 0.0344 & 0.1319 \\ 5338 & 12: 55: 21 & 0.0153 & 0.1461 \\ 5340 & 12: 55: 23 & 0.0380 & 0.1422 \\ 5342 & 12: 55: 25 & 0.0717 & 0.0551 \\ 5344 & 12: 55: 27 & 0.0938 & 0.0634 \\ 5346 & 12: 55: 29 & 0.0592 & 0.0651 \\ 5348 & 12: 55: 31 & 0.0892 & 0.1569 \\ 5350 & 12: 55: 33 & 0.0144 & 0.0380 \\ 5352 & 12: 55: 35 & 0.1029 & 0.1107 \\ 5354 & 12: 55: 37 & 0.0189 & 0.0523 \\ 5356 & 12: 55: 39 & 0.0389 & 0.2004 \\ 5358 & 12: 55: 41 & 0.0434 & 0.1248 \\ 5360 & 12: 55: 43 & 0.0472 & 0.0882 \\ 5362 & 12: 55: 45 & 0.1093 & 0.0957 \\ 5364 & 12: 55: 47 & 0.0521 & 0.1885 \\ 5366 & 12: 55: 49 & 0.0424 & 0.1890 \\ 5368 & 12: 55: 51 & 0.0199 & 0.1311 \\ 5370 & 12: 55: 53 & 0.0681 & 0.0972 \\ 5372 & 12: 55: 55 & 0.0946 & 0.2114 \\ 5374 & 12: 55: 57 & 0.1263 & 0.1655 \\ 5376 & 12: 55: 59 & 0.0681 & 0.0527 \\ 5378 & 12: 56: 01 & 0.0771 & 0.3412 \\ 5380 & 12: 56: 03 & 0.0575 & 0.2417 \\ 5382 & 12: 56: 05 & 0.0782 & 0.2253 \\ 5384 & 12: 56: 07 & 0.0233 & 0.1500 \\ 5386 & 12: 56: 09 & 0.0951 & 0.0967 \\ 5388 & 12: 56: 11 & 0.1348 & 0.0882 \\ 5390 & 12: 56: 13 & 0.1156 & 0.0680 \\ 5392 & 12: 56: 15 & 0.1178 & 0.1321 \\ 5394 & 12: 56: 17 & 0.1726 & 0.1930 \\ 5396 & 12: 56: 19 & 0.0188 & 0.0750 \\ 5398 & 12: 56: 21 & 0.0311 & 0.0665 \\ 5400 & 12: 56: 23 & 0.0142 & 0.2414 \\ 5402 & 12: 56: 25 & 0.0192 & 0.0359 \\ 5404 & 12: 56: 27 & 0.1927 & 0.1376\end{array}$




$\begin{array}{llll}5406 & 12: 56: 29 & 0.0503 & 0.4099 \\ 5408 & 12: 56: 31 & 0.0230 & 0.1055 \\ 5410 & 12: 56: 33 & 0.0660 & 0.1785 \\ 5412 & 12: 56: 35 & 0.0457 & 0.1104 \\ 5414 & 12: 56: 37 & 0.0185 & 0.0764 \\ 5416 & 12: 56: 39 & 0.0270 & 0.0962 \\ 5418 & 12: 56: 41 & 0.0387 & 0.1554 \\ 5420 & 12: 56: 43 & 0.0178 & 0.0571 \\ 5422 & 12: 56: 45 & 0.0833 & 0.1921 \\ 5424 & 12: 56: 47 & 0.0370 & 0.2222 \\ 5426 & 12: 56: 49 & 0.0669 & 0.2596 \\ 5428 & 12: 56: 51 & 0.0636 & 0.1757 \\ 5430 & 12: 56: 53 & 0.0395 & 0.2201 \\ 5432 & 12: 56: 55 & 0.0531 & 0.1050 \\ 5434 & 12: 56: 57 & 0.0916 & 0.2230 \\ 5436 & 12: 56: 59 & 0.0863 & 0.0946 \\ 5438 & 12: 57: 01 & 0.1110 & 0.0589 \\ 5440 & 12: 57: 03 & 0.0447 & 0.1076 \\ 5442 & 12: 57: 05 & 0.1117 & 0.0682 \\ 5444 & 12: 57: 07 & 0.0447 & 0.0452 \\ 5446 & 12: 57: 09 & 0.0112 & 0.0998 \\ 5448 & 12: 57: 11 & 0.0924 & 0.0836 \\ 5450 & 12: 57: 13 & 0.0267 & 0.1174 \\ 5452 & 12: 57: 15 & 0.0369 & 0.0844 \\ 5454 & 12: 57: 17 & 0.0244 & 0.1988 \\ 5456 & 12: 57: 19 & 0.0764 & 0.1446 \\ 5458 & 12: 57: 21 & 0.0885 & 0.0490 \\ 5460 & 12: 57: 23 & 0.0060 & 0.0327 \\ 5462 & 12: 57: 25 & 0.0332 & 0.1088 \\ 5464 & 12: 57: 27 & 0.0763 & 0.1287 \\ 5466 & 12: 57: 29 & 0.0447 & 0.1307 \\ 5468 & 12: 57: 31 & 0.0300 & 0.1158 \\ 5470 & 12: 57: 33 & 0.0300 & 0.1020 \\ 5472 & 12: 57: 35 & 0.0794 & 0.0388 \\ 5474 & 12: 57: 37 & 0.0574 & 0.0540 \\ 5476 & 12: 57: 39 & 0.0181 & 0.0849 \\ 5478 & 12: 57: 41 & 0.0771 & 0.1049 \\ 5480 & 12: 57: 43 & 0.0339 & 0.0339 \\ 5482 & 12: 57: 45 & 0.0151 & 0.0721 \\ 5484 & 12: 57: 47 & 0.0316 & 0.0837 \\ 5486 & 12: 57: 49 & 0.0352 & 0.0568 \\ 5488 & 12: 57: 51 & 0.0387 & 0.1340 \\ 5490 & 12: 57: 53 & 0.0958 & 0.1671 \\ 5492 & 12: 57: 55 & 0.0302 & 0.1324 \\ 5494 & 12: 57: 57 & 0.0449 & 0.1132 \\ 5496 & 12: 57: 59 & 0.1129 & 0.0732 \\ 5498 & 12: 58: 01 & 0.0224 & 0.1479 \\ 5500 & 12: 58: 03 & 0.0831 & 0.3078 \\ 5502 & 12: 58: 05 & 0.0699 & 0.0503 \\ 5504 & 12: 58: 07 & 0.0145 & 0.1048 \\ 5506 & 12: 58: 09 & 0.0478 & 0.0824 \\ 5508 & 12: 58: 11 & 0.0622 & 0.1738\end{array}$




$\begin{array}{llll}5510 & 12: 58: 13 & 0.1268 & 0.1374 \\ 5512 & 12: 58: 15 & 0.0150 & 0.0634 \\ 5514 & 12: 58: 17 & 0.0659 & 0.0981 \\ 5516 & 12: 58: 19 & 0.0670 & 0.1155 \\ 5518 & 12: 58: 21 & 0.0843 & 0.1277 \\ 5520 & 12: 58: 23 & 0.0837 & 0.0447 \\ 5522 & 12: 58: 25 & 0.0505 & 0.0291 \\ 5524 & 12: 58: 27 & 0.0958 & 0.1441 \\ 5526 & 12: 58: 29 & 0.0297 & 0.1568 \\ 5528 & 12: 58: 31 & 0.0808 & 0.0487 \\ 5530 & 12: 58: 33 & 0.0854 & 0.1255 \\ 5532 & 12: 58: 35 & 0.0346 & 0.1738 \\ 5534 & 12: 58: 37 & 0.0331 & 0.2327 \\ 5536 & 12: 58: 39 & 0.0264 & 0.0970 \\ 5538 & 12: 58: 41 & 0.0417 & 0.0844 \\ 5540 & 12: 58: 43 & 0.0383 & 0.1259 \\ 5542 & 12: 58: 45 & 0.0603 & 0.1330 \\ 5544 & 12: 58: 47 & 0.0666 & 0.0947 \\ 5546 & 12: 58: 49 & 0.0241 & 0.0609 \\ 5548 & 12: 58: 51 & 0.1090 & 0.0951 \\ 5550 & 12: 58: 53 & 0.0069 & 0.0595 \\ 5552 & 12: 58: 55 & 0.1321 & 0.0925 \\ 5554 & 12: 58: 57 & 0.1335 & 0.0910 \\ 5556 & 12: 58: 59 & 0.0613 & 0.1569 \\ 5558 & 12: 59: 01 & 0.1019 & 0.1392 \\ 5560 & 12: 59: 03 & 0.0643 & 0.1056 \\ 5562 & 12: 59: 05 & 0.1394 & 0.3901 \\ 5564 & 12: 59: 07 & 0.0235 & 0.1620 \\ 5566 & 12: 59: 09 & 0.0935 & 0.2162 \\ 5568 & 12: 59: 11 & 0.0764 & 0.1453 \\ 5570 & 12: 59: 13 & 0.0536 & 0.1859 \\ 5572 & 12: 59: 15 & 0.0958 & 0.1821 \\ 5574 & 12: 59: 17 & 0.1203 & 0.0535 \\ 5576 & 12: 59: 19 & 0.0316 & 0.0471 \\ 5578 & 12: 59: 21 & 0.0640 & 0.0830 \\ 5580 & 12: 59: 23 & 0.0669 & 0.1867 \\ 5582 & 12: 59: 25 & 0.0383 & 0.0684 \\ 5584 & 12: 59: 27 & 0.1618 & 0.0899 \\ 5586 & 12: 59: 29 & 0.0420 & 0.2371 \\ 5588 & 12: 59: 31 & 0.0685 & 0.1003 \\ 5590 & 12: 59: 33 & 0.0408 & 0.0904 \\ 5592 & 12: 59: 35 & 0.0876 & 0.1443 \\ 5594 & 12: 59: 37 & 0.0717 & 0.0431 \\ 5596 & 12: 59: 39 & 0.0448 & 0.0497 \\ 5598 & 12: 59: 41 & 0.0265 & 0.1279 \\ 5600 & 12: 59: 43 & 0.0208 & 0.1245 \\ 5602 & 12: 59: 45 & 0.0785 & 0.0705 \\ 5604 & 12: 59: 47 & 0.2409 & 0.2579 \\ 5606 & 12: 59: 49 & 0.1021 & 0.0800 \\ 5608 & 12: 59: 51 & 0.0518 & 0.1121 \\ 5610 & 12: 59: 53 & 0.0606 & 0.1427 \\ 5612 & 12: 59: 55 & 0.0260 & 0.2791\end{array}$




$\begin{array}{llll}5614 & 12: 59: 57 & 0.0299 & 0.1367 \\ 5616 & 12: 59: 59 & 0.0196 & 0.1101 \\ 5618 & 13: 00: 01 & 0.2048 & 0.0515 \\ 5620 & 13: 00: 03 & 0.0600 & 0.0402 \\ 5622 & 13: 00: 05 & 0.0184 & 0.1889 \\ 5624 & 13: 00: 07 & 0.0177 & 0.1141 \\ 5626 & 13: 00: 09 & 0.1040 & 0.0267 \\ 5628 & 13: 00: 11 & 0.0142 & 0.0752 \\ 5630 & 13: 00: 13 & 0.1719 & 0.0380 \\ 5632 & 13: 00: 15 & 0.0331 & 0.0896 \\ 5634 & 13: 00: 17 & 0.0127 & 0.0384 \\ 5636 & 13: 00: 19 & 0.0490 & 0.1334 \\ 5638 & 13: 00: 21 & 0.0331 & 0.0219 \\ 5640 & 13: 00: 23 & 0.0472 & 0.0652 \\ 5642 & 13: 00: 25 & 0.0495 & 0.0774 \\ 5644 & 13: 00: 27 & 0.0665 & 0.1357 \\ 5646 & 13: 00: 29 & 0.0400 & 0.1800 \\ 5648 & 13: 00: 31 & 0.0361 & 0.6082 \\ 5650 & 13: 00: 33 & 0.0524 & 0.2355 \\ 5652 & 13: 00: 35 & 0.1064 & 0.2008 \\ 5654 & 13: 00: 37 & 0.0492 & 0.1983 \\ 5656 & 13: 00: 39 & 0.0475 & 0.1236 \\ 5658 & 13: 00: 41 & 0.0059 & 0.0572 \\ 5660 & 13: 00: 43 & 0.0238 & 0.1803 \\ 5662 & 13: 00: 45 & 0.0168 & 0.0655 \\ 5664 & 13: 00: 47 & 0.0197 & 0.0703 \\ 5666 & 13: 00: 49 & 0.0994 & 0.1962 \\ 5668 & 13: 00: 51 & 0.0240 & 0.1983 \\ 5670 & 13: 00: 53 & 0.0523 & 0.1733 \\ 5672 & 13: 00: 55 & 0.0912 & 0.1526 \\ 5674 & 13: 00: 57 & 0.1124 & 0.2084 \\ 5676 & 13: 00: 59 & 0.0451 & 0.0853 \\ 5678 & 13: 01: 01 & 0.0431 & 0.3438 \\ 5680 & 13: 01: 03 & 0.0315 & 0.1375 \\ 5682 & 13: 01: 05 & 0.0544 & 0.1847 \\ 5684 & 13: 01: 07 & 0.0473 & 0.0646 \\ 5686 & 13: 01: 09 & 0.0481 & 0.1047 \\ 5688 & 13: 01: 11 & 0.0343 & 0.0582 \\ 5690 & 13: 01: 13 & 0.0450 & 0.1094 \\ 5692 & 13: 01: 15 & 0.0961 & 0.2677 \\ 5694 & 13: 01: 17 & 0.0343 & 0.0410 \\ 5696 & 13: 01: 19 & 0.0466 & 0.1632 \\ 5698 & 13: 01: 21 & 0.0164 & 0.0872 \\ 5700 & 13: 01: 23 & 0.0472 & 0.0743 \\ 5702 & 13: 01: 25 & 0.0427 & 0.1940 \\ 5704 & 13: 01: 27 & 0.1178 & 0.1806 \\ 5706 & 13: 01: 29 & 0.0429 & 0.1884 \\ 5708 & 13: 01: 31 & 0.0368 & 0.1844 \\ 5710 & 13: 01: 33 & 0.0491 & 0.1314 \\ 5712 & 13: 01: 35 & 0.0815 & 0.1511 \\ 5714 & 13: 01: 37 & 0.0290 & 0.1172 \\ 5716 & 13: 01: 39 & 0.0488 & 0.0393\end{array}$


Appendix B - Laser Data Test 1 NIST102505_1.xls

$\begin{array}{llll}5718 & 13: 01: 41 & 0.0322 & 0.0873 \\ 5720 & 13: 01: 43 & 0.0703 & 0.0822 \\ 5722 & 13: 01: 45 & 0.1014 & 0.0581 \\ 5724 & 13: 01: 47 & 0.0620 & 0.0484 \\ 5726 & 13: 01: 49 & 0.0461 & 0.0675 \\ 5728 & 13: 01: 51 & 0.0977 & 0.0780 \\ 5730 & 13: 01: 53 & 0.0913 & 0.0488 \\ 5732 & 13: 01: 55 & 0.0236 & 0.0600 \\ 5734 & 13: 01: 57 & 0.0528 & 0.2789 \\ 5736 & 13: 01: 59 & 0.0731 & 0.1403 \\ 5738 & 13: 02: 01 & 0.0560 & 0.1513 \\ 5740 & 13: 02: 03 & 0.0953 & 0.1582 \\ 5742 & 13: 02: 05 & 0.0388 & 0.0832 \\ 5744 & 13: 02: 07 & 0.0434 & 0.1623 \\ 5746 & 13: 02: 09 & 0.0609 & 0.1651 \\ 5748 & 13: 02: 11 & 0.1419 & 0.1097 \\ 5750 & 13: 02: 13 & 0.0620 & 0.1542 \\ 5752 & 13: 02: 15 & 0.0317 & 0.0575 \\ 5754 & 13: 02: 17 & 0.0213 & 0.0888 \\ 5756 & 13: 02: 19 & 0.1060 & 0.0436 \\ 5758 & 13: 02: 21 & 0.0713 & 0.0830 \\ 5760 & 13: 02: 23 & 0.0217 & 0.0751 \\ 5762 & 13: 02: 25 & 0.0244 & 0.0876 \\ 5764 & 13: 02: 27 & 0.0518 & 0.1715 \\ 5766 & 13: 02: 29 & 0.0736 & 0.0321 \\ 5768 & 13: 02: 31 & 0.0071 & 0.0943 \\ 5770 & 13: 02: 33 & 0.0311 & 0.1519 \\ 5772 & 13: 02: 35 & 0.0444 & 0.1340 \\ 5774 & 13: 02: 37 & 0.0509 & 0.0720 \\ 5776 & 13: 02: 39 & 0.0593 & 0.0626 \\ 5778 & 13: 02: 41 & 0.0081 & 0.0446 \\ 5780 & 13: 02: 43 & 0.0652 & 0.0649 \\ 5782 & 13: 02: 45 & 0.0793 & 0.1090 \\ 5784 & 13: 02: 47 & 0.0789 & 0.1590 \\ 5786 & 13: 02: 49 & 0.0458 & 0.0791 \\ 5788 & 13: 02: 51 & 0.0607 & 0.1178 \\ 5790 & 13: 02: 53 & 0.0934 & 0.0775 \\ 5792 & 13: 02: 55 & 0.1453 & 0.0234 \\ 5794 & 13: 02: 57 & 0.0616 & 0.0461 \\ 5796 & 13: 02: 59 & 0.1282 & 0.1498 \\ 5798 & 13: 03: 01 & 0.0596 & 0.0410 \\ 5800 & 13: 03: 03 & 0.0088 & 0.0452 \\ 5802 & 13: 03: 05 & 0.0806 & 0.0335 \\ 5804 & 13: 03: 07 & 0.0502 & 0.0930 \\ 5806 & 13: 03: 09 & 0.0320 & 0.0322 \\ 5808 & 13: 03: 11 & 0.0302 & 0.0500 \\ 5810 & 13: 03: 13 & 0.0629 & 0.0722 \\ 5812 & 13: 03: 15 & 0.0511 & 0.0598 \\ 5814 & 13: 03: 17 & 0.0720 & 0.0317 \\ 5816 & 13: 03: 19 & 0.0340 & 0.0613 \\ 5818 & 13: 03: 21 & 0.0406 & 0.0402 \\ 5820 & 13: 03: 23 & 0.1411 & 0.1041\end{array}$




$\begin{array}{llll}5822 & 13: 03: 25 & 0.0335 & 0.0600 \\ 5824 & 13: 03: 27 & 0.0407 & 0.0352 \\ 5826 & 13: 03: 29 & 0.0323 & 0.0179 \\ 5828 & 13: 03: 31 & 0.0078 & 0.0738 \\ 5830 & 13: 03: 33 & 0.0444 & 0.1648 \\ 5832 & 13: 03: 35 & 0.0489 & 0.1185 \\ 5834 & 13: 03: 37 & 0.1093 & 0.0736 \\ 5836 & 13: 03: 39 & 0.0069 & 0.0426 \\ 5838 & 13: 03: 41 & 0.0921 & 0.1661 \\ 5840 & 13: 03: 43 & 0.0569 & 0.1040 \\ 5842 & 13: 03: 45 & 0.0581 & 0.0841 \\ 5844 & 13: 03: 47 & 0.0343 & 0.1035 \\ 5846 & 13: 03: 49 & 0.0398 & 0.1571 \\ 5848 & 13: 03: 51 & 0.0476 & 0.2284 \\ 5850 & 13: 03: 53 & 0.1189 & 0.3248 \\ 5852 & 13: 03: 55 & 0.0841 & 0.1066 \\ 5854 & 13: 03: 57 & 0.0871 & 0.1851 \\ 5856 & 13: 03: 59 & 0.0298 & 0.1420 \\ 5858 & 13: 04: 01 & 0.0274 & 0.0816 \\ 5860 & 13: 04: 03 & 0.1524 & 0.1102 \\ 5862 & 13: 04: 05 & 0.0953 & 0.2102 \\ 5864 & 13: 04: 07 & 0.0355 & 0.2795 \\ 5866 & 13: 04: 09 & 0.0316 & 0.1630 \\ 5868 & 13: 04: 11 & 0.0427 & 0.0859 \\ 5870 & 13: 04: 13 & 0.0118 & 0.3313 \\ 5872 & 13: 04: 15 & 0.0345 & 0.2695 \\ 5874 & 13: 04: 17 & 0.1716 & 0.1089 \\ 5876 & 13: 04: 19 & 0.0751 & 0.1961 \\ 5878 & 13: 04: 21 & 0.0147 & 0.0904 \\ 5880 & 13: 04: 23 & 0.1012 & 0.1036 \\ 5882 & 13: 04: 25 & 0.1022 & 0.1097 \\ 5884 & 13: 04: 27 & 0.1750 & 0.1300 \\ 5886 & 13: 04: 29 & 0.0633 & 0.2107 \\ 5888 & 13: 04: 31 & 0.0617 & 0.0496 \\ 5890 & 13: 04: 33 & 0.1506 & 0.2226 \\ 5892 & 13: 04: 35 & 0.1736 & 0.1164 \\ 5894 & 13: 04: 37 & 0.0786 & 0.0704 \\ 5896 & 13: 04: 39 & 0.0311 & 0.1223 \\ 5898 & 13: 04: 41 & 0.1417 & 0.1928 \\ 5900 & 13: 04: 43 & 0.0496 & 0.0653 \\ 5902 & 13: 04: 45 & 0.1259 & 0.2647 \\ 5904 & 13: 04: 47 & 0.0784 & 0.0224 \\ 5906 & 13: 04: 49 & 0.1568 & 0.0915 \\ 5908 & 13: 04: 51 & 0.0528 & 0.1299 \\ 5910 & 13: 04: 53 & 0.0388 & 0.2432 \\ 5912 & 13: 04: 55 & 0.0515 & 0.1733 \\ 5914 & 13: 04: 57 & 0.0561 & 0.1155 \\ 5916 & 13: 04: 59 & 0.0187 & 0.1063 \\ 5918 & 13: 05: 01 & 0.0862 & 0.0285 \\ 5920 & 13: 05: 03 & 0.0463 & 0.0611 \\ 5922 & 13: 05: 05 & 0.0594 & 0.0801 \\ 5924 & 13: 05: 07 & 0.1735 & 0.0285\end{array}$




$\begin{array}{llll}5926 & 13: 05: 09 & 0.1251 & 0.0426 \\ 5928 & 13: 05: 11 & 0.1944 & 0.1093 \\ 5930 & 13: 05: 13 & 0.0356 & 0.0422 \\ 5932 & 13: 05: 15 & 0.0415 & 0.1488 \\ 5934 & 13: 05: 17 & 0.0399 & 0.0841 \\ 5936 & 13: 05: 19 & 0.0593 & 0.1245 \\ 5938 & 13: 05: 21 & 0.0857 & 0.2576 \\ 5940 & 13: 05: 23 & 0.0308 & 0.0412 \\ 5942 & 13: 05: 25 & 0.0230 & 0.0627 \\ 5944 & 13: 05: 27 & 0.2372 & 0.1270 \\ 5946 & 13: 05: 29 & 0.0992 & 0.1292 \\ 5948 & 13: 05: 31 & 0.1714 & 0.0379 \\ 5950 & 13: 05: 33 & 0.0933 & 0.1145 \\ 5952 & 13: 05: 35 & 0.1038 & 0.1264 \\ 5954 & 13: 05: 37 & 0.0280 & 0.0868 \\ 5956 & 13: 05: 39 & 0.1148 & 0.4145 \\ 5958 & 13: 05: 41 & 0.0863 & 0.0438 \\ 5960 & 13: 05: 43 & 0.0223 & 0.0705 \\ 5962 & 13: 05: 45 & 0.0504 & 0.1528 \\ 5964 & 13: 05: 47 & 0.0298 & 0.3372 \\ 5966 & 13: 05: 49 & 0.0555 & 0.2190 \\ 5968 & 13: 05: 51 & 0.0215 & 0.1252 \\ 5970 & 13: 05: 53 & 0.0631 & 0.1841 \\ 5972 & 13: 05: 55 & 0.1251 & 0.1217 \\ 5974 & 13: 05: 57 & 0.1112 & 0.1986 \\ 5976 & 13: 05: 59 & 0.0508 & 0.0820 \\ 5978 & 13: 06: 01 & 0.0737 & 0.1111 \\ 5980 & 13: 06: 03 & 0.0207 & 0.1453 \\ 5982 & 13: 06: 05 & 0.0296 & 0.1240 \\ 5984 & 13: 06: 07 & 0.0608 & 0.2547 \\ 5986 & 13: 06: 09 & 0.0819 & 0.2070 \\ 5988 & 13: 06: 11 & 0.0347 & 0.1434 \\ 5990 & 13: 06: 13 & 0.0164 & 0.0971 \\ 5992 & 13: 06: 15 & 0.1079 & 0.1925 \\ 5994 & 13: 06: 17 & 0.0122 & 0.1135 \\ 5996 & 13: 06: 19 & 0.0153 & 0.0795 \\ 5998 & 13: 06: 21 & 0.0236 & 0.1049 \\ 6000 & 13: 06: 23 & 0.0125 & 0.2879 \\ 6002 & 13: 06: 25 & 0.0124 & 0.1183 \\ 6004 & 13: 06: 27 & 0.0585 & 0.0989 \\ 6006 & 13: 06: 29 & 0.0339 & 0.2309 \\ 6008 & 13: 06: 31 & 0.1490 & 0.0675 \\ 6010 & 13: 06: 33 & 0.0309 & 0.1915 \\ 6012 & 13: 06: 35 & 0.0405 & 0.1418 \\ 6014 & 13: 06: 37 & 0.0216 & 0.1417 \\ 6016 & 13: 06: 39 & 0.0581 & 0.3479 \\ 6018 & 13: 06: 41 & 0.0501 & 0.1083 \\ 6020 & 13: 06: 43 & 0.0452 & 0.0803 \\ 6022 & 13: 06: 45 & 0.0867 & 0.2571 \\ 6024 & 13: 06: 47 & 0.0388 & 0.1130 \\ 6026 & 13: 06: 49 & 0.0701 & 0.1250 \\ 6028 & 13: 06: 51 & 0.0418 & 0.0744\end{array}$




$\begin{array}{llll}6030 & 13: 06: 53 & 0.1369 & 0.2081 \\ 6032 & 13: 06: 55 & 0.1054 & 0.3514 \\ 6034 & 13: 06: 57 & 0.0557 & 0.1492 \\ 6036 & 13: 06: 59 & 0.0945 & 0.1116 \\ 6038 & 13: 07: 01 & 0.0378 & 0.1978 \\ 6040 & 13: 07: 03 & 0.0184 & 0.0752 \\ 6042 & 13: 07: 05 & 0.0562 & 0.1293 \\ 6044 & 13: 07: 07 & 0.0538 & 0.1042 \\ 6046 & 13: 07: 09 & 0.0817 & 0.1115 \\ 6048 & 13: 07: 11 & 0.0609 & 0.1067 \\ 6050 & 13: 07: 13 & 0.0937 & 0.1633 \\ 6052 & 13: 07: 15 & 0.0393 & 0.2337 \\ 6054 & 13: 07: 17 & 0.0413 & 0.2300 \\ 6056 & 13: 07: 19 & 0.1119 & 0.1898 \\ 6058 & 13: 07: 21 & 0.0688 & 0.1680 \\ 6060 & 13: 07: 23 & 0.0387 & 0.1464 \\ 6062 & 13: 07: 25 & 0.0267 & 0.1073 \\ 6064 & 13: 07: 27 & 0.0258 & 0.1812 \\ 6066 & 13: 07: 29 & 0.0438 & 0.0739 \\ 6068 & 13: 07: 31 & 0.1003 & 0.1649 \\ 6070 & 13: 07: 33 & 0.0091 & 0.1160 \\ 6072 & 13: 07: 35 & 0.0273 & 0.1103 \\ 6074 & 13: 07: 37 & 0.0534 & 0.0914 \\ 6076 & 13: 07: 39 & 0.0316 & 0.1251 \\ 6078 & 13: 07: 41 & 0.0631 & 0.0548 \\ 6080 & 13: 07: 43 & 0.0301 & 0.0814 \\ 6082 & 13: 07: 45 & 0.0776 & 0.2211 \\ 6084 & 13: 07: 47 & 0.0351 & 0.1216 \\ 6086 & 13: 07: 49 & 0.0263 & 0.1745 \\ 6088 & 13: 07: 51 & 0.0169 & 0.1320 \\ 6090 & 13: 07: 53 & 0.0226 & 0.1422 \\ 6092 & 13: 07: 55 & 0.0447 & 0.4706 \\ 6094 & 13: 07: 57 & 0.0855 & 0.3554 \\ 6096 & 13: 07: 59 & 0.0491 & 0.0764 \\ 6098 & 13: 08: 01 & 0.0253 & 0.1647 \\ 6100 & 13: 08: 03 & 0.1173 & 0.1175 \\ 6102 & 13: 08: 05 & 0.0620 & 0.0681 \\ 6104 & 13: 08: 07 & 0.0704 & 0.1265 \\ 6106 & 13: 08: 09 & 0.1099 & 0.1467 \\ 6108 & 13: 08: 11 & 0.0309 & 0.0352 \\ 6110 & 13: 08: 13 & 0.0478 & 0.1170 \\ 6112 & 13: 08: 15 & 0.0275 & 0.0741 \\ 6114 & 13: 08: 17 & 0.0231 & 0.0530 \\ 6116 & 13: 08: 19 & 0.0641 & 0.1414 \\ 6118 & 13: 08: 21 & 0.0552 & 0.0833 \\ 6120 & 13: 08: 23 & 0.0283 & 0.1408 \\ 6122 & 13: 08: 25 & 0.0557 & 0.1627 \\ 6124 & 13: 08: 27 & 0.0131 & 0.0653 \\ 6126 & 13: 08: 29 & 0.0242 & 0.1535 \\ 6128 & 13: 08: 31 & 0.0495 & 0.2885 \\ 6130 & 13: 08: 33 & 0.0856 & 0.0206 \\ 6132 & 13: 08: 35 & 0.0357 & 0.0597\end{array}$




$\begin{array}{llll}6134 & 13: 08: 37 & 0.0208 & 0.1225 \\ 6136 & 13: 08: 39 & 0.0510 & 0.1396 \\ 6138 & 13: 08: 41 & 0.0451 & 0.1047 \\ 6140 & 13: 08: 43 & 0.0533 & 0.0532 \\ 6142 & 13: 08: 45 & 0.1089 & 0.0637 \\ 6144 & 13: 08: 47 & 0.0953 & 0.0775 \\ 6146 & 13: 08: 49 & 0.0199 & 0.0298 \\ 6148 & 13: 08: 51 & 0.0186 & 0.0358 \\ 6150 & 13: 08: 53 & 0.0758 & 0.1497 \\ 6152 & 13: 08: 55 & 0.0550 & 0.1397 \\ 6154 & 13: 08: 57 & 0.0230 & 0.1808 \\ 6156 & 13: 08: 59 & 0.0166 & 0.0908 \\ 6158 & 13: 09: 01 & 0.0570 & 0.2019 \\ 6160 & 13: 09: 03 & 0.1290 & 0.0969 \\ 6162 & 13: 09: 05 & 0.0276 & 0.1266 \\ 6164 & 13: 09: 07 & 0.1030 & 0.1345 \\ 6166 & 13: 09: 09 & 0.0495 & 0.2110 \\ 6168 & 13: 09: 11 & 0.0294 & 0.1214 \\ 6170 & 13: 09: 13 & 0.0747 & 0.1514 \\ 6172 & 13: 09: 15 & 0.0485 & 0.2159 \\ 6174 & 13: 09: 17 & 0.0542 & 0.0843 \\ 6176 & 13: 09: 19 & 0.0644 & 0.1770 \\ 6178 & 13: 09: 21 & 0.0222 & 0.2639 \\ 6180 & 13: 09: 23 & 0.0265 & 0.0480 \\ 6182 & 13: 09: 25 & 0.0486 & 0.1282 \\ 6184 & 13: 09: 27 & 0.0529 & 0.1298 \\ 6186 & 13: 09: 29 & 0.1023 & 0.1383 \\ 6188 & 13: 09: 31 & 0.0364 & 0.0265 \\ 6190 & 13: 09: 33 & 0.0466 & 0.0766 \\ 6192 & 13: 09: 35 & 0.0167 & 0.0514 \\ 6194 & 13: 09: 37 & 0.1442 & 0.1404 \\ 6196 & 13: 09: 39 & 0.0536 & 0.0286 \\ 6198 & 13: 09: 41 & 0.2384 & 0.0823 \\ 6200 & 13: 09: 43 & 0.0756 & 0.1319 \\ 6202 & 13: 09: 45 & 0.0962 & 0.0546 \\ 6204 & 13: 09: 47 & 0.1422 & 0.0944 \\ 6206 & 13: 09: 49 & 0.0238 & 0.1079 \\ 6208 & 13: 09: 51 & 0.0666 & 0.2201 \\ 6210 & 13: 09: 53 & 0.0633 & 0.0789 \\ 6212 & 13: 09: 55 & 0.0707 & 0.1797 \\ 6214 & 13: 09: 57 & 0.0902 & 0.0582 \\ 6216 & 13: 09: 59 & 0.0861 & 0.0777 \\ 6218 & 13: 10: 01 & 0.0882 & 0.1163 \\ 6220 & 13: 10: 03 & 0.0404 & 0.1037 \\ 6222 & 13: 10: 05 & 0.0242 & 0.1627 \\ 6224 & 13: 10: 07 & 0.0225 & 0.0468 \\ 6226 & 13: 10: 09 & 0.0495 & 0.1104 \\ 6228 & 13: 10: 11 & 0.0339 & 0.0929 \\ 6230 & 13: 10: 13 & 0.0885 & 0.0735 \\ 6232 & 13: 10: 15 & 0.0871 & 0.0743 \\ 6234 & 13: 10: 17 & 0.0871 & 0.0668 \\ 6236 & 13: 10: 19 & 0.0903 & 0.0720\end{array}$




$\begin{array}{llll}6238 & 13: 10: 21 & 0.0657 & 0.1377 \\ 6240 & 13: 10: 23 & 0.0671 & 0.0656 \\ 6242 & 13: 10: 25 & 0.1150 & 0.2151 \\ 6244 & 13: 10: 27 & 0.1453 & 0.1299 \\ 6246 & 13: 10: 29 & 0.0163 & 0.0770 \\ 6248 & 13: 10: 31 & 0.0516 & 0.1263 \\ 6250 & 13: 10: 33 & 0.0641 & 0.1281 \\ 6252 & 13: 10: 35 & 0.1779 & 0.0825 \\ 6254 & 13: 10: 37 & 0.0413 & 0.0417 \\ 6256 & 13: 10: 39 & 0.0158 & 0.0883 \\ 6258 & 13: 10: 41 & 0.0309 & 0.0933 \\ 6260 & 13: 10: 43 & 0.0111 & 0.1341 \\ 6262 & 13: 10: 45 & 0.0500 & 0.1168 \\ 6264 & 13: 10: 47 & 0.0532 & 0.1901 \\ 6266 & 13: 10: 49 & 0.0499 & 0.0932 \\ 6268 & 13: 10: 51 & 0.0341 & 0.1158 \\ 6270 & 13: 10: 53 & 0.0558 & 0.2144 \\ 6272 & 13: 10: 55 & 0.0334 & 0.1974 \\ 6274 & 13: 10: 57 & 0.0788 & 0.1962 \\ 6276 & 13: 10: 59 & 0.0657 & 0.1261 \\ 6278 & 13: 11: 01 & 0.0355 & 0.1187 \\ 6280 & 13: 11: 03 & 0.0395 & 0.1935 \\ 6282 & 13: 11: 05 & 0.1014 & 0.2396 \\ 6284 & 13: 11: 07 & 0.0983 & 0.0888 \\ 6286 & 13: 11: 09 & 0.0699 & 0.1274 \\ 6288 & 13: 11: 11 & 0.0231 & 0.0676 \\ 6290 & 13: 11: 13 & 0.0476 & 0.0454 \\ 6292 & 13: 11: 15 & 0.0732 & 0.2272 \\ 6294 & 13: 11: 17 & 0.0397 & 0.1121 \\ 6296 & 13: 11: 19 & 0.1953 & 0.2152 \\ 6298 & 13: 11: 21 & 0.0591 & 0.1245 \\ 6300 & 13: 11: 23 & 0.0720 & 0.1744 \\ 6302 & 13: 11: 25 & 0.1201 & 0.0667 \\ 6304 & 13: 11: 27 & 0.0652 & 0.2071 \\ 6306 & 13: 11: 29 & 0.0163 & 0.1095 \\ 6308 & 13: 11: 31 & 0.0581 & 0.1459 \\ 6310 & 13: 11: 33 & 0.0224 & 0.2061 \\ 6312 & 13: 11: 35 & 0.0365 & 0.2301 \\ 6314 & 13: 11: 37 & 0.0555 & 0.2368 \\ 6316 & 13: 11: 39 & 0.0829 & 0.2650 \\ 6318 & 13: 11: 41 & 0.0737 & 0.2032 \\ 6320 & 13: 11: 43 & 0.0988 & 0.2585 \\ 6322 & 13: 11: 45 & 0.0410 & 0.2005 \\ 6324 & 13: 11: 47 & 0.0282 & 0.2763 \\ 6326 & 13: 11: 49 & 0.0722 & 0.1558 \\ 6328 & 13: 11: 51 & 0.0565 & 0.1044 \\ 6330 & 13: 11: 53 & 0.0722 & 0.0988 \\ 6332 & 13: 11: 55 & 0.0693 & 0.0838 \\ 6334 & 13: 11: 57 & 0.0274 & 0.1190 \\ 6336 & 13: 11: 59 & 0.0187 & 0.2088 \\ 6338 & 13: 12: 01 & 0.0910 & 0.1012 \\ 6340 & 13: 12: 03 & 0.1527 & 0.2848\end{array}$




$\begin{array}{llll}6342 & 13: 12: 05 & 0.0768 & 0.1253 \\ 6344 & 13: 12: 07 & 0.1069 & 0.1212 \\ 6346 & 13: 12: 09 & 0.0708 & 0.1040 \\ 6348 & 13: 12: 11 & 0.0322 & 0.2341 \\ 6350 & 13: 12: 13 & 0.1278 & 0.0926 \\ 6352 & 13: 12: 15 & 0.0623 & 0.1813 \\ 6354 & 13: 12: 17 & 0.0791 & 0.0995 \\ 6356 & 13: 12: 19 & 0.0088 & 0.1276 \\ 6358 & 13: 12: 21 & 0.0438 & 0.0594 \\ 6360 & 13: 12: 23 & 0.0500 & 0.1474 \\ 6362 & 13: 12: 25 & 0.0699 & 0.2110 \\ 6364 & 13: 12: 27 & 0.0421 & 0.0413 \\ 6366 & 13: 12: 29 & 0.1192 & 0.1692 \\ 6368 & 13: 12: 31 & 0.0483 & 0.1481 \\ 6370 & 13: 12: 33 & 0.0490 & 0.2054 \\ 6372 & 13: 12: 35 & 0.0942 & 0.1285 \\ 6374 & 13: 12: 37 & 0.0418 & 0.0776 \\ 6376 & 13: 12: 39 & 0.0832 & 0.0404 \\ 6378 & 13: 12: 41 & 0.0957 & 0.0877 \\ 6380 & 13: 12: 43 & 0.0195 & 0.0638 \\ 6382 & 13: 12: 45 & 0.1137 & 0.0711 \\ 6384 & 13: 12: 47 & 0.1711 & 0.0610 \\ 6386 & 13: 12: 49 & 0.0383 & 0.0329 \\ 6388 & 13: 12: 51 & 0.0150 & 0.1270 \\ 6390 & 13: 12: 53 & 0.0366 & 0.0535 \\ 6392 & 13: 12: 55 & 0.0539 & 0.0856 \\ 6394 & 13: 12: 57 & 0.0993 & 0.0982 \\ 6396 & 13: 12: 59 & 0.0595 & 0.0570 \\ 6398 & 13: 13: 01 & 0.0280 & 0.1526 \\ 6400 & 13: 13: 03 & 0.0097 & 0.1486 \\ 6402 & 13: 13: 05 & 0.0586 & 0.0488 \\ 6404 & 13: 13: 07 & 0.0405 & 0.1889 \\ 6406 & 13: 13: 09 & 0.0186 & 0.2363 \\ 6408 & 13: 13: 11 & 0.0513 & 0.0767 \\ 6410 & 13: 13: 13 & 0.0670 & 0.0639 \\ 6412 & 13: 13: 15 & 0.2266 & 0.0758 \\ 6414 & 13: 13: 17 & 0.0763 & 0.1904 \\ 6416 & 13: 13: 19 & 0.2520 & 0.2318 \\ 6418 & 13: 13: 21 & 0.0333 & 0.1705 \\ 6420 & 13: 13: 23 & 0.0562 & 0.1149 \\ 6422 & 13: 13: 25 & 0.0113 & 0.0652 \\ 6424 & 13: 13: 27 & 0.0156 & 0.0473 \\ 6426 & 13: 13: 29 & 0.0415 & 0.1084 \\ 6428 & 13: 13: 31 & 0.0233 & 0.2653 \\ 6430 & 13: 13: 33 & 0.0363 & 0.1128 \\ 6432 & 13: 13: 35 & 0.0157 & 0.1945 \\ 6434 & 13: 13: 37 & 0.0531 & 0.1199 \\ 6436 & 13: 13: 39 & 0.0549 & 0.2732 \\ 6438 & 13: 13: 41 & 0.0366 & 0.1748 \\ 6440 & 13: 13: 43 & 0.0322 & 0.1275 \\ 6442 & 13: 13: 45 & 0.0744 & 0.1194 \\ 6444 & 13: 13: 47 & 0.0559 & 0.0525\end{array}$




$\begin{array}{llll}6446 & 13: 13: 49 & 0.0618 & 0.1405 \\ 6448 & 13: 13: 51 & 0.1210 & 0.0974 \\ 6450 & 13: 13: 53 & 0.0383 & 0.0603 \\ 6452 & 13: 13: 55 & 0.0410 & 0.1149 \\ 6454 & 13: 13: 57 & 0.0338 & 0.1024 \\ 6456 & 13: 13: 59 & 0.0245 & 0.1114 \\ 6458 & 13: 14: 01 & 0.0216 & 0.1693 \\ 6460 & 13: 14: 03 & 0.0426 & 0.1518 \\ 6462 & 13: 14: 05 & 0.0166 & 0.1266 \\ 6464 & 13: 14: 07 & 0.0143 & 0.1239 \\ 6466 & 13: 14: 09 & 0.0321 & 0.0778 \\ 6468 & 13: 14: 11 & 0.0958 & 0.1701 \\ 6470 & 13: 14: 13 & 0.0104 & 0.1166 \\ 6472 & 13: 14: 15 & 0.3294 & 0.1350 \\ 6474 & 13: 14: 17 & 0.0173 & 0.1838 \\ 6476 & 13: 14: 19 & 0.0251 & 0.0700 \\ 6478 & 13: 14: 21 & 0.0787 & 0.0620 \\ 6480 & 13: 14: 23 & 0.0190 & 0.1112 \\ 6482 & 13: 14: 25 & 0.0206 & 0.1311 \\ 6484 & 13: 14: 27 & 0.0675 & 0.1340 \\ 6486 & 13: 14: 29 & 0.0280 & 0.1512 \\ 6488 & 13: 14: 31 & 0.0319 & 0.1107 \\ 6490 & 13: 14: 33 & 0.0685 & 0.0743 \\ 6492 & 13: 14: 35 & 0.0430 & 0.0677 \\ 6494 & 13: 14: 37 & 0.0256 & 0.1910 \\ 6496 & 13: 14: 39 & 0.0248 & 0.1333 \\ 6498 & 13: 14: 41 & 0.0178 & 0.1256 \\ 6500 & 13: 14: 43 & 0.0455 & 0.0813 \\ 6502 & 13: 14: 45 & 0.0298 & 0.1119 \\ 6504 & 13: 14: 47 & 0.0275 & 0.1590 \\ 6506 & 13: 14: 49 & 0.0494 & 0.1110 \\ 6508 & 13: 14: 51 & 0.0344 & 0.0935 \\ 6510 & 13: 14: 53 & 0.0299 & 0.0960 \\ 6512 & 13: 14: 55 & 0.0734 & 0.2339 \\ 6514 & 13: 14: 57 & 0.0243 & 0.0781 \\ 6516 & 13: 14: 59 & 0.0546 & 0.1693 \\ 6518 & 13: 15: 01 & 0.0209 & 0.1015 \\ 6520 & 13: 15: 03 & 0.0151 & 0.2021 \\ 6522 & 13: 15: 05 & 0.1106 & 0.1774 \\ 6524 & 13: 15: 07 & 0.0413 & 0.1896 \\ 6526 & 13: 15: 09 & 0.0691 & 0.0777 \\ 6528 & 13: 15: 11 & 0.0661 & 0.1873 \\ 6530 & 13: 15: 13 & 0.0573 & 0.1992 \\ 6532 & 13: 15: 15 & 0.0190 & 0.1251 \\ 6534 & 13: 15: 17 & 0.0516 & 0.1194 \\ 6536 & 13: 15: 19 & 0.0489 & 0.1094 \\ 6538 & 13: 15: 21 & 0.0535 & 0.1251 \\ 6540 & 13: 15: 23 & 0.0541 & 0.1564 \\ 6542 & 13: 15: 25 & 0.0785 & 0.0775 \\ 6544 & 13: 15: 27 & 0.0552 & 0.1730 \\ 6546 & 13: 15: 29 & 0.1344 & 0.0658 \\ 6548 & 13: 15: 31 & 0.0474 & 0.0727\end{array}$




$\begin{array}{llll}6550 & 13: 15: 33 & 0.0567 & 0.0578 \\ 6552 & 13: 15: 35 & 0.0908 & 0.1126 \\ 6554 & 13: 15: 37 & 0.0652 & 0.0708 \\ 6556 & 13: 15: 39 & 0.0100 & 0.1022 \\ 6558 & 13: 15: 41 & 0.0589 & 0.0445 \\ 6560 & 13: 15: 43 & 0.0672 & 0.1167 \\ 6562 & 13: 15: 45 & 0.0241 & 0.1341 \\ 6564 & 13: 15: 47 & 0.0723 & 0.1279 \\ 6566 & 13: 15: 49 & 0.0426 & 0.0485 \\ 6568 & 13: 15: 51 & 0.1235 & 0.0661 \\ 6570 & 13: 15: 53 & 0.0098 & 0.0281 \\ 6572 & 13: 15: 55 & 0.0772 & 0.1141 \\ 6574 & 13: 15: 57 & 0.0551 & 0.1204 \\ 6576 & 13: 15: 59 & 0.0418 & 0.1307 \\ 6578 & 13: 16: 01 & 0.0580 & 0.1705 \\ 6580 & 13: 16: 03 & 0.0467 & 0.1079 \\ 6582 & 13: 16: 05 & 0.1464 & 0.0468 \\ 6584 & 13: 16: 07 & 0.1109 & 0.1665 \\ 6586 & 13: 16: 09 & 0.0390 & 0.1062 \\ 6588 & 13: 16: 11 & 0.1163 & 0.0696 \\ 6590 & 13: 16: 13 & 0.0400 & 0.0788 \\ 6592 & 13: 16: 15 & 0.0660 & 0.0817 \\ 6594 & 13: 16: 17 & 0.0423 & 0.0748 \\ 6596 & 13: 16: 19 & 0.0548 & 0.0572 \\ 6598 & 13: 16: 21 & 0.0658 & 0.1620 \\ 6600 & 13: 16: 23 & 0.0428 & 0.2349 \\ 6602 & 13: 16: 25 & 0.0777 & 0.3354 \\ 6604 & 13: 16: 27 & 0.0756 & 0.1052 \\ 6606 & 13: 16: 29 & 0.0387 & 0.1748 \\ 6608 & 13: 16: 31 & 0.0946 & 0.1963 \\ 6610 & 13: 16: 33 & 0.0557 & 0.1369 \\ 6612 & 13: 16: 35 & 0.0341 & 0.1999 \\ 6614 & 13: 16: 37 & 0.0776 & 0.4015 \\ 6616 & 13: 16: 39 & 0.0866 & 0.0703 \\ 6618 & 13: 16: 41 & 0.0174 & 0.1258 \\ 6620 & 13: 16: 43 & 0.0247 & 0.1420 \\ 6622 & 13: 16: 45 & 0.0410 & 0.2588 \\ 6624 & 13: 16: 47 & 0.0687 & 0.2561 \\ 6626 & 13: 16: 49 & 0.0645 & 0.1415 \\ 6628 & 13: 16: 51 & 0.0496 & 0.1191 \\ 6630 & 13: 16: 53 & 0.0292 & 0.1062 \\ 6632 & 13: 16: 55 & 0.0535 & 0.1815 \\ 6634 & 13: 16: 57 & 0.0255 & 0.0883 \\ 6636 & 13: 16: 59 & 0.0412 & 0.1704 \\ 6638 & 13: 17: 01 & 0.0864 & 0.0760 \\ 6640 & 13: 17: 03 & 0.0335 & 0.1696 \\ 6642 & 13: 17: 05 & 0.1043 & 0.1336 \\ 6644 & 13: 17: 07 & 0.0663 & 0.1534 \\ 6646 & 13: 17: 09 & 0.0537 & 0.1189 \\ 6648 & 13: 17: 11 & 0.1550 & 0.0718 \\ 6650 & 13: 17: 13 & 0.0934 & 0.1287 \\ 6652 & 13: 17: 15 & 0.1027 & 0.1697\end{array}$




$\begin{array}{llll}6654 & 13: 17: 17 & 0.0561 & 0.1392 \\ 6656 & 13: 17: 19 & 0.0408 & 0.1826 \\ 6658 & 13: 17: 21 & 0.0654 & 0.1905 \\ 6660 & 13: 17: 23 & 0.1069 & 0.1380 \\ 6662 & 13: 17: 25 & 0.0594 & 0.0970 \\ 6664 & 13: 17: 27 & 0.0192 & 0.1021 \\ 6666 & 13: 17: 29 & 0.1425 & 0.3664 \\ 6668 & 13: 17: 31 & 0.0676 & 0.0648 \\ 6670 & 13: 17: 33 & 0.0911 & 0.0735 \\ 6672 & 13: 17: 35 & 0.2564 & 0.0704 \\ 6674 & 13: 17: 37 & 0.1490 & 0.2274 \\ 6676 & 13: 17: 39 & 0.0647 & 0.1914 \\ 6678 & 13: 17: 41 & 0.0378 & 0.0508 \\ 6680 & 13: 17: 43 & 0.0100 & 0.0603 \\ 6682 & 13: 17: 45 & 0.0366 & 0.1172 \\ 6684 & 13: 17: 47 & 0.0147 & 0.0355 \\ 6686 & 13: 17: 49 & 0.0459 & 0.1281 \\ 6688 & 13: 17: 51 & 0.0160 & 0.1181 \\ 6690 & 13: 17: 53 & 0.0269 & 0.0714 \\ 6692 & 13: 17: 55 & 0.0405 & 0.1850 \\ 6694 & 13: 17: 57 & 0.0373 & 0.0864 \\ 6696 & 13: 17: 59 & 0.0461 & 0.0313 \\ 6698 & 13: 18: 01 & 0.0633 & 0.1105 \\ 6700 & 13: 18: 03 & 0.0768 & 0.1699 \\ 6702 & 13: 18: 05 & 0.0341 & 0.1432 \\ 6704 & 13: 18: 07 & 0.1123 & 0.1916 \\ 6706 & 13: 18: 09 & 0.1158 & 0.1574 \\ 6708 & 13: 18: 11 & 0.0662 & 0.1064 \\ 6710 & 13: 18: 13 & 0.0450 & 0.1531 \\ 6712 & 13: 18: 15 & 0.0464 & 0.1212 \\ 6714 & 13: 18: 17 & 0.0642 & 0.2930 \\ 6716 & 13: 18: 19 & 0.0326 & 0.1479 \\ 6718 & 13: 18: 21 & 0.0132 & 0.1206 \\ 6720 & 13: 18: 23 & 0.0523 & 0.3208 \\ 6722 & 13: 18: 25 & 0.0827 & 0.2032 \\ 6724 & 13: 18: 27 & 0.0092 & 0.1661 \\ 6726 & 13: 18: 29 & 0.0518 & 0.1698 \\ 6728 & 13: 18: 31 & 0.0385 & 0.0815 \\ 6730 & 13: 18: 33 & 0.0203 & 0.1212 \\ 6732 & 13: 18: 35 & 0.0558 & 0.1115 \\ 6734 & 13: 18: 37 & 0.0330 & 0.1227 \\ 6736 & 13: 18: 39 & 0.0802 & 0.0705 \\ 6738 & 13: 18: 41 & 0.0300 & 0.2019 \\ 6740 & 13: 18: 43 & 0.0270 & 0.0684 \\ 6742 & 13: 18: 45 & 0.0215 & 0.0699 \\ 6744 & 13: 18: 47 & 0.0382 & 0.1513 \\ 6746 & 13: 18: 49 & 0.0372 & 0.0596 \\ 6748 & 13: 18: 51 & 0.0720 & 0.0486 \\ 6750 & 13: 18: 53 & 0.0782 & 0.1084 \\ 6752 & 13: 18: 55 & 0.1392 & 0.1938 \\ 6754 & 13: 18: 57 & 0.0135 & 0.0728 \\ 6756 & 13: 18: 59 & 0.0150 & 0.1484\end{array}$




$\begin{array}{llll}6758 & 13: 19: 01 & 0.0481 & 0.1207 \\ 6760 & 13: 19: 03 & 0.0535 & 0.2253 \\ 6762 & 13: 19: 05 & 0.0245 & 0.0566 \\ 6764 & 13: 19: 07 & 0.0884 & 0.0789 \\ 6766 & 13: 19: 09 & 0.0459 & 0.0390 \\ 6768 & 13: 19: 11 & 0.0332 & 0.1685 \\ 6770 & 13: 19: 13 & 0.1200 & 0.0615 \\ 6772 & 13: 19: 15 & 0.0728 & 0.0404 \\ 6774 & 13: 19: 17 & 0.0239 & 0.0655 \\ 6776 & 13: 19: 19 & 0.0279 & 0.0736 \\ 6778 & 13: 19: 21 & 0.0829 & 0.0649 \\ 6780 & 13: 19: 23 & 0.0431 & 0.0922 \\ 6782 & 13: 19: 25 & 0.0566 & 0.1977 \\ 6784 & 13: 19: 27 & 0.0611 & 0.0414 \\ 6786 & 13: 19: 29 & 0.0544 & 0.2304 \\ 6788 & 13: 19: 31 & 0.0526 & 0.1110 \\ 6790 & 13: 19: 33 & 0.0319 & 0.1040 \\ 6792 & 13: 19: 35 & 0.0399 & 0.0700 \\ 6794 & 13: 19: 37 & 0.0185 & 0.1611 \\ 6796 & 13: 19: 39 & 0.0299 & 0.1142 \\ 6798 & 13: 19: 41 & 0.0599 & 0.0335 \\ 6800 & 13: 19: 43 & 0.0318 & 0.1414 \\ 6802 & 13: 19: 45 & 0.0281 & 0.2189 \\ 6804 & 13: 19: 47 & 0.0390 & 0.0790 \\ 6806 & 13: 19: 49 & 0.0249 & 0.1242 \\ 6808 & 13: 19: 51 & 0.1438 & 0.0927 \\ 6810 & 13: 19: 53 & 0.0306 & 0.1819 \\ 6812 & 13: 19: 55 & 0.0524 & 0.1543 \\ 6814 & 13: 19: 57 & 0.0276 & 0.1001 \\ 6816 & 13: 19: 59 & 0.0280 & 0.1160 \\ 6818 & 13: 20: 01 & 0.0253 & 0.0904 \\ 6820 & 13: 20: 03 & 0.0497 & 0.1276 \\ 6822 & 13: 20: 05 & 0.0630 & 0.1207 \\ 6824 & 13: 20: 07 & 0.0469 & 0.2253 \\ 6826 & 13: 20: 09 & 0.0444 & 0.1068 \\ 6828 & 13: 20: 11 & 0.0531 & 0.0909 \\ 6830 & 13: 20: 13 & 0.0423 & 0.1012 \\ 6832 & 13: 20: 15 & 0.0444 & 0.1699 \\ 6834 & 13: 20: 17 & 0.0524 & 0.0966 \\ 6836 & 13: 20: 19 & 0.0391 & 0.1712 \\ 6838 & 13: 20: 21 & 0.0321 & 0.0752 \\ 6840 & 13: 20: 23 & 0.1113 & 0.0435 \\ 6842 & 13: 20: 25 & 0.0102 & 0.0871 \\ 6844 & 13: 20: 27 & 0.0115 & 0.1484 \\ 6846 & 13: 20: 29 & 0.0707 & 0.0751 \\ 6848 & 13: 20: 31 & 0.0701 & 0.1076 \\ 6850 & 13: 20: 33 & 0.1274 & 0.1100 \\ 6852 & 13: 20: 35 & 0.0274 & 0.1131 \\ 6854 & 13: 20: 37 & 0.0303 & 0.1630 \\ 6856 & 13: 20: 39 & 0.0275 & 0.0729 \\ 6858 & 13: 20: 41 & 0.0599 & 0.1959 \\ 6860 & 13: 20: 43 & 0.0652 & 0.1418\end{array}$




$\begin{array}{llll}6862 & 13: 20: 45 & 0.0340 & 0.1317 \\ 6864 & 13: 20: 47 & 0.0380 & 0.1941 \\ 6866 & 13: 20: 49 & 0.0809 & 0.3932 \\ 6868 & 13: 20: 51 & 0.0204 & 0.0702 \\ 6870 & 13: 20: 53 & 0.0490 & 0.1310 \\ 6872 & 13: 20: 55 & 0.0518 & 0.1661 \\ 6874 & 13: 20: 57 & 0.0528 & 0.1095 \\ 6876 & 13: 20: 59 & 0.0322 & 0.0779 \\ 6878 & 13: 21: 01 & 0.0190 & 0.2407 \\ 6880 & 13: 21: 03 & 0.0135 & 0.1691 \\ 6882 & 13: 21: 05 & 0.1208 & 0.1649 \\ 6884 & 13: 21: 07 & 0.0258 & 0.0948 \\ 6886 & 13: 21: 09 & 0.0432 & 0.0580 \\ 6888 & 13: 21: 11 & 0.1058 & 0.0615 \\ 6890 & 13: 21: 13 & 0.0254 & 0.0615 \\ 6892 & 13: 21: 15 & 0.0305 & 0.0852 \\ 6894 & 13: 21: 17 & 0.0250 & 0.0989 \\ 6896 & 13: 21: 19 & 0.0375 & 0.1464 \\ 6898 & 13: 21: 21 & 0.0211 & 0.0672 \\ 6900 & 13: 21: 23 & 0.0528 & 0.1108 \\ 6902 & 13: 21: 25 & 0.0654 & 0.0605 \\ 6904 & 13: 21: 27 & 0.0472 & 0.1728 \\ 6906 & 13: 21: 29 & 0.0843 & 0.1493 \\ 6908 & 13: 21: 31 & 0.0375 & 0.0781 \\ 6910 & 13: 21: 33 & 0.0546 & 0.0850 \\ 6912 & 13: 21: 35 & 0.0470 & 0.1982 \\ 6914 & 13: 21: 37 & 0.0273 & 0.0783 \\ 6916 & 13: 21: 39 & 0.0751 & 0.2025 \\ 6918 & 13: 21: 41 & 0.0701 & 0.0801 \\ 6920 & 13: 21: 43 & 0.0152 & 0.2419 \\ 6922 & 13: 21: 45 & 0.0559 & 0.2295 \\ 6924 & 13: 21: 47 & 0.0185 & 0.2724 \\ 6926 & 13: 21: 49 & 0.0195 & 0.0746 \\ 6928 & 13: 21: 51 & 0.0472 & 0.3702 \\ 6930 & 13: 21: 53 & 0.0933 & 0.0760 \\ 6932 & 13: 21: 55 & 0.0333 & 0.1606 \\ 6934 & 13: 21: 57 & 0.0501 & 0.2383 \\ 6936 & 13: 21: 59 & 0.0311 & 0.1568 \\ 6938 & 13: 22: 01 & 0.1069 & 0.2070 \\ 6940 & 13: 22: 03 & 0.0372 & 0.1369 \\ 6942 & 13: 22: 05 & 0.0415 & 0.1046 \\ 6944 & 13: 22: 07 & 0.0342 & 0.1527 \\ 6946 & 13: 22: 09 & 0.0680 & 0.1557 \\ 6948 & 13: 22: 11 & 0.0171 & 0.2467 \\ 6950 & 13: 22: 13 & 0.0592 & 0.1862 \\ 6952 & 13: 22: 15 & 0.0372 & 0.1029 \\ 6954 & 13: 22: 17 & 0.0262 & 0.1106 \\ 6956 & 13: 22: 19 & 0.0588 & 0.2021 \\ 6958 & 13: 22: 21 & 0.0753 & 0.2359 \\ 6960 & 13: 22: 23 & 0.0307 & 0.0769 \\ 6962 & 13: 22: 25 & 0.0696 & 0.0909 \\ 6964 & 13: 22: 27 & 0.0478 & 0.1384\end{array}$




$\begin{array}{llll}6966 & 13: 22: 29 & 0.1178 & 0.1987 \\ 6968 & 13: 22: 31 & 0.0561 & 0.1774 \\ 6970 & 13: 22: 33 & 0.0307 & 0.0296 \\ 6972 & 13: 22: 35 & 0.0689 & 0.3910 \\ 6974 & 13: 22: 37 & 0.0233 & 0.1354 \\ 6976 & 13: 22: 39 & 0.0274 & 0.1610 \\ 6978 & 13: 22: 41 & 0.0854 & 0.1327 \\ 6980 & 13: 22: 43 & 0.0494 & 0.0706 \\ 6982 & 13: 22: 45 & 0.0140 & 0.0852 \\ 6984 & 13: 22: 47 & 0.0417 & 0.2108 \\ 6986 & 13: 22: 49 & 0.0067 & 0.1898 \\ 6988 & 13: 22: 51 & 0.0358 & 0.1153 \\ 6990 & 13: 22: 53 & 0.0584 & 0.1414 \\ 6992 & 13: 22: 55 & 0.0662 & 0.3054 \\ 6994 & 13: 22: 57 & 0.1344 & 0.1077 \\ 6996 & 13: 22: 59 & 0.0974 & 0.1828 \\ 6998 & 13: 23: 01 & 0.0835 & 0.1146 \\ 7000 & 13: 23: 03 & 0.0756 & 0.1138 \\ 7002 & 13: 23: 05 & 0.0389 & 0.0711 \\ 7004 & 13: 23: 07 & 0.0120 & 0.0731 \\ 7006 & 13: 23: 09 & 0.0127 & 0.1720 \\ 7008 & 13: 23: 11 & 0.0458 & 0.2541 \\ 7010 & 13: 23: 13 & 0.0150 & 0.1448 \\ 7012 & 13: 23: 15 & 0.0143 & 0.2022 \\ 7014 & 13: 23: 17 & 0.0108 & 0.0829 \\ 7016 & 13: 23: 19 & 0.0172 & 0.0946 \\ 7018 & 13: 23: 21 & 0.0185 & 0.0985 \\ 7020 & 13: 23: 23 & 0.0437 & 0.0443 \\ 7022 & 13: 23: 25 & 0.0506 & 0.1250 \\ 7024 & 13: 23: 27 & 0.0323 & 0.0594 \\ 7026 & 13: 23: 29 & 0.0567 & 0.1164 \\ 7028 & 13: 23: 31 & 0.0393 & 0.2094 \\ 7030 & 13: 23: 33 & 0.0090 & 0.1040 \\ 7032 & 13: 23: 35 & 0.0882 & 0.1578 \\ 7034 & 13: 23: 37 & 0.1018 & 0.0958 \\ 7036 & 13: 23: 39 & 0.0530 & 0.0925 \\ 7038 & 13: 23: 41 & 0.1904 & 0.1313 \\ 7040 & 13: 23: 43 & 0.0858 & 0.2845 \\ 7042 & 13: 23: 45 & 0.2251 & 0.0772 \\ 7044 & 13: 23: 47 & 0.1134 & 0.0443 \\ 7046 & 13: 23: 49 & 0.0729 & 0.0452 \\ 7048 & 13: 23: 51 & 0.0842 & 0.1663 \\ 7050 & 13: 23: 53 & 0.0386 & 0.1189 \\ 7052 & 13: 23: 55 & 0.0245 & 0.1019 \\ 7054 & 13: 23: 57 & 0.0559 & 0.1640 \\ 7056 & 13: 23: 59 & 0.0098 & 0.1471 \\ 7058 & 13: 24: 01 & 0.0708 & 0.2384 \\ 7060 & 13: 24: 03 & 0.0431 & 0.1408 \\ 7062 & 13: 24: 05 & 0.0911 & 0.1053 \\ 7064 & 13: 24: 07 & 0.0868 & 0.1579 \\ 7066 & 13: 24: 09 & 0.0905 & 0.0581 \\ 7068 & 13: 24: 11 & 0.1015 & 0.0513\end{array}$




$\begin{array}{llll}7070 & 13: 24: 13 & 0.0301 & 0.1645 \\ 7072 & 13: 24: 15 & 0.0351 & 0.0720 \\ 7074 & 13: 24: 17 & 0.0218 & 0.0802 \\ 7076 & 13: 24: 19 & 0.0448 & 0.1236 \\ 7078 & 13: 24: 21 & 0.0237 & 0.0808 \\ 7080 & 13: 24: 23 & 0.0407 & 0.2746 \\ 7082 & 13: 24: 25 & 0.0139 & 0.2037 \\ 7084 & 13: 24: 27 & 0.1147 & 0.1473 \\ 7086 & 13: 24: 29 & 0.0838 & 0.2897 \\ 7088 & 13: 24: 31 & 0.2079 & 0.0734 \\ 7090 & 13: 24: 33 & 0.0612 & 0.0759 \\ 7092 & 13: 24: 35 & 0.0554 & 0.2205 \\ 7094 & 13: 24: 37 & 0.0424 & 0.0810 \\ 7096 & 13: 24: 39 & 0.1203 & 0.2274 \\ 7098 & 13: 24: 41 & 0.0371 & 0.0922 \\ 7100 & 13: 24: 43 & 0.0507 & 0.0528 \\ 7102 & 13: 24: 45 & 0.0934 & 0.0861 \\ 7104 & 13: 24: 47 & 0.0776 & 0.2004 \\ 7106 & 13: 24: 49 & 0.0259 & 0.2048 \\ 7108 & 13: 24: 51 & 0.0590 & 0.1220 \\ 7110 & 13: 24: 53 & 0.0292 & 0.1184 \\ 7112 & 13: 24: 55 & 0.0473 & 0.0566 \\ 7114 & 13: 24: 57 & 0.0334 & 0.1220 \\ 7116 & 13: 24: 59 & 0.0533 & 0.1474 \\ 7118 & 13: 25: 01 & 0.0458 & 0.1917 \\ 7120 & 13: 25: 03 & 0.0295 & 0.1519 \\ 7122 & 13: 25: 05 & 0.0436 & 0.2258 \\ 7124 & 13: 25: 07 & 0.0203 & 0.2961 \\ 7126 & 13: 25: 09 & 0.0118 & 0.2048 \\ 7128 & 13: 25: 11 & 0.1124 & 0.3480 \\ 7130 & 13: 25: 13 & 0.0414 & 0.0909 \\ 7132 & 13: 25: 15 & 0.0311 & 0.2846 \\ 7134 & 13: 25: 17 & 0.0171 & 0.1943 \\ 7136 & 13: 25: 19 & 0.0600 & 0.0947 \\ 7138 & 13: 25: 21 & 0.0611 & 0.1861 \\ 7140 & 13: 25: 23 & 0.1165 & 0.1608 \\ 7142 & 13: 25: 25 & 0.0549 & 0.1427 \\ 7144 & 13: 25: 27 & 0.0804 & 0.1782 \\ 7146 & 13: 25: 29 & 0.0517 & 0.1718 \\ 7148 & 13: 25: 31 & 0.0735 & 0.0960 \\ 7150 & 13: 25: 33 & 0.0669 & 0.1169 \\ 7152 & 13: 25: 35 & 0.0855 & 0.1689 \\ 7154 & 13: 25: 37 & 0.0592 & 0.1514 \\ 7156 & 13: 25: 39 & 0.0097 & 0.0518 \\ 7158 & 13: 25: 41 & 0.0446 & 0.0680 \\ 7160 & 13: 25: 43 & 0.0108 & 0.1168 \\ 7162 & 13: 25: 45 & 0.0294 & 0.4001 \\ 7164 & 13: 25: 47 & 0.0268 & 0.0729 \\ 7166 & 13: 25: 49 & 0.0180 & 0.0718 \\ 7168 & 13: 25: 51 & 0.0354 & 0.2224 \\ 7170 & 13: 25: 53 & 0.0169 & 0.0597 \\ 7172 & 13: 25: 55 & 0.0243 & 0.1302\end{array}$




$\begin{array}{llll}7174 & 13: 25: 57 & 0.0826 & 0.3008 \\ 7176 & 13: 25: 59 & 0.0218 & 0.1486 \\ 7178 & 13: 26: 01 & 0.0417 & 0.0457 \\ 7180 & 13: 26: 03 & 0.0348 & 0.0777 \\ 7182 & 13: 26: 05 & 0.0382 & 0.2563 \\ 7184 & 13: 26: 07 & 0.1641 & 0.1081 \\ 7186 & 13: 26: 09 & 0.0575 & 0.0993 \\ 7188 & 13: 26: 11 & 0.1260 & 0.2285 \\ 7190 & 13: 26: 13 & 0.0805 & 0.0424 \\ 7192 & 13: 26: 15 & 0.1042 & 0.0499 \\ 7194 & 13: 26: 17 & 0.1402 & 0.1067 \\ 7196 & 13: 26: 19 & 0.0745 & 0.1043 \\ 7198 & 13: 26: 21 & 0.0877 & 0.1406 \\ 7200 & 13: 26: 23 & 0.0084 & 0.1495 \\ 7202 & 13: 26: 25 & 0.0180 & 0.0609 \\ 7204 & 13: 26: 27 & 0.0555 & 0.0900 \\ 7206 & 13: 26: 29 & 0.0556 & 0.0902 \\ 7208 & 13: 26: 31 & 0.0514 & 0.0739 \\ 7210 & 13: 26: 33 & 0.0608 & 0.1307 \\ 7212 & 13: 26: 35 & 0.1427 & 0.1055 \\ 7214 & 13: 26: 37 & 0.0696 & 0.0999 \\ 7216 & 13: 26: 39 & 0.0433 & 0.0796 \\ 7218 & 13: 26: 41 & 0.0250 & 0.1200 \\ 7220 & 13: 26: 43 & 0.0277 & 0.2275 \\ 7222 & 13: 26: 45 & 0.0554 & 0.1328 \\ 7224 & 13: 26: 47 & 0.0240 & 0.0785 \\ 7226 & 13: 26: 49 & 0.0239 & 0.1839 \\ 7228 & 13: 26: 51 & 0.0215 & 0.1232 \\ 7230 & 13: 26: 53 & 0.0251 & 0.2279 \\ 7232 & 13: 26: 55 & 0.0384 & 0.0885 \\ 7234 & 13: 26: 57 & 0.0352 & 0.0808 \\ 7236 & 13: 26: 59 & 0.0589 & 0.0951 \\ 7238 & 13: 27: 01 & 0.1596 & 0.0205 \\ 7240 & 13: 27: 03 & 0.0552 & 0.0159 \\ 7242 & 13: 27: 05 & 0.0308 & 0.0529 \\ 7244 & 13: 27: 07 & 0.0205 & 0.0933 \\ 7246 & 13: 27: 09 & 0.1182 & 0.0698 \\ 7248 & 13: 27: 11 & 0.0693 & 0.1168 \\ 7250 & 13: 27: 13 & 0.0236 & 0.1036 \\ 7252 & 13: 27: 15 & 0.0984 & 0.0715 \\ 7254 & 13: 27: 17 & 0.0391 & 0.0323 \\ 7256 & 13: 27: 19 & 0.0691 & 0.1410 \\ 7258 & 13: 27: 21 & 0.0327 & 0.0731 \\ 7260 & 13: 27: 23 & 0.0150 & 0.0287 \\ 7262 & 13: 27: 25 & 0.0489 & 0.0751 \\ 7264 & 13: 27: 27 & 0.0266 & 0.1167 \\ 7266 & 13: 27: 29 & 0.0869 & 0.1076 \\ 7268 & 13: 27: 31 & 0.1003 & 0.1432 \\ 7270 & 13: 27: 33 & 0.0536 & 0.1355 \\ 7272 & 13: 27: 35 & 0.0489 & 0.2095 \\ 7274 & 13: 27: 37 & 0.0364 & 0.1516 \\ 7276 & 13: 27: 39 & 0.0475 & 0.1954\end{array}$




$\begin{array}{llll}7278 & 13: 27: 41 & 0.0928 & 0.1253 \\ 7280 & 13: 27: 43 & 0.0249 & 0.1517 \\ 7282 & 13: 27: 45 & 0.0245 & 0.1090 \\ 7284 & 13: 27: 47 & 0.0167 & 0.2742 \\ 7286 & 13: 27: 49 & 0.0219 & 0.1060 \\ 7288 & 13: 27: 51 & 0.0748 & 0.1473 \\ 7290 & 13: 27: 53 & 0.0690 & 0.1696 \\ 7292 & 13: 27: 55 & 0.0476 & 0.1610 \\ 7294 & 13: 27: 57 & 0.1059 & 0.1666 \\ 7296 & 13: 27: 59 & 0.0609 & 0.1792 \\ 7298 & 13: 28: 01 & 0.0282 & 0.1948 \\ 7300 & 13: 28: 03 & 0.0255 & 0.1642 \\ 7302 & 13: 28: 05 & 0.0782 & 0.0837 \\ 7304 & 13: 28: 07 & 0.1396 & 0.0642 \\ 7306 & 13: 28: 09 & 0.0628 & 0.0672 \\ 7308 & 13: 28: 11 & 0.0733 & 0.0539 \\ 7310 & 13: 28: 13 & 0.0353 & 0.0494 \\ 7312 & 13: 28: 15 & 0.0246 & 0.0626 \\ 7314 & 13: 28: 17 & 0.0884 & 0.0483 \\ 7316 & 13: 28: 19 & 0.0720 & 0.0539 \\ 7318 & 13: 28: 21 & 0.0147 & 0.0354 \\ 7320 & 13: 28: 23 & 0.0477 & 0.0828 \\ 7322 & 13: 28: 25 & 0.0852 & 0.0910 \\ 7324 & 13: 28: 27 & 0.0419 & 0.0362 \\ 7326 & 13: 28: 29 & 0.1290 & 0.0518 \\ 7328 & 13: 28: 31 & 0.0762 & 0.1003 \\ 7330 & 13: 28: 33 & 0.0207 & 0.1227 \\ 7332 & 13: 28: 35 & 0.0474 & 0.0873 \\ 7334 & 13: 28: 37 & 0.0275 & 0.0761 \\ 7336 & 13: 28: 39 & 0.0778 & 0.3023 \\ 7338 & 13: 28: 41 & 0.0683 & 0.1417 \\ 7340 & 13: 28: 43 & 0.0350 & 0.2194 \\ 7342 & 13: 28: 45 & 0.0334 & 0.0762 \\ 7344 & 13: 28: 47 & 0.0754 & 0.2487 \\ 7346 & 13: 28: 49 & 0.1740 & 0.0866 \\ 7348 & 13: 28: 51 & 0.0599 & 0.1075 \\ 7350 & 13: 28: 53 & 0.2105 & 0.1297 \\ 7352 & 13: 28: 55 & 0.0193 & 0.0834 \\ 7354 & 13: 28: 57 & 0.0381 & 0.1031 \\ 7356 & 13: 28: 59 & 0.0658 & 0.1692 \\ 7358 & 13: 29: 01 & 0.1092 & 0.1434 \\ 7360 & 13: 29: 03 & 0.1045 & 0.1770 \\ 7362 & 13: 29: 05 & 0.0518 & 0.1150 \\ 7364 & 13: 29: 07 & 0.1479 & 0.2950 \\ 7366 & 13: 29: 09 & 0.2393 & 0.1233 \\ 7368 & 13: 29: 11 & 0.0573 & 0.1748 \\ 7370 & 13: 29: 13 & 0.0568 & 0.1449 \\ 7372 & 13: 29: 15 & 0.0868 & 0.1175 \\ 7374 & 13: 29: 17 & 0.1852 & 0.0903 \\ 7376 & 13: 29: 19 & 0.1113 & 0.1385 \\ 7378 & 13: 29: 21 & 0.0469 & 0.2011 \\ 7380 & 13: 29: 23 & 0.0494 & 0.1031\end{array}$


Appendix B - Laser Data Test 1 NIST102505_1.xls

$\begin{array}{llll}7382 & 13: 29: 25 & 0.0418 & 0.2184 \\ 7384 & 13: 29: 27 & 0.0905 & 0.2331 \\ 7386 & 13: 29: 29 & 0.1601 & 0.1665 \\ 7388 & 13: 29: 31 & 0.0180 & 0.2082 \\ 7390 & 13: 29: 33 & 0.0911 & 0.1003 \\ 7392 & 13: 29: 35 & 0.1018 & 0.1242 \\ 7394 & 13: 29: 37 & 0.0676 & 0.0498 \\ 7396 & 13: 29: 39 & 0.0484 & 0.1466 \\ 7398 & 13: 29: 41 & 0.0919 & 0.1043 \\ 7400 & 13: 29: 43 & 0.0564 & 0.2206 \\ 7402 & 13: 29: 45 & 0.0575 & 0.0454 \\ 7404 & 13: 29: 47 & 0.1009 & 0.2211 \\ 7406 & 13: 29: 49 & 0.0389 & 0.0884 \\ 7408 & 13: 29: 51 & 0.0453 & 0.1001 \\ 7410 & 13: 29: 53 & 0.0383 & 0.3028 \\ 7412 & 13: 29: 55 & 0.0277 & 0.1995 \\ 7414 & 13: 29: 57 & 0.0564 & 0.0857 \\ 7416 & 13: 29: 59 & 0.0141 & 0.0287 \\ 7418 & 13: 30: 01 & 0.0602 & 0.1703 \\ 7420 & 13: 30: 03 & 0.1048 & 0.0505 \\ 7422 & 13: 30: 05 & 0.0235 & 0.1224 \\ 7424 & 13: 30: 07 & 0.0259 & 0.1202 \\ 7426 & 13: 30: 09 & 0.0185 & 0.2039 \\ 7428 & 13: 30: 11 & 0.0260 & 0.1300 \\ 7430 & 13: 30: 13 & 0.0466 & 0.1399 \\ 7432 & 13: 30: 15 & 0.0296 & 0.1522 \\ 7434 & 13: 30: 17 & 0.0385 & 0.1714 \\ 7436 & 13: 30: 19 & 0.0563 & 0.1169 \\ 7438 & 13: 30: 21 & 0.0324 & 0.0987 \\ 7440 & 13: 30: 23 & 0.0209 & 0.0971 \\ 7442 & 13: 30: 25 & 0.0393 & 0.1725 \\ 7444 & 13: 30: 27 & 0.0254 & 0.2315 \\ 7446 & 13: 30: 29 & 0.0143 & 0.1908 \\ 7448 & 13: 30: 31 & 0.0950 & 0.0673 \\ 7450 & 13: 30: 33 & 0.0265 & 0.1519 \\ 7452 & 13: 30: 35 & 0.1002 & 0.1481 \\ 7454 & 13: 30: 37 & 0.0701 & 0.2165 \\ 7456 & 13: 30: 39 & 0.0499 & 0.1205 \\ 7458 & 13: 30: 41 & 0.0539 & 0.0808 \\ 7460 & 13: 30: 43 & 0.0214 & 0.1447 \\ 7462 & 13: 30: 45 & 0.0435 & 0.1499 \\ 7464 & 13: 30: 47 & 0.0330 & 0.1053 \\ 7466 & 13: 30: 49 & 0.1019 & 0.1380 \\ 7468 & 13: 30: 51 & 0.0282 & 0.3168 \\ 7470 & 13: 30: 53 & 0.0333 & 0.0652 \\ 7472 & 13: 30: 55 & 0.0244 & 0.1275 \\ 7474 & 13: 30: 57 & 0.0180 & 0.1005 \\ 7476 & 13: 30: 59 & 0.0381 & 0.0946 \\ 7478 & 13: 31: 01 & 0.0265 & 0.1569 \\ 7480 & 13: 31: 03 & 0.0704 & 0.1929 \\ 7482 & 13: 31: 05 & 0.0335 & 0.0834 \\ 7484 & 13: 31: 07 & 0.2233 & 0.1891\end{array}$




$\begin{array}{llll}7486 & 13: 31: 09 & 0.0238 & 0.1271 \\ 7488 & 13: 31: 11 & 0.0735 & 0.1375 \\ 7490 & 13: 31: 13 & 0.0556 & 0.2682 \\ 7492 & 13: 31: 15 & 0.0747 & 0.1964 \\ 7494 & 13: 31: 17 & 0.0904 & 0.2025 \\ 7496 & 13: 31: 19 & 0.0463 & 0.1169 \\ 7498 & 13: 31: 21 & 0.0486 & 0.1555 \\ 7500 & 13: 31: 23 & 0.0342 & 0.1933 \\ 7502 & 13: 31: 25 & 0.0487 & 0.1017 \\ 7504 & 13: 31: 27 & 0.1728 & 0.2229 \\ 7506 & 13: 31: 29 & 0.0947 & 0.1842 \\ 7508 & 13: 31: 31 & 0.0573 & 0.1345 \\ 7510 & 13: 31: 33 & 0.0881 & 0.0587 \\ 7512 & 13: 31: 35 & 0.0382 & 0.0702 \\ 7514 & 13: 31: 37 & 0.0249 & 0.1801 \\ 7516 & 13: 31: 39 & 0.0203 & 0.0681 \\ 7518 & 13: 31: 41 & 0.0320 & 0.0993 \\ 7520 & 13: 31: 43 & 0.0135 & 0.1420 \\ 7522 & 13: 31: 45 & 0.0697 & 0.2196 \\ 7524 & 13: 31: 47 & 0.0579 & 0.1946 \\ 7526 & 13: 31: 49 & 0.0544 & 0.0463 \\ 7528 & 13: 31: 51 & 0.0516 & 0.1023 \\ 7530 & 13: 31: 53 & 0.0309 & 0.1992 \\ 7532 & 13: 31: 55 & 0.0310 & 0.1026 \\ 7534 & 13: 31: 57 & 0.0139 & 0.0783 \\ 7536 & 13: 31: 59 & 0.0609 & 0.2343 \\ 7538 & 13: 32: 01 & 0.0401 & 0.2485 \\ 7540 & 13: 32: 03 & 0.1232 & 0.3378 \\ 7542 & 13: 32: 05 & 0.0168 & 0.1185 \\ 7544 & 13: 32: 07 & 0.0618 & 0.0848 \\ 7546 & 13: 32: 09 & 0.0486 & 0.2092 \\ 7548 & 13: 32: 11 & 0.0080 & 0.1186 \\ 7550 & 13: 32: 13 & 0.0442 & 0.1840 \\ 7552 & 13: 32: 15 & 0.0327 & 0.1064 \\ 7554 & 13: 32: 17 & 0.0371 & 0.2359 \\ 7556 & 13: 32: 19 & 0.0441 & 0.1354 \\ 7558 & 13: 32: 21 & 0.0136 & 0.1688 \\ 7560 & 13: 32: 23 & 0.0487 & 0.1231 \\ 7562 & 13: 32: 25 & 0.0597 & 0.1079 \\ 7564 & 13: 32: 27 & 0.0239 & 0.1292 \\ 7566 & 13: 32: 29 & 0.0385 & 0.1384 \\ 7568 & 13: 32: 31 & 0.0415 & 0.1455 \\ 7570 & 13: 32: 33 & 0.0824 & 0.0744 \\ 7572 & 13: 32: 35 & 0.0417 & 0.0741 \\ 7574 & 13: 32: 37 & 0.0199 & 0.0792 \\ 7576 & 13: 32: 39 & 0.0385 & 0.0964 \\ 7578 & 13: 32: 41 & 0.0222 & 0.1332 \\ 7580 & 13: 32: 43 & 0.0269 & 0.2226 \\ 7582 & 13: 32: 45 & 0.0231 & 0.0675 \\ 7584 & 13: 32: 47 & 0.1147 & 0.1649 \\ 7586 & 13: 32: 49 & 0.0544 & 0.1218 \\ 7588 & 13: 32: 51 & 0.0555 & 0.1438\end{array}$




$\begin{array}{llll}7590 & 13: 32: 53 & 0.0354 & 0.0535 \\ 7592 & 13: 32: 55 & 0.0449 & 0.2211 \\ 7594 & 13: 32: 57 & 0.1239 & 0.2229 \\ 7596 & 13: 32: 59 & 0.0591 & 0.2130 \\ 7598 & 13: 33: 01 & 0.0341 & 0.0684 \\ 7600 & 13: 33: 03 & 0.0860 & 0.0622 \\ 7602 & 13: 33: 05 & 0.0155 & 0.0907 \\ 7604 & 13: 33: 07 & 0.0920 & 0.0881 \\ 7606 & 13: 33: 09 & 0.0593 & 0.1049 \\ 7608 & 13: 33: 11 & 0.0555 & 0.0677 \\ 7610 & 13: 33: 13 & 0.0416 & 0.1801 \\ 7612 & 13: 33: 15 & 0.0699 & 0.0805 \\ 7614 & 13: 33: 17 & 0.0359 & 0.1748 \\ 7616 & 13: 33: 19 & 0.0676 & 0.0874 \\ 7618 & 13: 33: 21 & 0.0359 & 0.1449 \\ 7620 & 13: 33: 23 & 0.0735 & 0.1043 \\ 7622 & 13: 33: 25 & 0.0480 & 0.1324 \\ 7624 & 13: 33: 27 & 0.0271 & 0.1160 \\ 7626 & 13: 33: 29 & 0.0853 & 0.1468 \\ 7628 & 13: 33: 31 & 0.0520 & 0.1637 \\ 7630 & 13: 33: 33 & 0.0609 & 0.1687 \\ 7632 & 13: 33: 35 & 0.0723 & 0.0421 \\ 7634 & 13: 33: 37 & 0.0716 & 0.1187 \\ 7636 & 13: 33: 39 & 0.0577 & 0.1787 \\ 7638 & 13: 33: 41 & 0.0313 & 0.3156 \\ 7640 & 13: 33: 43 & 0.0489 & 0.1214 \\ 7642 & 13: 33: 45 & 0.0757 & 0.1654 \\ 7644 & 13: 33: 47 & 0.0234 & 0.0819 \\ 7646 & 13: 33: 49 & 0.0188 & 0.1329 \\ 7648 & 13: 33: 51 & 0.0222 & 0.1484 \\ 7650 & 13: 33: 53 & 0.1455 & 0.1872 \\ 7652 & 13: 33: 55 & 0.0780 & 0.2914 \\ 7654 & 13: 33: 57 & 0.0254 & 0.1045 \\ 7656 & 13: 33: 59 & 0.0383 & 0.2283 \\ 7658 & 13: 34: 01 & 0.0494 & 0.0340 \\ 7660 & 13: 34: 03 & 0.0735 & 0.0464 \\ 7662 & 13: 34: 05 & 0.0275 & 0.1288 \\ 7664 & 13: 34: 07 & 0.1099 & 0.1151 \\ 7666 & 13: 34: 09 & 0.1034 & 0.0659 \\ 7668 & 13: 34: 11 & 0.0332 & 0.0294 \\ 7670 & 13: 34: 13 & 0.1005 & 0.0963 \\ 7672 & 13: 34: 15 & 0.0436 & 0.1505 \\ 7674 & 13: 34: 17 & 0.0535 & 0.1248 \\ 7676 & 13: 34: 19 & 0.1843 & 0.0411 \\ 7678 & 13: 34: 21 & 0.0764 & 0.0492 \\ 7680 & 13: 34: 23 & 0.0478 & 0.0929 \\ 7682 & 13: 34: 25 & 0.0391 & 0.0831 \\ 7684 & 13: 34: 27 & 0.0143 & 0.1394 \\ 7686 & 13: 34: 29 & 0.0149 & 0.0845 \\ 7688 & 13: 34: 31 & 0.0271 & 0.1581 \\ 7690 & 13: 34: 33 & 0.0322 & 0.2677 \\ 7692 & 13: 34: 35 & 0.0654 & 0.2136\end{array}$




$\begin{array}{llll}7694 & 13: 34: 37 & 0.0375 & 0.1298 \\ 7696 & 13: 34: 39 & 0.2232 & 0.2256 \\ 7698 & 13: 34: 41 & 0.0208 & 0.1840 \\ 7700 & 13: 34: 43 & 0.0589 & 0.1901 \\ 7702 & 13: 34: 45 & 0.0578 & 0.0567 \\ 7704 & 13: 34: 47 & 0.0141 & 0.0917 \\ 7706 & 13: 34: 49 & 0.0263 & 0.1401 \\ 7708 & 13: 34: 51 & 0.0598 & 0.2445 \\ 7710 & 13: 34: 53 & 0.0480 & 0.1644 \\ 7712 & 13: 34: 55 & 0.0200 & 0.1598 \\ 7714 & 13: 34: 57 & 0.0385 & 0.1221 \\ 7716 & 13: 34: 59 & 0.0986 & 0.1503 \\ 7718 & 13: 35: 01 & 0.0667 & 0.4128 \\ 7720 & 13: 35: 03 & 0.0497 & 0.2005 \\ 7722 & 13: 35: 05 & 0.0820 & 0.0497 \\ 7724 & 13: 35: 07 & 0.0731 & 0.0507 \\ 7726 & 13: 35: 09 & 0.1121 & 0.0527 \\ 7728 & 13: 35: 11 & 0.0191 & 0.0738 \\ 7730 & 13: 35: 13 & 0.0244 & 0.0961 \\ 7732 & 13: 35: 15 & 0.0297 & 0.1311 \\ 7734 & 13: 35: 17 & 0.0402 & 0.0815 \\ 7736 & 13: 35: 19 & 0.0365 & 0.0557 \\ 7738 & 13: 35: 21 & 0.0059 & 0.1789 \\ 7740 & 13: 35: 23 & 0.1466 & 0.1480 \\ 7742 & 13: 35: 25 & 0.0290 & 0.1008 \\ 7744 & 13: 35: 27 & 0.1064 & 0.1514 \\ 7746 & 13: 35: 29 & 0.0081 & 0.1393 \\ 7748 & 13: 35: 31 & 0.0100 & 0.0775 \\ 7750 & 13: 35: 33 & 0.0600 & 0.1135 \\ 7752 & 13: 35: 35 & 0.1787 & 0.1225 \\ 7754 & 13: 35: 37 & 0.1660 & 0.2464 \\ 7756 & 13: 35: 39 & 0.0297 & 0.1152 \\ 7758 & 13: 35: 41 & 0.0378 & 0.0838 \\ 7760 & 13: 35: 43 & 0.0366 & 0.1055 \\ 7762 & 13: 35: 45 & 0.1447 & 0.0989 \\ 7764 & 13: 35: 47 & 0.0340 & 0.0832 \\ 7766 & 13: 35: 49 & 0.0354 & 0.1898 \\ 7768 & 13: 35: 51 & 0.0623 & 0.0585 \\ 7770 & 13: 35: 53 & 0.0948 & 0.1140 \\ 7772 & 13: 35: 55 & 0.1402 & 0.0714 \\ 7774 & 13: 35: 57 & 0.0779 & 0.1371 \\ 7776 & 13: 35: 59 & 0.0912 & 0.0776 \\ 7778 & 13: 36: 01 & 0.0471 & 0.1427 \\ 7780 & 13: 36: 03 & 0.0361 & 0.3811 \\ 7782 & 13: 36: 05 & 0.0339 & 0.1576 \\ 7784 & 13: 36: 07 & 0.0112 & 0.2223 \\ 7786 & 13: 36: 09 & 0.1137 & 0.1773 \\ 7788 & 13: 36: 11 & 0.0636 & 0.1252 \\ 7790 & 13: 36: 13 & 0.0590 & 0.0846 \\ 7792 & 13: 36: 15 & 0.0669 & 0.1751 \\ 7794 & 13: 36: 17 & 0.0335 & 0.1856 \\ 7796 & 13: 36: 19 & 0.0509 & 0.1795\end{array}$




$\begin{array}{llll}7798 & 13: 36: 21 & 0.0296 & 0.1761 \\ 7800 & 13: 36: 23 & 0.0176 & 0.1236 \\ 7802 & 13: 36: 25 & 0.0392 & 0.1435 \\ 7804 & 13: 36: 27 & 0.0747 & 0.1280 \\ 7806 & 13: 36: 29 & 0.0189 & 0.0508 \\ 7808 & 13: 36: 31 & 0.0254 & 0.2464 \\ 7810 & 13: 36: 33 & 0.0264 & 0.1706 \\ 7812 & 13: 36: 35 & 0.0287 & 0.1783 \\ 7814 & 13: 36: 37 & 0.0312 & 0.0807 \\ 7816 & 13: 36: 39 & 0.0246 & 0.1006 \\ 7818 & 13: 36: 41 & 0.0761 & 0.1940 \\ 7820 & 13: 36: 43 & 0.0383 & 0.2377 \\ 7822 & 13: 36: 45 & 0.0979 & 0.4374 \\ 7824 & 13: 36: 47 & 0.0377 & 0.0830 \\ 7826 & 13: 36: 49 & 0.0483 & 0.1308 \\ 7828 & 13: 36: 51 & 0.0514 & 0.2186 \\ 7830 & 13: 36: 53 & 0.0622 & 0.2876 \\ 7832 & 13: 36: 55 & 0.1269 & 0.1301 \\ 7834 & 13: 36: 57 & 0.0583 & 0.1288 \\ 7836 & 13: 36: 59 & 0.3052 & 0.3688 \\ 7838 & 13: 37: 01 & 0.0393 & 0.1551 \\ 7840 & 13: 37: 03 & 0.1768 & 0.0352 \\ 7842 & 13: 37: 05 & 0.0613 & 0.0929 \\ 7844 & 13: 37: 07 & 0.0445 & 0.1459 \\ 7846 & 13: 37: 09 & 0.0739 & 0.1589 \\ 7848 & 13: 37: 11 & 0.0953 & 0.0689 \\ 7850 & 13: 37: 13 & 0.1239 & 0.1071 \\ 7852 & 13: 37: 15 & 0.0293 & 0.1302 \\ 7854 & 13: 37: 17 & 0.0508 & 0.1111 \\ 7856 & 13: 37: 19 & 0.1735 & 0.0909 \\ 7858 & 13: 37: 21 & 0.0454 & 0.0630 \\ 7860 & 13: 37: 23 & 0.0486 & 0.1619 \\ 7862 & 13: 37: 25 & 0.0639 & 0.1066 \\ 7864 & 13: 37: 27 & 0.0535 & 0.0983 \\ 7866 & 13: 37: 29 & 0.0842 & 0.1344 \\ 7868 & 13: 37: 31 & 0.0496 & 0.1247 \\ 7870 & 13: 37: 33 & 0.0496 & 0.1701 \\ 7872 & 13: 37: 35 & 0.1211 & 0.2072 \\ 7874 & 13: 37: 37 & 0.0468 & 0.2322 \\ 7876 & 13: 37: 39 & 0.0538 & 0.1628 \\ 7878 & 13: 37: 41 & 0.0835 & 0.0668 \\ 7880 & 13: 37: 43 & 0.0437 & 0.1335 \\ 7882 & 13: 37: 45 & 0.1146 & 0.1076 \\ 7884 & 13: 37: 47 & 0.0305 & 0.1016 \\ 7886 & 13: 37: 49 & 0.0664 & 0.0877 \\ 7888 & 13: 37: 51 & 0.0655 & 0.1073 \\ 7890 & 13: 37: 53 & 0.1064 & 0.1026 \\ 7892 & 13: 37: 55 & 0.0104 & 0.0882 \\ 7894 & 13: 37: 57 & 0.0836 & 0.2426 \\ 7896 & 13: 37: 59 & 0.0173 & 0.3388 \\ 7898 & 13: 38: 01 & 0.0496 & 0.1675 \\ 7900 & 13: 38: 03 & 0.0433 & 0.2380\end{array}$




$\begin{array}{llll}7902 & 13: 38: 05 & 0.0562 & 0.1593 \\ 7904 & 13: 38: 07 & 0.0120 & 0.1191 \\ 7906 & 13: 38: 09 & 0.0419 & 0.0713 \\ 7908 & 13: 38: 11 & 0.0167 & 0.1429 \\ 7910 & 13: 38: 13 & 0.0261 & 0.1413 \\ 7912 & 13: 38: 15 & 0.0512 & 0.1555 \\ 7914 & 13: 38: 17 & 0.0642 & 0.1408 \\ 7916 & 13: 38: 19 & 0.0230 & 0.2617 \\ 7918 & 13: 38: 21 & 0.0390 & 0.5385 \\ 7920 & 13: 38: 23 & 0.0141 & 0.2880 \\ 7922 & 13: 38: 25 & 0.1030 & 0.0492 \\ 7924 & 13: 38: 27 & 0.0297 & 0.1285 \\ 7926 & 13: 38: 29 & 0.0330 & 0.1075 \\ 7928 & 13: 38: 31 & 0.0839 & 0.1040 \\ 7930 & 13: 38: 33 & 0.0176 & 0.0596 \\ 7932 & 13: 38: 35 & 0.0499 & 0.1499 \\ 7934 & 13: 38: 37 & 0.0509 & 0.0412 \\ 7936 & 13: 38: 39 & 0.0503 & 0.0761 \\ 7938 & 13: 38: 41 & 0.0239 & 0.2271 \\ 7940 & 13: 38: 43 & 0.0585 & 0.3675 \\ 7942 & 13: 38: 45 & 0.0881 & 0.0710 \\ 7944 & 13: 38: 47 & 0.0613 & 0.1597 \\ 7946 & 13: 38: 49 & 0.0095 & 0.1088 \\ 7948 & 13: 38: 51 & 0.0184 & 0.0769 \\ 7950 & 13: 38: 53 & 0.0365 & 0.1310 \\ 7952 & 13: 38: 55 & 0.0473 & 0.2566 \\ 7954 & 13: 38: 57 & 0.0423 & 0.1320 \\ 7956 & 13: 38: 59 & 0.0235 & 0.0436 \\ 7958 & 13: 39: 01 & 0.0505 & 0.2615 \\ 7960 & 13: 39: 03 & 0.0345 & 0.0653 \\ 7962 & 13: 39: 05 & 0.0232 & 0.2157 \\ 7964 & 13: 39: 07 & 0.0191 & 0.0463 \\ 7966 & 13: 39: 09 & 0.0266 & 0.3251 \\ 7968 & 13: 39: 11 & 0.0245 & 0.1267 \\ 7970 & 13: 39: 13 & 0.0181 & 0.0879 \\ 7972 & 13: 39: 15 & 0.0348 & 0.0658 \\ 7974 & 13: 39: 17 & 0.1011 & 0.1099 \\ 7976 & 13: 39: 19 & 0.0500 & 0.0779 \\ 7978 & 13: 39: 21 & 0.0242 & 0.0612 \\ 7980 & 13: 39: 23 & 0.1109 & 0.0594 \\ 7982 & 13: 39: 25 & 0.0202 & 0.1605 \\ 7984 & 13: 39: 27 & 0.0272 & 0.0818 \\ 7986 & 13: 39: 29 & 0.0805 & 0.0555 \\ 7988 & 13: 39: 31 & 0.0675 & 0.0477 \\ 7990 & 13: 39: 33 & 0.0785 & 0.2309 \\ 7992 & 13: 39: 35 & 0.0345 & 0.0876 \\ 7994 & 13: 39: 37 & 0.0488 & 0.0199 \\ 7996 & 13: 39: 39 & 0.0633 & 0.0997 \\ 7998 & 13: 39: 41 & 0.0813 & 0.1143 \\ 8000 & 13: 39: 43 & 0.0418 & 0.0989 \\ 8002 & 13: 39: 45 & 0.0323 & 0.0602 \\ 8004 & 13: 39: 47 & 0.0731 & 0.0557\end{array}$




$\begin{array}{llll}8006 & 13: 39: 49 & 0.0596 & 0.0372 \\ 8008 & 13: 39: 51 & 0.0763 & 0.1500 \\ 8010 & 13: 39: 53 & 0.1072 & 0.0686 \\ 8012 & 13: 39: 55 & 0.0512 & 0.2226 \\ 8014 & 13: 39: 57 & 0.0862 & 0.0535 \\ 8016 & 13: 39: 59 & 0.0069 & 0.0624 \\ 8018 & 13: 40: 01 & 0.0576 & 0.2038 \\ 8020 & 13: 40: 03 & 0.0700 & 0.0201 \\ 8022 & 13: 40: 05 & 0.0286 & 0.2770 \\ 8024 & 13: 40: 07 & 0.0531 & 0.2957 \\ 8026 & 13: 40: 09 & 0.0570 & 0.2091 \\ 8028 & 13: 40: 11 & 0.0471 & 0.0485 \\ 8030 & 13: 40: 13 & 0.1235 & 0.0617 \\ 8032 & 13: 40: 15 & 0.1191 & 0.0844 \\ 8034 & 13: 40: 17 & 0.0165 & 0.1536 \\ 8036 & 13: 40: 19 & 0.0647 & 0.0523 \\ 8038 & 13: 40: 21 & 0.0340 & 0.1091 \\ 8040 & 13: 40: 23 & 0.0475 & 0.1364 \\ 8042 & 13: 40: 25 & 0.0787 & 0.2811 \\ 8044 & 13: 40: 27 & 0.1507 & 0.2572 \\ 8046 & 13: 40: 29 & 0.0638 & 0.1442 \\ 8048 & 13: 40: 31 & 0.1356 & 0.2169 \\ 8050 & 13: 40: 33 & 0.0309 & 0.3136 \\ 8052 & 13: 40: 35 & 0.0238 & 0.0722 \\ 8054 & 13: 40: 37 & 0.0371 & 0.1626 \\ 8056 & 13: 40: 39 & 0.0157 & 0.1427 \\ 8058 & 13: 40: 41 & 0.0573 & 0.1124 \\ 8060 & 13: 40: 43 & 0.0470 & 0.0698 \\ 8062 & 13: 40: 45 & 0.1509 & 0.1031 \\ 8064 & 13: 40: 47 & 0.0431 & 0.0997 \\ 8066 & 13: 40: 49 & 0.1088 & 0.2536 \\ 8068 & 13: 40: 51 & 0.0650 & 0.2069 \\ 8070 & 13: 40: 53 & 0.0740 & 0.0481 \\ 8072 & 13: 40: 55 & 0.1334 & 0.1825 \\ 8074 & 13: 40: 57 & 0.1328 & 0.0924 \\ 8076 & 13: 40: 59 & 0.0612 & 0.1772 \\ 8078 & 13: 41: 01 & 0.0743 & 0.1381 \\ 8080 & 13: 41: 03 & 0.0989 & 0.1565 \\ 8082 & 13: 41: 05 & 0.0529 & 0.0834 \\ 8084 & 13: 41: 07 & 0.0729 & 0.1414 \\ 8086 & 13: 41: 09 & 0.0726 & 0.1153 \\ 8088 & 13: 41: 11 & 0.0444 & 0.1287 \\ 8090 & 13: 41: 13 & 0.0461 & 0.1645 \\ 8092 & 13: 41: 15 & 0.0422 & 0.3445 \\ 8094 & 13: 41: 17 & 0.0724 & 0.0694 \\ 8096 & 13: 41: 19 & 0.0419 & 0.2402 \\ 8098 & 13: 41: 21 & 0.1392 & 0.2196 \\ 8100 & 13: 41: 23 & 0.1482 & 0.1217 \\ 8102 & 13: 41: 25 & 0.0547 & 0.0414 \\ 8104 & 13: 41: 27 & 0.0717 & 0.1697 \\ 8106 & 13: 41: 29 & 0.1231 & 0.0871 \\ 8108 & 13: 41: 31 & 0.0455 & 0.1498\end{array}$




$\begin{array}{llll}8110 & 13: 41: 33 & 0.0124 & 0.1066 \\ 8112 & 13: 41: 35 & 0.0301 & 0.0896 \\ 8114 & 13: 41: 37 & 0.0416 & 0.1304 \\ 8116 & 13: 41: 39 & 0.0733 & 0.0293 \\ 8118 & 13: 41: 41 & 0.0179 & 0.1268 \\ 8120 & 13: 41: 43 & 0.1244 & 0.1165 \\ 8122 & 13: 41: 45 & 0.0456 & 0.2020 \\ 8124 & 13: 41: 47 & 0.0370 & 0.1199 \\ 8126 & 13: 41: 49 & 0.1025 & 0.0628 \\ 8128 & 13: 41: 51 & 0.0425 & 0.0948 \\ 8130 & 13: 41: 53 & 0.0597 & 0.0859 \\ 8132 & 13: 41: 55 & 0.0271 & 0.1674 \\ 8134 & 13: 41: 57 & 0.0874 & 0.1913 \\ 8136 & 13: 41: 59 & 0.0378 & 0.3536 \\ 8138 & 13: 42: 01 & 0.0442 & 0.1359 \\ 8140 & 13: 42: 03 & 0.0218 & 0.0631 \\ 8142 & 13: 42: 05 & 0.0140 & 0.0760 \\ 8144 & 13: 42: 07 & 0.0572 & 0.1788 \\ 8146 & 13: 42: 09 & 0.0199 & 0.0961 \\ 8148 & 13: 42: 11 & 0.0825 & 0.5235 \\ 8150 & 13: 42: 13 & 0.0407 & 0.1174 \\ 8152 & 13: 42: 15 & 0.0460 & 0.0984 \\ 8154 & 13: 42: 17 & 0.0464 & 0.6084 \\ 8156 & 13: 42: 19 & 0.0713 & 0.1757 \\ 8158 & 13: 42: 21 & 0.0123 & 0.1907 \\ 8160 & 13: 42: 23 & 0.0274 & 0.1257 \\ 8162 & 13: 42: 25 & 0.0342 & 0.2505 \\ 8164 & 13: 42: 27 & 0.0641 & 0.2108 \\ 8166 & 13: 42: 29 & 0.0419 & 0.1378 \\ 8168 & 13: 42: 31 & 0.0495 & 0.0926 \\ 8170 & 13: 42: 33 & 0.0404 & 0.1460 \\ 8172 & 13: 42: 35 & 0.1774 & 0.1282 \\ 8174 & 13: 42: 37 & 0.0370 & 0.1066 \\ 8176 & 13: 42: 39 & 0.0750 & 0.1229 \\ 8178 & 13: 42: 41 & 0.0202 & 0.0891 \\ 8180 & 13: 42: 43 & 0.0095 & 0.1233 \\ 8182 & 13: 42: 45 & 0.0244 & 0.1150 \\ 8184 & 13: 42: 47 & 0.0531 & 0.1333 \\ 8186 & 13: 42: 49 & 0.0666 & 0.1515 \\ 8188 & 13: 42: 51 & 0.0710 & 0.1479 \\ 8190 & 13: 42: 53 & 0.0601 & 0.0997 \\ 8192 & 13: 42: 55 & 0.0728 & 0.0348 \\ 8194 & 13: 42: 57 & 0.0399 & 0.0773 \\ 8196 & 13: 42: 59 & 0.0549 & 0.0327 \\ 8198 & 13: 43: 01 & 0.0332 & 0.1916 \\ 8200 & 13: 43: 03 & 0.0392 & 0.2899 \\ 8202 & 13: 43: 05 & 0.0448 & 0.0449 \\ 8204 & 13: 43: 07 & 0.1149 & 0.0890 \\ 8206 & 13: 43: 09 & 0.0337 & 0.0183 \\ 8208 & 13: 43: 11 & 0.1075 & 0.0636 \\ 8210 & 13: 43: 13 & 0.1471 & 0.0539 \\ 8212 & 13: 43: 15 & 0.0261 & 0.0672\end{array}$




$\begin{array}{llll}8214 & 13: 43: 17 & 0.0951 & 0.0584 \\ 8216 & 13: 43: 19 & 0.2184 & 0.0619 \\ 8218 & 13: 43: 21 & 0.1193 & 0.0380 \\ 8220 & 13: 43: 23 & 0.0695 & 0.0714 \\ 8222 & 13: 43: 25 & 0.0412 & 0.0863 \\ 8224 & 13: 43: 27 & 0.0345 & 0.1519 \\ 8226 & 13: 43: 29 & 0.0507 & 0.1457 \\ 8228 & 13: 43: 31 & 0.0289 & 0.1100 \\ 8230 & 13: 43: 33 & 0.0950 & 0.1340 \\ 8232 & 13: 43: 35 & 0.0968 & 0.0471 \\ 8234 & 13: 43: 37 & 0.0459 & 0.0690 \\ 8236 & 13: 43: 39 & 0.0307 & 0.0985 \\ 8238 & 13: 43: 41 & 0.0148 & 0.1441 \\ 8240 & 13: 43: 43 & 0.0280 & 0.1543 \\ 8242 & 13: 43: 45 & 0.0509 & 0.0627 \\ 8244 & 13: 43: 47 & 0.0534 & 0.1964 \\ 8246 & 13: 43: 49 & 0.0361 & 0.0740 \\ 8248 & 13: 43: 51 & 0.0494 & 0.2038 \\ 8250 & 13: 43: 53 & 0.0353 & 0.1923 \\ 8252 & 13: 43: 55 & 0.0122 & 0.2045 \\ 8254 & 13: 43: 57 & 0.0612 & 0.2012 \\ 8256 & 13: 43: 59 & 0.0094 & 0.0864 \\ 8258 & 13: 44: 01 & 0.0143 & 0.2078 \\ 8260 & 13: 44: 03 & 0.0367 & 0.1926 \\ 8262 & 13: 44: 05 & 0.0241 & 0.0509 \\ 8264 & 13: 44: 07 & 0.0334 & 0.1304 \\ 8266 & 13: 44: 09 & 0.0244 & 0.0558 \\ 8268 & 13: 44: 11 & 0.0569 & 0.1096 \\ 8270 & 13: 44: 13 & 0.0207 & 0.0933 \\ 8272 & 13: 44: 15 & 0.0229 & 0.0685 \\ 8274 & 13: 44: 17 & 0.1238 & 0.0821 \\ 8276 & 13: 44: 19 & 0.0346 & 0.0613 \\ 8278 & 13: 44: 21 & 0.0557 & 0.5073 \\ 8280 & 13: 44: 23 & 0.0188 & 0.1090 \\ 8282 & 13: 44: 25 & 0.1072 & 0.1095 \\ 8284 & 13: 44: 27 & 0.0337 & 0.0830 \\ 8286 & 13: 44: 29 & 0.0561 & 0.0546 \\ 8288 & 13: 44: 31 & 0.0853 & 0.0793 \\ 8290 & 13: 44: 33 & 0.0884 & 0.1610 \\ 8292 & 13: 44: 35 & 0.0755 & 0.1771 \\ 8294 & 13: 44: 37 & 0.0334 & 0.1055 \\ 8296 & 13: 44: 39 & 0.0146 & 0.0438 \\ 8298 & 13: 44: 41 & 0.0273 & 0.1273 \\ 8300 & 13: 44: 43 & 0.1045 & 0.1720 \\ 8302 & 13: 44: 45 & 0.0213 & 0.1418 \\ 8304 & 13: 44: 47 & 0.0233 & 0.1131 \\ 8306 & 13: 44: 49 & 0.0187 & 0.0471 \\ 8308 & 13: 44: 51 & 0.0190 & 0.0899 \\ 8310 & 13: 44: 53 & 0.0074 & 0.2438 \\ 8312 & 13: 44: 55 & 0.0281 & 0.1192 \\ 8314 & 13: 44: 57 & 0.0176 & 0.0529 \\ 8316 & 13: 44: 59 & 0.0506 & 0.0555\end{array}$




$\begin{array}{llll}8318 & 13: 45: 01 & 0.0934 & 0.1543 \\ 8320 & 13: 45: 03 & 0.0630 & 0.0159 \\ 8322 & 13: 45: 05 & 0.0426 & 0.0179 \\ 8324 & 13: 45: 07 & 0.0442 & 0.0605 \\ 8326 & 13: 45: 09 & 0.0128 & 0.0733 \\ 8328 & 13: 45: 11 & 0.0194 & 0.0837 \\ 8330 & 13: 45: 13 & 0.0361 & 0.0355 \\ 8332 & 13: 45: 15 & 0.0615 & 0.1191 \\ 8334 & 13: 45: 17 & 0.0369 & 0.1797 \\ 8336 & 13: 45: 19 & 0.0227 & 0.0257 \\ 8338 & 13: 45: 21 & 0.0774 & 0.2865 \\ 8340 & 13: 45: 23 & 0.0436 & 0.1006 \\ 8342 & 13: 45: 25 & 0.0465 & 0.0919 \\ 8344 & 13: 45: 27 & 0.0542 & 0.1363 \\ 8346 & 13: 45: 29 & 0.1838 & 0.0325 \\ 8348 & 13: 45: 31 & 0.0635 & 0.1774 \\ 8350 & 13: 45: 33 & 0.0224 & 0.0367 \\ 8352 & 13: 45: 35 & 0.0411 & 0.0616 \\ 8354 & 13: 45: 37 & 0.1253 & 0.3963 \\ 8356 & 13: 45: 39 & 0.0198 & 0.1488 \\ 8358 & 13: 45: 41 & 0.0274 & 0.0801 \\ 8360 & 13: 45: 43 & 0.0243 & 0.1302 \\ 8362 & 13: 45: 45 & 0.0220 & 0.0644 \\ 8364 & 13: 45: 47 & 0.0749 & 0.2032 \\ 8366 & 13: 45: 49 & 0.0159 & 0.1601 \\ 8368 & 13: 45: 51 & 0.0242 & 0.2216 \\ 8370 & 13: 45: 53 & 0.0229 & 0.1219 \\ 8372 & 13: 45: 55 & 0.0314 & 0.0932 \\ 8374 & 13: 45: 57 & 0.0403 & 0.0875 \\ 8376 & 13: 45: 59 & 0.0217 & 0.1194 \\ 8378 & 13: 46: 01 & 0.0899 & 0.2499 \\ 8380 & 13: 46: 03 & 0.0103 & 0.2140 \\ 8382 & 13: 46: 05 & 0.0532 & 0.1926 \\ 8384 & 13: 46: 07 & 0.0569 & 0.0618 \\ 8386 & 13: 46: 09 & 0.0546 & 0.3092 \\ 8388 & 13: 46: 11 & 0.0391 & 0.0771 \\ 8390 & 13: 46: 13 & 0.0480 & 0.1810 \\ 8392 & 13: 46: 15 & 0.0871 & 0.2072 \\ 8394 & 13: 46: 17 & 0.0121 & 0.0790 \\ 8396 & 13: 46: 19 & 0.1317 & 0.2207 \\ 8398 & 13: 46: 21 & 0.0671 & 0.0924 \\ 8400 & 13: 46: 23 & 0.1105 & 0.0981 \\ 8402 & 13: 46: 25 & 0.0607 & 0.2258 \\ 8404 & 13: 46: 27 & 0.0444 & 0.0974 \\ 8406 & 13: 46: 29 & 0.0220 & 0.1115 \\ 8408 & 13: 46: 31 & 0.0308 & 0.2581 \\ 8410 & 13: 46: 33 & 0.0286 & 0.2500 \\ 8412 & 13: 46: 35 & 0.0216 & 0.0983 \\ 8414 & 13: 46: 37 & 0.0402 & 0.1817 \\ 8416 & 13: 46: 39 & 0.1158 & 0.0796 \\ 8418 & 13: 46: 41 & 0.1072 & 0.1237 \\ 8420 & 13: 46: 43 & 0.0492 & 0.1163\end{array}$




$\begin{array}{llll}8422 & 13: 46: 45 & 0.0941 & 0.0814 \\ 8424 & 13: 46: 47 & 0.0601 & 0.1261 \\ 8426 & 13: 46: 49 & 0.0476 & 0.1424 \\ 8428 & 13: 46: 51 & 0.1182 & 0.1388 \\ 8430 & 13: 46: 53 & 0.0770 & 0.0614 \\ 8432 & 13: 46: 55 & 0.0198 & 0.0453 \\ 8434 & 13: 46: 57 & 0.0620 & 0.2136 \\ 8436 & 13: 46: 59 & 0.0597 & 0.1511 \\ 8438 & 13: 47: 01 & 0.0765 & 0.1924 \\ 8440 & 13: 47: 03 & 0.0478 & 0.1748 \\ 8442 & 13: 47: 05 & 0.0195 & 0.0668 \\ 8444 & 13: 47: 07 & 0.0412 & 0.1798 \\ 8446 & 13: 47: 09 & 0.0858 & 0.4014 \\ 8448 & 13: 47: 11 & 0.0242 & 0.1956 \\ 8450 & 13: 47: 13 & 0.0372 & 0.1046 \\ 8452 & 13: 47: 15 & 0.0721 & 0.2213 \\ 8454 & 13: 47: 17 & 0.0354 & 0.0995 \\ 8456 & 13: 47: 19 & 0.0892 & 0.1398 \\ 8458 & 13: 47: 21 & 0.0721 & 0.2475 \\ 8460 & 13: 47: 23 & 0.0424 & 0.1389 \\ 8462 & 13: 47: 25 & 0.0581 & 0.1313 \\ 8464 & 13: 47: 27 & 0.1393 & 0.1330 \\ 8466 & 13: 47: 29 & 0.0244 & 0.1935 \\ 8468 & 13: 47: 31 & 0.0315 & 0.1436 \\ 8470 & 13: 47: 33 & 0.1107 & 0.1614 \\ 8472 & 13: 47: 35 & 0.0269 & 0.1585 \\ 8474 & 13: 47: 37 & 0.0247 & 0.2015 \\ 8476 & 13: 47: 39 & 0.0235 & 0.0815 \\ 8478 & 13: 47: 41 & 0.0728 & 0.1355 \\ 8480 & 13: 47: 43 & 0.0503 & 0.1931 \\ 8482 & 13: 47: 45 & 0.0583 & 0.2079 \\ 8484 & 13: 47: 47 & 0.0980 & 0.1328 \\ 8486 & 13: 47: 49 & 0.1044 & 0.0708 \\ 8488 & 13: 47: 51 & 0.0512 & 0.0941 \\ 8490 & 13: 47: 53 & 0.0665 & 0.3035 \\ 8492 & 13: 47: 55 & 0.0504 & 0.0953 \\ 8494 & 13: 47: 57 & 0.0284 & 0.1373 \\ 8496 & 13: 47: 59 & 0.0503 & 0.0734 \\ 8498 & 13: 48: 01 & 0.0598 & 0.0945 \\ 8500 & 13: 48: 03 & 0.1835 & 0.1936 \\ 8502 & 13: 48: 05 & 0.0604 & 0.0614 \\ 8504 & 13: 48: 07 & 0.1304 & 0.0856 \\ 8506 & 13: 48: 09 & 0.0386 & 0.1286 \\ 8508 & 13: 48: 11 & 0.0325 & 0.0472 \\ 8510 & 13: 48: 13 & 0.0464 & 0.1135 \\ 8512 & 13: 48: 15 & 0.0167 & 0.0210 \\ 8514 & 13: 48: 17 & 0.0591 & 0.1499 \\ 8516 & 13: 48: 19 & 0.0442 & 0.0440 \\ 8518 & 13: 48: 21 & 0.0431 & 0.0955 \\ 8520 & 13: 48: 23 & 0.0517 & 0.0936 \\ 8522 & 13: 48: 25 & 0.1407 & 0.2000 \\ 8524 & 13: 48: 27 & 0.0375 & 0.3405\end{array}$




$\begin{array}{llll}8526 & 13: 48: 29 & 0.0265 & 0.1445 \\ 8528 & 13: 48: 31 & 0.1277 & 0.1259 \\ 8530 & 13: 48: 33 & 0.0646 & 0.0668 \\ 8532 & 13: 48: 35 & 0.1586 & 0.1164 \\ 8534 & 13: 48: 37 & 0.0985 & 0.0631 \\ 8536 & 13: 48: 39 & 0.0529 & 0.0289 \\ 8538 & 13: 48: 41 & 0.0992 & 0.2257 \\ 8540 & 13: 48: 43 & 0.0867 & 0.1531 \\ 8542 & 13: 48: 45 & 0.1521 & 0.2656 \\ 8544 & 13: 48: 47 & 0.0635 & 0.1009 \\ 8546 & 13: 48: 49 & 0.1066 & 0.1351 \\ 8548 & 13: 48: 51 & 0.0236 & 0.0764 \\ 8550 & 13: 48: 53 & 0.0640 & 0.0577 \\ 8552 & 13: 48: 55 & 0.1046 & 0.1254 \\ 8554 & 13: 48: 57 & 0.0374 & 0.1532 \\ 8556 & 13: 48: 59 & 0.0907 & 0.1672 \\ 8558 & 13: 49: 01 & 0.0301 & 0.1038 \\ 8560 & 13: 49: 03 & 0.0329 & 0.1267 \\ 8562 & 13: 49: 05 & 0.1466 & 0.1478 \\ 8564 & 13: 49: 07 & 0.0748 & 0.0393 \\ 8566 & 13: 49: 09 & 0.0683 & 0.3246 \\ 8568 & 13: 49: 11 & 0.0310 & 0.1013 \\ 8570 & 13: 49: 13 & 0.1128 & 0.2160 \\ 8572 & 13: 49: 15 & 0.1004 & 0.1107 \\ 8574 & 13: 49: 17 & 0.0122 & 0.5802 \\ 8576 & 13: 49: 19 & 0.0577 & 0.4688 \\ 8578 & 13: 49: 21 & 0.0523 & 0.1649 \\ 8580 & 13: 49: 23 & 0.0256 & 0.2022 \\ 8582 & 13: 49: 25 & 0.0413 & 0.1905 \\ 8584 & 13: 49: 27 & 0.0371 & 0.1348 \\ 8586 & 13: 49: 29 & 0.0423 & 0.2042 \\ 8588 & 13: 49: 31 & 0.0191 & 0.0714 \\ 8590 & 13: 49: 33 & 0.0174 & 0.0864 \\ 8592 & 13: 49: 35 & 0.0752 & 0.0876 \\ 8594 & 13: 49: 37 & 0.1022 & 0.0708 \\ 8596 & 13: 49: 39 & 0.0530 & 0.0345 \\ 8598 & 13: 49: 41 & 0.0526 & 0.0793 \\ 8600 & 13: 49: 43 & 0.0230 & 0.0656 \\ 8602 & 13: 49: 45 & 0.0179 & 0.0469 \\ 8604 & 13: 49: 47 & 0.0289 & 0.1253 \\ 8606 & 13: 49: 49 & 0.0144 & 0.1319 \\ 8608 & 13: 49: 51 & 0.0557 & 0.1979 \\ 8610 & 13: 49: 53 & 0.0554 & 0.0552 \\ 8612 & 13: 49: 55 & 0.0552 & 0.2924 \\ 8614 & 13: 49: 57 & 0.1266 & 0.3180 \\ 8616 & 13: 49: 59 & 0.0528 & 0.3020 \\ 8618 & 13: 50: 01 & 0.0540 & 0.0957 \\ 8620 & 13: 50: 03 & 0.0304 & 0.0416 \\ 8622 & 13: 50: 05 & 0.0942 & 0.1175 \\ 8624 & 13: 50: 07 & 0.0170 & 0.1310 \\ 8626 & 13: 50: 09 & 0.0401 & 0.0563 \\ 8628 & 13: 50: 11 & 0.0952 & 0.0808\end{array}$




$\begin{array}{llll}8630 & 13: 50: 13 & 0.0407 & 0.0898 \\ 8632 & 13: 50: 15 & 0.0512 & 0.2143 \\ 8634 & 13: 50: 17 & 0.0505 & 0.3291 \\ 8636 & 13: 50: 19 & 0.0842 & 0.1191 \\ 8638 & 13: 50: 21 & 0.0893 & 0.1091 \\ 8640 & 13: 50: 23 & 0.0448 & 0.0999 \\ 8642 & 13: 50: 25 & 0.1105 & 0.1126 \\ 8644 & 13: 50: 27 & 0.0261 & 0.0509 \\ 8646 & 13: 50: 29 & 0.0369 & 0.1370 \\ 8648 & 13: 50: 31 & 0.0390 & 0.2568 \\ 8650 & 13: 50: 33 & 0.0437 & 0.1144 \\ 8652 & 13: 50: 35 & 0.0399 & 0.1481 \\ 8654 & 13: 50: 37 & 0.0825 & 0.1668 \\ 8656 & 13: 50: 39 & 0.0669 & 0.1121 \\ 8658 & 13: 50: 41 & 0.0309 & 0.1124 \\ 8660 & 13: 50: 43 & 0.0533 & 0.0750 \\ 8662 & 13: 50: 45 & 0.0428 & 0.1671 \\ 8664 & 13: 50: 47 & 0.1563 & 0.0509 \\ 8666 & 13: 50: 49 & 0.0545 & 0.0649 \\ 8668 & 13: 50: 51 & 0.0375 & 0.0949 \\ 8670 & 13: 50: 53 & 0.0170 & 0.0444 \\ 8672 & 13: 50: 55 & 0.0270 & 0.0522 \\ 8674 & 13: 50: 57 & 0.0512 & 0.0988 \\ 8676 & 13: 50: 59 & 0.0868 & 0.1457 \\ 8678 & 13: 51: 01 & 0.1098 & 0.2926 \\ 8680 & 13: 51: 03 & 0.1538 & 0.2990 \\ 8682 & 13: 51: 05 & 0.0846 & 0.1238 \\ 8684 & 13: 51: 07 & 0.0547 & 0.1008 \\ 8686 & 13: 51: 09 & 0.1077 & 0.1099 \\ 8688 & 13: 51: 11 & 0.0417 & 0.1162 \\ 8690 & 13: 51: 13 & 0.0834 & 0.1331 \\ 8692 & 13: 51: 15 & 0.0709 & 0.1148 \\ 8694 & 13: 51: 17 & 0.0793 & 0.0440 \\ 8696 & 13: 51: 19 & 0.0057 & 0.0568 \\ 8698 & 13: 51: 21 & 0.0164 & 0.2046 \\ 8700 & 13: 51: 23 & 0.0405 & 0.2171 \\ 8702 & 13: 51: 25 & 0.0182 & 0.1149 \\ 8704 & 13: 51: 27 & 0.0356 & 0.0393 \\ 8706 & 13: 51: 29 & 0.0471 & 0.1109 \\ 8708 & 13: 51: 31 & 0.1105 & 0.0826 \\ 8710 & 13: 51: 33 & 0.0946 & 0.0766 \\ 8712 & 13: 51: 35 & 0.0383 & 0.1144 \\ 8714 & 13: 51: 37 & 0.1438 & 0.1298 \\ 8716 & 13: 51: 39 & 0.0871 & 0.0834 \\ 8718 & 13: 51: 41 & 0.0624 & 0.1322 \\ 8720 & 13: 51: 43 & 0.0506 & 0.0557 \\ 8722 & 13: 51: 45 & 0.0516 & 0.0510 \\ 8724 & 13: 51: 47 & 0.0267 & 0.0854 \\ 8726 & 13: 51: 49 & 0.0570 & 0.1881 \\ 8728 & 13: 51: 51 & 0.1467 & 0.0688 \\ 8730 & 13: 51: 53 & 0.1006 & 0.0531 \\ 8732 & 13: 51: 55 & 0.1860 & 0.2116\end{array}$




$\begin{array}{llll}8734 & 13: 51: 57 & 0.1468 & 0.2666 \\ 8736 & 13: 51: 59 & 0.0314 & 0.1168 \\ 8738 & 13: 52: 01 & 0.0561 & 0.3023 \\ 8740 & 13: 52: 03 & 0.0683 & 0.2353 \\ 8742 & 13: 52: 05 & 0.1341 & 0.2944 \\ 8744 & 13: 52: 07 & 0.0824 & 0.0729 \\ 8746 & 13: 52: 09 & 0.0946 & 0.1142 \\ 8748 & 13: 52: 11 & 0.0788 & 0.1502 \\ 8750 & 13: 52: 13 & 0.2337 & 0.2356 \\ 8752 & 13: 52: 15 & 0.0351 & 0.1449 \\ 8754 & 13: 52: 17 & 0.0461 & 0.0840 \\ 8756 & 13: 52: 19 & 0.1570 & 0.4454 \\ 8758 & 13: 52: 21 & 0.1170 & 0.1448 \\ 8760 & 13: 52: 23 & 0.0730 & 0.1855 \\ 8762 & 13: 52: 25 & 0.0376 & 0.1632 \\ 8764 & 13: 52: 27 & 0.1403 & 0.1065 \\ 8766 & 13: 52: 29 & 0.0512 & 0.2384 \\ 8768 & 13: 52: 31 & 0.0658 & 0.1834 \\ 8770 & 13: 52: 33 & 0.0791 & 0.1751 \\ 8772 & 13: 52: 35 & 0.0378 & 0.0955 \\ 8774 & 13: 52: 37 & 0.0380 & 0.2310 \\ 8776 & 13: 52: 39 & 0.0648 & 0.1428 \\ 8778 & 13: 52: 41 & 0.0988 & 0.3409 \\ 8780 & 13: 52: 43 & 0.0476 & 0.1389 \\ 8782 & 13: 52: 45 & 0.0261 & 0.1033 \\ 8784 & 13: 52: 47 & 0.0377 & 0.0659 \\ 8786 & 13: 52: 49 & 0.0344 & 0.1114 \\ 8788 & 13: 52: 51 & 0.1319 & 0.2641 \\ 8790 & 13: 52: 53 & 0.0489 & 0.2789 \\ 8792 & 13: 52: 55 & 0.0479 & 0.0793 \\ 8794 & 13: 52: 57 & 0.0529 & 0.1414 \\ 8796 & 13: 52: 59 & 0.0206 & 0.1195 \\ 8798 & 13: 53: 01 & 0.0074 & 0.0768 \\ 8800 & 13: 53: 03 & 0.0346 & 0.1590 \\ 8802 & 13: 53: 05 & 0.0507 & 0.0993 \\ 8804 & 13: 53: 07 & 0.0523 & 0.0223 \\ 8806 & 13: 53: 09 & 0.0352 & 0.1087 \\ 8808 & 13: 53: 11 & 0.0987 & 0.2754 \\ 8810 & 13: 53: 13 & 0.0394 & 0.0622 \\ 8812 & 13: 53: 15 & 0.0242 & 0.2269 \\ 8814 & 13: 53: 17 & 0.1648 & 0.0232 \\ 8816 & 13: 53: 19 & 0.0155 & 0.0457 \\ 8818 & 13: 53: 21 & 0.1298 & 0.1404 \\ 8820 & 13: 53: 23 & 0.0973 & 0.1195 \\ 8822 & 13: 53: 25 & 0.0672 & 0.1336 \\ 8824 & 13: 53: 27 & 0.0496 & 0.1715 \\ 8826 & 13: 53: 29 & 0.0672 & 0.1027 \\ 8828 & 13: 53: 31 & 0.1028 & 0.1007 \\ 8830 & 13: 53: 33 & 0.0671 & 0.1117 \\ 8832 & 13: 53: 35 & 0.0441 & 0.0616 \\ 8834 & 13: 53: 37 & 0.0440 & 0.1176 \\ 8836 & 13: 53: 39 & 0.0438 & 0.1314\end{array}$




$\begin{array}{llll}8838 & 13: 53: 41 & 0.0346 & 0.0950 \\ 8840 & 13: 53: 43 & 0.0139 & 0.0721 \\ 8842 & 13: 53: 45 & 0.1115 & 0.1235 \\ 8844 & 13: 53: 47 & 0.0259 & 0.0429 \\ 8846 & 13: 53: 49 & 0.0135 & 0.2258 \\ 8848 & 13: 53: 51 & 0.0344 & 0.0874 \\ 8850 & 13: 53: 53 & 0.0224 & 0.1580 \\ 8852 & 13: 53: 55 & 0.0802 & 0.1634 \\ 8854 & 13: 53: 57 & 0.0167 & 0.0496 \\ 8856 & 13: 53: 59 & 0.0842 & 0.2074 \\ 8858 & 13: 54: 01 & 0.0576 & 0.3111 \\ 8860 & 13: 54: 03 & 0.0669 & 0.2279 \\ 8862 & 13: 54: 05 & 0.0336 & 0.1737 \\ 8864 & 13: 54: 07 & 0.0146 & 0.1585 \\ 8866 & 13: 54: 09 & 0.1487 & 0.1802 \\ 8868 & 13: 54: 11 & 0.0556 & 0.2236 \\ 8870 & 13: 54: 13 & 0.0322 & 0.2322 \\ 8872 & 13: 54: 15 & 0.1555 & 0.0631 \\ 8874 & 13: 54: 17 & 0.0506 & 0.3953 \\ 8876 & 13: 54: 19 & 0.0896 & 0.1475 \\ 8878 & 13: 54: 21 & 0.0338 & 0.1562 \\ 8880 & 13: 54: 23 & 0.0375 & 0.1009 \\ 8882 & 13: 54: 25 & 0.0505 & 0.2260 \\ 8884 & 13: 54: 27 & 0.0724 & 0.3034 \\ 8886 & 13: 54: 29 & 0.1207 & 0.1646 \\ 8888 & 13: 54: 31 & 0.0705 & 0.1347 \\ 8890 & 13: 54: 33 & 0.0294 & 0.1610 \\ 8892 & 13: 54: 35 & 0.1118 & 0.3300 \\ 8894 & 13: 54: 37 & 0.0392 & 0.0857 \\ 8896 & 13: 54: 39 & 0.0713 & 0.0538 \\ 8898 & 13: 54: 41 & 0.0296 & 0.0859 \\ 8900 & 13: 54: 43 & 0.0879 & 0.1231 \\ 8902 & 13: 54: 45 & 0.0538 & 0.0652 \\ 8904 & 13: 54: 47 & 0.0276 & 0.0383 \\ 8906 & 13: 54: 49 & 0.0766 & 0.1508 \\ 8908 & 13: 54: 51 & 0.0715 & 0.1965 \\ 8910 & 13: 54: 53 & 0.0312 & 0.1052 \\ 8912 & 13: 54: 55 & 0.0740 & 0.1306 \\ 8914 & 13: 54: 57 & 0.0702 & 0.1165 \\ 8916 & 13: 54: 59 & 0.0443 & 0.1880 \\ 8918 & 13: 55: 01 & 0.0441 & 0.0851 \\ 8920 & 13: 55: 03 & 0.1339 & 0.0421 \\ 8922 & 13: 55: 05 & 0.0435 & 0.1238 \\ 8924 & 13: 55: 07 & 0.0515 & 0.1967 \\ 8926 & 13: 55: 09 & 0.0256 & 0.2073 \\ 8928 & 13: 55: 11 & 0.0266 & 0.0547 \\ 8930 & 13: 55: 13 & 0.1490 & 0.1426 \\ 8932 & 13: 55: 15 & 0.0272 & 0.0983 \\ 8934 & 13: 55: 17 & 0.0299 & 0.1139 \\ 8936 & 13: 55: 19 & 0.0268 & 0.1137 \\ 8938 & 13: 55: 21 & 0.0276 & 0.0758 \\ 8940 & 13: 55: 23 & 0.0302 & 0.1162\end{array}$




$\begin{array}{llll}8942 & 13: 55: 25 & 0.0348 & 0.2190 \\ 8944 & 13: 55: 27 & 0.0259 & 0.1339 \\ 8946 & 13: 55: 29 & 0.0960 & 0.1520 \\ 8948 & 13: 55: 31 & 0.0296 & 0.0594 \\ 8950 & 13: 55: 33 & 0.0323 & 0.1385 \\ 8952 & 13: 55: 35 & 0.1213 & 0.1970 \\ 8954 & 13: 55: 37 & 0.0865 & 0.0585 \\ 8956 & 13: 55: 39 & 0.0284 & 0.0738 \\ 8958 & 13: 55: 41 & 0.0324 & 0.2333 \\ 8960 & 13: 55: 43 & 0.0221 & 0.1280 \\ 8962 & 13: 55: 45 & 0.0401 & 0.1465 \\ 8964 & 13: 55: 47 & 0.0134 & 0.1313 \\ 8966 & 13: 55: 49 & 0.0345 & 0.2128 \\ 8968 & 13: 55: 51 & 0.1239 & 0.2624 \\ 8970 & 13: 55: 53 & 0.0636 & 0.2256 \\ 8972 & 13: 55: 55 & 0.0204 & 0.0745 \\ 8974 & 13: 55: 57 & 0.0278 & 0.1693 \\ 8976 & 13: 55: 59 & 0.0644 & 0.0758 \\ 8978 & 13: 56: 01 & 0.0374 & 0.3273 \\ 8980 & 13: 56: 03 & 0.0629 & 0.1561 \\ 8982 & 13: 56: 05 & 0.0429 & 0.4692 \\ 8984 & 13: 56: 07 & 0.1544 & 0.1269 \\ 8986 & 13: 56: 09 & 0.0693 & 0.2139 \\ 8988 & 13: 56: 11 & 0.0570 & 0.0803 \\ 8990 & 13: 56: 13 & 0.0790 & 0.1755 \\ 8992 & 13: 56: 15 & 0.0317 & 0.1158 \\ 8994 & 13: 56: 17 & 0.0386 & 0.4233 \\ 8996 & 13: 56: 19 & 0.0203 & 0.1544 \\ 8998 & 13: 56: 21 & 0.0247 & 0.1263 \\ 9000 & 13: 56: 23 & 0.0678 & 0.1278 \\ 9002 & 13: 56: 25 & 0.0396 & 0.0940 \\ 9004 & 13: 56: 27 & 0.0349 & 0.0518 \\ 9006 & 13: 56: 29 & 0.0559 & 0.0760 \\ 9008 & 13: 56: 31 & 0.1154 & 0.1105 \\ 9010 & 13: 56: 33 & 0.1184 & 0.0502 \\ 9012 & 13: 56: 35 & 0.0897 & 0.0683 \\ 9014 & 13: 56: 37 & 0.0411 & 0.1303 \\ 9016 & 13: 56: 39 & 0.0457 & 0.0607 \\ 9018 & 13: 56: 41 & 0.0261 & 0.0994 \\ 9020 & 13: 56: 43 & 0.0259 & 0.1586 \\ 9022 & 13: 56: 45 & 0.0307 & 0.1546 \\ 9024 & 13: 56: 47 & 0.0187 & 0.0740 \\ 9026 & 13: 56: 49 & 0.0984 & 0.0722 \\ 9028 & 13: 56: 51 & 0.0525 & 0.1222 \\ 9030 & 13: 56: 53 & 0.0599 & 0.1284 \\ 9032 & 13: 56: 55 & 0.0353 & 0.0523 \\ 9034 & 13: 56: 57 & 0.0170 & 0.0612 \\ 9036 & 13: 56: 59 & 0.0593 & 0.1269 \\ 9038 & 13: 57: 01 & 0.0381 & 0.2271 \\ 9040 & 13: 57: 03 & 0.0938 & 0.1696 \\ 9042 & 13: 57: 05 & 0.0258 & 0.1315 \\ 9044 & 13: 57: 07 & 0.0315 & 0.1188\end{array}$




\begin{tabular}{llll}
9046 & $13: 57: 09$ & 0.1634 & 0.1404 \\
9048 & $13: 57: 11$ & 0.0262 & 0.0836 \\
9050 & $13: 57: 13$ & 0.1547 & 0.1061 \\
9052 & $13: 57: 15$ & 0.0203 & 0.1550 \\
9054 & $13: 57: 17$ & 0.1569 & 0.1222 \\
9056 & $13: 57: 19$ & 0.1207 & 0.1377 \\
9058 & $13: 57: 21$ & 0.0483 & 0.0844 \\
9060 & $13: 57: 23$ & 0.0344 & 0.3534 \\
9062 & $13: 57: 25$ & 0.0412 & 0.1116 \\
9064 & $13: 57: 27$ & 0.0423 & 0.0930 \\
9066 & $13: 57: 29$ & 0.0521 & 0.1211 \\
9068 & $13: 57: 31$ & 0.0465 & 0.0477 \\
9070 & $13: 57: 33$ & 0.0133 & 0.0917 \\
9072 & $13: 57: 35$ & 0.0866 & 0.1333 \\
9074 & $13: 57: 37$ & 0.0332 & 0.1803 \\
9076 & $13: 57: 39$ & 0.0145 & 0.1647 \\
9078 & $13: 57: 41$ & 0.0634 & 0.0459 \\
9080 & $13: 57: 43$ & 0.0781 & 0.1768 \\
9082 & $13: 57: 45$ & 0.0461 & 0.0546 \\
9084 & $13: 57: 47$ & 0.1970 & 0.0478 \\
9086 & $13: 57: 49$ & 0.0883 & 0.1360 \\
9088 & $13: 57: 51$ & 0.0093 & 0.1179 \\
9090 & $13: 57: 53$ & 0.0384 & 0.1109 \\
9092 & $13: 57: 55$ & 0.0301 & 0.1247 \\
9094 & $13: 57: 57$ & 0.0592 & 0.4694 \\
9096 & $13: 57: 59$ & 0.0537 & 0.2127 \\
9098 & $13: 58: 01$ & 0.1243 & 0.1214 \\
9100 & $13: 58: 03$ & 0.0612 & 0.0964 \\
9102 & $13: 58: 05$ & 0.0262 & 0.0902 \\
9104 & $13: 58: 07$ & 0.0410 & 0.0926 \\
9106 & $13: 58: 09$ & 0.0602 & 0.3541 \\
9108 & $13: 58: 11$ & 0.0897 & 0.3178 \\
9110 & $13: 58: 13$ & 0.1050 & 0.1826 \\
9112 & $13: 58: 15$ & 0.0139 & 0.1427 \\
9114 & $13: 58: 17$ & 0.0129 & 0.0473 \\
9116 & $13: 58: 19$ & 0.0869 & 0.1513 \\
9118 & $13: 58: 21$ & 0.0907 & 0.1242 \\
9120 & $13: 58: 23$ & 0.0571 & 0.0557 \\
9122 & $13: 58: 25$ & 0.0183 & 0.0935 \\
9124 & $13: 58: 27$ & 0.0909 & 0.1688 \\
9126 & $13: 58: 29$ & 0.0714 & 0.1025 \\
9128 & $13: 58: 31$ & 0.0384 & 0.2345 \\
9130 & $13: 58: 33$ & 0.0334 & 0.3219 \\
9132 & $13: 58: 35$ & 0.0439 & 0.0936 \\
9134 & $13: 58: 37$ & 0.0193 & 0.0872 \\
9136 & $13: 58: 39$ & 0.0706 & 0.1308 \\
9138 & $13: 58: 41$ & 0.0172 & 0.0738 \\
9146 & $13: 58: 43$ & 0.0550 & 0.0831 \\
9148 & $13: 58: 45: 49$ & 0.0269 & 0.2941 \\
\hline & 0.0285 & 0.1455 \\
\hline 140 & 0.0377 & 0.0855
\end{tabular}




$\begin{array}{llll}9150 & 13: 58: 53 & 0.0510 & 0.1699 \\ 9152 & 13: 58: 55 & 0.0341 & 0.2212 \\ 9154 & 13: 58: 57 & 0.0331 & 0.1267 \\ 9156 & 13: 58: 59 & 0.0792 & 0.0450 \\ 9158 & 13: 59: 01 & 0.0646 & 0.1076 \\ 9160 & 13: 59: 03 & 0.0500 & 0.0794 \\ 9162 & 13: 59: 05 & 0.1466 & 0.2347 \\ 9164 & 13: 59: 07 & 0.1312 & 0.1711 \\ 9166 & 13: 59: 09 & 0.2526 & 0.4618 \\ 9168 & 13: 59: 11 & 0.0474 & 0.1271 \\ 9170 & 13: 59: 13 & 0.0297 & 0.0873 \\ 9172 & 13: 59: 15 & 0.0656 & 0.0648 \\ 9174 & 13: 59: 17 & 0.0413 & 0.1503 \\ 9176 & 13: 59: 19 & 0.0391 & 0.1314 \\ 9178 & 13: 59: 21 & 0.0912 & 0.0815 \\ 9180 & 13: 59: 23 & 0.0989 & 0.0892 \\ 9182 & 13: 59: 25 & 0.0594 & 0.3081 \\ 9184 & 13: 59: 27 & 0.3034 & 0.2191 \\ 9186 & 13: 59: 29 & 0.0756 & 0.1381 \\ 9188 & 13: 59: 31 & 0.0793 & 0.0815 \\ 9190 & 13: 59: 33 & 0.0836 & 0.1456 \\ 9192 & 13: 59: 35 & 0.1429 & 0.2000 \\ 9194 & 13: 59: 37 & 0.0639 & 0.0332 \\ 9196 & 13: 59: 39 & 0.1169 & 0.0423 \\ 9198 & 13: 59: 41 & 0.0961 & 0.0905 \\ 9200 & 13: 59: 43 & 0.0463 & 0.0989 \\ 9202 & 13: 59: 45 & 0.0251 & 0.0744 \\ 9204 & 13: 59: 47 & 0.0284 & 0.1161 \\ 9206 & 13: 59: 49 & 0.0097 & 0.0891 \\ 9208 & 13: 59: 51 & 0.0216 & 0.2264 \\ 9210 & 13: 59: 53 & 0.0674 & 0.0827 \\ 9212 & 13: 59: 55 & 0.0767 & 0.1798 \\ 9214 & 13: 59: 57 & 0.0488 & 0.0545 \\ 9216 & 13: 59: 59 & 0.0312 & 0.1298 \\ 9218 & 14: 00: 01 & 0.0890 & 0.0792 \\ 9220 & 14: 00: 03 & 0.0688 & 0.0544 \\ 9222 & 14: 00: 05 & 0.0972 & 0.0344 \\ 9224 & 14: 00: 07 & 0.0683 & 0.1839 \\ 9226 & 14: 00: 09 & 0.0493 & 0.0745 \\ 9228 & 14: 00: 11 & 0.0289 & 0.0609 \\ 9230 & 14: 00: 13 & 0.0687 & 0.2882 \\ 9232 & 14: 00: 15 & 0.0764 & 0.3941 \\ 9234 & 14: 00: 17 & 0.2389 & 0.1840 \\ 9236 & 14: 00: 19 & 0.1730 & 0.2343 \\ 9238 & 14: 00: 21 & 0.1228 & 0.3866 \\ 9240 & 14: 00: 23 & 0.0162 & 0.0944 \\ 9242 & 14: 00: 25 & 0.0143 & 0.1446 \\ 9244 & 14: 00: 27 & 0.0122 & 0.1145 \\ 9246 & 14: 00: 29 & 0.0729 & 0.1059 \\ 9248 & 14: 00: 31 & 0.0342 & 0.1264 \\ 9250 & 14: 00: 33 & 0.0649 & 0.1848 \\ 9252 & 14: 00: 35 & 0.0578 & 0.1844\end{array}$




$\begin{array}{llll}9254 & 14: 00: 37 & 0.0394 & 0.1201 \\ 9256 & 14: 00: 39 & 0.0167 & 0.1991 \\ 9258 & 14: 00: 41 & 0.0273 & 0.1117 \\ 9260 & 14: 00: 43 & 0.0894 & 0.2265 \\ 9262 & 14: 00: 45 & 0.1546 & 0.1199 \\ 9264 & 14: 00: 47 & 0.0646 & 0.1860 \\ 9266 & 14: 00: 49 & 0.0942 & 0.0400 \\ 9268 & 14: 00: 51 & 0.0459 & 0.0887 \\ 9270 & 14: 00: 53 & 0.1418 & 0.1229 \\ 9272 & 14: 00: 55 & 0.0540 & 0.0589 \\ 9274 & 14: 00: 57 & 0.0541 & 0.0947 \\ 9276 & 14: 00: 59 & 0.0214 & 0.1372 \\ 9278 & 14: 01: 01 & 0.0357 & 0.1984 \\ 9280 & 14: 01: 03 & 0.0592 & 0.1105 \\ 9282 & 14: 01: 05 & 0.0283 & 0.1489 \\ 9284 & 14: 01: 07 & 0.0533 & 0.1626 \\ 9286 & 14: 01: 09 & 0.0501 & 0.1930 \\ 9288 & 14: 01: 11 & 0.0143 & 0.1223 \\ 9290 & 14: 01: 13 & 0.1208 & 0.0840 \\ 9292 & 14: 01: 15 & 0.0406 & 0.1076 \\ 9294 & 14: 01: 17 & 0.0738 & 0.0535 \\ 9296 & 14: 01: 19 & 0.0280 & 0.0947 \\ 9298 & 14: 01: 21 & 0.0198 & 0.0428 \\ 9300 & 14: 01: 23 & 0.0595 & 0.1988 \\ 9302 & 14: 01: 25 & 0.0882 & 0.0540 \\ 9304 & 14: 01: 27 & 0.0698 & 0.1400 \\ 9306 & 14: 01: 29 & 0.0299 & 0.0997 \\ 9308 & 14: 01: 31 & 0.0468 & 0.0938 \\ 9310 & 14: 01: 33 & 0.0226 & 0.0764 \\ 9312 & 14: 01: 35 & 0.0138 & 0.1265 \\ 9314 & 14: 01: 37 & 0.1348 & 0.0315 \\ 9316 & 14: 01: 39 & 0.0698 & 0.0776 \\ 9318 & 14: 01: 41 & 0.0435 & 0.1118 \\ 9320 & 14: 01: 43 & 0.0535 & 0.2165 \\ 9322 & 14: 01: 45 & 0.0445 & 0.1202 \\ 9324 & 14: 01: 47 & 0.0347 & 0.1033 \\ 9326 & 14: 01: 49 & 0.0626 & 0.0925 \\ 9328 & 14: 01: 51 & 0.0221 & 0.1065 \\ 9330 & 14: 01: 53 & 0.0492 & 0.1015 \\ 9332 & 14: 01: 55 & 0.0383 & 0.0613 \\ 9334 & 14: 01: 57 & 0.0339 & 0.1148 \\ 9336 & 14: 01: 59 & 0.0615 & 0.2144 \\ 9338 & 14: 02: 01 & 0.0137 & 0.1087 \\ 9340 & 14: 02: 03 & 0.0199 & 0.2928 \\ 9342 & 14: 02: 05 & 0.0303 & 0.0776 \\ 9344 & 14: 02: 07 & 0.0536 & 0.2399 \\ 9346 & 14: 02: 09 & 0.0417 & 0.0720 \\ 9348 & 14: 02: 11 & 0.0392 & 0.1513 \\ 9350 & 14: 02: 13 & 0.0808 & 0.2383 \\ 9352 & 14: 02: 15 & 0.0384 & 0.1125 \\ 9354 & 14: 02: 17 & 0.0355 & 0.1508 \\ 9356 & 14: 02: 19 & 0.0094 & 0.1083\end{array}$




\begin{tabular}{|c|c|c|c|}
\hline & 4:02:21 & 0.0972 & \\
\hline 50 & $02: 23$ & 0.0169 & \\
\hline 362 & $14: 02: 25$ & 0006 & 285 \\
\hline & 4:02:27 & & \\
\hline 66 & $02: 29$ & & \\
\hline & 4:02:31 & & \\
\hline 70 & 4:02:33 & 48 & 10 \\
\hline 7 & $14: 02: 35$ & & \\
\hline 74 & $14: 02: 37$ & & \\
\hline 376 & 14:02:39 & 0.0265 & 09 \\
\hline 78 & $14: 02: 41$ & 0.0228 & .106 \\
\hline 80 & $14: 02: 43$ & 0.0596 & 0.06 \\
\hline 382 & $14: 02: 45$ & 0.0458 & 0.08 \\
\hline 384 & $14: 02: 47$ & & \\
\hline 38 & $14: 02: 49$ & $n$ & \\
\hline 8 & 14: & & 0 \\
\hline 90 & 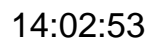 & & \\
\hline 92 & & & \\
\hline 94 & & & \\
\hline 96 & & & \\
\hline 98 & 14: & & \\
\hline 00 & 14: & & \\
\hline 102 & $14: 0$ & 87 & \\
\hline 104 & $14: 03: 07$ & & \\
\hline 406 & & & \\
\hline 0 & 14: & & .090 \\
\hline 10 & 14 & & \\
\hline 12 & 14 & & \\
\hline & & & \\
\hline & & & \\
\hline 8 & & & \\
\hline+20 & & & \\
\hline 22 & 14: & & \\
\hline 124 & 14:03:27 & & 112 \\
\hline 426 & 14:03:29 & & .091 \\
\hline & & & 147 \\
\hline 430 & & & .098 \\
\hline 32 & 14: & & 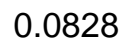 \\
\hline & & & \\
\hline & & & \\
\hline & & & \\
\hline & & & \\
\hline & & & \\
\hline & & & \\
\hline & & & \\
\hline שות & $14: 03: 51$ & & .036 \\
\hline $54 J 0$ & $14: 03: 53$ & 0.0612 & .116 \\
\hline ruc & 14:03:55 & 0.1582 & .182 \\
\hline & & & \\
\hline & & & \\
\hline & & & \\
\hline & $14: 0$ & 0.0158 & 0 \\
\hline
\end{tabular}




$\begin{array}{llll}9462 & 14: 04: 05 & 0.0193 & 0.1780 \\ 9464 & 14: 04: 07 & 0.0469 & 0.0661 \\ 9466 & 14: 04: 09 & 0.0703 & 0.2839 \\ 9468 & 14: 04: 11 & 0.0364 & 0.1659 \\ 9470 & 14: 04: 13 & 0.0193 & 0.1302 \\ 9472 & 14: 04: 15 & 0.0337 & 0.0902 \\ 9474 & 14: 04: 17 & 0.0465 & 0.0303 \\ 9476 & 14: 04: 19 & 0.0695 & 0.1336 \\ 9478 & 14: 04: 21 & 0.1109 & 0.2558 \\ 9480 & 14: 04: 23 & 0.0401 & 0.0542 \\ 9482 & 14: 04: 25 & 0.1144 & 0.1336 \\ 9484 & 14: 04: 27 & 0.1766 & 0.1885 \\ 9486 & 14: 04: 29 & 0.0468 & 0.0559 \\ 9488 & 14: 04: 31 & 0.1013 & 0.1879 \\ 9490 & 14: 04: 33 & 0.0725 & 0.1297 \\ 9492 & 14: 04: 35 & 0.0346 & 0.1782 \\ 9494 & 14: 04: 37 & 0.0173 & 0.1186 \\ 9496 & 14: 04: 39 & 0.0533 & 0.0574 \\ 9498 & 14: 04: 41 & 0.2366 & 0.1820 \\ 9500 & 14: 04: 43 & 0.0616 & 0.2658 \\ 9502 & 14: 04: 45 & 0.0833 & 0.0859 \\ 9504 & 14: 04: 47 & 0.0636 & 0.1066 \\ 9506 & 14: 04: 49 & 0.1284 & 0.1929 \\ 9508 & 14: 04: 51 & 0.0452 & 0.0346 \\ 9510 & 14: 04: 53 & 0.0775 & 0.0913 \\ 9512 & 14: 04: 55 & 0.0597 & 0.1379 \\ 9514 & 14: 04: 57 & 0.0543 & 0.3020 \\ 9516 & 14: 04: 59 & 0.0610 & 0.2861 \\ 9518 & 14: 05: 01 & 0.0445 & 0.1587 \\ 9520 & 14: 05: 03 & 0.0191 & 0.0759 \\ 9522 & 14: 05: 05 & 0.0534 & 0.0796 \\ 9524 & 14: 05: 07 & 0.0743 & 0.0557 \\ 9526 & 14: 05: 09 & 0.0181 & 0.0466 \\ 9528 & 14: 05: 11 & 0.0624 & 0.0909 \\ 9530 & 14: 05: 13 & 0.0496 & 0.0959 \\ 9532 & 14: 05: 15 & 0.0828 & 0.2623 \\ 9534 & 14: 05: 17 & 0.0185 & 0.1203 \\ 9536 & 14: 05: 19 & 0.0332 & 0.1000 \\ 9538 & 14: 05: 21 & 0.0548 & 0.1141 \\ 9540 & 14: 05: 23 & 0.0799 & 0.1824 \\ 9542 & 14: 05: 25 & 0.0560 & 0.3205 \\ 9544 & 14: 05: 27 & 0.0341 & 0.1044 \\ 9546 & 14: 05: 29 & 0.0340 & 0.3096 \\ 9548 & 14: 05: 31 & 0.0275 & 0.2195 \\ 9550 & 14: 05: 33 & 0.0167 & 0.1261 \\ 9552 & 14: 05: 35 & 0.0698 & 0.2149 \\ 9554 & 14: 05: 37 & 0.0661 & 0.1253 \\ 9556 & 14: 05: 39 & 0.0535 & 0.1291 \\ 9560 & 14: 05: 41 & 0.0240 & 0.2730 \\ 9562 & 14: 05: 43 & 0.0749 & 0.1229 \\ 9564 & 14: 05: 47 & 0.0383 & 0.0863 \\ & & 0.0745 & 0.1271\end{array}$




$\begin{array}{llll}9566 & 14: 05: 49 & 0.0661 & 0.1375 \\ 9568 & 14: 05: 51 & 0.0330 & 0.1634 \\ 9570 & 14: 05: 53 & 0.0221 & 0.1901 \\ 9572 & 14: 05: 55 & 0.0253 & 0.1593 \\ 9574 & 14: 05: 57 & 0.0606 & 0.0825 \\ 9576 & 14: 05: 59 & 0.0608 & 0.0124 \\ 9578 & 14: 06: 01 & 0.2362 & 0.1138 \\ 9580 & 14: 06: 03 & 0.0278 & 0.0522 \\ 9582 & 14: 06: 05 & 0.0177 & 0.0814 \\ 9584 & 14: 06: 07 & 0.0399 & 0.1207 \\ 9586 & 14: 06: 09 & 0.0657 & 0.1135 \\ 9588 & 14: 06: 11 & 0.0818 & 0.1158 \\ 9590 & 14: 06: 13 & 0.0552 & 0.1648 \\ 9592 & 14: 06: 15 & 0.0660 & 0.0911 \\ 9594 & 14: 06: 17 & 0.0083 & 0.2363 \\ 9596 & 14: 06: 19 & 0.1114 & 0.0905 \\ 9598 & 14: 06: 21 & 0.0173 & 0.1492 \\ 9600 & 14: 06: 23 & 0.0713 & 0.0934 \\ 9602 & 14: 06: 25 & 0.1128 & 0.0807 \\ 9604 & 14: 06: 27 & 0.0921 & 0.2056 \\ 9606 & 14: 06: 29 & 0.0666 & 0.0770 \\ 9608 & 14: 06: 31 & 0.0589 & 0.2066 \\ 9610 & 14: 06: 33 & 0.0278 & 0.0471 \\ 9612 & 14: 06: 35 & 0.0722 & 0.0538 \\ 9614 & 14: 06: 37 & 0.0928 & 0.0638 \\ 9616 & 14: 06: 39 & 0.1300 & 0.2030 \\ 9618 & 14: 06: 41 & 0.0281 & 0.1436 \\ 9620 & 14: 06: 43 & 0.0603 & 0.0598 \\ 9622 & 14: 06: 45 & 0.0120 & 0.1028 \\ 9624 & 14: 06: 47 & 0.1205 & 0.1862 \\ 9626 & 14: 06: 49 & 0.1145 & 0.2157 \\ 9628 & 14: 06: 51 & 0.0035 & 0.1110 \\ 9630 & 14: 06: 53 & 0.0453 & 0.3914 \\ 9632 & 14: 06: 55 & 0.0758 & 0.1426 \\ 9634 & 14: 06: 57 & 0.0512 & 0.0808 \\ 9636 & 14: 06: 59 & 0.0797 & 0.2217 \\ 9638 & 14: 07: 01 & 0.0466 & 0.1312 \\ 9640 & 14: 07: 03 & 0.0324 & 0.0674 \\ 9642 & 14: 07: 05 & 0.0268 & 0.0966 \\ 9644 & 14: 07: 07 & 0.0229 & 0.1931 \\ 9646 & 14: 07: 09 & 0.0619 & 0.0929 \\ 9648 & 14: 07: 11 & 0.0203 & 0.0819 \\ 9650 & 14: 07: 13 & 0.0963 & 0.2520 \\ 9652 & 14: 07: 15 & 0.0875 & 0.1499 \\ 9654 & 14: 07: 17 & 0.1369 & 0.2189 \\ 9656 & 14: 07: 19 & 0.0657 & 0.0949 \\ 9658 & 14: 07: 21 & 0.0975 & 0.0980 \\ 9660 & 14: 07: 23 & 0.0903 & 0.1383 \\ 9662 & 14: 07: 25 & 0.0957 & 0.1823 \\ 9666 & 14: 07: 27 & 0.0608 & 0.1663 \\ 9668 & 14: 07: 29 & 0.2378 & 0.0273 \\ & 14: 07: 31 & 0.1529 & 0.0791\end{array}$




\begin{tabular}{llll}
9670 & $14: 07: 33$ & 0.0904 & 0.1122 \\
9672 & $14: 07: 35$ & 0.1709 & 0.3677 \\
9674 & $14: 07: 37$ & 0.0385 & 0.0591 \\
9676 & $14: 07: 39$ & 0.0916 & 0.0697 \\
9678 & $14: 07: 41$ & 0.0173 & 0.2070 \\
9680 & $14: 07: 43$ & 0.0963 & 0.0541 \\
9682 & $14: 07: 45$ & 0.0378 & 0.0740 \\
9684 & $14: 07: 47$ & 0.0181 & 0.1506 \\
9686 & $14: 07: 49$ & 0.0621 & 0.2126 \\
9688 & $14: 07: 51$ & 0.0344 & 0.0890 \\
9690 & $14: 07: 53$ & 0.0331 & 0.0763 \\
9692 & $14: 07: 55$ & 0.0524 & 0.1423 \\
9694 & $14: 07: 57$ & 0.0578 & 0.1422 \\
9696 & $14: 07: 59$ & 0.0312 & 0.0859 \\
9698 & $14: 08: 01$ & 0.0242 & 0.0520 \\
9700 & $14: 08: 03$ & 0.0746 & 0.0989 \\
9702 & $14: 08: 05$ & 0.0600 & 0.1269 \\
9704 & $14: 08: 07$ & 0.0780 & 0.1220 \\
9706 & $14: 08: 09$ & 0.1270 & 0.0966 \\
9708 & $14: 08: 11$ & 0.0356 & 0.0824 \\
9710 & $14: 08: 13$ & 0.0392 & 0.1777 \\
9712 & $14: 08: 15$ & 0.0407 & 0.1090 \\
9714 & $14: 08: 17$ & 0.0380 & 0.2295 \\
9716 & $14: 08: 19$ & 0.0223 & 0.3557 \\
9718 & $14: 08: 21$ & 0.0545 & 0.0380 \\
9720 & $14: 08: 23$ & 0.0767 & 0.2122 \\
9722 & $14: 08: 25$ & 0.0689 & 0.1799 \\
9724 & $14: 08: 27$ & 0.0741 & 0.0830 \\
9726 & $14: 08: 29$ & 0.0440 & 0.1660 \\
9728 & $14: 08: 31$ & 0.1933 & 0.1309 \\
9730 & $14: 08: 33$ & 0.1523 & 0.2548 \\
9732 & $14: 08: 35$ & 0.0254 & 0.0925 \\
9734 & $14: 08: 37$ & 0.0833 & 0.1175 \\
9736 & $14: 08: 39$ & 0.0449 & 0.1640 \\
9738 & $14: 08: 41$ & 0.0815 & 0.0758 \\
9740 & $14: 08: 43$ & 0.0434 & 0.1309 \\
9742 & $14: 08: 45$ & 0.0643 & 0.1246 \\
9744 & $14: 08: 47$ & 0.0708 & 0.1572 \\
9746 & $14: 08: 49$ & 0.0217 & 0.1805 \\
9748 & $14: 08: 51$ & 0.0237 & 0.1078 \\
9750 & $14: 08: 53$ & 0.0132 & 0.0813 \\
9752 & $14: 08: 55$ & 0.0111 & 0.0729 \\
9754 & $14: 08: 57$ & 0.0326 & 0.1631 \\
9756 & $14: 08: 59$ & 0.0541 & 0.0745 \\
9758 & $14: 09: 01$ & 0.0374 & 0.1265 \\
9760 & $14: 09: 03$ & 0.0379 & 0.0881 \\
9762 & $14: 09: 05$ & 0.0388 & 0.1646 \\
9764 & $14: 09: 07$ & 0.0368 & 0.1616 \\
9770 & $14: 09: 09$ & 0.0724 & 0.0668 \\
9772 & $14: 09: 13$ & 0.1802 & 0.0511 \\
& & 0.0648 & 0.1053 \\
\hline
\end{tabular}




$\begin{array}{llll}9774 & 14: 09: 17 & 0.0329 & 0.1113 \\ 9776 & 14: 09: 19 & 0.0520 & 0.1248 \\ 9778 & 14: 09: 21 & 0.0451 & 0.0716 \\ 9780 & 14: 09: 23 & 0.0453 & 0.1409 \\ 9782 & 14: 09: 25 & 0.0357 & 0.1698 \\ 9784 & 14: 09: 27 & 0.1185 & 0.1539 \\ 9786 & 14: 09: 29 & 0.0360 & 0.1002 \\ 9788 & 14: 09: 31 & 0.1091 & 0.0883 \\ 9790 & 14: 09: 33 & 0.0417 & 0.0832 \\ 9792 & 14: 09: 35 & 0.0214 & 0.0774 \\ 9794 & 14: 09: 37 & 0.0783 & 0.1333 \\ 9796 & 14: 09: 39 & 0.0876 & 0.1259 \\ 9798 & 14: 09: 41 & 0.0335 & 0.1454 \\ 9800 & 14: 09: 43 & 0.0715 & 0.0541 \\ 9802 & 14: 09: 45 & 0.0693 & 0.3193 \\ 9804 & 14: 09: 47 & 0.0262 & 0.1218 \\ 9806 & 14: 09: 49 & 0.0168 & 0.0528 \\ 9808 & 14: 09: 51 & 0.0366 & 0.1306 \\ 9810 & 14: 09: 53 & 0.0680 & 0.0520 \\ 9812 & 14: 09: 55 & 0.1071 & 0.1544 \\ 9814 & 14: 09: 57 & 0.0712 & 0.1046 \\ 9816 & 14: 09: 59 & 0.0453 & 0.1075 \\ 9818 & 14: 10: 01 & 0.0538 & 0.1757 \\ 9820 & 14: 10: 03 & 0.0394 & 0.2981 \\ 9822 & 14: 10: 05 & 0.0698 & 0.1740 \\ 9824 & 14: 10: 07 & 0.0456 & 0.1000 \\ 9826 & 14: 10: 09 & 0.1561 & 0.1067 \\ 9828 & 14: 10: 11 & 0.0479 & 0.1786 \\ 9830 & 14: 10: 13 & 0.0280 & 0.1169 \\ 9832 & 14: 10: 15 & 0.0500 & 0.1639 \\ 9834 & 14: 10: 17 & 0.0507 & 0.1117 \\ 9836 & 14: 10: 19 & 0.0297 & 0.2211 \\ 9838 & 14: 10: 21 & 0.0342 & 0.1020 \\ 9840 & 14: 10: 23 & 0.0431 & 0.0504 \\ 9842 & 14: 10: 25 & 0.0297 & 0.1465 \\ 9844 & 14: 10: 27 & 0.0465 & 0.1803 \\ 9846 & 14: 10: 29 & 0.0915 & 0.2324 \\ 9848 & 14: 10: 31 & 0.0629 & 0.1341 \\ 9850 & 14: 10: 33 & 0.0444 & 0.0664 \\ 9852 & 14: 10: 35 & 0.0507 & 0.0383 \\ 9854 & 14: 10: 37 & 0.1056 & 0.0723 \\ 9856 & 14: 10: 39 & 0.1177 & 0.1529 \\ 9858 & 14: 10: 41 & 0.1391 & 0.1381 \\ 9860 & 14: 10: 43 & 0.0313 & 0.2585 \\ 9862 & 14: 10: 45 & 0.1077 & 0.3129 \\ 9864 & 14: 10: 47 & 0.0158 & 0.1448 \\ 9866 & 14: 10: 49 & 0.1119 & 0.3437 \\ 9868 & 14: 10: 51 & 0.0480 & 0.1089 \\ 9870 & 14: 10: 53 & 0.0231 & 0.3278 \\ 9872 & 14: 10: 55 & 0.0948 & 0.4048 \\ 9874 & 14: 10: 57 & 0.0628 & 0.2452 \\ 9876 & 14: 10: 59 & 0.1024 & 0.1979\end{array}$




$\begin{array}{llll}9878 & 14: 11: 01 & 0.1175 & 0.1269 \\ 9880 & 14: 11: 03 & 0.0206 & 0.1495 \\ 9882 & 14: 11: 05 & 0.0581 & 0.3598 \\ 9884 & 14: 11: 07 & 0.0517 & 0.1129 \\ 9886 & 14: 11: 09 & 0.0628 & 0.1792 \\ 9888 & 14: 11: 11 & 0.0615 & 0.1228 \\ 9890 & 14: 11: 13 & 0.0557 & 0.1298 \\ 9892 & 14: 11: 15 & 0.0358 & 0.1545 \\ 9894 & 14: 11: 17 & 0.0731 & 0.4183 \\ 9896 & 14: 11: 19 & 0.1179 & 0.1897 \\ 9898 & 14: 11: 21 & 0.0554 & 0.1434 \\ 9900 & 14: 11: 23 & 0.0817 & 0.2179 \\ 9902 & 14: 11: 25 & 0.0648 & 0.2472 \\ 9904 & 14: 11: 27 & 0.0463 & 0.1575 \\ 9906 & 14: 11: 29 & 0.0790 & 0.2418 \\ 9908 & 14: 11: 31 & 0.0569 & 0.1531 \\ 9910 & 14: 11: 33 & 0.0467 & 0.0877 \\ 9912 & 14: 11: 35 & 0.0959 & 0.1297 \\ 9914 & 14: 11: 37 & 0.0296 & 0.1020 \\ 9916 & 14: 11: 39 & 0.0461 & 0.1006 \\ 9918 & 14: 11: 41 & 0.0462 & 0.3225 \\ 9920 & 14: 11: 43 & 0.0556 & 0.1308 \\ 9922 & 14: 11: 45 & 0.0631 & 0.0709 \\ 9924 & 14: 11: 47 & 0.0427 & 0.1650 \\ 9926 & 14: 11: 49 & 0.0516 & 0.1242 \\ 9928 & 14: 11: 51 & 0.0866 & 0.2274 \\ 9930 & 14: 11: 53 & 0.1196 & 0.0946 \\ 9932 & 14: 11: 55 & 0.0575 & 0.1986 \\ 9934 & 14: 11: 57 & 0.0781 & 0.1340 \\ 9936 & 14: 11: 59 & 0.0492 & 0.1675 \\ 9938 & 14: 12: 01 & 0.0972 & 0.0379 \\ 9940 & 14: 12: 03 & 0.0957 & 0.1506 \\ 9942 & 14: 12: 05 & 0.1268 & 0.2711 \\ 9944 & 14: 12: 07 & 0.0509 & 0.2601 \\ 9946 & 14: 12: 09 & 0.0467 & 0.0434 \\ 9948 & 14: 12: 11 & 0.0263 & 0.0493 \\ 9950 & 14: 12: 13 & 0.0439 & 0.1450 \\ 9952 & 14: 12: 15 & 0.0907 & 0.1448 \\ 9954 & 14: 12: 17 & 0.0836 & 0.3227 \\ 9956 & 14: 12: 19 & 0.0693 & 0.0672 \\ 9958 & 14: 12: 21 & 0.0512 & 0.0423 \\ 9960 & 14: 12: 23 & 0.0455 & 0.1174 \\ 9962 & 14: 12: 25 & 0.1057 & 0.2542 \\ 9964 & 14: 12: 27 & 0.0427 & 0.1093 \\ 9966 & 14: 12: 29 & 0.0818 & 0.0999 \\ 9968 & 14: 12: 31 & 0.0456 & 0.1528 \\ 9970 & 14: 12: 33 & 0.0379 & 0.1303 \\ 9972 & 14: 12: 35 & 0.1574 & 0.2088 \\ 9974 & 14: 12: 37 & 0.0288 & 0.1542 \\ 9978 & 14: 12: 39 & 0.0616 & 0.1368 \\ 9980 & 14: 12: 43 & 0.0390 & 0.1327 \\ & 0.0566 & 0.1816\end{array}$




$\begin{array}{rlll}9982 & 14: 12: 45 & 0.0159 & 0.2207 \\ 9984 & 14: 12: 47 & 0.0322 & 0.1216 \\ 9986 & 14: 12: 49 & 0.0332 & 0.0652 \\ 9988 & 14: 12: 51 & 0.0588 & 0.2666 \\ 9990 & 14: 12: 53 & 0.0526 & 0.2137 \\ 9992 & 14: 12: 55 & 0.0431 & 0.2758 \\ 9994 & 14: 12: 57 & 0.0584 & 0.0625 \\ 9996 & 14: 12: 59 & 0.0498 & 0.1697 \\ 9998 & 14: 13: 01 & 0.0305 & 0.0757 \\ 10000 & 14: 13: 03 & 0.0434 & 0.1275 \\ 10002 & 14: 13: 05 & 0.0419 & 0.1913 \\ 10004 & 14: 13: 07 & 0.0412 & 0.0610 \\ 10006 & 14: 13: 09 & 0.0430 & 0.2104 \\ 10008 & 14: 13: 11 & 0.0151 & 0.1140 \\ 10010 & 14: 13: 13 & 0.1011 & 0.0831 \\ 10012 & 14: 13: 15 & 0.0711 & 0.1137 \\ 10014 & 14: 13: 17 & 0.0266 & 0.1050 \\ 10016 & 14: 13: 19 & 0.0485 & 0.1419 \\ 10018 & 14: 13: 21 & 0.0725 & 0.1671 \\ 10020 & 14: 13: 23 & 0.0639 & 0.0864 \\ 10022 & 14: 13: 25 & 0.0845 & 0.1005 \\ 10024 & 14: 13: 27 & 0.0277 & 0.0750 \\ 10026 & 14: 13: 29 & 0.0245 & 0.0820 \\ 10028 & 14: 13: 31 & 0.0869 & 0.0772 \\ 10030 & 14: 13: 33 & 0.0500 & 0.1946 \\ 10032 & 14: 13: 35 & 0.0594 & 0.1291 \\ 10034 & 14: 13: 37 & 0.0564 & 0.0649 \\ 10036 & 14: 13: 39 & 0.0342 & 0.0774 \\ 10038 & 14: 13: 41 & 0.0297 & 0.1325 \\ 10040 & 14: 13: 43 & 0.0357 & 0.3614 \\ 10042 & 14: 13: 45 & 0.0851 & 0.0961 \\ 10044 & 14: 13: 47 & 0.0370 & 0.1709 \\ 10046 & 14: 13: 49 & 0.1030 & 0.0954 \\ 10048 & 14: 13: 51 & 0.1154 & 0.0577 \\ 10050 & 14: 13: 53 & 0.0904 & 0.1337 \\ 10052 & 14: 13: 55 & 0.0161 & 0.0470 \\ 10054 & 14: 13: 57 & 0.0977 & 0.2528 \\ 10056 & 14: 13: 59 & 0.0484 & 0.2778 \\ 10058 & 14: 14: 01 & 0.0415 & 0.2233 \\ 10060 & 14: 14: 03 & 0.0245 & 0.1227 \\ 10062 & 14: 14: 05 & 0.0224 & 0.3179 \\ 10064 & 14: 14: 07 & 0.0404 & 0.1839 \\ 10066 & 14: 14: 09 & 0.0676 & 0.2524 \\ 10068 & 14: 14: 11 & 0.0174 & 0.1361 \\ 10070 & 14: 14: 13 & 0.0294 & 0.2153 \\ 10072 & 14: 14: 15 & 0.0459 & 0.1492 \\ 10074 & 14: 14: 17 & 0.0606 & 0.1431 \\ 10076 & 14: 14: 19 & 0.0645 & 0.1082 \\ 10078 & 14: 14: 21 & 0.0514 & 0.2587 \\ 10080 & 14: 14: 23 & 0.0622 & 0.0910 \\ 10082 & 14: 14: 25 & 0.1917 & 0.2508 \\ 10084 & 14: 14: 27 & 0.0080 & 0.1935\end{array}$




$\begin{array}{llll}10086 & 14: 14: 29 & 0.0979 & 0.1361 \\ 10088 & 14: 14: 31 & 0.0509 & 0.2000 \\ 10090 & 14: 14: 33 & 0.1823 & 0.0669 \\ 10092 & 14: 14: 35 & 0.0264 & 0.1890 \\ 10094 & 14: 14: 37 & 0.0248 & 0.1356 \\ 10096 & 14: 14: 39 & 0.0788 & 0.2398 \\ 10098 & 14: 14: 41 & 0.0749 & 0.1091 \\ 10100 & 14: 14: 43 & 0.0575 & 0.0701 \\ 10102 & 14: 14: 45 & 0.0490 & 0.4856 \\ 10104 & 14: 14: 47 & 0.0304 & 0.0886 \\ 10106 & 14: 14: 49 & 0.0815 & 0.0573 \\ 10108 & 14: 14: 51 & 0.1082 & 0.4622 \\ 10110 & 14: 14: 53 & 0.1567 & 0.1472 \\ 10112 & 14: 14: 55 & 0.1345 & 0.0923 \\ 10114 & 14: 14: 57 & 0.0933 & 0.1108 \\ 10116 & 14: 14: 59 & 0.1892 & 0.1100 \\ 10118 & 14: 15: 01 & 0.0553 & 0.1447 \\ 10120 & 14: 15: 03 & 0.1033 & 0.2220 \\ 10122 & 14: 15: 05 & 0.0532 & 0.0542 \\ 10124 & 14: 15: 07 & 0.1109 & 0.0512 \\ 10126 & 14: 15: 09 & 0.1105 & 0.3575 \\ 10128 & 14: 15: 11 & 0.0909 & 0.0771 \\ 10130 & 14: 15: 13 & 0.0219 & 0.0823 \\ 10132 & 14: 15: 15 & 0.0612 & 0.1269 \\ 10134 & 14: 15: 17 & 0.0428 & 0.1003 \\ 10136 & 14: 15: 19 & 0.0616 & 0.1135 \\ 10138 & 14: 15: 21 & 0.1142 & 0.1174 \\ 10140 & 14: 15: 23 & 0.0858 & 0.1961 \\ 10142 & 14: 15: 25 & 0.0567 & 0.2118 \\ 10144 & 14: 15: 27 & 0.0614 & 0.1701 \\ 10146 & 14: 15: 29 & 0.2585 & 0.4825 \\ 10148 & 14: 15: 31 & 0.0251 & 0.0537 \\ 10150 & 14: 15: 33 & 0.0261 & 0.1338 \\ 10152 & 14: 15: 35 & 0.0417 & 0.0834 \\ 10154 & 14: 15: 37 & 0.0603 & 0.1096 \\ 10156 & 14: 15: 39 & 0.0351 & 0.1644 \\ 10158 & 14: 15: 41 & 0.0701 & 0.1303 \\ 10160 & 14: 15: 43 & 0.0728 & 0.0651 \\ 10162 & 14: 15: 45 & 0.1178 & 0.1490 \\ 10164 & 14: 15: 47 & 0.1356 & 0.1904 \\ 10166 & 14: 15: 49 & 0.0896 & 0.0915 \\ 10168 & 14: 15: 51 & 0.0109 & 0.0730 \\ 10170 & 14: 15: 53 & 0.0522 & 0.2912 \\ 10172 & 14: 15: 55 & 0.0760 & 0.2575 \\ 10174 & 14: 15: 57 & 0.1737 & 0.0868 \\ 10176 & 14: 15: 59 & 0.0625 & 0.1222 \\ 10178 & 14: 16: 01 & 0.0175 & 0.2211 \\ 10180 & 14: 16: 03 & 0.0274 & 0.0545 \\ 10182 & 14: 16: 05 & 0.0539 & 0.1032 \\ 10184 & 14: 16: 07 & 0.0871 & 0.2485 \\ 10186 & 14: 16: 09 & 0.0170 & 0.1203 \\ 10188 & 14: 16: 11 & 0.0519 & 0.1302 \\ & & & \end{array}$




$\begin{array}{llll}10190 & 14: 16: 13 & 0.0530 & 0.1214 \\ 10192 & 14: 16: 15 & 0.0593 & 0.2350 \\ 10194 & 14: 16: 17 & 0.0864 & 0.0381 \\ 10196 & 14: 16: 19 & 0.0822 & 0.1199 \\ 10198 & 14: 16: 21 & 0.0552 & 0.1736 \\ 10200 & 14: 16: 23 & 0.0185 & 0.0830 \\ 10202 & 14: 16: 25 & 0.0723 & 0.0343 \\ 10204 & 14: 16: 27 & 0.0621 & 0.1834 \\ 10206 & 14: 16: 29 & 0.1130 & 0.1306 \\ 10208 & 14: 16: 31 & 0.0497 & 0.0486 \\ 10210 & 14: 16: 33 & 0.0893 & 0.1493 \\ 10212 & 14: 16: 35 & 0.0290 & 0.1130 \\ 10214 & 14: 16: 37 & 0.0355 & 0.1109 \\ 10216 & 14: 16: 39 & 0.0517 & 0.1332 \\ 10218 & 14: 16: 41 & 0.0890 & 0.0847 \\ 10220 & 14: 16: 43 & 0.0745 & 0.0826 \\ 10222 & 14: 16: 45 & 0.0812 & 0.0981 \\ 10224 & 14: 16: 47 & 0.0948 & 0.2032 \\ 10226 & 14: 16: 49 & 0.2588 & 0.1129 \\ 10228 & 14: 16: 51 & 0.0672 & 0.2222 \\ 10230 & 14: 16: 53 & 0.1308 & 0.2035 \\ 10232 & 14: 16: 55 & 0.0995 & 0.4495 \\ 10234 & 14: 16: 57 & 0.0946 & 0.1154 \\ 10236 & 14: 16: 59 & 0.0558 & 0.1144 \\ 10238 & 14: 17: 01 & 0.0732 & 0.0260 \\ 10240 & 14: 17: 03 & 0.0409 & 0.1834 \\ 10242 & 14: 17: 05 & 0.0500 & 0.2219 \\ 10244 & 14: 17: 07 & 0.1123 & 0.0469 \\ 10246 & 14: 17: 09 & 0.0732 & 0.0348 \\ 10248 & 14: 17: 11 & 0.0244 & 0.0278 \\ 10250 & 14: 17: 13 & 0.0606 & 0.1785 \\ 10252 & 14: 17: 15 & 0.0133 & 0.1308 \\ 10254 & 14: 17: 17 & 0.0129 & 0.0586 \\ 10256 & 14: 17: 19 & 0.0596 & 0.2732 \\ 10258 & 14: 17: 21 & 0.0644 & 0.0937 \\ 10260 & 14: 17: 23 & 0.0468 & 0.1115 \\ 10262 & 14: 17: 25 & 0.1313 & 0.0804 \\ 10264 & 14: 17: 27 & 0.0371 & 0.1209 \\ 10266 & 14: 17: 29 & 0.0490 & 0.0342 \\ 10268 & 14: 17: 31 & 0.0757 & 0.0296 \\ 10270 & 14: 17: 33 & 0.0274 & 0.1347 \\ 10272 & 14: 17: 35 & 0.0418 & 0.0604 \\ 10274 & 14: 17: 37 & 0.0231 & 0.1174 \\ 10276 & 14: 17: 39 & 0.0354 & 0.0287 \\ 10278 & 14: 17: 41 & 0.0268 & 0.0586 \\ 10280 & 14: 17: 43 & 0.0098 & 0.1232 \\ 10282 & 14: 17: 45 & 0.0482 & 0.0915 \\ 10284 & 14: 17: 47 & 0.0681 & 0.1466 \\ 10286 & 14: 17: 49 & 0.0412 & 0.0888 \\ 10288 & 14: 17: 51 & 0.0883 & 0.1059 \\ 10290 & 14: 17: 53 & 0.0407 & 0.0653 \\ 10292 & 14: 17: 55 & 0.0580 & 0.1109\end{array}$




$\begin{array}{llll}10294 & 14: 17: 57 & 0.0911 & 0.1421 \\ 10296 & 14: 17: 59 & 0.1579 & 0.1411 \\ 10298 & 14: 18: 01 & 0.0176 & 0.0750 \\ 10300 & 14: 18: 03 & 0.0891 & 0.1070 \\ 10302 & 14: 18: 05 & 0.0383 & 0.0808 \\ 10304 & 14: 18: 07 & 0.0577 & 0.1284 \\ 10306 & 14: 18: 09 & 0.0423 & 0.1080 \\ 10308 & 14: 18: 11 & 0.0265 & 0.0485 \\ 10310 & 14: 18: 13 & 0.0166 & 0.1886 \\ 10312 & 14: 18: 15 & 0.0555 & 0.0768 \\ 10314 & 14: 18: 17 & 0.1407 & 0.0905 \\ 10316 & 14: 18: 19 & 0.0482 & 0.0951 \\ 10318 & 14: 18: 21 & 0.1994 & 0.1023 \\ 10320 & 14: 18: 23 & 0.0556 & 0.1349 \\ 10322 & 14: 18: 25 & 0.0915 & 0.0779 \\ 10324 & 14: 18: 27 & 0.0350 & 0.1997 \\ 10326 & 14: 18: 29 & 0.1003 & 0.1665 \\ 10328 & 14: 18: 31 & 0.0456 & 0.0833 \\ 10330 & 14: 18: 33 & 0.0462 & 0.2011 \\ 10332 & 14: 18: 35 & 0.1907 & 0.2350 \\ 10334 & 14: 18: 37 & 0.0755 & 0.0533 \\ 10336 & 14: 18: 39 & 0.0234 & 0.0937 \\ 10338 & 14: 18: 41 & 0.0767 & 0.0791 \\ 10340 & 14: 18: 43 & 0.0359 & 0.0775 \\ 10342 & 14: 18: 45 & 0.0934 & 0.0914 \\ 10344 & 14: 18: 47 & 0.0659 & 0.1174 \\ 10346 & 14: 18: 49 & 0.0625 & 0.1045 \\ 10348 & 14: 18: 51 & 0.0222 & 0.1624 \\ 10350 & 14: 18: 53 & 0.0942 & 0.3688 \\ 10352 & 14: 18: 55 & 0.0403 & 0.1999 \\ 10354 & 14: 18: 57 & 0.0617 & 0.0906 \\ 10356 & 14: 18: 59 & 0.0518 & 0.2128 \\ 10358 & 14: 19: 01 & 0.0197 & 0.2332 \\ 10360 & 14: 19: 03 & 0.0356 & 0.1108 \\ 10362 & 14: 19: 05 & 0.1188 & 0.3583 \\ 10364 & 14: 19: 07 & 0.0337 & 0.1072 \\ 10366 & 14: 19: 09 & 0.0383 & 0.1071 \\ 10368 & 14: 19: 11 & 0.0187 & 0.0461 \\ 10370 & 14: 19: 13 & 0.0196 & 0.0396 \\ 10372 & 14: 19: 15 & 0.0616 & 0.0880 \\ 10374 & 14: 19: 17 & 0.0422 & 0.0614 \\ 10376 & 14: 19: 19 & 0.0908 & 0.1997 \\ 10378 & 14: 19: 21 & 0.0367 & 0.2584 \\ 10380 & 14: 19: 23 & 0.1387 & 0.0798 \\ 10382 & 14: 19: 25 & 0.0991 & 0.1624 \\ 10384 & 14: 19: 27 & 0.1102 & 0.2528 \\ 10386 & 14: 19: 29 & 0.0266 & 0.2423 \\ 10388 & 14: 19: 31 & 0.0129 & 0.2410 \\ 10390 & 14: 19: 33 & 0.0510 & 0.3409 \\ 10392 & 14: 19: 35 & 0.0753 & 0.1429 \\ 10394 & 14: 19: 37 & 0.0259 & 0.3780 \\ 10396 & 14: 19: 39 & 0.0681 & 0.1792\end{array}$




$\begin{array}{llll}10398 & 14: 19: 41 & 0.0654 & 0.1449 \\ 10400 & 14: 19: 43 & 0.0958 & 0.1943 \\ 10402 & 14: 19: 45 & 0.0378 & 0.0798 \\ 10404 & 14: 19: 47 & 0.0331 & 0.2925 \\ 10406 & 14: 19: 49 & 0.0313 & 0.1922 \\ 10408 & 14: 19: 51 & 0.0521 & 0.1673 \\ 10410 & 14: 19: 53 & 0.1363 & 0.2559 \\ 10412 & 14: 19: 55 & 0.1274 & 0.4722 \\ 10414 & 14: 19: 57 & 0.0564 & 0.1546 \\ 10416 & 14: 19: 59 & 0.0370 & 0.2389 \\ 10418 & 14: 20: 01 & 0.0589 & 0.1275 \\ 10420 & 14: 20: 03 & 0.1060 & 0.1037 \\ 10422 & 14: 20: 05 & 0.0535 & 0.0621 \\ 10424 & 14: 20: 07 & 0.0543 & 0.1654 \\ 10426 & 14: 20: 09 & 0.0460 & 0.0786 \\ 10428 & 14: 20: 11 & 0.0637 & 0.0710 \\ 10430 & 14: 20: 13 & 0.1105 & 0.4639 \\ 10432 & 14: 20: 15 & 0.0303 & 0.1547 \\ 10434 & 14: 20: 17 & 0.0401 & 0.0739 \\ 10436 & 14: 20: 19 & 0.0751 & 0.5465 \\ 10438 & 14: 20: 21 & 0.0172 & 0.1233 \\ 10440 & 14: 20: 23 & 0.1617 & 0.1364 \\ 10442 & 14: 20: 25 & 0.0830 & 0.2352 \\ 10444 & 14: 20: 27 & 0.0529 & 0.0977 \\ 10446 & 14: 20: 29 & 0.0162 & 0.2618 \\ 10448 & 14: 20: 31 & 0.0491 & 0.1191 \\ 10450 & 14: 20: 33 & 0.0398 & 0.0904 \\ 10452 & 14: 20: 35 & 0.0629 & 0.1139 \\ 10454 & 14: 20: 37 & 0.1075 & 0.0661 \\ 10456 & 14: 20: 39 & 0.1043 & 0.1516 \\ 10458 & 14: 20: 41 & 0.0767 & 0.1560 \\ 10460 & 14: 20: 43 & 0.0218 & 0.0806 \\ 10462 & 14: 20: 45 & 0.0552 & 0.0914 \\ 10464 & 14: 20: 47 & 0.2300 & 0.1749 \\ 10466 & 14: 20: 49 & 0.0230 & 0.2223 \\ 10468 & 14: 20: 51 & 0.0168 & 0.4055 \\ 10470 & 14: 20: 53 & 0.0326 & 0.1577 \\ 10472 & 14: 20: 55 & 0.0812 & 0.3132 \\ 10474 & 14: 20: 57 & 0.0940 & 0.1826 \\ 10476 & 14: 20: 59 & 0.0175 & 0.2211 \\ 10478 & 14: 21: 01 & 0.1167 & 0.1552 \\ 10480 & 14: 21: 03 & 0.0140 & 0.1426 \\ 10482 & 14: 21: 05 & 0.0979 & 0.1064 \\ 10484 & 14: 21: 07 & 0.0553 & 0.1634 \\ 10486 & 14: 21: 09 & 0.0642 & 0.0526 \\ 10488 & 14: 21: 11 & 0.0168 & 0.1881 \\ 10490 & 14: 21: 13 & 0.0189 & 0.2014 \\ 10492 & 14: 21: 15 & 0.0292 & 0.1267 \\ 10494 & 14: 21: 17 & 0.0361 & 0.3268 \\ 10496 & 14: 21: 19 & 0.0657 & 0.1343 \\ 10498 & 14: 21: 21 & 0.1309 & 0.2442 \\ 10500 & 14: 21: 23 & 0.0341 & 0.0944\end{array}$




$\begin{array}{llll}10502 & 14: 21: 25 & 0.0183 & 0.1329 \\ 10504 & 14: 21: 27 & 0.0264 & 0.1201 \\ 10506 & 14: 21: 29 & 0.2950 & 0.0968 \\ 10508 & 14: 21: 31 & 0.0429 & 0.2050 \\ 10510 & 14: 21: 33 & 0.0674 & 0.2201 \\ 10512 & 14: 21: 35 & 0.0287 & 0.2216 \\ 10514 & 14: 21: 37 & 0.1553 & 0.1655 \\ 10516 & 14: 21: 39 & 0.0707 & 0.1103 \\ 10518 & 14: 21: 41 & 0.0581 & 0.6818 \\ 10520 & 14: 21: 43 & 0.0358 & 0.1584 \\ 10522 & 14: 21: 45 & 0.1155 & 0.2703 \\ 10524 & 14: 21: 47 & 0.0201 & 0.0799 \\ 10526 & 14: 21: 49 & 0.0861 & 0.2794 \\ 10528 & 14: 21: 51 & 0.0909 & 0.3273 \\ 10530 & 14: 21: 53 & 0.0310 & 0.0364 \\ 10532 & 14: 21: 55 & 0.1401 & 0.1785 \\ 10534 & 14: 21: 57 & 0.0858 & 0.1649 \\ 10536 & 14: 21: 59 & 0.1320 & 0.0434 \\ 10538 & 14: 22: 01 & 0.0304 & 0.1137 \\ 10540 & 14: 22: 03 & 0.0317 & 0.0937 \\ 10542 & 14: 22: 05 & 0.0396 & 0.1629 \\ 10544 & 14: 22: 07 & 0.0169 & 0.0711 \\ 10546 & 14: 22: 09 & 0.0894 & 0.1049 \\ 10548 & 14: 22: 11 & 0.0454 & 0.1139 \\ 10550 & 14: 22: 13 & 0.0220 & 0.2016 \\ 10552 & 14: 22: 15 & 0.0635 & 0.0788 \\ 10554 & 14: 22: 17 & 0.0333 & 0.1385 \\ 10556 & 14: 22: 19 & 0.0492 & 0.1084 \\ 10558 & 14: 22: 21 & 0.0644 & 0.2916 \\ 10560 & 14: 22: 23 & 0.0836 & 0.1915 \\ 10562 & 14: 22: 25 & 0.0486 & 0.0664 \\ 10564 & 14: 22: 27 & 0.0283 & 0.1485 \\ 10566 & 14: 22: 29 & 0.0746 & 0.1068 \\ 10568 & 14: 22: 31 & 0.2040 & 0.1551 \\ 10570 & 14: 22: 33 & 0.0263 & 0.1415 \\ 10572 & 14: 22: 35 & 0.1165 & 0.3045 \\ 10574 & 14: 22: 37 & 0.0717 & 0.1213 \\ 10576 & 14: 22: 39 & 0.0707 & 0.2665 \\ 10578 & 14: 22: 41 & 0.0313 & 0.1787 \\ 10580 & 14: 22: 43 & 0.0851 & 0.2281 \\ 10582 & 14: 22: 45 & 0.0379 & 0.2435 \\ 10584 & 14: 22: 47 & 0.0628 & 0.1533 \\ 10586 & 14: 22: 49 & 0.0804 & 0.0864 \\ 10588 & 14: 22: 51 & 0.0250 & 0.0785 \\ 10590 & 14: 22: 53 & 0.1024 & 0.0910 \\ 10592 & 14: 22: 55 & 0.0351 & 0.0365 \\ 10594 & 14: 22: 57 & 0.0527 & 0.0875 \\ 10596 & 14: 22: 59 & 0.0317 & 0.0926 \\ 10598 & 14: 23: 01 & 0.0496 & 0.0599 \\ 10600 & 14: 23: 03 & 0.1430 & 0.0484 \\ 10602 & 14: 23: 05 & 0.0567 & 0.1004 \\ 10604 & 14: 23: 07 & 0.0136 & 0.0651\end{array}$




$\begin{array}{llll}10606 & 14: 23: 09 & 0.0185 & 0.0909 \\ 10608 & 14: 23: 11 & 0.0218 & 0.1739 \\ 10610 & 14: 23: 13 & 0.0413 & 0.1805 \\ 10612 & 14: 23: 15 & 0.0204 & 0.1067 \\ 10614 & 14: 23: 17 & 0.0390 & 0.0941 \\ 10616 & 14: 23: 19 & 0.0298 & 0.2305 \\ 10618 & 14: 23: 21 & 0.0193 & 0.0233 \\ 10620 & 14: 23: 23 & 0.0992 & 0.1046 \\ 10622 & 14: 23: 25 & 0.0582 & 0.2191 \\ 10624 & 14: 23: 27 & 0.0768 & 0.1927 \\ 10626 & 14: 23: 29 & 0.0408 & 0.1857 \\ 10628 & 14: 23: 31 & 0.0163 & 0.2660 \\ 10630 & 14: 23: 33 & 0.0804 & 0.3410 \\ 10632 & 14: 23: 35 & 0.0217 & 0.1669 \\ 10634 & 14: 23: 37 & 0.0860 & 0.2395 \\ 10636 & 14: 23: 39 & 0.0490 & 0.3037 \\ 10638 & 14: 23: 41 & 0.0793 & 0.4701 \\ 10640 & 14: 23: 43 & 0.0410 & 0.1148 \\ 10642 & 14: 23: 45 & 0.0471 & 0.1680 \\ 10644 & 14: 23: 47 & 0.0636 & 0.2582 \\ 10646 & 14: 23: 49 & 0.1290 & 0.2197 \\ 10648 & 14: 23: 51 & 0.0256 & 0.2552 \\ 10650 & 14: 23: 53 & 0.0632 & 0.2401 \\ 10652 & 14: 23: 55 & 0.0273 & 0.5081 \\ 10654 & 14: 23: 57 & 0.1182 & 0.2819 \\ 10656 & 14: 23: 59 & 0.0606 & 0.1248 \\ 10658 & 14: 24: 01 & 0.1009 & 0.1566 \\ 10660 & 14: 24: 03 & 0.1166 & 0.1883 \\ 10662 & 14: 24: 05 & 0.0908 & 0.1640 \\ 10664 & 14: 24: 07 & 0.0807 & 0.3059 \\ 10666 & 14: 24: 09 & 0.0696 & 0.2503 \\ 10668 & 14: 24: 11 & 0.0928 & 0.1607 \\ 10670 & 14: 24: 13 & 0.0375 & 0.1420 \\ 10672 & 14: 24: 15 & 0.0267 & 0.1264 \\ 10674 & 14: 24: 17 & 0.0629 & 0.3342 \\ 10676 & 14: 24: 19 & 0.1121 & 0.2284 \\ 10678 & 14: 24: 21 & 0.0157 & 0.1311 \\ 10680 & 14: 24: 23 & 0.0156 & 0.2559 \\ 10682 & 14: 24: 25 & 0.0203 & 0.1559 \\ 10684 & 14: 24: 27 & 0.0193 & 0.0767 \\ 10686 & 14: 24: 29 & 0.0579 & 0.1911 \\ 10688 & 14: 24: 31 & 0.0983 & 0.1143 \\ 10690 & 14: 24: 33 & 0.0485 & 0.1403 \\ 10692 & 14: 24: 35 & 0.0227 & 0.0438 \\ 10694 & 14: 24: 37 & 0.0428 & 0.0784 \\ 10696 & 14: 24: 39 & 0.0417 & 0.1628 \\ 10698 & 14: 24: 41 & 0.1269 & 0.2554 \\ 10700 & 14: 24: 43 & 0.0865 & 0.1632 \\ 10702 & 14: 24: 45 & 0.0600 & 0.1337 \\ 10704 & 14: 24: 47 & 0.1400 & 0.0615 \\ 10706 & 14: 24: 49 & 0.0191 & 0.1454 \\ 10708 & 14: 24: 51 & 0.2297 & 0.1205\end{array}$




$\begin{array}{llll}10710 & 14: 24: 53 & 0.0781 & 0.1156 \\ 10712 & 14: 24: 55 & 0.1282 & 0.0777 \\ 10714 & 14: 24: 57 & 0.0239 & 0.0493 \\ 10716 & 14: 24: 59 & 0.0615 & 0.1060 \\ 10718 & 14: 25: 01 & 0.1167 & 0.1829 \\ 10720 & 14: 25: 03 & 0.0792 & 0.1844 \\ 10722 & 14: 25: 05 & 0.0536 & 0.2190 \\ 10724 & 14: 25: 07 & 0.0471 & 0.1940 \\ 10726 & 14: 25: 09 & 0.0749 & 0.2713 \\ 10728 & 14: 25: 11 & 0.0304 & 0.2209 \\ 10730 & 14: 25: 13 & 0.0614 & 0.2918 \\ 10732 & 14: 25: 15 & 0.1528 & 0.1620 \\ 10734 & 14: 25: 17 & 0.0367 & 0.1711 \\ 10736 & 14: 25: 19 & 0.0263 & 0.0910 \\ 10738 & 14: 25: 21 & 0.0414 & 0.3437 \\ 10740 & 14: 25: 23 & 0.0155 & 0.1156 \\ 10742 & 14: 25: 25 & 0.0553 & 0.1560 \\ 10744 & 14: 25: 27 & 0.0402 & 0.1174 \\ 10746 & 14: 25: 29 & 0.0209 & 0.1375 \\ 10748 & 14: 25: 31 & 0.0824 & 0.1636 \\ 10750 & 14: 25: 33 & 0.0748 & 0.2262 \\ 10752 & 14: 25: 35 & 0.0738 & 0.2048 \\ 10754 & 14: 25: 37 & 0.0228 & 0.0937 \\ 10756 & 14: 25: 39 & 0.0683 & 0.1235 \\ 10758 & 14: 25: 41 & 0.2178 & 0.2084 \\ 10760 & 14: 25: 43 & 0.0306 & 0.1054 \\ 10762 & 14: 25: 45 & 0.1772 & 0.2079 \\ 10764 & 14: 25: 47 & 0.0424 & 0.0765 \\ 10766 & 14: 25: 49 & 0.0561 & 0.0948 \\ 10768 & 14: 25: 51 & 0.0305 & 0.0873 \\ 10770 & 14: 25: 53 & 0.0383 & 0.0629 \\ 10772 & 14: 25: 55 & 0.0498 & 0.3508 \\ 10774 & 14: 25: 57 & 0.0775 & 0.1017 \\ 10776 & 14: 25: 59 & 0.1087 & 0.1187 \\ 10778 & 14: 26: 01 & 0.0483 & 0.0914 \\ 10780 & 14: 26: 03 & 0.0798 & 0.3362 \\ 10782 & 14: 26: 05 & 0.0290 & 0.2296 \\ 10784 & 14: 26: 07 & 0.0588 & 0.0965 \\ 10786 & 14: 26: 09 & 0.0341 & 0.2162 \\ 10788 & 14: 26: 11 & 0.0586 & 0.1624 \\ 10790 & 14: 26: 13 & 0.0894 & 0.1674 \\ 10792 & 14: 26: 15 & 0.0418 & 0.0748 \\ 10794 & 14: 26: 17 & 0.0349 & 0.0983 \\ 10796 & 14: 26: 19 & 0.0488 & 0.1862 \\ 10798 & 14: 26: 21 & 0.0488 & 0.0834 \\ 10800 & 14: 26: 23 & 0.0439 & 0.0926 \\ 10802 & 14: 26: 25 & 0.0758 & 0.1638 \\ 10804 & 14: 26: 27 & 0.0425 & 0.0271 \\ 10806 & 14: 26: 29 & 0.0328 & 0.2128 \\ 10808 & 14: 26: 31 & 0.0561 & 0.0757 \\ 10810 & 14: 26: 33 & 0.0275 & 0.1915 \\ 10812 & 14: 26: 35 & 0.0719 & 0.2591\end{array}$




$\begin{array}{llll}10814 & 14: 26: 37 & 0.0634 & 0.1731 \\ 10816 & 14: 26: 39 & 0.1348 & 0.1269 \\ 10818 & 14: 26: 41 & 0.0886 & 0.1524 \\ 10820 & 14: 26: 43 & 0.0240 & 0.0908 \\ 10822 & 14: 26: 45 & 0.0296 & 0.1515 \\ 10824 & 14: 26: 47 & 0.0104 & 0.1647 \\ 10826 & 14: 26: 49 & 0.0414 & 0.0825 \\ 10828 & 14: 26: 51 & 0.0322 & 0.3938 \\ 10830 & 14: 26: 53 & 0.0843 & 0.2350 \\ 10832 & 14: 26: 55 & 0.0998 & 0.0883 \\ 10834 & 14: 26: 57 & 0.0893 & 0.2062 \\ 10836 & 14: 26: 59 & 0.0598 & 0.1487 \\ 10838 & 14: 27: 01 & 0.0240 & 0.0848 \\ 10840 & 14: 27: 03 & 0.0723 & 0.1931 \\ 10842 & 14: 27: 05 & 0.0114 & 0.1788 \\ 10844 & 14: 27: 07 & 0.0717 & 0.1334 \\ 10846 & 14: 27: 09 & 0.1045 & 0.1374 \\ 10848 & 14: 27: 11 & 0.0472 & 0.0941 \\ 10850 & 14: 27: 13 & 0.1665 & 0.0801 \\ 10852 & 14: 27: 15 & 0.0982 & 0.2810 \\ 10854 & 14: 27: 17 & 0.0440 & 0.0656 \\ 10856 & 14: 27: 19 & 0.0255 & 0.2037 \\ 10858 & 14: 27: 21 & 0.0780 & 0.0463 \\ 10860 & 14: 27: 23 & 0.0584 & 0.0986 \\ 10862 & 14: 27: 25 & 0.0272 & 0.1299 \\ 10864 & 14: 27: 27 & 0.0459 & 0.0311 \\ 10866 & 14: 27: 29 & 0.0269 & 0.0653 \\ 10868 & 14: 27: 31 & 0.1462 & 0.0736 \\ 10870 & 14: 27: 33 & 0.0377 & 0.0606 \\ 10872 & 14: 27: 35 & 0.0342 & 0.0752 \\ 10874 & 14: 27: 37 & 0.0382 & 0.1366 \\ 10876 & 14: 27: 39 & 0.0649 & 0.2026 \\ 10878 & 14: 27: 41 & 0.0422 & 0.1983 \\ 10880 & 14: 27: 43 & 0.0568 & 0.1997 \\ 10882 & 14: 27: 45 & 0.0772 & 0.1663 \\ 10884 & 14: 27: 47 & 0.1071 & 0.1098 \\ 10886 & 14: 27: 49 & 0.0325 & 0.2632 \\ 10888 & 14: 27: 51 & 0.0331 & 0.2681 \\ 10890 & 14: 27: 53 & 0.0579 & 0.3442 \\ 10892 & 14: 27: 55 & 0.1025 & 0.1752 \\ 10894 & 14: 27: 57 & 0.0315 & 0.2414 \\ 10896 & 14: 27: 59 & 0.0977 & 0.0769 \\ 10898 & 14: 28: 01 & 0.1742 & 0.1346 \\ 10900 & 14: 28: 03 & 0.0487 & 0.2034 \\ 10902 & 14: 28: 05 & 0.0316 & 0.1444 \\ 10904 & 14: 28: 07 & 0.1243 & 0.2438 \\ 10906 & 14: 28: 09 & 0.0760 & 0.4478 \\ 10908 & 14: 28: 11 & 0.0885 & 0.1286 \\ 10910 & 14: 28: 13 & 0.0791 & 0.1336 \\ 10912 & 14: 28: 15 & 0.0527 & 0.1173 \\ 10914 & 14: 28: 17 & 0.0830 & 0.1485 \\ 10916 & 14: 28: 19 & 0.0269 & 0.0526\end{array}$




$\begin{array}{llll}10918 & 14: 28: 21 & 0.2049 & 0.0575 \\ 10920 & 14: 28: 23 & 0.0388 & 0.1742 \\ 10922 & 14: 28: 25 & 0.0533 & 0.0547 \\ 10924 & 14: 28: 27 & 0.0610 & 0.1286 \\ 10926 & 14: 28: 29 & 0.0942 & 0.2340 \\ 10928 & 14: 28: 31 & 0.0425 & 0.0672 \\ 10930 & 14: 28: 33 & 0.0717 & 0.1946 \\ 10932 & 14: 28: 35 & 0.0657 & 0.1789 \\ 10934 & 14: 28: 37 & 0.0656 & 0.1562 \\ 10936 & 14: 28: 39 & 0.0664 & 0.0740 \\ 10938 & 14: 28: 41 & 0.0441 & 0.1226 \\ 10940 & 14: 28: 43 & 0.1155 & 0.1825 \\ 10942 & 14: 28: 45 & 0.0805 & 0.2300 \\ 10944 & 14: 28: 47 & 0.0265 & 0.1374 \\ 10946 & 14: 28: 49 & 0.0329 & 0.0813 \\ 10948 & 14: 28: 51 & 0.0934 & 0.2562 \\ 10950 & 14: 28: 53 & 0.1006 & 0.1838 \\ 10952 & 14: 28: 55 & 0.0529 & 0.0955 \\ 10954 & 14: 28: 57 & 0.1084 & 0.1323 \\ 10956 & 14: 28: 59 & 0.0587 & 0.3173 \\ 10958 & 14: 29: 01 & 0.0657 & 0.2624 \\ 10960 & 14: 29: 03 & 0.1284 & 0.2593 \\ 10962 & 14: 29: 05 & 0.0469 & 0.2229 \\ 10964 & 14: 29: 07 & 0.0927 & 0.1113 \\ 10966 & 14: 29: 09 & 0.0233 & 0.1728 \\ 10968 & 14: 29: 11 & 0.0490 & 0.2652 \\ 10970 & 14: 29: 13 & 0.1397 & 0.1922 \\ 10972 & 14: 29: 15 & 0.0342 & 0.2138 \\ 10974 & 14: 29: 17 & 0.2500 & 0.2053 \\ 10976 & 14: 29: 19 & 0.0519 & 0.1174 \\ 10978 & 14: 29: 21 & 0.0341 & 0.2567 \\ 10980 & 14: 29: 23 & 0.0498 & 0.1851 \\ 10982 & 14: 29: 25 & 0.0605 & 0.2503 \\ 10984 & 14: 29: 27 & 0.0279 & 0.2172 \\ 10986 & 14: 29: 29 & 0.0604 & 0.1361 \\ 10988 & 14: 29: 31 & 0.0801 & 0.1505 \\ 10990 & 14: 29: 33 & 0.0835 & 0.2086 \\ 10992 & 14: 29: 35 & 0.0695 & 0.0569 \\ 10994 & 14: 29: 37 & 0.0452 & 0.0955 \\ 10996 & 14: 29: 39 & 0.0576 & 0.1461 \\ 10998 & 14: 29: 41 & 0.0718 & 0.1472 \\ 11000 & 14: 29: 43 & 0.0127 & 0.2617 \\ 11002 & 14: 29: 45 & 0.0931 & 0.1333 \\ 11004 & 14: 29: 47 & 0.0842 & 0.0829 \\ 11006 & 14: 29: 49 & 0.1443 & 0.1383 \\ 11008 & 14: 29: 51 & 0.0363 & 0.0989 \\ 11010 & 14: 29: 53 & 0.0235 & 0.1825 \\ 11012 & 14: 29: 55 & 0.0259 & 0.0676 \\ 11014 & 14: 29: 57 & 0.0973 & 0.0987 \\ 11016 & 14: 29: 59 & 0.0568 & 0.0479 \\ 11018 & 14: 30: 01 & 0.0523 & 0.1523 \\ 11020 & 14: 30: 03 & 0.0702 & 0.2016\end{array}$




$\begin{array}{llll}11022 & 14: 30: 05 & 0.1249 & 0.3120 \\ 11024 & 14: 30: 07 & 0.1090 & 0.2837 \\ 11026 & 14: 30: 09 & 0.0562 & 0.1071 \\ 11028 & 14: 30: 11 & 0.1715 & 0.3053 \\ 11030 & 14: 30: 13 & 0.0796 & 0.1427 \\ 11032 & 14: 30: 15 & 0.0601 & 0.2676 \\ 11034 & 14: 30: 17 & 0.0585 & 0.1674 \\ 11036 & 14: 30: 19 & 0.0620 & 0.2389 \\ 11038 & 14: 30: 21 & 0.0249 & 0.1235 \\ 11040 & 14: 30: 23 & 0.0813 & 0.0503 \\ 11042 & 14: 30: 25 & 0.0313 & 0.1809 \\ 11044 & 14: 30: 27 & 0.1186 & 0.2616 \\ 11046 & 14: 30: 29 & 0.0562 & 0.1364 \\ 11048 & 14: 30: 31 & 0.1065 & 0.0530 \\ 11050 & 14: 30: 33 & 0.0879 & 0.1401 \\ 11052 & 14: 30: 35 & 0.0519 & 0.0803 \\ 11054 & 14: 30: 37 & 0.0465 & 0.2407 \\ 11056 & 14: 30: 39 & 0.0353 & 0.1388 \\ 11058 & 14: 30: 41 & 0.0599 & 0.3226 \\ 11060 & 14: 30: 43 & 0.0387 & 0.1510 \\ 11062 & 14: 30: 45 & 0.1201 & 0.1509 \\ 11064 & 14: 30: 47 & 0.0167 & 0.1339 \\ 11066 & 14: 30: 49 & 0.0532 & 0.1476 \\ 11068 & 14: 30: 51 & 0.0634 & 0.1915 \\ 11070 & 14: 30: 53 & 0.0146 & 0.0937 \\ 11072 & 14: 30: 55 & 0.0321 & 0.1836 \\ 11074 & 14: 30: 57 & 0.0099 & 0.1016 \\ 11076 & 14: 30: 59 & 0.0161 & 0.1202 \\ 11078 & 14: 31: 01 & 0.1093 & 0.3804 \\ 11080 & 14: 31: 03 & 0.0418 & 0.0669 \\ 11082 & 14: 31: 05 & 0.0621 & 0.1366 \\ 11084 & 14: 31: 07 & 0.0977 & 0.0850 \\ 11086 & 14: 31: 09 & 0.0437 & 0.1374 \\ 11088 & 14: 31: 11 & 0.0320 & 0.1021 \\ 11090 & 14: 31: 13 & 0.0294 & 0.2392 \\ 11092 & 14: 31: 15 & 0.0874 & 0.5007 \\ 11094 & 14: 31: 17 & 0.0179 & 0.2362 \\ 11096 & 14: 31: 19 & 0.0544 & 0.1432 \\ 11098 & 14: 31: 21 & 0.0533 & 0.2347 \\ 11100 & 14: 31: 23 & 0.0321 & 0.0383 \\ 11102 & 14: 31: 25 & 0.0377 & 0.0895 \\ 11104 & 14: 31: 27 & 0.0719 & 0.1084 \\ 11106 & 14: 31: 29 & 0.0938 & 0.1000 \\ 11108 & 14: 31: 31 & 0.0634 & 0.1038 \\ 11110 & 14: 31: 33 & 0.0715 & 0.0731 \\ 11112 & 14: 31: 35 & 0.0357 & 0.0427 \\ 11114 & 14: 31: 37 & 0.0386 & 0.3138 \\ 11116 & 14: 31: 39 & 0.0482 & 0.0824 \\ 11118 & 14: 31: 41 & 0.1674 & 0.0932 \\ 11120 & 14: 31: 43 & 0.0820 & 0.0384 \\ 11122 & 14: 31: 45 & 0.1225 & 0.0889 \\ 11124 & 14: 31: 47 & 0.0740 & 0.0519\end{array}$


Appendix B - Laser Data Test 1 NIST102505_1.xls

$\begin{array}{llll}11126 & 14: 31: 49 & 0.1359 & 0.6656 \\ 11128 & 14: 31: 51 & 0.1293 & 0.1406 \\ 11130 & 14: 31: 53 & 0.0478 & 0.3363 \\ 11132 & 14: 31: 55 & 0.0356 & 0.0896 \\ 11134 & 14: 31: 57 & 0.0396 & 0.0715 \\ 11136 & 14: 31: 59 & 0.0821 & 0.1070 \\ 11138 & 14: 32: 01 & 0.0616 & 0.0419 \\ 11140 & 14: 32: 03 & 0.2295 & 0.1798 \\ 11142 & 14: 32: 05 & 0.0780 & 0.2780 \\ 11144 & 14: 32: 07 & 0.0210 & 0.0976 \\ 11146 & 14: 32: 09 & 0.0967 & 0.3643 \\ 11148 & 14: 32: 11 & 0.0222 & 0.1714 \\ 11150 & 14: 32: 13 & 0.0939 & 0.0353 \\ 11152 & 14: 32: 15 & 0.0638 & 0.0290 \\ 11154 & 14: 32: 17 & 0.0676 & 0.0769 \\ 11156 & 14: 32: 19 & 0.0500 & 0.1115 \\ 11158 & 14: 32: 21 & 0.0879 & 0.1404 \\ 11160 & 14: 32: 23 & 0.1206 & 0.1684 \\ 11162 & 14: 32: 25 & 0.0496 & 0.1605 \\ 11164 & 14: 32: 27 & 0.0255 & 0.1283 \\ 11166 & 14: 32: 29 & 0.0581 & 0.1656 \\ 11168 & 14: 32: 31 & 0.1100 & 0.1133 \\ 11170 & 14: 32: 33 & 0.0411 & 0.1462 \\ 11172 & 14: 32: 35 & 0.0735 & 0.1181 \\ 11174 & 14: 32: 37 & 0.0891 & 0.0697 \\ 11176 & 14: 32: 39 & 0.1094 & 0.1032 \\ 11178 & 14: 32: 41 & 0.0622 & 0.1667 \\ 11180 & 14: 32: 43 & 0.0734 & 0.2115 \\ 11182 & 14: 32: 45 & 0.0223 & 0.0793 \\ 11184 & 14: 32: 47 & 0.1056 & 0.1594 \\ 11186 & 14: 32: 49 & 0.0721 & 0.1870 \\ 11188 & 14: 32: 51 & 0.1428 & 0.2088 \\ 11190 & 14: 32: 53 & 0.0437 & 0.0722 \\ 11192 & 14: 32: 55 & 0.0793 & 0.2096 \\ 11194 & 14: 32: 57 & 0.0364 & 0.2322 \\ 11196 & 14: 32: 59 & 0.0496 & 0.1575 \\ 11198 & 14: 33: 01 & 0.0610 & 0.2085 \\ 11200 & 14: 33: 03 & 0.0388 & 0.1057 \\ 11202 & 14: 33: 05 & 0.0343 & 0.1591 \\ 11204 & 14: 33: 07 & 0.0567 & 0.1076 \\ 11206 & 14: 33: 09 & 0.0507 & 0.1500 \\ 11208 & 14: 33: 11 & 0.0576 & 0.2337 \\ 11210 & 14: 33: 13 & 0.1157 & 0.1024 \\ 11212 & 14: 33: 15 & 0.0744 & 0.2107 \\ 11214 & 14: 33: 17 & 0.0528 & 0.0589 \\ 11216 & 14: 33: 19 & 0.0725 & 0.0725 \\ 11218 & 14: 33: 21 & 0.0776 & 0.2902 \\ 11220 & 14: 33: 23 & 0.0304 & 0.0380 \\ 11222 & 14: 33: 25 & 0.0744 & 0.1363 \\ 11224 & 14: 33: 27 & 0.0414 & 0.1014 \\ 11226 & 14: 33: 29 & 0.0071 & 0.0728 \\ 11228 & 14: 33: 31 & 0.0440 & 0.1418\end{array}$

B - 108 
Appendix B - Laser Data Test 1 NIST102505_1.xls

$\begin{array}{llll}11230 & 14: 33: 33 & 0.0944 & 0.0204 \\ 11232 & 14: 33: 35 & 0.0933 & 0.0656 \\ 11234 & 14: 33: 37 & 0.0326 & 0.0473 \\ 11236 & 14: 33: 39 & 0.0223 & 0.0787 \\ 11238 & 14: 33: 41 & 0.0309 & 0.0451 \\ 11240 & 14: 33: 43 & 0.0281 & 0.1675 \\ 11242 & 14: 33: 45 & 0.0957 & 0.1536 \\ 11244 & 14: 33: 47 & 0.0155 & 0.0762 \\ 11246 & 14: 33: 49 & 0.1686 & 0.6262 \\ 11248 & 14: 33: 51 & 0.0443 & 0.3688 \\ 11250 & 14: 33: 53 & 0.0453 & 0.0883 \\ 11252 & 14: 33: 55 & 0.1361 & 0.1043 \\ 11254 & 14: 33: 57 & 0.0516 & 0.1338 \\ 11256 & 14: 33: 59 & 0.0877 & 0.2348 \\ 11258 & 14: 34: 01 & 0.0536 & 0.2232 \\ 11260 & 14: 34: 03 & 0.0650 & 0.1812 \\ 11262 & 14: 34: 05 & 0.0696 & 0.1579 \\ 11264 & 14: 34: 07 & 0.0347 & 0.2042 \\ 11266 & 14: 34: 09 & 0.0710 & 0.1263 \\ 11268 & 14: 34: 11 & 0.0402 & 0.1544 \\ 11270 & 14: 34: 13 & 0.0152 & 0.1444 \\ 11272 & 14: 34: 15 & 0.0817 & 0.1019 \\ 11274 & 14: 34: 17 & 0.0231 & 0.2351 \\ 11276 & 14: 34: 19 & 0.1095 & 0.4282 \\ 11278 & 14: 34: 21 & 0.0528 & 0.0807 \\ 11280 & 14: 34: 23 & 0.1224 & 0.1292 \\ 11282 & 14: 34: 25 & 0.0501 & 0.4857 \\ 11284 & 14: 34: 27 & 0.0590 & 0.1638 \\ 11286 & 14: 34: 29 & 0.0640 & 0.0927 \\ 11288 & 14: 34: 31 & 0.0446 & 0.0777 \\ 11290 & 14: 34: 33 & 0.0311 & 0.1790 \\ 11292 & 14: 34: 35 & 0.0601 & 0.3061 \\ 11294 & 14: 34: 37 & 0.0592 & 0.1374 \\ 11296 & 14: 34: 39 & 0.0367 & 0.0874 \\ 11298 & 14: 34: 41 & 0.0317 & 0.1612 \\ 11300 & 14: 34: 43 & 0.0377 & 0.1766 \\ 11302 & 14: 34: 45 & 0.0232 & 0.1150 \\ 11304 & 14: 34: 47 & 0.0299 & 0.0691 \\ 11306 & 14: 34: 49 & 0.1168 & 0.2056 \\ 11308 & 14: 34: 51 & 0.0598 & 0.1132 \\ 11310 & 14: 34: 53 & 0.1897 & 0.1548 \\ 11312 & 14: 34: 55 & 0.0195 & 0.0865 \\ 11314 & 14: 34: 57 & 0.0226 & 0.1370 \\ 11316 & 14: 34: 59 & 0.0293 & 0.1832 \\ 11318 & 14: 35: 01 & 0.0519 & 0.1698 \\ 11320 & 14: 35: 03 & 0.0417 & 0.1561 \\ 11322 & 14: 35: 05 & 0.0582 & 0.1053 \\ 11324 & 14: 35: 07 & 0.0645 & 0.0602 \\ 11326 & 14: 35: 09 & 0.0668 & 0.1631 \\ 11328 & 14: 35: 11 & 0.0485 & 0.1784 \\ 11330 & 14: 35: 13 & 0.0418 & 0.2504 \\ 11332 & 14: 35: 15 & 0.1571 & 0.1869\end{array}$

B - 109 


$\begin{array}{llll}11334 & 14: 35: 17 & 0.0652 & 0.1533 \\ 11336 & 14: 35: 19 & 0.0369 & 0.2239 \\ 11338 & 14: 35: 21 & 0.0377 & 0.0956 \\ 11340 & 14: 35: 23 & 0.0561 & 0.1478 \\ 11342 & 14: 35: 25 & 0.2491 & 0.4539 \\ 11344 & 14: 35: 27 & 0.0226 & 0.4978 \\ 11346 & 14: 35: 29 & 0.0251 & 0.1285 \\ 11348 & 14: 35: 31 & 0.1213 & 0.1604 \\ 11350 & 14: 35: 33 & 0.1025 & 0.2405 \\ 11352 & 14: 35: 35 & 0.1052 & 0.2319 \\ 11354 & 14: 35: 37 & 0.0170 & 0.0769 \\ 11356 & 14: 35: 39 & 0.1797 & 0.2335 \\ 11358 & 14: 35: 41 & 0.0504 & 0.2589 \\ 11360 & 14: 35: 43 & 0.0481 & 0.2306 \\ 11362 & 14: 35: 45 & 0.0601 & 0.3543 \\ 11364 & 14: 35: 47 & 0.1412 & 0.3051 \\ 11366 & 14: 35: 49 & 0.0765 & 0.1125 \\ 11368 & 14: 35: 51 & 0.0562 & 0.1223 \\ 11370 & 14: 35: 53 & 0.2800 & 0.2578 \\ 11372 & 14: 35: 55 & 0.0732 & 0.0831 \\ 11374 & 14: 35: 57 & 0.0233 & 0.0532 \\ 11376 & 14: 35: 59 & 0.0592 & 0.1276 \\ 11378 & 14: 36: 01 & 0.0769 & 0.0861 \\ 11380 & 14: 36: 03 & 0.0245 & 0.0570 \\ 11382 & 14: 36: 05 & 0.0616 & 0.0851 \\ 11384 & 14: 36: 07 & 0.0736 & 0.0454 \\ 11386 & 14: 36: 09 & 0.0715 & 0.0876 \\ 11388 & 14: 36: 11 & 0.0460 & 0.0965 \\ 11390 & 14: 36: 13 & 0.0468 & 0.1138 \\ 11392 & 14: 36: 15 & 0.0171 & 0.4294 \\ 11394 & 14: 36: 17 & 0.0433 & 0.3706 \\ 11396 & 14: 36: 19 & 0.0595 & 0.1855 \\ 11398 & 14: 36: 21 & 0.0103 & 0.0716 \\ 11400 & 14: 36: 23 & 0.0263 & 0.0855 \\ 11402 & 14: 36: 25 & 0.1074 & 0.3052 \\ 11404 & 14: 36: 27 & 0.0962 & 0.2941 \\ 11406 & 14: 36: 29 & 0.0766 & 0.0843 \\ 11408 & 14: 36: 31 & 0.0531 & 0.2696 \\ 11410 & 14: 36: 33 & 0.0870 & 0.1027 \\ 11412 & 14: 36: 35 & 0.0175 & 0.1782 \\ 11414 & 14: 36: 37 & 0.1049 & 0.1282 \\ 11416 & 14: 36: 39 & 0.0233 & 0.1094 \\ 11418 & 14: 36: 41 & 0.1549 & 0.0668 \\ 11420 & 14: 36: 43 & 0.0141 & 0.0964 \\ 11422 & 14: 36: 45 & 0.0626 & 0.2123 \\ 11424 & 14: 36: 47 & 0.0209 & 0.1387 \\ 11426 & 14: 36: 49 & 0.1004 & 0.2629 \\ 11428 & 14: 36: 51 & 0.0672 & 0.1051 \\ 11430 & 14: 36: 53 & 0.0143 & 0.1520 \\ 11432 & 14: 36: 55 & 0.0514 & 0.1183 \\ 11434 & 14: 36: 57 & 0.0281 & 0.2362 \\ 11436 & 14: 36: 59 & 0.0959 & 0.0664\end{array}$




$\begin{array}{llll}11438 & 14: 37: 01 & 0.1495 & 0.1522 \\ 11440 & 14: 37: 03 & 0.0619 & 0.0624 \\ 11442 & 14: 37: 05 & 0.0840 & 0.1649 \\ 11444 & 14: 37: 07 & 0.0407 & 0.3666 \\ 11446 & 14: 37: 09 & 0.0362 & 0.2170 \\ 11448 & 14: 37: 11 & 0.1801 & 0.0948 \\ 11450 & 14: 37: 13 & 0.0778 & 0.4158 \\ 11452 & 14: 37: 15 & 0.0278 & 0.2697 \\ 11454 & 14: 37: 17 & 0.0434 & 0.1516 \\ 11456 & 14: 37: 19 & 0.0361 & 0.1430 \\ 11458 & 14: 37: 21 & 0.1289 & 0.1099 \\ 11460 & 14: 37: 23 & 0.0723 & 0.2552 \\ 11462 & 14: 37: 25 & 0.0225 & 0.2375 \\ 11464 & 14: 37: 27 & 0.0322 & 0.3487 \\ 11466 & 14: 37: 29 & 0.0557 & 0.2335 \\ 11468 & 14: 37: 31 & 0.0626 & 0.2214 \\ 11470 & 14: 37: 33 & 0.0626 & 0.0818 \\ 11472 & 14: 37: 35 & 0.0556 & 0.2299 \\ 11474 & 14: 37: 37 & 0.0893 & 0.2357 \\ 11476 & 14: 37: 39 & 0.1497 & 0.2826 \\ 11478 & 14: 37: 41 & 0.0655 & 0.3365 \\ 11480 & 14: 37: 43 & 0.0872 & 0.2551 \\ 11482 & 14: 37: 45 & 0.0430 & 0.2211 \\ 11484 & 14: 37: 47 & 0.0403 & 0.2948 \\ 11486 & 14: 37: 49 & 0.1029 & 0.2042 \\ 11488 & 14: 37: 51 & 0.0680 & 0.0880 \\ 11490 & 14: 37: 53 & 0.0600 & 0.5098 \\ 11492 & 14: 37: 55 & 0.1118 & 0.2561 \\ 11494 & 14: 37: 57 & 0.0493 & 0.1030 \\ 11496 & 14: 37: 59 & 0.0262 & 0.1642 \\ 11498 & 14: 38: 01 & 0.0694 & 0.1608 \\ 11500 & 14: 38: 03 & 0.0840 & 0.1969 \\ 11502 & 14: 38: 05 & 0.0855 & 0.0743 \\ 11504 & 14: 38: 07 & 0.0614 & 0.2220 \\ 11506 & 14: 38: 09 & 0.0486 & 0.1016 \\ 11508 & 14: 38: 11 & 0.0360 & 0.0339 \\ 11510 & 14: 38: 13 & 0.0187 & 0.1794 \\ 11512 & 14: 38: 15 & 0.0202 & 0.1036 \\ 11514 & 14: 38: 17 & 0.2222 & 0.3089 \\ 11516 & 14: 38: 19 & 0.1426 & 0.0373 \\ 11518 & 14: 38: 21 & 0.4239 & 0.3619 \\ 11520 & 14: 38: 23 & 0.0858 & 0.1631 \\ 11522 & 14: 38: 25 & 0.0440 & 0.1415 \\ 11524 & 14: 38: 27 & 0.0889 & 0.0597 \\ 11526 & 14: 38: 29 & 0.0371 & 0.0482 \\ 11528 & 14: 38: 31 & 0.0675 & 0.3311 \\ 11530 & 14: 38: 33 & 0.1291 & 0.1417 \\ 11532 & 14: 38: 35 & 0.0557 & 0.0571 \\ 11534 & 14: 38: 37 & 0.1012 & 0.1372 \\ 11536 & 14: 38: 39 & 0.0233 & 0.0516 \\ 11538 & 14: 38: 41 & 0.0800 & 0.1161 \\ 11540 & 14: 38: 43 & 0.0220 & 0.1806\end{array}$




$\begin{array}{llll}11542 & 14: 38: 45 & 0.0584 & 0.2393 \\ 11544 & 14: 38: 47 & 0.0679 & 0.2185 \\ 11546 & 14: 38: 49 & 0.0355 & 0.1509 \\ 11548 & 14: 38: 51 & 0.0616 & 0.0907 \\ 11550 & 14: 38: 53 & 0.0351 & 0.1348 \\ 11552 & 14: 38: 55 & 0.0306 & 0.1661 \\ 11554 & 14: 38: 57 & 0.0209 & 0.2448 \\ 11556 & 14: 38: 59 & 0.0686 & 0.1401 \\ 11558 & 14: 39: 01 & 0.0424 & 0.2342 \\ 11560 & 14: 39: 03 & 0.0394 & 0.2207 \\ 11562 & 14: 39: 05 & 0.0471 & 0.1602 \\ 11564 & 14: 39: 07 & 0.0520 & 0.2637 \\ 11566 & 14: 39: 09 & 0.0202 & 0.1307 \\ 11568 & 14: 39: 11 & 0.0604 & 0.1777 \\ 11570 & 14: 39: 13 & 0.0842 & 0.1750 \\ 11572 & 14: 39: 15 & 0.0510 & 0.1243 \\ 11574 & 14: 39: 17 & 0.0365 & 0.0738 \\ 11576 & 14: 39: 19 & 0.0715 & 0.0494 \\ 11578 & 14: 39: 21 & 0.1092 & 0.0115 \\ 11580 & 14: 39: 23 & 0.0382 & 0.1493 \\ 11582 & 14: 39: 25 & 0.0265 & 0.1200 \\ 11584 & 14: 39: 27 & 0.0395 & 0.0790 \\ 11586 & 14: 39: 29 & 0.0422 & 0.0421 \\ 11588 & 14: 39: 31 & 0.0474 & 0.1931 \\ 11590 & 14: 39: 33 & 0.0365 & 0.0903 \\ 11592 & 14: 39: 35 & 0.0869 & 0.1917 \\ 11594 & 14: 39: 37 & 0.0433 & 0.2226 \\ 11596 & 14: 39: 39 & 0.0261 & 0.1437 \\ 11598 & 14: 39: 41 & 0.0543 & 0.0744 \\ 11600 & 14: 39: 43 & 0.0441 & 0.2858 \\ 11602 & 14: 39: 45 & 0.0733 & 0.3476 \\ 11604 & 14: 39: 47 & 0.0463 & 0.1405 \\ 11606 & 14: 39: 49 & 0.0600 & 0.1158 \\ 11608 & 14: 39: 51 & 0.1061 & 0.0431 \\ 11610 & 14: 39: 53 & 0.0807 & 0.1826 \\ 11612 & 14: 39: 55 & 0.0340 & 0.2043 \\ 11614 & 14: 39: 57 & 0.0856 & 0.1258 \\ 11616 & 14: 39: 59 & 0.0279 & 0.1370 \\ 11618 & 14: 40: 01 & 0.0572 & 0.0960 \\ 11620 & 14: 40: 03 & 0.0455 & 0.1869 \\ 11622 & 14: 40: 05 & 0.0374 & 0.1492 \\ 11624 & 14: 40: 07 & 0.0748 & 0.2906 \\ 11626 & 14: 40: 09 & 0.0383 & 0.2037 \\ 11628 & 14: 40: 11 & 0.0363 & 0.0829 \\ 11630 & 14: 40: 13 & 0.0190 & 0.1005 \\ 11632 & 14: 40: 15 & 0.0765 & 0.2427 \\ 11634 & 14: 40: 17 & 0.0123 & 0.1918 \\ 11636 & 14: 40: 19 & 0.0225 & 0.1952 \\ 11638 & 14: 40: 21 & 0.0572 & 0.2611 \\ 11640 & 14: 40: 23 & 0.0216 & 0.1138 \\ 11642 & 14: 40: 25 & 0.0309 & 0.1330 \\ 11644 & 14: 40: 27 & 0.0341 & 0.1145\end{array}$




$\begin{array}{llll}11646 & 14: 40: 29 & 0.0374 & 0.0781 \\ 11648 & 14: 40: 31 & 0.0463 & 0.2140 \\ 11650 & 14: 40: 33 & 0.0640 & 0.3035 \\ 11652 & 14: 40: 35 & 0.1284 & 0.0532 \\ 11654 & 14: 40: 37 & 0.0518 & 0.1680 \\ 11656 & 14: 40: 39 & 0.0374 & 0.2194 \\ 11658 & 14: 40: 41 & 0.1130 & 0.1567 \\ 11660 & 14: 40: 43 & 0.1005 & 0.2544 \\ 11662 & 14: 40: 45 & 0.0767 & 0.1021 \\ 11664 & 14: 40: 47 & 0.0916 & 0.1851 \\ 11666 & 14: 40: 49 & 0.0968 & 0.1341 \\ 11668 & 14: 40: 51 & 0.0344 & 0.3428 \\ 11670 & 14: 40: 53 & 0.0510 & 0.1298 \\ 11672 & 14: 40: 55 & 0.0357 & 0.1308 \\ 11674 & 14: 40: 57 & 0.1396 & 0.1388 \\ 11676 & 14: 40: 59 & 0.0534 & 0.3271 \\ 11678 & 14: 41: 01 & 0.0519 & 0.2065 \\ 11680 & 14: 41: 03 & 0.0639 & 0.1243 \\ 11682 & 14: 41: 05 & 0.0609 & 0.1319 \\ 11684 & 14: 41: 07 & 0.0316 & 0.1557 \\ 11686 & 14: 41: 09 & 0.0525 & 0.1857 \\ 11688 & 14: 41: 11 & 0.0495 & 0.1908 \\ 11690 & 14: 41: 13 & 0.1090 & 0.1013 \\ 11692 & 14: 41: 15 & 0.0556 & 0.1644 \\ 11694 & 14: 41: 17 & 0.0530 & 0.1571 \\ 11696 & 14: 41: 19 & 0.0937 & 0.2041 \\ 11698 & 14: 41: 21 & 0.0642 & 0.2777 \\ 11700 & 14: 41: 23 & 0.0851 & 0.2476 \\ 11702 & 14: 41: 25 & 0.1288 & 0.2766 \\ 11704 & 14: 41: 27 & 0.0348 & 0.1717 \\ 11706 & 14: 41: 29 & 0.0482 & 0.1496 \\ 11708 & 14: 41: 31 & 0.1041 & 0.1093 \\ 11710 & 14: 41: 33 & 0.0114 & 0.3541 \\ 11712 & 14: 41: 35 & 0.0196 & 0.1707 \\ 11714 & 14: 41: 37 & 0.0467 & 0.1088 \\ 11716 & 14: 41: 39 & 0.0301 & 0.2702 \\ 11718 & 14: 41: 41 & 0.0519 & 0.2405 \\ 11720 & 14: 41: 43 & 0.0619 & 0.0874 \\ 11722 & 14: 41: 45 & 0.1272 & 0.2863 \\ 11724 & 14: 41: 47 & 0.1308 & 0.0876 \\ 11726 & 14: 41: 49 & 0.0723 & 0.1510 \\ 11728 & 14: 41: 51 & 0.0453 & 0.2007 \\ 11730 & 14: 41: 53 & 0.1541 & 0.0463 \\ 11732 & 14: 41: 55 & 0.0427 & 0.1519 \\ 11734 & 14: 41: 57 & 0.1302 & 0.1839 \\ 11736 & 14: 41: 59 & 0.0393 & 0.1323 \\ 11738 & 14: 42: 01 & 0.0321 & 0.0653 \\ 11740 & 14: 42: 03 & 0.0266 & 0.3341 \\ 11742 & 14: 42: 05 & 0.0252 & 0.1204 \\ 11744 & 14: 42: 07 & 0.0339 & 0.0511 \\ 11746 & 14: 42: 09 & 0.0667 & 0.0219 \\ 11748 & 14: 42: 11 & 0.0580 & 0.0702 \\ & & & \end{array}$




$\begin{array}{llll}11750 & 14: 42: 13 & 0.0231 & 0.0751 \\ 11752 & 14: 42: 15 & 0.0209 & 0.0586 \\ 11754 & 14: 42: 17 & 0.0817 & 0.1138 \\ 11756 & 14: 42: 19 & 0.0901 & 0.1365 \\ 11758 & 14: 42: 21 & 0.0907 & 0.5239 \\ 11760 & 14: 42: 23 & 0.0525 & 0.1663 \\ 11762 & 14: 42: 25 & 0.0312 & 0.1242 \\ 11764 & 14: 42: 27 & 0.1329 & 0.2091 \\ 11766 & 14: 42: 29 & 0.0257 & 0.1263 \\ 11768 & 14: 42: 31 & 0.0675 & 0.1180 \\ 11770 & 14: 42: 33 & 0.0306 & 0.1217 \\ 11772 & 14: 42: 35 & 0.0233 & 0.0450 \\ 11774 & 14: 42: 37 & 0.0330 & 0.0610 \\ 11776 & 14: 42: 39 & 0.0898 & 0.0784 \\ 11778 & 14: 42: 41 & 0.0550 & 0.1311 \\ 11780 & 14: 42: 43 & 0.0306 & 0.0504 \\ 11782 & 14: 42: 45 & 0.0445 & 0.1212 \\ 11784 & 14: 42: 47 & 0.0195 & 0.1320 \\ 11786 & 14: 42: 49 & 0.0747 & 0.1247 \\ 11788 & 14: 42: 51 & 0.0987 & 0.1311 \\ 11790 & 14: 42: 53 & 0.0557 & 0.0627 \\ 11792 & 14: 42: 55 & 0.0573 & 0.0938 \\ 11794 & 14: 42: 57 & 0.0846 & 0.2012 \\ 11796 & 14: 42: 59 & 0.0917 & 0.1935 \\ 11798 & 14: 43: 01 & 0.0465 & 0.0898 \\ 11800 & 14: 43: 03 & 0.0131 & 0.0964 \\ 11802 & 14: 43: 05 & 0.0450 & 0.0397 \\ 11804 & 14: 43: 07 & 0.0239 & 0.1868 \\ 11806 & 14: 43: 09 & 0.0234 & 0.1231 \\ 11808 & 14: 43: 11 & 0.0185 & 0.0744 \\ 11810 & 14: 43: 13 & 0.1142 & 0.2471 \\ 11812 & 14: 43: 15 & 0.0481 & 0.2410 \\ 11814 & 14: 43: 17 & 0.0170 & 0.1488 \\ 11816 & 14: 43: 19 & 0.0695 & 0.2765 \\ 11818 & 14: 43: 21 & 0.0532 & 0.1338 \\ 11820 & 14: 43: 23 & 0.0421 & 0.0798 \\ 11822 & 14: 43: 25 & 0.0302 & 0.1248 \\ 11824 & 14: 43: 27 & 0.1067 & 0.1480 \\ 11826 & 14: 43: 29 & 0.0242 & 0.1267 \\ 11828 & 14: 43: 31 & 0.0150 & 0.1078 \\ 11830 & 14: 43: 33 & 0.0255 & 0.1015 \\ 11832 & 14: 43: 35 & 0.0079 & 0.1812 \\ 11834 & 14: 43: 37 & 0.0823 & 0.1708 \\ 11836 & 14: 43: 39 & 0.0915 & 0.0977 \\ 11838 & 14: 43: 41 & 0.0808 & 0.0949 \\ 11840 & 14: 43: 43 & 0.0463 & 0.2541 \\ 11842 & 14: 43: 45 & 0.0235 & 0.1325 \\ 11844 & 14: 43: 47 & 0.0966 & 0.1329 \\ 11846 & 14: 43: 49 & 0.0177 & 0.1006 \\ 11848 & 14: 43: 51 & 0.0592 & 0.3509 \\ 11850 & 14: 43: 53 & 0.0528 & 0.2379 \\ 11852 & 14: 43: 55 & 0.0144 & 0.0969\end{array}$




$\begin{array}{llll}11854 & 14: 43: 57 & 0.1219 & 0.1800 \\ 11856 & 14: 43: 59 & 0.0657 & 0.1162 \\ 11858 & 14: 44: 01 & 0.0266 & 0.1571 \\ 11860 & 14: 44: 03 & 0.0761 & 0.1103 \\ 11862 & 14: 44: 05 & 0.0533 & 0.1181 \\ 11864 & 14: 44: 07 & 0.0628 & 0.1222 \\ 11866 & 14: 44: 09 & 0.0638 & 0.1165 \\ 11868 & 14: 44: 11 & 0.1774 & 0.1172 \\ 11870 & 14: 44: 13 & 0.0513 & 0.1106 \\ 11872 & 14: 44: 15 & 0.1160 & 0.1500 \\ 11874 & 14: 44: 17 & 0.0752 & 0.1027 \\ 11876 & 14: 44: 19 & 0.0406 & 0.2710 \\ 11878 & 14: 44: 21 & 0.0638 & 0.0590 \\ 11880 & 14: 44: 23 & 0.0458 & 0.2089 \\ 11882 & 14: 44: 25 & 0.0307 & 0.0529 \\ 11884 & 14: 44: 27 & 0.0476 & 0.0686 \\ 11886 & 14: 44: 29 & 0.0447 & 0.1305 \\ 11888 & 14: 44: 31 & 0.0507 & 0.1885 \\ 11890 & 14: 44: 33 & 0.0973 & 0.1252 \\ 11892 & 14: 44: 35 & 0.0888 & 0.0922 \\ 11894 & 14: 44: 37 & 0.0740 & 0.1240 \\ 11896 & 14: 44: 39 & 0.2837 & 0.5764 \\ 11898 & 14: 44: 41 & 0.0445 & 0.3177 \\ 11900 & 14: 44: 43 & 0.0359 & 0.1755 \\ 11902 & 14: 44: 45 & 0.0846 & 0.1692 \\ 11904 & 14: 44: 47 & 0.0916 & 0.1463 \\ 11906 & 14: 44: 49 & 0.0273 & 0.1648 \\ 11908 & 14: 44: 51 & 0.0572 & 0.0851 \\ 11910 & 14: 44: 53 & 0.0561 & 0.0753 \\ 11912 & 14: 44: 55 & 0.0814 & 0.3870 \\ 11914 & 14: 44: 57 & 0.0900 & 0.1181 \\ 11916 & 14: 44: 59 & 0.1517 & 0.4321 \\ 11918 & 14: 45: 01 & 0.0323 & 0.1959 \\ 11920 & 14: 45: 03 & 0.1274 & 0.1427 \\ 11922 & 14: 45: 05 & 0.0672 & 0.1319 \\ 11924 & 14: 45: 07 & 0.0185 & 0.0904 \\ 11926 & 14: 45: 09 & 0.1604 & 0.2107 \\ 11928 & 14: 45: 11 & 0.0363 & 0.1070 \\ 11930 & 14: 45: 13 & 0.0271 & 0.3323 \\ 11932 & 14: 45: 15 & 0.1677 & 0.0979 \\ 11934 & 14: 45: 17 & 0.0554 & 0.1006 \\ 11936 & 14: 45: 19 & 0.0775 & 0.2746 \\ 11938 & 14: 45: 21 & 0.0408 & 0.0805 \\ 11940 & 14: 45: 23 & 0.0742 & 0.1122 \\ 11942 & 14: 45: 25 & 0.1432 & 0.2824 \\ 11944 & 14: 45: 27 & 0.1055 & 0.2882 \\ 11946 & 14: 45: 29 & 0.1250 & 0.1595 \\ 11948 & 14: 45: 31 & 0.2291 & 0.4469 \\ 11950 & 14: 45: 33 & 0.0560 & 0.0911 \\ 11952 & 14: 45: 35 & 0.0659 & 0.1564 \\ 11954 & 14: 45: 37 & 0.0921 & 0.2784 \\ 11956 & 14: 45: 39 & 0.0616 & 0.0888\end{array}$




$\begin{array}{llll}11958 & 14: 45: 41 & 0.0477 & 0.1031 \\ 11960 & 14: 45: 43 & 0.0171 & 0.2025 \\ 11962 & 14: 45: 45 & 0.0719 & 0.2370 \\ 11964 & 14: 45: 47 & 0.0832 & 0.1609 \\ 11966 & 14: 45: 49 & 0.0372 & 0.1881 \\ 11968 & 14: 45: 51 & 0.0603 & 0.2844 \\ 11970 & 14: 45: 53 & 0.1153 & 0.1153 \\ 11972 & 14: 45: 55 & 0.0544 & 0.1982 \\ 11974 & 14: 45: 57 & 0.0398 & 0.0497 \\ 11976 & 14: 45: 59 & 0.0528 & 0.0670 \\ 11978 & 14: 46: 01 & 0.0965 & 0.1372 \\ 11980 & 14: 46: 03 & 0.0681 & 0.0803 \\ 11982 & 14: 46: 05 & 0.1351 & 0.1147 \\ 11984 & 14: 46: 07 & 0.0502 & 0.0686 \\ 11986 & 14: 46: 09 & 0.0461 & 0.2746 \\ 11988 & 14: 46: 11 & 0.0892 & 0.0830 \\ 11990 & 14: 46: 13 & 0.0746 & 0.0578 \\ 11992 & 14: 46: 15 & 0.0519 & 0.1224 \\ 11994 & 14: 46: 17 & 0.0749 & 0.1602 \\ 11996 & 14: 46: 19 & 0.0885 & 0.2743 \\ 11998 & 14: 46: 21 & 0.0367 & 0.0532 \\ 12000 & 14: 46: 23 & 0.1557 & 0.1901 \\ 12002 & 14: 46: 25 & 0.0425 & 0.0857 \\ 12004 & 14: 46: 27 & 0.0722 & 0.0767 \\ 12006 & 14: 46: 29 & 0.0458 & 0.1404 \\ 12008 & 14: 46: 31 & 0.0683 & 0.1958 \\ 12010 & 14: 46: 33 & 0.1027 & 0.5928 \\ 12012 & 14: 46: 35 & 0.0558 & 0.0526 \\ 12014 & 14: 46: 37 & 0.0333 & 0.1287 \\ 12016 & 14: 46: 39 & 0.0394 & 0.2911 \\ 12018 & 14: 46: 41 & 0.0550 & 0.1022 \\ 12020 & 14: 46: 43 & 0.0554 & 0.0740 \\ 12022 & 14: 46: 45 & 0.1430 & 0.2980 \\ 12024 & 14: 46: 47 & 0.1068 & 0.1666 \\ 12026 & 14: 46: 49 & 0.0667 & 0.1544 \\ 12028 & 14: 46: 51 & 0.1050 & 0.0396 \\ 12030 & 14: 46: 53 & 0.1357 & 0.0564 \\ 12032 & 14: 46: 55 & 0.1238 & 0.0597 \\ 12034 & 14: 46: 57 & 0.1059 & 0.2370 \\ 12036 & 14: 46: 59 & 0.0253 & 0.0489 \\ 12038 & 14: 47: 01 & 0.0520 & 0.1187 \\ 12040 & 14: 47: 03 & 0.0985 & 0.1242 \\ 12042 & 14: 47: 05 & 0.0552 & 0.0465 \\ 12044 & 14: 47: 07 & 0.0396 & 0.1461 \\ 12046 & 14: 47: 09 & 0.0598 & 0.1571 \\ 12048 & 14: 47: 11 & 0.0294 & 0.0927 \\ 12050 & 14: 47: 13 & 0.0454 & 0.1192 \\ 12052 & 14: 47: 15 & 0.3543 & 0.1705 \\ 12054 & 14: 47: 17 & 0.0501 & 0.1119 \\ 12056 & 14: 47: 19 & 0.0302 & 0.1244 \\ 12058 & 14: 47: 21 & 0.0301 & 0.0539 \\ 12060 & 14: 47: 23 & 0.0275 & 0.2836\end{array}$




$\begin{array}{llll}12062 & 14: 47: 25 & 0.0256 & 0.1197 \\ 12064 & 14: 47: 27 & 0.0340 & 0.0976 \\ 12066 & 14: 47: 29 & 0.0949 & 0.1723 \\ 12068 & 14: 47: 31 & 0.1551 & 0.2665 \\ 12070 & 14: 47: 33 & 0.0458 & 0.1373 \\ 12072 & 14: 47: 35 & 0.0550 & 0.3663 \\ 12074 & 14: 47: 37 & 0.0421 & 0.0787 \\ 12076 & 14: 47: 39 & 0.0472 & 0.2272 \\ 12078 & 14: 47: 41 & 0.0167 & 0.1692 \\ 12080 & 14: 47: 43 & 0.1055 & 0.3446 \\ 12082 & 14: 47: 45 & 0.1479 & 0.1638 \\ 12084 & 14: 47: 47 & 0.0838 & 0.2041 \\ 12086 & 14: 47: 49 & 0.0405 & 0.1415 \\ 12088 & 14: 47: 51 & 0.1119 & 0.2229 \\ 12090 & 14: 47: 53 & 0.0531 & 0.2045 \\ 12092 & 14: 47: 55 & 0.0389 & 0.1107 \\ 12094 & 14: 47: 57 & 0.0300 & 0.1087 \\ 12096 & 14: 47: 59 & 0.0886 & 0.1314 \\ 12098 & 14: 48: 01 & 0.0169 & 0.1321 \\ 12100 & 14: 48: 03 & 0.1010 & 0.2947 \\ 12102 & 14: 48: 05 & 0.0570 & 0.2711 \\ 12104 & 14: 48: 07 & 0.0471 & 0.1618 \\ 12106 & 14: 48: 09 & 0.0262 & 0.1083 \\ 12108 & 14: 48: 11 & 0.0631 & 0.2064 \\ 12110 & 14: 48: 13 & 0.0448 & 0.1229 \\ 12112 & 14: 48: 15 & 0.0382 & 0.1389 \\ 12114 & 14: 48: 17 & 0.0122 & 0.1108 \\ 12116 & 14: 48: 19 & 0.0255 & 0.3356 \\ 12118 & 14: 48: 21 & 0.0568 & 0.1139 \\ 12120 & 14: 48: 23 & 0.0400 & 0.1904 \\ 12122 & 14: 48: 25 & 0.0511 & 0.2013 \\ 12124 & 14: 48: 27 & 0.0299 & 0.0920 \\ 12126 & 14: 48: 29 & 0.1498 & 0.2816 \\ 12128 & 14: 48: 31 & 0.0418 & 0.2372 \\ 12130 & 14: 48: 33 & 0.0125 & 0.2426 \\ 12132 & 14: 48: 35 & 0.1035 & 0.2269 \\ 12134 & 14: 48: 37 & 0.0778 & 0.2260 \\ 12136 & 14: 48: 39 & 0.0369 & 0.2104 \\ 12138 & 14: 48: 41 & 0.0405 & 0.1547 \\ 12140 & 14: 48: 43 & 0.0731 & 0.0398 \\ 12142 & 14: 48: 45 & 0.0844 & 0.2295 \\ 12144 & 14: 48: 47 & 0.0831 & 0.0880 \\ 12146 & 14: 48: 49 & 0.0822 & 0.1133 \\ 12148 & 14: 48: 51 & 0.0932 & 0.0888 \\ 12150 & 14: 48: 53 & 0.1000 & 0.2360 \\ 12152 & 14: 48: 55 & 0.0856 & 0.0864 \\ 12154 & 14: 48: 57 & 0.0343 & 0.1574 \\ 12156 & 14: 48: 59 & 0.1206 & 0.2451 \\ 12158 & 14: 49: 01 & 0.0436 & 0.2850 \\ 12160 & 14: 49: 03 & 0.0109 & 0.0959 \\ 12162 & 14: 49: 05 & 0.0560 & 0.2207 \\ 12164 & 14: 49: 07 & 0.0327 & 0.0867\end{array}$




$\begin{array}{llll}12166 & 14: 49: 09 & 0.0351 & 0.0884 \\ 12168 & 14: 49: 11 & 0.1416 & 0.0900 \\ 12170 & 14: 49: 13 & 0.1397 & 0.0474 \\ 12172 & 14: 49: 15 & 0.1374 & 0.1345 \\ 12174 & 14: 49: 17 & 0.0843 & 0.0941 \\ 12176 & 14: 49: 19 & 0.0222 & 0.1885 \\ 12178 & 14: 49: 21 & 0.0372 & 0.0640 \\ 12180 & 14: 49: 23 & 0.0832 & 0.2920 \\ 12182 & 14: 49: 25 & 0.0335 & 0.0780 \\ 12184 & 14: 49: 27 & 0.0463 & 0.0589 \\ 12186 & 14: 49: 29 & 0.0254 & 0.0528 \\ 12188 & 14: 49: 31 & 0.0678 & 0.2247 \\ 12190 & 14: 49: 33 & 0.0586 & 0.0603 \\ 12192 & 14: 49: 35 & 0.0570 & 0.1304 \\ 12194 & 14: 49: 37 & 0.0112 & 0.3310 \\ 12196 & 14: 49: 39 & 0.0153 & 0.2375 \\ 12198 & 14: 49: 41 & 0.0589 & 0.2166 \\ 12200 & 14: 49: 43 & 0.1624 & 0.2029 \\ 12202 & 14: 49: 45 & 0.0649 & 0.2866 \\ 12204 & 14: 49: 47 & 0.0767 & 0.1382 \\ 12206 & 14: 49: 49 & 0.0423 & 0.2129 \\ 12208 & 14: 49: 51 & 0.0484 & 0.0560 \\ 12210 & 14: 49: 53 & 0.0170 & 0.1563 \\ 12212 & 14: 49: 55 & 0.0466 & 0.1823 \\ 12214 & 14: 49: 57 & 0.0305 & 0.1481 \\ 12216 & 14: 49: 59 & 0.0050 & 0.0784 \\ 12218 & 14: 50: 01 & 0.0817 & 0.2807 \\ 12220 & 14: 50: 03 & 0.0584 & 0.0918 \\ 12222 & 14: 50: 05 & 0.0431 & 0.2143 \\ 12224 & 14: 50: 07 & 0.0219 & 0.1695 \\ 12226 & 14: 50: 09 & 0.0888 & 0.4062 \\ 12228 & 14: 50: 11 & 0.0578 & 0.0986 \\ 12230 & 14: 50: 13 & 0.1352 & 0.0821 \\ 12232 & 14: 50: 15 & 0.0304 & 0.1101 \\ 12234 & 14: 50: 17 & 0.0684 & 0.0979 \\ 12236 & 14: 50: 19 & 0.0870 & 0.2363 \\ 12238 & 14: 50: 21 & 0.0378 & 0.1082 \\ 12240 & 14: 50: 23 & 0.0223 & 0.1223 \\ 12242 & 14: 50: 25 & 0.0914 & 0.3272 \\ 12244 & 14: 50: 27 & 0.1363 & 0.1648 \\ 12246 & 14: 50: 29 & 0.0400 & 0.2199 \\ 12248 & 14: 50: 31 & 0.0231 & 0.1092 \\ 12250 & 14: 50: 33 & 0.0292 & 0.4249 \\ 12252 & 14: 50: 35 & 0.0523 & 0.2156 \\ 12254 & 14: 50: 37 & 0.0526 & 0.3882 \\ 12256 & 14: 50: 39 & 0.0290 & 0.2070 \\ 12258 & 14: 50: 41 & 0.0969 & 0.2063 \\ 12260 & 14: 50: 43 & 0.0445 & 0.1544 \\ 12262 & 14: 50: 45 & 0.0204 & 0.0920 \\ 12264 & 14: 50: 47 & 0.0928 & 0.2689 \\ 12266 & 14: 50: 49 & 0.0452 & 0.1656 \\ 12268 & 14: 50: 51 & 0.1488 & 0.2933\end{array}$


Appendix B - Laser Data Test 1 NIST102505_1.xls

$\begin{array}{llll}12270 & 14: 50: 53 & 0.0970 & 0.1562 \\ 12272 & 14: 50: 55 & 0.0366 & 0.0936 \\ 12274 & 14: 50: 57 & 0.0615 & 0.1703 \\ 12276 & 14: 50: 59 & 0.1410 & 0.1991 \\ 12278 & 14: 51: 01 & 0.0804 & 0.1090 \\ 12280 & 14: 51: 03 & 0.0462 & 0.2062 \\ 12282 & 14: 51: 05 & 0.0173 & 0.0796 \\ 12284 & 14: 51: 07 & 0.0112 & 0.1163 \\ 12286 & 14: 51: 09 & 0.2286 & 0.3209 \\ 12288 & 14: 51: 11 & 0.0465 & 0.0870 \\ 12290 & 14: 51: 13 & 0.0692 & 0.1773 \\ 12292 & 14: 51: 15 & 0.0154 & 0.0579 \\ 12294 & 14: 51: 17 & 0.1685 & 0.1908 \\ 12296 & 14: 51: 19 & 0.1107 & 0.1833 \\ 12298 & 14: 51: 21 & 0.1062 & 0.1790 \\ 12300 & 14: 51: 23 & 0.0474 & 0.2227 \\ 12302 & 14: 51: 25 & 0.0492 & 0.0843 \\ 12304 & 14: 51: 27 & 0.0646 & 0.2723 \\ 12306 & 14: 51: 29 & 0.0181 & 0.1407 \\ 12308 & 14: 51: 31 & 0.0307 & 0.0630 \\ 12310 & 14: 51: 33 & 0.0393 & 0.1332 \\ 12312 & 14: 51: 35 & 0.0428 & 0.0791 \\ 12314 & 14: 51: 37 & 0.0591 & 0.2525 \\ 12316 & 14: 51: 39 & 0.0977 & 0.4569 \\ 12318 & 14: 51: 41 & 0.0303 & 0.0881 \\ 12320 & 14: 51: 43 & 0.0644 & 0.2339 \\ 12322 & 14: 51: 45 & 0.0461 & 0.1868 \\ 12324 & 14: 51: 47 & 0.1645 & 0.3029 \\ 12326 & 14: 51: 49 & 0.0965 & 0.1541 \\ 12328 & 14: 51: 51 & 0.0242 & 0.1422 \\ 12330 & 14: 51: 53 & 0.0977 & 0.2251 \\ 12332 & 14: 51: 55 & 0.0415 & 0.1406 \\ 12334 & 14: 51: 57 & 0.1025 & 0.2013 \\ 12336 & 14: 51: 59 & 0.0327 & 0.1324 \\ 12338 & 14: 52: 01 & 0.0219 & 0.1831 \\ 12340 & 14: 52: 03 & 0.1107 & 0.1020 \\ 12342 & 14: 52: 05 & 0.0407 & 0.2904 \\ 12344 & 14: 52: 07 & 0.0288 & 0.1458 \\ 12346 & 14: 52: 09 & 0.0862 & 0.1358 \\ 12348 & 14: 52: 11 & 0.0580 & 0.2793 \\ 12350 & 14: 52: 13 & 0.0253 & 0.2813 \\ 12352 & 14: 52: 15 & 0.0656 & 0.2535 \\ 12354 & 14: 52: 17 & 0.0558 & 0.1341 \\ 12356 & 14: 52: 19 & 0.0264 & 0.1112 \\ 12358 & 14: 52: 21 & 0.0405 & 0.2474 \\ 12360 & 14: 52: 23 & 0.2318 & 0.2506 \\ 12362 & 14: 52: 25 & 0.1348 & 0.0333 \\ 12364 & 14: 52: 27 & 0.1570 & 0.1932 \\ 12366 & 14: 52: 29 & 0.0846 & 0.0363 \\ 12368 & 14: 52: 31 & 0.0750 & 0.1114 \\ 12370 & 14: 52: 33 & 0.0683 & 0.1365 \\ 12372 & 14: 52: 35 & 0.0755 & 0.1912\end{array}$

B - 119 


$\begin{array}{llll}12374 & 14: 52: 37 & 0.0481 & 0.0383 \\ 12376 & 14: 52: 39 & 0.0250 & 0.2683 \\ 12378 & 14: 52: 41 & 0.0422 & 0.1582 \\ 12380 & 14: 52: 43 & 0.0523 & 0.1908 \\ 12382 & 14: 52: 45 & 0.0362 & 0.1342 \\ 12384 & 14: 52: 47 & 0.1039 & 0.0731 \\ 12386 & 14: 52: 49 & 0.0256 & 0.1109 \\ 12388 & 14: 52: 51 & 0.0211 & 0.3608 \\ 12390 & 14: 52: 53 & 0.0814 & 0.2182 \\ 12392 & 14: 52: 55 & 0.1028 & 0.1491 \\ 12394 & 14: 52: 57 & 0.0558 & 0.1498 \\ 12396 & 14: 52: 59 & 0.0484 & 0.4123 \\ 12398 & 14: 53: 01 & 0.0623 & 0.1950 \\ 12400 & 14: 53: 03 & 0.0770 & 0.0897 \\ 12402 & 14: 53: 05 & 0.0846 & 0.1680 \\ 12404 & 14: 53: 07 & 0.0914 & 0.1182 \\ 12406 & 14: 53: 09 & 0.1402 & 0.1235 \\ 12408 & 14: 53: 11 & 0.1119 & 0.2405 \\ 12410 & 14: 53: 13 & 0.1236 & 0.1359 \\ 12412 & 14: 53: 15 & 0.0678 & 0.1041 \\ 12414 & 14: 53: 17 & 0.0194 & 0.1209 \\ 12416 & 14: 53: 19 & 0.0575 & 0.2698 \\ 12418 & 14: 53: 21 & 0.0159 & 0.1662 \\ 12420 & 14: 53: 23 & 0.0444 & 0.2126 \\ 12422 & 14: 53: 25 & 0.0268 & 0.1816 \\ 12424 & 14: 53: 27 & 0.0348 & 0.2332 \\ 12426 & 14: 53: 29 & 0.0355 & 0.0835 \\ 12428 & 14: 53: 31 & 0.0323 & 0.1550 \\ 12430 & 14: 53: 33 & 0.0610 & 0.1066 \\ 12432 & 14: 53: 35 & 0.0501 & 0.1758 \\ 12434 & 14: 53: 37 & 0.0534 & 0.3626 \\ 12436 & 14: 53: 39 & 0.0731 & 0.1461 \\ 12438 & 14: 53: 41 & 0.0446 & 0.1338 \\ 12440 & 14: 53: 43 & 0.0540 & 0.2012 \\ 12442 & 14: 53: 45 & 0.0817 & 0.1851 \\ 12444 & 14: 53: 47 & 0.0405 & 0.2295 \\ 12446 & 14: 53: 49 & 0.0326 & 0.1555 \\ 12448 & 14: 53: 51 & 0.1036 & 0.1375 \\ 12450 & 14: 53: 53 & 0.0166 & 0.3086 \\ 12452 & 14: 53: 55 & 0.0106 & 0.0826 \\ 12454 & 14: 53: 57 & 0.0605 & 0.1586 \\ 12456 & 14: 53: 59 & 0.1237 & 0.0226 \\ 12458 & 14: 54: 01 & 0.0528 & 0.1064 \\ 12460 & 14: 54: 03 & 0.0411 & 0.2494 \\ 12462 & 14: 54: 05 & 0.1196 & 0.0444 \\ 12464 & 14: 54: 07 & 0.0345 & 0.2989 \\ 12466 & 14: 54: 09 & 0.0259 & 0.1119 \\ 12468 & 14: 54: 11 & 0.0256 & 0.0603 \\ 12470 & 14: 54: 13 & 0.0039 & 0.1056 \\ 12472 & 14: 54: 15 & 0.0764 & 0.2682 \\ 12474 & 14: 54: 17 & 0.0192 & 0.1183 \\ 12476 & 14: 54: 19 & 0.0510 & 0.2623\end{array}$




$\begin{array}{llll}12478 & 14: 54: 21 & 0.0442 & 0.1752 \\ 12480 & 14: 54: 23 & 0.0815 & 0.1929 \\ 12482 & 14: 54: 25 & 0.0370 & 0.1871 \\ 12484 & 14: 54: 27 & 0.0936 & 0.2292 \\ 12486 & 14: 54: 29 & 0.1336 & 0.2330 \\ 12488 & 14: 54: 31 & 0.0308 & 0.2366 \\ 12490 & 14: 54: 33 & 0.2854 & 0.3187 \\ 12492 & 14: 54: 35 & 0.1016 & 0.1068 \\ 12494 & 14: 54: 37 & 0.1098 & 0.0985 \\ 12496 & 14: 54: 39 & 0.0656 & 0.1021 \\ 12498 & 14: 54: 41 & 0.1224 & 0.1715 \\ 12500 & 14: 54: 43 & 0.0353 & 0.2591 \\ 12502 & 14: 54: 45 & 0.0870 & 0.1362 \\ 12504 & 14: 54: 47 & 0.0637 & 0.3297 \\ 12506 & 14: 54: 49 & 0.0472 & 0.0854 \\ 12508 & 14: 54: 51 & 0.0807 & 0.3453 \\ 12510 & 14: 54: 53 & 0.0450 & 0.1178 \\ 12512 & 14: 54: 55 & 0.1352 & 0.0713 \\ 12514 & 14: 54: 57 & 0.0579 & 0.1388 \\ 12516 & 14: 54: 59 & 0.0697 & 0.0941 \\ 12518 & 14: 55: 01 & 0.0384 & 0.1503 \\ 12520 & 14: 55: 03 & 0.0165 & 0.1100 \\ 12522 & 14: 55: 05 & 0.0835 & 0.1384 \\ 12524 & 14: 55: 07 & 0.0432 & 0.1058 \\ 12526 & 14: 55: 09 & 0.0169 & 0.1266 \\ 12528 & 14: 55: 11 & 0.1385 & 0.0944 \\ 12530 & 14: 55: 13 & 0.0260 & 0.1244 \\ 12532 & 14: 55: 15 & 0.0928 & 0.1360 \\ 12534 & 14: 55: 17 & 0.0569 & 0.0825 \\ 12536 & 14: 55: 19 & 0.1049 & 0.2203 \\ 12538 & 14: 55: 21 & 0.0596 & 0.0932 \\ 12540 & 14: 55: 23 & 0.0329 & 0.1431 \\ 12542 & 14: 55: 25 & 0.0160 & 0.0676 \\ 12544 & 14: 55: 27 & 0.0547 & 0.1880 \\ 12546 & 14: 55: 29 & 0.1264 & 0.1704 \\ 12548 & 14: 55: 31 & 0.0874 & 0.0760 \\ 12550 & 14: 55: 33 & 0.0425 & 0.0834 \\ 12552 & 14: 55: 35 & 0.0664 & 0.2384 \\ 12554 & 14: 55: 37 & 0.0588 & 0.1925 \\ 12556 & 14: 55: 39 & 0.0561 & 0.1854 \\ 12558 & 14: 55: 41 & 0.0253 & 0.1896 \\ 12560 & 14: 55: 43 & 0.0730 & 0.0753 \\ 12562 & 14: 55: 45 & 0.1196 & 0.1397 \\ 12564 & 14: 55: 47 & 0.0491 & 0.3166 \\ 12566 & 14: 55: 49 & 0.0431 & 0.1367 \\ 12568 & 14: 55: 51 & 0.0686 & 0.1201 \\ 12570 & 14: 55: 53 & 0.0464 & 0.1721 \\ 12572 & 14: 55: 55 & 0.0425 & 0.1634 \\ 12574 & 14: 55: 57 & 0.0397 & 0.0537 \\ 12576 & 14: 55: 59 & 0.0179 & 0.1348 \\ 12578 & 14: 56: 01 & 0.1739 & 0.1307 \\ 12580 & 14: 56: 03 & 0.0657 & 0.0629\end{array}$




$\begin{array}{llll}12582 & 14: 56: 05 & 0.0173 & 0.1660 \\ 12584 & 14: 56: 07 & 0.0423 & 0.1709 \\ 12586 & 14: 56: 09 & 0.0334 & 0.1490 \\ 12588 & 14: 56: 11 & 0.0549 & 0.0692 \\ 12590 & 14: 56: 13 & 0.0512 & 0.2285 \\ 12592 & 14: 56: 15 & 0.0167 & 0.1163 \\ 12594 & 14: 56: 17 & 0.0469 & 0.1669 \\ 12596 & 14: 56: 19 & 0.0476 & 0.1423 \\ 12598 & 14: 56: 21 & 0.0381 & 0.1466 \\ 12600 & 14: 56: 23 & 0.0626 & 0.0704 \\ 12602 & 14: 56: 25 & 0.1009 & 0.1208 \\ 12604 & 14: 56: 27 & 0.0413 & 0.1092 \\ 12606 & 14: 56: 29 & 0.0719 & 0.0534 \\ 12608 & 14: 56: 31 & 0.0511 & 0.0659 \\ 12610 & 14: 56: 33 & 0.0461 & 0.0634 \\ 12612 & 14: 56: 35 & 0.0769 & 0.0983 \\ 12614 & 14: 56: 37 & 0.0270 & 0.0878 \\ 12616 & 14: 56: 39 & 0.0414 & 0.1366 \\ 12618 & 14: 56: 41 & 0.0392 & 0.0633 \\ 12620 & 14: 56: 43 & 0.0595 & 0.2718 \\ 12622 & 14: 56: 45 & 0.0208 & 0.1239 \\ 12624 & 14: 56: 47 & 0.0214 & 0.1035 \\ 12626 & 14: 56: 49 & 0.0283 & 0.0560 \\ 12628 & 14: 56: 51 & 0.0136 & 0.2169 \\ 12630 & 14: 56: 53 & 0.0609 & 0.2899 \\ 12632 & 14: 56: 55 & 0.0521 & 0.0914 \\ 12634 & 14: 56: 57 & 0.0190 & 0.2402 \\ 12636 & 14: 56: 59 & 0.1146 & 0.1559 \\ 12638 & 14: 57: 01 & 0.1566 & 0.2035 \\ 12640 & 14: 57: 03 & 0.1260 & 0.1063 \\ 12642 & 14: 57: 05 & 0.1601 & 0.2002 \\ 12644 & 14: 57: 07 & 0.0688 & 0.0957 \\ 12646 & 14: 57: 09 & 0.0522 & 0.2098 \\ 12648 & 14: 57: 11 & 0.0560 & 0.0874 \\ 12650 & 14: 57: 13 & 0.0503 & 0.2237 \\ 12652 & 14: 57: 15 & 0.0938 & 0.1842 \\ 12654 & 14: 57: 17 & 0.0557 & 0.1724 \\ 12656 & 14: 57: 19 & 0.0350 & 0.2066 \\ 12658 & 14: 57: 21 & 0.0962 & 0.1622 \\ 12660 & 14: 57: 23 & 0.1145 & 0.3007 \\ 12662 & 14: 57: 25 & 0.0430 & 0.1874 \\ 12664 & 14: 57: 27 & 0.0831 & 0.2516 \\ 12666 & 14: 57: 29 & 0.1086 & 0.1356 \\ 12668 & 14: 57: 31 & 0.0941 & 0.1720 \\ 12670 & 14: 57: 33 & 0.0280 & 0.1990 \\ 12672 & 14: 57: 35 & 0.0451 & 0.1142 \\ 12674 & 14: 57: 37 & 0.0309 & 0.2488 \\ 12676 & 14: 57: 39 & 0.0352 & 0.2370 \\ 12678 & 14: 57: 41 & 0.2252 & 0.2571 \\ 12680 & 14: 57: 43 & 0.0737 & 0.3199 \\ 12682 & 14: 57: 45 & 0.1102 & 0.1143 \\ 12684 & 14: 57: 47 & 0.0428 & 0.0887\end{array}$




$\begin{array}{llll}12686 & 14: 57: 49 & 0.1220 & 0.0766 \\ 12688 & 14: 57: 51 & 0.0357 & 0.1907 \\ 12690 & 14: 57: 53 & 0.0586 & 0.0837 \\ 12692 & 14: 57: 55 & 0.0861 & 0.1509 \\ 12694 & 14: 57: 57 & 0.0402 & 0.1093 \\ 12696 & 14: 57: 59 & 0.0731 & 0.0765 \\ 12698 & 14: 58: 01 & 0.1120 & 0.0518 \\ 12700 & 14: 58: 03 & 0.1056 & 0.1455 \\ 12702 & 14: 58: 05 & 0.0260 & 0.2543 \\ 12704 & 14: 58: 07 & 0.0173 & 0.1775 \\ 12706 & 14: 58: 09 & 0.0711 & 0.0963 \\ 12708 & 14: 58: 11 & 0.0527 & 0.3668 \\ 12710 & 14: 58: 13 & 0.0478 & 0.2008 \\ 12712 & 14: 58: 15 & 0.0624 & 0.0750 \\ 12714 & 14: 58: 17 & 0.1006 & 0.2979 \\ 12716 & 14: 58: 19 & 0.0544 & 0.1959 \\ 12718 & 14: 58: 21 & 0.1723 & 0.1762 \\ 12720 & 14: 58: 23 & 0.0151 & 0.1618 \\ 12722 & 14: 58: 25 & 0.0334 & 0.2513 \\ 12724 & 14: 58: 27 & 0.1740 & 0.0933 \\ 12726 & 14: 58: 29 & 0.0426 & 0.1802 \\ 12728 & 14: 58: 31 & 0.0428 & 0.2254 \\ 12730 & 14: 58: 33 & 0.0225 & 0.2000 \\ 12732 & 14: 58: 35 & 0.0901 & 0.1741 \\ 12734 & 14: 58: 37 & 0.0384 & 0.2232 \\ 12736 & 14: 58: 39 & 0.0432 & 0.5588 \\ 12738 & 14: 58: 41 & 0.0350 & 0.1453 \\ 12740 & 14: 58: 43 & 0.0121 & 0.2988 \\ 12742 & 14: 58: 45 & 0.0288 & 0.2788 \\ 12744 & 14: 58: 47 & 0.0669 & 0.3085 \\ 12746 & 14: 58: 49 & 0.0434 & 0.1687 \\ 12748 & 14: 58: 51 & 0.0213 & 0.2171 \\ 12750 & 14: 58: 53 & 0.0158 & 0.1685 \\ 12752 & 14: 58: 55 & 0.0379 & 0.2734 \\ 12754 & 14: 58: 57 & 0.0398 & 0.1354 \\ 12756 & 14: 58: 59 & 0.1018 & 0.1620 \\ 12758 & 14: 59: 01 & 0.1073 & 0.5366 \\ 12760 & 14: 59: 03 & 0.0877 & 0.1732 \\ 12762 & 14: 59: 05 & 0.0745 & 0.3069 \\ 12764 & 14: 59: 07 & 0.1156 & 0.2241 \\ 12766 & 14: 59: 09 & 0.0831 & 0.1504 \\ 12768 & 14: 59: 11 & 0.0358 & 0.1200 \\ 12770 & 14: 59: 13 & 0.0941 & 0.2349 \\ 12772 & 14: 59: 15 & 0.0180 & 0.1191 \\ 12774 & 14: 59: 17 & 0.0831 & 0.2145 \\ 12776 & 14: 59: 19 & 0.0178 & 0.1406 \\ 12778 & 14: 59: 21 & 0.0360 & 0.2940 \\ 12780 & 14: 59: 23 & 0.0241 & 0.0959 \\ 12782 & 14: 59: 25 & 0.0658 & 0.2484 \\ 12784 & 14: 59: 27 & 0.0684 & 0.3957 \\ 12786 & 14: 59: 29 & 0.0233 & 0.1833 \\ 12788 & 14: 59: 31 & 0.0538 & 0.4699\end{array}$


Appendix B - Laser Data Test 1 NIST102505_1.xls

$\begin{array}{llll}12790 & 14: 59: 33 & 0.0636 & 0.3979 \\ 12792 & 14: 59: 35 & 0.0127 & 0.2589 \\ 12794 & 14: 59: 37 & 0.0333 & 0.2457 \\ 12796 & 14: 59: 39 & 0.0268 & 0.1833 \\ 12798 & 14: 59: 41 & 0.0717 & 0.1055 \\ 12800 & 14: 59: 43 & 0.0623 & 0.1327 \\ 12802 & 14: 59: 45 & 0.0483 & 0.1547 \\ 12804 & 14: 59: 47 & 0.0600 & 0.1938 \\ 12806 & 14: 59: 49 & 0.1110 & 0.0881 \\ 12808 & 14: 59: 51 & 0.1093 & 0.1172 \\ 12810 & 14: 59: 53 & 0.0170 & 0.1589 \\ 12812 & 14: 59: 55 & 0.0719 & 0.1308 \\ 12814 & 14: 59: 57 & 0.0392 & 0.0462 \\ 12816 & 14: 59: 59 & 0.0179 & 0.0755 \\ 12818 & 15: 00: 01 & 0.0800 & 0.0730 \\ 12820 & 15: 00: 03 & 0.0680 & 0.1492 \\ 12822 & 15: 00: 05 & 0.0452 & 0.0792 \\ 12824 & 15: 00: 07 & 0.0646 & 0.0799 \\ 12826 & 15: 00: 09 & 0.0188 & 0.0906 \\ 12828 & 15: 00: 11 & 0.1131 & 0.1213 \\ 12830 & 15: 00: 13 & 0.0626 & 0.0312 \\ 12832 & 15: 00: 15 & 0.0712 & 0.0590 \\ 12834 & 15: 00: 17 & 0.0417 & 0.1684 \\ 12836 & 15: 00: 19 & 0.0355 & 0.0902 \\ 12838 & 15: 00: 21 & 0.0509 & 0.0937 \\ 12840 & 15: 00: 23 & 0.2027 & 0.1317 \\ 12842 & 15: 00: 25 & 0.0399 & 0.0973 \\ 12844 & 15: 00: 27 & 0.0158 & 0.0977 \\ 12846 & 15: 00: 29 & 0.0345 & 0.1351 \\ 12848 & 15: 00: 31 & 0.0436 & 0.2128 \\ 12850 & 15: 00: 33 & 0.0731 & 0.1658 \\ 12852 & 15: 00: 35 & 0.0410 & 0.1236 \\ 12854 & 15: 00: 37 & 0.0838 & 0.2123 \\ 12856 & 15: 00: 39 & 0.0700 & 0.1613 \\ 12858 & 15: 00: 41 & 0.1316 & 0.0464 \\ 12860 & 15: 00: 43 & 0.0967 & 0.0696 \\ 12862 & 15: 00: 45 & 0.0938 & 0.0763 \\ 12864 & 15: 00: 47 & 0.0695 & 0.1048 \\ 12866 & 15: 00: 49 & 0.1199 & 0.0891 \\ 12868 & 15: 00: 51 & 0.0568 & 0.1743 \\ 12870 & 15: 00: 53 & 0.0276 & 0.0314 \\ 12872 & 15: 00: 55 & 0.0882 & 0.1502 \\ 12874 & 15: 00: 57 & 0.0115 & 0.2035 \\ 12876 & 15: 00: 59 & 0.0489 & 0.1647 \\ 12878 & 15: 01: 01 & 0.0608 & 0.0853 \\ 12880 & 15: 01: 03 & 0.0461 & 0.1993 \\ 12882 & 15: 01: 05 & 0.0969 & 0.0844 \\ 12884 & 15: 01: 07 & 0.0514 & 0.1625 \\ 12886 & 15: 01: 09 & 0.0404 & 0.1953 \\ 12888 & 15: 01: 11 & 0.0635 & 0.1398 \\ 12890 & 15: 01: 13 & 0.0375 & 0.1202 \\ 12892 & 15: 01: 15 & 0.0770 & 0.0833\end{array}$


Appendix B - Laser Data Test 1 NIST102505_1.xls

$\begin{array}{llll}12894 & 15: 01: 17 & 0.0844 & 0.0608 \\ 12896 & 15: 01: 19 & 0.0377 & 0.0504 \\ 12898 & 15: 01: 21 & 0.0364 & 0.1367 \\ 12900 & 15: 01: 23 & 0.0292 & 0.0679 \\ 12902 & 15: 01: 25 & 0.0237 & 0.1421 \\ 12904 & 15: 01: 27 & 0.0755 & 0.0809 \\ 12906 & 15: 01: 29 & 0.0370 & 0.4337 \\ 12908 & 15: 01: 31 & 0.0259 & 0.2844 \\ 12910 & 15: 01: 33 & 0.0494 & 0.3298 \\ 12912 & 15: 01: 35 & 0.1006 & 0.3474 \\ 12914 & 15: 01: 37 & 0.0231 & 0.1800 \\ 12916 & 15: 01: 39 & 0.0267 & 0.4816 \\ 12918 & 15: 01: 41 & 0.0198 & 0.0985 \\ 12920 & 15: 01: 43 & 0.0367 & 0.1168 \\ 12922 & 15: 01: 45 & 0.0527 & 0.1292 \\ 12924 & 15: 01: 47 & 0.0441 & 0.0937 \\ 12926 & 15: 01: 49 & 0.0587 & 0.1145 \\ 12928 & 15: 01: 51 & 0.0550 & 0.0612 \\ 12930 & 15: 01: 53 & 0.0610 & 0.1406 \\ 12932 & 15: 01: 55 & 0.0464 & 0.1440 \\ 12934 & 15: 01: 57 & 0.0762 & 0.1041 \\ 12936 & 15: 01: 59 & 0.0768 & 0.1976 \\ 12938 & 15: 02: 01 & 0.1254 & 0.2448 \\ 12940 & 15: 02: 03 & 0.0454 & 0.1251 \\ 12942 & 15: 02: 05 & 0.0693 & 0.1747 \\ 12944 & 15: 02: 07 & 0.0340 & 0.1044 \\ 12946 & 15: 02: 09 & 0.0529 & 0.1415 \\ 12948 & 15: 02: 11 & 0.1307 & 0.1767 \\ 12950 & 15: 02: 13 & 0.0835 & 0.3153 \\ 12952 & 15: 02: 15 & 0.1375 & 0.1979 \\ 12954 & 15: 02: 17 & 0.0896 & 0.1476 \\ 12956 & 15: 02: 19 & 0.0576 & 0.1534 \\ 12958 & 15: 02: 21 & 0.0639 & 0.1963 \\ 12960 & 15: 02: 23 & 0.0864 & 0.4322 \\ 12962 & 15: 02: 25 & 0.1079 & 0.2172 \\ 12964 & 15: 02: 27 & 0.0506 & 0.1392 \\ 12966 & 15: 02: 29 & 0.1681 & 0.2281 \\ 12968 & 15: 02: 31 & 0.0201 & 0.0325 \\ 12970 & 15: 02: 33 & 0.0334 & 0.1191 \\ 12972 & 15: 02: 35 & 0.0349 & 0.2181 \\ 12974 & 15: 02: 37 & 0.0659 & 0.1340 \\ 12976 & 15: 02: 39 & 0.0255 & 0.1245 \\ 12978 & 15: 02: 41 & 0.0843 & 0.3571 \\ 12980 & 15: 02: 43 & 0.0375 & 0.1294 \\ 12982 & 15: 02: 45 & 0.0251 & 0.0213 \\ 12984 & 15: 02: 47 & 0.1104 & 0.2074 \\ 12986 & 15: 02: 49 & 0.0257 & 0.1623 \\ 12988 & 15: 02: 51 & 0.0377 & 0.1171 \\ 12990 & 15: 02: 53 & 0.0845 & 0.1733 \\ 12992 & 15: 02: 55 & 0.0318 & 0.1911 \\ 12994 & 15: 02: 57 & 0.0233 & 0.3219 \\ 12996 & 15: 02: 59 & 0.0443 & 0.1599\end{array}$

B - 125 


$\begin{array}{llll}12998 & 15: 03: 01 & 0.0237 & 0.1370 \\ 13000 & 15: 03: 03 & 0.0268 & 0.1316 \\ 13002 & 15: 03: 05 & 0.0238 & 0.1536 \\ 13004 & 15: 03: 07 & 0.0417 & 0.0967 \\ 13006 & 15: 03: 09 & 0.0184 & 0.0797 \\ 13008 & 15: 03: 11 & 0.1067 & 0.1281 \\ 13010 & 15: 03: 13 & 0.0182 & 0.0504 \\ 13012 & 15: 03: 15 & 0.0477 & 0.2254 \\ 13014 & 15: 03: 17 & 0.0177 & 0.0797 \\ 13016 & 15: 03: 19 & 0.0330 & 0.2156 \\ 13018 & 15: 03: 21 & 0.0679 & 0.0480 \\ 13020 & 15: 03: 23 & 0.0627 & 0.1454 \\ 13022 & 15: 03: 25 & 0.0363 & 0.0957 \\ 13024 & 15: 03: 27 & 0.0507 & 0.1117 \\ 13026 & 15: 03: 29 & 0.0344 & 0.0650 \\ 13028 & 15: 03: 31 & 0.0937 & 0.1961 \\ 13030 & 15: 03: 33 & 0.0657 & 0.0550 \\ 13032 & 15: 03: 35 & 0.0684 & 0.1516 \\ 13034 & 15: 03: 37 & 0.0330 & 0.1444 \\ 13036 & 15: 03: 39 & 0.0307 & 0.0579 \\ 13038 & 15: 03: 41 & 0.0513 & 0.0964 \\ 13040 & 15: 03: 43 & 0.0666 & 0.1540 \\ 13042 & 15: 03: 45 & 0.0050 & 0.0886 \\ 13044 & 15: 03: 47 & 0.0293 & 0.0709 \\ 13046 & 15: 03: 49 & 0.0892 & 0.2528 \\ 13048 & 15: 03: 51 & 0.1189 & 0.1830 \\ 13050 & 15: 03: 53 & 0.0147 & 0.1578 \\ 13052 & 15: 03: 55 & 0.0716 & 0.0901 \\ 13054 & 15: 03: 57 & 0.0236 & 0.1211 \\ 13056 & 15: 03: 59 & 0.0282 & 0.1511 \\ 13058 & 15: 04: 01 & 0.0253 & 0.2307 \\ 13060 & 15: 04: 03 & 0.0258 & 0.1735 \\ 13062 & 15: 04: 05 & 0.1175 & 0.1301 \\ 13064 & 15: 04: 07 & 0.0665 & 0.1586 \\ 13066 & 15: 04: 09 & 0.0648 & 0.1850 \\ 13068 & 15: 04: 11 & 0.1104 & 0.1793 \\ 13070 & 15: 04: 13 & 0.0467 & 0.0957 \\ 13072 & 15: 04: 15 & 0.0796 & 0.1264 \\ 13074 & 15: 04: 17 & 0.2261 & 0.0663 \\ 13076 & 15: 04: 19 & 0.0598 & 0.0662 \\ 13078 & 15: 04: 21 & 0.0567 & 0.1143 \\ 13080 & 15: 04: 23 & 0.0526 & 0.0599 \\ 13082 & 15: 04: 25 & 0.1576 & 0.0943 \\ 13084 & 15: 04: 27 & 0.0760 & 0.1628 \\ 13086 & 15: 04: 29 & 0.0378 & 0.1449 \\ 13088 & 15: 04: 31 & 0.0814 & 0.0982 \\ 13090 & 15: 04: 33 & 0.0220 & 0.2684 \\ 13092 & 15: 04: 35 & 0.0580 & 0.1962 \\ 13094 & 15: 04: 37 & 0.0319 & 0.5120 \\ 13096 & 15: 04: 39 & 0.0529 & 0.1845 \\ 13098 & 15: 04: 41 & 0.0410 & 0.2568 \\ 13100 & 15: 04: 43 & 0.0198 & 0.2253\end{array}$


Appendix B - Laser Data Test 1 NIST102505_1.xls

$\begin{array}{llll}13102 & 15: 04: 45 & 0.0636 & 0.2207 \\ 13104 & 15: 04: 47 & 0.1646 & 0.1249 \\ 13106 & 15: 04: 49 & 0.1446 & 0.5340 \\ 13108 & 15: 04: 51 & 0.2419 & 0.1756 \\ 13110 & 15: 04: 53 & 0.0210 & 0.0964 \\ 13112 & 15: 04: 55 & 0.1098 & 0.2047 \\ 13114 & 15: 04: 57 & 0.1197 & 0.2619 \\ 13116 & 15: 04: 59 & 0.0517 & 0.1699 \\ 13118 & 15: 05: 01 & 0.1497 & 0.5848 \\ 13120 & 15: 05: 03 & 0.1728 & 0.3839 \\ 13122 & 15: 05: 05 & 0.0501 & 0.1540 \\ 13124 & 15: 05: 07 & 0.1082 & 0.3252 \\ 13126 & 15: 05: 09 & 0.0406 & 0.2402 \\ 13128 & 15: 05: 11 & 0.0636 & 0.2439 \\ 13130 & 15: 05: 13 & 0.0900 & 0.3884 \\ 13132 & 15: 05: 15 & 0.0326 & 0.2998 \\ 13134 & 15: 05: 17 & 0.0654 & 0.1342 \\ 13136 & 15: 05: 19 & 0.0431 & 0.3053 \\ 13138 & 15: 05: 21 & 0.0279 & 0.0937 \\ 13140 & 15: 05: 23 & 0.0655 & 0.2058 \\ 13142 & 15: 05: 25 & 0.0257 & 0.1481 \\ 13144 & 15: 05: 27 & 0.0465 & 0.2149 \\ 13146 & 15: 05: 29 & 0.0232 & 0.0849 \\ 13148 & 15: 05: 31 & 0.0571 & 0.3076 \\ 13150 & 15: 05: 33 & 0.0415 & 0.2884 \\ 13152 & 15: 05: 35 & 0.1176 & 0.0791 \\ 13154 & 15: 05: 37 & 0.0329 & 0.1768 \\ 13156 & 15: 05: 39 & 0.0466 & 0.0977 \\ 13158 & 15: 05: 41 & 0.0747 & 0.1334 \\ 13160 & 15: 05: 43 & 0.0729 & 0.1940 \\ 13162 & 15: 05: 45 & 0.0835 & 0.1698 \\ 13164 & 15: 05: 47 & 0.1045 & 0.1273 \\ 13166 & 15: 05: 49 & 0.0361 & 0.1286 \\ 13168 & 15: 05: 51 & 0.0636 & 0.0709 \\ 13170 & 15: 05: 53 & 0.0129 & 0.1675 \\ 13172 & 15: 05: 55 & 0.0394 & 0.1522 \\ 13174 & 15: 05: 57 & 0.0792 & 0.0979 \\ 13176 & 15: 05: 59 & 0.0328 & 0.1535 \\ 13178 & 15: 06: 01 & 0.1026 & 0.2920 \\ 13180 & 15: 06: 03 & 0.1959 & 0.0797 \\ 13182 & 15: 06: 05 & 0.0250 & 0.2001 \\ 13184 & 15: 06: 07 & 0.0197 & 0.2154 \\ 13186 & 15: 06: 09 & 0.0717 & 0.2606 \\ 13188 & 15: 06: 11 & 0.0531 & 0.2194 \\ 13190 & 15: 06: 13 & 0.0515 & 0.2501 \\ 13192 & 15: 06: 15 & 0.0256 & 0.2014 \\ 13194 & 15: 06: 17 & 0.0693 & 0.2321 \\ 13196 & 15: 06: 19 & 0.0195 & 0.2262 \\ 13198 & 15: 06: 21 & 0.0140 & 0.1181 \\ 13200 & 15: 06: 23 & 0.0431 & 0.1977 \\ 13202 & 15: 06: 25 & 0.0503 & 0.3604 \\ 13204 & 15: 06: 27 & 0.0371 & 0.2266\end{array}$




$\begin{array}{llll}13206 & 15: 06: 29 & 0.0227 & 0.1836 \\ 13208 & 15: 06: 31 & 0.0623 & 0.1201 \\ 13210 & 15: 06: 33 & 0.1303 & 0.1955 \\ 13212 & 15: 06: 35 & 0.0332 & 0.1999 \\ 13214 & 15: 06: 37 & 0.0595 & 0.1558 \\ 13216 & 15: 06: 39 & 0.1130 & 0.3136 \\ 13218 & 15: 06: 41 & 0.0630 & 0.1889 \\ 13220 & 15: 06: 43 & 0.0630 & 0.4702 \\ 13222 & 15: 06: 45 & 0.0621 & 0.1461 \\ 13224 & 15: 06: 47 & 0.0850 & 0.2132 \\ 13226 & 15: 06: 49 & 0.0207 & 0.2840 \\ 13228 & 15: 06: 51 & 0.0707 & 0.1402 \\ 13230 & 15: 06: 53 & 0.0543 & 0.1468 \\ 13232 & 15: 06: 55 & 0.0752 & 0.1471 \\ 13234 & 15: 06: 57 & 0.0436 & 0.2383 \\ 13236 & 15: 06: 59 & 0.0683 & 0.1683 \\ 13238 & 15: 07: 01 & 0.0722 & 0.1290 \\ 13240 & 15: 07: 03 & 0.0386 & 0.1825 \\ 13242 & 15: 07: 05 & 0.0313 & 0.0897 \\ 13244 & 15: 07: 07 & 0.1109 & 0.2175 \\ 13246 & 15: 07: 09 & 0.0384 & 0.1606 \\ 13248 & 15: 07: 11 & 0.0138 & 0.1193 \\ 13250 & 15: 07: 13 & 0.0926 & 0.1267 \\ 13252 & 15: 07: 15 & 0.0889 & 0.1160 \\ 13254 & 15: 07: 17 & 0.0516 & 0.0784 \\ 13256 & 15: 07: 19 & 0.0359 & 0.1754 \\ 13258 & 15: 07: 21 & 0.0227 & 0.1025 \\ 13260 & 15: 07: 23 & 0.0402 & 0.3050 \\ 13262 & 15: 07: 25 & 0.0598 & 0.1090 \\ 13264 & 15: 07: 27 & 0.1118 & 0.2923 \\ 13266 & 15: 07: 29 & 0.0165 & 0.2180 \\ 13268 & 15: 07: 31 & 0.0764 & 0.2694 \\ 13270 & 15: 07: 33 & 0.0146 & 0.1328 \\ 13272 & 15: 07: 35 & 0.0331 & 0.2234 \\ 13274 & 15: 07: 37 & 0.0570 & 0.0615 \\ 13276 & 15: 07: 39 & 0.1026 & 0.1231 \\ 13278 & 15: 07: 41 & 0.0368 & 0.1713 \\ 13280 & 15: 07: 43 & 0.0147 & 0.0760 \\ 13282 & 15: 07: 45 & 0.0496 & 0.1326 \\ 13284 & 15: 07: 47 & 0.0852 & 0.1043 \\ 13286 & 15: 07: 49 & 0.1263 & 0.1261 \\ 13288 & 15: 07: 51 & 0.0931 & 0.0596 \\ 13290 & 15: 07: 53 & 0.0737 & 0.0963 \\ 13292 & 15: 07: 55 & 0.1192 & 0.1195 \\ 13294 & 15: 07: 57 & 0.1126 & 0.2677 \\ 13296 & 15: 07: 59 & 0.0523 & 0.2429 \\ 13298 & 15: 08: 01 & 0.0260 & 0.3899 \\ 13300 & 15: 08: 03 & 0.0460 & 0.0693 \\ 13302 & 15: 08: 05 & 0.0786 & 0.1711 \\ 13304 & 15: 08: 07 & 0.0448 & 0.0553 \\ 13306 & 15: 08: 09 & 0.0507 & 0.1655 \\ 13308 & 15: 08: 11 & 0.0281 & 0.0657\end{array}$




$\begin{array}{llll}13310 & 15: 08: 13 & 0.0195 & 0.1200 \\ 13312 & 15: 08: 15 & 0.0480 & 0.1782 \\ 13314 & 15: 08: 17 & 0.0104 & 0.1444 \\ 13316 & 15: 08: 19 & 0.0114 & 0.0790 \\ 13318 & 15: 08: 21 & 0.0199 & 0.1460 \\ 13320 & 15: 08: 23 & 0.0233 & 0.1416 \\ 13322 & 15: 08: 25 & 0.0708 & 0.3678 \\ 13324 & 15: 08: 27 & 0.0420 & 0.2565 \\ 13326 & 15: 08: 29 & 0.0962 & 0.2077 \\ 13328 & 15: 08: 31 & 0.1042 & 0.1293 \\ 13330 & 15: 08: 33 & 0.0601 & 0.0825 \\ 13332 & 15: 08: 35 & 0.0681 & 0.0956 \\ 13334 & 15: 08: 37 & 0.0311 & 0.1742 \\ 13336 & 15: 08: 39 & 0.0363 & 0.2180 \\ 13338 & 15: 08: 41 & 0.0582 & 0.1699 \\ 13340 & 15: 08: 43 & 0.0503 & 0.1424 \\ 13342 & 15: 08: 45 & 0.0451 & 0.0338 \\ 13344 & 15: 08: 47 & 0.0375 & 0.0490 \\ 13346 & 15: 08: 49 & 0.1210 & 0.2544 \\ 13348 & 15: 08: 51 & 0.0890 & 0.0278 \\ 13350 & 15: 08: 53 & 0.0712 & 0.2372 \\ 13352 & 15: 08: 55 & 0.0128 & 0.1163 \\ 13354 & 15: 08: 57 & 0.0592 & 0.1391 \\ 13356 & 15: 08: 59 & 0.0514 & 0.1364 \\ 13358 & 15: 09: 01 & 0.0181 & 0.1009 \\ 13360 & 15: 09: 03 & 0.0558 & 0.1080 \\ 13362 & 15: 09: 05 & 0.0374 & 0.1513 \\ 13364 & 15: 09: 07 & 0.0369 & 0.0565 \\ 13366 & 15: 09: 09 & 0.0466 & 0.2240 \\ 13368 & 15: 09: 11 & 0.0416 & 0.0914 \\ 13370 & 15: 09: 13 & 0.0789 & 0.1539 \\ 13372 & 15: 09: 15 & 0.0035 & 0.1144 \\ 13374 & 15: 09: 17 & 0.0428 & 0.0742 \\ 13376 & 15: 09: 19 & 0.0399 & 0.1132 \\ 13378 & 15: 09: 21 & 0.0906 & 0.2759 \\ 13380 & 15: 09: 23 & 0.0409 & 0.1427 \\ 13382 & 15: 09: 25 & 0.0444 & 0.1704 \\ 13384 & 15: 09: 27 & 0.0136 & 0.1831 \\ 13386 & 15: 09: 29 & 0.0838 & 0.1745 \\ 13388 & 15: 09: 31 & 0.0297 & 0.1656 \\ 13390 & 15: 09: 33 & 0.0459 & 0.1633 \\ 13392 & 15: 09: 35 & 0.0897 & 0.1842 \\ 13394 & 15: 09: 37 & 0.0485 & 0.0997 \\ 13396 & 15: 09: 39 & 0.0134 & 0.2030 \\ 13398 & 15: 09: 41 & 0.0934 & 0.2626 \\ 13400 & 15: 09: 43 & 0.0612 & 0.3152 \\ 13402 & 15: 09: 45 & 0.0418 & 0.1196 \\ 13404 & 15: 09: 47 & 0.0741 & 0.1851 \\ 13406 & 15: 09: 49 & 0.0861 & 0.0871 \\ 13408 & 15: 09: 51 & 0.0887 & 0.1135 \\ 13410 & 15: 09: 53 & 0.0845 & 0.0875 \\ 13412 & 15: 09: 55 & 0.1037 & 0.2039\end{array}$




$\begin{array}{llll}13414 & 15: 09: 57 & 0.0326 & 0.0584 \\ 13416 & 15: 09: 59 & 0.0358 & 0.0849 \\ 13418 & 15: 10: 01 & 0.0493 & 0.2536 \\ 13420 & 15: 10: 03 & 0.0561 & 0.2368 \\ 13422 & 15: 10: 05 & 0.0614 & 0.0876 \\ 13424 & 15: 10: 07 & 0.0455 & 0.2653 \\ 13426 & 15: 10: 09 & 0.1037 & 0.1159 \\ 13428 & 15: 10: 11 & 0.1005 & 0.1078 \\ 13430 & 15: 10: 13 & 0.0811 & 0.1209 \\ 13432 & 15: 10: 15 & 0.1131 & 0.2170 \\ 13434 & 15: 10: 17 & 0.1013 & 0.0716 \\ 13436 & 15: 10: 19 & 0.1056 & 0.0923 \\ 13438 & 15: 10: 21 & 0.0300 & 0.0743 \\ 13440 & 15: 10: 23 & 0.0252 & 0.2761 \\ 13442 & 15: 10: 25 & 0.0304 & 0.5328 \\ 13444 & 15: 10: 27 & 0.0516 & 0.2029 \\ 13446 & 15: 10: 29 & 0.2175 & 0.3937 \\ 13448 & 15: 10: 31 & 0.0666 & 0.1553 \\ 13450 & 15: 10: 33 & 0.0520 & 0.2541 \\ 13452 & 15: 10: 35 & 0.0600 & 0.1560 \\ 13454 & 15: 10: 37 & 0.0648 & 0.2325 \\ 13456 & 15: 10: 39 & 0.0596 & 0.2398 \\ 13458 & 15: 10: 41 & 0.0506 & 0.1235 \\ 13460 & 15: 10: 43 & 0.0379 & 0.1103 \\ 13462 & 15: 10: 45 & 0.0344 & 0.2870 \\ 13464 & 15: 10: 47 & 0.0447 & 0.0943 \\ 13466 & 15: 10: 49 & 0.1018 & 0.1870 \\ 13468 & 15: 10: 51 & 0.0246 & 0.0894 \\ 13470 & 15: 10: 53 & 0.0395 & 0.1995 \\ 13472 & 15: 10: 55 & 0.0362 & 0.1207 \\ 13474 & 15: 10: 57 & 0.1070 & 0.1512 \\ 13476 & 15: 10: 59 & 0.0262 & 0.2651 \\ 13478 & 15: 11: 01 & 0.0430 & 0.0774 \\ 13480 & 15: 11: 03 & 0.0682 & 0.1190 \\ 13482 & 15: 11: 05 & 0.2066 & 0.1872 \\ 13484 & 15: 11: 07 & 0.0250 & 0.0893 \\ 13486 & 15: 11: 09 & 0.0860 & 0.2349 \\ 13488 & 15: 11: 11 & 0.2446 & 0.3524 \\ 13490 & 15: 11: 13 & 0.0730 & 0.1650 \\ 13492 & 15: 11: 15 & 0.1401 & 0.2159 \\ 13494 & 15: 11: 17 & 0.0883 & 0.2247 \\ 13496 & 15: 11: 19 & 0.0403 & 0.3695 \\ 13498 & 15: 11: 21 & 0.0695 & 0.1764 \\ 13500 & 15: 11: 23 & 0.0674 & 0.3240 \\ 13502 & 15: 11: 25 & 0.1411 & 0.4120 \\ 13504 & 15: 11: 27 & 0.0996 & 0.2979 \\ 13506 & 15: 11: 29 & 0.0464 & 0.1915 \\ 13508 & 15: 11: 31 & 0.0989 & 0.2051 \\ 13510 & 15: 11: 33 & 0.2657 & 0.0965 \\ 13512 & 15: 11: 35 & 0.0571 & 0.2508 \\ 13514 & 15: 11: 37 & 0.1607 & 0.1489 \\ 13516 & 15: 11: 39 & 0.0314 & 0.1859\end{array}$


Appendix B - Laser Data Test 1 NIST102505_1.xls

$\begin{array}{llll}13518 & 15: 11: 41 & 0.0597 & 0.1569 \\ 13520 & 15: 11: 43 & 0.1562 & 0.1784 \\ 13522 & 15: 11: 45 & 0.0201 & 0.1100 \\ 13524 & 15: 11: 47 & 0.0557 & 0.1259 \\ 13526 & 15: 11: 49 & 0.0515 & 0.1231 \\ 13528 & 15: 11: 51 & 0.0440 & 0.1338 \\ 13530 & 15: 11: 53 & 0.0839 & 0.1373 \\ 13532 & 15: 11: 55 & 0.1007 & 0.0897 \\ 13534 & 15: 11: 57 & 0.0713 & 0.1823 \\ 13536 & 15: 11: 59 & 0.1168 & 0.1327 \\ 13538 & 15: 12: 01 & 0.1117 & 0.2009 \\ 13540 & 15: 12: 03 & 0.0619 & 0.0916 \\ 13542 & 15: 12: 05 & 0.0372 & 0.1851 \\ 13544 & 15: 12: 07 & 0.0635 & 0.0996 \\ 13546 & 15: 12: 09 & 0.0358 & 0.1515 \\ 13548 & 15: 12: 11 & 0.0628 & 0.0955 \\ 13550 & 15: 12: 13 & 0.1697 & 0.0752 \\ 13552 & 15: 12: 15 & 0.1369 & 0.4272 \\ 13554 & 15: 12: 17 & 0.0947 & 0.0704 \\ 13556 & 15: 12: 19 & 0.1885 & 0.0801 \\ 13558 & 15: 12: 21 & 0.0640 & 0.0472 \\ 13560 & 15: 12: 23 & 0.0877 & 0.0403 \\ 13562 & 15: 12: 25 & 0.0322 & 0.1531 \\ 13564 & 15: 12: 27 & 0.0313 & 0.0599 \\ 13566 & 15: 12: 29 & 0.0178 & 0.1202 \\ 13568 & 15: 12: 31 & 0.0813 & 0.0979 \\ 13570 & 15: 12: 33 & 0.0494 & 0.1731 \\ 13572 & 15: 12: 35 & 0.0283 & 0.2842 \\ 13574 & 15: 12: 37 & 0.0453 & 0.0723 \\ 13576 & 15: 12: 39 & 0.0335 & 0.1516 \\ 13578 & 15: 12: 41 & 0.0347 & 0.0863 \\ 13580 & 15: 12: 43 & 0.0294 & 0.1579 \\ 13582 & 15: 12: 45 & 0.0271 & 0.2095 \\ 13584 & 15: 12: 47 & 0.0636 & 0.1256 \\ 13586 & 15: 12: 49 & 0.1098 & 0.0790 \\ 13588 & 15: 12: 51 & 0.2247 & 0.0425 \\ 13590 & 15: 12: 53 & 0.0809 & 0.0877 \\ 13592 & 15: 12: 55 & 0.0538 & 0.0558 \\ 13594 & 15: 12: 57 & 0.0509 & 0.0575 \\ 13596 & 15: 12: 59 & 0.0463 & 0.1080 \\ 13598 & 15: 13: 01 & 0.0931 & 0.1322 \\ 13600 & 15: 13: 03 & 0.0190 & 0.0928 \\ 13602 & 15: 13: 05 & 0.0367 & 0.1597 \\ 13604 & 15: 13: 07 & 0.0612 & 0.1502 \\ 13606 & 15: 13: 09 & 0.0314 & 0.1094 \\ 13608 & 15: 13: 11 & 0.0125 & 0.3979 \\ 13610 & 15: 13: 13 & 0.0590 & 0.1379 \\ 13612 & 15: 13: 15 & 0.0510 & 0.2052 \\ 13614 & 15: 13: 17 & 0.1786 & 0.3221 \\ 13616 & 15: 13: 19 & 0.0929 & 0.1576 \\ 13618 & 15: 13: 21 & 0.0761 & 0.1544 \\ 13620 & 15: 13: 23 & 0.0118 & 0.1620\end{array}$

B - 131 


$\begin{array}{llll}13622 & 15: 13: 25 & 0.0761 & 0.1924 \\ 13624 & 15: 13: 27 & 0.2296 & 0.1581 \\ 13626 & 15: 13: 29 & 0.0880 & 0.1341 \\ 13628 & 15: 13: 31 & 0.0310 & 0.1662 \\ 13630 & 15: 13: 33 & 0.0188 & 0.0588 \\ 13632 & 15: 13: 35 & 0.0461 & 0.1817 \\ 13634 & 15: 13: 37 & 0.0450 & 0.1713 \\ 13636 & 15: 13: 39 & 0.0398 & 0.1771 \\ 13638 & 15: 13: 41 & 0.0911 & 0.0802 \\ 13640 & 15: 13: 43 & 0.1060 & 0.1023 \\ 13642 & 15: 13: 45 & 0.0311 & 0.2447 \\ 13644 & 15: 13: 47 & 0.0484 & 0.2792 \\ 13646 & 15: 13: 49 & 0.1275 & 0.1730 \\ 13648 & 15: 13: 51 & 0.0345 & 0.0689 \\ 13650 & 15: 13: 53 & 0.0705 & 0.0939 \\ 13652 & 15: 13: 55 & 0.0495 & 0.1734 \\ 13654 & 15: 13: 57 & 0.0272 & 0.2346 \\ 13656 & 15: 13: 59 & 0.0468 & 0.2238 \\ 13658 & 15: 14: 01 & 0.0692 & 0.1731 \\ 13660 & 15: 14: 03 & 0.0678 & 0.1245 \\ 13662 & 15: 14: 05 & 0.0752 & 0.1674 \\ 13664 & 15: 14: 07 & 0.0344 & 0.0741 \\ 13666 & 15: 14: 09 & 0.1595 & 0.0400 \\ 13668 & 15: 14: 11 & 0.0247 & 0.0670 \\ 13670 & 15: 14: 13 & 0.0211 & 0.1341 \\ 13672 & 15: 14: 15 & 0.0557 & 0.1841 \\ 13674 & 15: 14: 17 & 0.0293 & 0.0950 \\ 13676 & 15: 14: 19 & 0.0650 & 0.1331 \\ 13678 & 15: 14: 21 & 0.1696 & 0.2326 \\ 13680 & 15: 14: 23 & 0.0649 & 0.0337 \\ 13682 & 15: 14: 25 & 0.0780 & 0.1322 \\ 13684 & 15: 14: 27 & 0.0913 & 0.1723 \\ 13686 & 15: 14: 29 & 0.0509 & 0.1711 \\ 13688 & 15: 14: 31 & 0.0421 & 0.1169 \\ 13690 & 15: 14: 33 & 0.0358 & 0.1311 \\ 13692 & 15: 14: 35 & 0.0587 & 0.2210 \\ 13694 & 15: 14: 37 & 0.1115 & 0.2551 \\ 13696 & 15: 14: 39 & 0.0163 & 0.1458 \\ 13698 & 15: 14: 41 & 0.0749 & 0.2312 \\ 13700 & 15: 14: 43 & 0.0917 & 0.1227 \\ 13702 & 15: 14: 45 & 0.0799 & 0.1066 \\ 13704 & 15: 14: 47 & 0.0614 & 0.1385 \\ 13706 & 15: 14: 49 & 0.2044 & 0.2359 \\ 13708 & 15: 14: 51 & 0.1288 & 0.1136 \\ 13710 & 15: 14: 53 & 0.0264 & 0.2137 \\ 13712 & 15: 14: 55 & 0.0175 & 0.2050 \\ 13714 & 15: 14: 57 & 0.0619 & 0.1734 \\ 13716 & 15: 14: 59 & 0.0211 & 0.2248 \\ 13718 & 15: 15: 01 & 0.0283 & 0.1608 \\ 13720 & 15: 15: 03 & 0.0999 & 0.1839 \\ 13722 & 15: 15: 05 & 0.0682 & 0.3365 \\ 13724 & 15: 15: 07 & 0.0928 & 0.3529\end{array}$




$\begin{array}{llll}13726 & 15: 15: 09 & 0.0247 & 0.1758 \\ 13728 & 15: 15: 11 & 0.2057 & 0.2146 \\ 13730 & 15: 15: 13 & 0.0609 & 0.1539 \\ 13732 & 15: 15: 15 & 0.0764 & 0.1307 \\ 13734 & 15: 15: 17 & 0.1432 & 0.0938 \\ 13736 & 15: 15: 19 & 0.0150 & 0.0226 \\ 13738 & 15: 15: 21 & 0.0319 & 0.1119 \\ 13740 & 15: 15: 23 & 0.0486 & 0.0414 \\ 13742 & 15: 15: 25 & 0.0136 & 0.1950 \\ 13744 & 15: 15: 27 & 0.0409 & 0.2295 \\ 13746 & 15: 15: 29 & 0.0536 & 0.1535 \\ 13748 & 15: 15: 31 & 0.0340 & 0.3015 \\ 13750 & 15: 15: 33 & 0.0454 & 0.1861 \\ 13752 & 15: 15: 35 & 0.1148 & 0.2555 \\ 13754 & 15: 15: 37 & 0.0602 & 0.1343 \\ 13756 & 15: 15: 39 & 0.1318 & 0.1354 \\ 13758 & 15: 15: 41 & 0.0586 & 0.0960 \\ 13760 & 15: 15: 43 & 0.0628 & 0.1716 \\ 13762 & 15: 15: 45 & 0.0454 & 0.1066 \\ 13764 & 15: 15: 47 & 0.0576 & 0.1596 \\ 13766 & 15: 15: 49 & 0.0992 & 0.1025 \\ 13768 & 15: 15: 51 & 0.0458 & 0.0953 \\ 13770 & 15: 15: 53 & 0.0752 & 0.0443 \\ 13772 & 15: 15: 55 & 0.1857 & 0.1002 \\ 13774 & 15: 15: 57 & 0.0493 & 0.1059 \\ 13776 & 15: 15: 59 & 0.0383 & 0.4165 \\ 13778 & 15: 16: 01 & 0.0718 & 0.1298 \\ 13780 & 15: 16: 03 & 0.1101 & 0.2376 \\ 13782 & 15: 16: 05 & 0.0514 & 0.1843 \\ 13784 & 15: 16: 07 & 0.0381 & 0.1617 \\ 13786 & 15: 16: 09 & 0.1021 & 0.2021 \\ 13788 & 15: 16: 11 & 0.1052 & 0.2157 \\ 13790 & 15: 16: 13 & 0.0415 & 0.0469 \\ 13792 & 15: 16: 15 & 0.0578 & 0.1460 \\ 13794 & 15: 16: 17 & 0.0444 & 0.1234 \\ 13796 & 15: 16: 19 & 0.0705 & 0.1206 \\ 13798 & 15: 16: 21 & 0.0255 & 0.0944 \\ 13800 & 15: 16: 23 & 0.0390 & 0.1388 \\ 13802 & 15: 16: 25 & 0.0601 & 0.1529 \\ 13804 & 15: 16: 27 & 0.0523 & 0.1574 \\ 13806 & 15: 16: 29 & 0.0139 & 0.2023 \\ 13808 & 15: 16: 31 & 0.0240 & 0.2334 \\ 13810 & 15: 16: 33 & 0.1275 & 0.1367 \\ 13812 & 15: 16: 35 & 0.0919 & 0.1920 \\ 13814 & 15: 16: 37 & 0.1234 & 0.2727 \\ 13816 & 15: 16: 39 & 0.0450 & 0.2148 \\ 13818 & 15: 16: 41 & 0.0127 & 0.1346 \\ 13820 & 15: 16: 43 & 0.0243 & 0.1492 \\ 13822 & 15: 16: 45 & 0.0547 & 0.1812 \\ 13824 & 15: 16: 47 & 0.0395 & 0.2243 \\ 13826 & 15: 16: 49 & 0.0379 & 0.1604 \\ 13828 & 15: 16: 51 & 0.0593 & 0.1834\end{array}$




$\begin{array}{llll}13830 & 15: 16: 53 & 0.0231 & 0.1494 \\ 13832 & 15: 16: 55 & 0.0994 & 0.2098 \\ 13834 & 15: 16: 57 & 0.0186 & 0.2108 \\ 13836 & 15: 16: 59 & 0.0300 & 0.3257 \\ 13838 & 15: 17: 01 & 0.0857 & 0.2160 \\ 13840 & 15: 17: 03 & 0.0209 & 0.1821 \\ 13842 & 15: 17: 05 & 0.0456 & 0.1627 \\ 13844 & 15: 17: 07 & 0.0361 & 0.0615 \\ 13846 & 15: 17: 09 & 0.0653 & 0.1426 \\ 13848 & 15: 17: 11 & 0.1724 & 0.2948 \\ 13850 & 15: 17: 13 & 0.0709 & 0.2780 \\ 13852 & 15: 17: 15 & 0.0701 & 0.1370 \\ 13854 & 15: 17: 17 & 0.1225 & 0.1540 \\ 13856 & 15: 17: 19 & 0.0840 & 0.1179 \\ 13858 & 15: 17: 21 & 0.0595 & 0.2033 \\ 13860 & 15: 17: 23 & 0.0558 & 0.1136 \\ 13862 & 15: 17: 25 & 0.0738 & 0.2232 \\ 13864 & 15: 17: 27 & 0.0962 & 0.2556 \\ 13866 & 15: 17: 29 & 0.0532 & 0.1617 \\ 13868 & 15: 17: 31 & 0.0467 & 0.1113 \\ 13870 & 15: 17: 33 & 0.0708 & 0.1741 \\ 13872 & 15: 17: 35 & 0.0344 & 0.0590 \\ 13874 & 15: 17: 37 & 0.0234 & 0.1454 \\ 13876 & 15: 17: 39 & 0.0388 & 0.1429 \\ 13878 & 15: 17: 41 & 0.0305 & 0.2867 \\ 13880 & 15: 17: 43 & 0.0376 & 0.1299 \\ 13882 & 15: 17: 45 & 0.0342 & 0.1691 \\ 13884 & 15: 17: 47 & 0.0335 & 0.2040 \\ 13886 & 15: 17: 49 & 0.0706 & 0.2763 \\ 13888 & 15: 17: 51 & 0.0498 & 0.1675 \\ 13890 & 15: 17: 53 & 0.0828 & 0.1599 \\ 13892 & 15: 17: 55 & 0.0241 & 0.2107 \\ 13894 & 15: 17: 57 & 0.0787 & 0.0979 \\ 13896 & 15: 17: 59 & 0.0520 & 0.1845 \\ 13898 & 15: 18: 01 & 0.0230 & 0.4527 \\ 13900 & 15: 18: 03 & 0.0481 & 0.0411 \\ 13902 & 15: 18: 05 & 0.0621 & 0.1028 \\ 13904 & 15: 18: 07 & 0.0679 & 0.1327 \\ 13906 & 15: 18: 09 & 0.0414 & 0.0768 \\ 13908 & 15: 18: 11 & 0.1539 & 0.1072 \\ 13910 & 15: 18: 13 & 0.0219 & 0.0887 \\ 13912 & 15: 18: 15 & 0.1306 & 0.1249 \\ 13914 & 15: 18: 17 & 0.1139 & 0.3935 \\ 13916 & 15: 18: 19 & 0.0388 & 0.1386 \\ 13918 & 15: 18: 21 & 0.0322 & 0.2919 \\ 13920 & 15: 18: 23 & 0.0472 & 0.2624 \\ 13922 & 15: 18: 25 & 0.0263 & 0.1986 \\ 13924 & 15: 18: 27 & 0.0120 & 0.1878 \\ 13926 & 15: 18: 29 & 0.0218 & 0.0828 \\ 13928 & 15: 18: 31 & 0.1112 & 0.1386 \\ 13930 & 15: 18: 33 & 0.0540 & 0.0801 \\ 13932 & 15: 18: 35 & 0.1252 & 0.1872\end{array}$




$\begin{array}{llll}13934 & 15: 18: 37 & 0.1806 & 0.1711 \\ 13936 & 15: 18: 39 & 0.0403 & 0.0983 \\ 13938 & 15: 18: 41 & 0.1012 & 0.0817 \\ 13940 & 15: 18: 43 & 0.0526 & 0.3451 \\ 13942 & 15: 18: 45 & 0.0437 & 0.1191 \\ 13944 & 15: 18: 47 & 0.1622 & 0.2158 \\ 13946 & 15: 18: 49 & 0.0492 & 0.0945 \\ 13948 & 15: 18: 51 & 0.0504 & 0.0443 \\ 13950 & 15: 18: 53 & 0.1129 & 0.3059 \\ 13952 & 15: 18: 55 & 0.0502 & 0.1948 \\ 13954 & 15: 18: 57 & 0.0588 & 0.2082 \\ 13956 & 15: 18: 59 & 0.0763 & 0.2162 \\ 13958 & 15: 19: 01 & 0.0552 & 0.1478 \\ 13960 & 15: 19: 03 & 0.1941 & 0.2822 \\ 13962 & 15: 19: 05 & 0.0535 & 0.2550 \\ 13964 & 15: 19: 07 & 0.0695 & 0.0920 \\ 13966 & 15: 19: 09 & 0.0607 & 0.1366 \\ 13968 & 15: 19: 11 & 0.0430 & 0.2973 \\ 13970 & 15: 19: 13 & 0.1706 & 0.1782 \\ 13972 & 15: 19: 15 & 0.0396 & 0.2010 \\ 13974 & 15: 19: 17 & 0.0315 & 0.1053 \\ 13976 & 15: 19: 19 & 0.0605 & 0.1649 \\ 13978 & 15: 19: 21 & 0.0165 & 0.3919 \\ 13980 & 15: 19: 23 & 0.0604 & 0.2546 \\ 13982 & 15: 19: 25 & 0.1429 & 0.2079 \\ 13984 & 15: 19: 27 & 0.0862 & 0.1034 \\ 13986 & 15: 19: 29 & 0.0551 & 0.2371 \\ 13988 & 15: 19: 31 & 0.0909 & 0.4636 \\ 13990 & 15: 19: 33 & 0.0500 & 0.0662 \\ 13992 & 15: 19: 35 & 0.0676 & 0.1487 \\ 13994 & 15: 19: 37 & 0.0446 & 0.4349 \\ 13996 & 15: 19: 39 & 0.0504 & 0.0725 \\ 13998 & 15: 19: 41 & 0.0995 & 0.0796 \\ 14000 & 15: 19: 43 & 0.0278 & 0.0810 \\ 14002 & 15: 19: 45 & 0.0930 & 0.1376 \\ 14004 & 15: 19: 47 & 0.0392 & 0.1423 \\ 14006 & 15: 19: 49 & 0.1570 & 0.1940 \\ 14008 & 15: 19: 51 & 0.0288 & 0.2188 \\ 14010 & 15: 19: 53 & 0.0800 & 0.2025 \\ 14012 & 15: 19: 55 & 0.0554 & 0.2921 \\ 14014 & 15: 19: 57 & 0.1325 & 0.1814 \\ 14016 & 15: 19: 59 & 0.1288 & 0.3110 \\ 14018 & 15: 20: 01 & 0.0065 & 0.1397 \\ 14020 & 15: 20: 03 & 0.1067 & 0.2024 \\ 14022 & 15: 20: 05 & 0.0288 & 0.1547 \\ 14024 & 15: 20: 07 & 0.2396 & 0.1270 \\ 14026 & 15: 20: 09 & 0.0564 & 0.2154 \\ 14028 & 15: 20: 11 & 0.0884 & 0.2902 \\ 14030 & 15: 20: 13 & 0.0767 & 0.1151 \\ 14032 & 15: 20: 15 & 0.0183 & 0.0959 \\ 14034 & 15: 20: 17 & 0.0319 & 0.0995 \\ 14036 & 15: 20: 19 & 0.1397 & 0.3331\end{array}$


Appendix B - Laser Data Test 1 NIST102505_1.xls

$\begin{array}{llll}14038 & 15: 20: 21 & 0.0799 & 0.0925 \\ 14040 & 15: 20: 23 & 0.1048 & 0.2894 \\ 14042 & 15: 20: 25 & 0.0352 & 0.1164 \\ 14044 & 15: 20: 27 & 0.0639 & 0.4204 \\ 14046 & 15: 20: 29 & 0.0480 & 0.1336 \\ 14048 & 15: 20: 31 & 0.1351 & 0.1462 \\ 14050 & 15: 20: 33 & 0.0124 & 0.1302 \\ 14052 & 15: 20: 35 & 0.0186 & 0.2219 \\ 14054 & 15: 20: 37 & 0.0592 & 0.1702 \\ 14056 & 15: 20: 39 & 0.0992 & 0.1929 \\ 14058 & 15: 20: 41 & 0.0455 & 0.2035 \\ 14060 & 15: 20: 43 & 0.0195 & 0.1688 \\ 14062 & 15: 20: 45 & 0.0154 & 0.2868 \\ 14064 & 15: 20: 47 & 0.0647 & 0.2451 \\ 14066 & 15: 20: 49 & 0.1033 & 0.1202 \\ 14068 & 15: 20: 51 & 0.1912 & 0.1906 \\ 14070 & 15: 20: 53 & 0.0892 & 0.1220 \\ 14072 & 15: 20: 55 & 0.0312 & 0.2429 \\ 14074 & 15: 20: 57 & 0.1409 & 0.1502 \\ 14076 & 15: 20: 59 & 0.0936 & 0.0988 \\ 14078 & 15: 21: 01 & 0.0692 & 0.2551 \\ 14080 & 15: 21: 03 & 0.0700 & 0.1338 \\ 14082 & 15: 21: 05 & 0.0154 & 0.2036 \\ 14084 & 15: 21: 07 & 0.1364 & 0.1937 \\ 14086 & 15: 21: 09 & 0.1880 & 0.1152 \\ 14088 & 15: 21: 11 & 0.2171 & 0.4358 \\ 14090 & 15: 21: 13 & 0.0656 & 0.1201 \\ 14092 & 15: 21: 15 & 0.0734 & 0.2034 \\ 14094 & 15: 21: 17 & 0.0702 & 0.5316 \\ 14096 & 15: 21: 19 & 0.0390 & 0.1885 \\ 14098 & 15: 21: 21 & 0.0326 & 0.2743 \\ 14100 & 15: 21: 23 & 0.1417 & 0.1774 \\ 14102 & 15: 21: 25 & 0.1014 & 0.1268 \\ 14104 & 15: 21: 27 & 0.0666 & 0.1434 \\ 14106 & 15: 21: 29 & 0.1551 & 0.1724 \\ 14108 & 15: 21: 31 & 0.0252 & 0.2081 \\ 14110 & 15: 21: 33 & 0.1298 & 0.2141 \\ 14112 & 15: 21: 35 & 0.0501 & 0.1273 \\ 14114 & 15: 21: 37 & 0.0961 & 0.1647 \\ 14116 & 15: 21: 39 & 0.0298 & 0.1148 \\ 14118 & 15: 21: 41 & 0.0571 & 0.4455 \\ 14120 & 15: 21: 43 & 0.1420 & 0.1853 \\ 14122 & 15: 21: 45 & 0.0434 & 0.1794 \\ 14124 & 15: 21: 47 & 0.1433 & 0.1676 \\ 14126 & 15: 21: 49 & 0.1205 & 0.1934 \\ 14128 & 15: 21: 51 & 0.0119 & 0.0681 \\ 14130 & 15: 21: 53 & 0.0339 & 0.2059 \\ 14132 & 15: 21: 55 & 0.0432 & 0.4132 \\ 14134 & 15: 21: 57 & 0.0253 & 0.1498 \\ 14136 & 15: 21: 59 & 0.0541 & 0.0880 \\ 14138 & 15: 22: 01 & 0.0147 & 0.2831 \\ 14140 & 15: 22: 03 & 0.0473 & 0.1045\end{array}$

B - 136 
Appendix B - Laser Data Test 1 NIST102505_1.xls

$\begin{array}{llll}14142 & 15: 22: 05 & 0.0532 & 0.3104 \\ 14144 & 15: 22: 07 & 0.0933 & 0.0975 \\ 14146 & 15: 22: 09 & 0.0461 & 0.2559 \\ 14148 & 15: 22: 11 & 0.2315 & 0.1243 \\ 14150 & 15: 22: 13 & 0.0321 & 0.2523 \\ 14152 & 15: 22: 15 & 0.1897 & 0.3621 \\ 14154 & 15: 22: 17 & 0.1211 & 0.2317 \\ 14156 & 15: 22: 19 & 0.0266 & 0.1310 \\ 14158 & 15: 22: 21 & 0.0419 & 0.1522 \\ 14160 & 15: 22: 23 & 0.0411 & 0.0964 \\ 14162 & 15: 22: 25 & 0.0345 & 0.1139 \\ 14164 & 15: 22: 27 & 0.0663 & 0.5330 \\ 14166 & 15: 22: 29 & 0.0838 & 0.1890 \\ 14168 & 15: 22: 31 & 0.0701 & 0.1353 \\ 14170 & 15: 22: 33 & 0.0435 & 0.0903 \\ 14172 & 15: 22: 35 & 0.0282 & 0.1138 \\ 14174 & 15: 22: 37 & 0.1073 & 0.4041 \\ 14176 & 15: 22: 39 & 0.0158 & 0.1387 \\ 14178 & 15: 22: 41 & 0.0867 & 0.3459 \\ 14180 & 15: 22: 43 & 0.1428 & 0.1582 \\ 14182 & 15: 22: 45 & 0.0407 & 0.1707 \\ 14184 & 15: 22: 47 & 0.1309 & 0.1405 \\ 14186 & 15: 22: 49 & 0.1274 & 0.1681 \\ 14188 & 15: 22: 51 & 0.0149 & 0.1138 \\ 14190 & 15: 22: 53 & 0.0541 & 0.2933 \\ 14192 & 15: 22: 55 & 0.0281 & 0.3829 \\ 14194 & 15: 22: 57 & 0.0521 & 0.1983 \\ 14196 & 15: 22: 59 & 0.0794 & 0.2187 \\ 14198 & 15: 23: 01 & 0.0774 & 0.1245 \\ 14200 & 15: 23: 03 & 0.0875 & 0.1704 \\ 14202 & 15: 23: 05 & 0.0937 & 0.1654 \\ 14204 & 15: 23: 07 & 0.1671 & 0.0733 \\ 14206 & 15: 23: 09 & 0.0313 & 0.0695 \\ 14208 & 15: 23: 11 & 0.0833 & 0.1643 \\ 14210 & 15: 23: 13 & 0.0538 & 0.1807 \\ 14212 & 15: 23: 15 & 0.0702 & 0.1772 \\ 14214 & 15: 23: 17 & 0.0844 & 0.1888 \\ 14216 & 15: 23: 19 & 0.0647 & 0.1286 \\ 14218 & 15: 23: 21 & 0.1641 & 0.1179 \\ 14220 & 15: 23: 23 & 0.0480 & 0.2203 \\ 14222 & 15: 23: 25 & 0.0568 & 0.0990 \\ 14224 & 15: 23: 27 & 0.0885 & 0.2532 \\ 14226 & 15: 23: 29 & 0.0542 & 0.1571 \\ 14228 & 15: 23: 31 & 0.0438 & 0.1525 \\ 14230 & 15: 23: 33 & 0.0309 & 0.2518 \\ 14232 & 15: 23: 35 & 0.0376 & 0.2054 \\ 14234 & 15: 23: 37 & 0.0639 & 0.2592 \\ 14236 & 15: 23: 39 & 0.0883 & 0.2113 \\ 14238 & 15: 23: 41 & 0.1021 & 0.1642 \\ 14240 & 15: 23: 43 & 0.1308 & 0.2108 \\ 14242 & 15: 23: 45 & 0.0516 & 0.1508 \\ 14244 & 15: 23: 47 & 0.1367 & 0.1809\end{array}$


Appendix B - Laser Data Test 1 NIST102505_1.xls

$\begin{array}{llll}14246 & 15: 23: 49 & 0.2274 & 0.2552 \\ 14248 & 15: 23: 51 & 0.1339 & 0.2278 \\ 14250 & 15: 23: 53 & 0.0950 & 0.1909 \\ 14252 & 15: 23: 55 & 0.0839 & 0.2399 \\ 14254 & 15: 23: 57 & 0.0858 & 0.3547 \\ 14256 & 15: 23: 59 & 0.1258 & 0.1139 \\ 14258 & 15: 24: 01 & 0.0231 & 0.1206 \\ 14260 & 15: 24: 03 & 0.0470 & 0.1455 \\ 14262 & 15: 24: 05 & 0.0728 & 0.2239 \\ 14264 & 15: 24: 07 & 0.0427 & 0.1912 \\ 14266 & 15: 24: 09 & 0.1192 & 0.3680 \\ 14268 & 15: 24: 11 & 0.0600 & 0.0712 \\ 14270 & 15: 24: 13 & 0.0891 & 0.2374 \\ 14272 & 15: 24: 15 & 0.0702 & 0.1184 \\ 14274 & 15: 24: 17 & 0.0643 & 0.1656 \\ 14276 & 15: 24: 19 & 0.0424 & 0.1003 \\ 14278 & 15: 24: 21 & 0.0431 & 0.1298 \\ 14280 & 15: 24: 23 & 0.0440 & 0.2029 \\ 14282 & 15: 24: 25 & 0.0618 & 0.3017 \\ 14284 & 15: 24: 27 & 0.0415 & 0.2393 \\ 14286 & 15: 24: 29 & 0.0235 & 0.1986 \\ 14288 & 15: 24: 31 & 0.0184 & 0.1924 \\ 14290 & 15: 24: 33 & 0.0263 & 0.2294 \\ 14292 & 15: 24: 35 & 0.0936 & 0.3392 \\ 14294 & 15: 24: 37 & 0.0467 & 0.3167 \\ 14296 & 15: 24: 39 & 0.0448 & 0.2260 \\ 14298 & 15: 24: 41 & 0.0319 & 0.1698 \\ 14300 & 15: 24: 43 & 0.0697 & 0.0911 \\ 14302 & 15: 24: 45 & 0.0174 & 0.1001 \\ 14304 & 15: 24: 47 & 0.0253 & 0.2550 \\ 14306 & 15: 24: 49 & 0.1368 & 0.0719 \\ 14308 & 15: 24: 51 & 0.0949 & 0.1334 \\ 14310 & 15: 24: 53 & 0.0518 & 0.1878 \\ 14312 & 15: 24: 55 & 0.0464 & 0.0867 \\ 14314 & 15: 24: 57 & 0.0141 & 0.2648 \\ 14316 & 15: 24: 59 & 0.0328 & 0.2560 \\ 14318 & 15: 25: 01 & 0.0503 & 0.1495 \\ 14320 & 15: 25: 03 & 0.0204 & 0.1391 \\ 14322 & 15: 25: 05 & 0.0544 & 0.2431 \\ 14324 & 15: 25: 07 & 0.0806 & 0.1937 \\ 14326 & 15: 25: 09 & 0.0853 & 0.2336 \\ 14328 & 15: 25: 11 & 0.0425 & 0.1049 \\ 14330 & 15: 25: 13 & 0.0706 & 0.1251 \\ 14332 & 15: 25: 15 & 0.0479 & 0.0716 \\ 14334 & 15: 25: 17 & 0.0836 & 0.1365 \\ 14336 & 15: 25: 19 & 0.0165 & 0.1625 \\ 14338 & 15: 25: 21 & 0.0494 & 0.2202 \\ 14340 & 15: 25: 23 & 0.0239 & 0.0550 \\ 14342 & 15: 25: 25 & 0.0152 & 0.1517 \\ 14344 & 15: 25: 27 & 0.0336 & 0.1376 \\ 14346 & 15: 25: 29 & 0.0558 & 0.2091 \\ 14348 & 15: 25: 31 & 0.0532 & 0.1018\end{array}$

B - 138 


$\begin{array}{llll}14350 & 15: 25: 33 & 0.0416 & 0.2170 \\ 14352 & 15: 25: 35 & 0.0361 & 0.1167 \\ 14354 & 15: 25: 37 & 0.0483 & 0.3706 \\ 14356 & 15: 25: 39 & 0.0427 & 0.2930 \\ 14358 & 15: 25: 41 & 0.0219 & 0.0951 \\ 14360 & 15: 25: 43 & 0.0369 & 0.1031 \\ 14362 & 15: 25: 45 & 0.0700 & 0.1795 \\ 14364 & 15: 25: 47 & 0.0596 & 0.1725 \\ 14366 & 15: 25: 49 & 0.0077 & 0.1908 \\ 14368 & 15: 25: 51 & 0.0366 & 0.1689 \\ 14370 & 15: 25: 53 & 0.1428 & 0.2885 \\ 14372 & 15: 25: 55 & 0.0955 & 0.1684 \\ 14374 & 15: 25: 57 & 0.0623 & 0.0979 \\ 14376 & 15: 25: 59 & 0.0845 & 0.0963 \\ 14378 & 15: 26: 01 & 0.0720 & 0.1806 \\ 14380 & 15: 26: 03 & 0.1103 & 0.1285 \\ 14382 & 15: 26: 05 & 0.1937 & 0.2107 \\ 14384 & 15: 26: 07 & 0.0199 & 0.1165 \\ 14386 & 15: 26: 09 & 0.0644 & 0.1703 \\ 14388 & 15: 26: 11 & 0.0502 & 0.1527 \\ 14390 & 15: 26: 13 & 0.1437 & 0.2781 \\ 14392 & 15: 26: 15 & 0.0343 & 0.1773 \\ 14394 & 15: 26: 17 & 0.0849 & 0.1835 \\ 14396 & 15: 26: 19 & 0.0265 & 0.1457 \\ 14398 & 15: 26: 21 & 0.0380 & 0.1422 \\ 14400 & 15: 26: 23 & 0.0845 & 0.2489 \\ 14402 & 15: 26: 25 & 0.0493 & 0.1444 \\ 14404 & 15: 26: 27 & 0.0267 & 0.1728 \\ 14406 & 15: 26: 29 & 0.0351 & 0.2033 \\ 14408 & 15: 26: 31 & 0.0149 & 0.2933 \\ 14410 & 15: 26: 33 & 0.0452 & 0.1144 \\ 14412 & 15: 26: 35 & 0.0629 & 0.4764 \\ 14414 & 15: 26: 37 & 0.1422 & 0.1011 \\ 14416 & 15: 26: 39 & 0.0682 & 0.1372 \\ 14418 & 15: 26: 41 & 0.0130 & 0.1531 \\ 14420 & 15: 26: 43 & 0.1667 & 0.3509 \\ 14422 & 15: 26: 45 & 0.0875 & 0.1426 \\ 14424 & 15: 26: 47 & 0.0806 & 0.2043 \\ 14426 & 15: 26: 49 & 0.0368 & 0.0744 \\ 14428 & 15: 26: 51 & 0.0358 & 0.1651 \\ 14430 & 15: 26: 53 & 0.0654 & 0.1059 \\ 14432 & 15: 26: 55 & 0.0187 & 0.1171 \\ 14434 & 15: 26: 57 & 0.1281 & 0.3129 \\ 14436 & 15: 26: 59 & 0.0347 & 0.3219 \\ 14438 & 15: 27: 01 & 0.1431 & 0.2151 \\ 14440 & 15: 27: 03 & 0.1164 & 0.1332 \\ 14442 & 15: 27: 05 & 0.1008 & 0.4282 \\ 14444 & 15: 27: 07 & 0.0263 & 0.2881 \\ 14446 & 15: 27: 09 & 0.0378 & 0.2033 \\ 14448 & 15: 27: 11 & 0.0720 & 0.0693 \\ 14450 & 15: 27: 13 & 0.0651 & 0.2337 \\ 14452 & 15: 27: 15 & 0.0732 & 0.2082 \\ & & & \end{array}$




$\begin{array}{llll}14454 & 15: 27: 17 & 0.0656 & 0.4103 \\ 14456 & 15: 27: 19 & 0.0155 & 0.3781 \\ 14458 & 15: 27: 21 & 0.0295 & 0.2155 \\ 14460 & 15: 27: 23 & 0.0791 & 0.2803 \\ 14462 & 15: 27: 25 & 0.1159 & 0.2722 \\ 14464 & 15: 27: 27 & 0.1440 & 0.1894 \\ 14466 & 15: 27: 29 & 0.0566 & 0.1258 \\ 14468 & 15: 27: 31 & 0.1138 & 0.0819 \\ 14470 & 15: 27: 33 & 0.0292 & 0.1598 \\ 14472 & 15: 27: 35 & 0.0478 & 0.1565 \\ 14474 & 15: 27: 37 & 0.0228 & 0.1228 \\ 14476 & 15: 27: 39 & 0.0484 & 0.1764 \\ 14478 & 15: 27: 41 & 0.0105 & 0.1837 \\ 14480 & 15: 27: 43 & 0.0284 & 0.1154 \\ 14482 & 15: 27: 45 & 0.0385 & 0.4903 \\ 14484 & 15: 27: 47 & 0.0285 & 0.2459 \\ 14486 & 15: 27: 49 & 0.1276 & 0.3284 \\ 14488 & 15: 27: 51 & 0.0381 & 0.2041 \\ 14490 & 15: 27: 53 & 0.0894 & 0.1682 \\ 14492 & 15: 27: 55 & 0.0457 & 0.3506 \\ 14494 & 15: 27: 57 & 0.0445 & 0.0809 \\ 14496 & 15: 27: 59 & 0.1110 & 0.1695 \\ 14498 & 15: 28: 01 & 0.0773 & 0.1338 \\ 14500 & 15: 28: 03 & 0.0762 & 0.0877 \\ 14502 & 15: 28: 05 & 0.0582 & 0.1148 \\ 14504 & 15: 28: 07 & 0.0381 & 0.1047 \\ 14506 & 15: 28: 09 & 0.0330 & 0.1204 \\ 14508 & 15: 28: 11 & 0.0976 & 0.0897 \\ 14510 & 15: 28: 13 & 0.0430 & 0.0723 \\ 14512 & 15: 28: 15 & 0.1113 & 0.1436 \\ 14514 & 15: 28: 17 & 0.0554 & 0.2471 \\ 14516 & 15: 28: 19 & 0.0408 & 0.1521 \\ 14518 & 15: 28: 21 & 0.0215 & 0.2115 \\ 14520 & 15: 28: 23 & 0.1952 & 0.3939 \\ 14522 & 15: 28: 25 & 0.0412 & 0.1819 \\ 14524 & 15: 28: 27 & 0.0275 & 0.1099 \\ 14526 & 15: 28: 29 & 0.0818 & 0.2311 \\ 14528 & 15: 28: 31 & 0.1052 & 0.3641 \\ 14530 & 15: 28: 33 & 0.0205 & 0.2665 \\ 14532 & 15: 28: 35 & 0.0338 & 0.2729 \\ 14534 & 15: 28: 37 & 0.0518 & 0.2044 \\ 14536 & 15: 28: 39 & 0.0752 & 0.2594 \\ 14538 & 15: 28: 41 & 0.0270 & 0.1930 \\ 14540 & 15: 28: 43 & 0.1182 & 0.4152 \\ 14542 & 15: 28: 45 & 0.0519 & 0.2656 \\ 14544 & 15: 28: 47 & 0.0588 & 0.2562 \\ 14546 & 15: 28: 49 & 0.1518 & 0.4310 \\ 14548 & 15: 28: 51 & 0.0531 & 0.1512 \\ 14550 & 15: 28: 53 & 0.0296 & 0.1333 \\ 14552 & 15: 28: 55 & 0.0564 & 0.2594 \\ 14554 & 15: 28: 57 & 0.0748 & 0.1448 \\ 14556 & 15: 28: 59 & 0.1642 & 0.2259\end{array}$




$\begin{array}{llll}14558 & 15: 29: 01 & 0.0958 & 0.2489 \\ 14560 & 15: 29: 03 & 0.0601 & 0.1674 \\ 14562 & 15: 29: 05 & 0.1212 & 0.1275 \\ 14564 & 15: 29: 07 & 0.1125 & 0.2174 \\ 14566 & 15: 29: 09 & 0.0636 & 0.1035 \\ 14568 & 15: 29: 11 & 0.0691 & 0.2365 \\ 14570 & 15: 29: 13 & 0.0303 & 0.1959 \\ 14572 & 15: 29: 15 & 0.0341 & 0.0717 \\ 14574 & 15: 29: 17 & 0.0991 & 0.1951 \\ 14576 & 15: 29: 19 & 0.0501 & 0.1819 \\ 14578 & 15: 29: 21 & 0.0256 & 0.1392 \\ 14580 & 15: 29: 23 & 0.0896 & 0.1041 \\ 14582 & 15: 29: 25 & 0.0160 & 0.1741 \\ 14584 & 15: 29: 27 & 0.0195 & 0.1015 \\ 14586 & 15: 29: 29 & 0.0348 & 0.1409 \\ 14588 & 15: 29: 31 & 0.0737 & 0.0379 \\ 14590 & 15: 29: 33 & 0.0633 & 0.1112 \\ 14592 & 15: 29: 35 & 0.0300 & 0.1471 \\ 14594 & 15: 29: 37 & 0.1406 & 0.1848 \\ 14596 & 15: 29: 39 & 0.1464 & 0.1518 \\ 14598 & 15: 29: 41 & 0.0157 & 0.0527 \\ 14600 & 15: 29: 43 & 0.0640 & 0.1123 \\ 14602 & 15: 29: 45 & 0.1145 & 0.1260 \\ 14604 & 15: 29: 47 & 0.0210 & 0.0719 \\ 14606 & 15: 29: 49 & 0.0220 & 0.1713 \\ 14608 & 15: 29: 51 & 0.0745 & 0.3215 \\ 14610 & 15: 29: 53 & 0.0187 & 0.3348 \\ 14612 & 15: 29: 55 & 0.0721 & 0.1242 \\ 14614 & 15: 29: 57 & 0.0366 & 0.0920 \\ 14616 & 15: 29: 59 & 0.0349 & 0.2323 \\ 14618 & 15: 30: 01 & 0.0382 & 0.2047 \\ 14620 & 15: 30: 03 & 0.0336 & 0.1373 \\ 14622 & 15: 30: 05 & 0.0446 & 0.1850 \\ 14624 & 15: 30: 07 & 0.0867 & 0.2056 \\ 14626 & 15: 30: 09 & 0.0378 & 0.2232 \\ 14628 & 15: 30: 11 & 0.0494 & 0.1775 \\ 14630 & 15: 30: 13 & 0.0072 & 0.1868 \\ 14632 & 15: 30: 15 & 0.0863 & 0.0696 \\ 14634 & 15: 30: 17 & 0.2172 & 0.1051 \\ 14636 & 15: 30: 19 & 0.0761 & 0.3960 \\ 14638 & 15: 30: 21 & 0.0547 & 0.1363 \\ 14640 & 15: 30: 23 & 0.0441 & 0.1472 \\ 14642 & 15: 30: 25 & 0.1342 & 0.1663 \\ 14644 & 15: 30: 27 & 0.1124 & 0.2880 \\ 14646 & 15: 30: 29 & 0.0178 & 0.2403 \\ 14648 & 15: 30: 31 & 0.0557 & 0.2521 \\ 14650 & 15: 30: 33 & 0.0443 & 0.1807 \\ 14652 & 15: 30: 35 & 0.0501 & 0.5293 \\ 14654 & 15: 30: 37 & 0.0629 & 0.2757 \\ 14656 & 15: 30: 39 & 0.0744 & 0.1247 \\ 14658 & 15: 30: 41 & 0.1460 & 0.3225 \\ 14660 & 15: 30: 43 & 0.1085 & 0.3113\end{array}$


Appendix B - Laser Data Test 1 NIST102505_1.xls

$\begin{array}{llll}14662 & 15: 30: 45 & 0.0220 & 0.2007 \\ 14664 & 15: 30: 47 & 0.0146 & 0.2075 \\ 14666 & 15: 30: 49 & 0.0184 & 0.1098 \\ 14668 & 15: 30: 51 & 0.0550 & 0.3734 \\ 14670 & 15: 30: 53 & 0.0511 & 0.1124 \\ 14672 & 15: 30: 55 & 0.0552 & 0.2533 \\ 14674 & 15: 30: 57 & 0.0335 & 0.1677 \\ 14676 & 15: 30: 59 & 0.0300 & 0.1085 \\ 14678 & 15: 31: 01 & 0.0578 & 0.1208 \\ 14680 & 15: 31: 03 & 0.0211 & 0.1790 \\ 14682 & 15: 31: 05 & 0.0385 & 0.2255 \\ 14684 & 15: 31: 07 & 0.0723 & 0.4699 \\ 14686 & 15: 31: 09 & 0.0699 & 0.2019 \\ 14688 & 15: 31: 11 & 0.0478 & 0.1925 \\ 14690 & 15: 31: 13 & 0.0152 & 0.2600 \\ 14692 & 15: 31: 15 & 0.0792 & 0.1012 \\ 14694 & 15: 31: 17 & 0.0375 & 0.1785 \\ 14696 & 15: 31: 19 & 0.0866 & 0.1445 \\ 14698 & 15: 31: 21 & 0.0581 & 0.1743 \\ 14700 & 15: 31: 23 & 0.0732 & 0.1181 \\ 14702 & 15: 31: 25 & 0.0334 & 0.0907 \\ 14704 & 15: 31: 27 & 0.0437 & 0.2298 \\ 14706 & 15: 31: 29 & 0.0792 & 0.0769 \\ 14708 & 15: 31: 31 & 0.0887 & 0.1157 \\ 14710 & 15: 31: 33 & 0.0718 & 0.1336 \\ 14712 & 15: 31: 35 & 0.0606 & 0.1872 \\ 14714 & 15: 31: 37 & 0.0274 & 0.1306 \\ 14716 & 15: 31: 39 & 0.2067 & 0.1029 \\ 14718 & 15: 31: 41 & 0.1223 & 0.1083 \\ 14720 & 15: 31: 43 & 0.0728 & 0.0714 \\ 14722 & 15: 31: 45 & 0.0394 & 0.1020 \\ 14724 & 15: 31: 47 & 0.0484 & 0.0394 \\ 14726 & 15: 31: 49 & 0.0614 & 0.0844 \\ 14728 & 15: 31: 51 & 0.1334 & 0.1856 \\ 14730 & 15: 31: 53 & 0.0573 & 0.0436 \\ 14732 & 15: 31: 55 & 0.0502 & 0.0662 \\ 14734 & 15: 31: 57 & 0.0441 & 0.1188 \\ 14736 & 15: 31: 59 & 0.0296 & 0.1843 \\ 14738 & 15: 32: 01 & 0.0443 & 0.1870 \\ 14740 & 15: 32: 03 & 0.1512 & 0.2519 \\ 14742 & 15: 32: 05 & 0.0247 & 0.0878 \\ 14744 & 15: 32: 07 & 0.0737 & 0.1074 \\ 14746 & 15: 32: 09 & 0.0644 & 0.2200 \\ 14748 & 15: 32: 11 & 0.1849 & 0.2191 \\ 14750 & 15: 32: 13 & 0.0907 & 0.1043 \\ 14752 & 15: 32: 15 & 0.0375 & 0.2248 \\ 14754 & 15: 32: 17 & 0.0972 & 0.2180 \\ 14756 & 15: 32: 19 & 0.0928 & 0.1605 \\ 14758 & 15: 32: 21 & 0.0701 & 0.2769 \\ 14760 & 15: 32: 23 & 0.0358 & 0.1442 \\ 14762 & 15: 32: 25 & 0.0456 & 0.2056 \\ 14764 & 15: 32: 27 & 0.0338 & 0.1008\end{array}$

B - 142 


$\begin{array}{llll}14766 & 15: 32: 29 & 0.0652 & 0.1853 \\ 14768 & 15: 32: 31 & 0.0218 & 0.1084 \\ 14770 & 15: 32: 33 & 0.0614 & 0.1841 \\ 14772 & 15: 32: 35 & 0.0373 & 0.0843 \\ 14774 & 15: 32: 37 & 0.0423 & 0.0371 \\ 14776 & 15: 32: 39 & 0.1635 & 0.0935 \\ 14778 & 15: 32: 41 & 0.0452 & 0.1111 \\ 14780 & 15: 32: 43 & 0.0437 & 0.1368 \\ 14782 & 15: 32: 45 & 0.0327 & 0.1195 \\ 14784 & 15: 32: 47 & 0.1296 & 0.1287 \\ 14786 & 15: 32: 49 & 0.0581 & 0.0812 \\ 14788 & 15: 32: 51 & 0.0909 & 0.1531 \\ 14790 & 15: 32: 53 & 0.0547 & 0.2548 \\ 14792 & 15: 32: 55 & 0.0406 & 0.1344 \\ 14794 & 15: 32: 57 & 0.0407 & 0.1696 \\ 14796 & 15: 32: 59 & 0.0184 & 0.0674 \\ 14798 & 15: 33: 01 & 0.0515 & 0.1367 \\ 14800 & 15: 33: 03 & 0.0768 & 0.1385 \\ 14802 & 15: 33: 05 & 0.0333 & 0.1877 \\ 14804 & 15: 33: 07 & 0.1372 & 0.5919 \\ 14806 & 15: 33: 09 & 0.1084 & 0.2665 \\ 14808 & 15: 33: 11 & 0.1016 & 0.1089 \\ 14810 & 15: 33: 13 & 0.0414 & 0.1991 \\ 14812 & 15: 33: 15 & 0.1218 & 0.2168 \\ 14814 & 15: 33: 17 & 0.0247 & 0.1650 \\ 14816 & 15: 33: 19 & 0.1189 & 0.3335 \\ 14818 & 15: 33: 21 & 0.0410 & 0.0884 \\ 14820 & 15: 33: 23 & 0.0228 & 0.2295 \\ 14822 & 15: 33: 25 & 0.0177 & 0.1405 \\ 14824 & 15: 33: 27 & 0.0186 & 0.1590 \\ 14826 & 15: 33: 29 & 0.0983 & 0.2417 \\ 14828 & 15: 33: 31 & 0.0441 & 0.1248 \\ 14830 & 15: 33: 33 & 0.0534 & 0.2310 \\ 14832 & 15: 33: 35 & 0.0741 & 0.1981 \\ 14834 & 15: 33: 37 & 0.0343 & 0.2399 \\ 14836 & 15: 33: 39 & 0.2053 & 0.1239 \\ 14838 & 15: 33: 41 & 0.0649 & 0.1750 \\ 14840 & 15: 33: 43 & 0.0701 & 0.1341 \\ 14842 & 15: 33: 45 & 0.1040 & 0.1397 \\ 14844 & 15: 33: 47 & 0.0573 & 0.2754 \\ 14846 & 15: 33: 49 & 0.0573 & 0.0798 \\ 14848 & 15: 33: 51 & 0.0994 & 0.1959 \\ 14850 & 15: 33: 53 & 0.0993 & 0.1585 \\ 14852 & 15: 33: 55 & 0.1457 & 0.1945 \\ 14854 & 15: 33: 57 & 0.2954 & 0.3127 \\ 14856 & 15: 33: 59 & 0.0821 & 0.0883 \\ 14858 & 15: 34: 01 & 0.0972 & 0.0945 \\ 14860 & 15: 34: 03 & 0.0575 & 0.3405 \\ 14862 & 15: 34: 05 & 0.0514 & 0.4089 \\ 14864 & 15: 34: 07 & 0.1078 & 0.2690 \\ 14866 & 15: 34: 09 & 0.2713 & 0.2489 \\ 14868 & 15: 34: 11 & 0.0524 & 0.1393\end{array}$




$\begin{array}{llll}14870 & 15: 34: 13 & 0.0126 & 0.1870 \\ 14872 & 15: 34: 15 & 0.0235 & 0.2197 \\ 14874 & 15: 34: 17 & 0.1073 & 0.2970 \\ 14876 & 15: 34: 19 & 0.0297 & 0.1996 \\ 14878 & 15: 34: 21 & 0.0583 & 0.0649 \\ 14880 & 15: 34: 23 & 0.0729 & 0.3006 \\ 14882 & 15: 34: 25 & 0.0529 & 0.1613 \\ 14884 & 15: 34: 27 & 0.0168 & 0.1334 \\ 14886 & 15: 34: 29 & 0.0465 & 0.0496 \\ 14888 & 15: 34: 31 & 0.0310 & 0.0961 \\ 14890 & 15: 34: 33 & 0.0359 & 0.0980 \\ 14892 & 15: 34: 35 & 0.0235 & 0.1104 \\ 14894 & 15: 34: 37 & 0.0179 & 0.2671 \\ 14896 & 15: 34: 39 & 0.0425 & 0.0570 \\ 14898 & 15: 34: 41 & 0.0741 & 0.1866 \\ 14900 & 15: 34: 43 & 0.0299 & 0.1948 \\ 14902 & 15: 34: 45 & 0.0786 & 0.0898 \\ 14904 & 15: 34: 47 & 0.1210 & 0.3337 \\ 14906 & 15: 34: 49 & 0.0764 & 0.2528 \\ 14908 & 15: 34: 51 & 0.0459 & 0.1936 \\ 14910 & 15: 34: 53 & 0.0789 & 0.1807 \\ 14912 & 15: 34: 55 & 0.1266 & 0.2275 \\ 14914 & 15: 34: 57 & 0.0400 & 0.1832 \\ 14916 & 15: 34: 59 & 0.0550 & 0.1323 \\ 14918 & 15: 35: 01 & 0.0694 & 0.2752 \\ 14920 & 15: 35: 03 & 0.0550 & 0.2754 \\ 14922 & 15: 35: 05 & 0.0981 & 0.2025 \\ 14924 & 15: 35: 07 & 0.0389 & 0.2760 \\ 14926 & 15: 35: 09 & 0.0566 & 0.2264 \\ 14928 & 15: 35: 11 & 0.0389 & 0.0951 \\ 14930 & 15: 35: 13 & 0.1053 & 0.1193 \\ 14932 & 15: 35: 15 & 0.1019 & 0.1230 \\ 14934 & 15: 35: 17 & 0.0475 & 0.2993 \\ 14936 & 15: 35: 19 & 0.0279 & 0.1405 \\ 14938 & 15: 35: 21 & 0.0299 & 0.1867 \\ 14940 & 15: 35: 23 & 0.0882 & 0.1840 \\ 14942 & 15: 35: 25 & 0.0528 & 0.2046 \\ 14944 & 15: 35: 27 & 0.0731 & 0.1788 \\ 14946 & 15: 35: 29 & 0.0490 & 0.4934 \\ 14948 & 15: 35: 31 & 0.1177 & 0.3249 \\ 14950 & 15: 35: 33 & 0.0239 & 0.1062 \\ 14952 & 15: 35: 35 & 0.3338 & 0.3472 \\ 14954 & 15: 35: 37 & 0.0537 & 0.4898 \\ 14956 & 15: 35: 39 & 0.1429 & 0.3360 \\ 14958 & 15: 35: 41 & 0.0389 & 0.1496 \\ 14960 & 15: 35: 43 & 0.0163 & 0.0546 \\ 14962 & 15: 35: 45 & 0.1151 & 0.3752 \\ 14964 & 15: 35: 47 & 0.0322 & 0.1822 \\ 14966 & 15: 35: 49 & 0.0715 & 0.0771 \\ 14968 & 15: 35: 51 & 0.0281 & 0.1473 \\ 14970 & 15: 35: 53 & 0.0620 & 0.1547 \\ 14972 & 15: 35: 55 & 0.0838 & 0.0953\end{array}$




$\begin{array}{llll}14974 & 15: 35: 57 & 0.0714 & 0.1471 \\ 14976 & 15: 35: 59 & 0.0518 & 0.2526 \\ 14978 & 15: 36: 01 & 0.0234 & 0.1169 \\ 14980 & 15: 36: 03 & 0.0954 & 0.2443 \\ 14982 & 15: 36: 05 & 0.1958 & 0.1079 \\ 14984 & 15: 36: 07 & 0.1144 & 0.1051 \\ 14986 & 15: 36: 09 & 0.0379 & 0.0279 \\ 14988 & 15: 36: 11 & 0.1762 & 0.1924 \\ 14990 & 15: 36: 13 & 0.0510 & 0.1246 \\ 14992 & 15: 36: 15 & 0.1351 & 0.1449 \\ 14994 & 15: 36: 17 & 0.0510 & 0.1547 \\ 14996 & 15: 36: 19 & 0.0438 & 0.2306 \\ 14998 & 15: 36: 21 & 0.0609 & 0.2015 \\ 15000 & 15: 36: 23 & 0.0368 & 0.2237 \\ 15002 & 15: 36: 25 & 0.0922 & 0.1370 \\ 15004 & 15: 36: 27 & 0.0340 & 0.1302 \\ 15006 & 15: 36: 29 & 0.0252 & 0.1059 \\ 15008 & 15: 36: 31 & 0.0512 & 0.1456 \\ 15010 & 15: 36: 33 & 0.0710 & 0.1328 \\ 15012 & 15: 36: 35 & 0.0484 & 0.2129 \\ 15014 & 15: 36: 37 & 0.0244 & 0.1368 \\ 15016 & 15: 36: 39 & 0.0348 & 0.2200 \\ 15018 & 15: 36: 41 & 0.0394 & 0.1420 \\ 15020 & 15: 36: 43 & 0.0475 & 0.1066 \\ 15022 & 15: 36: 45 & 0.0473 & 0.1723 \\ 15024 & 15: 36: 47 & 0.0862 & 0.1556 \\ 15026 & 15: 36: 49 & 0.0316 & 0.1068 \\ 15028 & 15: 36: 51 & 0.0411 & 0.0603 \\ 15030 & 15: 36: 53 & 0.0378 & 0.1605 \\ 15032 & 15: 36: 55 & 0.0983 & 0.0956 \\ 15034 & 15: 36: 57 & 0.0386 & 0.0966 \\ 15036 & 15: 36: 59 & 0.0209 & 0.1831 \\ 15038 & 15: 37: 01 & 0.0680 & 0.3522 \\ 15040 & 15: 37: 03 & 0.0966 & 0.1847 \\ 15042 & 15: 37: 05 & 0.0530 & 0.1970 \\ 15044 & 15: 37: 07 & 0.0720 & 0.2206 \\ 15046 & 15: 37: 09 & 0.0901 & 0.0617 \\ 15048 & 15: 37: 11 & 0.0469 & 0.2019 \\ 15050 & 15: 37: 13 & 0.0569 & 0.2046 \\ 15052 & 15: 37: 15 & 0.0391 & 0.1474 \\ 15054 & 15: 37: 17 & 0.0183 & 0.1159 \\ 15056 & 15: 37: 19 & 0.1019 & 0.1768 \\ 15058 & 15: 37: 21 & 0.0997 & 0.1276 \\ 15060 & 15: 37: 23 & 0.0921 & 0.1013 \\ 15062 & 15: 37: 25 & 0.2071 & 0.2584 \\ 15064 & 15: 37: 27 & 0.0667 & 0.3397 \\ 15066 & 15: 37: 29 & 0.0545 & 0.4524 \\ 15068 & 15: 37: 31 & 0.0675 & 0.1653 \\ 15070 & 15: 37: 33 & 0.1224 & 0.1667 \\ 15072 & 15: 37: 35 & 0.0463 & 0.3356 \\ 15074 & 15: 37: 37 & 0.0434 & 0.1282 \\ 15076 & 15: 37: 39 & 0.0477 & 0.1836\end{array}$


Appendix B - Laser Data Test 1 NIST102505_1.xls

$\begin{array}{llll}15078 & 15: 37: 41 & 0.0863 & 0.3103 \\ 15080 & 15: 37: 43 & 0.0570 & 0.1235 \\ 15082 & 15: 37: 45 & 0.0823 & 0.1350 \\ 15084 & 15: 37: 47 & 0.0717 & 0.1401 \\ 15086 & 15: 37: 49 & 0.1033 & 0.2858 \\ 15088 & 15: 37: 51 & 0.0311 & 0.1648 \\ 15090 & 15: 37: 53 & 0.0854 & 0.1761 \\ 15092 & 15: 37: 55 & 0.0376 & 0.1725 \\ 15094 & 15: 37: 57 & 0.0453 & 0.1625 \\ 15096 & 15: 37: 59 & 0.0745 & 0.1426 \\ 15098 & 15: 38: 01 & 0.0609 & 0.3128 \\ 15100 & 15: 38: 03 & 0.0813 & 0.5297 \\ 15102 & 15: 38: 05 & 0.0399 & 0.1486 \\ 15104 & 15: 38: 07 & 0.0428 & 0.3880 \\ 15106 & 15: 38: 09 & 0.0943 & 0.1757 \\ 15108 & 15: 38: 11 & 0.0300 & 0.1260 \\ 15110 & 15: 38: 13 & 0.0893 & 0.0839 \\ 15112 & 15: 38: 15 & 0.0528 & 0.1288 \\ 15114 & 15: 38: 17 & 0.0705 & 0.2693 \\ 15116 & 15: 38: 19 & 0.1039 & 0.0698 \\ 15118 & 15: 38: 21 & 0.1285 & 0.2515 \\ 15120 & 15: 38: 23 & 0.0468 & 0.0743 \\ 15122 & 15: 38: 25 & 0.0632 & 0.1263 \\ 15124 & 15: 38: 27 & 0.0371 & 0.1111 \\ 15126 & 15: 38: 29 & 0.0506 & 0.0969 \\ 15128 & 15: 38: 31 & 0.0387 & 0.3614 \\ 15130 & 15: 38: 33 & 0.0837 & 0.1322 \\ 15132 & 15: 38: 35 & 0.0783 & 0.1078 \\ 15134 & 15: 38: 37 & 0.0368 & 0.1388 \\ 15136 & 15: 38: 39 & 0.0400 & 0.2622 \\ 15138 & 15: 38: 41 & 0.1283 & 0.1901 \\ 15140 & 15: 38: 43 & 0.0380 & 0.2433 \\ 15142 & 15: 38: 45 & 0.0215 & 0.1376 \\ 15144 & 15: 38: 47 & 0.0548 & 0.2405 \\ 15146 & 15: 38: 49 & 0.0515 & 0.1869 \\ 15148 & 15: 38: 51 & 0.1145 & 0.1526 \\ 15150 & 15: 38: 53 & 0.1820 & 0.1257 \\ 15152 & 15: 38: 55 & 0.0439 & 0.0761 \\ 15154 & 15: 38: 57 & 0.1414 & 0.1376 \\ 15156 & 15: 38: 59 & 0.0618 & 0.1560 \\ 15158 & 15: 39: 01 & 0.0721 & 0.2475 \\ 15160 & 15: 39: 03 & 0.0220 & 0.1288 \\ 15162 & 15: 39: 05 & 0.0155 & 0.2587 \\ 15164 & 15: 39: 07 & 0.1358 & 0.4888 \\ 15166 & 15: 39: 09 & 0.0553 & 0.1833 \\ 15168 & 15: 39: 11 & 0.0505 & 0.1023 \\ 15170 & 15: 39: 13 & 0.1050 & 0.0888 \\ 15172 & 15: 39: 15 & 0.0741 & 0.1270 \\ 15174 & 15: 39: 17 & 0.0440 & 0.2679 \\ 15176 & 15: 39: 19 & 0.0730 & 0.0810 \\ 15178 & 15: 39: 21 & 0.0381 & 0.0907 \\ 15180 & 15: 39: 23 & 0.0845 & 0.1333\end{array}$

B - 146 


$\begin{array}{llll}15182 & 15: 39: 25 & 0.0590 & 0.1017 \\ 15184 & 15: 39: 27 & 0.1001 & 0.2273 \\ 15186 & 15: 39: 29 & 0.0315 & 0.1857 \\ 15188 & 15: 39: 31 & 0.1113 & 0.0730 \\ 15190 & 15: 39: 33 & 0.0916 & 0.1606 \\ 15192 & 15: 39: 35 & 0.0273 & 0.0683 \\ 15194 & 15: 39: 37 & 0.0217 & 0.1518 \\ 15196 & 15: 39: 39 & 0.0445 & 0.1180 \\ 15198 & 15: 39: 41 & 0.0386 & 0.1638 \\ 15200 & 15: 39: 43 & 0.1155 & 0.1271 \\ 15202 & 15: 39: 45 & 0.0613 & 0.1290 \\ 15204 & 15: 39: 47 & 0.1261 & 0.4554 \\ 15206 & 15: 39: 49 & 0.1220 & 0.0513 \\ 15208 & 15: 39: 51 & 0.0472 & 0.0850 \\ 15210 & 15: 39: 53 & 0.1055 & 0.2100 \\ 15212 & 15: 39: 55 & 0.0589 & 0.0664 \\ 15214 & 15: 39: 57 & 0.0263 & 0.2667 \\ 15216 & 15: 39: 59 & 0.0849 & 0.1245 \\ 15218 & 15: 40: 01 & 0.0239 & 0.0263 \\ 15220 & 15: 40: 03 & 0.0538 & 0.1742 \\ 15222 & 15: 40: 05 & 0.0435 & 0.1539 \\ 15224 & 15: 40: 07 & 0.0231 & 0.0914 \\ 15226 & 15: 40: 09 & 0.1025 & 0.1849 \\ 15228 & 15: 40: 11 & 0.0521 & 0.1782 \\ 15230 & 15: 40: 13 & 0.0693 & 0.1704 \\ 15232 & 15: 40: 15 & 0.0699 & 0.1887 \\ 15234 & 15: 40: 17 & 0.0888 & 0.0993 \\ 15236 & 15: 40: 19 & 0.0286 & 0.0657 \\ 15238 & 15: 40: 21 & 0.0709 & 0.0652 \\ 15240 & 15: 40: 23 & 0.0462 & 0.0697 \\ 15242 & 15: 40: 25 & 0.1107 & 0.0638 \\ 15244 & 15: 40: 27 & 0.0448 & 0.1933 \\ 15246 & 15: 40: 29 & 0.1321 & 0.1045 \\ 15248 & 15: 40: 31 & 0.0583 & 0.0818 \\ 15250 & 15: 40: 33 & 0.0478 & 0.2285 \\ 15252 & 15: 40: 35 & 0.0856 & 0.1292 \\ 15254 & 15: 40: 37 & 0.0600 & 0.1964 \\ 15256 & 15: 40: 39 & 0.1009 & 0.2102 \\ 15258 & 15: 40: 41 & 0.1970 & 0.0815 \\ 15260 & 15: 40: 43 & 0.0503 & 0.2847 \\ 15262 & 15: 40: 45 & 0.0847 & 0.1918 \\ 15264 & 15: 40: 47 & 0.0094 & 0.1602 \\ 15266 & 15: 40: 49 & 0.0232 & 0.1468 \\ 15268 & 15: 40: 51 & 0.1278 & 0.1621 \\ 15270 & 15: 40: 53 & 0.0318 & 0.0746 \\ 15272 & 15: 40: 55 & 0.0610 & 0.0944 \\ 15274 & 15: 40: 57 & 0.0945 & 0.0878 \\ 15276 & 15: 40: 59 & 0.0671 & 0.0737 \\ 15278 & 15: 41: 01 & 0.1471 & 0.2265 \\ 15280 & 15: 41: 03 & 0.0535 & 0.1830 \\ 15282 & 15: 41: 05 & 0.1668 & 0.0558 \\ 15284 & 15: 41: 07 & 0.1348 & 0.0592\end{array}$




$\begin{array}{llll}15286 & 15: 41: 09 & 0.1192 & 0.3904 \\ 15288 & 15: 41: 11 & 0.1060 & 0.3106 \\ 15290 & 15: 41: 13 & 0.0598 & 0.2597 \\ 15292 & 15: 41: 15 & 0.0227 & 0.1960 \\ 15294 & 15: 41: 17 & 0.1279 & 0.2122 \\ 15296 & 15: 41: 19 & 0.0726 & 0.2043 \\ 15298 & 15: 41: 21 & 0.0373 & 0.1244 \\ 15300 & 15: 41: 23 & 0.0705 & 0.2945 \\ 15302 & 15: 41: 25 & 0.1113 & 0.2231 \\ 15304 & 15: 41: 27 & 0.0826 & 0.1363 \\ 15306 & 15: 41: 29 & 0.0187 & 0.1527 \\ 15308 & 15: 41: 31 & 0.0334 & 0.1961 \\ 15310 & 15: 41: 33 & 0.0376 & 0.1963 \\ 15312 & 15: 41: 35 & 0.0721 & 0.1606 \\ 15314 & 15: 41: 37 & 0.0217 & 0.1320 \\ 15316 & 15: 41: 39 & 0.0480 & 0.3607 \\ 15318 & 15: 41: 41 & 0.0578 & 0.2956 \\ 15320 & 15: 41: 43 & 0.0691 & 0.4188 \\ 15322 & 15: 41: 45 & 0.0714 & 0.2591 \\ 15324 & 15: 41: 47 & 0.0249 & 0.0729 \\ 15326 & 15: 41: 49 & 0.0803 & 0.0772 \\ 15328 & 15: 41: 51 & 0.0603 & 0.2620 \\ 15330 & 15: 41: 53 & 0.0494 & 0.2559 \\ 15332 & 15: 41: 55 & 0.0885 & 0.2036 \\ 15334 & 15: 41: 57 & 0.0514 & 0.1183 \\ 15336 & 15: 41: 59 & 0.0808 & 0.1025 \\ 15338 & 15: 42: 01 & 0.0846 & 0.3222 \\ 15340 & 15: 42: 03 & 0.0378 & 0.2652 \\ 15342 & 15: 42: 05 & 0.0132 & 0.2526 \\ 15344 & 15: 42: 07 & 0.0628 & 0.1659 \\ 15346 & 15: 42: 09 & 0.0892 & 0.4185 \\ 15348 & 15: 42: 11 & 0.0631 & 0.2830 \\ 15350 & 15: 42: 13 & 0.0861 & 0.2419 \\ 15352 & 15: 42: 15 & 0.1058 & 0.2212 \\ 15354 & 15: 42: 17 & 0.0706 & 0.0635 \\ 15356 & 15: 42: 19 & 0.1350 & 0.1792 \\ 15358 & 15: 42: 21 & 0.0285 & 0.2511 \\ 15360 & 15: 42: 23 & 0.0560 & 0.2864 \\ 15362 & 15: 42: 25 & 0.1755 & 0.3113 \\ 15364 & 15: 42: 27 & 0.0918 & 0.3342 \\ 15366 & 15: 42: 29 & 0.0272 & 0.1072 \\ 15368 & 15: 42: 31 & 0.0557 & 0.0799 \\ 15370 & 15: 42: 33 & 0.1426 & 0.0719 \\ 15372 & 15: 42: 35 & 0.0895 & 0.2010 \\ 15374 & 15: 42: 37 & 0.0450 & 0.1526 \\ 15376 & 15: 42: 39 & 0.0670 & 0.1733 \\ 15378 & 15: 42: 41 & 0.0401 & 0.1363 \\ 15380 & 15: 42: 43 & 0.0423 & 0.2570 \\ 15382 & 15: 42: 45 & 0.0450 & 0.2695 \\ 15384 & 15: 42: 47 & 0.1144 & 0.2316 \\ 15386 & 15: 42: 49 & 0.0668 & 0.2828 \\ 15388 & 15: 42: 51 & 0.0621 & 0.2077\end{array}$




$\begin{array}{llll}15390 & 15: 42: 53 & 0.0178 & 0.1944 \\ 15392 & 15: 42: 55 & 0.0469 & 0.2296 \\ 15394 & 15: 42: 57 & 0.0360 & 0.1098 \\ 15396 & 15: 42: 59 & 0.0252 & 0.1411 \\ 15398 & 15: 43: 01 & 0.0719 & 0.3804 \\ 15400 & 15: 43: 03 & 0.0889 & 0.3874 \\ 15402 & 15: 43: 05 & 0.0462 & 0.1926 \\ 15404 & 15: 43: 07 & 0.1001 & 0.3084 \\ 15406 & 15: 43: 09 & 0.0757 & 0.1994 \\ 15408 & 15: 43: 11 & 0.0199 & 0.1240 \\ 15410 & 15: 43: 13 & 0.0448 & 0.0943 \\ 15412 & 15: 43: 15 & 0.0590 & 0.3753 \\ 15414 & 15: 43: 17 & 0.0979 & 0.2383 \\ 15416 & 15: 43: 19 & 0.0514 & 0.2856 \\ 15418 & 15: 43: 21 & 0.0979 & 0.1923 \\ 15420 & 15: 43: 23 & 0.0406 & 0.2624 \\ 15422 & 15: 43: 25 & 0.0667 & 0.1833 \\ 15424 & 15: 43: 27 & 0.0114 & 0.2631 \\ 15426 & 15: 43: 29 & 0.2739 & 0.2519 \\ 15428 & 15: 43: 31 & 0.0193 & 0.2109 \\ 15430 & 15: 43: 33 & 0.0438 & 0.2282 \\ 15432 & 15: 43: 35 & 0.0329 & 0.1384 \\ 15434 & 15: 43: 37 & 0.0319 & 0.2038 \\ 15436 & 15: 43: 39 & 0.0688 & 0.3423 \\ 15438 & 15: 43: 41 & 0.0318 & 0.1807 \\ 15440 & 15: 43: 43 & 0.0353 & 0.1534 \\ 15442 & 15: 43: 45 & 0.0139 & 0.1221 \\ 15444 & 15: 43: 47 & 0.0411 & 0.1190 \\ 15446 & 15: 43: 49 & 0.0161 & 0.2718 \\ 15448 & 15: 43: 51 & 0.0697 & 0.1095 \\ 15450 & 15: 43: 53 & 0.0711 & 0.1476 \\ 15452 & 15: 43: 55 & 0.0519 & 0.1947 \\ 15454 & 15: 43: 57 & 0.1609 & 0.1800 \\ 15456 & 15: 43: 59 & 0.0581 & 0.1880 \\ 15458 & 15: 44: 01 & 0.0571 & 0.1493 \\ 15460 & 15: 44: 03 & 0.1352 & 0.2929 \\ 15462 & 15: 44: 05 & 0.0163 & 0.2238 \\ 15464 & 15: 44: 07 & 0.0448 & 0.1869 \\ 15466 & 15: 44: 09 & 0.0637 & 0.2528 \\ 15468 & 15: 44: 11 & 0.0600 & 0.1188 \\ 15470 & 15: 44: 13 & 0.0270 & 0.1594 \\ 15472 & 15: 44: 15 & 0.0336 & 0.1702 \\ 15474 & 15: 44: 17 & 0.0562 & 0.3573 \\ 15476 & 15: 44: 19 & 0.1290 & 0.3010 \\ 15478 & 15: 44: 21 & 0.0321 & 0.1963 \\ 15480 & 15: 44: 23 & 0.0676 & 0.1039 \\ 15482 & 15: 44: 25 & 0.0279 & 0.2653 \\ 15484 & 15: 44: 27 & 0.0230 & 0.1063 \\ 15486 & 15: 44: 29 & 0.1364 & 0.2245 \\ 15488 & 15: 44: 31 & 0.0865 & 0.4087 \\ 15490 & 15: 44: 33 & 0.0245 & 0.0665 \\ 15492 & 15: 44: 35 & 0.0380 & 0.2138\end{array}$




$\begin{array}{llll}15494 & 15: 44: 37 & 0.0399 & 0.2487 \\ 15496 & 15: 44: 39 & 0.0245 & 0.0593 \\ 15498 & 15: 44: 41 & 0.0770 & 0.4143 \\ 15500 & 15: 44: 43 & 0.0102 & 0.1825 \\ 15502 & 15: 44: 45 & 0.0431 & 0.1993 \\ 15504 & 15: 44: 47 & 0.0070 & 0.1095 \\ 15506 & 15: 44: 49 & 0.0380 & 0.1989 \\ 15508 & 15: 44: 51 & 0.0173 & 0.1842 \\ 15510 & 15: 44: 53 & 0.0367 & 0.0997 \\ 15512 & 15: 44: 55 & 0.0671 & 0.0595 \\ 15514 & 15: 44: 57 & 0.0132 & 0.1194 \\ 15516 & 15: 44: 59 & 0.0121 & 0.1014 \\ 15518 & 15: 45: 01 & 0.0638 & 0.2247 \\ 15520 & 15: 45: 03 & 0.0797 & 0.2282 \\ 15522 & 15: 45: 05 & 0.1355 & 0.1182 \\ 15524 & 15: 45: 07 & 0.0722 & 0.1138 \\ 15526 & 15: 45: 09 & 0.0290 & 0.0415 \\ 15528 & 15: 45: 11 & 0.0660 & 0.1093 \\ 15530 & 15: 45: 13 & 0.0674 & 0.1430 \\ 15532 & 15: 45: 15 & 0.1816 & 0.2790 \\ 15534 & 15: 45: 17 & 0.0376 & 0.0868 \\ 15536 & 15: 45: 19 & 0.0225 & 0.1881 \\ 15538 & 15: 45: 21 & 0.0326 & 0.2571 \\ 15540 & 15: 45: 23 & 0.0411 & 0.1904 \\ 15542 & 15: 45: 25 & 0.0410 & 0.1485 \\ 15544 & 15: 45: 27 & 0.0287 & 0.0846 \\ 15546 & 15: 45: 29 & 0.0537 & 0.2780 \\ 15548 & 15: 45: 31 & 0.1408 & 0.1905 \\ 15550 & 15: 45: 33 & 0.0630 & 0.1101 \\ 15552 & 15: 45: 35 & 0.0550 & 0.1987 \\ 15554 & 15: 45: 37 & 0.0174 & 0.0447 \\ 15556 & 15: 45: 39 & 0.0193 & 0.0803 \\ 15558 & 15: 45: 41 & 0.1387 & 0.1865 \\ 15560 & 15: 45: 43 & 0.0540 & 0.1235 \\ 15562 & 15: 45: 45 & 0.0242 & 0.1878 \\ 15564 & 15: 45: 47 & 0.0459 & 0.1025 \\ 15566 & 15: 45: 49 & 0.0229 & 0.0584 \\ 15568 & 15: 45: 51 & 0.0276 & 0.2055 \\ 15570 & 15: 45: 53 & 0.0186 & 0.1287 \\ 15572 & 15: 45: 55 & 0.0385 & 0.1272 \\ 15574 & 15: 45: 57 & 0.0982 & 0.0660 \\ 15576 & 15: 45: 59 & 0.0518 & 0.0903 \\ 15578 & 15: 46: 01 & 0.1229 & 0.1201 \\ 15580 & 15: 46: 03 & 0.0343 & 0.1692 \\ 15582 & 15: 46: 05 & 0.0498 & 0.1628 \\ 15584 & 15: 46: 07 & 0.0590 & 0.2234 \\ 15586 & 15: 46: 09 & 0.0448 & 0.2563 \\ 15588 & 15: 46: 11 & 0.0146 & 0.1619 \\ 15590 & 15: 46: 13 & 0.0188 & 0.1611 \\ 15592 & 15: 46: 15 & 0.0202 & 0.0732 \\ 15594 & 15: 46: 17 & 0.0666 & 0.1228 \\ 15596 & 15: 46: 19 & 0.0346 & 0.1662\end{array}$




$\begin{array}{llll}15598 & 15: 46: 21 & 0.0884 & 0.0846 \\ 15600 & 15: 46: 23 & 0.0332 & 0.2004 \\ 15602 & 15: 46: 25 & 0.0809 & 0.2118 \\ 15604 & 15: 46: 27 & 0.0782 & 0.2084 \\ 15606 & 15: 46: 29 & 0.1399 & 0.2452 \\ 15608 & 15: 46: 31 & 0.1283 & 0.2578 \\ 15610 & 15: 46: 33 & 0.0097 & 0.1733 \\ 15612 & 15: 46: 35 & 0.0322 & 0.1208 \\ 15614 & 15: 46: 37 & 0.0495 & 0.3719 \\ 15616 & 15: 46: 39 & 0.0930 & 0.1006 \\ 15618 & 15: 46: 41 & 0.1018 & 0.2868 \\ 15620 & 15: 46: 43 & 0.0404 & 0.2653 \\ 15622 & 15: 46: 45 & 0.0456 & 0.3230 \\ 15624 & 15: 46: 47 & 0.1507 & 0.0683 \\ 15626 & 15: 46: 49 & 0.0887 & 0.2136 \\ 15628 & 15: 46: 51 & 0.1404 & 0.1901 \\ 15630 & 15: 46: 53 & 0.0331 & 0.1148 \\ 15632 & 15: 46: 55 & 0.0827 & 0.3866 \\ 15634 & 15: 46: 57 & 0.0607 & 0.1053 \\ 15636 & 15: 46: 59 & 0.0515 & 0.1548 \\ 15638 & 15: 47: 01 & 0.0233 & 0.1250 \\ 15640 & 15: 47: 03 & 0.0303 & 0.2740 \\ 15642 & 15: 47: 05 & 0.0442 & 0.1348 \\ 15644 & 15: 47: 07 & 0.0494 & 0.1838 \\ 15646 & 15: 47: 09 & 0.0499 & 0.2521 \\ 15648 & 15: 47: 11 & 0.0811 & 0.2057 \\ 15650 & 15: 47: 13 & 0.0322 & 0.1707 \\ 15652 & 15: 47: 15 & 0.0941 & 0.1464 \\ 15654 & 15: 47: 17 & 0.0551 & 0.1883 \\ 15656 & 15: 47: 19 & 0.0614 & 0.0407 \\ 15658 & 15: 47: 21 & 0.0734 & 0.1337 \\ 15660 & 15: 47: 23 & 0.0737 & 0.0656 \\ 15662 & 15: 47: 25 & 0.0351 & 0.1624 \\ 15664 & 15: 47: 27 & 0.0388 & 0.2118 \\ 15666 & 15: 47: 29 & 0.0687 & 0.2413 \\ 15668 & 15: 47: 31 & 0.0441 & 0.2319 \\ 15670 & 15: 47: 33 & 0.0273 & 0.0853 \\ 15672 & 15: 47: 35 & 0.0358 & 0.2911 \\ 15674 & 15: 47: 37 & 0.0204 & 0.0824 \\ 15676 & 15: 47: 39 & 0.0719 & 0.1264 \\ 15678 & 15: 47: 41 & 0.0230 & 0.1238 \\ 15680 & 15: 47: 43 & 0.0339 & 0.2219 \\ 15682 & 15: 47: 45 & 0.0190 & 0.1413 \\ 15684 & 15: 47: 47 & 0.0371 & 0.1491 \\ 15686 & 15: 47: 49 & 0.0292 & 0.3050 \\ 15688 & 15: 47: 51 & 0.0312 & 0.1589 \\ 15690 & 15: 47: 53 & 0.0352 & 0.2622 \\ 15692 & 15: 47: 55 & 0.0215 & 0.1514 \\ 15694 & 15: 47: 57 & 0.0176 & 0.1645 \\ 15696 & 15: 47: 59 & 0.0426 & 0.0732 \\ 15698 & 15: 48: 01 & 0.1191 & 0.1583 \\ 15700 & 15: 48: 03 & 0.0397 & 0.1282\end{array}$




$\begin{array}{llll}15702 & 15: 48: 05 & 0.1049 & 0.0777 \\ 15704 & 15: 48: 07 & 0.0678 & 0.1764 \\ 15706 & 15: 48: 09 & 0.0272 & 0.2075 \\ 15708 & 15: 48: 11 & 0.0728 & 0.5390 \\ 15710 & 15: 48: 13 & 0.0175 & 0.3174 \\ 15712 & 15: 48: 15 & 0.1143 & 0.2484 \\ 15714 & 15: 48: 17 & 0.0716 & 0.1350 \\ 15716 & 15: 48: 19 & 0.0397 & 0.1371 \\ 15718 & 15: 48: 21 & 0.0232 & 0.0995 \\ 15720 & 15: 48: 23 & 0.0339 & 0.2307 \\ 15722 & 15: 48: 25 & 0.0569 & 0.0867 \\ 15724 & 15: 48: 27 & 0.0634 & 0.1450 \\ 15726 & 15: 48: 29 & 0.2639 & 0.1998 \\ 15728 & 15: 48: 31 & 0.0371 & 0.1708 \\ 15730 & 15: 48: 33 & 0.0270 & 0.1920 \\ 15732 & 15: 48: 35 & 0.1046 & 0.4625 \\ 15734 & 15: 48: 37 & 0.0653 & 0.1223 \\ 15736 & 15: 48: 39 & 0.0088 & 0.1467 \\ 15738 & 15: 48: 41 & 0.0587 & 0.2055 \\ 15740 & 15: 48: 43 & 0.0532 & 0.0924 \\ 15742 & 15: 48: 45 & 0.0404 & 0.1329 \\ 15744 & 15: 48: 47 & 0.0620 & 0.0449 \\ 15746 & 15: 48: 49 & 0.0885 & 0.2749 \\ 15748 & 15: 48: 51 & 0.0668 & 0.0967 \\ 15750 & 15: 48: 53 & 0.0526 & 0.3116 \\ 15752 & 15: 48: 55 & 0.0431 & 0.1726 \\ 15754 & 15: 48: 57 & 0.0455 & 0.1510 \\ 15756 & 15: 48: 59 & 0.1240 & 0.0650 \\ 15758 & 15: 49: 01 & 0.1996 & 0.3643 \\ 15760 & 15: 49: 03 & 0.0971 & 0.1117 \\ 15762 & 15: 49: 05 & 0.0505 & 0.3007 \\ 15764 & 15: 49: 07 & 0.0628 & 0.1259 \\ 15766 & 15: 49: 09 & 0.1253 & 0.1448 \\ 15768 & 15: 49: 11 & 0.2063 & 0.1683 \\ 15770 & 15: 49: 13 & 0.0245 & 0.1044 \\ 15772 & 15: 49: 15 & 0.0921 & 0.1588 \\ 15774 & 15: 49: 17 & 0.0979 & 0.1033 \\ 15776 & 15: 49: 19 & 0.0464 & 0.1658 \\ 15778 & 15: 49: 21 & 0.0375 & 0.2123 \\ 15780 & 15: 49: 23 & 0.1630 & 0.2263 \\ 15782 & 15: 49: 25 & 0.0557 & 0.1845 \\ 15784 & 15: 49: 27 & 0.0651 & 0.2264 \\ 15786 & 15: 49: 29 & 0.0942 & 0.4785 \\ 15788 & 15: 49: 31 & 0.0399 & 0.1526 \\ 15790 & 15: 49: 33 & 0.0756 & 0.2052 \\ 15792 & 15: 49: 35 & 0.0251 & 0.1124 \\ 15794 & 15: 49: 37 & 0.0309 & 0.1186 \\ 15796 & 15: 49: 39 & 0.0357 & 0.1707 \\ 15798 & 15: 49: 41 & 0.0909 & 0.3056 \\ 15800 & 15: 49: 43 & 0.0834 & 0.0732 \\ 15802 & 15: 49: 45 & 0.0602 & 0.2256 \\ 15804 & 15: 49: 47 & 0.1080 & 0.2803\end{array}$




$\begin{array}{llll}15806 & 15: 49: 49 & 0.0706 & 0.1061 \\ 15808 & 15: 49: 51 & 0.0802 & 0.3394 \\ 15810 & 15: 49: 53 & 0.0267 & 0.1090 \\ 15812 & 15: 49: 55 & 0.0872 & 0.2912 \\ 15814 & 15: 49: 57 & 0.1113 & 0.2305 \\ 15816 & 15: 49: 59 & 0.0863 & 0.1253 \\ 15818 & 15: 50: 01 & 0.0951 & 0.1269 \\ 15820 & 15: 50: 03 & 0.0616 & 0.1784 \\ 15822 & 15: 50: 05 & 0.0928 & 0.1133 \\ 15824 & 15: 50: 07 & 0.2270 & 0.0867 \\ 15826 & 15: 50: 09 & 0.0427 & 0.0613 \\ 15828 & 15: 50: 11 & 0.0359 & 0.2802 \\ 15830 & 15: 50: 13 & 0.0698 & 0.0997 \\ 15832 & 15: 50: 15 & 0.1780 & 0.2325 \\ 15834 & 15: 50: 17 & 0.0306 & 0.2229 \\ 15836 & 15: 50: 19 & 0.0667 & 0.3463 \\ 15838 & 15: 50: 21 & 0.0501 & 0.1625 \\ 15840 & 15: 50: 23 & 0.1149 & 0.1552 \\ 15842 & 15: 50: 25 & 0.2569 & 0.2063 \\ 15844 & 15: 50: 27 & 0.1080 & 0.1918 \\ 15846 & 15: 50: 29 & 0.0637 & 0.1274 \\ 15848 & 15: 50: 31 & 0.0565 & 0.2451 \\ 15850 & 15: 50: 33 & 0.0744 & 0.1469 \\ 15852 & 15: 50: 35 & 0.0309 & 0.1865 \\ 15854 & 15: 50: 37 & 0.0219 & 0.2338 \\ 15856 & 15: 50: 39 & 0.1525 & 0.2590 \\ 15858 & 15: 50: 41 & 0.0483 & 0.1774 \\ 15860 & 15: 50: 43 & 0.0981 & 0.1296 \\ 15862 & 15: 50: 45 & 0.1094 & 0.0940 \\ 15864 & 15: 50: 47 & 0.0823 & 0.2172 \\ 15866 & 15: 50: 49 & 0.0390 & 0.1121 \\ 15868 & 15: 50: 51 & 0.0404 & 0.1841 \\ 15870 & 15: 50: 53 & 0.1639 & 0.1706 \\ 15872 & 15: 50: 55 & 0.0331 & 0.1229 \\ 15874 & 15: 50: 57 & 0.0771 & 0.1943 \\ 15876 & 15: 50: 59 & 0.0778 & 0.1243 \\ 15878 & 15: 51: 01 & 0.0629 & 0.1602 \\ 15880 & 15: 51: 03 & 0.0341 & 0.3348 \\ 15882 & 15: 51: 05 & 0.0858 & 0.0855 \\ 15884 & 15: 51: 07 & 0.1683 & 0.0927 \\ 15886 & 15: 51: 09 & 0.0945 & 0.1641 \\ 15888 & 15: 51: 11 & 0.0720 & 0.1032 \\ 15890 & 15: 51: 13 & 0.0234 & 0.0982 \\ 15892 & 15: 51: 15 & 0.0513 & 0.1469 \\ 15894 & 15: 51: 17 & 0.0436 & 0.2122 \\ 15896 & 15: 51: 19 & 0.0220 & 0.2972 \\ 15898 & 15: 51: 21 & 0.0363 & 0.1807 \\ 15900 & 15: 51: 23 & 0.0810 & 0.1763 \\ 15902 & 15: 51: 25 & 0.0758 & 0.2654 \\ 15904 & 15: 51: 27 & 0.0636 & 0.2448 \\ 15906 & 15: 51: 29 & 0.0639 & 0.3985 \\ 15908 & 15: 51: 31 & 0.0646 & 0.1901\end{array}$




$\begin{array}{llll}15910 & 15: 51: 33 & 0.0815 & 0.1304 \\ 15912 & 15: 51: 35 & 0.0424 & 0.1146 \\ 15914 & 15: 51: 37 & 0.0820 & 0.0887 \\ 15916 & 15: 51: 39 & 0.0738 & 0.2791 \\ 15918 & 15: 51: 41 & 0.1511 & 0.1848 \\ 15920 & 15: 51: 43 & 0.1047 & 0.2528 \\ 15922 & 15: 51: 45 & 0.0497 & 0.0986 \\ 15924 & 15: 51: 47 & 0.0701 & 0.2608 \\ 15926 & 15: 51: 49 & 0.0669 & 0.1712 \\ 15928 & 15: 51: 51 & 0.1059 & 0.3582 \\ 15930 & 15: 51: 53 & 0.0506 & 0.0936 \\ 15932 & 15: 51: 55 & 0.1499 & 0.2171 \\ 15934 & 15: 51: 57 & 0.0224 & 0.2587 \\ 15936 & 15: 51: 59 & 0.0275 & 0.1712 \\ 15938 & 15: 52: 01 & 0.0225 & 0.3725 \\ 15940 & 15: 52: 03 & 0.0709 & 0.0890 \\ 15942 & 15: 52: 05 & 0.1862 & 0.1095 \\ 15944 & 15: 52: 07 & 0.0789 & 0.1091 \\ 15946 & 15: 52: 09 & 0.1287 & 0.1506 \\ 15948 & 15: 52: 11 & 0.0975 & 0.0922 \\ 15950 & 15: 52: 13 & 0.0993 & 0.0581 \\ 15952 & 15: 52: 15 & 0.1413 & 0.1087 \\ 15954 & 15: 52: 17 & 0.0614 & 0.1521 \\ 15956 & 15: 52: 19 & 0.1151 & 0.1413 \\ 15958 & 15: 52: 21 & 0.0363 & 0.2454 \\ 15960 & 15: 52: 23 & 0.0350 & 0.1242 \\ 15962 & 15: 52: 25 & 0.0598 & 0.1304 \\ 15964 & 15: 52: 27 & 0.0170 & 0.1360 \\ 15966 & 15: 52: 29 & 0.0165 & 0.1441 \\ 15968 & 15: 52: 31 & 0.0675 & 0.4965 \\ 15970 & 15: 52: 33 & 0.1199 & 0.1878 \\ 15972 & 15: 52: 35 & 0.2874 & 0.1579 \\ 15974 & 15: 52: 37 & 0.2655 & 0.1308 \\ 15976 & 15: 52: 39 & 0.1876 & 0.2561 \\ 15978 & 15: 52: 41 & 0.1133 & 0.2121 \\ 15980 & 15: 52: 43 & 0.0519 & 0.1108 \\ 15982 & 15: 52: 45 & 0.0784 & 0.2522 \\ 15984 & 15: 52: 47 & 0.1057 & 0.2450 \\ 15986 & 15: 52: 49 & 0.1407 & 0.1868 \\ 15988 & 15: 52: 51 & 0.0376 & 0.1835 \\ 15990 & 15: 52: 53 & 0.0677 & 0.1978 \\ 15992 & 15: 52: 55 & 0.0436 & 0.2249 \\ 15994 & 15: 52: 57 & 0.1198 & 0.0775 \\ 15996 & 15: 52: 59 & 0.1382 & 0.1668 \\ 15998 & 15: 53: 01 & 0.0861 & 0.3879 \\ 16000 & 15: 53: 03 & 0.0719 & 0.1742 \\ 16002 & 15: 53: 05 & 0.0543 & 0.1112 \\ 16004 & 15: 53: 07 & 0.0331 & 0.1990 \\ 16006 & 15: 53: 09 & 0.0193 & 0.2204 \\ 16008 & 15: 53: 11 & 0.1774 & 0.3246 \\ 16010 & 15: 53: 13 & 0.1076 & 0.1955 \\ 16012 & 15: 53: 15 & 0.0387 & 0.1083\end{array}$




$\begin{array}{llll}16014 & 15: 53: 17 & 0.1659 & 0.2805 \\ 16016 & 15: 53: 19 & 0.0721 & 0.1396 \\ 16018 & 15: 53: 21 & 0.0825 & 0.0673 \\ 16020 & 15: 53: 23 & 0.1458 & 0.1281 \\ 16022 & 15: 53: 25 & 0.0686 & 0.2975 \\ 16024 & 15: 53: 27 & 0.0387 & 0.1095 \\ 16026 & 15: 53: 29 & 0.0346 & 0.3026 \\ 16028 & 15: 53: 31 & 0.1203 & 0.1810 \\ 16030 & 15: 53: 33 & 0.0693 & 0.2985 \\ 16032 & 15: 53: 35 & 0.0431 & 0.2705 \\ 16034 & 15: 53: 37 & 0.1288 & 0.1743 \\ 16036 & 15: 53: 39 & 0.1062 & 0.1585 \\ 16038 & 15: 53: 41 & 0.0701 & 0.1138 \\ 16040 & 15: 53: 43 & 0.0252 & 0.0913 \\ 16042 & 15: 53: 45 & 0.0953 & 0.0567 \\ 16044 & 15: 53: 47 & 0.0715 & 0.2358 \\ 16046 & 15: 53: 49 & 0.0415 & 0.3727 \\ 16048 & 15: 53: 51 & 0.0352 & 0.1686 \\ 16050 & 15: 53: 53 & 0.0648 & 0.1933 \\ 16052 & 15: 53: 55 & 0.0341 & 0.1192 \\ 16054 & 15: 53: 57 & 0.1186 & 0.2229 \\ 16056 & 15: 53: 59 & 0.0888 & 0.2893 \\ 16058 & 15: 54: 01 & 0.0242 & 0.4511 \\ 16060 & 15: 54: 03 & 0.0357 & 0.1317 \\ 16062 & 15: 54: 05 & 0.1104 & 0.1439 \\ 16064 & 15: 54: 07 & 0.0461 & 0.1361 \\ 16066 & 15: 54: 09 & 0.0696 & 0.1370 \\ 16068 & 15: 54: 11 & 0.1028 & 0.2889 \\ 16070 & 15: 54: 13 & 0.0898 & 0.2145 \\ 16072 & 15: 54: 15 & 0.0349 & 0.1887 \\ 16074 & 15: 54: 17 & 0.0642 & 0.1605 \\ 16076 & 15: 54: 19 & 0.1179 & 0.1303 \\ 16078 & 15: 54: 21 & 0.0927 & 0.0513 \\ 16080 & 15: 54: 23 & 0.1270 & 0.1204 \\ 16082 & 15: 54: 25 & 0.1606 & 0.1302 \\ 16084 & 15: 54: 27 & 0.1592 & 0.4590 \\ 16086 & 15: 54: 29 & 0.0833 & 0.3555 \\ 16088 & 15: 54: 31 & 0.0265 & 0.0900 \\ 16090 & 15: 54: 33 & 0.0324 & 0.1175 \\ 16092 & 15: 54: 35 & 0.0502 & 0.1105 \\ 16094 & 15: 54: 37 & 0.0897 & 0.2224 \\ 16096 & 15: 54: 39 & 0.2370 & 0.2573 \\ 16098 & 15: 54: 41 & 0.2853 & 0.5253 \\ 16100 & 15: 54: 43 & 0.0499 & 0.1507 \\ 16102 & 15: 54: 45 & 0.2062 & 0.1845 \\ 16104 & 15: 54: 47 & 0.0425 & 0.2025 \\ 16106 & 15: 54: 49 & 0.0503 & 0.1924 \\ 16108 & 15: 54: 51 & 0.1103 & 0.0261 \\ 16110 & 15: 54: 53 & 0.1061 & 0.2469 \\ 16112 & 15: 54: 55 & 0.0623 & 0.1222 \\ 16114 & 15: 54: 57 & 0.1565 & 0.3357 \\ 16116 & 15: 54: 59 & 0.0299 & 0.1699\end{array}$




$\begin{array}{llll}16118 & 15: 55: 01 & 0.1260 & 0.2292 \\ 16120 & 15: 55: 03 & 0.1178 & 0.1799 \\ 16122 & 15: 55: 05 & 0.1064 & 0.1281 \\ 16124 & 15: 55: 07 & 0.0270 & 0.2165 \\ 16126 & 15: 55: 09 & 0.0696 & 0.0740 \\ 16128 & 15: 55: 11 & 0.0221 & 0.0890 \\ 16130 & 15: 55: 13 & 0.0799 & 0.1788 \\ 16132 & 15: 55: 15 & 0.0179 & 0.2596 \\ 16134 & 15: 55: 17 & 0.0607 & 0.1452 \\ 16136 & 15: 55: 19 & 0.0490 & 0.1123 \\ 16138 & 15: 55: 21 & 0.0383 & 0.1978 \\ 16140 & 15: 55: 23 & 0.0930 & 0.2484 \\ 16142 & 15: 55: 25 & 0.0487 & 0.1447 \\ 16144 & 15: 55: 27 & 0.0811 & 0.1564 \\ 16146 & 15: 55: 29 & 0.0513 & 0.0657 \\ 16148 & 15: 55: 31 & 0.0729 & 0.2850 \\ 16150 & 15: 55: 33 & 0.0471 & 0.1220 \\ 16152 & 15: 55: 35 & 0.0452 & 0.1460 \\ 16154 & 15: 55: 37 & 0.1255 & 0.3411 \\ 16156 & 15: 55: 39 & 0.1152 & 0.2090 \\ 16158 & 15: 55: 41 & 0.1331 & 0.0899 \\ 16160 & 15: 55: 43 & 0.0194 & 0.0579 \\ 16162 & 15: 55: 45 & 0.0599 & 0.1189 \\ 16164 & 15: 55: 47 & 0.1149 & 0.1477 \\ 16166 & 15: 55: 49 & 0.0778 & 0.1166 \\ 16168 & 15: 55: 51 & 0.0565 & 0.4111 \\ 16170 & 15: 55: 53 & 0.0576 & 0.1493 \\ 16172 & 15: 55: 55 & 0.0128 & 0.0296 \\ 16174 & 15: 55: 57 & 0.0945 & 0.1406 \\ 16176 & 15: 55: 59 & 0.0428 & 0.2071 \\ 16178 & 15: 56: 01 & 0.0185 & 0.0318 \\ 16180 & 15: 56: 03 & 0.1037 & 0.2033 \\ 16182 & 15: 56: 05 & 0.0545 & 0.1131 \\ 16184 & 15: 56: 07 & 0.1331 & 0.2264 \\ 16186 & 15: 56: 09 & 0.1287 & 0.2849 \\ 16188 & 15: 56: 11 & 0.0446 & 0.2253 \\ 16190 & 15: 56: 13 & 0.1281 & 0.0687 \\ 16192 & 15: 56: 15 & 0.1457 & 0.1393 \\ 16194 & 15: 56: 17 & 0.1122 & 0.3237 \\ 16196 & 15: 56: 19 & 0.0623 & 0.1956 \\ 16198 & 15: 56: 21 & 0.0718 & 0.0970 \\ 16200 & 15: 56: 23 & 0.0233 & 0.1262 \\ 16202 & 15: 56: 25 & 0.0380 & 0.0887 \\ 16204 & 15: 56: 27 & 0.0173 & 0.1256 \\ 16206 & 15: 56: 29 & 0.0156 & 0.2069 \\ 16208 & 15: 56: 31 & 0.0381 & 0.1525 \\ 16210 & 15: 56: 33 & 0.0312 & 0.1007 \\ 16212 & 15: 56: 35 & 0.0628 & 0.0793 \\ 16214 & 15: 56: 37 & 0.0087 & 0.1244 \\ 16216 & 15: 56: 39 & 0.0202 & 0.0832 \\ 16218 & 15: 56: 41 & 0.0170 & 0.0995 \\ 16220 & 15: 56: 43 & 0.0519 & 0.2085\end{array}$




$\begin{array}{llll}16222 & 15: 56: 45 & 0.0306 & 0.2125 \\ 16224 & 15: 56: 47 & 0.0676 & 0.1307 \\ 16226 & 15: 56: 49 & 0.0568 & 0.2879 \\ 16228 & 15: 56: 51 & 0.0322 & 0.0621 \\ 16230 & 15: 56: 53 & 0.0233 & 0.1527 \\ 16232 & 15: 56: 55 & 0.0948 & 0.1441 \\ 16234 & 15: 56: 57 & 0.0869 & 0.1408 \\ 16236 & 15: 56: 59 & 0.0711 & 0.2439 \\ 16238 & 15: 57: 01 & 0.0885 & 0.0867 \\ 16240 & 15: 57: 03 & 0.0424 & 0.0755 \\ 16242 & 15: 57: 05 & 0.0432 & 0.2118 \\ 16244 & 15: 57: 07 & 0.0443 & 0.1079 \\ 16246 & 15: 57: 09 & 0.0377 & 0.0973 \\ 16248 & 15: 57: 11 & 0.0325 & 0.1182 \\ 16250 & 15: 57: 13 & 0.0639 & 0.2313 \\ 16252 & 15: 57: 15 & 0.0653 & 0.1257 \\ 16254 & 15: 57: 17 & 0.0697 & 0.1292 \\ 16256 & 15: 57: 19 & 0.0425 & 0.0723 \\ 16258 & 15: 57: 21 & 0.0728 & 0.2475 \\ 16260 & 15: 57: 23 & 0.0806 & 0.1868 \\ 16262 & 15: 57: 25 & 0.0666 & 0.3676 \\ 16264 & 15: 57: 27 & 0.0330 & 0.2336 \\ 16266 & 15: 57: 29 & 0.0152 & 0.1961 \\ 16268 & 15: 57: 31 & 0.0743 & 0.1507 \\ 16270 & 15: 57: 33 & 0.0388 & 0.2539 \\ 16272 & 15: 57: 35 & 0.0741 & 0.1164 \\ 16274 & 15: 57: 37 & 0.0712 & 0.1355 \\ 16276 & 15: 57: 39 & 0.0339 & 0.2444 \\ 16278 & 15: 57: 41 & 0.0989 & 0.3351 \\ 16280 & 15: 57: 43 & 0.1296 & 0.1858 \\ 16282 & 15: 57: 45 & 0.1192 & 0.2155 \\ 16284 & 15: 57: 47 & 0.1092 & 0.0932 \\ 16286 & 15: 57: 49 & 0.1005 & 0.0879 \\ 16288 & 15: 57: 51 & 0.0553 & 0.2972 \\ 16290 & 15: 57: 53 & 0.0918 & 0.1363 \\ 16292 & 15: 57: 55 & 0.0340 & 0.0760 \\ 16294 & 15: 57: 57 & 0.1744 & 0.2210 \\ 16296 & 15: 57: 59 & 0.0213 & 0.1602 \\ 16298 & 15: 58: 01 & 0.0431 & 0.2410 \\ 16300 & 15: 58: 03 & 0.0618 & 0.1460 \\ 16302 & 15: 58: 05 & 0.0397 & 0.1761 \\ 16304 & 15: 58: 07 & 0.0232 & 0.1583 \\ 16306 & 15: 58: 09 & 0.1117 & 0.3058 \\ 16308 & 15: 58: 11 & 0.1410 & 0.1894 \\ 16310 & 15: 58: 13 & 0.0693 & 0.1907 \\ 16312 & 15: 58: 15 & 0.0837 & 0.2007 \\ 16314 & 15: 58: 17 & 0.0746 & 0.1510 \\ 16316 & 15: 58: 19 & 0.0531 & 0.1033 \\ 16318 & 15: 58: 21 & 0.0571 & 0.2636 \\ 16320 & 15: 58: 23 & 0.0615 & 0.1793 \\ 16322 & 15: 58: 25 & 0.0336 & 0.2397 \\ 16324 & 15: 58: 27 & 0.0466 & 0.2593\end{array}$




$\begin{array}{llll}16326 & 15: 58: 29 & 0.0394 & 0.1108 \\ 16328 & 15: 58: 31 & 0.0420 & 0.0947 \\ 16330 & 15: 58: 33 & 0.1015 & 0.1624 \\ 16332 & 15: 58: 35 & 0.1387 & 0.2566 \\ 16334 & 15: 58: 37 & 0.0818 & 0.1894 \\ 16336 & 15: 58: 39 & 0.0244 & 0.0687 \\ 16338 & 15: 58: 41 & 0.0579 & 0.0650 \\ 16340 & 15: 58: 43 & 0.0516 & 0.1149 \\ 16342 & 15: 58: 45 & 0.0313 & 0.0631 \\ 16344 & 15: 58: 47 & 0.0501 & 0.2382 \\ 16346 & 15: 58: 49 & 0.0490 & 0.2882 \\ 16348 & 15: 58: 51 & 0.0403 & 0.4163 \\ 16350 & 15: 58: 53 & 0.0856 & 0.2598 \\ 16352 & 15: 58: 55 & 0.0525 & 0.1659 \\ 16354 & 15: 58: 57 & 0.0602 & 0.3796 \\ 16356 & 15: 58: 59 & 0.0507 & 0.1188 \\ 16358 & 15: 59: 01 & 0.0742 & 0.1551 \\ 16360 & 15: 59: 03 & 0.1290 & 0.2214 \\ 16362 & 15: 59: 05 & 0.0324 & 0.2099 \\ 16364 & 15: 59: 07 & 0.0885 & 0.1962 \\ 16366 & 15: 59: 09 & 0.0582 & 0.0367 \\ 16368 & 15: 59: 11 & 0.1087 & 0.1147 \\ 16370 & 15: 59: 13 & 0.0445 & 0.2044 \\ 16372 & 15: 59: 15 & 0.0203 & 0.0966 \\ 16374 & 15: 59: 17 & 0.1198 & 0.0880 \\ 16376 & 15: 59: 19 & 0.0845 & 0.1755 \\ 16378 & 15: 59: 21 & 0.0354 & 0.1845 \\ 16380 & 15: 59: 23 & 0.0453 & 0.2319 \\ 16382 & 15: 59: 25 & 0.0109 & 0.1427 \\ 16384 & 15: 59: 27 & 0.0453 & 0.3908 \\ 16386 & 15: 59: 29 & 0.0427 & 0.1635 \\ 16388 & 15: 59: 31 & 0.0307 & 0.1012 \\ 16390 & 15: 59: 33 & 0.0174 & 0.3120 \\ 16392 & 15: 59: 35 & 0.0340 & 0.0572 \\ 16394 & 15: 59: 37 & 0.0297 & 0.1543 \\ 16396 & 15: 59: 39 & 0.0677 & 0.2011 \\ 16398 & 15: 59: 41 & 0.0199 & 0.3252 \\ 16400 & 15: 59: 43 & 0.0841 & 0.2936 \\ 16402 & 15: 59: 45 & 0.0334 & 0.0876 \\ 16404 & 15: 59: 47 & 0.0136 & 0.2482 \\ 16406 & 15: 59: 49 & 0.1377 & 0.1593 \\ 16408 & 15: 59: 51 & 0.0514 & 0.2131 \\ 16410 & 15: 59: 53 & 0.0729 & 0.2336 \\ 16412 & 15: 59: 55 & 0.0472 & 0.1695 \\ 16414 & 15: 59: 57 & 0.1209 & 0.3125 \\ 16416 & 15: 59: 59 & 0.0662 & 0.3308 \\ 16418 & 16: 00: 01 & 0.1454 & 0.1895 \\ 16420 & 16: 00: 03 & 0.1096 & 0.2225 \\ 16422 & 16: 00: 05 & 0.0768 & 0.2199 \\ 16424 & 16: 00: 07 & 0.0572 & 0.1996 \\ 16426 & 16: 00: 09 & 0.0475 & 0.1210 \\ 16428 & 16: 00: 11 & 0.0791 & 0.2171\end{array}$




$\begin{array}{llll}16430 & 16: 00: 13 & 0.0679 & 0.2115 \\ 16432 & 16: 00: 15 & 0.0567 & 0.1396 \\ 16434 & 16: 00: 17 & 0.0525 & 0.2891 \\ 16436 & 16: 00: 19 & 0.0326 & 0.1422 \\ 16438 & 16: 00: 21 & 0.0725 & 0.1547 \\ 16440 & 16: 00: 23 & 0.0838 & 0.1643 \\ 16442 & 16: 00: 25 & 0.0305 & 0.0724 \\ 16444 & 16: 00: 27 & 0.0524 & 0.1232 \\ 16446 & 16: 00: 29 & 0.2393 & 0.1653 \\ 16448 & 16: 00: 31 & 0.0253 & 0.2205 \\ 16450 & 16: 00: 33 & 0.0884 & 0.0726 \\ 16452 & 16: 00: 35 & 0.0182 & 0.0758 \\ 16454 & 16: 00: 37 & 0.1323 & 0.1880 \\ 16456 & 16: 00: 39 & 0.0786 & 0.1801 \\ 16458 & 16: 00: 41 & 0.0659 & 0.1416 \\ 16460 & 16: 00: 43 & 0.1044 & 0.3384 \\ 16462 & 16: 00: 45 & 0.0478 & 0.1597 \\ 16464 & 16: 00: 47 & 0.0390 & 0.1596 \\ 16466 & 16: 00: 49 & 0.0599 & 0.2136 \\ 16468 & 16: 00: 51 & 0.0745 & 0.2417 \\ 16470 & 16: 00: 53 & 0.0159 & 0.0823 \\ 16472 & 16: 00: 55 & 0.0392 & 0.1671 \\ 16474 & 16: 00: 57 & 0.0411 & 0.2013 \\ 16476 & 16: 00: 59 & 0.0974 & 0.2816 \\ 16478 & 16: 01: 01 & 0.2233 & 0.3028 \\ 16480 & 16: 01: 03 & 0.0758 & 0.3181 \\ 16482 & 16: 01: 05 & 0.0506 & 0.3100 \\ 16484 & 16: 01: 07 & 0.0832 & 0.1755 \\ 16486 & 16: 01: 09 & 0.0401 & 0.1806 \\ 16488 & 16: 01: 11 & 0.0182 & 0.1271 \\ 16490 & 16: 01: 13 & 0.0380 & 0.2023 \\ 16492 & 16: 01: 15 & 0.0748 & 0.2262 \\ 16494 & 16: 01: 17 & 0.0158 & 0.0790 \\ 16496 & 16: 01: 19 & 0.0208 & 0.2525 \\ 16498 & 16: 01: 21 & 0.0151 & 0.2003 \\ 16500 & 16: 01: 23 & 0.1521 & 0.6445 \\ 16502 & 16: 01: 25 & 0.1152 & 0.0984 \\ 16504 & 16: 01: 27 & 0.0302 & 0.1252 \\ 16506 & 16: 01: 29 & 0.0565 & 0.1807 \\ 16508 & 16: 01: 31 & 0.0260 & 0.1292 \\ 16510 & 16: 01: 33 & 0.0355 & 0.2104 \\ 16512 & 16: 01: 35 & 0.0502 & 0.3355 \\ 16514 & 16: 01: 37 & 0.0724 & 0.1632 \\ 16516 & 16: 01: 39 & 0.2913 & 0.1471 \\ 16518 & 16: 01: 41 & 0.0604 & 0.2209 \\ 16520 & 16: 01: 43 & 0.0462 & 0.1034 \\ 16522 & 16: 01: 45 & 0.0898 & 0.0938 \\ 16524 & 16: 01: 47 & 0.1260 & 0.1425 \\ 16526 & 16: 01: 49 & 0.1454 & 0.1368 \\ 16528 & 16: 01: 51 & 0.1048 & 0.1153 \\ 16530 & 16: 01: 53 & 0.0491 & 0.3460 \\ 16532 & 16: 01: 55 & 0.1069 & 0.2149\end{array}$




$\begin{array}{llll}16534 & 16: 01: 57 & 0.0531 & 0.2309 \\ 16536 & 16: 01: 59 & 0.1602 & 0.1186 \\ 16538 & 16: 02: 01 & 0.0239 & 0.2267 \\ 16540 & 16: 02: 03 & 0.1126 & 0.2209 \\ 16542 & 16: 02: 05 & 0.0550 & 0.1149 \\ 16544 & 16: 02: 07 & 0.0581 & 0.0938 \\ 16546 & 16: 02: 09 & 0.0807 & 0.1186 \\ 16548 & 16: 02: 11 & 0.0842 & 0.1429 \\ 16550 & 16: 02: 13 & 0.3174 & 0.1267 \\ 16552 & 16: 02: 15 & 0.1340 & 0.4344 \\ 16554 & 16: 02: 17 & 0.0577 & 0.1290 \\ 16556 & 16: 02: 19 & 0.2206 & 0.2206 \\ 16558 & 16: 02: 21 & 0.0800 & 0.3147 \\ 16560 & 16: 02: 23 & 0.0531 & 0.1838 \\ 16562 & 16: 02: 25 & 0.1123 & 0.1936 \\ 16564 & 16: 02: 27 & 0.0559 & 0.2979 \\ 16566 & 16: 02: 29 & 0.0544 & 0.1639 \\ 16568 & 16: 02: 31 & 0.0524 & 0.0969 \\ 16570 & 16: 02: 33 & 0.0507 & 0.2224 \\ 16572 & 16: 02: 35 & 0.0556 & 0.0801 \\ 16574 & 16: 02: 37 & 0.0470 & 0.2392 \\ 16576 & 16: 02: 39 & 0.0975 & 0.1669 \\ 16578 & 16: 02: 41 & 0.0351 & 0.1886 \\ 16580 & 16: 02: 43 & 0.0535 & 0.1805 \\ 16582 & 16: 02: 45 & 0.0332 & 0.2860 \\ 16584 & 16: 02: 47 & 0.0337 & 0.3893 \\ 16586 & 16: 02: 49 & 0.1052 & 0.1539 \\ 16588 & 16: 02: 51 & 0.1278 & 0.1665 \\ 16590 & 16: 02: 53 & 0.1040 & 0.0737 \\ 16592 & 16: 02: 55 & 0.0724 & 0.1189 \\ 16594 & 16: 02: 57 & 0.0303 & 0.0712 \\ 16596 & 16: 02: 59 & 0.0662 & 0.2647 \\ 16598 & 16: 03: 01 & 0.1327 & 0.2114 \\ 16600 & 16: 03: 03 & 0.0257 & 0.0622 \\ 16602 & 16: 03: 05 & 0.0631 & 0.1480 \\ 16604 & 16: 03: 07 & 0.0690 & 0.0551 \\ 16606 & 16: 03: 09 & 0.1477 & 0.1774 \\ 16608 & 16: 03: 11 & 0.0501 & 0.1467 \\ 16610 & 16: 03: 13 & 0.0247 & 0.1766 \\ 16612 & 16: 03: 15 & 0.0697 & 0.1634 \\ 16614 & 16: 03: 17 & 0.1079 & 0.2960 \\ 16616 & 16: 03: 19 & 0.1152 & 0.2193 \\ 16618 & 16: 03: 21 & 0.0577 & 0.2806 \\ 16620 & 16: 03: 23 & 0.0492 & 0.0952 \\ 16622 & 16: 03: 25 & 0.0944 & 0.1294 \\ 16624 & 16: 03: 27 & 0.1477 & 0.1972 \\ 16626 & 16: 03: 29 & 0.0671 & 0.0718 \\ 16628 & 16: 03: 31 & 0.0402 & 0.1798 \\ 16630 & 16: 03: 33 & 0.0273 & 0.1176 \\ 16632 & 16: 03: 35 & 0.0529 & 0.4355 \\ 16634 & 16: 03: 37 & 0.0567 & 0.1771 \\ 16636 & 16: 03: 39 & 0.0387 & 0.1580\end{array}$




$\begin{array}{llll}16638 & 16: 03: 41 & 0.0584 & 0.0721 \\ 16640 & 16: 03: 43 & 0.0720 & 0.3303 \\ 16642 & 16: 03: 45 & 0.0529 & 0.2443 \\ 16644 & 16: 03: 47 & 0.1065 & 0.2571 \\ 16646 & 16: 03: 49 & 0.1609 & 0.0697 \\ 16648 & 16: 03: 51 & 0.0619 & 0.1885 \\ 16650 & 16: 03: 53 & 0.0868 & 0.4965 \\ 16652 & 16: 03: 55 & 0.1565 & 0.2757 \\ 16654 & 16: 03: 57 & 0.1756 & 0.2210 \\ 16656 & 16: 03: 59 & 0.1019 & 0.2662 \\ 16658 & 16: 04: 01 & 0.0423 & 0.1488 \\ 16660 & 16: 04: 03 & 0.2693 & 0.2348 \\ 16662 & 16: 04: 05 & 0.0586 & 0.1453 \\ 16664 & 16: 04: 07 & 0.0533 & 0.2267 \\ 16666 & 16: 04: 09 & 0.1054 & 0.2775 \\ 16668 & 16: 04: 11 & 0.0901 & 0.1121 \\ 16670 & 16: 04: 13 & 0.0125 & 0.2383 \\ 16672 & 16: 04: 15 & 0.1093 & 0.1026 \\ 16674 & 16: 04: 17 & 0.1607 & 0.3734 \\ 16676 & 16: 04: 19 & 0.1051 & 0.1155 \\ 16678 & 16: 04: 21 & 0.0146 & 0.0974 \\ 16680 & 16: 04: 23 & 0.1382 & 0.1729 \\ 16682 & 16: 04: 25 & 0.0971 & 0.0569 \\ 16684 & 16: 04: 27 & 0.0666 & 0.3222 \\ 16686 & 16: 04: 29 & 0.0940 & 0.2618 \\ 16688 & 16: 04: 31 & 0.0408 & 0.1602 \\ 16690 & 16: 04: 33 & 0.0438 & 0.2318 \\ 16692 & 16: 04: 35 & 0.0509 & 0.1269 \\ 16694 & 16: 04: 37 & 0.0476 & 0.2089 \\ 16696 & 16: 04: 39 & 0.0178 & 0.1218 \\ 16698 & 16: 04: 41 & 0.0494 & 0.0957 \\ 16700 & 16: 04: 43 & 0.0604 & 0.1766 \\ 16702 & 16: 04: 45 & 0.0907 & 0.2929 \\ 16704 & 16: 04: 47 & 0.0345 & 0.1401 \\ 16706 & 16: 04: 49 & 0.0232 & 0.1875 \\ 16708 & 16: 04: 51 & 0.0300 & 0.2744 \\ 16710 & 16: 04: 53 & 0.0336 & 0.1479 \\ 16712 & 16: 04: 55 & 0.1109 & 0.2407 \\ 16714 & 16: 04: 57 & 0.0892 & 0.1453 \\ 16716 & 16: 04: 59 & 0.0618 & 0.1531 \\ 16718 & 16: 05: 01 & 0.1455 & 0.3303 \\ 16720 & 16: 05: 03 & 0.0151 & 0.2048 \\ 16722 & 16: 05: 05 & 0.0869 & 0.2443 \\ 16724 & 16: 05: 07 & 0.1888 & 0.1826 \\ 16726 & 16: 05: 09 & 0.0305 & 0.2427 \\ 16728 & 16: 05: 11 & 0.0241 & 0.1124 \\ 16730 & 16: 05: 13 & 0.0651 & 0.2032 \\ 16732 & 16: 05: 15 & 0.0890 & 0.0578 \\ 16734 & 16: 05: 17 & 0.0582 & 0.1620 \\ 16736 & 16: 05: 19 & 0.0545 & 0.2519 \\ 16738 & 16: 05: 21 & 0.0570 & 0.2049 \\ 16740 & 16: 05: 23 & 0.1121 & 0.1366\end{array}$




$\begin{array}{llll}16742 & 16: 05: 25 & 0.0258 & 0.0663 \\ 16744 & 16: 05: 27 & 0.2713 & 0.1431 \\ 16746 & 16: 05: 29 & 0.1073 & 0.0948 \\ 16748 & 16: 05: 31 & 0.0702 & 0.0889 \\ 16750 & 16: 05: 33 & 0.1573 & 0.1098 \\ 16752 & 16: 05: 35 & 0.0499 & 0.1371 \\ 16754 & 16: 05: 37 & 0.0659 & 0.3332 \\ 16756 & 16: 05: 39 & 0.0674 & 0.1864 \\ 16758 & 16: 05: 41 & 0.0761 & 0.1210 \\ 16760 & 16: 05: 43 & 0.1484 & 0.1837 \\ 16762 & 16: 05: 45 & 0.1434 & 0.1206 \\ 16764 & 16: 05: 47 & 0.1350 & 0.0232 \\ 16766 & 16: 05: 49 & 0.0902 & 0.0489 \\ 16768 & 16: 05: 51 & 0.0572 & 0.0511 \\ 16770 & 16: 05: 53 & 0.0228 & 0.1122 \\ 16772 & 16: 05: 55 & 0.0734 & 0.1570 \\ 16774 & 16: 05: 57 & 0.0456 & 0.1214 \\ 16776 & 16: 05: 59 & 0.0193 & 0.2340 \\ 16778 & 16: 06: 01 & 0.0812 & 0.2199 \\ 16780 & 16: 06: 03 & 0.1173 & 0.1783 \\ 16782 & 16: 06: 05 & 0.0348 & 0.0978 \\ 16784 & 16: 06: 07 & 0.0370 & 0.1165 \\ 16786 & 16: 06: 09 & 0.0144 & 0.2182 \\ 16788 & 16: 06: 11 & 0.0650 & 0.1659 \\ 16790 & 16: 06: 13 & 0.0759 & 0.1871 \\ 16792 & 16: 06: 15 & 0.0394 & 0.1684 \\ 16794 & 16: 06: 17 & 0.0570 & 0.0857 \\ 16796 & 16: 06: 19 & 0.0689 & 0.2103 \\ 16798 & 16: 06: 21 & 0.0286 & 0.3163 \\ 16800 & 16: 06: 23 & 0.0397 & 0.1350 \\ 16802 & 16: 06: 25 & 0.0416 & 0.0896 \\ 16804 & 16: 06: 27 & 0.0947 & 0.1816 \\ 16806 & 16: 06: 29 & 0.1810 & 0.1020 \\ 16808 & 16: 06: 31 & 0.0975 & 0.0813 \\ 16810 & 16: 06: 33 & 0.0344 & 0.1298 \\ 16812 & 16: 06: 35 & 0.0494 & 0.1204 \\ 16814 & 16: 06: 37 & 0.0580 & 0.1976 \\ 16816 & 16: 06: 39 & 0.0833 & 0.0689 \\ 16818 & 16: 06: 41 & 0.0133 & 0.2155 \\ 16820 & 16: 06: 43 & 0.0720 & 0.1950 \\ 16822 & 16: 06: 45 & 0.0870 & 0.1768 \\ 16824 & 16: 06: 47 & 0.0292 & 0.1851 \\ 16826 & 16: 06: 49 & 0.0335 & 0.1155 \\ 16828 & 16: 06: 51 & 0.0273 & 0.1567 \\ 16830 & 16: 06: 53 & 0.0553 & 0.2198 \\ 16832 & 16: 06: 55 & 0.0450 & 0.2365 \\ 16834 & 16: 06: 57 & 0.0320 & 0.1658 \\ 16836 & 16: 06: 59 & 0.1141 & 0.3966 \\ 16838 & 16: 07: 01 & 0.0204 & 0.3035 \\ 16840 & 16: 07: 03 & 0.0478 & 0.2092 \\ 16842 & 16: 07: 05 & 0.1227 & 0.1949 \\ 16844 & 16: 07: 07 & 0.0701 & 0.2260\end{array}$




$\begin{array}{llll}16846 & 16: 07: 09 & 0.0790 & 0.0873 \\ 16848 & 16: 07: 11 & 0.0341 & 0.1468 \\ 16850 & 16: 07: 13 & 0.0332 & 0.1437 \\ 16852 & 16: 07: 15 & 0.1006 & 0.2453 \\ 16854 & 16: 07: 17 & 0.0426 & 0.1322 \\ 16856 & 16: 07: 19 & 0.0483 & 0.1279 \\ 16858 & 16: 07: 21 & 0.0404 & 0.2053 \\ 16860 & 16: 07: 23 & 0.0502 & 0.1377 \\ 16862 & 16: 07: 25 & 0.0789 & 0.1324 \\ 16864 & 16: 07: 27 & 0.0491 & 0.1960 \\ 16866 & 16: 07: 29 & 0.0568 & 0.0782 \\ 16868 & 16: 07: 31 & 0.1458 & 0.1260 \\ 16870 & 16: 07: 33 & 0.0369 & 0.3471 \\ 16872 & 16: 07: 35 & 0.0330 & 0.0462 \\ 16874 & 16: 07: 37 & 0.0370 & 0.0494 \\ 16876 & 16: 07: 39 & 0.0929 & 0.1529 \\ 16878 & 16: 07: 41 & 0.0520 & 0.0578 \\ 16880 & 16: 07: 43 & 0.1460 & 0.2012 \\ 16882 & 16: 07: 45 & 0.0496 & 0.1213 \\ 16884 & 16: 07: 47 & 0.1116 & 0.1368 \\ 16886 & 16: 07: 49 & 0.2175 & 0.0955 \\ 16888 & 16: 07: 51 & 0.1716 & 0.0345 \\ 16890 & 16: 07: 53 & 0.1441 & 0.1147 \\ 16892 & 16: 07: 55 & 0.0946 & 0.0549 \\ 16894 & 16: 07: 57 & 0.0686 & 0.1454 \\ 16896 & 16: 07: 59 & 0.1323 & 0.0896 \\ 16898 & 16: 08: 01 & 0.0431 & 0.1820 \\ 16900 & 16: 08: 03 & 0.0252 & 0.0910 \\ 16902 & 16: 08: 05 & 0.0739 & 0.1076 \\ 16904 & 16: 08: 07 & 0.0826 & 0.1044 \\ 16906 & 16: 08: 09 & 0.0442 & 0.2212 \\ 16908 & 16: 08: 11 & 0.0765 & 0.2732 \\ 16910 & 16: 08: 13 & 0.0447 & 0.1754 \\ 16912 & 16: 08: 15 & 0.0237 & 0.1281 \\ 16914 & 16: 08: 17 & 0.0436 & 0.1119 \\ 16916 & 16: 08: 19 & 0.1398 & 0.2185 \\ 16918 & 16: 08: 21 & 0.1158 & 0.2129 \\ 16920 & 16: 08: 23 & 0.1553 & 0.2292 \\ 16922 & 16: 08: 25 & 0.0837 & 0.1697 \\ 16924 & 16: 08: 27 & 0.0934 & 0.2168 \\ 16926 & 16: 08: 29 & 0.0529 & 0.1696 \\ 16928 & 16: 08: 31 & 0.0938 & 0.1085 \\ 16930 & 16: 08: 33 & 0.0487 & 0.1114 \\ 16932 & 16: 08: 35 & 0.1910 & 0.1472 \\ 16934 & 16: 08: 37 & 0.0198 & 0.1288 \\ 16936 & 16: 08: 39 & 0.1759 & 0.3388 \\ 16938 & 16: 08: 41 & 0.0941 & 0.2136 \\ 16940 & 16: 08: 43 & 0.1311 & 0.1349 \\ 16942 & 16: 08: 45 & 0.0723 & 0.2077 \\ 16944 & 16: 08: 47 & 0.0856 & 0.2279 \\ 16946 & 16: 08: 49 & 0.1277 & 0.0454 \\ 16948 & 16: 08: 51 & 0.0709 & 0.1579\end{array}$




$\begin{array}{llll}16950 & 16: 08: 53 & 0.0406 & 0.0820 \\ 16952 & 16: 08: 55 & 0.0360 & 0.1073 \\ 16954 & 16: 08: 57 & 0.0969 & 0.1809 \\ 16956 & 16: 08: 59 & 0.0940 & 0.2916 \\ 16958 & 16: 09: 01 & 0.0663 & 0.1741 \\ 16960 & 16: 09: 03 & 0.0746 & 0.2376 \\ 16962 & 16: 09: 05 & 0.0491 & 0.0826 \\ 16964 & 16: 09: 07 & 0.0706 & 0.1961 \\ 16966 & 16: 09: 09 & 0.1272 & 0.1084 \\ 16968 & 16: 09: 11 & 0.0255 & 0.3153 \\ 16970 & 16: 09: 13 & 0.0790 & 0.1599 \\ 16972 & 16: 09: 15 & 0.0551 & 0.2120 \\ 16974 & 16: 09: 17 & 0.0230 & 0.3135 \\ 16976 & 16: 09: 19 & 0.0700 & 0.1442 \\ 16978 & 16: 09: 21 & 0.0672 & 0.2645 \\ 16980 & 16: 09: 23 & 0.0962 & 0.2748 \\ 16982 & 16: 09: 25 & 0.0400 & 0.2209 \\ 16984 & 16: 09: 27 & 0.0104 & 0.0718 \\ 16986 & 16: 09: 29 & 0.0445 & 0.3375 \\ 16988 & 16: 09: 31 & 0.0546 & 0.2317 \\ 16990 & 16: 09: 33 & 0.1410 & 0.2966 \\ 16992 & 16: 09: 35 & 0.0746 & 0.1521 \\ 16994 & 16: 09: 37 & 0.0871 & 0.1752 \\ 16996 & 16: 09: 39 & 0.1385 & 0.3602 \\ 16998 & 16: 09: 41 & 0.0729 & 0.1715 \\ 17000 & 16: 09: 43 & 0.0969 & 0.0575 \\ 17002 & 16: 09: 45 & 0.0488 & 0.1109 \\ 17004 & 16: 09: 47 & 0.1255 & 0.1886 \\ 17006 & 16: 09: 49 & 0.0699 & 0.1451 \\ 17008 & 16: 09: 51 & 0.0927 & 0.1331 \\ 17010 & 16: 09: 53 & 0.0478 & 0.3448 \\ 17012 & 16: 09: 55 & 0.1546 & 0.2411 \\ 17014 & 16: 09: 57 & 0.0758 & 0.1200 \\ 17016 & 16: 09: 59 & 0.1681 & 0.2596 \\ 17018 & 16: 10: 01 & 0.1164 & 0.0995 \\ 17020 & 16: 10: 03 & 0.0674 & 0.1423 \\ 17022 & 16: 10: 05 & 0.0554 & 0.1376 \\ 17024 & 16: 10: 07 & 0.0988 & 0.0806 \\ 17026 & 16: 10: 09 & 0.1287 & 0.1345 \\ 17028 & 16: 10: 11 & 0.1120 & 0.1791 \\ 17030 & 16: 10: 13 & 0.0296 & 0.2718 \\ 17032 & 16: 10: 15 & 0.0727 & 0.1929 \\ 17034 & 16: 10: 17 & 0.0461 & 0.2583 \\ 17036 & 16: 10: 19 & 0.0467 & 0.2437 \\ 17038 & 16: 10: 21 & 0.0445 & 0.2394 \\ 17040 & 16: 10: 23 & 0.0506 & 0.1563 \\ 17042 & 16: 10: 25 & 0.0621 & 0.1849 \\ 17044 & 16: 10: 27 & 0.0274 & 0.2530 \\ 17046 & 16: 10: 29 & 0.0363 & 0.2283 \\ 17048 & 16: 10: 31 & 0.1255 & 0.4532 \\ 17050 & 16: 10: 33 & 0.0948 & 0.1692 \\ 17052 & 16: 10: 35 & 0.1338 & 0.1585\end{array}$




$\begin{array}{llll}17054 & 16: 10: 37 & 0.0512 & 0.2403 \\ 17056 & 16: 10: 39 & 0.1425 & 0.2494 \\ 17058 & 16: 10: 41 & 0.0741 & 0.2096 \\ 17060 & 16: 10: 43 & 0.1152 & 0.1293 \\ 17062 & 16: 10: 45 & 0.0215 & 0.0691 \\ 17064 & 16: 10: 47 & 0.0624 & 0.1537 \\ 17066 & 16: 10: 49 & 0.0489 & 0.2683 \\ 17068 & 16: 10: 51 & 0.0916 & 0.1641 \\ 17070 & 16: 10: 53 & 0.1042 & 0.1792 \\ 17072 & 16: 10: 55 & 0.0439 & 0.1190 \\ 17074 & 16: 10: 57 & 0.0348 & 0.2628 \\ 17076 & 16: 10: 59 & 0.1472 & 0.2347 \\ 17078 & 16: 11: 01 & 0.0775 & 0.2850 \\ 17080 & 16: 11: 03 & 0.0812 & 0.3574 \\ 17082 & 16: 11: 05 & 0.0555 & 0.2381 \\ 17084 & 16: 11: 07 & 0.0844 & 0.3396 \\ 17086 & 16: 11: 09 & 0.0559 & 0.1267 \\ 17088 & 16: 11: 11 & 0.0453 & 0.2682 \\ 17090 & 16: 11: 13 & 0.0527 & 0.1930 \\ 17092 & 16: 11: 15 & 0.0554 & 0.1798 \\ 17094 & 16: 11: 17 & 0.1208 & 0.3522 \\ 17096 & 16: 11: 19 & 0.1051 & 0.0571 \\ 17098 & 16: 11: 21 & 0.0433 & 0.0660 \\ 17100 & 16: 11: 23 & 0.1043 & 0.1724 \\ 17102 & 16: 11: 25 & 0.0270 & 0.0758 \\ 17104 & 16: 11: 27 & 0.0133 & 0.2344 \\ 17106 & 16: 11: 29 & 0.0920 & 0.1953 \\ 17108 & 16: 11: 31 & 0.1420 & 0.2189 \\ 17110 & 16: 11: 33 & 0.0381 & 0.0937 \\ 17112 & 16: 11: 35 & 0.0697 & 0.0852 \\ 17114 & 16: 11: 37 & 0.0791 & 0.2925 \\ 17116 & 16: 11: 39 & 0.1747 & 0.1268 \\ 17118 & 16: 11: 41 & 0.1519 & 0.1334 \\ 17120 & 16: 11: 43 & 0.0655 & 0.1745 \\ 17122 & 16: 11: 45 & 0.0402 & 0.1532 \\ 17124 & 16: 11: 47 & 0.0427 & 0.1453 \\ 17126 & 16: 11: 49 & 0.0604 & 0.1025 \\ 17128 & 16: 11: 51 & 0.2208 & 0.4344 \\ 17130 & 16: 11: 53 & 0.1462 & 0.3576 \\ 17132 & 16: 11: 55 & 0.0282 & 0.1561 \\ 17134 & 16: 11: 57 & 0.0663 & 0.2982 \\ 17136 & 16: 11: 59 & 0.0770 & 0.1410 \\ 17138 & 16: 12: 01 & 0.2048 & 0.1052 \\ 17140 & 16: 12: 03 & 0.0299 & 0.0718 \\ 17142 & 16: 12: 05 & 0.0490 & 0.1574 \\ 17144 & 16: 12: 07 & 0.0721 & 0.1440 \\ 17146 & 16: 12: 09 & 0.0717 & 0.3240 \\ 17148 & 16: 12: 11 & 0.0231 & 0.1559 \\ 17150 & 16: 12: 13 & 0.0321 & 0.2748 \\ 17152 & 16: 12: 15 & 0.0714 & 0.1139 \\ 17154 & 16: 12: 17 & 0.1355 & 0.2374 \\ 17156 & 16: 12: 19 & 0.2014 & 0.0856\end{array}$


Appendix B - Laser Data Test 1 NIST102505_1.xls

$17158 \quad 16: 12: 21 \quad 0.0562 \quad 0.1439$

B -166 
APPENDIX C. - Fuel Data Test 2 NIST 102605

Appendix $\mathrm{C}$ is fuel data from Test $\mathbf{2}$ in tabular format and is approximately 171 pages in length. Fuel data is included with electronic version of report on compact disk, but not routinely included with paper version of report. 
Appendix C - Fuel Data Test 2 NIST102605_1.xls

Time from Ignition, s EDT Time

$0 \quad 9: 47: 01$

$1 \quad 9: 47: 02$

$2 \quad 9: 47: 03$

$3 \quad 9: 47: 04$

$4 \quad 9: 47: 05$

$5 \quad 9: 47: 06$

$6 \quad 9: 47: 07$

$7 \quad 9: 47: 08$

$8 \quad 9: 47: 09$

$9 \quad 9: 47: 10$

$10 \quad 9: 47: 11$

$11 \quad 9: 47: 12$

$12 \quad 9: 47: 13$

$13 \quad 9: 47: 14$

$14 \quad 9: 47: 15$

$15 \quad 9: 47: 16$

$16 \quad 9: 47: 17$

$17 \quad 9: 47: 18$

$18 \quad 9: 47: 19$

$19 \quad 9: 47: 20$

$20 \quad 9: 47: 21$

$21 \quad 9: 47: 22$

$22 \quad 9: 47: 23$

$23 \quad 9: 47: 24$

24 9:47:25

25 9:47:26

$26 \quad 9: 47: 27$

$27 \quad 9: 47: 28$

28 9:47:29

$29 \quad 9: 47: 30$

$30 \quad 9: 47: 31$

$31 \quad 9: 47: 32$

$32 \quad 9: 47: 33$

$33 \quad 9: 47: 34$

$34 \quad 9: 47: 35$

$35 \quad 9: 47: 36$

$36 \quad 9: 47: 37$

$37 \quad 9: 47: 38$

$38 \quad 9: 47: 39$

$39 \quad 9: 47: 40$

$40 \quad 9: 47: 41$

$41 \quad 9: 47: 42$

$42 \quad 9: 47: 43$

$43 \quad 9: 47: 44$

$44 \quad 9: 47: 45$

$45 \quad 9: 47: 46$

$46 \quad 9: 47: 47$

$47 \quad 9: 47: 48$

$48 \quad 9: 47: 49$

$49 \quad 9: 47: 50$
Liq Flow, I/min

$$
0.4418
$$

0.4446

0.4453

0.4450

0.4436

0.4434

0.4419

0.4448

0.4439

0.4466

0.4450

0.4476

0.4508

0.4471

0.4497

0.4486

0.4493

0.4491

0.4474

0.4472

0.4446

0.4478

0.4474

0.4487

0.4468

0.4436

0.4476

0.4462

0.4488

0.4478

0.4483

0.4461

0.4505

0.4503

0.4492

0.4512

0.4489

0.4504

0.4462

0.4494

0.4489

0.4459

0.4455

0.4445

0.4498

0.4462

0.4470

0.4481

0.4501

0.4537 
Appendix C - Fuel Data Test 2 NIST102605_1.xIs

\begin{tabular}{|c|c|c|}
\hline 50 & $9: 47: 51$ & 0.4507 \\
\hline 51 & $9: 47: 52$ & 0.4535 \\
\hline 52 & $9: 47: 53$ & 0.4509 \\
\hline 53 & $9: 47: 54$ & 0.4512 \\
\hline 54 & $9: 47: 55$ & 0.4524 \\
\hline 55 & $9: 47: 56$ & 0.4487 \\
\hline 56 & $9: 47: 57$ & 0.4490 \\
\hline 57 & $9: 47: 58$ & 0.4484 \\
\hline 58 & $9: 47: 59$ & 0.4490 \\
\hline 59 & $9: 48: 00$ & 0.4457 \\
\hline 60 & $9: 48: 01$ & 0.4478 \\
\hline 61 & 9:48:02 & 0.4453 \\
\hline 62 & $9: 48: 03$ & 0.4502 \\
\hline 63 & $9: 48: 04$ & 0.4481 \\
\hline 64 & $9: 48: 05$ & 0.4492 \\
\hline 65 & $9: 48: 06$ & 0.4441 \\
\hline 66 & 9:48:07 & 0.4460 \\
\hline 67 & 9:48:08 & 0.4475 \\
\hline 68 & $9: 48: 09$ & 0.4484 \\
\hline 69 & $9: 48: 10$ & 0.4501 \\
\hline 70 & $9: 48: 11$ & 0.4470 \\
\hline 71 & $9: 48: 12$ & 0.4494 \\
\hline 72 & $9: 48: 13$ & 0.4502 \\
\hline 73 & $9: 48: 14$ & 0.4489 \\
\hline 74 & $9: 48: 15$ & 0.4498 \\
\hline 75 & $9: 48: 16$ & 0.4465 \\
\hline 76 & $9: 48: 17$ & 0.4484 \\
\hline 77 & $9: 48: 18$ & 0.4466 \\
\hline 78 & $9: 48: 19$ & 0.4475 \\
\hline 79 & $9: 48: 20$ & 0.4458 \\
\hline 80 & $9: 48: 21$ & 0.4447 \\
\hline 81 & $9: 48: 22$ & 0.4450 \\
\hline 82 & $9: 48: 23$ & 0.4438 \\
\hline 83 & $9: 48: 24$ & 0.4463 \\
\hline 84 & $9: 48: 25$ & 0.4432 \\
\hline 85 & $9: 48: 26$ & 0.4474 \\
\hline 86 & $9: 48: 27$ & 0.4466 \\
\hline 87 & 9:48:28 & 0.4483 \\
\hline 88 & $9: 48: 29$ & 0.4507 \\
\hline 89 & $9: 48: 30$ & 0.4499 \\
\hline 90 & $9: 48: 31$ & 0.4507 \\
\hline 91 & $9: 48: 32$ & 0.4469 \\
\hline 92 & $9: 48: 33$ & 0.4481 \\
\hline 93 & $9: 48: 34$ & 0.4460 \\
\hline 94 & $9: 48: 35$ & 0.4479 \\
\hline 95 & $9: 48: 36$ & 0.4471 \\
\hline 96 & $9: 48: 37$ & 0.4486 \\
\hline 97 & $9: 48: 38$ & 0.4465 \\
\hline 98 & $9: 48: 39$ & 0.4442 \\
\hline 99 & $9: 48: 40$ & 0.4460 \\
\hline 00 & $9: 48: 41$ & 0.4445 \\
\hline
\end{tabular}


Appendix C - Fuel Data Test 2 NIST102605_1.xIs

\begin{tabular}{|c|c|c|}
\hline 101 & $9: 48: 42$ & 0.4480 \\
\hline 102 & $9: 48: 43$ & 0.4455 \\
\hline 103 & $9: 48: 44$ & 0.4474 \\
\hline 104 & $9: 48: 45$ & 0.4439 \\
\hline 105 & $9: 48: 46$ & 0.4464 \\
\hline 106 & $9: 48: 47$ & 0.4471 \\
\hline 107 & $9: 48: 48$ & 0.4485 \\
\hline 108 & $9: 48: 49$ & 0.4474 \\
\hline 109 & $9: 48: 50$ & 0.4470 \\
\hline 110 & $9: 48: 51$ & 0.4479 \\
\hline 111 & $9: 48: 52$ & 0.4449 \\
\hline 112 & $9: 48: 53$ & 0.4477 \\
\hline 113 & $9: 48: 54$ & 0.4454 \\
\hline 114 & $9: 48: 55$ & 0.4470 \\
\hline 115 & $9: 48: 56$ & 0.4467 \\
\hline 116 & $9: 48: 57$ & 0.4471 \\
\hline 117 & $9: 48: 58$ & 0.4455 \\
\hline 118 & 9:48:59 & 0.4435 \\
\hline 119 & $9: 49: 00$ & 0.4441 \\
\hline 120 & $9: 49: 01$ & 0.4433 \\
\hline 121 & 9:49:02 & 0.4459 \\
\hline 122 & $9: 49: 03$ & 0.4429 \\
\hline 123 & 9:49:04 & 0.4436 \\
\hline 124 & 9:49:05 & 0.4429 \\
\hline 125 & 9:49:06 & 0.4450 \\
\hline 126 & $9: 49: 07$ & 0.4473 \\
\hline 127 & $9: 49: 08$ & 0.4485 \\
\hline 128 & 9:49:09 & 0.4502 \\
\hline 129 & $9: 49: 10$ & 0.4474 \\
\hline 130 & $9: 49: 11$ & 0.4482 \\
\hline 131 & $9: 49: 12$ & 0.4440 \\
\hline 132 & $9: 49: 13$ & 0.4469 \\
\hline 133 & $9: 49: 14$ & 0.4458 \\
\hline 134 & $9: 49: 15$ & 0.4469 \\
\hline 135 & $9: 49: 16$ & 0.4460 \\
\hline 136 & $9: 49: 17$ & 0.4425 \\
\hline 137 & $9: 49: 18$ & 0.4460 \\
\hline 138 & $9: 49: 19$ & 0.4456 \\
\hline 139 & $9: 49: 20$ & 0.4470 \\
\hline 140 & $9: 49: 21$ & 0.4437 \\
\hline 141 & $9: 49: 22$ & 0.4434 \\
\hline 142 & $9: 49: 23$ & 0.4435 \\
\hline 143 & $9: 49: 24$ & 0.4432 \\
\hline 144 & $9: 49: 25$ & 0.4448 \\
\hline 145 & $9: 49: 26$ & 0.4455 \\
\hline 146 & $9: 49: 27$ & 0.4477 \\
\hline 147 & $9: 49: 28$ & 0.4453 \\
\hline 148 & $9: 49: 29$ & 0.4468 \\
\hline 149 & $9: 49: 30$ & 0.4446 \\
\hline 150 & $9: 49: 31$ & 0.4462 \\
\hline 151 & 9:49:32 & 0.445 \\
\hline
\end{tabular}


Appendix C - Fuel Data Test 2 NIST102605_1.xIs

\begin{tabular}{|c|c|c|}
\hline 152 & $9: 49: 33$ & 0.4471 \\
\hline 153 & $9: 49: 34$ & 0.4478 \\
\hline 154 & $9: 49: 35$ & 0.4460 \\
\hline 155 & $9: 49: 36$ & 0.4469 \\
\hline 156 & $9: 49: 37$ & 0.4438 \\
\hline 157 & $9: 49: 38$ & 0.4458 \\
\hline 158 & $9: 49: 39$ & 0.4435 \\
\hline 159 & $9: 49: 40$ & 0.4434 \\
\hline 160 & $9: 49: 41$ & 0.4440 \\
\hline 161 & $9: 49: 42$ & 0.4431 \\
\hline 162 & $9: 49: 43$ & 0.4450 \\
\hline 163 & $9: 49: 44$ & 0.4419 \\
\hline 164 & $9: 49: 45$ & 0.4445 \\
\hline 165 & $9: 49: 46$ & 0.4435 \\
\hline 166 & $9: 49: 47$ & 0.4461 \\
\hline 167 & $9: 49: 48$ & 0.4485 \\
\hline 168 & $9: 49: 49$ & 0.4474 \\
\hline 169 & $9: 49: 50$ & 0.4488 \\
\hline 170 & $9: 49: 51$ & 0.4443 \\
\hline 171 & $9: 49: 52$ & 0.4464 \\
\hline 172 & $9: 49: 53$ & 0.4457 \\
\hline 173 & $9: 49: 54$ & 0.4459 \\
\hline 174 & $9: 49: 55$ & 0.4453 \\
\hline 175 & $9: 49: 56$ & 0.4456 \\
\hline 176 & $9: 49: 57$ & 0.4459 \\
\hline 177 & $9: 49: 58$ & 0.4422 \\
\hline 178 & $9: 49: 59$ & 0.4457 \\
\hline 179 & $9: 50: 00$ & 0.4446 \\
\hline 180 & $9: 50: 01$ & 0.4447 \\
\hline 181 & $9: 50: 02$ & 0.4429 \\
\hline 182 & $9: 50: 03$ & 0.4421 \\
\hline 183 & $9: 50: 04$ & 0.4459 \\
\hline 184 & 9:50:05 & 0.4442 \\
\hline 185 & 9:50:06 & 0.4469 \\
\hline 186 & $9: 50: 07$ & 0.4448 \\
\hline 187 & $9: 50: 08$ & 0.4463 \\
\hline 188 & 9:50:09 & 0.4447 \\
\hline 189 & $9: 50: 10$ & 0.4461 \\
\hline 190 & $9: 50: 11$ & 0.4477 \\
\hline 191 & $9: 50: 12$ & 0.4462 \\
\hline 192 & $9: 50: 13$ & 0.4468 \\
\hline 193 & $9: 50: 14$ & 0.4430 \\
\hline 194 & $9: 50: 15$ & 0.4451 \\
\hline 195 & $9: 50: 16$ & 0.4432 \\
\hline 196 & $9: 50: 17$ & 0.4463 \\
\hline 197 & $9: 50: 18$ & 0.4441 \\
\hline 198 & $9: 50: 19$ & 0.4451 \\
\hline 199 & $9: 50: 20$ & 0.4421 \\
\hline 200 & $9: 50: 21$ & 0.4408 \\
\hline 201 & $9: 50: 22$ & 0.4419 \\
\hline 202 & $9: 50: 23$ & 0.441 \\
\hline
\end{tabular}


Appendix C - Fuel Data Test 2 NIST102605_1.xIs

$\begin{array}{lll}203 & 9: 50: 24 & 0.4439 \\ 204 & 9: 50: 25 & 0.4418 \\ 205 & 9: 50: 26 & 0.4453 \\ 206 & 9: 50: 27 & 0.4444 \\ 207 & 9: 50: 28 & 0.4458 \\ 208 & 9: 50: 29 & 0.4479 \\ 209 & 9: 50: 30 & 0.4438 \\ 210 & 9: 50: 31 & 0.4460 \\ 211 & 9: 50: 32 & 0.4446 \\ 212 & 9: 50: 33 & 0.4453 \\ 213 & 9: 50: 34 & 0.4450 \\ 214 & 9: 50: 35 & 0.4416 \\ 215 & 9: 50: 36 & 0.4441 \\ 216 & 9: 50: 37 & 0.4416 \\ 217 & 9: 50: 38 & 0.4448 \\ 218 & 9: 50: 39 & 0.4441 \\ 219 & 9: 50: 40 & 0.4440 \\ 220 & 9: 50: 41 & 0.4428 \\ 221 & 9: 50: 42 & 0.4399 \\ 222 & 9: 50: 43 & 0.4427 \\ 223 & 9: 50: 44 & 0.4422 \\ 224 & 9: 50: 45 & 0.4455 \\ 225 & 9: 50: 46 & 0.4426 \\ 226 & 9: 50: 47 & 0.4446 \\ 227 & 9: 50: 48 & 0.4442 \\ 228 & 9: 50: 49 & 0.4458 \\ 229 & 9: 50: 50 & 0.4474 \\ 230 & 9: 50: 51 & 0.4443 \\ 231 & 9: 50: 52 & 0.4459 \\ 232 & 9: 50: 53 & 0.4429 \\ 233 & 9: 50: 54 & 0.4451 \\ 234 & 9: 50: 55 & 0.4451 \\ 235 & 9: 50: 56 & 0.4428 \\ 236 & 9: 50: 57 & 0.4453 \\ 237 & 9: 50: 58 & 0.4441 \\ 238 & 9: 50: 59 & 0.4448 \\ 239 & 9: 51: 00 & 0.4408 \\ 240 & 9: 51: 01 & 0.4407 \\ 241 & 9: 51: 02 & 0.4415 \\ 242 & 9: 51: 03 & 0.4421 \\ 243 & 9: 51: 04 & 0.4427 \\ 244 & 9: 51: 05 & 0.4428 \\ 245 & 9: 51: 06 & 0.4473 \\ 246 & 9: 51: 07 & 0.4471 \\ 247 & 9: 51: 08 & 0.4489 \\ 248 & 9: 51: 09 & 0.4438 \\ 249 & 9: 51: 10 & 0.4452 \\ 250 & 9: 51: 11 & 0.4432 \\ 251 & 9: 51: 12 & \\ 252 & 9: 51: 13 & 9459 \\ 253 & 9: 51: 14 & \end{array}$


Appendix C - Fuel Data Test 2 NIST102605_1.xIs

\begin{tabular}{|c|c|c|}
\hline 254 & $9: 51: 15$ & 0.4448 \\
\hline 255 & $9: 51: 16$ & 0.4436 \\
\hline 256 & $9: 51: 17$ & 0.4436 \\
\hline 257 & $9: 51: 18$ & 0.4408 \\
\hline 258 & $9: 51: 19$ & 0.4453 \\
\hline 259 & $9: 51: 20$ & 0.4415 \\
\hline 260 & $9: 51: 21$ & 0.4446 \\
\hline 261 & $9: 51: 22$ & 0.4432 \\
\hline 262 & $9: 51: 23$ & 0.4435 \\
\hline 263 & $9: 51: 24$ & 0.4409 \\
\hline 264 & $9: 51: 25$ & 0.4419 \\
\hline 265 & $9: 51: 26$ & 0.4452 \\
\hline 266 & $9: 51: 27$ & 0.4427 \\
\hline 267 & $9: 51: 28$ & 0.4450 \\
\hline 268 & $9: 51: 29$ & 0.4464 \\
\hline 269 & $9: 51: 30$ & 0.4462 \\
\hline 270 & $9: 51: 31$ & 0.4464 \\
\hline 271 & $9: 51: 32$ & 0.4432 \\
\hline 272 & $9: 51: 33$ & 0.4450 \\
\hline 273 & $9: 51: 34$ & 0.4416 \\
\hline 274 & $9: 51: 35$ & 0.4447 \\
\hline 275 & $9: 51: 36$ & 0.4441 \\
\hline 276 & $9: 51: 37$ & 0.4454 \\
\hline 277 & $9: 51: 38$ & 0.4430 \\
\hline 278 & $9: 51: 39$ & 0.4410 \\
\hline 279 & $9: 51: 40$ & 0.4436 \\
\hline 280 & $9: 51: 41$ & 0.4422 \\
\hline 281 & $9: 51: 42$ & 0.4433 \\
\hline 282 & $9: 51: 43$ & 0.4402 \\
\hline 283 & $9: 51: 44$ & 0.4427 \\
\hline 284 & $9: 51: 45$ & 0.4428 \\
\hline 285 & $9: 51: 46$ & 0.4446 \\
\hline 286 & $9: 51: 47$ & 0.4485 \\
\hline 287 & $9: 51: 48$ & 0.4441 \\
\hline 288 & $9: 51: 49$ & 0.4456 \\
\hline 289 & $9: 51: 50$ & 0.4436 \\
\hline 290 & $9: 51: 51$ & 0.4454 \\
\hline 291 & $9: 51: 52$ & 0.4469 \\
\hline 292 & $9: 51: 53$ & 0.4442 \\
\hline 293 & $9: 51: 54$ & 0.4435 \\
\hline 294 & $9: 51: 55$ & 0.4410 \\
\hline 295 & $9: 51: 56$ & 0.4444 \\
\hline 296 & $9: 51: 57$ & 0.4419 \\
\hline 297 & $9: 51: 58$ & 0.4451 \\
\hline 298 & $9: 51: 59$ & 0.4449 \\
\hline 299 & $9: 52: 00$ & 0.4408 \\
\hline 300 & $9: 52: 01$ & 0.4429 \\
\hline 301 & $9: 52: 02$ & 0.4403 \\
\hline 302 & $9: 52: 03$ & 0.4440 \\
\hline 303 & $9: 52: 04$ & 0.442 \\
\hline 304 & $9: 52: 05$ & 0.445 \\
\hline
\end{tabular}


Appendix C - Fuel Data Test 2 NIST102605_1.xls

\begin{tabular}{|c|c|c|}
\hline 305 & 9:52:06 & 0.4433 \\
\hline 306 & 9:52:07 & 0.4443 \\
\hline 307 & 9:52:08 & 0.4442 \\
\hline 308 & 9:52:09 & 0.4452 \\
\hline 309 & $9: 52: 10$ & 0.4478 \\
\hline 310 & $9: 52: 11$ & 0.4427 \\
\hline 311 & 9:52:12 & 0.4444 \\
\hline 312 & $9: 52: 13$ & 0.4429 \\
\hline 313 & $9: 52: 14$ & 0.4451 \\
\hline 314 & $9: 52: 15$ & 0.4451 \\
\hline 315 & $9: 52: 16$ & 0.4434 \\
\hline 316 & $9: 52: 17$ & 0.4442 \\
\hline 317 & $9: 52: 18$ & 0.4402 \\
\hline 318 & 9:52:19 & 0.4423 \\
\hline 319 & 9:52:20 & 0.4414 \\
\hline 320 & 9:52:21 & 0.4441 \\
\hline 321 & 9:52:22 & 0.4404 \\
\hline 322 & 9:52:23 & 0.4409 \\
\hline 323 & $9: 52: 24$ & 0.4408 \\
\hline 324 & $9: 52: 25$ & 0.4425 \\
\hline 325 & $9: 52: 26$ & 0.4462 \\
\hline 326 & $9: 52: 27$ & 0.4450 \\
\hline 327 & $9: 52: 28$ & 0.4484 \\
\hline 328 & $9: 52: 29$ & 0.4440 \\
\hline 329 & $9: 52: 30$ & 0.4453 \\
\hline 330 & 9:52:31 & 0.4456 \\
\hline 331 & 9:52:32 & 0.4455 \\
\hline 332 & $9: 52: 33$ & 0.4473 \\
\hline 333 & 9:52:34 & 0.4439 \\
\hline 334 & $9: 52: 35$ & 0.4441 \\
\hline 335 & 9:52:36 & 0.4414 \\
\hline 336 & 9:52:37 & 0.4446 \\
\hline 337 & 9:52:38 & 0.4436 \\
\hline 338 & $9: 52: 39$ & 0.4434 \\
\hline 339 & $9: 52: 40$ & 0.4431 \\
\hline 340 & $9: 52: 41$ & 0.4402 \\
\hline 341 & $9: 52: 42$ & 0.4441 \\
\hline 342 & $9: 52: 43$ & 0.4414 \\
\hline 343 & $9: 52: 44$ & 0.4422 \\
\hline 344 & 9:52:45 & 0.4400 \\
\hline 345 & 9:52:46 & 0.4423 \\
\hline 346 & $9: 52: 47$ & 0.4425 \\
\hline 347 & $9: 52: 48$ & 0.4439 \\
\hline 348 & $9: 52: 49$ & 0.4472 \\
\hline 349 & $9: 52: 50$ & 0.4452 \\
\hline 350 & $9: 52: 51$ & 0.4461 \\
\hline 351 & 9:52:52 & 0.4410 \\
\hline 352 & $9: 52: 53$ & 0.4438 \\
\hline 353 & $9: 52: 54$ & 0.4445 \\
\hline 354 & $9: 52: 55$ & 0.4448 \\
\hline 355 & $9: 52: 56$ & 0.444 \\
\hline
\end{tabular}


Appendix C - Fuel Data Test 2 NIST102605_1.xIs

$\begin{array}{lll}356 & 9: 52: 57 & 0.4407 \\ 357 & 9: 52: 58 & 0.4431 \\ 358 & 9: 52: 59 & 0.4411 \\ 359 & 9: 53: 00 & 0.4442 \\ 360 & 9: 53: 01 & 0.4424 \\ 361 & 9: 53: 02 & 0.4422 \\ 362 & 9: 53: 03 & 0.4395 \\ 363 & 9: 53: 04 & 0.4400 \\ 364 & 9: 53: 05 & 0.4411 \\ 365 & 9: 53: 06 & 0.4429 \\ 366 & 9: 53: 07 & 0.4462 \\ 367 & 9: 53: 08 & 0.4450 \\ 368 & 9: 53: 09 & 0.4480 \\ 369 & 9: 53: 10 & 0.4438 \\ 370 & 9: 53: 11 & 0.4455 \\ 371 & 9: 53: 12 & 0.4438 \\ 372 & 9: 53: 13 & 0.4461 \\ 373 & 9: 53: 14 & 0.4460 \\ 374 & 9: 53: 15 & 0.4431 \\ 375 & 9: 53: 16 & 0.4429 \\ 376 & 9: 53: 17 & 0.4409 \\ 377 & 9: 53: 18 & 0.4448 \\ 378 & 9: 53: 19 & 0.4433 \\ 379 & 9: 53: 20 & 0.4435 \\ 380 & 9: 53: 21 & 0.4426 \\ 381 & 9: 53: 22 & 0.4399 \\ 382 & 9: 53: 23 & 0.4402 \\ 383 & 9: 53: 24 & 0.4407 \\ 384 & 9: 53: 25 & 0.4435 \\ 385 & 9: 53: 26 & 0.4416 \\ 386 & 9: 53: 27 & 0.4440 \\ 387 & 9: 53: 28 & 0.4420 \\ 388 & 9: 53: 29 & 0.4453 \\ 389 & 9: 53: 30 & 0.4431 \\ 390 & 9: 53: 31 & 0.4453 \\ 391 & 9: 53: 32 & 0.4444 \\ 392 & 9: 53: 33 & 0.4438 \\ 393 & 9: 53: 34 & 0.4447 \\ 394 & 9: 53: 35 & 0.4413 \\ 395 & 9: 53: 36 & 0.4445 \\ 396 & 9: 53: 37 & 0.4423 \\ 397 & 9: 53: 38 & 0.4430 \\ 398 & 9: 53: 39 & 0.4424 \\ 399 & 9: 53: 40 & 0.4411 \\ 400 & 9: 53: 41 & 0.4445 \\ 401 & 9: 53: 42 & 0.4414 \\ 402 & 9: 53: 43 & 0.4416 \\ 403 & 9: 53: 44 & 0.4399 \\ 404 & 9: 53: 45 & \\ 405 & 9: 53: 46 & 9439 \\ 406 & 9: 53: 47 & \end{array}$


Appendix C - Fuel Data Test 2 NIST102605_1.xIs

$\begin{array}{lll}407 & 9: 53: 48 & 0.4464 \\ 408 & 9: 53: 49 & 0.4432 \\ 409 & 9: 53: 50 & 0.4452 \\ 410 & 9: 53: 51 & 0.4444 \\ 411 & 9: 53: 52 & 0.4442 \\ 412 & 9: 53: 53 & 0.4467 \\ 413 & 9: 53: 54 & 0.4459 \\ 414 & 9: 53: 55 & 0.4443 \\ 415 & 9: 53: 56 & 0.4407 \\ 416 & 9: 53: 57 & 0.4426 \\ 417 & 9: 53: 58 & 0.4424 \\ 418 & 9: 53: 59 & 0.4425 \\ 419 & 9: 54: 00 & 0.4386 \\ 420 & 9: 54: 01 & 0.4446 \\ 421 & 9: 54: 02 & 0.4420 \\ 422 & 9: 54: 03 & 0.4424 \\ 423 & 9: 54: 04 & 0.4423 \\ 424 & 9: 54: 05 & 0.4414 \\ 425 & 9: 54: 06 & 0.4444 \\ 426 & 9: 54: 07 & 0.4414 \\ 427 & 9: 54: 08 & 0.4425 \\ 428 & 9: 54: 09 & 0.4419 \\ 429 & 9: 54: 10 & 0.4437 \\ 430 & 9: 54: 11 & 0.4458 \\ 431 & 9: 54: 12 & 0.4453 \\ 432 & 9: 54: 13 & 0.4465 \\ 433 & 9: 54: 14 & 0.4409 \\ 434 & 9: 54: 15 & 0.4440 \\ 435 & 9: 54: 16 & 0.4438 \\ 436 & 9: 54: 17 & 0.4433 \\ 437 & 9: 54: 18 & 0.4437 \\ 438 & 9: 54: 19 & 0.4416 \\ 439 & 9: 54: 20 & 0.4431 \\ 440 & 9: 54: 21 & 0.4408 \\ 441 & 9: 54: 22 & 0.4422 \\ 442 & 9: 54: 23 & 0.4401 \\ 443 & 9: 54: 24 & 0.4407 \\ 444 & 9: 54: 25 & 0.4410 \\ 445 & 9: 54: 26 & 0.4405 \\ 446 & 9: 54: 27 & 0.4447 \\ 447 & 9: 54: 28 & 0.4421 \\ 448 & 9: 54: 29 & 0.4452 \\ 449 & 9: 54: 30 & 0.4472 \\ 450 & 9: 54: 31 & 0.4453 \\ 451 & 9: 54: 32 & 0.4466 \\ 452 & 9: 54: 33 & 0.4432 \\ 453 & 9: 54: 34 & 0.4447 \\ 454 & 9: 54: 35 & 0.4442 \\ 455 & 9: 54: 36 & 0.4425 \\ 456 & 9: 54: 37 & 9408 \\ 457 & 9: 54: 38 & \end{array}$


Appendix C - Fuel Data Test 2 NIST102605_1.xIs

$\begin{array}{lll}458 & 9: 54: 39 & 0.4433 \\ 459 & 9: 54: 40 & 0.4397 \\ 460 & 9: 54: 41 & 0.4449 \\ 461 & 9: 54: 42 & 0.4426 \\ 462 & 9: 54: 43 & 0.4424 \\ 463 & 9: 54: 44 & 0.4397 \\ 464 & 9: 54: 45 & 0.4392 \\ 465 & 9: 54: 46 & 0.4398 \\ 466 & 9: 54: 47 & 0.4403 \\ 467 & 9: 54: 48 & 0.4448 \\ 468 & 9: 54: 49 & 0.4431 \\ 469 & 9: 54: 50 & 0.4473 \\ 470 & 9: 54: 51 & 0.4447 \\ 471 & 9: 54: 52 & 0.4453 \\ 472 & 9: 54: 53 & 0.4460 \\ 473 & 9: 54: 54 & 0.4425 \\ 474 & 9: 54: 55 & 0.4444 \\ 475 & 9: 54: 56 & 0.4424 \\ 476 & 9: 54: 57 & 0.4449 \\ 477 & 9: 54: 58 & 0.4419 \\ 478 & 9: 54: 59 & 0.4431 \\ 479 & 9: 55: 00 & 0.4428 \\ 480 & 9: 55: 01 & 0.4432 \\ 481 & 9: 55: 02 & 0.4404 \\ 482 & 9: 55: 03 & 0.4394 \\ 483 & 9: 55: 04 & 0.4394 \\ 484 & 9: 55: 05 & 0.4405 \\ 485 & 9: 55: 06 & 0.4402 \\ 486 & 9: 55: 07 & 0.4398 \\ 487 & 9: 55: 08 & 0.4447 \\ 488 & 9: 55: 09 & 0.4429 \\ 489 & 9: 55: 10 & 0.4458 \\ 490 & 9: 55: 11 & 0.4435 \\ 491 & 9: 55: 12 & 0.4464 \\ 492 & 9: 55: 13 & 0.4456 \\ 493 & 9: 55: 14 & 0.4443 \\ 494 & 9: 55: 15 & 0.4446 \\ 495 & 9: 55: 16 & 0.4431 \\ 496 & 9: 55: 17 & 0.4435 \\ 497 & 9: 55: 18 & 0.4395 \\ 498 & 9: 55: 19 & 0.4421 \\ 499 & 9: 55: 20 & 0.4398 \\ 500 & 9: 55: 21 & 0.4441 \\ 501 & 9: 55: 22 & 0.4425 \\ 502 & 9: 55: 23 & 0.4429 \\ 503 & 9: 55: 24 & 0.4416 \\ 504 & 9: 55: 25 & 0.4394 \\ 505 & 9: 55: 26 & 0.4426 \\ 506 & 9: 55: 27 & \\ 507 & 9: 55: 28 & 9431 \\ 508 & 9: 55: 29 & \end{array}$

C - 10 
Appendix C - Fuel Data Test 2 NIST102605_1.xIs

\begin{tabular}{|c|c|c|}
\hline 509 & $9: 55: 30$ & 0.4447 \\
\hline 510 & $9: 55: 31$ & 0.4472 \\
\hline 511 & $9: 55: 32$ & 0.4422 \\
\hline 512 & $9: 55: 33$ & 0.4432 \\
\hline 514 & $9: 55: 35$ & 0.4415 \\
\hline 514 & $9: 55: 35$ & 0.4452 \\
\hline 515 & $9: 55: 36$ & 0.4449 \\
\hline 516 & 9:55:37 & 0.4405 \\
\hline 517 & $9: 55: 38$ & 0.4433 \\
\hline 518 & $9: 55: 39$ & 0.4415 \\
\hline 519 & $9: 55: 40$ & 0.4438 \\
\hline 520 & $9: 55: 41$ & 0.4427 \\
\hline 521 & $9: 55: 42$ & 0.4434 \\
\hline 522 & $9: 55: 43$ & 0.4399 \\
\hline 523 & $9: 55: 44$ & 0.4389 \\
\hline 524 & $9: 55: 45$ & 0.4400 \\
\hline 525 & $9: 55: 46$ & 0.4402 \\
\hline 526 & $9: 55: 47$ & 0.4437 \\
\hline 527 & $9: 55: 48$ & 0.4405 \\
\hline 528 & $9: 55: 49$ & 0.4432 \\
\hline 529 & $9: 55: 50$ & 0.4451 \\
\hline 530 & $9: 55: 51$ & 0.4444 \\
\hline 531 & $9: 55: 52$ & 0.4469 \\
\hline 532 & $9: 55: 53$ & 0.4454 \\
\hline 533 & $9: 55: 54$ & 0.4452 \\
\hline 534 & $9: 55: 55$ & 0.4402 \\
\hline 535 & $9: 55: 56$ & 0.4436 \\
\hline 536 & $9: 55: 57$ & 0.4434 \\
\hline 537 & $9: 55: 58$ & 0.4443 \\
\hline 538 & $9: 55: 59$ & 0.4433 \\
\hline 539 & $9: 56: 00$ & 0.4397 \\
\hline 540 & $9: 56: 01$ & 0.4418 \\
\hline 541 & $9: 56: 02$ & 0.4395 \\
\hline 542 & $9: 56: 03$ & 0.4444 \\
\hline 543 & $9: 56: 04$ & 0.4420 \\
\hline 544 & $9: 56: 05$ & 0.4426 \\
\hline 545 & $9: 56: 06$ & 0.4416 \\
\hline 546 & $9: 56: 07$ & 0.4410 \\
\hline 547 & $9: 56: 08$ & 0.4435 \\
\hline 548 & $9: 56: 09$ & 0.4435 \\
\hline 549 & $9: 56: 10$ & 0.4459 \\
\hline 550 & $9: 56: 11$ & 0.4438 \\
\hline 551 & $9: 56: 12$ & 0.4466 \\
\hline 552 & $9: 56: 13$ & 0.4432 \\
\hline 553 & $9: 56: 14$ & 0.4428 \\
\hline 554 & $9: 56: 15$ & 0.4458 \\
\hline 555 & $9: 56: 16$ & 0.4418 \\
\hline 556 & $9: 56: 17$ & 0.4454 \\
\hline 557 & $9: 56: 18$ & 0.4447 \\
\hline 558 & $9: 56: 19$ & 0.442 \\
\hline 559 & $9: 56: 20$ & 0.441 \\
\hline
\end{tabular}

C - 11 
Appendix C - Fuel Data Test 2 NIST102605_1.xIs

\begin{tabular}{|c|c|c|}
\hline 560 & 9:56:21 & 0.4404 \\
\hline 561 & 9:56:22 & 0.4430 \\
\hline 562 & 9:56:23 & 0.4415 \\
\hline 563 & $9: 56: 24$ & 0.4437 \\
\hline 564 & $9: 56: 25$ & 0.4405 \\
\hline 565 & 9:56:26 & 0.4416 \\
\hline 566 & 9:56:27 & 0.4393 \\
\hline 567 & $9: 56: 28$ & 0.4410 \\
\hline 568 & $9: 56: 29$ & 0.4427 \\
\hline 569 & 9:56:30 & 0.4450 \\
\hline 570 & $9: 56: 31$ & 0.4471 \\
\hline 571 & 9:56:32 & 0.4437 \\
\hline 572 & $9: 56: 33$ & 0.4465 \\
\hline 573 & 9:56:34 & 0.4457 \\
\hline 574 & 9:56:35 & 0.4449 \\
\hline 575 & 9:56:36 & 0.4458 \\
\hline 576 & 9:56:37 & 0.4427 \\
\hline 577 & 9:56:38 & 0.4439 \\
\hline 578 & 9:56:39 & 0.4403 \\
\hline 579 & 9:56:40 & 0.4444 \\
\hline 580 & 9:56:41 & 0.4416 \\
\hline 581 & 9:56:42 & 0.4417 \\
\hline 582 & $9: 56: 43$ & 0.4411 \\
\hline 583 & $9: 56: 44$ & 0.4418 \\
\hline 584 & 9:56:45 & 0.4431 \\
\hline 585 & 9:56:46 & 0.4398 \\
\hline 586 & $9: 56: 47$ & 0.4409 \\
\hline 587 & 9:56:48 & 0.4403 \\
\hline 588 & 9:56:49 & 0.4449 \\
\hline 589 & 9:56:50 & 0.4418 \\
\hline 590 & $9: 56: 51$ & 0.4446 \\
\hline 591 & $9: 56: 52$ & 0.4461 \\
\hline 592 & $9: 56: 53$ & 0.4446 \\
\hline 593 & 9:56:54 & 0.4458 \\
\hline 594 & $9: 56: 55$ & 0.4427 \\
\hline 595 & $9: 56: 56$ & 0.4462 \\
\hline 596 & $9: 56: 57$ & 0.4419 \\
\hline 597 & $9: 56: 58$ & 0.4429 \\
\hline 598 & 9:56:59 & 0.4413 \\
\hline 599 & $9: 57: 00$ & 0.4423 \\
\hline 600 & $9: 57: 01$ & 0.4418 \\
\hline 601 & 9:57:02 & 0.4391 \\
\hline 602 & $9: 57: 03$ & 0.4436 \\
\hline 603 & $9: 57: 04$ & 0.4421 \\
\hline 604 & $9: 57: 05$ & 0.443 \\
\hline 605 & $9: 57: 06$ & 0.4395 \\
\hline 606 & $9: 57: 07$ & 0.4421 \\
\hline 607 & $9: 57: 08$ & 0.4410 \\
\hline 608 & $9: 57: 09$ & 0.4417 \\
\hline 609 & $9: 57: 10$ & 0.446 \\
\hline 610 & $9: 57: 11$ & 0.445 \\
\hline
\end{tabular}

C -12 
Appendix C - Fuel Data Test 2 NIST102605_1.xIs

$\begin{array}{lll}611 & 9: 57: 12 & 0.4470 \\ 612 & 9: 57: 13 & 0.4428 \\ 613 & 9: 57: 14 & 0.4451 \\ 614 & 9: 57: 15 & 0.4464 \\ 615 & 9: 57: 16 & 0.4430 \\ 616 & 9: 57: 17 & 0.4448 \\ 617 & 9: 57: 18 & 0.4427 \\ 618 & 9: 57: 19 & 0.4454 \\ 619 & 9: 57: 20 & 0.4433 \\ 620 & 9: 57: 21 & 0.4448 \\ 621 & 9: 57: 22 & 0.4440 \\ 622 & 9: 57: 23 & 0.4392 \\ 623 & 9: 57: 24 & 0.4441 \\ 624 & 9: 57: 25 & 0.4411 \\ 625 & 9: 57: 26 & 0.4408 \\ 626 & 9: 57: 27 & 0.4409 \\ 627 & 9: 57: 28 & 0.4416 \\ 628 & 9: 57: 29 & 0.4432 \\ 629 & 9: 57: 30 & 0.4433 \\ 630 & 9: 57: 31 & 0.4461 \\ 631 & 9: 57: 32 & 0.4432 \\ 632 & 9: 57: 33 & 0.4454 \\ 633 & 9: 57: 34 & 0.4443 \\ 634 & 9: 57: 35 & 0.4442 \\ 635 & 9: 57: 36 & 0.4462 \\ 636 & 9: 57: 37 & 0.4420 \\ 637 & 9: 57: 38 & 0.4434 \\ 638 & 9: 57: 39 & 0.4419 \\ 639 & 9: 57: 40 & 0.4435 \\ 640 & 9: 57: 41 & 0.4432 \\ 641 & 9: 57: 42 & 0.4417 \\ 642 & 9: 57: 43 & 0.4416 \\ 643 & 9: 57: 44 & 0.4403 \\ 644 & 9: 57: 45 & 0.4425 \\ 645 & 9: 57: 46 & 0.4412 \\ 646 & 9: 57: 47 & 0.4436 \\ 647 & 9: 57: 48 & 0.4397 \\ 648 & 9: 57: 49 & 0.4412 \\ 649 & 9: 57: 50 & 0.4415 \\ 650 & 9: 57: 51 & 0.4443 \\ 651 & 9: 57: 52 & 0.4476 \\ 652 & 9: 57: 53 & 0.4434 \\ 653 & 9: 57: 54 & 0.4461 \\ 654 & 9: 57: 55 & 0.4438 \\ 655 & 9: 57: 56 & 0.4432 \\ 656 & 9: 57: 57 & 0.4459 \\ 657 & 9: 57: 58 & 0.4448 \\ 658 & 9: 57: 59 & 0.4450 \\ 659 & 9: 58: 00 & 9: 58: 01 \\ 660 & 9: 53 \\ 661 & 9: 58: 02 & \end{array}$

C -13 
Appendix C - Fuel Data Test 2 NIST102605_1.xIs

$\begin{array}{lll}662 & 9: 58: 03 & 0.4435 \\ 663 & 9: 58: 04 & 0.4423 \\ 664 & 9: 58: 05 & 0.4415 \\ 665 & 9: 58: 06 & 0.4419 \\ 666 & 9: 58: 07 & 0.4393 \\ 667 & 9: 58: 08 & 0.4429 \\ 668 & 9: 58: 09 & 0.4413 \\ 669 & 9: 58: 10 & 0.4440 \\ 670 & 9: 58: 11 & 0.4417 \\ 671 & 9: 58: 12 & 0.4433 \\ 672 & 9: 58: 13 & 0.4445 \\ 673 & 9: 58: 14 & 0.4458 \\ 674 & 9: 58: 15 & 0.4455 \\ 675 & 9: 58: 16 & 0.4418 \\ 676 & 9: 58: 17 & 0.4458 \\ 677 & 9: 58: 18 & 0.4424 \\ 678 & 9: 58: 19 & 0.4437 \\ 679 & 9: 58: 20 & 0.4446 \\ 680 & 9: 58: 21 & 0.4440 \\ 681 & 9: 58: 22 & 0.4436 \\ 682 & 9: 58: 23 & 0.4397 \\ 683 & 9: 58: 24 & 0.4426 \\ 684 & 9: 58: 25 & 0.4404 \\ 685 & 9: 58: 26 & 0.4404 \\ 686 & 9: 58: 27 & 0.4409 \\ 687 & 9: 58: 28 & 0.4402 \\ 688 & 9: 58: 29 & 0.4426 \\ 689 & 9: 58: 30 & 0.4402 \\ 690 & 9: 58: 31 & 0.4439 \\ 691 & 9: 58: 32 & 0.4435 \\ 692 & 9: 58: 33 & 0.4431 \\ 693 & 9: 58: 34 & 0.4477 \\ 694 & 9: 58: 35 & 0.4461 \\ 695 & 9: 58: 36 & 0.4459 \\ 696 & 9: 58: 37 & 0.4400 \\ 697 & 9: 58: 38 & 0.4435 \\ 698 & 9: 58: 39 & 0.4426 \\ 699 & 9: 58: 40 & 0.4432 \\ 700 & 9: 58: 41 & 0.4424 \\ 701 & 9: 58: 42 & 0.4404 \\ 702 & 9: 58: 43 & 0.4407 \\ 703 & 9: 58: 44 & 0.4381 \\ 704 & 9: 58: 45 & 0.4432 \\ 705 & 9: 58: 46 & 0.4414 \\ 706 & 9: 58: 47 & 0.4423 \\ 707 & 9: 58: 48 & 0.4415 \\ 708 & 9: 58: 49 & 0.4403 \\ 709 & 9: 58: 50 & 0.4429 \\ 710 & 9: 58: 51 & \\ 711 & 9: 58: 52 & 9403 \\ 712 & 9: 58: 53 & \end{array}$

C -14 
Appendix C - Fuel Data Test 2 NIST102605_1.xIs

$\begin{array}{lll}713 & 9: 58: 54 & 0.4438 \\ 714 & 9: 58: 55 & 0.4453 \\ 715 & 9: 58: 56 & 0.4431 \\ 716 & 9: 58: 57 & 0.4452 \\ 717 & 9: 58: 58 & 0.4420 \\ 718 & 9: 58: 59 & 0.4446 \\ 719 & 9: 59: 00 & 0.4446 \\ 720 & 9: 59: 01 & 0.4436 \\ 721 & 9: 59: 02 & 0.4428 \\ 722 & 9: 59: 03 & 0.4402 \\ 723 & 9: 59: 04 & 0.4425 \\ 724 & 9: 59: 05 & 0.4412 \\ 725 & 9: 59: 06 & 0.4444 \\ 726 & 9: 59: 07 & 0.4403 \\ 727 & 9: 59: 08 & 0.4409 \\ 728 & 9: 59: 09 & 0.4404 \\ 729 & 9: 59: 10 & 0.4395 \\ 730 & 9: 59: 11 & 0.4459 \\ 731 & 9: 59: 12 & 0.4455 \\ 732 & 9: 59: 13 & 0.4468 \\ 733 & 9: 59: 14 & 0.4429 \\ 734 & 9: 59: 15 & 0.4456 \\ 735 & 9: 59: 16 & 0.4435 \\ 736 & 9: 59: 17 & 0.4453 \\ 737 & 9: 59: 18 & 0.4420 \\ 738 & 9: 59: 19 & 0.4437 \\ 739 & 9: 59: 20 & 0.4428 \\ 740 & 9: 59: 21 & 0.4444 \\ 741 & 9: 59: 22 & 0.4427 \\ 742 & 9: 59: 23 & 0.4425 \\ 743 & 9: 59: 24 & 0.4417 \\ 744 & 9: 59: 25 & 0.4395 \\ 745 & 9: 59: 26 & 0.4425 \\ 746 & 9: 59: 27 & 0.4408 \\ 747 & 9: 59: 28 & 0.4435 \\ 748 & 9: 59: 29 & 0.4408 \\ 749 & 9: 59: 30 & 0.4428 \\ 750 & 9: 59: 31 & 0.4405 \\ 751 & 9: 59: 32 & 0.4437 \\ 752 & 9: 59: 33 & 0.4427 \\ 753 & 9: 59: 34 & 0.4448 \\ 754 & 9: 59: 35 & 0.4473 \\ 755 & 9: 59: 36 & 0.4445 \\ 756 & 9: 59: 37 & 0.4453 \\ 757 & 9: 59: 38 & 0.4413 \\ 758 & 9: 59: 39 & 0.4447 \\ 759 & 9: 59: 40 & 0.4427 \\ 760 & 9: 59: 41 & 0.4446 \\ 761 & 9: 59: 42 & 0.4432 \\ 762 & 9: 59: 43 & \\ 763 & 9: 59: 44 & \end{array}$


Appendix C - Fuel Data Test 2 NIST102605_1.xIs

$\begin{array}{lrl}764 & 9: 59: 45 & 0.4409 \\ 765 & 9: 59: 46 & 0.4413 \\ 766 & 9: 59: 47 & 0.4410 \\ 767 & 9: 59: 48 & 0.4420 \\ 768 & 9: 59: 49 & 0.4388 \\ 769 & 9: 59: 50 & 0.4438 \\ 770 & 9: 59: 51 & 0.4399 \\ 771 & 9: 59: 52 & 0.4415 \\ 772 & 9: 59: 53 & 0.4407 \\ 773 & 9: 59: 54 & 0.4444 \\ 774 & 9: 59: 55 & 0.4467 \\ 775 & 9: 59: 56 & 0.4457 \\ 776 & 9: 59: 57 & 0.4446 \\ 777 & 9: 59: 58 & 0.4438 \\ 778 & 9: 59: 59 & 0.4453 \\ 779 & 10: 00: 00 & 0.4435 \\ 780 & 10: 00: 01 & 0.4434 \\ 781 & 10: 00: 02 & 0.4402 \\ 782 & 10: 00: 03 & 0.4430 \\ 783 & 10: 00: 04 & 0.4405 \\ 784 & 10: 00: 05 & 0.4444 \\ 785 & 10: 00: 06 & 0.4427 \\ 786 & 10: 00: 07 & 0.4407 \\ 787 & 10: 00: 08 & 0.4409 \\ 788 & 10: 00: 09 & 0.4394 \\ 789 & 10: 00: 10 & 0.4431 \\ 790 & 10: 00: 11 & 0.4400 \\ 791 & 10: 00: 12 & 0.4416 \\ 792 & 10: 00: 13 & 0.4448 \\ 793 & 10: 00: 14 & 0.4439 \\ 794 & 10: 00: 15 & 0.4452 \\ 795 & 10: 00: 16 & 0.4423 \\ 796 & 10: 00: 17 & 0.4458 \\ 797 & 10: 00: 18 & 0.4439 \\ 798 & 10: 00: 19 & 0.4454 \\ 799 & 10: 00: 20 & 0.4457 \\ 800 & 10: 00: 21 & 0.4425 \\ 801 & 10: 00: 22 & 0.4432 \\ 802 & 10: 00: 23 & 0.4412 \\ 803 & 10: 00: 24 & 0.4444 \\ 804 & 10: 00: 25 & 0.4410 \\ 805 & 10: 00: 26 & 0.4428 \\ 806 & 10: 00: 27 & 0.4421 \\ 807 & 10: 00: 28 & 0.4431 \\ 808 & 10: 00: 29 & 0.4417 \\ 809 & 10: 00: 30 & 0.4404 \\ 810 & 10: 00: 31 & 0.4415 \\ 811 & 10: 00: 32 & 0.4415 \\ 812 & 10: 00: 33 & \\ 813 & 10: 00: 34 & 0.4449 \\ 814 & 10: 00: 35 & \end{array}$

C - 16 
Appendix C - Fuel Data Test 2 NIST102605_1.xIs

\begin{tabular}{|c|c|c|}
\hline 815 & 10:00:36 & 0.4435 \\
\hline 816 & $10: 00: 37$ & 0.4439 \\
\hline 817 & $10: 00: 38$ & 0.4418 \\
\hline 818 & 10:00:39 & 0.4452 \\
\hline 819 & $10: 00: 40$ & 0.4460 \\
\hline 820 & $10: 00: 41$ & 0.4422 \\
\hline 821 & $10: 00: 42$ & 0.4431 \\
\hline 822 & $10: 00: 43$ & 0.4403 \\
\hline 823 & $10: 00: 44$ & 0.4438 \\
\hline 824 & $10: 00: 45$ & 0.4416 \\
\hline 825 & $10: 00: 46$ & 0.4439 \\
\hline 826 & $10: 00: 47$ & 0.4421 \\
\hline 827 & $10: 00: 48$ & 0.4397 \\
\hline 828 & 10:00:49 & 0.4434 \\
\hline 829 & $10: 00: 50$ & 0.4418 \\
\hline 830 & $10: 00: 51$ & 0.4443 \\
\hline 831 & $10: 00: 52$ & 0.4399 \\
\hline 832 & $10: 00: 53$ & 0.4412 \\
\hline 833 & $10: 00: 54$ & 0.4419 \\
\hline 834 & $10: 00: 55$ & 0.4444 \\
\hline 835 & $10: 00: 56$ & 0.4448 \\
\hline 836 & $10: 00: 57$ & 0.4450 \\
\hline 837 & $10: 00: 58$ & 0.4460 \\
\hline 838 & 10:00:59 & 0.4415 \\
\hline 839 & 10:01:00 & 0.4432 \\
\hline 840 & 10:01:01 & 0.4410 \\
\hline 841 & $10: 01: 02$ & 0.4448 \\
\hline 842 & $10: 01: 03$ & 0.4442 \\
\hline 843 & $10: 01: 04$ & 0.4404 \\
\hline 844 & 10:01:05 & 0.4415 \\
\hline 845 & 10:01:06 & 0.4407 \\
\hline 846 & $10: 01: 07$ & 0.4419 \\
\hline 847 & $10: 01: 08$ & 0.4408 \\
\hline 848 & 10:01:09 & 0.4421 \\
\hline 849 & $10: 01: 10$ & 0.4405 \\
\hline 850 & $10: 01: 11$ & 0.4429 \\
\hline 851 & $10: 01: 12$ & 0.4410 \\
\hline 852 & $10: 01: 13$ & 0.4428 \\
\hline 853 & $10: 01: 14$ & 0.4429 \\
\hline 854 & $10: 01: 15$ & 0.4436 \\
\hline 855 & $10: 01: 16$ & 0.4472 \\
\hline 856 & $10: 01: 17$ & 0.4436 \\
\hline 857 & 10:01:18 & 0.4460 \\
\hline 858 & $10: 01: 19$ & 0.4442 \\
\hline 859 & $10: 01: 20$ & 0.4442 \\
\hline 860 & $10: 01: 21$ & 0.4448 \\
\hline 861 & 10:01:22 & 0.4418 \\
\hline 862 & $10: 01: 23$ & 0.4429 \\
\hline 863 & $10: 01: 24$ & 0.4407 \\
\hline 864 & $10: 01: 25$ & 0.444 \\
\hline 865 & $10: 01: 26$ & $0.44 C$ \\
\hline
\end{tabular}

C -17 
Appendix C - Fuel Data Test 2 NIST102605_1.xIs

\begin{tabular}{|c|c|c|}
\hline 866 & $10: 01: 27$ & 0.4414 \\
\hline 867 & $10: 01: 28$ & 0.4414 \\
\hline 868 & 10:01:29 & 0.4395 \\
\hline 869 & $10: 01: 30$ & 0.4428 \\
\hline 870 & 10:01:31 & 0.4415 \\
\hline 871 & 10:01:32 & 0.4442 \\
\hline 872 & $10: 01: 33$ & 0.4405 \\
\hline 873 & $10: 01: 34$ & 0.4421 \\
\hline 874 & $10: 01: 35$ & 0.4434 \\
\hline 875 & $10: 01: 36$ & 0.4449 \\
\hline 876 & $10: 01: 37$ & 0.4439 \\
\hline 877 & $10: 01: 38$ & 0.4438 \\
\hline 878 & $10: 01: 39$ & 0.4457 \\
\hline 879 & $10: 01: 40$ & 0.4408 \\
\hline 880 & $10: 01: 41$ & 0.4440 \\
\hline 881 & $10: 01: 42$ & 0.4442 \\
\hline 882 & $10: 01: 43$ & 0.4429 \\
\hline 883 & $10: 01: 44$ & 0.4429 \\
\hline 884 & $10: 01: 45$ & 0.4406 \\
\hline 885 & $10: 01: 46$ & 0.4437 \\
\hline 886 & $10: 01: 47$ & 0.4424 \\
\hline 887 & $10: 01: 48$ & 0.4429 \\
\hline 888 & $10: 01: 49$ & 0.4415 \\
\hline 889 & 10:01:50 & 0.4396 \\
\hline 890 & $10: 01: 51$ & 0.4420 \\
\hline 891 & $10: 01: 52$ & 0.4388 \\
\hline 892 & $10: 01: 53$ & 0.4416 \\
\hline 893 & $10: 01: 54$ & 0.4437 \\
\hline 894 & $10: 01: 55$ & 0.4435 \\
\hline 895 & $10: 01: 56$ & 0.4468 \\
\hline 896 & $10: 01: 57$ & 0.4432 \\
\hline 897 & $10: 01: 58$ & 0.4446 \\
\hline 898 & $10: 01: 59$ & 0.4414 \\
\hline 899 & $10: 02: 00$ & 0.4439 \\
\hline 900 & $10: 02: 01$ & 0.4428 \\
\hline 901 & 10:02:02 & 0.4433 \\
\hline 902 & $10: 02: 03$ & 0.4434 \\
\hline 903 & $10: 02: 04$ & 0.4402 \\
\hline 904 & $10: 02: 05$ & 0.4444 \\
\hline 905 & $10: 02: 06$ & 0.4425 \\
\hline 906 & $10: 02: 07$ & 0.4435 \\
\hline 907 & $10: 02: 08$ & 0.4418 \\
\hline 908 & 10:02:09 & 0.4393 \\
\hline 909 & $10: 02: 10$ & 0.4419 \\
\hline 910 & $10: 02: 11$ & 0.4413 \\
\hline 911 & $10: 02: 12$ & 0.4430 \\
\hline 912 & $10: 02: 13$ & 0.4393 \\
\hline 913 & $10: 02: 14$ & 0.4435 \\
\hline 914 & $10: 02: 15$ & 0.4420 \\
\hline 915 & $10: 02: 16$ & 0.444 \\
\hline 916 & $10: 02: 17$ & 0.443 \\
\hline
\end{tabular}

C - 18 
Appendix C - Fuel Data Test 2 NIST102605_1.xIs

\begin{tabular}{|c|c|c|}
\hline 917 & 10:02:18 & 0.4445 \\
\hline 918 & $10: 02: 19$ & 0.4461 \\
\hline 919 & $10: 02: 20$ & 0.4417 \\
\hline 920 & $10: 02: 21$ & 0.4429 \\
\hline 921 & $10: 02: 22$ & 0.4411 \\
\hline 922 & 10:02:23 & 0.4449 \\
\hline 923 & $10: 02: 24$ & 0.4422 \\
\hline 924 & 10:02:25 & 0.4435 \\
\hline 925 & $10: 02: 26$ & 0.4436 \\
\hline 926 & $10: 02: 27$ & 0.4432 \\
\hline 927 & 10:02:28 & 0.4425 \\
\hline 928 & $10: 02: 29$ & 0.4406 \\
\hline 929 & $10: 02: 30$ & 0.4431 \\
\hline 930 & $10: 02: 31$ & 0.4398 \\
\hline 931 & 10:02:32 & 0.4429 \\
\hline 932 & $10: 02: 33$ & 0.4393 \\
\hline 933 & $10: 02: 34$ & 0.4430 \\
\hline 934 & $10: 02: 35$ & 0.4440 \\
\hline 935 & $10: 02: 36$ & 0.4422 \\
\hline 936 & $10: 02: 37$ & 0.4474 \\
\hline 937 & $10: 02: 38$ & 0.4439 \\
\hline 938 & $10: 02: 39$ & 0.4453 \\
\hline 939 & $10: 02: 40$ & 0.4455 \\
\hline 940 & $10: 02: 41$ & 0.4445 \\
\hline 941 & $10: 02: 42$ & 0.4450 \\
\hline 942 & $10: 02: 43$ & 0.4417 \\
\hline 943 & $10: 02: 44$ & 0.4423 \\
\hline 944 & $10: 02: 45$ & 0.4408 \\
\hline 945 & $10: 02: 46$ & 0.4427 \\
\hline 946 & $10: 02: 47$ & 0.4384 \\
\hline 947 & $10: 02: 48$ & 0.4424 \\
\hline 948 & $10: 02: 49$ & 0.4410 \\
\hline 949 & 10:02:50 & 0.4445 \\
\hline 950 & $10: 02: 51$ & 0.4406 \\
\hline 951 & $10: 02: 52$ & 0.4395 \\
\hline 952 & $10: 02: 53$ & 0.4422 \\
\hline 953 & $10: 02: 54$ & 0.4407 \\
\hline 954 & $10: 02: 55$ & 0.4448 \\
\hline 955 & $10: 02: 56$ & 0.4439 \\
\hline 956 & $10: 02: 57$ & 0.4461 \\
\hline 957 & $10: 02: 58$ & 0.4416 \\
\hline 958 & $10: 02: 59$ & 0.4429 \\
\hline 959 & $10: 03: 00$ & 0.4432 \\
\hline 960 & $10: 03: 01$ & 0.4431 \\
\hline 961 & 10:03:02 & 0.4445 \\
\hline 962 & 10:03:03 & 0.4410 \\
\hline 963 & $10: 03: 04$ & 0.4438 \\
\hline 964 & $10: 03: 05$ & 0.4407 \\
\hline 965 & $10: 03: 06$ & 0.4436 \\
\hline 966 & 10:03:07 & 0.443 \\
\hline 967 & $10: 03: 08$ & 0.443 \\
\hline
\end{tabular}

C - 19 
Appendix C - Fuel Data Test 2 NIST102605_1.xIs

\begin{tabular}{|c|c|c|}
\hline 968 & 10:03:09 & 0.4425 \\
\hline 969 & $10: 03: 10$ & 0.4406 \\
\hline 970 & 10:03:11 & 0.4436 \\
\hline 971 & 10:03:12 & 0.4390 \\
\hline 972 & $10: 03: 13$ & 0.4407 \\
\hline 973 & $10: 03: 14$ & 0.4408 \\
\hline 974 & $10: 03: 15$ & 0.4428 \\
\hline 975 & 10:03:16 & 0.4450 \\
\hline 976 & 10:03:17 & 0.4433 \\
\hline 977 & 10:03:18 & 0.4464 \\
\hline 978 & $10: 03: 19$ & 0.4432 \\
\hline 979 & 10:03:20 & 0.4456 \\
\hline 980 & $10: 03: 21$ & 0.4450 \\
\hline 981 & $10: 03: 22$ & 0.4423 \\
\hline 982 & $10: 03: 23$ & 0.4431 \\
\hline 983 & $10: 03: 24$ & 0.4397 \\
\hline 984 & 10:03:25 & 0.4429 \\
\hline 985 & 10:03:26 & 0.4417 \\
\hline 986 & $10: 03: 27$ & 0.4443 \\
\hline 987 & $10: 03: 28$ & 0.4410 \\
\hline 988 & 10:03:29 & 0.4409 \\
\hline 989 & 10:03:30 & 0.4427 \\
\hline 990 & $10: 03: 31$ & 0.4400 \\
\hline 991 & 10:03:32 & 0.4437 \\
\hline 992 & $10: 03: 33$ & 0.4399 \\
\hline 993 & $10: 03: 34$ & 0.4394 \\
\hline 994 & 10:03:35 & 0.4420 \\
\hline 995 & 10:03:36 & 0.4426 \\
\hline 996 & $10: 03: 37$ & 0.4458 \\
\hline 997 & 10:03:38 & 0.4410 \\
\hline 998 & 10:03:39 & 0.4437 \\
\hline 999 & $10: 03: 40$ & 0.4444 \\
\hline 1000 & $10: 03: 41$ & 0.4445 \\
\hline 1001 & $10: 03: 42$ & 0.4445 \\
\hline 1002 & 10:03:43 & 0.4416 \\
\hline 1003 & $10: 03: 44$ & 0.4449 \\
\hline 1004 & $10: 03: 45$ & 0.4442 \\
\hline 1005 & $10: 03: 46$ & 0.4399 \\
\hline 1006 & $10: 03: 47$ & 0.4420 \\
\hline 1007 & $10: 03: 48$ & 0.4399 \\
\hline 1008 & $10: 03: 49$ & 0.4430 \\
\hline 1009 & $10: 03: 50$ & 0.4422 \\
\hline 1010 & $10: 03: 51$ & 0.4428 \\
\hline 1011 & 10:03:52 & 0.4388 \\
\hline 1012 & 10:03:53 & 0.4393 \\
\hline 1013 & $10: 03: 54$ & 0.4422 \\
\hline 1014 & 10:03:55 & 0.4415 \\
\hline 1015 & $10: 03: 56$ & 0.4449 \\
\hline 1016 & $10: 03: 57$ & 0.4418 \\
\hline 1017 & 10:03:58 & 0.444 \\
\hline 1018 & 10:03:59 & 0.44 \\
\hline
\end{tabular}


Appendix C - Fuel Data Test 2 NIST102605_1.xIs

\begin{tabular}{|c|c|c|}
\hline 1019 & $10: 04: 00$ & 0.4452 \\
\hline 1020 & 10:04:01 & 0.4444 \\
\hline 1021 & $10: 04: 02$ & 0.4449 \\
\hline 1022 & 10:04:03 & 0.4451 \\
\hline 1023 & $10: 04: 04$ & 0.4409 \\
\hline 1024 & 10:04:05 & 0.4427 \\
\hline 1025 & 10:04:06 & 0.4407 \\
\hline 1026 & $10: 04: 07$ & 0.4440 \\
\hline 1027 & 10:04:08 & 0.4411 \\
\hline 1028 & 10:04:09 & 0.4394 \\
\hline 1029 & 10:04:10 & 0.4427 \\
\hline 1030 & 10:04:11 & 0.4387 \\
\hline 1031 & 10:04:12 & 0.4419 \\
\hline 1032 & $10: 04: 13$ & 0.4405 \\
\hline 1033 & $10: 04: 14$ & 0.4396 \\
\hline 1034 & 10:04:15 & 0.4433 \\
\hline 1035 & $10: 04: 16$ & 0.4418 \\
\hline 1036 & $10: 04: 17$ & 0.4440 \\
\hline 1037 & $10: 04: 18$ & 0.4408 \\
\hline 1038 & $10: 04: 19$ & 0.4431 \\
\hline 1039 & 10:04:20 & 0.4448 \\
\hline 1040 & 10:04:21 & 0.4454 \\
\hline 1041 & 10:04:22 & 0.4455 \\
\hline 1042 & $10: 04: 23$ & 0.4416 \\
\hline 1043 & 10:04:24 & 0.4443 \\
\hline 1044 & 10:04:25 & 0.4414 \\
\hline 1045 & 10:04:26 & 0.4440 \\
\hline 1046 & $10: 04: 27$ & 0.4440 \\
\hline 1047 & 10:04:28 & 0.4420 \\
\hline 1048 & 10:04:29 & 0.4399 \\
\hline 1049 & $10: 04: 30$ & 0.4390 \\
\hline 1050 & $10: 04: 31$ & 0.4423 \\
\hline 1051 & 10:04:32 & 0.4415 \\
\hline 1052 & $10: 04: 33$ & 0.4416 \\
\hline 1053 & $10: 04: 34$ & 0.4378 \\
\hline 1054 & 10:04:35 & 0.4427 \\
\hline 1055 & 10:04:36 & 0.4402 \\
\hline 1056 & $10: 04: 37$ & 0.4420 \\
\hline 1057 & 10:04:38 & 0.4450 \\
\hline 1058 & 10:04:39 & 0.4455 \\
\hline 1059 & $10: 04: 40$ & 0.4470 \\
\hline 1060 & $10: 04: 41$ & 0.4414 \\
\hline 1061 & $10: 04: 42$ & 0.4445 \\
\hline 1062 & 10:04:43 & 0.4431 \\
\hline 1063 & $10: 04: 44$ & 0.4438 \\
\hline 1064 & $10: 04: 45$ & 0.4447 \\
\hline 1065 & 10:04:46 & 0.4412 \\
\hline 1066 & $10: 04: 47$ & 0.4435 \\
\hline 1067 & $10: 04: 48$ & 0.4401 \\
\hline 1068 & $10: 04: 49$ & 0.4425 \\
\hline 1069 & $10: 04: 50$ & 0.4420 \\
\hline
\end{tabular}


Appendix C - Fuel Data Test 2 NIST102605_1.xIs

\begin{tabular}{|c|c|c|}
\hline 1070 & $10: 04: 51$ & 0.4394 \\
\hline 1071 & $10: 04: 52$ & 0.4428 \\
\hline 1072 & $10: 04: 53$ & 0.4405 \\
\hline 1073 & $10: 04: 54$ & 0.4436 \\
\hline 1074 & $10: 04: 55$ & 0.4415 \\
\hline 1075 & 10:04:56 & 0.4442 \\
\hline 1076 & 10:04:57 & 0.4421 \\
\hline 1077 & $10: 04: 58$ & 0.4443 \\
\hline 1078 & $10: 04: 59$ & 0.4415 \\
\hline 1079 & $10: 05: 00$ & 0.4440 \\
\hline 1080 & 10:05:01 & 0.4445 \\
\hline 1081 & 10:05:02 & 0.4451 \\
\hline 1082 & $10: 05: 03$ & 0.4447 \\
\hline 1083 & $10: 05: 04$ & 0.4421 \\
\hline 1084 & 10:05:05 & 0.4449 \\
\hline 1085 & 10:05:06 & 0.4420 \\
\hline 1086 & $10: 05: 07$ & 0.4451 \\
\hline 1087 & 10:05:08 & 0.4433 \\
\hline 1088 & 10:05:09 & 0.4422 \\
\hline 1089 & $10: 05: 10$ & 0.4409 \\
\hline 1090 & 10:05:11 & 0.4389 \\
\hline 1091 & $10: 05: 12$ & 0.4421 \\
\hline 1092 & $10: 05: 13$ & 0.4405 \\
\hline 1093 & $10: 05: 14$ & 0.4422 \\
\hline 1094 & 10:05:15 & 0.4388 \\
\hline 1095 & $10: 05: 16$ & 0.4414 \\
\hline 1096 & $10: 05: 17$ & 0.4450 \\
\hline 1097 & 10:05:18 & 0.4436 \\
\hline 1098 & $10: 05: 19$ & 0.4464 \\
\hline 1099 & $10: 05: 20$ & 0.4422 \\
\hline 1100 & $10: 05: 21$ & 0.4437 \\
\hline 1101 & 10:05:22 & 0.4408 \\
\hline 1102 & $10: 05: 23$ & 0.4445 \\
\hline 1103 & 10:05:24 & 0.4432 \\
\hline 1104 & $10: 05: 25$ & 0.4436 \\
\hline 1105 & $10: 05: 26$ & 0.4431 \\
\hline 1106 & $10: 05: 27$ & 0.4418 \\
\hline 1107 & $10: 05: 28$ & 0.4432 \\
\hline 1108 & 10:05:29 & 0.4390 \\
\hline 1109 & $10: 05: 30$ & 0.4435 \\
\hline 1110 & $10: 05: 31$ & 0.4413 \\
\hline 1111 & 10:05:32 & 0.4426 \\
\hline 1112 & $10: 05: 33$ & 0.4400 \\
\hline 1113 & $10: 05: 34$ & 0.4385 \\
\hline 1114 & $10: 05: 35$ & 0.4421 \\
\hline 1115 & $10: 05: 36$ & 0.4395 \\
\hline 1116 & $10: 05: 37$ & 0.4423 \\
\hline 1117 & $10: 05: 38$ & 0.4409 \\
\hline 1118 & $10: 05: 39$ & 0.4450 \\
\hline 1119 & $10: 05: 40$ & 0.4460 \\
\hline 1120 & $10: 05: 41$ & 0.4433 \\
\hline
\end{tabular}


Appendix C - Fuel Data Test 2 NIST102605_1.xIs

\begin{tabular}{|c|c|c|}
\hline 1121 & $10: 05: 42$ & 0.4450 \\
\hline 1122 & $10: 05: 43$ & 0.4404 \\
\hline 1123 & $10: 05: 44$ & 0.4418 \\
\hline 1124 & $10: 05: 45$ & 0.4413 \\
\hline 1125 & 10:05:46 & 0.4448 \\
\hline 1126 & $10: 05: 47$ & 0.4435 \\
\hline 1127 & $10: 05: 48$ & 0.4413 \\
\hline 1128 & $10: 05: 49$ & 0.4427 \\
\hline 1129 & $10: 05: 50$ & 0.4394 \\
\hline 1130 & $10: 05: 51$ & 0.4435 \\
\hline 1131 & $10: 05: 52$ & 0.4420 \\
\hline 1132 & $10: 05: 53$ & 0.4437 \\
\hline 1133 & $10: 05: 54$ & 0.4385 \\
\hline 1134 & 10:05:55 & 0.4386 \\
\hline 1135 & $10: 05: 56$ & 0.4409 \\
\hline 1136 & $10: 05: 57$ & 0.4419 \\
\hline 1137 & $10: 05: 58$ & 0.4447 \\
\hline 1138 & $10: 05: 59$ & 0.4425 \\
\hline 1139 & $10: 06: 00$ & 0.4462 \\
\hline 1140 & 10:06:01 & 0.4434 \\
\hline 1141 & 10:06:02 & 0.4456 \\
\hline 1142 & $10: 06: 03$ & 0.4443 \\
\hline 1143 & 10:06:04 & 0.4450 \\
\hline 1144 & 10:06:05 & 0.4445 \\
\hline 1145 & 10:06:06 & 0.4410 \\
\hline 1146 & $10: 06: 07$ & 0.4427 \\
\hline 1147 & $10: 06: 08$ & 0.4403 \\
\hline 1148 & $10: 06: 09$ & 0.4420 \\
\hline 1149 & $10: 06: 10$ & 0.4381 \\
\hline 1150 & $10: 06: 11$ & 0.4440 \\
\hline 1151 & 10:06:12 & 0.4422 \\
\hline 1152 & 10:06:13 & 0.4426 \\
\hline 1153 & 10:06:14 & 0.4420 \\
\hline 1154 & $10: 06: 15$ & 0.4401 \\
\hline 1155 & $10: 06: 16$ & 0.4424 \\
\hline 1156 & $10: 06: 17$ & 0.4388 \\
\hline 1157 & $10: 06: 18$ & 0.4416 \\
\hline 1158 & $10: 06: 19$ & 0.4408 \\
\hline 1159 & $10: 06: 20$ & 0.4448 \\
\hline 1160 & $10: 06: 21$ & 0.4427 \\
\hline 1161 & 10:06:22 & 0.4432 \\
\hline 1162 & 10:06:23 & 0.4437 \\
\hline 1163 & $10: 06: 24$ & 0.4454 \\
\hline 1164 & $10: 06: 25$ & 0.4447 \\
\hline 1165 & $10: 06: 26$ & 0.4422 \\
\hline 1166 & $10: 06: 27$ & 0.4446 \\
\hline 1167 & 10:06:28 & 0.4400 \\
\hline 1168 & $10: 06: 29$ & 0.4423 \\
\hline 1169 & $10: 06: 30$ & 0.4428 \\
\hline 1170 & 10:06:31 & 0.4424 \\
\hline 1171 & 10:06:32 & 0.4431 \\
\hline
\end{tabular}


Appendix C - Fuel Data Test 2 NIST102605_1.xIs

\begin{tabular}{|c|c|c|}
\hline 1172 & $10: 06: 33$ & 0.4412 \\
\hline 1173 & $10: 06: 34$ & 0.4435 \\
\hline 1174 & $10: 06: 35$ & 0.4389 \\
\hline 1175 & $10: 06: 36$ & 0.4397 \\
\hline 1176 & $10: 06: 37$ & 0.4399 \\
\hline 1177 & $10: 06: 38$ & 0.4419 \\
\hline 1178 & 10:06:39 & 0.4451 \\
\hline 1179 & $10: 06: 40$ & 0.4430 \\
\hline 1180 & $10: 06: 41$ & 0.4458 \\
\hline 1181 & $10: 06: 42$ & 0.4430 \\
\hline 1182 & $10: 06: 43$ & 0.4456 \\
\hline 1183 & $10: 06: 44$ & 0.4448 \\
\hline 1184 & $10: 06: 45$ & 0.4432 \\
\hline 1185 & $10: 06: 46$ & 0.4436 \\
\hline 1186 & $10: 06: 47$ & 0.4403 \\
\hline 1187 & $10: 06: 48$ & 0.4439 \\
\hline 1188 & $10: 06: 49$ & 0.4424 \\
\hline 1189 & $10: 06: 50$ & 0.4426 \\
\hline 1190 & $10: 06: 51$ & 0.4425 \\
\hline 1191 & $10: 06: 52$ & 0.4397 \\
\hline 1192 & $10: 06: 53$ & 0.4433 \\
\hline 1193 & $10: 06: 54$ & 0.4406 \\
\hline 1194 & $10: 06: 55$ & 0.4402 \\
\hline 1195 & 10:06:56 & 0.4411 \\
\hline 1196 & $10: 06: 57$ & 0.4402 \\
\hline 1197 & $10: 06: 58$ & 0.4417 \\
\hline 1198 & $10: 06: 59$ & 0.4423 \\
\hline 1199 & $10: 07: 00$ & 0.4458 \\
\hline 1200 & 10:07:01 & 0.4435 \\
\hline 1201 & 10:07:02 & 0.4458 \\
\hline 1202 & $10: 07: 03$ & 0.4436 \\
\hline 1203 & $10: 07: 04$ & 0.4446 \\
\hline 1204 & 10:07:05 & 0.4453 \\
\hline 1205 & 10:07:06 & 0.4415 \\
\hline 1206 & $10: 07: 07$ & 0.4441 \\
\hline 1207 & $10: 07: 08$ & 0.4424 \\
\hline 1208 & 10:07:09 & 0.4433 \\
\hline 1209 & $10: 07: 10$ & 0.4442 \\
\hline 1210 & 10:07:11 & 0.4401 \\
\hline 1211 & $10: 07: 12$ & 0.4399 \\
\hline 1212 & $10: 07: 13$ & 0.4387 \\
\hline 1213 & 10:07:14 & 0.4441 \\
\hline 1214 & 10:07:15 & 0.4402 \\
\hline 1215 & $10: 07: 16$ & 0.4388 \\
\hline 1216 & $10: 07: 17$ & 0.4399 \\
\hline 1217 & $10: 07: 18$ & 0.4404 \\
\hline 1218 & $10: 07: 19$ & 0.4445 \\
\hline 1219 & $10: 07: 20$ & 0.4416 \\
\hline 1220 & $10: 07: 21$ & 0.4454 \\
\hline 1221 & $10: 07: 22$ & 0.4460 \\
\hline 1222 & $10: 07: 23$ & 0.4444 \\
\hline
\end{tabular}


Appendix C - Fuel Data Test 2 NIST102605_1.xIs

\begin{tabular}{|c|c|c|}
\hline 1223 & $10: 07: 24$ & 0.4462 \\
\hline 1224 & $10: 07: 25$ & 0.4421 \\
\hline 1225 & $10: 07: 26$ & 0.4444 \\
\hline 1226 & $10: 07: 27$ & 0.4419 \\
\hline 1227 & $10: 07: 28$ & 0.4450 \\
\hline 1228 & $10: 07: 29$ & 0.4429 \\
\hline 1229 & $10: 07: 30$ & 0.4414 \\
\hline 1230 & $10: 07: 31$ & 0.4418 \\
\hline 1231 & $10: 07: 32$ & 0.4393 \\
\hline 1232 & $10: 07: 33$ & 0.4443 \\
\hline 1233 & $10: 07: 34$ & 0.4404 \\
\hline 1234 & $10: 07: 35$ & 0.4404 \\
\hline 1235 & $10: 07: 36$ & 0.4426 \\
\hline 1236 & $10: 07: 37$ & 0.4386 \\
\hline 1237 & 10:07:38 & 0.4408 \\
\hline 1238 & 10:07:39 & 0.4400 \\
\hline 1239 & $10: 07: 40$ & 0.4420 \\
\hline 1240 & $10: 07: 41$ & 0.4405 \\
\hline 1241 & $10: 07: 42$ & 0.4433 \\
\hline 1242 & $10: 07: 43$ & 0.4426 \\
\hline 1243 & $10: 07: 44$ & 0.4452 \\
\hline 1244 & $10: 07: 45$ & 0.4449 \\
\hline 1245 & $10: 07: 46$ & 0.4443 \\
\hline 1246 & $10: 07: 47$ & 0.4452 \\
\hline 1247 & $10: 07: 48$ & 0.4417 \\
\hline 1248 & $10: 07: 49$ & 0.4425 \\
\hline 1249 & $10: 07: 50$ & 0.4399 \\
\hline 1250 & $10: 07: 51$ & 0.4442 \\
\hline 1251 & $10: 07: 52$ & 0.4422 \\
\hline 1252 & $10: 07: 53$ & 0.4418 \\
\hline 1253 & $10: 07: 54$ & 0.4401 \\
\hline 1254 & $10: 07: 55$ & 0.4413 \\
\hline 1255 & 10:07:56 & 0.4429 \\
\hline 1256 & $10: 07: 57$ & 0.4399 \\
\hline 1257 & $10: 07: 58$ & 0.4427 \\
\hline 1258 & $10: 07: 59$ & 0.4394 \\
\hline 1259 & 10:08:00 & 0.4421 \\
\hline 1260 & 10:08:01 & 0.4435 \\
\hline 1261 & 10:08:02 & 0.4449 \\
\hline 1262 & 10:08:03 & 0.4468 \\
\hline 1263 & 10:08:04 & 0.4433 \\
\hline 1264 & 10:08:05 & 0.4453 \\
\hline 1265 & 10:08:06 & 0.4418 \\
\hline 1266 & 10:08:07 & 0.4443 \\
\hline 1267 & $10: 08: 08$ & 0.4430 \\
\hline 1268 & 10:08:09 & 0.4444 \\
\hline 1269 & $10: 08: 10$ & 0.4419 \\
\hline 1270 & 10:08:11 & 0.4398 \\
\hline 1271 & 10:08:12 & 0.4413 \\
\hline 1272 & 10:08:13 & 0.4396 \\
\hline 1273 & 10:08:14 & 0.4435 \\
\hline
\end{tabular}


Appendix C - Fuel Data Test 2 NIST102605_1.xIs

\begin{tabular}{|c|c|c|}
\hline 1274 & 10:08:15 & 0.4398 \\
\hline 1275 & 10:08:16 & 0.4429 \\
\hline 1276 & $10: 08: 17$ & 0.4409 \\
\hline 1277 & 10:08:18 & 0.4400 \\
\hline 1278 & 10:08:19 & 0.4422 \\
\hline 1279 & 10:08:20 & 0.4418 \\
\hline 1280 & 10:08:21 & 0.4448 \\
\hline 1281 & 10:08:22 & 0.4432 \\
\hline 1282 & 10:08:23 & 0.4448 \\
\hline 1283 & 10:08:24 & 0.4426 \\
\hline 1284 & 10:08:25 & 0.4448 \\
\hline 1285 & 10:08:26 & 0.4445 \\
\hline 1286 & 10:08:27 & 0.4438 \\
\hline 1287 & 10:08:28 & 0.4455 \\
\hline 1288 & 10:08:29 & 0.4430 \\
\hline 1289 & 10:08:30 & 0.4424 \\
\hline 1290 & 10:08:31 & 0.4403 \\
\hline 1291 & 10:08:32 & 0.4431 \\
\hline 1292 & 10:08:33 & 0.4405 \\
\hline 1293 & 10:08:34 & 0.4417 \\
\hline 1294 & 10:08:35 & 0.4400 \\
\hline 1295 & 10:08:36 & 0.4430 \\
\hline 1296 & 10:08:37 & 0.4385 \\
\hline 1297 & 10:08:38 & 0.4398 \\
\hline 1298 & 10:08:39 & 0.4400 \\
\hline 1299 & 10:08:40 & 0.4415 \\
\hline 1300 & $10: 08: 41$ & 0.4452 \\
\hline 1301 & 10:08:42 & 0.4424 \\
\hline 1302 & $10: 08: 43$ & 0.4468 \\
\hline 1303 & 10:08:44 & 0.4434 \\
\hline 1304 & 10:08:45 & 0.4443 \\
\hline 1305 & 10:08:46 & 0.4445 \\
\hline 1306 & 10:08:47 & 0.4450 \\
\hline 1307 & 10:08:48 & 0.4459 \\
\hline 1308 & 10:08:49 & 0.4434 \\
\hline 1309 & 10:08:50 & 0.4434 \\
\hline 1310 & 10:08:51 & 0.4401 \\
\hline 1311 & 10:08:52 & 0.4427 \\
\hline 1312 & 10:08:53 & 0.4387 \\
\hline 1313 & 10:08:54 & 0.4435 \\
\hline 1314 & 10:08:55 & 0.4417 \\
\hline 1315 & 10:08:56 & 0.4390 \\
\hline 1316 & 10:08:57 & 0.4394 \\
\hline 1317 & 10:08:58 & 0.4392 \\
\hline 1318 & 10:08:59 & 0.4432 \\
\hline 1319 & 10:09:00 & 0.4406 \\
\hline 1320 & 10:09:01 & 0.4444 \\
\hline 1321 & 10:09:02 & 0.4431 \\
\hline 1322 & 10:09:03 & 0.4454 \\
\hline 1323 & 10:09:04 & 0.4419 \\
\hline 1324 & 10:09:05 & 0.4438 \\
\hline
\end{tabular}


Appendix C - Fuel Data Test 2 NIST102605_1.xIs

\begin{tabular}{|c|c|c|}
\hline 1325 & 10:09:06 & 0.4427 \\
\hline 1326 & 10:09:07 & 0.4436 \\
\hline 1327 & 10:09:08 & 0.4440 \\
\hline 1328 & 10:09:09 & 0.4418 \\
\hline 1329 & 10:09:10 & 0.4450 \\
\hline 1330 & 10:09:11 & 0.4420 \\
\hline 1331 & 10:09:12 & 0.4434 \\
\hline 1332 & 10:09:13 & 0.4425 \\
\hline 1333 & $10: 09: 14$ & 0.4417 \\
\hline 1334 & 10:09:15 & 0.4412 \\
\hline 1335 & 10:09:16 & 0.4399 \\
\hline 1336 & 10:09:17 & 0.4412 \\
\hline 1337 & 10:09:18 & 0.4394 \\
\hline 1338 & 10:09:19 & 0.4423 \\
\hline 1339 & 10:09:20 & 0.4401 \\
\hline 1340 & 10:09:21 & 0.4440 \\
\hline 1341 & 10:09:22 & 0.4425 \\
\hline 1342 & 10:09:23 & 0.4434 \\
\hline 1343 & 10:09:24 & 0.4465 \\
\hline 1344 & 10:09:25 & 0.4465 \\
\hline 1345 & 10:09:26 & 0.4474 \\
\hline 1346 & 10:09:27 & 0.4414 \\
\hline 1347 & 10:09:28 & 0.4437 \\
\hline 1348 & 10:09:29 & 0.4435 \\
\hline 1349 & 10:09:30 & 0.4442 \\
\hline 1350 & 10:09:31 & 0.4425 \\
\hline 1351 & 10:09:32 & 0.4435 \\
\hline 1352 & 10:09:33 & 0.4443 \\
\hline 1353 & 10:09:34 & 0.4395 \\
\hline 1354 & 10:09:35 & 0.4446 \\
\hline 1355 & 10:09:36 & 0.4421 \\
\hline 1356 & 10:09:37 & 0.4398 \\
\hline 1357 & 10:09:38 & 0.4420 \\
\hline 1358 & 10:09:39 & 0.4411 \\
\hline 1359 & $10: 09: 40$ & 0.4428 \\
\hline 1360 & $10: 09: 41$ & 0.4384 \\
\hline 1361 & 10:09:42 & 0.4412 \\
\hline 1362 & $10: 09: 43$ & 0.4433 \\
\hline 1363 & 10:09:44 & 0.4448 \\
\hline 1364 & $10: 09: 45$ & 0.4449 \\
\hline 1365 & 10:09:46 & 0.4449 \\
\hline 1366 & 10:09:47 & 0.4461 \\
\hline 1367 & 10:09:48 & 0.4414 \\
\hline 1368 & 10:09:49 & 0.4431 \\
\hline 1369 & 10:09:50 & 0.4404 \\
\hline 1370 & $10: 09: 51$ & 0.4442 \\
\hline 1371 & $10: 09: 52$ & 0.4415 \\
\hline 1372 & 10:09:53 & 0.4432 \\
\hline 1373 & $10: 09: 54$ & 0.4419 \\
\hline 1374 & 10:09:55 & 0.4426 \\
\hline 1375 & 10:09:56 & 0.4415 \\
\hline
\end{tabular}


Appendix C - Fuel Data Test 2 NIST102605_1.xIs

\begin{tabular}{|c|c|c|}
\hline 1376 & 10:09:57 & 0.4392 \\
\hline 1377 & 10:09:58 & 0.4394 \\
\hline 1378 & 10:09:59 & 0.4401 \\
\hline 1379 & $10: 10: 00$ & 0.4434 \\
\hline 1380 & 10:10:01 & 0.4399 \\
\hline 1381 & 10:10:02 & 0.4438 \\
\hline 1382 & $10: 10: 03$ & 0.4413 \\
\hline 1383 & $10: 10: 04$ & 0.4434 \\
\hline 1384 & 10:10:05 & 0.4472 \\
\hline 1385 & 10:10:06 & 0.4470 \\
\hline 1386 & $10: 10: 07$ & 0.4470 \\
\hline 1387 & 10:10:08 & 0.4423 \\
\hline 1388 & 10:10:09 & 0.4446 \\
\hline 1389 & $10: 10: 10$ & 0.4415 \\
\hline 1390 & $10: 10: 11$ & 0.4429 \\
\hline 1391 & $10: 10: 12$ & 0.4404 \\
\hline 1392 & $10: 10: 13$ & 0.4433 \\
\hline 1393 & $10: 10: 14$ & 0.4424 \\
\hline 1394 & $10: 10: 15$ & 0.4400 \\
\hline 1395 & $10: 10: 16$ & 0.4448 \\
\hline 1396 & 10:10:17 & 0.4422 \\
\hline 1397 & 10:10:18 & 0.4422 \\
\hline 1398 & $10: 10: 19$ & 0.4402 \\
\hline 1399 & $10: 10: 20$ & 0.4393 \\
\hline 1400 & $10: 10: 21$ & 0.4423 \\
\hline 1401 & $10: 10: 22$ & 0.4408 \\
\hline 1402 & $10: 10: 23$ & 0.4437 \\
\hline 1403 & $10: 10: 24$ & 0.4435 \\
\hline 1404 & $10: 10: 25$ & 0.4448 \\
\hline 1405 & 10:10:26 & 0.4458 \\
\hline 1406 & $10: 10: 27$ & 0.4426 \\
\hline 1407 & $10: 10: 28$ & 0.4448 \\
\hline 1408 & $10: 10: 29$ & 0.4402 \\
\hline 1409 & $10: 10: 30$ & 0.4437 \\
\hline 1410 & $10: 10: 31$ & 0.4447 \\
\hline 1411 & $10: 10: 32$ & 0.4444 \\
\hline 1412 & $10: 10: 33$ & 0.4419 \\
\hline 1413 & 10:10:34 & 0.4411 \\
\hline 1414 & 10:10:35 & 0.4420 \\
\hline 1415 & $10: 10: 36$ & 0.4422 \\
\hline 1416 & $10: 10: 37$ & 0.4418 \\
\hline 1417 & $10: 10: 38$ & 0.4402 \\
\hline 1418 & $10: 10: 39$ & 0.4432 \\
\hline 1419 & $10: 10: 40$ & 0.4400 \\
\hline 1420 & $10: 10: 41$ & 0.4432 \\
\hline 1421 & $10: 10: 42$ & 0.4404 \\
\hline 1422 & $10: 10: 43$ & 0.4427 \\
\hline 1423 & $10: 10: 44$ & 0.4438 \\
\hline 1424 & $10: 10: 45$ & 0.4437 \\
\hline 1425 & $10: 10: 46$ & 0.4471 \\
\hline 1426 & $10: 10: 47$ & 0.4437 \\
\hline
\end{tabular}


Appendix C - Fuel Data Test 2 NIST102605_1.xls

\begin{tabular}{|c|c|c|}
\hline 1427 & $10: 10: 48$ & 0.4452 \\
\hline 1428 & $10: 10: 49$ & 0.4424 \\
\hline 1429 & $10: 10: 50$ & 0.4450 \\
\hline 1430 & $10: 10: 51$ & 0.4449 \\
\hline 1431 & $10: 10: 52$ & 0.4430 \\
\hline 1432 & $10: 10: 53$ & 0.4436 \\
\hline 1433 & $10: 10: 54$ & 0.4410 \\
\hline 1434 & $10: 10: 55$ & 0.4433 \\
\hline 1435 & $10: 10: 56$ & 0.4403 \\
\hline 1436 & $10: 10: 57$ & 0.4436 \\
\hline 1437 & $10: 10: 58$ & 0.4423 \\
\hline 1438 & $10: 10: 59$ & 0.4402 \\
\hline 1439 & $10: 11: 00$ & 0.4425 \\
\hline 1440 & 10:11:01 & 0.4411 \\
\hline 1441 & 10:11:02 & 0.4435 \\
\hline 1442 & $10: 11: 03$ & 0.4412 \\
\hline 1443 & $10: 11: 04$ & 0.4433 \\
\hline 1444 & $10: 11: 05$ & 0.4431 \\
\hline 1445 & $10: 11: 06$ & 0.4446 \\
\hline 1446 & $10: 11: 07$ & 0.4452 \\
\hline 1447 & $10: 11: 08$ & 0.4439 \\
\hline 1448 & 10:11:09 & 0.4458 \\
\hline 1449 & 10:11:10 & 0.4422 \\
\hline 1450 & 10:11:11 & 0.4443 \\
\hline 1451 & $10: 11: 12$ & 0.4445 \\
\hline 1452 & $10: 11: 13$ & 0.4423 \\
\hline 1453 & $10: 11: 14$ & 0.4436 \\
\hline 1454 & $10: 11: 15$ & 0.4417 \\
\hline 1455 & $10: 11: 16$ & 0.4439 \\
\hline 1456 & $10: 11: 17$ & 0.4417 \\
\hline 1457 & $10: 11: 18$ & 0.4425 \\
\hline 1458 & 10:11:19 & 0.4403 \\
\hline 1459 & 10:11:20 & 0.4399 \\
\hline 1460 & $10: 11: 21$ & 0.4384 \\
\hline 1461 & $10: 11: 22$ & 0.4403 \\
\hline 1462 & $10: 11: 23$ & 0.4420 \\
\hline 1463 & $10: 11: 24$ & 0.4418 \\
\hline 1464 & $10: 11: 25$ & 0.4455 \\
\hline 1465 & $10: 11: 26$ & 0.4432 \\
\hline 1466 & $10: 11: 27$ & 0.4451 \\
\hline 1467 & 10:11:28 & 0.4424 \\
\hline 1468 & 10:11:29 & 0.4441 \\
\hline 1469 & $10: 11: 30$ & 0.4451 \\
\hline 1470 & $10: 11: 31$ & 0.4444 \\
\hline 1471 & $10: 11: 32$ & 0.4445 \\
\hline 1472 & $10: 11: 33$ & 0.4423 \\
\hline 1473 & $10: 11: 34$ & 0.4418 \\
\hline 1474 & $10: 11: 35$ & 0.4401 \\
\hline 1475 & $10: 11: 36$ & 0.4439 \\
\hline 1476 & $10: 11: 37$ & 0.4398 \\
\hline 1477 & $10: 11: 38$ & 0.4437 \\
\hline
\end{tabular}


Appendix C - Fuel Data Test 2 NIST102605_1.xIs

\begin{tabular}{|c|c|c|}
\hline 1478 & 10:11:39 & 0.4408 \\
\hline 1479 & $10: 11: 40$ & 0.4415 \\
\hline 1480 & $10: 11: 41$ & 0.4394 \\
\hline 1481 & $10: 11: 42$ & 0.4391 \\
\hline 1482 & $10: 11: 43$ & 0.4402 \\
\hline 1483 & $10: 11: 44$ & 0.4415 \\
\hline 1484 & $10: 11: 45$ & 0.4443 \\
\hline 1485 & $10: 11: 46$ & 0.4447 \\
\hline 1486 & $10: 11: 47$ & 0.4463 \\
\hline 1487 & $10: 11: 48$ & 0.4414 \\
\hline 1488 & $10: 11: 49$ & 0.4438 \\
\hline 1489 & $10: 11: 50$ & 0.4421 \\
\hline 1490 & $10: 11: 51$ & 0.4449 \\
\hline 1491 & $10: 11: 52$ & 0.4435 \\
\hline 1492 & $10: 11: 53$ & 0.4431 \\
\hline 1493 & $10: 11: 54$ & 0.4439 \\
\hline 1494 & $10: 11: 55$ & 0.4402 \\
\hline 1495 & $10: 11: 56$ & 0.4436 \\
\hline 1496 & $10: 11: 57$ & 0.4417 \\
\hline 1497 & $10: 11: 58$ & 0.4438 \\
\hline 1498 & $10: 11: 59$ & 0.4405 \\
\hline 1499 & $10: 12: 00$ & 0.4394 \\
\hline 1500 & 10:12:01 & 0.4398 \\
\hline 1501 & $10: 12: 02$ & 0.4390 \\
\hline 1502 & $10: 12: 03$ & 0.4426 \\
\hline 1503 & $10: 12: 04$ & 0.4394 \\
\hline 1504 & $10: 12: 05$ & 0.4434 \\
\hline 1505 & $10: 12: 06$ & 0.4429 \\
\hline 1506 & $10: 12: 07$ & 0.4440 \\
\hline 1507 & $10: 12: 08$ & 0.4472 \\
\hline 1508 & $10: 12: 09$ & 0.4459 \\
\hline 1509 & $10: 12: 10$ & 0.4463 \\
\hline 1510 & $10: 12: 11$ & 0.4415 \\
\hline 1511 & $10: 12: 12$ & 0.4434 \\
\hline 1512 & $10: 12: 13$ & 0.4413 \\
\hline 1513 & $10: 12: 14$ & 0.4449 \\
\hline 1514 & $10: 12: 15$ & 0.4424 \\
\hline 1515 & $10: 12: 16$ & 0.4422 \\
\hline 1516 & $10: 12: 17$ & 0.4416 \\
\hline 1517 & $10: 12: 18$ & 0.4379 \\
\hline 1518 & $10: 12: 19$ & 0.4437 \\
\hline 1519 & $10: 12: 20$ & 0.4413 \\
\hline 1520 & $10: 12: 21$ & 0.4397 \\
\hline 1521 & $10: 12: 22$ & 0.4416 \\
\hline 1522 & $10: 12: 23$ & 0.4397 \\
\hline 1523 & $10: 12: 24$ & 0.4417 \\
\hline 1524 & $10: 12: 25$ & 0.4412 \\
\hline 1525 & $10: 12: 26$ & 0.4434 \\
\hline 1526 & $10: 12: 27$ & 0.4432 \\
\hline 1527 & $10: 12: 28$ & 0.4452 \\
\hline 1528 & $10: 12: 29$ & 0.4458 \\
\hline
\end{tabular}


Appendix C - Fuel Data Test 2 NIST102605_1.xIs

\begin{tabular}{|c|c|c|}
\hline 1529 & $10: 12: 30$ & 0.4435 \\
\hline 1530 & $10: 12: 31$ & 0.4456 \\
\hline 1531 & $10: 12: 32$ & 0.4415 \\
\hline 1532 & $10: 12: 33$ & 0.4451 \\
\hline 1533 & $10: 12: 34$ & 0.4445 \\
\hline 1534 & $10: 12: 35$ & 0.4433 \\
\hline 1535 & $10: 12: 36$ & 0.4434 \\
\hline 1536 & $10: 12: 37$ & 0.4406 \\
\hline 1537 & $10: 12: 38$ & 0.4427 \\
\hline 1538 & $10: 12: 39$ & 0.4417 \\
\hline 1539 & $10: 12: 40$ & 0.4435 \\
\hline 1540 & $10: 12: 41$ & 0.4400 \\
\hline 1541 & $10: 12: 42$ & 0.4403 \\
\hline 1542 & $10: 12: 43$ & 0.4423 \\
\hline 1543 & $10: 12: 44$ & 0.4388 \\
\hline 1544 & $10: 12: 45$ & 0.4424 \\
\hline 1545 & $10: 12: 46$ & 0.4434 \\
\hline 1546 & $10: 12: 47$ & 0.4450 \\
\hline 1547 & $10: 12: 48$ & 0.4460 \\
\hline 1548 & $10: 12: 49$ & 0.4451 \\
\hline 1549 & $10: 12: 50$ & 0.4456 \\
\hline 1550 & $10: 12: 51$ & 0.4435 \\
\hline 1551 & $10: 12: 52$ & 0.4454 \\
\hline 1552 & $10: 12: 53$ & 0.4418 \\
\hline 1553 & $10: 12: 54$ & 0.4435 \\
\hline 1554 & $10: 12: 55$ & 0.4406 \\
\hline 1555 & $10: 12: 56$ & 0.4427 \\
\hline 1556 & $10: 12: 57$ & 0.4422 \\
\hline 1557 & $10: 12: 58$ & 0.4388 \\
\hline 1558 & $10: 12: 59$ & 0.4427 \\
\hline 1559 & $10: 13: 00$ & 0.4408 \\
\hline 1560 & $10: 13: 01$ & 0.4432 \\
\hline 1561 & 10:13:02 & 0.4403 \\
\hline 1562 & $10: 13: 03$ & 0.4413 \\
\hline 1563 & $10: 13: 04$ & 0.4398 \\
\hline 1564 & $10: 13: 05$ & 0.4410 \\
\hline 1565 & $10: 13: 06$ & 0.4435 \\
\hline 1566 & $10: 13: 07$ & 0.4416 \\
\hline 1567 & $10: 13: 08$ & 0.4451 \\
\hline 1568 & 10:13:09 & 0.4434 \\
\hline 1569 & $10: 13: 10$ & 0.4454 \\
\hline 1570 & $10: 13: 11$ & 0.4450 \\
\hline 1571 & $10: 13: 12$ & 0.4426 \\
\hline 1572 & $10: 13: 13$ & 0.4435 \\
\hline 1573 & $10: 13: 14$ & 0.4410 \\
\hline 1574 & $10: 13: 15$ & 0.4440 \\
\hline 1575 & $10: 13: 16$ & 0.4437 \\
\hline 1576 & $10: 13: 17$ & 0.4403 \\
\hline 1577 & $10: 13: 18$ & 0.4414 \\
\hline 1578 & $10: 13: 19$ & 0.4398 \\
\hline 1579 & $10: 13: 20$ & 0.4423 \\
\hline
\end{tabular}


Appendix C - Fuel Data Test 2 NIST102605_1.xIs

\begin{tabular}{|c|c|c|}
\hline 1580 & $10: 13: 21$ & 0.4415 \\
\hline 1581 & 10:13:22 & 0.4428 \\
\hline 1582 & $10: 13: 23$ & 0.4398 \\
\hline 1583 & $10: 13: 24$ & 0.4420 \\
\hline 1584 & $10: 13: 25$ & 0.4393 \\
\hline 1585 & $10: 13: 26$ & 0.4431 \\
\hline 1586 & $10: 13: 27$ & 0.4441 \\
\hline 1587 & $10: 13: 28$ & 0.4441 \\
\hline 1588 & 10:13:29 & 0.4468 \\
\hline 1589 & $10: 13: 30$ & 0.4440 \\
\hline 1590 & $10: 13: 31$ & 0.4464 \\
\hline 1591 & $10: 13: 32$ & 0.4432 \\
\hline 1592 & $10: 13: 33$ & 0.4445 \\
\hline 1593 & $10: 13: 34$ & 0.4439 \\
\hline 1594 & $10: 13: 35$ & 0.4403 \\
\hline 1595 & $10: 13: 36$ & 0.4442 \\
\hline 1596 & $10: 13: 37$ & 0.4423 \\
\hline 1597 & 10:13:38 & 0.4441 \\
\hline 1598 & $10: 13: 39$ & 0.4409 \\
\hline 1599 & $10: 13: 40$ & 0.4401 \\
\hline 1600 & $10: 13: 41$ & 0.4428 \\
\hline 1601 & $10: 13: 42$ & 0.4398 \\
\hline 1602 & $10: 13: 43$ & 0.4424 \\
\hline 1603 & $10: 13: 44$ & 0.4408 \\
\hline 1604 & $10: 13: 45$ & 0.4428 \\
\hline 1605 & $10: 13: 46$ & 0.4404 \\
\hline 1606 & $10: 13: 47$ & 0.4422 \\
\hline 1607 & $10: 13: 48$ & 0.4423 \\
\hline 1608 & $10: 13: 49$ & 0.4457 \\
\hline 1609 & $10: 13: 50$ & 0.4437 \\
\hline 1610 & $10: 13: 51$ & 0.4453 \\
\hline 1611 & $10: 13: 52$ & 0.4468 \\
\hline 1612 & $10: 13: 53$ & 0.4418 \\
\hline 1613 & $10: 13: 54$ & 0.4430 \\
\hline 1614 & 10:13:55 & 0.4410 \\
\hline 1615 & $10: 13: 56$ & 0.4444 \\
\hline 1616 & $10: 13: 57$ & 0.4420 \\
\hline 1617 & $10: 13: 58$ & 0.4420 \\
\hline 1618 & $10: 13: 59$ & 0.4415 \\
\hline 1619 & $10: 14: 00$ & 0.4417 \\
\hline 1620 & $10: 14: 01$ & 0.4419 \\
\hline 1621 & $10: 14: 02$ & 0.4396 \\
\hline 1622 & $10: 14: 03$ & 0.4429 \\
\hline 1623 & $10: 14: 04$ & 0.4393 \\
\hline 1624 & $10: 14: 05$ & 0.4415 \\
\hline 1625 & $10: 14: 06$ & 0.4398 \\
\hline 1626 & $10: 14: 07$ & 0.4428 \\
\hline 1627 & $10: 14: 08$ & 0.4415 \\
\hline 1628 & $10: 14: 09$ & 0.4441 \\
\hline 1629 & $10: 14: 10$ & 0.4458 \\
\hline 1630 & $10: 14: 11$ & 0.4433 \\
\hline
\end{tabular}

C - 32 
Appendix C - Fuel Data Test 2 NIST102605_1.xIs

\begin{tabular}{|c|c|c|}
\hline 1631 & $10: 14: 12$ & 0.4457 \\
\hline 1632 & $10: 14: 13$ & 0.4429 \\
\hline 1633 & $10: 14: 14$ & 0.4437 \\
\hline 1634 & $10: 14: 15$ & 0.4435 \\
\hline 1635 & $10: 14: 16$ & 0.4411 \\
\hline 1636 & $10: 14: 17$ & 0.4428 \\
\hline 1637 & $10: 14: 18$ & 0.4405 \\
\hline 1638 & $10: 14: 19$ & 0.4444 \\
\hline 1639 & $10: 14: 20$ & 0.4400 \\
\hline 1640 & $10: 14: 21$ & 0.4406 \\
\hline 1641 & $10: 14: 22$ & 0.4407 \\
\hline 1642 & $10: 14: 23$ & 0.4388 \\
\hline 1643 & $10: 14: 24$ & 0.4425 \\
\hline 1644 & $10: 14: 25$ & 0.4413 \\
\hline 1645 & 10:14:26 & 0.4434 \\
\hline 1646 & $10: 14: 27$ & 0.4401 \\
\hline 1647 & $10: 14: 28$ & 0.4425 \\
\hline 1648 & $10: 14: 29$ & 0.4429 \\
\hline 1649 & $10: 14: 30$ & 0.4447 \\
\hline 1650 & $10: 14: 31$ & 0.4441 \\
\hline 1651 & $10: 14: 32$ & 0.4412 \\
\hline 1652 & $10: 14: 33$ & 0.4437 \\
\hline 1653 & $10: 14: 34$ & 0.4418 \\
\hline 1654 & 10:14:35 & 0.4448 \\
\hline 1655 & $10: 14: 36$ & 0.4441 \\
\hline 1656 & $10: 14: 37$ & 0.4429 \\
\hline 1657 & $10: 14: 38$ & 0.4417 \\
\hline 1658 & $10: 14: 39$ & 0.4405 \\
\hline 1659 & $10: 14: 40$ & 0.4427 \\
\hline 1660 & $10: 14: 41$ & 0.4413 \\
\hline 1661 & $10: 14: 42$ & 0.4437 \\
\hline 1662 & $10: 14: 43$ & 0.4396 \\
\hline 1663 & $10: 14: 44$ & 0.4404 \\
\hline 1664 & $10: 14: 45$ & 0.4386 \\
\hline 1665 & $10: 14: 46$ & 0.4407 \\
\hline 1666 & $10: 14: 47$ & 0.4430 \\
\hline 1667 & $10: 14: 48$ & 0.4427 \\
\hline 1668 & $10: 14: 49$ & 0.4451 \\
\hline 1669 & $10: 14: 50$ & 0.4420 \\
\hline 1670 & $10: 14: 51$ & 0.4455 \\
\hline 1671 & $10: 14: 52$ & 0.4451 \\
\hline 1672 & $10: 14: 53$ & 0.4444 \\
\hline 1673 & $10: 14: 54$ & 0.4416 \\
\hline 1674 & $10: 14: 55$ & 0.4442 \\
\hline 1675 & $10: 14: 56$ & 0.4451 \\
\hline 1676 & $10: 14: 57$ & 0.4444 \\
\hline 1677 & $10: 14: 58$ & 0.4421 \\
\hline 1678 & $10: 14: 59$ & 0.4396 \\
\hline 1679 & $10: 15: 00$ & 0.4444 \\
\hline 1680 & $10: 15: 01$ & 0.4409 \\
\hline 1681 & $10: 15: 02$ & 0.4414 \\
\hline
\end{tabular}


Appendix C - Fuel Data Test 2 NIST102605_1.xIs

\begin{tabular}{|c|c|c|}
\hline 1682 & $10: 15: 03$ & 0.4420 \\
\hline 1683 & $10: 15: 04$ & 0.4390 \\
\hline 1684 & $10: 15: 05$ & 0.4424 \\
\hline 1685 & 10:15:06 & 0.4411 \\
\hline 1686 & $10: 15: 07$ & 0.4435 \\
\hline 1687 & 10:15:08 & 0.4407 \\
\hline 1688 & 10:15:09 & 0.4425 \\
\hline 1689 & $10: 15: 10$ & 0.4418 \\
\hline 1690 & $10: 15: 11$ & 0.4459 \\
\hline 1691 & $10: 15: 12$ & 0.4446 \\
\hline 1692 & $10: 15: 13$ & 0.4450 \\
\hline 1693 & 10:15:14 & 0.4456 \\
\hline 1694 & 10:15:15 & 0.4419 \\
\hline 1695 & $10: 15: 16$ & 0.4432 \\
\hline 1696 & $10: 15: 17$ & 0.4412 \\
\hline 1697 & $10: 15: 18$ & 0.4450 \\
\hline 1698 & $10: 15: 19$ & 0.4435 \\
\hline 1699 & $10: 15: 20$ & 0.4419 \\
\hline 1700 & $10: 15: 21$ & 0.4419 \\
\hline 1701 & $10: 15: 22$ & 0.4401 \\
\hline 1702 & $10: 15: 23$ & 0.4438 \\
\hline 1703 & 10:15:24 & 0.4411 \\
\hline 1704 & $10: 15: 25$ & 0.4410 \\
\hline 1705 & $10: 15: 26$ & 0.4383 \\
\hline 1706 & $10: 15: 27$ & 0.4389 \\
\hline 1707 & $10: 15: 28$ & 0.4406 \\
\hline 1708 & $10: 15: 29$ & 0.4444 \\
\hline 1709 & $10: 15: 30$ & 0.4458 \\
\hline 1710 & 10:15:31 & 0.4430 \\
\hline 1711 & 10:15:32 & 0.4463 \\
\hline 1712 & $10: 15: 33$ & 0.4435 \\
\hline 1713 & $10: 15: 34$ & 0.4444 \\
\hline 1714 & $10: 15: 35$ & 0.4451 \\
\hline 1715 & $10: 15: 36$ & 0.4437 \\
\hline 1716 & 10:15:37 & 0.4440 \\
\hline 1717 & $10: 15: 38$ & 0.4400 \\
\hline 1718 & $10: 15: 39$ & 0.4415 \\
\hline 1719 & $10: 15: 40$ & 0.4403 \\
\hline 1720 & $10: 15: 41$ & 0.4432 \\
\hline 1721 & $10: 15: 42$ & 0.4393 \\
\hline 1722 & $10: 15: 43$ & 0.4443 \\
\hline 1723 & $10: 15: 44$ & 0.4426 \\
\hline 1724 & $10: 15: 45$ & 0.4426 \\
\hline 1725 & $10: 15: 46$ & 0.4417 \\
\hline 1726 & $10: 15: 47$ & 0.4407 \\
\hline 1727 & $10: 15: 48$ & 0.4430 \\
\hline 1728 & $10: 15: 49$ & 0.4400 \\
\hline 1729 & $10: 15: 50$ & 0.4429 \\
\hline 1730 & $10: 15: 51$ & 0.4446 \\
\hline 1731 & $10: 15: 52$ & 0.4430 \\
\hline 1732 & $10: 15: 53$ & 0.4444 \\
\hline
\end{tabular}


Appendix C - Fuel Data Test 2 NIST102605_1.xls

\begin{tabular}{|c|c|c|}
\hline 1733 & $10: 15: 54$ & 0.4421 \\
\hline 1734 & $10: 15: 55$ & 0.4450 \\
\hline 1735 & $10: 15: 56$ & 0.4444 \\
\hline 1736 & $10: 15: 57$ & 0.4414 \\
\hline 1737 & $10: 15: 58$ & 0.4431 \\
\hline 1738 & $10: 15: 59$ & 0.4401 \\
\hline 1739 & 10:16:00 & 0.4434 \\
\hline 1740 & $10: 16: 01$ & 0.4420 \\
\hline 1741 & $10: 16: 02$ & 0.4447 \\
\hline 1742 & $10: 16: 03$ & 0.4411 \\
\hline 1743 & $10: 16: 04$ & 0.4403 \\
\hline 1744 & $10: 16: 05$ & 0.4420 \\
\hline 1745 & $10: 16: 06$ & 0.4398 \\
\hline 1746 & $10: 16: 07$ & 0.4430 \\
\hline 1747 & 10:16:08 & 0.4392 \\
\hline 1748 & 10:16:09 & 0.4415 \\
\hline 1749 & $10: 16: 10$ & 0.4411 \\
\hline 1750 & $10: 16: 11$ & 0.4432 \\
\hline 1751 & $10: 16: 12$ & 0.4464 \\
\hline 1752 & $10: 16: 13$ & 0.4456 \\
\hline 1753 & $10: 16: 14$ & 0.4458 \\
\hline 1754 & $10: 16: 15$ & 0.4412 \\
\hline 1755 & $10: 16: 16$ & 0.4446 \\
\hline 1756 & $10: 16: 17$ & 0.4444 \\
\hline 1757 & $10: 16: 18$ & 0.4452 \\
\hline 1758 & $10: 16: 19$ & 0.4433 \\
\hline 1759 & $10: 16: 20$ & 0.4397 \\
\hline 1760 & $10: 16: 21$ & 0.4418 \\
\hline 1761 & $10: 16: 22$ & 0.4390 \\
\hline 1762 & $10: 16: 23$ & 0.4444 \\
\hline 1763 & $10: 16: 24$ & 0.4421 \\
\hline 1764 & $10: 16: 25$ & 0.4421 \\
\hline 1765 & 10:16:26 & 0.4396 \\
\hline 1766 & $10: 16: 27$ & 0.4404 \\
\hline 1767 & $10: 16: 28$ & 0.4406 \\
\hline 1768 & $10: 16: 29$ & 0.4385 \\
\hline 1769 & $10: 16: 30$ & 0.4400 \\
\hline 1770 & $10: 16: 31$ & 0.4424 \\
\hline 1771 & $10: 16: 32$ & 0.4453 \\
\hline 1772 & $10: 16: 33$ & 0.4422 \\
\hline 1773 & $10: 16: 34$ & 0.4435 \\
\hline 1774 & 10:16:35 & 0.4416 \\
\hline 1775 & $10: 16: 36$ & 0.4456 \\
\hline 1776 & $10: 16: 37$ & 0.4435 \\
\hline 1777 & $10: 16: 38$ & 0.4428 \\
\hline 1778 & $10: 16: 39$ & 0.4460 \\
\hline 1779 & $10: 16: 40$ & 0.4411 \\
\hline 1780 & $10: 16: 41$ & 0.4438 \\
\hline 1781 & $10: 16: 42$ & 0.4426 \\
\hline 1782 & $10: 16: 43$ & 0.4415 \\
\hline 1783 & $10: 16: 44$ & 0.4412 \\
\hline
\end{tabular}


Appendix C - Fuel Data Test 2 NIST102605_1.xls

\begin{tabular}{|c|c|c|}
\hline 1784 & $10: 16: 45$ & 0.4392 \\
\hline 1785 & $10: 16: 46$ & 0.4433 \\
\hline 1786 & $10: 16: 47$ & 0.4389 \\
\hline 1787 & $10: 16: 48$ & 0.4391 \\
\hline 1788 & $10: 16: 49$ & 0.4405 \\
\hline 1789 & $10: 16: 50$ & 0.4416 \\
\hline 1790 & $10: 16: 51$ & 0.4445 \\
\hline 1791 & $10: 16: 52$ & 0.4435 \\
\hline 1792 & $10: 16: 53$ & 0.4478 \\
\hline 1793 & $10: 16: 54$ & 0.4431 \\
\hline 1794 & $10: 16: 55$ & 0.4440 \\
\hline 1795 & $10: 16: 56$ & 0.4416 \\
\hline 1796 & $10: 16: 57$ & 0.4455 \\
\hline 1797 & $10: 16: 58$ & 0.4457 \\
\hline 1798 & $10: 16: 59$ & 0.4421 \\
\hline 1799 & $10: 17: 00$ & 0.4421 \\
\hline 1800 & $10: 17: 01$ & 0.4417 \\
\hline 1801 & $10: 17: 02$ & 0.4442 \\
\hline 1802 & $10: 17: 03$ & 0.4399 \\
\hline 1803 & $10: 17: 04$ & 0.4432 \\
\hline 1804 & $10: 17: 05$ & 0.4413 \\
\hline 1805 & $10: 17: 06$ & 0.4396 \\
\hline 1806 & $10: 17: 07$ & 0.4413 \\
\hline 1807 & 10:17:08 & 0.4404 \\
\hline 1808 & $10: 17: 09$ & 0.4406 \\
\hline 1809 & $10: 17: 10$ & 0.4395 \\
\hline 1810 & $10: 17: 11$ & 0.4424 \\
\hline 1811 & $10: 17: 12$ & 0.4431 \\
\hline 1812 & $10: 17: 13$ & 0.4457 \\
\hline 1813 & $10: 17: 14$ & 0.4415 \\
\hline 1814 & $10: 17: 15$ & 0.4429 \\
\hline 1815 & $10: 17: 16$ & 0.4430 \\
\hline 1816 & $10: 17: 17$ & 0.4452 \\
\hline 1817 & $10: 17: 18$ & 0.4438 \\
\hline 1818 & $10: 17: 19$ & 0.4415 \\
\hline 1819 & $10: 17: 20$ & 0.4429 \\
\hline 1820 & $10: 17: 21$ & 0.4415 \\
\hline 1821 & $10: 17: 22$ & 0.4417 \\
\hline 1822 & $10: 17: 23$ & 0.4402 \\
\hline 1823 & $10: 17: 24$ & 0.4431 \\
\hline 1824 & $10: 17: 25$ & 0.4420 \\
\hline 1825 & $10: 17: 26$ & 0.4437 \\
\hline 1826 & $10: 17: 27$ & 0.4420 \\
\hline 1827 & $10: 17: 28$ & 0.4438 \\
\hline 1828 & $10: 17: 29$ & 0.4397 \\
\hline 1829 & $10: 17: 30$ & 0.4415 \\
\hline 1830 & $10: 17: 31$ & 0.4405 \\
\hline 1831 & $10: 17: 32$ & 0.4435 \\
\hline 1832 & $10: 17: 33$ & 0.4457 \\
\hline 1833 & $10: 17: 34$ & 0.4432 \\
\hline 1834 & $10: 17: 35$ & 0.4462 \\
\hline
\end{tabular}


Appendix C - Fuel Data Test 2 NIST102605_1.xIs

\begin{tabular}{|c|c|c|}
\hline 1835 & $10: 17: 36$ & 0.4443 \\
\hline 1836 & $10: 17: 37$ & 0.4459 \\
\hline 1837 & $10: 17: 38$ & 0.4413 \\
\hline 1838 & $10: 17: 39$ & 0.4446 \\
\hline 1839 & $10: 17: 40$ & 0.4429 \\
\hline 1840 & $10: 17: 41$ & 0.4446 \\
\hline 1841 & $10: 17: 42$ & 0.4420 \\
\hline 1842 & $10: 17: 43$ & 0.4441 \\
\hline 1843 & $10: 17: 44$ & 0.4423 \\
\hline 1844 & $10: 17: 45$ & 0.4402 \\
\hline 1845 & $10: 17: 46$ & 0.4408 \\
\hline 1846 & $10: 17: 47$ & 0.4393 \\
\hline 1847 & $10: 17: 48$ & 0.4433 \\
\hline 1848 & $10: 17: 49$ & 0.4403 \\
\hline 1849 & 10:17:50 & 0.4402 \\
\hline 1850 & 10:17:51 & 0.4392 \\
\hline 1851 & $10: 17: 52$ & 0.4418 \\
\hline 1852 & $10: 17: 53$ & 0.4417 \\
\hline 1853 & $10: 17: 54$ & 0.4438 \\
\hline 1854 & $10: 17: 55$ & 0.4452 \\
\hline 1855 & $10: 17: 56$ & 0.4439 \\
\hline 1856 & $10: 17: 57$ & 0.4453 \\
\hline 1857 & $10: 17: 58$ & 0.4445 \\
\hline 1858 & 10:17:59 & 0.4458 \\
\hline 1859 & 10:18:00 & 0.4411 \\
\hline 1860 & 10:18:01 & 0.4440 \\
\hline 1861 & 10:18:02 & 0.4424 \\
\hline 1862 & 10:18:03 & 0.4444 \\
\hline 1863 & $10: 18: 04$ & 0.4435 \\
\hline 1864 & 10:18:05 & 0.4418 \\
\hline 1865 & 10:18:06 & 0.4414 \\
\hline 1866 & $10: 18: 07$ & 0.4409 \\
\hline 1867 & 10:18:08 & 0.4421 \\
\hline 1868 & 10:18:09 & 0.4396 \\
\hline 1869 & 10:18:10 & 0.4431 \\
\hline 1870 & $10: 18: 11$ & 0.4397 \\
\hline 1871 & 10:18:12 & 0.4407 \\
\hline 1872 & $10: 18: 13$ & 0.4428 \\
\hline 1873 & $10: 18: 14$ & 0.4430 \\
\hline 1874 & $10: 18: 15$ & 0.4461 \\
\hline 1875 & 10:18:16 & 0.4434 \\
\hline 1876 & 10:18:17 & 0.4464 \\
\hline 1877 & 10:18:18 & 0.4466 \\
\hline 1878 & 10:18:19 & 0.4433 \\
\hline 1879 & $10: 18: 20$ & 0.4443 \\
\hline 1880 & $10: 18: 21$ & 0.4421 \\
\hline 1881 & 10:18:22 & 0.4452 \\
\hline 1882 & $10: 18: 23$ & 0.4411 \\
\hline 1883 & 10:18:24 & 0.4420 \\
\hline 1884 & 10:18:25 & 0.4411 \\
\hline 1885 & 10:18:26 & 0.4388 \\
\hline
\end{tabular}


Appendix C - Fuel Data Test 2 NIST102605_1.xIs

\begin{tabular}{|c|c|c|}
\hline 1886 & $10: 18: 27$ & 0.4436 \\
\hline 1887 & $10: 18: 28$ & 0.4422 \\
\hline 1888 & 10:18:29 & 0.4431 \\
\hline 1889 & $10: 18: 30$ & 0.4401 \\
\hline 1890 & 10:18:31 & 0.4394 \\
\hline 1891 & 10:18:32 & 0.4435 \\
\hline 1892 & $10: 18: 33$ & 0.4423 \\
\hline 1893 & 10:18:34 & 0.4448 \\
\hline 1894 & 10:18:35 & 0.4433 \\
\hline 1895 & $10: 18: 36$ & 0.4453 \\
\hline 1896 & 10:18:37 & 0.4424 \\
\hline 1897 & $10: 18: 38$ & 0.4446 \\
\hline 1898 & 10:18:39 & 0.4444 \\
\hline 1899 & 10:18:40 & 0.4438 \\
\hline 1900 & 10:18:41 & 0.4442 \\
\hline 1901 & $10: 18: 42$ & 0.4407 \\
\hline 1902 & 10:18:43 & 0.4443 \\
\hline 1903 & 10:18:44 & 0.4434 \\
\hline 1904 & $10: 18: 45$ & 0.4426 \\
\hline 1905 & $10: 18: 46$ & 0.4428 \\
\hline 1906 & $10: 18: 47$ & 0.4407 \\
\hline 1907 & $10: 18: 48$ & 0.4424 \\
\hline 1908 & $10: 18: 49$ & 0.4401 \\
\hline 1909 & 10:18:50 & 0.4442 \\
\hline 1910 & $10: 18: 51$ & 0.4389 \\
\hline 1911 & $10: 18: 52$ & 0.4402 \\
\hline 1912 & $10: 18: 53$ & 0.4392 \\
\hline 1913 & $10: 18: 54$ & 0.4424 \\
\hline 1914 & $10: 18: 55$ & 0.4457 \\
\hline 1915 & $10: 18: 56$ & 0.4449 \\
\hline 1916 & $10: 18: 57$ & 0.4475 \\
\hline 1917 & 10:18:58 & 0.4434 \\
\hline 1918 & $10: 18: 59$ & 0.4447 \\
\hline 1919 & 10:19:00 & 0.4420 \\
\hline 1920 & 10:19:01 & 0.4449 \\
\hline 1921 & 10:19:02 & 0.4448 \\
\hline 1922 & 10:19:03 & 0.4446 \\
\hline 1923 & 10:19:04 & 0.4430 \\
\hline 1924 & 10:19:05 & 0.4415 \\
\hline 1925 & 10:19:06 & 0.4434 \\
\hline 1926 & 10:19:07 & 0.4394 \\
\hline 1927 & 10:19:08 & 0.4439 \\
\hline 1928 & 10:19:09 & 0.4415 \\
\hline 1929 & 10:19:10 & 0.4429 \\
\hline 1930 & 10:19:11 & 0.4396 \\
\hline 1931 & $10: 19: 12$ & 0.4400 \\
\hline 1932 & $10: 19: 13$ & 0.4401 \\
\hline 1933 & $10: 19: 14$ & 0.4396 \\
\hline 1934 & 10:19:15 & 0.4438 \\
\hline 1935 & $10: 19: 16$ & 0.4448 \\
\hline 1936 & $10: 19: 17$ & 0.4467 \\
\hline
\end{tabular}


Appendix C - Fuel Data Test 2 NIST102605_1.xIs

\begin{tabular}{|c|c|c|}
\hline 1937 & 10:19:18 & 0.4425 \\
\hline 1938 & 10:19:19 & 0.4437 \\
\hline 1939 & $10: 19: 20$ & 0.4431 \\
\hline 1940 & 10:19:21 & 0.4422 \\
\hline 1941 & 10:19:22 & 0.4450 \\
\hline 1942 & $10: 19: 23$ & 0.4429 \\
\hline 1943 & 10:19:24 & 0.4449 \\
\hline 1944 & 10:19:25 & 0.4435 \\
\hline 1945 & $10: 19: 26$ & 0.4419 \\
\hline 1946 & $10: 19: 27$ & 0.4416 \\
\hline 1947 & $10: 19: 28$ & 0.4393 \\
\hline 1948 & 10:19:29 & 0.4439 \\
\hline 1949 & 10:19:30 & 0.4427 \\
\hline 1950 & 10:19:31 & 0.4437 \\
\hline 1951 & 10:19:32 & 0.4381 \\
\hline 1952 & 10:19:33 & 0.4389 \\
\hline 1953 & $10: 19: 34$ & 0.4409 \\
\hline 1954 & $10: 19: 35$ & 0.4431 \\
\hline 1955 & $10: 19: 36$ & 0.4454 \\
\hline 1956 & $10: 19: 37$ & 0.4424 \\
\hline 1957 & 10:19:38 & 0.4453 \\
\hline 1958 & 10:19:39 & 0.4430 \\
\hline 1959 & $10: 19: 40$ & 0.4454 \\
\hline 1960 & $10: 19: 41$ & 0.4444 \\
\hline 1961 & 10:19:42 & 0.4456 \\
\hline 1962 & $10: 19: 43$ & 0.4455 \\
\hline 1963 & $10: 19: 44$ & 0.4415 \\
\hline 1964 & $10: 19: 45$ & 0.4441 \\
\hline 1965 & $10: 19: 46$ & 0.4432 \\
\hline 1966 & $10: 19: 47$ & 0.4449 \\
\hline 1967 & $10: 19: 48$ & 0.4424 \\
\hline 1968 & $10: 19: 49$ & 0.4407 \\
\hline 1969 & 10:19:50 & 0.4435 \\
\hline 1970 & 10:19:51 & 0.4390 \\
\hline 1971 & $10: 19: 52$ & 0.4445 \\
\hline 1972 & $10: 19: 53$ & 0.4417 \\
\hline 1973 & $10: 19: 54$ & 0.4418 \\
\hline 1974 & $10: 19: 55$ & 0.4382 \\
\hline 1975 & 10:19:56 & 0.4409 \\
\hline 1976 & $10: 19: 57$ & 0.4449 \\
\hline 1977 & 10:19:58 & 0.4441 \\
\hline 1978 & 10:19:59 & 0.4454 \\
\hline 1979 & $10: 20: 00$ & 0.4424 \\
\hline 1980 & 10:20:01 & 0.4453 \\
\hline 1981 & $10: 20: 02$ & 0.4452 \\
\hline 1982 & $10: 20: 03$ & 0.4419 \\
\hline 1983 & $10: 20: 04$ & 0.4441 \\
\hline 1984 & $10: 20: 05$ & 0.4407 \\
\hline 1985 & $10: 20: 06$ & 0.4441 \\
\hline 1986 & $10: 20: 07$ & 0.4421 \\
\hline 1987 & $10: 20: 08$ & 0.4438 \\
\hline
\end{tabular}


Appendix C - Fuel Data Test 2 NIST102605_1.xIs

\begin{tabular}{|c|c|c|}
\hline 1988 & $10: 20: 09$ & 0.4413 \\
\hline 1989 & $10: 20: 10$ & 0.4398 \\
\hline 1990 & $10: 20: 11$ & 0.4416 \\
\hline 1991 & $10: 20: 12$ & 0.4410 \\
\hline 1992 & $10: 20: 13$ & 0.4397 \\
\hline 1993 & $10: 20: 14$ & 0.4373 \\
\hline 1994 & $10: 20: 15$ & 0.4441 \\
\hline 1995 & $10: 20: 16$ & 0.4422 \\
\hline 1996 & $10: 20: 17$ & 0.4448 \\
\hline 1997 & $10: 20: 18$ & 0.4434 \\
\hline 1998 & $10: 20: 19$ & 0.4454 \\
\hline 1999 & 10:20:20 & 0.4473 \\
\hline 2000 & $10: 20: 21$ & 0.4441 \\
\hline 2001 & $10: 20: 22$ & 0.4466 \\
\hline 2002 & $10: 20: 23$ & 0.4422 \\
\hline 2003 & $10: 20: 24$ & 0.4429 \\
\hline 2004 & $10: 20: 25$ & 0.4411 \\
\hline 2005 & $10: 20: 26$ & 0.4432 \\
\hline 2006 & $10: 20: 27$ & 0.4426 \\
\hline 2007 & $10: 20: 28$ & 0.4413 \\
\hline 2008 & $10: 20: 29$ & 0.4435 \\
\hline 2009 & $10: 20: 30$ & 0.4411 \\
\hline 2010 & $10: 20: 31$ & 0.4438 \\
\hline 2011 & $10: 20: 32$ & 0.4404 \\
\hline 2012 & $10: 20: 33$ & 0.4415 \\
\hline 2013 & $10: 20: 34$ & 0.4404 \\
\hline 2014 & $10: 20: 35$ & 0.4398 \\
\hline 2015 & $10: 20: 36$ & 0.4403 \\
\hline 2016 & $10: 20: 37$ & 0.4412 \\
\hline 2017 & $10: 20: 38$ & 0.4445 \\
\hline 2018 & 10:20:39 & 0.4432 \\
\hline 2019 & $10: 20: 40$ & 0.4453 \\
\hline 2020 & $10: 20: 41$ & 0.4424 \\
\hline 2021 & $10: 20: 42$ & 0.4455 \\
\hline 2022 & $10: 20: 43$ & 0.4426 \\
\hline 2023 & $10: 20: 44$ & 0.4434 \\
\hline 2024 & $10: 20: 45$ & 0.4456 \\
\hline 2025 & $10: 20: 46$ & 0.4442 \\
\hline 2026 & $10: 20: 47$ & 0.4438 \\
\hline 2027 & $10: 20: 48$ & 0.4407 \\
\hline 2028 & $10: 20: 49$ & 0.4430 \\
\hline 2029 & $10: 20: 50$ & 0.4412 \\
\hline 2030 & $10: 20: 51$ & 0.4444 \\
\hline 2031 & $10: 20: 52$ & 0.4416 \\
\hline 2032 & $10: 20: 53$ & 0.4420 \\
\hline 2033 & $10: 20: 54$ & 0.4383 \\
\hline 2034 & $10: 20: 55$ & 0.4390 \\
\hline 2035 & 10:20:56 & 0.4415 \\
\hline 2036 & $10: 20: 57$ & 0.4420 \\
\hline 2037 & $10: 20: 58$ & 0.4452 \\
\hline 2038 & $10: 20: 59$ & 0.4423 \\
\hline
\end{tabular}


Appendix C - Fuel Data Test 2 NIST102605_1.xIs

\begin{tabular}{|c|c|c|}
\hline 2039 & $10: 21: 00$ & 0.4464 \\
\hline 2040 & $10: 21: 01$ & 0.4437 \\
\hline 2041 & $10: 21: 02$ & 0.4459 \\
\hline 2042 & $10: 21: 03$ & 0.4446 \\
\hline 2043 & $10: 21: 04$ & 0.4453 \\
\hline 2044 & 10:21:05 & 0.4456 \\
\hline 2045 & $10: 21: 06$ & 0.4433 \\
\hline 2046 & $10: 21: 07$ & 0.4433 \\
\hline 2047 & $10: 21: 08$ & 0.4398 \\
\hline 2048 & $10: 21: 09$ & 0.4423 \\
\hline 2049 & $10: 21: 10$ & 0.4394 \\
\hline 2050 & $10: 21: 11$ & 0.4437 \\
\hline 2051 & $10: 21: 12$ & 0.4429 \\
\hline 2052 & $10: 21: 13$ & 0.4424 \\
\hline 2053 & $10: 21: 14$ & 0.4406 \\
\hline 2054 & $10: 21: 15$ & 0.4394 \\
\hline 2055 & $10: 21: 16$ & 0.4419 \\
\hline 2056 & $10: 21: 17$ & 0.4411 \\
\hline 2057 & $10: 21: 18$ & 0.4434 \\
\hline 2058 & $10: 21: 19$ & 0.4419 \\
\hline 2059 & 10:21:20 & 0.4448 \\
\hline 2060 & $10: 21: 21$ & 0.4411 \\
\hline 2061 & 10:21:22 & 0.4448 \\
\hline 2062 & $10: 21: 23$ & 0.4472 \\
\hline 2063 & $10: 21: 24$ & 0.4445 \\
\hline 2064 & $10: 21: 25$ & 0.4455 \\
\hline 2065 & $10: 21: 26$ & 0.4440 \\
\hline 2066 & $10: 21: 27$ & 0.4450 \\
\hline 2067 & $10: 21: 28$ & 0.4428 \\
\hline 2068 & 10:21:29 & 0.4412 \\
\hline 2069 & $10: 21: 30$ & 0.4435 \\
\hline 2070 & $10: 21: 31$ & 0.4412 \\
\hline 2071 & $10: 21: 32$ & 0.4429 \\
\hline 2072 & $10: 21: 33$ & 0.4416 \\
\hline 2073 & $10: 21: 34$ & 0.4448 \\
\hline 2074 & $10: 21: 35$ & 0.4412 \\
\hline 2075 & $10: 21: 36$ & 0.4430 \\
\hline 2076 & $10: 21: 37$ & 0.4399 \\
\hline 2077 & $10: 21: 38$ & 0.4410 \\
\hline 2078 & $10: 21: 39$ & 0.4405 \\
\hline 2079 & $10: 21: 40$ & 0.4440 \\
\hline 2080 & $10: 21: 41$ & 0.4464 \\
\hline 2081 & $10: 21: 42$ & 0.4442 \\
\hline 2082 & $10: 21: 43$ & 0.4458 \\
\hline 2083 & $10: 21: 44$ & 0.4423 \\
\hline 2084 & $10: 21: 45$ & 0.4450 \\
\hline 2085 & $10: 21: 46$ & 0.4434 \\
\hline 2086 & $10: 21: 47$ & 0.4451 \\
\hline 2087 & $10: 21: 48$ & 0.4425 \\
\hline 2088 & $10: 21: 49$ & 0.4447 \\
\hline 2089 & $10: 21: 50$ & 0.4435 \\
\hline
\end{tabular}


Appendix C - Fuel Data Test 2 NIST102605_1.xls

\begin{tabular}{|c|c|c|}
\hline 2090 & $10: 21: 51$ & 0.4416 \\
\hline 2091 & $10: 21: 52$ & 0.4425 \\
\hline 2092 & $10: 21: 53$ & 0.4399 \\
\hline 2093 & $10: 21: 54$ & 0.4449 \\
\hline 2094 & $10: 21: 55$ & 0.4406 \\
\hline 2095 & $10: 21: 56$ & 0.4423 \\
\hline 2096 & $10: 21: 57$ & 0.4418 \\
\hline 2097 & $10: 21: 58$ & 0.4406 \\
\hline 2098 & $10: 21: 59$ & 0.4437 \\
\hline 2099 & $10: 22: 00$ & 0.4410 \\
\hline 2100 & $10: 22: 01$ & 0.4432 \\
\hline 2101 & $10: 22: 02$ & 0.4412 \\
\hline 2102 & $10: 22: 03$ & 0.4450 \\
\hline 2103 & $10: 22: 04$ & 0.4453 \\
\hline 2104 & 10:22:05 & 0.4437 \\
\hline 2105 & 10:22:06 & 0.4440 \\
\hline 2106 & $10: 22: 07$ & 0.4419 \\
\hline 2107 & $10: 22: 08$ & 0.4450 \\
\hline 2108 & $10: 22: 09$ & 0.4424 \\
\hline 2109 & $10: 22: 10$ & 0.4442 \\
\hline 2110 & $10: 22: 11$ & 0.4438 \\
\hline 2111 & $10: 22: 12$ & 0.4410 \\
\hline 2112 & $10: 22: 13$ & 0.4435 \\
\hline 2113 & $10: 22: 14$ & 0.4420 \\
\hline 2114 & $10: 22: 15$ & 0.4451 \\
\hline 2115 & $10: 22: 16$ & 0.4410 \\
\hline 2116 & $10: 22: 17$ & 0.4403 \\
\hline 2117 & $10: 22: 18$ & 0.4388 \\
\hline 2118 & $10: 22: 19$ & 0.4414 \\
\hline 2119 & $10: 22: 20$ & 0.4445 \\
\hline 2120 & $10: 22: 21$ & 0.4417 \\
\hline 2121 & $10: 22: 22$ & 0.4459 \\
\hline 2122 & $10: 22: 23$ & 0.4422 \\
\hline 2123 & $10: 22: 24$ & 0.4450 \\
\hline 2124 & $10: 22: 25$ & 0.4475 \\
\hline 2125 & $10: 22: 26$ & 0.4461 \\
\hline 2126 & $10: 22: 27$ & 0.4469 \\
\hline 2127 & $10: 22: 28$ & 0.4425 \\
\hline 2128 & $10: 22: 29$ & 0.4454 \\
\hline 2129 & $10: 22: 30$ & 0.4441 \\
\hline 2130 & $10: 22: 31$ & 0.4416 \\
\hline 2131 & $10: 22: 32$ & 0.4430 \\
\hline 2132 & $10: 22: 33$ & 0.4403 \\
\hline 2133 & $10: 22: 34$ & 0.4438 \\
\hline 2134 & $10: 22: 35$ & 0.4410 \\
\hline 2135 & $10: 22: 36$ & 0.4429 \\
\hline 2136 & $10: 22: 37$ & 0.4408 \\
\hline 2137 & $10: 22: 38$ & 0.4407 \\
\hline 2138 & $10: 22: 39$ & 0.4416 \\
\hline 2139 & $10: 22: 40$ & 0.4406 \\
\hline 2140 & $10: 22: 41$ & 0.4444 \\
\hline
\end{tabular}


Appendix C - Fuel Data Test 2 NIST102605_1.xIs

\begin{tabular}{|c|c|c|}
\hline 2141 & $10: 22: 42$ & 0.4429 \\
\hline 2142 & $10: 22: 43$ & 0.4453 \\
\hline 2143 & $10: 22: 44$ & 0.4425 \\
\hline 2144 & $10: 22: 45$ & 0.4435 \\
\hline 2145 & $10: 22: 46$ & 0.4418 \\
\hline 2146 & $10: 22: 47$ & 0.4445 \\
\hline 2147 & $10: 22: 48$ & 0.4469 \\
\hline 2148 & $10: 22: 49$ & 0.4422 \\
\hline 2149 & $10: 22: 50$ & 0.4446 \\
\hline 2150 & $10: 22: 51$ & 0.4428 \\
\hline 2151 & $10: 22: 52$ & 0.4453 \\
\hline 2152 & $10: 22: 53$ & 0.4422 \\
\hline 2153 & $10: 22: 54$ & 0.4402 \\
\hline 2154 & $10: 22: 55$ & 0.4424 \\
\hline 2155 & $10: 22: 56$ & 0.4409 \\
\hline 2156 & $10: 22: 57$ & 0.4429 \\
\hline 2157 & $10: 22: 58$ & 0.4395 \\
\hline 2158 & $10: 22: 59$ & 0.4422 \\
\hline 2159 & $10: 23: 00$ & 0.4399 \\
\hline 2160 & $10: 23: 01$ & 0.4419 \\
\hline 2161 & 10:23:02 & 0.4428 \\
\hline 2162 & $10: 23: 03$ & 0.4441 \\
\hline 2163 & $10: 23: 04$ & 0.4467 \\
\hline 2164 & 10:23:05 & 0.4441 \\
\hline 2165 & $10: 23: 06$ & 0.4468 \\
\hline 2166 & $10: 23: 07$ & 0.4433 \\
\hline 2167 & $10: 23: 08$ & 0.4434 \\
\hline 2168 & 10:23:09 & 0.4411 \\
\hline 2169 & $10: 23: 10$ & 0.4448 \\
\hline 2170 & 10:23:11 & 0.4446 \\
\hline 2171 & $10: 23: 12$ & 0.4401 \\
\hline 2172 & $10: 23: 13$ & 0.4433 \\
\hline 2173 & $10: 23: 14$ & 0.4412 \\
\hline 2174 & $10: 23: 15$ & 0.4431 \\
\hline 2175 & $10: 23: 16$ & 0.4413 \\
\hline 2176 & $10: 23: 17$ & 0.4423 \\
\hline 2177 & 10:23:18 & 0.4442 \\
\hline 2178 & $10: 23: 19$ & 0.4395 \\
\hline 2179 & $10: 23: 20$ & 0.4429 \\
\hline 2180 & $10: 23: 21$ & 0.4410 \\
\hline 2181 & $10: 23: 22$ & 0.4410 \\
\hline 2182 & $10: 23: 23$ & 0.4435 \\
\hline 2183 & $10: 23: 24$ & 0.4440 \\
\hline 2184 & $10: 23: 25$ & 0.4468 \\
\hline 2185 & $10: 23: 26$ & 0.4444 \\
\hline 2186 & $10: 23: 27$ & 0.4445 \\
\hline 2187 & $10: 23: 28$ & 0.4416 \\
\hline 2188 & $10: 23: 29$ & 0.4452 \\
\hline 2189 & $10: 23: 30$ & 0.4437 \\
\hline 2190 & $10: 23: 31$ & 0.4427 \\
\hline 2191 & $10: 23: 32$ & 0.4451 \\
\hline
\end{tabular}


Appendix C - Fuel Data Test 2 NIST102605_1.xls

\begin{tabular}{|c|c|c|}
\hline 2192 & $10: 23: 33$ & 0.4404 \\
\hline 2193 & $10: 23: 34$ & 0.4421 \\
\hline 2194 & $10: 23: 35$ & 0.4405 \\
\hline 2195 & $10: 23: 36$ & 0.4436 \\
\hline 2196 & $10: 23: 37$ & 0.4422 \\
\hline 2197 & $10: 23: 38$ & 0.4420 \\
\hline 2198 & $10: 23: 39$ & 0.4417 \\
\hline 2199 & $10: 23: 40$ & 0.4392 \\
\hline 2200 & $10: 23: 41$ & 0.4413 \\
\hline 2201 & $10: 23: 42$ & 0.4395 \\
\hline 2202 & $10: 23: 43$ & 0.4425 \\
\hline 2203 & $10: 23: 44$ & 0.4442 \\
\hline 2204 & $10: 23: 45$ & 0.4445 \\
\hline 2205 & $10: 23: 46$ & 0.4474 \\
\hline 2206 & $10: 23: 47$ & 0.4429 \\
\hline 2207 & $10: 23: 48$ & 0.4446 \\
\hline 2208 & $10: 23: 49$ & 0.4432 \\
\hline 2209 & $10: 23: 50$ & 0.4457 \\
\hline 2210 & $10: 23: 51$ & 0.4461 \\
\hline 2211 & $10: 23: 52$ & 0.4419 \\
\hline 2212 & $10: 23: 53$ & 0.4425 \\
\hline 2213 & $10: 23: 54$ & 0.4399 \\
\hline 2214 & $10: 23: 55$ & 0.4448 \\
\hline 2215 & $10: 23: 56$ & 0.4410 \\
\hline 2216 & $10: 23: 57$ & 0.4402 \\
\hline 2217 & $10: 23: 58$ & 0.4439 \\
\hline 2218 & $10: 23: 59$ & 0.4396 \\
\hline 2219 & $10: 24: 00$ & 0.4402 \\
\hline 2220 & $10: 24: 01$ & 0.4392 \\
\hline 2221 & $10: 24: 02$ & 0.4400 \\
\hline 2222 & $10: 24: 03$ & 0.4419 \\
\hline 2223 & $10: 24: 04$ & 0.4436 \\
\hline 2224 & $10: 24: 05$ & 0.4458 \\
\hline 2225 & $10: 24: 06$ & 0.4411 \\
\hline 2226 & $10: 24: 07$ & 0.4443 \\
\hline 2227 & $10: 24: 08$ & 0.4422 \\
\hline 2228 & $10: 24: 09$ & 0.4451 \\
\hline 2229 & $10: 24: 10$ & 0.4442 \\
\hline 2230 & $10: 24: 11$ & 0.4414 \\
\hline 2231 & $10: 24: 12$ & 0.4442 \\
\hline 2232 & $10: 24: 13$ & 0.4407 \\
\hline 2233 & $10: 24: 14$ & 0.4438 \\
\hline 2234 & $10: 24: 15$ & 0.4437 \\
\hline 2235 & $10: 24: 16$ & 0.4452 \\
\hline 2236 & $10: 24: 17$ & 0.4421 \\
\hline 2237 & $10: 24: 18$ & 0.4416 \\
\hline 2238 & $10: 24: 19$ & 0.4398 \\
\hline 2239 & $10: 24: 20$ & 0.4405 \\
\hline 2240 & $10: 24: 21$ & 0.4430 \\
\hline 2241 & $10: 24: 22$ & 0.4403 \\
\hline 2242 & $10: 24: 23$ & 0.4442 \\
\hline
\end{tabular}


Appendix C - Fuel Data Test 2 NIST102605_1.xIs

\begin{tabular}{|c|c|c|}
\hline 2243 & $10: 24: 24$ & 0.4403 \\
\hline 2244 & $10: 24: 25$ & 0.4427 \\
\hline 2245 & $10: 24: 26$ & 0.4434 \\
\hline 2246 & $10: 24: 27$ & 0.4463 \\
\hline 2247 & $10: 24: 28$ & 0.4465 \\
\hline 2248 & $10: 24: 29$ & 0.4441 \\
\hline 2249 & $10: 24: 30$ & 0.4457 \\
\hline 2250 & $10: 24: 31$ & 0.4420 \\
\hline 2251 & $10: 24: 32$ & 0.4446 \\
\hline 2252 & $10: 24: 33$ & 0.4442 \\
\hline 2253 & $10: 24: 34$ & 0.4452 \\
\hline 2254 & $10: 24: 35$ & 0.4433 \\
\hline 2255 & $10: 24: 36$ & 0.4396 \\
\hline 2256 & $10: 24: 37$ & 0.4419 \\
\hline 2257 & $10: 24: 38$ & 0.4403 \\
\hline 2258 & $10: 24: 39$ & 0.4447 \\
\hline 2259 & $10: 24: 40$ & 0.4417 \\
\hline 2260 & $10: 24: 41$ & 0.4437 \\
\hline 2261 & $10: 24: 42$ & 0.4406 \\
\hline 2262 & $10: 24: 43$ & 0.4411 \\
\hline 2263 & $10: 24: 44$ & 0.4392 \\
\hline 2264 & $10: 24: 45$ & 0.4423 \\
\hline 2265 & $10: 24: 46$ & 0.4448 \\
\hline 2266 & $10: 24: 47$ & 0.4442 \\
\hline 2267 & $10: 24: 48$ & 0.4459 \\
\hline 2268 & $10: 24: 49$ & 0.4424 \\
\hline 2269 & $10: 24: 50$ & 0.4469 \\
\hline 2270 & $10: 24: 51$ & 0.4451 \\
\hline 2271 & $10: 24: 52$ & 0.4453 \\
\hline 2272 & $10: 24: 53$ & 0.4463 \\
\hline 2273 & $10: 24: 54$ & 0.4405 \\
\hline 2274 & $10: 24: 55$ & 0.4432 \\
\hline 2275 & $10: 24: 56$ & 0.4422 \\
\hline 2276 & $10: 24: 57$ & 0.4427 \\
\hline 2277 & $10: 24: 58$ & 0.4408 \\
\hline 2278 & $10: 24: 59$ & 0.4402 \\
\hline 2279 & $10: 25: 00$ & 0.4421 \\
\hline 2280 & $10: 25: 01$ & 0.4415 \\
\hline 2281 & $10: 25: 02$ & 0.4435 \\
\hline 2282 & $10: 25: 03$ & 0.4391 \\
\hline 2283 & $10: 25: 04$ & 0.4406 \\
\hline 2284 & $10: 25: 05$ & 0.4429 \\
\hline 2285 & $10: 25: 06$ & 0.4431 \\
\hline 2286 & $10: 25: 07$ & 0.4467 \\
\hline 2287 & $10: 25: 08$ & 0.4439 \\
\hline 2288 & $10: 25: 09$ & 0.4456 \\
\hline 2289 & $10: 25: 10$ & 0.4426 \\
\hline 2290 & $10: 25: 11$ & 0.4463 \\
\hline 2291 & $10: 25: 12$ & 0.4453 \\
\hline 2292 & $10: 25: 13$ & 0.4445 \\
\hline 2293 & $10: 25: 14$ & 0.4443 \\
\hline
\end{tabular}


Appendix C - Fuel Data Test 2 NIST102605_1.xls

\begin{tabular}{|c|c|c|}
\hline 2294 & $10: 25: 15$ & 0.4421 \\
\hline 2295 & $10: 25: 16$ & 0.4447 \\
\hline 2296 & $10: 25: 17$ & 0.4418 \\
\hline 2297 & $10: 25: 18$ & 0.4439 \\
\hline 2298 & $10: 25: 19$ & 0.4427 \\
\hline 2299 & $10: 25: 20$ & 0.4398 \\
\hline 2300 & $10: 25: 21$ & 0.4451 \\
\hline 2301 & $10: 25: 22$ & 0.4418 \\
\hline 2302 & $10: 25: 23$ & 0.4438 \\
\hline 2303 & $10: 25: 24$ & 0.4402 \\
\hline 2304 & $10: 25: 25$ & 0.4397 \\
\hline 2305 & $10: 25: 26$ & 0.4410 \\
\hline 2306 & $10: 25: 27$ & 0.4426 \\
\hline 2307 & $10: 25: 28$ & 0.4455 \\
\hline 2308 & $10: 25: 29$ & 0.4444 \\
\hline 2309 & $10: 25: 30$ & 0.4465 \\
\hline 2310 & $10: 25: 31$ & 0.4424 \\
\hline 2311 & $10: 25: 32$ & 0.4454 \\
\hline 2312 & $10: 25: 33$ & 0.4448 \\
\hline 2313 & $10: 25: 34$ & 0.4458 \\
\hline 2314 & $10: 25: 35$ & 0.4446 \\
\hline 2315 & $10: 25: 36$ & 0.4412 \\
\hline 2316 & $10: 25: 37$ & 0.4431 \\
\hline 2317 & $10: 25: 38$ & 0.4409 \\
\hline 2318 & $10: 25: 39$ & 0.4436 \\
\hline 2319 & $10: 25: 40$ & 0.4431 \\
\hline 2320 & $10: 25: 41$ & 0.4442 \\
\hline 2321 & $10: 25: 42$ & 0.4427 \\
\hline 2322 & $10: 25: 43$ & 0.4437 \\
\hline 2323 & $10: 25: 44$ & 0.4399 \\
\hline 2324 & $10: 25: 45$ & 0.4428 \\
\hline 2325 & $10: 25: 46$ & 0.4398 \\
\hline 2326 & $10: 25: 47$ & 0.4415 \\
\hline 2327 & $10: 25: 48$ & 0.4419 \\
\hline 2328 & $10: 25: 49$ & 0.4429 \\
\hline 2329 & $10: 25: 50$ & 0.4462 \\
\hline 2330 & $10: 25: 51$ & 0.4431 \\
\hline 2331 & $10: 25: 52$ & 0.4460 \\
\hline 2332 & $10: 25: 53$ & 0.4446 \\
\hline 2333 & $10: 25: 54$ & 0.4434 \\
\hline 2334 & $10: 25: 55$ & 0.4451 \\
\hline 2335 & $10: 25: 56$ & 0.4442 \\
\hline 2336 & $10: 25: 57$ & 0.4446 \\
\hline 2337 & $10: 25: 58$ & 0.4400 \\
\hline 2338 & $10: 25: 59$ & 0.4434 \\
\hline 2339 & $10: 26: 00$ & 0.4423 \\
\hline 2340 & $10: 26: 01$ & 0.4432 \\
\hline 2341 & $10: 26: 02$ & 0.4410 \\
\hline 2342 & $10: 26: 03$ & 0.4399 \\
\hline 2343 & $10: 26: 04$ & 0.4434 \\
\hline 2344 & $10: 26: 05$ & 0.4399 \\
\hline
\end{tabular}


Appendix C - Fuel Data Test 2 NIST102605_1.xIs

\begin{tabular}{|c|c|c|}
\hline 2345 & $10: 26: 06$ & 0.4412 \\
\hline 2346 & $10: 26: 07$ & 0.4400 \\
\hline 2347 & $10: 26: 08$ & 0.4423 \\
\hline 2348 & 10:26:09 & 0.4425 \\
\hline 2349 & $10: 26: 10$ & 0.4440 \\
\hline 2350 & $10: 26: 11$ & 0.4462 \\
\hline 2351 & $10: 26: 12$ & 0.4434 \\
\hline 2352 & $10: 26: 13$ & 0.4456 \\
\hline 2353 & $10: 26: 14$ & 0.4431 \\
\hline 2354 & $10: 26: 15$ & 0.4450 \\
\hline 2355 & $10: 26: 16$ & 0.4420 \\
\hline 2356 & $10: 26: 17$ & 0.4442 \\
\hline 2357 & $10: 26: 18$ & 0.4447 \\
\hline 2358 & $10: 26: 19$ & 0.4436 \\
\hline 2359 & 10:26:20 & 0.4422 \\
\hline 2360 & $10: 26: 21$ & 0.4386 \\
\hline 2361 & 10:26:22 & 0.4424 \\
\hline 2362 & $10: 26: 23$ & 0.4426 \\
\hline 2363 & $10: 26: 24$ & 0.4434 \\
\hline 2364 & $10: 26: 25$ & 0.4391 \\
\hline 2365 & $10: 26: 26$ & 0.4404 \\
\hline 2366 & $10: 26: 27$ & 0.4430 \\
\hline 2367 & $10: 26: 28$ & 0.4398 \\
\hline 2368 & $10: 26: 29$ & 0.4438 \\
\hline 2369 & $10: 26: 30$ & 0.4414 \\
\hline 2370 & $10: 26: 31$ & 0.4445 \\
\hline 2371 & $10: 26: 32$ & 0.4458 \\
\hline 2372 & $10: 26: 33$ & 0.4455 \\
\hline 2373 & $10: 26: 34$ & 0.4458 \\
\hline 2374 & $10: 26: 35$ & 0.4421 \\
\hline 2375 & $10: 26: 36$ & 0.4451 \\
\hline 2376 & $10: 26: 37$ & 0.4428 \\
\hline 2377 & $10: 26: 38$ & 0.4436 \\
\hline 2378 & $10: 26: 39$ & 0.4390 \\
\hline 2379 & $10: 26: 40$ & 0.4429 \\
\hline 2380 & $10: 26: 41$ & 0.4431 \\
\hline 2381 & $10: 26: 42$ & 0.4427 \\
\hline 2382 & $10: 26: 43$ & 0.4424 \\
\hline 2383 & $10: 26: 44$ & 0.4398 \\
\hline 2384 & $10: 26: 45$ & 0.4442 \\
\hline 2385 & $10: 26: 46$ & 0.4411 \\
\hline 2386 & $10: 26: 47$ & 0.4410 \\
\hline 2387 & $10: 26: 48$ & 0.4413 \\
\hline 2388 & $10: 26: 49$ & 0.4418 \\
\hline 2389 & $10: 26: 50$ & 0.4444 \\
\hline 2390 & $10: 26: 51$ & 0.4421 \\
\hline 2391 & $10: 26: 52$ & 0.4445 \\
\hline 2392 & $10: 26: 53$ & 0.4419 \\
\hline 2393 & $10: 26: 54$ & 0.4454 \\
\hline 2394 & $10: 26: 55$ & 0.4447 \\
\hline 2395 & $10: 26: 56$ & 0.4436 \\
\hline
\end{tabular}


Appendix C - Fuel Data Test 2 NIST102605_1.xIs

\begin{tabular}{|c|c|c|}
\hline 2396 & $10: 26: 57$ & 0.4437 \\
\hline 2397 & $10: 26: 58$ & 0.4438 \\
\hline 2398 & $10: 26: 59$ & 0.4455 \\
\hline 2399 & $10: 27: 00$ & 0.4410 \\
\hline 2400 & $10: 27: 01$ & 0.4411 \\
\hline 2401 & $10: 27: 02$ & 0.4392 \\
\hline 2402 & $10: 27: 03$ & 0.4436 \\
\hline 2403 & $10: 27: 04$ & 0.4426 \\
\hline 2404 & $10: 27: 05$ & 0.4428 \\
\hline 2405 & $10: 27: 06$ & 0.4411 \\
\hline 2406 & $10: 27: 07$ & 0.4400 \\
\hline 2407 & $10: 27: 08$ & 0.4432 \\
\hline 2408 & 10:27:09 & 0.4402 \\
\hline 2409 & $10: 27: 10$ & 0.4429 \\
\hline 2410 & $10: 27: 11$ & 0.4424 \\
\hline 2411 & $10: 27: 12$ & 0.4437 \\
\hline 2412 & $10: 27: 13$ & 0.4469 \\
\hline 2413 & $10: 27: 14$ & 0.4438 \\
\hline 2414 & $10: 27: 15$ & 0.4456 \\
\hline 2415 & $10: 27: 16$ & 0.4427 \\
\hline 2416 & $10: 27: 17$ & 0.4454 \\
\hline 2417 & $10: 27: 18$ & 0.4438 \\
\hline 2418 & $10: 27: 19$ & 0.4441 \\
\hline 2419 & $10: 27: 20$ & 0.4439 \\
\hline 2420 & $10: 27: 21$ & 0.4445 \\
\hline 2421 & $10: 27: 22$ & 0.4441 \\
\hline 2422 & $10: 27: 23$ & 0.4386 \\
\hline 2423 & $10: 27: 24$ & 0.4439 \\
\hline 2424 & $10: 27: 25$ & 0.4423 \\
\hline 2425 & $10: 27: 26$ & 0.4421 \\
\hline 2426 & $10: 27: 27$ & 0.4400 \\
\hline 2427 & $10: 27: 28$ & 0.4398 \\
\hline 2428 & $10: 27: 29$ & 0.4421 \\
\hline 2429 & $10: 27: 30$ & 0.4400 \\
\hline 2430 & $10: 27: 31$ & 0.4438 \\
\hline 2431 & $10: 27: 32$ & 0.4423 \\
\hline 2432 & $10: 27: 33$ & 0.4442 \\
\hline 2433 & $10: 27: 34$ & 0.4419 \\
\hline 2434 & $10: 27: 35$ & 0.4437 \\
\hline 2435 & $10: 27: 36$ & 0.4457 \\
\hline 2436 & $10: 27: 37$ & 0.4458 \\
\hline 2437 & $10: 27: 38$ & 0.4456 \\
\hline 2438 & $10: 27: 39$ & 0.4432 \\
\hline 2439 & $10: 27: 40$ & 0.4433 \\
\hline 2440 & $10: 27: 41$ & 0.4407 \\
\hline 2441 & $10: 27: 42$ & 0.4445 \\
\hline 2442 & $10: 27: 43$ & 0.4439 \\
\hline 2443 & $10: 27: 44$ & 0.4449 \\
\hline 2444 & $10: 27: 45$ & 0.4414 \\
\hline 2445 & $10: 27: 46$ & 0.4400 \\
\hline 2446 & $10: 27: 47$ & 0.4427 \\
\hline
\end{tabular}


Appendix C - Fuel Data Test 2 NIST102605_1.xIs

\begin{tabular}{|c|c|c|}
\hline 2447 & $10: 27: 48$ & 0.4393 \\
\hline 2448 & $10: 27: 49$ & 0.4402 \\
\hline 2449 & $10: 27: 50$ & 0.4410 \\
\hline 2450 & $10: 27: 51$ & 0.4424 \\
\hline 2451 & $10: 27: 52$ & 0.4455 \\
\hline 2452 & $10: 27: 53$ & 0.4418 \\
\hline 2453 & $10: 27: 54$ & 0.4449 \\
\hline 2454 & $10: 27: 55$ & 0.4430 \\
\hline 2455 & $10: 27: 56$ & 0.4455 \\
\hline 2456 & $10: 27: 57$ & 0.4465 \\
\hline 2457 & $10: 27: 58$ & 0.4443 \\
\hline 2458 & $10: 27: 59$ & 0.4449 \\
\hline 2459 & 10:28:00 & 0.4409 \\
\hline 2460 & $10: 28: 01$ & 0.4451 \\
\hline 2461 & 10:28:02 & 0.4439 \\
\hline 2462 & $10: 28: 03$ & 0.4393 \\
\hline 2463 & $10: 28: 04$ & 0.4440 \\
\hline 2464 & $10: 28: 05$ & 0.4420 \\
\hline 2465 & $10: 28: 06$ & 0.4438 \\
\hline 2466 & $10: 28: 07$ & 0.4404 \\
\hline 2467 & $10: 28: 08$ & 0.4396 \\
\hline 2468 & 10:28:09 & 0.4432 \\
\hline 2469 & $10: 28: 10$ & 0.4417 \\
\hline 2470 & 10:28:11 & 0.4432 \\
\hline 2471 & $10: 28: 12$ & 0.4413 \\
\hline 2472 & $10: 28: 13$ & 0.4447 \\
\hline 2473 & $10: 28: 14$ & 0.4450 \\
\hline 2474 & $10: 28: 15$ & 0.4458 \\
\hline 2475 & $10: 28: 16$ & 0.4422 \\
\hline 2476 & $10: 28: 17$ & 0.4450 \\
\hline 2477 & 10:28:18 & 0.4422 \\
\hline 2478 & $10: 28: 19$ & 0.4455 \\
\hline 2479 & $10: 28: 20$ & 0.4459 \\
\hline 2480 & $10: 28: 21$ & 0.4428 \\
\hline 2481 & 10:28:22 & 0.4432 \\
\hline 2482 & $10: 28: 23$ & 0.4415 \\
\hline 2483 & $10: 28: 24$ & 0.4458 \\
\hline 2484 & $10: 28: 25$ & 0.4435 \\
\hline 2485 & 10:28:26 & 0.4444 \\
\hline 2486 & $10: 28: 27$ & 0.4416 \\
\hline 2487 & 10:28:28 & 0.4414 \\
\hline 2488 & $10: 28: 29$ & 0.4392 \\
\hline 2489 & $10: 28: 30$ & 0.4388 \\
\hline 2490 & 10:28:31 & 0.4428 \\
\hline 2491 & $10: 28: 32$ & 0.4402 \\
\hline 2492 & $10: 28: 33$ & 0.4428 \\
\hline 2493 & $10: 28: 34$ & 0.4426 \\
\hline 2494 & $10: 28: 35$ & 0.4441 \\
\hline 2495 & $10: 28: 36$ & 0.4423 \\
\hline 2496 & $10: 28: 37$ & 0.4443 \\
\hline 2497 & $10: 28: 38$ & 0.4467 \\
\hline
\end{tabular}


Appendix C - Fuel Data Test 2 NIST102605_1.xIs

\begin{tabular}{|c|c|c|}
\hline 2498 & $10: 28: 39$ & 0.4468 \\
\hline 2499 & $10: 28: 40$ & 0.4461 \\
\hline 2500 & $10: 28: 41$ & 0.4420 \\
\hline 2501 & $10: 28: 42$ & 0.4440 \\
\hline 2502 & $10: 28: 43$ & 0.4415 \\
\hline 2503 & $10: 28: 44$ & 0.4438 \\
\hline 2504 & $10: 28: 45$ & 0.4403 \\
\hline 2505 & $10: 28: 46$ & 0.4417 \\
\hline 2506 & $10: 28: 47$ & 0.4425 \\
\hline 2507 & $10: 28: 48$ & 0.4401 \\
\hline 2508 & $10: 28: 49$ & 0.4440 \\
\hline 2509 & $10: 28: 50$ & 0.4420 \\
\hline 2510 & $10: 28: 51$ & 0.4437 \\
\hline 2511 & $10: 28: 52$ & 0.4391 \\
\hline 2512 & $10: 28: 53$ & 0.4405 \\
\hline 2513 & $10: 28: 54$ & 0.4421 \\
\hline 2514 & $10: 28: 55$ & 0.4439 \\
\hline 2515 & $10: 28: 56$ & 0.4456 \\
\hline 2516 & $10: 28: 57$ & 0.4430 \\
\hline 2517 & $10: 28: 58$ & 0.4457 \\
\hline 2518 & $10: 28: 59$ & 0.4428 \\
\hline 2519 & $10: 29: 00$ & 0.4457 \\
\hline 2520 & 10:29:01 & 0.4430 \\
\hline 2521 & 10:29:02 & 0.4440 \\
\hline 2522 & $10: 29: 03$ & 0.4436 \\
\hline 2523 & $10: 29: 04$ & 0.4414 \\
\hline 2524 & $10: 29: 05$ & 0.4447 \\
\hline 2525 & 10:29:06 & 0.4421 \\
\hline 2526 & $10: 29: 07$ & 0.4435 \\
\hline 2527 & 10:29:08 & 0.4430 \\
\hline 2528 & 10:29:09 & 0.4406 \\
\hline 2529 & $10: 29: 10$ & 0.4416 \\
\hline 2530 & 10:29:11 & 0.4401 \\
\hline 2531 & 10:29:12 & 0.4433 \\
\hline 2532 & $10: 29: 13$ & 0.4406 \\
\hline 2533 & $10: 29: 14$ & 0.4418 \\
\hline 2534 & 10:29:15 & 0.4445 \\
\hline 2535 & $10: 29: 16$ & 0.4411 \\
\hline 2536 & $10: 29: 17$ & 0.4441 \\
\hline 2537 & 10:29:18 & 0.4458 \\
\hline 2538 & 10:29:19 & 0.4449 \\
\hline 2539 & $10: 29: 20$ & 0.4465 \\
\hline 2540 & $10: 29: 21$ & 0.4422 \\
\hline 2541 & 10:29:22 & 0.4443 \\
\hline 2542 & $10: 29: 23$ & 0.4423 \\
\hline 2543 & $10: 29: 24$ & 0.4441 \\
\hline 2544 & 10:29:25 & 0.4433 \\
\hline 2545 & 10:29:26 & 0.4451 \\
\hline 2546 & $10: 29: 27$ & 0.4437 \\
\hline 2547 & 10:29:28 & 0.4404 \\
\hline 2548 & $10: 29: 29$ & 0.4414 \\
\hline
\end{tabular}


Appendix C - Fuel Data Test 2 NIST102605_1.xIs

\begin{tabular}{|c|c|c|}
\hline 2549 & $10: 29: 30$ & 0.4401 \\
\hline 2550 & $10: 29: 31$ & 0.4434 \\
\hline 2551 & $10: 29: 32$ & 0.4397 \\
\hline 2552 & $10: 29: 33$ & 0.4420 \\
\hline 2553 & $10: 29: 34$ & 0.4414 \\
\hline 2554 & $10: 29: 35$ & 0.4449 \\
\hline 2555 & $10: 29: 36$ & 0.4429 \\
\hline 2556 & $10: 29: 37$ & 0.4442 \\
\hline 2557 & $10: 29: 38$ & 0.4423 \\
\hline 2558 & 10:29:39 & 0.4461 \\
\hline 2559 & $10: 29: 40$ & 0.4436 \\
\hline 2560 & $10: 29: 41$ & 0.4441 \\
\hline 2561 & $10: 29: 42$ & 0.4465 \\
\hline 2562 & $10: 29: 43$ & 0.4413 \\
\hline 2563 & $10: 29: 44$ & 0.4448 \\
\hline 2564 & $10: 29: 45$ & 0.4437 \\
\hline 2565 & $10: 29: 46$ & 0.4447 \\
\hline 2566 & $10: 29: 47$ & 0.4439 \\
\hline 2567 & $10: 29: 48$ & 0.4402 \\
\hline 2568 & $10: 29: 49$ & 0.4433 \\
\hline 2569 & $10: 29: 50$ & 0.4416 \\
\hline 2570 & $10: 29: 51$ & 0.4435 \\
\hline 2571 & $10: 29: 52$ & 0.4413 \\
\hline 2572 & $10: 29: 53$ & 0.4441 \\
\hline 2573 & $10: 29: 54$ & 0.4394 \\
\hline 2574 & $10: 29: 55$ & 0.4394 \\
\hline 2575 & $10: 29: 56$ & 0.4405 \\
\hline 2576 & $10: 29: 57$ & 0.4431 \\
\hline 2577 & $10: 29: 58$ & 0.4469 \\
\hline 2578 & 10:29:59 & 0.4419 \\
\hline 2579 & $10: 30: 00$ & 0.4448 \\
\hline 2580 & 10:30:01 & 0.4427 \\
\hline 2581 & 10:30:02 & 0.4455 \\
\hline 2582 & $10: 30: 03$ & 0.4430 \\
\hline 2583 & $10: 30: 04$ & 0.4442 \\
\hline 2584 & $10: 30: 05$ & 0.4427 \\
\hline 2585 & $10: 30: 06$ & 0.4443 \\
\hline 2586 & $10: 30: 07$ & 0.4432 \\
\hline 2587 & $10: 30: 08$ & 0.4437 \\
\hline 2588 & 10:30:09 & 0.4439 \\
\hline 2589 & 10:30:10 & 0.4393 \\
\hline 2590 & $10: 30: 11$ & 0.4440 \\
\hline 2591 & $10: 30: 12$ & 0.4420 \\
\hline 2592 & $10: 30: 13$ & 0.4420 \\
\hline 2593 & $10: 30: 14$ & 0.4402 \\
\hline 2594 & $10: 30: 15$ & 0.4400 \\
\hline 2595 & $10: 30: 16$ & 0.4417 \\
\hline 2596 & $10: 30: 17$ & 0.4408 \\
\hline 2597 & 10:30:18 & 0.4449 \\
\hline 2598 & $10: 30: 19$ & 0.4441 \\
\hline 2599 & $10: 30: 20$ & 0.4463 \\
\hline
\end{tabular}

C - 51 
Appendix C - Fuel Data Test 2 NIST102605_1.xIs

\begin{tabular}{|c|c|c|}
\hline 2600 & $10: 30: 21$ & 0.4422 \\
\hline 2601 & $10: 30: 22$ & 0.4441 \\
\hline 2602 & $10: 30: 23$ & 0.4428 \\
\hline 2603 & $10: 30: 24$ & 0.4439 \\
\hline 2604 & $10: 30: 25$ & 0.4448 \\
\hline 2605 & $10: 30: 26$ & 0.4423 \\
\hline 2606 & $10: 30: 27$ & 0.4448 \\
\hline 2607 & $10: 30: 28$ & 0.4422 \\
\hline 2608 & $10: 30: 29$ & 0.4446 \\
\hline 2609 & $10: 30: 30$ & 0.4425 \\
\hline 2610 & $10: 30: 31$ & 0.4403 \\
\hline 2611 & $10: 30: 32$ & 0.4424 \\
\hline 2612 & $10: 30: 33$ & 0.4416 \\
\hline 2613 & $10: 30: 34$ & 0.4441 \\
\hline 2614 & $10: 30: 35$ & 0.4389 \\
\hline 2615 & $10: 30: 36$ & 0.4401 \\
\hline 2616 & $10: 30: 37$ & 0.4390 \\
\hline 2617 & $10: 30: 38$ & 0.4424 \\
\hline 2618 & $10: 30: 39$ & 0.4439 \\
\hline 2619 & $10: 30: 40$ & 0.4439 \\
\hline 2620 & $10: 30: 41$ & 0.4462 \\
\hline 2621 & $10: 30: 42$ & 0.4438 \\
\hline 2622 & $10: 30: 43$ & 0.4463 \\
\hline 2623 & $10: 30: 44$ & 0.4438 \\
\hline 2624 & $10: 30: 45$ & 0.4441 \\
\hline 2625 & $10: 30: 46$ & 0.4414 \\
\hline 2626 & $10: 30: 47$ & 0.4431 \\
\hline 2627 & $10: 30: 48$ & 0.4408 \\
\hline 2628 & $10: 30: 49$ & 0.4427 \\
\hline 2629 & $10: 30: 50$ & 0.4402 \\
\hline 2630 & $10: 30: 51$ & 0.4424 \\
\hline 2631 & $10: 30: 52$ & 0.4392 \\
\hline 2632 & $10: 30: 53$ & 0.4451 \\
\hline 2633 & $10: 30: 54$ & 0.4424 \\
\hline 2634 & $10: 30: 55$ & 0.4430 \\
\hline 2635 & $10: 30: 56$ & 0.4395 \\
\hline 2636 & $10: 30: 57$ & 0.4429 \\
\hline 2637 & $10: 30: 58$ & 0.4404 \\
\hline 2638 & $10: 30: 59$ & 0.4416 \\
\hline 2639 & $10: 31: 00$ & 0.4408 \\
\hline 2640 & $10: 31: 01$ & 0.4429 \\
\hline 2641 & $10: 31: 02$ & 0.4447 \\
\hline 2642 & $10: 31: 03$ & 0.4458 \\
\hline 2643 & $10: 31: 04$ & 0.4460 \\
\hline 2644 & $10: 31: 05$ & 0.4436 \\
\hline 2645 & $10: 31: 06$ & 0.4449 \\
\hline 2646 & $10: 31: 07$ & 0.4415 \\
\hline 2647 & $10: 31: 08$ & 0.4449 \\
\hline 2648 & 10:31:09 & 0.4448 \\
\hline 2649 & $10: 31: 10$ & 0.4447 \\
\hline 2650 & $10: 31: 11$ & 0.4438 \\
\hline
\end{tabular}

C - 52 
Appendix C - Fuel Data Test 2 NIST102605_1.xIs

\begin{tabular}{|c|c|c|}
\hline 2651 & $10: 31: 12$ & 0.4404 \\
\hline 2652 & $10: 31: 13$ & 0.4441 \\
\hline 2653 & $10: 31: 14$ & 0.4417 \\
\hline 2654 & $10: 31: 15$ & 0.4432 \\
\hline 2655 & $10: 31: 16$ & 0.4411 \\
\hline 2656 & $10: 31: 17$ & 0.4400 \\
\hline 2657 & $10: 31: 18$ & 0.4401 \\
\hline 2658 & $10: 31: 19$ & 0.4388 \\
\hline 2659 & $10: 31: 20$ & 0.4445 \\
\hline 2660 & $10: 31: 21$ & 0.4416 \\
\hline 2661 & 10:31:22 & 0.4446 \\
\hline 2662 & $10: 31: 23$ & 0.4453 \\
\hline 2663 & $10: 31: 24$ & 0.4453 \\
\hline 2664 & $10: 31: 25$ & 0.4466 \\
\hline 2665 & 10:31:26 & 0.4435 \\
\hline 2666 & $10: 31: 27$ & 0.4455 \\
\hline 2667 & $10: 31: 28$ & 0.4418 \\
\hline 2668 & $10: 31: 29$ & 0.4421 \\
\hline 2669 & $10: 31: 30$ & 0.4398 \\
\hline 2670 & $10: 31: 31$ & 0.4435 \\
\hline 2671 & $10: 31: 32$ & 0.4426 \\
\hline 2672 & $10: 31: 33$ & 0.4415 \\
\hline 2673 & $10: 31: 34$ & 0.4415 \\
\hline 2674 & 10:31:35 & 0.4398 \\
\hline 2675 & $10: 31: 36$ & 0.4436 \\
\hline 2676 & $10: 31: 37$ & 0.4411 \\
\hline 2677 & $10: 31: 38$ & 0.4434 \\
\hline 2678 & $10: 31: 39$ & 0.4411 \\
\hline 2679 & $10: 31: 40$ & 0.4433 \\
\hline 2680 & $10: 31: 41$ & 0.4408 \\
\hline 2681 & $10: 31: 42$ & 0.4434 \\
\hline 2682 & $10: 31: 43$ & 0.4424 \\
\hline 2683 & $10: 31: 44$ & 0.4437 \\
\hline 2684 & $10: 31: 45$ & 0.4467 \\
\hline 2685 & $10: 31: 46$ & 0.4439 \\
\hline 2686 & $10: 31: 47$ & 0.4458 \\
\hline 2687 & $10: 31: 48$ & 0.4447 \\
\hline 2688 & $10: 31: 49$ & 0.4408 \\
\hline 2689 & $10: 31: 50$ & 0.4435 \\
\hline 2690 & $10: 31: 51$ & 0.4410 \\
\hline 2691 & $10: 31: 52$ & 0.4442 \\
\hline 2692 & $10: 31: 53$ & 0.4436 \\
\hline 2693 & $10: 31: 54$ & 0.4438 \\
\hline 2694 & $10: 31: 55$ & 0.4405 \\
\hline 2695 & $10: 31: 56$ & 0.4404 \\
\hline 2696 & $10: 31: 57$ & 0.4419 \\
\hline 2697 & $10: 31: 58$ & 0.4410 \\
\hline 2698 & $10: 31: 59$ & 0.4433 \\
\hline 2699 & $10: 32: 00$ & 0.4388 \\
\hline 2700 & $10: 32: 01$ & 0.4413 \\
\hline 2701 & $10: 32: 02$ & 0.4403 \\
\hline
\end{tabular}

C - 53 
Appendix C - Fuel Data Test 2 NIST102605_1.xIs

\begin{tabular}{|c|c|c|}
\hline 2702 & $10: 32: 03$ & 0.4433 \\
\hline 2703 & $10: 32: 04$ & 0.4451 \\
\hline 2704 & $10: 32: 05$ & 0.4457 \\
\hline 2705 & $10: 32: 06$ & 0.4471 \\
\hline 2706 & $10: 32: 07$ & 0.4453 \\
\hline 2707 & $10: 32: 08$ & 0.4471 \\
\hline 2708 & 10:32:09 & 0.4461 \\
\hline 2709 & $10: 32: 10$ & 0.4465 \\
\hline 2710 & $10: 32: 11$ & 0.4406 \\
\hline 2711 & $10: 32: 12$ & 0.4428 \\
\hline 2712 & $10: 32: 13$ & 0.4406 \\
\hline 2713 & $10: 32: 14$ & 0.4440 \\
\hline 2714 & $10: 32: 15$ & 0.4424 \\
\hline 2715 & $10: 32: 16$ & 0.4424 \\
\hline 2716 & $10: 32: 17$ & 0.4423 \\
\hline 2717 & 10:32:18 & 0.4393 \\
\hline 2718 & $10: 32: 19$ & 0.4407 \\
\hline 2719 & $10: 32: 20$ & 0.4406 \\
\hline 2720 & $10: 32: 21$ & 0.4440 \\
\hline 2721 & $10: 32: 22$ & 0.4392 \\
\hline 2722 & $10: 32: 23$ & 0.4410 \\
\hline 2723 & $10: 32: 24$ & 0.4415 \\
\hline 2724 & $10: 32: 25$ & 0.4450 \\
\hline 2725 & 10:32:26 & 0.4438 \\
\hline 2726 & $10: 32: 27$ & 0.4458 \\
\hline 2727 & $10: 32: 28$ & 0.4464 \\
\hline 2728 & $10: 32: 29$ & 0.4439 \\
\hline 2729 & $10: 32: 30$ & 0.4453 \\
\hline 2730 & $10: 32: 31$ & 0.4411 \\
\hline 2731 & $10: 32: 32$ & 0.4448 \\
\hline 2732 & $10: 32: 33$ & 0.4440 \\
\hline 2733 & $10: 32: 34$ & 0.4428 \\
\hline 2734 & $10: 32: 35$ & 0.4429 \\
\hline 2735 & $10: 32: 36$ & 0.4423 \\
\hline 2736 & $10: 32: 37$ & 0.4447 \\
\hline 2737 & $10: 32: 38$ & 0.4414 \\
\hline 2738 & $10: 32: 39$ & 0.4413 \\
\hline 2739 & $10: 32: 40$ & 0.4387 \\
\hline 2740 & $10: 32: 41$ & 0.4391 \\
\hline 2741 & $10: 32: 42$ & 0.4391 \\
\hline 2742 & $10: 32: 43$ & 0.4407 \\
\hline 2743 & $10: 32: 44$ & 0.4452 \\
\hline 2744 & $10: 32: 45$ & 0.4423 \\
\hline 2745 & $10: 32: 46$ & 0.4455 \\
\hline 2746 & $10: 32: 47$ & 0.4440 \\
\hline 2747 & $10: 32: 48$ & 0.4474 \\
\hline 2748 & $10: 32: 49$ & 0.4459 \\
\hline 2749 & $10: 32: 50$ & 0.4464 \\
\hline 2750 & $10: 32: 51$ & 0.4456 \\
\hline 2751 & $10: 32: 52$ & 0.4420 \\
\hline 2752 & $10: 32: 53$ & 0.4453 \\
\hline
\end{tabular}

C - 54 
Appendix C - Fuel Data Test 2 NIST102605_1.xIs

\begin{tabular}{|c|c|c|}
\hline 2753 & $10: 32: 54$ & 0.4440 \\
\hline 2754 & $10: 32: 55$ & 0.4445 \\
\hline 2755 & $10: 32: 56$ & 0.4429 \\
\hline 2756 & $10: 32: 57$ & 0.4413 \\
\hline 2757 & $10: 32: 58$ & 0.4424 \\
\hline 2758 & $10: 32: 59$ & 0.4393 \\
\hline 2759 & 10:33:00 & 0.4447 \\
\hline 2760 & 10:33:01 & 0.4418 \\
\hline 2761 & $10: 33: 02$ & 0.4419 \\
\hline 2762 & $10: 33: 03$ & 0.4393 \\
\hline 2763 & $10: 33: 04$ & 0.4393 \\
\hline 2764 & 10:33:05 & 0.4436 \\
\hline 2765 & 10:33:06 & 0.4430 \\
\hline 2766 & $10: 33: 07$ & 0.4444 \\
\hline 2767 & 10:33:08 & 0.4425 \\
\hline 2768 & 10:33:09 & 0.4458 \\
\hline 2769 & 10:33:10 & 0.4461 \\
\hline 2770 & 10:33:11 & 0.4442 \\
\hline 2771 & $10: 33: 12$ & 0.4475 \\
\hline 2772 & $10: 33: 13$ & 0.4428 \\
\hline 2773 & $10: 33: 14$ & 0.4445 \\
\hline 2774 & 10:33:15 & 0.4446 \\
\hline 2775 & 10:33:16 & 0.4402 \\
\hline 2776 & 10:33:17 & 0.4411 \\
\hline 2777 & 10:33:18 & 0.4402 \\
\hline 2778 & 10:33:19 & 0.4454 \\
\hline 2779 & $10: 33: 20$ & 0.4426 \\
\hline 2780 & $10: 33: 21$ & 0.4419 \\
\hline 2781 & $10: 33: 22$ & 0.4406 \\
\hline 2782 & $10: 33: 23$ & 0.4397 \\
\hline 2783 & $10: 33: 24$ & 0.4425 \\
\hline 2784 & 10:33:25 & 0.4412 \\
\hline 2785 & 10:33:26 & 0.4447 \\
\hline 2786 & $10: 33: 27$ & 0.4413 \\
\hline 2787 & 10:33:28 & 0.4445 \\
\hline 2788 & 10:33:29 & 0.4473 \\
\hline 2789 & $10: 33: 30$ & 0.4454 \\
\hline 2790 & $10: 33: 31$ & 0.4471 \\
\hline 2791 & $10: 33: 32$ & 0.4430 \\
\hline 2792 & $10: 33: 33$ & 0.4460 \\
\hline 2793 & $10: 33: 34$ & 0.4448 \\
\hline 2794 & 10:33:35 & 0.4440 \\
\hline 2795 & $10: 33: 36$ & 0.4436 \\
\hline 2796 & $10: 33: 37$ & 0.4416 \\
\hline 2797 & $10: 33: 38$ & 0.4446 \\
\hline 2798 & 10:33:39 & 0.4405 \\
\hline 2799 & $10: 33: 40$ & 0.4454 \\
\hline 2800 & $10: 33: 41$ & 0.4429 \\
\hline 2801 & $10: 33: 42$ & 0.4429 \\
\hline 2802 & $10: 33: 43$ & 0.4402 \\
\hline 2803 & $10: 33: 44$ & 0.4395 \\
\hline
\end{tabular}

C - 55 
Appendix C - Fuel Data Test 2 NIST102605_1.xIs

\begin{tabular}{|c|c|c|}
\hline 2804 & $10: 33: 45$ & 0.4400 \\
\hline 2805 & $10: 33: 46$ & 0.4402 \\
\hline 2806 & $10: 33: 47$ & 0.4436 \\
\hline 2807 & $10: 33: 48$ & 0.4424 \\
\hline 2808 & $10: 33: 49$ & 0.4461 \\
\hline 2809 & $10: 33: 50$ & 0.4440 \\
\hline 2810 & $10: 33: 51$ & 0.4463 \\
\hline 2811 & $10: 33: 52$ & 0.4463 \\
\hline 2812 & $10: 33: 53$ & 0.4450 \\
\hline 2813 & $10: 33: 54$ & 0.4457 \\
\hline 2814 & 10:33:55 & 0.4414 \\
\hline 2815 & $10: 33: 56$ & 0.4443 \\
\hline 2816 & $10: 33: 57$ & 0.4422 \\
\hline 2817 & 10:33:58 & 0.4441 \\
\hline 2818 & $10: 33: 59$ & 0.4441 \\
\hline 2819 & $10: 34: 00$ & 0.4430 \\
\hline 2820 & 10:34:01 & 0.4411 \\
\hline 2821 & $10: 34: 02$ & 0.4396 \\
\hline 2822 & $10: 34: 03$ & 0.4435 \\
\hline 2823 & 10:34:04 & 0.4424 \\
\hline 2824 & $10: 34: 05$ & 0.4435 \\
\hline 2825 & $10: 34: 06$ & 0.4384 \\
\hline 2826 & $10: 34: 07$ & 0.4408 \\
\hline 2827 & $10: 34: 08$ & 0.4416 \\
\hline 2828 & 10:34:09 & 0.4428 \\
\hline 2829 & $10: 34: 10$ & 0.4464 \\
\hline 2830 & $10: 34: 11$ & 0.4447 \\
\hline 2831 & $10: 34: 12$ & 0.4461 \\
\hline 2832 & $10: 34: 13$ & 0.4428 \\
\hline 2833 & $10: 34: 14$ & 0.4465 \\
\hline 2834 & $10: 34: 15$ & 0.4465 \\
\hline 2835 & $10: 34: 16$ & 0.4439 \\
\hline 2836 & $10: 34: 17$ & 0.4438 \\
\hline 2837 & $10: 34: 18$ & 0.4415 \\
\hline 2838 & $10: 34: 19$ & 0.4423 \\
\hline 2839 & $10: 34: 20$ & 0.4389 \\
\hline 2840 & $10: 34: 21$ & 0.4422 \\
\hline 2841 & $10: 34: 22$ & 0.4404 \\
\hline 2842 & $10: 34: 23$ & 0.4450 \\
\hline 2843 & 10:34:24 & 0.4418 \\
\hline 2844 & $10: 34: 25$ & 0.4404 \\
\hline 2845 & $10: 34: 26$ & 0.4413 \\
\hline 2846 & $10: 34: 27$ & 0.4398 \\
\hline 2847 & $10: 34: 28$ & 0.4403 \\
\hline 2848 & $10: 34: 29$ & 0.4417 \\
\hline 2849 & $10: 34: 30$ & 0.4461 \\
\hline 2850 & $10: 34: 31$ & 0.4431 \\
\hline 2851 & $10: 34: 32$ & 0.4447 \\
\hline 2852 & 10:34:33 & 0.4430 \\
\hline 2853 & $10: 34: 34$ & 0.4444 \\
\hline 2854 & $10: 34: 35$ & 0.4457 \\
\hline
\end{tabular}


Appendix C - Fuel Data Test 2 NIST102605_1.xIs

\begin{tabular}{|c|c|c|}
\hline 2855 & $10: 34: 36$ & 0.4421 \\
\hline 2856 & $10: 34: 37$ & 0.4446 \\
\hline 2857 & $10: 34: 38$ & 0.4408 \\
\hline 2858 & $10: 34: 39$ & 0.4434 \\
\hline 2859 & $10: 34: 40$ & 0.4437 \\
\hline 2860 & $10: 34: 41$ & 0.4416 \\
\hline 2861 & $10: 34: 42$ & 0.4421 \\
\hline 2862 & $10: 34: 43$ & 0.4421 \\
\hline 2863 & $10: 34: 44$ & 0.4422 \\
\hline 2864 & $10: 34: 45$ & 0.4408 \\
\hline 2865 & $10: 34: 46$ & 0.4399 \\
\hline 2866 & $10: 34: 47$ & 0.4379 \\
\hline 2867 & $10: 34: 48$ & 0.4438 \\
\hline 2868 & $10: 34: 49$ & 0.4419 \\
\hline 2869 & $10: 34: 50$ & 0.4443 \\
\hline 2870 & $10: 34: 51$ & 0.4415 \\
\hline 2871 & $10: 34: 52$ & 0.4433 \\
\hline 2872 & $10: 34: 53$ & 0.4467 \\
\hline 2873 & $10: 34: 54$ & 0.4439 \\
\hline 2874 & $10: 34: 55$ & 0.4463 \\
\hline 2875 & $10: 34: 56$ & 0.4438 \\
\hline 2876 & $10: 34: 57$ & 0.4453 \\
\hline 2877 & $10: 34: 58$ & 0.4448 \\
\hline 2878 & $10: 34: 59$ & 0.4436 \\
\hline 2879 & $10: 35: 00$ & 0.4444 \\
\hline 2880 & $10: 35: 01$ & 0.4423 \\
\hline 2881 & $10: 35: 02$ & 0.4422 \\
\hline 2882 & $10: 35: 03$ & 0.4404 \\
\hline 2883 & $10: 35: 04$ & 0.4441 \\
\hline 2884 & $10: 35: 05$ & 0.4415 \\
\hline 2885 & 10:35:06 & 0.4426 \\
\hline 2886 & 10:35:07 & 0.4434 \\
\hline 2887 & $10: 35: 08$ & 0.4395 \\
\hline 2888 & $10: 35: 09$ & 0.4430 \\
\hline 2889 & $10: 35: 10$ & 0.4394 \\
\hline 2890 & $10: 35: 11$ & 0.4414 \\
\hline 2891 & $10: 35: 12$ & 0.4414 \\
\hline 2892 & $10: 35: 13$ & 0.4439 \\
\hline 2893 & $10: 35: 14$ & 0.4450 \\
\hline 2894 & 10:35:15 & 0.4443 \\
\hline 2895 & 10:35:16 & 0.4453 \\
\hline 2896 & $10: 35: 17$ & 0.4429 \\
\hline 2897 & $10: 35: 18$ & 0.4455 \\
\hline 2898 & $10: 35: 19$ & 0.4427 \\
\hline 2899 & $10: 35: 20$ & 0.4443 \\
\hline 2900 & $10: 35: 21$ & 0.4446 \\
\hline 2901 & $10: 35: 22$ & 0.4410 \\
\hline 2902 & $10: 35: 23$ & 0.4425 \\
\hline 2903 & $10: 35: 24$ & 0.4410 \\
\hline 2904 & $10: 35: 25$ & 0.4451 \\
\hline 2905 & $10: 35: 26$ & 0.4422 \\
\hline
\end{tabular}


Appendix C - Fuel Data Test 2 NIST102605_1.xIs

\begin{tabular}{|c|c|c|}
\hline 2906 & $10: 35: 27$ & 0.4416 \\
\hline 2907 & $10: 35: 28$ & 0.4396 \\
\hline 2908 & $10: 35: 29$ & 0.4394 \\
\hline 2909 & $10: 35: 30$ & 0.4406 \\
\hline 2910 & $10: 35: 31$ & 0.4401 \\
\hline 2911 & $10: 35: 32$ & 0.4435 \\
\hline 2912 & $10: 35: 33$ & 0.4430 \\
\hline 2913 & $10: 35: 34$ & 0.4461 \\
\hline 2914 & $10: 35: 35$ & 0.4446 \\
\hline 2915 & $10: 35: 36$ & 0.4475 \\
\hline 2916 & $10: 35: 37$ & 0.4432 \\
\hline 2917 & $10: 35: 38$ & 0.4442 \\
\hline 2918 & $10: 35: 39$ & 0.4422 \\
\hline 2919 & $10: 35: 40$ & 0.4447 \\
\hline 2920 & $10: 35: 41$ & 0.4417 \\
\hline 2921 & $10: 35: 42$ & 0.4428 \\
\hline 2922 & $10: 35: 43$ & 0.4395 \\
\hline 2923 & $10: 35: 44$ & 0.4448 \\
\hline 2924 & $10: 35: 45$ & 0.4425 \\
\hline 2925 & $10: 35: 46$ & 0.4448 \\
\hline 2926 & $10: 35: 47$ & 0.4426 \\
\hline 2927 & $10: 35: 48$ & 0.4403 \\
\hline 2928 & $10: 35: 49$ & 0.4435 \\
\hline 2929 & $10: 35: 50$ & 0.4401 \\
\hline 2930 & $10: 35: 51$ & 0.4434 \\
\hline 2931 & $10: 35: 52$ & 0.4410 \\
\hline 2932 & $10: 35: 53$ & 0.4401 \\
\hline 2933 & $10: 35: 54$ & 0.4442 \\
\hline 2934 & $10: 35: 55$ & 0.4436 \\
\hline 2935 & $10: 35: 56$ & 0.4460 \\
\hline 2936 & $10: 35: 57$ & 0.4424 \\
\hline 2937 & $10: 35: 58$ & 0.4446 \\
\hline 2938 & $10: 35: 59$ & 0.4436 \\
\hline 2939 & $10: 36: 00$ & 0.4455 \\
\hline 2940 & 10:36:01 & 0.4450 \\
\hline 2941 & $10: 36: 02$ & 0.4425 \\
\hline 2942 & $10: 36: 03$ & 0.4454 \\
\hline 2943 & $10: 36: 04$ & 0.4427 \\
\hline 2944 & $10: 36: 05$ & 0.4448 \\
\hline 2945 & $10: 36: 06$ & 0.4435 \\
\hline 2946 & $10: 36: 07$ & 0.4409 \\
\hline 2947 & $10: 36: 08$ & 0.4403 \\
\hline 2948 & $10: 36: 09$ & 0.4398 \\
\hline 2949 & $10: 36: 10$ & 0.4425 \\
\hline 2950 & $10: 36: 11$ & 0.4400 \\
\hline 2951 & $10: 36: 12$ & 0.4425 \\
\hline 2952 & $10: 36: 13$ & 0.4388 \\
\hline 2953 & $10: 36: 14$ & 0.4428 \\
\hline 2954 & $10: 36: 15$ & 0.4441 \\
\hline 2955 & $10: 36: 16$ & 0.4417 \\
\hline 2956 & $10: 36: 17$ & 0.4464 \\
\hline
\end{tabular}

C - 58 
Appendix C - Fuel Data Test 2 NIST102605_1.xIs

\begin{tabular}{|c|c|c|}
\hline 2957 & $10: 36: 18$ & 0.4438 \\
\hline 2958 & $10: 36: 19$ & 0.4450 \\
\hline 2959 & $10: 36: 20$ & 0.4458 \\
\hline 2960 & $10: 36: 21$ & 0.4435 \\
\hline 2961 & $10: 36: 22$ & 0.4453 \\
\hline 2962 & $10: 36: 23$ & 0.4430 \\
\hline 2963 & $10: 36: 24$ & 0.4457 \\
\hline 2964 & $10: 36: 25$ & 0.4433 \\
\hline 2965 & $10: 36: 26$ & 0.4420 \\
\hline 2966 & $10: 36: 27$ & 0.4417 \\
\hline 2967 & $10: 36: 28$ & 0.4403 \\
\hline 2968 & $10: 36: 29$ & 0.4443 \\
\hline 2969 & $10: 36: 30$ & 0.4413 \\
\hline 2970 & $10: 36: 31$ & 0.4436 \\
\hline 2971 & $10: 36: 32$ & 0.4409 \\
\hline 2972 & $10: 36: 33$ & 0.4411 \\
\hline 2973 & $10: 36: 34$ & 0.4393 \\
\hline 2974 & $10: 36: 35$ & 0.4407 \\
\hline 2975 & $10: 36: 36$ & 0.4448 \\
\hline 2976 & $10: 36: 37$ & 0.4429 \\
\hline 2977 & $10: 36: 38$ & 0.4444 \\
\hline 2978 & $10: 36: 39$ & 0.4416 \\
\hline 2979 & $10: 36: 40$ & 0.4445 \\
\hline 2980 & $10: 36: 41$ & 0.4439 \\
\hline 2981 & $10: 36: 42$ & 0.4445 \\
\hline 2982 & $10: 36: 43$ & 0.4440 \\
\hline 2983 & $10: 36: 44$ & 0.4428 \\
\hline 2984 & $10: 36: 45$ & 0.4435 \\
\hline 2985 & $10: 36: 46$ & 0.4411 \\
\hline 2986 & $10: 36: 47$ & 0.4448 \\
\hline 2987 & $10: 36: 48$ & 0.4429 \\
\hline 2988 & $10: 36: 49$ & 0.4416 \\
\hline 2989 & $10: 36: 50$ & 0.4419 \\
\hline 2990 & $10: 36: 51$ & 0.4411 \\
\hline 2991 & $10: 36: 52$ & 0.4425 \\
\hline 2992 & $10: 36: 53$ & 0.4402 \\
\hline 2993 & $10: 36: 54$ & 0.4429 \\
\hline 2994 & $10: 36: 55$ & 0.4395 \\
\hline 2995 & $10: 36: 56$ & 0.4411 \\
\hline 2996 & $10: 36: 57$ & 0.4408 \\
\hline 2997 & $10: 36: 58$ & 0.4450 \\
\hline 2998 & $10: 36: 59$ & 0.4454 \\
\hline 2999 & $10: 37: 00$ & 0.4450 \\
\hline 3000 & $10: 37: 01$ & 0.4476 \\
\hline 3001 & $10: 37: 02$ & 0.4431 \\
\hline 3002 & $10: 37: 03$ & 0.4456 \\
\hline 3003 & $10: 37: 04$ & 0.4448 \\
\hline 3004 & $10: 37: 05$ & 0.4455 \\
\hline 3005 & $10: 37: 06$ & 0.4443 \\
\hline 3006 & $10: 37: 07$ & 0.4399 \\
\hline 3007 & $10: 37: 08$ & 0.4426 \\
\hline
\end{tabular}

C - 59 
Appendix C - Fuel Data Test 2 NIST102605_1.xIs

\begin{tabular}{|c|c|c|}
\hline 3008 & $10: 37: 09$ & 0.4412 \\
\hline 3009 & $10: 37: 10$ & 0.4440 \\
\hline 3010 & $10: 37: 11$ & 0.4417 \\
\hline 3011 & $10: 37: 12$ & 0.4401 \\
\hline 3012 & $10: 37: 13$ & 0.4421 \\
\hline 3013 & $10: 37: 14$ & 0.4396 \\
\hline 3014 & $10: 37: 15$ & 0.4434 \\
\hline 3015 & $10: 37: 16$ & 0.4422 \\
\hline 3016 & $10: 37: 17$ & 0.4452 \\
\hline 3017 & $10: 37: 18$ & 0.4416 \\
\hline 3018 & $10: 37: 19$ & 0.4428 \\
\hline 3019 & $10: 37: 20$ & 0.4437 \\
\hline 3020 & $10: 37: 21$ & 0.4463 \\
\hline 3021 & $10: 37: 22$ & 0.4457 \\
\hline 3022 & $10: 37: 23$ & 0.4444 \\
\hline 3023 & $10: 37: 24$ & 0.4457 \\
\hline 3024 & $10: 37: 25$ & 0.4440 \\
\hline 3025 & $10: 37: 26$ & 0.4450 \\
\hline 3026 & $10: 37: 27$ & 0.4415 \\
\hline 3027 & $10: 37: 28$ & 0.4437 \\
\hline 3028 & $10: 37: 29$ & 0.4408 \\
\hline 3029 & $10: 37: 30$ & 0.4437 \\
\hline 3030 & $10: 37: 31$ & 0.4431 \\
\hline 3031 & $10: 37: 32$ & 0.4402 \\
\hline 3032 & $10: 37: 33$ & 0.4432 \\
\hline 3033 & $10: 37: 34$ & 0.4428 \\
\hline 3034 & $10: 37: 35$ & 0.4450 \\
\hline 3035 & $10: 37: 36$ & 0.4404 \\
\hline 3036 & $10: 37: 37$ & 0.4416 \\
\hline 3037 & $10: 37: 38$ & 0.4405 \\
\hline 3038 & $10: 37: 39$ & 0.4420 \\
\hline 3039 & $10: 37: 40$ & 0.4464 \\
\hline 3040 & $10: 37: 41$ & 0.4432 \\
\hline 3041 & $10: 37: 42$ & 0.4459 \\
\hline 3042 & $10: 37: 43$ & 0.4454 \\
\hline 3043 & $10: 37: 44$ & 0.4461 \\
\hline 3044 & $10: 37: 45$ & 0.4464 \\
\hline 3045 & $10: 37: 46$ & 0.4460 \\
\hline 3046 & $10: 37: 47$ & 0.4465 \\
\hline 3047 & $10: 37: 48$ & 0.4423 \\
\hline 3048 & $10: 37: 49$ & 0.4428 \\
\hline 3049 & $10: 37: 50$ & 0.4404 \\
\hline 3050 & $10: 37: 51$ & 0.4446 \\
\hline 3051 & $10: 37: 52$ & 0.4405 \\
\hline 3052 & $10: 37: 53$ & 0.4421 \\
\hline 3053 & $10: 37: 54$ & 0.4413 \\
\hline 3054 & $10: 37: 55$ & 0.4397 \\
\hline 3055 & $10: 37: 56$ & 0.4420 \\
\hline 3056 & $10: 37: 57$ & 0.4415 \\
\hline 3057 & $10: 37: 58$ & 0.4427 \\
\hline 3058 & $10: 37: 59$ & 0.4393 \\
\hline
\end{tabular}

C - 60 
Appendix C - Fuel Data Test 2 NIST102605_1.xIs

\begin{tabular}{|c|c|c|}
\hline 3059 & $10: 38: 00$ & 0.4439 \\
\hline 3060 & 10:38:01 & 0.4450 \\
\hline 3061 & $10: 38: 02$ & 0.4469 \\
\hline 3062 & 10:38:03 & 0.4418 \\
\hline 3063 & $10: 38: 04$ & 0.4440 \\
\hline 3064 & 10:38:05 & 0.4454 \\
\hline 3065 & 10:38:06 & 0.4452 \\
\hline 3066 & 10:38:07 & 0.4451 \\
\hline 3067 & 10:38:08 & 0.4413 \\
\hline 3068 & 10:38:09 & 0.4433 \\
\hline 3069 & $10: 38: 10$ & 0.4410 \\
\hline 3070 & 10:38:11 & 0.4432 \\
\hline 3071 & 10:38:12 & 0.4420 \\
\hline 3072 & $10: 38: 13$ & 0.4446 \\
\hline 3073 & $10: 38: 14$ & 0.4415 \\
\hline 3074 & 10:38:15 & 0.4409 \\
\hline 3075 & 10:38:16 & 0.4408 \\
\hline 3076 & $10: 38: 17$ & 0.4410 \\
\hline 3077 & 10:38:18 & 0.4421 \\
\hline 3078 & $10: 38: 19$ & 0.4392 \\
\hline 3079 & 10:38:20 & 0.4430 \\
\hline 3080 & 10:38:21 & 0.4419 \\
\hline 3081 & 10:38:22 & 0.4418 \\
\hline 3082 & $10: 38: 23$ & 0.4462 \\
\hline 3083 & 10:38:24 & 0.4432 \\
\hline 3084 & $10: 38: 25$ & 0.4466 \\
\hline 3085 & $10: 38: 26$ & 0.4448 \\
\hline 3086 & $10: 38: 27$ & 0.4450 \\
\hline 3087 & $10: 38: 28$ & 0.4457 \\
\hline 3088 & 10:38:29 & 0.4429 \\
\hline 3089 & $10: 38: 30$ & 0.4441 \\
\hline 3090 & 10:38:31 & 0.4417 \\
\hline 3091 & 10:38:32 & 0.4429 \\
\hline 3092 & $10: 38: 33$ & 0.4390 \\
\hline 3093 & $10: 38: 34$ & 0.4435 \\
\hline 3094 & $10: 38: 35$ & 0.4426 \\
\hline 3095 & 10:38:36 & 0.4441 \\
\hline 3096 & $10: 38: 37$ & 0.4407 \\
\hline 3097 & 10:38:38 & 0.4410 \\
\hline 3098 & 10:38:39 & 0.4419 \\
\hline 3099 & $10: 38: 40$ & 0.4400 \\
\hline 3100 & $10: 38: 41$ & 0.4436 \\
\hline 3101 & $10: 38: 42$ & 0.4421 \\
\hline 3102 & $10: 38: 43$ & 0.4432 \\
\hline 3103 & $10: 38: 44$ & 0.4437 \\
\hline 3104 & $10: 38: 45$ & 0.4440 \\
\hline 3105 & $10: 38: 46$ & 0.4466 \\
\hline 3106 & $10: 38: 47$ & 0.4427 \\
\hline 3107 & $10: 38: 48$ & 0.4449 \\
\hline 3108 & $10: 38: 49$ & 0.4424 \\
\hline 3109 & $10: 38: 50$ & 0.4443 \\
\hline
\end{tabular}

C -61 
Appendix C - Fuel Data Test 2 NIST102605_1.xls

\begin{tabular}{|c|c|c|}
\hline 3110 & $10: 38: 51$ & 0.4445 \\
\hline 3111 & $10: 38: 52$ & 0.4410 \\
\hline 3112 & $10: 38: 53$ & 0.4453 \\
\hline 3113 & $10: 38: 54$ & 0.4433 \\
\hline 3114 & $10: 38: 55$ & 0.4426 \\
\hline 3115 & 10:38:56 & 0.4419 \\
\hline 3116 & $10: 38: 57$ & 0.4409 \\
\hline 3117 & $10: 38: 58$ & 0.4427 \\
\hline 3118 & $10: 38: 59$ & 0.4410 \\
\hline 3119 & $10: 39: 00$ & 0.4440 \\
\hline 3120 & 10:39:01 & 0.4392 \\
\hline 3121 & $10: 39: 02$ & 0.4412 \\
\hline 3122 & 10:39:03 & 0.4418 \\
\hline 3123 & 10:39:04 & 0.4419 \\
\hline 3124 & 10:39:05 & 0.4457 \\
\hline 3125 & 10:39:06 & 0.4432 \\
\hline 3126 & $10: 39: 07$ & 0.4459 \\
\hline 3127 & $10: 39: 08$ & 0.4429 \\
\hline 3128 & $10: 39: 09$ & 0.4452 \\
\hline 3129 & $10: 39: 10$ & 0.4455 \\
\hline 3130 & $10: 39: 11$ & 0.4449 \\
\hline 3131 & $10: 39: 12$ & 0.4446 \\
\hline 3132 & $10: 39: 13$ & 0.4406 \\
\hline 3133 & 10:39:14 & 0.4434 \\
\hline 3134 & 10:39:15 & 0.4408 \\
\hline 3135 & $10: 39: 16$ & 0.4446 \\
\hline 3136 & $10: 39: 17$ & 0.4418 \\
\hline 3137 & $10: 39: 18$ & 0.4411 \\
\hline 3138 & $10: 39: 19$ & 0.4423 \\
\hline 3139 & $10: 39: 20$ & 0.4388 \\
\hline 3140 & $10: 39: 21$ & 0.4422 \\
\hline 3141 & 10:39:22 & 0.4399 \\
\hline 3142 & $10: 39: 23$ & 0.4407 \\
\hline 3143 & $10: 39: 24$ & 0.4446 \\
\hline 3144 & $10: 39: 25$ & 0.4436 \\
\hline 3145 & $10: 39: 26$ & 0.4454 \\
\hline 3146 & $10: 39: 27$ & 0.4422 \\
\hline 3147 & $10: 39: 28$ & 0.4446 \\
\hline 3148 & $10: 39: 29$ & 0.4450 \\
\hline 3149 & $10: 39: 30$ & 0.4455 \\
\hline 3150 & $10: 39: 31$ & 0.4457 \\
\hline 3151 & 10:39:32 & 0.4419 \\
\hline 3152 & $10: 39: 33$ & 0.4430 \\
\hline 3153 & $10: 39: 34$ & 0.4410 \\
\hline 3154 & $10: 39: 35$ & 0.4443 \\
\hline 3155 & $10: 39: 36$ & 0.4427 \\
\hline 3156 & $10: 39: 37$ & 0.4442 \\
\hline 3157 & $10: 39: 38$ & 0.4407 \\
\hline 3158 & $10: 39: 39$ & 0.4408 \\
\hline 3159 & $10: 39: 40$ & 0.4406 \\
\hline 3160 & $10: 39: 41$ & 0.4410 \\
\hline
\end{tabular}

C - 62 
Appendix C - Fuel Data Test 2 NIST102605_1.xIs

\begin{tabular}{|c|c|c|}
\hline 3161 & $10: 39: 42$ & 0.4407 \\
\hline 3162 & $10: 39: 43$ & 0.4388 \\
\hline 3163 & 10:39:44 & 0.4438 \\
\hline 3164 & $10: 39: 45$ & 0.4415 \\
\hline 3165 & $10: 39: 46$ & 0.4447 \\
\hline 3166 & $10: 39: 47$ & 0.4436 \\
\hline 3167 & $10: 39: 48$ & 0.4457 \\
\hline 3168 & 10:39:49 & 0.4473 \\
\hline 3169 & 10:39:50 & 0.4426 \\
\hline 3170 & $10: 39: 51$ & 0.4455 \\
\hline 3171 & 10:39:52 & 0.4437 \\
\hline 3172 & $10: 39: 53$ & 0.4452 \\
\hline 3173 & $10: 39: 54$ & 0.4423 \\
\hline 3174 & $10: 39: 55$ & 0.4434 \\
\hline 3175 & $10: 39: 56$ & 0.4422 \\
\hline 3176 & $10: 39: 57$ & 0.4393 \\
\hline 3177 & $10: 39: 58$ & 0.4438 \\
\hline 3178 & 10:39:59 & 0.4418 \\
\hline 3179 & $10: 40: 00$ & 0.4442 \\
\hline 3180 & $10: 40: 01$ & 0.4413 \\
\hline 3181 & 10:40:02 & 0.4437 \\
\hline 3182 & $10: 40: 03$ & 0.4422 \\
\hline 3183 & $10: 40: 04$ & 0.4423 \\
\hline 3184 & $10: 40: 05$ & 0.4387 \\
\hline 3185 & $10: 40: 06$ & 0.4412 \\
\hline 3186 & $10: 40: 07$ & 0.4446 \\
\hline 3187 & $10: 40: 08$ & 0.4457 \\
\hline 3188 & $10: 40: 09$ & 0.4440 \\
\hline 3189 & $10: 40: 10$ & 0.4448 \\
\hline 3190 & $10: 40: 11$ & 0.4461 \\
\hline 3191 & $10: 40: 12$ & 0.4439 \\
\hline 3192 & $10: 40: 13$ & 0.4451 \\
\hline 3193 & $10: 40: 14$ & 0.4419 \\
\hline 3194 & $10: 40: 15$ & 0.4449 \\
\hline 3195 & $10: 40: 16$ & 0.4434 \\
\hline 3196 & $10: 40: 17$ & 0.4436 \\
\hline 3197 & $10: 40: 18$ & 0.4438 \\
\hline 3198 & $10: 40: 19$ & 0.4422 \\
\hline 3199 & $10: 40: 20$ & 0.4400 \\
\hline 3200 & $10: 40: 21$ & 0.4400 \\
\hline 3201 & $10: 40: 22$ & 0.4413 \\
\hline 3202 & $10: 40: 23$ & 0.4411 \\
\hline 3203 & $10: 40: 24$ & 0.4424 \\
\hline 3204 & $10: 40: 25$ & 0.4391 \\
\hline 3205 & $10: 40: 26$ & 0.4434 \\
\hline 3206 & $10: 40: 27$ & 0.4411 \\
\hline 3207 & $10: 40: 28$ & 0.4431 \\
\hline 3208 & $10: 40: 29$ & 0.4463 \\
\hline 3209 & $10: 40: 30$ & 0.4449 \\
\hline 3210 & $10: 40: 31$ & 0.4462 \\
\hline 3211 & 10:40:32 & 0.4419 \\
\hline
\end{tabular}

C - 63 
Appendix C - Fuel Data Test 2 NIST102605_1.xIs

\begin{tabular}{|c|c|c|}
\hline 3212 & $10: 40: 33$ & 0.4456 \\
\hline 3213 & $10: 40: 34$ & 0.4461 \\
\hline 3214 & $10: 40: 35$ & 0.4456 \\
\hline 3215 & $10: 40: 36$ & 0.4451 \\
\hline 3216 & $10: 40: 37$ & 0.4434 \\
\hline 3217 & $10: 40: 38$ & 0.4426 \\
\hline 3218 & $10: 40: 39$ & 0.4386 \\
\hline 3219 & $10: 40: 40$ & 0.4438 \\
\hline 3220 & $10: 40: 41$ & 0.4420 \\
\hline 3221 & $10: 40: 42$ & 0.4437 \\
\hline 3222 & $10: 40: 43$ & 0.4416 \\
\hline 3223 & $10: 40: 44$ & 0.4397 \\
\hline 3224 & $10: 40: 45$ & 0.4406 \\
\hline 3225 & $10: 40: 46$ & 0.4395 \\
\hline 3226 & $10: 40: 47$ & 0.4432 \\
\hline 3227 & $10: 40: 48$ & 0.4433 \\
\hline 3228 & $10: 40: 49$ & 0.4457 \\
\hline 3229 & $10: 40: 50$ & 0.4416 \\
\hline 3230 & $10: 40: 51$ & 0.4433 \\
\hline 3231 & $10: 40: 52$ & 0.4420 \\
\hline 3232 & $10: 40: 53$ & 0.4452 \\
\hline 3233 & $10: 40: 54$ & 0.4420 \\
\hline 3234 & $10: 40: 55$ & 0.4445 \\
\hline 3235 & $10: 40: 56$ & 0.4448 \\
\hline 3236 & $10: 40: 57$ & 0.4437 \\
\hline 3237 & $10: 40: 58$ & 0.4440 \\
\hline 3238 & $10: 40: 59$ & 0.4404 \\
\hline 3239 & $10: 41: 00$ & 0.4435 \\
\hline 3240 & $10: 41: 01$ & 0.4410 \\
\hline 3241 & $10: 41: 02$ & 0.4432 \\
\hline 3242 & $10: 41: 03$ & 0.4415 \\
\hline 3243 & 10:41:04 & 0.4417 \\
\hline 3244 & 10:41:05 & 0.4426 \\
\hline 3245 & $10: 41: 06$ & 0.4397 \\
\hline 3246 & $10: 41: 07$ & 0.4430 \\
\hline 3247 & $10: 41: 08$ & 0.4400 \\
\hline 3248 & $10: 41: 09$ & 0.4435 \\
\hline 3249 & $10: 41: 10$ & 0.4420 \\
\hline 3250 & $10: 41: 11$ & 0.4449 \\
\hline 3251 & $10: 41: 12$ & 0.4466 \\
\hline 3252 & $10: 41: 13$ & 0.4431 \\
\hline 3253 & $10: 41: 14$ & 0.4461 \\
\hline 3254 & $10: 41: 15$ & 0.4429 \\
\hline 3255 & $10: 41: 16$ & 0.4451 \\
\hline 3256 & $10: 41: 17$ & 0.4437 \\
\hline 3257 & $10: 41: 18$ & 0.4439 \\
\hline 3258 & $10: 41: 19$ & 0.4433 \\
\hline 3259 & $10: 41: 20$ & 0.4405 \\
\hline 3260 & $10: 41: 21$ & 0.4429 \\
\hline 3261 & $10: 41: 22$ & 0.4415 \\
\hline 3262 & $10: 41: 23$ & 0.4445 \\
\hline
\end{tabular}

C - 64 
Appendix C - Fuel Data Test 2 NIST102605_1.xIs

\begin{tabular}{|c|c|c|}
\hline 3263 & $10: 41: 24$ & 0.4412 \\
\hline 3264 & $10: 41: 25$ & 0.4415 \\
\hline 3265 & $10: 41: 26$ & 0.4413 \\
\hline 3266 & $10: 41: 27$ & 0.4412 \\
\hline 3267 & $10: 41: 28$ & 0.4405 \\
\hline 3268 & $10: 41: 29$ & 0.4398 \\
\hline 3269 & $10: 41: 30$ & 0.4425 \\
\hline 3270 & $10: 41: 31$ & 0.4446 \\
\hline 3271 & $10: 41: 32$ & 0.4454 \\
\hline 3272 & $10: 41: 33$ & 0.4434 \\
\hline 3273 & $10: 41: 34$ & 0.4463 \\
\hline 3274 & $10: 41: 35$ & 0.4420 \\
\hline 3275 & $10: 41: 36$ & 0.4439 \\
\hline 3276 & $10: 41: 37$ & 0.4453 \\
\hline 3277 & $10: 41: 38$ & 0.4428 \\
\hline 3278 & $10: 41: 39$ & 0.4448 \\
\hline 3279 & $10: 41: 40$ & 0.4428 \\
\hline 3280 & $10: 41: 41$ & 0.4448 \\
\hline 3281 & $10: 41: 42$ & 0.4429 \\
\hline 3282 & $10: 41: 43$ & 0.4399 \\
\hline 3283 & $10: 41: 44$ & 0.4441 \\
\hline 3284 & $10: 41: 45$ & 0.4419 \\
\hline 3285 & $10: 41: 46$ & 0.4423 \\
\hline 3286 & $10: 41: 47$ & 0.4407 \\
\hline 3287 & $10: 41: 48$ & 0.4406 \\
\hline 3288 & $10: 41: 49$ & 0.4427 \\
\hline 3289 & $10: 41: 50$ & 0.4393 \\
\hline 3290 & $10: 41: 51$ & 0.4431 \\
\hline 3291 & $10: 41: 52$ & 0.4421 \\
\hline 3292 & $10: 41: 53$ & 0.4453 \\
\hline 3293 & $10: 41: 54$ & 0.4437 \\
\hline 3294 & $10: 41: 55$ & 0.4459 \\
\hline 3295 & $10: 41: 56$ & 0.4470 \\
\hline 3296 & $10: 41: 57$ & 0.4424 \\
\hline 3297 & $10: 41: 58$ & 0.4446 \\
\hline 3298 & $10: 41: 59$ & 0.4422 \\
\hline 3299 & $10: 42: 00$ & 0.4432 \\
\hline 3300 & $10: 42: 01$ & 0.4407 \\
\hline 3301 & $10: 42: 02$ & 0.4439 \\
\hline 3302 & $10: 42: 03$ & 0.4420 \\
\hline 3303 & $10: 42: 04$ & 0.4438 \\
\hline 3304 & 10:42:05 & 0.4424 \\
\hline 3305 & $10: 42: 06$ & 0.4406 \\
\hline 3306 & $10: 42: 07$ & 0.4409 \\
\hline 3307 & $10: 42: 08$ & 0.4394 \\
\hline 3308 & $10: 42: 09$ & 0.4424 \\
\hline 3309 & $10: 42: 10$ & 0.4413 \\
\hline 3310 & $10: 42: 11$ & 0.4435 \\
\hline 3311 & $10: 42: 12$ & 0.4429 \\
\hline 3312 & $10: 42: 13$ & 0.4457 \\
\hline 3313 & $10: 42: 14$ & 0.4425 \\
\hline
\end{tabular}

C - 65 
Appendix C - Fuel Data Test 2 NIST102605_1.xIs

\begin{tabular}{|c|c|c|}
\hline 3314 & $10: 42: 15$ & 0.4454 \\
\hline 3315 & $10: 42: 16$ & 0.4443 \\
\hline 3316 & $10: 42: 17$ & 0.4433 \\
\hline 3317 & $10: 42: 18$ & 0.4459 \\
\hline 3318 & $10: 42: 19$ & 0.4413 \\
\hline 3319 & $10: 42: 20$ & 0.4433 \\
\hline 3320 & $10: 42: 21$ & 0.4445 \\
\hline 3321 & $10: 42: 22$ & 0.4423 \\
\hline 3322 & $10: 42: 23$ & 0.4434 \\
\hline 3323 & $10: 42: 24$ & 0.4414 \\
\hline 3324 & $10: 42: 25$ & 0.4439 \\
\hline 3325 & $10: 42: 26$ & 0.4415 \\
\hline 3326 & $10: 42: 27$ & 0.4437 \\
\hline 3327 & $10: 42: 28$ & 0.4414 \\
\hline 3328 & $10: 42: 29$ & 0.4404 \\
\hline 3329 & $10: 42: 30$ & 0.4383 \\
\hline 3330 & $10: 42: 31$ & 0.4401 \\
\hline 3331 & $10: 42: 32$ & 0.4409 \\
\hline 3332 & $10: 42: 33$ & 0.4429 \\
\hline 3333 & $10: 42: 34$ & 0.4456 \\
\hline 3334 & $10: 42: 35$ & 0.4431 \\
\hline 3335 & $10: 42: 36$ & 0.4465 \\
\hline 3336 & $10: 42: 37$ & 0.4437 \\
\hline 3337 & $10: 42: 38$ & 0.4455 \\
\hline 3338 & $10: 42: 39$ & 0.4420 \\
\hline 3339 & $10: 42: 40$ & 0.4447 \\
\hline 3340 & $10: 42: 41$ & 0.4446 \\
\hline 3341 & $10: 42: 42$ & 0.4421 \\
\hline 3342 & $10: 42: 43$ & 0.4450 \\
\hline 3343 & $10: 42: 44$ & 0.4433 \\
\hline 3344 & $10: 42: 45$ & 0.4448 \\
\hline 3345 & $10: 42: 46$ & 0.4425 \\
\hline 3346 & $10: 42: 47$ & 0.4409 \\
\hline 3347 & $10: 42: 48$ & 0.4421 \\
\hline 3348 & $10: 42: 49$ & 0.4391 \\
\hline 3349 & $10: 42: 50$ & 0.4434 \\
\hline 3350 & $10: 42: 51$ & 0.4398 \\
\hline 3351 & $10: 42: 52$ & 0.4389 \\
\hline 3352 & $10: 42: 53$ & 0.4382 \\
\hline 3353 & $10: 42: 54$ & 0.4410 \\
\hline 3354 & $10: 42: 55$ & 0.4441 \\
\hline 3355 & $10: 42: 56$ & 0.4433 \\
\hline 3356 & $10: 42: 57$ & 0.4449 \\
\hline 3357 & $10: 42: 58$ & 0.4426 \\
\hline 3358 & $10: 42: 59$ & 0.4454 \\
\hline 3359 & $10: 43: 00$ & 0.4422 \\
\hline 3360 & $10: 43: 01$ & 0.4449 \\
\hline 3361 & $10: 43: 02$ & 0.4451 \\
\hline 3362 & $10: 43: 03$ & 0.4424 \\
\hline 3363 & $10: 43: 04$ & 0.4425 \\
\hline 3364 & $10: 43: 05$ & 0.4422 \\
\hline
\end{tabular}

C - 66 
Appendix C - Fuel Data Test 2 NIST102605_1.xIs

\begin{tabular}{|c|c|c|}
\hline 3365 & $10: 43: 06$ & 0.4444 \\
\hline 3366 & $10: 43: 07$ & 0.4413 \\
\hline 3367 & 10:43:08 & 0.4438 \\
\hline 3368 & 10:43:09 & 0.4420 \\
\hline 3369 & $10: 43: 10$ & 0.4421 \\
\hline 3370 & $10: 43: 11$ & 0.4413 \\
\hline 3371 & $10: 43: 12$ & 0.4401 \\
\hline 3372 & $10: 43: 13$ & 0.4431 \\
\hline 3373 & 10:43:14 & 0.4398 \\
\hline 3374 & $10: 43: 15$ & 0.4426 \\
\hline 3375 & $10: 43: 16$ & 0.4411 \\
\hline 3376 & $10: 43: 17$ & 0.4453 \\
\hline 3377 & $10: 43: 18$ & 0.4456 \\
\hline 3378 & $10: 43: 19$ & 0.4434 \\
\hline 3379 & 10:43:20 & 0.4470 \\
\hline 3380 & $10: 43: 21$ & 0.4446 \\
\hline 3381 & 10:43:22 & 0.4429 \\
\hline 3382 & $10: 43: 23$ & 0.4449 \\
\hline 3383 & $10: 43: 24$ & 0.4415 \\
\hline 3384 & $10: 43: 25$ & 0.4433 \\
\hline 3385 & $10: 43: 26$ & 0.4394 \\
\hline 3386 & $10: 43: 27$ & 0.4435 \\
\hline 3387 & 10:43:28 & 0.4419 \\
\hline 3388 & 10:43:29 & 0.4416 \\
\hline 3389 & 10:43:30 & 0.4420 \\
\hline 3390 & $10: 43: 31$ & 0.4402 \\
\hline 3391 & $10: 43: 32$ & 0.4413 \\
\hline 3392 & $10: 43: 33$ & 0.4399 \\
\hline 3393 & $10: 43: 34$ & 0.4437 \\
\hline 3394 & $10: 43: 35$ & 0.4418 \\
\hline 3395 & $10: 43: 36$ & 0.4449 \\
\hline 3396 & $10: 43: 37$ & 0.4413 \\
\hline 3397 & 10:43:38 & 0.4438 \\
\hline 3398 & 10:43:39 & 0.4461 \\
\hline 3399 & $10: 43: 40$ & 0.4445 \\
\hline 3400 & $10: 43: 41$ & 0.4458 \\
\hline 3401 & $10: 43: 42$ & 0.4434 \\
\hline 3402 & $10: 43: 43$ & 0.4458 \\
\hline 3403 & $10: 43: 44$ & 0.4435 \\
\hline 3404 & $10: 43: 45$ & 0.4427 \\
\hline 3405 & $10: 43: 46$ & 0.4444 \\
\hline 3406 & $10: 43: 47$ & 0.4433 \\
\hline 3407 & $10: 43: 48$ & 0.4413 \\
\hline 3408 & $10: 43: 49$ & 0.4394 \\
\hline 3409 & 10:43:50 & 0.4433 \\
\hline 3410 & $10: 43: 51$ & 0.4428 \\
\hline 3411 & $10: 43: 52$ & 0.4438 \\
\hline 3412 & $10: 43: 53$ & 0.4404 \\
\hline 3413 & $10: 43: 54$ & 0.4415 \\
\hline 3414 & 10:43:55 & 0.4407 \\
\hline 3415 & 10:43:56 & 0.4445 \\
\hline
\end{tabular}

C - 67 
Appendix C - Fuel Data Test 2 NIST102605_1.xIs

\begin{tabular}{|c|c|c|}
\hline 3416 & $10: 43: 57$ & 0.4417 \\
\hline 3417 & $10: 43: 58$ & 0.4440 \\
\hline 3418 & $10: 43: 59$ & 0.4421 \\
\hline 3419 & $10: 44: 00$ & 0.4449 \\
\hline 3420 & $10: 44: 01$ & 0.4460 \\
\hline 3421 & $10: 44: 02$ & 0.4476 \\
\hline 3422 & $10: 44: 03$ & 0.4469 \\
\hline 3423 & $10: 44: 04$ & 0.4429 \\
\hline 3424 & $10: 44: 05$ & 0.4441 \\
\hline 3425 & $10: 44: 06$ & 0.4420 \\
\hline 3426 & $10: 44: 07$ & 0.4438 \\
\hline 3427 & $10: 44: 08$ & 0.4405 \\
\hline 3428 & $10: 44: 09$ & 0.4438 \\
\hline 3429 & $10: 44: 10$ & 0.4422 \\
\hline 3430 & $10: 44: 11$ & 0.4401 \\
\hline 3431 & $10: 44: 12$ & 0.4409 \\
\hline 3432 & $10: 44: 13$ & 0.4402 \\
\hline 3433 & $10: 44: 14$ & 0.4443 \\
\hline 3434 & $10: 44: 15$ & 0.4396 \\
\hline 3435 & $10: 44: 16$ & 0.4396 \\
\hline 3436 & $10: 44: 17$ & 0.4402 \\
\hline 3437 & $10: 44: 18$ & 0.4421 \\
\hline 3438 & $10: 44: 19$ & 0.4452 \\
\hline 3439 & $10: 44: 20$ & 0.4442 \\
\hline 3440 & $10: 44: 21$ & 0.4466 \\
\hline 3441 & $10: 44: 22$ & 0.4416 \\
\hline 3442 & $10: 44: 23$ & 0.4452 \\
\hline 3443 & $10: 44: 24$ & 0.4446 \\
\hline 3444 & $10: 44: 25$ & 0.4447 \\
\hline 3445 & $10: 44: 26$ & 0.4450 \\
\hline 3446 & $10: 44: 27$ & 0.4406 \\
\hline 3447 & $10: 44: 28$ & 0.4430 \\
\hline 3448 & $10: 44: 29$ & 0.4409 \\
\hline 3449 & $10: 44: 30$ & 0.4442 \\
\hline 3450 & $10: 44: 31$ & 0.4432 \\
\hline 3451 & $10: 44: 32$ & 0.4459 \\
\hline 3452 & $10: 44: 33$ & 0.4415 \\
\hline 3453 & $10: 44: 34$ & 0.4412 \\
\hline 3454 & $10: 44: 35$ & 0.4410 \\
\hline 3455 & $10: 44: 36$ & 0.4410 \\
\hline 3456 & $10: 44: 37$ & 0.4429 \\
\hline 3457 & $10: 44: 38$ & 0.4391 \\
\hline 3458 & $10: 44: 39$ & 0.4430 \\
\hline 3459 & $10: 44: 40$ & 0.4441 \\
\hline 3460 & $10: 44: 41$ & 0.4440 \\
\hline 3461 & $10: 44: 42$ & 0.4473 \\
\hline 3462 & $10: 44: 43$ & 0.4441 \\
\hline 3463 & $10: 44: 44$ & 0.4458 \\
\hline 3464 & $10: 44: 45$ & 0.4433 \\
\hline 3465 & $10: 44: 46$ & 0.4456 \\
\hline 3466 & $10: 44: 47$ & 0.4454 \\
\hline
\end{tabular}

C - 68 
Appendix C - Fuel Data Test 2 NIST102605_1.xIs

\begin{tabular}{|c|c|c|}
\hline 3467 & $10: 44: 48$ & 0.4442 \\
\hline 3468 & $10: 44: 49$ & 0.4443 \\
\hline 3469 & $10: 44: 50$ & 0.4407 \\
\hline 3470 & $10: 44: 51$ & 0.4435 \\
\hline 3471 & $10: 44: 52$ & 0.4412 \\
\hline 3472 & $10: 44: 53$ & 0.4444 \\
\hline 3473 & $10: 44: 54$ & 0.4433 \\
\hline 3474 & $10: 44: 55$ & 0.4423 \\
\hline 3475 & $10: 44: 56$ & 0.4398 \\
\hline 3476 & $10: 44: 57$ & 0.4393 \\
\hline 3477 & $10: 44: 58$ & 0.4420 \\
\hline 3478 & $10: 44: 59$ & 0.4412 \\
\hline 3479 & $10: 45: 00$ & 0.4443 \\
\hline 3480 & $10: 45: 01$ & 0.4417 \\
\hline 3481 & $10: 45: 02$ & 0.4439 \\
\hline 3482 & $10: 45: 03$ & 0.4417 \\
\hline 3483 & $10: 45: 04$ & 0.4438 \\
\hline 3484 & $10: 45: 05$ & 0.4458 \\
\hline 3485 & $10: 45: 06$ & 0.4445 \\
\hline 3486 & $10: 45: 07$ & 0.4443 \\
\hline 3487 & $10: 45: 08$ & 0.4405 \\
\hline 3488 & $10: 45: 09$ & 0.4437 \\
\hline 3489 & $10: 45: 10$ & 0.4404 \\
\hline 3490 & $10: 45: 11$ & 0.4439 \\
\hline 3491 & $10: 45: 12$ & 0.4438 \\
\hline 3492 & $10: 45: 13$ & 0.4423 \\
\hline 3493 & $10: 45: 14$ & 0.4418 \\
\hline 3494 & $10: 45: 15$ & 0.4404 \\
\hline 3495 & $10: 45: 16$ & 0.4430 \\
\hline 3496 & $10: 45: 17$ & 0.4406 \\
\hline 3497 & $10: 45: 18$ & 0.4419 \\
\hline 3498 & $10: 45: 19$ & 0.4397 \\
\hline 3499 & $10: 45: 20$ & 0.4422 \\
\hline 3500 & $10: 45: 21$ & 0.4428 \\
\hline 3501 & $10: 45: 22$ & 0.4427 \\
\hline 3502 & $10: 45: 23$ & 0.4472 \\
\hline 3503 & $10: 45: 24$ & 0.4456 \\
\hline 3504 & $10: 45: 25$ & 0.4475 \\
\hline 3505 & $10: 45: 26$ & 0.4437 \\
\hline 3506 & $10: 45: 27$ & 0.4461 \\
\hline 3507 & $10: 45: 28$ & 0.4450 \\
\hline 3508 & $10: 45: 29$ & 0.4458 \\
\hline 3509 & $10: 45: 30$ & 0.4443 \\
\hline 3510 & $10: 45: 31$ & 0.4444 \\
\hline 3511 & $10: 45: 32$ & 0.4421 \\
\hline 3512 & $10: 45: 33$ & 0.4384 \\
\hline 3513 & $10: 45: 34$ & 0.4453 \\
\hline 3514 & $10: 45: 35$ & 0.4428 \\
\hline 3515 & $10: 45: 36$ & 0.4435 \\
\hline 3516 & $10: 45: 37$ & 0.4396 \\
\hline 3517 & $10: 45: 38$ & 0.4391 \\
\hline
\end{tabular}

C - 69 
Appendix C - Fuel Data Test 2 NIST102605_1.xIs

\begin{tabular}{|c|c|c|}
\hline 3518 & $10: 45: 39$ & 0.4405 \\
\hline 3519 & $10: 45: 40$ & 0.4393 \\
\hline 3520 & $10: 45: 41$ & 0.4429 \\
\hline 3521 & $10: 45: 42$ & 0.4424 \\
\hline 3522 & $10: 45: 43$ & 0.4460 \\
\hline 3523 & $10: 45: 44$ & 0.4425 \\
\hline 3524 & $10: 45: 45$ & 0.4439 \\
\hline 3525 & $10: 45: 46$ & 0.4422 \\
\hline 3526 & $10: 45: 47$ & 0.4435 \\
\hline 3527 & $10: 45: 48$ & 0.4459 \\
\hline 3528 & $10: 45: 49$ & 0.4423 \\
\hline 3529 & $10: 45: 50$ & 0.4452 \\
\hline 3530 & $10: 45: 51$ & 0.4434 \\
\hline 3531 & $10: 45: 52$ & 0.4436 \\
\hline 3532 & $10: 45: 53$ & 0.4442 \\
\hline 3533 & $10: 45: 54$ & 0.4450 \\
\hline 3534 & $10: 45: 55$ & 0.4415 \\
\hline 3535 & $10: 45: 56$ & 0.4400 \\
\hline 3536 & $10: 45: 57$ & 0.4413 \\
\hline 3537 & $10: 45: 58$ & 0.4414 \\
\hline 3538 & $10: 45: 59$ & 0.4434 \\
\hline 3539 & $10: 46: 00$ & 0.4388 \\
\hline 3540 & $10: 46: 01$ & 0.4423 \\
\hline 3541 & $10: 46: 02$ & 0.4397 \\
\hline 3542 & $10: 46: 03$ & 0.4416 \\
\hline 3543 & $10: 46: 04$ & 0.4473 \\
\hline 3544 & $10: 46: 05$ & 0.4446 \\
\hline 3545 & $10: 46: 06$ & 0.4471 \\
\hline 3546 & $10: 46: 07$ & 0.4454 \\
\hline 3547 & $10: 46: 08$ & 0.4458 \\
\hline 3548 & $10: 46: 09$ & 0.4453 \\
\hline 3549 & $10: 46: 10$ & 0.4424 \\
\hline 3550 & $10: 46: 11$ & 0.4452 \\
\hline 3551 & $10: 46: 12$ & 0.4422 \\
\hline 3552 & $10: 46: 13$ & 0.4442 \\
\hline 3553 & $10: 46: 14$ & 0.4430 \\
\hline 3554 & $10: 46: 15$ & 0.4410 \\
\hline 3555 & $10: 46: 16$ & 0.4426 \\
\hline 3556 & $10: 46: 17$ & 0.4385 \\
\hline 3557 & $10: 46: 18$ & 0.4449 \\
\hline 3558 & $10: 46: 19$ & 0.4413 \\
\hline 3559 & $10: 46: 20$ & 0.4394 \\
\hline 3560 & $10: 46: 21$ & 0.4411 \\
\hline 3561 & $10: 46: 22$ & 0.4409 \\
\hline 3562 & $10: 46: 23$ & 0.4447 \\
\hline 3563 & $10: 46: 24$ & 0.4429 \\
\hline 3564 & $10: 46: 25$ & 0.4442 \\
\hline 3565 & $10: 46: 26$ & 0.4435 \\
\hline 3566 & $10: 46: 27$ & 0.4443 \\
\hline 3567 & $10: 46: 28$ & 0.4452 \\
\hline 3568 & $10: 46: 29$ & 0.4421 \\
\hline
\end{tabular}


Appendix C - Fuel Data Test 2 NIST102605_1.xIs

\begin{tabular}{|c|c|c|}
\hline 3569 & $10: 46: 30$ & 0.4449 \\
\hline 3570 & $10: 46: 31$ & 0.4418 \\
\hline 3571 & $10: 46: 32$ & 0.4443 \\
\hline 3572 & $10: 46: 33$ & 0.4438 \\
\hline 3573 & $10: 46: 34$ & 0.4422 \\
\hline 3574 & 10:46:35 & 0.4429 \\
\hline 3575 & $10: 46: 36$ & 0.4408 \\
\hline 3576 & $10: 46: 37$ & 0.4455 \\
\hline 3577 & $10: 46: 38$ & 0.4425 \\
\hline 3578 & $10: 46: 39$ & 0.4404 \\
\hline 3579 & $10: 46: 40$ & 0.4404 \\
\hline 3580 & $10: 46: 41$ & 0.4394 \\
\hline 3581 & $10: 46: 42$ & 0.4431 \\
\hline 3582 & $10: 46: 43$ & 0.4400 \\
\hline 3583 & $10: 46: 44$ & 0.4437 \\
\hline 3584 & $10: 46: 45$ & 0.4405 \\
\hline 3585 & $10: 46: 46$ & 0.4427 \\
\hline 3586 & $10: 46: 47$ & 0.4461 \\
\hline 3587 & $10: 46: 48$ & 0.4449 \\
\hline 3588 & $10: 46: 49$ & 0.4470 \\
\hline 3589 & $10: 46: 50$ & 0.4429 \\
\hline 3590 & $10: 46: 51$ & 0.4449 \\
\hline 3591 & $10: 46: 52$ & 0.4427 \\
\hline 3592 & $10: 46: 53$ & 0.4450 \\
\hline 3593 & $10: 46: 54$ & 0.4440 \\
\hline 3594 & $10: 46: 55$ & 0.4458 \\
\hline 3595 & $10: 46: 56$ & 0.4435 \\
\hline 3596 & $10: 46: 57$ & 0.4409 \\
\hline 3597 & $10: 46: 58$ & 0.4412 \\
\hline 3598 & $10: 46: 59$ & 0.4396 \\
\hline 3599 & $10: 47: 00$ & 0.4424 \\
\hline 3600 & $10: 47: 01$ & 0.4390 \\
\hline 3601 & $10: 47: 02$ & 0.4424 \\
\hline 3602 & $10: 47: 03$ & 0.4409 \\
\hline 3603 & $10: 47: 04$ & 0.4435 \\
\hline 3604 & $10: 47: 05$ & 0.4394 \\
\hline 3605 & $10: 47: 06$ & 0.4418 \\
\hline 3606 & $10: 47: 07$ & 0.4421 \\
\hline 3607 & $10: 47: 08$ & 0.4442 \\
\hline 3608 & $10: 47: 09$ & 0.4466 \\
\hline 3609 & $10: 47: 10$ & 0.4436 \\
\hline 3610 & $10: 47: 11$ & 0.4452 \\
\hline 3611 & $10: 47: 12$ & 0.4406 \\
\hline 3612 & $10: 47: 13$ & 0.4446 \\
\hline 3613 & $10: 47: 14$ & 0.4443 \\
\hline 3614 & $10: 47: 15$ & 0.4437 \\
\hline 3615 & $10: 47: 16$ & 0.4436 \\
\hline 3616 & $10: 47: 17$ & 0.4409 \\
\hline 3617 & $10: 47: 18$ & 0.4428 \\
\hline 3618 & $10: 47: 19$ & 0.4404 \\
\hline 3619 & $10: 47: 20$ & 0.4437 \\
\hline
\end{tabular}


Appendix C - Fuel Data Test 2 NIST102605_1.xIs

\begin{tabular}{|c|c|c|}
\hline 3620 & $10: 47: 21$ & 0.4418 \\
\hline 3621 & $10: 47: 22$ & 0.4409 \\
\hline 3622 & $10: 47: 23$ & 0.4403 \\
\hline 3623 & $10: 47: 24$ & 0.4382 \\
\hline 3624 & $10: 47: 25$ & 0.4433 \\
\hline 3625 & $10: 47: 26$ & 0.4412 \\
\hline 3626 & $10: 47: 27$ & 0.4429 \\
\hline 3627 & $10: 47: 28$ & 0.4464 \\
\hline 3628 & $10: 47: 29$ & 0.4435 \\
\hline 3629 & $10: 47: 30$ & 0.4453 \\
\hline 3630 & $10: 47: 31$ & 0.4431 \\
\hline 3631 & $10: 47: 32$ & 0.4461 \\
\hline 3632 & $10: 47: 33$ & 0.4456 \\
\hline 3633 & $10: 47: 34$ & 0.4444 \\
\hline 3634 & $10: 47: 35$ & 0.4451 \\
\hline 3635 & $10: 47: 36$ & 0.4414 \\
\hline 3636 & $10: 47: 37$ & 0.4423 \\
\hline 3637 & $10: 47: 38$ & 0.4381 \\
\hline 3638 & $10: 47: 39$ & 0.4443 \\
\hline 3639 & $10: 47: 40$ & 0.4430 \\
\hline 3640 & $10: 47: 41$ & 0.4454 \\
\hline 3641 & $10: 47: 42$ & 0.4415 \\
\hline 3642 & $10: 47: 43$ & 0.4394 \\
\hline 3643 & $10: 47: 44$ & 0.4428 \\
\hline 3644 & $10: 47: 45$ & 0.4406 \\
\hline 3645 & $10: 47: 46$ & 0.4438 \\
\hline 3646 & $10: 47: 47$ & 0.4421 \\
\hline 3647 & $10: 47: 48$ & 0.4446 \\
\hline 3648 & $10: 47: 49$ & 0.4428 \\
\hline 3649 & $10: 47: 50$ & 0.4449 \\
\hline 3650 & $10: 47: 51$ & 0.4425 \\
\hline 3651 & $10: 47: 52$ & 0.4444 \\
\hline 3652 & $10: 47: 53$ & 0.4463 \\
\hline 3653 & $10: 47: 54$ & 0.4416 \\
\hline 3654 & $10: 47: 55$ & 0.4454 \\
\hline 3655 & $10: 47: 56$ & 0.4436 \\
\hline 3656 & $10: 47: 57$ & 0.4428 \\
\hline 3657 & $10: 47: 58$ & 0.4438 \\
\hline 3658 & $10: 47: 59$ & 0.4403 \\
\hline 3659 & $10: 48: 00$ & 0.4405 \\
\hline 3660 & $10: 48: 01$ & 0.4392 \\
\hline 3661 & $10: 48: 02$ & 0.4432 \\
\hline 3662 & $10: 48: 03$ & 0.4422 \\
\hline 3663 & $10: 48: 04$ & 0.4441 \\
\hline 3664 & $10: 48: 05$ & 0.4391 \\
\hline 3665 & $10: 48: 06$ & 0.4399 \\
\hline 3666 & $10: 48: 07$ & 0.4400 \\
\hline 3667 & $10: 48: 08$ & 0.4415 \\
\hline 3668 & $10: 48: 09$ & 0.4447 \\
\hline 3669 & $10: 48: 10$ & 0.4430 \\
\hline 3670 & $10: 48: 11$ & 0.4455 \\
\hline
\end{tabular}

C - 72 
Appendix C - Fuel Data Test 2 NIST102605_1.xIs

\begin{tabular}{|c|c|c|}
\hline 3671 & $10: 48: 12$ & 0.4427 \\
\hline 3672 & $10: 48: 13$ & 0.4459 \\
\hline 3673 & $10: 48: 14$ & 0.4458 \\
\hline 3674 & $10: 48: 15$ & 0.4460 \\
\hline 3675 & $10: 48: 16$ & 0.4455 \\
\hline 3676 & $10: 48: 17$ & 0.4420 \\
\hline 3677 & 10:48:18 & 0.4424 \\
\hline 3678 & 10:48:19 & 0.4401 \\
\hline 3679 & 10:48:20 & 0.4427 \\
\hline 3680 & $10: 48: 21$ & 0.4412 \\
\hline 3681 & $10: 48: 22$ & 0.4443 \\
\hline 3682 & $10: 48: 23$ & 0.4404 \\
\hline 3683 & 10:48:24 & 0.4390 \\
\hline 3684 & $10: 48: 25$ & 0.4433 \\
\hline 3685 & $10: 48: 26$ & 0.4406 \\
\hline 3686 & $10: 48: 27$ & 0.4422 \\
\hline 3687 & $10: 48: 28$ & 0.4397 \\
\hline 3688 & $10: 48: 29$ & 0.4409 \\
\hline 3689 & $10: 48: 30$ & 0.4427 \\
\hline 3690 & $10: 48: 31$ & 0.4421 \\
\hline 3691 & $10: 48: 32$ & 0.4455 \\
\hline 3692 & 10:48:33 & 0.4427 \\
\hline 3693 & $10: 48: 34$ & 0.4443 \\
\hline 3694 & $10: 48: 35$ & 0.4433 \\
\hline 3695 & $10: 48: 36$ & 0.4451 \\
\hline 3696 & $10: 48: 37$ & 0.4441 \\
\hline 3697 & $10: 48: 38$ & 0.4449 \\
\hline 3698 & $10: 48: 39$ & 0.4448 \\
\hline 3699 & $10: 48: 40$ & 0.4413 \\
\hline 3700 & $10: 48: 41$ & 0.4412 \\
\hline 3701 & $10: 48: 42$ & 0.4408 \\
\hline 3702 & $10: 48: 43$ & 0.4444 \\
\hline 3703 & $10: 48: 44$ & 0.4417 \\
\hline 3704 & $10: 48: 45$ & 0.4432 \\
\hline 3705 & $10: 48: 46$ & 0.4399 \\
\hline 3706 & $10: 48: 47$ & 0.4397 \\
\hline 3707 & $10: 48: 48$ & 0.4412 \\
\hline 3708 & $10: 48: 49$ & 0.4405 \\
\hline 3709 & $10: 48: 50$ & 0.4446 \\
\hline 3710 & $10: 48: 51$ & 0.4409 \\
\hline 3711 & $10: 48: 52$ & 0.4435 \\
\hline 3712 & $10: 48: 53$ & 0.4425 \\
\hline 3713 & $10: 48: 54$ & 0.4434 \\
\hline 3714 & $10: 48: 55$ & 0.4464 \\
\hline 3715 & $10: 48: 56$ & 0.4434 \\
\hline 3716 & $10: 48: 57$ & 0.4463 \\
\hline 3717 & $10: 48: 58$ & 0.4448 \\
\hline 3718 & $10: 48: 59$ & 0.4434 \\
\hline 3719 & $10: 49: 00$ & 0.4434 \\
\hline 3720 & 10:49:01 & 0.4410 \\
\hline 3721 & $10: 49: 02$ & 0.4441 \\
\hline
\end{tabular}


Appendix C - Fuel Data Test 2 NIST102605_1.xIs

\begin{tabular}{|c|c|c|}
\hline 3722 & $10: 49: 03$ & 0.4409 \\
\hline 3723 & $10: 49: 04$ & 0.4442 \\
\hline 3724 & $10: 49: 05$ & 0.4422 \\
\hline 3725 & $10: 49: 06$ & 0.4407 \\
\hline 3726 & $10: 49: 07$ & 0.4416 \\
\hline 3727 & $10: 49: 08$ & 0.4405 \\
\hline 3728 & 10:49:09 & 0.4429 \\
\hline 3729 & $10: 49: 10$ & 0.4388 \\
\hline 3730 & $10: 49: 11$ & 0.4403 \\
\hline 3731 & $10: 49: 12$ & 0.4414 \\
\hline 3732 & $10: 49: 13$ & 0.4435 \\
\hline 3733 & $10: 49: 14$ & 0.4465 \\
\hline 3734 & $10: 49: 15$ & 0.4433 \\
\hline 3735 & $10: 49: 16$ & 0.4451 \\
\hline 3736 & $10: 49: 17$ & 0.4415 \\
\hline 3737 & 10:49:18 & 0.4450 \\
\hline 3738 & $10: 49: 19$ & 0.4455 \\
\hline 3739 & $10: 49: 20$ & 0.4437 \\
\hline 3740 & $10: 49: 21$ & 0.4429 \\
\hline 3741 & $10: 49: 22$ & 0.4403 \\
\hline 3742 & $10: 49: 23$ & 0.4418 \\
\hline 3743 & $10: 49: 24$ & 0.4404 \\
\hline 3744 & $10: 49: 25$ & 0.4442 \\
\hline 3745 & $10: 49: 26$ & 0.4423 \\
\hline 3746 & $10: 49: 27$ & 0.4436 \\
\hline 3747 & $10: 49: 28$ & 0.4400 \\
\hline 3748 & $10: 49: 29$ & 0.4403 \\
\hline 3749 & $10: 49: 30$ & 0.4414 \\
\hline 3750 & $10: 49: 31$ & 0.4413 \\
\hline 3751 & $10: 49: 32$ & 0.4442 \\
\hline 3752 & $10: 49: 33$ & 0.4411 \\
\hline 3753 & $10: 49: 34$ & 0.4441 \\
\hline 3754 & 10:49:35 & 0.4428 \\
\hline 3755 & 10:49:36 & 0.4449 \\
\hline 3756 & $10: 49: 37$ & 0.4480 \\
\hline 3757 & $10: 49: 38$ & 0.4446 \\
\hline 3758 & 10:49:39 & 0.4444 \\
\hline 3759 & $10: 49: 40$ & 0.4399 \\
\hline 3760 & $10: 49: 41$ & 0.4447 \\
\hline 3761 & $10: 49: 42$ & 0.4438 \\
\hline 3762 & $10: 49: 43$ & 0.4446 \\
\hline 3763 & $10: 49: 44$ & 0.4417 \\
\hline 3764 & $10: 49: 45$ & 0.4408 \\
\hline 3765 & $10: 49: 46$ & 0.4434 \\
\hline 3766 & $10: 49: 47$ & 0.4385 \\
\hline 3767 & $10: 49: 48$ & 0.4432 \\
\hline 3768 & $10: 49: 49$ & 0.4422 \\
\hline 3769 & $10: 49: 50$ & 0.4429 \\
\hline 3770 & $10: 49: 51$ & 0.4410 \\
\hline 3771 & $10: 49: 52$ & 0.4402 \\
\hline 3772 & $10: 49: 53$ & 0.4436 \\
\hline
\end{tabular}


Appendix C - Fuel Data Test 2 NIST102605_1.xIs

\begin{tabular}{|c|c|c|}
\hline 3773 & $10: 49: 54$ & 0.4417 \\
\hline 3774 & $10: 49: 55$ & 0.4447 \\
\hline 3775 & $10: 49: 56$ & 0.4425 \\
\hline 3776 & $10: 49: 57$ & 0.4451 \\
\hline 3777 & $10: 49: 58$ & 0.4445 \\
\hline 3778 & $10: 49: 59$ & 0.4427 \\
\hline 3779 & $10: 50: 00$ & 0.4459 \\
\hline 3780 & 10:50:01 & 0.4416 \\
\hline 3781 & $10: 50: 02$ & 0.4450 \\
\hline 3782 & $10: 50: 03$ & 0.4440 \\
\hline 3783 & $10: 50: 04$ & 0.4425 \\
\hline 3784 & 10:50:05 & 0.4420 \\
\hline 3785 & 10:50:06 & 0.4403 \\
\hline 3786 & $10: 50: 07$ & 0.4455 \\
\hline 3787 & 10:50:08 & 0.4431 \\
\hline 3788 & 10:50:09 & 0.4430 \\
\hline 3789 & $10: 50: 10$ & 0.4405 \\
\hline 3790 & $10: 50: 11$ & 0.4399 \\
\hline 3791 & $10: 50: 12$ & 0.4414 \\
\hline 3792 & $10: 50: 13$ & 0.4387 \\
\hline 3793 & $10: 50: 14$ & 0.4434 \\
\hline 3794 & $10: 50: 15$ & 0.4429 \\
\hline 3795 & $10: 50: 16$ & 0.4430 \\
\hline 3796 & $10: 50: 17$ & 0.4475 \\
\hline 3797 & $10: 50: 18$ & 0.4432 \\
\hline 3798 & $10: 50: 19$ & 0.4449 \\
\hline 3799 & $10: 50: 20$ & 0.4438 \\
\hline 3800 & $10: 50: 21$ & 0.4466 \\
\hline 3801 & $10: 50: 22$ & 0.4456 \\
\hline 3802 & $10: 50: 23$ & 0.4425 \\
\hline 3803 & $10: 50: 24$ & 0.4430 \\
\hline 3804 & $10: 50: 25$ & 0.4401 \\
\hline 3805 & $10: 50: 26$ & 0.4442 \\
\hline 3806 & $10: 50: 27$ & 0.4422 \\
\hline 3807 & $10: 50: 28$ & 0.4435 \\
\hline 3808 & $10: 50: 29$ & 0.4422 \\
\hline 3809 & $10: 50: 30$ & 0.4398 \\
\hline 3810 & $10: 50: 31$ & 0.4431 \\
\hline 3811 & $10: 50: 32$ & 0.4400 \\
\hline 3812 & $10: 50: 33$ & 0.4406 \\
\hline 3813 & $10: 50: 34$ & 0.4411 \\
\hline 3814 & $10: 50: 35$ & 0.4413 \\
\hline 3815 & $10: 50: 36$ & 0.4448 \\
\hline 3816 & $10: 50: 37$ & 0.4427 \\
\hline 3817 & $10: 50: 38$ & 0.4445 \\
\hline 3818 & $10: 50: 39$ & 0.4421 \\
\hline 3819 & $10: 50: 40$ & 0.4454 \\
\hline 3820 & $10: 50: 41$ & 0.4448 \\
\hline 3821 & $10: 50: 42$ & 0.4434 \\
\hline 3822 & $10: 50: 43$ & 0.4450 \\
\hline 3823 & $10: 50: 44$ & 0.4412 \\
\hline
\end{tabular}


Appendix C - Fuel Data Test 2 NIST102605_1.xIs

\begin{tabular}{|c|c|c|}
\hline 3824 & $10: 50: 45$ & 0.4440 \\
\hline 3825 & $10: 50: 46$ & 0.4422 \\
\hline 3826 & $10: 50: 47$ & 0.4448 \\
\hline 3827 & $10: 50: 48$ & 0.4418 \\
\hline 3828 & $10: 50: 49$ & 0.4411 \\
\hline 3829 & $10: 50: 50$ & 0.4418 \\
\hline 3830 & $10: 50: 51$ & 0.4412 \\
\hline 3831 & $10: 50: 52$ & 0.4430 \\
\hline 3832 & $10: 50: 53$ & 0.4397 \\
\hline 3833 & $10: 50: 54$ & 0.4425 \\
\hline 3834 & $10: 50: 55$ & 0.4390 \\
\hline 3835 & $10: 50: 56$ & 0.4417 \\
\hline 3836 & $10: 50: 57$ & 0.4415 \\
\hline 3837 & $10: 50: 58$ & 0.4441 \\
\hline 3838 & $10: 50: 59$ & 0.4432 \\
\hline 3839 & $10: 51: 00$ & 0.4439 \\
\hline 3840 & $10: 51: 01$ & 0.4478 \\
\hline 3841 & $10: 51: 02$ & 0.4442 \\
\hline 3842 & $10: 51: 03$ & 0.4448 \\
\hline 3843 & $10: 51: 04$ & 0.4409 \\
\hline 3844 & $10: 51: 05$ & 0.4436 \\
\hline 3845 & $10: 51: 06$ & 0.4438 \\
\hline 3846 & $10: 51: 07$ & 0.4419 \\
\hline 3847 & 10:51:08 & 0.4431 \\
\hline 3848 & 10:51:09 & 0.4407 \\
\hline 3849 & $10: 51: 10$ & 0.4451 \\
\hline 3850 & $10: 51: 11$ & 0.4413 \\
\hline 3851 & $10: 51: 12$ & 0.4422 \\
\hline 3852 & $10: 51: 13$ & 0.4431 \\
\hline 3853 & $10: 51: 14$ & 0.4400 \\
\hline 3854 & $10: 51: 15$ & 0.4430 \\
\hline 3855 & $10: 51: 16$ & 0.4398 \\
\hline 3856 & $10: 51: 17$ & 0.4392 \\
\hline 3857 & $10: 51: 18$ & 0.4424 \\
\hline 3858 & $10: 51: 19$ & 0.4441 \\
\hline 3859 & $10: 51: 20$ & 0.4470 \\
\hline 3860 & $10: 51: 21$ & 0.4429 \\
\hline 3861 & $10: 51: 22$ & 0.4441 \\
\hline 3862 & $10: 51: 23$ & 0.4420 \\
\hline 3863 & $10: 51: 24$ & 0.4455 \\
\hline 3864 & $10: 51: 25$ & 0.4441 \\
\hline 3865 & $10: 51: 26$ & 0.4438 \\
\hline 3866 & $10: 51: 27$ & 0.4447 \\
\hline 3867 & $10: 51: 28$ & 0.4399 \\
\hline 3868 & $10: 51: 29$ & 0.4432 \\
\hline 3869 & $10: 51: 30$ & 0.4425 \\
\hline 3870 & $10: 51: 31$ & 0.4450 \\
\hline 3871 & $10: 51: 32$ & 0.4416 \\
\hline 3872 & $10: 51: 33$ & 0.4407 \\
\hline 3873 & $10: 51: 34$ & 0.4394 \\
\hline 3874 & $10: 51: 35$ & 0.4409 \\
\hline
\end{tabular}


Appendix C - Fuel Data Test 2 NIST102605_1.xIs

\begin{tabular}{|c|c|c|}
\hline 3875 & $10: 51: 36$ & 0.4407 \\
\hline 3876 & $10: 51: 37$ & 0.4406 \\
\hline 3877 & $10: 51: 38$ & 0.4443 \\
\hline 3878 & $10: 51: 39$ & 0.4407 \\
\hline 3879 & $10: 51: 40$ & 0.4438 \\
\hline 3880 & $10: 51: 41$ & 0.4429 \\
\hline 3881 & $10: 51: 42$ & 0.4462 \\
\hline 3882 & $10: 51: 43$ & 0.4467 \\
\hline 3883 & $10: 51: 44$ & 0.4436 \\
\hline 3884 & $10: 51: 45$ & 0.4447 \\
\hline 3885 & $10: 51: 46$ & 0.4442 \\
\hline 3886 & $10: 51: 47$ & 0.4464 \\
\hline 3887 & $10: 51: 48$ & 0.4412 \\
\hline 3888 & $10: 51: 49$ & 0.4444 \\
\hline 3889 & $10: 51: 50$ & 0.4420 \\
\hline 3890 & $10: 51: 51$ & 0.4400 \\
\hline 3891 & $10: 51: 52$ & 0.4432 \\
\hline 3892 & $10: 51: 53$ & 0.4409 \\
\hline 3893 & $10: 51: 54$ & 0.4453 \\
\hline 3894 & $10: 51: 55$ & 0.4401 \\
\hline 3895 & $10: 51: 56$ & 0.4398 \\
\hline 3896 & $10: 51: 57$ & 0.4395 \\
\hline 3897 & $10: 51: 58$ & 0.4396 \\
\hline 3898 & $10: 51: 59$ & 0.4425 \\
\hline 3899 & $10: 52: 00$ & 0.4430 \\
\hline 3900 & $10: 52: 01$ & 0.4456 \\
\hline 3901 & $10: 52: 02$ & 0.4413 \\
\hline 3902 & $10: 52: 03$ & 0.4449 \\
\hline 3903 & $10: 52: 04$ & 0.4444 \\
\hline 3904 & $10: 52: 05$ & 0.4463 \\
\hline 3905 & $10: 52: 06$ & 0.4448 \\
\hline 3906 & $10: 52: 07$ & 0.4430 \\
\hline 3907 & 10:52:08 & 0.4453 \\
\hline 3908 & 10:52:09 & 0.4425 \\
\hline 3909 & $10: 52: 10$ & 0.4439 \\
\hline 3910 & $10: 52: 11$ & 0.4413 \\
\hline 3911 & $10: 52: 12$ & 0.4443 \\
\hline 3912 & $10: 52: 13$ & 0.4418 \\
\hline 3913 & $10: 52: 14$ & 0.4424 \\
\hline 3914 & $10: 52: 15$ & 0.4427 \\
\hline 3915 & $10: 52: 16$ & 0.4411 \\
\hline 3916 & $10: 52: 17$ & 0.4436 \\
\hline 3917 & $10: 52: 18$ & 0.4391 \\
\hline 3918 & $10: 52: 19$ & 0.4420 \\
\hline 3919 & $10: 52: 20$ & 0.4415 \\
\hline 3920 & $10: 52: 21$ & 0.4398 \\
\hline 3921 & $10: 52: 22$ & 0.4453 \\
\hline 3922 & $10: 52: 23$ & 0.4446 \\
\hline 3923 & $10: 52: 24$ & 0.4464 \\
\hline 3924 & $10: 52: 25$ & 0.4423 \\
\hline 3925 & $10: 52: 26$ & 0.4450 \\
\hline
\end{tabular}


Appendix C - Fuel Data Test 2 NIST102605_1.xIs

\begin{tabular}{|c|c|c|}
\hline 3926 & $10: 52: 27$ & 0.4434 \\
\hline 3927 & $10: 52: 28$ & 0.4449 \\
\hline 3928 & $10: 52: 29$ & 0.4446 \\
\hline 3929 & $10: 52: 30$ & 0.4455 \\
\hline 3930 & $10: 52: 31$ & 0.4442 \\
\hline 3931 & $10: 52: 32$ & 0.4433 \\
\hline 3932 & $10: 52: 33$ & 0.4417 \\
\hline 3933 & $10: 52: 34$ & 0.4407 \\
\hline 3934 & $10: 52: 35$ & 0.4421 \\
\hline 3935 & $10: 52: 36$ & 0.4388 \\
\hline 3936 & $10: 52: 37$ & 0.4444 \\
\hline 3937 & $10: 52: 38$ & 0.4422 \\
\hline 3938 & $10: 52: 39$ & 0.4420 \\
\hline 3939 & $10: 52: 40$ & 0.4384 \\
\hline 3940 & $10: 52: 41$ & 0.4406 \\
\hline 3941 & $10: 52: 42$ & 0.4413 \\
\hline 3942 & $10: 52: 43$ & 0.4430 \\
\hline 3943 & $10: 52: 44$ & 0.4419 \\
\hline 3944 & $10: 52: 45$ & 0.4451 \\
\hline 3945 & $10: 52: 46$ & 0.4464 \\
\hline 3946 & $10: 52: 47$ & 0.4455 \\
\hline 3947 & $10: 52: 48$ & 0.4458 \\
\hline 3948 & $10: 52: 49$ & 0.4415 \\
\hline 3949 & $10: 52: 50$ & 0.4449 \\
\hline 3950 & $10: 52: 51$ & 0.4425 \\
\hline 3951 & $10: 52: 52$ & 0.4434 \\
\hline 3952 & $10: 52: 53$ & 0.4438 \\
\hline 3953 & $10: 52: 54$ & 0.4421 \\
\hline 3954 & $10: 52: 55$ & 0.4428 \\
\hline 3955 & $10: 52: 56$ & 0.4405 \\
\hline 3956 & $10: 52: 57$ & 0.4443 \\
\hline 3957 & $10: 52: 58$ & 0.4419 \\
\hline 3958 & $10: 52: 59$ & 0.4424 \\
\hline 3959 & $10: 53: 00$ & 0.4403 \\
\hline 3960 & 10:53:01 & 0.4402 \\
\hline 3961 & $10: 53: 02$ & 0.4428 \\
\hline 3962 & $10: 53: 03$ & 0.4418 \\
\hline 3963 & $10: 53: 04$ & 0.4460 \\
\hline 3964 & 10:53:05 & 0.4428 \\
\hline 3965 & 10:53:06 & 0.4454 \\
\hline 3966 & $10: 53: 07$ & 0.4447 \\
\hline 3967 & 10:53:08 & 0.4448 \\
\hline 3968 & 10:53:09 & 0.4452 \\
\hline 3969 & $10: 53: 10$ & 0.4410 \\
\hline 3970 & $10: 53: 11$ & 0.4449 \\
\hline 3971 & $10: 53: 12$ & 0.4439 \\
\hline 3972 & $10: 53: 13$ & 0.4446 \\
\hline 3973 & $10: 53: 14$ & 0.4422 \\
\hline 3974 & $10: 53: 15$ & 0.4401 \\
\hline 3975 & 10:53:16 & 0.4435 \\
\hline 3976 & $10: 53: 17$ & 0.4396 \\
\hline
\end{tabular}


Appendix C - Fuel Data Test 2 NIST102605_1.xIs

\begin{tabular}{|c|c|c|}
\hline 3977 & $10: 53: 18$ & 0.4451 \\
\hline 3978 & $10: 53: 19$ & 0.4417 \\
\hline 3979 & $10: 53: 20$ & 0.4393 \\
\hline 3980 & $10: 53: 21$ & 0.4390 \\
\hline 3981 & $10: 53: 22$ & 0.4399 \\
\hline 3982 & $10: 53: 23$ & 0.4417 \\
\hline 3983 & 10:53:24 & 0.4420 \\
\hline 3984 & $10: 53: 25$ & 0.4440 \\
\hline 3985 & $10: 53: 26$ & 0.4429 \\
\hline 3986 & $10: 53: 27$ & 0.4460 \\
\hline 3987 & $10: 53: 28$ & 0.4424 \\
\hline 3988 & $10: 53: 29$ & 0.4458 \\
\hline 3989 & $10: 53: 30$ & 0.4453 \\
\hline 3990 & $10: 53: 31$ & 0.4452 \\
\hline 3991 & 10:53:32 & 0.4451 \\
\hline 3992 & 10:53:33 & 0.4405 \\
\hline 3993 & $10: 53: 34$ & 0.4443 \\
\hline 3994 & $10: 53: 35$ & 0.4438 \\
\hline 3995 & $10: 53: 36$ & 0.4430 \\
\hline 3996 & $10: 53: 37$ & 0.4430 \\
\hline 3997 & 10:53:38 & 0.4408 \\
\hline 3998 & $10: 53: 39$ & 0.4413 \\
\hline 3999 & $10: 53: 40$ & 0.4396 \\
\hline 4000 & $10: 53: 41$ & 0.4444 \\
\hline 4001 & $10: 53: 42$ & 0.4408 \\
\hline 4002 & $10: 53: 43$ & 0.4419 \\
\hline 4003 & $10: 53: 44$ & 0.4383 \\
\hline 4004 & $10: 53: 45$ & 0.4423 \\
\hline 4005 & $10: 53: 46$ & 0.4422 \\
\hline 4006 & $10: 53: 47$ & 0.4424 \\
\hline 4007 & $10: 53: 48$ & 0.4474 \\
\hline 4008 & $10: 53: 49$ & 0.4431 \\
\hline 4009 & 10:53:50 & 0.4445 \\
\hline 4010 & $10: 53: 51$ & 0.4416 \\
\hline 4011 & $10: 53: 52$ & 0.4452 \\
\hline 4012 & $10: 53: 53$ & 0.4462 \\
\hline 4013 & $10: 53: 54$ & 0.4437 \\
\hline 4014 & $10: 53: 55$ & 0.4436 \\
\hline 4015 & $10: 53: 56$ & 0.4402 \\
\hline 4016 & $10: 53: 57$ & 0.4436 \\
\hline 4017 & $10: 53: 58$ & 0.4399 \\
\hline 4018 & 10:53:59 & 0.4426 \\
\hline 4019 & $10: 54: 00$ & 0.4416 \\
\hline 4020 & $10: 54: 01$ & 0.4398 \\
\hline 4021 & $10: 54: 02$ & 0.4424 \\
\hline 4022 & $10: 54: 03$ & 0.4401 \\
\hline 4023 & $10: 54: 04$ & 0.4424 \\
\hline 4024 & $10: 54: 05$ & 0.4389 \\
\hline 4025 & $10: 54: 06$ & 0.4423 \\
\hline 4026 & $10: 54: 07$ & 0.4427 \\
\hline 4027 & $10: 54: 08$ & 0.4438 \\
\hline
\end{tabular}


Appendix C - Fuel Data Test 2 NIST102605_1.xIs

\begin{tabular}{|c|c|c|}
\hline 4028 & $10: 54: 09$ & 0.4459 \\
\hline 4029 & $10: 54: 10$ & 0.4424 \\
\hline 4030 & $10: 54: 11$ & 0.4444 \\
\hline 4031 & $10: 54: 12$ & 0.4443 \\
\hline 4032 & $10: 54: 13$ & 0.4420 \\
\hline 4033 & $10: 54: 14$ & 0.4465 \\
\hline 4034 & $10: 54: 15$ & 0.4425 \\
\hline 4035 & $10: 54: 16$ & 0.4432 \\
\hline 4036 & $10: 54: 17$ & 0.4406 \\
\hline 4037 & $10: 54: 18$ & 0.4411 \\
\hline 4038 & $10: 54: 19$ & 0.4429 \\
\hline 4039 & $10: 54: 20$ & 0.4407 \\
\hline 4040 & $10: 54: 21$ & 0.4450 \\
\hline 4041 & $10: 54: 22$ & 0.4419 \\
\hline 4042 & $10: 54: 23$ & 0.4425 \\
\hline 4043 & $10: 54: 24$ & 0.4385 \\
\hline 4044 & $10: 54: 25$ & 0.4411 \\
\hline 4045 & $10: 54: 26$ & 0.4389 \\
\hline 4046 & $10: 54: 27$ & 0.4413 \\
\hline 4047 & $10: 54: 28$ & 0.4433 \\
\hline 4048 & $10: 54: 29$ & 0.4450 \\
\hline 4049 & $10: 54: 30$ & 0.4466 \\
\hline 4050 & $10: 54: 31$ & 0.4458 \\
\hline 4051 & $10: 54: 32$ & 0.4479 \\
\hline 4052 & $10: 54: 33$ & 0.4427 \\
\hline 4053 & $10: 54: 34$ & 0.4442 \\
\hline 4054 & $10: 54: 35$ & 0.4428 \\
\hline 4055 & $10: 54: 36$ & 0.4451 \\
\hline 4056 & $10: 54: 37$ & 0.4448 \\
\hline 4057 & $10: 54: 38$ & 0.4409 \\
\hline 4058 & $10: 54: 39$ & 0.4422 \\
\hline 4059 & $10: 54: 40$ & 0.4408 \\
\hline 4060 & $10: 54: 41$ & 0.4442 \\
\hline 4061 & $10: 54: 42$ & 0.4419 \\
\hline 4062 & $10: 54: 43$ & 0.4447 \\
\hline 4063 & $10: 54: 44$ & 0.4412 \\
\hline 4064 & $10: 54: 45$ & 0.4395 \\
\hline 4065 & $10: 54: 46$ & 0.4394 \\
\hline 4066 & $10: 54: 47$ & 0.4407 \\
\hline 4067 & $10: 54: 48$ & 0.4449 \\
\hline 4068 & $10: 54: 49$ & 0.4418 \\
\hline 4069 & $10: 54: 50$ & 0.4438 \\
\hline 4070 & $10: 54: 51$ & 0.4425 \\
\hline 4071 & $10: 54: 52$ & 0.4444 \\
\hline 4072 & $10: 54: 53$ & 0.4421 \\
\hline 4073 & $10: 54: 54$ & 0.4447 \\
\hline 4074 & $10: 54: 55$ & 0.4465 \\
\hline 4075 & $10: 54: 56$ & 0.4429 \\
\hline 4076 & $10: 54: 57$ & 0.4443 \\
\hline 4077 & $10: 54: 58$ & 0.4419 \\
\hline 4078 & $10: 54: 59$ & 0.4447 \\
\hline
\end{tabular}


Appendix C - Fuel Data Test 2 NIST102605_1.xIs

\begin{tabular}{|c|c|c|}
\hline 4079 & $10: 55: 00$ & 0.4437 \\
\hline 4080 & $10: 55: 01$ & 0.4438 \\
\hline 4081 & $10: 55: 02$ & 0.4434 \\
\hline 4082 & $10: 55: 03$ & 0.4407 \\
\hline 4083 & $10: 55: 04$ & 0.4443 \\
\hline 4084 & $10: 55: 05$ & 0.4406 \\
\hline 4085 & $10: 55: 06$ & 0.4415 \\
\hline 4086 & $10: 55: 07$ & 0.4391 \\
\hline 4087 & 10:55:08 & 0.4409 \\
\hline 4088 & $10: 55: 09$ & 0.4417 \\
\hline 4089 & $10: 55: 10$ & 0.4414 \\
\hline 4090 & 10:55:11 & 0.4452 \\
\hline 4091 & $10: 55: 12$ & 0.4418 \\
\hline 4092 & $10: 55: 13$ & 0.4458 \\
\hline 4093 & $10: 55: 14$ & 0.4471 \\
\hline 4094 & $10: 55: 15$ & 0.4461 \\
\hline 4095 & $10: 55: 16$ & 0.4464 \\
\hline 4096 & $10: 55: 17$ & 0.4426 \\
\hline 4097 & $10: 55: 18$ & 0.4441 \\
\hline 4098 & $10: 55: 19$ & 0.4403 \\
\hline 4099 & $10: 55: 20$ & 0.4440 \\
\hline 4100 & $10: 55: 21$ & 0.4416 \\
\hline 4101 & $10: 55: 22$ & 0.4432 \\
\hline 4102 & $10: 55: 23$ & 0.4417 \\
\hline 4103 & $10: 55: 24$ & 0.4397 \\
\hline 4104 & $10: 55: 25$ & 0.4441 \\
\hline 4105 & $10: 55: 26$ & 0.4410 \\
\hline 4106 & $10: 55: 27$ & 0.4447 \\
\hline 4107 & $10: 55: 28$ & 0.4414 \\
\hline 4108 & $10: 55: 29$ & 0.4421 \\
\hline 4109 & $10: 55: 30$ & 0.4384 \\
\hline 4110 & $10: 55: 31$ & 0.4413 \\
\hline 4111 & $10: 55: 32$ & 0.4449 \\
\hline 4112 & $10: 55: 33$ & 0.4451 \\
\hline 4113 & $10: 55: 34$ & 0.4464 \\
\hline 4114 & $10: 55: 35$ & 0.4421 \\
\hline 4115 & $10: 55: 36$ & 0.4455 \\
\hline 4116 & $10: 55: 37$ & 0.4435 \\
\hline 4117 & 10:55:38 & 0.4423 \\
\hline 4118 & $10: 55: 39$ & 0.4451 \\
\hline 4119 & $10: 55: 40$ & 0.4397 \\
\hline 4120 & $10: 55: 41$ & 0.4429 \\
\hline 4121 & $10: 55: 42$ & 0.4425 \\
\hline 4122 & $10: 55: 43$ & 0.4429 \\
\hline 4123 & $10: 55: 44$ & 0.4412 \\
\hline 4124 & $10: 55: 45$ & 0.4403 \\
\hline 4125 & $10: 55: 46$ & 0.4439 \\
\hline 4126 & $10: 55: 47$ & 0.4421 \\
\hline 4127 & $10: 55: 48$ & 0.4413 \\
\hline 4128 & $10: 55: 49$ & 0.4386 \\
\hline 29 & $10: 55: 50$ & 0.4403 \\
\hline
\end{tabular}


Appendix C - Fuel Data Test 2 NIST102605_1.xIs

\begin{tabular}{|c|c|c|}
\hline 4130 & $10: 55: 51$ & 0.4422 \\
\hline 4131 & $10: 55: 52$ & 0.4405 \\
\hline 4132 & $10: 55: 53$ & 0.4458 \\
\hline 4133 & $10: 55: 54$ & 0.4433 \\
\hline 4134 & $10: 55: 55$ & 0.4446 \\
\hline 4135 & $10: 55: 56$ & 0.4463 \\
\hline 4136 & $10: 55: 57$ & 0.4459 \\
\hline 4137 & $10: 55: 58$ & 0.4467 \\
\hline 4138 & 10:55:59 & 0.4414 \\
\hline 4139 & $10: 56: 00$ & 0.4450 \\
\hline 4140 & $10: 56: 01$ & 0.4433 \\
\hline 4141 & $10: 56: 02$ & 0.4437 \\
\hline 4142 & $10: 56: 03$ & 0.4425 \\
\hline 4143 & $10: 56: 04$ & 0.4405 \\
\hline 4144 & $10: 56: 05$ & 0.4441 \\
\hline 4145 & $10: 56: 06$ & 0.4402 \\
\hline 4146 & $10: 56: 07$ & 0.4449 \\
\hline 4147 & 10:56:08 & 0.4416 \\
\hline 4148 & $10: 56: 09$ & 0.4403 \\
\hline 4149 & $10: 56: 10$ & 0.4404 \\
\hline 4150 & $10: 56: 11$ & 0.4409 \\
\hline 4151 & $10: 56: 12$ & 0.4421 \\
\hline 4152 & $10: 56: 13$ & 0.4416 \\
\hline 4153 & $10: 56: 14$ & 0.4451 \\
\hline 4154 & $10: 56: 15$ & 0.4456 \\
\hline 4155 & $10: 56: 16$ & 0.4474 \\
\hline 4156 & $10: 56: 17$ & 0.4428 \\
\hline 4157 & $10: 56: 18$ & 0.4454 \\
\hline 4158 & $10: 56: 19$ & 0.4450 \\
\hline 4159 & $10: 56: 20$ & 0.4439 \\
\hline 4160 & $10: 56: 21$ & 0.4451 \\
\hline 4161 & $10: 56: 22$ & 0.4417 \\
\hline 4162 & $10: 56: 23$ & 0.4447 \\
\hline 4163 & $10: 56: 24$ & 0.4431 \\
\hline 4164 & $10: 56: 25$ & 0.4438 \\
\hline 4165 & $10: 56: 26$ & 0.4433 \\
\hline 4166 & $10: 56: 27$ & 0.4410 \\
\hline 4167 & $10: 56: 28$ & 0.4431 \\
\hline 4168 & $10: 56: 29$ & 0.4413 \\
\hline 4169 & $10: 56: 30$ & 0.4438 \\
\hline 4170 & $10: 56: 31$ & 0.4394 \\
\hline 4171 & $10: 56: 32$ & 0.4410 \\
\hline 4172 & $10: 56: 33$ & 0.4400 \\
\hline 4173 & 10:56:34 & 0.4427 \\
\hline 4174 & $10: 56: 35$ & 0.4454 \\
\hline 4175 & $10: 56: 36$ & 0.4426 \\
\hline 4176 & $10: 56: 37$ & 0.4461 \\
\hline 4177 & $10: 56: 38$ & 0.4428 \\
\hline 4178 & $10: 56: 39$ & 0.4454 \\
\hline 4179 & $10: 56: 40$ & 0.4455 \\
\hline 4180 & $10: 56: 41$ & 0.4421 \\
\hline
\end{tabular}

C - 82 
Appendix C - Fuel Data Test 2 NIST102605_1.xls

\begin{tabular}{|c|c|c|}
\hline 4181 & $10: 56: 42$ & 0.4452 \\
\hline 4182 & $10: 56: 43$ & 0.4424 \\
\hline 4183 & $10: 56: 44$ & 0.4440 \\
\hline 4184 & $10: 56: 45$ & 0.4416 \\
\hline 4185 & $10: 56: 46$ & 0.4391 \\
\hline 4186 & $10: 56: 47$ & 0.4419 \\
\hline 4187 & $10: 56: 48$ & 0.4406 \\
\hline 4188 & $10: 56: 49$ & 0.4427 \\
\hline 4189 & $10: 56: 50$ & 0.4395 \\
\hline 4190 & $10: 56: 51$ & 0.4427 \\
\hline 4191 & $10: 56: 52$ & 0.4405 \\
\hline 4192 & $10: 56: 53$ & 0.4398 \\
\hline 4193 & $10: 56: 54$ & 0.4429 \\
\hline 4194 & $10: 56: 55$ & 0.4418 \\
\hline 4195 & $10: 56: 56$ & 0.4452 \\
\hline 4196 & $10: 56: 57$ & 0.4408 \\
\hline 4197 & $10: 56: 58$ & 0.4430 \\
\hline 4198 & $10: 56: 59$ & 0.4438 \\
\hline 4199 & $10: 57: 00$ & 0.4450 \\
\hline 4200 & $10: 57: 01$ & 0.4464 \\
\hline 4201 & $10: 57: 02$ & 0.4414 \\
\hline 4202 & $10: 57: 03$ & 0.4443 \\
\hline 4203 & 10:57:04 & 0.4442 \\
\hline 4204 & $10: 57: 05$ & 0.4406 \\
\hline 4205 & $10: 57: 06$ & 0.4447 \\
\hline 4206 & $10: 57: 07$ & 0.4429 \\
\hline 4207 & $10: 57: 08$ & 0.4436 \\
\hline 4208 & $10: 57: 09$ & 0.4430 \\
\hline 4209 & $10: 57: 10$ & 0.4405 \\
\hline 4210 & $10: 57: 11$ & 0.4428 \\
\hline 4211 & $10: 57: 12$ & 0.4399 \\
\hline 4212 & $10: 57: 13$ & 0.4419 \\
\hline 4213 & $10: 57: 14$ & 0.4396 \\
\hline 4214 & $10: 57: 15$ & 0.4384 \\
\hline 4215 & $10: 57: 16$ & 0.4446 \\
\hline 4216 & $10: 57: 17$ & 0.4423 \\
\hline 4217 & $10: 57: 18$ & 0.4456 \\
\hline 4218 & $10: 57: 19$ & 0.4452 \\
\hline 4219 & $10: 57: 20$ & 0.4448 \\
\hline 4220 & $10: 57: 21$ & 0.4464 \\
\hline 4221 & $10: 57: 22$ & 0.4420 \\
\hline 4222 & $10: 57: 23$ & 0.4459 \\
\hline 4223 & $10: 57: 24$ & 0.4452 \\
\hline 4224 & $10: 57: 25$ & 0.4443 \\
\hline 4225 & $10: 57: 26$ & 0.4440 \\
\hline 4226 & $10: 57: 27$ & 0.4415 \\
\hline 4227 & $10: 57: 28$ & 0.4422 \\
\hline 4228 & $10: 57: 29$ & 0.4384 \\
\hline 4229 & $10: 57: 30$ & 0.4443 \\
\hline 4230 & $10: 57: 31$ & 0.4420 \\
\hline 4231 & $10: 57: 32$ & 0.4439 \\
\hline
\end{tabular}

C - 83 
Appendix C - Fuel Data Test 2 NIST102605_1.xIs

\begin{tabular}{|c|c|c|}
\hline 4232 & $10: 57: 33$ & 0.4403 \\
\hline 4233 & $10: 57: 34$ & 0.4394 \\
\hline 4234 & $10: 57: 35$ & 0.4418 \\
\hline 4235 & $10: 57: 36$ & 0.4409 \\
\hline 4236 & $10: 57: 37$ & 0.4426 \\
\hline 4237 & $10: 57: 38$ & 0.4414 \\
\hline 4238 & $10: 57: 39$ & 0.4453 \\
\hline 4239 & $10: 57: 40$ & 0.4430 \\
\hline 4240 & $10: 57: 41$ & 0.4452 \\
\hline 4241 & $10: 57: 42$ & 0.4445 \\
\hline 4242 & $10: 57: 43$ & 0.4431 \\
\hline 4243 & $10: 57: 44$ & 0.4441 \\
\hline 4244 & $10: 57: 45$ & 0.4411 \\
\hline 4245 & $10: 57: 46$ & 0.4454 \\
\hline 4246 & $10: 57: 47$ & 0.4434 \\
\hline 4247 & $10: 57: 48$ & 0.4434 \\
\hline 4248 & $10: 57: 49$ & 0.4437 \\
\hline 4249 & $10: 57: 50$ & 0.4409 \\
\hline 4250 & $10: 57: 51$ & 0.4402 \\
\hline 4251 & $10: 57: 52$ & 0.4390 \\
\hline 4252 & $10: 57: 53$ & 0.4440 \\
\hline 4253 & $10: 57: 54$ & 0.4421 \\
\hline 4254 & $10: 57: 55$ & 0.4424 \\
\hline 4255 & $10: 57: 56$ & 0.4381 \\
\hline 4256 & $10: 57: 57$ & 0.4404 \\
\hline 4257 & $10: 57: 58$ & 0.4397 \\
\hline 4258 & $10: 57: 59$ & 0.4419 \\
\hline 4259 & $10: 58: 00$ & 0.4432 \\
\hline 4260 & $10: 58: 01$ & 0.4452 \\
\hline 4261 & 10:58:02 & 0.4463 \\
\hline 4262 & $10: 58: 03$ & 0.4461 \\
\hline 4263 & $10: 58: 04$ & 0.4476 \\
\hline 4264 & 10:58:05 & 0.4458 \\
\hline 4265 & 10:58:06 & 0.4453 \\
\hline 4266 & $10: 58: 07$ & 0.4429 \\
\hline 4267 & $10: 58: 08$ & 0.4444 \\
\hline 4268 & 10:58:09 & 0.4411 \\
\hline 4269 & $10: 58: 10$ & 0.4412 \\
\hline 4270 & 10:58:11 & 0.4379 \\
\hline 4271 & 10:58:12 & 0.4434 \\
\hline 4272 & $10: 58: 13$ & 0.4417 \\
\hline 4273 & 10:58:14 & 0.4408 \\
\hline 4274 & $10: 58: 15$ & 0.4438 \\
\hline 4275 & $10: 58: 16$ & 0.4408 \\
\hline 4276 & $10: 58: 17$ & 0.4418 \\
\hline 4277 & 10:58:18 & 0.4385 \\
\hline 4278 & $10: 58: 19$ & 0.4397 \\
\hline 4279 & 10:58:20 & 0.4438 \\
\hline 4280 & $10: 58: 21$ & 0.4435 \\
\hline 4281 & 10:58:22 & 0.4461 \\
\hline 4282 & $10: 58: 23$ & 0.4420 \\
\hline
\end{tabular}


Appendix C - Fuel Data Test 2 NIST102605_1.xIs

\begin{tabular}{|c|c|c|}
\hline 4283 & $10: 58: 24$ & 0.4439 \\
\hline 4284 & $10: 58: 25$ & 0.4426 \\
\hline 4285 & $10: 58: 26$ & 0.4451 \\
\hline 4286 & $10: 58: 27$ & 0.4456 \\
\hline 4287 & $10: 58: 28$ & 0.4424 \\
\hline 4288 & 10:58:29 & 0.4432 \\
\hline 4289 & 10:58:30 & 0.4410 \\
\hline 4290 & 10:58:31 & 0.4442 \\
\hline 4291 & $10: 58: 32$ & 0.4418 \\
\hline 4292 & $10: 58: 33$ & 0.4437 \\
\hline 4293 & $10: 58: 34$ & 0.4411 \\
\hline 4294 & 10:58:35 & 0.4436 \\
\hline 4295 & $10: 58: 36$ & 0.4407 \\
\hline 4296 & $10: 58: 37$ & 0.4412 \\
\hline 4297 & 10:58:38 & 0.4409 \\
\hline 4298 & 10:58:39 & 0.4412 \\
\hline 4299 & $10: 58: 40$ & 0.4438 \\
\hline 4300 & $10: 58: 41$ & 0.4406 \\
\hline 4301 & $10: 58: 42$ & 0.4444 \\
\hline 4302 & $10: 58: 43$ & 0.4418 \\
\hline 4303 & $10: 58: 44$ & 0.4450 \\
\hline 4304 & $10: 58: 45$ & 0.4428 \\
\hline 4305 & $10: 58: 46$ & 0.4458 \\
\hline 4306 & $10: 58: 47$ & 0.4469 \\
\hline 4307 & $10: 58: 48$ & 0.4435 \\
\hline 4308 & $10: 58: 49$ & 0.4449 \\
\hline 4309 & $10: 58: 50$ & 0.4420 \\
\hline 4310 & $10: 58: 51$ & 0.4448 \\
\hline 4311 & $10: 58: 52$ & 0.4404 \\
\hline 4312 & $10: 58: 53$ & 0.4422 \\
\hline 4313 & $10: 58: 54$ & 0.4424 \\
\hline 4314 & $10: 58: 55$ & 0.4390 \\
\hline 4315 & 10:58:56 & 0.4429 \\
\hline 4316 & $10: 58: 57$ & 0.4412 \\
\hline 4317 & $10: 58: 58$ & 0.4436 \\
\hline 4318 & $10: 58: 59$ & 0.4402 \\
\hline 4319 & 10:59:00 & 0.4423 \\
\hline 4320 & 10:59:01 & 0.4395 \\
\hline 4321 & 10:59:02 & 0.4411 \\
\hline 4322 & 10:59:03 & 0.4423 \\
\hline 4323 & 10:59:04 & 0.4425 \\
\hline 4324 & 10:59:05 & 0.4457 \\
\hline 4325 & 10:59:06 & 0.4414 \\
\hline 4326 & $10: 59: 07$ & 0.4449 \\
\hline 4327 & 10:59:08 & 0.4448 \\
\hline 4328 & $10: 59: 09$ & 0.4453 \\
\hline 4329 & $10: 59: 10$ & 0.4453 \\
\hline 4330 & 10:59:11 & 0.4412 \\
\hline 4331 & $10: 59: 12$ & 0.4445 \\
\hline 4332 & $10: 59: 13$ & 0.4425 \\
\hline 4333 & $10: 59: 14$ & 0.4447 \\
\hline
\end{tabular}


Appendix C - Fuel Data Test 2 NIST102605_1.xIs

\begin{tabular}{|c|c|c|}
\hline 4334 & $10: 59: 15$ & 0.4426 \\
\hline 4335 & $10: 59: 16$ & 0.4414 \\
\hline 4336 & $10: 59: 17$ & 0.4414 \\
\hline 4337 & 10:59:18 & 0.4395 \\
\hline 4338 & $10: 59: 19$ & 0.4444 \\
\hline 4339 & 10:59:20 & 0.4405 \\
\hline 4340 & 10:59:21 & 0.4425 \\
\hline 4341 & 10:59:22 & 0.4400 \\
\hline 4342 & $10: 59: 23$ & 0.4418 \\
\hline 4343 & $10: 59: 24$ & 0.4443 \\
\hline 4344 & $10: 59: 25$ & 0.4421 \\
\hline 4345 & 10:59:26 & 0.4467 \\
\hline 4346 & $10: 59: 27$ & 0.4437 \\
\hline 4347 & 10:59:28 & 0.4445 \\
\hline 4348 & 10:59:29 & 0.4434 \\
\hline 4349 & 10:59:30 & 0.4441 \\
\hline 4350 & $10: 59: 31$ & 0.4452 \\
\hline 4351 & $10: 59: 32$ & 0.4408 \\
\hline 4352 & $10: 59: 33$ & 0.4453 \\
\hline 4353 & $10: 59: 34$ & 0.4430 \\
\hline 4354 & $10: 59: 35$ & 0.4422 \\
\hline 4355 & $10: 59: 36$ & 0.4410 \\
\hline 4356 & $10: 59: 37$ & 0.4400 \\
\hline 4357 & 10:59:38 & 0.4452 \\
\hline 4358 & 10:59:39 & 0.4406 \\
\hline 4359 & $10: 59: 40$ & 0.4440 \\
\hline 4360 & $10: 59: 41$ & 0.4407 \\
\hline 4361 & $10: 59: 42$ & 0.4403 \\
\hline 4362 & $10: 59: 43$ & 0.4385 \\
\hline 4363 & $10: 59: 44$ & 0.4399 \\
\hline 4364 & $10: 59: 45$ & 0.4442 \\
\hline 4365 & $10: 59: 46$ & 0.4423 \\
\hline 4366 & $10: 59: 47$ & 0.4439 \\
\hline 4367 & $10: 59: 48$ & 0.4423 \\
\hline 4368 & $10: 59: 49$ & 0.4464 \\
\hline 4369 & $10: 59: 50$ & 0.4430 \\
\hline 4370 & $10: 59: 51$ & 0.4448 \\
\hline 4371 & $10: 59: 52$ & 0.4438 \\
\hline 4372 & $10: 59: 53$ & 0.4424 \\
\hline 4373 & $10: 59: 54$ & 0.4427 \\
\hline 4374 & $10: 59: 55$ & 0.4395 \\
\hline 4375 & $10: 59: 56$ & 0.4426 \\
\hline 4376 & $10: 59: 57$ & 0.4402 \\
\hline 4377 & 10:59:58 & 0.4431 \\
\hline 4378 & $10: 59: 59$ & 0.4430 \\
\hline 4379 & 11:00:00 & 0.4442 \\
\hline 4380 & $11: 00: 01$ & 0.4411 \\
\hline 4381 & 11:00:02 & 0.4398 \\
\hline 4382 & $11: 00: 03$ & 0.4422 \\
\hline 4383 & $11: 00: 04$ & 0.4384 \\
\hline 4384 & $11: 00: 05$ & 0.4410 \\
\hline
\end{tabular}


Appendix C - Fuel Data Test 2 NIST102605_1.xIs

\begin{tabular}{|c|c|c|}
\hline 4385 & $11: 00: 06$ & 0.4410 \\
\hline 4386 & $11: 00: 07$ & 0.4421 \\
\hline 4387 & $11: 00: 08$ & 0.4458 \\
\hline 4388 & 11:00:09 & 0.4430 \\
\hline 4389 & $11: 00: 10$ & 0.4452 \\
\hline 4390 & $11: 00: 11$ & 0.4421 \\
\hline 4391 & $11: 00: 12$ & 0.4446 \\
\hline 4392 & $11: 00: 13$ & 0.4432 \\
\hline 4393 & $11: 00: 14$ & 0.4441 \\
\hline 4394 & $11: 00: 15$ & 0.4450 \\
\hline 4395 & $11: 00: 16$ & 0.4405 \\
\hline 4396 & $11: 00: 17$ & 0.4428 \\
\hline 4397 & $11: 00: 18$ & 0.4425 \\
\hline 4398 & $11: 00: 19$ & 0.4395 \\
\hline 4399 & $11: 00: 20$ & 0.4413 \\
\hline 4400 & $11: 00: 21$ & 0.4403 \\
\hline 4401 & 11:00:22 & 0.4444 \\
\hline 4402 & $11: 00: 23$ & 0.4390 \\
\hline 4403 & $11: 00: 24$ & 0.4390 \\
\hline 4404 & $11: 00: 25$ & 0.4395 \\
\hline 4405 & $11: 00: 26$ & 0.4393 \\
\hline 4406 & $11: 00: 27$ & 0.4438 \\
\hline 4407 & $11: 00: 28$ & 0.4424 \\
\hline 4408 & $11: 00: 29$ & 0.4450 \\
\hline 4409 & $11: 00: 30$ & 0.4415 \\
\hline 4410 & $11: 00: 31$ & 0.4440 \\
\hline 4411 & 11:00:32 & 0.4431 \\
\hline 4412 & $11: 00: 33$ & 0.4459 \\
\hline 4413 & $11: 00: 34$ & 0.4436 \\
\hline 4414 & $11: 00: 35$ & 0.4427 \\
\hline 4415 & 11:00:36 & 0.4452 \\
\hline 4416 & $11: 00: 37$ & 0.4403 \\
\hline 4417 & $11: 00: 38$ & 0.4441 \\
\hline 4418 & $11: 00: 39$ & 0.4438 \\
\hline 4419 & $11: 00: 40$ & 0.4442 \\
\hline 4420 & $11: 00: 41$ & 0.4410 \\
\hline 4421 & $11: 00: 42$ & 0.4391 \\
\hline 4422 & $11: 00: 43$ & 0.4419 \\
\hline 4423 & $11: 00: 44$ & 0.4416 \\
\hline 4424 & $11: 00: 45$ & 0.4432 \\
\hline 4425 & $11: 00: 46$ & 0.4387 \\
\hline 4426 & $11: 00: 47$ & 0.4425 \\
\hline 4427 & $11: 00: 48$ & 0.4411 \\
\hline 4428 & $11: 00: 49$ & 0.4419 \\
\hline 4429 & $11: 00: 50$ & 0.4468 \\
\hline 4430 & $11: 00: 51$ & 0.4451 \\
\hline 4431 & $11: 00: 52$ & 0.4463 \\
\hline 4432 & $11: 00: 53$ & 0.4424 \\
\hline 4433 & $11: 00: 54$ & 0.4453 \\
\hline 4434 & $11: 00: 55$ & 0.4427 \\
\hline 4435 & $11: 00: 56$ & 0.4427 \\
\hline
\end{tabular}

C - 87 
Appendix C - Fuel Data Test 2 NIST102605_1.xIs

\begin{tabular}{|c|c|c|}
\hline 4436 & $11: 00: 57$ & 0.4449 \\
\hline 4437 & $11: 00: 58$ & 0.4421 \\
\hline 4438 & $11: 00: 59$ & 0.4433 \\
\hline 4439 & 11:01:00 & 0.4389 \\
\hline 4440 & $11: 01: 01$ & 0.4420 \\
\hline 4441 & 11:01:02 & 0.4413 \\
\hline 4442 & 11:01:03 & 0.4393 \\
\hline 4443 & $11: 01: 04$ & 0.4414 \\
\hline 4444 & 11:01:05 & 0.4403 \\
\hline 4445 & $11: 01: 06$ & 0.4434 \\
\hline 4446 & $11: 01: 07$ & 0.4397 \\
\hline 4447 & 11:01:08 & 0.4413 \\
\hline 4448 & 11:01:09 & 0.4392 \\
\hline 4449 & $11: 01: 10$ & 0.4427 \\
\hline 4450 & 11:01:11 & 0.4440 \\
\hline 4451 & 11:01:12 & 0.4434 \\
\hline 4452 & $11: 01: 13$ & 0.4460 \\
\hline 4453 & $11: 01: 14$ & 0.4411 \\
\hline 4454 & $11: 01: 15$ & 0.4440 \\
\hline 4455 & $11: 01: 16$ & 0.4462 \\
\hline 4456 & $11: 01: 17$ & 0.4433 \\
\hline 4457 & $11: 01: 18$ & 0.4444 \\
\hline 4458 & $11: 01: 19$ & 0.4415 \\
\hline 4459 & 11:01:20 & 0.4436 \\
\hline 4460 & $11: 01: 21$ & 0.4418 \\
\hline 4461 & $11: 01: 22$ & 0.4403 \\
\hline 4462 & $11: 01: 23$ & 0.4425 \\
\hline 4463 & $11: 01: 24$ & 0.4417 \\
\hline 4464 & $11: 01: 25$ & 0.4442 \\
\hline 4465 & $11: 01: 26$ & 0.4395 \\
\hline 4466 & $11: 01: 27$ & 0.4429 \\
\hline 4467 & $11: 01: 28$ & 0.4400 \\
\hline 4468 & 11:01:29 & 0.4416 \\
\hline 4469 & 11:01:30 & 0.4398 \\
\hline 4470 & $11: 01: 31$ & 0.4424 \\
\hline 4471 & $11: 01: 32$ & 0.4458 \\
\hline 4472 & $11: 01: 33$ & 0.4425 \\
\hline 4473 & $11: 01: 34$ & 0.4467 \\
\hline 4474 & $11: 01: 35$ & 0.4425 \\
\hline 4475 & $11: 01: 36$ & 0.4431 \\
\hline 4476 & $11: 01: 37$ & 0.4409 \\
\hline 4477 & 11:01:38 & 0.4431 \\
\hline 4478 & 11:01:39 & 0.4444 \\
\hline 4479 & $11: 01: 40$ & 0.4418 \\
\hline 4480 & $11: 01: 41$ & 0.4428 \\
\hline 4481 & $11: 01: 42$ & 0.4397 \\
\hline 4482 & $11: 01: 43$ & 0.4442 \\
\hline 4483 & $11: 01: 44$ & 0.4409 \\
\hline 4484 & $11: 01: 45$ & 0.4409 \\
\hline 4485 & $11: 01: 46$ & 0.4426 \\
\hline 4486 & $11: 01: 47$ & 0.4388 \\
\hline
\end{tabular}

C - 88 
Appendix C - Fuel Data Test 2 NIST102605_1.xIs

\begin{tabular}{|c|c|c|}
\hline 4487 & $11: 01: 48$ & 0.4430 \\
\hline 4488 & $11: 01: 49$ & 0.4394 \\
\hline 4489 & 11:01:50 & 0.4400 \\
\hline 4490 & $11: 01: 51$ & 0.4378 \\
\hline 4491 & $11: 01: 52$ & 0.4414 \\
\hline 4492 & 11:01:53 & 0.4459 \\
\hline 4493 & $11: 01: 54$ & 0.4438 \\
\hline 4494 & 11:01:55 & 0.4437 \\
\hline 4495 & $11: 01: 56$ & 0.4424 \\
\hline 4496 & $11: 01: 57$ & 0.4453 \\
\hline 4497 & 11:01:58 & 0.4404 \\
\hline 4498 & $11: 01: 59$ & 0.4436 \\
\hline 4499 & $11: 02: 00$ & 0.4418 \\
\hline 4500 & 11:02:01 & 0.4443 \\
\hline 4501 & 11:02:02 & 0.4436 \\
\hline 4502 & $11: 02: 03$ & 0.4425 \\
\hline 4503 & $11: 02: 04$ & 0.4430 \\
\hline 4504 & $11: 02: 05$ & 0.4408 \\
\hline 4505 & $11: 02: 06$ & 0.4434 \\
\hline 4506 & $11: 02: 07$ & 0.4401 \\
\hline 4507 & $11: 02: 08$ & 0.4407 \\
\hline 4508 & $11: 02: 09$ & 0.4389 \\
\hline 4509 & $11: 02: 10$ & 0.4397 \\
\hline 4510 & 11:02:11 & 0.4413 \\
\hline 4511 & $11: 02: 12$ & 0.4405 \\
\hline 4512 & $11: 02: 13$ & 0.4445 \\
\hline 4513 & $11: 02: 14$ & 0.4425 \\
\hline 4514 & $11: 02: 15$ & 0.4458 \\
\hline 4515 & $11: 02: 16$ & 0.4433 \\
\hline 4516 & $11: 02: 17$ & 0.4441 \\
\hline 4517 & $11: 02: 18$ & 0.4461 \\
\hline 4518 & $11: 02: 19$ & 0.4416 \\
\hline 4519 & $11: 02: 20$ & 0.4442 \\
\hline 4520 & $11: 02: 21$ & 0.4455 \\
\hline 4521 & 11:02:22 & 0.4421 \\
\hline 4522 & $11: 02: 23$ & 0.4446 \\
\hline 4523 & $11: 02: 24$ & 0.4411 \\
\hline 4524 & $11: 02: 25$ & 0.4440 \\
\hline 4525 & $11: 02: 26$ & 0.4419 \\
\hline 4526 & $11: 02: 27$ & 0.4404 \\
\hline 4527 & 11:02:28 & 0.4428 \\
\hline 4528 & $11: 02: 29$ & 0.4405 \\
\hline 4529 & $11: 02: 30$ & 0.4424 \\
\hline 4530 & $11: 02: 31$ & 0.4400 \\
\hline 4531 & $11: 02: 32$ & 0.4400 \\
\hline 4532 & $11: 02: 33$ & 0.4438 \\
\hline 4533 & $11: 02: 34$ & 0.4400 \\
\hline 4534 & $11: 02: 35$ & 0.4418 \\
\hline 4535 & 11:02:36 & 0.4425 \\
\hline 4536 & $11: 02: 37$ & 0.4450 \\
\hline 4537 & $11: 02: 38$ & 0.4456 \\
\hline
\end{tabular}

C - 89 
Appendix C - Fuel Data Test 2 NIST102605_1.xIs

\begin{tabular}{|c|c|c|}
\hline 4538 & $11: 02: 39$ & 0.4415 \\
\hline 4539 & $11: 02: 40$ & 0.4437 \\
\hline 4540 & $11: 02: 41$ & 0.4403 \\
\hline 4541 & $11: 02: 42$ & 0.4444 \\
\hline 4542 & $11: 02: 43$ & 0.4435 \\
\hline 4543 & $11: 02: 44$ & 0.4446 \\
\hline 4544 & $11: 02: 45$ & 0.4428 \\
\hline 4545 & $11: 02: 46$ & 0.4419 \\
\hline 4546 & $11: 02: 47$ & 0.4437 \\
\hline 4547 & $11: 02: 48$ & 0.4401 \\
\hline 4548 & $11: 02: 49$ & 0.4434 \\
\hline 4549 & $11: 02: 50$ & 0.4412 \\
\hline 4550 & $11: 02: 51$ & 0.4403 \\
\hline 4551 & $11: 02: 52$ & 0.4387 \\
\hline 4552 & $11: 02: 53$ & 0.4387 \\
\hline 4553 & $11: 02: 54$ & 0.4429 \\
\hline 4554 & $11: 02: 55$ & 0.4411 \\
\hline 4555 & $11: 02: 56$ & 0.4431 \\
\hline 4556 & $11: 02: 57$ & 0.4408 \\
\hline 4557 & $11: 02: 58$ & 0.4447 \\
\hline 4558 & $11: 02: 59$ & 0.4472 \\
\hline 4559 & 11:03:00 & 0.4450 \\
\hline 4560 & 11:03:01 & 0.4459 \\
\hline 4561 & 11:03:02 & 0.4412 \\
\hline 4562 & 11:03:03 & 0.4439 \\
\hline 4563 & $11: 03: 04$ & 0.4448 \\
\hline 4564 & $11: 03: 05$ & 0.4411 \\
\hline 4565 & 11:03:06 & 0.4446 \\
\hline 4566 & $11: 03: 07$ & 0.4423 \\
\hline 4567 & $11: 03: 08$ & 0.4446 \\
\hline 4568 & 11:03:09 & 0.4433 \\
\hline 4569 & $11: 03: 10$ & 0.4384 \\
\hline 4570 & 11:03:11 & 0.4425 \\
\hline 4571 & 11:03:12 & 0.4402 \\
\hline 4572 & $11: 03: 13$ & 0.4413 \\
\hline 4573 & $11: 03: 14$ & 0.4388 \\
\hline 4574 & $11: 03: 15$ & 0.4406 \\
\hline 4575 & $11: 03: 16$ & 0.4394 \\
\hline 4576 & $11: 03: 17$ & 0.4420 \\
\hline 4577 & $11: 03: 18$ & 0.4441 \\
\hline 4578 & $11: 03: 19$ & 0.4426 \\
\hline 4579 & 11:03:20 & 0.4468 \\
\hline 4580 & $11: 03: 21$ & 0.4415 \\
\hline 4581 & $11: 03: 22$ & 0.4448 \\
\hline 4582 & $11: 03: 23$ & 0.4455 \\
\hline 4583 & $11: 03: 24$ & 0.4409 \\
\hline 4584 & $11: 03: 25$ & 0.4440 \\
\hline 4585 & 11:03:26 & 0.4426 \\
\hline 4586 & $11: 03: 27$ & 0.4452 \\
\hline 4587 & $11: 03: 28$ & 0.4431 \\
\hline 4588 & $11: 03: 29$ & 0.4415 \\
\hline
\end{tabular}


Appendix C - Fuel Data Test 2 NIST102605_1.xIs

\begin{tabular}{|c|c|c|}
\hline 4589 & $11: 03: 30$ & 0.4416 \\
\hline 4590 & $11: 03: 31$ & 0.4402 \\
\hline 4591 & 11:03:32 & 0.4431 \\
\hline 4592 & $11: 03: 33$ & 0.4416 \\
\hline 4593 & $11: 03: 34$ & 0.4430 \\
\hline 4594 & $11: 03: 35$ & 0.4390 \\
\hline 4595 & 11:03:36 & 0.4414 \\
\hline 4596 & $11: 03: 37$ & 0.4399 \\
\hline 4597 & $11: 03: 38$ & 0.4426 \\
\hline 4598 & 11:03:39 & 0.4455 \\
\hline 4599 & $11: 03: 40$ & 0.4415 \\
\hline 4600 & 11:03:41 & 0.4448 \\
\hline 4601 & $11: 03: 42$ & 0.4427 \\
\hline 4602 & $11: 03: 43$ & 0.4463 \\
\hline 4603 & $11: 03: 44$ & 0.4452 \\
\hline 4604 & 11:03:45 & 0.4436 \\
\hline 4605 & $11: 03: 46$ & 0.4442 \\
\hline 4606 & $11: 03: 47$ & 0.4401 \\
\hline 4607 & $11: 03: 48$ & 0.4445 \\
\hline 4608 & $11: 03: 49$ & 0.4422 \\
\hline 4609 & $11: 03: 50$ & 0.4429 \\
\hline 4610 & $11: 03: 51$ & 0.4421 \\
\hline 4611 & $11: 03: 52$ & 0.4400 \\
\hline 4612 & $11: 03: 53$ & 0.4413 \\
\hline 4613 & $11: 03: 54$ & 0.4391 \\
\hline 4614 & $11: 03: 55$ & 0.4434 \\
\hline 4615 & $11: 03: 56$ & 0.4403 \\
\hline 4616 & $11: 03: 57$ & 0.4413 \\
\hline 4617 & $11: 03: 58$ & 0.4396 \\
\hline 4618 & $11: 03: 59$ & 0.4402 \\
\hline 4619 & $11: 04: 00$ & 0.4425 \\
\hline 4620 & $11: 04: 01$ & 0.4436 \\
\hline 4621 & 11:04:02 & 0.4455 \\
\hline 4622 & 11:04:03 & 0.4417 \\
\hline 4623 & $11: 04: 04$ & 0.4442 \\
\hline 4624 & $11: 04: 05$ & 0.4417 \\
\hline 4625 & $11: 04: 06$ & 0.4450 \\
\hline 4626 & $11: 04: 07$ & 0.4446 \\
\hline 4627 & $11: 04: 08$ & 0.4439 \\
\hline 4628 & 11:04:09 & 0.4438 \\
\hline 4629 & $11: 04: 10$ & 0.4404 \\
\hline 4630 & 11:04:11 & 0.4436 \\
\hline 4631 & $11: 04: 12$ & 0.4414 \\
\hline 4632 & $11: 04: 13$ & 0.4438 \\
\hline 4633 & $11: 04: 14$ & 0.4420 \\
\hline 4634 & $11: 04: 15$ & 0.4439 \\
\hline 4635 & $11: 04: 16$ & 0.4386 \\
\hline 4636 & $11: 04: 17$ & 0.4379 \\
\hline 4637 & $11: 04: 18$ & 0.4391 \\
\hline 4638 & $11: 04: 19$ & 0.4402 \\
\hline 4639 & $11: 04: 20$ & 0.4447 \\
\hline
\end{tabular}


Appendix C - Fuel Data Test 2 NIST102605_1.xIs

\begin{tabular}{|c|c|c|}
\hline 4640 & $11: 04: 21$ & 0.4404 \\
\hline 4641 & $11: 04: 22$ & 0.4435 \\
\hline 4642 & $11: 04: 23$ & 0.4425 \\
\hline 4643 & $11: 04: 24$ & 0.4445 \\
\hline 4644 & $11: 04: 25$ & 0.4467 \\
\hline 4645 & $11: 04: 26$ & 0.4447 \\
\hline 4646 & $11: 04: 27$ & 0.4450 \\
\hline 4647 & $11: 04: 28$ & 0.4406 \\
\hline 4648 & $11: 04: 29$ & 0.4451 \\
\hline 4649 & $11: 04: 30$ & 0.4442 \\
\hline 4650 & $11: 04: 31$ & 0.4444 \\
\hline 4651 & 11:04:32 & 0.4429 \\
\hline 4652 & $11: 04: 33$ & 0.4400 \\
\hline 4653 & $11: 04: 34$ & 0.4431 \\
\hline 4654 & 11:04:35 & 0.4395 \\
\hline 4655 & 11:04:36 & 0.4430 \\
\hline 4656 & $11: 04: 37$ & 0.4411 \\
\hline 4657 & $11: 04: 38$ & 0.4418 \\
\hline 4658 & $11: 04: 39$ & 0.4383 \\
\hline 4659 & $11: 04: 40$ & 0.4388 \\
\hline 4660 & $11: 04: 41$ & 0.4415 \\
\hline 4661 & $11: 04: 42$ & 0.4434 \\
\hline 4662 & $11: 04: 43$ & 0.4437 \\
\hline 4663 & $11: 04: 44$ & 0.4430 \\
\hline 4664 & $11: 04: 45$ & 0.4458 \\
\hline 4665 & $11: 04: 46$ & 0.4409 \\
\hline 4666 & $11: 04: 47$ & 0.4452 \\
\hline 4667 & $11: 04: 48$ & 0.4454 \\
\hline 4668 & $11: 04: 49$ & 0.4447 \\
\hline 4669 & $11: 04: 50$ & 0.4453 \\
\hline 4670 & $11: 04: 51$ & 0.4408 \\
\hline 4671 & $11: 04: 52$ & 0.4445 \\
\hline 4672 & $11: 04: 53$ & 0.4427 \\
\hline 4673 & $11: 04: 54$ & 0.4436 \\
\hline 4674 & $11: 04: 55$ & 0.4437 \\
\hline 4675 & $11: 04: 56$ & 0.4404 \\
\hline 4676 & $11: 04: 57$ & 0.4410 \\
\hline 4677 & $11: 04: 58$ & 0.4381 \\
\hline 4678 & $11: 04: 59$ & 0.4430 \\
\hline 4679 & $11: 05: 00$ & 0.4391 \\
\hline 4680 & $11: 05: 01$ & 0.4397 \\
\hline 4681 & 11:05:02 & 0.4430 \\
\hline 4682 & $11: 05: 03$ & 0.4413 \\
\hline 4683 & $11: 05: 04$ & 0.4446 \\
\hline 4684 & $11: 05: 05$ & 0.4418 \\
\hline 4685 & 11:05:06 & 0.4458 \\
\hline 4686 & $11: 05: 07$ & 0.4436 \\
\hline 4687 & $11: 05: 08$ & 0.4449 \\
\hline 4688 & 11:05:09 & 0.4436 \\
\hline 4689 & $11: 05: 10$ & 0.4438 \\
\hline 4690 & $11: 05: 11$ & 0.4450 \\
\hline
\end{tabular}

C - 92 
Appendix C - Fuel Data Test 2 NIST102605_1.xIs

\begin{tabular}{|c|c|c|}
\hline 4691 & $11: 05: 12$ & 0.4427 \\
\hline 4692 & $11: 05: 13$ & 0.4429 \\
\hline 4693 & $11: 05: 14$ & 0.4397 \\
\hline 4694 & $11: 05: 15$ & 0.4429 \\
\hline 4695 & 11:05:16 & 0.4410 \\
\hline 4696 & $11: 05: 17$ & 0.4432 \\
\hline 4697 & 11:05:18 & 0.4420 \\
\hline 4698 & $11: 05: 19$ & 0.4395 \\
\hline 4699 & $11: 05: 20$ & 0.4408 \\
\hline 4700 & $11: 05: 21$ & 0.4395 \\
\hline 4701 & 11:05:22 & 0.4437 \\
\hline 4702 & $11: 05: 23$ & 0.4399 \\
\hline 4703 & $11: 05: 24$ & 0.4408 \\
\hline 4704 & 11:05:25 & 0.4412 \\
\hline 4705 & 11:05:26 & 0.4429 \\
\hline 4706 & $11: 05: 27$ & 0.4452 \\
\hline 4707 & $11: 05: 28$ & 0.4447 \\
\hline 4708 & $11: 05: 29$ & 0.4459 \\
\hline 4709 & $11: 05: 30$ & 0.4413 \\
\hline 4710 & $11: 05: 31$ & 0.4445 \\
\hline 4711 & 11:05:32 & 0.4432 \\
\hline 4712 & $11: 05: 33$ & 0.4435 \\
\hline 4713 & $11: 05: 34$ & 0.4430 \\
\hline 4714 & 11:05:35 & 0.4399 \\
\hline 4715 & $11: 05: 36$ & 0.4434 \\
\hline 4716 & $11: 05: 37$ & 0.4406 \\
\hline 4717 & $11: 05: 38$ & 0.4423 \\
\hline 4718 & $11: 05: 39$ & 0.4416 \\
\hline 4719 & $11: 05: 40$ & 0.4421 \\
\hline 4720 & $11: 05: 41$ & 0.4442 \\
\hline 4721 & $11: 05: 42$ & 0.4396 \\
\hline 4722 & $11: 05: 43$ & 0.4417 \\
\hline 4723 & $11: 05: 44$ & 0.4388 \\
\hline 4724 & $11: 05: 45$ & 0.4407 \\
\hline 4725 & $11: 05: 46$ & 0.4438 \\
\hline 4726 & $11: 05: 47$ & 0.4430 \\
\hline 4727 & $11: 05: 48$ & 0.4459 \\
\hline 4728 & $11: 05: 49$ & 0.4422 \\
\hline 4729 & $11: 05: 50$ & 0.4455 \\
\hline 4730 & $11: 05: 51$ & 0.4441 \\
\hline 4731 & $11: 05: 52$ & 0.4439 \\
\hline 4732 & $11: 05: 53$ & 0.4455 \\
\hline 4733 & $11: 05: 54$ & 0.4459 \\
\hline 4734 & $11: 05: 55$ & 0.4443 \\
\hline 4735 & $11: 05: 56$ & 0.4397 \\
\hline 4736 & $11: 05: 57$ & 0.4417 \\
\hline 4737 & $11: 05: 58$ & 0.4399 \\
\hline 4738 & $11: 05: 59$ & 0.4438 \\
\hline 4739 & 11:06:00 & 0.4396 \\
\hline 4740 & $11: 06: 01$ & 0.4441 \\
\hline 4741 & $11: 06: 02$ & 0.4412 \\
\hline
\end{tabular}

C - 93 
Appendix C - Fuel Data Test 2 NIST102605_1.xls

\begin{tabular}{|c|c|c|}
\hline 4742 & $11: 06: 03$ & 0.4426 \\
\hline 4743 & $11: 06: 04$ & 0.4392 \\
\hline 4744 & $11: 06: 05$ & 0.4392 \\
\hline 4745 & $11: 06: 06$ & 0.4397 \\
\hline 4746 & $11: 06: 07$ & 0.4410 \\
\hline 4747 & $11: 06: 08$ & 0.4439 \\
\hline 4748 & $11: 06: 09$ & 0.4450 \\
\hline 4749 & $11: 06: 10$ & 0.4460 \\
\hline 4750 & $11: 06: 11$ & 0.4429 \\
\hline 4751 & $11: 06: 12$ & 0.4455 \\
\hline 4752 & $11: 06: 13$ & 0.4428 \\
\hline 4753 & $11: 06: 14$ & 0.4436 \\
\hline 4754 & $11: 06: 15$ & 0.4446 \\
\hline 4755 & $11: 06: 16$ & 0.4412 \\
\hline 4756 & $11: 06: 17$ & 0.4442 \\
\hline 4757 & $11: 06: 18$ & 0.4426 \\
\hline 4758 & $11: 06: 19$ & 0.4392 \\
\hline 4759 & $11: 06: 20$ & 0.4427 \\
\hline 4760 & $11: 06: 21$ & 0.4402 \\
\hline 4761 & $11: 06: 22$ & 0.4420 \\
\hline 4762 & $11: 06: 23$ & 0.4409 \\
\hline 4763 & $11: 06: 24$ & 0.4399 \\
\hline 4764 & $11: 06: 25$ & 0.4384 \\
\hline 4765 & $11: 06: 26$ & 0.4389 \\
\hline 4766 & $11: 06: 27$ & 0.4433 \\
\hline 4767 & 11:06:28 & 0.4417 \\
\hline 4768 & $11: 06: 29$ & 0.4443 \\
\hline 4769 & $11: 06: 30$ & 0.4415 \\
\hline 4770 & $11: 06: 31$ & 0.4452 \\
\hline 4771 & $11: 06: 32$ & 0.4430 \\
\hline 4772 & $11: 06: 33$ & 0.4445 \\
\hline 4773 & $11: 06: 34$ & 0.4415 \\
\hline 4774 & $11: 06: 35$ & 0.4446 \\
\hline 4775 & $11: 06: 36$ & 0.4434 \\
\hline 4776 & $11: 06: 37$ & 0.4454 \\
\hline 4777 & $11: 06: 38$ & 0.4429 \\
\hline 4778 & $11: 06: 39$ & 0.4404 \\
\hline 4779 & $11: 06: 40$ & 0.4419 \\
\hline 4780 & $11: 06: 41$ & 0.4390 \\
\hline 4781 & $11: 06: 42$ & 0.4451 \\
\hline 4782 & $11: 06: 43$ & 0.4429 \\
\hline 4783 & $11: 06: 44$ & 0.4439 \\
\hline 4784 & $11: 06: 45$ & 0.4399 \\
\hline 4785 & $11: 06: 46$ & 0.4404 \\
\hline 4786 & $11: 06: 47$ & 0.4406 \\
\hline 4787 & $11: 06: 48$ & 0.4394 \\
\hline 4788 & $11: 06: 49$ & 0.4396 \\
\hline 4789 & $11: 06: 50$ & 0.4411 \\
\hline 4790 & $11: 06: 51$ & 0.4443 \\
\hline 4791 & $11: 06: 52$ & 0.4420 \\
\hline 4792 & $11: 06: 53$ & 0.4449 \\
\hline
\end{tabular}

C - 94 
Appendix C - Fuel Data Test 2 NIST102605_1.xIs

\begin{tabular}{|c|c|c|}
\hline 4793 & $11: 06: 54$ & 0.4416 \\
\hline 4794 & $11: 06: 55$ & 0.4450 \\
\hline 4795 & $11: 06: 56$ & 0.4454 \\
\hline 4796 & $11: 06: 57$ & 0.4438 \\
\hline 4797 & 11:06:58 & 0.4463 \\
\hline 4798 & $11: 06: 59$ & 0.4409 \\
\hline 4799 & 11:07:00 & 0.4432 \\
\hline 4800 & $11: 07: 01$ & 0.4413 \\
\hline 4801 & $11: 07: 02$ & 0.4433 \\
\hline 4802 & $11: 07: 03$ & 0.4403 \\
\hline 4803 & $11: 07: 04$ & 0.4401 \\
\hline 4804 & 11:07:05 & 0.4427 \\
\hline 4805 & $11: 07: 06$ & 0.4416 \\
\hline 4806 & $11: 07: 07$ & 0.4437 \\
\hline 4807 & 11:07:08 & 0.4387 \\
\hline 4808 & 11:07:09 & 0.4404 \\
\hline 4809 & $11: 07: 10$ & 0.4398 \\
\hline 4810 & $11: 07: 11$ & 0.4434 \\
\hline 4811 & $11: 07: 12$ & 0.4441 \\
\hline 4812 & $11: 07: 13$ & 0.4431 \\
\hline 4813 & $11: 07: 14$ & 0.4457 \\
\hline 4814 & $11: 07: 15$ & 0.4421 \\
\hline 4815 & $11: 07: 16$ & 0.4455 \\
\hline 4816 & $11: 07: 17$ & 0.4428 \\
\hline 4817 & 11:07:18 & 0.4442 \\
\hline 4818 & $11: 07: 19$ & 0.4421 \\
\hline 4819 & $11: 07: 20$ & 0.4448 \\
\hline 4820 & $11: 07: 21$ & 0.4440 \\
\hline 4821 & $11: 07: 22$ & 0.4438 \\
\hline 4822 & $11: 07: 23$ & 0.4392 \\
\hline 4823 & $11: 07: 24$ & 0.4429 \\
\hline 4824 & $11: 07: 25$ & 0.4426 \\
\hline 4825 & 11:07:26 & 0.4386 \\
\hline 4826 & $11: 07: 27$ & 0.4444 \\
\hline 4827 & $11: 07: 28$ & 0.4408 \\
\hline 4828 & $11: 07: 29$ & 0.4406 \\
\hline 4829 & $11: 07: 30$ & 0.4395 \\
\hline 4830 & $11: 07: 31$ & 0.4397 \\
\hline 4831 & $11: 07: 32$ & 0.4436 \\
\hline 4832 & $11: 07: 33$ & 0.4439 \\
\hline 4833 & $11: 07: 34$ & 0.4453 \\
\hline 4834 & 11:07:35 & 0.4425 \\
\hline 4835 & $11: 07: 36$ & 0.4452 \\
\hline 4836 & $11: 07: 37$ & 0.4426 \\
\hline 4837 & $11: 07: 38$ & 0.4442 \\
\hline 4838 & $11: 07: 39$ & 0.4458 \\
\hline 4839 & $11: 07: 40$ & 0.4444 \\
\hline 4840 & $11: 07: 41$ & 0.4426 \\
\hline 4841 & $11: 07: 42$ & 0.4402 \\
\hline 4842 & $11: 07: 43$ & 0.4435 \\
\hline 4843 & $11: 07: 44$ & 0.4405 \\
\hline
\end{tabular}


Appendix C - Fuel Data Test 2 NIST102605_1.xIs

\begin{tabular}{|c|c|c|}
\hline 4844 & $11: 07: 45$ & 0.4429 \\
\hline 4845 & $11: 07: 46$ & 0.4427 \\
\hline 4846 & $11: 07: 47$ & 0.4402 \\
\hline 4847 & $11: 07: 48$ & 0.4390 \\
\hline 4848 & $11: 07: 49$ & 0.4395 \\
\hline 4849 & $11: 07: 50$ & 0.4422 \\
\hline 4850 & $11: 07: 51$ & 0.4408 \\
\hline 4851 & $11: 07: 52$ & 0.4436 \\
\hline 4852 & $11: 07: 53$ & 0.4402 \\
\hline 4853 & $11: 07: 54$ & 0.4443 \\
\hline 4854 & $11: 07: 55$ & 0.4414 \\
\hline 4855 & $11: 07: 56$ & 0.4443 \\
\hline 4856 & $11: 07: 57$ & 0.4464 \\
\hline 4857 & $11: 07: 58$ & 0.4450 \\
\hline 4858 & $11: 07: 59$ & 0.4457 \\
\hline 4859 & $11: 08: 00$ & 0.4419 \\
\hline 4860 & 11:08:01 & 0.4445 \\
\hline 4861 & 11:08:02 & 0.4414 \\
\hline 4862 & $11: 08: 03$ & 0.4429 \\
\hline 4863 & $11: 08: 04$ & 0.4402 \\
\hline 4864 & $11: 08: 05$ & 0.4456 \\
\hline 4865 & $11: 08: 06$ & 0.4430 \\
\hline 4866 & $11: 08: 07$ & 0.4433 \\
\hline 4867 & $11: 08: 08$ & 0.4414 \\
\hline 4868 & 11:08:09 & 0.4399 \\
\hline 4869 & 11:08:10 & 0.4397 \\
\hline 4870 & $11: 08: 11$ & 0.4385 \\
\hline 4871 & $11: 08: 12$ & 0.4396 \\
\hline 4872 & $11: 08: 13$ & 0.4399 \\
\hline 4873 & $11: 08: 14$ & 0.4434 \\
\hline 4874 & 11:08:15 & 0.4407 \\
\hline 4875 & $11: 08: 16$ & 0.4432 \\
\hline 4876 & $11: 08: 17$ & 0.4425 \\
\hline 4877 & $11: 08: 18$ & 0.4449 \\
\hline 4878 & 11:08:19 & 0.4438 \\
\hline 4879 & $11: 08: 20$ & 0.4421 \\
\hline 4880 & $11: 08: 21$ & 0.4457 \\
\hline 4881 & $11: 08: 22$ & 0.4427 \\
\hline 4882 & $11: 08: 23$ & 0.4450 \\
\hline 4883 & $11: 08: 24$ & 0.4444 \\
\hline 4884 & $11: 08: 25$ & 0.4417 \\
\hline 4885 & $11: 08: 26$ & 0.4430 \\
\hline 4886 & $11: 08: 27$ & 0.4403 \\
\hline 4887 & 11:08:28 & 0.4437 \\
\hline 4888 & 11:08:29 & 0.4414 \\
\hline 4889 & $11: 08: 30$ & 0.4396 \\
\hline 4890 & $11: 08: 31$ & 0.4399 \\
\hline 4891 & $11: 08: 32$ & 0.4401 \\
\hline 4892 & $11: 08: 33$ & 0.4378 \\
\hline 4893 & $11: 08: 34$ & 0.4393 \\
\hline 4894 & $11: 08: 35$ & 0.4418 \\
\hline
\end{tabular}


Appendix C - Fuel Data Test 2 NIST102605_1.xIs

\begin{tabular}{|c|c|c|}
\hline 4895 & $11: 08: 36$ & 0.4423 \\
\hline 4896 & $11: 08: 37$ & 0.4457 \\
\hline 4897 & $11: 08: 38$ & 0.4413 \\
\hline 4898 & $11: 08: 39$ & 0.4448 \\
\hline 4899 & $11: 08: 40$ & 0.4454 \\
\hline 4900 & $11: 08: 41$ & 0.4449 \\
\hline 4901 & $11: 08: 42$ & 0.4470 \\
\hline 4902 & $11: 08: 43$ & 0.4414 \\
\hline 4903 & $11: 08: 44$ & 0.4437 \\
\hline 4904 & $11: 08: 45$ & 0.4434 \\
\hline 4905 & $11: 08: 46$ & 0.4451 \\
\hline 4906 & $11: 08: 47$ & 0.4438 \\
\hline 4907 & $11: 08: 48$ & 0.4432 \\
\hline 4908 & $11: 08: 49$ & 0.4414 \\
\hline 4909 & $11: 08: 50$ & 0.4410 \\
\hline 4910 & $11: 08: 51$ & 0.4441 \\
\hline 4911 & 11:08:52 & 0.4399 \\
\hline 4912 & $11: 08: 53$ & 0.4426 \\
\hline 4913 & $11: 08: 54$ & 0.4416 \\
\hline 4914 & $11: 08: 55$ & 0.4393 \\
\hline 4915 & $11: 08: 56$ & 0.4431 \\
\hline 4916 & $11: 08: 57$ & 0.4412 \\
\hline 4917 & $11: 08: 58$ & 0.4448 \\
\hline 4918 & $11: 08: 59$ & 0.4417 \\
\hline 4919 & $11: 09: 00$ & 0.4444 \\
\hline 4920 & 11:09:01 & 0.4437 \\
\hline 4921 & 11:09:02 & 0.4440 \\
\hline 4922 & $11: 09: 03$ & 0.4453 \\
\hline 4923 & $11: 09: 04$ & 0.4417 \\
\hline 4924 & $11: 09: 05$ & 0.4457 \\
\hline 4925 & 11:09:06 & 0.4428 \\
\hline 4926 & $11: 09: 07$ & 0.4451 \\
\hline 4927 & $11: 09: 08$ & 0.4441 \\
\hline 4928 & 11:09:09 & 0.4423 \\
\hline 4929 & 11:09:10 & 0.4413 \\
\hline 4930 & 11:09:11 & 0.4392 \\
\hline 4931 & $11: 09: 12$ & 0.4430 \\
\hline 4932 & $11: 09: 13$ & 0.4408 \\
\hline 4933 & $11: 09: 14$ & 0.4422 \\
\hline 4934 & $11: 09: 15$ & 0.4395 \\
\hline 4935 & $11: 09: 16$ & 0.4430 \\
\hline 4936 & $11: 09: 17$ & 0.4405 \\
\hline 4937 & $11: 09: 18$ & 0.4423 \\
\hline 4938 & 11:09:19 & 0.4404 \\
\hline 4939 & $11: 09: 20$ & 0.4434 \\
\hline 4940 & $11: 09: 21$ & 0.4454 \\
\hline 4941 & 11:09:22 & 0.4425 \\
\hline 4942 & $11: 09: 23$ & 0.4462 \\
\hline 4943 & 11:09:24 & 0.4463 \\
\hline 4944 & $11: 09: 25$ & 0.4462 \\
\hline 4945 & $11: 09: 26$ & 0.4431 \\
\hline
\end{tabular}

C - 97 
Appendix C - Fuel Data Test 2 NIST102605_1.xIs

\begin{tabular}{|c|c|c|}
\hline 4946 & $11: 09: 27$ & 0.4444 \\
\hline 4947 & $11: 09: 28$ & 0.4450 \\
\hline 4948 & $11: 09: 29$ & 0.4411 \\
\hline 4949 & $11: 09: 30$ & 0.4445 \\
\hline 4950 & $11: 09: 31$ & 0.4418 \\
\hline 4951 & $11: 09: 32$ & 0.4437 \\
\hline 4952 & $11: 09: 33$ & 0.4414 \\
\hline 4953 & $11: 09: 34$ & 0.4402 \\
\hline 4954 & 11:09:35 & 0.4440 \\
\hline 4955 & $11: 09: 36$ & 0.4407 \\
\hline 4956 & $11: 09: 37$ & 0.4425 \\
\hline 4957 & 11:09:38 & 0.4395 \\
\hline 4958 & 11:09:39 & 0.4402 \\
\hline 4959 & $11: 09: 40$ & 0.4397 \\
\hline 4960 & $11: 09: 41$ & 0.4427 \\
\hline 4961 & $11: 09: 42$ & 0.4460 \\
\hline 4962 & 11:09:43 & 0.4426 \\
\hline 4963 & 11:09:44 & 0.4447 \\
\hline 4964 & $11: 09: 45$ & 0.4419 \\
\hline 4965 & $11: 09: 46$ & 0.4448 \\
\hline 4966 & $11: 09: 47$ & 0.4454 \\
\hline 4967 & $11: 09: 48$ & 0.4433 \\
\hline 4968 & $11: 09: 49$ & 0.4449 \\
\hline 4969 & $11: 09: 50$ & 0.4407 \\
\hline 4970 & $11: 09: 51$ & 0.4429 \\
\hline 4971 & 11:09:52 & 0.4419 \\
\hline 4972 & $11: 09: 53$ & 0.4457 \\
\hline 4973 & $11: 09: 54$ & 0.4423 \\
\hline 4974 & $11: 09: 55$ & 0.4421 \\
\hline 4975 & $11: 09: 56$ & 0.4412 \\
\hline 4976 & $11: 09: 57$ & 0.4408 \\
\hline 4977 & $11: 09: 58$ & 0.4404 \\
\hline 4978 & $11: 09: 59$ & 0.4388 \\
\hline 4979 & $11: 10: 00$ & 0.4436 \\
\hline 4980 & 11:10:01 & 0.4422 \\
\hline 4981 & $11: 10: 02$ & 0.4447 \\
\hline 4982 & $11: 10: 03$ & 0.4413 \\
\hline 4983 & $11: 10: 04$ & 0.4441 \\
\hline 4984 & $11: 10: 05$ & 0.4472 \\
\hline 4985 & 11:10:06 & 0.4451 \\
\hline 4986 & $11: 10: 07$ & 0.4471 \\
\hline 4987 & $11: 10: 08$ & 0.4415 \\
\hline 4988 & $11: 10: 09$ & 0.4446 \\
\hline 4989 & $11: 10: 10$ & 0.4439 \\
\hline 4990 & $11: 10: 11$ & 0.4419 \\
\hline 4991 & $11: 10: 12$ & 0.4425 \\
\hline 4992 & $11: 10: 13$ & 0.4392 \\
\hline 4993 & $11: 10: 14$ & 0.4439 \\
\hline 4994 & $11: 10: 15$ & 0.4419 \\
\hline 4995 & $11: 10: 16$ & 0.4431 \\
\hline 4996 & $11: 10: 17$ & 0.4407 \\
\hline
\end{tabular}


Appendix C - Fuel Data Test 2 NIST102605_1.xls

\begin{tabular}{|c|c|c|}
\hline 4997 & $11: 10: 18$ & 0.4399 \\
\hline 4998 & $11: 10: 19$ & 0.4422 \\
\hline 4999 & $11: 10: 20$ & 0.4400 \\
\hline 5000 & $11: 10: 21$ & 0.4429 \\
\hline 5001 & $11: 10: 22$ & 0.4400 \\
\hline 5002 & $11: 10: 23$ & 0.4431 \\
\hline 5003 & $11: 10: 24$ & 0.4410 \\
\hline 5004 & $11: 10: 25$ & 0.4443 \\
\hline 5005 & $11: 10: 26$ & 0.4454 \\
\hline 5006 & $11: 10: 27$ & 0.4453 \\
\hline 5007 & $11: 10: 28$ & 0.4460 \\
\hline 5008 & $11: 10: 29$ & 0.4426 \\
\hline 5009 & $11: 10: 30$ & 0.4449 \\
\hline 5010 & $11: 10: 31$ & 0.4436 \\
\hline 5011 & $11: 10: 32$ & 0.4445 \\
\hline 5012 & $11: 10: 33$ & 0.4448 \\
\hline 5013 & $11: 10: 34$ & 0.4425 \\
\hline 5014 & $11: 10: 35$ & 0.4422 \\
\hline 5015 & $11: 10: 36$ & 0.4424 \\
\hline 5016 & $11: 10: 37$ & 0.4433 \\
\hline 5017 & $11: 10: 38$ & 0.4402 \\
\hline 5018 & $11: 10: 39$ & 0.4393 \\
\hline 5019 & $11: 10: 40$ & 0.4393 \\
\hline 5020 & $11: 10: 41$ & 0.4415 \\
\hline 5021 & $11: 10: 42$ & 0.4417 \\
\hline 5022 & $11: 10: 43$ & 0.4413 \\
\hline 5023 & $11: 10: 44$ & 0.4388 \\
\hline 5024 & $11: 10: 45$ & 0.4445 \\
\hline 5025 & $11: 10: 46$ & 0.4419 \\
\hline 5026 & $11: 10: 47$ & 0.4436 \\
\hline 5027 & $11: 10: 48$ & 0.4469 \\
\hline 5028 & $11: 10: 49$ & 0.4429 \\
\hline 5029 & $11: 10: 50$ & 0.4451 \\
\hline 5030 & $11: 10: 51$ & 0.4423 \\
\hline 5031 & $11: 10: 52$ & 0.4455 \\
\hline 5032 & $11: 10: 53$ & 0.4457 \\
\hline 5033 & $11: 10: 54$ & 0.4435 \\
\hline 5034 & $11: 10: 55$ & 0.4431 \\
\hline 5035 & $11: 10: 56$ & 0.4400 \\
\hline 5036 & $11: 10: 57$ & 0.4417 \\
\hline 5037 & $11: 10: 58$ & 0.4391 \\
\hline 5038 & $11: 10: 59$ & 0.4452 \\
\hline 5039 & $11: 11: 00$ & 0.4426 \\
\hline 5040 & $11: 11: 01$ & 0.4419 \\
\hline 5041 & $11: 11: 02$ & 0.4396 \\
\hline 5042 & $11: 11: 03$ & 0.4400 \\
\hline 5043 & $11: 11: 04$ & 0.4419 \\
\hline 5044 & $11: 11: 05$ & 0.4409 \\
\hline 5045 & $11: 11: 06$ & 0.4420 \\
\hline 5046 & $11: 11: 07$ & 0.4415 \\
\hline 5047 & 11:11:08 & 0.4454 \\
\hline
\end{tabular}

C - 99 
Appendix C - Fuel Data Test 2 NIST102605_1.xIs

\begin{tabular}{|c|c|c|}
\hline 5048 & 11:11:09 & 0.4428 \\
\hline 5049 & $11: 11: 10$ & 0.4453 \\
\hline 5050 & $11: 11: 11$ & 0.4443 \\
\hline 5051 & $11: 11: 12$ & 0.4455 \\
\hline 5052 & $11: 11: 13$ & 0.4454 \\
\hline 5053 & $11: 11: 14$ & 0.4445 \\
\hline 5054 & 11:11:15 & 0.4450 \\
\hline 5055 & $11: 11: 16$ & 0.4405 \\
\hline 5056 & $11: 11: 17$ & 0.4426 \\
\hline 5057 & 11:11:18 & 0.4402 \\
\hline 5058 & $11: 11: 19$ & 0.4441 \\
\hline 5059 & 11:11:20 & 0.4425 \\
\hline 5060 & $11: 11: 21$ & 0.4440 \\
\hline 5061 & $11: 11: 22$ & 0.4402 \\
\hline 5062 & 11:11:23 & 0.4411 \\
\hline 5063 & 11:11:24 & 0.4411 \\
\hline 5064 & $11: 11: 25$ & 0.4393 \\
\hline 5065 & $11: 11: 26$ & 0.4433 \\
\hline 5066 & $11: 11: 27$ & 0.4397 \\
\hline 5067 & $11: 11: 28$ & 0.4421 \\
\hline 5068 & 11:11:29 & 0.4410 \\
\hline 5069 & $11: 11: 30$ & 0.4444 \\
\hline 5070 & $11: 11: 31$ & 0.4472 \\
\hline 5071 & 11:11:32 & 0.4443 \\
\hline 5072 & 11:11:33 & 0.4471 \\
\hline 5073 & $11: 11: 34$ & 0.4425 \\
\hline 5074 & $11: 11: 35$ & 0.4440 \\
\hline 5075 & $11: 11: 36$ & 0.4406 \\
\hline 5076 & $11: 11: 37$ & 0.4458 \\
\hline 5077 & $11: 11: 38$ & 0.4435 \\
\hline 5078 & $11: 11: 39$ & 0.4446 \\
\hline 5079 & $11: 11: 40$ & 0.4424 \\
\hline 5080 & $11: 11: 41$ & 0.4401 \\
\hline 5081 & 11:11:42 & 0.4431 \\
\hline 5082 & $11: 11: 43$ & 0.4400 \\
\hline 5083 & $11: 11: 44$ & 0.4441 \\
\hline 5084 & $11: 11: 45$ & 0.4406 \\
\hline 5085 & $11: 11: 46$ & 0.4390 \\
\hline 5086 & $11: 11: 47$ & 0.4388 \\
\hline 5087 & $11: 11: 48$ & 0.4403 \\
\hline 5088 & $11: 11: 49$ & 0.4445 \\
\hline 5089 & 11:11:50 & 0.4406 \\
\hline 5090 & $11: 11: 51$ & 0.4433 \\
\hline 5091 & $11: 11: 52$ & 0.4451 \\
\hline 5092 & $11: 11: 53$ & 0.4448 \\
\hline 5093 & $11: 11: 54$ & 0.4446 \\
\hline 5094 & $11: 11: 55$ & 0.4425 \\
\hline 5095 & 11:11:56 & 0.4463 \\
\hline 5096 & $11: 11: 57$ & 0.4428 \\
\hline 5097 & $11: 11: 58$ & 0.4445 \\
\hline 5098 & $11: 11: 59$ & 0.4454 \\
\hline
\end{tabular}


Appendix C - Fuel Data Test 2 NIST102605_1.xls

\begin{tabular}{|c|c|c|}
\hline 5099 & $11: 12: 00$ & 0.4400 \\
\hline 5100 & $11: 12: 01$ & 0.4413 \\
\hline 5101 & $11: 12: 02$ & 0.4395 \\
\hline 5102 & $11: 12: 03$ & 0.4440 \\
\hline 5103 & $11: 12: 04$ & 0.4423 \\
\hline 5104 & $11: 12: 05$ & 0.4423 \\
\hline 5105 & $11: 12: 06$ & 0.4412 \\
\hline 5106 & $11: 12: 07$ & 0.4408 \\
\hline 5107 & 11:12:08 & 0.4431 \\
\hline 5108 & $11: 12: 09$ & 0.4400 \\
\hline 5109 & $11: 12: 10$ & 0.4445 \\
\hline 5110 & $11: 12: 11$ & 0.4402 \\
\hline 5111 & $11: 12: 12$ & 0.4424 \\
\hline 5112 & $11: 12: 13$ & 0.4448 \\
\hline 5113 & $11: 12: 14$ & 0.4444 \\
\hline 5114 & $11: 12: 15$ & 0.4465 \\
\hline 5115 & $11: 12: 16$ & 0.4435 \\
\hline 5116 & $11: 12: 17$ & 0.4468 \\
\hline 5117 & $11: 12: 18$ & 0.4435 \\
\hline 5118 & $11: 12: 19$ & 0.4441 \\
\hline 5119 & $11: 12: 20$ & 0.4429 \\
\hline 5120 & $11: 12: 21$ & 0.4423 \\
\hline 5121 & $11: 12: 22$ & 0.4426 \\
\hline 5122 & $11: 12: 23$ & 0.4393 \\
\hline 5123 & $11: 12: 24$ & 0.4446 \\
\hline 5124 & $11: 12: 25$ & 0.4423 \\
\hline 5125 & $11: 12: 26$ & 0.4421 \\
\hline 5126 & $11: 12: 27$ & 0.4424 \\
\hline 5127 & $11: 12: 28$ & 0.4397 \\
\hline 5128 & $11: 12: 29$ & 0.4427 \\
\hline 5129 & $11: 12: 30$ & 0.4391 \\
\hline 5130 & $11: 12: 31$ & 0.4397 \\
\hline 5131 & $11: 12: 32$ & 0.4413 \\
\hline 5132 & $11: 12: 33$ & 0.4416 \\
\hline 5133 & $11: 12: 34$ & 0.4453 \\
\hline 5134 & $11: 12: 35$ & 0.4430 \\
\hline 5135 & $11: 12: 36$ & 0.4461 \\
\hline 5136 & $11: 12: 37$ & 0.4426 \\
\hline 5137 & $11: 12: 38$ & 0.4456 \\
\hline 5138 & $11: 12: 39$ & 0.4453 \\
\hline 5139 & $11: 12: 40$ & 0.4438 \\
\hline 5140 & $11: 12: 41$ & 0.4443 \\
\hline 5141 & $11: 12: 42$ & 0.4417 \\
\hline 5142 & $11: 12: 43$ & 0.4452 \\
\hline 5143 & $11: 12: 44$ & 0.4415 \\
\hline 5144 & $11: 12: 45$ & 0.4419 \\
\hline 5145 & $11: 12: 46$ & 0.4400 \\
\hline 5146 & $11: 12: 47$ & 0.4447 \\
\hline 5147 & $11: 12: 48$ & 0.4416 \\
\hline 5148 & $11: 12: 49$ & 0.4400 \\
\hline 5149 & $11: 12: 50$ & 0.4410 \\
\hline
\end{tabular}


Appendix C - Fuel Data Test 2 NIST102605_1.xIs

\begin{tabular}{|c|c|c|}
\hline 5150 & $11: 12: 51$ & 0.4398 \\
\hline 5151 & $11: 12: 52$ & 0.4415 \\
\hline 5152 & $11: 12: 53$ & 0.4385 \\
\hline 5153 & $11: 12: 54$ & 0.4431 \\
\hline 5154 & $11: 12: 55$ & 0.4433 \\
\hline 5155 & $11: 12: 56$ & 0.4436 \\
\hline 5156 & $11: 12: 57$ & 0.4445 \\
\hline 5157 & $11: 12: 58$ & 0.4427 \\
\hline 5158 & $11: 12: 59$ & 0.4447 \\
\hline 5159 & 11:13:00 & 0.4418 \\
\hline 5160 & $11: 13: 01$ & 0.4458 \\
\hline 5161 & 11:13:02 & 0.4457 \\
\hline 5162 & $11: 13: 03$ & 0.4451 \\
\hline 5163 & $11: 13: 04$ & 0.4446 \\
\hline 5164 & 11:13:05 & 0.4415 \\
\hline 5165 & 11:13:06 & 0.4428 \\
\hline 5166 & $11: 13: 07$ & 0.4385 \\
\hline 5167 & $11: 13: 08$ & 0.4447 \\
\hline 5168 & 11:13:09 & 0.4419 \\
\hline 5169 & $11: 13: 10$ & 0.4401 \\
\hline 5170 & 11:13:11 & 0.4408 \\
\hline 5171 & $11: 13: 12$ & 0.4392 \\
\hline 5172 & $11: 13: 13$ & 0.4428 \\
\hline 5173 & $11: 13: 14$ & 0.4417 \\
\hline 5174 & 11:13:15 & 0.4439 \\
\hline 5175 & $11: 13: 16$ & 0.4412 \\
\hline 5176 & $11: 13: 17$ & 0.4440 \\
\hline 5177 & $11: 13: 18$ & 0.4422 \\
\hline 5178 & $11: 13: 19$ & 0.4449 \\
\hline 5179 & $11: 13: 20$ & 0.4434 \\
\hline 5180 & $11: 13: 21$ & 0.4452 \\
\hline 5181 & $11: 13: 22$ & 0.4459 \\
\hline 5182 & 11:13:23 & 0.4419 \\
\hline 5183 & 11:13:24 & 0.4464 \\
\hline 5184 & $11: 13: 25$ & 0.4441 \\
\hline 5185 & $11: 13: 26$ & 0.4444 \\
\hline 5186 & $11: 13: 27$ & 0.4449 \\
\hline 5187 & $11: 13: 28$ & 0.4402 \\
\hline 5188 & 11:13:29 & 0.4417 \\
\hline 5189 & $11: 13: 30$ & 0.4398 \\
\hline 5190 & $11: 13: 31$ & 0.4455 \\
\hline 5191 & $11: 13: 32$ & 0.4423 \\
\hline 5192 & $11: 13: 33$ & 0.4422 \\
\hline 5193 & $11: 13: 34$ & 0.4375 \\
\hline 5194 & $11: 13: 35$ & 0.4409 \\
\hline 5195 & $11: 13: 36$ & 0.4412 \\
\hline 5196 & $11: 13: 37$ & 0.4402 \\
\hline 5197 & 11:13:38 & 0.4457 \\
\hline 5198 & $11: 13: 39$ & 0.4411 \\
\hline 5199 & $11: 13: 40$ & 0.4437 \\
\hline 5200 & $11: 13: 41$ & 0.4439 \\
\hline
\end{tabular}


Appendix C - Fuel Data Test 2 NIST102605_1.xls

\begin{tabular}{|c|c|c|}
\hline 5201 & $11: 13: 42$ & 0.4453 \\
\hline 5202 & $11: 13: 43$ & 0.4464 \\
\hline 5203 & $11: 13: 44$ & 0.4428 \\
\hline 5204 & $11: 13: 45$ & 0.4455 \\
\hline 5205 & $11: 13: 46$ & 0.4428 \\
\hline 5206 & $11: 13: 47$ & 0.4442 \\
\hline 5207 & $11: 13: 48$ & 0.4439 \\
\hline 5208 & $11: 13: 49$ & 0.4408 \\
\hline 5209 & $11: 13: 50$ & 0.4417 \\
\hline 5210 & $11: 13: 51$ & 0.4386 \\
\hline 5211 & $11: 13: 52$ & 0.4427 \\
\hline 5212 & $11: 13: 53$ & 0.4416 \\
\hline 5213 & $11: 13: 54$ & 0.4438 \\
\hline 5214 & $11: 13: 55$ & 0.4404 \\
\hline 5215 & $11: 13: 56$ & 0.4405 \\
\hline 5216 & $11: 13: 57$ & 0.4423 \\
\hline 5217 & 11:13:58 & 0.4391 \\
\hline 5218 & $11: 13: 59$ & 0.4418 \\
\hline 5219 & $11: 14: 00$ & 0.4415 \\
\hline 5220 & $11: 14: 01$ & 0.4432 \\
\hline 5221 & $11: 14: 02$ & 0.4450 \\
\hline 5222 & $11: 14: 03$ & 0.4462 \\
\hline 5223 & $11: 14: 04$ & 0.4467 \\
\hline 5224 & $11: 14: 05$ & 0.4409 \\
\hline 5225 & $11: 14: 06$ & 0.4439 \\
\hline 5226 & $11: 14: 07$ & 0.4426 \\
\hline 5227 & $11: 14: 08$ & 0.4459 \\
\hline 5228 & $11: 14: 09$ & 0.4428 \\
\hline 5229 & $11: 14: 10$ & 0.4425 \\
\hline 5230 & $11: 14: 11$ & 0.4439 \\
\hline 5231 & $11: 14: 12$ & 0.4405 \\
\hline 5232 & $11: 14: 13$ & 0.4435 \\
\hline 5233 & $11: 14: 14$ & 0.4420 \\
\hline 5234 & $11: 14: 15$ & 0.4440 \\
\hline 5235 & $11: 14: 16$ & 0.4403 \\
\hline 5236 & $11: 14: 17$ & 0.4392 \\
\hline 5237 & $11: 14: 18$ & 0.4407 \\
\hline 5238 & $11: 14: 19$ & 0.4400 \\
\hline 5239 & $11: 14: 20$ & 0.4436 \\
\hline 5240 & $11: 14: 21$ & 0.4405 \\
\hline 5241 & $11: 14: 22$ & 0.4438 \\
\hline 5242 & $11: 14: 23$ & 0.4429 \\
\hline 5243 & $11: 14: 24$ & 0.4443 \\
\hline 5244 & $11: 14: 25$ & 0.4480 \\
\hline 5245 & $11: 14: 26$ & 0.4467 \\
\hline 5246 & $11: 14: 27$ & 0.4471 \\
\hline 5247 & $11: 14: 28$ & 0.4419 \\
\hline 5248 & $11: 14: 29$ & 0.4451 \\
\hline 5249 & $11: 14: 30$ & 0.4435 \\
\hline 5250 & $11: 14: 31$ & 0.4425 \\
\hline 5251 & $11: 14: 32$ & 0.4415 \\
\hline
\end{tabular}


Appendix C - Fuel Data Test 2 NIST102605_1.xIs

\begin{tabular}{|c|c|c|}
\hline 5252 & $11: 14: 33$ & 0.4402 \\
\hline 5253 & $11: 14: 34$ & 0.4427 \\
\hline 5254 & $11: 14: 35$ & 0.4389 \\
\hline 5255 & $11: 14: 36$ & 0.4452 \\
\hline 5256 & $11: 14: 37$ & 0.4424 \\
\hline 5257 & $11: 14: 38$ & 0.4428 \\
\hline 5258 & 11:14:39 & 0.4407 \\
\hline 5259 & $11: 14: 40$ & 0.4402 \\
\hline 5260 & $11: 14: 41$ & 0.4424 \\
\hline 5261 & $11: 14: 42$ & 0.4414 \\
\hline 5262 & $11: 14: 43$ & 0.4452 \\
\hline 5263 & $11: 14: 44$ & 0.4449 \\
\hline 5264 & $11: 14: 45$ & 0.4466 \\
\hline 5265 & $11: 14: 46$ & 0.4412 \\
\hline 5266 & $11: 14: 47$ & 0.4453 \\
\hline 5267 & $11: 14: 48$ & 0.4453 \\
\hline 5268 & $11: 14: 49$ & 0.4446 \\
\hline 5269 & $11: 14: 50$ & 0.4462 \\
\hline 5270 & $11: 14: 51$ & 0.4420 \\
\hline 5271 & $11: 14: 52$ & 0.4449 \\
\hline 5272 & $11: 14: 53$ & 0.4432 \\
\hline 5273 & $11: 14: 54$ & 0.4429 \\
\hline 5274 & $11: 14: 55$ & 0.4438 \\
\hline 5275 & $11: 14: 56$ & 0.4430 \\
\hline 5276 & $11: 14: 57$ & 0.4413 \\
\hline 5277 & $11: 14: 58$ & 0.4399 \\
\hline 5278 & $11: 14: 59$ & 0.4435 \\
\hline 5279 & $11: 15: 00$ & 0.4419 \\
\hline 5280 & $11: 15: 01$ & 0.4432 \\
\hline 5281 & 11:15:02 & 0.4376 \\
\hline 5282 & $11: 15: 03$ & 0.4417 \\
\hline 5283 & $11: 15: 04$ & 0.4400 \\
\hline 5284 & 11:15:05 & 0.4438 \\
\hline 5285 & 11:15:06 & 0.4426 \\
\hline 5286 & $11: 15: 07$ & 0.4449 \\
\hline 5287 & $11: 15: 08$ & 0.4434 \\
\hline 5288 & $11: 15: 09$ & 0.4454 \\
\hline 5289 & $11: 15: 10$ & 0.4472 \\
\hline 5290 & $11: 15: 11$ & 0.4466 \\
\hline 5291 & $11: 15: 12$ & 0.4457 \\
\hline 5292 & $11: 15: 13$ & 0.4406 \\
\hline 5293 & $11: 15: 14$ & 0.4442 \\
\hline 5294 & $11: 15: 15$ & 0.4437 \\
\hline 5295 & $11: 15: 16$ & 0.4436 \\
\hline 5296 & $11: 15: 17$ & 0.4423 \\
\hline 5297 & $11: 15: 18$ & 0.4403 \\
\hline 5298 & $11: 15: 19$ & 0.4424 \\
\hline 5299 & 11:15:20 & 0.4387 \\
\hline 5300 & $11: 15: 21$ & 0.4444 \\
\hline 5301 & $11: 15: 22$ & 0.4420 \\
\hline 5302 & $11: 15: 23$ & 0.4428 \\
\hline
\end{tabular}


Appendix C - Fuel Data Test 2 NIST102605_1.xIs

\begin{tabular}{|c|c|c|}
\hline 5303 & $11: 15: 24$ & 0.4387 \\
\hline 5304 & $11: 15: 25$ & 0.4413 \\
\hline 5305 & $11: 15: 26$ & 0.4415 \\
\hline 5306 & $11: 15: 27$ & 0.4432 \\
\hline 5307 & $11: 15: 28$ & 0.4464 \\
\hline 5308 & 11:15:29 & 0.4437 \\
\hline 5309 & $11: 15: 30$ & 0.4457 \\
\hline 5310 & $11: 15: 31$ & 0.4419 \\
\hline 5311 & 11:15:32 & 0.4451 \\
\hline 5312 & $11: 15: 33$ & 0.4464 \\
\hline 5313 & 11:15:34 & 0.4435 \\
\hline 5314 & $11: 15: 35$ & 0.4453 \\
\hline 5315 & $11: 15: 36$ & 0.4428 \\
\hline 5316 & $11: 15: 37$ & 0.4454 \\
\hline 5317 & 11:15:38 & 0.4426 \\
\hline 5318 & $11: 15: 39$ & 0.4405 \\
\hline 5319 & $11: 15: 40$ & 0.4436 \\
\hline 5320 & $11: 15: 41$ & 0.4418 \\
\hline 5321 & $11: 15: 42$ & 0.4429 \\
\hline 5322 & $11: 15: 43$ & 0.4403 \\
\hline 5323 & $11: 15: 44$ & 0.4394 \\
\hline 5324 & $11: 15: 45$ & 0.4426 \\
\hline 5325 & 11:15:46 & 0.4395 \\
\hline 5326 & $11: 15: 47$ & 0.4431 \\
\hline 5327 & $11: 15: 48$ & 0.4405 \\
\hline 5328 & $11: 15: 49$ & 0.4436 \\
\hline 5329 & $11: 15: 50$ & 0.4473 \\
\hline 5330 & $11: 15: 51$ & 0.4445 \\
\hline 5331 & $11: 15: 52$ & 0.4459 \\
\hline 5332 & $11: 15: 53$ & 0.4416 \\
\hline 5333 & $11: 15: 54$ & 0.4460 \\
\hline 5334 & 11:15:55 & 0.4463 \\
\hline 5335 & 11:15:56 & 0.4452 \\
\hline 5336 & $11: 15: 57$ & 0.4444 \\
\hline 5337 & $11: 15: 58$ & 0.4413 \\
\hline 5338 & $11: 15: 59$ & 0.4427 \\
\hline 5339 & $11: 16: 00$ & 0.4385 \\
\hline 5340 & $11: 16: 01$ & 0.4442 \\
\hline 5341 & $11: 16: 02$ & 0.4426 \\
\hline 5342 & $11: 16: 03$ & 0.4440 \\
\hline 5343 & 11:16:04 & 0.4404 \\
\hline 5344 & $11: 16: 05$ & 0.4406 \\
\hline 5345 & $11: 16: 06$ & 0.4400 \\
\hline 5346 & $11: 16: 07$ & 0.4399 \\
\hline 5347 & $11: 16: 08$ & 0.4415 \\
\hline 5348 & $11: 16: 09$ & 0.4425 \\
\hline 5349 & $11: 16: 10$ & 0.4447 \\
\hline 5350 & $11: 16: 11$ & 0.4415 \\
\hline 5351 & $11: 16: 12$ & 0.4438 \\
\hline 5352 & $11: 16: 13$ & 0.4428 \\
\hline 5353 & $11: 16: 14$ & 0.4460 \\
\hline
\end{tabular}


Appendix C - Fuel Data Test 2 NIST102605_1.xls

\begin{tabular}{|c|c|c|}
\hline 5354 & $11: 16: 15$ & 0.4433 \\
\hline 5355 & $11: 16: 16$ & 0.4433 \\
\hline 5356 & $11: 16: 17$ & 0.4464 \\
\hline 5357 & $11: 16: 18$ & 0.4413 \\
\hline 5358 & $11: 16: 19$ & 0.4453 \\
\hline 5359 & $11: 16: 20$ & 0.4441 \\
\hline 5360 & $11: 16: 21$ & 0.4431 \\
\hline 5361 & $11: 16: 22$ & 0.4430 \\
\hline 5362 & $11: 16: 23$ & 0.4399 \\
\hline 5363 & $11: 16: 24$ & 0.4441 \\
\hline 5364 & $11: 16: 25$ & 0.4422 \\
\hline 5365 & $11: 16: 26$ & 0.4426 \\
\hline 5366 & $11: 16: 27$ & 0.4394 \\
\hline 5367 & $11: 16: 28$ & 0.4403 \\
\hline 5368 & $11: 16: 29$ & 0.4421 \\
\hline 5369 & $11: 16: 30$ & 0.4389 \\
\hline 5370 & $11: 16: 31$ & 0.4444 \\
\hline 5371 & $11: 16: 32$ & 0.4410 \\
\hline 5372 & $11: 16: 33$ & 0.4440 \\
\hline 5373 & $11: 16: 34$ & 0.4437 \\
\hline 5374 & $11: 16: 35$ & 0.4462 \\
\hline 5375 & $11: 16: 36$ & 0.4478 \\
\hline 5376 & $11: 16: 37$ & 0.4448 \\
\hline 5377 & $11: 16: 38$ & 0.4462 \\
\hline 5378 & $11: 16: 39$ & 0.4422 \\
\hline 5379 & $11: 16: 40$ & 0.4444 \\
\hline 5380 & $11: 16: 41$ & 0.4413 \\
\hline 5381 & $11: 16: 42$ & 0.4442 \\
\hline 5382 & $11: 16: 43$ & 0.4433 \\
\hline 5383 & $11: 16: 44$ & 0.4421 \\
\hline 5384 & $11: 16: 45$ & 0.4412 \\
\hline 5385 & $11: 16: 46$ & 0.4396 \\
\hline 5386 & $11: 16: 47$ & 0.4443 \\
\hline 5387 & $11: 16: 48$ & 0.4408 \\
\hline 5388 & $11: 16: 49$ & 0.4418 \\
\hline 5389 & $11: 16: 50$ & 0.4407 \\
\hline 5390 & $11: 16: 51$ & 0.4396 \\
\hline 5391 & $11: 16: 52$ & 0.4404 \\
\hline 5392 & $11: 16: 53$ & 0.4434 \\
\hline 5393 & $11: 16: 54$ & 0.4467 \\
\hline 5394 & $11: 16: 55$ & 0.4445 \\
\hline 5395 & $11: 16: 56$ & 0.4457 \\
\hline 5396 & $11: 16: 57$ & 0.4427 \\
\hline 5397 & $11: 16: 58$ & 0.4453 \\
\hline 5398 & $11: 16: 59$ & 0.4456 \\
\hline 5399 & $11: 17: 00$ & 0.4427 \\
\hline 5400 & $11: 17: 01$ & 0.4452 \\
\hline 5401 & $11: 17: 02$ & 0.4408 \\
\hline 5402 & $11: 17: 03$ & 0.4438 \\
\hline 5403 & $11: 17: 04$ & 0.4431 \\
\hline 5404 & $11: 17: 05$ & 0.4436 \\
\hline
\end{tabular}


Appendix C - Fuel Data Test 2 NIST102605_1.xls

\begin{tabular}{|c|c|c|}
\hline 5405 & $11: 17: 06$ & 0.4416 \\
\hline 5406 & $11: 17: 07$ & 0.4405 \\
\hline 5407 & $11: 17: 08$ & 0.4418 \\
\hline 5408 & $11: 17: 09$ & 0.4409 \\
\hline 5409 & $11: 17: 10$ & 0.4438 \\
\hline 5410 & $11: 17: 11$ & 0.4405 \\
\hline 5411 & $11: 17: 12$ & 0.4423 \\
\hline 5412 & $11: 17: 13$ & 0.4393 \\
\hline 5413 & $11: 17: 14$ & 0.4409 \\
\hline 5414 & $11: 17: 15$ & 0.4420 \\
\hline 5415 & $11: 17: 16$ & 0.4452 \\
\hline 5416 & $11: 17: 17$ & 0.4470 \\
\hline 5417 & $11: 17: 18$ & 0.4454 \\
\hline 5418 & $11: 17: 19$ & 0.4474 \\
\hline 5419 & $11: 17: 20$ & 0.4428 \\
\hline 5420 & $11: 17: 21$ & 0.4448 \\
\hline 5421 & 11:17:22 & 0.4419 \\
\hline 5422 & $11: 17: 23$ & 0.4459 \\
\hline 5423 & $11: 17: 24$ & 0.4449 \\
\hline 5424 & $11: 17: 25$ & 0.4429 \\
\hline 5425 & $11: 17: 26$ & 0.4422 \\
\hline 5426 & $11: 17: 27$ & 0.4400 \\
\hline 5427 & $11: 17: 28$ & 0.4448 \\
\hline 5428 & $11: 17: 29$ & 0.4411 \\
\hline 5429 & $11: 17: 30$ & 0.4449 \\
\hline 5430 & $11: 17: 31$ & 0.4402 \\
\hline 5431 & $11: 17: 32$ & 0.4402 \\
\hline 5432 & $11: 17: 33$ & 0.4403 \\
\hline 5433 & $11: 17: 34$ & 0.4418 \\
\hline 5434 & $11: 17: 35$ & 0.4426 \\
\hline 5435 & $11: 17: 36$ & 0.4420 \\
\hline 5436 & $11: 17: 37$ & 0.4447 \\
\hline 5437 & $11: 17: 38$ & 0.4423 \\
\hline 5438 & $11: 17: 39$ & 0.4463 \\
\hline 5439 & $11: 17: 40$ & 0.4429 \\
\hline 5440 & $11: 17: 41$ & 0.4456 \\
\hline 5441 & $11: 17: 42$ & 0.4458 \\
\hline 5442 & $11: 17: 43$ & 0.4438 \\
\hline 5443 & $11: 17: 44$ & 0.4444 \\
\hline 5444 & $11: 17: 45$ & 0.4403 \\
\hline 5445 & $11: 17: 46$ & 0.4448 \\
\hline 5446 & $11: 17: 47$ & 0.4424 \\
\hline 5447 & $11: 17: 48$ & 0.4437 \\
\hline 5448 & $11: 17: 49$ & 0.4424 \\
\hline 5449 & $11: 17: 50$ & 0.4421 \\
\hline 5450 & $11: 17: 51$ & 0.4426 \\
\hline 5451 & $11: 17: 52$ & 0.4409 \\
\hline 5452 & $11: 17: 53$ & 0.4430 \\
\hline 5453 & $11: 17: 54$ & 0.4387 \\
\hline 5454 & $11: 17: 55$ & 0.4407 \\
\hline 5455 & $11: 17: 56$ & 0.4389 \\
\hline
\end{tabular}


Appendix C - Fuel Data Test 2 NIST102605_1.xIs

\begin{tabular}{|c|c|c|}
\hline 5456 & $11: 17: 57$ & 0.4423 \\
\hline 5457 & $11: 17: 58$ & 0.4441 \\
\hline 5458 & $11: 17: 59$ & 0.4414 \\
\hline 5459 & $11: 18: 00$ & 0.4462 \\
\hline 5460 & $11: 18: 01$ & 0.4439 \\
\hline 5461 & $11: 18: 02$ & 0.4445 \\
\hline 5462 & $11: 18: 03$ & 0.4464 \\
\hline 5463 & $11: 18: 04$ & 0.4433 \\
\hline 5464 & 11:18:05 & 0.4464 \\
\hline 5465 & $11: 18: 06$ & 0.4428 \\
\hline 5466 & $11: 18: 07$ & 0.4448 \\
\hline 5467 & $11: 18: 08$ & 0.4439 \\
\hline 5468 & $11: 18: 09$ & 0.4411 \\
\hline 5469 & $11: 18: 10$ & 0.4437 \\
\hline 5470 & $11: 18: 11$ & 0.4418 \\
\hline 5471 & $11: 18: 12$ & 0.4455 \\
\hline 5472 & 11:18:13 & 0.4426 \\
\hline 5473 & 11:18:14 & 0.4404 \\
\hline 5474 & $11: 18: 15$ & 0.4430 \\
\hline 5475 & $11: 18: 16$ & 0.4401 \\
\hline 5476 & $11: 18: 17$ & 0.4429 \\
\hline 5477 & $11: 18: 18$ & 0.4404 \\
\hline 5478 & $11: 18: 19$ & 0.4410 \\
\hline 5479 & $11: 18: 20$ & 0.4405 \\
\hline 5480 & $11: 18: 21$ & 0.4448 \\
\hline 5481 & 11:18:22 & 0.4464 \\
\hline 5482 & $11: 18: 23$ & 0.4458 \\
\hline 5483 & $11: 18: 24$ & 0.4460 \\
\hline 5484 & $11: 18: 25$ & 0.4427 \\
\hline 5485 & $11: 18: 26$ & 0.4454 \\
\hline 5486 & $11: 18: 27$ & 0.4422 \\
\hline 5487 & $11: 18: 28$ & 0.4437 \\
\hline 5488 & $11: 18: 29$ & 0.4456 \\
\hline 5489 & $11: 18: 30$ & 0.4407 \\
\hline 5490 & 11:18:31 & 0.4421 \\
\hline 5491 & $11: 18: 32$ & 0.4403 \\
\hline 5492 & $11: 18: 33$ & 0.4438 \\
\hline 5493 & $11: 18: 34$ & 0.4426 \\
\hline 5494 & $11: 18: 35$ & 0.4436 \\
\hline 5495 & $11: 18: 36$ & 0.4406 \\
\hline 5496 & $11: 18: 37$ & 0.4409 \\
\hline 5497 & $11: 18: 38$ & 0.4382 \\
\hline 5498 & $11: 18: 39$ & 0.4387 \\
\hline 5499 & $11: 18: 40$ & 0.4438 \\
\hline 5500 & $11: 18: 41$ & 0.4426 \\
\hline 5501 & $11: 18: 42$ & 0.4449 \\
\hline 5502 & $11: 18: 43$ & 0.4413 \\
\hline 5503 & $11: 18: 44$ & 0.4448 \\
\hline 5504 & $11: 18: 45$ & 0.4456 \\
\hline 5505 & $11: 18: 46$ & 0.4436 \\
\hline 5506 & $11: 18: 47$ & 0.4476 \\
\hline
\end{tabular}


Appendix C - Fuel Data Test 2 NIST102605_1.xls

\begin{tabular}{|c|c|c|}
\hline 5507 & $11: 18: 48$ & 0.4445 \\
\hline 5508 & $11: 18: 49$ & 0.4452 \\
\hline 5509 & $11: 18: 50$ & 0.4455 \\
\hline 5510 & $11: 18: 51$ & 0.4424 \\
\hline 5511 & $11: 18: 52$ & 0.4425 \\
\hline 5512 & $11: 18: 53$ & 0.4397 \\
\hline 5513 & $11: 18: 54$ & 0.4448 \\
\hline 5514 & $11: 18: 55$ & 0.4415 \\
\hline 5515 & 11:18:56 & 0.4422 \\
\hline 5516 & $11: 18: 57$ & 0.4402 \\
\hline 5517 & $11: 18: 58$ & 0.4392 \\
\hline 5518 & $11: 18: 59$ & 0.4434 \\
\hline 5519 & $11: 19: 00$ & 0.4410 \\
\hline 5520 & $11: 19: 01$ & 0.4444 \\
\hline 5521 & 11:19:02 & 0.4409 \\
\hline 5522 & $11: 19: 03$ & 0.4425 \\
\hline 5523 & $11: 19: 04$ & 0.4404 \\
\hline 5524 & 11:19:05 & 0.4428 \\
\hline 5525 & $11: 19: 06$ & 0.4453 \\
\hline 5526 & $11: 19: 07$ & 0.4425 \\
\hline 5527 & $11: 19: 08$ & 0.4459 \\
\hline 5528 & $11: 19: 09$ & 0.4451 \\
\hline 5529 & $11: 19: 10$ & 0.4453 \\
\hline 5530 & $11: 19: 11$ & 0.4442 \\
\hline 5531 & $11: 19: 12$ & 0.4418 \\
\hline 5532 & 11:19:13 & 0.4449 \\
\hline 5533 & $11: 19: 14$ & 0.4411 \\
\hline 5534 & $11: 19: 15$ & 0.4429 \\
\hline 5535 & $11: 19: 16$ & 0.4413 \\
\hline 5536 & $11: 19: 17$ & 0.4430 \\
\hline 5537 & $11: 19: 18$ & 0.4405 \\
\hline 5538 & $11: 19: 19$ & 0.4407 \\
\hline 5539 & $11: 19: 20$ & 0.4434 \\
\hline 5540 & $11: 19: 21$ & 0.4404 \\
\hline 5541 & 11:19:22 & 0.4417 \\
\hline 5542 & $11: 19: 23$ & 0.4386 \\
\hline 5543 & $11: 19: 24$ & 0.4422 \\
\hline 5544 & $11: 19: 25$ & 0.4438 \\
\hline 5545 & $11: 19: 26$ & 0.4418 \\
\hline 5546 & $11: 19: 27$ & 0.4467 \\
\hline 5547 & $11: 19: 28$ & 0.4436 \\
\hline 5548 & $11: 19: 29$ & 0.4456 \\
\hline 5549 & $11: 19: 30$ & 0.4470 \\
\hline 5550 & $11: 19: 31$ & 0.4462 \\
\hline 5551 & $11: 19: 32$ & 0.4465 \\
\hline 5552 & $11: 19: 33$ & 0.4421 \\
\hline 5553 & $11: 19: 34$ & 0.4441 \\
\hline 5554 & $11: 19: 35$ & 0.4413 \\
\hline 5555 & $11: 19: 36$ & 0.4429 \\
\hline 5556 & $11: 19: 37$ & 0.4413 \\
\hline 5557 & $11: 19: 38$ & 0.4407 \\
\hline
\end{tabular}


Appendix C - Fuel Data Test 2 NIST102605_1.xIs

\begin{tabular}{|c|c|c|}
\hline 5558 & $11: 19: 39$ & 0.4446 \\
\hline 5559 & $11: 19: 40$ & 0.4410 \\
\hline 5560 & $11: 19: 41$ & 0.4444 \\
\hline 5561 & $11: 19: 42$ & 0.4401 \\
\hline 5562 & $11: 19: 43$ & 0.4405 \\
\hline 5563 & $11: 19: 44$ & 0.4393 \\
\hline 5564 & $11: 19: 45$ & 0.4414 \\
\hline 5565 & $11: 19: 46$ & 0.4413 \\
\hline 5566 & $11: 19: 47$ & 0.4423 \\
\hline 5567 & $11: 19: 48$ & 0.4459 \\
\hline 5568 & $11: 19: 49$ & 0.4423 \\
\hline 5569 & $11: 19: 50$ & 0.4460 \\
\hline 5570 & $11: 19: 51$ & 0.4453 \\
\hline 5571 & $11: 19: 52$ & 0.4458 \\
\hline 5572 & $11: 19: 53$ & 0.4474 \\
\hline 5573 & $11: 19: 54$ & 0.4407 \\
\hline 5574 & $11: 19: 55$ & 0.4439 \\
\hline 5575 & $11: 19: 56$ & 0.4445 \\
\hline 5576 & $11: 19: 57$ & 0.4407 \\
\hline 5577 & $11: 19: 58$ & 0.4445 \\
\hline 5578 & $11: 19: 59$ & 0.4433 \\
\hline 5579 & $11: 20: 00$ & 0.4436 \\
\hline 5580 & $11: 20: 01$ & 0.4416 \\
\hline 5581 & $11: 20: 02$ & 0.4401 \\
\hline 5582 & $11: 20: 03$ & 0.4422 \\
\hline 5583 & $11: 20: 04$ & 0.4399 \\
\hline 5584 & $11: 20: 05$ & 0.4418 \\
\hline 5585 & $11: 20: 06$ & 0.4399 \\
\hline 5586 & $11: 20: 07$ & 0.4433 \\
\hline 5587 & $11: 20: 08$ & 0.4397 \\
\hline 5588 & $11: 20: 09$ & 0.4424 \\
\hline 5589 & $11: 20: 10$ & 0.4457 \\
\hline 5590 & $11: 20: 11$ & 0.4445 \\
\hline 5591 & $11: 20: 12$ & 0.4468 \\
\hline 5592 & $11: 20: 13$ & 0.4432 \\
\hline 5593 & $11: 20: 14$ & 0.4452 \\
\hline 5594 & $11: 20: 15$ & 0.4424 \\
\hline 5595 & $11: 20: 16$ & 0.4453 \\
\hline 5596 & $11: 20: 17$ & 0.4445 \\
\hline 5597 & $11: 20: 18$ & 0.4426 \\
\hline 5598 & $11: 20: 19$ & 0.4440 \\
\hline 5599 & $11: 20: 20$ & 0.4399 \\
\hline 5600 & $11: 20: 21$ & 0.4418 \\
\hline 5601 & $11: 20: 22$ & 0.4401 \\
\hline 5602 & $11: 20: 23$ & 0.4433 \\
\hline 5603 & $11: 20: 24$ & 0.4417 \\
\hline 5604 & $11: 20: 25$ & 0.4402 \\
\hline 5605 & $11: 20: 26$ & 0.4430 \\
\hline 5606 & $11: 20: 27$ & 0.4400 \\
\hline 5607 & $11: 20: 28$ & 0.4438 \\
\hline 5608 & $11: 20: 29$ & 0.4420 \\
\hline
\end{tabular}


Appendix C - Fuel Data Test 2 NIST102605_1.xIs

\begin{tabular}{|c|c|c|}
\hline 5609 & $11: 20: 30$ & 0.4448 \\
\hline 5610 & $11: 20: 31$ & 0.4415 \\
\hline 5611 & $11: 20: 32$ & 0.4431 \\
\hline 5612 & $11: 20: 33$ & 0.4425 \\
\hline 5613 & $11: 20: 34$ & 0.4459 \\
\hline 5614 & $11: 20: 35$ & 0.4443 \\
\hline 5615 & $11: 20: 36$ & 0.4447 \\
\hline 5616 & $11: 20: 37$ & 0.4477 \\
\hline 5617 & $11: 20: 38$ & 0.4417 \\
\hline 5618 & $11: 20: 39$ & 0.4430 \\
\hline 5619 & $11: 20: 40$ & 0.4407 \\
\hline 5620 & $11: 20: 41$ & 0.4449 \\
\hline 5621 & $11: 20: 42$ & 0.4440 \\
\hline 5622 & $11: 20: 43$ & 0.4440 \\
\hline 5623 & $11: 20: 44$ & 0.4416 \\
\hline 5624 & $11: 20: 45$ & 0.4405 \\
\hline 5625 & $11: 20: 46$ & 0.4431 \\
\hline 5626 & $11: 20: 47$ & 0.4415 \\
\hline 5627 & $11: 20: 48$ & 0.4433 \\
\hline 5628 & $11: 20: 49$ & 0.4389 \\
\hline 5629 & $11: 20: 50$ & 0.4404 \\
\hline 5630 & $11: 20: 51$ & 0.4403 \\
\hline 5631 & $11: 20: 52$ & 0.4438 \\
\hline 5632 & $11: 20: 53$ & 0.4464 \\
\hline 5633 & $11: 20: 54$ & 0.4428 \\
\hline 5634 & $11: 20: 55$ & 0.4468 \\
\hline 5635 & $11: 20: 56$ & 0.4434 \\
\hline 5636 & $11: 20: 57$ & 0.4445 \\
\hline 5637 & $11: 20: 58$ & 0.4465 \\
\hline 5638 & $11: 20: 59$ & 0.4469 \\
\hline 5639 & $11: 21: 00$ & 0.4457 \\
\hline 5640 & $11: 21: 01$ & 0.4420 \\
\hline 5641 & $11: 21: 02$ & 0.4428 \\
\hline 5642 & $11: 21: 03$ & 0.4399 \\
\hline 5643 & $11: 21: 04$ & 0.4435 \\
\hline 5644 & $11: 21: 05$ & 0.4405 \\
\hline 5645 & $11: 21: 06$ & 0.4415 \\
\hline 5646 & $11: 21: 07$ & 0.4409 \\
\hline 5647 & $11: 21: 08$ & 0.4390 \\
\hline 5648 & $11: 21: 09$ & 0.4439 \\
\hline 5649 & $11: 21: 10$ & 0.4416 \\
\hline 5650 & $11: 21: 11$ & 0.4433 \\
\hline 5651 & $11: 21: 12$ & 0.4389 \\
\hline 5652 & $11: 21: 13$ & 0.4407 \\
\hline 5653 & $11: 21: 14$ & 0.4432 \\
\hline 5654 & $11: 21: 15$ & 0.4427 \\
\hline 5655 & $11: 21: 16$ & 0.4450 \\
\hline 5656 & $11: 21: 17$ & 0.4427 \\
\hline 5657 & $11: 21: 18$ & 0.4457 \\
\hline 5658 & $11: 21: 19$ & 0.4412 \\
\hline 5659 & $11: 21: 20$ & 0.4461 \\
\hline
\end{tabular}


Appendix C - Fuel Data Test 2 NIST102605_1.xIs

\begin{tabular}{|c|c|c|}
\hline 5660 & $11: 21: 21$ & 0.4458 \\
\hline 5661 & $11: 21: 22$ & 0.4464 \\
\hline 5662 & $11: 21: 23$ & 0.4442 \\
\hline 5663 & $11: 21: 24$ & 0.4410 \\
\hline 5664 & $11: 21: 25$ & 0.4425 \\
\hline 5665 & $11: 21: 26$ & 0.4402 \\
\hline 5666 & $11: 21: 27$ & 0.4435 \\
\hline 5667 & $11: 21: 28$ & 0.4411 \\
\hline 5668 & $11: 21: 29$ & 0.4436 \\
\hline 5669 & $11: 21: 30$ & 0.4408 \\
\hline 5670 & $11: 21: 31$ & 0.4418 \\
\hline 5671 & $11: 21: 32$ & 0.4399 \\
\hline 5672 & $11: 21: 33$ & 0.4402 \\
\hline 5673 & $11: 21: 34$ & 0.4414 \\
\hline 5674 & $11: 21: 35$ & 0.4384 \\
\hline 5675 & $11: 21: 36$ & 0.4441 \\
\hline 5676 & $11: 21: 37$ & 0.4420 \\
\hline 5677 & $11: 21: 38$ & 0.4441 \\
\hline 5678 & $11: 21: 39$ & 0.4470 \\
\hline 5679 & $11: 21: 40$ & 0.4470 \\
\hline 5680 & $11: 21: 41$ & 0.4469 \\
\hline 5681 & $11: 21: 42$ & 0.4412 \\
\hline 5682 & $11: 21: 43$ & 0.4445 \\
\hline 5683 & $11: 21: 44$ & 0.4435 \\
\hline 5684 & $11: 21: 45$ & 0.4456 \\
\hline 5685 & $11: 21: 46$ & 0.4435 \\
\hline 5686 & $11: 21: 47$ & 0.4454 \\
\hline 5687 & $11: 21: 48$ & 0.4433 \\
\hline 5688 & $11: 21: 49$ & 0.4423 \\
\hline 5689 & $11: 21: 50$ & 0.4414 \\
\hline 5690 & $11: 21: 51$ & 0.4404 \\
\hline 5691 & $11: 21: 52$ & 0.4426 \\
\hline 5692 & $11: 21: 53$ & 0.4394 \\
\hline 5693 & $11: 21: 54$ & 0.4438 \\
\hline 5694 & $11: 21: 55$ & 0.4414 \\
\hline 5695 & $11: 21: 56$ & 0.4426 \\
\hline 5696 & $11: 21: 57$ & 0.4397 \\
\hline 5697 & $11: 21: 58$ & 0.4431 \\
\hline 5698 & $11: 21: 59$ & 0.4450 \\
\hline 5699 & $11: 22: 00$ & 0.4444 \\
\hline 5700 & $11: 22: 01$ & 0.4461 \\
\hline 5701 & $11: 22: 02$ & 0.4426 \\
\hline 5702 & $11: 22: 03$ & 0.4464 \\
\hline 5703 & $11: 22: 04$ & 0.4432 \\
\hline 5704 & $11: 22: 05$ & 0.4445 \\
\hline 5705 & $11: 22: 06$ & 0.4448 \\
\hline 5706 & $11: 22: 07$ & 0.4415 \\
\hline 5707 & $11: 22: 08$ & 0.4449 \\
\hline 5708 & $11: 22: 09$ & 0.4439 \\
\hline 5709 & $11: 22: 10$ & 0.4454 \\
\hline 5710 & $11: 22: 11$ & 0.4419 \\
\hline
\end{tabular}


Appendix C - Fuel Data Test 2 NIST102605_1.xls

\begin{tabular}{|c|c|c|}
\hline 5711 & $11: 22: 12$ & 0.4417 \\
\hline 5712 & $11: 22: 13$ & 0.4412 \\
\hline 5713 & $11: 22: 14$ & 0.4412 \\
\hline 5714 & $11: 22: 15$ & 0.4428 \\
\hline 5715 & $11: 22: 16$ & 0.4391 \\
\hline 5716 & $11: 22: 17$ & 0.4428 \\
\hline 5717 & $11: 22: 18$ & 0.4387 \\
\hline 5718 & $11: 22: 19$ & 0.4426 \\
\hline 5719 & $11: 22: 20$ & 0.4445 \\
\hline 5720 & $11: 22: 21$ & 0.4454 \\
\hline 5721 & $11: 22: 22$ & 0.4469 \\
\hline 5722 & $11: 22: 23$ & 0.4425 \\
\hline 5723 & $11: 22: 24$ & 0.4465 \\
\hline 5724 & $11: 22: 25$ & 0.4451 \\
\hline 5725 & $11: 22: 26$ & 0.4460 \\
\hline 5726 & $11: 22: 27$ & 0.4446 \\
\hline 5727 & $11: 22: 28$ & 0.4405 \\
\hline 5728 & $11: 22: 29$ & 0.4441 \\
\hline 5729 & $11: 22: 30$ & 0.4420 \\
\hline 5730 & $11: 22: 31$ & 0.4444 \\
\hline 5731 & $11: 22: 32$ & 0.4428 \\
\hline 5732 & $11: 22: 33$ & 0.4415 \\
\hline 5733 & $11: 22: 34$ & 0.4416 \\
\hline 5734 & $11: 22: 35$ & 0.4402 \\
\hline 5735 & $11: 22: 36$ & 0.4431 \\
\hline 5736 & $11: 22: 37$ & 0.4406 \\
\hline 5737 & $11: 22: 38$ & 0.4437 \\
\hline 5738 & $11: 22: 39$ & 0.4407 \\
\hline 5739 & $11: 22: 40$ & 0.4421 \\
\hline 5740 & $11: 22: 41$ & 0.4398 \\
\hline 5741 & $11: 22: 42$ & 0.4439 \\
\hline 5742 & $11: 22: 43$ & 0.4459 \\
\hline 5743 & $11: 22: 44$ & 0.4455 \\
\hline 5744 & $11: 22: 45$ & 0.4458 \\
\hline 5745 & $11: 22: 46$ & 0.4447 \\
\hline 5746 & $11: 22: 47$ & 0.4467 \\
\hline 5747 & $11: 22: 48$ & 0.4418 \\
\hline 5748 & $11: 22: 49$ & 0.4439 \\
\hline 5749 & $11: 22: 50$ & 0.4407 \\
\hline 5750 & $11: 22: 51$ & 0.4444 \\
\hline 5751 & $11: 22: 52$ & 0.4438 \\
\hline 5752 & $11: 22: 53$ & 0.4418 \\
\hline 5753 & $11: 22: 54$ & 0.4429 \\
\hline 5754 & $11: 22: 55$ & 0.4399 \\
\hline 5755 & $11: 22: 56$ & 0.4427 \\
\hline 5756 & $11: 22: 57$ & 0.4411 \\
\hline 5757 & $11: 22: 58$ & 0.4434 \\
\hline 5758 & $11: 22: 59$ & 0.4387 \\
\hline 5759 & $11: 23: 00$ & 0.4396 \\
\hline 5760 & $11: 23: 01$ & 0.4403 \\
\hline 5761 & $11: 23: 02$ & 0.4409 \\
\hline
\end{tabular}


Appendix C - Fuel Data Test 2 NIST102605_1.xIs

\begin{tabular}{|c|c|c|}
\hline 5762 & $11: 23: 03$ & 0.4440 \\
\hline 5763 & $11: 23: 04$ & 0.4411 \\
\hline 5764 & $11: 23: 05$ & 0.4454 \\
\hline 5765 & $11: 23: 06$ & 0.4460 \\
\hline 5766 & $11: 23: 07$ & 0.4443 \\
\hline 5767 & $11: 23: 08$ & 0.4473 \\
\hline 5768 & 11:23:09 & 0.4422 \\
\hline 5769 & $11: 23: 10$ & 0.4441 \\
\hline 5770 & $11: 23: 11$ & 0.4410 \\
\hline 5771 & $11: 23: 12$ & 0.4444 \\
\hline 5772 & $11: 23: 13$ & 0.4444 \\
\hline 5773 & $11: 23: 14$ & 0.4417 \\
\hline 5774 & $11: 23: 15$ & 0.4420 \\
\hline 5775 & $11: 23: 16$ & 0.4401 \\
\hline 5776 & $11: 23: 17$ & 0.4432 \\
\hline 5777 & 11:23:18 & 0.4408 \\
\hline 5778 & $11: 23: 19$ & 0.4448 \\
\hline 5779 & $11: 23: 20$ & 0.4415 \\
\hline 5780 & $11: 23: 21$ & 0.4425 \\
\hline 5781 & $11: 23: 22$ & 0.4394 \\
\hline 5782 & $11: 23: 23$ & 0.4431 \\
\hline 5783 & $11: 23: 24$ & 0.4411 \\
\hline 5784 & $11: 23: 25$ & 0.4420 \\
\hline 5785 & 11:23:26 & 0.4421 \\
\hline 5786 & $11: 23: 27$ & 0.4434 \\
\hline 5787 & $11: 23: 28$ & 0.4467 \\
\hline 5788 & $11: 23: 29$ & 0.4424 \\
\hline 5789 & $11: 23: 30$ & 0.4460 \\
\hline 5790 & $11: 23: 31$ & 0.4448 \\
\hline 5791 & $11: 23: 32$ & 0.4460 \\
\hline 5792 & $11: 23: 33$ & 0.4419 \\
\hline 5793 & $11: 23: 34$ & 0.4437 \\
\hline 5794 & 11:23:35 & 0.4432 \\
\hline 5795 & $11: 23: 36$ & 0.4426 \\
\hline 5796 & $11: 23: 37$ & 0.4443 \\
\hline 5797 & $11: 23: 38$ & 0.4419 \\
\hline 5798 & $11: 23: 39$ & 0.4450 \\
\hline 5799 & $11: 23: 40$ & 0.4420 \\
\hline 5800 & $11: 23: 41$ & 0.4431 \\
\hline 5801 & $11: 23: 42$ & 0.4409 \\
\hline 5802 & $11: 23: 43$ & 0.4407 \\
\hline 5803 & $11: 23: 44$ & 0.4389 \\
\hline 5804 & $11: 23: 45$ & 0.4391 \\
\hline 5805 & $11: 23: 46$ & 0.4433 \\
\hline 5806 & $11: 23: 47$ & 0.4409 \\
\hline 5807 & $11: 23: 48$ & 0.4451 \\
\hline 5808 & $11: 23: 49$ & 0.4453 \\
\hline 5809 & $11: 23: 50$ & 0.4465 \\
\hline 5810 & $11: 23: 51$ & 0.4466 \\
\hline 5811 & $11: 23: 52$ & 0.4453 \\
\hline 5812 & $11: 23: 53$ & 0.4468 \\
\hline
\end{tabular}


Appendix C - Fuel Data Test 2 NIST102605_1.xIs

\begin{tabular}{|c|c|c|}
\hline 5813 & $11: 23: 54$ & 0.4419 \\
\hline 5814 & $11: 23: 55$ & 0.4442 \\
\hline 5815 & $11: 23: 56$ & 0.4407 \\
\hline 5816 & $11: 23: 57$ & 0.4443 \\
\hline 5817 & $11: 23: 58$ & 0.4427 \\
\hline 5818 & $11: 23: 59$ & 0.4414 \\
\hline 5819 & $11: 24: 00$ & 0.4426 \\
\hline 5820 & $11: 24: 01$ & 0.4404 \\
\hline 5821 & $11: 24: 02$ & 0.4449 \\
\hline 5822 & $11: 24: 03$ & 0.4402 \\
\hline 5823 & $11: 24: 04$ & 0.4404 \\
\hline 5824 & $11: 24: 05$ & 0.4424 \\
\hline 5825 & $11: 24: 06$ & 0.4397 \\
\hline 5826 & $11: 24: 07$ & 0.4439 \\
\hline 5827 & $11: 24: 08$ & 0.4409 \\
\hline 5828 & $11: 24: 09$ & 0.4419 \\
\hline 5829 & $11: 24: 10$ & 0.4425 \\
\hline 5830 & $11: 24: 11$ & 0.4447 \\
\hline 5831 & $11: 24: 12$ & 0.4470 \\
\hline 5832 & $11: 24: 13$ & 0.4425 \\
\hline 5833 & $11: 24: 14$ & 0.4448 \\
\hline 5834 & $11: 24: 15$ & 0.4421 \\
\hline 5835 & $11: 24: 16$ & 0.4453 \\
\hline 5836 & $11: 24: 17$ & 0.4439 \\
\hline 5837 & $11: 24: 18$ & 0.4440 \\
\hline 5838 & $11: 24: 19$ & 0.4445 \\
\hline 5839 & $11: 24: 20$ & 0.4410 \\
\hline 5840 & $11: 24: 21$ & 0.4415 \\
\hline 5841 & $11: 24: 22$ & 0.4402 \\
\hline 5842 & $11: 24: 23$ & 0.4448 \\
\hline 5843 & $11: 24: 24$ & 0.4421 \\
\hline 5844 & $11: 24: 25$ & 0.4412 \\
\hline 5845 & $11: 24: 26$ & 0.4400 \\
\hline 5846 & $11: 24: 27$ & 0.4396 \\
\hline 5847 & $11: 24: 28$ & 0.4431 \\
\hline 5848 & $11: 24: 29$ & 0.4390 \\
\hline 5849 & $11: 24: 30$ & 0.4441 \\
\hline 5850 & $11: 24: 31$ & 0.4420 \\
\hline 5851 & $11: 24: 32$ & 0.4436 \\
\hline 5852 & $11: 24: 33$ & 0.4459 \\
\hline 5853 & $11: 24: 34$ & 0.4461 \\
\hline 5854 & $11: 24: 35$ & 0.4478 \\
\hline 5855 & $11: 24: 36$ & 0.4424 \\
\hline 5856 & $11: 24: 37$ & 0.4444 \\
\hline 5857 & $11: 24: 38$ & 0.4412 \\
\hline 5858 & $11: 24: 39$ & 0.4445 \\
\hline 5859 & $11: 24: 40$ & 0.4443 \\
\hline 5860 & $11: 24: 41$ & 0.4439 \\
\hline 5861 & $11: 24: 42$ & 0.4432 \\
\hline 5862 & $11: 24: 43$ & 0.4390 \\
\hline 5863 & $11: 24: 44$ & 0.4461 \\
\hline
\end{tabular}


Appendix C - Fuel Data Test 2 NIST102605_1.xIs

\begin{tabular}{|c|c|c|}
\hline 5864 & $11: 24: 45$ & 0.4421 \\
\hline 5865 & $11: 24: 46$ & 0.4419 \\
\hline 5866 & $11: 24: 47$ & 0.4416 \\
\hline 5867 & $11: 24: 48$ & 0.4407 \\
\hline 5868 & $11: 24: 49$ & 0.4436 \\
\hline 5869 & $11: 24: 50$ & 0.4400 \\
\hline 5870 & $11: 24: 51$ & 0.4430 \\
\hline 5871 & $11: 24: 52$ & 0.4416 \\
\hline 5872 & $11: 24: 53$ & 0.4450 \\
\hline 5873 & $11: 24: 54$ & 0.4417 \\
\hline 5874 & $11: 24: 55$ & 0.4441 \\
\hline 5875 & $11: 24: 56$ & 0.4438 \\
\hline 5876 & $11: 24: 57$ & 0.4433 \\
\hline 5877 & $11: 24: 58$ & 0.4453 \\
\hline 5878 & $11: 24: 59$ & 0.4429 \\
\hline 5879 & $11: 25: 00$ & 0.4467 \\
\hline 5880 & $11: 25: 01$ & 0.4409 \\
\hline 5881 & $11: 25: 02$ & 0.4431 \\
\hline 5882 & $11: 25: 03$ & 0.4407 \\
\hline 5883 & $11: 25: 04$ & 0.4431 \\
\hline 5884 & $11: 25: 05$ & 0.4423 \\
\hline 5885 & $11: 25: 06$ & 0.4392 \\
\hline 5886 & $11: 25: 07$ & 0.4410 \\
\hline 5887 & $11: 25: 08$ & 0.4408 \\
\hline 5888 & $11: 25: 09$ & 0.4433 \\
\hline 5889 & $11: 25: 10$ & 0.4409 \\
\hline 5890 & $11: 25: 11$ & 0.4440 \\
\hline 5891 & $11: 25: 12$ & 0.4396 \\
\hline 5892 & $11: 25: 13$ & 0.4413 \\
\hline 5893 & $11: 25: 14$ & 0.4415 \\
\hline 5894 & $11: 25: 15$ & 0.4422 \\
\hline 5895 & $11: 25: 16$ & 0.4452 \\
\hline 5896 & $11: 25: 17$ & 0.4420 \\
\hline 5897 & $11: 25: 18$ & 0.4460 \\
\hline 5898 & $11: 25: 19$ & 0.4454 \\
\hline 5899 & $11: 25: 20$ & 0.4449 \\
\hline 5900 & $11: 25: 21$ & 0.4456 \\
\hline 5901 & $11: 25: 22$ & 0.4428 \\
\hline 5902 & $11: 25: 23$ & 0.4449 \\
\hline 5903 & $11: 25: 24$ & 0.4413 \\
\hline 5904 & $11: 25: 25$ & 0.4444 \\
\hline 5905 & $11: 25: 26$ & 0.4415 \\
\hline 5906 & $11: 25: 27$ & 0.4440 \\
\hline 5907 & $11: 25: 28$ & 0.4416 \\
\hline 5908 & $11: 25: 29$ & 0.4409 \\
\hline 5909 & $11: 25: 30$ & 0.4423 \\
\hline 5910 & $11: 25: 31$ & 0.4392 \\
\hline 5911 & $11: 25: 32$ & 0.4444 \\
\hline 5912 & $11: 25: 33$ & 0.4416 \\
\hline 5913 & $11: 25: 34$ & 0.4433 \\
\hline 5914 & $11: 25: 35$ & 0.4392 \\
\hline
\end{tabular}


Appendix C - Fuel Data Test 2 NIST102605_1.xIs

\begin{tabular}{|c|c|c|}
\hline 5915 & $11: 25: 36$ & 0.4412 \\
\hline 5916 & $11: 25: 37$ & 0.4412 \\
\hline 5917 & $11: 25: 38$ & 0.4441 \\
\hline 5918 & $11: 25: 39$ & 0.4450 \\
\hline 5919 & $11: 25: 40$ & 0.4435 \\
\hline 5920 & $11: 25: 41$ & 0.4465 \\
\hline 5921 & $11: 25: 42$ & 0.4420 \\
\hline 5922 & $11: 25: 43$ & 0.4438 \\
\hline 5923 & $11: 25: 44$ & 0.4423 \\
\hline 5924 & $11: 25: 45$ & 0.4460 \\
\hline 5925 & $11: 25: 46$ & 0.4430 \\
\hline 5926 & $11: 25: 47$ & 0.4434 \\
\hline 5927 & $11: 25: 48$ & 0.4440 \\
\hline 5928 & $11: 25: 49$ & 0.4403 \\
\hline 5929 & $11: 25: 50$ & 0.4435 \\
\hline 5930 & $11: 25: 51$ & 0.4423 \\
\hline 5931 & $11: 25: 52$ & 0.4443 \\
\hline 5932 & $11: 25: 53$ & 0.4393 \\
\hline 5933 & $11: 25: 54$ & 0.4390 \\
\hline 5934 & $11: 25: 55$ & 0.4401 \\
\hline 5935 & $11: 25: 56$ & 0.4414 \\
\hline 5936 & $11: 25: 57$ & 0.4443 \\
\hline 5937 & $11: 25: 58$ & 0.4395 \\
\hline 5938 & $11: 25: 59$ & 0.4437 \\
\hline 5939 & $11: 26: 00$ & 0.4420 \\
\hline 5940 & $11: 26: 01$ & 0.4445 \\
\hline 5941 & $11: 26: 02$ & 0.4472 \\
\hline 5942 & $11: 26: 03$ & 0.4437 \\
\hline 5943 & $11: 26: 04$ & 0.4466 \\
\hline 5944 & $11: 26: 05$ & 0.4433 \\
\hline 5945 & $11: 26: 06$ & 0.4444 \\
\hline 5946 & $11: 26: 07$ & 0.4434 \\
\hline 5947 & $11: 26: 08$ & 0.4431 \\
\hline 5948 & $11: 26: 09$ & 0.4446 \\
\hline 5949 & $11: 26: 10$ & 0.4418 \\
\hline 5950 & $11: 26: 11$ & 0.4442 \\
\hline 5951 & $11: 26: 12$ & 0.4410 \\
\hline 5952 & $11: 26: 13$ & 0.4431 \\
\hline 5953 & $11: 26: 14$ & 0.4426 \\
\hline 5954 & $11: 26: 15$ & 0.4394 \\
\hline 5955 & $11: 26: 16$ & 0.4438 \\
\hline 5956 & $11: 26: 17$ & 0.4419 \\
\hline 5957 & $11: 26: 18$ & 0.4440 \\
\hline 5958 & $11: 26: 19$ & 0.4393 \\
\hline 5959 & $11: 26: 20$ & 0.4420 \\
\hline 5960 & $11: 26: 21$ & 0.4423 \\
\hline 5961 & $11: 26: 22$ & 0.4445 \\
\hline 5962 & $11: 26: 23$ & 0.4453 \\
\hline 5963 & $11: 26: 24$ & 0.4439 \\
\hline 5964 & $11: 26: 25$ & 0.4465 \\
\hline 5965 & $11: 26: 26$ & 0.4420 \\
\hline
\end{tabular}


Appendix C - Fuel Data Test 2 NIST102605_1.xIs

\begin{tabular}{|c|c|c|}
\hline 5966 & $11: 26: 27$ & 0.4456 \\
\hline 5967 & $11: 26: 28$ & 0.4456 \\
\hline 5968 & $11: 26: 29$ & 0.4431 \\
\hline 5969 & $11: 26: 30$ & 0.4425 \\
\hline 5970 & $11: 26: 31$ & 0.4401 \\
\hline 5971 & $11: 26: 32$ & 0.4451 \\
\hline 5972 & $11: 26: 33$ & 0.4434 \\
\hline 5973 & $11: 26: 34$ & 0.4439 \\
\hline 5974 & $11: 26: 35$ & 0.4421 \\
\hline 5975 & $11: 26: 36$ & 0.4413 \\
\hline 5976 & $11: 26: 37$ & 0.4390 \\
\hline 5977 & $11: 26: 38$ & 0.4379 \\
\hline 5978 & $11: 26: 39$ & 0.4420 \\
\hline 5979 & $11: 26: 40$ & 0.4383 \\
\hline 5980 & $11: 26: 41$ & 0.4413 \\
\hline 5981 & $11: 26: 42$ & 0.4441 \\
\hline 5982 & $11: 26: 43$ & 0.4437 \\
\hline 5983 & $11: 26: 44$ & 0.4464 \\
\hline 5984 & $11: 26: 45$ & 0.4424 \\
\hline 5985 & $11: 26: 46$ & 0.4464 \\
\hline 5986 & $11: 26: 47$ & 0.4446 \\
\hline 5987 & $11: 26: 48$ & 0.4453 \\
\hline 5988 & $11: 26: 49$ & 0.4451 \\
\hline 5989 & $11: 26: 50$ & 0.4407 \\
\hline 5990 & $11: 26: 51$ & 0.4443 \\
\hline 5991 & $11: 26: 52$ & 0.4432 \\
\hline 5992 & $11: 26: 53$ & 0.4438 \\
\hline 5993 & $11: 26: 54$ & 0.4431 \\
\hline 5994 & $11: 26: 55$ & 0.4404 \\
\hline 5995 & $11: 26: 56$ & 0.4445 \\
\hline 5996 & $11: 26: 57$ & 0.4407 \\
\hline 5997 & $11: 26: 58$ & 0.4436 \\
\hline 5998 & $11: 26: 59$ & 0.4410 \\
\hline 5999 & $11: 27: 00$ & 0.4428 \\
\hline 6000 & $11: 27: 01$ & 0.4396 \\
\hline 6001 & $11: 27: 02$ & 0.4404 \\
\hline 6002 & $11: 27: 03$ & 0.4390 \\
\hline 6003 & $11: 27: 04$ & 0.4423 \\
\hline 6004 & $11: 27: 05$ & 0.4434 \\
\hline 6005 & $11: 27: 06$ & 0.4444 \\
\hline 6006 & $11: 27: 07$ & 0.4441 \\
\hline 6007 & $11: 27: 08$ & 0.4443 \\
\hline 6008 & $11: 27: 09$ & 0.4466 \\
\hline 6009 & $11: 27: 10$ & 0.4413 \\
\hline 6010 & $11: 27: 11$ & 0.4445 \\
\hline 6011 & $11: 27: 12$ & 0.4419 \\
\hline 6012 & $11: 27: 13$ & 0.4438 \\
\hline 6013 & $11: 27: 14$ & 0.4427 \\
\hline 6014 & $11: 27: 15$ & 0.4421 \\
\hline 6015 & $11: 27: 16$ & 0.4437 \\
\hline 6016 & $11: 27: 17$ & 0.4411 \\
\hline
\end{tabular}


Appendix C - Fuel Data Test 2 NIST102605_1.xIs

\begin{tabular}{|c|c|c|}
\hline 6017 & $11: 27: 18$ & 0.4407 \\
\hline 6018 & $11: 27: 19$ & 0.4395 \\
\hline 6019 & $11: 27: 20$ & 0.4441 \\
\hline 6020 & $11: 27: 21$ & 0.4424 \\
\hline 6021 & $11: 27: 22$ & 0.4426 \\
\hline 6022 & $11: 27: 23$ & 0.4378 \\
\hline 6023 & $11: 27: 24$ & 0.4412 \\
\hline 6024 & $11: 27: 25$ & 0.4429 \\
\hline 6025 & $11: 27: 26$ & 0.4424 \\
\hline 6026 & $11: 27: 27$ & 0.4471 \\
\hline 6027 & $11: 27: 28$ & 0.4430 \\
\hline 6028 & $11: 27: 29$ & 0.4449 \\
\hline 6029 & $11: 27: 30$ & 0.4475 \\
\hline 6030 & $11: 27: 31$ & 0.4428 \\
\hline 6031 & $11: 27: 32$ & 0.4447 \\
\hline 6032 & $11: 27: 33$ & 0.4419 \\
\hline 6033 & $11: 27: 34$ & 0.4456 \\
\hline 6034 & $11: 27: 35$ & 0.4435 \\
\hline 6035 & $11: 27: 36$ & 0.4433 \\
\hline 6036 & $11: 27: 37$ & 0.4428 \\
\hline 6037 & $11: 27: 38$ & 0.4412 \\
\hline 6038 & $11: 27: 39$ & 0.4431 \\
\hline 6039 & $11: 27: 40$ & 0.4396 \\
\hline 6040 & $11: 27: 41$ & 0.4444 \\
\hline 6041 & $11: 27: 42$ & 0.4414 \\
\hline 6042 & $11: 27: 43$ & 0.4421 \\
\hline 6043 & $11: 27: 44$ & 0.4401 \\
\hline 6044 & $11: 27: 45$ & 0.4402 \\
\hline 6045 & $11: 27: 46$ & 0.4402 \\
\hline 6046 & $11: 27: 47$ & 0.4397 \\
\hline 6047 & $11: 27: 48$ & 0.4448 \\
\hline 6048 & $11: 27: 49$ & 0.4436 \\
\hline 6049 & $11: 27: 50$ & 0.4469 \\
\hline 6050 & $11: 27: 51$ & 0.4431 \\
\hline 6051 & $11: 27: 52$ & 0.4450 \\
\hline 6052 & $11: 27: 53$ & 0.4458 \\
\hline 6053 & $11: 27: 54$ & 0.4440 \\
\hline 6054 & $11: 27: 55$ & 0.4446 \\
\hline 6055 & $11: 27: 56$ & 0.4419 \\
\hline 6056 & $11: 27: 57$ & 0.4462 \\
\hline 6057 & $11: 27: 58$ & 0.4432 \\
\hline 6058 & $11: 27: 59$ & 0.4440 \\
\hline 6059 & $11: 28: 00$ & 0.4431 \\
\hline 6060 & $11: 28: 01$ & 0.4406 \\
\hline 6061 & $11: 28: 02$ & 0.4405 \\
\hline 6062 & $11: 28: 03$ & 0.4402 \\
\hline 6063 & $11: 28: 04$ & 0.4445 \\
\hline 6064 & $11: 28: 05$ & 0.4420 \\
\hline 6065 & $11: 28: 06$ & 0.4419 \\
\hline 6066 & $11: 28: 07$ & 0.4379 \\
\hline 6067 & $11: 28: 08$ & 0.4408 \\
\hline
\end{tabular}


Appendix C - Fuel Data Test 2 NIST102605_1.xIs

\begin{tabular}{|c|c|c|}
\hline 6068 & $11: 28: 09$ & 0.4419 \\
\hline 6069 & $11: 28: 10$ & 0.4413 \\
\hline 6070 & $11: 28: 11$ & 0.4470 \\
\hline 6071 & $11: 28: 12$ & 0.4413 \\
\hline 6072 & $11: 28: 13$ & 0.4447 \\
\hline 6073 & $11: 28: 14$ & 0.4464 \\
\hline 6074 & $11: 28: 15$ & 0.4471 \\
\hline 6075 & $11: 28: 16$ & 0.4479 \\
\hline 6076 & $11: 28: 17$ & 0.4420 \\
\hline 6077 & $11: 28: 18$ & 0.4456 \\
\hline 6078 & $11: 28: 19$ & 0.4426 \\
\hline 6079 & $11: 28: 20$ & 0.4436 \\
\hline 6080 & $11: 28: 21$ & 0.4416 \\
\hline 6081 & $11: 28: 22$ & 0.4454 \\
\hline 6082 & $11: 28: 23$ & 0.4437 \\
\hline 6083 & $11: 28: 24$ & 0.4427 \\
\hline 6084 & $11: 28: 25$ & 0.4427 \\
\hline 6085 & $11: 28: 26$ & 0.4405 \\
\hline 6086 & $11: 28: 27$ & 0.4439 \\
\hline 6087 & $11: 28: 28$ & 0.4408 \\
\hline 6088 & $11: 28: 29$ & 0.4416 \\
\hline 6089 & $11: 28: 30$ & 0.4418 \\
\hline 6090 & $11: 28: 31$ & 0.4410 \\
\hline 6091 & $11: 28: 32$ & 0.4450 \\
\hline 6092 & $11: 28: 33$ & 0.4428 \\
\hline 6093 & $11: 28: 34$ & 0.4453 \\
\hline 6094 & $11: 28: 35$ & 0.4418 \\
\hline 6095 & $11: 28: 36$ & 0.4457 \\
\hline 6096 & $11: 28: 37$ & 0.4455 \\
\hline 6097 & $11: 28: 38$ & 0.4445 \\
\hline 6098 & $11: 28: 39$ & 0.4448 \\
\hline 6099 & $11: 28: 40$ & 0.4424 \\
\hline 6100 & $11: 28: 41$ & 0.4461 \\
\hline 6101 & $11: 28: 42$ & 0.4416 \\
\hline 6102 & $11: 28: 43$ & 0.4433 \\
\hline 6103 & $11: 28: 44$ & 0.4416 \\
\hline 6104 & $11: 28: 45$ & 0.4447 \\
\hline 6105 & $11: 28: 46$ & 0.4429 \\
\hline 6106 & $11: 28: 47$ & 0.4437 \\
\hline 6107 & $11: 28: 48$ & 0.4418 \\
\hline 6108 & $11: 28: 49$ & 0.4402 \\
\hline 6109 & $11: 28: 50$ & 0.4428 \\
\hline 6110 & $11: 28: 51$ & 0.4386 \\
\hline 6111 & $11: 28: 52$ & 0.4412 \\
\hline 6112 & $11: 28: 53$ & 0.4434 \\
\hline 6113 & $11: 28: 54$ & 0.4421 \\
\hline 6114 & $11: 28: 55$ & 0.4464 \\
\hline 6115 & $11: 28: 56$ & 0.4419 \\
\hline 6116 & $11: 28: 57$ & 0.4438 \\
\hline 6117 & $11: 28: 58$ & 0.4423 \\
\hline 6118 & $11: 28: 59$ & 0.4475 \\
\hline
\end{tabular}


Appendix C - Fuel Data Test 2 NIST102605_1.xls

\begin{tabular}{|c|c|c|}
\hline 6119 & $11: 29: 00$ & 0.4462 \\
\hline 6120 & $11: 29: 01$ & 0.4434 \\
\hline 6121 & $11: 29: 02$ & 0.4447 \\
\hline 6122 & $11: 29: 03$ & 0.4418 \\
\hline 6123 & $11: 29: 04$ & 0.4436 \\
\hline 6124 & $11: 29: 05$ & 0.4401 \\
\hline 6125 & $11: 29: 06$ & 0.4442 \\
\hline 6126 & $11: 29: 07$ & 0.4413 \\
\hline 6127 & $11: 29: 08$ & 0.4416 \\
\hline 6128 & $11: 29: 09$ & 0.4411 \\
\hline 6129 & $11: 29: 10$ & 0.4400 \\
\hline 6130 & $11: 29: 11$ & 0.4436 \\
\hline 6131 & $11: 29: 12$ & 0.4405 \\
\hline 6132 & $11: 29: 13$ & 0.4427 \\
\hline 6133 & $11: 29: 14$ & 0.4392 \\
\hline 6134 & $11: 29: 15$ & 0.4419 \\
\hline 6135 & $11: 29: 16$ & 0.4425 \\
\hline 6136 & $11: 29: 17$ & 0.4427 \\
\hline 6137 & $11: 29: 18$ & 0.4457 \\
\hline 6138 & $11: 29: 19$ & 0.4414 \\
\hline 6139 & $11: 29: 20$ & 0.4433 \\
\hline 6140 & $11: 29: 21$ & 0.4416 \\
\hline 6141 & $11: 29: 22$ & 0.4460 \\
\hline 6142 & $11: 29: 23$ & 0.4446 \\
\hline 6143 & $11: 29: 24$ & 0.4425 \\
\hline 6144 & $11: 29: 25$ & 0.4442 \\
\hline 6145 & $11: 29: 26$ & 0.4409 \\
\hline 6146 & $11: 29: 27$ & 0.4444 \\
\hline 6147 & $11: 29: 28$ & 0.4426 \\
\hline 6148 & $11: 29: 29$ & 0.4442 \\
\hline 6149 & $11: 29: 30$ & 0.4400 \\
\hline 6150 & $11: 29: 31$ & 0.4398 \\
\hline 6151 & $11: 29: 32$ & 0.4432 \\
\hline 6152 & $11: 29: 33$ & 0.4413 \\
\hline 6153 & $11: 29: 34$ & 0.4425 \\
\hline 6154 & $11: 29: 35$ & 0.4391 \\
\hline 6155 & $11: 29: 36$ & 0.4414 \\
\hline 6156 & $11: 29: 37$ & 0.4392 \\
\hline 6157 & $11: 29: 38$ & 0.4412 \\
\hline 6158 & $11: 29: 39$ & 0.4434 \\
\hline 6159 & $11: 29: 40$ & 0.4454 \\
\hline 6160 & $11: 29: 41$ & 0.4466 \\
\hline 6161 & $11: 29: 42$ & 0.4413 \\
\hline 6162 & $11: 29: 43$ & 0.4463 \\
\hline 6163 & $11: 29: 44$ & 0.4463 \\
\hline 6164 & $11: 29: 45$ & 0.4459 \\
\hline 6165 & $11: 29: 46$ & 0.4454 \\
\hline 6166 & $11: 29: 47$ & 0.4417 \\
\hline 6167 & $11: 29: 48$ & 0.4442 \\
\hline 6168 & $11: 29: 49$ & 0.4404 \\
\hline 6169 & $11: 29: 50$ & 0.4434 \\
\hline
\end{tabular}


Appendix C - Fuel Data Test 2 NIST102605_1.xIs

\begin{tabular}{|c|c|c|}
\hline 6170 & $11: 29: 51$ & 0.4434 \\
\hline 6171 & $11: 29: 52$ & 0.4437 \\
\hline 6172 & $11: 29: 53$ & 0.4417 \\
\hline 6173 & $11: 29: 54$ & 0.4404 \\
\hline 6174 & $11: 29: 55$ & 0.4417 \\
\hline 6175 & $11: 29: 56$ & 0.4399 \\
\hline 6176 & $11: 29: 57$ & 0.4434 \\
\hline 6177 & $11: 29: 58$ & 0.4404 \\
\hline 6178 & $11: 29: 59$ & 0.4427 \\
\hline 6179 & $11: 30: 00$ & 0.4401 \\
\hline 6180 & $11: 30: 01$ & 0.4432 \\
\hline 6181 & $11: 30: 02$ & 0.4447 \\
\hline 6182 & $11: 30: 03$ & 0.4445 \\
\hline 6183 & $11: 30: 04$ & 0.4460 \\
\hline 6184 & 11:30:05 & 0.4426 \\
\hline 6185 & $11: 30: 06$ & 0.4445 \\
\hline 6186 & $11: 30: 07$ & 0.4446 \\
\hline 6187 & $11: 30: 08$ & 0.4434 \\
\hline 6188 & $11: 30: 09$ & 0.4459 \\
\hline 6189 & $11: 30: 10$ & 0.4411 \\
\hline 6190 & $11: 30: 11$ & 0.4429 \\
\hline 6191 & $11: 30: 12$ & 0.4407 \\
\hline 6192 & $11: 30: 13$ & 0.4449 \\
\hline 6193 & $11: 30: 14$ & 0.4427 \\
\hline 6194 & $11: 30: 15$ & 0.4429 \\
\hline 6195 & $11: 30: 16$ & 0.4406 \\
\hline 6196 & $11: 30: 17$ & 0.4406 \\
\hline 6197 & $11: 30: 18$ & 0.4424 \\
\hline 6198 & $11: 30: 19$ & 0.4397 \\
\hline 6199 & $11: 30: 20$ & 0.4442 \\
\hline 6200 & $11: 30: 21$ & 0.4386 \\
\hline 6201 & $11: 30: 22$ & 0.4415 \\
\hline 6202 & $11: 30: 23$ & 0.4399 \\
\hline 6203 & $11: 30: 24$ & 0.4452 \\
\hline 6204 & $11: 30: 25$ & 0.4477 \\
\hline 6205 & $11: 30: 26$ & 0.4437 \\
\hline 6206 & $11: 30: 27$ & 0.4459 \\
\hline 6207 & $11: 30: 28$ & 0.4422 \\
\hline 6208 & $11: 30: 29$ & 0.4448 \\
\hline 6209 & $11: 30: 30$ & 0.4437 \\
\hline 6210 & $11: 30: 31$ & 0.4456 \\
\hline 6211 & $11: 30: 32$ & 0.4438 \\
\hline 6212 & $11: 30: 33$ & 0.4397 \\
\hline 6213 & $11: 30: 34$ & 0.4425 \\
\hline 6214 & $11: 30: 35$ & 0.4409 \\
\hline 6215 & $11: 30: 36$ & 0.4446 \\
\hline 6216 & $11: 30: 37$ & 0.4413 \\
\hline 6217 & $11: 30: 38$ & 0.4418 \\
\hline 6218 & $11: 30: 39$ & 0.4403 \\
\hline 6219 & $11: 30: 40$ & 0.4384 \\
\hline 6220 & $11: 30: 41$ & 0.4402 \\
\hline
\end{tabular}


Appendix C - Fuel Data Test 2 NIST102605_1.xls

\begin{tabular}{|c|c|c|}
\hline 6221 & $11: 30: 42$ & 0.4418 \\
\hline 6222 & $11: 30: 43$ & 0.4444 \\
\hline 6223 & $11: 30: 44$ & 0.4408 \\
\hline 6224 & $11: 30: 45$ & 0.4435 \\
\hline 6225 & $11: 30: 46$ & 0.4421 \\
\hline 6226 & $11: 30: 47$ & 0.4443 \\
\hline 6227 & $11: 30: 48$ & 0.4460 \\
\hline 6228 & $11: 30: 49$ & 0.4422 \\
\hline 6229 & $11: 30: 50$ & 0.4458 \\
\hline 6230 & $11: 30: 51$ & 0.4429 \\
\hline 6231 & $11: 30: 52$ & 0.4446 \\
\hline 6232 & $11: 30: 53$ & 0.4445 \\
\hline 6233 & $11: 30: 54$ & 0.4414 \\
\hline 6234 & $11: 30: 55$ & 0.4443 \\
\hline 6235 & $11: 30: 56$ & 0.4425 \\
\hline 6236 & $11: 30: 57$ & 0.4441 \\
\hline 6237 & $11: 30: 58$ & 0.4416 \\
\hline 6238 & $11: 30: 59$ & 0.4395 \\
\hline 6239 & $11: 31: 00$ & 0.4458 \\
\hline 6240 & $11: 31: 01$ & 0.4407 \\
\hline 6241 & $11: 31: 02$ & 0.4402 \\
\hline 6242 & $11: 31: 03$ & 0.4401 \\
\hline 6243 & $11: 31: 04$ & 0.4404 \\
\hline 6244 & $11: 31: 05$ & 0.4433 \\
\hline 6245 & $11: 31: 06$ & 0.4397 \\
\hline 6246 & $11: 31: 07$ & 0.4439 \\
\hline 6247 & $11: 31: 08$ & 0.4465 \\
\hline 6248 & $11: 31: 09$ & 0.4451 \\
\hline 6249 & $11: 31: 10$ & 0.4485 \\
\hline 6250 & $11: 31: 11$ & 0.4430 \\
\hline 6251 & $11: 31: 12$ & 0.4452 \\
\hline 6252 & $11: 31: 13$ & 0.4444 \\
\hline 6253 & $11: 31: 14$ & 0.4440 \\
\hline 6254 & $11: 31: 15$ & 0.4436 \\
\hline 6255 & $11: 31: 16$ & 0.4392 \\
\hline 6256 & $11: 31: 17$ & 0.4448 \\
\hline 6257 & $11: 31: 18$ & 0.4418 \\
\hline 6258 & $11: 31: 19$ & 0.4430 \\
\hline 6259 & $11: 31: 20$ & 0.4389 \\
\hline 6260 & $11: 31: 21$ & 0.4434 \\
\hline 6261 & $11: 31: 22$ & 0.4415 \\
\hline 6262 & $11: 31: 23$ & 0.4427 \\
\hline 6263 & $11: 31: 24$ & 0.4408 \\
\hline 6264 & $11: 31: 25$ & 0.4394 \\
\hline 6265 & $11: 31: 26$ & 0.4426 \\
\hline 6266 & $11: 31: 27$ & 0.4402 \\
\hline 6267 & $11: 31: 28$ & 0.4442 \\
\hline 6268 & $11: 31: 29$ & 0.4423 \\
\hline 6269 & $11: 31: 30$ & 0.4441 \\
\hline 6270 & $11: 31: 31$ & 0.4416 \\
\hline 6271 & $11: 31: 32$ & 0.4448 \\
\hline
\end{tabular}


Appendix C - Fuel Data Test 2 NIST102605_1.xIs

\begin{tabular}{|c|c|c|}
\hline 6272 & $11: 31: 33$ & 0.4450 \\
\hline 6273 & $11: 31: 34$ & 0.4451 \\
\hline 6274 & $11: 31: 35$ & 0.4448 \\
\hline 6275 & $11: 31: 36$ & 0.4402 \\
\hline 6276 & $11: 31: 37$ & 0.4448 \\
\hline 6277 & $11: 31: 38$ & 0.4422 \\
\hline 6278 & $11: 31: 39$ & 0.4443 \\
\hline 6279 & $11: 31: 40$ & 0.4435 \\
\hline 6280 & $11: 31: 41$ & 0.4414 \\
\hline 6281 & $11: 31: 42$ & 0.4393 \\
\hline 6282 & $11: 31: 43$ & 0.4394 \\
\hline 6283 & $11: 31: 44$ & 0.4419 \\
\hline 6284 & $11: 31: 45$ & 0.4420 \\
\hline 6285 & $11: 31: 46$ & 0.4401 \\
\hline 6286 & $11: 31: 47$ & 0.4385 \\
\hline 6287 & $11: 31: 48$ & 0.4436 \\
\hline 6288 & $11: 31: 49$ & 0.4397 \\
\hline 6289 & $11: 31: 50$ & 0.4434 \\
\hline 6290 & $11: 31: 51$ & 0.4460 \\
\hline 6291 & $11: 31: 52$ & 0.4442 \\
\hline 6292 & $11: 31: 53$ & 0.4457 \\
\hline 6293 & $11: 31: 54$ & 0.4441 \\
\hline 6294 & $11: 31: 55$ & 0.4468 \\
\hline 6295 & $11: 31: 56$ & 0.4426 \\
\hline 6296 & $11: 31: 57$ & 0.4439 \\
\hline 6297 & $11: 31: 58$ & 0.4406 \\
\hline 6298 & $11: 31: 59$ & 0.4462 \\
\hline 6299 & $11: 32: 00$ & 0.4443 \\
\hline 6300 & $11: 32: 01$ & 0.4426 \\
\hline 6301 & $11: 32: 02$ & 0.4419 \\
\hline 6302 & $11: 32: 03$ & 0.4397 \\
\hline 6303 & $11: 32: 04$ & 0.4440 \\
\hline 6304 & $11: 32: 05$ & 0.4404 \\
\hline 6305 & $11: 32: 06$ & 0.4394 \\
\hline 6306 & $11: 32: 07$ & 0.4440 \\
\hline 6307 & $11: 32: 08$ & 0.4417 \\
\hline 6308 & $11: 32: 09$ & 0.4421 \\
\hline 6309 & $11: 32: 10$ & 0.4398 \\
\hline 6310 & $11: 32: 11$ & 0.4437 \\
\hline 6311 & $11: 32: 12$ & 0.4415 \\
\hline 6312 & $11: 32: 13$ & 0.4444 \\
\hline 6313 & $11: 32: 14$ & 0.4456 \\
\hline 6314 & $11: 32: 15$ & 0.4437 \\
\hline 6315 & $11: 32: 16$ & 0.4446 \\
\hline 6316 & $11: 32: 17$ & 0.4429 \\
\hline 6317 & $11: 32: 18$ & 0.4472 \\
\hline 6318 & $11: 32: 19$ & 0.4423 \\
\hline 6319 & $11: 32: 20$ & 0.4445 \\
\hline 6320 & $11: 32: 21$ & 0.4439 \\
\hline 6321 & $11: 32: 22$ & 0.4411 \\
\hline 6322 & $11: 32: 23$ & 0.4437 \\
\hline
\end{tabular}


Appendix C - Fuel Data Test 2 NIST102605_1.xIs

\begin{tabular}{|c|c|c|}
\hline 6323 & $11: 32: 24$ & 0.4419 \\
\hline 6324 & $11: 32: 25$ & 0.4453 \\
\hline 6325 & $11: 32: 26$ & 0.4429 \\
\hline 6326 & $11: 32: 27$ & 0.4427 \\
\hline 6327 & $11: 32: 28$ & 0.4400 \\
\hline 6328 & $11: 32: 29$ & 0.4395 \\
\hline 6329 & $11: 32: 30$ & 0.4432 \\
\hline 6330 & $11: 32: 31$ & 0.4400 \\
\hline 6331 & $11: 32: 32$ & 0.4436 \\
\hline 6332 & $11: 32: 33$ & 0.4392 \\
\hline 6333 & $11: 32: 34$ & 0.4417 \\
\hline 6334 & $11: 32: 35$ & 0.4417 \\
\hline 6335 & $11: 32: 36$ & 0.4446 \\
\hline 6336 & $11: 32: 37$ & 0.4467 \\
\hline 6337 & $11: 32: 38$ & 0.4424 \\
\hline 6338 & $11: 32: 39$ & 0.4457 \\
\hline 6339 & $11: 32: 40$ & 0.4436 \\
\hline 6340 & $11: 32: 41$ & 0.4442 \\
\hline 6341 & $11: 32: 42$ & 0.4454 \\
\hline 6342 & $11: 32: 43$ & 0.4416 \\
\hline 6343 & $11: 32: 44$ & 0.4428 \\
\hline 6344 & $11: 32: 45$ & 0.4388 \\
\hline 6345 & $11: 32: 46$ & 0.4431 \\
\hline 6346 & $11: 32: 47$ & 0.4424 \\
\hline 6347 & $11: 32: 48$ & 0.4444 \\
\hline 6348 & $11: 32: 49$ & 0.4408 \\
\hline 6349 & $11: 32: 50$ & 0.4411 \\
\hline 6350 & $11: 32: 51$ & 0.4396 \\
\hline 6351 & $11: 32: 52$ & 0.4397 \\
\hline 6352 & $11: 32: 53$ & 0.4420 \\
\hline 6353 & $11: 32: 54$ & 0.4419 \\
\hline 6354 & $11: 32: 55$ & 0.4448 \\
\hline 6355 & $11: 32: 56$ & 0.4399 \\
\hline 6356 & $11: 32: 57$ & 0.4428 \\
\hline 6357 & $11: 32: 58$ & 0.4446 \\
\hline 6358 & $11: 32: 59$ & 0.4428 \\
\hline 6359 & $11: 33: 00$ & 0.4439 \\
\hline 6360 & $11: 33: 01$ & 0.4424 \\
\hline 6361 & $11: 33: 02$ & 0.4464 \\
\hline 6362 & $11: 33: 03$ & 0.4453 \\
\hline 6363 & $11: 33: 04$ & 0.4405 \\
\hline 6364 & $11: 33: 05$ & 0.4439 \\
\hline 6365 & $11: 33: 06$ & 0.4407 \\
\hline 6366 & $11: 33: 07$ & 0.4444 \\
\hline 6367 & $11: 33: 08$ & 0.4435 \\
\hline 6368 & $11: 33: 09$ & 0.4447 \\
\hline 6369 & $11: 33: 10$ & 0.4422 \\
\hline 6370 & $11: 33: 11$ & 0.4402 \\
\hline 6371 & $11: 33: 12$ & 0.4425 \\
\hline 6372 & $11: 33: 13$ & 0.4420 \\
\hline 6373 & $11: 33: 14$ & 0.4423 \\
\hline
\end{tabular}


Appendix C - Fuel Data Test 2 NIST102605_1.xIs

\begin{tabular}{|c|c|c|}
\hline 6374 & $11: 33: 15$ & 0.4389 \\
\hline 6375 & $11: 33: 16$ & 0.4428 \\
\hline 6376 & $11: 33: 17$ & 0.4395 \\
\hline 6377 & $11: 33: 18$ & 0.4396 \\
\hline 6378 & $11: 33: 19$ & 0.4454 \\
\hline 6379 & $11: 33: 20$ & 0.4434 \\
\hline 6380 & $11: 33: 21$ & 0.4456 \\
\hline 6381 & $11: 33: 22$ & 0.4425 \\
\hline 6382 & $11: 33: 23$ & 0.4462 \\
\hline 6383 & $11: 33: 24$ & 0.4461 \\
\hline 6384 & $11: 33: 25$ & 0.4454 \\
\hline 6385 & $11: 33: 26$ & 0.4449 \\
\hline 6386 & $11: 33: 27$ & 0.4460 \\
\hline 6387 & $11: 33: 28$ & 0.4443 \\
\hline 6388 & $11: 33: 29$ & 0.4395 \\
\hline 6389 & $11: 33: 30$ & 0.4418 \\
\hline 6390 & $11: 33: 31$ & 0.4401 \\
\hline 6391 & $11: 33: 32$ & 0.4444 \\
\hline 6392 & $11: 33: 33$ & 0.4403 \\
\hline 6393 & $11: 33: 34$ & 0.4401 \\
\hline 6394 & $11: 33: 35$ & 0.4408 \\
\hline 6395 & $11: 33: 36$ & 0.4390 \\
\hline 6396 & $11: 33: 37$ & 0.4433 \\
\hline 6397 & $11: 33: 38$ & 0.4417 \\
\hline 6398 & $11: 33: 39$ & 0.4439 \\
\hline 6399 & $11: 33: 40$ & 0.4405 \\
\hline 6400 & $11: 33: 41$ & 0.4425 \\
\hline 6401 & $11: 33: 42$ & 0.4417 \\
\hline 6402 & $11: 33: 43$ & 0.4448 \\
\hline 6403 & $11: 33: 44$ & 0.4449 \\
\hline 6404 & $11: 33: 45$ & 0.4445 \\
\hline 6405 & $11: 33: 46$ & 0.4470 \\
\hline 6406 & $11: 33: 47$ & 0.4409 \\
\hline 6407 & $11: 33: 48$ & 0.4436 \\
\hline 6408 & $11: 33: 49$ & 0.4412 \\
\hline 6409 & $11: 33: 50$ & 0.4450 \\
\hline 6410 & $11: 33: 51$ & 0.4437 \\
\hline 6411 & $11: 33: 52$ & 0.4413 \\
\hline 6412 & $11: 33: 53$ & 0.4420 \\
\hline 6413 & $11: 33: 54$ & 0.4409 \\
\hline 6414 & $11: 33: 55$ & 0.4419 \\
\hline 6415 & $11: 33: 56$ & 0.4406 \\
\hline 6416 & $11: 33: 57$ & 0.4435 \\
\hline 6417 & $11: 33: 58$ & 0.4378 \\
\hline 6418 & $11: 33: 59$ & 0.4396 \\
\hline 6419 & $11: 34: 00$ & 0.4393 \\
\hline 6420 & $11: 34: 01$ & 0.4420 \\
\hline 6421 & $11: 34: 02$ & 0.4443 \\
\hline 6422 & $11: 34: 03$ & 0.4405 \\
\hline 6423 & $11: 34: 04$ & 0.4455 \\
\hline 6424 & $11: 34: 05$ & 0.442 \\
\hline
\end{tabular}


Appendix C - Fuel Data Test 2 NIST102605_1.xIs

\begin{tabular}{|c|c|c|}
\hline 6425 & $11: 34: 06$ & 0.4456 \\
\hline 6426 & $11: 34: 07$ & 0.4457 \\
\hline 6427 & $11: 34: 08$ & 0.4474 \\
\hline 6428 & 11:34:09 & 0.4462 \\
\hline 6429 & $11: 34: 10$ & 0.4413 \\
\hline 6430 & $11: 34: 11$ & 0.4440 \\
\hline 6431 & $11: 34: 12$ & 0.4416 \\
\hline 6432 & $11: 34: 13$ & 0.4447 \\
\hline 6433 & $11: 34: 14$ & 0.4426 \\
\hline 6434 & $11: 34: 15$ & 0.4398 \\
\hline 6435 & $11: 34: 16$ & 0.4419 \\
\hline 6436 & $11: 34: 17$ & 0.4392 \\
\hline 6437 & $11: 34: 18$ & 0.4444 \\
\hline 6438 & $11: 34: 19$ & 0.4411 \\
\hline 6439 & 11:34:20 & 0.4418 \\
\hline 6440 & 11:34:21 & 0.4390 \\
\hline 6441 & $11: 34: 22$ & 0.4401 \\
\hline 6442 & $11: 34: 23$ & 0.4431 \\
\hline 6443 & $11: 34: 24$ & 0.4426 \\
\hline 6444 & $11: 34: 25$ & 0.4449 \\
\hline 6445 & $11: 34: 26$ & 0.4427 \\
\hline 6446 & $11: 34: 27$ & 0.4454 \\
\hline 6447 & $11: 34: 28$ & 0.4418 \\
\hline 6448 & 11:34:29 & 0.4454 \\
\hline 6449 & 11:34:30 & 0.4445 \\
\hline 6450 & $11: 34: 31$ & 0.4454 \\
\hline 6451 & $11: 34: 32$ & 0.4441 \\
\hline 6452 & $11: 34: 33$ & 0.4410 \\
\hline 6453 & $11: 34: 34$ & 0.4452 \\
\hline 6454 & $11: 34: 35$ & 0.4418 \\
\hline 6455 & $11: 34: 36$ & 0.4440 \\
\hline 6456 & $11: 34: 37$ & 0.4429 \\
\hline 6457 & $11: 34: 38$ & 0.4402 \\
\hline 6458 & 11:34:39 & 0.4398 \\
\hline 6459 & $11: 34: 40$ & 0.4401 \\
\hline 6460 & $11: 34: 41$ & 0.4422 \\
\hline 6461 & $11: 34: 42$ & 0.4416 \\
\hline 6462 & $11: 34: 43$ & 0.4424 \\
\hline 6463 & $11: 34: 44$ & 0.4371 \\
\hline 6464 & $11: 34: 45$ & 0.4415 \\
\hline 6465 & $11: 34: 46$ & 0.4397 \\
\hline 6466 & $11: 34: 47$ & 0.4425 \\
\hline 6467 & $11: 34: 48$ & 0.4462 \\
\hline 6468 & $11: 34: 49$ & 0.4460 \\
\hline 6469 & $11: 34: 50$ & 0.4468 \\
\hline 6470 & $11: 34: 51$ & 0.4417 \\
\hline 6471 & $11: 34: 52$ & 0.4459 \\
\hline 6472 & $11: 34: 53$ & 0.4431 \\
\hline 6473 & $11: 34: 54$ & 0.4445 \\
\hline 6474 & $11: 34: 55$ & 0.4420 \\
\hline 6475 & $11: 34: 56$ & 0.4443 \\
\hline
\end{tabular}


Appendix C - Fuel Data Test 2 NIST102605_1.xIs

\begin{tabular}{|c|c|c|}
\hline 6476 & $11: 34: 57$ & 0.4432 \\
\hline 6477 & $11: 34: 58$ & 0.4411 \\
\hline 6478 & $11: 34: 59$ & 0.4453 \\
\hline 6479 & $11: 35: 00$ & 0.4427 \\
\hline 6480 & $11: 35: 01$ & 0.4431 \\
\hline 6481 & $11: 35: 02$ & 0.4405 \\
\hline 6482 & $11: 35: 03$ & 0.4392 \\
\hline 6483 & $11: 35: 04$ & 0.4422 \\
\hline 6484 & $11: 35: 05$ & 0.4394 \\
\hline 6485 & $11: 35: 06$ & 0.4431 \\
\hline 6486 & $11: 35: 07$ & 0.4407 \\
\hline 6487 & $11: 35: 08$ & 0.4404 \\
\hline 6488 & $11: 35: 09$ & 0.4440 \\
\hline 6489 & $11: 35: 10$ & 0.4442 \\
\hline 6490 & $11: 35: 11$ & 0.4472 \\
\hline 6491 & $11: 35: 12$ & 0.4424 \\
\hline 6492 & $11: 35: 13$ & 0.4455 \\
\hline 6493 & $11: 35: 14$ & 0.4452 \\
\hline 6494 & $11: 35: 15$ & 0.4415 \\
\hline 6495 & $11: 35: 16$ & 0.4446 \\
\hline 6496 & $11: 35: 17$ & 0.4408 \\
\hline 6497 & $11: 35: 18$ & 0.4431 \\
\hline 6498 & $11: 35: 19$ & 0.4432 \\
\hline 6499 & $11: 35: 20$ & 0.4411 \\
\hline 6500 & $11: 35: 21$ & 0.4420 \\
\hline 6501 & $11: 35: 22$ & 0.4404 \\
\hline 6502 & $11: 35: 23$ & 0.4438 \\
\hline 6503 & $11: 35: 24$ & 0.4413 \\
\hline 6504 & $11: 35: 25$ & 0.4437 \\
\hline 6505 & $11: 35: 26$ & 0.4413 \\
\hline 6506 & $11: 35: 27$ & 0.4390 \\
\hline 6507 & $11: 35: 28$ & 0.4438 \\
\hline 6508 & $11: 35: 29$ & 0.4396 \\
\hline 6509 & $11: 35: 30$ & 0.4418 \\
\hline 6510 & $11: 35: 31$ & 0.4439 \\
\hline 6511 & $11: 35: 32$ & 0.4439 \\
\hline 6512 & $11: 35: 33$ & 0.4470 \\
\hline 6513 & $11: 35: 34$ & 0.4430 \\
\hline 6514 & $11: 35: 35$ & 0.4468 \\
\hline 6515 & $11: 35: 36$ & 0.4457 \\
\hline 6516 & $11: 35: 37$ & 0.4443 \\
\hline 6517 & $11: 35: 38$ & 0.4460 \\
\hline 6518 & $11: 35: 39$ & 0.4427 \\
\hline 6519 & $11: 35: 40$ & 0.4457 \\
\hline 6520 & $11: 35: 41$ & 0.4424 \\
\hline 6521 & $11: 35: 42$ & 0.4452 \\
\hline 6522 & $11: 35: 43$ & 0.4422 \\
\hline 6523 & $11: 35: 44$ & 0.4401 \\
\hline 6524 & $11: 35: 45$ & 0.4402 \\
\hline 6525 & $11: 35: 46$ & 0.4399 \\
\hline 6526 & $11: 35: 47$ & 0.4425 \\
\hline
\end{tabular}


Appendix C - Fuel Data Test 2 NIST102605_1.xIs

\begin{tabular}{|c|c|c|}
\hline 6527 & $11: 35: 48$ & 0.4393 \\
\hline 6528 & $11: 35: 49$ & 0.4436 \\
\hline 6529 & $11: 35: 50$ & 0.4407 \\
\hline 6530 & $11: 35: 51$ & 0.4432 \\
\hline 6531 & $11: 35: 52$ & 0.4396 \\
\hline 6532 & $11: 35: 53$ & 0.4421 \\
\hline 6533 & $11: 35: 54$ & 0.4437 \\
\hline 6534 & $11: 35: 55$ & 0.4445 \\
\hline 6535 & $11: 35: 56$ & 0.4466 \\
\hline 6536 & $11: 35: 57$ & 0.4456 \\
\hline 6537 & $11: 35: 58$ & 0.4474 \\
\hline 6538 & $11: 35: 59$ & 0.4414 \\
\hline 6539 & $11: 36: 00$ & 0.4456 \\
\hline 6540 & $11: 36: 01$ & 0.4444 \\
\hline 6541 & $11: 36: 02$ & 0.4439 \\
\hline 6542 & $11: 36: 03$ & 0.4452 \\
\hline 6543 & $11: 36: 04$ & 0.4417 \\
\hline 6544 & $11: 36: 05$ & 0.4446 \\
\hline 6545 & $11: 36: 06$ & 0.4418 \\
\hline 6546 & $11: 36: 07$ & 0.4400 \\
\hline 6547 & $11: 36: 08$ & 0.4422 \\
\hline 6548 & $11: 36: 09$ & 0.4429 \\
\hline 6549 & $11: 36: 10$ & 0.4429 \\
\hline 6550 & $11: 36: 11$ & 0.4399 \\
\hline 6551 & $11: 36: 12$ & 0.4439 \\
\hline 6552 & $11: 36: 13$ & 0.4409 \\
\hline 6553 & $11: 36: 14$ & 0.4444 \\
\hline 6554 & $11: 36: 15$ & 0.4402 \\
\hline 6555 & $11: 36: 16$ & 0.4429 \\
\hline 6556 & $11: 36: 17$ & 0.4423 \\
\hline 6557 & $11: 36: 18$ & 0.4445 \\
\hline 6558 & $11: 36: 19$ & 0.4476 \\
\hline 6559 & $11: 36: 20$ & 0.4439 \\
\hline 6560 & $11: 36: 21$ & 0.4467 \\
\hline 6561 & $11: 36: 22$ & 0.4440 \\
\hline 6562 & $11: 36: 23$ & 0.4443 \\
\hline 6563 & $11: 36: 24$ & 0.4441 \\
\hline 6564 & $11: 36: 25$ & 0.4401 \\
\hline 6565 & $11: 36: 26$ & 0.4439 \\
\hline 6566 & $11: 36: 27$ & 0.4421 \\
\hline 6567 & $11: 36: 28$ & 0.4435 \\
\hline 6568 & $11: 36: 29$ & 0.4430 \\
\hline 6569 & $11: 36: 30$ & 0.4401 \\
\hline 6570 & $11: 36: 31$ & 0.4434 \\
\hline 6571 & $11: 36: 32$ & 0.4402 \\
\hline 6572 & $11: 36: 33$ & 0.4413 \\
\hline 6573 & $11: 36: 34$ & 0.4398 \\
\hline 6574 & $11: 36: 35$ & 0.4393 \\
\hline 6575 & $11: 36: 36$ & 0.4383 \\
\hline 6576 & $11: 36: 37$ & 0.4414 \\
\hline 6577 & $11: 36: 38$ & 0.4447 \\
\hline
\end{tabular}


Appendix C - Fuel Data Test 2 NIST102605_1.xIs

\begin{tabular}{|c|c|c|}
\hline 6578 & $11: 36: 39$ & 0.4445 \\
\hline 6579 & $11: 36: 40$ & 0.4455 \\
\hline 6580 & $11: 36: 41$ & 0.4418 \\
\hline 6581 & $11: 36: 42$ & 0.4461 \\
\hline 6582 & $11: 36: 43$ & 0.4441 \\
\hline 6583 & $11: 36: 44$ & 0.4453 \\
\hline 6584 & $11: 36: 45$ & 0.4461 \\
\hline 6585 & $11: 36: 46$ & 0.4409 \\
\hline 6586 & $11: 36: 47$ & 0.4419 \\
\hline 6587 & $11: 36: 48$ & 0.4394 \\
\hline 6588 & $11: 36: 49$ & 0.4446 \\
\hline 6589 & $11: 36: 50$ & 0.4431 \\
\hline 6590 & $11: 36: 51$ & 0.4421 \\
\hline 6591 & $11: 36: 52$ & 0.4413 \\
\hline 6592 & $11: 36: 53$ & 0.4397 \\
\hline 6593 & $11: 36: 54$ & 0.4392 \\
\hline 6594 & $11: 36: 55$ & 0.4388 \\
\hline 6595 & $11: 36: 56$ & 0.4428 \\
\hline 6596 & $11: 36: 57$ & 0.4408 \\
\hline 6597 & $11: 36: 58$ & 0.4429 \\
\hline 6598 & $11: 36: 59$ & 0.4394 \\
\hline 6599 & $11: 37: 00$ & 0.4445 \\
\hline 6600 & $11: 37: 01$ & 0.4416 \\
\hline 6601 & $11: 37: 02$ & 0.4446 \\
\hline 6602 & $11: 37: 03$ & 0.4440 \\
\hline 6603 & $11: 37: 04$ & 0.4457 \\
\hline 6604 & $11: 37: 05$ & 0.4470 \\
\hline 6605 & $11: 37: 06$ & 0.4419 \\
\hline 6606 & $11: 37: 07$ & 0.4447 \\
\hline 6607 & $11: 37: 08$ & 0.4428 \\
\hline 6608 & $11: 37: 09$ & 0.4426 \\
\hline 6609 & $11: 37: 10$ & 0.4427 \\
\hline 6610 & $11: 37: 11$ & 0.4396 \\
\hline 6611 & $11: 37: 12$ & 0.4442 \\
\hline 6612 & $11: 37: 13$ & 0.4419 \\
\hline 6613 & $11: 37: 14$ & 0.4428 \\
\hline 6614 & $11: 37: 15$ & 0.4415 \\
\hline 6615 & $11: 37: 16$ & 0.4390 \\
\hline 6616 & $11: 37: 17$ & 0.4414 \\
\hline 6617 & $11: 37: 18$ & 0.4403 \\
\hline 6618 & $11: 37: 19$ & 0.4438 \\
\hline 6619 & $11: 37: 20$ & 0.4406 \\
\hline 6620 & $11: 37: 21$ & 0.4421 \\
\hline 6621 & $11: 37: 22$ & 0.4409 \\
\hline 6622 & $11: 37: 23$ & 0.4441 \\
\hline 6623 & $11: 37: 24$ & 0.4432 \\
\hline 6624 & $11: 37: 25$ & 0.4443 \\
\hline 6625 & $11: 37: 26$ & 0.4471 \\
\hline 6626 & $11: 37: 27$ & 0.4414 \\
\hline 6627 & $11: 37: 28$ & 0.4445 \\
\hline 6628 & $11: 37: 29$ & 0.4416 \\
\hline
\end{tabular}


Appendix C - Fuel Data Test 2 NIST102605_1.xIs

\begin{tabular}{|c|c|c|}
\hline 6629 & $11: 37: 30$ & 0.4458 \\
\hline 6630 & $11: 37: 31$ & 0.4444 \\
\hline 6631 & $11: 37: 32$ & 0.4411 \\
\hline 6632 & $11: 37: 33$ & 0.4448 \\
\hline 6633 & $11: 37: 34$ & 0.4421 \\
\hline 6634 & $11: 37: 35$ & 0.4443 \\
\hline 6635 & $11: 37: 36$ & 0.4426 \\
\hline 6636 & $11: 37: 37$ & 0.4432 \\
\hline 6637 & $11: 37: 38$ & 0.4411 \\
\hline 6638 & $11: 37: 39$ & 0.4398 \\
\hline 6639 & $11: 37: 40$ & 0.4435 \\
\hline 6640 & $11: 37: 41$ & 0.4380 \\
\hline 6641 & $11: 37: 42$ & 0.4410 \\
\hline 6642 & $11: 37: 43$ & 0.4408 \\
\hline 6643 & $11: 37: 44$ & 0.4434 \\
\hline 6644 & $11: 37: 45$ & 0.4454 \\
\hline 6645 & $11: 37: 46$ & 0.4414 \\
\hline 6646 & $11: 37: 47$ & 0.4453 \\
\hline 6647 & $11: 37: 48$ & 0.4425 \\
\hline 6648 & $11: 37: 49$ & 0.4456 \\
\hline 6649 & $11: 37: 50$ & 0.4458 \\
\hline 6650 & $11: 37: 51$ & 0.4464 \\
\hline 6651 & $11: 37: 52$ & 0.4451 \\
\hline 6652 & $11: 37: 53$ & 0.4407 \\
\hline 6653 & $11: 37: 54$ & 0.4428 \\
\hline 6654 & $11: 37: 55$ & 0.4395 \\
\hline 6655 & $11: 37: 56$ & 0.4425 \\
\hline 6656 & $11: 37: 57$ & 0.4395 \\
\hline 6657 & $11: 37: 58$ & 0.4446 \\
\hline 6658 & $11: 37: 59$ & 0.4415 \\
\hline 6659 & $11: 38: 00$ & 0.4398 \\
\hline 6660 & 11:38:01 & 0.4442 \\
\hline 6661 & $11: 38: 02$ & 0.4410 \\
\hline 6662 & $11: 38: 03$ & 0.4422 \\
\hline 6663 & $11: 38: 04$ & 0.4396 \\
\hline 6664 & $11: 38: 05$ & 0.4401 \\
\hline 6665 & $11: 38: 06$ & 0.4433 \\
\hline 6666 & $11: 38: 07$ & 0.4419 \\
\hline 6667 & $11: 38: 08$ & 0.4444 \\
\hline 6668 & $11: 38: 09$ & 0.4438 \\
\hline 6669 & $11: 38: 10$ & 0.4457 \\
\hline 6670 & $11: 38: 11$ & 0.4455 \\
\hline 6671 & $11: 38: 12$ & 0.4433 \\
\hline 6672 & $11: 38: 13$ & 0.4452 \\
\hline 6673 & $11: 38: 14$ & 0.4410 \\
\hline 6674 & $11: 38: 15$ & 0.4444 \\
\hline 6675 & $11: 38: 16$ & 0.4427 \\
\hline 6676 & $11: 38: 17$ & 0.4445 \\
\hline 6677 & $11: 38: 18$ & 0.4418 \\
\hline 6678 & $11: 38: 19$ & 0.4413 \\
\hline 6679 & $11: 38: 20$ & 0.4419 \\
\hline
\end{tabular}


Appendix C - Fuel Data Test 2 NIST102605_1.xIs

\begin{tabular}{|c|c|c|}
\hline 6680 & $11: 38: 21$ & 0.4410 \\
\hline 6681 & $11: 38: 22$ & 0.4409 \\
\hline 6682 & $11: 38: 23$ & 0.4401 \\
\hline 6683 & $11: 38: 24$ & 0.4451 \\
\hline 6684 & $11: 38: 25$ & 0.4409 \\
\hline 6685 & $11: 38: 26$ & 0.4436 \\
\hline 6686 & $11: 38: 27$ & 0.4387 \\
\hline 6687 & $11: 38: 28$ & 0.4418 \\
\hline 6688 & 11:38:29 & 0.4410 \\
\hline 6689 & $11: 38: 30$ & 0.4435 \\
\hline 6690 & $11: 38: 31$ & 0.4468 \\
\hline 6691 & $11: 38: 32$ & 0.4441 \\
\hline 6692 & $11: 38: 33$ & 0.4462 \\
\hline 6693 & $11: 38: 34$ & 0.4425 \\
\hline 6694 & $11: 38: 35$ & 0.4467 \\
\hline 6695 & $11: 38: 36$ & 0.4456 \\
\hline 6696 & $11: 38: 37$ & 0.4461 \\
\hline 6697 & $11: 38: 38$ & 0.4435 \\
\hline 6698 & $11: 38: 39$ & 0.4413 \\
\hline 6699 & $11: 38: 40$ & 0.4421 \\
\hline 6700 & $11: 38: 41$ & 0.4407 \\
\hline 6701 & $11: 38: 42$ & 0.4453 \\
\hline 6702 & $11: 38: 43$ & 0.4415 \\
\hline 6703 & $11: 38: 44$ & 0.4426 \\
\hline 6704 & $11: 38: 45$ & 0.4398 \\
\hline 6705 & $11: 38: 46$ & 0.4396 \\
\hline 6706 & $11: 38: 47$ & 0.4425 \\
\hline 6707 & $11: 38: 48$ & 0.4407 \\
\hline 6708 & $11: 38: 49$ & 0.4441 \\
\hline 6709 & $11: 38: 50$ & 0.4401 \\
\hline 6710 & $11: 38: 51$ & 0.4421 \\
\hline 6711 & $11: 38: 52$ & 0.4414 \\
\hline 6712 & $11: 38: 53$ & 0.4453 \\
\hline 6713 & $11: 38: 54$ & 0.4456 \\
\hline 6714 & $11: 38: 55$ & 0.4433 \\
\hline 6715 & $11: 38: 56$ & 0.4446 \\
\hline 6716 & $11: 38: 57$ & 0.4418 \\
\hline 6717 & $11: 38: 58$ & 0.4461 \\
\hline 6718 & $11: 38: 59$ & 0.4418 \\
\hline 6719 & $11: 39: 00$ & 0.4446 \\
\hline 6720 & $11: 39: 01$ & 0.4450 \\
\hline 6721 & $11: 39: 02$ & 0.4397 \\
\hline 6722 & $11: 39: 03$ & 0.4414 \\
\hline 6723 & $11: 39: 04$ & 0.4397 \\
\hline 6724 & $11: 39: 05$ & 0.4445 \\
\hline 6725 & $11: 39: 06$ & 0.4413 \\
\hline 6726 & $11: 39: 07$ & 0.4408 \\
\hline 6727 & $11: 39: 08$ & 0.4419 \\
\hline 6728 & $11: 39: 09$ & 0.4400 \\
\hline 6729 & $11: 39: 10$ & 0.4423 \\
\hline 6730 & $11: 39: 11$ & 0.4387 \\
\hline
\end{tabular}


Appendix C - Fuel Data Test 2 NIST102605_1.xls

\begin{tabular}{|c|c|c|}
\hline 6731 & $11: 39: 12$ & 0.4423 \\
\hline 6732 & $11: 39: 13$ & 0.4449 \\
\hline 6733 & $11: 39: 14$ & 0.4443 \\
\hline 6734 & $11: 39: 15$ & 0.4468 \\
\hline 6735 & $11: 39: 16$ & 0.4425 \\
\hline 6736 & $11: 39: 17$ & 0.4446 \\
\hline 6737 & $11: 39: 18$ & 0.4421 \\
\hline 6738 & $11: 39: 19$ & 0.4459 \\
\hline 6739 & $11: 39: 20$ & 0.4446 \\
\hline 6740 & $11: 39: 21$ & 0.4431 \\
\hline 6741 & $11: 39: 22$ & 0.4432 \\
\hline 6742 & $11: 39: 23$ & 0.4399 \\
\hline 6743 & $11: 39: 24$ & 0.4445 \\
\hline 6744 & $11: 39: 25$ & 0.4415 \\
\hline 6745 & $11: 39: 26$ & 0.4421 \\
\hline 6746 & $11: 39: 27$ & 0.4435 \\
\hline 6747 & 11:39:28 & 0.4399 \\
\hline 6748 & $11: 39: 29$ & 0.4433 \\
\hline 6749 & $11: 39: 30$ & 0.4409 \\
\hline 6750 & $11: 39: 31$ & 0.4427 \\
\hline 6751 & $11: 39: 32$ & 0.4394 \\
\hline 6752 & $11: 39: 33$ & 0.4407 \\
\hline 6753 & $11: 39: 34$ & 0.4391 \\
\hline 6754 & $11: 39: 35$ & 0.4426 \\
\hline 6755 & $11: 39: 36$ & 0.4443 \\
\hline 6756 & $11: 39: 37$ & 0.4437 \\
\hline 6757 & $11: 39: 38$ & 0.4473 \\
\hline 6758 & $11: 39: 39$ & 0.4425 \\
\hline 6759 & $11: 39: 40$ & 0.4448 \\
\hline 6760 & $11: 39: 41$ & 0.4477 \\
\hline 6761 & $11: 39: 42$ & 0.4420 \\
\hline 6762 & $11: 39: 43$ & 0.4449 \\
\hline 6763 & $11: 39: 44$ & 0.4427 \\
\hline 6764 & $11: 39: 45$ & 0.4421 \\
\hline 6765 & $11: 39: 46$ & 0.4431 \\
\hline 6766 & $11: 39: 47$ & 0.4410 \\
\hline 6767 & $11: 39: 48$ & 0.4454 \\
\hline 6768 & $11: 39: 49$ & 0.4416 \\
\hline 6769 & $11: 39: 50$ & 0.4413 \\
\hline 6770 & $11: 39: 51$ & 0.4398 \\
\hline 6771 & $11: 39: 52$ & 0.4413 \\
\hline 6772 & $11: 39: 53$ & 0.4434 \\
\hline 6773 & $11: 39: 54$ & 0.4381 \\
\hline 6774 & $11: 39: 55$ & 0.4416 \\
\hline 6775 & $11: 39: 56$ & 0.4381 \\
\hline 6776 & $11: 39: 57$ & 0.4413 \\
\hline 6777 & $11: 39: 58$ & 0.4429 \\
\hline 6778 & $11: 39: 59$ & 0.4464 \\
\hline 6779 & $11: 40: 00$ & 0.4468 \\
\hline 6780 & $11: 40: 01$ & 0.4439 \\
\hline 6781 & $11: 40: 02$ & 0.4468 \\
\hline
\end{tabular}


Appendix C - Fuel Data Test 2 NIST102605_1.xls

\begin{tabular}{|c|c|c|}
\hline 6782 & $11: 40: 03$ & 0.4429 \\
\hline 6783 & $11: 40: 04$ & 0.4450 \\
\hline 6784 & $11: 40: 05$ & 0.4411 \\
\hline 6785 & $11: 40: 06$ & 0.4445 \\
\hline 6786 & $11: 40: 07$ & 0.4435 \\
\hline 6787 & $11: 40: 08$ & 0.4445 \\
\hline 6788 & $11: 40: 09$ & 0.4412 \\
\hline 6789 & $11: 40: 10$ & 0.4445 \\
\hline 6790 & $11: 40: 11$ & 0.4417 \\
\hline 6791 & $11: 40: 12$ & 0.4409 \\
\hline 6792 & $11: 40: 13$ & 0.4424 \\
\hline 6793 & $11: 40: 14$ & 0.4409 \\
\hline 6794 & $11: 40: 15$ & 0.4433 \\
\hline 6795 & $11: 40: 16$ & 0.4388 \\
\hline 6796 & $11: 40: 17$ & 0.4420 \\
\hline 6797 & $11: 40: 18$ & 0.4400 \\
\hline 6798 & $11: 40: 19$ & 0.4431 \\
\hline 6799 & $11: 40: 20$ & 0.4431 \\
\hline 6800 & $11: 40: 21$ & 0.4440 \\
\hline 6801 & $11: 40: 22$ & 0.4465 \\
\hline 6802 & $11: 40: 23$ & 0.4416 \\
\hline 6803 & $11: 40: 24$ & 0.4458 \\
\hline 6804 & $11: 40: 25$ & 0.4456 \\
\hline 6805 & $11: 40: 26$ & 0.4463 \\
\hline 6806 & $11: 40: 27$ & 0.4442 \\
\hline 6807 & $11: 40: 28$ & 0.4404 \\
\hline 6808 & $11: 40: 29$ & 0.4438 \\
\hline 6809 & $11: 40: 30$ & 0.4402 \\
\hline 6810 & $11: 40: 31$ & 0.4444 \\
\hline 6811 & $11: 40: 32$ & 0.4436 \\
\hline 6812 & $11: 40: 33$ & 0.4421 \\
\hline 6813 & $11: 40: 34$ & 0.4433 \\
\hline 6814 & $11: 40: 35$ & 0.4413 \\
\hline 6815 & $11: 40: 36$ & 0.4445 \\
\hline 6816 & $11: 40: 37$ & 0.4394 \\
\hline 6817 & $11: 40: 38$ & 0.4409 \\
\hline 6818 & $11: 40: 39$ & 0.4404 \\
\hline 6819 & $11: 40: 40$ & 0.4417 \\
\hline 6820 & $11: 40: 41$ & 0.4444 \\
\hline 6821 & $11: 40: 42$ & 0.4409 \\
\hline 6822 & $11: 40: 43$ & 0.4451 \\
\hline 6823 & $11: 40: 44$ & 0.4422 \\
\hline 6824 & $11: 40: 45$ & 0.4446 \\
\hline 6825 & $11: 40: 46$ & 0.4471 \\
\hline 6826 & $11: 40: 47$ & 0.4427 \\
\hline 6827 & $11: 40: 48$ & 0.4452 \\
\hline 6828 & $11: 40: 49$ & 0.4422 \\
\hline 6829 & $11: 40: 50$ & 0.4450 \\
\hline 6830 & $11: 40: 51$ & 0.4439 \\
\hline 6831 & $11: 40: 52$ & 0.4415 \\
\hline 6832 & $11: 40: 53$ & 0.4439 \\
\hline
\end{tabular}


Appendix C - Fuel Data Test 2 NIST102605_1.xIs

\begin{tabular}{|c|c|c|}
\hline 6833 & $11: 40: 54$ & 0.4405 \\
\hline 6834 & $11: 40: 55$ & 0.4447 \\
\hline 6835 & $11: 40: 56$ & 0.4410 \\
\hline 6836 & $11: 40: 57$ & 0.4418 \\
\hline 6837 & $11: 40: 58$ & 0.4411 \\
\hline 6838 & $11: 40: 59$ & 0.4388 \\
\hline 6839 & $11: 41: 00$ & 0.4410 \\
\hline 6840 & $11: 41: 01$ & 0.4397 \\
\hline 6841 & $11: 41: 02$ & 0.4437 \\
\hline 6842 & $11: 41: 03$ & 0.4418 \\
\hline 6843 & $11: 41: 04$ & 0.4447 \\
\hline 6844 & $11: 41: 05$ & 0.4421 \\
\hline 6845 & $11: 41: 06$ & 0.4446 \\
\hline 6846 & $11: 41: 07$ & 0.4454 \\
\hline 6847 & $11: 41: 08$ & 0.4415 \\
\hline 6848 & $11: 41: 09$ & 0.4460 \\
\hline 6849 & $11: 41: 10$ & 0.4442 \\
\hline 6850 & $11: 41: 11$ & 0.4451 \\
\hline 6851 & $11: 41: 12$ & 0.4452 \\
\hline 6852 & $11: 41: 13$ & 0.4408 \\
\hline 6853 & $11: 41: 14$ & 0.4426 \\
\hline 6854 & $11: 41: 15$ & 0.4398 \\
\hline 6855 & $11: 41: 16$ & 0.4435 \\
\hline 6856 & $11: 41: 17$ & 0.4424 \\
\hline 6857 & $11: 41: 18$ & 0.4394 \\
\hline 6858 & $11: 41: 19$ & 0.4424 \\
\hline 6859 & $11: 41: 20$ & 0.4390 \\
\hline 6860 & $11: 41: 21$ & 0.4408 \\
\hline 6861 & $11: 41: 22$ & 0.4410 \\
\hline 6862 & $11: 41: 23$ & 0.4436 \\
\hline 6863 & $11: 41: 24$ & 0.4393 \\
\hline 6864 & $11: 41: 25$ & 0.4419 \\
\hline 6865 & $11: 41: 26$ & 0.4406 \\
\hline 6866 & $11: 41: 27$ & 0.4439 \\
\hline 6867 & $11: 41: 28$ & 0.4472 \\
\hline 6868 & $11: 41: 29$ & 0.4424 \\
\hline 6869 & $11: 41: 30$ & 0.4461 \\
\hline 6870 & $11: 41: 31$ & 0.4427 \\
\hline 6871 & $11: 41: 32$ & 0.4431 \\
\hline 6872 & $11: 41: 33$ & 0.4452 \\
\hline 6873 & $11: 41: 34$ & 0.4411 \\
\hline 6874 & $11: 41: 35$ & 0.4448 \\
\hline 6875 & $11: 41: 36$ & 0.4424 \\
\hline 6876 & $11: 41: 37$ & 0.4409 \\
\hline 6877 & $11: 41: 38$ & 0.4419 \\
\hline 6878 & $11: 41: 39$ & 0.4386 \\
\hline 6879 & $11: 41: 40$ & 0.4442 \\
\hline 6880 & $11: 41: 41$ & 0.4422 \\
\hline 6881 & $11: 41: 42$ & 0.4431 \\
\hline 6882 & $11: 41: 43$ & 0.4432 \\
\hline 6883 & $11: 41: 44$ & 0.4406 \\
\hline
\end{tabular}


Appendix C - Fuel Data Test 2 NIST102605_1.xIs

\begin{tabular}{|c|c|c|}
\hline 6884 & $11: 41: 45$ & 0.4426 \\
\hline 6885 & $11: 41: 46$ & 0.4391 \\
\hline 6886 & $11: 41: 47$ & 0.4422 \\
\hline 6887 & $11: 41: 48$ & 0.4433 \\
\hline 6888 & $11: 41: 49$ & 0.4450 \\
\hline 6889 & $11: 41: 50$ & 0.4465 \\
\hline 6890 & $11: 41: 51$ & 0.4425 \\
\hline 6891 & $11: 41: 52$ & 0.4446 \\
\hline 6892 & $11: 41: 53$ & 0.4414 \\
\hline 6893 & $11: 41: 54$ & 0.4454 \\
\hline 6894 & $11: 41: 55$ & 0.4452 \\
\hline 6895 & $11: 41: 56$ & 0.4433 \\
\hline 6896 & $11: 41: 57$ & 0.4434 \\
\hline 6897 & $11: 41: 58$ & 0.4402 \\
\hline 6898 & $11: 41: 59$ & 0.4448 \\
\hline 6899 & $11: 42: 00$ & 0.4428 \\
\hline 6900 & 11:42:01 & 0.4429 \\
\hline 6901 & $11: 42: 02$ & 0.4415 \\
\hline 6902 & $11: 42: 03$ & 0.4401 \\
\hline 6903 & $11: 42: 04$ & 0.4391 \\
\hline 6904 & $11: 42: 05$ & 0.4389 \\
\hline 6905 & $11: 42: 06$ & 0.4435 \\
\hline 6906 & $11: 42: 07$ & 0.4405 \\
\hline 6907 & $11: 42: 08$ & 0.4426 \\
\hline 6908 & $11: 42: 09$ & 0.4392 \\
\hline 6909 & $11: 42: 10$ & 0.4430 \\
\hline 6910 & $11: 42: 11$ & 0.4446 \\
\hline 6911 & $11: 42: 12$ & 0.4432 \\
\hline 6912 & $11: 42: 13$ & 0.4468 \\
\hline 6913 & $11: 42: 14$ & 0.4437 \\
\hline 6914 & $11: 42: 15$ & 0.4468 \\
\hline 6915 & $11: 42: 16$ & 0.4447 \\
\hline 6916 & $11: 42: 17$ & 0.4444 \\
\hline 6917 & $11: 42: 18$ & 0.4445 \\
\hline 6918 & $11: 42: 19$ & 0.4410 \\
\hline 6919 & $11: 42: 20$ & 0.4447 \\
\hline 6920 & $11: 42: 21$ & 0.4425 \\
\hline 6921 & $11: 42: 22$ & 0.4432 \\
\hline 6922 & $11: 42: 23$ & 0.4418 \\
\hline 6923 & $11: 42: 24$ & 0.4394 \\
\hline 6924 & $11: 42: 25$ & 0.4444 \\
\hline 6925 & $11: 42: 26$ & 0.4406 \\
\hline 6926 & $11: 42: 27$ & 0.4403 \\
\hline 6927 & $11: 42: 28$ & 0.4410 \\
\hline 6928 & $11: 42: 29$ & 0.4393 \\
\hline 6929 & $11: 42: 30$ & 0.4422 \\
\hline 6930 & $11: 42: 31$ & 0.4394 \\
\hline 6931 & $11: 42: 32$ & 0.4429 \\
\hline 6932 & $11: 42: 33$ & 0.4411 \\
\hline 6933 & $11: 42: 34$ & 0.4440 \\
\hline 6934 & $11: 42: 35$ & 0.4453 \\
\hline
\end{tabular}


Appendix C - Fuel Data Test 2 NIST102605_1.xIs

\begin{tabular}{|c|c|c|}
\hline 6935 & $11: 42: 36$ & 0.4443 \\
\hline 6936 & $11: 42: 37$ & 0.4454 \\
\hline 6937 & $11: 42: 38$ & 0.4424 \\
\hline 6938 & $11: 42: 39$ & 0.4452 \\
\hline 6939 & $11: 42: 40$ & 0.4451 \\
\hline 6940 & $11: 42: 41$ & 0.4404 \\
\hline 6941 & $11: 42: 42$ & 0.4435 \\
\hline 6942 & $11: 42: 43$ & 0.4400 \\
\hline 6943 & $11: 42: 44$ & 0.4442 \\
\hline 6944 & $11: 42: 45$ & 0.4435 \\
\hline 6945 & $11: 42: 46$ & 0.4439 \\
\hline 6946 & $11: 42: 47$ & 0.4415 \\
\hline 6947 & $11: 42: 48$ & 0.4405 \\
\hline 6948 & $11: 42: 49$ & 0.4401 \\
\hline 6949 & $11: 42: 50$ & 0.4398 \\
\hline 6950 & $11: 42: 51$ & 0.4421 \\
\hline 6951 & $11: 42: 52$ & 0.4399 \\
\hline 6952 & $11: 42: 53$ & 0.4446 \\
\hline 6953 & $11: 42: 54$ & 0.4405 \\
\hline 6954 & $11: 42: 55$ & 0.4433 \\
\hline 6955 & $11: 42: 56$ & 0.4412 \\
\hline 6956 & $11: 42: 57$ & 0.4456 \\
\hline 6957 & $11: 42: 58$ & 0.4446 \\
\hline 6958 & $11: 42: 59$ & 0.4448 \\
\hline 6959 & $11: 43: 00$ & 0.4462 \\
\hline 6960 & 11:43:01 & 0.4413 \\
\hline 6961 & $11: 43: 02$ & 0.4451 \\
\hline 6962 & $11: 43: 03$ & 0.4438 \\
\hline 6963 & $11: 43: 04$ & 0.4422 \\
\hline 6964 & $11: 43: 05$ & 0.4434 \\
\hline 6965 & $11: 43: 06$ & 0.4398 \\
\hline 6966 & $11: 43: 07$ & 0.4449 \\
\hline 6967 & $11: 43: 08$ & 0.4418 \\
\hline 6968 & $11: 43: 09$ & 0.4435 \\
\hline 6969 & $11: 43: 10$ & 0.4420 \\
\hline 6970 & $11: 43: 11$ & 0.4402 \\
\hline 6971 & $11: 43: 12$ & 0.4430 \\
\hline 6972 & $11: 43: 13$ & 0.4393 \\
\hline 6973 & $11: 43: 14$ & 0.4407 \\
\hline 6974 & $11: 43: 15$ & 0.4400 \\
\hline 6975 & $11: 43: 16$ & 0.4415 \\
\hline 6976 & $11: 43: 17$ & 0.4448 \\
\hline 6977 & $11: 43: 18$ & 0.4444 \\
\hline 6978 & $11: 43: 19$ & 0.4472 \\
\hline 6979 & $11: 43: 20$ & 0.4417 \\
\hline 6980 & $11: 43: 21$ & 0.4447 \\
\hline 6981 & $11: 43: 22$ & 0.4457 \\
\hline 6982 & $11: 43: 23$ & 0.4420 \\
\hline 6983 & $11: 43: 24$ & 0.4465 \\
\hline 6984 & $11: 43: 25$ & 0.4440 \\
\hline 6985 & $11: 43: 26$ & 0.4433 \\
\hline
\end{tabular}


Appendix C - Fuel Data Test 2 NIST102605_1.xIs

\begin{tabular}{|c|c|c|}
\hline 6986 & $11: 43: 27$ & 0.4424 \\
\hline 6987 & $11: 43: 28$ & 0.4397 \\
\hline 6988 & $11: 43: 29$ & 0.4439 \\
\hline 6989 & $11: 43: 30$ & 0.4428 \\
\hline 6990 & $11: 43: 31$ & 0.4441 \\
\hline 6991 & $11: 43: 32$ & 0.4407 \\
\hline 6992 & $11: 43: 33$ & 0.4426 \\
\hline 6993 & $11: 43: 34$ & 0.4396 \\
\hline 6994 & $11: 43: 35$ & 0.4387 \\
\hline 6995 & $11: 43: 36$ & 0.4432 \\
\hline 6996 & $11: 43: 37$ & 0.4413 \\
\hline 6997 & $11: 43: 38$ & 0.4447 \\
\hline 6998 & $11: 43: 39$ & 0.4408 \\
\hline 6999 & $11: 43: 40$ & 0.4439 \\
\hline 7000 & $11: 43: 41$ & 0.4446 \\
\hline 7001 & $11: 43: 42$ & 0.4457 \\
\hline 7002 & $11: 43: 43$ & 0.4463 \\
\hline 7003 & $11: 43: 44$ & 0.4432 \\
\hline 7004 & $11: 43: 45$ & 0.4454 \\
\hline 7005 & $11: 43: 46$ & 0.4412 \\
\hline 7006 & $11: 43: 47$ & 0.4454 \\
\hline 7007 & $11: 43: 48$ & 0.4448 \\
\hline 7008 & $11: 43: 49$ & 0.4399 \\
\hline 7009 & $11: 43: 50$ & 0.4418 \\
\hline 7010 & $11: 43: 51$ & 0.4398 \\
\hline 7011 & $11: 43: 52$ & 0.4436 \\
\hline 7012 & $11: 43: 53$ & 0.4409 \\
\hline 7013 & $11: 43: 54$ & 0.4439 \\
\hline 7014 & $11: 43: 55$ & 0.4408 \\
\hline 7015 & $11: 43: 56$ & 0.4404 \\
\hline 7016 & $11: 43: 57$ & 0.4408 \\
\hline 7017 & $11: 43: 58$ & 0.4404 \\
\hline 7018 & $11: 43: 59$ & 0.4443 \\
\hline 7019 & $11: 44: 00$ & 0.4410 \\
\hline 7020 & $11: 44: 01$ & 0.4436 \\
\hline 7021 & $11: 44: 02$ & 0.4419 \\
\hline 7022 & $11: 44: 03$ & 0.4431 \\
\hline 7023 & $11: 44: 04$ & 0.4447 \\
\hline 7024 & $11: 44: 05$ & 0.4428 \\
\hline 7025 & $11: 44: 06$ & 0.4468 \\
\hline 7026 & $11: 44: 07$ & 0.4426 \\
\hline 7027 & $11: 44: 08$ & 0.4443 \\
\hline 7028 & $11: 44: 09$ & 0.4421 \\
\hline 7029 & $11: 44: 10$ & 0.4419 \\
\hline 7030 & $11: 44: 11$ & 0.4427 \\
\hline 7031 & $11: 44: 12$ & 0.4392 \\
\hline 7032 & $11: 44: 13$ & 0.4436 \\
\hline 7033 & $11: 44: 14$ & 0.4418 \\
\hline 7034 & $11: 44: 15$ & 0.4420 \\
\hline 7035 & $11: 44: 16$ & 0.4418 \\
\hline 7036 & $11: 44: 17$ & 0.4402 \\
\hline
\end{tabular}


Appendix C - Fuel Data Test 2 NIST102605_1.xIs

\begin{tabular}{|c|c|c|}
\hline 7037 & $11: 44: 18$ & 0.4428 \\
\hline 7038 & $11: 44: 19$ & 0.4405 \\
\hline 7039 & $11: 44: 20$ & 0.4432 \\
\hline 7040 & $11: 44: 21$ & 0.4383 \\
\hline 7041 & $11: 44: 22$ & 0.4414 \\
\hline 7042 & $11: 44: 23$ & 0.4390 \\
\hline 7043 & $11: 44: 24$ & 0.4435 \\
\hline 7044 & $11: 44: 25$ & 0.4444 \\
\hline 7045 & $11: 44: 26$ & 0.4419 \\
\hline 7046 & $11: 44: 27$ & 0.4464 \\
\hline 7047 & $11: 44: 28$ & 0.4438 \\
\hline 7048 & $11: 44: 29$ & 0.4450 \\
\hline 7049 & $11: 44: 30$ & 0.4462 \\
\hline 7050 & $11: 44: 31$ & 0.4456 \\
\hline 7051 & $11: 44: 32$ & 0.4459 \\
\hline 7052 & $11: 44: 33$ & 0.4395 \\
\hline 7053 & $11: 44: 34$ & 0.4436 \\
\hline 7054 & $11: 44: 35$ & 0.4424 \\
\hline 7055 & $11: 44: 36$ & 0.4426 \\
\hline 7056 & $11: 44: 37$ & 0.4414 \\
\hline 7057 & $11: 44: 38$ & 0.4398 \\
\hline 7058 & $11: 44: 39$ & 0.4434 \\
\hline 7059 & $11: 44: 40$ & 0.4382 \\
\hline 7060 & $11: 44: 41$ & 0.4407 \\
\hline 7061 & $11: 44: 42$ & 0.4393 \\
\hline 7062 & $11: 44: 43$ & 0.4388 \\
\hline 7063 & $11: 44: 44$ & 0.4416 \\
\hline 7064 & $11: 44: 45$ & 0.4412 \\
\hline 7065 & $11: 44: 46$ & 0.4441 \\
\hline 7066 & $11: 44: 47$ & 0.4405 \\
\hline 7067 & $11: 44: 48$ & 0.4443 \\
\hline 7068 & $11: 44: 49$ & 0.4440 \\
\hline 7069 & $11: 44: 50$ & 0.4461 \\
\hline 7070 & $11: 44: 51$ & 0.4453 \\
\hline 7071 & $11: 44: 52$ & 0.4427 \\
\hline 7072 & $11: 44: 53$ & 0.4449 \\
\hline 7073 & $11: 44: 54$ & 0.4414 \\
\hline 7074 & $11: 44: 55$ & 0.4432 \\
\hline 7075 & $11: 44: 56$ & 0.4405 \\
\hline 7076 & $11: 44: 57$ & 0.4442 \\
\hline 7077 & $11: 44: 58$ & 0.4425 \\
\hline 7078 & $11: 44: 59$ & 0.4440 \\
\hline 7079 & $11: 45: 00$ & 0.4422 \\
\hline 7080 & $11: 45: 01$ & 0.4397 \\
\hline 7081 & $11: 45: 02$ & 0.4412 \\
\hline 7082 & $11: 45: 03$ & 0.4398 \\
\hline 7083 & $11: 45: 04$ & 0.4425 \\
\hline 7084 & $11: 45: 05$ & 0.4404 \\
\hline 7085 & $11: 45: 06$ & 0.4429 \\
\hline 7086 & $11: 45: 07$ & 0.4395 \\
\hline 7087 & $11: 45: 08$ & 0.4434 \\
\hline
\end{tabular}


Appendix C - Fuel Data Test 2 NIST102605_1.xIs

\begin{tabular}{|c|c|c|}
\hline 7088 & $11: 45: 09$ & 0.4421 \\
\hline 7089 & $11: 45: 10$ & 0.4430 \\
\hline 7090 & $11: 45: 11$ & 0.4472 \\
\hline 7091 & $11: 45: 12$ & 0.4426 \\
\hline 7092 & $11: 45: 13$ & 0.4455 \\
\hline 7093 & $11: 45: 14$ & 0.4422 \\
\hline 7094 & $11: 45: 15$ & 0.4449 \\
\hline 7095 & $11: 45: 16$ & 0.4449 \\
\hline 7096 & $11: 45: 17$ & 0.4454 \\
\hline 7097 & $11: 45: 18$ & 0.4437 \\
\hline 7098 & $11: 45: 19$ & 0.4405 \\
\hline 7099 & $11: 45: 20$ & 0.4418 \\
\hline 7100 & $11: 45: 21$ & 0.4387 \\
\hline 7101 & $11: 45: 22$ & 0.4434 \\
\hline 7102 & $11: 45: 23$ & 0.4414 \\
\hline 7103 & $11: 45: 24$ & 0.4435 \\
\hline 7104 & $11: 45: 25$ & 0.4393 \\
\hline 7105 & $11: 45: 26$ & 0.4399 \\
\hline 7106 & $11: 45: 27$ & 0.4408 \\
\hline 7107 & $11: 45: 28$ & 0.4389 \\
\hline 7108 & $11: 45: 29$ & 0.4432 \\
\hline 7109 & $11: 45: 30$ & 0.4399 \\
\hline 7110 & $11: 45: 31$ & 0.4429 \\
\hline 7111 & $11: 45: 32$ & 0.4430 \\
\hline 7112 & $11: 45: 33$ & 0.4444 \\
\hline 7113 & $11: 45: 34$ & 0.4457 \\
\hline 7114 & $11: 45: 35$ & 0.4423 \\
\hline 7115 & $11: 45: 36$ & 0.4454 \\
\hline 7116 & $11: 45: 37$ & 0.4424 \\
\hline 7117 & $11: 45: 38$ & 0.4456 \\
\hline 7118 & $11: 45: 39$ & 0.4442 \\
\hline 7119 & $11: 45: 40$ & 0.4406 \\
\hline 7120 & $11: 45: 41$ & 0.4426 \\
\hline 7121 & $11: 45: 42$ & 0.4397 \\
\hline 7122 & $11: 45: 43$ & 0.4442 \\
\hline 7123 & $11: 45: 44$ & 0.4423 \\
\hline 7124 & $11: 45: 45$ & 0.4436 \\
\hline 7125 & $11: 45: 46$ & 0.4401 \\
\hline 7126 & $11: 45: 47$ & 0.4393 \\
\hline 7127 & $11: 45: 48$ & 0.4439 \\
\hline 7128 & $11: 45: 49$ & 0.4412 \\
\hline 7129 & $11: 45: 50$ & 0.4434 \\
\hline 7130 & $11: 45: 51$ & 0.4380 \\
\hline 7131 & $11: 45: 52$ & 0.4417 \\
\hline 7132 & $11: 45: 53$ & 0.4402 \\
\hline 7133 & $11: 45: 54$ & 0.4430 \\
\hline 7134 & $11: 45: 55$ & 0.4459 \\
\hline 7135 & $11: 45: 56$ & 0.4447 \\
\hline 7136 & $11: 45: 57$ & 0.4456 \\
\hline 7137 & $11: 45: 58$ & 0.4425 \\
\hline 7138 & $11: 45: 59$ & 0.4457 \\
\hline
\end{tabular}


Appendix C - Fuel Data Test 2 NIST102605_1.xls

\begin{tabular}{|c|c|c|}
\hline 7139 & $11: 46: 00$ & 0.4419 \\
\hline 7140 & $11: 46: 01$ & 0.4429 \\
\hline 7141 & $11: 46: 02$ & 0.4388 \\
\hline 7142 & $11: 46: 03$ & 0.4435 \\
\hline 7143 & $11: 46: 04$ & 0.4423 \\
\hline 7144 & $11: 46: 05$ & 0.4434 \\
\hline 7145 & $11: 46: 06$ & 0.4401 \\
\hline 7146 & $11: 46: 07$ & 0.4415 \\
\hline 7147 & $11: 46: 08$ & 0.4420 \\
\hline 7148 & $11: 46: 09$ & 0.4379 \\
\hline 7149 & $11: 46: 10$ & 0.4432 \\
\hline 7150 & $11: 46: 11$ & 0.4403 \\
\hline 7151 & $11: 46: 12$ & 0.4396 \\
\hline 7152 & $11: 46: 13$ & 0.4403 \\
\hline 7153 & $11: 46: 14$ & 0.4403 \\
\hline 7154 & $11: 46: 15$ & 0.4438 \\
\hline 7155 & $11: 46: 16$ & 0.4416 \\
\hline 7156 & $11: 46: 17$ & 0.4445 \\
\hline 7157 & $11: 46: 18$ & 0.4424 \\
\hline 7158 & $11: 46: 19$ & 0.4452 \\
\hline 7159 & $11: 46: 20$ & 0.4442 \\
\hline 7160 & $11: 46: 21$ & 0.4424 \\
\hline 7161 & $11: 46: 22$ & 0.4449 \\
\hline 7162 & $11: 46: 23$ & 0.4403 \\
\hline 7163 & $11: 46: 24$ & 0.4443 \\
\hline 7164 & $11: 46: 25$ & 0.4425 \\
\hline 7165 & $11: 46: 26$ & 0.4427 \\
\hline 7166 & $11: 46: 27$ & 0.4411 \\
\hline 7167 & $11: 46: 28$ & 0.4400 \\
\hline 7168 & $11: 46: 29$ & 0.4430 \\
\hline 7169 & $11: 46: 30$ & 0.4413 \\
\hline 7170 & $11: 46: 31$ & 0.4438 \\
\hline 7171 & $11: 46: 32$ & 0.4392 \\
\hline 7172 & $11: 46: 33$ & 0.4411 \\
\hline 7173 & $11: 46: 34$ & 0.4378 \\
\hline 7174 & $11: 46: 35$ & 0.4404 \\
\hline 7175 & $11: 46: 36$ & 0.4401 \\
\hline 7176 & $11: 46: 37$ & 0.4428 \\
\hline 7177 & $11: 46: 38$ & 0.4465 \\
\hline 7178 & $11: 46: 39$ & 0.4413 \\
\hline 7179 & $11: 46: 40$ & 0.4441 \\
\hline 7180 & $11: 46: 41$ & 0.4426 \\
\hline 7181 & $11: 46: 42$ & 0.4456 \\
\hline 7182 & $11: 46: 43$ & 0.4466 \\
\hline 7183 & $11: 46: 44$ & 0.4439 \\
\hline 7184 & $11: 46: 45$ & 0.4442 \\
\hline 7185 & $11: 46: 46$ & 0.4404 \\
\hline 7186 & $11: 46: 47$ & 0.4449 \\
\hline 7187 & $11: 46: 48$ & 0.4423 \\
\hline 7188 & $11: 46: 49$ & 0.4441 \\
\hline 7189 & $11: 46: 50$ & 0.4408 \\
\hline
\end{tabular}


Appendix C - Fuel Data Test 2 NIST102605_1.xls

\begin{tabular}{|c|c|c|}
\hline 7190 & $11: 46: 51$ & 0.4394 \\
\hline 7191 & $11: 46: 52$ & 0.4421 \\
\hline 7192 & $11: 46: 53$ & 0.4386 \\
\hline 7193 & $11: 46: 54$ & 0.4442 \\
\hline 7194 & $11: 46: 55$ & 0.4411 \\
\hline 7195 & $11: 46: 56$ & 0.4409 \\
\hline 7196 & $11: 46: 57$ & 0.4409 \\
\hline 7197 & $11: 46: 58$ & 0.4406 \\
\hline 7198 & $11: 46: 59$ & 0.4442 \\
\hline 7199 & $11: 47: 00$ & 0.4412 \\
\hline 7200 & $11: 47: 01$ & 0.4440 \\
\hline 7201 & $11: 47: 02$ & 0.4419 \\
\hline 7202 & $11: 47: 03$ & 0.4447 \\
\hline 7203 & $11: 47: 04$ & 0.4417 \\
\hline 7204 & 11:47:05 & 0.4435 \\
\hline 7205 & $11: 47: 06$ & 0.4452 \\
\hline 7206 & $11: 47: 07$ & 0.4430 \\
\hline 7207 & $11: 47: 08$ & 0.4447 \\
\hline 7208 & $11: 47: 09$ & 0.4412 \\
\hline 7209 & $11: 47: 10$ & 0.4450 \\
\hline 7210 & $11: 47: 11$ & 0.4422 \\
\hline 7211 & $11: 47: 12$ & 0.4421 \\
\hline 7212 & $11: 47: 13$ & 0.4423 \\
\hline 7213 & $11: 47: 14$ & 0.4414 \\
\hline 7214 & $11: 47: 15$ & 0.4412 \\
\hline 7215 & $11: 47: 16$ & 0.4396 \\
\hline 7216 & $11: 47: 17$ & 0.4414 \\
\hline 7217 & $11: 47: 18$ & 0.4374 \\
\hline 7218 & $11: 47: 19$ & 0.4395 \\
\hline 7219 & $11: 47: 20$ & 0.4411 \\
\hline 7220 & $11: 47: 21$ & 0.4421 \\
\hline 7221 & $11: 47: 22$ & 0.4442 \\
\hline 7222 & $11: 47: 23$ & 0.4414 \\
\hline 7223 & $11: 47: 24$ & 0.4455 \\
\hline 7224 & $11: 47: 25$ & 0.4421 \\
\hline 7225 & $11: 47: 26$ & 0.4456 \\
\hline 7226 & $11: 47: 27$ & 0.4441 \\
\hline 7227 & $11: 47: 28$ & 0.4465 \\
\hline 7228 & $11: 47: 29$ & 0.4454 \\
\hline 7229 & $11: 47: 30$ & 0.4400 \\
\hline 7230 & $11: 47: 31$ & 0.4428 \\
\hline 7231 & $11: 47: 32$ & 0.4404 \\
\hline 7232 & $11: 47: 33$ & 0.4441 \\
\hline 7233 & $11: 47: 34$ & 0.4419 \\
\hline 7234 & $11: 47: 35$ & 0.4443 \\
\hline 7235 & $11: 47: 36$ & 0.4415 \\
\hline 7236 & $11: 47: 37$ & 0.4395 \\
\hline 7237 & $11: 47: 38$ & 0.4395 \\
\hline 7238 & $11: 47: 39$ & 0.4395 \\
\hline 7239 & $11: 47: 40$ & 0.4432 \\
\hline 7240 & $11: 47: 41$ & 0.4395 \\
\hline
\end{tabular}


Appendix C - Fuel Data Test 2 NIST102605_1.xIs

\begin{tabular}{|c|c|c|}
\hline 7241 & $11: 47: 42$ & 0.4424 \\
\hline 7242 & $11: 47: 43$ & 0.4394 \\
\hline 7243 & $11: 47: 44$ & 0.4405 \\
\hline 7244 & $11: 47: 45$ & 0.4441 \\
\hline 7245 & $11: 47: 46$ & 0.4430 \\
\hline 7246 & $11: 47: 47$ & 0.4458 \\
\hline 7247 & $11: 47: 48$ & 0.4434 \\
\hline 7248 & $11: 47: 49$ & 0.4452 \\
\hline 7249 & $11: 47: 50$ & 0.4463 \\
\hline 7250 & $11: 47: 51$ & 0.4410 \\
\hline 7251 & $11: 47: 52$ & 0.4427 \\
\hline 7252 & $11: 47: 53$ & 0.4401 \\
\hline 7253 & $11: 47: 54$ & 0.4448 \\
\hline 7254 & $11: 47: 55$ & 0.4427 \\
\hline 7255 & $11: 47: 56$ & 0.4429 \\
\hline 7256 & $11: 47: 57$ & 0.4419 \\
\hline 7257 & $11: 47: 58$ & 0.4403 \\
\hline 7258 & $11: 47: 59$ & 0.4412 \\
\hline 7259 & $11: 48: 00$ & 0.4408 \\
\hline 7260 & $11: 48: 01$ & 0.4421 \\
\hline 7261 & $11: 48: 02$ & 0.4394 \\
\hline 7262 & $11: 48: 03$ & 0.4423 \\
\hline 7263 & $11: 48: 04$ & 0.4390 \\
\hline 7264 & 11:48:05 & 0.4407 \\
\hline 7265 & $11: 48: 06$ & 0.4446 \\
\hline 7266 & $11: 48: 07$ & 0.4395 \\
\hline 7267 & $11: 48: 08$ & 0.4442 \\
\hline 7268 & $11: 48: 09$ & 0.4465 \\
\hline 7269 & $11: 48: 10$ & 0.4430 \\
\hline 7270 & $11: 48: 11$ & 0.4467 \\
\hline 7271 & $11: 48: 12$ & 0.4441 \\
\hline 7272 & $11: 48: 13$ & 0.4443 \\
\hline 7273 & $11: 48: 14$ & 0.4403 \\
\hline 7274 & $11: 48: 15$ & 0.4451 \\
\hline 7275 & $11: 48: 16$ & 0.4436 \\
\hline 7276 & $11: 48: 17$ & 0.4441 \\
\hline 7277 & $11: 48: 18$ & 0.4405 \\
\hline 7278 & $11: 48: 19$ & 0.4400 \\
\hline 7279 & $11: 48: 20$ & 0.4412 \\
\hline 7280 & $11: 48: 21$ & 0.4406 \\
\hline 7281 & $11: 48: 22$ & 0.4411 \\
\hline 7282 & $11: 48: 23$ & 0.4393 \\
\hline 7283 & $11: 48: 24$ & 0.4412 \\
\hline 7284 & $11: 48: 25$ & 0.4384 \\
\hline 7285 & $11: 48: 26$ & 0.4431 \\
\hline 7286 & $11: 48: 27$ & 0.4399 \\
\hline 7287 & $11: 48: 28$ & 0.4414 \\
\hline 7288 & $11: 48: 29$ & 0.4413 \\
\hline 7289 & $11: 48: 30$ & 0.4426 \\
\hline 7290 & $11: 48: 31$ & 0.4450 \\
\hline 7291 & $11: 48: 32$ & 0.4415 \\
\hline
\end{tabular}


Appendix C - Fuel Data Test 2 NIST102605_1.xls

\begin{tabular}{|c|c|c|}
\hline 7292 & $11: 48: 33$ & 0.4459 \\
\hline 7293 & $11: 48: 34$ & 0.4455 \\
\hline 7294 & $11: 48: 35$ & 0.4454 \\
\hline 7295 & $11: 48: 36$ & 0.4446 \\
\hline 7296 & $11: 48: 37$ & 0.4407 \\
\hline 7297 & $11: 48: 38$ & 0.4444 \\
\hline 7298 & $11: 48: 39$ & 0.4419 \\
\hline 7299 & $11: 48: 40$ & 0.4449 \\
\hline 7300 & $11: 48: 41$ & 0.4434 \\
\hline 7301 & $11: 48: 42$ & 0.4439 \\
\hline 7302 & $11: 48: 43$ & 0.4414 \\
\hline 7303 & $11: 48: 44$ & 0.4393 \\
\hline 7304 & $11: 48: 45$ & 0.4422 \\
\hline 7305 & $11: 48: 46$ & 0.4414 \\
\hline 7306 & $11: 48: 47$ & 0.4426 \\
\hline 7307 & $11: 48: 48$ & 0.4371 \\
\hline 7308 & $11: 48: 49$ & 0.4406 \\
\hline 7309 & $11: 48: 50$ & 0.4385 \\
\hline 7310 & $11: 48: 51$ & 0.4421 \\
\hline 7311 & $11: 48: 52$ & 0.4431 \\
\hline 7312 & $11: 48: 53$ & 0.4458 \\
\hline 7313 & $11: 48: 54$ & 0.4462 \\
\hline 7314 & $11: 48: 55$ & 0.4431 \\
\hline 7315 & $11: 48: 56$ & 0.4465 \\
\hline 7316 & $11: 48: 57$ & 0.4436 \\
\hline 7317 & $11: 48: 58$ & 0.4458 \\
\hline 7318 & $11: 48: 59$ & 0.4423 \\
\hline 7319 & $11: 49: 00$ & 0.4442 \\
\hline 7320 & $11: 49: 01$ & 0.4432 \\
\hline 7321 & $11: 49: 02$ & 0.4394 \\
\hline 7322 & $11: 49: 03$ & 0.4424 \\
\hline 7323 & $11: 49: 04$ & 0.4406 \\
\hline 7324 & 11:49:05 & 0.4451 \\
\hline 7325 & $11: 49: 06$ & 0.4388 \\
\hline 7326 & $11: 49: 07$ & 0.4388 \\
\hline 7327 & $11: 49: 08$ & 0.4425 \\
\hline 7328 & $11: 49: 09$ & 0.4399 \\
\hline 7329 & $11: 49: 10$ & 0.4434 \\
\hline 7330 & $11: 49: 11$ & 0.4418 \\
\hline 7331 & $11: 49: 12$ & 0.4430 \\
\hline 7332 & $11: 49: 13$ & 0.4392 \\
\hline 7333 & $11: 49: 14$ & 0.4424 \\
\hline 7334 & $11: 49: 15$ & 0.4446 \\
\hline 7335 & $11: 49: 16$ & 0.4448 \\
\hline 7336 & $11: 49: 17$ & 0.4460 \\
\hline 7337 & $11: 49: 18$ & 0.4415 \\
\hline 7338 & $11: 49: 19$ & 0.4443 \\
\hline 7339 & $11: 49: 20$ & 0.4408 \\
\hline 7340 & $11: 49: 21$ & 0.4448 \\
\hline 7341 & $11: 49: 22$ & 0.4448 \\
\hline 7342 & $11: 49: 23$ & 0.4417 \\
\hline
\end{tabular}


Appendix C - Fuel Data Test 2 NIST102605_1.xIs

\begin{tabular}{|c|c|c|}
\hline 7343 & $11: 49: 24$ & 0.4442 \\
\hline 7344 & $11: 49: 25$ & 0.4415 \\
\hline 7345 & $11: 49: 26$ & 0.4446 \\
\hline 7346 & $11: 49: 27$ & 0.4418 \\
\hline 7347 & $11: 49: 28$ & 0.4403 \\
\hline 7348 & $11: 49: 29$ & 0.4421 \\
\hline 7349 & $11: 49: 30$ & 0.4405 \\
\hline 7350 & $11: 49: 31$ & 0.4426 \\
\hline 7351 & $11: 49: 32$ & 0.4389 \\
\hline 7352 & $11: 49: 33$ & 0.4416 \\
\hline 7353 & $11: 49: 34$ & 0.4384 \\
\hline 7354 & $11: 49: 35$ & 0.4409 \\
\hline 7355 & $11: 49: 36$ & 0.4441 \\
\hline 7356 & $11: 49: 37$ & 0.4447 \\
\hline 7357 & $11: 49: 38$ & 0.4461 \\
\hline 7358 & $11: 49: 39$ & 0.4422 \\
\hline 7359 & $11: 49: 40$ & 0.4465 \\
\hline 7360 & $11: 49: 41$ & 0.4446 \\
\hline 7361 & $11: 49: 42$ & 0.4447 \\
\hline 7362 & $11: 49: 43$ & 0.4426 \\
\hline 7363 & $11: 49: 44$ & 0.4429 \\
\hline 7364 & $11: 49: 45$ & 0.4441 \\
\hline 7365 & $11: 49: 46$ & 0.4400 \\
\hline 7366 & $11: 49: 47$ & 0.4439 \\
\hline 7367 & $11: 49: 48$ & 0.4413 \\
\hline 7368 & $11: 49: 49$ & 0.4434 \\
\hline 7369 & $11: 49: 50$ & 0.4407 \\
\hline 7370 & $11: 49: 51$ & 0.4399 \\
\hline 7371 & $11: 49: 52$ & 0.4411 \\
\hline 7372 & $11: 49: 53$ & 0.4393 \\
\hline 7373 & $11: 49: 54$ & 0.4422 \\
\hline 7374 & $11: 49: 55$ & 0.4408 \\
\hline 7375 & $11: 49: 56$ & 0.4427 \\
\hline 7376 & $11: 49: 57$ & 0.4404 \\
\hline 7377 & $11: 49: 58$ & 0.4439 \\
\hline 7378 & $11: 49: 59$ & 0.4407 \\
\hline 7379 & $11: 50: 00$ & 0.4445 \\
\hline 7380 & $11: 50: 01$ & 0.4446 \\
\hline 7381 & $11: 50: 02$ & 0.4425 \\
\hline 7382 & $11: 50: 03$ & 0.4457 \\
\hline 7383 & $11: 50: 04$ & 0.4408 \\
\hline 7384 & 11:50:05 & 0.4448 \\
\hline 7385 & $11: 50: 06$ & 0.4452 \\
\hline 7386 & $11: 50: 07$ & 0.4440 \\
\hline 7387 & $11: 50: 08$ & 0.4439 \\
\hline 7388 & 11:50:09 & 0.4400 \\
\hline 7389 & $11: 50: 10$ & 0.4435 \\
\hline 7390 & $11: 50: 11$ & 0.4412 \\
\hline 7391 & $11: 50: 12$ & 0.4427 \\
\hline 7392 & $11: 50: 13$ & 0.4419 \\
\hline 7393 & $11: 50: 14$ & 0.4401 \\
\hline
\end{tabular}


Appendix C - Fuel Data Test 2 NIST102605_1.xls

\begin{tabular}{|c|c|c|}
\hline 7394 & $11: 50: 15$ & 0.4430 \\
\hline 7395 & $11: 50: 16$ & 0.4379 \\
\hline 7396 & $11: 50: 17$ & 0.4408 \\
\hline 7397 & $11: 50: 18$ & 0.4373 \\
\hline 7398 & $11: 50: 19$ & 0.4396 \\
\hline 7399 & $11: 50: 20$ & 0.4420 \\
\hline 7400 & $11: 50: 21$ & 0.4414 \\
\hline 7401 & $11: 50: 22$ & 0.4451 \\
\hline 7402 & $11: 50: 23$ & 0.4414 \\
\hline 7403 & $11: 50: 24$ & 0.4454 \\
\hline 7404 & $11: 50: 25$ & 0.4453 \\
\hline 7405 & $11: 50: 26$ & 0.4464 \\
\hline 7406 & $11: 50: 27$ & 0.4464 \\
\hline 7407 & $11: 50: 28$ & 0.4418 \\
\hline 7408 & $11: 50: 29$ & 0.4432 \\
\hline 7409 & $11: 50: 30$ & 0.4395 \\
\hline 7410 & $11: 50: 31$ & 0.4449 \\
\hline 7411 & $11: 50: 32$ & 0.4429 \\
\hline 7412 & $11: 50: 33$ & 0.4419 \\
\hline 7413 & $11: 50: 34$ & 0.4408 \\
\hline 7414 & $11: 50: 35$ & 0.4402 \\
\hline 7415 & $11: 50: 36$ & 0.4434 \\
\hline 7416 & $11: 50: 37$ & 0.4388 \\
\hline 7417 & $11: 50: 38$ & 0.4419 \\
\hline 7418 & $11: 50: 39$ & 0.4403 \\
\hline 7419 & $11: 50: 40$ & 0.4387 \\
\hline 7420 & $11: 50: 41$ & 0.4424 \\
\hline 7421 & $11: 50: 42$ & 0.4417 \\
\hline 7422 & $11: 50: 43$ & 0.4458 \\
\hline 7423 & $11: 50: 44$ & 0.4430 \\
\hline 7424 & $11: 50: 45$ & 0.4444 \\
\hline 7425 & $11: 50: 46$ & 0.4426 \\
\hline 7426 & $11: 50: 47$ & 0.4442 \\
\hline 7427 & $11: 50: 48$ & 0.4448 \\
\hline 7428 & $11: 50: 49$ & 0.4427 \\
\hline 7429 & $11: 50: 50$ & 0.4464 \\
\hline 7430 & $11: 50: 51$ & 0.4416 \\
\hline 7431 & $11: 50: 52$ & 0.4450 \\
\hline 7432 & $11: 50: 53$ & 0.4438 \\
\hline 7433 & $11: 50: 54$ & 0.4429 \\
\hline 7434 & $11: 50: 55$ & 0.4424 \\
\hline 7435 & $11: 50: 56$ & 0.4401 \\
\hline 7436 & $11: 50: 57$ & 0.4416 \\
\hline 7437 & $11: 50: 58$ & 0.4398 \\
\hline 7438 & $11: 50: 59$ & 0.4419 \\
\hline 7439 & $11: 51: 00$ & 0.4402 \\
\hline 7440 & $11: 51: 01$ & 0.4428 \\
\hline 7441 & $11: 51: 02$ & 0.4378 \\
\hline 7442 & $11: 51: 03$ & 0.4402 \\
\hline 7443 & $11: 51: 04$ & 0.4427 \\
\hline 7444 & $11: 51: 05$ & 0.4428 \\
\hline
\end{tabular}


Appendix C - Fuel Data Test 2 NIST102605_1.xIs

\begin{tabular}{|c|c|c|}
\hline 7445 & $11: 51: 06$ & 0.4463 \\
\hline 7446 & $11: 51: 07$ & 0.4414 \\
\hline 7447 & $11: 51: 08$ & 0.4445 \\
\hline 7448 & $11: 51: 09$ & 0.4437 \\
\hline 7449 & $11: 51: 10$ & 0.4454 \\
\hline 7450 & $11: 51: 11$ & 0.4476 \\
\hline 7451 & $11: 51: 12$ & 0.4465 \\
\hline 7452 & $11: 51: 13$ & 0.4460 \\
\hline 7453 & $11: 51: 14$ & 0.4403 \\
\hline 7454 & $11: 51: 15$ & 0.4449 \\
\hline 7455 & $11: 51: 16$ & 0.4431 \\
\hline 7456 & $11: 51: 17$ & 0.4408 \\
\hline 7457 & $11: 51: 18$ & 0.4425 \\
\hline 7458 & $11: 51: 19$ & 0.4402 \\
\hline 7459 & $11: 51: 20$ & 0.4434 \\
\hline 7460 & $11: 51: 21$ & 0.4408 \\
\hline 7461 & $11: 51: 22$ & 0.4397 \\
\hline 7462 & $11: 51: 23$ & 0.4411 \\
\hline 7463 & $11: 51: 24$ & 0.4386 \\
\hline 7464 & $11: 51: 25$ & 0.4443 \\
\hline 7465 & $11: 51: 26$ & 0.4399 \\
\hline 7466 & $11: 51: 27$ & 0.4411 \\
\hline 7467 & $11: 51: 28$ & 0.4410 \\
\hline 7468 & $11: 51: 29$ & 0.4433 \\
\hline 7469 & $11: 51: 30$ & 0.4455 \\
\hline 7470 & $11: 51: 31$ & 0.4434 \\
\hline 7471 & $11: 51: 32$ & 0.4453 \\
\hline 7472 & $11: 51: 33$ & 0.4422 \\
\hline 7473 & $11: 51: 34$ & 0.4458 \\
\hline 7474 & $11: 51: 35$ & 0.4424 \\
\hline 7475 & $11: 51: 36$ & 0.4407 \\
\hline 7476 & $11: 51: 37$ & 0.4436 \\
\hline 7477 & $11: 51: 38$ & 0.4400 \\
\hline 7478 & $11: 51: 39$ & 0.4443 \\
\hline 7479 & $11: 51: 40$ & 0.4434 \\
\hline 7480 & $11: 51: 41$ & 0.4418 \\
\hline 7481 & $11: 51: 42$ & 0.4427 \\
\hline 7482 & $11: 51: 43$ & 0.4408 \\
\hline 7483 & $11: 51: 44$ & 0.4447 \\
\hline 7484 & $11: 51: 45$ & 0.4411 \\
\hline 7485 & $11: 51: 46$ & 0.4424 \\
\hline 7486 & $11: 51: 47$ & 0.4386 \\
\hline 7487 & $11: 51: 48$ & 0.4402 \\
\hline 7488 & $11: 51: 49$ & 0.4384 \\
\hline 7489 & $11: 51: 50$ & 0.4400 \\
\hline 7490 & $11: 51: 51$ & 0.4457 \\
\hline 7491 & $11: 51: 52$ & 0.4435 \\
\hline 7492 & $11: 51: 53$ & 0.4458 \\
\hline 7493 & $11: 51: 54$ & 0.4419 \\
\hline 7494 & $11: 51: 55$ & 0.4456 \\
\hline 7495 & $11: 51: 56$ & 0.4461 \\
\hline
\end{tabular}


Appendix C - Fuel Data Test 2 NIST102605_1.xIs

\begin{tabular}{|c|c|c|}
\hline 7496 & $11: 51: 57$ & 0.4439 \\
\hline 7497 & $11: 51: 58$ & 0.4451 \\
\hline 7498 & $11: 51: 59$ & 0.4416 \\
\hline 7499 & $11: 52: 00$ & 0.4426 \\
\hline 7500 & $11: 52: 01$ & 0.4390 \\
\hline 7501 & $11: 52: 02$ & 0.4448 \\
\hline 7502 & $11: 52: 03$ & 0.4419 \\
\hline 7503 & $11: 52: 04$ & 0.4454 \\
\hline 7504 & 11:52:05 & 0.4401 \\
\hline 7505 & $11: 52: 06$ & 0.4409 \\
\hline 7506 & $11: 52: 07$ & 0.4404 \\
\hline 7507 & $11: 52: 08$ & 0.4386 \\
\hline 7508 & $11: 52: 09$ & 0.4430 \\
\hline 7509 & $11: 52: 10$ & 0.4410 \\
\hline 7510 & $11: 52: 11$ & 0.4426 \\
\hline 7511 & $11: 52: 12$ & 0.4381 \\
\hline 7512 & $11: 52: 13$ & 0.4424 \\
\hline 7513 & $11: 52: 14$ & 0.4424 \\
\hline 7514 & $11: 52: 15$ & 0.4451 \\
\hline 7515 & $11: 52: 16$ & 0.4448 \\
\hline 7516 & $11: 52: 17$ & 0.4421 \\
\hline 7517 & $11: 52: 18$ & 0.4450 \\
\hline 7518 & $11: 52: 19$ & 0.4407 \\
\hline 7519 & $11: 52: 20$ & 0.4450 \\
\hline 7520 & $11: 52: 21$ & 0.4434 \\
\hline 7521 & $11: 52: 22$ & 0.4436 \\
\hline 7522 & $11: 52: 23$ & 0.4436 \\
\hline 7523 & $11: 52: 24$ & 0.4408 \\
\hline 7524 & $11: 52: 25$ & 0.4456 \\
\hline 7525 & $11: 52: 26$ & 0.4427 \\
\hline 7526 & $11: 52: 27$ & 0.4410 \\
\hline 7527 & $11: 52: 28$ & 0.4412 \\
\hline 7528 & $11: 52: 29$ & 0.4399 \\
\hline 7529 & $11: 52: 30$ & 0.4423 \\
\hline 7530 & $11: 52: 31$ & 0.4396 \\
\hline 7531 & $11: 52: 32$ & 0.4431 \\
\hline 7532 & $11: 52: 33$ & 0.4387 \\
\hline 7533 & $11: 52: 34$ & 0.4405 \\
\hline 7534 & $11: 52: 35$ & 0.4422 \\
\hline 7535 & $11: 52: 36$ & 0.4399 \\
\hline 7536 & $11: 52: 37$ & 0.4447 \\
\hline 7537 & $11: 52: 38$ & 0.4441 \\
\hline 7538 & $11: 52: 39$ & 0.4452 \\
\hline 7539 & $11: 52: 40$ & 0.4469 \\
\hline 7540 & $11: 52: 41$ & 0.4443 \\
\hline 7541 & $11: 52: 42$ & 0.4453 \\
\hline 7542 & $11: 52: 43$ & 0.4403 \\
\hline 7543 & $11: 52: 44$ & 0.4448 \\
\hline 7544 & $11: 52: 45$ & 0.4425 \\
\hline 7545 & $11: 52: 46$ & 0.4448 \\
\hline 7546 & $11: 52: 47$ & 0.4426 \\
\hline
\end{tabular}


Appendix C - Fuel Data Test 2 NIST102605_1.xls

\begin{tabular}{|c|c|c|}
\hline 7547 & $11: 52: 48$ & 0.4397 \\
\hline 7548 & $11: 52: 49$ & 0.4438 \\
\hline 7549 & $11: 52: 50$ & 0.4418 \\
\hline 7550 & $11: 52: 51$ & 0.4436 \\
\hline 7551 & $11: 52: 52$ & 0.4405 \\
\hline 7552 & $11: 52: 53$ & 0.4396 \\
\hline 7553 & $11: 52: 54$ & 0.4412 \\
\hline 7554 & $11: 52: 55$ & 0.4389 \\
\hline 7555 & $11: 52: 56$ & 0.4434 \\
\hline 7556 & $11: 52: 57$ & 0.4404 \\
\hline 7557 & $11: 52: 58$ & 0.4428 \\
\hline 7558 & $11: 52: 59$ & 0.4415 \\
\hline 7559 & $11: 53: 00$ & 0.4437 \\
\hline 7560 & 11:53:01 & 0.4444 \\
\hline 7561 & 11:53:02 & 0.4459 \\
\hline 7562 & $11: 53: 03$ & 0.4457 \\
\hline 7563 & 11:53:04 & 0.4429 \\
\hline 7564 & 11:53:05 & 0.4463 \\
\hline 7565 & 11:53:06 & 0.4407 \\
\hline 7566 & $11: 53: 07$ & 0.4440 \\
\hline 7567 & 11:53:08 & 0.4454 \\
\hline 7568 & $11: 53: 09$ & 0.4398 \\
\hline 7569 & 11:53:10 & 0.4443 \\
\hline 7570 & 11:53:11 & 0.4422 \\
\hline 7571 & $11: 53: 12$ & 0.4439 \\
\hline 7572 & $11: 53: 13$ & 0.4404 \\
\hline 7573 & $11: 53: 14$ & 0.4410 \\
\hline 7574 & $11: 53: 15$ & 0.4420 \\
\hline 7575 & $11: 53: 16$ & 0.4409 \\
\hline 7576 & $11: 53: 17$ & 0.4441 \\
\hline 7577 & $11: 53: 18$ & 0.4391 \\
\hline 7578 & 11:53:19 & 0.4410 \\
\hline 7579 & $11: 53: 20$ & 0.4426 \\
\hline 7580 & $11: 53: 21$ & 0.4410 \\
\hline 7581 & 11:53:22 & 0.4469 \\
\hline 7582 & $11: 53: 23$ & 0.4443 \\
\hline 7583 & 11:53:24 & 0.4448 \\
\hline 7584 & $11: 53: 25$ & 0.4485 \\
\hline 7585 & $11: 53: 26$ & 0.4433 \\
\hline 7586 & 11:53:27 & 0.4441 \\
\hline 7587 & 11:53:28 & 0.4406 \\
\hline 7588 & $11: 53: 29$ & 0.4456 \\
\hline 7589 & 11:53:30 & 0.4448 \\
\hline 7590 & $11: 53: 31$ & 0.4423 \\
\hline 7591 & $11: 53: 32$ & 0.4412 \\
\hline 7592 & $11: 53: 33$ & 0.4399 \\
\hline 7593 & $11: 53: 34$ & 0.4438 \\
\hline 7594 & 11:53:35 & 0.4393 \\
\hline 7595 & 11:53:36 & 0.4438 \\
\hline 7596 & $11: 53: 37$ & 0.4426 \\
\hline 7597 & $11: 53: 38$ & 0.4395 \\
\hline
\end{tabular}


Appendix C - Fuel Data Test 2 NIST102605_1.xIs

\begin{tabular}{|c|c|c|}
\hline 7598 & $11: 53: 39$ & 0.4427 \\
\hline 7599 & $11: 53: 40$ & 0.4389 \\
\hline 7600 & $11: 53: 41$ & 0.4409 \\
\hline 7601 & $11: 53: 42$ & 0.4406 \\
\hline 7602 & $11: 53: 43$ & 0.4431 \\
\hline 7603 & $11: 53: 44$ & 0.4462 \\
\hline 7604 & $11: 53: 45$ & 0.4445 \\
\hline 7605 & $11: 53: 46$ & 0.4445 \\
\hline 7606 & $11: 53: 47$ & 0.4419 \\
\hline 7607 & $11: 53: 48$ & 0.4464 \\
\hline 7608 & $11: 53: 49$ & 0.4434 \\
\hline 7609 & $11: 53: 50$ & 0.4450 \\
\hline 7610 & $11: 53: 51$ & 0.4459 \\
\hline 7611 & $11: 53: 52$ & 0.4411 \\
\hline 7612 & $11: 53: 53$ & 0.4427 \\
\hline 7613 & $11: 53: 54$ & 0.4402 \\
\hline 7614 & 11:53:55 & 0.4457 \\
\hline 7615 & $11: 53: 56$ & 0.4438 \\
\hline 7616 & $11: 53: 57$ & 0.4451 \\
\hline 7617 & $11: 53: 58$ & 0.4410 \\
\hline 7618 & $11: 53: 59$ & 0.4413 \\
\hline 7619 & $11: 54: 00$ & 0.4392 \\
\hline 7620 & $11: 54: 01$ & 0.4393 \\
\hline 7621 & $11: 54: 02$ & 0.4428 \\
\hline 7622 & $11: 54: 03$ & 0.4409 \\
\hline 7623 & $11: 54: 04$ & 0.4433 \\
\hline 7624 & $11: 54: 05$ & 0.4403 \\
\hline 7625 & $11: 54: 06$ & 0.4454 \\
\hline 7626 & $11: 54: 07$ & 0.4417 \\
\hline 7627 & $11: 54: 08$ & 0.4439 \\
\hline 7628 & $11: 54: 09$ & 0.4431 \\
\hline 7629 & $11: 54: 10$ & 0.4445 \\
\hline 7630 & $11: 54: 11$ & 0.4460 \\
\hline 7631 & $11: 54: 12$ & 0.4416 \\
\hline 7632 & $11: 54: 13$ & 0.4462 \\
\hline 7633 & $11: 54: 14$ & 0.4454 \\
\hline 7634 & $11: 54: 15$ & 0.4430 \\
\hline 7635 & $11: 54: 16$ & 0.4420 \\
\hline 7636 & $11: 54: 17$ & 0.4403 \\
\hline 7637 & $11: 54: 18$ & 0.4440 \\
\hline 7638 & $11: 54: 19$ & 0.4402 \\
\hline 7639 & $11: 54: 20$ & 0.4431 \\
\hline 7640 & $11: 54: 21$ & 0.4412 \\
\hline 7641 & $11: 54: 22$ & 0.4391 \\
\hline 7642 & $11: 54: 23$ & 0.4402 \\
\hline 7643 & $11: 54: 24$ & 0.4392 \\
\hline 7644 & $11: 54: 25$ & 0.4434 \\
\hline 7645 & $11: 54: 26$ & 0.4390 \\
\hline 7646 & $11: 54: 27$ & 0.4399 \\
\hline 7647 & $11: 54: 28$ & 0.4423 \\
\hline 7648 & $11: 54: 29$ & 0.4429 \\
\hline
\end{tabular}


Appendix C - Fuel Data Test 2 NIST102605_1.xIs

\begin{tabular}{|c|c|c|}
\hline 7649 & $11: 54: 30$ & 0.4453 \\
\hline 7650 & $11: 54: 31$ & 0.4430 \\
\hline 7651 & $11: 54: 32$ & 0.4469 \\
\hline 7652 & $11: 54: 33$ & 0.4418 \\
\hline 7653 & $11: 54: 34$ & 0.4443 \\
\hline 7654 & $11: 54: 35$ & 0.4425 \\
\hline 7655 & $11: 54: 36$ & 0.4464 \\
\hline 7656 & $11: 54: 37$ & 0.4442 \\
\hline 7657 & $11: 54: 38$ & 0.4413 \\
\hline 7658 & $11: 54: 39$ & 0.4445 \\
\hline 7659 & $11: 54: 40$ & 0.4410 \\
\hline 7660 & $11: 54: 41$ & 0.4433 \\
\hline 7661 & $11: 54: 42$ & 0.4424 \\
\hline 7662 & $11: 54: 43$ & 0.4404 \\
\hline 7663 & $11: 54: 44$ & 0.4446 \\
\hline 7664 & $11: 54: 45$ & 0.4400 \\
\hline 7665 & $11: 54: 46$ & 0.4422 \\
\hline 7666 & $11: 54: 47$ & 0.4400 \\
\hline 7667 & $11: 54: 48$ & 0.4381 \\
\hline 7668 & $11: 54: 49$ & 0.4438 \\
\hline 7669 & $11: 54: 50$ & 0.4401 \\
\hline 7670 & $11: 54: 51$ & 0.4425 \\
\hline 7671 & $11: 54: 52$ & 0.4418 \\
\hline 7672 & $11: 54: 53$ & 0.4466 \\
\hline 7673 & $11: 54: 54$ & 0.4472 \\
\hline 7674 & $11: 54: 55$ & 0.4427 \\
\hline 7675 & $11: 54: 56$ & 0.4461 \\
\hline 7676 & $11: 54: 57$ & 0.4433 \\
\hline 7677 & $11: 54: 58$ & 0.4453 \\
\hline 7678 & $11: 54: 59$ & 0.4448 \\
\hline 7679 & $11: 55: 00$ & 0.4445 \\
\hline 7680 & $11: 55: 01$ & 0.4434 \\
\hline 7681 & 11:55:02 & 0.4392 \\
\hline 7682 & $11: 55: 03$ & 0.4424 \\
\hline 7683 & $11: 55: 04$ & 0.4411 \\
\hline 7684 & $11: 55: 05$ & 0.4453 \\
\hline 7685 & $11: 55: 06$ & 0.4393 \\
\hline 7686 & $11: 55: 07$ & 0.4399 \\
\hline 7687 & $11: 55: 08$ & 0.4407 \\
\hline 7688 & 11:55:09 & 0.4388 \\
\hline 7689 & $11: 55: 10$ & 0.4433 \\
\hline 7690 & $11: 55: 11$ & 0.4403 \\
\hline 7691 & $11: 55: 12$ & 0.4428 \\
\hline 7692 & $11: 55: 13$ & 0.4427 \\
\hline 7693 & $11: 55: 14$ & 0.4428 \\
\hline 7694 & $11: 55: 15$ & 0.4456 \\
\hline 7695 & $11: 55: 16$ & 0.4422 \\
\hline 7696 & $11: 55: 17$ & 0.4455 \\
\hline 7697 & $11: 55: 18$ & 0.4438 \\
\hline 7698 & $11: 55: 19$ & 0.4451 \\
\hline 7699 & $11: 55: 20$ & 0.4443 \\
\hline
\end{tabular}


Appendix C - Fuel Data Test 2 NIST102605_1.xIs

\begin{tabular}{|c|c|c|}
\hline 7700 & $11: 55: 21$ & 0.4411 \\
\hline 7701 & $11: 55: 22$ & 0.4462 \\
\hline 7702 & $11: 55: 23$ & 0.4431 \\
\hline 7703 & $11: 55: 24$ & 0.4430 \\
\hline 7704 & $11: 55: 25$ & 0.4433 \\
\hline 7705 & $11: 55: 26$ & 0.4403 \\
\hline 7706 & $11: 55: 27$ & 0.4401 \\
\hline 7707 & $11: 55: 28$ & 0.4390 \\
\hline 7708 & $11: 55: 29$ & 0.4432 \\
\hline 7709 & $11: 55: 30$ & 0.4417 \\
\hline 7710 & $11: 55: 31$ & 0.4430 \\
\hline 7711 & $11: 55: 32$ & 0.4376 \\
\hline 7712 & $11: 55: 33$ & 0.4411 \\
\hline 7713 & $11: 55: 34$ & 0.4416 \\
\hline 7714 & 11:55:35 & 0.4418 \\
\hline 7715 & $11: 55: 36$ & 0.4466 \\
\hline 7716 & $11: 55: 37$ & 0.4410 \\
\hline 7717 & $11: 55: 38$ & 0.4443 \\
\hline 7718 & $11: 55: 39$ & 0.4428 \\
\hline 7719 & $11: 55: 40$ & 0.4469 \\
\hline 7720 & $11: 55: 41$ & 0.4478 \\
\hline 7721 & $11: 55: 42$ & 0.4433 \\
\hline 7722 & $11: 55: 43$ & 0.4457 \\
\hline 7723 & $11: 55: 44$ & 0.4427 \\
\hline 7724 & $11: 55: 45$ & 0.4448 \\
\hline 7725 & $11: 55: 46$ & 0.4418 \\
\hline 7726 & $11: 55: 47$ & 0.4450 \\
\hline 7727 & $11: 55: 48$ & 0.4423 \\
\hline 7728 & $11: 55: 49$ & 0.4393 \\
\hline 7729 & $11: 55: 50$ & 0.4420 \\
\hline 7730 & $11: 55: 51$ & 0.4400 \\
\hline 7731 & $11: 55: 52$ & 0.4436 \\
\hline 7732 & $11: 55: 53$ & 0.4400 \\
\hline 7733 & $11: 55: 54$ & 0.4404 \\
\hline 7734 & $11: 55: 55$ & 0.4389 \\
\hline 7735 & $11: 55: 56$ & 0.4392 \\
\hline 7736 & $11: 55: 57$ & 0.4417 \\
\hline 7737 & $11: 55: 58$ & 0.4412 \\
\hline 7738 & $11: 55: 59$ & 0.4455 \\
\hline 7739 & $11: 56: 00$ & 0.4437 \\
\hline 7740 & $11: 56: 01$ & 0.4447 \\
\hline 7741 & $11: 56: 02$ & 0.4461 \\
\hline 7742 & $11: 56: 03$ & 0.4407 \\
\hline 7743 & $11: 56: 04$ & 0.4433 \\
\hline 7744 & $11: 56: 05$ & 0.4415 \\
\hline 7745 & $11: 56: 06$ & 0.4459 \\
\hline 7746 & $11: 56: 07$ & 0.4423 \\
\hline 7747 & $11: 56: 08$ & 0.4435 \\
\hline 7748 & $11: 56: 09$ & 0.4432 \\
\hline 7749 & $11: 56: 10$ & 0.4434 \\
\hline 7750 & $11: 56: 11$ & 0.4423 \\
\hline
\end{tabular}


Appendix C - Fuel Data Test 2 NIST102605_1.xIs

\begin{tabular}{|c|c|c|}
\hline 7751 & $11: 56: 12$ & 0.4395 \\
\hline 7752 & $11: 56: 13$ & 0.4411 \\
\hline 7753 & $11: 56: 14$ & 0.4407 \\
\hline 7754 & $11: 56: 15$ & 0.4442 \\
\hline 7755 & $11: 56: 16$ & 0.4394 \\
\hline 7756 & $11: 56: 17$ & 0.4398 \\
\hline 7757 & $11: 56: 18$ & 0.4406 \\
\hline 7758 & $11: 56: 19$ & 0.4401 \\
\hline 7759 & $11: 56: 20$ & 0.4447 \\
\hline 7760 & $11: 56: 21$ & 0.4413 \\
\hline 7761 & $11: 56: 22$ & 0.4450 \\
\hline 7762 & $11: 56: 23$ & 0.4416 \\
\hline 7763 & $11: 56: 24$ & 0.4462 \\
\hline 7764 & $11: 56: 25$ & 0.4453 \\
\hline 7765 & 11:56:26 & 0.4440 \\
\hline 7766 & $11: 56: 27$ & 0.4450 \\
\hline 7767 & $11: 56: 28$ & 0.4417 \\
\hline 7768 & $11: 56: 29$ & 0.4452 \\
\hline 7769 & $11: 56: 30$ & 0.4426 \\
\hline 7770 & $11: 56: 31$ & 0.4437 \\
\hline 7771 & $11: 56: 32$ & 0.4430 \\
\hline 7772 & $11: 56: 33$ & 0.4384 \\
\hline 7773 & $11: 56: 34$ & 0.4446 \\
\hline 7774 & 11:56:35 & 0.4406 \\
\hline 7775 & $11: 56: 36$ & 0.4420 \\
\hline 7776 & $11: 56: 37$ & 0.4409 \\
\hline 7777 & $11: 56: 38$ & 0.4404 \\
\hline 7778 & $11: 56: 39$ & 0.4433 \\
\hline 7779 & $11: 56: 40$ & 0.4396 \\
\hline 7780 & $11: 56: 41$ & 0.4415 \\
\hline 7781 & $11: 56: 42$ & 0.4397 \\
\hline 7782 & $11: 56: 43$ & 0.4432 \\
\hline 7783 & $11: 56: 44$ & 0.4453 \\
\hline 7784 & $11: 56: 45$ & 0.4457 \\
\hline 7785 & $11: 56: 46$ & 0.4474 \\
\hline 7786 & $11: 56: 47$ & 0.4416 \\
\hline 7787 & $11: 56: 48$ & 0.4437 \\
\hline 7788 & $11: 56: 49$ & 0.4412 \\
\hline 7789 & $11: 56: 50$ & 0.4428 \\
\hline 7790 & $11: 56: 51$ & 0.4431 \\
\hline 7791 & $11: 56: 52$ & 0.4404 \\
\hline 7792 & $11: 56: 53$ & 0.4450 \\
\hline 7793 & $11: 56: 54$ & 0.4425 \\
\hline 7794 & $11: 56: 55$ & 0.4443 \\
\hline 7795 & $11: 56: 56$ & 0.4422 \\
\hline 7796 & $11: 56: 57$ & 0.4426 \\
\hline 7797 & $11: 56: 58$ & 0.4414 \\
\hline 7798 & $11: 56: 59$ & 0.4397 \\
\hline 7799 & $11: 57: 00$ & 0.4426 \\
\hline 7800 & $11: 57: 01$ & 0.4387 \\
\hline 7801 & $11: 57: 02$ & 0.4399 \\
\hline
\end{tabular}


Appendix C - Fuel Data Test 2 NIST102605_1.xIs

\begin{tabular}{|c|c|c|}
\hline 7802 & $11: 57: 03$ & 0.4399 \\
\hline 7803 & $11: 57: 04$ & 0.4421 \\
\hline 7804 & $11: 57: 05$ & 0.4455 \\
\hline 7805 & $11: 57: 06$ & 0.4409 \\
\hline 7806 & $11: 57: 07$ & 0.4447 \\
\hline 7807 & $11: 57: 08$ & 0.4423 \\
\hline 7808 & 11:57:09 & 0.4446 \\
\hline 7809 & $11: 57: 10$ & 0.4471 \\
\hline 7810 & $11: 57: 11$ & 0.4427 \\
\hline 7811 & $11: 57: 12$ & 0.4452 \\
\hline 7812 & $11: 57: 13$ & 0.4431 \\
\hline 7813 & $11: 57: 14$ & 0.4464 \\
\hline 7814 & $11: 57: 15$ & 0.4438 \\
\hline 7815 & $11: 57: 16$ & 0.4410 \\
\hline 7816 & $11: 57: 17$ & 0.4417 \\
\hline 7817 & 11:57:18 & 0.4401 \\
\hline 7818 & $11: 57: 19$ & 0.4444 \\
\hline 7819 & $11: 57: 20$ & 0.4406 \\
\hline 7820 & $11: 57: 21$ & 0.4441 \\
\hline 7821 & $11: 57: 22$ & 0.4403 \\
\hline 7822 & $11: 57: 23$ & 0.4404 \\
\hline 7823 & $11: 57: 24$ & 0.4391 \\
\hline 7824 & $11: 57: 25$ & 0.4406 \\
\hline 7825 & $11: 57: 26$ & 0.4429 \\
\hline 7826 & $11: 57: 27$ & 0.4421 \\
\hline 7827 & $11: 57: 28$ & 0.4452 \\
\hline 7828 & $11: 57: 29$ & 0.4447 \\
\hline 7829 & $11: 57: 30$ & 0.4469 \\
\hline 7830 & $11: 57: 31$ & 0.4423 \\
\hline 7831 & $11: 57: 32$ & 0.4459 \\
\hline 7832 & $11: 57: 33$ & 0.4450 \\
\hline 7833 & $11: 57: 34$ & 0.4435 \\
\hline 7834 & $11: 57: 35$ & 0.4441 \\
\hline 7835 & $11: 57: 36$ & 0.4397 \\
\hline 7836 & $11: 57: 37$ & 0.4449 \\
\hline 7837 & $11: 57: 38$ & 0.4428 \\
\hline 7838 & $11: 57: 39$ & 0.4423 \\
\hline 7839 & $11: 57: 40$ & 0.4436 \\
\hline 7840 & $11: 57: 41$ & 0.4402 \\
\hline 7841 & $11: 57: 42$ & 0.4447 \\
\hline 7842 & $11: 57: 43$ & 0.4415 \\
\hline 7843 & $11: 57: 44$ & 0.4407 \\
\hline 7844 & $11: 57: 45$ & 0.4397 \\
\hline 7845 & $11: 57: 46$ & 0.4391 \\
\hline 7846 & $11: 57: 47$ & 0.4426 \\
\hline 7847 & $11: 57: 48$ & 0.4385 \\
\hline 7848 & $11: 57: 49$ & 0.4429 \\
\hline 7849 & $11: 57: 50$ & 0.4444 \\
\hline 7850 & $11: 57: 51$ & 0.4456 \\
\hline 7851 & $11: 57: 52$ & 0.4467 \\
\hline 7852 & $11: 57: 53$ & 0.4419 \\
\hline
\end{tabular}


Appendix C - Fuel Data Test 2 NIST102605_1.xIs

\begin{tabular}{|c|c|c|}
\hline 7853 & $11: 57: 54$ & 0.4458 \\
\hline 7854 & $11: 57: 55$ & 0.4453 \\
\hline 7855 & $11: 57: 56$ & 0.4451 \\
\hline 7856 & $11: 57: 57$ & 0.4455 \\
\hline 7857 & $11: 57: 58$ & 0.4408 \\
\hline 7858 & $11: 57: 59$ & 0.4427 \\
\hline 7859 & 11:58:00 & 0.4391 \\
\hline 7860 & 11:58:01 & 0.4435 \\
\hline 7861 & 11:58:02 & 0.4420 \\
\hline 7862 & $11: 58: 03$ & 0.4398 \\
\hline 7863 & $11: 58: 04$ & 0.4434 \\
\hline 7864 & 11:58:05 & 0.4412 \\
\hline 7865 & 11:58:06 & 0.4436 \\
\hline 7866 & $11: 58: 07$ & 0.4393 \\
\hline 7867 & 11:58:08 & 0.4395 \\
\hline 7868 & 11:58:09 & 0.4424 \\
\hline 7869 & $11: 58: 10$ & 0.4408 \\
\hline 7870 & $11: 58: 11$ & 0.4436 \\
\hline 7871 & $11: 58: 12$ & 0.4438 \\
\hline 7872 & $11: 58: 13$ & 0.4462 \\
\hline 7873 & $11: 58: 14$ & 0.4410 \\
\hline 7874 & 11:58:15 & 0.4443 \\
\hline 7875 & $11: 58: 16$ & 0.4447 \\
\hline 7876 & $11: 58: 17$ & 0.4456 \\
\hline 7877 & 11:58:18 & 0.4456 \\
\hline 7878 & 11:58:19 & 0.4416 \\
\hline 7879 & $11: 58: 20$ & 0.4442 \\
\hline 7880 & $11: 58: 21$ & 0.4409 \\
\hline 7881 & $11: 58: 22$ & 0.4452 \\
\hline 7882 & $11: 58: 23$ & 0.4437 \\
\hline 7883 & $11: 58: 24$ & 0.4413 \\
\hline 7884 & 11:58:25 & 0.4434 \\
\hline 7885 & 11:58:26 & 0.4413 \\
\hline 7886 & $11: 58: 27$ & 0.4440 \\
\hline 7887 & 11:58:28 & 0.4413 \\
\hline 7888 & $11: 58: 29$ & 0.4417 \\
\hline 7889 & $11: 58: 30$ & 0.4405 \\
\hline 7890 & $11: 58: 31$ & 0.4405 \\
\hline 7891 & $11: 58: 32$ & 0.4395 \\
\hline 7892 & $11: 58: 33$ & 0.4388 \\
\hline 7893 & $11: 58: 34$ & 0.4434 \\
\hline 7894 & 11:58:35 & 0.4422 \\
\hline 7895 & $11: 58: 36$ & 0.4464 \\
\hline 7896 & $11: 58: 37$ & 0.4443 \\
\hline 7897 & $11: 58: 38$ & 0.4443 \\
\hline 7898 & $11: 58: 39$ & 0.4463 \\
\hline 7899 & $11: 58: 40$ & 0.4422 \\
\hline 7900 & $11: 58: 41$ & 0.4461 \\
\hline 7901 & $11: 58: 42$ & 0.4421 \\
\hline 7902 & $11: 58: 43$ & 0.4439 \\
\hline 7903 & $11: 58: 44$ & 0.4417 \\
\hline
\end{tabular}


Appendix C - Fuel Data Test 2 NIST102605_1.xls

\begin{tabular}{|c|c|c|}
\hline 7904 & $11: 58: 45$ & 0.4431 \\
\hline 7905 & $11: 58: 46$ & 0.4418 \\
\hline 7906 & $11: 58: 47$ & 0.4395 \\
\hline 7907 & $11: 58: 48$ & 0.4438 \\
\hline 7908 & $11: 58: 49$ & 0.4406 \\
\hline 7909 & $11: 58: 50$ & 0.4447 \\
\hline 7910 & $11: 58: 51$ & 0.4406 \\
\hline 7911 & $11: 58: 52$ & 0.4434 \\
\hline 7912 & 11:58:53 & 0.4406 \\
\hline 7913 & $11: 58: 54$ & 0.4402 \\
\hline 7914 & $11: 58: 55$ & 0.4380 \\
\hline 7915 & $11: 58: 56$ & 0.4391 \\
\hline 7916 & $11: 58: 57$ & 0.4434 \\
\hline 7917 & $11: 58: 58$ & 0.4442 \\
\hline 7918 & $11: 58: 59$ & 0.4458 \\
\hline 7919 & $11: 59: 00$ & 0.4429 \\
\hline 7920 & 11:59:01 & 0.4456 \\
\hline 7921 & 11:59:02 & 0.4419 \\
\hline 7922 & $11: 59: 03$ & 0.4429 \\
\hline 7923 & $11: 59: 04$ & 0.4465 \\
\hline 7924 & $11: 59: 05$ & 0.4410 \\
\hline 7925 & $11: 59: 06$ & 0.4456 \\
\hline 7926 & $11: 59: 07$ & 0.4444 \\
\hline 7927 & $11: 59: 08$ & 0.4454 \\
\hline 7928 & $11: 59: 09$ & 0.4445 \\
\hline 7929 & 11:59:10 & 0.4409 \\
\hline 7930 & $11: 59: 11$ & 0.4446 \\
\hline 7931 & $11: 59: 12$ & 0.4427 \\
\hline 7932 & $11: 59: 13$ & 0.4445 \\
\hline 7933 & $11: 59: 14$ & 0.4402 \\
\hline 7934 & $11: 59: 15$ & 0.4426 \\
\hline 7935 & $11: 59: 16$ & 0.4399 \\
\hline 7936 & $11: 59: 17$ & 0.4372 \\
\hline 7937 & $11: 59: 18$ & 0.4430 \\
\hline 7938 & 11:59:19 & 0.4400 \\
\hline 7939 & $11: 59: 20$ & 0.4445 \\
\hline 7940 & $11: 59: 21$ & 0.4457 \\
\hline 7941 & $11: 59: 22$ & 0.4426 \\
\hline 7942 & $11: 59: 23$ & 0.4454 \\
\hline 7943 & $11: 59: 24$ & 0.4414 \\
\hline 7944 & $11: 59: 25$ & 0.4458 \\
\hline 7945 & $11: 59: 26$ & 0.4454 \\
\hline 7946 & $11: 59: 27$ & 0.4448 \\
\hline 7947 & $11: 59: 28$ & 0.4419 \\
\hline 7948 & $11: 59: 29$ & 0.4421 \\
\hline 7949 & $11: 59: 30$ & 0.4419 \\
\hline 7950 & $11: 59: 31$ & 0.4381 \\
\hline 7951 & $11: 59: 32$ & 0.4439 \\
\hline 7952 & $11: 59: 33$ & 0.4407 \\
\hline 7953 & $11: 59: 34$ & 0.4416 \\
\hline 7954 & $11: 59: 35$ & 0.4398 \\
\hline
\end{tabular}


Appendix C - Fuel Data Test 2 NIST102605_1.xIs

\begin{tabular}{|c|c|c|}
\hline 7955 & $11: 59: 36$ & 0.4387 \\
\hline 7956 & $11: 59: 37$ & 0.4432 \\
\hline 7957 & $11: 59: 38$ & 0.4409 \\
\hline 7958 & 11:59:39 & 0.4437 \\
\hline 7959 & $11: 59: 40$ & 0.4401 \\
\hline 7960 & $11: 59: 41$ & 0.4422 \\
\hline 7961 & $11: 59: 42$ & 0.4411 \\
\hline 7962 & $11: 59: 43$ & 0.4438 \\
\hline 7963 & $11: 59: 44$ & 0.4434 \\
\hline 7964 & $11: 59: 45$ & 0.4449 \\
\hline 7965 & $11: 59: 46$ & 0.4457 \\
\hline 7966 & $11: 59: 47$ & 0.4415 \\
\hline 7967 & $11: 59: 48$ & 0.4464 \\
\hline 7968 & $11: 59: 49$ & 0.4439 \\
\hline 7969 & 11:59:50 & 0.4450 \\
\hline 7970 & $11: 59: 51$ & 0.4438 \\
\hline 7971 & $11: 59: 52$ & 0.4407 \\
\hline 7972 & $11: 59: 53$ & 0.4431 \\
\hline 7973 & $11: 59: 54$ & 0.4414 \\
\hline 7974 & $11: 59: 55$ & 0.4455 \\
\hline 7975 & $11: 59: 56$ & 0.4419 \\
\hline 7976 & $11: 59: 57$ & 0.4418 \\
\hline 7977 & $11: 59: 58$ & 0.4395 \\
\hline 7978 & $11: 59: 59$ & 0.4399 \\
\hline 7979 & 12:00:00 & 0.4421 \\
\hline 7980 & 12:00:01 & 0.4405 \\
\hline 7981 & $12: 00: 02$ & 0.4416 \\
\hline 7982 & $12: 00: 03$ & 0.4392 \\
\hline 7983 & $12: 00: 04$ & 0.4440 \\
\hline 7984 & $12: 00: 05$ & 0.4433 \\
\hline 7985 & 12:00:06 & 0.4462 \\
\hline 7986 & $12: 00: 07$ & 0.4467 \\
\hline 7987 & 12:00:08 & 0.4434 \\
\hline 7988 & $12: 00: 09$ & 0.4464 \\
\hline 7989 & $12: 00: 10$ & 0.4417 \\
\hline 7990 & $12: 00: 11$ & 0.4453 \\
\hline 7991 & $12: 00: 12$ & 0.4450 \\
\hline 7992 & $12: 00: 13$ & 0.4452 \\
\hline 7993 & $12: 00: 14$ & 0.4436 \\
\hline 7994 & $12: 00: 15$ & 0.4401 \\
\hline 7995 & $12: 00: 16$ & 0.4428 \\
\hline 7996 & $12: 00: 17$ & 0.4416 \\
\hline 7997 & $12: 00: 18$ & 0.4439 \\
\hline 7998 & $12: 00: 19$ & 0.4386 \\
\hline 7999 & $12: 00: 20$ & 0.4436 \\
\hline 8000 & $12: 00: 21$ & 0.4411 \\
\hline 8001 & $12: 00: 22$ & 0.4421 \\
\hline 8002 & $12: 00: 23$ & 0.4396 \\
\hline 8003 & $12: 00: 24$ & 0.4398 \\
\hline 8004 & $12: 00: 25$ & 0.4428 \\
\hline 8005 & $12: 00: 26$ & 0.4414 \\
\hline
\end{tabular}


Appendix C - Fuel Data Test 2 NIST102605_1.xIs

\begin{tabular}{|c|c|c|}
\hline 8006 & $12: 00: 27$ & 0.4447 \\
\hline 8007 & $12: 00: 28$ & 0.4411 \\
\hline 8008 & $12: 00: 29$ & 0.4447 \\
\hline 8009 & $12: 00: 30$ & 0.4423 \\
\hline 8010 & $12: 00: 31$ & 0.4447 \\
\hline 8011 & $12: 00: 32$ & 0.4459 \\
\hline 8012 & $12: 00: 33$ & 0.4425 \\
\hline 8013 & $12: 00: 34$ & 0.4445 \\
\hline 8014 & $12: 00: 35$ & 0.4417 \\
\hline 8015 & $12: 00: 36$ & 0.4456 \\
\hline 8016 & $12: 00: 37$ & 0.4428 \\
\hline 8017 & 12:00:38 & 0.4401 \\
\hline 8018 & $12: 00: 39$ & 0.4450 \\
\hline 8019 & $12: 00: 40$ & 0.4436 \\
\hline 8020 & $12: 00: 41$ & 0.4445 \\
\hline 8021 & $12: 00: 42$ & 0.4412 \\
\hline 8022 & $12: 00: 43$ & 0.4417 \\
\hline 8023 & $12: 00: 44$ & 0.4391 \\
\hline 8024 & $12: 00: 45$ & 0.4395 \\
\hline 8025 & $12: 00: 46$ & 0.4411 \\
\hline 8026 & $12: 00: 47$ & 0.4407 \\
\hline 8027 & $12: 00: 48$ & 0.4430 \\
\hline 8028 & $12: 00: 49$ & 0.4387 \\
\hline 8029 & $12: 00: 50$ & 0.4442 \\
\hline 8030 & 12:00:51 & 0.4433 \\
\hline 8031 & $12: 00: 52$ & 0.4444 \\
\hline 8032 & $12: 00: 53$ & 0.4480 \\
\hline 8033 & $12: 00: 54$ & 0.4430 \\
\hline 8034 & $12: 00: 55$ & 0.4457 \\
\hline 8035 & $12: 00: 56$ & 0.4441 \\
\hline 8036 & $12: 00: 57$ & 0.4409 \\
\hline 8037 & $12: 00: 58$ & 0.4434 \\
\hline 8038 & $12: 00: 59$ & 0.4396 \\
\hline 8039 & 12:01:00 & 0.4458 \\
\hline 8040 & $12: 01: 01$ & 0.4432 \\
\hline 8041 & 12:01:02 & 0.4441 \\
\hline 8042 & $12: 01: 03$ & 0.4417 \\
\hline 8043 & $12: 01: 04$ & 0.4403 \\
\hline 8044 & $12: 01: 05$ & 0.4426 \\
\hline 8045 & $12: 01: 06$ & 0.4396 \\
\hline 8046 & $12: 01: 07$ & 0.4438 \\
\hline 8047 & 12:01:08 & 0.4398 \\
\hline 8048 & 12:01:09 & 0.4401 \\
\hline 8049 & $12: 01: 10$ & 0.4383 \\
\hline 8050 & $12: 01: 11$ & 0.4413 \\
\hline 8051 & $12: 01: 12$ & 0.4454 \\
\hline 8052 & $12: 01: 13$ & 0.4456 \\
\hline 8053 & $12: 01: 14$ & 0.4456 \\
\hline 8054 & $12: 01: 15$ & 0.4419 \\
\hline 8055 & $12: 01: 16$ & 0.4454 \\
\hline 8056 & $12: 01: 17$ & 0.4410 \\
\hline
\end{tabular}


Appendix C - Fuel Data Test 2 NIST102605_1.xIs

\begin{tabular}{|c|c|c|}
\hline 8057 & $12: 01: 18$ & 0.4456 \\
\hline 8058 & $12: 01: 19$ & 0.4432 \\
\hline 8059 & 12:01:20 & 0.4461 \\
\hline 8060 & $12: 01: 21$ & 0.4428 \\
\hline 8061 & $12: 01: 22$ & 0.4445 \\
\hline 8062 & 12:01:23 & 0.4438 \\
\hline 8063 & $12: 01: 24$ & 0.4402 \\
\hline 8064 & $12: 01: 25$ & 0.4420 \\
\hline 8065 & $12: 01: 26$ & 0.4397 \\
\hline 8066 & $12: 01: 27$ & 0.4439 \\
\hline 8067 & $12: 01: 28$ & 0.4416 \\
\hline 8068 & 12:01:29 & 0.4421 \\
\hline 8069 & $12: 01: 30$ & 0.4382 \\
\hline 8070 & 12:01:31 & 0.4397 \\
\hline 8071 & 12:01:32 & 0.4379 \\
\hline 8072 & $12: 01: 33$ & 0.4407 \\
\hline 8073 & $12: 01: 34$ & 0.4454 \\
\hline 8074 & $12: 01: 35$ & 0.4417 \\
\hline 8075 & $12: 01: 36$ & 0.4450 \\
\hline 8076 & $12: 01: 37$ & 0.4409 \\
\hline 8077 & $12: 01: 38$ & 0.4460 \\
\hline 8078 & 12:01:39 & 0.4476 \\
\hline 8079 & 12:01:40 & 0.4458 \\
\hline 8080 & $12: 01: 41$ & 0.4464 \\
\hline 8081 & $12: 01: 42$ & 0.4412 \\
\hline 8082 & $12: 01: 43$ & 0.4437 \\
\hline 8083 & $12: 01: 44$ & 0.4403 \\
\hline 8084 & $12: 01: 45$ & 0.4440 \\
\hline 8085 & $12: 01: 46$ & 0.4425 \\
\hline 8086 & $12: 01: 47$ & 0.4405 \\
\hline 8087 & $12: 01: 48$ & 0.4422 \\
\hline 8088 & $12: 01: 49$ & 0.4395 \\
\hline 8089 & $12: 01: 50$ & 0.4444 \\
\hline 8090 & $12: 01: 51$ & 0.4404 \\
\hline 8091 & $12: 01: 52$ & 0.4401 \\
\hline 8092 & $12: 01: 53$ & 0.4434 \\
\hline 8093 & $12: 01: 54$ & 0.4399 \\
\hline 8094 & $12: 01: 55$ & 0.4425 \\
\hline 8095 & $12: 01: 56$ & 0.4397 \\
\hline 8096 & $12: 01: 57$ & 0.4433 \\
\hline 8097 & 12:01:58 & 0.4434 \\
\hline 8098 & $12: 01: 59$ & 0.4452 \\
\hline 8099 & $12: 02: 00$ & 0.4463 \\
\hline 8100 & 12:02:01 & 0.4427 \\
\hline 8101 & $12: 02: 02$ & 0.4447 \\
\hline 8102 & $12: 02: 03$ & 0.4417 \\
\hline 8103 & $12: 02: 04$ & 0.4462 \\
\hline 8104 & $12: 02: 05$ & 0.4434 \\
\hline 8105 & $12: 02: 06$ & 0.4453 \\
\hline 8106 & 12:02:07 & 0.4439 \\
\hline 8107 & $12: 02: 08$ & 0.4422 \\
\hline
\end{tabular}


Appendix C - Fuel Data Test 2 NIST102605_1.xls

\begin{tabular}{|c|c|c|}
\hline 8108 & $12: 02: 09$ & 0.4433 \\
\hline 8109 & $12: 02: 10$ & 0.4422 \\
\hline 8110 & $12: 02: 11$ & 0.4426 \\
\hline 8111 & $12: 02: 12$ & 0.4407 \\
\hline 8112 & $12: 02: 13$ & 0.4438 \\
\hline 8113 & $12: 02: 14$ & 0.4402 \\
\hline 8114 & $12: 02: 15$ & 0.4427 \\
\hline 8115 & $12: 02: 16$ & 0.4398 \\
\hline 8116 & $12: 02: 17$ & 0.4396 \\
\hline 8117 & $12: 02: 18$ & 0.4404 \\
\hline 8118 & $12: 02: 19$ & 0.4423 \\
\hline 8119 & $12: 02: 20$ & 0.4455 \\
\hline 8120 & $12: 02: 21$ & 0.4418 \\
\hline 8121 & $12: 02: 22$ & 0.4458 \\
\hline 8122 & $12: 02: 23$ & 0.4436 \\
\hline 8123 & $12: 02: 24$ & 0.4455 \\
\hline 8124 & $12: 02: 25$ & 0.4471 \\
\hline 8125 & $12: 02: 26$ & 0.4447 \\
\hline 8126 & $12: 02: 27$ & 0.4451 \\
\hline 8127 & $12: 02: 28$ & 0.4408 \\
\hline 8128 & $12: 02: 29$ & 0.4436 \\
\hline 8129 & $12: 02: 30$ & 0.4415 \\
\hline 8130 & $12: 02: 31$ & 0.4431 \\
\hline 8131 & $12: 02: 32$ & 0.4418 \\
\hline 8132 & $12: 02: 33$ & 0.4402 \\
\hline 8133 & 12:02:34 & 0.4439 \\
\hline 8134 & $12: 02: 35$ & 0.4391 \\
\hline 8135 & $12: 02: 36$ & 0.4432 \\
\hline 8136 & $12: 02: 37$ & 0.4406 \\
\hline 8137 & $12: 02: 38$ & 0.4404 \\
\hline 8138 & $12: 02: 39$ & 0.4421 \\
\hline 8139 & $12: 02: 40$ & 0.4414 \\
\hline 8140 & $12: 02: 41$ & 0.4445 \\
\hline 8141 & $12: 02: 42$ & 0.4407 \\
\hline 8142 & $12: 02: 43$ & 0.4427 \\
\hline 8143 & $12: 02: 44$ & 0.4422 \\
\hline 8144 & $12: 02: 45$ & 0.4450 \\
\hline 8145 & $12: 02: 46$ & 0.4447 \\
\hline 8146 & $12: 02: 47$ & 0.4411 \\
\hline 8147 & $12: 02: 48$ & 0.4446 \\
\hline 8148 & $12: 02: 49$ & 0.4406 \\
\hline 8149 & $12: 02: 50$ & 0.4457 \\
\hline 8150 & $12: 02: 51$ & 0.4448 \\
\hline 8151 & $12: 02: 52$ & 0.4461 \\
\hline 8152 & $12: 02: 53$ & 0.4429 \\
\hline 8153 & $12: 02: 54$ & 0.4418 \\
\hline 8154 & $12: 02: 55$ & 0.4442 \\
\hline 8155 & $12: 02: 56$ & 0.4412 \\
\hline 8156 & $12: 02: 57$ & 0.4443 \\
\hline 8157 & $12: 02: 58$ & 0.4406 \\
\hline 8158 & $12: 02: 59$ & 0.4435 \\
\hline
\end{tabular}


Appendix C - Fuel Data Test 2 NIST102605_1.xls

\begin{tabular}{|c|c|c|}
\hline 8159 & $12: 03: 00$ & 0.4389 \\
\hline 8160 & $12: 03: 01$ & 0.4395 \\
\hline 8161 & 12:03:02 & 0.4434 \\
\hline 8162 & $12: 03: 03$ & 0.4387 \\
\hline 8163 & $12: 03: 04$ & 0.4420 \\
\hline 8164 & $12: 03: 05$ & 0.4433 \\
\hline 8165 & $12: 03: 06$ & 0.4433 \\
\hline 8166 & $12: 03: 07$ & 0.4454 \\
\hline 8167 & 12:03:08 & 0.4443 \\
\hline 8168 & 12:03:09 & 0.4472 \\
\hline 8169 & $12: 03: 10$ & 0.4437 \\
\hline 8170 & $12: 03: 11$ & 0.4448 \\
\hline 8171 & $12: 03: 12$ & 0.4404 \\
\hline 8172 & $12: 03: 13$ & 0.4463 \\
\hline 8173 & $12: 03: 14$ & 0.4453 \\
\hline 8174 & $12: 03: 15$ & 0.4435 \\
\hline 8175 & $12: 03: 16$ & 0.4428 \\
\hline 8176 & $12: 03: 17$ & 0.4396 \\
\hline 8177 & $12: 03: 18$ & 0.4430 \\
\hline 8178 & $12: 03: 19$ & 0.4405 \\
\hline 8179 & $12: 03: 20$ & 0.4409 \\
\hline 8180 & $12: 03: 21$ & 0.4435 \\
\hline 8181 & $12: 03: 22$ & 0.4396 \\
\hline 8182 & $12: 03: 23$ & 0.4442 \\
\hline 8183 & $12: 03: 24$ & 0.4402 \\
\hline 8184 & $12: 03: 25$ & 0.4419 \\
\hline 8185 & $12: 03: 26$ & 0.4402 \\
\hline 8186 & $12: 03: 27$ & 0.4427 \\
\hline 8187 & $12: 03: 28$ & 0.4450 \\
\hline 8188 & $12: 03: 29$ & 0.4440 \\
\hline 8189 & $12: 03: 30$ & 0.4459 \\
\hline 8190 & $12: 03: 31$ & 0.4414 \\
\hline 8191 & 12:03:32 & 0.4459 \\
\hline 8192 & $12: 03: 33$ & 0.4435 \\
\hline 8193 & $12: 03: 34$ & 0.4439 \\
\hline 8194 & $12: 03: 35$ & 0.4478 \\
\hline 8195 & $12: 03: 36$ & 0.4417 \\
\hline 8196 & $12: 03: 37$ & 0.4438 \\
\hline 8197 & $12: 03: 38$ & 0.4407 \\
\hline 8198 & $12: 03: 39$ & 0.4431 \\
\hline 8199 & $12: 03: 40$ & 0.4427 \\
\hline 8200 & $12: 03: 41$ & 0.4412 \\
\hline 8201 & $12: 03: 42$ & 0.4443 \\
\hline 8202 & $12: 03: 43$ & 0.4419 \\
\hline 8203 & $12: 03: 44$ & 0.4418 \\
\hline 8204 & $12: 03: 45$ & 0.4396 \\
\hline 8205 & $12: 03: 46$ & 0.4396 \\
\hline 8206 & $12: 03: 47$ & 0.4418 \\
\hline 8207 & $12: 03: 48$ & 0.4402 \\
\hline 8208 & $12: 03: 49$ & 0.4448 \\
\hline 8209 & $12: 03: 50$ & 0.4386 \\
\hline
\end{tabular}


Appendix C - Fuel Data Test 2 NIST102605_1.xIs

\begin{tabular}{|c|c|c|}
\hline 8210 & $12: 03: 51$ & 0.4430 \\
\hline 8211 & $12: 03: 52$ & 0.4443 \\
\hline 8212 & $12: 03: 53$ & 0.4461 \\
\hline 8213 & $12: 03: 54$ & 0.4470 \\
\hline 8214 & $12: 03: 55$ & 0.4427 \\
\hline 8215 & $12: 03: 56$ & 0.4445 \\
\hline 8216 & $12: 03: 57$ & 0.4414 \\
\hline 8217 & $12: 03: 58$ & 0.4445 \\
\hline 8218 & 12:03:59 & 0.4422 \\
\hline 8219 & $12: 04: 00$ & 0.4455 \\
\hline 8220 & $12: 04: 01$ & 0.4432 \\
\hline 8221 & $12: 04: 02$ & 0.4414 \\
\hline 8222 & $12: 04: 03$ & 0.4411 \\
\hline 8223 & $12: 04: 04$ & 0.4401 \\
\hline 8224 & $12: 04: 05$ & 0.4417 \\
\hline 8225 & $12: 04: 06$ & 0.4389 \\
\hline 8226 & $12: 04: 07$ & 0.4425 \\
\hline 8227 & $12: 04: 08$ & 0.4403 \\
\hline 8228 & $12: 04: 09$ & 0.4432 \\
\hline 8229 & $12: 04: 10$ & 0.4391 \\
\hline 8230 & $12: 04: 11$ & 0.4406 \\
\hline 8231 & $12: 04: 12$ & 0.4400 \\
\hline 8232 & $12: 04: 13$ & 0.4427 \\
\hline 8233 & $12: 04: 14$ & 0.4452 \\
\hline 8234 & $12: 04: 15$ & 0.4449 \\
\hline 8235 & 12:04:16 & 0.4459 \\
\hline 8236 & $12: 04: 17$ & 0.4413 \\
\hline 8237 & $12: 04: 18$ & 0.4437 \\
\hline 8238 & $12: 04: 19$ & 0.4410 \\
\hline 8239 & $12: 04: 20$ & 0.4460 \\
\hline 8240 & $12: 04: 21$ & 0.4420 \\
\hline 8241 & $12: 04: 22$ & 0.4440 \\
\hline 8242 & $12: 04: 23$ & 0.4439 \\
\hline 8243 & $12: 04: 24$ & 0.4443 \\
\hline 8244 & $12: 04: 25$ & 0.4437 \\
\hline 8245 & $12: 04: 26$ & 0.4410 \\
\hline 8246 & $12: 04: 27$ & 0.4405 \\
\hline 8247 & $12: 04: 28$ & 0.4404 \\
\hline 8248 & $12: 04: 29$ & 0.4417 \\
\hline 8249 & $12: 04: 30$ & 0.4413 \\
\hline 8250 & $12: 04: 31$ & 0.4431 \\
\hline 8251 & $12: 04: 32$ & 0.4398 \\
\hline 8252 & $12: 04: 33$ & 0.4424 \\
\hline 8253 & $12: 04: 34$ & 0.4406 \\
\hline 8254 & $12: 04: 35$ & 0.4456 \\
\hline 8255 & $12: 04: 36$ & 0.4418 \\
\hline 8256 & $12: 04: 37$ & 0.4443 \\
\hline 8257 & $12: 04: 38$ & 0.4458 \\
\hline 8258 & $12: 04: 39$ & 0.4437 \\
\hline 8259 & $12: 04: 40$ & 0.4461 \\
\hline 8260 & $12: 04: 41$ & 0.4423 \\
\hline
\end{tabular}


Appendix C - Fuel Data Test 2 NIST102605_1.xIs

\begin{tabular}{|c|c|c|}
\hline 8261 & $12: 04: 42$ & 0.4477 \\
\hline 8262 & $12: 04: 43$ & 0.4448 \\
\hline 8263 & $12: 04: 44$ & 0.4444 \\
\hline 8264 & $12: 04: 45$ & 0.4424 \\
\hline 8265 & $12: 04: 46$ & 0.4400 \\
\hline 8266 & $12: 04: 47$ & 0.4427 \\
\hline 8267 & 12:04:48 & 0.4402 \\
\hline 8268 & $12: 04: 49$ & 0.4443 \\
\hline 8269 & $12: 04: 50$ & 0.4408 \\
\hline 8270 & $12: 04: 51$ & 0.4447 \\
\hline 8271 & $12: 04: 52$ & 0.4408 \\
\hline 8272 & $12: 04: 53$ & 0.4407 \\
\hline 8273 & $12: 04: 54$ & 0.4410 \\
\hline 8274 & $12: 04: 55$ & 0.4397 \\
\hline 8275 & 12:04:56 & 0.4433 \\
\hline 8276 & $12: 04: 57$ & 0.4401 \\
\hline 8277 & $12: 04: 58$ & 0.4428 \\
\hline 8278 & $12: 04: 59$ & 0.4414 \\
\hline 8279 & $12: 05: 00$ & 0.4448 \\
\hline 8280 & $12: 05: 01$ & 0.4446 \\
\hline 8281 & $12: 05: 02$ & 0.4459 \\
\hline 8282 & $12: 05: 03$ & 0.4451 \\
\hline 8283 & $12: 05: 04$ & 0.4440 \\
\hline 8284 & 12:05:05 & 0.4474 \\
\hline 8285 & 12:05:06 & 0.4408 \\
\hline 8286 & $12: 05: 07$ & 0.4450 \\
\hline 8287 & $12: 05: 08$ & 0.4439 \\
\hline 8288 & $12: 05: 09$ & 0.4423 \\
\hline 8289 & $12: 05: 10$ & 0.4426 \\
\hline 8290 & $12: 05: 11$ & 0.4408 \\
\hline 8291 & $12: 05: 12$ & 0.4452 \\
\hline 8292 & $12: 05: 13$ & 0.4420 \\
\hline 8293 & $12: 05: 14$ & 0.4417 \\
\hline 8294 & $12: 05: 15$ & 0.4402 \\
\hline 8295 & $12: 05: 16$ & 0.4396 \\
\hline 8296 & $12: 05: 17$ & 0.4410 \\
\hline 8297 & $12: 05: 18$ & 0.4406 \\
\hline 8298 & $12: 05: 19$ & 0.4452 \\
\hline 8299 & $12: 05: 20$ & 0.4403 \\
\hline 8300 & $12: 05: 21$ & 0.4437 \\
\hline 8301 & $12: 05: 22$ & 0.4462 \\
\hline 8302 & $12: 05: 23$ & 0.4426 \\
\hline 8303 & $12: 05: 24$ & 0.4450 \\
\hline 8304 & $12: 05: 25$ & 0.4422 \\
\hline 8305 & $12: 05: 26$ & 0.4468 \\
\hline 8306 & $12: 05: 27$ & 0.4457 \\
\hline 8307 & $12: 05: 28$ & 0.4459 \\
\hline 8308 & $12: 05: 29$ & 0.4456 \\
\hline 8309 & $12: 05: 30$ & 0.4459 \\
\hline 8310 & $12: 05: 31$ & 0.4445 \\
\hline 8311 & $12: 05: 32$ & 0.4447 \\
\hline
\end{tabular}


Appendix C - Fuel Data Test 2 NIST102605_1.xIs

\begin{tabular}{|c|c|c|}
\hline 8312 & $12: 05: 33$ & 0.4414 \\
\hline 8313 & $12: 05: 34$ & 0.4420 \\
\hline 8314 & $12: 05: 35$ & 0.4438 \\
\hline 8315 & $12: 05: 36$ & 0.4398 \\
\hline 8316 & $12: 05: 37$ & 0.4445 \\
\hline 8317 & $12: 05: 38$ & 0.4400 \\
\hline 8318 & $12: 05: 39$ & 0.4393 \\
\hline 8319 & $12: 05: 40$ & 0.4413 \\
\hline 8320 & $12: 05: 41$ & 0.4417 \\
\hline 8321 & $12: 05: 42$ & 0.4449 \\
\hline 8322 & $12: 05: 43$ & 0.4410 \\
\hline 8323 & $12: 05: 44$ & 0.4433 \\
\hline 8324 & $12: 05: 45$ & 0.4417 \\
\hline 8325 & $12: 05: 46$ & 0.4449 \\
\hline 8326 & $12: 05: 47$ & 0.4445 \\
\hline 8327 & $12: 05: 48$ & 0.4457 \\
\hline 8328 & $12: 05: 49$ & 0.4458 \\
\hline 8329 & $12: 05: 50$ & 0.4446 \\
\hline 8330 & $12: 05: 51$ & 0.4445 \\
\hline 8331 & $12: 05: 52$ & 0.4414 \\
\hline 8332 & $12: 05: 53$ & 0.4442 \\
\hline 8333 & $12: 05: 54$ & 0.4409 \\
\hline 8334 & $12: 05: 55$ & 0.4451 \\
\hline 8335 & $12: 05: 56$ & 0.4431 \\
\hline 8336 & $12: 05: 57$ & 0.4421 \\
\hline 8337 & 12:05:58 & 0.4438 \\
\hline 8338 & $12: 05: 59$ & 0.4424 \\
\hline 8339 & $12: 06: 00$ & 0.4434 \\
\hline 8340 & $12: 06: 01$ & 0.4410 \\
\hline 8341 & $12: 06: 02$ & 0.4424 \\
\hline 8342 & $12: 06: 03$ & 0.4386 \\
\hline 8343 & $12: 06: 04$ & 0.4418 \\
\hline 8344 & $12: 06: 05$ & 0.4413 \\
\hline 8345 & $12: 06: 06$ & 0.4411 \\
\hline 8346 & $12: 06: 07$ & 0.4455 \\
\hline 8347 & $12: 06: 08$ & 0.4423 \\
\hline 8348 & $12: 06: 09$ & 0.4463 \\
\hline 8349 & $12: 06: 10$ & 0.4434 \\
\hline 8350 & $12: 06: 11$ & 0.4465 \\
\hline 8351 & $12: 06: 12$ & 0.4472 \\
\hline 8352 & $12: 06: 13$ & 0.4435 \\
\hline 8353 & $12: 06: 14$ & 0.4445 \\
\hline 8354 & $12: 06: 15$ & 0.4409 \\
\hline 8355 & $12: 06: 16$ & 0.4440 \\
\hline 8356 & $12: 06: 17$ & 0.4396 \\
\hline 8357 & $12: 06: 18$ & 0.4428 \\
\hline 8358 & $12: 06: 19$ & 0.4415 \\
\hline 8359 & $12: 06: 20$ & 0.4409 \\
\hline 8360 & $12: 06: 21$ & 0.4433 \\
\hline 8361 & $12: 06: 22$ & 0.4409 \\
\hline 8362 & $12: 06: 23$ & 0.4442 \\
\hline
\end{tabular}


Appendix C - Fuel Data Test 2 NIST102605_1.xls

\begin{tabular}{|c|c|c|}
\hline 8363 & $12: 06: 24$ & 0.4392 \\
\hline 8364 & $12: 06: 25$ & 0.4408 \\
\hline 8365 & $12: 06: 26$ & 0.4417 \\
\hline 8366 & $12: 06: 27$ & 0.4411 \\
\hline 8367 & $12: 06: 28$ & 0.4428 \\
\hline 8368 & 12:06:29 & 0.4416 \\
\hline 8369 & 12:06:30 & 0.4451 \\
\hline 8370 & $12: 06: 31$ & 0.4406 \\
\hline 8371 & $12: 06: 32$ & 0.4454 \\
\hline 8372 & $12: 06: 33$ & 0.4449 \\
\hline 8373 & $12: 06: 34$ & 0.4453 \\
\hline 8374 & 12:06:35 & 0.4423 \\
\hline 8375 & $12: 06: 36$ & 0.4442 \\
\hline 8376 & $12: 06: 37$ & 0.4445 \\
\hline 8377 & 12:06:38 & 0.4402 \\
\hline 8378 & 12:06:39 & 0.4433 \\
\hline 8379 & $12: 06: 40$ & 0.4405 \\
\hline 8380 & $12: 06: 41$ & 0.4444 \\
\hline 8381 & $12: 06: 42$ & 0.4428 \\
\hline 8382 & $12: 06: 43$ & 0.4436 \\
\hline 8383 & $12: 06: 44$ & 0.4416 \\
\hline 8384 & $12: 06: 45$ & 0.4409 \\
\hline 8385 & $12: 06: 46$ & 0.4413 \\
\hline 8386 & $12: 06: 47$ & 0.4370 \\
\hline 8387 & $12: 06: 48$ & 0.4422 \\
\hline 8388 & $12: 06: 49$ & 0.4383 \\
\hline 8389 & $12: 06: 50$ & 0.4408 \\
\hline 8390 & $12: 06: 51$ & 0.4425 \\
\hline 8391 & $12: 06: 52$ & 0.4420 \\
\hline 8392 & $12: 06: 53$ & 0.4453 \\
\hline 8393 & $12: 06: 54$ & 0.4416 \\
\hline 8394 & $12: 06: 55$ & 0.4466 \\
\hline 8395 & $12: 06: 56$ & 0.4456 \\
\hline 8396 & $12: 06: 57$ & 0.4455 \\
\hline 8397 & $12: 06: 58$ & 0.4446 \\
\hline 8398 & $12: 06: 59$ & 0.4445 \\
\hline 8399 & $12: 07: 00$ & 0.4445 \\
\hline 8400 & $12: 07: 01$ & 0.4404 \\
\hline 8401 & $12: 07: 02$ & 0.4422 \\
\hline 8402 & $12: 07: 03$ & 0.4397 \\
\hline 8403 & $12: 07: 04$ & 0.4446 \\
\hline 8404 & 12:07:05 & 0.4406 \\
\hline 8405 & $12: 07: 06$ & 0.4452 \\
\hline 8406 & $12: 07: 07$ & 0.4414 \\
\hline 8407 & $12: 07: 08$ & 0.4386 \\
\hline 8408 & $12: 07: 09$ & 0.4413 \\
\hline 8409 & $12: 07: 10$ & 0.4395 \\
\hline 8410 & $12: 07: 11$ & 0.4426 \\
\hline 8411 & $12: 07: 12$ & 0.4394 \\
\hline 8412 & $12: 07: 13$ & 0.4407 \\
\hline 8413 & $12: 07: 14$ & 0.4408 \\
\hline
\end{tabular}


Appendix C - Fuel Data Test 2 NIST102605_1.xIs

\begin{tabular}{|c|c|c|}
\hline 8414 & $12: 07: 15$ & 0.4433 \\
\hline 8415 & $12: 07: 16$ & 0.4430 \\
\hline 8416 & $12: 07: 17$ & 0.4453 \\
\hline 8417 & $12: 07: 18$ & 0.4471 \\
\hline 8418 & $12: 07: 19$ & 0.4416 \\
\hline 8419 & 12:07:20 & 0.4444 \\
\hline 8420 & $12: 07: 21$ & 0.4432 \\
\hline 8421 & $12: 07: 22$ & 0.4457 \\
\hline 8422 & $12: 07: 23$ & 0.4438 \\
\hline 8423 & $12: 07: 24$ & 0.4430 \\
\hline 8424 & $12: 07: 25$ & 0.4430 \\
\hline 8425 & $12: 07: 26$ & 0.4437 \\
\hline 8426 & $12: 07: 27$ & 0.4426 \\
\hline 8427 & 12:07:28 & 0.4405 \\
\hline 8428 & $12: 07: 29$ & 0.4403 \\
\hline 8429 & $12: 07: 30$ & 0.4401 \\
\hline 8430 & $12: 07: 31$ & 0.4404 \\
\hline 8431 & $12: 07: 32$ & 0.4405 \\
\hline 8432 & $12: 07: 33$ & 0.4417 \\
\hline 8433 & $12: 07: 34$ & 0.4400 \\
\hline 8434 & $12: 07: 35$ & 0.4425 \\
\hline 8435 & $12: 07: 36$ & 0.4393 \\
\hline 8436 & 12:07:37 & 0.4445 \\
\hline 8437 & $12: 07: 38$ & 0.4430 \\
\hline 8438 & $12: 07: 39$ & 0.4433 \\
\hline 8439 & $12: 07: 40$ & 0.4467 \\
\hline 8440 & $12: 07: 41$ & 0.4416 \\
\hline 8441 & $12: 07: 42$ & 0.4460 \\
\hline 8442 & $12: 07: 43$ & 0.4455 \\
\hline 8443 & $12: 07: 44$ & 0.4461 \\
\hline 8444 & $12: 07: 45$ & 0.4452 \\
\hline 8445 & 12:07:46 & 0.4413 \\
\hline 8446 & $12: 07: 47$ & 0.4430 \\
\hline 8447 & $12: 07: 48$ & 0.4396 \\
\hline 8448 & $12: 07: 49$ & 0.4444 \\
\hline 8449 & $12: 07: 50$ & 0.4400 \\
\hline 8450 & 12:07:51 & 0.4418 \\
\hline 8451 & $12: 07: 52$ & 0.4422 \\
\hline 8452 & $12: 07: 53$ & 0.4378 \\
\hline 8453 & $12: 07: 54$ & 0.4437 \\
\hline 8454 & 12:07:55 & 0.4411 \\
\hline 8455 & $12: 07: 56$ & 0.4429 \\
\hline 8456 & $12: 07: 57$ & 0.4389 \\
\hline 8457 & 12:07:58 & 0.4397 \\
\hline 8458 & $12: 07: 59$ & 0.4410 \\
\hline 8459 & 12:08:00 & 0.4434 \\
\hline 8460 & $12: 08: 01$ & 0.4450 \\
\hline 8461 & 12:08:02 & 0.4411 \\
\hline 8462 & $12: 08: 03$ & 0.4439 \\
\hline 8463 & 12:08:04 & 0.4429 \\
\hline 8464 & $12: 08: 05$ & 0.4448 \\
\hline
\end{tabular}


Appendix C - Fuel Data Test 2 NIST102605_1.xIs

\begin{tabular}{|c|c|c|}
\hline 8465 & 12:08:06 & 0.4419 \\
\hline 8466 & $12: 08: 07$ & 0.4443 \\
\hline 8467 & 12:08:08 & 0.4409 \\
\hline 8468 & 12:08:09 & 0.4444 \\
\hline 8469 & $12: 08: 10$ & 0.4443 \\
\hline 8470 & 12:08:11 & 0.4394 \\
\hline 8471 & $12: 08: 12$ & 0.4408 \\
\hline 8472 & 12:08:13 & 0.4394 \\
\hline 8473 & 12:08:14 & 0.4441 \\
\hline 8474 & 12:08:15 & 0.4431 \\
\hline 8475 & $12: 08: 16$ & 0.4434 \\
\hline 8476 & 12:08:17 & 0.4398 \\
\hline 8477 & $12: 08: 18$ & 0.4434 \\
\hline 8478 & 12:08:19 & 0.4399 \\
\hline 8479 & 12:08:20 & 0.4417 \\
\hline 8480 & $12: 08: 21$ & 0.4419 \\
\hline 8481 & 12:08:22 & 0.4410 \\
\hline 8482 & 12:08:23 & 0.4447 \\
\hline 8483 & $12: 08: 24$ & 0.4406 \\
\hline 8484 & $12: 08: 25$ & 0.4458 \\
\hline 8485 & $12: 08: 26$ & 0.4463 \\
\hline 8486 & $12: 08: 27$ & 0.4447 \\
\hline 8487 & 12:08:28 & 0.4467 \\
\hline 8488 & $12: 08: 29$ & 0.4423 \\
\hline 8489 & $12: 08: 30$ & 0.4439 \\
\hline 8490 & 12:08:31 & 0.4415 \\
\hline 8491 & $12: 08: 32$ & 0.4452 \\
\hline 8492 & $12: 08: 33$ & 0.4425 \\
\hline 8493 & $12: 08: 34$ & 0.4422 \\
\hline 8494 & 12:08:35 & 0.4418 \\
\hline 8495 & $12: 08: 36$ & 0.4428 \\
\hline 8496 & 12:08:37 & 0.4419 \\
\hline 8497 & $12: 08: 38$ & 0.4390 \\
\hline 8498 & 12:08:39 & 0.4439 \\
\hline 8499 & $12: 08: 40$ & 0.4410 \\
\hline 8500 & $12: 08: 41$ & 0.4442 \\
\hline 8501 & $12: 08: 42$ & 0.4396 \\
\hline 8502 & $12: 08: 43$ & 0.4399 \\
\hline 8503 & $12: 08: 44$ & 0.4425 \\
\hline 8504 & $12: 08: 45$ & 0.4419 \\
\hline 8505 & 12:08:46 & 0.4447 \\
\hline 8506 & $12: 08: 47$ & 0.4428 \\
\hline 8507 & $12: 08: 48$ & 0.4460 \\
\hline 8508 & $12: 08: 49$ & 0.4429 \\
\hline 8509 & $12: 08: 50$ & 0.4449 \\
\hline 8510 & 12:08:51 & 0.4461 \\
\hline 8511 & $12: 08: 52$ & 0.4429 \\
\hline 8512 & $12: 08: 53$ & 0.4450 \\
\hline 8513 & $12: 08: 54$ & 0.4418 \\
\hline 8514 & 12:08:55 & 0.4445 \\
\hline 8515 & $12: 08: 56$ & 0.4426 \\
\hline
\end{tabular}


Appendix C - Fuel Data Test 2 NIST102605_1.xls

\begin{tabular}{|c|c|c|}
\hline 8516 & $12: 08: 57$ & 0.4393 \\
\hline 8517 & $12: 08: 58$ & 0.4453 \\
\hline 8518 & $12: 08: 59$ & 0.4427 \\
\hline 8519 & $12: 09: 00$ & 0.4431 \\
\hline 8520 & $12: 09: 01$ & 0.4410 \\
\hline 8521 & 12:09:02 & 0.4402 \\
\hline 8522 & $12: 09: 03$ & 0.4402 \\
\hline 8523 & $12: 09: 04$ & 0.4383 \\
\hline 8524 & 12:09:05 & 0.4430 \\
\hline 8525 & 12:09:06 & 0.4384 \\
\hline 8526 & $12: 09: 07$ & 0.4423 \\
\hline 8527 & $12: 09: 08$ & 0.4402 \\
\hline 8528 & 12:09:09 & 0.4437 \\
\hline 8529 & $12: 09: 10$ & 0.4464 \\
\hline 8530 & 12:09:11 & 0.4457 \\
\hline 8531 & $12: 09: 12$ & 0.4474 \\
\hline 8532 & 12:09:13 & 0.4424 \\
\hline 8533 & $12: 09: 14$ & 0.4446 \\
\hline 8534 & $12: 09: 15$ & 0.4414 \\
\hline 8535 & $12: 09: 16$ & 0.4460 \\
\hline 8536 & $12: 09: 17$ & 0.4454 \\
\hline 8537 & 12:09:18 & 0.4424 \\
\hline 8538 & $12: 09: 19$ & 0.4411 \\
\hline 8539 & 12:09:20 & 0.4404 \\
\hline 8540 & $12: 09: 21$ & 0.4437 \\
\hline 8541 & 12:09:22 & 0.4390 \\
\hline 8542 & $12: 09: 23$ & 0.4435 \\
\hline 8543 & $12: 09: 24$ & 0.4420 \\
\hline 8544 & $12: 09: 25$ & 0.4390 \\
\hline 8545 & $12: 09: 26$ & 0.4432 \\
\hline 8546 & $12: 09: 27$ & 0.4393 \\
\hline 8547 & $12: 09: 28$ & 0.4411 \\
\hline 8548 & $12: 09: 29$ & 0.4389 \\
\hline 8549 & 12:09:30 & 0.4423 \\
\hline 8550 & 12:09:31 & 0.4445 \\
\hline 8551 & 12:09:32 & 0.4445 \\
\hline 8552 & $12: 09: 33$ & 0.4452 \\
\hline 8553 & $12: 09: 34$ & 0.4446 \\
\hline 8554 & $12: 09: 35$ & 0.4463 \\
\hline 8555 & 12:09:36 & 0.4416 \\
\hline 8556 & $12: 09: 37$ & 0.4441 \\
\hline 8557 & $12: 09: 38$ & 0.4417 \\
\hline 8558 & $12: 09: 39$ & 0.4464 \\
\hline 8559 & $12: 09: 40$ & 0.4428 \\
\hline 8560 & $12: 09: 41$ & 0.4452 \\
\hline 8561 & $12: 09: 42$ & 0.4440 \\
\hline 8562 & $12: 09: 43$ & 0.4408 \\
\hline 8563 & $12: 09: 44$ & 0.4437 \\
\hline 8564 & $12: 09: 45$ & 0.4406 \\
\hline 8565 & $12: 09: 46$ & 0.4441 \\
\hline 8566 & $12: 09: 47$ & 0.4425 \\
\hline
\end{tabular}


Appendix C - Fuel Data Test 2 NIST102605_1.xIs

\begin{tabular}{|c|c|c|}
\hline 8567 & $12: 09: 48$ & 0.4424 \\
\hline 8568 & $12: 09: 49$ & 0.4387 \\
\hline 8569 & $12: 09: 50$ & 0.4393 \\
\hline 8570 & 12:09:51 & 0.4431 \\
\hline 8571 & 12:09:52 & 0.4412 \\
\hline 8572 & $12: 09: 53$ & 0.4452 \\
\hline 8573 & 12:09:54 & 0.4401 \\
\hline 8574 & 12:09:55 & 0.4434 \\
\hline 8575 & $12: 09: 56$ & 0.4450 \\
\hline 8576 & 12:09:57 & 0.4452 \\
\hline 8577 & $12: 09: 58$ & 0.4473 \\
\hline 8578 & 12:09:59 & 0.4432 \\
\hline 8579 & $12: 10: 00$ & 0.4466 \\
\hline 8580 & $12: 10: 01$ & 0.4447 \\
\hline 8581 & 12:10:02 & 0.4447 \\
\hline 8582 & $12: 10: 03$ & 0.4449 \\
\hline 8583 & $12: 10: 04$ & 0.4402 \\
\hline 8584 & $12: 10: 05$ & 0.4432 \\
\hline 8585 & $12: 10: 06$ & 0.4409 \\
\hline 8586 & $12: 10: 07$ & 0.4423 \\
\hline 8587 & 12:10:08 & 0.4430 \\
\hline 8588 & $12: 10: 09$ & 0.4394 \\
\hline 8589 & $12: 10: 10$ & 0.4425 \\
\hline 8590 & 12:10:11 & 0.4396 \\
\hline 8591 & $12: 10: 12$ & 0.4420 \\
\hline 8592 & $12: 10: 13$ & 0.4399 \\
\hline 8593 & $12: 10: 14$ & 0.4414 \\
\hline 8594 & $12: 10: 15$ & 0.4433 \\
\hline 8595 & $12: 10: 16$ & 0.4420 \\
\hline 8596 & $12: 10: 17$ & 0.4448 \\
\hline 8597 & $12: 10: 18$ & 0.4425 \\
\hline 8598 & $12: 10: 19$ & 0.4465 \\
\hline 8599 & $12: 10: 20$ & 0.4420 \\
\hline 8600 & $12: 10: 21$ & 0.4449 \\
\hline 8601 & $12: 10: 22$ & 0.4438 \\
\hline 8602 & $12: 10: 23$ & 0.4429 \\
\hline 8603 & $12: 10: 24$ & 0.4437 \\
\hline 8604 & $12: 10: 25$ & 0.4404 \\
\hline 8605 & $12: 10: 26$ & 0.4455 \\
\hline 8606 & $12: 10: 27$ & 0.4421 \\
\hline 8607 & $12: 10: 28$ & 0.4428 \\
\hline 8608 & $12: 10: 29$ & 0.4421 \\
\hline 8609 & $12: 10: 30$ & 0.4415 \\
\hline 8610 & $12: 10: 31$ & 0.4430 \\
\hline 8611 & $12: 10: 32$ & 0.4407 \\
\hline 8612 & $12: 10: 33$ & 0.4421 \\
\hline 8613 & $12: 10: 34$ & 0.4384 \\
\hline 8614 & $12: 10: 35$ & 0.4407 \\
\hline 8615 & $12: 10: 36$ & 0.4394 \\
\hline 8616 & $12: 10: 37$ & 0.4403 \\
\hline 8617 & $12: 10: 38$ & 0.4440 \\
\hline
\end{tabular}


Appendix C - Fuel Data Test 2 NIST102605_1.xIs

\begin{tabular}{|c|c|c|}
\hline 8618 & $12: 10: 39$ & 0.4399 \\
\hline 8619 & $12: 10: 40$ & 0.4447 \\
\hline 8620 & $12: 10: 41$ & 0.4439 \\
\hline 8621 & $12: 10: 42$ & 0.4438 \\
\hline 8622 & $12: 10: 43$ & 0.4470 \\
\hline 8623 & $12: 10: 44$ & 0.4429 \\
\hline 8624 & $12: 10: 45$ & 0.4442 \\
\hline 8625 & $12: 10: 46$ & 0.4399 \\
\hline 8626 & $12: 10: 47$ & 0.4442 \\
\hline 8627 & $12: 10: 48$ & 0.4436 \\
\hline 8628 & $12: 10: 49$ & 0.4453 \\
\hline 8629 & $12: 10: 50$ & 0.4415 \\
\hline 8630 & $12: 10: 51$ & 0.4449 \\
\hline 8631 & $12: 10: 52$ & 0.4429 \\
\hline 8632 & $12: 10: 53$ & 0.4445 \\
\hline 8633 & $12: 10: 54$ & 0.4384 \\
\hline 8634 & $12: 10: 55$ & 0.4401 \\
\hline 8635 & $12: 10: 56$ & 0.4398 \\
\hline 8636 & $12: 10: 57$ & 0.4406 \\
\hline 8637 & $12: 10: 58$ & 0.4433 \\
\hline 8638 & $12: 10: 59$ & 0.4408 \\
\hline 8639 & $12: 11: 00$ & 0.4427 \\
\hline 8640 & $12: 11: 01$ & 0.4403 \\
\hline 8641 & $12: 11: 02$ & 0.4434 \\
\hline 8642 & $12: 11: 03$ & 0.4459 \\
\hline 8643 & 12:11:04 & 0.4441 \\
\hline 8644 & $12: 11: 05$ & 0.4460 \\
\hline 8645 & $12: 11: 06$ & 0.4421 \\
\hline 8646 & $12: 11: 07$ & 0.4454 \\
\hline 8647 & $12: 11: 08$ & 0.4436 \\
\hline 8648 & 12:11:09 & 0.4447 \\
\hline 8649 & $12: 11: 10$ & 0.4450 \\
\hline 8650 & $12: 11: 11$ & 0.4442 \\
\hline 8651 & $12: 11: 12$ & 0.4427 \\
\hline 8652 & $12: 11: 13$ & 0.4399 \\
\hline 8653 & $12: 11: 14$ & 0.4437 \\
\hline 8654 & $12: 11: 15$ & 0.4411 \\
\hline 8655 & $12: 11: 16$ & 0.4428 \\
\hline 8656 & $12: 11: 17$ & 0.4429 \\
\hline 8657 & $12: 11: 18$ & 0.4409 \\
\hline 8658 & $12: 11: 19$ & 0.4425 \\
\hline 8659 & $12: 11: 20$ & 0.4399 \\
\hline 8660 & $12: 11: 21$ & 0.4436 \\
\hline 8661 & $12: 11: 22$ & 0.4422 \\
\hline 8662 & $12: 11: 23$ & 0.4442 \\
\hline 8663 & $12: 11: 24$ & 0.4396 \\
\hline 8664 & $12: 11: 25$ & 0.4444 \\
\hline 8665 & $12: 11: 26$ & 0.4428 \\
\hline 8666 & $12: 11: 27$ & 0.4452 \\
\hline 8667 & $12: 11: 28$ & 0.4459 \\
\hline 8668 & $12: 11: 29$ & 0.4438 \\
\hline
\end{tabular}


Appendix C - Fuel Data Test 2 NIST102605_1.xls

$\begin{array}{lll}8669 & 12: 11: 30 & 0.4454 \\ 8670 & 12: 11: 31 & 0.4412 \\ 8671 & 12: 11: 32 & 0.4454 \\ 8672 & 12: 11: 33 & 0.4449 \\ 8673 & 12: 11: 34 & 0.4407 \\ 8674 & 12: 11: 35 & 0.4444 \\ 8675 & 12: 11: 36 & 0.4406 \\ 8676 & 12: 11: 37 & 0.4426 \\ 8677 & 12: 11: 38 & 0.4407 \\ 8678 & 12: 11: 39 & 0.4408 \\ 8679 & 12: 11: 40 & 0.4435 \\ 8680 & 12: 11: 41 & 0.4386 \\ 8681 & 12: 11: 42 & 0.4413 \\ 8682 & 12: 11: 43 & 0.4405 \\ 8683 & 12: 11: 44 & 0.4386 \\ 8684 & 12: 11: 45 & 0.4436 \\ 8685 & 12: 11: 46 & 0.4424 \\ 8686 & 12: 11: 47 & 0.4460 \\ 8687 & 12: 11: 48 & 0.4423 \\ 8688 & 12: 11: 49 & 0.4451 \\ 8689 & 12: 11: 50 & 0.4456 \\ 8690 & 12: 11: 51 & 0.4425 \\ 8691 & 12: 11: 52 & 0.4446 \\ 8692 & 12: 11: 53 & 0.3990\end{array}$


APPENDIX D. - Laser Data Test 2 NIST 102605

Appendix $D$ is laser data from Test 2 in tabular format and is approximately 86 pages in length. Fuel data is included with electronic version of report on compact disk, but not routinely included with paper version of report. 
Appendix D - Laser Data Test 2 NIST102605_1.xls

Time from Ignition, s EDT Time $\quad$ //lo East $\quad$ //lo West

$\begin{array}{rlll}0 & 9: 47: 01 & 0.9451 & 0.9067 \\ 2 & 9: 47: 03 & 0.9634 & 0.9940 \\ 4 & 9: 47: 05 & 0.9841 & 0.9787 \\ 6 & 9: 47: 07 & 0.9688 & 0.9968 \\ 8 & 9: 47: 09 & 0.8790 & 0.8860 \\ 10 & 9: 47: 11 & 0.8429 & 0.8160 \\ 12 & 9: 47: 13 & 0.8600 & 0.8138 \\ 14 & 9: 47: 15 & 0.8005 & 0.8126 \\ 16 & 9: 47: 17 & 0.8608 & 0.7385 \\ 18 & 9: 47: 19 & 0.8357 & 0.7566 \\ 20 & 9: 47: 21 & 0.8078 & 0.7547 \\ 22 & 9: 47: 23 & 0.7477 & 0.6506 \\ 24 & 9: 47: 25 & 0.6460 & 0.6884 \\ 26 & 9: 47: 27 & 0.6907 & 0.6107 \\ 28 & 9: 47: 29 & 0.7496 & 0.4375 \\ 30 & 9: 47: 31 & 0.5889 & 0.4412 \\ 32 & 9: 47: 33 & 0.4084 & 0.5070 \\ 34 & 9: 47: 35 & 0.4166 & 0.3844 \\ 36 & 9: 47: 37 & 0.3285 & 0.3904 \\ 38 & 9: 47: 39 & 0.2326 & 0.4368 \\ 40 & 9: 47: 41 & 0.2056 & 0.3339 \\ 42 & 9: 47: 43 & 0.1822 & 0.2496 \\ 44 & 9: 47: 45 & 0.2516 & 0.2425 \\ 46 & 9: 47: 47 & 0.1751 & 0.2294 \\ 48 & 9: 47: 49 & 0.1646 & 0.2195 \\ 50 & 9: 47: 51 & 0.4637 & 0.3033 \\ 52 & 9: 47: 53 & 0.1485 & 0.1553 \\ 54 & 9: 47: 55 & 0.2192 & 0.1149 \\ 56 & 9: 47: 57 & 0.6582 & 0.2145 \\ 58 & 9: 47: 59 & 0.1906 & 0.1165 \\ 60 & 9: 48: 01 & 0.1386 & 0.1407 \\ 62 & 9: 48: 03 & 0.1197 & 0.2374 \\ 64 & 9: 48: 05 & 0.1081 & 0.2682 \\ 66 & 9: 48: 07 & 0.1167 & 0.1492 \\ 68 & 9: 48: 09 & 0.0626 & 0.0868 \\ 70 & 9: 48: 11 & 0.1329 & 0.1603 \\ 72 & 9: 48: 13 & 0.1029 & 0.1222 \\ 74 & 9: 48: 15 & 0.1053 & 0.1236 \\ 76 & 9: 48: 17 & 0.0339 & 0.1302 \\ 78 & 9: 48: 19 & 0.0762 & 0.1905 \\ 80 & 9: 48: 21 & 0.1182 & 0.1979 \\ 82 & 9: 48: 23 & 0.0769 & 0.1867 \\ 84 & 9: 48: 25 & 0.0461 & 0.0576 \\ 86 & 9: 48: 27 & 0.2399 & 0.1542 \\ 88 & 9: 48: 29 & 0.0357 & 0.0626 \\ 90 & 9: 48: 31 & 0.0242 & 0.0871 \\ 92 & 9: 48: 33 & 0.0308 & 0.1004 \\ 94 & 9: 48: 35 & 0.0318 & 0.0545 \\ 96 & 9: 48: 37 & 0.0435 & 0.0271 \\ 98 & 9: 48: 39 & 0.0230 & 0.0438\end{array}$


Appendix D - Laser Data Test 2 NIST102605_1.xls

$\begin{array}{llll}100 & 9: 48: 41 & 0.0148 & 0.0131 \\ 102 & 9: 48: 43 & 0.0134 & 0.0072 \\ 104 & 9: 48: 45 & 0.0088 & 0.0570 \\ 106 & 9: 48: 47 & 0.0376 & 0.0414 \\ 108 & 9: 48: 49 & 0.0233 & 0.0345 \\ 110 & 9: 48: 51 & 0.0216 & 0.0231 \\ 112 & 9: 48: 53 & 0.0073 & 0.0380 \\ 114 & 9: 48: 55 & 0.0036 & 0.0062 \\ 116 & 9: 48: 57 & 0.0168 & 0.0323 \\ 118 & 9: 48: 59 & 0.0080 & 0.0114 \\ 120 & 9: 49: 01 & 0.0012 & 0.0245 \\ 122 & 9: 49: 03 & 0.0078 & 0.0179 \\ 124 & 9: 49: 05 & 0.0024 & 0.0360 \\ 126 & 9: 49: 07 & 0.0003 & 0.0148 \\ 128 & 9: 49: 09 & 0.0011 & 0.0149 \\ 130 & 9: 49: 11 & 0.0011 & 0.0151 \\ 132 & 9: 49: 13 & 0.0022 & 0.0037 \\ 134 & 9: 49: 15 & 0.0019 & 0.0064 \\ 136 & 9: 49: 17 & 0.0010 & 0.0168 \\ 138 & 9: 49: 19 & 0.0042 & 0.0127 \\ 140 & 9: 49: 21 & 0.0020 & 0.0013 \\ 142 & 9: 49: 23 & 0.0016 & 0.0071 \\ 144 & 9: 49: 25 & 0.0029 & 0.0064 \\ 146 & 9: 49: 27 & 0.0006 & 0.0166 \\ 148 & 9: 49: 29 & 0.0004 & 0.0034 \\ 150 & 9: 49: 31 & 0.0004 & 0.0015 \\ 152 & 9: 49: 33 & 0.0026 & 0.0009 \\ 154 & 9: 49: 35 & 0.0026 & 0.0119 \\ 156 & 9: 49: 37 & 0.0002 & 0.0086 \\ 158 & 9: 49: 39 & 0.0009 & 0.0071 \\ 160 & 9: 49: 41 & 0.0008 & 0.0035 \\ 162 & 9: 49: 43 & 0.0014 & 0.0006 \\ 164 & 9: 49: 45 & 0.0021 & 0.0025 \\ 166 & 9: 49: 47 & 0.0023 & 0.0024 \\ 168 & 9: 49: 49 & 0.0016 & 0.0010 \\ 170 & 9: 49: 51 & 0.0047 & 0.0022 \\ 172 & 9: 49: 53 & 0.0021 & 0.0023 \\ 174 & 9: 49: 55 & 0.0016 & 0.0025 \\ 176 & 9: 49: 57 & 0.0003 & 0.0075 \\ 178 & 9: 49: 59 & 0.0037 & 0.0489 \\ 180 & 9: 50: 01 & 0.0019 & 0.0091 \\ 182 & 9: 50: 03 & 0.0008 & 0.0099 \\ 184 & 9: 50: 05 & 0.0025 & 0.0013 \\ 186 & 9: 50: 07 & 0.0007 & 0.0041 \\ 188 & 9: 50: 09 & 0.0003 & 0.0010 \\ 190 & 9: 50: 11 & 0.0005 & 0.0007 \\ 192 & 9: 50: 13 & 0.0016 & 0.0085 \\ 194 & 9: 50: 15 & 0.0029 & 0.0121 \\ 196 & 9: 50: 17 & 0.0148 & 0.0046 \\ 198 & 9: 50: 19 & 0.0016 & 0.0034 \\ 200 & 9: 50: 21 & 0.0132 & 0.0044\end{array}$


Appendix D - Laser Data Test 2 NIST102605_1.xls

$\begin{array}{llll}202 & 9: 50: 23 & 0.0052 & 0.0049 \\ 204 & 9: 50: 25 & 0.0006 & 0.0013 \\ 206 & 9: 50: 27 & 0.0016 & 0.0014 \\ 208 & 9: 50: 29 & 0.0009 & 0.0119 \\ 210 & 9: 50: 31 & 0.0058 & 0.0079 \\ 212 & 9: 50: 33 & 0.0053 & 0.0014 \\ 214 & 9: 50: 35 & 0.0005 & 0.0128 \\ 216 & 9: 50: 37 & 0.0003 & 0.0091 \\ 218 & 9: 50: 39 & 0.0009 & 0.0014 \\ 220 & 9: 50: 41 & 0.0015 & 0.0014 \\ 222 & 9: 50: 43 & 0.0051 & 0.0165 \\ 224 & 9: 50: 45 & 0.0008 & 0.0060 \\ 226 & 9: 50: 47 & 0.0037 & 0.0015 \\ 228 & 9: 50: 49 & 0.0019 & 0.0062 \\ 230 & 9: 50: 51 & 0.0005 & 0.0141 \\ 232 & 9: 50: 53 & 0.0013 & 0.0038 \\ 234 & 9: 50: 55 & 0.0016 & 0.0047 \\ 236 & 9: 50: 57 & 0.0115 & 0.0010 \\ 238 & 9: 50: 59 & 0.0023 & 0.0017 \\ 240 & 9: 51: 01 & 0.0021 & 0.0019 \\ 242 & 9: 51: 03 & 0.0015 & 0.0033 \\ 244 & 9: 51: 05 & 0.0002 & 0.0012 \\ 246 & 9: 51: 07 & 0.0087 & 0.0067 \\ 248 & 9: 51: 09 & 0.0044 & 0.0032 \\ 250 & 9: 51: 11 & 0.0009 & 0.0041 \\ 252 & 9: 51: 13 & 0.0068 & 0.0015 \\ 254 & 9: 51: 15 & 0.0017 & 0.0016 \\ 256 & 9: 51: 17 & 0.0039 & 0.0126 \\ 258 & 9: 51: 19 & 0.0006 & 0.0153 \\ 260 & 9: 51: 21 & 0.0040 & 0.0012 \\ 262 & 9: 51: 23 & 0.0061 & 0.0660 \\ 264 & 9: 51: 25 & 0.0052 & 0.0488 \\ 266 & 9: 51: 27 & 0.0019 & 0.0042 \\ 268 & 9: 51: 29 & 0.0023 & 0.0031 \\ 270 & 9: 51: 31 & 0.0096 & 0.0062 \\ 272 & 9: 51: 33 & 0.0025 & 0.0010 \\ 274 & 9: 51: 35 & 0.0029 & 0.0017 \\ 276 & 9: 51: 37 & 0.0006 & 0.0017 \\ 278 & 9: 51: 39 & 0.0053 & 0.0014 \\ 280 & 9: 51: 41 & 0.0037 & 0.0023 \\ 282 & 9: 51: 43 & 0.0003 & 0.0058 \\ 284 & 9: 51: 45 & 0.0040 & 0.0052 \\ 286 & 9: 51: 47 & 0.0025 & 0.0017 \\ 288 & 9: 51: 49 & 0.0016 & 0.0893 \\ 290 & 9: 51: 51 & 0.0079 & 0.0108 \\ 292 & 9: 51: 53 & 0.0019 & 0.0102 \\ 294 & 9: 51: 55 & 0.0009 & 0.0127 \\ 296 & 9: 51: 57 & 0.0002 & 0.0117 \\ 298 & 9: 51: 59 & 0.0004 & 0.0112 \\ 300 & 9: 52: 01 & 0.0008 & 0.0086 \\ 302 & 9: 52: 03 & 0.0069 & 0.0200\end{array}$

D - 3 
Appendix D - Laser Data Test 2 NIST102605_1.xls

$\begin{array}{llll}304 & 9: 52: 05 & 0.0002 & 0.0020 \\ 306 & 9: 52: 07 & 0.0017 & 0.0173 \\ 308 & 9: 52: 09 & 0.0096 & 0.0032 \\ 310 & 9: 52: 11 & 0.0037 & 0.0025 \\ 312 & 9: 52: 13 & 0.0022 & 0.0023 \\ 314 & 9: 52: 15 & 0.0012 & 0.0017 \\ 316 & 9: 52: 17 & 0.0022 & 0.0021 \\ 318 & 9: 52: 19 & 0.0026 & 0.0609 \\ 320 & 9: 52: 21 & 0.0058 & 0.0226 \\ 322 & 9: 52: 23 & 0.0003 & 0.0187 \\ 324 & 9: 52: 25 & 0.0011 & 0.0044 \\ 326 & 9: 52: 27 & 0.0007 & 0.0011 \\ 328 & 9: 52: 29 & 0.0034 & 0.0052 \\ 330 & 9: 52: 31 & 0.0009 & 0.0045 \\ 332 & 9: 52: 33 & 0.0014 & 0.0019 \\ 334 & 9: 52: 35 & 0.0009 & 0.0004 \\ 336 & 9: 52: 37 & 0.0002 & 0.0069 \\ 338 & 9: 52: 39 & 0.0044 & 0.0038 \\ 340 & 9: 52: 41 & 0.0002 & 0.0262 \\ 342 & 9: 52: 43 & 0.0013 & 0.0103 \\ 344 & 9: 52: 45 & 0.0023 & 0.0021 \\ 346 & 9: 52: 47 & 0.0274 & 0.0088 \\ 348 & 9: 52: 49 & 0.0009 & 0.0098 \\ 350 & 9: 52: 51 & 0.0008 & 0.0090 \\ 352 & 9: 52: 53 & 0.0005 & 0.0112 \\ 354 & 9: 52: 55 & 0.0008 & 0.0519 \\ 356 & 9: 52: 57 & 0.0011 & 0.0228 \\ 358 & 9: 52: 59 & 0.0002 & 0.0012 \\ 360 & 9: 53: 01 & 0.0031 & 0.0045 \\ 362 & 9: 53: 03 & 0.0045 & 0.0014 \\ 364 & 9: 53: 05 & 0.0022 & 0.0013 \\ 366 & 9: 53: 07 & 0.0003 & 0.0265 \\ 368 & 9: 53: 09 & 0.0014 & 0.0015 \\ 370 & 9: 53: 11 & 0.0041 & 0.0094 \\ 372 & 9: 53: 13 & 0.0002 & 0.0029 \\ 374 & 9: 53: 15 & 0.0040 & 0.0018 \\ 376 & 9: 53: 17 & 0.0119 & 0.0018 \\ 378 & 9: 53: 19 & 0.0034 & 0.0053 \\ 380 & 9: 53: 21 & 0.0047 & 0.0045 \\ 382 & 9: 53: 23 & 0.0588 & 0.0294 \\ 384 & 9: 53: 25 & 0.0012 & 0.0147 \\ 386 & 9: 53: 27 & 0.0008 & 0.0064 \\ 388 & 9: 53: 29 & 0.0011 & 0.0173 \\ 390 & 9: 53: 31 & 0.0008 & 0.0141 \\ 392 & 9: 53: 33 & 0.0062 & 0.0178 \\ 394 & 9: 53: 35 & 0.0002 & 0.0190 \\ 396 & 9: 53: 37 & 0.0014 & 0.0129 \\ 398 & 9: 53: 39 & 0.0059 & 0.0094 \\ 400 & 9: 53: 41 & 0.0009 & 0.0114 \\ 402 & 9: 53: 43 & 0.0009 & 0.0069 \\ 404 & 9: 53: 45 & 0.0023 & 0.0017\end{array}$


Appendix D - Laser Data Test 2 NIST102605_1.xls

$\begin{array}{llll}406 & 9: 53: 47 & 0.0009 & 0.0071 \\ 408 & 9: 53: 49 & 0.0005 & 0.0576 \\ 410 & 9: 53: 51 & 0.0027 & 0.0091 \\ 412 & 9: 53: 53 & 0.0058 & 0.0065 \\ 414 & 9: 53: 55 & 0.0006 & 0.0174 \\ 416 & 9: 53: 57 & 0.0008 & 0.0024 \\ 418 & 9: 53: 59 & 0.0035 & 0.0221 \\ 420 & 9: 54: 01 & 0.0019 & 0.0162 \\ 422 & 9: 54: 03 & 0.0004 & 0.0018 \\ 424 & 9: 54: 05 & 0.0017 & 0.0313 \\ 426 & 9: 54: 07 & 0.0013 & 0.0021 \\ 428 & 9: 54: 09 & 0.0007 & 0.0089 \\ 430 & 9: 54: 11 & 0.0001 & 0.0174 \\ 432 & 9: 54: 13 & 0.0027 & 0.0166 \\ 434 & 9: 54: 15 & 0.0037 & 0.0125 \\ 436 & 9: 54: 17 & 0.0011 & 0.0165 \\ 438 & 9: 54: 19 & 0.0002 & 0.0084 \\ 440 & 9: 54: 21 & 0.0004 & 0.0069 \\ 442 & 9: 54: 23 & 0.0004 & 0.0468 \\ 444 & 9: 54: 25 & 0.0032 & 0.0127 \\ 446 & 9: 54: 27 & 0.0008 & 0.0206 \\ 448 & 9: 54: 29 & 0.0030 & 0.0058 \\ 450 & 9: 54: 31 & 0.0125 & 0.0046 \\ 452 & 9: 54: 33 & 0.0003 & 0.0034 \\ 454 & 9: 54: 35 & 0.0009 & 0.0064 \\ 456 & 9: 54: 37 & 0.0005 & 0.0128 \\ 458 & 9: 54: 39 & 0.0007 & 0.0228 \\ 460 & 9: 54: 41 & 0.0020 & 0.0096 \\ 462 & 9: 54: 43 & 0.0056 & 0.0332 \\ 464 & 9: 54: 45 & 0.0007 & 0.0409 \\ 466 & 9: 54: 47 & 0.0205 & 0.0119 \\ 468 & 9: 54: 49 & 0.0058 & 0.0164 \\ 470 & 9: 54: 51 & 0.0020 & 0.0075 \\ 472 & 9: 54: 53 & 0.0081 & 0.0080 \\ 474 & 9: 54: 55 & 0.0005 & 0.0050 \\ 476 & 9: 54: 57 & 0.0021 & 0.0136 \\ 478 & 9: 54: 59 & 0.0029 & 0.0038 \\ 480 & 9: 55: 01 & 0.0074 & 0.0723 \\ 482 & 9: 55: 03 & 0.0025 & 0.0009 \\ 484 & 9: 55: 05 & 0.0122 & 0.0041 \\ 486 & 9: 55: 07 & 0.0035 & 0.0072 \\ 488 & 9: 55: 09 & 0.0014 & 0.0020 \\ 490 & 9: 55: 11 & 0.0128 & 0.0127 \\ 492 & 9: 55: 13 & 0.0017 & 0.0013 \\ 494 & 9: 55: 15 & 0.0047 & 0.0445 \\ 496 & 9: 55: 17 & 0.0094 & 0.0025 \\ 498 & 9: 55: 19 & 0.0041 & 0.0070 \\ 500 & 9: 55: 21 & 0.0014 & 0.0071 \\ 502 & 9: 55: 23 & 0.0100 & 0.0035 \\ 504 & 9: 55: 25 & 0.0036 & 0.0142 \\ 506 & 9: 55: 27 & 0.0175 & 0.0323\end{array}$


Appendix D - Laser Data Test 2 NIST102605_1.xls

$\begin{array}{llll}508 & 9: 55: 29 & 0.0030 & 0.0186 \\ 510 & 9: 55: 31 & 0.0062 & 0.0055 \\ 512 & 9: 55: 33 & 0.0088 & 0.0031 \\ 514 & 9: 55: 35 & 0.0088 & 0.0149 \\ 516 & 9: 55: 37 & 0.0072 & 0.0037 \\ 518 & 9: 55: 39 & 0.0120 & 0.0224 \\ 520 & 9: 55: 41 & 0.0117 & 0.0014 \\ 522 & 9: 55: 43 & 0.0213 & 0.0074 \\ 524 & 9: 55: 45 & 0.0117 & 0.0134 \\ 526 & 9: 55: 47 & 0.0060 & 0.0289 \\ 528 & 9: 55: 49 & 0.0046 & 0.0296 \\ 530 & 9: 55: 51 & 0.0270 & 0.0139 \\ 532 & 9: 55: 53 & 0.0054 & 0.0143 \\ 534 & 9: 55: 55 & 0.0355 & 0.0108 \\ 536 & 9: 55: 57 & 0.0073 & 0.0122 \\ 538 & 9: 55: 59 & 0.0121 & 0.0077 \\ 540 & 9: 56: 01 & 0.0082 & 0.0097 \\ 542 & 9: 56: 03 & 0.0036 & 0.0046 \\ 544 & 9: 56: 05 & 0.0078 & 0.0030 \\ 546 & 9: 56: 07 & 0.0076 & 0.0045 \\ 548 & 9: 56: 09 & 0.0014 & 0.0021 \\ 550 & 9: 56: 11 & 0.0023 & 0.0089 \\ 552 & 9: 56: 13 & 0.0014 & 0.0055 \\ 554 & 9: 56: 15 & 0.0078 & 0.0008 \\ 556 & 9: 56: 17 & 0.0026 & 0.0061 \\ 558 & 9: 56: 19 & 0.0275 & 0.0150 \\ 560 & 9: 56: 21 & 0.0025 & 0.0039 \\ 562 & 9: 56: 23 & 0.0020 & 0.0104 \\ 564 & 9: 56: 25 & 0.0008 & 0.0059 \\ 566 & 9: 56: 27 & 0.0031 & 0.0031 \\ 568 & 9: 56: 29 & 0.0071 & 0.0048 \\ 570 & 9: 56: 31 & 0.0050 & 0.0178 \\ 572 & 9: 56: 33 & 0.0013 & 0.0027 \\ 574 & 9: 56: 35 & 0.0022 & 0.0275 \\ 576 & 9: 56: 37 & 0.0043 & 0.0167 \\ 578 & 9: 56: 39 & 0.0045 & 0.0022 \\ 580 & 9: 56: 41 & 0.0058 & 0.0026 \\ 582 & 9: 56: 43 & 0.0160 & 0.0045 \\ 584 & 9: 56: 45 & 0.0164 & 0.0165 \\ 586 & 9: 56: 47 & 0.0322 & 0.0019 \\ 588 & 9: 56: 49 & 0.0023 & 0.0133 \\ 590 & 9: 56: 51 & 0.0005 & 0.0222 \\ 592 & 9: 56: 53 & 0.0053 & 0.0147 \\ 594 & 9: 56: 55 & 0.0002 & 0.0092 \\ 596 & 9: 56: 57 & 0.0036 & 0.0036 \\ 598 & 9: 56: 59 & 0.0064 & 0.0638 \\ 600 & 9: 57: 01 & 0.0037 & 0.0304 \\ 602 & 9: 57: 03 & 0.0031 & 0.0194 \\ 604 & 9: 57: 05 & 0.0034 & 0.0027 \\ 606 & 9: 57: 07 & 0.0008 & 0.0081 \\ 608 & 9: 57: 09 & 0.0024 & 0.0052\end{array}$


Appendix D - Laser Data Test 2 NIST102605_1.xls

$\begin{array}{llll}610 & 9: 57: 11 & 0.0264 & 0.0033 \\ 612 & 9: 57: 13 & 0.0038 & 0.0076 \\ 614 & 9: 57: 15 & 0.0013 & 0.0029 \\ 616 & 9: 57: 17 & 0.0159 & 0.0388 \\ 618 & 9: 57: 19 & 0.0005 & 0.0151 \\ 620 & 9: 57: 21 & 0.0002 & 0.0305 \\ 622 & 9: 57: 23 & 0.0007 & 0.0073 \\ 624 & 9: 57: 25 & 0.0027 & 0.0204 \\ 626 & 9: 57: 27 & 0.0045 & 0.0157 \\ 628 & 9: 57: 29 & 0.0012 & 0.0073 \\ 630 & 9: 57: 31 & 0.0029 & 0.0062 \\ 632 & 9: 57: 33 & 0.0143 & 0.0114 \\ 634 & 9: 57: 35 & 0.0115 & 0.0090 \\ 636 & 9: 57: 37 & 0.0035 & 0.0095 \\ 638 & 9: 57: 39 & 0.0030 & 0.0031 \\ 640 & 9: 57: 41 & 0.0015 & 0.0226 \\ 642 & 9: 57: 43 & 0.0011 & 0.0397 \\ 644 & 9: 57: 45 & 0.0002 & 0.0285 \\ 646 & 9: 57: 47 & 0.0004 & 0.0130 \\ 648 & 9: 57: 49 & 0.0020 & 0.0137 \\ 650 & 9: 57: 51 & 0.0005 & 0.0021 \\ 652 & 9: 57: 53 & 0.0002 & 0.0161 \\ 654 & 9: 57: 55 & 0.0019 & 0.0069 \\ 656 & 9: 57: 57 & 0.0006 & 0.0119 \\ 658 & 9: 57: 59 & 0.0065 & 0.0133 \\ 660 & 9: 58: 01 & 0.0042 & 0.0188 \\ 662 & 9: 58: 03 & 0.0020 & 0.0159 \\ 664 & 9: 58: 05 & 0.0028 & 0.0081 \\ 666 & 9: 58: 07 & 0.0003 & 0.0222 \\ 668 & 9: 58: 09 & 0.0005 & 0.0135 \\ 670 & 9: 58: 11 & 0.0005 & 0.0607 \\ 672 & 9: 58: 13 & 0.0022 & 0.0111 \\ 674 & 9: 58: 15 & 0.0013 & 0.0025 \\ 676 & 9: 58: 17 & 0.0009 & 0.0477 \\ 678 & 9: 58: 19 & 0.0011 & 0.0084 \\ 680 & 9: 58: 21 & 0.0009 & 0.0061 \\ 682 & 9: 58: 23 & 0.0053 & 0.0033 \\ 684 & 9: 58: 25 & 0.0027 & 0.0293 \\ 686 & 9: 58: 27 & 0.0020 & 0.0048 \\ 688 & 9: 58: 29 & 0.0005 & 0.0019 \\ 690 & 9: 58: 31 & 0.0002 & 0.0053 \\ 692 & 9: 58: 33 & 0.0006 & 0.0013 \\ 694 & 9: 58: 35 & 0.0022 & 0.0041 \\ 696 & 9: 58: 37 & 0.0035 & 0.0128 \\ 698 & 9: 58: 39 & 0.0040 & 0.0014 \\ 700 & 9: 58: 41 & 0.0007 & 0.0050 \\ 702 & 9: 58: 43 & 0.0039 & 0.0032 \\ 704 & 9: 58: 45 & 0.0017 & 0.0041 \\ 706 & 9: 58: 47 & 0.0058 & 0.0050 \\ 708 & 9: 58: 49 & 0.0027 & 0.0053 \\ 710 & 9: 58: 51 & 0.0009 & 0.0279\end{array}$


Appendix D - Laser Data Test 2 NIST102605_1.xls

$\begin{array}{lrrr}712 & 9: 58: 53 & 0.0013 & 0.0184 \\ 714 & 9: 58: 55 & 0.0013 & 0.0212 \\ 716 & 9: 58: 57 & 0.0006 & 0.0087 \\ 718 & 9: 58: 59 & 0.0003 & 0.2295 \\ 720 & 9: 59: 01 & 0.0159 & 0.0089 \\ 722 & 9: 59: 03 & 0.0179 & 0.0030 \\ 724 & 9: 59: 05 & 0.0006 & 0.0226 \\ 726 & 9: 59: 07 & 0.0013 & 0.0111 \\ 728 & 9: 59: 09 & 0.0014 & 0.0079 \\ 730 & 9: 59: 11 & 0.0249 & 0.0134 \\ 732 & 9: 59: 13 & 0.0022 & 0.0160 \\ 734 & 9: 59: 15 & 0.0006 & 0.0045 \\ 736 & 9: 59: 17 & 0.0050 & 0.0029 \\ 738 & 9: 59: 19 & 0.0070 & 0.0128 \\ 740 & 9: 59: 21 & 0.0009 & 0.0160 \\ 742 & 9: 59: 23 & 0.0120 & 0.0053 \\ 744 & 9: 59: 25 & 0.0026 & 0.0018 \\ 746 & 9: 59: 27 & 0.0032 & 0.0045 \\ 748 & 9: 59: 29 & 0.0013 & 0.0013 \\ 750 & 9: 59: 31 & 0.0025 & 0.0028 \\ 752 & 9: 59: 33 & 0.0014 & 0.0009 \\ 754 & 9: 59: 35 & 0.0037 & 0.0185 \\ 756 & 9: 59: 37 & 0.0091 & 0.0035 \\ 758 & 9: 59: 39 & 0.0193 & 0.0085 \\ 760 & 9: 59: 41 & 0.0080 & 0.0015 \\ 762 & 9: 59: 43 & 0.0061 & 0.0047 \\ 764 & 9: 59: 45 & 0.0091 & 0.0226 \\ 766 & 9: 59: 47 & 0.0016 & 0.0038 \\ 768 & 9: 59: 49 & 0.0035 & 0.0087 \\ 770 & 9: 59: 51 & 0.0102 & 0.0102 \\ 772 & 9: 59: 53 & 0.0143 & 0.0563 \\ 774 & 9: 59: 55 & 0.0025 & 0.0049 \\ 776 & 9: 59: 57 & 0.0126 & 0.0117 \\ 778 & 9: 59: 59 & 0.0147 & 0.0107 \\ 780 & 10: 00: 01 & 0.0009 & 0.0100 \\ 782 & 10: 00: 03 & 0.0035 & 0.0075 \\ 784 & 10: 00: 05 & 0.0030 & 0.0338 \\ 786 & 10: 00: 07 & 0.0068 & 0.0060 \\ 788 & 10: 00: 09 & 0.0036 & 0.0008 \\ 790 & 10: 00: 11 & 0.0046 & 0.0119 \\ 792 & 10: 00: 13 & 0.0059 & 0.0534 \\ 794 & 10: 00: 15 & 0.0003 & 0.0014 \\ 796 & 10: 00: 17 & 0.0004 & 0.0030 \\ 798 & 10: 00: 19 & 0.0009 & 0.0019 \\ 800 & 10: 00: 21 & 0.0009 & 0.0125 \\ 802 & 10: 00: 23 & 0.0017 & 0.0009 \\ 804 & 10: 00: 25 & 0.0010 & 0.0086 \\ 806 & 10: 00: 27 & 0.0002 & 0.0221 \\ 808 & 10: 00: 29 & 0.0004 & 0.0061 \\ 810 & 10: 00: 31 & 0.0009 & 0.0054 \\ 812 & 10: 00: 33 & 0.0018 & 0.0021\end{array}$

D - 8 
Appendix D - Laser Data Test 2 NIST102605_1.xls

$\begin{array}{llll}814 & 10: 00: 35 & 0.0043 & 0.0024 \\ 816 & 10: 00: 37 & 0.0021 & 0.0093 \\ 818 & 10: 00: 39 & 0.0020 & 0.0061 \\ 820 & 10: 00: 41 & 0.0013 & 0.0061 \\ 822 & 10: 00: 43 & 0.0013 & 0.0053 \\ 824 & 10: 00: 45 & 0.0002 & 0.0073 \\ 826 & 10: 00: 47 & 0.0036 & 0.0067 \\ 828 & 10: 00: 49 & 0.0012 & 0.0028 \\ 830 & 10: 00: 51 & 0.0057 & 0.0070 \\ 832 & 10: 00: 53 & 0.0045 & 0.0155 \\ 834 & 10: 00: 55 & 0.0017 & 0.0061 \\ 836 & 10: 00: 57 & 0.0083 & 0.0007 \\ 838 & 10: 00: 59 & 0.0043 & 0.0045 \\ 840 & 10: 01: 01 & 0.0017 & 0.0139 \\ 842 & 10: 01: 03 & 0.0002 & 0.0226 \\ 844 & 10: 01: 05 & 0.0026 & 0.0046 \\ 846 & 10: 01: 07 & 0.0049 & 0.0377 \\ 848 & 10: 01: 09 & 0.0021 & 0.0109 \\ 850 & 10: 01: 11 & 0.0011 & 0.0035 \\ 852 & 10: 01: 13 & 0.0010 & 0.0006 \\ 854 & 10: 01: 15 & 0.0061 & 0.0276 \\ 856 & 10: 01: 17 & 0.0018 & 0.0067 \\ 858 & 10: 01: 19 & 0.0011 & 0.0430 \\ 860 & 10: 01: 21 & 0.0005 & 0.0132 \\ 862 & 10: 01: 23 & 0.0040 & 0.0046 \\ 864 & 10: 01: 25 & 0.0036 & 0.0149 \\ 866 & 10: 01: 27 & 0.0006 & 0.0042 \\ 868 & 10: 01: 29 & 0.0001 & 0.0044 \\ 870 & 10: 01: 31 & 0.0005 & 0.0050 \\ 872 & 10: 01: 33 & 0.0010 & 0.0003 \\ 874 & 10: 01: 35 & 0.0003 & 0.0026 \\ 876 & 10: 01: 37 & 0.0033 & 0.0042 \\ 878 & 10: 01: 39 & 0.0024 & 0.2651 \\ 880 & 10: 01: 41 & 0.0006 & 0.0024 \\ 882 & 10: 01: 43 & 0.0009 & 0.0032 \\ 884 & 10: 01: 45 & 0.0092 & 0.0033 \\ 886 & 10: 01: 47 & 0.0130 & 0.0042 \\ 888 & 10: 01: 49 & 0.0120 & 0.0026 \\ 890 & 10: 01: 51 & 0.0063 & 0.0003 \\ 892 & 10: 01: 53 & 0.0045 & 0.0012 \\ 894 & 10: 01: 55 & 0.0069 & 0.0053 \\ 896 & 10: 01: 57 & 0.0016 & 0.0028 \\ 898 & 10: 01: 59 & 0.0022 & 0.0025 \\ 900 & 10: 02: 01 & 0.0244 & 0.0067 \\ 902 & 10: 02: 03 & 0.0039 & 0.0042 \\ 904 & 10: 02: 05 & 0.0158 & 0.0091 \\ 906 & 10: 02: 07 & 0.0096 & 0.0151 \\ 908 & 10: 02: 09 & 0.0220 & 0.0022 \\ 910 & 10: 02: 11 & 0.0091 & 0.0145 \\ 912 & 10: 02: 13 & 0.0062 & 0.0066 \\ 914 & 10: 02: 15 & 0.0013 & 0.0169\end{array}$


Appendix D - Laser Data Test 2 NIST102605_1.xls

\begin{tabular}{|c|c|c|c|}
\hline & $10: 02: 17$ & 0.0088 & . \\
\hline & $10: 02: 19$ & 0.0138 & 0.0117 \\
\hline & $10: 02: 21$ & 0015 & 00 \\
\hline & $10: 02: 23$ & 0040 & 0058 \\
\hline 24 & 10:02:25 & 0.0021 & 0018 \\
\hline 6 & $10: 02: 27$ & 0.0059 & .0120 \\
\hline 28 & $10: 02: 29$ & 0.0273 & 0.0701 \\
\hline 30 & $10: 02: 31$ & 0.0180 & 0.0042 \\
\hline 32 & $10: 02: 33$ & 0.0145 & 0.0727 \\
\hline 34 & $10: 02: 35$ & 0.0027 & 0.0135 \\
\hline & $10: 02: 37$ & 0.0006 & .0056 \\
\hline & $10: 02: 39$ & 0.0006 & .0047 \\
\hline & $10: 02: 41$ & & 0.0024 \\
\hline 42 & $10: 02: 43$ & 0.0 & 0.0050 \\
\hline 14 & $10: 02: 45$ & & 0.0086 \\
\hline 46 & $10: 02: 47$ & 0.0 & 0.0296 \\
\hline 8 & $10: 02: 49$ & & 0.0014 \\
\hline 0 & $10: 02: 51$ & 0.0 & 0.0028 \\
\hline 52 & $10: 02: 53$ & 0.0 & 0.0014 \\
\hline 54 & $10:$ & & .0082 \\
\hline J & 10: & & .0046 \\
\hline 8 & 10: & & 0.0020 \\
\hline 60 & 10:03:01 & & 0.0081 \\
\hline 32 & 10: & & 0.0327 \\
\hline 54 & 10:03:05 & 0.0 & 0.0044 \\
\hline 66 & $10: 03: 07$ & & 0.0424 \\
\hline 68 & 10:03:09 & 0.0 & 0.0041 \\
\hline 70 & 10: & & 0.0157 \\
\hline 972 & $10:($ & & .0 \\
\hline 74 & 10: & 0.0 & 0.0020 \\
\hline 76 & 10 & 0.0 & 0.0305 \\
\hline 8 & 10:03:19 & 0.0 & 0.0098 \\
\hline 80 & 10:03:21 & & 0.0113 \\
\hline 982 & $10: 03: 23$ & 0.0 & 0.0060 \\
\hline 984 & $10: 03: 25$ & & 0.0086 \\
\hline 986 & $10: 03: 27$ & & 0.0087 \\
\hline 988 & $10:($ & & 86 \\
\hline חa & $10: 03: 31$ & 0.0 & 80 \\
\hline 92 & 10: & 0.0 & .0356 \\
\hline T & 10: & 0.0 & .0106 \\
\hline 996 & $10: 03: 37$ & & 0.0400 \\
\hline 998 & 10:03:39 & & .0325 \\
\hline 00 & $10: 03: 41$ & 0.0027 & .0045 \\
\hline 02 & $10: 03: 43$ & 0.0 & .0166 \\
\hline & $10: 03: 45$ & 0.0 & 0.004 \\
\hline & $10: 03: 47$ & & 0.0266 \\
\hline & $10: 03: 49$ & & 015 \\
\hline & $10: 03: 51$ & 0.0292 & 0.0288 \\
\hline & $10: 03: 53$ & 0.0158 & 0.0078 \\
\hline & $10: 03: 55$ & 0.0015 & 0.0046 \\
\hline & 10:03: & $0.011 \varepsilon$ & 0.01 \\
\hline
\end{tabular}

D - 10 
Appendix D - Laser Data Test 2 NIST102605_1.xls

$\begin{array}{llll}1018 & 10: 03: 59 & 0.0228 & 0.0036 \\ 1020 & 10: 04: 01 & 0.0350 & 0.0128 \\ 1022 & 10: 04: 03 & 0.0101 & 0.0066 \\ 1024 & 10: 04: 05 & 0.0119 & 0.0034 \\ 1026 & 10: 04: 07 & 0.0138 & 0.0030 \\ 1028 & 10: 04: 09 & 0.0077 & 0.0025 \\ 1030 & 10: 04: 11 & 0.0050 & 0.0034 \\ 1032 & 10: 04: 13 & 0.0156 & 0.0079 \\ 1034 & 10: 04: 15 & 0.0067 & 0.0221 \\ 1036 & 10: 04: 17 & 0.0060 & 0.0158 \\ 1038 & 10: 04: 19 & 0.0094 & 0.0068 \\ 1040 & 10: 04: 21 & 0.0064 & 0.0034 \\ 1042 & 10: 04: 23 & 0.0036 & 0.0055 \\ 1044 & 10: 04: 25 & 0.0032 & 0.0393 \\ 1046 & 10: 04: 27 & 0.0091 & 0.0241 \\ 1048 & 10: 04: 29 & 0.0014 & 0.0030 \\ 1050 & 10: 04: 31 & 0.0103 & 0.0156 \\ 1052 & 10: 04: 33 & 0.0225 & 0.0464 \\ 1054 & 10: 04: 35 & 0.0059 & 0.0076 \\ 1056 & 10: 04: 37 & 0.0287 & 0.0318 \\ 1058 & 10: 04: 39 & 0.0063 & 0.0226 \\ 1060 & 10: 04: 41 & 0.0054 & 0.0065 \\ 1062 & 10: 04: 43 & 0.0189 & 0.0125 \\ 1064 & 10: 04: 45 & 0.0021 & 0.0239 \\ 1066 & 10: 04: 47 & 0.0054 & 0.0086 \\ 1068 & 10: 04: 49 & 0.0076 & 0.0274 \\ 1070 & 10: 04: 51 & 0.0121 & 0.0034 \\ 1072 & 10: 04: 53 & 0.0066 & 0.0115 \\ 1074 & 10: 04: 55 & 0.0085 & 0.0093 \\ 1076 & 10: 04: 57 & 0.0053 & 0.0148 \\ 1078 & 10: 04: 59 & 0.0017 & 0.0065 \\ 1080 & 10: 05: 01 & 0.0006 & 0.0009 \\ 1082 & 10: 05: 03 & 0.0013 & 0.0025 \\ 1084 & 10: 05: 05 & 0.0021 & 0.0154 \\ 1086 & 10: 05: 07 & 0.0025 & 0.0124 \\ 1088 & 10: 05: 09 & 0.0055 & 0.0040 \\ 1090 & 10: 05: 11 & 0.0027 & 0.0120 \\ 1092 & 10: 05: 13 & 0.0025 & 0.0045 \\ 1094 & 10: 05: 15 & 0.0086 & 0.0150 \\ 1096 & 10: 05: 17 & 0.0013 & 0.0167 \\ 1098 & 10: 05: 19 & 0.0027 & 0.0099 \\ 1100 & 10: 05: 21 & 0.0084 & 0.0330 \\ 1102 & 10: 05: 23 & 0.0223 & 0.0035 \\ 1104 & 10: 05: 25 & 0.0020 & 0.0202 \\ 1106 & 10: 05: 27 & 0.0022 & 0.0219 \\ 1108 & 10: 05: 29 & 0.0149 & 0.0046 \\ 1110 & 10: 05: 31 & 0.0009 & 0.0110 \\ 1112 & 10: 05: 33 & 0.0037 & 0.0078 \\ 1114 & 10: 05: 35 & 0.0003 & 0.0038 \\ 1116 & 10: 05: 37 & 0.0017 & 0.0079 \\ 1118 & 10: 05: 39 & 0.0013 & 0.0148\end{array}$


Appendix D - Laser Data Test 2 NIST102605_1.xls

$\begin{array}{llll}1120 & 10: 05: 41 & 0.0018 & 0.0321 \\ 1122 & 10: 05: 43 & 0.0019 & 0.0223 \\ 1124 & 10: 05: 45 & 0.0005 & 0.0038 \\ 1126 & 10: 05: 47 & 0.0035 & 0.0308 \\ 1128 & 10: 05: 49 & 0.0017 & 0.0182 \\ 1130 & 10: 05: 51 & 0.0017 & 0.0177 \\ 1132 & 10: 05: 53 & 0.0016 & 0.0321 \\ 1134 & 10: 05: 55 & 0.0029 & 0.0248 \\ 1136 & 10: 05: 57 & 0.0038 & 0.0070 \\ 1138 & 10: 05: 59 & 0.0019 & 0.0038 \\ 1140 & 10: 06: 01 & 0.0009 & 0.0067 \\ 1142 & 10: 06: 03 & 0.0032 & 0.0095 \\ 1144 & 10: 06: 05 & 0.0048 & 0.0013 \\ 1146 & 10: 06: 07 & 0.0102 & 0.0010 \\ 1148 & 10: 06: 09 & 0.0016 & 0.0009 \\ 1150 & 10: 06: 11 & 0.0029 & 0.0108 \\ 1152 & 10: 06: 13 & 0.0078 & 0.0065 \\ 1154 & 10: 06: 15 & 0.0170 & 0.0017 \\ 1156 & 10: 06: 17 & 0.0028 & 0.0056 \\ 1158 & 10: 06: 19 & 0.0276 & 0.0019 \\ 1160 & 10: 06: 21 & 0.0038 & 0.0116 \\ 1162 & 10: 06: 23 & 0.0051 & 0.0052 \\ 1164 & 10: 06: 25 & 0.0105 & 0.0162 \\ 1166 & 10: 06: 27 & 0.0017 & 0.0005 \\ 1168 & 10: 06: 29 & 0.0232 & 0.0037 \\ 1170 & 10: 06: 31 & 0.0029 & 0.0360 \\ 1172 & 10: 06: 33 & 0.0137 & 0.0479 \\ 1174 & 10: 06: 35 & 0.0099 & 0.0018 \\ 1176 & 10: 06: 37 & 0.0072 & 0.0058 \\ 1178 & 10: 06: 39 & 0.0064 & 0.0061 \\ 1180 & 10: 06: 41 & 0.0042 & 0.0097 \\ 1182 & 10: 06: 43 & 0.0032 & 0.0016 \\ 1184 & 10: 06: 45 & 0.0155 & 0.0138 \\ 1186 & 10: 06: 47 & 0.0117 & 0.0045 \\ 1188 & 10: 06: 49 & 0.0125 & 0.0290 \\ 1190 & 10: 06: 51 & 0.0057 & 0.0449 \\ 1192 & 10: 06: 53 & 0.0044 & 0.0014 \\ 1194 & 10: 06: 55 & 0.0160 & 0.0024 \\ 1196 & 10: 06: 57 & 0.0025 & 0.0053 \\ 1198 & 10: 06: 59 & 0.0137 & 0.0111 \\ 1200 & 10: 07: 01 & 0.0180 & 0.0033 \\ 1202 & 10: 07: 03 & 0.0048 & 0.0572 \\ 1204 & 10: 07: 05 & 0.0017 & 0.0134 \\ 1206 & 10: 07: 07 & 0.0087 & 0.0010 \\ 1208 & 10: 07: 09 & 0.0078 & 0.0021 \\ 1210 & 10: 07: 11 & 0.0049 & 0.0056 \\ 1212 & 10: 07: 13 & 0.0029 & 0.0179 \\ 1214 & 10: 07: 15 & 0.0133 & 0.0086 \\ 1216 & 10: 07: 17 & 0.0021 & 0.0043 \\ 1218 & 10: 07: 19 & 0.0058 & 0.0044 \\ 1220 & 10: 07: 21 & 0.0006 & 0.0127\end{array}$

D - 12 
Appendix D - Laser Data Test 2 NIST102605_1.xls

$\begin{array}{llll}1222 & 10: 07: 23 & 0.0094 & 0.0054 \\ 1224 & 10: 07: 25 & 0.0048 & 0.0018 \\ 1226 & 10: 07: 27 & 0.0120 & 0.0031 \\ 1228 & 10: 07: 29 & 0.0080 & 0.0050 \\ 1230 & 10: 07: 31 & 0.0127 & 0.0057 \\ 1232 & 10: 07: 33 & 0.0140 & 0.0084 \\ 1234 & 10: 07: 35 & 0.0085 & 0.0332 \\ 1236 & 10: 07: 37 & 0.0105 & 0.0206 \\ 1238 & 10: 07: 39 & 0.0279 & 0.0084 \\ 1240 & 10: 07: 41 & 0.0175 & 0.0031 \\ 1242 & 10: 07: 43 & 0.0061 & 0.0020 \\ 1244 & 10: 07: 45 & 0.0025 & 0.0042 \\ 1246 & 10: 07: 47 & 0.0037 & 0.0062 \\ 1248 & 10: 07: 49 & 0.0021 & 0.0021 \\ 1250 & 10: 07: 51 & 0.0006 & 0.0076 \\ 1252 & 10: 07: 53 & 0.0447 & 0.0078 \\ 1254 & 10: 07: 55 & 0.0076 & 0.0106 \\ 1256 & 10: 07: 57 & 0.0045 & 0.0088 \\ 1258 & 10: 07: 59 & 0.0040 & 0.0260 \\ 1260 & 10: 08: 01 & 0.0100 & 0.0066 \\ 1262 & 10: 08: 03 & 0.0749 & 0.0076 \\ 1264 & 10: 08: 05 & 0.0135 & 0.0109 \\ 1266 & 10: 08: 07 & 0.0048 & 0.0053 \\ 1268 & 10: 08: 09 & 0.0122 & 0.0043 \\ 1270 & 10: 08: 11 & 0.0011 & 0.0005 \\ 1272 & 10: 08: 13 & 0.0043 & 0.0091 \\ 1274 & 10: 08: 15 & 0.0044 & 0.0046 \\ 1276 & 10: 08: 17 & 0.0053 & 0.0773 \\ 1278 & 10: 08: 19 & 0.0159 & 0.0425 \\ 1280 & 10: 08: 21 & 0.0045 & 0.0148 \\ 1282 & 10: 08: 23 & 0.0027 & 0.0090 \\ 1284 & 10: 08: 25 & 0.0014 & 0.0163 \\ 1286 & 10: 08: 27 & 0.0114 & 0.0186 \\ 1288 & 10: 08: 29 & 0.0057 & 0.0045 \\ 1290 & 10: 08: 31 & 0.0037 & 0.0086 \\ 1292 & 10: 08: 33 & 0.0007 & 0.0030 \\ 1294 & 10: 08: 35 & 0.0011 & 0.0036 \\ 1296 & 10: 08: 37 & 0.0029 & 0.0193 \\ 1298 & 10: 08: 39 & 0.0041 & 0.0041 \\ 1300 & 10: 08: 41 & 0.0006 & 0.0054 \\ 1302 & 10: 08: 43 & 0.0007 & 0.0026 \\ 1304 & 10: 08: 45 & 0.0059 & 0.0062 \\ 1306 & 10: 08: 47 & 0.0035 & 0.0375 \\ 1308 & 10: 08: 49 & 0.0069 & 0.0055 \\ 1310 & 10: 08: 51 & 0.0021 & 0.0044 \\ 1312 & 10: 08: 53 & 0.0151 & 0.0106 \\ 1314 & 10: 08: 55 & 0.0044 & 0.0098 \\ 1316 & 10: 08: 57 & 0.0001 & 0.0047 \\ 1318 & 10: 08: 59 & 0.0023 & 0.0021 \\ 1320 & 10: 09: 01 & 0.0002 & 0.0006 \\ 1322 & 10: 09: 03 & 0.0009 & 0.0008\end{array}$

D - 13 
Appendix D - Laser Data Test 2 NIST102605_1.xls

$\begin{array}{llll}1324 & 10: 09: 05 & 0.0020 & 0.0014 \\ 1326 & 10: 09: 07 & 0.0014 & 0.0110 \\ 1328 & 10: 09: 09 & 0.0042 & 0.0061 \\ 1330 & 10: 09: 11 & 0.0005 & 0.0035 \\ 1332 & 10: 09: 13 & 0.0011 & 0.0095 \\ 1334 & 10: 09: 15 & 0.0024 & 0.0103 \\ 1336 & 10: 09: 17 & 0.0042 & 0.0017 \\ 1338 & 10: 09: 19 & 0.0081 & 0.0021 \\ 1340 & 10: 09: 21 & 0.0123 & 0.0085 \\ 1342 & 10: 09: 23 & 0.0074 & 0.0008 \\ 1344 & 10: 09: 25 & 0.0148 & 0.0044 \\ 1346 & 10: 09: 27 & 0.0026 & 0.0063 \\ 1348 & 10: 09: 29 & 0.0018 & 0.0095 \\ 1350 & 10: 09: 31 & 0.0002 & 0.0135 \\ 1352 & 10: 09: 33 & 0.0005 & 0.0002 \\ 1354 & 10: 09: 35 & 0.0026 & 0.0063 \\ 1356 & 10: 09: 37 & 0.0058 & 0.0039 \\ 1358 & 10: 09: 39 & 0.0033 & 0.0606 \\ 1360 & 10: 09: 41 & 0.0022 & 0.0030 \\ 1362 & 10: 09: 43 & 0.0034 & 0.0342 \\ 1364 & 10: 09: 45 & 0.0025 & 0.0139 \\ 1366 & 10: 09: 47 & 0.0033 & 0.0176 \\ 1368 & 10: 09: 49 & 0.0066 & 0.0025 \\ 1370 & 10: 09: 51 & 0.0002 & 0.0041 \\ 1372 & 10: 09: 53 & 0.0094 & 0.0159 \\ 1374 & 10: 09: 55 & 0.0125 & 0.0132 \\ 1376 & 10: 09: 57 & 0.0016 & 0.0030 \\ 1378 & 10: 09: 59 & 0.0132 & 0.0026 \\ 1380 & 10: 10: 01 & 0.0011 & 0.0064 \\ 1382 & 10: 10: 03 & 0.0024 & 0.0042 \\ 1384 & 10: 10: 05 & 0.0017 & 0.0172 \\ 1386 & 10: 10: 07 & 0.0012 & 0.0072 \\ 1388 & 10: 10: 09 & 0.0026 & 0.0172 \\ 1390 & 10: 10: 11 & 0.0005 & 0.0011 \\ 1392 & 10: 10: 13 & 0.0006 & 0.0147 \\ 1394 & 10: 10: 15 & 0.0015 & 0.0025 \\ 1396 & 10: 10: 17 & 0.0018 & 0.0108 \\ 1398 & 10: 10: 19 & 0.0061 & 0.0029 \\ 1400 & 10: 10: 21 & 0.0013 & 0.0045 \\ 1402 & 10: 10: 23 & 0.0038 & 0.0009 \\ 1404 & 10: 10: 25 & 0.0068 & 0.0309 \\ 1406 & 10: 10: 27 & 0.0472 & 0.0160 \\ 1408 & 10: 10: 29 & 0.0019 & 0.0131 \\ 1410 & 10: 10: 31 & 0.0053 & 0.0027 \\ 1412 & 10: 10: 33 & 0.0006 & 0.0225 \\ 1414 & 10: 10: 35 & 0.0006 & 0.0068 \\ 1416 & 10: 10: 37 & 0.0122 & 0.0051 \\ 1418 & 10: 10: 39 & 0.0081 & 0.0008 \\ 1420 & 10: 10: 41 & 0.0133 & 0.0038 \\ 1422 & 10: 10: 43 & 0.0018 & 0.0167 \\ 1424 & 10: 10: 45 & 0.0242 & 0.0207\end{array}$

D - 14 
Appendix D - Laser Data Test 2 NIST102605_1.xls

$\begin{array}{llll}1426 & 10: 10: 47 & 0.0001 & 0.0184 \\ 1428 & 10: 10: 49 & 0.0016 & 0.0099 \\ 1430 & 10: 10: 51 & 0.0044 & 0.0392 \\ 1432 & 10: 10: 53 & 0.0052 & 0.0098 \\ 1434 & 10: 10: 55 & 0.0057 & 0.0050 \\ 1436 & 10: 10: 57 & 0.0010 & 0.0260 \\ 1438 & 10: 10: 59 & 0.0005 & 0.0246 \\ 1440 & 10: 11: 01 & 0.0011 & 0.0022 \\ 1442 & 10: 11: 03 & 0.0006 & 0.0018 \\ 1444 & 10: 11: 05 & 0.0020 & 0.0055 \\ 1446 & 10: 11: 07 & 0.0068 & 0.0043 \\ 1448 & 10: 11: 09 & 0.0017 & 0.0099 \\ 1450 & 10: 11: 11 & 0.0064 & 0.0291 \\ 1452 & 10: 11: 13 & 0.0014 & 0.0015 \\ 1454 & 10: 11: 15 & 0.0014 & 0.0044 \\ 1456 & 10: 11: 17 & 0.0010 & 0.0084 \\ 1458 & 10: 11: 19 & 0.0022 & 0.0051 \\ 1460 & 10: 11: 21 & 0.0143 & 0.0010 \\ 1462 & 10: 11: 23 & 0.0102 & 0.0213 \\ 1464 & 10: 11: 25 & 0.0139 & 0.0092 \\ 1466 & 10: 11: 27 & 0.0071 & 0.0098 \\ 1468 & 10: 11: 29 & 0.0019 & 0.0158 \\ 1470 & 10: 11: 31 & 0.0009 & 0.0170 \\ 1472 & 10: 11: 33 & 0.0026 & 0.0012 \\ 1474 & 10: 11: 35 & 0.0005 & 0.0040 \\ 1476 & 10: 11: 37 & 0.0010 & 0.0045 \\ 1478 & 10: 11: 39 & 0.0037 & 0.0034 \\ 1480 & 10: 11: 41 & 0.0025 & 0.0065 \\ 1482 & 10: 11: 43 & 0.0021 & 0.0055 \\ 1484 & 10: 11: 45 & 0.0003 & 0.0020 \\ 1486 & 10: 11: 47 & 0.0104 & 0.0019 \\ 1488 & 10: 11: 49 & 0.0010 & 0.0012 \\ 1490 & 10: 11: 51 & 0.0010 & 0.0026 \\ 1492 & 10: 11: 53 & 0.0005 & 0.0047 \\ 1494 & 10: 11: 55 & 0.0007 & 0.0055 \\ 1496 & 10: 11: 57 & 0.0027 & 0.0128 \\ 1498 & 10: 11: 59 & 0.0039 & 0.0253 \\ 1500 & 10: 12: 01 & 0.0175 & 0.0233 \\ 1502 & 10: 12: 03 & 0.0012 & 0.0019 \\ 1504 & 10: 12: 05 & 0.0015 & 0.0157 \\ 1506 & 10: 12: 07 & 0.0036 & 0.0022 \\ 1508 & 10: 12: 09 & 0.0009 & 0.0024 \\ 1510 & 10: 12: 11 & 0.0026 & 0.0513 \\ 1512 & 10: 12: 13 & 0.0056 & 0.0012 \\ 1514 & 10: 12: 15 & 0.0046 & 0.0035 \\ 1516 & 10: 12: 17 & 0.0007 & 0.0058 \\ 1518 & 10: 12: 19 & 0.0075 & 0.0063 \\ 1520 & 10: 12: 21 & 0.0011 & 0.0053 \\ 1522 & 10: 12: 23 & 0.0222 & 0.0117 \\ 1524 & 10: 12: 25 & 0.0020 & 0.0059 \\ 1526 & 10: 12: 27 & 0.0040 & 0.0055\end{array}$

D - 15 
Appendix D - Laser Data Test 2 NIST102605_1.xls

$\begin{array}{llll}1528 & 10: 12: 29 & 0.0147 & 0.0077 \\ 1530 & 10: 12: 31 & 0.0019 & 0.0121 \\ 1532 & 10: 12: 33 & 0.0091 & 0.0016 \\ 1534 & 10: 12: 35 & 0.0002 & 0.0057 \\ 1536 & 10: 12: 37 & 0.0004 & 0.0051 \\ 1538 & 10: 12: 39 & 0.0021 & 0.0072 \\ 1540 & 10: 12: 41 & 0.0260 & 0.0026 \\ 1542 & 10: 12: 43 & 0.0006 & 0.0020 \\ 1544 & 10: 12: 45 & 0.0061 & 0.0029 \\ 1546 & 10: 12: 47 & 0.0066 & 0.0037 \\ 1548 & 10: 12: 49 & 0.0416 & 0.0077 \\ 1550 & 10: 12: 51 & 0.0519 & 0.0130 \\ 1552 & 10: 12: 53 & 0.0262 & 0.0167 \\ 1554 & 10: 12: 55 & 0.0305 & 0.0191 \\ 1556 & 10: 12: 57 & 0.0120 & 0.0210 \\ 1558 & 10: 12: 59 & 0.0038 & 0.0086 \\ 1560 & 10: 13: 01 & 0.0006 & 0.0031 \\ 1562 & 10: 13: 03 & 0.0121 & 0.0160 \\ 1564 & 10: 13: 05 & 0.0019 & 0.0413 \\ 1566 & 10: 13: 07 & 0.0002 & 0.0046 \\ 1568 & 10: 13: 09 & 0.0014 & 0.0017 \\ 1570 & 10: 13: 11 & 0.0007 & 0.0065 \\ 1572 & 10: 13: 13 & 0.0036 & 0.0078 \\ 1574 & 10: 13: 15 & 0.0116 & 0.0091 \\ 1576 & 10: 13: 17 & 0.0030 & 0.0644 \\ 1578 & 10: 13: 19 & 0.0004 & 0.0125 \\ 1580 & 10: 13: 21 & 0.0013 & 0.0139 \\ 1582 & 10: 13: 23 & 0.0017 & 0.0145 \\ 1584 & 10: 13: 25 & 0.0037 & 0.0090 \\ 1586 & 10: 13: 27 & 0.0009 & 0.0571 \\ 1588 & 10: 13: 29 & 0.0012 & 0.0039 \\ 1590 & 10: 13: 31 & 0.0047 & 0.0068 \\ 1592 & 10: 13: 33 & 0.0099 & 0.0100 \\ 1594 & 10: 13: 35 & 0.0004 & 0.0026 \\ 1596 & 10: 13: 37 & 0.0023 & 0.0068 \\ 1598 & 10: 13: 39 & 0.0074 & 0.0304 \\ 1600 & 10: 13: 41 & 0.0010 & 0.0166 \\ 1602 & 10: 13: 43 & 0.0058 & 0.0432 \\ 1604 & 10: 13: 45 & 0.0023 & 0.0715 \\ 1606 & 10: 13: 47 & 0.0123 & 0.0050 \\ 1608 & 10: 13: 49 & 0.0004 & 0.0347 \\ 1610 & 10: 13: 51 & 0.0028 & 0.0124 \\ 1612 & 10: 13: 53 & 0.0017 & 0.1018 \\ 1614 & 10: 13: 55 & 0.0038 & 0.0269 \\ 1616 & 10: 13: 57 & 0.0043 & 0.0062 \\ 1618 & 10: 13: 59 & 0.0008 & 0.0198 \\ 1620 & 10: 14: 01 & 0.0169 & 0.0065 \\ 1622 & 10: 14: 03 & 0.0018 & 0.0057 \\ 1624 & 10: 14: 05 & 0.0011 & 0.0215 \\ 1626 & 10: 14: 07 & 0.0002 & 0.0251 \\ 1628 & 10: 14: 09 & 0.0059 & 0.0234\end{array}$


Appendix D - Laser Data Test 2 NIST102605_1.xls

$\begin{array}{llll}1630 & 10: 14: 11 & 0.0031 & 0.0090 \\ 1632 & 10: 14: 13 & 0.0058 & 0.0311 \\ 1634 & 10: 14: 15 & 0.0070 & 0.0058 \\ 1636 & 10: 14: 17 & 0.0020 & 0.0059 \\ 1638 & 10: 14: 19 & 0.0227 & 0.0153 \\ 1640 & 10: 14: 21 & 0.0012 & 0.0127 \\ 1642 & 10: 14: 23 & 0.0044 & 0.0150 \\ 1644 & 10: 14: 25 & 0.0009 & 0.0297 \\ 1646 & 10: 14: 27 & 0.0003 & 0.0393 \\ 1648 & 10: 14: 29 & 0.0010 & 0.0182 \\ 1650 & 10: 14: 31 & 0.0026 & 0.0006 \\ 1652 & 10: 14: 33 & 0.0010 & 0.0070 \\ 1654 & 10: 14: 35 & 0.0004 & 0.0020 \\ 1656 & 10: 14: 37 & 0.0006 & 0.0027 \\ 1658 & 10: 14: 39 & 0.0009 & 0.0008 \\ 1660 & 10: 14: 41 & 0.0036 & 0.0017 \\ 1662 & 10: 14: 43 & 0.0006 & 0.0035 \\ 1664 & 10: 14: 45 & 0.0038 & 0.0186 \\ 1666 & 10: 14: 47 & 0.0007 & 0.0036 \\ 1668 & 10: 14: 49 & 0.0006 & 0.0046 \\ 1670 & 10: 14: 51 & 0.0016 & 0.0133 \\ 1672 & 10: 14: 53 & 0.0068 & 0.0114 \\ 1674 & 10: 14: 55 & 0.0081 & 0.0309 \\ 1676 & 10: 14: 57 & 0.0022 & 0.0034 \\ 1678 & 10: 14: 59 & 0.0109 & 0.0045 \\ 1680 & 10: 15: 01 & 0.0008 & 0.0364 \\ 1682 & 10: 15: 03 & 0.0022 & 0.0174 \\ 1684 & 10: 15: 05 & 0.0011 & 0.0205 \\ 1686 & 10: 15: 07 & 0.0006 & 0.0082 \\ 1688 & 10: 15: 09 & 0.0013 & 0.0033 \\ 1690 & 10: 15: 11 & 0.0048 & 0.0186 \\ 1692 & 10: 15: 13 & 0.0134 & 0.0033 \\ 1694 & 10: 15: 15 & 0.0328 & 0.0067 \\ 1696 & 10: 15: 17 & 0.0031 & 0.0042 \\ 1698 & 10: 15: 19 & 0.0051 & 0.0059 \\ 1700 & 10: 15: 21 & 0.0232 & 0.0018 \\ 1702 & 10: 15: 23 & 0.0164 & 0.0047 \\ 1704 & 10: 15: 25 & 0.0032 & 0.0026 \\ 1706 & 10: 15: 27 & 0.0170 & 0.0096 \\ 1708 & 10: 15: 29 & 0.0010 & 0.0010 \\ 1710 & 10: 15: 31 & 0.0043 & 0.0083 \\ 1712 & 10: 15: 33 & 0.0049 & 0.0044 \\ 1714 & 10: 15: 35 & 0.0056 & 0.0064 \\ 1716 & 10: 15: 37 & 0.0066 & 0.0064 \\ 1718 & 10: 15: 39 & 0.0068 & 0.0020 \\ 1720 & 10: 15: 41 & 0.0032 & 0.0453 \\ 1722 & 10: 15: 43 & 0.0221 & 0.0598 \\ 1724 & 10: 15: 45 & 0.0249 & 0.1780 \\ 1726 & 10: 15: 47 & 0.0010 & 0.0127 \\ 1728 & 10: 15: 49 & 0.0010 & 0.0028 \\ 1730 & 10: 15: 51 & 0.0048 & 0.0034\end{array}$


Appendix D - Laser Data Test 2 NIST102605_1.xls

$\begin{array}{llll}1732 & 10: 15: 53 & 0.0050 & 0.0283 \\ 1734 & 10: 15: 55 & 0.0177 & 0.0045 \\ 1736 & 10: 15: 57 & 0.0167 & 0.0006 \\ 1738 & 10: 15: 59 & 0.0016 & 0.0047 \\ 1740 & 10: 16: 01 & 0.0209 & 0.0015 \\ 1742 & 10: 16: 03 & 0.0074 & 0.0042 \\ 1744 & 10: 16: 05 & 0.0425 & 0.0093 \\ 1746 & 10: 16: 07 & 0.0012 & 0.0077 \\ 1748 & 10: 16: 09 & 0.0039 & 0.0260 \\ 1750 & 10: 16: 11 & 0.0006 & 0.0075 \\ 1752 & 10: 16: 13 & 0.0262 & 0.0111 \\ 1754 & 10: 16: 15 & 0.0101 & 0.0235 \\ 1756 & 10: 16: 17 & 0.0138 & 0.0027 \\ 1758 & 10: 16: 19 & 0.0023 & 0.0057 \\ 1760 & 10: 16: 21 & 0.0107 & 0.0138 \\ 1762 & 10: 16: 23 & 0.0093 & 0.0078 \\ 1764 & 10: 16: 25 & 0.0106 & 0.0232 \\ 1766 & 10: 16: 27 & 0.0141 & 0.0079 \\ 1768 & 10: 16: 29 & 0.0011 & 0.0232 \\ 1770 & 10: 16: 31 & 0.0045 & 0.0073 \\ 1772 & 10: 16: 33 & 0.0020 & 0.0509 \\ 1774 & 10: 16: 35 & 0.0114 & 0.0048 \\ 1776 & 10: 16: 37 & 0.0100 & 0.0084 \\ 1778 & 10: 16: 39 & 0.0085 & 0.0152 \\ 1780 & 10: 16: 41 & 0.0069 & 0.0042 \\ 1782 & 10: 16: 43 & 0.0078 & 0.0025 \\ 1784 & 10: 16: 45 & 0.0048 & 0.0072 \\ 1786 & 10: 16: 47 & 0.0038 & 0.0172 \\ 1788 & 10: 16: 49 & 0.0041 & 0.0273 \\ 1790 & 10: 16: 51 & 0.0106 & 0.0037 \\ 1792 & 10: 16: 53 & 0.0056 & 0.0025 \\ 1794 & 10: 16: 55 & 0.0139 & 0.0045 \\ 1796 & 10: 16: 57 & 0.0042 & 0.0039 \\ 1798 & 10: 16: 59 & 0.0044 & 0.0121 \\ 1800 & 10: 17: 01 & 0.0077 & 0.0024 \\ 1802 & 10: 17: 03 & 0.0098 & 0.0022 \\ 1804 & 10: 17: 05 & 0.0161 & 0.0101 \\ 1806 & 10: 17: 07 & 0.0198 & 0.0089 \\ 1808 & 10: 17: 09 & 0.0080 & 0.0265 \\ 1810 & 10: 17: 11 & 0.0045 & 0.0027 \\ 1812 & 10: 17: 13 & 0.0667 & 0.0014 \\ 1814 & 10: 17: 15 & 0.0034 & 0.0088 \\ 1816 & 10: 17: 17 & 0.0108 & 0.0085 \\ 1818 & 10: 17: 19 & 0.0102 & 0.0169 \\ 1820 & 10: 17: 21 & 0.0071 & 0.0036 \\ 1822 & 10: 17: 23 & 0.0074 & 0.0139 \\ 1824 & 10: 17: 25 & 0.0375 & 0.0253 \\ 1826 & 10: 17: 27 & 0.0066 & 0.0018 \\ 1828 & 10: 17: 29 & 0.0059 & 0.0034 \\ 1830 & 10: 17: 31 & 0.0102 & 0.0049 \\ 1832 & 10: 17: 33 & 0.0059 & 0.0335\end{array}$

D - 18 
Appendix D - Laser Data Test 2 NIST102605_1.xls

$\begin{array}{llll}1834 & 10: 17: 35 & 0.0055 & 0.0083 \\ 1836 & 10: 17: 37 & 0.0072 & 0.0028 \\ 1838 & 10: 17: 39 & 0.0026 & 0.0009 \\ 1840 & 10: 17: 41 & 0.0227 & 0.0008 \\ 1842 & 10: 17: 43 & 0.0031 & 0.0323 \\ 1844 & 10: 17: 45 & 0.0063 & 0.0047 \\ 1846 & 10: 17: 47 & 0.0065 & 0.0035 \\ 1848 & 10: 17: 49 & 0.0006 & 0.0108 \\ 1850 & 10: 17: 51 & 0.0104 & 0.0117 \\ 1852 & 10: 17: 53 & 0.0076 & 0.0188 \\ 1854 & 10: 17: 55 & 0.0036 & 0.0059 \\ 1856 & 10: 17: 57 & 0.0008 & 0.0051 \\ 1858 & 10: 17: 59 & 0.0031 & 0.0228 \\ 1860 & 10: 18: 01 & 0.0023 & 0.0041 \\ 1862 & 10: 18: 03 & 0.0194 & 0.0035 \\ 1864 & 10: 18: 05 & 0.0010 & 0.0006 \\ 1866 & 10: 18: 07 & 0.0172 & 0.0327 \\ 1868 & 10: 18: 09 & 0.0073 & 0.0027 \\ 1870 & 10: 18: 11 & 0.0014 & 0.0090 \\ 1872 & 10: 18: 13 & 0.0091 & 0.0030 \\ 1874 & 10: 18: 15 & 0.0048 & 0.0266 \\ 1876 & 10: 18: 17 & 0.0021 & 0.0025 \\ 1878 & 10: 18: 19 & 0.0050 & 0.0147 \\ 1880 & 10: 18: 21 & 0.0127 & 0.0380 \\ 1882 & 10: 18: 23 & 0.0034 & 0.0649 \\ 1884 & 10: 18: 25 & 0.0002 & 0.0153 \\ 1886 & 10: 18: 27 & 0.0008 & 0.0365 \\ 1888 & 10: 18: 29 & 0.0060 & 0.0012 \\ 1890 & 10: 18: 31 & 0.0006 & 0.0015 \\ 1892 & 10: 18: 33 & 0.0004 & 0.0021 \\ 1894 & 10: 18: 35 & 0.0015 & 0.0055 \\ 1896 & 10: 18: 37 & 0.0088 & 0.0033 \\ 1898 & 10: 18: 39 & 0.0017 & 0.0043 \\ 1900 & 10: 18: 41 & 0.0006 & 0.0288 \\ 1902 & 10: 18: 43 & 0.0043 & 0.0075 \\ 1904 & 10: 18: 45 & 0.0019 & 0.0080 \\ 1906 & 10: 18: 47 & 0.0010 & 0.0032 \\ 1908 & 10: 18: 49 & 0.0064 & 0.0705 \\ 1910 & 10: 18: 51 & 0.0002 & 0.0089 \\ 1912 & 10: 18: 53 & 0.0009 & 0.0063 \\ 1914 & 10: 18: 55 & 0.0021 & 0.0208 \\ 1916 & 10: 18: 57 & 0.0008 & 0.0024 \\ 1918 & 10: 18: 59 & 0.0009 & 0.0108 \\ 1920 & 10: 19: 01 & 0.0009 & 0.0042 \\ 1922 & 10: 19: 03 & 0.0019 & 0.0042 \\ 1924 & 10: 19: 05 & 0.0037 & 0.0031 \\ 1926 & 10: 19: 07 & 0.0022 & 0.0146 \\ 1928 & 10: 19: 09 & 0.0009 & 0.0593 \\ 1930 & 10: 19: 11 & 0.0044 & 0.0019 \\ 1932 & 10: 19: 13 & 0.0006 & 0.0035 \\ 1934 & 10: 19: 15 & 0.0066 & 0.0275\end{array}$

D - 19 
Appendix D - Laser Data Test 2 NIST102605_1.xls

$\begin{array}{llll}1936 & 10: 19: 17 & 0.0078 & 0.0126 \\ 1938 & 10: 19: 19 & 0.0053 & 0.0025 \\ 1940 & 10: 19: 21 & 0.0006 & 0.0017 \\ 1942 & 10: 19: 23 & 0.0027 & 0.0058 \\ 1944 & 10: 19: 25 & 0.0015 & 0.0057 \\ 1946 & 10: 19: 27 & 0.0006 & 0.0159 \\ 1948 & 10: 19: 29 & 0.0036 & 0.0019 \\ 1950 & 10: 19: 31 & 0.0012 & 0.0057 \\ 1952 & 10: 19: 33 & 0.0025 & 0.0024 \\ 1954 & 10: 19: 35 & 0.0002 & 0.0060 \\ 1956 & 10: 19: 37 & 0.0081 & 0.0098 \\ 1958 & 10: 19: 39 & 0.0045 & 0.0064 \\ 1960 & 10: 19: 41 & 0.0005 & 0.0092 \\ 1962 & 10: 19: 43 & 0.0054 & 0.0217 \\ 1964 & 10: 19: 45 & 0.0006 & 0.0028 \\ 1966 & 10: 19: 47 & 0.0002 & 0.0357 \\ 1968 & 10: 19: 49 & 0.0028 & 0.0134 \\ 1970 & 10: 19: 51 & 0.0006 & 0.0121 \\ 1972 & 10: 19: 53 & 0.0004 & 0.0048 \\ 1974 & 10: 19: 55 & 0.0006 & 0.0129 \\ 1976 & 10: 19: 57 & 0.0007 & 0.0190 \\ 1978 & 10: 19: 59 & 0.0018 & 0.0137 \\ 1980 & 10: 20: 01 & 0.0002 & 0.0356 \\ 1982 & 10: 20: 03 & 0.0047 & 0.0109 \\ 1984 & 10: 20: 05 & 0.0019 & 0.0210 \\ 1986 & 10: 20: 07 & 0.0005 & 0.0154 \\ 1988 & 10: 20: 09 & 0.0002 & 0.0044 \\ 1990 & 10: 20: 11 & 0.0014 & 0.0034 \\ 1992 & 10: 20: 13 & 0.0022 & 0.0061 \\ 1994 & 10: 20: 15 & 0.0036 & 0.0221 \\ 1996 & 10: 20: 17 & 0.0011 & 0.0105 \\ 1998 & 10: 20: 19 & 0.0018 & 0.0052 \\ 2000 & 10: 20: 21 & 0.0003 & 0.0002 \\ 2002 & 10: 20: 23 & 0.0017 & 0.0080 \\ 2004 & 10: 20: 25 & 0.0062 & 0.0048 \\ 2006 & 10: 20: 27 & 0.0011 & 0.0054 \\ 2008 & 10: 20: 29 & 0.0026 & 0.0095 \\ 2010 & 10: 20: 31 & 0.0152 & 0.0061 \\ 2012 & 10: 20: 33 & 0.0126 & 0.0035 \\ 2014 & 10: 20: 35 & 0.0180 & 0.0042 \\ 2016 & 10: 20: 37 & 0.0034 & 0.0090 \\ 2018 & 10: 20: 39 & 0.0021 & 0.0143 \\ 2020 & 10: 20: 41 & 0.0002 & 0.0145 \\ 2022 & 10: 20: 43 & 0.0034 & 0.0484 \\ 2024 & 10: 20: 45 & 0.0120 & 0.0131 \\ 2026 & 10: 20: 47 & 0.0082 & 0.0756 \\ 2028 & 10: 20: 49 & 0.0009 & 0.0160 \\ 2030 & 10: 20: 51 & 0.0004 & 0.0018 \\ 2032 & 10: 20: 53 & 0.0036 & 0.0234 \\ 2034 & 10: 20: 55 & 0.0002 & 0.0012 \\ 2036 & 10: 20: 57 & 0.0081 & 0.0286\end{array}$


Appendix D - Laser Data Test 2 NIST102605_1.xls

$\begin{array}{llll}2038 & 10: 20: 59 & 0.0033 & 0.0008 \\ 2040 & 10: 21: 01 & 0.0075 & 0.0263 \\ 2042 & 10: 21: 03 & 0.0004 & 0.0056 \\ 2044 & 10: 21: 05 & 0.0004 & 0.0189 \\ 2046 & 10: 21: 07 & 0.0004 & 0.0077 \\ 2048 & 10: 21: 09 & 0.0102 & 0.0166 \\ 2050 & 10: 21: 11 & 0.0015 & 0.0015 \\ 2052 & 10: 21: 13 & 0.0084 & 0.0102 \\ 2054 & 10: 21: 15 & 0.0115 & 0.0341 \\ 2056 & 10: 21: 17 & 0.0045 & 0.0043 \\ 2058 & 10: 21: 19 & 0.0018 & 0.0058 \\ 2060 & 10: 21: 21 & 0.0023 & 0.0155 \\ 2062 & 10: 21: 23 & 0.0039 & 0.0023 \\ 2064 & 10: 21: 25 & 0.0054 & 0.0075 \\ 2066 & 10: 21: 27 & 0.0046 & 0.0160 \\ 2068 & 10: 21: 29 & 0.0004 & 0.0056 \\ 2070 & 10: 21: 31 & 0.0118 & 0.0060 \\ 2072 & 10: 21: 33 & 0.0080 & 0.0062 \\ 2074 & 10: 21: 35 & 0.0019 & 0.0087 \\ 2076 & 10: 21: 37 & 0.0037 & 0.0023 \\ 2078 & 10: 21: 39 & 0.0084 & 0.0093 \\ 2080 & 10: 21: 41 & 0.0006 & 0.0181 \\ 2082 & 10: 21: 43 & 0.0019 & 0.0036 \\ 2084 & 10: 21: 45 & 0.0011 & 0.0246 \\ 2086 & 10: 21: 47 & 0.0075 & 0.0575 \\ 2088 & 10: 21: 49 & 0.0080 & 0.0340 \\ 2090 & 10: 21: 51 & 0.0040 & 0.0029 \\ 2092 & 10: 21: 53 & 0.0098 & 0.0121 \\ 2094 & 10: 21: 55 & 0.0010 & 0.0114 \\ 2096 & 10: 21: 57 & 0.0005 & 0.0112 \\ 2098 & 10: 21: 59 & 0.0020 & 0.0114 \\ 2100 & 10: 22: 01 & 0.0035 & 0.0048 \\ 2102 & 10: 22: 03 & 0.0009 & 0.0079 \\ 2104 & 10: 22: 05 & 0.0039 & 0.0141 \\ 2106 & 10: 22: 07 & 0.0005 & 0.0082 \\ 2108 & 10: 22: 09 & 0.0025 & 0.0046 \\ 2110 & 10: 22: 11 & 0.0050 & 0.0167 \\ 2112 & 10: 22: 13 & 0.0071 & 0.0040 \\ 2114 & 10: 22: 15 & 0.0002 & 0.0201 \\ 2116 & 10: 22: 17 & 0.0023 & 0.0059 \\ 2118 & 10: 22: 19 & 0.0338 & 0.0110 \\ 2120 & 10: 22: 21 & 0.0246 & 0.0276 \\ 2122 & 10: 22: 23 & 0.0004 & 0.0085 \\ 2124 & 10: 22: 25 & 0.0007 & 0.0297 \\ 2126 & 10: 22: 27 & 0.0013 & 0.0118 \\ 2128 & 10: 22: 29 & 0.0007 & 0.0046 \\ 2130 & 10: 22: 31 & 0.0013 & 0.0035 \\ 2132 & 10: 22: 33 & 0.0029 & 0.0056 \\ 2134 & 10: 22: 35 & 0.0038 & 0.0028 \\ 2136 & 10: 22: 37 & 0.0035 & 0.0135 \\ 2138 & 10: 22: 39 & 0.0018 & 0.0231\end{array}$


Appendix D - Laser Data Test 2 NIST102605_1.xls

$\begin{array}{llll}2140 & 10: 22: 41 & 0.0005 & 0.0044 \\ 2142 & 10: 22: 43 & 0.0004 & 0.0164 \\ 2144 & 10: 22: 45 & 0.0073 & 0.0188 \\ 2146 & 10: 22: 47 & 0.0062 & 0.0131 \\ 2148 & 10: 22: 49 & 0.0097 & 0.0108 \\ 2150 & 10: 22: 51 & 0.0025 & 0.0054 \\ 2152 & 10: 22: 53 & 0.0101 & 0.0227 \\ 2154 & 10: 22: 55 & 0.0628 & 0.0306 \\ 2156 & 10: 22: 57 & 0.0151 & 0.0185 \\ 2158 & 10: 22: 59 & 0.0068 & 0.0230 \\ 2160 & 10: 23: 01 & 0.0045 & 0.0044 \\ 2162 & 10: 23: 03 & 0.0005 & 0.0080 \\ 2164 & 10: 23: 05 & 0.0002 & 0.0048 \\ 2166 & 10: 23: 07 & 0.0014 & 0.0033 \\ 2168 & 10: 23: 09 & 0.0123 & 0.0024 \\ 2170 & 10: 23: 11 & 0.0018 & 0.0015 \\ 2172 & 10: 23: 13 & 0.0011 & 0.0083 \\ 2174 & 10: 23: 15 & 0.0005 & 0.0011 \\ 2176 & 10: 23: 17 & 0.0002 & 0.0019 \\ 2178 & 10: 23: 19 & 0.0006 & 0.0033 \\ 2180 & 10: 23: 21 & 0.0011 & 0.0203 \\ 2182 & 10: 23: 23 & 0.0015 & 0.0156 \\ 2184 & 10: 23: 25 & 0.0014 & 0.0063 \\ 2186 & 10: 23: 27 & 0.0028 & 0.0373 \\ 2188 & 10: 23: 29 & 0.0030 & 0.0094 \\ 2190 & 10: 23: 31 & 0.0013 & 0.0027 \\ 2192 & 10: 23: 33 & 0.0022 & 0.0048 \\ 2194 & 10: 23: 35 & 0.0013 & 0.0051 \\ 2196 & 10: 23: 37 & 0.0033 & 0.0123 \\ 2198 & 10: 23: 39 & 0.0005 & 0.0015 \\ 2200 & 10: 23: 41 & 0.0048 & 0.0032 \\ 2202 & 10: 23: 43 & 0.0050 & 0.0016 \\ 2204 & 10: 23: 45 & 0.0053 & 0.0195 \\ 2206 & 10: 23: 47 & 0.0063 & 0.0018 \\ 2208 & 10: 23: 49 & 0.0035 & 0.0056 \\ 2210 & 10: 23: 51 & 0.0020 & 0.0044 \\ 2212 & 10: 23: 53 & 0.0053 & 0.0035 \\ 2214 & 10: 23: 55 & 0.0120 & 0.0192 \\ 2216 & 10: 23: 57 & 0.0034 & 0.0048 \\ 2218 & 10: 23: 59 & 0.0011 & 0.0067 \\ 2220 & 10: 24: 01 & 0.0028 & 0.0227 \\ 2222 & 10: 24: 03 & 0.0011 & 0.0045 \\ 2224 & 10: 24: 05 & 0.0010 & 0.0257 \\ 2226 & 10: 24: 07 & 0.0064 & 0.0041 \\ 2228 & 10: 24: 09 & 0.0068 & 0.0127 \\ 2230 & 10: 24: 11 & 0.0354 & 0.0124 \\ 2232 & 10: 24: 13 & 0.0102 & 0.0044 \\ 2234 & 10: 24: 15 & 0.0008 & 0.0019 \\ 2236 & 10: 24: 17 & 0.0021 & 0.0171 \\ 2238 & 10: 24: 19 & 0.0036 & 0.0210 \\ 2240 & 10: 24: 21 & 0.0180 & 0.0038\end{array}$


Appendix D - Laser Data Test 2 NIST102605_1.xls

$\begin{array}{llll}2242 & 10: 24: 23 & 0.0002 & 0.0132 \\ 2244 & 10: 24: 25 & 0.0023 & 0.0051 \\ 2246 & 10: 24: 27 & 0.0010 & 0.0067 \\ 2248 & 10: 24: 29 & 0.0011 & 0.0096 \\ 2250 & 10: 24: 31 & 0.0025 & 0.0159 \\ 2252 & 10: 24: 33 & 0.0193 & 0.0032 \\ 2254 & 10: 24: 35 & 0.0123 & 0.0085 \\ 2256 & 10: 24: 37 & 0.0015 & 0.0188 \\ 2258 & 10: 24: 39 & 0.0029 & 0.0028 \\ 2260 & 10: 24: 41 & 0.0016 & 0.0024 \\ 2262 & 10: 24: 43 & 0.0009 & 0.0014 \\ 2264 & 10: 24: 45 & 0.0007 & 0.0042 \\ 2266 & 10: 24: 47 & 0.0007 & 0.0118 \\ 2268 & 10: 24: 49 & 0.0007 & 0.0048 \\ 2270 & 10: 24: 51 & 0.0012 & 0.0153 \\ 2272 & 10: 24: 53 & 0.0127 & 0.0086 \\ 2274 & 10: 24: 55 & 0.0018 & 0.0188 \\ 2276 & 10: 24: 57 & 0.0074 & 0.0034 \\ 2278 & 10: 24: 59 & 0.0009 & 0.0660 \\ 2280 & 10: 25: 01 & 0.0044 & 0.0019 \\ 2282 & 10: 25: 03 & 0.0060 & 0.0467 \\ 2284 & 10: 25: 05 & 0.0235 & 0.0021 \\ 2286 & 10: 25: 07 & 0.0156 & 0.0068 \\ 2288 & 10: 25: 09 & 0.0145 & 0.0217 \\ 2290 & 10: 25: 11 & 0.0161 & 0.0053 \\ 2292 & 10: 25: 13 & 0.0028 & 0.0129 \\ 2294 & 10: 25: 15 & 0.0018 & 0.0124 \\ 2296 & 10: 25: 17 & 0.0006 & 0.0098 \\ 2298 & 10: 25: 19 & 0.0203 & 0.0059 \\ 2300 & 10: 25: 21 & 0.0027 & 0.0079 \\ 2302 & 10: 25: 23 & 0.0003 & 0.0015 \\ 2304 & 10: 25: 25 & 0.0030 & 0.0016 \\ 2306 & 10: 25: 27 & 0.0013 & 0.0016 \\ 2308 & 10: 25: 29 & 0.0003 & 0.0016 \\ 2310 & 10: 25: 31 & 0.0011 & 0.0091 \\ 2312 & 10: 25: 33 & 0.0028 & 0.0300 \\ 2314 & 10: 25: 35 & 0.0035 & 0.0027 \\ 2316 & 10: 25: 37 & 0.0067 & 0.0005 \\ 2318 & 10: 25: 39 & 0.0023 & 0.0086 \\ 2320 & 10: 25: 41 & 0.0198 & 0.0316 \\ 2322 & 10: 25: 43 & 0.0189 & 0.0061 \\ 2324 & 10: 25: 45 & 0.0081 & 0.0235 \\ 2326 & 10: 25: 47 & 0.0048 & 0.0078 \\ 2328 & 10: 25: 49 & 0.0078 & 0.0220 \\ 2330 & 10: 25: 51 & 0.0011 & 0.0140 \\ 2332 & 10: 25: 53 & 0.0109 & 0.0040 \\ 2334 & 10: 25: 55 & 0.0008 & 0.0078 \\ 2336 & 10: 25: 57 & 0.0084 & 0.0022 \\ 2338 & 10: 25: 59 & 0.0007 & 0.0075 \\ 2340 & 10: 26: 01 & 0.0016 & 0.0090 \\ 2342 & 10: 26: 03 & 0.0033 & 0.0037\end{array}$


Appendix D - Laser Data Test 2 NIST102605_1.xls

$\begin{array}{llll}2344 & 10: 26: 05 & 0.0006 & 0.0031 \\ 2346 & 10: 26: 07 & 0.0091 & 0.0069 \\ 2348 & 10: 26: 09 & 0.0053 & 0.0027 \\ 2350 & 10: 26: 11 & 0.0016 & 0.0091 \\ 2352 & 10: 26: 13 & 0.0193 & 0.0082 \\ 2354 & 10: 26: 15 & 0.0070 & 0.0499 \\ 2356 & 10: 26: 17 & 0.0042 & 0.0019 \\ 2358 & 10: 26: 19 & 0.0019 & 0.0111 \\ 2360 & 10: 26: 21 & 0.0081 & 0.0086 \\ 2362 & 10: 26: 23 & 0.0044 & 0.0093 \\ 2364 & 10: 26: 25 & 0.0007 & 0.0134 \\ 2366 & 10: 26: 27 & 0.0056 & 0.0030 \\ 2368 & 10: 26: 29 & 0.0112 & 0.0036 \\ 2370 & 10: 26: 31 & 0.0045 & 0.0044 \\ 2372 & 10: 26: 33 & 0.0026 & 0.0012 \\ 2374 & 10: 26: 35 & 0.0027 & 0.0312 \\ 2376 & 10: 26: 37 & 0.0034 & 0.0069 \\ 2378 & 10: 26: 39 & 0.0025 & 0.0061 \\ 2380 & 10: 26: 41 & 0.0067 & 0.0151 \\ 2382 & 10: 26: 43 & 0.0151 & 0.0117 \\ 2384 & 10: 26: 45 & 0.0089 & 0.0031 \\ 2386 & 10: 26: 47 & 0.0224 & 0.0040 \\ 2388 & 10: 26: 49 & 0.0046 & 0.0294 \\ 2390 & 10: 26: 51 & 0.0066 & 0.0157 \\ 2392 & 10: 26: 53 & 0.0108 & 0.0187 \\ 2394 & 10: 26: 55 & 0.0360 & 0.0051 \\ 2396 & 10: 26: 57 & 0.0024 & 0.0041 \\ 2398 & 10: 26: 59 & 0.0164 & 0.0385 \\ 2400 & 10: 27: 01 & 0.0015 & 0.0069 \\ 2402 & 10: 27: 03 & 0.0032 & 0.0024 \\ 2404 & 10: 27: 05 & 0.0020 & 0.0071 \\ 2406 & 10: 27: 07 & 0.0010 & 0.0130 \\ 2408 & 10: 27: 09 & 0.0022 & 0.0042 \\ 2410 & 10: 27: 11 & 0.0069 & 0.0037 \\ 2412 & 10: 27: 13 & 0.0097 & 0.0022 \\ 2414 & 10: 27: 15 & 0.0026 & 0.0085 \\ 2416 & 10: 27: 17 & 0.0034 & 0.0052 \\ 2418 & 10: 27: 19 & 0.0002 & 0.0007 \\ 2420 & 10: 27: 21 & 0.0004 & 0.0119 \\ 2422 & 10: 27: 23 & 0.0038 & 0.0107 \\ 2424 & 10: 27: 25 & 0.0008 & 0.0149 \\ 2426 & 10: 27: 27 & 0.0061 & 0.0661 \\ 2428 & 10: 27: 29 & 0.0070 & 0.0051 \\ 2430 & 10: 27: 31 & 0.0041 & 0.0253 \\ 2432 & 10: 27: 33 & 0.0023 & 0.0014 \\ 2434 & 10: 27: 35 & 0.0025 & 0.0087 \\ 2436 & 10: 27: 37 & 0.0019 & 0.0016 \\ 2438 & 10: 27: 39 & 0.0043 & 0.0013 \\ 2440 & 10: 27: 41 & 0.0080 & 0.0042 \\ 2442 & 10: 27: 43 & 0.0009 & 0.0010 \\ 2444 & 10: 27: 45 & 0.0083 & 0.0263\end{array}$


Appendix D - Laser Data Test 2 NIST102605_1.xls

$\begin{array}{llll}2446 & 10: 27: 47 & 0.0027 & 0.0078 \\ 2448 & 10: 27: 49 & 0.0025 & 0.0061 \\ 2450 & 10: 27: 51 & 0.0151 & 0.0158 \\ 2452 & 10: 27: 53 & 0.0068 & 0.0106 \\ 2454 & 10: 27: 55 & 0.0011 & 0.0005 \\ 2456 & 10: 27: 57 & 0.0018 & 0.0183 \\ 2458 & 10: 27: 59 & 0.0436 & 0.0203 \\ 2460 & 10: 28: 01 & 0.0005 & 0.0147 \\ 2462 & 10: 28: 03 & 0.0069 & 0.0149 \\ 2464 & 10: 28: 05 & 0.0097 & 0.0111 \\ 2466 & 10: 28: 07 & 0.0016 & 0.0201 \\ 2468 & 10: 28: 09 & 0.0111 & 0.0086 \\ 2470 & 10: 28: 11 & 0.0093 & 0.0034 \\ 2472 & 10: 28: 13 & 0.0048 & 0.0081 \\ 2474 & 10: 28: 15 & 0.0173 & 0.0076 \\ 2476 & 10: 28: 17 & 0.0018 & 0.0328 \\ 2478 & 10: 28: 19 & 0.0025 & 0.0118 \\ 2480 & 10: 28: 21 & 0.0024 & 0.0029 \\ 2482 & 10: 28: 23 & 0.0073 & 0.0015 \\ 2484 & 10: 28: 25 & 0.0029 & 0.0018 \\ 2486 & 10: 28: 27 & 0.0025 & 0.0019 \\ 2488 & 10: 28: 29 & 0.0061 & 0.0065 \\ 2490 & 10: 28: 31 & 0.0092 & 0.0048 \\ 2492 & 10: 28: 33 & 0.0112 & 0.0017 \\ 2494 & 10: 28: 35 & 0.0032 & 0.0144 \\ 2496 & 10: 28: 37 & 0.0018 & 0.0028 \\ 2498 & 10: 28: 39 & 0.0150 & 0.0157 \\ 2500 & 10: 28: 41 & 0.0271 & 0.0007 \\ 2502 & 10: 28: 43 & 0.0087 & 0.0070 \\ 2504 & 10: 28: 45 & 0.0135 & 0.0023 \\ 2506 & 10: 28: 47 & 0.0148 & 0.0006 \\ 2508 & 10: 28: 49 & 0.0157 & 0.0011 \\ 2510 & 10: 28: 51 & 0.0088 & 0.0010 \\ 2512 & 10: 28: 53 & 0.0051 & 0.0011 \\ 2514 & 10: 28: 55 & 0.0061 & 0.0069 \\ 2516 & 10: 28: 57 & 0.0025 & 0.0014 \\ 2518 & 10: 28: 59 & 0.0064 & 0.0019 \\ 2520 & 10: 29: 01 & 0.0079 & 0.0028 \\ 2522 & 10: 29: 03 & 0.0075 & 0.0027 \\ 2524 & 10: 29: 05 & 0.0080 & 0.0142 \\ 2526 & 10: 29: 07 & 0.0130 & 0.0112 \\ 2528 & 10: 29: 09 & 0.0035 & 0.0106 \\ 2530 & 10: 29: 11 & 0.0271 & 0.0015 \\ 2532 & 10: 29: 13 & 0.0035 & 0.0019 \\ 2534 & 10: 29: 15 & 0.0143 & 0.0010 \\ 2536 & 10: 29: 17 & 0.0044 & 0.0020 \\ 2538 & 10: 29: 19 & 0.0041 & 0.0094 \\ 2540 & 10: 29: 21 & 0.0153 & 0.0059 \\ 2542 & 10: 29: 23 & 0.0026 & 0.0043 \\ 2544 & 10: 29: 25 & 0.0139 & 0.0131 \\ 2546 & 10: 29: 27 & 0.0016 & 0.0038\end{array}$


Appendix D - Laser Data Test 2 NIST102605_1.xls

$\begin{array}{llll}2548 & 10: 29: 29 & 0.0030 & 0.0331 \\ 2550 & 10: 29: 31 & 0.0089 & 0.0033 \\ 2552 & 10: 29: 33 & 0.0110 & 0.0043 \\ 2554 & 10: 29: 35 & 0.0093 & 0.0074 \\ 2556 & 10: 29: 37 & 0.0032 & 0.0146 \\ 2558 & 10: 29: 39 & 0.0171 & 0.0006 \\ 2560 & 10: 29: 41 & 0.0140 & 0.0026 \\ 2562 & 10: 29: 43 & 0.0087 & 0.0033 \\ 2564 & 10: 29: 45 & 0.0083 & 0.0036 \\ 2566 & 10: 29: 47 & 0.0031 & 0.0018 \\ 2568 & 10: 29: 49 & 0.0050 & 0.0032 \\ 2570 & 10: 29: 51 & 0.0113 & 0.0083 \\ 2572 & 10: 29: 53 & 0.0040 & 0.0125 \\ 2574 & 10: 29: 55 & 0.0080 & 0.0031 \\ 2576 & 10: 29: 57 & 0.0083 & 0.0029 \\ 2578 & 10: 29: 59 & 0.0006 & 0.0417 \\ 2580 & 10: 30: 01 & 0.0009 & 0.0024 \\ 2582 & 10: 30: 03 & 0.0020 & 0.0045 \\ 2584 & 10: 30: 05 & 0.0007 & 0.0375 \\ 2586 & 10: 30: 07 & 0.0062 & 0.0085 \\ 2588 & 10: 30: 09 & 0.0014 & 0.0440 \\ 2590 & 10: 30: 11 & 0.0025 & 0.0234 \\ 2592 & 10: 30: 13 & 0.0016 & 0.0128 \\ 2594 & 10: 30: 15 & 0.0016 & 0.0042 \\ 2596 & 10: 30: 17 & 0.0016 & 0.0152 \\ 2598 & 10: 30: 19 & 0.0015 & 0.0137 \\ 2600 & 10: 30: 21 & 0.0007 & 0.0044 \\ 2602 & 10: 30: 23 & 0.0030 & 0.0513 \\ 2604 & 10: 30: 25 & 0.0056 & 0.0133 \\ 2606 & 10: 30: 27 & 0.0057 & 0.0025 \\ 2608 & 10: 30: 29 & 0.0026 & 0.0057 \\ 2610 & 10: 30: 31 & 0.0072 & 0.0132 \\ 2612 & 10: 30: 33 & 0.0002 & 0.0064 \\ 2614 & 10: 30: 35 & 0.0060 & 0.0288 \\ 2616 & 10: 30: 37 & 0.0007 & 0.0115 \\ 2618 & 10: 30: 39 & 0.0023 & 0.0197 \\ 2620 & 10: 30: 41 & 0.0007 & 0.0055 \\ 2622 & 10: 30: 43 & 0.0012 & 0.0025 \\ 2624 & 10: 30: 45 & 0.0035 & 0.0387 \\ 2626 & 10: 30: 47 & 0.0007 & 0.0042 \\ 2628 & 10: 30: 49 & 0.0015 & 0.0067 \\ 2630 & 10: 30: 51 & 0.0151 & 0.0033 \\ 2632 & 10: 30: 53 & 0.0190 & 0.0062 \\ 2634 & 10: 30: 55 & 0.0092 & 0.0015 \\ 2636 & 10: 30: 57 & 0.0030 & 0.0062 \\ 2638 & 10: 30: 59 & 0.0024 & 0.0076 \\ 2640 & 10: 31: 01 & 0.0090 & 0.0086 \\ 2642 & 10: 31: 03 & 0.0083 & 0.0083 \\ 2644 & 10: 31: 05 & 0.0044 & 0.0054 \\ 2646 & 10: 31: 07 & 0.0130 & 0.0041 \\ 2648 & 10: 31: 09 & 0.0013 & 0.0047\end{array}$


Appendix D - Laser Data Test 2 NIST102605_1.xls

$\begin{array}{llll}2650 & 10: 31: 11 & 0.0014 & 0.0064 \\ 2652 & 10: 31: 13 & 0.0002 & 0.0135 \\ 2654 & 10: 31: 15 & 0.0081 & 0.0241 \\ 2656 & 10: 31: 17 & 0.0182 & 0.0042 \\ 2658 & 10: 31: 19 & 0.0007 & 0.0100 \\ 2660 & 10: 31: 21 & 0.0079 & 0.0085 \\ 2662 & 10: 31: 23 & 0.0025 & 0.0081 \\ 2664 & 10: 31: 25 & 0.0012 & 0.0075 \\ 2666 & 10: 31: 27 & 0.0014 & 0.0056 \\ 2668 & 10: 31: 29 & 0.0141 & 0.0032 \\ 2670 & 10: 31: 31 & 0.0039 & 0.0285 \\ 2672 & 10: 31: 33 & 0.0030 & 0.0204 \\ 2674 & 10: 31: 35 & 0.0063 & 0.0154 \\ 2676 & 10: 31: 37 & 0.0122 & 0.0018 \\ 2678 & 10: 31: 39 & 0.0022 & 0.0045 \\ 2680 & 10: 31: 41 & 0.0022 & 0.0088 \\ 2682 & 10: 31: 43 & 0.0022 & 0.0061 \\ 2684 & 10: 31: 45 & 0.0013 & 0.0174 \\ 2686 & 10: 31: 47 & 0.0038 & 0.0036 \\ 2688 & 10: 31: 49 & 0.0016 & 0.0032 \\ 2690 & 10: 31: 51 & 0.0018 & 0.0045 \\ 2692 & 10: 31: 53 & 0.0099 & 0.0089 \\ 2694 & 10: 31: 55 & 0.0063 & 0.0057 \\ 2696 & 10: 31: 57 & 0.0265 & 0.0096 \\ 2698 & 10: 31: 59 & 0.0006 & 0.0074 \\ 2700 & 10: 32: 01 & 0.0053 & 0.0085 \\ 2702 & 10: 32: 03 & 0.0004 & 0.0023 \\ 2704 & 10: 32: 05 & 0.0002 & 0.0035 \\ 2706 & 10: 32: 07 & 0.0037 & 0.0479 \\ 2708 & 10: 32: 09 & 0.0050 & 0.0046 \\ 2710 & 10: 32: 11 & 0.0016 & 0.0300 \\ 2712 & 10: 32: 13 & 0.0016 & 0.0059 \\ 2714 & 10: 32: 15 & 0.0072 & 0.0112 \\ 2716 & 10: 32: 17 & 0.0123 & 0.0041 \\ 2718 & 10: 32: 19 & 0.0069 & 0.0074 \\ 2720 & 10: 32: 21 & 0.0061 & 0.0632 \\ 2722 & 10: 32: 23 & 0.0054 & 0.0183 \\ 2724 & 10: 32: 25 & 0.0004 & 0.0113 \\ 2726 & 10: 32: 27 & 0.0059 & 0.0040 \\ 2728 & 10: 32: 29 & 0.0013 & 0.0040 \\ 2730 & 10: 32: 31 & 0.0012 & 0.0292 \\ 2732 & 10: 32: 33 & 0.0043 & 0.0121 \\ 2734 & 10: 32: 35 & 0.0031 & 0.0072 \\ 2736 & 10: 32: 37 & 0.0015 & 0.0020 \\ 2738 & 10: 32: 39 & 0.0011 & 0.0047 \\ 2740 & 10: 32: 41 & 0.0050 & 0.0047 \\ 2742 & 10: 32: 43 & 0.0009 & 0.0028 \\ 2744 & 10: 32: 45 & 0.0030 & 0.0021 \\ 2746 & 10: 32: 47 & 0.0009 & 0.0151 \\ 2748 & 10: 32: 49 & 0.0004 & 0.0066 \\ 2750 & 10: 32: 51 & 0.0101 & 0.0014\end{array}$


Appendix D - Laser Data Test 2 NIST102605_1.xls

$\begin{array}{llll}2752 & 10: 32: 53 & 0.0037 & 0.0114 \\ 2754 & 10: 32: 55 & 0.0153 & 0.0006 \\ 2756 & 10: 32: 57 & 0.0049 & 0.0172 \\ 2758 & 10: 32: 59 & 0.0059 & 0.0151 \\ 2760 & 10: 33: 01 & 0.0017 & 0.0024 \\ 2762 & 10: 33: 03 & 0.0001 & 0.0029 \\ 2764 & 10: 33: 05 & 0.0014 & 0.0044 \\ 2766 & 10: 33: 07 & 0.0003 & 0.0033 \\ 2768 & 10: 33: 09 & 0.0021 & 0.0126 \\ 2770 & 10: 33: 11 & 0.0080 & 0.0075 \\ 2772 & 10: 33: 13 & 0.0006 & 0.0054 \\ 2774 & 10: 33: 15 & 0.0170 & 0.0249 \\ 2776 & 10: 33: 17 & 0.0032 & 0.0142 \\ 2778 & 10: 33: 19 & 0.0021 & 0.0129 \\ 2780 & 10: 33: 21 & 0.0113 & 0.0122 \\ 2782 & 10: 33: 23 & 0.0064 & 0.0062 \\ 2784 & 10: 33: 25 & 0.0002 & 0.0035 \\ 2786 & 10: 33: 27 & 0.0101 & 0.0043 \\ 2788 & 10: 33: 29 & 0.0011 & 0.0057 \\ 2790 & 10: 33: 31 & 0.0055 & 0.0026 \\ 2792 & 10: 33: 33 & 0.0050 & 0.0164 \\ 2794 & 10: 33: 35 & 0.0014 & 0.0058 \\ 2796 & 10: 33: 37 & 0.0014 & 0.0023 \\ 2798 & 10: 33: 39 & 0.0032 & 0.0089 \\ 2800 & 10: 33: 41 & 0.0071 & 0.0066 \\ 2802 & 10: 33: 43 & 0.0064 & 0.0395 \\ 2804 & 10: 33: 45 & 0.0034 & 0.0012 \\ 2806 & 10: 33: 47 & 0.0097 & 0.0208 \\ 2808 & 10: 33: 49 & 0.0053 & 0.0151 \\ 2810 & 10: 33: 51 & 0.0012 & 0.0081 \\ 2812 & 10: 33: 53 & 0.0048 & 0.0643 \\ 2814 & 10: 33: 55 & 0.0003 & 0.0158 \\ 2816 & 10: 33: 57 & 0.0010 & 0.0032 \\ 2818 & 10: 33: 59 & 0.0059 & 0.0009 \\ 2820 & 10: 34: 01 & 0.0104 & 0.0038 \\ 2822 & 10: 34: 03 & 0.0124 & 0.0261 \\ 2824 & 10: 34: 05 & 0.0052 & 0.0011 \\ 2826 & 10: 34: 07 & 0.0045 & 0.0028 \\ 2828 & 10: 34: 09 & 0.0213 & 0.0021 \\ 2830 & 10: 34: 11 & 0.0066 & 0.0019 \\ 2832 & 10: 34: 13 & 0.0021 & 0.0031 \\ 2834 & 10: 34: 15 & 0.0107 & 0.0114 \\ 2836 & 10: 34: 17 & 0.0073 & 0.0013 \\ 2838 & 10: 34: 19 & 0.0026 & 0.0129 \\ 2840 & 10: 34: 21 & 0.0006 & 0.0227 \\ 2842 & 10: 34: 23 & 0.0221 & 0.0208 \\ 2844 & 10: 34: 25 & 0.0012 & 0.0093 \\ 2846 & 10: 34: 27 & 0.0032 & 0.0130 \\ 2848 & 10: 34: 29 & 0.0007 & 0.0143 \\ 2850 & 10: 34: 31 & 0.0341 & 0.0062 \\ 2852 & 10: 34: 33 & 0.0032 & 0.0068\end{array}$


Appendix D - Laser Data Test 2 NIST102605_1.xls

$\begin{array}{llll}2854 & 10: 34: 35 & 0.0021 & 0.0197 \\ 2856 & 10: 34: 37 & 0.0068 & 0.0053 \\ 2858 & 10: 34: 39 & 0.0031 & 0.0056 \\ 2860 & 10: 34: 41 & 0.0026 & 0.0024 \\ 2862 & 10: 34: 43 & 0.0021 & 0.0124 \\ 2864 & 10: 34: 45 & 0.0000 & 0.0105 \\ 2866 & 10: 34: 47 & 0.0141 & 0.0130 \\ 2868 & 10: 34: 49 & 0.0026 & 0.0084 \\ 2870 & 10: 34: 51 & 0.0008 & 0.0077 \\ 2872 & 10: 34: 53 & 0.0002 & 0.0021 \\ 2874 & 10: 34: 55 & 0.0022 & 0.0025 \\ 2876 & 10: 34: 57 & 0.0079 & 0.0027 \\ 2878 & 10: 34: 59 & 0.0012 & 0.0029 \\ 2880 & 10: 35: 01 & 0.0017 & 0.0113 \\ 2882 & 10: 35: 03 & 0.0045 & 0.0210 \\ 2884 & 10: 35: 05 & 0.0044 & 0.0187 \\ 2886 & 10: 35: 07 & 0.0017 & 0.0237 \\ 2888 & 10: 35: 09 & 0.0012 & 0.0020 \\ 2890 & 10: 35: 11 & 0.0009 & 0.0031 \\ 2892 & 10: 35: 13 & 0.0018 & 0.0179 \\ 2894 & 10: 35: 15 & 0.0017 & 0.0031 \\ 2896 & 10: 35: 17 & 0.0098 & 0.0103 \\ 2898 & 10: 35: 19 & 0.0007 & 0.0118 \\ 2900 & 10: 35: 21 & 0.0030 & 0.0103 \\ 2902 & 10: 35: 23 & 0.0060 & 0.0091 \\ 2904 & 10: 35: 25 & 0.0026 & 0.0055 \\ 2906 & 10: 35: 27 & 0.0011 & 0.0115 \\ 2908 & 10: 35: 29 & 0.0086 & 0.0042 \\ 2910 & 10: 35: 31 & 0.0032 & 0.0019 \\ 2912 & 10: 35: 33 & 0.0046 & 0.0007 \\ 2914 & 10: 35: 35 & 0.0031 & 0.0055 \\ 2916 & 10: 35: 37 & 0.0012 & 0.0022 \\ 2918 & 10: 35: 39 & 0.0007 & 0.0032 \\ 2920 & 10: 35: 41 & 0.0026 & 0.0105 \\ 2922 & 10: 35: 43 & 0.0018 & 0.0075 \\ 2924 & 10: 35: 45 & 0.0055 & 0.0058 \\ 2926 & 10: 35: 47 & 0.0002 & 0.0055 \\ 2928 & 10: 35: 49 & 0.0022 & 0.0035 \\ 2930 & 10: 35: 51 & 0.0023 & 0.0075 \\ 2932 & 10: 35: 53 & 0.0042 & 0.0036 \\ 2934 & 10: 35: 55 & 0.0008 & 0.0055 \\ 2936 & 10: 35: 57 & 0.0067 & 0.0012 \\ 2938 & 10: 35: 59 & 0.0005 & 0.0031 \\ 2940 & 10: 36: 01 & 0.0017 & 0.0110 \\ 2942 & 10: 36: 03 & 0.0002 & 0.0033 \\ 2944 & 10: 36: 05 & 0.0026 & 0.0374 \\ 2946 & 10: 36: 07 & 0.0064 & 0.0014 \\ 2948 & 10: 36: 09 & 0.0066 & 0.0180 \\ 2950 & 10: 36: 11 & 0.0144 & 0.0522 \\ 2952 & 10: 36: 13 & 0.0235 & 0.0006 \\ 2954 & 10: 36: 15 & 0.0071 & 0.0130\end{array}$


Appendix D - Laser Data Test 2 NIST102605_1.xls

$\begin{array}{llll}2956 & 10: 36: 17 & 0.0009 & 0.0044 \\ 2958 & 10: 36: 19 & 0.0094 & 0.0013 \\ 2960 & 10: 36: 21 & 0.0040 & 0.0105 \\ 2962 & 10: 36: 23 & 0.0012 & 0.0025 \\ 2964 & 10: 36: 25 & 0.0017 & 0.0051 \\ 2966 & 10: 36: 27 & 0.0075 & 0.0226 \\ 2968 & 10: 36: 29 & 0.0026 & 0.0177 \\ 2970 & 10: 36: 31 & 0.0016 & 0.0246 \\ 2972 & 10: 36: 33 & 0.0003 & 0.0043 \\ 2974 & 10: 36: 35 & 0.0133 & 0.0140 \\ 2976 & 10: 36: 37 & 0.0039 & 0.0049 \\ 2978 & 10: 36: 39 & 0.0005 & 0.0015 \\ 2980 & 10: 36: 41 & 0.0106 & 0.0535 \\ 2982 & 10: 36: 43 & 0.0022 & 0.0033 \\ 2984 & 10: 36: 45 & 0.0017 & 0.0084 \\ 2986 & 10: 36: 47 & 0.0048 & 0.0070 \\ 2988 & 10: 36: 49 & 0.0046 & 0.0065 \\ 2990 & 10: 36: 51 & 0.0109 & 0.0475 \\ 2992 & 10: 36: 53 & 0.0049 & 0.0359 \\ 2994 & 10: 36: 55 & 0.0120 & 0.0043 \\ 2996 & 10: 36: 57 & 0.0024 & 0.0029 \\ 2998 & 10: 36: 59 & 0.0004 & 0.0080 \\ 3000 & 10: 37: 01 & 0.0011 & 0.0113 \\ 3002 & 10: 37: 03 & 0.0116 & 0.0094 \\ 3004 & 10: 37: 05 & 0.0005 & 0.0145 \\ 3006 & 10: 37: 07 & 0.0017 & 0.0099 \\ 3008 & 10: 37: 09 & 0.0046 & 0.0034 \\ 3010 & 10: 37: 11 & 0.0086 & 0.0062 \\ 3012 & 10: 37: 13 & 0.0042 & 0.0283 \\ 3014 & 10: 37: 15 & 0.0099 & 0.0184 \\ 3016 & 10: 37: 17 & 0.0018 & 0.0150 \\ 3018 & 10: 37: 19 & 0.0017 & 0.0178 \\ 3020 & 10: 37: 21 & 0.0062 & 0.0532 \\ 3022 & 10: 37: 23 & 0.0041 & 0.0054 \\ 3024 & 10: 37: 25 & 0.0012 & 0.0248 \\ 3026 & 10: 37: 27 & 0.0044 & 0.0114 \\ 3028 & 10: 37: 29 & 0.0018 & 0.0069 \\ 3030 & 10: 37: 31 & 0.0009 & 0.0136 \\ 3032 & 10: 37: 33 & 0.0012 & 0.0062 \\ 3034 & 10: 37: 35 & 0.0117 & 0.0014 \\ 3036 & 10: 37: 37 & 0.0082 & 0.0011 \\ 3038 & 10: 37: 39 & 0.0077 & 0.0209 \\ 3040 & 10: 37: 41 & 0.0024 & 0.0007 \\ 3042 & 10: 37: 43 & 0.0012 & 0.0091 \\ 3044 & 10: 37: 45 & 0.0010 & 0.0007 \\ 3046 & 10: 37: 47 & 0.0014 & 0.0011 \\ 3048 & 10: 37: 49 & 0.0091 & 0.0187 \\ 3050 & 10: 37: 51 & 0.0058 & 0.0056 \\ 3052 & 10: 37: 53 & 0.0003 & 0.0089 \\ 3054 & 10: 37: 55 & 0.0003 & 0.0117 \\ 3056 & 10: 37: 57 & 0.0014 & 0.0050\end{array}$


Appendix D - Laser Data Test 2 NIST102605_1.xls

$\begin{array}{llll}3058 & 10: 37: 59 & 0.0017 & 0.0051 \\ 3060 & 10: 38: 01 & 0.0011 & 0.0106 \\ 3062 & 10: 38: 03 & 0.0005 & 0.0005 \\ 3064 & 10: 38: 05 & 0.0141 & 0.0023 \\ 3066 & 10: 38: 07 & 0.0003 & 0.0060 \\ 3068 & 10: 38: 09 & 0.0066 & 0.0003 \\ 3070 & 10: 38: 11 & 0.0042 & 0.0061 \\ 3072 & 10: 38: 13 & 0.0028 & 0.0028 \\ 3074 & 10: 38: 15 & 0.0084 & 0.0239 \\ 3076 & 10: 38: 17 & 0.0007 & 0.0019 \\ 3078 & 10: 38: 19 & 0.0052 & 0.0539 \\ 3080 & 10: 38: 21 & 0.0017 & 0.0013 \\ 3082 & 10: 38: 23 & 0.0204 & 0.0029 \\ 3084 & 10: 38: 25 & 0.0201 & 0.0010 \\ 3086 & 10: 38: 27 & 0.0015 & 0.0007 \\ 3088 & 10: 38: 29 & 0.0140 & 0.0004 \\ 3090 & 10: 38: 31 & 0.0115 & 0.0019 \\ 3092 & 10: 38: 33 & 0.0071 & 0.0016 \\ 3094 & 10: 38: 35 & 0.0016 & 0.0276 \\ 3096 & 10: 38: 37 & 0.0006 & 0.0094 \\ 3098 & 10: 38: 39 & 0.0008 & 0.0159 \\ 3100 & 10: 38: 41 & 0.0010 & 0.0063 \\ 3102 & 10: 38: 43 & 0.0027 & 0.0066 \\ 3104 & 10: 38: 45 & 0.0007 & 0.0041 \\ 3106 & 10: 38: 47 & 0.0017 & 0.0009 \\ 3108 & 10: 38: 49 & 0.0054 & 0.0036 \\ 3110 & 10: 38: 51 & 0.0237 & 0.0037 \\ 3112 & 10: 38: 53 & 0.0070 & 0.0037 \\ 3114 & 10: 38: 55 & 0.0022 & 0.0109 \\ 3116 & 10: 38: 57 & 0.0024 & 0.0038 \\ 3118 & 10: 38: 59 & 0.0030 & 0.0053 \\ 3120 & 10: 39: 01 & 0.0012 & 0.0547 \\ 3122 & 10: 39: 03 & 0.0003 & 0.0102 \\ 3124 & 10: 39: 05 & 0.0025 & 0.0048 \\ 3126 & 10: 39: 07 & 0.0045 & 0.0053 \\ 3128 & 10: 39: 09 & 0.0028 & 0.0281 \\ 3130 & 10: 39: 11 & 0.0046 & 0.0028 \\ 3132 & 10: 39: 13 & 0.0027 & 0.0083 \\ 3134 & 10: 39: 15 & 0.0138 & 0.0143 \\ 3136 & 10: 39: 17 & 0.0064 & 0.0031 \\ 3138 & 10: 39: 19 & 0.0012 & 0.0069 \\ 3140 & 10: 39: 21 & 0.0011 & 0.0005 \\ 3142 & 10: 39: 23 & 0.0125 & 0.0168 \\ 3144 & 10: 39: 25 & 0.0017 & 0.0030 \\ 3146 & 10: 39: 27 & 0.0032 & 0.0134 \\ 3148 & 10: 39: 29 & 0.0044 & 0.0028 \\ 3150 & 10: 39: 31 & 0.0051 & 0.0166 \\ 3152 & 10: 39: 33 & 0.0072 & 0.0026 \\ 3154 & 10: 39: 35 & 0.0109 & 0.0116 \\ 3156 & 10: 39: 37 & 0.0011 & 0.0057 \\ 3158 & 10: 39: 39 & 0.0034 & 0.0062\end{array}$


Appendix D - Laser Data Test 2 NIST102605_1.xls

$\begin{array}{llll}3160 & 10: 39: 41 & 0.0041 & 0.0039 \\ 3162 & 10: 39: 43 & 0.0059 & 0.0075 \\ 3164 & 10: 39: 45 & 0.0075 & 0.0143 \\ 3166 & 10: 39: 47 & 0.0026 & 0.0034 \\ 3168 & 10: 39: 49 & 0.0047 & 0.0276 \\ 3170 & 10: 39: 51 & 0.0078 & 0.0054 \\ 3172 & 10: 39: 53 & 0.0033 & 0.0062 \\ 3174 & 10: 39: 55 & 0.0050 & 0.0033 \\ 3176 & 10: 39: 57 & 0.0072 & 0.0024 \\ 3178 & 10: 39: 59 & 0.0112 & 0.0313 \\ 3180 & 10: 40: 01 & 0.0009 & 0.0027 \\ 3182 & 10: 40: 03 & 0.0043 & 0.0376 \\ 3184 & 10: 40: 05 & 0.0025 & 0.0128 \\ 3186 & 10: 40: 07 & 0.0030 & 0.0046 \\ 3188 & 10: 40: 09 & 0.0063 & 0.0480 \\ 3190 & 10: 40: 11 & 0.0025 & 0.0042 \\ 3192 & 10: 40: 13 & 0.0057 & 0.0623 \\ 3194 & 10: 40: 15 & 0.0045 & 0.0105 \\ 3196 & 10: 40: 17 & 0.0014 & 0.0069 \\ 3198 & 10: 40: 19 & 0.0005 & 0.0093 \\ 3200 & 10: 40: 21 & 0.0088 & 0.0020 \\ 3202 & 10: 40: 23 & 0.0058 & 0.0088 \\ 3204 & 10: 40: 25 & 0.0018 & 0.0073 \\ 3206 & 10: 40: 27 & 0.0014 & 0.0050 \\ 3208 & 10: 40: 29 & 0.0069 & 0.0106 \\ 3210 & 10: 40: 31 & 0.0060 & 0.0089 \\ 3212 & 10: 40: 33 & 0.0127 & 0.0030 \\ 3214 & 10: 40: 35 & 0.0066 & 0.0043 \\ 3216 & 10: 40: 37 & 0.0013 & 0.0140 \\ 3218 & 10: 40: 39 & 0.0030 & 0.0161 \\ 3220 & 10: 40: 41 & 0.0016 & 0.0024 \\ 3222 & 10: 40: 43 & 0.0038 & 0.0002 \\ 3224 & 10: 40: 45 & 0.0038 & 0.0017 \\ 3226 & 10: 40: 47 & 0.0154 & 0.0024 \\ 3228 & 10: 40: 49 & 0.0024 & 0.0010 \\ 3230 & 10: 40: 51 & 0.0071 & 0.0018 \\ 3232 & 10: 40: 53 & 0.0047 & 0.0023 \\ 3234 & 10: 40: 55 & 0.0010 & 0.0128 \\ 3236 & 10: 40: 57 & 0.0041 & 0.0064 \\ 3238 & 10: 40: 59 & 0.0221 & 0.0088 \\ 3240 & 10: 41: 01 & 0.0013 & 0.0054 \\ 3242 & 10: 41: 03 & 0.0063 & 0.0028 \\ 3244 & 10: 41: 05 & 0.0058 & 0.0141 \\ 3246 & 10: 41: 07 & 0.0073 & 0.0015 \\ 3248 & 10: 41: 09 & 0.0068 & 0.0052 \\ 3250 & 10: 41: 11 & 0.0076 & 0.0068 \\ 3252 & 10: 41: 13 & 0.0103 & 0.0023 \\ 3254 & 10: 41: 15 & 0.0070 & 0.0033 \\ 3256 & 10: 41: 17 & 0.0028 & 0.0029 \\ 3258 & 10: 41: 19 & 0.0015 & 0.0010 \\ 3260 & 10: 41: 21 & 0.0009 & 0.0011\end{array}$


Appendix D - Laser Data Test 2 NIST102605_1.xls

$\begin{array}{llll}3262 & 10: 41: 23 & 0.0018 & 0.0024 \\ 3264 & 10: 41: 25 & 0.0044 & 0.0006 \\ 3266 & 10: 41: 27 & 0.0077 & 0.0033 \\ 3268 & 10: 41: 29 & 0.0126 & 0.0026 \\ 3270 & 10: 41: 31 & 0.0056 & 0.0168 \\ 3272 & 10: 41: 33 & 0.0013 & 0.0041 \\ 3274 & 10: 41: 35 & 0.0159 & 0.0021 \\ 3276 & 10: 41: 37 & 0.0081 & 0.0074 \\ 3278 & 10: 41: 39 & 0.0077 & 0.0004 \\ 3280 & 10: 41: 41 & 0.0010 & 0.0014 \\ 3282 & 10: 41: 43 & 0.0013 & 0.0060 \\ 3284 & 10: 41: 45 & 0.0108 & 0.0063 \\ 3286 & 10: 41: 47 & 0.0166 & 0.0032 \\ 3288 & 10: 41: 49 & 0.0182 & 0.0146 \\ 3290 & 10: 41: 51 & 0.0028 & 0.0068 \\ 3292 & 10: 41: 53 & 0.0015 & 0.0040 \\ 3294 & 10: 41: 55 & 0.0008 & 0.0008 \\ 3296 & 10: 41: 57 & 0.0035 & 0.0027 \\ 3298 & 10: 41: 59 & 0.0206 & 0.0019 \\ 3300 & 10: 42: 01 & 0.0213 & 0.0100 \\ 3302 & 10: 42: 03 & 0.0097 & 0.0098 \\ 3304 & 10: 42: 05 & 0.0048 & 0.0034 \\ 3306 & 10: 42: 07 & 0.0059 & 0.0061 \\ 3308 & 10: 42: 09 & 0.0111 & 0.0102 \\ 3310 & 10: 42: 11 & 0.0145 & 0.0120 \\ 3312 & 10: 42: 13 & 0.0086 & 0.0059 \\ 3314 & 10: 42: 15 & 0.0015 & 0.0050 \\ 3316 & 10: 42: 17 & 0.0032 & 0.0072 \\ 3318 & 10: 42: 19 & 0.0030 & 0.0024 \\ 3320 & 10: 42: 21 & 0.0053 & 0.0109 \\ 3322 & 10: 42: 23 & 0.0013 & 0.0106 \\ 3324 & 10: 42: 25 & 0.0061 & 0.0191 \\ 3326 & 10: 42: 27 & 0.0016 & 0.0058 \\ 3328 & 10: 42: 29 & 0.0126 & 0.0109 \\ 3330 & 10: 42: 31 & 0.0025 & 0.0052 \\ 3332 & 10: 42: 33 & 0.0005 & 0.0181 \\ 3334 & 10: 42: 35 & 0.0043 & 0.0058 \\ 3336 & 10: 42: 37 & 0.0032 & 0.0042 \\ 3338 & 10: 42: 39 & 0.0033 & 0.0141 \\ 3340 & 10: 42: 41 & 0.0008 & 0.0036 \\ 3342 & 10: 42: 43 & 0.0011 & 0.0059 \\ 3344 & 10: 42: 45 & 0.0018 & 0.0011 \\ 3346 & 10: 42: 47 & 0.0134 & 0.0023 \\ 3348 & 10: 42: 49 & 0.0191 & 0.0073 \\ 3350 & 10: 42: 51 & 0.0178 & 0.0062 \\ 3352 & 10: 42: 53 & 0.0231 & 0.0208 \\ 3354 & 10: 42: 55 & 0.0059 & 0.0020 \\ 3356 & 10: 42: 57 & 0.0213 & 0.0098 \\ 3358 & 10: 42: 59 & 0.0105 & 0.0173 \\ 3360 & 10: 43: 01 & 0.0004 & 0.0034 \\ 3362 & 10: 43: 03 & 0.0024 & 0.0104\end{array}$


Appendix D - Laser Data Test 2 NIST102605_1.xls

$\begin{array}{llll}3364 & 10: 43: 05 & 0.0058 & 0.0011 \\ 3366 & 10: 43: 07 & 0.0060 & 0.0043 \\ 3368 & 10: 43: 09 & 0.0049 & 0.0199 \\ 3370 & 10: 43: 11 & 0.0004 & 0.0083 \\ 3372 & 10: 43: 13 & 0.0025 & 0.0046 \\ 3374 & 10: 43: 15 & 0.0030 & 0.0015 \\ 3376 & 10: 43: 17 & 0.0105 & 0.0015 \\ 3378 & 10: 43: 19 & 0.0015 & 0.0020 \\ 3380 & 10: 43: 21 & 0.0076 & 0.0036 \\ 3382 & 10: 43: 23 & 0.0128 & 0.0016 \\ 3384 & 10: 43: 25 & 0.0010 & 0.0095 \\ 3386 & 10: 43: 27 & 0.0004 & 0.0059 \\ 3388 & 10: 43: 29 & 0.0012 & 0.0111 \\ 3390 & 10: 43: 31 & 0.0066 & 0.0142 \\ 3392 & 10: 43: 33 & 0.0036 & 0.0094 \\ 3394 & 10: 43: 35 & 0.0014 & 0.0146 \\ 3396 & 10: 43: 37 & 0.0125 & 0.0094 \\ 3398 & 10: 43: 39 & 0.0075 & 0.0011 \\ 3400 & 10: 43: 41 & 0.0068 & 0.0022 \\ 3402 & 10: 43: 43 & 0.0024 & 0.0060 \\ 3404 & 10: 43: 45 & 0.0075 & 0.0046 \\ 3406 & 10: 43: 47 & 0.0075 & 0.0029 \\ 3408 & 10: 43: 49 & 0.0007 & 0.0029 \\ 3410 & 10: 43: 51 & 0.0121 & 0.0606 \\ 3412 & 10: 43: 53 & 0.0121 & 0.0021 \\ 3414 & 10: 43: 55 & 0.0098 & 0.0179 \\ 3416 & 10: 43: 57 & 0.0034 & 0.0259 \\ 3418 & 10: 43: 59 & 0.0032 & 0.0011 \\ 3420 & 10: 44: 01 & 0.0068 & 0.0016 \\ 3422 & 10: 44: 03 & 0.0039 & 0.0034 \\ 3424 & 10: 44: 05 & 0.0066 & 0.0058 \\ 3426 & 10: 44: 07 & 0.0143 & 0.0027 \\ 3428 & 10: 44: 09 & 0.0110 & 0.0064 \\ 3430 & 10: 44: 11 & 0.0134 & 0.0146 \\ 3432 & 10: 44: 13 & 0.0032 & 0.0529 \\ 3434 & 10: 44: 15 & 0.0120 & 0.0032 \\ 3436 & 10: 44: 17 & 0.0077 & 0.0152 \\ 3438 & 10: 44: 19 & 0.0027 & 0.0052 \\ 3440 & 10: 44: 21 & 0.0021 & 0.0050 \\ 3442 & 10: 44: 23 & 0.0005 & 0.0441 \\ 3444 & 10: 44: 25 & 0.0013 & 0.0021 \\ 3446 & 10: 44: 27 & 0.0003 & 0.0075 \\ 3448 & 10: 44: 29 & 0.0029 & 0.0422 \\ 3450 & 10: 44: 31 & 0.0179 & 0.0118 \\ 3452 & 10: 44: 33 & 0.0293 & 0.0098 \\ 3454 & 10: 44: 35 & 0.0089 & 0.0008 \\ 3456 & 10: 44: 37 & 0.0038 & 0.0160 \\ 3458 & 10: 44: 39 & 0.0055 & 0.0002 \\ 3460 & 10: 44: 41 & 0.0051 & 0.0113 \\ 3462 & 10: 44: 43 & 0.0007 & 0.0089 \\ 3464 & 10: 44: 45 & 0.0132 & 0.0015\end{array}$

D - 34 
Appendix D - Laser Data Test 2 NIST102605_1.xls

$\begin{array}{llll}3466 & 10: 44: 47 & 0.0085 & 0.0290 \\ 3468 & 10: 44: 49 & 0.0060 & 0.0077 \\ 3470 & 10: 44: 51 & 0.0029 & 0.0065 \\ 3472 & 10: 44: 53 & 0.0019 & 0.0033 \\ 3474 & 10: 44: 55 & 0.0031 & 0.0134 \\ 3476 & 10: 44: 57 & 0.0062 & 0.0144 \\ 3478 & 10: 44: 59 & 0.0014 & 0.0336 \\ 3480 & 10: 45: 01 & 0.0068 & 0.0017 \\ 3482 & 10: 45: 03 & 0.0021 & 0.0108 \\ 3484 & 10: 45: 05 & 0.0003 & 0.0007 \\ 3486 & 10: 45: 07 & 0.0292 & 0.0441 \\ 3488 & 10: 45: 09 & 0.0005 & 0.0029 \\ 3490 & 10: 45: 11 & 0.0081 & 0.0156 \\ 3492 & 10: 45: 13 & 0.0032 & 0.0127 \\ 3494 & 10: 45: 15 & 0.0105 & 0.0203 \\ 3496 & 10: 45: 17 & 0.0101 & 0.0192 \\ 3498 & 10: 45: 19 & 0.0043 & 0.0016 \\ 3500 & 10: 45: 21 & 0.0017 & 0.0005 \\ 3502 & 10: 45: 23 & 0.0039 & 0.0023 \\ 3504 & 10: 45: 25 & 0.0058 & 0.0029 \\ 3506 & 10: 45: 27 & 0.0007 & 0.0054 \\ 3508 & 10: 45: 29 & 0.0110 & 0.0015 \\ 3510 & 10: 45: 31 & 0.0026 & 0.0228 \\ 3512 & 10: 45: 33 & 0.0013 & 0.0070 \\ 3514 & 10: 45: 35 & 0.0027 & 0.0043 \\ 3516 & 10: 45: 37 & 0.0011 & 0.0112 \\ 3518 & 10: 45: 39 & 0.0055 & 0.0077 \\ 3520 & 10: 45: 41 & 0.0029 & 0.0074 \\ 3522 & 10: 45: 43 & 0.0004 & 0.0183 \\ 3524 & 10: 45: 45 & 0.0024 & 0.0041 \\ 3526 & 10: 45: 47 & 0.0021 & 0.0063 \\ 3528 & 10: 45: 49 & 0.0112 & 0.0023 \\ 3530 & 10: 45: 51 & 0.0008 & 0.0021 \\ 3532 & 10: 45: 53 & 0.0024 & 0.0073 \\ 3534 & 10: 45: 55 & 0.0055 & 0.0073 \\ 3536 & 10: 45: 57 & 0.0094 & 0.0269 \\ 3538 & 10: 45: 59 & 0.0159 & 0.0036 \\ 3540 & 10: 46: 01 & 0.0006 & 0.0096 \\ 3542 & 10: 46: 03 & 0.0013 & 0.0038 \\ 3544 & 10: 46: 05 & 0.0116 & 0.0125 \\ 3546 & 10: 46: 07 & 0.0203 & 0.0021 \\ 3548 & 10: 46: 09 & 0.0070 & 0.0031 \\ 3550 & 10: 46: 11 & 0.0243 & 0.0032 \\ 3552 & 10: 46: 13 & 0.1015 & 0.0081 \\ 3554 & 10: 46: 15 & 0.0065 & 0.0072 \\ 3556 & 10: 46: 17 & 0.0077 & 0.0015 \\ 3558 & 10: 46: 19 & 0.0034 & 0.0018 \\ 3560 & 10: 46: 21 & 0.0046 & 0.0111 \\ 3562 & 10: 46: 23 & 0.0028 & 0.0005 \\ 3564 & 10: 46: 25 & 0.0018 & 0.0020 \\ 3566 & 10: 46: 27 & 0.0020 & 0.0020\end{array}$


Appendix D - Laser Data Test 2 NIST102605_1.xls

$\begin{array}{llll}3568 & 10: 46: 29 & 0.0006 & 0.0043 \\ 3570 & 10: 46: 31 & 0.0020 & 0.0035 \\ 3572 & 10: 46: 33 & 0.0010 & 0.0070 \\ 3574 & 10: 46: 35 & 0.0003 & 0.0090 \\ 3576 & 10: 46: 37 & 0.0087 & 0.0183 \\ 3578 & 10: 46: 39 & 0.0006 & 0.0308 \\ 3580 & 10: 46: 41 & 0.0135 & 0.0059 \\ 3582 & 10: 46: 43 & 0.0066 & 0.0026 \\ 3584 & 10: 46: 45 & 0.0008 & 0.0204 \\ 3586 & 10: 46: 47 & 0.0037 & 0.0051 \\ 3588 & 10: 46: 49 & 0.0029 & 0.0170 \\ 3590 & 10: 46: 51 & 0.0058 & 0.0101 \\ 3592 & 10: 46: 53 & 0.0023 & 0.0182 \\ 3594 & 10: 46: 55 & 0.0046 & 0.0058 \\ 3596 & 10: 46: 57 & 0.0052 & 0.0130 \\ 3598 & 10: 46: 59 & 0.0138 & 0.0002 \\ 3600 & 10: 47: 01 & 0.0060 & 0.0010 \\ 3602 & 10: 47: 03 & 0.0114 & 0.0116 \\ 3604 & 10: 47: 05 & 0.0329 & 0.0028 \\ 3606 & 10: 47: 07 & 0.0068 & 0.0011 \\ 3608 & 10: 47: 09 & 0.0104 & 0.0008 \\ 3610 & 10: 47: 11 & 0.0140 & 0.0017 \\ 3612 & 10: 47: 13 & 0.0034 & 0.0041 \\ 3614 & 10: 47: 15 & 0.0129 & 0.0002 \\ 3616 & 10: 47: 17 & 0.0024 & 0.0064 \\ 3618 & 10: 47: 19 & 0.0010 & 0.0012 \\ 3620 & 10: 47: 21 & 0.0174 & 0.0012 \\ 3622 & 10: 47: 23 & 0.0286 & 0.0069 \\ 3624 & 10: 47: 25 & 0.0024 & 0.0166 \\ 3626 & 10: 47: 27 & 0.0179 & 0.0021 \\ 3628 & 10: 47: 29 & 0.0008 & 0.0110 \\ 3630 & 10: 47: 31 & 0.0019 & 0.0166 \\ 3632 & 10: 47: 33 & 0.0006 & 0.0510 \\ 3634 & 10: 47: 35 & 0.0024 & 0.0350 \\ 3636 & 10: 47: 37 & 0.0176 & 0.0606 \\ 3638 & 10: 47: 39 & 0.0030 & 0.0176 \\ 3640 & 10: 47: 41 & 0.0037 & 0.0040 \\ 3642 & 10: 47: 43 & 0.0015 & 0.0137 \\ 3644 & 10: 47: 45 & 0.0007 & 0.0249 \\ 3646 & 10: 47: 47 & 0.0004 & 0.0028 \\ 3648 & 10: 47: 49 & 0.0004 & 0.0465 \\ 3650 & 10: 47: 51 & 0.0014 & 0.0002 \\ 3652 & 10: 47: 53 & 0.0020 & 0.0048 \\ 3654 & 10: 47: 55 & 0.0031 & 0.0012 \\ 3656 & 10: 47: 57 & 0.0021 & 0.0241 \\ 3658 & 10: 47: 59 & 0.0014 & 0.0054 \\ 3660 & 10: 48: 01 & 0.0021 & 0.0020 \\ 3662 & 10: 48: 03 & 0.0072 & 0.0015 \\ 3664 & 10: 48: 05 & 0.0023 & 0.0038 \\ 3666 & 10: 48: 07 & 0.0068 & 0.0134 \\ 3668 & 10: 48: 09 & 0.0446 & 0.0253\end{array}$


Appendix D - Laser Data Test 2 NIST102605_1.xls

$\begin{array}{llll}3670 & 10: 48: 11 & 0.0083 & 0.0017 \\ 3672 & 10: 48: 13 & 0.0022 & 0.0006 \\ 3674 & 10: 48: 15 & 0.0086 & 0.0071 \\ 3676 & 10: 48: 17 & 0.0315 & 0.0082 \\ 3678 & 10: 48: 19 & 0.0022 & 0.0025 \\ 3680 & 10: 48: 21 & 0.0040 & 0.0011 \\ 3682 & 10: 48: 23 & 0.0050 & 0.0033 \\ 3684 & 10: 48: 25 & 0.0060 & 0.0038 \\ 3686 & 10: 48: 27 & 0.0256 & 0.0032 \\ 3688 & 10: 48: 29 & 0.0065 & 0.0097 \\ 3690 & 10: 48: 31 & 0.0186 & 0.0042 \\ 3692 & 10: 48: 33 & 0.0082 & 0.0082 \\ 3694 & 10: 48: 35 & 0.0009 & 0.0054 \\ 3696 & 10: 48: 37 & 0.0009 & 0.0031 \\ 3698 & 10: 48: 39 & 0.0232 & 0.0108 \\ 3700 & 10: 48: 41 & 0.0013 & 0.0058 \\ 3702 & 10: 48: 43 & 0.0008 & 0.0080 \\ 3704 & 10: 48: 45 & 0.0011 & 0.0053 \\ 3706 & 10: 48: 47 & 0.0068 & 0.0121 \\ 3708 & 10: 48: 49 & 0.0026 & 0.0091 \\ 3710 & 10: 48: 51 & 0.0023 & 0.0034 \\ 3712 & 10: 48: 53 & 0.0019 & 0.0105 \\ 3714 & 10: 48: 55 & 0.0018 & 0.0727 \\ 3716 & 10: 48: 57 & 0.0127 & 0.0106 \\ 3718 & 10: 48: 59 & 0.0013 & 0.0043 \\ 3720 & 10: 49: 01 & 0.0070 & 0.0390 \\ 3722 & 10: 49: 03 & 0.0001 & 0.0343 \\ 3724 & 10: 49: 05 & 0.0024 & 0.0370 \\ 3726 & 10: 49: 07 & 0.0002 & 0.0091 \\ 3728 & 10: 49: 09 & 0.0052 & 0.0815 \\ 3730 & 10: 49: 11 & 0.0014 & 0.0039 \\ 3732 & 10: 49: 13 & 0.0010 & 0.0209 \\ 3734 & 10: 49: 15 & 0.0044 & 0.0021 \\ 3736 & 10: 49: 17 & 0.0006 & 0.0247 \\ 3738 & 10: 49: 19 & 0.0009 & 0.0201 \\ 3740 & 10: 49: 21 & 0.0008 & 0.0060 \\ 3742 & 10: 49: 23 & 0.0034 & 0.0118 \\ 3744 & 10: 49: 25 & 0.0051 & 0.0208 \\ 3746 & 10: 49: 27 & 0.0282 & 0.0033 \\ 3748 & 10: 49: 29 & 0.0036 & 0.0138 \\ 3750 & 10: 49: 31 & 0.0065 & 0.0091 \\ 3752 & 10: 49: 33 & 0.0084 & 0.0064 \\ 3754 & 10: 49: 35 & 0.0068 & 0.0038 \\ 3756 & 10: 49: 37 & 0.0112 & 0.0076 \\ 3758 & 10: 49: 39 & 0.0365 & 0.0066 \\ 3760 & 10: 49: 41 & 0.0020 & 0.0077 \\ 3762 & 10: 49: 43 & 0.0030 & 0.0192 \\ 3764 & 10: 49: 45 & 0.0016 & 0.0092 \\ 3766 & 10: 49: 47 & 0.0059 & 0.0051 \\ 3768 & 10: 49: 49 & 0.0088 & 0.0001 \\ 3770 & 10: 49: 51 & 0.0082 & 0.0005\end{array}$


Appendix D - Laser Data Test 2 NIST102605_1.xls

$\begin{array}{llll}3772 & 10: 49: 53 & 0.0106 & 0.0024 \\ 3774 & 10: 49: 55 & 0.0567 & 0.0017 \\ 3776 & 10: 49: 57 & 0.0050 & 0.0027 \\ 3778 & 10: 49: 59 & 0.0056 & 0.0057 \\ 3780 & 10: 50: 01 & 0.0098 & 0.0057 \\ 3782 & 10: 50: 03 & 0.0008 & 0.0011 \\ 3784 & 10: 50: 05 & 0.0001 & 0.0013 \\ 3786 & 10: 50: 07 & 0.0033 & 0.0020 \\ 3788 & 10: 50: 09 & 0.0046 & 0.0200 \\ 3790 & 10: 50: 11 & 0.0002 & 0.0074 \\ 3792 & 10: 50: 13 & 0.0523 & 0.0317 \\ 3794 & 10: 50: 15 & 0.0048 & 0.0061 \\ 3796 & 10: 50: 17 & 0.0121 & 0.0078 \\ 3798 & 10: 50: 19 & 0.0336 & 0.0036 \\ 3800 & 10: 50: 21 & 0.0003 & 0.0199 \\ 3802 & 10: 50: 23 & 0.0022 & 0.0282 \\ 3804 & 10: 50: 25 & 0.0041 & 0.0103 \\ 3806 & 10: 50: 27 & 0.0224 & 0.0017 \\ 3808 & 10: 50: 29 & 0.0046 & 0.0124 \\ 3810 & 10: 50: 31 & 0.0181 & 0.0053 \\ 3812 & 10: 50: 33 & 0.0003 & 0.0007 \\ 3814 & 10: 50: 35 & 0.0025 & 0.0078 \\ 3816 & 10: 50: 37 & 0.0063 & 0.0156 \\ 3818 & 10: 50: 39 & 0.0003 & 0.0029 \\ 3820 & 10: 50: 41 & 0.0014 & 0.0937 \\ 3822 & 10: 50: 43 & 0.0015 & 0.0014 \\ 3824 & 10: 50: 45 & 0.0010 & 0.0017 \\ 3826 & 10: 50: 47 & 0.0012 & 0.0363 \\ 3828 & 10: 50: 49 & 0.0060 & 0.0283 \\ 3830 & 10: 50: 51 & 0.0010 & 0.0109 \\ 3832 & 10: 50: 53 & 0.0070 & 0.0208 \\ 3834 & 10: 50: 55 & 0.0062 & 0.0158 \\ 3836 & 10: 50: 57 & 0.0069 & 0.0327 \\ 3838 & 10: 50: 59 & 0.0138 & 0.0636 \\ 3840 & 10: 51: 01 & 0.0030 & 0.0056 \\ 3842 & 10: 51: 03 & 0.0071 & 0.0343 \\ 3844 & 10: 51: 05 & 0.0008 & 0.0252 \\ 3846 & 10: 51: 07 & 0.0043 & 0.0041 \\ 3848 & 10: 51: 09 & 0.0034 & 0.0078 \\ 3850 & 10: 51: 11 & 0.0019 & 0.0025 \\ 3852 & 10: 51: 13 & 0.0010 & 0.0136 \\ 3854 & 10: 51: 15 & 0.0004 & 0.0086 \\ 3856 & 10: 51: 17 & 0.0022 & 0.0130 \\ 3858 & 10: 51: 19 & 0.0025 & 0.0095 \\ 3860 & 10: 51: 21 & 0.0092 & 0.0176 \\ 3862 & 10: 51: 23 & 0.0124 & 0.0098 \\ 3864 & 10: 51: 25 & 0.0017 & 0.0043 \\ 3866 & 10: 51: 27 & 0.0039 & 0.0186 \\ 3868 & 10: 51: 29 & 0.0042 & 0.0112 \\ 3870 & 10: 51: 31 & 0.0030 & 0.0048 \\ 3872 & 10: 51: 33 & 0.0158 & 0.0002\end{array}$


Appendix D - Laser Data Test 2 NIST102605_1.xls

$\begin{array}{llll}3874 & 10: 51: 35 & 0.0486 & 0.0011 \\ 3876 & 10: 51: 37 & 0.0120 & 0.0014 \\ 3878 & 10: 51: 39 & 0.0170 & 0.0033 \\ 3880 & 10: 51: 41 & 0.0097 & 0.0114 \\ 3882 & 10: 51: 43 & 0.0197 & 0.0016 \\ 3884 & 10: 51: 45 & 0.0352 & 0.0074 \\ 3886 & 10: 51: 47 & 0.0075 & 0.0020 \\ 3888 & 10: 51: 49 & 0.0108 & 0.0012 \\ 3890 & 10: 51: 51 & 0.0066 & 0.0069 \\ 3892 & 10: 51: 53 & 0.0170 & 0.0014 \\ 3894 & 10: 51: 55 & 0.0085 & 0.0045 \\ 3896 & 10: 51: 57 & 0.0034 & 0.0052 \\ 3898 & 10: 51: 59 & 0.0089 & 0.0034 \\ 3900 & 10: 52: 01 & 0.0042 & 0.0034 \\ 3902 & 10: 52: 03 & 0.0060 & 0.0046 \\ 3904 & 10: 52: 05 & 0.0062 & 0.0029 \\ 3906 & 10: 52: 07 & 0.0090 & 0.0031 \\ 3908 & 10: 52: 09 & 0.0047 & 0.0053 \\ 3910 & 10: 52: 11 & 0.0042 & 0.0555 \\ 3912 & 10: 52: 13 & 0.0279 & 0.0058 \\ 3914 & 10: 52: 15 & 0.0016 & 0.0080 \\ 3916 & 10: 52: 17 & 0.0092 & 0.0020 \\ 3918 & 10: 52: 19 & 0.0014 & 0.0014 \\ 3920 & 10: 52: 21 & 0.0016 & 0.0017 \\ 3922 & 10: 52: 23 & 0.0053 & 0.0154 \\ 3924 & 10: 52: 25 & 0.0024 & 0.0101 \\ 3926 & 10: 52: 27 & 0.0014 & 0.0006 \\ 3928 & 10: 52: 29 & 0.0052 & 0.0122 \\ 3930 & 10: 52: 31 & 0.0004 & 0.0040 \\ 3932 & 10: 52: 33 & 0.0014 & 0.0074 \\ 3934 & 10: 52: 35 & 0.0013 & 0.0069 \\ 3936 & 10: 52: 37 & 0.0021 & 0.0024 \\ 3938 & 10: 52: 39 & 0.0009 & 0.0195 \\ 3940 & 10: 52: 41 & 0.0042 & 0.0166 \\ 3942 & 10: 52: 43 & 0.0093 & 0.0027 \\ 3944 & 10: 52: 45 & 0.0005 & 0.0179 \\ 3946 & 10: 52: 47 & 0.0084 & 0.0036 \\ 3948 & 10: 52: 49 & 0.0014 & 0.0048 \\ 3950 & 10: 52: 51 & 0.0008 & 0.0147 \\ 3952 & 10: 52: 53 & 0.0013 & 0.0182 \\ 3954 & 10: 52: 55 & 0.0023 & 0.0034 \\ 3956 & 10: 52: 57 & 0.0031 & 0.0065 \\ 3958 & 10: 52: 59 & 0.0025 & 0.0015 \\ 3960 & 10: 53: 01 & 0.0145 & 0.0013 \\ 3962 & 10: 53: 03 & 0.0017 & 0.0010 \\ 3964 & 10: 53: 05 & 0.0055 & 0.0026 \\ 3966 & 10: 53: 07 & 0.0025 & 0.0057 \\ 3968 & 10: 53: 09 & 0.0023 & 0.0018 \\ 3970 & 10: 53: 11 & 0.0080 & 0.0055 \\ 3972 & 10: 53: 13 & 0.0070 & 0.0044 \\ 3974 & 10: 53: 15 & 0.0006 & 0.0150\end{array}$


Appendix D - Laser Data Test 2 NIST102605_1.xls

$\begin{array}{llll}3976 & 10: 53: 17 & 0.0007 & 0.0017 \\ 3978 & 10: 53: 19 & 0.0010 & 0.0082 \\ 3980 & 10: 53: 21 & 0.0030 & 0.0041 \\ 3982 & 10: 53: 23 & 0.0179 & 0.0338 \\ 3984 & 10: 53: 25 & 0.0025 & 0.0037 \\ 3986 & 10: 53: 27 & 0.0153 & 0.0024 \\ 3988 & 10: 53: 29 & 0.0086 & 0.0016 \\ 3990 & 10: 53: 31 & 0.0008 & 0.0020 \\ 3992 & 10: 53: 33 & 0.0003 & 0.0124 \\ 3994 & 10: 53: 35 & 0.0020 & 0.0047 \\ 3996 & 10: 53: 37 & 0.0014 & 0.0056 \\ 3998 & 10: 53: 39 & 0.0089 & 0.0058 \\ 4000 & 10: 53: 41 & 0.0135 & 0.0007 \\ 4002 & 10: 53: 43 & 0.0143 & 0.0057 \\ 4004 & 10: 53: 45 & 0.0009 & 0.0036 \\ 4006 & 10: 53: 47 & 0.0030 & 0.0057 \\ 4008 & 10: 53: 49 & 0.0044 & 0.0108 \\ 4010 & 10: 53: 51 & 0.0106 & 0.0176 \\ 4012 & 10: 53: 53 & 0.0093 & 0.0824 \\ 4014 & 10: 53: 55 & 0.0080 & 0.0244 \\ 4016 & 10: 53: 57 & 0.0047 & 0.0138 \\ 4018 & 10: 53: 59 & 0.0028 & 0.0012 \\ 4020 & 10: 54: 01 & 0.0017 & 0.0034 \\ 4022 & 10: 54: 03 & 0.0020 & 0.0130 \\ 4024 & 10: 54: 05 & 0.0087 & 0.0753 \\ 4026 & 10: 54: 07 & 0.0097 & 0.0041 \\ 4028 & 10: 54: 09 & 0.0018 & 0.0144 \\ 4030 & 10: 54: 11 & 0.0208 & 0.0056 \\ 4032 & 10: 54: 13 & 0.0037 & 0.0137 \\ 4034 & 10: 54: 15 & 0.0083 & 0.0065 \\ 4036 & 10: 54: 17 & 0.0050 & 0.0056 \\ 4038 & 10: 54: 19 & 0.0004 & 0.0123 \\ 4040 & 10: 54: 21 & 0.0024 & 0.0761 \\ 4042 & 10: 54: 23 & 0.0004 & 0.0064 \\ 4044 & 10: 54: 25 & 0.0016 & 0.0234 \\ 4046 & 10: 54: 27 & 0.0011 & 0.0203 \\ 4048 & 10: 54: 29 & 0.0010 & 0.0188 \\ 4050 & 10: 54: 31 & 0.0023 & 0.0088 \\ 4052 & 10: 54: 33 & 0.0099 & 0.0038 \\ 4054 & 10: 54: 35 & 0.0010 & 0.0211 \\ 4056 & 10: 54: 37 & 0.0003 & 0.0070 \\ 4058 & 10: 54: 39 & 0.0043 & 0.0270 \\ 4060 & 10: 54: 41 & 0.0048 & 0.0101 \\ 4062 & 10: 54: 43 & 0.0020 & 0.0011 \\ 4064 & 10: 54: 45 & 0.0085 & 0.0142 \\ 4066 & 10: 54: 47 & 0.0006 & 0.0055 \\ 4068 & 10: 54: 49 & 0.0136 & 0.0030 \\ 4070 & 10: 54: 51 & 0.0069 & 0.0167 \\ 4072 & 10: 54: 53 & 0.0075 & 0.0049 \\ 4074 & 10: 54: 55 & 0.0260 & 0.0297 \\ 4076 & 10: 54: 57 & 0.0018 & 0.0038\end{array}$


Appendix D - Laser Data Test 2 NIST102605_1.xls

$\begin{array}{llll}4078 & 10: 54: 59 & 0.0020 & 0.0142 \\ 4080 & 10: 55: 01 & 0.0083 & 0.0091 \\ 4082 & 10: 55: 03 & 0.0004 & 0.0047 \\ 4084 & 10: 55: 05 & 0.0025 & 0.0061 \\ 4086 & 10: 55: 07 & 0.0051 & 0.0162 \\ 4088 & 10: 55: 09 & 0.0020 & 0.0050 \\ 4090 & 10: 55: 11 & 0.0040 & 0.0019 \\ 4092 & 10: 55: 13 & 0.0005 & 0.0011 \\ 4094 & 10: 55: 15 & 0.0029 & 0.0067 \\ 4096 & 10: 55: 17 & 0.0002 & 0.0070 \\ 4098 & 10: 55: 19 & 0.0009 & 0.0103 \\ 4100 & 10: 55: 21 & 0.0055 & 0.0029 \\ 4102 & 10: 55: 23 & 0.0020 & 0.0038 \\ 4104 & 10: 55: 25 & 0.0004 & 0.0286 \\ 4106 & 10: 55: 27 & 0.0147 & 0.0090 \\ 4108 & 10: 55: 29 & 0.0076 & 0.0050 \\ 4110 & 10: 55: 31 & 0.0049 & 0.0029 \\ 4112 & 10: 55: 33 & 0.0029 & 0.0112 \\ 4114 & 10: 55: 35 & 0.0013 & 0.0351 \\ 4116 & 10: 55: 37 & 0.0030 & 0.0054 \\ 4118 & 10: 55: 39 & 0.0056 & 0.0049 \\ 4120 & 10: 55: 41 & 0.0026 & 0.0101 \\ 4122 & 10: 55: 43 & 0.0018 & 0.0027 \\ 4124 & 10: 55: 45 & 0.0007 & 0.0069 \\ 4126 & 10: 55: 47 & 0.0009 & 0.0032 \\ 4128 & 10: 55: 49 & 0.0019 & 0.0037 \\ 4130 & 10: 55: 51 & 0.0052 & 0.0029 \\ 4132 & 10: 55: 53 & 0.0047 & 0.0058 \\ 4134 & 10: 55: 55 & 0.0030 & 0.0288 \\ 4136 & 10: 55: 57 & 0.0014 & 0.0312 \\ 4138 & 10: 55: 59 & 0.0073 & 0.0593 \\ 4140 & 10: 56: 01 & 0.0036 & 0.0071 \\ 4142 & 10: 56: 03 & 0.0093 & 0.0038 \\ 4144 & 10: 56: 05 & 0.0016 & 0.0267 \\ 4146 & 10: 56: 07 & 0.0087 & 0.0156 \\ 4148 & 10: 56: 09 & 0.0052 & 0.0044 \\ 4150 & 10: 56: 11 & 0.0021 & 0.0007 \\ 4152 & 10: 56: 13 & 0.0141 & 0.0032 \\ 4154 & 10: 56: 15 & 0.0028 & 0.0020 \\ 4156 & 10: 56: 17 & 0.0030 & 0.0109 \\ 4158 & 10: 56: 19 & 0.0099 & 0.0043 \\ 4160 & 10: 56: 21 & 0.0141 & 0.0025 \\ 4162 & 10: 56: 23 & 0.0016 & 0.0021 \\ 4164 & 10: 56: 25 & 0.0125 & 0.0165 \\ 4166 & 10: 56: 27 & 0.0010 & 0.0161 \\ 4168 & 10: 56: 29 & 0.0003 & 0.0032 \\ 4170 & 10: 56: 31 & 0.0074 & 0.0019 \\ 4172 & 10: 56: 33 & 0.0022 & 0.0071 \\ 4174 & 10: 56: 35 & 0.0015 & 0.0031 \\ 4176 & 10: 56: 37 & 0.0153 & 0.0037 \\ 4178 & 10: 56: 39 & 0.0024 & 0.0017\end{array}$


Appendix D - Laser Data Test 2 NIST102605_1.xls

$\begin{array}{llll}4180 & 10: 56: 41 & 0.0042 & 0.0065 \\ 4182 & 10: 56: 43 & 0.0190 & 0.0124 \\ 4184 & 10: 56: 45 & 0.0054 & 0.0013 \\ 4186 & 10: 56: 47 & 0.0133 & 0.0023 \\ 4188 & 10: 56: 49 & 0.0169 & 0.0014 \\ 4190 & 10: 56: 51 & 0.0028 & 0.0009 \\ 4192 & 10: 56: 53 & 0.0541 & 0.0052 \\ 4194 & 10: 56: 55 & 0.0095 & 0.0062 \\ 4196 & 10: 56: 57 & 0.0100 & 0.0387 \\ 4198 & 10: 56: 59 & 0.0006 & 0.0059 \\ 4200 & 10: 57: 01 & 0.0026 & 0.0155 \\ 4202 & 10: 57: 03 & 0.0030 & 0.0001 \\ 4204 & 10: 57: 05 & 0.0026 & 0.0032 \\ 4206 & 10: 57: 07 & 0.0027 & 0.0020 \\ 4208 & 10: 57: 09 & 0.0010 & 0.0064 \\ 4210 & 10: 57: 11 & 0.0044 & 0.0043 \\ 4212 & 10: 57: 13 & 0.0005 & 0.0174 \\ 4214 & 10: 57: 15 & 0.0012 & 0.0014 \\ 4216 & 10: 57: 17 & 0.0069 & 0.0156 \\ 4218 & 10: 57: 19 & 0.0021 & 0.0520 \\ 4220 & 10: 57: 21 & 0.0079 & 0.0070 \\ 4222 & 10: 57: 23 & 0.0021 & 0.0259 \\ 4224 & 10: 57: 25 & 0.0025 & 0.0161 \\ 4226 & 10: 57: 27 & 0.0061 & 0.0033 \\ 4228 & 10: 57: 29 & 0.0261 & 0.0093 \\ 4230 & 10: 57: 31 & 0.0012 & 0.0011 \\ 4232 & 10: 57: 33 & 0.0081 & 0.0052 \\ 4234 & 10: 57: 35 & 0.0026 & 0.0170 \\ 4236 & 10: 57: 37 & 0.0070 & 0.0033 \\ 4238 & 10: 57: 39 & 0.0153 & 0.0041 \\ 4240 & 10: 57: 41 & 0.0128 & 0.0251 \\ 4242 & 10: 57: 43 & 0.0046 & 0.0282 \\ 4244 & 10: 57: 45 & 0.0162 & 0.0030 \\ 4246 & 10: 57: 47 & 0.0012 & 0.0016 \\ 4248 & 10: 57: 49 & 0.0032 & 0.0030 \\ 4250 & 10: 57: 51 & 0.0002 & 0.0097 \\ 4252 & 10: 57: 53 & 0.0027 & 0.0057 \\ 4254 & 10: 57: 55 & 0.0144 & 0.0328 \\ 4256 & 10: 57: 57 & 0.0037 & 0.0022 \\ 4258 & 10: 57: 59 & 0.0046 & 0.0025 \\ 4260 & 10: 58: 01 & 0.0068 & 0.0075 \\ 4262 & 10: 58: 03 & 0.0152 & 0.0308 \\ 4264 & 10: 58: 05 & 0.0024 & 0.0048 \\ 4266 & 10: 58: 07 & 0.0148 & 0.0128 \\ 4268 & 10: 58: 09 & 0.0027 & 0.0029 \\ 4270 & 10: 58: 11 & 0.0014 & 0.0033 \\ 4272 & 10: 58: 13 & 0.0099 & 0.0165 \\ 4274 & 10: 58: 15 & 0.0097 & 0.0004 \\ 4276 & 10: 58: 17 & 0.0027 & 0.0051 \\ 4278 & 10: 58: 19 & 0.0001 & 0.0008 \\ 4280 & 10: 58: 21 & 0.0086 & 0.0058\end{array}$


Appendix D - Laser Data Test 2 NIST102605_1.xls

$\begin{array}{llll}4282 & 10: 58: 23 & 0.0176 & 0.0056 \\ 4284 & 10: 58: 25 & 0.0016 & 0.0053 \\ 4286 & 10: 58: 27 & 0.0067 & 0.0040 \\ 4288 & 10: 58: 29 & 0.0002 & 0.0107 \\ 4290 & 10: 58: 31 & 0.0059 & 0.0047 \\ 4292 & 10: 58: 33 & 0.0020 & 0.0102 \\ 4294 & 10: 58: 35 & 0.0124 & 0.0071 \\ 4296 & 10: 58: 37 & 0.0473 & 0.0009 \\ 4298 & 10: 58: 39 & 0.0100 & 0.0066 \\ 4300 & 10: 58: 41 & 0.0090 & 0.0009 \\ 4302 & 10: 58: 43 & 0.0011 & 0.0023 \\ 4304 & 10: 58: 45 & 0.0014 & 0.0027 \\ 4306 & 10: 58: 47 & 0.0009 & 0.0064 \\ 4308 & 10: 58: 49 & 0.0009 & 0.0007 \\ 4310 & 10: 58: 51 & 0.0104 & 0.0013 \\ 4312 & 10: 58: 53 & 0.0172 & 0.0018 \\ 4314 & 10: 58: 55 & 0.0015 & 0.0021 \\ 4316 & 10: 58: 57 & 0.0045 & 0.0011 \\ 4318 & 10: 58: 59 & 0.0059 & 0.0052 \\ 4320 & 10: 59: 01 & 0.0006 & 0.0066 \\ 4322 & 10: 59: 03 & 0.0033 & 0.0049 \\ 4324 & 10: 59: 05 & 0.0054 & 0.0011 \\ 4326 & 10: 59: 07 & 0.0093 & 0.0055 \\ 4328 & 10: 59: 09 & 0.0009 & 0.0011 \\ 4330 & 10: 59: 11 & 0.0008 & 0.0034 \\ 4332 & 10: 59: 13 & 0.0125 & 0.0015 \\ 4334 & 10: 59: 15 & 0.0015 & 0.0300 \\ 4336 & 10: 59: 17 & 0.0059 & 0.0050 \\ 4338 & 10: 59: 19 & 0.0010 & 0.0130 \\ 4340 & 10: 59: 21 & 0.0014 & 0.0010 \\ 4342 & 10: 59: 23 & 0.0058 & 0.0015 \\ 4344 & 10: 59: 25 & 0.0053 & 0.0102 \\ 4346 & 10: 59: 27 & 0.0066 & 0.0218 \\ 4348 & 10: 59: 29 & 0.0021 & 0.0053 \\ 4350 & 10: 59: 31 & 0.0040 & 0.0137 \\ 4352 & 10: 59: 33 & 0.0027 & 0.0021 \\ 4354 & 10: 59: 35 & 0.0014 & 0.0076 \\ 4356 & 10: 59: 37 & 0.0051 & 0.0071 \\ 4358 & 10: 59: 39 & 0.0039 & 0.0007 \\ 4360 & 10: 59: 41 & 0.0071 & 0.0064 \\ 4362 & 10: 59: 43 & 0.0049 & 0.0132 \\ 4364 & 10: 59: 45 & 0.0268 & 0.0002 \\ 4366 & 10: 59: 47 & 0.0075 & 0.0011 \\ 4368 & 10: 59: 49 & 0.0023 & 0.0053 \\ 4370 & 10: 59: 51 & 0.0224 & 0.0007 \\ 4372 & 10: 59: 53 & 0.0282 & 0.0075 \\ 4374 & 10: 59: 55 & 0.0298 & 0.0015 \\ 4376 & 10: 59: 57 & 0.0255 & 0.0006 \\ 4378 & 10: 59: 59 & 0.0021 & 0.0056 \\ 4380 & 11: 00: 01 & 0.0075 & 0.0011 \\ 4382 & 11: 00: 03 & 0.0027 & 0.0112\end{array}$


Appendix D - Laser Data Test 2 NIST102605_1.xls

$\begin{array}{llll}4384 & 11: 00: 05 & 0.0183 & 0.0040 \\ 4386 & 11: 00: 07 & 0.0091 & 0.0046 \\ 4388 & 11: 00: 09 & 0.0021 & 0.0013 \\ 4390 & 11: 00: 11 & 0.0641 & 0.0068 \\ 4392 & 11: 00: 13 & 0.0105 & 0.0012 \\ 4394 & 11: 00: 15 & 0.0018 & 0.0008 \\ 4396 & 11: 00: 17 & 0.0041 & 0.0017 \\ 4398 & 11: 00: 19 & 0.0045 & 0.0223 \\ 4400 & 11: 00: 21 & 0.0203 & 0.0054 \\ 4402 & 11: 00: 23 & 0.0047 & 0.0038 \\ 4404 & 11: 00: 25 & 0.0026 & 0.0007 \\ 4406 & 11: 00: 27 & 0.0068 & 0.0093 \\ 4408 & 11: 00: 29 & 0.0244 & 0.0008 \\ 4410 & 11: 00: 31 & 0.0125 & 0.0225 \\ 4412 & 11: 00: 33 & 0.0094 & 0.0089 \\ 4414 & 11: 00: 35 & 0.0238 & 0.0026 \\ 4416 & 11: 00: 37 & 0.0106 & 0.0025 \\ 4418 & 11: 00: 39 & 0.0062 & 0.0497 \\ 4420 & 11: 00: 41 & 0.0220 & 0.0039 \\ 4422 & 11: 00: 43 & 0.0137 & 0.0043 \\ 4424 & 11: 00: 45 & 0.0015 & 0.0352 \\ 4426 & 11: 00: 47 & 0.0052 & 0.0577 \\ 4428 & 11: 00: 49 & 0.0018 & 0.0014 \\ 4430 & 11: 00: 51 & 0.0115 & 0.0042 \\ 4432 & 11: 00: 53 & 0.0097 & 0.0060 \\ 4434 & 11: 00: 55 & 0.0015 & 0.0034 \\ 4436 & 11: 00: 57 & 0.0180 & 0.0131 \\ 4438 & 11: 00: 59 & 0.0058 & 0.0078 \\ 4440 & 11: 01: 01 & 0.0319 & 0.0322 \\ 4442 & 11: 01: 03 & 0.0068 & 0.0219 \\ 4444 & 11: 01: 05 & 0.0065 & 0.0141 \\ 4446 & 11: 01: 07 & 0.0105 & 0.0052 \\ 4448 & 11: 01: 09 & 0.0051 & 0.0302 \\ 4450 & 11: 01: 11 & 0.0054 & 0.0130 \\ 4452 & 11: 01: 13 & 0.0026 & 0.0025 \\ 4454 & 11: 01: 15 & 0.0037 & 0.0117 \\ 4456 & 11: 01: 17 & 0.0036 & 0.0096 \\ 4458 & 11: 01: 19 & 0.0058 & 0.0096 \\ 4460 & 11: 01: 21 & 0.0079 & 0.0033 \\ 4462 & 11: 01: 23 & 0.0094 & 0.0025 \\ 4464 & 11: 01: 25 & 0.0053 & 0.0029 \\ 4466 & 11: 01: 27 & 0.0484 & 0.0502 \\ 4468 & 11: 01: 29 & 0.0541 & 0.0048 \\ 4470 & 11: 01: 31 & 0.0157 & 0.0007 \\ 4472 & 11: 01: 33 & 0.0089 & 0.0062 \\ 4474 & 11: 01: 35 & 0.0012 & 0.0010 \\ 4476 & 11: 01: 37 & 0.0698 & 0.0008 \\ 4478 & 11: 01: 39 & 0.0057 & 0.0019 \\ 4480 & 11: 01: 41 & 0.0089 & 0.0020 \\ 4482 & 11: 01: 43 & 0.0165 & 0.0048 \\ 4484 & 11: 01: 45 & 0.0241 & 0.0058\end{array}$


Appendix D - Laser Data Test 2 NIST102605_1.xls

$\begin{array}{llll}4486 & 11: 01: 47 & 0.0335 & 0.0021 \\ 4488 & 11: 01: 49 & 0.0090 & 0.0025 \\ 4490 & 11: 01: 51 & 0.0081 & 0.0056 \\ 4492 & 11: 01: 53 & 0.0022 & 0.0094 \\ 4494 & 11: 01: 55 & 0.0043 & 0.0012 \\ 4496 & 11: 01: 57 & 0.0172 & 0.0037 \\ 4498 & 11: 01: 59 & 0.0089 & 0.0061 \\ 4500 & 11: 02: 01 & 0.0120 & 0.0026 \\ 4502 & 11: 02: 03 & 0.0046 & 0.0009 \\ 4504 & 11: 02: 05 & 0.0077 & 0.0087 \\ 4506 & 11: 02: 07 & 0.0201 & 0.0103 \\ 4508 & 11: 02: 09 & 0.0071 & 0.0088 \\ 4510 & 11: 02: 11 & 0.0222 & 0.0149 \\ 4512 & 11: 02: 13 & 0.0099 & 0.0016 \\ 4514 & 11: 02: 15 & 0.0325 & 0.0237 \\ 4516 & 11: 02: 17 & 0.0213 & 0.0009 \\ 4518 & 11: 02: 19 & 0.0065 & 0.0039 \\ 4520 & 11: 02: 21 & 0.0090 & 0.0309 \\ 4522 & 11: 02: 23 & 0.0248 & 0.0009 \\ 4524 & 11: 02: 25 & 0.0201 & 0.0039 \\ 4526 & 11: 02: 27 & 0.0078 & 0.0067 \\ 4528 & 11: 02: 29 & 0.0141 & 0.0013 \\ 4530 & 11: 02: 31 & 0.0024 & 0.0048 \\ 4532 & 11: 02: 33 & 0.0070 & 0.0082 \\ 4534 & 11: 02: 35 & 0.0179 & 0.0158 \\ 4536 & 11: 02: 37 & 0.0270 & 0.0089 \\ 4538 & 11: 02: 39 & 0.0071 & 0.0043 \\ 4540 & 11: 02: 41 & 0.0065 & 0.0130 \\ 4542 & 11: 02: 43 & 0.0246 & 0.0022 \\ 4544 & 11: 02: 45 & 0.0047 & 0.0338 \\ 4546 & 11: 02: 47 & 0.0023 & 0.0039 \\ 4548 & 11: 02: 49 & 0.0008 & 0.0008 \\ 4550 & 11: 02: 51 & 0.0136 & 0.0071 \\ 4552 & 11: 02: 53 & 0.0010 & 0.0019 \\ 4554 & 11: 02: 55 & 0.0038 & 0.0009 \\ 4556 & 11: 02: 57 & 0.0002 & 0.0013 \\ 4558 & 11: 02: 59 & 0.0039 & 0.0148 \\ 4560 & 11: 03: 01 & 0.0019 & 0.0025 \\ 4562 & 11: 03: 03 & 0.0045 & 0.0038 \\ 4564 & 11: 03: 05 & 0.0182 & 0.0055 \\ 4566 & 11: 03: 07 & 0.0043 & 0.0011 \\ 4568 & 11: 03: 09 & 0.0015 & 0.0011 \\ 4570 & 11: 03: 11 & 0.0033 & 0.0135 \\ 4572 & 11: 03: 13 & 0.0076 & 0.0053 \\ 4574 & 11: 03: 15 & 0.0017 & 0.0024 \\ 4576 & 11: 03: 17 & 0.0111 & 0.0110 \\ 4578 & 11: 03: 19 & 0.0111 & 0.0015 \\ 4580 & 11: 03: 21 & 0.0066 & 0.0066 \\ 4582 & 11: 03: 23 & 0.0189 & 0.0011 \\ 4584 & 11: 03: 25 & 0.0062 & 0.0012 \\ 4586 & 11: 03: 27 & 0.0466 & 0.0068\end{array}$


Appendix D - Laser Data Test 2 NIST102605_1.xls

$\begin{array}{llll}4588 & 11: 03: 29 & 0.0328 & 0.0011 \\ 4590 & 11: 03: 31 & 0.0051 & 0.0062 \\ 4592 & 11: 03: 33 & 0.0090 & 0.0027 \\ 4594 & 11: 03: 35 & 0.0044 & 0.0065 \\ 4596 & 11: 03: 37 & 0.0024 & 0.0038 \\ 4598 & 11: 03: 39 & 0.0041 & 0.0030 \\ 4600 & 11: 03: 41 & 0.0034 & 0.0059 \\ 4602 & 11: 03: 43 & 0.0153 & 0.0135 \\ 4604 & 11: 03: 45 & 0.0298 & 0.0221 \\ 4606 & 11: 03: 47 & 0.0091 & 0.0036 \\ 4608 & 11: 03: 49 & 0.0053 & 0.0243 \\ 4610 & 11: 03: 51 & 0.0034 & 0.0045 \\ 4612 & 11: 03: 53 & 0.0010 & 0.0159 \\ 4614 & 11: 03: 55 & 0.0023 & 0.0017 \\ 4616 & 11: 03: 57 & 0.0069 & 0.0154 \\ 4618 & 11: 03: 59 & 0.0191 & 0.0039 \\ 4620 & 11: 04: 01 & 0.0040 & 0.0053 \\ 4622 & 11: 04: 03 & 0.0036 & 0.0012 \\ 4624 & 11: 04: 05 & 0.0287 & 0.0036 \\ 4626 & 11: 04: 07 & 0.0091 & 0.0037 \\ 4628 & 11: 04: 09 & 0.0017 & 0.0078 \\ 4630 & 11: 04: 11 & 0.0012 & 0.0165 \\ 4632 & 11: 04: 13 & 0.0046 & 0.0029 \\ 4634 & 11: 04: 15 & 0.0029 & 0.0233 \\ 4636 & 11: 04: 17 & 0.0068 & 0.0177 \\ 4638 & 11: 04: 19 & 0.0005 & 0.0324 \\ 4640 & 11: 04: 21 & 0.0022 & 0.0085 \\ 4642 & 11: 04: 23 & 0.0086 & 0.0108 \\ 4644 & 11: 04: 25 & 0.0052 & 0.0205 \\ 4646 & 11: 04: 27 & 0.0017 & 0.0025 \\ 4648 & 11: 04: 29 & 0.0178 & 0.0035 \\ 4650 & 11: 04: 31 & 0.0115 & 0.0053 \\ 4652 & 11: 04: 33 & 0.0019 & 0.0025 \\ 4654 & 11: 04: 35 & 0.0048 & 0.0394 \\ 4656 & 11: 04: 37 & 0.0077 & 0.0030 \\ 4658 & 11: 04: 39 & 0.0037 & 0.0047 \\ 4660 & 11: 04: 41 & 0.0007 & 0.0058 \\ 4662 & 11: 04: 43 & 0.0112 & 0.0130 \\ 4664 & 11: 04: 45 & 0.0371 & 0.0069 \\ 4666 & 11: 04: 47 & 0.0279 & 0.0141 \\ 4668 & 11: 04: 49 & 0.0005 & 0.0320 \\ 4670 & 11: 04: 51 & 0.0077 & 0.0078 \\ 4672 & 11: 04: 53 & 0.0042 & 0.0241 \\ 4674 & 11: 04: 55 & 0.0035 & 0.0052 \\ 4676 & 11: 04: 57 & 0.0025 & 0.0014 \\ 4678 & 11: 04: 59 & 0.0026 & 0.0005 \\ 4680 & 11: 05: 01 & 0.0219 & 0.0042 \\ 4682 & 11: 05: 03 & 0.0008 & 0.0072 \\ 4684 & 11: 05: 05 & 0.0003 & 0.0226 \\ 4686 & 11: 05: 07 & 0.0016 & 0.0316 \\ 4688 & 11: 05: 09 & 0.0008 & 0.0010\end{array}$


Appendix D - Laser Data Test 2 NIST102605_1.xls

$\begin{array}{llll}4690 & 11: 05: 11 & 0.0010 & 0.0003 \\ 4692 & 11: 05: 13 & 0.0022 & 0.0028 \\ 4694 & 11: 05: 15 & 0.0008 & 0.0072 \\ 4696 & 11: 05: 17 & 0.0013 & 0.0016 \\ 4698 & 11: 05: 19 & 0.0207 & 0.0116 \\ 4700 & 11: 05: 21 & 0.0131 & 0.0094 \\ 4702 & 11: 05: 23 & 0.0057 & 0.0007 \\ 4704 & 11: 05: 25 & 0.0421 & 0.0560 \\ 4706 & 11: 05: 27 & 0.0415 & 0.0035 \\ 4708 & 11: 05: 29 & 0.0077 & 0.0210 \\ 4710 & 11: 05: 31 & 0.0029 & 0.0007 \\ 4712 & 11: 05: 33 & 0.0084 & 0.0145 \\ 4714 & 11: 05: 35 & 0.0216 & 0.0071 \\ 4716 & 11: 05: 37 & 0.0067 & 0.0007 \\ 4718 & 11: 05: 39 & 0.0019 & 0.0099 \\ 4720 & 11: 05: 41 & 0.0072 & 0.0030 \\ 4722 & 11: 05: 43 & 0.0184 & 0.0012 \\ 4724 & 11: 05: 45 & 0.0031 & 0.0083 \\ 4726 & 11: 05: 47 & 0.0006 & 0.0136 \\ 4728 & 11: 05: 49 & 0.0025 & 0.0080 \\ 4730 & 11: 05: 51 & 0.0015 & 0.0137 \\ 4732 & 11: 05: 53 & 0.0034 & 0.0048 \\ 4734 & 11: 05: 55 & 0.0061 & 0.0086 \\ 4736 & 11: 05: 57 & 0.0046 & 0.0149 \\ 4738 & 11: 05: 59 & 0.0094 & 0.0018 \\ 4740 & 11: 06: 01 & 0.0024 & 0.0004 \\ 4742 & 11: 06: 03 & 0.0078 & 0.0093 \\ 4744 & 11: 06: 05 & 0.0074 & 0.0020 \\ 4746 & 11: 06: 07 & 0.0458 & 0.0079 \\ 4748 & 11: 06: 09 & 0.0281 & 0.0124 \\ 4750 & 11: 06: 11 & 0.0192 & 0.0026 \\ 4752 & 11: 06: 13 & 0.0219 & 0.0028 \\ 4754 & 11: 06: 15 & 0.0037 & 0.0067 \\ 4756 & 11: 06: 17 & 0.0043 & 0.0007 \\ 4758 & 11: 06: 19 & 0.0019 & 0.0122 \\ 4760 & 11: 06: 21 & 0.0036 & 0.0067 \\ 4762 & 11: 06: 23 & 0.0042 & 0.0037 \\ 4764 & 11: 06: 25 & 0.0123 & 0.0027 \\ 4766 & 11: 06: 27 & 0.0061 & 0.0013 \\ 4768 & 11: 06: 29 & 0.0056 & 0.0039 \\ 4770 & 11: 06: 31 & 0.0085 & 0.0037 \\ 4772 & 11: 06: 33 & 0.0049 & 0.0028 \\ 4774 & 11: 06: 35 & 0.0096 & 0.0120 \\ 4776 & 11: 06: 37 & 0.0112 & 0.0137 \\ 4778 & 11: 06: 39 & 0.0312 & 0.0090 \\ 4780 & 11: 06: 41 & 0.0078 & 0.0090 \\ 4782 & 11: 06: 43 & 0.0054 & 0.0067 \\ 4784 & 11: 06: 45 & 0.0211 & 0.0006 \\ 4786 & 11: 06: 47 & 0.0101 & 0.0070 \\ 4788 & 11: 06: 49 & 0.0094 & 0.0038 \\ 4790 & 11: 06: 51 & 0.0102 & 0.0055\end{array}$


Appendix D - Laser Data Test 2 NIST102605_1.xls

$\begin{array}{llll}4792 & 11: 06: 53 & 0.0245 & 0.0040 \\ 4794 & 11: 06: 55 & 0.0118 & 0.0020 \\ 4796 & 11: 06: 57 & 0.0654 & 0.0749 \\ 4798 & 11: 06: 59 & 0.0335 & 0.0090 \\ 4800 & 11: 07: 01 & 0.0058 & 0.0114 \\ 4802 & 11: 07: 03 & 0.0107 & 0.0213 \\ 4804 & 11: 07: 05 & 0.0157 & 0.0025 \\ 4806 & 11: 07: 07 & 0.0016 & 0.0086 \\ 4808 & 11: 07: 09 & 0.0015 & 0.0014 \\ 4810 & 11: 07: 11 & 0.0183 & 0.0039 \\ 4812 & 11: 07: 13 & 0.0013 & 0.0104 \\ 4814 & 11: 07: 15 & 0.0045 & 0.0020 \\ 4816 & 11: 07: 17 & 0.0015 & 0.0030 \\ 4818 & 11: 07: 19 & 0.0271 & 0.0033 \\ 4820 & 11: 07: 21 & 0.0093 & 0.0031 \\ 4822 & 11: 07: 23 & 0.0024 & 0.0022 \\ 4824 & 11: 07: 25 & 0.0065 & 0.0198 \\ 4826 & 11: 07: 27 & 0.0142 & 0.0143 \\ 4828 & 11: 07: 29 & 0.0043 & 0.0062 \\ 4830 & 11: 07: 31 & 0.0078 & 0.0007 \\ 4832 & 11: 07: 33 & 0.0125 & 0.0107 \\ 4834 & 11: 07: 35 & 0.0301 & 0.0033 \\ 4836 & 11: 07: 37 & 0.0250 & 0.0055 \\ 4838 & 11: 07: 39 & 0.0153 & 0.0026 \\ 4840 & 11: 07: 41 & 0.0093 & 0.0144 \\ 4842 & 11: 07: 43 & 0.0033 & 0.0152 \\ 4844 & 11: 07: 45 & 0.0014 & 0.0146 \\ 4846 & 11: 07: 47 & 0.0051 & 0.0060 \\ 4848 & 11: 07: 49 & 0.1056 & 0.0039 \\ 4850 & 11: 07: 51 & 0.0062 & 0.0089 \\ 4852 & 11: 07: 53 & 0.0276 & 0.0061 \\ 4854 & 11: 07: 55 & 0.0134 & 0.0044 \\ 4856 & 11: 07: 57 & 0.0126 & 0.0016 \\ 4858 & 11: 07: 59 & 0.0010 & 0.0064 \\ 4860 & 11: 08: 01 & 0.0063 & 0.0038 \\ 4862 & 11: 08: 03 & 0.0016 & 0.0119 \\ 4864 & 11: 08: 05 & 0.0010 & 0.0033 \\ 4866 & 11: 08: 07 & 0.0001 & 0.0016 \\ 4868 & 11: 08: 09 & 0.0006 & 0.0133 \\ 4870 & 11: 08: 11 & 0.0023 & 0.0036 \\ 4872 & 11: 08: 13 & 0.0062 & 0.0016 \\ 4874 & 11: 08: 15 & 0.0020 & 0.0043 \\ 4876 & 11: 08: 17 & 0.0001 & 0.0049 \\ 4878 & 11: 08: 19 & 0.0003 & 0.0207 \\ 4880 & 11: 08: 21 & 0.0039 & 0.0111 \\ 4882 & 11: 08: 23 & 0.0023 & 0.0105 \\ 4884 & 11: 08: 25 & 0.0050 & 0.0030 \\ 4886 & 11: 08: 27 & 0.0040 & 0.0165 \\ 4888 & 11: 08: 29 & 0.0029 & 0.0124 \\ 4890 & 11: 08: 31 & 0.0050 & 0.0155 \\ 4892 & 11: 08: 33 & 0.0051 & 0.0767\end{array}$


Appendix D - Laser Data Test 2 NIST102605_1.xls

$\begin{array}{llll}4894 & 11: 08: 35 & 0.0038 & 0.0066 \\ 4896 & 11: 08: 37 & 0.0017 & 0.0026 \\ 4898 & 11: 08: 39 & 0.0006 & 0.0194 \\ 4900 & 11: 08: 41 & 0.0006 & 0.0017 \\ 4902 & 11: 08: 43 & 0.0010 & 0.0086 \\ 4904 & 11: 08: 45 & 0.0028 & 0.0029 \\ 4906 & 11: 08: 47 & 0.0022 & 0.0120 \\ 4908 & 11: 08: 49 & 0.0016 & 0.0022 \\ 4910 & 11: 08: 51 & 0.0017 & 0.0277 \\ 4912 & 11: 08: 53 & 0.0079 & 0.0046 \\ 4914 & 11: 08: 55 & 0.0004 & 0.0023 \\ 4916 & 11: 08: 57 & 0.0023 & 0.0510 \\ 4918 & 11: 08: 59 & 0.0003 & 0.0053 \\ 4920 & 11: 09: 01 & 0.0044 & 0.0240 \\ 4922 & 11: 09: 03 & 0.0049 & 0.0165 \\ 4924 & 11: 09: 05 & 0.0012 & 0.0019 \\ 4926 & 11: 09: 07 & 0.0127 & 0.0044 \\ 4928 & 11: 09: 09 & 0.0017 & 0.0068 \\ 4930 & 11: 09: 11 & 0.0023 & 0.0244 \\ 4932 & 11: 09: 13 & 0.0033 & 0.0036 \\ 4934 & 11: 09: 15 & 0.0029 & 0.0133 \\ 4936 & 11: 09: 17 & 0.0050 & 0.0006 \\ 4938 & 11: 09: 19 & 0.0030 & 0.0012 \\ 4940 & 11: 09: 21 & 0.0007 & 0.0005 \\ 4942 & 11: 09: 23 & 0.0085 & 0.0063 \\ 4944 & 11: 09: 25 & 0.0472 & 0.0016 \\ 4946 & 11: 09: 27 & 0.0328 & 0.0007 \\ 4948 & 11: 09: 29 & 0.0052 & 0.0087 \\ 4950 & 11: 09: 31 & 0.0113 & 0.0056 \\ 4952 & 11: 09: 33 & 0.0111 & 0.0008 \\ 4954 & 11: 09: 35 & 0.0085 & 0.0107 \\ 4956 & 11: 09: 37 & 0.0054 & 0.0153 \\ 4958 & 11: 09: 39 & 0.0116 & 0.0371 \\ 4960 & 11: 09: 41 & 0.0193 & 0.0104 \\ 4962 & 11: 09: 43 & 0.0078 & 0.0002 \\ 4964 & 11: 09: 45 & 0.0024 & 0.0011 \\ 4966 & 11: 09: 47 & 0.0071 & 0.0100 \\ 4968 & 11: 09: 49 & 0.0058 & 0.0029 \\ 4970 & 11: 09: 51 & 0.0051 & 0.0078 \\ 4972 & 11: 09: 53 & 0.0071 & 0.0117 \\ 4974 & 11: 09: 55 & 0.0145 & 0.0171 \\ 4976 & 11: 09: 57 & 0.0199 & 0.0047 \\ 4978 & 11: 09: 59 & 0.0109 & 0.0075 \\ 4980 & 11: 10: 01 & 0.0304 & 0.0392 \\ 4982 & 11: 10: 03 & 0.0155 & 0.0015 \\ 4984 & 11: 10: 05 & 0.0020 & 0.0019 \\ 4986 & 11: 10: 07 & 0.0251 & 0.0068 \\ 4988 & 11: 10: 09 & 0.0176 & 0.0482 \\ 4990 & 11: 10: 11 & 0.0156 & 0.0028 \\ 4992 & 11: 10: 13 & 0.0134 & 0.0295 \\ 4994 & 11: 10: 15 & 0.0023 & 0.0077\end{array}$


Appendix D - Laser Data Test 2 NIST102605_1.xls

$\begin{array}{llll}4996 & 11: 10: 17 & 0.0016 & 0.0056 \\ 4998 & 11: 10: 19 & 0.0206 & 0.0068 \\ 5000 & 11: 10: 21 & 0.0024 & 0.0270 \\ 5002 & 11: 10: 23 & 0.0675 & 0.0052 \\ 5004 & 11: 10: 25 & 0.0108 & 0.0077 \\ 5006 & 11: 10: 27 & 0.0052 & 0.0162 \\ 5008 & 11: 10: 29 & 0.0018 & 0.0030 \\ 5010 & 11: 10: 31 & 0.0095 & 0.0118 \\ 5012 & 11: 10: 33 & 0.0112 & 0.0077 \\ 5014 & 11: 10: 35 & 0.0015 & 0.0258 \\ 5016 & 11: 10: 37 & 0.0031 & 0.0065 \\ 5018 & 11: 10: 39 & 0.0009 & 0.0068 \\ 5020 & 11: 10: 41 & 0.0024 & 0.0058 \\ 5022 & 11: 10: 43 & 0.0004 & 0.0032 \\ 5024 & 11: 10: 45 & 0.0037 & 0.0078 \\ 5026 & 11: 10: 47 & 0.0029 & 0.0056 \\ 5028 & 11: 10: 49 & 0.0010 & 0.0112 \\ 5030 & 11: 10: 51 & 0.0019 & 0.0008 \\ 5032 & 11: 10: 53 & 0.0002 & 0.0046 \\ 5034 & 11: 10: 55 & 0.0014 & 0.0304 \\ 5036 & 11: 10: 57 & 0.0135 & 0.0051 \\ 5038 & 11: 10: 59 & 0.0074 & 0.0073 \\ 5040 & 11: 11: 01 & 0.0342 & 0.0293 \\ 5042 & 11: 11: 03 & 0.0017 & 0.0389 \\ 5044 & 11: 11: 05 & 0.0005 & 0.0041 \\ 5046 & 11: 11: 07 & 0.0092 & 0.0282 \\ 5048 & 11: 11: 09 & 0.0009 & 0.0190 \\ 5050 & 11: 11: 11 & 0.0031 & 0.0040 \\ 5052 & 11: 11: 13 & 0.0011 & 0.0013 \\ 5054 & 11: 11: 15 & 0.0031 & 0.0132 \\ 5056 & 11: 11: 17 & 0.0239 & 0.0038 \\ 5058 & 11: 11: 19 & 0.0123 & 0.0018 \\ 5060 & 11: 11: 21 & 0.0022 & 0.0110 \\ 5062 & 11: 11: 23 & 0.0099 & 0.0081 \\ 5064 & 11: 11: 25 & 0.0100 & 0.0340 \\ 5066 & 11: 11: 27 & 0.0215 & 0.0037 \\ 5068 & 11: 11: 29 & 0.0099 & 0.0013 \\ 5070 & 11: 11: 31 & 0.0018 & 0.0039 \\ 5072 & 11: 11: 33 & 0.0312 & 0.0004 \\ 5074 & 11: 11: 35 & 0.0233 & 0.0085 \\ 5076 & 11: 11: 37 & 0.0197 & 0.0130 \\ 5078 & 11: 11: 39 & 0.0145 & 0.0104 \\ 5080 & 11: 11: 41 & 0.0061 & 0.0021 \\ 5082 & 11: 11: 43 & 0.0039 & 0.0293 \\ 5084 & 11: 11: 45 & 0.0017 & 0.0054 \\ 5086 & 11: 11: 47 & 0.0084 & 0.0071 \\ 5088 & 11: 11: 49 & 0.0100 & 0.0012 \\ 5090 & 11: 11: 51 & 0.0018 & 0.0196 \\ 5092 & 11: 11: 53 & 0.0064 & 0.0027 \\ 5094 & 11: 11: 55 & 0.0005 & 0.0053 \\ 5096 & 11: 11: 57 & 0.0007 & 0.0505\end{array}$

D - 50 
Appendix D - Laser Data Test 2 NIST102605_1.xls

$\begin{array}{llll}5098 & 11: 11: 59 & 0.0532 & 0.0236 \\ 5100 & 11: 12: 01 & 0.0181 & 0.0260 \\ 5102 & 11: 12: 03 & 0.0550 & 0.0177 \\ 5104 & 11: 12: 05 & 0.0003 & 0.0032 \\ 5106 & 11: 12: 07 & 0.0051 & 0.0023 \\ 5108 & 11: 12: 09 & 0.0036 & 0.0147 \\ 5110 & 11: 12: 11 & 0.0012 & 0.0037 \\ 5112 & 11: 12: 13 & 0.0026 & 0.0085 \\ 5114 & 11: 12: 15 & 0.0017 & 0.0021 \\ 5116 & 11: 12: 17 & 0.0011 & 0.0086 \\ 5118 & 11: 12: 19 & 0.0017 & 0.0053 \\ 5120 & 11: 12: 21 & 0.0024 & 0.0158 \\ 5122 & 11: 12: 23 & 0.0004 & 0.0067 \\ 5124 & 11: 12: 25 & 0.0031 & 0.0062 \\ 5126 & 11: 12: 27 & 0.0070 & 0.0100 \\ 5128 & 11: 12: 29 & 0.0203 & 0.0005 \\ 5130 & 11: 12: 31 & 0.0028 & 0.0162 \\ 5132 & 11: 12: 33 & 0.0068 & 0.0069 \\ 5134 & 11: 12: 35 & 0.0025 & 0.0012 \\ 5136 & 11: 12: 37 & 0.0038 & 0.0010 \\ 5138 & 11: 12: 39 & 0.0072 & 0.0012 \\ 5140 & 11: 12: 41 & 0.0111 & 0.0072 \\ 5142 & 11: 12: 43 & 0.0504 & 0.0061 \\ 5144 & 11: 12: 45 & 0.0178 & 0.0006 \\ 5146 & 11: 12: 47 & 0.0022 & 0.0005 \\ 5148 & 11: 12: 49 & 0.0034 & 0.0072 \\ 5150 & 11: 12: 51 & 0.0136 & 0.0040 \\ 5152 & 11: 12: 53 & 0.0120 & 0.0062 \\ 5154 & 11: 12: 55 & 0.0239 & 0.0029 \\ 5156 & 11: 12: 57 & 0.0061 & 0.0135 \\ 5158 & 11: 12: 59 & 0.0508 & 0.0006 \\ 5160 & 11: 13: 01 & 0.0109 & 0.0031 \\ 5162 & 11: 13: 03 & 0.0049 & 0.0051 \\ 5164 & 11: 13: 05 & 0.0230 & 0.0016 \\ 5166 & 11: 13: 07 & 0.0101 & 0.0630 \\ 5168 & 11: 13: 09 & 0.0051 & 0.0046 \\ 5170 & 11: 13: 11 & 0.0130 & 0.0066 \\ 5172 & 11: 13: 13 & 0.0022 & 0.0025 \\ 5174 & 11: 13: 15 & 0.0079 & 0.0026 \\ 5176 & 11: 13: 17 & 0.0279 & 0.0156 \\ 5178 & 11: 13: 19 & 0.0232 & 0.0141 \\ 5180 & 11: 13: 21 & 0.0109 & 0.0019 \\ 5182 & 11: 13: 23 & 0.0023 & 0.0063 \\ 5184 & 11: 13: 25 & 0.0024 & 0.0447 \\ 5186 & 11: 13: 27 & 0.0151 & 0.0126 \\ 5188 & 11: 13: 29 & 0.0268 & 0.0021 \\ 5190 & 11: 13: 31 & 0.0179 & 0.0060 \\ 5192 & 11: 13: 33 & 0.0112 & 0.0035 \\ 5194 & 11: 13: 35 & 0.0044 & 0.0061 \\ 5196 & 11: 13: 37 & 0.0119 & 0.0146 \\ 5198 & 11: 13: 39 & 0.0236 & 0.0271\end{array}$


Appendix D - Laser Data Test 2 NIST102605_1.xls

$\begin{array}{llll}5200 & 11: 13: 41 & 0.0038 & 0.0040 \\ 5202 & 11: 13: 43 & 0.0019 & 0.0168 \\ 5204 & 11: 13: 45 & 0.0086 & 0.0068 \\ 5206 & 11: 13: 47 & 0.0009 & 0.0090 \\ 5208 & 11: 13: 49 & 0.0033 & 0.0113 \\ 5210 & 11: 13: 51 & 0.0079 & 0.0056 \\ 5212 & 11: 13: 53 & 0.0027 & 0.0080 \\ 5214 & 11: 13: 55 & 0.0077 & 0.0074 \\ 5216 & 11: 13: 57 & 0.0057 & 0.0078 \\ 5218 & 11: 13: 59 & 0.0033 & 0.0462 \\ 5220 & 11: 14: 01 & 0.0015 & 0.0069 \\ 5222 & 11: 14: 03 & 0.0039 & 0.0026 \\ 5224 & 11: 14: 05 & 0.0072 & 0.0025 \\ 5226 & 11: 14: 07 & 0.0027 & 0.0011 \\ 5228 & 11: 14: 09 & 0.0240 & 0.0019 \\ 5230 & 11: 14: 11 & 0.0248 & 0.0012 \\ 5232 & 11: 14: 13 & 0.0126 & 0.0076 \\ 5234 & 11: 14: 15 & 0.0020 & 0.0028 \\ 5236 & 11: 14: 17 & 0.0045 & 0.0012 \\ 5238 & 11: 14: 19 & 0.0261 & 0.0009 \\ 5240 & 11: 14: 21 & 0.0160 & 0.0073 \\ 5242 & 11: 14: 23 & 0.0188 & 0.0135 \\ 5244 & 11: 14: 25 & 0.0335 & 0.0073 \\ 5246 & 11: 14: 27 & 0.0096 & 0.0114 \\ 5248 & 11: 14: 29 & 0.0029 & 0.0007 \\ 5250 & 11: 14: 31 & 0.0190 & 0.0043 \\ 5252 & 11: 14: 33 & 0.0016 & 0.0014 \\ 5254 & 11: 14: 35 & 0.0091 & 0.0358 \\ 5256 & 11: 14: 37 & 0.0580 & 0.0034 \\ 5258 & 11: 14: 39 & 0.0495 & 0.0073 \\ 5260 & 11: 14: 41 & 0.0611 & 0.0032 \\ 5262 & 11: 14: 43 & 0.0153 & 0.0116 \\ 5264 & 11: 14: 45 & 0.0070 & 0.0064 \\ 5266 & 11: 14: 47 & 0.0053 & 0.0041 \\ 5268 & 11: 14: 49 & 0.0206 & 0.0027 \\ 5270 & 11: 14: 51 & 0.0061 & 0.0066 \\ 5272 & 11: 14: 53 & 0.0161 & 0.0013 \\ 5274 & 11: 14: 55 & 0.0151 & 0.0064 \\ 5276 & 11: 14: 57 & 0.0104 & 0.0088 \\ 5278 & 11: 14: 59 & 0.0060 & 0.0192 \\ 5280 & 11: 15: 01 & 0.0028 & 0.0427 \\ 5282 & 11: 15: 03 & 0.0096 & 0.0036 \\ 5284 & 11: 15: 05 & 0.0019 & 0.0058 \\ 5286 & 11: 15: 07 & 0.0104 & 0.0030 \\ 5288 & 11: 15: 09 & 0.0185 & 0.0330 \\ 5290 & 11: 15: 11 & 0.0190 & 0.0049 \\ 5292 & 11: 15: 13 & 0.0047 & 0.0007 \\ 5294 & 11: 15: 15 & 0.0166 & 0.0033 \\ 5296 & 11: 15: 17 & 0.0050 & 0.0069 \\ 5298 & 11: 15: 19 & 0.0053 & 0.0327 \\ 5300 & 11: 15: 21 & 0.0110 & 0.0067\end{array}$

D - 52 
Appendix D - Laser Data Test 2 NIST102605_1.xls

$\begin{array}{llll}5302 & 11: 15: 23 & 0.0339 & 0.0073 \\ 5304 & 11: 15: 25 & 0.0515 & 0.0091 \\ 5306 & 11: 15: 27 & 0.0205 & 0.0010 \\ 5308 & 11: 15: 29 & 0.0438 & 0.0075 \\ 5310 & 11: 15: 31 & 0.0143 & 0.0091 \\ 5312 & 11: 15: 33 & 0.0160 & 0.0009 \\ 5314 & 11: 15: 35 & 0.0206 & 0.0007 \\ 5316 & 11: 15: 37 & 0.0082 & 0.0007 \\ 5318 & 11: 15: 39 & 0.0133 & 0.0090 \\ 5320 & 11: 15: 41 & 0.0049 & 0.0045 \\ 5322 & 11: 15: 43 & 0.0043 & 0.0086 \\ 5324 & 11: 15: 45 & 0.0096 & 0.0044 \\ 5326 & 11: 15: 47 & 0.0048 & 0.0012 \\ 5328 & 11: 15: 49 & 0.0154 & 0.0019 \\ 5330 & 11: 15: 51 & 0.0112 & 0.0008 \\ 5332 & 11: 15: 53 & 0.0065 & 0.0019 \\ 5334 & 11: 15: 55 & 0.0015 & 0.0013 \\ 5336 & 11: 15: 57 & 0.0018 & 0.0015 \\ 5338 & 11: 15: 59 & 0.0054 & 0.0102 \\ 5340 & 11: 16: 01 & 0.0009 & 0.0009 \\ 5342 & 11: 16: 03 & 0.0037 & 0.0020 \\ 5344 & 11: 16: 05 & 0.0150 & 0.0016 \\ 5346 & 11: 16: 07 & 0.0317 & 0.0068 \\ 5348 & 11: 16: 09 & 0.0081 & 0.0014 \\ 5350 & 11: 16: 11 & 0.0218 & 0.0051 \\ 5352 & 11: 16: 13 & 0.0123 & 0.0002 \\ 5354 & 11: 16: 15 & 0.0018 & 0.0036 \\ 5356 & 11: 16: 17 & 0.0051 & 0.0036 \\ 5358 & 11: 16: 19 & 0.0095 & 0.0197 \\ 5360 & 11: 16: 21 & 0.0069 & 0.0025 \\ 5362 & 11: 16: 23 & 0.0197 & 0.0273 \\ 5364 & 11: 16: 25 & 0.0095 & 0.0073 \\ 5366 & 11: 16: 27 & 0.0120 & 0.0040 \\ 5368 & 11: 16: 29 & 0.0086 & 0.0039 \\ 5370 & 11: 16: 31 & 0.0238 & 0.0028 \\ 5372 & 11: 16: 33 & 0.0151 & 0.0007 \\ 5374 & 11: 16: 35 & 0.0134 & 0.0017 \\ 5376 & 11: 16: 37 & 0.0019 & 0.0012 \\ 5378 & 11: 16: 39 & 0.0325 & 0.0030 \\ 5380 & 11: 16: 41 & 0.0277 & 0.0008 \\ 5382 & 11: 16: 43 & 0.0161 & 0.0091 \\ 5384 & 11: 16: 45 & 0.0240 & 0.0079 \\ 5386 & 11: 16: 47 & 0.0125 & 0.0063 \\ 5388 & 11: 16: 49 & 0.0120 & 0.0048 \\ 5390 & 11: 16: 51 & 0.0062 & 0.0159 \\ 5392 & 11: 16: 53 & 0.0056 & 0.0012 \\ 5394 & 11: 16: 55 & 0.0170 & 0.0031 \\ 5396 & 11: 16: 57 & 0.0239 & 0.0027 \\ 5398 & 11: 16: 59 & 0.0245 & 0.0030 \\ 5400 & 11: 17: 01 & 0.0253 & 0.0140 \\ 5402 & 11: 17: 03 & 0.0298 & 0.0061\end{array}$

D - 53 
Appendix D - Laser Data Test 2 NIST102605_1.xls

$\begin{array}{llll}5404 & 11: 17: 05 & 0.0105 & 0.0024 \\ 5406 & 11: 17: 07 & 0.0058 & 0.0050 \\ 5408 & 11: 17: 09 & 0.0071 & 0.0032 \\ 5410 & 11: 17: 11 & 0.0077 & 0.0063 \\ 5412 & 11: 17: 13 & 0.0095 & 0.0012 \\ 5414 & 11: 17: 15 & 0.0026 & 0.0007 \\ 5416 & 11: 17: 17 & 0.0101 & 0.0047 \\ 5418 & 11: 17: 19 & 0.0068 & 0.0011 \\ 5420 & 11: 17: 21 & 0.0265 & 0.0017 \\ 5422 & 11: 17: 23 & 0.0291 & 0.0042 \\ 5424 & 11: 17: 25 & 0.0105 & 0.0020 \\ 5426 & 11: 17: 27 & 0.0091 & 0.0026 \\ 5428 & 11: 17: 29 & 0.0008 & 0.0004 \\ 5430 & 11: 17: 31 & 0.0018 & 0.0007 \\ 5432 & 11: 17: 33 & 0.0039 & 0.0026 \\ 5434 & 11: 17: 35 & 0.0215 & 0.0095 \\ 5436 & 11: 17: 37 & 0.0144 & 0.0206 \\ 5438 & 11: 17: 39 & 0.0032 & 0.0007 \\ 5440 & 11: 17: 41 & 0.0024 & 0.0018 \\ 5442 & 11: 17: 43 & 0.0070 & 0.0040 \\ 5444 & 11: 17: 45 & 0.0313 & 0.0004 \\ 5446 & 11: 17: 47 & 0.0036 & 0.0014 \\ 5448 & 11: 17: 49 & 0.0115 & 0.0045 \\ 5450 & 11: 17: 51 & 0.0060 & 0.0077 \\ 5452 & 11: 17: 53 & 0.0036 & 0.0152 \\ 5454 & 11: 17: 55 & 0.0054 & 0.0002 \\ 5456 & 11: 17: 57 & 0.0078 & 0.0103 \\ 5458 & 11: 17: 59 & 0.0038 & 0.0032 \\ 5460 & 11: 18: 01 & 0.0105 & 0.0091 \\ 5462 & 11: 18: 03 & 0.0130 & 0.0034 \\ 5464 & 11: 18: 05 & 0.0155 & 0.0029 \\ 5466 & 11: 18: 07 & 0.0057 & 0.0016 \\ 5468 & 11: 18: 09 & 0.0553 & 0.0114 \\ 5470 & 11: 18: 11 & 0.0038 & 0.0079 \\ 5472 & 11: 18: 13 & 0.0175 & 0.0591 \\ 5474 & 11: 18: 15 & 0.0116 & 0.0037 \\ 5476 & 11: 18: 17 & 0.0028 & 0.0092 \\ 5478 & 11: 18: 19 & 0.0131 & 0.0053 \\ 5480 & 11: 18: 21 & 0.0033 & 0.0022 \\ 5482 & 11: 18: 23 & 0.0017 & 0.0046 \\ 5484 & 11: 18: 25 & 0.0018 & 0.0270 \\ 5486 & 11: 18: 27 & 0.0007 & 0.0007 \\ 5488 & 11: 18: 29 & 0.0014 & 0.0728 \\ 5490 & 11: 18: 31 & 0.0103 & 0.0040 \\ 5492 & 11: 18: 33 & 0.0194 & 0.0034 \\ 5494 & 11: 18: 35 & 0.0161 & 0.0049 \\ 5496 & 11: 18: 37 & 0.0162 & 0.0098 \\ 5498 & 11: 18: 39 & 0.0133 & 0.0021 \\ 5500 & 11: 18: 41 & 0.0057 & 0.0017 \\ 5502 & 11: 18: 43 & 0.0025 & 0.0017 \\ 5504 & 11: 18: 45 & 0.0082 & 0.0032\end{array}$


Appendix D - Laser Data Test 2 NIST102605_1.xls

$\begin{array}{llll}5506 & 11: 18: 47 & 0.0067 & 0.0042 \\ 5508 & 11: 18: 49 & 0.0036 & 0.0010 \\ 5510 & 11: 18: 51 & 0.0100 & 0.0015 \\ 5512 & 11: 18: 53 & 0.0219 & 0.0012 \\ 5514 & 11: 18: 55 & 0.0142 & 0.0011 \\ 5516 & 11: 18: 57 & 0.0099 & 0.0022 \\ 5518 & 11: 18: 59 & 0.0131 & 0.0002 \\ 5520 & 11: 19: 01 & 0.0065 & 0.0068 \\ 5522 & 11: 19: 03 & 0.0029 & 0.0017 \\ 5524 & 11: 19: 05 & 0.0003 & 0.0198 \\ 5526 & 11: 19: 07 & 0.0051 & 0.0029 \\ 5528 & 11: 19: 09 & 0.0027 & 0.0074 \\ 5530 & 11: 19: 11 & 0.0039 & 0.0048 \\ 5532 & 11: 19: 13 & 0.0022 & 0.0106 \\ 5534 & 11: 19: 15 & 0.0055 & 0.0177 \\ 5536 & 11: 19: 17 & 0.0088 & 0.0112 \\ 5538 & 11: 19: 19 & 0.0033 & 0.0187 \\ 5540 & 11: 19: 21 & 0.0102 & 0.0056 \\ 5542 & 11: 19: 23 & 0.0005 & 0.0048 \\ 5544 & 11: 19: 25 & 0.0061 & 0.0363 \\ 5546 & 11: 19: 27 & 0.0168 & 0.0031 \\ 5548 & 11: 19: 29 & 0.0009 & 0.0048 \\ 5550 & 11: 19: 31 & 0.0019 & 0.0034 \\ 5552 & 11: 19: 33 & 0.0023 & 0.0026 \\ 5554 & 11: 19: 35 & 0.0032 & 0.0064 \\ 5556 & 11: 19: 37 & 0.0161 & 0.0041 \\ 5558 & 11: 19: 39 & 0.0017 & 0.0032 \\ 5560 & 11: 19: 41 & 0.0004 & 0.0080 \\ 5562 & 11: 19: 43 & 0.0036 & 0.0083 \\ 5564 & 11: 19: 45 & 0.0063 & 0.0095 \\ 5566 & 11: 19: 47 & 0.0019 & 0.0118 \\ 5568 & 11: 19: 49 & 0.0057 & 0.0124 \\ 5570 & 11: 19: 51 & 0.0006 & 0.0015 \\ 5572 & 11: 19: 53 & 0.0017 & 0.0017 \\ 5574 & 11: 19: 55 & 0.0110 & 0.0042 \\ 5576 & 11: 19: 57 & 0.0010 & 0.0055 \\ 5578 & 11: 19: 59 & 0.0012 & 0.0043 \\ 5580 & 11: 20: 01 & 0.0081 & 0.0047 \\ 5582 & 11: 20: 03 & 0.0009 & 0.0019 \\ 5584 & 11: 20: 05 & 0.0013 & 0.0063 \\ 5586 & 11: 20: 07 & 0.0157 & 0.0137 \\ 5588 & 11: 20: 09 & 0.0030 & 0.0244 \\ 5590 & 11: 20: 11 & 0.0134 & 0.0060 \\ 5592 & 11: 20: 13 & 0.0038 & 0.0197 \\ 5594 & 11: 20: 15 & 0.0047 & 0.0103 \\ 5596 & 11: 20: 17 & 0.0029 & 0.0026 \\ 5598 & 11: 20: 19 & 0.0010 & 0.0059 \\ 5600 & 11: 20: 21 & 0.0013 & 0.0064 \\ 5602 & 11: 20: 23 & 0.0010 & 0.0081 \\ 5604 & 11: 20: 25 & 0.0007 & 0.0060 \\ 5606 & 11: 20: 27 & 0.0065 & 0.0072\end{array}$

D - 55 
Appendix D - Laser Data Test 2 NIST102605_1.xls

$\begin{array}{llll}5608 & 11: 20: 29 & 0.0008 & 0.0049 \\ 5610 & 11: 20: 31 & 0.0057 & 0.0377 \\ 5612 & 11: 20: 33 & 0.0039 & 0.0041 \\ 5614 & 11: 20: 35 & 0.0265 & 0.0104 \\ 5616 & 11: 20: 37 & 0.0037 & 0.0377 \\ 5618 & 11: 20: 39 & 0.0045 & 0.0080 \\ 5620 & 11: 20: 41 & 0.0049 & 0.0031 \\ 5622 & 11: 20: 43 & 0.0080 & 0.0163 \\ 5624 & 11: 20: 45 & 0.0007 & 0.0151 \\ 5626 & 11: 20: 47 & 0.0030 & 0.0036 \\ 5628 & 11: 20: 49 & 0.0004 & 0.0054 \\ 5630 & 11: 20: 51 & 0.0065 & 0.0043 \\ 5632 & 11: 20: 53 & 0.0053 & 0.0057 \\ 5634 & 11: 20: 55 & 0.0014 & 0.0070 \\ 5636 & 11: 20: 57 & 0.0109 & 0.0116 \\ 5638 & 11: 20: 59 & 0.0059 & 0.0038 \\ 5640 & 11: 21: 01 & 0.0049 & 0.0439 \\ 5642 & 11: 21: 03 & 0.0029 & 0.0046 \\ 5644 & 11: 21: 05 & 0.0017 & 0.0038 \\ 5646 & 11: 21: 07 & 0.0082 & 0.0108 \\ 5648 & 11: 21: 09 & 0.0099 & 0.0323 \\ 5650 & 11: 21: 11 & 0.0043 & 0.0065 \\ 5652 & 11: 21: 13 & 0.0066 & 0.0185 \\ 5654 & 11: 21: 15 & 0.0013 & 0.0144 \\ 5656 & 11: 21: 17 & 0.0122 & 0.0023 \\ 5658 & 11: 21: 19 & 0.0003 & 0.0022 \\ 5660 & 11: 21: 21 & 0.0009 & 0.0062 \\ 5662 & 11: 21: 23 & 0.0095 & 0.0074 \\ 5664 & 11: 21: 25 & 0.0010 & 0.0255 \\ 5666 & 11: 21: 27 & 0.0033 & 0.0152 \\ 5668 & 11: 21: 29 & 0.0009 & 0.0240 \\ 5670 & 11: 21: 31 & 0.0008 & 0.0089 \\ 5672 & 11: 21: 33 & 0.0010 & 0.0072 \\ 5674 & 11: 21: 35 & 0.0010 & 0.0031 \\ 5676 & 11: 21: 37 & 0.0020 & 0.0032 \\ 5678 & 11: 21: 39 & 0.0009 & 0.0075 \\ 5680 & 11: 21: 41 & 0.0009 & 0.0011 \\ 5682 & 11: 21: 43 & 0.0196 & 0.0012 \\ 5684 & 11: 21: 45 & 0.0094 & 0.0036 \\ 5686 & 11: 21: 47 & 0.0057 & 0.0089 \\ 5688 & 11: 21: 49 & 0.0062 & 0.0079 \\ 5690 & 11: 21: 51 & 0.0023 & 0.0053 \\ 5692 & 11: 21: 53 & 0.0005 & 0.0202 \\ 5694 & 11: 21: 55 & 0.0109 & 0.0095 \\ 5696 & 11: 21: 57 & 0.0012 & 0.0298 \\ 5698 & 11: 21: 59 & 0.0004 & 0.0094 \\ 5700 & 11: 22: 01 & 0.0014 & 0.0073 \\ 5702 & 11: 22: 03 & 0.0003 & 0.0016 \\ 5704 & 11: 22: 05 & 0.0010 & 0.0010 \\ 5706 & 11: 22: 07 & 0.0425 & 0.0075 \\ 5708 & 11: 22: 09 & 0.0082 & 0.0019\end{array}$


Appendix D - Laser Data Test 2 NIST102605_1.xls

$\begin{array}{llll}5710 & 11: 22: 11 & 0.0004 & 0.0036 \\ 5712 & 11: 22: 13 & 0.0009 & 0.0029 \\ 5714 & 11: 22: 15 & 0.0099 & 0.0047 \\ 5716 & 11: 22: 17 & 0.0041 & 0.0018 \\ 5718 & 11: 22: 19 & 0.0021 & 0.0080 \\ 5720 & 11: 22: 21 & 0.0003 & 0.0046 \\ 5722 & 11: 22: 23 & 0.0004 & 0.0061 \\ 5724 & 11: 22: 25 & 0.0009 & 0.0254 \\ 5726 & 11: 22: 27 & 0.0051 & 0.0359 \\ 5728 & 11: 22: 29 & 0.0136 & 0.0095 \\ 5730 & 11: 22: 31 & 0.0032 & 0.0041 \\ 5732 & 11: 22: 33 & 0.0062 & 0.0027 \\ 5734 & 11: 22: 35 & 0.0055 & 0.0125 \\ 5736 & 11: 22: 37 & 0.0003 & 0.0065 \\ 5738 & 11: 22: 39 & 0.0051 & 0.0208 \\ 5740 & 11: 22: 41 & 0.0009 & 0.0033 \\ 5742 & 11: 22: 43 & 0.0123 & 0.0027 \\ 5744 & 11: 22: 45 & 0.0044 & 0.0242 \\ 5746 & 11: 22: 47 & 0.0022 & 0.0041 \\ 5748 & 11: 22: 49 & 0.0005 & 0.0027 \\ 5750 & 11: 22: 51 & 0.0055 & 0.0049 \\ 5752 & 11: 22: 53 & 0.0268 & 0.0024 \\ 5754 & 11: 22: 55 & 0.0037 & 0.0069 \\ 5756 & 11: 22: 57 & 0.0076 & 0.0052 \\ 5758 & 11: 22: 59 & 0.0014 & 0.0052 \\ 5760 & 11: 23: 01 & 0.0080 & 0.0012 \\ 5762 & 11: 23: 03 & 0.0180 & 0.0520 \\ 5764 & 11: 23: 05 & 0.0005 & 0.0192 \\ 5766 & 11: 23: 07 & 0.0030 & 0.0046 \\ 5768 & 11: 23: 09 & 0.0011 & 0.0057 \\ 5770 & 11: 23: 11 & 0.0012 & 0.0274 \\ 5772 & 11: 23: 13 & 0.0003 & 0.0047 \\ 5774 & 11: 23: 15 & 0.0004 & 0.0187 \\ 5776 & 11: 23: 17 & 0.0189 & 0.0066 \\ 5778 & 11: 23: 19 & 0.0045 & 0.0079 \\ 5780 & 11: 23: 21 & 0.0071 & 0.0240 \\ 5782 & 11: 23: 23 & 0.0252 & 0.0269 \\ 5784 & 11: 23: 25 & 0.0016 & 0.0038 \\ 5786 & 11: 23: 27 & 0.0028 & 0.0250 \\ 5788 & 11: 23: 29 & 0.0003 & 0.0002 \\ 5790 & 11: 23: 31 & 0.0101 & 0.0015 \\ 5792 & 11: 23: 33 & 0.0020 & 0.0116 \\ 5794 & 11: 23: 35 & 0.0066 & 0.0070 \\ 5796 & 11: 23: 37 & 0.0021 & 0.0089 \\ 5798 & 11: 23: 39 & 0.0030 & 0.0152 \\ 5800 & 11: 23: 41 & 0.0004 & 0.0011 \\ 5802 & 11: 23: 43 & 0.0050 & 0.0134 \\ 5804 & 11: 23: 45 & 0.0023 & 0.0216 \\ 5806 & 11: 23: 47 & 0.0005 & 0.0037 \\ 5808 & 11: 23: 49 & 0.0031 & 0.0172 \\ 5810 & 11: 23: 51 & 0.0140 & 0.0178\end{array}$

D - 57 
Appendix D - Laser Data Test 2 NIST102605_1.xls

$\begin{array}{llll}5812 & 11: 23: 53 & 0.0209 & 0.0052 \\ 5814 & 11: 23: 55 & 0.0209 & 0.0037 \\ 5816 & 11: 23: 57 & 0.0016 & 0.0104 \\ 5818 & 11: 23: 59 & 0.0019 & 0.0293 \\ 5820 & 11: 24: 01 & 0.0020 & 0.0059 \\ 5822 & 11: 24: 03 & 0.0033 & 0.0155 \\ 5824 & 11: 24: 05 & 0.0069 & 0.0031 \\ 5826 & 11: 24: 07 & 0.0030 & 0.0018 \\ 5828 & 11: 24: 09 & 0.0178 & 0.0103 \\ 5830 & 11: 24: 11 & 0.0056 & 0.0040 \\ 5832 & 11: 24: 13 & 0.0017 & 0.0139 \\ 5834 & 11: 24: 15 & 0.0071 & 0.0103 \\ 5836 & 11: 24: 17 & 0.0009 & 0.0050 \\ 5838 & 11: 24: 19 & 0.0066 & 0.0127 \\ 5840 & 11: 24: 21 & 0.0031 & 0.0327 \\ 5842 & 11: 24: 23 & 0.0060 & 0.0076 \\ 5844 & 11: 24: 25 & 0.0500 & 0.0051 \\ 5846 & 11: 24: 27 & 0.0265 & 0.0059 \\ 5848 & 11: 24: 29 & 0.0231 & 0.0116 \\ 5850 & 11: 24: 31 & 0.0099 & 0.0154 \\ 5852 & 11: 24: 33 & 0.0087 & 0.0074 \\ 5854 & 11: 24: 35 & 0.0048 & 0.0132 \\ 5856 & 11: 24: 37 & 0.0094 & 0.0069 \\ 5858 & 11: 24: 39 & 0.0029 & 0.0686 \\ 5860 & 11: 24: 41 & 0.0149 & 0.0094 \\ 5862 & 11: 24: 43 & 0.0072 & 0.0009 \\ 5864 & 11: 24: 45 & 0.0011 & 0.0085 \\ 5866 & 11: 24: 47 & 0.0087 & 0.0004 \\ 5868 & 11: 24: 49 & 0.0019 & 0.0151 \\ 5870 & 11: 24: 51 & 0.0005 & 0.0165 \\ 5872 & 11: 24: 53 & 0.0010 & 0.0098 \\ 5874 & 11: 24: 55 & 0.0034 & 0.0156 \\ 5876 & 11: 24: 57 & 0.0040 & 0.0074 \\ 5878 & 11: 24: 59 & 0.0015 & 0.0177 \\ 5880 & 11: 25: 01 & 0.0031 & 0.0017 \\ 5882 & 11: 25: 03 & 0.0176 & 0.0003 \\ 5884 & 11: 25: 05 & 0.0012 & 0.0026 \\ 5886 & 11: 25: 07 & 0.0023 & 0.0050 \\ 5888 & 11: 25: 09 & 0.0054 & 0.0057 \\ 5890 & 11: 25: 11 & 0.0007 & 0.0004 \\ 5892 & 11: 25: 13 & 0.0021 & 0.0025 \\ 5894 & 11: 25: 15 & 0.0039 & 0.0090 \\ 5896 & 11: 25: 17 & 0.0008 & 0.0079 \\ 5898 & 11: 25: 19 & 0.0019 & 0.0157 \\ 5900 & 11: 25: 21 & 0.0012 & 0.0075 \\ 5902 & 11: 25: 23 & 0.0005 & 0.0082 \\ 5904 & 11: 25: 25 & 0.0011 & 0.0066 \\ 5906 & 11: 25: 27 & 0.0050 & 0.0275 \\ 5908 & 11: 25: 29 & 0.0086 & 0.0114 \\ 5910 & 11: 25: 31 & 0.0029 & 0.0034 \\ 5912 & 11: 25: 33 & 0.0021 & 0.0027\end{array}$

D - 58 
Appendix D - Laser Data Test 2 NIST102605_1.xls

$\begin{array}{llll}5914 & 11: 25: 35 & 0.0049 & 0.0323 \\ 5916 & 11: 25: 37 & 0.0078 & 0.0017 \\ 5918 & 11: 25: 39 & 0.0065 & 0.0002 \\ 5920 & 11: 25: 41 & 0.0110 & 0.0056 \\ 5922 & 11: 25: 43 & 0.0046 & 0.0041 \\ 5924 & 11: 25: 45 & 0.0035 & 0.0002 \\ 5926 & 11: 25: 47 & 0.0469 & 0.0035 \\ 5928 & 11: 25: 49 & 0.0112 & 0.0014 \\ 5930 & 11: 25: 51 & 0.0157 & 0.0061 \\ 5932 & 11: 25: 53 & 0.0010 & 0.0014 \\ 5934 & 11: 25: 55 & 0.0058 & 0.0049 \\ 5936 & 11: 25: 57 & 0.0043 & 0.0030 \\ 5938 & 11: 25: 59 & 0.0021 & 0.0022 \\ 5940 & 11: 26: 01 & 0.0048 & 0.0102 \\ 5942 & 11: 26: 03 & 0.0003 & 0.0067 \\ 5944 & 11: 26: 05 & 0.0009 & 0.0015 \\ 5946 & 11: 26: 07 & 0.0004 & 0.0037 \\ 5948 & 11: 26: 09 & 0.0068 & 0.0012 \\ 5950 & 11: 26: 11 & 0.0016 & 0.0297 \\ 5952 & 11: 26: 13 & 0.0299 & 0.0061 \\ 5954 & 11: 26: 15 & 0.0072 & 0.0043 \\ 5956 & 11: 26: 17 & 0.0009 & 0.0172 \\ 5958 & 11: 26: 19 & 0.0021 & 0.0017 \\ 5960 & 11: 26: 21 & 0.0070 & 0.0044 \\ 5962 & 11: 26: 23 & 0.0022 & 0.0032 \\ 5964 & 11: 26: 25 & 0.0019 & 0.0048 \\ 5966 & 11: 26: 27 & 0.0015 & 0.0054 \\ 5968 & 11: 26: 29 & 0.0013 & 0.0143 \\ 5970 & 11: 26: 31 & 0.0010 & 0.0027 \\ 5972 & 11: 26: 33 & 0.0011 & 0.0129 \\ 5974 & 11: 26: 35 & 0.0009 & 0.0134 \\ 5976 & 11: 26: 37 & 0.0027 & 0.0504 \\ 5978 & 11: 26: 39 & 0.0079 & 0.0273 \\ 5980 & 11: 26: 41 & 0.0013 & 0.0173 \\ 5982 & 11: 26: 43 & 0.0018 & 0.0022 \\ 5984 & 11: 26: 45 & 0.0206 & 0.0251 \\ 5986 & 11: 26: 47 & 0.0013 & 0.0131 \\ 5988 & 11: 26: 49 & 0.0008 & 0.0090 \\ 5990 & 11: 26: 51 & 0.0006 & 0.0058 \\ 5992 & 11: 26: 53 & 0.0160 & 0.0209 \\ 5994 & 11: 26: 55 & 0.0172 & 0.0076 \\ 5996 & 11: 26: 57 & 0.0096 & 0.0055 \\ 5998 & 11: 26: 59 & 0.0070 & 0.0073 \\ 6000 & 11: 27: 01 & 0.0090 & 0.0015 \\ 6002 & 11: 27: 03 & 0.0004 & 0.0047 \\ 6004 & 11: 27: 05 & 0.0010 & 0.0365 \\ 6006 & 11: 27: 07 & 0.0028 & 0.0257 \\ 6008 & 11: 27: 09 & 0.0024 & 0.0081 \\ 6010 & 11: 27: 11 & 0.0041 & 0.0034 \\ 6012 & 11: 27: 13 & 0.0021 & 0.0011 \\ 6014 & 11: 27: 15 & 0.0153 & 0.0066\end{array}$

D - 59 
Appendix D - Laser Data Test 2 NIST102605_1.xls

$\begin{array}{llll}6016 & 11: 27: 17 & 0.0024 & 0.0023 \\ 6018 & 11: 27: 19 & 0.0007 & 0.0354 \\ 6020 & 11: 27: 21 & 0.0146 & 0.0022 \\ 6022 & 11: 27: 23 & 0.0058 & 0.0104 \\ 6024 & 11: 27: 25 & 0.0047 & 0.0031 \\ 6026 & 11: 27: 27 & 0.0039 & 0.0069 \\ 6028 & 11: 27: 29 & 0.0092 & 0.0025 \\ 6030 & 11: 27: 31 & 0.0071 & 0.0053 \\ 6032 & 11: 27: 33 & 0.0018 & 0.0011 \\ 6034 & 11: 27: 35 & 0.0011 & 0.0048 \\ 6036 & 11: 27: 37 & 0.0564 & 0.0029 \\ 6038 & 11: 27: 39 & 0.0035 & 0.0069 \\ 6040 & 11: 27: 41 & 0.0132 & 0.0051 \\ 6042 & 11: 27: 43 & 0.0135 & 0.0016 \\ 6044 & 11: 27: 45 & 0.0107 & 0.0019 \\ 6046 & 11: 27: 47 & 0.0008 & 0.0083 \\ 6048 & 11: 27: 49 & 0.0024 & 0.0025 \\ 6050 & 11: 27: 51 & 0.0093 & 0.0048 \\ 6052 & 11: 27: 53 & 0.0035 & 0.0029 \\ 6054 & 11: 27: 55 & 0.0032 & 0.0064 \\ 6056 & 11: 27: 57 & 0.0155 & 0.0041 \\ 6058 & 11: 27: 59 & 0.0119 & 0.0593 \\ 6060 & 11: 28: 01 & 0.0004 & 0.0017 \\ 6062 & 11: 28: 03 & 0.0061 & 0.0021 \\ 6064 & 11: 28: 05 & 0.0104 & 0.0025 \\ 6066 & 11: 28: 07 & 0.0032 & 0.0014 \\ 6068 & 11: 28: 09 & 0.0129 & 0.0071 \\ 6070 & 11: 28: 11 & 0.0185 & 0.0037 \\ 6072 & 11: 28: 13 & 0.0064 & 0.0064 \\ 6074 & 11: 28: 15 & 0.0160 & 0.0097 \\ 6076 & 11: 28: 17 & 0.0172 & 0.0009 \\ 6078 & 11: 28: 19 & 0.0088 & 0.0234 \\ 6080 & 11: 28: 21 & 0.0056 & 0.0039 \\ 6082 & 11: 28: 23 & 0.0004 & 0.0051 \\ 6084 & 11: 28: 25 & 0.0073 & 0.0090 \\ 6086 & 11: 28: 27 & 0.0019 & 0.0181 \\ 6088 & 11: 28: 29 & 0.0031 & 0.0027 \\ 6090 & 11: 28: 31 & 0.0027 & 0.0014 \\ 6092 & 11: 28: 33 & 0.0002 & 0.0027 \\ 6094 & 11: 28: 35 & 0.0009 & 0.0042 \\ 6096 & 11: 28: 37 & 0.0030 & 0.0010 \\ 6098 & 11: 28: 39 & 0.0022 & 0.0098 \\ 6100 & 11: 28: 41 & 0.0102 & 0.0067 \\ 6102 & 11: 28: 43 & 0.0022 & 0.0028 \\ 6104 & 11: 28: 45 & 0.0043 & 0.0033 \\ 6106 & 11: 28: 47 & 0.0023 & 0.0124 \\ 6108 & 11: 28: 49 & 0.0064 & 0.0017 \\ 6110 & 11: 28: 51 & 0.0079 & 0.0104 \\ 6112 & 11: 28: 53 & 0.0243 & 0.0182 \\ 6114 & 11: 28: 55 & 0.0048 & 0.0350 \\ 6116 & 11: 28: 57 & 0.0042 & 0.0135\end{array}$


Appendix D - Laser Data Test 2 NIST102605_1.xls

$\begin{array}{llll}6118 & 11: 28: 59 & 0.0059 & 0.0032 \\ 6120 & 11: 29: 01 & 0.0028 & 0.0184 \\ 6122 & 11: 29: 03 & 0.0039 & 0.0192 \\ 6124 & 11: 29: 05 & 0.0013 & 0.0140 \\ 6126 & 11: 29: 07 & 0.0006 & 0.0152 \\ 6128 & 11: 29: 09 & 0.0023 & 0.0305 \\ 6130 & 11: 29: 11 & 0.0072 & 0.0035 \\ 6132 & 11: 29: 13 & 0.0022 & 0.0070 \\ 6134 & 11: 29: 15 & 0.0030 & 0.0027 \\ 6136 & 11: 29: 17 & 0.0011 & 0.0309 \\ 6138 & 11: 29: 19 & 0.0121 & 0.0007 \\ 6140 & 11: 29: 21 & 0.0069 & 0.0289 \\ 6142 & 11: 29: 23 & 0.0114 & 0.0041 \\ 6144 & 11: 29: 25 & 0.0006 & 0.0318 \\ 6146 & 11: 29: 27 & 0.0048 & 0.0104 \\ 6148 & 11: 29: 29 & 0.0022 & 0.0037 \\ 6150 & 11: 29: 31 & 0.0174 & 0.0046 \\ 6152 & 11: 29: 33 & 0.0094 & 0.0085 \\ 6154 & 11: 29: 35 & 0.0137 & 0.0135 \\ 6156 & 11: 29: 37 & 0.0081 & 0.0032 \\ 6158 & 11: 29: 39 & 0.0022 & 0.0287 \\ 6160 & 11: 29: 41 & 0.0043 & 0.0028 \\ 6162 & 11: 29: 43 & 0.0022 & 0.0241 \\ 6164 & 11: 29: 45 & 0.0101 & 0.0067 \\ 6166 & 11: 29: 47 & 0.0014 & 0.0116 \\ 6168 & 11: 29: 49 & 0.0084 & 0.0051 \\ 6170 & 11: 29: 51 & 0.0003 & 0.0388 \\ 6172 & 11: 29: 53 & 0.0021 & 0.0373 \\ 6174 & 11: 29: 55 & 0.0306 & 0.0022 \\ 6176 & 11: 29: 57 & 0.0010 & 0.0109 \\ 6178 & 11: 29: 59 & 0.0082 & 0.0030 \\ 6180 & 11: 30: 01 & 0.0013 & 0.0048 \\ 6182 & 11: 30: 03 & 0.0005 & 0.0090 \\ 6184 & 11: 30: 05 & 0.0033 & 0.0003 \\ 6186 & 11: 30: 07 & 0.0019 & 0.0170 \\ 6188 & 11: 30: 09 & 0.0071 & 0.0003 \\ 6190 & 11: 30: 11 & 0.0045 & 0.0353 \\ 6192 & 11: 30: 13 & 0.0013 & 0.0020 \\ 6194 & 11: 30: 15 & 0.0044 & 0.0019 \\ 6196 & 11: 30: 17 & 0.0164 & 0.0188 \\ 6198 & 11: 30: 19 & 0.0109 & 0.0149 \\ 6200 & 11: 30: 21 & 0.0271 & 0.0166 \\ 6202 & 11: 30: 23 & 0.0193 & 0.1058 \\ 6204 & 11: 30: 25 & 0.0112 & 0.0007 \\ 6206 & 11: 30: 27 & 0.0088 & 0.0083 \\ 6208 & 11: 30: 29 & 0.0157 & 0.0145 \\ 6210 & 11: 30: 31 & 0.0035 & 0.0156 \\ 6212 & 11: 30: 33 & 0.0035 & 0.0093 \\ 6214 & 11: 30: 35 & 0.0007 & 0.0012 \\ 6216 & 11: 30: 37 & 0.0011 & 0.0187 \\ 6218 & 11: 30: 39 & 0.0071 & 0.0018\end{array}$


Appendix D - Laser Data Test 2 NIST102605_1.xls

$\begin{array}{llll}6220 & 11: 30: 41 & 0.0086 & 0.0085 \\ 6222 & 11: 30: 43 & 0.0064 & 0.0064 \\ 6224 & 11: 30: 45 & 0.0048 & 0.0082 \\ 6226 & 11: 30: 47 & 0.0118 & 0.0283 \\ 6228 & 11: 30: 49 & 0.0085 & 0.0061 \\ 6230 & 11: 30: 51 & 0.0137 & 0.0114 \\ 6232 & 11: 30: 53 & 0.0030 & 0.0156 \\ 6234 & 11: 30: 55 & 0.0041 & 0.0151 \\ 6236 & 11: 30: 57 & 0.0017 & 0.0035 \\ 6238 & 11: 30: 59 & 0.0147 & 0.0111 \\ 6240 & 11: 31: 01 & 0.0105 & 0.0043 \\ 6242 & 11: 31: 03 & 0.0025 & 0.0067 \\ 6244 & 11: 31: 05 & 0.0109 & 0.0216 \\ 6246 & 11: 31: 07 & 0.0012 & 0.0108 \\ 6248 & 11: 31: 09 & 0.0003 & 0.0033 \\ 6250 & 11: 31: 11 & 0.0087 & 0.0236 \\ 6252 & 11: 31: 13 & 0.0157 & 0.0178 \\ 6254 & 11: 31: 15 & 0.0116 & 0.0015 \\ 6256 & 11: 31: 17 & 0.0031 & 0.0038 \\ 6258 & 11: 31: 19 & 0.0051 & 0.0103 \\ 6260 & 11: 31: 21 & 0.0009 & 0.0018 \\ 6262 & 11: 31: 23 & 0.0106 & 0.0080 \\ 6264 & 11: 31: 25 & 0.0008 & 0.0038 \\ 6266 & 11: 31: 27 & 0.0009 & 0.0101 \\ 6268 & 11: 31: 29 & 0.0003 & 0.0024 \\ 6270 & 11: 31: 31 & 0.0054 & 0.0037 \\ 6272 & 11: 31: 33 & 0.0059 & 0.0054 \\ 6274 & 11: 31: 35 & 0.0081 & 0.0468 \\ 6276 & 11: 31: 37 & 0.0026 & 0.0093 \\ 6278 & 11: 31: 39 & 0.0015 & 0.0048 \\ 6280 & 11: 31: 41 & 0.0154 & 0.0012 \\ 6282 & 11: 31: 43 & 0.0031 & 0.0093 \\ 6284 & 11: 31: 45 & 0.0070 & 0.0091 \\ 6286 & 11: 31: 47 & 0.0109 & 0.0027 \\ 6288 & 11: 31: 49 & 0.0059 & 0.0010 \\ 6290 & 11: 31: 51 & 0.0047 & 0.0042 \\ 6292 & 11: 31: 53 & 0.0061 & 0.0095 \\ 6294 & 11: 31: 55 & 0.0074 & 0.0207 \\ 6296 & 11: 31: 57 & 0.0002 & 0.0246 \\ 6298 & 11: 31: 59 & 0.0044 & 0.0011 \\ 6300 & 11: 32: 01 & 0.0016 & 0.0150 \\ 6302 & 11: 32: 03 & 0.0040 & 0.0030 \\ 6304 & 11: 32: 05 & 0.0087 & 0.0115 \\ 6306 & 11: 32: 07 & 0.0012 & 0.0020 \\ 6308 & 11: 32: 09 & 0.0002 & 0.0019 \\ 6310 & 11: 32: 11 & 0.0007 & 0.0023 \\ 6312 & 11: 32: 13 & 0.0009 & 0.0163 \\ 6314 & 11: 32: 15 & 0.0006 & 0.0693 \\ 6316 & 11: 32: 17 & 0.0005 & 0.0071 \\ 6318 & 11: 32: 19 & 0.0007 & 0.0255 \\ 6320 & 11: 32: 21 & 0.0005 & 0.0003\end{array}$

D - 62 
Appendix D - Laser Data Test 2 NIST102605_1.xls

$\begin{array}{llll}6322 & 11: 32: 23 & 0.0023 & 0.0017 \\ 6324 & 11: 32: 25 & 0.0025 & 0.0142 \\ 6326 & 11: 32: 27 & 0.0790 & 0.0063 \\ 6328 & 11: 32: 29 & 0.0005 & 0.0123 \\ 6330 & 11: 32: 31 & 0.0006 & 0.0027 \\ 6332 & 11: 32: 33 & 0.0062 & 0.0032 \\ 6334 & 11: 32: 35 & 0.0190 & 0.0152 \\ 6336 & 11: 32: 37 & 0.0049 & 0.0063 \\ 6338 & 11: 32: 39 & 0.0085 & 0.0430 \\ 6340 & 11: 32: 41 & 0.0026 & 0.0092 \\ 6342 & 11: 32: 43 & 0.0019 & 0.0068 \\ 6344 & 11: 32: 45 & 0.0010 & 0.0009 \\ 6346 & 11: 32: 47 & 0.0012 & 0.0022 \\ 6348 & 11: 32: 49 & 0.0058 & 0.0187 \\ 6350 & 11: 32: 51 & 0.0053 & 0.0039 \\ 6352 & 11: 32: 53 & 0.0057 & 0.0090 \\ 6354 & 11: 32: 55 & 0.0021 & 0.0084 \\ 6356 & 11: 32: 57 & 0.0003 & 0.0132 \\ 6358 & 11: 32: 59 & 0.0018 & 0.0046 \\ 6360 & 11: 33: 01 & 0.0022 & 0.0023 \\ 6362 & 11: 33: 03 & 0.0220 & 0.0088 \\ 6364 & 11: 33: 05 & 0.0157 & 0.0380 \\ 6366 & 11: 33: 07 & 0.0021 & 0.0024 \\ 6368 & 11: 33: 09 & 0.0005 & 0.0312 \\ 6370 & 11: 33: 11 & 0.0045 & 0.0023 \\ 6372 & 11: 33: 13 & 0.0039 & 0.0152 \\ 6374 & 11: 33: 15 & 0.0119 & 0.0177 \\ 6376 & 11: 33: 17 & 0.0095 & 0.1118 \\ 6378 & 11: 33: 19 & 0.0087 & 0.0175 \\ 6380 & 11: 33: 21 & 0.0007 & 0.0068 \\ 6382 & 11: 33: 23 & 0.0151 & 0.0185 \\ 6384 & 11: 33: 25 & 0.0112 & 0.0269 \\ 6386 & 11: 33: 27 & 0.0079 & 0.0284 \\ 6388 & 11: 33: 29 & 0.0011 & 0.0016 \\ 6390 & 11: 33: 31 & 0.0011 & 0.0189 \\ 6392 & 11: 33: 33 & 0.0019 & 0.0167 \\ 6394 & 11: 33: 35 & 0.0079 & 0.0036 \\ 6396 & 11: 33: 37 & 0.0070 & 0.0052 \\ 6398 & 11: 33: 39 & 0.0248 & 0.0128 \\ 6400 & 11: 33: 41 & 0.0025 & 0.0027 \\ 6402 & 11: 33: 43 & 0.0142 & 0.0026 \\ 6404 & 11: 33: 45 & 0.0186 & 0.0119 \\ 6406 & 11: 33: 47 & 0.0020 & 0.0012 \\ 6408 & 11: 33: 49 & 0.0040 & 0.0096 \\ 6410 & 11: 33: 51 & 0.0047 & 0.0010 \\ 6412 & 11: 33: 53 & 0.0100 & 0.0431 \\ 6414 & 11: 33: 55 & 0.0085 & 0.0022 \\ 6416 & 11: 33: 57 & 0.0503 & 0.0061 \\ 6418 & 11: 33: 59 & 0.0192 & 0.0022 \\ 6420 & 11: 34: 01 & 0.0018 & 0.0007 \\ 6422 & 11: 34: 03 & 0.0025 & 0.0021\end{array}$

D - 63 
Appendix D - Laser Data Test 2 NIST102605_1.xls

$\begin{array}{llll}6424 & 11: 34: 05 & 0.0070 & 0.0051 \\ 6426 & 11: 34: 07 & 0.0123 & 0.0041 \\ 6428 & 11: 34: 09 & 0.0071 & 0.0034 \\ 6430 & 11: 34: 11 & 0.0049 & 0.0027 \\ 6432 & 11: 34: 13 & 0.0662 & 0.0007 \\ 6434 & 11: 34: 15 & 0.0381 & 0.0107 \\ 6436 & 11: 34: 17 & 0.0107 & 0.0031 \\ 6438 & 11: 34: 19 & 0.0069 & 0.0092 \\ 6440 & 11: 34: 21 & 0.0071 & 0.0077 \\ 6442 & 11: 34: 23 & 0.0069 & 0.0027 \\ 6444 & 11: 34: 25 & 0.0939 & 0.0111 \\ 6446 & 11: 34: 27 & 0.0043 & 0.0012 \\ 6448 & 11: 34: 29 & 0.0037 & 0.0009 \\ 6450 & 11: 34: 31 & 0.0160 & 0.0272 \\ 6452 & 11: 34: 33 & 0.0066 & 0.0049 \\ 6454 & 11: 34: 35 & 0.0140 & 0.0142 \\ 6456 & 11: 34: 37 & 0.0232 & 0.0106 \\ 6458 & 11: 34: 39 & 0.0334 & 0.0201 \\ 6460 & 11: 34: 41 & 0.0129 & 0.0010 \\ 6462 & 11: 34: 43 & 0.0213 & 0.0018 \\ 6464 & 11: 34: 45 & 0.0019 & 0.0147 \\ 6466 & 11: 34: 47 & 0.0144 & 0.0064 \\ 6468 & 11: 34: 49 & 0.0074 & 0.0148 \\ 6470 & 11: 34: 51 & 0.0054 & 0.0013 \\ 6472 & 11: 34: 53 & 0.0044 & 0.0043 \\ 6474 & 11: 34: 55 & 0.0603 & 0.0048 \\ 6476 & 11: 34: 57 & 0.0159 & 0.0035 \\ 6478 & 11: 34: 59 & 0.0106 & 0.0044 \\ 6480 & 11: 35: 01 & 0.0119 & 0.0044 \\ 6482 & 11: 35: 03 & 0.0203 & 0.0013 \\ 6484 & 11: 35: 05 & 0.0315 & 0.0033 \\ 6486 & 11: 35: 07 & 0.0232 & 0.0098 \\ 6488 & 11: 35: 09 & 0.0074 & 0.0143 \\ 6490 & 11: 35: 11 & 0.0060 & 0.0062 \\ 6492 & 11: 35: 13 & 0.0051 & 0.0029 \\ 6494 & 11: 35: 15 & 0.0310 & 0.0026 \\ 6496 & 11: 35: 17 & 0.0213 & 0.0023 \\ 6498 & 11: 35: 19 & 0.0474 & 0.0088 \\ 6500 & 11: 35: 21 & 0.0119 & 0.0010 \\ 6502 & 11: 35: 23 & 0.0334 & 0.0023 \\ 6504 & 11: 35: 25 & 0.0089 & 0.0099 \\ 6506 & 11: 35: 27 & 0.0070 & 0.0082 \\ 6508 & 11: 35: 29 & 0.0092 & 0.0033 \\ 6510 & 11: 35: 31 & 0.0106 & 0.0037 \\ 6512 & 11: 35: 33 & 0.0097 & 0.0030 \\ 6514 & 11: 35: 35 & 0.0117 & 0.0032 \\ 6516 & 11: 35: 37 & 0.0148 & 0.0005 \\ 6518 & 11: 35: 39 & 0.0017 & 0.0028 \\ 6520 & 11: 35: 41 & 0.0052 & 0.0025 \\ 6522 & 11: 35: 43 & 0.0078 & 0.0008 \\ 6524 & 11: 35: 45 & 0.0261 & 0.0042\end{array}$

D - 64 
Appendix D - Laser Data Test 2 NIST102605_1.xls

$\begin{array}{llll}6526 & 11: 35: 47 & 0.0005 & 0.0015 \\ 6528 & 11: 35: 49 & 0.0080 & 0.0017 \\ 6530 & 11: 35: 51 & 0.0053 & 0.0092 \\ 6532 & 11: 35: 53 & 0.0038 & 0.0103 \\ 6534 & 11: 35: 55 & 0.0016 & 0.0039 \\ 6536 & 11: 35: 57 & 0.0023 & 0.0034 \\ 6538 & 11: 35: 59 & 0.0147 & 0.0079 \\ 6540 & 11: 36: 01 & 0.0209 & 0.0028 \\ 6542 & 11: 36: 03 & 0.0162 & 0.0037 \\ 6544 & 11: 36: 05 & 0.0127 & 0.0027 \\ 6546 & 11: 36: 07 & 0.0127 & 0.0048 \\ 6548 & 11: 36: 09 & 0.0112 & 0.0011 \\ 6550 & 11: 36: 11 & 0.0126 & 0.0007 \\ 6552 & 11: 36: 13 & 0.0431 & 0.0033 \\ 6554 & 11: 36: 15 & 0.0138 & 0.0506 \\ 6556 & 11: 36: 17 & 0.0170 & 0.0098 \\ 6558 & 11: 36: 19 & 0.0063 & 0.0033 \\ 6560 & 11: 36: 21 & 0.0093 & 0.0136 \\ 6562 & 11: 36: 23 & 0.0073 & 0.0008 \\ 6564 & 11: 36: 25 & 0.0284 & 0.0013 \\ 6566 & 11: 36: 27 & 0.0047 & 0.0047 \\ 6568 & 11: 36: 29 & 0.0078 & 0.0023 \\ 6570 & 11: 36: 31 & 0.0299 & 0.0368 \\ 6572 & 11: 36: 33 & 0.0043 & 0.0093 \\ 6574 & 11: 36: 35 & 0.0029 & 0.0018 \\ 6576 & 11: 36: 37 & 0.0030 & 0.0010 \\ 6578 & 11: 36: 39 & 0.0234 & 0.0029 \\ 6580 & 11: 36: 41 & 0.0027 & 0.0073 \\ 6582 & 11: 36: 43 & 0.0211 & 0.0058 \\ 6584 & 11: 36: 45 & 0.0305 & 0.0049 \\ 6586 & 11: 36: 47 & 0.0064 & 0.0147 \\ 6588 & 11: 36: 49 & 0.0234 & 0.0017 \\ 6590 & 11: 36: 51 & 0.0372 & 0.0116 \\ 6592 & 11: 36: 53 & 0.0017 & 0.0028 \\ 6594 & 11: 36: 55 & 0.0109 & 0.0045 \\ 6596 & 11: 36: 57 & 0.0048 & 0.0028 \\ 6598 & 11: 36: 59 & 0.0198 & 0.0030 \\ 6600 & 11: 37: 01 & 0.0448 & 0.0024 \\ 6602 & 11: 37: 03 & 0.0058 & 0.0019 \\ 6604 & 11: 37: 05 & 0.0064 & 0.0016 \\ 6606 & 11: 37: 07 & 0.0236 & 0.0019 \\ 6608 & 11: 37: 09 & 0.0154 & 0.0071 \\ 6610 & 11: 37: 11 & 0.0031 & 0.0013 \\ 6612 & 11: 37: 13 & 0.0013 & 0.0053 \\ 6614 & 11: 37: 15 & 0.0020 & 0.0004 \\ 6616 & 11: 37: 17 & 0.0023 & 0.0007 \\ 6618 & 11: 37: 19 & 0.0223 & 0.0015 \\ 6620 & 11: 37: 21 & 0.0080 & 0.0013 \\ 6622 & 11: 37: 23 & 0.0041 & 0.0012 \\ 6624 & 11: 37: 25 & 0.0294 & 0.0002 \\ 6626 & 11: 37: 27 & 0.0119 & 0.0001\end{array}$

D - 65 
Appendix D - Laser Data Test 2 NIST102605_1.xls

$\begin{array}{llll}6628 & 11: 37: 29 & 0.0028 & 0.0007 \\ 6630 & 11: 37: 31 & 0.0158 & 0.0025 \\ 6632 & 11: 37: 33 & 0.0014 & 0.0019 \\ 6634 & 11: 37: 35 & 0.0015 & 0.0016 \\ 6636 & 11: 37: 37 & 0.0049 & 0.0215 \\ 6638 & 11: 37: 39 & 0.0047 & 0.0284 \\ 6640 & 11: 37: 41 & 0.0067 & 0.0015 \\ 6642 & 11: 37: 43 & 0.0055 & 0.0025 \\ 6644 & 11: 37: 45 & 0.0024 & 0.0027 \\ 6646 & 11: 37: 47 & 0.0043 & 0.0089 \\ 6648 & 11: 37: 49 & 0.0175 & 0.0107 \\ 6650 & 11: 37: 51 & 0.0101 & 0.0022 \\ 6652 & 11: 37: 53 & 0.0068 & 0.0026 \\ 6654 & 11: 37: 55 & 0.0058 & 0.0004 \\ 6656 & 11: 37: 57 & 0.0045 & 0.0357 \\ 6658 & 11: 37: 59 & 0.0065 & 0.0026 \\ 6660 & 11: 38: 01 & 0.0065 & 0.0019 \\ 6662 & 11: 38: 03 & 0.0284 & 0.0051 \\ 6664 & 11: 38: 05 & 0.0065 & 0.0058 \\ 6666 & 11: 38: 07 & 0.0025 & 0.0026 \\ 6668 & 11: 38: 09 & 0.0115 & 0.0044 \\ 6670 & 11: 38: 11 & 0.0429 & 0.0056 \\ 6672 & 11: 38: 13 & 0.0057 & 0.0021 \\ 6674 & 11: 38: 15 & 0.0136 & 0.0048 \\ 6676 & 11: 38: 17 & 0.0195 & 0.0107 \\ 6678 & 11: 38: 19 & 0.0072 & 0.0067 \\ 6680 & 11: 38: 21 & 0.0110 & 0.0127 \\ 6682 & 11: 38: 23 & 0.0073 & 0.0088 \\ 6684 & 11: 38: 25 & 0.0147 & 0.0004 \\ 6686 & 11: 38: 27 & 0.0188 & 0.0007 \\ 6688 & 11: 38: 29 & 0.0555 & 0.0028 \\ 6690 & 11: 38: 31 & 0.0113 & 0.0183 \\ 6692 & 11: 38: 33 & 0.0026 & 0.0229 \\ 6694 & 11: 38: 35 & 0.0089 & 0.0009 \\ 6696 & 11: 38: 37 & 0.0188 & 0.0027 \\ 6698 & 11: 38: 39 & 0.0043 & 0.0012 \\ 6700 & 11: 38: 41 & 0.0113 & 0.0090 \\ 6702 & 11: 38: 43 & 0.0025 & 0.0030 \\ 6704 & 11: 38: 45 & 0.0046 & 0.0017 \\ 6706 & 11: 38: 47 & 0.0046 & 0.0038 \\ 6708 & 11: 38: 49 & 0.0018 & 0.0020 \\ 6710 & 11: 38: 51 & 0.0006 & 0.0327 \\ 6712 & 11: 38: 53 & 0.0033 & 0.0044 \\ 6714 & 11: 38: 55 & 0.0205 & 0.0094 \\ 6716 & 11: 38: 57 & 0.0187 & 0.0025 \\ 6718 & 11: 38: 59 & 0.0058 & 0.0013 \\ 6720 & 11: 39: 01 & 0.0013 & 0.0011 \\ 6722 & 11: 39: 03 & 0.0026 & 0.0030 \\ 6724 & 11: 39: 05 & 0.0056 & 0.0047 \\ 6726 & 11: 39: 07 & 0.0157 & 0.0010 \\ 6728 & 11: 39: 09 & 0.0076 & 0.0053\end{array}$


Appendix D - Laser Data Test 2 NIST102605_1.xls

$\begin{array}{llll}6730 & 11: 39: 11 & 0.0204 & 0.0043 \\ 6732 & 11: 39: 13 & 0.0046 & 0.0043 \\ 6734 & 11: 39: 15 & 0.0664 & 0.0072 \\ 6736 & 11: 39: 17 & 0.0027 & 0.0032 \\ 6738 & 11: 39: 19 & 0.0093 & 0.0067 \\ 6740 & 11: 39: 21 & 0.0649 & 0.0146 \\ 6742 & 11: 39: 23 & 0.0048 & 0.0138 \\ 6744 & 11: 39: 25 & 0.0046 & 0.0029 \\ 6746 & 11: 39: 27 & 0.0089 & 0.0028 \\ 6748 & 11: 39: 29 & 0.0273 & 0.0196 \\ 6750 & 11: 39: 31 & 0.0199 & 0.0025 \\ 6752 & 11: 39: 33 & 0.0024 & 0.0008 \\ 6754 & 11: 39: 35 & 0.0040 & 0.0360 \\ 6756 & 11: 39: 37 & 0.0038 & 0.0228 \\ 6758 & 11: 39: 39 & 0.0021 & 0.0014 \\ 6760 & 11: 39: 41 & 0.0042 & 0.0448 \\ 6762 & 11: 39: 43 & 0.0198 & 0.0008 \\ 6764 & 11: 39: 45 & 0.0265 & 0.0005 \\ 6766 & 11: 39: 47 & 0.0057 & 0.0324 \\ 6768 & 11: 39: 49 & 0.0124 & 0.0495 \\ 6770 & 11: 39: 51 & 0.0225 & 0.0006 \\ 6772 & 11: 39: 53 & 0.0192 & 0.0350 \\ 6774 & 11: 39: 55 & 0.0493 & 0.0064 \\ 6776 & 11: 39: 57 & 0.0220 & 0.0106 \\ 6778 & 11: 39: 59 & 0.0047 & 0.0104 \\ 6780 & 11: 40: 01 & 0.0150 & 0.0195 \\ 6782 & 11: 40: 03 & 0.0114 & 0.0628 \\ 6784 & 11: 40: 05 & 0.0039 & 0.0162 \\ 6786 & 11: 40: 07 & 0.0292 & 0.0091 \\ 6788 & 11: 40: 09 & 0.0342 & 0.0037 \\ 6790 & 11: 40: 11 & 0.0276 & 0.0057 \\ 6792 & 11: 40: 13 & 0.0015 & 0.0029 \\ 6794 & 11: 40: 15 & 0.0109 & 0.0008 \\ 6796 & 11: 40: 17 & 0.0018 & 0.0159 \\ 6798 & 11: 40: 19 & 0.0074 & 0.0035 \\ 6800 & 11: 40: 21 & 0.0199 & 0.0173 \\ 6802 & 11: 40: 23 & 0.0066 & 0.0282 \\ 6804 & 11: 40: 25 & 0.0119 & 0.0021 \\ 6806 & 11: 40: 27 & 0.0011 & 0.0073 \\ 6808 & 11: 40: 29 & 0.0142 & 0.0031 \\ 6810 & 11: 40: 31 & 0.0110 & 0.0025 \\ 6812 & 11: 40: 33 & 0.0123 & 0.0072 \\ 6814 & 11: 40: 35 & 0.0141 & 0.0031 \\ 6816 & 11: 40: 37 & 0.0062 & 0.0027 \\ 6818 & 11: 40: 39 & 0.0105 & 0.0016 \\ 6820 & 11: 40: 41 & 0.0123 & 0.0011 \\ 6822 & 11: 40: 43 & 0.0058 & 0.0309 \\ 6824 & 11: 40: 45 & 0.0070 & 0.0294 \\ 6826 & 11: 40: 47 & 0.0051 & 0.0125 \\ 6828 & 11: 40: 49 & 0.0028 & 0.0044 \\ 6830 & 11: 40: 51 & 0.0217 & 0.0186\end{array}$


Appendix D - Laser Data Test 2 NIST102605_1.xls

$\begin{array}{llll}6832 & 11: 40: 53 & 0.0034 & 0.0024 \\ 6834 & 11: 40: 55 & 0.0030 & 0.0034 \\ 6836 & 11: 40: 57 & 0.0047 & 0.0152 \\ 6838 & 11: 40: 59 & 0.0058 & 0.0049 \\ 6840 & 11: 41: 01 & 0.0016 & 0.0099 \\ 6842 & 11: 41: 03 & 0.0045 & 0.0033 \\ 6844 & 11: 41: 05 & 0.0045 & 0.0007 \\ 6846 & 11: 41: 07 & 0.0348 & 0.0038 \\ 6848 & 11: 41: 09 & 0.0065 & 0.0013 \\ 6850 & 11: 41: 11 & 0.0229 & 0.0092 \\ 6852 & 11: 41: 13 & 0.0151 & 0.0018 \\ 6854 & 11: 41: 15 & 0.0482 & 0.0032 \\ 6856 & 11: 41: 17 & 0.0163 & 0.0091 \\ 6858 & 11: 41: 19 & 0.0133 & 0.0079 \\ 6860 & 11: 41: 21 & 0.0074 & 0.0043 \\ 6862 & 11: 41: 23 & 0.0026 & 0.0175 \\ 6864 & 11: 41: 25 & 0.0347 & 0.0066 \\ 6866 & 11: 41: 27 & 0.0175 & 0.0129 \\ 6868 & 11: 41: 29 & 0.0222 & 0.0022 \\ 6870 & 11: 41: 31 & 0.0086 & 0.0212 \\ 6872 & 11: 41: 33 & 0.0033 & 0.0024 \\ 6874 & 11: 41: 35 & 0.0207 & 0.0185 \\ 6876 & 11: 41: 37 & 0.0442 & 0.0093 \\ 6878 & 11: 41: 39 & 0.0025 & 0.0052 \\ 6880 & 11: 41: 41 & 0.0011 & 0.0015 \\ 6882 & 11: 41: 43 & 0.0070 & 0.0047 \\ 6884 & 11: 41: 45 & 0.0020 & 0.0039 \\ 6886 & 11: 41: 47 & 0.0024 & 0.0224 \\ 6888 & 11: 41: 49 & 0.0074 & 0.0287 \\ 6890 & 11: 41: 51 & 0.0145 & 0.0108 \\ 6892 & 11: 41: 53 & 0.0035 & 0.0022 \\ 6894 & 11: 41: 55 & 0.0013 & 0.0132 \\ 6896 & 11: 41: 57 & 0.0097 & 0.0005 \\ 6898 & 11: 41: 59 & 0.0179 & 0.0009 \\ 6900 & 11: 42: 01 & 0.0065 & 0.0013 \\ 6902 & 11: 42: 03 & 0.0102 & 0.0155 \\ 6904 & 11: 42: 05 & 0.0177 & 0.0008 \\ 6906 & 11: 42: 07 & 0.0294 & 0.0064 \\ 6908 & 11: 42: 09 & 0.0080 & 0.0023 \\ 6910 & 11: 42: 11 & 0.0157 & 0.0033 \\ 6912 & 11: 42: 13 & 0.0376 & 0.0033 \\ 6914 & 11: 42: 15 & 0.0037 & 0.0023 \\ 6916 & 11: 42: 17 & 0.0007 & 0.0076 \\ 6918 & 11: 42: 19 & 0.0190 & 0.0022 \\ 6920 & 11: 42: 21 & 0.0155 & 0.0086 \\ 6922 & 11: 42: 23 & 0.0049 & 0.0047 \\ 6924 & 11: 42: 25 & 0.0125 & 0.0008 \\ 6926 & 11: 42: 27 & 0.0033 & 0.0021 \\ 6928 & 11: 42: 29 & 0.0015 & 0.0004 \\ 6930 & 11: 42: 31 & 0.0212 & 0.0090 \\ 6932 & 11: 42: 33 & 0.0239 & 0.0063\end{array}$


Appendix D - Laser Data Test 2 NIST102605_1.xls

$\begin{array}{llll}6934 & 11: 42: 35 & 0.0044 & 0.0024 \\ 6936 & 11: 42: 37 & 0.0098 & 0.0026 \\ 6938 & 11: 42: 39 & 0.0242 & 0.0015 \\ 6940 & 11: 42: 41 & 0.0488 & 0.0504 \\ 6942 & 11: 42: 43 & 0.0140 & 0.0095 \\ 6944 & 11: 42: 45 & 0.0126 & 0.0043 \\ 6946 & 11: 42: 47 & 0.0069 & 0.0030 \\ 6948 & 11: 42: 49 & 0.0138 & 0.0061 \\ 6950 & 11: 42: 51 & 0.0181 & 0.0072 \\ 6952 & 11: 42: 53 & 0.0059 & 0.0036 \\ 6954 & 11: 42: 55 & 0.0018 & 0.0061 \\ 6956 & 11: 42: 57 & 0.0048 & 0.0105 \\ 6958 & 11: 42: 59 & 0.0071 & 0.0294 \\ 6960 & 11: 43: 01 & 0.0134 & 0.0053 \\ 6962 & 11: 43: 03 & 0.0082 & 0.0058 \\ 6964 & 11: 43: 05 & 0.0344 & 0.0018 \\ 6966 & 11: 43: 07 & 0.0278 & 0.0164 \\ 6968 & 11: 43: 09 & 0.0037 & 0.0125 \\ 6970 & 11: 43: 11 & 0.0011 & 0.0084 \\ 6972 & 11: 43: 13 & 0.0027 & 0.0213 \\ 6974 & 11: 43: 15 & 0.0070 & 0.0038 \\ 6976 & 11: 43: 17 & 0.0044 & 0.0050 \\ 6978 & 11: 43: 19 & 0.0060 & 0.0248 \\ 6980 & 11: 43: 21 & 0.0086 & 0.0052 \\ 6982 & 11: 43: 23 & 0.0264 & 0.0156 \\ 6984 & 11: 43: 25 & 0.0055 & 0.0027 \\ 6986 & 11: 43: 27 & 0.0064 & 0.0029 \\ 6988 & 11: 43: 29 & 0.0023 & 0.0033 \\ 6990 & 11: 43: 31 & 0.0020 & 0.0030 \\ 6992 & 11: 43: 33 & 0.0050 & 0.0062 \\ 6994 & 11: 43: 35 & 0.0005 & 0.0039 \\ 6996 & 11: 43: 37 & 0.0006 & 0.0008 \\ 6998 & 11: 43: 39 & 0.0174 & 0.0095 \\ 7000 & 11: 43: 41 & 0.0263 & 0.0040 \\ 7002 & 11: 43: 43 & 0.0042 & 0.0218 \\ 7004 & 11: 43: 45 & 0.0017 & 0.0056 \\ 7006 & 11: 43: 47 & 0.0021 & 0.0053 \\ 7008 & 11: 43: 49 & 0.0069 & 0.0117 \\ 7010 & 11: 43: 51 & 0.0010 & 0.1481 \\ 7012 & 11: 43: 53 & 0.0019 & 0.0040 \\ 7014 & 11: 43: 55 & 0.0009 & 0.0170 \\ 7016 & 11: 43: 57 & 0.0038 & 0.0067 \\ 7018 & 11: 43: 59 & 0.0021 & 0.0021 \\ 7020 & 11: 44: 01 & 0.0129 & 0.0105 \\ 7022 & 11: 44: 03 & 0.0020 & 0.0041 \\ 7024 & 11: 44: 05 & 0.0022 & 0.0236 \\ 7026 & 11: 44: 07 & 0.0019 & 0.0139 \\ 7028 & 11: 44: 09 & 0.0017 & 0.0223 \\ 7030 & 11: 44: 11 & 0.0011 & 0.0438 \\ 7032 & 11: 44: 13 & 0.0039 & 0.0133 \\ 7034 & 11: 44: 15 & 0.0028 & 0.0179\end{array}$

D - 69 
Appendix D - Laser Data Test 2 NIST102605_1.xls

$\begin{array}{llll}7036 & 11: 44: 17 & 0.0058 & 0.0055 \\ 7038 & 11: 44: 19 & 0.0017 & 0.0239 \\ 7040 & 11: 44: 21 & 0.0054 & 0.0016 \\ 7042 & 11: 44: 23 & 0.0050 & 0.0086 \\ 7044 & 11: 44: 25 & 0.0023 & 0.0010 \\ 7046 & 11: 44: 27 & 0.0028 & 0.0024 \\ 7048 & 11: 44: 29 & 0.0081 & 0.0077 \\ 7050 & 11: 44: 31 & 0.0050 & 0.0125 \\ 7052 & 11: 44: 33 & 0.0070 & 0.0122 \\ 7054 & 11: 44: 35 & 0.0088 & 0.0044 \\ 7056 & 11: 44: 37 & 0.0125 & 0.0167 \\ 7058 & 11: 44: 39 & 0.0055 & 0.0075 \\ 7060 & 11: 44: 41 & 0.0493 & 0.0086 \\ 7062 & 11: 44: 43 & 0.0103 & 0.0021 \\ 7064 & 11: 44: 45 & 0.0051 & 0.0031 \\ 7066 & 11: 44: 47 & 0.0040 & 0.0030 \\ 7068 & 11: 44: 49 & 0.0018 & 0.0049 \\ 7070 & 11: 44: 51 & 0.0190 & 0.0488 \\ 7072 & 11: 44: 53 & 0.0136 & 0.0018 \\ 7074 & 11: 44: 55 & 0.0660 & 0.0016 \\ 7076 & 11: 44: 57 & 0.0089 & 0.0051 \\ 7078 & 11: 44: 59 & 0.0028 & 0.0144 \\ 7080 & 11: 45: 01 & 0.0087 & 0.0098 \\ 7082 & 11: 45: 03 & 0.0145 & 0.0274 \\ 7084 & 11: 45: 05 & 0.0023 & 0.0076 \\ 7086 & 11: 45: 07 & 0.0214 & 0.0076 \\ 7088 & 11: 45: 09 & 0.0068 & 0.0024 \\ 7090 & 11: 45: 11 & 0.0050 & 0.0044 \\ 7092 & 11: 45: 13 & 0.0041 & 0.0041 \\ 7094 & 11: 45: 15 & 0.0062 & 0.0031 \\ 7096 & 11: 45: 17 & 0.0093 & 0.0011 \\ 7098 & 11: 45: 19 & 0.0152 & 0.0224 \\ 7100 & 11: 45: 21 & 0.0003 & 0.0846 \\ 7102 & 11: 45: 23 & 0.0063 & 0.0106 \\ 7104 & 11: 45: 25 & 0.0027 & 0.0023 \\ 7106 & 11: 45: 27 & 0.0029 & 0.0069 \\ 7108 & 11: 45: 29 & 0.0037 & 0.0296 \\ 7110 & 11: 45: 31 & 0.0028 & 0.0288 \\ 7112 & 11: 45: 33 & 0.0004 & 0.0127 \\ 7114 & 11: 45: 35 & 0.0044 & 0.0163 \\ 7116 & 11: 45: 37 & 0.0040 & 0.0356 \\ 7118 & 11: 45: 39 & 0.0014 & 0.0102 \\ 7120 & 11: 45: 41 & 0.0007 & 0.0058 \\ 7122 & 11: 45: 43 & 0.0002 & 0.0061 \\ 7124 & 11: 45: 45 & 0.0006 & 0.0051 \\ 7126 & 11: 45: 47 & 0.0065 & 0.0106 \\ 7128 & 11: 45: 49 & 0.0069 & 0.0343 \\ 7130 & 11: 45: 51 & 0.0062 & 0.0164 \\ 7132 & 11: 45: 53 & 0.0143 & 0.0054 \\ 7134 & 11: 45: 55 & 0.0006 & 0.0066 \\ 7136 & 11: 45: 57 & 0.0201 & 0.0072\end{array}$


Appendix D - Laser Data Test 2 NIST102605_1.xls

$\begin{array}{llll}7138 & 11: 45: 59 & 0.0040 & 0.0118 \\ 7140 & 11: 46: 01 & 0.0005 & 0.0075 \\ 7142 & 11: 46: 03 & 0.0053 & 0.0134 \\ 7144 & 11: 46: 05 & 0.0057 & 0.0212 \\ 7146 & 11: 46: 07 & 0.0034 & 0.0232 \\ 7148 & 11: 46: 09 & 0.0026 & 0.0024 \\ 7150 & 11: 46: 11 & 0.0030 & 0.0021 \\ 7152 & 11: 46: 13 & 0.0018 & 0.0062 \\ 7154 & 11: 46: 15 & 0.0110 & 0.0018 \\ 7156 & 11: 46: 17 & 0.0053 & 0.0082 \\ 7158 & 11: 46: 19 & 0.0101 & 0.0141 \\ 7160 & 11: 46: 21 & 0.0063 & 0.0201 \\ 7162 & 11: 46: 23 & 0.0098 & 0.0019 \\ 7164 & 11: 46: 25 & 0.0011 & 0.0034 \\ 7166 & 11: 46: 27 & 0.0082 & 0.0023 \\ 7168 & 11: 46: 29 & 0.0042 & 0.0115 \\ 7170 & 11: 46: 31 & 0.0032 & 0.0040 \\ 7172 & 11: 46: 33 & 0.0038 & 0.0116 \\ 7174 & 11: 46: 35 & 0.0020 & 0.0018 \\ 7176 & 11: 46: 37 & 0.0055 & 0.0288 \\ 7178 & 11: 46: 39 & 0.0018 & 0.0115 \\ 7180 & 11: 46: 41 & 0.0556 & 0.0233 \\ 7182 & 11: 46: 43 & 0.0019 & 0.0091 \\ 7184 & 11: 46: 45 & 0.0137 & 0.0210 \\ 7186 & 11: 46: 47 & 0.0032 & 0.0020 \\ 7188 & 11: 46: 49 & 0.0061 & 0.0026 \\ 7190 & 11: 46: 51 & 0.0020 & 0.0038 \\ 7192 & 11: 46: 53 & 0.0002 & 0.0085 \\ 7194 & 11: 46: 55 & 0.0024 & 0.0137 \\ 7196 & 11: 46: 57 & 0.0010 & 0.0168 \\ 7198 & 11: 46: 59 & 0.0055 & 0.0025 \\ 7200 & 11: 47: 01 & 0.0109 & 0.0053 \\ 7202 & 11: 47: 03 & 0.0024 & 0.0090 \\ 7204 & 11: 47: 05 & 0.0119 & 0.0079 \\ 7206 & 11: 47: 07 & 0.0032 & 0.0013 \\ 7208 & 11: 47: 09 & 0.0169 & 0.0085 \\ 7210 & 11: 47: 11 & 0.0027 & 0.0103 \\ 7212 & 11: 47: 13 & 0.0003 & 0.0047 \\ 7214 & 11: 47: 15 & 0.0243 & 0.0028 \\ 7216 & 11: 47: 17 & 0.0038 & 0.0018 \\ 7218 & 11: 47: 19 & 0.0010 & 0.0082 \\ 7220 & 11: 47: 21 & 0.0034 & 0.0585 \\ 7222 & 11: 47: 23 & 0.0739 & 0.0086 \\ 7224 & 11: 47: 25 & 0.0044 & 0.0133 \\ 7226 & 11: 47: 27 & 0.0022 & 0.0007 \\ 7228 & 11: 47: 29 & 0.0054 & 0.0009 \\ 7230 & 11: 47: 31 & 0.0005 & 0.0056 \\ 7232 & 11: 47: 33 & 0.0017 & 0.0044 \\ 7234 & 11: 47: 35 & 0.0055 & 0.0068 \\ 7236 & 11: 47: 37 & 0.0042 & 0.0111 \\ 7238 & 11: 47: 39 & 0.0022 & 0.0080\end{array}$


Appendix D - Laser Data Test 2 NIST102605_1.xls

$\begin{array}{llll}7240 & 11: 47: 41 & 0.0027 & 0.0272 \\ 7242 & 11: 47: 43 & 0.0041 & 0.0076 \\ 7244 & 11: 47: 45 & 0.0030 & 0.0228 \\ 7246 & 11: 47: 47 & 0.0120 & 0.0109 \\ 7248 & 11: 47: 49 & 0.0011 & 0.0122 \\ 7250 & 11: 47: 51 & 0.0003 & 0.0140 \\ 7252 & 11: 47: 53 & 0.0018 & 0.0101 \\ 7254 & 11: 47: 55 & 0.0101 & 0.1542 \\ 7256 & 11: 47: 57 & 0.0050 & 0.0050 \\ 7258 & 11: 47: 59 & 0.0009 & 0.0042 \\ 7260 & 11: 48: 01 & 0.0030 & 0.0177 \\ 7262 & 11: 48: 03 & 0.0059 & 0.0302 \\ 7264 & 11: 48: 05 & 0.0128 & 0.0234 \\ 7266 & 11: 48: 07 & 0.0032 & 0.0013 \\ 7268 & 11: 48: 09 & 0.0089 & 0.0133 \\ 7270 & 11: 48: 11 & 0.0267 & 0.0112 \\ 7272 & 11: 48: 13 & 0.0027 & 0.0176 \\ 7274 & 11: 48: 15 & 0.0067 & 0.0080 \\ 7276 & 11: 48: 17 & 0.0135 & 0.0028 \\ 7278 & 11: 48: 19 & 0.0327 & 0.0039 \\ 7280 & 11: 48: 21 & 0.0294 & 0.0007 \\ 7282 & 11: 48: 23 & 0.0333 & 0.0060 \\ 7284 & 11: 48: 25 & 0.0051 & 0.0004 \\ 7286 & 11: 48: 27 & 0.0068 & 0.0048 \\ 7288 & 11: 48: 29 & 0.0167 & 0.0309 \\ 7290 & 11: 48: 31 & 0.0055 & 0.0054 \\ 7292 & 11: 48: 33 & 0.0042 & 0.0080 \\ 7294 & 11: 48: 35 & 0.0169 & 0.0071 \\ 7296 & 11: 48: 37 & 0.0120 & 0.0062 \\ 7298 & 11: 48: 39 & 0.0044 & 0.0082 \\ 7300 & 11: 48: 41 & 0.0104 & 0.0229 \\ 7302 & 11: 48: 43 & 0.0029 & 0.0055 \\ 7304 & 11: 48: 45 & 0.0049 & 0.0065 \\ 7306 & 11: 48: 47 & 0.0021 & 0.0025 \\ 7308 & 11: 48: 49 & 0.0023 & 0.0031 \\ 7310 & 11: 48: 51 & 0.0006 & 0.0030 \\ 7312 & 11: 48: 53 & 0.0006 & 0.0036 \\ 7314 & 11: 48: 55 & 0.0003 & 0.0040 \\ 7316 & 11: 48: 57 & 0.0013 & 0.0036 \\ 7318 & 11: 48: 59 & 0.0038 & 0.0178 \\ 7320 & 11: 49: 01 & 0.0052 & 0.0061 \\ 7322 & 11: 49: 03 & 0.0053 & 0.0168 \\ 7324 & 11: 49: 05 & 0.0044 & 0.0133 \\ 7326 & 11: 49: 07 & 0.0046 & 0.0065 \\ 7328 & 11: 49: 09 & 0.0347 & 0.0208 \\ 7330 & 11: 49: 11 & 0.0037 & 0.0039 \\ 7332 & 11: 49: 13 & 0.0060 & 0.0497 \\ 7334 & 11: 49: 15 & 0.0018 & 0.0127 \\ 7336 & 11: 49: 17 & 0.0033 & 0.0130 \\ 7338 & 11: 49: 19 & 0.0009 & 0.0035 \\ 7340 & 11: 49: 21 & 0.0008 & 0.0028\end{array}$

D - 72 
Appendix D - Laser Data Test 2 NIST102605_1.xls

$\begin{array}{llll}7342 & 11: 49: 23 & 0.0032 & 0.0216 \\ 7344 & 11: 49: 25 & 0.0088 & 0.0086 \\ 7346 & 11: 49: 27 & 0.0022 & 0.0050 \\ 7348 & 11: 49: 29 & 0.0027 & 0.0131 \\ 7350 & 11: 49: 31 & 0.0010 & 0.0036 \\ 7352 & 11: 49: 33 & 0.0050 & 0.0056 \\ 7354 & 11: 49: 35 & 0.0013 & 0.0047 \\ 7356 & 11: 49: 37 & 0.0006 & 0.0075 \\ 7358 & 11: 49: 39 & 0.0031 & 0.0595 \\ 7360 & 11: 49: 41 & 0.0045 & 0.0047 \\ 7362 & 11: 49: 43 & 0.0009 & 0.0034 \\ 7364 & 11: 49: 45 & 0.0152 & 0.0023 \\ 7366 & 11: 49: 47 & 0.0296 & 0.0039 \\ 7368 & 11: 49: 49 & 0.0038 & 0.0035 \\ 7370 & 11: 49: 51 & 0.0070 & 0.0011 \\ 7372 & 11: 49: 53 & 0.0042 & 0.0279 \\ 7374 & 11: 49: 55 & 0.0017 & 0.0008 \\ 7376 & 11: 49: 57 & 0.0069 & 0.0036 \\ 7378 & 11: 49: 59 & 0.0295 & 0.0087 \\ 7380 & 11: 50: 01 & 0.0138 & 0.0024 \\ 7382 & 11: 50: 03 & 0.0012 & 0.0067 \\ 7384 & 11: 50: 05 & 0.0025 & 0.0125 \\ 7386 & 11: 50: 07 & 0.0158 & 0.0049 \\ 7388 & 11: 50: 09 & 0.0045 & 0.0761 \\ 7390 & 11: 50: 11 & 0.0050 & 0.0025 \\ 7392 & 11: 50: 13 & 0.0013 & 0.0008 \\ 7394 & 11: 50: 15 & 0.0193 & 0.0032 \\ 7396 & 11: 50: 17 & 0.0043 & 0.0055 \\ 7398 & 11: 50: 19 & 0.0204 & 0.0071 \\ 7400 & 11: 50: 21 & 0.0211 & 0.0082 \\ 7402 & 11: 50: 23 & 0.0048 & 0.0077 \\ 7404 & 11: 50: 25 & 0.0044 & 0.0018 \\ 7406 & 11: 50: 27 & 0.0016 & 0.0092 \\ 7408 & 11: 50: 29 & 0.0024 & 0.0208 \\ 7410 & 11: 50: 31 & 0.0035 & 0.0087 \\ 7412 & 11: 50: 33 & 0.0084 & 0.0262 \\ 7414 & 11: 50: 35 & 0.0173 & 0.0112 \\ 7416 & 11: 50: 37 & 0.0238 & 0.0051 \\ 7418 & 11: 50: 39 & 0.0037 & 0.0038 \\ 7420 & 11: 50: 41 & 0.0048 & 0.0139 \\ 7422 & 11: 50: 43 & 0.0119 & 0.0081 \\ 7424 & 11: 50: 45 & 0.0017 & 0.0060 \\ 7426 & 11: 50: 47 & 0.0252 & 0.0176 \\ 7428 & 11: 50: 49 & 0.0070 & 0.0015 \\ 7430 & 11: 50: 51 & 0.0031 & 0.0117 \\ 7432 & 11: 50: 53 & 0.0071 & 0.0191 \\ 7434 & 11: 50: 55 & 0.0032 & 0.0100 \\ 7436 & 11: 50: 57 & 0.0021 & 0.0029 \\ 7438 & 11: 50: 59 & 0.0009 & 0.0051 \\ 7440 & 11: 51: 01 & 0.0008 & 0.0050 \\ 7442 & 11: 51: 03 & 0.0300 & 0.0098\end{array}$

D - 73 
Appendix D - Laser Data Test 2 NIST102605_1.xls

$\begin{array}{llll}7444 & 11: 51: 05 & 0.0009 & 0.0033 \\ 7446 & 11: 51: 07 & 0.0007 & 0.0012 \\ 7448 & 11: 51: 09 & 0.0034 & 0.0246 \\ 7450 & 11: 51: 11 & 0.0007 & 0.0125 \\ 7452 & 11: 51: 13 & 0.0007 & 0.0052 \\ 7454 & 11: 51: 15 & 0.0059 & 0.0254 \\ 7456 & 11: 51: 17 & 0.0033 & 0.0009 \\ 7458 & 11: 51: 19 & 0.0007 & 0.0008 \\ 7460 & 11: 51: 21 & 0.0022 & 0.0108 \\ 7462 & 11: 51: 23 & 0.0153 & 0.0146 \\ 7464 & 11: 51: 25 & 0.0317 & 0.0066 \\ 7466 & 11: 51: 27 & 0.0033 & 0.0045 \\ 7468 & 11: 51: 29 & 0.0002 & 0.0108 \\ 7470 & 11: 51: 31 & 0.0022 & 0.0517 \\ 7472 & 11: 51: 33 & 0.0121 & 0.0141 \\ 7474 & 11: 51: 35 & 0.0031 & 0.0192 \\ 7476 & 11: 51: 37 & 0.0046 & 0.0029 \\ 7478 & 11: 51: 39 & 0.0015 & 0.0025 \\ 7480 & 11: 51: 41 & 0.0011 & 0.0035 \\ 7482 & 11: 51: 43 & 0.0010 & 0.0048 \\ 7484 & 11: 51: 45 & 0.0008 & 0.0164 \\ 7486 & 11: 51: 47 & 0.0223 & 0.0019 \\ 7488 & 11: 51: 49 & 0.0479 & 0.0013 \\ 7490 & 11: 51: 51 & 0.0373 & 0.0011 \\ 7492 & 11: 51: 53 & 0.0343 & 0.0024 \\ 7494 & 11: 51: 55 & 0.0520 & 0.0006 \\ 7496 & 11: 51: 57 & 0.0267 & 0.0025 \\ 7498 & 11: 51: 59 & 0.0013 & 0.0007 \\ 7500 & 11: 52: 01 & 0.0285 & 0.0037 \\ 7502 & 11: 52: 03 & 0.0233 & 0.0416 \\ 7504 & 11: 52: 05 & 0.0143 & 0.0018 \\ 7506 & 11: 52: 07 & 0.0039 & 0.0068 \\ 7508 & 11: 52: 09 & 0.0025 & 0.0074 \\ 7510 & 11: 52: 11 & 0.0054 & 0.0025 \\ 7512 & 11: 52: 13 & 0.0032 & 0.0045 \\ 7514 & 11: 52: 15 & 0.0048 & 0.0141 \\ 7516 & 11: 52: 17 & 0.0013 & 0.0136 \\ 7518 & 11: 52: 19 & 0.0129 & 0.0733 \\ 7520 & 11: 52: 21 & 0.0026 & 0.0091 \\ 7522 & 11: 52: 23 & 0.0030 & 0.0126 \\ 7524 & 11: 52: 25 & 0.0007 & 0.0289 \\ 7526 & 11: 52: 27 & 0.0030 & 0.0137 \\ 7528 & 11: 52: 29 & 0.0043 & 0.0112 \\ 7530 & 11: 52: 31 & 0.0034 & 0.0197 \\ 7532 & 11: 52: 33 & 0.0025 & 0.0133 \\ 7534 & 11: 52: 35 & 0.0443 & 0.0123 \\ 7536 & 11: 52: 37 & 0.0253 & 0.0008 \\ 7538 & 11: 52: 39 & 0.1986 & 0.0034 \\ 7540 & 11: 52: 41 & 0.0286 & 0.0043 \\ 7542 & 11: 52: 43 & 0.0272 & 0.0116 \\ 7544 & 11: 52: 45 & 0.0368 & 0.0081\end{array}$

D -74 
Appendix D - Laser Data Test 2 NIST102605_1.xls

$\begin{array}{llll}7546 & 11: 52: 47 & 0.0049 & 0.0102 \\ 7548 & 11: 52: 49 & 0.0276 & 0.0119 \\ 7550 & 11: 52: 51 & 0.0181 & 0.0127 \\ 7552 & 11: 52: 53 & 0.0089 & 0.0149 \\ 7554 & 11: 52: 55 & 0.0247 & 0.0029 \\ 7556 & 11: 52: 57 & 0.0068 & 0.0061 \\ 7558 & 11: 52: 59 & 0.0273 & 0.0033 \\ 7560 & 11: 53: 01 & 0.0078 & 0.0079 \\ 7562 & 11: 53: 03 & 0.0037 & 0.0076 \\ 7564 & 11: 53: 05 & 0.0560 & 0.0014 \\ 7566 & 11: 53: 07 & 0.0055 & 0.0050 \\ 7568 & 11: 53: 09 & 0.0060 & 0.0424 \\ 7570 & 11: 53: 11 & 0.0091 & 0.0096 \\ 7572 & 11: 53: 13 & 0.0121 & 0.0028 \\ 7574 & 11: 53: 15 & 0.0026 & 0.0077 \\ 7576 & 11: 53: 17 & 0.0109 & 0.0071 \\ 7578 & 11: 53: 19 & 0.0003 & 0.0024 \\ 7580 & 11: 53: 21 & 0.0060 & 0.0424 \\ 7582 & 11: 53: 23 & 0.0029 & 0.0037 \\ 7584 & 11: 53: 25 & 0.0020 & 0.0040 \\ 7586 & 11: 53: 27 & 0.0018 & 0.0079 \\ 7588 & 11: 53: 29 & 0.0024 & 0.0240 \\ 7590 & 11: 53: 31 & 0.0211 & 0.0041 \\ 7592 & 11: 53: 33 & 0.0082 & 0.0030 \\ 7594 & 11: 53: 35 & 0.0096 & 0.0057 \\ 7596 & 11: 53: 37 & 0.0021 & 0.0145 \\ 7598 & 11: 53: 39 & 0.0085 & 0.0136 \\ 7600 & 11: 53: 41 & 0.0009 & 0.0115 \\ 7602 & 11: 53: 43 & 0.0076 & 0.0042 \\ 7604 & 11: 53: 45 & 0.0097 & 0.0034 \\ 7606 & 11: 53: 47 & 0.0056 & 0.0026 \\ 7608 & 11: 53: 49 & 0.0148 & 0.0013 \\ 7610 & 11: 53: 51 & 0.0008 & 0.0006 \\ 7612 & 11: 53: 53 & 0.0030 & 0.0099 \\ 7614 & 11: 53: 55 & 0.0083 & 0.0062 \\ 7616 & 11: 53: 57 & 0.0007 & 0.0067 \\ 7618 & 11: 53: 59 & 0.0039 & 0.0064 \\ 7620 & 11: 54: 01 & 0.0028 & 0.0065 \\ 7622 & 11: 54: 03 & 0.0135 & 0.0017 \\ 7624 & 11: 54: 05 & 0.0021 & 0.0078 \\ 7626 & 11: 54: 07 & 0.0209 & 0.0048 \\ 7628 & 11: 54: 09 & 0.0053 & 0.0017 \\ 7630 & 11: 54: 11 & 0.0229 & 0.0024 \\ 7632 & 11: 54: 13 & 0.0245 & 0.0019 \\ 7634 & 11: 54: 15 & 0.0060 & 0.0059 \\ 7636 & 11: 54: 17 & 0.0083 & 0.0151 \\ 7638 & 11: 54: 19 & 0.0121 & 0.0173 \\ 7640 & 11: 54: 21 & 0.0114 & 0.0036 \\ 7642 & 11: 54: 23 & 0.0213 & 0.0050 \\ 7644 & 11: 54: 25 & 0.0035 & 0.0333 \\ 7646 & 11: 54: 27 & 0.0112 & 0.0145\end{array}$

D - 75 
Appendix D - Laser Data Test 2 NIST102605_1.xls

$\begin{array}{llll}7648 & 11: 54: 29 & 0.0359 & 0.0088 \\ 7650 & 11: 54: 31 & 0.0230 & 0.0094 \\ 7652 & 11: 54: 33 & 0.0076 & 0.0021 \\ 7654 & 11: 54: 35 & 0.0404 & 0.0091 \\ 7656 & 11: 54: 37 & 0.0231 & 0.0117 \\ 7658 & 11: 54: 39 & 0.0267 & 0.0025 \\ 7660 & 11: 54: 41 & 0.0305 & 0.0017 \\ 7662 & 11: 54: 43 & 0.0212 & 0.0017 \\ 7664 & 11: 54: 45 & 0.0149 & 0.0072 \\ 7666 & 11: 54: 47 & 0.1515 & 0.0562 \\ 7668 & 11: 54: 49 & 0.0063 & 0.0057 \\ 7670 & 11: 54: 51 & 0.0164 & 0.0009 \\ 7672 & 11: 54: 53 & 0.0053 & 0.0042 \\ 7674 & 11: 54: 55 & 0.0135 & 0.0018 \\ 7676 & 11: 54: 57 & 0.0052 & 0.0052 \\ 7678 & 11: 54: 59 & 0.0534 & 0.0034 \\ 7680 & 11: 55: 01 & 0.0293 & 0.0087 \\ 7682 & 11: 55: 03 & 0.0250 & 0.0112 \\ 7684 & 11: 55: 05 & 0.0062 & 0.0082 \\ 7686 & 11: 55: 07 & 0.0049 & 0.0171 \\ 7688 & 11: 55: 09 & 0.0062 & 0.0037 \\ 7690 & 11: 55: 11 & 0.0043 & 0.0057 \\ 7692 & 11: 55: 13 & 0.0061 & 0.0066 \\ 7694 & 11: 55: 15 & 0.0319 & 0.0006 \\ 7696 & 11: 55: 17 & 0.0280 & 0.0105 \\ 7698 & 11: 55: 19 & 0.0055 & 0.0430 \\ 7700 & 11: 55: 21 & 0.0105 & 0.0029 \\ 7702 & 11: 55: 23 & 0.0136 & 0.0048 \\ 7704 & 11: 55: 25 & 0.0053 & 0.0029 \\ 7706 & 11: 55: 27 & 0.0180 & 0.0055 \\ 7708 & 11: 55: 29 & 0.0140 & 0.0026 \\ 7710 & 11: 55: 31 & 0.0108 & 0.0017 \\ 7712 & 11: 55: 33 & 0.0175 & 0.0039 \\ 7714 & 11: 55: 35 & 0.0059 & 0.0025 \\ 7716 & 11: 55: 37 & 0.0079 & 0.0021 \\ 7718 & 11: 55: 39 & 0.0075 & 0.0226 \\ 7720 & 11: 55: 41 & 0.0100 & 0.0171 \\ 7722 & 11: 55: 43 & 0.0083 & 0.0108 \\ 7724 & 11: 55: 45 & 0.0136 & 0.0093 \\ 7726 & 11: 55: 47 & 0.0040 & 0.0348 \\ 7728 & 11: 55: 49 & 0.0061 & 0.0066 \\ 7730 & 11: 55: 51 & 0.0455 & 0.0019 \\ 7732 & 11: 55: 53 & 0.0226 & 0.0032 \\ 7734 & 11: 55: 55 & 0.0051 & 0.0064 \\ 7736 & 11: 55: 57 & 0.0359 & 0.0251 \\ 7738 & 11: 55: 59 & 0.0081 & 0.0004 \\ 7740 & 11: 56: 01 & 0.0151 & 0.0025 \\ 7742 & 11: 56: 03 & 0.0066 & 0.0009 \\ 7744 & 11: 56: 05 & 0.0505 & 0.0066 \\ 7746 & 11: 56: 07 & 0.0136 & 0.0035 \\ 7748 & 11: 56: 09 & 0.0081 & 0.0078\end{array}$


Appendix D - Laser Data Test 2 NIST102605_1.xls

$\begin{array}{llll}7750 & 11: 56: 11 & 0.0030 & 0.0042 \\ 7752 & 11: 56: 13 & 0.0354 & 0.0033 \\ 7754 & 11: 56: 15 & 0.0065 & 0.0021 \\ 7756 & 11: 56: 17 & 0.0024 & 0.0067 \\ 7758 & 11: 56: 19 & 0.0063 & 0.0061 \\ 7760 & 11: 56: 21 & 0.0060 & 0.0009 \\ 7762 & 11: 56: 23 & 0.0021 & 0.0046 \\ 7764 & 11: 56: 25 & 0.0034 & 0.0038 \\ 7766 & 11: 56: 27 & 0.0044 & 0.0020 \\ 7768 & 11: 56: 29 & 0.0015 & 0.0064 \\ 7770 & 11: 56: 31 & 0.0006 & 0.0327 \\ 7772 & 11: 56: 33 & 0.0022 & 0.0097 \\ 7774 & 11: 56: 35 & 0.0113 & 0.0078 \\ 7776 & 11: 56: 37 & 0.0078 & 0.0426 \\ 7778 & 11: 56: 39 & 0.0165 & 0.0085 \\ 7780 & 11: 56: 41 & 0.0093 & 0.0173 \\ 7782 & 11: 56: 43 & 0.0064 & 0.0080 \\ 7784 & 11: 56: 45 & 0.0094 & 0.0091 \\ 7786 & 11: 56: 47 & 0.0032 & 0.0014 \\ 7788 & 11: 56: 49 & 0.0084 & 0.0017 \\ 7790 & 11: 56: 51 & 0.0075 & 0.0007 \\ 7792 & 11: 56: 53 & 0.0030 & 0.0151 \\ 7794 & 11: 56: 55 & 0.0247 & 0.0040 \\ 7796 & 11: 56: 57 & 0.0230 & 0.0034 \\ 7798 & 11: 56: 59 & 0.0361 & 0.0026 \\ 7800 & 11: 57: 01 & 0.0093 & 0.0018 \\ 7802 & 11: 57: 03 & 0.0021 & 0.0087 \\ 7804 & 11: 57: 05 & 0.0134 & 0.0164 \\ 7806 & 11: 57: 07 & 0.0366 & 0.0083 \\ 7808 & 11: 57: 09 & 0.0198 & 0.0042 \\ 7810 & 11: 57: 11 & 0.0101 & 0.0195 \\ 7812 & 11: 57: 13 & 0.0121 & 0.0025 \\ 7814 & 11: 57: 15 & 0.0021 & 0.0073 \\ 7816 & 11: 57: 17 & 0.0224 & 0.0111 \\ 7818 & 11: 57: 19 & 0.0217 & 0.0294 \\ 7820 & 11: 57: 21 & 0.0066 & 0.0020 \\ 7822 & 11: 57: 23 & 0.0057 & 0.0008 \\ 7824 & 11: 57: 25 & 0.0186 & 0.0113 \\ 7826 & 11: 57: 27 & 0.0254 & 0.0022 \\ 7828 & 11: 57: 29 & 0.0224 & 0.0142 \\ 7830 & 11: 57: 31 & 0.0143 & 0.0055 \\ 7832 & 11: 57: 33 & 0.0030 & 0.0022 \\ 7834 & 11: 57: 35 & 0.0075 & 0.0018 \\ 7836 & 11: 57: 37 & 0.0079 & 0.0063 \\ 7838 & 11: 57: 39 & 0.0025 & 0.0214 \\ 7840 & 11: 57: 41 & 0.0020 & 0.0045 \\ 7842 & 11: 57: 43 & 0.0115 & 0.0032 \\ 7844 & 11: 57: 45 & 0.0023 & 0.0024 \\ 7846 & 11: 57: 47 & 0.0129 & 0.0066 \\ 7848 & 11: 57: 49 & 0.0151 & 0.0142 \\ 7850 & 11: 57: 51 & 0.0022 & 0.0062\end{array}$


Appendix D - Laser Data Test 2 NIST102605_1.xls

$\begin{array}{llll}7852 & 11: 57: 53 & 0.0024 & 0.0035 \\ 7854 & 11: 57: 55 & 0.0080 & 0.0164 \\ 7856 & 11: 57: 57 & 0.0136 & 0.0467 \\ 7858 & 11: 57: 59 & 0.0395 & 0.0037 \\ 7860 & 11: 58: 01 & 0.0138 & 0.0036 \\ 7862 & 11: 58: 03 & 0.0094 & 0.0016 \\ 7864 & 11: 58: 05 & 0.0189 & 0.0081 \\ 7866 & 11: 58: 07 & 0.0118 & 0.0163 \\ 7868 & 11: 58: 09 & 0.0207 & 0.0265 \\ 7870 & 11: 58: 11 & 0.0271 & 0.0186 \\ 7872 & 11: 58: 13 & 0.0099 & 0.0147 \\ 7874 & 11: 58: 15 & 0.0170 & 0.0085 \\ 7876 & 11: 58: 17 & 0.0074 & 0.0055 \\ 7878 & 11: 58: 19 & 0.0176 & 0.0059 \\ 7880 & 11: 58: 21 & 0.0070 & 0.0125 \\ 7882 & 11: 58: 23 & 0.0314 & 0.0035 \\ 7884 & 11: 58: 25 & 0.0046 & 0.0149 \\ 7886 & 11: 58: 27 & 0.0244 & 0.0126 \\ 7888 & 11: 58: 29 & 0.0109 & 0.0027 \\ 7890 & 11: 58: 31 & 0.0166 & 0.0179 \\ 7892 & 11: 58: 33 & 0.0055 & 0.0367 \\ 7894 & 11: 58: 35 & 0.0023 & 0.0067 \\ 7896 & 11: 58: 37 & 0.0920 & 0.0064 \\ 7898 & 11: 58: 39 & 0.0133 & 0.0186 \\ 7900 & 11: 58: 41 & 0.0500 & 0.0287 \\ 7902 & 11: 58: 43 & 0.0090 & 0.0183 \\ 7904 & 11: 58: 45 & 0.0048 & 0.0063 \\ 7906 & 11: 58: 47 & 0.0047 & 0.0018 \\ 7908 & 11: 58: 49 & 0.0048 & 0.0004 \\ 7910 & 11: 58: 51 & 0.0053 & 0.0046 \\ 7912 & 11: 58: 53 & 0.0079 & 0.0083 \\ 7914 & 11: 58: 55 & 0.0129 & 0.0013 \\ 7916 & 11: 58: 57 & 0.0230 & 0.0034 \\ 7918 & 11: 58: 59 & 0.0066 & 0.0137 \\ 7920 & 11: 59: 01 & 0.0196 & 0.0027 \\ 7922 & 11: 59: 03 & 0.0078 & 0.0139 \\ 7924 & 11: 59: 05 & 0.0040 & 0.0030 \\ 7926 & 11: 59: 07 & 0.0012 & 0.0060 \\ 7928 & 11: 59: 09 & 0.0071 & 0.0024 \\ 7930 & 11: 59: 11 & 0.0070 & 0.0110 \\ 7932 & 11: 59: 13 & 0.0327 & 0.0064 \\ 7934 & 11: 59: 15 & 0.0118 & 0.0777 \\ 7936 & 11: 59: 17 & 0.0028 & 0.0009 \\ 7938 & 11: 59: 19 & 0.0055 & 0.0081 \\ 7940 & 11: 59: 21 & 0.0206 & 0.0033 \\ 7942 & 11: 59: 23 & 0.0183 & 0.0067 \\ 7944 & 11: 59: 25 & 0.0040 & 0.0043 \\ 7946 & 11: 59: 27 & 0.0270 & 0.0013 \\ 7948 & 11: 59: 29 & 0.0102 & 0.0033 \\ 7950 & 11: 59: 31 & 0.0133 & 0.0015 \\ 7952 & 11: 59: 33 & 0.0234 & 0.0109\end{array}$


Appendix D - Laser Data Test 2 NIST102605_1.xls

$\begin{array}{llll}7954 & 11: 59: 35 & 0.0262 & 0.0049 \\ 7956 & 11: 59: 37 & 0.0187 & 0.0059 \\ 7958 & 11: 59: 39 & 0.0269 & 0.0052 \\ 7960 & 11: 59: 41 & 0.0105 & 0.0228 \\ 7962 & 11: 59: 43 & 0.0090 & 0.0021 \\ 7964 & 11: 59: 45 & 0.0185 & 0.0150 \\ 7966 & 11: 59: 47 & 0.0226 & 0.0062 \\ 7968 & 11: 59: 49 & 0.0185 & 0.0013 \\ 7970 & 11: 59: 51 & 0.0186 & 0.0008 \\ 7972 & 11: 59: 53 & 0.0163 & 0.0062 \\ 7974 & 11: 59: 55 & 0.0149 & 0.0018 \\ 7976 & 11: 59: 57 & 0.0134 & 0.0073 \\ 7978 & 11: 59: 59 & 0.0087 & 0.0049 \\ 7980 & 12: 00: 01 & 0.0088 & 0.0309 \\ 7982 & 12: 00: 03 & 0.0245 & 0.0054 \\ 7984 & 12: 00: 05 & 0.0022 & 0.0046 \\ 7986 & 12: 00: 07 & 0.0120 & 0.0037 \\ 7988 & 12: 00: 09 & 0.0277 & 0.0047 \\ 7990 & 12: 00: 11 & 0.0154 & 0.0019 \\ 7992 & 12: 00: 13 & 0.0081 & 0.0078 \\ 7994 & 12: 00: 15 & 0.0047 & 0.0334 \\ 7996 & 12: 00: 17 & 0.0571 & 0.0511 \\ 7998 & 12: 00: 19 & 0.0012 & 0.0105 \\ 8000 & 12: 00: 21 & 0.0028 & 0.0003 \\ 8002 & 12: 00: 23 & 0.0056 & 0.0046 \\ 8004 & 12: 00: 25 & 0.0206 & 0.0071 \\ 8006 & 12: 00: 27 & 0.0137 & 0.0043 \\ 8008 & 12: 00: 29 & 0.0236 & 0.0067 \\ 8010 & 12: 00: 31 & 0.0128 & 0.0003 \\ 8012 & 12: 00: 33 & 0.0054 & 0.0030 \\ 8014 & 12: 00: 35 & 0.0198 & 0.0033 \\ 8016 & 12: 00: 37 & 0.0049 & 0.0392 \\ 8018 & 12: 00: 39 & 0.0019 & 0.0032 \\ 8020 & 12: 00: 41 & 0.0045 & 0.0035 \\ 8022 & 12: 00: 43 & 0.0121 & 0.0035 \\ 8024 & 12: 00: 45 & 0.0036 & 0.0030 \\ 8026 & 12: 00: 47 & 0.0074 & 0.0324 \\ 8028 & 12: 00: 49 & 0.0093 & 0.0044 \\ 8030 & 12: 00: 51 & 0.0135 & 0.0033 \\ 8032 & 12: 00: 53 & 0.0062 & 0.0024 \\ 8034 & 12: 00: 55 & 0.0155 & 0.0078 \\ 8036 & 12: 00: 57 & 0.0282 & 0.0116 \\ 8038 & 12: 00: 59 & 0.0208 & 0.0008 \\ 8040 & 12: 01: 01 & 0.0264 & 0.0038 \\ 8042 & 12: 01: 03 & 0.0210 & 0.0024 \\ 8044 & 12: 01: 05 & 0.0095 & 0.0056 \\ 8046 & 12: 01: 07 & 0.0014 & 0.0504 \\ 8048 & 12: 01: 09 & 0.0202 & 0.0011 \\ 8050 & 12: 01: 11 & 0.0040 & 0.0051 \\ 8052 & 12: 01: 13 & 0.0073 & 0.0047 \\ 8054 & 12: 01: 15 & 0.0064 & 0.0436\end{array}$


Appendix D - Laser Data Test 2 NIST102605_1.xls

$\begin{array}{llll}8056 & 12: 01: 17 & 0.0220 & 0.0017 \\ 8058 & 12: 01: 19 & 0.0108 & 0.0056 \\ 8060 & 12: 01: 21 & 0.0172 & 0.0017 \\ 8062 & 12: 01: 23 & 0.0087 & 0.0034 \\ 8064 & 12: 01: 25 & 0.0115 & 0.0008 \\ 8066 & 12: 01: 27 & 0.0131 & 0.0032 \\ 8068 & 12: 01: 29 & 0.0090 & 0.0051 \\ 8070 & 12: 01: 31 & 0.0277 & 0.0231 \\ 8072 & 12: 01: 33 & 0.0064 & 0.0054 \\ 8074 & 12: 01: 35 & 0.0172 & 0.0105 \\ 8076 & 12: 01: 37 & 0.0017 & 0.0014 \\ 8078 & 12: 01: 39 & 0.0016 & 0.0362 \\ 8080 & 12: 01: 41 & 0.0043 & 0.0098 \\ 8082 & 12: 01: 43 & 0.0068 & 0.0076 \\ 8084 & 12: 01: 45 & 0.0079 & 0.0998 \\ 8086 & 12: 01: 47 & 0.0016 & 0.0063 \\ 8088 & 12: 01: 49 & 0.0007 & 0.0059 \\ 8090 & 12: 01: 51 & 0.0055 & 0.0229 \\ 8092 & 12: 01: 53 & 0.0076 & 0.0131 \\ 8094 & 12: 01: 55 & 0.0017 & 0.0017 \\ 8096 & 12: 01: 57 & 0.0055 & 0.0907 \\ 8098 & 12: 01: 59 & 0.0030 & 0.0585 \\ 8100 & 12: 02: 01 & 0.0019 & 0.0117 \\ 8102 & 12: 02: 03 & 0.0017 & 0.0037 \\ 8104 & 12: 02: 05 & 0.0075 & 0.0051 \\ 8106 & 12: 02: 07 & 0.0007 & 0.0013 \\ 8108 & 12: 02: 09 & 0.0025 & 0.0079 \\ 8110 & 12: 02: 11 & 0.0011 & 0.0029 \\ 8112 & 12: 02: 13 & 0.0116 & 0.0064 \\ 8114 & 12: 02: 15 & 0.0054 & 0.0058 \\ 8116 & 12: 02: 17 & 0.0008 & 0.0395 \\ 8118 & 12: 02: 19 & 0.0026 & 0.0190 \\ 8120 & 12: 02: 21 & 0.0004 & 0.0047 \\ 8122 & 12: 02: 23 & 0.0009 & 0.0404 \\ 8124 & 12: 02: 25 & 0.0020 & 0.0099 \\ 8126 & 12: 02: 27 & 0.0009 & 0.0068 \\ 8128 & 12: 02: 29 & 0.0007 & 0.0083 \\ 8130 & 12: 02: 31 & 0.0043 & 0.0038 \\ 8132 & 12: 02: 33 & 0.0062 & 0.0133 \\ 8134 & 12: 02: 35 & 0.0010 & 0.0113 \\ 8136 & 12: 02: 37 & 0.0034 & 0.0257 \\ 8138 & 12: 02: 39 & 0.0134 & 0.0944 \\ 8140 & 12: 02: 41 & 0.0211 & 0.0231 \\ 8142 & 12: 02: 43 & 0.0088 & 0.0063 \\ 8144 & 12: 02: 45 & 0.0022 & 0.0038 \\ 8146 & 12: 02: 47 & 0.0017 & 0.0187 \\ 8148 & 12: 02: 49 & 0.0071 & 0.0045 \\ 8150 & 12: 02: 51 & 0.0013 & 0.0138 \\ 8152 & 12: 02: 53 & 0.0009 & 0.0055 \\ 8154 & 12: 02: 55 & 0.0016 & 0.0089 \\ 8156 & 12: 02: 57 & 0.0020 & 0.0022\end{array}$


Appendix D - Laser Data Test 2 NIST102605_1.xls

$\begin{array}{llll}8158 & 12: 02: 59 & 0.0026 & 0.0173 \\ 8160 & 12: 03: 01 & 0.0007 & 0.0052 \\ 8162 & 12: 03: 03 & 0.0011 & 0.0446 \\ 8164 & 12: 03: 05 & 0.0114 & 0.0073 \\ 8166 & 12: 03: 07 & 0.0003 & 0.0340 \\ 8168 & 12: 03: 09 & 0.0081 & 0.0067 \\ 8170 & 12: 03: 11 & 0.0029 & 0.0005 \\ 8172 & 12: 03: 13 & 0.0076 & 0.0067 \\ 8174 & 12: 03: 15 & 0.0099 & 0.0448 \\ 8176 & 12: 03: 17 & 0.0108 & 0.0203 \\ 8178 & 12: 03: 19 & 0.0117 & 0.0026 \\ 8180 & 12: 03: 21 & 0.0037 & 0.0155 \\ 8182 & 12: 03: 23 & 0.0134 & 0.0791 \\ 8184 & 12: 03: 25 & 0.0059 & 0.0142 \\ 8186 & 12: 03: 27 & 0.0089 & 0.0296 \\ 8188 & 12: 03: 29 & 0.0129 & 0.0049 \\ 8190 & 12: 03: 31 & 0.0002 & 0.0019 \\ 8192 & 12: 03: 33 & 0.0013 & 0.0099 \\ 8194 & 12: 03: 35 & 0.0053 & 0.0090 \\ 8196 & 12: 03: 37 & 0.0020 & 0.0133 \\ 8198 & 12: 03: 39 & 0.0019 & 0.0214 \\ 8200 & 12: 03: 41 & 0.0075 & 0.0098 \\ 8202 & 12: 03: 43 & 0.0038 & 0.0172 \\ 8204 & 12: 03: 45 & 0.0004 & 0.0032 \\ 8206 & 12: 03: 47 & 0.0009 & 0.0117 \\ 8208 & 12: 03: 49 & 0.0058 & 0.0336 \\ 8210 & 12: 03: 51 & 0.0049 & 0.0318 \\ 8212 & 12: 03: 53 & 0.0010 & 0.0704 \\ 8214 & 12: 03: 55 & 0.0025 & 0.0030 \\ 8216 & 12: 03: 57 & 0.0072 & 0.0147 \\ 8218 & 12: 03: 59 & 0.0028 & 0.0051 \\ 8220 & 12: 04: 01 & 0.0048 & 0.0057 \\ 8222 & 12: 04: 03 & 0.0018 & 0.0008 \\ 8224 & 12: 04: 05 & 0.0046 & 0.0144 \\ 8226 & 12: 04: 07 & 0.0002 & 0.0020 \\ 8228 & 12: 04: 09 & 0.0031 & 0.0025 \\ 8230 & 12: 04: 11 & 0.0019 & 0.0070 \\ 8232 & 12: 04: 13 & 0.0042 & 0.0028 \\ 8234 & 12: 04: 15 & 0.0019 & 0.0101 \\ 8236 & 12: 04: 17 & 0.0125 & 0.0033 \\ 8238 & 12: 04: 19 & 0.0014 & 0.0228 \\ 8240 & 12: 04: 21 & 0.0023 & 0.0029 \\ 8242 & 12: 04: 23 & 0.0017 & 0.0055 \\ 8244 & 12: 04: 25 & 0.0019 & 0.0034 \\ 8246 & 12: 04: 27 & 0.0011 & 0.0385 \\ 8248 & 12: 04: 29 & 0.0022 & 0.0057 \\ 8250 & 12: 04: 31 & 0.0050 & 0.0058 \\ 8252 & 12: 04: 33 & 0.0094 & 0.0031 \\ 8254 & 12: 04: 35 & 0.0027 & 0.0031 \\ 8256 & 12: 04: 37 & 0.0017 & 0.0020 \\ 8258 & 12: 04: 39 & 0.0027 & 0.0055\end{array}$


Appendix D - Laser Data Test 2 NIST102605_1.xls

$\begin{array}{llll}8260 & 12: 04: 41 & 0.0050 & 0.0065 \\ 8262 & 12: 04: 43 & 0.0015 & 0.0173 \\ 8264 & 12: 04: 45 & 0.0006 & 0.0247 \\ 8266 & 12: 04: 47 & 0.0066 & 0.0069 \\ 8268 & 12: 04: 49 & 0.0002 & 0.0020 \\ 8270 & 12: 04: 51 & 0.0003 & 0.0052 \\ 8272 & 12: 04: 53 & 0.0009 & 0.0111 \\ 8274 & 12: 04: 55 & 0.0005 & 0.0056 \\ 8276 & 12: 04: 57 & 0.0014 & 0.0030 \\ 8278 & 12: 04: 59 & 0.0079 & 0.0042 \\ 8280 & 12: 05: 01 & 0.0083 & 0.0482 \\ 8282 & 12: 05: 03 & 0.0057 & 0.0063 \\ 8284 & 12: 05: 05 & 0.0041 & 0.0274 \\ 8286 & 12: 05: 07 & 0.0041 & 0.0183 \\ 8288 & 12: 05: 09 & 0.0097 & 0.0166 \\ 8290 & 12: 05: 11 & 0.0199 & 0.0118 \\ 8292 & 12: 05: 13 & 0.0094 & 0.0321 \\ 8294 & 12: 05: 15 & 0.0044 & 0.0144 \\ 8296 & 12: 05: 17 & 0.0014 & 0.0145 \\ 8298 & 12: 05: 19 & 0.0048 & 0.0104 \\ 8300 & 12: 05: 21 & 0.0057 & 0.0030 \\ 8302 & 12: 05: 23 & 0.0017 & 0.0062 \\ 8304 & 12: 05: 25 & 0.0003 & 0.0041 \\ 8306 & 12: 05: 27 & 0.0020 & 0.0025 \\ 8308 & 12: 05: 29 & 0.0081 & 0.0041 \\ 8310 & 12: 05: 31 & 0.0028 & 0.0073 \\ 8312 & 12: 05: 33 & 0.0067 & 0.0139 \\ 8314 & 12: 05: 35 & 0.0345 & 0.0024 \\ 8316 & 12: 05: 37 & 0.0041 & 0.0027 \\ 8318 & 12: 05: 39 & 0.0020 & 0.0012 \\ 8320 & 12: 05: 41 & 0.0174 & 0.0046 \\ 8322 & 12: 05: 43 & 0.0010 & 0.0108 \\ 8324 & 12: 05: 45 & 0.0068 & 0.0099 \\ 8326 & 12: 05: 47 & 0.0048 & 0.0023 \\ 8328 & 12: 05: 49 & 0.0044 & 0.0031 \\ 8330 & 12: 05: 51 & 0.0020 & 0.0014 \\ 8332 & 12: 05: 53 & 0.0044 & 0.0109 \\ 8334 & 12: 05: 55 & 0.0123 & 0.0189 \\ 8336 & 12: 05: 57 & 0.0194 & 0.0005 \\ 8338 & 12: 05: 59 & 0.0163 & 0.0134 \\ 8340 & 12: 06: 01 & 0.0175 & 0.0061 \\ 8342 & 12: 06: 03 & 0.0228 & 0.0027 \\ 8344 & 12: 06: 05 & 0.0326 & 0.0019 \\ 8346 & 12: 06: 07 & 0.0036 & 0.0029 \\ 8348 & 12: 06: 09 & 0.0086 & 0.0011 \\ 8350 & 12: 06: 11 & 0.0220 & 0.0150 \\ 8352 & 12: 06: 13 & 0.0095 & 0.0062 \\ 8354 & 12: 06: 15 & 0.0062 & 0.0166 \\ 8356 & 12: 06: 17 & 0.0167 & 0.0084 \\ 8358 & 12: 06: 19 & 0.0208 & 0.0023 \\ 8360 & 12: 06: 21 & 0.0151 & 0.0091\end{array}$

D - 82 
Appendix D - Laser Data Test 2 NIST102605_1.xls

$\begin{array}{llll}8362 & 12: 06: 23 & 0.0045 & 0.0167 \\ 8364 & 12: 06: 25 & 0.0170 & 0.0013 \\ 8366 & 12: 06: 27 & 0.0056 & 0.0030 \\ 8368 & 12: 06: 29 & 0.0060 & 0.0071 \\ 8370 & 12: 06: 31 & 0.0092 & 0.0266 \\ 8372 & 12: 06: 33 & 0.0069 & 0.0009 \\ 8374 & 12: 06: 35 & 0.0082 & 0.0014 \\ 8376 & 12: 06: 37 & 0.0132 & 0.0155 \\ 8378 & 12: 06: 39 & 0.0054 & 0.0012 \\ 8380 & 12: 06: 41 & 0.0075 & 0.0003 \\ 8382 & 12: 06: 43 & 0.0103 & 0.0018 \\ 8384 & 12: 06: 45 & 0.0029 & 0.0110 \\ 8386 & 12: 06: 47 & 0.0070 & 0.0063 \\ 8388 & 12: 06: 49 & 0.0118 & 0.0004 \\ 8390 & 12: 06: 51 & 0.0055 & 0.0054 \\ 8392 & 12: 06: 53 & 0.0952 & 0.0044 \\ 8394 & 12: 06: 55 & 0.0047 & 0.0133 \\ 8396 & 12: 06: 57 & 0.0015 & 0.0047 \\ 8398 & 12: 06: 59 & 0.0045 & 0.0208 \\ 8400 & 12: 07: 01 & 0.0163 & 0.0459 \\ 8402 & 12: 07: 03 & 0.0032 & 0.0262 \\ 8404 & 12: 07: 05 & 0.0116 & 0.0031 \\ 8406 & 12: 07: 07 & 0.0316 & 0.0140 \\ 8408 & 12: 07: 09 & 0.0045 & 0.0381 \\ 8410 & 12: 07: 11 & 0.0064 & 0.0074 \\ 8412 & 12: 07: 13 & 0.0223 & 0.0144 \\ 8414 & 12: 07: 15 & 0.0292 & 0.0098 \\ 8416 & 12: 07: 17 & 0.0068 & 0.0400 \\ 8418 & 12: 07: 19 & 0.0004 & 0.0256 \\ 8420 & 12: 07: 21 & 0.0116 & 0.0237 \\ 8422 & 12: 07: 23 & 0.0288 & 0.0018 \\ 8424 & 12: 07: 25 & 0.0067 & 0.0214 \\ 8426 & 12: 07: 27 & 0.0022 & 0.0357 \\ 8428 & 12: 07: 29 & 0.0335 & 0.0745 \\ 8430 & 12: 07: 31 & 0.0100 & 0.0184 \\ 8432 & 12: 07: 33 & 0.0009 & 0.0017 \\ 8434 & 12: 07: 35 & 0.0037 & 0.0043 \\ 8436 & 12: 07: 37 & 0.0167 & 0.0063 \\ 8438 & 12: 07: 39 & 0.0122 & 0.0050 \\ 8440 & 12: 07: 41 & 0.0023 & 0.0043 \\ 8442 & 12: 07: 43 & 0.0128 & 0.0547 \\ 8444 & 12: 07: 45 & 0.0007 & 0.0051 \\ 8446 & 12: 07: 47 & 0.0181 & 0.0576 \\ 8448 & 12: 07: 49 & 0.0010 & 0.0059 \\ 8450 & 12: 07: 51 & 0.0019 & 0.0047 \\ 8452 & 12: 07: 53 & 0.0019 & 0.0088 \\ 8454 & 12: 07: 55 & 0.0003 & 0.0025 \\ 8456 & 12: 07: 57 & 0.0034 & 0.0192 \\ 8458 & 12: 07: 59 & 0.0018 & 0.0015 \\ 8460 & 12: 08: 01 & 0.0002 & 0.0011 \\ 8462 & 12: 08: 03 & 0.0006 & 0.0039\end{array}$

D - 83 
Appendix D - Laser Data Test 2 NIST102605_1.xls

$\begin{array}{llll}8464 & 12: 08: 05 & 0.0007 & 0.0025 \\ 8466 & 12: 08: 07 & 0.0115 & 0.0310 \\ 8468 & 12: 08: 09 & 0.0064 & 0.0073 \\ 8470 & 12: 08: 11 & 0.0011 & 0.0078 \\ 8472 & 12: 08: 13 & 0.0106 & 0.0064 \\ 8474 & 12: 08: 15 & 0.0029 & 0.0069 \\ 8476 & 12: 08: 17 & 0.0218 & 0.0013 \\ 8478 & 12: 08: 19 & 0.0032 & 0.0102 \\ 8480 & 12: 08: 21 & 0.0012 & 0.0086 \\ 8482 & 12: 08: 23 & 0.0157 & 0.0009 \\ 8484 & 12: 08: 25 & 0.0252 & 0.0096 \\ 8486 & 12: 08: 27 & 0.0054 & 0.0032 \\ 8488 & 12: 08: 29 & 0.0235 & 0.0156 \\ 8490 & 12: 08: 31 & 0.0118 & 0.0019 \\ 8492 & 12: 08: 33 & 0.0420 & 0.0079 \\ 8494 & 12: 08: 35 & 0.0220 & 0.0183 \\ 8496 & 12: 08: 37 & 0.0140 & 0.0022 \\ 8498 & 12: 08: 39 & 0.0252 & 0.0103 \\ 8500 & 12: 08: 41 & 0.0247 & 0.0086 \\ 8502 & 12: 08: 43 & 0.0051 & 0.0028 \\ 8504 & 12: 08: 45 & 0.0039 & 0.0069 \\ 8506 & 12: 08: 47 & 0.0090 & 0.0026 \\ 8508 & 12: 08: 49 & 0.0121 & 0.0087 \\ 8510 & 12: 08: 51 & 0.0145 & 0.0027 \\ 8512 & 12: 08: 53 & 0.0103 & 0.0222 \\ 8514 & 12: 08: 55 & 0.0014 & 0.0242 \\ 8516 & 12: 08: 57 & 0.0111 & 0.0013 \\ 8518 & 12: 08: 59 & 0.0395 & 0.0051 \\ 8520 & 12: 09: 01 & 0.0097 & 0.0076 \\ 8522 & 12: 09: 03 & 0.0178 & 0.0057 \\ 8524 & 12: 09: 05 & 0.0150 & 0.0147 \\ 8526 & 12: 09: 07 & 0.0155 & 0.0004 \\ 8528 & 12: 09: 09 & 0.0051 & 0.0028 \\ 8530 & 12: 09: 11 & 0.0118 & 0.0064 \\ 8532 & 12: 09: 13 & 0.0084 & 0.0042 \\ 8534 & 12: 09: 15 & 0.0205 & 0.0135 \\ 8536 & 12: 09: 17 & 0.0188 & 0.0146 \\ 8538 & 12: 09: 19 & 0.0169 & 0.0114 \\ 8540 & 12: 09: 21 & 0.1119 & 0.0017 \\ 8542 & 12: 09: 23 & 0.0188 & 0.0053 \\ 8544 & 12: 09: 25 & 0.0164 & 0.0030 \\ 8546 & 12: 09: 27 & 0.0050 & 0.0024 \\ 8548 & 12: 09: 29 & 0.0139 & 0.0034 \\ 8550 & 12: 09: 31 & 0.0099 & 0.0080 \\ 8552 & 12: 09: 33 & 0.0106 & 0.0050 \\ 8554 & 12: 09: 35 & 0.0022 & 0.0062 \\ 8556 & 12: 09: 37 & 0.0059 & 0.0045 \\ 8558 & 12: 09: 39 & 0.0034 & 0.0032 \\ 8560 & 12: 09: 41 & 0.0055 & 0.0025 \\ 8562 & 12: 09: 43 & 0.0081 & 0.0019 \\ 8564 & 12: 09: 45 & 0.0083 & 0.0014\end{array}$

D - 84 
Appendix D - Laser Data Test 2 NIST102605_1.xls

$\begin{array}{llll}8566 & 12: 09: 47 & 0.0174 & 0.0231 \\ 8568 & 12: 09: 49 & 0.0321 & 0.0125 \\ 8570 & 12: 09: 51 & 0.0090 & 0.0047 \\ 8572 & 12: 09: 53 & 0.0182 & 0.0078 \\ 8574 & 12: 09: 55 & 0.0480 & 0.0352 \\ 8576 & 12: 09: 57 & 0.0074 & 0.0094 \\ 8578 & 12: 09: 59 & 0.0048 & 0.0015 \\ 8580 & 12: 10: 01 & 0.0170 & 0.0041 \\ 8582 & 12: 10: 03 & 0.0215 & 0.0865 \\ 8584 & 12: 10: 05 & 0.0082 & 0.0042 \\ 8586 & 12: 10: 07 & 0.0130 & 0.0006 \\ 8588 & 12: 10: 09 & 0.0088 & 0.0036 \\ 8590 & 12: 10: 11 & 0.0124 & 0.0024 \\ 8592 & 12: 10: 13 & 0.0270 & 0.0031 \\ 8594 & 12: 10: 15 & 0.0120 & 0.0059 \\ 8596 & 12: 10: 17 & 0.0047 & 0.0058 \\ 8598 & 12: 10: 19 & 0.0042 & 0.0039 \\ 8600 & 12: 10: 21 & 0.0120 & 0.0004 \\ 8602 & 12: 10: 23 & 0.0107 & 0.0023 \\ 8604 & 12: 10: 25 & 0.0260 & 0.0063 \\ 8606 & 12: 10: 27 & 0.0235 & 0.0058 \\ 8608 & 12: 10: 29 & 0.0245 & 0.0022 \\ 8610 & 12: 10: 31 & 0.0043 & 0.0359 \\ 8612 & 12: 10: 33 & 0.0160 & 0.0058 \\ 8614 & 12: 10: 35 & 0.0161 & 0.0091 \\ 8616 & 12: 10: 37 & 0.0103 & 0.0022 \\ 8618 & 12: 10: 39 & 0.0160 & 0.0133 \\ 8620 & 12: 10: 41 & 0.0179 & 0.0069 \\ 8622 & 12: 10: 43 & 0.0624 & 0.0248 \\ 8624 & 12: 10: 45 & 0.0051 & 0.0163 \\ 8626 & 12: 10: 47 & 0.0233 & 0.0018 \\ 8628 & 12: 10: 49 & 0.0120 & 0.0243 \\ 8630 & 12: 10: 51 & 0.0116 & 0.0031 \\ 8632 & 12: 10: 53 & 0.0187 & 0.0177 \\ 8634 & 12: 10: 55 & 0.0107 & 0.0164 \\ 8636 & 12: 10: 57 & 0.0009 & 0.0193 \\ 8638 & 12: 10: 59 & 0.1380 & 0.0103 \\ 8640 & 12: 11: 01 & 0.0080 & 0.0025 \\ 8642 & 12: 11: 03 & 0.0014 & 0.0056 \\ 8644 & 12: 11: 05 & 0.0051 & 0.0114 \\ 8646 & 12: 11: 07 & 0.0189 & 0.0276 \\ 8648 & 12: 11: 09 & 0.0103 & 0.0031 \\ 8650 & 12: 11: 11 & 0.0598 & 0.0020 \\ 8652 & 12: 11: 13 & 0.0083 & 0.0014 \\ 8654 & 12: 11: 15 & 0.0191 & 0.0004 \\ 8656 & 12: 11: 17 & 0.0159 & 0.0053 \\ 8658 & 12: 11: 19 & 0.0185 & 0.0043 \\ 8660 & 12: 11: 21 & 0.0080 & 0.0033 \\ 8662 & 12: 11: 23 & 0.0044 & 0.0004 \\ 8664 & 12: 11: 25 & 0.0341 & 0.0288 \\ 8666 & 12: 11: 27 & 0.0042 & 0.0016\end{array}$


Appendix D - Laser Data Test 2 NIST102605_1.xls

$\begin{array}{llll}8668 & 12: 11: 29 & 0.0080 & 0.0020 \\ 8670 & 12: 11: 31 & 0.0144 & 0.0023 \\ 8672 & 12: 11: 33 & 0.0141 & 0.0010 \\ 8674 & 12: 11: 35 & 0.0023 & 0.0036 \\ 8676 & 12: 11: 37 & 0.0273 & 0.0018 \\ 8678 & 12: 11: 39 & 0.0096 & 0.0073 \\ 8680 & 12: 11: 41 & 0.0189 & 0.0021 \\ 8682 & 12: 11: 43 & 0.0058 & 0.0017 \\ 8684 & 12: 11: 45 & 0.0294 & 0.0012 \\ 8686 & 12: 11: 47 & 0.0114 & 0.0047 \\ 8688 & 12: 11: 49 & 0.0044 & 0.0053 \\ 8690 & 12: 11: 51 & 0.0044 & 0.0067 \\ 8692 & 12: 11: 53 & 0.0161 & 0.0014\end{array}$

D - 86 
APPENDIX E. - Fuel Data Test 3 NIST 102605

Appendix $E$ is fuel data from Test 3 in tabular format and is approximately 114 pages in length. Fuel data is included with electronic version of report on compact disk, but not routinely included with paper version of report. 
Appendix E -Fuel Data Test 3 NIST102605_1.xls

Time from Ignition, s EDT Time

0 14.57.20

1 14:57:21

2 14:57:22

3 14:57:23

4 14:57:24

5 14:57:25

6 14:57:26

$\begin{array}{lll}7 & 14: 57: 27\end{array}$

8 14:57:28

9 14:57:29

10 14:57:30

11 14:57:31

12 14:57:32

13 14:57:33

14 14:57:34

15 14:57:35

$16 \quad 14: 57: 36$

17 14:57:37

18 14:57:38

19 14:57:39

20 14:57:40

$21 \quad 14: 57: 41$

22 14:57:42

23 14:57:43

$24 \quad 14: 57: 44$

25 14:57:45

$26 \quad 14: 57: 46$

$27 \quad 14: 57: 47$

28 14:57:48

29 14:57:49

30 14:57:50

31 14:57:51

32 14:57:52

33 14:57:53

34 14:57:54

35 14:57:55

36 14:57:56

37 14:57:57

38 14:57:58

39 14:57:59

40 14:58:00

41 14:58:01

42 14:58:02

43 14:58:03

44 14:58:04

45 14:58:05

46 14:58:06

47 14:58:07

48 14:58:08

49 14:58:09
Liq Flow, I/min

$$
0.4266
$$

0.4219

0.4177

0.4238

0.4192

0.4244

0.4176

0.4217

0.4264

0.4222

0.4264

0.4173

0.4208

0.4155

0.4230

0.4197

0.4186

0.4161

0.4142

0.4150

0.4099

0.4129

0.4120

0.4186

0.4096

0.4149

0.4143

0.4170

0.4179

0.4130

0.4234

0.4120

0.4166

0.4138

0.4193

0.4142

0.4147

0.4158

0.4127

0.4159

0.4074

0.4185

0.4130

0.4103

0.4217

0.4127

0.4155

0.4165

0.4144

0.4207 
Appendix E -Fuel Data Test 3 NIST102605_1.xls

\begin{tabular}{|c|c|c|}
\hline 50 & $14: 58: 10$ & 0.4134 \\
\hline 51 & $14: 58: 11$ & 0.4221 \\
\hline 52 & $14: 58: 12$ & 0.4198 \\
\hline 53 & $14: 58: 13$ & 0.4142 \\
\hline 54 & $14: 58: 14$ & 0.4168 \\
\hline 55 & $14: 58: 15$ & 0.4120 \\
\hline 56 & $14: 58: 16$ & 0.4202 \\
\hline 57 & $14: 58: 17$ & 0.4153 \\
\hline 58 & 14:58:18 & 0.4195 \\
\hline 59 & $14: 58: 19$ & 0.4140 \\
\hline 60 & $14: 58: 20$ & 0.4138 \\
\hline 61 & 14:58:21 & 0.4195 \\
\hline 62 & 14:58:22 & 0.4175 \\
\hline 63 & $14: 58: 23$ & 0.4260 \\
\hline 64 & $14: 58: 24$ & 0.4136 \\
\hline 65 & $14: 58: 25$ & 0.4172 \\
\hline 66 & 14:58:26 & 0.4189 \\
\hline 67 & $14: 58: 27$ & 0.4190 \\
\hline 68 & $14: 58: 28$ & 0.4222 \\
\hline 69 & $14: 58: 29$ & 0.4168 \\
\hline 70 & $14: 58: 30$ & 0.4203 \\
\hline 71 & $14: 58: 31$ & 0.4081 \\
\hline 72 & $14: 58: 32$ & 0.4110 \\
\hline 73 & $14: 58: 33$ & 0.4038 \\
\hline 74 & $14: 58: 34$ & 0.4106 \\
\hline 75 & $14: 58: 35$ & 0.3983 \\
\hline 76 & $14: 58: 36$ & 0.4059 \\
\hline 77 & $14: 58: 37$ & 0.3940 \\
\hline 78 & $14: 58: 38$ & 0.3962 \\
\hline 79 & $14: 58: 39$ & 0.3929 \\
\hline 80 & $14: 58: 40$ & 0.3894 \\
\hline 81 & $14: 58: 41$ & 0.3912 \\
\hline 82 & $14: 58: 42$ & 0.3882 \\
\hline 83 & $14: 58: 43$ & 0.3907 \\
\hline 84 & $14: 58: 44$ & 0.3763 \\
\hline 85 & $14: 58: 45$ & 0.3883 \\
\hline 86 & $14: 58: 46$ & 0.3837 \\
\hline 87 & $14: 58: 47$ & 0.3834 \\
\hline 88 & $14: 58: 48$ & 0.3776 \\
\hline 89 & $14: 58: 49$ & 0.3783 \\
\hline 90 & $14: 58: 50$ & 0.3783 \\
\hline 91 & $14: 58: 51$ & 0.3766 \\
\hline 92 & $14: 58: 52$ & 0.3832 \\
\hline 93 & $14: 58: 53$ & 0.3727 \\
\hline 94 & $14: 58: 54$ & 0.3822 \\
\hline 95 & $14: 58: 55$ & 0.3718 \\
\hline 96 & $14: 58: 56$ & 0.3724 \\
\hline 97 & $14: 58: 57$ & 0.3709 \\
\hline 98 & 14:58:58 & 0.3712 \\
\hline 99 & $14: 58: 59$ & 0.3676 \\
\hline 00 & 14:59:00 & 0.3663 \\
\hline
\end{tabular}

E - 2 
Appendix E -Fuel Data Test 3 NIST102605_1.xls

\begin{tabular}{|c|c|c|}
\hline 101 & $14: 59: 01$ & 0.3679 \\
\hline 102 & $14: 59: 02$ & 0.3676 \\
\hline 103 & $14: 59: 03$ & 0.3694 \\
\hline 104 & $14: 59: 04$ & 0.3660 \\
\hline 105 & $14: 59: 05$ & 0.3723 \\
\hline 106 & $14: 59: 06$ & 0.3684 \\
\hline 107 & $14: 59: 07$ & 0.3702 \\
\hline 108 & $14: 59: 08$ & 0.3612 \\
\hline 109 & 14:59:09 & 0.3685 \\
\hline 110 & $14: 59: 10$ & 0.3638 \\
\hline 111 & $14: 59: 11$ & 0.3679 \\
\hline 112 & $14: 59: 12$ & 0.3623 \\
\hline 113 & $14: 59: 13$ & 0.3699 \\
\hline 114 & $14: 59: 14$ & 0.3666 \\
\hline 115 & $14: 59: 15$ & 0.3731 \\
\hline 116 & $14: 59: 16$ & 0.3611 \\
\hline 117 & $14: 59: 17$ & 0.3621 \\
\hline 118 & 14:59:18 & 0.3630 \\
\hline 119 & $14: 59: 19$ & 0.3628 \\
\hline 120 & $14: 59: 20$ & 0.3632 \\
\hline 121 & $14: 59: 21$ & 0.3551 \\
\hline 122 & $14: 59: 22$ & 0.3611 \\
\hline 123 & $14: 59: 23$ & 0.3548 \\
\hline 124 & $14: 59: 24$ & 0.3615 \\
\hline 125 & $14: 59: 25$ & 0.3547 \\
\hline 126 & $14: 59: 26$ & 0.3626 \\
\hline 127 & $14: 59: 27$ & 0.3554 \\
\hline 128 & $14: 59: 28$ & 0.3574 \\
\hline 129 & $14: 59: 29$ & 0.3512 \\
\hline 130 & $14: 59: 30$ & 0.3573 \\
\hline 131 & $14: 59: 31$ & 0.3541 \\
\hline 132 & $14: 59: 32$ & 0.3532 \\
\hline 133 & $14: 59: 33$ & 0.3498 \\
\hline 134 & $14: 59: 34$ & 0.3532 \\
\hline 135 & $14: 59: 35$ & 0.3570 \\
\hline 136 & $14: 59: 36$ & 0.3536 \\
\hline 137 & $14: 59: 37$ & 0.3565 \\
\hline 138 & $14: 59: 38$ & 0.3473 \\
\hline 139 & $14: 59: 39$ & 0.3531 \\
\hline 140 & $14: 59: 40$ & 0.3452 \\
\hline 141 & $14: 59: 41$ & 0.3524 \\
\hline 142 & $14: 59: 42$ & 0.3428 \\
\hline 143 & $14: 59: 43$ & 0.3541 \\
\hline 144 & $14: 59: 44$ & 0.3484 \\
\hline 145 & $14: 59: 45$ & 0.3502 \\
\hline 146 & $14: 59: 46$ & 0.3546 \\
\hline 147 & $14: 59: 47$ & 0.3551 \\
\hline 148 & $14: 59: 48$ & 0.3560 \\
\hline 149 & $14: 59: 49$ & 0.3436 \\
\hline 150 & $14: 59: 50$ & 0.3497 \\
\hline 151 & $14: 59: 51$ & 0.3447 \\
\hline
\end{tabular}

$E-3$ 
Appendix E -Fuel Data Test 3 NIST102605_1.xls

\begin{tabular}{|c|c|c|}
\hline 152 & $14: 59: 52$ & 0.3507 \\
\hline 153 & $14: 59: 53$ & 0.3461 \\
\hline 154 & $14: 59: 54$ & 0.3533 \\
\hline 155 & $14: 59: 55$ & 0.3446 \\
\hline 156 & $14: 59: 56$ & 0.3504 \\
\hline 157 & $14: 59: 57$ & 0.3463 \\
\hline 158 & $14: 59: 58$ & 0.3444 \\
\hline 159 & $14: 59: 59$ & 0.3456 \\
\hline 160 & $15: 00: 00$ & 0.3447 \\
\hline 161 & 15:00:01 & 0.3455 \\
\hline 162 & $15: 00: 02$ & 0.3383 \\
\hline 163 & $15: 00: 03$ & 0.3499 \\
\hline 164 & 15:00:04 & 0.3436 \\
\hline 165 & 15:00:05 & 0.3499 \\
\hline 166 & 15:00:06 & 0.3419 \\
\hline 167 & $15: 00: 07$ & 0.3490 \\
\hline 168 & $15: 00: 08$ & 0.3452 \\
\hline 169 & 15:00:09 & 0.3453 \\
\hline 170 & $15: 00: 10$ & 0.3388 \\
\hline 171 & $15: 00: 11$ & 0.3412 \\
\hline 172 & $15: 00: 12$ & 0.3429 \\
\hline 173 & $15: 00: 13$ & 0.3446 \\
\hline 174 & $15: 00: 14$ & 0.3388 \\
\hline 175 & $15: 00: 15$ & 0.3434 \\
\hline 176 & $15: 00: 16$ & 0.3417 \\
\hline 177 & $15: 00: 17$ & 0.3463 \\
\hline 178 & $15: 00: 18$ & 0.3424 \\
\hline 179 & $15: 00: 19$ & 0.3436 \\
\hline 180 & $15: 00: 20$ & 0.3445 \\
\hline 181 & $15: 00: 21$ & 0.3413 \\
\hline 182 & $15: 00: 22$ & 0.3417 \\
\hline 183 & $15: 00: 23$ & 0.3352 \\
\hline 184 & $15: 00: 24$ & 0.3445 \\
\hline 185 & $15: 00: 25$ & 0.3418 \\
\hline 186 & $15: 00: 26$ & 0.3451 \\
\hline 187 & $15: 00: 27$ & 0.3375 \\
\hline 188 & $15: 00: 28$ & 0.3438 \\
\hline 189 & $15: 00: 29$ & 0.3375 \\
\hline 190 & $15: 00: 30$ & 0.3399 \\
\hline 191 & $15: 00: 31$ & 0.3348 \\
\hline 192 & $15: 00: 32$ & 0.3402 \\
\hline 193 & $15: 00: 33$ & 0.3403 \\
\hline 194 & $15: 00: 34$ & 0.3411 \\
\hline 195 & $15: 00: 35$ & 0.3476 \\
\hline 196 & $15: 00: 36$ & 0.3354 \\
\hline 197 & $15: 00: 37$ & 0.3411 \\
\hline 198 & $15: 00: 38$ & 0.3330 \\
\hline 199 & 15:00:39 & 0.3418 \\
\hline 200 & $15: 00: 40$ & 0.3358 \\
\hline 201 & $15: 00: 41$ & 0.3443 \\
\hline 202 & $15: 00: 42$ & 0.3362 \\
\hline
\end{tabular}

E - 4 
Appendix E -Fuel Data Test 3 NIST102605_1.xls

\begin{tabular}{|c|c|c|}
\hline 203 & $15: 00: 43$ & 0.3378 \\
\hline 204 & $15: 00: 44$ & 0.3383 \\
\hline 205 & $15: 00: 45$ & 0.3425 \\
\hline 206 & $15: 00: 46$ & 0.3425 \\
\hline 207 & $15: 00: 47$ & 0.3381 \\
\hline 208 & $15: 00: 48$ & 0.3415 \\
\hline 209 & $15: 00: 49$ & 0.3373 \\
\hline 210 & $15: 00: 50$ & 0.3372 \\
\hline 211 & $15: 00: 51$ & 0.3342 \\
\hline 212 & $15: 00: 52$ & 0.3368 \\
\hline 213 & $15: 00: 53$ & 0.3360 \\
\hline 214 & $15: 00: 54$ & 0.3459 \\
\hline 215 & $15: 00: 55$ & 0.3347 \\
\hline 216 & $15: 00: 56$ & 0.3397 \\
\hline 217 & $15: 00: 57$ & 0.3318 \\
\hline 218 & $15: 00: 58$ & 0.3403 \\
\hline 219 & $15: 00: 59$ & 0.3291 \\
\hline 220 & $15: 01: 00$ & 0.3393 \\
\hline 221 & 15:01:01 & 0.3342 \\
\hline 222 & 15:01:02 & 0.3396 \\
\hline 223 & $15: 01: 03$ & 0.3384 \\
\hline 224 & $15: 01: 04$ & 0.3380 \\
\hline 225 & 15:01:05 & 0.3421 \\
\hline 226 & 15:01:06 & 0.3385 \\
\hline 227 & $15: 01: 07$ & 0.3437 \\
\hline 228 & 15:01:08 & 0.3304 \\
\hline 229 & 15:01:09 & 0.3404 \\
\hline 230 & $15: 01: 10$ & 0.3375 \\
\hline 231 & $15: 01: 11$ & 0.3488 \\
\hline 232 & $15: 01: 12$ & 0.3347 \\
\hline 233 & $15: 01: 13$ & 0.3382 \\
\hline 234 & $15: 01: 14$ & 0.3345 \\
\hline 235 & $15: 01: 15$ & 0.3385 \\
\hline 236 & $15: 01: 16$ & 0.3348 \\
\hline 237 & $15: 01: 17$ & 0.3356 \\
\hline 238 & $15: 01: 18$ & 0.3364 \\
\hline 239 & $15: 01: 19$ & 0.3282 \\
\hline 240 & $15: 01: 20$ & 0.3398 \\
\hline 241 & $15: 01: 21$ & 0.3340 \\
\hline 242 & 15:01:22 & 0.3412 \\
\hline 243 & $15: 01: 23$ & 0.3327 \\
\hline 244 & $15: 01: 24$ & 0.3378 \\
\hline 245 & $15: 01: 25$ & 0.3320 \\
\hline 246 & 15:01:26 & 0.3366 \\
\hline 247 & $15: 01: 27$ & 0.3356 \\
\hline 248 & $15: 01: 28$ & 0.3374 \\
\hline 249 & $15: 01: 29$ & 0.3332 \\
\hline 250 & $15: 01: 30$ & 0.3370 \\
\hline 251 & $15: 01: 31$ & 0.3356 \\
\hline 252 & $15: 01: 32$ & 0.3351 \\
\hline & $15: 01: 33$ & 0.3373 \\
\hline
\end{tabular}

E - 5 
Appendix E -Fuel Data Test 3 NIST102605_1.xls

\begin{tabular}{|c|c|c|}
\hline 254 & $15: 01: 34$ & 0.3301 \\
\hline 255 & $15: 01: 35$ & 0.3384 \\
\hline 256 & $15: 01: 36$ & 0.3308 \\
\hline 257 & $15: 01: 37$ & 0.3390 \\
\hline 258 & $15: 01: 38$ & 0.3290 \\
\hline 259 & $15: 01: 39$ & 0.3408 \\
\hline 260 & $15: 01: 40$ & 0.3365 \\
\hline 261 & $15: 01: 41$ & 0.3365 \\
\hline 262 & 15:01:42 & 0.3375 \\
\hline 263 & $15: 01: 43$ & 0.3393 \\
\hline 264 & $15: 01: 44$ & 0.3416 \\
\hline 265 & $15: 01: 45$ & 0.3290 \\
\hline 266 & $15: 01: 46$ & 0.3347 \\
\hline 267 & $15: 01: 47$ & 0.3319 \\
\hline 268 & $15: 01: 48$ & 0.3410 \\
\hline 269 & $15: 01: 49$ & 0.3347 \\
\hline 270 & $15: 01: 50$ & 0.3420 \\
\hline 271 & 15:01:51 & 0.3307 \\
\hline 272 & $15: 01: 52$ & 0.3421 \\
\hline 273 & $15: 01: 53$ & 0.3300 \\
\hline 274 & 15:01:54 & 0.3328 \\
\hline 275 & $15: 01: 55$ & 0.3358 \\
\hline 276 & $15: 01: 56$ & 0.3287 \\
\hline 277 & $15: 01: 57$ & 0.3379 \\
\hline 278 & $15: 01: 58$ & 0.3312 \\
\hline 279 & $15: 01: 59$ & 0.3388 \\
\hline 280 & $15: 02: 00$ & 0.3334 \\
\hline 281 & $15: 02: 01$ & 0.3375 \\
\hline 282 & $15: 02: 02$ & 0.3315 \\
\hline 283 & $15: 02: 03$ & 0.3369 \\
\hline 284 & $15: 02: 04$ & 0.3331 \\
\hline 285 & $15: 02: 05$ & 0.3314 \\
\hline 286 & $15: 02: 06$ & 0.3297 \\
\hline 287 & $15: 02: 07$ & 0.3338 \\
\hline 288 & 15:02:08 & 0.3375 \\
\hline 289 & $15: 02: 09$ & 0.3322 \\
\hline 290 & $15: 02: 10$ & 0.3429 \\
\hline 291 & $15: 02: 11$ & 0.3314 \\
\hline 292 & $15: 02: 12$ & 0.3408 \\
\hline 293 & $15: 02: 13$ & 0.3305 \\
\hline 294 & $15: 02: 14$ & 0.3382 \\
\hline 295 & $15: 02: 15$ & 0.3310 \\
\hline 296 & $15: 02: 16$ & 0.3399 \\
\hline 297 & $15: 02: 17$ & 0.3354 \\
\hline 298 & $15: 02: 18$ & 0.3320 \\
\hline 299 & $15: 02: 19$ & 0.3416 \\
\hline 300 & $15: 02: 20$ & 0.3402 \\
\hline 301 & $15: 02: 21$ & 0.3382 \\
\hline 302 & $15: 02: 22$ & 0.3317 \\
\hline 303 & $15: 02: 23$ & 0.3350 \\
\hline & $15: 02: 24$ & 0.3308 \\
\hline
\end{tabular}

$E-6$ 
Appendix E -Fuel Data Test 3 NIST102605_1.xls

\begin{tabular}{|c|c|c|}
\hline 305 & $15: 02: 25$ & 0.3378 \\
\hline 306 & $15: 02: 26$ & 0.3296 \\
\hline 307 & $15: 02: 27$ & 0.3431 \\
\hline 308 & $15: 02: 28$ & 0.3354 \\
\hline 309 & $15: 02: 29$ & 0.3402 \\
\hline 310 & $15: 02: 30$ & 0.3348 \\
\hline 311 & $15: 02: 31$ & 0.3341 \\
\hline 312 & 15:02:32 & 0.3386 \\
\hline 313 & $15: 02: 33$ & 0.3274 \\
\hline 314 & $15: 02: 34$ & 0.3355 \\
\hline 315 & $15: 02: 35$ & 0.3270 \\
\hline 316 & $15: 02: 36$ & 0.3392 \\
\hline 317 & $15: 02: 37$ & 0.3349 \\
\hline 318 & $15: 02: 38$ & 0.3394 \\
\hline 319 & $15: 02: 39$ & 0.3333 \\
\hline 320 & $15: 02: 40$ & 0.3371 \\
\hline 321 & $15: 02: 41$ & 0.3328 \\
\hline 322 & $15: 02: 42$ & 0.3337 \\
\hline 323 & $15: 02: 43$ & 0.3318 \\
\hline 324 & $15: 02: 44$ & 0.3340 \\
\hline 325 & $15: 02: 45$ & 0.3349 \\
\hline 326 & $15: 02: 46$ & 0.3343 \\
\hline 327 & $15: 02: 47$ & 0.3357 \\
\hline 328 & $15: 02: 48$ & 0.3321 \\
\hline 329 & $15: 02: 49$ & 0.3392 \\
\hline 330 & $15: 02: 50$ & 0.3308 \\
\hline 331 & $15: 02: 51$ & 0.3347 \\
\hline 332 & $15: 02: 52$ & 0.3258 \\
\hline 333 & $15: 02: 53$ & 0.3395 \\
\hline 334 & $15: 02: 54$ & 0.3325 \\
\hline 335 & $15: 02: 55$ & 0.3373 \\
\hline 336 & $15: 02: 56$ & 0.3307 \\
\hline 337 & $15: 02: 57$ & 0.3368 \\
\hline 338 & $15: 02: 58$ & 0.3309 \\
\hline 339 & $15: 02: 59$ & 0.3378 \\
\hline 340 & $15: 03: 00$ & 0.3308 \\
\hline 341 & $15: 03: 01$ & 0.3352 \\
\hline 342 & 15:03:02 & 0.3339 \\
\hline 343 & $15: 03: 03$ & 0.3348 \\
\hline 344 & 15:03:04 & 0.3327 \\
\hline 345 & 15:03:05 & 0.3336 \\
\hline 346 & 15:03:06 & 0.3371 \\
\hline 347 & $15: 03: 07$ & 0.3312 \\
\hline 348 & 15:03:08 & 0.3360 \\
\hline 349 & 15:03:09 & 0.3309 \\
\hline 350 & $15: 03: 10$ & 0.3358 \\
\hline 351 & $15: 03: 11$ & 0.3287 \\
\hline 352 & $15: 03: 12$ & 0.3335 \\
\hline 353 & $15: 03: 13$ & 0.3280 \\
\hline 354 & $15: 03: 14$ & 0.3397 \\
\hline & $15: 03: 15$ & 0.3384 \\
\hline
\end{tabular}


Appendix E -Fuel Data Test 3 NIST102605_1.xls

\begin{tabular}{|c|c|c|}
\hline 356 & $15: 03: 16$ & 0.3413 \\
\hline 357 & $15: 03: 17$ & 0.3296 \\
\hline 358 & 15:03:18 & 0.3394 \\
\hline 359 & 15:03:19 & 0.3328 \\
\hline 360 & 15:03:20 & 0.3366 \\
\hline 361 & 15:03:21 & 0.3269 \\
\hline 362 & 15:03:22 & 0.3360 \\
\hline 363 & $15: 03: 23$ & 0.3378 \\
\hline 364 & $15: 03: 24$ & 0.3436 \\
\hline 365 & 15:03:25 & 0.3284 \\
\hline 366 & 15:03:26 & 0.3348 \\
\hline 367 & 15:03:27 & 0.3294 \\
\hline 368 & 15:03:28 & 0.3371 \\
\hline 369 & 15:03:29 & 0.3343 \\
\hline 370 & 15:03:30 & 0.3297 \\
\hline 371 & 15:03:31 & 0.3356 \\
\hline 372 & 15:03:32 & 0.3326 \\
\hline 373 & $15: 03: 33$ & 0.3394 \\
\hline 374 & 15:03:34 & 0.3336 \\
\hline 375 & $15: 03: 35$ & 0.3410 \\
\hline 376 & $15: 03: 36$ & 0.3328 \\
\hline 377 & 15:03:37 & 0.3374 \\
\hline 378 & 15:03:38 & 0.3285 \\
\hline 379 & 15:03:39 & 0.3342 \\
\hline 380 & $15: 03: 40$ & 0.3347 \\
\hline 381 & $15: 03: 41$ & 0.3402 \\
\hline 382 & $15: 03: 42$ & 0.3305 \\
\hline 383 & $15: 03: 43$ & 0.3325 \\
\hline 384 & $15: 03: 44$ & 0.3391 \\
\hline 385 & $15: 03: 45$ & 0.3312 \\
\hline 386 & $15: 03: 46$ & 0.3348 \\
\hline 387 & 15:03:47 & 0.3269 \\
\hline 388 & 15:03:48 & 0.3357 \\
\hline 389 & $15: 03: 49$ & 0.3283 \\
\hline 390 & 15:03:50 & 0.3371 \\
\hline 391 & 15:03:51 & 0.3307 \\
\hline 392 & 15:03:52 & 0.3388 \\
\hline 393 & $15: 03: 53$ & 0.3349 \\
\hline 394 & $15: 03: 54$ & 0.3370 \\
\hline 395 & $15: 03: 55$ & 0.3283 \\
\hline 396 & 15:03:56 & 0.3371 \\
\hline 397 & $15: 03: 57$ & 0.3310 \\
\hline 398 & 15:03:58 & 0.3345 \\
\hline 399 & $15: 03: 59$ & 0.3296 \\
\hline 400 & $15: 04: 00$ & 0.3386 \\
\hline 401 & 15:04:01 & 0.3359 \\
\hline 402 & $15: 04: 02$ & 0.3431 \\
\hline 403 & $15: 04: 03$ & 0.3282 \\
\hline 404 & $15: 04: 04$ & 0.3330 \\
\hline 405 & 15:04:05 & 0.3327 \\
\hline 406 & $15: 04: 06$ & 0.335 \\
\hline
\end{tabular}

$E-8$ 
Appendix E -Fuel Data Test 3 NIST102605_1.xls

\begin{tabular}{|c|c|c|}
\hline 407 & $15: 04: 07$ & 0.3314 \\
\hline 408 & $15: 04: 08$ & 0.3281 \\
\hline 409 & 15:04:09 & 0.3334 \\
\hline 410 & $15: 04: 10$ & 0.3352 \\
\hline 411 & $15: 04: 11$ & 0.3372 \\
\hline 412 & $15: 04: 12$ & 0.3332 \\
\hline 413 & $15: 04: 13$ & 0.3378 \\
\hline 414 & $15: 04: 14$ & 0.3300 \\
\hline 415 & $15: 04: 15$ & 0.3348 \\
\hline 416 & $15: 04: 16$ & 0.3287 \\
\hline 417 & $15: 04: 17$ & 0.3679 \\
\hline 418 & $15: 04: 18$ & 0.4100 \\
\hline 419 & $15: 04: 19$ & 0.4377 \\
\hline 420 & $15: 04: 20$ & 0.4704 \\
\hline 421 & $15: 04: 21$ & 0.5133 \\
\hline 422 & $15: 04: 22$ & 0.5340 \\
\hline 423 & $15: 04: 23$ & 0.5711 \\
\hline 424 & $15: 04: 24$ & 0.6073 \\
\hline 425 & $15: 04: 25$ & 0.6300 \\
\hline 426 & $15: 04: 26$ & 0.6547 \\
\hline 427 & $15: 04: 27$ & 0.6713 \\
\hline 428 & $15: 04: 28$ & 0.6808 \\
\hline 429 & $15: 04: 29$ & 0.6849 \\
\hline 430 & $15: 04: 30$ & 0.6844 \\
\hline 431 & $15: 04: 31$ & 0.6840 \\
\hline 432 & $15: 04: 32$ & 0.6837 \\
\hline 433 & $15: 04: 33$ & 0.6835 \\
\hline 434 & $15: 04: 34$ & 0.6868 \\
\hline 435 & $15: 04: 35$ & 0.6851 \\
\hline 436 & $15: 04: 36$ & 0.6857 \\
\hline 437 & $15: 04: 37$ & 0.6850 \\
\hline 438 & $15: 04: 38$ & 0.6844 \\
\hline 439 & 15:04:39 & 0.6852 \\
\hline 440 & 15:04:40 & 0.6845 \\
\hline 441 & $15: 04: 41$ & 0.6843 \\
\hline 442 & $15: 04: 42$ & 0.6853 \\
\hline 443 & $15: 04: 43$ & 0.6844 \\
\hline 444 & $15: 04: 44$ & 0.6834 \\
\hline 445 & $15: 04: 45$ & 0.6842 \\
\hline 446 & $15: 04: 46$ & 0.6838 \\
\hline 447 & $15: 04: 47$ & 0.6838 \\
\hline 448 & $15: 04: 48$ & 0.6848 \\
\hline 449 & $15: 04: 49$ & 0.6876 \\
\hline 450 & $15: 04: 50$ & 0.6852 \\
\hline 451 & $15: 04: 51$ & 0.6868 \\
\hline 452 & $15: 04: 52$ & 0.6875 \\
\hline 453 & $15: 04: 53$ & 0.6861 \\
\hline 454 & $15: 04: 54$ & 0.6860 \\
\hline 455 & $15: 04: 55$ & 0.6878 \\
\hline 456 & $15: 04: 56$ & 0.6877 \\
\hline 457 & $15: 04: 57$ & 0.6895 \\
\hline
\end{tabular}


Appendix E -Fuel Data Test 3 NIST102605_1.xls

\begin{tabular}{|c|c|c|}
\hline 458 & 15:04:58 & 0.6896 \\
\hline 459 & $15: 04: 59$ & 0.6869 \\
\hline 460 & $15: 05: 00$ & 0.6871 \\
\hline 461 & $15: 05: 01$ & 0.6853 \\
\hline 462 & $15: 05: 02$ & 0.6856 \\
\hline 463 & $15: 05: 03$ & 0.6883 \\
\hline 464 & $15: 05: 04$ & 0.6897 \\
\hline 465 & $15: 05: 05$ & 0.6876 \\
\hline 466 & $15: 05: 06$ & 0.6866 \\
\hline 467 & $15: 05: 07$ & 0.6870 \\
\hline 468 & $15: 05: 08$ & 0.6864 \\
\hline 469 & $15: 05: 09$ & 0.6850 \\
\hline 470 & $15: 05: 10$ & 0.6860 \\
\hline 471 & $15: 05: 11$ & 0.6873 \\
\hline 472 & $15: 05: 12$ & 0.6878 \\
\hline 473 & $15: 05: 13$ & 0.6844 \\
\hline 474 & $15: 05: 14$ & 0.6856 \\
\hline 475 & $15: 05: 15$ & 0.6855 \\
\hline 476 & $15: 05: 16$ & 0.6847 \\
\hline 477 & $15: 05: 17$ & 0.6838 \\
\hline 478 & $15: 05: 18$ & 0.6879 \\
\hline 479 & $15: 05: 19$ & 0.6866 \\
\hline 480 & 15:05:20 & 0.6857 \\
\hline 481 & $15: 05: 21$ & 0.6884 \\
\hline 482 & $15: 05: 22$ & 0.6857 \\
\hline 483 & $15: 05: 23$ & 0.6841 \\
\hline 484 & $15: 05: 24$ & 0.6856 \\
\hline 485 & $15: 05: 25$ & 0.6874 \\
\hline 486 & $15: 05: 26$ & 0.6863 \\
\hline 487 & $15: 05: 27$ & 0.6850 \\
\hline 488 & $15: 05: 28$ & 0.6856 \\
\hline 489 & $15: 05: 29$ & 0.6874 \\
\hline 490 & 15:05:30 & 0.6859 \\
\hline 491 & 15:05:31 & 0.6858 \\
\hline 492 & $15: 05: 32$ & 0.6859 \\
\hline 493 & $15: 05: 33$ & 0.6875 \\
\hline 494 & $15: 05: 34$ & 0.6887 \\
\hline 495 & $15: 05: 35$ & 0.6874 \\
\hline 496 & $15: 05: 36$ & 0.6848 \\
\hline 497 & $15: 05: 37$ & 0.6859 \\
\hline 498 & $15: 05: 38$ & 0.6860 \\
\hline 499 & $15: 05: 39$ & 0.6886 \\
\hline 500 & $15: 05: 40$ & 0.6876 \\
\hline 501 & $15: 05: 41$ & 0.6865 \\
\hline 502 & $15: 05: 42$ & 0.6849 \\
\hline 503 & $15: 05: 43$ & 0.6863 \\
\hline 504 & $15: 05: 44$ & 0.6885 \\
\hline 505 & $15: 05: 45$ & 0.6878 \\
\hline 506 & $15: 05: 46$ & 0.6858 \\
\hline 507 & $15: 05: 47$ & 0.6879 \\
\hline 508 & $15: 05: 48$ & 0.68 \\
\hline
\end{tabular}


Appendix E -Fuel Data Test 3 NIST102605_1.xls

\begin{tabular}{|c|c|c|}
\hline 509 & $15: 05: 49$ & 0.6870 \\
\hline 510 & $15: 05: 50$ & 0.6897 \\
\hline 511 & $15: 05: 51$ & 0.6860 \\
\hline 512 & $15: 05: 52$ & 0.6847 \\
\hline 513 & $15: 05: 53$ & 0.6863 \\
\hline 514 & $15: 05: 54$ & 0.6895 \\
\hline 515 & $15: 05: 55$ & 0.6883 \\
\hline 516 & $15: 05: 56$ & 0.6859 \\
\hline 517 & $15: 05: 57$ & 0.6859 \\
\hline 518 & $15: 05: 58$ & 0.6853 \\
\hline 519 & $15: 05: 59$ & 0.6858 \\
\hline 520 & $15: 06: 00$ & 0.6872 \\
\hline 521 & $15: 06: 01$ & 0.6870 \\
\hline 522 & $15: 06: 02$ & 0.6898 \\
\hline 523 & $15: 06: 03$ & 0.6882 \\
\hline 524 & $15: 06: 04$ & 0.6890 \\
\hline 525 & 15:06:05 & 0.6876 \\
\hline 526 & $15: 06: 06$ & 0.6865 \\
\hline 527 & $15: 06: 07$ & 0.6863 \\
\hline 528 & $15: 06: 08$ & 0.6908 \\
\hline 529 & 15:06:09 & 0.6869 \\
\hline 530 & $15: 06: 10$ & 0.6849 \\
\hline 531 & $15: 06: 11$ & 0.6839 \\
\hline 532 & $15: 06: 12$ & 0.6853 \\
\hline 533 & $15: 06: 13$ & 0.6854 \\
\hline 534 & $15: 06: 14$ & 0.6847 \\
\hline 535 & $15: 06: 15$ & 0.6841 \\
\hline 536 & $15: 06: 16$ & 0.6883 \\
\hline 537 & $15: 06: 17$ & 0.6881 \\
\hline 538 & $15: 06: 18$ & 0.6860 \\
\hline 539 & $15: 06: 19$ & 0.6862 \\
\hline 540 & $15: 06: 20$ & 0.6856 \\
\hline 541 & $15: 06: 21$ & 0.6849 \\
\hline 542 & $15: 06: 22$ & 0.6846 \\
\hline 543 & $15: 06: 23$ & 0.6887 \\
\hline 544 & $15: 06: 24$ & 0.6881 \\
\hline 545 & $15: 06: 25$ & 0.6881 \\
\hline 546 & $15: 06: 26$ & 0.6864 \\
\hline 547 & $15: 06: 27$ & 0.6881 \\
\hline 548 & $15: 06: 28$ & 0.6869 \\
\hline 549 & $15: 06: 29$ & 0.6872 \\
\hline 550 & $15: 06: 30$ & 0.6862 \\
\hline 551 & $15: 06: 31$ & 0.6902 \\
\hline 552 & $15: 06: 32$ & 0.6897 \\
\hline 553 & $15: 06: 33$ & 0.6890 \\
\hline 554 & $15: 06: 34$ & 0.6869 \\
\hline 555 & $15: 06: 35$ & 0.6875 \\
\hline 556 & $15: 06: 36$ & 0.6857 \\
\hline 557 & $15: 06: 37$ & 0.6870 \\
\hline 558 & $15: 06: 38$ & 0.6889 \\
\hline$y$ & $15: 06: 39$ & 0.6888 \\
\hline
\end{tabular}


Appendix E -Fuel Data Test 3 NIST102605_1.xls

\begin{tabular}{|c|c|c|}
\hline 560 & $15: 06: 40$ & 0.6856 \\
\hline 561 & $15: 06: 41$ & 0.6880 \\
\hline 562 & $15: 06: 42$ & 0.6864 \\
\hline 563 & $15: 06: 43$ & 0.6869 \\
\hline 564 & $15: 06: 44$ & 0.6856 \\
\hline 565 & $15: 06: 45$ & 0.6876 \\
\hline 566 & $15: 06: 46$ & 0.6896 \\
\hline 567 & 15:06:47 & 0.6898 \\
\hline 568 & 15:06:48 & 0.6885 \\
\hline 569 & $15: 06: 49$ & 0.6899 \\
\hline 570 & 15:06:50 & 0.6872 \\
\hline 571 & $15: 06: 51$ & 0.6899 \\
\hline 572 & $15: 06: 52$ & 0.6912 \\
\hline 573 & $15: 06: 53$ & 0.6907 \\
\hline 574 & $15: 06: 54$ & 0.6902 \\
\hline 575 & $15: 06: 55$ & 0.6899 \\
\hline 576 & 15:06:56 & 0.6875 \\
\hline 577 & 15:06:57 & 0.6875 \\
\hline 578 & $15: 06: 58$ & 0.6866 \\
\hline 579 & $15: 06: 59$ & 0.6906 \\
\hline 580 & 15:07:00 & 0.6895 \\
\hline 581 & 15:07:01 & 0.6887 \\
\hline 582 & 15:07:02 & 0.6883 \\
\hline 583 & 15:07:03 & 0.6873 \\
\hline 584 & 15:07:04 & 0.6882 \\
\hline 585 & 15:07:05 & 0.6897 \\
\hline 586 & 15:07:06 & 0.6896 \\
\hline 587 & $15: 07: 07$ & 0.6900 \\
\hline 588 & $15: 07: 08$ & 0.6905 \\
\hline 589 & 15:07:09 & 0.6882 \\
\hline 590 & 15:07:10 & 0.6901 \\
\hline 591 & 15:07:11 & 0.6891 \\
\hline 592 & 15:07:12 & 0.6892 \\
\hline 593 & $15: 07: 13$ & 0.6909 \\
\hline 594 & 15:07:14 & 0.6931 \\
\hline 595 & 15:07:15 & 0.6909 \\
\hline 596 & 15:07:16 & 0.6893 \\
\hline 597 & $15: 07: 17$ & 0.6886 \\
\hline 598 & 15:07:18 & 0.6903 \\
\hline 599 & $15: 07: 19$ & 0.6899 \\
\hline 600 & 15:07:20 & 0.6956 \\
\hline 601 & $15: 07: 21$ & 0.6913 \\
\hline 602 & 15:07:22 & 0.6901 \\
\hline 603 & 15:07:23 & 0.6871 \\
\hline 604 & $15: 07: 24$ & 0.6891 \\
\hline 605 & 15:07:25 & 0.6892 \\
\hline 606 & $15: 07: 26$ & 0.6898 \\
\hline 607 & $15: 07: 27$ & 0.6915 \\
\hline 608 & 15:07:28 & 0.6941 \\
\hline 609 & $15: 07: 29$ & 0.6925 \\
\hline 610 & 15:07:30 & 0.691 \\
\hline
\end{tabular}


Appendix E -Fuel Data Test 3 NIST102605_1.xls

\begin{tabular}{|c|c|c|}
\hline 611 & $15: 07: 31$ & 0.6899 \\
\hline 612 & $15: 07: 32$ & 0.6916 \\
\hline 613 & $15: 07: 33$ & 0.6906 \\
\hline 614 & $15: 07: 34$ & 0.6967 \\
\hline 615 & $15: 07: 35$ & 0.6940 \\
\hline 616 & $15: 07: 36$ & 0.6946 \\
\hline 617 & $15: 07: 37$ & 0.6916 \\
\hline 618 & $15: 07: 38$ & 0.6949 \\
\hline 619 & $15: 07: 39$ & 0.7081 \\
\hline 620 & $15: 07: 40$ & 0.7250 \\
\hline 621 & $15: 07: 41$ & 0.7381 \\
\hline 622 & $15: 07: 42$ & 0.7566 \\
\hline 623 & $15: 07: 43$ & 0.7616 \\
\hline 624 & $15: 07: 44$ & 0.7646 \\
\hline 625 & $15: 07: 45$ & 0.7617 \\
\hline 626 & $15: 07: 46$ & 0.7620 \\
\hline 627 & $15: 07: 47$ & 0.7628 \\
\hline 628 & $15: 07: 48$ & 0.7645 \\
\hline 629 & $15: 07: 49$ & 0.7652 \\
\hline 630 & $15: 07: 50$ & 0.7629 \\
\hline 631 & $15: 07: 51$ & 0.7652 \\
\hline 632 & $15: 07: 52$ & 0.7684 \\
\hline 633 & $15: 07: 53$ & 0.7686 \\
\hline 634 & $15: 07: 54$ & 0.7679 \\
\hline 635 & $15: 07: 55$ & 0.7665 \\
\hline 636 & $15: 07: 56$ & 0.7695 \\
\hline 637 & $15: 07: 57$ & 0.7696 \\
\hline 638 & $15: 07: 58$ & 0.7643 \\
\hline 639 & $15: 07: 59$ & 0.7615 \\
\hline 640 & 15:08:00 & 0.7620 \\
\hline 641 & $15: 08: 01$ & 0.7657 \\
\hline 642 & 15:08:02 & 0.7659 \\
\hline 643 & $15: 08: 03$ & 0.7677 \\
\hline 644 & $15: 08: 04$ & 0.7690 \\
\hline 645 & 15:08:05 & 0.7671 \\
\hline 646 & 15:08:06 & 0.7678 \\
\hline 647 & $15: 08: 07$ & 0.7717 \\
\hline 648 & 15:08:08 & 0.7795 \\
\hline 649 & 15:08:09 & 0.7810 \\
\hline 650 & $15: 08: 10$ & 0.7858 \\
\hline 651 & $15: 08: 11$ & 0.7911 \\
\hline 652 & $15: 08: 12$ & 0.7942 \\
\hline 653 & $15: 08: 13$ & 0.7951 \\
\hline 654 & $15: 08: 14$ & 0.7960 \\
\hline 655 & $15: 08: 15$ & 0.7995 \\
\hline 656 & $15: 08: 16$ & 0.7985 \\
\hline 657 & $15: 08: 17$ & 0.8030 \\
\hline 658 & $15: 08: 18$ & 0.8066 \\
\hline 659 & $15: 08: 19$ & 0.8042 \\
\hline 660 & $15: 08: 20$ & 0.8042 \\
\hline 661 & $15: 08: 21$ & 0.8071 \\
\hline
\end{tabular}


Appendix E -Fuel Data Test 3 NIST102605_1.xls

\begin{tabular}{|c|c|c|}
\hline 662 & $15: 08: 22$ & 0.8108 \\
\hline 663 & $15: 08: 23$ & 0.8145 \\
\hline 664 & $15: 08: 24$ & 0.8154 \\
\hline 665 & $15: 08: 25$ & 0.8172 \\
\hline 666 & $15: 08: 26$ & 0.8141 \\
\hline 667 & $15: 08: 27$ & 0.8166 \\
\hline 668 & $15: 08: 28$ & 0.8148 \\
\hline 669 & $15: 08: 29$ & 0.8159 \\
\hline 670 & $15: 08: 30$ & 0.8213 \\
\hline 671 & $15: 08: 31$ & 0.8226 \\
\hline 672 & $15: 08: 32$ & 0.8200 \\
\hline 673 & $15: 08: 33$ & 0.8206 \\
\hline 674 & $15: 08: 34$ & 0.8185 \\
\hline 675 & $15: 08: 35$ & 0.8200 \\
\hline 676 & $15: 08: 36$ & 0.8196 \\
\hline 677 & $15: 08: 37$ & 0.8208 \\
\hline 678 & $15: 08: 38$ & 0.8229 \\
\hline 679 & 15:08:39 & 0.8224 \\
\hline 680 & $15: 08: 40$ & 0.8228 \\
\hline 681 & $15: 08: 41$ & 0.8257 \\
\hline 682 & $15: 08: 42$ & 0.8237 \\
\hline 683 & $15: 08: 43$ & 0.8227 \\
\hline 684 & $15: 08: 44$ & 0.8250 \\
\hline 685 & $15: 08: 45$ & 0.8262 \\
\hline 686 & $15: 08: 46$ & 0.8250 \\
\hline 687 & $15: 08: 47$ & 0.8236 \\
\hline 688 & $15: 08: 48$ & 0.8239 \\
\hline 689 & $15: 08: 49$ & 0.8247 \\
\hline 690 & $15: 08: 50$ & 0.8268 \\
\hline 691 & $15: 08: 51$ & 0.8256 \\
\hline 692 & $15: 08: 52$ & 0.8233 \\
\hline 693 & $15: 08: 53$ & 0.8229 \\
\hline 694 & $15: 08: 54$ & 0.8235 \\
\hline 695 & $15: 08: 55$ & 0.8230 \\
\hline 696 & $15: 08: 56$ & 0.8218 \\
\hline 697 & $15: 08: 57$ & 0.8225 \\
\hline 698 & $15: 08: 58$ & 0.8271 \\
\hline 699 & $15: 08: 59$ & 0.8300 \\
\hline 700 & $15: 09: 00$ & 0.8281 \\
\hline 701 & 15:09:01 & 0.8265 \\
\hline 702 & $15: 09: 02$ & 0.8255 \\
\hline 703 & $15: 09: 03$ & 0.8273 \\
\hline 704 & $15: 09: 04$ & 0.8260 \\
\hline 705 & 15:09:05 & 0.8260 \\
\hline 706 & $15: 09: 06$ & 0.8290 \\
\hline 707 & $15: 09: 07$ & 0.8281 \\
\hline 708 & $15: 09: 08$ & 0.8294 \\
\hline 709 & $15: 09: 09$ & 0.8300 \\
\hline 710 & $15: 09: 10$ & 0.8268 \\
\hline 711 & $15: 09: 11$ & 0.8227 \\
\hline 712 & $15: 09: 12$ & 0.8228 \\
\hline
\end{tabular}


Appendix E -Fuel Data Test 3 NIST102605_1.xls

\begin{tabular}{|c|c|c|}
\hline 713 & $15: 09: 13$ & 0.8288 \\
\hline 714 & $15: 09: 14$ & 0.8298 \\
\hline 715 & $15: 09: 15$ & 0.8299 \\
\hline 716 & $15: 09: 16$ & 0.8248 \\
\hline 717 & $15: 09: 17$ & 0.8287 \\
\hline 718 & $15: 09: 18$ & 0.8266 \\
\hline 719 & $15: 09: 19$ & 0.8246 \\
\hline 720 & $15: 09: 20$ & 0.8260 \\
\hline 721 & $15: 09: 21$ & 0.8285 \\
\hline 722 & 15:09:22 & 0.8323 \\
\hline 723 & $15: 09: 23$ & 0.8284 \\
\hline 724 & $15: 09: 24$ & 0.8275 \\
\hline 725 & $15: 09: 25$ & 0.8281 \\
\hline 726 & $15: 09: 26$ & 0.8257 \\
\hline 727 & $15: 09: 27$ & 0.8306 \\
\hline 728 & 15:09:28 & 0.8320 \\
\hline 729 & 15:09:29 & 0.8307 \\
\hline 730 & 15:09:30 & 0.8290 \\
\hline 731 & 15:09:31 & 0.8284 \\
\hline 732 & 15:09:32 & 0.8308 \\
\hline 733 & $15: 09: 33$ & 0.8309 \\
\hline 734 & $15: 09: 34$ & 0.8299 \\
\hline 735 & 15:09:35 & 0.8273 \\
\hline 736 & 15:09:36 & 0.8280 \\
\hline 737 & $15: 09: 37$ & 0.8292 \\
\hline 738 & 15:09:38 & 0.8284 \\
\hline 739 & 15:09:39 & 0.8302 \\
\hline 740 & $15: 09: 40$ & 0.8297 \\
\hline 741 & $15: 09: 41$ & 0.8293 \\
\hline 742 & $15: 09: 42$ & 0.8312 \\
\hline 743 & $15: 09: 43$ & 0.8298 \\
\hline 744 & $15: 09: 44$ & 0.8295 \\
\hline 745 & $15: 09: 45$ & 0.8254 \\
\hline 746 & $15: 09: 46$ & 0.8284 \\
\hline 747 & $15: 09: 47$ & 0.8324 \\
\hline 748 & $15: 09: 48$ & 0.8311 \\
\hline 749 & $15: 09: 49$ & 0.8321 \\
\hline 750 & 15:09:50 & 0.8326 \\
\hline 751 & $15: 09: 51$ & 0.8327 \\
\hline 752 & 15:09:52 & 0.8314 \\
\hline 753 & $15: 09: 53$ & 0.8272 \\
\hline 754 & $15: 09: 54$ & 0.8254 \\
\hline 755 & 15:09:55 & 0.8252 \\
\hline 756 & $15: 09: 56$ & 0.8282 \\
\hline 757 & $15: 09: 57$ & 0.8296 \\
\hline 758 & $15: 09: 58$ & 0.8283 \\
\hline 759 & 15:09:59 & 0.8298 \\
\hline 760 & $15: 10: 00$ & 0.8294 \\
\hline 761 & $15: 10: 01$ & 0.8305 \\
\hline 762 & $15: 10: 02$ & 0.8312 \\
\hline 763 & $15: 10: 03$ & 0.8326 \\
\hline
\end{tabular}

E - 15 
Appendix E -Fuel Data Test 3 NIST102605_1.xls

\begin{tabular}{|c|c|c|}
\hline 764 & $15: 10: 04$ & 0.8321 \\
\hline 765 & $15: 10: 05$ & 0.8332 \\
\hline 766 & $15: 10: 06$ & 0.8312 \\
\hline 767 & $15: 10: 07$ & 0.8341 \\
\hline 768 & $15: 10: 08$ & 0.8274 \\
\hline 769 & 15:10:09 & 0.8291 \\
\hline 770 & $15: 10: 10$ & 0.8347 \\
\hline 771 & 15:10:11 & 0.8376 \\
\hline 772 & $15: 10: 12$ & 0.8374 \\
\hline 773 & $15: 10: 13$ & 0.8361 \\
\hline 774 & $15: 10: 14$ & 0.8334 \\
\hline 775 & $15: 10: 15$ & 0.8323 \\
\hline 776 & 15:10:16 & 0.8337 \\
\hline 777 & $15: 10: 17$ & 0.8368 \\
\hline 778 & 15:10:18 & 0.8342 \\
\hline 779 & $15: 10: 19$ & 0.8333 \\
\hline 780 & 15:10:20 & 0.8351 \\
\hline 781 & $15: 10: 21$ & 0.8346 \\
\hline 782 & $15: 10: 22$ & 0.8344 \\
\hline 783 & $15: 10: 23$ & 0.8305 \\
\hline 784 & $15: 10: 24$ & 0.8331 \\
\hline 785 & $15: 10: 25$ & 0.8391 \\
\hline 786 & 15:10:26 & 0.8383 \\
\hline 787 & $15: 10: 27$ & 0.8371 \\
\hline 788 & $15: 10: 28$ & 0.8339 \\
\hline 789 & $15: 10: 29$ & 0.8401 \\
\hline 790 & $15: 10: 30$ & 0.8384 \\
\hline 791 & $15: 10: 31$ & 0.8366 \\
\hline 792 & $15: 10: 32$ & 0.8355 \\
\hline 793 & $15: 10: 33$ & 0.8409 \\
\hline 794 & $15: 10: 34$ & 0.8406 \\
\hline 795 & $15: 10: 35$ & 0.8387 \\
\hline 796 & $15: 10: 36$ & 0.8439 \\
\hline 797 & $15: 10: 37$ & 0.8422 \\
\hline 798 & $15: 10: 38$ & 0.8410 \\
\hline 799 & $15: 10: 39$ & 0.8407 \\
\hline 800 & $15: 10: 40$ & 0.8422 \\
\hline 801 & $15: 10: 41$ & 0.8454 \\
\hline 802 & $15: 10: 42$ & 0.8455 \\
\hline 803 & $15: 10: 43$ & 0.8426 \\
\hline 804 & $15: 10: 44$ & 0.8455 \\
\hline 805 & $15: 10: 45$ & 0.8457 \\
\hline 8061 & $15: 10: 46$ & 0.8438 \\
\hline 8071 & $15: 10: 47$ & 0.8479 \\
\hline 8081 & $15: 10: 48$ & 0.8486 \\
\hline 8091 & $15: 10: 49$ & 0.8498 \\
\hline 8101 & $15: 10: 50$ & 0.8471 \\
\hline 8111 & $15: 10: 51$ & 0.8433 \\
\hline 8121 & $15: 10: 52$ & 0.8485 \\
\hline 8131 & $15: 10: 53$ & 0.8471 \\
\hline 814 & $15: 10: 54$ & 0.8501 \\
\hline
\end{tabular}


Appendix E -Fuel Data Test 3 NIST102605_1.xls

\begin{tabular}{|c|c|c|}
\hline 815 & $15: 10: 55$ & 0.8510 \\
\hline 816 & $15: 10: 56$ & 0.8498 \\
\hline 817 & $15: 10: 57$ & 0.8494 \\
\hline 818 & $15: 10: 58$ & 0.8469 \\
\hline 819 & $15: 10: 59$ & 0.8463 \\
\hline 820 & $15: 11: 00$ & 0.8466 \\
\hline 821 & $15: 11: 01$ & 0.8474 \\
\hline 822 & $15: 11: 02$ & 0.8477 \\
\hline 823 & $15: 11: 03$ & 0.8525 \\
\hline 824 & $15: 11: 04$ & 0.8538 \\
\hline 825 & $15: 11: 05$ & 0.8525 \\
\hline 826 & $15: 11: 06$ & 0.8511 \\
\hline 827 & $15: 11: 07$ & 0.8481 \\
\hline 828 & $15: 11: 08$ & 0.8459 \\
\hline 829 & 15:11:09 & 0.8497 \\
\hline 830 & $15: 11: 10$ & 0.8518 \\
\hline 831 & $15: 11: 11$ & 0.8562 \\
\hline 832 & $15: 11: 12$ & 0.8540 \\
\hline 833 & $15: 11: 13$ & 0.8527 \\
\hline 834 & $15: 11: 14$ & 0.8519 \\
\hline 835 & $15: 11: 15$ & 0.8539 \\
\hline 836 & $15: 11: 16$ & 0.8509 \\
\hline 837 & $15: 11: 17$ & 0.8491 \\
\hline 838 & $15: 11: 18$ & 0.8514 \\
\hline 839 & $15: 11: 19$ & 0.8542 \\
\hline 840 & $15: 11: 20$ & 0.8500 \\
\hline 841 & $15: 11: 21$ & 0.8483 \\
\hline 842 & $15: 11: 22$ & 0.8465 \\
\hline 843 & $15: 11: 23$ & 0.8529 \\
\hline 844 & $15: 11: 24$ & 0.8561 \\
\hline 845 & $15: 11: 25$ & 0.8569 \\
\hline 846 & $15: 11: 26$ & 0.8511 \\
\hline 847 & $15: 11: 27$ & 0.8506 \\
\hline 848 & $15: 11: 28$ & 0.8513 \\
\hline 849 & $15: 11: 29$ & 0.8491 \\
\hline 850 & $15: 11: 30$ & 0.8534 \\
\hline 851 & $15: 11: 31$ & 0.8543 \\
\hline 852 & $15: 11: 32$ & 0.8543 \\
\hline 853 & $15: 11: 33$ & 0.8549 \\
\hline 854 & $15: 11: 34$ & 0.8546 \\
\hline 855 & $15: 11: 35$ & 0.8552 \\
\hline 856 & $15: 11: 36$ & 0.8535 \\
\hline 857 & $15: 11: 37$ & 0.8531 \\
\hline 858 & $15: 11: 38$ & 0.8534 \\
\hline 859 & $15: 11: 39$ & 0.8550 \\
\hline 860 & $15: 11: 40$ & 0.8578 \\
\hline 861 & $15: 11: 41$ & 0.8528 \\
\hline 862 & $15: 11: 42$ & 0.8553 \\
\hline 863 & $15: 11: 43$ & 0.8550 \\
\hline 864 & $15: 11: 44$ & 0.8546 \\
\hline 865 & $15: 11: 45$ & 0.8520 \\
\hline
\end{tabular}


Appendix E -Fuel Data Test 3 NIST102605_1.xls

\begin{tabular}{|c|c|c|}
\hline 866 & $15: 11: 46$ & 0.8558 \\
\hline 867 & $15: 11: 47$ & 0.8585 \\
\hline 868 & $15: 11: 48$ & 0.8572 \\
\hline 869 & $15: 11: 49$ & 0.8575 \\
\hline 870 & $15: 11: 50$ & 0.8556 \\
\hline 871 & $15: 11: 51$ & 0.8553 \\
\hline 872 & $15: 11: 52$ & 0.8531 \\
\hline 873 & $15: 11: 53$ & 0.8566 \\
\hline 874 & $15: 11: 54$ & 0.8546 \\
\hline 875 & $15: 11: 55$ & 0.8579 \\
\hline 876 & $15: 11: 56$ & 0.8589 \\
\hline 877 & $15: 11: 57$ & 0.8546 \\
\hline 878 & $15: 11: 58$ & 0.8572 \\
\hline 879 & $15: 11: 59$ & 0.8581 \\
\hline 880 & $15: 12: 00$ & 0.8590 \\
\hline 881 & $15: 12: 01$ & 0.8584 \\
\hline 882 & $15: 12: 02$ & 0.8603 \\
\hline 883 & $15: 12: 03$ & 0.8583 \\
\hline 884 & $15: 12: 04$ & 0.8542 \\
\hline 885 & $15: 12: 05$ & 0.8538 \\
\hline 886 & $15: 12: 06$ & 0.8606 \\
\hline 887 & $15: 12: 07$ & 0.8596 \\
\hline 888 & $15: 12: 08$ & 0.8580 \\
\hline 889 & $15: 12: 09$ & 0.8600 \\
\hline 890 & $15: 12: 10$ & 0.8605 \\
\hline 891 & $15: 12: 11$ & 0.8574 \\
\hline 892 & $15: 12: 12$ & 0.8548 \\
\hline 893 & $15: 12: 13$ & 0.8571 \\
\hline 894 & $15: 12: 14$ & 0.8591 \\
\hline 895 & $15: 12: 15$ & 0.8608 \\
\hline 896 & $15: 12: 16$ & 0.8570 \\
\hline 897 & $15: 12: 17$ & 0.8556 \\
\hline 898 & $15: 12: 18$ & 0.8613 \\
\hline 899 & $15: 12: 19$ & 0.8614 \\
\hline 900 & $15: 12: 20$ & 0.8607 \\
\hline 901 & $15: 12: 21$ & 0.8570 \\
\hline 902 & $15: 12: 22$ & 0.8548 \\
\hline 903 & $15: 12: 23$ & 0.8587 \\
\hline 904 & $15: 12: 24$ & 0.8596 \\
\hline 905 & $15: 12: 25$ & 0.8584 \\
\hline 906 & $15: 12: 26$ & 0.8576 \\
\hline 907 & $15: 12: 27$ & 0.8558 \\
\hline 908 & $15: 12: 28$ & 0.8536 \\
\hline 909 & $15: 12: 29$ & 0.8545 \\
\hline 910 & $15: 12: 30$ & 0.8546 \\
\hline 911 & $15: 12: 31$ & 0.8574 \\
\hline 912 & $15: 12: 32$ & 0.8638 \\
\hline 913 & $15: 12: 33$ & 0.8595 \\
\hline 914 & $15: 12: 34$ & 0.8619 \\
\hline 915 & $15: 12: 35$ & 0.8636 \\
\hline & $15: 12: 36$ & 0.858 \\
\hline
\end{tabular}


Appendix E -Fuel Data Test 3 NIST102605_1.xls

\begin{tabular}{|c|c|c|}
\hline 917 & $15: 12: 37$ & 0.8596 \\
\hline 918 & $15: 12: 38$ & 0.8616 \\
\hline 919 & $15: 12: 39$ & 0.8598 \\
\hline 920 & $15: 12: 40$ & 0.8585 \\
\hline 921 & $15: 12: 41$ & 0.8570 \\
\hline 922 & $15: 12: 42$ & 0.8559 \\
\hline 923 & $15: 12: 43$ & 0.8563 \\
\hline 924 & $15: 12: 44$ & 0.8610 \\
\hline 925 & $15: 12: 45$ & 0.8620 \\
\hline 926 & $15: 12: 46$ & 0.8611 \\
\hline 927 & $15: 12: 47$ & 0.8618 \\
\hline 928 & $15: 12: 48$ & 0.8617 \\
\hline 929 & $15: 12: 49$ & 0.8636 \\
\hline 930 & $15: 12: 50$ & 0.8604 \\
\hline 931 & $15: 12: 51$ & 0.8577 \\
\hline 932 & $15: 12: 52$ & 0.8574 \\
\hline 933 & $15: 12: 53$ & 0.8592 \\
\hline 934 & $15: 12: 54$ & 0.8649 \\
\hline 935 & $15: 12: 55$ & 0.8645 \\
\hline 936 & $15: 12: 56$ & 0.8635 \\
\hline 937 & $15: 12: 57$ & 0.8605 \\
\hline 938 & $15: 12: 58$ & 0.8580 \\
\hline 939 & $15: 12: 59$ & 0.8612 \\
\hline 940 & $15: 13: 00$ & 0.8601 \\
\hline 941 & $15: 13: 01$ & 0.8580 \\
\hline 942 & $15: 13: 02$ & 0.8624 \\
\hline 943 & 15:13:03 & 0.8644 \\
\hline 944 & $15: 13: 04$ & 0.8613 \\
\hline 945 & $15: 13: 05$ & 0.8591 \\
\hline 946 & $15: 13: 06$ & 0.8619 \\
\hline 947 & $15: 13: 07$ & 0.8614 \\
\hline 948 & $15: 13: 08$ & 0.8610 \\
\hline 949 & $15: 13: 09$ & 0.8649 \\
\hline 950 & $15: 13: 10$ & 0.8664 \\
\hline 951 & $15: 13: 11$ & 0.8621 \\
\hline 952 & $15: 13: 12$ & 0.8588 \\
\hline 953 & $15: 13: 13$ & 0.8620 \\
\hline 954 & $15: 13: 14$ & 0.8594 \\
\hline 955 & $15: 13: 15$ & 0.8567 \\
\hline 956 & $15: 13: 16$ & 0.8594 \\
\hline 957 & $15: 13: 17$ & 0.8634 \\
\hline 958 & $15: 13: 18$ & 0.8650 \\
\hline 959 & $15: 13: 19$ & 0.8608 \\
\hline 960 & 15:13:20 & 0.8601 \\
\hline 961 & $15: 13: 21$ & 0.8599 \\
\hline 962 & $15: 13: 22$ & 0.8575 \\
\hline 963 & $15: 13: 23$ & 0.8594 \\
\hline 964 & $15: 13: 24$ & 0.8655 \\
\hline 965 & $15: 13: 25$ & 0.8656 \\
\hline 966 & $15: 13: 26$ & 0.8643 \\
\hline 967 & $15: 13: 27$ & 0.8622 \\
\hline
\end{tabular}


Appendix E -Fuel Data Test 3 NIST102605_1.xls

\begin{tabular}{|c|c|c|}
\hline 968 & $15: 13: 28$ & 0.8667 \\
\hline 969 & $15: 13: 29$ & 0.8646 \\
\hline 970 & $15: 13: 30$ & 0.8645 \\
\hline 971 & $15: 13: 31$ & 0.8646 \\
\hline 972 & 15:13:32 & 0.8654 \\
\hline 973 & 15:13:33 & 0.8627 \\
\hline 974 & 15:13:34 & 0.8609 \\
\hline 975 & $15: 13: 35$ & 0.8594 \\
\hline 976 & $15: 13: 36$ & 0.8599 \\
\hline 977 & $15: 13: 37$ & 0.8579 \\
\hline 9781 & $15: 13: 38$ & 0.8598 \\
\hline 9791 & $15: 13: 39$ & 0.8625 \\
\hline 9801 & $15: 13: 40$ & 0.8628 \\
\hline 9811 & $15: 13: 41$ & 0.8594 \\
\hline 9821 & 15:13:42 & 0.8577 \\
\hline 9831 & $15: 13: 43$ & 0.8651 \\
\hline 9841 & $15: 13: 44$ & 0.8647 \\
\hline 9851 & $15: 13: 45$ & 0.8637 \\
\hline 986 & $15: 13: 46$ & 0.8628 \\
\hline 987 & $15: 13: 47$ & 0.8657 \\
\hline 9881 & $15: 13: 48$ & 0.8658 \\
\hline 9891 & $15: 13: 49$ & 0.8647 \\
\hline 9901 & $15: 13: 50$ & 0.8602 \\
\hline 9911 & $15: 13: 51$ & 0.8612 \\
\hline 9921 & 15:13:52 & 0.8636 \\
\hline 9931 & $15: 13: 53$ & 0.8645 \\
\hline 9941 & $15: 13: 54$ & 0.8648 \\
\hline 9951 & $15: 13: 55$ & 0.8675 \\
\hline 9961 & 15:13:56 & 0.8650 \\
\hline 9971 & $15: 13: 57$ & 0.8625 \\
\hline 9981 & 15:13:58 & 0.8615 \\
\hline 9991 & $15: 13: 59$ & 0.8638 \\
\hline 10001 & $15: 14: 00$ & 0.8618 \\
\hline 10011 & 15:14:01 & 0.8622 \\
\hline 10021 & $15: 14: 02$ & 0.8676 \\
\hline 10031 & $15: 14: 03$ & 0.8663 \\
\hline 10041 & $15: 14: 04$ & 0.8655 \\
\hline 10051 & $15: 14: 05$ & 0.8648 \\
\hline 10061 & $15: 14: 06$ & 0.8649 \\
\hline 10071 & $15: 14: 07$ & 0.8653 \\
\hline 10081 & $15: 14: 08$ & 0.8666 \\
\hline 10091 & $15: 14: 09$ & 0.8686 \\
\hline 10101 & $15: 14: 10$ & 0.8673 \\
\hline 10111 & $15: 14: 11$ & 0.8660 \\
\hline 10121 & $15: 14: 12$ & 0.8666 \\
\hline 10131 & $15: 14: 13$ & 0.8669 \\
\hline 10141 & $15: 14: 14$ & 0.8657 \\
\hline 10151 & $15: 14: 15$ & 0.8629 \\
\hline 10161 & $15: 14: 16$ & 0.8658 \\
\hline 10171 & $15: 14: 17$ & 0.8663 \\
\hline 10181 & $15: 14: 18$ & 0.867 \\
\hline
\end{tabular}


Appendix E -Fuel Data Test 3 NIST102605_1.xls

\begin{tabular}{|c|c|c|}
\hline 1019 & $15: 14: 19$ & 0.8665 \\
\hline 1020 & $15: 14: 20$ & 0.8619 \\
\hline 1021 & $15: 14: 21$ & 0.8630 \\
\hline 1022 & $15: 14: 22$ & 0.8652 \\
\hline 1023 & $15: 14: 23$ & 0.8641 \\
\hline 1024 & $15: 14: 24$ & 0.8637 \\
\hline 1025 & $15: 14: 25$ & 0.8609 \\
\hline 1026 & $15: 14: 26$ & 0.8596 \\
\hline 1027 & $15: 14: 27$ & 0.8631 \\
\hline 1028 & $15: 14: 28$ & 0.8664 \\
\hline 1029 & $15: 14: 29$ & 0.8647 \\
\hline 1030 & $15: 14: 30$ & 0.8606 \\
\hline 1031 & $15: 14: 31$ & 0.8637 \\
\hline 1032 & $15: 14: 32$ & 0.8643 \\
\hline 1033 & $15: 14: 33$ & 0.8625 \\
\hline 1034 & $15: 14: 34$ & 0.8649 \\
\hline 1035 & $15: 14: 35$ & 0.8647 \\
\hline 1036 & $15: 14: 36$ & 0.8637 \\
\hline 1037 & $15: 14: 37$ & 0.8628 \\
\hline 1038 & $15: 14: 38$ & 0.8634 \\
\hline 1039 & $15: 14: 39$ & 0.8660 \\
\hline 1040 & $15: 14: 40$ & 0.8659 \\
\hline 1041 & $15: 14: 41$ & 0.8640 \\
\hline 1042 & $15: 14: 42$ & 0.8629 \\
\hline 1043 & $15: 14: 43$ & 0.8668 \\
\hline 1044 & $15: 14: 44$ & 0.8658 \\
\hline 1045 & $15: 14: 45$ & 0.8645 \\
\hline 1046 & $15: 14: 46$ & 0.8643 \\
\hline 1047 & $15: 14: 47$ & 0.8635 \\
\hline 1048 & $15: 14: 48$ & 0.8629 \\
\hline 1049 & $15: 14: 49$ & 0.8635 \\
\hline 1050 & $15: 14: 50$ & 0.8635 \\
\hline 1051 & $15: 14: 51$ & 0.8595 \\
\hline 1052 & $15: 14: 52$ & 0.8638 \\
\hline 1053 & $15: 14: 53$ & 0.8671 \\
\hline 1054 & $15: 14: 54$ & 0.8639 \\
\hline 1055 & $15: 14: 55$ & 0.8658 \\
\hline 1056 & $15: 14: 56$ & 0.8644 \\
\hline 1057 & $15: 14: 57$ & 0.8630 \\
\hline 1058 & $15: 14: 58$ & 0.8618 \\
\hline 1059 & $15: 14: 59$ & 0.8599 \\
\hline 1060 & $15: 15: 00$ & 0.8596 \\
\hline 1061 & $15: 15: 01$ & 0.8609 \\
\hline 1062 & $15: 15: 02$ & 0.8649 \\
\hline 1063 & $15: 15: 03$ & 0.8650 \\
\hline 1064 & $15: 15: 04$ & 0.8610 \\
\hline 1065 & $15: 15: 05$ & 0.8590 \\
\hline 1066 & $15: 15: 06$ & 0.8601 \\
\hline 1067 & $15: 15: 07$ & 0.8631 \\
\hline 1068 & $15: 15: 08$ & 0.8647 \\
\hline 1069 & $15: 15: 09$ & 0.867 \\
\hline
\end{tabular}


Appendix E -Fuel Data Test 3 NIST102605_1.xls

\begin{tabular}{|c|c|c|}
\hline 1070 & $15: 15: 10$ & 0.8642 \\
\hline 1071 & $15: 15: 11$ & 0.8624 \\
\hline 1072 & $15: 15: 12$ & 0.8621 \\
\hline 1073 & $15: 15: 13$ & 0.8601 \\
\hline 1074 & $15: 15: 14$ & 0.8585 \\
\hline 1075 & $15: 15: 15$ & 0.8627 \\
\hline 1076 & $15: 15: 16$ & 0.8668 \\
\hline 1077 & $15: 15: 17$ & 0.8679 \\
\hline 10781 & $15: 15: 18$ & 0.8652 \\
\hline 1079 & $15: 15: 19$ & 0.8614 \\
\hline 1080 & $15: 15: 20$ & 0.8606 \\
\hline 1081 & $15: 15: 21$ & 0.8614 \\
\hline 1082 & $15: 15: 22$ & 0.8626 \\
\hline 1083 & $15: 15: 23$ & 0.8624 \\
\hline 1084 & $15: 15: 24$ & 0.8635 \\
\hline 1085 & $15: 15: 25$ & 0.8664 \\
\hline 1086 & $15: 15: 26$ & 0.8655 \\
\hline 1087 & $15: 15: 27$ & 0.8686 \\
\hline 1088 & $15: 15: 28$ & 0.8657 \\
\hline 1089 & $15: 15: 29$ & 0.8656 \\
\hline 1090 & $15: 15: 30$ & 0.8657 \\
\hline 1091 & $15: 15: 31$ & 0.8631 \\
\hline 1092 & $15: 15: 32$ & 0.8680 \\
\hline 1093 & $15: 15: 33$ & 0.8677 \\
\hline 1094 & $15: 15: 34$ & 0.8675 \\
\hline 10951 & $15: 15: 35$ & 0.8670 \\
\hline 10961 & $15: 15: 36$ & 0.8630 \\
\hline 10971 & $15: 15: 37$ & 0.8611 \\
\hline 10981 & $15: 15: 38$ & 0.8651 \\
\hline 10991 & $15: 15: 39$ & 0.8658 \\
\hline 11001 & $15: 15: 40$ & 0.8627 \\
\hline 11011 & $15: 15: 41$ & 0.8686 \\
\hline 11021 & 15:15:42 & 0.8807 \\
\hline 11031 & $15: 15: 43$ & 0.8915 \\
\hline 11041 & $15: 15: 44$ & 0.8855 \\
\hline 11051 & $15: 15: 45$ & 0.8924 \\
\hline 11061 & $15: 15: 46$ & 0.8995 \\
\hline 11071 & $15: 15: 47$ & 0.8998 \\
\hline 11081 & $15: 15: 48$ & 0.8991 \\
\hline 11091 & $15: 15: 49$ & 0.8974 \\
\hline 11101 & $15: 15: 50$ & 0.8963 \\
\hline 11111 & 15:15:51 & 0.8994 \\
\hline 11121 & $15: 15: 52$ & 0.9001 \\
\hline 11131 & $15: 15: 53$ & 0.9160 \\
\hline 11141 & $15: 15: 54$ & 0.9250 \\
\hline 11151 & $15: 15: 55$ & 0.9330 \\
\hline 11161 & $15: 15: 56$ & 0.9500 \\
\hline 11171 & $15: 15: 57$ & 0.9569 \\
\hline 11181 & $15: 15: 58$ & 0.9625 \\
\hline 11191 & $15: 15: 59$ & 0.963 \\
\hline 11201 & $15: 16: 00$ & 0.96 \\
\hline
\end{tabular}


Appendix E -Fuel Data Test 3 NIST102605_1.xls

\begin{tabular}{|c|c|c|}
\hline 1121 & $15: 16: 01$ & 0.9694 \\
\hline 1122 & $15: 16: 02$ & 0.9711 \\
\hline 1123 & $15: 16: 03$ & 0.9721 \\
\hline 1124 & $15: 16: 04$ & 0.9692 \\
\hline 1125 & $15: 16: 05$ & 0.9707 \\
\hline 1126 & $15: 16: 06$ & 0.9737 \\
\hline 1127 & $15: 16: 07$ & 0.9794 \\
\hline 1128 & $15: 16: 08$ & 0.9783 \\
\hline 1129 & 15:16:09 & 0.9785 \\
\hline 1130 & $15: 16: 10$ & 0.9774 \\
\hline 1131 & $15: 16: 11$ & 0.9763 \\
\hline 1132 & $15: 16: 12$ & 0.9748 \\
\hline 1133 & $15: 16: 13$ & 0.9777 \\
\hline 1134 & $15: 16: 14$ & 0.9767 \\
\hline 1135 & $15: 16: 15$ & 0.9748 \\
\hline 1136 & $15: 16: 16$ & 0.9758 \\
\hline 1137 & $15: 16: 17$ & 0.9824 \\
\hline 1138 & $15: 16: 18$ & 0.9846 \\
\hline 1139 & $15: 16: 19$ & 0.9845 \\
\hline 1140 & $15: 16: 20$ & 0.9833 \\
\hline 1141 & $15: 16: 21$ & 0.9786 \\
\hline 1142 & $15: 16: 22$ & 0.9805 \\
\hline 1143 & $15: 16: 23$ & 0.9824 \\
\hline 1144 & $15: 16: 24$ & 0.9867 \\
\hline 1145 & $15: 16: 25$ & 0.9860 \\
\hline 1146 & $15: 16: 26$ & 0.9876 \\
\hline 1147 & $15: 16: 27$ & 0.9874 \\
\hline 1148 & $15: 16: 28$ & 0.9869 \\
\hline 1149 & $15: 16: 29$ & 0.9843 \\
\hline 1150 & $15: 16: 30$ & 0.9847 \\
\hline 1151 & $15: 16: 31$ & 0.9821 \\
\hline 1152 & $15: 16: 32$ & 0.9858 \\
\hline 1153 & $15: 16: 33$ & 0.9863 \\
\hline 1154 & $15: 16: 34$ & 0.9866 \\
\hline 1155 & $15: 16: 35$ & 0.9888 \\
\hline 1156 & $15: 16: 36$ & 0.9882 \\
\hline 1157 & $15: 16: 37$ & 0.9873 \\
\hline 1158 & $15: 16: 38$ & 0.9879 \\
\hline 1159 & $15: 16: 39$ & 0.9864 \\
\hline 1160 & $15: 16: 40$ & 0.9865 \\
\hline 1161 & $15: 16: 41$ & 0.9869 \\
\hline 1162 & $15: 16: 42$ & 0.9890 \\
\hline 1163 & $15: 16: 43$ & 0.9904 \\
\hline 1164 & $15: 16: 44$ & 0.9894 \\
\hline 1165 & $15: 16: 45$ & 0.9880 \\
\hline 1166 & $15: 16: 46$ & 0.9887 \\
\hline 1167 & $15: 16: 47$ & 0.9886 \\
\hline 1168 & $15: 16: 48$ & 0.9895 \\
\hline 1169 & $15: 16: 49$ & 0.9901 \\
\hline 1170 & $15: 16: 50$ & 0.9953 \\
\hline 1171 & $15: 16: 51$ & 0.9943 \\
\hline
\end{tabular}


Appendix E -Fuel Data Test 3 NIST102605_1.xls

\begin{tabular}{|c|c|c|}
\hline 1172 & $15: 16: 52$ & 0.9898 \\
\hline 1173 & $15: 16: 53$ & 0.9875 \\
\hline 1174 & $15: 16: 54$ & 0.9891 \\
\hline 1175 & $15: 16: 55$ & 0.9915 \\
\hline 1176 & 15:16:56 & 0.9947 \\
\hline 1177 & $15: 16: 57$ & 0.9941 \\
\hline 1178 & $15: 16: 58$ & 0.9915 \\
\hline 1179 & $15: 16: 59$ & 0.9938 \\
\hline 1180 & $15: 17: 00$ & 0.9921 \\
\hline 1181 & $15: 17: 01$ & 0.9944 \\
\hline 1182 & 15:17:02 & 0.9926 \\
\hline 1183 & $15: 17: 03$ & 0.9933 \\
\hline 1184 & $15: 17: 04$ & 0.9944 \\
\hline 1185 & $15: 17: 05$ & 0.9935 \\
\hline 1186 & 15:17:06 & 0.9920 \\
\hline 1187 & $15: 17: 07$ & 0.9901 \\
\hline 1188 & $15: 17: 08$ & 0.9899 \\
\hline 1189 & 15:17:09 & 0.9933 \\
\hline 1190 & $15: 17: 10$ & 0.9964 \\
\hline 1191 & $15: 17: 11$ & 0.9951 \\
\hline 1192 & $15: 17: 12$ & 0.9931 \\
\hline 1193 & $15: 17: 13$ & 0.9896 \\
\hline 1194 & $15: 17: 14$ & 0.9895 \\
\hline 1195 & 15:17:15 & 0.9942 \\
\hline 1196 & $15: 17: 16$ & 0.9932 \\
\hline 1197 & $15: 17: 17$ & 0.9955 \\
\hline 1198 & $15: 17: 18$ & 0.9946 \\
\hline 1199 & $15: 17: 19$ & 0.9942 \\
\hline 1200 & $15: 17: 20$ & 0.9929 \\
\hline 1201 & $15: 17: 21$ & 0.9932 \\
\hline 1202 & $15: 17: 22$ & 0.9928 \\
\hline 1203 & $15: 17: 23$ & 0.9928 \\
\hline 1204 & 15:17:24 & 0.9924 \\
\hline 1205 & $15: 17: 25$ & 0.9955 \\
\hline 1206 & $15: 17: 26$ & 0.9968 \\
\hline 1207 & $15: 17: 27$ & 0.9946 \\
\hline 1208 & $15: 17: 28$ & 0.9946 \\
\hline 1209 & $15: 17: 29$ & 0.9927 \\
\hline 1210 & $15: 17: 30$ & 0.9914 \\
\hline 1211 & $15: 17: 31$ & 0.9929 \\
\hline 1212 & $15: 17: 32$ & 0.9923 \\
\hline 1213 & 15:17:33 & 0.9892 \\
\hline 1214 & $15: 17: 34$ & 0.9907 \\
\hline 1215 & $15: 17: 35$ & 0.9914 \\
\hline 1216 & $15: 17: 36$ & 0.9918 \\
\hline 1217 & $15: 17: 37$ & 0.9959 \\
\hline 1218 & $15: 17: 38$ & 0.9976 \\
\hline 1219 & $15: 17: 39$ & 0.9936 \\
\hline 1220 & $15: 17: 40$ & 0.9935 \\
\hline 1221 & $15: 17: 41$ & 0.993 \\
\hline 1222 & $15: 17: 42$ & 0.99 \\
\hline
\end{tabular}


Appendix E -Fuel Data Test 3 NIST102605_1.xls

\begin{tabular}{|c|c|c|}
\hline 1223 & $15: 17: 43$ & 0.9929 \\
\hline 1224 & $15: 17: 44$ & 0.9948 \\
\hline 1225 & $15: 17: 45$ & 0.9951 \\
\hline 1226 & $15: 17: 46$ & 0.9962 \\
\hline 1227 & $15: 17: 47$ & 0.9915 \\
\hline 1228 & $15: 17: 48$ & 0.9912 \\
\hline 1229 & 15:17:49 & 0.9916 \\
\hline 1230 & $15: 17: 50$ & 0.9901 \\
\hline 1231 & $15: 17: 51$ & 0.9916 \\
\hline 1232 & 15:17:52 & 0.9930 \\
\hline 1233 & $15: 17: 53$ & 0.9967 \\
\hline 1234 & 15:17:54 & 0.9966 \\
\hline 1235 & $15: 17: 55$ & 0.9970 \\
\hline 1236 & $15: 17: 56$ & 0.9979 \\
\hline 1237 & 15:17:57 & 0.9948 \\
\hline 1238 & 15:17:58 & 0.9920 \\
\hline 1239 & $15: 17: 59$ & 0.9891 \\
\hline 1240 & $15: 18: 00$ & 0.9939 \\
\hline 1241 & 15:18:01 & 0.9932 \\
\hline 1242 & $15: 18: 02$ & 0.9965 \\
\hline 1243 & $15: 18: 03$ & 0.9949 \\
\hline 1244 & $15: 18: 04$ & 0.9959 \\
\hline 1245 & 15:18:05 & 0.9967 \\
\hline 1246 & 15:18:06 & 0.9948 \\
\hline 1247 & 15:18:07 & 0.9943 \\
\hline 1248 & 15:18:08 & 0.9957 \\
\hline 1249 & 15:18:09 & 0.9959 \\
\hline 1250 & $15: 18: 10$ & 0.9973 \\
\hline 1251 & $15: 18: 11$ & 0.9978 \\
\hline 1252 & 15:18:12 & 0.9983 \\
\hline 1253 & $15: 18: 13$ & 0.9945 \\
\hline 1254 & $15: 18: 14$ & 0.9971 \\
\hline 1255 & $15: 18: 15$ & 0.9977 \\
\hline 1256 & 15:18:16 & 0.9977 \\
\hline 1257 & $15: 18: 17$ & 0.9950 \\
\hline 1258 & $15: 18: 18$ & 0.9920 \\
\hline 1259 & $15: 18: 19$ & 0.9926 \\
\hline 1260 & 15:18:20 & 0.9936 \\
\hline 1261 & 15:18:21 & 0.9945 \\
\hline 1262 & 15:18:22 & 0.9971 \\
\hline 1263 & $15: 18: 23$ & 0.9992 \\
\hline 1264 & $15: 18: 24$ & 0.9978 \\
\hline 1265 & 15:18:25 & 0.9986 \\
\hline 1266 & $15: 18: 26$ & 0.9964 \\
\hline 1267 & $15: 18: 27$ & 0.9969 \\
\hline 1268 & $15: 18: 28$ & 0.9971 \\
\hline 1269 & 15:18:29 & 0.9965 \\
\hline 1270 & 15:18:30 & 0.9937 \\
\hline 1271 & 15:18:31 & 0.9924 \\
\hline 1272 & 15:18:32 & 0.9925 \\
\hline 1273 & $15: 18: 33$ & 0.993 \\
\hline
\end{tabular}


Appendix E -Fuel Data Test 3 NIST102605_1.xls

\begin{tabular}{|c|c|c|}
\hline 1274 & $15: 18: 34$ & 0.9923 \\
\hline 1275 & $15: 18: 35$ & 0.9953 \\
\hline 1276 & $15: 18: 36$ & 0.9957 \\
\hline 1277 & $15: 18: 37$ & 0.9981 \\
\hline 1278 & $15: 18: 38$ & 0.9971 \\
\hline 1279 & $15: 18: 39$ & 0.9958 \\
\hline 1280 & $15: 18: 40$ & 0.9942 \\
\hline 1281 & $15: 18: 41$ & 0.9980 \\
\hline 1282 & 15:18:42 & 0.9977 \\
\hline 1283 & $15: 18: 43$ & 0.9981 \\
\hline 1284 & $15: 18: 44$ & 0.9971 \\
\hline 1285 & $15: 18: 45$ & 0.9946 \\
\hline 1286 & $15: 18: 46$ & 0.9954 \\
\hline 1287 & $15: 18: 47$ & 0.9963 \\
\hline 1288 & $15: 18: 48$ & 0.9941 \\
\hline 1289 & $15: 18: 49$ & 0.9956 \\
\hline 1290 & $15: 18: 50$ & 0.9937 \\
\hline 1291 & 15:18:51 & 0.9915 \\
\hline 1292 & $15: 18: 52$ & 0.9956 \\
\hline 1293 & $15: 18: 53$ & 0.9957 \\
\hline 1294 & $15: 18: 54$ & 0.9986 \\
\hline 1295 & $15: 18: 55$ & 0.9976 \\
\hline 1296 & $15: 18: 56$ & 0.9970 \\
\hline 1297 & $15: 18: 57$ & 0.9962 \\
\hline 1298 & $15: 18: 58$ & 0.9987 \\
\hline 1299 & $15: 18: 59$ & 0.9979 \\
\hline 1300 & 15:19:00 & 0.9966 \\
\hline 1301 & $15: 19: 01$ & 0.9959 \\
\hline 1302 & $15: 19: 02$ & 0.9973 \\
\hline 1303 & $15: 19: 03$ & 0.9975 \\
\hline 1304 & $15: 19: 04$ & 0.9953 \\
\hline 1305 & $15: 19: 05$ & 0.9961 \\
\hline 1306 & $15: 19: 06$ & 0.9974 \\
\hline 1307 & $15: 19: 07$ & 0.9975 \\
\hline 1308 & $15: 19: 08$ & 0.9941 \\
\hline 1309 & 15:19:09 & 0.9945 \\
\hline 1310 & $15: 19: 10$ & 0.9959 \\
\hline 1311 & $15: 19: 11$ & 0.9960 \\
\hline 1312 & $15: 19: 12$ & 0.9995 \\
\hline 1313 & $15: 19: 13$ & 0.9979 \\
\hline 1314 & $15: 19: 14$ & 0.9957 \\
\hline 1315 & $15: 19: 15$ & 0.9943 \\
\hline 1316 & $15: 19: 16$ & 0.9967 \\
\hline 1317 & $15: 19: 17$ & 0.9981 \\
\hline 1318 & $15: 19: 18$ & 0.9952 \\
\hline 1319 & $15: 19: 19$ & 0.9955 \\
\hline 1320 & $15: 19: 20$ & 0.9922 \\
\hline 1321 & $15: 19: 21$ & 0.9927 \\
\hline 1322 & $15: 19: 22$ & 0.9935 \\
\hline 1323 & $15: 19: 23$ & 0.995 \\
\hline 1324 & $15: 19: 24$ & 0.993 \\
\hline
\end{tabular}


Appendix E -Fuel Data Test 3 NIST102605_1.xls

\begin{tabular}{|c|c|c|}
\hline 1325 & $15: 19: 25$ & 0.9956 \\
\hline 1326 & $15: 19: 26$ & 0.9980 \\
\hline 1327 & $15: 19: 27$ & 1.0001 \\
\hline 1328 & $15: 19: 28$ & 0.9986 \\
\hline 1329 & $15: 19: 29$ & 0.9951 \\
\hline 1330 & $15: 19: 30$ & 0.9939 \\
\hline 1331 & $15: 19: 31$ & 0.9931 \\
\hline 1332 & $15: 19: 32$ & 0.9971 \\
\hline 1333 & 15:19:33 & 0.9972 \\
\hline 1334 & $15: 19: 34$ & 0.9982 \\
\hline 1335 & $15: 19: 35$ & 0.9961 \\
\hline 1336 & $15: 19: 36$ & 0.9959 \\
\hline 1337 & $15: 19: 37$ & 0.9959 \\
\hline 1338 & $15: 19: 38$ & 0.9943 \\
\hline 1339 & $15: 19: 39$ & 0.9965 \\
\hline 1340 & $15: 19: 40$ & 0.9991 \\
\hline 1341 & $15: 19: 41$ & 1.0000 \\
\hline 1342 & $15: 19: 42$ & 0.9984 \\
\hline 1343 & $15: 19: 43$ & 0.9998 \\
\hline 1344 & $15: 19: 44$ & 0.9984 \\
\hline 1345 & $15: 19: 45$ & 0.9973 \\
\hline 1346 & $15: 19: 46$ & 0.9975 \\
\hline 1347 & $15: 19: 47$ & 0.9987 \\
\hline 1348 & $15: 19: 48$ & 0.9992 \\
\hline 1349 & $15: 19: 49$ & 0.9998 \\
\hline 1350 & $15: 19: 50$ & 0.9993 \\
\hline 1351 & $15: 19: 51$ & 0.9992 \\
\hline 1352 & $15: 19: 52$ & 0.9953 \\
\hline 1353 & $15: 19: 53$ & 0.9920 \\
\hline 1354 & $15: 19: 54$ & 0.9928 \\
\hline 1355 & $15: 19: 55$ & 0.9970 \\
\hline 1356 & $15: 19: 56$ & 0.9979 \\
\hline 1357 & $15: 19: 57$ & 0.9990 \\
\hline 1358 & $15: 19: 58$ & 1.0002 \\
\hline 1359 & $15: 19: 59$ & 1.0012 \\
\hline 1360 & $15: 20: 00$ & 1.0022 \\
\hline 1361 & $15: 20: 01$ & 1.0008 \\
\hline 1362 & $15: 20: 02$ & 1.0000 \\
\hline 1363 & $15: 20: 03$ & 0.9966 \\
\hline 1364 & $15: 20: 04$ & 0.9984 \\
\hline 1365 & $15: 20: 05$ & 0.9983 \\
\hline 1366 & $15: 20: 06$ & 0.9972 \\
\hline 1367 & $15: 20: 07$ & 0.9980 \\
\hline 1368 & 15:20:08 & 0.9960 \\
\hline 1369 & $15: 20: 09$ & 0.9972 \\
\hline 1370 & $15: 20: 10$ & 0.9979 \\
\hline 1371 & $15: 20: 11$ & 0.9976 \\
\hline 1372 & $15: 20: 12$ & 0.9970 \\
\hline 1373 & $15: 20: 13$ & 0.9959 \\
\hline 1374 & $15: 20: 14$ & 0.996 \\
\hline 1375 & $15: 20: 15$ & 0.996 \\
\hline
\end{tabular}


Appendix E -Fuel Data Test 3 NIST102605_1.xls

\begin{tabular}{|c|c|c|}
\hline 1376 & $15: 20: 16$ & 0.9968 \\
\hline 1377 & $15: 20: 17$ & 0.9957 \\
\hline 1378 & $15: 20: 18$ & 0.9965 \\
\hline 1379 & $15: 20: 19$ & 0.9985 \\
\hline 1380 & $15: 20: 20$ & 0.9989 \\
\hline 1381 & $15: 20: 21$ & 0.9994 \\
\hline 1382 & $15: 20: 22$ & 0.9972 \\
\hline 1383 & $15: 20: 23$ & 0.9985 \\
\hline 1384 & $15: 20: 24$ & 0.9952 \\
\hline 1385 & $15: 20: 25$ & 0.9939 \\
\hline 1386 & $15: 20: 26$ & 0.9950 \\
\hline 1387 & $15: 20: 27$ & 0.9971 \\
\hline 1388 & $15: 20: 28$ & 0.9983 \\
\hline 1389 & $15: 20: 29$ & 0.9990 \\
\hline 1390 & $15: 20: 30$ & 0.9982 \\
\hline 1391 & $15: 20: 31$ & 0.9992 \\
\hline 1392 & $15: 20: 32$ & 0.9998 \\
\hline 1393 & $15: 20: 33$ & 1.0014 \\
\hline 1394 & $15: 20: 34$ & 1.0010 \\
\hline 1395 & $15: 20: 35$ & 0.9985 \\
\hline 1396 & $15: 20: 36$ & 0.9953 \\
\hline 1397 & $15: 20: 37$ & 0.9936 \\
\hline 1398 & $15: 20: 38$ & 0.9937 \\
\hline 1399 & $15: 20: 39$ & 0.9969 \\
\hline 1400 & $15: 20: 40$ & 0.9983 \\
\hline 1401 & $15: 20: 41$ & 0.9967 \\
\hline 1402 & $15: 20: 42$ & 0.9949 \\
\hline 1403 & $15: 20: 43$ & 0.9965 \\
\hline 1404 & $15: 20: 44$ & 0.9967 \\
\hline 1405 & $15: 20: 45$ & 0.9974 \\
\hline 1406 & $15: 20: 46$ & 1.0000 \\
\hline 1407 & $15: 20: 47$ & 1.0003 \\
\hline 1408 & $15: 20: 48$ & 0.9998 \\
\hline 1409 & $15: 20: 49$ & 0.9995 \\
\hline 1410 & $15: 20: 50$ & 0.9980 \\
\hline 1411 & $15: 20: 51$ & 0.9970 \\
\hline 1412 & $15: 20: 52$ & 0.9967 \\
\hline 1413 & $15: 20: 53$ & 0.9985 \\
\hline 1414 & $15: 20: 54$ & 0.9985 \\
\hline 1415 & $15: 20: 55$ & 0.9993 \\
\hline 1416 & $15: 20: 56$ & 0.9954 \\
\hline 1417 & $15: 20: 57$ & 0.9943 \\
\hline 1418 & $15: 20: 58$ & 0.9936 \\
\hline 1419 & $15: 20: 59$ & 0.9929 \\
\hline 1420 & $15: 21: 00$ & 0.9956 \\
\hline 1421 & $15: 21: 01$ & 0.9969 \\
\hline 1422 & $15: 21: 02$ & 0.9968 \\
\hline 1423 & $15: 21: 03$ & 0.9967 \\
\hline 1424 & $15: 21: 04$ & 0.9971 \\
\hline 1425 & $15: 21: 05$ & 0.997 \\
\hline 1426 & $15: 21: 06$ & 0.999 \\
\hline
\end{tabular}


Appendix E -Fuel Data Test 3 NIST102605_1.xls

\begin{tabular}{|c|c|c|}
\hline 1427 & $15: 21: 07$ & 0.9961 \\
\hline 1428 & $15: 21: 08$ & 0.9956 \\
\hline 1429 & 15:21:09 & 0.9936 \\
\hline 1430 & $15: 21: 10$ & 0.9925 \\
\hline 1431 & $15: 21: 11$ & 0.9936 \\
\hline 1432 & $15: 21: 12$ & 0.9929 \\
\hline 1433 & $15: 21: 13$ & 0.9891 \\
\hline 1434 & $15: 21: 14$ & 0.9936 \\
\hline 1435 & $15: 21: 15$ & 0.9947 \\
\hline 1436 & $15: 21: 16$ & 0.9959 \\
\hline 1437 & $15: 21: 17$ & 0.9958 \\
\hline 1438 & $15: 21: 18$ & 0.9967 \\
\hline 1439 & $15: 21: 19$ & 0.9981 \\
\hline 1440 & $15: 21: 20$ & 0.9969 \\
\hline 1441 & $15: 21: 21$ & 0.9945 \\
\hline 1442 & $15: 21: 22$ & 0.9970 \\
\hline 1443 & $15: 21: 23$ & 0.9960 \\
\hline 1444 & $15: 21: 24$ & 0.9961 \\
\hline 1445 & $15: 21: 25$ & 0.9963 \\
\hline 1446 & $15: 21: 26$ & 0.9945 \\
\hline 1447 & $15: 21: 27$ & 0.9926 \\
\hline 1448 & $15: 21: 28$ & 0.9932 \\
\hline 1449 & $15: 21: 29$ & 0.9916 \\
\hline 1450 & $15: 21: 30$ & 0.9909 \\
\hline 1451 & $15: 21: 31$ & 0.9902 \\
\hline 1452 & $15: 21: 32$ & 0.9937 \\
\hline 1453 & $15: 21: 33$ & 0.9936 \\
\hline 1454 & $15: 21: 34$ & 0.9946 \\
\hline 1455 & $15: 21: 35$ & 0.9945 \\
\hline 1456 & $15: 21: 36$ & 0.9956 \\
\hline 1457 & $15: 21: 37$ & 0.9959 \\
\hline 1458 & $15: 21: 38$ & 0.9936 \\
\hline 1459 & $15: 21: 39$ & 0.9907 \\
\hline 1460 & $15: 21: 40$ & 0.9891 \\
\hline 1461 & $15: 21: 41$ & 0.9888 \\
\hline 1462 & $15: 21: 42$ & 0.9886 \\
\hline 1463 & $15: 21: 43$ & 0.9926 \\
\hline 1464 & $15: 21: 44$ & 0.9905 \\
\hline 1465 & $15: 21: 45$ & 0.9881 \\
\hline 1466 & $15: 21: 46$ & 0.9896 \\
\hline 1467 & $15: 21: 47$ & 0.9928 \\
\hline 1468 & $15: 21: 48$ & 0.9928 \\
\hline 1469 & $15: 21: 49$ & 0.9958 \\
\hline 1470 & $15: 21: 50$ & 0.9960 \\
\hline 1471 & $15: 21: 51$ & 0.9960 \\
\hline 1472 & $15: 21: 52$ & 0.9946 \\
\hline 1473 & $15: 21: 53$ & 0.9919 \\
\hline 1474 & $15: 21: 54$ & 0.9902 \\
\hline 1475 & $15: 21: 55$ & 0.9891 \\
\hline 1476 & $15: 21: 56$ & 0.988 \\
\hline 1477 & $15: 21: 57$ & 0.992 \\
\hline
\end{tabular}


Appendix E -Fuel Data Test 3 NIST102605_1.xls

\begin{tabular}{|c|c|c|}
\hline 1478 & $15: 21: 58$ & 0.9903 \\
\hline 1479 & $15: 21: 59$ & 0.9878 \\
\hline 1480 & $15: 22: 00$ & 0.9903 \\
\hline 1481 & $15: 22: 01$ & 0.9903 \\
\hline 1482 & $15: 22: 02$ & 0.9904 \\
\hline 1483 & $15: 22: 03$ & 0.9920 \\
\hline 1484 & $15: 22: 04$ & 0.9943 \\
\hline 1485 & 15:22:05 & 0.9953 \\
\hline 1486 & $15: 22: 06$ & 0.9970 \\
\hline 1487 & $15: 22: 07$ & 0.9942 \\
\hline 1488 & $15: 22: 08$ & 0.9930 \\
\hline 1489 & 15:22:09 & 0.9915 \\
\hline 1490 & $15: 22: 10$ & 0.9954 \\
\hline 1491 & $15: 22: 11$ & 0.9936 \\
\hline 1492 & $15: 22: 12$ & 0.9913 \\
\hline 1493 & $15: 22: 13$ & 0.9904 \\
\hline 1494 & $15: 22: 14$ & 0.9897 \\
\hline 1495 & $15: 22: 15$ & 0.9922 \\
\hline 1496 & $15: 22: 16$ & 0.9912 \\
\hline 1497 & $15: 22: 17$ & 0.9912 \\
\hline 1498 & $15: 22: 18$ & 0.9914 \\
\hline 1499 & $15: 22: 19$ & 0.9876 \\
\hline 1500 & $15: 22: 20$ & 0.9875 \\
\hline 1501 & $15: 22: 21$ & 0.9884 \\
\hline 1502 & $15: 22: 22$ & 0.9917 \\
\hline 1503 & $15: 22: 23$ & 0.9913 \\
\hline 1504 & $15: 22: 24$ & 0.9889 \\
\hline 1505 & $15: 22: 25$ & 0.9905 \\
\hline 1506 & $15: 22: 26$ & 0.9894 \\
\hline 1507 & $15: 22: 27$ & 0.9896 \\
\hline 1508 & $15: 22: 28$ & 0.9893 \\
\hline 1509 & $15: 22: 29$ & 0.9908 \\
\hline 1510 & $15: 22: 30$ & 0.9901 \\
\hline 1511 & $15: 22: 31$ & 0.9885 \\
\hline 1512 & $15: 22: 32$ & 0.9876 \\
\hline 1513 & $15: 22: 33$ & 0.9854 \\
\hline 1514 & $15: 22: 34$ & 0.9857 \\
\hline 1515 & $15: 22: 35$ & 0.9853 \\
\hline 1516 & $15: 22: 36$ & 0.9832 \\
\hline 1517 & $15: 22: 37$ & 0.9829 \\
\hline 1518 & $15: 22: 38$ & 0.9834 \\
\hline 1519 & $15: 22: 39$ & 0.9838 \\
\hline 1520 & $15: 22: 40$ & 0.9800 \\
\hline 1521 & $15: 22: 41$ & 0.9791 \\
\hline 1522 & $15: 22: 42$ & 0.9781 \\
\hline 1523 & $15: 22: 43$ & 0.9741 \\
\hline 1524 & $15: 22: 44$ & 0.9775 \\
\hline 1525 & $15: 22: 45$ & 0.9769 \\
\hline 1526 & $15: 22: 46$ & 0.9762 \\
\hline 1527 & $15: 22: 47$ & 0.9736 \\
\hline 1528 & $15: 22: 48$ & 0.975 \\
\hline
\end{tabular}


Appendix E -Fuel Data Test 3 NIST102605_1.xls

\begin{tabular}{|c|c|c|}
\hline 1529 & $15: 22: 49$ & 0.9745 \\
\hline 1530 & $15: 22: 50$ & 0.9746 \\
\hline 1531 & $15: 22: 51$ & 0.9738 \\
\hline 1532 & $15: 22: 52$ & 0.9739 \\
\hline 1533 & $15: 22: 53$ & 0.9776 \\
\hline 1534 & $15: 22: 54$ & 0.9772 \\
\hline 1535 & $15: 22: 55$ & 0.9760 \\
\hline 1536 & $15: 22: 56$ & 0.9763 \\
\hline 1537 & $15: 22: 57$ & 0.9744 \\
\hline 1538 & $15: 22: 58$ & 0.9721 \\
\hline 1539 & $15: 22: 59$ & 0.9701 \\
\hline 1540 & $15: 23: 00$ & 0.9726 \\
\hline 1541 & $15: 23: 01$ & 0.9734 \\
\hline 1542 & $15: 23: 02$ & 0.9712 \\
\hline 1543 & $15: 23: 03$ & 0.9695 \\
\hline 1544 & $15: 23: 04$ & 0.9690 \\
\hline 1545 & 15:23:05 & 0.9696 \\
\hline 1546 & $15: 23: 06$ & 0.9689 \\
\hline 1547 & $15: 23: 07$ & 0.9721 \\
\hline 1548 & $15: 23: 08$ & 0.9724 \\
\hline 1549 & 15:23:09 & 0.9725 \\
\hline 1550 & $15: 23: 10$ & 0.9718 \\
\hline 1551 & $15: 23: 11$ & 0.9706 \\
\hline 1552 & $15: 23: 12$ & 0.9678 \\
\hline 1553 & $15: 23: 13$ & 0.9672 \\
\hline 1554 & $15: 23: 14$ & 0.9688 \\
\hline 1555 & $15: 23: 15$ & 0.9691 \\
\hline 1556 & $15: 23: 16$ & 0.9670 \\
\hline 1557 & $15: 23: 17$ & 0.9658 \\
\hline 1558 & $15: 23: 18$ & 0.9655 \\
\hline 1559 & $15: 23: 19$ & 0.9647 \\
\hline 1560 & $15: 23: 20$ & 0.9647 \\
\hline 1561 & $15: 23: 21$ & 0.9682 \\
\hline 1562 & $15: 23: 22$ & 0.9682 \\
\hline 1563 & $15: 23: 23$ & 0.9684 \\
\hline 1564 & $15: 23: 24$ & 0.9675 \\
\hline 1565 & $15: 23: 25$ & 0.9654 \\
\hline 1566 & $15: 23: 26$ & 0.9641 \\
\hline 1567 & $15: 23: 27$ & 0.9634 \\
\hline 1568 & $15: 23: 28$ & 0.9610 \\
\hline 1569 & $15: 23: 29$ & 0.9651 \\
\hline 1570 & $15: 23: 30$ & 0.9645 \\
\hline 1571 & $15: 23: 31$ & 0.9633 \\
\hline 1572 & $15: 23: 32$ & 0.9647 \\
\hline 1573 & $15: 23: 33$ & 0.9649 \\
\hline 1574 & $15: 23: 34$ & 0.9630 \\
\hline 1575 & $15: 23: 35$ & 0.9625 \\
\hline 1576 & $15: 23: 36$ & 0.9590 \\
\hline 1577 & $15: 23: 37$ & 0.9608 \\
\hline 1578 & $15: 23: 38$ & 0.9630 \\
\hline 1579 & $15: 23: 39$ & 0.9615 \\
\hline
\end{tabular}


Appendix E -Fuel Data Test 3 NIST102605_1.xls

\begin{tabular}{|c|c|c|}
\hline 1580 & $15: 23: 40$ & 0.9622 \\
\hline 1581 & $15: 23: 41$ & 0.9609 \\
\hline 1582 & $15: 23: 42$ & 0.9617 \\
\hline 1583 & $15: 23: 43$ & 0.9622 \\
\hline 1584 & $15: 23: 44$ & 0.9600 \\
\hline 1585 & $15: 23: 45$ & 0.9600 \\
\hline 1586 & $15: 23: 46$ & 0.9591 \\
\hline 1587 & $15: 23: 47$ & 0.9626 \\
\hline 1588 & $15: 23: 48$ & 0.9615 \\
\hline 1589 & $15: 23: 49$ & 0.9570 \\
\hline 1590 & $15: 23: 50$ & 0.9549 \\
\hline 1591 & $15: 23: 51$ & 0.9555 \\
\hline 1592 & $15: 23: 52$ & 0.9547 \\
\hline 1593 & $15: 23: 53$ & 0.9581 \\
\hline 1594 & $15: 23: 54$ & 0.9589 \\
\hline 1595 & $15: 23: 55$ & 0.9592 \\
\hline 1596 & $15: 23: 56$ & 0.9579 \\
\hline 1597 & $15: 23: 57$ & 0.9568 \\
\hline 1598 & $15: 23: 58$ & 0.9548 \\
\hline 1599 & $15: 23: 59$ & 0.9564 \\
\hline 1600 & $15: 24: 00$ & 0.9559 \\
\hline 1601 & $15: 24: 01$ & 0.9556 \\
\hline 1602 & $15: 24: 02$ & 0.9578 \\
\hline 1603 & $15: 24: 03$ & 0.9537 \\
\hline 1604 & $15: 24: 04$ & 0.9513 \\
\hline 1605 & $15: 24: 05$ & 0.9519 \\
\hline 1606 & $15: 24: 06$ & 0.9526 \\
\hline 1607 & $15: 24: 07$ & 0.9557 \\
\hline 1608 & $15: 24: 08$ & 0.9553 \\
\hline 1609 & $15: 24: 09$ & 0.9562 \\
\hline 1610 & $15: 24: 10$ & 0.9571 \\
\hline 1611 & $15: 24: 11$ & 0.9533 \\
\hline 1612 & $15: 24: 12$ & 0.9498 \\
\hline 1613 & $15: 24: 13$ & 0.9504 \\
\hline 1614 & $15: 24: 14$ & 0.9508 \\
\hline 1615 & $15: 24: 15$ & 0.9489 \\
\hline 1616 & $15: 24: 16$ & 0.9477 \\
\hline 1617 & $15: 24: 17$ & 0.9485 \\
\hline 1618 & $15: 24: 18$ & 0.9477 \\
\hline 1619 & $15: 24: 19$ & 0.9462 \\
\hline 1620 & $15: 24: 20$ & 0.9487 \\
\hline 1621 & $15: 24: 21$ & 0.9463 \\
\hline 1622 & $15: 24: 22$ & 0.9471 \\
\hline 1623 & $15: 24: 23$ & 0.9478 \\
\hline 1624 & $15: 24: 24$ & 0.9498 \\
\hline 1625 & $15: 24: 25$ & 0.9471 \\
\hline 1626 & $15: 24: 26$ & 0.9470 \\
\hline 1627 & $15: 24: 27$ & 0.9474 \\
\hline 1628 & $15: 24: 28$ & 0.9449 \\
\hline 1629 & $15: 24: 29$ & 0.9441 \\
\hline 1630 & $15: 24: 30$ & 0.943 \\
\hline
\end{tabular}


Appendix E -Fuel Data Test 3 NIST102605_1.xls

\begin{tabular}{|c|c|c|}
\hline 1631 & $15: 24: 31$ & 0.9447 \\
\hline 1632 & $15: 24: 32$ & 0.9455 \\
\hline 1633 & $15: 24: 33$ & 0.9442 \\
\hline 1634 & $15: 24: 34$ & 0.9422 \\
\hline 1635 & $15: 24: 35$ & 0.9385 \\
\hline 1636 & $15: 24: 36$ & 0.9388 \\
\hline 1637 & $15: 24: 37$ & 0.9386 \\
\hline 1638 & $15: 24: 38$ & 0.9430 \\
\hline 1639 & $15: 24: 39$ & 0.9418 \\
\hline 1640 & $15: 24: 40$ & 0.9451 \\
\hline 1641 & $15: 24: 41$ & 0.9432 \\
\hline 1642 & $15: 24: 42$ & 0.9431 \\
\hline 1643 & $15: 24: 43$ & 0.9419 \\
\hline 1644 & $15: 24: 44$ & 0.9416 \\
\hline 1645 & $15: 24: 45$ & 0.9398 \\
\hline 1646 & $15: 24: 46$ & 0.9384 \\
\hline 1647 & $15: 24: 47$ & 0.9397 \\
\hline 1648 & $15: 24: 48$ & 0.9358 \\
\hline 1649 & $15: 24: 49$ & 0.9351 \\
\hline 1650 & $15: 24: 50$ & 0.9367 \\
\hline 1651 & $15: 24: 51$ & 0.9368 \\
\hline 1652 & $15: 24: 52$ & 0.9394 \\
\hline 1653 & $15: 24: 53$ & 0.9403 \\
\hline 1654 & $15: 24: 54$ & 0.9375 \\
\hline 1655 & $15: 24: 55$ & 0.9396 \\
\hline 1656 & $15: 24: 56$ & 0.9376 \\
\hline 1657 & $15: 24: 57$ & 0.9374 \\
\hline 1658 & $15: 24: 58$ & 0.9357 \\
\hline 1659 & $15: 24: 59$ & 0.9349 \\
\hline 1660 & $15: 25: 00$ & 0.9352 \\
\hline 1661 & $15: 25: 01$ & 0.9355 \\
\hline 1662 & $15: 25: 02$ & 0.9367 \\
\hline 1663 & $15: 25: 03$ & 0.9389 \\
\hline 1664 & $15: 25: 04$ & 0.9382 \\
\hline 1665 & $15: 25: 05$ & 0.9356 \\
\hline 1666 & $15: 25: 06$ & 0.9347 \\
\hline 1667 & $15: 25: 07$ & 0.9345 \\
\hline 1668 & $15: 25: 08$ & 0.9386 \\
\hline 1669 & $15: 25: 09$ & 0.9385 \\
\hline 1670 & $15: 25: 10$ & 0.9373 \\
\hline 1671 & $15: 25: 11$ & 0.9344 \\
\hline 1672 & $15: 25: 12$ & 0.9331 \\
\hline 1673 & $15: 25: 13$ & 0.9335 \\
\hline 1674 & $15: 25: 14$ & 0.9324 \\
\hline 1675 & $15: 25: 15$ & 0.9322 \\
\hline 1676 & $15: 25: 16$ & 0.9366 \\
\hline 1677 & $15: 25: 17$ & 0.9374 \\
\hline 1678 & $15: 25: 18$ & 0.9347 \\
\hline 1679 & $15: 25: 19$ & 0.9352 \\
\hline 1680 & $15: 25: 20$ & 0.9370 \\
\hline 1681 & $15: 25: 21$ & 0.934 \\
\hline
\end{tabular}


Appendix E -Fuel Data Test 3 NIST102605_1.xls

\begin{tabular}{|c|c|c|}
\hline 1682 & $15: 25: 22$ & 0.9347 \\
\hline 1683 & $15: 25: 23$ & 0.9331 \\
\hline 1684 & $15: 25: 24$ & 0.9316 \\
\hline 1685 & $15: 25: 25$ & 0.9308 \\
\hline 1686 & $15: 25: 26$ & 0.9326 \\
\hline 1687 & $15: 25: 27$ & 0.9359 \\
\hline 1688 & $15: 25: 28$ & 0.9338 \\
\hline 1689 & $15: 25: 29$ & 0.9332 \\
\hline 1690 & $15: 25: 30$ & 0.9316 \\
\hline 1691 & $15: 25: 31$ & 0.9327 \\
\hline 1692 & $15: 25: 32$ & 0.9321 \\
\hline 1693 & $15: 25: 33$ & 0.9320 \\
\hline 1694 & $15: 25: 34$ & 0.9339 \\
\hline 1695 & $15: 25: 35$ & 0.9364 \\
\hline 1696 & $15: 25: 36$ & 0.9355 \\
\hline 1697 & $15: 25: 37$ & 0.9370 \\
\hline 1698 & $15: 25: 38$ & 0.9355 \\
\hline 1699 & $15: 25: 39$ & 0.9342 \\
\hline 1700 & $15: 25: 40$ & 0.9311 \\
\hline 1701 & $15: 25: 41$ & 0.9313 \\
\hline 1702 & $15: 25: 42$ & 0.9310 \\
\hline 1703 & $15: 25: 43$ & 0.9313 \\
\hline 1704 & $15: 25: 44$ & 0.9338 \\
\hline 1705 & $15: 25: 45$ & 0.9338 \\
\hline 1706 & $15: 25: 46$ & 0.9331 \\
\hline 1707 & $15: 25: 47$ & 0.9308 \\
\hline 1708 & $15: 25: 48$ & 0.9351 \\
\hline 1709 & $15: 25: 49$ & 0.9340 \\
\hline 1710 & $15: 25: 50$ & 0.9327 \\
\hline 1711 & $15: 25: 51$ & 0.9315 \\
\hline 1712 & $15: 25: 52$ & 0.9321 \\
\hline 1713 & $15: 25: 53$ & 0.9327 \\
\hline 1714 & $15: 25: 54$ & 0.9322 \\
\hline 1715 & $15: 25: 55$ & 0.9307 \\
\hline 1716 & $15: 25: 56$ & 0.9331 \\
\hline 1717 & $15: 25: 57$ & 0.9311 \\
\hline 1718 & $15: 25: 58$ & 0.9274 \\
\hline 1719 & $15: 25: 59$ & 0.9286 \\
\hline 1720 & $15: 26: 00$ & 0.9299 \\
\hline 1721 & $15: 26: 01$ & 0.9337 \\
\hline 1722 & $15: 26: 02$ & 0.9335 \\
\hline 1723 & $15: 26: 03$ & 0.9332 \\
\hline 1724 & $15: 26: 04$ & 0.9335 \\
\hline 1725 & $15: 26: 05$ & 0.9335 \\
\hline 1726 & $15: 26: 06$ & 0.9316 \\
\hline 1727 & $15: 26: 07$ & 0.9301 \\
\hline 1728 & $15: 26: 08$ & 0.9303 \\
\hline 1729 & $15: 26: 09$ & 0.9288 \\
\hline 1730 & $15: 26: 10$ & 0.9293 \\
\hline 1731 & $15: 26: 11$ & 0.9318 \\
\hline 1732 & $15: 26: 12$ & 0.9296 \\
\hline
\end{tabular}


Appendix E -Fuel Data Test 3 NIST102605_1.xls

\begin{tabular}{|c|c|c|}
\hline 1733 & $15: 26: 13$ & 0.9289 \\
\hline 1734 & $15: 26: 14$ & 0.9293 \\
\hline 1735 & $15: 26: 15$ & 0.9335 \\
\hline 1736 & $15: 26: 16$ & 0.9330 \\
\hline 1737 & $15: 26: 17$ & 0.9300 \\
\hline 1738 & 15:26:18 & 0.9302 \\
\hline 1739 & $15: 26: 19$ & 0.9338 \\
\hline 1740 & 15:26:20 & 0.9324 \\
\hline 1741 & $15: 26: 21$ & 0.9296 \\
\hline 1742 & $15: 26: 22$ & 0.9284 \\
\hline 1743 & $15: 26: 23$ & 0.9283 \\
\hline 1744 & $15: 26: 24$ & 0.9294 \\
\hline 1745 & $15: 26: 25$ & 0.9276 \\
\hline 1746 & $15: 26: 26$ & 0.9287 \\
\hline 1747 & $15: 26: 27$ & 0.9284 \\
\hline 1748 & $15: 26: 28$ & 0.9291 \\
\hline 1749 & $15: 26: 29$ & 0.9311 \\
\hline 1750 & $15: 26: 30$ & 0.9323 \\
\hline 1751 & $15: 26: 31$ & 0.9344 \\
\hline 1752 & $15: 26: 32$ & 0.9334 \\
\hline 1753 & $15: 26: 33$ & 0.9307 \\
\hline 1754 & $15: 26: 34$ & 0.9294 \\
\hline 1755 & $15: 26: 35$ & 0.9290 \\
\hline 1756 & $15: 26: 36$ & 0.9315 \\
\hline 1757 & $15: 26: 37$ & 0.9302 \\
\hline 17581 & $15: 26: 38$ & 0.9288 \\
\hline 17591 & 15:26:39 & 0.9262 \\
\hline 17601 & $15: 26: 40$ & 0.9253 \\
\hline 17611 & $15: 26: 41$ & 0.9269 \\
\hline 17621 & $15: 26: 42$ & 0.9296 \\
\hline 17631 & $15: 26: 43$ & 0.9309 \\
\hline 17641 & 15:26:44 & 0.9295 \\
\hline 17651 & $15: 26: 45$ & 0.9275 \\
\hline 17661 & $15: 26: 46$ & 0.9268 \\
\hline 17671 & $15: 26: 47$ & 0.9297 \\
\hline 17681 & $15: 26: 48$ & 0.9292 \\
\hline 17691 & $15: 26: 49$ & 0.9288 \\
\hline 17701 & $15: 26: 50$ & 0.9279 \\
\hline 17711 & $15: 26: 51$ & 0.9283 \\
\hline 17721 & $15: 26: 52$ & 0.9264 \\
\hline 17731 & $15: 26: 53$ & 0.9263 \\
\hline 17741 & $15: 26: 54$ & 0.9296 \\
\hline 17751 & $15: 26: 55$ & 0.9288 \\
\hline 17761 & $15: 26: 56$ & 0.9297 \\
\hline 17771 & $15: 26: 57$ & 0.9303 \\
\hline 17781 & 15:26:58 & 0.9287 \\
\hline 17791 & $15: 26: 59$ & 0.928 \\
\hline 17801 & $15: 27: 00$ & $0.926 \varepsilon$ \\
\hline 17811 & 15:27:01 & 0.9267 \\
\hline 17821 & 15:27:02 & 0.9250 \\
\hline 17831 & $15: 27: 03$ & 00 \\
\hline
\end{tabular}


Appendix E -Fuel Data Test 3 NIST102605_1.xls

\begin{tabular}{|c|c|c|}
\hline 17841 & $15: 27: 04$ & 0.9269 \\
\hline 17851 & $15: 27: 05$ & 0.9250 \\
\hline 17861 & 15:27:06 & 0.9262 \\
\hline 17871 & $15: 27: 07$ & 0.9275 \\
\hline 17881 & $15: 27: 08$ & 0.9285 \\
\hline 17891 & 15:27:09 & 0.9295 \\
\hline 17901 & $15: 27: 10$ & 0.9305 \\
\hline 17911 & $15: 27: 11$ & 0.9306 \\
\hline 17921 & $15: 27: 12$ & 0.9285 \\
\hline 17931 & $15: 27: 13$ & 0.9278 \\
\hline 17941 & $15: 27: 14$ & 0.9294 \\
\hline 17951 & $15: 27: 15$ & 0.9278 \\
\hline 17961 & $15: 27: 16$ & 0.9299 \\
\hline 17971 & $15: 27: 17$ & 0.9294 \\
\hline 17981 & $15: 27: 18$ & 0.9259 \\
\hline 17991 & $15: 27: 19$ & 0.9239 \\
\hline 18001 & 15:27:20 & 0.9228 \\
\hline 18011 & $15: 27: 21$ & 0.9224 \\
\hline 18021 & $15: 27: 22$ & 0.9267 \\
\hline 18031 & $15: 27: 23$ & 0.9278 \\
\hline 18041 & $15: 27: 24$ & 0.9269 \\
\hline 18051 & $15: 27: 25$ & 0.9284 \\
\hline 18061 & $15: 27: 26$ & 0.9299 \\
\hline 18071 & 15:27:27 & 0.9278 \\
\hline 18081 & 15:27:28 & 0.9282 \\
\hline 18091 & $15: 27: 29$ & 0.9270 \\
\hline 18101 & $15: 27: 30$ & 0.9273 \\
\hline 18111 & $15: 27: 31$ & 0.9279 \\
\hline 18121 & $15: 27: 32$ & 0.9232 \\
\hline 18131 & $15: 27: 33$ & 0.9232 \\
\hline 18141 & $15: 27: 34$ & 0.9240 \\
\hline 18151 & $15: 27: 35$ & 0.9246 \\
\hline 18161 & 15:27:36 & 0.9262 \\
\hline 18171 & $15: 27: 37$ & 0.9262 \\
\hline 18181 & $15: 27: 38$ & 0.9256 \\
\hline 18191 & 15:27:39 & 0.9277 \\
\hline 18201 & $15: 27: 40$ & 0.9273 \\
\hline 18211 & $15: 27: 41$ & 0.9268 \\
\hline 18221 & $15: 27: 42$ & 0.9277 \\
\hline 18231 & $15: 27: 43$ & 0.9291 \\
\hline 18241 & $15: 27: 44$ & 0.9299 \\
\hline 18251 & $15: 27: 45$ & 0.9268 \\
\hline 18261 & $15: 27: 46$ & 0.9241 \\
\hline 18271 & $15: 27: 47$ & 0.9222 \\
\hline 18281 & $15: 27: 48$ & 0.9215 \\
\hline 18291 & $15: 27: 49$ & 0.9243 \\
\hline 18301 & $15: 27: 50$ & 0.9266 \\
\hline 18311 & $15: 27: 51$ & 0.926 \\
\hline 18321 & $15: 27: 52$ & 0.9276 \\
\hline 18331 & 15:27:53 & 0.9282 \\
\hline 18341 & $15: 27: 54$ & 0.92 \\
\hline
\end{tabular}


Appendix E -Fuel Data Test 3 NIST102605_1.xls

\begin{tabular}{|c|c|c|}
\hline 1835 & $15: 27: 55$ & 0.9279 \\
\hline 1836 & $15: 27: 56$ & 0.9275 \\
\hline 1837 & $15: 27: 57$ & 0.9281 \\
\hline 1838 & $15: 27: 58$ & 0.9278 \\
\hline 1839 & $15: 27: 59$ & 0.9247 \\
\hline 1840 & 15:28:00 & 0.9223 \\
\hline 1841 & 15:28:01 & 0.9222 \\
\hline 1842 & 15:28:02 & 0.9243 \\
\hline 1843 & 15:28:03 & 0.9278 \\
\hline 1844 & 15:28:04 & 0.9287 \\
\hline 1845 & 15:28:05 & 0.9279 \\
\hline 1846 & 15:28:06 & 0.9295 \\
\hline 1847 & 15:28:07 & 0.9282 \\
\hline 1848 & 15:28:08 & 0.9285 \\
\hline 1849 & 15:28:09 & 0.9261 \\
\hline 1850 & $15: 28: 10$ & 0.9267 \\
\hline 1851 & 15:28:11 & 0.9273 \\
\hline 18521 & $15: 28: 12$ & 0.9265 \\
\hline 1853 & $15: 28: 13$ & 0.9263 \\
\hline 1854 & $15: 28: 14$ & 0.9244 \\
\hline 1855 & $15: 28: 15$ & 0.9246 \\
\hline 1856 & 15:28:16 & 0.9265 \\
\hline 1857 & $15: 28: 17$ & 0.9277 \\
\hline 1858 & 15:28:18 & 0.9288 \\
\hline 1859 & $15: 28: 19$ & 0.9288 \\
\hline 18601 & 15:28:20 & 0.9275 \\
\hline 18611 & $15: 28: 21$ & 0.9268 \\
\hline 18621 & 15:28:22 & 0.9263 \\
\hline 18631 & 15:28:23 & 0.9267 \\
\hline 18641 & 15:28:24 & 0.9245 \\
\hline 18651 & 15:28:25 & 0.9261 \\
\hline 18661 & 15:28:26 & 0.9268 \\
\hline 18671 & 15:28:27 & 0.9251 \\
\hline 18681 & 15:28:28 & 0.9244 \\
\hline 18691 & 15:28:29 & 0.9248 \\
\hline 18701 & 15:28:30 & 0.9264 \\
\hline 18711 & 15:28:31 & 0.9274 \\
\hline 18721 & 15:28:32 & 0.9248 \\
\hline 18731 & 15:28:33 & 0.9276 \\
\hline 18741 & 15:28:34 & 0.9268 \\
\hline 18751 & 15:28:35 & 0.9261 \\
\hline 18761 & 15:28:36 & 0.9266 \\
\hline 18771 & $15: 28: 37$ & 0.9243 \\
\hline 18781 & 15:28:38 & 0.9237 \\
\hline 18791 & 15:28:39 & 0.9224 \\
\hline 18801 & $15: 28: 40$ & 0.9243 \\
\hline 18811 & $15: 28: 41$ & 0.9234 \\
\hline 18821 & 15:28:42 & 0.9238 \\
\hline 18831 & $15: 28: 43$ & 0.9242 \\
\hline 18841 & 15:28:44 & 0.9274 \\
\hline 18851 & $15: 28: 45$ & 0.927 \\
\hline
\end{tabular}


Appendix E -Fuel Data Test 3 NIST102605_1.xls

\begin{tabular}{|c|c|c|}
\hline 1886 & $15: 28: 46$ & 0.9284 \\
\hline 1887 & $15: 28: 47$ & 0.9295 \\
\hline 1888 & $15: 28: 48$ & 0.9287 \\
\hline 1889 & $15: 28: 49$ & 0.9270 \\
\hline 1890 & $15: 28: 50$ & 0.9266 \\
\hline 1891 & $15: 28: 51$ & 0.9248 \\
\hline 1892 & $15: 28: 52$ & 0.9241 \\
\hline 1893 & $15: 28: 53$ & 0.9257 \\
\hline 1894 & $15: 28: 54$ & 0.9235 \\
\hline 1895 & $15: 28: 55$ & 0.9248 \\
\hline 1896 & $15: 28: 56$ & 0.9240 \\
\hline 1897 & $15: 28: 57$ & 0.9233 \\
\hline 1898 & $15: 28: 58$ & 0.9235 \\
\hline 1899 & $15: 28: 59$ & 0.9267 \\
\hline 1900 & $15: 29: 00$ & 0.9251 \\
\hline 1901 & $15: 29: 01$ & 0.9237 \\
\hline 1902 & $15: 29: 02$ & 0.9234 \\
\hline 1903 & $15: 29: 03$ & 0.9234 \\
\hline 1904 & $15: 29: 04$ & 0.9252 \\
\hline 1905 & $15: 29: 05$ & 0.9251 \\
\hline 1906 & $15: 29: 06$ & 0.9257 \\
\hline 1907 & $15: 29: 07$ & 0.9234 \\
\hline 1908 & $15: 29: 08$ & 0.9225 \\
\hline 1909 & $15: 29: 09$ & 0.9219 \\
\hline 1910 & $15: 29: 10$ & 0.9218 \\
\hline 1911 & $15: 29: 11$ & 0.9211 \\
\hline 1912 & $15: 29: 12$ & 0.9243 \\
\hline 1913 & $15: 29: 13$ & 0.9270 \\
\hline 1914 & $15: 29: 14$ & 0.9278 \\
\hline 1915 & $15: 29: 15$ & 0.9277 \\
\hline 1916 & $15: 29: 16$ & 0.9279 \\
\hline 1917 & $15: 29: 17$ & 0.9272 \\
\hline 1918 & $15: 29: 18$ & 0.9259 \\
\hline 1919 & $15: 29: 19$ & 0.9259 \\
\hline 1920 & $15: 29: 20$ & 0.9239 \\
\hline 1921 & $15: 29: 21$ & 0.9207 \\
\hline 1922 & $15: 29: 22$ & 0.9232 \\
\hline 1923 & $15: 29: 23$ & 0.9239 \\
\hline 1924 & $15: 29: 24$ & 0.9237 \\
\hline 1925 & $15: 29: 25$ & 0.9254 \\
\hline 1926 & $15: 29: 26$ & 0.9254 \\
\hline 1927 & $15: 29: 27$ & 0.9259 \\
\hline 1928 & $15: 29: 28$ & 0.9263 \\
\hline 1929 & 15:29:29 & 0.9262 \\
\hline 1930 & $15: 29: 30$ & 0.9234 \\
\hline 1931 & $15: 29: 31$ & 0.9248 \\
\hline 1932 & $15: 29: 32$ & 0.9278 \\
\hline 1933 & $15: 29: 33$ & 0.9274 \\
\hline 1934 & $15: 29: 34$ & 0.9230 \\
\hline 1935 & $15: 29: 35$ & 0.9216 \\
\hline 1936 & 15:29:36 & 0.920 \\
\hline
\end{tabular}


Appendix E -Fuel Data Test 3 NIST102605_1.xls

\begin{tabular}{|c|c|c|}
\hline 1937 & $15: 29: 37$ & 0.9204 \\
\hline 1938 & $15: 29: 38$ & 0.9240 \\
\hline 1939 & $15: 29: 39$ & 0.9239 \\
\hline 1940 & $15: 29: 40$ & 0.9252 \\
\hline 1941 & $15: 29: 41$ & 0.9277 \\
\hline 1942 & $15: 29: 42$ & 0.9286 \\
\hline 1943 & $15: 29: 43$ & 0.9267 \\
\hline 1944 & $15: 29: 44$ & 0.9264 \\
\hline 1945 & $15: 29: 45$ & 0.9263 \\
\hline 1946 & $15: 29: 46$ & 0.9259 \\
\hline 1947 & $15: 29: 47$ & 0.9225 \\
\hline 1948 & $15: 29: 48$ & 0.9195 \\
\hline 1949 & $15: 29: 49$ & 0.9209 \\
\hline 1950 & $15: 29: 50$ & 0.9220 \\
\hline 1951 & $15: 29: 51$ & 0.9242 \\
\hline 1952 & $15: 29: 52$ & 0.9253 \\
\hline 1953 & $15: 29: 53$ & 0.9257 \\
\hline 1954 & $15: 29: 54$ & 0.9266 \\
\hline 1955 & $15: 29: 55$ & 0.9262 \\
\hline 1956 & $15: 29: 56$ & 0.9259 \\
\hline 1957 & $15: 29: 57$ & 0.9259 \\
\hline 1958 & $15: 29: 58$ & 0.9254 \\
\hline 1959 & $15: 29: 59$ & 0.9251 \\
\hline 1960 & $15: 30: 00$ & 0.9266 \\
\hline 1961 & $15: 30: 01$ & 0.9255 \\
\hline 1962 & $15: 30: 02$ & 0.9249 \\
\hline 1963 & $15: 30: 03$ & 0.9232 \\
\hline 1964 & $15: 30: 04$ & 0.9215 \\
\hline 1965 & $15: 30: 05$ & 0.9226 \\
\hline 1966 & $15: 30: 06$ & 0.9242 \\
\hline 1967 & $15: 30: 07$ & 0.9248 \\
\hline 1968 & $15: 30: 08$ & 0.9273 \\
\hline 1969 & $15: 30: 09$ & 0.9271 \\
\hline 1970 & $15: 30: 10$ & 0.9266 \\
\hline 1971 & $15: 30: 11$ & 0.9247 \\
\hline 1972 & $15: 30: 12$ & 0.9247 \\
\hline 1973 & $15: 30: 13$ & 0.9264 \\
\hline 1974 & $15: 30: 14$ & 0.9249 \\
\hline 1975 & $15: 30: 15$ & 0.9251 \\
\hline 1976 & $15: 30: 16$ & 0.9234 \\
\hline 1977 & $15: 30: 17$ & 0.9213 \\
\hline 1978 & $15: 30: 18$ & 0.9221 \\
\hline 1979 & $15: 30: 19$ & 0.9232 \\
\hline 1980 & $15: 30: 20$ & 0.9274 \\
\hline 1981 & $15: 30: 21$ & 0.9271 \\
\hline 1982 & $15: 30: 22$ & 0.9249 \\
\hline 1983 & $15: 30: 23$ & 0.9237 \\
\hline 1984 & $15: 30: 24$ & 0.9259 \\
\hline 1985 & $15: 30: 25$ & 0.9246 \\
\hline 1986 & $15: 30: 26$ & 0.9226 \\
\hline 1987 & $15: 30: 27$ & 0.9247 \\
\hline
\end{tabular}


Appendix E -Fuel Data Test 3 NIST102605_1.xls

\begin{tabular}{|c|c|c|}
\hline 1988 & $15: 30: 28$ & 0.9269 \\
\hline 1989 & 15:30:29 & 0.9253 \\
\hline 1990 & $15: 30: 30$ & 0.9229 \\
\hline 1991 & $15: 30: 31$ & 0.9242 \\
\hline 1992 & 15:30:32 & 0.9234 \\
\hline 1993 & $15: 30: 33$ & 0.9244 \\
\hline 1994 & $15: 30: 34$ & 0.9261 \\
\hline 1995 & 15:30:35 & 0.9257 \\
\hline 1996 & 15:30:36 & 0.9242 \\
\hline 1997 & $15: 30: 37$ & 0.9277 \\
\hline 1998 & 15:30:38 & 0.9254 \\
\hline 1999 & 15:30:39 & 0.9269 \\
\hline 2000 & $15: 30: 40$ & 0.9243 \\
\hline 2001 & $15: 30: 41$ & 0.9234 \\
\hline 2002 & $15: 30: 42$ & 0.9225 \\
\hline 2003 & $15: 30: 43$ & 0.9207 \\
\hline 2004 & $15: 30: 44$ & 0.9219 \\
\hline 2005 & $15: 30: 45$ & 0.9223 \\
\hline 2006 & $15: 30: 46$ & 0.9223 \\
\hline 2007 & $15: 30: 47$ & 0.9261 \\
\hline 2008 & $15: 30: 48$ & 0.9262 \\
\hline 2009 & $15: 30: 49$ & 0.9265 \\
\hline 2010 & $15: 30: 50$ & 0.9232 \\
\hline 2011 & $15: 30: 51$ & 0.9249 \\
\hline 2012 & $15: 30: 52$ & 0.9241 \\
\hline 2013 & $15: 30: 53$ & 0.9256 \\
\hline 2014 & $15: 30: 54$ & 0.9228 \\
\hline 2015 & $15: 30: 55$ & 0.9230 \\
\hline 2016 & $15: 30: 56$ & 0.9232 \\
\hline 2017 & $15: 30: 57$ & 0.9208 \\
\hline 2018 & $15: 30: 58$ & 0.9200 \\
\hline 2019 & $15: 30: 59$ & 0.9205 \\
\hline 2020 & $15: 31: 00$ & 0.9204 \\
\hline 2021 & $15: 31: 01$ & 0.9257 \\
\hline 2022 & 15:31:02 & 0.9260 \\
\hline 2023 & 15:31:03 & 0.9275 \\
\hline 2024 & 15:31:04 & 0.9263 \\
\hline 2025 & $15: 31: 05$ & 0.9275 \\
\hline 2026 & 15:31:06 & 0.9253 \\
\hline 2027 & 15:31:07 & 0.922 \\
\hline 2028 & 15:31:08 & 0.9210 \\
\hline 2029 & $15: 31: 09$ & 0.9190 \\
\hline 2030 & $15: 31: 10$ & 0.9231 \\
\hline 2031 & 15:31:11 & 0.9230 \\
\hline 2032 & $15: 31: 12$ & 0.923 \\
\hline 2033 & $15: 31: 13$ & 0.9248 \\
\hline 2034 & $15: 31: 14$ & 0.925 \\
\hline 2035 & $15: 31: 15$ & 0.926 \\
\hline 2036 & 15:31:16 & 0.9262 \\
\hline 2037 & $15: 31: 17$ & 0.926 \\
\hline 2038 & 15:31:18 & 0.92 \\
\hline
\end{tabular}


Appendix E -Fuel Data Test 3 NIST102605_1.xls

\begin{tabular}{|c|c|c|}
\hline 2039 & $15: 31: 19$ & 0.9241 \\
\hline 2040 & $15: 31: 20$ & 0.9251 \\
\hline 2041 & $15: 31: 21$ & 0.9253 \\
\hline 2042 & $15: 31: 22$ & 0.9269 \\
\hline 2043 & $15: 31: 23$ & 0.9252 \\
\hline 2044 & $15: 31: 24$ & 0.9244 \\
\hline 2045 & $15: 31: 25$ & 0.9233 \\
\hline 2046 & $15: 31: 26$ & 0.9220 \\
\hline 2047 & $15: 31: 27$ & 0.9246 \\
\hline 2048 & $15: 31: 28$ & 0.9249 \\
\hline 2049 & $15: 31: 29$ & 0.9251 \\
\hline 2050 & $15: 31: 30$ & 0.9252 \\
\hline 2051 & $15: 31: 31$ & 0.9271 \\
\hline 2052 & $15: 31: 32$ & 0.9253 \\
\hline 2053 & $15: 31: 33$ & 0.9248 \\
\hline 2054 & $15: 31: 34$ & 0.9219 \\
\hline 2055 & $15: 31: 35$ & 0.9229 \\
\hline 2056 & $15: 31: 36$ & 0.9249 \\
\hline 2057 & $15: 31: 37$ & 0.9218 \\
\hline 2058 & $15: 31: 38$ & 0.9206 \\
\hline 2059 & $15: 31: 39$ & 0.9199 \\
\hline 2060 & $15: 31: 40$ & 0.9254 \\
\hline 2061 & $15: 31: 41$ & 0.9249 \\
\hline 2062 & $15: 31: 42$ & 0.9255 \\
\hline 2063 & $15: 31: 43$ & 0.9257 \\
\hline 2064 & $15: 31: 44$ & 0.9265 \\
\hline 2065 & $15: 31: 45$ & 0.9259 \\
\hline 2066 & $15: 31: 46$ & 0.9241 \\
\hline 2067 & $15: 31: 47$ & 0.9233 \\
\hline 2068 & $15: 31: 48$ & 0.9211 \\
\hline 2069 & $15: 31: 49$ & 0.9243 \\
\hline 2070 & $15: 31: 50$ & 0.9241 \\
\hline 2071 & $15: 31: 51$ & 0.9227 \\
\hline 2072 & $15: 31: 52$ & 0.9236 \\
\hline 2073 & $15: 31: 53$ & 0.9235 \\
\hline 2074 & $15: 31: 54$ & 0.9236 \\
\hline 2075 & $15: 31: 55$ & 0.9241 \\
\hline 2076 & $15: 31: 56$ & 0.9252 \\
\hline 2077 & $15: 31: 57$ & 0.9248 \\
\hline 2078 & $15: 31: 58$ & 0.9241 \\
\hline 2079 & $15: 31: 59$ & 0.9242 \\
\hline 2080 & $15: 32: 00$ & 0.9234 \\
\hline 2081 & $15: 32: 01$ & 0.9208 \\
\hline 2082 & 15:32:02 & 0.9233 \\
\hline 2083 & $15: 32: 03$ & 0.9230 \\
\hline 2084 & $15: 32: 04$ & 0.9221 \\
\hline 2085 & $15: 32: 05$ & 0.9217 \\
\hline 2086 & $15: 32: 06$ & 0.9212 \\
\hline 2087 & $15: 32: 07$ & 0.9225 \\
\hline 2088 & $15: 32: 08$ & 0.9272 \\
\hline 2089 & 15:32:09 & 0.9262 \\
\hline
\end{tabular}


Appendix E -Fuel Data Test 3 NIST102605_1.xls

\begin{tabular}{|c|c|c|}
\hline 2090 & $15: 32: 10$ & 0.9260 \\
\hline 2091 & $15: 32: 11$ & 0.9240 \\
\hline 2092 & $15: 32: 12$ & 0.9240 \\
\hline 2093 & $15: 32: 13$ & 0.9215 \\
\hline 2094 & $15: 32: 14$ & 0.9214 \\
\hline 2095 & 15:32:15 & 0.9204 \\
\hline 2096 & 15:32:16 & 0.9241 \\
\hline 2097 & $15: 32: 17$ & 0.9238 \\
\hline 2098 & $15: 32: 18$ & 0.9218 \\
\hline 2099 & 15:32:19 & 0.9219 \\
\hline 2100 & 15:32:20 & 0.9218 \\
\hline 2101 & 15:32:21 & 0.9219 \\
\hline 2102 & 15:32:22 & 0.9237 \\
\hline 2103 & 15:32:23 & 0.9261 \\
\hline 2104 & 15:32:24 & 0.9283 \\
\hline 2105 & 15:32:25 & 0.9293 \\
\hline 2106 & 15:32:26 & 0.9270 \\
\hline 2107 & $15: 32: 27$ & 0.9234 \\
\hline 2108 & $15: 32: 28$ & 0.9242 \\
\hline 2109 & 15:32:29 & 0.9230 \\
\hline 2110 & $15: 32: 30$ & 0.9252 \\
\hline 2111 & 15:32:31 & 0.9253 \\
\hline 2112 & 15:32:32 & 0.9223 \\
\hline 2113 & 15:32:33 & 0.9218 \\
\hline 2114 & $15: 32: 34$ & 0.9236 \\
\hline 2115 & 15:32:35 & 0.9264 \\
\hline 2116 & 15:32:36 & 0.925 \\
\hline 2117 & $15: 32: 37$ & 0.9239 \\
\hline 2118 & 15:32:38 & 0.9238 \\
\hline 2119 & 15:32:39 & 0.9241 \\
\hline 2120 & $15: 32: 40$ & 0.9219 \\
\hline 2121 & $15: 32: 41$ & 0.9211 \\
\hline 2122 & 15:32:42 & 0.9208 \\
\hline 2123 & $15: 32: 43$ & 0.9225 \\
\hline 2124 & $15: 32: 44$ & 0.9233 \\
\hline 2125 & $15: 32: 45$ & 0.9224 \\
\hline 2126 & $15: 32: 46$ & 0.9227 \\
\hline 2127 & $15: 32: 47$ & 0.9208 \\
\hline 2128 & 15:32:48 & 0.9209 \\
\hline 2129 & $15: 32: 49$ & 0.9217 \\
\hline 2130 & $15: 32: 50$ & 0.9239 \\
\hline 2131 & 15:32:51 & 0.9252 \\
\hline 2132 & 15:32:52 & 0.9263 \\
\hline 2133 & $15: 32: 53$ & 0.9260 \\
\hline 2134 & 15:32:54 & 0.925 \\
\hline 2135 & 15:32:55 & 0.925 \\
\hline 2136 & $15: 32: 56$ & 0.924 \\
\hline 2137 & $15: 32: 57$ & 0.922 \\
\hline 2138 & 15:32:58 & 0.924 \\
\hline 2139 & 15:32:59 & 0.921 \\
\hline 2140 & 15:33:00 & 0.92 \\
\hline
\end{tabular}


Appendix E -Fuel Data Test 3 NIST102605_1.xls

\begin{tabular}{|c|c|c|}
\hline 2141 & 15:33:01 & 0.9222 \\
\hline 2142 & 15:33:02 & 0.9226 \\
\hline 2143 & 15:33:03 & 0.9246 \\
\hline 2144 & 15:33:04 & 0.9242 \\
\hline 2145 & 15:33:05 & 0.9247 \\
\hline 2146 & 15:33:06 & 0.9241 \\
\hline 2147 & 15:33:07 & 0.9239 \\
\hline 2148 & 15:33:08 & 0.9245 \\
\hline 2149 & 15:33:09 & 0.9236 \\
\hline 2151 & 15:33:11 & 0.9260 \\
\hline 2152 & 15:33:12 & 0.9229 \\
\hline 2152 & 15:33:12 & 0.9211 \\
\hline 2153 & 15:33:13 & 0.9210 \\
\hline 2154 & $15: 33: 14$ & 0.9186 \\
\hline 2155 & $15: 33: 15$ & 0.9217 \\
\hline 2156 & 15:33:16 & 0.9245 \\
\hline 2157 & 15:33:17 & 0.9250 \\
\hline 2158 & 15:33:18 & 0.9284 \\
\hline 2159 & 15:33:19 & 0.9281 \\
\hline 2160 & 15:33:20 & 0.9272 \\
\hline 2161 & 15:33:21 & 0.9256 \\
\hline 2162 & 15:33:22 & 0.9185 \\
\hline 2163 & 15:33:23 & 0.9175 \\
\hline 2164 & 15:33:24 & 0.9136 \\
\hline 2165 & 15:33:25 & 0.9169 \\
\hline 2166 & 15:33:26 & 0.9166 \\
\hline 2167 & 15:33:27 & 0.915 \\
\hline 2168 & 15:33:28 & 0.9167 \\
\hline 2169 & 15:33:29 & 0.9198 \\
\hline 2170 & 15:33:30 & 0.9221 \\
\hline 2171 & 15:33:31 & 0.9213 \\
\hline 2172 & 15:33:32 & 0.9189 \\
\hline 2173 & 15:33:33 & 0.9184 \\
\hline 2174 & 15:33:34 & 0.9174 \\
\hline 2175 & 15:33:35 & 0.9191 \\
\hline 2176 & 15:33:36 & 0.9170 \\
\hline 2177 & 15:33:37 & 0.9173 \\
\hline 2178 & 15:33:38 & 0.9201 \\
\hline 2179 & 15:33:39 & 0.9172 \\
\hline 2180 & 15:33:40 & 0.9179 \\
\hline 2181 & 15:33:41 & 0.9163 \\
\hline 2182 & 15:33:42 & 0.9184 \\
\hline 2183 & $15: 33: 43$ & 0.9205 \\
\hline 2184 & 15:33:44 & 0.9216 \\
\hline 2185 & 15:33:45 & 0.921 \\
\hline 2186 & 15:33:46 & 0.9237 \\
\hline 2187 & $15: 33: 47$ & 0.923 \\
\hline 2188 & 15:33:48 & 0.918 \\
\hline 2189 & 15:33:49 & 0.918 \\
\hline 2190 & 15:33:50 & 0.918 \\
\hline 2191 & 15:33:51 & 0.914 \\
\hline
\end{tabular}


Appendix E -Fuel Data Test 3 NIST102605_1.xls

\begin{tabular}{|c|c|c|}
\hline 2192 & $15: 33: 52$ & 0.9177 \\
\hline 2193 & 15:33:53 & 0.9165 \\
\hline 2194 & 15:33:54 & 0.9179 \\
\hline 2195 & 15:33:55 & 0.9197 \\
\hline 2196 & 15:33:56 & 0.9200 \\
\hline 2197 & 15:33:57 & 0.9185 \\
\hline 2198 & 15:33:58 & 0.9202 \\
\hline 2199 & 15:33:59 & 0.9209 \\
\hline 2200 & $15: 34: 00$ & 0.9192 \\
\hline 2201 & 15:34:01 & 0.9196 \\
\hline 2202 & $15: 34: 02$ & 0.9185 \\
\hline 2203 & 15:34:03 & 0.9175 \\
\hline 2204 & $15: 34: 04$ & 0.9215 \\
\hline 2205 & $15: 34: 05$ & 0.9201 \\
\hline 2206 & 15:34:06 & 0.9184 \\
\hline 2207 & 15:34:07 & 0.9189 \\
\hline 2208 & 15:34:08 & 0.9190 \\
\hline 2209 & 15:34:09 & 0.9198 \\
\hline 2210 & $15: 34: 10$ & 0.9193 \\
\hline 2211 & $15: 34: 11$ & 0.9210 \\
\hline 2212 & $15: 34: 12$ & 0.9208 \\
\hline 2213 & $15: 34: 13$ & 0.9230 \\
\hline 2214 & $15: 34: 14$ & 0.9223 \\
\hline 2215 & $15: 34: 15$ & 0.9182 \\
\hline 2216 & 15:34:16 & 0.9170 \\
\hline 2217 & $15: 34: 17$ & 0.9176 \\
\hline 2218 & $15: 34: 18$ & 0.9171 \\
\hline 2219 & $15: 34: 19$ & 0.9184 \\
\hline 2220 & 15:34:20 & 0.9182 \\
\hline 2221 & $15: 34: 21$ & 0.9197 \\
\hline 2222 & $15: 34: 22$ & 0.9203 \\
\hline 2223 & $15: 34: 23$ & 0.9205 \\
\hline 2224 & $15: 34: 24$ & 0.9214 \\
\hline 2225 & $15: 34: 25$ & 0.9193 \\
\hline 2226 & $15: 34: 26$ & 0.9174 \\
\hline 2227 & $15: 34: 27$ & 0.9155 \\
\hline 2228 & $15: 34: 28$ & 0.9168 \\
\hline 2229 & $15: 34: 29$ & 0.9181 \\
\hline 2230 & 15:34:30 & 0.9197 \\
\hline 2231 & $15: 34: 31$ & 0.9184 \\
\hline 2232 & $15: 34: 32$ & 0.9181 \\
\hline 2233 & $15: 34: 33$ & 0.9138 \\
\hline 2234 & $15: 34: 34$ & 0.9159 \\
\hline 2235 & 15:34:35 & 0.9168 \\
\hline 2236 & $15: 34: 36$ & 0.9187 \\
\hline 2237 & $15: 34: 37$ & 0.9206 \\
\hline 2238 & $15: 34: 38$ & 0.922 \\
\hline 2239 & $15: 34: 39$ & 0.922 \\
\hline 2240 & $15: 34: 40$ & 0.919 \\
\hline 2241 & $15: 34: 41$ & 0.917 \\
\hline 2242 & $15: 34: 42$ & 0.917 \\
\hline
\end{tabular}


Appendix E -Fuel Data Test 3 NIST102605_1.xls

\begin{tabular}{|c|c|c|}
\hline 2243 & $15: 34: 43$ & 0.9184 \\
\hline 2244 & $15: 34: 44$ & 0.9165 \\
\hline 2245 & $15: 34: 45$ & 0.9145 \\
\hline 2246 & $15: 34: 46$ & 0.9144 \\
\hline 2247 & $15: 34: 47$ & 0.9147 \\
\hline 2248 & $15: 34: 48$ & 0.9180 \\
\hline 2249 & $15: 34: 49$ & 0.9169 \\
\hline 2250 & $15: 34: 50$ & 0.9187 \\
\hline 2251 & $15: 34: 51$ & 0.9203 \\
\hline 2252 & $15: 34: 52$ & 0.9192 \\
\hline 2253 & $15: 34: 53$ & 0.9203 \\
\hline 2254 & $15: 34: 54$ & 0.9194 \\
\hline 2255 & $15: 34: 55$ & 0.9208 \\
\hline 2256 & $15: 34: 56$ & 0.9211 \\
\hline 2257 & $15: 34: 57$ & 0.9171 \\
\hline 2258 & 15:34:58 & 0.9179 \\
\hline 2259 & $15: 34: 59$ & 0.9170 \\
\hline 2260 & $15: 35: 00$ & 0.9141 \\
\hline 2261 & $15: 35: 01$ & 0.9172 \\
\hline 2262 & $15: 35: 02$ & 0.9160 \\
\hline 2263 & $15: 35: 03$ & 0.9187 \\
\hline 2264 & $15: 35: 04$ & 0.9209 \\
\hline 2265 & $15: 35: 05$ & 0.9209 \\
\hline 2266 & 15:35:06 & 0.9230 \\
\hline 2267 & 15:35:07 & 0.9193 \\
\hline 2268 & $15: 35: 08$ & 0.9185 \\
\hline 2269 & $15: 35: 09$ & 0.9171 \\
\hline 2270 & $15: 35: 10$ & 0.9144 \\
\hline 2271 & $15: 35: 11$ & 0.9177 \\
\hline 2272 & $15: 35: 12$ & 0.9172 \\
\hline 2273 & $15: 35: 13$ & 0.9166 \\
\hline 2274 & $15: 35: 14$ & 0.9174 \\
\hline 2275 & $15: 35: 15$ & 0.9161 \\
\hline 2276 & $15: 35: 16$ & 0.9166 \\
\hline 2277 & $15: 35: 17$ & 0.9173 \\
\hline 2278 & $15: 35: 18$ & 0.9210 \\
\hline 2279 & $15: 35: 19$ & 0.9193 \\
\hline 2280 & $15: 35: 20$ & 0.9186 \\
\hline 2281 & $15: 35: 21$ & 0.9169 \\
\hline 2282 & $15: 35: 22$ & 0.9149 \\
\hline 2283 & $15: 35: 23$ & 0.9153 \\
\hline 2284 & $15: 35: 24$ & 0.9145 \\
\hline 2285 & $15: 35: 25$ & 0.9141 \\
\hline 2286 & $15: 35: 26$ & 0.9152 \\
\hline 2287 & $15: 35: 27$ & 0.9132 \\
\hline 2288 & $15: 35: 28$ & 0.9141 \\
\hline 2289 & $15: 35: 29$ & 0.9163 \\
\hline 2290 & $15: 35: 30$ & 0.9192 \\
\hline 2291 & $15: 35: 31$ & 0.9188 \\
\hline 2292 & $15: 35: 32$ & 0.9207 \\
\hline 2293 & $15: 35: 33$ & 0.919 \\
\hline
\end{tabular}


Appendix E -Fuel Data Test 3 NIST102605_1.xls

\begin{tabular}{|c|c|c|}
\hline 2294 & $15: 35: 34$ & 0.9192 \\
\hline 2295 & $15: 35: 35$ & 0.9183 \\
\hline 2296 & $15: 35: 36$ & 0.9173 \\
\hline 2297 & $15: 35: 37$ & 0.9176 \\
\hline 2298 & $15: 35: 38$ & 0.9161 \\
\hline 2299 & 15:35:39 & 0.9145 \\
\hline 2300 & 15:35:40 & 0.9149 \\
\hline 2301 & $15: 35: 41$ & 0.9175 \\
\hline 2302 & $15: 35: 42$ & 0.9167 \\
\hline 2303 & $15: 35: 43$ & 0.9161 \\
\hline 2304 & $15: 35: 44$ & 0.9179 \\
\hline 2305 & 15:35:45 & 0.9191 \\
\hline 2306 & $15: 35: 46$ & 0.9184 \\
\hline 2307 & $15: 35: 47$ & 0.9160 \\
\hline 2308 & 15:35:48 & 0.9140 \\
\hline 2309 & 15:35:49 & 0.9135 \\
\hline 2310 & $15: 35: 50$ & 0.9181 \\
\hline 2311 & $15: 35: 51$ & 0.9177 \\
\hline 2312 & $15: 35: 52$ & 0.9146 \\
\hline 2313 & $15: 35: 53$ & 0.9147 \\
\hline 2314 & $15: 35: 54$ & 0.9148 \\
\hline 2315 & $15: 35: 55$ & 0.9154 \\
\hline 2316 & $15: 35: 56$ & 0.9213 \\
\hline 2317 & 15:35:57 & 0.9205 \\
\hline 2318 & 15:35:58 & 0.9215 \\
\hline 2319 & $15: 35: 59$ & 0.9206 \\
\hline 2320 & $15: 36: 00$ & 0.9164 \\
\hline 2321 & $15: 36: 01$ & 0.9153 \\
\hline 2322 & 15:36:02 & 0.9171 \\
\hline 2323 & 15:36:03 & 0.9175 \\
\hline 2324 & 15:36:04 & 0.9167 \\
\hline 2325 & 15:36:05 & 0.9167 \\
\hline 2326 & 15:36:06 & 0.9150 \\
\hline 2327 & 15:36:07 & 0.9165 \\
\hline 2328 & 15:36:08 & 0.9178 \\
\hline 2329 & 15:36:09 & 0.9188 \\
\hline 2330 & $15: 36: 10$ & 0.9191 \\
\hline 2331 & $15: 36: 11$ & 0.9187 \\
\hline 2332 & $15: 36: 12$ & 0.9169 \\
\hline 2333 & $15: 36: 13$ & 0.9197 \\
\hline 2334 & $15: 36: 14$ & 0.9188 \\
\hline 2335 & $15: 36: 15$ & 0.9203 \\
\hline 2336 & $15: 36: 16$ & 0.9207 \\
\hline 2337 & $15: 36: 17$ & 0.9182 \\
\hline 2338 & 15:36:18 & 0.9178 \\
\hline 2339 & $15: 36: 19$ & 0.9177 \\
\hline 2340 & 15:36:20 & 0.9148 \\
\hline 2341 & 15:36:21 & 0.9145 \\
\hline 2342 & 15:36:22 & 0.915 \\
\hline 2343 & $15: 36: 23$ & 0.918 \\
\hline 2344 & 15:36:24 & 0.919 \\
\hline
\end{tabular}


Appendix E -Fuel Data Test 3 NIST102605_1.xls

\begin{tabular}{|c|c|c|}
\hline 2345 & $15: 36: 25$ & 0.9210 \\
\hline 2346 & 15:36:26 & 0.9224 \\
\hline 2347 & $15: 36: 27$ & 0.9204 \\
\hline 2348 & 15:36:28 & 0.9191 \\
\hline 2349 & $15: 36: 29$ & 0.9194 \\
\hline 2350 & 15:36:30 & 0.9176 \\
\hline 2351 & 15:36:31 & 0.9187 \\
\hline 2352 & 15:36:32 & 0.9169 \\
\hline 2353 & 15:36:33 & 0.9149 \\
\hline 2354 & 15:36:34 & 0.9161 \\
\hline 2355 & 15:36:35 & 0.9176 \\
\hline 2356 & 15:36:36 & 0.9172 \\
\hline 2357 & 15:36:37 & 0.9185 \\
\hline 2358 & $15: 36: 38$ & 0.9189 \\
\hline 2359 & 15:36:39 & 0.9166 \\
\hline 2360 & 15:36:40 & 0.9162 \\
\hline 2361 & $15: 36: 41$ & 0.9181 \\
\hline 2362 & $15: 36: 42$ & 0.9176 \\
\hline 2363 & $15: 36: 43$ & 0.9210 \\
\hline 2364 & $15: 36: 44$ & 0.9202 \\
\hline 2365 & $15: 36: 45$ & 0.9194 \\
\hline 2366 & $15: 36: 46$ & 0.9178 \\
\hline 2367 & $15: 36: 47$ & 0.9148 \\
\hline 2368 & 15:36:48 & 0.9161 \\
\hline 2369 & 15:36:49 & 0.9183 \\
\hline 2370 & $15: 36: 50$ & 0.9192 \\
\hline 2371 & $15: 36: 51$ & 0.9172 \\
\hline 2372 & $15: 36: 52$ & 0.9186 \\
\hline 2373 & $15: 36: 53$ & 0.9195 \\
\hline 2374 & $15: 36: 54$ & 0.9209 \\
\hline 2375 & 15:36:55 & 0.9169 \\
\hline 2376 & 15:36:56 & 0.9162 \\
\hline 2377 & $15: 36: 57$ & 0.9128 \\
\hline 2378 & 15:36:58 & 0.9126 \\
\hline 2379 & $15: 36: 59$ & 0.9157 \\
\hline 2380 & 15:37:00 & 0.9177 \\
\hline 2381 & 15:37:01 & 0.9177 \\
\hline 2382 & 15:37:02 & 0.9201 \\
\hline 2383 & 15:37:03 & 0.9212 \\
\hline 2384 & $15: 37: 04$ & 0.9211 \\
\hline 2385 & 15:37:05 & 0.9187 \\
\hline 2386 & 15:37:06 & 0.9162 \\
\hline 2387 & 15:37:07 & 0.9172 \\
\hline 2388 & $15: 37: 08$ & 0.9169 \\
\hline 2389 & 15:37:09 & 0.9182 \\
\hline 2390 & $15: 37: 10$ & 0.9216 \\
\hline 2391 & $15: 37: 11$ & 0.919 \\
\hline 2392 & 15:37:12 & 0.9182 \\
\hline 2393 & $15: 37: 13$ & 0.9181 \\
\hline 2394 & $15: 37: 14$ & 0.918 \\
\hline 2395 & 15:37:15 & 0.917 \\
\hline
\end{tabular}


Appendix E -Fuel Data Test 3 NIST102605_1.xls

\begin{tabular}{|c|c|c|}
\hline 2396 & $15: 37: 16$ & 0.9220 \\
\hline 2397 & $15: 37: 17$ & 0.9227 \\
\hline 2398 & 15:37:18 & 0.9238 \\
\hline 2399 & $15: 37: 19$ & 0.9246 \\
\hline 2400 & 15:37:20 & 0.9220 \\
\hline 2401 & 15:37:21 & 0.9211 \\
\hline 2402 & 15:37:22 & 0.9206 \\
\hline 2403 & $15: 37: 23$ & 0.9197 \\
\hline 2404 & 15:37:24 & 0.9211 \\
\hline 2405 & $15: 37: 25$ & 0.9172 \\
\hline 2406 & $15: 37: 26$ & 0.9161 \\
\hline 2407 & $15: 37: 27$ & 0.9191 \\
\hline 2408 & 15:37:28 & 0.9189 \\
\hline 2409 & 15:37:29 & 0.9189 \\
\hline 2410 & 15:37:30 & 0.9192 \\
\hline 2411 & $15: 37: 31$ & 0.9203 \\
\hline 2412 & 15:37:32 & 0.9196 \\
\hline 2413 & 15:37:33 & 0.9207 \\
\hline 2414 & $15: 37: 34$ & 0.9210 \\
\hline 2415 & $15: 37: 35$ & 0.9211 \\
\hline 2416 & $15: 37: 36$ & 0.9208 \\
\hline 2417 & $15: 37: 37$ & 0.9206 \\
\hline 2418 & $15: 37: 38$ & 0.9197 \\
\hline 2419 & 15:37:39 & 0.9174 \\
\hline 2420 & $15: 37: 40$ & 0.9156 \\
\hline 2421 & $15: 37: 41$ & 0.9160 \\
\hline 2422 & $15: 37: 42$ & 0.9178 \\
\hline 2423 & $15: 37: 43$ & 0.9206 \\
\hline 2424 & $15: 37: 44$ & 0.9229 \\
\hline 2425 & $15: 37: 45$ & 0.9250 \\
\hline 2426 & $15: 37: 46$ & 0.9241 \\
\hline 2427 & $15: 37: 47$ & 0.9242 \\
\hline 2428 & 15:37:48 & 0.9211 \\
\hline 2429 & $15: 37: 49$ & 0.9178 \\
\hline 2430 & $15: 37: 50$ & 0.9193 \\
\hline 2431 & 15:37:51 & 0.9215 \\
\hline 2432 & 15:37:52 & 0.9191 \\
\hline 2433 & $15: 37: 53$ & 0.9165 \\
\hline 2434 & $15: 37: 54$ & 0.9183 \\
\hline 2435 & $15: 37: 55$ & 0.9194 \\
\hline 2436 & 15:37:56 & 0.9182 \\
\hline 2437 & 15:37:57 & 0.9182 \\
\hline 2438 & $15: 37: 58$ & 0.9195 \\
\hline 2439 & 15:37:59 & 0.9178 \\
\hline 2440 & 15:38:00 & 0.9198 \\
\hline 2441 & 15:38:01 & 0.921 \\
\hline 2442 & 15:38:02 & 0.919 \\
\hline 2443 & 15:38:03 & 0.920 \\
\hline 2444 & 15:38:04 & 0.920 \\
\hline 2445 & 15:38:05 & 0.919 \\
\hline 2446 & 15:38:06 & 0.920 \\
\hline
\end{tabular}


Appendix E -Fuel Data Test 3 NIST102605_1.xls

\begin{tabular}{|c|c|c|}
\hline 2447 & $15: 38: 07$ & 0.9190 \\
\hline 2448 & 15:38:08 & 0.9188 \\
\hline 2449 & 15:38:09 & 0.9200 \\
\hline 2450 & 15:38:10 & 0.9221 \\
\hline 2451 & $15: 38: 11$ & 0.9225 \\
\hline 2452 & 15:38:12 & 0.9245 \\
\hline 2453 & 15:38:13 & 0.9257 \\
\hline 2454 & $15: 38: 14$ & 0.9251 \\
\hline 2455 & 15:38:15 & 0.9196 \\
\hline 2456 & 15:38:16 & 0.9182 \\
\hline 2457 & $15: 38: 17$ & 0.9182 \\
\hline 2458 & 15:38:18 & 0.9207 \\
\hline 2459 & 15:38:19 & 0.9204 \\
\hline 2460 & 15:38:20 & 0.9176 \\
\hline 2461 & 15:38:21 & 0.9194 \\
\hline 2462 & 15:38:22 & 0.9224 \\
\hline 2463 & 15:38:23 & 0.9221 \\
\hline 2464 & 15:38:24 & 0.9212 \\
\hline 2465 & $15: 38: 25$ & 0.9203 \\
\hline 2466 & 15:38:26 & 0.9187 \\
\hline 2467 & 15:38:27 & 0.9184 \\
\hline 2468 & 15:38:28 & 0.9194 \\
\hline 2469 & 15:38:29 & 0.9198 \\
\hline 2470 & 15:38:30 & 0.9188 \\
\hline 2471 & 15:38:31 & 0.9211 \\
\hline 2472 & 15:38:32 & 0.9206 \\
\hline 2473 & 15:38:33 & 0.9191 \\
\hline 2474 & 15:38:34 & 0.9173 \\
\hline 2475 & 15:38:35 & 0.9178 \\
\hline 2476 & 15:38:36 & 0.9198 \\
\hline 2477 & 15:38:37 & 0.9198 \\
\hline 2478 & 15:38:38 & 0.9221 \\
\hline 2479 & 15:38:39 & 0.9243 \\
\hline 2480 & $15: 38: 40$ & 0.9222 \\
\hline 2481 & 15:38:41 & 0.9220 \\
\hline 2482 & 15:38:42 & 0.920 \\
\hline 2483 & $15: 38: 43$ & 0.9184 \\
\hline 2484 & $15: 38: 44$ & 0.9179 \\
\hline 2485 & 15:38:45 & 0.9164 \\
\hline 2486 & 15:38:46 & 0.9177 \\
\hline 2487 & 15:38:47 & 0.9186 \\
\hline 2488 & 15:38:48 & 0.9178 \\
\hline 2489 & 15:38:49 & 0.9191 \\
\hline 2490 & 15:38:50 & 0.9222 \\
\hline 2491 & 15:38:51 & 0.923 \\
\hline 2492 & 15:38:52 & 0.9231 \\
\hline 2493 & 15:38:53 & 0.921 \\
\hline 2494 & 15:38:54 & 0.919 \\
\hline 2495 & 15:38:55 & 0.919 \\
\hline 2496 & 15:38:56 & 0.917 \\
\hline 2497 & 15:38:57 & 0.916 \\
\hline
\end{tabular}


Appendix E -Fuel Data Test 3 NIST102605_1.xls

\begin{tabular}{|c|c|c|}
\hline 2498 & $15: 38: 58$ & 0.9189 \\
\hline 2499 & 15:38:59 & 0.9167 \\
\hline 2500 & 15:39:00 & 0.9168 \\
\hline 2501 & 15:39:01 & 0.9171 \\
\hline 2502 & 15:39:02 & 0.9164 \\
\hline 2503 & 15:39:03 & 0.9170 \\
\hline 2504 & 15:39:04 & 0.9197 \\
\hline 2505 & 15:39:05 & 0.9220 \\
\hline 2506 & 15:39:06 & 0.9233 \\
\hline 2507 & 15:39:07 & 0.9233 \\
\hline 2508 & 15:39:08 & 0.9239 \\
\hline 2509 & 15:39:09 & 0.9215 \\
\hline 2510 & 15:39:10 & 0.9180 \\
\hline 2511 & 15:39:11 & 0.9177 \\
\hline 2512 & 15:39:12 & 0.9155 \\
\hline 2513 & 15:39:13 & 0.9192 \\
\hline 2514 & $15: 39: 14$ & 0.9206 \\
\hline 2515 & $15: 39: 15$ & 0.9204 \\
\hline 2516 & 15:39:16 & 0.9210 \\
\hline 2517 & $15: 39: 17$ & 0.9175 \\
\hline 2518 & 15:39:18 & 0.9174 \\
\hline 2519 & 15:39:19 & 0.9139 \\
\hline 2520 & 15:39:20 & 0.9140 \\
\hline 2521 & 15:39:21 & 0.9105 \\
\hline 2522 & 15:39:22 & 0.9093 \\
\hline 2523 & 15:39:23 & 0.9068 \\
\hline 2524 & 15:39:24 & 0.9081 \\
\hline 2525 & $15: 39: 25$ & 0.9092 \\
\hline 2526 & 15:39:26 & 0.9089 \\
\hline 2527 & 15:39:27 & 0.9065 \\
\hline 2528 & 15:39:28 & 0.9051 \\
\hline 2529 & 15:39:29 & 0.9056 \\
\hline 2530 & 15:39:30 & 0.9069 \\
\hline 2531 & 15:39:31 & 0.9079 \\
\hline 2532 & 15:39:32 & 0.9083 \\
\hline 2533 & 15:39:33 & 0.9099 \\
\hline 2534 & 15:39:34 & 0.9064 \\
\hline 2535 & 15:39:35 & 0.9030 \\
\hline 2536 & 15:39:36 & 0.9029 \\
\hline 2537 & 15:39:37 & 0.9014 \\
\hline 2538 & 15:39:38 & 0.9005 \\
\hline 2539 & 15:39:39 & 0.9007 \\
\hline 2540 & $15: 39: 40$ & 0.8985 \\
\hline 2541 & 15:39:41 & 0.9012 \\
\hline 2542 & 15:39:42 & 0.9000 \\
\hline 2543 & 15:39:43 & 0.9015 \\
\hline 2544 & $15: 39: 44$ & 0.8977 \\
\hline 2545 & 15:39:45 & 0.8933 \\
\hline 2546 & 15:39:46 & 0.8914 \\
\hline 2547 & 15:39:47 & 0.894 \\
\hline 2548 & 15:39:48 & 0.891 \\
\hline
\end{tabular}


Appendix E -Fuel Data Test 3 NIST102605_1.xls

\begin{tabular}{|c|c|c|}
\hline 2549 & $15: 39: 49$ & 0.8918 \\
\hline 2550 & $15: 39: 50$ & 0.8909 \\
\hline 2551 & $15: 39: 51$ & 0.8897 \\
\hline 2552 & $15: 39: 52$ & 0.8889 \\
\hline 2553 & $15: 39: 53$ & 0.8892 \\
\hline 2554 & $15: 39: 54$ & 0.8928 \\
\hline 2555 & $15: 39: 55$ & 0.8942 \\
\hline 2556 & $15: 39: 56$ & 0.8954 \\
\hline 2557 & 15:39:57 & 0.8967 \\
\hline 2558 & $15: 39: 58$ & 0.8973 \\
\hline 2559 & $15: 39: 59$ & 0.8987 \\
\hline 2560 & $15: 40: 00$ & 0.8954 \\
\hline 2561 & $15: 40: 01$ & 0.8934 \\
\hline 2562 & $15: 40: 02$ & 0.8930 \\
\hline 2563 & $15: 40: 03$ & 0.8927 \\
\hline 2564 & $15: 40: 04$ & 0.8928 \\
\hline 2565 & $15: 40: 05$ & 0.8990 \\
\hline 2566 & $15: 40: 06$ & 0.8996 \\
\hline 2567 & $15: 40: 07$ & 0.8989 \\
\hline 2568 & $15: 40: 08$ & 0.9040 \\
\hline 2569 & $15: 40: 09$ & 0.9039 \\
\hline 2570 & $15: 40: 10$ & 0.9005 \\
\hline 2571 & $15: 40: 11$ & 0.8974 \\
\hline 2572 & $15: 40: 12$ & 0.8980 \\
\hline 2573 & $15: 40: 13$ & 0.8985 \\
\hline 2574 & $15: 40: 14$ & 0.9005 \\
\hline 2575 & $15: 40: 15$ & 0.9031 \\
\hline 2576 & $15: 40: 16$ & 0.9033 \\
\hline 2577 & $15: 40: 17$ & 0.9015 \\
\hline 2578 & $15: 40: 18$ & 0.9001 \\
\hline 2579 & $15: 40: 19$ & 0.9001 \\
\hline 2580 & $15: 40: 20$ & 0.9034 \\
\hline 2581 & $15: 40: 21$ & 0.9035 \\
\hline 2582 & $15: 40: 22$ & 0.9036 \\
\hline 2583 & $15: 40: 23$ & 0.9042 \\
\hline 2584 & $15: 40: 24$ & 0.9032 \\
\hline 2585 & $15: 40: 25$ & 0.9049 \\
\hline 2586 & $15: 40: 26$ & 0.9050 \\
\hline 2587 & $15: 40: 27$ & 0.9017 \\
\hline 2588 & $15: 40: 28$ & 0.9013 \\
\hline 2589 & $15: 40: 29$ & 0.9031 \\
\hline 2590 & $15: 40: 30$ & 0.9055 \\
\hline 2591 & $15: 40: 31$ & 0.9049 \\
\hline 2592 & 15:40:32 & 0.9052 \\
\hline 2593 & $15: 40: 33$ & 0.9029 \\
\hline 2594 & $15: 40: 34$ & 0.9037 \\
\hline 2595 & $15: 40: 35$ & 0.903 \\
\hline 2596 & $15: 40: 36$ & 0.905 \\
\hline 2597 & $15: 40: 37$ & 0.9039 \\
\hline 2598 & $15: 40: 38$ & 0.905 \\
\hline 2599 & $15: 40: 39$ & 0.904 \\
\hline
\end{tabular}


Appendix E -Fuel Data Test 3 NIST102605_1.xls

\begin{tabular}{|c|c|c|}
\hline 2600 & $15: 40: 40$ & 0.9080 \\
\hline 2601 & $15: 40: 41$ & 0.9095 \\
\hline 2602 & $15: 40: 42$ & 0.9055 \\
\hline 2603 & $15: 40: 43$ & 0.9042 \\
\hline 2604 & $15: 40: 44$ & 0.9046 \\
\hline 2605 & $15: 40: 45$ & 0.9025 \\
\hline 2606 & $15: 40: 46$ & 0.9022 \\
\hline 2607 & $15: 40: 47$ & 0.9038 \\
\hline 2608 & $15: 40: 48$ & 0.9046 \\
\hline 2609 & $15: 40: 49$ & 0.9072 \\
\hline 2610 & $15: 40: 50$ & 0.9093 \\
\hline 2611 & $15: 40: 51$ & 0.9063 \\
\hline 2612 & $15: 40: 52$ & 0.9043 \\
\hline 2613 & $15: 40: 53$ & 0.9057 \\
\hline 2614 & $15: 40: 54$ & 0.9073 \\
\hline 2615 & $15: 40: 55$ & 0.9033 \\
\hline 2616 & $15: 40: 56$ & 0.9010 \\
\hline 2617 & $15: 40: 57$ & 0.9023 \\
\hline 2618 & $15: 40: 58$ & 0.9091 \\
\hline 2619 & $15: 40: 59$ & 0.9073 \\
\hline 2620 & $15: 41: 00$ & 0.9068 \\
\hline 2621 & $15: 41: 01$ & 0.9082 \\
\hline 2622 & $15: 41: 02$ & 0.9081 \\
\hline 2623 & $15: 41: 03$ & 0.9098 \\
\hline 2624 & $15: 41: 04$ & 0.9082 \\
\hline 2625 & $15: 41: 05$ & 0.9071 \\
\hline 2626 & $15: 41: 06$ & 0.9056 \\
\hline 2627 & $15: 41: 07$ & 0.9067 \\
\hline 2628 & $15: 41: 08$ & 0.9061 \\
\hline 2629 & $15: 41: 09$ & 0.9049 \\
\hline 2630 & $15: 41: 10$ & 0.9042 \\
\hline 2631 & $15: 41: 11$ & 0.9047 \\
\hline 2632 & $15: 41: 12$ & 0.9048 \\
\hline 2633 & $15: 41: 13$ & 0.9073 \\
\hline 2634 & $15: 41: 14$ & 0.9050 \\
\hline 2635 & $15: 41: 15$ & 0.9087 \\
\hline 2636 & $15: 41: 16$ & 0.9094 \\
\hline 2637 & $15: 41: 17$ & 0.9121 \\
\hline 2638 & $15: 41: 18$ & 0.9105 \\
\hline 2639 & $15: 41: 19$ & 0.9095 \\
\hline 2640 & $15: 41: 20$ & 0.9084 \\
\hline 2641 & $15: 41: 21$ & 0.9066 \\
\hline 2642 & $15: 41: 22$ & 0.9043 \\
\hline 2643 & $15: 41: 23$ & 0.9042 \\
\hline 2644 & $15: 41: 24$ & 0.9074 \\
\hline 2645 & $15: 41: 25$ & 0.9057 \\
\hline 2646 & $15: 41: 26$ & 0.9079 \\
\hline 2647 & $15: 41: 27$ & 0.9090 \\
\hline 2648 & $15: 41: 28$ & 0.9107 \\
\hline 2649 & $15: 41: 29$ & 0.911 \\
\hline 2650 & $15: 41: 30$ & 0.912 \\
\hline
\end{tabular}

$E-52$ 
Appendix E -Fuel Data Test 3 NIST102605_1.xls

\begin{tabular}{|c|c|c|}
\hline 2651 & $15: 41: 31$ & 0.9089 \\
\hline 2652 & $15: 41: 32$ & 0.9072 \\
\hline 2653 & $15: 41: 33$ & 0.9056 \\
\hline 2654 & $15: 41: 34$ & 0.9054 \\
\hline 2655 & $15: 41: 35$ & 0.9055 \\
\hline 2656 & $15: 41: 36$ & 0.9052 \\
\hline 2657 & 15:41:37 & 0.9034 \\
\hline 2658 & $15: 41: 38$ & 0.9052 \\
\hline 2659 & 15:41:39 & 0.9063 \\
\hline 2660 & $15: 41: 40$ & 0.9060 \\
\hline 2661 & $15: 41: 41$ & 0.9077 \\
\hline 2662 & 15:41:42 & 0.9104 \\
\hline 2663 & $15: 41: 43$ & 0.9117 \\
\hline 2664 & $15: 41: 44$ & 0.9128 \\
\hline 2665 & $15: 41: 45$ & 0.9127 \\
\hline 2666 & $15: 41: 46$ & 0.9102 \\
\hline 2667 & $15: 41: 47$ & 0.9106 \\
\hline 2668 & $15: 41: 48$ & 0.9071 \\
\hline 2669 & $15: 41: 49$ & 0.9043 \\
\hline 2670 & $15: 41: 50$ & 0.9030 \\
\hline 2671 & $15: 41: 51$ & 0.9062 \\
\hline 2672 & $15: 41: 52$ & 0.9076 \\
\hline 2673 & $15: 41: 53$ & 0.9076 \\
\hline 2674 & 15:41:54 & 0.9102 \\
\hline 2675 & $15: 41: 55$ & 0.9096 \\
\hline 2676 & $15: 41: 56$ & 0.9095 \\
\hline 2677 & $15: 41: 57$ & 0.9110 \\
\hline 2678 & $15: 41: 58$ & 0.9120 \\
\hline 2679 & 15:41:59 & 0.9090 \\
\hline 2680 & $15: 42: 00$ & 0.9103 \\
\hline 2681 & $15: 42: 01$ & 0.9103 \\
\hline 2682 & $15: 42: 02$ & 0.9122 \\
\hline 2683 & $15: 42: 03$ & 0.9094 \\
\hline 2684 & 15:42:04 & 0.9071 \\
\hline 2685 & $15: 42: 05$ & 0.9060 \\
\hline 2686 & $15: 42: 06$ & 0.9045 \\
\hline 2687 & $15: 42: 07$ & 0.9055 \\
\hline 2688 & $15: 42: 08$ & 0.9069 \\
\hline 2689 & 15:42:09 & 0.9081 \\
\hline 2690 & $15: 42: 10$ & 0.9122 \\
\hline 2691 & $15: 42: 11$ & 0.9099 \\
\hline 2692 & 15:42:12 & 0.9109 \\
\hline 2693 & $15: 42: 13$ & 0.9119 \\
\hline 2694 & $15: 42: 14$ & 0.9089 \\
\hline 2695 & $15: 42: 15$ & 0.9078 \\
\hline 2696 & $15: 42: 16$ & 0.909 \\
\hline 2697 & $15: 42: 17$ & 0.9091 \\
\hline 2698 & $15: 42: 18$ & 0.907 \\
\hline 2699 & $15: 42: 19$ & 0.905 \\
\hline 2700 & $15: 42: 20$ & 0.906 \\
\hline & $15: 42: 21$ & 0.908 \\
\hline
\end{tabular}

E - 53 
Appendix E -Fuel Data Test 3 NIST102605_1.xls

\begin{tabular}{|c|c|c|}
\hline 2702 & $15: 42: 22$ & 0.9087 \\
\hline 2703 & $15: 42: 23$ & 0.9120 \\
\hline 2704 & $15: 42: 24$ & 0.9085 \\
\hline 2705 & $15: 42: 25$ & 0.9088 \\
\hline 2706 & $15: 42: 26$ & 0.9120 \\
\hline 2707 & $15: 42: 27$ & 0.9106 \\
\hline 2708 & $15: 42: 28$ & 0.9093 \\
\hline 2709 & $15: 42: 29$ & 0.9117 \\
\hline 2710 & $15: 42: 30$ & 0.9122 \\
\hline 2711 & $15: 42: 31$ & 0.9103 \\
\hline 2712 & $15: 42: 32$ & 0.9057 \\
\hline 2713 & $15: 42: 33$ & 0.9076 \\
\hline 2714 & $15: 42: 34$ & 0.9064 \\
\hline 2715 & $15: 42: 35$ & 0.9087 \\
\hline 2716 & $15: 42: 36$ & 0.9102 \\
\hline 2717 & $15: 42: 37$ & 0.9121 \\
\hline 2718 & $15: 42: 38$ & 0.9140 \\
\hline 2719 & $15: 42: 39$ & 0.9127 \\
\hline 2720 & $15: 42: 40$ & 0.9120 \\
\hline 2721 & $15: 42: 41$ & 0.9089 \\
\hline 2722 & $15: 42: 42$ & 0.9075 \\
\hline 2723 & $15: 42: 43$ & 0.9058 \\
\hline 2724 & $15: 42: 44$ & 0.9053 \\
\hline 2725 & $15: 42: 45$ & 0.9063 \\
\hline 2726 & $15: 42: 46$ & 0.9079 \\
\hline 2727 & $15: 42: 47$ & 0.9093 \\
\hline 2728 & $15: 42: 48$ & 0.9123 \\
\hline 2729 & $15: 42: 49$ & 0.9132 \\
\hline 2730 & $15: 42: 50$ & 0.9096 \\
\hline 2731 & $15: 42: 51$ & 0.9086 \\
\hline 2732 & $15: 42: 52$ & 0.9071 \\
\hline 2733 & $15: 42: 53$ & 0.9109 \\
\hline 2734 & $15: 42: 54$ & 0.9096 \\
\hline 2735 & $15: 42: 55$ & 0.9125 \\
\hline 2736 & $15: 42: 56$ & 0.9124 \\
\hline 2737 & $15: 42: 57$ & 0.9118 \\
\hline 2738 & $15: 42: 58$ & 0.9100 \\
\hline 2739 & $15: 42: 59$ & 0.9068 \\
\hline 2740 & $15: 43: 00$ & 0.9050 \\
\hline 2741 & 15:43:01 & 0.9050 \\
\hline 2742 & $15: 43: 02$ & 0.9097 \\
\hline 2743 & $15: 43: 03$ & 0.9099 \\
\hline 2744 & $15: 43: 04$ & 0.9119 \\
\hline 2745 & 15:43:05 & 0.9134 \\
\hline 2746 & $15: 43: 06$ & 0.913 \\
\hline 2747 & $15: 43: 07$ & 0.9119 \\
\hline 2748 & 15:43:08 & 0.9093 \\
\hline 2749 & 15:43:09 & 0.908 \\
\hline 2750 & $15: 43: 10$ & 0.910 \\
\hline 2751 & $15: 43: 11$ & 0.910 \\
\hline 2752 & $15: 43: 12$ & 0.906 \\
\hline
\end{tabular}


Appendix E -Fuel Data Test 3 NIST102605_1.xls

\begin{tabular}{|c|c|c|}
\hline 2753 & $15: 43: 13$ & 0.9056 \\
\hline 2754 & $15: 43: 14$ & 0.9064 \\
\hline 2755 & $15: 43: 15$ & 0.9050 \\
\hline 2756 & $15: 43: 16$ & 0.9089 \\
\hline 2757 & $15: 43: 17$ & 0.9111 \\
\hline 2758 & $15: 43: 18$ & 0.9112 \\
\hline 2759 & $15: 43: 19$ & 0.9105 \\
\hline 2760 & $15: 43: 20$ & 0.9133 \\
\hline 2761 & $15: 43: 21$ & 0.9123 \\
\hline 2762 & $15: 43: 22$ & 0.9130 \\
\hline 2763 & $15: 43: 23$ & 0.9120 \\
\hline 2764 & $15: 43: 24$ & 0.9081 \\
\hline 2765 & $15: 43: 25$ & 0.9064 \\
\hline 2766 & $15: 43: 26$ & 0.9087 \\
\hline 2767 & $15: 43: 27$ & 0.9084 \\
\hline 2768 & 15:43:28 & 0.9066 \\
\hline 2769 & 15:43:29 & 0.9103 \\
\hline 2770 & $15: 43: 30$ & 0.9129 \\
\hline 2771 & $15: 43: 31$ & 0.9141 \\
\hline 2772 & $15: 43: 32$ & 0.9153 \\
\hline 2773 & $15: 43: 33$ & 0.9148 \\
\hline 2774 & $15: 43: 34$ & 0.9089 \\
\hline 2775 & $15: 43: 35$ & 0.9091 \\
\hline 2776 & $15: 43: 36$ & 0.9069 \\
\hline 2777 & 15:43:37 & 0.9067 \\
\hline 2778 & $15: 43: 38$ & 0.9080 \\
\hline 2779 & $15: 43: 39$ & 0.9086 \\
\hline 2780 & $15: 43: 40$ & 0.9107 \\
\hline 2781 & $15: 43: 41$ & 0.9113 \\
\hline 2782 & $15: 43: 42$ & 0.9105 \\
\hline 2783 & $15: 43: 43$ & 0.9115 \\
\hline 2784 & $15: 43: 44$ & 0.9095 \\
\hline 2785 & $15: 43: 45$ & 0.9092 \\
\hline 2786 & 15:43:46 & 0.9097 \\
\hline 2787 & $15: 43: 47$ & 0.9090 \\
\hline 2788 & $15: 43: 48$ & 0.9109 \\
\hline 2789 & $15: 43: 49$ & 0.9092 \\
\hline 2790 & $15: 43: 50$ & 0.9092 \\
\hline 2791 & $15: 43: 51$ & 0.9097 \\
\hline 2792 & $15: 43: 52$ & 0.9096 \\
\hline 2793 & $15: 43: 53$ & 0.9113 \\
\hline 2794 & $15: 43: 54$ & 0.9091 \\
\hline 2795 & $15: 43: 55$ & 0.9096 \\
\hline 2796 & $15: 43: 56$ & 0.9105 \\
\hline 2797 & $15: 43: 57$ & 0.9078 \\
\hline 2798 & $15: 43: 58$ & 0.9114 \\
\hline 2799 & $15: 43: 59$ & 0.9125 \\
\hline 2800 & $15: 44: 00$ & 0.9091 \\
\hline 2801 & $15: 44: 01$ & 0.9095 \\
\hline 2802 & $15: 44: 02$ & 0.908 \\
\hline 2803 & $15: 44: 03$ & 0.907 \\
\hline
\end{tabular}

E - 55 
Appendix E -Fuel Data Test 3 NIST102605_1.xls

\begin{tabular}{|c|c|c|}
\hline 2804 & $15: 44: 04$ & 0.9052 \\
\hline 2805 & $15: 44: 05$ & 0.9080 \\
\hline 2806 & $15: 44: 06$ & 0.9100 \\
\hline 2807 & $15: 44: 07$ & 0.9077 \\
\hline 2808 & $15: 44: 08$ & 0.9092 \\
\hline 2809 & $15: 44: 09$ & 0.9112 \\
\hline 2810 & 15:44:10 & 0.9140 \\
\hline 2811 & $15: 44: 11$ & 0.9117 \\
\hline 2812 & $15: 44: 12$ & 0.9087 \\
\hline 2813 & $15: 44: 13$ & 0.9075 \\
\hline 2814 & $15: 44: 14$ & 0.9069 \\
\hline 2815 & $15: 44: 15$ & 0.9081 \\
\hline 2816 & $15: 44: 16$ & 0.9083 \\
\hline 2817 & $15: 44: 17$ & 0.9088 \\
\hline 2818 & 15:44:18 & 0.9100 \\
\hline 2819 & 15:44:19 & 0.9093 \\
\hline 2820 & $15: 44: 20$ & 0.9086 \\
\hline 2821 & $15: 44: 21$ & 0.9099 \\
\hline 2822 & $15: 44: 22$ & 0.9105 \\
\hline 2823 & $15: 44: 23$ & 0.9120 \\
\hline 2824 & $15: 44: 24$ & 0.9108 \\
\hline 2825 & $15: 44: 25$ & 0.9105 \\
\hline 2826 & $15: 44: 26$ & 0.9097 \\
\hline 2827 & $15: 44: 27$ & 0.9120 \\
\hline 2828 & $15: 44: 28$ & 0.9108 \\
\hline 2829 & $15: 44: 29$ & 0.9114 \\
\hline 2830 & $15: 44: 30$ & 0.9090 \\
\hline 2831 & $15: 44: 31$ & 0.9116 \\
\hline 2832 & $15: 44: 32$ & 0.9115 \\
\hline 2833 & $15: 44: 33$ & 0.9075 \\
\hline 2834 & $15: 44: 34$ & 0.9089 \\
\hline 2835 & $15: 44: 35$ & 0.9116 \\
\hline 2836 & 15:44:36 & 0.9143 \\
\hline 2837 & $15: 44: 37$ & 0.9111 \\
\hline 2838 & $15: 44: 38$ & 0.9101 \\
\hline 2839 & 15:44:39 & 0.9091 \\
\hline 2840 & $15: 44: 40$ & 0.9120 \\
\hline 2841 & $15: 44: 41$ & 0.9107 \\
\hline 2842 & $15: 44: 42$ & 0.9110 \\
\hline 2843 & $15: 44: 43$ & 0.9112 \\
\hline 2844 & $15: 44: 44$ & 0.9128 \\
\hline 2845 & $15: 44: 45$ & 0.9127 \\
\hline 2846 & $15: 44: 46$ & 0.9077 \\
\hline 2847 & $15: 44: 47$ & 0.9063 \\
\hline 2848 & $15: 44: 48$ & 0.906 \\
\hline 2849 & $15: 44: 49$ & 0.9055 \\
\hline 2850 & $15: 44: 50$ & 0.909 \\
\hline 2851 & $15: 44: 51$ & 0.911 \\
\hline 2852 & $15: 44: 52$ & 0.9103 \\
\hline 2853 & $15: 44: 53$ & 0.912 \\
\hline & $15: 44: 54$ & 0.913 \\
\hline
\end{tabular}


Appendix E -Fuel Data Test 3 NIST102605_1.xls

\begin{tabular}{|c|c|c|}
\hline 2855 & $15: 44: 55$ & 0.9148 \\
\hline 2856 & $15: 44: 56$ & 0.9088 \\
\hline 2857 & $15: 44: 57$ & 0.9072 \\
\hline 2858 & $15: 44: 58$ & 0.9056 \\
\hline 2859 & $15: 44: 59$ & 0.9090 \\
\hline 2860 & $15: 45: 00$ & 0.9085 \\
\hline 2861 & 15:45:01 & 0.9082 \\
\hline 2862 & $15: 45: 02$ & 0.9090 \\
\hline 2863 & $15: 45: 03$ & 0.9112 \\
\hline 2864 & $15: 45: 04$ & 0.9139 \\
\hline 2865 & $15: 45: 05$ & 0.9127 \\
\hline 2866 & 15:45:06 & 0.9110 \\
\hline 2867 & $15: 45: 07$ & 0.9101 \\
\hline 2868 & $15: 45: 08$ & 0.9100 \\
\hline 2869 & $15: 45: 09$ & 0.9103 \\
\hline 2870 & $15: 45: 10$ & 0.9107 \\
\hline 2871 & $15: 45: 11$ & 0.9119 \\
\hline 2872 & $15: 45: 12$ & 0.9113 \\
\hline 2873 & $15: 45: 13$ & 0.9107 \\
\hline 2874 & $15: 45: 14$ & 0.9094 \\
\hline 2875 & $15: 45: 15$ & 0.9083 \\
\hline 2876 & $15: 45: 16$ & 0.9100 \\
\hline 2877 & $15: 45: 17$ & 0.912 \\
\hline 2878 & $15: 45: 18$ & 0.9137 \\
\hline 2879 & $15: 45: 19$ & 0.9108 \\
\hline 2880 & $15: 45: 20$ & 0.9109 \\
\hline 2881 & $15: 45: 21$ & 0.9111 \\
\hline 2882 & $15: 45: 22$ & 0.9143 \\
\hline 2883 & $15: 45: 23$ & 0.9093 \\
\hline 2884 & $15: 45: 24$ & 0.9110 \\
\hline 2885 & $15: 45: 25$ & 0.9106 \\
\hline 2886 & $15: 45: 26$ & 0.9097 \\
\hline 2887 & $15: 45: 27$ & 0.9092 \\
\hline 2888 & $15: 45: 28$ & 0.9094 \\
\hline 2889 & $15: 45: 29$ & 0.9096 \\
\hline 2890 & $15: 45: 30$ & 0.9083 \\
\hline 2891 & $15: 45: 31$ & 0.9114 \\
\hline 2892 & $15: 45: 32$ & 0.9121 \\
\hline 2893 & $15: 45: 33$ & 0.9105 \\
\hline 2894 & $15: 45: 34$ & 0.9123 \\
\hline 2895 & $15: 45: 35$ & 0.9117 \\
\hline 2896 & $15: 45: 36$ & 0.9098 \\
\hline 2897 & $15: 45: 37$ & 0.9133 \\
\hline 2898 & $15: 45: 38$ & 0.9129 \\
\hline 2899 & $15: 45: 39$ & 0.9128 \\
\hline 2900 & $15: 45: 40$ & 0.9101 \\
\hline 2901 & $15: 45: 41$ & 0.909 \\
\hline 2902 & $15: 45: 42$ & 0.908 \\
\hline 2903 & $15: 45: 43$ & 0.910 \\
\hline 2904 & $15: 45: 44$ & 0.911 \\
\hline & $15: 45: 45$ & 0.915 \\
\hline
\end{tabular}


Appendix E -Fuel Data Test 3 NIST102605_1.xls

\begin{tabular}{|c|c|c|}
\hline 2906 & $15: 45: 46$ & 0.9148 \\
\hline 2907 & $15: 45: 47$ & 0.9165 \\
\hline 2908 & $15: 45: 48$ & 0.9158 \\
\hline 2909 & $15: 45: 49$ & 0.9139 \\
\hline 2910 & $15: 45: 50$ & 0.9108 \\
\hline 2911 & 15:45:51 & 0.9105 \\
\hline 2912 & 15:45:52 & 0.9105 \\
\hline 2913 & $15: 45: 53$ & 0.9099 \\
\hline 2914 & $15: 45: 54$ & 0.9083 \\
\hline 2915 & $15: 45: 55$ & 0.9088 \\
\hline 2916 & $15: 45: 56$ & 0.9128 \\
\hline 2917 & $15: 45: 57$ & 0.9126 \\
\hline 2918 & $15: 45: 58$ & 0.9102 \\
\hline 2919 & $15: 45: 59$ & 0.9105 \\
\hline 2920 & 15:46:00 & 0.9138 \\
\hline 2921 & 15:46:01 & 0.9132 \\
\hline 2922 & $15: 46: 02$ & 0.9123 \\
\hline 2923 & $15: 46: 03$ & 0.9105 \\
\hline 2924 & $15: 46: 04$ & 0.9109 \\
\hline 2925 & $15: 46: 05$ & 0.9144 \\
\hline 2926 & $15: 46: 06$ & 0.9136 \\
\hline 2927 & $15: 46: 07$ & 0.9119 \\
\hline 2928 & $15: 46: 08$ & 0.9109 \\
\hline 2929 & 15:46:09 & 0.9121 \\
\hline 2930 & $15: 46: 10$ & 0.9122 \\
\hline 2931 & $15: 46: 11$ & 0.9160 \\
\hline 2932 & $15: 46: 12$ & 0.9152 \\
\hline 2933 & $15: 46: 13$ & 0.9172 \\
\hline 2934 & $15: 46: 14$ & 0.9172 \\
\hline 2935 & $15: 46: 15$ & 0.9177 \\
\hline 2936 & $15: 46: 16$ & 0.9121 \\
\hline 2937 & $15: 46: 17$ & 0.9108 \\
\hline 2938 & 15:46:18 & 0.9114 \\
\hline 2939 & $15: 46: 19$ & 0.9118 \\
\hline 2940 & 15:46:20 & 0.9096 \\
\hline 2941 & $15: 46: 21$ & 0.9100 \\
\hline 2942 & $15: 46: 22$ & 0.9101 \\
\hline 2943 & $15: 46: 23$ & 0.9119 \\
\hline 2944 & $15: 46: 24$ & 0.9146 \\
\hline 2945 & $15: 46: 25$ & 0.9162 \\
\hline 2946 & 15:46:26 & 0.9133 \\
\hline 2947 & $15: 46: 27$ & 0.9128 \\
\hline 2948 & $15: 46: 28$ & 0.9104 \\
\hline 2949 & $15: 46: 29$ & 0.9141 \\
\hline 2950 & $15: 46: 30$ & 0.9134 \\
\hline 2951 & $15: 46: 31$ & 0.9134 \\
\hline 2952 & $15: 46: 32$ & 0.9132 \\
\hline 2953 & $15: 46: 33$ & 0.9170 \\
\hline 2954 & $15: 46: 34$ & 0.9144 \\
\hline 2955 & $15: 46: 35$ & 0.910 \\
\hline 2956 & $15: 46: 36$ & 0.910 \\
\hline
\end{tabular}


Appendix E -Fuel Data Test 3 NIST102605_1.xls

\begin{tabular}{|c|c|c|}
\hline 2957 & $15: 46: 37$ & 0.9101 \\
\hline 2958 & $15: 46: 38$ & 0.9144 \\
\hline 2959 & $15: 46: 39$ & 0.9150 \\
\hline 2960 & $15: 46: 40$ & 0.9152 \\
\hline 2961 & $15: 46: 41$ & 0.9155 \\
\hline 2962 & 15:46:42 & 0.9152 \\
\hline 2963 & $15: 46: 43$ & 0.9143 \\
\hline 2964 & $15: 46: 44$ & 0.9143 \\
\hline 2965 & $15: 46: 45$ & 0.9153 \\
\hline 2966 & $15: 46: 46$ & 0.9161 \\
\hline 2967 & $15: 46: 47$ & 0.9157 \\
\hline 2968 & 15:46:48 & 0.9115 \\
\hline 2969 & $15: 46: 49$ & 0.9106 \\
\hline 2970 & $15: 46: 50$ & 0.9122 \\
\hline 2971 & 15:46:51 & 0.9145 \\
\hline 2972 & 15:46:52 & 0.9153 \\
\hline 2973 & $15: 46: 53$ & 0.9150 \\
\hline 2974 & $15: 46: 54$ & 0.9161 \\
\hline 2975 & $15: 46: 55$ & 0.9137 \\
\hline 2976 & $15: 46: 56$ & 0.9151 \\
\hline 2977 & $15: 46: 57$ & 0.9162 \\
\hline 2978 & $15: 46: 58$ & 0.9148 \\
\hline 2979 & $15: 46: 59$ & 0.9159 \\
\hline 2980 & 15:47:00 & 0.9138 \\
\hline 2981 & $15: 47: 01$ & 0.9098 \\
\hline 2982 & $15: 47: 02$ & 0.9104 \\
\hline 2983 & $15: 47: 03$ & 0.9112 \\
\hline 2984 & $15: 47: 04$ & 0.9112 \\
\hline 2985 & $15: 47: 05$ & 0.9149 \\
\hline 2986 & 15:47:06 & 0.9161 \\
\hline 2987 & $15: 47: 07$ & 0.9184 \\
\hline 2988 & 15:47:08 & 0.9166 \\
\hline 2989 & 15:47:09 & 0.9164 \\
\hline 2990 & $15: 47: 10$ & 0.9113 \\
\hline 2991 & $15: 47: 11$ & 0.9107 \\
\hline 2992 & $15: 47: 12$ & 0.9103 \\
\hline 2993 & $15: 47: 13$ & 0.9079 \\
\hline 2994 & $15: 47: 14$ & 0.9123 \\
\hline 2995 & $15: 47: 15$ & 0.9126 \\
\hline 2996 & $15: 47: 16$ & 0.9153 \\
\hline 2997 & $15: 47: 17$ & 0.9142 \\
\hline 2998 & $15: 47: 18$ & 0.9153 \\
\hline 2999 & $15: 47: 19$ & 0.9138 \\
\hline 3000 & $15: 47: 20$ & 0.9141 \\
\hline 3001 & $15: 47: 21$ & 0.9123 \\
\hline 3002 & $15: 47: 22$ & 0.9105 \\
\hline 3003 & $15: 47: 23$ & 0.9110 \\
\hline 3004 & $15: 47: 24$ & 0.9149 \\
\hline 3005 & $15: 47: 25$ & 0.9142 \\
\hline 3006 & $15: 47: 26$ & 0.912 \\
\hline 3007 & $15: 47: 27$ & 0.913 \\
\hline
\end{tabular}

E - 59 
Appendix E -Fuel Data Test 3 NIST102605_1.xls

\begin{tabular}{|c|c|c|}
\hline 3008 & $15: 47: 28$ & 0.9117 \\
\hline 3009 & $15: 47: 29$ & 0.9117 \\
\hline 3010 & $15: 47: 30$ & 0.9149 \\
\hline 3011 & $15: 47: 31$ & 0.9169 \\
\hline 3012 & $15: 47: 32$ & 0.9166 \\
\hline 3013 & $15: 47: 33$ & 0.9178 \\
\hline 3014 & $15: 47: 34$ & 0.9160 \\
\hline 3015 & $15: 47: 35$ & 0.9174 \\
\hline 3016 & $15: 47: 36$ & 0.9164 \\
\hline 3017 & $15: 47: 37$ & 0.9146 \\
\hline 3018 & $15: 47: 38$ & 0.9123 \\
\hline 3019 & $15: 47: 39$ & 0.9096 \\
\hline 3020 & $15: 47: 40$ & 0.9088 \\
\hline 3021 & $15: 47: 41$ & 0.9093 \\
\hline 3022 & $15: 47: 42$ & 0.9110 \\
\hline 3023 & $15: 47: 43$ & 0.9130 \\
\hline 3024 & $15: 47: 44$ & 0.9118 \\
\hline 3025 & $15: 47: 45$ & 0.9140 \\
\hline 3026 & $15: 47: 46$ & 0.9129 \\
\hline 3027 & $15: 47: 47$ & 0.9156 \\
\hline 3028 & $15: 47: 48$ & 0.9160 \\
\hline 3029 & $15: 47: 49$ & 0.9137 \\
\hline 3030 & $15: 47: 50$ & 0.9133 \\
\hline 3031 & $15: 47: 51$ & 0.9149 \\
\hline 3032 & $15: 47: 52$ & 0.9156 \\
\hline 3033 & $15: 47: 53$ & 0.9167 \\
\hline 3034 & $15: 47: 54$ & 0.9132 \\
\hline 3035 & $15: 47: 55$ & 0.9123 \\
\hline 3036 & $15: 47: 56$ & 0.9148 \\
\hline 3037 & $15: 47: 57$ & 0.9152 \\
\hline 3038 & $15: 47: 58$ & 0.9183 \\
\hline 3039 & $15: 47: 59$ & 0.9181 \\
\hline 3040 & $15: 48: 00$ & 0.9149 \\
\hline 3041 & 15:48:01 & 0.9152 \\
\hline 3042 & $15: 48: 02$ & 0.9162 \\
\hline 3043 & $15: 48: 03$ & 0.9142 \\
\hline 3044 & $15: 48: 04$ & 0.9128 \\
\hline 3045 & $15: 48: 05$ & 0.9147 \\
\hline 3046 & $15: 48: 06$ & 0.9147 \\
\hline 3047 & $15: 48: 07$ & 0.9143 \\
\hline 3048 & $15: 48: 08$ & 0.9136 \\
\hline 3049 & $15: 48: 09$ & 0.9165 \\
\hline 3050 & $15: 48: 10$ & 0.9167 \\
\hline 3051 & $15: 48: 11$ & 0.9168 \\
\hline 3052 & $15: 48: 12$ & 0.9164 \\
\hline 3053 & $15: 48: 13$ & 0.9164 \\
\hline 3054 & $15: 48: 14$ & 0.9137 \\
\hline 3055 & $15: 48: 15$ & 0.9129 \\
\hline 3056 & $15: 48: 16$ & 0.9128 \\
\hline 3057 & $15: 48: 17$ & 0.915 \\
\hline 3058 & $15: 48: 18$ & 0.912 \\
\hline
\end{tabular}


Appendix E -Fuel Data Test 3 NIST102605_1.xls

\begin{tabular}{|c|c|c|}
\hline 3059 & $15: 48: 19$ & 0.9115 \\
\hline 3060 & $15: 48: 20$ & 0.9111 \\
\hline 3061 & $15: 48: 21$ & 0.9123 \\
\hline 3062 & 15:48:22 & 0.9111 \\
\hline 3063 & $15: 48: 23$ & 0.9125 \\
\hline 3064 & $15: 48: 24$ & 0.9156 \\
\hline 3065 & $15: 48: 25$ & 0.9188 \\
\hline 3066 & 15:48:26 & 0.9180 \\
\hline 3067 & $15: 48: 27$ & 0.9148 \\
\hline 3068 & $15: 48: 28$ & 0.9142 \\
\hline 3069 & $15: 48: 29$ & 0.9125 \\
\hline 3070 & 15:48:30 & 0.9112 \\
\hline 3071 & $15: 48: 31$ & 0.9127 \\
\hline 3072 & $15: 48: 32$ & 0.9102 \\
\hline 3073 & $15: 48: 33$ & 0.9126 \\
\hline 3074 & 15:48:34 & 0.9134 \\
\hline 3075 & $15: 48: 35$ & 0.9131 \\
\hline 3076 & $15: 48: 36$ & 0.9148 \\
\hline 3077 & $15: 48: 37$ & 0.9160 \\
\hline 3078 & $15: 48: 38$ & 0.9147 \\
\hline 3079 & $15: 48: 39$ & 0.9158 \\
\hline 3080 & $15: 48: 40$ & 0.9144 \\
\hline 3081 & $15: 48: 41$ & 0.9144 \\
\hline 3082 & 15:48:42 & 0.9129 \\
\hline 3083 & $15: 48: 43$ & 0.9145 \\
\hline 3084 & $15: 48: 44$ & 0.9140 \\
\hline 3085 & $15: 48: 45$ & 0.9129 \\
\hline 3086 & $15: 48: 46$ & 0.9125 \\
\hline 3087 & $15: 48: 47$ & 0.9129 \\
\hline 3088 & 15:48:48 & 0.9135 \\
\hline 3089 & $15: 48: 49$ & 0.9131 \\
\hline 3090 & $15: 48: 50$ & 0.9131 \\
\hline 3091 & $15: 48: 51$ & 0.9135 \\
\hline 3092 & $15: 48: 52$ & 0.9139 \\
\hline 3093 & $15: 48: 53$ & 0.9136 \\
\hline 3094 & $15: 48: 54$ & 0.9154 \\
\hline 3095 & $15: 48: 55$ & 0.9141 \\
\hline 3096 & $15: 48: 56$ & 0.9110 \\
\hline 3097 & $15: 48: 57$ & 0.9128 \\
\hline 3098 & $15: 48: 58$ & 0.9132 \\
\hline 3099 & $15: 48: 59$ & 0.9152 \\
\hline 3100 & $15: 49: 00$ & 0.9140 \\
\hline 3101 & $15: 49: 01$ & 0.9161 \\
\hline 3102 & $15: 49: 02$ & 0.9167 \\
\hline 3103 & $15: 49: 03$ & 0.9159 \\
\hline 3104 & $15: 49: 04$ & 0.9128 \\
\hline 3105 & $15: 49: 05$ & 0.9132 \\
\hline 3106 & 15:49:06 & 0.9107 \\
\hline 3107 & $15: 49: 07$ & 0.9148 \\
\hline 3108 & $15: 49: 08$ & 0.9163 \\
\hline 3109 & $15: 49: 09$ & 0.915 \\
\hline
\end{tabular}


Appendix E -Fuel Data Test 3 NIST102605_1.xls

\begin{tabular}{|c|c|c|}
\hline 3110 & $15: 49: 10$ & 0.9179 \\
\hline 3111 & $15: 49: 11$ & 0.9157 \\
\hline 3112 & $15: 49: 12$ & 0.9132 \\
\hline 3113 & $15: 49: 13$ & 0.9120 \\
\hline 3114 & $15: 49: 14$ & 0.9126 \\
\hline 3115 & $15: 49: 15$ & 0.9129 \\
\hline 3116 & $15: 49: 16$ & 0.9138 \\
\hline 3117 & $15: 49: 17$ & 0.9185 \\
\hline 3118 & $15: 49: 18$ & 0.9165 \\
\hline 3119 & $15: 49: 19$ & 0.9166 \\
\hline 3120 & $15: 49: 20$ & 0.9176 \\
\hline 3121 & 15:49:21 & 0.9152 \\
\hline 3122 & $15: 49: 22$ & 0.9116 \\
\hline 3123 & $15: 49: 23$ & 0.9107 \\
\hline 3124 & $15: 49: 24$ & 0.9092 \\
\hline 3125 & $15: 49: 25$ & 0.9112 \\
\hline 3126 & $15: 49: 26$ & 0.9138 \\
\hline 3127 & $15: 49: 27$ & 0.9138 \\
\hline 3128 & $15: 49: 28$ & 0.9129 \\
\hline 3129 & $15: 49: 29$ & 0.9120 \\
\hline 3130 & 15:49:30 & 0.9142 \\
\hline 3131 & $15: 49: 31$ & 0.9136 \\
\hline 3132 & 15:49:32 & 0.9133 \\
\hline 3133 & $15: 49: 33$ & 0.9141 \\
\hline 3134 & 15:49:34 & 0.9136 \\
\hline 3135 & $15: 49: 35$ & 0.9146 \\
\hline 3136 & $15: 49: 36$ & 0.9153 \\
\hline 3137 & $15: 49: 37$ & 0.9130 \\
\hline 3138 & $15: 49: 38$ & 0.9146 \\
\hline 3139 & 15:49:39 & 0.9134 \\
\hline 3140 & $15: 49: 40$ & 0.9122 \\
\hline 3141 & $15: 49: 41$ & 0.9073 \\
\hline 3142 & $15: 49: 42$ & 0.9095 \\
\hline 3143 & $15: 49: 43$ & 0.9138 \\
\hline 3144 & $15: 49: 44$ & 0.9148 \\
\hline 3145 & $15: 49: 45$ & 0.9158 \\
\hline 3146 & $15: 49: 46$ & 0.9187 \\
\hline 3147 & $15: 49: 47$ & 0.9189 \\
\hline 3148 & $15: 49: 48$ & 0.9161 \\
\hline 3149 & $15: 49: 49$ & 0.9151 \\
\hline 3150 & $15: 49: 50$ & 0.9150 \\
\hline 3151 & $15: 49: 51$ & 0.9137 \\
\hline 3152 & $15: 49: 52$ & 0.9117 \\
\hline 3153 & $15: 49: 53$ & 0.9097 \\
\hline 3154 & $15: 49: 54$ & 0.9096 \\
\hline 3155 & $15: 49: 55$ & 0.9119 \\
\hline 3156 & $15: 49: 56$ & 0.9133 \\
\hline 3157 & $15: 49: 57$ & 0.9123 \\
\hline 3158 & $15: 49: 58$ & 0.9133 \\
\hline 3159 & $15: 49: 59$ & 0.9136 \\
\hline 3160 & $15: 50: 00$ & 0.914 \\
\hline
\end{tabular}


Appendix E -Fuel Data Test 3 NIST102605_1.xls

\begin{tabular}{|c|c|c|}
\hline 3161 & $15: 50: 01$ & 0.9143 \\
\hline 3162 & $15: 50: 02$ & 0.9123 \\
\hline 3163 & $15: 50: 03$ & 0.9138 \\
\hline 3164 & $15: 50: 04$ & 0.9134 \\
\hline 3165 & $15: 50: 05$ & 0.9125 \\
\hline 3166 & $15: 50: 06$ & 0.9153 \\
\hline 3167 & 15:50:07 & 0.9129 \\
\hline 3168 & $15: 50: 08$ & 0.9120 \\
\hline 3169 & $15: 50: 09$ & 0.9131 \\
\hline 3170 & $15: 50: 10$ & 0.9145 \\
\hline 3171 & $15: 50: 11$ & 0.9135 \\
\hline 3172 & $15: 50: 12$ & 0.9153 \\
\hline 3173 & $15: 50: 13$ & 0.9164 \\
\hline 3174 & $15: 50: 14$ & 0.9151 \\
\hline 3175 & $15: 50: 15$ & 0.9139 \\
\hline 3176 & $15: 50: 16$ & 0.9157 \\
\hline 3177 & $15: 50: 17$ & 0.9132 \\
\hline 3178 & $15: 50: 18$ & 0.9136 \\
\hline 3179 & $15: 50: 19$ & 0.9132 \\
\hline 3180 & $15: 50: 20$ & 0.9112 \\
\hline 3181 & $15: 50: 21$ & 0.9105 \\
\hline 3182 & $15: 50: 22$ & 0.9109 \\
\hline 3183 & $15: 50: 23$ & 0.9101 \\
\hline 3184 & $15: 50: 24$ & 0.9158 \\
\hline 3185 & $15: 50: 25$ & 0.9163 \\
\hline 3186 & $15: 50: 26$ & 0.9171 \\
\hline 3187 & $15: 50: 27$ & 0.9153 \\
\hline 3188 & $15: 50: 28$ & 0.9154 \\
\hline 3189 & $15: 50: 29$ & 0.9151 \\
\hline 3190 & $15: 50: 30$ & 0.9135 \\
\hline 3191 & $15: 50: 31$ & 0.9163 \\
\hline 3192 & $15: 50: 32$ & 0.9157 \\
\hline 3193 & $15: 50: 33$ & 0.9131 \\
\hline 3194 & 15:50:34 & 0.9111 \\
\hline 3195 & $15: 50: 35$ & 0.9101 \\
\hline 3196 & $15: 50: 36$ & 0.9106 \\
\hline 3197 & $15: 50: 37$ & 0.9151 \\
\hline 3198 & $15: 50: 38$ & 0.9160 \\
\hline 3199 & $15: 50: 39$ & 0.9169 \\
\hline 3200 & $15: 50: 40$ & 0.9182 \\
\hline 3201 & $15: 50: 41$ & 0.9173 \\
\hline 3202 & $15: 50: 42$ & 0.9166 \\
\hline 3203 & $15: 50: 43$ & 0.9154 \\
\hline 3204 & $15: 50: 44$ & 0.9115 \\
\hline 3205 & $15: 50: 45$ & 0.9091 \\
\hline 3206 & $15: 50: 46$ & 0.9089 \\
\hline 3207 & $15: 50: 47$ & 0.9115 \\
\hline 3208 & $15: 50: 48$ & 0.9092 \\
\hline 3209 & $15: 50: 49$ & 0.9131 \\
\hline 3210 & $15: 50: 50$ & 0.9118 \\
\hline 3211 & $15: 50: 51$ & 0.914 \\
\hline
\end{tabular}


Appendix E -Fuel Data Test 3 NIST102605_1.xls

\begin{tabular}{|c|c|c|}
\hline 3212 & $15: 50: 52$ & 0.9124 \\
\hline 3213 & $15: 50: 53$ & 0.9128 \\
\hline 3214 & $15: 50: 54$ & 0.9120 \\
\hline 3215 & $15: 50: 55$ & 0.9129 \\
\hline 3216 & $15: 50: 56$ & 0.9127 \\
\hline 3217 & $15: 50: 57$ & 0.9156 \\
\hline 3218 & $15: 50: 58$ & 0.9150 \\
\hline 3219 & $15: 50: 59$ & 0.9101 \\
\hline 3220 & $15: 51: 00$ & 0.9080 \\
\hline 3221 & $15: 51: 01$ & 0.9095 \\
\hline 3222 & $15: 51: 02$ & 0.9139 \\
\hline 3223 & $15: 51: 03$ & 0.9128 \\
\hline 3224 & $15: 51: 04$ & 0.9155 \\
\hline 3225 & $15: 51: 05$ & 0.9157 \\
\hline 3226 & $15: 51: 06$ & 0.9148 \\
\hline 3227 & 15:51:07 & 0.9147 \\
\hline 3228 & $15: 51: 08$ & 0.9131 \\
\hline 3229 & $15: 51: 09$ & 0.9111 \\
\hline 3230 & $15: 51: 10$ & 0.9142 \\
\hline 3231 & $15: 51: 11$ & 0.9120 \\
\hline 3232 & $15: 51: 12$ & 0.9094 \\
\hline 3233 & $15: 51: 13$ & 0.9107 \\
\hline 3234 & $15: 51: 14$ & 0.9116 \\
\hline 3235 & $15: 51: 15$ & 0.9111 \\
\hline 3236 & $15: 51: 16$ & 0.9142 \\
\hline 3237 & $15: 51: 17$ & 0.9148 \\
\hline 3238 & $15: 51: 18$ & 0.9149 \\
\hline 3239 & $15: 51: 19$ & 0.9151 \\
\hline 3240 & $15: 51: 20$ & 0.9162 \\
\hline 3241 & $15: 51: 21$ & 0.9149 \\
\hline 3242 & $15: 51: 22$ & 0.9129 \\
\hline 3243 & $15: 51: 23$ & 0.9130 \\
\hline 3244 & $15: 51: 24$ & 0.9114 \\
\hline 3245 & $15: 51: 25$ & 0.9125 \\
\hline 3246 & $15: 51: 26$ & 0.9101 \\
\hline 3247 & $15: 51: 27$ & 0.9089 \\
\hline 3248 & $15: 51: 28$ & 0.9092 \\
\hline 3249 & $15: 51: 29$ & 0.9111 \\
\hline 3250 & $15: 51: 30$ & 0.9138 \\
\hline 3251 & $15: 51: 31$ & 0.9178 \\
\hline 3252 & $15: 51: 32$ & 0.9195 \\
\hline 3253 & $15: 51: 33$ & 0.9179 \\
\hline 3254 & $15: 51: 34$ & 0.9159 \\
\hline 3255 & $15: 51: 35$ & 0.9160 \\
\hline 3256 & $15: 51: 36$ & 0.9126 \\
\hline 3257 & $15: 51: 37$ & 0.9109 \\
\hline 3258 & $15: 51: 38$ & 0.9111 \\
\hline 3259 & $15: 51: 39$ & 0.9100 \\
\hline 3260 & $15: 51: 40$ & 0.9123 \\
\hline 3261 & $15: 51: 41$ & 0.9135 \\
\hline 3262 & $15: 51: 42$ & 0.912 \\
\hline
\end{tabular}


Appendix E -Fuel Data Test 3 NIST102605_1.xls

\begin{tabular}{|c|c|c|}
\hline 3263 & $15: 51: 43$ & 0.9167 \\
\hline 3264 & $15: 51: 44$ & 0.9156 \\
\hline 3265 & $15: 51: 45$ & 0.9148 \\
\hline 3266 & $15: 51: 46$ & 0.9159 \\
\hline 3267 & $15: 51: 47$ & 0.9135 \\
\hline 3268 & $15: 51: 48$ & 0.9143 \\
\hline 3269 & $15: 51: 49$ & 0.9135 \\
\hline 3270 & $15: 51: 50$ & 0.9113 \\
\hline 3271 & $15: 51: 51$ & 0.9099 \\
\hline 3272 & $15: 51: 52$ & 0.9103 \\
\hline 3273 & $15: 51: 53$ & 0.9098 \\
\hline 3274 & $15: 51: 54$ & 0.9090 \\
\hline 3275 & $15: 51: 55$ & 0.9124 \\
\hline 3276 & $15: 51: 56$ & 0.9127 \\
\hline 3277 & $15: 51: 57$ & 0.9158 \\
\hline 3278 & $15: 51: 58$ & 0.9177 \\
\hline 3279 & $15: 51: 59$ & 0.9155 \\
\hline 3280 & $15: 52: 00$ & 0.9174 \\
\hline 3281 & $15: 52: 01$ & 0.9146 \\
\hline 3282 & $15: 52: 02$ & 0.9132 \\
\hline 3283 & $15: 52: 03$ & 0.9112 \\
\hline 3284 & $15: 52: 04$ & 0.9090 \\
\hline 3285 & $15: 52: 05$ & 0.9097 \\
\hline 3286 & $15: 52: 06$ & 0.9086 \\
\hline 3287 & 15:52:07 & 0.9125 \\
\hline 3288 & $15: 52: 08$ & 0.9135 \\
\hline 3289 & $15: 52: 09$ & 0.9142 \\
\hline 3290 & $15: 52: 10$ & 0.9146 \\
\hline 3291 & $15: 52: 11$ & 0.9151 \\
\hline 3292 & $15: 52: 12$ & 0.9140 \\
\hline 3293 & $15: 52: 13$ & 0.9156 \\
\hline 3294 & $15: 52: 14$ & 0.9157 \\
\hline 3295 & $15: 52: 15$ & 0.9141 \\
\hline 3296 & $15: 52: 16$ & 0.9125 \\
\hline 3297 & $15: 52: 17$ & 0.9112 \\
\hline 3298 & $15: 52: 18$ & 0.9096 \\
\hline 3299 & $15: 52: 19$ & 0.9095 \\
\hline 3300 & $15: 52: 20$ & 0.9114 \\
\hline 3301 & $15: 52: 21$ & 0.9112 \\
\hline 3302 & $15: 52: 22$ & 0.9144 \\
\hline 3303 & $15: 52: 23$ & 0.9154 \\
\hline 3304 & $15: 52: 24$ & 0.9155 \\
\hline 3305 & $15: 52: 25$ & 0.9165 \\
\hline 3306 & $15: 52: 26$ & 0.9156 \\
\hline 3307 & $15: 52: 27$ & 0.9163 \\
\hline 3308 & $15: 52: 28$ & 0.9148 \\
\hline 3309 & $15: 52: 29$ & 0.9127 \\
\hline 3310 & $15: 52: 30$ & 0.9132 \\
\hline 3311 & $15: 52: 31$ & 0.9115 \\
\hline 3312 & $15: 52: 32$ & 0.9127 \\
\hline & $15: 52: 33$ & 0.913 \\
\hline
\end{tabular}


Appendix E -Fuel Data Test 3 NIST102605_1.xls

\begin{tabular}{|c|c|c|}
\hline 3314 & $15: 52: 34$ & 0.9107 \\
\hline 3315 & $15: 52: 35$ & 0.9124 \\
\hline 3316 & $15: 52: 36$ & 0.9117 \\
\hline 3317 & $15: 52: 37$ & 0.9136 \\
\hline 3318 & $15: 52: 38$ & 0.9139 \\
\hline 3319 & $15: 52: 39$ & 0.9131 \\
\hline 3320 & 15:52:40 & 0.9159 \\
\hline 3321 & $15: 52: 41$ & 0.9164 \\
\hline 3322 & $15: 52: 42$ & 0.9173 \\
\hline 3323 & $15: 52: 43$ & 0.9175 \\
\hline 3324 & $15: 52: 44$ & 0.9130 \\
\hline 3325 & $15: 52: 45$ & 0.9096 \\
\hline 3326 & $15: 52: 46$ & 0.9061 \\
\hline 3327 & $15: 52: 47$ & 0.9115 \\
\hline 3328 & $15: 52: 48$ & 0.9123 \\
\hline 3329 & 15:52:49 & 0.9139 \\
\hline 3330 & $15: 52: 50$ & 0.9151 \\
\hline 3331 & $15: 52: 51$ & 0.9172 \\
\hline 3332 & $15: 52: 52$ & 0.9157 \\
\hline 3333 & $15: 52: 53$ & 0.9130 \\
\hline 3334 & $15: 52: 54$ & 0.9105 \\
\hline 3335 & $15: 52: 55$ & 0.9115 \\
\hline 3336 & $15: 52: 56$ & 0.9131 \\
\hline 3337 & $15: 52: 57$ & 0.9112 \\
\hline 3338 & $15: 52: 58$ & 0.9107 \\
\hline 3339 & $15: 52: 59$ & 0.9127 \\
\hline 3340 & $15: 53: 00$ & 0.9147 \\
\hline 3341 & $15: 53: 01$ & 0.9168 \\
\hline 3342 & $15: 53: 02$ & 0.9172 \\
\hline 3343 & $15: 53: 03$ & 0.9150 \\
\hline 3344 & $15: 53: 04$ & 0.9155 \\
\hline 3345 & 15:53:05 & 0.9160 \\
\hline 3346 & 15:53:06 & 0.9164 \\
\hline 3347 & 15:53:07 & 0.9156 \\
\hline 3348 & 15:53:08 & 0.9137 \\
\hline 3349 & 15:53:09 & 0.9118 \\
\hline 3350 & $15: 53: 10$ & 0.9125 \\
\hline 3351 & $15: 53: 11$ & 0.9129 \\
\hline 3352 & 15:53:12 & 0.9098 \\
\hline 3353 & $15: 53: 13$ & 0.9090 \\
\hline 3354 & $15: 53: 14$ & 0.9102 \\
\hline 3355 & $15: 53: 15$ & 0.9127 \\
\hline 3356 & $15: 53: 16$ & 0.9144 \\
\hline 3357 & $15: 53: 17$ & 0.9149 \\
\hline 3358 & $15: 53: 18$ & 0.9161 \\
\hline 3359 & $15: 53: 19$ & 0.9170 \\
\hline 3360 & $15: 53: 20$ & 0.9170 \\
\hline 3361 & 15:53:21 & 0.9124 \\
\hline 3362 & $15: 53: 22$ & 0.9089 \\
\hline 3363 & $15: 53: 23$ & 0.910 \\
\hline 3 & $15: 53: 24$ & 0.914 \\
\hline
\end{tabular}


Appendix E -Fuel Data Test 3 NIST102605_1.xls

\begin{tabular}{|c|c|c|}
\hline 3365 & $15: 53: 25$ & 0.9111 \\
\hline 3366 & $15: 53: 26$ & 0.9113 \\
\hline 3367 & $15: 53: 27$ & 0.9119 \\
\hline 3368 & $15: 53: 28$ & 0.9124 \\
\hline 3369 & $15: 53: 29$ & 0.9161 \\
\hline 3370 & $15: 53: 30$ & 0.9171 \\
\hline 3371 & $15: 53: 31$ & 0.9159 \\
\hline 3372 & $15: 53: 32$ & 0.9130 \\
\hline 3373 & 15:53:33 & 0.9121 \\
\hline 3374 & $15: 53: 34$ & 0.9114 \\
\hline 3375 & $15: 53: 35$ & 0.9111 \\
\hline 3376 & $15: 53: 36$ & 0.9143 \\
\hline 3377 & $15: 53: 37$ & 0.9147 \\
\hline 3378 & $15: 53: 38$ & 0.9125 \\
\hline 3379 & $15: 53: 39$ & 0.9107 \\
\hline 3380 & $15: 53: 40$ & 0.9123 \\
\hline 3381 & $15: 53: 41$ & 0.9154 \\
\hline 3382 & 15:53:42 & 0.9126 \\
\hline 3383 & $15: 53: 43$ & 0.9135 \\
\hline 3384 & $15: 53: 44$ & 0.9129 \\
\hline 3385 & $15: 53: 45$ & 0.9179 \\
\hline 3386 & $15: 53: 46$ & 0.9157 \\
\hline 3387 & $15: 53: 47$ & 0.9128 \\
\hline 3388 & $15: 53: 48$ & 0.9129 \\
\hline 3389 & $15: 53: 49$ & 0.9127 \\
\hline 3390 & $15: 53: 50$ & 0.9120 \\
\hline 3391 & $15: 53: 51$ & 0.9147 \\
\hline 3392 & $15: 53: 52$ & 0.9140 \\
\hline 3393 & $15: 53: 53$ & 0.9145 \\
\hline 3394 & $15: 53: 54$ & 0.9152 \\
\hline 3395 & $15: 53: 55$ & 0.9127 \\
\hline 3396 & $15: 53: 56$ & 0.9122 \\
\hline 3397 & $15: 53: 57$ & 0.9103 \\
\hline 3398 & $15: 53: 58$ & 0.9146 \\
\hline 3399 & 15:53:59 & 0.9157 \\
\hline 3400 & $15: 54: 00$ & 0.9133 \\
\hline 3401 & $15: 54: 01$ & 0.9143 \\
\hline 3402 & $15: 54: 02$ & 0.9133 \\
\hline 3403 & $15: 54: 03$ & 0.9125 \\
\hline 3404 & $15: 54: 04$ & 0.9133 \\
\hline 3405 & $15: 54: 05$ & 0.9110 \\
\hline 3406 & $15: 54: 06$ & 0.9121 \\
\hline 3407 & $15: 54: 07$ & 0.9124 \\
\hline 3408 & 15:54:08 & 0.9157 \\
\hline 3409 & $15: 54: 09$ & 0.9164 \\
\hline 3410 & $15: 54: 10$ & 0.9181 \\
\hline 3411 & $15: 54: 11$ & 0.9164 \\
\hline 3412 & $15: 54: 12$ & 0.9160 \\
\hline 3413 & $15: 54: 13$ & 0.9119 \\
\hline 3414 & $15: 54: 14$ & 0.9098 \\
\hline 3415 & $15: 54: 15$ & 0.9086 \\
\hline
\end{tabular}


Appendix E -Fuel Data Test 3 NIST102605_1.xls

\begin{tabular}{|c|c|c|}
\hline 3416 & $15: 54: 16$ & 0.9130 \\
\hline 3417 & $15: 54: 17$ & 0.9117 \\
\hline 3418 & $15: 54: 18$ & 0.9101 \\
\hline 3419 & $15: 54: 19$ & 0.9142 \\
\hline 3420 & $15: 54: 20$ & 0.9127 \\
\hline 3421 & $15: 54: 21$ & 0.9129 \\
\hline 3422 & $15: 54: 22$ & 0.9159 \\
\hline 3423 & $15: 54: 23$ & 0.9176 \\
\hline 3424 & $15: 54: 24$ & 0.9169 \\
\hline 3425 & $15: 54: 25$ & 0.9152 \\
\hline 3426 & $15: 54: 26$ & 0.9156 \\
\hline 3427 & $15: 54: 27$ & 0.9147 \\
\hline 3428 & $15: 54: 28$ & 0.9142 \\
\hline 3429 & $15: 54: 29$ & 0.9123 \\
\hline 3430 & $15: 54: 30$ & 0.9103 \\
\hline 3431 & 15:54:31 & 0.9091 \\
\hline 3432 & $15: 54: 32$ & 0.9079 \\
\hline 3433 & $15: 54: 33$ & 0.9111 \\
\hline 3434 & $15: 54: 34$ & 0.9122 \\
\hline 3435 & $15: 54: 35$ & 0.9131 \\
\hline 3436 & $15: 54: 36$ & 0.9140 \\
\hline 3437 & $15: 54: 37$ & 0.9156 \\
\hline 3438 & $15: 54: 38$ & 0.9161 \\
\hline 3439 & $15: 54: 39$ & 0.9149 \\
\hline 3440 & $15: 54: 40$ & 0.9152 \\
\hline 3441 & $15: 54: 41$ & 0.9133 \\
\hline 3442 & $15: 54: 42$ & 0.9140 \\
\hline 3443 & $15: 54: 43$ & 0.9110 \\
\hline 3444 & $15: 54: 44$ & 0.9093 \\
\hline 3445 & $15: 54: 45$ & 0.9103 \\
\hline 3446 & $15: 54: 46$ & 0.9106 \\
\hline 3447 & $15: 54: 47$ & 0.9142 \\
\hline 3448 & $15: 54: 48$ & 0.9160 \\
\hline 3449 & $15: 54: 49$ & 0.9143 \\
\hline 3450 & $15: 54: 50$ & 0.9130 \\
\hline 3451 & $15: 54: 51$ & 0.9142 \\
\hline 3452 & $15: 54: 52$ & 0.9136 \\
\hline 3453 & $15: 54: 53$ & 0.9126 \\
\hline 3454 & $15: 54: 54$ & 0.9129 \\
\hline 3455 & $15: 54: 55$ & 0.9124 \\
\hline 3456 & $15: 54: 56$ & 0.9099 \\
\hline 3457 & $15: 54: 57$ & 0.9091 \\
\hline 3458 & $15: 54: 58$ & 0.9095 \\
\hline 3459 & $15: 54: 59$ & 0.9101 \\
\hline 3460 & $15: 55: 00$ & 0.9124 \\
\hline 3461 & $15: 55: 01$ & 0.9163 \\
\hline 3462 & $15: 55: 02$ & 0.9174 \\
\hline 3463 & $15: 55: 03$ & 0.9182 \\
\hline 3464 & $15: 55: 04$ & 0.9146 \\
\hline 3465 & $15: 55: 05$ & $0.911 \mathrm{C}$ \\
\hline 3466 & $15: 55: 06$ & 0.910 \\
\hline
\end{tabular}


Appendix E -Fuel Data Test 3 NIST102605_1.xls

\begin{tabular}{|c|c|c|}
\hline 3467 & $15: 55: 07$ & 0.9094 \\
\hline 3468 & $15: 55: 08$ & 0.9106 \\
\hline 3469 & $15: 55: 09$ & 0.9114 \\
\hline 3470 & $15: 55: 10$ & 0.9129 \\
\hline 3471 & $15: 55: 11$ & 0.9154 \\
\hline 3472 & $15: 55: 12$ & 0.9131 \\
\hline 3473 & $15: 55: 13$ & 0.9106 \\
\hline 3474 & $15: 55: 14$ & 0.9096 \\
\hline 3475 & $15: 55: 15$ & 0.9114 \\
\hline 3476 & $15: 55: 16$ & 0.9144 \\
\hline 3477 & $15: 55: 17$ & 0.9152 \\
\hline 3478 & $15: 55: 18$ & 0.9150 \\
\hline 3479 & $15: 55: 19$ & 0.9161 \\
\hline 3480 & $15: 55: 20$ & 0.9158 \\
\hline 3481 & $15: 55: 21$ & 0.9136 \\
\hline 3482 & $15: 55: 22$ & 0.9114 \\
\hline 3483 & $15: 55: 23$ & 0.9092 \\
\hline 3484 & $15: 55: 24$ & 0.9094 \\
\hline 3485 & $15: 55: 25$ & 0.9089 \\
\hline 3486 & $15: 55: 26$ & 0.9084 \\
\hline 3487 & $15: 55: 27$ & 0.9102 \\
\hline 3488 & $15: 55: 28$ & 0.9153 \\
\hline 3489 & $15: 55: 29$ & 0.9154 \\
\hline 3490 & $15: 55: 30$ & 0.9150 \\
\hline 3491 & 15:55:31 & 0.9130 \\
\hline 3492 & $15: 55: 32$ & 0.9160 \\
\hline 3493 & $15: 55: 33$ & 0.9135 \\
\hline 3494 & $15: 55: 34$ & 0.9124 \\
\hline 3495 & $15: 55: 35$ & 0.9101 \\
\hline 3496 & $15: 55: 36$ & 0.9089 \\
\hline 3497 & $15: 55: 37$ & 0.9092 \\
\hline 3498 & $15: 55: 38$ & 0.9099 \\
\hline 3499 & $15: 55: 39$ & 0.9125 \\
\hline 3500 & $15: 55: 40$ & 0.9132 \\
\hline 3501 & $15: 55: 41$ & 0.9144 \\
\hline 3502 & $15: 55: 42$ & 0.9152 \\
\hline 3503 & $15: 55: 43$ & 0.9135 \\
\hline 3504 & $15: 55: 44$ & 0.9122 \\
\hline 3505 & $15: 55: 45$ & 0.9137 \\
\hline 3506 & $15: 55: 46$ & 0.9145 \\
\hline 3507 & $15: 55: 47$ & 0.9151 \\
\hline 3508 & $15: 55: 48$ & 0.9139 \\
\hline 3509 & $15: 55: 49$ & 0.9121 \\
\hline 3510 & $15: 55: 50$ & 0.9117 \\
\hline 3511 & $15: 55: 51$ & 0.9100 \\
\hline 3512 & $15: 55: 52$ & 0.9119 \\
\hline 3513 & $15: 55: 53$ & 0.9122 \\
\hline 3514 & $15: 55: 54$ & 0.9117 \\
\hline 3515 & $15: 55: 55$ & 0.9153 \\
\hline 3516 & $15: 55: 56$ & 0.9166 \\
\hline 3517 & $15: 55: 57$ & 0.916 \\
\hline
\end{tabular}


Appendix E -Fuel Data Test 3 NIST102605_1.xls

\begin{tabular}{|c|c|c|}
\hline 3518 & $15: 55: 58$ & 0.9162 \\
\hline 3519 & $15: 55: 59$ & 0.9099 \\
\hline 3520 & $15: 56: 00$ & 0.9091 \\
\hline 3521 & $15: 56: 01$ & 0.9104 \\
\hline 3522 & $15: 56: 02$ & 0.9091 \\
\hline 3523 & $15: 56: 03$ & 0.9113 \\
\hline 3524 & 15:56:04 & 0.9122 \\
\hline 3525 & $15: 56: 05$ & 0.9135 \\
\hline 3526 & $15: 56: 06$ & 0.9149 \\
\hline 3527 & $15: 56: 07$ & 0.9151 \\
\hline 3528 & $15: 56: 08$ & 0.9151 \\
\hline 3529 & 15:56:09 & 0.9150 \\
\hline 3530 & $15: 56: 10$ & 0.9163 \\
\hline 3531 & $15: 56: 11$ & 0.9153 \\
\hline 3532 & $15: 56: 12$ & 0.9108 \\
\hline 3533 & $15: 56: 13$ & 0.9109 \\
\hline 3534 & $15: 56: 14$ & 0.9131 \\
\hline 3535 & $15: 56: 15$ & 0.9136 \\
\hline 3536 & $15: 56: 16$ & 0.9105 \\
\hline 3537 & $15: 56: 17$ & 0.9103 \\
\hline 3538 & $15: 56: 18$ & 0.9130 \\
\hline 3539 & $15: 56: 19$ & 0.9124 \\
\hline 3540 & $15: 56: 20$ & 0.9137 \\
\hline 3541 & $15: 56: 21$ & 0.9180 \\
\hline 3542 & $15: 56: 22$ & 0.9186 \\
\hline 3543 & $15: 56: 23$ & 0.9179 \\
\hline 3544 & $15: 56: 24$ & 0.9142 \\
\hline 3545 & $15: 56: 25$ & 0.9107 \\
\hline 3546 & $15: 56: 26$ & 0.9098 \\
\hline 3547 & $15: 56: 27$ & 0.9124 \\
\hline 3548 & $15: 56: 28$ & 0.9122 \\
\hline 3549 & $15: 56: 29$ & 0.9100 \\
\hline 3550 & $15: 56: 30$ & 0.9092 \\
\hline 3551 & 15:56:31 & 0.9146 \\
\hline 3552 & $15: 56: 32$ & 0.9161 \\
\hline 3553 & $15: 56: 33$ & 0.9165 \\
\hline 3554 & $15: 56: 34$ & 0.9157 \\
\hline 3555 & $15: 56: 35$ & 0.9132 \\
\hline 3556 & $15: 56: 36$ & 0.9112 \\
\hline 3557 & $15: 56: 37$ & 0.9117 \\
\hline 3558 & $15: 56: 38$ & 0.9136 \\
\hline 3559 & $15: 56: 39$ & 0.9111 \\
\hline 3560 & $15: 56: 40$ & 0.9146 \\
\hline 3561 & $15: 56: 41$ & 0.9139 \\
\hline 3562 & $15: 56: 42$ & 0.9106 \\
\hline 3563 & $15: 56: 43$ & 0.9097 \\
\hline 3564 & $15: 56: 44$ & 0.9137 \\
\hline 3565 & $15: 56: 45$ & 0.9126 \\
\hline 3566 & $15: 56: 46$ & 0.9139 \\
\hline 3567 & $15: 56: 47$ & 0.9156 \\
\hline 3568 & $15: 56: 48$ & 0.914 \\
\hline
\end{tabular}


Appendix E -Fuel Data Test 3 NIST102605_1.xls

\begin{tabular}{|c|c|c|}
\hline 3569 & $15: 56: 49$ & 0.9146 \\
\hline 3570 & $15: 56: 50$ & 0.9130 \\
\hline 3571 & 15:56:51 & 0.9102 \\
\hline 3572 & $15: 56: 52$ & 0.9131 \\
\hline 3573 & $15: 56: 53$ & 0.9118 \\
\hline 3574 & 15:56:54 & 0.9102 \\
\hline 3575 & $15: 56: 55$ & 0.9101 \\
\hline 3576 & 15:56:56 & 0.9112 \\
\hline 3577 & $15: 56: 57$ & 0.9120 \\
\hline 3578 & $15: 56: 58$ & 0.9109 \\
\hline 3579 & $15: 56: 59$ & 0.9131 \\
\hline 3580 & $15: 57: 00$ & 0.9151 \\
\hline 3581 & $15: 57: 01$ & 0.9140 \\
\hline 3582 & $15: 57: 02$ & 0.9138 \\
\hline 3583 & 15:57:03 & 0.9160 \\
\hline 3584 & $15: 57: 04$ & 0.9160 \\
\hline 3585 & $15: 57: 05$ & 0.9151 \\
\hline 3586 & 15:57:06 & 0.9136 \\
\hline 3587 & $15: 57: 07$ & 0.9125 \\
\hline 3588 & $15: 57: 08$ & 0.9100 \\
\hline 3589 & $15: 57: 09$ & 0.9103 \\
\hline 3590 & $15: 57: 10$ & 0.9113 \\
\hline 3591 & $15: 57: 11$ & 0.9122 \\
\hline 3592 & 15:57:12 & 0.9147 \\
\hline 3593 & $15: 57: 13$ & 0.9162 \\
\hline 3594 & $15: 57: 14$ & 0.9173 \\
\hline 3595 & $15: 57: 15$ & 0.9192 \\
\hline 3596 & $15: 57: 16$ & 0.9173 \\
\hline 3597 & $15: 57: 17$ & 0.9126 \\
\hline 3598 & $15: 57: 18$ & 0.9115 \\
\hline 3599 & $15: 57: 19$ & 0.9125 \\
\hline 3600 & $15: 57: 20$ & 0.9115 \\
\hline 3601 & 15:57:21 & 0.9119 \\
\hline 3602 & $15: 57: 22$ & 0.9092 \\
\hline 3603 & $15: 57: 23$ & 0.9119 \\
\hline 3604 & 15:57:24 & 0.9132 \\
\hline 3605 & $15: 57: 25$ & 0.9125 \\
\hline 3606 & $15: 57: 26$ & 0.9136 \\
\hline 3607 & $15: 57: 27$ & 0.9131 \\
\hline 3608 & $15: 57: 28$ & 0.9125 \\
\hline 3609 & $15: 57: 29$ & 0.9142 \\
\hline 3610 & 15:57:30 & 0.9140 \\
\hline 3611 & $15: 57: 31$ & 0.9124 \\
\hline 3612 & 15:57:32 & 0.9119 \\
\hline 3613 & 15:57:33 & 0.9146 \\
\hline 3614 & $15: 57: 34$ & 0.9143 \\
\hline 3615 & $15: 57: 35$ & 0.9120 \\
\hline 3616 & $15: 57: 36$ & 0.907 \\
\hline 3617 & $15: 57: 37$ & 0.907 \\
\hline 3618 & 15:57:38 & 0.909 \\
\hline 3619 & 15:57:39 & 0.912 \\
\hline
\end{tabular}


Appendix E -Fuel Data Test 3 NIST102605_1.xls

\begin{tabular}{|c|c|c|}
\hline 3620 & $15: 57: 40$ & 0.9142 \\
\hline 3621 & $15: 57: 41$ & 0.9148 \\
\hline 3622 & $15: 57: 42$ & 0.9172 \\
\hline 3623 & $15: 57: 43$ & 0.9174 \\
\hline 3624 & $15: 57: 44$ & 0.9165 \\
\hline 3625 & $15: 57: 45$ & 0.9153 \\
\hline 3626 & $15: 57: 46$ & 0.9139 \\
\hline 3627 & $15: 57: 47$ & 0.9110 \\
\hline 3628 & $15: 57: 48$ & 0.9109 \\
\hline 3629 & $15: 57: 49$ & 0.9096 \\
\hline 3630 & $15: 57: 50$ & 0.9096 \\
\hline 3631 & $15: 57: 51$ & 0.9107 \\
\hline 3632 & $15: 57: 52$ & 0.9126 \\
\hline 3633 & $15: 57: 53$ & 0.9117 \\
\hline 3634 & $15: 57: 54$ & 0.9126 \\
\hline 3635 & $15: 57: 55$ & 0.9126 \\
\hline 3636 & $15: 57: 56$ & 0.9125 \\
\hline 3637 & 15:57:57 & 0.9145 \\
\hline 3638 & $15: 57: 58$ & 0.9156 \\
\hline 3639 & $15: 57: 59$ & 0.9133 \\
\hline 3640 & $15: 58: 00$ & 0.9110 \\
\hline 3641 & $15: 58: 01$ & 0.9104 \\
\hline 3642 & $15: 58: 02$ & 0.9119 \\
\hline 3643 & $15: 58: 03$ & 0.9106 \\
\hline 3644 & $15: 58: 04$ & 0.9094 \\
\hline 3645 & 15:58:05 & 0.9122 \\
\hline 3646 & $15: 58: 06$ & 0.9158 \\
\hline 3647 & $15: 58: 07$ & 0.9151 \\
\hline 3648 & $15: 58: 08$ & 0.9163 \\
\hline 3649 & $15: 58: 09$ & 0.9165 \\
\hline 3650 & $15: 58: 10$ & 0.9172 \\
\hline 3651 & $15: 58: 11$ & 0.9146 \\
\hline 3652 & $15: 58: 12$ & 0.9124 \\
\hline 3653 & $15: 58: 13$ & 0.9093 \\
\hline 3654 & 15:58:14 & 0.9090 \\
\hline 3655 & $15: 58: 15$ & 0.9087 \\
\hline 3656 & $15: 58: 16$ & 0.9105 \\
\hline 3657 & $15: 58: 17$ & 0.9110 \\
\hline 3658 & $15: 58: 18$ & 0.9134 \\
\hline 3659 & 15:58:19 & 0.9140 \\
\hline 3660 & $15: 58: 20$ & 0.9142 \\
\hline 3661 & $15: 58: 21$ & 0.9120 \\
\hline 3662 & $15: 58: 22$ & 0.9117 \\
\hline 3663 & 15:58:23 & 0.9129 \\
\hline 3664 & $15: 58: 24$ & 0.9134 \\
\hline 3665 & $15: 58: 25$ & 0.9163 \\
\hline 3666 & $15: 58: 26$ & 0.9154 \\
\hline 3667 & $15: 58: 27$ & 0.9109 \\
\hline 3668 & 15:58:28 & 0.9104 \\
\hline 3669 & $15: 58: 29$ & 0.909 \\
\hline 3670 & $15: 58: 30$ & 0.908 \\
\hline
\end{tabular}


Appendix E -Fuel Data Test 3 NIST102605_1.xls

\begin{tabular}{|c|c|c|}
\hline 3671 & $15: 58: 31$ & 0.9117 \\
\hline 3672 & 15:58:32 & 0.9123 \\
\hline 3673 & 15:58:33 & 0.9143 \\
\hline 3674 & 15:58:34 & 0.9171 \\
\hline 3675 & 15:58:35 & 0.9145 \\
\hline 3676 & 15:58:36 & 0.9153 \\
\hline 3677 & 15:58:37 & 0.9171 \\
\hline 3678 & 15:58:38 & 0.9148 \\
\hline 3679 & 15:58:39 & 0.9145 \\
\hline 3680 & 15:58:40 & 0.9142 \\
\hline 3681 & $15: 58: 41$ & 0.9136 \\
\hline 3682 & 15:58:42 & 0.9114 \\
\hline 3683 & $15: 58: 43$ & 0.9098 \\
\hline 3684 & $15: 58: 44$ & 0.9110 \\
\hline 3685 & $15: 58: 45$ & 0.9132 \\
\hline 3686 & 15:58:46 & 0.9164 \\
\hline 3687 & $15: 58: 47$ & 0.9121 \\
\hline 3688 & 15:58:48 & 0.9111 \\
\hline 3689 & $15: 58: 49$ & 0.9130 \\
\hline 3690 & $15: 58: 50$ & 0.9154 \\
\hline 3691 & 15:58:51 & 0.9126 \\
\hline 3692 & 15:58:52 & 0.9105 \\
\hline 3693 & 15:58:53 & 0.9101 \\
\hline 3694 & 15:58:54 & 0.9091 \\
\hline 3695 & 15:58:55 & 0.9069 \\
\hline 3696 & 15:58:56 & 0.9080 \\
\hline 3697 & $15: 58: 57$ & 0.9098 \\
\hline 3698 & 15:58:58 & 0.9115 \\
\hline 3699 & 15:58:59 & 0.9158 \\
\hline 3700 & 15:59:00 & 0.9161 \\
\hline 3701 & 15:59:01 & 0.9155 \\
\hline 3702 & 15:59:02 & 0.9158 \\
\hline 3703 & 15:59:03 & 0.9137 \\
\hline 3704 & 15:59:04 & 0.9137 \\
\hline 3705 & 15:59:05 & 0.9132 \\
\hline 3706 & 15:59:06 & 0.9108 \\
\hline 3707 & 15:59:07 & 0.9093 \\
\hline 3708 & 15:59:08 & 0.9111 \\
\hline 3709 & 15:59:09 & 0.9120 \\
\hline 3710 & 15:59:10 & 0.9097 \\
\hline 3711 & 15:59:11 & 0.9118 \\
\hline 3712 & 15:59:12 & 0.9174 \\
\hline 3713 & 15:59:13 & 0.9160 \\
\hline 3714 & 15:59:14 & 0.9134 \\
\hline 3715 & 15:59:15 & 0.9114 \\
\hline 3716 & 15:59:16 & 0.9110 \\
\hline 3717 & $15: 59: 17$ & 0.9120 \\
\hline 3718 & 15:59:18 & 0.9121 \\
\hline 3719 & 15:59:19 & 0.913 \\
\hline 3720 & 15:59:20 & 0.910 \\
\hline 3721 & 15:59:21 & 0.911 \\
\hline
\end{tabular}


Appendix E -Fuel Data Test 3 NIST102605_1.xls

\begin{tabular}{|c|c|c|}
\hline 3722 & $15: 59: 22$ & 0.9093 \\
\hline 3723 & $15: 59: 23$ & 0.9090 \\
\hline 3724 & $15: 59: 24$ & 0.9117 \\
\hline 3725 & 15:59:25 & 0.9142 \\
\hline 3726 & $15: 59: 26$ & 0.9177 \\
\hline 3727 & $15: 59: 27$ & 0.9161 \\
\hline 3728 & 15:59:28 & 0.9152 \\
\hline 3729 & 15:59:29 & 0.9144 \\
\hline 3730 & $15: 59: 30$ & 0.9132 \\
\hline 3731 & $15: 59: 31$ & 0.9147 \\
\hline 3732 & $15: 59: 32$ & 0.9128 \\
\hline 3733 & 15:59:33 & 0.9113 \\
\hline 3734 & $15: 59: 34$ & 0.9097 \\
\hline 3735 & $15: 59: 35$ & 0.9094 \\
\hline 3736 & $15: 59: 36$ & 0.9092 \\
\hline 3737 & 15:59:37 & 0.9141 \\
\hline 3738 & 15:59:38 & 0.9129 \\
\hline 3739 & $15: 59: 39$ & 0.9151 \\
\hline 3740 & $15: 59: 40$ & 0.9145 \\
\hline 3741 & $15: 59: 41$ & 0.9154 \\
\hline 3742 & 15:59:42 & 0.9159 \\
\hline 3743 & $15: 59: 43$ & 0.9116 \\
\hline 3744 & $15: 59: 44$ & 0.9109 \\
\hline 3745 & $15: 59: 45$ & 0.9113 \\
\hline 3746 & 15:59:46 & 0.9093 \\
\hline 3747 & $15: 59: 47$ & 0.9109 \\
\hline 3748 & $15: 59: 48$ & 0.9101 \\
\hline 3749 & $15: 59: 49$ & 0.9099 \\
\hline 3750 & $15: 59: 50$ & 0.9095 \\
\hline 3751 & 15:59:51 & 0.9145 \\
\hline 3752 & $15: 59: 52$ & 0.9164 \\
\hline 3753 & $15: 59: 53$ & 0.9145 \\
\hline 3754 & $15: 59: 54$ & 0.9139 \\
\hline 3755 & 15:59:55 & 0.9142 \\
\hline 3756 & $15: 59: 56$ & 0.9119 \\
\hline 3757 & $15: 59: 57$ & 0.9114 \\
\hline 3758 & $15: 59: 58$ & 0.9124 \\
\hline 3759 & $15: 59: 59$ & 0.9099 \\
\hline 3760 & $16: 00: 00$ & 0.9101 \\
\hline 3761 & $16: 00: 01$ & 0.9126 \\
\hline 3762 & $16: 00: 02$ & 0.9134 \\
\hline 3763 & $16: 00: 03$ & 0.9136 \\
\hline 3764 & $16: 00: 04$ & 0.9131 \\
\hline 3765 & $16: 00: 05$ & 0.9130 \\
\hline 3766 & $16: 00: 06$ & 0.9129 \\
\hline 3767 & $16: 00: 07$ & 0.9114 \\
\hline 3768 & $16: 00: 08$ & 0.9119 \\
\hline 3769 & 16:00:09 & 0.9112 \\
\hline 3770 & $16: 00: 10$ & 0.9153 \\
\hline 3771 & $16: 00: 11$ & 0.915 \\
\hline 3772 & $16: 00: 12$ & 0.915 \\
\hline
\end{tabular}


Appendix E -Fuel Data Test 3 NIST102605_1.xls

\begin{tabular}{|c|c|c|}
\hline 3773 & $16: 00: 13$ & 0.9149 \\
\hline 3774 & $16: 00: 14$ & 0.9120 \\
\hline 3775 & $16: 00: 15$ & 0.9124 \\
\hline 3776 & $16: 00: 16$ & 0.9112 \\
\hline 3777 & $16: 00: 17$ & 0.9102 \\
\hline 3778 & 16:00:18 & 0.9116 \\
\hline 3779 & $16: 00: 19$ & 0.9164 \\
\hline 3780 & $16: 00: 20$ & 0.9165 \\
\hline 3781 & $16: 00: 21$ & 0.9156 \\
\hline 3782 & $16: 00: 22$ & 0.9114 \\
\hline 3783 & $16: 00: 23$ & 0.9118 \\
\hline 3784 & $16: 00: 24$ & 0.9113 \\
\hline 3785 & $16: 00: 25$ & 0.9109 \\
\hline 3786 & $16: 00: 26$ & 0.9103 \\
\hline 3787 & $16: 00: 27$ & 0.9139 \\
\hline 3788 & $16: 00: 28$ & 0.9145 \\
\hline 3789 & 16:00:29 & 0.9118 \\
\hline 3790 & $16: 00: 30$ & 0.9116 \\
\hline 3791 & $16: 00: 31$ & 0.9143 \\
\hline 3792 & $16: 00: 32$ & 0.9122 \\
\hline 3793 & $16: 00: 33$ & 0.9102 \\
\hline 3794 & $16: 00: 34$ & 0.9126 \\
\hline 3795 & $16: 00: 35$ & 0.9104 \\
\hline 3796 & $16: 00: 36$ & 0.9129 \\
\hline 3797 & $16: 00: 37$ & 0.9146 \\
\hline 3798 & $16: 00: 38$ & 0.9150 \\
\hline 3799 & $16: 00: 39$ & 0.9143 \\
\hline 3800 & $16: 00: 40$ & 0.9114 \\
\hline 3801 & $16: 00: 41$ & 0.9119 \\
\hline 3802 & $16: 00: 42$ & 0.9119 \\
\hline 3803 & $16: 00: 43$ & 0.9127 \\
\hline 3804 & $16: 00: 44$ & 0.9127 \\
\hline 3805 & $16: 00: 45$ & 0.9121 \\
\hline 3806 & $16: 00: 46$ & 0.9114 \\
\hline 3807 & $16: 00: 47$ & 0.9147 \\
\hline 3808 & $16: 00: 48$ & 0.9143 \\
\hline 3809 & $16: 00: 49$ & 0.9142 \\
\hline 3810 & $16: 00: 50$ & 0.9136 \\
\hline 3811 & $16: 00: 51$ & 0.9132 \\
\hline 3812 & $16: 00: 52$ & 0.9121 \\
\hline 3813 & $16: 00: 53$ & 0.9106 \\
\hline 3814 & $16: 00: 54$ & 0.9094 \\
\hline 3815 & $16: 00: 55$ & 0.9113 \\
\hline 3816 & $16: 00: 56$ & 0.9110 \\
\hline 3817 & $16: 00: 57$ & 0.9141 \\
\hline 3818 & $16: 00: 58$ & 0.9137 \\
\hline 3819 & $16: 00: 59$ & 0.9132 \\
\hline 3820 & $16: 01: 00$ & 0.9149 \\
\hline 3821 & $16: 01: 01$ & 0.9137 \\
\hline 3822 & $16: 01: 02$ & 0.913 \\
\hline 3823 & $16: 01: 03$ & 0.909 \\
\hline
\end{tabular}


Appendix E -Fuel Data Test 3 NIST102605_1.xls

\begin{tabular}{|c|c|c|}
\hline 3824 & $16: 01: 04$ & 0.9108 \\
\hline 3825 & 16:01:05 & 0.9132 \\
\hline 3826 & $16: 01: 06$ & 0.9120 \\
\hline 3827 & $16: 01: 07$ & 0.9110 \\
\hline 3828 & $16: 01: 08$ & 0.9078 \\
\hline 3829 & 16:01:09 & 0.9094 \\
\hline 3830 & 16:01:10 & 0.9104 \\
\hline 3831 & $16: 01: 11$ & 0.9137 \\
\hline 3832 & $16: 01: 12$ & 0.9152 \\
\hline 3833 & $16: 01: 13$ & 0.9148 \\
\hline 3834 & $16: 01: 14$ & 0.9157 \\
\hline 3835 & 16:01:15 & 0.9142 \\
\hline 3836 & $16: 01: 16$ & 0.9133 \\
\hline 3837 & $16: 01: 17$ & 0.9082 \\
\hline 3838 & 16:01:18 & 0.9088 \\
\hline 3839 & 16:01:19 & 0.9089 \\
\hline 3840 & 16:01:20 & 0.9074 \\
\hline 3841 & $16: 01: 21$ & 0.9099 \\
\hline 3842 & $16: 01: 22$ & 0.9100 \\
\hline 3843 & $16: 01: 23$ & 0.9138 \\
\hline 3844 & 16:01:24 & 0.9157 \\
\hline 3845 & 16:01:25 & 0.9157 \\
\hline 3846 & 16:01:26 & 0.9119 \\
\hline 3847 & $16: 01: 27$ & 0.9100 \\
\hline 3848 & 16:01:28 & 0.9123 \\
\hline 3849 & 16:01:29 & 0.9140 \\
\hline 3850 & $16: 01: 30$ & 0.913 \\
\hline 3851 & $16: 01: 31$ & 0.9141 \\
\hline 3852 & $16: 01: 32$ & 0.9163 \\
\hline 3853 & $16: 01: 33$ & 0.9134 \\
\hline 3854 & $16: 01: 34$ & 0.9113 \\
\hline 3855 & $16: 01: 35$ & 0.9115 \\
\hline 3856 & $16: 01: 36$ & 0.9111 \\
\hline 3857 & $16: 01: 37$ & 0.9128 \\
\hline 3858 & $16: 01: 38$ & 0.9124 \\
\hline 3859 & $16: 01: 39$ & 0.9168 \\
\hline 3860 & $16: 01: 40$ & 0.9155 \\
\hline 3861 & $16: 01: 41$ & 0.9155 \\
\hline 3862 & 16:01:42 & 0.9146 \\
\hline 3863 & $16: 01: 43$ & 0.9113 \\
\hline 3864 & $16: 01: 44$ & 0.9105 \\
\hline 3865 & $16: 01: 45$ & 0.9110 \\
\hline 3866 & $16: 01: 46$ & 0.9113 \\
\hline 3867 & $16: 01: 47$ & 0.9128 \\
\hline 3868 & $16: 01: 48$ & 0.912 \\
\hline 3869 & $16: 01: 49$ & 0.9149 \\
\hline 3870 & $16: 01: 50$ & 0.9130 \\
\hline 3871 & $16: 01: 51$ & 0.9128 \\
\hline 3872 & $16: 01: 52$ & 0.9117 \\
\hline 3873 & $16: 01: 53$ & 0.912 \\
\hline 3874 & $16: 01: 54$ & 0.911 \\
\hline
\end{tabular}


Appendix E -Fuel Data Test 3 NIST102605_1.xls

\begin{tabular}{|c|c|c|}
\hline 3875 & $16: 01: 55$ & 0.9117 \\
\hline 3876 & 16:01:56 & 0.9108 \\
\hline 3877 & $16: 01: 57$ & 0.9127 \\
\hline 3878 & 16:01:58 & 0.9120 \\
\hline 3879 & 16:01:59 & 0.9090 \\
\hline 3880 & $16: 02: 00$ & 0.9106 \\
\hline 3881 & 16:02:01 & 0.9087 \\
\hline 3882 & 16:02:02 & 0.9106 \\
\hline 3883 & 16:02:03 & 0.9132 \\
\hline 3884 & 16:02:04 & 0.9130 \\
\hline 3885 & 16:02:05 & 0.9171 \\
\hline 3886 & 16:02:06 & 0.9151 \\
\hline 3887 & 16:02:07 & 0.9151 \\
\hline 3888 & 16:02:08 & 0.9170 \\
\hline 3889 & 16:02:09 & 0.9116 \\
\hline 3890 & 16:02:10 & 0.9084 \\
\hline 3891 & $16: 02: 11$ & 0.9086 \\
\hline 3892 & $16: 02: 12$ & 0.9109 \\
\hline 3893 & $16: 02: 13$ & 0.9107 \\
\hline 3894 & $16: 02: 14$ & 0.9110 \\
\hline 3895 & $16: 02: 15$ & 0.9145 \\
\hline 3896 & $16: 02: 16$ & 0.9153 \\
\hline 3897 & $16: 02: 17$ & 0.9163 \\
\hline 3898 & $16: 02: 18$ & 0.9145 \\
\hline 3899 & 16:02:19 & 0.9125 \\
\hline 3900 & 16:02:20 & 0.9122 \\
\hline 3901 & $16: 02: 21$ & 0.9107 \\
\hline 3902 & 16:02:22 & 0.9134 \\
\hline 3903 & $16: 02: 23$ & 0.9142 \\
\hline 3904 & 16:02:24 & 0.9165 \\
\hline 3905 & $16: 02: 25$ & 0.9130 \\
\hline 3906 & 16:02:26 & 0.9115 \\
\hline 3907 & $16: 02: 27$ & 0.9098 \\
\hline 3908 & 16:02:28 & 0.9070 \\
\hline 3909 & 16:02:29 & 0.9110 \\
\hline 3910 & 16:02:30 & 0.9118 \\
\hline 3911 & $16: 02: 31$ & 0.9162 \\
\hline 3912 & 16:02:32 & 0.9177 \\
\hline 3913 & 16:02:33 & 0.9165 \\
\hline 3914 & 16:02:34 & 0.9153 \\
\hline 3915 & 16:02:35 & 0.9104 \\
\hline 3916 & 16:02:36 & 0.9087 \\
\hline 3917 & 16:02:37 & 0.9058 \\
\hline 3918 & 16:02:38 & 0.9104 \\
\hline 3919 & 16:02:39 & 0.911 \\
\hline 3920 & $16: 02: 40$ & 0.9132 \\
\hline 3921 & $16: 02: 41$ & 0.913 \\
\hline 3922 & $16: 02: 42$ & 0.914 \\
\hline 3923 & $16: 02: 43$ & 0.9140 \\
\hline 3924 & $16: 02: 44$ & 0.912 \\
\hline 3925 & $16: 02: 45$ & 0.912 \\
\hline
\end{tabular}


Appendix E -Fuel Data Test 3 NIST102605_1.xls

\begin{tabular}{|c|c|c|}
\hline 3926 & $16: 02: 46$ & 0.9122 \\
\hline 3927 & $16: 02: 47$ & 0.9120 \\
\hline 3928 & $16: 02: 48$ & 0.9106 \\
\hline 3929 & $16: 02: 49$ & 0.9101 \\
\hline 3930 & $16: 02: 50$ & 0.9102 \\
\hline 3931 & $16: 02: 51$ & 0.9090 \\
\hline 3932 & 16:02:52 & 0.9084 \\
\hline 3933 & $16: 02: 53$ & 0.9096 \\
\hline 3934 & $16: 02: 54$ & 0.9099 \\
\hline 3935 & $16: 02: 55$ & 0.9125 \\
\hline 3936 & $16: 02: 56$ & 0.9146 \\
\hline 3937 & $16: 02: 57$ & 0.9148 \\
\hline 3938 & $16: 02: 58$ & 0.9161 \\
\hline 3939 & $16: 02: 59$ & 0.9166 \\
\hline 3940 & $16: 03: 00$ & 0.9134 \\
\hline 3941 & 16:03:01 & 0.9140 \\
\hline 3942 & 16:03:02 & 0.9107 \\
\hline 3943 & $16: 03: 03$ & 0.9078 \\
\hline 3944 & $16: 03: 04$ & 0.9077 \\
\hline 3945 & $16: 03: 05$ & 0.9130 \\
\hline 3946 & 16:03:06 & 0.9116 \\
\hline 3947 & $16: 03: 07$ & 0.9136 \\
\hline 3948 & 16:03:08 & 0.9137 \\
\hline 3949 & 16:03:09 & 0.9152 \\
\hline 3950 & 16:03:10 & 0.9139 \\
\hline 3951 & $16: 03: 11$ & 0.9141 \\
\hline 3952 & $16: 03: 12$ & 0.9109 \\
\hline 3953 & $16: 03: 13$ & 0.9104 \\
\hline 3954 & $16: 03: 14$ & 0.9108 \\
\hline 3955 & $16: 03: 15$ & 0.9115 \\
\hline 3956 & $16: 03: 16$ & 0.9108 \\
\hline 3957 & $16: 03: 17$ & 0.9130 \\
\hline 3958 & $16: 03: 18$ & 0.9106 \\
\hline 3959 & 16:03:19 & 0.9087 \\
\hline 3960 & 16:03:20 & 0.9083 \\
\hline 3961 & 16:03:21 & 0.9097 \\
\hline 3962 & 16:03:22 & 0.9106 \\
\hline 3963 & $16: 03: 23$ & 0.9107 \\
\hline 3964 & 16:03:24 & 0.9145 \\
\hline 3965 & 16:03:25 & 0.9154 \\
\hline 3966 & 16:03:26 & 0.9157 \\
\hline 3967 & $16: 03: 27$ & 0.9153 \\
\hline 3968 & 16:03:28 & 0.9137 \\
\hline 3969 & 16:03:29 & 0.9115 \\
\hline 3970 & 16:03:30 & 0.912 \\
\hline 3971 & 16:03:31 & 0.9129 \\
\hline 3972 & $16: 03: 32$ & 0.9117 \\
\hline 3973 & 16:03:33 & 0.911 \\
\hline 3974 & $16: 03: 34$ & 0.912 \\
\hline 3975 & 16:03:35 & 0.912 \\
\hline 3976 & 16:03:36 & 0.913 \\
\hline
\end{tabular}


Appendix E -Fuel Data Test 3 NIST102605_1.xls

\begin{tabular}{|c|c|c|}
\hline 3977 & $16: 03: 37$ & 0.9153 \\
\hline 3978 & 16:03:38 & 0.9143 \\
\hline 3979 & 16:03:39 & 0.9128 \\
\hline 3980 & 16:03:40 & 0.9126 \\
\hline 3981 & $16: 03: 41$ & 0.9125 \\
\hline 3982 & 16:03:42 & 0.9125 \\
\hline 3983 & 16:03:43 & 0.9126 \\
\hline 3984 & 16:03:44 & 0.9108 \\
\hline 3985 & $16: 03: 45$ & 0.9088 \\
\hline 3986 & 16:03:46 & 0.9062 \\
\hline 3987 & $16: 03: 47$ & 0.9106 \\
\hline 3988 & 16:03:48 & 0.9109 \\
\hline 3989 & 16:03:49 & 0.9118 \\
\hline 3990 & 16:03:50 & 0.9153 \\
\hline 3991 & 16:03:51 & 0.9152 \\
\hline 3992 & 16:03:52 & 0.9164 \\
\hline 3993 & 16:03:53 & 0.9141 \\
\hline 3994 & $16: 03: 54$ & 0.9149 \\
\hline 3995 & $16: 03: 55$ & 0.9140 \\
\hline 3996 & 16:03:56 & 0.9136 \\
\hline 3997 & 16:03:57 & 0.9096 \\
\hline 3998 & 16:03:58 & 0.9080 \\
\hline 3999 & 16:03:59 & 0.9083 \\
\hline 4000 & $16: 04: 00$ & 0.9102 \\
\hline 4001 & $16: 04: 01$ & 0.9099 \\
\hline 4002 & 16:04:02 & 0.9118 \\
\hline 4003 & $16: 04: 03$ & 0.9118 \\
\hline 4004 & $16: 04: 04$ & 0.9135 \\
\hline 4005 & 16:04:05 & 0.9133 \\
\hline 4006 & 16:04:06 & 0.9131 \\
\hline 4007 & $16: 04: 07$ & 0.9146 \\
\hline 4008 & 16:04:08 & 0.9164 \\
\hline 4009 & 16:04:09 & 0.9147 \\
\hline 4010 & $16: 04: 10$ & 0.9162 \\
\hline 4011 & $16: 04: 11$ & 0.9126 \\
\hline 4012 & 16:04:12 & 0.9084 \\
\hline 4013 & $16: 04: 13$ & 0.9058 \\
\hline 4014 & $16: 04: 14$ & 0.9107 \\
\hline 4015 & $16: 04: 15$ & 0.9129 \\
\hline 4016 & $16: 04: 16$ & 0.9130 \\
\hline 4017 & $16: 04: 17$ & 0.9152 \\
\hline 4018 & $16: 04: 18$ & 0.9181 \\
\hline 4019 & $16: 04: 19$ & 0.9173 \\
\hline 4020 & 16:04:20 & 0.9149 \\
\hline 4021 & 16:04:21 & 0.9110 \\
\hline 4022 & 16:04:22 & 0.9088 \\
\hline 4023 & $16: 04: 23$ & 0.907 \\
\hline 4024 & $16: 04: 24$ & 0.908 \\
\hline 4025 & $16: 04: 25$ & 0.9100 \\
\hline 4026 & $16: 04: 26$ & 0.913 \\
\hline 4027 & $16: 04: 27$ & 0.915 \\
\hline
\end{tabular}


Appendix E -Fuel Data Test 3 NIST102605_1.xls

\begin{tabular}{|c|c|c|}
\hline 4028 & $16: 04: 28$ & 0.9154 \\
\hline 4029 & 16:04:29 & 0.9148 \\
\hline 4030 & $16: 04: 30$ & 0.9143 \\
\hline 4031 & 16:04:31 & 0.9119 \\
\hline 4032 & $16: 04: 32$ & 0.9097 \\
\hline 4033 & $16: 04: 33$ & 0.9134 \\
\hline 4034 & 16:04:34 & 0.9116 \\
\hline 4035 & $16: 04: 35$ & 0.9148 \\
\hline 4036 & $16: 04: 36$ & 0.9139 \\
\hline 4037 & $16: 04: 37$ & 0.9140 \\
\hline 4038 & 16:04:38 & 0.9122 \\
\hline 4039 & 16:04:39 & 0.9064 \\
\hline 4040 & $16: 04: 40$ & 0.9072 \\
\hline 4041 & $16: 04: 41$ & 0.9109 \\
\hline 4042 & $16: 04: 42$ & 0.9140 \\
\hline 4043 & $16: 04: 43$ & 0.9128 \\
\hline 4044 & $16: 04: 44$ & 0.9138 \\
\hline 4045 & $16: 04: 45$ & 0.9145 \\
\hline 4046 & $16: 04: 46$ & 0.9122 \\
\hline 4047 & $16: 04: 47$ & 0.9112 \\
\hline 4048 & 16:04:48 & 0.9099 \\
\hline 4049 & $16: 04: 49$ & 0.9111 \\
\hline 4050 & $16: 04: 50$ & 0.9110 \\
\hline 4051 & 16:04:51 & 0.9103 \\
\hline 4052 & 16:04:52 & 0.9120 \\
\hline 4053 & $16: 04: 53$ & 0.9146 \\
\hline 4054 & $16: 04: 54$ & 0.9157 \\
\hline 4055 & $16: 04: 55$ & 0.9168 \\
\hline 4056 & $16: 04: 56$ & 0.9134 \\
\hline 4057 & 16:04:57 & 0.9130 \\
\hline 4058 & $16: 04: 58$ & 0.9138 \\
\hline 4059 & $16: 04: 59$ & 0.9103 \\
\hline 4060 & 16:05:00 & 0.9092 \\
\hline 4061 & 16:05:01 & 0.9111 \\
\hline 4062 & $16: 05: 02$ & 0.9108 \\
\hline 4063 & $16: 05: 03$ & 0.9134 \\
\hline 4064 & $16: 05: 04$ & 0.9109 \\
\hline 4065 & $16: 05: 05$ & 0.9110 \\
\hline 4066 & $16: 05: 06$ & 0.9098 \\
\hline 4067 & $16: 05: 07$ & 0.9126 \\
\hline 4068 & $16: 05: 08$ & 0.9141 \\
\hline 4069 & $16: 05: 09$ & 0.9159 \\
\hline 4070 & $16: 05: 10$ & 0.9169 \\
\hline 4071 & $16: 05: 11$ & 0.9163 \\
\hline 4072 & $16: 05: 12$ & 0.9175 \\
\hline 4073 & $16: 05: 13$ & 0.9122 \\
\hline 4074 & $16: 05: 14$ & 0.9088 \\
\hline 4075 & $16: 05: 15$ & 0.9083 \\
\hline 4076 & $16: 05: 16$ & 0.9124 \\
\hline 4077 & $16: 05: 17$ & 0.9110 \\
\hline 4078 & $16: 05: 18$ & 0.911 \\
\hline
\end{tabular}


Appendix E -Fuel Data Test 3 NIST102605_1.xls

\begin{tabular}{|c|c|c|}
\hline 4079 & $16: 05: 19$ & 0.9108 \\
\hline 4080 & $16: 05: 20$ & 0.9105 \\
\hline 4081 & $16: 05: 21$ & 0.9133 \\
\hline 4082 & $16: 05: 22$ & 0.9149 \\
\hline 4083 & $16: 05: 23$ & 0.9152 \\
\hline 4084 & $16: 05: 24$ & 0.9150 \\
\hline 4085 & $16: 05: 25$ & 0.9152 \\
\hline 4086 & 16:05:26 & 0.9161 \\
\hline 4087 & $16: 05: 27$ & 0.9144 \\
\hline 4088 & $16: 05: 28$ & 0.9122 \\
\hline 4089 & $16: 05: 29$ & 0.9110 \\
\hline 4090 & 16:05:30 & 0.9127 \\
\hline 4091 & $16: 05: 31$ & 0.9109 \\
\hline 4092 & $16: 05: 32$ & 0.9098 \\
\hline 4093 & $16: 05: 33$ & 0.9108 \\
\hline 4094 & 16:05:34 & 0.9142 \\
\hline 4095 & $16: 05: 35$ & 0.9138 \\
\hline 4096 & $16: 05: 36$ & 0.9145 \\
\hline 4097 & $16: 05: 37$ & 0.9183 \\
\hline 4098 & $16: 05: 38$ & 0.9153 \\
\hline 4099 & 16:05:39 & 0.9136 \\
\hline 4100 & $16: 05: 40$ & 0.9107 \\
\hline 4101 & $16: 05: 41$ & 0.9082 \\
\hline 4102 & 16:05:42 & 0.9075 \\
\hline 4103 & $16: 05: 43$ & 0.9090 \\
\hline 4104 & $16: 05: 44$ & 0.9103 \\
\hline 4105 & $16: 05: 45$ & 0.9106 \\
\hline 4106 & $16: 05: 46$ & 0.9099 \\
\hline 4107 & $16: 05: 47$ & 0.9117 \\
\hline 4108 & 16:05:48 & 0.9104 \\
\hline 4109 & $16: 05: 49$ & 0.9141 \\
\hline 4110 & $16: 05: 50$ & 0.9134 \\
\hline 4111 & $16: 05: 51$ & 0.9121 \\
\hline 4112 & 16:05:52 & 0.9133 \\
\hline 4113 & $16: 05: 53$ & 0.9160 \\
\hline 4114 & $16: 05: 54$ & 0.9148 \\
\hline 4115 & $16: 05: 55$ & 0.9162 \\
\hline 4116 & $16: 05: 56$ & 0.9128 \\
\hline 4117 & $16: 05: 57$ & 0.9099 \\
\hline 4118 & $16: 05: 58$ & 0.9078 \\
\hline 4119 & $16: 05: 59$ & 0.9067 \\
\hline 4120 & $16: 06: 00$ & 0.9099 \\
\hline 4121 & 16:06:01 & 0.9131 \\
\hline 4122 & $16: 06: 02$ & 0.9135 \\
\hline 4123 & $16: 06: 03$ & 0.9150 \\
\hline 4124 & $16: 06: 04$ & 0.9147 \\
\hline 4125 & $16: 06: 05$ & 0.9152 \\
\hline 4126 & 16:06:06 & 0.9125 \\
\hline 4127 & $16: 06: 07$ & 0.9120 \\
\hline 4128 & 16:06:08 & 0.9127 \\
\hline 4129 & $16: 06: 09$ & 0.911 \\
\hline
\end{tabular}


Appendix E -Fuel Data Test 3 NIST102605_1.xls

\begin{tabular}{|c|c|c|}
\hline 4130 & $16: 06: 10$ & 0.9086 \\
\hline 4131 & $16: 06: 11$ & 0.9084 \\
\hline 4132 & $16: 06: 12$ & 0.9090 \\
\hline 4133 & $16: 06: 13$ & 0.9110 \\
\hline 4134 & $16: 06: 14$ & 0.9147 \\
\hline 4135 & $16: 06: 15$ & 0.9141 \\
\hline 4136 & $16: 06: 16$ & 0.9128 \\
\hline 4137 & $16: 06: 17$ & 0.9140 \\
\hline 4138 & $16: 06: 18$ & 0.9134 \\
\hline 4139 & $16: 06: 19$ & 0.9135 \\
\hline 4140 & 16:06:20 & 0.9144 \\
\hline 4141 & 16:06:21 & 0.9150 \\
\hline 4142 & 16:06:22 & 0.9149 \\
\hline 4143 & 16:06:23 & 0.9117 \\
\hline 4144 & $16: 06: 24$ & 0.9090 \\
\hline 4145 & 16:06:25 & 0.9072 \\
\hline 4146 & 16:06:26 & 0.9092 \\
\hline 4147 & $16: 06: 27$ & 0.9118 \\
\hline 4148 & $16: 06: 28$ & 0.9137 \\
\hline 4149 & $16: 06: 29$ & 0.9152 \\
\hline 4150 & 16:06:30 & 0.9172 \\
\hline 4151 & $16: 06: 31$ & 0.9153 \\
\hline 4152 & $16: 06: 32$ & 0.9124 \\
\hline 4153 & $16: 06: 33$ & 0.9141 \\
\hline 4154 & 16:06:34 & 0.9107 \\
\hline 4155 & $16: 06: 35$ & 0.9104 \\
\hline 4156 & $16: 06: 36$ & 0.9112 \\
\hline 4157 & $16: 06: 37$ & 0.9108 \\
\hline 4158 & $16: 06: 38$ & 0.9118 \\
\hline 4159 & 16:06:39 & 0.9108 \\
\hline 4160 & $16: 06: 40$ & 0.9124 \\
\hline 4161 & $16: 06: 41$ & 0.9138 \\
\hline 4162 & $16: 06: 42$ & 0.9138 \\
\hline 4163 & $16: 06: 43$ & 0.9110 \\
\hline 4164 & $16: 06: 44$ & 0.9106 \\
\hline 4165 & $16: 06: 45$ & 0.9102 \\
\hline 4166 & $16: 06: 46$ & 0.9129 \\
\hline 4167 & $16: 06: 47$ & 0.9123 \\
\hline 4168 & $16: 06: 48$ & 0.9111 \\
\hline 4169 & $16: 06: 49$ & 0.9153 \\
\hline 4170 & $16: 06: 50$ & 0.9127 \\
\hline 4171 & $16: 06: 51$ & 0.9118 \\
\hline 4172 & $16: 06: 52$ & 0.9107 \\
\hline 4173 & $16: 06: 53$ & 0.9084 \\
\hline 4174 & $16: 06: 54$ & 0.9114 \\
\hline 4175 & $16: 06: 55$ & 0.9147 \\
\hline 4176 & $16: 06: 56$ & 0.9147 \\
\hline 4177 & $16: 06: 57$ & 0.9164 \\
\hline 4178 & $16: 06: 58$ & 0.9127 \\
\hline 4179 & $16: 06: 59$ & 0.9109 \\
\hline 4180 & $16: 07: 00$ & 0.9117 \\
\hline
\end{tabular}


Appendix E -Fuel Data Test 3 NIST102605_1.xls

\begin{tabular}{|c|c|c|}
\hline 4181 & $16: 07: 01$ & 0.9105 \\
\hline 4182 & $16: 07: 02$ & 0.9128 \\
\hline 4183 & $16: 07: 03$ & 0.9136 \\
\hline 4184 & $16: 07: 04$ & 0.9146 \\
\hline 4185 & $16: 07: 05$ & 0.9143 \\
\hline 4186 & 16:07:06 & 0.9119 \\
\hline 4187 & 16:07:07 & 0.9111 \\
\hline 4188 & 16:07:08 & 0.9117 \\
\hline 4189 & 16:07:09 & 0.9103 \\
\hline 4190 & 16:07:10 & 0.9128 \\
\hline 4191 & $16: 07: 11$ & 0.9128 \\
\hline 4192 & 16:07:12 & 0.9141 \\
\hline 4193 & $16: 07: 13$ & 0.9156 \\
\hline 4194 & $16: 07: 14$ & 0.9157 \\
\hline 4195 & 16:07:15 & 0.9150 \\
\hline 4196 & 16:07:16 & 0.9092 \\
\hline 4197 & $16: 07: 17$ & 0.9093 \\
\hline 4198 & $16: 07: 18$ & 0.9097 \\
\hline 4199 & $16: 07: 19$ & 0.9082 \\
\hline 4200 & 16:07:20 & 0.9115 \\
\hline 4201 & 16:07:21 & 0.9129 \\
\hline 4202 & 16:07:22 & 0.9140 \\
\hline 4203 & $16: 07: 23$ & 0.9166 \\
\hline 4204 & $16: 07: 24$ & 0.9129 \\
\hline 4205 & 16:07:25 & 0.9106 \\
\hline 42061 & $16: 07: 26$ & 0.9072 \\
\hline 42071 & $16: 07: 27$ & 0.9096 \\
\hline 42081 & $16: 07: 28$ & 0.9100 \\
\hline 42091 & $16: 07: 29$ & 0.9100 \\
\hline 42101 & $16: 07: 30$ & 0.9108 \\
\hline 42111 & $16: 07: 31$ & 0.9139 \\
\hline 42121 & 16:07:32 & 0.9152 \\
\hline 42131 & $16: 07: 33$ & 0.9163 \\
\hline 42141 & 16:07:34 & 0.9145 \\
\hline 42151 & $16: 07: 35$ & 0.9129 \\
\hline 42161 & $16: 07: 36$ & 0.9128 \\
\hline $4217 \quad 1$ & $16: 07: 37$ & 0.9119 \\
\hline 42181 & $16: 07: 38$ & 0.9098 \\
\hline 42191 & $16: 07: 39$ & 0.9095 \\
\hline 42201 & $16: 07: 40$ & 0.9103 \\
\hline $4221 \quad 1$ & $16: 07: 41$ & 0.9096 \\
\hline 42221 & $16: 07: 42$ & 0.9098 \\
\hline 42231 & $16: 07: 43$ & 0.9087 \\
\hline 42241 & $16: 07: 44$ & 0.9089 \\
\hline 42251 & $16: 07: 45$ & 0.9098 \\
\hline 42261 & $16: 07: 46$ & 0.9140 \\
\hline $4227 \quad 1$ & $16: 07: 47$ & 0.9154 \\
\hline 42281 & $16: 07: 48$ & 0.9153 \\
\hline 42291 & $16: 07: 49$ & 0.915 \\
\hline 42301 & $16: 07: 50$ & 0.913 \\
\hline 42311 & 16:07:51 & 0.912 \\
\hline
\end{tabular}

E - 83 
Appendix E -Fuel Data Test 3 NIST102605_1.xls

\begin{tabular}{|c|c|c|}
\hline 4232 & $16: 07: 52$ & 0.9120 \\
\hline 4233 & $16: 07: 53$ & 0.9095 \\
\hline 4234 & $16: 07: 54$ & 0.9088 \\
\hline 4235 & 16:07:55 & 0.9088 \\
\hline 4236 & $16: 07: 56$ & 0.9082 \\
\hline 4237 & $16: 07: 57$ & 0.9118 \\
\hline 4238 & 16:07:58 & 0.9117 \\
\hline 4239 & 16:07:59 & 0.9127 \\
\hline 4240 & 16:08:00 & 0.9110 \\
\hline 4241 & 16:08:01 & 0.9127 \\
\hline 4242 & 16:08:02 & 0.9143 \\
\hline 4243 & 16:08:03 & 0.9129 \\
\hline 4244 & 16:08:04 & 0.9110 \\
\hline 4245 & 16:08:05 & 0.9136 \\
\hline 4246 & 16:08:06 & 0.9130 \\
\hline 4247 & 16:08:07 & 0.9138 \\
\hline 4248 & 16:08:08 & 0.9142 \\
\hline 4249 & 16:08:09 & 0.9131 \\
\hline 4250 & 16:08:10 & 0.9118 \\
\hline 4251 & 16:08:11 & 0.9117 \\
\hline 4252 & 16:08:12 & 0.9133 \\
\hline 4253 & 16:08:13 & 0.9109 \\
\hline 4254 & 16:08:14 & 0.9142 \\
\hline 4255 & 16:08:15 & 0.9158 \\
\hline 4256 & 16:08:16 & 0.9156 \\
\hline 4257 & $16: 08: 17$ & 0.9138 \\
\hline 4258 & 16:08:18 & 0.9148 \\
\hline 4259 & 16:08:19 & 0.9122 \\
\hline 4260 & 16:08:20 & 0.9118 \\
\hline 4261 & 16:08:21 & 0.9098 \\
\hline 4262 & 16:08:22 & 0.9078 \\
\hline 4263 & 16:08:23 & 0.9091 \\
\hline 4264 & 16:08:24 & 0.9120 \\
\hline 4265 & 16:08:25 & 0.9115 \\
\hline 4266 & 16:08:26 & 0.9130 \\
\hline 4267 & 16:08:27 & 0.9128 \\
\hline 4268 & 16:08:28 & 0.9137 \\
\hline 4269 & 16:08:29 & 0.9112 \\
\hline 4270 & 16:08:30 & 0.9116 \\
\hline 4271 & 16:08:31 & 0.9116 \\
\hline 4272 & 16:08:32 & 0.9144 \\
\hline 4273 & 16:08:33 & 0.9131 \\
\hline 4274 & 16:08:34 & 0.9146 \\
\hline 4275 & 16:08:35 & 0.9145 \\
\hline 4276 & 16:08:36 & 0.913 \\
\hline 4277 & $16: 08: 37$ & 0.9093 \\
\hline 4278 & 16:08:38 & 0.9103 \\
\hline 4279 & 16:08:39 & 0.9128 \\
\hline 4280 & 16:08:40 & 0.917 \\
\hline 4281 & 16:08:41 & 0.916 \\
\hline 4282 & 16:08:42 & 0.914 \\
\hline
\end{tabular}


Appendix E -Fuel Data Test 3 NIST102605_1.xls

\begin{tabular}{|c|c|c|}
\hline 4283 & $16: 08: 43$ & 0.9138 \\
\hline 4284 & $16: 08: 44$ & 0.9126 \\
\hline 4285 & $16: 08: 45$ & 0.9100 \\
\hline 4286 & 16:08:46 & 0.9098 \\
\hline 4287 & $16: 08: 47$ & 0.9106 \\
\hline 4288 & 16:08:48 & 0.9108 \\
\hline 4289 & 16:08:49 & 0.9106 \\
\hline 4290 & 16:08:50 & 0.9107 \\
\hline 4291 & 16:08:51 & 0.9099 \\
\hline 4292 & 16:08:52 & 0.9158 \\
\hline 4293 & 16:08:53 & 0.9133 \\
\hline 4294 & 16:08:54 & 0.9116 \\
\hline 4295 & 16:08:55 & 0.9110 \\
\hline 4296 & 16:08:56 & 0.9116 \\
\hline 4297 & 16:08:57 & 0.9115 \\
\hline 4298 & 16:08:58 & 0.9109 \\
\hline 4299 & 16:08:59 & 0.9143 \\
\hline 4300 & $16: 09: 00$ & 0.9143 \\
\hline 4301 & $16: 09: 01$ & 0.9109 \\
\hline 4302 & 16:09:02 & 0.9109 \\
\hline 4303 & 16:09:03 & 0.9116 \\
\hline 4304 & 16:09:04 & 0.9103 \\
\hline 4305 & 16:09:05 & 0.9090 \\
\hline 4306 & 16:09:06 & 0.9110 \\
\hline 4307 & 16:09:07 & 0.9138 \\
\hline 4308 & 16:09:08 & 0.9132 \\
\hline 4309 & 16:09:09 & 0.9155 \\
\hline 4310 & $16: 09: 10$ & 0.9124 \\
\hline 4311 & $16: 09: 11$ & 0.9125 \\
\hline 4312 & 16:09:12 & 0.9125 \\
\hline 4313 & $16: 09: 13$ & 0.9117 \\
\hline 4314 & $16: 09: 14$ & 0.9128 \\
\hline 4315 & 16:09:15 & 0.9107 \\
\hline 4316 & 16:09:16 & 0.9098 \\
\hline 4317 & $16: 09: 17$ & 0.9114 \\
\hline 4318 & $16: 09: 18$ & 0.9159 \\
\hline 4319 & $16: 09: 19$ & 0.9147 \\
\hline 4320 & 16:09:20 & 0.9155 \\
\hline 4321 & 16:09:21 & 0.9154 \\
\hline 4322 & 16:09:22 & 0.9159 \\
\hline 4323 & 16:09:23 & 0.9141 \\
\hline 4324 & $16: 09: 24$ & 0.9106 \\
\hline 4325 & 16:09:25 & 0.9101 \\
\hline 4326 & 16:09:26 & 0.9140 \\
\hline 4327 & $16: 09: 27$ & 0.9123 \\
\hline 4328 & $16: 09: 28$ & 0.9111 \\
\hline 4329 & 16:09:29 & 0.9093 \\
\hline 4330 & 16:09:30 & 0.9101 \\
\hline 4331 & 16:09:31 & 0.9086 \\
\hline 4332 & 16:09:32 & $0.910 \varepsilon$ \\
\hline 43 & $16: 09: 33$ & 0.913 \\
\hline
\end{tabular}


Appendix E -Fuel Data Test 3 NIST102605_1.xls

\begin{tabular}{|c|c|c|}
\hline 4334 & $16: 09: 34$ & 0.9142 \\
\hline 4335 & $16: 09: 35$ & 0.9129 \\
\hline 4336 & $16: 09: 36$ & 0.9131 \\
\hline 4337 & 16:09:37 & 0.9104 \\
\hline 4338 & $16: 09: 38$ & 0.9108 \\
\hline 4339 & 16:09:39 & 0.9124 \\
\hline 4340 & 16:09:40 & 0.9103 \\
\hline 4341 & $16: 09: 41$ & 0.9103 \\
\hline 4342 & $16: 09: 42$ & 0.9108 \\
\hline 4343 & $16: 09: 43$ & 0.9108 \\
\hline 4344 & $16: 09: 44$ & 0.9122 \\
\hline 4345 & 16:09:45 & 0.9156 \\
\hline 4346 & $16: 09: 46$ & 0.9142 \\
\hline 4347 & $16: 09: 47$ & 0.9152 \\
\hline 4348 & $16: 09: 48$ & 0.9152 \\
\hline 4349 & 16:09:49 & 0.9105 \\
\hline 4350 & 16:09:50 & 0.9126 \\
\hline 4351 & $16: 09: 51$ & 0.9114 \\
\hline 4352 & 16:09:52 & 0.9120 \\
\hline 4353 & $16: 09: 53$ & 0.9150 \\
\hline 4354 & 16:09:54 & 0.9147 \\
\hline 4355 & 16:09:55 & 0.9106 \\
\hline 4356 & 16:09:56 & 0.9078 \\
\hline 4357 & 16:09:57 & 0.9070 \\
\hline 4358 & 16:09:58 & 0.9085 \\
\hline 4359 & 16:09:59 & 0.9146 \\
\hline 4360 & $16: 10: 00$ & 0.9129 \\
\hline 4361 & $16: 10: 01$ & 0.9142 \\
\hline 4362 & $16: 10: 02$ & 0.9160 \\
\hline 4363 & $16: 10: 03$ & 0.9153 \\
\hline 4364 & $16: 10: 04$ & 0.9124 \\
\hline 4365 & $16: 10: 05$ & 0.9131 \\
\hline 4366 & $16: 10: 06$ & 0.9123 \\
\hline 4367 & $16: 10: 07$ & 0.9130 \\
\hline 4368 & $16: 10: 08$ & 0.9124 \\
\hline 4369 & 16:10:09 & 0.9088 \\
\hline 4370 & $16: 10: 10$ & 0.9100 \\
\hline 4371 & $16: 10: 11$ & 0.9126 \\
\hline 4372 & $16: 10: 12$ & 0.9122 \\
\hline 4373 & $16: 10: 13$ & 0.9105 \\
\hline 4374 & $16: 10: 14$ & 0.9103 \\
\hline 4375 & $16: 10: 15$ & 0.9080 \\
\hline 4376 & $16: 10: 16$ & 0.9134 \\
\hline 4377 & $16: 10: 17$ & 0.9142 \\
\hline 4378 & $16: 10: 18$ & 0.9117 \\
\hline 4379 & $16: 10: 19$ & 0.9111 \\
\hline 4380 & $16: 10: 20$ & 0.9122 \\
\hline 4381 & $16: 10: 21$ & 0.9116 \\
\hline 4382 & $16: 10: 22$ & 0.9134 \\
\hline 4383 & $16: 10: 23$ & 0.911 \\
\hline 4384 & $16: 10: 24$ & 0.912 \\
\hline
\end{tabular}


Appendix E -Fuel Data Test 3 NIST102605_1.xls

\begin{tabular}{|c|c|c|}
\hline 4385 & $16: 10: 25$ & 0.9131 \\
\hline 4386 & $16: 10: 26$ & 0.9147 \\
\hline 4387 & $16: 10: 27$ & 0.9144 \\
\hline 4388 & $16: 10: 28$ & 0.9180 \\
\hline 4389 & $16: 10: 29$ & 0.9171 \\
\hline 4390 & $16: 10: 30$ & 0.9164 \\
\hline 4391 & $16: 10: 31$ & 0.9112 \\
\hline 4392 & $16: 10: 32$ & 0.9080 \\
\hline 4393 & $16: 10: 33$ & 0.9089 \\
\hline 4394 & $16: 10: 34$ & 0.9113 \\
\hline 4395 & $16: 10: 35$ & 0.9110 \\
\hline 4396 & $16: 10: 36$ & 0.9102 \\
\hline 4397 & $16: 10: 37$ & 0.9116 \\
\hline 4398 & $16: 10: 38$ & 0.9131 \\
\hline 4399 & $16: 10: 39$ & 0.9134 \\
\hline 4400 & $16: 10: 40$ & 0.9141 \\
\hline 4401 & $16: 10: 41$ & 0.9132 \\
\hline 4402 & $16: 10: 42$ & 0.9120 \\
\hline 4403 & $16: 10: 43$ & 0.9131 \\
\hline 4404 & $16: 10: 44$ & 0.9151 \\
\hline 4405 & $16: 10: 45$ & 0.9145 \\
\hline 4406 & $16: 10: 46$ & 0.9109 \\
\hline 4407 & $16: 10: 47$ & 0.9115 \\
\hline 4408 & $16: 10: 48$ & 0.9126 \\
\hline 4409 & $16: 10: 49$ & 0.9100 \\
\hline 4410 & $16: 10: 50$ & 0.9083 \\
\hline 4411 & $16: 10: 51$ & 0.9104 \\
\hline 4412 & $16: 10: 52$ & 0.9170 \\
\hline 4413 & $16: 10: 53$ & 0.9168 \\
\hline 4414 & $16: 10: 54$ & 0.9173 \\
\hline 4415 & $16: 10: 55$ & 0.9176 \\
\hline 4416 & $16: 10: 56$ & 0.9133 \\
\hline 4417 & $16: 10: 57$ & 0.9103 \\
\hline 4418 & $16: 10: 58$ & 0.9085 \\
\hline 4419 & $16: 10: 59$ & 0.9079 \\
\hline 4420 & $16: 11: 00$ & 0.9119 \\
\hline 4421 & $16: 11: 01$ & 0.9105 \\
\hline 4422 & $16: 11: 02$ & 0.9109 \\
\hline 4423 & $16: 11: 03$ & 0.9100 \\
\hline 4424 & $16: 11: 04$ & 0.9145 \\
\hline 4425 & $16: 11: 05$ & 0.9132 \\
\hline 4426 & 16:11:06 & 0.9132 \\
\hline 4427 & $16: 11: 07$ & 0.9129 \\
\hline 4428 & $16: 11: 08$ & 0.9129 \\
\hline 4429 & 16:11:09 & 0.9138 \\
\hline 4430 & $16: 11: 10$ & 0.9144 \\
\hline 4431 & $16: 11: 11$ & 0.9146 \\
\hline 4432 & $16: 11: 12$ & 0.9139 \\
\hline 4433 & $16: 11: 13$ & 0.9150 \\
\hline 4434 & $16: 11: 14$ & 0.9147 \\
\hline 4435 & $16: 11: 15$ & 0.9133 \\
\hline
\end{tabular}


Appendix E -Fuel Data Test 3 NIST102605_1.xls

\begin{tabular}{|c|c|c|}
\hline 4436 & $16: 11: 16$ & 0.9119 \\
\hline 4437 & $16: 11: 17$ & 0.9123 \\
\hline 4438 & $16: 11: 18$ & 0.9144 \\
\hline 4439 & $16: 11: 19$ & 0.9171 \\
\hline 4440 & $16: 11: 20$ & 0.9152 \\
\hline 4441 & $16: 11: 21$ & 0.9146 \\
\hline 4442 & 16:11:22 & 0.9150 \\
\hline 4443 & $16: 11: 23$ & 0.9126 \\
\hline 4444 & $16: 11: 24$ & 0.9128 \\
\hline 4445 & $16: 11: 25$ & 0.9119 \\
\hline 4446 & $16: 11: 26$ & 0.9096 \\
\hline 4447 & $16: 11: 27$ & 0.9116 \\
\hline 4448 & $16: 11: 28$ & 0.9112 \\
\hline 4449 & $16: 11: 29$ & 0.9117 \\
\hline 4450 & $16: 11: 30$ & 0.9114 \\
\hline 4451 & 16:11:31 & 0.9129 \\
\hline 4452 & $16: 11: 32$ & 0.9158 \\
\hline 4453 & $16: 11: 33$ & 0.9165 \\
\hline 4454 & $16: 11: 34$ & 0.9145 \\
\hline 4455 & $16: 11: 35$ & 0.9119 \\
\hline 4456 & 16:11:36 & 0.9134 \\
\hline 4457 & $16: 11: 37$ & 0.9103 \\
\hline 4458 & $16: 11: 38$ & 0.9105 \\
\hline 4459 & $16: 11: 39$ & 0.9120 \\
\hline 4460 & $16: 11: 40$ & 0.9133 \\
\hline 4461 & $16: 11: 41$ & 0.9107 \\
\hline 4462 & $16: 11: 42$ & 0.9103 \\
\hline 4463 & $16: 11: 43$ & 0.9092 \\
\hline 4464 & $16: 11: 44$ & 0.9103 \\
\hline 4465 & $16: 11: 45$ & 0.9127 \\
\hline 4466 & $16: 11: 46$ & 0.9172 \\
\hline 4467 & $16: 11: 47$ & 0.9192 \\
\hline 4468 & $16: 11: 48$ & 0.9187 \\
\hline 4469 & $16: 11: 49$ & 0.9145 \\
\hline 4470 & $16: 11: 50$ & 0.9102 \\
\hline 4471 & $16: 11: 51$ & 0.9091 \\
\hline 4472 & $16: 11: 52$ & 0.9104 \\
\hline 4473 & $16: 11: 53$ & 0.9103 \\
\hline 4474 & $16: 11: 54$ & 0.9112 \\
\hline 4475 & $16: 11: 55$ & 0.9132 \\
\hline 4476 & $16: 11: 56$ & 0.9115 \\
\hline 4477 & $16: 11: 57$ & 0.9109 \\
\hline 4478 & 16:11:58 & 0.9115 \\
\hline 4479 & $16: 11: 59$ & 0.9132 \\
\hline 4480 & $16: 12: 00$ & 0.9163 \\
\hline 4481 & $16: 12: 01$ & 0.9155 \\
\hline 4482 & $16: 12: 02$ & 0.9116 \\
\hline 4483 & 16:12:03 & 0.9107 \\
\hline 4484 & $16: 12: 04$ & 0.9122 \\
\hline 4485 & $16: 12: 05$ & $0.911<$ \\
\hline 4486 & $16: 12: 06$ & 0.913 \\
\hline
\end{tabular}


Appendix E -Fuel Data Test 3 NIST102605_1.xls

\begin{tabular}{|c|c|c|}
\hline 4487 & $16: 12: 07$ & 0.9111 \\
\hline 4488 & $16: 12: 08$ & 0.9133 \\
\hline 4489 & $16: 12: 09$ & 0.9118 \\
\hline 4490 & $16: 12: 10$ & 0.9115 \\
\hline 4491 & $16: 12: 11$ & 0.9111 \\
\hline 4492 & $16: 12: 12$ & 0.9110 \\
\hline 4493 & $16: 12: 13$ & 0.9141 \\
\hline 4494 & $16: 12: 14$ & 0.9146 \\
\hline 4495 & $16: 12: 15$ & 0.9123 \\
\hline 4496 & $16: 12: 16$ & 0.9139 \\
\hline 4497 & $16: 12: 17$ & 0.9109 \\
\hline 4498 & $16: 12: 18$ & 0.9142 \\
\hline 4499 & $16: 12: 19$ & 0.9143 \\
\hline 4500 & $16: 12: 20$ & 0.9135 \\
\hline 4501 & $16: 12: 21$ & 0.9140 \\
\hline 4502 & 16:12:22 & 0.9133 \\
\hline 4503 & $16: 12: 23$ & 0.9132 \\
\hline 4504 & $16: 12: 24$ & 0.9136 \\
\hline 4505 & $16: 12: 25$ & 0.9115 \\
\hline 4506 & $16: 12: 26$ & 0.9113 \\
\hline 4507 & $16: 12: 27$ & 0.9115 \\
\hline 4508 & $16: 12: 28$ & 0.9096 \\
\hline 4509 & $16: 12: 29$ & 0.9119 \\
\hline 4510 & $16: 12: 30$ & 0.9128 \\
\hline 4511 & 16:12:31 & 0.9127 \\
\hline 4512 & $16: 12: 32$ & 0.9137 \\
\hline 4513 & $16: 12: 33$ & 0.9128 \\
\hline 4514 & $16: 12: 34$ & 0.9117 \\
\hline 4515 & $16: 12: 35$ & 0.9108 \\
\hline 4516 & $16: 12: 36$ & 0.9127 \\
\hline 4517 & $16: 12: 37$ & 0.9124 \\
\hline 4518 & $16: 12: 38$ & 0.9154 \\
\hline 4519 & $16: 12: 39$ & 0.9163 \\
\hline 4520 & $16: 12: 40$ & 0.9184 \\
\hline 4521 & $16: 12: 41$ & 0.9182 \\
\hline 4522 & $16: 12: 42$ & 0.9132 \\
\hline 4523 & $16: 12: 43$ & 0.9116 \\
\hline 4524 & $16: 12: 44$ & 0.9112 \\
\hline 4525 & $16: 12: 45$ & 0.9097 \\
\hline 4526 & $16: 12: 46$ & 0.9111 \\
\hline 4527 & $16: 12: 47$ & 0.9107 \\
\hline 4528 & $16: 12: 48$ & 0.9094 \\
\hline 4529 & $16: 12: 49$ & 0.9134 \\
\hline 4530 & $16: 12: 50$ & 0.9136 \\
\hline 4531 & $16: 12: 51$ & 0.9144 \\
\hline 4532 & $16: 12: 52$ & 0.9137 \\
\hline 4533 & $16: 12: 53$ & 0.9129 \\
\hline 4534 & $16: 12: 54$ & 0.9141 \\
\hline 4535 & $16: 12: 55$ & 0.9160 \\
\hline 4536 & $16: 12: 56$ & 0.914 \\
\hline 4537 & $16: 12: 57$ & 0.915 \\
\hline
\end{tabular}


Appendix E -Fuel Data Test 3 NIST102605_1.xls

\begin{tabular}{|c|c|c|}
\hline 4538 & $16: 12: 58$ & 0.9162 \\
\hline 4539 & $16: 12: 59$ & 0.9112 \\
\hline 4540 & $16: 13: 00$ & 0.9098 \\
\hline 4541 & $16: 13: 01$ & 0.9068 \\
\hline 4542 & $16: 13: 02$ & 0.9086 \\
\hline 4543 & $16: 13: 03$ & 0.9101 \\
\hline 4544 & 16:13:04 & 0.9112 \\
\hline 4545 & $16: 13: 05$ & 0.9122 \\
\hline 4546 & $16: 13: 06$ & 0.9158 \\
\hline 4547 & $16: 13: 07$ & 0.9177 \\
\hline 4548 & $16: 13: 08$ & 0.9161 \\
\hline 4549 & 16:13:09 & 0.9131 \\
\hline 4550 & $16: 13: 10$ & 0.9144 \\
\hline 4551 & $16: 13: 11$ & 0.9122 \\
\hline 4552 & $16: 13: 12$ & 0.9119 \\
\hline 4553 & $16: 13: 13$ & 0.9105 \\
\hline 4554 & $16: 13: 14$ & 0.9117 \\
\hline 4555 & $16: 13: 15$ & 0.9139 \\
\hline 4556 & $16: 13: 16$ & 0.9110 \\
\hline 4557 & $16: 13: 17$ & 0.9108 \\
\hline 4558 & 16:13:18 & 0.9114 \\
\hline 4559 & $16: 13: 19$ & 0.9150 \\
\hline 4560 & $16: 13: 20$ & 0.9130 \\
\hline 4561 & $16: 13: 21$ & 0.9166 \\
\hline 4562 & 16:13:22 & 0.9124 \\
\hline 4563 & $16: 13: 23$ & 0.9105 \\
\hline 4564 & $16: 13: 24$ & 0.9116 \\
\hline 4565 & $16: 13: 25$ & 0.9139 \\
\hline 4566 & $16: 13: 26$ & 0.9139 \\
\hline 4567 & $16: 13: 27$ & 0.9140 \\
\hline 4568 & $16: 13: 28$ & 0.9110 \\
\hline 4569 & $16: 13: 29$ & 0.9085 \\
\hline 4570 & $16: 13: 30$ & 0.9099 \\
\hline 4571 & 16:13:31 & 0.9156 \\
\hline 4572 & $16: 13: 32$ & 0.9163 \\
\hline 4573 & $16: 13: 33$ & 0.9173 \\
\hline 4574 & $16: 13: 34$ & 0.9187 \\
\hline 4575 & $16: 13: 35$ & 0.9138 \\
\hline 4576 & 16:13:36 & 0.9120 \\
\hline 4577 & $16: 13: 37$ & 0.9105 \\
\hline 4578 & $16: 13: 38$ & 0.9106 \\
\hline 4579 & $16: 13: 39$ & 0.9096 \\
\hline 4580 & $16: 13: 40$ & 0.9092 \\
\hline 4581 & $16: 13: 41$ & 0.9082 \\
\hline 4582 & $16: 13: 42$ & 0.9127 \\
\hline 4583 & $16: 13: 43$ & 0.9130 \\
\hline 4584 & $16: 13: 44$ & 0.9134 \\
\hline 4585 & $16: 13: 45$ & 0.9159 \\
\hline 4586 & $16: 13: 46$ & 0.9163 \\
\hline 4587 & $16: 13: 47$ & 0.9150 \\
\hline 4588 & $16: 13: 48$ & 0.915 \\
\hline
\end{tabular}


Appendix E -Fuel Data Test 3 NIST102605_1.xls

\begin{tabular}{|c|c|c|}
\hline 4589 & $16: 13: 49$ & 0.9138 \\
\hline 4590 & $16: 13: 50$ & 0.9114 \\
\hline 4591 & $16: 13: 51$ & 0.9107 \\
\hline 4592 & $16: 13: 52$ & 0.9112 \\
\hline 4593 & $16: 13: 53$ & 0.9106 \\
\hline 4594 & $16: 13: 54$ & 0.9081 \\
\hline 4595 & 16:13:55 & 0.9113 \\
\hline 4596 & $16: 13: 56$ & 0.9114 \\
\hline 4597 & $16: 13: 57$ & 0.9109 \\
\hline 4598 & $16: 13: 58$ & 0.9145 \\
\hline 4599 & $16: 13: 59$ & 0.9157 \\
\hline 4600 & $16: 14: 00$ & 0.9188 \\
\hline 4601 & $16: 14: 01$ & 0.9179 \\
\hline 4602 & $16: 14: 02$ & 0.9151 \\
\hline 4603 & $16: 14: 03$ & 0.9115 \\
\hline 4604 & 16:14:04 & 0.9137 \\
\hline 4605 & $16: 14: 05$ & 0.9125 \\
\hline 4606 & $16: 14: 06$ & 0.9109 \\
\hline 4607 & $16: 14: 07$ & 0.9123 \\
\hline 4608 & $16: 14: 08$ & 0.9099 \\
\hline 4609 & 16:14:09 & 0.9092 \\
\hline 4610 & $16: 14: 10$ & 0.9101 \\
\hline 4611 & $16: 14: 11$ & 0.9136 \\
\hline 4612 & $16: 14: 12$ & 0.9135 \\
\hline 4613 & $16: 14: 13$ & 0.9115 \\
\hline 4614 & $16: 14: 14$ & 0.9127 \\
\hline 4615 & $16: 14: 15$ & 0.9130 \\
\hline 4616 & $16: 14: 16$ & 0.9132 \\
\hline 4617 & $16: 14: 17$ & 0.9138 \\
\hline 4618 & $16: 14: 18$ & 0.9146 \\
\hline 4619 & $16: 14: 19$ & 0.9155 \\
\hline 4620 & $16: 14: 20$ & 0.9139 \\
\hline 4621 & $16: 14: 21$ & 0.9128 \\
\hline 4622 & $16: 14: 22$ & 0.9120 \\
\hline 4623 & $16: 14: 23$ & 0.9091 \\
\hline 4624 & $16: 14: 24$ & 0.9105 \\
\hline 4625 & $16: 14: 25$ & 0.9127 \\
\hline 4626 & $16: 14: 26$ & 0.9141 \\
\hline 4627 & $16: 14: 27$ & 0.9146 \\
\hline 4628 & $16: 14: 28$ & 0.9135 \\
\hline 4629 & $16: 14: 29$ & 0.9099 \\
\hline 4630 & $16: 14: 30$ & 0.9111 \\
\hline 4631 & $16: 14: 31$ & 0.9099 \\
\hline 4632 & $16: 14: 32$ & 0.9099 \\
\hline 4633 & $16: 14: 33$ & 0.9140 \\
\hline 4634 & $16: 14: 34$ & 0.9112 \\
\hline 4635 & $16: 14: 35$ & 0.9106 \\
\hline 4636 & $16: 14: 36$ & 0.9109 \\
\hline 4637 & $16: 14: 37$ & 0.9142 \\
\hline 4638 & $16: 14: 38$ & 0.913 \\
\hline 4639 & $16: 14: 39$ & 0.914 \\
\hline
\end{tabular}


Appendix E -Fuel Data Test 3 NIST102605_1.xls

\begin{tabular}{|c|c|c|}
\hline 4640 & $16: 14: 40$ & 0.9147 \\
\hline 4641 & $16: 14: 41$ & 0.9152 \\
\hline 4642 & $16: 14: 42$ & 0.9142 \\
\hline 4643 & $16: 14: 43$ & 0.9143 \\
\hline 4644 & $16: 14: 44$ & 0.9157 \\
\hline 4645 & $16: 14: 45$ & 0.9158 \\
\hline 4646 & $16: 14: 46$ & 0.9153 \\
\hline 4647 & $16: 14: 47$ & 0.9093 \\
\hline 4648 & $16: 14: 48$ & 0.9075 \\
\hline 4649 & $16: 14: 49$ & 0.9066 \\
\hline 4650 & $16: 14: 50$ & 0.9118 \\
\hline 4651 & $16: 14: 51$ & 0.9138 \\
\hline 4652 & $16: 14: 52$ & 0.9158 \\
\hline 4653 & $16: 14: 53$ & 0.9172 \\
\hline 4654 & $16: 14: 54$ & 0.9166 \\
\hline 4655 & 16:14:55 & 0.9144 \\
\hline 4656 & $16: 14: 56$ & 0.9147 \\
\hline 4657 & $16: 14: 57$ & 0.9137 \\
\hline 4658 & $16: 14: 58$ & 0.9117 \\
\hline 4659 & $16: 14: 59$ & 0.9102 \\
\hline 4660 & $16: 15: 00$ & 0.9116 \\
\hline 4661 & $16: 15: 01$ & 0.9106 \\
\hline 4662 & $16: 15: 02$ & 0.9094 \\
\hline 4663 & $16: 15: 03$ & 0.9099 \\
\hline 4664 & 16:15:04 & 0.9133 \\
\hline 4665 & $16: 15: 05$ & 0.9148 \\
\hline 4666 & $16: 15: 06$ & 0.9136 \\
\hline 4667 & $16: 15: 07$ & 0.9136 \\
\hline 4668 & $16: 15: 08$ & 0.9142 \\
\hline 4669 & 16:15:09 & 0.9130 \\
\hline 4670 & $16: 15: 10$ & 0.9114 \\
\hline 4671 & $16: 15: 11$ & 0.9155 \\
\hline 4672 & $16: 15: 12$ & 0.9148 \\
\hline 4673 & $16: 15: 13$ & 0.9140 \\
\hline 4674 & $16: 15: 14$ & 0.9119 \\
\hline 4675 & $16: 15: 15$ & 0.9122 \\
\hline 4676 & $16: 15: 16$ & 0.9106 \\
\hline 4677 & $16: 15: 17$ & 0.9133 \\
\hline 4678 & $16: 15: 18$ & 0.9157 \\
\hline 4679 & $16: 15: 19$ & 0.9139 \\
\hline 4680 & $16: 15: 20$ & 0.9142 \\
\hline 4681 & $16: 15: 21$ & 0.9142 \\
\hline 4682 & $16: 15: 22$ & 0.9111 \\
\hline 4683 & $16: 15: 23$ & 0.9123 \\
\hline 4684 & $16: 15: 24$ & 0.9112 \\
\hline 4685 & $16: 15: 25$ & 0.9114 \\
\hline 4686 & $16: 15: 26$ & 0.9117 \\
\hline 4687 & $16: 15: 27$ & 0.9108 \\
\hline 4688 & $16: 15: 28$ & 0.9091 \\
\hline 4689 & $16: 15: 29$ & 0.911 \\
\hline 4690 & $16: 15: 30$ & 0.911 \\
\hline
\end{tabular}


Appendix E -Fuel Data Test 3 NIST102605_1.xls

\begin{tabular}{|c|c|c|}
\hline 4691 & $16: 15: 31$ & 0.9113 \\
\hline 4692 & $16: 15: 32$ & 0.9142 \\
\hline 4693 & $16: 15: 33$ & 0.9143 \\
\hline 4694 & $16: 15: 34$ & 0.9138 \\
\hline 4695 & $16: 15: 35$ & 0.9136 \\
\hline 4696 & $16: 15: 36$ & 0.9134 \\
\hline 4697 & 16:15:37 & 0.9127 \\
\hline 4698 & $16: 15: 38$ & 0.9149 \\
\hline 4699 & $16: 15: 39$ & 0.9154 \\
\hline 4700 & $16: 15: 40$ & 0.9137 \\
\hline 4701 & $16: 15: 41$ & 0.9110 \\
\hline 4702 & $16: 15: 42$ & 0.9080 \\
\hline 4703 & $16: 15: 43$ & 0.9105 \\
\hline 4704 & $16: 15: 44$ & 0.9133 \\
\hline 4705 & $16: 15: 45$ & 0.9135 \\
\hline 4706 & $16: 15: 46$ & 0.9182 \\
\hline 4707 & $16: 15: 47$ & 0.9147 \\
\hline 4708 & $16: 15: 48$ & 0.9163 \\
\hline 4709 & $16: 15: 49$ & 0.9161 \\
\hline 4710 & $16: 15: 50$ & 0.9135 \\
\hline 4711 & $16: 15: 51$ & 0.9094 \\
\hline 4712 & $16: 15: 52$ & 0.9079 \\
\hline 4713 & $16: 15: 53$ & 0.9115 \\
\hline 4714 & $16: 15: 54$ & 0.9111 \\
\hline 4715 & 16:15:55 & 0.9109 \\
\hline 4716 & $16: 15: 56$ & 0.9108 \\
\hline 4717 & $16: 15: 57$ & 0.9148 \\
\hline 4718 & $16: 15: 58$ & 0.9149 \\
\hline 4719 & $16: 15: 59$ & 0.9142 \\
\hline 4720 & $16: 16: 00$ & 0.9126 \\
\hline 4721 & $16: 16: 01$ & 0.9125 \\
\hline 4722 & $16: 16: 02$ & 0.9109 \\
\hline 4723 & $16: 16: 03$ & 0.9134 \\
\hline 4724 & 16:16:04 & 0.9139 \\
\hline 4725 & $16: 16: 05$ & 0.9136 \\
\hline 4726 & $16: 16: 06$ & 0.9119 \\
\hline 4727 & $16: 16: 07$ & 0.9121 \\
\hline 4728 & $16: 16: 08$ & 0.9105 \\
\hline 4729 & $16: 16: 09$ & 0.9101 \\
\hline 4730 & $16: 16: 10$ & 0.9128 \\
\hline 4731 & $16: 16: 11$ & 0.9126 \\
\hline 4732 & $16: 16: 12$ & 0.9156 \\
\hline 4733 & $16: 16: 13$ & 0.9174 \\
\hline 4734 & $16: 16: 14$ & 0.9179 \\
\hline 4735 & $16: 16: 15$ & 0.9129 \\
\hline 4736 & $16: 16: 16$ & 0.9114 \\
\hline 4737 & $16: 16: 17$ & 0.9115 \\
\hline 4738 & $16: 16: 18$ & 0.9088 \\
\hline 4739 & $16: 16: 19$ & 0.9100 \\
\hline 4740 & $16: 16: 20$ & 0.910 \\
\hline 4741 & $16: 16: 21$ & 0.906 \\
\hline
\end{tabular}


Appendix E -Fuel Data Test 3 NIST102605_1.xls

\begin{tabular}{|c|c|c|}
\hline 4742 & $16: 16: 22$ & 0.9098 \\
\hline 4743 & $16: 16: 23$ & 0.9124 \\
\hline 4744 & $16: 16: 24$ & 0.9152 \\
\hline 4745 & $16: 16: 25$ & 0.9144 \\
\hline 4746 & 16:16:26 & 0.9133 \\
\hline 4747 & 16:16:27 & 0.9118 \\
\hline 4748 & 16:16:28 & 0.9130 \\
\hline 4749 & 16:16:29 & 0.9120 \\
\hline 4750 & 16:16:30 & 0.9121 \\
\hline 4751 & $16: 16: 31$ & 0.9096 \\
\hline 4752 & 16:16:32 & 0.9106 \\
\hline 4753 & $16: 16: 33$ & 0.9113 \\
\hline 4754 & $16: 16: 34$ & 0.9093 \\
\hline 4755 & $16: 16: 35$ & 0.9087 \\
\hline 4756 & 16:16:36 & 0.9109 \\
\hline 4757 & $16: 16: 37$ & 0.9124 \\
\hline 47581 & 16:16:38 & 0.9147 \\
\hline 4759 & 16:16:39 & 0.9176 \\
\hline 4760 & $16: 16: 40$ & 0.9156 \\
\hline 4761 & $16: 16: 41$ & 0.9157 \\
\hline 4762 & $16: 16: 42$ & 0.9147 \\
\hline 4763 & $16: 16: 43$ & 0.9148 \\
\hline 4764 & 16:16:44 & 0.9125 \\
\hline 47651 & 16:16:45 & 0.9105 \\
\hline 4766 & $16: 16: 46$ & 0.9091 \\
\hline $4767 \quad 1$ & $16: 16: 47$ & 0.9090 \\
\hline 47681 & $16: 16: 48$ & 0.9083 \\
\hline 47691 & $16: 16: 49$ & 0.9105 \\
\hline 47701 & $16: 16: 50$ & 0.9126 \\
\hline $4771 \quad 1$ & $16: 16: 51$ & 0.9127 \\
\hline 47721 & $16: 16: 52$ & 0.9116 \\
\hline 47731 & $16: 16: 53$ & 0.9156 \\
\hline $4774 \quad 1$ & 16:16:54 & 0.9144 \\
\hline 47751 & $16: 16: 55$ & 0.9140 \\
\hline 47761 & 16:16:56 & 0.9117 \\
\hline 47771 & $16: 16: 57$ & 0.9100 \\
\hline $4778 \quad 1$ & 16:16:58 & 0.9122 \\
\hline 47791 & $16: 16: 59$ & 0.9126 \\
\hline 47801 & $16: 17: 00$ & 0.9115 \\
\hline $4781 \quad 1$ & $16: 17: 01$ & 0.9088 \\
\hline 47821 & 16:17:02 & 0.9091 \\
\hline 47831 & $16: 17: 03$ & 0.9084 \\
\hline $4784 \quad 1$ & $16: 17: 04$ & 0.9144 \\
\hline 47851 & $16: 17: 05$ & 0.9159 \\
\hline 47861 & $16: 17: 06$ & 0.9168 \\
\hline 47871 & 16:17:07 & 0.9176 \\
\hline 47881 & $16: 17: 08$ & 0.9156 \\
\hline 47891 & $16: 17: 09$ & 0.9143 \\
\hline 47901 & $16: 17: 10$ & 0.912 \\
\hline $4791 \quad 1$ & 16:17:11 & 0.9075 \\
\hline 47921 & $16: 17: 12$ & 0.906 \\
\hline
\end{tabular}


Appendix E -Fuel Data Test 3 NIST102605_1.xls

\begin{tabular}{|c|c|c|}
\hline 4793 & $16: 17: 13$ & 0.9085 \\
\hline 4794 & $16: 17: 14$ & 0.9119 \\
\hline 4795 & $16: 17: 15$ & 0.9109 \\
\hline 4796 & $16: 17: 16$ & 0.9111 \\
\hline 4797 & $16: 17: 17$ & 0.9137 \\
\hline 4798 & $16: 17: 18$ & 0.9148 \\
\hline 4799 & $16: 17: 19$ & 0.9146 \\
\hline 4800 & $16: 17: 20$ & 0.9120 \\
\hline 4801 & $16: 17: 21$ & 0.9111 \\
\hline 4802 & $16: 17: 22$ & 0.9115 \\
\hline 4803 & $16: 17: 23$ & 0.9120 \\
\hline 4804 & 16:17:24 & 0.9135 \\
\hline 4805 & $16: 17: 25$ & 0.9124 \\
\hline 4806 & $16: 17: 26$ & 0.9124 \\
\hline 4807 & $16: 17: 27$ & 0.9130 \\
\hline 4808 & 16:17:28 & 0.9117 \\
\hline 4809 & $16: 17: 29$ & 0.9114 \\
\hline 4810 & $16: 17: 30$ & 0.9091 \\
\hline 4811 & $16: 17: 31$ & 0.9110 \\
\hline 4812 & $16: 17: 32$ & 0.9141 \\
\hline 4813 & $16: 17: 33$ & 0.9145 \\
\hline 4814 & $16: 17: 34$ & 0.9119 \\
\hline 4815 & $16: 17: 35$ & 0.9108 \\
\hline 4816 & $16: 17: 36$ & 0.9082 \\
\hline 4817 & 16:17:37 & 0.9115 \\
\hline 4818 & $16: 17: 38$ & 0.9108 \\
\hline 4819 & $16: 17: 39$ & 0.9090 \\
\hline 4820 & $16: 17: 40$ & 0.9104 \\
\hline 4821 & $16: 17: 41$ & 0.9123 \\
\hline 4822 & 16:17:42 & 0.9124 \\
\hline 4823 & $16: 17: 43$ & 0.9127 \\
\hline 4824 & $16: 17: 44$ & 0.9140 \\
\hline 4825 & $16: 17: 45$ & 0.9147 \\
\hline 4826 & $16: 17: 46$ & 0.9124 \\
\hline 4827 & $16: 17: 47$ & 0.9116 \\
\hline 4828 & $16: 17: 48$ & 0.9118 \\
\hline 4829 & $16: 17: 49$ & 0.9141 \\
\hline 4830 & $16: 17: 50$ & 0.9133 \\
\hline 4831 & $16: 17: 51$ & 0.9133 \\
\hline 4832 & $16: 17: 52$ & 0.9101 \\
\hline 4833 & $16: 17: 53$ & 0.8999 \\
\hline 4834 & $16: 17: 54$ & 0.8534 \\
\hline 4835 & $16: 17: 55$ & 0.7567 \\
\hline 4836 & $16: 17: 56$ & 0.6445 \\
\hline 4837 & $16: 17: 57$ & 0.5886 \\
\hline 4838 & $16: 17: 58$ & 0.5653 \\
\hline 4839 & $16: 17: 59$ & 0.5066 \\
\hline 4840 & 16:18:00 & 0.4465 \\
\hline 4841 & $16: 18: 01$ & 0.4332 \\
\hline 4842 & $16: 18: 02$ & 0.4207 \\
\hline 4843 & $16: 18: 03$ & $0.42 C$ \\
\hline
\end{tabular}


Appendix E -Fuel Data Test 3 NIST102605_1.xls

\begin{tabular}{|c|c|c|}
\hline 4844 & $16: 18: 04$ & 0.4198 \\
\hline 4845 & $16: 18: 05$ & 0.4128 \\
\hline 4846 & $16: 18: 06$ & 0.4238 \\
\hline 4847 & $16: 18: 07$ & 0.4190 \\
\hline 4848 & $16: 18: 08$ & 0.4132 \\
\hline 4849 & 16:18:09 & 0.4194 \\
\hline 4850 & $16: 18: 10$ & 0.4107 \\
\hline 4851 & $16: 18: 11$ & 0.4194 \\
\hline 4852 & $16: 18: 12$ & 0.4146 \\
\hline 4853 & $16: 18: 13$ & 0.4200 \\
\hline 4854 & $16: 18: 14$ & 0.4206 \\
\hline 4855 & $16: 18: 15$ & 0.4175 \\
\hline 4856 & $16: 18: 16$ & 0.4271 \\
\hline 4857 & $16: 18: 17$ & 0.4183 \\
\hline 4858 & $16: 18: 18$ & 0.4221 \\
\hline 4859 & $16: 18: 19$ & 0.4138 \\
\hline 4860 & 16:18:20 & 0.4222 \\
\hline 4861 & $16: 18: 21$ & 0.4190 \\
\hline 4862 & $16: 18: 22$ & 0.4163 \\
\hline 4863 & $16: 18: 23$ & 0.4194 \\
\hline 4864 & $16: 18: 24$ & 0.4158 \\
\hline 4865 & $16: 18: 25$ & 0.4202 \\
\hline 4866 & $16: 18: 26$ & 0.4143 \\
\hline 4867 & $16: 18: 27$ & 0.4220 \\
\hline 4868 & 16:18:28 & 0.4149 \\
\hline 4869 & 16:18:29 & 0.4148 \\
\hline 4870 & $16: 18: 30$ & 0.4115 \\
\hline 4871 & $16: 18: 31$ & 0.4146 \\
\hline 4872 & $16: 18: 32$ & 0.4233 \\
\hline 4873 & $16: 18: 33$ & 0.4173 \\
\hline 4874 & $16: 18: 34$ & 0.4254 \\
\hline 4875 & $16: 18: 35$ & 0.4181 \\
\hline 4876 & $16: 18: 36$ & 0.4245 \\
\hline 4877 & 16:18:37 & 0.4146 \\
\hline 4878 & 16:18:38 & 0.4196 \\
\hline 4879 & $16: 18: 39$ & 0.4211 \\
\hline 4880 & $16: 18: 40$ & 0.4215 \\
\hline 4881 & $16: 18: 41$ & 0.4228 \\
\hline 4882 & $16: 18: 42$ & 0.4126 \\
\hline 4883 & $16: 18: 43$ & 0.4216 \\
\hline 4884 & $16: 18: 44$ & 0.4131 \\
\hline 4885 & $16: 18: 45$ & 0.4186 \\
\hline 4886 & 16:18:46 & 0.4159 \\
\hline 4887 & $16: 18: 47$ & 0.4151 \\
\hline 4888 & $16: 18: 48$ & 0.4164 \\
\hline 4889 & $16: 18: 49$ & 0.4116 \\
\hline 4890 & $16: 18: 50$ & 0.4205 \\
\hline 4891 & $16: 18: 51$ & 0.4148 \\
\hline 4892 & $16: 18: 52$ & 0.4215 \\
\hline 4893 & $16: 18: 53$ & 0.414 \\
\hline 4894 & $16: 18: 54$ & 0.42 \\
\hline
\end{tabular}


Appendix E -Fuel Data Test 3 NIST102605_1.xls

\begin{tabular}{|c|c|c|}
\hline 4895 & $16: 18: 55$ & 0.4219 \\
\hline 4896 & $16: 18: 56$ & 0.4210 \\
\hline 4897 & $16: 18: 57$ & 0.4200 \\
\hline 4898 & $16: 18: 58$ & 0.4166 \\
\hline 4899 & $16: 18: 59$ & 0.4212 \\
\hline 4900 & 16:19:00 & 0.4102 \\
\hline 4901 & 16:19:01 & 0.4192 \\
\hline 4902 & $16: 19: 02$ & 0.4154 \\
\hline 4903 & $16: 19: 03$ & 0.4204 \\
\hline 4904 & 16:19:04 & 0.4153 \\
\hline 4905 & $16: 19: 05$ & 0.4180 \\
\hline 4906 & $16: 19: 06$ & 0.4180 \\
\hline 4907 & $16: 19: 07$ & 0.4165 \\
\hline 4908 & $16: 19: 08$ & 0.4188 \\
\hline 4909 & 16:19:09 & 0.4122 \\
\hline 4910 & $16: 19: 10$ & 0.4243 \\
\hline 4911 & $16: 19: 11$ & 0.4160 \\
\hline 4912 & $16: 19: 12$ & 0.4207 \\
\hline 4913 & $16: 19: 13$ & 0.4203 \\
\hline 4914 & $16: 19: 14$ & 0.4222 \\
\hline 4915 & $16: 19: 15$ & 0.4144 \\
\hline 4916 & $16: 19: 16$ & 0.4187 \\
\hline 4917 & $16: 19: 17$ & 0.4185 \\
\hline 4918 & $16: 19: 18$ & 0.4219 \\
\hline 4919 & $16: 19: 19$ & 0.4199 \\
\hline 4920 & 16:19:20 & 0.4142 \\
\hline 4921 & $16: 19: 21$ & 0.4170 \\
\hline 4922 & $16: 19: 22$ & 0.4124 \\
\hline 4923 & $16: 19: 23$ & 0.4231 \\
\hline 4924 & $16: 19: 24$ & 0.4152 \\
\hline 4925 & $16: 19: 25$ & 0.4234 \\
\hline 4926 & $16: 19: 26$ & 0.4147 \\
\hline 4927 & $16: 19: 27$ & 0.4176 \\
\hline 4928 & 16:19:28 & 0.4137 \\
\hline 4929 & 16:19:29 & 0.4231 \\
\hline 4930 & $16: 19: 30$ & 0.4240 \\
\hline 4931 & $16: 19: 31$ & 0.4182 \\
\hline 4932 & $16: 19: 32$ & 0.4250 \\
\hline 4933 & $16: 19: 33$ & 0.4148 \\
\hline 4934 & $16: 19: 34$ & 0.4221 \\
\hline 4935 & $16: 19: 35$ & 0.4186 \\
\hline 4936 & $16: 19: 36$ & 0.4150 \\
\hline 4937 & 16:19:37 & 0.4224 \\
\hline 4938 & 16:19:38 & 0.4133 \\
\hline 4939 & $16: 19: 39$ & 0.4186 \\
\hline 4940 & $16: 19: 40$ & 0.4134 \\
\hline 4941 & $16: 19: 41$ & 0.4173 \\
\hline 4942 & $16: 19: 42$ & 0.4134 \\
\hline 4943 & $16: 19: 43$ & 0.4151 \\
\hline 4944 & $16: 19: 44$ & 0.4235 \\
\hline 49 & $16: 19: 45$ & 0.414 \\
\hline
\end{tabular}


Appendix E -Fuel Data Test 3 NIST102605_1.xls

\begin{tabular}{|c|c|c|}
\hline 4946 & $16: 19: 46$ & 0.4213 \\
\hline 4947 & $16: 19: 47$ & 0.4171 \\
\hline 4948 & $16: 19: 48$ & 0.4242 \\
\hline 4949 & $16: 19: 49$ & 0.4223 \\
\hline 4950 & $16: 19: 50$ & 0.4193 \\
\hline 4951 & $16: 19: 51$ & 0.4260 \\
\hline 4952 & 16:19:52 & 0.4143 \\
\hline 4953 & $16: 19: 53$ & 0.4194 \\
\hline 4954 & $16: 19: 54$ & 0.4142 \\
\hline 4955 & $16: 19: 55$ & 0.4190 \\
\hline 4956 & $16: 19: 56$ & 0.4215 \\
\hline 4957 & $16: 19: 57$ & 0.4142 \\
\hline 4958 & $16: 19: 58$ & 0.4244 \\
\hline 4959 & $16: 19: 59$ & 0.4133 \\
\hline 4960 & $16: 20: 00$ & 0.4154 \\
\hline 4961 & 16:20:01 & 0.4141 \\
\hline 4962 & $16: 20: 02$ & 0.4139 \\
\hline 4963 & $16: 20: 03$ & 0.4235 \\
\hline 4964 & $16: 20: 04$ & 0.4175 \\
\hline 4965 & $16: 20: 05$ & 0.4265 \\
\hline 4966 & $16: 20: 06$ & 0.4183 \\
\hline 4967 & $16: 20: 07$ & 0.4209 \\
\hline 4968 & $16: 20: 08$ & 0.4219 \\
\hline 4969 & $16: 20: 09$ & 0.4202 \\
\hline 4970 & $16: 20: 10$ & 0.4217 \\
\hline 4971 & $16: 20: 11$ & 0.4133 \\
\hline 4972 & $16: 20: 12$ & 0.4196 \\
\hline 4973 & $16: 20: 13$ & 0.4135 \\
\hline 4974 & $16: 20: 14$ & 0.4230 \\
\hline 4975 & $16: 20: 15$ & 0.4173 \\
\hline 4976 & $16: 20: 16$ & 0.4223 \\
\hline 4977 & $16: 20: 17$ & 0.4166 \\
\hline 4978 & $16: 20: 18$ & 0.4185 \\
\hline 4979 & $16: 20: 19$ & 0.4154 \\
\hline 4980 & $16: 20: 20$ & 0.4141 \\
\hline 4981 & $16: 20: 21$ & 0.4217 \\
\hline 4982 & $16: 20: 22$ & 0.4174 \\
\hline 4983 & $16: 20: 23$ & 0.4242 \\
\hline 4984 & $16: 20: 24$ & 0.4184 \\
\hline 4985 & $16: 20: 25$ & 0.4261 \\
\hline 4986 & $16: 20: 26$ & 0.4138 \\
\hline 4987 & $16: 20: 27$ & 0.4188 \\
\hline 4988 & $16: 20: 28$ & 0.4193 \\
\hline 4989 & $16: 20: 29$ & 0.4215 \\
\hline 4990 & $16: 20: 30$ & 0.4214 \\
\hline 4991 & $16: 20: 31$ & 0.4140 \\
\hline 4992 & $16: 20: 32$ & 0.4237 \\
\hline 4993 & $16: 20: 33$ & 0.4157 \\
\hline 4994 & $16: 20: 34$ & 0.4158 \\
\hline 4995 & $16: 20: 35$ & $0.417 €$ \\
\hline 4996 & $16: 20: 36$ & 0.411 \\
\hline
\end{tabular}


Appendix E -Fuel Data Test 3 NIST102605_1.xls

\begin{tabular}{|c|c|c|}
\hline 4997 & $16: 20: 37$ & 0.4200 \\
\hline 4998 & $16: 20: 38$ & 0.4121 \\
\hline 4999 & $16: 20: 39$ & 0.4229 \\
\hline 5000 & $16: 20: 40$ & 0.4160 \\
\hline 5001 & $16: 20: 41$ & 0.4179 \\
\hline 5002 & $16: 20: 42$ & 0.4219 \\
\hline 5003 & $16: 20: 43$ & 0.4229 \\
\hline 5004 & $16: 20: 44$ & 0.4268 \\
\hline 5005 & $16: 20: 45$ & 0.4155 \\
\hline 5006 & $16: 20: 46$ & 0.4224 \\
\hline 5007 & $16: 20: 47$ & 0.4179 \\
\hline 5008 & $16: 20: 48$ & 0.4192 \\
\hline 5009 & $16: 20: 49$ & 0.4216 \\
\hline 5010 & $16: 20: 50$ & 0.4172 \\
\hline 5011 & $16: 20: 51$ & 0.4224 \\
\hline 5012 & $16: 20: 52$ & 0.4134 \\
\hline 5013 & $16: 20: 53$ & 0.4179 \\
\hline 5014 & $16: 20: 54$ & 0.4157 \\
\hline 5015 & $16: 20: 55$ & 0.4234 \\
\hline 5016 & $16: 20: 56$ & 0.4147 \\
\hline 5017 & $16: 20: 57$ & 0.4183 \\
\hline 5018 & $16: 20: 58$ & 0.4221 \\
\hline 5019 & $16: 20: 59$ & 0.4168 \\
\hline 5020 & $16: 21: 00$ & 0.4242 \\
\hline 5021 & 16:21:01 & 0.4169 \\
\hline 5022 & $16: 21: 02$ & 0.4235 \\
\hline 5023 & $16: 21: 03$ & 0.4251 \\
\hline 5024 & $16: 21: 04$ & 0.4200 \\
\hline 5025 & $16: 21: 05$ & 0.4252 \\
\hline 5026 & $16: 21: 06$ & 0.4134 \\
\hline 5027 & $16: 21: 07$ & 0.4192 \\
\hline 5028 & $16: 21: 08$ & 0.4129 \\
\hline 5029 & $16: 21: 09$ & 0.4231 \\
\hline 5030 & $16: 21: 10$ & 0.4189 \\
\hline 5031 & $16: 21: 11$ & 0.4165 \\
\hline 5032 & $16: 21: 12$ & 0.4242 \\
\hline 5033 & $16: 21: 13$ & 0.4138 \\
\hline 5034 & $16: 21: 14$ & 0.4147 \\
\hline 5035 & $16: 21: 15$ & 0.4142 \\
\hline 5036 & $16: 21: 16$ & 0.4152 \\
\hline 5037 & $16: 21: 17$ & 0.4215 \\
\hline 5038 & $16: 21: 18$ & 0.4169 \\
\hline 5039 & $16: 21: 19$ & 0.4269 \\
\hline 5040 & 16:21:20 & 0.4181 \\
\hline 5041 & $16: 21: 21$ & 0.4209 \\
\hline 5042 & $16: 21: 22$ & 0.4226 \\
\hline 5043 & $16: 21: 23$ & 0.4198 \\
\hline 5044 & $16: 21: 24$ & 0.4239 \\
\hline 5045 & $16: 21: 25$ & 0.4127 \\
\hline 5046 & $16: 21: 26$ & 0.422 \\
\hline 5047 & $16: 21: 27$ & 0.418 \\
\hline
\end{tabular}


Appendix E -Fuel Data Test 3 NIST102605_1.xls

\begin{tabular}{|c|c|c|}
\hline 5048 & $16: 21: 28$ & 0.4161 \\
\hline 5049 & $16: 21: 29$ & 0.4164 \\
\hline 5050 & $16: 21: 30$ & 0.4128 \\
\hline 5051 & $16: 21: 31$ & 0.4238 \\
\hline 5052 & $16: 21: 32$ & 0.4158 \\
\hline 5053 & $16: 21: 33$ & 0.4225 \\
\hline 5054 & $16: 21: 34$ & 0.4173 \\
\hline 5055 & $16: 21: 35$ & 0.4188 \\
\hline 5056 & $16: 21: 36$ & 0.4133 \\
\hline 5057 & $16: 21: 37$ & 0.4182 \\
\hline 5058 & $16: 21: 38$ & 0.4210 \\
\hline 5059 & $16: 21: 39$ & 0.4206 \\
\hline 5060 & $16: 21: 40$ & 0.4243 \\
\hline 5061 & $16: 21: 41$ & 0.4140 \\
\hline 5062 & $16: 21: 42$ & 0.4258 \\
\hline 5063 & $16: 21: 43$ & 0.4221 \\
\hline 5064 & $16: 21: 44$ & 0.4172 \\
\hline 5065 & $16: 21: 45$ & 0.4245 \\
\hline 5066 & $16: 21: 46$ & 0.4134 \\
\hline 5067 & $16: 21: 47$ & 0.4208 \\
\hline 5068 & $16: 21: 48$ & 0.4161 \\
\hline 5069 & $16: 21: 49$ & 0.4128 \\
\hline 5070 & $16: 21: 50$ & 0.4220 \\
\hline 5071 & $16: 21: 51$ & 0.4142 \\
\hline 5072 & $16: 21: 52$ & 0.4231 \\
\hline 5073 & $16: 21: 53$ & 0.4161 \\
\hline 5074 & $16: 21: 54$ & 0.4191 \\
\hline 5075 & $16: 21: 55$ & 0.4145 \\
\hline 5076 & $16: 21: 56$ & 0.4231 \\
\hline 5077 & $16: 21: 57$ & 0.4214 \\
\hline 5078 & $16: 21: 58$ & 0.4218 \\
\hline 5079 & $16: 21: 59$ & 0.4208 \\
\hline 5080 & $16: 22: 00$ & 0.4194 \\
\hline 5081 & $16: 22: 01$ & 0.4226 \\
\hline 5082 & $16: 22: 02$ & 0.4118 \\
\hline 5083 & $16: 22: 03$ & 0.4218 \\
\hline 5084 & $16: 22: 04$ & 0.4182 \\
\hline 5085 & $16: 22: 05$ & 0.4146 \\
\hline 5086 & $16: 22: 06$ & 0.4166 \\
\hline 5087 & $16: 22: 07$ & 0.4143 \\
\hline 5088 & $16: 22: 08$ & 0.4223 \\
\hline 5089 & $16: 22: 09$ & 0.4149 \\
\hline 5090 & $16: 22: 10$ & 0.4211 \\
\hline 5091 & $16: 22: 11$ & 0.4156 \\
\hline 5092 & $16: 22: 12$ & 0.4183 \\
\hline 5093 & $16: 22: 13$ & 0.4139 \\
\hline 5094 & $16: 22: 14$ & 0.4187 \\
\hline 5095 & $16: 22: 15$ & 0.4253 \\
\hline 5096 & $16: 22: 16$ & 0.4202 \\
\hline 5097 & $16: 22: 17$ & 0.424 \\
\hline 5098 & $16: 22: 18$ & 0.417 \\
\hline
\end{tabular}


Appendix E -Fuel Data Test 3 NIST102605_1.xls

\begin{tabular}{|c|c|c|}
\hline 5099 & $16: 22: 19$ & 0.4249 \\
\hline 5100 & $16: 22: 20$ & 0.4132 \\
\hline 5101 & $16: 22: 21$ & 0.4199 \\
\hline 5102 & $16: 22: 22$ & 0.4148 \\
\hline 5103 & $16: 22: 23$ & 0.4230 \\
\hline 5104 & $16: 22: 24$ & 0.4200 \\
\hline 5105 & $16: 22: 25$ & 0.4139 \\
\hline 5106 & $16: 22: 26$ & 0.4222 \\
\hline 5107 & $16: 22: 27$ & 0.4136 \\
\hline 5108 & $16: 22: 28$ & 0.4214 \\
\hline 5109 & $16: 22: 29$ & 0.4121 \\
\hline 5110 & $16: 22: 30$ & 0.4167 \\
\hline 5111 & $16: 22: 31$ & 0.4171 \\
\hline 5112 & $16: 22: 32$ & 0.4165 \\
\hline 5113 & $16: 22: 33$ & 0.4251 \\
\hline 5114 & $16: 22: 34$ & 0.4187 \\
\hline 5115 & $16: 22: 35$ & 0.4239 \\
\hline 5116 & $16: 22: 36$ & 0.4148 \\
\hline 5117 & $16: 22: 37$ & 0.4216 \\
\hline 5118 & $16: 22: 38$ & 0.4176 \\
\hline 5119 & $16: 22: 39$ & 0.4213 \\
\hline 5120 & $16: 22: 40$ & 0.4189 \\
\hline 5121 & $16: 22: 41$ & 0.4174 \\
\hline 5122 & $16: 22: 42$ & 0.4225 \\
\hline 5123 & $16: 22: 43$ & 0.4132 \\
\hline 5124 & $16: 22: 44$ & 0.4167 \\
\hline 5125 & $16: 22: 45$ & 0.4126 \\
\hline 5126 & $16: 22: 46$ & 0.4203 \\
\hline 5127 & $16: 22: 47$ & 0.4155 \\
\hline 5128 & $16: 22: 48$ & 0.4160 \\
\hline 5129 & $16: 22: 49$ & 0.4211 \\
\hline 5130 & $16: 22: 50$ & 0.4147 \\
\hline 5131 & $16: 22: 51$ & 0.4235 \\
\hline 5132 & $16: 22: 52$ & 0.4161 \\
\hline 5133 & $16: 22: 53$ & 0.4254 \\
\hline 5134 & $16: 22: 54$ & 0.4219 \\
\hline 5135 & $16: 22: 55$ & 0.4180 \\
\hline 5136 & $16: 22: 56$ & 0.4241 \\
\hline 5137 & $16: 22: 57$ & 0.4132 \\
\hline 5138 & $16: 22: 58$ & 0.4205 \\
\hline 5139 & $16: 22: 59$ & 0.4168 \\
\hline 5140 & $16: 23: 00$ & 0.4240 \\
\hline 5141 & $16: 23: 01$ & 0.4187 \\
\hline 5142 & $16: 23: 02$ & 0.4134 \\
\hline 5143 & $16: 23: 03$ & 0.4155 \\
\hline 5144 & $16: 23: 04$ & 0.4177 \\
\hline 5145 & $16: 23: 05$ & 0.4219 \\
\hline 5146 & $16: 23: 06$ & 0.4125 \\
\hline 5147 & $16: 23: 07$ & 0.4225 \\
\hline 5148 & $16: 23: 08$ & 0.4140 \\
\hline 514 & $16: 23: 09$ & 0.418 \\
\hline
\end{tabular}


Appendix E -Fuel Data Test 3 NIST102605_1.xls

\begin{tabular}{|c|c|c|}
\hline 5150 & $16: 23: 10$ & 0.4178 \\
\hline 5151 & $16: 23: 11$ & 0.4212 \\
\hline 5152 & $16: 23: 12$ & 0.4255 \\
\hline 5153 & $16: 23: 13$ & 0.4221 \\
\hline 5154 & $16: 23: 14$ & 0.4223 \\
\hline 5155 & $16: 23: 15$ & 0.4168 \\
\hline 5156 & $16: 23: 16$ & 0.4228 \\
\hline 5157 & $16: 23: 17$ & 0.4145 \\
\hline 5158 & $16: 23: 18$ & 0.4218 \\
\hline 5159 & $16: 23: 19$ & 0.4198 \\
\hline 5160 & $16: 23: 20$ & 0.4182 \\
\hline 5161 & $16: 23: 21$ & 0.4166 \\
\hline 5162 & $16: 23: 22$ & 0.4121 \\
\hline 5163 & $16: 23: 23$ & 0.4236 \\
\hline 5164 & $16: 23: 24$ & 0.4133 \\
\hline 5165 & $16: 23: 25$ & 0.4190 \\
\hline 5166 & $16: 23: 26$ & 0.4172 \\
\hline 5167 & $16: 23: 27$ & 0.4178 \\
\hline 5168 & $16: 23: 28$ & 0.4132 \\
\hline 5169 & $16: 23: 29$ & 0.4177 \\
\hline 5170 & $16: 23: 30$ & 0.4210 \\
\hline 5171 & $16: 23: 31$ & 0.4204 \\
\hline 5172 & 16:23:32 & 0.4247 \\
\hline 5173 & $16: 23: 33$ & 0.4163 \\
\hline 5174 & $16: 23: 34$ & 0.4233 \\
\hline 5175 & $16: 23: 35$ & 0.4132 \\
\hline 5176 & $16: 23: 36$ & 0.4193 \\
\hline 5177 & $16: 23: 37$ & 0.4191 \\
\hline 5178 & $16: 23: 38$ & 0.4230 \\
\hline 5179 & $16: 23: 39$ & 0.4224 \\
\hline 5180 & $16: 23: 40$ & 0.4116 \\
\hline 5181 & $16: 23: 41$ & 0.4235 \\
\hline 5182 & $16: 23: 42$ & 0.4141 \\
\hline 5183 & $16: 23: 43$ & 0.4171 \\
\hline 5184 & $16: 23: 44$ & 0.4158 \\
\hline 5185 & $16: 23: 45$ & 0.4183 \\
\hline 5186 & $16: 23: 46$ & 0.4220 \\
\hline 5187 & $16: 23: 47$ & 0.4141 \\
\hline 5188 & $16: 23: 48$ & 0.4245 \\
\hline 5189 & $16: 23: 49$ & 0.4166 \\
\hline 5190 & $16: 23: 50$ & 0.4207 \\
\hline 5191 & $16: 23: 51$ & 0.4189 \\
\hline 5192 & $16: 23: 52$ & 0.4224 \\
\hline 5193 & $16: 23: 53$ & 0.4234 \\
\hline 5194 & $16: 23: 54$ & 0.4134 \\
\hline 5195 & $16: 23: 55$ & 0.4186 \\
\hline 5196 & $16: 23: 56$ & 0.4161 \\
\hline 5197 & $16: 23: 57$ & 0.4209 \\
\hline 5198 & $16: 23: 58$ & 0.413 \\
\hline 5199 & $16: 23: 59$ & 0.418 \\
\hline 5200 & $16: 24: 00$ & 0.416 \\
\hline
\end{tabular}


Appendix E -Fuel Data Test 3 NIST102605_1.xls

\begin{tabular}{|c|c|c|}
\hline 5201 & $16: 24: 01$ & 0.4153 \\
\hline 5202 & $16: 24: 02$ & 0.4174 \\
\hline 5203 & $16: 24: 03$ & 0.4158 \\
\hline 5204 & $16: 24: 04$ & 0.4249 \\
\hline 5205 & $16: 24: 05$ & 0.4139 \\
\hline 5206 & $16: 24: 06$ & 0.4214 \\
\hline 5207 & $16: 24: 07$ & 0.4187 \\
\hline 5208 & $16: 24: 08$ & 0.4216 \\
\hline 5209 & $16: 24: 09$ & 0.4219 \\
\hline 5210 & $16: 24: 10$ & 0.4140 \\
\hline 5211 & $16: 24: 11$ & 0.4230 \\
\hline 5212 & $16: 24: 12$ & 0.4124 \\
\hline 5213 & $16: 24: 13$ & 0.4192 \\
\hline 5214 & $16: 24: 14$ & 0.4191 \\
\hline 5215 & $16: 24: 15$ & 0.4188 \\
\hline 5216 & $16: 24: 16$ & 0.4218 \\
\hline 5217 & $16: 24: 17$ & 0.4128 \\
\hline 5218 & $16: 24: 18$ & 0.4224 \\
\hline 5219 & $16: 24: 19$ & 0.4135 \\
\hline 5220 & $16: 24: 20$ & 0.4153 \\
\hline 5221 & $16: 24: 21$ & 0.4167 \\
\hline 5222 & 16:24:22 & 0.4166 \\
\hline 5223 & $16: 24: 23$ & 0.4229 \\
\hline 5224 & $16: 24: 24$ & 0.4149 \\
\hline 5225 & $16: 24: 25$ & 0.4241 \\
\hline 5226 & $16: 24: 26$ & 0.4185 \\
\hline 5227 & $16: 24: 27$ & 0.4182 \\
\hline 5228 & $16: 24: 28$ & 0.4238 \\
\hline 5229 & $16: 24: 29$ & 0.4164 \\
\hline 5230 & $16: 24: 30$ & 0.4231 \\
\hline 5231 & $16: 24: 31$ & 0.4146 \\
\hline 5232 & $16: 24: 32$ & 0.4214 \\
\hline 5233 & $16: 24: 33$ & 0.4191 \\
\hline 5234 & $16: 24: 34$ & 0.4173 \\
\hline 5235 & $16: 24: 35$ & 0.4167 \\
\hline 5236 & $16: 24: 36$ & 0.4149 \\
\hline 5237 & $16: 24: 37$ & 0.4220 \\
\hline 5238 & $16: 24: 38$ & 0.4123 \\
\hline 5239 & $16: 24: 39$ & 0.4160 \\
\hline 5240 & $16: 24: 40$ & 0.4160 \\
\hline 5241 & $16: 24: 41$ & 0.423 \\
\hline 5242 & $16: 24: 42$ & 0.4204 \\
\hline 5243 & $16: 24: 43$ & 0.4195 \\
\hline 5244 & $16: 24: 44$ & 0.4260 \\
\hline 5245 & $16: 24: 45$ & 0.4177 \\
\hline 5246 & $16: 24: 46$ & 0.4230 \\
\hline 5247 & $16: 24: 47$ & 0.4155 \\
\hline 5248 & $16: 24: 48$ & 0.4230 \\
\hline 5249 & $16: 24: 49$ & 0.419 \\
\hline 5250 & $16: 24: 50$ & 0.416 \\
\hline 5251 & $16: 24: 51$ & 0.422 \\
\hline
\end{tabular}


Appendix E -Fuel Data Test 3 NIST102605_1.xls

\begin{tabular}{|c|c|c|}
\hline 5252 & $16: 24: 52$ & 0.4116 \\
\hline 5253 & $16: 24: 53$ & 0.4222 \\
\hline 5254 & $16: 24: 54$ & 0.4197 \\
\hline 5255 & $16: 24: 55$ & 0.4229 \\
\hline 5256 & $16: 24: 56$ & 0.4176 \\
\hline 5257 & $16: 24: 57$ & 0.4112 \\
\hline 5258 & $16: 24: 58$ & 0.4203 \\
\hline 5259 & $16: 24: 59$ & 0.4127 \\
\hline 5260 & $16: 25: 00$ & 0.4186 \\
\hline 5261 & $16: 25: 01$ & 0.4186 \\
\hline 5262 & $16: 25: 02$ & 0.4212 \\
\hline 5263 & $16: 25: 03$ & 0.4252 \\
\hline 5264 & $16: 25: 04$ & 0.4150 \\
\hline 5265 & $16: 25: 05$ & 0.4224 \\
\hline 5266 & $16: 25: 06$ & 0.4152 \\
\hline 5267 & $16: 25: 07$ & 0.4188 \\
\hline 5268 & $16: 25: 08$ & 0.4199 \\
\hline 5269 & $16: 25: 09$ & 0.4225 \\
\hline 5270 & $16: 25: 10$ & 0.4210 \\
\hline 5271 & $16: 25: 11$ & 0.4138 \\
\hline 5272 & $16: 25: 12$ & 0.4159 \\
\hline 5273 & $16: 25: 13$ & 0.4117 \\
\hline 5274 & $16: 25: 14$ & 0.4201 \\
\hline 5275 & $16: 25: 15$ & 0.4153 \\
\hline 5276 & $16: 25: 16$ & 0.4158 \\
\hline 5277 & $16: 25: 17$ & 0.4168 \\
\hline 5278 & $16: 25: 18$ & 0.4194 \\
\hline 5279 & $16: 25: 19$ & 0.4198 \\
\hline 5280 & $16: 25: 20$ & 0.4186 \\
\hline 5281 & $16: 25: 21$ & 0.4277 \\
\hline 5282 & $16: 25: 22$ & 0.4140 \\
\hline 5283 & $16: 25: 23$ & 0.4189 \\
\hline 5284 & $16: 25: 24$ & 0.4156 \\
\hline 5285 & $16: 25: 25$ & 0.4211 \\
\hline 5286 & $16: 25: 26$ & 0.4206 \\
\hline 5287 & $16: 25: 27$ & 0.4152 \\
\hline 5288 & $16: 25: 28$ & 0.4235 \\
\hline 5289 & $16: 25: 29$ & 0.4141 \\
\hline 5290 & $16: 25: 30$ & 0.4214 \\
\hline 5291 & $16: 25: 31$ & 0.4143 \\
\hline 5292 & $16: 25: 32$ & 0.4211 \\
\hline 5293 & $16: 25: 33$ & 0.4153 \\
\hline 5294 & $16: 25: 34$ & 0.4104 \\
\hline 5295 & $16: 25: 35$ & 0.4131 \\
\hline 5296 & $16: 25: 36$ & 0.4159 \\
\hline 5297 & $16: 25: 37$ & 0.4220 \\
\hline 5298 & $16: 25: 38$ & 0.4141 \\
\hline 5299 & $16: 25: 39$ & 0.4250 \\
\hline 5300 & $16: 25: 40$ & 0.4175 \\
\hline 5301 & $16: 25: 41$ & 0.4218 \\
\hline 5302 & $16: 25: 42$ & 0.4212 \\
\hline
\end{tabular}


Appendix E -Fuel Data Test 3 NIST102605_1.xls

\begin{tabular}{|c|c|c|}
\hline 5303 & $16: 25: 43$ & 0.4218 \\
\hline 5304 & $16: 25: 44$ & 0.4230 \\
\hline 5305 & $16: 25: 45$ & 0.4106 \\
\hline 5306 & $16: 25: 46$ & 0.4178 \\
\hline 5307 & $16: 25: 47$ & 0.4136 \\
\hline 5308 & $16: 25: 48$ & 0.4237 \\
\hline 5309 & $16: 25: 49$ & 0.4169 \\
\hline 5310 & $16: 25: 50$ & 0.4192 \\
\hline 5311 & $16: 25: 51$ & 0.4174 \\
\hline 5312 & $16: 25: 52$ & 0.4124 \\
\hline 5313 & $16: 25: 53$ & 0.4151 \\
\hline 5314 & $16: 25: 54$ & 0.4151 \\
\hline 5315 & $16: 25: 55$ & 0.4250 \\
\hline 5316 & $16: 25: 56$ & 0.4151 \\
\hline 5317 & $16: 25: 57$ & 0.4220 \\
\hline 5318 & $16: 25: 58$ & 0.4198 \\
\hline 5319 & $16: 25: 59$ & 0.4186 \\
\hline 5320 & $16: 26: 00$ & 0.4236 \\
\hline 5321 & $16: 26: 01$ & 0.4179 \\
\hline 5322 & $16: 26: 02$ & 0.4249 \\
\hline 5323 & $16: 26: 03$ & 0.4120 \\
\hline 5324 & $16: 26: 04$ & 0.4186 \\
\hline 5325 & $16: 26: 05$ & 0.4120 \\
\hline 5326 & $16: 26: 06$ & 0.4194 \\
\hline 5327 & $16: 26: 07$ & 0.4195 \\
\hline 5328 & $16: 26: 08$ & 0.4117 \\
\hline 5329 & $16: 26: 09$ & 0.4244 \\
\hline 5330 & $16: 26: 10$ & 0.4156 \\
\hline 5331 & $16: 26: 11$ & 0.4155 \\
\hline 5332 & $16: 26: 12$ & 0.4144 \\
\hline 5333 & $16: 26: 13$ & 0.4173 \\
\hline 5334 & $16: 26: 14$ & 0.4209 \\
\hline 5335 & $16: 26: 15$ & 0.4148 \\
\hline 5336 & $16: 26: 16$ & 0.4265 \\
\hline 5337 & $16: 26: 17$ & 0.4177 \\
\hline 5338 & $16: 26: 18$ & 0.4198 \\
\hline 5339 & $16: 26: 19$ & 0.4233 \\
\hline 5340 & $16: 26: 20$ & 0.4170 \\
\hline 5341 & $16: 26: 21$ & 0.4188 \\
\hline 5342 & $16: 26: 22$ & 0.4118 \\
\hline 5343 & $16: 26: 23$ & 0.4193 \\
\hline 5344 & $16: 26: 24$ & 0.4128 \\
\hline 5345 & $16: 26: 25$ & 0.4190 \\
\hline 5346 & $16: 26: 26$ & 0.4114 \\
\hline 5347 & $16: 26: 27$ & 0.4184 \\
\hline 5348 & $16: 26: 28$ & 0.4191 \\
\hline 5349 & $16: 26: 29$ & 0.4121 \\
\hline 5350 & $16: 26: 30$ & 0.4210 \\
\hline 5351 & $16: 26: 31$ & 0.4180 \\
\hline 5352 & $16: 26: 32$ & 0.425 \\
\hline 5353 & $16: 26: 33$ & 0.410 \\
\hline
\end{tabular}


Appendix E -Fuel Data Test 3 NIST102605_1.xls

\begin{tabular}{|c|c|c|}
\hline 5354 & $16: 26: 34$ & 0.4166 \\
\hline 5355 & $16: 26: 35$ & 0.4246 \\
\hline 5356 & $16: 26: 36$ & 0.4212 \\
\hline 5357 & $16: 26: 37$ & 0.4244 \\
\hline 5358 & $16: 26: 38$ & 0.4155 \\
\hline 5359 & $16: 26: 39$ & 0.4231 \\
\hline 5360 & $16: 26: 40$ & 0.4151 \\
\hline 5361 & $16: 26: 41$ & 0.4192 \\
\hline 5362 & $16: 26: 42$ & 0.4166 \\
\hline 5363 & $16: 26: 43$ & 0.4180 \\
\hline 5364 & $16: 26: 44$ & 0.4189 \\
\hline 5365 & $16: 26: 45$ & 0.4142 \\
\hline 5366 & $16: 26: 46$ & 0.4181 \\
\hline 5367 & $16: 26: 47$ & 0.4113 \\
\hline 5368 & $16: 26: 48$ & 0.4221 \\
\hline 5369 & $16: 26: 49$ & 0.4124 \\
\hline 5370 & $16: 26: 50$ & 0.4209 \\
\hline 5371 & $16: 26: 51$ & 0.4207 \\
\hline 5372 & $16: 26: 52$ & 0.4142 \\
\hline 5373 & $16: 26: 53$ & 0.4254 \\
\hline 5374 & $16: 26: 54$ & 0.4178 \\
\hline 5375 & $16: 26: 55$ & 0.4204 \\
\hline 5376 & $16: 26: 56$ & 0.4172 \\
\hline 5377 & $16: 26: 57$ & 0.4236 \\
\hline 5378 & $16: 26: 58$ & 0.4244 \\
\hline 5379 & $16: 26: 59$ & 0.4127 \\
\hline 5380 & $16: 27: 00$ & 0.4182 \\
\hline 5381 & $16: 27: 01$ & 0.4145 \\
\hline 5382 & $16: 27: 02$ & 0.4205 \\
\hline 5383 & $16: 27: 03$ & 0.4170 \\
\hline 5384 & $16: 27: 04$ & 0.4221 \\
\hline 5385 & $16: 27: 05$ & 0.4168 \\
\hline 5386 & $16: 27: 06$ & 0.4152 \\
\hline 5387 & $16: 27: 07$ & 0.4133 \\
\hline 5388 & $16: 27: 08$ & 0.4151 \\
\hline 5389 & $16: 27: 09$ & 0.4205 \\
\hline 5390 & $16: 27: 10$ & 0.4174 \\
\hline 5391 & $16: 27: 11$ & 0.4239 \\
\hline 5392 & $16: 27: 12$ & 0.4117 \\
\hline 5393 & $16: 27: 13$ & 0.4212 \\
\hline 5394 & $16: 27: 14$ & 0.4238 \\
\hline 5395 & $16: 27: 15$ & 0.4156 \\
\hline 5396 & $16: 27: 16$ & 0.4243 \\
\hline 5397 & $16: 27: 17$ & 0.4161 \\
\hline 5398 & $16: 27: 18$ & 0.4189 \\
\hline 5399 & $16: 27: 19$ & 0.4209 \\
\hline 5400 & $16: 27: 20$ & 0.4222 \\
\hline 5401 & $16: 27: 21$ & 0.4224 \\
\hline 5402 & $16: 27: 22$ & 0.4111 \\
\hline 5403 & $16: 27: 23$ & 0.4235 \\
\hline 5404 & $16: 27: 24$ & 0.414 \\
\hline
\end{tabular}


Appendix E -Fuel Data Test 3 NIST102605_1.xls

\begin{tabular}{|c|c|c|}
\hline 5405 & $16: 27: 25$ & 0.4151 \\
\hline 5406 & $16: 27: 26$ & 0.4157 \\
\hline 5407 & $16: 27: 27$ & 0.4147 \\
\hline 5408 & $16: 27: 28$ & 0.4204 \\
\hline 5409 & 16:27:29 & 0.4143 \\
\hline 5410 & $16: 27: 30$ & 0.4233 \\
\hline 5411 & $16: 27: 31$ & 0.4170 \\
\hline 5412 & 16:27:32 & 0.4250 \\
\hline 5413 & $16: 27: 33$ & 0.4204 \\
\hline 5414 & $16: 27: 34$ & 0.4240 \\
\hline 5415 & $16: 27: 35$ & 0.4201 \\
\hline 5416 & $16: 27: 36$ & 0.4190 \\
\hline 5417 & $16: 27: 37$ & 0.4197 \\
\hline 5418 & $16: 27: 38$ & 0.4194 \\
\hline 5419 & $16: 27: 39$ & 0.4209 \\
\hline 5420 & $16: 27: 40$ & 0.4099 \\
\hline 5421 & $16: 27: 41$ & 0.4167 \\
\hline 5422 & $16: 27: 42$ & 0.4137 \\
\hline 5423 & $16: 27: 43$ & 0.4217 \\
\hline 5424 & $16: 27: 44$ & 0.4158 \\
\hline 5425 & $16: 27: 45$ & 0.4215 \\
\hline 5426 & $16: 27: 46$ & 0.4161 \\
\hline 5427 & $16: 27: 47$ & 0.4208 \\
\hline 5428 & $16: 27: 48$ & 0.4116 \\
\hline 5429 & $16: 27: 49$ & 0.4165 \\
\hline 5430 & $16: 27: 50$ & 0.4219 \\
\hline 5431 & $16: 27: 51$ & 0.4211 \\
\hline 5432 & 16:27:52 & 0.4217 \\
\hline 5433 & $16: 27: 53$ & 0.4167 \\
\hline 5434 & $16: 27: 54$ & 0.4246 \\
\hline 5435 & $16: 27: 55$ & 0.4150 \\
\hline 5436 & $16: 27: 56$ & 0.4217 \\
\hline 5437 & $16: 27: 57$ & 0.4175 \\
\hline 5438 & 16:27:58 & 0.4238 \\
\hline 5439 & 16:27:59 & 0.4177 \\
\hline 5440 & 16:28:00 & 0.4128 \\
\hline 5441 & 16:28:01 & 0.4158 \\
\hline 5442 & 16:28:02 & 0.4113 \\
\hline 5443 & 16:28:03 & 0.4199 \\
\hline 5444 & 16:28:04 & 0.4119 \\
\hline 5445 & 16:28:05 & 0.4212 \\
\hline 5446 & $16: 28: 06$ & 0.415 \\
\hline 5447 & $16: 28: 07$ & 0.4189 \\
\hline 5448 & 16:28:08 & 0.4216 \\
\hline 5449 & 16:28:09 & 0.420 \\
\hline 5450 & 16:28:10 & 0.425 \\
\hline 5451 & $16: 28: 11$ & 0.4132 \\
\hline 5452 & $16: 28: 12$ & 0.4210 \\
\hline 5453 & $16: 28: 13$ & 0.416 \\
\hline 5454 & $16: 28: 14$ & 0.423 \\
\hline 5455 & $16: 28: 15$ & 0.419 \\
\hline
\end{tabular}


Appendix E -Fuel Data Test 3 NIST102605_1.xls

\begin{tabular}{|c|c|c|}
\hline 5456 & $16: 28: 16$ & 0.4154 \\
\hline 5457 & $16: 28: 17$ & 0.4194 \\
\hline 5458 & $16: 28: 18$ & 0.4114 \\
\hline 5459 & $16: 28: 19$ & 0.4180 \\
\hline 5460 & $16: 28: 20$ & 0.4122 \\
\hline 5461 & $16: 28: 21$ & 0.4175 \\
\hline 5462 & $16: 28: 22$ & 0.4141 \\
\hline 5463 & $16: 28: 23$ & 0.4183 \\
\hline 5464 & $16: 28: 24$ & 0.4180 \\
\hline 5465 & $16: 28: 25$ & 0.4181 \\
\hline 5466 & $16: 28: 26$ & 0.4207 \\
\hline 5467 & $16: 28: 27$ & 0.4157 \\
\hline 5468 & $16: 28: 28$ & 0.4269 \\
\hline 5469 & $16: 28: 29$ & 0.4153 \\
\hline 5470 & $16: 28: 30$ & 0.4212 \\
\hline 5471 & $16: 28: 31$ & 0.4201 \\
\hline 5472 & $16: 28: 32$ & 0.4215 \\
\hline 5473 & $16: 28: 33$ & 0.4209 \\
\hline 5474 & $16: 28: 34$ & 0.4135 \\
\hline 5475 & $16: 28: 35$ & 0.4222 \\
\hline 5476 & $16: 28: 36$ & 0.4138 \\
\hline 5477 & $16: 28: 37$ & 0.4178 \\
\hline 5478 & $16: 28: 38$ & 0.4187 \\
\hline 5479 & $16: 28: 39$ & 0.4122 \\
\hline 5480 & $16: 28: 40$ & 0.4186 \\
\hline 5481 & $16: 28: 41$ & 0.4094 \\
\hline 5482 & $16: 28: 42$ & 0.4210 \\
\hline 5483 & $16: 28: 43$ & 0.4149 \\
\hline 5484 & $16: 28: 44$ & 0.4150 \\
\hline 5485 & $16: 28: 45$ & 0.4247 \\
\hline 5486 & $16: 28: 46$ & 0.4169 \\
\hline 5487 & $16: 28: 47$ & 0.4241 \\
\hline 5488 & $16: 28: 48$ & 0.4169 \\
\hline 5489 & $16: 28: 49$ & 0.423 \\
\hline 5490 & $16: 28: 50$ & 0.4248 \\
\hline 5491 & $16: 28: 51$ & 0.4135 \\
\hline 5492 & $16: 28: 52$ & 0.4186 \\
\hline 5493 & $16: 28: 53$ & 0.4153 \\
\hline 5494 & $16: 28: 54$ & 0.4160 \\
\hline 5495 & $16: 28: 55$ & 0.4171 \\
\hline 5496 & $16: 28: 56$ & 0.4133 \\
\hline 5497 & $16: 28: 57$ & 0.4194 \\
\hline 5498 & $16: 28: 58$ & 0.4112 \\
\hline 5499 & $16: 28: 59$ & 0.4203 \\
\hline 5500 & $16: 29: 00$ & 0.4175 \\
\hline 5501 & $16: 29: 01$ & 0.4236 \\
\hline 5502 & $16: 29: 02$ & 0.4147 \\
\hline 5503 & $16: 29: 03$ & 0.4171 \\
\hline 5504 & $16: 29: 04$ & 0.4247 \\
\hline 5505 & $16: 29: 05$ & 0.415 \\
\hline 5506 & $16: 29: 06$ & 0.422 \\
\hline
\end{tabular}


Appendix E -Fuel Data Test 3 NIST102605_1.xls

\begin{tabular}{|c|c|c|}
\hline 5507 & $16: 29: 07$ & 0.4143 \\
\hline 5508 & $16: 29: 08$ & 0.4209 \\
\hline 5509 & $16: 29: 09$ & 0.4144 \\
\hline 5510 & $16: 29: 10$ & 0.4199 \\
\hline 5511 & $16: 29: 11$ & 0.4187 \\
\hline 5512 & $16: 29: 12$ & 0.4223 \\
\hline 5513 & $16: 29: 13$ & 0.4214 \\
\hline 5514 & $16: 29: 14$ & 0.4137 \\
\hline 5515 & $16: 29: 15$ & 0.4230 \\
\hline 5516 & $16: 29: 16$ & 0.4132 \\
\hline 5517 & $16: 29: 17$ & 0.4172 \\
\hline 5518 & $16: 29: 18$ & 0.4122 \\
\hline 5519 & $16: 29: 19$ & 0.4151 \\
\hline 5520 & $16: 29: 20$ & 0.4224 \\
\hline 5521 & $16: 29: 21$ & 0.4161 \\
\hline 5522 & $16: 29: 22$ & 0.4256 \\
\hline 5523 & $16: 29: 23$ & 0.4164 \\
\hline 5524 & $16: 29: 24$ & 0.4203 \\
\hline 5525 & $16: 29: 25$ & 0.4143 \\
\hline 5526 & $16: 29: 26$ & 0.4221 \\
\hline 5527 & $16: 29: 27$ & 0.4222 \\
\hline 5528 & $16: 29: 28$ & 0.4144 \\
\hline 5529 & 16:29:29 & 0.4216 \\
\hline 5530 & $16: 29: 30$ & 0.4150 \\
\hline 5531 & $16: 29: 31$ & 0.4148 \\
\hline 5532 & $16: 29: 32$ & 0.4151 \\
\hline 5533 & $16: 29: 33$ & 0.4170 \\
\hline 5534 & $16: 29: 34$ & 0.4196 \\
\hline 5535 & $16: 29: 35$ & 0.4132 \\
\hline 5536 & $16: 29: 36$ & 0.4158 \\
\hline 5537 & $16: 29: 37$ & 0.4133 \\
\hline 5538 & $16: 29: 38$ & 0.4232 \\
\hline 5539 & $16: 29: 39$ & 0.4145 \\
\hline 5540 & $16: 29: 40$ & 0.4208 \\
\hline 5541 & $16: 29: 41$ & 0.4195 \\
\hline 5542 & $16: 29: 42$ & 0.4201 \\
\hline 5543 & $16: 29: 43$ & 0.4244 \\
\hline 5544 & $16: 29: 44$ & 0.4164 \\
\hline 5545 & $16: 29: 45$ & 0.4238 \\
\hline 5546 & $16: 29: 46$ & 0.4122 \\
\hline 5547 & $16: 29: 47$ & 0.4180 \\
\hline 5548 & $16: 29: 48$ & 0.4119 \\
\hline 5549 & $16: 29: 49$ & 0.4234 \\
\hline 5550 & $16: 29: 50$ & 0.4183 \\
\hline 5551 & $16: 29: 51$ & 0.4181 \\
\hline 5552 & $16: 29: 52$ & 0.4171 \\
\hline 5553 & $16: 29: 53$ & 0.4127 \\
\hline 5554 & $16: 29: 54$ & 0.4167 \\
\hline 5555 & $16: 29: 55$ & 0.4082 \\
\hline 5556 & $16: 29: 56$ & 0.4203 \\
\hline 5557 & $16: 29: 57$ & 0.416 \\
\hline
\end{tabular}


Appendix E -Fuel Data Test 3 NIST102605_1.xls

\begin{tabular}{|c|c|c|}
\hline 5558 & $16: 29: 58$ & 0.4210 \\
\hline 5559 & $16: 29: 59$ & 0.4197 \\
\hline 5560 & $16: 30: 00$ & 0.4170 \\
\hline 5561 & $16: 30: 01$ & 0.4256 \\
\hline 5562 & $16: 30: 02$ & 0.4157 \\
\hline 5563 & $16: 30: 03$ & 0.4235 \\
\hline 5564 & 16:30:04 & 0.4222 \\
\hline 5565 & $16: 30: 05$ & 0.4176 \\
\hline 5566 & $16: 30: 06$ & 0.4205 \\
\hline 5567 & $16: 30: 07$ & 0.4125 \\
\hline 5568 & $16: 30: 08$ & 0.4197 \\
\hline 5569 & $16: 30: 09$ & 0.4168 \\
\hline 5570 & $16: 30: 10$ & 0.4206 \\
\hline 5571 & $16: 30: 11$ & 0.4181 \\
\hline 5572 & $16: 30: 12$ & 0.4141 \\
\hline 5573 & $16: 30: 13$ & 0.4159 \\
\hline 5574 & $16: 30: 14$ & 0.4124 \\
\hline 5575 & $16: 30: 15$ & 0.4234 \\
\hline 5576 & $16: 30: 16$ & 0.4134 \\
\hline 5577 & $16: 30: 17$ & 0.4183 \\
\hline 5578 & $16: 30: 18$ & 0.4229 \\
\hline 5579 & $16: 30: 19$ & 0.4186 \\
\hline 5580 & $16: 30: 20$ & 0.4244 \\
\hline 5581 & $16: 30: 21$ & 0.4135 \\
\hline 5582 & 16:30:22 & 0.4261 \\
\hline 5583 & $16: 30: 23$ & 0.4215 \\
\hline 5584 & $16: 30: 24$ & 0.4201 \\
\hline 5585 & $16: 30: 25$ & 0.4196 \\
\hline 5586 & $16: 30: 26$ & 0.4114 \\
\hline 5587 & $16: 30: 27$ & 0.4178 \\
\hline 5588 & $16: 30: 28$ & 0.4110 \\
\hline 5589 & $16: 30: 29$ & 0.4242 \\
\hline 5590 & $16: 30: 30$ & 0.4144 \\
\hline 5591 & 16:30:31 & 0.4181 \\
\hline 5592 & $16: 30: 32$ & 0.4150 \\
\hline 5593 & $16: 30: 33$ & 0.4138 \\
\hline 5594 & $16: 30: 34$ & 0.4209 \\
\hline 5595 & $16: 30: 35$ & 0.4150 \\
\hline 5596 & $16: 30: 36$ & 0.4249 \\
\hline 5597 & $16: 30: 37$ & 0.4152 \\
\hline 5598 & $16: 30: 38$ & 0.4200 \\
\hline 5599 & $16: 30: 39$ & 0.4163 \\
\hline 5600 & $16: 30: 40$ & 0.4193 \\
\hline 5601 & $16: 30: 41$ & 0.4233 \\
\hline 5602 & $16: 30: 42$ & 0.4160 \\
\hline 5603 & $16: 30: 43$ & 0.4219 \\
\hline 5604 & $16: 30: 44$ & 0.4134 \\
\hline 5605 & $16: 30: 45$ & 0.418 \\
\hline 5606 & $16: 30: 46$ & 0.4130 \\
\hline 5607 & $16: 30: 47$ & 0.421 \\
\hline 5608 & $16: 30: 48$ & 0.417 \\
\hline
\end{tabular}


Appendix E -Fuel Data Test 3 NIST102605_1.xls

\begin{tabular}{|c|c|c|}
\hline 5609 & $16: 30: 49$ & 0.4149 \\
\hline 5610 & $16: 30: 50$ & 0.4152 \\
\hline 5611 & $16: 30: 51$ & 0.4120 \\
\hline 5612 & $16: 30: 52$ & 0.4228 \\
\hline 5613 & $16: 30: 53$ & 0.4142 \\
\hline 5614 & $16: 30: 54$ & 0.4223 \\
\hline 5615 & $16: 30: 55$ & 0.4166 \\
\hline 5616 & $16: 30: 56$ & 0.4200 \\
\hline 5617 & $16: 30: 57$ & 0.4154 \\
\hline 5618 & $16: 30: 58$ & 0.4170 \\
\hline 5619 & $16: 30: 59$ & 0.4234 \\
\hline 5620 & $16: 31: 00$ & 0.4174 \\
\hline 5621 & $16: 31: 01$ & 0.4228 \\
\hline 5622 & $16: 31: 02$ & 0.4143 \\
\hline 5623 & $16: 31: 03$ & 0.4182 \\
\hline 5624 & $16: 31: 04$ & 0.4196 \\
\hline 5625 & $16: 31: 05$ & 0.4130 \\
\hline 5626 & $16: 31: 06$ & 0.4196 \\
\hline 5627 & $16: 31: 07$ & 0.4130 \\
\hline 5628 & $16: 31: 08$ & 0.4168 \\
\hline 5629 & 16:31:09 & 0.4094 \\
\hline 5630 & $16: 31: 10$ & 0.4201 \\
\hline 5631 & $16: 31: 11$ & 0.4136 \\
\hline 5632 & $16: 31: 12$ & 0.4210 \\
\hline 5633 & $16: 31: 13$ & 0.4159 \\
\hline 5634 & $16: 31: 14$ & 0.4216 \\
\hline 5635 & $16: 31: 15$ & 0.4173 \\
\hline 5636 & $16: 31: 16$ & 0.4212 \\
\hline 5637 & $16: 31: 17$ & 0.4193 \\
\hline 5638 & $16: 31: 18$ & 0.4230 \\
\hline 5639 & $16: 31: 19$ & 0.4240 \\
\hline 5640 & $16: 31: 20$ & 0.4107 \\
\hline 5641 & $16: 31: 21$ & 0.4187 \\
\hline 5642 & $16: 31: 22$ & 0.4150 \\
\hline 5643 & $16: 31: 23$ & 0.4178 \\
\hline 5644 & $16: 31: 24$ & 0.4174 \\
\hline 5645 & $16: 31: 25$ & 0.4148 \\
\hline 5646 & $16: 31: 26$ & 0.4172 \\
\hline 5647 & $16: 31: 27$ & 0.4139 \\
\hline 5648 & $16: 31: 28$ & 0.4170 \\
\hline 5649 & $16: 31: 29$ & 0.4117 \\
\hline 5650 & $16: 31: 30$ & 0.4221 \\
\hline 5651 & $16: 31: 31$ & 0.4138 \\
\hline 5652 & $16: 31: 32$ & 0.4171 \\
\hline 5653 & $16: 31: 33$ & 0.4204 \\
\hline 5654 & $16: 31: 34$ & 0.4201 \\
\hline 5655 & $16: 31: 35$ & 0.4247 \\
\hline 5656 & $16: 31: 36$ & 0.4172 \\
\hline 5657 & $16: 31: 37$ & 0.4256 \\
\hline 5658 & $16: 31: 38$ & 0.413 \\
\hline 5659 & $16: 31: 39$ & 0.417 \\
\hline
\end{tabular}


Appendix E -Fuel Data Test 3 NIST102605_1.xls

\begin{tabular}{|c|c|c|}
\hline 5660 & $16: 31: 40$ & 0.4203 \\
\hline 5661 & $16: 31: 41$ & 0.4145 \\
\hline 5662 & $16: 31: 42$ & 0.4180 \\
\hline 5663 & $16: 31: 43$ & 0.4121 \\
\hline 5664 & $16: 31: 44$ & 0.4234 \\
\hline 5665 & $16: 31: 45$ & 0.4125 \\
\hline 5666 & $16: 31: 46$ & 0.4196 \\
\hline 5667 & $16: 31: 47$ & 0.4128 \\
\hline 5668 & $16: 31: 48$ & 0.4194 \\
\hline 5669 & $16: 31: 49$ & 0.4142 \\
\hline 5670 & $16: 31: 50$ & 0.4164 \\
\hline 5671 & $16: 31: 51$ & 0.4168 \\
\hline 5672 & $16: 31: 52$ & 0.4188 \\
\hline 5673 & $16: 31: 53$ & 0.4258 \\
\hline 5674 & $16: 31: 54$ & 0.4148 \\
\hline 5675 & $16: 31: 55$ & 0.4218 \\
\hline 5676 & $16: 31: 56$ & 0.4147 \\
\hline 5677 & $16: 31: 57$ & 0.4206 \\
\hline 5678 & $16: 31: 58$ & 0.4149 \\
\hline 5679 & $16: 31: 59$ & 0.4190 \\
\hline 5680 & $16: 32: 00$ & 0.4179 \\
\hline 5681 & $16: 32: 01$ & 0.4129 \\
\hline 5682 & $16: 32: 02$ & 0.4163 \\
\hline 5683 & $16: 32: 03$ & 0.4139 \\
\hline 5684 & $16: 32: 04$ & 0.4212 \\
\hline 5685 & $16: 32: 05$ & 0.4118 \\
\hline 5686 & 16:32:06 & 0.4149 \\
\hline 5687 & $16: 32: 07$ & 0.4155 \\
\hline 5688 & $16: 32: 08$ & 0.4160 \\
\hline 5689 & $16: 32: 09$ & 0.4220 \\
\hline 5690 & $16: 32: 10$ & 0.4156 \\
\hline 5691 & $16: 32: 11$ & 0.4289 \\
\hline 5692 & $16: 32: 12$ & 0.4157 \\
\hline 5693 & $16: 32: 13$ & 0.4170 \\
\hline 5694 & $16: 32: 14$ & 0.4192 \\
\hline 5695 & $16: 32: 15$ & 0.4145 \\
\hline 5696 & $16: 32: 16$ & 0.4209 \\
\hline 5697 & $16: 32: 17$ & 0.4139 \\
\hline 5698 & $16: 32: 18$ & 0.4193 \\
\hline 5699 & $16: 32: 19$ & 0.4186 \\
\hline 5700 & $16: 32: 20$ & 0.4125 \\
\hline 5701 & $16: 32: 21$ & 0.4209 \\
\hline 5702 & $16: 32: 22$ & 0.4108 \\
\hline 5703 & $16: 32: 23$ & 0.4170 \\
\hline 5704 & $16: 32: 24$ & 0.4142 \\
\hline 5705 & $16: 32: 25$ & 0.4149 \\
\hline 5706 & $16: 32: 26$ & 0.4172 \\
\hline 5707 & $16: 32: 27$ & 0.4153 \\
\hline 5708 & $16: 32: 28$ & 0.4224 \\
\hline 5709 & $16: 32: 29$ & 0.416 \\
\hline 571 & $16: 32: 30$ & 0.422 \\
\hline
\end{tabular}


Appendix E -Fuel Data Test 3 NIST102605_1.xls

\begin{tabular}{|c|c|c|}
\hline 5711 & $16: 32: 31$ & 0.4143 \\
\hline 5712 & $16: 32: 32$ & 0.4225 \\
\hline 5713 & $16: 32: 33$ & 0.4240 \\
\hline 5714 & $16: 32: 34$ & 0.4161 \\
\hline 5715 & $16: 32: 35$ & 0.4180 \\
\hline 5716 & $16: 32: 36$ & 0.4155 \\
\hline 5717 & $16: 32: 37$ & 0.4229 \\
\hline 5718 & $16: 32: 38$ & 0.4136 \\
\hline 5719 & $16: 32: 39$ & 0.4195 \\
\hline 5720 & $16: 32: 40$ & 0.4156 \\
\hline 5721 & $16: 32: 41$ & 0.4133 \\
\hline 5722 & $16: 32: 42$ & 0.4136 \\
\hline 5723 & $16: 32: 43$ & 0.4120 \\
\hline 5724 & $16: 32: 44$ & 0.4217 \\
\hline 5725 & $16: 32: 45$ & 0.4153 \\
\hline 5726 & $16: 32: 46$ & 0.4216 \\
\hline 5727 & $16: 32: 47$ & 0.4161 \\
\hline 5728 & $16: 32: 48$ & 0.4190 \\
\hline 5729 & $16: 32: 49$ & 0.4154 \\
\hline 5730 & $16: 32: 50$ & 0.4154 \\
\hline 5731 & $16: 32: 51$ & 0.4248 \\
\hline 5732 & $16: 32: 52$ & 0.4162 \\
\hline 5733 & $16: 32: 53$ & 0.4203 \\
\hline 5734 & $16: 32: 54$ & 0.4154 \\
\hline 5735 & $16: 32: 55$ & 0.4226 \\
\hline 5736 & $16: 32: 56$ & 0.4139 \\
\hline 5737 & $16: 32: 57$ & 0.4158 \\
\hline 5738 & $16: 32: 58$ & 0.4161 \\
\hline 5739 & $16: 32: 59$ & 0.4101 \\
\hline 5740 & $16: 33: 00$ & 0.4189 \\
\hline 5741 & 16:33:01 & 0.4134 \\
\hline 5742 & 16:33:02 & 0.4214 \\
\hline 5743 & $16: 33: 03$ & 0.4152 \\
\hline 5744 & 16:33:04 & 0.4215 \\
\hline 5745 & $16: 33: 05$ & 0.4123 \\
\hline 5746 & $16: 33: 06$ & 0.4207 \\
\hline 5747 & $16: 33: 07$ & 0.4185 \\
\hline 5748 & $16: 33: 08$ & 0.4165 \\
\hline 5749 & $16: 33: 09$ & 0.4229 \\
\hline 5750 & $16: 33: 10$ & 0.4169 \\
\hline 5751 & $16: 33: 11$ & 0.4200 \\
\hline 5752 & $16: 33: 12$ & 0.4077 \\
\hline 5753 & $16: 33: 13$ & 0.4182 \\
\hline 5754 & $16: 33: 14$ & 0.4152 \\
\hline 5755 & $16: 33: 15$ & 0.4180 \\
\hline 5756 & $16: 33: 16$ & 0.4161 \\
\hline 5757 & $16: 33: 17$ & 0.4119 \\
\hline 5758 & 16:33:18 & 0.4237 \\
\hline 5759 & $16: 33: 19$ & 0.4139 \\
\hline 5760 & $16: 33: 20$ & 0.4149 \\
\hline 5761 & $16: 33: 21$ & 0.417 \\
\hline
\end{tabular}


Appendix E -Fuel Data Test 3 NIST102605_1.xls

\begin{tabular}{lll}
5762 & $16: 33: 22$ & 0.4148 \\
5763 & $16: 33: 23$ & 0.4228 \\
5764 & $16: 33: 24$ & 0.4123 \\
5765 & $16: 33: 25$ & 0.4193 \\
5766 & $16: 33: 26$ & 0.4171 \\
5767 & $16: 33: 27$ & 0.4168 \\
5768 & $16: 33: 28$ & 0.4237 \\
5769 & $16: 33: 29$ & 0.4179 \\
5770 & $16: 33: 30$ & 0.4210 \\
5771 & $16: 33: 31$ & 0.4122 \\
5772 & $16: 33: 32$ & 0.4219 \\
5773 & $16: 33: 33$ & 0.4139 \\
5774 & $16: 33: 34$ & 0.4178 \\
5775 & $16: 33: 35$ & 0.4165 \\
5776 & $16: 33: 36$ & 0.4186 \\
5777 & $16: 33: 37$ & 0.4176 \\
5778 & $16: 33: 38$ & 0.4097 \\
5779 & $16: 33: 39$ & 0.4216 \\
5780 & $16: 33: 40$ & 0.4134 \\
5781 & $16: 33: 41$ & 0.4193 \\
5782 & $16: 33: 42$ & 0.4185 \\
5783 & $16: 33: 43$ & 0.4185 \\
5784 & $16: 33: 44$ & 0.4277 \\
5785 & $16: 33: 45$ & 0.4188 \\
5786 & $16: 33: 46$ & 0.4213 \\
5787 & $16: 33: 47$ & 0.4252 \\
5788 & $16: 33: 48$ & 0.4112 \\
5789 & $16: 33: 49$ & 0.4192 \\
5790 & $16: 33: 50$ & 0.4148 \\
5791 & $16: 33: 51$ & 0.4156 \\
5792 & $16: 33: 52$ & 0.4169 \\
5793 & $16: 33: 53$ & 0.4141 \\
5794 & $16: 33: 54$ & 0.4175 \\
5795 & $16: 33: 55$ & 0.4094 \\
5796 & $16: 33: 56$ & 0.4142 \\
5797 & $16: 33: 57$ & 0.2861 \\
5798 & $16: 33: 58$ & 0.0792 \\
& & \\
\hline
\end{tabular}


APPENDIX F. - Laser Data Test 3 NIST 102605

Appendix $\mathrm{F}$ is laser data from Test 3 in tabular format and is approximately 57 pages in length. Fuel data is included with electronic version of report on compact disk, but not routinely included with paper version of report. 
Appendix F - Laser Data Test 3 NIST102605_1.xls

Time from Ignition, s EDT Time $\quad$ //lo East $\quad$ //lo West

\begin{tabular}{rlll}
0 & $14: 57: 20$ & 1.0234 & 0.9917 \\
2 & $14: 57: 22$ & 1.0084 & 0.9870 \\
4 & $14: 57: 24$ & 0.9760 & 0.8723 \\
6 & $14: 57: 26$ & 0.8878 & 0.9385 \\
8 & $14: 57: 28$ & 0.8200 & 0.8716 \\
10 & $14: 57: 30$ & 0.8676 & 0.8390 \\
12 & $14: 57: 32$ & 0.8012 & 0.6838 \\
14 & $14: 57: 34$ & 0.7114 & 0.7196 \\
16 & $14: 57: 36$ & 0.8123 & 0.7518 \\
18 & $14: 57: 38$ & 0.7224 & 0.7272 \\
20 & $14: 57: 40$ & 0.4975 & 0.4821 \\
22 & $14: 57: 42$ & 0.5737 & 0.5865 \\
24 & $14: 57: 44$ & 0.4977 & 0.4429 \\
26 & $14: 57: 46$ & 0.6795 & 0.7785 \\
28 & $14: 57: 48$ & 0.4713 & 0.6699 \\
30 & $14: 57: 50$ & 0.4754 & 0.4635 \\
32 & $14: 57: 52$ & 0.3699 & 0.3951 \\
34 & $14: 57: 54$ & 0.3890 & 0.4210 \\
36 & $14: 57: 56$ & 0.4641 & 0.3506 \\
38 & $14: 57: 58$ & 0.3097 & 0.4283 \\
40 & $14: 58: 00$ & 0.3958 & 0.3012 \\
42 & $14: 58: 02$ & 0.3433 & 0.2966 \\
44 & $14: 58: 04$ & 0.3862 & 0.2399 \\
46 & $14: 58: 06$ & 0.1482 & 0.2430 \\
48 & $14: 58: 08$ & 0.1942 & 0.2264 \\
50 & $14: 58: 10$ & 0.1338 & 0.2130 \\
52 & $14: 58: 12$ & 0.1914 & 0.2003 \\
54 & $14: 58: 14$ & 0.3217 & 0.3218 \\
56 & $14: 58: 16$ & 0.1693 & 0.3260 \\
58 & $14: 58: 18$ & 0.2158 & 0.1566 \\
60 & $14: 58: 20$ & 0.1234 & 0.1464 \\
62 & $14: 58: 22$ & 0.1504 & 0.0758 \\
64 & $14: 58: 24$ & 0.2482 & 0.1393 \\
66 & $14: 58: 26$ & 0.2047 & 0.0673 \\
68 & $14: 58: 28$ & 0.0875 & 0.2664 \\
70 & $14: 58: 30$ & 0.1022 & 0.2049 \\
72 & $14: 58: 32$ & 0.1180 & 0.1244 \\
74 & $14: 58: 34$ & 0.1367 & 0.2251 \\
76 & $14: 58: 36$ & 0.1968 & 0.1538 \\
78 & $14: 58: 38$ & 0.0549 & 0.1005 \\
80 & $14: 58: 40$ & 0.1222 & 0.0774 \\
82 & $14: 58: 42$ & 0.0751 & 0.1769 \\
84 & $14: 58: 44$ & 0.0866 & 0.1140 \\
86 & $14: 58: 46$ & 0.1257 & 0.1224 \\
88 & $14: 58: 48$ & 0.0127 & 0.1484 \\
90 & $14: 58: 50$ & 0.0671 & 0.0750 \\
92 & $14: 58: 52$ & 0.1366 & 0.0492 \\
94 & $14: 58: 54$ & 0.2407 & 0.1666 \\
98 & $14: 58: 56$ & 0.0896 & 0.1397 \\
& $14: 58: 58$ & 0.1543 & 0.0662 \\
& & & \\
\hline
\end{tabular}


Appendix F - Laser Data Test 3 NIST102605_1.xls

$\begin{array}{llll}100 & 14: 59: 00 & 0.0528 & 0.0295 \\ 102 & 14: 59: 02 & 0.0370 & 0.0328 \\ 104 & 14: 59: 04 & 0.0163 & 0.0311 \\ 106 & 14: 59: 06 & 0.0353 & 0.0193 \\ 108 & 14: 59: 08 & 0.0592 & 0.0300 \\ 110 & 14: 59: 10 & 0.0590 & 0.0746 \\ 112 & 14: 59: 12 & 0.0249 & 0.0137 \\ 114 & 14: 59: 14 & 0.0702 & 0.0362 \\ 116 & 14: 59: 16 & 0.0259 & 0.2175 \\ 118 & 14: 59: 18 & 0.1005 & 0.1986 \\ 120 & 14: 59: 20 & 0.0199 & 0.0806 \\ 122 & 14: 59: 22 & 0.0360 & 0.0548 \\ 124 & 14: 59: 24 & 0.0089 & 0.0121 \\ 126 & 14: 59: 26 & 0.0079 & 0.0592 \\ 128 & 14: 59: 28 & 0.0122 & 0.0102 \\ 130 & 14: 59: 30 & 0.0172 & 0.0178 \\ 132 & 14: 59: 32 & 0.0465 & 0.0094 \\ 134 & 14: 59: 34 & 0.0154 & 0.0125 \\ 136 & 14: 59: 36 & 0.0150 & 0.0196 \\ 138 & 14: 59: 38 & 0.0035 & 0.0037 \\ 140 & 14: 59: 40 & 0.0433 & 0.0079 \\ 142 & 14: 59: 42 & 0.0044 & 0.0138 \\ 144 & 14: 59: 44 & 0.0049 & 0.1280 \\ 146 & 14: 59: 46 & 0.0141 & 0.0089 \\ 148 & 14: 59: 48 & 0.0120 & 0.0234 \\ 150 & 14: 59: 50 & 0.0041 & 0.0150 \\ 152 & 14: 59: 52 & 0.0102 & 0.0040 \\ 154 & 14: 59: 54 & 0.0056 & 0.0034 \\ 156 & 14: 59: 56 & 0.0261 & 0.0069 \\ 158 & 14: 59: 58 & 0.0122 & 0.0101 \\ 160 & 15: 00: 00 & 0.0015 & 0.0069 \\ 162 & 15: 00: 02 & 0.0040 & 0.0480 \\ 164 & 15: 00: 04 & 0.0065 & 0.0042 \\ 166 & 15: 00: 06 & 0.0025 & 0.0013 \\ 168 & 15: 00: 08 & 0.0095 & 0.0091 \\ 170 & 15: 00: 10 & 0.0389 & 0.0044 \\ 172 & 15: 00: 12 & 0.0037 & 0.0096 \\ 174 & 15: 00: 14 & 0.0499 & 0.0084 \\ 176 & 15: 00: 16 & 0.0158 & 0.0047 \\ 178 & 15: 00: 18 & 0.0170 & 0.0019 \\ 180 & 15: 00: 20 & 0.0103 & 0.0027 \\ 182 & 15: 00: 22 & 0.0026 & 0.0056 \\ 184 & 15: 00: 24 & 0.0161 & 0.0138 \\ 186 & 15: 00: 26 & 0.0045 & 0.0008 \\ 188 & 15: 00: 28 & 0.0029 & 0.0006 \\ 190 & 15: 00: 30 & 0.0146 & 0.0072 \\ 192 & 15: 00: 32 & 0.0020 & 0.0007 \\ 194 & 15: 00: 34 & 0.0041 & 0.0012 \\ 196 & 15: 00: 36 & 0.0092 & 0.0057 \\ 198 & 15: 00: 38 & 0.0180 & 0.0970 \\ 200 & 15: 00: 40 & 0.0097 & 0.1144\end{array}$

F - 2 
Appendix F - Laser Data Test 3 NIST102605_1.xls

$\begin{array}{llll}202 & 15: 00: 42 & 0.0018 & 0.0086 \\ 204 & 15: 00: 44 & 0.0025 & 0.0064 \\ 206 & 15: 00: 46 & 0.0146 & 0.0072 \\ 208 & 15: 00: 48 & 0.0013 & 0.0028 \\ 210 & 15: 00: 50 & 0.0004 & 0.0036 \\ 212 & 15: 00: 52 & 0.0003 & 0.0012 \\ 214 & 15: 00: 54 & 0.0061 & 0.0026 \\ 216 & 15: 00: 56 & 0.0073 & 0.0304 \\ 218 & 15: 00: 58 & 0.0089 & 0.0020 \\ 220 & 15: 01: 00 & 0.0124 & 0.0039 \\ 222 & 15: 01: 02 & 0.0024 & 0.0111 \\ 224 & 15: 01: 04 & 0.0050 & 0.0060 \\ 226 & 15: 01: 06 & 0.0017 & 0.0037 \\ 228 & 15: 01: 08 & 0.0179 & 0.0019 \\ 230 & 15: 01: 10 & 0.0314 & 0.0046 \\ 232 & 15: 01: 12 & 0.0252 & 0.0017 \\ 234 & 15: 01: 14 & 0.0155 & 0.0022 \\ 236 & 15: 01: 16 & 0.0063 & 0.0039 \\ 238 & 15: 01: 18 & 0.0078 & 0.0060 \\ 240 & 15: 01: 20 & 0.0008 & 0.0057 \\ 242 & 15: 01: 22 & 0.0115 & 0.0071 \\ 244 & 15: 01: 24 & 0.0092 & 0.0002 \\ 246 & 15: 01: 26 & 0.0481 & 0.0030 \\ 248 & 15: 01: 28 & 0.0092 & 0.0022 \\ 250 & 15: 01: 30 & 0.0008 & 0.0012 \\ 252 & 15: 01: 32 & 0.0076 & 0.0082 \\ 254 & 15: 01: 34 & 0.0098 & 0.0004 \\ 256 & 15: 01: 36 & 0.0089 & 0.0211 \\ 258 & 15: 01: 38 & 0.0099 & 0.0031 \\ 260 & 15: 01: 40 & 0.0141 & 0.0067 \\ 262 & 15: 01: 42 & 0.0269 & 0.0181 \\ 264 & 15: 01: 44 & 0.0033 & 0.0012 \\ 266 & 15: 01: 46 & 0.0081 & 0.0122 \\ 268 & 15: 01: 48 & 0.0363 & 0.0071 \\ 270 & 15: 01: 50 & 0.0213 & 0.0031 \\ 272 & 15: 01: 52 & 0.0078 & 0.0022 \\ 274 & 15: 01: 54 & 0.0093 & 0.0002 \\ 276 & 15: 01: 56 & 0.0021 & 0.0040 \\ 278 & 15: 01: 58 & 0.0070 & 0.0135 \\ 280 & 15: 02: 00 & 0.0115 & 0.0015 \\ 282 & 15: 02: 02 & 0.0213 & 0.0062 \\ 284 & 15: 02: 04 & 0.0120 & 0.0052 \\ 286 & 15: 02: 06 & 0.0045 & 0.0070 \\ 288 & 15: 02: 08 & 0.0212 & 0.0127 \\ 290 & 15: 02: 10 & 0.0456 & 0.0003 \\ 292 & 15: 02: 12 & 0.0183 & 0.0030 \\ 294 & 15: 02: 14 & 0.1079 & 0.0091 \\ 296 & 15: 02: 16 & 0.0171 & 0.0012 \\ 298 & 15: 02: 18 & 0.0006 & 0.0222 \\ 300 & 15: 02: 20 & 0.0131 & 0.0042 \\ 302 & 15: 02: 22 & 0.0745 & 0.0055\end{array}$


Appendix F - Laser Data Test 3 NIST102605_1.xls

$\begin{array}{llll}304 & 15: 02: 24 & 0.0289 & 0.0051 \\ 306 & 15: 02: 26 & 0.0960 & 0.0157 \\ 308 & 15: 02: 28 & 0.0194 & 0.0057 \\ 310 & 15: 02: 30 & 0.0096 & 0.0020 \\ 312 & 15: 02: 32 & 0.1297 & 0.0405 \\ 314 & 15: 02: 34 & 0.0085 & 0.0061 \\ 316 & 15: 02: 36 & 0.0008 & 0.0071 \\ 318 & 15: 02: 38 & 0.0425 & 0.0040 \\ 320 & 15: 02: 40 & 0.0208 & 0.0383 \\ 322 & 15: 02: 42 & 0.0268 & 0.0061 \\ 324 & 15: 02: 44 & 0.0501 & 0.0226 \\ 326 & 15: 02: 46 & 0.0074 & 0.0070 \\ 328 & 15: 02: 48 & 0.0193 & 0.0010 \\ 330 & 15: 02: 50 & 0.0320 & 0.0138 \\ 332 & 15: 02: 52 & 0.0518 & 0.0049 \\ 334 & 15: 02: 54 & 0.0302 & 0.0046 \\ 336 & 15: 02: 56 & 0.0189 & 0.0139 \\ 338 & 15: 02: 58 & 0.0314 & 0.0031 \\ 340 & 15: 03: 00 & 0.0349 & 0.0136 \\ 342 & 15: 03: 02 & 0.0012 & 0.0008 \\ 344 & 15: 03: 04 & 0.0101 & 0.0013 \\ 346 & 15: 03: 06 & 0.0396 & 0.0293 \\ 348 & 15: 03: 08 & 0.0048 & 0.0083 \\ 350 & 15: 03: 10 & 0.0049 & 0.0053 \\ 352 & 15: 03: 12 & 0.0114 & 0.0018 \\ 354 & 15: 03: 14 & 0.0139 & 0.0068 \\ 356 & 15: 03: 16 & 0.0298 & 0.0051 \\ 358 & 15: 03: 18 & 0.0236 & 0.0388 \\ 360 & 15: 03: 20 & 0.0055 & 0.0066 \\ 362 & 15: 03: 22 & 0.0206 & 0.0079 \\ 364 & 15: 03: 24 & 0.0170 & 0.0159 \\ 366 & 15: 03: 26 & 0.0035 & 0.0139 \\ 368 & 15: 03: 28 & 0.0104 & 0.0307 \\ 370 & 15: 03: 30 & 0.0184 & 0.0029 \\ 372 & 15: 03: 32 & 0.0288 & 0.0021 \\ 374 & 15: 03: 34 & 0.0132 & 0.0073 \\ 376 & 15: 03: 36 & 0.0252 & 0.0033 \\ 378 & 15: 03: 38 & 0.0173 & 0.0349 \\ 380 & 15: 03: 40 & 0.0207 & 0.0159 \\ 382 & 15: 03: 42 & 0.0023 & 0.0069 \\ 384 & 15: 03: 44 & 0.0145 & 0.0160 \\ 386 & 15: 03: 46 & 0.0136 & 0.0076 \\ 388 & 15: 03: 48 & 0.0219 & 0.0041 \\ 390 & 15: 03: 50 & 0.0191 & 0.0278 \\ 392 & 15: 03: 52 & 0.0279 & 0.0312 \\ 394 & 15: 03: 54 & 0.0243 & 0.0154 \\ 396 & 15: 03: 56 & 0.0095 & 0.0689 \\ 398 & 15: 03: 58 & 0.0219 & 0.0059 \\ 400 & 15: 04: 00 & 0.0148 & 0.0038 \\ 402 & 15: 04: 02 & 0.0550 & 0.0065 \\ 404 & 15: 04: 04 & 0.0191 & 0.0031\end{array}$


Appendix F - Laser Data Test 3 NIST102605_1.xIs

$\begin{array}{llll}406 & 15: 04: 06 & 0.1017 & 0.0155 \\ 408 & 15: 04: 08 & 0.0129 & 0.0060 \\ 410 & 15: 04: 10 & 0.0148 & 0.0067 \\ 412 & 15: 04: 12 & 0.0106 & 0.0398 \\ 414 & 15: 04: 14 & 0.1068 & 0.0107 \\ 416 & 15: 04: 16 & 0.0170 & 0.0120 \\ 418 & 15: 04: 18 & 0.0321 & 0.0225 \\ 420 & 15: 04: 20 & 0.0080 & 0.0072 \\ 422 & 15: 04: 22 & 0.0075 & 0.0017 \\ 424 & 15: 04: 24 & 0.0120 & 0.0246 \\ 426 & 15: 04: 26 & 0.0186 & 0.0029 \\ 428 & 15: 04: 28 & 0.0116 & 0.0162 \\ 430 & 15: 04: 30 & 0.0047 & 0.0013 \\ 432 & 15: 04: 32 & 0.0154 & 0.0265 \\ 434 & 15: 04: 34 & 0.0075 & 0.0061 \\ 436 & 15: 04: 36 & 0.0048 & 0.0004 \\ 438 & 15: 04: 38 & 0.0051 & 0.0008 \\ 440 & 15: 04: 40 & 0.0105 & 0.0034 \\ 442 & 15: 04: 42 & 0.0036 & 0.0016 \\ 444 & 15: 04: 44 & 0.0061 & 0.0144 \\ 446 & 15: 04: 46 & 0.0049 & 0.0339 \\ 448 & 15: 04: 48 & 0.0003 & 0.0147 \\ 450 & 15: 04: 50 & 0.0008 & 0.0048 \\ 452 & 15: 04: 52 & 0.0044 & 0.0019 \\ 454 & 15: 04: 54 & 0.0038 & 0.0022 \\ 456 & 15: 04: 56 & 0.0222 & 0.0084 \\ 458 & 15: 04: 58 & 0.0093 & 0.0253 \\ 460 & 15: 05: 00 & 0.0003 & 0.0002 \\ 462 & 15: 05: 02 & 0.0040 & 0.0184 \\ 464 & 15: 05: 04 & 0.0007 & 0.0045 \\ 466 & 15: 05: 06 & 0.0011 & 0.0039 \\ 468 & 15: 05: 08 & 0.0017 & 0.0142 \\ 470 & 15: 05: 10 & 0.0139 & 0.0028 \\ 472 & 15: 05: 12 & 0.0078 & 0.0018 \\ 474 & 15: 05: 14 & 0.0056 & 0.0026 \\ 476 & 15: 05: 16 & 0.0010 & 0.0069 \\ 478 & 15: 05: 18 & 0.0016 & 0.0007 \\ 480 & 15: 05: 20 & 0.0003 & 0.0010 \\ 482 & 15: 05: 22 & 0.0010 & 0.0095 \\ 484 & 15: 05: 24 & 0.0014 & 0.0077 \\ 486 & 15: 05: 26 & 0.0009 & 0.0016 \\ 488 & 15: 05: 28 & 0.0017 & 0.0097 \\ 490 & 15: 05: 30 & 0.0036 & 0.0030 \\ 492 & 15: 05: 32 & 0.0013 & 0.0047 \\ 494 & 15: 05: 34 & 0.0020 & 0.0036 \\ 496 & 15: 05: 36 & 0.0008 & 0.0006 \\ 498 & 15: 05: 38 & 0.0002 & 0.0099 \\ 500 & 15: 05: 40 & 0.0006 & 0.0056 \\ 502 & 15: 05: 42 & 0.0009 & 0.0062 \\ 504 & 15: 05: 44 & 0.0054 & 0.0015 \\ 506 & 15: 05: 46 & 0.0108 & 0.0006\end{array}$


Appendix F - Laser Data Test 3 NIST102605_1.xIs

$\begin{array}{llll}508 & 15: 05: 48 & 0.0015 & 0.0031 \\ 510 & 15: 05: 50 & 0.0054 & 0.0013 \\ 512 & 15: 05: 52 & 0.0083 & 0.0008 \\ 514 & 15: 05: 54 & 0.0024 & 0.0020 \\ 516 & 15: 05: 56 & 0.0003 & 0.0047 \\ 518 & 15: 05: 58 & 0.0012 & 0.0117 \\ 520 & 15: 06: 00 & 0.0003 & 0.0008 \\ 522 & 15: 06: 02 & 0.0001 & 0.0006 \\ 524 & 15: 06: 04 & 0.0003 & 0.0043 \\ 526 & 15: 06: 06 & 0.0029 & 0.0044 \\ 528 & 15: 06: 08 & 0.0081 & 0.0038 \\ 530 & 15: 06: 10 & 0.0100 & 0.0012 \\ 532 & 15: 06: 12 & 0.0021 & 0.0008 \\ 534 & 15: 06: 14 & 0.0009 & 0.0237 \\ 536 & 15: 06: 16 & 0.0032 & 0.0045 \\ 538 & 15: 06: 18 & 0.0026 & 0.0021 \\ 540 & 15: 06: 20 & 0.0001 & 0.0003 \\ 542 & 15: 06: 22 & 0.0091 & 0.0043 \\ 544 & 15: 06: 24 & 0.0010 & 0.0022 \\ 546 & 15: 06: 26 & 0.0014 & 0.0013 \\ 548 & 15: 06: 28 & 0.0003 & 0.0003 \\ 550 & 15: 06: 30 & 0.0008 & 0.0008 \\ 552 & 15: 06: 32 & 0.0038 & 0.0014 \\ 554 & 15: 06: 34 & 0.0024 & 0.0001 \\ 556 & 15: 06: 36 & 0.0061 & 0.0007 \\ 558 & 15: 06: 38 & 0.0068 & 0.0029 \\ 560 & 15: 06: 40 & 0.0196 & 0.0003 \\ 562 & 15: 06: 42 & 0.0014 & 0.0042 \\ 564 & 15: 06: 44 & 0.0003 & 0.0033 \\ 566 & 15: 06: 46 & 0.0003 & 0.0003 \\ 568 & 15: 06: 48 & 0.0015 & 0.0011 \\ 570 & 15: 06: 50 & 0.0027 & 0.0049 \\ 572 & 15: 06: 52 & 0.0015 & 0.0010 \\ 574 & 15: 06: 54 & 0.0013 & 0.0004 \\ 576 & 15: 06: 56 & 0.0015 & 0.0038 \\ 578 & 15: 06: 58 & 0.0032 & 0.0078 \\ 580 & 15: 07: 00 & 0.0025 & 0.0002 \\ 582 & 15: 07: 02 & 0.0151 & 0.0001 \\ 584 & 15: 07: 04 & 0.0070 & 0.0003 \\ 586 & 15: 07: 06 & 0.0016 & 0.0003 \\ 588 & 15: 07: 08 & 0.0005 & 0.0025 \\ 590 & 15: 07: 10 & 0.0025 & 0.0023 \\ 592 & 15: 07: 12 & 0.0004 & 0.0004 \\ 594 & 15: 07: 14 & 0.0023 & 0.0003 \\ 596 & 15: 07: 16 & 0.0015 & 0.0005 \\ 598 & 15: 07: 18 & 0.0086 & 0.0007 \\ 600 & 15: 07: 20 & 0.0011 & 0.0003 \\ 602 & 15: 07: 22 & 0.0006 & 0.0009 \\ 604 & 15: 07: 24 & 0.0163 & 0.0045 \\ 606 & 15: 07: 26 & 0.0026 & 0.0073 \\ 608 & 15: 07: 28 & 0.0016 & 0.0004\end{array}$


Appendix F - Laser Data Test 3 NIST102605_1.xls

$\begin{array}{llll}610 & 15: 07: 30 & 0.0004 & 0.0004 \\ 612 & 15: 07: 32 & 0.1202 & 0.0020 \\ 614 & 15: 07: 34 & 0.0011 & 0.0007 \\ 616 & 15: 07: 36 & 0.0019 & 0.0014 \\ 618 & 15: 07: 38 & 0.0009 & 0.0676 \\ 620 & 15: 07: 40 & 0.0003 & 0.0003 \\ 622 & 15: 07: 42 & 0.0020 & 0.0008 \\ 624 & 15: 07: 44 & 0.0007 & 0.0006 \\ 626 & 15: 07: 46 & 0.0074 & 0.0016 \\ 628 & 15: 07: 48 & 0.0011 & 0.0019 \\ 630 & 15: 07: 50 & 0.0068 & 0.0011 \\ 632 & 15: 07: 52 & 0.0020 & 0.0010 \\ 634 & 15: 07: 54 & 0.0010 & 0.0009 \\ 636 & 15: 07: 56 & 0.0020 & 0.0210 \\ 638 & 15: 07: 58 & 0.0061 & 0.0014 \\ 640 & 15: 08: 00 & 0.0004 & 0.0004 \\ 642 & 15: 08: 02 & 0.0175 & 0.0044 \\ 644 & 15: 08: 04 & 0.0028 & 0.0000 \\ 646 & 15: 08: 06 & 0.0008 & 0.0013 \\ 648 & 15: 08: 08 & 0.0089 & 0.0034 \\ 650 & 15: 08: 10 & 0.0021 & 0.0022 \\ 652 & 15: 08: 12 & 0.0023 & 0.0046 \\ 654 & 15: 08: 14 & 0.0059 & 0.0008 \\ 656 & 15: 08: 16 & 0.0037 & 0.0008 \\ 658 & 15: 08: 18 & 0.0096 & 0.0090 \\ 660 & 15: 08: 20 & 0.0006 & 0.0008 \\ 662 & 15: 08: 22 & 0.0010 & 0.0034 \\ 664 & 15: 08: 24 & 0.0005 & 0.0001 \\ 666 & 15: 08: 26 & 0.0013 & 0.0020 \\ 668 & 15: 08: 28 & 0.0011 & 0.0003 \\ 670 & 15: 08: 30 & 0.0031 & 0.0011 \\ 672 & 15: 08: 32 & 0.0029 & 0.0003 \\ 674 & 15: 08: 34 & 0.0033 & 0.0001 \\ 676 & 15: 08: 36 & 0.0001 & 0.0010 \\ 678 & 15: 08: 38 & 0.0005 & 0.0066 \\ 680 & 15: 08: 40 & 0.0037 & 0.0181 \\ 682 & 15: 08: 42 & 0.0015 & 0.0022 \\ 684 & 15: 08: 44 & 0.0035 & 0.0005 \\ 686 & 15: 08: 46 & 0.0003 & 0.0009 \\ 688 & 15: 08: 48 & 0.0029 & 0.0003 \\ 690 & 15: 08: 50 & 0.0005 & 0.0003 \\ 692 & 15: 08: 52 & 0.0028 & 0.0010 \\ 694 & 15: 08: 54 & 0.0073 & 0.0002 \\ 696 & 15: 08: 56 & 0.0087 & 0.0008 \\ 698 & 15: 08: 58 & 0.0032 & 0.0017 \\ 700 & 15: 09: 00 & 0.0009 & 0.0003 \\ 702 & 15: 09: 02 & 0.0047 & 0.0018 \\ 704 & 15: 09: 04 & 0.0203 & 0.0025 \\ 706 & 15: 09: 06 & 0.0010 & 0.0003 \\ 708 & 15: 09: 08 & 0.0017 & 0.0012 \\ 710 & 15: 09: 10 & 0.0024 & 0.0005\end{array}$


Appendix F - Laser Data Test 3 NIST102605_1.xls

$\begin{array}{llll}712 & 15: 09: 12 & 0.0045 & 0.0181 \\ 714 & 15: 09: 14 & 0.0310 & 0.0010 \\ 716 & 15: 09: 16 & 0.0010 & 0.0083 \\ 718 & 15: 09: 18 & 0.0001 & 0.0003 \\ 720 & 15: 09: 20 & 0.0033 & 0.0019 \\ 722 & 15: 09: 22 & 0.0010 & 0.0005 \\ 724 & 15: 09: 24 & 0.0002 & 0.0011 \\ 726 & 15: 09: 26 & 0.0003 & 0.0139 \\ 728 & 15: 09: 28 & 0.0003 & 0.0001 \\ 730 & 15: 09: 30 & 0.0005 & 0.0015 \\ 732 & 15: 09: 32 & 0.0001 & 0.0044 \\ 734 & 15: 09: 34 & 0.0004 & 0.0001 \\ 736 & 15: 09: 36 & 0.0030 & 0.0030 \\ 738 & 15: 09: 38 & 0.0020 & 0.0009 \\ 740 & 15: 09: 40 & 0.0007 & 0.0018 \\ 742 & 15: 09: 42 & 0.0052 & 0.0018 \\ 744 & 15: 09: 44 & 0.0033 & 0.0003 \\ 746 & 15: 09: 46 & 0.0102 & 0.0004 \\ 748 & 15: 09: 48 & 0.0029 & 0.0008 \\ 750 & 15: 09: 50 & 0.0013 & 0.0010 \\ 752 & 15: 09: 52 & 0.0003 & 0.0004 \\ 754 & 15: 09: 54 & 0.0083 & 0.0014 \\ 756 & 15: 09: 56 & 0.0076 & 0.0004 \\ 758 & 15: 09: 58 & 0.0013 & 0.0008 \\ 760 & 15: 10: 00 & 0.0009 & 0.0010 \\ 762 & 15: 10: 02 & 0.0004 & 0.0005 \\ 764 & 15: 10: 04 & 0.0033 & 0.0140 \\ 766 & 15: 10: 06 & 0.0029 & 0.0073 \\ 768 & 15: 10: 08 & 0.0004 & 0.0002 \\ 770 & 15: 10: 10 & 0.0003 & 0.0010 \\ 772 & 15: 10: 12 & 0.0018 & 0.0006 \\ 774 & 15: 10: 14 & 0.0005 & 0.0008 \\ 776 & 15: 10: 16 & 0.0012 & 0.0009 \\ 778 & 15: 10: 18 & 0.0037 & 0.0021 \\ 780 & 15: 10: 20 & 0.0002 & 0.0003 \\ 782 & 15: 10: 22 & 0.0006 & 0.0014 \\ 784 & 15: 10: 24 & 0.0002 & 0.0004 \\ 786 & 15: 10: 26 & 0.0001 & 0.0002 \\ 788 & 15: 10: 28 & 0.0000 & 0.0007 \\ 790 & 15: 10: 30 & 0.0002 & 0.0029 \\ 792 & 15: 10: 32 & 0.0002 & 0.0002 \\ 794 & 15: 10: 34 & 0.0002 & 0.0008 \\ 796 & 15: 10: 36 & 0.0005 & 0.0045 \\ 798 & 15: 10: 38 & 0.0001 & 0.0004 \\ 800 & 15: 10: 40 & 0.0002 & 0.0004 \\ 802 & 15: 10: 42 & 0.0002 & 0.0002 \\ 804 & 15: 10: 44 & 0.0015 & 0.0003 \\ 806 & 15: 10: 46 & 0.0016 & 0.0047 \\ 808 & 15: 10: 48 & 0.0064 & 0.0024 \\ 810 & 15: 10: 50 & 0.0028 & 0.0004 \\ 812 & 15: 10: 52 & 0.0015 & 0.0006\end{array}$


Appendix F - Laser Data Test 3 NIST102605_1.xls

$\begin{array}{llll}814 & 15: 10: 54 & 0.0014 & 0.0038 \\ 816 & 15: 10: 56 & 0.0005 & 0.0013 \\ 818 & 15: 10: 58 & 0.0012 & 0.0028 \\ 820 & 15: 11: 00 & 0.0031 & 0.0013 \\ 822 & 15: 11: 02 & 0.0006 & 0.0008 \\ 824 & 15: 11: 04 & 0.0053 & 0.0004 \\ 826 & 15: 11: 06 & 0.0025 & 0.0003 \\ 828 & 15: 11: 08 & 0.0022 & 0.0003 \\ 830 & 15: 11: 10 & 0.0122 & 0.0045 \\ 832 & 15: 11: 12 & 0.0079 & 0.0058 \\ 834 & 15: 11: 14 & 0.0022 & 0.0010 \\ 836 & 15: 11: 16 & 0.0011 & 0.0026 \\ 838 & 15: 11: 18 & 0.0016 & 0.0033 \\ 840 & 15: 11: 20 & 0.0004 & 0.0477 \\ 842 & 15: 11: 22 & 0.0025 & 0.0146 \\ 844 & 15: 11: 24 & 0.0077 & 0.0144 \\ 846 & 15: 11: 26 & 0.0226 & 0.0024 \\ 848 & 15: 11: 28 & 0.0013 & 0.0041 \\ 850 & 15: 11: 30 & 0.0011 & 0.0050 \\ 852 & 15: 11: 32 & 0.0008 & 0.0047 \\ 854 & 15: 11: 34 & 0.0059 & 0.0048 \\ 856 & 15: 11: 36 & 0.0022 & 0.0018 \\ 858 & 15: 11: 38 & 0.0031 & 0.0058 \\ 860 & 15: 11: 40 & 0.0088 & 0.0020 \\ 862 & 15: 11: 42 & 0.0358 & 0.0048 \\ 864 & 15: 11: 44 & 0.0130 & 0.0062 \\ 866 & 15: 11: 46 & 0.0350 & 0.0247 \\ 868 & 15: 11: 48 & 0.0059 & 0.0071 \\ 870 & 15: 11: 50 & 0.0175 & 0.0115 \\ 872 & 15: 11: 52 & 0.0255 & 0.0092 \\ 874 & 15: 11: 54 & 0.0796 & 0.0275 \\ 876 & 15: 11: 56 & 0.0422 & 0.0009 \\ 878 & 15: 11: 58 & 0.0322 & 0.0209 \\ 880 & 15: 12: 00 & 0.0363 & 0.0451 \\ 882 & 15: 12: 02 & 0.0068 & 0.0739 \\ 884 & 15: 12: 04 & 0.0376 & 0.0156 \\ 886 & 15: 12: 06 & 0.0268 & 0.0409 \\ 888 & 15: 12: 08 & 0.0165 & 0.0295 \\ 890 & 15: 12: 10 & 0.0295 & 0.0115 \\ 892 & 15: 12: 12 & 0.0492 & 0.0452 \\ 894 & 15: 12: 14 & 0.0333 & 0.0207 \\ 896 & 15: 12: 16 & 0.0267 & 0.0454 \\ 898 & 15: 12: 18 & 0.0237 & 0.0195 \\ 900 & 15: 12: 20 & 0.0155 & 0.0475 \\ 902 & 15: 12: 22 & 0.0222 & 0.0544 \\ 904 & 15: 12: 24 & 0.0169 & 0.0343 \\ 906 & 15: 12: 26 & 0.0582 & 0.0435 \\ 908 & 15: 12: 28 & 0.0277 & 0.0358 \\ 910 & 15: 12: 30 & 0.0244 & 0.0601 \\ 912 & 15: 12: 32 & 0.1130 & 0.0401 \\ 914 & 15: 12: 34 & 0.0278 & 0.0456\end{array}$


Appendix F - Laser Data Test 3 NIST102605_1.xls

$\begin{array}{rlll}916 & 15: 12: 36 & 0.1950 & 0.0333 \\ 918 & 15: 12: 38 & 0.0256 & 0.0614 \\ 920 & 15: 12: 40 & 0.0554 & 0.0613 \\ 922 & 15: 12: 42 & 0.1845 & 0.0428 \\ 924 & 15: 12: 44 & 0.0758 & 0.0790 \\ 926 & 15: 12: 46 & 0.1404 & 0.2090 \\ 928 & 15: 12: 48 & 0.0470 & 0.0450 \\ 930 & 15: 12: 50 & 0.1254 & 0.0590 \\ 932 & 15: 12: 52 & 0.0897 & 0.0424 \\ 934 & 15: 12: 54 & 0.0543 & 0.0812 \\ 936 & 15: 12: 56 & 0.0742 & 0.0994 \\ 938 & 15: 12: 58 & 0.0803 & 0.0360 \\ 940 & 15: 13: 00 & 0.0447 & 0.0278 \\ 942 & 15: 13: 02 & 0.1197 & 0.0694 \\ 944 & 15: 13: 04 & 0.0732 & 0.0904 \\ 946 & 15: 13: 06 & 0.0616 & 0.0988 \\ 948 & 15: 13: 08 & 0.0358 & 0.0358 \\ 950 & 15: 13: 10 & 0.0763 & 0.0709 \\ 952 & 15: 13: 12 & 0.0862 & 0.0328 \\ 954 & 15: 13: 14 & 0.0639 & 0.0881 \\ 956 & 15: 13: 16 & 0.0441 & 0.1476 \\ 958 & 15: 13: 18 & 0.1914 & 0.1868 \\ 960 & 15: 13: 20 & 0.2392 & 0.1275 \\ 962 & 15: 13: 22 & 0.1136 & 0.1396 \\ 964 & 15: 13: 24 & 0.1522 & 0.0369 \\ 966 & 15: 13: 26 & 0.0751 & 0.0828 \\ 968 & 15: 13: 28 & 0.0464 & 0.0664 \\ 970 & 15: 13: 30 & 0.1533 & 0.0432 \\ 972 & 15: 13: 32 & 0.0641 & 0.0396 \\ 974 & 15: 13: 34 & 0.0365 & 0.0536 \\ 976 & 15: 13: 36 & 0.1446 & 0.0438 \\ 978 & 15: 13: 38 & 0.0738 & 0.1283 \\ 980 & 15: 13: 40 & 0.1178 & 0.1508 \\ 982 & 15: 13: 42 & 0.0683 & 0.1646 \\ 984 & 15: 13: 44 & 0.0570 & 0.0712 \\ 986 & 15: 13: 46 & 0.0660 & 0.1228 \\ 988 & 15: 13: 48 & 0.1273 & 0.0431 \\ 990 & 15: 13: 50 & 0.1607 & 0.0900 \\ 992 & 15: 13: 52 & 0.1710 & 0.1582 \\ 994 & 15: 13: 54 & 0.3068 & 0.0745 \\ 996 & 15: 13: 56 & 0.1491 & 0.0831 \\ 998 & 15: 13: 58 & 0.1247 & 0.0900 \\ 1000 & 15: 14: 00 & 0.1444 & 0.1929 \\ 1002 & 15: 14: 02 & 0.1602 & 0.0493 \\ 1004 & 15: 14: 04 & 0.0819 & 0.1559 \\ 1006 & 15: 14: 06 & 0.0586 & 0.2375 \\ 1008 & 15: 14: 08 & 0.0574 & 0.1533 \\ 1010 & 15: 14: 10 & 0.0395 & 0.1351 \\ 1012 & 15: 14: 12 & 0.0432 & 0.1203 \\ 1014 & 15: 14: 14 & 0.0927 & 0.1840 \\ 1016 & 15: 14: 16 & 0.1324 & 0.2834\end{array}$


Appendix F - Laser Data Test 3 NIST102605_1.xls

$\begin{array}{llll}1018 & 15: 14: 18 & 0.0900 & 0.1635 \\ 1020 & 15: 14: 20 & 0.1737 & 0.1218 \\ 1022 & 15: 14: 22 & 0.1283 & 0.1857 \\ 1024 & 15: 14: 24 & 0.1114 & 0.1170 \\ 1026 & 15: 14: 26 & 0.1562 & 0.2045 \\ 1028 & 15: 14: 28 & 0.1504 & 0.0832 \\ 1030 & 15: 14: 30 & 0.0969 & 0.0937 \\ 1032 & 15: 14: 32 & 0.0940 & 0.0914 \\ 1034 & 15: 14: 34 & 0.0801 & 0.1199 \\ 1036 & 15: 14: 36 & 0.1748 & 0.0862 \\ 1038 & 15: 14: 38 & 0.1366 & 0.0875 \\ 1040 & 15: 14: 40 & 0.1340 & 0.0803 \\ 1042 & 15: 14: 42 & 0.1308 & 0.1946 \\ 1044 & 15: 14: 44 & 0.1186 & 0.1244 \\ 1046 & 15: 14: 46 & 0.1562 & 0.1904 \\ 1048 & 15: 14: 48 & 0.1341 & 0.1109 \\ 1050 & 15: 14: 50 & 0.1702 & 0.0595 \\ 1052 & 15: 14: 52 & 0.1920 & 0.1126 \\ 1054 & 15: 14: 54 & 0.2209 & 0.1152 \\ 1056 & 15: 14: 56 & 0.0701 & 0.1210 \\ 1058 & 15: 14: 58 & 0.1162 & 0.0607 \\ 1060 & 15: 15: 00 & 0.1945 & 0.1125 \\ 1062 & 15: 15: 02 & 0.1733 & 0.0620 \\ 1064 & 15: 15: 04 & 0.1935 & 0.1165 \\ 1066 & 15: 15: 06 & 0.1386 & 0.1058 \\ 1068 & 15: 15: 08 & 0.1699 & 0.0636 \\ 1070 & 15: 15: 10 & 0.1892 & 0.0339 \\ 1072 & 15: 15: 12 & 0.0333 & 0.1004 \\ 1074 & 15: 15: 14 & 0.1756 & 0.0532 \\ 1076 & 15: 15: 16 & 0.1849 & 0.0812 \\ 1078 & 15: 15: 18 & 0.1430 & 0.1203 \\ 1080 & 15: 15: 20 & 0.1521 & 0.2164 \\ 1082 & 15: 15: 22 & 0.1513 & 0.1444 \\ 1084 & 15: 15: 24 & 0.1345 & 0.0692 \\ 1086 & 15: 15: 26 & 0.1082 & 0.1638 \\ 1088 & 15: 15: 28 & 0.1867 & 0.2352 \\ 1090 & 15: 15: 30 & 0.1919 & 0.1633 \\ 1092 & 15: 15: 32 & 0.0366 & 0.0762 \\ 1094 & 15: 15: 34 & 0.2926 & 0.1719 \\ 1096 & 15: 15: 36 & 0.1992 & 0.1075 \\ 1098 & 15: 15: 38 & 0.1296 & 0.1767 \\ 1100 & 15: 15: 40 & 0.1275 & 0.2735 \\ 1102 & 15: 15: 42 & 0.2061 & 0.0778 \\ 1104 & 15: 15: 44 & 0.0662 & 0.0830 \\ 1106 & 15: 15: 46 & 0.1261 & 0.1505 \\ 1108 & 15: 15: 48 & 0.1571 & 0.1822 \\ 1110 & 15: 15: 50 & 0.2450 & 0.0491 \\ 1112 & 15: 15: 52 & 0.1866 & 0.2029 \\ 1114 & 15: 15: 54 & 0.1825 & 0.1820 \\ 1116 & 15: 15: 56 & 0.1315 & 0.1106 \\ 1118 & 15: 15: 58 & 0.2040 & 0.1020\end{array}$


Appendix F - Laser Data Test 3 NIST102605_1.xls

$\begin{array}{llll}1120 & 15: 16: 00 & 0.1455 & 0.0765 \\ 1122 & 15: 16: 02 & 0.0578 & 0.1443 \\ 1124 & 15: 16: 04 & 0.2555 & 0.1606 \\ 1126 & 15: 16: 06 & 0.0652 & 0.1596 \\ 1128 & 15: 16: 08 & 0.1637 & 0.0803 \\ 1130 & 15: 16: 10 & 0.2984 & 0.1313 \\ 1132 & 15: 16: 12 & 0.1838 & 0.1956 \\ 1134 & 15: 16: 14 & 0.2174 & 0.2236 \\ 1136 & 15: 16: 16 & 0.2918 & 0.2842 \\ 1138 & 15: 16: 18 & 0.0898 & 0.0703 \\ 1140 & 15: 16: 20 & 0.1761 & 0.1062 \\ 1142 & 15: 16: 22 & 0.1261 & 0.1831 \\ 1144 & 15: 16: 24 & 0.2012 & 0.1594 \\ 1146 & 15: 16: 26 & 0.2697 & 0.1511 \\ 1148 & 15: 16: 28 & 0.1200 & 0.2062 \\ 1150 & 15: 16: 30 & 0.1460 & 0.1809 \\ 1152 & 15: 16: 32 & 0.1693 & 0.1164 \\ 1154 & 15: 16: 34 & 0.2159 & 0.1528 \\ 1156 & 15: 16: 36 & 0.2455 & 0.1113 \\ 1158 & 15: 16: 38 & 0.2042 & 0.3026 \\ 1160 & 15: 16: 40 & 0.1898 & 0.1351 \\ 1162 & 15: 16: 42 & 0.3404 & 0.0694 \\ 1164 & 15: 16: 44 & 0.1638 & 0.0618 \\ 1166 & 15: 16: 46 & 0.2640 & 0.1373 \\ 1168 & 15: 16: 48 & 0.2081 & 0.1570 \\ 1170 & 15: 16: 50 & 0.3004 & 0.1654 \\ 1172 & 15: 16: 52 & 0.2322 & 0.2202 \\ 1174 & 15: 16: 54 & 0.0756 & 0.1503 \\ 1176 & 15: 16: 56 & 0.1798 & 0.1480 \\ 1178 & 15: 16: 58 & 0.1464 & 0.1327 \\ 1180 & 15: 17: 00 & 0.2033 & 0.1124 \\ 1182 & 15: 17: 02 & 0.1061 & 0.1967 \\ 1184 & 15: 17: 04 & 0.1488 & 0.2232 \\ 1186 & 15: 17: 06 & 0.1557 & 0.2279 \\ 1188 & 15: 17: 08 & 0.2413 & 0.2529 \\ 1190 & 15: 17: 10 & 0.2564 & 0.0386 \\ 1192 & 15: 17: 12 & 0.1405 & 0.1140 \\ 1194 & 15: 17: 14 & 0.1725 & 0.2800 \\ 1196 & 15: 17: 16 & 0.2100 & 0.1427 \\ 1198 & 15: 17: 18 & 0.1942 & 0.2463 \\ 1200 & 15: 17: 20 & 0.1648 & 0.2444 \\ 1202 & 15: 17: 22 & 0.1069 & 0.2338 \\ 1204 & 15: 17: 24 & 0.4068 & 0.1160 \\ 1206 & 15: 17: 26 & 0.3539 & 0.1159 \\ 1208 & 15: 17: 28 & 0.1418 & 0.1721 \\ 1210 & 15: 17: 30 & 0.0794 & 0.1852 \\ 1212 & 15: 17: 32 & 0.2608 & 0.2896 \\ 1214 & 15: 17: 34 & 0.3490 & 0.2159 \\ 1216 & 15: 17: 36 & 0.1452 & 0.2022 \\ 1218 & 15: 17: 38 & 0.2168 & 0.1903 \\ 1220 & 15: 17: 40 & 0.2344 & 0.0759\end{array}$


Appendix F - Laser Data Test 3 NIST102605_1.xls

$\begin{array}{llll}1222 & 15: 17: 42 & 0.2498 & 0.1985 \\ 1224 & 15: 17: 44 & 0.1352 & 0.2230 \\ 1226 & 15: 17: 46 & 0.2073 & 0.2252 \\ 1228 & 15: 17: 48 & 0.0942 & 0.1630 \\ 1230 & 15: 17: 50 & 0.1829 & 0.2008 \\ 1232 & 15: 17: 52 & 0.3109 & 0.2888 \\ 1234 & 15: 17: 54 & 0.2595 & 0.1642 \\ 1236 & 15: 17: 56 & 0.3300 & 0.2047 \\ 1238 & 15: 17: 58 & 0.1377 & 0.1781 \\ 1240 & 15: 18: 00 & 0.3085 & 0.2247 \\ 1242 & 15: 18: 02 & 0.2118 & 0.3464 \\ 1244 & 15: 18: 04 & 0.1512 & 0.2759 \\ 1246 & 15: 18: 06 & 0.2306 & 0.1225 \\ 1248 & 15: 18: 08 & 0.4100 & 0.2018 \\ 1250 & 15: 18: 10 & 0.4620 & 0.0896 \\ 1252 & 15: 18: 12 & 0.3516 & 0.2129 \\ 1254 & 15: 18: 14 & 0.2278 & 0.1387 \\ 1256 & 15: 18: 16 & 0.3826 & 0.1590 \\ 1258 & 15: 18: 18 & 0.2875 & 0.0868 \\ 1260 & 15: 18: 20 & 0.2256 & 0.1001 \\ 1262 & 15: 18: 22 & 0.2938 & 0.1048 \\ 1264 & 15: 18: 24 & 0.2500 & 0.1663 \\ 1266 & 15: 18: 26 & 0.1860 & 0.2688 \\ 1268 & 15: 18: 28 & 0.1610 & 0.2769 \\ 1270 & 15: 18: 30 & 0.2158 & 0.3387 \\ 1272 & 15: 18: 32 & 0.2995 & 0.1224 \\ 1274 & 15: 18: 34 & 0.2402 & 0.2106 \\ 1276 & 15: 18: 36 & 0.2610 & 0.3484 \\ 1278 & 15: 18: 38 & 0.2035 & 0.1352 \\ 1280 & 15: 18: 40 & 0.2993 & 0.1306 \\ 1282 & 15: 18: 42 & 0.3448 & 0.3196 \\ 1284 & 15: 18: 44 & 0.1431 & 0.3587 \\ 1286 & 15: 18: 46 & 0.3849 & 0.2473 \\ 1288 & 15: 18: 48 & 0.1429 & 0.1359 \\ 1290 & 15: 18: 50 & 0.1998 & 0.1575 \\ 1292 & 15: 18: 52 & 0.1372 & 0.2929 \\ 1294 & 15: 18: 54 & 0.1564 & 0.1523 \\ 1296 & 15: 18: 56 & 0.3476 & 0.1535 \\ 1298 & 15: 18: 58 & 0.3244 & 0.1308 \\ 1300 & 15: 19: 00 & 0.1816 & 0.2158 \\ 1302 & 15: 19: 02 & 0.4129 & 0.2469 \\ 1304 & 15: 19: 04 & 0.4532 & 0.1355 \\ 1306 & 15: 19: 06 & 0.1567 & 0.1762 \\ 1308 & 15: 19: 08 & 0.2862 & 0.2149 \\ 1310 & 15: 19: 10 & 0.3340 & 0.2159 \\ 1312 & 15: 19: 12 & 0.2017 & 0.1780 \\ 1314 & 15: 19: 14 & 0.2634 & 0.0376 \\ 1316 & 15: 19: 16 & 0.0945 & 0.2205 \\ 1318 & 15: 19: 18 & 0.1386 & 0.1754 \\ 1320 & 15: 19: 20 & 0.3854 & 0.1490 \\ 1322 & 15: 19: 22 & 0.3300 & 0.2151\end{array}$


Appendix F - Laser Data Test 3 NIST102605_1.xls

$\begin{array}{llll}1324 & 15: 19: 24 & 0.2237 & 0.2331 \\ 1326 & 15: 19: 26 & 0.2135 & 0.1942 \\ 1328 & 15: 19: 28 & 0.1007 & 0.3917 \\ 1330 & 15: 19: 30 & 0.1687 & 0.2312 \\ 1332 & 15: 19: 32 & 0.2358 & 0.2687 \\ 1334 & 15: 19: 34 & 0.2947 & 0.3213 \\ 1336 & 15: 19: 36 & 0.2695 & 0.2454 \\ 1338 & 15: 19: 38 & 0.2517 & 0.0982 \\ 1340 & 15: 19: 40 & 0.3201 & 0.1659 \\ 1342 & 15: 19: 42 & 0.2618 & 0.0916 \\ 1344 & 15: 19: 44 & 0.0443 & 0.4716 \\ 1346 & 15: 19: 46 & 0.2099 & 0.2096 \\ 1348 & 15: 19: 48 & 0.4546 & 0.2054 \\ 1350 & 15: 19: 50 & 0.1452 & 0.2717 \\ 1352 & 15: 19: 52 & 0.1603 & 0.2261 \\ 1354 & 15: 19: 54 & 0.3231 & 0.1543 \\ 1356 & 15: 19: 56 & 0.3886 & 0.3142 \\ 1358 & 15: 19: 58 & 0.2611 & 0.2504 \\ 1360 & 15: 20: 00 & 0.1756 & 0.2293 \\ 1362 & 15: 20: 02 & 0.3174 & 0.0671 \\ 1364 & 15: 20: 04 & 0.2821 & 0.3135 \\ 1366 & 15: 20: 06 & 0.2954 & 0.1223 \\ 1368 & 15: 20: 08 & 0.2507 & 0.2760 \\ 1370 & 15: 20: 10 & 0.2902 & 0.2406 \\ 1372 & 15: 20: 12 & 0.3930 & 0.2443 \\ 1374 & 15: 20: 14 & 0.1900 & 0.0971 \\ 1376 & 15: 20: 16 & 0.3404 & 0.2414 \\ 1378 & 15: 20: 18 & 0.3419 & 0.3647 \\ 1380 & 15: 20: 20 & 0.2519 & 0.2761 \\ 1382 & 15: 20: 22 & 0.4557 & 0.2150 \\ 1384 & 15: 20: 24 & 0.1204 & 0.2295 \\ 1386 & 15: 20: 26 & 0.3284 & 0.2418 \\ 1388 & 15: 20: 28 & 0.2924 & 0.3802 \\ 1390 & 15: 20: 30 & 0.2313 & 0.1953 \\ 1392 & 15: 20: 32 & 0.2304 & 0.0927 \\ 1394 & 15: 20: 34 & 0.2724 & 0.2698 \\ 1396 & 15: 20: 36 & 0.3457 & 0.1549 \\ 1398 & 15: 20: 38 & 0.2695 & 0.2473 \\ 1400 & 15: 20: 40 & 0.4262 & 0.1457 \\ 1402 & 15: 20: 42 & 0.4016 & 0.2197 \\ 1404 & 15: 20: 44 & 0.3388 & 0.2598 \\ 1406 & 15: 20: 46 & 0.3063 & 0.2623 \\ 1408 & 15: 20: 48 & 0.2091 & 0.2328 \\ 1410 & 15: 20: 50 & 0.3106 & 0.4010 \\ 1412 & 15: 20: 52 & 0.2752 & 0.2425 \\ 1414 & 15: 20: 54 & 0.4405 & 0.3807 \\ 1416 & 15: 20: 56 & 0.2590 & 0.1814 \\ 1418 & 15: 20: 58 & 0.2216 & 0.3565 \\ 1420 & 15: 21: 00 & 0.1508 & 0.1960 \\ 1422 & 15: 21: 02 & 0.2244 & 0.3735 \\ 1424 & 15: 21: 04 & 0.2567 & 0.3130\end{array}$


Appendix F - Laser Data Test 3 NIST102605_1.xls

$\begin{array}{llll}1426 & 15: 21: 06 & 0.3851 & 0.0914 \\ 1428 & 15: 21: 08 & 0.3799 & 0.4662 \\ 1430 & 15: 21: 10 & 0.4179 & 0.1566 \\ 1432 & 15: 21: 12 & 0.1227 & 0.3051 \\ 1434 & 15: 21: 14 & 0.2468 & 0.3807 \\ 1436 & 15: 21: 16 & 0.2663 & 0.3035 \\ 1438 & 15: 21: 18 & 0.1085 & 0.4044 \\ 1440 & 15: 21: 20 & 0.0700 & 0.1038 \\ 1442 & 15: 21: 22 & 0.3562 & 0.2716 \\ 1444 & 15: 21: 24 & 0.4931 & 0.3188 \\ 1446 & 15: 21: 26 & 0.2844 & 0.1970 \\ 1448 & 15: 21: 28 & 0.2071 & 0.1214 \\ 1450 & 15: 21: 30 & 0.2361 & 0.2421 \\ 1452 & 15: 21: 32 & 0.2576 & 0.2960 \\ 1454 & 15: 21: 34 & 0.3790 & 0.2047 \\ 1456 & 15: 21: 36 & 0.4333 & 0.2522 \\ 1458 & 15: 21: 38 & 0.5252 & 0.1368 \\ 1460 & 15: 21: 40 & 0.2106 & 0.3422 \\ 1462 & 15: 21: 42 & 0.3076 & 0.1584 \\ 1464 & 15: 21: 44 & 0.1662 & 0.2238 \\ 1466 & 15: 21: 46 & 0.2835 & 0.2828 \\ 1468 & 15: 21: 48 & 0.1450 & 0.3229 \\ 1470 & 15: 21: 50 & 0.2748 & 0.1425 \\ 1472 & 15: 21: 52 & 0.3212 & 0.1324 \\ 1474 & 15: 21: 54 & 0.2073 & 0.2170 \\ 1476 & 15: 21: 56 & 0.3995 & 0.0860 \\ 1478 & 15: 21: 58 & 0.2614 & 0.2218 \\ 1480 & 15: 22: 00 & 0.1168 & 0.2593 \\ 1482 & 15: 22: 02 & 0.2736 & 0.2033 \\ 1484 & 15: 22: 04 & 0.3215 & 0.4426 \\ 1486 & 15: 22: 06 & 0.2090 & 0.3132 \\ 1488 & 15: 22: 08 & 0.3790 & 0.1341 \\ 1490 & 15: 22: 10 & 0.1480 & 0.1774 \\ 1492 & 15: 22: 12 & 0.3044 & 0.1938 \\ 1494 & 15: 22: 14 & 0.2491 & 0.3014 \\ 1496 & 15: 22: 16 & 0.2718 & 0.2186 \\ 1498 & 15: 22: 18 & 0.2213 & 0.2530 \\ 1500 & 15: 22: 20 & 0.3449 & 0.0992 \\ 1502 & 15: 22: 22 & 0.0721 & 0.2712 \\ 1504 & 15: 22: 24 & 0.4727 & 0.2073 \\ 1506 & 15: 22: 26 & 0.3608 & 0.1596 \\ 1508 & 15: 22: 28 & 0.1260 & 0.1130 \\ 1510 & 15: 22: 30 & 0.3279 & 0.3283 \\ 1512 & 15: 22: 32 & 0.3701 & 0.3046 \\ 1514 & 15: 22: 34 & 0.1752 & 0.3107 \\ 1516 & 15: 22: 36 & 0.3691 & 0.3095 \\ 1518 & 15: 22: 38 & 0.2785 & 0.1560 \\ 1520 & 15: 22: 40 & 0.2171 & 0.1854 \\ 1522 & 15: 22: 42 & 0.2662 & 0.2030 \\ 1524 & 15: 22: 44 & 0.1827 & 0.2250 \\ 1526 & 15: 22: 46 & 0.0829 & 0.1914\end{array}$


Appendix F - Laser Data Test 3 NIST102605_1.xls

$\begin{array}{llll}1528 & 15: 22: 48 & 0.3717 & 0.1486 \\ 1530 & 15: 22: 50 & 0.2375 & 0.3301 \\ 1532 & 15: 22: 52 & 0.2691 & 0.2534 \\ 1534 & 15: 22: 54 & 0.4090 & 0.4908 \\ 1536 & 15: 22: 56 & 0.1135 & 0.1073 \\ 1538 & 15: 22: 58 & 0.1656 & 0.1602 \\ 1540 & 15: 23: 00 & 0.3750 & 0.2333 \\ 1542 & 15: 23: 02 & 0.3476 & 0.2456 \\ 1544 & 15: 23: 04 & 0.3707 & 0.2627 \\ 1546 & 15: 23: 06 & 0.3024 & 0.3669 \\ 1548 & 15: 23: 08 & 0.2114 & 0.3458 \\ 1550 & 15: 23: 10 & 0.0771 & 0.2237 \\ 1552 & 15: 23: 12 & 0.3309 & 0.2559 \\ 1554 & 15: 23: 14 & 0.3765 & 0.2966 \\ 1556 & 15: 23: 16 & 0.1755 & 0.3176 \\ 1558 & 15: 23: 18 & 0.3630 & 0.3782 \\ 1560 & 15: 23: 20 & 0.2849 & 0.1919 \\ 1562 & 15: 23: 22 & 0.3997 & 0.3214 \\ 1564 & 15: 23: 24 & 0.2464 & 0.3167 \\ 1566 & 15: 23: 26 & 0.2104 & 0.3413 \\ 1568 & 15: 23: 28 & 0.1626 & 0.3680 \\ 1570 & 15: 23: 30 & 0.2033 & 0.4296 \\ 1572 & 15: 23: 32 & 0.2731 & 0.2796 \\ 1574 & 15: 23: 34 & 0.2136 & 0.3917 \\ 1576 & 15: 23: 36 & 0.1636 & 0.2645 \\ 1578 & 15: 23: 38 & 0.2378 & 0.2143 \\ 1580 & 15: 23: 40 & 0.3451 & 0.1661 \\ 1582 & 15: 23: 42 & 0.1124 & 0.1964 \\ 1584 & 15: 23: 44 & 0.2406 & 0.3529 \\ 1586 & 15: 23: 46 & 0.2852 & 0.2040 \\ 1588 & 15: 23: 48 & 0.2007 & 0.1800 \\ 1590 & 15: 23: 50 & 0.2125 & 0.1489 \\ 1592 & 15: 23: 52 & 0.2000 & 0.1761 \\ 1594 & 15: 23: 54 & 0.1357 & 0.3032 \\ 1596 & 15: 23: 56 & 0.1748 & 0.2945 \\ 1598 & 15: 23: 58 & 0.0618 & 0.1757 \\ 1600 & 15: 24: 00 & 0.2507 & 0.1247 \\ 1602 & 15: 24: 02 & 0.0695 & 0.1912 \\ 1604 & 15: 24: 04 & 0.1633 & 0.1156 \\ 1606 & 15: 24: 06 & 0.1144 & 0.1441 \\ 1608 & 15: 24: 08 & 0.1371 & 0.1345 \\ 1610 & 15: 24: 10 & 0.0825 & 0.0586 \\ 1612 & 15: 24: 12 & 0.0717 & 0.0666 \\ 1614 & 15: 24: 14 & 0.0244 & 0.0464 \\ 1616 & 15: 24: 16 & 0.0394 & 0.0562 \\ 1618 & 15: 24: 18 & 0.0457 & 0.0252 \\ 1620 & 15: 24: 20 & 0.1024 & 0.0608 \\ 1622 & 15: 24: 22 & 0.0137 & 0.0288 \\ 1624 & 15: 24: 24 & 0.0321 & 0.0341 \\ 1626 & 15: 24: 26 & 0.0838 & 0.0125 \\ 1628 & 15: 24: 28 & 0.0594 & 0.0099\end{array}$


Appendix F - Laser Data Test 3 NIST102605_1.xIs

$\begin{array}{llll}1630 & 15: 24: 30 & 0.0634 & 0.0103 \\ 1632 & 15: 24: 32 & 0.0160 & 0.0027 \\ 1634 & 15: 24: 34 & 0.0065 & 0.0020 \\ 1636 & 15: 24: 36 & 0.0188 & 0.0040 \\ 1638 & 15: 24: 38 & 0.0348 & 0.0087 \\ 1640 & 15: 24: 40 & 0.0119 & 0.0010 \\ 1642 & 15: 24: 42 & 0.0074 & 0.0004 \\ 1644 & 15: 24: 44 & 0.0072 & 0.0139 \\ 1646 & 15: 24: 46 & 0.0108 & 0.0013 \\ 1648 & 15: 24: 48 & 0.0093 & 0.0051 \\ 1650 & 15: 24: 50 & 0.0033 & 0.0010 \\ 1652 & 15: 24: 52 & 0.0009 & 0.0020 \\ 1654 & 15: 24: 54 & 0.0131 & 0.0009 \\ 1656 & 15: 24: 56 & 0.0075 & 0.0018 \\ 1658 & 15: 24: 58 & 0.0016 & 0.0021 \\ 1660 & 15: 25: 00 & 0.0099 & 0.0023 \\ 1662 & 15: 25: 02 & 0.0087 & 0.0010 \\ 1664 & 15: 25: 04 & 0.0105 & 0.0068 \\ 1666 & 15: 25: 06 & 0.0054 & 0.0061 \\ 1668 & 15: 25: 08 & 0.0027 & 0.0004 \\ 1670 & 15: 25: 10 & 0.0119 & 0.0204 \\ 1672 & 15: 25: 12 & 0.0043 & 0.0057 \\ 1674 & 15: 25: 14 & 0.0017 & 0.0007 \\ 1676 & 15: 25: 16 & 0.0017 & 0.0031 \\ 1678 & 15: 25: 18 & 0.0147 & 0.0009 \\ 1680 & 15: 25: 20 & 0.0013 & 0.0051 \\ 1682 & 15: 25: 22 & 0.0012 & 0.0018 \\ 1684 & 15: 25: 24 & 0.0024 & 0.0015 \\ 1686 & 15: 25: 26 & 0.0133 & 0.0036 \\ 1688 & 15: 25: 28 & 0.0025 & 0.0009 \\ 1690 & 15: 25: 30 & 0.0056 & 0.0014 \\ 1692 & 15: 25: 32 & 0.0091 & 0.0024 \\ 1694 & 15: 25: 34 & 0.0004 & 0.0000 \\ 1696 & 15: 25: 36 & 0.0015 & 0.0012 \\ 1698 & 15: 25: 38 & 0.0102 & 0.0014 \\ 1700 & 15: 25: 40 & 0.0062 & 0.0072 \\ 1702 & 15: 25: 42 & 0.0039 & 0.0012 \\ 1704 & 15: 25: 44 & 0.0018 & 0.0011 \\ 1706 & 15: 25: 46 & 0.0099 & 0.0003 \\ 1708 & 15: 25: 48 & 0.0031 & 0.0020 \\ 1710 & 15: 25: 50 & 0.0047 & 0.0024 \\ 1712 & 15: 25: 52 & 0.0153 & 0.0006 \\ 1714 & 15: 25: 54 & 0.0153 & 0.0038 \\ 1716 & 15: 25: 56 & 0.0195 & 0.0011 \\ 1718 & 15: 25: 58 & 0.0064 & 0.0032 \\ 1720 & 15: 26: 00 & 0.0045 & 0.0013 \\ 1722 & 15: 26: 02 & 0.0030 & 0.0014 \\ 1724 & 15: 26: 04 & 0.0359 & 0.0017 \\ 1726 & 15: 26: 06 & 0.0196 & 0.0003 \\ 1728 & 15: 26: 08 & 0.0058 & 0.0035 \\ 1730 & 15: 26: 10 & 0.0014 & 0.0001\end{array}$


Appendix F - Laser Data Test 3 NIST102605_1.xIs

$\begin{array}{llll}1732 & 15: 26: 12 & 0.0082 & 0.0026 \\ 1734 & 15: 26: 14 & 0.0035 & 0.0093 \\ 1736 & 15: 26: 16 & 0.0102 & 0.0015 \\ 1738 & 15: 26: 18 & 0.0152 & 0.0015 \\ 1740 & 15: 26: 20 & 0.0078 & 0.0097 \\ 1742 & 15: 26: 22 & 0.0013 & 0.0003 \\ 1744 & 15: 26: 24 & 0.0024 & 0.0006 \\ 1746 & 15: 26: 26 & 0.0036 & 0.0003 \\ 1748 & 15: 26: 28 & 0.0100 & 0.0016 \\ 1750 & 15: 26: 30 & 0.0327 & 0.0029 \\ 1752 & 15: 26: 32 & 0.0044 & 0.0010 \\ 1754 & 15: 26: 34 & 0.0031 & 0.0003 \\ 1756 & 15: 26: 36 & 0.0054 & 0.0003 \\ 1758 & 15: 26: 38 & 0.0032 & 0.0019 \\ 1760 & 15: 26: 40 & 0.0008 & 0.0251 \\ 1762 & 15: 26: 42 & 0.0059 & 0.0015 \\ 1764 & 15: 26: 44 & 0.0027 & 0.0028 \\ 1766 & 15: 26: 46 & 0.0003 & 0.0002 \\ 1768 & 15: 26: 48 & 0.0022 & 0.0000 \\ 1770 & 15: 26: 50 & 0.0065 & 0.0008 \\ 1772 & 15: 26: 52 & 0.0077 & 0.0016 \\ 1774 & 15: 26: 54 & 0.0036 & 0.0073 \\ 1776 & 15: 26: 56 & 0.0037 & 0.0008 \\ 1778 & 15: 26: 58 & 0.0042 & 0.0040 \\ 1780 & 15: 27: 00 & 0.0031 & 0.0027 \\ 1782 & 15: 27: 02 & 0.0005 & 0.0003 \\ 1784 & 15: 27: 04 & 0.0073 & 0.0018 \\ 1786 & 15: 27: 06 & 0.0112 & 0.0014 \\ 1788 & 15: 27: 08 & 0.0011 & 0.0143 \\ 1790 & 15: 27: 10 & 0.0009 & 0.0078 \\ 1792 & 15: 27: 12 & 0.0006 & 0.0072 \\ 1794 & 15: 27: 14 & 0.0005 & 0.0002 \\ 1796 & 15: 27: 16 & 0.0040 & 0.0002 \\ 1798 & 15: 27: 18 & 0.0006 & 0.0003 \\ 1800 & 15: 27: 20 & 0.0003 & 0.0003 \\ 1802 & 15: 27: 22 & 0.0002 & 0.0003 \\ 1804 & 15: 27: 24 & 0.0013 & 0.0007 \\ 1806 & 15: 27: 26 & 0.0056 & 0.0032 \\ 1808 & 15: 27: 28 & 0.0007 & 0.0059 \\ 1810 & 15: 27: 30 & 0.0079 & 0.0019 \\ 1812 & 15: 27: 32 & 0.0031 & 0.0003 \\ 1814 & 15: 27: 34 & 0.0062 & 0.0002 \\ 1816 & 15: 27: 36 & 0.0043 & 0.0034 \\ 1818 & 15: 27: 38 & 0.0140 & 0.0016 \\ 1820 & 15: 27: 40 & 0.0130 & 0.0021 \\ 1822 & 15: 27: 42 & 0.0054 & 0.0006 \\ 1824 & 15: 27: 44 & 0.0013 & 0.0020 \\ 1826 & 15: 27: 46 & 0.0016 & 0.0022 \\ 1828 & 15: 27: 48 & 0.0034 & 0.0028 \\ 1830 & 15: 27: 50 & 0.0014 & 0.0001 \\ 1832 & 15: 27: 52 & 0.0028 & 0.0012\end{array}$


Appendix F - Laser Data Test 3 NIST102605_1.xIs

$\begin{array}{llll}1834 & 15: 27: 54 & 0.0005 & 0.0003 \\ 1836 & 15: 27: 56 & 0.0007 & 0.0018 \\ 1838 & 15: 27: 58 & 0.0005 & 0.0011 \\ 1840 & 15: 28: 00 & 0.0102 & 0.0016 \\ 1842 & 15: 28: 02 & 0.0096 & 0.0002 \\ 1844 & 15: 28: 04 & 0.0005 & 0.0002 \\ 1846 & 15: 28: 06 & 0.0011 & 0.0026 \\ 1848 & 15: 28: 08 & 0.0025 & 0.0058 \\ 1850 & 15: 28: 10 & 0.0053 & 0.0009 \\ 1852 & 15: 28: 12 & 0.0012 & 0.0011 \\ 1854 & 15: 28: 14 & 0.0018 & 0.0015 \\ 1856 & 15: 28: 16 & 0.0023 & 0.0003 \\ 1858 & 15: 28: 18 & 0.0020 & 0.0007 \\ 1860 & 15: 28: 20 & 0.0039 & 0.0003 \\ 1862 & 15: 28: 22 & 0.0038 & 0.0015 \\ 1864 & 15: 28: 24 & 0.0005 & 0.0007 \\ 1866 & 15: 28: 26 & 0.0012 & 0.0067 \\ 1868 & 15: 28: 28 & 0.0033 & 0.0119 \\ 1870 & 15: 28: 30 & 0.0008 & 0.0038 \\ 1872 & 15: 28: 32 & 0.0074 & 0.0016 \\ 1874 & 15: 28: 34 & 0.0007 & 0.0020 \\ 1876 & 15: 28: 36 & 0.0009 & 0.0139 \\ 1878 & 15: 28: 38 & 0.0077 & 0.0010 \\ 1880 & 15: 28: 40 & 0.0006 & 0.0015 \\ 1882 & 15: 28: 42 & 0.0034 & 0.0009 \\ 1884 & 15: 28: 44 & 0.0008 & 0.0000 \\ 1886 & 15: 28: 46 & 0.0005 & 0.0015 \\ 1888 & 15: 28: 48 & 0.0026 & 0.0079 \\ 1890 & 15: 28: 50 & 0.0034 & 0.0005 \\ 1892 & 15: 28: 52 & 0.0006 & 0.0024 \\ 1894 & 15: 28: 54 & 0.0004 & 0.0012 \\ 1896 & 15: 28: 56 & 0.0014 & 0.0019 \\ 1898 & 15: 28: 58 & 0.0017 & 0.0039 \\ 1900 & 15: 29: 00 & 0.0053 & 0.0001 \\ 1902 & 15: 29: 02 & 0.0024 & 0.0011 \\ 1904 & 15: 29: 04 & 0.0013 & 0.0009 \\ 1906 & 15: 29: 06 & 0.0046 & 0.0004 \\ 1908 & 15: 29: 08 & 0.0005 & 0.0023 \\ 1910 & 15: 29: 10 & 0.0014 & 0.0008 \\ 1912 & 15: 29: 12 & 0.0023 & 0.0003 \\ 1914 & 15: 29: 14 & 0.0014 & 0.0009 \\ 1916 & 15: 29: 16 & 0.0019 & 0.0026 \\ 1918 & 15: 29: 18 & 0.0009 & 0.0041 \\ 1920 & 15: 29: 20 & 0.0039 & 0.0013 \\ 1922 & 15: 29: 22 & 0.0003 & 0.0000 \\ 1924 & 15: 29: 24 & 0.0002 & 0.0001 \\ 1926 & 15: 29: 26 & 0.0004 & 0.0003 \\ 1928 & 15: 29: 28 & 0.0003 & 0.0004 \\ 1930 & 15: 29: 30 & 0.0050 & 0.0006 \\ 1932 & 15: 29: 32 & 0.0012 & 0.0002 \\ 1934 & 15: 29: 34 & 0.0020 & 0.0005\end{array}$


Appendix F - Laser Data Test 3 NIST102605_1.xIs

$\begin{array}{llll}1936 & 15: 29: 36 & 0.0058 & 0.0013 \\ 1938 & 15: 29: 38 & 0.0126 & 0.0011 \\ 1940 & 15: 29: 40 & 0.0034 & 0.0006 \\ 1942 & 15: 29: 42 & 0.0002 & 0.0000 \\ 1944 & 15: 29: 44 & 0.0022 & 0.0002 \\ 1946 & 15: 29: 46 & 0.0062 & 0.0003 \\ 1948 & 15: 29: 48 & 0.0018 & 0.0005 \\ 1950 & 15: 29: 50 & 0.0004 & 0.0003 \\ 1952 & 15: 29: 52 & 0.0007 & 0.0003 \\ 1954 & 15: 29: 54 & 0.0006 & 0.0003 \\ 1956 & 15: 29: 56 & 0.0011 & 0.0002 \\ 1958 & 15: 29: 58 & 0.0006 & 0.0001 \\ 1960 & 15: 30: 00 & 0.0005 & 0.0001 \\ 1962 & 15: 30: 02 & 0.0011 & 0.0005 \\ 1964 & 15: 30: 04 & 0.0036 & 0.0011 \\ 1966 & 15: 30: 06 & 0.0005 & 0.0058 \\ 1968 & 15: 30: 08 & 0.0000 & 0.0006 \\ 1970 & 15: 30: 10 & 0.0008 & 0.0003 \\ 1972 & 15: 30: 12 & 0.0058 & 0.0296 \\ 1974 & 15: 30: 14 & 0.0012 & 0.0012 \\ 1976 & 15: 30: 16 & 0.0052 & 0.0006 \\ 1978 & 15: 30: 18 & 0.0025 & 0.0002 \\ 1980 & 15: 30: 20 & 0.0301 & 0.0000 \\ 1982 & 15: 30: 22 & 0.0239 & 0.0005 \\ 1984 & 15: 30: 24 & 0.0008 & 0.0003 \\ 1986 & 15: 30: 26 & 0.0024 & 0.0002 \\ 1988 & 15: 30: 28 & 0.0017 & 0.0003 \\ 1990 & 15: 30: 30 & 0.0158 & 0.0003 \\ 1992 & 15: 30: 32 & 0.0079 & 0.0003 \\ 1994 & 15: 30: 34 & 0.0021 & 0.0001 \\ 1996 & 15: 30: 36 & 0.0007 & 0.0038 \\ 1998 & 15: 30: 38 & 0.0007 & 0.0000 \\ 2000 & 15: 30: 40 & 0.0002 & 0.0017 \\ 2002 & 15: 30: 42 & 0.0069 & 0.0027 \\ 2004 & 15: 30: 44 & 0.0022 & 0.0001 \\ 2006 & 15: 30: 46 & 0.0005 & 0.0014 \\ 2008 & 15: 30: 48 & 0.0005 & 0.0001 \\ 2010 & 15: 30: 50 & 0.0007 & 0.0002 \\ 2012 & 15: 30: 52 & 0.0036 & 0.0036 \\ 2014 & 15: 30: 54 & 0.0007 & 0.0039 \\ 2016 & 15: 30: 56 & 0.0005 & 0.0003 \\ 2018 & 15: 30: 58 & 0.0008 & 0.0005 \\ 2020 & 15: 31: 00 & 0.0008 & 0.0002 \\ 2022 & 15: 31: 02 & 0.0039 & 0.0001 \\ 2024 & 15: 31: 04 & 0.0015 & 0.0033 \\ 2026 & 15: 31: 06 & 0.0005 & 0.0046 \\ 2028 & 15: 31: 08 & 0.0045 & 0.0006 \\ 2030 & 15: 31: 10 & 0.0005 & 0.0032 \\ 2032 & 15: 31: 12 & 0.0040 & 0.0001 \\ 2034 & 15: 31: 14 & 0.0009 & 0.0002 \\ 2036 & 15: 31: 16 & 0.0039 & 0.0010\end{array}$


Appendix F - Laser Data Test 3 NIST102605_1.xls

$\begin{array}{llll}2038 & 15: 31: 18 & 0.0002 & 0.0013 \\ 2040 & 15: 31: 20 & 0.0026 & 0.0005 \\ 2042 & 15: 31: 22 & 0.0002 & 0.0002 \\ 2044 & 15: 31: 24 & 0.0025 & 0.0003 \\ 2046 & 15: 31: 26 & 0.0038 & 0.0002 \\ 2048 & 15: 31: 28 & 0.0040 & 0.0002 \\ 2050 & 15: 31: 30 & 0.0004 & 0.0004 \\ 2052 & 15: 31: 32 & 0.0069 & 0.0000 \\ 2054 & 15: 31: 34 & 0.0025 & 0.0009 \\ 2056 & 15: 31: 36 & 0.0009 & 0.0001 \\ 2058 & 15: 31: 38 & 0.0092 & 0.0004 \\ 2060 & 15: 31: 40 & 0.0024 & 0.0002 \\ 2062 & 15: 31: 42 & 0.0001 & 0.0012 \\ 2064 & 15: 31: 44 & 0.0002 & 0.0000 \\ 2066 & 15: 31: 46 & 0.0003 & 0.0001 \\ 2068 & 15: 31: 48 & 0.0007 & 0.0004 \\ 2070 & 15: 31: 50 & 0.0020 & 0.0001 \\ 2072 & 15: 31: 52 & 0.0002 & 0.0016 \\ 2074 & 15: 31: 54 & 0.0005 & 0.0011 \\ 2076 & 15: 31: 56 & 0.0033 & 0.0001 \\ 2078 & 15: 31: 58 & 0.0067 & 0.0027 \\ 2080 & 15: 32: 00 & 0.0018 & 0.0003 \\ 2082 & 15: 32: 02 & 0.0027 & 0.0032 \\ 2084 & 15: 32: 04 & 0.0121 & 0.0076 \\ 2086 & 15: 32: 06 & 0.0030 & 0.0005 \\ 2088 & 15: 32: 08 & 0.0005 & 0.0003 \\ 2090 & 15: 32: 10 & 0.0005 & 0.0007 \\ 2092 & 15: 32: 12 & 0.0005 & 0.0001 \\ 2094 & 15: 32: 14 & 0.0014 & 0.0005 \\ 2096 & 15: 32: 16 & 0.0067 & 0.0003 \\ 2098 & 15: 32: 18 & 0.0027 & 0.0000 \\ 2100 & 15: 32: 20 & 0.0048 & 0.0004 \\ 2102 & 15: 32: 22 & 0.0466 & 0.0004 \\ 2104 & 15: 32: 24 & 0.0017 & 0.0003 \\ 2106 & 15: 32: 26 & 0.0033 & 0.0059 \\ 2108 & 15: 32: 28 & 0.0018 & 0.0003 \\ 2110 & 15: 32: 30 & 0.0003 & 0.0004 \\ 2112 & 15: 32: 32 & 0.0002 & 0.0007 \\ 2114 & 15: 32: 34 & 0.0007 & 0.0011 \\ 2116 & 15: 32: 36 & 0.0013 & 0.0117 \\ 2118 & 15: 32: 38 & 0.0079 & 0.0032 \\ 2120 & 15: 32: 40 & 0.0082 & 0.0003 \\ 2122 & 15: 32: 42 & 0.0002 & 0.0001 \\ 2124 & 15: 32: 44 & 0.0007 & 0.0000 \\ 2126 & 15: 32: 46 & 0.0012 & 0.0001 \\ 2128 & 15: 32: 48 & 0.0002 & 0.0002 \\ 2130 & 15: 32: 50 & 0.0004 & 0.0002 \\ 2132 & 15: 32: 52 & 0.0001 & 0.0003 \\ 2134 & 15: 32: 54 & 0.0001 & 0.0000 \\ 2136 & 15: 32: 56 & 0.0052 & 0.0000 \\ 2138 & 15: 32: 58 & 0.0005 & 0.0002\end{array}$


Appendix F - Laser Data Test 3 NIST102605_1.xls

$\begin{array}{llll}2140 & 15: 33: 00 & 0.0006 & 0.0001 \\ 2142 & 15: 33: 02 & 0.0007 & 0.0002 \\ 2144 & 15: 33: 04 & 0.0001 & 0.0070 \\ 2146 & 15: 33: 06 & 0.0002 & 0.0003 \\ 2148 & 15: 33: 08 & 0.0005 & 0.0003 \\ 2150 & 15: 33: 10 & 0.0006 & 0.0008 \\ 2152 & 15: 33: 12 & 0.0004 & 0.0029 \\ 2154 & 15: 33: 14 & 0.0003 & 0.0004 \\ 2156 & 15: 33: 16 & 0.0007 & 0.0002 \\ 2158 & 15: 33: 18 & 0.0013 & 0.0001 \\ 2160 & 15: 33: 20 & 0.0010 & 0.0018 \\ 2162 & 15: 33: 22 & 0.0023 & 0.0034 \\ 2164 & 15: 33: 24 & 0.0007 & 0.0000 \\ 2166 & 15: 33: 26 & 0.0002 & 0.0007 \\ 2168 & 15: 33: 28 & 0.0000 & 0.0000 \\ 2170 & 15: 33: 30 & 0.0006 & 0.0002 \\ 2172 & 15: 33: 32 & 0.0002 & 0.0002 \\ 2174 & 15: 33: 34 & 0.0008 & 0.0000 \\ 2176 & 15: 33: 36 & 0.0003 & 0.0003 \\ 2178 & 15: 33: 38 & 0.0044 & 0.0002 \\ 2180 & 15: 33: 40 & 0.0018 & 0.0000 \\ 2182 & 15: 33: 42 & 0.0017 & 0.0031 \\ 2184 & 15: 33: 44 & 0.0056 & 0.0017 \\ 2186 & 15: 33: 46 & 0.0008 & 0.0003 \\ 2188 & 15: 33: 48 & 0.0002 & 0.0000 \\ 2190 & 15: 33: 50 & 0.0005 & 0.0000 \\ 2192 & 15: 33: 52 & 0.0001 & 0.0000 \\ 2194 & 15: 33: 54 & 0.0004 & 0.0029 \\ 2196 & 15: 33: 56 & 0.0031 & 0.0022 \\ 2198 & 15: 33: 58 & 0.0064 & 0.0005 \\ 2200 & 15: 34: 00 & 0.0005 & 0.0006 \\ 2202 & 15: 34: 02 & 0.0023 & 0.0006 \\ 2204 & 15: 34: 04 & 0.0097 & 0.0004 \\ 2206 & 15: 34: 06 & 0.0040 & 0.0004 \\ 2208 & 15: 34: 08 & 0.0043 & 0.0012 \\ 2210 & 15: 34: 10 & 0.0005 & 0.0010 \\ 2212 & 15: 34: 12 & 0.0011 & 0.0001 \\ 2214 & 15: 34: 14 & 0.0007 & 0.0002 \\ 2216 & 15: 34: 16 & 0.0005 & 0.0003 \\ 2218 & 15: 34: 18 & 0.0283 & 0.0020 \\ 2220 & 15: 34: 20 & 0.0022 & 0.0006 \\ 2222 & 15: 34: 22 & 0.0089 & 0.0001 \\ 2224 & 15: 34: 24 & 0.0024 & 0.0003 \\ 2226 & 15: 34: 26 & 0.0006 & 0.0007 \\ 2228 & 15: 34: 28 & 0.0075 & 0.0015 \\ 2230 & 15: 34: 30 & 0.0017 & 0.0003 \\ 2232 & 15: 34: 32 & 0.0039 & 0.0000 \\ 2234 & 15: 34: 34 & 0.0033 & 0.0002 \\ 2236 & 15: 34: 36 & 0.0006 & 0.0001 \\ 2238 & 15: 34: 38 & 0.0025 & 0.0013 \\ 2240 & 15: 34: 40 & 0.0109 & 0.0003\end{array}$


Appendix F - Laser Data Test 3 NIST102605_1.xIs

$\begin{array}{llll}2242 & 15: 34: 42 & 0.0045 & 0.0039 \\ 2244 & 15: 34: 44 & 0.0118 & 0.0004 \\ 2246 & 15: 34: 46 & 0.0036 & 0.0004 \\ 2248 & 15: 34: 48 & 0.0014 & 0.0006 \\ 2250 & 15: 34: 50 & 0.0006 & 0.0003 \\ 2252 & 15: 34: 52 & 0.0009 & 0.0005 \\ 2254 & 15: 34: 54 & 0.0130 & 0.0005 \\ 2256 & 15: 34: 56 & 0.0007 & 0.0003 \\ 2258 & 15: 34: 58 & 0.0017 & 0.0015 \\ 2260 & 15: 35: 00 & 0.0010 & 0.0002 \\ 2262 & 15: 35: 02 & 0.0133 & 0.0043 \\ 2264 & 15: 35: 04 & 0.0002 & 0.0001 \\ 2266 & 15: 35: 06 & 0.0002 & 0.0002 \\ 2268 & 15: 35: 08 & 0.0006 & 0.0001 \\ 2270 & 15: 35: 10 & 0.0003 & 0.0001 \\ 2272 & 15: 35: 12 & 0.0012 & 0.0007 \\ 2274 & 15: 35: 14 & 0.0015 & 0.0002 \\ 2276 & 15: 35: 16 & 0.0002 & 0.0000 \\ 2278 & 15: 35: 18 & 0.0073 & 0.0002 \\ 2280 & 15: 35: 20 & 0.0006 & 0.0005 \\ 2282 & 15: 35: 22 & -0.0001 & 0.0028 \\ 2284 & 15: 35: 24 & 0.0000 & 0.0000 \\ 2286 & 15: 35: 26 & 0.0016 & 0.0001 \\ 2288 & 15: 35: 28 & 0.0000 & 0.0000 \\ 2290 & 15: 35: 30 & 0.0056 & 0.0025 \\ 2292 & 15: 35: 32 & 0.0007 & 0.0002 \\ 2294 & 15: 35: 34 & 0.0039 & 0.0011 \\ 2296 & 15: 35: 36 & 0.0007 & 0.0000 \\ 2298 & 15: 35: 38 & 0.0028 & 0.0011 \\ 2300 & 15: 35: 40 & 0.0006 & 0.0000 \\ 2302 & 15: 35: 42 & 0.0016 & 0.0001 \\ 2304 & 15: 35: 44 & 0.0010 & 0.0000 \\ 2306 & 15: 35: 46 & 0.0008 & 0.0001 \\ 2308 & 15: 35: 48 & 0.0011 & 0.0000 \\ 2310 & 15: 35: 50 & 0.0021 & 0.0003 \\ 2312 & 15: 35: 52 & 0.0009 & 0.0003 \\ 2314 & 15: 35: 54 & 0.0027 & 0.0011 \\ 2316 & 15: 35: 56 & 0.0025 & 0.0000 \\ 2318 & 15: 35: 58 & 0.0003 & 0.0003 \\ 2320 & 15: 36: 00 & 0.0030 & 0.0003 \\ 2322 & 15: 36: 02 & 0.0026 & 0.0011 \\ 2324 & 15: 36: 04 & 0.0003 & 0.0001 \\ 2326 & 15: 36: 06 & 0.0041 & 0.0004 \\ 2328 & 15: 36: 08 & 0.0010 & 0.0047 \\ 2330 & 15: 36: 10 & 0.0003 & 0.0002 \\ 2332 & 15: 36: 12 & 0.0076 & 0.0005 \\ 2334 & 15: 36: 14 & 0.0031 & 0.0027 \\ 2336 & 15: 36: 16 & 0.0029 & 0.0028 \\ 2338 & 15: 36: 18 & 0.0000 & 0.0005 \\ 2340 & 15: 36: 20 & 0.0020 & -0.0001 \\ 2342 & 15: 36: 22 & 0.0002 & 0.0008\end{array}$


Appendix F - Laser Data Test 3 NIST102605_1.xIs

$\begin{array}{llll}2344 & 15: 36: 24 & 0.0004 & 0.0003 \\ 2346 & 15: 36: 26 & 0.0028 & 0.0041 \\ 2348 & 15: 36: 28 & 0.0011 & 0.0009 \\ 2350 & 15: 36: 30 & 0.0000 & 0.0034 \\ 2352 & 15: 36: 32 & 0.0001 & 0.0150 \\ 2354 & 15: 36: 34 & 0.0000 & 0.0000 \\ 2356 & 15: 36: 36 & 0.0001 & 0.0003 \\ 2358 & 15: 36: 38 & 0.0003 & 0.0000 \\ 2360 & 15: 36: 40 & 0.0054 & 0.0005 \\ 2362 & 15: 36: 42 & 0.0064 & 0.0002 \\ 2364 & 15: 36: 44 & 0.0236 & 0.0027 \\ 2366 & 15: 36: 46 & 0.0018 & 0.0007 \\ 2368 & 15: 36: 48 & 0.0006 & 0.0003 \\ 2370 & 15: 36: 50 & 0.0005 & 0.0001 \\ 2372 & 15: 36: 52 & 0.0016 & 0.0005 \\ 2374 & 15: 36: 54 & 0.0002 & 0.0013 \\ 2376 & 15: 36: 56 & 0.0002 & 0.0047 \\ 2378 & 15: 36: 58 & 0.0000 & 0.0001 \\ 2380 & 15: 37: 00 & 0.0005 & 0.0002 \\ 2382 & 15: 37: 02 & 0.0045 & 0.0003 \\ 2384 & 15: 37: 04 & 0.0050 & 0.0002 \\ 2386 & 15: 37: 06 & 0.0012 & 0.0003 \\ 2388 & 15: 37: 08 & 0.0009 & 0.0000 \\ 2390 & 15: 37: 10 & 0.0058 & 0.0000 \\ 2392 & 15: 37: 12 & 0.0009 & 0.0005 \\ 2394 & 15: 37: 14 & 0.0006 & 0.0003 \\ 2396 & 15: 37: 16 & 0.0092 & 0.0000 \\ 2398 & 15: 37: 18 & 0.0007 & 0.0027 \\ 2400 & 15: 37: 20 & 0.0007 & 0.0012 \\ 2402 & 15: 37: 22 & 0.0009 & 0.0000 \\ 2404 & 15: 37: 24 & 0.0102 & 0.0001 \\ 2406 & 15: 37: 26 & 0.0079 & 0.0005 \\ 2408 & 15: 37: 28 & 0.0002 & 0.0002 \\ 2410 & 15: 37: 30 & 0.0021 & 0.0002 \\ 2412 & 15: 37: 32 & 0.0020 & 0.0001 \\ 2414 & 15: 37: 34 & 0.0016 & 0.0058 \\ 2416 & 15: 37: 36 & 0.0002 & 0.0003 \\ 2418 & 15: 37: 38 & 0.0011 & 0.0000 \\ 2420 & 15: 37: 40 & 0.0002 & 0.0007 \\ 2422 & 15: 37: 42 & 0.0051 & 0.0001 \\ 2424 & 15: 37: 44 & 0.0003 & 0.0000 \\ 2426 & 15: 37: 46 & 0.0019 & 0.0000 \\ 2428 & 15: 37: 48 & 0.0010 & 0.0000 \\ 2430 & 15: 37: 50 & 0.0002 & 0.0084 \\ 2432 & 15: 37: 52 & 0.0002 & 0.0001 \\ 2434 & 15: 37: 54 & 0.0001 & 0.0000 \\ 2436 & 15: 37: 56 & 0.0009 & 0.0003 \\ 2438 & 15: 37: 58 & 0.0013 & 0.0022 \\ 2440 & 15: 38: 00 & 0.0005 & 0.0021 \\ 2442 & 15: 38: 02 & 0.0172 & 0.0010 \\ 2444 & 15: 38: 04 & 0.0001 & 0.0216\end{array}$


Appendix F - Laser Data Test 3 NIST102605_1.xIs

$\begin{array}{llll}2446 & 15: 38: 06 & 0.0003 & 0.0132 \\ 2448 & 15: 38: 08 & 0.0020 & 0.0002 \\ 2450 & 15: 38: 10 & 0.0014 & 0.0002 \\ 2452 & 15: 38: 12 & 0.0012 & 0.0001 \\ 2454 & 15: 38: 14 & 0.0065 & 0.0003 \\ 2456 & 15: 38: 16 & 0.0007 & 0.0042 \\ 2458 & 15: 38: 18 & 0.0079 & 0.0032 \\ 2460 & 15: 38: 20 & 0.0035 & 0.0017 \\ 2462 & 15: 38: 22 & 0.0002 & 0.0024 \\ 2464 & 15: 38: 24 & 0.0001 & 0.0057 \\ 2466 & 15: 38: 26 & 0.0029 & 0.0008 \\ 2468 & 15: 38: 28 & 0.0003 & 0.0010 \\ 2470 & 15: 38: 30 & 0.0010 & 0.0042 \\ 2472 & 15: 38: 32 & 0.0002 & 0.0004 \\ 2474 & 15: 38: 34 & 0.0003 & 0.0003 \\ 2476 & 15: 38: 36 & 0.0032 & 0.0017 \\ 2478 & 15: 38: 38 & 0.0022 & 0.0011 \\ 2480 & 15: 38: 40 & 0.0005 & 0.0009 \\ 2482 & 15: 38: 42 & 0.0104 & 0.0017 \\ 2484 & 15: 38: 44 & 0.0006 & 0.0007 \\ 2486 & 15: 38: 46 & 0.0047 & 0.0005 \\ 2488 & 15: 38: 48 & 0.0004 & 0.0002 \\ 2490 & 15: 38: 50 & 0.0002 & 0.0003 \\ 2492 & 15: 38: 52 & 0.0033 & 0.0003 \\ 2494 & 15: 38: 54 & 0.0020 & 0.0001 \\ 2496 & 15: 38: 56 & 0.0005 & 0.0000 \\ 2498 & 15: 38: 58 & 0.0003 & 0.0003 \\ 2500 & 15: 39: 00 & 0.0003 & 0.0000 \\ 2502 & 15: 39: 02 & 0.0015 & 0.0000 \\ 2504 & 15: 39: 04 & 0.0008 & 0.0009 \\ 2506 & 15: 39: 06 & 0.0003 & 0.0008 \\ 2508 & 15: 39: 08 & 0.0012 & 0.0000 \\ 2510 & 15: 39: 10 & 0.0004 & 0.0008 \\ 2512 & 15: 39: 12 & 0.0008 & 0.0000 \\ 2514 & 15: 39: 14 & 0.0004 & 0.0004 \\ 2516 & 15: 39: 16 & 0.0002 & 0.0079 \\ 2518 & 15: 39: 18 & 0.0041 & 0.0003 \\ 2520 & 15: 39: 20 & 0.0019 & 0.0015 \\ 2522 & 15: 39: 22 & 0.0001 & 0.0013 \\ 2524 & 15: 39: 24 & 0.0001 & 0.0003 \\ 2526 & 15: 39: 26 & 0.0036 & 0.0013 \\ 2528 & 15: 39: 28 & 0.0007 & 0.0003 \\ 2530 & 15: 39: 30 & 0.0013 & 0.0003 \\ 2532 & 15: 39: 32 & 0.0000 & 0.0001 \\ 2534 & 15: 39: 34 & 0.0005 & 0.0008 \\ 2536 & 15: 39: 36 & 0.0013 & 0.0002 \\ 2538 & 15: 39: 38 & 0.0076 & 0.0002 \\ 2540 & 15: 39: 40 & 0.0067 & 0.0003 \\ 2542 & 15: 39: 42 & 0.0008 & 0.0003 \\ 2544 & 15: 39: 44 & 0.0001 & 0.0116 \\ 2546 & 15: 39: 46 & 0.0000 & 0.0002\end{array}$


Appendix F - Laser Data Test 3 NIST102605_1.xIs

$\begin{array}{llll}2548 & 15: 39: 48 & 0.0016 & 0.0003 \\ 2550 & 15: 39: 50 & 0.0001 & 0.0003 \\ 2552 & 15: 39: 52 & 0.0005 & 0.0004 \\ 2554 & 15: 39: 54 & 0.0010 & 0.0009 \\ 2556 & 15: 39: 56 & 0.0039 & 0.0025 \\ 2558 & 15: 39: 58 & 0.0006 & 0.0057 \\ 2560 & 15: 40: 00 & 0.0054 & 0.0000 \\ 2562 & 15: 40: 02 & 0.0261 & 0.0003 \\ 2564 & 15: 40: 04 & 0.0032 & 0.0001 \\ 2566 & 15: 40: 06 & 0.0438 & 0.0127 \\ 2568 & 15: 40: 08 & 0.0179 & 0.0105 \\ 2570 & 15: 40: 10 & 0.0020 & 0.0001 \\ 2572 & 15: 40: 12 & 0.0019 & 0.0003 \\ 2574 & 15: 40: 14 & 0.0001 & 0.0002 \\ 2576 & 15: 40: 16 & 0.0002 & 0.0000 \\ 2578 & 15: 40: 18 & 0.0018 & 0.0003 \\ 2580 & 15: 40: 20 & 0.0005 & 0.0001 \\ 2582 & 15: 40: 22 & 0.0013 & 0.0001 \\ 2584 & 15: 40: 24 & 0.0006 & 0.0010 \\ 2586 & 15: 40: 26 & 0.0091 & 0.0062 \\ 2588 & 15: 40: 28 & 0.0029 & 0.0002 \\ 2590 & 15: 40: 30 & 0.0037 & 0.0033 \\ 2592 & 15: 40: 32 & 0.0085 & 0.0002 \\ 2594 & 15: 40: 34 & 0.0194 & 0.0002 \\ 2596 & 15: 40: 36 & 0.0019 & 0.0007 \\ 2598 & 15: 40: 38 & 0.0007 & 0.0006 \\ 2600 & 15: 40: 40 & 0.0011 & 0.0020 \\ 2602 & 15: 40: 42 & 0.0013 & 0.0007 \\ 2604 & 15: 40: 44 & 0.0021 & 0.0008 \\ 2606 & 15: 40: 46 & 0.0044 & 0.0003 \\ 2608 & 15: 40: 48 & 0.0129 & 0.0006 \\ 2610 & 15: 40: 50 & 0.0035 & 0.0045 \\ 2612 & 15: 40: 52 & 0.0067 & 0.0016 \\ 2614 & 15: 40: 54 & 0.0003 & 0.0129 \\ 2616 & 15: 40: 56 & 0.0004 & 0.0005 \\ 2618 & 15: 40: 58 & 0.0004 & 0.0041 \\ 2620 & 15: 41: 00 & 0.0074 & 0.0011 \\ 2622 & 15: 41: 02 & 0.0001 & 0.0000 \\ 2624 & 15: 41: 04 & 0.0024 & 0.0002 \\ 2626 & 15: 41: 06 & 0.0005 & 0.0002 \\ 2628 & 15: 41: 08 & 0.0004 & 0.0004 \\ 2630 & 15: 41: 10 & 0.0003 & 0.0028 \\ 2632 & 15: 41: 12 & 0.0006 & 0.0003 \\ 2634 & 15: 41: 14 & 0.0020 & 0.0001 \\ 2636 & 15: 41: 16 & 0.0026 & 0.0002 \\ 2638 & 15: 41: 18 & 0.0002 & 0.0000 \\ 2640 & 15: 41: 20 & 0.0009 & 0.0001 \\ 2642 & 15: 41: 22 & 0.0001 & 0.0002 \\ 2644 & 15: 41: 24 & 0.0005 & 0.0009 \\ 2646 & 15: 41: 26 & 0.0056 & 0.0000 \\ 2648 & 15: 41: 28 & 0.0010 & 0.0013\end{array}$


Appendix F - Laser Data Test 3 NIST102605_1.xls

$\begin{array}{llll}2650 & 15: 41: 30 & 0.0010 & 0.0016 \\ 2652 & 15: 41: 32 & 0.0038 & 0.0002 \\ 2654 & 15: 41: 34 & 0.0046 & 0.0001 \\ 2656 & 15: 41: 36 & 0.0047 & 0.0003 \\ 2658 & 15: 41: 38 & 0.0015 & 0.0007 \\ 2660 & 15: 41: 40 & 0.0162 & 0.0104 \\ 2662 & 15: 41: 42 & 0.0003 & 0.0021 \\ 2664 & 15: 41: 44 & 0.0002 & 0.0001 \\ 2666 & 15: 41: 46 & 0.0004 & 0.0005 \\ 2668 & 15: 41: 48 & 0.0001 & 0.0000 \\ 2670 & 15: 41: 50 & 0.0001 & 0.0002 \\ 2672 & 15: 41: 52 & 0.0022 & 0.0010 \\ 2674 & 15: 41: 54 & 0.0011 & 0.0006 \\ 2676 & 15: 41: 56 & 0.0017 & 0.0060 \\ 2678 & 15: 41: 58 & 0.0039 & 0.0003 \\ 2680 & 15: 42: 00 & 0.0001 & 0.0013 \\ 2682 & 15: 42: 02 & 0.0002 & 0.0003 \\ 2684 & 15: 42: 04 & 0.0001 & 0.0003 \\ 2686 & 15: 42: 06 & 0.0023 & 0.0000 \\ 2688 & 15: 42: 08 & 0.0003 & 0.0002 \\ 2690 & 15: 42: 10 & 0.0000 & 0.0001 \\ 2692 & 15: 42: 12 & 0.0000 & 0.0003 \\ 2694 & 15: 42: 14 & 0.0000 & 0.0003 \\ 2696 & 15: 42: 16 & 0.0020 & 0.0002 \\ 2698 & 15: 42: 18 & 0.0012 & 0.0003 \\ 2700 & 15: 42: 20 & 0.0016 & 0.0003 \\ 2702 & 15: 42: 22 & 0.0106 & 0.0001 \\ 2704 & 15: 42: 24 & 0.0002 & -0.0001 \\ 2706 & 15: 42: 26 & 0.0005 & 0.0018 \\ 2708 & 15: 42: 28 & 0.0128 & 0.0000 \\ 2710 & 15: 42: 30 & 0.0008 & 0.0052 \\ 2712 & 15: 42: 32 & 0.0009 & 0.0003 \\ 2714 & 15: 42: 34 & 0.0005 & 0.0034 \\ 2716 & 15: 42: 36 & 0.0006 & 0.0024 \\ 2718 & 15: 42: 38 & 0.0001 & 0.0004 \\ 2720 & 15: 42: 40 & 0.0017 & 0.0001 \\ 2722 & 15: 42: 42 & 0.0008 & 0.0003 \\ 2724 & 15: 42: 44 & 0.0000 & 0.0006 \\ 2726 & 15: 42: 46 & 0.0001 & 0.0040 \\ 2728 & 15: 42: 48 & 0.0003 & 0.0003 \\ 2730 & 15: 42: 50 & 0.0002 & 0.0002 \\ 2732 & 15: 42: 52 & 0.0009 & 0.0001 \\ 2734 & 15: 42: 54 & 0.0011 & 0.0003 \\ 2736 & 15: 42: 56 & 0.0002 & 0.0001 \\ 2738 & 15: 42: 58 & 0.0002 & 0.0003 \\ 2740 & 15: 43: 00 & 0.0003 & 0.0024 \\ 2742 & 15: 43: 02 & 0.0004 & 0.0076 \\ 2744 & 15: 43: 04 & 0.0001 & 0.0000 \\ 2746 & 15: 43: 06 & 0.0019 & 0.0010 \\ 2748 & 15: 43: 08 & 0.0004 & 0.0002 \\ 2750 & 15: 43: 10 & 0.0001 & 0.0010\end{array}$


Appendix F - Laser Data Test 3 NIST102605_1.xls

$\begin{array}{llll}2752 & 15: 43: 12 & 0.0014 & 0.0002 \\ 2754 & 15: 43: 14 & 0.0018 & 0.0007 \\ 2756 & 15: 43: 16 & 0.0008 & 0.0000 \\ 2758 & 15: 43: 18 & 0.0009 & 0.0000 \\ 2760 & 15: 43: 20 & 0.0020 & 0.0010 \\ 2762 & 15: 43: 22 & 0.0012 & 0.0000 \\ 2764 & 15: 43: 24 & 0.0107 & 0.0003 \\ 2766 & 15: 43: 26 & 0.0003 & 0.0004 \\ 2768 & 15: 43: 28 & 0.0008 & 0.0006 \\ 2770 & 15: 43: 30 & 0.0002 & 0.0000 \\ 2772 & 15: 43: 32 & 0.0002 & 0.0004 \\ 2774 & 15: 43: 34 & 0.0003 & 0.0003 \\ 2776 & 15: 43: 36 & 0.0003 & 0.0015 \\ 2778 & 15: 43: 38 & 0.0002 & 0.0001 \\ 2780 & 15: 43: 40 & 0.0018 & 0.0004 \\ 2782 & 15: 43: 42 & 0.0020 & 0.0002 \\ 2784 & 15: 43: 44 & 0.0009 & 0.0042 \\ 2786 & 15: 43: 46 & 0.0001 & 0.0004 \\ 2788 & 15: 43: 48 & 0.0004 & -0.0001 \\ 2790 & 15: 43: 50 & 0.0012 & 0.0003 \\ 2792 & 15: 43: 52 & 0.0001 & 0.0003 \\ 2794 & 15: 43: 54 & 0.0048 & 0.0003 \\ 2796 & 15: 43: 56 & 0.0149 & 0.0000 \\ 2798 & 15: 43: 58 & 0.0024 & 0.0056 \\ 2800 & 15: 44: 00 & 0.0042 & 0.0002 \\ 2802 & 15: 44: 02 & 0.0058 & 0.0000 \\ 2804 & 15: 44: 04 & 0.0022 & 0.0001 \\ 2806 & 15: 44: 06 & 0.0010 & 0.0004 \\ 2808 & 15: 44: 08 & 0.0023 & 0.0003 \\ 2810 & 15: 44: 10 & 0.0030 & 0.0006 \\ 2812 & 15: 44: 12 & 0.0004 & 0.0002 \\ 2814 & 15: 44: 14 & 0.0025 & 0.0001 \\ 2816 & 15: 44: 16 & 0.0002 & 0.0001 \\ 2818 & 15: 44: 18 & 0.0010 & 0.0000 \\ 2820 & 15: 44: 20 & 0.0043 & 0.0000 \\ 2822 & 15: 44: 22 & 0.0039 & 0.0014 \\ 2824 & 15: 44: 24 & 0.0025 & 0.0004 \\ 2826 & 15: 44: 26 & 0.0013 & 0.0005 \\ 2828 & 15: 44: 28 & 0.0005 & 0.0023 \\ 2830 & 15: 44: 30 & 0.0003 & 0.0003 \\ 2832 & 15: 44: 32 & 0.0005 & 0.0011 \\ 2834 & 15: 44: 34 & 0.0028 & 0.0004 \\ 2836 & 15: 44: 36 & 0.0003 & 0.0000 \\ 2838 & 15: 44: 38 & 0.0008 & 0.0007 \\ 2840 & 15: 44: 40 & 0.0010 & 0.0002 \\ 2842 & 15: 44: 42 & 0.0005 & 0.0001 \\ 2844 & 15: 44: 44 & 0.0008 & 0.0008 \\ 2846 & 15: 44: 46 & 0.0040 & 0.0003 \\ 2848 & 15: 44: 48 & 0.0009 & 0.0003 \\ 2850 & 15: 44: 50 & 0.0011 & 0.0002 \\ 2852 & 15: 44: 52 & 0.0001 & 0.0003\end{array}$


Appendix F - Laser Data Test 3 NIST102605_1.xls

$\begin{array}{llll}2854 & 15: 44: 54 & 0.0003 & 0.0003 \\ 2856 & 15: 44: 56 & 0.0001 & 0.0001 \\ 2858 & 15: 44: 58 & 0.0019 & 0.0001 \\ 2860 & 15: 45: 00 & 0.0001 & 0.0002 \\ 2862 & 15: 45: 02 & 0.0008 & 0.0003 \\ 2864 & 15: 45: 04 & 0.0021 & 0.0003 \\ 2866 & 15: 45: 06 & 0.0007 & 0.0003 \\ 2868 & 15: 45: 08 & 0.0009 & 0.0002 \\ 2870 & 15: 45: 10 & 0.0002 & 0.0001 \\ 2872 & 15: 45: 12 & 0.0027 & 0.0007 \\ 2874 & 15: 45: 14 & 0.0051 & 0.0012 \\ 2876 & 15: 45: 16 & 0.0008 & 0.0002 \\ 2878 & 15: 45: 18 & 0.0002 & 0.0005 \\ 2880 & 15: 45: 20 & 0.0002 & 0.0003 \\ 2882 & 15: 45: 22 & 0.0004 & 0.0001 \\ 2884 & 15: 45: 24 & 0.0000 & 0.0020 \\ 2886 & 15: 45: 26 & 0.0009 & 0.0004 \\ 2888 & 15: 45: 28 & 0.0009 & 0.0001 \\ 2890 & 15: 45: 30 & 0.0011 & 0.0000 \\ 2892 & 15: 45: 32 & 0.0002 & 0.0005 \\ 2894 & 15: 45: 34 & 0.0043 & 0.0001 \\ 2896 & 15: 45: 36 & 0.0009 & 0.0000 \\ 2898 & 15: 45: 38 & 0.0009 & 0.0002 \\ 2900 & 15: 45: 40 & 0.0032 & 0.0012 \\ 2902 & 15: 45: 42 & 0.0025 & 0.0003 \\ 2904 & 15: 45: 44 & 0.0009 & 0.0015 \\ 2906 & 15: 45: 46 & 0.0263 & 0.0011 \\ 2908 & 15: 45: 48 & 0.0031 & 0.0000 \\ 2910 & 15: 45: 50 & 0.0005 & 0.0003 \\ 2912 & 15: 45: 52 & 0.0006 & 0.0033 \\ 2914 & 15: 45: 54 & 0.0004 & 0.0143 \\ 2916 & 15: 45: 56 & 0.0001 & 0.0024 \\ 2918 & 15: 45: 58 & 0.0004 & 0.0015 \\ 2920 & 15: 46: 00 & 0.0001 & 0.0016 \\ 2922 & 15: 46: 02 & 0.0004 & 0.0000 \\ 2924 & 15: 46: 04 & 0.0007 & 0.0000 \\ 2926 & 15: 46: 06 & 0.0012 & 0.0004 \\ 2928 & 15: 46: 08 & 0.0100 & 0.0012 \\ 2930 & 15: 46: 10 & 0.0002 & 0.0006 \\ 2932 & 15: 46: 12 & 0.0093 & 0.0000 \\ 2934 & 15: 46: 14 & 0.0001 & 0.0001 \\ 2936 & 15: 46: 16 & 0.0028 & 0.0002 \\ 2938 & 15: 46: 18 & 0.0001 & 0.0027 \\ 2940 & 15: 46: 20 & 0.0003 & 0.0003 \\ 2942 & 15: 46: 22 & 0.0027 & 0.0003 \\ 2944 & 15: 46: 24 & 0.0021 & 0.0005 \\ 2946 & 15: 46: 26 & 0.0007 & 0.0005 \\ 2948 & 15: 46: 28 & 0.0009 & 0.0007 \\ 2950 & 15: 46: 30 & 0.0030 & 0.0003 \\ 2952 & 15: 46: 32 & 0.0005 & 0.0027 \\ 2954 & 15: 46: 34 & 0.0017 & 0.0006\end{array}$


Appendix F - Laser Data Test 3 NIST102605_1.xls

$\begin{array}{llll}2956 & 15: 46: 36 & 0.0002 & 0.0022 \\ 2958 & 15: 46: 38 & 0.0002 & 0.0010 \\ 2960 & 15: 46: 40 & 0.0008 & 0.0030 \\ 2962 & 15: 46: 42 & 0.0017 & 0.0004 \\ 2964 & 15: 46: 44 & 0.0009 & 0.0002 \\ 2966 & 15: 46: 46 & 0.0027 & 0.0006 \\ 2968 & 15: 46: 48 & 0.0005 & 0.0005 \\ 2970 & 15: 46: 50 & 0.0007 & 0.0007 \\ 2972 & 15: 46: 52 & 0.0001 & 0.0002 \\ 2974 & 15: 46: 54 & 0.0001 & 0.0002 \\ 2976 & 15: 46: 56 & 0.0001 & 0.0014 \\ 2978 & 15: 46: 58 & 0.0009 & 0.0017 \\ 2980 & 15: 47: 00 & 0.0019 & -0.0001 \\ 2982 & 15: 47: 02 & 0.0010 & 0.0008 \\ 2984 & 15: 47: 04 & 0.0001 & 0.0003 \\ 2986 & 15: 47: 06 & 0.0015 & 0.0014 \\ 2988 & 15: 47: 08 & 0.0002 & 0.0012 \\ 2990 & 15: 47: 10 & 0.0015 & 0.0010 \\ 2992 & 15: 47: 12 & 0.0003 & 0.0008 \\ 2994 & 15: 47: 14 & 0.0001 & 0.0005 \\ 2996 & 15: 47: 16 & 0.0001 & 0.0002 \\ 2998 & 15: 47: 18 & 0.0002 & 0.0000 \\ 3000 & 15: 47: 20 & 0.0008 & 0.0002 \\ 3002 & 15: 47: 22 & 0.0002 & 0.0003 \\ 3004 & 15: 47: 24 & 0.0002 & 0.0049 \\ 3006 & 15: 47: 26 & 0.0056 & 0.0000 \\ 3008 & 15: 47: 28 & 0.0004 & 0.0004 \\ 3010 & 15: 47: 30 & 0.0019 & 0.0002 \\ 3012 & 15: 47: 32 & 0.0010 & 0.0001 \\ 3014 & 15: 47: 34 & 0.0070 & 0.0019 \\ 3016 & 15: 47: 36 & 0.0010 & 0.0010 \\ 3018 & 15: 47: 38 & 0.0008 & 0.0013 \\ 3020 & 15: 47: 40 & 0.0000 & 0.0028 \\ 3022 & 15: 47: 42 & 0.0041 & 0.0010 \\ 3024 & 15: 47: 44 & 0.0002 & 0.0006 \\ 3026 & 15: 47: 46 & 0.0019 & 0.0000 \\ 3028 & 15: 47: 48 & 0.0056 & 0.0004 \\ 3030 & 15: 47: 50 & 0.0047 & 0.0001 \\ 3032 & 15: 47: 52 & 0.0112 & 0.0019 \\ 3034 & 15: 47: 54 & 0.0025 & 0.0003 \\ 3036 & 15: 47: 56 & 0.0004 & 0.0010 \\ 3038 & 15: 47: 58 & 0.0009 & 0.0002 \\ 3040 & 15: 48: 00 & 0.0006 & 0.0009 \\ 3042 & 15: 48: 02 & 0.0020 & 0.0021 \\ 3044 & 15: 48: 04 & 0.0020 & 0.0017 \\ 3046 & 15: 48: 06 & 0.0010 & 0.0003 \\ 3048 & 15: 48: 08 & 0.0003 & 0.0083 \\ 3050 & 15: 48: 10 & 0.0006 & 0.0040 \\ 3052 & 15: 48: 12 & 0.0002 & 0.0003 \\ 3054 & 15: 48: 14 & 0.0051 & 0.0024 \\ 3056 & 15: 48: 16 & 0.0005 & 0.0008\end{array}$


Appendix F - Laser Data Test 3 NIST102605_1.xls

$\begin{array}{llll}3058 & 15: 48: 18 & 0.0065 & 0.0000 \\ 3060 & 15: 48: 20 & 0.0023 & 0.0000 \\ 3062 & 15: 48: 22 & 0.0004 & 0.0001 \\ 3064 & 15: 48: 24 & 0.0072 & 0.0013 \\ 3066 & 15: 48: 26 & 0.0133 & 0.0004 \\ 3068 & 15: 48: 28 & 0.0017 & 0.0003 \\ 3070 & 15: 48: 30 & 0.0009 & 0.0001 \\ 3072 & 15: 48: 32 & 0.0013 & 0.0000 \\ 3074 & 15: 48: 34 & 0.0015 & 0.0007 \\ 3076 & 15: 48: 36 & 0.0013 & 0.0006 \\ 3078 & 15: 48: 38 & 0.0001 & 0.0001 \\ 3080 & 15: 48: 40 & 0.0000 & 0.0047 \\ 3082 & 15: 48: 42 & 0.0000 & 0.0007 \\ 3084 & 15: 48: 44 & 0.0000 & 0.0003 \\ 3086 & 15: 48: 46 & 0.0001 & 0.0017 \\ 3088 & 15: 48: 48 & 0.0006 & 0.0002 \\ 3090 & 15: 48: 50 & 0.0000 & 0.0011 \\ 3092 & 15: 48: 52 & 0.0002 & 0.0001 \\ 3094 & 15: 48: 54 & 0.0015 & 0.0000 \\ 3096 & 15: 48: 56 & 0.0019 & 0.0014 \\ 3098 & 15: 48: 58 & 0.0005 & 0.0001 \\ 3100 & 15: 49: 00 & 0.0018 & 0.0002 \\ 3102 & 15: 49: 02 & 0.0017 & 0.0004 \\ 3104 & 15: 49: 04 & 0.0006 & 0.0003 \\ 3106 & 15: 49: 06 & 0.0008 & 0.0032 \\ 3108 & 15: 49: 08 & 0.0060 & 0.0661 \\ 3110 & 15: 49: 10 & 0.0002 & 0.0001 \\ 3112 & 15: 49: 12 & 0.0005 & 0.0009 \\ 3114 & 15: 49: 14 & 0.0006 & 0.0004 \\ 3116 & 15: 49: 16 & 0.0023 & 0.0007 \\ 3118 & 15: 49: 18 & 0.0016 & 0.0010 \\ 3120 & 15: 49: 20 & 0.0002 & 0.0010 \\ 3122 & 15: 49: 22 & 0.0002 & 0.0005 \\ 3124 & 15: 49: 24 & 0.0001 & 0.0001 \\ 3126 & 15: 49: 26 & 0.0001 & 0.0000 \\ 3128 & 15: 49: 28 & 0.0004 & 0.0018 \\ 3130 & 15: 49: 30 & 0.0246 & 0.0002 \\ 3132 & 15: 49: 32 & 0.0001 & 0.0005 \\ 3134 & 15: 49: 34 & 0.0002 & 0.0003 \\ 3136 & 15: 49: 36 & 0.0001 & 0.0006 \\ 3138 & 15: 49: 38 & 0.0002 & 0.0025 \\ 3140 & 15: 49: 40 & 0.0004 & 0.0037 \\ 3142 & 15: 49: 42 & 0.0008 & 0.0017 \\ 3144 & 15: 49: 44 & 0.0006 & 0.0030 \\ 3146 & 15: 49: 46 & 0.0036 & 0.0133 \\ 3148 & 15: 49: 48 & 0.0012 & 0.0004 \\ 3150 & 15: 49: 50 & 0.0015 & 0.0019 \\ 3152 & 15: 49: 52 & 0.0006 & 0.0034 \\ 3154 & 15: 49: 54 & 0.0135 & 0.0022 \\ 3156 & 15: 49: 56 & 0.0005 & 0.0001 \\ 3158 & 15: 49: 58 & 0.0064 & 0.0003\end{array}$


Appendix F - Laser Data Test 3 NIST102605_1.xIs

$\begin{array}{llll}3160 & 15: 50: 00 & 0.0093 & 0.0010 \\ 3162 & 15: 50: 02 & 0.0003 & 0.0000 \\ 3164 & 15: 50: 04 & 0.0037 & 0.0011 \\ 3166 & 15: 50: 06 & 0.0001 & 0.0072 \\ 3168 & 15: 50: 08 & 0.0078 & 0.0003 \\ 3170 & 15: 50: 10 & 0.0154 & 0.0003 \\ 3172 & 15: 50: 12 & 0.0011 & 0.0002 \\ 3174 & 15: 50: 14 & 0.0010 & 0.0046 \\ 3176 & 15: 50: 16 & 0.0007 & 0.0123 \\ 3178 & 15: 50: 18 & 0.0026 & 0.0017 \\ 3180 & 15: 50: 20 & 0.0012 & 0.0002 \\ 3182 & 15: 50: 22 & 0.0011 & 0.0001 \\ 3184 & 15: 50: 24 & 0.0002 & 0.0000 \\ 3186 & 15: 50: 26 & 0.0008 & 0.0000 \\ 3188 & 15: 50: 28 & 0.0001 & 0.0000 \\ 3190 & 15: 50: 30 & 0.0001 & 0.0001 \\ 3192 & 15: 50: 32 & 0.0005 & 0.0000 \\ 3194 & 15: 50: 34 & 0.0006 & 0.0013 \\ 3196 & 15: 50: 36 & 0.0012 & 0.0035 \\ 3198 & 15: 50: 38 & 0.0002 & 0.0001 \\ 3200 & 15: 50: 40 & 0.0001 & 0.0077 \\ 3202 & 15: 50: 42 & 0.0006 & 0.0000 \\ 3204 & 15: 50: 44 & 0.0002 & 0.0001 \\ 3206 & 15: 50: 46 & 0.0046 & 0.0002 \\ 3208 & 15: 50: 48 & 0.0022 & 0.0001 \\ 3210 & 15: 50: 50 & 0.0013 & 0.0003 \\ 3212 & 15: 50: 52 & 0.0008 & 0.0000 \\ 3214 & 15: 50: 54 & 0.0005 & 0.0013 \\ 3216 & 15: 50: 56 & 0.0020 & 0.0003 \\ 3218 & 15: 50: 58 & 0.0191 & 0.0013 \\ 3220 & 15: 51: 00 & 0.0045 & 0.0005 \\ 3222 & 15: 51: 02 & 0.0002 & 0.0015 \\ 3224 & 15: 51: 04 & 0.0006 & 0.0004 \\ 3226 & 15: 51: 06 & 0.0015 & 0.0018 \\ 3228 & 15: 51: 08 & 0.0033 & 0.0002 \\ 3230 & 15: 51: 10 & 0.0016 & 0.0091 \\ 3232 & 15: 51: 12 & 0.0004 & 0.0015 \\ 3234 & 15: 51: 14 & 0.0065 & 0.0002 \\ 3236 & 15: 51: 16 & 0.0006 & 0.0000 \\ 3238 & 15: 51: 18 & 0.0017 & 0.0002 \\ 3240 & 15: 51: 20 & 0.0158 & 0.0000 \\ 3242 & 15: 51: 22 & 0.0030 & 0.0002 \\ 3244 & 15: 51: 24 & 0.0032 & 0.0003 \\ 3246 & 15: 51: 26 & 0.0027 & 0.0005 \\ 3248 & 15: 51: 28 & 0.0039 & 0.0003 \\ 3250 & 15: 51: 30 & 0.0043 & 0.0004 \\ 3252 & 15: 51: 32 & 0.0020 & 0.0052 \\ 3254 & 15: 51: 34 & 0.0046 & 0.0070 \\ 3256 & 15: 51: 36 & 0.0149 & 0.0045 \\ 3258 & 15: 51: 38 & 0.0077 & 0.0009 \\ 3260 & 15: 51: 40 & 0.0063 & 0.0007\end{array}$


Appendix F - Laser Data Test 3 NIST102605_1.xls

$\begin{array}{llll}3262 & 15: 51: 42 & 0.0015 & 0.0004 \\ 3264 & 15: 51: 44 & 0.0014 & 0.0004 \\ 3266 & 15: 51: 46 & 0.0040 & 0.0055 \\ 3268 & 15: 51: 48 & 0.0091 & 0.0008 \\ 3270 & 15: 51: 50 & 0.0010 & 0.0010 \\ 3272 & 15: 51: 52 & 0.0002 & 0.0016 \\ 3274 & 15: 51: 54 & 0.0011 & 0.0002 \\ 3276 & 15: 51: 56 & 0.0011 & 0.0002 \\ 3278 & 15: 51: 58 & 0.0025 & 0.0003 \\ 3280 & 15: 52: 00 & 0.0025 & 0.0046 \\ 3282 & 15: 52: 02 & 0.0002 & 0.0010 \\ 3284 & 15: 52: 04 & 0.0200 & 0.0006 \\ 3286 & 15: 52: 06 & 0.0008 & 0.0004 \\ 3288 & 15: 52: 08 & 0.0012 & 0.0030 \\ 3290 & 15: 52: 10 & 0.0020 & 0.0020 \\ 3292 & 15: 52: 12 & 0.0034 & 0.0142 \\ 3294 & 15: 52: 14 & 0.0015 & 0.0099 \\ 3296 & 15: 52: 16 & 0.0057 & 0.0010 \\ 3298 & 15: 52: 18 & 0.0156 & 0.0176 \\ 3300 & 15: 52: 20 & 0.0029 & 0.0009 \\ 3302 & 15: 52: 22 & 0.0042 & 0.0018 \\ 3304 & 15: 52: 24 & 0.0014 & 0.0002 \\ 3306 & 15: 52: 26 & 0.0015 & 0.0006 \\ 3308 & 15: 52: 28 & 0.0009 & 0.0005 \\ 3310 & 15: 52: 30 & 0.0011 & 0.0006 \\ 3312 & 15: 52: 32 & 0.0013 & 0.0018 \\ 3314 & 15: 52: 34 & 0.0001 & 0.0012 \\ 3316 & 15: 52: 36 & 0.0005 & 0.0008 \\ 3318 & 15: 52: 38 & 0.0001 & 0.0002 \\ 3320 & 15: 52: 40 & 0.0001 & 0.0001 \\ 3322 & 15: 52: 42 & 0.0001 & 0.0035 \\ 3324 & 15: 52: 44 & 0.0008 & 0.0006 \\ 3326 & 15: 52: 46 & 0.0002 & 0.0016 \\ 3328 & 15: 52: 48 & 0.0003 & 0.0061 \\ 3330 & 15: 52: 50 & 0.0002 & 0.0005 \\ 3332 & 15: 52: 52 & 0.0023 & 0.0001 \\ 3334 & 15: 52: 54 & 0.0038 & 0.0006 \\ 3336 & 15: 52: 56 & 0.0090 & 0.0013 \\ 3338 & 15: 52: 58 & 0.0013 & 0.0036 \\ 3340 & 15: 53: 00 & 0.0035 & 0.0004 \\ 3342 & 15: 53: 02 & 0.0010 & 0.0124 \\ 3344 & 15: 53: 04 & 0.0006 & 0.0023 \\ 3346 & 15: 53: 06 & 0.0002 & 0.0028 \\ 3348 & 15: 53: 08 & 0.0024 & 0.0047 \\ 3350 & 15: 53: 10 & 0.0003 & 0.0006 \\ 3352 & 15: 53: 12 & 0.0002 & 0.0007 \\ 3354 & 15: 53: 14 & 0.0048 & 0.0018 \\ 3356 & 15: 53: 16 & 0.0011 & 0.0024 \\ 3358 & 15: 53: 18 & 0.0004 & 0.0047 \\ 3360 & 15: 53: 20 & 0.0001 & 0.0001 \\ 3362 & 15: 53: 22 & 0.0000 & 0.0017\end{array}$


Appendix F - Laser Data Test 3 NIST102605_1.xls

$\begin{array}{llll}3364 & 15: 53: 24 & 0.0001 & 0.0005 \\ 3366 & 15: 53: 26 & 0.0000 & 0.0037 \\ 3368 & 15: 53: 28 & 0.0017 & 0.0005 \\ 3370 & 15: 53: 30 & 0.0011 & 0.0001 \\ 3372 & 15: 53: 32 & 0.0001 & 0.0001 \\ 3374 & 15: 53: 34 & 0.0004 & 0.0000 \\ 3376 & 15: 53: 36 & 0.0002 & 0.0018 \\ 3378 & 15: 53: 38 & 0.0001 & 0.0004 \\ 3380 & 15: 53: 40 & 0.0009 & 0.0076 \\ 3382 & 15: 53: 42 & 0.0003 & 0.0001 \\ 3384 & 15: 53: 44 & 0.0045 & 0.0011 \\ 3386 & 15: 53: 46 & 0.0018 & 0.0001 \\ 3388 & 15: 53: 48 & 0.0024 & 0.0004 \\ 3390 & 15: 53: 50 & 0.0069 & 0.0041 \\ 3392 & 15: 53: 52 & 0.0641 & 0.0019 \\ 3394 & 15: 53: 54 & 0.0021 & 0.0053 \\ 3396 & 15: 53: 56 & 0.0170 & 0.0013 \\ 3398 & 15: 53: 58 & 0.0187 & 0.0004 \\ 3400 & 15: 54: 00 & 0.0035 & 0.0001 \\ 3402 & 15: 54: 02 & 0.0002 & 0.0003 \\ 3404 & 15: 54: 04 & 0.0026 & 0.0005 \\ 3406 & 15: 54: 06 & 0.0010 & 0.0028 \\ 3408 & 15: 54: 08 & 0.0016 & 0.0000 \\ 3410 & 15: 54: 10 & 0.0001 & 0.0001 \\ 3412 & 15: 54: 12 & 0.0178 & 0.0004 \\ 3414 & 15: 54: 14 & 0.0015 & 0.0040 \\ 3416 & 15: 54: 16 & 0.0001 & 0.0143 \\ 3418 & 15: 54: 18 & 0.0007 & 0.0018 \\ 3420 & 15: 54: 20 & 0.0008 & 0.0002 \\ 3422 & 15: 54: 22 & 0.0063 & 0.0001 \\ 3424 & 15: 54: 24 & 0.0006 & 0.0026 \\ 3426 & 15: 54: 26 & 0.0121 & 0.0058 \\ 3428 & 15: 54: 28 & 0.0013 & 0.0005 \\ 3430 & 15: 54: 30 & 0.0017 & 0.0002 \\ 3432 & 15: 54: 32 & 0.0067 & 0.0022 \\ 3434 & 15: 54: 34 & 0.0017 & 0.0052 \\ 3436 & 15: 54: 36 & 0.0129 & 0.0006 \\ 3438 & 15: 54: 38 & 0.0006 & 0.0076 \\ 3440 & 15: 54: 40 & 0.0015 & 0.0054 \\ 3442 & 15: 54: 42 & 0.0003 & 0.0002 \\ 3444 & 15: 54: 44 & 0.0023 & 0.0014 \\ 3446 & 15: 54: 46 & 0.0749 & 0.0002 \\ 3448 & 15: 54: 48 & 0.0081 & 0.0015 \\ 3450 & 15: 54: 50 & 0.0002 & 0.0032 \\ 3452 & 15: 54: 52 & 0.0043 & 0.0001 \\ 3454 & 15: 54: 54 & 0.0122 & 0.0003 \\ 3456 & 15: 54: 56 & 0.0039 & 0.0011 \\ 3458 & 15: 54: 58 & 0.0005 & 0.0001 \\ 3460 & 15: 55: 00 & 0.0004 & 0.0006 \\ 3462 & 15: 55: 02 & 0.0016 & 0.0012 \\ 3464 & 15: 55: 04 & 0.0001 & 0.0004\end{array}$


Appendix F - Laser Data Test 3 NIST102605_1.xls

$\begin{array}{llll}3466 & 15: 55: 06 & 0.0047 & 0.0000 \\ 3468 & 15: 55: 08 & 0.0005 & 0.0052 \\ 3470 & 15: 55: 10 & 0.0015 & 0.0003 \\ 3472 & 15: 55: 12 & 0.0034 & 0.0021 \\ 3474 & 15: 55: 14 & 0.0027 & 0.0001 \\ 3476 & 15: 55: 16 & 0.0031 & 0.0004 \\ 3478 & 15: 55: 18 & 0.0049 & 0.0003 \\ 3480 & 15: 55: 20 & 0.0008 & 0.0004 \\ 3482 & 15: 55: 22 & 0.0023 & 0.0004 \\ 3484 & 15: 55: 24 & 0.0002 & 0.0069 \\ 3486 & 15: 55: 26 & 0.0002 & 0.0039 \\ 3488 & 15: 55: 28 & 0.0030 & 0.0017 \\ 3490 & 15: 55: 30 & 0.0031 & 0.0010 \\ 3492 & 15: 55: 32 & 0.0025 & 0.0001 \\ 3494 & 15: 55: 34 & 0.0003 & 0.0005 \\ 3496 & 15: 55: 36 & 0.0002 & 0.0029 \\ 3498 & 15: 55: 38 & 0.0015 & 0.0012 \\ 3500 & 15: 55: 40 & 0.0004 & 0.0002 \\ 3502 & 15: 55: 42 & 0.0006 & 0.0041 \\ 3504 & 15: 55: 44 & 0.0005 & 0.0019 \\ 3506 & 15: 55: 46 & 0.0016 & 0.0000 \\ 3508 & 15: 55: 48 & 0.0006 & 0.0004 \\ 3510 & 15: 55: 50 & 0.0052 & 0.0006 \\ 3512 & 15: 55: 52 & 0.0013 & 0.0004 \\ 3514 & 15: 55: 54 & 0.0003 & 0.0000 \\ 3516 & 15: 55: 56 & 0.0007 & 0.0001 \\ 3518 & 15: 55: 58 & 0.0009 & 0.0000 \\ 3520 & 15: 56: 00 & 0.0006 & 0.0004 \\ 3522 & 15: 56: 02 & 0.0016 & 0.0001 \\ 3524 & 15: 56: 04 & 0.0019 & 0.0000 \\ 3526 & 15: 56: 06 & 0.0011 & 0.0077 \\ 3528 & 15: 56: 08 & 0.0008 & 0.0018 \\ 3530 & 15: 56: 10 & 0.0030 & 0.0067 \\ 3532 & 15: 56: 12 & 0.0026 & 0.0002 \\ 3534 & 15: 56: 14 & 0.0008 & 0.0000 \\ 3536 & 15: 56: 16 & 0.0011 & 0.0031 \\ 3538 & 15: 56: 18 & 0.0040 & 0.0094 \\ 3540 & 15: 56: 20 & 0.0009 & 0.0002 \\ 3542 & 15: 56: 22 & 0.0026 & 0.0012 \\ 3544 & 15: 56: 24 & 0.0034 & 0.0012 \\ 3546 & 15: 56: 26 & 0.0004 & 0.0002 \\ 3548 & 15: 56: 28 & 0.0009 & 0.0054 \\ 3550 & 15: 56: 30 & 0.0010 & 0.0002 \\ 3552 & 15: 56: 32 & 0.0057 & -0.0001 \\ 3554 & 15: 56: 34 & 0.0007 & 0.0049 \\ 3556 & 15: 56: 36 & 0.0252 & 0.0009 \\ 3558 & 15: 56: 38 & 0.0004 & 0.0003 \\ 3560 & 15: 56: 40 & 0.0001 & 0.0004 \\ 3562 & 15: 56: 42 & 0.0003 & 0.0011 \\ 3564 & 15: 56: 44 & 0.0006 & 0.0029 \\ 3566 & 15: 56: 46 & 0.0002 & 0.0001\end{array}$


Appendix F - Laser Data Test 3 NIST102605_1.xIs

$\begin{array}{llll}3568 & 15: 56: 48 & 0.0003 & 0.0003 \\ 3570 & 15: 56: 50 & 0.0007 & 0.0009 \\ 3572 & 15: 56: 52 & 0.0021 & 0.0004 \\ 3574 & 15: 56: 54 & 0.0026 & 0.0026 \\ 3576 & 15: 56: 56 & 0.0079 & 0.0008 \\ 3578 & 15: 56: 58 & 0.0021 & 0.0012 \\ 3580 & 15: 57: 00 & 0.0002 & 0.0000 \\ 3582 & 15: 57: 02 & 0.0031 & 0.0023 \\ 3584 & 15: 57: 04 & 0.0006 & 0.0007 \\ 3586 & 15: 57: 06 & 0.0017 & 0.0084 \\ 3588 & 15: 57: 08 & 0.0004 & 0.0004 \\ 3590 & 15: 57: 10 & 0.0011 & 0.0065 \\ 3592 & 15: 57: 12 & 0.0006 & 0.0004 \\ 3594 & 15: 57: 14 & 0.0017 & 0.0003 \\ 3596 & 15: 57: 16 & 0.0008 & 0.0003 \\ 3598 & 15: 57: 18 & 0.0000 & 0.0004 \\ 3600 & 15: 57: 20 & 0.0005 & 0.0048 \\ 3602 & 15: 57: 22 & 0.0005 & 0.0016 \\ 3604 & 15: 57: 24 & 0.0003 & 0.0001 \\ 3606 & 15: 57: 26 & 0.0000 & 0.0020 \\ 3608 & 15: 57: 28 & 0.0012 & 0.0013 \\ 3610 & 15: 57: 30 & 0.0013 & 0.0011 \\ 3612 & 15: 57: 32 & 0.0010 & 0.0008 \\ 3614 & 15: 57: 34 & 0.0009 & 0.0014 \\ 3616 & 15: 57: 36 & 0.0036 & 0.0149 \\ 3618 & 15: 57: 38 & 0.0062 & 0.0011 \\ 3620 & 15: 57: 40 & 0.0006 & 0.0003 \\ 3622 & 15: 57: 42 & 0.0013 & 0.0020 \\ 3624 & 15: 57: 44 & 0.0000 & 0.0035 \\ 3626 & 15: 57: 46 & 0.0010 & 0.0000 \\ 3628 & 15: 57: 48 & 0.0000 & 0.0009 \\ 3630 & 15: 57: 50 & 0.0011 & 0.0027 \\ 3632 & 15: 57: 52 & 0.0049 & 0.0004 \\ 3634 & 15: 57: 54 & 0.0020 & 0.0022 \\ 3636 & 15: 57: 56 & 0.0025 & 0.0009 \\ 3638 & 15: 57: 58 & 0.0018 & 0.0004 \\ 3640 & 15: 58: 00 & 0.0030 & 0.0002 \\ 3642 & 15: 58: 02 & 0.0003 & 0.0011 \\ 3644 & 15: 58: 04 & 0.0016 & 0.0004 \\ 3646 & 15: 58: 06 & 0.0153 & 0.0012 \\ 3648 & 15: 58: 08 & 0.0011 & 0.0023 \\ 3650 & 15: 58: 10 & 0.0012 & 0.0019 \\ 3652 & 15: 58: 12 & 0.0019 & 0.0004 \\ 3654 & 15: 58: 14 & 0.0022 & 0.0039 \\ 3656 & 15: 58: 16 & 0.0017 & 0.0002 \\ 3658 & 15: 58: 18 & 0.0031 & 0.0056 \\ 3660 & 15: 58: 20 & 0.0010 & 0.0004 \\ 3662 & 15: 58: 22 & 0.0033 & 0.0039 \\ 3664 & 15: 58: 24 & 0.0001 & 0.0006 \\ 3666 & 15: 58: 26 & 0.0014 & 0.0105 \\ 3668 & 15: 58: 28 & 0.0002 & 0.0007\end{array}$


Appendix F - Laser Data Test 3 NIST102605_1.xls

$\begin{array}{llll}3670 & 15: 58: 30 & 0.0002 & 0.0079 \\ 3672 & 15: 58: 32 & 0.0005 & 0.0004 \\ 3674 & 15: 58: 34 & 0.0018 & 0.0000 \\ 3676 & 15: 58: 36 & 0.0005 & 0.0001 \\ 3678 & 15: 58: 38 & 0.0007 & 0.0002 \\ 3680 & 15: 58: 40 & 0.0008 & 0.0064 \\ 3682 & 15: 58: 42 & 0.0002 & 0.0007 \\ 3684 & 15: 58: 44 & 0.0000 & 0.0002 \\ 3686 & 15: 58: 46 & 0.0005 & 0.0001 \\ 3688 & 15: 58: 48 & 0.0004 & 0.0013 \\ 3690 & 15: 58: 50 & 0.0093 & 0.0000 \\ 3692 & 15: 58: 52 & 0.0063 & 0.0005 \\ 3694 & 15: 58: 54 & 0.0008 & 0.0000 \\ 3696 & 15: 58: 56 & 0.0123 & 0.0002 \\ 3698 & 15: 58: 58 & 0.0013 & 0.0001 \\ 3700 & 15: 59: 00 & 0.0077 & 0.0049 \\ 3702 & 15: 59: 02 & 0.0044 & 0.0008 \\ 3704 & 15: 59: 04 & 0.0004 & 0.0002 \\ 3706 & 15: 59: 06 & 0.0020 & 0.0004 \\ 3708 & 15: 59: 08 & 0.0047 & 0.0085 \\ 3710 & 15: 59: 10 & 0.0022 & 0.0076 \\ 3712 & 15: 59: 12 & 0.0004 & 0.0003 \\ 3714 & 15: 59: 14 & 0.0018 & 0.0002 \\ 3716 & 15: 59: 16 & 0.0023 & 0.0057 \\ 3718 & 15: 59: 18 & 0.0011 & 0.0049 \\ 3720 & 15: 59: 20 & 0.0012 & 0.0006 \\ 3722 & 15: 59: 22 & 0.0044 & 0.0009 \\ 3724 & 15: 59: 24 & 0.0004 & 0.0010 \\ 3726 & 15: 59: 26 & 0.0002 & 0.0002 \\ 3728 & 15: 59: 28 & 0.0002 & 0.0006 \\ 3730 & 15: 59: 30 & 0.0000 & 0.0002 \\ 3732 & 15: 59: 32 & 0.0000 & 0.0047 \\ 3734 & 15: 59: 34 & 0.0002 & 0.0046 \\ 3736 & 15: 59: 36 & 0.0007 & 0.0187 \\ 3738 & 15: 59: 38 & 0.0005 & 0.0001 \\ 3740 & 15: 59: 40 & 0.0019 & 0.0004 \\ 3742 & 15: 59: 42 & 0.0141 & 0.0015 \\ 3744 & 15: 59: 44 & 0.0022 & 0.0015 \\ 3746 & 15: 59: 46 & 0.0010 & 0.0000 \\ 3748 & 15: 59: 48 & 0.0015 & 0.0004 \\ 3750 & 15: 59: 50 & 0.0000 & 0.0012 \\ 3752 & 15: 59: 52 & 0.0001 & 0.0004 \\ 3754 & 15: 59: 54 & 0.0006 & 0.0027 \\ 3756 & 15: 59: 56 & 0.0014 & 0.0018 \\ 3758 & 15: 59: 58 & 0.0001 & 0.0001 \\ 3760 & 16: 00: 00 & 0.0004 & 0.0003 \\ 3762 & 16: 00: 02 & 0.0038 & 0.0004 \\ 3764 & 16: 00: 04 & 0.0002 & 0.0025 \\ 3766 & 16: 00: 06 & 0.0015 & 0.0194 \\ 3768 & 16: 00: 08 & 0.0012 & 0.0035 \\ 3770 & 16: 00: 10 & 0.0016 & 0.0002\end{array}$


Appendix F - Laser Data Test 3 NIST102605_1.xIs

$\begin{array}{llll}3772 & 16: 00: 12 & 0.0000 & 0.0016 \\ 3774 & 16: 00: 14 & 0.0058 & 0.0024 \\ 3776 & 16: 00: 16 & 0.0007 & 0.0003 \\ 3778 & 16: 00: 18 & 0.0001 & 0.0001 \\ 3780 & 16: 00: 20 & 0.0067 & 0.0035 \\ 3782 & 16: 00: 22 & 0.0003 & 0.0018 \\ 3784 & 16: 00: 24 & 0.0001 & 0.0065 \\ 3786 & 16: 00: 26 & 0.0003 & 0.0003 \\ 3788 & 16: 00: 28 & 0.0001 & 0.0009 \\ 3790 & 16: 00: 30 & 0.0003 & 0.0008 \\ 3792 & 16: 00: 32 & 0.0002 & 0.0006 \\ 3794 & 16: 00: 34 & 0.0007 & 0.0020 \\ 3796 & 16: 00: 36 & 0.0026 & 0.0002 \\ 3798 & 16: 00: 38 & 0.0019 & 0.0000 \\ 3800 & 16: 00: 40 & 0.0004 & 0.0003 \\ 3802 & 16: 00: 42 & 0.0015 & 0.0016 \\ 3804 & 16: 00: 44 & 0.0072 & 0.0001 \\ 3806 & 16: 00: 46 & 0.0018 & 0.0004 \\ 3808 & 16: 00: 48 & 0.0014 & 0.0003 \\ 3810 & 16: 00: 50 & 0.0056 & 0.0031 \\ 3812 & 16: 00: 52 & 0.0033 & 0.0012 \\ 3814 & 16: 00: 54 & 0.0425 & 0.0012 \\ 3816 & 16: 00: 56 & 0.0044 & 0.0004 \\ 3818 & 16: 00: 58 & 0.0098 & 0.0003 \\ 3820 & 16: 01: 00 & 0.0351 & 0.0004 \\ 3822 & 16: 01: 02 & 0.0018 & 0.0031 \\ 3824 & 16: 01: 04 & 0.0025 & 0.0001 \\ 3826 & 16: 01: 06 & 0.0003 & 0.0018 \\ 3828 & 16: 01: 08 & 0.0017 & 0.0011 \\ 3830 & 16: 01: 10 & 0.0014 & 0.0000 \\ 3832 & 16: 01: 12 & 0.0001 & 0.0000 \\ 3834 & 16: 01: 14 & 0.0005 & 0.0000 \\ 3836 & 16: 01: 16 & 0.0010 & 0.0016 \\ 3838 & 16: 01: 18 & 0.0119 & 0.0019 \\ 3840 & 16: 01: 20 & 0.0044 & 0.0001 \\ 3842 & 16: 01: 22 & 0.0408 & 0.0069 \\ 3844 & 16: 01: 24 & 0.0019 & 0.0001 \\ 3846 & 16: 01: 26 & 0.0015 & 0.0018 \\ 3848 & 16: 01: 28 & 0.0042 & 0.0002 \\ 3850 & 16: 01: 30 & 0.0009 & 0.0010 \\ 3852 & 16: 01: 32 & 0.0005 & 0.0010 \\ 3854 & 16: 01: 34 & 0.0009 & 0.0043 \\ 3856 & 16: 01: 36 & 0.0021 & 0.0002 \\ 3858 & 16: 01: 38 & 0.0023 & 0.0003 \\ 3860 & 16: 01: 40 & 0.0018 & 0.0001 \\ 3862 & 16: 01: 42 & 0.0000 & 0.0002 \\ 3864 & 16: 01: 44 & 0.0134 & 0.0005 \\ 3866 & 16: 01: 46 & 0.0009 & 0.0004 \\ 3868 & 16: 01: 48 & 0.0190 & 0.0003 \\ 3870 & 16: 01: 50 & 0.0017 & 0.0000 \\ 3872 & 16: 01: 52 & 0.0011 & 0.0003\end{array}$


Appendix F - Laser Data Test 3 NIST102605_1.xls

$\begin{array}{llll}3874 & 16: 01: 54 & 0.0018 & 0.0003 \\ 3876 & 16: 01: 56 & 0.0004 & 0.0005 \\ 3878 & 16: 01: 58 & 0.0000 & 0.0028 \\ 3880 & 16: 02: 00 & 0.0001 & 0.0014 \\ 3882 & 16: 02: 02 & 0.0011 & 0.0004 \\ 3884 & 16: 02: 04 & 0.0034 & 0.0002 \\ 3886 & 16: 02: 06 & 0.0061 & 0.0006 \\ 3888 & 16: 02: 08 & 0.0008 & 0.0003 \\ 3890 & 16: 02: 10 & 0.0030 & 0.0051 \\ 3892 & 16: 02: 12 & 0.0038 & 0.0003 \\ 3894 & 16: 02: 14 & 0.0024 & 0.0004 \\ 3896 & 16: 02: 16 & 0.0014 & 0.0003 \\ 3898 & 16: 02: 18 & 0.0018 & 0.0000 \\ 3900 & 16: 02: 20 & 0.0029 & 0.0026 \\ 3902 & 16: 02: 22 & 0.0048 & 0.0003 \\ 3904 & 16: 02: 24 & 0.0014 & 0.0000 \\ 3906 & 16: 02: 26 & 0.0002 & 0.0002 \\ 3908 & 16: 02: 28 & 0.0002 & 0.0001 \\ 3910 & 16: 02: 30 & 0.0047 & 0.0002 \\ 3912 & 16: 02: 32 & 0.0002 & 0.0003 \\ 3914 & 16: 02: 34 & 0.0005 & 0.0005 \\ 3916 & 16: 02: 36 & 0.0171 & 0.0041 \\ 3918 & 16: 02: 38 & 0.0011 & 0.0002 \\ 3920 & 16: 02: 40 & 0.0111 & 0.0038 \\ 3922 & 16: 02: 42 & 0.0003 & 0.0018 \\ 3924 & 16: 02: 44 & 0.0012 & 0.0028 \\ 3926 & 16: 02: 46 & 0.0029 & 0.0005 \\ 3928 & 16: 02: 48 & 0.0035 & 0.0001 \\ 3930 & 16: 02: 50 & 0.0061 & 0.0004 \\ 3932 & 16: 02: 52 & 0.0011 & 0.0003 \\ 3934 & 16: 02: 54 & 0.0002 & 0.0004 \\ 3936 & 16: 02: 56 & 0.0002 & 0.0019 \\ 3938 & 16: 02: 58 & 0.0001 & 0.0001 \\ 3940 & 16: 03: 00 & 0.0006 & 0.0076 \\ 3942 & 16: 03: 02 & 0.0080 & 0.0010 \\ 3944 & 16: 03: 04 & 0.0041 & 0.0021 \\ 3946 & 16: 03: 06 & 0.0016 & 0.0048 \\ 3948 & 16: 03: 08 & 0.0034 & 0.0002 \\ 3950 & 16: 03: 10 & 0.0011 & 0.0035 \\ 3952 & 16: 03: 12 & 0.0004 & 0.0039 \\ 3954 & 16: 03: 14 & 0.0003 & 0.0012 \\ 3956 & 16: 03: 16 & 0.0003 & 0.0001 \\ 3958 & 16: 03: 18 & 0.0029 & 0.0009 \\ 3960 & 16: 03: 20 & 0.0006 & 0.0096 \\ 3962 & 16: 03: 22 & 0.0034 & 0.0012 \\ 3964 & 16: 03: 24 & 0.0008 & 0.0036 \\ 3966 & 16: 03: 26 & 0.0020 & 0.0006 \\ 3968 & 16: 03: 28 & 0.0000 & 0.0036 \\ 3970 & 16: 03: 30 & 0.0001 & 0.0023 \\ 3974 & 16: 03: 32 & 0.0001 & 0.0007 \\ & 16: 03: 34 & 0.0004 & 0.0001\end{array}$


Appendix F - Laser Data Test 3 NIST102605_1.xls

$\begin{array}{llll}3976 & 16: 03: 36 & 0.0007 & 0.0052 \\ 3978 & 16: 03: 38 & 0.0035 & 0.0002 \\ 3980 & 16: 03: 40 & 0.0018 & 0.0083 \\ 3982 & 16: 03: 42 & 0.0174 & 0.0006 \\ 3984 & 16: 03: 44 & 0.0027 & 0.0024 \\ 3986 & 16: 03: 46 & 0.0016 & 0.0015 \\ 3988 & 16: 03: 48 & 0.0019 & 0.0005 \\ 3990 & 16: 03: 50 & 0.0109 & 0.0009 \\ 3992 & 16: 03: 52 & 0.0008 & 0.0001 \\ 3994 & 16: 03: 54 & 0.0050 & 0.0034 \\ 3996 & 16: 03: 56 & 0.0004 & 0.0066 \\ 3998 & 16: 03: 58 & 0.0003 & 0.0006 \\ 4000 & 16: 04: 00 & 0.0004 & 0.0001 \\ 4002 & 16: 04: 02 & 0.0020 & 0.0011 \\ 4004 & 16: 04: 04 & 0.0003 & 0.0001 \\ 4006 & 16: 04: 06 & 0.0018 & 0.0002 \\ 4008 & 16: 04: 08 & 0.0030 & 0.0008 \\ 4010 & 16: 04: 10 & 0.0013 & 0.0006 \\ 4012 & 16: 04: 12 & 0.0018 & 0.0040 \\ 4014 & 16: 04: 14 & 0.0038 & 0.0006 \\ 4016 & 16: 04: 16 & 0.0007 & 0.0003 \\ 4018 & 16: 04: 18 & 0.0017 & 0.0001 \\ 4020 & 16: 04: 20 & 0.0071 & 0.0002 \\ 4022 & 16: 04: 22 & 0.0058 & 0.0004 \\ 4024 & 16: 04: 24 & 0.0019 & 0.0001 \\ 4026 & 16: 04: 26 & 0.0009 & 0.0002 \\ 4028 & 16: 04: 28 & 0.0047 & 0.0002 \\ 4030 & 16: 04: 30 & 0.0032 & 0.0005 \\ 4032 & 16: 04: 32 & 0.0074 & 0.0030 \\ 4034 & 16: 04: 34 & 0.0006 & 0.0003 \\ 4036 & 16: 04: 36 & 0.0013 & 0.0003 \\ 4038 & 16: 04: 38 & 0.0024 & 0.0001 \\ 4040 & 16: 04: 40 & 0.0037 & 0.0197 \\ 4042 & 16: 04: 42 & 0.0003 & 0.0228 \\ 4044 & 16: 04: 44 & 0.0056 & 0.0000 \\ 4046 & 16: 04: 46 & 0.0002 & 0.0005 \\ 4048 & 16: 04: 48 & 0.0010 & 0.0001 \\ 4050 & 16: 04: 50 & 0.0051 & 0.0002 \\ 4052 & 16: 04: 52 & 0.0004 & 0.0003 \\ 4054 & 16: 04: 54 & 0.0007 & 0.0008 \\ 4056 & 16: 04: 56 & 0.0023 & 0.0002 \\ 4058 & 16: 04: 58 & 0.0518 & 0.0001 \\ 4060 & 16: 05: 00 & 0.0003 & 0.0037 \\ 4062 & 16: 05: 02 & 0.0077 & 0.0048 \\ 4064 & 16: 05: 04 & 0.0006 & 0.0001 \\ 4066 & 16: 05: 06 & 0.0067 & 0.0004 \\ 4068 & 16: 05: 08 & 0.0005 & 0.0002 \\ 4070 & 16: 05: 10 & 0.0040 & 0.0002 \\ 4072 & 16: 05: 12 & 0.0022 & 0.0002 \\ 4074 & 16: 05: 14 & 0.0033 & 0.0011 \\ 4076 & 16: 05: 16 & 0.0052 & 0.0008\end{array}$


Appendix F - Laser Data Test 3 NIST102605_1.xls

$\begin{array}{llll}4078 & 16: 05: 18 & 0.0005 & 0.0003 \\ 4080 & 16: 05: 20 & 0.0005 & 0.0002 \\ 4082 & 16: 05: 22 & 0.0000 & 0.0002 \\ 4084 & 16: 05: 24 & 0.0023 & 0.0005 \\ 4086 & 16: 05: 26 & 0.0032 & 0.0003 \\ 4088 & 16: 05: 28 & 0.0018 & 0.0005 \\ 4090 & 16: 05: 30 & 0.0053 & 0.0074 \\ 4092 & 16: 05: 32 & 0.0019 & 0.0023 \\ 4094 & 16: 05: 34 & 0.0007 & 0.0008 \\ 4096 & 16: 05: 36 & 0.0002 & 0.0003 \\ 4098 & 16: 05: 38 & 0.0002 & 0.0006 \\ 4100 & 16: 05: 40 & 0.0011 & 0.0001 \\ 4102 & 16: 05: 42 & 0.0030 & 0.0007 \\ 4104 & 16: 05: 44 & 0.0006 & 0.0001 \\ 4106 & 16: 05: 46 & 0.0003 & 0.0004 \\ 4108 & 16: 05: 48 & 0.0011 & 0.0004 \\ 4110 & 16: 05: 50 & 0.0010 & 0.0017 \\ 4112 & 16: 05: 52 & 0.0007 & 0.0005 \\ 4114 & 16: 05: 54 & 0.0014 & 0.0000 \\ 4116 & 16: 05: 56 & 0.0008 & 0.0007 \\ 4118 & 16: 05: 58 & 0.0019 & 0.0000 \\ 4120 & 16: 06: 00 & 0.0009 & 0.0002 \\ 4122 & 16: 06: 02 & 0.0003 & 0.0017 \\ 4124 & 16: 06: 04 & 0.0013 & 0.0011 \\ 4126 & 16: 06: 06 & 0.0008 & 0.0004 \\ 4128 & 16: 06: 08 & 0.0001 & 0.0035 \\ 4130 & 16: 06: 10 & 0.0042 & 0.0003 \\ 4132 & 16: 06: 12 & 0.0112 & 0.0063 \\ 4134 & 16: 06: 14 & 0.0022 & 0.0002 \\ 4136 & 16: 06: 16 & 0.0024 & 0.0035 \\ 4138 & 16: 06: 18 & 0.0023 & 0.0018 \\ 4140 & 16: 06: 20 & 0.0002 & 0.0012 \\ 4142 & 16: 06: 22 & 0.0057 & 0.0004 \\ 4144 & 16: 06: 24 & 0.0005 & 0.0002 \\ 4146 & 16: 06: 26 & 0.0002 & 0.0001 \\ 4148 & 16: 06: 28 & 0.0197 & 0.0006 \\ 4150 & 16: 06: 30 & 0.0007 & 0.0000 \\ 4152 & 16: 06: 32 & 0.0037 & 0.0000 \\ 4154 & 16: 06: 34 & 0.0002 & 0.0015 \\ 4156 & 16: 06: 36 & 0.0047 & 0.0012 \\ 4158 & 16: 06: 38 & 0.0037 & 0.0007 \\ 4160 & 16: 06: 40 & 0.0014 & 0.0009 \\ 4162 & 16: 06: 42 & 0.0007 & 0.0014 \\ 4164 & 16: 06: 44 & 0.0046 & 0.0023 \\ 4166 & 16: 06: 46 & 0.0028 & 0.0000 \\ 4168 & 16: 06: 48 & 0.0021 & 0.0006 \\ 4170 & 16: 06: 50 & 0.0021 & 0.0015 \\ 4172 & 16: 06: 52 & 0.0012 & 0.0001 \\ 4174 & 16: 06: 54 & 0.0073 & 0.0044 \\ 4176 & 16: 06: 56 & 0.0007 & 0.0001 \\ 4178 & 16: 06: 58 & 0.0164 & 0.0000\end{array}$


Appendix F - Laser Data Test 3 NIST102605_1.xls

$\begin{array}{llll}4180 & 16: 07: 00 & 0.0003 & 0.0001 \\ 4182 & 16: 07: 02 & 0.0010 & 0.0000 \\ 4184 & 16: 07: 04 & 0.0076 & 0.0013 \\ 4186 & 16: 07: 06 & 0.0020 & 0.0020 \\ 4188 & 16: 07: 08 & 0.0003 & 0.0087 \\ 4190 & 16: 07: 10 & 0.0002 & 0.0005 \\ 4192 & 16: 07: 12 & 0.0125 & 0.0061 \\ 4194 & 16: 07: 14 & 0.0021 & 0.0004 \\ 4196 & 16: 07: 16 & 0.0007 & 0.0005 \\ 4198 & 16: 07: 18 & 0.0011 & 0.0004 \\ 4200 & 16: 07: 20 & 0.0039 & 0.0023 \\ 4202 & 16: 07: 22 & 0.0039 & 0.0041 \\ 4204 & 16: 07: 24 & 0.0003 & 0.0003 \\ 4206 & 16: 07: 26 & 0.0019 & 0.0005 \\ 4208 & 16: 07: 28 & 0.0008 & 0.0032 \\ 4210 & 16: 07: 30 & 0.0002 & 0.0006 \\ 4212 & 16: 07: 32 & 0.0002 & 0.0042 \\ 4214 & 16: 07: 34 & 0.0040 & 0.0038 \\ 4216 & 16: 07: 36 & 0.0007 & 0.0004 \\ 4218 & 16: 07: 38 & 0.0006 & 0.0069 \\ 4220 & 16: 07: 40 & 0.0005 & 0.0008 \\ 4222 & 16: 07: 42 & 0.0003 & 0.0182 \\ 4224 & 16: 07: 44 & 0.0005 & 0.0001 \\ 4226 & 16: 07: 46 & 0.0002 & 0.0004 \\ 4228 & 16: 07: 48 & 0.0002 & 0.0010 \\ 4230 & 16: 07: 50 & 0.0002 & 0.0024 \\ 4232 & 16: 07: 52 & 0.0002 & 0.0002 \\ 4234 & 16: 07: 54 & 0.0002 & 0.0004 \\ 4236 & 16: 07: 56 & 0.0011 & 0.0018 \\ 4238 & 16: 07: 58 & 0.0007 & 0.0004 \\ 4240 & 16: 08: 00 & 0.0001 & 0.0015 \\ 4242 & 16: 08: 02 & 0.0001 & 0.0022 \\ 4244 & 16: 08: 04 & 0.0012 & 0.0010 \\ 4246 & 16: 08: 06 & 0.0003 & 0.0005 \\ 4248 & 16: 08: 08 & 0.0006 & 0.0003 \\ 4250 & 16: 08: 10 & 0.0007 & 0.0002 \\ 4252 & 16: 08: 12 & 0.0011 & 0.0023 \\ 4254 & 16: 08: 14 & 0.0019 & 0.0003 \\ 4256 & 16: 08: 16 & 0.0045 & 0.0010 \\ 4258 & 16: 08: 18 & 0.0046 & 0.0021 \\ 4260 & 16: 08: 20 & 0.0002 & 0.0003 \\ 4262 & 16: 08: 22 & 0.0002 & 0.0013 \\ 4264 & 16: 08: 24 & 0.0002 & 0.0010 \\ 4266 & 16: 08: 26 & 0.0002 & 0.0012 \\ 4268 & 16: 08: 28 & 0.0010 & 0.0002 \\ 4270 & 16: 08: 30 & 0.0064 & 0.0024 \\ 4272 & 16: 08: 32 & 0.0086 & 0.0007 \\ 4274 & 16: 08: 34 & 0.0001 & 0.0016 \\ 4276 & 16: 08: 36 & 0.0002 & 0.0004 \\ 4278 & 16: 08: 38 & 0.0005 & 0.0005 \\ 4280 & 16: 08: 40 & 0.0006 & 0.0004\end{array}$


Appendix F - Laser Data Test 3 NIST102605_1.xls

$\begin{array}{llll}4282 & 16: 08: 42 & 0.0002 & 0.0011 \\ 4284 & 16: 08: 44 & 0.0002 & 0.0007 \\ 4286 & 16: 08: 46 & 0.0001 & 0.0015 \\ 4288 & 16: 08: 48 & 0.0094 & 0.0019 \\ 4290 & 16: 08: 50 & 0.0031 & 0.0003 \\ 4292 & 16: 08: 52 & 0.0026 & 0.0026 \\ 4294 & 16: 08: 54 & 0.0008 & 0.0004 \\ 4296 & 16: 08: 56 & 0.0011 & 0.0014 \\ 4298 & 16: 08: 58 & 0.0006 & 0.0003 \\ 4300 & 16: 09: 00 & 0.0009 & 0.0006 \\ 4302 & 16: 09: 02 & 0.0085 & 0.0000 \\ 4304 & 16: 09: 04 & 0.0138 & 0.0004 \\ 4306 & 16: 09: 06 & 0.0018 & 0.0004 \\ 4308 & 16: 09: 08 & 0.0027 & 0.0046 \\ 4310 & 16: 09: 10 & 0.0002 & 0.0003 \\ 4312 & 16: 09: 12 & 0.0006 & 0.0005 \\ 4314 & 16: 09: 14 & 0.0006 & 0.0017 \\ 4316 & 16: 09: 16 & 0.0010 & 0.0014 \\ 4318 & 16: 09: 18 & 0.0010 & 0.0096 \\ 4320 & 16: 09: 20 & 0.0001 & 0.0070 \\ 4322 & 16: 09: 22 & 0.0002 & 0.0079 \\ 4324 & 16: 09: 24 & 0.0002 & 0.0046 \\ 4326 & 16: 09: 26 & 0.0017 & 0.0002 \\ 4328 & 16: 09: 28 & 0.0047 & 0.0006 \\ 4330 & 16: 09: 30 & 0.0045 & 0.0002 \\ 4332 & 16: 09: 32 & 0.0005 & 0.0021 \\ 4334 & 16: 09: 34 & 0.0013 & 0.0014 \\ 4336 & 16: 09: 36 & 0.0072 & 0.0007 \\ 4338 & 16: 09: 38 & 0.0006 & 0.0016 \\ 4340 & 16: 09: 40 & 0.0008 & 0.0018 \\ 4342 & 16: 09: 42 & 0.0012 & 0.0003 \\ 4344 & 16: 09: 44 & 0.0044 & 0.0011 \\ 4346 & 16: 09: 46 & 0.0140 & 0.0016 \\ 4348 & 16: 09: 48 & 0.0002 & 0.0002 \\ 4350 & 16: 09: 50 & 0.0004 & 0.0004 \\ 4352 & 16: 09: 52 & 0.0013 & 0.0034 \\ 4354 & 16: 09: 54 & 0.0012 & 0.0019 \\ 4356 & 16: 09: 56 & 0.0007 & 0.0116 \\ 4358 & 16: 09: 58 & 0.0007 & 0.0206 \\ 4360 & 16: 10: 00 & 0.0014 & 0.0083 \\ 4362 & 16: 10: 02 & 0.0003 & 0.0006 \\ 4364 & 16: 10: 04 & 0.0003 & 0.0116 \\ 4366 & 16: 10: 06 & 0.0005 & 0.0130 \\ 4368 & 16: 10: 08 & 0.0012 & 0.0140 \\ 4370 & 16: 10: 10 & 0.0017 & 0.0314 \\ 4372 & 16: 10: 12 & 0.0004 & 0.0008 \\ 4374 & 16: 10: 14 & 0.0007 & 0.0069 \\ 4376 & 16: 10: 16 & 0.0002 & 0.0015 \\ 4378 & 16: 10: 18 & 0.0013 & 0.0030 \\ 4380 & 16: 10: 20 & 0.0026 & 0.0016 \\ 4382 & 16: 10: 22 & 0.0032 & 0.0021\end{array}$


Appendix F - Laser Data Test 3 NIST102605_1.xls

$\begin{array}{llll}4384 & 16: 10: 24 & 0.0002 & 0.0093 \\ 4386 & 16: 10: 26 & 0.0029 & 0.0067 \\ 4388 & 16: 10: 28 & 0.0009 & 0.0043 \\ 4390 & 16: 10: 30 & 0.0015 & 0.0006 \\ 4392 & 16: 10: 32 & 0.0035 & 0.0033 \\ 4394 & 16: 10: 34 & 0.0146 & 0.0009 \\ 4396 & 16: 10: 36 & 0.0036 & 0.0019 \\ 4398 & 16: 10: 38 & 0.0008 & 0.0065 \\ 4400 & 16: 10: 40 & 0.0030 & 0.0044 \\ 4402 & 16: 10: 42 & 0.0010 & 0.0011 \\ 4404 & 16: 10: 44 & 0.0007 & 0.0005 \\ 4406 & 16: 10: 46 & 0.0001 & 0.0021 \\ 4408 & 16: 10: 48 & 0.0002 & 0.0001 \\ 4410 & 16: 10: 50 & 0.0001 & 0.0012 \\ 4412 & 16: 10: 52 & 0.0068 & 0.0304 \\ 4414 & 16: 10: 54 & 0.0035 & 0.0211 \\ 4416 & 16: 10: 56 & 0.0064 & 0.0156 \\ 4418 & 16: 10: 58 & 0.0016 & 0.0004 \\ 4420 & 16: 11: 00 & 0.0001 & 0.0061 \\ 4422 & 16: 11: 02 & 0.0002 & 0.0004 \\ 4424 & 16: 11: 04 & 0.0024 & 0.0023 \\ 4426 & 16: 11: 06 & 0.0006 & 0.0044 \\ 4428 & 16: 11: 08 & 0.0002 & 0.0026 \\ 4430 & 16: 11: 10 & 0.0013 & 0.0004 \\ 4432 & 16: 11: 12 & 0.0034 & 0.0046 \\ 4434 & 16: 11: 14 & 0.0010 & 0.0021 \\ 4436 & 16: 11: 16 & 0.0037 & 0.0101 \\ 4438 & 16: 11: 18 & 0.0022 & 0.0027 \\ 4440 & 16: 11: 20 & 0.0007 & 0.0013 \\ 4442 & 16: 11: 22 & 0.0002 & 0.0021 \\ 4444 & 16: 11: 24 & 0.0003 & 0.0055 \\ 4446 & 16: 11: 26 & 0.0001 & 0.0013 \\ 4448 & 16: 11: 28 & 0.0003 & 0.0021 \\ 4450 & 16: 11: 30 & 0.0001 & 0.0047 \\ 4452 & 16: 11: 32 & 0.0004 & 0.0052 \\ 4454 & 16: 11: 34 & 0.0008 & 0.0785 \\ 4456 & 16: 11: 36 & 0.0008 & 0.0814 \\ 4458 & 16: 11: 38 & 0.0004 & 0.0103 \\ 4460 & 16: 11: 40 & 0.0039 & 0.0011 \\ 4462 & 16: 11: 42 & 0.0006 & 0.0011 \\ 4464 & 16: 11: 44 & 0.0013 & 0.0007 \\ 4466 & 16: 11: 46 & 0.0002 & 0.0001 \\ 4468 & 16: 11: 48 & 0.0016 & 0.0008 \\ 4470 & 16: 11: 50 & 0.0037 & 0.0015 \\ 4472 & 16: 11: 52 & 0.0023 & 0.0000 \\ 4474 & 16: 11: 54 & 0.0014 & 0.0072 \\ 4476 & 16: 11: 56 & 0.0011 & 0.0018 \\ 4478 & 16: 11: 58 & 0.0002 & 0.0078 \\ 4480 & 16: 12: 00 & 0.0013 & 0.0016 \\ 4482 & 16: 12: 02 & 0.0001 & 0.0019 \\ 4484 & 16: 12: 04 & 0.0002 & 0.0027\end{array}$


Appendix F - Laser Data Test 3 NIST102605_1.xls

$\begin{array}{llll}4486 & 16: 12: 06 & 0.0016 & 0.0028 \\ 4488 & 16: 12: 08 & 0.0008 & 0.0006 \\ 4490 & 16: 12: 10 & 0.0007 & 0.0008 \\ 4492 & 16: 12: 12 & 0.0014 & 0.0001 \\ 4494 & 16: 12: 14 & 0.0019 & 0.0016 \\ 4496 & 16: 12: 16 & 0.0039 & 0.0006 \\ 4498 & 16: 12: 18 & 0.0016 & 0.0011 \\ 4500 & 16: 12: 20 & 0.0002 & 0.0004 \\ 4502 & 16: 12: 22 & 0.0004 & 0.0003 \\ 4504 & 16: 12: 24 & 0.0003 & 0.0004 \\ 4506 & 16: 12: 26 & 0.0034 & 0.0010 \\ 4508 & 16: 12: 28 & 0.0004 & 0.0052 \\ 4510 & 16: 12: 30 & 0.0016 & 0.0021 \\ 4512 & 16: 12: 32 & 0.0015 & 0.0030 \\ 4514 & 16: 12: 34 & 0.0008 & 0.0144 \\ 4516 & 16: 12: 36 & 0.0014 & 0.0001 \\ 4518 & 16: 12: 38 & 0.0023 & 0.0033 \\ 4520 & 16: 12: 40 & 0.0006 & 0.0020 \\ 4522 & 16: 12: 42 & 0.0052 & 0.0000 \\ 4524 & 16: 12: 44 & 0.0033 & 0.0015 \\ 4526 & 16: 12: 46 & 0.0000 & 0.0001 \\ 4528 & 16: 12: 48 & 0.0002 & 0.0005 \\ 4530 & 16: 12: 50 & 0.0002 & 0.0022 \\ 4532 & 16: 12: 52 & 0.0026 & 0.0014 \\ 4534 & 16: 12: 54 & 0.0138 & 0.0131 \\ 4536 & 16: 12: 56 & 0.0030 & 0.0005 \\ 4538 & 16: 12: 58 & 0.0053 & 0.0001 \\ 4540 & 16: 13: 00 & 0.0018 & 0.0004 \\ 4542 & 16: 13: 02 & 0.0019 & 0.0004 \\ 4544 & 16: 13: 04 & 0.0030 & 0.0039 \\ 4546 & 16: 13: 06 & 0.0036 & 0.0017 \\ 4548 & 16: 13: 08 & 0.0008 & 0.0020 \\ 4550 & 16: 13: 10 & 0.0018 & 0.0086 \\ 4552 & 16: 13: 12 & 0.0000 & 0.0019 \\ 4554 & 16: 13: 14 & 0.0009 & 0.0020 \\ 4556 & 16: 13: 16 & 0.0010 & 0.0010 \\ 4558 & 16: 13: 18 & 0.0009 & 0.0002 \\ 4560 & 16: 13: 20 & 0.0004 & 0.0001 \\ 4562 & 16: 13: 22 & 0.0007 & 0.0004 \\ 4564 & 16: 13: 24 & 0.0001 & 0.0004 \\ 4566 & 16: 13: 26 & 0.0020 & 0.0006 \\ 4568 & 16: 13: 28 & 0.0045 & 0.0008 \\ 4570 & 16: 13: 30 & 0.0024 & 0.0014 \\ 4572 & 16: 13: 32 & 0.0070 & 0.0022 \\ 4574 & 16: 13: 34 & 0.0005 & 0.0023 \\ 4576 & 16: 13: 36 & 0.0003 & 0.0014 \\ 4578 & 16: 13: 38 & 0.0086 & 0.0059 \\ 4580 & 16: 13: 40 & 0.0014 & 0.0007 \\ 4582 & 16: 13: 42 & 0.0011 & 0.0001 \\ 4584 & 16: 13: 44 & 0.0106 & 0.0022 \\ 4586 & 16: 13: 46 & 0.0074 & 0.0005\end{array}$


Appendix F - Laser Data Test 3 NIST102605_1.xls

$\begin{array}{llll}4588 & 16: 13: 48 & 0.0186 & 0.0061 \\ 4590 & 16: 13: 50 & 0.0027 & 0.0196 \\ 4592 & 16: 13: 52 & 0.0015 & 0.0007 \\ 4594 & 16: 13: 54 & 0.0023 & 0.0004 \\ 4596 & 16: 13: 56 & 0.0198 & 0.0000 \\ 4598 & 16: 13: 58 & 0.0010 & 0.0035 \\ 4600 & 16: 14: 00 & 0.0021 & 0.0007 \\ 4602 & 16: 14: 02 & 0.0004 & 0.0027 \\ 4604 & 16: 14: 04 & 0.0002 & 0.0040 \\ 4606 & 16: 14: 06 & 0.0005 & 0.0013 \\ 4608 & 16: 14: 08 & 0.0013 & 0.0008 \\ 4610 & 16: 14: 10 & 0.0034 & 0.0068 \\ 4612 & 16: 14: 12 & 0.0095 & 0.0004 \\ 4614 & 16: 14: 14 & 0.0016 & 0.0003 \\ 4616 & 16: 14: 16 & 0.0052 & 0.0000 \\ 4618 & 16: 14: 18 & 0.0013 & 0.0033 \\ 4620 & 16: 14: 20 & 0.0008 & 0.0001 \\ 4622 & 16: 14: 22 & 0.0003 & 0.0033 \\ 4624 & 16: 14: 24 & 0.0003 & 0.0004 \\ 4626 & 16: 14: 26 & 0.0025 & 0.0011 \\ 4628 & 16: 14: 28 & 0.0006 & 0.0008 \\ 4630 & 16: 14: 30 & 0.0138 & 0.0057 \\ 4632 & 16: 14: 32 & 0.0048 & 0.0001 \\ 4634 & 16: 14: 34 & 0.0006 & 0.0004 \\ 4636 & 16: 14: 36 & 0.0084 & 0.0008 \\ 4638 & 16: 14: 38 & 0.0089 & 0.0004 \\ 4640 & 16: 14: 40 & 0.0016 & 0.0004 \\ 4642 & 16: 14: 42 & 0.0439 & 0.0003 \\ 4644 & 16: 14: 44 & 0.0041 & 0.0013 \\ 4646 & 16: 14: 46 & 0.0016 & 0.0003 \\ 4648 & 16: 14: 48 & 0.0032 & 0.0017 \\ 4650 & 16: 14: 50 & 0.0007 & 0.0020 \\ 4652 & 16: 14: 52 & 0.0006 & 0.0004 \\ 4654 & 16: 14: 54 & 0.0002 & 0.0016 \\ 4656 & 16: 14: 56 & 0.0002 & 0.0153 \\ 4658 & 16: 14: 58 & 0.0001 & 0.0039 \\ 4660 & 16: 15: 00 & 0.0009 & 0.0001 \\ 4662 & 16: 15: 02 & 0.0063 & 0.0036 \\ 4664 & 16: 15: 04 & 0.0010 & 0.0025 \\ 4666 & 16: 15: 06 & 0.0004 & 0.0025 \\ 4668 & 16: 15: 08 & 0.0058 & 0.0015 \\ 4670 & 16: 15: 10 & 0.0004 & 0.0001 \\ 4672 & 16: 15: 12 & 0.0007 & 0.0014 \\ 4674 & 16: 15: 14 & 0.0007 & 0.0004 \\ 4676 & 16: 15: 16 & 0.0025 & 0.0013 \\ 4678 & 16: 15: 18 & 0.0045 & 0.0005 \\ 4680 & 16: 15: 20 & 0.0003 & 0.0291 \\ 4682 & 16: 15: 22 & 0.0010 & 0.0003 \\ 4684 & 16: 15: 24 & 0.0011 & 0.0004 \\ 4686 & 16: 15: 26 & 0.0008 & 0.0003 \\ 4688 & 16: 15: 28 & 0.0009 & 0.0003\end{array}$


Appendix F - Laser Data Test 3 NIST102605_1.xls

$\begin{array}{llll}4690 & 16: 15: 30 & 0.0043 & 0.0003 \\ 4692 & 16: 15: 32 & 0.0005 & 0.0015 \\ 4694 & 16: 15: 34 & 0.0024 & 0.0050 \\ 4696 & 16: 15: 36 & 0.0012 & 0.0011 \\ 4698 & 16: 15: 38 & 0.0035 & 0.0010 \\ 4700 & 16: 15: 40 & 0.0005 & 0.0004 \\ 4702 & 16: 15: 42 & 0.0002 & 0.0023 \\ 4704 & 16: 15: 44 & 0.0005 & 0.0019 \\ 4706 & 16: 15: 46 & 0.0070 & 0.0008 \\ 4708 & 16: 15: 48 & 0.0016 & 0.0002 \\ 4710 & 16: 15: 50 & 0.0006 & 0.0016 \\ 4712 & 16: 15: 52 & 0.0010 & 0.0002 \\ 4714 & 16: 15: 54 & 0.0006 & 0.0001 \\ 4716 & 16: 15: 56 & 0.0017 & 0.0002 \\ 4718 & 16: 15: 58 & 0.0019 & 0.0002 \\ 4720 & 16: 16: 00 & 0.0026 & 0.0048 \\ 4722 & 16: 16: 02 & 0.0017 & 0.0005 \\ 4724 & 16: 16: 04 & 0.0010 & 0.0023 \\ 4726 & 16: 16: 06 & 0.0003 & 0.0032 \\ 4728 & 16: 16: 08 & 0.0012 & 0.0076 \\ 4730 & 16: 16: 10 & 0.0010 & 0.0005 \\ 4732 & 16: 16: 12 & 0.0004 & 0.0040 \\ 4734 & 16: 16: 14 & 0.0003 & 0.0034 \\ 4736 & 16: 16: 16 & 0.0036 & 0.0004 \\ 4738 & 16: 16: 18 & 0.0056 & 0.0103 \\ 4740 & 16: 16: 20 & 0.0004 & 0.0150 \\ 4742 & 16: 16: 22 & 0.0005 & 0.0033 \\ 4744 & 16: 16: 24 & 0.0008 & 0.0238 \\ 4746 & 16: 16: 26 & 0.0001 & 0.0090 \\ 4748 & 16: 16: 28 & 0.0045 & 0.0007 \\ 4750 & 16: 16: 30 & 0.0002 & 0.0124 \\ 4752 & 16: 16: 32 & 0.0002 & 0.0037 \\ 4754 & 16: 16: 34 & 0.0001 & 0.0182 \\ 4756 & 16: 16: 36 & 0.0009 & 0.0058 \\ 4758 & 16: 16: 38 & 0.0003 & 0.0011 \\ 4760 & 16: 16: 40 & 0.0003 & 0.0008 \\ 4762 & 16: 16: 42 & 0.0007 & 0.0004 \\ 4764 & 16: 16: 44 & 0.0003 & 0.0077 \\ 4766 & 16: 16: 46 & 0.0002 & 0.0005 \\ 4768 & 16: 16: 48 & 0.0001 & 0.0016 \\ 4770 & 16: 16: 50 & 0.0002 & 0.0111 \\ 4772 & 16: 16: 52 & 0.0005 & 0.0081 \\ 4774 & 16: 16: 54 & 0.0009 & 0.0047 \\ 4776 & 16: 16: 56 & 0.0002 & 0.0054 \\ 4778 & 16: 16: 58 & 0.0015 & 0.0052 \\ 4780 & 16: 17: 00 & 0.0009 & 0.0082 \\ 4782 & 16: 17: 02 & 0.0015 & 0.0071 \\ 4784 & 16: 17: 04 & 0.0012 & 0.0006 \\ 4786 & 16: 17: 06 & 0.0016 & 0.0125 \\ 4788 & 16: 17: 08 & 0.0000 & 0.0010 \\ 4790 & 16: 17: 10 & 0.0006 & 0.0057\end{array}$


Appendix F - Laser Data Test 3 NIST102605_1.xls

$\begin{array}{llll}4792 & 16: 17: 12 & 0.0000 & 0.0098 \\ 4794 & 16: 17: 14 & 0.0000 & 0.0016 \\ 4796 & 16: 17: 16 & 0.0009 & 0.0004 \\ 4798 & 16: 17: 18 & 0.0068 & 0.0030 \\ 4800 & 16: 17: 20 & 0.0005 & 0.0051 \\ 4802 & 16: 17: 22 & 0.0002 & 0.0009 \\ 4804 & 16: 17: 24 & 0.0015 & 0.0028 \\ 4806 & 16: 17: 26 & 0.0006 & 0.0006 \\ 4808 & 16: 17: 28 & 0.0106 & 0.0005 \\ 4810 & 16: 17: 30 & 0.0007 & 0.0006 \\ 4812 & 16: 17: 32 & 0.0001 & 0.0006 \\ 4814 & 16: 17: 34 & 0.0071 & 0.0005 \\ 4816 & 16: 17: 36 & 0.0002 & 0.0052 \\ 4818 & 16: 17: 38 & 0.0004 & 0.0023 \\ 4820 & 16: 17: 40 & 0.0001 & 0.0098 \\ 4822 & 16: 17: 42 & 0.0006 & 0.0385 \\ 4824 & 16: 17: 44 & 0.0003 & 0.0059 \\ 4826 & 16: 17: 46 & 0.0007 & 0.0084 \\ 4828 & 16: 17: 48 & 0.0007 & 0.0088 \\ 4830 & 16: 17: 50 & 0.0080 & 0.0023 \\ 4832 & 16: 17: 52 & 0.0009 & 0.0204 \\ 4834 & 16: 17: 54 & 0.0050 & 0.0011 \\ 4836 & 16: 17: 56 & 0.0135 & 0.0040 \\ 4838 & 16: 17: 58 & 0.0027 & 0.0005 \\ 4840 & 16: 18: 00 & 0.0005 & 0.0020 \\ 4842 & 16: 18: 02 & 0.0027 & 0.0014 \\ 4844 & 16: 18: 04 & 0.0004 & 0.0054 \\ 4846 & 16: 18: 06 & 0.0019 & 0.0123 \\ 4848 & 16: 18: 08 & 0.0037 & 0.0013 \\ 4850 & 16: 18: 10 & 0.0047 & 0.0028 \\ 4852 & 16: 18: 12 & 0.0081 & 0.0183 \\ 4854 & 16: 18: 14 & 0.0155 & 0.0068 \\ 4856 & 16: 18: 16 & 0.0102 & 0.0153 \\ 4858 & 16: 18: 18 & 0.0063 & 0.0423 \\ 4860 & 16: 18: 20 & 0.0099 & 0.0056 \\ 4862 & 16: 18: 22 & 0.0032 & 0.0233 \\ 4864 & 16: 18: 24 & 0.0072 & 0.0055 \\ 4866 & 16: 18: 26 & 0.0046 & 0.0350 \\ 4868 & 16: 18: 28 & 0.0063 & 0.0059 \\ 4870 & 16: 18: 30 & 0.0019 & 0.0317 \\ 4872 & 16: 18: 32 & 0.0047 & 0.0217 \\ 4874 & 16: 18: 34 & 0.0419 & 0.0051 \\ 4876 & 16: 18: 36 & 0.0062 & 0.0015 \\ 4878 & 16: 18: 38 & 0.0015 & 0.0078 \\ 4880 & 16: 18: 40 & 0.0069 & 0.0116 \\ 4882 & 16: 18: 42 & 0.0204 & 0.0183 \\ 4884 & 16: 18: 44 & 0.0021 & 0.0164 \\ 4886 & 16: 18: 46 & 0.0002 & 0.0069 \\ 4888 & 16: 18: 48 & 0.0014 & 0.0358 \\ 4890 & 16: 18: 50 & 0.0112 & 0.0110 \\ 4892 & 16: 18: 52 & 0.0247 & 0.0089\end{array}$


Appendix F - Laser Data Test 3 NIST102605_1.xls

$\begin{array}{llll}4894 & 16: 18: 54 & 0.0035 & 0.0136 \\ 4896 & 16: 18: 56 & 0.0052 & 0.0089 \\ 4898 & 16: 18: 58 & 0.0048 & 0.0010 \\ 4900 & 16: 19: 00 & 0.0009 & 0.0265 \\ 4902 & 16: 19: 02 & 0.0006 & 0.0114 \\ 4904 & 16: 19: 04 & 0.0013 & 0.0210 \\ 4906 & 16: 19: 06 & 0.0136 & 0.0117 \\ 4908 & 16: 19: 08 & 0.0107 & 0.0089 \\ 4910 & 16: 19: 10 & 0.0042 & 0.0014 \\ 4912 & 16: 19: 12 & 0.0155 & 0.0111 \\ 4914 & 16: 19: 14 & 0.0071 & 0.0041 \\ 4916 & 16: 19: 16 & 0.0036 & 0.0053 \\ 4918 & 16: 19: 18 & 0.0106 & 0.0307 \\ 4920 & 16: 19: 20 & 0.0078 & 0.0274 \\ 4922 & 16: 19: 22 & 0.0015 & 0.0181 \\ 4924 & 16: 19: 24 & 0.0251 & 0.0084 \\ 4926 & 16: 19: 26 & 0.0018 & 0.0333 \\ 4928 & 16: 19: 28 & 0.0039 & 0.0009 \\ 4930 & 16: 19: 30 & 0.0460 & 0.0120 \\ 4932 & 16: 19: 32 & 0.0295 & 0.0120 \\ 4934 & 16: 19: 34 & 0.0312 & 0.0071 \\ 4936 & 16: 19: 36 & 0.0033 & 0.0153 \\ 4938 & 16: 19: 38 & 0.0037 & 0.0174 \\ 4940 & 16: 19: 40 & 0.0029 & 0.0203 \\ 4942 & 16: 19: 42 & 0.0094 & 0.0151 \\ 4944 & 16: 19: 44 & 0.0024 & 0.0292 \\ 4946 & 16: 19: 46 & 0.0082 & 0.0581 \\ 4948 & 16: 19: 48 & 0.0087 & 0.0161 \\ 4950 & 16: 19: 50 & 0.0439 & 0.0048 \\ 4952 & 16: 19: 52 & 0.0034 & 0.0606 \\ 4954 & 16: 19: 54 & 0.0149 & 0.0081 \\ 4956 & 16: 19: 56 & 0.0133 & 0.0829 \\ 4958 & 16: 19: 58 & 0.0030 & 0.0076 \\ 4960 & 16: 20: 00 & 0.0074 & 0.0179 \\ 4962 & 16: 20: 02 & 0.0109 & 0.0178 \\ 4964 & 16: 20: 04 & 0.0049 & 0.0085 \\ 4966 & 16: 20: 06 & 0.0023 & 0.0051 \\ 4968 & 16: 20: 08 & 0.0089 & 0.0319 \\ 4970 & 16: 20: 10 & 0.0046 & 0.0042 \\ 4972 & 16: 20: 12 & 0.0042 & 0.0306 \\ 4974 & 16: 20: 14 & 0.0027 & 0.0288 \\ 4976 & 16: 20: 16 & 0.0101 & 0.1093 \\ 4978 & 16: 20: 18 & 0.0027 & 0.0216 \\ 4980 & 16: 20: 20 & 0.0015 & 0.0857 \\ 4982 & 16: 20: 22 & 0.0196 & 0.0707 \\ 4984 & 16: 20: 24 & 0.0162 & 0.0090 \\ 4986 & 16: 20: 26 & 0.1211 & 0.0190 \\ 4988 & 16: 20: 28 & 0.0176 & 0.0307 \\ 4990 & 16: 20: 30 & 0.0115 & 0.0286 \\ 4992 & 16: 20: 32 & 0.0343 & 0.0076 \\ 4994 & 16: 20: 34 & 0.0017 & 0.0016\end{array}$


Appendix F - Laser Data Test 3 NIST102605_1.xIs

$\begin{array}{llll}4996 & 16: 20: 36 & 0.0037 & 0.0138 \\ 4998 & 16: 20: 38 & 0.0021 & 0.0220 \\ 5000 & 16: 20: 40 & 0.0045 & 0.0127 \\ 5002 & 16: 20: 42 & 0.0049 & 0.0079 \\ 5004 & 16: 20: 44 & 0.0017 & 0.0018 \\ 5006 & 16: 20: 46 & 0.0077 & 0.0159 \\ 5008 & 16: 20: 48 & 0.0078 & 0.0071 \\ 5010 & 16: 20: 50 & 0.0033 & 0.0371 \\ 5012 & 16: 20: 52 & 0.0022 & 0.0180 \\ 5014 & 16: 20: 54 & 0.0007 & 0.0110 \\ 5016 & 16: 20: 56 & 0.0058 & 0.0033 \\ 5018 & 16: 20: 58 & 0.0045 & 0.0374 \\ 5020 & 16: 21: 00 & 0.0095 & 0.0137 \\ 5022 & 16: 21: 02 & 0.0239 & 0.0013 \\ 5024 & 16: 21: 04 & 0.0028 & 0.0587 \\ 5026 & 16: 21: 06 & 0.0042 & 0.0023 \\ 5028 & 16: 21: 08 & 0.0155 & 0.0180 \\ 5030 & 16: 21: 10 & 0.0023 & 0.0329 \\ 5032 & 16: 21: 12 & 0.0069 & 0.0126 \\ 5034 & 16: 21: 14 & 0.0114 & 0.0880 \\ 5036 & 16: 21: 16 & 0.0034 & 0.0079 \\ 5038 & 16: 21: 18 & 0.0461 & 0.0355 \\ 5040 & 16: 21: 20 & 0.0027 & 0.0314 \\ 5042 & 16: 21: 22 & 0.0035 & 0.0370 \\ 5044 & 16: 21: 24 & 0.0037 & 0.0331 \\ 5046 & 16: 21: 26 & 0.0035 & 0.0445 \\ 5048 & 16: 21: 28 & 0.0087 & 0.0454 \\ 5050 & 16: 21: 30 & 0.0045 & 0.0357 \\ 5052 & 16: 21: 32 & 0.0084 & 0.0323 \\ 5054 & 16: 21: 34 & 0.0129 & 0.0437 \\ 5056 & 16: 21: 36 & 0.0021 & 0.0063 \\ 5058 & 16: 21: 38 & 0.0009 & 0.0071 \\ 5060 & 16: 21: 40 & 0.0041 & 0.0170 \\ 5062 & 16: 21: 42 & 0.0023 & 0.0618 \\ 5064 & 16: 21: 44 & 0.0099 & 0.0050 \\ 5066 & 16: 21: 46 & 0.0019 & 0.0529 \\ 5068 & 16: 21: 48 & 0.0013 & 0.0039 \\ 5070 & 16: 21: 50 & 0.0099 & 0.0154 \\ 5072 & 16: 21: 52 & 0.0009 & 0.0054 \\ 5074 & 16: 21: 54 & 0.0043 & 0.0019 \\ 5076 & 16: 21: 56 & 0.0043 & 0.0412 \\ 5078 & 16: 21: 58 & 0.0031 & 0.0100 \\ 5080 & 16: 22: 00 & 0.0076 & 0.0083 \\ 5082 & 16: 22: 02 & 0.0154 & 0.0210 \\ 5084 & 16: 22: 04 & 0.0007 & 0.0128 \\ 5086 & 16: 22: 06 & 0.0080 & 0.0148 \\ 5088 & 16: 22: 08 & 0.0131 & 0.0144 \\ 5090 & 16: 22: 10 & 0.0307 & 0.0029 \\ 5092 & 16: 22: 12 & 0.0192 & 0.0024 \\ 5094 & 16: 22: 14 & 0.0198 & 0.0286 \\ 5096 & 16: 22: 16 & 0.0056 & 0.0049\end{array}$


Appendix F - Laser Data Test 3 NIST102605_1.xIs

$\begin{array}{llll}5098 & 16: 22: 18 & 0.0158 & 0.0059 \\ 5100 & 16: 22: 20 & 0.0061 & 0.0195 \\ 5102 & 16: 22: 22 & 0.0226 & 0.0240 \\ 5104 & 16: 22: 24 & 0.0126 & 0.0087 \\ 5106 & 16: 22: 26 & 0.0020 & 0.0326 \\ 5108 & 16: 22: 28 & 0.0031 & 0.0154 \\ 5110 & 16: 22: 30 & 0.0049 & 0.0101 \\ 5112 & 16: 22: 32 & 0.0034 & 0.0187 \\ 5114 & 16: 22: 34 & 0.0024 & 0.0154 \\ 5116 & 16: 22: 36 & 0.0014 & 0.0430 \\ 5118 & 16: 22: 38 & 0.0016 & 0.0387 \\ 5120 & 16: 22: 40 & 0.0403 & 0.0282 \\ 5122 & 16: 22: 42 & 0.0055 & 0.0149 \\ 5124 & 16: 22: 44 & 0.0008 & 0.0258 \\ 5126 & 16: 22: 46 & 0.0114 & 0.0188 \\ 5128 & 16: 22: 48 & 0.0046 & 0.0157 \\ 5130 & 16: 22: 50 & 0.0296 & 0.0274 \\ 5132 & 16: 22: 52 & 0.0058 & 0.0218 \\ 5134 & 16: 22: 54 & 0.0049 & 0.0091 \\ 5136 & 16: 22: 56 & 0.0013 & 0.0055 \\ 5138 & 16: 22: 58 & 0.0316 & 0.0311 \\ 5140 & 16: 23: 00 & 0.0064 & 0.0043 \\ 5142 & 16: 23: 02 & 0.0226 & 0.0134 \\ 5144 & 16: 23: 04 & 0.0067 & 0.0145 \\ 5146 & 16: 23: 06 & 0.0224 & 0.0192 \\ 5148 & 16: 23: 08 & 0.0055 & 0.0208 \\ 5150 & 16: 23: 10 & 0.0193 & 0.0050 \\ 5152 & 16: 23: 12 & 0.0393 & 0.0044 \\ 5154 & 16: 23: 14 & 0.0060 & 0.0132 \\ 5156 & 16: 23: 16 & 0.0031 & 0.0171 \\ 5158 & 16: 23: 18 & 0.0021 & 0.0115 \\ 5160 & 16: 23: 20 & 0.0030 & 0.0242 \\ 5162 & 16: 23: 22 & 0.0021 & 0.0073 \\ 5164 & 16: 23: 24 & 0.0165 & 0.0199 \\ 5166 & 16: 23: 26 & 0.0428 & 0.0068 \\ 5168 & 16: 23: 28 & 0.0084 & 0.0346 \\ 5170 & 16: 23: 30 & 0.0005 & 0.0097 \\ 5172 & 16: 23: 32 & 0.0066 & 0.0137 \\ 5174 & 16: 23: 34 & 0.0158 & 0.0523 \\ 5176 & 16: 23: 36 & 0.0110 & 0.0120 \\ 5178 & 16: 23: 38 & 0.0046 & 0.0528 \\ 5180 & 16: 23: 40 & 0.0121 & 0.0850 \\ 5182 & 16: 23: 42 & 0.0582 & 0.0506 \\ 5184 & 16: 23: 44 & 0.0034 & 0.0091 \\ 5186 & 16: 23: 46 & 0.0029 & 0.0152 \\ 5188 & 16: 23: 48 & 0.0030 & 0.0103 \\ 5190 & 16: 23: 50 & 0.0067 & 0.0613 \\ 5192 & 16: 23: 52 & 0.0076 & 0.0473 \\ 5194 & 16: 23: 54 & 0.0024 & 0.0223 \\ 5196 & 16: 23: 56 & 0.0072 & 0.0397 \\ 5198 & 16: 23: 58 & 0.0070 & 0.0175\end{array}$


Appendix F - Laser Data Test 3 NIST102605_1.xls

$\begin{array}{llll}5200 & 16: 24: 00 & 0.0056 & 0.0140 \\ 5202 & 16: 24: 02 & 0.0015 & 0.0219 \\ 5204 & 16: 24: 04 & 0.0174 & 0.0098 \\ 5206 & 16: 24: 06 & 0.0050 & 0.0279 \\ 5208 & 16: 24: 08 & 0.0133 & 0.0057 \\ 5210 & 16: 24: 10 & 0.0067 & 0.0052 \\ 5212 & 16: 24: 12 & 0.0070 & 0.0179 \\ 5214 & 16: 24: 14 & 0.0079 & 0.0770 \\ 5216 & 16: 24: 16 & 0.0114 & 0.0126 \\ 5218 & 16: 24: 18 & 0.0038 & 0.0072 \\ 5220 & 16: 24: 20 & 0.0030 & 0.0083 \\ 5222 & 16: 24: 22 & 0.0021 & 0.0740 \\ 5224 & 16: 24: 24 & 0.0058 & 0.0151 \\ 5226 & 16: 24: 26 & 0.0126 & 0.0164 \\ 5228 & 16: 24: 28 & 0.0195 & 0.0081 \\ 5230 & 16: 24: 30 & 0.0035 & 0.0009 \\ 5232 & 16: 24: 32 & 0.0027 & 0.0383 \\ 5234 & 16: 24: 34 & 0.0205 & 0.0133 \\ 5236 & 16: 24: 36 & 0.0071 & 0.0084 \\ 5238 & 16: 24: 38 & 0.0027 & 0.0049 \\ 5240 & 16: 24: 40 & 0.0136 & 0.0168 \\ 5242 & 16: 24: 42 & 0.0024 & 0.0221 \\ 5244 & 16: 24: 44 & 0.0058 & 0.0054 \\ 5246 & 16: 24: 46 & 0.0212 & 0.0632 \\ 5248 & 16: 24: 48 & 0.0075 & 0.0159 \\ 5250 & 16: 24: 50 & 0.0104 & 0.0081 \\ 5252 & 16: 24: 52 & 0.0034 & 0.1087 \\ 5254 & 16: 24: 54 & 0.0015 & 0.0225 \\ 5256 & 16: 24: 56 & 0.0041 & 0.0500 \\ 5258 & 16: 24: 58 & 0.0149 & 0.0791 \\ 5260 & 16: 25: 00 & 0.0094 & 0.0248 \\ 5262 & 16: 25: 02 & 0.0173 & 0.0223 \\ 5264 & 16: 25: 04 & 0.0038 & 0.0148 \\ 5266 & 16: 25: 06 & 0.0033 & 0.0053 \\ 5268 & 16: 25: 08 & 0.0169 & 0.0261 \\ 5270 & 16: 25: 10 & 0.0192 & 0.0071 \\ 5272 & 16: 25: 12 & 0.0116 & 0.0544 \\ 5274 & 16: 25: 14 & 0.0014 & 0.0286 \\ 5276 & 16: 25: 16 & 0.0197 & 0.0125 \\ 5278 & 16: 25: 18 & 0.0061 & 0.0542 \\ 5280 & 16: 25: 20 & 0.0066 & 0.0271 \\ 5282 & 16: 25: 22 & 0.0034 & 0.0510 \\ 5284 & 16: 25: 24 & 0.0229 & 0.0226 \\ 5286 & 16: 25: 26 & 0.0611 & 0.0165 \\ 5288 & 16: 25: 28 & 0.0038 & 0.0158 \\ 5290 & 16: 25: 30 & 0.0042 & 0.0331 \\ 5292 & 16: 25: 32 & 0.0069 & 0.0326 \\ 5294 & 16: 25: 34 & 0.0135 & 0.0504 \\ 5296 & 16: 25: 36 & 0.0200 & 0.0199 \\ 5298 & 16: 25: 38 & 0.0067 & 0.0144 \\ 5300 & 16: 25: 40 & 0.0057 & 0.0152 \\ & & & \end{array}$


Appendix F - Laser Data Test 3 NIST102605_1.xls

$\begin{array}{llll}5302 & 16: 25: 42 & 0.0093 & 0.0392 \\ 5304 & 16: 25: 44 & 0.0058 & 0.0099 \\ 5306 & 16: 25: 46 & 0.0007 & 0.0187 \\ 5308 & 16: 25: 48 & 0.0046 & 0.0049 \\ 5310 & 16: 25: 50 & 0.0119 & 0.0459 \\ 5312 & 16: 25: 52 & 0.0032 & 0.0105 \\ 5314 & 16: 25: 54 & 0.0122 & 0.0131 \\ 5316 & 16: 25: 56 & 0.0004 & 0.0280 \\ 5318 & 16: 25: 58 & 0.0020 & 0.0075 \\ 5320 & 16: 26: 00 & 0.0061 & 0.0056 \\ 5322 & 16: 26: 02 & 0.0064 & 0.0125 \\ 5324 & 16: 26: 04 & 0.0321 & 0.0163 \\ 5326 & 16: 26: 06 & 0.0098 & 0.0273 \\ 5328 & 16: 26: 08 & 0.0058 & 0.0185 \\ 5330 & 16: 26: 10 & 0.0026 & 0.0299 \\ 5332 & 16: 26: 12 & 0.0063 & 0.0134 \\ 5334 & 16: 26: 14 & 0.0044 & 0.0094 \\ 5336 & 16: 26: 16 & 0.0022 & 0.0145 \\ 5338 & 16: 26: 18 & 0.0087 & 0.0105 \\ 5340 & 16: 26: 20 & 0.0389 & 0.0553 \\ 5342 & 16: 26: 22 & 0.0314 & 0.0658 \\ 5344 & 16: 26: 24 & 0.0043 & 0.0126 \\ 5346 & 16: 26: 26 & 0.0155 & 0.0096 \\ 5348 & 16: 26: 28 & 0.0007 & 0.0295 \\ 5350 & 16: 26: 30 & 0.0154 & 0.0113 \\ 5352 & 16: 26: 32 & 0.0652 & 0.0086 \\ 5354 & 16: 26: 34 & 0.0156 & 0.0068 \\ 5356 & 16: 26: 36 & 0.0153 & 0.0169 \\ 5358 & 16: 26: 38 & 0.0291 & 0.0178 \\ 5360 & 16: 26: 40 & 0.0045 & 0.0257 \\ 5362 & 16: 26: 42 & 0.0015 & 0.0564 \\ 5364 & 16: 26: 44 & 0.0116 & 0.0393 \\ 5366 & 16: 26: 46 & 0.0013 & 0.0164 \\ 5368 & 16: 26: 48 & 0.0248 & 0.0325 \\ 5370 & 16: 26: 50 & 0.0018 & 0.0255 \\ 5372 & 16: 26: 52 & 0.0026 & 0.0178 \\ 5374 & 16: 26: 54 & 0.0019 & 0.0016 \\ 5376 & 16: 26: 56 & 0.0033 & 0.0144 \\ 5378 & 16: 26: 58 & 0.0079 & 0.0137 \\ 5380 & 16: 27: 00 & 0.0061 & 0.0046 \\ 5382 & 16: 27: 02 & 0.0042 & 0.0122 \\ 5384 & 16: 27: 04 & 0.0005 & 0.0258 \\ 5386 & 16: 27: 06 & 0.0061 & 0.0085 \\ 5388 & 16: 27: 08 & 0.0085 & 0.0023 \\ 5390 & 16: 27: 10 & 0.0025 & 0.0059 \\ 5392 & 16: 27: 12 & 0.0035 & 0.0024 \\ 5394 & 16: 27: 14 & 0.0006 & 0.0108 \\ 5396 & 16: 27: 16 & 0.0110 & 0.0150 \\ 5398 & 16: 27: 18 & 0.0223 & 0.0224 \\ 5400 & 16: 27: 20 & 0.0046 & 0.0270 \\ 5402 & 16: 27: 22 & 0.0019 & 0.0056\end{array}$


Appendix F - Laser Data Test 3 NIST102605_1.xls

$\begin{array}{llll}5404 & 16: 27: 24 & 0.0115 & 0.0370 \\ 5406 & 16: 27: 26 & 0.0064 & 0.0243 \\ 5408 & 16: 27: 28 & 0.0337 & 0.0097 \\ 5410 & 16: 27: 30 & 0.0014 & 0.0074 \\ 5412 & 16: 27: 32 & 0.0071 & 0.0185 \\ 5414 & 16: 27: 34 & 0.0013 & 0.1009 \\ 5416 & 16: 27: 36 & 0.0127 & 0.0066 \\ 5418 & 16: 27: 38 & 0.0039 & 0.0031 \\ 5420 & 16: 27: 40 & 0.0040 & 0.0283 \\ 5422 & 16: 27: 42 & 0.0010 & 0.0325 \\ 5424 & 16: 27: 44 & 0.0203 & 0.0053 \\ 5426 & 16: 27: 46 & 0.0195 & 0.0207 \\ 5428 & 16: 27: 48 & 0.0073 & 0.0125 \\ 5430 & 16: 27: 50 & 0.0223 & 0.0288 \\ 5432 & 16: 27: 52 & 0.0040 & 0.0020 \\ 5434 & 16: 27: 54 & 0.0043 & 0.0047 \\ 5436 & 16: 27: 56 & 0.0177 & 0.0402 \\ 5438 & 16: 27: 58 & 0.0349 & 0.0710 \\ 5440 & 16: 28: 00 & 0.0037 & 0.0474 \\ 5442 & 16: 28: 02 & 0.0175 & 0.0128 \\ 5444 & 16: 28: 04 & 0.0016 & 0.0982 \\ 5446 & 16: 28: 06 & 0.0041 & 0.0038 \\ 5448 & 16: 28: 08 & 0.0071 & 0.0016 \\ 5450 & 16: 28: 10 & 0.0045 & 0.0115 \\ 5452 & 16: 28: 12 & 0.0063 & 0.0010 \\ 5454 & 16: 28: 14 & 0.0054 & 0.0185 \\ 5456 & 16: 28: 16 & 0.0037 & 0.0071 \\ 5458 & 16: 28: 18 & 0.0168 & 0.0032 \\ 5460 & 16: 28: 20 & 0.0251 & 0.0327 \\ 5462 & 16: 28: 22 & 0.0067 & 0.0064 \\ 5464 & 16: 28: 24 & 0.0070 & 0.0105 \\ 5466 & 16: 28: 26 & 0.0162 & 0.0201 \\ 5468 & 16: 28: 28 & 0.0028 & 0.0142 \\ 5470 & 16: 28: 30 & 0.0188 & 0.0072 \\ 5472 & 16: 28: 32 & 0.0037 & 0.0109 \\ 5474 & 16: 28: 34 & 0.0036 & 0.0073 \\ 5476 & 16: 28: 36 & 0.0056 & 0.0067 \\ 5478 & 16: 28: 38 & 0.0014 & 0.0063 \\ 5480 & 16: 28: 40 & 0.0090 & 0.0028 \\ 5482 & 16: 28: 42 & 0.0029 & 0.0199 \\ 5484 & 16: 28: 44 & 0.0012 & 0.0089 \\ 5486 & 16: 28: 46 & 0.0037 & 0.0497 \\ 5488 & 16: 28: 48 & 0.0037 & 0.0155 \\ 5490 & 16: 28: 50 & 0.0011 & 0.0391 \\ 5492 & 16: 28: 52 & 0.0014 & 0.0067 \\ 5494 & 16: 28: 54 & 0.0006 & 0.0813 \\ 5496 & 16: 28: 56 & 0.0131 & 0.0618 \\ 5498 & 16: 28: 58 & 0.0008 & 0.0163 \\ 5500 & 16: 29: 00 & 0.0088 & 0.0089 \\ 5502 & 16: 29: 02 & 0.0161 & 0.0479 \\ 5504 & 16: 29: 04 & 0.0100 & 0.0119\end{array}$


Appendix F - Laser Data Test 3 NIST102605_1.xIs

$\begin{array}{llll}5506 & 16: 29: 06 & 0.0254 & 0.0268 \\ 5508 & 16: 29: 08 & 0.0066 & 0.0539 \\ 5510 & 16: 29: 10 & 0.0353 & 0.0905 \\ 5512 & 16: 29: 12 & 0.0159 & 0.0142 \\ 5514 & 16: 29: 14 & 0.0066 & 0.0178 \\ 5516 & 16: 29: 16 & 0.0166 & 0.0026 \\ 5518 & 16: 29: 18 & 0.0066 & 0.0477 \\ 5520 & 16: 29: 20 & 0.0020 & 0.0017 \\ 5522 & 16: 29: 22 & 0.0013 & 0.0039 \\ 5524 & 16: 29: 24 & 0.0022 & 0.0126 \\ 5526 & 16: 29: 26 & 0.0097 & 0.0647 \\ 5528 & 16: 29: 28 & 0.0013 & 0.0080 \\ 5530 & 16: 29: 30 & 0.0060 & 0.0809 \\ 5532 & 16: 29: 32 & 0.0004 & 0.0046 \\ 5534 & 16: 29: 34 & 0.0187 & 0.0207 \\ 5536 & 16: 29: 36 & 0.0003 & 0.0285 \\ 5538 & 16: 29: 38 & 0.0134 & 0.0127 \\ 5540 & 16: 29: 40 & 0.0008 & 0.0026 \\ 5542 & 16: 29: 42 & 0.0044 & 0.0122 \\ 5544 & 16: 29: 44 & 0.0172 & 0.0054 \\ 5546 & 16: 29: 46 & 0.0043 & 0.0138 \\ 5548 & 16: 29: 48 & 0.0280 & 0.0244 \\ 5550 & 16: 29: 50 & 0.0011 & 0.0080 \\ 5552 & 16: 29: 52 & 0.0230 & 0.0406 \\ 5554 & 16: 29: 54 & 0.0011 & 0.0061 \\ 5556 & 16: 29: 56 & 0.0144 & 0.0412 \\ 5558 & 16: 29: 58 & 0.0451 & 0.0129 \\ 5560 & 16: 30: 00 & 0.0043 & 0.0047 \\ 5562 & 16: 30: 02 & 0.0038 & 0.0066 \\ 5564 & 16: 30: 04 & 0.0025 & 0.0112 \\ 5566 & 16: 30: 06 & 0.0045 & 0.0151 \\ 5568 & 16: 30: 08 & 0.0116 & 0.0058 \\ 5570 & 16: 30: 10 & 0.0066 & 0.0066 \\ 5572 & 16: 30: 12 & 0.0173 & 0.0023 \\ 5574 & 16: 30: 14 & 0.0035 & 0.0187 \\ 5576 & 16: 30: 16 & 0.0006 & 0.0136 \\ 5578 & 16: 30: 18 & 0.0037 & 0.0160 \\ 5580 & 16: 30: 20 & 0.0281 & 0.0518 \\ 5582 & 16: 30: 22 & 0.0086 & 0.0464 \\ 5584 & 16: 30: 24 & 0.0023 & 0.0084 \\ 5586 & 16: 30: 26 & 0.0181 & 0.0093 \\ 5588 & 16: 30: 28 & 0.0162 & 0.0090 \\ 5590 & 16: 30: 30 & 0.0019 & 0.0196 \\ 5592 & 16: 30: 32 & 0.0029 & 0.0531 \\ 5594 & 16: 30: 34 & 0.0067 & 0.0190 \\ 5596 & 16: 30: 36 & 0.0064 & 0.0359 \\ 5598 & 16: 30: 38 & 0.0027 & 0.1174 \\ 5600 & 16: 30: 40 & 0.0042 & 0.0164 \\ 5602 & 16: 30: 42 & 0.0114 & 0.0112 \\ 5604 & 16: 30: 44 & 0.0024 & 0.0215 \\ 5606 & 16: 30: 46 & 0.0316 & 0.0042 \\ & & & \end{array}$


Appendix F - Laser Data Test 3 NIST102605_1.xIs

$\begin{array}{llll}5608 & 16: 30: 48 & 0.0040 & 0.0161 \\ 5610 & 16: 30: 50 & 0.0088 & 0.0038 \\ 5612 & 16: 30: 52 & 0.0081 & 0.0067 \\ 5614 & 16: 30: 54 & 0.0404 & 0.0113 \\ 5616 & 16: 30: 56 & 0.0260 & 0.0237 \\ 5618 & 16: 30: 58 & 0.0062 & 0.0017 \\ 5620 & 16: 31: 00 & 0.0409 & 0.0027 \\ 5622 & 16: 31: 02 & 0.0302 & 0.0167 \\ 5624 & 16: 31: 04 & 0.0051 & 0.0421 \\ 5626 & 16: 31: 06 & 0.0187 & 0.0172 \\ 5628 & 16: 31: 08 & 0.0307 & 0.0065 \\ 5630 & 16: 31: 10 & 0.0377 & 0.0072 \\ 5632 & 16: 31: 12 & 0.0405 & 0.0060 \\ 5634 & 16: 31: 14 & 0.0124 & 0.0080 \\ 5636 & 16: 31: 16 & 0.0074 & 0.0334 \\ 5638 & 16: 31: 18 & 0.0744 & 0.0102 \\ 5640 & 16: 31: 20 & 0.0127 & 0.0058 \\ 5642 & 16: 31: 22 & 0.0113 & 0.0527 \\ 5644 & 16: 31: 24 & 0.0157 & 0.0059 \\ 5646 & 16: 31: 26 & 0.0078 & 0.0019 \\ 5648 & 16: 31: 28 & 0.0151 & 0.0012 \\ 5650 & 16: 31: 30 & 0.0365 & 0.0067 \\ 5652 & 16: 31: 32 & 0.0315 & 0.0349 \\ 5654 & 16: 31: 34 & 0.0318 & 0.0135 \\ 5656 & 16: 31: 36 & 0.0261 & 0.0070 \\ 5658 & 16: 31: 38 & 0.0186 & 0.0041 \\ 5660 & 16: 31: 40 & 0.0109 & 0.0019 \\ 5662 & 16: 31: 42 & 0.0130 & 0.0007 \\ 5664 & 16: 31: 44 & 0.0116 & 0.0100 \\ 5666 & 16: 31: 46 & 0.0664 & 0.0133 \\ 5668 & 16: 31: 48 & 0.0175 & 0.0167 \\ 5670 & 16: 31: 50 & 0.0089 & 0.0537 \\ 5672 & 16: 31: 52 & 0.0370 & 0.0120 \\ 5674 & 16: 31: 54 & 0.0456 & 0.0137 \\ 5676 & 16: 31: 56 & 0.0145 & 0.0012 \\ 5678 & 16: 31: 58 & 0.0318 & 0.0028 \\ 5680 & 16: 32: 00 & 0.0245 & 0.0474 \\ 5682 & 16: 32: 02 & 0.0065 & 0.0230 \\ 5684 & 16: 32: 04 & 0.0152 & 0.0075 \\ 5686 & 16: 32: 06 & 0.0116 & 0.0236 \\ 5688 & 16: 32: 08 & 0.0049 & 0.0088 \\ 5690 & 16: 32: 10 & 0.0100 & 0.0007 \\ 5692 & 16: 32: 12 & 0.0163 & 0.0295 \\ 5694 & 16: 32: 14 & 0.0039 & 0.0249 \\ 5696 & 16: 32: 16 & 0.0486 & 0.0226 \\ 5698 & 16: 32: 18 & 0.0541 & 0.0232 \\ 5700 & 16: 32: 20 & 0.0232 & 0.0408 \\ 5702 & 16: 32: 22 & 0.0096 & 0.0079 \\ 5704 & 16: 32: 24 & 0.0376 & 0.0060 \\ 5706 & 16: 32: 26 & 0.0039 & 0.0097 \\ 5708 & 16: 32: 28 & 0.0123 & 0.0488\end{array}$


Appendix F - Laser Data Test 3 NIST102605_1.xls

$\begin{array}{llll}5710 & 16: 32: 30 & 0.0021 & 0.0211 \\ 5712 & 16: 32: 32 & 0.0063 & 0.0026 \\ 5714 & 16: 32: 34 & 0.0095 & 0.0107 \\ 5716 & 16: 32: 36 & 0.0062 & 0.0122 \\ 5718 & 16: 32: 38 & 0.0059 & 0.0210 \\ 5720 & 16: 32: 40 & 0.0096 & 0.0409 \\ 5722 & 16: 32: 42 & 0.0180 & 0.0340 \\ 5724 & 16: 32: 44 & 0.0047 & 0.0108 \\ 5726 & 16: 32: 46 & 0.0108 & 0.0198 \\ 5728 & 16: 32: 48 & 0.0079 & 0.0533 \\ 5730 & 16: 32: 50 & 0.0132 & 0.0466 \\ 5732 & 16: 32: 52 & 0.0029 & 0.0172 \\ 5734 & 16: 32: 54 & 0.0005 & 0.0066 \\ 5736 & 16: 32: 56 & 0.0043 & 0.0147 \\ 5738 & 16: 32: 58 & 0.0029 & 0.0124 \\ 5740 & 16: 33: 00 & 0.0084 & 0.0027 \\ 5742 & 16: 33: 02 & 0.0013 & 0.0049 \\ 5744 & 16: 33: 04 & 0.0143 & 0.1306 \\ 5746 & 16: 33: 06 & 0.0085 & 0.0170 \\ 5748 & 16: 33: 08 & 0.0020 & 0.0329 \\ 5750 & 16: 33: 10 & 0.0173 & 0.0071 \\ 5752 & 16: 33: 12 & 0.0014 & 0.0047 \\ 5754 & 16: 33: 14 & 0.0544 & 0.0077 \\ 5756 & 16: 33: 16 & 0.0020 & 0.0324 \\ 5758 & 16: 33: 18 & 0.0040 & 0.0064 \\ 5760 & 16: 33: 20 & 0.0497 & 0.0131 \\ 5762 & 16: 33: 22 & 0.0097 & 0.0192 \\ 5764 & 16: 33: 24 & 0.0121 & 0.0051 \\ 5766 & 16: 33: 26 & 0.0047 & 0.0189 \\ 5768 & 16: 33: 28 & 0.0095 & 0.0289 \\ 5770 & 16: 33: 30 & 0.0323 & 0.0309 \\ 5772 & 16: 33: 32 & 0.0011 & 0.0159 \\ 5774 & 16: 33: 34 & 0.0030 & 0.0437 \\ 5776 & 16: 33: 36 & 0.0118 & 0.0246 \\ 5778 & 16: 33: 38 & 0.0222 & 0.0438 \\ 5780 & 16: 33: 40 & 0.0279 & 0.0225 \\ 5782 & 16: 33: 42 & 0.0055 & 0.0156 \\ 5784 & 16: 33: 44 & 0.0096 & 0.0179 \\ 5786 & 16: 33: 46 & 0.0161 & 0.0069 \\ 5788 & 16: 33: 48 & 0.0101 & 0.0358 \\ 5790 & 16: 33: 50 & 0.0227 & 0.0677 \\ 5792 & 16: 33: 52 & 0.0019 & 0.0065 \\ 5794 & 16: 33: 54 & 0.0086 & 0.0692 \\ 5796 & 16: 33: 56 & 0.0184 & 0.0391 \\ 5798 & 16: 33: 58 & 0.0049 & 0.0064\end{array}$


APPENDIX G. - Fuel Data Test 4 NIST 102705

Appendix $G$ is fuel data from Test 4 in tabular format and is approximately 107 pages in length. Fuel data is included with electronic version of report on compact disk, but not routinely included with paper version of report. 
Appendix G - Fuel Data Test 4 NIST102705_1.xls

Time from Ignition, s EDT Time Liq Flow, $1 / \mathrm{min}$

$\begin{array}{rll}0 & 14: 20: 46 & 0.8179 \\ 1 & 14: 20: 47 & 0.8220 \\ 2 & 14: 20: 48 & 0.8202 \\ 3 & 14: 20: 49 & 0.8190 \\ 4 & 14: 20: 50 & 0.8166 \\ 5 & 14: 20: 51 & 0.8221 \\ 6 & 14: 20: 52 & 0.8205 \\ 7 & 14: 20: 53 & 0.8194 \\ 8 & 14: 20: 54 & 0.8226 \\ 9 & 14: 20: 55 & 0.8172 \\ 10 & 14: 20: 56 & 0.8182 \\ 11 & 14: 20: 57 & 0.8148 \\ 12 & 14: 20: 58 & 0.8119 \\ 13 & 14: 20: 59 & 0.8155 \\ 14 & 14: 21: 00 & 0.8176 \\ 15 & 14: 21: 01 & 0.8202 \\ 16 & 14: 21: 02 & 0.8157 \\ 17 & 14: 21: 03 & 0.8104 \\ 18 & 14: 21: 04 & 0.8065 \\ 19 & 14: 21: 05 & 0.8079 \\ 20 & 14: 21: 06 & 0.8094 \\ 21 & 14: 21: 07 & 0.8106 \\ 23 & 14: 21: 09 & 0.8134 \\ 23 & 14: 21: 09 & 0.8108 \\ 24 & 14: 21: 10 & 0.8100 \\ 25 & 14: 21: 11 & 0.8090 \\ 26 & 14: 21: 12 & 0.8207 \\ 27 & 14: 21: 13 & 0.8133 \\ 29 & 14: 21: 15 & 0.8109 \\ 29 & 14: 21: 15 & 0.8181 \\ 30 & 14: 21: 16 & 0.8157 \\ 31 & 14: 21: 17 & 0.8178 \\ 32 & 14: 21: 18 & 0.8210 \\ 33 & 14: 21: 19 & 0.8117 \\ 34 & 14: 21: 20 & 0.8108 \\ 35 & 14: 21: 21 & 0.8097 \\ 36 & 14: 21: 22 & 0.8154 \\ 37 & 14: 21: 23 & 0.8218 \\ 38 & 14: 21: 24 & 0.8204 \\ 39 & 14: 21: 25 & 0.8232 \\ 40 & 14: 21: 26 & 0.8211 \\ 41 & 14: 21: 27 & 0.8183 \\ 42 & 14: 21: 28 & 0.8193 \\ 43 & 14: 21: 29 & 0.8198 \\ 44 & 14: 21: 30 & 0.8164 \\ 45 & 14: 21: 31 & 0.8139 \\ 46 & 14: 21: 32 & 0.8214 \\ 47 & 14: 21: 33 & 0.8201 \\ 48 & 14: 21: 34 & 0.8224 \\ 49 & 14: 21: 35 & 0.8239\end{array}$

G - 1 
Appendix G - Fuel Data Test 4 NIST102705_1.xls

\begin{tabular}{rll}
50 & $14: 21: 36$ & 0.8185 \\
51 & $14: 21: 37$ & 0.8175 \\
52 & $14: 21: 38$ & 0.8170 \\
53 & $14: 21: 39$ & 0.8181 \\
54 & $14: 21: 40$ & 0.8274 \\
55 & $14: 21: 41$ & 0.8239 \\
56 & $14: 21: 42$ & 0.8209 \\
57 & $14: 21: 43$ & 0.8244 \\
58 & $14: 21: 44$ & 0.8258 \\
59 & $14: 21: 45$ & 0.8236 \\
60 & $14: 21: 46$ & 0.8144 \\
61 & $14: 21: 47$ & 0.8119 \\
62 & $14: 21: 48$ & 0.8172 \\
63 & $14: 21: 49$ & 0.8228 \\
64 & $14: 21: 50$ & 0.8266 \\
65 & $14: 21: 51$ & 0.8249 \\
66 & $14: 21: 52$ & 0.8227 \\
67 & $14: 21: 53$ & 0.8173 \\
68 & $14: 21: 54$ & 0.8187 \\
69 & $14: 21: 55$ & 0.8198 \\
70 & $14: 21: 56$ & 0.8187 \\
71 & $14: 21: 57$ & 0.8159 \\
72 & $14: 21: 58$ & 0.8195 \\
73 & $14: 21: 59$ & 0.8227 \\
74 & $14: 22: 00$ & 0.8174 \\
75 & $14: 22: 01$ & 0.8165 \\
76 & $14: 22: 02$ & 0.8174 \\
77 & $14: 22: 03$ & 0.8177 \\
78 & $14: 22: 04$ & 0.8153 \\
79 & $14: 22: 05$ & 0.8179 \\
80 & $14: 22: 06$ & 0.8221 \\
81 & $14: 22: 07$ & 0.8227 \\
82 & $14: 22: 08$ & 0.8261 \\
83 & $14: 22: 09$ & 0.8213 \\
84 & $14: 22: 10$ & 0.8142 \\
85 & $14: 22: 11$ & 0.8163 \\
86 & $14: 22: 12$ & 0.8219 \\
87 & $14: 22: 13$ & 0.8196 \\
88 & $14: 22: 14$ & 0.8151 \\
89 & $14: 22: 15$ & 0.8193 \\
90 & $14: 22: 16$ & 0.8242 \\
91 & $14: 22: 17$ & 0.8228 \\
92 & $14: 22: 18$ & 0.8188 \\
93 & $14: 22: 19$ & 0.8170 \\
94 & $14: 22: 20$ & 0.8189 \\
95 & $14: 22: 21$ & 0.8184 \\
96 & $14: 22: 22$ & 0.8165 \\
98 & $14: 22: 24$ & 0.8150 \\
98 & $14: 22: 24$ & 0.8208 \\
100 & $14: 22: 25$ & 0.8292 \\
\hline $9: 22: 26$ & 0.8247
\end{tabular}


Appendix G - Fuel Data Test 4 NIST102705_1.xls

$\begin{array}{lll}101 & 14: 22: 27 & 0.8214 \\ 102 & 14: 22: 28 & 0.8225 \\ 103 & 14: 22: 29 & 0.8163 \\ 104 & 14: 22: 30 & 0.8199 \\ 105 & 14: 22: 31 & 0.8168 \\ 106 & 14: 22: 32 & 0.8138 \\ 107 & 14: 22: 33 & 0.8154 \\ 108 & 14: 22: 34 & 0.8156 \\ 109 & 14: 22: 35 & 0.8201 \\ 110 & 14: 22: 36 & 0.8156 \\ 111 & 14: 22: 37 & 0.8154 \\ 112 & 14: 22: 38 & 0.8163 \\ 113 & 14: 22: 39 & 0.8180 \\ 114 & 14: 22: 40 & 0.8136 \\ 115 & 14: 22: 41 & 0.8165 \\ 116 & 14: 22: 42 & 0.8227 \\ 117 & 14: 22: 43 & 0.8320 \\ 118 & 14: 22: 44 & 0.8259 \\ 119 & 14: 22: 45 & 0.8204 \\ 120 & 14: 22: 46 & 0.8130 \\ 121 & 14: 22: 47 & 0.8121 \\ 122 & 14: 22: 48 & 0.8183 \\ 123 & 14: 22: 49 & 0.8180 \\ 124 & 14: 22: 50 & 0.8186 \\ 125 & 14: 22: 51 & 0.8164 \\ 126 & 14: 22: 52 & 0.8184 \\ 127 & 14: 22: 53 & 0.8177 \\ 128 & 14: 22: 54 & 0.8169 \\ 129 & 14: 22: 55 & 0.8221 \\ 130 & 14: 22: 56 & 0.8209 \\ 131 & 14: 22: 57 & 0.8184 \\ 132 & 14: 22: 58 & 0.8136 \\ 133 & 14: 22: 59 & 0.8187 \\ 134 & 14: 23: 00 & 0.8222 \\ 135 & 14: 23: 01 & 0.8282 \\ 136 & 14: 23: 02 & 0.8271 \\ 137 & 14: 23: 03 & 0.8222 \\ 138 & 14: 23: 04 & 0.8174 \\ 139 & 14: 23: 05 & 0.8191 \\ 140 & 14: 23: 06 & 0.8152 \\ 141 & 14: 23: 07 & 0.8144 \\ 142 & 14: 23: 08 & 0.8150 \\ 143 & 14: 23: 09 & 0.8150 \\ 144 & 14: 23: 10 & 0.8174 \\ 145 & 14: 23: 11 & 0.8210 \\ 146 & 14: 23: 12 & 0.8196 \\ 147 & 14: 23: 13 & 0.8206 \\ 148 & 14: 23: 14 & 0.8162 \\ 149 & 14: 23: 15 & 0.8169 \\ 150 & 14: 23: 16 & 0.8162 \\ 151 & 14: 23: 17 & 0.8218\end{array}$


Appendix G - Fuel Data Test 4 NIST102705_1.xls

$\begin{array}{lll}152 & 14: 23: 18 & 0.8264 \\ 153 & 14: 23: 19 & 0.8242 \\ 154 & 14: 23: 20 & 0.8227 \\ 155 & 14: 23: 21 & 0.8167 \\ 156 & 14: 23: 22 & 0.8239 \\ 157 & 14: 23: 23 & 0.8236 \\ 158 & 14: 23: 24 & 0.8197 \\ 159 & 14: 23: 25 & 0.8161 \\ 160 & 14: 23: 26 & 0.8176 \\ 161 & 14: 23: 27 & 0.8228 \\ 162 & 14: 23: 28 & 0.8223 \\ 163 & 14: 23: 29 & 0.8162 \\ 164 & 14: 23: 30 & 0.8193 \\ 165 & 14: 23: 31 & 0.8162 \\ 166 & 14: 23: 32 & 0.8122 \\ 167 & 14: 23: 33 & 0.8175 \\ 168 & 14: 23: 34 & 0.8196 \\ 169 & 14: 23: 35 & 0.8207 \\ 170 & 14: 23: 36 & 0.8199 \\ 171 & 14: 23: 37 & 0.8155 \\ 172 & 14: 23: 38 & 0.8123 \\ 173 & 14: 23: 39 & 0.8125 \\ 174 & 14: 23: 40 & 0.8198 \\ 175 & 14: 23: 41 & 0.8148 \\ 176 & 14: 23: 42 & 0.8188 \\ 177 & 14: 23: 43 & 0.8240 \\ 178 & 14: 23: 44 & 0.8177 \\ 179 & 14: 23: 45 & 0.8165 \\ 180 & 14: 23: 46 & 0.8212 \\ 181 & 14: 23: 47 & 0.8197 \\ 182 & 14: 23: 48 & 0.8169 \\ 183 & 14: 23: 49 & 0.8141 \\ 184 & 14: 23: 50 & 0.8116 \\ 185 & 14: 23: 51 & 0.8147 \\ 186 & 14: 23: 52 & 0.8155 \\ 187 & 14: 23: 53 & 0.8235 \\ 188 & 14: 23: 54 & 0.8207 \\ 189 & 14: 23: 55 & 0.8208 \\ 190 & 14: 23: 56 & 0.8213 \\ 191 & 14: 23: 57 & 0.8191 \\ 192 & 14: 23: 58 & 0.8159 \\ 193 & 14: 23: 59 & 0.8181 \\ 194 & 14: 24: 00 & 0.8229 \\ 195 & 14: 24: 01 & 0.8170 \\ 196 & 14: 24: 02 & 0.8194 \\ 197 & 14: 24: 03 & 0.8236 \\ 198 & 14: 24: 04 & 0.8222 \\ 199 & 14: 24: 05 & 0.8186 \\ 200 & 14: 24: 06 & 0.8177 \\ 201 & 14: 24: 07 & 0.8199 \\ 202 & 14: 24: 08 & 0.8159\end{array}$


Appendix G - Fuel Data Test 4 NIST102705_1.xls

$\begin{array}{lll}203 & 14: 24: 09 & 0.8205 \\ 204 & 14: 24: 10 & 0.8256 \\ 205 & 14: 24: 11 & 0.8250 \\ 206 & 14: 24: 12 & 0.8217 \\ 207 & 14: 24: 13 & 0.8241 \\ 208 & 14: 24: 14 & 0.8195 \\ 209 & 14: 24: 15 & 0.8173 \\ 210 & 14: 24: 16 & 0.8187 \\ 211 & 14: 24: 17 & 0.8214 \\ 212 & 14: 24: 18 & 0.8366 \\ 213 & 14: 24: 19 & 0.8986 \\ 214 & 14: 24: 20 & 1.0146 \\ 215 & 14: 24: 21 & 1.0791 \\ 216 & 14: 24: 22 & 1.1142 \\ 217 & 14: 24: 23 & 1.1515 \\ 218 & 14: 24: 24 & 1.1684 \\ 219 & 14: 24: 25 & 1.1716 \\ 220 & 14: 24: 26 & 1.1645 \\ 221 & 14: 24: 27 & 1.1578 \\ 222 & 14: 24: 28 & 1.1634 \\ 223 & 14: 24: 29 & 1.1625 \\ 224 & 14: 24: 30 & 1.1556 \\ 225 & 14: 24: 31 & 1.1530 \\ 226 & 14: 24: 32 & 1.1574 \\ 227 & 14: 24: 33 & 1.1637 \\ 228 & 14: 24: 34 & 1.1687 \\ 229 & 14: 24: 35 & 1.1679 \\ 230 & 14: 24: 36 & 1.1681 \\ 231 & 14: 24: 37 & 1.1670 \\ 232 & 14: 24: 38 & 1.1636 \\ 233 & 14: 24: 39 & 1.1608 \\ 234 & 14: 24: 40 & 1.1591 \\ 235 & 14: 24: 41 & 1.1666 \\ 236 & 14: 24: 42 & 1.1647 \\ 237 & 14: 24: 43 & 1.1528 \\ 238 & 14: 24: 44 & 1.1489 \\ 239 & 14: 24: 45 & 1.1518 \\ 240 & 14: 24: 46 & 1.1548 \\ 241 & 14: 24: 47 & 1.1566 \\ 242 & 14: 24: 48 & 1.1645 \\ 243 & 14: 24: 49 & 1.1709 \\ 244 & 14: 24: 50 & 1.1722 \\ 245 & 14: 24: 51 & 1.1634 \\ 246 & 14: 24: 52 & 1.1609 \\ 247 & 14: 24: 53 & 1.1604 \\ 248 & 14: 24: 54 & 1.1649 \\ 249 & 14: 24: 55 & 1.1665 \\ 250 & 14: 24: 56 & 1.1690 \\ 251 & 14: 24: 57 & 1.1600 \\ 252 & 14: 24: 58 & 1.1515 \\ 253 & 14: 24: 59 & 1.1527\end{array}$

G - 5 
Appendix G - Fuel Data Test 4 NIST102705_1.xls

$\begin{array}{lll}254 & 14: 25: 00 & 1.1550 \\ 255 & 14: 25: 01 & 1.1634 \\ 256 & 14: 25: 02 & 1.1685 \\ 257 & 14: 25: 03 & 1.1696 \\ 258 & 14: 25: 04 & 1.1546 \\ 259 & 14: 25: 05 & 1.1490 \\ 260 & 14: 25: 06 & 1.1524 \\ 261 & 14: 25: 07 & 1.1545 \\ 263 & 14: 25: 09 & 1.1578 \\ 263 & 14: 25: 09 & 1.1629 \\ 264 & 14: 25: 10 & 1.1512 \\ 265 & 14: 25: 11 & 1.1499 \\ 266 & 14: 25: 12 & 1.1595 \\ 267 & 14: 25: 13 & 1.1682 \\ 268 & 14: 25: 14 & 1.1664 \\ 269 & 14: 25: 15 & 1.1629 \\ 270 & 14: 25: 16 & 1.1594 \\ 271 & 14: 25: 17 & 1.1636 \\ 272 & 14: 25: 18 & 1.1668 \\ 273 & 14: 25: 19 & 1.1603 \\ 274 & 14: 25: 20 & 1.1535 \\ 275 & 14: 25: 21 & 1.1606 \\ 276 & 14: 25: 22 & 1.1668 \\ 277 & 14: 25: 23 & 1.1605 \\ 278 & 14: 25: 24 & 1.1629 \\ 279 & 14: 25: 25 & 1.1615 \\ 280 & 14: 25: 26 & 1.1667 \\ 281 & 14: 25: 27 & 1.1719 \\ 282 & 14: 25: 28 & 1.1693 \\ 283 & 14: 25: 29 & 1.1615 \\ 284 & 14: 25: 30 & 1.1590 \\ 285 & 14: 25: 31 & 1.1610 \\ 286 & 14: 25: 32 & 1.1618 \\ 287 & 14: 25: 33 & 1.1603 \\ 288 & 14: 25: 34 & 1.1612 \\ 289 & 14: 25: 35 & 1.1578 \\ 290 & 14: 25: 36 & 1.1586 \\ 291 & 14: 25: 37 & 1.1555 \\ 292 & 14: 25: 38 & 1.1541 \\ 293 & 14: 25: 39 & 1.1581 \\ 294 & 14: 25: 40 & 1.1608 \\ 295 & 14: 25: 41 & 1.1594 \\ 296 & 14: 25: 42 & 1.1600 \\ 297 & 14: 25: 43 & 1.1613 \\ 298 & 14: 25: 44 & 1.1608 \\ 299 & 14: 25: 45 & 1.1607 \\ 300 & 14: 25: 46 & 1.1579 \\ 301 & 14: 25: 47 & 1.1584 \\ 302 & 14: 25: 48 & 1.1584 \\ 303 & 14: 25: 49 & 1.1604 \\ 304 & 14: 25: 50 & 1.1604\end{array}$


Appendix G - Fuel Data Test 4 NIST102705_1.xls

$\begin{array}{lll}305 & 14: 25: 51 & 1.1654 \\ 306 & 14: 25: 52 & 1.1654 \\ 307 & 14: 25: 53 & 1.1621 \\ 308 & 14: 25: 54 & 1.1592 \\ 309 & 14: 25: 55 & 1.1602 \\ 310 & 14: 25: 56 & 1.1595 \\ 311 & 14: 25: 57 & 1.1511 \\ 312 & 14: 25: 58 & 1.1610 \\ 313 & 14: 25: 59 & 1.1702 \\ 314 & 14: 26: 00 & 1.1742 \\ 315 & 14: 26: 01 & 1.1723 \\ 316 & 14: 26: 02 & 1.1626 \\ 317 & 14: 26: 03 & 1.1657 \\ 318 & 14: 26: 04 & 1.1699 \\ 319 & 14: 26: 05 & 1.1615 \\ 320 & 14: 26: 06 & 1.1615 \\ 321 & 14: 26: 07 & 1.1607 \\ 322 & 14: 26: 08 & 1.1646 \\ 323 & 14: 26: 09 & 1.1644 \\ 324 & 14: 26: 10 & 1.1569 \\ 325 & 14: 26: 11 & 1.1537 \\ 326 & 14: 26: 12 & 1.1580 \\ 327 & 14: 26: 13 & 1.1637 \\ 328 & 14: 26: 14 & 1.1699 \\ 329 & 14: 26: 15 & 1.1733 \\ 330 & 14: 26: 16 & 1.1697 \\ 331 & 14: 26: 17 & 1.1841 \\ 332 & 14: 26: 18 & 1.2531 \\ 333 & 14: 26: 19 & 1.3009 \\ 334 & 14: 26: 20 & 1.3261 \\ 335 & 14: 26: 21 & 1.3555 \\ 336 & 14: 26: 22 & 1.3639 \\ 337 & 14: 26: 23 & 1.3657 \\ 338 & 14: 26: 24 & 1.3678 \\ 339 & 14: 26: 25 & 1.3700 \\ 340 & 14: 26: 26 & 1.3711 \\ 341 & 14: 26: 27 & 1.3658 \\ 342 & 14: 26: 28 & 1.3676 \\ 343 & 14: 26: 29 & 1.3669 \\ 344 & 14: 26: 30 & 1.3651 \\ 345 & 14: 26: 31 & 1.3690 \\ 346 & 14: 26: 32 & 1.3685 \\ 347 & 14: 26: 33 & 1.3664 \\ 348 & 14: 26: 34 & 1.3648 \\ 349 & 14: 26: 35 & 1.3680 \\ 350 & 14: 26: 36 & 1.3658 \\ 351 & 14: 26: 37 & 1.3649 \\ 352 & 14: 26: 38 & 1.3642 \\ 353 & 14: 26: 39 & 1.3637 \\ 354 & 14: 26: 40 & 1.3714 \\ 355 & 14: 26: 41 & 1.3737\end{array}$


Appendix G - Fuel Data Test 4 NIST102705_1.xls

$\begin{array}{lll}356 & 14: 26: 42 & 1.3704 \\ 357 & 14: 26: 43 & 1.3703 \\ 358 & 14: 26: 44 & 1.3703 \\ 359 & 14: 26: 45 & 1.3689 \\ 360 & 14: 26: 46 & 1.3650 \\ 361 & 14: 26: 47 & 1.3683 \\ 362 & 14: 26: 48 & 1.3684 \\ 363 & 14: 26: 49 & 1.3650 \\ 364 & 14: 26: 50 & 1.3669 \\ 365 & 14: 26: 51 & 1.3663 \\ 366 & 14: 26: 52 & 1.3656 \\ 367 & 14: 26: 53 & 1.3664 \\ 368 & 14: 26: 54 & 1.3693 \\ 369 & 14: 26: 55 & 1.3728 \\ 370 & 14: 26: 56 & 1.3701 \\ 371 & 14: 26: 57 & 1.3647 \\ 372 & 14: 26: 58 & 1.3653 \\ 373 & 14: 26: 59 & 1.3693 \\ 374 & 14: 27: 00 & 1.3682 \\ 375 & 14: 27: 01 & 1.3646 \\ 376 & 14: 27: 02 & 1.3685 \\ 377 & 14: 27: 03 & 1.3698 \\ 378 & 14: 27: 04 & 1.3683 \\ 379 & 14: 27: 05 & 1.3670 \\ 380 & 14: 27: 06 & 1.3682 \\ 381 & 14: 27: 07 & 1.3692 \\ 382 & 14: 27: 08 & 1.3652 \\ 383 & 14: 27: 09 & 1.3661 \\ 384 & 14: 27: 10 & 1.3664 \\ 385 & 14: 27: 11 & 1.3671 \\ 386 & 14: 27: 12 & 1.3679 \\ 387 & 14: 27: 13 & 1.3680 \\ 388 & 14: 27: 14 & 1.3686 \\ 389 & 14: 27: 15 & 1.3653 \\ 390 & 14: 27: 16 & 1.3654 \\ 391 & 14: 27: 17 & 1.3671 \\ 392 & 14: 27: 18 & 1.3683 \\ 393 & 14: 27: 19 & 1.3702 \\ 394 & 14: 27: 20 & 1.3687 \\ 395 & 14: 27: 21 & 1.3698 \\ 396 & 14: 27: 22 & 1.3654 \\ 397 & 14: 27: 23 & 1.3652 \\ 398 & 14: 27: 24 & 1.3684 \\ 399 & 14: 27: 25 & 1.3680 \\ 400 & 14: 27: 26 & 1.3675 \\ 401 & 14: 27: 27 & 1.3683 \\ 402 & 14: 27: 28 & 1.3648 \\ 403 & 14: 27: 29 & 1.3666 \\ 404 & 14: 27: 30 & 1.3692 \\ 405 & 14: 27: 31 & 1.3690 \\ 406 & 14: 27: 32 & 1.3710\end{array}$


Appendix G - Fuel Data Test 4 NIST102705_1.xls

$\begin{array}{lll}407 & 14: 27: 33 & 1.3659 \\ 408 & 14: 27: 34 & 1.3657 \\ 409 & 14: 27: 35 & 1.3700 \\ 410 & 14: 27: 36 & 1.3679 \\ 411 & 14: 27: 37 & 1.3648 \\ 412 & 14: 27: 38 & 1.3681 \\ 413 & 14: 27: 39 & 1.3637 \\ 414 & 14: 27: 40 & 1.3672 \\ 415 & 14: 27: 41 & 1.3702 \\ 416 & 14: 27: 42 & 1.3686 \\ 417 & 14: 27: 43 & 1.3640 \\ 418 & 14: 27: 44 & 1.3656 \\ 419 & 14: 27: 45 & 1.3674 \\ 420 & 14: 27: 46 & 1.3698 \\ 421 & 14: 27: 47 & 1.3662 \\ 422 & 14: 27: 48 & 1.3627 \\ 423 & 14: 27: 49 & 1.3667 \\ 424 & 14: 27: 50 & 1.3642 \\ 425 & 14: 27: 51 & 1.3664 \\ 426 & 14: 27: 52 & 1.3675 \\ 427 & 14: 27: 53 & 1.3645 \\ 428 & 14: 27: 54 & 1.3673 \\ 429 & 14: 27: 55 & 1.3697 \\ 430 & 14: 27: 56 & 1.3700 \\ 431 & 14: 27: 57 & 1.3709 \\ 432 & 14: 27: 58 & 1.3672 \\ 433 & 14: 27: 59 & 1.3676 \\ 434 & 14: 28: 00 & 1.3697 \\ 435 & 14: 28: 01 & 1.3680 \\ 436 & 14: 28: 02 & 1.3688 \\ 437 & 14: 28: 03 & 1.3692 \\ 438 & 14: 28: 04 & 1.3694 \\ 439 & 14: 28: 05 & 1.3676 \\ 440 & 14: 28: 06 & 1.3677 \\ 441 & 14: 28: 07 & 1.3659 \\ 442 & 14: 28: 08 & 1.3654 \\ 443 & 14: 28: 09 & 1.3701 \\ 444 & 14: 28: 10 & 1.3673 \\ 445 & 14: 28: 11 & 1.3629 \\ 446 & 14: 28: 12 & 1.3661 \\ 447 & 14: 28: 13 & 1.3676 \\ 448 & 14: 28: 14 & 1.3664 \\ 449 & 14: 28: 15 & 1.3682 \\ 450 & 14: 28: 16 & 1.3654 \\ 451 & 14: 28: 17 & 1.3653 \\ 452 & 14: 28: 18 & 1.3695 \\ 453 & 14: 28: 19 & 1.3668 \\ 454 & 14: 28: 20 & 1.3669 \\ 455 & 14: 28: 21 & 1.3666 \\ 456 & 14: 28: 22 & 1.3679 \\ 457 & 14: 28: 23 & 1.3674\end{array}$


Appendix G - Fuel Data Test 4 NIST102705_1.xls

$\begin{array}{lll}458 & 14: 28: 24 & 1.3658 \\ 459 & 14: 28: 25 & 1.3682 \\ 460 & 14: 28: 26 & 1.3680 \\ 461 & 14: 28: 27 & 1.3650 \\ 462 & 14: 28: 28 & 1.3658 \\ 463 & 14: 28: 29 & 1.3681 \\ 464 & 14: 28: 30 & 1.3705 \\ 465 & 14: 28: 31 & 1.3731 \\ 466 & 14: 28: 32 & 1.3694 \\ 467 & 14: 28: 33 & 1.3675 \\ 468 & 14: 28: 34 & 1.3682 \\ 469 & 14: 28: 35 & 1.3686 \\ 470 & 14: 28: 36 & 1.3674 \\ 471 & 14: 28: 37 & 1.3662 \\ 472 & 14: 28: 38 & 1.3692 \\ 473 & 14: 28: 39 & 1.3685 \\ 474 & 14: 28: 40 & 1.3700 \\ 475 & 14: 28: 41 & 1.3691 \\ 476 & 14: 28: 42 & 1.3668 \\ 477 & 14: 28: 43 & 1.3681 \\ 478 & 14: 28: 44 & 1.3669 \\ 479 & 14: 28: 45 & 1.3681 \\ 480 & 14: 28: 46 & 1.3713 \\ 481 & 14: 28: 47 & 1.3683 \\ 482 & 14: 28: 48 & 1.3662 \\ 483 & 14: 28: 49 & 1.3697 \\ 484 & 14: 28: 50 & 1.3678 \\ 485 & 14: 28: 51 & 1.3673 \\ 486 & 14: 28: 52 & 1.3698 \\ 487 & 14: 28: 53 & 1.3682 \\ 488 & 14: 28: 54 & 1.3683 \\ 489 & 14: 28: 55 & 1.3666 \\ 490 & 14: 28: 56 & 1.3715 \\ 491 & 14: 28: 57 & 1.3731 \\ 492 & 14: 28: 58 & 1.3716 \\ 493 & 14: 28: 59 & 1.3720 \\ 494 & 14: 29: 00 & 1.3708 \\ 495 & 14: 29: 01 & 1.3700 \\ 496 & 14: 29: 02 & 1.3703 \\ 497 & 14: 29: 03 & 1.3689 \\ 498 & 14: 29: 04 & 1.3691 \\ 499 & 14: 29: 05 & 1.3731 \\ 500 & 14: 29: 06 & 1.3707 \\ 501 & 14: 29: 07 & 1.3660 \\ 502 & 14: 29: 08 & 1.3680 \\ 503 & 14: 29: 09 & 1.3691 \\ 504 & 14: 29: 10 & 1.3700 \\ 505 & 14: 29: 11 & 1.3690 \\ 506 & 14: 29: 12 & 1.3663 \\ 507 & 14: 29: 13 & 1.3665 \\ 508 & 14: 29: 14 & 1.3682\end{array}$

G - 10 
Appendix G - Fuel Data Test 4 NIST102705_1.xls

$\begin{array}{lll}509 & 14: 29: 15 & 1.3694 \\ 510 & 14: 29: 16 & 1.3693 \\ 511 & 14: 29: 17 & 1.3687 \\ 512 & 14: 29: 18 & 1.3694 \\ 513 & 14: 29: 19 & 1.3684 \\ 514 & 14: 29: 20 & 1.3692 \\ 515 & 14: 29: 21 & 1.3705 \\ 516 & 14: 29: 22 & 1.3686 \\ 517 & 14: 29: 23 & 1.3675 \\ 518 & 14: 29: 24 & 1.3688 \\ 519 & 14: 29: 25 & 1.3682 \\ 520 & 14: 29: 26 & 1.3678 \\ 521 & 14: 29: 27 & 1.3688 \\ 522 & 14: 29: 28 & 1.3687 \\ 523 & 14: 29: 29 & 1.3698 \\ 524 & 14: 29: 30 & 1.3705 \\ 525 & 14: 29: 31 & 1.3706 \\ 526 & 14: 29: 32 & 1.3695 \\ 527 & 14: 29: 33 & 1.3649 \\ 528 & 14: 29: 34 & 1.3675 \\ 529 & 14: 29: 35 & 1.3714 \\ 530 & 14: 29: 36 & 1.3714 \\ 531 & 14: 29: 37 & 1.3686 \\ 532 & 14: 29: 38 & 1.3692 \\ 533 & 14: 29: 39 & 1.3698 \\ 534 & 14: 29: 40 & 1.3686 \\ 535 & 14: 29: 41 & 1.3695 \\ 536 & 14: 29: 42 & 1.3682 \\ 537 & 14: 29: 43 & 1.3688 \\ 538 & 14: 29: 44 & 1.3686 \\ 539 & 14: 29: 45 & 1.3685 \\ 540 & 14: 29: 46 & 1.3696 \\ 541 & 14: 29: 47 & 1.3679 \\ 542 & 14: 29: 48 & 1.3663 \\ 543 & 14: 29: 49 & 1.3661 \\ 544 & 14: 29: 50 & 1.3688 \\ 545 & 14: 29: 51 & 1.3696 \\ 546 & 14: 29: 52 & 1.3707 \\ 547 & 14: 29: 53 & 1.3710 \\ 548 & 14: 29: 54 & 1.3714 \\ 549 & 14: 29: 55 & 1.3701 \\ 550 & 14: 29: 56 & 1.3671 \\ 551 & 14: 29: 57 & 1.3666 \\ 552 & 14: 29: 58 & 1.3698 \\ 553 & 14: 29: 59 & 1.3673 \\ 554 & 14: 30: 00 & 1.3674 \\ 555 & 14: 30: 01 & 1.3683 \\ 556 & 14: 30: 02 & 1.3654 \\ 557 & 14: 30: 03 & 1.3672 \\ 558 & 14: 30: 04 & 1.3704 \\ 559 & 14: 30: 05 & 1.3668\end{array}$

$G-11$ 
Appendix G - Fuel Data Test 4 NIST102705_1.xls

$\begin{array}{lll}560 & 14: 30: 06 & 1.3654 \\ 561 & 14: 30: 07 & 1.3694 \\ 562 & 14: 30: 08 & 1.3725 \\ 563 & 14: 30: 09 & 1.3697 \\ 564 & 14: 30: 10 & 1.3697 \\ 565 & 14: 30: 11 & 1.3684 \\ 566 & 14: 30: 12 & 1.3700 \\ 567 & 14: 30: 13 & 1.3716 \\ 568 & 14: 30: 14 & 1.3695 \\ 569 & 14: 30: 15 & 1.3653 \\ 570 & 14: 30: 16 & 1.3689 \\ 571 & 14: 30: 17 & 1.3685 \\ 572 & 14: 30: 18 & 1.3687 \\ 573 & 14: 30: 19 & 1.3710 \\ 574 & 14: 30: 20 & 1.3677 \\ 575 & 14: 30: 21 & 1.3670 \\ 576 & 14: 30: 22 & 1.3630 \\ 577 & 14: 30: 23 & 1.3649 \\ 578 & 14: 30: 24 & 1.3700 \\ 579 & 14: 30: 25 & 1.3692 \\ 580 & 14: 30: 26 & 1.3689 \\ 581 & 14: 30: 27 & 1.3671 \\ 582 & 14: 30: 28 & 1.3712 \\ 583 & 14: 30: 29 & 1.3690 \\ 584 & 14: 30: 30 & 1.3680 \\ 585 & 14: 30: 31 & 1.3682 \\ 586 & 14: 30: 32 & 1.3709 \\ 587 & 14: 30: 33 & 1.3678 \\ 588 & 14: 30: 34 & 1.3698 \\ 589 & 14: 30: 35 & 1.3687 \\ 590 & 14: 30: 36 & 1.3607 \\ 591 & 14: 30: 37 & 1.3292 \\ 592 & 14: 30: 38 & 1.2557 \\ 593 & 14: 30: 39 & 1.1265 \\ 594 & 14: 30: 40 & 0.8948 \\ 595 & 14: 30: 41 & 0.4657 \\ 596 & 14: 30: 42 & 0.3070 \\ 597 & 14: 30: 43 & 0.3130 \\ 598 & 14: 30: 44 & 0.1714 \\ 599 & 14: 30: 45 & 0.0533 \\ 600 & 14: 30: 46 & 0.0248 \\ 601 & 14: 30: 47 & 0.0192 \\ 602 & 14: 30: 48 & 0.0165 \\ 603 & 14: 30: 49 & 0.0170 \\ 604 & 14: 30: 50 & 0.0182 \\ 605 & 14: 30: 51 & 0.0175 \\ 606 & 14: 30: 52 & 0.0153 \\ 607 & 14: 30: 53 & 0.0127 \\ 608 & 14: 30: 54 & 0.0117 \\ 609 & 14: 30: 55 & 0.0139 \\ 610 & 14: 30: 56 & 0.0141\end{array}$

G - 12 
Appendix G - Fuel Data Test 4 NIST102705_1.xls

$\begin{array}{lll}611 & 14: 30: 57 & 0.0135 \\ 612 & 14: 30: 58 & 0.0137 \\ 613 & 14: 30: 59 & 0.0140 \\ 614 & 14: 31: 00 & 0.0136 \\ 615 & 14: 31: 01 & 0.0117 \\ 616 & 14: 31: 02 & 0.0106 \\ 617 & 14: 31: 03 & 0.0129 \\ 618 & 14: 31: 04 & 0.0127 \\ 619 & 14: 31: 05 & 0.0119 \\ 620 & 14: 31: 06 & 0.0120 \\ 621 & 14: 31: 07 & 0.0116 \\ 622 & 14: 31: 08 & 0.0069 \\ 623 & 14: 31: 09 & 0.0144 \\ 624 & 14: 31: 10 & 0.0214 \\ 625 & 14: 31: 11 & 0.0203 \\ 626 & 14: 31: 12 & 0.0181 \\ 627 & 14: 31: 13 & 0.0177 \\ 628 & 14: 31: 14 & 0.0121 \\ 629 & 14: 31: 15 & 0.0115 \\ 630 & 14: 31: 16 & 0.0169 \\ 631 & 14: 31: 17 & 0.0168 \\ 632 & 14: 31: 18 & 0.0136 \\ 633 & 14: 31: 19 & 0.0114 \\ 634 & 14: 31: 20 & 0.0066 \\ 635 & 14: 31: 21 & 0.0131 \\ 636 & 14: 31: 22 & 0.0180 \\ 637 & 14: 31: 23 & 0.0174 \\ 638 & 14: 31: 24 & 0.0125 \\ 639 & 14: 31: 25 & 0.0122 \\ 640 & 14: 31: 26 & 0.0072 \\ 641 & 14: 31: 27 & 0.0061 \\ 642 & 14: 31: 28 & 0.0119 \\ 643 & 14: 31: 29 & 0.0158 \\ 644 & 14: 31: 30 & 0.0168 \\ 645 & 14: 31: 31 & 0.0165 \\ 646 & 14: 31: 32 & 0.0119 \\ 647 & 14: 31: 33 & 0.0158 \\ 648 & 14: 31: 34 & 0.0220 \\ 649 & 14: 31: 35 & 0.0156 \\ 650 & 14: 31: 36 & 0.0062 \\ 651 & 14: 31: 37 & 0.0081 \\ 652 & 14: 31: 38 & 0.0150 \\ 653 & 14: 31: 39 & 0.0140 \\ 654 & 14: 31: 40 & 0.0106 \\ 655 & 14: 31: 41 & 0.0106 \\ 656 & 14: 31: 42 & 0.0106 \\ 657 & 14: 31: 43 & 0.0137 \\ 658 & 14: 31: 44 & 0.0172 \\ 659 & 14: 31: 45 & 0.0190 \\ 660 & 14: 31: 46 & 0.0182 \\ 661 & 14: 31: 47 & 0.0162\end{array}$

G - 13 
Appendix G - Fuel Data Test 4 NIST102705_1.xls

$\begin{array}{lll}662 & 14: 31: 48 & 0.0130 \\ 663 & 14: 31: 49 & 0.0113 \\ 664 & 14: 31: 50 & 0.0183 \\ 665 & 14: 31: 51 & 0.0143 \\ 666 & 14: 31: 52 & 0.0081 \\ 667 & 14: 31: 53 & 0.0155 \\ 668 & 14: 31: 54 & 0.0203 \\ 669 & 14: 31: 55 & 0.0129 \\ 670 & 14: 31: 56 & 0.0074 \\ 671 & 14: 31: 57 & 0.0164 \\ 672 & 14: 31: 58 & 0.0167 \\ 673 & 14: 31: 59 & 0.0130 \\ 674 & 14: 32: 00 & 0.0154 \\ 675 & 14: 32: 01 & 0.0137 \\ 676 & 14: 32: 02 & 0.0090 \\ 677 & 14: 32: 03 & 0.0118 \\ 678 & 14: 32: 04 & 0.0188 \\ 679 & 14: 32: 05 & 0.0187 \\ 680 & 14: 32: 06 & 0.0105 \\ 681 & 14: 32: 07 & 0.0083 \\ 682 & 14: 32: 08 & 0.0143 \\ 683 & 14: 32: 09 & 0.0165 \\ 684 & 14: 32: 10 & 0.0135 \\ 685 & 14: 32: 11 & 0.0051 \\ 686 & 14: 32: 12 & 0.0008 \\ 687 & 14: 32: 13 & 0.0014 \\ 688 & 14: 32: 14 & 0.0082 \\ 689 & 14: 32: 15 & 0.0083 \\ 690 & 14: 32: 16 & 0.0075 \\ 691 & 14: 32: 17 & 0.0075 \\ 692 & 14: 32: 18 & 0.0072 \\ 693 & 14: 32: 19 & 0.0046 \\ 694 & 14: 32: 20 & 0.0084 \\ 695 & 14: 32: 21 & 0.0063 \\ 696 & 14: 32: 22 & 0.0041 \\ 697 & 14: 32: 23 & 0.0008 \\ 698 & 14: 32: 24 & 0.0071 \\ 699 & 14: 32: 25 & 0.0074 \\ 700 & 14: 32: 26 & 0.0010 \\ 701 & 14: 32: 27 & 0.0010 \\ 702 & 14: 32: 28 & 0.0054 \\ 703 & 14: 32: 29 & 0.0055 \\ 704 & 14: 32: 30 & 0.0025 \\ 705 & 14: 32: 31 & 0.0079 \\ 706 & 14: 32: 32 & 0.0125 \\ 707 & 14: 32: 33 & 0.0078 \\ 708 & 14: 32: 34 & 0.0026 \\ 709 & 14: 32: 35 & 0.0003 \\ 710 & 14: 32: 36 & 0.0003 \\ 711 & 14: 32: 37 & 0.0001 \\ 712 & 14: 32: 38 & 0.0024\end{array}$

$G-14$ 
Appendix G - Fuel Data Test 4 NIST102705_1.xls

$\begin{array}{lll}713 & 14: 32: 39 & 0.0050 \\ 714 & 14: 32: 40 & 0.0029 \\ 715 & 14: 32: 41 & 0.0002 \\ 716 & 14: 32: 42 & 0.0013 \\ 717 & 14: 32: 43 & 0.0074 \\ 718 & 14: 32: 44 & 0.0074 \\ 719 & 14: 32: 45 & 0.0007 \\ 720 & 14: 32: 46 & 0.0001 \\ 721 & 14: 32: 47 & 0.0001 \\ 722 & 14: 32: 48 & 0.0061 \\ 723 & 14: 32: 49 & 0.0073 \\ 724 & 14: 32: 50 & 0.0038 \\ 725 & 14: 32: 51 & 0.0001 \\ 726 & 14: 32: 52 & 0.0001 \\ 727 & 14: 32: 53 & 0.0004 \\ 728 & 14: 32: 54 & 0.0002 \\ 729 & 14: 32: 55 & 0.0001 \\ 730 & 14: 32: 56 & 0.0000 \\ 731 & 14: 32: 57 & 0.0002 \\ 732 & 14: 32: 58 & 0.0003 \\ 733 & 14: 32: 59 & 0.0005 \\ 734 & 14: 33: 00 & 0.0068 \\ 735 & 14: 33: 01 & 0.0071 \\ 736 & 14: 33: 02 & 0.0010 \\ 737 & 14: 33: 03 & 0.0003 \\ 738 & 14: 33: 04 & 0.0001 \\ 739 & 14: 33: 05 & 0.0040 \\ 740 & 14: 33: 06 & 0.0096 \\ 741 & 14: 33: 07 & 0.0089 \\ 742 & 14: 33: 08 & 0.0028 \\ 743 & 14: 33: 09 & 0.0001 \\ 744 & 14: 33: 10 & 0.0001 \\ 745 & 14: 33: 11 & 0.0053 \\ 746 & 14: 33: 12 & 0.0062 \\ 747 & 14: 33: 13 & 0.0022 \\ 748 & 14: 33: 14 & 0.0001 \\ 749 & 14: 33: 15 & 0.0003 \\ 750 & 14: 33: 16 & 0.0003 \\ 751 & 14: 33: 17 & 0.0002 \\ 752 & 14: 33: 18 & 0.0001 \\ 753 & 14: 33: 19 & 0.0001 \\ 754 & 14: 33: 20 & 0.0005 \\ 755 & 14: 33: 21 & 0.0062 \\ 756 & 14: 33: 22 & 0.0081 \\ 757 & 14: 33: 23 & 0.0031 \\ 758 & 14: 33: 24 & 0.0003 \\ 759 & 14: 33: 25 & 0.0004 \\ 760 & 14: 33: 26 & 0.0002 \\ 761 & 14: 33: 27 & 0.0001 \\ 762 & 14: 33: 28 & 0.0001 \\ 763 & 14: 33: 29 & 0.0046\end{array}$


Appendix G - Fuel Data Test 4 NIST102705_1.xls

$\begin{array}{lll}764 & 14: 33: 30 & 0.0068 \\ 765 & 14: 33: 31 & 0.0032 \\ 766 & 14: 33: 32 & 0.0016 \\ 767 & 14: 33: 33 & 0.0072 \\ 768 & 14: 33: 34 & 0.0065 \\ 769 & 14: 33: 35 & 0.0004 \\ 770 & 14: 33: 36 & 0.0049 \\ 771 & 14: 33: 37 & 0.0081 \\ 772 & 14: 33: 38 & 0.0046 \\ 773 & 14: 33: 39 & 0.0003 \\ 774 & 14: 33: 40 & 0.0000 \\ 775 & 14: 33: 41 & 0.0012 \\ 776 & 14: 33: 42 & 0.0077 \\ 777 & 14: 33: 43 & 0.0076 \\ 778 & 14: 33: 44 & 0.0006 \\ 779 & 14: 33: 45 & 0.0000 \\ 780 & 14: 33: 46 & 0.0002 \\ 781 & 14: 33: 47 & 0.0003 \\ 782 & 14: 33: 48 & 0.0002 \\ 783 & 14: 33: 49 & 0.0001 \\ 784 & 14: 33: 50 & 0.0001 \\ 785 & 14: 33: 51 & 0.0004 \\ 786 & 14: 33: 52 & 0.0004 \\ 787 & 14: 33: 53 & 0.0001 \\ 788 & 14: 33: 54 & 0.0024 \\ 789 & 14: 33: 55 & 0.0087 \\ 790 & 14: 33: 56 & 0.0123 \\ 791 & 14: 33: 57 & 0.0071 \\ 792 & 14: 33: 58 & 0.0004 \\ 793 & 14: 33: 59 & 0.0001 \\ 794 & 14: 34: 00 & 0.0003 \\ 795 & 14: 34: 01 & 0.0036 \\ 796 & 14: 34: 02 & 0.0041 \\ 797 & 14: 34: 03 & 0.0011 \\ 798 & 14: 34: 04 & 0.0002 \\ 799 & 14: 34: 05 & 0.0004 \\ 800 & 14: 34: 06 & 0.0003 \\ 801 & 14: 34: 07 & 0.0001 \\ 802 & 14: 34: 08 & 0.0001 \\ 803 & 14: 34: 09 & 0.0003 \\ 804 & 14: 34: 10 & 0.0003 \\ 805 & 14: 34: 11 & 0.0001 \\ 806 & 14: 34: 12 & 0.0001 \\ 807 & 14: 34: 13 & 0.0002 \\ 808 & 14: 34: 14 & 0.0004 \\ 809 & 14: 34: 15 & 0.0002 \\ 810 & 14: 34: 16 & 0.0000 \\ 811 & 14: 34: 17 & 0.0002 \\ 812 & 14: 34: 18 & 0.0004 \\ 813 & 14: 34: 19 & 0.0070 \\ 814 & 14: 34: 20 & 0.0075\end{array}$

G - 16 
Appendix G - Fuel Data Test 4 NIST102705_1.xls

$\begin{array}{lll}815 & 14: 34: 21 & 0.0014 \\ 816 & 14: 34: 22 & 0.0002 \\ 817 & 14: 34: 23 & 0.0004 \\ 818 & 14: 34: 24 & 0.0001 \\ 819 & 14: 34: 25 & 0.0012 \\ 820 & 14: 34: 26 & 0.0054 \\ 821 & 14: 34: 27 & 0.0053 \\ 822 & 14: 34: 28 & 0.0006 \\ 823 & 14: 34: 29 & 0.0001 \\ 824 & 14: 34: 30 & 0.0001 \\ 825 & 14: 34: 31 & 0.0002 \\ 826 & 14: 34: 32 & 0.0004 \\ 827 & 14: 34: 33 & 0.0006 \\ 828 & 14: 34: 34 & 0.0062 \\ 829 & 14: 34: 35 & 0.0067 \\ 830 & 14: 34: 36 & 0.0012 \\ 831 & 14: 34: 37 & 0.0003 \\ 832 & 14: 34: 38 & 0.0001 \\ 833 & 14: 34: 39 & 0.0001 \\ 834 & 14: 34: 40 & 0.0002 \\ 835 & 14: 34: 41 & 0.0004 \\ 836 & 14: 34: 42 & 0.0003 \\ 837 & 14: 34: 43 & 0.0001 \\ 838 & 14: 34: 44 & 0.0001 \\ 839 & 14: 34: 45 & 0.0003 \\ 840 & 14: 34: 46 & 0.0002 \\ 841 & 14: 34: 47 & 0.0001 \\ 842 & 14: 34: 48 & 0.0000 \\ 843 & 14: 34: 49 & 0.0002 \\ 844 & 14: 34: 50 & 0.0004 \\ 845 & 14: 34: 51 & 0.0002 \\ 846 & 14: 34: 52 & 0.0001 \\ 847 & 14: 34: 53 & 0.0001 \\ 848 & 14: 34: 54 & 0.0003 \\ 849 & 14: 34: 55 & 0.0003 \\ 850 & 14: 34: 56 & 0.0001 \\ 851 & 14: 34: 57 & 0.0001 \\ 852 & 14: 34: 58 & 0.0002 \\ 853 & 14: 34: 59 & 0.0004 \\ 854 & 14: 35: 00 & 0.0001 \\ 855 & 14: 35: 01 & 0.0001 \\ 856 & 14: 35: 02 & 0.0001 \\ 857 & 14: 35: 03 & 0.0003 \\ 858 & 14: 35: 04 & 0.0041 \\ 859 & 14: 35: 05 & 0.0128 \\ 860 & 14: 35: 06 & 0.0096 \\ 861 & 14: 35: 07 & 0.0030 \\ 862 & 14: 35: 08 & 0.0004 \\ 863 & 14: 35: 09 & 0.0001 \\ 864 & 14: 35: 10 & 0.0194 \\ 865 & 14: 35: 11 & 0.0291\end{array}$


Appendix G - Fuel Data Test 4 NIST102705_1.xls

$\begin{array}{lll}866 & 14: 35: 12 & 0.0585 \\ 867 & 14: 35: 13 & 0.0794 \\ 868 & 14: 35: 14 & 0.0316 \\ 869 & 14: 35: 15 & 0.0046 \\ 870 & 14: 35: 16 & 0.0003 \\ 871 & 14: 35: 17 & 0.0004 \\ 872 & 14: 35: 18 & 0.0002 \\ 873 & 14: 35: 19 & 0.0000 \\ 874 & 14: 35: 20 & 0.0002 \\ 875 & 14: 35: 21 & 0.0011 \\ 876 & 14: 35: 22 & 0.0069 \\ 877 & 14: 35: 23 & 0.0122 \\ 878 & 14: 35: 24 & 0.0068 \\ 879 & 14: 35: 25 & 0.0014 \\ 880 & 14: 35: 26 & 0.0004 \\ 881 & 14: 35: 27 & 0.0002 \\ 882 & 14: 35: 28 & 0.0001 \\ 883 & 14: 35: 29 & 0.0001 \\ 884 & 14: 35: 30 & 0.0004 \\ 885 & 14: 35: 31 & 0.0002 \\ 886 & 14: 35: 32 & 0.0000 \\ 887 & 14: 35: 33 & 0.0002 \\ 888 & 14: 35: 34 & 0.0002 \\ 889 & 14: 35: 35 & 0.0004 \\ 890 & 14: 35: 36 & 0.0001 \\ 891 & 14: 35: 37 & 0.0000 \\ 892 & 14: 35: 38 & 0.0001 \\ 893 & 14: 35: 39 & 0.0004 \\ 894 & 14: 35: 40 & 0.0002 \\ 895 & 14: 35: 41 & 0.0001 \\ 896 & 14: 35: 42 & 0.0000 \\ 897 & 14: 35: 43 & 0.0003 \\ 898 & 14: 35: 44 & 0.0003 \\ 899 & 14: 35: 45 & 0.0001 \\ 900 & 14: 35: 46 & 0.0001 \\ 901 & 14: 35: 47 & 0.0001 \\ 902 & 14: 35: 48 & 0.0004 \\ 903 & 14: 35: 49 & 0.0003 \\ 904 & 14: 35: 50 & 0.0001 \\ 905 & 14: 35: 51 & 0.0001 \\ 906 & 14: 35: 52 & 0.0003 \\ 907 & 14: 35: 53 & 0.0004 \\ 908 & 14: 35: 54 & 0.0002 \\ 909 & 14: 35: 55 & 0.0001 \\ 910 & 14: 35: 56 & 0.0002 \\ 911 & 14: 35: 57 & 0.0004 \\ 912 & 14: 35: 58 & 0.0003 \\ 913 & 14: 35: 59 & 0.0035 \\ 914 & 14: 36: 00 & 0.0050 \\ 915 & 14: 36: 01 & 0.0044 \\ 916 & 14: 36: 02 & 0.0016\end{array}$


Appendix G - Fuel Data Test 4 NIST102705_1.xls

$\begin{array}{lll}917 & 14: 36: 03 & 0.0071 \\ 918 & 14: 36: 04 & 0.0128 \\ 919 & 14: 36: 05 & 0.0073 \\ 920 & 14: 36: 06 & 0.0025 \\ 921 & 14: 36: 07 & 0.0003 \\ 922 & 14: 36: 08 & 0.0001 \\ 923 & 14: 36: 09 & 0.0001 \\ 924 & 14: 36: 10 & 0.0054 \\ 925 & 14: 36: 11 & 0.0063 \\ 926 & 14: 36: 12 & 0.0020 \\ 927 & 14: 36: 13 & 0.0001 \\ 928 & 14: 36: 14 & 0.0002 \\ 929 & 14: 36: 15 & 0.0004 \\ 930 & 14: 36: 16 & 0.0002 \\ 931 & 14: 36: 17 & 0.0001 \\ 932 & 14: 36: 18 & 0.0001 \\ 933 & 14: 36: 19 & 0.0003 \\ 934 & 14: 36: 20 & 0.0004 \\ 935 & 14: 36: 21 & 0.0045 \\ 936 & 14: 36: 22 & 0.0066 \\ 937 & 14: 36: 23 & 0.0039 \\ 938 & 14: 36: 24 & 0.0004 \\ 939 & 14: 36: 25 & 0.0002 \\ 940 & 14: 36: 26 & 0.0001 \\ 941 & 14: 36: 27 & 0.0001 \\ 942 & 14: 36: 28 & 0.0003 \\ 943 & 14: 36: 29 & 0.0004 \\ 944 & 14: 36: 30 & 0.0002 \\ 945 & 14: 36: 31 & 0.0000 \\ 946 & 14: 36: 32 & 0.0002 \\ 947 & 14: 36: 33 & 0.0004 \\ 948 & 14: 36: 34 & 0.0003 \\ 949 & 14: 36: 35 & 0.0001 \\ 950 & 14: 36: 36 & 0.0021 \\ 951 & 14: 36: 37 & 0.0066 \\ 952 & 14: 36: 38 & 0.0053 \\ 953 & 14: 36: 39 & 0.0003 \\ 954 & 14: 36: 40 & 0.0001 \\ 955 & 14: 36: 41 & 0.0002 \\ 956 & 14: 36: 42 & 0.0004 \\ 957 & 14: 36: 43 & 0.0002 \\ 958 & 14: 36: 44 & 0.0001 \\ 959 & 14: 36: 45 & 0.0001 \\ 960 & 14: 36: 46 & 0.0004 \\ 961 & 14: 36: 47 & 0.0003 \\ 962 & 14: 36: 48 & 0.0001 \\ 963 & 14: 36: 49 & 0.0001 \\ 964 & 14: 36: 50 & 0.0002 \\ 965 & 14: 36: 51 & 0.0003 \\ 966 & 14: 36: 52 & 0.0002 \\ 967 & 14: 36: 53 & 0.0001\end{array}$

G - 19 
Appendix G - Fuel Data Test 4 NIST102705_1.xls

$\begin{array}{rll}968 & 14: 36: 54 & 0.0001 \\ 969 & 14: 36: 55 & 0.0003 \\ 970 & 14: 36: 56 & 0.0003 \\ 971 & 14: 36: 57 & 0.0001 \\ 972 & 14: 36: 58 & 0.0001 \\ 973 & 14: 36: 59 & 0.0002 \\ 974 & 14: 37: 00 & 0.0003 \\ 975 & 14: 37: 01 & 0.0051 \\ 976 & 14: 37: 02 & 0.0069 \\ 977 & 14: 37: 03 & 0.0058 \\ 978 & 14: 37: 04 & 0.0005 \\ 979 & 14: 37: 05 & 0.0003 \\ 980 & 14: 37: 06 & 0.0001 \\ 981 & 14: 37: 07 & 0.0037 \\ 982 & 14: 37: 08 & 0.0048 \\ 983 & 14: 37: 09 & 0.0017 \\ 984 & 14: 37: 10 & 0.0002 \\ 985 & 14: 37: 11 & 0.0001 \\ 986 & 14: 37: 12 & 0.0001 \\ 987 & 14: 37: 13 & 0.0004 \\ 988 & 14: 37: 14 & 0.0003 \\ 989 & 14: 37: 15 & 0.0001 \\ 990 & 14: 37: 16 & 0.0001 \\ 991 & 14: 37: 17 & 0.0002 \\ 992 & 14: 37: 18 & 0.0004 \\ 993 & 14: 37: 19 & 0.0002 \\ 994 & 14: 37: 20 & 0.0001 \\ 995 & 14: 37: 21 & 0.0001 \\ 996 & 14: 37: 22 & 0.0019 \\ 997 & 14: 37: 23 & 0.0051 \\ 998 & 14: 37: 24 & 0.0041 \\ 999 & 14: 37: 25 & 0.0002 \\ 1000 & 14: 37: 26 & 0.0002 \\ 1001 & 14: 37: 27 & 0.0004 \\ 1002 & 14: 37: 28 & 0.0010 \\ 1003 & 14: 37: 29 & 0.0035 \\ 1004 & 14: 37: 30 & 0.0034 \\ 1005 & 14: 37: 31 & 0.0006 \\ 1006 & 14: 37: 32 & 0.0003 \\ 1007 & 14: 37: 33 & 0.0002 \\ 1008 & 14: 37: 34 & 0.0001 \\ 1009 & 14: 37: 35 & 0.0003 \\ 1010 & 14: 37: 36 & 0.0021 \\ 1011 & 14: 37: 37 & 0.0042 \\ 1012 & 14: 37: 38 & 0.0031 \\ 1013 & 14: 37: 39 & 0.0001 \\ 1014 & 14: 37: 40 & 0.0004 \\ 1015 & 14: 37: 41 & 0.0003 \\ 1016 & 14: 37: 42 & 0.0001 \\ 1017 & 14: 37: 43 & 0.0001 \\ 1018 & 14: 37: 44 & 0.0003\end{array}$


Appendix G - Fuel Data Test 4 NIST102705_1.xls

$\begin{array}{lll}1019 & 14: 37: 45 & 0.0004 \\ 1020 & 14: 37: 46 & 0.0001 \\ 1021 & 14: 37: 47 & 0.0001 \\ 1022 & 14: 37: 48 & 0.0001 \\ 1023 & 14: 37: 49 & 0.0004 \\ 1024 & 14: 37: 50 & 0.0002 \\ 1025 & 14: 37: 51 & 0.0001 \\ 1026 & 14: 37: 52 & 0.0001 \\ 1027 & 14: 37: 53 & 0.0003 \\ 1028 & 14: 37: 54 & 0.0004 \\ 1029 & 14: 37: 55 & 0.0002 \\ 1030 & 14: 37: 56 & 0.0001 \\ 1031 & 14: 37: 57 & 0.0002 \\ 1032 & 14: 37: 58 & 0.0003 \\ 1033 & 14: 37: 59 & 0.0002 \\ 1034 & 14: 38: 00 & 0.0001 \\ 1035 & 14: 38: 01 & 0.0001 \\ 1036 & 14: 38: 02 & 0.0003 \\ 1037 & 14: 38: 03 & 0.0004 \\ 1038 & 14: 38: 04 & 0.0001 \\ 1039 & 14: 38: 05 & 0.0001 \\ 1040 & 14: 38: 06 & 0.0002 \\ 1041 & 14: 38: 07 & 0.0004 \\ 1042 & 14: 38: 08 & 0.0003 \\ 1043 & 14: 38: 09 & 0.0001 \\ 1044 & 14: 38: 10 & 0.0001 \\ 1045 & 14: 38: 11 & 0.0003 \\ 1046 & 14: 38: 12 & 0.0004 \\ 1047 & 14: 38: 13 & 0.0001 \\ 1048 & 14: 38: 14 & 0.0001 \\ 1049 & 14: 38: 15 & 0.0002 \\ 1050 & 14: 38: 16 & 0.0004 \\ 1051 & 14: 38: 17 & 0.0002 \\ 1052 & 14: 38: 18 & 0.0001 \\ 1053 & 14: 38: 19 & 0.0001 \\ 1054 & 14: 38: 20 & 0.0003 \\ 1055 & 14: 38: 21 & 0.0003 \\ 1056 & 14: 38: 22 & 0.0001 \\ 1057 & 14: 38: 23 & 0.0001 \\ 1058 & 14: 38: 24 & 0.0001 \\ 1059 & 14: 38: 25 & 0.0003 \\ 1060 & 14: 38: 26 & 0.0002 \\ 1061 & 14: 38: 27 & 0.0001 \\ 1062 & 14: 38: 28 & 0.0001 \\ 1063 & 14: 38: 29 & 0.0003 \\ 1064 & 14: 38: 30 & 0.0003 \\ 1065 & 14: 38: 31 & 0.0002 \\ 1066 & 14: 38: 32 & 0.0001 \\ 1067 & 14: 38: 33 & 0.0002 \\ 1068 & 14: 38: 34 & 0.0003 \\ 1069 & 14: 38: 35 & 0.0002\end{array}$


Appendix G - Fuel Data Test 4 NIST102705_1.xls

$\begin{array}{lll}1070 & 14: 38: 36 & 0.0001 \\ 1071 & 14: 38: 37 & 0.0001 \\ 1072 & 14: 38: 38 & 0.0003 \\ 1073 & 14: 38: 39 & 0.0004 \\ 1074 & 14: 38: 40 & 0.0001 \\ 1075 & 14: 38: 41 & 0.0001 \\ 1076 & 14: 38: 42 & 0.0002 \\ 1077 & 14: 38: 43 & 0.0004 \\ 1078 & 14: 38: 44 & 0.0003 \\ 1079 & 14: 38: 45 & 0.0000 \\ 1080 & 14: 38: 46 & 0.0001 \\ 1081 & 14: 38: 47 & 0.0003 \\ 1082 & 14: 38: 48 & 0.0003 \\ 1083 & 14: 38: 49 & 0.0001 \\ 1084 & 14: 38: 50 & 0.0001 \\ 1085 & 14: 38: 51 & 0.0001 \\ 1086 & 14: 38: 52 & 0.0005 \\ 1087 & 14: 38: 53 & 0.0002 \\ 1088 & 14: 38: 54 & 0.0001 \\ 1089 & 14: 38: 55 & 0.0001 \\ 1090 & 14: 38: 56 & 0.0003 \\ 1091 & 14: 38: 57 & 0.0003 \\ 1092 & 14: 38: 58 & 0.0002 \\ 1093 & 14: 38: 59 & 0.0000 \\ 1094 & 14: 39: 00 & 0.0002 \\ 1095 & 14: 39: 01 & 0.0004 \\ 1096 & 14: 39: 02 & 0.0002 \\ 1097 & 14: 39: 03 & 0.0001 \\ 1098 & 14: 39: 04 & 0.0002 \\ 1099 & 14: 39: 05 & 0.0004 \\ 1100 & 14: 39: 06 & 0.0003 \\ 1101 & 14: 39: 07 & 0.0001 \\ 1102 & 14: 39: 08 & 0.0000 \\ 1103 & 14: 39: 09 & 0.0003 \\ 1104 & 14: 39: 10 & 0.0003 \\ 1105 & 14: 39: 11 & 0.0002 \\ 1106 & 14: 39: 12 & 0.0001 \\ 1107 & 14: 39: 13 & 0.0002 \\ 1108 & 14: 39: 14 & 0.0004 \\ 1109 & 14: 39: 15 & 0.0003 \\ 1110 & 14: 39: 16 & 0.0002 \\ 1111 & 14: 39: 17 & 0.0000 \\ 1112 & 14: 39: 18 & 0.0002 \\ 1113 & 14: 39: 19 & 0.0003 \\ 1114 & 14: 39: 20 & 0.0002 \\ 1115 & 14: 39: 21 & 0.0001 \\ 1116 & 14: 39: 22 & 0.0001 \\ 1117 & 14: 39: 23 & 0.0003 \\ 1118 & 14: 39: 24 & 0.0003 \\ 1119 & 14: 39: 25 & 0.0001 \\ 1120 & 14: 39: 26 & 0.0000\end{array}$


Appendix G - Fuel Data Test 4 NIST102705_1.xls

$\begin{array}{lll}1121 & 14: 39: 27 & 0.0003 \\ 1122 & 14: 39: 28 & 0.0004 \\ 1123 & 14: 39: 29 & 0.0002 \\ 1124 & 14: 39: 30 & 0.0001 \\ 1125 & 14: 39: 31 & 0.0001 \\ 1126 & 14: 39: 32 & 0.0003 \\ 1127 & 14: 39: 33 & 0.0003 \\ 1128 & 14: 39: 34 & 0.0001 \\ 1129 & 14: 39: 35 & 0.0001 \\ 1130 & 14: 39: 36 & 0.0002 \\ 1131 & 14: 39: 37 & 0.0004 \\ 1132 & 14: 39: 38 & 0.0002 \\ 1133 & 14: 39: 39 & 0.0000 \\ 1134 & 14: 39: 40 & 0.0001 \\ 1135 & 14: 39: 41 & 0.0004 \\ 1136 & 14: 39: 42 & 0.0003 \\ 1137 & 14: 39: 43 & 0.0002 \\ 1138 & 14: 39: 44 & 0.0001 \\ 1139 & 14: 39: 45 & 0.0003 \\ 1140 & 14: 39: 46 & 0.0003 \\ 1141 & 14: 39: 47 & 0.0001 \\ 1142 & 14: 39: 48 & 0.0001 \\ 1143 & 14: 39: 49 & 0.0002 \\ 1144 & 14: 39: 50 & 0.0004 \\ 1145 & 14: 39: 51 & 0.0003 \\ 1146 & 14: 39: 52 & 0.0001 \\ 1147 & 14: 39: 53 & 0.0001 \\ 1148 & 14: 39: 54 & 0.0002 \\ 1149 & 14: 39: 55 & 0.0003 \\ 1150 & 14: 39: 56 & 0.0002 \\ 1151 & 14: 39: 57 & 0.0001 \\ 1152 & 14: 39: 58 & 0.0001 \\ 1153 & 14: 39: 59 & 0.0004 \\ 1154 & 14: 40: 00 & 0.0003 \\ 1155 & 14: 40: 01 & 0.0001 \\ 1156 & 14: 40: 02 & 0.0001 \\ 1157 & 14: 40: 03 & 0.0003 \\ 1158 & 14: 40: 04 & 0.0003 \\ 1159 & 14: 40: 05 & 0.0002 \\ 1160 & 14: 40: 06 & 0.0000 \\ 1161 & 14: 40: 07 & 0.0001 \\ 1162 & 14: 40: 08 & 0.0004 \\ 1163 & 14: 40: 09 & 0.0002 \\ 1164 & 14: 40: 10 & 0.0001 \\ 1165 & 14: 40: 11 & 0.0001 \\ 1166 & 14: 40: 12 & 0.0003 \\ 1167 & 14: 40: 13 & 0.0003 \\ 1168 & 14: 40: 14 & 0.0002 \\ 1169 & 14: 40: 15 & 0.0001 \\ 1170 & 14: 40: 16 & 0.0001 \\ 1171 & 14: 40: 17 & 0.0003\end{array}$


Appendix G - Fuel Data Test 4 NIST102705_1.xls

$\begin{array}{lll}1172 & 14: 40: 18 & 0.0002 \\ 1173 & 14: 40: 19 & 0.0000 \\ 1174 & 14: 40: 20 & 0.0001 \\ 1175 & 14: 40: 21 & 0.0002 \\ 1176 & 14: 40: 22 & 0.0003 \\ 1177 & 14: 40: 23 & 0.0002 \\ 1178 & 14: 40: 24 & 0.0000 \\ 1179 & 14: 40: 25 & 0.0002 \\ 1180 & 14: 40: 26 & 0.0004 \\ 1181 & 14: 40: 27 & 0.0002 \\ 1182 & 14: 40: 28 & 0.0001 \\ 1183 & 14: 40: 29 & 0.0001 \\ 1184 & 14: 40: 30 & 0.0004 \\ 1185 & 14: 40: 31 & 0.0004 \\ 1186 & 14: 40: 32 & 0.0001 \\ 1187 & 14: 40: 33 & 0.0001 \\ 1188 & 14: 40: 34 & 0.0002 \\ 1189 & 14: 40: 35 & 0.0004 \\ 1190 & 14: 40: 36 & 0.0002 \\ 1191 & 14: 40: 37 & 0.0001 \\ 1192 & 14: 40: 38 & 0.0001 \\ 1193 & 14: 40: 39 & 0.0003 \\ 1194 & 14: 40: 40 & 0.0003 \\ 1195 & 14: 40: 41 & 0.0001 \\ 1196 & 14: 40: 42 & 0.0001 \\ 1197 & 14: 40: 43 & 0.0002 \\ 1198 & 14: 40: 44 & 0.0004 \\ 1199 & 14: 40: 45 & 0.0002 \\ 1200 & 14: 40: 46 & 0.0001 \\ 1201 & 14: 40: 47 & 0.0001 \\ 1202 & 14: 40: 48 & 0.0003 \\ 1203 & 14: 40: 49 & 0.1508 \\ 1204 & 14: 40: 50 & 0.3847 \\ 1205 & 14: 40: 51 & 0.3880 \\ 1206 & 14: 40: 52 & 0.3811 \\ 1207 & 14: 40: 53 & 0.3927 \\ 1208 & 14: 40: 54 & 0.3805 \\ 1209 & 14: 40: 55 & 0.3667 \\ 1210 & 14: 40: 56 & 0.3822 \\ 1211 & 14: 40: 57 & 0.3659 \\ 1212 & 14: 40: 58 & 0.3846 \\ 1213 & 14: 40: 59 & 0.3700 \\ 1214 & 14: 41: 00 & 0.3711 \\ 1215 & 14: 41: 01 & 0.3813 \\ 1216 & 14: 41: 02 & 0.3835 \\ 1217 & 14: 41: 03 & 0.4831 \\ 1218 & 14: 41: 04 & 0.5473 \\ 1219 & 14: 41: 05 & 0.5631 \\ 1220 & 14: 41: 06 & 0.6128 \\ 1221 & 14: 41: 07 & 0.7135 \\ 1222 & 14: 41: 08 & 0.7434\end{array}$


Appendix G - Fuel Data Test 4 NIST102705_1.xls

$\begin{array}{lll}1223 & 14: 41: 09 & 0.7395 \\ 1224 & 14: 41: 10 & 0.7452 \\ 1225 & 14: 41: 11 & 0.7389 \\ 1226 & 14: 41: 12 & 0.7543 \\ 1227 & 14: 41: 13 & 0.7760 \\ 1228 & 14: 41: 14 & 0.7892 \\ 1229 & 14: 41: 15 & 0.7892 \\ 1230 & 14: 41: 16 & 0.7831 \\ 1231 & 14: 41: 17 & 0.7839 \\ 1232 & 14: 41: 18 & 0.7904 \\ 1233 & 14: 41: 19 & 0.7935 \\ 1234 & 14: 41: 20 & 0.7937 \\ 1235 & 14: 41: 21 & 0.7887 \\ 1236 & 14: 41: 22 & 0.7827 \\ 1237 & 14: 41: 23 & 0.7779 \\ 1238 & 14: 41: 24 & 0.7629 \\ 1239 & 14: 41: 25 & 0.7454 \\ 1240 & 14: 41: 26 & 0.5136 \\ 1241 & 14: 41: 27 & 0.2089 \\ 1242 & 14: 41: 28 & 0.1941 \\ 1243 & 14: 41: 29 & 0.1911 \\ 1244 & 14: 41: 30 & 0.1909 \\ 1245 & 14: 41: 31 & 0.1999 \\ 1246 & 14: 41: 32 & 0.1875 \\ 1247 & 14: 41: 33 & 0.1839 \\ 1248 & 14: 41: 34 & 0.1809 \\ 1249 & 14: 41: 35 & 0.1776 \\ 1250 & 14: 41: 36 & 0.3916 \\ 1251 & 14: 41: 37 & 0.7228 \\ 1252 & 14: 41: 38 & 0.7727 \\ 1253 & 14: 41: 39 & 0.7677 \\ 1254 & 14: 41: 40 & 0.7728 \\ 1255 & 14: 41: 41 & 0.7611 \\ 1256 & 14: 41: 42 & 0.7739 \\ 1257 & 14: 41: 43 & 0.8320 \\ 1258 & 14: 41: 44 & 0.8679 \\ 1259 & 14: 41: 45 & 0.8894 \\ 1260 & 14: 41: 46 & 0.8957 \\ 1261 & 14: 41: 47 & 0.9022 \\ 1262 & 14: 41: 48 & 0.8938 \\ 1263 & 14: 41: 49 & 0.8960 \\ 1264 & 14: 41: 50 & 0.8943 \\ 1265 & 14: 41: 51 & 0.8945 \\ 1266 & 14: 41: 52 & 0.8954 \\ 1267 & 14: 41: 53 & 0.8919 \\ 1268 & 14: 41: 54 & 0.9088 \\ 1269 & 14: 41: 55 & 0.9509 \\ 1270 & 14: 41: 56 & 1.0017 \\ 1271 & 14: 41: 57 & 1.0226 \\ 1272 & 14: 41: 58 & 1.0213 \\ 1273 & 14: 41: 59 & 1.0256\end{array}$


Appendix G - Fuel Data Test 4 NIST102705_1.xls

$\begin{array}{lll}1274 & 14: 42: 00 & 1.0454 \\ 1275 & 14: 42: 01 & 1.0595 \\ 1276 & 14: 42: 02 & 1.1071 \\ 1277 & 14: 42: 03 & 1.1635 \\ 1278 & 14: 42: 04 & 1.1841 \\ 1279 & 14: 42: 05 & 1.1913 \\ 1280 & 14: 42: 06 & 1.2004 \\ 1281 & 14: 42: 07 & 1.2003 \\ 1282 & 14: 42: 08 & 1.2065 \\ 1283 & 14: 42: 09 & 1.2416 \\ 1284 & 14: 42: 10 & 1.2536 \\ 1285 & 14: 42: 11 & 1.2463 \\ 1286 & 14: 42: 12 & 1.2516 \\ 1287 & 14: 42: 13 & 1.2651 \\ 1288 & 14: 42: 14 & 1.2841 \\ 1289 & 14: 42: 15 & 1.2984 \\ 1290 & 14: 42: 16 & 1.3123 \\ 1291 & 14: 42: 17 & 1.3300 \\ 1292 & 14: 42: 18 & 1.3472 \\ 1293 & 14: 42: 19 & 1.3499 \\ 1294 & 14: 42: 20 & 1.3506 \\ 1295 & 14: 42: 21 & 1.3544 \\ 1296 & 14: 42: 22 & 1.3518 \\ 1297 & 14: 42: 23 & 1.3547 \\ 1298 & 14: 42: 24 & 1.3565 \\ 1299 & 14: 42: 25 & 1.3557 \\ 1300 & 14: 42: 26 & 1.3541 \\ 1301 & 14: 42: 27 & 1.3556 \\ 1302 & 14: 42: 28 & 1.3535 \\ 1303 & 14: 42: 29 & 1.3552 \\ 1304 & 14: 42: 30 & 1.3511 \\ 1305 & 14: 42: 31 & 1.3524 \\ 1306 & 14: 42: 32 & 1.3594 \\ 1307 & 14: 42: 33 & 1.3617 \\ 1308 & 14: 42: 34 & 1.3580 \\ 1309 & 14: 42: 35 & 1.3596 \\ 1310 & 14: 42: 36 & 1.3509 \\ 1311 & 14: 42: 37 & 1.3515 \\ 1312 & 14: 42: 38 & 1.3577 \\ 1313 & 14: 42: 39 & 1.3598 \\ 1314 & 14: 42: 40 & 1.3580 \\ 1315 & 14: 42: 41 & 1.3562 \\ 1316 & 14: 42: 42 & 1.3552 \\ 1317 & 14: 42: 43 & 1.3566 \\ 1318 & 14: 42: 44 & 1.3584 \\ 1319 & 14: 42: 45 & 1.3499 \\ 1320 & 14: 42: 46 & 1.3540 \\ 1321 & 14: 42: 47 & 1.3591 \\ 1322 & 14: 42: 48 & 1.3518 \\ 1323 & 14: 42: 49 & 1.3535 \\ 1324 & 14: 42: 50 & 1.3577\end{array}$


Appendix G - Fuel Data Test 4 NIST102705_1.xls

$\begin{array}{lll}1325 & 14: 42: 51 & 1.3561 \\ 1326 & 14: 42: 52 & 1.3501 \\ 1327 & 14: 42: 53 & 1.3539 \\ 1328 & 14: 42: 54 & 1.3570 \\ 1329 & 14: 42: 55 & 1.3623 \\ 1330 & 14: 42: 56 & 1.3536 \\ 1331 & 14: 42: 57 & 1.3482 \\ 1332 & 14: 42: 58 & 1.3521 \\ 1333 & 14: 42: 59 & 1.3527 \\ 1334 & 14: 43: 00 & 1.3554 \\ 1335 & 14: 43: 01 & 1.3547 \\ 1336 & 14: 43: 02 & 1.3472 \\ 1337 & 14: 43: 03 & 1.3520 \\ 1338 & 14: 43: 04 & 1.3564 \\ 1339 & 14: 43: 05 & 1.3519 \\ 1340 & 14: 43: 06 & 1.3507 \\ 1341 & 14: 43: 07 & 1.3554 \\ 1342 & 14: 43: 08 & 1.3518 \\ 1343 & 14: 43: 09 & 1.3520 \\ 1344 & 14: 43: 10 & 1.3532 \\ 1345 & 14: 43: 11 & 1.3508 \\ 1346 & 14: 43: 12 & 1.3545 \\ 1347 & 14: 43: 13 & 1.3515 \\ 1348 & 14: 43: 14 & 1.3509 \\ 1349 & 14: 43: 15 & 1.3574 \\ 1350 & 14: 43: 16 & 1.3560 \\ 1351 & 14: 43: 17 & 1.3537 \\ 1352 & 14: 43: 18 & 1.3544 \\ 1353 & 14: 43: 19 & 1.3553 \\ 1354 & 14: 43: 20 & 1.3557 \\ 1355 & 14: 43: 21 & 1.3558 \\ 1356 & 14: 43: 22 & 1.3552 \\ 1357 & 14: 43: 23 & 1.3521 \\ 1358 & 14: 43: 24 & 1.3567 \\ 1359 & 14: 43: 25 & 1.3564 \\ 1360 & 14: 43: 26 & 1.3521 \\ 1361 & 14: 43: 27 & 1.3523 \\ 1362 & 14: 43: 28 & 1.3555 \\ 1363 & 14: 43: 29 & 1.3536 \\ 1364 & 14: 43: 30 & 1.3573 \\ 1365 & 14: 43: 31 & 1.3590 \\ 1366 & 14: 43: 32 & 1.3527 \\ 1367 & 14: 43: 33 & 1.3558 \\ 1368 & 14: 43: 34 & 1.3585 \\ 1369 & 14: 43: 35 & 1.3564 \\ 1370 & 14: 43: 36 & 1.3554 \\ 1371 & 14: 43: 37 & 1.3545 \\ 1372 & 14: 43: 38 & 1.3550 \\ 1373 & 14: 43: 39 & 1.3546 \\ 1374 & 14: 43: 40 & 1.3555 \\ 1375 & 14: 43: 41 & 1.3535\end{array}$


Appendix G - Fuel Data Test 4 NIST102705_1.xls

$\begin{array}{lll}1376 & 14: 43: 42 & 1.3519 \\ 1377 & 14: 43: 43 & 1.3523 \\ 1378 & 14: 43: 44 & 1.3511 \\ 1379 & 14: 43: 45 & 1.3556 \\ 1380 & 14: 43: 46 & 1.3539 \\ 1381 & 14: 43: 47 & 1.3517 \\ 1382 & 14: 43: 48 & 1.3581 \\ 1383 & 14: 43: 49 & 1.3545 \\ 1384 & 14: 43: 50 & 1.3522 \\ 1385 & 14: 43: 51 & 1.3526 \\ 1386 & 14: 43: 52 & 1.3527 \\ 1387 & 14: 43: 53 & 1.3539 \\ 1388 & 14: 43: 54 & 1.3545 \\ 1389 & 14: 43: 55 & 1.3569 \\ 1390 & 14: 43: 56 & 1.3588 \\ 1391 & 14: 43: 57 & 1.3530 \\ 1392 & 14: 43: 58 & 1.3484 \\ 1393 & 14: 43: 59 & 1.3536 \\ 1394 & 14: 44: 00 & 1.3553 \\ 1395 & 14: 44: 01 & 1.3548 \\ 1396 & 14: 44: 02 & 1.3565 \\ 1397 & 14: 44: 03 & 1.3560 \\ 1398 & 14: 44: 04 & 1.3554 \\ 1399 & 14: 44: 05 & 1.3504 \\ 1400 & 14: 44: 06 & 1.3527 \\ 1401 & 14: 44: 07 & 1.3528 \\ 1402 & 14: 44: 08 & 1.3507 \\ 1403 & 14: 44: 09 & 1.3548 \\ 1404 & 14: 44: 10 & 1.3560 \\ 1405 & 14: 44: 11 & 1.3541 \\ 1406 & 14: 44: 12 & 1.3538 \\ 1407 & 14: 44: 13 & 1.3538 \\ 1408 & 14: 44: 14 & 1.3520 \\ 1409 & 14: 44: 15 & 1.3556 \\ 1410 & 14: 44: 16 & 1.3565 \\ 1411 & 14: 44: 17 & 1.3526 \\ 1412 & 14: 44: 18 & 1.3539 \\ 1413 & 14: 44: 19 & 1.3558 \\ 1414 & 14: 44: 20 & 1.3517 \\ 1415 & 14: 44: 21 & 1.3531 \\ 1416 & 14: 44: 22 & 1.3509 \\ 1417 & 14: 44: 23 & 1.3523 \\ 1418 & 14: 44: 24 & 1.3540 \\ 1419 & 14: 44: 25 & 1.3509 \\ 1420 & 14: 44: 26 & 1.3535 \\ 1421 & 14: 44: 27 & 1.3583 \\ 1422 & 14: 44: 28 & 1.3548 \\ 1423 & 14: 44: 29 & 1.3521 \\ 1424 & 14: 44: 30 & 1.3536 \\ 1425 & 14: 44: 31 & 1.3536 \\ 1426 & 14: 44: 32 & 1.3538\end{array}$


Appendix G - Fuel Data Test 4 NIST102705_1.xls

$\begin{array}{lll}1427 & 14: 44: 33 & 1.3539 \\ 1428 & 14: 44: 34 & 1.3556 \\ 1429 & 14: 44: 35 & 1.3573 \\ 1430 & 14: 44: 36 & 1.3524 \\ 1431 & 14: 44: 37 & 1.3554 \\ 1432 & 14: 44: 38 & 1.3526 \\ 1433 & 14: 44: 39 & 1.3501 \\ 1434 & 14: 44: 40 & 1.3540 \\ 1435 & 14: 44: 41 & 1.3537 \\ 1436 & 14: 44: 42 & 1.3521 \\ 1437 & 14: 44: 43 & 1.3548 \\ 1438 & 14: 44: 44 & 1.3542 \\ 1439 & 14: 44: 45 & 1.3549 \\ 1440 & 14: 44: 46 & 1.3532 \\ 1441 & 14: 44: 47 & 1.3545 \\ 1442 & 14: 44: 48 & 1.3532 \\ 1443 & 14: 44: 49 & 1.3513 \\ 1444 & 14: 44: 50 & 1.3589 \\ 1445 & 14: 44: 51 & 1.3551 \\ 1446 & 14: 44: 52 & 1.3544 \\ 1447 & 14: 44: 53 & 1.3592 \\ 1448 & 14: 44: 54 & 1.3587 \\ 1449 & 14: 44: 55 & 1.3573 \\ 1450 & 14: 44: 56 & 1.3504 \\ 1451 & 14: 44: 57 & 1.3500 \\ 1452 & 14: 44: 58 & 1.3550 \\ 1453 & 14: 44: 59 & 1.3544 \\ 1454 & 14: 45: 00 & 1.3522 \\ 1455 & 14: 45: 01 & 1.3552 \\ 1456 & 14: 45: 02 & 1.3523 \\ 1457 & 14: 45: 03 & 1.3556 \\ 1458 & 14: 45: 04 & 1.3552 \\ 1459 & 14: 45: 05 & 1.3524 \\ 1460 & 14: 45: 06 & 1.3536 \\ 1461 & 14: 45: 07 & 1.3547 \\ 1462 & 14: 45: 08 & 1.3568 \\ 1463 & 14: 45: 09 & 1.3554 \\ 1464 & 14: 45: 10 & 1.3530 \\ 1465 & 14: 45: 11 & 1.3552 \\ 1466 & 14: 45: 12 & 1.3593 \\ 1467 & 14: 45: 13 & 1.3524 \\ 1468 & 14: 45: 14 & 1.3553 \\ 1469 & 14: 45: 15 & 1.3562 \\ 1470 & 14: 45: 16 & 1.3519 \\ 1471 & 14: 45: 17 & 1.3545 \\ 1472 & 14: 45: 18 & 1.3547 \\ 1473 & 14: 45: 19 & 1.3541 \\ 1474 & 14: 45: 20 & 1.3511 \\ 1475 & 14: 45: 21 & 1.3493 \\ 1476 & 14: 45: 22 & 1.3516 \\ 1477 & 14: 45: 23 & 1.3536\end{array}$


Appendix G - Fuel Data Test 4 NIST102705_1.xls

$\begin{array}{lll}1478 & 14: 45: 24 & 1.3540 \\ 1479 & 14: 45: 25 & 1.3519 \\ 1480 & 14: 45: 26 & 1.3518 \\ 1481 & 14: 45: 27 & 1.3546 \\ 1482 & 14: 45: 28 & 1.3543 \\ 1483 & 14: 45: 29 & 1.3546 \\ 1484 & 14: 45: 30 & 1.3513 \\ 1485 & 14: 45: 31 & 1.3553 \\ 1486 & 14: 45: 32 & 1.3561 \\ 1487 & 14: 45: 33 & 1.3547 \\ 1488 & 14: 45: 34 & 1.3581 \\ 1489 & 14: 45: 35 & 1.3585 \\ 1490 & 14: 45: 36 & 1.3554 \\ 1491 & 14: 45: 37 & 1.3575 \\ 1492 & 14: 45: 38 & 1.3553 \\ 1493 & 14: 45: 39 & 1.3551 \\ 1494 & 14: 45: 40 & 1.3517 \\ 1495 & 14: 45: 41 & 1.3372 \\ 1496 & 14: 45: 42 & 1.2787 \\ 1497 & 14: 45: 43 & 1.1770 \\ 1498 & 14: 45: 44 & 1.0262 \\ 1499 & 14: 45: 45 & 0.7330 \\ 1500 & 14: 45: 46 & 0.4859 \\ 1501 & 14: 45: 47 & 0.4368 \\ 1502 & 14: 45: 48 & 0.4195 \\ 1503 & 14: 45: 49 & 0.4244 \\ 1504 & 14: 45: 50 & 0.4487 \\ 1505 & 14: 45: 51 & 0.4586 \\ 1506 & 14: 45: 52 & 0.4608 \\ 1507 & 14: 45: 53 & 0.4589 \\ 1508 & 14: 45: 54 & 0.4545 \\ 1509 & 14: 45: 55 & 0.4531 \\ 1510 & 14: 45: 56 & 0.4460 \\ 1511 & 14: 45: 57 & 0.4431 \\ 1512 & 14: 45: 58 & 0.4305 \\ 1513 & 14: 45: 59 & 0.4237 \\ 1514 & 14: 46: 00 & 0.4201 \\ 1515 & 14: 46: 01 & 0.4326 \\ 1516 & 14: 46: 02 & 0.4369 \\ 1517 & 14: 46: 03 & 0.4382 \\ 1518 & 14: 46: 04 & 0.4428 \\ 1519 & 14: 46: 05 & 0.4458 \\ 1520 & 14: 46: 06 & 0.4636 \\ 1521 & 14: 46: 07 & 0.4702 \\ 1522 & 14: 46: 08 & 0.4633 \\ 1523 & 14: 46: 09 & 0.4502 \\ 1524 & 14: 46: 10 & 0.4422 \\ 1525 & 14: 46: 11 & 0.4401 \\ 1526 & 14: 46: 12 & 0.4348 \\ 1527 & 14: 46: 13 & 0.4193 \\ 1528 & 14: 46: 14 & 0.4268\end{array}$


Appendix G - Fuel Data Test 4 NIST102705_1.xls

$\begin{array}{lll}1529 & 14: 46: 15 & 0.4383 \\ 1530 & 14: 46: 16 & 0.4571 \\ 1531 & 14: 46: 17 & 0.4654 \\ 1532 & 14: 46: 18 & 0.4619 \\ 1533 & 14: 46: 19 & 0.4519 \\ 1534 & 14: 46: 20 & 0.4344 \\ 1535 & 14: 46: 21 & 0.4137 \\ 1536 & 14: 46: 22 & 0.4186 \\ 1537 & 14: 46: 23 & 0.4418 \\ 1538 & 14: 46: 24 & 0.4627 \\ 1539 & 14: 46: 25 & 0.4633 \\ 1540 & 14: 46: 26 & 0.4592 \\ 1541 & 14: 46: 27 & 0.4261 \\ 1542 & 14: 46: 28 & 0.3488 \\ 1543 & 14: 46: 29 & 0.3434 \\ 1544 & 14: 46: 30 & 0.3446 \\ 1545 & 14: 46: 31 & 0.2847 \\ 1546 & 14: 46: 32 & 0.3006 \\ 1547 & 14: 46: 33 & 0.2657 \\ 1548 & 14: 46: 34 & 0.2811 \\ 1549 & 14: 46: 35 & 0.2520 \\ 1550 & 14: 46: 36 & 0.2488 \\ 1551 & 14: 46: 37 & 0.2452 \\ 1552 & 14: 46: 38 & 0.2607 \\ 1553 & 14: 46: 39 & 0.2604 \\ 1554 & 14: 46: 40 & 0.2583 \\ 1555 & 14: 46: 41 & 0.2519 \\ 1556 & 14: 46: 42 & 0.2433 \\ 1557 & 14: 46: 43 & 0.2569 \\ 1558 & 14: 46: 44 & 0.2534 \\ 1559 & 14: 46: 45 & 0.2727 \\ 1560 & 14: 46: 46 & 0.2549 \\ 1561 & 14: 46: 47 & 0.2647 \\ 1562 & 14: 46: 48 & 0.2490 \\ 1563 & 14: 46: 49 & 0.2687 \\ 1564 & 14: 46: 50 & 0.2663 \\ 1565 & 14: 46: 51 & 0.2624 \\ 1566 & 14: 46: 52 & 0.2680 \\ 1567 & 14: 46: 53 & 0.2567 \\ 1568 & 14: 46: 54 & 0.2820 \\ 1569 & 14: 46: 55 & 0.2656 \\ 1570 & 14: 46: 56 & 0.2619 \\ 1571 & 14: 46: 57 & 0.2434 \\ 1572 & 14: 46: 58 & 0.2645 \\ 1573 & 14: 46: 59 & 0.2569 \\ 1574 & 14: 47: 00 & 0.2574 \\ 1575 & 14: 47: 01 & 0.2573 \\ 1576 & 14: 47: 02 & 0.2525 \\ 1577 & 14: 47: 03 & 0.2725 \\ 1578 & 14: 47: 04 & 0.2475 \\ 1579 & 14: 47: 05 & 0.2589\end{array}$


Appendix G - Fuel Data Test 4 NIST102705_1.xls

$\begin{array}{lll}1580 & 14: 47: 06 & 0.2417 \\ 1581 & 14: 47: 07 & 0.2658 \\ 1582 & 14: 47: 08 & 0.2526 \\ 1583 & 14: 47: 09 & 0.2637 \\ 1584 & 14: 47: 10 & 0.2498 \\ 1585 & 14: 47: 11 & 0.2533 \\ 1586 & 14: 47: 12 & 0.2673 \\ 1587 & 14: 47: 13 & 0.2696 \\ 1588 & 14: 47: 14 & 0.2614 \\ 1589 & 14: 47: 15 & 0.2452 \\ 1590 & 14: 47: 16 & 0.2551 \\ 1591 & 14: 47: 17 & 0.2510 \\ 1592 & 14: 47: 18 & 0.2685 \\ 1593 & 14: 47: 19 & 0.2673 \\ 1594 & 14: 47: 20 & 0.2713 \\ 1595 & 14: 47: 21 & 0.2599 \\ 1596 & 14: 47: 22 & 0.2460 \\ 1597 & 14: 47: 23 & 0.2580 \\ 1598 & 14: 47: 24 & 0.2522 \\ 1599 & 14: 47: 25 & 0.2597 \\ 1600 & 14: 47: 26 & 0.2488 \\ 1601 & 14: 47: 27 & 0.2583 \\ 1602 & 14: 47: 28 & 0.2654 \\ 1603 & 14: 47: 29 & 0.2597 \\ 1604 & 14: 47: 30 & 0.2739 \\ 1605 & 14: 47: 31 & 0.2697 \\ 1606 & 14: 47: 32 & 0.2796 \\ 1607 & 14: 47: 33 & 0.2480 \\ 1608 & 14: 47: 34 & 0.2540 \\ 1609 & 14: 47: 35 & 0.2513 \\ 1610 & 14: 47: 36 & 0.2695 \\ 1611 & 14: 47: 37 & 0.2720 \\ 1612 & 14: 47: 38 & 0.2789 \\ 1613 & 14: 47: 39 & 0.2597 \\ 1614 & 14: 47: 40 & 0.2402 \\ 1615 & 14: 47: 41 & 0.2693 \\ 1616 & 14: 47: 42 & 0.2623 \\ 1617 & 14: 47: 43 & 0.2733 \\ 1618 & 14: 47: 44 & 0.2496 \\ 1619 & 14: 47: 45 & 0.2510 \\ 1620 & 14: 47: 46 & 0.2498 \\ 1621 & 14: 47: 47 & 0.2655 \\ 1622 & 14: 47: 48 & 0.2619 \\ 1623 & 14: 47: 49 & 0.2705 \\ 1624 & 14: 47: 50 & 0.2514 \\ 1625 & 14: 47: 51 & 0.2446 \\ 1626 & 14: 47: 52 & 0.2488 \\ 1627 & 14: 47: 53 & 0.2392 \\ 1628 & 14: 47: 54 & 0.1830 \\ 1629 & 14: 47: 55 & 0.0780 \\ 1630 & 14: 47: 56 & 0.0569\end{array}$


Appendix G - Fuel Data Test 4 NIST102705_1.xls

$\begin{array}{lll}1631 & 14: 47: 57 & 0.0533 \\ 1632 & 14: 47: 58 & 0.0644 \\ 1633 & 14: 47: 59 & 0.1109 \\ 1634 & 14: 48: 00 & 0.1809 \\ 1635 & 14: 48: 01 & 0.1907 \\ 1636 & 14: 48: 02 & 0.2144 \\ 1637 & 14: 48: 03 & 0.2186 \\ 1638 & 14: 48: 04 & 0.2253 \\ 1639 & 14: 48: 05 & 0.2474 \\ 1640 & 14: 48: 06 & 0.2582 \\ 1641 & 14: 48: 07 & 0.2645 \\ 1642 & 14: 48: 08 & 0.2537 \\ 1643 & 14: 48: 09 & 0.2663 \\ 1644 & 14: 48: 10 & 0.2524 \\ 1645 & 14: 48: 11 & 0.2631 \\ 1646 & 14: 48: 12 & 0.2606 \\ 1647 & 14: 48: 13 & 0.2501 \\ 1648 & 14: 48: 14 & 0.2508 \\ 1649 & 14: 48: 15 & 0.2364 \\ 1650 & 14: 48: 16 & 0.2588 \\ 1651 & 14: 48: 17 & 0.2556 \\ 1652 & 14: 48: 18 & 0.2705 \\ 1653 & 14: 48: 19 & 0.2572 \\ 1654 & 14: 48: 20 & 0.2663 \\ 1655 & 14: 48: 21 & 0.2585 \\ 1656 & 14: 48: 22 & 0.2713 \\ 1657 & 14: 48: 23 & 0.2719 \\ 1658 & 14: 48: 24 & 0.2528 \\ 1659 & 14: 48: 25 & 0.2598 \\ 1660 & 14: 48: 26 & 0.2430 \\ 1661 & 14: 48: 27 & 0.2718 \\ 1662 & 14: 48: 28 & 0.2671 \\ 1663 & 14: 48: 29 & 0.2808 \\ 1664 & 14: 48: 30 & 0.2581 \\ 1665 & 14: 48: 31 & 0.2698 \\ 1666 & 14: 48: 32 & 0.2527 \\ 1667 & 14: 48: 33 & 0.2703 \\ 1668 & 14: 48: 34 & 0.2629 \\ 1669 & 14: 48: 35 & 0.2644 \\ 1670 & 14: 48: 36 & 0.2592 \\ 1671 & 14: 48: 37 & 0.2466 \\ 1672 & 14: 48: 38 & 0.2647 \\ 1673 & 14: 48: 39 & 0.2690 \\ 1674 & 14: 48: 40 & 0.2731 \\ 1675 & 14: 48: 41 & 0.2493 \\ 1676 & 14: 48: 42 & 0.2502 \\ 1677 & 14: 48: 43 & 0.2504 \\ 1678 & 14: 48: 44 & 0.2481 \\ 1679 & 14: 48: 45 & 0.2581 \\ 1680 & 14: 48: 46 & 0.2531 \\ 1681 & 14: 48: 47 & 0.2706\end{array}$


Appendix G - Fuel Data Test 4 NIST102705_1.xls

$\begin{array}{lll}1682 & 14: 48: 48 & 0.2528 \\ 1683 & 14: 48: 49 & 0.2740 \\ 1684 & 14: 48: 50 & 0.2558 \\ 1685 & 14: 48: 51 & 0.2666 \\ 1686 & 14: 48: 52 & 0.2497 \\ 1687 & 14: 48: 53 & 0.2686 \\ 1688 & 14: 48: 54 & 0.2523 \\ 1689 & 14: 48: 55 & 0.2635 \\ 1690 & 14: 48: 56 & 0.2630 \\ 1691 & 14: 48: 57 & 0.2682 \\ 1692 & 14: 48: 58 & 0.2673 \\ 1693 & 14: 48: 59 & 0.2509 \\ 1694 & 14: 49: 00 & 0.2578 \\ 1695 & 14: 49: 01 & 0.2401 \\ 1696 & 14: 49: 02 & 0.2598 \\ 1697 & 14: 49: 03 & 0.2610 \\ 1698 & 14: 49: 04 & 0.2558 \\ 1699 & 14: 49: 05 & 0.2707 \\ 1700 & 14: 49: 06 & 0.2573 \\ 1701 & 14: 49: 07 & 0.2689 \\ 1702 & 14: 49: 08 & 0.2608 \\ 1703 & 14: 49: 09 & 0.2667 \\ 1704 & 14: 49: 10 & 0.2541 \\ 1705 & 14: 49: 11 & 0.2609 \\ 1706 & 14: 49: 12 & 0.2527 \\ 1707 & 14: 49: 13 & 0.2700 \\ 1708 & 14: 49: 14 & 0.2674 \\ 1709 & 14: 49: 15 & 0.2656 \\ 1710 & 14: 49: 16 & 0.2683 \\ 1711 & 14: 49: 17 & 0.2570 \\ 1712 & 14: 49: 18 & 0.2573 \\ 1713 & 14: 49: 19 & 0.2474 \\ 1714 & 14: 49: 20 & 0.2523 \\ 1715 & 14: 49: 21 & 0.2622 \\ 1716 & 14: 49: 22 & 0.2481 \\ 1717 & 14: 49: 23 & 0.2558 \\ 1718 & 14: 49: 24 & 0.2485 \\ 1719 & 14: 49: 25 & 0.2644 \\ 1720 & 14: 49: 26 & 0.2620 \\ 1721 & 14: 49: 27 & 0.2751 \\ 1722 & 14: 49: 28 & 0.2556 \\ 1723 & 14: 49: 29 & 0.2591 \\ 1724 & 14: 49: 30 & 0.2483 \\ 1725 & 14: 49: 31 & 0.2678 \\ 1726 & 14: 49: 32 & 0.2734 \\ 1727 & 14: 49: 33 & 0.2670 \\ 1728 & 14: 49: 34 & 0.2655 \\ 1729 & 14: 49: 35 & 0.2557 \\ 1730 & 14: 49: 36 & 0.2762 \\ 1731 & 14: 49: 37 & 0.2602 \\ 1732 & 14: 49: 38 & 0.2745\end{array}$


Appendix G - Fuel Data Test 4 NIST102705_1.xls

$\begin{array}{lll}1733 & 14: 49: 39 & 0.2451 \\ 1734 & 14: 49: 40 & 0.2547 \\ 1735 & 14: 49: 41 & 0.2478 \\ 1736 & 14: 49: 42 & 0.2672 \\ 1737 & 14: 49: 43 & 0.2582 \\ 1738 & 14: 49: 44 & 0.2580 \\ 1739 & 14: 49: 45 & 0.2558 \\ 1740 & 14: 49: 46 & 0.2672 \\ 1741 & 14: 49: 47 & 0.2559 \\ 1742 & 14: 49: 48 & 0.2716 \\ 1743 & 14: 49: 49 & 0.2443 \\ 1744 & 14: 49: 50 & 0.2546 \\ 1745 & 14: 49: 51 & 0.2468 \\ 1746 & 14: 49: 52 & 0.2727 \\ 1747 & 14: 49: 53 & 0.2585 \\ 1748 & 14: 49: 54 & 0.2836 \\ 1749 & 14: 49: 55 & 0.2520 \\ 1750 & 14: 49: 56 & 0.2761 \\ 1751 & 14: 49: 57 & 0.2562 \\ 1752 & 14: 49: 58 & 0.2729 \\ 1753 & 14: 49: 59 & 0.2541 \\ 1754 & 14: 50: 00 & 0.2626 \\ 1755 & 14: 50: 01 & 0.2508 \\ 1756 & 14: 50: 02 & 0.2699 \\ 1757 & 14: 50: 03 & 0.2604 \\ 1758 & 14: 50: 04 & 0.2754 \\ 1759 & 14: 50: 05 & 0.2460 \\ 1760 & 14: 50: 06 & 0.2672 \\ 1761 & 14: 50: 07 & 0.2481 \\ 1762 & 14: 50: 08 & 0.2654 \\ 1763 & 14: 50: 09 & 0.2556 \\ 1764 & 14: 50: 10 & 0.2602 \\ 1765 & 14: 50: 11 & 0.2501 \\ 1766 & 14: 50: 12 & 0.2657 \\ 1767 & 14: 50: 13 & 0.2573 \\ 1768 & 14: 50: 14 & 0.2610 \\ 1769 & 14: 50: 15 & 0.2684 \\ 1770 & 14: 50: 16 & 0.2521 \\ 1771 & 14: 50: 17 & 0.2575 \\ 1772 & 14: 50: 18 & 0.2470 \\ 1773 & 14: 50: 19 & 0.2685 \\ 1774 & 14: 50: 20 & 0.2665 \\ 1775 & 14: 50: 21 & 0.2719 \\ 1776 & 14: 50: 22 & 0.2608 \\ 1777 & 14: 50: 23 & 0.2656 \\ 1778 & 14: 50: 24 & 0.2526 \\ 1779 & 14: 50: 25 & 0.2598 \\ 1780 & 14: 50: 26 & 0.2675 \\ 1781 & 14: 50: 27 & 0.2507 \\ 1782 & 14: 50: 28 & 0.2601 \\ 1783 & 14: 50: 29 & 0.2420\end{array}$


Appendix G - Fuel Data Test 4 NIST102705_1.xls

$\begin{array}{lll}1784 & 14: 50: 30 & 0.2697 \\ 1785 & 14: 50: 31 & 0.2636 \\ 1786 & 14: 50: 32 & 0.2716 \\ 1787 & 14: 50: 33 & 0.2529 \\ 1788 & 14: 50: 34 & 0.2583 \\ 1789 & 14: 50: 35 & 0.2618 \\ 1790 & 14: 50: 36 & 0.2676 \\ 1791 & 14: 50: 37 & 0.2705 \\ 1792 & 14: 50: 38 & 0.2529 \\ 1793 & 14: 50: 39 & 0.2814 \\ 1794 & 14: 50: 40 & 0.3051 \\ 1795 & 14: 50: 41 & 0.4163 \\ 1796 & 14: 50: 42 & 0.5017 \\ 1797 & 14: 50: 43 & 0.5354 \\ 1798 & 14: 50: 44 & 0.5873 \\ 1799 & 14: 50: 45 & 0.6699 \\ 1800 & 14: 50: 46 & 0.8077 \\ 1801 & 14: 50: 47 & 0.9278 \\ 1802 & 14: 50: 48 & 0.9382 \\ 1803 & 14: 50: 49 & 0.9414 \\ 1804 & 14: 50: 50 & 0.9803 \\ 1805 & 14: 50: 51 & 1.0398 \\ 1806 & 14: 50: 52 & 1.0722 \\ 1807 & 14: 50: 53 & 1.0979 \\ 1808 & 14: 50: 54 & 1.1283 \\ 1809 & 14: 50: 55 & 1.1424 \\ 1810 & 14: 50: 56 & 1.1504 \\ 1811 & 14: 50: 57 & 1.1421 \\ 1812 & 14: 50: 58 & 1.1434 \\ 1813 & 14: 50: 59 & 1.1486 \\ 1814 & 14: 51: 00 & 1.1513 \\ 1815 & 14: 51: 01 & 1.1497 \\ 1816 & 14: 51: 02 & 1.1575 \\ 1817 & 14: 51: 03 & 1.1611 \\ 1818 & 14: 51: 04 & 1.1575 \\ 1819 & 14: 51: 05 & 1.1594 \\ 1820 & 14: 51: 06 & 1.1581 \\ 1821 & 14: 51: 07 & 1.1542 \\ 1822 & 14: 51: 08 & 1.1609 \\ 1823 & 14: 51: 09 & 1.1683 \\ 1824 & 14: 51: 10 & 1.1670 \\ 1825 & 14: 51: 11 & 1.1647 \\ 1826 & 14: 51: 12 & 1.1633 \\ 1827 & 14: 51: 13 & 1.1610 \\ 1828 & 14: 51: 14 & 1.1571 \\ 1829 & 14: 51: 15 & 1.1501 \\ 1830 & 14: 51: 16 & 1.1504 \\ 1831 & 14: 51: 17 & 1.1625 \\ 1832 & 14: 51: 18 & 1.1615 \\ 1833 & 14: 51: 19 & 1.1582 \\ 1834 & 14: 51: 20 & 1.1571\end{array}$


Appendix G - Fuel Data Test 4 NIST102705_1.xls

$\begin{array}{lll}1835 & 14: 51: 21 & 1.1621 \\ 1836 & 14: 51: 22 & 1.1591 \\ 1837 & 14: 51: 23 & 1.1489 \\ 1838 & 14: 51: 24 & 1.1539 \\ 1839 & 14: 51: 25 & 1.1581 \\ 1840 & 14: 51: 26 & 1.1588 \\ 1841 & 14: 51: 27 & 1.1501 \\ 1842 & 14: 51: 28 & 1.1528 \\ 1843 & 14: 51: 29 & 1.1603 \\ 1844 & 14: 51: 30 & 1.1626 \\ 1845 & 14: 51: 31 & 1.1665 \\ 1846 & 14: 51: 32 & 1.1624 \\ 1847 & 14: 51: 33 & 1.1486 \\ 1848 & 14: 51: 34 & 1.1528 \\ 1849 & 14: 51: 35 & 1.1523 \\ 1850 & 14: 51: 36 & 1.1503 \\ 1851 & 14: 51: 37 & 1.1512 \\ 1852 & 14: 51: 38 & 1.1636 \\ 1853 & 14: 51: 39 & 1.1713 \\ 1854 & 14: 51: 40 & 1.1684 \\ 1855 & 14: 51: 41 & 1.1618 \\ 1856 & 14: 51: 42 & 1.1580 \\ 1857 & 14: 51: 43 & 1.1609 \\ 1858 & 14: 51: 44 & 1.1544 \\ 1859 & 14: 51: 45 & 1.1406 \\ 1860 & 14: 51: 46 & 1.1272 \\ 1861 & 14: 51: 47 & 1.1217 \\ 1862 & 14: 51: 48 & 1.1466 \\ 1863 & 14: 51: 49 & 1.1618 \\ 1864 & 14: 51: 50 & 1.1675 \\ 1865 & 14: 51: 51 & 1.1647 \\ 1866 & 14: 51: 52 & 1.1526 \\ 1867 & 14: 51: 53 & 1.1443 \\ 1868 & 14: 51: 54 & 1.1631 \\ 1869 & 14: 51: 55 & 1.1649 \\ 1870 & 14: 51: 56 & 1.1690 \\ 1871 & 14: 51: 57 & 1.1628 \\ 1872 & 14: 51: 58 & 1.1557 \\ 1873 & 14: 51: 59 & 1.1458 \\ 1874 & 14: 52: 00 & 1.1463 \\ 1875 & 14: 52: 01 & 1.1486 \\ 1876 & 14: 52: 02 & 1.1605 \\ 1877 & 14: 52: 03 & 1.1610 \\ 1878 & 14: 52: 04 & 1.1568 \\ 1879 & 14: 52: 05 & 1.1622 \\ 1880 & 14: 52: 06 & 1.1511 \\ 1881 & 14: 52: 07 & 1.1523 \\ 1882 & 14: 52: 08 & 1.1634 \\ 1883 & 14: 52: 09 & 1.1696 \\ 1884 & 14: 52: 10 & 1.1623 \\ 1885 & 14: 52: 11 & 1.1518\end{array}$


Appendix G - Fuel Data Test 4 NIST102705_1.xls

$\begin{array}{lll}1886 & 14: 52: 12 & 1.1539 \\ 1887 & 14: 52: 13 & 1.1578 \\ 1888 & 14: 52: 14 & 1.1600 \\ 1889 & 14: 52: 15 & 1.1577 \\ 1890 & 14: 52: 16 & 1.1494 \\ 1891 & 14: 52: 17 & 1.1514 \\ 1892 & 14: 52: 18 & 1.1465 \\ 1893 & 14: 52: 19 & 1.1541 \\ 1894 & 14: 52: 20 & 1.1608 \\ 1895 & 14: 52: 21 & 1.1660 \\ 1896 & 14: 52: 22 & 1.1550 \\ 1897 & 14: 52: 23 & 1.1467 \\ 1898 & 14: 52: 24 & 1.1457 \\ 1899 & 14: 52: 25 & 1.1506 \\ 1900 & 14: 52: 26 & 1.1590 \\ 1901 & 14: 52: 27 & 1.1662 \\ 1902 & 14: 52: 28 & 1.1681 \\ 1903 & 14: 52: 29 & 1.1637 \\ 1904 & 14: 52: 30 & 1.1560 \\ 1905 & 14: 52: 31 & 1.1455 \\ 1906 & 14: 52: 32 & 1.1451 \\ 1907 & 14: 52: 33 & 1.1479 \\ 1908 & 14: 52: 34 & 1.1541 \\ 1909 & 14: 52: 35 & 1.1624 \\ 1910 & 14: 52: 36 & 1.1601 \\ 1911 & 14: 52: 37 & 1.1579 \\ 1912 & 14: 52: 38 & 1.1592 \\ 1913 & 14: 52: 39 & 1.1563 \\ 1914 & 14: 52: 40 & 1.1538 \\ 1915 & 14: 52: 41 & 1.1586 \\ 1916 & 14: 52: 42 & 1.1608 \\ 1917 & 14: 52: 43 & 1.1533 \\ 1918 & 14: 52: 44 & 1.1444 \\ 1919 & 14: 52: 45 & 1.1547 \\ 1920 & 14: 52: 46 & 1.1628 \\ 1921 & 14: 52: 47 & 1.1634 \\ 1922 & 14: 52: 48 & 1.1618 \\ 1923 & 14: 52: 49 & 1.1527 \\ 1924 & 14: 52: 50 & 1.1514 \\ 1925 & 14: 52: 51 & 1.1551 \\ 1926 & 14: 52: 52 & 1.1554 \\ 1927 & 14: 52: 53 & 1.1527 \\ 1928 & 14: 52: 54 & 1.1558 \\ 1929 & 14: 52: 55 & 1.1524 \\ 1930 & 14: 52: 56 & 1.1746 \\ 1931 & 14: 52: 57 & 1.2452 \\ 1932 & 14: 52: 58 & 1.3213 \\ 1933 & 14: 52: 59 & 1.3441 \\ 1934 & 14: 53: 00 & 1.3523 \\ 1935 & 14: 53: 01 & 1.3549 \\ 1936 & 14: 53: 02 & 1.3536\end{array}$


Appendix G - Fuel Data Test 4 NIST102705_1.xls

$\begin{array}{lll}1937 & 14: 53: 03 & 1.3565 \\ 1938 & 14: 53: 04 & 1.3535 \\ 1939 & 14: 53: 05 & 1.3567 \\ 1940 & 14: 53: 06 & 1.3576 \\ 1941 & 14: 53: 07 & 1.3538 \\ 1942 & 14: 53: 08 & 1.3551 \\ 1943 & 14: 53: 09 & 1.3549 \\ 1944 & 14: 53: 10 & 1.3513 \\ 1945 & 14: 53: 11 & 1.3530 \\ 1946 & 14: 53: 12 & 1.3525 \\ 1947 & 14: 53: 13 & 1.3536 \\ 1948 & 14: 53: 14 & 1.3541 \\ 1949 & 14: 53: 15 & 1.3524 \\ 1950 & 14: 53: 16 & 1.3532 \\ 1951 & 14: 53: 17 & 1.3534 \\ 1952 & 14: 53: 18 & 1.3528 \\ 1953 & 14: 53: 19 & 1.3557 \\ 1954 & 14: 53: 20 & 1.3548 \\ 1955 & 14: 53: 21 & 1.3528 \\ 1956 & 14: 53: 22 & 1.3492 \\ 1957 & 14: 53: 23 & 1.3496 \\ 1958 & 14: 53: 24 & 1.3550 \\ 1959 & 14: 53: 25 & 1.3531 \\ 1960 & 14: 53: 26 & 1.3530 \\ 1961 & 14: 53: 27 & 1.3536 \\ 1962 & 14: 53: 28 & 1.3510 \\ 1963 & 14: 53: 29 & 1.3567 \\ 1964 & 14: 53: 30 & 1.3544 \\ 1965 & 14: 53: 31 & 1.3546 \\ 1966 & 14: 53: 32 & 1.3557 \\ 1967 & 14: 53: 33 & 1.3563 \\ 1968 & 14: 53: 34 & 1.3524 \\ 1969 & 14: 53: 35 & 1.3529 \\ 1970 & 14: 53: 36 & 1.3533 \\ 1971 & 14: 53: 37 & 1.3500 \\ 1972 & 14: 53: 38 & 1.3510 \\ 1973 & 14: 53: 39 & 1.3567 \\ 1974 & 14: 53: 40 & 1.3562 \\ 1975 & 14: 53: 41 & 1.3554 \\ 1976 & 14: 53: 42 & 1.3535 \\ 1977 & 14: 53: 43 & 1.3512 \\ 1978 & 14: 53: 44 & 1.3548 \\ 1979 & 14: 53: 45 & 1.3517 \\ 1980 & 14: 53: 46 & 1.3503 \\ 1981 & 14: 53: 47 & 1.3510 \\ 1982 & 14: 53: 48 & 1.3555 \\ 1983 & 14: 53: 49 & 1.3541 \\ 1984 & 14: 53: 50 & 1.3522 \\ 1985 & 14: 53: 51 & 1.3543 \\ 1986 & 14: 53: 52 & 1.3519 \\ 1987 & 14: 53: 53 & 1.3548\end{array}$


Appendix G - Fuel Data Test 4 NIST102705_1.xls

$\begin{array}{lll}1988 & 14: 53: 54 & 1.3558 \\ 1989 & 14: 53: 55 & 1.3520 \\ 1990 & 14: 53: 56 & 1.3531 \\ 1991 & 14: 53: 57 & 1.3538 \\ 1992 & 14: 53: 58 & 1.3515 \\ 1993 & 14: 53: 59 & 1.3516 \\ 1994 & 14: 54: 00 & 1.3563 \\ 1995 & 14: 54: 01 & 1.3572 \\ 1996 & 14: 54: 02 & 1.3481 \\ 1997 & 14: 54: 03 & 1.3495 \\ 1998 & 14: 54: 04 & 1.3544 \\ 1999 & 14: 54: 05 & 1.3503 \\ 2000 & 14: 54: 06 & 1.3544 \\ 2001 & 14: 54: 07 & 1.3565 \\ 2002 & 14: 54: 08 & 1.3520 \\ 2003 & 14: 54: 09 & 1.3534 \\ 2004 & 14: 54: 10 & 1.3564 \\ 2005 & 14: 54: 11 & 1.3552 \\ 2006 & 14: 54: 12 & 1.3498 \\ 2007 & 14: 54: 13 & 1.3480 \\ 2008 & 14: 54: 14 & 1.3494 \\ 2009 & 14: 54: 15 & 1.3556 \\ 2010 & 14: 54: 16 & 1.3547 \\ 2011 & 14: 54: 17 & 1.3532 \\ 2012 & 14: 54: 18 & 1.3543 \\ 2013 & 14: 54: 19 & 1.3542 \\ 2014 & 14: 54: 20 & 1.3540 \\ 2015 & 14: 54: 21 & 1.3527 \\ 2016 & 14: 54: 22 & 1.3536 \\ 2017 & 14: 54: 23 & 1.3514 \\ 2018 & 14: 54: 24 & 1.3527 \\ 2019 & 14: 54: 25 & 1.3549 \\ 2020 & 14: 54: 26 & 1.3568 \\ 2021 & 14: 54: 27 & 1.3541 \\ 2022 & 14: 54: 28 & 1.3519 \\ 2023 & 14: 54: 29 & 1.3531 \\ 2024 & 14: 54: 30 & 1.3499 \\ 2025 & 14: 54: 31 & 1.3525 \\ 2026 & 14: 54: 32 & 1.3526 \\ 2027 & 14: 54: 33 & 1.3525 \\ 2028 & 14: 54: 34 & 1.3520 \\ 2029 & 14: 54: 35 & 1.3581 \\ 2030 & 14: 54: 36 & 1.3612 \\ 2031 & 14: 54: 37 & 1.3547 \\ 2032 & 14: 54: 38 & 1.3531 \\ 2033 & 14: 54: 39 & 1.3504 \\ 2034 & 14: 54: 40 & 1.3466 \\ 2035 & 14: 54: 41 & 1.3492 \\ 2036 & 14: 54: 42 & 1.3548 \\ 2037 & 14: 54: 43 & 1.3535 \\ 2038 & 14: 54: 44 & 1.3511\end{array}$


Appendix G - Fuel Data Test 4 NIST102705_1.xls

$\begin{array}{lll}2039 & 14: 54: 45 & 1.3529 \\ 2040 & 14: 54: 46 & 1.3553 \\ 2041 & 14: 54: 47 & 1.3548 \\ 2042 & 14: 54: 48 & 1.3553 \\ 2043 & 14: 54: 49 & 1.3548 \\ 2044 & 14: 54: 50 & 1.3509 \\ 2045 & 14: 54: 51 & 1.3524 \\ 2046 & 14: 54: 52 & 1.3518 \\ 2047 & 14: 54: 53 & 1.3516 \\ 2048 & 14: 54: 54 & 1.3548 \\ 2049 & 14: 54: 55 & 1.3543 \\ 2050 & 14: 54: 56 & 1.3520 \\ 2051 & 14: 54: 57 & 1.3520 \\ 2052 & 14: 54: 58 & 1.3555 \\ 2053 & 14: 54: 59 & 1.3495 \\ 2054 & 14: 55: 00 & 1.3522 \\ 2055 & 14: 55: 01 & 1.3516 \\ 2056 & 14: 55: 02 & 1.3511 \\ 2057 & 14: 55: 03 & 1.3536 \\ 2058 & 14: 55: 04 & 1.3484 \\ 2059 & 14: 55: 05 & 1.3453 \\ 2060 & 14: 55: 06 & 1.3509 \\ 2061 & 14: 55: 07 & 1.3517 \\ 2062 & 14: 55: 08 & 1.3552 \\ 2063 & 14: 55: 09 & 1.3596 \\ 2064 & 14: 55: 10 & 1.3575 \\ 2065 & 14: 55: 11 & 1.3543 \\ 2066 & 14: 55: 12 & 1.3535 \\ 2067 & 14: 55: 13 & 1.3539 \\ 2068 & 14: 55: 14 & 1.3528 \\ 2069 & 14: 55: 15 & 1.3528 \\ 2070 & 14: 55: 16 & 1.3545 \\ 2071 & 14: 55: 17 & 1.3560 \\ 2072 & 14: 55: 18 & 1.3529 \\ 2073 & 14: 55: 19 & 1.3505 \\ 2074 & 14: 55: 20 & 1.3540 \\ 2075 & 14: 55: 21 & 1.3536 \\ 2076 & 14: 55: 22 & 1.3533 \\ 2077 & 14: 55: 23 & 1.3548 \\ 2078 & 14: 55: 24 & 1.3500 \\ 2079 & 14: 55: 25 & 1.3489 \\ 2080 & 14: 55: 26 & 1.3561 \\ 2081 & 14: 55: 27 & 1.3521 \\ 2082 & 14: 55: 28 & 1.3486 \\ 2083 & 14: 55: 29 & 1.3522 \\ 2084 & 14: 55: 30 & 1.3525 \\ 2085 & 14: 55: 31 & 1.3505 \\ 2086 & 14: 55: 32 & 1.3539 \\ 2087 & 14: 55: 33 & 1.3519 \\ 2088 & 14: 55: 34 & 1.3506 \\ & 14: 55: 35 & 1.3528\end{array}$


Appendix G - Fuel Data Test 4 NIST102705_1.xls

$\begin{array}{lll}2090 & 14: 55: 36 & 1.3484 \\ 2091 & 14: 55: 37 & 1.3491 \\ 2092 & 14: 55: 38 & 1.3549 \\ 2093 & 14: 55: 39 & 1.3498 \\ 2094 & 14: 55: 40 & 1.3508 \\ 2095 & 14: 55: 41 & 1.3508 \\ 2096 & 14: 55: 42 & 1.3531 \\ 2097 & 14: 55: 43 & 1.3572 \\ 2098 & 14: 55: 44 & 1.3525 \\ 2099 & 14: 55: 45 & 1.3519 \\ 2100 & 14: 55: 46 & 1.3503 \\ 2101 & 14: 55: 47 & 1.3487 \\ 2102 & 14: 55: 48 & 1.3502 \\ 2103 & 14: 55: 49 & 1.3503 \\ 2104 & 14: 55: 50 & 1.3553 \\ 2105 & 14: 55: 51 & 1.3546 \\ 2106 & 14: 55: 52 & 1.3525 \\ 2107 & 14: 55: 53 & 1.3514 \\ 2108 & 14: 55: 54 & 1.3542 \\ 2109 & 14: 55: 55 & 1.3525 \\ 2110 & 14: 55: 56 & 1.3524 \\ 2111 & 14: 55: 57 & 1.3528 \\ 2112 & 14: 55: 58 & 1.3508 \\ 2113 & 14: 55: 59 & 1.3524 \\ 2114 & 14: 56: 00 & 1.3536 \\ 2115 & 14: 56: 01 & 1.3525 \\ 2116 & 14: 56: 02 & 1.3511 \\ 2117 & 14: 56: 03 & 1.3559 \\ 2118 & 14: 56: 04 & 1.3576 \\ 2119 & 14: 56: 05 & 1.3530 \\ 2120 & 14: 56: 06 & 1.3505 \\ 2121 & 14: 56: 07 & 1.3498 \\ 2122 & 14: 56: 08 & 1.3557 \\ 2123 & 14: 56: 09 & 1.3537 \\ 2124 & 14: 56: 10 & 1.3526 \\ 2125 & 14: 56: 11 & 1.3524 \\ 2126 & 14: 56: 12 & 1.3495 \\ 2127 & 14: 56: 13 & 1.3529 \\ 2128 & 14: 56: 14 & 1.3578 \\ 2129 & 14: 56: 15 & 1.3555 \\ 2130 & 14: 56: 16 & 1.3539 \\ 2131 & 14: 56: 17 & 1.3531 \\ 2132 & 14: 56: 18 & 1.3548 \\ 2133 & 14: 56: 19 & 1.3542 \\ 2134 & 14: 56: 20 & 1.3540 \\ 2135 & 14: 56: 21 & 1.3504 \\ 2136 & 14: 56: 22 & 1.3509 \\ 2137 & 14: 56: 23 & 1.3528 \\ 2138 & 14: 56: 24 & 1.3494 \\ 2139 & 14: 56: 25 & 1.3534 \\ 2140 & 14: 56: 26 & 1.3538\end{array}$


Appendix G - Fuel Data Test 4 NIST102705_1.xls

$\begin{array}{lll}2141 & 14: 56: 27 & 1.3509 \\ 2142 & 14: 56: 28 & 1.3541 \\ 2143 & 14: 56: 29 & 1.3542 \\ 2144 & 14: 56: 30 & 1.3509 \\ 2145 & 14: 56: 31 & 1.3554 \\ 2146 & 14: 56: 32 & 1.3549 \\ 2147 & 14: 56: 33 & 1.3509 \\ 2148 & 14: 56: 34 & 1.3539 \\ 2149 & 14: 56: 35 & 1.3537 \\ 2150 & 14: 56: 36 & 1.3495 \\ 2151 & 14: 56: 37 & 1.3490 \\ 2152 & 14: 56: 38 & 1.3563 \\ 2153 & 14: 56: 39 & 1.3566 \\ 2154 & 14: 56: 40 & 1.3543 \\ 2155 & 14: 56: 41 & 1.3545 \\ 2156 & 14: 56: 42 & 1.3527 \\ 2157 & 14: 56: 43 & 1.3526 \\ 2158 & 14: 56: 44 & 1.3540 \\ 2159 & 14: 56: 45 & 1.3531 \\ 2160 & 14: 56: 46 & 1.3513 \\ 2161 & 14: 56: 47 & 1.3535 \\ 2162 & 14: 56: 48 & 1.3518 \\ 2163 & 14: 56: 49 & 1.3536 \\ 2164 & 14: 56: 50 & 1.3538 \\ 2165 & 14: 56: 51 & 1.3517 \\ 2166 & 14: 56: 52 & 1.3530 \\ 2167 & 14: 56: 53 & 1.3553 \\ 2168 & 14: 56: 54 & 1.3548 \\ 2169 & 14: 56: 55 & 1.3559 \\ 2170 & 14: 56: 56 & 1.3534 \\ 2171 & 14: 56: 57 & 1.3542 \\ 2172 & 14: 56: 58 & 1.3532 \\ 2173 & 14: 56: 59 & 1.3551 \\ 2174 & 14: 57: 00 & 1.3561 \\ 2175 & 14: 57: 01 & 1.3554 \\ 2176 & 14: 57: 02 & 1.3521 \\ 2177 & 14: 57: 03 & 1.3477 \\ 2178 & 14: 57: 04 & 1.3551 \\ 2179 & 14: 57: 05 & 1.3563 \\ 2180 & 14: 57: 06 & 1.3545 \\ 2181 & 14: 57: 07 & 1.3540 \\ 2182 & 14: 57: 08 & 1.3546 \\ 2183 & 14: 57: 09 & 1.3536 \\ 2184 & 14: 57: 10 & 1.3544 \\ 2185 & 14: 57: 11 & 1.3543 \\ 2186 & 14: 57: 12 & 1.3529 \\ 2187 & 14: 57: 13 & 1.3523 \\ 2188 & 14: 57: 14 & 1.3524 \\ 2189 & 14: 57: 15 & 1.3551 \\ 2190 & 14: 57: 16 & 1.3556 \\ 2191 & 14: 57: 17 & 1.3520\end{array}$


Appendix G - Fuel Data Test 4 NIST102705_1.xls

$\begin{array}{lll}2192 & 14: 57: 18 & 1.3479 \\ 2193 & 14: 57: 19 & 1.3553 \\ 2194 & 14: 57: 20 & 1.3560 \\ 2195 & 14: 57: 21 & 1.3541 \\ 2196 & 14: 57: 22 & 1.3543 \\ 2197 & 14: 57: 23 & 1.3563 \\ 2198 & 14: 57: 24 & 1.3521 \\ 2199 & 14: 57: 25 & 1.3534 \\ 2200 & 14: 57: 26 & 1.3557 \\ 2201 & 14: 57: 27 & 1.3550 \\ 2202 & 14: 57: 28 & 1.3536 \\ 2203 & 14: 57: 29 & 1.3535 \\ 2204 & 14: 57: 30 & 1.3517 \\ 2205 & 14: 57: 31 & 1.3551 \\ 2206 & 14: 57: 32 & 1.3563 \\ 2207 & 14: 57: 33 & 1.3533 \\ 2208 & 14: 57: 34 & 1.3496 \\ 2209 & 14: 57: 35 & 1.3522 \\ 2210 & 14: 57: 36 & 1.3525 \\ 2211 & 14: 57: 37 & 1.3528 \\ 2212 & 14: 57: 38 & 1.3507 \\ 2213 & 14: 57: 39 & 1.3482 \\ 2214 & 14: 57: 40 & 1.3512 \\ 2215 & 14: 57: 41 & 1.3539 \\ 2216 & 14: 57: 42 & 1.3537 \\ 2217 & 14: 57: 43 & 1.3529 \\ 2218 & 14: 57: 44 & 1.3530 \\ 2219 & 14: 57: 45 & 1.3532 \\ 2220 & 14: 57: 46 & 1.3524 \\ 2221 & 14: 57: 47 & 1.3522 \\ 2222 & 14: 57: 48 & 1.3563 \\ 2223 & 14: 57: 49 & 1.3566 \\ 2224 & 14: 57: 50 & 1.3580 \\ 2225 & 14: 57: 51 & 1.3537 \\ 2226 & 14: 57: 52 & 1.3538 \\ 2227 & 14: 57: 53 & 1.3524 \\ 2228 & 14: 57: 54 & 1.3506 \\ 2229 & 14: 57: 55 & 1.3514 \\ 2230 & 14: 57: 56 & 1.3508 \\ 2231 & 14: 57: 57 & 1.3504 \\ 2232 & 14: 57: 58 & 1.3539 \\ 2233 & 14: 57: 59 & 1.3523 \\ 2234 & 14: 58: 00 & 1.3516 \\ 2235 & 14: 58: 01 & 1.3552 \\ 2236 & 14: 58: 02 & 1.3569 \\ 2237 & 14: 58: 03 & 1.3545 \\ 2238 & 14: 58: 04 & 1.3538 \\ 2239 & 14: 58: 05 & 1.3563 \\ 2240 & 14: 58: 06 & 1.3543 \\ 2241 & 14: 58: 07 & 1.3530 \\ 2242 & 14: 58: 08 & 1.3542 \\ & & \end{array}$


Appendix G - Fuel Data Test 4 NIST102705_1.xls

$\begin{array}{lll}2243 & 14: 58: 09 & 1.3526 \\ 2244 & 14: 58: 10 & 1.3509 \\ 2245 & 14: 58: 11 & 1.3548 \\ 2246 & 14: 58: 12 & 1.3551 \\ 2247 & 14: 58: 13 & 1.3519 \\ 2248 & 14: 58: 14 & 1.3530 \\ 2249 & 14: 58: 15 & 1.3505 \\ 2250 & 14: 58: 16 & 1.3540 \\ 2251 & 14: 58: 17 & 1.3554 \\ 2252 & 14: 58: 18 & 1.3507 \\ 2253 & 14: 58: 19 & 1.3521 \\ 2254 & 14: 58: 20 & 1.3536 \\ 2255 & 14: 58: 21 & 1.3513 \\ 2256 & 14: 58: 22 & 1.3532 \\ 2257 & 14: 58: 23 & 1.3519 \\ 2258 & 14: 58: 24 & 1.3491 \\ 2259 & 14: 58: 25 & 1.3529 \\ 2260 & 14: 58: 26 & 1.3522 \\ 2261 & 14: 58: 27 & 1.3531 \\ 2262 & 14: 58: 28 & 1.3543 \\ 2263 & 14: 58: 29 & 1.3521 \\ 2264 & 14: 58: 30 & 1.3534 \\ 2265 & 14: 58: 31 & 1.3557 \\ 2266 & 14: 58: 32 & 1.3527 \\ 2267 & 14: 58: 33 & 1.3521 \\ 2268 & 14: 58: 34 & 1.3525 \\ 2269 & 14: 58: 35 & 1.3526 \\ 2270 & 14: 58: 36 & 1.3528 \\ 2271 & 14: 58: 37 & 1.3530 \\ 2272 & 14: 58: 38 & 1.3540 \\ 2273 & 14: 58: 39 & 1.3562 \\ 2274 & 14: 58: 40 & 1.3534 \\ 2275 & 14: 58: 41 & 1.3478 \\ 2276 & 14: 58: 42 & 1.3534 \\ 2277 & 14: 58: 43 & 1.3547 \\ 2278 & 14: 58: 44 & 1.3515 \\ 2279 & 14: 58: 45 & 1.3523 \\ 2280 & 14: 58: 46 & 1.3500 \\ 2281 & 14: 58: 47 & 1.3526 \\ 2282 & 14: 58: 48 & 1.3571 \\ 2283 & 14: 58: 49 & 1.3539 \\ 2284 & 14: 58: 50 & 1.3527 \\ 2285 & 14: 58: 51 & 1.3542 \\ 2286 & 14: 58: 52 & 1.3564 \\ 2287 & 14: 58: 53 & 1.3537 \\ 2288 & 14: 58: 54 & 1.3506 \\ 2289 & 14: 58: 55 & 1.3523 \\ 2290 & 14: 58: 56 & 1.3541 \\ 2291 & 14: 58: 57 & 1.3551 \\ 2292 & 14: 58: 58 & 1.3539 \\ 2293 & 14: 58: 59 & 1.3547\end{array}$


Appendix G - Fuel Data Test 4 NIST102705_1.xls

$\begin{array}{lll}2294 & 14: 59: 00 & 1.3512 \\ 2295 & 14: 59: 01 & 1.3510 \\ 2296 & 14: 59: 02 & 1.3526 \\ 2297 & 14: 59: 03 & 1.3543 \\ 2298 & 14: 59: 04 & 1.3545 \\ 2299 & 14: 59: 05 & 1.3529 \\ 2300 & 14: 59: 06 & 1.3534 \\ 2301 & 14: 59: 07 & 1.3515 \\ 2302 & 14: 59: 08 & 1.3522 \\ 2303 & 14: 59: 09 & 1.3537 \\ 2304 & 14: 59: 10 & 1.3530 \\ 2305 & 14: 59: 11 & 1.3501 \\ 2306 & 14: 59: 12 & 1.3505 \\ 2307 & 14: 59: 13 & 1.3548 \\ 2308 & 14: 59: 14 & 1.3552 \\ 2309 & 14: 59: 15 & 1.3527 \\ 2310 & 14: 59: 16 & 1.3532 \\ 2311 & 14: 59: 17 & 1.3508 \\ 2312 & 14: 59: 18 & 1.3528 \\ 2313 & 14: 59: 19 & 1.3538 \\ 2314 & 14: 59: 20 & 1.3504 \\ 2315 & 14: 59: 21 & 1.3521 \\ 2316 & 14: 59: 22 & 1.3537 \\ 2317 & 14: 59: 23 & 1.3551 \\ 2318 & 14: 59: 24 & 1.3531 \\ 2319 & 14: 59: 25 & 1.3506 \\ 2320 & 14: 59: 26 & 1.3542 \\ 2321 & 14: 59: 27 & 1.3562 \\ 2322 & 14: 59: 28 & 1.3520 \\ 2323 & 14: 59: 29 & 1.3548 \\ 2324 & 14: 59: 30 & 1.3567 \\ 2325 & 14: 59: 31 & 1.3525 \\ 2326 & 14: 59: 32 & 1.3509 \\ 2327 & 14: 59: 33 & 1.3538 \\ 2328 & 14: 59: 34 & 1.3513 \\ 2329 & 14: 59: 35 & 1.3527 \\ 2330 & 14: 59: 36 & 1.3542 \\ 2331 & 14: 59: 37 & 1.3542 \\ 2332 & 14: 59: 38 & 1.3520 \\ 2333 & 14: 59: 39 & 1.3510 \\ 2334 & 14: 59: 40 & 1.3528 \\ 2335 & 14: 59: 41 & 1.3537 \\ 2336 & 14: 59: 42 & 1.3526 \\ 2337 & 14: 59: 43 & 1.3527 \\ 2338 & 14: 59: 44 & 1.3530 \\ 2339 & 14: 59: 45 & 1.3527 \\ 2340 & 14: 59: 46 & 1.3529 \\ 2341 & 14: 59: 47 & 1.3533 \\ 2342 & 14: 59: 48 & 1.3559 \\ 2344 & 14: 59: 49 & 1.3560 \\ & 14: 59: 50 & 1.3546\end{array}$


Appendix G - Fuel Data Test 4 NIST102705_1.xls

$\begin{array}{lll}2345 & 14: 59: 51 & 1.3561 \\ 2346 & 14: 59: 52 & 1.3514 \\ 2347 & 14: 59: 53 & 1.3499 \\ 2348 & 14: 59: 54 & 1.3526 \\ 2349 & 14: 59: 55 & 1.3534 \\ 2350 & 14: 59: 56 & 1.3493 \\ 2351 & 14: 59: 57 & 1.3520 \\ 2352 & 14: 59: 58 & 1.3539 \\ 2353 & 14: 59: 59 & 1.3505 \\ 2354 & 15: 00: 00 & 1.3524 \\ 2355 & 15: 00: 01 & 1.3509 \\ 2356 & 15: 00: 02 & 1.3484 \\ 2357 & 15: 00: 03 & 1.3524 \\ 2358 & 15: 00: 04 & 1.3538 \\ 2359 & 15: 00: 05 & 1.3515 \\ 2360 & 15: 00: 06 & 1.3518 \\ 2361 & 15: 00: 07 & 1.3539 \\ 2362 & 15: 00: 08 & 1.3526 \\ 2363 & 15: 00: 09 & 1.3518 \\ 2364 & 15: 00: 10 & 1.3564 \\ 2365 & 15: 00: 11 & 1.3551 \\ 2366 & 15: 00: 12 & 1.3531 \\ 2367 & 15: 00: 13 & 1.3520 \\ 2368 & 15: 00: 14 & 1.3505 \\ 2369 & 15: 00: 15 & 1.3519 \\ 2370 & 15: 00: 16 & 1.3549 \\ 2371 & 15: 00: 17 & 1.3525 \\ 2372 & 15: 00: 18 & 1.3534 \\ 2373 & 15: 00: 19 & 1.3543 \\ 2374 & 15: 00: 20 & 1.3526 \\ 2375 & 15: 00: 21 & 1.3535 \\ 2376 & 15: 00: 22 & 1.3514 \\ 2377 & 15: 00: 23 & 1.3484 \\ 2378 & 15: 00: 24 & 1.3505 \\ 2379 & 15: 00: 25 & 1.3562 \\ 2380 & 15: 00: 26 & 1.3534 \\ 2381 & 15: 00: 27 & 1.3520 \\ 2382 & 15: 00: 28 & 1.3531 \\ 2383 & 15: 00: 29 & 1.3533 \\ 2384 & 15: 00: 30 & 1.3530 \\ 2385 & 15: 00: 31 & 1.3538 \\ 2386 & 15: 00: 32 & 1.3551 \\ 2387 & 15: 00: 33 & 1.3538 \\ 2388 & 15: 00: 34 & 1.3525 \\ 2389 & 15: 00: 35 & 1.3513 \\ 2390 & 15: 00: 36 & 1.3581 \\ 2391 & 15: 00: 37 & 1.3552 \\ 2392 & 15: 00: 38 & 1.3512 \\ 2395 & 15: 00: 39 & 1.3509 \\ & 15: 00: 40 & 1.3514 \\ & & 1.3536\end{array}$


Appendix G - Fuel Data Test 4 NIST102705_1.xls

$\begin{array}{lll}2396 & 15: 00: 42 & 1.3547 \\ 2397 & 15: 00: 43 & 1.3521 \\ 2398 & 15: 00: 44 & 1.3485 \\ 2399 & 15: 00: 45 & 1.3514 \\ 2400 & 15: 00: 46 & 1.3548 \\ 2401 & 15: 00: 47 & 1.3529 \\ 2402 & 15: 00: 48 & 1.3542 \\ 2403 & 15: 00: 49 & 1.3572 \\ 2404 & 15: 00: 50 & 1.3553 \\ 2405 & 15: 00: 51 & 1.3536 \\ 2406 & 15: 00: 52 & 1.3543 \\ 2407 & 15: 00: 53 & 1.3536 \\ 2408 & 15: 00: 54 & 1.3548 \\ 2409 & 15: 00: 55 & 1.3534 \\ 2410 & 15: 00: 56 & 1.3514 \\ 2411 & 15: 00: 57 & 1.3504 \\ 2412 & 15: 00: 58 & 1.3526 \\ 2413 & 15: 00: 59 & 1.3554 \\ 2414 & 15: 01: 00 & 1.3531 \\ 2415 & 15: 01: 01 & 1.3524 \\ 2416 & 15: 01: 02 & 1.3549 \\ 2417 & 15: 01: 03 & 1.3539 \\ 2418 & 15: 01: 04 & 1.3535 \\ 2419 & 15: 01: 05 & 1.3525 \\ 2420 & 15: 01: 06 & 1.3513 \\ 2421 & 15: 01: 07 & 1.3523 \\ 2422 & 15: 01: 08 & 1.3514 \\ 2423 & 15: 01: 09 & 1.3488 \\ 2424 & 15: 01: 10 & 1.3515 \\ 2425 & 15: 01: 11 & 1.3567 \\ 2426 & 15: 01: 12 & 1.3540 \\ 2427 & 15: 01: 13 & 1.3538 \\ 2428 & 15: 01: 14 & 1.3540 \\ 2429 & 15: 01: 15 & 1.3529 \\ 2430 & 15: 01: 16 & 1.3520 \\ 2431 & 15: 01: 17 & 1.3555 \\ 2432 & 15: 01: 18 & 1.3546 \\ 2433 & 15: 01: 19 & 1.3511 \\ 2434 & 15: 01: 20 & 1.3530 \\ 2435 & 15: 01: 21 & 1.3538 \\ 2436 & 15: 01: 22 & 1.3556 \\ 2437 & 15: 01: 23 & 1.3562 \\ 2438 & 15: 01: 24 & 1.3541 \\ 2439 & 15: 01: 25 & 1.3531 \\ 2440 & 15: 01: 26 & 1.3517 \\ 2441 & 15: 01: 27 & 1.3535 \\ 2442 & 15: 01: 28 & 1.3587 \\ 2443 & 15: 01: 29 & 1.3551 \\ 2444 & 15: 01: 30 & 1.3526 \\ 2445 & 15: 01: 31 & 1.3530 \\ 2446 & 15: 01: 32 & 1.3525\end{array}$


Appendix G - Fuel Data Test 4 NIST102705_1.xls

$\begin{array}{lll}2447 & 15: 01: 33 & 1.3532 \\ 2448 & 15: 01: 34 & 1.3533 \\ 2449 & 15: 01: 35 & 1.3541 \\ 2450 & 15: 01: 36 & 1.3503 \\ 2451 & 15: 01: 37 & 1.3547 \\ 2452 & 15: 01: 38 & 1.3564 \\ 2453 & 15: 01: 39 & 1.3481 \\ 2454 & 15: 01: 40 & 1.3493 \\ 2455 & 15: 01: 41 & 1.3536 \\ 2456 & 15: 01: 42 & 1.3493 \\ 2457 & 15: 01: 43 & 1.3521 \\ 2458 & 15: 01: 44 & 1.3551 \\ 2459 & 15: 01: 45 & 1.3538 \\ 2460 & 15: 01: 46 & 1.3524 \\ 2461 & 15: 01: 47 & 1.3550 \\ 2462 & 15: 01: 48 & 1.3562 \\ 2463 & 15: 01: 49 & 1.3559 \\ 2464 & 15: 01: 50 & 1.3537 \\ 2465 & 15: 01: 51 & 1.3511 \\ 2466 & 15: 01: 52 & 1.3526 \\ 2467 & 15: 01: 53 & 1.3512 \\ 2468 & 15: 01: 54 & 1.3536 \\ 2469 & 15: 01: 55 & 1.3572 \\ 2470 & 15: 01: 56 & 1.3530 \\ 2471 & 15: 01: 57 & 1.3548 \\ 2472 & 15: 01: 58 & 1.3557 \\ 2473 & 15: 01: 59 & 1.3584 \\ 2474 & 15: 02: 00 & 1.3562 \\ 2475 & 15: 02: 01 & 1.3534 \\ 2476 & 15: 02: 02 & 1.3503 \\ 2477 & 15: 02: 03 & 1.3523 \\ 2478 & 15: 02: 04 & 1.3547 \\ 2479 & 15: 02: 05 & 1.3558 \\ 2480 & 15: 02: 06 & 1.3538 \\ 2481 & 15: 02: 07 & 1.3517 \\ 2482 & 15: 02: 08 & 1.3534 \\ 2483 & 15: 02: 09 & 1.3516 \\ 2484 & 15: 02: 10 & 1.3535 \\ 2485 & 15: 02: 11 & 1.3524 \\ 2486 & 15: 02: 12 & 1.3540 \\ 2487 & 15: 02: 13 & 1.3540 \\ 2488 & 15: 02: 14 & 1.3526 \\ 2489 & 15: 02: 15 & 1.3534 \\ 2490 & 15: 02: 16 & 1.3577 \\ 2491 & 15: 02: 17 & 1.3566 \\ 2492 & 15: 02: 18 & 1.3511 \\ 2493 & 15: 02: 19 & 1.3555 \\ 2494 & 15: 02: 20 & 1.3569 \\ 2495 & 15: 02: 21 & 1.3538 \\ 2496 & 15: 02: 22 & 1.3511 \\ 2497 & 15: 02: 23 & 1.3480\end{array}$


Appendix G - Fuel Data Test 4 NIST102705_1.xls

$\begin{array}{lll}2498 & 15: 02: 24 & 1.3502 \\ 2499 & 15: 02: 25 & 1.3547 \\ 2500 & 15: 02: 26 & 1.3531 \\ 2501 & 15: 02: 27 & 1.3509 \\ 2502 & 15: 02: 28 & 1.3494 \\ 2503 & 15: 02: 29 & 1.3495 \\ 2504 & 15: 02: 30 & 1.3536 \\ 2505 & 15: 02: 31 & 1.3560 \\ 2506 & 15: 02: 32 & 1.3519 \\ 2507 & 15: 02: 33 & 1.3508 \\ 2508 & 15: 02: 34 & 1.3534 \\ 2509 & 15: 02: 35 & 1.3558 \\ 2510 & 15: 02: 36 & 1.3542 \\ 2511 & 15: 02: 37 & 1.3539 \\ 2512 & 15: 02: 38 & 1.3533 \\ 2513 & 15: 02: 39 & 1.3545 \\ 2514 & 15: 02: 40 & 1.3566 \\ 2515 & 15: 02: 41 & 1.3541 \\ 2516 & 15: 02: 42 & 1.3503 \\ 2517 & 15: 02: 43 & 1.3529 \\ 2518 & 15: 02: 44 & 1.3527 \\ 2519 & 15: 02: 45 & 1.3513 \\ 2520 & 15: 02: 46 & 1.3542 \\ 2521 & 15: 02: 47 & 1.3528 \\ 2522 & 15: 02: 48 & 1.3519 \\ 2523 & 15: 02: 49 & 1.3556 \\ 2524 & 15: 02: 50 & 1.3526 \\ 2525 & 15: 02: 51 & 1.3511 \\ 2526 & 15: 02: 52 & 1.3550 \\ 2527 & 15: 02: 53 & 1.3558 \\ 2528 & 15: 02: 54 & 1.3509 \\ 2529 & 15: 02: 55 & 1.3525 \\ 2530 & 15: 02: 56 & 1.3535 \\ 2531 & 15: 02: 57 & 1.3530 \\ 2532 & 15: 02: 58 & 1.3526 \\ 2533 & 15: 02: 59 & 1.3520 \\ 2534 & 15: 03: 00 & 1.3531 \\ 2535 & 15: 03: 01 & 1.3545 \\ 2536 & 15: 03: 02 & 1.3514 \\ 2537 & 15: 03: 03 & 1.3516 \\ 2538 & 15: 03: 04 & 1.3540 \\ 2539 & 15: 03: 05 & 1.3542 \\ 2540 & 15: 03: 06 & 1.3569 \\ 2541 & 15: 03: 07 & 1.3539 \\ 2542 & 15: 03: 08 & 1.3520 \\ 2543 & 15: 03: 09 & 1.3528 \\ 2544 & 15: 03: 10 & 1.3559 \\ 2545 & 15: 03: 11 & 1.3548 \\ 2546 & 15: 03: 12 & 1.3505 \\ 2547 & 15: 03: 13 & 1.3535 \\ 2548 & 15: 03: 14 & 1.3547\end{array}$

G - 50 
Appendix G - Fuel Data Test 4 NIST102705_1.xls

\begin{tabular}{lll}
2549 & $15: 03: 15$ & 1.3484 \\
2550 & $15: 03: 16$ & 1.3542 \\
2551 & $15: 03: 17$ & 1.3565 \\
2552 & $15: 03: 18$ & 1.3533 \\
2553 & $15: 03: 19$ & 1.3505 \\
2554 & $15: 03: 20$ & 1.3527 \\
2555 & $15: 03: 21$ & 1.3517 \\
2556 & $15: 03: 22$ & 1.3524 \\
2557 & $15: 03: 23$ & 1.3550 \\
2558 & $15: 03: 24$ & 1.3538 \\
2559 & $15: 03: 25$ & 1.3516 \\
2560 & $15: 03: 26$ & 1.3504 \\
2561 & $15: 03: 27$ & 1.3536 \\
2562 & $15: 03: 28$ & 1.3546 \\
2563 & $15: 03: 29$ & 1.3534 \\
2564 & $15: 03: 30$ & 1.3513 \\
2565 & $15: 03: 31$ & 1.3535 \\
2566 & $15: 03: 32$ & 1.3527 \\
2567 & $15: 03: 33$ & 1.3527 \\
2568 & $15: 03: 34$ & 1.3526 \\
2569 & $15: 03: 35$ & 1.3515 \\
2570 & $15: 03: 36$ & 1.3517 \\
2571 & $15: 03: 37$ & 1.3529 \\
2572 & $15: 03: 38$ & 1.3514 \\
2573 & $15: 03: 39$ & 1.3518 \\
2574 & $15: 03: 40$ & 1.3492 \\
2575 & $15: 03: 41$ & 1.3520 \\
2576 & $15: 03: 42$ & 1.3571 \\
2577 & $15: 03: 43$ & 1.3525 \\
2578 & $15: 03: 44$ & 1.3538 \\
2579 & $15: 03: 45$ & 1.3553 \\
2580 & $15: 03: 46$ & 1.3536 \\
2581 & $15: 03: 47$ & 1.3521 \\
2582 & $15: 03: 48$ & 1.3553 \\
2583 & $15: 03: 49$ & 1.3552 \\
2584 & $15: 03: 50$ & 1.3520 \\
2585 & $15: 03: 51$ & 1.3511 \\
2586 & $15: 03: 52$ & 1.3543 \\
2587 & $15: 03: 53$ & 1.3540 \\
2588 & $15: 03: 54$ & 1.3525 \\
2589 & $15: 03: 55$ & 1.3518 \\
2590 & $15: 03: 56$ & 1.3518 \\
2591 & $15: 03: 57$ & 1.3552 \\
2592 & $15: 03: 58$ & 1.3565 \\
2593 & $15: 03: 59$ & 1.3514 \\
2594 & $15: 04: 00$ & 1.3487 \\
2595 & $15: 04: 01$ & 1.3492 \\
2596 & $15: 04: 02$ & 1.3560 \\
2597 & $15: 04: 03$ & 1.3553 \\
2598 & $15: 04: 04$ & 1.3527 \\
\hline & $15: 04: 05$ & 1.3527
\end{tabular}

G - 51 
Appendix G - Fuel Data Test 4 NIST102705_1.xls

$\begin{array}{lll}2600 & 15: 04: 06 & 1.3498 \\ 2601 & 15: 04: 07 & 1.3507 \\ 2602 & 15: 04: 08 & 1.3526 \\ 2603 & 15: 04: 09 & 1.3531 \\ 2604 & 15: 04: 10 & 1.3517 \\ 2605 & 15: 04: 11 & 1.3516 \\ 2606 & 15: 04: 12 & 1.3521 \\ 2607 & 15: 04: 13 & 1.3548 \\ 2608 & 15: 04: 14 & 1.3521 \\ 2609 & 15: 04: 15 & 1.3505 \\ 2610 & 15: 04: 16 & 1.3538 \\ 2611 & 15: 04: 17 & 1.3541 \\ 2612 & 15: 04: 18 & 1.3553 \\ 2613 & 15: 04: 19 & 1.3575 \\ 2614 & 15: 04: 20 & 1.3533 \\ 2615 & 15: 04: 21 & 1.3496 \\ 2616 & 15: 04: 22 & 1.3520 \\ 2617 & 15: 04: 23 & 1.3543 \\ 2618 & 15: 04: 24 & 1.3539 \\ 2619 & 15: 04: 25 & 1.3513 \\ 2620 & 15: 04: 26 & 1.3480 \\ 2621 & 15: 04: 27 & 1.3513 \\ 2622 & 15: 04: 28 & 1.3548 \\ 2623 & 15: 04: 29 & 1.3554 \\ 2624 & 15: 04: 30 & 1.3544 \\ 2625 & 15: 04: 31 & 1.3521 \\ 2626 & 15: 04: 32 & 1.3508 \\ 2627 & 15: 04: 33 & 1.3507 \\ 2628 & 15: 04: 34 & 1.3517 \\ 2629 & 15: 04: 35 & 1.3527 \\ 2630 & 15: 04: 36 & 1.3514 \\ 2631 & 15: 04: 37 & 1.3553 \\ 2632 & 15: 04: 38 & 1.3542 \\ 2633 & 15: 04: 39 & 1.3541 \\ 2634 & 15: 04: 40 & 1.3561 \\ 2635 & 15: 04: 41 & 1.3543 \\ 2636 & 15: 04: 42 & 1.3500 \\ 2637 & 15: 04: 43 & 1.3504 \\ 2638 & 15: 04: 44 & 1.3539 \\ 2639 & 15: 04: 45 & 1.3527 \\ 2640 & 15: 04: 46 & 1.3509 \\ 2641 & 15: 04: 47 & 1.3523 \\ 2642 & 15: 04: 48 & 1.3497 \\ 2643 & 15: 04: 49 & 1.3508 \\ 2644 & 15: 04: 50 & 1.3513 \\ 2645 & 15: 04: 51 & 1.3550 \\ 2646 & 15: 04: 52 & 1.3545 \\ 2647 & 15: 04: 53 & 1.3491 \\ 2648 & 15: 04: 54 & 1.3517 \\ 2649 & 15: 04: 55 & 1.3530 \\ 2650 & 15: 04: 56 & 1.3541\end{array}$

G - 52 
Appendix G - Fuel Data Test 4 NIST102705_1.xls

$\begin{array}{lll}2651 & 15: 04: 57 & 1.3522 \\ 2652 & 15: 04: 58 & 1.3525 \\ 2653 & 15: 04: 59 & 1.3532 \\ 2654 & 15: 05: 00 & 1.3554 \\ 2655 & 15: 05: 01 & 1.3565 \\ 2656 & 15: 05: 02 & 1.3530 \\ 2657 & 15: 05: 03 & 1.3523 \\ 2658 & 15: 05: 04 & 1.3536 \\ 2659 & 15: 05: 05 & 1.3534 \\ 2660 & 15: 05: 06 & 1.3540 \\ 2661 & 15: 05: 07 & 1.3519 \\ 2662 & 15: 05: 08 & 1.3523 \\ 2663 & 15: 05: 09 & 1.3509 \\ 2664 & 15: 05: 10 & 1.3528 \\ 2665 & 15: 05: 11 & 1.3544 \\ 2666 & 15: 05: 12 & 1.3535 \\ 2667 & 15: 05: 13 & 1.3498 \\ 2668 & 15: 05: 14 & 1.3548 \\ 2669 & 15: 05: 15 & 1.3561 \\ 2670 & 15: 05: 16 & 1.3537 \\ 2671 & 15: 05: 17 & 1.3522 \\ 2672 & 15: 05: 18 & 1.3550 \\ 2673 & 15: 05: 19 & 1.3561 \\ 2674 & 15: 05: 20 & 1.3561 \\ 2675 & 15: 05: 21 & 1.3554 \\ 2676 & 15: 05: 22 & 1.3541 \\ 2677 & 15: 05: 23 & 1.3525 \\ 2678 & 15: 05: 24 & 1.3534 \\ 2679 & 15: 05: 25 & 1.3542 \\ 2680 & 15: 05: 26 & 1.3510 \\ 2681 & 15: 05: 27 & 1.3525 \\ 2682 & 15: 05: 28 & 1.3526 \\ 2683 & 15: 05: 29 & 1.3514 \\ 2684 & 15: 05: 30 & 1.3540 \\ 2685 & 15: 05: 31 & 1.3552 \\ 2686 & 15: 05: 32 & 1.3547 \\ 2687 & 15: 05: 33 & 1.3549 \\ 2688 & 15: 05: 34 & 1.3547 \\ 2689 & 15: 05: 35 & 1.3568 \\ 2690 & 15: 05: 36 & 1.3562 \\ 2691 & 15: 05: 37 & 1.3518 \\ 2692 & 15: 05: 38 & 1.3536 \\ 2693 & 15: 05: 39 & 1.3549 \\ 2694 & 15: 05: 40 & 1.3549 \\ 2695 & 15: 05: 41 & 1.3547 \\ 2696 & 15: 05: 42 & 1.3551 \\ 2697 & 15: 05: 43 & 1.3512 \\ 2698 & 15: 05: 44 & 1.3507 \\ 2699 & 15: 05: 45 & 1.3526 \\ 2700 & 15: 05: 46 & 1.3548 \\ 2701 & 15: 05: 47 & 1.3547\end{array}$

G - 53 
Appendix G - Fuel Data Test 4 NIST102705_1.xls

\begin{tabular}{lll}
2702 & $15: 05: 48$ & 1.3535 \\
2703 & $15: 05: 49$ & 1.3516 \\
2704 & $15: 05: 50$ & 1.3514 \\
2705 & $15: 05: 51$ & 1.3543 \\
2706 & $15: 05: 52$ & 1.3517 \\
2707 & $15: 05: 53$ & 1.3510 \\
2708 & $15: 05: 54$ & 1.3542 \\
2709 & $15: 05: 55$ & 1.3532 \\
2710 & $15: 05: 56$ & 1.3549 \\
2711 & $15: 05: 57$ & 1.3550 \\
2712 & $15: 05: 58$ & 1.3549 \\
2713 & $15: 05: 59$ & 1.3556 \\
2714 & $15: 06: 00$ & 1.3526 \\
2715 & $15: 06: 01$ & 1.3521 \\
2716 & $15: 06: 02$ & 1.3544 \\
2717 & $15: 06: 03$ & 1.3535 \\
2718 & $15: 06: 04$ & 1.3528 \\
2719 & $15: 06: 05$ & 1.3526 \\
2720 & $15: 06: 06$ & 1.3527 \\
2721 & $15: 06: 07$ & 1.3536 \\
2722 & $15: 06: 08$ & 1.3553 \\
2723 & $15: 06: 09$ & 1.3553 \\
2724 & $15: 06: 10$ & 1.3535 \\
2725 & $15: 06: 11$ & 1.3534 \\
2726 & $15: 06: 12$ & 1.3543 \\
2727 & $15: 06: 13$ & 1.3545 \\
2728 & $15: 06: 14$ & 1.3520 \\
2729 & $15: 06: 15$ & 1.3514 \\
2730 & $15: 06: 16$ & 1.3549 \\
2731 & $15: 06: 17$ & 1.3568 \\
2732 & $15: 06: 18$ & 1.3555 \\
2733 & $15: 06: 19$ & 1.3512 \\
2734 & $15: 06: 20$ & 1.3524 \\
2735 & $15: 06: 21$ & 1.3530 \\
2736 & $15: 06: 22$ & 1.3534 \\
2737 & $15: 06: 23$ & 1.3567 \\
2738 & $15: 06: 24$ & 1.3532 \\
2739 & $15: 06: 25$ & 1.3510 \\
2740 & $15: 06: 26$ & 1.3526 \\
2741 & $15: 06: 27$ & 1.3534 \\
2742 & $15: 06: 28$ & 1.3528 \\
2743 & $15: 06: 29$ & 1.3518 \\
2744 & $15: 06: 30$ & 1.3504 \\
2745 & $15: 06: 31$ & 1.3519 \\
2746 & $15: 06: 32$ & 1.3539 \\
2747 & $15: 06: 33$ & 1.3539 \\
2748 & $15: 06: 34$ & 1.3541 \\
2749 & $15: 06: 35$ & 1.3537 \\
2750 & $15: 06: 36$ & 1.3551 \\
2752 & $15: 06: 37$ & 1.3540 \\
\hline & $15: 06: 38$ & 1.3515
\end{tabular}

G - 54 
Appendix G - Fuel Data Test 4 NIST102705_1.xls

$\begin{array}{lll}2753 & 15: 06: 39 & 1.3527 \\ 2754 & 15: 06: 40 & 1.3536 \\ 2755 & 15: 06: 41 & 1.3520 \\ 2756 & 15: 06: 42 & 1.3522 \\ 2757 & 15: 06: 43 & 1.3521 \\ 2758 & 15: 06: 44 & 1.3538 \\ 2759 & 15: 06: 45 & 1.3547 \\ 2760 & 15: 06: 46 & 1.3502 \\ 2761 & 15: 06: 47 & 1.3511 \\ 2762 & 15: 06: 48 & 1.3560 \\ 2763 & 15: 06: 49 & 1.3542 \\ 2764 & 15: 06: 50 & 1.3516 \\ 2765 & 15: 06: 51 & 1.3531 \\ 2766 & 15: 06: 52 & 1.3532 \\ 2767 & 15: 06: 53 & 1.3524 \\ 2768 & 15: 06: 54 & 1.3535 \\ 2769 & 15: 06: 55 & 1.3503 \\ 2770 & 15: 06: 56 & 1.3514 \\ 2771 & 15: 06: 57 & 1.3539 \\ 2772 & 15: 06: 58 & 1.3534 \\ 2773 & 15: 06: 59 & 1.3532 \\ 2774 & 15: 07: 00 & 1.3520 \\ 2775 & 15: 07: 01 & 1.3522 \\ 2776 & 15: 07: 02 & 1.3542 \\ 2777 & 15: 07: 03 & 1.3536 \\ 2778 & 15: 07: 04 & 1.3530 \\ 2779 & 15: 07: 05 & 1.3541 \\ 2780 & 15: 07: 06 & 1.3522 \\ 2781 & 15: 07: 07 & 1.3529 \\ 2782 & 15: 07: 08 & 1.3544 \\ 2783 & 15: 07: 09 & 1.3521 \\ 2784 & 15: 07: 10 & 1.3497 \\ 2785 & 15: 07: 11 & 1.3514 \\ 2786 & 15: 07: 12 & 1.3532 \\ 2787 & 15: 07: 13 & 1.3530 \\ 2788 & 15: 07: 14 & 1.3531 \\ 2789 & 15: 07: 15 & 1.3544 \\ 2790 & 15: 07: 16 & 1.3527 \\ 2791 & 15: 07: 17 & 1.3517 \\ 2792 & 15: 07: 18 & 1.3518 \\ 2793 & 15: 07: 19 & 1.3515 \\ 2794 & 15: 07: 20 & 1.3536 \\ 2795 & 15: 07: 21 & 1.3552 \\ 2796 & 15: 07: 22 & 1.3512 \\ 2797 & 15: 07: 23 & 1.3513 \\ 2798 & 15: 07: 24 & 1.3535 \\ 2799 & 15: 07: 25 & 1.3566 \\ 2800 & 15: 07: 26 & 1.3542 \\ 2801 & 15: 07: 27 & 1.3521 \\ 2802 & 15: 07: 28 & 1.3506 \\ 2803 & 15: 07: 29 & 1.3533\end{array}$


Appendix G - Fuel Data Test 4 NIST102705_1.xls

\begin{tabular}{lll}
2804 & $15: 07: 30$ & 1.3540 \\
2805 & $15: 07: 31$ & 1.3517 \\
2806 & $15: 07: 32$ & 1.3494 \\
2807 & $15: 07: 33$ & 1.3533 \\
2808 & $15: 07: 34$ & 1.3525 \\
2809 & $15: 07: 35$ & 1.3542 \\
2810 & $15: 07: 36$ & 1.3553 \\
2811 & $15: 07: 37$ & 1.3546 \\
2812 & $15: 07: 38$ & 1.3524 \\
2813 & $15: 07: 39$ & 1.3549 \\
2814 & $15: 07: 40$ & 1.3560 \\
2815 & $15: 07: 41$ & 1.3537 \\
2816 & $15: 07: 42$ & 1.3519 \\
2817 & $15: 07: 43$ & 1.3505 \\
2818 & $15: 07: 44$ & 1.3511 \\
2819 & $15: 07: 45$ & 1.3522 \\
2820 & $15: 07: 46$ & 1.3522 \\
2821 & $15: 07: 47$ & 1.3535 \\
2822 & $15: 07: 48$ & 1.3529 \\
2823 & $15: 07: 49$ & 1.3523 \\
2824 & $15: 07: 50$ & 1.3534 \\
2825 & $15: 07: 51$ & 1.3545 \\
2826 & $15: 07: 52$ & 1.3515 \\
2827 & $15: 07: 53$ & 1.3508 \\
2828 & $15: 07: 54$ & 1.3508 \\
2829 & $15: 07: 55$ & 1.3503 \\
2830 & $15: 07: 56$ & 1.3510 \\
2831 & $15: 07: 57$ & 1.3560 \\
2832 & $15: 07: 58$ & 1.3543 \\
2833 & $15: 07: 59$ & 1.3514 \\
2834 & $15: 08: 00$ & 1.3536 \\
2835 & $15: 08: 01$ & 1.3549 \\
2836 & $15: 08: 02$ & 1.3514 \\
2837 & $15: 08: 03$ & 1.3500 \\
2838 & $15: 08: 04$ & 1.3514 \\
2839 & $15: 08: 05$ & 1.3522 \\
2840 & $15: 08: 06$ & 1.3570 \\
2841 & $15: 08: 07$ & 1.3554 \\
2842 & $15: 08: 08$ & 1.3531 \\
2843 & $15: 08: 09$ & 1.3534 \\
2844 & $15: 08: 10$ & 1.3523 \\
2845 & $15: 08: 11$ & 1.3559 \\
2846 & $15: 08: 12$ & 1.3548 \\
2847 & $15: 08: 13$ & 1.3527 \\
2848 & $15: 08: 14$ & 1.3532 \\
2849 & $15: 08: 15$ & 1.3523 \\
2850 & $15: 08: 16$ & 1.3522 \\
2851 & $15: 08: 17$ & 1.3535 \\
2853 & $15: 08: 18$ & 1.3563 \\
$15: 08: 19$ & 1.3551 \\
\hline & $15: 08: 20$ & 1.3524
\end{tabular}


Appendix G - Fuel Data Test 4 NIST102705_1.xls

$\begin{array}{lll}2855 & 15: 08: 21 & 1.3526 \\ 2856 & 15: 08: 22 & 1.3547 \\ 2857 & 15: 08: 23 & 1.3531 \\ 2858 & 15: 08: 24 & 1.3515 \\ 2859 & 15: 08: 25 & 1.3555 \\ 2860 & 15: 08: 26 & 1.3526 \\ 2861 & 15: 08: 27 & 1.3518 \\ 2862 & 15: 08: 28 & 1.3544 \\ 2863 & 15: 08: 29 & 1.3543 \\ 2864 & 15: 08: 30 & 1.3504 \\ 2865 & 15: 08: 31 & 1.3521 \\ 2866 & 15: 08: 32 & 1.3511 \\ 2867 & 15: 08: 33 & 1.3488 \\ 2868 & 15: 08: 34 & 1.3519 \\ 2869 & 15: 08: 35 & 1.3529 \\ 2870 & 15: 08: 36 & 1.3529 \\ 2871 & 15: 08: 37 & 1.3520 \\ 2872 & 15: 08: 38 & 1.3525 \\ 2873 & 15: 08: 39 & 1.3511 \\ 2874 & 15: 08: 40 & 1.3505 \\ 2875 & 15: 08: 41 & 1.3516 \\ 2876 & 15: 08: 42 & 1.3528 \\ 2877 & 15: 08: 43 & 1.3526 \\ 2878 & 15: 08: 44 & 1.3501 \\ 2879 & 15: 08: 45 & 1.3528 \\ 2880 & 15: 08: 46 & 1.3544 \\ 2881 & 15: 08: 47 & 1.3510 \\ 2882 & 15: 08: 48 & 1.3534 \\ 2883 & 15: 08: 49 & 1.3544 \\ 2884 & 15: 08: 50 & 1.3533 \\ 2885 & 15: 08: 51 & 1.3519 \\ 2886 & 15: 08: 52 & 1.3533 \\ 2887 & 15: 08: 53 & 1.3534 \\ 2888 & 15: 08: 54 & 1.3541 \\ 2889 & 15: 08: 55 & 1.3539 \\ 2890 & 15: 08: 56 & 1.3503 \\ 2891 & 15: 08: 57 & 1.3520 \\ 2892 & 15: 08: 58 & 1.3552 \\ 2893 & 15: 08: 59 & 1.3517 \\ 2894 & 15: 09: 00 & 1.3520 \\ 2895 & 15: 09: 01 & 1.3539 \\ 2896 & 15: 09: 02 & 1.3538 \\ 2897 & 15: 09: 03 & 1.3542 \\ 2898 & 15: 09: 04 & 1.3536 \\ 2899 & 15: 09: 05 & 1.3526 \\ 2900 & 15: 09: 06 & 1.3543 \\ 2901 & 15: 09: 07 & 1.3548 \\ 2902 & 15: 09: 08 & 1.3551 \\ 2903 & 15: 09: 09 & 1.3546 \\ 2905 & 15: 09: 10 & 1.3525 \\ & 15: 09: 11 & 1.3527\end{array}$

G - 57 
Appendix G - Fuel Data Test 4 NIST102705_1.xls

$\begin{array}{lll}2906 & 15: 09: 12 & 1.3548 \\ 2907 & 15: 09: 13 & 1.3539 \\ 2908 & 15: 09: 14 & 1.3538 \\ 2909 & 15: 09: 15 & 1.3548 \\ 2910 & 15: 09: 16 & 1.3522 \\ 2911 & 15: 09: 17 & 1.3526 \\ 2912 & 15: 09: 18 & 1.3544 \\ 2913 & 15: 09: 19 & 1.3531 \\ 2914 & 15: 09: 20 & 1.3546 \\ 2915 & 15: 09: 21 & 1.3550 \\ 2916 & 15: 09: 22 & 1.3508 \\ 2917 & 15: 09: 23 & 1.3519 \\ 2918 & 15: 09: 24 & 1.3549 \\ 2919 & 15: 09: 25 & 1.3544 \\ 2920 & 15: 09: 26 & 1.3523 \\ 2921 & 15: 09: 27 & 1.3519 \\ 2922 & 15: 09: 28 & 1.3522 \\ 2923 & 15: 09: 29 & 1.3542 \\ 2924 & 15: 09: 30 & 1.3558 \\ 2925 & 15: 09: 31 & 1.3529 \\ 2926 & 15: 09: 32 & 1.3531 \\ 2927 & 15: 09: 33 & 1.3507 \\ 2928 & 15: 09: 34 & 1.3537 \\ 2929 & 15: 09: 35 & 1.3562 \\ 2930 & 15: 09: 36 & 1.3546 \\ 2931 & 15: 09: 37 & 1.3533 \\ 2932 & 15: 09: 38 & 1.3508 \\ 2933 & 15: 09: 39 & 1.3525 \\ 2934 & 15: 09: 40 & 1.3550 \\ 2935 & 15: 09: 41 & 1.3519 \\ 2936 & 15: 09: 42 & 1.3489 \\ 2937 & 15: 09: 43 & 1.3523 \\ 2938 & 15: 09: 44 & 1.3547 \\ 2939 & 15: 09: 45 & 1.3531 \\ 2940 & 15: 09: 46 & 1.3505 \\ 2941 & 15: 09: 47 & 1.3529 \\ 2942 & 15: 09: 48 & 1.3533 \\ 2943 & 15: 09: 49 & 1.3502 \\ 2944 & 15: 09: 50 & 1.3503 \\ 2945 & 15: 09: 51 & 1.3497 \\ 2946 & 15: 09: 52 & 1.3509 \\ 2947 & 15: 09: 53 & 1.3517 \\ 2948 & 15: 09: 54 & 1.3530 \\ 2949 & 15: 09: 55 & 1.3532 \\ 2950 & 15: 09: 56 & 1.3525 \\ 2951 & 15: 09: 57 & 1.3551 \\ 2952 & 15: 09: 58 & 1.3537 \\ 2953 & 15: 09: 59 & 1.3537 \\ 2954 & 15: 10: 00 & 1.3533 \\ 2956 & 15: 10: 01 & 1.3515 \\ 15: 10: 02 & 1.3531\end{array}$


Appendix G - Fuel Data Test 4 NIST102705_1.xls

$\begin{array}{lll}2957 & 15: 10: 03 & 1.3516 \\ 2958 & 15: 10: 04 & 1.3545 \\ 2959 & 15: 10: 05 & 1.3545 \\ 2960 & 15: 10: 06 & 1.3535 \\ 2961 & 15: 10: 07 & 1.3500 \\ 2962 & 15: 10: 08 & 1.3522 \\ 2963 & 15: 10: 09 & 1.3553 \\ 2964 & 15: 10: 10 & 1.3537 \\ 2965 & 15: 10: 11 & 1.3555 \\ 2966 & 15: 10: 12 & 1.3539 \\ 2967 & 15: 10: 13 & 1.3478 \\ 2968 & 15: 10: 14 & 1.3500 \\ 2969 & 15: 10: 15 & 1.3543 \\ 2970 & 15: 10: 16 & 1.3518 \\ 2971 & 15: 10: 17 & 1.3511 \\ 2972 & 15: 10: 18 & 1.3494 \\ 2973 & 15: 10: 19 & 1.3495 \\ 2974 & 15: 10: 20 & 1.3524 \\ 2975 & 15: 10: 21 & 1.3519 \\ 2976 & 15: 10: 22 & 1.3529 \\ 2977 & 15: 10: 23 & 1.3534 \\ 2978 & 15: 10: 24 & 1.3529 \\ 2979 & 15: 10: 25 & 1.3536 \\ 2980 & 15: 10: 26 & 1.3552 \\ 2981 & 15: 10: 27 & 1.3503 \\ 2982 & 15: 10: 28 & 1.3512 \\ 2983 & 15: 10: 29 & 1.3549 \\ 2984 & 15: 10: 30 & 1.3517 \\ 2985 & 15: 10: 31 & 1.3510 \\ 2986 & 15: 10: 32 & 1.3538 \\ 2987 & 15: 10: 33 & 1.3537 \\ 2988 & 15: 10: 34 & 1.3502 \\ 2989 & 15: 10: 35 & 1.3501 \\ 2990 & 15: 10: 36 & 1.3542 \\ 2991 & 15: 10: 37 & 1.3547 \\ 2992 & 15: 10: 38 & 1.3538 \\ 2993 & 15: 10: 39 & 1.3540 \\ 2994 & 15: 10: 40 & 1.3506 \\ 2995 & 15: 10: 41 & 1.3529 \\ 2996 & 15: 10: 42 & 1.3516 \\ 2997 & 15: 10: 43 & 1.3532 \\ 2998 & 15: 10: 44 & 1.3533 \\ 2999 & 15: 10: 45 & 1.3494 \\ 3000 & 15: 10: 46 & 1.3528 \\ 3001 & 15: 10: 47 & 1.3520 \\ 3002 & 15: 10: 48 & 1.3487 \\ 3003 & 15: 10: 49 & 1.3525 \\ 3004 & 15: 10: 50 & 1.3504 \\ 3005 & 15: 10: 51 & 1.3505 \\ 3006 & 15: 10: 52 & 1.3543 \\ 3007 & 15: 10: 53 & 1.3520\end{array}$

G - 59 
Appendix G - Fuel Data Test 4 NIST102705_1.xls

$\begin{array}{lll}3008 & 15: 10: 54 & 1.3521 \\ 3009 & 15: 10: 55 & 1.3526 \\ 3010 & 15: 10: 56 & 1.3512 \\ 3011 & 15: 10: 57 & 1.3532 \\ 3012 & 15: 10: 58 & 1.3546 \\ 3013 & 15: 10: 59 & 1.3519 \\ 3014 & 15: 11: 00 & 1.3522 \\ 3015 & 15: 11: 01 & 1.3525 \\ 3016 & 15: 11: 02 & 1.3538 \\ 3017 & 15: 11: 03 & 1.3538 \\ 3018 & 15: 11: 04 & 1.3540 \\ 3019 & 15: 11: 05 & 1.3511 \\ 3020 & 15: 11: 06 & 1.3508 \\ 3021 & 15: 11: 07 & 1.3528 \\ 3022 & 15: 11: 08 & 1.3539 \\ 3023 & 15: 11: 09 & 1.3532 \\ 3024 & 15: 11: 10 & 1.3535 \\ 3025 & 15: 11: 11 & 1.3542 \\ 3026 & 15: 11: 12 & 1.3540 \\ 3027 & 15: 11: 13 & 1.3559 \\ 3028 & 15: 11: 14 & 1.3569 \\ 3029 & 15: 11: 15 & 1.3531 \\ 3030 & 15: 11: 16 & 1.3517 \\ 3031 & 15: 11: 17 & 1.3521 \\ 3032 & 15: 11: 18 & 1.3511 \\ 3033 & 15: 11: 19 & 1.3510 \\ 3034 & 15: 11: 20 & 1.3543 \\ 3035 & 15: 11: 21 & 1.3531 \\ 3036 & 15: 11: 22 & 1.3524 \\ 3037 & 15: 11: 23 & 1.3563 \\ 3038 & 15: 11: 24 & 1.3516 \\ 3039 & 15: 11: 25 & 1.3512 \\ 3040 & 15: 11: 26 & 1.3532 \\ 3041 & 15: 11: 27 & 1.3528 \\ 3042 & 15: 11: 28 & 1.3526 \\ 3043 & 15: 11: 29 & 1.3517 \\ 3044 & 15: 11: 30 & 1.3523 \\ 3045 & 15: 11: 31 & 1.3544 \\ 3046 & 15: 11: 32 & 1.3533 \\ 3047 & 15: 11: 33 & 1.3518 \\ 3048 & 15: 11: 34 & 1.3528 \\ 3049 & 15: 11: 35 & 1.3557 \\ 3050 & 15: 11: 36 & 1.3530 \\ 3051 & 15: 11: 37 & 1.3519 \\ 3052 & 15: 11: 38 & 1.3531 \\ 3053 & 15: 11: 39 & 1.3537 \\ 3054 & 15: 11: 40 & 1.3538 \\ 3055 & 15: 11: 41 & 1.3536 \\ 3056 & 15: 11: 42 & 1.3517 \\ 3057 & 15: 11: 43 & 1.3537 \\ 3058 & 15: 11: 44 & 1.3540\end{array}$


Appendix G - Fuel Data Test 4 NIST102705_1.xls

$\begin{array}{lll}3059 & 15: 11: 45 & 1.3511 \\ 3060 & 15: 11: 46 & 1.3502 \\ 3061 & 15: 11: 47 & 1.3488 \\ 3062 & 15: 11: 48 & 1.3512 \\ 3063 & 15: 11: 49 & 1.3582 \\ 3064 & 15: 11: 50 & 1.3529 \\ 3065 & 15: 11: 51 & 1.3509 \\ 3066 & 15: 11: 52 & 1.3515 \\ 3067 & 15: 11: 53 & 1.3484 \\ 3068 & 15: 11: 54 & 1.3534 \\ 3069 & 15: 11: 55 & 1.3564 \\ 3070 & 15: 11: 56 & 1.3501 \\ 3071 & 15: 11: 57 & 1.3499 \\ 3072 & 15: 11: 58 & 1.3515 \\ 3073 & 15: 11: 59 & 1.3518 \\ 3074 & 15: 12: 00 & 1.3509 \\ 3075 & 15: 12: 01 & 1.3526 \\ 3076 & 15: 12: 02 & 1.3526 \\ 3077 & 15: 12: 03 & 1.3523 \\ 3078 & 15: 12: 04 & 1.3522 \\ 3079 & 15: 12: 05 & 1.3520 \\ 3080 & 15: 12: 06 & 1.3521 \\ 3081 & 15: 12: 07 & 1.3525 \\ 3082 & 15: 12: 08 & 1.3527 \\ 3083 & 15: 12: 09 & 1.3563 \\ 3084 & 15: 12: 10 & 1.3554 \\ 3085 & 15: 12: 11 & 1.3516 \\ 3086 & 15: 12: 12 & 1.3526 \\ 3087 & 15: 12: 13 & 1.3530 \\ 3088 & 15: 12: 14 & 1.3534 \\ 3089 & 15: 12: 15 & 1.3511 \\ 3090 & 15: 12: 16 & 1.3521 \\ 3091 & 15: 12: 17 & 1.3530 \\ 3092 & 15: 12: 18 & 1.3522 \\ 3093 & 15: 12: 19 & 1.3520 \\ 3094 & 15: 12: 20 & 1.3538 \\ 3095 & 15: 12: 21 & 1.3539 \\ 3096 & 15: 12: 22 & 1.3534 \\ 3097 & 15: 12: 23 & 1.3488 \\ 3098 & 15: 12: 24 & 1.3509 \\ 3099 & 15: 12: 25 & 1.3535 \\ 3100 & 15: 12: 26 & 1.3532 \\ 3101 & 15: 12: 27 & 1.3504 \\ 3102 & 15: 12: 28 & 1.3509 \\ 3103 & 15: 12: 29 & 1.3506 \\ 3104 & 15: 12: 30 & 1.3520 \\ 3105 & 15: 12: 31 & 1.3522 \\ 3106 & 15: 12: 32 & 1.3501 \\ 3107 & 15: 12: 33 & 1.3504 \\ 3108 & 15: 12: 34 & 1.3509 \\ 3109 & 15: 12: 35 & 1.3513\end{array}$

$G-61$ 
Appendix G - Fuel Data Test 4 NIST102705_1.xls

$\begin{array}{lll}3110 & 15: 12: 36 & 1.3527 \\ 3111 & 15: 12: 37 & 1.3533 \\ 3112 & 15: 12: 38 & 1.3522 \\ 3113 & 15: 12: 39 & 1.3510 \\ 3114 & 15: 12: 40 & 1.3535 \\ 3115 & 15: 12: 41 & 1.3542 \\ 3116 & 15: 12: 42 & 1.3504 \\ 3117 & 15: 12: 43 & 1.3533 \\ 3118 & 15: 12: 44 & 1.3534 \\ 3119 & 15: 12: 45 & 1.3530 \\ 3120 & 15: 12: 46 & 1.3560 \\ 3121 & 15: 12: 47 & 1.3545 \\ 3122 & 15: 12: 48 & 1.3546 \\ 3123 & 15: 12: 49 & 1.3533 \\ 3124 & 15: 12: 50 & 1.3528 \\ 3125 & 15: 12: 51 & 1.3555 \\ 3126 & 15: 12: 52 & 1.3519 \\ 3127 & 15: 12: 53 & 1.3501 \\ 3128 & 15: 12: 54 & 1.3531 \\ 3129 & 15: 12: 55 & 1.3509 \\ 3130 & 15: 12: 56 & 1.3531 \\ 3131 & 15: 12: 57 & 1.3552 \\ 3132 & 15: 12: 58 & 1.3528 \\ 3133 & 15: 12: 59 & 1.3508 \\ 3134 & 15: 13: 00 & 1.3512 \\ 3135 & 15: 13: 01 & 1.3541 \\ 3136 & 15: 13: 02 & 1.3546 \\ 3137 & 15: 13: 03 & 1.3519 \\ 3138 & 15: 13: 04 & 1.3515 \\ 3139 & 15: 13: 05 & 1.3535 \\ 3140 & 15: 13: 06 & 1.3544 \\ 3141 & 15: 13: 07 & 1.3533 \\ 3142 & 15: 13: 08 & 1.3522 \\ 3143 & 15: 13: 09 & 1.3545 \\ 3144 & 15: 13: 10 & 1.3511 \\ 3145 & 15: 13: 11 & 1.3527 \\ 3146 & 15: 13: 12 & 1.3539 \\ 3147 & 15: 13: 13 & 1.3515 \\ 3148 & 15: 13: 14 & 1.3540 \\ 3149 & 15: 13: 15 & 1.3520 \\ 3150 & 15: 13: 16 & 1.3491 \\ 3151 & 15: 13: 17 & 1.3520 \\ 3152 & 15: 13: 18 & 1.3540 \\ 3153 & 15: 13: 19 & 1.3526 \\ 3154 & 15: 13: 20 & 1.3521 \\ 3155 & 15: 13: 21 & 1.3527 \\ 3156 & 15: 13: 22 & 1.3525 \\ 3157 & 15: 13: 23 & 1.3509 \\ 3158 & 15: 13: 24 & 1.3536 \\ 3159 & 15: 13: 25 & 1.3543 \\ 3160 & 15: 13: 26 & 1.3529\end{array}$

$G-62$ 
Appendix G - Fuel Data Test 4 NIST102705_1.xls

$\begin{array}{lll}3161 & 15: 13: 27 & 1.3517 \\ 3162 & 15: 13: 28 & 1.3540 \\ 3163 & 15: 13: 29 & 1.3499 \\ 3164 & 15: 13: 30 & 1.3515 \\ 3165 & 15: 13: 31 & 1.3566 \\ 3166 & 15: 13: 32 & 1.3517 \\ 3167 & 15: 13: 33 & 1.3500 \\ 3168 & 15: 13: 34 & 1.3503 \\ 3169 & 15: 13: 35 & 1.3511 \\ 3170 & 15: 13: 36 & 1.3516 \\ 3171 & 15: 13: 37 & 1.3519 \\ 3172 & 15: 13: 38 & 1.3498 \\ 3173 & 15: 13: 39 & 1.3548 \\ 3174 & 15: 13: 40 & 1.3531 \\ 3175 & 15: 13: 41 & 1.3503 \\ 3176 & 15: 13: 42 & 1.3518 \\ 3177 & 15: 13: 43 & 1.3569 \\ 3178 & 15: 13: 44 & 1.3533 \\ 3179 & 15: 13: 45 & 1.3498 \\ 3180 & 15: 13: 46 & 1.3521 \\ 3181 & 15: 13: 47 & 1.3541 \\ 3182 & 15: 13: 48 & 1.3523 \\ 3183 & 15: 13: 49 & 1.3522 \\ 3184 & 15: 13: 50 & 1.3536 \\ 3185 & 15: 13: 51 & 1.3495 \\ 3186 & 15: 13: 52 & 1.3502 \\ 3187 & 15: 13: 53 & 1.3547 \\ 3188 & 15: 13: 54 & 1.3539 \\ 3189 & 15: 13: 55 & 1.3516 \\ 3190 & 15: 13: 56 & 1.3521 \\ 3191 & 15: 13: 57 & 1.3524 \\ 3192 & 15: 13: 58 & 1.3561 \\ 3193 & 15: 13: 59 & 1.3568 \\ 3194 & 15: 14: 00 & 1.3521 \\ 3195 & 15: 14: 01 & 1.3526 \\ 3196 & 15: 14: 02 & 1.3515 \\ 3197 & 15: 14: 03 & 1.3509 \\ 3198 & 15: 14: 04 & 1.3532 \\ 3199 & 15: 14: 05 & 1.3512 \\ 3200 & 15: 14: 06 & 1.3489 \\ 3201 & 15: 14: 07 & 1.3507 \\ 3202 & 15: 14: 08 & 1.3536 \\ 3203 & 15: 14: 09 & 1.3539 \\ 3204 & 15: 14: 10 & 1.3528 \\ 3205 & 15: 14: 11 & 1.3513 \\ 3206 & 15: 14: 12 & 1.3511 \\ 3207 & 15: 14: 13 & 1.3527 \\ 3208 & 15: 14: 14 & 1.3563 \\ 3209 & 15: 14: 15 & 1.3542 \\ 3210 & 15: 14: 16 & 1.3530 \\ 3211 & 15: 14: 17 & 1.3539\end{array}$

$G-63$ 
Appendix G - Fuel Data Test 4 NIST102705_1.xls

$\begin{array}{lll}3212 & 15: 14: 18 & 1.3528 \\ 3213 & 15: 14: 19 & 1.3540 \\ 3214 & 15: 14: 20 & 1.3557 \\ 3215 & 15: 14: 21 & 1.3534 \\ 3216 & 15: 14: 22 & 1.3537 \\ 3217 & 15: 14: 23 & 1.3536 \\ 3218 & 15: 14: 24 & 1.3547 \\ 3219 & 15: 14: 25 & 1.3547 \\ 3220 & 15: 14: 26 & 1.3526 \\ 3221 & 15: 14: 27 & 1.3536 \\ 3222 & 15: 14: 28 & 1.3530 \\ 3223 & 15: 14: 29 & 1.3522 \\ 3224 & 15: 14: 30 & 1.3537 \\ 3225 & 15: 14: 31 & 1.3520 \\ 3226 & 15: 14: 32 & 1.3504 \\ 3227 & 15: 14: 33 & 1.3504 \\ 3228 & 15: 14: 34 & 1.3579 \\ 3229 & 15: 14: 35 & 1.3559 \\ 3230 & 15: 14: 36 & 1.3528 \\ 3231 & 15: 14: 37 & 1.3528 \\ 3232 & 15: 14: 38 & 1.3505 \\ 3233 & 15: 14: 39 & 1.3496 \\ 3234 & 15: 14: 40 & 1.3526 \\ 3235 & 15: 14: 41 & 1.3532 \\ 3236 & 15: 14: 42 & 1.3520 \\ 3237 & 15: 14: 43 & 1.3514 \\ 3238 & 15: 14: 44 & 1.3502 \\ 3239 & 15: 14: 45 & 1.3515 \\ 3240 & 15: 14: 46 & 1.3503 \\ 3241 & 15: 14: 47 & 1.3490 \\ 3242 & 15: 14: 48 & 1.3521 \\ 3243 & 15: 14: 49 & 1.3515 \\ 3244 & 15: 14: 50 & 1.3503 \\ 3245 & 15: 14: 51 & 1.3523 \\ 3246 & 15: 14: 52 & 1.3533 \\ 3247 & 15: 14: 53 & 1.3519 \\ 3248 & 15: 14: 54 & 1.3513 \\ 3249 & 15: 14: 55 & 1.3525 \\ 3250 & 15: 14: 56 & 1.3507 \\ 3251 & 15: 14: 57 & 1.3504 \\ 3252 & 15: 14: 58 & 1.3529 \\ 3253 & 15: 14: 59 & 1.3511 \\ 3254 & 15: 15: 00 & 1.3494 \\ 3255 & 15: 15: 01 & 1.3533 \\ 3256 & 15: 15: 02 & 1.3534 \\ 3257 & 15: 15: 03 & 1.3502 \\ 3258 & 15: 15: 04 & 1.3513 \\ 3259 & 15: 15: 05 & 1.3536 \\ 3260 & 15: 15: 06 & 1.3546 \\ 3261 & 15: 15: 07 & 1.3522 \\ 3262 & 15: 15: 08 & 1.3502 \\ & & \end{array}$

$G-64$ 
Appendix G - Fuel Data Test 4 NIST102705_1.xls

$\begin{array}{lll}3263 & 15: 15: 09 & 1.3517 \\ 3264 & 15: 15: 10 & 1.3505 \\ 3265 & 15: 15: 11 & 1.3532 \\ 3266 & 15: 15: 12 & 1.3547 \\ 3267 & 15: 15: 13 & 1.3553 \\ 3268 & 15: 15: 14 & 1.3499 \\ 3269 & 15: 15: 15 & 1.3523 \\ 3270 & 15: 15: 16 & 1.3524 \\ 3271 & 15: 15: 17 & 1.3517 \\ 3272 & 15: 15: 18 & 1.3516 \\ 3273 & 15: 15: 19 & 1.3527 \\ 3274 & 15: 15: 20 & 1.3512 \\ 3275 & 15: 15: 21 & 1.3514 \\ 3276 & 15: 15: 22 & 1.3517 \\ 3277 & 15: 15: 23 & 1.3539 \\ 3278 & 15: 15: 24 & 1.3551 \\ 3279 & 15: 15: 25 & 1.3519 \\ 3280 & 15: 15: 26 & 1.3505 \\ 3281 & 15: 15: 27 & 1.3538 \\ 3282 & 15: 15: 28 & 1.3550 \\ 3283 & 15: 15: 29 & 1.3540 \\ 3284 & 15: 15: 30 & 1.3511 \\ 3285 & 15: 15: 31 & 1.3487 \\ 3286 & 15: 15: 32 & 1.3523 \\ 3287 & 15: 15: 33 & 1.3544 \\ 3288 & 15: 15: 34 & 1.3517 \\ 3289 & 15: 15: 35 & 1.3495 \\ 3290 & 15: 15: 36 & 1.3520 \\ 3291 & 15: 15: 37 & 1.3534 \\ 3292 & 15: 15: 38 & 1.3509 \\ 3293 & 15: 15: 39 & 1.3513 \\ 3294 & 15: 15: 40 & 1.3526 \\ 3295 & 15: 15: 41 & 1.3496 \\ 3296 & 15: 15: 42 & 1.3514 \\ 3297 & 15: 15: 43 & 1.3519 \\ 3298 & 15: 15: 44 & 1.3522 \\ 3299 & 15: 15: 45 & 1.3505 \\ 3300 & 15: 15: 46 & 1.3483 \\ 3301 & 15: 15: 47 & 1.3510 \\ 3302 & 15: 15: 48 & 1.3532 \\ 3303 & 15: 15: 49 & 1.3556 \\ 3304 & 15: 15: 50 & 1.3564 \\ 3305 & 15: 15: 51 & 1.3540 \\ 3306 & 15: 15: 52 & 1.3511 \\ 3307 & 15: 15: 53 & 1.3529 \\ 3308 & 15: 15: 54 & 1.3542 \\ 3309 & 15: 15: 55 & 1.3541 \\ 3310 & 15: 15: 56 & 1.3523 \\ 3311 & 15: 15: 57 & 1.3514 \\ 3312 & 15: 15: 58 & 1.3514 \\ 3313 & 15: 15: 59 & 1.3539\end{array}$

$G-65$ 
Appendix G - Fuel Data Test 4 NIST102705_1.xls

$\begin{array}{lll}3314 & 15: 16: 00 & 1.3525 \\ 3315 & 15: 16: 01 & 1.3518 \\ 3316 & 15: 16: 02 & 1.3525 \\ 3317 & 15: 16: 03 & 1.3523 \\ 3318 & 15: 16: 04 & 1.3545 \\ 3319 & 15: 16: 05 & 1.3541 \\ 3320 & 15: 16: 06 & 1.3505 \\ 3321 & 15: 16: 07 & 1.3513 \\ 3322 & 15: 16: 08 & 1.3544 \\ 3323 & 15: 16: 09 & 1.3553 \\ 3324 & 15: 16: 10 & 1.3530 \\ 3325 & 15: 16: 11 & 1.3526 \\ 3326 & 15: 16: 12 & 1.3515 \\ 3327 & 15: 16: 13 & 1.3524 \\ 3328 & 15: 16: 14 & 1.3556 \\ 3329 & 15: 16: 15 & 1.3524 \\ 3330 & 15: 16: 16 & 1.3512 \\ 3331 & 15: 16: 17 & 1.3475 \\ 3332 & 15: 16: 18 & 1.3466 \\ 3333 & 15: 16: 19 & 1.3498 \\ 3334 & 15: 16: 20 & 1.3505 \\ 3335 & 15: 16: 21 & 1.3546 \\ 3336 & 15: 16: 22 & 1.3532 \\ 3337 & 15: 16: 23 & 1.3509 \\ 3338 & 15: 16: 24 & 1.3513 \\ 3339 & 15: 16: 25 & 1.3520 \\ 3340 & 15: 16: 26 & 1.3535 \\ 3341 & 15: 16: 27 & 1.3533 \\ 3342 & 15: 16: 28 & 1.3528 \\ 3343 & 15: 16: 29 & 1.3532 \\ 3344 & 15: 16: 30 & 1.3516 \\ 3345 & 15: 16: 31 & 1.3511 \\ 3346 & 15: 16: 32 & 1.3512 \\ 3347 & 15: 16: 33 & 1.3502 \\ 3348 & 15: 16: 34 & 1.3520 \\ 3349 & 15: 16: 35 & 1.3521 \\ 3350 & 15: 16: 36 & 1.3544 \\ 3351 & 15: 16: 37 & 1.3520 \\ 3352 & 15: 16: 38 & 1.3514 \\ 3353 & 15: 16: 39 & 1.3541 \\ 3354 & 15: 16: 40 & 1.3542 \\ 3355 & 15: 16: 41 & 1.3529 \\ 3356 & 15: 16: 42 & 1.3524 \\ 3357 & 15: 16: 43 & 1.3518 \\ 3358 & 15: 16: 44 & 1.3526 \\ 3359 & 15: 16: 45 & 1.3528 \\ 3360 & 15: 16: 46 & 1.3533 \\ 3361 & 15: 16: 47 & 1.3517 \\ 3362 & 15: 16: 48 & 1.3494 \\ 3363 & 15: 16: 49 & 1.3527 \\ 3364 & 15: 16: 50 & 1.3545\end{array}$


Appendix G - Fuel Data Test 4 NIST102705_1.xls

$\begin{array}{lll}3365 & 15: 16: 51 & 1.3514 \\ 3366 & 15: 16: 52 & 1.3503 \\ 3367 & 15: 16: 53 & 1.3507 \\ 3368 & 15: 16: 54 & 1.3529 \\ 3369 & 15: 16: 55 & 1.3524 \\ 3370 & 15: 16: 56 & 1.3527 \\ 3371 & 15: 16: 57 & 1.3501 \\ 3372 & 15: 16: 58 & 1.3502 \\ 3373 & 15: 16: 59 & 1.3535 \\ 3374 & 15: 17: 00 & 1.3530 \\ 3375 & 15: 17: 01 & 1.3521 \\ 3376 & 15: 17: 02 & 1.3533 \\ 3377 & 15: 17: 03 & 1.3506 \\ 3378 & 15: 17: 04 & 1.3504 \\ 3379 & 15: 17: 05 & 1.3532 \\ 3380 & 15: 17: 06 & 1.3516 \\ 3381 & 15: 17: 07 & 1.3518 \\ 3382 & 15: 17: 08 & 1.3515 \\ 3383 & 15: 17: 09 & 1.3544 \\ 3384 & 15: 17: 10 & 1.3532 \\ 3385 & 15: 17: 11 & 1.3519 \\ 3386 & 15: 17: 12 & 1.3521 \\ 3387 & 15: 17: 13 & 1.3503 \\ 3388 & 15: 17: 14 & 1.3492 \\ 3389 & 15: 17: 15 & 1.3501 \\ 3390 & 15: 17: 16 & 1.3544 \\ 3391 & 15: 17: 17 & 1.3535 \\ 3392 & 15: 17: 18 & 1.3515 \\ 3393 & 15: 17: 19 & 1.3508 \\ 3394 & 15: 17: 20 & 1.3495 \\ 3395 & 15: 17: 21 & 1.3480 \\ 3396 & 15: 17: 22 & 1.3526 \\ 3397 & 15: 17: 23 & 1.3535 \\ 3398 & 15: 17: 24 & 1.3531 \\ 3399 & 15: 17: 25 & 1.3506 \\ 3400 & 15: 17: 26 & 1.3522 \\ 3401 & 15: 17: 27 & 1.3547 \\ 3402 & 15: 17: 28 & 1.3523 \\ 3403 & 15: 17: 29 & 1.3529 \\ 3404 & 15: 17: 30 & 1.3542 \\ 3405 & 15: 17: 31 & 1.3538 \\ 3406 & 15: 17: 32 & 1.3523 \\ 3407 & 15: 17: 33 & 1.3504 \\ 3408 & 15: 17: 34 & 1.3522 \\ 3409 & 15: 17: 35 & 1.3494 \\ 3410 & 15: 17: 36 & 1.3514 \\ 3411 & 15: 17: 37 & 1.3533 \\ 3412 & 15: 17: 38 & 1.3509 \\ 3413 & 15: 17: 39 & 1.3487 \\ 3414 & 15: 17: 40 & 1.3513 \\ 3415 & 15: 17: 41 & 1.3525\end{array}$

G - 67 
Appendix G - Fuel Data Test 4 NIST102705_1.xls

$\begin{array}{lll}3416 & 15: 17: 42 & 1.3551 \\ 3417 & 15: 17: 43 & 1.3519 \\ 3418 & 15: 17: 44 & 1.3491 \\ 3419 & 15: 17: 45 & 1.3511 \\ 3420 & 15: 17: 46 & 1.3505 \\ 3421 & 15: 17: 47 & 1.3525 \\ 3422 & 15: 17: 48 & 1.3531 \\ 3423 & 15: 17: 49 & 1.3506 \\ 3424 & 15: 17: 50 & 1.3497 \\ 3425 & 15: 17: 51 & 1.3519 \\ 3426 & 15: 17: 52 & 1.3529 \\ 3427 & 15: 17: 53 & 1.3526 \\ 3428 & 15: 17: 54 & 1.3520 \\ 3429 & 15: 17: 55 & 1.3538 \\ 3430 & 15: 17: 56 & 1.3540 \\ 3431 & 15: 17: 57 & 1.3525 \\ 3432 & 15: 17: 58 & 1.3495 \\ 3433 & 15: 17: 59 & 1.3501 \\ 3434 & 15: 18: 00 & 1.3493 \\ 3435 & 15: 18: 01 & 1.3504 \\ 3436 & 15: 18: 02 & 1.3537 \\ 3437 & 15: 18: 03 & 1.3541 \\ 3438 & 15: 18: 04 & 1.3507 \\ 3439 & 15: 18: 05 & 1.3562 \\ 3440 & 15: 18: 06 & 1.3569 \\ 3441 & 15: 18: 07 & 1.3526 \\ 3442 & 15: 18: 08 & 1.3534 \\ 3443 & 15: 18: 09 & 1.3513 \\ 3444 & 15: 18: 10 & 1.3510 \\ 3445 & 15: 18: 11 & 1.3495 \\ 3446 & 15: 18: 12 & 1.3482 \\ 3447 & 15: 18: 13 & 1.3482 \\ 3448 & 15: 18: 14 & 1.3502 \\ 3449 & 15: 18: 15 & 1.3487 \\ 3450 & 15: 18: 16 & 1.3508 \\ 3451 & 15: 18: 17 & 1.3538 \\ 3452 & 15: 18: 18 & 1.3532 \\ 3453 & 15: 18: 19 & 1.3518 \\ 3454 & 15: 18: 20 & 1.3520 \\ 3455 & 15: 18: 21 & 1.3492 \\ 3456 & 15: 18: 22 & 1.3531 \\ 3457 & 15: 18: 23 & 1.3542 \\ 3458 & 15: 18: 24 & 1.3526 \\ 3459 & 15: 18: 25 & 1.3511 \\ 3460 & 15: 18: 26 & 1.3522 \\ 3461 & 15: 18: 27 & 1.3508 \\ 3462 & 15: 18: 28 & 1.3497 \\ 3463 & 15: 18: 29 & 1.3525 \\ 3464 & 15: 18: 30 & 1.3527 \\ 3465 & 15: 18: 31 & 1.3498 \\ 3466 & 15: 18: 32 & 1.3525\end{array}$


Appendix G - Fuel Data Test 4 NIST102705_1.xls

$\begin{array}{lll}3467 & 15: 18: 33 & 1.3527 \\ 3468 & 15: 18: 34 & 1.3495 \\ 3469 & 15: 18: 35 & 1.3507 \\ 3470 & 15: 18: 36 & 1.3492 \\ 3471 & 15: 18: 37 & 1.3479 \\ 3472 & 15: 18: 38 & 1.3519 \\ 3473 & 15: 18: 39 & 1.3545 \\ 3474 & 15: 18: 40 & 1.3530 \\ 3475 & 15: 18: 41 & 1.3501 \\ 3476 & 15: 18: 42 & 1.3481 \\ 3477 & 15: 18: 43 & 1.3479 \\ 3478 & 15: 18: 44 & 1.3505 \\ 3479 & 15: 18: 45 & 1.3519 \\ 3480 & 15: 18: 46 & 1.3523 \\ 3481 & 15: 18: 47 & 1.3517 \\ 3482 & 15: 18: 48 & 1.3497 \\ 3483 & 15: 18: 49 & 1.3502 \\ 3484 & 15: 18: 50 & 1.3515 \\ 3485 & 15: 18: 51 & 1.3479 \\ 3486 & 15: 18: 52 & 1.3513 \\ 3487 & 15: 18: 53 & 1.3513 \\ 3488 & 15: 18: 54 & 1.3513 \\ 3489 & 15: 18: 55 & 1.3490 \\ 3490 & 15: 18: 56 & 1.3478 \\ 3491 & 15: 18: 57 & 1.3533 \\ 3492 & 15: 18: 58 & 1.3523 \\ 3493 & 15: 18: 59 & 1.3518 \\ 3494 & 15: 19: 00 & 1.3534 \\ 3495 & 15: 19: 01 & 1.3520 \\ 3496 & 15: 19: 02 & 1.3506 \\ 3497 & 15: 19: 03 & 1.3519 \\ 3498 & 15: 19: 04 & 1.3525 \\ 3499 & 15: 19: 05 & 1.3517 \\ 3500 & 15: 19: 06 & 1.3517 \\ 3501 & 15: 19: 07 & 1.3517 \\ 3502 & 15: 19: 08 & 1.3504 \\ 3503 & 15: 19: 09 & 1.3487 \\ 3504 & 15: 19: 10 & 1.3503 \\ 3505 & 15: 19: 11 & 1.3527 \\ 3506 & 15: 19: 12 & 1.3511 \\ 3507 & 15: 19: 13 & 1.3507 \\ 3508 & 15: 19: 14 & 1.3500 \\ 3509 & 15: 19: 15 & 1.3507 \\ 3510 & 15: 19: 16 & 1.3507 \\ 3511 & 15: 19: 17 & 1.3492 \\ 3512 & 15: 19: 18 & 1.3513 \\ 3513 & 15: 19: 19 & 1.3513 \\ 3514 & 15: 19: 20 & 1.3517 \\ 3515 & 15: 19: 21 & 1.3518 \\ 3516 & 15: 19: 22 & 1.3501 \\ 3517 & 15: 19: 23 & 1.3500\end{array}$

G - 69 
Appendix G - Fuel Data Test 4 NIST102705_1.xIs

$\begin{array}{lll}3518 & 15: 19: 24 & 1.3528 \\ 3519 & 15: 19: 25 & 1.3534 \\ 3520 & 15: 19: 26 & 1.3513 \\ 3521 & 15: 19: 27 & 1.3510 \\ 3522 & 15: 19: 28 & 1.3545 \\ 3523 & 15: 19: 29 & 1.3521 \\ 3524 & 15: 19: 30 & 1.3518 \\ 3525 & 15: 19: 31 & 1.3522 \\ 3526 & 15: 19: 32 & 1.3510 \\ 3527 & 15: 19: 33 & 1.3506 \\ 3528 & 15: 19: 34 & 1.3521 \\ 3529 & 15: 19: 35 & 1.3526 \\ 3530 & 15: 19: 36 & 1.3533 \\ 3531 & 15: 19: 37 & 1.3516 \\ 3532 & 15: 19: 38 & 1.3515 \\ 3533 & 15: 19: 39 & 1.3521 \\ 3534 & 15: 19: 40 & 1.3522 \\ 3535 & 15: 19: 41 & 1.3533 \\ 3536 & 15: 19: 42 & 1.3541 \\ 3537 & 15: 19: 43 & 1.3498 \\ 3538 & 15: 19: 44 & 1.3504 \\ 3539 & 15: 19: 45 & 1.3527 \\ 3540 & 15: 19: 46 & 1.3521 \\ 3541 & 15: 19: 47 & 1.3500 \\ 3542 & 15: 19: 48 & 1.3542 \\ 3543 & 15: 19: 49 & 1.3547 \\ 3544 & 15: 19: 50 & 1.3545 \\ 3545 & 15: 19: 51 & 1.3532 \\ 3546 & 15: 19: 52 & 1.3512 \\ 3547 & 15: 19: 53 & 1.3505 \\ 3548 & 15: 19: 54 & 1.3489 \\ 3549 & 15: 19: 55 & 1.3502 \\ 3550 & 15: 19: 56 & 1.3528 \\ 3551 & 15: 19: 57 & 1.3525 \\ 3552 & 15: 19: 58 & 1.3510 \\ 3553 & 15: 19: 59 & 1.3523 \\ 3554 & 15: 20: 00 & 1.3495 \\ 3555 & 15: 20: 01 & 1.3512 \\ 3556 & 15: 20: 02 & 1.3535 \\ 3557 & 15: 20: 03 & 1.3509 \\ 3558 & 15: 20: 04 & 1.3498 \\ 3559 & 15: 20: 05 & 1.3510 \\ 3560 & 15: 20: 06 & 1.3523 \\ 3561 & 15: 20: 07 & 1.3497 \\ 3562 & 15: 20: 08 & 1.3519 \\ 3563 & 15: 20: 09 & 1.3530 \\ 3564 & 15: 20: 10 & 1.3499 \\ 3565 & 15: 20: 11 & 1.3510 \\ 3566 & 15: 20: 12 & 1.3508 \\ 3567 & 15: 20: 13 & 1.3502 \\ 3568 & 15: 20: 14 & 1.3513\end{array}$


Appendix G - Fuel Data Test 4 NIST102705_1.xls

$\begin{array}{lll}3569 & 15: 20: 15 & 1.3496 \\ 3570 & 15: 20: 16 & 1.3529 \\ 3571 & 15: 20: 17 & 1.3518 \\ 3572 & 15: 20: 18 & 1.3479 \\ 3573 & 15: 20: 19 & 1.3510 \\ 3574 & 15: 20: 20 & 1.3517 \\ 3575 & 15: 20: 21 & 1.3489 \\ 3576 & 15: 20: 22 & 1.3525 \\ 3577 & 15: 20: 23 & 1.3539 \\ 3578 & 15: 20: 24 & 1.3518 \\ 3579 & 15: 20: 25 & 1.3508 \\ 3580 & 15: 20: 26 & 1.3508 \\ 3581 & 15: 20: 27 & 1.3501 \\ 3582 & 15: 20: 28 & 1.3515 \\ 3583 & 15: 20: 29 & 1.3524 \\ 3584 & 15: 20: 30 & 1.3521 \\ 3585 & 15: 20: 31 & 1.3501 \\ 3586 & 15: 20: 32 & 1.3502 \\ 3587 & 15: 20: 33 & 1.3519 \\ 3588 & 15: 20: 34 & 1.3509 \\ 3589 & 15: 20: 35 & 1.3474 \\ 3590 & 15: 20: 36 & 1.3489 \\ 3591 & 15: 20: 37 & 1.3515 \\ 3592 & 15: 20: 38 & 1.3500 \\ 3593 & 15: 20: 39 & 1.3508 \\ 3594 & 15: 20: 40 & 1.3522 \\ 3595 & 15: 20: 41 & 1.3529 \\ 3596 & 15: 20: 42 & 1.3532 \\ 3597 & 15: 20: 43 & 1.3539 \\ 3598 & 15: 20: 44 & 1.3505 \\ 3599 & 15: 20: 45 & 1.3491 \\ 3600 & 15: 20: 46 & 1.3505 \\ 3601 & 15: 20: 47 & 1.3541 \\ 3602 & 15: 20: 48 & 1.3533 \\ 3603 & 15: 20: 49 & 1.3529 \\ 3604 & 15: 20: 50 & 1.3517 \\ 3605 & 15: 20: 51 & 1.3531 \\ 3606 & 15: 20: 52 & 1.3538 \\ 3607 & 15: 20: 53 & 1.3513 \\ 3608 & 15: 20: 54 & 1.3526 \\ 3609 & 15: 20: 55 & 1.3540 \\ 3610 & 15: 20: 56 & 1.3503 \\ 3611 & 15: 20: 57 & 1.3491 \\ 3612 & 15: 20: 58 & 1.3517 \\ 3613 & 15: 20: 59 & 1.3498 \\ 3614 & 15: 21: 00 & 1.3515 \\ 3615 & 15: 21: 01 & 1.3538 \\ 3616 & 15: 21: 02 & 1.3538 \\ 3617 & 15: 21: 03 & 1.3509 \\ 3618 & 15: 21: 04 & 1.3516 \\ 3619 & 15: 21: 05 & 1.3514\end{array}$


Appendix G - Fuel Data Test 4 NIST102705_1.xls

$\begin{array}{lll}3620 & 15: 21: 06 & 1.3494 \\ 3621 & 15: 21: 07 & 1.3486 \\ 3622 & 15: 21: 08 & 1.3535 \\ 3623 & 15: 21: 09 & 1.3537 \\ 3624 & 15: 21: 10 & 1.3545 \\ 3625 & 15: 21: 11 & 1.3517 \\ 3626 & 15: 21: 12 & 1.3501 \\ 3627 & 15: 21: 13 & 1.3528 \\ 3628 & 15: 21: 14 & 1.3533 \\ 3629 & 15: 21: 15 & 1.3518 \\ 3630 & 15: 21: 16 & 1.3507 \\ 3631 & 15: 21: 17 & 1.3504 \\ 3632 & 15: 21: 18 & 1.3507 \\ 3633 & 15: 21: 19 & 1.3540 \\ 3634 & 15: 21: 20 & 1.3503 \\ 3635 & 15: 21: 21 & 1.3513 \\ 3636 & 15: 21: 22 & 1.3515 \\ 3637 & 15: 21: 23 & 1.3528 \\ 3638 & 15: 21: 24 & 1.3570 \\ 3639 & 15: 21: 25 & 1.3533 \\ 3640 & 15: 21: 26 & 1.3507 \\ 3641 & 15: 21: 27 & 1.3520 \\ 3642 & 15: 21: 28 & 1.3537 \\ 3643 & 15: 21: 29 & 1.3549 \\ 3644 & 15: 21: 30 & 1.3525 \\ 3645 & 15: 21: 31 & 1.3514 \\ 3646 & 15: 21: 32 & 1.3526 \\ 3647 & 15: 21: 33 & 1.3519 \\ 3648 & 15: 21: 34 & 1.3517 \\ 3649 & 15: 21: 35 & 1.3538 \\ 3650 & 15: 21: 36 & 1.3531 \\ 3651 & 15: 21: 37 & 1.3510 \\ 3652 & 15: 21: 38 & 1.3501 \\ 3653 & 15: 21: 39 & 1.3531 \\ 3654 & 15: 21: 40 & 1.3530 \\ 3655 & 15: 21: 41 & 1.3520 \\ 3656 & 15: 21: 42 & 1.3524 \\ 3657 & 15: 21: 43 & 1.3520 \\ 3658 & 15: 21: 44 & 1.3519 \\ 3659 & 15: 21: 45 & 1.3532 \\ 3660 & 15: 21: 46 & 1.3533 \\ 3661 & 15: 21: 47 & 1.3501 \\ 3662 & 15: 21: 48 & 1.3521 \\ 3663 & 15: 21: 49 & 1.3543 \\ 3664 & 15: 21: 50 & 1.3535 \\ 3665 & 15: 21: 51 & 1.3512 \\ 3666 & 15: 21: 52 & 1.3510 \\ 3667 & 15: 21: 53 & 1.3513 \\ 3668 & 15: 21: 54 & 1.3507 \\ 3669 & 15: 21: 55 & 1.3501 \\ 3670 & 15: 21: 56 & 1.3528\end{array}$


Appendix G - Fuel Data Test 4 NIST102705_1.xls

$\begin{array}{lll}3671 & 15: 21: 57 & 1.3520 \\ 3672 & 15: 21: 58 & 1.3486 \\ 3673 & 15: 21: 59 & 1.3519 \\ 3674 & 15: 22: 00 & 1.3561 \\ 3675 & 15: 22: 01 & 1.3522 \\ 3676 & 15: 22: 02 & 1.3511 \\ 3677 & 15: 22: 03 & 1.3493 \\ 3678 & 15: 22: 04 & 1.3480 \\ 3679 & 15: 22: 05 & 1.3537 \\ 3680 & 15: 22: 06 & 1.3544 \\ 3681 & 15: 22: 07 & 1.3509 \\ 3682 & 15: 22: 08 & 1.3516 \\ 3683 & 15: 22: 09 & 1.3529 \\ 3684 & 15: 22: 10 & 1.3543 \\ 3685 & 15: 22: 11 & 1.3552 \\ 3686 & 15: 22: 12 & 1.3524 \\ 3687 & 15: 22: 13 & 1.3513 \\ 3688 & 15: 22: 14 & 1.3479 \\ 3689 & 15: 22: 15 & 1.3496 \\ 3690 & 15: 22: 16 & 1.3548 \\ 3691 & 15: 22: 17 & 1.3544 \\ 3692 & 15: 22: 18 & 1.3507 \\ 3693 & 15: 22: 19 & 1.3493 \\ 3694 & 15: 22: 20 & 1.3498 \\ 3695 & 15: 22: 21 & 1.3525 \\ 3696 & 15: 22: 22 & 1.3516 \\ 3697 & 15: 22: 23 & 1.3517 \\ 3698 & 15: 22: 24 & 1.3526 \\ 3699 & 15: 22: 25 & 1.3499 \\ 3700 & 15: 22: 26 & 1.3519 \\ 3701 & 15: 22: 27 & 1.3527 \\ 3702 & 15: 22: 28 & 1.3508 \\ 3703 & 15: 22: 29 & 1.3512 \\ 3704 & 15: 22: 30 & 1.3514 \\ 3705 & 15: 22: 31 & 1.3502 \\ 3706 & 15: 22: 32 & 1.3511 \\ 3707 & 15: 22: 33 & 1.3511 \\ 3708 & 15: 22: 34 & 1.3515 \\ 3709 & 15: 22: 35 & 1.3515 \\ 3710 & 15: 22: 36 & 1.3521 \\ 3711 & 15: 22: 37 & 1.3541 \\ 3712 & 15: 22: 38 & 1.3526 \\ 3713 & 15: 22: 39 & 1.3509 \\ 3714 & 15: 22: 40 & 1.3520 \\ 3715 & 15: 22: 41 & 1.3533 \\ 3716 & 15: 22: 42 & 1.3521 \\ 3717 & 15: 22: 43 & 1.3516 \\ 3718 & 15: 22: 44 & 1.3539 \\ 3719 & 15: 22: 45 & 1.3531 \\ 3720 & 15: 22: 46 & 1.3519 \\ 3721 & 15: 22: 47 & 1.3540\end{array}$


Appendix G - Fuel Data Test 4 NIST102705_1.xls

$\begin{array}{lll}3722 & 15: 22: 48 & 1.3517 \\ 3723 & 15: 22: 49 & 1.3476 \\ 3724 & 15: 22: 50 & 1.3518 \\ 3725 & 15: 22: 51 & 1.3522 \\ 3726 & 15: 22: 52 & 1.3511 \\ 3727 & 15: 22: 53 & 1.3511 \\ 3728 & 15: 22: 54 & 1.3522 \\ 3729 & 15: 22: 55 & 1.3493 \\ 3730 & 15: 22: 56 & 1.3474 \\ 3731 & 15: 22: 57 & 1.3503 \\ 3732 & 15: 22: 58 & 1.3532 \\ 3733 & 15: 22: 59 & 1.3526 \\ 3734 & 15: 23: 00 & 1.3523 \\ 3735 & 15: 23: 01 & 1.3515 \\ 3736 & 15: 23: 02 & 1.3536 \\ 3737 & 15: 23: 03 & 1.3529 \\ 3738 & 15: 23: 04 & 1.3494 \\ 3739 & 15: 23: 05 & 1.3500 \\ 3740 & 15: 23: 06 & 1.3519 \\ 3741 & 15: 23: 07 & 1.3500 \\ 3742 & 15: 23: 08 & 1.3521 \\ 3743 & 15: 23: 09 & 1.3503 \\ 3744 & 15: 23: 10 & 1.3505 \\ 3745 & 15: 23: 11 & 1.3524 \\ 3746 & 15: 23: 12 & 1.3523 \\ 3747 & 15: 23: 13 & 1.3492 \\ 3748 & 15: 23: 14 & 1.3504 \\ 3749 & 15: 23: 15 & 1.3524 \\ 3750 & 15: 23: 16 & 1.3508 \\ 3751 & 15: 23: 17 & 1.3515 \\ 3752 & 15: 23: 18 & 1.3520 \\ 3753 & 15: 23: 19 & 1.3510 \\ 3754 & 15: 23: 20 & 1.3518 \\ 3755 & 15: 23: 21 & 1.3502 \\ 3756 & 15: 23: 22 & 1.3498 \\ 3757 & 15: 23: 23 & 1.3516 \\ 3758 & 15: 23: 24 & 1.3501 \\ 3759 & 15: 23: 25 & 1.3489 \\ 3760 & 15: 23: 26 & 1.3504 \\ 3761 & 15: 23: 27 & 1.3502 \\ 3762 & 15: 23: 28 & 1.3510 \\ 3763 & 15: 23: 29 & 1.3523 \\ 3764 & 15: 23: 30 & 1.3508 \\ 3765 & 15: 23: 31 & 1.3504 \\ 3766 & 15: 23: 32 & 1.3507 \\ 3767 & 15: 23: 33 & 1.3534 \\ 3768 & 15: 23: 34 & 1.3519 \\ 3769 & 15: 23: 35 & 1.3489 \\ 3770 & 15: 23: 36 & 1.3493 \\ 3771 & 15: 23: 37 & 1.3521 \\ 3772 & 15: 23: 38 & 1.3495\end{array}$


Appendix G - Fuel Data Test 4 NIST102705_1.xls

$\begin{array}{lll}3773 & 15: 23: 39 & 1.3512 \\ 3774 & 15: 23: 40 & 1.3539 \\ 3775 & 15: 23: 41 & 1.3529 \\ 3776 & 15: 23: 42 & 1.3495 \\ 3777 & 15: 23: 43 & 1.3513 \\ 3778 & 15: 23: 44 & 1.3526 \\ 3779 & 15: 23: 45 & 1.3501 \\ 3780 & 15: 23: 46 & 1.3520 \\ 3781 & 15: 23: 47 & 1.3498 \\ 3782 & 15: 23: 48 & 1.3484 \\ 3783 & 15: 23: 49 & 1.3515 \\ 3784 & 15: 23: 50 & 1.3520 \\ 3785 & 15: 23: 51 & 1.3512 \\ 3786 & 15: 23: 52 & 1.3508 \\ 3787 & 15: 23: 53 & 1.3509 \\ 3788 & 15: 23: 54 & 1.3529 \\ 3789 & 15: 23: 55 & 1.3538 \\ 3790 & 15: 23: 56 & 1.3507 \\ 3791 & 15: 23: 57 & 1.3502 \\ 3792 & 15: 23: 58 & 1.3528 \\ 3793 & 15: 23: 59 & 1.3528 \\ 3794 & 15: 24: 00 & 1.3518 \\ 3795 & 15: 24: 01 & 1.3515 \\ 3796 & 15: 24: 02 & 1.3479 \\ 3797 & 15: 24: 03 & 1.3491 \\ 3798 & 15: 24: 04 & 1.3542 \\ 3799 & 15: 24: 05 & 1.3523 \\ 3800 & 15: 24: 06 & 1.3483 \\ 3801 & 15: 24: 07 & 1.3524 \\ 3802 & 15: 24: 08 & 1.3549 \\ 3803 & 15: 24: 09 & 1.3517 \\ 3804 & 15: 24: 10 & 1.3517 \\ 3805 & 15: 24: 11 & 1.3533 \\ 3806 & 15: 24: 12 & 1.3494 \\ 3807 & 15: 24: 13 & 1.3504 \\ 3808 & 15: 24: 14 & 1.3538 \\ 3809 & 15: 24: 15 & 1.3527 \\ 3810 & 15: 24: 16 & 1.3508 \\ 3811 & 15: 24: 17 & 1.3494 \\ 3812 & 15: 24: 18 & 1.3479 \\ 3813 & 15: 24: 19 & 1.3492 \\ 3814 & 15: 24: 20 & 1.3514 \\ 3815 & 15: 24: 21 & 1.3511 \\ 3816 & 15: 24: 22 & 1.3518 \\ 3817 & 15: 24: 23 & 1.3487 \\ 3818 & 15: 24: 24 & 1.3497 \\ 3819 & 15: 24: 25 & 1.3535 \\ 3820 & 15: 24: 26 & 1.3518 \\ 3821 & 15: 24: 27 & 1.3510 \\ 3822 & 15: 24: 28 & 1.3519 \\ 3823 & 15: 24: 29 & 1.3497\end{array}$


Appendix G - Fuel Data Test 4 NIST102705_1.xls

$\begin{array}{lll}3824 & 15: 24: 30 & 1.3519 \\ 3825 & 15: 24: 31 & 1.3557 \\ 3826 & 15: 24: 32 & 1.3515 \\ 3827 & 15: 24: 33 & 1.3492 \\ 3828 & 15: 24: 34 & 1.3489 \\ 3829 & 15: 24: 35 & 1.3506 \\ 3830 & 15: 24: 36 & 1.3524 \\ 3831 & 15: 24: 37 & 1.3515 \\ 3832 & 15: 24: 38 & 1.3540 \\ 3833 & 15: 24: 39 & 1.3518 \\ 3834 & 15: 24: 40 & 1.3513 \\ 3835 & 15: 24: 41 & 1.3533 \\ 3836 & 15: 24: 42 & 1.3518 \\ 3837 & 15: 24: 43 & 1.3501 \\ 3838 & 15: 24: 44 & 1.3520 \\ 3839 & 15: 24: 45 & 1.3518 \\ 3840 & 15: 24: 46 & 1.3532 \\ 3841 & 15: 24: 47 & 1.3533 \\ 3842 & 15: 24: 48 & 1.3523 \\ 3843 & 15: 24: 49 & 1.3510 \\ 3844 & 15: 24: 50 & 1.3509 \\ 3845 & 15: 24: 51 & 1.3546 \\ 3846 & 15: 24: 52 & 1.3513 \\ 3847 & 15: 24: 53 & 1.3462 \\ 3848 & 15: 24: 54 & 1.3527 \\ 3849 & 15: 24: 55 & 1.3524 \\ 3850 & 15: 24: 56 & 1.3520 \\ 3851 & 15: 24: 57 & 1.3522 \\ 3852 & 15: 24: 58 & 1.3524 \\ 3853 & 15: 24: 59 & 1.3522 \\ 3854 & 15: 25: 00 & 1.3526 \\ 3855 & 15: 25: 01 & 1.3508 \\ 3856 & 15: 25: 02 & 1.3555 \\ 3857 & 15: 25: 03 & 1.3570 \\ 3858 & 15: 25: 04 & 1.3516 \\ 3859 & 15: 25: 05 & 1.3511 \\ 3860 & 15: 25: 06 & 1.3536 \\ 3861 & 15: 25: 07 & 1.3516 \\ 3862 & 15: 25: 08 & 1.3515 \\ 3863 & 15: 25: 09 & 1.3509 \\ 3864 & 15: 25: 10 & 1.3494 \\ 3865 & 15: 25: 11 & 1.3494 \\ 3866 & 15: 25: 12 & 1.3508 \\ 3867 & 15: 25: 13 & 1.3535 \\ 3868 & 15: 25: 14 & 1.3523 \\ 3869 & 15: 25: 15 & 1.3510 \\ 3870 & 15: 25: 16 & 1.3523 \\ 3871 & 15: 25: 17 & 1.3525 \\ 3872 & 15: 25: 18 & 1.3520 \\ 3873 & 15: 25: 19 & 1.3502 \\ 3874 & 15: 25: 20 & 1.3488\end{array}$


Appendix G - Fuel Data Test 4 NIST102705_1.xls

$\begin{array}{lll}3875 & 15: 25: 21 & 1.3524 \\ 3876 & 15: 25: 22 & 1.3515 \\ 3877 & 15: 25: 23 & 1.3517 \\ 3878 & 15: 25: 24 & 1.3516 \\ 3879 & 15: 25: 25 & 1.3491 \\ 3880 & 15: 25: 26 & 1.3499 \\ 3881 & 15: 25: 27 & 1.3529 \\ 3882 & 15: 25: 28 & 1.3540 \\ 3883 & 15: 25: 29 & 1.3542 \\ 3884 & 15: 25: 30 & 1.3506 \\ 3885 & 15: 25: 31 & 1.3485 \\ 3886 & 15: 25: 32 & 1.3522 \\ 3887 & 15: 25: 33 & 1.3555 \\ 3888 & 15: 25: 34 & 1.3527 \\ 3889 & 15: 25: 35 & 1.3523 \\ 3890 & 15: 25: 36 & 1.3537 \\ 3891 & 15: 25: 37 & 1.3535 \\ 3892 & 15: 25: 38 & 1.3522 \\ 3893 & 15: 25: 39 & 1.3495 \\ 3894 & 15: 25: 40 & 1.3503 \\ 3895 & 15: 25: 41 & 1.3501 \\ 3896 & 15: 25: 42 & 1.3496 \\ 3897 & 15: 25: 43 & 1.3554 \\ 3898 & 15: 25: 44 & 1.3534 \\ 3899 & 15: 25: 45 & 1.3506 \\ 3900 & 15: 25: 46 & 1.3513 \\ 3901 & 15: 25: 47 & 1.3512 \\ 3902 & 15: 25: 48 & 1.3537 \\ 3903 & 15: 25: 49 & 1.3541 \\ 3904 & 15: 25: 50 & 1.3528 \\ 3905 & 15: 25: 51 & 1.3522 \\ 3906 & 15: 25: 52 & 1.3511 \\ 3907 & 15: 25: 53 & 1.3514 \\ 3908 & 15: 25: 54 & 1.3524 \\ 3909 & 15: 25: 55 & 1.3530 \\ 3910 & 15: 25: 56 & 1.3536 \\ 3911 & 15: 25: 57 & 1.3562 \\ 3912 & 15: 25: 58 & 1.3536 \\ 3913 & 15: 25: 59 & 1.3497 \\ 3914 & 15: 26: 00 & 1.3508 \\ 3915 & 15: 26: 01 & 1.3530 \\ 3916 & 15: 26: 02 & 1.3520 \\ 3917 & 15: 26: 03 & 1.3506 \\ 3918 & 15: 26: 04 & 1.3501 \\ 3919 & 15: 26: 05 & 1.3509 \\ 3920 & 15: 26: 06 & 1.3499 \\ 3921 & 15: 26: 07 & 1.3519 \\ 3922 & 15: 26: 08 & 1.3527 \\ 3923 & 15: 26: 09 & 1.3532 \\ 3924 & 15: 26: 10 & 1.3516 \\ 3925 & 15: 26: 11 & 1.3517\end{array}$


Appendix G - Fuel Data Test 4 NIST102705_1.xls

$\begin{array}{lll}3926 & 15: 26: 12 & 1.3510 \\ 3927 & 15: 26: 13 & 1.3506 \\ 3928 & 15: 26: 14 & 1.3510 \\ 3929 & 15: 26: 15 & 1.3509 \\ 3930 & 15: 26: 16 & 1.3515 \\ 3931 & 15: 26: 17 & 1.3510 \\ 3932 & 15: 26: 18 & 1.3493 \\ 3933 & 15: 26: 19 & 1.3518 \\ 3934 & 15: 26: 20 & 1.3510 \\ 3935 & 15: 26: 21 & 1.3502 \\ 3936 & 15: 26: 22 & 1.3495 \\ 3937 & 15: 26: 23 & 1.3510 \\ 3938 & 15: 26: 24 & 1.3525 \\ 3939 & 15: 26: 25 & 1.3534 \\ 3940 & 15: 26: 26 & 1.3507 \\ 3941 & 15: 26: 27 & 1.3474 \\ 3942 & 15: 26: 28 & 1.3491 \\ 3943 & 15: 26: 29 & 1.3532 \\ 3944 & 15: 26: 30 & 1.3519 \\ 3945 & 15: 26: 31 & 1.3509 \\ 3946 & 15: 26: 32 & 1.3519 \\ 3947 & 15: 26: 33 & 1.3489 \\ 3948 & 15: 26: 34 & 1.3502 \\ 3949 & 15: 26: 35 & 1.3542 \\ 3950 & 15: 26: 36 & 1.3520 \\ 3951 & 15: 26: 37 & 1.3515 \\ 3952 & 15: 26: 38 & 1.3498 \\ 3953 & 15: 26: 39 & 1.3500 \\ 3954 & 15: 26: 40 & 1.3518 \\ 3955 & 15: 26: 41 & 1.3481 \\ 3956 & 15: 26: 42 & 1.3489 \\ 3957 & 15: 26: 43 & 1.3513 \\ 3958 & 15: 26: 44 & 1.3521 \\ 3959 & 15: 26: 45 & 1.3524 \\ 3960 & 15: 26: 46 & 1.3543 \\ 3961 & 15: 26: 47 & 1.3525 \\ 3962 & 15: 26: 48 & 1.3508 \\ 3963 & 15: 26: 49 & 1.3510 \\ 3964 & 15: 26: 50 & 1.3537 \\ 3965 & 15: 26: 51 & 1.3509 \\ 3966 & 15: 26: 52 & 1.3490 \\ 3967 & 15: 26: 53 & 1.3513 \\ 3968 & 15: 26: 54 & 1.3508 \\ 3969 & 15: 26: 55 & 1.3526 \\ 3970 & 15: 26: 56 & 1.3541 \\ 3971 & 15: 26: 57 & 1.3518 \\ 3972 & 15: 26: 58 & 1.3505 \\ 3973 & 15: 26: 59 & 1.3502 \\ 3974 & 15: 27: 00 & 1.3544 \\ 3975 & 15: 27: 01 & 1.3556 \\ 3976 & 15: 27: 02 & 1.3504\end{array}$


Appendix G - Fuel Data Test 4 NIST102705_1.xls

$\begin{array}{lll}3977 & 15: 27: 03 & 1.3518 \\ 3978 & 15: 27: 04 & 1.3515 \\ 3979 & 15: 27: 05 & 1.3501 \\ 3980 & 15: 27: 06 & 1.3552 \\ 3981 & 15: 27: 07 & 1.3548 \\ 3982 & 15: 27: 08 & 1.3527 \\ 3983 & 15: 27: 09 & 1.3509 \\ 3984 & 15: 27: 10 & 1.3505 \\ 3985 & 15: 27: 11 & 1.3542 \\ 3986 & 15: 27: 12 & 1.3524 \\ 3987 & 15: 27: 13 & 1.3502 \\ 3988 & 15: 27: 14 & 1.3500 \\ 3989 & 15: 27: 15 & 1.3514 \\ 3990 & 15: 27: 16 & 1.3528 \\ 3991 & 15: 27: 17 & 1.3503 \\ 3992 & 15: 27: 18 & 1.3472 \\ 3993 & 15: 27: 19 & 1.3512 \\ 3994 & 15: 27: 20 & 1.3524 \\ 3995 & 15: 27: 21 & 1.3511 \\ 3996 & 15: 27: 22 & 1.3486 \\ 3997 & 15: 27: 23 & 1.3480 \\ 3998 & 15: 27: 24 & 1.3522 \\ 3999 & 15: 27: 25 & 1.3511 \\ 4000 & 15: 27: 26 & 1.3513 \\ 4001 & 15: 27: 27 & 1.3529 \\ 4002 & 15: 27: 28 & 1.3494 \\ 4003 & 15: 27: 29 & 1.3495 \\ 4004 & 15: 27: 30 & 1.3513 \\ 4005 & 15: 27: 31 & 1.3515 \\ 4006 & 15: 27: 32 & 1.3519 \\ 4007 & 15: 27: 33 & 1.3518 \\ 4008 & 15: 27: 34 & 1.3489 \\ 4009 & 15: 27: 35 & 1.3501 \\ 4010 & 15: 27: 36 & 1.3520 \\ 4011 & 15: 27: 37 & 1.3549 \\ 4012 & 15: 27: 38 & 1.3491 \\ 4013 & 15: 27: 39 & 1.3485 \\ 4014 & 15: 27: 40 & 1.3485 \\ 4015 & 15: 27: 41 & 1.3482 \\ 4016 & 15: 27: 42 & 1.3525 \\ 4017 & 15: 27: 43 & 1.3518 \\ 4018 & 15: 27: 44 & 1.3494 \\ 4019 & 15: 27: 45 & 1.3504 \\ 4020 & 15: 27: 46 & 1.3501 \\ 4021 & 15: 27: 47 & 1.3513 \\ 4022 & 15: 27: 48 & 1.3530 \\ 4023 & 15: 27: 49 & 1.3517 \\ 4024 & 15: 27: 50 & 1.3510 \\ 4025 & 15: 27: 51 & 1.3509 \\ 4026 & 15: 27: 52 & 1.3505 \\ 4027 & 15: 27: 53 & 1.3518\end{array}$


Appendix G - Fuel Data Test 4 NIST102705_1.xls

$\begin{array}{lll}4028 & 15: 27: 54 & 1.3540 \\ 4029 & 15: 27: 55 & 1.3546 \\ 4030 & 15: 27: 56 & 1.3507 \\ 4031 & 15: 27: 57 & 1.3510 \\ 4032 & 15: 27: 58 & 1.3541 \\ 4033 & 15: 27: 59 & 1.3536 \\ 4034 & 15: 28: 00 & 1.3538 \\ 4035 & 15: 28: 01 & 1.3520 \\ 4036 & 15: 28: 02 & 1.3509 \\ 4037 & 15: 28: 03 & 1.3489 \\ 4038 & 15: 28: 04 & 1.3481 \\ 4039 & 15: 28: 05 & 1.3507 \\ 4040 & 15: 28: 06 & 1.3546 \\ 4041 & 15: 28: 07 & 1.3508 \\ 4042 & 15: 28: 08 & 1.3509 \\ 4043 & 15: 28: 09 & 1.3517 \\ 4044 & 15: 28: 10 & 1.3499 \\ 4045 & 15: 28: 11 & 1.3485 \\ 4046 & 15: 28: 12 & 1.3499 \\ 4047 & 15: 28: 13 & 1.3507 \\ 4048 & 15: 28: 14 & 1.3494 \\ 4049 & 15: 28: 15 & 1.3535 \\ 4050 & 15: 28: 16 & 1.3533 \\ 4051 & 15: 28: 17 & 1.3494 \\ 4052 & 15: 28: 18 & 1.3502 \\ 4053 & 15: 28: 19 & 1.3482 \\ 4054 & 15: 28: 20 & 1.3491 \\ 4055 & 15: 28: 21 & 1.3507 \\ 4056 & 15: 28: 22 & 1.3502 \\ 4057 & 15: 28: 23 & 1.3513 \\ 4058 & 15: 28: 24 & 1.3487 \\ 4059 & 15: 28: 25 & 1.3479 \\ 4060 & 15: 28: 26 & 1.3495 \\ 4061 & 15: 28: 27 & 1.3511 \\ 4062 & 15: 28: 28 & 1.3542 \\ 4063 & 15: 28: 29 & 1.3560 \\ 4064 & 15: 28: 30 & 1.3535 \\ 4065 & 15: 28: 31 & 1.3497 \\ 4066 & 15: 28: 32 & 1.3497 \\ 4067 & 15: 28: 33 & 1.3498 \\ 4068 & 15: 28: 34 & 1.3503 \\ 4069 & 15: 28: 35 & 1.3491 \\ 4070 & 15: 28: 36 & 1.3525 \\ 4071 & 15: 28: 37 & 1.3507 \\ 4072 & 15: 28: 38 & 1.3509 \\ 4073 & 15: 28: 39 & 1.3521 \\ 4074 & 15: 28: 40 & 1.3516 \\ 4075 & 15: 28: 41 & 1.3513 \\ 4076 & 15: 28: 42 & 1.3511 \\ 4077 & 15: 28: 43 & 1.3525 \\ 4078 & 15: 28: 44 & 1.3531\end{array}$


Appendix G - Fuel Data Test 4 NIST102705_1.xls

$\begin{array}{lll}4079 & 15: 28: 45 & 1.3515 \\ 4080 & 15: 28: 46 & 1.3482 \\ 4081 & 15: 28: 47 & 1.3499 \\ 4082 & 15: 28: 48 & 1.3534 \\ 4083 & 15: 28: 49 & 1.3521 \\ 4084 & 15: 28: 50 & 1.3523 \\ 4085 & 15: 28: 51 & 1.3519 \\ 4086 & 15: 28: 52 & 1.3515 \\ 4087 & 15: 28: 53 & 1.3506 \\ 4088 & 15: 28: 54 & 1.3533 \\ 4089 & 15: 28: 55 & 1.3527 \\ 4090 & 15: 28: 56 & 1.3506 \\ 4091 & 15: 28: 57 & 1.3497 \\ 4092 & 15: 28: 58 & 1.3509 \\ 4093 & 15: 28: 59 & 1.3526 \\ 4094 & 15: 29: 00 & 1.3546 \\ 4095 & 15: 29: 01 & 1.3516 \\ 4096 & 15: 29: 02 & 1.3531 \\ 4097 & 15: 29: 03 & 1.3539 \\ 4098 & 15: 29: 04 & 1.3530 \\ 4099 & 15: 29: 05 & 1.3524 \\ 4100 & 15: 29: 06 & 1.3511 \\ 4101 & 15: 29: 07 & 1.3530 \\ 4102 & 15: 29: 08 & 1.3529 \\ 4103 & 15: 29: 09 & 1.3524 \\ 4104 & 15: 29: 10 & 1.3518 \\ 4105 & 15: 29: 11 & 1.3546 \\ 4106 & 15: 29: 12 & 1.3526 \\ 4107 & 15: 29: 13 & 1.3471 \\ 4108 & 15: 29: 14 & 1.3514 \\ 4109 & 15: 29: 15 & 1.3536 \\ 4110 & 15: 29: 16 & 1.3523 \\ 4111 & 15: 29: 17 & 1.3480 \\ 4112 & 15: 29: 18 & 1.3505 \\ 4113 & 15: 29: 19 & 1.3519 \\ 4114 & 15: 29: 20 & 1.3527 \\ 4115 & 15: 29: 21 & 1.3525 \\ 4116 & 15: 29: 22 & 1.3521 \\ 4117 & 15: 29: 23 & 1.3493 \\ 4118 & 15: 29: 24 & 1.3497 \\ 4119 & 15: 29: 25 & 1.3537 \\ 4120 & 15: 29: 26 & 1.3565 \\ 4121 & 15: 29: 27 & 1.3516 \\ 4122 & 15: 29: 28 & 1.3500 \\ 4123 & 15: 29: 29 & 1.3523 \\ 4124 & 15: 29: 30 & 1.3502 \\ 4125 & 15: 29: 31 & 1.3524 \\ 4126 & 15: 29: 32 & 1.3537 \\ 4127 & 15: 29: 33 & 1.3513 \\ 4128 & 15: 29: 34 & 1.3502 \\ 4129 & 15: 29: 35 & 1.3517\end{array}$

$G-81$ 
Appendix G - Fuel Data Test 4 NIST102705_1.xls

$\begin{array}{lll}4130 & 15: 29: 36 & 1.3563 \\ 4131 & 15: 29: 37 & 1.3525 \\ 4132 & 15: 29: 38 & 1.3498 \\ 4133 & 15: 29: 39 & 1.3495 \\ 4134 & 15: 29: 40 & 1.3471 \\ 4135 & 15: 29: 41 & 1.3503 \\ 4136 & 15: 29: 42 & 1.3518 \\ 4137 & 15: 29: 43 & 1.3531 \\ 4138 & 15: 29: 44 & 1.3518 \\ 4139 & 15: 29: 45 & 1.3538 \\ 4140 & 15: 29: 46 & 1.3527 \\ 4141 & 15: 29: 47 & 1.3525 \\ 4142 & 15: 29: 48 & 1.3489 \\ 4143 & 15: 29: 49 & 1.3477 \\ 4144 & 15: 29: 50 & 1.3508 \\ 4145 & 15: 29: 51 & 1.3514 \\ 4146 & 15: 29: 52 & 1.3520 \\ 4147 & 15: 29: 53 & 1.3529 \\ 4148 & 15: 29: 54 & 1.3512 \\ 4149 & 15: 29: 55 & 1.3495 \\ 4150 & 15: 29: 56 & 1.3493 \\ 4151 & 15: 29: 57 & 1.3532 \\ 4152 & 15: 29: 58 & 1.3543 \\ 4153 & 15: 29: 59 & 1.3499 \\ 4154 & 15: 30: 00 & 1.3493 \\ 4155 & 15: 30: 01 & 1.3530 \\ 4156 & 15: 30: 02 & 1.3540 \\ 4157 & 15: 30: 03 & 1.3534 \\ 4158 & 15: 30: 04 & 1.3521 \\ 4159 & 15: 30: 05 & 1.3478 \\ 4160 & 15: 30: 06 & 1.3501 \\ 4161 & 15: 30: 07 & 1.3519 \\ 4162 & 15: 30: 08 & 1.3537 \\ 4163 & 15: 30: 09 & 1.3513 \\ 4164 & 15: 30: 10 & 1.3494 \\ 4165 & 15: 30: 11 & 1.3493 \\ 4166 & 15: 30: 12 & 1.3504 \\ 4167 & 15: 30: 13 & 1.3525 \\ 4168 & 15: 30: 14 & 1.3512 \\ 4169 & 15: 30: 15 & 1.3502 \\ 4170 & 15: 30: 16 & 1.3539 \\ 4171 & 15: 30: 17 & 1.3554 \\ 4172 & 15: 30: 18 & 1.3558 \\ 4173 & 15: 30: 19 & 1.3517 \\ 4174 & 15: 30: 20 & 1.3486 \\ 4175 & 15: 30: 21 & 1.3507 \\ 4176 & 15: 30: 22 & 1.3525 \\ 4177 & 15: 30: 23 & 1.3520 \\ 4178 & 15: 30: 24 & 1.3529 \\ 4179 & 15: 30: 25 & 1.3507 \\ 4180 & 15: 30: 26 & 1.3474\end{array}$


Appendix G - Fuel Data Test 4 NIST102705_1.xls

$\begin{array}{lll}4181 & 15: 30: 27 & 1.3520 \\ 4182 & 15: 30: 28 & 1.3530 \\ 4183 & 15: 30: 29 & 1.3505 \\ 4184 & 15: 30: 30 & 1.3496 \\ 4185 & 15: 30: 31 & 1.3526 \\ 4186 & 15: 30: 32 & 1.3511 \\ 4187 & 15: 30: 33 & 1.3516 \\ 4188 & 15: 30: 34 & 1.3545 \\ 4189 & 15: 30: 35 & 1.3514 \\ 4190 & 15: 30: 36 & 1.3520 \\ 4191 & 15: 30: 37 & 1.3541 \\ 4192 & 15: 30: 38 & 1.3535 \\ 4193 & 15: 30: 39 & 1.3521 \\ 4194 & 15: 30: 40 & 1.3522 \\ 4195 & 15: 30: 41 & 1.3506 \\ 4196 & 15: 30: 42 & 1.3501 \\ 4197 & 15: 30: 43 & 1.3490 \\ 4198 & 15: 30: 44 & 1.3495 \\ 4199 & 15: 30: 45 & 1.3521 \\ 4200 & 15: 30: 46 & 1.3529 \\ 4201 & 15: 30: 47 & 1.3489 \\ 4202 & 15: 30: 48 & 1.3510 \\ 4203 & 15: 30: 49 & 1.3539 \\ 4204 & 15: 30: 50 & 1.3519 \\ 4205 & 15: 30: 51 & 1.3508 \\ 4206 & 15: 30: 52 & 1.3515 \\ 4207 & 15: 30: 53 & 1.3503 \\ 4208 & 15: 30: 54 & 1.3534 \\ 4209 & 15: 30: 55 & 1.3543 \\ 4210 & 15: 30: 56 & 1.3513 \\ 4211 & 15: 30: 57 & 1.3499 \\ 4212 & 15: 30: 58 & 1.3513 \\ 4213 & 15: 30: 59 & 1.3534 \\ 4214 & 15: 31: 00 & 1.3525 \\ 4215 & 15: 31: 01 & 1.3514 \\ 4216 & 15: 31: 02 & 1.3521 \\ 4217 & 15: 31: 03 & 1.3509 \\ 4218 & 15: 31: 04 & 1.3514 \\ 4219 & 15: 31: 05 & 1.3526 \\ 4220 & 15: 31: 06 & 1.3536 \\ 4221 & 15: 31: 07 & 1.3490 \\ 4222 & 15: 31: 08 & 1.3511 \\ 4223 & 15: 31: 09 & 1.3527 \\ 4224 & 15: 31: 10 & 1.3529 \\ 4225 & 15: 31: 11 & 1.3492 \\ 4226 & 15: 31: 12 & 1.3505 \\ 4227 & 15: 31: 13 & 1.3499 \\ 4228 & 15: 31: 14 & 1.3480 \\ 4229 & 15: 31: 15 & 1.3508 \\ 4230 & 15: 31: 16 & 1.3519 \\ 4231 & 15: 31: 17 & 1.3513\end{array}$


Appendix G - Fuel Data Test 4 NIST102705_1.xls

$\begin{array}{lll}4232 & 15: 31: 18 & 1.3494 \\ 4233 & 15: 31: 19 & 1.3501 \\ 4234 & 15: 31: 20 & 1.3506 \\ 4235 & 15: 31: 21 & 1.3503 \\ 4236 & 15: 31: 22 & 1.3512 \\ 4237 & 15: 31: 23 & 1.3533 \\ 4238 & 15: 31: 24 & 1.3524 \\ 4239 & 15: 31: 25 & 1.3508 \\ 4240 & 15: 31: 26 & 1.3507 \\ 4241 & 15: 31: 27 & 1.3515 \\ 4242 & 15: 31: 28 & 1.3503 \\ 4243 & 15: 31: 29 & 1.3481 \\ 4244 & 15: 31: 30 & 1.3527 \\ 4245 & 15: 31: 31 & 1.3545 \\ 4246 & 15: 31: 32 & 1.3525 \\ 4247 & 15: 31: 33 & 1.3520 \\ 4248 & 15: 31: 34 & 1.3519 \\ 4249 & 15: 31: 35 & 1.3508 \\ 4250 & 15: 31: 36 & 1.3513 \\ 4251 & 15: 31: 37 & 1.3512 \\ 4252 & 15: 31: 38 & 1.3508 \\ 4253 & 15: 31: 39 & 1.3472 \\ 4254 & 15: 31: 40 & 1.3529 \\ 4255 & 15: 31: 41 & 1.3541 \\ 4256 & 15: 31: 42 & 1.3513 \\ 4257 & 15: 31: 43 & 1.3505 \\ 4258 & 15: 31: 44 & 1.3535 \\ 4259 & 15: 31: 45 & 1.3504 \\ 4260 & 15: 31: 46 & 1.3501 \\ 4261 & 15: 31: 47 & 1.3539 \\ 4262 & 15: 31: 48 & 1.3522 \\ 4263 & 15: 31: 49 & 1.3543 \\ 4264 & 15: 31: 50 & 1.3522 \\ 4265 & 15: 31: 51 & 1.3500 \\ 4266 & 15: 31: 52 & 1.3504 \\ 4267 & 15: 31: 53 & 1.3501 \\ 4268 & 15: 31: 54 & 1.3499 \\ 4269 & 15: 31: 55 & 1.3515 \\ 4270 & 15: 31: 56 & 1.3514 \\ 4271 & 15: 31: 57 & 1.3495 \\ 4272 & 15: 31: 58 & 1.3502 \\ 4273 & 15: 31: 59 & 1.3505 \\ 4274 & 15: 32: 00 & 1.3489 \\ 4275 & 15: 32: 01 & 1.3487 \\ 4276 & 15: 32: 02 & 1.3518 \\ 4277 & 15: 32: 03 & 1.3520 \\ 4278 & 15: 32: 04 & 1.3524 \\ 4279 & 15: 32: 05 & 1.3511 \\ 4280 & 15: 32: 06 & 1.3506 \\ 4281 & 15: 32: 07 & 1.3501 \\ 4282 & 15: 32: 08 & 1.3528\end{array}$

$G-84$ 
Appendix G - Fuel Data Test 4 NIST102705_1.xls

$\begin{array}{lll}4283 & 15: 32: 09 & 1.3540 \\ 4284 & 15: 32: 10 & 1.3514 \\ 4285 & 15: 32: 11 & 1.3501 \\ 4286 & 15: 32: 12 & 1.3501 \\ 4287 & 15: 32: 13 & 1.3486 \\ 4288 & 15: 32: 14 & 1.3482 \\ 4289 & 15: 32: 15 & 1.3494 \\ 4290 & 15: 32: 16 & 1.3483 \\ 4291 & 15: 32: 17 & 1.3485 \\ 4292 & 15: 32: 18 & 1.3506 \\ 4293 & 15: 32: 19 & 1.3495 \\ 4294 & 15: 32: 20 & 1.3490 \\ 4295 & 15: 32: 21 & 1.3494 \\ 4296 & 15: 32: 22 & 1.3513 \\ 4297 & 15: 32: 23 & 1.3528 \\ 4298 & 15: 32: 24 & 1.3502 \\ 4299 & 15: 32: 25 & 1.3499 \\ 4300 & 15: 32: 26 & 1.3507 \\ 4301 & 15: 32: 27 & 1.3499 \\ 4302 & 15: 32: 28 & 1.3503 \\ 4303 & 15: 32: 29 & 1.3517 \\ 4304 & 15: 32: 30 & 1.3494 \\ 4305 & 15: 32: 31 & 1.3480 \\ 4306 & 15: 32: 32 & 1.3489 \\ 4307 & 15: 32: 33 & 1.3504 \\ 4308 & 15: 32: 34 & 1.3509 \\ 4309 & 15: 32: 35 & 1.3486 \\ 4310 & 15: 32: 36 & 1.3498 \\ 4311 & 15: 32: 37 & 1.3507 \\ 4312 & 15: 32: 38 & 1.3509 \\ 4313 & 15: 32: 39 & 1.3513 \\ 4314 & 15: 32: 40 & 1.3528 \\ 4315 & 15: 32: 41 & 1.3513 \\ 4316 & 15: 32: 42 & 1.3486 \\ 4317 & 15: 32: 43 & 1.3511 \\ 4318 & 15: 32: 44 & 1.3533 \\ 4319 & 15: 32: 45 & 1.3536 \\ 4320 & 15: 32: 46 & 1.3539 \\ 4321 & 15: 32: 47 & 1.3519 \\ 4322 & 15: 32: 48 & 1.3489 \\ 4323 & 15: 32: 49 & 1.3500 \\ 4324 & 15: 32: 50 & 1.3483 \\ 4325 & 15: 32: 51 & 1.3469 \\ 4326 & 15: 32: 52 & 1.3500 \\ 4327 & 15: 32: 53 & 1.3512 \\ 4328 & 15: 32: 54 & 1.3505 \\ 4329 & 15: 32: 55 & 1.3517 \\ 4330 & 15: 32: 56 & 1.3478 \\ 4331 & 15: 32: 57 & 1.3480 \\ 4332 & 15: 32: 58 & 1.3503 \\ 4333 & 15: 32: 59 & 1.3492 \\ & & \end{array}$

G - 85 
Appendix G - Fuel Data Test 4 NIST102705_1.xls

$\begin{array}{lll}4334 & 15: 33: 00 & 1.3514 \\ 4335 & 15: 33: 01 & 1.3521 \\ 4336 & 15: 33: 02 & 1.3496 \\ 4337 & 15: 33: 03 & 1.3467 \\ 4338 & 15: 33: 04 & 1.3534 \\ 4339 & 15: 33: 05 & 1.3538 \\ 4341 & 15: 33: 07 & 1.3492 \\ 4342 & 15: 33: 07 & 1.3510 \\ 4342 & 15: 33: 08 & 1.3506 \\ 4343 & 15: 33: 09 & 1.3487 \\ 4344 & 15: 33: 10 & 1.3490 \\ 4345 & 15: 33: 11 & 1.3484 \\ 4346 & 15: 33: 12 & 1.3511 \\ 4347 & 15: 33: 13 & 1.3508 \\ 4348 & 15: 33: 14 & 1.3505 \\ 4349 & 15: 33: 15 & 1.3518 \\ 4350 & 15: 33: 16 & 1.3516 \\ 4351 & 15: 33: 17 & 1.3531 \\ 4352 & 15: 33: 18 & 1.3530 \\ 4353 & 15: 33: 19 & 1.3513 \\ 4354 & 15: 33: 20 & 1.3508 \\ 4355 & 15: 33: 21 & 1.3490 \\ 4356 & 15: 33: 22 & 1.3464 \\ 4357 & 15: 33: 23 & 1.3502 \\ 4358 & 15: 33: 24 & 1.3514 \\ 4359 & 15: 33: 25 & 1.3493 \\ 4360 & 15: 33: 26 & 1.3509 \\ 4361 & 15: 33: 27 & 1.3509 \\ 4362 & 15: 33: 28 & 1.3515 \\ 4363 & 15: 33: 29 & 1.3535 \\ 4364 & 15: 33: 30 & 1.3504 \\ 4365 & 15: 33: 31 & 1.3491 \\ 4366 & 15: 33: 32 & 1.3517 \\ 4367 & 15: 33: 33 & 1.3498 \\ 4368 & 15: 33: 34 & 1.3460 \\ 4369 & 15: 33: 35 & 1.3477 \\ 4370 & 15: 33: 36 & 1.3492 \\ 4371 & 15: 33: 37 & 1.3509 \\ 4372 & 15: 33: 38 & 1.3516 \\ 4373 & 15: 33: 39 & 1.3502 \\ 4374 & 15: 33: 40 & 1.3491 \\ 4375 & 15: 33: 41 & 1.3493 \\ 4376 & 15: 33: 42 & 1.3490 \\ 4377 & 15: 33: 43 & 1.3528 \\ 4378 & 15: 33: 44 & 1.3512 \\ 4379 & 15: 33: 45 & 1.3444 \\ 4380 & 15: 33: 46 & 1.3504 \\ 4381 & 15: 33: 47 & 1.3525 \\ 4382 & 15: 33: 48 & 1.3497 \\ 4383 & 15: 33: 49 & 1.3494 \\ 4384 & 15: 33: 50 & 1.3493\end{array}$


Appendix G - Fuel Data Test 4 NIST102705_1.xls

$\begin{array}{lll}4385 & 15: 33: 51 & 1.3490 \\ 4386 & 15: 33: 52 & 1.3504 \\ 4387 & 15: 33: 53 & 1.3497 \\ 4388 & 15: 33: 54 & 1.3503 \\ 4389 & 15: 33: 55 & 1.3471 \\ 4390 & 15: 33: 56 & 1.3491 \\ 4391 & 15: 33: 57 & 1.3530 \\ 4392 & 15: 33: 58 & 1.3494 \\ 4393 & 15: 33: 59 & 1.3487 \\ 4394 & 15: 34: 00 & 1.3520 \\ 4395 & 15: 34: 01 & 1.3485 \\ 4396 & 15: 34: 02 & 1.3485 \\ 4397 & 15: 34: 03 & 1.3508 \\ 4398 & 15: 34: 04 & 1.3462 \\ 4399 & 15: 34: 05 & 1.3457 \\ 4400 & 15: 34: 06 & 1.3483 \\ 4401 & 15: 34: 07 & 1.3519 \\ 4402 & 15: 34: 08 & 1.3549 \\ 4403 & 15: 34: 09 & 1.3509 \\ 4404 & 15: 34: 10 & 1.3483 \\ 4405 & 15: 34: 11 & 1.3512 \\ 4406 & 15: 34: 12 & 1.3513 \\ 4407 & 15: 34: 13 & 1.3476 \\ 4408 & 15: 34: 14 & 1.3534 \\ 4409 & 15: 34: 15 & 1.3523 \\ 4410 & 15: 34: 16 & 1.3486 \\ 4411 & 15: 34: 17 & 1.3502 \\ 4412 & 15: 34: 18 & 1.3552 \\ 4413 & 15: 34: 19 & 1.3532 \\ 4414 & 15: 34: 20 & 1.3513 \\ 4415 & 15: 34: 21 & 1.3507 \\ 4416 & 15: 34: 22 & 1.3493 \\ 4417 & 15: 34: 23 & 1.3511 \\ 4418 & 15: 34: 24 & 1.3516 \\ 4419 & 15: 34: 25 & 1.3510 \\ 4420 & 15: 34: 26 & 1.3511 \\ 4421 & 15: 34: 27 & 1.3522 \\ 4422 & 15: 34: 28 & 1.3507 \\ 4423 & 15: 34: 29 & 1.3509 \\ 4424 & 15: 34: 30 & 1.3491 \\ 4425 & 15: 34: 31 & 1.3501 \\ 4426 & 15: 34: 32 & 1.3498 \\ 4427 & 15: 34: 33 & 1.3492 \\ 4428 & 15: 34: 34 & 1.3514 \\ 4429 & 15: 34: 35 & 1.3477 \\ 4430 & 15: 34: 36 & 1.3490 \\ 4431 & 15: 34: 37 & 1.3491 \\ 4432 & 15: 34: 38 & 1.3490 \\ 4433 & 15: 34: 39 & 1.3509 \\ 4434 & 15: 34: 40 & 1.3499 \\ 4435 & 15: 34: 41 & 1.3489\end{array}$


Appendix G - Fuel Data Test 4 NIST102705_1.xls

$\begin{array}{lll}4436 & 15: 34: 42 & 1.3526 \\ 4437 & 15: 34: 43 & 1.3530 \\ 4438 & 15: 34: 44 & 1.3524 \\ 4439 & 15: 34: 45 & 1.3533 \\ 4440 & 15: 34: 46 & 1.3517 \\ 4441 & 15: 34: 47 & 1.3475 \\ 4442 & 15: 34: 48 & 1.3488 \\ 4443 & 15: 34: 49 & 1.3499 \\ 4444 & 15: 34: 50 & 1.3488 \\ 4445 & 15: 34: 51 & 1.3476 \\ 4446 & 15: 34: 52 & 1.3482 \\ 4447 & 15: 34: 53 & 1.3496 \\ 4448 & 15: 34: 54 & 1.3526 \\ 4449 & 15: 34: 55 & 1.3522 \\ 4450 & 15: 34: 56 & 1.3523 \\ 4451 & 15: 34: 57 & 1.3502 \\ 4452 & 15: 34: 58 & 1.3498 \\ 4453 & 15: 34: 59 & 1.3520 \\ 4454 & 15: 35: 00 & 1.3506 \\ 4455 & 15: 35: 01 & 1.3495 \\ 4456 & 15: 35: 02 & 1.3501 \\ 4457 & 15: 35: 03 & 1.3504 \\ 4458 & 15: 35: 04 & 1.3501 \\ 4459 & 15: 35: 05 & 1.3514 \\ 4460 & 15: 35: 06 & 1.3520 \\ 4461 & 15: 35: 07 & 1.3494 \\ 4462 & 15: 35: 08 & 1.3487 \\ 4463 & 15: 35: 09 & 1.3504 \\ 4464 & 15: 35: 10 & 1.3504 \\ 4465 & 15: 35: 11 & 1.3503 \\ 4466 & 15: 35: 12 & 1.3506 \\ 4467 & 15: 35: 13 & 1.3500 \\ 4468 & 15: 35: 14 & 1.3495 \\ 4469 & 15: 35: 15 & 1.3489 \\ 4470 & 15: 35: 16 & 1.3461 \\ 4471 & 15: 35: 17 & 1.3483 \\ 4472 & 15: 35: 18 & 1.3461 \\ 4473 & 15: 35: 19 & 1.3452 \\ 4474 & 15: 35: 20 & 1.3520 \\ 4475 & 15: 35: 21 & 1.3514 \\ 4476 & 15: 35: 22 & 1.3485 \\ 4477 & 15: 35: 23 & 1.3505 \\ 4478 & 15: 35: 24 & 1.3474 \\ 4479 & 15: 35: 25 & 1.3493 \\ 4480 & 15: 35: 26 & 1.3506 \\ 4481 & 15: 35: 27 & 1.3486 \\ 4482 & 15: 35: 28 & 1.3504 \\ 4483 & 15: 35: 29 & 1.3530 \\ 4484 & 15: 35: 30 & 1.3493 \\ 4485 & 15: 35: 31 & 1.3490 \\ 4486 & 15: 35: 32 & 1.3492\end{array}$


Appendix G - Fuel Data Test 4 NIST102705_1.xls

$\begin{array}{lll}4487 & 15: 35: 33 & 1.3487 \\ 4488 & 15: 35: 34 & 1.3471 \\ 4489 & 15: 35: 35 & 1.3510 \\ 4490 & 15: 35: 36 & 1.3537 \\ 4491 & 15: 35: 37 & 1.3508 \\ 4492 & 15: 35: 38 & 1.3507 \\ 4493 & 15: 35: 39 & 1.3513 \\ 4494 & 15: 35: 40 & 1.3505 \\ 4495 & 15: 35: 41 & 1.3522 \\ 4496 & 15: 35: 42 & 1.3522 \\ 4497 & 15: 35: 43 & 1.3499 \\ 4498 & 15: 35: 44 & 1.3511 \\ 4499 & 15: 35: 45 & 1.3498 \\ 4500 & 15: 35: 46 & 1.3480 \\ 4501 & 15: 35: 47 & 1.3512 \\ 4502 & 15: 35: 48 & 1.3504 \\ 4503 & 15: 35: 49 & 1.3481 \\ 4504 & 15: 35: 50 & 1.3478 \\ 4505 & 15: 35: 51 & 1.3503 \\ 4506 & 15: 35: 52 & 1.3516 \\ 4507 & 15: 35: 53 & 1.3499 \\ 4508 & 15: 35: 54 & 1.3491 \\ 4509 & 15: 35: 55 & 1.3493 \\ 4510 & 15: 35: 56 & 1.3498 \\ 4511 & 15: 35: 57 & 1.3501 \\ 4512 & 15: 35: 58 & 1.3511 \\ 4513 & 15: 35: 59 & 1.3505 \\ 4514 & 15: 36: 00 & 1.3451 \\ 4515 & 15: 36: 01 & 1.3482 \\ 4516 & 15: 36: 02 & 1.3527 \\ 4517 & 15: 36: 03 & 1.3505 \\ 4518 & 15: 36: 04 & 1.3504 \\ 4519 & 15: 36: 05 & 1.3467 \\ 4520 & 15: 36: 06 & 1.3475 \\ 4521 & 15: 36: 07 & 1.3511 \\ 4522 & 15: 36: 08 & 1.3535 \\ 4523 & 15: 36: 09 & 1.3518 \\ 4524 & 15: 36: 10 & 1.3488 \\ 4525 & 15: 36: 11 & 1.3500 \\ 4526 & 15: 36: 12 & 1.3492 \\ 4527 & 15: 36: 13 & 1.3472 \\ 4528 & 15: 36: 14 & 1.3494 \\ 4529 & 15: 36: 15 & 1.3522 \\ 4530 & 15: 36: 16 & 1.3487 \\ 4531 & 15: 36: 17 & 1.3494 \\ 4532 & 15: 36: 18 & 1.3495 \\ 4533 & 15: 36: 19 & 1.3530 \\ 4534 & 15: 36: 20 & 1.3526 \\ 4535 & 15: 36: 21 & 1.3488 \\ 4536 & 15: 36: 22 & 1.3506 \\ 4537 & 15: 36: 23 & 1.3507\end{array}$

G - 89 
Appendix G - Fuel Data Test 4 NIST102705_1.xls

$\begin{array}{lll}4538 & 15: 36: 24 & 1.3520 \\ 4539 & 15: 36: 25 & 1.3528 \\ 4540 & 15: 36: 26 & 1.3492 \\ 4541 & 15: 36: 27 & 1.3466 \\ 4542 & 15: 36: 28 & 1.3505 \\ 4543 & 15: 36: 29 & 1.3470 \\ 4544 & 15: 36: 30 & 1.3451 \\ 4545 & 15: 36: 31 & 1.3498 \\ 4546 & 15: 36: 32 & 1.3500 \\ 4547 & 15: 36: 33 & 1.3506 \\ 4548 & 15: 36: 34 & 1.3472 \\ 4549 & 15: 36: 35 & 1.3467 \\ 4550 & 15: 36: 36 & 1.3495 \\ 4551 & 15: 36: 37 & 1.3498 \\ 4552 & 15: 36: 38 & 1.3507 \\ 4553 & 15: 36: 39 & 1.3507 \\ 4554 & 15: 36: 40 & 1.3502 \\ 4555 & 15: 36: 41 & 1.3483 \\ 4556 & 15: 36: 42 & 1.3507 \\ 4557 & 15: 36: 43 & 1.3530 \\ 4558 & 15: 36: 44 & 1.3512 \\ 4559 & 15: 36: 45 & 1.3473 \\ 4560 & 15: 36: 46 & 1.3489 \\ 4561 & 15: 36: 47 & 1.3508 \\ 4562 & 15: 36: 48 & 1.3489 \\ 4563 & 15: 36: 49 & 1.3502 \\ 4564 & 15: 36: 50 & 1.3515 \\ 4565 & 15: 36: 51 & 1.3509 \\ 4566 & 15: 36: 52 & 1.3491 \\ 4567 & 15: 36: 53 & 1.3488 \\ 4568 & 15: 36: 54 & 1.3491 \\ 4569 & 15: 36: 55 & 1.3477 \\ 4570 & 15: 36: 56 & 1.3485 \\ 4571 & 15: 36: 57 & 1.3498 \\ 4572 & 15: 36: 58 & 1.3502 \\ 4573 & 15: 36: 59 & 1.3514 \\ 4574 & 15: 37: 00 & 1.3525 \\ 4575 & 15: 37: 01 & 1.3531 \\ 4576 & 15: 37: 02 & 1.3504 \\ 4577 & 15: 37: 03 & 1.3481 \\ 4578 & 15: 37: 04 & 1.3505 \\ 4579 & 15: 37: 05 & 1.3498 \\ 4580 & 15: 37: 06 & 1.3497 \\ 4581 & 15: 37: 07 & 1.3502 \\ 4582 & 15: 37: 08 & 1.3478 \\ 4583 & 15: 37: 09 & 1.3502 \\ 4584 & 15: 37: 10 & 1.3510 \\ 4585 & 15: 37: 11 & 1.3460 \\ 4586 & 15: 37: 12 & 1.3466 \\ 4587 & 15: 37: 13 & 1.3487 \\ 4588 & 15: 37: 14 & 1.3488\end{array}$


Appendix G - Fuel Data Test 4 NIST102705_1.xls

$\begin{array}{lll}4589 & 15: 37: 15 & 1.3518 \\ 4590 & 15: 37: 16 & 1.3498 \\ 4591 & 15: 37: 17 & 1.3479 \\ 4592 & 15: 37: 18 & 1.3492 \\ 4593 & 15: 37: 19 & 1.3521 \\ 4594 & 15: 37: 20 & 1.3522 \\ 4595 & 15: 37: 21 & 1.3504 \\ 4596 & 15: 37: 22 & 1.3490 \\ 4597 & 15: 37: 23 & 1.3480 \\ 4598 & 15: 37: 24 & 1.3470 \\ 4599 & 15: 37: 25 & 1.3518 \\ 4600 & 15: 37: 26 & 1.3494 \\ 4601 & 15: 37: 27 & 1.3480 \\ 4602 & 15: 37: 28 & 1.3521 \\ 4603 & 15: 37: 29 & 1.3517 \\ 4604 & 15: 37: 30 & 1.3529 \\ 4605 & 15: 37: 31 & 1.3526 \\ 4606 & 15: 37: 32 & 1.3508 \\ 4607 & 15: 37: 33 & 1.3499 \\ 4608 & 15: 37: 34 & 1.3501 \\ 4609 & 15: 37: 35 & 1.3508 \\ 4610 & 15: 37: 36 & 1.3504 \\ 4611 & 15: 37: 37 & 1.3459 \\ 4612 & 15: 37: 38 & 1.3496 \\ 4613 & 15: 37: 39 & 1.3499 \\ 4614 & 15: 37: 40 & 1.3484 \\ 4615 & 15: 37: 41 & 1.3501 \\ 4616 & 15: 37: 42 & 1.3499 \\ 4617 & 15: 37: 43 & 1.3463 \\ 4618 & 15: 37: 44 & 1.3496 \\ 4619 & 15: 37: 45 & 1.3508 \\ 4620 & 15: 37: 46 & 1.3513 \\ 4621 & 15: 37: 47 & 1.3511 \\ 4622 & 15: 37: 48 & 1.3482 \\ 4623 & 15: 37: 49 & 1.3483 \\ 4624 & 15: 37: 50 & 1.3500 \\ 4625 & 15: 37: 51 & 1.3499 \\ 4626 & 15: 37: 52 & 1.3531 \\ 4627 & 15: 37: 53 & 1.3516 \\ 4628 & 15: 37: 54 & 1.3472 \\ 4629 & 15: 37: 55 & 1.3487 \\ 4630 & 15: 37: 56 & 1.3512 \\ 4631 & 15: 37: 57 & 1.3512 \\ 4632 & 15: 37: 58 & 1.3488 \\ 4633 & 15: 37: 59 & 1.3496 \\ 4634 & 15: 38: 00 & 1.3490 \\ 4635 & 15: 38: 01 & 1.3488 \\ 4636 & 15: 38: 02 & 1.3509 \\ 4637 & 15: 38: 03 & 1.3514 \\ 4638 & 15: 38: 04 & 1.3514 \\ 4639 & 15: 38: 05 & 1.3483\end{array}$

G - 91 
Appendix G - Fuel Data Test 4 NIST102705_1.xls

$\begin{array}{lll}4640 & 15: 38: 06 & 1.3484 \\ 4641 & 15: 38: 07 & 1.3524 \\ 4642 & 15: 38: 08 & 1.3515 \\ 4643 & 15: 38: 09 & 1.3512 \\ 4644 & 15: 38: 10 & 1.3490 \\ 4645 & 15: 38: 11 & 1.3481 \\ 4646 & 15: 38: 12 & 1.3508 \\ 4647 & 15: 38: 13 & 1.3498 \\ 4648 & 15: 38: 14 & 1.3498 \\ 4649 & 15: 38: 15 & 1.3482 \\ 4650 & 15: 38: 16 & 1.3500 \\ 4651 & 15: 38: 17 & 1.3520 \\ 4652 & 15: 38: 18 & 1.3503 \\ 4653 & 15: 38: 19 & 1.3496 \\ 4654 & 15: 38: 20 & 1.3541 \\ 4655 & 15: 38: 21 & 1.3507 \\ 4656 & 15: 38: 22 & 1.3479 \\ 4657 & 15: 38: 23 & 1.3533 \\ 4658 & 15: 38: 24 & 1.3536 \\ 4659 & 15: 38: 25 & 1.3508 \\ 4660 & 15: 38: 26 & 1.3474 \\ 4661 & 15: 38: 27 & 1.3466 \\ 4662 & 15: 38: 28 & 1.3504 \\ 4663 & 15: 38: 29 & 1.3501 \\ 4664 & 15: 38: 30 & 1.3506 \\ 4665 & 15: 38: 31 & 1.3509 \\ 4666 & 15: 38: 32 & 1.3498 \\ 4667 & 15: 38: 33 & 1.3503 \\ 4668 & 15: 38: 34 & 1.3521 \\ 4669 & 15: 38: 35 & 1.3503 \\ 4670 & 15: 38: 36 & 1.3487 \\ 4671 & 15: 38: 37 & 1.3541 \\ 4672 & 15: 38: 38 & 1.3522 \\ 4673 & 15: 38: 39 & 1.3482 \\ 4674 & 15: 38: 40 & 1.3506 \\ 4675 & 15: 38: 41 & 1.3525 \\ 4676 & 15: 38: 42 & 1.3504 \\ 4677 & 15: 38: 43 & 1.3486 \\ 4678 & 15: 38: 44 & 1.3462 \\ 4679 & 15: 38: 45 & 1.3448 \\ 4680 & 15: 38: 46 & 1.3463 \\ 4681 & 15: 38: 47 & 1.3453 \\ 4682 & 15: 38: 48 & 1.3488 \\ 4683 & 15: 38: 49 & 1.3517 \\ 4684 & 15: 38: 50 & 1.3498 \\ 4685 & 15: 38: 51 & 1.3504 \\ 4686 & 15: 38: 52 & 1.3505 \\ 4687 & 15: 38: 53 & 1.3494 \\ 4688 & 15: 38: 54 & 1.3503 \\ 4689 & 15: 38: 55 & 1.3498 \\ 4690 & 15: 38: 56 & 1.3495\end{array}$


Appendix G - Fuel Data Test 4 NIST102705_1.xls

$\begin{array}{lll}4691 & 15: 38: 57 & 1.3488 \\ 4692 & 15: 38: 58 & 1.3477 \\ 4693 & 15: 38: 59 & 1.3497 \\ 4694 & 15: 39: 00 & 1.3507 \\ 4695 & 15: 39: 01 & 1.3505 \\ 4696 & 15: 39: 02 & 1.3512 \\ 4697 & 15: 39: 03 & 1.3499 \\ 4698 & 15: 39: 04 & 1.3495 \\ 4699 & 15: 39: 05 & 1.3501 \\ 4700 & 15: 39: 06 & 1.3457 \\ 4701 & 15: 39: 07 & 1.3454 \\ 4702 & 15: 39: 08 & 1.3486 \\ 4703 & 15: 39: 09 & 1.3513 \\ 4704 & 15: 39: 10 & 1.3513 \\ 4705 & 15: 39: 11 & 1.3468 \\ 4706 & 15: 39: 12 & 1.3491 \\ 4707 & 15: 39: 13 & 1.3530 \\ 4708 & 15: 39: 14 & 1.3525 \\ 4709 & 15: 39: 15 & 1.3513 \\ 4710 & 15: 39: 16 & 1.3502 \\ 4711 & 15: 39: 17 & 1.3483 \\ 4712 & 15: 39: 18 & 1.3486 \\ 4713 & 15: 39: 19 & 1.3504 \\ 4714 & 15: 39: 20 & 1.3528 \\ 4715 & 15: 39: 21 & 1.3514 \\ 4716 & 15: 39: 22 & 1.3495 \\ 4717 & 15: 39: 23 & 1.3492 \\ 4718 & 15: 39: 24 & 1.3502 \\ 4719 & 15: 39: 25 & 1.3499 \\ 4720 & 15: 39: 26 & 1.3513 \\ 4721 & 15: 39: 27 & 1.3509 \\ 4722 & 15: 39: 28 & 1.3490 \\ 4723 & 15: 39: 29 & 1.3483 \\ 4724 & 15: 39: 30 & 1.3493 \\ 4725 & 15: 39: 31 & 1.3492 \\ 4726 & 15: 39: 32 & 1.3505 \\ 4727 & 15: 39: 33 & 1.3496 \\ 4728 & 15: 39: 34 & 1.3443 \\ 4729 & 15: 39: 35 & 1.3485 \\ 4730 & 15: 39: 36 & 1.3523 \\ 4731 & 15: 39: 37 & 1.3499 \\ 4732 & 15: 39: 38 & 1.3489 \\ 4733 & 15: 39: 39 & 1.3465 \\ 4734 & 15: 39: 40 & 1.3472 \\ 4735 & 15: 39: 41 & 1.3518 \\ 4736 & 15: 39: 42 & 1.3496 \\ 4737 & 15: 39: 43 & 1.3460 \\ 4738 & 15: 39: 44 & 1.3480 \\ 4739 & 15: 39: 45 & 1.3504 \\ 4740 & 15: 39: 46 & 1.3505 \\ 4741 & 15: 39: 47 & 1.3496\end{array}$

G - 93 
Appendix G - Fuel Data Test 4 NIST102705_1.xls

\begin{tabular}{lll}
4742 & $15: 39: 48$ & 1.3481 \\
4743 & $15: 39: 49$ & 1.3487 \\
4744 & $15: 39: 50$ & 1.3492 \\
4745 & $15: 39: 51$ & 1.3494 \\
4746 & $15: 39: 52$ & 1.3475 \\
4747 & $15: 39: 53$ & 1.3493 \\
4748 & $15: 39: 54$ & 1.3539 \\
4749 & $15: 39: 55$ & 1.3500 \\
4750 & $15: 39: 56$ & 1.3481 \\
4751 & $15: 39: 57$ & 1.3485 \\
4752 & $15: 39: 58$ & 1.3494 \\
4753 & $15: 39: 59$ & 1.3484 \\
4754 & $15: 40: 00$ & 1.3477 \\
4755 & $15: 40: 01$ & 1.3506 \\
4756 & $15: 40: 02$ & 1.3508 \\
4757 & $15: 40: 03$ & 1.3467 \\
4758 & $15: 40: 04$ & 1.3493 \\
4759 & $15: 40: 05$ & 1.3500 \\
4760 & $15: 40: 06$ & 1.3480 \\
4761 & $15: 40: 07$ & 1.3508 \\
4762 & $15: 40: 08$ & 1.3516 \\
4763 & $15: 40: 09$ & 1.3515 \\
4764 & $15: 40: 10$ & 1.3491 \\
4765 & $15: 40: 11$ & 1.3493 \\
4766 & $15: 40: 12$ & 1.3494 \\
4767 & $15: 40: 13$ & 1.3498 \\
4768 & $15: 40: 14$ & 1.3474 \\
4769 & $15: 40: 15$ & 1.3505 \\
4770 & $15: 40: 16$ & 1.3518 \\
4771 & $15: 40: 17$ & 1.3514 \\
4772 & $15: 40: 18$ & 1.3533 \\
4773 & $15: 40: 19$ & 1.3486 \\
4774 & $15: 40: 20$ & 1.3489 \\
4775 & $15: 40: 21$ & 1.3484 \\
4776 & $15: 40: 22$ & 1.3491 \\
4777 & $15: 40: 23$ & 1.3515 \\
4778 & $15: 40: 24$ & 1.3494 \\
4779 & $15: 40: 25$ & 1.3488 \\
4780 & $15: 40: 26$ & 1.3486 \\
4781 & $15: 40: 27$ & 1.3469 \\
4782 & $15: 40: 28$ & 1.3493 \\
4783 & $15: 40: 29$ & 1.3503 \\
4784 & $15: 40: 30$ & 1.3470 \\
4785 & $15: 40: 31$ & 1.3488 \\
4786 & $15: 40: 32$ & 1.3485 \\
4787 & $15: 40: 33$ & 1.3473 \\
4788 & $15: 40: 34$ & 1.3494 \\
4789 & $15: 40: 35$ & 1.3507 \\
4790 & $15: 40: 36$ & 1.3509 \\
4791 & $15: 40: 37$ & 1.3499 \\
4792 & $15: 40: 38$ & 1.3502 \\
& & \\
\hline
\end{tabular}


Appendix G - Fuel Data Test 4 NIST102705_1.xls

$\begin{array}{lll}4793 & 15: 40: 39 & 1.3496 \\ 4794 & 15: 40: 40 & 1.3510 \\ 4795 & 15: 40: 41 & 1.3491 \\ 4796 & 15: 40: 42 & 1.3454 \\ 4797 & 15: 40: 43 & 1.3509 \\ 4798 & 15: 40: 44 & 1.3510 \\ 4799 & 15: 40: 45 & 1.3453 \\ 4800 & 15: 40: 46 & 1.3502 \\ 4801 & 15: 40: 47 & 1.3504 \\ 4802 & 15: 40: 48 & 1.3478 \\ 4803 & 15: 40: 49 & 1.3502 \\ 4804 & 15: 40: 50 & 1.3525 \\ 4805 & 15: 40: 51 & 1.3499 \\ 4806 & 15: 40: 52 & 1.3471 \\ 4807 & 15: 40: 53 & 1.3513 \\ 4808 & 15: 40: 54 & 1.3521 \\ 4809 & 15: 40: 55 & 1.3524 \\ 4810 & 15: 40: 56 & 1.3519 \\ 4811 & 15: 40: 57 & 1.3495 \\ 4812 & 15: 40: 58 & 1.3494 \\ 4813 & 15: 40: 59 & 1.3520 \\ 4814 & 15: 41: 00 & 1.3504 \\ 4815 & 15: 41: 01 & 1.3505 \\ 4816 & 15: 41: 02 & 1.3499 \\ 4817 & 15: 41: 03 & 1.3496 \\ 4818 & 15: 41: 04 & 1.3503 \\ 4819 & 15: 41: 05 & 1.3497 \\ 4820 & 15: 41: 06 & 1.3498 \\ 4821 & 15: 41: 07 & 1.3534 \\ 4822 & 15: 41: 08 & 1.3513 \\ 4823 & 15: 41: 09 & 1.3495 \\ 4824 & 15: 41: 10 & 1.3532 \\ 4825 & 15: 41: 11 & 1.3523 \\ 4826 & 15: 41: 12 & 1.3481 \\ 4827 & 15: 41: 13 & 1.3484 \\ 4828 & 15: 41: 14 & 1.3513 \\ 4829 & 15: 41: 15 & 1.3525 \\ 4830 & 15: 41: 16 & 1.3491 \\ 4831 & 15: 41: 17 & 1.3503 \\ 4832 & 15: 41: 18 & 1.3495 \\ 4833 & 15: 41: 19 & 1.3516 \\ 4834 & 15: 41: 20 & 1.3533 \\ 4835 & 15: 41: 21 & 1.3521 \\ 4836 & 15: 41: 22 & 1.3502 \\ 4837 & 15: 41: 23 & 1.3485 \\ 4838 & 15: 41: 24 & 1.3484 \\ 4839 & 15: 41: 25 & 1.3487 \\ 4840 & 15: 41: 26 & 1.3501 \\ 4841 & 15: 41: 27 & 1.3502 \\ 4842 & 15: 41: 28 & 1.3512 \\ 4843 & 15: 41: 29 & 1.3517\end{array}$

G - 95 
Appendix G - Fuel Data Test 4 NIST102705_1.xls

$\begin{array}{lll}4844 & 15: 41: 30 & 1.3531 \\ 4845 & 15: 41: 31 & 1.3536 \\ 4846 & 15: 41: 32 & 1.3503 \\ 4847 & 15: 41: 33 & 1.3488 \\ 4848 & 15: 41: 34 & 1.3463 \\ 4849 & 15: 41: 35 & 1.3488 \\ 4850 & 15: 41: 36 & 1.3512 \\ 4851 & 15: 41: 37 & 1.3485 \\ 4852 & 15: 41: 38 & 1.3503 \\ 4853 & 15: 41: 39 & 1.3505 \\ 4854 & 15: 41: 40 & 1.3489 \\ 4855 & 15: 41: 41 & 1.3510 \\ 4856 & 15: 41: 42 & 1.3521 \\ 4857 & 15: 41: 43 & 1.3492 \\ 4858 & 15: 41: 44 & 1.3477 \\ 4859 & 15: 41: 45 & 1.3502 \\ 4860 & 15: 41: 46 & 1.3511 \\ 4861 & 15: 41: 47 & 1.3519 \\ 4862 & 15: 41: 48 & 1.3483 \\ 4863 & 15: 41: 49 & 1.3468 \\ 4864 & 15: 41: 50 & 1.3508 \\ 4865 & 15: 41: 51 & 1.3508 \\ 4866 & 15: 41: 52 & 1.3507 \\ 4867 & 15: 41: 53 & 1.3494 \\ 4868 & 15: 41: 54 & 1.3488 \\ 4869 & 15: 41: 55 & 1.3461 \\ 4870 & 15: 41: 56 & 1.3499 \\ 4871 & 15: 41: 57 & 1.3537 \\ 4872 & 15: 41: 58 & 1.3500 \\ 4873 & 15: 41: 59 & 1.3470 \\ 4874 & 15: 42: 00 & 1.3502 \\ 4875 & 15: 42: 01 & 1.3513 \\ 4876 & 15: 42: 02 & 1.3494 \\ 4877 & 15: 42: 03 & 1.3499 \\ 4878 & 15: 42: 04 & 1.3501 \\ 4879 & 15: 42: 05 & 1.3510 \\ 4880 & 15: 42: 06 & 1.3505 \\ 4881 & 15: 42: 07 & 1.3490 \\ 4882 & 15: 42: 08 & 1.3461 \\ 4883 & 15: 42: 09 & 1.3502 \\ 4884 & 15: 42: 10 & 1.3475 \\ 4885 & 15: 42: 11 & 1.3459 \\ 4886 & 15: 42: 12 & 1.3504 \\ 4887 & 15: 42: 13 & 1.3519 \\ 4888 & 15: 42: 14 & 1.3505 \\ 4889 & 15: 42: 15 & 1.3490 \\ 4890 & 15: 42: 16 & 1.3482 \\ 4891 & 15: 42: 17 & 1.3515 \\ 4892 & 15: 42: 18 & 1.3522 \\ 4893 & 15: 42: 19 & 1.3510 \\ 4894 & 15: 42: 20 & 1.3494\end{array}$


Appendix G - Fuel Data Test 4 NIST102705_1.xls

$\begin{array}{lll}4895 & 15: 42: 21 & 1.3487 \\ 4896 & 15: 42: 22 & 1.3495 \\ 4897 & 15: 42: 23 & 1.3493 \\ 4898 & 15: 42: 24 & 1.3473 \\ 4899 & 15: 42: 25 & 1.3478 \\ 4900 & 15: 42: 26 & 1.3492 \\ 4901 & 15: 42: 27 & 1.3517 \\ 4902 & 15: 42: 28 & 1.3516 \\ 4903 & 15: 42: 29 & 1.3496 \\ 4904 & 15: 42: 30 & 1.3486 \\ 4905 & 15: 42: 31 & 1.3529 \\ 4906 & 15: 42: 32 & 1.3521 \\ 4907 & 15: 42: 33 & 1.3513 \\ 4908 & 15: 42: 34 & 1.3515 \\ 4909 & 15: 42: 35 & 1.3490 \\ 4910 & 15: 42: 36 & 1.3495 \\ 4911 & 15: 42: 37 & 1.3513 \\ 4912 & 15: 42: 38 & 1.3486 \\ 4913 & 15: 42: 39 & 1.3465 \\ 4914 & 15: 42: 40 & 1.3511 \\ 4915 & 15: 42: 41 & 1.3519 \\ 4916 & 15: 42: 42 & 1.3504 \\ 4917 & 15: 42: 43 & 1.3514 \\ 4918 & 15: 42: 44 & 1.3505 \\ 4919 & 15: 42: 45 & 1.3511 \\ 4920 & 15: 42: 46 & 1.3503 \\ 4921 & 15: 42: 47 & 1.3477 \\ 4922 & 15: 42: 48 & 1.3493 \\ 4923 & 15: 42: 49 & 1.3506 \\ 4924 & 15: 42: 50 & 1.3498 \\ 4925 & 15: 42: 51 & 1.3486 \\ 4926 & 15: 42: 52 & 1.3493 \\ 4927 & 15: 42: 53 & 1.3483 \\ 4928 & 15: 42: 54 & 1.3499 \\ 4929 & 15: 42: 55 & 1.3527 \\ 4930 & 15: 42: 56 & 1.3471 \\ 4931 & 15: 42: 57 & 1.3482 \\ 4932 & 15: 42: 58 & 1.3518 \\ 4933 & 15: 42: 59 & 1.3509 \\ 4934 & 15: 43: 00 & 1.3487 \\ 4935 & 15: 43: 01 & 1.3488 \\ 4936 & 15: 43: 02 & 1.3463 \\ 4937 & 15: 43: 03 & 1.3499 \\ 4938 & 15: 43: 04 & 1.3488 \\ 4939 & 15: 43: 05 & 1.3475 \\ 4940 & 15: 43: 06 & 1.3486 \\ 4941 & 15: 43: 07 & 1.3512 \\ 4942 & 15: 43: 08 & 1.3508 \\ 4943 & 15: 43: 09 & 1.3496 \\ 4944 & 15: 43: 10 & 1.3497 \\ 4945 & 15: 43: 11 & 1.3496\end{array}$


Appendix G - Fuel Data Test 4 NIST102705_1.xls

$\begin{array}{lll}4946 & 15: 43: 12 & 1.3485 \\ 4947 & 15: 43: 13 & 1.3460 \\ 4948 & 15: 43: 14 & 1.3498 \\ 4949 & 15: 43: 15 & 1.3516 \\ 4950 & 15: 43: 16 & 1.3492 \\ 4951 & 15: 43: 17 & 1.3455 \\ 4952 & 15: 43: 18 & 1.3462 \\ 4953 & 15: 43: 19 & 1.3505 \\ 4954 & 15: 43: 20 & 1.3526 \\ 4955 & 15: 43: 21 & 1.3463 \\ 4956 & 15: 43: 22 & 1.3491 \\ 4957 & 15: 43: 23 & 1.3503 \\ 4958 & 15: 43: 24 & 1.3490 \\ 4959 & 15: 43: 25 & 1.3527 \\ 4960 & 15: 43: 26 & 1.3515 \\ 4961 & 15: 43: 27 & 1.3501 \\ 4962 & 15: 43: 28 & 1.3481 \\ 4963 & 15: 43: 29 & 1.3502 \\ 4964 & 15: 43: 30 & 1.3528 \\ 4965 & 15: 43: 31 & 1.3541 \\ 4966 & 15: 43: 32 & 1.3489 \\ 4967 & 15: 43: 33 & 1.3481 \\ 4968 & 15: 43: 34 & 1.3483 \\ 4969 & 15: 43: 35 & 1.3513 \\ 4970 & 15: 43: 36 & 1.3529 \\ 4971 & 15: 43: 37 & 1.3474 \\ 4972 & 15: 43: 38 & 1.3453 \\ 4973 & 15: 43: 39 & 1.3478 \\ 4974 & 15: 43: 40 & 1.3514 \\ 4975 & 15: 43: 41 & 1.3483 \\ 4976 & 15: 43: 42 & 1.3465 \\ 4977 & 15: 43: 43 & 1.3491 \\ 4978 & 15: 43: 44 & 1.3480 \\ 4979 & 15: 43: 45 & 1.3496 \\ 4980 & 15: 43: 46 & 1.3522 \\ 4981 & 15: 43: 47 & 1.3522 \\ 4982 & 15: 43: 48 & 1.3484 \\ 4983 & 15: 43: 49 & 1.3450 \\ 4984 & 15: 43: 50 & 1.3483 \\ 4985 & 15: 43: 51 & 1.3504 \\ 4986 & 15: 43: 52 & 1.3496 \\ 4987 & 15: 43: 53 & 1.3476 \\ 4988 & 15: 43: 54 & 1.3459 \\ 4989 & 15: 43: 55 & 1.3479 \\ 4990 & 15: 43: 56 & 1.3502 \\ 4991 & 15: 43: 57 & 1.3496 \\ 4992 & 15: 43: 58 & 1.3486 \\ 4993 & 15: 43: 59 & 1.3473 \\ 4994 & 15: 44: 00 & 1.3482 \\ 4995 & 15: 44: 01 & 1.3514 \\ 4996 & 15: 44: 02 & 1.3516\end{array}$


Appendix G - Fuel Data Test 4 NIST102705_1.xls

$\begin{array}{lll}4997 & 15: 44: 03 & 1.3481 \\ 4998 & 15: 44: 04 & 1.3496 \\ 4999 & 15: 44: 05 & 1.3508 \\ 5000 & 15: 44: 06 & 1.3502 \\ 5001 & 15: 44: 07 & 1.3510 \\ 5002 & 15: 44: 08 & 1.3474 \\ 5003 & 15: 44: 09 & 1.3470 \\ 5004 & 15: 44: 10 & 1.3482 \\ 5005 & 15: 44: 11 & 1.3491 \\ 5006 & 15: 44: 12 & 1.3540 \\ 5007 & 15: 44: 13 & 1.3513 \\ 5008 & 15: 44: 14 & 1.3462 \\ 5009 & 15: 44: 15 & 1.3462 \\ 5010 & 15: 44: 16 & 1.3484 \\ 5011 & 15: 44: 17 & 1.3491 \\ 5012 & 15: 44: 18 & 1.3502 \\ 5013 & 15: 44: 19 & 1.3482 \\ 5014 & 15: 44: 20 & 1.3473 \\ 5015 & 15: 44: 21 & 1.3503 \\ 5016 & 15: 44: 22 & 1.3488 \\ 5017 & 15: 44: 23 & 1.3471 \\ 5018 & 15: 44: 24 & 1.3520 \\ 5019 & 15: 44: 25 & 1.3522 \\ 5020 & 15: 44: 26 & 1.3476 \\ 5021 & 15: 44: 27 & 1.3494 \\ 5022 & 15: 44: 28 & 1.3524 \\ 5023 & 15: 44: 29 & 1.3525 \\ 5024 & 15: 44: 30 & 1.3515 \\ 5025 & 15: 44: 31 & 1.3483 \\ 5026 & 15: 44: 32 & 1.3469 \\ 5027 & 15: 44: 33 & 1.3523 \\ 5028 & 15: 44: 34 & 1.3501 \\ 5029 & 15: 44: 35 & 1.3529 \\ 5030 & 15: 44: 36 & 1.3514 \\ 5031 & 15: 44: 37 & 1.3484 \\ 5032 & 15: 44: 38 & 1.3502 \\ 5033 & 15: 44: 39 & 1.3502 \\ 5034 & 15: 44: 40 & 1.3504 \\ 5035 & 15: 44: 41 & 1.3492 \\ 5036 & 15: 44: 42 & 1.3517 \\ 5037 & 15: 44: 43 & 1.3557 \\ 5038 & 15: 44: 44 & 1.3511 \\ 5039 & 15: 44: 45 & 1.3474 \\ 5040 & 15: 44: 46 & 1.3460 \\ 5041 & 15: 44: 47 & 1.3479 \\ 5042 & 15: 44: 48 & 1.3476 \\ 5043 & 15: 44: 49 & 1.3514 \\ 5044 & 15: 44: 50 & 1.3525 \\ 5045 & 15: 44: 51 & 1.3493 \\ 5046 & 15: 44: 52 & 1.3489 \\ 5047 & 15: 44: 53 & 1.3508\end{array}$


Appendix G - Fuel Data Test 4 NIST102705_1.xls

$\begin{array}{lll}5048 & 15: 44: 54 & 1.3504 \\ 5049 & 15: 44: 55 & 1.3500 \\ 5050 & 15: 44: 56 & 1.3497 \\ 5051 & 15: 44: 57 & 1.3467 \\ 5052 & 15: 44: 58 & 1.3501 \\ 5053 & 15: 44: 59 & 1.3515 \\ 5054 & 15: 45: 00 & 1.3499 \\ 5055 & 15: 45: 01 & 1.3468 \\ 5056 & 15: 45: 02 & 1.3424 \\ 5057 & 15: 45: 03 & 1.3472 \\ 5058 & 15: 45: 04 & 1.3508 \\ 5059 & 15: 45: 05 & 1.3487 \\ 5060 & 15: 45: 06 & 1.3491 \\ 5061 & 15: 45: 07 & 1.3507 \\ 5062 & 15: 45: 08 & 1.3503 \\ 5063 & 15: 45: 09 & 1.3498 \\ 5064 & 15: 45: 10 & 1.3504 \\ 5065 & 15: 45: 11 & 1.3497 \\ 5066 & 15: 45: 12 & 1.3469 \\ 5067 & 15: 45: 13 & 1.3476 \\ 5068 & 15: 45: 14 & 1.3492 \\ 5069 & 15: 45: 15 & 1.3467 \\ 5070 & 15: 45: 16 & 1.3472 \\ 5071 & 15: 45: 17 & 1.3460 \\ 5072 & 15: 45: 18 & 1.3492 \\ 5073 & 15: 45: 19 & 1.3524 \\ 5074 & 15: 45: 20 & 1.3521 \\ 5075 & 15: 45: 21 & 1.3487 \\ 5076 & 15: 45: 22 & 1.3471 \\ 5077 & 15: 45: 23 & 1.3454 \\ 5078 & 15: 45: 24 & 1.3480 \\ 5079 & 15: 45: 25 & 1.3489 \\ 5080 & 15: 45: 26 & 1.3461 \\ 5081 & 15: 45: 27 & 1.3461 \\ 5082 & 15: 45: 28 & 1.3476 \\ 5083 & 15: 45: 29 & 1.3496 \\ 5084 & 15: 45: 30 & 1.3496 \\ 5085 & 15: 45: 31 & 1.3506 \\ 5086 & 15: 45: 32 & 1.3495 \\ 5087 & 15: 45: 33 & 1.3480 \\ 5088 & 15: 45: 34 & 1.3496 \\ 5089 & 15: 45: 35 & 1.3481 \\ 5090 & 15: 45: 36 & 1.3512 \\ 5091 & 15: 45: 37 & 1.3499 \\ 5092 & 15: 45: 38 & 1.3473 \\ 5093 & 15: 45: 39 & 1.3487 \\ 5094 & 15: 45: 40 & 1.3473 \\ 5095 & 15: 45: 41 & 1.3493 \\ 5096 & 15: 45: 42 & 1.3512 \\ 5097 & 15: 45: 43 & 1.3471 \\ 5098 & 15: 45: 44 & 1.3440\end{array}$


Appendix G - Fuel Data Test 4 NIST102705_1.xls

$\begin{array}{lll}5099 & 15: 45: 45 & 1.3479 \\ 5100 & 15: 45: 46 & 1.3493 \\ 5101 & 15: 45: 47 & 1.3503 \\ 5102 & 15: 45: 48 & 1.3501 \\ 5103 & 15: 45: 49 & 1.3472 \\ 5104 & 15: 45: 50 & 1.3471 \\ 5105 & 15: 45: 51 & 1.3517 \\ 5106 & 15: 45: 52 & 1.3500 \\ 5107 & 15: 45: 53 & 1.3489 \\ 5108 & 15: 45: 54 & 1.3487 \\ 5109 & 15: 45: 55 & 1.3477 \\ 5110 & 15: 45: 56 & 1.3522 \\ 5111 & 15: 45: 57 & 1.3517 \\ 5112 & 15: 45: 58 & 1.3484 \\ 5113 & 15: 45: 59 & 1.3494 \\ 5114 & 15: 46: 00 & 1.3508 \\ 5115 & 15: 46: 01 & 1.3492 \\ 5116 & 15: 46: 02 & 1.3516 \\ 5117 & 15: 46: 03 & 1.3507 \\ 5118 & 15: 46: 04 & 1.3488 \\ 5119 & 15: 46: 05 & 1.3484 \\ 5120 & 15: 46: 06 & 1.3501 \\ 5121 & 15: 46: 07 & 1.3512 \\ 5122 & 15: 46: 08 & 1.3504 \\ 5123 & 15: 46: 09 & 1.3494 \\ 5124 & 15: 46: 10 & 1.3496 \\ 5125 & 15: 46: 11 & 1.3506 \\ 5126 & 15: 46: 12 & 1.3514 \\ 5127 & 15: 46: 13 & 1.3518 \\ 5128 & 15: 46: 14 & 1.3486 \\ 5129 & 15: 46: 15 & 1.3486 \\ 5130 & 15: 46: 16 & 1.3496 \\ 5131 & 15: 46: 17 & 1.3480 \\ 5132 & 15: 46: 18 & 1.3498 \\ 5133 & 15: 46: 19 & 1.3496 \\ 5134 & 15: 46: 20 & 1.3490 \\ 5135 & 15: 46: 21 & 1.3492 \\ 5136 & 15: 46: 22 & 1.3492 \\ 5137 & 15: 46: 23 & 1.3501 \\ 5138 & 15: 46: 24 & 1.3497 \\ 5139 & 15: 46: 25 & 1.3469 \\ 5140 & 15: 46: 26 & 1.3502 \\ 5141 & 15: 46: 27 & 1.3510 \\ 5142 & 15: 46: 28 & 1.3497 \\ 5143 & 15: 46: 29 & 1.3466 \\ 5144 & 15: 46: 30 & 1.3472 \\ 5145 & 15: 46: 31 & 1.3504 \\ 5146 & 15: 46: 32 & 1.3502 \\ 5147 & 15: 46: 33 & 1.3494 \\ 5148 & 15: 46: 34 & 1.3498 \\ 5149 & 15: 46: 35 & 1.3475\end{array}$


Appendix G - Fuel Data Test 4 NIST102705_1.xls

$\begin{array}{lll}5150 & 15: 46: 36 & 1.3487 \\ 5151 & 15: 46: 37 & 1.3506 \\ 5152 & 15: 46: 38 & 1.3532 \\ 5153 & 15: 46: 39 & 1.3508 \\ 5154 & 15: 46: 40 & 1.3492 \\ 5155 & 15: 46: 41 & 1.3484 \\ 5156 & 15: 46: 42 & 1.3466 \\ 5157 & 15: 46: 43 & 1.3484 \\ 5158 & 15: 46: 44 & 1.3504 \\ 5159 & 15: 46: 45 & 1.3501 \\ 5160 & 15: 46: 46 & 1.3494 \\ 5161 & 15: 46: 47 & 1.3469 \\ 5162 & 15: 46: 48 & 1.3493 \\ 5163 & 15: 46: 49 & 1.3513 \\ 5164 & 15: 46: 50 & 1.3490 \\ 5165 & 15: 46: 51 & 1.3482 \\ 5166 & 15: 46: 52 & 1.3490 \\ 5167 & 15: 46: 53 & 1.3490 \\ 5168 & 15: 46: 54 & 1.3498 \\ 5169 & 15: 46: 55 & 1.3502 \\ 5170 & 15: 46: 56 & 1.3502 \\ 5171 & 15: 46: 57 & 1.3508 \\ 5172 & 15: 46: 58 & 1.3497 \\ 5173 & 15: 46: 59 & 1.3512 \\ 5174 & 15: 47: 00 & 1.3479 \\ 5175 & 15: 47: 01 & 1.3473 \\ 5176 & 15: 47: 02 & 1.3485 \\ 5177 & 15: 47: 03 & 1.3478 \\ 5178 & 15: 47: 04 & 1.3492 \\ 5179 & 15: 47: 05 & 1.3510 \\ 5180 & 15: 47: 06 & 1.3509 \\ 5181 & 15: 47: 07 & 1.3502 \\ 5182 & 15: 47: 08 & 1.3505 \\ 5183 & 15: 47: 09 & 1.3487 \\ 5184 & 15: 47: 10 & 1.3490 \\ 5185 & 15: 47: 11 & 1.3490 \\ 5186 & 15: 47: 12 & 1.3490 \\ 5187 & 15: 47: 13 & 1.3483 \\ 5188 & 15: 47: 14 & 1.3500 \\ 5189 & 15: 47: 15 & 1.3523 \\ 5190 & 15: 47: 16 & 1.3491 \\ 5191 & 15: 47: 17 & 1.3455 \\ 5192 & 15: 47: 18 & 1.3460 \\ 5193 & 15: 47: 19 & 1.3504 \\ 5194 & 15: 47: 20 & 1.3505 \\ 5195 & 15: 47: 21 & 1.3510 \\ 5196 & 15: 47: 22 & 1.3506 \\ 5197 & 15: 47: 23 & 1.3462 \\ 5198 & 15: 47: 24 & 1.3461 \\ 5199 & 15: 47: 25 & 1.3509 \\ 5200 & 15: 47: 26 & 1.3496\end{array}$


Appendix G - Fuel Data Test 4 NIST102705_1.xls

$\begin{array}{lll}5201 & 15: 47: 27 & 1.3496 \\ 5202 & 15: 47: 28 & 1.3478 \\ 5203 & 15: 47: 29 & 1.3493 \\ 5204 & 15: 47: 30 & 1.3514 \\ 5205 & 15: 47: 31 & 1.3484 \\ 5206 & 15: 47: 32 & 1.3486 \\ 5207 & 15: 47: 33 & 1.3499 \\ 5208 & 15: 47: 34 & 1.3479 \\ 5209 & 15: 47: 35 & 1.3490 \\ 5210 & 15: 47: 36 & 1.3506 \\ 5211 & 15: 47: 37 & 1.3493 \\ 5212 & 15: 47: 38 & 1.3490 \\ 5213 & 15: 47: 39 & 1.3453 \\ 5214 & 15: 47: 40 & 1.3487 \\ 5215 & 15: 47: 41 & 1.3531 \\ 5216 & 15: 47: 42 & 1.3500 \\ 5217 & 15: 47: 43 & 1.3465 \\ 5218 & 15: 47: 44 & 1.3487 \\ 5219 & 15: 47: 45 & 1.3499 \\ 5220 & 15: 47: 46 & 1.3497 \\ 5221 & 15: 47: 47 & 1.3505 \\ 5222 & 15: 47: 48 & 1.3485 \\ 5223 & 15: 47: 49 & 1.3484 \\ 5224 & 15: 47: 50 & 1.3523 \\ 5225 & 15: 47: 51 & 1.3501 \\ 5226 & 15: 47: 52 & 1.3458 \\ 5227 & 15: 47: 53 & 1.3502 \\ 5228 & 15: 47: 54 & 1.3491 \\ 5229 & 15: 47: 55 & 1.3464 \\ 5230 & 15: 47: 56 & 1.3490 \\ 5231 & 15: 47: 57 & 1.3504 \\ 5232 & 15: 47: 58 & 1.3500 \\ 5233 & 15: 47: 59 & 1.3489 \\ 5234 & 15: 48: 00 & 1.3515 \\ 5235 & 15: 48: 01 & 1.3517 \\ 5236 & 15: 48: 02 & 1.3503 \\ 5237 & 15: 48: 03 & 1.3488 \\ 5238 & 15: 48: 04 & 1.3496 \\ 5239 & 15: 48: 05 & 1.3500 \\ 5240 & 15: 48: 06 & 1.3490 \\ 5241 & 15: 48: 07 & 1.3492 \\ 5242 & 15: 48: 08 & 1.3471 \\ 5243 & 15: 48: 09 & 1.3485 \\ 5244 & 15: 48: 10 & 1.3493 \\ 5245 & 15: 48: 11 & 1.3499 \\ 5246 & 15: 48: 12 & 1.3524 \\ 5247 & 15: 48: 13 & 1.3503 \\ 5248 & 15: 48: 14 & 1.3477 \\ 5249 & 15: 48: 15 & 1.3479 \\ 5250 & 15: 48: 16 & 1.3455 \\ 5251 & 15: 48: 17 & 1.3481\end{array}$


Appendix G - Fuel Data Test 4 NIST102705_1.xIs

$\begin{array}{lll}5252 & 15: 48: 18 & 1.3518 \\ 5253 & 15: 48: 19 & 1.3515 \\ 5254 & 15: 48: 20 & 1.3474 \\ 5256 & 15: 48: 22 & 1.3512 \\ 5256 & 15: 48: 22 & 1.3522 \\ 5257 & 15: 48: 23 & 1.3483 \\ 5258 & 15: 48: 24 & 1.3484 \\ 5259 & 15: 48: 25 & 1.3483 \\ 5260 & 15: 48: 26 & 1.3471 \\ 5261 & 15: 48: 27 & 1.3502 \\ 5262 & 15: 48: 28 & 1.3508 \\ 5263 & 15: 48: 29 & 1.3491 \\ 5264 & 15: 48: 30 & 1.3480 \\ 5265 & 15: 48: 31 & 1.3492 \\ 5266 & 15: 48: 32 & 1.3541 \\ 5267 & 15: 48: 33 & 1.3536 \\ 5268 & 15: 48: 34 & 1.3517 \\ 5269 & 15: 48: 35 & 1.3498 \\ 5270 & 15: 48: 36 & 1.3479 \\ 5271 & 15: 48: 37 & 1.3484 \\ 5272 & 15: 48: 38 & 1.3509 \\ 5273 & 15: 48: 39 & 1.3501 \\ 5274 & 15: 48: 40 & 1.3510 \\ 5275 & 15: 48: 41 & 1.3482 \\ 5276 & 15: 48: 42 & 1.3484 \\ 5277 & 15: 48: 43 & 1.3505 \\ 5278 & 15: 48: 44 & 1.3492 \\ 5279 & 15: 48: 45 & 1.3456 \\ 5280 & 15: 48: 46 & 1.3463 \\ 5281 & 15: 48: 47 & 1.3479 \\ 5282 & 15: 48: 48 & 1.3496 \\ 5283 & 15: 48: 49 & 1.3522 \\ 5284 & 15: 48: 50 & 1.3484 \\ 5285 & 15: 48: 51 & 1.3460 \\ 5286 & 15: 48: 52 & 1.3498 \\ 5287 & 15: 48: 53 & 1.3509 \\ 5288 & 15: 48: 54 & 1.3506 \\ 5289 & 15: 48: 55 & 1.3483 \\ 5290 & 15: 48: 56 & 1.3488 \\ 5291 & 15: 48: 57 & 1.3496 \\ 5292 & 15: 48: 58 & 1.3494 \\ 5293 & 15: 48: 59 & 1.3475 \\ 5294 & 15: 49: 00 & 1.3495 \\ 5295 & 15: 49: 01 & 1.3519 \\ 5296 & 15: 49: 02 & 1.3495 \\ 5297 & 15: 49: 03 & 1.3497 \\ 5298 & 15: 49: 04 & 1.3488 \\ 5299 & 15: 49: 05 & 1.3472 \\ 5300 & 15: 49: 06 & 1.3499 \\ 5301 & 15: 49: 07 & 1.3497 \\ 5302 & 15: 49: 08 & 1.3476\end{array}$


Appendix G - Fuel Data Test 4 NIST102705_1.xls

$\begin{array}{lll}5303 & 15: 49: 09 & 1.3498 \\ 5304 & 15: 49: 10 & 1.3470 \\ 5305 & 15: 49: 11 & 1.3512 \\ 5306 & 15: 49: 12 & 1.3522 \\ 5307 & 15: 49: 13 & 1.3473 \\ 5308 & 15: 49: 14 & 1.3497 \\ 5309 & 15: 49: 15 & 1.3519 \\ 5310 & 15: 49: 16 & 1.3488 \\ 5311 & 15: 49: 17 & 1.3474 \\ 5312 & 15: 49: 18 & 1.3470 \\ 5313 & 15: 49: 19 & 1.3465 \\ 5314 & 15: 49: 20 & 1.3524 \\ 5315 & 15: 49: 21 & 1.3494 \\ 5316 & 15: 49: 22 & 1.3483 \\ 5317 & 15: 49: 23 & 1.3474 \\ 5318 & 15: 49: 24 & 1.3485 \\ 5319 & 15: 49: 25 & 1.3509 \\ 5320 & 15: 49: 26 & 1.3502 \\ 5321 & 15: 49: 27 & 1.3493 \\ 5322 & 15: 49: 28 & 1.3477 \\ 5323 & 15: 49: 29 & 1.3477 \\ 5324 & 15: 49: 30 & 1.3477 \\ 5325 & 15: 49: 31 & 1.3506 \\ 5326 & 15: 49: 32 & 1.3499 \\ 5327 & 15: 49: 33 & 1.3493 \\ 5328 & 15: 49: 34 & 1.3499 \\ 5329 & 15: 49: 35 & 1.3511 \\ 5330 & 15: 49: 36 & 1.3496 \\ 5331 & 15: 49: 37 & 1.3457 \\ 5332 & 15: 49: 38 & 1.3464 \\ 5333 & 15: 49: 39 & 1.3485 \\ 5334 & 15: 49: 40 & 1.3491 \\ 5335 & 15: 49: 41 & 1.3493 \\ 5336 & 15: 49: 42 & 1.3501 \\ 5337 & 15: 49: 43 & 1.3487 \\ 5338 & 15: 49: 44 & 1.3459 \\ 5339 & 15: 49: 45 & 1.3464 \\ 5340 & 15: 49: 46 & 1.3283 \\ 5341 & 15: 49: 47 & 1.2869 \\ 5342 & 15: 49: 48 & 1.2611 \\ 5343 & 15: 49: 49 & 1.2304 \\ 5344 & 15: 49: 50 & 1.1894 \\ 5345 & 15: 49: 51 & 1.1686 \\ 5346 & 15: 49: 52 & 1.1500 \\ 5347 & 15: 49: 53 & 1.1430 \\ 5348 & 15: 49: 54 & 1.1376 \\ 5349 & 15: 49: 55 & 1.1408 \\ 5350 & 15: 49: 56 & 1.1452 \\ 5351 & 15: 49: 57 & 1.1447 \\ 5352 & 15: 49: 58 & 1.1386 \\ 5353 & 15: 49: 59 & 1.1374\end{array}$


Appendix G - Fuel Data Test 4 NIST102705_1.xls

$\begin{array}{lll}5354 & 15: 50: 00 & 1.1393 \\ 5355 & 15: 50: 01 & 1.1449 \\ 5356 & 15: 50: 02 & 1.1498 \\ 5357 & 15: 50: 03 & 1.1521 \\ 5358 & 15: 50: 04 & 1.1519 \\ 5359 & 15: 50: 05 & 1.1439 \\ 5360 & 15: 50: 06 & 1.1361 \\ 5361 & 15: 50: 07 & 1.1332 \\ 5362 & 15: 50: 08 & 1.1409 \\ 5363 & 15: 50: 09 & 1.1508 \\ 5364 & 15: 50: 10 & 1.1503 \\ 5365 & 15: 50: 11 & 1.1493 \\ 5366 & 15: 50: 12 & 1.1448 \\ 5367 & 15: 50: 13 & 1.1376 \\ 5368 & 15: 50: 14 & 1.1331 \\ 5369 & 15: 50: 15 & 1.1376 \\ 5370 & 15: 50: 16 & 1.1452 \\ 5371 & 15: 50: 17 & 1.1518 \\ 5372 & 15: 50: 18 & 1.1503 \\ 5373 & 15: 50: 19 & 1.1438 \\ 5374 & 15: 50: 20 & 1.1380 \\ 5375 & 15: 50: 21 & 1.1387 \\ 5376 & 15: 50: 22 & 1.1406 \\ 5377 & 15: 50: 23 & 1.1492 \\ 5378 & 15: 50: 24 & 1.1512 \\ 5379 & 15: 50: 25 & 1.1490 \\ 5380 & 15: 50: 26 & 1.1460 \\ 5381 & 15: 50: 27 & 1.1468 \\ 5382 & 15: 50: 28 & 1.1402 \\ 5383 & 15: 50: 29 & 1.1399 \\ 5384 & 15: 50: 30 & 1.1453 \\ 5385 & 15: 50: 31 & 1.1453 \\ 5386 & 15: 50: 32 & 1.1424 \\ 5387 & 15: 50: 33 & 1.1423 \\ 5388 & 15: 50: 34 & 1.1487 \\ 5389 & 15: 50: 35 & 1.1498 \\ 5390 & 15: 50: 36 & 1.1516 \\ 5391 & 15: 50: 37 & 1.1506 \\ 5392 & 15: 50: 38 & 1.1419 \\ 5393 & 15: 50: 39 & 1.1326 \\ 5394 & 15: 50: 40 & 1.1336 \\ 5395 & 15: 50: 41 & 1.1382 \\ 5396 & 15: 50: 42 & 1.1426 \\ 5397 & 15: 50: 43 & 1.1452 \\ 5398 & 15: 50: 44 & 1.1491 \\ 5399 & 15: 50: 45 & 1.1416 \\ 5400 & 15: 50: 46 & 1.1215 \\ 5401 & 15: 50: 47 & 1.0784 \\ 5402 & 15: 50: 48 & 1.0160 \\ 5403 & 15: 50: 49 & 0.9830 \\ 5404 & 15: 50: 50 & 0.9653\end{array}$


Appendix G - Fuel Data Test 4 NIST102705_1.xls

$\begin{array}{lll}5405 & 15: 50: 51 & 0.9528 \\ 5406 & 15: 50: 52 & 0.9512 \\ 5407 & 15: 50: 53 & 0.9383 \\ 5408 & 15: 50: 54 & 0.9376 \\ 5409 & 15: 50: 55 & 0.9390 \\ 5410 & 15: 50: 56 & 0.9250 \\ 5411 & 15: 50: 57 & 0.9313 \\ 5412 & 15: 50: 58 & 0.9342 \\ 5413 & 15: 50: 59 & 0.9247 \\ 5414 & 15: 51: 00 & 0.9347 \\ 5415 & 15: 51: 01 & 0.9342 \\ 5416 & 15: 51: 02 & 0.9256 \\ 5417 & 15: 51: 03 & 0.9399 \\ 5418 & 15: 51: 04 & 0.9348 \\ 5419 & 15: 51: 05 & 0.9320 \\ 5420 & 15: 51: 06 & 0.9310 \\ 5421 & 15: 51: 07 & 0.9251 \\ 5422 & 15: 51: 08 & 0.9375 \\ 5423 & 15: 51: 09 & 0.9349 \\ 5424 & 15: 51: 10 & 0.9260 \\ 5425 & 15: 51: 11 & 0.9309 \\ 5426 & 15: 51: 12 & 0.9397 \\ 5427 & 15: 51: 13 & 0.9299 \\ 5428 & 15: 51: 14 & 0.9291 \\ 5429 & 15: 51: 15 & 0.9415 \\ 5430 & 15: 51: 16 & 0.9391 \\ 5431 & 15: 51: 17 & 0.9342 \\ 5432 & 15: 51: 18 & 0.9345 \\ 5433 & 15: 51: 19 & 0.9273 \\ 5434 & 15: 51: 20 & 0.9373 \\ 5435 & 15: 51: 21 & 0.9350 \\ 5436 & 15: 51: 22 & 0.9257 \\ 5437 & 15: 51: 23 & 0.9395 \\ 5438 & 15: 51: 24 & 0.9362 \\ 5439 & 15: 51: 25 & 0.9311 \\ 5440 & 15: 51: 26 & 0.9133 \\ 5441 & 15: 51: 27 & 0.4298 \\ 5442 & 15: 51: 28 & 0.0659\end{array}$


APPENDIX H. - Laser Data Test 4 NIST 102705

Appendix $\mathrm{H}$ is laser data from Test 4 in tabular format and is approximately 54 pages in length. Fuel data is included with electronic version of report on compact disk, but not routinely included with paper version of report. 
Time from Ignition, s EDT Time $\quad$ //lo East $\quad$ //lo West

$\begin{array}{rlll}0 & 14: 20: 46 & 1.0281 & 0.6049 \\ 2 & 14: 20: 48 & 0.7031 & 0.3299 \\ 4 & 14: 20: 50 & 1.0692 & 0.3666 \\ 6 & 14: 20: 52 & 0.6321 & 0.3942 \\ 8 & 14: 20: 54 & 0.9382 & 0.5388 \\ 10 & 14: 20: 56 & 0.9550 & 0.3544 \\ 12 & 14: 20: 58 & 1.0623 & 0.2188 \\ 14 & 14: 21: 00 & 0.7891 & 0.3325 \\ 16 & 14: 21: 02 & 0.7643 & 0.1038 \\ 18 & 14: 21: 04 & 0.2016 & 0.4772 \\ 20 & 14: 21: 06 & 0.2578 & 0.7654 \\ 22 & 14: 21: 08 & 0.0350 & 0.7432 \\ 24 & 14: 21: 10 & 0.2274 & 0.5445 \\ 26 & 14: 21: 12 & 0.6577 & 0.2248 \\ 28 & 14: 21: 14 & 0.2482 & 0.0776 \\ 30 & 14: 21: 16 & 0.1066 & 0.0342 \\ 32 & 14: 21: 18 & 0.2649 & 0.0202 \\ 34 & 14: 21: 20 & 0.3436 & 0.0264 \\ 36 & 14: 21: 22 & 0.1726 & 0.0536 \\ 38 & 14: 21: 24 & 0.6192 & 0.0355 \\ 40 & 14: 21: 26 & 0.0299 & 0.0065 \\ 42 & 14: 21: 28 & 0.1884 & 0.0389 \\ 44 & 14: 21: 30 & 0.0688 & 0.0099 \\ 46 & 14: 21: 32 & 0.0166 & 0.0418 \\ 48 & 14: 21: 34 & 1.0249 & 0.0197 \\ 50 & 14: 21: 36 & 0.6962 & 0.0537 \\ 52 & 14: 21: 38 & 0.1978 & 0.0420 \\ 54 & 14: 21: 40 & 0.0532 & 0.0037 \\ 56 & 14: 21: 42 & 0.0020 & 0.0413 \\ 58 & 14: 21: 44 & 0.0367 & 0.2400 \\ 60 & 14: 21: 46 & 0.0009 & 0.1885 \\ 62 & 14: 21: 48 & 0.0034 & 0.0367 \\ 64 & 14: 21: 50 & 0.0016 & 0.0150 \\ 66 & 14: 21: 52 & 0.0002 & 0.0100 \\ 68 & 14: 21: 54 & 0.0004 & 0.0059 \\ 70 & 14: 21: 56 & 0.0008 & 0.0224 \\ 72 & 14: 21: 58 & 0.0002 & 0.0131 \\ 74 & 14: 22: 00 & 0.0069 & 0.0789 \\ 76 & 14: 22: 02 & 0.0005 & 0.0285 \\ 78 & 14: 22: 04 & 0.0009 & 0.1458 \\ 80 & 14: 22: 06 & 0.0002 & 0.0054 \\ 82 & 14: 22: 08 & 0.0002 & 0.0357 \\ 84 & 14: 22: 10 & 0.0004 & 0.0016 \\ 86 & 14: 22: 12 & 0.0004 & 0.0303 \\ 88 & 14: 22: 14 & 0.0002 & 0.0078 \\ 90 & 14: 22: 16 & 0.0004 & 0.0006 \\ 92 & 14: 22: 18 & 0.0002 & 0.0141 \\ 94 & 14: 22: 20 & 0.0002 & 0.0112 \\ 96 & 14: 22: 22 & 0.0002 & 0.0016 \\ 98 & 14: 22: 24 & 0.0007 & 0.0153\end{array}$


Appendix H - Laser Data Test 4 NIST102705_1.xls

$\begin{array}{llll}100 & 14: 22: 26 & 0.0028 & 0.2194 \\ 102 & 14: 22: 28 & 0.0002 & 0.0287 \\ 104 & 14: 22: 30 & 0.0161 & 0.0365 \\ 106 & 14: 22: 32 & 0.0013 & 0.0047 \\ 108 & 14: 22: 34 & 0.0053 & 0.2055 \\ 110 & 14: 22: 36 & 0.0268 & 0.0300 \\ 112 & 14: 22: 38 & 0.0008 & 0.0731 \\ 114 & 14: 22: 40 & 0.0001 & 0.0127 \\ 116 & 14: 22: 42 & 0.0160 & 0.0107 \\ 118 & 14: 22: 44 & 0.0078 & 0.3451 \\ 120 & 14: 22: 46 & 0.0003 & 0.0020 \\ 122 & 14: 22: 48 & 0.0026 & 0.0556 \\ 124 & 14: 22: 50 & 0.0042 & 0.1038 \\ 126 & 14: 22: 52 & 0.0026 & 0.3042 \\ 128 & 14: 22: 54 & 0.0014 & 0.1806 \\ 130 & 14: 22: 56 & 0.0027 & 0.0152 \\ 132 & 14: 22: 58 & 0.0002 & 0.0217 \\ 134 & 14: 23: 00 & 0.0004 & 0.7179 \\ 136 & 14: 23: 02 & 0.0004 & 0.2708 \\ 138 & 14: 23: 04 & 0.0004 & 0.0140 \\ 140 & 14: 23: 06 & 0.0161 & 0.0211 \\ 142 & 14: 23: 08 & 0.0008 & 0.0170 \\ 144 & 14: 23: 10 & 0.0002 & 0.0355 \\ 146 & 14: 23: 12 & 0.0002 & 0.0100 \\ 148 & 14: 23: 14 & 0.0039 & 0.0890 \\ 150 & 14: 23: 16 & 0.0002 & 0.0125 \\ 152 & 14: 23: 18 & 0.0059 & 0.0198 \\ 154 & 14: 23: 20 & 0.0002 & 0.0021 \\ 156 & 14: 23: 22 & 0.0002 & 0.0006 \\ 158 & 14: 23: 24 & 0.0087 & 0.0016 \\ 160 & 14: 23: 26 & 0.0147 & 0.0028 \\ 162 & 14: 23: 28 & 0.0020 & 0.0091 \\ 164 & 14: 23: 30 & 0.0005 & 0.0067 \\ 166 & 14: 23: 32 & 0.0055 & 0.0510 \\ 168 & 14: 23: 34 & 0.0010 & 0.0255 \\ 170 & 14: 23: 36 & 0.0035 & 0.1525 \\ 172 & 14: 23: 38 & 0.0002 & 0.0211 \\ 174 & 14: 23: 40 & 0.0006 & 0.0583 \\ 176 & 14: 23: 42 & 0.0017 & 0.0119 \\ 178 & 14: 23: 44 & 0.0002 & 0.0004 \\ 180 & 14: 23: 46 & 0.0002 & 0.0013 \\ 182 & 14: 23: 48 & 0.0008 & 0.0065 \\ 184 & 14: 23: 50 & 0.0003 & 0.0207 \\ 186 & 14: 23: 52 & 0.0003 & 0.0003 \\ 188 & 14: 23: 54 & 0.0002 & 0.0016 \\ 190 & 14: 23: 56 & 0.0002 & 0.0006 \\ 192 & 14: 23: 58 & 0.0003 & 0.0002 \\ 194 & 14: 24: 00 & 0.0004 & 0.0409 \\ 196 & 14: 24: 02 & 0.0029 & 0.0011 \\ 198 & 14: 24: 04 & 0.0002 & 0.0107 \\ 200 & 14: 24: 06 & 0.0011 & 0.0004\end{array}$


Appendix H - Laser Data Test 4 NIST102705_1.xls

$\begin{array}{llll}202 & 14: 24: 08 & 0.0013 & 0.0018 \\ 204 & 14: 24: 10 & 0.0009 & 0.0137 \\ 206 & 14: 24: 12 & 0.0003 & 0.0028 \\ 208 & 14: 24: 14 & 0.0017 & 0.0036 \\ 210 & 14: 24: 16 & 0.0003 & 0.0011 \\ 212 & 14: 24: 18 & 0.0042 & 0.0037 \\ 214 & 14: 24: 20 & 0.0003 & 0.0201 \\ 216 & 14: 24: 22 & 0.0004 & 0.0157 \\ 218 & 14: 24: 24 & 0.0002 & 0.0376 \\ 220 & 14: 24: 26 & 0.0002 & 0.0209 \\ 222 & 14: 24: 28 & 0.0004 & 0.0217 \\ 224 & 14: 24: 30 & 0.0002 & 0.0112 \\ 226 & 14: 24: 32 & 0.0002 & 0.0004 \\ 228 & 14: 24: 34 & 0.0013 & 0.0007 \\ 230 & 14: 24: 36 & 0.0007 & 0.0002 \\ 232 & 14: 24: 38 & 0.0015 & 0.0002 \\ 234 & 14: 24: 40 & 0.0007 & 0.0002 \\ 236 & 14: 24: 42 & 0.0014 & 0.0957 \\ 238 & 14: 24: 44 & 0.0002 & 0.0095 \\ 240 & 14: 24: 46 & 0.0002 & 0.0022 \\ 242 & 14: 24: 48 & 0.0003 & 0.0649 \\ 244 & 14: 24: 50 & 0.0004 & 0.2837 \\ 246 & 14: 24: 52 & 0.0002 & 0.0034 \\ 248 & 14: 24: 54 & 0.0007 & 0.0054 \\ 250 & 14: 24: 56 & 0.0002 & 0.0016 \\ 252 & 14: 24: 58 & 0.0002 & 0.0014 \\ 254 & 14: 25: 00 & 0.0004 & 0.0001 \\ 256 & 14: 25: 02 & 0.0002 & 0.0014 \\ 258 & 14: 25: 04 & 0.0002 & 0.0048 \\ 260 & 14: 25: 06 & 0.0004 & 0.0277 \\ 262 & 14: 25: 08 & 0.0003 & 0.0037 \\ 264 & 14: 25: 10 & 0.0004 & 0.0094 \\ 266 & 14: 25: 12 & 0.0002 & 0.0050 \\ 268 & 14: 25: 14 & 0.0004 & 0.0410 \\ 270 & 14: 25: 16 & 0.0023 & 0.0115 \\ 272 & 14: 25: 18 & 0.0004 & 0.0019 \\ 274 & 14: 25: 20 & 0.0002 & 0.1864 \\ 276 & 14: 25: 22 & 0.0030 & 0.0002 \\ 278 & 14: 25: 24 & 0.0002 & 0.0309 \\ 280 & 14: 25: 26 & 0.0003 & 0.0053 \\ 282 & 14: 25: 28 & 0.0004 & 0.0002 \\ 284 & 14: 25: 30 & 0.0002 & 0.0010 \\ 286 & 14: 25: 32 & 0.0002 & 0.0037 \\ 288 & 14: 25: 34 & 0.0002 & 0.0003 \\ 290 & 14: 25: 36 & 0.0002 & 0.0143 \\ 292 & 14: 25: 38 & 0.0002 & 0.0444 \\ 294 & 14: 25: 40 & 0.0002 & 0.0106 \\ 296 & 14: 25: 42 & 0.0002 & 0.0244 \\ 298 & 14: 25: 44 & 0.0035 & 0.0001 \\ 300 & 14: 25: 46 & 0.0025 & 0.0001 \\ 302 & 14: 25: 48 & 0.0005 & 0.0679\end{array}$


Appendix H - Laser Data Test 4 NIST102705_1.xls

$\begin{array}{llll}304 & 14: 25: 50 & 0.0002 & 0.0001 \\ 306 & 14: 25: 52 & 0.0035 & 0.0012 \\ 308 & 14: 25: 54 & 0.0008 & 0.0126 \\ 310 & 14: 25: 56 & 0.0003 & 0.0002 \\ 312 & 14: 25: 58 & 0.0004 & 0.0057 \\ 314 & 14: 26: 00 & 0.0002 & 0.0006 \\ 316 & 14: 26: 02 & 0.0002 & 0.0002 \\ 318 & 14: 26: 04 & 0.0075 & 0.0006 \\ 320 & 14: 26: 06 & 0.0005 & 0.0347 \\ 322 & 14: 26: 08 & 0.0002 & 0.0318 \\ 324 & 14: 26: 10 & 0.0017 & 0.0002 \\ 326 & 14: 26: 12 & 0.0046 & 0.0037 \\ 328 & 14: 26: 14 & 0.0010 & 0.0313 \\ 330 & 14: 26: 16 & 0.0003 & 0.0003 \\ 332 & 14: 26: 18 & 0.0001 & 0.0059 \\ 334 & 14: 26: 20 & 0.0002 & 0.3160 \\ 336 & 14: 26: 22 & 0.0006 & 0.0007 \\ 338 & 14: 26: 24 & 0.0003 & 0.0772 \\ 340 & 14: 26: 26 & 0.0004 & 0.0003 \\ 342 & 14: 26: 28 & 0.0221 & 0.0085 \\ 344 & 14: 26: 30 & 0.0050 & 0.0002 \\ 346 & 14: 26: 32 & 0.0484 & 0.0010 \\ 348 & 14: 26: 34 & 0.0032 & 0.0009 \\ 350 & 14: 26: 36 & 0.0002 & 0.0017 \\ 352 & 14: 26: 38 & 0.0002 & 0.0016 \\ 354 & 14: 26: 40 & 0.0002 & 0.0071 \\ 356 & 14: 26: 42 & 0.0002 & 0.0033 \\ 358 & 14: 26: 44 & 0.0007 & 0.0013 \\ 360 & 14: 26: 46 & 0.0014 & 0.0002 \\ 362 & 14: 26: 48 & 0.0002 & 0.0002 \\ 364 & 14: 26: 50 & 0.0002 & 0.0003 \\ 366 & 14: 26: 52 & 0.0002 & 0.0002 \\ 368 & 14: 26: 54 & 0.0002 & 0.0002 \\ 370 & 14: 26: 56 & 0.0017 & 0.0087 \\ 372 & 14: 26: 58 & 0.0002 & 0.0003 \\ 374 & 14: 27: 00 & 0.0005 & 0.0002 \\ 376 & 14: 27: 02 & 0.0009 & 0.0039 \\ 378 & 14: 27: 04 & 0.0002 & 0.0009 \\ 380 & 14: 27: 06 & 0.0002 & 0.0055 \\ 382 & 14: 27: 08 & 0.0005 & 0.0005 \\ 384 & 14: 27: 10 & 0.0002 & 0.0059 \\ 386 & 14: 27: 12 & 0.0003 & 0.0366 \\ 388 & 14: 27: 14 & 0.0003 & 0.0085 \\ 390 & 14: 27: 16 & 0.0015 & 0.0052 \\ 392 & 14: 27: 18 & 0.0004 & 0.0001 \\ 394 & 14: 27: 20 & 0.0003 & 0.0002 \\ 396 & 14: 27: 22 & 0.0009 & 0.0002 \\ 398 & 14: 27: 24 & 0.0064 & 0.0086 \\ 400 & 14: 27: 26 & 0.0006 & 0.1457 \\ 402 & 14: 27: 28 & 0.0002 & 0.0002 \\ 404 & 14: 27: 30 & 0.0002 & 0.0004\end{array}$


Appendix H - Laser Data Test 4 NIST102705_1.xls

$\begin{array}{llll}406 & 14: 27: 32 & 0.0004 & 0.0002 \\ 408 & 14: 27: 34 & 0.0005 & 0.0002 \\ 410 & 14: 27: 36 & 0.0009 & 0.0002 \\ 412 & 14: 27: 38 & 0.0002 & 0.0026 \\ 414 & 14: 27: 40 & 0.0002 & 0.0039 \\ 416 & 14: 27: 42 & 0.0009 & 0.0002 \\ 418 & 14: 27: 44 & 0.0003 & 0.0009 \\ 420 & 14: 27: 46 & 0.0001 & 0.0001 \\ 422 & 14: 27: 48 & 0.0002 & 0.0285 \\ 424 & 14: 27: 50 & 0.0005 & 0.0083 \\ 426 & 14: 27: 52 & 0.0002 & 0.0097 \\ 428 & 14: 27: 54 & 0.0002 & 0.0002 \\ 430 & 14: 27: 56 & 0.0002 & 0.0017 \\ 432 & 14: 27: 58 & 0.0096 & 0.0112 \\ 434 & 14: 28: 00 & 0.0255 & 0.0475 \\ 436 & 14: 28: 02 & 0.0002 & 0.0001 \\ 438 & 14: 28: 04 & 0.0007 & 0.0007 \\ 440 & 14: 28: 06 & 0.0032 & 0.1126 \\ 442 & 14: 28: 08 & 0.0002 & 0.0005 \\ 444 & 14: 28: 10 & 0.0002 & 0.0003 \\ 446 & 14: 28: 12 & 0.0006 & 0.0001 \\ 448 & 14: 28: 14 & 0.0003 & 0.0002 \\ 450 & 14: 28: 16 & 0.0006 & 0.0002 \\ 452 & 14: 28: 18 & 0.0009 & 0.0013 \\ 454 & 14: 28: 20 & 0.0006 & 0.0017 \\ 456 & 14: 28: 22 & 0.0083 & 0.0002 \\ 458 & 14: 28: 24 & 0.0056 & 0.0002 \\ 460 & 14: 28: 26 & 0.0011 & 0.0012 \\ 462 & 14: 28: 28 & 0.0059 & 0.0002 \\ 464 & 14: 28: 30 & 0.0005 & 0.0002 \\ 466 & 14: 28: 32 & 0.0003 & 0.0001 \\ 468 & 14: 28: 34 & 0.0002 & 0.0002 \\ 470 & 14: 28: 36 & 0.0004 & 0.0087 \\ 472 & 14: 28: 38 & 0.0002 & 0.0165 \\ 474 & 14: 28: 40 & 0.0006 & 0.0003 \\ 476 & 14: 28: 42 & 0.0011 & 0.0002 \\ 478 & 14: 28: 44 & 0.0311 & 0.0016 \\ 480 & 14: 28: 46 & 0.0014 & 0.0183 \\ 482 & 14: 28: 48 & 0.0015 & 0.0736 \\ 484 & 14: 28: 50 & 0.0002 & 0.1138 \\ 486 & 14: 28: 52 & 0.0017 & 0.0006 \\ 488 & 14: 28: 54 & 0.0002 & 0.0002 \\ 490 & 14: 28: 56 & 0.0002 & 0.0002 \\ 492 & 14: 28: 58 & 0.0002 & 0.0003 \\ 494 & 14: 29: 00 & 0.0005 & 0.0183 \\ 496 & 14: 29: 02 & 0.0008 & 0.0015 \\ 498 & 14: 29: 04 & 0.0004 & 0.0045 \\ 500 & 14: 29: 06 & 0.0002 & 0.0003 \\ 502 & 14: 29: 08 & 0.0013 & 0.0094 \\ 504 & 14: 29: 10 & 0.0005 & 0.0227 \\ 506 & 14: 29: 12 & 0.0014 & 0.0007\end{array}$


Appendix H - Laser Data Test 4 NIST102705_1.xls

$\begin{array}{llll}508 & 14: 29: 14 & 0.0004 & 0.0003 \\ 510 & 14: 29: 16 & 0.1330 & 0.0001 \\ 512 & 14: 29: 18 & 0.0026 & 0.0001 \\ 514 & 14: 29: 20 & 0.0112 & 0.0001 \\ 516 & 14: 29: 22 & 0.0003 & 0.0003 \\ 518 & 14: 29: 24 & 0.0009 & 0.0067 \\ 520 & 14: 29: 26 & 0.0002 & 0.0001 \\ 522 & 14: 29: 28 & 0.0028 & 0.0004 \\ 524 & 14: 29: 30 & 0.0095 & 0.0030 \\ 526 & 14: 29: 32 & 0.0020 & 0.0223 \\ 528 & 14: 29: 34 & 0.0002 & 0.0005 \\ 530 & 14: 29: 36 & 0.0005 & 0.0002 \\ 532 & 14: 29: 38 & 0.0008 & 0.0002 \\ 534 & 14: 29: 40 & 0.0048 & 0.0002 \\ 536 & 14: 29: 42 & 0.0031 & 0.0002 \\ 538 & 14: 29: 44 & 0.0002 & 0.0001 \\ 540 & 14: 29: 46 & 0.0003 & 0.0247 \\ 542 & 14: 29: 48 & 0.0001 & 0.0017 \\ 544 & 14: 29: 50 & 0.0313 & 0.0007 \\ 546 & 14: 29: 52 & 0.0002 & 0.0006 \\ 548 & 14: 29: 54 & 0.0014 & 0.0002 \\ 550 & 14: 29: 56 & 0.0099 & 0.0001 \\ 552 & 14: 29: 58 & 0.0073 & 0.0001 \\ 554 & 14: 30: 00 & 0.0002 & 0.0001 \\ 556 & 14: 30: 02 & 0.0099 & 0.0002 \\ 558 & 14: 30: 04 & 0.0004 & 0.0002 \\ 560 & 14: 30: 06 & 0.0004 & 0.0009 \\ 562 & 14: 30: 08 & 0.0002 & 0.0408 \\ 564 & 14: 30: 10 & 0.0004 & 0.0003 \\ 566 & 14: 30: 12 & 0.0008 & 0.0003 \\ 568 & 14: 30: 14 & 0.0002 & 0.0002 \\ 570 & 14: 30: 16 & 0.0009 & 0.0002 \\ 572 & 14: 30: 18 & 0.0002 & 0.0002 \\ 574 & 14: 30: 20 & 0.0008 & 0.0003 \\ 576 & 14: 30: 22 & 0.0002 & 0.0023 \\ 578 & 14: 30: 24 & 0.0006 & 0.1297 \\ 580 & 14: 30: 26 & 0.0040 & 0.0009 \\ 582 & 14: 30: 28 & 0.0002 & 0.0003 \\ 584 & 14: 30: 30 & 0.0081 & 0.0110 \\ 586 & 14: 30: 32 & 0.0002 & 0.0007 \\ 588 & 14: 30: 34 & 0.0002 & 0.0545 \\ 590 & 14: 30: 36 & 0.0002 & 0.0098 \\ 592 & 14: 30: 38 & 0.0002 & 0.0007 \\ 594 & 14: 30: 40 & 0.0043 & 0.3813 \\ 596 & 14: 30: 42 & 0.0002 & 0.0311 \\ 598 & 14: 30: 44 & 0.0001 & 0.3833 \\ 600 & 14: 30: 46 & 0.0027 & 0.1322 \\ 602 & 14: 30: 48 & 0.0153 & 0.1121 \\ 604 & 14: 30: 50 & 0.0158 & 0.1234 \\ 606 & 14: 30: 52 & 0.1292 & 0.6575 \\ 608 & 14: 30: 54 & 0.0545 & 0.1502\end{array}$




$\begin{array}{llll}610 & 14: 30: 56 & 0.1463 & 0.1523 \\ 612 & 14: 30: 58 & 0.3115 & 0.1272 \\ 614 & 14: 31: 00 & 0.2468 & 0.2697 \\ 616 & 14: 31: 02 & 0.2538 & 0.2735 \\ 618 & 14: 31: 04 & 0.3765 & 0.5917 \\ 620 & 14: 31: 06 & 0.4847 & 0.6338 \\ 622 & 14: 31: 08 & 0.5907 & 0.8466 \\ 624 & 14: 31: 10 & 0.8194 & 0.6663 \\ 626 & 14: 31: 12 & 0.8024 & 0.7623 \\ 628 & 14: 31: 14 & 0.6973 & 0.8903 \\ 630 & 14: 31: 16 & 0.7558 & 0.6349 \\ 632 & 14: 31: 18 & 0.6871 & 0.6834 \\ 634 & 14: 31: 20 & 0.7855 & 0.7296 \\ 636 & 14: 31: 22 & 0.8364 & 0.9197 \\ 638 & 14: 31: 24 & 0.6586 & 0.8995 \\ 640 & 14: 31: 26 & 0.8597 & 0.6298 \\ 642 & 14: 31: 28 & 0.7901 & 0.7736 \\ 644 & 14: 31: 30 & 0.6584 & 0.9010 \\ 646 & 14: 31: 32 & 0.8023 & 0.7777 \\ 648 & 14: 31: 34 & 0.7273 & 0.7711 \\ 650 & 14: 31: 36 & 0.6235 & 0.8671 \\ 652 & 14: 31: 38 & 0.8590 & 0.6421 \\ 654 & 14: 31: 40 & 0.7144 & 0.8872 \\ 656 & 14: 31: 42 & 0.8271 & 0.8764 \\ 658 & 14: 31: 44 & 0.7983 & 0.8944 \\ 660 & 14: 31: 46 & 0.7288 & 0.6812 \\ 662 & 14: 31: 48 & 0.8075 & 0.9290 \\ 664 & 14: 31: 50 & 0.8109 & 1.0559 \\ 666 & 14: 31: 52 & 0.7395 & 0.8664 \\ 668 & 14: 31: 54 & 0.8276 & 0.6873 \\ 670 & 14: 31: 56 & 0.8822 & 0.9206 \\ 672 & 14: 31: 58 & 0.7668 & 0.7756 \\ 674 & 14: 32: 00 & 0.8043 & 0.8490 \\ 676 & 14: 32: 02 & 0.8112 & 0.8772 \\ 678 & 14: 32: 04 & 0.6815 & 0.9455 \\ 680 & 14: 32: 06 & 0.7072 & 0.8601 \\ 682 & 14: 32: 08 & 0.8272 & 1.0274 \\ 684 & 14: 32: 10 & 0.7548 & 0.9808 \\ 686 & 14: 32: 12 & 0.7909 & 1.0167 \\ 688 & 14: 32: 14 & 0.8410 & 0.9472 \\ 690 & 14: 32: 16 & 0.7810 & 0.9796 \\ 692 & 14: 32: 18 & 0.8695 & 1.0662 \\ 694 & 14: 32: 20 & 0.8625 & 0.9510 \\ 696 & 14: 32: 22 & 0.8123 & 0.9823 \\ 698 & 14: 32: 24 & 0.9510 & 0.9025 \\ 700 & 14: 32: 26 & 0.8838 & 0.9222 \\ 702 & 14: 32: 28 & 0.8870 & 0.9938 \\ 704 & 14: 32: 30 & 0.8770 & 0.9294 \\ 706 & 14: 32: 32 & 0.9109 & 0.9257 \\ 708 & 14: 32: 34 & 0.8316 & 1.0034 \\ 710 & 14: 32: 36 & 0.8341 & 0.9674\end{array}$


Appendix H - Laser Data Test 4 NIST102705_1.xls

$\begin{array}{llll}712 & 14: 32: 38 & 0.8672 & 1.0427 \\ 714 & 14: 32: 40 & 0.8686 & 1.0678 \\ 716 & 14: 32: 42 & 0.8064 & 0.9793 \\ 718 & 14: 32: 44 & 0.9707 & 1.0081 \\ 720 & 14: 32: 46 & 0.8827 & 0.9239 \\ 722 & 14: 32: 48 & 0.9337 & 1.0149 \\ 724 & 14: 32: 50 & 0.8506 & 0.9245 \\ 726 & 14: 32: 52 & 0.9567 & 0.9866 \\ 728 & 14: 32: 54 & 0.8876 & 0.9276 \\ 730 & 14: 32: 56 & 0.8375 & 0.9557 \\ 732 & 14: 32: 58 & 0.8028 & 1.0149 \\ 734 & 14: 33: 00 & 0.8151 & 0.9942 \\ 736 & 14: 33: 02 & 0.8175 & 0.9475 \\ 738 & 14: 33: 04 & 0.8103 & 1.0038 \\ 740 & 14: 33: 06 & 0.8786 & 1.0940 \\ 742 & 14: 33: 08 & 0.8645 & 0.9318 \\ 744 & 14: 33: 10 & 0.9574 & 0.8817 \\ 746 & 14: 33: 12 & 0.8381 & 0.9314 \\ 748 & 14: 33: 14 & 0.8883 & 0.9172 \\ 750 & 14: 33: 16 & 0.8726 & 1.0966 \\ 752 & 14: 33: 18 & 0.8662 & 1.0240 \\ 754 & 14: 33: 20 & 0.7996 & 0.9833 \\ 756 & 14: 33: 22 & 0.8434 & 0.9520 \\ 758 & 14: 33: 24 & 0.8870 & 1.0655 \\ 760 & 14: 33: 26 & 0.8943 & 0.9650 \\ 762 & 14: 33: 28 & 0.8712 & 1.0094 \\ 764 & 14: 33: 30 & 0.8579 & 1.0774 \\ 766 & 14: 33: 32 & 0.9142 & 0.9574 \\ 768 & 14: 33: 34 & 0.8333 & 1.0431 \\ 770 & 14: 33: 36 & 0.8114 & 0.8593 \\ 772 & 14: 33: 38 & 0.6957 & 0.9525 \\ 774 & 14: 33: 40 & 0.9055 & 0.8454 \\ 776 & 14: 33: 42 & 0.8983 & 0.9106 \\ 778 & 14: 33: 44 & 0.8329 & 0.9095 \\ 780 & 14: 33: 46 & 0.9393 & 0.9622 \\ 782 & 14: 33: 48 & 0.8891 & 0.8577 \\ 784 & 14: 33: 50 & 0.9219 & 0.9622 \\ 786 & 14: 33: 52 & 0.8564 & 0.9235 \\ 788 & 14: 33: 54 & 0.9278 & 0.9203 \\ 790 & 14: 33: 56 & 0.9249 & 1.0537 \\ 792 & 14: 33: 58 & 0.8599 & 1.2004 \\ 794 & 14: 34: 00 & 0.8755 & 0.8259 \\ 796 & 14: 34: 02 & 0.8398 & 0.9831 \\ 798 & 14: 34: 04 & 0.8183 & 0.9715 \\ 800 & 14: 34: 06 & 0.8663 & 0.9889 \\ 802 & 14: 34: 08 & 0.9185 & 0.9466 \\ 804 & 14: 34: 10 & 0.8187 & 0.9966 \\ 806 & 14: 34: 12 & 0.8963 & 1.0198 \\ 808 & 14: 34: 14 & 0.8685 & 0.9731 \\ 810 & 14: 34: 16 & 0.7646 & 0.9623 \\ 812 & 14: 34: 18 & 0.9097 & 0.9645\end{array}$


Appendix H - Laser Data Test 4 NIST102705_1.xls

$\begin{array}{llll}814 & 14: 34: 20 & 0.9682 & 0.9914 \\ 816 & 14: 34: 22 & 0.8771 & 0.9666 \\ 818 & 14: 34: 24 & 0.9033 & 1.0671 \\ 820 & 14: 34: 26 & 0.8691 & 0.9969 \\ 822 & 14: 34: 28 & 0.8633 & 1.0128 \\ 824 & 14: 34: 30 & 0.8642 & 1.0107 \\ 826 & 14: 34: 32 & 0.8960 & 1.0044 \\ 828 & 14: 34: 34 & 0.8996 & 0.9889 \\ 830 & 14: 34: 36 & 0.8855 & 0.9829 \\ 832 & 14: 34: 38 & 0.9230 & 0.9273 \\ 834 & 14: 34: 40 & 0.8949 & 0.9164 \\ 836 & 14: 34: 42 & 0.9475 & 0.9069 \\ 838 & 14: 34: 44 & 0.8941 & 0.9940 \\ 840 & 14: 34: 46 & 0.8872 & 0.9277 \\ 842 & 14: 34: 48 & 0.9095 & 1.0464 \\ 844 & 14: 34: 50 & 0.8903 & 0.9947 \\ 846 & 14: 34: 52 & 0.8027 & 0.8473 \\ 848 & 14: 34: 54 & 0.8923 & 1.0615 \\ 850 & 14: 34: 56 & 0.8483 & 0.9628 \\ 852 & 14: 34: 58 & 0.8938 & 1.0051 \\ 854 & 14: 35: 00 & 1.0118 & 1.0227 \\ 856 & 14: 35: 02 & 0.9445 & 0.9635 \\ 858 & 14: 35: 04 & 0.9398 & 1.0023 \\ 860 & 14: 35: 06 & 0.9251 & 0.9974 \\ 862 & 14: 35: 08 & 0.8688 & 0.9753 \\ 864 & 14: 35: 10 & 0.9031 & 0.9846 \\ 866 & 14: 35: 12 & 0.8496 & 0.9885 \\ 868 & 14: 35: 14 & 0.9256 & 0.9678 \\ 870 & 14: 35: 16 & 0.9068 & 0.9699 \\ 872 & 14: 35: 18 & 0.8343 & 1.0512 \\ 874 & 14: 35: 20 & 0.8979 & 1.0014 \\ 876 & 14: 35: 22 & 0.9455 & 1.0048 \\ 878 & 14: 35: 24 & 1.0073 & 0.9364 \\ 880 & 14: 35: 26 & 0.8942 & 1.0423 \\ 882 & 14: 35: 28 & 0.8798 & 0.9918 \\ 884 & 14: 35: 30 & 0.9555 & 0.9565 \\ 886 & 14: 35: 32 & 0.9356 & 1.0121 \\ 888 & 14: 35: 34 & 0.9177 & 0.9398 \\ 890 & 14: 35: 36 & 0.8988 & 0.9413 \\ 892 & 14: 35: 38 & 0.8689 & 0.9188 \\ 894 & 14: 35: 40 & 0.8786 & 0.9104 \\ 896 & 14: 35: 42 & 0.8915 & 0.9918 \\ 898 & 14: 35: 44 & 0.9063 & 0.9585 \\ 900 & 14: 35: 46 & 0.9256 & 1.0560 \\ 902 & 14: 35: 48 & 0.8921 & 0.9686 \\ 904 & 14: 35: 50 & 0.9587 & 0.9555 \\ 906 & 14: 35: 52 & 0.8944 & 0.9490 \\ 908 & 14: 35: 54 & 0.9440 & 0.8782 \\ 910 & 14: 35: 56 & 0.8886 & 1.0299 \\ 912 & 14: 35: 58 & 0.9165 & 0.9583 \\ 914 & 14: 36: 00 & 1.0021 & 1.0230\end{array}$




$\begin{array}{rlll}916 & 14: 36: 02 & 0.9002 & 1.0069 \\ 918 & 14: 36: 04 & 0.9595 & 0.8994 \\ 920 & 14: 36: 06 & 0.8589 & 1.0328 \\ 922 & 14: 36: 08 & 0.9195 & 1.0130 \\ 924 & 14: 36: 10 & 0.8968 & 0.8933 \\ 926 & 14: 36: 12 & 0.8602 & 0.8440 \\ 928 & 14: 36: 14 & 0.9416 & 0.8537 \\ 930 & 14: 36: 16 & 0.9116 & 0.9492 \\ 932 & 14: 36: 18 & 0.8885 & 0.9750 \\ 934 & 14: 36: 20 & 0.9278 & 1.0052 \\ 936 & 14: 36: 22 & 0.9442 & 0.9814 \\ 938 & 14: 36: 24 & 0.8737 & 0.9583 \\ 940 & 14: 36: 26 & 0.8815 & 0.9909 \\ 942 & 14: 36: 28 & 0.9690 & 0.9762 \\ 944 & 14: 36: 30 & 0.9777 & 1.0436 \\ 946 & 14: 36: 32 & 0.9611 & 0.9514 \\ 948 & 14: 36: 34 & 0.8908 & 1.0192 \\ 950 & 14: 36: 36 & 0.9372 & 0.9784 \\ 952 & 14: 36: 38 & 0.9877 & 1.0397 \\ 954 & 14: 36: 40 & 0.9804 & 0.9702 \\ 956 & 14: 36: 42 & 0.9598 & 0.9938 \\ 958 & 14: 36: 44 & 0.8768 & 1.0429 \\ 960 & 14: 36: 46 & 0.8444 & 1.0334 \\ 962 & 14: 36: 48 & 0.8955 & 0.9932 \\ 964 & 14: 36: 50 & 0.9476 & 0.9737 \\ 966 & 14: 36: 52 & 0.8222 & 0.9907 \\ 968 & 14: 36: 54 & 0.9451 & 0.9118 \\ 970 & 14: 36: 56 & 0.8928 & 0.8957 \\ 972 & 14: 36: 58 & 0.8993 & 1.0449 \\ 974 & 14: 37: 00 & 0.9331 & 0.8887 \\ 976 & 14: 37: 02 & 0.8968 & 0.9305 \\ 978 & 14: 37: 04 & 0.9370 & 0.9443 \\ 980 & 14: 37: 06 & 0.9572 & 0.9772 \\ 982 & 14: 37: 08 & 0.9380 & 1.0092 \\ 984 & 14: 37: 10 & 0.9153 & 0.9160 \\ 986 & 14: 37: 12 & 0.9794 & 0.9659 \\ 988 & 14: 37: 14 & 0.9285 & 0.9615 \\ 990 & 14: 37: 16 & 0.8586 & 0.9715 \\ 992 & 14: 37: 18 & 0.9445 & 0.9774 \\ 994 & 14: 37: 20 & 0.9151 & 0.9106 \\ 996 & 14: 37: 22 & 0.9256 & 0.9793 \\ 998 & 14: 37: 24 & 1.0249 & 0.9266 \\ 1000 & 14: 37: 26 & 0.9981 & 1.0507 \\ 1002 & 14: 37: 28 & 0.9173 & 0.9888 \\ 1004 & 14: 37: 30 & 0.9023 & 0.9863 \\ 1006 & 14: 37: 32 & 0.9310 & 1.0037 \\ 1008 & 14: 37: 34 & 0.8746 & 0.9996 \\ 1010 & 14: 37: 36 & 0.9000 & 0.9839 \\ 1012 & 14: 37: 38 & 0.9239 & 0.9819 \\ 1014 & 14: 37: 40 & 0.9946 & 1.0111 \\ 1016 & 14: 37: 42 & 0.9376 & 1.0015\end{array}$


Appendix H - Laser Data Test 4 NIST102705_1.xls

$\begin{array}{llll}1018 & 14: 37: 44 & 0.9084 & 1.0605 \\ 1020 & 14: 37: 46 & 0.9087 & 0.8645 \\ 1022 & 14: 37: 48 & 0.9169 & 1.1254 \\ 1024 & 14: 37: 50 & 0.9476 & 0.9750 \\ 1026 & 14: 37: 52 & 0.9630 & 1.0618 \\ 1028 & 14: 37: 54 & 0.9177 & 1.0930 \\ 1030 & 14: 37: 56 & 0.9326 & 1.0259 \\ 1032 & 14: 37: 58 & 1.0219 & 1.0145 \\ 1034 & 14: 38: 00 & 0.9545 & 0.9524 \\ 1036 & 14: 38: 02 & 0.9298 & 1.0371 \\ 1038 & 14: 38: 04 & 0.9488 & 0.9678 \\ 1040 & 14: 38: 06 & 0.8879 & 1.0169 \\ 1042 & 14: 38: 08 & 0.9003 & 1.0201 \\ 1044 & 14: 38: 10 & 0.9208 & 0.9846 \\ 1046 & 14: 38: 12 & 0.8687 & 0.9425 \\ 1048 & 14: 38: 14 & 0.8538 & 0.9105 \\ 1050 & 14: 38: 16 & 0.9087 & 0.9607 \\ 1052 & 14: 38: 18 & 0.8560 & 0.9418 \\ 1054 & 14: 38: 20 & 0.8847 & 0.9689 \\ 1056 & 14: 38: 22 & 0.9342 & 1.0304 \\ 1058 & 14: 38: 24 & 0.9567 & 0.9577 \\ 1060 & 14: 38: 26 & 0.9233 & 0.9552 \\ 1062 & 14: 38: 28 & 0.9553 & 0.9707 \\ 1064 & 14: 38: 30 & 0.9362 & 0.9794 \\ 1066 & 14: 38: 32 & 0.9131 & 0.9904 \\ 1068 & 14: 38: 34 & 0.8785 & 0.9628 \\ 1070 & 14: 38: 36 & 0.9399 & 1.0158 \\ 1072 & 14: 38: 38 & 0.8789 & 0.9957 \\ 1074 & 14: 38: 40 & 0.8968 & 0.9734 \\ 1076 & 14: 38: 42 & 0.9285 & 1.0138 \\ 1078 & 14: 38: 44 & 0.8503 & 1.0019 \\ 1080 & 14: 38: 46 & 0.8434 & 1.0020 \\ 1082 & 14: 38: 48 & 0.9209 & 0.8849 \\ 1084 & 14: 38: 50 & 0.9337 & 0.9417 \\ 1086 & 14: 38: 52 & 0.9107 & 1.0791 \\ 1088 & 14: 38: 54 & 0.8953 & 0.9938 \\ 1090 & 14: 38: 56 & 0.9376 & 0.9706 \\ 1092 & 14: 38: 58 & 0.8982 & 0.9525 \\ 1094 & 14: 39: 00 & 0.9373 & 0.9757 \\ 1096 & 14: 39: 02 & 0.8713 & 0.9600 \\ 1098 & 14: 39: 04 & 0.9853 & 0.9924 \\ 1100 & 14: 39: 06 & 0.8918 & 0.9857 \\ 1102 & 14: 39: 08 & 0.9033 & 1.0371 \\ 1104 & 14: 39: 10 & 0.9509 & 1.0064 \\ 1106 & 14: 39: 12 & 0.9542 & 0.9982 \\ 1108 & 14: 39: 14 & 0.9403 & 1.0137 \\ 1110 & 14: 39: 16 & 0.8808 & 0.9782 \\ 1112 & 14: 39: 18 & 0.9094 & 0.9555 \\ 1114 & 14: 39: 20 & 0.9656 & 0.9660 \\ 1116 & 14: 39: 22 & 0.9581 & 0.9507 \\ 1118 & 14: 39: 24 & 0.9215 & 1.0112\end{array}$




$\begin{array}{llll}1120 & 14: 39: 26 & 0.8978 & 1.0000 \\ 1122 & 14: 39: 28 & 0.9420 & 0.9446 \\ 1124 & 14: 39: 30 & 0.8756 & 1.0008 \\ 1126 & 14: 39: 32 & 0.9708 & 0.9449 \\ 1128 & 14: 39: 34 & 0.9090 & 1.0447 \\ 1130 & 14: 39: 36 & 0.9617 & 0.9625 \\ 1132 & 14: 39: 38 & 0.9012 & 0.9363 \\ 1134 & 14: 39: 40 & 0.9512 & 0.9623 \\ 1136 & 14: 39: 42 & 0.8958 & 1.0168 \\ 1138 & 14: 39: 44 & 0.9319 & 1.0428 \\ 1140 & 14: 39: 46 & 0.9778 & 1.0128 \\ 1142 & 14: 39: 48 & 0.9362 & 0.9851 \\ 1144 & 14: 39: 50 & 0.9480 & 0.9415 \\ 1146 & 14: 39: 52 & 0.9430 & 1.0053 \\ 1148 & 14: 39: 54 & 0.9545 & 0.9491 \\ 1150 & 14: 39: 56 & 0.9332 & 0.9654 \\ 1152 & 14: 39: 58 & 0.9003 & 1.0136 \\ 1154 & 14: 40: 00 & 0.9407 & 0.9843 \\ 1156 & 14: 40: 02 & 0.9587 & 0.9309 \\ 1158 & 14: 40: 04 & 0.9162 & 0.9695 \\ 1160 & 14: 40: 06 & 0.9693 & 0.9719 \\ 1162 & 14: 40: 08 & 0.8903 & 1.0145 \\ 1164 & 14: 40: 10 & 0.9386 & 1.0215 \\ 1166 & 14: 40: 12 & 0.9552 & 0.9632 \\ 1168 & 14: 40: 14 & 0.9472 & 0.9572 \\ 1170 & 14: 40: 16 & 0.9902 & 0.9907 \\ 1172 & 14: 40: 18 & 0.9274 & 0.9641 \\ 1174 & 14: 40: 20 & 0.8868 & 0.9387 \\ 1176 & 14: 40: 22 & 0.8888 & 1.0233 \\ 1178 & 14: 40: 24 & 0.9183 & 0.9726 \\ 1180 & 14: 40: 26 & 0.9277 & 0.9962 \\ 1182 & 14: 40: 28 & 0.9122 & 1.0061 \\ 1184 & 14: 40: 30 & 0.9259 & 0.8977 \\ 1186 & 14: 40: 32 & 0.9204 & 0.9759 \\ 1188 & 14: 40: 34 & 0.9452 & 0.9668 \\ 1190 & 14: 40: 36 & 0.9366 & 0.9679 \\ 1192 & 14: 40: 38 & 0.9489 & 0.9980 \\ 1194 & 14: 40: 40 & 0.9650 & 0.9646 \\ 1196 & 14: 40: 42 & 0.8833 & 1.0109 \\ 1198 & 14: 40: 44 & 1.0333 & 1.0148 \\ 1200 & 14: 40: 46 & 0.9591 & 1.0259 \\ 1202 & 14: 40: 48 & 0.9345 & 0.9870 \\ 1204 & 14: 40: 50 & 0.9743 & 0.9663 \\ 1206 & 14: 40: 52 & 0.9718 & 0.9880 \\ 1208 & 14: 40: 54 & 0.9640 & 1.0110 \\ 1210 & 14: 40: 56 & 0.8358 & 0.8121 \\ 1212 & 14: 40: 58 & 0.9529 & 0.8856 \\ 1214 & 14: 41: 00 & 0.9652 & 0.9563 \\ 1216 & 14: 41: 02 & 1.0492 & 0.9583 \\ 1218 & 14: 41: 04 & 0.9489 & 0.9983 \\ 1220 & 14: 41: 06 & 0.9482 & 1.0556\end{array}$


Appendix H - Laser Data Test 4 NIST102705_1.xls

$\begin{array}{llll}1222 & 14: 41: 08 & 0.8553 & 1.0661 \\ 1224 & 14: 41: 10 & 0.9642 & 0.9962 \\ 1226 & 14: 41: 12 & 0.9402 & 0.9714 \\ 1228 & 14: 41: 14 & 0.7531 & 0.9666 \\ 1230 & 14: 41: 16 & 0.9747 & 0.9502 \\ 1232 & 14: 41: 18 & 0.9506 & 0.9717 \\ 1234 & 14: 41: 20 & 0.9596 & 1.0365 \\ 1236 & 14: 41: 22 & 1.0163 & 0.9886 \\ 1238 & 14: 41: 24 & 1.0903 & 0.9419 \\ 1240 & 14: 41: 26 & 1.0008 & 0.7556 \\ 1242 & 14: 41: 28 & 0.9844 & 0.9392 \\ 1244 & 14: 41: 30 & 1.0552 & 1.1718 \\ 1246 & 14: 41: 32 & 0.9513 & 0.9738 \\ 1248 & 14: 41: 34 & 0.9445 & 1.1038 \\ 1250 & 14: 41: 36 & 1.0105 & 0.9631 \\ 1252 & 14: 41: 38 & 0.9002 & 0.9868 \\ 1254 & 14: 41: 40 & 0.9344 & 1.0104 \\ 1256 & 14: 41: 42 & 1.0014 & 1.0466 \\ 1258 & 14: 41: 44 & 1.0243 & 0.9703 \\ 1260 & 14: 41: 46 & 0.9646 & 0.3163 \\ 1262 & 14: 41: 48 & 0.9638 & 0.9322 \\ 1264 & 14: 41: 50 & 0.9340 & 0.7151 \\ 1266 & 14: 41: 52 & 0.7583 & 0.3292 \\ 1268 & 14: 41: 54 & 0.5042 & 0.4735 \\ 1270 & 14: 41: 56 & 0.3773 & 0.2271 \\ 1272 & 14: 41: 58 & 0.0984 & 0.2415 \\ 1274 & 14: 42: 00 & 0.0502 & 0.2242 \\ 1276 & 14: 42: 02 & 0.1164 & 0.2143 \\ 1278 & 14: 42: 04 & 0.0535 & 0.0665 \\ 1280 & 14: 42: 06 & 0.2048 & 0.5431 \\ 1282 & 14: 42: 08 & 0.0798 & 0.0372 \\ 1284 & 14: 42: 10 & 0.9795 & 0.0387 \\ 1286 & 14: 42: 12 & 1.0624 & 0.0036 \\ 1288 & 14: 42: 14 & 0.7087 & 0.5395 \\ 1290 & 14: 42: 16 & 0.9636 & 0.6111 \\ 1292 & 14: 42: 18 & 0.7287 & 0.8532 \\ 1294 & 14: 42: 20 & 0.2301 & 0.3743 \\ 1296 & 14: 42: 22 & 0.0454 & 0.3091 \\ 1298 & 14: 42: 24 & 0.0018 & 0.7200 \\ 1300 & 14: 42: 26 & 0.0084 & 0.0005 \\ 1302 & 14: 42: 28 & 0.0331 & 0.0028 \\ 1304 & 14: 42: 30 & 0.0002 & 0.0002 \\ 1306 & 14: 42: 32 & 0.0005 & 0.0022 \\ 1308 & 14: 42: 34 & 0.0002 & 0.0002 \\ 1310 & 14: 42: 36 & 0.0003 & 0.0003 \\ 1312 & 14: 42: 38 & 0.0028 & 0.0016 \\ 1314 & 14: 42: 40 & 0.0002 & 0.0006 \\ 1316 & 14: 42: 42 & 0.0011 & 0.0011 \\ 1318 & 14: 42: 44 & 0.0002 & 0.0168 \\ 1320 & 14: 42: 46 & 0.0003 & 0.0114 \\ 1322 & 14: 42: 48 & 0.0002 & 0.0002\end{array}$


Appendix H - Laser Data Test 4 NIST102705_1.xls

$\begin{array}{llll}1324 & 14: 42: 50 & 0.0022 & 0.0014 \\ 1326 & 14: 42: 52 & 0.0002 & 0.0015 \\ 1328 & 14: 42: 54 & 0.0036 & 0.0002 \\ 1330 & 14: 42: 56 & 0.0031 & 0.4755 \\ 1332 & 14: 42: 58 & 0.0024 & 0.1529 \\ 1334 & 14: 43: 00 & 0.0009 & 0.0237 \\ 1336 & 14: 43: 02 & 0.0004 & 0.0005 \\ 1338 & 14: 43: 04 & 0.0004 & 0.0059 \\ 1340 & 14: 43: 06 & 0.0002 & 0.0002 \\ 1342 & 14: 43: 08 & 0.0082 & 0.0020 \\ 1344 & 14: 43: 10 & 0.1114 & 0.0726 \\ 1346 & 14: 43: 12 & 0.0058 & 0.0010 \\ 1348 & 14: 43: 14 & 0.0002 & 0.0002 \\ 1350 & 14: 43: 16 & 0.0003 & 0.0001 \\ 1352 & 14: 43: 18 & 0.0027 & 0.0022 \\ 1354 & 14: 43: 20 & 0.0006 & 0.0006 \\ 1356 & 14: 43: 22 & 0.0003 & 0.0001 \\ 1358 & 14: 43: 24 & 0.0008 & 0.0002 \\ 1360 & 14: 43: 26 & 0.0003 & 0.0004 \\ 1362 & 14: 43: 28 & 0.0002 & 0.0003 \\ 1364 & 14: 43: 30 & 0.0002 & 0.0002 \\ 1366 & 14: 43: 32 & 0.0039 & 0.0525 \\ 1368 & 14: 43: 34 & 0.0003 & 0.2045 \\ 1370 & 14: 43: 36 & 0.0005 & 0.0007 \\ 1372 & 14: 43: 38 & 0.0002 & 0.0020 \\ 1374 & 14: 43: 40 & 0.0002 & 0.2702 \\ 1376 & 14: 43: 42 & 0.0108 & 0.0127 \\ 1378 & 14: 43: 44 & 0.0137 & 0.0602 \\ 1380 & 14: 43: 46 & 0.0018 & 0.0282 \\ 1382 & 14: 43: 48 & 0.0003 & 0.0154 \\ 1384 & 14: 43: 50 & 0.0002 & 0.1762 \\ 1386 & 14: 43: 52 & 0.0051 & 0.0004 \\ 1388 & 14: 43: 54 & 0.0007 & 0.0005 \\ 1390 & 14: 43: 56 & 0.0002 & 0.9063 \\ 1392 & 14: 43: 58 & 0.0002 & 0.1442 \\ 1394 & 14: 44: 00 & 0.0094 & 0.0974 \\ 1396 & 14: 44: 02 & 0.4179 & 0.0101 \\ 1398 & 14: 44: 04 & 0.0131 & 0.0071 \\ 1400 & 14: 44: 06 & 0.0040 & 0.1366 \\ 1402 & 14: 44: 08 & 0.0002 & 0.3574 \\ 1404 & 14: 44: 10 & 0.0050 & 0.9982 \\ 1406 & 14: 44: 12 & 0.0060 & 0.3883 \\ 1408 & 14: 44: 14 & 0.0007 & 0.2281 \\ 1410 & 14: 44: 16 & 0.0051 & 0.0150 \\ 1412 & 14: 44: 18 & 0.0501 & 0.0046 \\ 1414 & 14: 44: 20 & 0.0194 & 0.0737 \\ 1416 & 14: 44: 22 & 0.0018 & 0.3930 \\ 1418 & 14: 44: 24 & 0.0086 & 0.0246 \\ 1420 & 14: 44: 26 & 0.0028 & 0.1820 \\ 1422 & 14: 44: 28 & 0.0366 & 0.0179 \\ 1424 & 14: 44: 30 & 0.0010 & 0.0600\end{array}$


Appendix H - Laser Data Test 4 NIST102705_1.xls

$\begin{array}{llll}1426 & 14: 44: 32 & 0.0005 & 0.0121 \\ 1428 & 14: 44: 34 & 0.0059 & 0.0083 \\ 1430 & 14: 44: 36 & 0.0014 & 0.0023 \\ 1432 & 14: 44: 38 & 0.0083 & 0.0055 \\ 1434 & 14: 44: 40 & 0.0024 & 0.0043 \\ 1436 & 14: 44: 42 & 0.0007 & 0.0135 \\ 1438 & 14: 44: 44 & 0.0017 & 0.2693 \\ 1440 & 14: 44: 46 & 0.0085 & 0.4905 \\ 1442 & 14: 44: 48 & 0.0146 & 0.0624 \\ 1444 & 14: 44: 50 & 0.0017 & 0.6511 \\ 1446 & 14: 44: 52 & 0.0073 & 0.0591 \\ 1448 & 14: 44: 54 & 0.0005 & 0.0573 \\ 1450 & 14: 44: 56 & 0.0060 & 0.0780 \\ 1452 & 14: 44: 58 & 0.0127 & 0.2537 \\ 1454 & 14: 45: 00 & 0.0067 & 0.0026 \\ 1456 & 14: 45: 02 & 0.0124 & 0.5290 \\ 1458 & 14: 45: 04 & 0.0018 & 0.0307 \\ 1460 & 14: 45: 06 & 0.0186 & 0.4768 \\ 1462 & 14: 45: 08 & 0.0003 & 0.1803 \\ 1464 & 14: 45: 10 & 0.0002 & 0.0039 \\ 1466 & 14: 45: 12 & 0.0007 & 0.1462 \\ 1468 & 14: 45: 14 & 0.0003 & 0.0343 \\ 1470 & 14: 45: 16 & 0.0056 & 0.1017 \\ 1472 & 14: 45: 18 & 0.0002 & 0.0002 \\ 1474 & 14: 45: 20 & 0.0018 & 0.0037 \\ 1476 & 14: 45: 22 & 0.0416 & 0.0330 \\ 1478 & 14: 45: 24 & 0.0058 & 0.0072 \\ 1480 & 14: 45: 26 & 0.0048 & 0.0433 \\ 1482 & 14: 45: 28 & 0.0108 & 0.0002 \\ 1484 & 14: 45: 30 & 0.0028 & 0.0133 \\ 1486 & 14: 45: 32 & 0.0038 & 0.0156 \\ 1488 & 14: 45: 34 & 0.0025 & 0.0010 \\ 1490 & 14: 45: 36 & 0.0756 & 0.0003 \\ 1492 & 14: 45: 38 & 0.0031 & 0.0001 \\ 1494 & 14: 45: 40 & 0.0002 & 0.0131 \\ 1496 & 14: 45: 42 & 0.0002 & 0.0010 \\ 1498 & 14: 45: 44 & 0.0003 & 0.0037 \\ 1500 & 14: 45: 46 & 0.0005 & 0.0862 \\ 1502 & 14: 45: 48 & 0.0911 & 0.0517 \\ 1504 & 14: 45: 50 & 0.0040 & 0.9115 \\ 1506 & 14: 45: 52 & 0.0035 & 0.8459 \\ 1508 & 14: 45: 54 & 0.0090 & 0.8672 \\ 1510 & 14: 45: 56 & 0.0300 & 0.3300 \\ 1512 & 14: 45: 58 & 0.0441 & 0.2226 \\ 1514 & 14: 46: 00 & 0.0108 & 0.4650 \\ 1516 & 14: 46: 02 & 0.0137 & 0.2501 \\ 1518 & 14: 46: 04 & 0.0048 & 0.4380 \\ 1520 & 14: 46: 06 & 0.0022 & 0.5581 \\ 1522 & 14: 46: 08 & 0.0092 & 0.4085 \\ 1524 & 14: 46: 10 & 0.0225 & 0.6681 \\ 1526 & 14: 46: 12 & 0.0302 & 1.0982\end{array}$


Appendix H - Laser Data Test 4 NIST102705_1.xls

$\begin{array}{llll}1528 & 14: 46: 14 & 0.0012 & 0.5808 \\ 1530 & 14: 46: 16 & 0.0154 & 0.0399 \\ 1532 & 14: 46: 18 & 0.0162 & 0.1155 \\ 1534 & 14: 46: 20 & 0.0028 & 0.1123 \\ 1536 & 14: 46: 22 & 0.0012 & 0.0245 \\ 1538 & 14: 46: 24 & 0.0003 & 0.1956 \\ 1540 & 14: 46: 26 & 0.0002 & 0.0101 \\ 1542 & 14: 46: 28 & 0.0118 & 0.3875 \\ 1544 & 14: 46: 30 & 0.0084 & 0.0049 \\ 1546 & 14: 46: 32 & 0.0083 & 0.0280 \\ 1548 & 14: 46: 34 & 0.0790 & 0.4031 \\ 1550 & 14: 46: 36 & 0.0418 & 0.0022 \\ 1552 & 14: 46: 38 & 0.1721 & 0.1157 \\ 1554 & 14: 46: 40 & 0.0301 & 0.2716 \\ 1556 & 14: 46: 42 & 0.0152 & 0.1182 \\ 1558 & 14: 46: 44 & 0.0134 & 0.0027 \\ 1560 & 14: 46: 46 & 0.0085 & 0.0136 \\ 1562 & 14: 46: 48 & 0.0130 & 0.0286 \\ 1564 & 14: 46: 50 & 0.0197 & 0.4231 \\ 1566 & 14: 46: 52 & 0.0067 & 0.0366 \\ 1568 & 14: 46: 54 & 0.0004 & 0.0090 \\ 1570 & 14: 46: 56 & 0.0100 & 0.0546 \\ 1572 & 14: 46: 58 & 0.0075 & 0.4034 \\ 1574 & 14: 47: 00 & 0.0047 & 0.2272 \\ 1576 & 14: 47: 02 & 0.0014 & 0.0872 \\ 1578 & 14: 47: 04 & 0.0025 & 0.0159 \\ 1580 & 14: 47: 06 & 0.0431 & 0.0126 \\ 1582 & 14: 47: 08 & 0.0095 & 0.0900 \\ 1584 & 14: 47: 10 & 0.0037 & 0.3935 \\ 1586 & 14: 47: 12 & 0.0485 & 0.2278 \\ 1588 & 14: 47: 14 & 0.2841 & 0.6558 \\ 1590 & 14: 47: 16 & 0.0088 & 0.1187 \\ 1592 & 14: 47: 18 & 0.1789 & 0.3230 \\ 1594 & 14: 47: 20 & 0.0559 & 0.1592 \\ 1596 & 14: 47: 22 & 0.1636 & 0.1993 \\ 1598 & 14: 47: 24 & 0.0198 & 0.4944 \\ 1600 & 14: 47: 26 & 0.1517 & 0.0196 \\ 1602 & 14: 47: 28 & 0.0294 & 0.0412 \\ 1604 & 14: 47: 30 & 0.0036 & 0.0596 \\ 1606 & 14: 47: 32 & 0.0012 & 0.0437 \\ 1608 & 14: 47: 34 & 0.0007 & 0.0116 \\ 1610 & 14: 47: 36 & 0.0373 & 0.7191 \\ 1612 & 14: 47: 38 & 0.0218 & 0.1172 \\ 1614 & 14: 47: 40 & 0.0259 & 0.1203 \\ 1616 & 14: 47: 42 & 0.0738 & 0.2684 \\ 1618 & 14: 47: 44 & 0.1042 & 0.0182 \\ 1620 & 14: 47: 46 & 0.0147 & 0.0053 \\ 1622 & 14: 47: 48 & 0.0031 & 0.0303 \\ 1624 & 14: 47: 50 & 0.0520 & 0.0575 \\ 1626 & 14: 47: 52 & 0.0022 & 0.0081 \\ 1628 & 14: 47: 54 & 0.0031 & 0.0220\end{array}$


Appendix H - Laser Data Test 4 NIST102705_1.xls

$\begin{array}{llll}1630 & 14: 47: 56 & 0.0364 & 0.0401 \\ 1632 & 14: 47: 58 & 0.0313 & 0.3679 \\ 1634 & 14: 48: 00 & 0.0283 & 0.0515 \\ 1636 & 14: 48: 02 & 0.0127 & 0.2917 \\ 1638 & 14: 48: 04 & 0.2261 & 0.3478 \\ 1640 & 14: 48: 06 & 0.0181 & 0.5679 \\ 1642 & 14: 48: 08 & 0.0172 & 0.3289 \\ 1644 & 14: 48: 10 & 0.4535 & 0.0047 \\ 1646 & 14: 48: 12 & 0.0784 & 0.0146 \\ 1648 & 14: 48: 14 & 0.7912 & 0.0082 \\ 1650 & 14: 48: 16 & 0.0395 & 0.0301 \\ 1652 & 14: 48: 18 & 0.0191 & 0.0763 \\ 1654 & 14: 48: 20 & 0.0043 & 0.2362 \\ 1656 & 14: 48: 22 & 0.0157 & 0.2522 \\ 1658 & 14: 48: 24 & 0.0151 & 0.0279 \\ 1660 & 14: 48: 26 & 0.1197 & 0.1034 \\ 1662 & 14: 48: 28 & 0.1455 & 0.0865 \\ 1664 & 14: 48: 30 & 0.0574 & 0.0256 \\ 1666 & 14: 48: 32 & 0.0016 & 0.1517 \\ 1668 & 14: 48: 34 & 0.2680 & 0.0525 \\ 1670 & 14: 48: 36 & 0.0027 & 0.0370 \\ 1672 & 14: 48: 38 & 0.0357 & 0.8183 \\ 1674 & 14: 48: 40 & 0.0849 & 0.4046 \\ 1676 & 14: 48: 42 & 0.1801 & 0.3248 \\ 1678 & 14: 48: 44 & 0.1681 & 0.2707 \\ 1680 & 14: 48: 46 & 0.6874 & 0.0769 \\ 1682 & 14: 48: 48 & 0.0318 & 0.0031 \\ 1684 & 14: 48: 50 & 0.0103 & 0.1287 \\ 1686 & 14: 48: 52 & 0.0079 & 0.7293 \\ 1688 & 14: 48: 54 & 0.3533 & 0.7227 \\ 1690 & 14: 48: 56 & 0.1053 & 0.2860 \\ 1692 & 14: 48: 58 & 0.0411 & 0.7953 \\ 1694 & 14: 49: 00 & 0.1153 & 0.3504 \\ 1696 & 14: 49: 02 & 0.3344 & 0.4747 \\ 1698 & 14: 49: 04 & 0.0107 & 0.0125 \\ 1700 & 14: 49: 06 & 0.0160 & 0.0610 \\ 1702 & 14: 49: 08 & 0.0782 & 0.0647 \\ 1704 & 14: 49: 10 & 0.0194 & 0.0087 \\ 1706 & 14: 49: 12 & 0.0262 & 0.2088 \\ 1708 & 14: 49: 14 & 0.0316 & 0.5334 \\ 1710 & 14: 49: 16 & 0.1586 & 0.1358 \\ 1712 & 14: 49: 18 & 0.0022 & 0.0209 \\ 1714 & 14: 49: 20 & 0.1109 & 0.0206 \\ 1716 & 14: 49: 22 & 0.1942 & 0.0312 \\ 1718 & 14: 49: 24 & 0.0029 & 0.0801 \\ 1720 & 14: 49: 26 & 0.0081 & 0.1277 \\ 1722 & 14: 49: 28 & 0.0015 & 0.1272 \\ 1724 & 14: 49: 30 & 0.0964 & 0.8270 \\ 1726 & 14: 49: 32 & 0.2058 & 0.4579 \\ 1728 & 14: 49: 34 & 0.0281 & 0.0102 \\ 1730 & 14: 49: 36 & 0.4446 & 0.0148\end{array}$


Appendix H - Laser Data Test 4 NIST102705_1.xls

$\begin{array}{llll}1732 & 14: 49: 38 & 0.0344 & 0.0313 \\ 1734 & 14: 49: 40 & 0.0145 & 0.0530 \\ 1736 & 14: 49: 42 & 0.0134 & 0.0051 \\ 1738 & 14: 49: 44 & 0.1069 & 0.0104 \\ 1740 & 14: 49: 46 & 0.0056 & 0.1129 \\ 1742 & 14: 49: 48 & 0.3207 & 0.4000 \\ 1744 & 14: 49: 50 & 0.2059 & 0.0307 \\ 1746 & 14: 49: 52 & 0.0275 & 0.0771 \\ 1748 & 14: 49: 54 & 0.0101 & 0.0008 \\ 1750 & 14: 49: 56 & 0.0232 & 0.0828 \\ 1752 & 14: 49: 58 & 0.0703 & 0.0093 \\ 1754 & 14: 50: 00 & 0.0758 & 0.0486 \\ 1756 & 14: 50: 02 & 0.0724 & 0.0841 \\ 1758 & 14: 50: 04 & 0.0639 & 0.0650 \\ 1760 & 14: 50: 06 & 0.0329 & 0.0243 \\ 1762 & 14: 50: 08 & 0.0629 & 0.0765 \\ 1764 & 14: 50: 10 & 0.4277 & 0.0212 \\ 1766 & 14: 50: 12 & 0.2392 & 0.0448 \\ 1768 & 14: 50: 14 & 0.3375 & 0.0476 \\ 1770 & 14: 50: 16 & 0.0312 & 0.1277 \\ 1772 & 14: 50: 18 & 0.0699 & 0.5228 \\ 1774 & 14: 50: 20 & 0.0097 & 0.2476 \\ 1776 & 14: 50: 22 & 0.0364 & 0.1446 \\ 1778 & 14: 50: 24 & 0.0725 & 0.0190 \\ 1780 & 14: 50: 26 & 0.4306 & 0.0834 \\ 1782 & 14: 50: 28 & 0.0241 & 0.0605 \\ 1784 & 14: 50: 30 & 0.0350 & 0.9327 \\ 1786 & 14: 50: 32 & 0.5447 & 0.2008 \\ 1788 & 14: 50: 34 & 0.0767 & 0.7160 \\ 1790 & 14: 50: 36 & 0.0664 & 0.2027 \\ 1792 & 14: 50: 38 & 0.3169 & 0.3242 \\ 1794 & 14: 50: 40 & 0.0133 & 0.7713 \\ 1796 & 14: 50: 42 & 0.0020 & 0.4365 \\ 1798 & 14: 50: 44 & 0.0027 & 0.0377 \\ 1800 & 14: 50: 46 & 0.0098 & 0.0071 \\ 1802 & 14: 50: 48 & 0.0012 & 0.8404 \\ 1804 & 14: 50: 50 & 0.0005 & 0.3929 \\ 1806 & 14: 50: 52 & 0.0002 & 0.0953 \\ 1808 & 14: 50: 54 & 0.0072 & 0.1105 \\ 1810 & 14: 50: 56 & 0.0015 & 0.0780 \\ 1812 & 14: 50: 58 & 0.0035 & 0.0027 \\ 1814 & 14: 51: 00 & 0.0004 & 0.0011 \\ 1816 & 14: 51: 02 & 0.0002 & 0.0098 \\ 1818 & 14: 51: 04 & 0.0002 & 0.0002 \\ 1820 & 14: 51: 06 & 0.0002 & 0.0031 \\ 1822 & 14: 51: 08 & 0.0505 & 0.0014 \\ 1824 & 14: 51: 10 & 0.0012 & 0.0011 \\ 1826 & 14: 51: 12 & 0.0005 & 0.0012 \\ 1828 & 14: 51: 14 & 0.0193 & 0.0724 \\ 1830 & 14: 51: 16 & 0.0045 & 0.0321 \\ 1832 & 14: 51: 18 & 0.0002 & 0.0049\end{array}$


Appendix H - Laser Data Test 4 NIST102705_1.xls

$\begin{array}{llll}1834 & 14: 51: 20 & 0.0740 & 0.0033 \\ 1836 & 14: 51: 22 & 0.0004 & 0.4139 \\ 1838 & 14: 51: 24 & 0.0175 & 0.6339 \\ 1840 & 14: 51: 26 & 0.0004 & 0.2466 \\ 1842 & 14: 51: 28 & 0.0003 & 0.0091 \\ 1844 & 14: 51: 30 & 0.0017 & 0.0155 \\ 1846 & 14: 51: 32 & 0.0002 & 0.0824 \\ 1848 & 14: 51: 34 & 0.0002 & 0.0185 \\ 1850 & 14: 51: 36 & 0.0026 & 0.1489 \\ 1852 & 14: 51: 38 & 0.1381 & 0.4197 \\ 1854 & 14: 51: 40 & 0.0005 & 0.0261 \\ 1856 & 14: 51: 42 & 0.0026 & 0.2722 \\ 1858 & 14: 51: 44 & 0.5477 & 0.0098 \\ 1860 & 14: 51: 46 & 0.0632 & 0.0033 \\ 1862 & 14: 51: 48 & 0.0390 & 0.0069 \\ 1864 & 14: 51: 50 & 0.0098 & 0.0440 \\ 1866 & 14: 51: 52 & 0.0057 & 0.0522 \\ 1868 & 14: 51: 54 & 0.0140 & 0.0162 \\ 1870 & 14: 51: 56 & 0.0002 & 0.0906 \\ 1872 & 14: 51: 58 & 0.0007 & 0.1190 \\ 1874 & 14: 52: 00 & 0.0056 & 0.0724 \\ 1876 & 14: 52: 02 & 0.0006 & 0.0034 \\ 1878 & 14: 52: 04 & 0.0023 & 0.0034 \\ 1880 & 14: 52: 06 & 0.0004 & 0.0012 \\ 1882 & 14: 52: 08 & 0.0002 & 0.0320 \\ 1884 & 14: 52: 10 & 0.0003 & 0.0547 \\ 1886 & 14: 52: 12 & 0.0009 & 0.1243 \\ 1888 & 14: 52: 14 & 0.0003 & 0.0003 \\ 1890 & 14: 52: 16 & 0.0002 & 0.0865 \\ 1892 & 14: 52: 18 & 0.0028 & 0.2642 \\ 1894 & 14: 52: 20 & 0.0225 & 0.0004 \\ 1896 & 14: 52: 22 & 0.0175 & 0.0033 \\ 1898 & 14: 52: 24 & 0.0006 & 0.0975 \\ 1900 & 14: 52: 26 & 0.0356 & 0.0012 \\ 1902 & 14: 52: 28 & 0.0002 & 0.0030 \\ 1904 & 14: 52: 30 & 0.0022 & 0.0010 \\ 1906 & 14: 52: 32 & 0.0002 & 0.0129 \\ 1908 & 14: 52: 34 & 0.0002 & 0.7591 \\ 1910 & 14: 52: 36 & 0.0002 & 0.0043 \\ 1912 & 14: 52: 38 & 0.0017 & 0.0102 \\ 1914 & 14: 52: 40 & 0.0027 & 0.1483 \\ 1916 & 14: 52: 42 & 0.0077 & 0.0002 \\ 1918 & 14: 52: 44 & 0.0004 & 0.0002 \\ 1920 & 14: 52: 46 & 0.0002 & 0.0002 \\ 1922 & 14: 52: 48 & 0.0017 & 0.0142 \\ 1924 & 14: 52: 50 & 0.0035 & 0.0160 \\ 1926 & 14: 52: 52 & 0.0015 & 0.0009 \\ 1928 & 14: 52: 54 & 0.0004 & 0.0014 \\ 1930 & 14: 52: 56 & 0.0017 & 0.0016 \\ 1932 & 14: 52: 58 & 0.0013 & 0.0016 \\ 1934 & 14: 53: 00 & 0.0002 & 0.0008\end{array}$


Appendix H - Laser Data Test 4 NIST102705_1.xls

$\begin{array}{llll}1936 & 14: 53: 02 & 0.0002 & 0.0032 \\ 1938 & 14: 53: 04 & 0.0002 & 0.0031 \\ 1940 & 14: 53: 06 & 0.0038 & 0.0014 \\ 1942 & 14: 53: 08 & 0.0037 & 0.0005 \\ 1944 & 14: 53: 10 & 0.0002 & 0.0183 \\ 1946 & 14: 53: 12 & 0.0002 & 0.0003 \\ 1948 & 14: 53: 14 & 0.0201 & 0.0068 \\ 1950 & 14: 53: 16 & 0.0005 & 0.0093 \\ 1952 & 14: 53: 18 & 0.0004 & 0.0002 \\ 1954 & 14: 53: 20 & 0.0004 & 0.0019 \\ 1956 & 14: 53: 22 & 0.0019 & 0.4077 \\ 1958 & 14: 53: 24 & 0.1446 & 0.0003 \\ 1960 & 14: 53: 26 & 0.1188 & 0.0082 \\ 1962 & 14: 53: 28 & 0.0015 & 0.0003 \\ 1964 & 14: 53: 30 & 0.0002 & 0.0558 \\ 1966 & 14: 53: 32 & 0.0006 & 0.0045 \\ 1968 & 14: 53: 34 & 0.0016 & 0.0008 \\ 1970 & 14: 53: 36 & 0.0071 & 0.0056 \\ 1972 & 14: 53: 38 & 0.0341 & 0.0002 \\ 1974 & 14: 53: 40 & 0.0004 & 0.0033 \\ 1976 & 14: 53: 42 & 0.0003 & 0.0042 \\ 1978 & 14: 53: 44 & 0.0010 & 0.0005 \\ 1980 & 14: 53: 46 & 0.0002 & 0.0025 \\ 1982 & 14: 53: 48 & 0.0003 & 0.0121 \\ 1984 & 14: 53: 50 & 0.0007 & 0.0010 \\ 1986 & 14: 53: 52 & 0.0003 & 0.0010 \\ 1988 & 14: 53: 54 & 0.0014 & 0.0002 \\ 1990 & 14: 53: 56 & 0.0002 & 0.0003 \\ 1992 & 14: 53: 58 & 0.0057 & 0.0080 \\ 1994 & 14: 54: 00 & 0.0034 & 0.0006 \\ 1996 & 14: 54: 02 & 0.0003 & 0.0002 \\ 1998 & 14: 54: 04 & 0.0015 & 0.0885 \\ 2000 & 14: 54: 06 & 0.0005 & 0.0769 \\ 2002 & 14: 54: 08 & 0.0331 & 0.1370 \\ 2004 & 14: 54: 10 & 0.0515 & 0.0672 \\ 2006 & 14: 54: 12 & 0.0005 & 0.0035 \\ 2008 & 14: 54: 14 & 0.0011 & 0.0019 \\ 2010 & 14: 54: 16 & 0.0051 & 0.1882 \\ 2012 & 14: 54: 18 & 0.0018 & 0.0055 \\ 2014 & 14: 54: 20 & 0.0159 & 0.0275 \\ 2016 & 14: 54: 22 & 0.0007 & 0.0006 \\ 2018 & 14: 54: 24 & 0.0002 & 0.0034 \\ 2020 & 14: 54: 26 & 0.0005 & 0.0004 \\ 2022 & 14: 54: 28 & 0.0006 & 0.0004 \\ 2024 & 14: 54: 30 & 0.0011 & 0.0002 \\ 2026 & 14: 54: 32 & 0.0002 & 0.0004 \\ 2028 & 14: 54: 34 & 0.0002 & 0.0026 \\ 2030 & 14: 54: 36 & 0.0002 & 0.0019 \\ 2032 & 14: 54: 38 & 0.0035 & 0.0330 \\ 2034 & 14: 54: 40 & 0.0020 & 0.2799 \\ 2036 & 14: 54: 42 & 0.0002 & 0.0066\end{array}$


Appendix H - Laser Data Test 4 NIST102705_1.xls

$\begin{array}{llll}2038 & 14: 54: 44 & 0.0025 & 0.0008 \\ 2040 & 14: 54: 46 & 0.0002 & 0.0003 \\ 2042 & 14: 54: 48 & 0.0002 & 0.0002 \\ 2044 & 14: 54: 50 & 0.0002 & 0.0001 \\ 2046 & 14: 54: 52 & 0.0004 & 0.0000 \\ 2048 & 14: 54: 54 & 0.0009 & 0.0002 \\ 2050 & 14: 54: 56 & 0.0063 & 0.0019 \\ 2052 & 14: 54: 58 & 0.0007 & 0.0075 \\ 2054 & 14: 55: 00 & 0.0002 & 0.0014 \\ 2056 & 14: 55: 02 & 0.0002 & 1.0677 \\ 2058 & 14: 55: 04 & 0.0005 & 0.0032 \\ 2060 & 14: 55: 06 & 0.0005 & 0.0006 \\ 2062 & 14: 55: 08 & 0.0033 & 0.0001 \\ 2064 & 14: 55: 10 & 0.0008 & 0.0033 \\ 2066 & 14: 55: 12 & 0.0003 & 0.0249 \\ 2068 & 14: 55: 14 & 0.0010 & 0.0193 \\ 2070 & 14: 55: 16 & 0.0002 & 0.2933 \\ 2072 & 14: 55: 18 & 0.0089 & 0.0003 \\ 2074 & 14: 55: 20 & 0.0002 & 0.0002 \\ 2076 & 14: 55: 22 & 0.0019 & 0.0066 \\ 2078 & 14: 55: 24 & 0.0026 & 0.0006 \\ 2080 & 14: 55: 26 & 0.0004 & 0.0002 \\ 2082 & 14: 55: 28 & 0.0010 & 0.0015 \\ 2084 & 14: 55: 30 & 0.0002 & 0.0004 \\ 2086 & 14: 55: 32 & 0.0009 & 0.0181 \\ 2088 & 14: 55: 34 & 0.0003 & 0.0004 \\ 2090 & 14: 55: 36 & 0.0002 & 0.0002 \\ 2092 & 14: 55: 38 & 0.0019 & 0.0382 \\ 2094 & 14: 55: 40 & 0.0002 & 0.0012 \\ 2096 & 14: 55: 42 & 0.0002 & 0.1310 \\ 2098 & 14: 55: 44 & 0.0009 & 0.0083 \\ 2100 & 14: 55: 46 & 0.0010 & 0.0410 \\ 2102 & 14: 55: 48 & 0.0003 & 0.0239 \\ 2104 & 14: 55: 50 & 0.0024 & 0.0021 \\ 2106 & 14: 55: 52 & 0.0083 & 0.0013 \\ 2108 & 14: 55: 54 & 0.0156 & 0.0002 \\ 2110 & 14: 55: 56 & 0.0002 & 0.0027 \\ 2112 & 14: 55: 58 & 0.0002 & 0.0001 \\ 2114 & 14: 56: 00 & 0.0002 & 0.0006 \\ 2116 & 14: 56: 02 & 0.0003 & 0.0001 \\ 2118 & 14: 56: 04 & 0.0002 & 0.0007 \\ 2120 & 14: 56: 06 & 0.0004 & 0.0083 \\ 2122 & 14: 56: 08 & 0.0064 & 0.0576 \\ 2124 & 14: 56: 10 & 0.0067 & 0.0196 \\ 2126 & 14: 56: 12 & 0.0002 & 0.0010 \\ 2128 & 14: 56: 14 & 0.0003 & 0.0042 \\ 2130 & 14: 56: 16 & 0.0003 & 0.0656 \\ 2132 & 14: 56: 18 & 0.0003 & 0.0003 \\ 2134 & 14: 56: 20 & 0.0210 & 0.0000 \\ 2136 & 14: 56: 22 & 0.0002 & 0.0010 \\ 2138 & 14: 56: 24 & 0.0002 & 0.0014\end{array}$


Appendix H - Laser Data Test 4 NIST102705_1.xls

$\begin{array}{llll}2140 & 14: 56: 26 & 0.0002 & 0.0003 \\ 2142 & 14: 56: 28 & 0.0002 & 0.0003 \\ 2144 & 14: 56: 30 & 0.0002 & 0.0888 \\ 2146 & 14: 56: 32 & 0.0014 & 0.0002 \\ 2148 & 14: 56: 34 & 0.0002 & 0.0035 \\ 2150 & 14: 56: 36 & 0.0005 & 0.0002 \\ 2152 & 14: 56: 38 & 0.0002 & 0.0002 \\ 2154 & 14: 56: 40 & 0.0004 & 0.0008 \\ 2156 & 14: 56: 42 & 0.0002 & 0.0190 \\ 2158 & 14: 56: 44 & 0.0002 & 0.0003 \\ 2160 & 14: 56: 46 & 0.0002 & 0.0011 \\ 2162 & 14: 56: 48 & 0.0002 & 0.0010 \\ 2164 & 14: 56: 50 & 0.0158 & 0.0166 \\ 2166 & 14: 56: 52 & 0.0002 & 0.0028 \\ 2168 & 14: 56: 54 & 0.0144 & 0.0022 \\ 2170 & 14: 56: 56 & 0.0002 & 0.0018 \\ 2172 & 14: 56: 58 & 0.0002 & 0.0002 \\ 2174 & 14: 57: 00 & 0.0072 & 0.0002 \\ 2176 & 14: 57: 02 & 0.0012 & 0.0004 \\ 2178 & 14: 57: 04 & 0.0004 & 0.0009 \\ 2180 & 14: 57: 06 & 0.0051 & 0.0013 \\ 2182 & 14: 57: 08 & 0.0004 & 0.0080 \\ 2184 & 14: 57: 10 & 0.0372 & 0.0002 \\ 2186 & 14: 57: 12 & 0.0003 & 0.0002 \\ 2188 & 14: 57: 14 & 0.0002 & 0.0004 \\ 2190 & 14: 57: 16 & 0.0047 & 0.0017 \\ 2192 & 14: 57: 18 & 0.0015 & 0.1104 \\ 2194 & 14: 57: 20 & 0.0004 & 0.0030 \\ 2196 & 14: 57: 22 & 0.0003 & 0.0083 \\ 2198 & 14: 57: 24 & 0.0002 & 0.0027 \\ 2200 & 14: 57: 26 & 0.0073 & 0.6730 \\ 2202 & 14: 57: 28 & 0.0217 & 0.0096 \\ 2204 & 14: 57: 30 & 0.0127 & 0.0066 \\ 2206 & 14: 57: 32 & 0.0019 & 0.0026 \\ 2208 & 14: 57: 34 & 0.0147 & 0.0222 \\ 2210 & 14: 57: 36 & 0.0010 & 0.0062 \\ 2212 & 14: 57: 38 & 0.0002 & 0.0009 \\ 2214 & 14: 57: 40 & 0.0048 & 0.0001 \\ 2216 & 14: 57: 42 & 0.0002 & 0.0001 \\ 2218 & 14: 57: 44 & 0.0128 & 0.0004 \\ 2220 & 14: 57: 46 & 0.0002 & 0.0024 \\ 2222 & 14: 57: 48 & 0.0005 & 0.0016 \\ 2224 & 14: 57: 50 & 0.0034 & 0.0003 \\ 2226 & 14: 57: 52 & 0.0007 & 0.0027 \\ 2228 & 14: 57: 54 & 0.0012 & 0.0002 \\ 2230 & 14: 57: 56 & 0.0010 & 0.0002 \\ 2232 & 14: 57: 58 & 0.2442 & 0.0140 \\ 2234 & 14: 58: 00 & 0.0132 & 0.0003 \\ 2236 & 14: 58: 02 & 0.0022 & 0.0008 \\ 2238 & 14: 58: 04 & 0.0023 & 0.0068 \\ 2240 & 14: 58: 06 & 0.0306 & 0.0007\end{array}$


Appendix H - Laser Data Test 4 NIST102705_1.xls

$\begin{array}{llll}2242 & 14: 58: 08 & 0.0153 & 0.0003 \\ 2244 & 14: 58: 10 & 0.0002 & 0.0461 \\ 2246 & 14: 58: 12 & 0.0005 & 0.0011 \\ 2248 & 14: 58: 14 & 0.0036 & 0.1669 \\ 2250 & 14: 58: 16 & 0.0032 & 0.0355 \\ 2252 & 14: 58: 18 & 0.0025 & 0.0003 \\ 2254 & 14: 58: 20 & 0.0013 & 0.0007 \\ 2256 & 14: 58: 22 & 0.0040 & 0.0002 \\ 2258 & 14: 58: 24 & 0.0005 & 0.0065 \\ 2260 & 14: 58: 26 & 0.0003 & 0.0018 \\ 2262 & 14: 58: 28 & 0.0024 & 0.0234 \\ 2264 & 14: 58: 30 & 0.0151 & 0.0002 \\ 2266 & 14: 58: 32 & 0.0002 & 0.0027 \\ 2268 & 14: 58: 34 & 0.0014 & 0.0013 \\ 2270 & 14: 58: 36 & 0.0004 & 0.0162 \\ 2272 & 14: 58: 38 & 0.0077 & 0.0008 \\ 2274 & 14: 58: 40 & 0.0024 & 0.0002 \\ 2276 & 14: 58: 42 & 0.0002 & 0.0002 \\ 2278 & 14: 58: 44 & 0.0002 & 0.0001 \\ 2280 & 14: 58: 46 & 0.0038 & 0.0027 \\ 2282 & 14: 58: 48 & 0.0003 & 0.0004 \\ 2284 & 14: 58: 50 & 0.0005 & 0.0040 \\ 2286 & 14: 58: 52 & 0.0003 & 0.0239 \\ 2288 & 14: 58: 54 & 0.0015 & 0.0033 \\ 2290 & 14: 58: 56 & 0.0075 & 0.0076 \\ 2292 & 14: 58: 58 & 0.0002 & 0.0109 \\ 2294 & 14: 59: 00 & 0.0005 & 0.0003 \\ 2296 & 14: 59: 02 & 0.0002 & 0.0036 \\ 2298 & 14: 59: 04 & 0.0028 & 0.0055 \\ 2300 & 14: 59: 06 & 0.0023 & 0.0240 \\ 2302 & 14: 59: 08 & 0.0005 & 0.0002 \\ 2304 & 14: 59: 10 & 0.0068 & 0.0031 \\ 2306 & 14: 59: 12 & 0.0003 & 0.0059 \\ 2308 & 14: 59: 14 & 0.0068 & 0.0002 \\ 2310 & 14: 59: 16 & 0.0004 & 0.0008 \\ 2312 & 14: 59: 18 & 0.0068 & 0.0018 \\ 2314 & 14: 59: 20 & 0.1552 & 0.0007 \\ 2316 & 14: 59: 22 & 0.0137 & 0.0086 \\ 2318 & 14: 59: 24 & 0.0110 & 0.0064 \\ 2320 & 14: 59: 26 & 0.0003 & 0.0377 \\ 2322 & 14: 59: 28 & 0.0006 & 0.0003 \\ 2324 & 14: 59: 30 & 0.0002 & 0.0708 \\ 2326 & 14: 59: 32 & 0.0012 & 0.0102 \\ 2328 & 14: 59: 34 & 0.0016 & 0.1467 \\ 2330 & 14: 59: 36 & 0.0002 & 0.0240 \\ 2332 & 14: 59: 38 & 0.0306 & 0.0063 \\ 2334 & 14: 59: 40 & 0.0008 & 0.0002 \\ 2336 & 14: 59: 42 & 0.0509 & 0.0002 \\ 2338 & 14: 59: 44 & 0.0061 & 0.0002 \\ 2340 & 14: 59: 46 & 0.0010 & 0.0002 \\ 2342 & 14: 59: 48 & 0.0082 & 0.0002\end{array}$


Appendix H - Laser Data Test 4 NIST102705_1.xls

$\begin{array}{llll}2344 & 14: 59: 50 & 0.1442 & 0.0002 \\ 2346 & 14: 59: 52 & 0.0228 & 0.0011 \\ 2348 & 14: 59: 54 & 0.0394 & 0.0022 \\ 2350 & 14: 59: 56 & 0.0040 & 0.0017 \\ 2352 & 14: 59: 58 & 0.0786 & 0.0002 \\ 2354 & 15: 00: 00 & 0.0019 & 0.0072 \\ 2356 & 15: 00: 02 & 0.0030 & 0.0002 \\ 2358 & 15: 00: 04 & 0.0015 & 0.0004 \\ 2360 & 15: 00: 06 & 0.0013 & 0.0137 \\ 2362 & 15: 00: 08 & 0.0007 & 0.0002 \\ 2364 & 15: 00: 10 & 0.0013 & 0.0049 \\ 2366 & 15: 00: 12 & 0.0002 & 0.0015 \\ 2368 & 15: 00: 14 & 0.0003 & 0.0002 \\ 2370 & 15: 00: 16 & 0.0002 & 0.0003 \\ 2372 & 15: 00: 18 & 0.0005 & 0.0002 \\ 2374 & 15: 00: 20 & 0.0198 & 0.0002 \\ 2376 & 15: 00: 22 & 0.0013 & 0.0002 \\ 2378 & 15: 00: 24 & 0.0015 & 0.0004 \\ 2380 & 15: 00: 26 & 0.0002 & 0.0002 \\ 2382 & 15: 00: 28 & 0.0863 & 0.0006 \\ 2384 & 15: 00: 30 & 0.0579 & 0.0002 \\ 2386 & 15: 00: 32 & 0.0066 & 0.0047 \\ 2388 & 15: 00: 34 & 0.0335 & 0.0009 \\ 2390 & 15: 00: 36 & 0.0011 & 0.0002 \\ 2392 & 15: 00: 38 & 0.0015 & 0.0006 \\ 2394 & 15: 00: 40 & 0.0540 & 0.0002 \\ 2396 & 15: 00: 42 & 0.0232 & 0.0017 \\ 2398 & 15: 00: 44 & 0.0144 & 0.0031 \\ 2400 & 15: 00: 46 & 0.0002 & 0.0009 \\ 2402 & 15: 00: 48 & 0.0568 & 0.0007 \\ 2404 & 15: 00: 50 & 0.0003 & 0.0116 \\ 2406 & 15: 00: 52 & 0.1018 & 0.0003 \\ 2408 & 15: 00: 54 & 0.0003 & 0.0017 \\ 2410 & 15: 00: 56 & 0.0020 & 0.0002 \\ 2412 & 15: 00: 58 & 0.1498 & 0.0016 \\ 2414 & 15: 01: 00 & 0.1890 & 0.0002 \\ 2416 & 15: 01: 02 & 0.0432 & 0.0012 \\ 2418 & 15: 01: 04 & 0.0080 & 0.0002 \\ 2420 & 15: 01: 06 & 0.1753 & 0.0002 \\ 2422 & 15: 01: 08 & 0.0999 & 0.0094 \\ 2424 & 15: 01: 10 & 0.0059 & 0.0002 \\ 2426 & 15: 01: 12 & 0.0004 & 0.0003 \\ 2428 & 15: 01: 14 & 0.0014 & 0.0002 \\ 2430 & 15: 01: 16 & 0.0522 & 0.0004 \\ 2432 & 15: 01: 18 & 0.1194 & 0.0003 \\ 2434 & 15: 01: 20 & 0.0159 & 0.0002 \\ 2436 & 15: 01: 22 & 0.0229 & 0.0001 \\ 2438 & 15: 01: 24 & 0.0321 & 0.0002 \\ 2440 & 15: 01: 26 & 0.0562 & 0.0002 \\ 2442 & 15: 01: 28 & 0.0557 & 0.0002 \\ 2444 & 15: 01: 30 & 0.0013 & 0.0001\end{array}$


Appendix H - Laser Data Test 4 NIST102705_1.xls

$\begin{array}{llll}2446 & 15: 01: 32 & 0.0318 & 0.0002 \\ 2448 & 15: 01: 34 & 0.0008 & 0.0025 \\ 2450 & 15: 01: 36 & 0.0206 & 0.0001 \\ 2452 & 15: 01: 38 & 0.0736 & 0.0001 \\ 2454 & 15: 01: 40 & 0.0769 & 0.0001 \\ 2456 & 15: 01: 42 & 0.0647 & 0.0010 \\ 2458 & 15: 01: 44 & 0.0030 & 0.0001 \\ 2460 & 15: 01: 46 & 0.0012 & 0.0002 \\ 2462 & 15: 01: 48 & 0.0012 & 0.0546 \\ 2464 & 15: 01: 50 & 0.0214 & 0.0002 \\ 2466 & 15: 01: 52 & 0.0674 & 0.0002 \\ 2468 & 15: 01: 54 & 0.0225 & 0.0115 \\ 2470 & 15: 01: 56 & 0.4206 & 0.0002 \\ 2472 & 15: 01: 58 & 0.0475 & 0.0002 \\ 2474 & 15: 02: 00 & 0.0314 & 0.0002 \\ 2476 & 15: 02: 02 & 0.0023 & 0.0002 \\ 2478 & 15: 02: 04 & 0.1790 & 0.0002 \\ 2480 & 15: 02: 06 & 0.0005 & 0.0002 \\ 2482 & 15: 02: 08 & 0.0071 & 0.0002 \\ 2484 & 15: 02: 10 & 0.0182 & 0.0068 \\ 2486 & 15: 02: 12 & 0.0003 & 0.0003 \\ 2488 & 15: 02: 14 & 0.0005 & 0.0003 \\ 2490 & 15: 02: 16 & 0.0002 & 0.0173 \\ 2492 & 15: 02: 18 & 0.0125 & 0.0003 \\ 2494 & 15: 02: 20 & 0.0111 & 0.0002 \\ 2496 & 15: 02: 22 & 0.0832 & 0.0133 \\ 2498 & 15: 02: 24 & 0.0617 & 0.0002 \\ 2500 & 15: 02: 26 & 0.2695 & 0.0035 \\ 2502 & 15: 02: 28 & 0.2141 & 0.0002 \\ 2504 & 15: 02: 30 & 0.0682 & 0.0003 \\ 2506 & 15: 02: 32 & 0.2594 & 0.0011 \\ 2508 & 15: 02: 34 & 0.0778 & 0.0005 \\ 2510 & 15: 02: 36 & 0.2918 & 0.0003 \\ 2512 & 15: 02: 38 & 0.2653 & 0.0002 \\ 2514 & 15: 02: 40 & 0.0751 & 0.0002 \\ 2516 & 15: 02: 42 & 0.0672 & 0.0002 \\ 2518 & 15: 02: 44 & 0.3000 & 0.0015 \\ 2520 & 15: 02: 46 & 0.1241 & 0.0002 \\ 2522 & 15: 02: 48 & 0.0593 & 0.0002 \\ 2524 & 15: 02: 50 & 0.5163 & 0.0002 \\ 2526 & 15: 02: 52 & 0.0093 & 0.0011 \\ 2528 & 15: 02: 54 & 0.0159 & 0.0002 \\ 2530 & 15: 02: 56 & 0.0523 & 0.0002 \\ 2532 & 15: 02: 58 & 0.0221 & 0.0001 \\ 2534 & 15: 03: 00 & 0.0005 & 0.0002 \\ 2536 & 15: 03: 02 & 0.0072 & 0.0002 \\ 2538 & 15: 03: 04 & 0.0090 & 0.0135 \\ 2540 & 15: 03: 06 & 0.0003 & 0.0007 \\ 2542 & 15: 03: 08 & 0.0002 & 0.0050 \\ 2544 & 15: 03: 10 & 0.0038 & 0.0002 \\ 2546 & 15: 03: 12 & 0.0734 & 0.0001\end{array}$


Appendix H - Laser Data Test 4 NIST102705_1.xls

$\begin{array}{llll}2548 & 15: 03: 14 & 0.0029 & 0.0006 \\ 2550 & 15: 03: 16 & 0.0224 & 0.0001 \\ 2552 & 15: 03: 18 & 0.0434 & 0.0001 \\ 2554 & 15: 03: 20 & 0.0010 & 0.0001 \\ 2556 & 15: 03: 22 & 0.0998 & 0.0005 \\ 2558 & 15: 03: 24 & 0.0146 & 0.0002 \\ 2560 & 15: 03: 26 & 0.0088 & 0.0002 \\ 2562 & 15: 03: 28 & 0.0008 & 0.0002 \\ 2564 & 15: 03: 30 & 0.0022 & 0.0005 \\ 2566 & 15: 03: 32 & 0.0004 & 0.0092 \\ 2568 & 15: 03: 34 & 0.0017 & 0.0002 \\ 2570 & 15: 03: 36 & 0.0002 & 0.0012 \\ 2572 & 15: 03: 38 & 0.0050 & 0.0016 \\ 2574 & 15: 03: 40 & 0.0558 & 0.0001 \\ 2576 & 15: 03: 42 & 0.0110 & 0.0002 \\ 2578 & 15: 03: 44 & 0.0061 & 0.0003 \\ 2580 & 15: 03: 46 & 0.0027 & 0.0002 \\ 2582 & 15: 03: 48 & 0.2738 & 0.0002 \\ 2584 & 15: 03: 50 & 0.1138 & 0.0046 \\ 2586 & 15: 03: 52 & 0.0012 & 0.0005 \\ 2588 & 15: 03: 54 & 0.0010 & 0.0263 \\ 2590 & 15: 03: 56 & 0.0071 & 0.0001 \\ 2592 & 15: 03: 58 & 0.0047 & 0.0000 \\ 2594 & 15: 04: 00 & 0.0470 & 0.0001 \\ 2596 & 15: 04: 02 & 0.0115 & 0.0001 \\ 2598 & 15: 04: 04 & 0.0472 & 0.0001 \\ 2600 & 15: 04: 06 & 0.0901 & 0.0001 \\ 2602 & 15: 04: 08 & 0.0183 & 0.0006 \\ 2604 & 15: 04: 10 & 0.0107 & 0.0002 \\ 2606 & 15: 04: 12 & 0.0493 & 0.0002 \\ 2608 & 15: 04: 14 & 0.4324 & 0.0002 \\ 2610 & 15: 04: 16 & 0.5873 & 0.0001 \\ 2612 & 15: 04: 18 & 0.0052 & 0.0000 \\ 2614 & 15: 04: 20 & 0.0133 & 0.0001 \\ 2616 & 15: 04: 22 & 0.0002 & 0.0107 \\ 2618 & 15: 04: 24 & 0.0002 & 0.0158 \\ 2620 & 15: 04: 26 & 0.0066 & 0.0006 \\ 2622 & 15: 04: 28 & 0.0004 & 0.0042 \\ 2624 & 15: 04: 30 & 0.0002 & 0.0005 \\ 2626 & 15: 04: 32 & 0.0054 & 0.0015 \\ 2628 & 15: 04: 34 & 0.0002 & 0.0017 \\ 2630 & 15: 04: 36 & 0.0092 & 0.0008 \\ 2632 & 15: 04: 38 & 0.0016 & 0.0002 \\ 2634 & 15: 04: 40 & 0.0059 & 0.0171 \\ 2636 & 15: 04: 42 & 0.0314 & 0.0002 \\ 2638 & 15: 04: 44 & 0.0089 & 0.0035 \\ 2640 & 15: 04: 46 & 0.0003 & 0.0003 \\ 2642 & 15: 04: 48 & 0.0003 & 0.0075 \\ 2644 & 15: 04: 50 & 0.0120 & 0.0292 \\ 2646 & 15: 04: 52 & 0.0016 & 0.0163 \\ 2648 & 15: 04: 54 & 0.0022 & 0.0003\end{array}$


Appendix H - Laser Data Test 4 NIST102705_1.xls

$\begin{array}{llll}2650 & 15: 04: 56 & 0.0028 & 0.0018 \\ 2652 & 15: 04: 58 & 0.0057 & 0.0002 \\ 2654 & 15: 05: 00 & 0.0345 & 0.0012 \\ 2656 & 15: 05: 02 & 0.0005 & 0.0016 \\ 2658 & 15: 05: 04 & 0.0358 & 0.0002 \\ 2660 & 15: 05: 06 & 0.0011 & 0.0003 \\ 2662 & 15: 05: 08 & 0.0017 & 0.0025 \\ 2664 & 15: 05: 10 & 0.0047 & 0.0001 \\ 2666 & 15: 05: 12 & 0.0374 & 0.0011 \\ 2668 & 15: 05: 14 & 0.0221 & 0.0007 \\ 2670 & 15: 05: 16 & 0.0004 & 0.0002 \\ 2672 & 15: 05: 18 & 0.0004 & 0.0123 \\ 2674 & 15: 05: 20 & 0.0003 & 0.0042 \\ 2676 & 15: 05: 22 & 0.0002 & 0.0005 \\ 2678 & 15: 05: 24 & 0.0005 & 0.0003 \\ 2680 & 15: 05: 26 & 0.0002 & 0.0002 \\ 2682 & 15: 05: 28 & 0.0085 & 0.0003 \\ 2684 & 15: 05: 30 & 0.0111 & 0.0002 \\ 2686 & 15: 05: 32 & 0.0003 & 0.0009 \\ 2688 & 15: 05: 34 & 0.0048 & 0.0002 \\ 2690 & 15: 05: 36 & 0.0276 & 0.0001 \\ 2692 & 15: 05: 38 & 0.0033 & 0.0002 \\ 2694 & 15: 05: 40 & 0.2481 & 0.0002 \\ 2696 & 15: 05: 42 & 0.0018 & 0.0002 \\ 2698 & 15: 05: 44 & 0.0008 & 0.0002 \\ 2700 & 15: 05: 46 & 0.0023 & 0.0002 \\ 2702 & 15: 05: 48 & 0.1583 & 0.0003 \\ 2704 & 15: 05: 50 & 0.0210 & 0.0003 \\ 2706 & 15: 05: 52 & 0.1792 & 0.0013 \\ 2708 & 15: 05: 54 & 0.0178 & 0.0048 \\ 2710 & 15: 05: 56 & 0.0003 & 0.0002 \\ 2712 & 15: 05: 58 & 0.0006 & 0.0007 \\ 2714 & 15: 06: 00 & 0.0004 & 0.0003 \\ 2716 & 15: 06: 02 & 0.0007 & 0.0002 \\ 2718 & 15: 06: 04 & 0.0135 & 0.0001 \\ 2720 & 15: 06: 06 & 0.0126 & 0.0002 \\ 2722 & 15: 06: 08 & 0.0098 & 0.0002 \\ 2724 & 15: 06: 10 & 0.0079 & 0.0002 \\ 2726 & 15: 06: 12 & 0.0218 & 0.0001 \\ 2728 & 15: 06: 14 & 0.0192 & 0.0002 \\ 2730 & 15: 06: 16 & 0.0208 & 0.0016 \\ 2732 & 15: 06: 18 & 0.1939 & 0.0002 \\ 2734 & 15: 06: 20 & 0.0209 & 0.0235 \\ 2736 & 15: 06: 22 & 0.0058 & 0.0003 \\ 2738 & 15: 06: 24 & 0.0008 & 0.0254 \\ 2740 & 15: 06: 26 & 0.0255 & 0.0008 \\ 2742 & 15: 06: 28 & 0.0021 & 0.0002 \\ 2744 & 15: 06: 30 & 0.0055 & 0.0016 \\ 2746 & 15: 06: 32 & 0.0067 & 0.0001 \\ 2748 & 15: 06: 34 & 0.0002 & 0.0005 \\ 2750 & 15: 06: 36 & 0.0049 & 0.0001\end{array}$


Appendix H - Laser Data Test 4 NIST102705_1.xls

$\begin{array}{llll}2752 & 15: 06: 38 & 0.0526 & 0.0006 \\ 2754 & 15: 06: 40 & 0.0015 & 0.0002 \\ 2756 & 15: 06: 42 & 0.0006 & 0.0003 \\ 2758 & 15: 06: 44 & 0.0330 & 0.0138 \\ 2760 & 15: 06: 46 & 0.0089 & 0.0056 \\ 2762 & 15: 06: 48 & 0.0048 & 0.0058 \\ 2764 & 15: 06: 50 & 0.0003 & 0.0002 \\ 2766 & 15: 06: 52 & 0.0002 & 0.0066 \\ 2768 & 15: 06: 54 & 0.0031 & 0.0332 \\ 2770 & 15: 06: 56 & 0.0379 & 0.0054 \\ 2772 & 15: 06: 58 & 0.0014 & 0.0016 \\ 2774 & 15: 07: 00 & 0.0007 & 0.0005 \\ 2776 & 15: 07: 02 & 0.0257 & 0.0074 \\ 2778 & 15: 07: 04 & 0.0003 & 0.0006 \\ 2780 & 15: 07: 06 & 0.0004 & 0.0021 \\ 2782 & 15: 07: 08 & 0.0897 & 0.0029 \\ 2784 & 15: 07: 10 & 0.0021 & 0.0085 \\ 2786 & 15: 07: 12 & 0.0089 & 0.0002 \\ 2788 & 15: 07: 14 & 0.0002 & 0.0001 \\ 2790 & 15: 07: 16 & 0.0169 & 0.0017 \\ 2792 & 15: 07: 18 & 0.0002 & 0.0011 \\ 2794 & 15: 07: 20 & 0.0026 & 0.0475 \\ 2796 & 15: 07: 22 & 0.0002 & 0.0054 \\ 2798 & 15: 07: 24 & 0.0003 & 0.0002 \\ 2800 & 15: 07: 26 & 0.0002 & 0.0002 \\ 2802 & 15: 07: 28 & 0.0002 & 0.0029 \\ 2804 & 15: 07: 30 & 0.0084 & 0.0138 \\ 2806 & 15: 07: 32 & 0.0003 & 0.0103 \\ 2808 & 15: 07: 34 & 0.0034 & 0.0055 \\ 2810 & 15: 07: 36 & 0.0014 & 0.0065 \\ 2812 & 15: 07: 38 & 0.0429 & 0.0305 \\ 2814 & 15: 07: 40 & 0.0015 & 0.0057 \\ 2816 & 15: 07: 42 & 0.0187 & 0.0138 \\ 2818 & 15: 07: 44 & 0.0009 & 0.0493 \\ 2820 & 15: 07: 46 & 0.0003 & 0.0318 \\ 2822 & 15: 07: 48 & 0.0012 & 0.0006 \\ 2824 & 15: 07: 50 & 0.0002 & 0.0002 \\ 2826 & 15: 07: 52 & 0.0002 & 0.0003 \\ 2828 & 15: 07: 54 & 0.0009 & 0.0002 \\ 2830 & 15: 07: 56 & 0.0004 & 0.0005 \\ 2832 & 15: 07: 58 & 0.0002 & 0.0003 \\ 2834 & 15: 08: 00 & 0.0011 & 0.1201 \\ 2836 & 15: 08: 02 & 0.0004 & 0.0002 \\ 2838 & 15: 08: 04 & 0.0031 & 0.0002 \\ 2840 & 15: 08: 06 & 0.0002 & 0.0001 \\ 2842 & 15: 08: 08 & 0.0088 & 0.0014 \\ 2844 & 15: 08: 10 & 0.0004 & 0.0007 \\ 2846 & 15: 08: 12 & 0.0002 & 0.0005 \\ 2848 & 15: 08: 14 & 0.0002 & 0.0010 \\ 2850 & 15: 08: 16 & 0.0005 & 0.0002 \\ 2852 & 15: 08: 18 & 0.0052 & 0.0651\end{array}$




$\begin{array}{llll}2854 & 15: 08: 20 & 0.0074 & 0.0103 \\ 2856 & 15: 08: 22 & 0.0009 & 0.0002 \\ 2858 & 15: 08: 24 & 0.0112 & 0.0006 \\ 2860 & 15: 08: 26 & 0.0057 & 0.0003 \\ 2862 & 15: 08: 28 & 0.0494 & 0.0006 \\ 2864 & 15: 08: 30 & 0.5351 & 0.0095 \\ 2866 & 15: 08: 32 & 0.1148 & 0.0019 \\ 2868 & 15: 08: 34 & 0.0047 & 0.0025 \\ 2870 & 15: 08: 36 & 0.0012 & 0.0018 \\ 2872 & 15: 08: 38 & 0.0003 & 0.0143 \\ 2874 & 15: 08: 40 & 0.0009 & 0.0001 \\ 2876 & 15: 08: 42 & 0.0002 & 0.0001 \\ 2878 & 15: 08: 44 & 0.0005 & 0.0011 \\ 2880 & 15: 08: 46 & 0.0004 & 0.0002 \\ 2882 & 15: 08: 48 & 0.5433 & 0.0014 \\ 2884 & 15: 08: 50 & 0.0076 & 0.0002 \\ 2886 & 15: 08: 52 & 0.0036 & 0.0002 \\ 2888 & 15: 08: 54 & 0.0908 & 0.0002 \\ 2890 & 15: 08: 56 & 0.0762 & 0.0002 \\ 2892 & 15: 08: 58 & 0.0059 & 0.0002 \\ 2894 & 15: 09: 00 & 0.0064 & 0.0007 \\ 2896 & 15: 09: 02 & 0.0018 & 0.0003 \\ 2898 & 15: 09: 04 & 0.0146 & 0.0015 \\ 2900 & 15: 09: 06 & 0.0002 & 0.0002 \\ 2902 & 15: 09: 08 & 0.0241 & 0.0014 \\ 2904 & 15: 09: 10 & 0.0239 & 0.0031 \\ 2906 & 15: 09: 12 & 0.0003 & 0.0002 \\ 2908 & 15: 09: 14 & 0.0002 & 0.0029 \\ 2910 & 15: 09: 16 & 0.0006 & 0.0007 \\ 2912 & 15: 09: 18 & 0.0013 & 0.0003 \\ 2914 & 15: 09: 20 & 0.0005 & 0.0002 \\ 2916 & 15: 09: 22 & 0.0003 & 0.0002 \\ 2918 & 15: 09: 24 & 0.0002 & 0.0002 \\ 2920 & 15: 09: 26 & 0.0002 & 0.0040 \\ 2922 & 15: 09: 28 & 0.0002 & 0.0030 \\ 2924 & 15: 09: 30 & 0.0002 & 0.0003 \\ 2926 & 15: 09: 32 & 0.0013 & 0.0012 \\ 2928 & 15: 09: 34 & 0.0003 & 0.0035 \\ 2930 & 15: 09: 36 & 0.0042 & 0.0059 \\ 2932 & 15: 09: 38 & 0.0006 & 0.0307 \\ 2934 & 15: 09: 40 & 0.0003 & 0.0025 \\ 2936 & 15: 09: 42 & 0.0137 & 0.0005 \\ 2938 & 15: 09: 44 & 0.0003 & 0.0005 \\ 2940 & 15: 09: 46 & 0.0006 & 0.0002 \\ 2942 & 15: 09: 48 & 0.0031 & 0.0017 \\ 2944 & 15: 09: 50 & 0.0002 & 0.0007 \\ 2946 & 15: 09: 52 & 0.0086 & 0.0007 \\ 2948 & 15: 09: 54 & 0.0003 & 0.0016 \\ 2950 & 15: 09: 56 & 0.0002 & 0.0003 \\ 2952 & 15: 09: 58 & 0.0080 & 0.0007 \\ 2954 & 15: 10: 00 & 0.0002 & 0.0007\end{array}$


Appendix H - Laser Data Test 4 NIST102705_1.xls

$\begin{array}{llll}2956 & 15: 10: 02 & 0.0009 & 0.0002 \\ 2958 & 15: 10: 04 & 0.0015 & 0.0003 \\ 2960 & 15: 10: 06 & 0.0017 & 0.0002 \\ 2962 & 15: 10: 08 & 0.0122 & 0.0002 \\ 2964 & 15: 10: 10 & 0.0198 & 0.0008 \\ 2966 & 15: 10: 12 & 0.0049 & 0.0002 \\ 2968 & 15: 10: 14 & 0.0350 & 0.0053 \\ 2970 & 15: 10: 16 & 0.0008 & 0.0002 \\ 2972 & 15: 10: 18 & 0.0009 & 0.0002 \\ 2974 & 15: 10: 20 & 0.0002 & 0.0031 \\ 2976 & 15: 10: 22 & 0.0053 & 0.0060 \\ 2978 & 15: 10: 24 & 0.0202 & 0.0002 \\ 2980 & 15: 10: 26 & 0.0003 & 0.0001 \\ 2982 & 15: 10: 28 & 0.0301 & 0.0002 \\ 2984 & 15: 10: 30 & 0.0010 & 0.0002 \\ 2986 & 15: 10: 32 & 0.0055 & 0.0003 \\ 2988 & 15: 10: 34 & 0.0002 & 0.0001 \\ 2990 & 15: 10: 36 & 0.0012 & 0.0001 \\ 2992 & 15: 10: 38 & 0.0731 & 0.0005 \\ 2994 & 15: 10: 40 & 0.0638 & 0.0001 \\ 2996 & 15: 10: 42 & 0.0046 & 0.0001 \\ 2998 & 15: 10: 44 & 0.0676 & 0.0017 \\ 3000 & 15: 10: 46 & 0.0009 & 0.0036 \\ 3002 & 15: 10: 48 & 0.0118 & 0.0006 \\ 3004 & 15: 10: 50 & 0.0005 & 0.0002 \\ 3006 & 15: 10: 52 & 0.0053 & 0.0006 \\ 3008 & 15: 10: 54 & 0.0111 & 0.0002 \\ 3010 & 15: 10: 56 & 0.0742 & 0.0003 \\ 3012 & 15: 10: 58 & 0.0230 & 0.0012 \\ 3014 & 15: 11: 00 & 0.0008 & 0.0002 \\ 3016 & 15: 11: 02 & 0.0006 & 0.0073 \\ 3018 & 15: 11: 04 & 0.0551 & 0.0049 \\ 3020 & 15: 11: 06 & 0.0002 & 0.0007 \\ 3022 & 15: 11: 08 & 0.2026 & 0.0002 \\ 3024 & 15: 11: 10 & 0.0032 & 0.0002 \\ 3026 & 15: 11: 12 & 0.0224 & 0.0002 \\ 3028 & 15: 11: 14 & 0.0663 & 0.0003 \\ 3030 & 15: 11: 16 & 0.0050 & 0.0005 \\ 3032 & 15: 11: 18 & 0.0011 & 0.0002 \\ 3034 & 15: 11: 20 & 0.0018 & 0.0009 \\ 3036 & 15: 11: 22 & 0.0745 & 0.0742 \\ 3038 & 15: 11: 24 & 0.0005 & 0.0311 \\ 3040 & 15: 11: 26 & 0.0162 & 0.0010 \\ 3042 & 15: 11: 28 & 0.0377 & 0.0005 \\ 3044 & 15: 11: 30 & 0.0050 & 0.0003 \\ 3046 & 15: 11: 32 & 0.2241 & 0.0069 \\ 3048 & 15: 11: 34 & 0.0034 & 0.0001 \\ 3050 & 15: 11: 36 & 0.0003 & 0.0002 \\ 3052 & 15: 11: 38 & 0.0019 & 0.0002 \\ 3054 & 15: 11: 40 & 0.0002 & 0.0002 \\ 3056 & 15: 11: 42 & 0.0011 & 0.0003\end{array}$


Appendix H - Laser Data Test 4 NIST102705_1.xls

$\begin{array}{llll}3058 & 15: 11: 44 & 0.0012 & 0.0005 \\ 3060 & 15: 11: 46 & 0.0005 & 0.0002 \\ 3062 & 15: 11: 48 & 0.1051 & 0.0008 \\ 3064 & 15: 11: 50 & 0.0460 & 0.0001 \\ 3066 & 15: 11: 52 & 0.0034 & 0.0002 \\ 3068 & 15: 11: 54 & 0.0010 & 0.0002 \\ 3070 & 15: 11: 56 & 0.0067 & 0.0002 \\ 3072 & 15: 11: 58 & 0.0106 & 0.0002 \\ 3074 & 15: 12: 00 & 0.1465 & 0.0002 \\ 3076 & 15: 12: 02 & 0.0308 & 0.0002 \\ 3078 & 15: 12: 04 & 0.0018 & 0.0002 \\ 3080 & 15: 12: 06 & 0.0190 & 0.0002 \\ 3082 & 15: 12: 08 & 0.0008 & 0.0002 \\ 3084 & 15: 12: 10 & 0.0014 & 0.0001 \\ 3086 & 15: 12: 12 & 0.0026 & 0.0002 \\ 3088 & 15: 12: 14 & 0.0002 & 0.0001 \\ 3090 & 15: 12: 16 & 0.1629 & 0.0002 \\ 3092 & 15: 12: 18 & 0.0002 & 0.0005 \\ 3094 & 15: 12: 20 & 0.0002 & 0.0002 \\ 3096 & 15: 12: 22 & 0.0037 & 0.0012 \\ 3098 & 15: 12: 24 & 0.0146 & 0.0002 \\ 3100 & 15: 12: 26 & 0.0030 & 0.0005 \\ 3102 & 15: 12: 28 & 0.0004 & 0.0002 \\ 3104 & 15: 12: 30 & 0.0510 & 0.0002 \\ 3106 & 15: 12: 32 & 0.0005 & 0.0002 \\ 3108 & 15: 12: 34 & 0.0005 & 0.0010 \\ 3110 & 15: 12: 36 & 0.0007 & 0.0002 \\ 3112 & 15: 12: 38 & 0.0027 & 0.0005 \\ 3114 & 15: 12: 40 & 0.0075 & 0.0003 \\ 3116 & 15: 12: 42 & 0.0296 & 0.0003 \\ 3118 & 15: 12: 44 & 0.1755 & 0.0002 \\ 3120 & 15: 12: 46 & 0.0121 & 0.0003 \\ 3122 & 15: 12: 48 & 0.0005 & 0.0001 \\ 3124 & 15: 12: 50 & 0.0023 & 0.0006 \\ 3126 & 15: 12: 52 & 0.0215 & 0.0016 \\ 3128 & 15: 12: 54 & 0.0242 & 0.0002 \\ 3130 & 15: 12: 56 & 0.0003 & 0.0010 \\ 3132 & 15: 12: 58 & 0.0022 & 0.0030 \\ 3134 & 15: 13: 00 & 0.0011 & 0.0002 \\ 3136 & 15: 13: 02 & 0.0031 & 0.0005 \\ 3138 & 15: 13: 04 & 0.0013 & 0.0002 \\ 3140 & 15: 13: 06 & 0.0062 & 0.0003 \\ 3142 & 15: 13: 08 & 0.0002 & 0.0003 \\ 3144 & 15: 13: 10 & 0.0762 & 0.0009 \\ 3146 & 15: 13: 12 & 0.0380 & 0.0014 \\ 3148 & 15: 13: 14 & 0.0325 & 0.0006 \\ 3150 & 15: 13: 16 & 0.2816 & 0.0001 \\ 3152 & 15: 13: 18 & 0.0048 & 0.0010 \\ 3154 & 15: 13: 20 & 0.0043 & 0.0002 \\ 3156 & 15: 13: 22 & 0.0188 & 0.0002 \\ 3158 & 15: 13: 24 & 0.0565 & 0.0009\end{array}$


Appendix H - Laser Data Test 4 NIST102705_1.xls

$\begin{array}{llll}3160 & 15: 13: 26 & 0.1854 & 0.0049 \\ 3162 & 15: 13: 28 & 0.0013 & 0.0042 \\ 3164 & 15: 13: 30 & 0.0015 & 0.0002 \\ 3166 & 15: 13: 32 & 0.0028 & 0.0002 \\ 3168 & 15: 13: 34 & 0.0003 & 0.0024 \\ 3170 & 15: 13: 36 & 0.0019 & 0.0005 \\ 3172 & 15: 13: 38 & 0.0752 & 0.0002 \\ 3174 & 15: 13: 40 & 0.0024 & 0.0079 \\ 3176 & 15: 13: 42 & 0.0096 & 0.0002 \\ 3178 & 15: 13: 44 & 0.0011 & 0.0001 \\ 3180 & 15: 13: 46 & 0.0003 & 0.0002 \\ 3182 & 15: 13: 48 & 0.2361 & 0.0007 \\ 3184 & 15: 13: 50 & 0.0113 & 0.0002 \\ 3186 & 15: 13: 52 & 0.0022 & 0.0002 \\ 3188 & 15: 13: 54 & 0.0051 & 0.0003 \\ 3190 & 15: 13: 56 & 0.0102 & 0.0009 \\ 3192 & 15: 13: 58 & 0.0003 & 0.0010 \\ 3194 & 15: 14: 00 & 0.0071 & 0.0003 \\ 3196 & 15: 14: 02 & 0.0011 & 0.0001 \\ 3198 & 15: 14: 04 & 0.0002 & 0.0082 \\ 3200 & 15: 14: 06 & 0.0021 & 0.0048 \\ 3202 & 15: 14: 08 & 0.0191 & 0.0002 \\ 3204 & 15: 14: 10 & 0.0080 & 0.0003 \\ 3206 & 15: 14: 12 & 0.0135 & 0.0006 \\ 3208 & 15: 14: 14 & 0.0002 & 0.0007 \\ 3210 & 15: 14: 16 & 0.0052 & 0.0002 \\ 3212 & 15: 14: 18 & 0.0027 & 0.0014 \\ 3214 & 15: 14: 20 & 0.0004 & 0.0002 \\ 3216 & 15: 14: 22 & 0.0012 & 0.0002 \\ 3218 & 15: 14: 24 & 0.0029 & 0.0003 \\ 3220 & 15: 14: 26 & 0.0004 & 0.0062 \\ 3222 & 15: 14: 28 & 0.0002 & 0.0126 \\ 3224 & 15: 14: 30 & 0.0003 & 0.0002 \\ 3226 & 15: 14: 32 & 0.0002 & 0.0002 \\ 3228 & 15: 14: 34 & 0.0008 & 0.0002 \\ 3230 & 15: 14: 36 & 0.0002 & 0.0106 \\ 3232 & 15: 14: 38 & 0.0015 & 0.0002 \\ 3234 & 15: 14: 40 & 0.0002 & 0.0002 \\ 3236 & 15: 14: 42 & 0.0023 & 0.0005 \\ 3238 & 15: 14: 44 & 0.0037 & 0.0022 \\ 3240 & 15: 14: 46 & 0.0004 & 0.0002 \\ 3242 & 15: 14: 48 & 0.0002 & 0.0005 \\ 3244 & 15: 14: 50 & 0.0003 & 0.0079 \\ 3246 & 15: 14: 52 & 0.0335 & 0.0002 \\ 3248 & 15: 14: 54 & 0.0007 & 0.0001 \\ 3250 & 15: 14: 56 & 0.0004 & 0.0004 \\ 3252 & 15: 14: 58 & 0.0206 & 0.0009 \\ 3254 & 15: 15: 00 & 0.0002 & 0.0004 \\ 3256 & 15: 15: 02 & 0.0020 & 0.0075 \\ 3258 & 15: 15: 04 & 0.0010 & 0.0075 \\ 3260 & 15: 15: 06 & 0.0349 & 0.0036\end{array}$


Appendix H - Laser Data Test 4 NIST102705_1.xls

$\begin{array}{llll}3262 & 15: 15: 08 & 0.0018 & 0.0004 \\ 3264 & 15: 15: 10 & 0.0002 & 0.0002 \\ 3266 & 15: 15: 12 & 0.0252 & 0.0028 \\ 3268 & 15: 15: 14 & 0.0624 & 0.0019 \\ 3270 & 15: 15: 16 & 0.0002 & 0.0035 \\ 3272 & 15: 15: 18 & 0.0150 & 0.0002 \\ 3274 & 15: 15: 20 & 0.0014 & 0.0171 \\ 3276 & 15: 15: 22 & 0.0035 & 0.0007 \\ 3278 & 15: 15: 24 & 0.0058 & 0.0004 \\ 3280 & 15: 15: 26 & 0.0004 & 0.0049 \\ 3282 & 15: 15: 28 & 0.0067 & 0.0012 \\ 3284 & 15: 15: 30 & 0.0002 & 0.0002 \\ 3286 & 15: 15: 32 & 0.0002 & 0.0002 \\ 3288 & 15: 15: 34 & 0.0003 & 0.0077 \\ 3290 & 15: 15: 36 & 0.0169 & 0.0008 \\ 3292 & 15: 15: 38 & 0.0004 & 0.0004 \\ 3294 & 15: 15: 40 & 0.0003 & 0.0002 \\ 3296 & 15: 15: 42 & 0.0094 & 0.0002 \\ 3298 & 15: 15: 44 & 0.0002 & 0.0001 \\ 3300 & 15: 15: 46 & 0.0040 & 0.0004 \\ 3302 & 15: 15: 48 & 0.0002 & 0.0005 \\ 3304 & 15: 15: 50 & 0.0004 & 0.0015 \\ 3306 & 15: 15: 52 & 0.0002 & 0.0001 \\ 3308 & 15: 15: 54 & 0.0021 & 0.0001 \\ 3310 & 15: 15: 56 & 0.0445 & 0.0004 \\ 3312 & 15: 15: 58 & 0.0004 & 0.0001 \\ 3314 & 15: 16: 00 & 0.0127 & 0.0006 \\ 3316 & 15: 16: 02 & 0.0050 & 0.0028 \\ 3318 & 15: 16: 04 & 0.0002 & 0.0018 \\ 3320 & 15: 16: 06 & 0.0002 & 0.0002 \\ 3322 & 15: 16: 08 & 0.0008 & 0.0004 \\ 3324 & 15: 16: 10 & 0.0027 & 0.0006 \\ 3326 & 15: 16: 12 & 0.0052 & 0.0002 \\ 3328 & 15: 16: 14 & 0.0065 & 0.0165 \\ 3330 & 15: 16: 16 & 0.0002 & 0.0001 \\ 3332 & 15: 16: 18 & 0.0002 & 0.0001 \\ 3334 & 15: 16: 20 & 0.0013 & 0.0134 \\ 3336 & 15: 16: 22 & 0.0006 & 0.0005 \\ 3338 & 15: 16: 24 & 0.0017 & 0.0036 \\ 3340 & 15: 16: 26 & 0.0002 & 0.0033 \\ 3342 & 15: 16: 28 & 0.0002 & 0.0464 \\ 3344 & 15: 16: 30 & 0.0003 & 0.0006 \\ 3346 & 15: 16: 32 & 0.0018 & 0.0002 \\ 3348 & 15: 16: 34 & 0.0002 & 0.0006 \\ 3350 & 15: 16: 36 & 0.0002 & 0.0013 \\ 3352 & 15: 16: 38 & 0.0051 & 0.0020 \\ 3354 & 15: 16: 40 & 0.0030 & 0.0552 \\ 3356 & 15: 16: 42 & 0.0002 & 0.0002 \\ 3358 & 15: 16: 44 & 0.0002 & 0.0066 \\ 3360 & 15: 16: 46 & 0.0002 & 0.0031 \\ 3362 & 15: 16: 48 & 0.0006 & 0.0018\end{array}$


Appendix H - Laser Data Test 4 NIST102705_1.xls

$\begin{array}{llll}3364 & 15: 16: 50 & 0.0147 & 0.0006 \\ 3366 & 15: 16: 52 & 0.0002 & 0.0002 \\ 3368 & 15: 16: 54 & 0.0002 & 0.0002 \\ 3370 & 15: 16: 56 & 0.0042 & 0.0032 \\ 3372 & 15: 16: 58 & 0.0002 & 0.0049 \\ 3374 & 15: 17: 00 & 0.0003 & 0.0061 \\ 3376 & 15: 17: 02 & 0.0002 & 0.0019 \\ 3378 & 15: 17: 04 & 0.0012 & 0.0005 \\ 3380 & 15: 17: 06 & 0.0008 & 0.0058 \\ 3382 & 15: 17: 08 & 0.0053 & 0.0024 \\ 3384 & 15: 17: 10 & 0.0004 & 0.0041 \\ 3386 & 15: 17: 12 & 0.0059 & 0.0049 \\ 3388 & 15: 17: 14 & 0.0003 & 0.0001 \\ 3390 & 15: 17: 16 & 0.0012 & 0.0007 \\ 3392 & 15: 17: 18 & 0.0007 & 0.0005 \\ 3394 & 15: 17: 20 & 0.0040 & 0.0002 \\ 3396 & 15: 17: 22 & 0.0006 & 0.0004 \\ 3398 & 15: 17: 24 & 0.0411 & 0.0039 \\ 3400 & 15: 17: 26 & 0.0143 & 0.0002 \\ 3402 & 15: 17: 28 & 0.3889 & 0.0011 \\ 3404 & 15: 17: 30 & 0.1486 & 0.0005 \\ 3406 & 15: 17: 32 & 0.0270 & 0.0002 \\ 3408 & 15: 17: 34 & 0.3091 & 0.0025 \\ 3410 & 15: 17: 36 & 0.0002 & 0.0008 \\ 3412 & 15: 17: 38 & 0.0006 & 0.0002 \\ 3414 & 15: 17: 40 & 0.2248 & 0.0009 \\ 3416 & 15: 17: 42 & 0.0013 & 0.0006 \\ 3418 & 15: 17: 44 & 0.0019 & 0.0018 \\ 3420 & 15: 17: 46 & 0.0154 & 0.0032 \\ 3422 & 15: 17: 48 & 0.1020 & 0.0002 \\ 3424 & 15: 17: 50 & 0.1037 & 0.0002 \\ 3426 & 15: 17: 52 & 0.0020 & 0.0021 \\ 3428 & 15: 17: 54 & 0.0009 & 0.0002 \\ 3430 & 15: 17: 56 & 0.0511 & 0.0011 \\ 3432 & 15: 17: 58 & 0.0010 & 0.0005 \\ 3434 & 15: 18: 00 & 0.0010 & 0.0005 \\ 3436 & 15: 18: 02 & 0.0008 & 0.0002 \\ 3438 & 15: 18: 04 & 0.0002 & 0.0007 \\ 3440 & 15: 18: 06 & 0.6002 & 0.0002 \\ 3442 & 15: 18: 08 & 0.0004 & 0.0002 \\ 3444 & 15: 18: 10 & 0.0233 & 0.0002 \\ 3446 & 15: 18: 12 & 0.0002 & 0.0002 \\ 3448 & 15: 18: 14 & 0.0010 & 0.0002 \\ 3450 & 15: 18: 16 & 0.0041 & 0.0002 \\ 3452 & 15: 18: 18 & 0.0009 & 0.0001 \\ 3454 & 15: 18: 20 & 0.0352 & 0.0001 \\ 3456 & 15: 18: 22 & 0.0025 & 0.0102 \\ 3458 & 15: 18: 24 & 0.0006 & 0.0001 \\ 3460 & 15: 18: 26 & 0.1136 & 0.0001 \\ 3462 & 15: 18: 28 & 0.0948 & 0.0015 \\ 3464 & 15: 18: 30 & 0.0068 & 0.0001\end{array}$


Appendix H - Laser Data Test 4 NIST102705_1.xls

$\begin{array}{llll}3466 & 15: 18: 32 & 0.0130 & 0.0045 \\ 3468 & 15: 18: 34 & 0.1408 & 0.0001 \\ 3470 & 15: 18: 36 & 0.0150 & 0.0000 \\ 3472 & 15: 18: 38 & 0.0003 & 0.0002 \\ 3474 & 15: 18: 40 & 0.0002 & 0.0002 \\ 3476 & 15: 18: 42 & 0.0003 & 0.0002 \\ 3478 & 15: 18: 44 & 0.0555 & 0.0002 \\ 3480 & 15: 18: 46 & 0.1631 & 0.0002 \\ 3482 & 15: 18: 48 & 0.0056 & 0.0002 \\ 3484 & 15: 18: 50 & 0.0187 & 0.0001 \\ 3486 & 15: 18: 52 & 0.0346 & 0.0001 \\ 3488 & 15: 18: 54 & 0.1360 & 0.0002 \\ 3490 & 15: 18: 56 & 0.0924 & 0.0002 \\ 3492 & 15: 18: 58 & 0.0344 & 0.0002 \\ 3494 & 15: 19: 00 & 0.0308 & 0.0004 \\ 3496 & 15: 19: 02 & 0.0003 & 0.0001 \\ 3498 & 15: 19: 04 & 0.0002 & 0.0002 \\ 3500 & 15: 19: 06 & 0.0187 & 0.0004 \\ 3502 & 15: 19: 08 & 0.0007 & 0.0002 \\ 3504 & 15: 19: 10 & 0.0010 & 0.0002 \\ 3506 & 15: 19: 12 & 0.0025 & 0.0002 \\ 3508 & 15: 19: 14 & 0.0083 & 0.0021 \\ 3510 & 15: 19: 16 & 0.0021 & 0.0069 \\ 3512 & 15: 19: 18 & 0.0010 & 0.0034 \\ 3514 & 15: 19: 20 & 0.0013 & 0.0026 \\ 3516 & 15: 19: 22 & 0.0002 & 0.0008 \\ 3518 & 15: 19: 24 & 0.0007 & 0.0020 \\ 3520 & 15: 19: 26 & 0.0015 & 0.0015 \\ 3522 & 15: 19: 28 & 0.0223 & 0.0002 \\ 3524 & 15: 19: 30 & 0.0007 & 0.0002 \\ 3526 & 15: 19: 32 & 0.0155 & 0.0011 \\ 3528 & 15: 19: 34 & 0.0171 & 0.0002 \\ 3530 & 15: 19: 36 & 0.0067 & 0.0002 \\ 3532 & 15: 19: 38 & 0.0003 & 0.0002 \\ 3534 & 15: 19: 40 & 0.0129 & 0.0001 \\ 3536 & 15: 19: 42 & 0.0217 & 0.0023 \\ 3538 & 15: 19: 44 & 0.0972 & 0.0004 \\ 3540 & 15: 19: 46 & 0.0671 & 0.0006 \\ 3542 & 15: 19: 48 & 0.0081 & 0.0006 \\ 3544 & 15: 19: 50 & 0.0112 & 0.0024 \\ 3546 & 15: 19: 52 & 0.0241 & 0.0006 \\ 3548 & 15: 19: 54 & 0.0083 & 0.0002 \\ 3550 & 15: 19: 56 & 0.0204 & 0.0002 \\ 3552 & 15: 19: 58 & 0.0003 & 0.0004 \\ 3554 & 15: 20: 00 & 0.1600 & 0.0049 \\ 3556 & 15: 20: 02 & 0.1566 & 0.0002 \\ 3558 & 15: 20: 04 & 0.0296 & 0.0002 \\ 3560 & 15: 20: 06 & 0.0113 & 0.0004 \\ 3562 & 15: 20: 08 & 0.0023 & 0.0126 \\ 3564 & 15: 20: 10 & 0.0010 & 0.0013 \\ 3566 & 15: 20: 12 & 0.0111 & 0.0002\end{array}$


Appendix H - Laser Data Test 4 NIST102705_1.xls

$\begin{array}{llll}3568 & 15: 20: 14 & 0.0111 & 0.0002 \\ 3570 & 15: 20: 16 & 0.0003 & 0.0015 \\ 3572 & 15: 20: 18 & 0.0274 & 0.0002 \\ 3574 & 15: 20: 20 & 0.0003 & 0.0017 \\ 3576 & 15: 20: 22 & 0.0011 & 0.0005 \\ 3578 & 15: 20: 24 & 0.0006 & 0.0001 \\ 3580 & 15: 20: 26 & 0.0065 & 0.0004 \\ 3582 & 15: 20: 28 & 0.0933 & 0.0001 \\ 3584 & 15: 20: 30 & 0.0086 & 0.0064 \\ 3586 & 15: 20: 32 & 0.0759 & 0.0006 \\ 3588 & 15: 20: 34 & 0.0012 & 0.0006 \\ 3590 & 15: 20: 36 & 0.0077 & 0.0002 \\ 3592 & 15: 20: 38 & 0.0647 & 0.0002 \\ 3594 & 15: 20: 40 & 0.0019 & 0.0005 \\ 3596 & 15: 20: 42 & 0.0112 & 0.0002 \\ 3598 & 15: 20: 44 & 0.0011 & 0.0002 \\ 3600 & 15: 20: 46 & 0.0024 & 0.0001 \\ 3602 & 15: 20: 48 & 0.0111 & 0.0011 \\ 3604 & 15: 20: 50 & 0.0002 & 0.0006 \\ 3606 & 15: 20: 52 & 0.0154 & 0.0002 \\ 3608 & 15: 20: 54 & 0.0020 & 0.0004 \\ 3610 & 15: 20: 56 & 0.0003 & 0.0007 \\ 3612 & 15: 20: 58 & 0.0049 & 0.0086 \\ 3614 & 15: 21: 00 & 0.0020 & 0.0002 \\ 3616 & 15: 21: 02 & 0.0024 & 0.0023 \\ 3618 & 15: 21: 04 & 0.0144 & 0.0001 \\ 3620 & 15: 21: 06 & 0.0012 & 0.0002 \\ 3622 & 15: 21: 08 & 0.2948 & 0.0006 \\ 3624 & 15: 21: 10 & 0.0002 & 0.0002 \\ 3626 & 15: 21: 12 & 0.0063 & 0.0004 \\ 3628 & 15: 21: 14 & 0.0010 & 0.0002 \\ 3630 & 15: 21: 16 & 0.0036 & 0.0001 \\ 3632 & 15: 21: 18 & 0.0027 & 0.0002 \\ 3634 & 15: 21: 20 & 0.0046 & 0.0004 \\ 3636 & 15: 21: 22 & 0.0200 & 0.0023 \\ 3638 & 15: 21: 24 & 0.0033 & 0.0002 \\ 3640 & 15: 21: 26 & 0.0002 & 0.0002 \\ 3642 & 15: 21: 28 & 0.0002 & 0.0004 \\ 3644 & 15: 21: 30 & 0.0029 & 0.0018 \\ 3646 & 15: 21: 32 & 0.0043 & 0.0002 \\ 3648 & 15: 21: 34 & 0.0040 & 0.0001 \\ 3650 & 15: 21: 36 & 0.0012 & 0.0002 \\ 3652 & 15: 21: 38 & 0.0324 & 0.0029 \\ 3654 & 15: 21: 40 & 0.0003 & 0.0011 \\ 3656 & 15: 21: 42 & 0.0002 & 0.0005 \\ 3658 & 15: 21: 44 & 0.0003 & 0.0013 \\ 3660 & 15: 21: 46 & 0.0096 & 0.0061 \\ 3662 & 15: 21: 48 & 0.0140 & 0.0006 \\ 3664 & 15: 21: 50 & 0.0170 & 0.0001 \\ 3666 & 15: 21: 52 & 0.0018 & 0.0002 \\ 3668 & 15: 21: 54 & 0.0121 & 0.0026\end{array}$


Appendix H - Laser Data Test 4 NIST102705_1.xls

$\begin{array}{llll}3670 & 15: 21: 56 & 0.0002 & 0.0002 \\ 3672 & 15: 21: 58 & 0.0018 & 0.0262 \\ 3674 & 15: 22: 00 & 0.0002 & 0.0002 \\ 3676 & 15: 22: 02 & 0.0023 & 0.0010 \\ 3678 & 15: 22: 04 & 0.0006 & 0.0006 \\ 3680 & 15: 22: 06 & 0.0002 & 0.0004 \\ 3682 & 15: 22: 08 & 0.0002 & 0.0002 \\ 3684 & 15: 22: 10 & 0.0006 & 0.0004 \\ 3686 & 15: 22: 12 & 0.0039 & 0.0002 \\ 3688 & 15: 22: 14 & 0.0003 & 0.0007 \\ 3690 & 15: 22: 16 & 0.0002 & 0.0002 \\ 3692 & 15: 22: 18 & 0.0057 & 0.0002 \\ 3694 & 15: 22: 20 & 0.0034 & 0.0002 \\ 3696 & 15: 22: 22 & 0.0056 & 0.0736 \\ 3698 & 15: 22: 24 & 0.0003 & 0.0002 \\ 3700 & 15: 22: 26 & 0.0002 & 0.0002 \\ 3702 & 15: 22: 28 & 0.0002 & 0.0004 \\ 3704 & 15: 22: 30 & 0.0002 & 0.0001 \\ 3706 & 15: 22: 32 & 0.0007 & 0.0020 \\ 3708 & 15: 22: 34 & 0.0264 & 0.0002 \\ 3710 & 15: 22: 36 & 0.0169 & 0.0002 \\ 3712 & 15: 22: 38 & 0.0673 & 0.0002 \\ 3714 & 15: 22: 40 & 0.0005 & 0.0005 \\ 3716 & 15: 22: 42 & 0.0008 & 0.0091 \\ 3718 & 15: 22: 44 & 0.0014 & 0.0032 \\ 3720 & 15: 22: 46 & 0.0002 & 0.0230 \\ 3722 & 15: 22: 48 & 0.0018 & 0.0036 \\ 3724 & 15: 22: 50 & 0.0002 & 0.3542 \\ 3726 & 15: 22: 52 & 0.0184 & 0.0683 \\ 3728 & 15: 22: 54 & 0.0003 & 0.0008 \\ 3730 & 15: 22: 56 & 0.0002 & 0.0011 \\ 3732 & 15: 22: 58 & 0.0002 & 0.0002 \\ 3734 & 15: 23: 00 & 0.0017 & 0.0002 \\ 3736 & 15: 23: 02 & 0.0216 & 0.0004 \\ 3738 & 15: 23: 04 & 0.0081 & 0.0018 \\ 3740 & 15: 23: 06 & 0.0620 & 0.0000 \\ 3742 & 15: 23: 08 & 0.0053 & 0.0006 \\ 3744 & 15: 23: 10 & 0.1124 & 0.0005 \\ 3746 & 15: 23: 12 & 0.0002 & 0.0002 \\ 3748 & 15: 23: 14 & 0.0002 & 0.0008 \\ 3750 & 15: 23: 16 & 0.0002 & 0.0007 \\ 3752 & 15: 23: 18 & 0.0016 & 0.3774 \\ 3754 & 15: 23: 20 & 0.0014 & 0.0010 \\ 3756 & 15: 23: 22 & 0.0042 & 0.0004 \\ 3758 & 15: 23: 24 & 0.0003 & 0.0002 \\ 3760 & 15: 23: 26 & 0.0006 & 0.0001 \\ 3762 & 15: 23: 28 & 0.0002 & 0.0018 \\ 3764 & 15: 23: 30 & 0.0002 & 0.0002 \\ 3766 & 15: 23: 32 & 0.0011 & 0.0024 \\ 3768 & 15: 23: 34 & 0.0003 & 0.0001 \\ 3770 & 15: 23: 36 & 0.0002 & 0.0055\end{array}$


Appendix H - Laser Data Test 4 NIST102705_1.xls

$\begin{array}{llll}3772 & 15: 23: 38 & 0.0015 & 0.0013 \\ 3774 & 15: 23: 40 & 0.0008 & 0.0008 \\ 3776 & 15: 23: 42 & 0.0003 & 0.0116 \\ 3778 & 15: 23: 44 & 0.0002 & 0.0140 \\ 3780 & 15: 23: 46 & 0.0003 & 0.0002 \\ 3782 & 15: 23: 48 & 0.0006 & 0.0001 \\ 3784 & 15: 23: 50 & 0.0102 & 0.0001 \\ 3786 & 15: 23: 52 & 0.0026 & 0.0042 \\ 3788 & 15: 23: 54 & 0.0002 & 0.0143 \\ 3790 & 15: 23: 56 & 0.0646 & 0.0072 \\ 3792 & 15: 23: 58 & 0.0008 & 0.0001 \\ 3794 & 15: 24: 00 & 0.0002 & 0.0002 \\ 3796 & 15: 24: 02 & 0.0056 & 0.0005 \\ 3798 & 15: 24: 04 & 0.0003 & 0.0005 \\ 3800 & 15: 24: 06 & 0.0023 & 0.0002 \\ 3802 & 15: 24: 08 & 0.0085 & 0.0004 \\ 3804 & 15: 24: 10 & 0.0002 & 0.0002 \\ 3806 & 15: 24: 12 & 0.0037 & 0.0002 \\ 3808 & 15: 24: 14 & 0.0067 & 0.0034 \\ 3810 & 15: 24: 16 & 0.0115 & 0.0002 \\ 3812 & 15: 24: 18 & 0.0111 & 0.0053 \\ 3814 & 15: 24: 20 & 0.0013 & 0.0014 \\ 3816 & 15: 24: 22 & 0.0256 & 0.0005 \\ 3818 & 15: 24: 24 & 0.0040 & 0.0025 \\ 3820 & 15: 24: 26 & 0.0017 & 0.0078 \\ 3822 & 15: 24: 28 & 0.0002 & 0.0013 \\ 3824 & 15: 24: 30 & 0.0002 & 0.0004 \\ 3826 & 15: 24: 32 & 0.0002 & 0.0016 \\ 3828 & 15: 24: 34 & 0.0007 & 0.0061 \\ 3830 & 15: 24: 36 & 0.0003 & 0.0059 \\ 3832 & 15: 24: 38 & 0.0002 & 0.0020 \\ 3834 & 15: 24: 40 & 0.0015 & 0.0006 \\ 3836 & 15: 24: 42 & 0.0014 & 0.0002 \\ 3838 & 15: 24: 44 & 0.0015 & 0.0156 \\ 3840 & 15: 24: 46 & 0.0087 & 0.0004 \\ 3842 & 15: 24: 48 & 0.0013 & 0.0002 \\ 3844 & 15: 24: 50 & 0.0002 & 0.0077 \\ 3846 & 15: 24: 52 & 0.0006 & 0.0004 \\ 3848 & 15: 24: 54 & 0.0002 & 0.0004 \\ 3850 & 15: 24: 56 & 0.0039 & 0.0002 \\ 3852 & 15: 24: 58 & 0.0002 & 0.0002 \\ 3854 & 15: 25: 00 & 0.0002 & 0.0005 \\ 3856 & 15: 25: 02 & 0.0002 & 0.0017 \\ 3858 & 15: 25: 04 & 0.0006 & 0.1345 \\ 3860 & 15: 25: 06 & 0.0017 & 0.0002 \\ 3862 & 15: 25: 08 & 0.0002 & 0.0005 \\ 3864 & 15: 25: 10 & 0.0002 & 0.0005 \\ 3866 & 15: 25: 12 & 0.0104 & 0.0048 \\ 3868 & 15: 25: 14 & 0.0014 & 0.0016 \\ 3870 & 15: 25: 16 & 0.0059 & 0.0001 \\ 3872 & 15: 25: 18 & 0.0010 & 0.0001\end{array}$


Appendix H - Laser Data Test 4 NIST102705_1.xls

$\begin{array}{llll}3874 & 15: 25: 20 & 0.0008 & 0.0007 \\ 3876 & 15: 25: 22 & 0.0002 & 0.0004 \\ 3878 & 15: 25: 24 & 0.0520 & 0.0004 \\ 3880 & 15: 25: 26 & 0.0002 & 0.0012 \\ 3882 & 15: 25: 28 & 0.0024 & 0.0025 \\ 3884 & 15: 25: 30 & 0.0025 & 0.0024 \\ 3886 & 15: 25: 32 & 0.1524 & 0.0010 \\ 3888 & 15: 25: 34 & 0.0232 & 0.0002 \\ 3890 & 15: 25: 36 & 0.0015 & 0.0004 \\ 3892 & 15: 25: 38 & 0.0263 & 0.0006 \\ 3894 & 15: 25: 40 & 0.0023 & 0.0002 \\ 3896 & 15: 25: 42 & 0.0223 & 0.0008 \\ 3898 & 15: 25: 44 & 0.0097 & 0.0006 \\ 3900 & 15: 25: 46 & 0.0003 & 0.0002 \\ 3902 & 15: 25: 48 & 0.0096 & 0.0002 \\ 3904 & 15: 25: 50 & 0.0108 & 0.0013 \\ 3906 & 15: 25: 52 & 0.0002 & 0.0021 \\ 3908 & 15: 25: 54 & 0.0551 & 0.0101 \\ 3910 & 15: 25: 56 & 0.0074 & 0.0002 \\ 3912 & 15: 25: 58 & 0.0003 & 0.0002 \\ 3914 & 15: 26: 00 & 0.1201 & 0.0002 \\ 3916 & 15: 26: 02 & 0.0017 & 0.0002 \\ 3918 & 15: 26: 04 & 0.0027 & 0.0002 \\ 3920 & 15: 26: 06 & 0.5041 & 0.0006 \\ 3922 & 15: 26: 08 & 0.0127 & 0.0002 \\ 3924 & 15: 26: 10 & 0.0183 & 0.0001 \\ 3926 & 15: 26: 12 & 0.0037 & 0.0002 \\ 3928 & 15: 26: 14 & 0.0002 & 0.0002 \\ 3930 & 15: 26: 16 & 0.0408 & 0.0005 \\ 3932 & 15: 26: 18 & 0.0024 & 0.0002 \\ 3934 & 15: 26: 20 & 0.0090 & 0.0002 \\ 3936 & 15: 26: 22 & 0.0021 & 0.0002 \\ 3938 & 15: 26: 24 & 0.0410 & 0.0002 \\ 3940 & 15: 26: 26 & 0.0042 & 0.0001 \\ 3942 & 15: 26: 28 & 0.0023 & 0.0001 \\ 3944 & 15: 26: 30 & 0.2833 & 0.0004 \\ 3946 & 15: 26: 32 & 0.1556 & 0.0001 \\ 3948 & 15: 26: 34 & 0.0026 & 0.0004 \\ 3950 & 15: 26: 36 & 0.0156 & 0.0001 \\ 3952 & 15: 26: 38 & 0.0520 & 0.0005 \\ 3954 & 15: 26: 40 & 0.2577 & 0.0002 \\ 3956 & 15: 26: 42 & 0.3057 & 0.0002 \\ 3958 & 15: 26: 44 & 0.0646 & 0.0001 \\ 3960 & 15: 26: 46 & 0.0962 & 0.0005 \\ 3962 & 15: 26: 48 & 0.0060 & 0.0000 \\ 3964 & 15: 26: 50 & 0.2354 & 0.0001 \\ 3966 & 15: 26: 52 & 0.0480 & 0.0001 \\ 3968 & 15: 26: 54 & 0.0522 & 0.0001 \\ 3970 & 15: 26: 56 & 0.0108 & 0.0002 \\ 3972 & 15: 26: 58 & 0.0002 & 0.0001 \\ 3974 & 15: 27: 00 & 0.0037 & 0.0002\end{array}$


Appendix H - Laser Data Test 4 NIST102705_1.xls

$\begin{array}{llll}3976 & 15: 27: 02 & 0.0007 & 0.0012 \\ 3978 & 15: 27: 04 & 0.1188 & 0.0001 \\ 3980 & 15: 27: 06 & 0.4310 & 0.0001 \\ 3982 & 15: 27: 08 & 0.1218 & 0.0002 \\ 3984 & 15: 27: 10 & 0.0503 & 0.0006 \\ 3986 & 15: 27: 12 & 0.6028 & 0.0001 \\ 3988 & 15: 27: 14 & 0.0580 & 0.0000 \\ 3990 & 15: 27: 16 & 0.3167 & 0.0002 \\ 3992 & 15: 27: 18 & 0.9469 & 0.0001 \\ 3994 & 15: 27: 20 & 0.0724 & 0.0001 \\ 3996 & 15: 27: 22 & 0.2390 & 0.0001 \\ 3998 & 15: 27: 24 & 0.0019 & 0.0001 \\ 4000 & 15: 27: 26 & 0.0964 & 0.0001 \\ 4002 & 15: 27: 28 & 0.0711 & 0.0002 \\ 4004 & 15: 27: 30 & 0.0120 & 0.0001 \\ 4006 & 15: 27: 32 & 0.0466 & 0.0001 \\ 4008 & 15: 27: 34 & 0.0160 & 0.0002 \\ 4010 & 15: 27: 36 & 0.0578 & 0.0002 \\ 4012 & 15: 27: 38 & 0.0003 & 0.0002 \\ 4014 & 15: 27: 40 & 0.0700 & 0.0002 \\ 4016 & 15: 27: 42 & 0.0088 & 0.0002 \\ 4018 & 15: 27: 44 & 0.0107 & 0.0002 \\ 4020 & 15: 27: 46 & 0.0004 & 0.0006 \\ 4022 & 15: 27: 48 & 0.0029 & 0.0002 \\ 4024 & 15: 27: 50 & 0.0004 & 0.0002 \\ 4026 & 15: 27: 52 & 0.0032 & 0.0010 \\ 4028 & 15: 27: 54 & 0.0002 & 0.0002 \\ 4030 & 15: 27: 56 & 0.0006 & 0.0002 \\ 4032 & 15: 27: 58 & 0.0102 & 0.0010 \\ 4034 & 15: 28: 00 & 0.0002 & 0.0002 \\ 4036 & 15: 28: 02 & 0.0004 & 0.0005 \\ 4038 & 15: 28: 04 & 0.0006 & 0.0004 \\ 4040 & 15: 28: 06 & 0.0028 & 0.0005 \\ 4042 & 15: 28: 08 & 0.0096 & 0.0023 \\ 4044 & 15: 28: 10 & 0.0018 & 0.0010 \\ 4046 & 15: 28: 12 & 0.0115 & 0.0018 \\ 4048 & 15: 28: 14 & 0.0006 & 0.0016 \\ 4050 & 15: 28: 16 & 0.0005 & 0.0016 \\ 4052 & 15: 28: 18 & 0.0005 & 0.0009 \\ 4054 & 15: 28: 20 & 0.0004 & 0.0005 \\ 4056 & 15: 28: 22 & 0.0448 & 0.0013 \\ 4058 & 15: 28: 24 & 0.0009 & 0.0001 \\ 4060 & 15: 28: 26 & 0.0054 & 0.0022 \\ 4062 & 15: 28: 28 & 0.0002 & 0.0002 \\ 4064 & 15: 28: 30 & 0.0108 & 0.0015 \\ 4066 & 15: 28: 32 & 0.0018 & 0.0005 \\ 4068 & 15: 28: 34 & 0.0002 & 0.0004 \\ 4070 & 15: 28: 36 & 0.0037 & 0.0004 \\ 4072 & 15: 28: 38 & 0.0008 & 0.0002 \\ 4074 & 15: 28: 40 & 0.0333 & 0.0001 \\ 4076 & 15: 28: 42 & 0.0033 & 0.0001\end{array}$


Appendix H - Laser Data Test 4 NIST102705_1.xls

$\begin{array}{llll}4078 & 15: 28: 44 & 0.0004 & 0.0004 \\ 4080 & 15: 28: 46 & 0.0505 & 0.0004 \\ 4082 & 15: 28: 48 & 0.0004 & 0.0002 \\ 4084 & 15: 28: 50 & 0.0002 & 0.0005 \\ 4086 & 15: 28: 52 & 0.0054 & 0.0004 \\ 4088 & 15: 28: 54 & 0.0790 & 0.0045 \\ 4090 & 15: 28: 56 & 0.0014 & 0.0002 \\ 4092 & 15: 28: 58 & 0.0002 & 0.0002 \\ 4094 & 15: 29: 00 & 0.0002 & 0.0002 \\ 4096 & 15: 29: 02 & 0.0002 & 0.0013 \\ 4098 & 15: 29: 04 & 0.0004 & 0.0004 \\ 4100 & 15: 29: 06 & 0.0002 & 0.0002 \\ 4102 & 15: 29: 08 & 0.0002 & 0.0002 \\ 4104 & 15: 29: 10 & 0.2369 & 0.0011 \\ 4106 & 15: 29: 12 & 0.0072 & 0.0006 \\ 4108 & 15: 29: 14 & 0.0022 & 0.0004 \\ 4110 & 15: 29: 16 & 0.0101 & 0.0063 \\ 4112 & 15: 29: 18 & 0.0008 & 0.0018 \\ 4114 & 15: 29: 20 & 0.0005 & 0.0046 \\ 4116 & 15: 29: 22 & 0.0035 & 0.0002 \\ 4118 & 15: 29: 24 & 0.0025 & 0.0002 \\ 4120 & 15: 29: 26 & 0.0002 & 0.0002 \\ 4122 & 15: 29: 28 & 0.0005 & 0.0004 \\ 4124 & 15: 29: 30 & 0.0004 & 0.0007 \\ 4126 & 15: 29: 32 & 0.0006 & 0.1144 \\ 4128 & 15: 29: 34 & 0.0016 & 0.0005 \\ 4130 & 15: 29: 36 & 0.0005 & 0.0001 \\ 4132 & 15: 29: 38 & 0.0022 & 0.0002 \\ 4134 & 15: 29: 40 & 0.0005 & 0.0004 \\ 4136 & 15: 29: 42 & 0.0025 & 0.0002 \\ 4138 & 15: 29: 44 & 0.0079 & 0.0270 \\ 4140 & 15: 29: 46 & 0.0002 & 0.0002 \\ 4142 & 15: 29: 48 & 0.0002 & 0.0002 \\ 4144 & 15: 29: 50 & 0.0026 & 0.0073 \\ 4146 & 15: 29: 52 & 0.0035 & 0.0017 \\ 4148 & 15: 29: 54 & 0.2696 & 0.0002 \\ 4150 & 15: 29: 56 & 0.0008 & 0.0002 \\ 4152 & 15: 29: 58 & 0.0013 & 0.0004 \\ 4154 & 15: 30: 00 & 0.0027 & 0.0095 \\ 4156 & 15: 30: 02 & 0.0002 & 0.0002 \\ 4158 & 15: 30: 04 & 0.0006 & 0.0002 \\ 4160 & 15: 30: 06 & 0.0057 & 0.0002 \\ 4162 & 15: 30: 08 & 0.0275 & 0.0005 \\ 4164 & 15: 30: 10 & 0.0005 & 0.0010 \\ 4166 & 15: 30: 12 & 0.0012 & 0.0002 \\ 4168 & 15: 30: 14 & 0.0211 & 0.0002 \\ 4170 & 15: 30: 16 & 0.0007 & 0.0054 \\ 4172 & 15: 30: 18 & 0.0230 & 0.0002 \\ 4174 & 15: 30: 20 & 0.0006 & 0.0004 \\ 4176 & 15: 30: 22 & 0.0040 & 0.0031 \\ 4178 & 15: 30: 24 & 0.0019 & 0.0078\end{array}$


Appendix H - Laser Data Test 4 NIST102705_1.xls

$\begin{array}{llll}4180 & 15: 30: 26 & 0.0006 & 0.0001 \\ 4182 & 15: 30: 28 & 0.0008 & 0.0005 \\ 4184 & 15: 30: 30 & 0.0002 & 0.0006 \\ 4186 & 15: 30: 32 & 0.0002 & 0.0006 \\ 4188 & 15: 30: 34 & 0.0212 & 0.0021 \\ 4190 & 15: 30: 36 & 0.0019 & 0.0001 \\ 4192 & 15: 30: 38 & 0.0002 & 0.0001 \\ 4194 & 15: 30: 40 & 0.0092 & 0.0002 \\ 4196 & 15: 30: 42 & 0.0004 & 0.0004 \\ 4198 & 15: 30: 44 & 0.0008 & 0.0009 \\ 4200 & 15: 30: 46 & 0.0943 & 0.0002 \\ 4202 & 15: 30: 48 & 0.0006 & 0.0002 \\ 4204 & 15: 30: 50 & 0.0005 & 0.0006 \\ 4206 & 15: 30: 52 & 0.0017 & 0.0034 \\ 4208 & 15: 30: 54 & 0.0002 & 0.0009 \\ 4210 & 15: 30: 56 & 0.0015 & 0.0002 \\ 4212 & 15: 30: 58 & 0.0002 & 0.0002 \\ 4214 & 15: 31: 00 & 0.0002 & 0.0004 \\ 4216 & 15: 31: 02 & 0.0046 & 0.0025 \\ 4218 & 15: 31: 04 & 0.0008 & 0.0002 \\ 4220 & 15: 31: 06 & 0.0139 & 0.0021 \\ 4222 & 15: 31: 08 & 0.0012 & 0.0045 \\ 4224 & 15: 31: 10 & 0.0002 & 0.0032 \\ 4226 & 15: 31: 12 & 0.0002 & 0.0005 \\ 4228 & 15: 31: 14 & 0.0279 & 0.0002 \\ 4230 & 15: 31: 16 & 0.0006 & 0.0001 \\ 4232 & 15: 31: 18 & 0.0043 & 0.0006 \\ 4234 & 15: 31: 20 & 0.0014 & 0.0002 \\ 4236 & 15: 31: 22 & 0.0089 & 0.0025 \\ 4238 & 15: 31: 24 & 0.0108 & 0.0009 \\ 4240 & 15: 31: 26 & 0.0088 & 0.0004 \\ 4242 & 15: 31: 28 & 0.0002 & 0.0002 \\ 4244 & 15: 31: 30 & 0.0057 & 0.0002 \\ 4246 & 15: 31: 32 & 0.0420 & 0.0002 \\ 4248 & 15: 31: 34 & 0.0554 & 0.0002 \\ 4250 & 15: 31: 36 & 0.1221 & 0.0002 \\ 4252 & 15: 31: 38 & 0.0075 & 0.0001 \\ 4254 & 15: 31: 40 & 0.0019 & 0.0001 \\ 4256 & 15: 31: 42 & 0.0004 & 0.0002 \\ 4258 & 15: 31: 44 & 0.0030 & 0.0002 \\ 4260 & 15: 31: 46 & 0.0131 & 0.0080 \\ 4262 & 15: 31: 48 & 0.0023 & 0.0002 \\ 4264 & 15: 31: 50 & 0.0004 & 0.0004 \\ 4266 & 15: 31: 52 & 0.0005 & 0.0004 \\ 4268 & 15: 31: 54 & 0.0002 & 0.0042 \\ 4270 & 15: 31: 56 & 0.0008 & 0.0005 \\ 4272 & 15: 31: 58 & 0.0002 & 0.0002 \\ 4274 & 15: 32: 00 & 0.0002 & 0.0002 \\ 4276 & 15: 32: 02 & 0.0007 & 0.0002 \\ 4278 & 15: 32: 04 & 0.0139 & 0.0002 \\ 4280 & 15: 32: 06 & 0.0340 & 0.0030\end{array}$


Appendix H - Laser Data Test 4 NIST102705_1.xls

$\begin{array}{llll}4282 & 15: 32: 08 & 0.0002 & 0.0002 \\ 4284 & 15: 32: 10 & 0.0397 & 0.0002 \\ 4286 & 15: 32: 12 & 0.0242 & 0.0015 \\ 4288 & 15: 32: 14 & 0.0050 & 0.0001 \\ 4290 & 15: 32: 16 & 0.0007 & 0.0002 \\ 4292 & 15: 32: 18 & 0.0004 & 0.0025 \\ 4294 & 15: 32: 20 & 0.0002 & 0.0018 \\ 4296 & 15: 32: 22 & 0.0002 & 0.0009 \\ 4298 & 15: 32: 24 & 0.0002 & 0.0005 \\ 4300 & 15: 32: 26 & 0.0021 & 0.0025 \\ 4302 & 15: 32: 28 & 0.0027 & 0.0006 \\ 4304 & 15: 32: 30 & 0.0002 & 0.0005 \\ 4306 & 15: 32: 32 & 0.0011 & 0.0004 \\ 4308 & 15: 32: 34 & 0.0004 & 0.0002 \\ 4310 & 15: 32: 36 & 0.0010 & 0.0002 \\ 4312 & 15: 32: 38 & 0.0042 & 0.0002 \\ 4314 & 15: 32: 40 & 0.0002 & 0.0002 \\ 4316 & 15: 32: 42 & 0.0032 & 0.0002 \\ 4318 & 15: 32: 44 & 0.0069 & 0.0002 \\ 4320 & 15: 32: 46 & 0.0045 & 0.0002 \\ 4322 & 15: 32: 48 & 0.0002 & 0.0002 \\ 4324 & 15: 32: 50 & 0.0023 & 0.0002 \\ 4326 & 15: 32: 52 & 0.0057 & 0.0009 \\ 4328 & 15: 32: 54 & 0.0061 & 0.0002 \\ 4330 & 15: 32: 56 & 0.0030 & 0.0011 \\ 4332 & 15: 32: 58 & 0.0035 & 0.0037 \\ 4334 & 15: 33: 00 & 0.0028 & 0.0002 \\ 4336 & 15: 33: 02 & 0.0002 & 0.0002 \\ 4338 & 15: 33: 04 & 0.0005 & 0.0001 \\ 4340 & 15: 33: 06 & 0.0018 & 0.0026 \\ 4342 & 15: 33: 08 & 0.0559 & 0.0026 \\ 4344 & 15: 33: 10 & 0.0473 & 0.0002 \\ 4346 & 15: 33: 12 & 0.0022 & 0.0002 \\ 4348 & 15: 33: 14 & 0.0055 & 0.0043 \\ 4350 & 15: 33: 16 & 0.0035 & 0.0023 \\ 4352 & 15: 33: 18 & 0.2251 & 0.0004 \\ 4354 & 15: 33: 20 & 0.0125 & 0.0002 \\ 4356 & 15: 33: 22 & 0.0047 & 0.0093 \\ 4358 & 15: 33: 24 & 0.0004 & 0.0070 \\ 4360 & 15: 33: 26 & 0.0010 & 0.0010 \\ 4362 & 15: 33: 28 & 0.0012 & 0.0004 \\ 4364 & 15: 33: 30 & 0.0026 & 0.0009 \\ 4366 & 15: 33: 32 & 0.0071 & 0.0002 \\ 4368 & 15: 33: 34 & 0.0013 & 0.0002 \\ 4370 & 15: 33: 36 & 0.5637 & 0.0004 \\ 4372 & 15: 33: 38 & 0.0013 & 0.0002 \\ 4374 & 15: 33: 40 & 0.2437 & 0.1006 \\ 4376 & 15: 33: 42 & 0.0225 & 0.0002 \\ 4378 & 15: 33: 44 & 0.0326 & 0.0002 \\ 4380 & 15: 33: 46 & 0.0002 & 0.0022 \\ 4382 & 15: 33: 48 & 0.0059 & 0.0002\end{array}$


Appendix H - Laser Data Test 4 NIST102705_1.xls

$\begin{array}{llll}4384 & 15: 33: 50 & 0.0089 & 0.0004 \\ 4386 & 15: 33: 52 & 0.0016 & 0.0004 \\ 4388 & 15: 33: 54 & 0.0106 & 0.0005 \\ 4390 & 15: 33: 56 & 0.0103 & 0.0002 \\ 4392 & 15: 33: 58 & 0.0838 & 0.0002 \\ 4394 & 15: 34: 00 & 0.0433 & 0.0002 \\ 4396 & 15: 34: 02 & 0.0085 & 0.0062 \\ 4398 & 15: 34: 04 & 0.8005 & 0.0005 \\ 4400 & 15: 34: 06 & 0.0166 & 0.0002 \\ 4402 & 15: 34: 08 & 0.0537 & 0.0007 \\ 4404 & 15: 34: 10 & 0.0022 & 0.0004 \\ 4406 & 15: 34: 12 & 0.0004 & 0.0002 \\ 4408 & 15: 34: 14 & 0.0108 & 0.0019 \\ 4410 & 15: 34: 16 & 0.4565 & 0.0002 \\ 4412 & 15: 34: 18 & 0.0080 & 0.0004 \\ 4414 & 15: 34: 20 & 0.0011 & 0.0001 \\ 4416 & 15: 34: 22 & 0.0577 & 0.0001 \\ 4418 & 15: 34: 24 & 0.0469 & 0.0002 \\ 4420 & 15: 34: 26 & 0.0016 & 0.0001 \\ 4422 & 15: 34: 28 & 0.0006 & 0.0022 \\ 4424 & 15: 34: 30 & 0.0007 & 0.0000 \\ 4426 & 15: 34: 32 & 0.0058 & 0.0002 \\ 4428 & 15: 34: 34 & 0.0042 & 0.0002 \\ 4430 & 15: 34: 36 & 0.0074 & 0.0002 \\ 4432 & 15: 34: 38 & 0.0695 & 0.0004 \\ 4434 & 15: 34: 40 & 0.0543 & 0.0649 \\ 4436 & 15: 34: 42 & 0.0736 & 0.0002 \\ 4438 & 15: 34: 44 & 0.0592 & 0.0001 \\ 4440 & 15: 34: 46 & 0.0012 & 0.0002 \\ 4442 & 15: 34: 48 & 0.1208 & 0.0035 \\ 4444 & 15: 34: 50 & 0.1777 & 0.0001 \\ 4446 & 15: 34: 52 & 0.0109 & 0.0002 \\ 4448 & 15: 34: 54 & 0.0013 & 0.0001 \\ 4450 & 15: 34: 56 & 0.0537 & 0.0007 \\ 4452 & 15: 34: 58 & 0.0068 & 0.0427 \\ 4454 & 15: 35: 00 & 0.0308 & 0.0002 \\ 4456 & 15: 35: 02 & 0.0654 & 0.0015 \\ 4458 & 15: 35: 04 & 0.1993 & 0.0002 \\ 4460 & 15: 35: 06 & 0.3114 & 0.0002 \\ 4462 & 15: 35: 08 & 0.2541 & 0.0004 \\ 4464 & 15: 35: 10 & 0.0805 & 0.0010 \\ 4466 & 15: 35: 12 & 0.1477 & 0.0007 \\ 4468 & 15: 35: 14 & 0.0357 & 0.0001 \\ 4470 & 15: 35: 16 & 0.0027 & 0.0000 \\ 4472 & 15: 35: 18 & 0.1267 & 0.0000 \\ 4474 & 15: 35: 20 & 0.0263 & 0.0002 \\ 4476 & 15: 35: 22 & 0.0039 & 0.0002 \\ 4478 & 15: 35: 24 & 0.6149 & 0.0002 \\ 4480 & 15: 35: 26 & 0.0005 & 0.0002 \\ 4482 & 15: 35: 28 & 0.0837 & 0.0002 \\ 4484 & 15: 35: 30 & 0.0131 & 0.0001\end{array}$


Appendix H - Laser Data Test 4 NIST102705_1.xls

$\begin{array}{llll}4486 & 15: 35: 32 & 0.0064 & 0.0004 \\ 4488 & 15: 35: 34 & 0.0223 & 0.0001 \\ 4490 & 15: 35: 36 & 0.0262 & 0.0004 \\ 4492 & 15: 35: 38 & 0.0025 & 0.0007 \\ 4494 & 15: 35: 40 & 0.1103 & 0.0007 \\ 4496 & 15: 35: 42 & 0.1474 & 0.0002 \\ 4498 & 15: 35: 44 & 0.3074 & 0.0002 \\ 4500 & 15: 35: 46 & 0.3626 & 0.0002 \\ 4502 & 15: 35: 48 & 0.0934 & 0.0002 \\ 4504 & 15: 35: 50 & 0.1149 & 0.0002 \\ 4506 & 15: 35: 52 & 0.0027 & 0.0001 \\ 4508 & 15: 35: 54 & 0.3910 & 0.0002 \\ 4510 & 15: 35: 56 & 0.0785 & 0.0001 \\ 4512 & 15: 35: 58 & 0.0186 & 0.0001 \\ 4514 & 15: 36: 00 & 0.0412 & 0.0001 \\ 4516 & 15: 36: 02 & 0.0096 & 0.0001 \\ 4518 & 15: 36: 04 & 0.0084 & 0.0002 \\ 4520 & 15: 36: 06 & 0.0002 & 0.0001 \\ 4522 & 15: 36: 08 & 0.0050 & 0.0000 \\ 4524 & 15: 36: 10 & 0.0657 & 0.0001 \\ 4526 & 15: 36: 12 & 0.2378 & 0.0002 \\ 4528 & 15: 36: 14 & 0.0153 & 0.0002 \\ 4530 & 15: 36: 16 & 0.1011 & 0.0000 \\ 4532 & 15: 36: 18 & 0.0021 & 0.0002 \\ 4534 & 15: 36: 20 & 0.0422 & 0.0002 \\ 4536 & 15: 36: 22 & 0.0144 & 0.0002 \\ 4538 & 15: 36: 24 & 0.0292 & 0.0002 \\ 4540 & 15: 36: 26 & 0.0287 & 0.0266 \\ 4542 & 15: 36: 28 & 0.1422 & 0.0001 \\ 4544 & 15: 36: 30 & 0.0641 & 0.0001 \\ 4546 & 15: 36: 32 & 0.0027 & 0.0001 \\ 4548 & 15: 36: 34 & 0.0043 & 0.0000 \\ 4550 & 15: 36: 36 & 0.0211 & 0.0002 \\ 4552 & 15: 36: 38 & 0.0407 & 0.0002 \\ 4554 & 15: 36: 40 & 0.0088 & 0.0001 \\ 4556 & 15: 36: 42 & 0.0568 & 0.0004 \\ 4558 & 15: 36: 44 & 0.0249 & 0.0002 \\ 4560 & 15: 36: 46 & 0.0090 & 0.0002 \\ 4562 & 15: 36: 48 & 0.0071 & 0.0002 \\ 4564 & 15: 36: 50 & 0.0392 & 0.0002 \\ 4566 & 15: 36: 52 & 0.0015 & 0.0001 \\ 4568 & 15: 36: 54 & 0.0010 & 0.0001 \\ 4570 & 15: 36: 56 & 0.0002 & 0.0002 \\ 4572 & 15: 36: 58 & 0.0443 & 0.0002 \\ 4574 & 15: 37: 00 & 0.2223 & 0.0001 \\ 4576 & 15: 37: 02 & 0.0011 & 0.0001 \\ 4578 & 15: 37: 04 & 0.0381 & 0.0002 \\ 4580 & 15: 37: 06 & 0.3732 & 0.0056 \\ 4582 & 15: 37: 08 & 0.1167 & 0.0002 \\ 4584 & 15: 37: 10 & 0.2843 & 0.0001 \\ 4586 & 15: 37: 12 & 0.0143 & 0.0001\end{array}$


Appendix H - Laser Data Test 4 NIST102705_1.xls

$\begin{array}{llll}4588 & 15: 37: 14 & 0.0556 & 0.0003 \\ 4590 & 15: 37: 16 & 0.0378 & 0.0001 \\ 4592 & 15: 37: 18 & 0.0002 & 0.0001 \\ 4594 & 15: 37: 20 & 0.0004 & 0.0040 \\ 4596 & 15: 37: 22 & 0.0002 & 0.0003 \\ 4598 & 15: 37: 24 & 0.0267 & 0.0004 \\ 4600 & 15: 37: 26 & 0.0239 & 0.0003 \\ 4602 & 15: 37: 28 & 0.0060 & 0.0003 \\ 4604 & 15: 37: 30 & 0.0096 & 0.0003 \\ 4606 & 15: 37: 32 & 0.0678 & 0.0003 \\ 4608 & 15: 37: 34 & 0.0129 & 0.0005 \\ 4610 & 15: 37: 36 & 0.2533 & 0.0008 \\ 4612 & 15: 37: 38 & 0.0005 & 0.0006 \\ 4614 & 15: 37: 40 & 0.0135 & 0.0004 \\ 4616 & 15: 37: 42 & 0.0441 & 0.0004 \\ 4618 & 15: 37: 44 & 0.0254 & 0.0003 \\ 4620 & 15: 37: 46 & 0.2218 & 0.0003 \\ 4622 & 15: 37: 48 & 0.0505 & 0.0003 \\ 4624 & 15: 37: 50 & 0.0827 & 0.0005 \\ 4626 & 15: 37: 52 & 0.0564 & 0.0003 \\ 4628 & 15: 37: 54 & 0.0558 & 0.0003 \\ 4630 & 15: 37: 56 & 0.0309 & 0.0004 \\ 4632 & 15: 37: 58 & 0.0061 & 0.0013 \\ 4634 & 15: 38: 00 & 0.0079 & 0.0003 \\ 4636 & 15: 38: 02 & 0.0111 & 0.0004 \\ 4638 & 15: 38: 04 & 0.0023 & 0.0003 \\ 4640 & 15: 38: 06 & 0.0025 & 0.0003 \\ 4642 & 15: 38: 08 & 0.0045 & 0.0001 \\ 4644 & 15: 38: 10 & 0.0097 & 0.0003 \\ 4646 & 15: 38: 12 & 0.0192 & 0.0095 \\ 4648 & 15: 38: 14 & 0.0017 & 0.0006 \\ 4650 & 15: 38: 16 & 0.0012 & 0.0003 \\ 4652 & 15: 38: 18 & 0.0004 & 0.0003 \\ 4654 & 15: 38: 20 & 0.0002 & 0.0001 \\ 4656 & 15: 38: 22 & 0.0016 & 0.0003 \\ 4658 & 15: 38: 24 & 0.0036 & 0.0003 \\ 4660 & 15: 38: 26 & 0.0005 & 0.0003 \\ 4662 & 15: 38: 28 & 0.0169 & 0.0001 \\ 4664 & 15: 38: 30 & 0.0041 & 0.0034 \\ 4666 & 15: 38: 32 & 0.0561 & 0.0003 \\ 4668 & 15: 38: 34 & 0.0021 & 0.0001 \\ 4670 & 15: 38: 36 & 0.0046 & 0.0000 \\ 4672 & 15: 38: 38 & 0.0042 & 0.0003 \\ 4674 & 15: 38: 40 & 0.0004 & 0.0003 \\ 4676 & 15: 38: 42 & 0.0005 & 0.0009 \\ 4678 & 15: 38: 44 & 0.0147 & 0.0001 \\ 4680 & 15: 38: 46 & 0.0260 & 0.0006 \\ 4682 & 15: 38: 48 & 0.0017 & 0.0001 \\ 4684 & 15: 38: 50 & 0.0067 & 0.0166 \\ 4686 & 15: 38: 52 & 0.0016 & 0.0003 \\ 4688 & 15: 38: 54 & 0.0444 & 0.0001\end{array}$


Appendix H - Laser Data Test 4 NIST102705_1.xls

$\begin{array}{llll}4690 & 15: 38: 56 & 0.0022 & 0.0003 \\ 4692 & 15: 38: 58 & 0.0183 & 0.0008 \\ 4694 & 15: 39: 00 & 0.0589 & 0.0003 \\ 4696 & 15: 39: 02 & 0.0991 & 0.0003 \\ 4698 & 15: 39: 04 & 0.1962 & 0.0018 \\ 4700 & 15: 39: 06 & 0.3671 & 0.0003 \\ 4702 & 15: 39: 08 & 0.0105 & 0.0003 \\ 4704 & 15: 39: 10 & 0.0020 & 0.0003 \\ 4706 & 15: 39: 12 & 0.0309 & 0.0063 \\ 4708 & 15: 39: 14 & 0.0015 & 0.0004 \\ 4710 & 15: 39: 16 & 0.0009 & 0.0003 \\ 4712 & 15: 39: 18 & 0.0303 & 0.0004 \\ 4714 & 15: 39: 20 & 0.0021 & 0.0003 \\ 4716 & 15: 39: 22 & 0.0089 & 0.0003 \\ 4718 & 15: 39: 24 & 0.0027 & 0.0001 \\ 4720 & 15: 39: 26 & 0.0718 & 0.0035 \\ 4722 & 15: 39: 28 & 0.0022 & 0.0003 \\ 4724 & 15: 39: 30 & 0.0038 & 0.0003 \\ 4726 & 15: 39: 32 & 0.0002 & 0.0003 \\ 4728 & 15: 39: 34 & 0.0202 & 0.0006 \\ 4730 & 15: 39: 36 & 0.0087 & 0.0006 \\ 4732 & 15: 39: 38 & 0.0062 & 0.0008 \\ 4734 & 15: 39: 40 & 0.0004 & 0.0003 \\ 4736 & 15: 39: 42 & 0.0011 & 0.0003 \\ 4738 & 15: 39: 44 & 0.0303 & 0.0001 \\ 4740 & 15: 39: 46 & 0.0002 & 0.0016 \\ 4742 & 15: 39: 48 & 0.0092 & 0.0003 \\ 4744 & 15: 39: 50 & 0.0004 & 0.0015 \\ 4746 & 15: 39: 52 & 0.0020 & 0.0006 \\ 4748 & 15: 39: 54 & 0.0069 & 0.0003 \\ 4750 & 15: 39: 56 & 0.0009 & 0.0004 \\ 4752 & 15: 39: 58 & 0.0021 & 0.0003 \\ 4754 & 15: 40: 00 & 0.0010 & 0.0003 \\ 4756 & 15: 40: 02 & 0.0005 & 0.0185 \\ 4758 & 15: 40: 04 & 0.0031 & 0.0006 \\ 4760 & 15: 40: 06 & 0.0105 & 0.0001 \\ 4762 & 15: 40: 08 & 0.0184 & 0.0438 \\ 4764 & 15: 40: 10 & 0.0006 & 0.0003 \\ 4766 & 15: 40: 12 & 0.0002 & 0.0001 \\ 4768 & 15: 40: 14 & 0.1042 & 0.0003 \\ 4770 & 15: 40: 16 & 0.0036 & 0.0008 \\ 4772 & 15: 40: 18 & 0.0004 & 0.0006 \\ 4774 & 15: 40: 20 & 0.0062 & 0.0019 \\ 4776 & 15: 40: 22 & 0.0335 & 0.0003 \\ 4778 & 15: 40: 24 & 0.0165 & 0.0287 \\ 4780 & 15: 40: 26 & 0.0004 & 0.0003 \\ 4782 & 15: 40: 28 & 0.0367 & 0.0005 \\ 4784 & 15: 40: 30 & 0.0101 & 0.0052 \\ 4786 & 15: 40: 32 & 0.1258 & 0.0004 \\ 4788 & 15: 40: 34 & 0.0002 & 0.0003 \\ 4790 & 15: 40: 36 & 0.0091 & 0.0003\end{array}$


Appendix H - Laser Data Test 4 NIST102705_1.xls

$\begin{array}{llll}4792 & 15: 40: 38 & 0.0071 & 0.0005 \\ 4794 & 15: 40: 40 & 0.0040 & 0.0003 \\ 4796 & 15: 40: 42 & 0.0853 & 0.0003 \\ 4798 & 15: 40: 44 & 0.0068 & 0.0003 \\ 4800 & 15: 40: 46 & 0.0692 & 0.0003 \\ 4802 & 15: 40: 48 & 0.0054 & 0.0004 \\ 4804 & 15: 40: 50 & 0.0177 & 0.0009 \\ 4806 & 15: 40: 52 & 0.0571 & 0.0003 \\ 4808 & 15: 40: 54 & 0.0217 & 0.0003 \\ 4810 & 15: 40: 56 & 0.0002 & 0.0004 \\ 4812 & 15: 40: 58 & 0.0184 & 0.0006 \\ 4814 & 15: 41: 00 & 0.0102 & 0.0014 \\ 4816 & 15: 41: 02 & 0.0010 & 0.0004 \\ 4818 & 15: 41: 04 & 0.0015 & 0.0004 \\ 4820 & 15: 41: 06 & 0.0126 & 0.0003 \\ 4822 & 15: 41: 08 & 0.0002 & 0.0003 \\ 4824 & 15: 41: 10 & 0.2241 & 0.0003 \\ 4826 & 15: 41: 12 & 0.0428 & 0.0003 \\ 4828 & 15: 41: 14 & 0.0045 & 0.0003 \\ 4830 & 15: 41: 16 & 0.0042 & 0.0528 \\ 4832 & 15: 41: 18 & 0.0016 & 0.0003 \\ 4834 & 15: 41: 20 & 0.0002 & 0.0003 \\ 4836 & 15: 41: 22 & 0.1912 & 0.0005 \\ 4838 & 15: 41: 24 & 0.0002 & 0.0003 \\ 4840 & 15: 41: 26 & 0.0014 & 0.0003 \\ 4842 & 15: 41: 28 & 0.0109 & 0.0005 \\ 4844 & 15: 41: 30 & 0.0004 & 0.0044 \\ 4846 & 15: 41: 32 & 0.0007 & 0.0001 \\ 4848 & 15: 41: 34 & 0.0003 & 0.0005 \\ 4850 & 15: 41: 36 & 0.0003 & 0.0003 \\ 4852 & 15: 41: 38 & 0.1306 & 0.0003 \\ 4854 & 15: 41: 40 & 0.0015 & 0.0003 \\ 4856 & 15: 41: 42 & 0.0211 & 0.0001 \\ 4858 & 15: 41: 44 & 0.0005 & 0.0001 \\ 4860 & 15: 41: 46 & 0.0004 & 0.0001 \\ 4862 & 15: 41: 48 & 0.0003 & 0.0008 \\ 4864 & 15: 41: 50 & 0.0003 & 0.0013 \\ 4866 & 15: 41: 52 & 0.0005 & 0.0003 \\ 4868 & 15: 41: 54 & 0.0038 & 0.0003 \\ 4870 & 15: 41: 56 & 0.0003 & 0.0005 \\ 4872 & 15: 41: 58 & 0.0005 & 0.0003 \\ 4874 & 15: 42: 00 & 0.0060 & 0.0003 \\ 4876 & 15: 42: 02 & 0.0242 & 0.0001 \\ 4878 & 15: 42: 04 & 0.1024 & 0.0001 \\ 4880 & 15: 42: 06 & 0.0039 & 0.0009 \\ 4882 & 15: 42: 08 & 0.0594 & 0.0001 \\ 4884 & 15: 42: 10 & 0.1082 & 0.0003 \\ 4886 & 15: 42: 12 & 0.0352 & 0.0001 \\ 4888 & 15: 42: 14 & 0.0462 & 0.0001 \\ 4890 & 15: 42: 16 & 0.0011 & 0.0006 \\ 4892 & 15: 42: 18 & 0.0005 & 0.0003\end{array}$


Appendix H - Laser Data Test 4 NIST102705_1.xls

$\begin{array}{llll}4894 & 15: 42: 20 & 0.0003 & 0.0464 \\ 4896 & 15: 42: 22 & 0.0013 & 0.0142 \\ 4898 & 15: 42: 24 & 0.0359 & 0.0155 \\ 4900 & 15: 42: 26 & 0.0005 & 0.0003 \\ 4902 & 15: 42: 28 & 0.1286 & 0.0189 \\ 4904 & 15: 42: 30 & 0.0005 & 0.0004 \\ 4906 & 15: 42: 32 & 0.0384 & 0.0041 \\ 4908 & 15: 42: 34 & 0.0013 & 0.0003 \\ 4910 & 15: 42: 36 & 0.0293 & 0.0003 \\ 4912 & 15: 42: 38 & 0.0003 & 0.0003 \\ 4914 & 15: 42: 40 & 0.0074 & 0.0005 \\ 4916 & 15: 42: 42 & 0.0092 & 0.0011 \\ 4918 & 15: 42: 44 & 0.1661 & 0.0003 \\ 4920 & 15: 42: 46 & 0.0003 & 0.0008 \\ 4922 & 15: 42: 48 & 0.1082 & 0.0027 \\ 4924 & 15: 42: 50 & 0.0459 & 0.0004 \\ 4926 & 15: 42: 52 & 0.0009 & 0.0003 \\ 4928 & 15: 42: 54 & 0.2260 & 0.0004 \\ 4930 & 15: 42: 56 & 0.1010 & 0.0004 \\ 4932 & 15: 42: 58 & 0.0045 & 0.0029 \\ 4934 & 15: 43: 00 & 0.0097 & 0.0005 \\ 4936 & 15: 43: 02 & 0.0008 & 0.0014 \\ 4938 & 15: 43: 04 & 0.0004 & 0.0003 \\ 4940 & 15: 43: 06 & 0.0030 & 0.0003 \\ 4942 & 15: 43: 08 & 0.0011 & 0.0003 \\ 4944 & 15: 43: 10 & 0.0010 & 0.0017 \\ 4946 & 15: 43: 12 & 0.0004 & 0.0003 \\ 4948 & 15: 43: 14 & 0.0030 & 0.0003 \\ 4950 & 15: 43: 16 & 0.0192 & 0.0735 \\ 4952 & 15: 43: 18 & 0.0494 & 0.0003 \\ 4954 & 15: 43: 20 & 0.0003 & 0.0003 \\ 4956 & 15: 43: 22 & 0.0074 & 0.0003 \\ 4958 & 15: 43: 24 & 0.0015 & 0.0003 \\ 4960 & 15: 43: 26 & 0.0048 & 0.0003 \\ 4962 & 15: 43: 28 & 0.0891 & 0.0003 \\ 4964 & 15: 43: 30 & 0.0009 & 0.0003 \\ 4966 & 15: 43: 32 & 0.0052 & 0.0003 \\ 4968 & 15: 43: 34 & 0.0037 & 0.0003 \\ 4970 & 15: 43: 36 & 0.0135 & 0.0003 \\ 4972 & 15: 43: 38 & 0.5536 & 0.0003 \\ 4974 & 15: 43: 40 & 0.0212 & 0.0003 \\ 4976 & 15: 43: 42 & 0.0884 & 0.0040 \\ 4978 & 15: 43: 44 & 0.0654 & 0.0001 \\ 4980 & 15: 43: 46 & 0.0091 & 0.0004 \\ 4982 & 15: 43: 48 & 0.0777 & 0.0027 \\ 4984 & 15: 43: 50 & 0.0014 & 0.0001 \\ 4986 & 15: 43: 52 & 0.0221 & 0.0006 \\ 4988 & 15: 43: 54 & 0.0418 & 0.0003 \\ 4990 & 15: 43: 56 & 0.3568 & 0.0001 \\ 4992 & 15: 43: 58 & 0.0951 & 0.0049 \\ 4994 & 15: 44: 00 & 0.0081 & 0.0005\end{array}$


Appendix H - Laser Data Test 4 NIST102705_1.xls

$\begin{array}{llll}4996 & 15: 44: 02 & 0.0491 & 0.0003 \\ 4998 & 15: 44: 04 & 0.0415 & 0.0004 \\ 5000 & 15: 44: 06 & 0.0193 & 0.0006 \\ 5002 & 15: 44: 08 & 0.1202 & 0.0069 \\ 5004 & 15: 44: 10 & 0.7831 & 0.0003 \\ 5006 & 15: 44: 12 & 0.1251 & 0.0003 \\ 5008 & 15: 44: 14 & 0.4978 & 0.0003 \\ 5010 & 15: 44: 16 & 0.4578 & 0.0001 \\ 5012 & 15: 44: 18 & 0.0491 & 0.0000 \\ 5014 & 15: 44: 20 & 0.5450 & 0.0003 \\ 5016 & 15: 44: 22 & 0.0046 & 0.0001 \\ 5018 & 15: 44: 24 & 0.1855 & 0.0032 \\ 5020 & 15: 44: 26 & 0.0036 & 0.0003 \\ 5022 & 15: 44: 28 & 0.0463 & 0.0005 \\ 5024 & 15: 44: 30 & 0.2815 & 0.0003 \\ 5026 & 15: 44: 32 & 0.2059 & 0.0003 \\ 5028 & 15: 44: 34 & 0.1090 & 0.0008 \\ 5030 & 15: 44: 36 & 0.0423 & 0.0003 \\ 5032 & 15: 44: 38 & 0.1864 & 0.0003 \\ 5034 & 15: 44: 40 & 0.0328 & 0.0142 \\ 5036 & 15: 44: 42 & 0.0268 & 0.0000 \\ 5038 & 15: 44: 44 & 0.0361 & 0.0003 \\ 5040 & 15: 44: 46 & 0.2977 & 0.0003 \\ 5042 & 15: 44: 48 & 0.2365 & 0.0003 \\ 5044 & 15: 44: 50 & 0.0207 & 0.0003 \\ 5046 & 15: 44: 52 & 0.1178 & 0.0003 \\ 5048 & 15: 44: 54 & 0.0298 & 0.0001 \\ 5050 & 15: 44: 56 & 0.0084 & 0.0000 \\ 5052 & 15: 44: 58 & 0.0335 & 0.0004 \\ 5054 & 15: 45: 00 & 0.0024 & 0.0001 \\ 5056 & 15: 45: 02 & 0.0989 & 0.0001 \\ 5058 & 15: 45: 04 & 0.0048 & 0.0001 \\ 5060 & 15: 45: 06 & 0.0041 & 0.0144 \\ 5062 & 15: 45: 08 & 0.0762 & 0.0076 \\ 5064 & 15: 45: 10 & 0.0623 & 0.0003 \\ 5066 & 15: 45: 12 & 0.0640 & 0.0003 \\ 5068 & 15: 45: 14 & 0.1913 & 0.0012 \\ 5070 & 15: 45: 16 & 0.0257 & 0.0003 \\ 5072 & 15: 45: 18 & 0.0376 & 0.0005 \\ 5074 & 15: 45: 20 & 0.0031 & 0.0009 \\ 5076 & 15: 45: 22 & 0.0649 & 0.0001 \\ 5078 & 15: 45: 24 & 0.2224 & 0.0004 \\ 5080 & 15: 45: 26 & 0.0194 & 0.0017 \\ 5082 & 15: 45: 28 & 0.0018 & 0.0001 \\ 5084 & 15: 45: 30 & 0.0017 & 0.0005 \\ 5086 & 15: 45: 32 & 0.0009 & 0.0001 \\ 5088 & 15: 45: 34 & 0.0449 & 0.0003 \\ 5090 & 15: 45: 36 & 0.0056 & 0.0003 \\ 5092 & 15: 45: 38 & 0.0005 & 0.0003 \\ 5094 & 15: 45: 40 & 0.0235 & 0.0001 \\ 5096 & 15: 45: 42 & 0.0609 & 0.0003\end{array}$


Appendix H - Laser Data Test 4 NIST102705_1.xls

$\begin{array}{llll}5098 & 15: 45: 44 & 0.0795 & 0.0003 \\ 5100 & 15: 45: 46 & 0.0078 & 0.0003 \\ 5102 & 15: 45: 48 & 0.0006 & 0.0126 \\ 5104 & 15: 45: 50 & 0.0219 & 0.0003 \\ 5106 & 15: 45: 52 & 0.0024 & 0.0001 \\ 5108 & 15: 45: 54 & 0.0093 & 0.0001 \\ 5110 & 15: 45: 56 & 0.0095 & 0.0001 \\ 5112 & 15: 45: 58 & 0.0003 & 0.0000 \\ 5114 & 15: 46: 00 & 0.0114 & 0.0001 \\ 5116 & 15: 46: 02 & 0.0983 & 0.0001 \\ 5118 & 15: 46: 04 & 0.0804 & 0.0003 \\ 5120 & 15: 46: 06 & 0.0022 & 0.0003 \\ 5122 & 15: 46: 08 & 0.0018 & 0.0003 \\ 5124 & 15: 46: 10 & 0.1912 & 0.0003 \\ 5126 & 15: 46: 12 & 0.0297 & 0.0003 \\ 5128 & 15: 46: 14 & 0.2895 & 0.0003 \\ 5130 & 15: 46: 16 & 0.0047 & 0.0003 \\ 5132 & 15: 46: 18 & 0.1543 & 0.0003 \\ 5134 & 15: 46: 20 & 0.0135 & 0.0003 \\ 5136 & 15: 46: 22 & 0.1446 & 0.0003 \\ 5138 & 15: 46: 24 & 0.0003 & 0.0003 \\ 5140 & 15: 46: 26 & 0.2353 & 0.0003 \\ 5142 & 15: 46: 28 & 0.0063 & 0.0001 \\ 5144 & 15: 46: 30 & 0.1538 & 0.0000 \\ 5146 & 15: 46: 32 & 0.1523 & 0.0001 \\ 5148 & 15: 46: 34 & 0.0610 & 0.0003 \\ 5150 & 15: 46: 36 & 0.0915 & 0.0001 \\ 5152 & 15: 46: 38 & 0.0148 & 0.0015 \\ 5154 & 15: 46: 40 & 0.0194 & 0.0001 \\ 5156 & 15: 46: 42 & 0.0100 & 0.0001 \\ 5158 & 15: 46: 44 & 0.0172 & 0.0001 \\ 5160 & 15: 46: 46 & 0.1615 & 0.0000 \\ 5162 & 15: 46: 48 & 0.1001 & 0.0000 \\ 5164 & 15: 46: 50 & 0.0003 & 0.0006 \\ 5166 & 15: 46: 52 & 0.0653 & 0.0003 \\ 5168 & 15: 46: 54 & 0.0143 & 0.0001 \\ 5170 & 15: 46: 56 & 0.0431 & 0.0048 \\ 5172 & 15: 46: 58 & 0.0066 & 0.0001 \\ 5174 & 15: 47: 00 & 0.0015 & 0.0001 \\ 5176 & 15: 47: 02 & 0.0091 & 0.0003 \\ 5178 & 15: 47: 04 & 0.1737 & 0.0004 \\ 5180 & 15: 47: 06 & 0.0759 & 0.0001 \\ 5182 & 15: 47: 08 & 0.2164 & 0.0063 \\ 5184 & 15: 47: 10 & 0.0028 & 0.0000 \\ 5186 & 15: 47: 12 & 0.0104 & 0.0003 \\ 5188 & 15: 47: 14 & 0.0417 & 0.0003 \\ 5190 & 15: 47: 16 & 0.1170 & 0.0003 \\ 5192 & 15: 47: 18 & 0.0687 & 0.0003 \\ 5194 & 15: 47: 20 & 0.0201 & 0.0003 \\ 5196 & 15: 47: 22 & 0.0771 & 0.0003 \\ 5198 & 15: 47: 24 & 0.3841 & 0.0003\end{array}$


Appendix H - Laser Data Test 4 NIST102705_1.xls

$\begin{array}{llll}5200 & 15: 47: 26 & 0.0438 & 0.0001 \\ 5202 & 15: 47: 28 & 0.0318 & 0.0003 \\ 5204 & 15: 47: 30 & 0.0312 & 0.0018 \\ 5206 & 15: 47: 32 & 0.0003 & 0.0003 \\ 5208 & 15: 47: 34 & 0.0311 & 0.0018 \\ 5210 & 15: 47: 36 & 0.0009 & 0.0001 \\ 5212 & 15: 47: 38 & 0.0110 & 0.0004 \\ 5214 & 15: 47: 40 & 0.1017 & 0.0003 \\ 5216 & 15: 47: 42 & 0.1371 & 0.0003 \\ 5218 & 15: 47: 44 & 0.0030 & 0.0003 \\ 5220 & 15: 47: 46 & 0.2094 & 0.0003 \\ 5222 & 15: 47: 48 & 0.0047 & 0.0003 \\ 5224 & 15: 47: 50 & 0.0016 & 0.0010 \\ 5226 & 15: 47: 52 & 0.0102 & 0.0003 \\ 5228 & 15: 47: 54 & 0.0100 & 0.0003 \\ 5230 & 15: 47: 56 & 0.0022 & 0.0001 \\ 5232 & 15: 47: 58 & 0.0167 & 0.0003 \\ 5234 & 15: 48: 00 & 0.0027 & 0.0003 \\ 5236 & 15: 48: 02 & 0.0780 & 0.0003 \\ 5238 & 15: 48: 04 & 0.2077 & 0.0003 \\ 5240 & 15: 48: 06 & 0.0044 & 0.0003 \\ 5242 & 15: 48: 08 & 0.0499 & 0.0003 \\ 5244 & 15: 48: 10 & 0.0003 & 0.0003 \\ 5246 & 15: 48: 12 & 0.0616 & 0.0006 \\ 5248 & 15: 48: 14 & 0.0012 & 0.0001 \\ 5250 & 15: 48: 16 & 0.0852 & 0.0001 \\ 5252 & 15: 48: 18 & 0.0082 & 0.0001 \\ 5254 & 15: 48: 20 & 0.0013 & 0.0001 \\ 5256 & 15: 48: 22 & 0.0991 & 0.0001 \\ 5258 & 15: 48: 24 & 0.0023 & 0.0003 \\ 5260 & 15: 48: 26 & 0.0004 & 0.0012 \\ 5262 & 15: 48: 28 & 0.0106 & 0.0069 \\ 5264 & 15: 48: 30 & 0.0016 & 0.0003 \\ 5266 & 15: 48: 32 & 0.0079 & 0.0001 \\ 5268 & 15: 48: 34 & 0.0139 & 0.0000 \\ 5270 & 15: 48: 36 & 0.0023 & 0.0004 \\ 5272 & 15: 48: 38 & 0.0113 & 0.0001 \\ 5274 & 15: 48: 40 & 0.0040 & 0.0001 \\ 5276 & 15: 48: 42 & 0.0016 & 0.0003 \\ 5278 & 15: 48: 44 & 0.1015 & 0.0003 \\ 5280 & 15: 48: 46 & 0.0186 & 0.0003 \\ 5282 & 15: 48: 48 & 0.0544 & 0.0003 \\ 5284 & 15: 48: 50 & 0.0087 & 0.0003 \\ 5286 & 15: 48: 52 & 0.0366 & 0.0003 \\ 5288 & 15: 48: 54 & 0.0122 & 0.0001 \\ 5290 & 15: 48: 56 & 0.0075 & 0.0003 \\ 5292 & 15: 48: 58 & 0.0009 & 0.0001 \\ 5294 & 15: 49: 00 & 0.0095 & 0.0001 \\ 5296 & 15: 49: 02 & 0.0194 & 0.0001 \\ 5298 & 15: 49: 04 & 0.0005 & 0.0001 \\ 5300 & 15: 49: 06 & 0.0040 & 0.0001\end{array}$

$\mathrm{H}-52$ 
Appendix H - Laser Data Test 4 NIST102705_1.xls

$\begin{array}{llll}5302 & 15: 49: 08 & 0.0029 & 0.0003 \\ 5304 & 15: 49: 10 & 0.1006 & 0.0158 \\ 5306 & 15: 49: 12 & 0.0014 & 0.0004 \\ 5308 & 15: 49: 14 & 0.0023 & 0.0003 \\ 5310 & 15: 49: 16 & 0.0060 & 0.0001 \\ 5312 & 15: 49: 18 & 0.0529 & 0.0000 \\ 5314 & 15: 49: 20 & 0.0672 & 0.0001 \\ 5316 & 15: 49: 22 & 0.1219 & 0.0001 \\ 5318 & 15: 49: 24 & 0.0840 & 0.0001 \\ 5320 & 15: 49: 26 & 0.0022 & 0.0018 \\ 5322 & 15: 49: 28 & 0.0009 & 0.0003 \\ 5324 & 15: 49: 30 & 0.0107 & 0.0013 \\ 5326 & 15: 49: 32 & 0.0007 & 0.0003 \\ 5328 & 15: 49: 34 & 0.0119 & 0.0003 \\ 5330 & 15: 49: 36 & 0.1493 & 0.0001 \\ 5332 & 15: 49: 38 & 0.1362 & 0.0003 \\ 5334 & 15: 49: 40 & 0.1940 & 0.0003 \\ 5336 & 15: 49: 42 & 0.0163 & 0.0005 \\ 5338 & 15: 49: 44 & 0.0010 & 0.0001 \\ 5340 & 15: 49: 46 & 0.0025 & 0.0003 \\ 5342 & 15: 49: 48 & 0.0470 & 0.0001 \\ 5344 & 15: 49: 50 & 0.0138 & 0.0003 \\ 5346 & 15: 49: 52 & 0.0107 & 0.0003 \\ 5348 & 15: 49: 54 & 0.0103 & 0.0003 \\ 5350 & 15: 49: 56 & 0.0184 & 0.0009 \\ 5352 & 15: 49: 58 & 0.0098 & 0.0003 \\ 5354 & 15: 50: 00 & 0.0209 & 0.0184 \\ 5356 & 15: 50: 02 & 0.0048 & 0.0018 \\ 5358 & 15: 50: 04 & 0.0009 & 0.0001 \\ 5360 & 15: 50: 06 & 0.0220 & 0.0001 \\ 5362 & 15: 50: 08 & 0.0046 & 0.0007 \\ 5364 & 15: 50: 10 & 0.1828 & 0.0009 \\ 5366 & 15: 50: 12 & 0.0425 & 0.0003 \\ 5368 & 15: 50: 14 & 0.2110 & 0.0003 \\ 5370 & 15: 50: 16 & 0.0034 & 0.0001 \\ 5372 & 15: 50: 18 & 0.0271 & 0.0001 \\ 5374 & 15: 50: 20 & 0.0786 & 0.0003 \\ 5376 & 15: 50: 22 & 0.1527 & 0.0003 \\ 5378 & 15: 50: 24 & 0.0553 & 0.0003 \\ 5380 & 15: 50: 26 & 0.1568 & 0.0003 \\ 5382 & 15: 50: 28 & 0.1492 & 0.0017 \\ 5384 & 15: 50: 30 & 0.0157 & 0.0010 \\ 5386 & 15: 50: 32 & 0.4825 & 0.0005 \\ 5388 & 15: 50: 34 & 0.0291 & 0.0003 \\ 5390 & 15: 50: 36 & 0.0690 & 0.0001 \\ 5392 & 15: 50: 38 & 0.0066 & 0.0001 \\ 5394 & 15: 50: 40 & 0.0028 & 0.0001 \\ 5396 & 15: 50: 42 & 0.0913 & 0.0001 \\ 5398 & 15: 50: 44 & 0.0566 & 0.0001 \\ 5400 & 15: 50: 46 & 0.4468 & 0.0020 \\ 5402 & 15: 50: 48 & 0.2217 & 0.0001\end{array}$

$\mathrm{H}-53$ 
Appendix H - Laser Data Test 4 NIST102705_1.xls

$\begin{array}{llll}5404 & 15: 50: 50 & 0.0818 & 0.0004 \\ 5406 & 15: 50: 52 & 0.0344 & 0.0000 \\ 5408 & 15: 50: 54 & 0.0449 & 0.0016 \\ 5410 & 15: 50: 56 & 0.0478 & 0.0004 \\ 5412 & 15: 50: 58 & 0.3754 & 0.0001 \\ 5414 & 15: 51: 00 & 0.2016 & 0.0001 \\ 5416 & 15: 51: 02 & 0.0105 & 0.0001 \\ 5418 & 15: 51: 04 & 0.1572 & 0.0001 \\ 5420 & 15: 51: 06 & 0.0818 & 0.0001 \\ 5422 & 15: 51: 08 & 0.4094 & 0.0000 \\ 5424 & 15: 51: 10 & 0.0192 & 0.0004 \\ 5426 & 15: 51: 12 & 0.0003 & 0.0013 \\ 5428 & 15: 51: 14 & 0.0003 & 0.0088 \\ 5430 & 15: 51: 16 & 0.0004 & 0.0162 \\ 5432 & 15: 51: 18 & 0.1289 & 0.0005 \\ 5434 & 15: 51: 20 & 0.2031 & 0.0001 \\ 5436 & 15: 51: 22 & 0.2017 & 0.0003 \\ 5438 & 15: 51: 24 & 0.0980 & 0.0003 \\ 5440 & 15: 51: 26 & 0.1495 & 0.0001 \\ 5442 & 15: 51: 28 & 0.3221 & 0.0030\end{array}$

